

\section{Greetings from the General Chair}

Welcome to the 2012 Solid State Sensors, Actuators, and Microsystems Workshop; the fifteenth in the biennial series of regional meetings that alternate with the International Transducers Conference. As with previous meetings, we have maintained the open atmosphere of discussion, collaboration and interaction, which has been its hallmark since the beginning in 1984. Our fundamental goal with the Workshop is to expose new ideas and research in a manner such that we also encourage the building of relationships among the research community in attendance. The combination of ideas, opportunities and relationships has always been the objective of this meeting and we've worked hard to continue in this tradition.

The Workshop schedule remains organized around single session oral presentations with several poster presentations. Mehran Mehregany has assembled and led a strong, active, and opinionated program committee through a paper selection process resulting in the program published in this digest. Four very exciting plenary presentations by the leading academics and industrial pioneers in the field will kick-off each day, followed by the oral and poster sessions from contributed authors. Oral papers were selected on the basis of high quality content and likely broad interest to the meeting audience. Poster papers were selected on the basis of high quality as well, and for their probable high interest to a subset of the meeting attendees and will be introduced by their authors in the popular "shotgun" format. We've also included Late News Oral and Late News Poster papers based on a selection process that occurred in mid-March. The program committee is to be congratulated for their efforts in reading, studying, ranking, debating, re-ranking, and selecting from the nearly 300 submitted abstracts - a number and quality level of abstracts that remains remarkably high given the expansion of the field.

We also have significant unscheduled time for informal discussions among friends and colleagues. Technical brainstorming, collaborative ideas, job offers and business plans are usually topics for these breaks. We hope the relaxed environment here on the ocean helps to stimulate such interaction and creativity.

Several changes have been introduced this year in an attempt to keep the meeting fresh. First, we have expanded our trend of inviting authors who are alumni from the Workshop, even if they have gone on in their careers to work outside of the Americas. We have also tried to tastefully expand the role of exhibitors and commercial support in the Workshop. Within the open poster session, we have invited two well-known supporters of the community from Asia and Europe to provide some global perspective on research in the field. In addition, we again have something new and exciting planned for the period formally known as the "Rump Session."

A tremendous "thank you" goes to Mehran and the entire Technical Program Committee for their efforts in creating this program. Additionally, this year, we had three folks working tirelessly on the development side of the meeting. James Walker served as Chair for Commercial Support. He brought new and innovative ideas to the meeting, and at the time this went to press, had lined up 25 Exhibitors/Sponsors. Alyssa Fitzgerald served as the Chair for Workshop Promotions, to expand our marketing communications efforts and introduce the Workshop to social media tools. Liwei Lin served as the Chair for Student Support, coordinating with government agencies to provide student travels support. These are valuable and essential features of the Hilton Head Workshop, and I am most thankful to have had outstanding people taking the lead on these tasks.

Thanks also goes to the Transducers Research Foundation for their continued support of travel for presenting student authors, thus enabling the meeting to continue the invigoration of our community with young talent, new ideas, and enthusiasm for the future of our community. Mark Allen has continued to provide excellent support of the local arrangements and thanks to Mehran Mehregany for his oversight of our finances. Katharine Cline and her team at Preferred Meeting Management, Inc., above all, deserve special recognition and thanks for all of their hard work and superb organization in pulling this meeting together and for making it run so smoothly. The work they do "behind the scenes" to make this meeting special is difficult to comprehend and our community and our meeting would suffer without them.

Finally, this meeting of course, belongs to all of you; thank you for your enthusiastic participation. I would appreciate hearing your opinion regarding the changes that have been made this year as well as your suggestions for changes to future meetings so we can keep this meeting special. As well, I sincerely hope you all have a great time.

David J. Monk

General Chair 


\section{Organizing Committee}

\section{General Chair}

Technical Program Chair \& Treasurer

Local Arrangements Chair

Travel Grant Chair

Promotions Chair

Commercial Development and Exhibition Chair
David J. Monk, Freescale Semiconductor, Inc.

Mehran Mehregany, Case Western Reserve University

Mark G. Allen, Georgia Institute of Technology

Liwei Lin, University of California, Berkeley

Alissa Fitzgerald, AM Fitzgerald \& Associates

James Walker, Demont \& Breyer Patent Law

\section{Technical Program Committee Members}

Farrokh Ayazi

Georgia Institute of Technology, USA

Sarah Bergbreiter

University of Maryland, USA

Jonathan Bernstein

Draper Laboratory, USA

Rob Candler

University of California, Los Angeles, USA

Eugene Chow

Palo Alto Research Center, USA

Alissa Fitzgerald

AM Fitzgerald \& Associates, USA

Luc Fréchette

University of Sherbrooke, CANADA

Xiao-An Fu

University of Louisville, USA

Jonathan Hammond

RFMD, USA

Hal Jerman

Coherent, USA

Houri Johari

Analog Devices, USA

Hanseup Kim

University of Utah, USA
Jae Wan Kwon

University of Missouri, USA

Pete Loeppert

Knowles Electronics, Inc.

Markus Lutz

SiTime, USA

Mehran Mehregany

Case Western Reserve University, USA

Dave Monk

Freescale Semiconductor, Inc., USA

Dennis Polla

DNI/IARPA, USA

Michael Post

National Research Council, CANADA

Mina Rais-Zadeh

University of Michigan, USA

George Skidmore

RSTA - DRS Technologies, USA

Srinivas Tadigadapa

Pennsylvania State University, USA

Dana Weinstein

Massachusetts Institute of Technology, USA

Maryam Ziaei-Moayyed

Sandia National Laboratory, USA 


\section{Technical Program Committee}
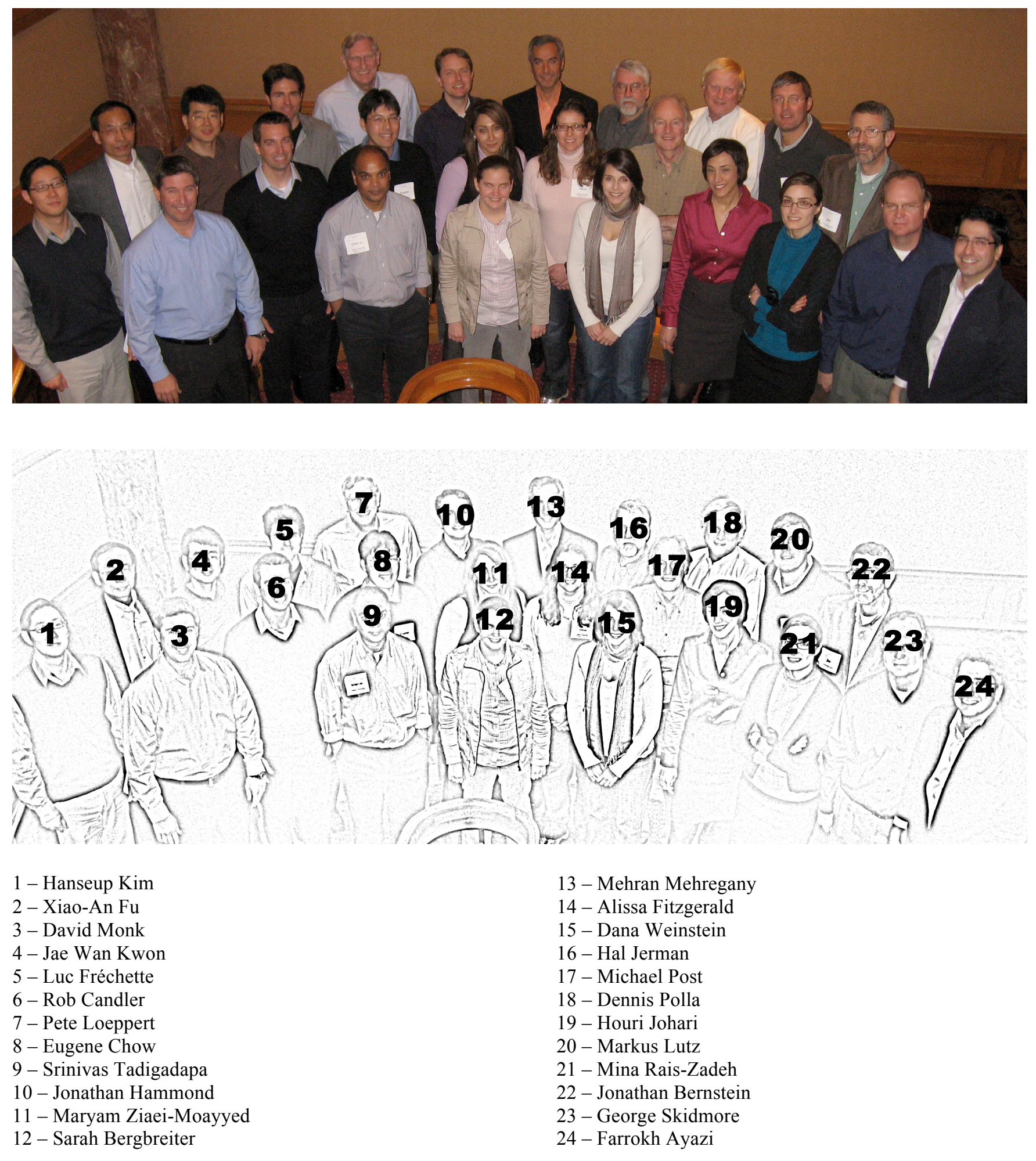


\section{Acknowledgements}

Special acknowledgement to the Transducer Research Foundation, Inc. and the National Science Foundation (NSF) for their educational grant funding support of this Workshop.

\section{Exhibitors and Benefactors}

We gratefully acknowledge the support of this Workshop from the following companies and institutions as of the printing of May 16, 2012:

Case Western Reserve University

Coventor

DeMont \& Breyer Patent Law

Digital Health group on LinkedIn

FEMTO Science Inc.

Freescale

Inspectrology LLC

IntelliSense

Labtec Sales Partners LLC

LioniX, BV

MEMS and Nanotechnology Exchange

MEMS Industry Group

MEMS Journal, Inc.

MEMSCAP

memsstar Limited

MicroFab Technologies, Inc.

OxFord Instruments

Polytec, Inc.

Sandia National Laboratories

Silex Microsystems, Inc.

SoftMEMS LLC

Tousimis

WIMS $^{2}$; Wireless Integrated Microsensing \& Systems

XACTIX 


\section{Table of Contents}

Scroll to the title and select a Blue link to open a paper. After viewing the paper, use the bookmarks to the left to return to the beginning of the Table of Contents.

\section{Sunday, June 3}

6:00 p.m. - Registration and Welcome Reception

9:00 p.m.

\section{Monday, June 4}

7:00 a.m. - Registration and Breakfast

7:45 a.m. - Welcome and Introduction

David J. Monk, Freescale Semiconductor, Inc., USA

Mehran Mehregany, Case Western Reserve University, USA

PLENARY SPEAKER 1

Session Chair: M. Mehregany, Case Western Reserve University, USA

8:15 a.m.

SIMPLE BIOANALYSIS

1

George M. Whitesides

Harvard University, USA

SESSION 1 - Chemistry/Biology Sensors

Session Chair: J.W. Kwon, University of Missouri, USA

9:00 a.m. LONG-TERM STABLE ION SENSITIVE FIELD EFFECT TRANSISTOR SENSORS BASED ON MICROFLUIDICS INDUCED TUNABLE GRAPHENE FILMS

B. Zhang and T. Cui

University of Minnesota, USA

9:25 a.m. OPEN-CHANNEL MICROFLUIDICS: FREE-STANDING HYDROGEL MICROARRAYS FOR PROTEIN ELECTROPHORESIS

T.A. Duncombe ${ }^{1}$, T.M. Tran ${ }^{1}$, F. Benito-Lopez ${ }^{2}$, D. Diamond ${ }^{2}$, and A.E. Herr ${ }^{1}$

${ }^{1}$ University of California, Berkeley, USA and ${ }^{2}$ Dublin City University, IRELAND

9:50 a.m. SELF-ASSEMBLED NANOMACHINES FOR PROGRAMMABLE SENSING AND ACTUATION 10

G. Lavella and M.M. Maharbiz

University of California, Berkeley, USA

10:15 a.m. Break and Tabletop Exhibits 
SESSION 2 - Pumps \& Flow Actuators

Session Chair: H. Kim, University of Utah, USA

10:40 a.m. A MONOLITHIC 162-STAGE TWO-PART KNUDSEN

PUMP FOR HIGH COMPRESSION RATIO ................................................................. 14

S. An, N.K. Gupta, and Y.B. Gianchandani

University of Michigan, USA

11:05 a.m. A LEAK-TIGHT AND ZERO-DEAD-VOLUME MICRO GAS PUMP INTEGRATED WITH ELECTROKINETIC LIQUID PISTONS

J. Jiao, R. Almeida, and J.W. Kwon

University of Missouri, USA

11:30 a.m. MECHANO-FLUIDIC CHARACTERIZATION OF MICROSCALE DIELECTRIC BARRIER DISCHARGE PLASMA ACTUATORS

J.C. Zito, R.J. Durscher, J. Soni, S. Roy, and D.P. Arnold

University of Florida, USA

12:00 p.m. POSTER PREVIEW SESSION 1

Session Chairs: E. Chow, Palo Alto Research Center, USA and D. Polla, DNI/IARPA, USA

1:00 p.m. - Networking Lunch

2:30 p.m.

2:30 p.m. - POSTER SESSION 1 - Contributed and Late News

4:00 p.m. Session Chairs: M. Post, National Research Council, CANADA and H. Johari, Analog Devices, Inc., USA

Contributed and Late News Posters for Session 1

4:00 p.m. - POSTER SESSION 2 - Contributed and Late News

5:30 p.m. Session Chairs: J. Hammond, RFMD, USA and J. Bernstein, Draper Laboratory, USA

Contributed and Late News Posters for Session 2 


\section{Tuesday, June 5}

7:30 a.m. - Breakfast

PLENARY SPEAKER 2

Session Chair: F. Ayazi, Georgia Institute of Technology, USA

8:15 a.m. WIRELESS IMPLANTABLE MICROSYSTEMS:

CREATING A REVOLUTION IN HEALTH CARE .................................................... 26

Kensall D. Wise

University of Michigan, USA

SESSION 3 - Implantable Biomedical Devices

Session Chair: A. Fitzgerald, AM Fitzgerald \& Associates, USA

9:00 a.m. FABRICATION OF 3D PARYLENE SHEATH PROBES FOR RELIABLE NEUROPROSTHETIC RECORDINGS

J.T.W. Kuo ${ }^{1}$, B.J. Kim ${ }^{1}$, S.A. Hara ${ }^{1}$, C. Lee ${ }^{1}$, C.A. Gutierrez ${ }^{2}$,

T. Hoang ${ }^{1}$, and E. Meng ${ }^{1}$

${ }^{1}$ University of Southern California, USA and ${ }^{2}$ Independent Consultant, USA

9:25 a.m. ULTRASONIC NEURAL PROBE FOR REAL TIME

ELECTROMECHANICAL HISTOLOGY OF NEURAL INTERFACES

P.C. Chen, C.J. Shen, J.C. Kuo, C.G. Clark, B. Nijjar, C. Schaffer,

R.F. Gilmour, Jr., and A. Lal

Cornell University, USA

9:50 a.m. A MICROFABRICATED WIRELESS RF PRESSURE SENSOR MADE COMPLETELY OF BIODEGRADABLE MATERIALS

M. Luo, C. Song, F. Herrault, and M.G. Allen

Georgia Institute of Technology, USA

10:15 a.m. Break and Tabletop Exhibits 
SESSION 4 - Physical Sensors

Session Chair: M. Ziaei-Moayyed, Sandia National Laboratories, USA

10:40 a.m. A NOVEL MAGNETOMETER EMPLOYING MAGNETOVISCOUS EFFECT OF FERROFLUIDS

G. Hatipoglu and S. Tadigadapa

Pennsylvania State University, USA

11:05 a.m. SENSITIVE UNCOOLED IR DETECTORS USING GALLIUM NITRIDE RESONATORS AND SILICON NITRIDE ABSORBERS

V.J. Gokhale ${ }^{1}$, J. Roberts ${ }^{2}$, and M. Rais-Zadeh ${ }^{1}$

${ }^{1}$ University of Michigan, USA and ${ }^{2}$ Nitronex Corporation, USA

11:30 a.m. IN-AIR ULTRASONIC RANGEFINDING AND ANGLE ESTIMATION USING AN ARRAY OF ALN MICROMACHINED TRANSDUCERS

R.J. Przybyla ${ }^{1}$, S.E. Shelton ${ }^{2}$, A. Guedes ${ }^{2}$, R. Krigel ${ }^{1}$, D.A. Horsley ${ }^{2}$, and B.E. Boser ${ }^{1}$

${ }^{1}$ University of California, Berkeley, USA and ${ }^{2}$ University of California, Davis, USA

11:55 a.m. A NOVEL, HIGH-RESOLUTION RESONANT THERMOMETER USED FOR TEMPERATURE COMPENSATION OF A

COFABRICATED PRESSURE SENSOR

C.-F. Chiang ${ }^{1}$, A.B. Graham ${ }^{2}$, E.J. Ng ${ }^{1}$, C.H. Ahn ${ }^{1}$, G.J. O'Brien ${ }^{2}$, and T.W. Kenny ${ }^{1}$

${ }^{1}$ Stanford University, USA and ${ }^{2}$ Robert Bosch RTC, USA

12:20 p.m. - Networking Lunch

2:00 p.m.

7:00 p.m. - Banquet

9:00 p.m. 


\section{Wednesday, June 6}

7:30 a.m. - Breakfast

PLENARY SPEAKER 3

Session Chair: D. Monk, Freescale Semiconductor, Inc., USA

8:15 a.m. IT MAKES SENSE: HOW EXTREME ANALOG AND

SENSING WILL CHANGE THE WORLD ..........................................................58

Benedetto Vigna

STMicroelectronics, SWITZERLAND

\section{SESSION 5 - Resonators}

Session Chair: D. Weinstein, Massachusetts Institute of Technology, USA

9:00 a.m. MEMS ENABLES OSCILLATORS WITH SUB-PPM

FREQUENCY STABILITY AND SUB-PS JITTER

R. Melamud, P.M. Hagelin, C.M. Arft, C. Grosjean, N. Arumugam, P. Gupta,

G. Hill, M. Lutz, A. Partridge, and F. Assaderaghi

SiTime Corporation, USA

9:25 a.m. MAGNETOELECTRIC RESONANT GATE TRANSISTOR

F. Li, R. Misra, Z. Fang, C. Curwen, Y. Wu, Q.M. Zhang, P. Schiffer,

S. Tadigadapa, and S. Datta

Pennsylvania State University, USA

9:50 a.m. A SELF-CONTROLLED FREQUENCY TRIMMING TECHNIQUE FOR MICROMECHANICAL RESONATORS

A. Hajjam, K. Dietrich, A. Rahafrooz, and S. Pourkamali

University of Denver, USA

10:15 a.m. Break and Tabletop Exhibits 
SESSION 6 - Rotary Actuators \& Motors

Session Chair: L. Fréchette, Université de Sherbrooke, CANADA

10:40 a.m. ELECTROSTATICALLY DRIVEN ROTOR ON

CONDUCTIVE LIQUID RING BEARINGS ................................................................... 78

G. Sun ${ }^{1}$, T. Liu ${ }^{1}$, P. Sen ${ }^{2}$, W. Shen ${ }^{2}$, C. Gudeman ${ }^{2}$, and C.-J. Kim ${ }^{1}$

${ }^{1}$ University of California, Los Angeles, USA and

${ }^{2}$ Innovative Micro Technology (IMT), USA

11:05 a.m. NANOGAP ULTRASONIC ACTUATOR FOR NON-CONTACT

CONTROL OF LEVITATED INERTIAL SENSOR ROTOR

S. Piratla, M. Pandey, and A. Lal

Cornell University, USA

11:30 a.m. MINIATURE WIRELESS RESONANT ROTARY MOTOR

ACTUATED BY LITHOGRAPHICALLY MICROMACHINED

MAGNETOELASTIC FOIL

J. Tang, S.R. Green, and Y.B. Gianchandani

University of Michigan, USA

11:55 a.m. - Networking Lunch

1:30 p.m. 
SESSION 7 - Late News Orals

Session Chair: G. Skidmore, DRS Technologies, USA

1:30 p.m. A VIRTUAL MICROFLUIDIC CHANNEL WITH OIL-REPELLANT SURFACES FOR GUIDING LOW SURFACE TENSION LIQUIDS

R. Almeida and J.W. Kwon

University of Missouri, USA

1:45 p.m. ACOUSTO-OPTIC FREQUENCY MODULATOR

S. Tallur and S.A. Bhave

Cornell University, USA

2:00 p.m. AUTONOMOUS MICROSYSTEMS FOR ASTROBIOLOGY:

DEVELOPMENT OF, AND SPACEFLIGHT RESULTS

FROM, THE O/OREOS NANOSATELLITE

A.J. Ricco ${ }^{1}$, P. Ehrenfreund ${ }^{2}$, D. Squires ${ }^{1}$, M. Piccini ${ }^{1}$, C. Beasley ${ }^{1}$, N. Bramall ${ }^{1}$,

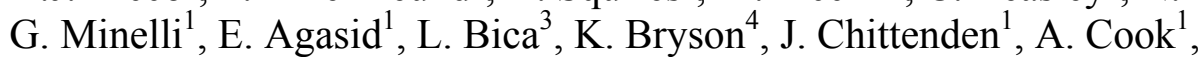

M. Diaz-Aguado ${ }^{1}$, C. Friedericks ${ }^{1}$, M. Henschke ${ }^{1}$, J.W. Hines ${ }^{1}$, C. Kitts ${ }^{3}$, D. Landis ${ }^{5}$,

E. Luzzi ${ }^{1}$, D. Ly $^{1}$, N. Mai ${ }^{1}$, R. Mancinelli ${ }^{4}$, A. Mattioda ${ }^{1}$, M. McIntyre ${ }^{1}$, M. Neumann ${ }^{3}$,

W. Nicholson ${ }^{6}$, M. Parra ${ }^{1}$, R. Quinn ${ }^{7}$, R. Rasay ${ }^{3}$, R. Ricks ${ }^{1}$, O. Santos ${ }^{1}$, A. Schooley ${ }^{1}$,

E. Stackpole ${ }^{1}$, L. Timucin ${ }^{1}$, B. Yost ${ }^{1}$, and A. Young ${ }^{3}$

${ }^{1}$ NASA Ames Research Center, USA, ${ }^{2}$ Space Policy Institute, USA,

${ }^{3}$ Santa Clara University, USA, ${ }^{4}$ Bay Area Environmental Research Institute, USA,

${ }^{5}$ Draper Laboratory, USA, ${ }^{6}$ University of Florida, USA, and ${ }^{7}$ SETI Institute, USA

2:15 p.m. MICROFLUIDIC OPTOMECHANICAL OSCILLATORS

G. Bahl, K.H. Kim, W. Lee, J. Liu, X. Fan, and T. Carmon

University of Michigan, USA

2:30 p.m. RECONFIGURABLE SIBAR FILTERS WITH SIDEWALL

ALUMINUM NITRIDE SIGNAL TRANSDUCTION

R. Tabrizian and F. Ayazi

Georgia Institute of Technology, USA

2:45 p.m. THREE-DIMENSIONAL HIERARCHICAL MICROBATTERY

ELECTRODES FOR TUNABLE HIGH ENERGY AND

POWER DENSITY BATTERIES

K. Gerasopoulos ${ }^{1}$, E. Pomerantseva ${ }^{1}$, A. Brown ${ }^{1}$, C. Wang ${ }^{1}$, J. Culver ${ }^{1}$, M. McCarthy ${ }^{2}$, and R. Ghodssi ${ }^{1}$

${ }^{1}$ University of Maryland, USA and ${ }^{2}$ Drexel University, USA 
3:00 p.m. POSTER PREVIEW SESSION 2

3:30 p.m. Session Chair: R. Candler, University of California, Los Angeles, USA

5:00 p.m. - POSTER SESSION 3 - Contributed

6:30 p.m. Session Chairs: P. Loeppert, Knowles Electronics, Inc., USA and M. Lutz, SiTime, USA

Contributed Posters for Session 3

6:30 p.m. - POSTER SESSION 4 - Commercial \& Open

8:00 p.m. $\quad$ Session Chairs: S. Bergbreiter, University of Maryland, USA and

X.-A. Fu, University of Louisville, USA

Papers not included in the Technical Digest

8:00 p.m. - Rump Session - Qualcomm \$10M Tricorder XPrize: Microsystems is the Word!

9:30 p.m. $\quad$ Session Chair: M. Mehregany, Case Western University, USA

The Rump Session is not sponsored by the Qualcomm Tricorder X PRIZE or the X PRIZE Foundation.

For official information regarding the prize, please visit www.qualcommtricorderxprize.org. 


\section{Thursday, June 7}

7:30 a.m. - Breakfast

PLENARY SPEAKER 4

Session Chair: H. Jerman, Coherent, USA

8:15 a.m. HOW WIRELESS HEALTH WILL

CHANGE HEALTH CARE DELIVERY

102

Mark J. Zdeblick

Proteus Biomedical, Inc., USA

\section{SESSION 8 - RF MEMS}

Session Chair: M. Rais-Zadeh, University of Michigan, USA

9:00 a.m. RF MEMS CAPACITOR INTEGRATION

A.K. Stamper ${ }^{1}$, S.J. Cunningham ${ }^{3}$, D.R. DeReus ${ }^{3}$, C. Gillman ${ }^{1}$, M.D. Gordon ${ }^{1}$, Z.-X. He ${ }^{1}$, C.V. Jahnes ${ }^{2}$, N. Lai ${ }^{1}$, S.L. Luce ${ }^{1}$, J. Maling ${ }^{1}$, A.S. Morris ${ }^{3}$, W.J. Murphy ${ }^{1}$, I. Vitomirov ${ }^{3}$, E.J. White ${ }^{1}$, and B.K. Wong ${ }^{1}$ ${ }^{1} I B M$, Essex Junction, VT, USA, ${ }^{2} I B M$, Inc. Yorktown Heights, NY, USA and

${ }^{3}$ Wispry, Inc., USA

9:25 a.m. TUNABLE MOBILE HANDSET ANTENNA IMPEDANCE MATCH BASED ON RF MEMS BI-STATE CAPACITORS X. Zhu ${ }^{1}$, A. Morris ${ }^{3}$, G. Hutcheson ${ }^{1}$, M. Brobston ${ }^{1}$, Y.J. Shin ${ }^{2}$, S. Cunningham ${ }^{3}$, J.H. Lee ${ }^{2}$, and J. Hilbert ${ }^{3}$

${ }^{1}$ Samsung Telecommunications America, USA, ${ }^{2}$ Samsung Electronics, SOUTH KOREA, and ${ }^{3}$ WiSpry, Inc., USA

9:50 a.m. ULTRA-MINIATURIZED POWER CONVERTER MODULES USING MICROMACHINED COPPER SCAFFOLDS

C.D. Meyer ${ }^{1,2}$, S.S. Bedair ${ }^{1}$, B.C. Morgan ${ }^{1}$, and D.P. Arnold ${ }^{2}$

${ }^{1}$ U.S. Army Research Laboratory, USA and ${ }^{2}$ University of Florida, USA

10:15 a.m. Break and Tabletop Exhibits 
SESSION 9 - Emerging Technologies

Session Chair: S. Tadigadapa, Pennsylvania State University, USA

10:40 a.m. CHAOTIC BEHAVIOUR OF A MEMBRANE RESONATOR WITH NANOWIRE ARRAY

Y. Lu, M. Pandey, and A. Lal

Cornell University, USA

11:05 a.m. LARGE AREA SEMI-PERMEABLE ENCAPSULATION MEMBRANES USING CARBON NANOTUBE COMPOSITES

A. Mahajerin and L. Lin

University of California, Berkeley, USA

11:30 a.m. DIRECT-WRITE SELF-ASSEMBLY OF 3D

COLLOIDAL MICROSTRUCTURES

J. Beroz, M. Bedewy, and A.J. Hart

University of Michigan, USA

11:55 a.m. A FERROFLUID-BASED PRESSURE SENSOR FOR BIOMEDICAL APPLICATIONS

G. Chitnis and B. Ziaie

Purdue University, USA

12:20 p.m. - Networking Lunch and Workshop Adjourns

2:00 p.m. 


\section{POSTER SESSION 1 \\ Contributed and Late News \\ Monday, June 4 \\ 2:30 p.m. $-4: 00$ p.m.}

\section{Actuators}

P1 HYDROGEL CHECK VALVE WITH NON-ZERO CRACKING

PRESSURE FOR USE AS A POTENTIAL ALTERNATIVE

HYDROCEPHALUS TREATMENT METHOD

H.N. Schwerdt ${ }^{1}$, R. Bristol ${ }^{2}$, and J. Chae ${ }^{1}$

${ }^{1}$ Arizona State University, USA and ${ }^{2}$ Phoenix Children's Hospital, USA

P2 A LARGE PISTON DISPLACEMENT MEMS MIRROR WITH ELECTROTHERMAL LADDER ACTUATOR ARRAYS FOR FOURIER TRANSFORM SPECTROSCOPY APPLICATIONS

S.R. Samuelson and, H. Xie

University of Florida, USA

P3 GENERATION OF PRESSURE BY EWOD-ACTUATED DROPLETS

J. Jenkins and C.-J. Kim

University of California, Los Angeles, USA

Bio-Inspiration and Biomedical Devices

P4 A HIGHLY DENSE NANONEEDLE ARRAY FOR INTRACELLULAR GENE DELIVERY

S.-J. Paik, S. Park, V. Zarnitsyn, S. Choi, X.D. Guo, M.R. Prausnitz, and M.G. Allen

Georgia Institute of Technology, USA

P5 CHRONICALLY IMPLANTABLE, 121-CHANNEL, POLYMER MICROELECTRODE ARRAY WITH HERMETICALLY-SEALED

WIRELESS INTERFACE

A. Tooker, K.G. Shah, V. Tolosa, H. Sheth, S. Felix, T. Delima, and S. Pannu

Lawrence Livermore National Laboratory, USA

P6 FLIP-CHIP BASED PACKAGING FOR LINEAR RATCHETING MICROACTUATORS ENABLES 3D STACKS OF MOVEBLE MICROELECTRODES FOR THE BRAIN

J. Sutanto ${ }^{1,2}$, S. Anand ${ }^{1}$, R. Korb ${ }^{1}$, L. Zhou ${ }^{1}$, M. Okandan ${ }^{3}$, M. Baker ${ }^{3}$, and J. Muthuswamy ${ }^{1}$

${ }^{1}$ Arizona State University, USA, ${ }^{2}$ Amkor Technology, USA, and

${ }^{3}$ Sandia National Laboratory, USA 
C. Lee, L. Jug, and E. Meng

University of Southern California, USA

P8 MEMS FORCE PROBES FOR CELL MECHANOBIOLOGY

AT THE MICROSECOND SCALE 165

J.C. Doll and B.L. Pruitt

Stanford University, USA

Biological Diagnostics, Systems, and Analysis

P9

A 1.5 MICRO-LITER MICROBIAL FUEL CELL ARRAY FOR

RAPID SCREENING OF EXOELECTROGENIC BACTERIA

S. Choi ${ }^{1}$, S. Mukherjee ${ }^{1}$, S. Su${ }^{2}$, W. Panmanee ${ }^{1}$, R.T. Irvin ${ }^{2,3}$, and D.J. Hassett ${ }^{1,3,4}$

${ }^{1}$ University of Cincinnati, USA, ${ }^{2}$ University of Alberta, CANADA, ${ }^{3}$ Pilus Energy LLC, USA, and ${ }^{4}$ Cincinnati VA Hospital, USA

P10 A MICROMACHINED CHEMICAL SENSOR FOR SEA FLOOR ENVIRONMENTS: INITIAL RESULTS

T. $\mathrm{Li}^{1}$, K. Ding ${ }^{2}$, W.E. Seyfried, Jr. ${ }^{2}$, and Y.B. Gianchandani ${ }^{1}$

${ }^{1}$ University of Michigan, USA and ${ }^{2}$ University of Minnesota, USA

P11 A SMART BIOCHIP WITH HAND-HELD ANALYZER FOR MONITORING SPECIFIC ENVIRONMENTAL BIOMARKERS

J. Han ${ }^{1}$, J. Kai ${ }^{1}$, S.H. Lee ${ }^{1}$, A. Puntambekar ${ }^{1}$, J.-Y. Lim ${ }^{2}$, D. Sul ${ }^{2}$, and C. Ahn ${ }^{1,3}$

${ }^{1}$ Siloam Biosciences, USA, ${ }^{2}$ Korea University, SOUTH KOREA, and

${ }^{3}$ University of Cincinnati, USA

P12 ADVANCING NEXT-GENERATION PROTEOMICS: A POLYMER-PATTERNED MICROCHAMBER ENABLES

INTEGRATION OF THE DISTINCT PROTEIN SEPARATIONS COMPRISING TWO-DIMENSIONAL ELECTROPHORESIS

A.M. Tentori, A.J. Hughes, and A.E. Herr

University of California, Berkeley and University of California, San Francisco

Joint Graduate Group, USA

P13 MULTICOLOR QUANTUM DOT-BASED FLUORESCENCE

EXCITATION FOR CELLULAR MICRO-ARRAY SCREENING

K. Hoshino, S. Diong, A. Gopal, E. Ng, and X.J. Zhang

University of Texas, USA

P14 SYNTHESIS OF DIVERSE TRACERS ON EWOD MICRODEVICE FOR POSITRON EMISSION TOMOGRAPHY (PET)

S. Chen, R. Javed, J. Lei, H.-K. Kim, G. Flores, R.M. van Dam, P.Y. Keng, and C.-J. Kim University of California, Los Angeles, USA 
H. Ben-Yoav, A.D. Brown, E. Pomerantseva, D.L. Kelly, J.N. Culver, and R. Ghodssi

University of Maryland, USA

Characterization, Fabrication and Materials

P16 HIGH-DENSITY, BIO-COMPATIBLE, AND HERMETIC ELECTRICAL FEEDTHROUGHS USING EXTRUDED METAL VIAS

K.G. Shah, T. DeLima, S. Felix, H. Sheth, V. Tolosa, A. Tooker, and S. Pannu

Lawrence Livermore National Laboratory, USA

P17 HIGHLY STABLE SURFACE FUNCTIONALIZATION OF HIGH-ASPECT-RATIO MICROGAS CHROMATOGRAPHY COLUMNS USING LAYER-BY-LAYER SELF-ASSEMBLY $\mathrm{OF} \mathrm{SiO}_{2}$ NANOPARTICLES

D. Wang ${ }^{1}$, H. Shakeel ${ }^{1}$, R. Montazami ${ }^{2}$, G. Rice ${ }^{3}$, J.R. Heflin ${ }^{1}$, and M. Agah ${ }^{1}$

${ }^{1}$ Virginia Tech, USA, ${ }^{2}$ Iowa State University, USA, and ${ }^{3}$ College of William \& Mary, USA

P18 ION-SENSITIVE FIELD EFFECT TRANSISTOR SENSORS USING SUSPENDED GRAPHENE NANORIBBON PATTERNED BY SHRINK LITHOGRAPHY

B. Zhang and T. Cui

University of Minnesota, USA

P19 NANOPOROUS-BASED HYDROPHILIC POLYMER MICROCHANNEL USING A SPRAY LAYER-BY-LAYER ELECTROSTATIC NANO-ASSEMBLY

K.K. Lee and C.H. Ahn

University of Cincinnati, USA

P20 RAPID AND FACILE FABRICATION OF 3D-NETWORKED

CIRCULAR MICROFLUIDIC CHANNELS IN PDMS

J. Lee, J. Paek, and J. Kim

Iowa State University, USA

P21 THREE-DIMENSIONALLY-PRINTED (3DP) MICROFLUIDIC DEVICES

O.H. Paydar, C.N. Paredes, and R.N. Candler

University of California, Los Angeles, USA

\section{Gas Sensors}

P22 A MICRO GAS CHROMATOGRAPHY COLUMN WITH AN EMBEDDED OUT-OF-PLANE THERMAL CONDUCTIVITY DETECTOR

S. Narayanan and M. Agah

Virginia Tech, USA 


\section{Microfluidics}

P23 3D FOCUSING IN PARALLEL MICROCHANNELS FOR HIGH-THROUGHPUT SYNTHESIS OF POLYMERIC NANOPARTICLES 225 J.-M. Lim ${ }^{1,2}$, P.M. Valencia ${ }^{1}$, M. Rhee ${ }^{1,2}$, R.S. Langer ${ }^{1}$, O.C. Farokhzad ${ }^{2}$, and R. Karnik ${ }^{1}$ ${ }^{1}$ Massachusetts Institute of Technology, USA and

${ }^{2}$ Brigham and Women's Hospital - Harvard Medical School, USA

P24 AUTONOMOUS RAILING AND TRAPPING OF MICROBEADS FOR CONTINUOUS FLOW MULTI-STAGE MICROFLUIDIC PROCESSES

R.D. Sochol, W.E.R. Krieger, L.P. Lee, and L. Lin

University of California, Berkeley, USA

P25 MICROFLUIDIC BIOFILM OBSERVATION, ANALYSIS

AND TREATMENT (MICRO-BOAT) PLATFORM

Y.W. Kim, M.P. Mosteller, M.T. Meyer, H. Ben-Yoav, W.E. Bentley, and R. Ghodssi

University of Maryland, USA

P26 MICROFLUIDIC DEVICES FOR RAPID LABEL-FREE SEPARATION

OF CELLS AND POINT-OF-CARE DIAGNOSTICS

S. Bose ${ }^{1}$, M.-H. Hollatz ${ }^{1}$, C.-H. Lee ${ }^{1}$, J.M. Karp ${ }^{1,2}$, and R. Karnik ${ }^{1}$

${ }^{1}$ Massachusetts Institute of Technology, USA and ${ }^{2}$ Harvard Medical School, USA

P27 MICROSCALE SURFACE ENERGY PROPERTIES FOR

ENHANCED SURFACE CONDENSATION AND

SAMPLING OF EXHALED BREATH METABOLITES

H.K. Bardaweel ${ }^{1}$, K. Zamuruyev ${ }^{1}$, J.-P. Delplanque ${ }^{1}$, N.J. Kenyon ${ }^{1}$, C. Carron ${ }^{2}$,

O. Brand ${ }^{2}$, and C.E. Davis ${ }^{1}$

${ }^{1}$ University of California, Davis, USA and ${ }^{2}$ Georgia Institute of Technology, USA

P28 SELF-FORMED, NATURALLY-OPTIMIZED MICROFLUIDIC

CHANNELS IN POLYDIMETHYLSILOXANE (PDMS)

A. Vinson and J.A. Potkay

Department of Veterans Affairs Medical Center, USA and

Case Western Reserve University, USA

\section{Late News}

P29 DELAYED LEIDENFROST PHENOMENA ON BIOTEMPLATED NANOSTRUCTURED SURFACES

S. King, M. Rahman, E. Olceroglu, and M. McCarthy

Drexel University, USA

P30 HIGH PERFORMANCE CARBON NANOTUBE/SILICON HETEROJUNCTION SOLAR CELLS

X. Li, Y. Jung, Y. Sun, M.A. Reed, and A.D. Taylor

Yale University, USA 
P31 LOW COST LOCALIZED BONDING OF PMMA

MICROFLUIDICS USING MICROWAVE SUSCEPTORS

A. Toossi, D. Sameoto, and M. Daneshmand

University of Alberta, CANADA

P32 PHOTORESIST-DERIVED POROUS CARBON FOR

INTEGRATED ON-CHIP ENERGY STORAGE

B.I. Hsia, M. Vincent, M.S. Kim, C. Carraro, and R. Maboudian

${ }^{1}$ University of California, Berkeley, USA

P33 WET ETCHING \& UNIFORM WAFER-LEVEL THINNING OF

BULK PIEZOELECTRIC CERAMICS ON SILICON

E.E. Aktakka, R.L. Peterson, and K. Najafi

University of Michigan, Ann Arbor, USA

\author{
POSTER SESSION 2 \\ Contributed and Late News \\ Monday, June 4 \\ 4:00 p.m. - 5:30 p.m.
}

Characterization, Fabrication and Materials

P34 HIGH PERFORMANCE SPUTTERED THERMOELECTRIC

JUNCTIONS FOR MICROMECHANICAL DEVICES ................................................. 258

R.P. Shea, A.S. Gawarikar, and J.J. Talghader

University of Minnesota, USA

P35 CHARACTERIZATION AND PRODUCTION CONTROL FOR DRIE

OF MEMS ACCELEROMETERS USING OPTICAL DISPLACEMENT

TRANSDUCER MEASUREMENTS

B. Keese ${ }^{1}$, B. Homeijer ${ }^{1}$, J.L. Williams ${ }^{1}$, and P. Knutrud ${ }^{2}$

${ }^{1}$ Hewlett-Packard, USA and ${ }^{2}$ Inspectrology, USA

P36 TITANIA SILICATE / FUSED QUARTZ GLASSBLOWING FOR 3-D FABRICATION OF LOW INTERNAL LOSS

WINEGLASS MICRO-STRUCTURES

D. Senkal, C.R. Raum, A.A. Trusov, and A.M. Shkel

University of California, Irvine, USA

P37 ULTRA-STABLE EPITAXIAL POLYSILICON RESONATORS

E.J. Ng ${ }^{1}$, S. Wang ${ }^{1}$, D. Buchman ${ }^{1}$, C.-F. Chiang ${ }^{1}$, T.W. Kenny ${ }^{1}$, H. Muenzel ${ }^{2}$,

M. Fuertsch ${ }^{3}$, J. Marek ${ }^{3}$, U.M. Gomez ${ }^{3}$, G. Yama ${ }^{2}$, and G. O'Brien ${ }^{2}$

${ }^{1}$ Stanford University, USA, ${ }^{2}$ Robert Bosch RTC, USA, and ${ }^{3}$ Robert Bosch GmbH, GERMANY 
P. Shao, L.D. Sorenson, X. Gao, and F. Ayazi

Georgia Institute of Technology, USA

Physical and Optical Sensors and Devices

P39 A MEMS CONTROLLED CAVITY OPTOMECHANICAL SENSING SYSTEM

H. Miao ${ }^{1,2}$, K. Srinivasan ${ }^{1}$, and V.A. Aksyuk ${ }^{1}$

${ }^{1}$ National Institute of Standards and Technology, USA and ${ }^{2}$ University of Maryland, USA

P40 A SINGLE-CHIP CMOS-MEMS MICROGRAVITY ACCELEROMETER

283

Y. Zhang, G. Meng, and N. Yazdi

Evigia Systems, Inc., USA

P41 ACHIEVING LONG-TERM BIAS STABILITY IN HIGH-Q

INERTIAL MEMS BY TEMPERATURE SELF-SENSING

WITH A 0.5 MILLICELCIUS PRECISION

I.P. Prikhodko, A.A. Trusov, and A.M. Shkel

University of California, Irvine, USA

P42 ALUMINUM NITRIDE PIEZOELECTRIC MICROMACHINED

ULTRASOUND TRANSDUCER ARRAYS

S. Shelton ${ }^{1}$, A. Guedes ${ }^{1}$, R. Przybyla ${ }^{2}$, R. Krigel ${ }^{2}$, B. Boser ${ }^{2}$, and D.A. Horsley ${ }^{1}$

${ }^{1}$ University of California, Davis, USA and ${ }^{2}$ University of California, Berkeley, USA

P43 CHARGED PARTICLE DETECTION USING A

MICROMECHANICAL ELECTROMETER

G. Jaramillo ${ }^{1}$, M. Li ${ }^{1}$, C. Buffa ${ }^{2}$, F.J. Brechtel ${ }^{3}$, and D.A. Horsley ${ }^{1}$

${ }^{1}$ University of California, Davis, USA, ${ }^{2}$ Politecnico di Milano, ITALY, and

${ }^{3}$ Brechtel Manufacturing Inc., USA

P44 DROPLET-BETWEEN-ELECTRODES FOR ULTRAHIGH

INTERFACIAL CAPACITIVE SENSING

B. Nie, S. Xing, J.D. Brandt, and T. Pan

University of California, Davis, USA

P45 EXPERIMENTAL VERIFICATION OF A CAPACITIVE SHEAR STRESS

SENSOR FOR LOW-SPEED WIND TUNNEL APPLICATIONS

J. Meloy, J. Sells, V. Chandrasekharan, J. Griffin, L.N. Cattafesta, D.P. Arnold, and M. Sheplak

University of Florida, USA

P46 FORCED OSCILLATION AND HIGHER HARMONIC DETECTION IN AN INTEGRATED CMOS-MEMS SCANNING PROBE MICROSCOPE 307

N. Sarkar, R.R. Mansour, and K. Trainor

University of Waterloo, CANADA 
P47 HIGHLY SENSITIVE III-V NITRIDE BASED PIEZORESISTIVE MICROCANTILEVER USING EMBEDDED ALGAN/GAN HFET

A. Talukdar and G. Koley

University of South Carolina, USA

P48 MEMS AIR-MICROFLUIDIC SENSOR FOR PORTABLE MONITORING OF AIRBORNE PARTICULATES

F.L. Doering, I. Paprotny, and R.M. White

University of California, Berkeley, USA

P49 MEMS AND METAMATERIAL-BASED TERAHERTZ DETECTOR

H. Tao ${ }^{1}$, E.A. Kadlec ${ }^{2}$, A.C. Strikwerda ${ }^{1}$, K. Fan ${ }^{1}$, W.J. Padilla ${ }^{3}$, R.D. Averitt ${ }^{1}$,

E.A. Shaner ${ }^{2}$, and X. Zhang ${ }^{1}$

${ }^{1}$ Boston University, USA, ${ }^{2}$ Sandia National Laboratory, USA, and ${ }^{3}$ Boston College, USA

P50 MEMS-ENABLED RETARDING POTENTIAL ANALYZERS FOR

HYPERSONIC IN-FLIGHT PLASMA DIAGNOSTICS

E.V. Heubel, A.I. Akinwande, and L.F. Velásquez-García

Massachusetts Institute of Technology, USA

P51 NOVEL READOUT SCHEME FOR MEMS VIBRATORY

GYROSCOPES BASED ON SIGNAL PHASE SHIFT

M. Dalal, A.N. Shirazi, W.K. Sung, G. Casinovi, and F. Ayazi

Georgia Institute of Technology, USA

P52 PARYLENE SUPPORTED UNCOOLED THERMOELECTRIC

INFRARED DETECTOR WITH UMBRELLA LIKE ABSORBER

M.J. Modarres-Zadeh, Z.S. Carpenter, and R. Abdolvand

Oklahoma State University, USA

P53 SIDEWALL SILICON CARBIDE EMITTERS FOR TERAHERTZ VACUUM ELECTRONICS

J.P. Snapp ${ }^{1}$, J.-H. Lee ${ }^{1}$, J Provine ${ }^{1}$, I. Bargatin ${ }^{1}$, R. Maboudian ${ }^{2}$, T.H. Lee ${ }^{1}$, and R.T. Howe ${ }^{1}$

${ }^{1}$ Stanford University, USA and ${ }^{2}$ University of California at Berkeley, USA

P54 STRESS SENSITIVE CAPACITIVE TRANSDUCERS

TO VERIFY PACKAGE MODELS

A. McNeil and G. Li

Freescale Semiconductor Inc., USA

P55 ULTRA HIGH SENSITIVITY GRAPHENE

PIEZORESISTIVE PRESSURE SENSOR 343

H. Hosseinzadegan, M. Pandey, and A. Lal

Cornell University, USA 
Resonant Devices

P56 970 MHZ OVENIZED OSCILLATOR BASED ON AN

ALN MEMS RESONATOR WITH MONOLITICALLY

INTEGRATED SUSPENDED NANO HOT PLATE

M. Rinaldi ${ }^{1}$, A. Tazzoli ${ }^{2}$, C. Zuniga ${ }^{2}$, and G. Piazza ${ }^{2,3}$

${ }^{1}$ Northeastern University, USA, ${ }^{2}$ University of Pennsylvania, USA, and

${ }^{3}$ Carnegie Mellon University, USA

P57 APODIZATION TECHNIQUES FOR SPURIOUS MODE SUPPRESSION IN 900 MHZ ALUMINUM NITRIDE CONTOUR-MODE RESONATORS M. Giovannini ${ }^{1,2}$, S. Yazici ${ }^{1}$, N.-K. Kuo ${ }^{1}$, and G. Piazza ${ }^{3}$

${ }^{1}$ University of Pennsylvania, USA, ${ }^{2}$ Politecnico of Milan, ITALY,

${ }^{3}$ Canegie Mellon University, USA

P58 MICROMACHINED POLYCRYSTALLINE DIAMOND HEMISPHERICAL SHELL RESONATORS 355

M.L. Chan ${ }^{1}$, J. Xie ${ }^{2}$, P. Fonda ${ }^{1}$, H. Najar ${ }^{1}$, K. Yamazaki ${ }^{1}$, L. Lin ${ }^{2}$, and D.A. Horsley ${ }^{1}$

${ }^{1}$ University of California, Davis, USA and ${ }^{2}$ University of California Berkeley, USA

P59 PIEZOELECTIRC ROTATIONAL MODE DISK RESONATORS FOR LIQUID VISCOSITY MONITORING

E. Mehdizadeh ${ }^{1}$, J. Gonzales ${ }^{2}$, A. Rahafrooz ${ }^{1}$, R. Abdolvand ${ }^{2}$, and S. Pourkamali ${ }^{1}$

${ }^{1}$ University of Denver, USA and ${ }^{2}$ Oklahoma State University, USA

P60 RADIO-FREQUENCY MULTI-MODE MICROMECHANICAL DISK RESONATORS IN 500nm THIN SILICON CARBIDE (SiC) 363

H. Zamani, J. Lee, S. Rajgopal, C.A. Zorman, M. Mehregany, and P.X.-L. Feng

Case Western Reserve University, USA

P61 SINGLE TRANSISTOR OSCILLATOR BASED ON PIEZOELECTRIC RESONANT NANOCHANNEL FOR OPERATION IN LIQUIDS

C. Zuniga ${ }^{1}$, M. Rinaldi ${ }^{2}$, A. Tazzoli ${ }^{1}$, and G. Piazza ${ }^{3}$

${ }^{1}$ University of Pennsylvania, USA, ${ }^{2}$ Northeastern University, USA, and

${ }^{3}$ Canergie Mellon University, USA

P62 TEMPERATURE-COMPENSATED EXTENSIONAL-MODE MEMS

RESONATORS ON HIGHLY N-TYPE DOPED SILICON SUBSTRATES

M. Shahmohammadi, B.P. Harrington, J. Gonzales, and R. Abdolvand

Oklahoma State University, USA

P63 ZERO BIAS OPERATION OF THERMAL-PIEZORESISTIVE MICROMECHANICAL RESONATORS VIA INTERNAL

ELECTROMECHANICAL MIXING

A. Rahafrooz and S. Pourkamali

University of Denver, USA 
Late News

P64 A WIDEBAND THERMO-ACOUSTIC SOUND GENERATOR ON NANOPOROUS POLYMERIC SUBSTRATE

A. Kim, G. Chitnis, S.H. Song, and B. Ziaie

Purdue University, USA

P65 AN INTEGRATED FIBER-OPTIC MICROFLUIDIC DEVICE FOR DETECTION OF MULTICELLULAR ORGANISM MECHANICS

P. Liu, D. Mao, R.J. Martin, and L. Dong

Iowa State University, USA

P66 SENSITIVE AND SELECTIVE DETECTION OF A CANCER BIOMARKER, THYROGLOBULIN, IN UNDILUTED HUMAN SERUM USING COMPETITIVE ADSORPTION OF PROTEINS AND PATTERN RECOGNITION 383

R. Wang, S. Huang, J. Li, and J. Chae Arizona State University, USA

\section{POSTER SESSION 3 \\ Contributed \\ Wednesday, June 6 5:00 p.m. - 6:30 p.m.}

\section{Actuators}

P67 A HIGH ON/OFF RATIO MEMS CAPACITIVE SWITCH WITH APPLICATIONS IN SOLAR ENERGY HARVESTING

V. Thakar, Z. Wu, and M. Rais-Zadeh

University of Michigan, USA

P68 ANALOG RF MEMS ATTENUATOR WITH PZT ACTUATORS

T.G. Ivanov, R.M. Proie, J.S. Pulskamp, R.G. Polcawich, and A. Zaghloul US Army Research Laboratory, USA

P69 HIGH-FREQUENCY LARGE-DEFLECTION ELECTROSTATIC DIAPHRAGM ACTUATORS WITH MAXIMIZED VOLUME DISPLACEMENT

S.Y. Yee, R.L. Peterson, L.P. Bernal, and K. Najafi

University of Michigan, USA

P70 HISTOLOGY-EQUIVALENT VERTICAL OPTICAL SECTIONING USING A 2-AXIS MAGNETIC CONFOCAL MICROSCANNER H. Mansoor ${ }^{1}$, H. Zeng ${ }^{2}$, K. Chen ${ }^{1}$, Y. Yu ${ }^{2}$, J. Zhao ${ }^{2}$, and M. Chiao ${ }^{1}$

${ }^{1}$ University of British Columbia, CANADA and

${ }^{2}$ British Columbia Cancer Agency Research Centre, CANADA 
P71 IN-PLANE MEMS-BASED FABRY-PEROT FILTER FOR HIGH-SPEED WAVELENGTH-SWEPT SEMICONDUCTOR LASER

A. Poulin, R. St-Gelais, N. Goulamhoussen, G. Zhu, C. Boudoux, and Y.-A. Peter

École Polytechnique de Montréal, CANADA

P72 SOFTWARE-DEFINED MICROSTRIP ANTENNAS ENABLED THROUGH

LARGE VERTICAL DISPLACEMENT ZIPPER MICROACTUATORS

J. Felder ${ }^{1,2}$, E. Lee $^{2}$, E. Walton ${ }^{3}$, and D.L. DeVoe ${ }^{1}$

${ }^{1}$ University of Maryland, USA, ${ }^{2}$ Syntonics LLC, USA, and ${ }^{3}$ Ohio State University, USA

P73 LARGE STROKE ELECTROSTATIC COMB-DRIVE ACTUATORS

BASED ON A NOVEL FLEXURE MECHANISM

M. Olfatnia ${ }^{1}$, S. Sood ${ }^{1}$, J.J. Gorman ${ }^{2}$, and S. Awtar ${ }^{1}$

${ }^{1}$ University of Michigan, USA and ${ }^{2}$ National Institute of Standards and Technology, USA

P74 MEMS BASED SINGLE DEVICE "XOR" AND "AND" LOGIC GATES

FOR LSI MECHANICAL PROCESSORS OPERATING IN HIGH

RADIATION ENVIRONMENTS

F.K. Chowdhury ${ }^{1}$, S. Han ${ }^{2}$, D. Saab ${ }^{2}$, and M. Tabib-Azar ${ }^{1}$

${ }^{1}$ University of Utah, USA and ${ }^{2}$ Case Western Reserve University, USA

P75 TRAVELING WAVE ULTRASONIC MOTOR USING THIN-FILM PIEZOELECTRICS

R.Q. Rudy ${ }^{1,2}$, G.L. Smith ${ }^{1}$, D.L. DeVoe ${ }^{2}$, and R.G. Polcawich ${ }^{1}$

${ }^{1}$ U.S. Army Research Laboratory, USA and ${ }^{2}$ University of Maryland, USA

Characterization, Fabrication and Materials

P76 3D INTEGRATION USING SELF-ASSEMBLY AT AIR-WATER INTERFACE 421 K.S. Park ${ }^{1}$, C. Varel $^{1}$, J.H. Hoo ${ }^{1}$, R. Baskaran ${ }^{1,2}$, and K.F. Böhringer ${ }^{1}$

${ }^{1}$ University of Washington, USA and ${ }^{2}$ Intel Corporation, USA

P77 A NOVEL HIGH-DENSITY CAPACITOR DESIGN AND ITS FABRICATION TECHNIQUE BASED ON SELECTIVE ETCHING V.F.-G. Tseng ${ }^{1}$, K. $\mathrm{Ngo}^{2}$, and H. Xie

${ }^{1}$ University of Florida, USA and ${ }^{2}$ Virginia Polytechnic Institute and State University, USA

P78 A SIMPLE SOI-CMOS COMPATIBLE TECHNOLOGY

TO MAKE FLEXIBLE ELECTRONICS

$\mathrm{H}$. Tu and Y. Xu

Wayne State University, USA

P79 CHARACTERIZING METAL NANOPARTICLE FILMS: A METHODOLOGY USING SURFACE MICROMACHINING BY DIRECT PRINTING 433

E.W. Lam and M.A. Schmidt

Massachusetts Institute of Technology, USA 
P80 CVD HAFNIUM DIBORIDE AS A CONTACT MATERIAL FOR

NANOELECTROMECHANICAL SWITCHES

W.S. Lee ${ }^{1}$, A.N. Cloud ${ }^{2}$, J. Provine ${ }^{1}$, N. Tayebi ${ }^{1}$, R. Parsa ${ }^{1}$, S. Mitra ${ }^{1}$, H.-S.P. Wong ${ }^{1}$, J.R. Abelson ${ }^{2}$, and R.T. Howe ${ }^{1}$

${ }^{1}$ Stanford University, USA and ${ }^{2}$ University of Illinois, Urbana-Champaign, USA

P81 DIRECT-WRITE NANOLITHOGRAPHY ON FLEXIBLE SUBSTRATE

J. Chang ${ }^{1}$, M. Dommer ${ }^{2}$, B.Y. Lee ${ }^{1}$, and L. Lin ${ }^{1}$

${ }^{1}$ University of California, Berkeley, USA and

${ }^{2}$ École Polytechnique Fédérale de Lausanne (EPFL), SWITZERLAND

P82 GROWTH OF CARBON NANOTUBES DIRECTLY FROM NATURAL PROTEINS

H.-C. Hsieh ${ }^{1}$, M.M. Rahman ${ }^{1}$, T.C. Shen ${ }^{2}$, and H. Kim ${ }^{1}$

${ }^{1}$ University of Utah, USA and ${ }^{2}$ Utah State University, USA

P83 HIGH FLUX THERMAL MANAGEMENT WITH CMOS COMPATIBLE MICROSTRUCTURED SURFACES

K.-H. Chu ${ }^{1}$, R. Enright ${ }^{1,2}$, and E.N. Wang ${ }^{1}$

${ }^{I}$ Massachusetts Institute of Technology, USA and ${ }^{2}$ University of Limerick, IRELAND

P84 HIGH-STRESS FATIGUE EXPERIMENTS ON SINGLE

CRYSTAL SILICON IN AN OXYGEN-FREE ENVIRONMENT

V.A. Hong, S. Yoneoka, M.W. Messana, A.B. Graham, J.C. Salvia,

T.T. Branchflower, E.J. Ng, and T.W. Kenny

Stanford University, USA

P85 LOW POWER STRAIN SENSING USING TUNNELING

CURRENT THROUGH MOS CAPACITORS

L. Zhu and S. McNamara

University of Louisville, USA

P86 MICROFLUIDIC FABRICATION OF MONODISPERSE MICROCAPSULES FOR SELF HEALING MATERIAL 461

R.J. Lemmens and D.D. Meng

Michigan Technological University, USA

P87 NONLINEAR DAMPING FOR SHOCK PROTECTION OF

MEMS DEVICES USING SHEAR THICKENING FLUID 465

S. Iyer ${ }^{1}$, H. Lee ${ }^{1}$, M. Liger ${ }^{2}$, J. Judy ${ }^{1}$, and R. Candler ${ }^{1}$

${ }^{1}$ University of California, Los Angeles, USA and ${ }^{2}$ Google, USA

P88 OIL-INFUSED SUPERHYDROPHOBIC SILICON SURFACES FOR ENHANCED WATER CONDENSATION HEAT TRANSFER R. Xiao ${ }^{1}$, R. Enright ${ }^{1,2}$, N. Miljkovic ${ }^{1}$, and E.N. Wang ${ }^{1}$

${ }^{I}$ Massachusetts Institute of Technology, USA and ${ }^{2}$ University of Limerick, IRELAND 
P89 A HIGH DYNAMIC RESTORING FORCE ELECTROSTATIC ACTUATOR

A. Gupta ${ }^{1,2}$, M. Fletcher ${ }^{1}$, D. Agrawal ${ }^{1}$, C. McMillen ${ }^{1}$, and J.-B. Lee ${ }^{2}$

${ }^{1}$ Texas Instruments, Inc., USA and ${ }^{2}$ University of Texas at Dallas, USA

P90 USING DRIE SILICON AS A CUTTING TOOL FOR HIGH

PRECISION MICROMACHINING OF METAL ALLOYS

T. $\mathrm{Li}^{1}, \mathrm{Q}$. Bai ${ }^{2}$, and Y.B. Gianchandani ${ }^{1}$

${ }^{1}$ University of Michigan, USA and ${ }^{2}$ Agilent Research Laboratories, USA

P91 UV-LED LITHOGRAPHY FOR 3-D HIGH ASPECT

RATIO MICROSTRUCTURE PATTERNING 481

J.K. Kim, S.-J. Paik, F. Herrault, and M.G. Allen

Georgia Institute of Technology, USA

\section{Modeling}

P92 MODELING OF THE NEUTRAL AXES OF A CIRCULAR PIEZOELECTRIC MICROMACHINED TRANSDUCER IN TRANSMIT AND RECEIVE MODE . F. Sammoura, ${ }^{1,2}$ and S.-G. Kim ${ }^{1}$

${ }^{1}$ Masdar Institute of Science and Technology, UAE and

${ }^{2}$ Massachusetts Institute of Technology, USA

P93 APPLICATION OF A PREDICTION METHODOLOGY FOR MEMS RELIABILITY IN SHOCK ENVIRONMENTS

M. Naumann', D. Lin ${ }^{2}$, A. McNeil ${ }^{2}$, G. Li ${ }^{2}$, and J. Mehner ${ }^{1}$

${ }^{1}$ Chemnitz University of Technology, GERMANY and ${ }^{2}$ Freescale Semiconductor, Inc., USA

Power Generation and Management

P94 ENCAPSULATED THERMIONIC ENERGY CONVERTER

WITH STIFFENED SUSPENSION

J.H. Lee ${ }^{1}$, I. Bargatin ${ }^{1}$, K. Iwami ${ }^{1,2}$, K.A. Littau ${ }^{1}$, M. Vincent ${ }^{3}$, R. Maboudian ${ }^{3}$, Z.-X. Shen ${ }^{1}$, N.A. Melosh ${ }^{1}$, and R.T. Howe ${ }^{1}$

${ }^{1}$ Stanford University, USA, ${ }^{2}$ Tokyo University of Agriculture and Technology, JAPAN, and

${ }^{3}$ University of California, Berkeley, USA

P95 HYBRID ENERGY HARVESTING SYSTEM BASED ON MICROFLUIDICS WITH INTERFACE CIRCUITRY

A. Bramhanand ${ }^{1}$, M.M. Rahman ${ }^{1}$, Y.-C. Bae ${ }^{2}$, and H. Kim ${ }^{1}$

${ }^{1}$ University of Utah, USA and ${ }^{2}$ Chonnam National University, SOUTH KOREA

P96 SCALING EFFECT ON MEMS-BASED MICROBIAL FUEL CELLS: TOWARD A CARBON-NEUTRAL MINIATURIZED POWER SOURCE

H. Ren and J. Chae

Arizona State University, USA 
P97 SIC/ALN PIEZOELECTRIC ENERGY HARVESTERS FOR PULSED PRESSURE SOURCES IN HARSH ENVIRONMENT APPLICATIONS Y.-J. Lai ${ }^{1}$, W.-C. Li ${ }^{1}$, C.-M. Lin ${ }^{1}$, V.V. Felmetsger ${ }^{2}$, D.G. Senesky ${ }^{3}$, and A.P. Pisano ${ }^{1}$

${ }^{1}$ University of California, Berkeley, USA, ${ }^{2}$ OEM Group Incorporated, USA, and

${ }^{3}$ Stanford University, USA 


\title{
SIMPLE BIOANALYSIS
}

\author{
George M. Whitesides \\ Department of Chemistry and Chemical Biology Diagnostics for All \\ Wyss Institute for Biologically Inspired Engineering \\ Harvard University, Cambridge, MA, USA
}

\begin{abstract}
Diagnosis of disease starts with observation and measurement. Biomedical analyses intended to aid diagnosis are important in determining treatment; they are also important in determining the cost of treatment (for better or worse). The cost of diagnostics is usually hidden from patients (and largely, from doctors), but they can be significant. When diagnostics developed for use in the developed world are transferred into the developing world, where cost is often a key constraint, the issue of cost can be critical in allowing or denying their use to patients. Cost is also important in applications in public health, agriculture and veterinary medicine, and a variety of others.

We are exploring bioanalytical methods that have the characteristics that they provide accurate information that is useful in diagnosis, and do so at costs that are acceptable in the developing world. The core technology, which is being developed in a collaboration between Harvard University and Diagnostics for All (a not-for-profit company focused on engineering development of low-cost diagnostic devices) used paper patterned into hydrophobic and hydrophilic regions, to achieve control of microfluidic flows similar to (or in some cases superior to those generated in) open-channel microfluidic systems. There are also a number of highly developed, low-cost analytical technologies that can be re-purposed for use in lowcost diagnostics: we have focused our developments on glucometers, which provide a convenient method of conducting electroanalyses.
\end{abstract}

The methods being explored to generate new types of lowcost diagnostics also extend to less familiar types of analyses (for example, two-phase aqueous polymer systems, and magnetic levitation, for density-based separations; 3D mammalian cell growth in gels supported in paper and mesh sheets). The early consideration of "cost" and "simplicity" as parameters in the design of all these systems is, in fact, a very useful stimulus to consider solutions of types not necessarily immediately obvious in an academic environment.
Another key component of this area of diagnostic technology concerns the communication of results to doctors or other capable to carrying out diagnostics, since doctors are also in very limited supply in the developing world. A key initial step in the solution of this problem is the coupling of diagnostic results to (probably, initially, low-capability) cell-phones. We have also carried out initial steps in this area.

Some of this work is described in these papers:

[1] "Paper-Based ELISA". Cheng, C., Martinez, A. W., Gong, J., Mace, C. R., Phillips, S. T., Carrilho, E., Mirica, K. A. \& Whitesides, G. M. Angew. Chem. . 2010, 122, 4881-4884

[2] "Programmable Diagnostic Devices Made from Paper and Tape". Martinez, A. W., Phillips, S. T., Nie, Z., Cheng, C., Carrilho, E., Wiley, B. J. \& Whitesides, G. M. LOC. 2010, $10,2499-2504$

[3] "Integration of Paper-Based Microfluidic Devices with Commercial Electrochemical Readers". Nie, Z., Deiss, F., Liu, X., Akbulut, O. \& Whitesides, G. M. LOC. 2010, 10, 3163-3169 PMC3060706

[4] "Diagnostics for the Developing World: Microfluidic Paper-Based Analytical Devices". Martinez, A. W., Phillips, S. T., Whitesides, G. M. \& Carrilho, E. Anal. Chem. 2010, $82,3-10$

[5] "Paper-Based Piezoresistive MEMS Sensors". Liu, X., Mwangi, M., Li, X., O'Brien, M. \& Whitesides, G. M. LOC. 2011, 11, 2189-2196

[6] "Measuring Markers of Liver Function Using a Micropatterned Paper Device Designed for Blood from a Fingerstick". Vella, S. J., Beattie, P., Cademartiri, R., Laromaine, A., Martinez, A. W., Phillips, S. T., Mirica, K. A. \& Whitesides, G. M. Anal. Chem. 2012, 84, 2883-2891

[7] "Measuring Binding of Protein to Gel-Bound Ligands Using Magnetic Levitation". Shapiro, N. D., Mirica, K. A., Soh, S., Phillips, S. T., Taran, O., Mace, C. R., Shevkoplyas, S. S. \& Whitesides, G. M. J. Amer. Chem. Soc. 2012, 134, 5637-5646 


\title{
LONG-TERM STABLE ION SENSITIVE FIELD EFFECT TRANSISTOR SENSORS BASED ON MICROFLUIDICS INDUCED TUNABLE GRAPHENE FILMS
}

\author{
B. Zhang and T. Cui* \\ University of Minnesota, Minneapolis, USA
}

\begin{abstract}
The ion sensitive field effect transistor (ISFET) sensor based on microfluidics induced films is reported in this paper. The graphene film demonstrates controllably tunable thickness different from the conventional graphene composites, and the sensor has a long-term stability in chemical detection. Using capillarity to introduce a graphene suspension solution to a microfluidic system, the graphene film patterns were confined and formed in microchannels. SEM, AFM and Raman spectroscopy were used to analyze the graphene films, demonstrating the tunable thickness of the graphene films in the microchannels. Ambipolar characteristics of the graphene ISFET were also presented. The long-term stability of the ISFET was investigated, and compared with graphene/polymer composites under the same patterning and measurement conditions.
\end{abstract}

\section{INTRODUCTION}

Graphene, a natural two-dimensional structure with only oneatom thick, has attracted more and more attention due to its unique electrical, chemical, and mechanical properties [1-4]. Recently, graphene ion sensitive field effect transistor (ISFET) has been employed to detect various biomolecules, offering advantages including large detection area, relatively low $1 / \mathrm{f}$ noise [5], tunable ambipolar field-effect characteristics, and biocompatibility [6]. Several graphene synthesis approaches were developed, including mechanical exfoliation of graphite [7], epitaxial growth on $\mathrm{SiC}$ or metals [8,9], and chemical vapor deposition growth on metal substrates [10]. However current techniques proposed to generate graphene usually rely on low-yield serial processes typically involving the transfer of optically identified exfoliated graphene or graphene grown on metal substrates, which requires highly trained professionals and high cost. After the synthesis and preparation, graphene films have been extensively integrated with other materials such as polymers to obtain various structures with superior strength, flexible transparent and conductive films [11] to extend their good properties for specific applications. However, most of these graphene composites are not capable of turning or controlling their structures owing to their fixed structural synthesis processes. In addition, the integrated materials in the graphene composite structures damage the electrical stability of pure graphene, introducing an electrical drift in sensing applications. To overcome these hurdles of previous graphene synthesis and applications, we present a simple and low-cost approach to form graphene films, using microfluidics to introduce, confine and pattern the graphene films for ISFETs. This developed graphene films were tunable in thickness due to the controllable height of microchannels. The graphene was demonstrated with long-term stability in ISFET sensors, compared with the graphene/polymer composite with the same patterns and measurement conditions. Therefore, this tunable microfluidics induced graphene films are expected to offer more controllable and flexible applications to chemical detection and microfluidics.

\section{DESIGN AND FABRICATION}

The fabrication process of microfluidics induced graphene films is illustrated in Fig. 1. Microfabrication was utilized to build the microchannels, and microfluidics was used to deposit the graphene films. First, a layer of photoresist (Shipley S1813) was spin coated on silicon wafers with silicon dioxide $300 \mathrm{~nm}$ thick, followed by a patterning of photoresist with photolithography. Subsequently, the patterned substrate was immersed into HF buffer solution (10:1) to obtain the microchannel array in the silicon dioxide layer. To control the etching time, different channel heights from $50 \mathrm{~nm}$ to $250 \mathrm{~nm}$ were generated. After striping the photoresist, research grade graphene (PureSheets ${ }^{\mathrm{TM}}$, Nanointegris Inc, $0.25 \mathrm{mg} / \mathrm{ml}$ ) suspension solution was introduced into the reservoir. Due to the hydrophilic property of silicon dioxide, the capillarity induced the graphene suspension into the microchannel array. Next, the whole system was placed in a vacuum oven (Model 280A) for $30 \mathrm{~min}$ to degas and dry up the solution at room temperature under vacuum condition. The extra graphene films in the reservoir were gently scraped off by a blade. Chromium/gold layers $10 / 100 \mathrm{~nm}$ thick were deposited at the two ends of the microchannels with an AJA sputter system (Model ATC 2000), and sensor electrodes were patterned by photolithograph with the same photoresist.
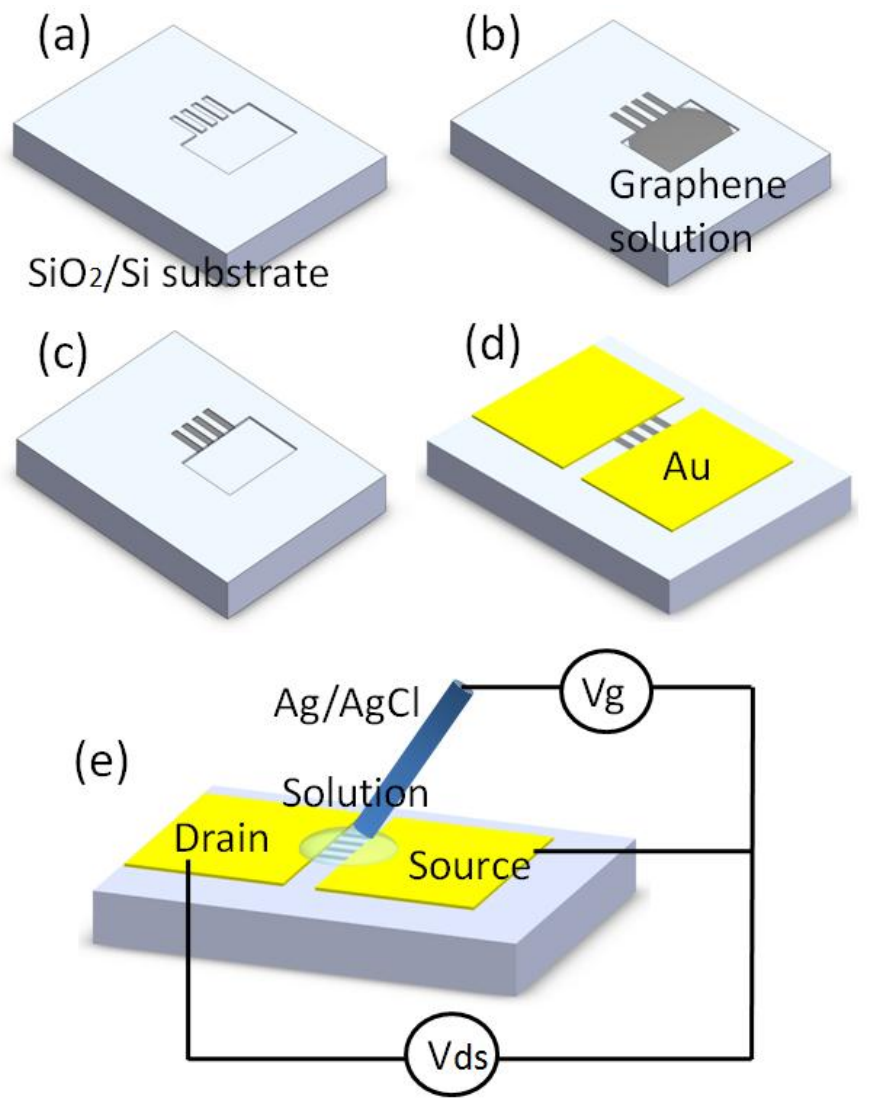

Figure 1: (a) Microchannels were patterned on a substrate; (b) Introduce graphene solution; (c) Remove the extra graphene films in reservoir; (d) Deposit and pattern electrodes; (e) Sketch of the microfluidics induced graphene ISFET. Target solutions were introduced onto the graphene films, and an $\mathrm{Ag} / \mathrm{AgCl}$ reference electrode was immersed to apply a desired gate. 
Fig. 1(e) demonstrates the structure of the microfluidics induced graphene ISFET. Target solutions were introduced onto the graphene films, and an $\mathrm{Ag} / \mathrm{AgCl}$ reference electrode was immersed to apply a desired gate voltage. Electrical measurements were carried out using a semiconductor device analyzer (HP 4145B). The microfluidics induced graphene films were also inspected by scanning electron microscope (SEM) and atomic force microscope (AFM). As shown in Fig. 2(a), the microchannel array was fabricated in the silicon dioxide layer on a silicon substrate. Two electrodes were deposited in the microchannel to form the ISFET structure, as shown in Fig. 2(b). Fig. 2(c) and 2(d) present the porous surface profile of microfluidics induced graphene, and the graphene nanoplatelets were deposited randomly across the channel of two electrodes. Besides, the samples of graphene films deposited in microchannels were studied by Raman mapping $(100 \times 100$ pixels, Witec Alpha300R), and G peak and 2D band mapping results were presented in Fig 3(a) and 3(b), respectively. The intensity difference of the $G$ peak and $2 D$ band was clearly shown between internal and external channels, which proved that the graphene film patterns were successfully deposited and confined by microchannels.
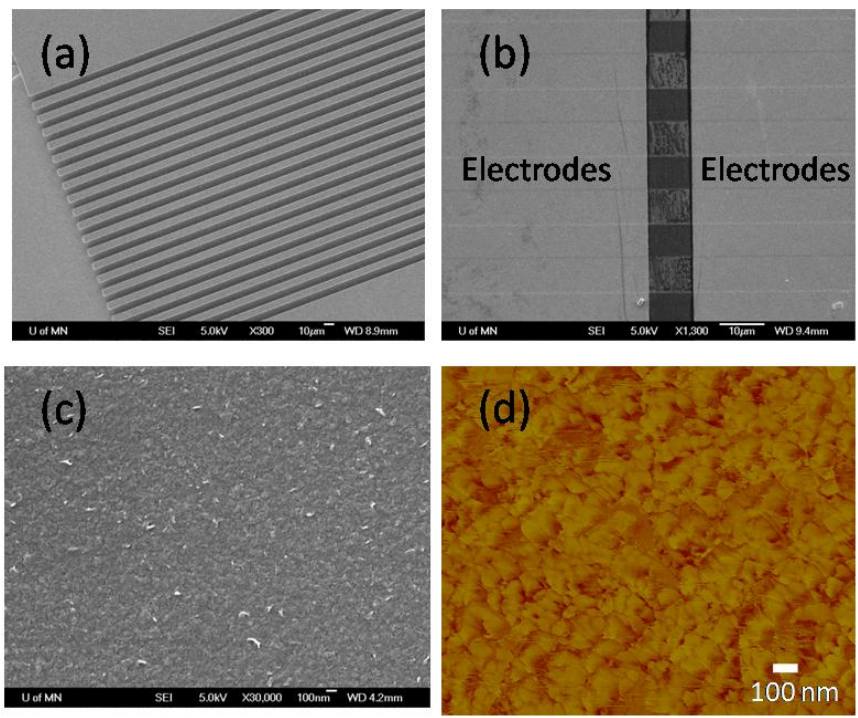

Figure 2: SEM images of (a) microchannel array, (b) ISFET structure with two electrons deposited at both ends of the microchannels, and (c) porous surface profile of microfluidics induced graphene films. (d) AFM image of the graphene nanoplatelets deposited randomly across the channel of two electrodes.

\section{RESULTS AND DISCUSSION}

AFM was used to characterize the thickness of graphene films in different microchannels with various heights. To control the etching time of silicon dioxide in HF buffer solution, the heights of microchannels were obtained from $65 \mathrm{~nm}$ to $221 \mathrm{~nm}$. After degasing and drying up in a vacuum oven, the thickness of the deposited graphene films was measured in the microchannels with different heights. As shown in Fig. 4, the thickness of the graphene films were raising with the increase of the microchannel heights. This can be explained that different amounts of graphene suspension were introduced by capillarity with various channel heights. As a result, the thickness of the graphene films is capable of tuning by adjusting the height of microchannel.
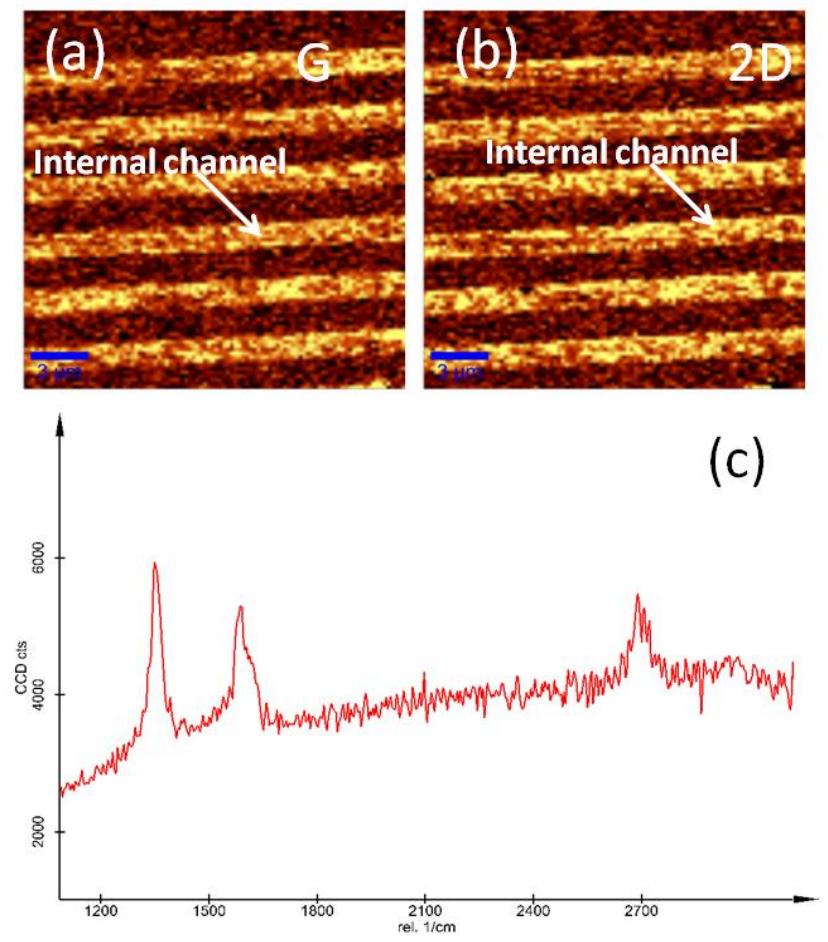

Figure 3: (a) $G$ peak and (b) 2D band Raman spectroscopy mapping results. (c) Typical Raman spectra of microfluidics induced graphene films. The intensity difference of the $G$ peak and $2 D$ band was clearly shown between internal and external channels, which proved that the graphene film patterns were successfully deposited and confined by microchannels.

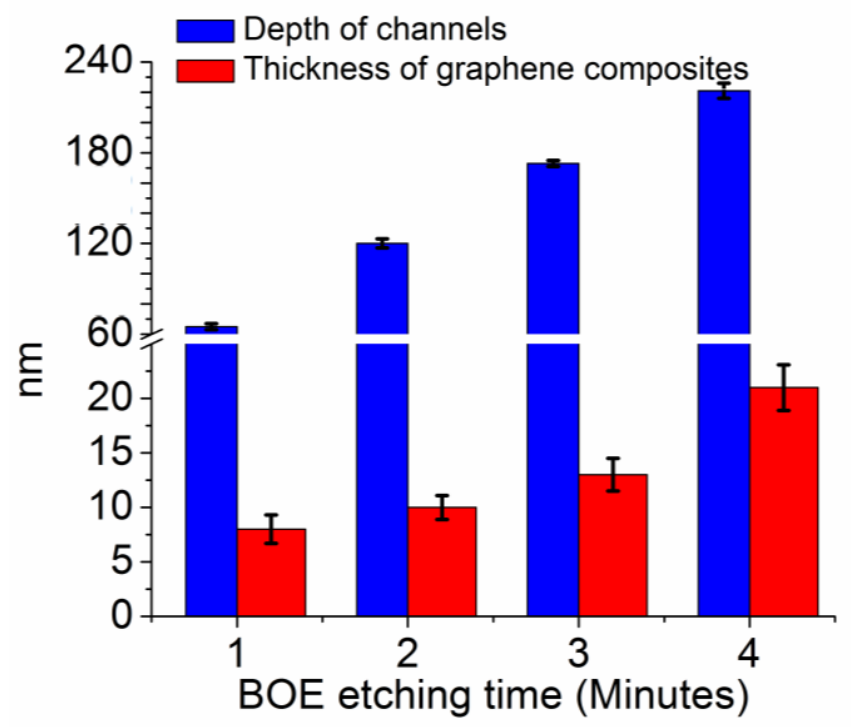

Figure 4: AFM was used to characterize the thickness of graphene films in different microchannels with various heights. The thickness of the graphene films increases with the increase of the microchannel heights.

Drain-to-source current versus solution gate voltage were recorded to investigate the electrical properties of microfluidic induced graphene ISFET. Fig. 5(a) presents ambipolar characteristics of the graphene ISFET measured in PBS buffer solution (Dulbecco's phosphate buffered saline, Invitrogen Inc.) at 
room temperature, showing the transition from $\mathrm{p}$-type region to $\mathrm{n}$ type region at the Dirac point. The electrolyte gate response of the ISFET's drain-to-source current $\left(\mathrm{I}_{\mathrm{d}}\right)$ in different $\mathrm{pH}$ solutions were also recorded. As shown in Fig. 5(b), solutions from $\mathrm{pH} 6$ to $\mathrm{pH} 9$ were delivered to ISFET sequentially. While the ISFET was biased at $\mathrm{V}_{\mathrm{ds}}$ at $2 \mathrm{~V}$, and the gate voltage was applied from -4.5 to $4.5 \mathrm{~V}$. The Dirac point of graphene ISFET was obviously observed to shift positively from $\mathrm{pH} 5$ to $\mathrm{pH} 9$, suggesting that $\mathrm{H}^{+}$ ions act as n-type dopants to the graphene. Adsorption of a positively charged $\mathrm{H}^{+}$induces additional negative charge in the graphene, thus n-type doping the graphene and shifting the Dirac point toward more negative gate voltages. The graphene films behave as a p-type material when negative gate potential is applied, and the $I_{d}$ of the ISFET increases with the increase of $\mathrm{pH}$ values. On the contrary, when the gate potential is switched positive, transition from $\mathrm{p}$-type region to n-type region occurs, and the $I_{d}$ of the ISFET decreases with increased $\mathrm{pH}$.
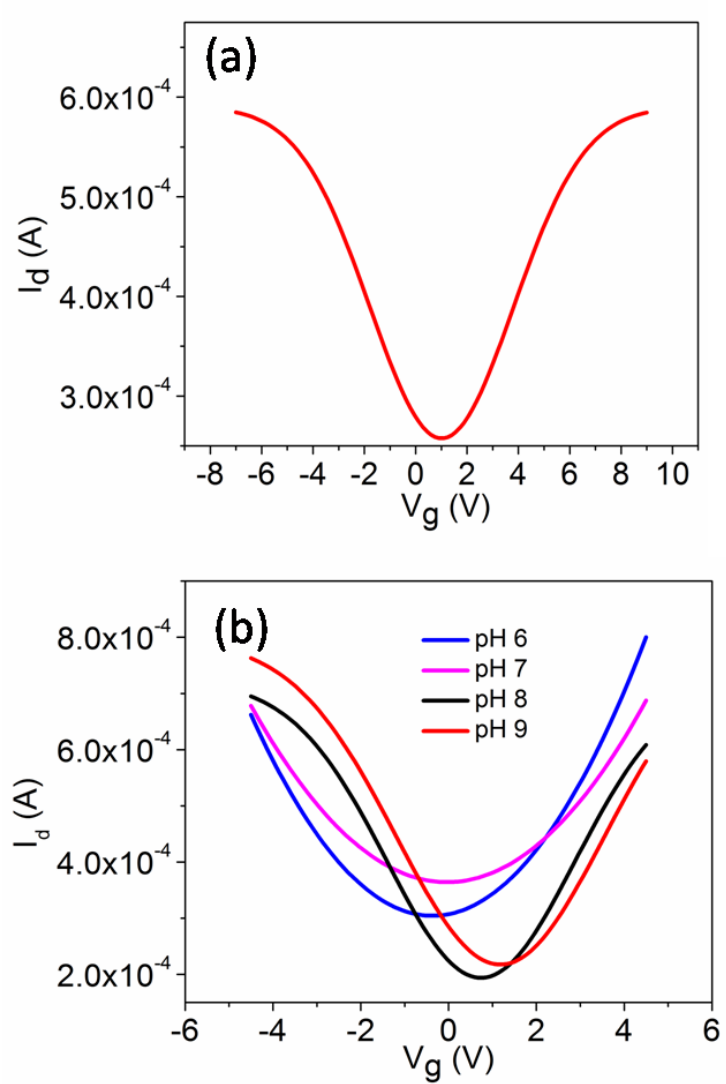

Figure 5: (a) Ambipolar characteristics of the graphene ISFET measured in a PBS buffer solution at room temperature; (b) Solutions from $\mathrm{pH} 6$ to $\mathrm{pH} 9$ were delivered to ISFET sequentially. Adsorption of a positively charged $H^{+}$induces additional negative charges in the graphene, thus n-type the graphene and shifting the Dirac point toward more negative gate voltages.

The ISFET was also verified for functionality and detect limitation of glucose sensing. After the graphene ISFET was fabricated, glucose oxidase (GOx) was immobilized on the sensing region. The GOx on the surface reacted with the glucose and created hydrogen ions as such:

$$
\begin{aligned}
& \beta-D-\text { glucose }+\mathrm{O}_{2} \stackrel{G O x}{\longrightarrow} D-\text { glucono }-\delta \text {-lactone }+\mathrm{H}_{2} \mathrm{O}_{2} \\
& D-\text { glucono- } \delta \text {-lactone }+\mathrm{H}_{2} \mathrm{O} \rightarrow D-\text { gluconate }^{-}+H^{+}
\end{aligned}
$$

As demonstrated previously, the graphene ISFET was capable of responding to the $\mathrm{H}^{+}$. Different concentrations of glucose can cause different local pH. As shown in Fig. 6(a), different concentrations of glucose solutions were delivered to the graphene ISFET. The $\mathrm{V}_{\mathrm{ds}}$ was biased at $2 \mathrm{~V}$, and the gate voltage was kept at $4 \mathrm{~V}$. The current from source to drain was measured at different concentrations of glucose, demonstrating the detection limits of graphene ISFET down to $400 \mathrm{pM}$.
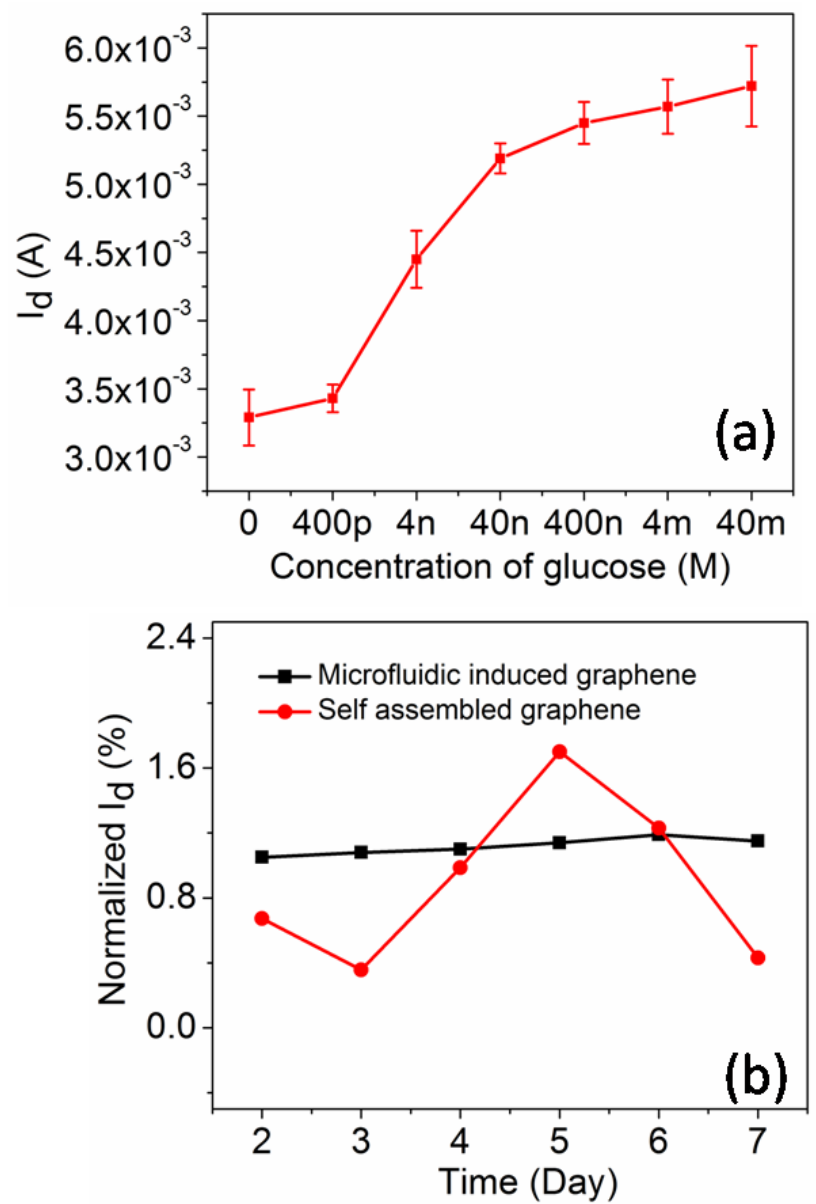

Figure 6: (a) Glucose detection was characterized on an ISFET modified by GOx, presenting a detection limit down to $400 \mathrm{pM}$. (b) Microfluidics induced graphene ISFET was much more stable than the self assembled graphene ISFET. The absence of polymer or other materials in the microfluidics induced graphene may be less sensitive to the disturbance of the environments, compared with the composite graphene structures.

The long-term stability of the graphene ISFET was investigated, and compared with layer-by-layer self assembled graphene ISFET. The fabrication processes of self assembled graphene were reported in our previous work [12]. Two types of graphene ISFET under same patterning and testing conditions were used to measure the response of $I_{d}$ for $\mathrm{pH} 7$ solutions in 7 days continuously. During the idle time, the ISFETs were kept in DI water to prevent from contamination and disturbance. Both of the ISFETs were biased at $\mathrm{V}_{\mathrm{ds}}$ of $1 \mathrm{~V}$, and the gate voltage was fixed at $2 \mathrm{~V}$. To obtain more clear results, a normalized $I_{d}$ was introduced. $I_{d}$ in the first day result was used as an initial current $I_{0}$. Normalized $I_{d}$ was represented as the ratio of the following day result to the initial current $\left(I_{d} / I_{0}\right)$. As shown in Fig. 6, the microfluidics induced graphene ISFET was much more stable than 
the self assembled graphene ISFET. The absence of polymer or other materials in the microfluidics induced graphene may be less sensitive to the disturbance of the environments, compared with the graphene composite structures.

\section{CONCLUSIONS}

In summary, the microfluidics induced graphene films offer a number of advantages over the current graphene composites. Due to controllable microchannel heights, the graphene thickness is tunable, superior to that of general macroscopic graphene composites with fixed structures. Using a microfluidic system as a fabrication platform, the graphene ISFET should be low cost, and easy to integrate with microfluidic system for many biomedical applications. In addition, owing to the absence of other materials in its structure, this graphene ISFET exhibits long-term stability for various chemical detections. This microfluidics induced graphene films can be a general strategy to fabricate a broad class of macroscopic graphene structures with tunable properties and longterm stability.

\section{ACKNOWLEDGEMENTS}

The authors acknowledge the assistance of fabrication and characterization from the Nanofabrication Center and the Characterization Facility at the University of Minnesota.

\section{REFERENCES}

[1] K. S. Novoselov, A. K. Geim, S. V. Morozov, D. Jiang, Y. Zhang, S. V. Dubonos, I. V. Grigorieva, A. A. Firsov, "Electric Field Effect in Atomically Thin Carbon Films", Science, 306, 666 (2004).

[2] K. S. Novoselov, A. K. Geim, S. V. Morozov, D. Jiang, M. I. Katsnelson, I. V. Grigorieva, S. V. Dubonos, A. A. Firsov, "Twodimensional Gas of Massless Dirac Fermions in Graphene", Nature, 438, 197 ( 2005).
[3] A. A. Balandin, S. Ghosh, W. Bao, I. Calizo, D. Teweldebrhan, F. Miao, C. Lau, "Superior Thermal Conductivity of Single-Layer Graphene", Nano Letters. 8, 902 (2008).

[4] C. Lee, X. D. Wei, J. W. Kysar, J. Hone, "Measurement of the Elastic Properties and Intrinsic Strength of Monolayer Graphene", Science, 321, 385 (2008).

[5] K. Ratinac, W. Yang, S. Ringer, F. Braet, "Toward Ubiquitous Environmental Gas Sensors-Capitalizing on the Promise of Graphene", Environmental Science Technology 44, 1167 (2010).

[6] S. Agarwal, X. Z. Zhou, F. Ye, Q. Y. He, G. C. K. Chen, J. Soo, F. Boey, H. Zhang, P. Chen, "Interfacing Live Cells with Nanocarbon Substrates", Langmuir, 26, 2244 (2010).

[7] P. Li, Z. You, G. Haugstad, T. Cui, "Graphene Fixed-End Beam Arrays based on Mechanical Exfoliation", Applied Physics Letter, 98, 253105 (2011).

[8] K. S. Kim, Y. Zhao, H. Jang, S. Y. Lee, J. M. Kim, K. S. Kim, J.-H. Ahn, P. Kim, J.-Y. Choi, B. H. Hong, "Large-Scale Pattern Growth of Graphene Films for Stretchable Transparent Electrodes", Nature 457, 706 (2009).

[9] C. Berger, Z. M. Song, X. B. Li, S. Wu, X. N. Brown, C. Naud, D. Mayou, T. B. Li, J. Hass, A. N. Marchenkov, "Electronic Confinement and Coherence in Patterned Epitaxial Graphene", Science, 312, 1191 (2006).

[10] M. P. Levendorf, C. S. Ruiz-Vargas, S. Garg, J. Park, "Transfer-Free Batch Fabrication of Single Layer Graphene Transistors", Nano Letters. 9, 4479 (2009).

[11] Z. Chen,W. Ren, L.Gao, B. Liu, S. Pei, H.Cheng, "ThreeDimensional Flexible and Conductive Interconnected Graphene Networks Grown by Chemical Vapour Deposition", Nature Materials, 10, 424, (2011).

[12] B. Zhang, T. Cui, "An Ultra-Sensitive and Low-Cost Graphene Sensor Based on Layer-by-Layer Nano Self-Assembly", Applied Physics Letter, 98, 073116 (2011).

\section{CONTACT}

*T. Cui, tel: +1-612-626-1636; tcui@me.umn.edu 


\title{
OPEN-CHANNEL MICROFLUIDICS: FREE-STANDING HYDROGEL MICROARRAYS FOR PROTEIN ELECTROPHORESIS
}

\author{
T.A. Duncombe ${ }^{1}$, T.M. Tran ${ }^{1}$, F. Benito-Lopez ${ }^{2}$, D. Diamond ${ }^{2}$, and A.E. Herr 1,3* \\ ${ }^{1}$ UC Berkeley / UC San Francisco Joint Graduate Group in Bioengineering, USA \\ ${ }^{2}$ CLARITY: Centre for Sensor Web Technologies, National Centre for Sensor Research, School of Chemical \\ Sciences, Dublin City University, Dublin 9, IRELAND \\ ${ }^{3}$ University of California, Berkeley, CA 94720, USA
}

\begin{abstract}
We report a new open-channel architecture that enables the utility of microfluidics without sacrificing accessibility for high performance protein electrophoresis. We utilize photo-patterned free-standing polyacrylamide gels as both an open-microchannel and a sieving matrix for protein sizing. The open format allows for easy downstream sample access for immunoblotting after rapid microfluidic protein separations are performed. For the purposes of multiplexing, the assay footprint is minimized to a single channel through the implementation of moving boundary electrophoresis protein separations.
\end{abstract}

\section{INTRODUCTION}

Contemporary microfluidic technologies are realized as miniaturized platforms comprising enclosed microchannels to exploit the unique physical attributes of the microscale. In microfluidic electrochromatography, the high surface area to volume ratio results in efficient heat dissipation enabling the application of high electric fields for rapid separations. To date, a principle limitation of microfluidics in closed channel formats is the inability for extraction and downstream processing of the sample. While digital microfluidics (e.g., EWOD) has remedied this for discrete droplet reactors [1], such advances have been attempted [2] but have not matured in separation science.

Consequently, we report here for the first time a new open-channel architecture that enables the utility of microfluidics without sacrificing accessibility - for high performance protein electrophoresis. Owing to its open architecture, this technology is optimized for interfacing with automated robotic controllers and downstream processing (i.e., sample spotters, immunological detection, mass spectroscopy). The unmatched accessibility makes this platform uniquely well-suited to massively parallelized proteomics, a major unrealized goal from bioanalytical technology.

In a notable departure from protein micro-separations approaches [3-6], the present study introduces a unique open-channel format for protein electrophoresis (Figure 1). Here we demonstrate a photo-patterning technique to realize free-standing polyacrylamide gel microchannels. A protein separation is demonstrated in a single free-standing microchannel by moving boundary electrophoresis (MBE). In MBE the moving boundary of analytes are analyzed, as opposed to discrete zones, thus eliminating the need for an injection channel. Polyacrylamide gel MBE enables rapid protein separations in short single channels [3]. The MBE format allows separations to be realized on a small device footprint and with low power consumption, both important when implementing a multiplexed array.

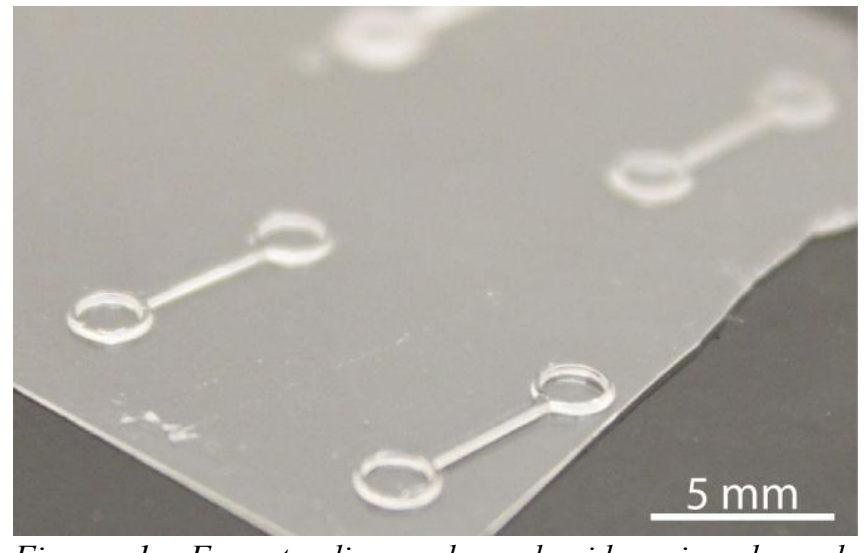

Figure 1: Free-standing polyacrylamide microchannels arrays are a fully accessible microfluidic platform easily interfaced with automated robotic fluid handling and operation.

\section{MATERIALS AND METHODS \\ Materials}

Solutions of 30\% (29:1) acrylamide/bis-acrylamide, 3(trimethoxysilyl)-propyl methacrylate $(98 \%)$, glacial acetic acid, methanol and glass coverslips were purchased from Sigma Aldrich (St. Louis, MO). Photoinitiator 2,2-azobis[2methyl-N-(2-hydroxyethyl) propionamide] (VA-086) was purchased from Wako Chemical (Richmond, VA). Alexa Fluor 488 conjugated Trypsin Inhibitor (TI), Ovalubmin (OVA), and Bovine Serum Albumin (BSA) were purchased from Sigma. Tris-glycine (10x) native electrophoresis buffer was purchased from Bio-Rad Laboratories (Hercules, CA).

\section{Coverslip Functionalization}

A glass coverslip is cleaned in a $1 \mathrm{M} \mathrm{NaOH}$ solution for 30 minutes and then washed with DI water and dried with nitrogen. A 20 L drop of 2:3:5 (v/v/v) mixture of 3(trimethoxysilyl)-propyl methacrylate, glacial acetic acid and DI water is sandwiched between a petri dish and a cleaned coverslip. The petri dish is sealed and placed in refrigerator at $4^{\circ} \mathrm{C}$ overnight. Afterwards the coverslip is rinsed with methanol and DI water and stored dry until it is used. 

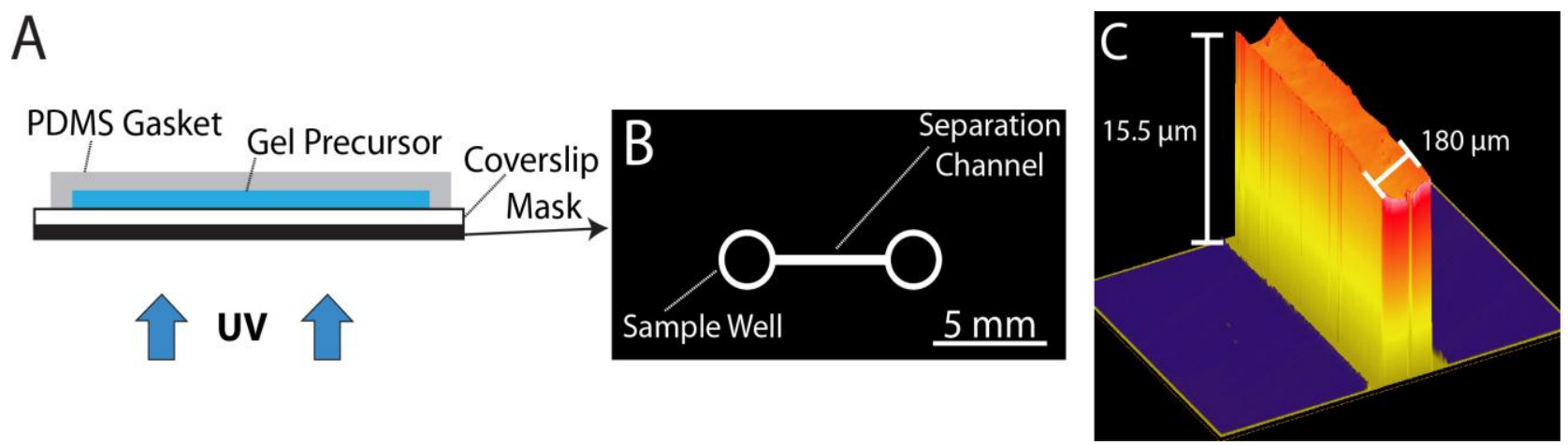

Figure 2: Free-standing polyacrylamide gel microchannels are fabricated using a (A) mask based photolithography technique. A reservoir of gel precursor is contained above a methacrylate functionalized coverslip. The desired micropattern is defined by a photo-mask, which determines the portions of the reservoir that are polymerized by a UV light source. Our design (B) consists of a single channel connecting two fluid reservoirs. (C) Optical profilometry shows well defined free-standing hydrogel with dimensions and uniformity appropriate for supporting electrokinetic protein separations.

\section{Free-Standing Gel Fabrication}

Gel precursor consisted of a $10 \%(\mathrm{w} / \mathrm{v})$ acrylamide concentration with a bis-acrylamide crosslinker ratio of $3 \%$ $(\mathrm{w} / \mathrm{w})$, and 1\% (w/v) VA-086 dissolved in DI water. After degassing, the precursor solution is placed in a polydimethylsiloxane gasket atop a glass coverslip that has been functionalized with methacrylate (Figure 2A). A BlakRay ${ }^{\circledR}$ UV lamp at $10 \mathrm{~mW} / \mathrm{cm}^{2}$ is exposed through a Mylar photo-mask (Figure 2B) on the back side of the coverslip for 5 minutes. After polymerization, the gasket is removed and excess precursor is washed away leaving only the freestanding gel microstructure behind. We establish fluid reservoirs by fabricating circular rings of gel, with the capacity to hold 2 L of sample delivered with a pipette.

Optical profilometry is performed to confirm a high level of controllability in the free-standing structure with a channel cross section of $180 \mathrm{~m}$ wide by $15.5 \mathrm{~m}$ tall in its hydrated state (Figure 2C). The gels are dehydrated for storage. They can be rehydrated in any aqueous buffer of interest and ready for experimentation in minutes.

\section{EXPERIMENTAL}

\section{Environmental Chamber}

A key hurdle to realizing electrokinetic separations in the open-channel format is evaporation. In ambient conditions we observed rapid evaporation that resulted in unsteady electrical current over the course of the separation. To alleviate the problem we utilized a small environmental chamber, consisting of an inverted petri dish and a moist $\mathrm{KIMWIPE}^{\circledR}$. Using this technique a stable electrical current is established.

\section{Testing Procedure}

Free-standing gels were rehydrated in a large droplet of $1 \mathrm{x}$ tris/glycine for 5 minutes. After gel hydration the excess buffer was removed from gel using a KIMWIPE ${ }^{\circledR}$. The sample of interest is pipetted into the sample well and a run buffer is pipetted in to the opposite, sample wash well.
Platinum electrodes are aligned and inserted from above into the two wells and electrophoresis is immediately initiated by a Caliper high voltage power supply.

Protein fronts are visualized using an inverted epiflourescence microscope (Olympus IX-70) equipped with a $100 \mathrm{~V}$ mercury arc lamp, a 10x objective, and a Peltiercooled charge-coupled device (CCD) camera (CoolSNAP HQ2, Roper Scientific, Trenton, NJ). Images are recorded using MetaMorph ${ }^{\circledR}$ acquisition software and post-processing was done in Image $(\mathrm{NIH})$ and $\mathrm{MATLAB}^{\circledR}$.

\section{Separation Efficiency}

The ability to resolve two analytes is quantified through 'separation resolution' (SR, the mean distance between neighboring peaks normalized by the average peak width). A $\mathrm{SR}>1$ indicates a successful separation. In this study we monitor the 'critical separation length', or the migration distance required to realize a $\mathrm{SR}>1$. As the critical separation length is reduced, so does the required channel length.

\section{RESULTS}

A protein separation was performed in the free-standing polyacrylamide gel format and is displayed in Figure 3. A fluorescently labeled sample of $250 \mathrm{~nm}$ BSA, $250 \mathrm{~nm}$ OVA, and $250 \mathrm{~nm}$ TI in $1 \mathrm{x}$ tris/glycine is baseline resolved in 2 minutes and in the first $250 \mathrm{~m}$ of migration. In Figure $3 \mathrm{~A}$ the protein moving boundaries are clearly visible several millimeters along the separation channel. Figure 3B displays the first $250 \mathrm{~m}$ of migration at the 130 seconds into the separation. The image is false colored such that the TI, OVA, and BSA fronts can be clearly distinguished. The corresponding fluorescence intensity plot is shown in Figure $3 \mathrm{C}$ and is overlaid with the derivative of the intensity over location $(\mathrm{dI} / \mathrm{dx})$. In the $\mathrm{dI} / \mathrm{dx}$ plot the unique protein species can be clearly identified. 

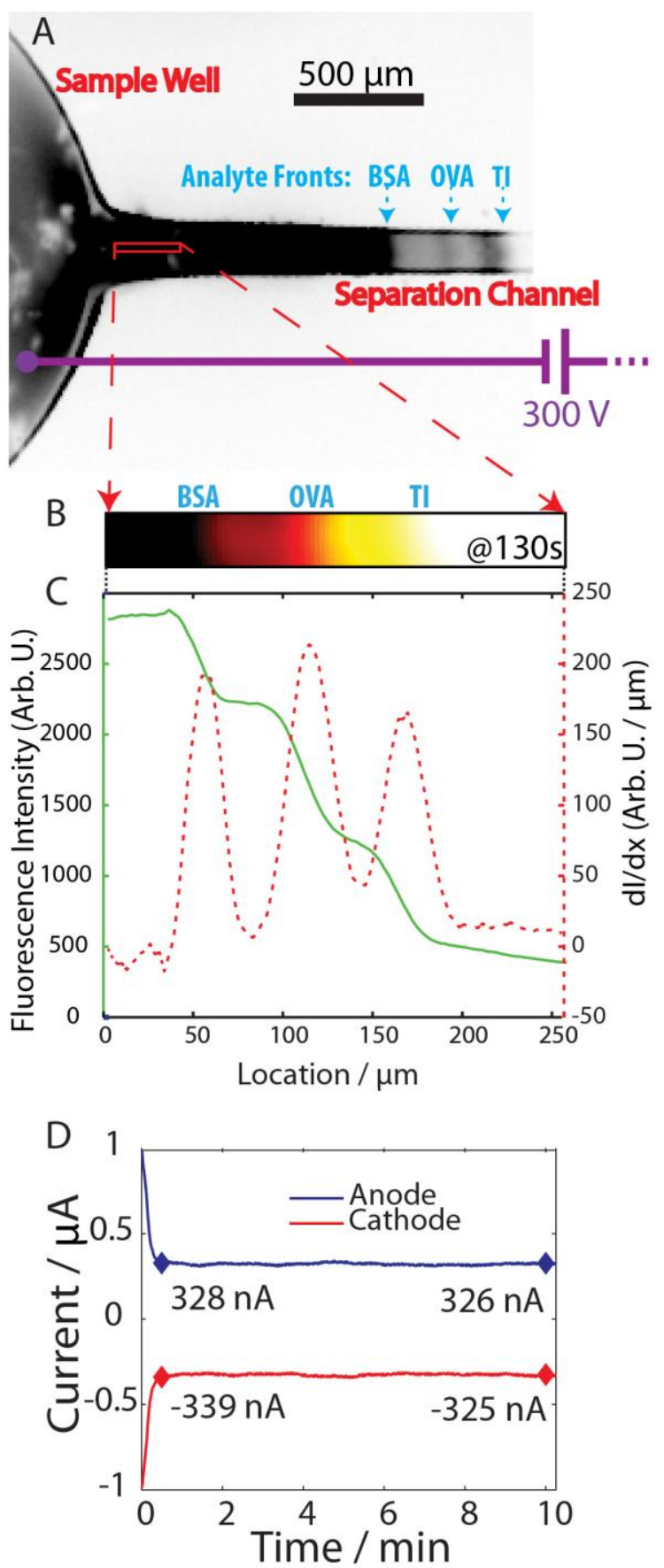

Figure 3: A free-standing 10\% (w/v) acrylamide gel acts as both the microchannel and sieving matrix for a native $M B E$ separation (A). 250 nM fluorescently labeled protein ladder consisting of Trypsin Inhibitor (TI), Ovalbumin (OVA) and Bovine Serum Albumin (BSA) is captured in an epifluorescence image. (B) A false color image of the first 250 $\mu m$ of the separation channel at 130s clearly shows well separated proteins, a $2.7 x$ reduction in separation length as compared to on-chip [3]. The corresponding (C) intensity profile and the derivative of the front $d I / d x$ are overlaid. (D) The environmental chamber prevents appreciable current reduction.

To visualize the separation in location and time, $\mathrm{dI} / \mathrm{dx}$ is plotted at 10 second intervals along the first $250 \mathrm{~m}$ of migration in Figure 4A. As the separation progresses in time and location the resolution of proteins improves. An automated Matlab Gaussian curve fitting program is used to determine the mean location and the dispersion of the protein species. The separation resolution is plotted as a function of time in Figure 4B. All three separations surpass the critical SR in under 50 seconds as compared to hours that would be required for a conventional benchtop slab-gel separation.
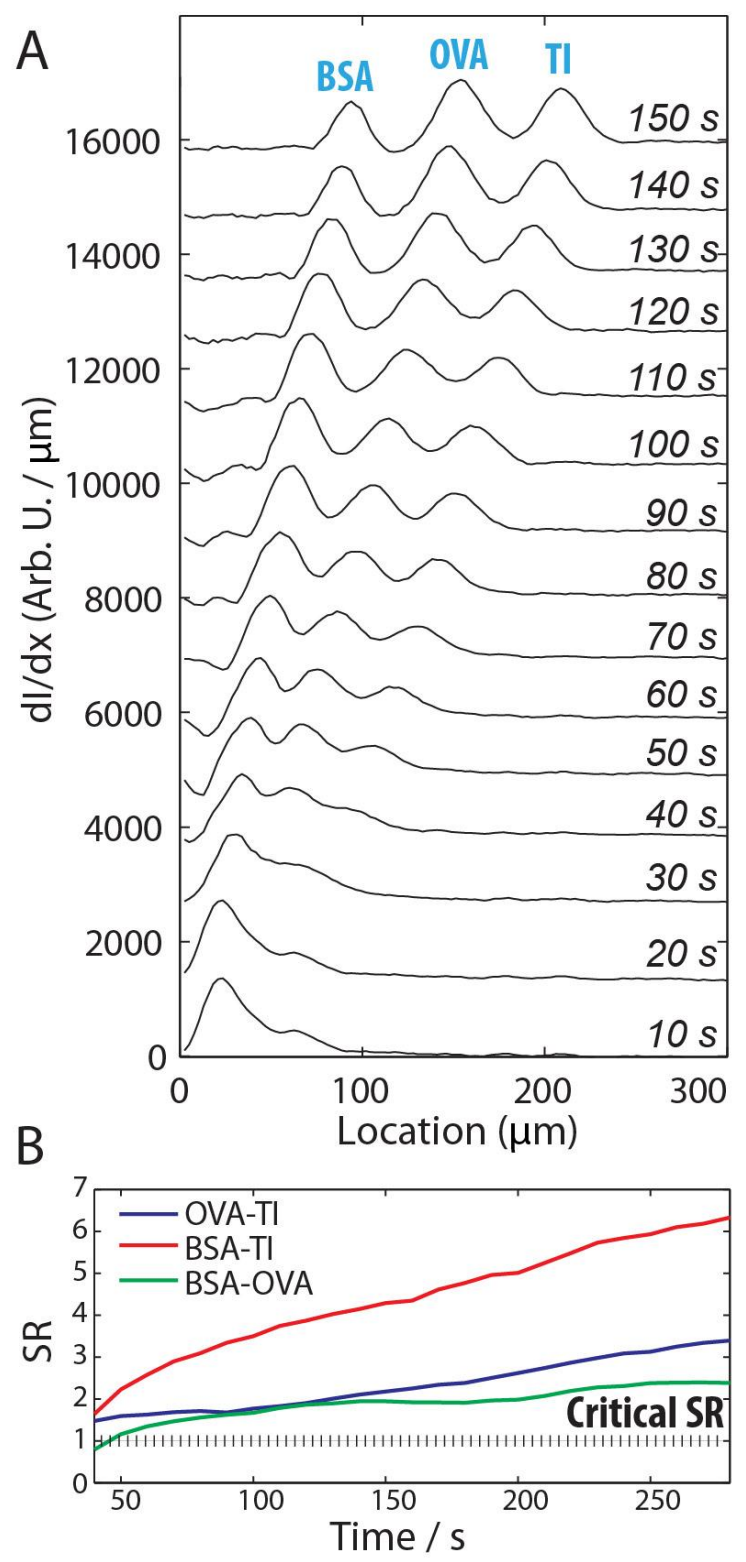

Figure 4: The MBE free-standing gel separation of a three protein ladder is completed in 50s and in the first $150 \mu \mathrm{m}$. (A) $d I / d x$ is staggered in time to visualize the protein migration with the $(B)$ separation resolution plotted for each protein separation from the Figure 3 experiment. 


\section{DISCUSSION}

We introduce a novel free standing polyacrylamide gel microarray platform for protein electrophoresis. Each microgel acts as both a microchannel and a sieving matrix for rapid protein analysis. The accessible microfluidic format is appropriate for massive scale up into large arrays, offering facile integration with automated robotics for highthroughput proteomics.

\section{ACKNOWLEDGEMENTS}

The authors gratefully acknowledge members of The Herr Laboratory at UC Berkeley for assistance and helpful discussions. The authors acknowledge financial support from the US National Science Foundation (NSF) in the form of both Graduate Research Fellowships (TAD, TMT) and CAREER Award (AEH, grant \# 1056035). Additional support was provided by the Science Foundation of Ireland (FBL, DD, grant 07/CE/I1147) and the Short Term Travel Fellowship Supplement 2011 (FBL). Partial conference travel support was provided by the Transducer Research Foundation. Partial infrastructure support was provided by the UC Berkeley/QB3 Biomolecular Nanofabrication Center (BNC). A.E.H. is an Alfred P. Sloan research fellow in chemistry.

\section{REFERENCES}

[1] Abdelgawad, M.; Freire, S. L. S.; Yang, H.; Wheeler, A. R., All-terrain droplet actuation. Lab on a Chip 2008, 8, 672-677.

[2] Liu, J.; Tseng, K.; Garcia, B.; Lebrilla, C. B.; Mukerjee, E.; Collins, S.; Smith, R., Electrophoresis Separation in Open Microchannels. A Method for Coupling Electrophoresis with MALDI-MS. Analytical Chemistry 2001, 73 (9), 2147-2151.

[3] Duncombe, T. A.; Tentori, A. M.; Hou, C.; Herr, A. E., From Bench-To-Bedside: Realizing On-Chip Electrophoretic Immunoassays For Protein Biomarkers In Serum Using a Standard 9V Battery. In IEEE MEMS Conference, 2012.

[4] Apori, A. A.; Herr, A. E., Homogeneous immunosubtraction integrated with sample preparation enabled by a microfluidic format. Anal Chem 2011, 83 (7), 2691-8

[5] Hughes, A. J.; Herr, A. E., Quantitative enzyme activity determination with zeptomole sensitivity by microfluidic gradient-gel zymography. Anal Chem 2010, 82 (9), 3803-11.

[6] Hou, C.; Herr, A. E., Ultrashort separation length homogeneous electrophoretic immunoassays using onchip discontinuous polyacrylamide gels. Anal Chem 2010, 82 (8), 3343-51.

[7] Khetan, S.; Burdick, J. A., Patterning hydrogels in three dimensions towards controlling cellular interactions. Soft Matter 2011, 7 (3).

\section{CONTACT}

*A.E. Herr, tel: +1-510-666-3396; aeh@berkeley.ed 


\section{SELF-ASSEMBLED NANOMACHINES FOR PROGRAMMABLE SENSING AND ACTUATION \\ Gabriel Lavella and Michel M. Maharbiz \\ University of California, Berkeley, California, USA}

\begin{abstract}
We demonstrate a Brownian ratchet architecture that can be used to create molecular machines capable of sensing and actuation. The machines are programmable and autonomous in the sense that the chemomechanical functionality and energy source can be readily tailored. Machines were self-assembled in a one pot reaction from a set of oligonucleotides and hapten molecules using honeycomb array DNA origami.[1], [2] This technique, allowed us to obtain a sophisticated level of control over the structural topology and consequently the energy landscapes that give rise to Brownian ratchet transduction phenomena. The self-assembled devices measure $\sim 100 \mathrm{~nm}$ in length and we set to transduce the detection of a streptavidin ligand into mechanical motion.
\end{abstract}

\section{INTRODUCTION}

Brownian ratcheting is a mechanism that can be used to create mechanically functional and autonomous molecular machines. Evidence for this exists in the multitudes of chemomechanical ratchets in nature and the wide range of tasks they perform[3], [4]. Well known examples include the actin ratchets used in filopodia protrusion[5], [6] and Lysteria monocytogenes locomotion[7], [8]. They distinguish themselves from molecular motors, such as myosin and kinesin, which operate on mechanochemical cycles, and acquire energy for work from the hydrolysis of ATP[9]. Instead, ratchets are non-cyclic, obtain energy from polymerization reactions, and achieve directed motion by rectifying random substrate movements with asymmetries in the energy landscape[10], [11]. While many natural ratchets have been studied in the context of their biological function, the technological pursuit of synthetic chemomechanical ratchets has garnered little attention. By comparison, molecular motors are being heavily pursued with applications in nanoscale transport in mind[10], [12]. Several synthetic motors have been produced; many of these are walking devices that move along artificial tracks and are fueled in artificial environments with DNA strands through hydrolysis[13], [14] or hybridization[15], [16]. The most ambitious efforts toward synthetic chemomechanical machine development focus on understanding how to design machines from scratch through direct engineering of macromolecules; ideally designing for function, operating environment and energy source. Nevertheless, before this vision is realized a multitude of challenges in molecular structure[17], [18] and function prediction[19]must be overcome.

Here we demonstrate a molecular machine based on a synthetic Brownian ratchet architecture that senses, mechanically reacts and extracts energy from a streptavidin ligand. Our architecture avoids the complexities of macromolecule design by using molecular assemblies. In addition it can allow user selection of the chemomechanical functionality and the energy source. These two characteristics result in a machine that can be adapted for the desired task and the application environment, respectively. We achieve this through topology, tailoring of energy landscapes, and a cascade of ligand-receptor binding events that serve as the Brownian ratchet polymerization reaction. This is distinguishes our architecture from natural systems, where the polymerization species is often structural and enables the chemomechanical functionality to be chosen during design by selecting a ligandreceptor pair.
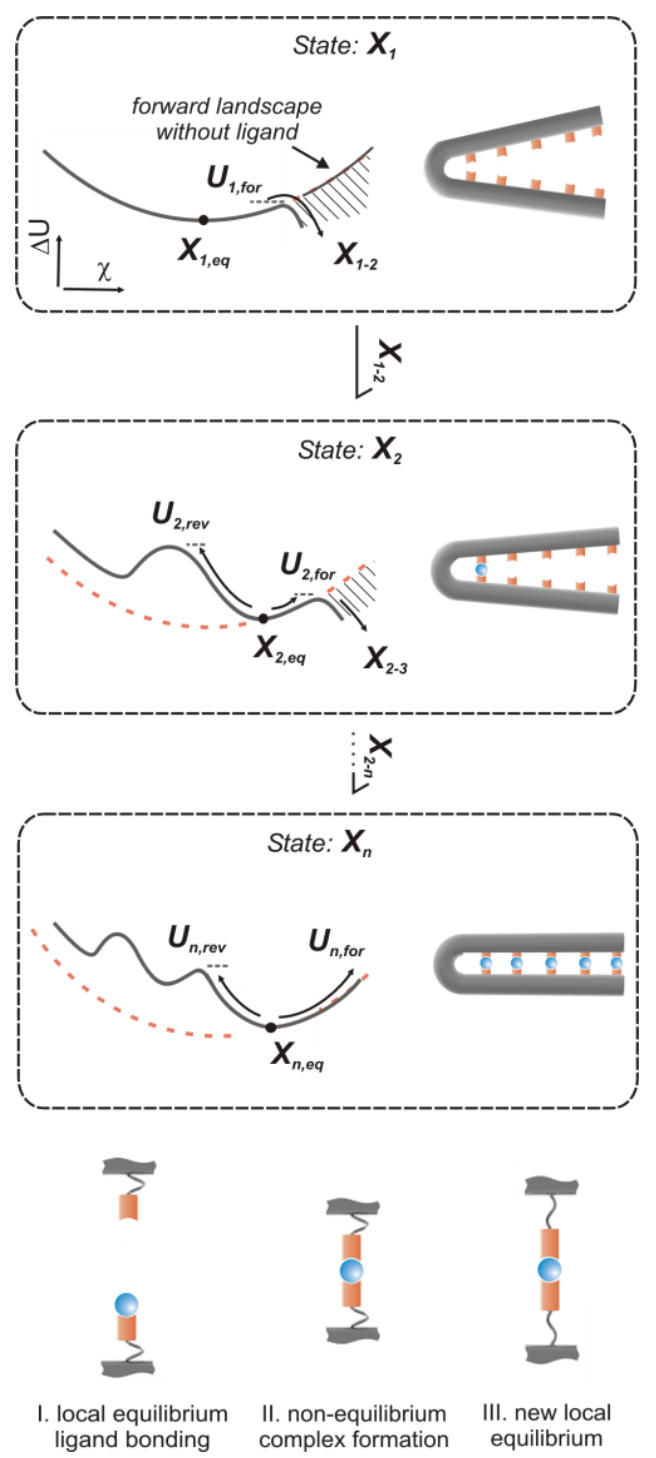

Figure 1: Device topology and operational mechanism. The backbone is shown in grey, receptors molecules are shown in orange, and the sensed ligand is shown in blue. (State $X_{l}$ ) device and notional energy landscape prior to encountering a ligand; (State $X_{2}$ ) after ligand introduction and sandwich bond formation; (State $X_{n}$ ) device in a completely ratcheted state. (bottom) Transition between local equilibriums at the level of a single sandwich bond complex.

\section{THEORY}

Our architecture consists of a clip shaped backbone (shown in grey, Fig. 1) conjugated with receptor molecules (orange, Fig. 1). Before the introduction of ligand molecules, this structure thermally vibrates in accord with the flexure characteristics of the backbone and the temperature of the bath (Fig. 1, State $\mathrm{X}_{1}$ ). The 
thermal vibrations, introduce potential energy shifts, $\Delta \mathrm{U}$, originating from the strain energy in the backbone and stretched molecular complexes. These changes cause the ratchet to move along the Brownian coordinate, $\chi$, which we have defined as the symmetric displacement of the tips; $\chi$ increases as the device moves toward a closed state.

An alternative path in the energy landscape is created though the bonding of a ligand to one of the receptors from the first pair This event enables a transition between states, $X_{1-2}$ by presenting the possibility of the formation of a sandwich bond (such as in Fig. 1, III). For this transition to occur the random thermal input must surmount the forward energy barrier, $U_{1, \text { for }}$ In addition a bound/unbound receptor configuration exists between opposing receptors. These configurations arise when the reaction rate for sandwich bond formation is sufficiently higher than the reaction rate for ligand-receptor binding.

Upon the a new potential well, State $\mathrm{X}_{2}$ is created, with a new equilibrium position, $\chi_{2, \text { eq, }}$ advanced in the closing direction and

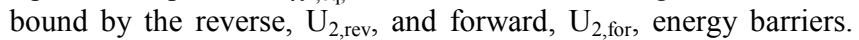
Energy is extracted for each of these transitions from the ligandreceptor binding free energy. This extraction can be accounted for with an additional term in the Gibbs free energy equation [20-22] and occurs during the simultaneous relaxation of the backbone and tensioning of the sandwich bond (Fig 1, bottom). As a result of the distribution of random thermal vibrations, not all receptor pairs need to form bonds, ratcheting can proceed by leapfrogging. Sequential transitions proceed until the ratchet is closed (Fig. 1, State $X_{n}$ ). In essence the ratchet moves though a progression towards a lower global equilibrium with the aid of thermal vibrations.

\section{SELF-ASSEMBLY AND DEVICE TOPOLOGY}

We created devices with similar topologies to those presented in Fig. 1. Device were made using the honeycomb array DNA origami[1], [2] wherein a backbone was formed from $10 \mathrm{~B}$-form DNA helices stressed by base pair insertions and deletions (Fig. 2, top). Each helices was joined to its three neighbors through Holiday junction crossovers every $21 \mathrm{bp}$. The backbone design was modified by eliminating sequences along the center helix and inserting toeholds strands (44 bp long) 5 ' modified with biotin (receptors).

Self-assembly reactions were performed in a $100 \mu$ reaction composed of $5 \mathrm{mM}$ Tris, $1 \mathrm{mM}$ EDTA and $18 \mathrm{mM}$ of $\mathrm{MgCl}_{2}$ and contained $200 \mathrm{nM}$ of all staple and toehold strands and $20 \mathrm{nM}$ of the scaffold strand[23]. The reaction was thermally annealed (Applied Biosystems 2720, Thermal Cycler) over a linear temperature gradient from $80^{\circ} \mathrm{C}$ to $60^{\circ} \mathrm{C}$ for two hours and over linear gradient from $60^{\circ} \mathrm{C}$ to $24^{\circ} \mathrm{C}$ for 156 hours.

Negative stain TEM images of self-assembled device ensembles (Fig 2, middle) show correctly formed devices. These devices were extracted from the gel band highlighted in (Fig 2, middle). Extraction was conducted by cutting the band from the gel, pesteling, and conducting freeze and squeeze purification. The purified devices could then be stored for several months and later used in the experiments.

Using this approach, four device variants with different receptor/toehold configurations were assembled; two active device sets, and two controls. The active device, with receptor spacings of $7 \mathrm{~nm}$ and $14 \mathrm{~nm}$ (Fig. 2, bottom) were expected to ratchet. While the two control sets (Fig. 2, bottom), possessed a high forward energy barrier such that closing could not occur in the absence of ratcheting phenomena. In control $\mathrm{A}$, the middle 6 receptors were eliminated, while in control B we removed the first nine receptor pairs.
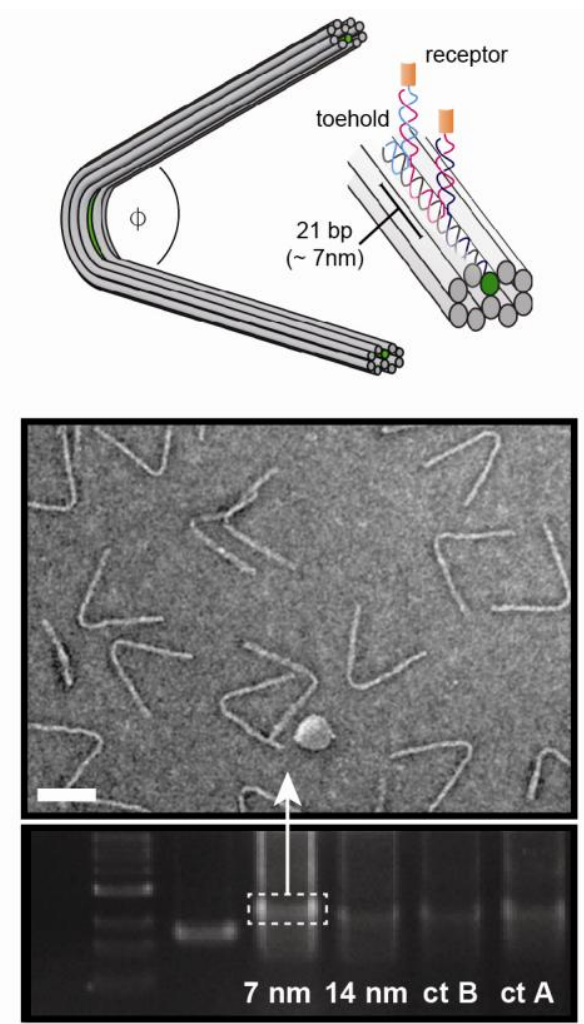

active devices

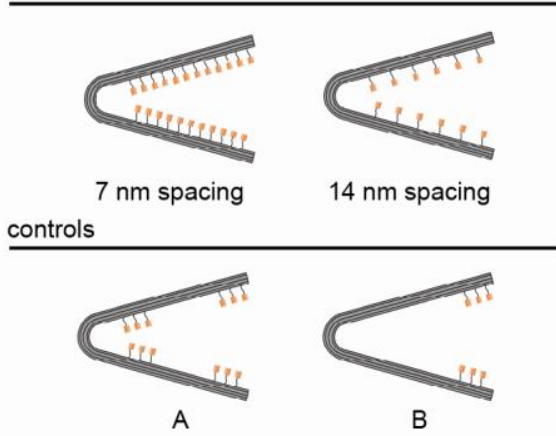

Figure 2: Molecular topology and receptor configurations for device variants. (top) Backbone topology schematic showing Bform DNA helix configuration and placement of toeholds/receptors; (middle) TEM of fabricated device ensemble, excised from gel and negatively stained, scale bar $=100 \mathrm{~nm}$ and agarose gel characterization of all device variants before ligand introduction; (bottom) device variant receptor/toehold configurations

\section{RESULTS}

In order to investigate the response we introduced the tetravalent streptavidin ligand into a solution containing devices in a ratio of $1: 10^{3}$ (device:streptavidin). The reaction was carried out in a solution containing $0.25 \mathrm{x}$ TBE and $0.5 \mathrm{x}$ PBS pH 7.4; the temperature was controlled at $25^{\circ} \mathrm{C}$ and was allowed to proceed for twenty minutes before aliquots were removed placed on a TEM grid for inspection. Negative stain TEM images show three primarly reaction products; ratcheted devices, devices which did not ratchet and devices which formed dimers (Fig. 3, bottom). In all devices the tetravalent streptavidin is visibly bound to the 
$1 \mathrm{~kb} \quad \mathrm{~m} 13 \quad 7 \mathrm{~nm} 14 \mathrm{~nm}$ cntl A cntl B
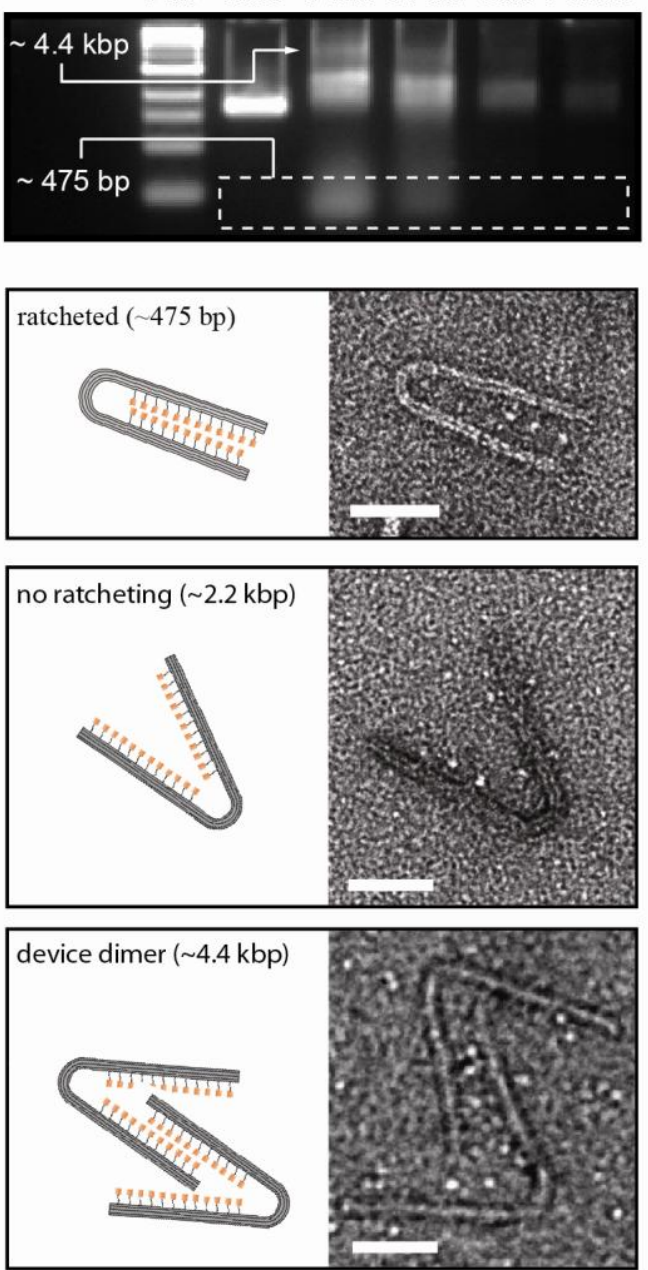

Figure 3: Analysis of device response to streptavidin ligand. Concentration ratios (device:ligand) were $1: 10^{3}$ (top) agarose gel image of reaction products for all device variants, the ratcheted band is highlighted (bottom) Negative stain TEM images of reaction products: ratcheted devices $(\sim 475 \mathrm{bp})$, devices that failed to ratchet $(\sim 2.2 \mathrm{kbp})$, and device dimers $(\sim 4.4 \mathrm{kbp})$ all scale bars are $50 \mathrm{~nm}$. In all images the ligand can be seen as a white dot.

toehold linked receptors. On the ratcheted device, streptavidin can be seen down the centerline.

Following identification of the products, gel analysis was performed on the reaction solution. Gels contained 1.75\% Agarose and $11 \mathrm{mM} \mathrm{MgCL} 2$. We used a $0.5 \mathrm{x}$ Tris/Borate/EDTA $(45 \mathrm{mM}$ Tris-borate, $1 \mathrm{mM} \mathrm{Na}{ }_{2}$ EDTA) running buffer containing $11 \mathrm{mM}$ $\mathrm{MgCl}_{2}$. Electrophoresis was run for 2.5 hours at $4 \mathrm{~V} / \mathrm{cm}$ (Thermo Scientific Owl B2 EasyCast Mini Gel System). After electrophoresis, the gel was removed and then stained using SYBR GREEN II (Invitrogen | Molecular probes) and imaged.

Inspection of the gel shows the primary product bands for the device variants with $7 \mathrm{~nm}$ and $14 \mathrm{~nm}$ receptor spacings. The dimer band $(\sim 4.4 \mathrm{kbp})$ can be seen at approximately twice the position of the unreacted band $(\sim 2.2 \mathrm{kbp})$; a smearing of this band occurs as the presence of streptavidin slows the device migration in the gel. The ratcheted device band ( $\sim 75 \mathrm{bp})$ migrates at a significantly faster speed in the gel. This occurs as a result of the streamlined configuration of the ratchet device. We excised and imaged this
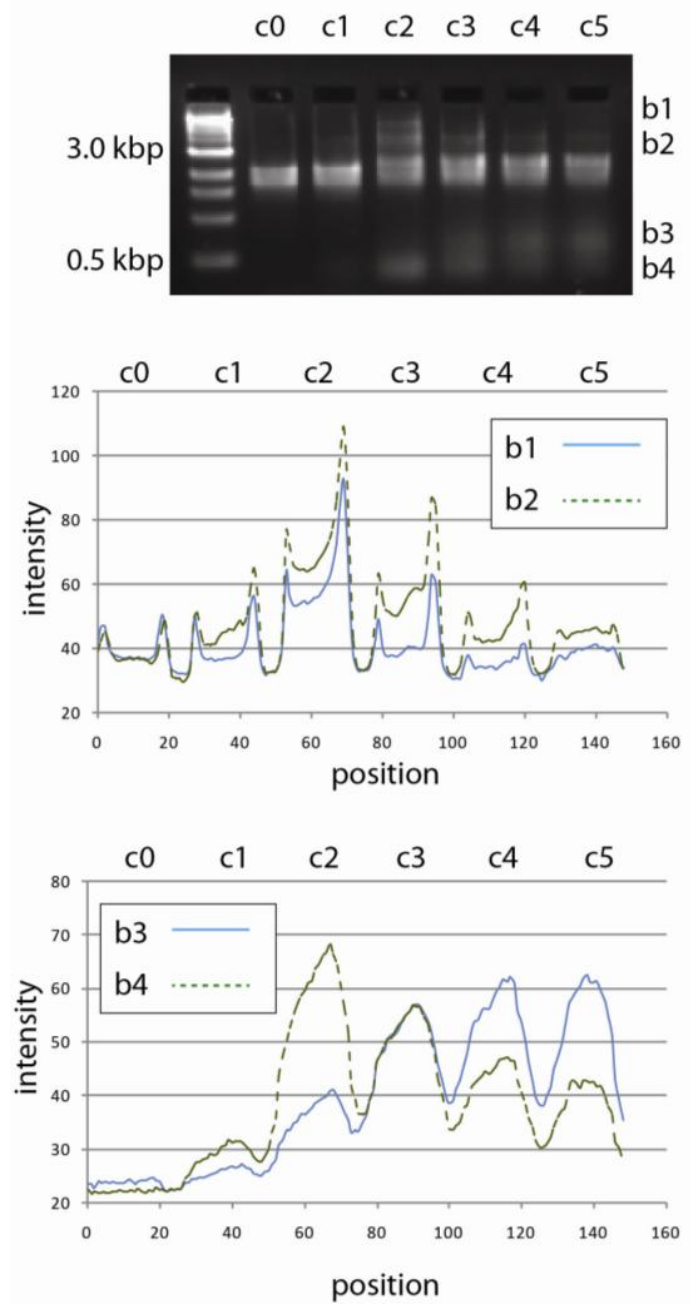

Figure 4: Agarose gel analysis of reacted devices with $7 \mathrm{~nm}$ receptor spacing over a range of concentration ratios (ligands:devices) from 1:10 to $1: 10^{5}$ (top) gel image; (middle) intensity analysis for trimer band, b1, and dimer band, b2; (bottom) intensity analysis for ratcheted bands $b 3$ and $b 4$.

band and confirmed the presence of ratchet devices. Control devices, which presented high forward energy barriers, did not ratchet as indicated by the lack of a forward band. The absence of closed devices for the controls strongly indicates that ratcheting must present for device actuation.

We subsequently inspected the $7 \mathrm{~nm}$ device response over a logarithmic concentration ratio range from 1:10 (Fig 4, lane $c 1$ ) to $1: 10^{5}$ (Fig 4, lane $c 5$ ). The device response is compared to the reference lane, $c 0$, for which the reaction contained no streptavidin. The aggregate of the ratcheted bands, b4 + b3 (more heavily bound with streptavidin), reaches a maximum at $1: 10^{3}$ and then remains relatively constant at higher concentrations. This result demonstrates a robustness of the device operation even in the presence of a high ligand concentration. The expectation that large quantities of ligand might cause receptor saturation may be offset by faster reaction kinetics from sandwich bond formation than the kinetics of ligand binding from the bulk solution. Interestingly, the contrary condition would conceivably result in a device that operated over a concentration range. Dimers, band b2, and trimers, band $\mathrm{b} 3$, peak and gradually from concentrations of $1: 10^{2}$. 


\section{DISCUSSION}

Ratchet designs, such as these, can become more sophisticated with improvements in our ability to model the energy landscapes and control molecular assemblies. There applications are apparent when considering the sensing and actuation can be coupled to technologically useful outputs. For instance, the coupling of a secondary molecule release can yield new technologies such as programmed artificial chemical to chemical transduction; a space which holds the promise of chemical logic, artificial signaling between cells, signal amplification, and new forms of diagnostics.

\section{REFERENCES}

[1] H. Dietz, S. M. Douglas, and W. M. Shih, "Folding DNA into twisted and curved nanoscale shapes.," Science (New York, N.Y.), vol. 325, no. 5941, pp. 725-30, Aug. 2009.

[2] S. M. Douglas, H. Dietz, T. Liedl, B. Högberg, F. Graf, and W. M. Shih, "Self-assembly of DNA into nanoscale three-dimensional shapes.," Nature, vol. 459, no. 7245, pp. 414-8, May 2009.

[3] S. Cruz and C. Biology, "Ratchets, power strokes, and molecular motors," vol. 323, pp. 315-323, 2002.

[4] L. Mahadevan, "Motility Powered by Supramolecular Springs and Ratchets," Science, vol. 95, no. 2000, 2008.

[5] C. S. Peskin, G. M. Odell, and G. F. Oster, "Cellular motions and thermal fluctuations: the Brownian ratchet.," Biophysical journal, vol. 65, no. 1, pp. 316-24, Jul. 1993.

[6] a. Fiasconaro, W. Ebeling, and E. Gudowska-Nowak, "Active Brownian motion models and applications to ratchets," The European Physical Journal B, vol. 65, no. 3, pp. 403-414, Jul. 2008.

[7] J. a Theriot, T. J. Mitchison, L. G. Tilney, and D. a Portnoy, "The rate of actin-based motility of intracellular Listeria monocytogenes equals the rate of actin polymerization.," Nature, vol. 357, no. 6375. pp. 257-60, 21-May-1992.

[8] L. G. Tilney and D. a Portnoy, "Actin filaments and the growth, movement, and spread of the intracellular bacterial parasite, Listeria monocytogenes.," The Journal of cell biology, vol. 109, no. 4 Pt 1, pp. 1597-608, Oct. 1989.

[9] E. R. Kay, D. A. Leigh, and F. Zerbetto, Molecular Devices Synthetic Molecular Motors and Mechanical Machines Angewandte. 2007, pp. 72 - 191.

[10] P. Hänggi, "Artificial Brownian motors: Controlling transport on the nanoscale," Reviews of Modern Physics, vol. 81, no. 1, pp. 387-442, Mar. 2009.

[11] L. Mahadevan, "Motility Powered by Supramolecular Springs and Ratchets," Science, vol. 288, no. 5463, pp. 95-99, Apr. 2000.

[12] H. Hess, H. Hess, G. D. Bachand, and V. Vogel, "Powering Nanodevices with Biomolecular Motors," 2004.
[13] Y. Tian, Y. He, Y. Chen, P. Yin, and C. Mao, "A DNAzyme that walks processively and autonomously along a one-dimensional track.," Angewandte Chemie (International ed. in English), vol. 44, no. 28, pp. 4355-8, Jul. 2005 .

[14] J. Bath, S. J. Green, and A. J. Turberfield, “A free-running DNA motor powered by a nicking enzyme.," Angewandte Chemie (International ed. in English), vol. 44, no. 28, pp. 4358-61, Jul. 2005.

[15] W. B. Sherman and N. C. Seeman, "A Precisely Controlled DNA Biped Walking Device," Nano Letters, vol. 4, no. 7, pp. 1203-1207, Jul. 2004.

[16] T. Omabegho, R. Sha, and N. C. Seeman, "A bipedal DNA Brownian motor with coordinated legs.," Science (New York, N.Y.), vol. 324, no. 5923, pp. 67-71, Apr. 2009.

[17] C. a. Floudas, H. K. Fung, S. R. McAllister, M. Mönnigmann, and R. Rajgaria, "Advances in protein structure prediction and de novo protein design: A review," Chemical Engineering Science, vol. 61, no. 3, pp. 966-988, Feb. 2006.

[18] a K. Dunker et al., "The unfoldomics decade: an update on intrinsically disordered proteins.," BMC genomics, vol. 9 Suppl 2, p. S1, Jan. 2008.

[19] S. M. Lippow and B. Tidor, "Progress in computational protein design.," Current opinion in biotechnology, vol. 18, no. 4, pp. 305-11, Aug. 2007.

[20] C. Bustamante, Y. R. Chemla, N. R. Forde, and D. Izhaky, "Mechanical processes in biochemistry.," Annual review of biochemistry, vol. 73, pp. 705-48, Jan. 2004.

[21] I. Tinoco and C. Bustamante, "The effect of force on thermodynamics and kinetics of single molecule reactions.," Biophysical chemistry, vol. 101-102, pp. 51333, Dec. 2002.

[22] D. Keller and C. Bustamante, "The Mechanochemistry of Molecular Motors STOCHASTIC MACHINES," Biophysical Journal, vol. 78, no. February, pp. 541-556, 2000.

[23] C. E. Castro et al., "a primer to scaffolded DNa origami," Nature Methods, vol. 8, no. 3, pp. 221-229, 2011.

\section{CONTACT}

*Gabriel J. Lavella, tel: 510.664.4144; glavella@eecs.berkeley.edu 


\title{
A MONOLITHIC 162-STAGE TWO-PART KNUDSEN PUMP FOR HIGH COMPRESSION RATIO
}

\author{
S. An*, N.K. Gupta, and Y.B. Gianchandani \\ Center for Wireless Integrated MicroSensing and Systems $\left(\mathrm{WIMS}^{2}\right)$, University of Michigan, \\ Ann Arbor, Michigan 48109, USA
}

\begin{abstract}
This paper reports on a 162-stage Knudsen pump, which is a motionless, thermomolecular pump, monolithically integrated into a single chip. The microchip is fabricated by a five mask silicon wafer process and has a footprint of $12 \times 15 \mathrm{~mm}^{2}$. It performs a self-evacuation of on-chip cavities, reducing pressures from 760 Torr to $\approx 7$ Torr. This is achieved with a power of $\approx 0.44 \mathrm{~W}$. The compression ratio of 109 is a $2 \times$ improvement over our previous best report, with a $3 \times$ reduction in power consumption. This performance is made possible by using a two-part design, which separately customizes stages for high and low pressure levels.
\end{abstract}

\section{INTRODUCTION}

First proposed and demonstrated by Knudsen in 1909 [1], the Knudsen pump, activated with thermal molecular pressure, has long been an appealing method for a vacuum pump. First, it has no moving parts [2]. Thermal gradient in a flow channel is the only driving force, so there is neither frictional loss nor mechanical failure. This greatly improves reliability and ensures long term operation. Second, it can potentially have small form factor. Using the miniaturization techniques of microelectromechanical systems (MEMS), compact Knudsen pumps have been reported [2-6].

Knudsen pumping is based on the phenomenon of thermal transpiration which occurs in a rarefied gas flow channel. If the hydraulic diameter of a flow channel is no larger than the mean free path (MFP) of gas molecules [2], i.e., the flow in the channel is confined to the free-molecular or transitional flow regimes, a thermal gradient results in flow from the cold end to the hot end. A monolithic Si implementation that used one such flow channel was reported in 2005, demonstrated a compression ratio of $\approx 2$ [2]. Because the compression ratio of a single-stage is limited by the temperature differential between the cold and hot ends, higher compression ratios can be achieved by multi-stage implementation. Mesoscale multistage Knudsen pumps have been reported [3, 5] in the past, but allow only a limited number of stages to be used.

In 2012 [6], our group reported a highly-packed multistage implementation, leveraging state-of-the-art silicon micromachining technology. It had 48 repetitions of a single type of stage for high compression ratio. The fabricated chip demonstrated the self-evacuation of on-chip cavities from 760 Torr to $<50$ Torr. However, this one-part approach is inadequate for a target vacuum level below about 50 Torr. The small height of the flow channel, where Knudsen pumping occurs, in the design $(0.15 \mu \mathrm{m})$ is suitable for initiating pumping from atmospheric pressure. However, when pumping is initiated from low pressure, the larger height of the channel can also provide similar compression with higher pumping speed, because the flow remains in free-molecular or transitional regime even for deeper flow channels that provide lower hydraulic resistance.

In this paper, we propose an improved, two-part design, which separately customizes stages for high and low pressure levels. Compression ratio and pumping speed are addressed in relation to the variation of narrow and wide channels at both pressure levels. Parametric design of a heater using finite element analysis (FEA) for low power heating is also described.

\section{DESIGN CONCEPT \\ Knudsen Pump Theory}

Thermal transpiration in a rarefied gas flow channel is described by thermal creep flow and viscous backflow [7]. The thermal gradient results in thermal creep flow along the channel walls from the cold end to the hot end; away from the walls, viscous backflow is caused by the pressure differential induced by the thermal creep flow. When the characteristic height of the flow channel is no larger than the MFP, the thermal creep flow is dominant over the viscous backflow; this establishes a pressure gradient in the same direction as the thermal gradient. In contrast, when the characteristic height of the flow channel is considerably larger than the MFP, the viscous backflow dominates, diminishing or eliminating the pressure gradient.

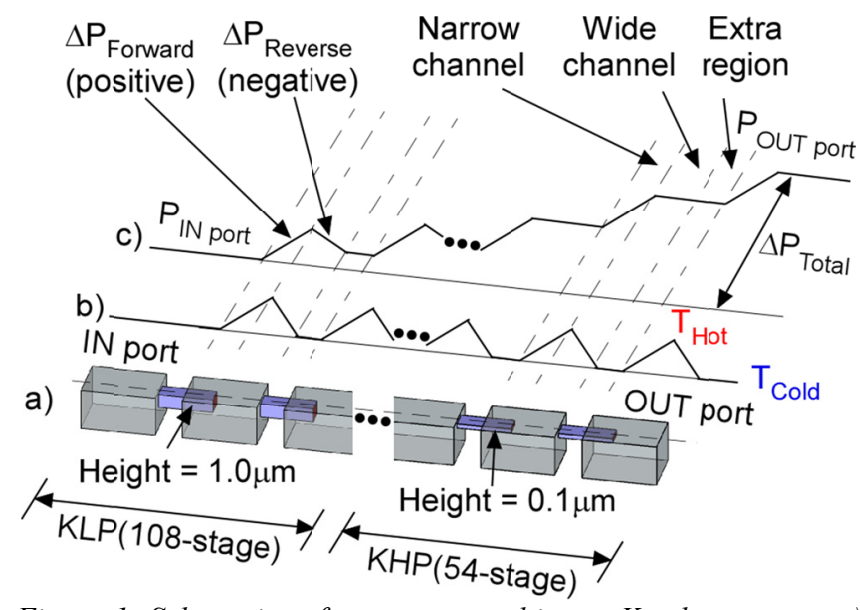

Figure 1: Schematics of a two-part multistage Knudsen pump. a) Serial cascading of KLP and KHP stages. b) Temperature profile along the dashed line in (a). c) Equilibrium pressure profile along the dashed line in (a). The dot-dash lines between (a)-(c) indicate simultaneous occurrences. KHP is from stage \#1 (OUT port) to \#54 and KLP is from stage \#55 to \#162 (IN port).

To achieve a large total pressure differential $\left(\Delta P_{\text {Total }}\right)$, multiple single-stage Knudsen pumps are serially cascaded (Fig. 1). Each stage includes a narrow channel and wide channel (Fig. 1a). The narrow channel, where the thermal creep flow is designed to be dominant, generates a forward pressure differential $\left(\Delta P_{\text {Forward }}\right)$ (Fig. 1c) under the applied temperature gradient along the intended forward path (Fig. 1b). In contrast, the wide channel, where the viscous backflow is designed to be dominant, is intended to restore the temperature from $\mathrm{T}_{\mathrm{Hot}}$ to $\mathrm{T}_{\text {Cold }}$ without a change in pressure. However, at low pressure, because the MFP increases to the extent that is comparable to the hydraulic diameter of the wide channel, a reverse pressure differential $\left(\Delta P_{\text {Reverse }}\right)$ is established in the wide channel, thereby reducing the net pressure differential $\left(\Delta P_{\text {Net }}=\Delta P_{\text {Forward }}-\Delta P_{\text {Reverse }}\right)$. This is mitigated by maximizing the dimension of the wide channel and by increasing the number of stages.

For the analytical design of micromachined Knudsen pumps, the Sharipov model [8] is an appropriate representative model for 
direct simulation Monte Carlo (DSMC) [9]. In a rectangular channel from the cold end to the hot end, the Sharipov model calculates the average mass flow rate of gas as:

$$
\frac{d M_{\text {avg }}}{d t}=\left(\frac{Q_{T} \Delta T}{T_{\text {avg }}}-\frac{Q_{P} \Delta P}{P_{\text {avg }}}\right) \frac{a^{2} b P_{\text {avg }}}{l} \sqrt{\frac{m}{2 k_{b} T_{\text {avg }}}}
$$

where $l$ is the length of the channel, $a$ is the height of the channel, $b$ is the width of the channel, $\Delta T$ is the temperature difference, $T_{a v g}$ is the average temperature, $\Delta P$ is the pressure difference, $P_{a v g}$ is the average of the pressure, $m$ is the mass of a single gas molecule, $k_{b}$ is the Boltzmann constant and $Q_{T}$ and $Q_{P}$ are, respectively, the thermal creep flow and Poiseuille flow (pressure-driven flow) coefficients that depend on the rarefaction parameter (inverse of Knudsen number). Over time, the gas flow approaches equilibrium, where there is no net mass flow, and the established pressure is given by:

$$
\Delta P_{\text {equilibrum }}=\frac{Q_{T}}{Q_{P}} \frac{\Delta T}{T_{\text {avg }}} P_{\text {avg }}
$$

$Q_{T}$ and $Q_{P}$ for each height/width ratio and rarefaction parameter can be found directly from the table or from an interpolation of the tabulated values. At a given pressure, the decrease of the channel height is accompanied by the increase of the $\Delta P_{\text {equilibrium }}$ and the decrease of the average mass flow rate, and vice versa.

Table I. The designed channel dimensions for KHP and KLP stages respectively, and the corresponding $M F P$ of $N_{2}$ gas at each pressure,

\begin{tabular}{|c|c|c|c|c|}
\hline $\begin{array}{c}\text { Two-part } \\
\text { architecture }\end{array}$ & $\begin{array}{c}\text { Narrow } \\
\text { channel } \\
\text { height } \\
(\mu \mathrm{m})\end{array}$ & $\begin{array}{c}\text { Wide } \\
\text { channel } \\
\text { height } \\
(\mu \mathrm{m})\end{array}$ & $\begin{array}{l}\text { Pressure } \\
\text { (Torr) }\end{array}$ & $\begin{array}{l}\text { MFP } \\
(\mu \mathrm{m})\end{array}$ \\
\hline \multirow{3}{*}{$\begin{array}{c}\text { KHP } \\
\text { (760Torr } \\
\text {-50Torr) }\end{array}$} & \multirow{3}{*}{0.1} & \multirow{3}{*}{$>30$} & 760 & 0.07 \\
\hline & & & 200 & 0.26 \\
\hline & & & 50 & 1.02 \\
\hline $\begin{array}{c}\text { KLP } \\
\text { ( } \leq 50 \text { Torr })\end{array}$ & 1.0 & $>50$ & $\frac{50}{1}$ & $\begin{array}{c}1.02 \\
51.10\end{array}$ \\
\hline
\end{tabular}
calculated at $300 \mathrm{~K}$.

\section{Two-part Architecture}

Due to the increase in the MFP resulting from the decrease in pressure level, the narrow channel can be enlarged at low pressure with an insignificant reduction of $\Delta P_{\text {Forward }}$, as long as its height is no larger than the MFP. Pumping speed can be considerably improved with an enlarged channel height, because mass flow rate is quadratically proportional to the channel height as shown in Eq. (1). In addition, the wide channel height needs to be significantly larger than the MFP at both high and low pressures to minimize $\Delta P_{\text {Reverse }}$. Hence, to enhance pumping speed and compression ratio at both high and low pressures, a two-part architecture (Fig. 1) is used: the Knudsen high-pressure part (KHP), located downstream, is designed for operating near atmospheric pressure, and the Knudsen low-pressure part (KLP), located upstream, is designed for $\leq 50$ Torr, as summarized in Table I. In the KHP, which pumps down from 760 Torr to 50 Torr, the narrow channel height is $0.1 \mu \mathrm{m}$, which is comparable to an MFP of $0.07 \mu \mathrm{m}$ at 760 Torr. In the KLP, which pumps down from 50 Torr to sub-Torr, the narrow channel height is $1.0 \mu \mathrm{m}$, which is comparable to the MFP of $1.0 \mu \mathrm{m}$ at 50 Torr. The wide channel height of the KHP is larger than $30 \mu \mathrm{m}$ for minimum $\Delta P_{\text {Reverse }}$ from 760 Torr to 50 Torr. The wide channel height of the KLP is maximized to be as deep as fabrication permits. In this generation of Knudsen pumps, the wide channel height of both KHP and KLP is equal to $\approx 50 \mu \mathrm{m}$ for the simplification of fabrication and mask design.

\section{Parametric Design of Heater Structure}

The length of the narrow channel in the device structure (Fig. $2 \mathrm{f}$ ) is one of the dominant factors determining the thermal isolation from the heater to the thermally ground. By varying structural parameters such as width, length, and thickness, the dominant dimensional parameters for low power heating are extracted and fine-tuned using FEA. Parametric FEA enables lower power operation in this generation of Knudsen pumps.

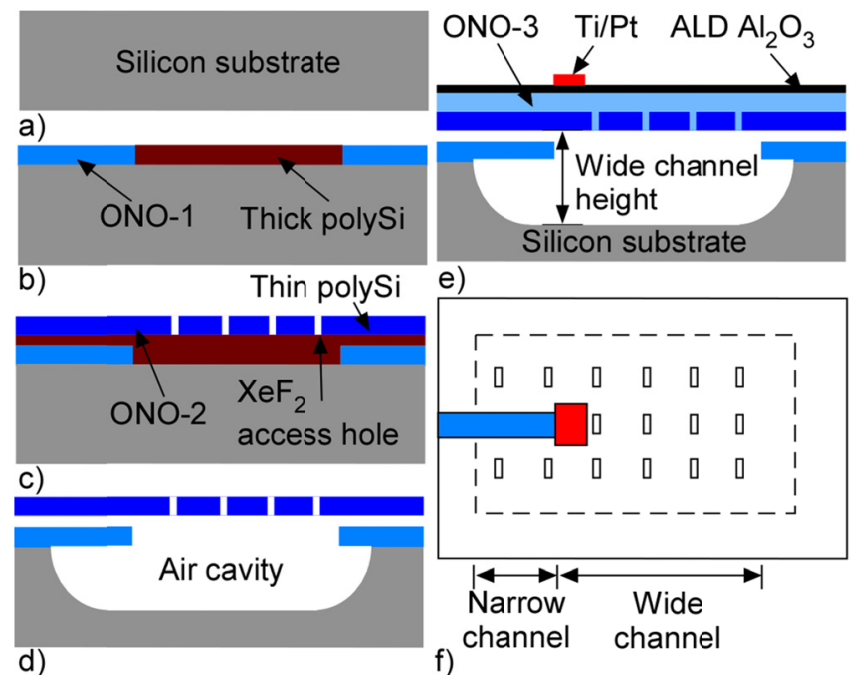

Figure 2: The five mask fabrication process and sectional views of structure. a-e) Side view of each fabrication process step: a) silicon wafer, b) (Mask I) deposition and patterning of ONO-1 and (Mask II) deposition and patterning of thick polySi for KLP narrow channel, c) (Mask III) deposition \& patterning of thin polySi for KHP narrow channel and (Mask IV) deposition and patterning of $\mathrm{ONO}-2$, d) $\mathrm{XeF}_{2}$ etch of polySi and air cavity, and e) deposition of ONO-3 and atomic layer deposited (ALD) $\mathrm{Al}_{2} \mathrm{O}_{3}$ for sealing and (Mask V) lift-off of Ti/Pt heater and leads. f) Top view of complete structure.

\section{FABRICATION}

\section{Microfabrication Process}

To implement the multistage Knudsen pump, a simple and high yield single wafer process is used; it uses five masking steps (Fig. 2). Important features in the channel fabrication are: 1) thin (sacrificial) polysilicon is used to define narrow channels in the KHP stages (Fig. 2c); 2) both thin and thick (sacrificial) polysilicon layers are used to define narrow channels for the KLP stages; 3) a cavity is etched in bulk silicon to increase the height of the wide channel (Fig. 2e); and 4) the cavity, in the left side under the heater, also defines the length of the narrow channel (Fig. 2f); and 5) stress compensated oxide-nitride-oxide (ONO) layers, ONO-1 and ONO-2, are used to define the walls of the narrow channel. A Ti/Pt heater, located at the end of the narrow channel, generates the intended temperature gradient for the $\Delta P_{\text {Forward }}$ in the narrow channel. The temperature gradient in the wide channel occurs, because the wide channel is linking the hot end of one narrow channel and the cold end of the next narrow channel. Increasing the height of the wide channel minimizes $\Delta P_{\text {Reverse }}$ at low pressures. At each step in the process, the thickness and residual stress of each layer are monitored (Table 2). ONO layers are designed to have a mild tensile stress to avoid a buckling of the suspended diaphragm due to compressive residual stress. 
Table 2: Summary of the approximate values of the thickness and residual stress of each layer.

\begin{tabular}{|c|c|c|}
\hline Layer & $\begin{array}{c}\text { Thickness } \\
(\boldsymbol{\mu m})\end{array}$ & $\begin{array}{c}\text { Residual stress } \\
(\mathbf{M P a})\end{array}$ \\
\hline $\mathrm{ONO}-1$ & $0.5 / 0.2 / 0.5$ & +42.5 \\
\hline $\mathrm{ONO}-2$ & $0.5 / 0.2 / 0.5$ & +42.5 \\
\hline $\mathrm{ONO}-3$ & $0.7 / 0.5 / 0.7$ & +42.1 \\
\hline $\mathrm{Thin} /$ thick poly & $0.1 / 0.7$ & - \\
\hline $\mathrm{ALD} \mathrm{Al} \mathrm{O}_{3}$ & 0.2 & +304 \\
\hline $\mathrm{Ti} / \mathrm{Pt}$ & $0.025 / 0.1$ & - \\
\hline
\end{tabular}

Additional challenges in microfabrication compared to our Knudsen pump in [6] are as follows: increased wide channel height using a higher density of $\mathrm{XeF}_{2}$ access holes for reduced $\Delta P_{\text {Reverse }}$; a reduced thickness of sealing layers for higher thermal isolation; and a decreased Ti/Pt-heater size by reducing winding-pattern linewidth, also for higher thermal isolation.

a)
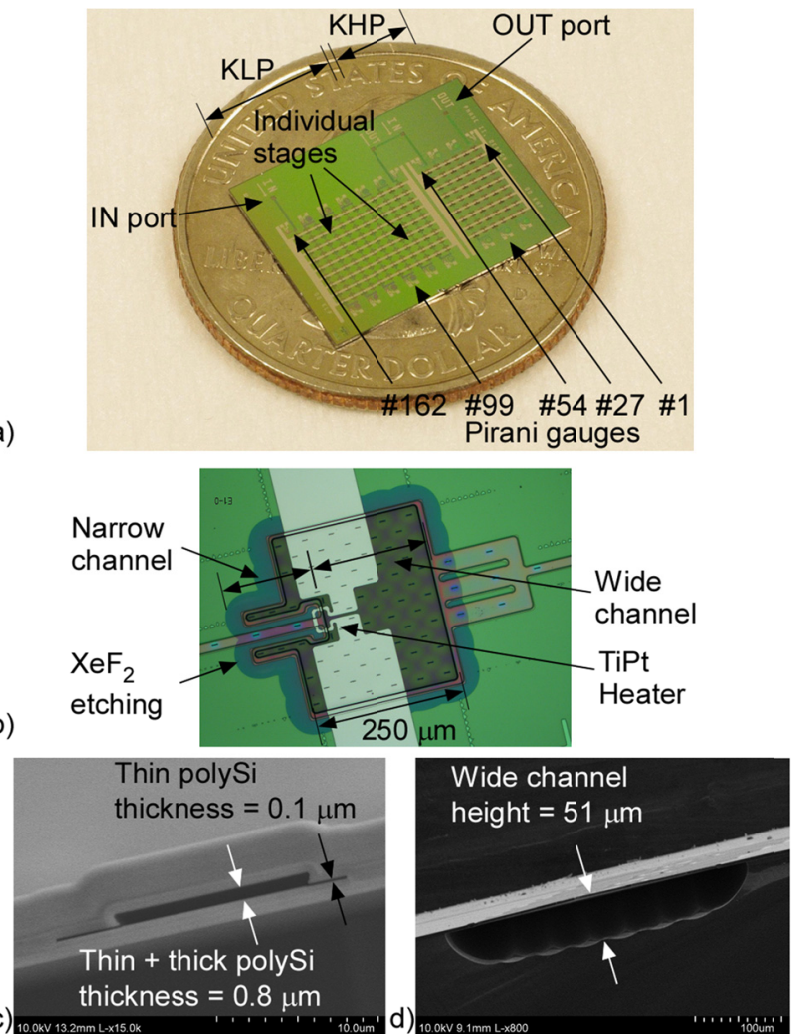

Figure 3: Photographs of the Knudsen pump as fabricated. a) The full die. b) Top view of one stage. c) SEM of the cross section of a narrow channel in the KLP. d) SEM of the cross section of a wide channel in both KLP and KHP.

\section{Fabrication Results}

The fabricated chip (Fig. 3a) has a footprint of $12 \times 15 \mathrm{~mm}^{2}$. A typical yield is $60-80 \%$. The KHP and KLP are hydraulically connected, using sacrificially etched polysilicon layers. Pirani gauges for the in situ measurement of vacuum levels are located on both sides of the pumping stages.

The length of the narrow channel with a temperature gradient is defined by $\mathrm{XeF}_{2}$ gas etching of the bulk silicon (Fig. 3b). The resistive heaters, required for the Knudsen pumping stages, are shaped with winding patterns of $5 \mu \mathrm{m}$ width.

Narrow channels (Fig.3c) are formed by etching sacrificial polySi layers. For the narrow channel of the KHP, only a thin polySi layer of $0.1 \mu \mathrm{m}$ thickness is used. However, for the narrow channel of the KLP, both thin and thick polySi layers of $0.8 \mu \mathrm{m}$ are used as sacrificial layers.

The wide channel height (Fig. 3d) is defined by the etching depth of the bulk silicon, using $\mathrm{XeF}_{2}$ gas. The density of the $\mathrm{XeF}_{2}$ access holes (Fig. 2c) and etching time determine the height of the wide channel. The $\mathrm{XeF}_{2}$ access hole is designed to be $1 \mu \mathrm{m} \times 10$ $\mu \mathrm{m}$ in size and is hermetically sealed by PECVD ONO-3 and ALD $\mathrm{Al}_{2} \mathrm{O}_{3}$ layers.

\section{TEST RESULTS AND DISCUSSION Test Preparation}

The fabricated chip is wire-bonded for electrical connections. The base of the Knudsen pump is placed in direct contact with a test chamber for the thermal grounding of the silicon substrate to the room temperature laboratory environment. The operating medium gas is air. Prior to the activation of the Knudsen pump, the five Pirani gauges $(\approx 125 \Omega)$, including the IN port and OUT port, are calibrated from high to low pressure levels (Fig. 4a). The IN port of the KLP is sealed, while the OUT port of the KHP is opened and vented to ambient pressure. The ambient pressure in the test chamber is controlled externally. By applying a constant current source of $4 \mathrm{~mA}$, the fractional changes in the resistance of each Pirani gauge are recorded at various pressure levels, representing the vacuum levels in nearby pumping stages upon pumping operation [6].

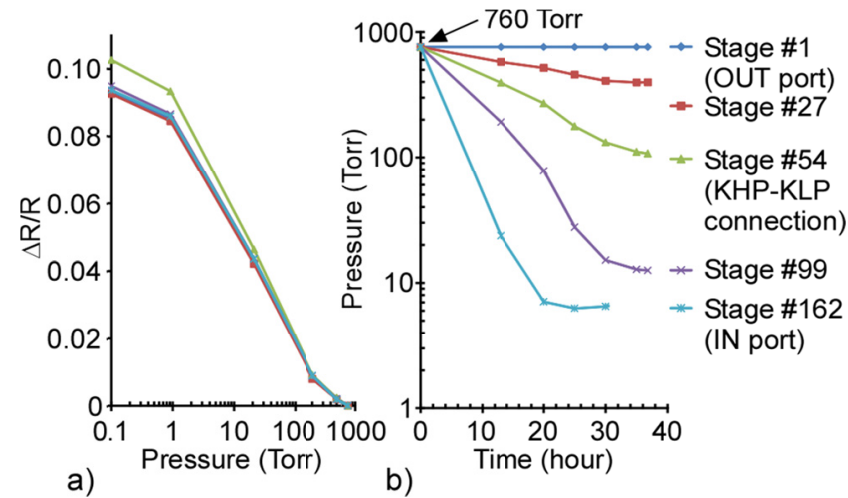

Figure 4: Pirani gauge calibration and time response of pressure a) Variation in the fractional change of the resistance of the five Pirani gauges. b) Measured time response of pressure levels in each stage, pumping down from 760 Torr, using an applied total power of $\approx 0.44 \mathrm{~W}$ to both KHP and KLP.

\section{Pumping Performance}

The pressure at the IN port approaches saturation in about 20 hours (Fig. 4b). In the case of the KHP-KLP connection stage, pressure saturation takes about 40 hours.

When only the KLP operates, pressure is reduced from 50 Torr to $\approx 2$ Torr, with an applied power of $\approx 0.3 \mathrm{~W}$ (Fig. $5 \mathrm{a}$ ). When both KLP and the KHP operate, pressure is reduced from 760 Torr to $\approx 7$ Torr, with an applied power of $\approx 0.44 \mathrm{~W}$ (Fig. 5b). This pump achieves a compression ratio of 109 when both KLP and the KHP are operating at 760 Torr.

\section{Discussion}

The KLP is composed of 108 stages while the KHP is composed of 54 stages. Due to the large height of the narrow channel in the KLP compared to the MFP at 760 Torr, the pressure is only reduced from 760 Torr to 150 Torr with KLP-only operation (Fig. 5a). In contrast, as shown in the simultaneous operation of KHP and KLP, the KHP can pump down from 760 Torr to about 
100 Torr (Fig. 5b), using a smaller number of stages than the KLP. In this two-part approach, the KHP, using the smaller height of the narrow channel suitable for initiation of pumping from atmospheric pressure, allows a greater compression ratio per stage and consequently operates with a smaller number of stages than the KLP at high pressure.

The KHP can also pump down from 50 Torr to less than 10 Torr (Fig. 5b). However, due to the dependence of mass flow rate upon the narrow channel height, the pumping time is longer. Using the larger narrow channel of KLP, mass flow rate is increased by about $64 \times$, which is simply the square of the ratio of the fabricated narrow channel height of KLP and KHP, as shown in Eq. (1). This is one of the reasons why the pressure at the IN port saturates twice as fast as the pressure at the KHP-KLP connection stage (Fig. 4b).

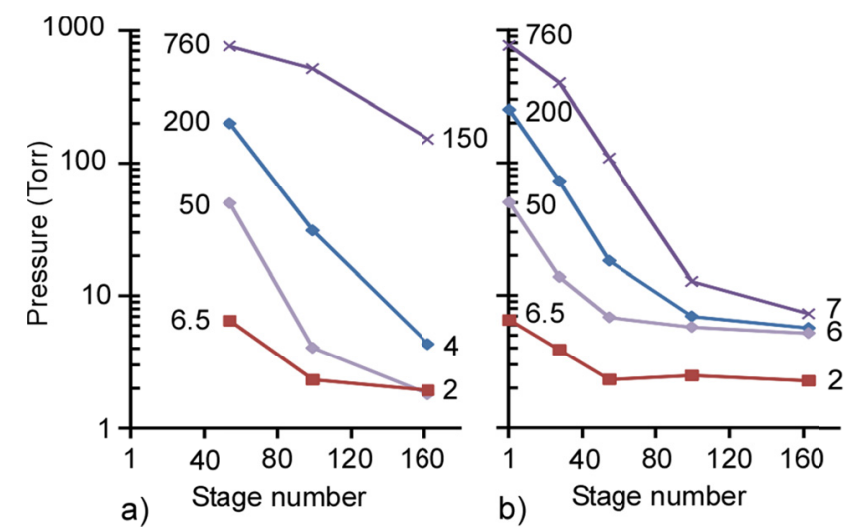

Figure 5: Measured pressure distribution in the selected five stages. a) Only KLP operating for pumping. b) Both KLP and KHP operating for pumping. The IN port is sealed while the OUT port is open to a test chamber whose pressure is controlled.

\section{Benchmarking}

Benchmarking shows that the resulting compression ratio of 109 exceeds the highest [6] by a factor of $2 \times$, with $3 \times$ power efficient (Fig. 6). The integration of 162 stages into one silicon chip, using the simple silicon-based micromachining, and two-part approach, separately customizing KHP/KLP stages, enabled this substantially higher performance.

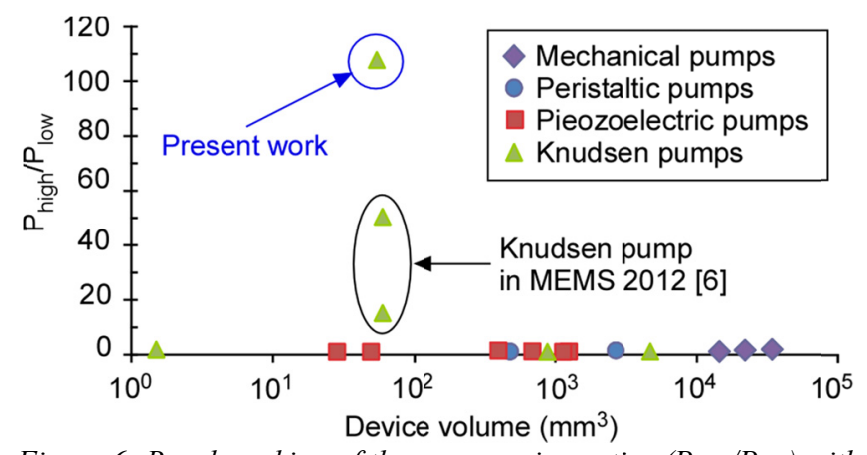

Figure 6: Benchmarking of the compression ratios $\left(P_{\text {high }} / P_{\text {low }}\right)$ with other pumps reported in literature to-date [2, 6, 10-11]. The present work achieves 760 Torr/7 Torr using both KHP and KLP.

\section{CONCLUSION}

A 162-stage Knudsen pump for high compression ratio, with an improved two-part (KHP and KLP) approach, is studied. Using a monolithic integration with a single-wafer microfabrication process, the designed highly-packed stages are serially cascaded in a small footprint of $12 \times 15 \mathrm{~mm}^{2}$. The pumping performance is measured in situ at five stages including the IN port and OUT port, and at various ambient pressures, using the calibration of Pirani gauges. Using KHP and KLP together, the fabricated Knudsen pump demonstrated the self-evacuation of on-chip cavities from 760 Torr to $\approx 7$ Torr, with an input power of $\approx 0.44 \mathrm{~W}$. Compared to our last reported micropump [6], this shows a $2 \times$ improvement in compression ratio, using $3 \times$ smaller power consumption. The substantial contributions are from the two-part Knudsen pumping architecture together with the monolithic integration of the increased number of stages by silicon-based micromachining technology. In the future, the further refinement of the heater and flow-channel structure, along with the microfabrication process, could enhance the pumping performance to higher vacuum levels with even lower power consumption in a smaller footprint.

\section{ACKNOWLEDGEMENT}

The study is supported in part by the Microsystems Technology Office of the Defense Advanced Research Projects Agency High-Vacuum Program (DARPA Hi-Vac). Facilities used for this research include the Lurie Nanofabrication Facility (LNF) operated by the Solid-State Electronics Laboratory (SSEL) and the University of Michigan.

\section{REFERENCES}

[1] M. Knudsen, "Eine Revision der Gleichgewichtsbedingung der Gase. Thermische Molekularstromung," Annalen der Physik, Leipzig, vol. 336, no. 1, pp. 205-229, 1909. (in German).

[2] S. McNamara, and Y.B. Gianchandani, "On-Chip Vacuum Generated by a Micromachined Knudsen Pump," $J$. Microelectromech. Syst., vol. 14, no. 4, pp. 741-746, 2005.

[3] M. Young, Y.L. Han, E.P. Muntz, and G. Shiflett, "Characterization of a Radiantly Driven Multistage Knudsen Compressor," Proc. ASME Int. Mech. Engin. Congress (IMECE'03), pp. 393-400, 2003.

[4] K. Pharas, and S. McNamara, "Knudsen Pump Driven by a Thermoelectric Material," J. Micromech. Microeng, vol. 20, no. 12, pp. 125032, 2010.

[5] N.K. Gupta, and Y.B. Gianchandani, "Porous Ceramics for Multistage Knudsen Micropumps - Modeling Approach and Experimental Evaluation," J. Micromech. Microeng, vol. 21, no. 9, pp. 095029, 2011.

[6] N.K. Gupta, S. An, and Y.B. Gianchandani, "A Monolithic 48-Stage Si-micromachined Knudsen Pump for High Compression Ratios," Proc. IEEE Micro Electro Mechanical Systems (MEMS'12), pp. 152-155, 2012.

[7] L. Loeb, The Kinetic Theory of Gases, McGraw Hill, pp. 355-359, 1934.

[8] F. Sharipov, "Non-isothermal Gas Flow through Rectangular Microchannels," J. Micromech. Microeng., vol. 9, no. 4, pp. 394-401, 1999.

[9] N.K. Gupta, N. D. Masters, W. Ye, Y.B. Gianchandani, "Gas Flow in Nano-Channels: Thermal Transpiration Models with Application to a Si-Micromachined Knudsen Pump," Proc. IEEE Transducers '07, Lyon, France, pp. 2329-2332, 2007.

[10] H. Kim, A.A. Astle, K. Najafi, L.P. Bernal, and P.D. Washabaugh, "A Fully Integrated High-Efficiency Peristaltic 18-Stage Gas Micropump with Active Microvalves," Proc. IEEE Micro Electro Mechanical Systems (MEMS'07), pp. 127-130, 2007.

[11] D.J. Laser, and J.G. Santiago, “A Review of Micropumps,” J. Micromech. Microeng., vol. 14, no. 6, pp. R35-R64, 2004.

\section{CONTACT}

*S. An, tel: +1-734-717-7853; sdan@umich.com 


\section{A LEAK-TIGHT AND ZERO-DEAD-VOLUME MICRO GAS PUMP INTEGRATED WITH ELECTROKINETIC LIQUID PISTONS \\ J.Jiao $^{* 1}$, R. Almeida ${ }^{l}$, and J.W. Kwon ${ }^{l}$ \\ ${ }^{1}$ Department of Electrical and Computer Engineering, University of Missouri, Columbia, MO, U.S.A.}

\begin{abstract}
This paper presents a novel micromachined gas pump utilizing electrokinetically driven liquid pistons in a microfluidic channel. As a result of our effort in improving the leakage problem of micro gas pumps, we have successfully utilized a low contact angle liquid droplet as a piston to move the gas phase medium. Because of the excellent wettability of the liquid piston, the contacting inner surfaces of the microfluidic channel get completely wetted, which seals any possible tiny leaky spots. From our newly developed micro pump, by circulating the liquid pistons along the microfluidic channel, a leak-tight air pumping without dead-volume has been clearly observed.
\end{abstract}

\section{INTRODUCTION}

A low backflow in a pump indicates high pumping efficiency, which is desirable especially for a micro gas pump. Though many scientists have been working on various types of micro gas pumps[1], a micro gas pump without backflow has hardly been achieved. The differently unique properties of gas as compared to liquids have made obtaining high pumping efficiencies extremely hard. Especially, it is very difficult to overcome the high compressibility of gases[2].

There are various types of micro gas pumps utilizing differently generated forces. A pressure driven mechanical force exerted in the plunger (solid, liquid or gas) can be used to interact with the target gas. Based on the phase of the plunger material for gas pumping, micro gas pumping with the solid plunger has been normally achieved through large diaphragm-based plunging interfaces actuated piezoelectrically[3], electrostatically[4], thermally[5], pneumatically[6, 7] and electromagnetically[8]. In addition, a gas pump with the gas-phase plunger utilizing vapor jet [9] has been introduced. Unlike many conventional methods, we adopted a different approach to achieve an effective gas pumping method with a simple structure. So far, the use of the liquid plunger for gas pumping has not been fully explored yet. Although the formerly used ferromagnetic fluid (liquid plunger) with large multiple external magnets has been used for liquid pumping application, it is not appropriate for gas pumping[10]. For our new gas pump, many interesting properties of low surface energy fluids have been considered because high surface energy fluids cannot wet the corner of the microfluidic channel but always leave some void spots causing gas leakage. The low surface energy fluid can effectively wet all the corners of the microfluidic channel very easily and completely[11]. Thus, it can be an effective plunger material for the gas pumping in the microfluidic channel. Moreover, the size of our pump has a great potential to be truly miniaturized since it requires no external large magnets but small electrodes.

\section{DESIGN AND THEORY}

Oil, which is one of the most common low surface energy liquids, was looked into and silicone oil was chosen as the liquid piston material. To drive the silicone oil droplet (a good dielectric material) in a microfluidic channel, we utilized the dielectrophoresis (DEP) force which can be directly imposed to the droplet. The dielectrophoretic body force is generated by the electric field and dominantly contributes to the motion of the dielectric liquid piston in the microfluidic channel. The motion of the liquid can also be further explained by the Navier-Stokes equation. In the equation, the body force density induced by the external electric field term can be expressed by the KortewegHelmholtz electric force density $[12,13]$ :

$$
\mathbf{f}_{\mathbf{b}}=\rho_{\mathrm{f}} \mathbf{E}-\frac{1}{2} \mathrm{E}^{2} \nabla \varepsilon+\nabla\left(\frac{1}{2} \mathbf{E} \cdot \mathbf{E} \frac{\partial \varepsilon}{\partial \rho}\right)
$$

where $\varepsilon$ is the fluid permittivity, $\rho$ is the density of the fluid, $\rho_{\mathrm{f}}$ is the free electric charge density, and $\mathrm{E}$ is the electric field. By ignoring the electrostriction and assuming the ideal dielectric conditions for the liquid piston, the body force can be further simplified as a proportional expression:

$$
\mathbf{f}_{\mathbf{b}} \propto E^{2} \nabla \varepsilon
$$

From this equation, we can notice that the DEP force increases especially at the region where the permittivity gradient is maximized. In addition, with an increase of the electric field applied across the gradient areas, the body force density will also be increased. Since we have used a fixed channel height in our design, the electrical field is strongly correlated to the actuation voltages.

\section{FABRICATION AND EXPERIMENT Single Liquid Piston}

To begin with, a single liquid piston in a straight microfluidic channel was tested for characterizing the pumping capability. As illustrated in Fig. 1, the straight channel device consists of two main parts: 1) A PDMS microfluidic channel, and 2) Parallel-plate electrodes for providing the electric field along the microfluidic channel. Fabrication of the device was very simple. First, a thin aluminum film was sputter-deposited and patterned to form electrodes. Along the electrode pads, a microfluidic channel was built by using patternable PDMS. A thin indium-tin-oxide (ITO) coated glass substrate was used as the top cover because it can provide clear visibility for careful observation of the liquid piston behavior from various tests. Finally, the bonding was achieved between top and bottom structures by oxygen plasma treatment on the PDMS surface.
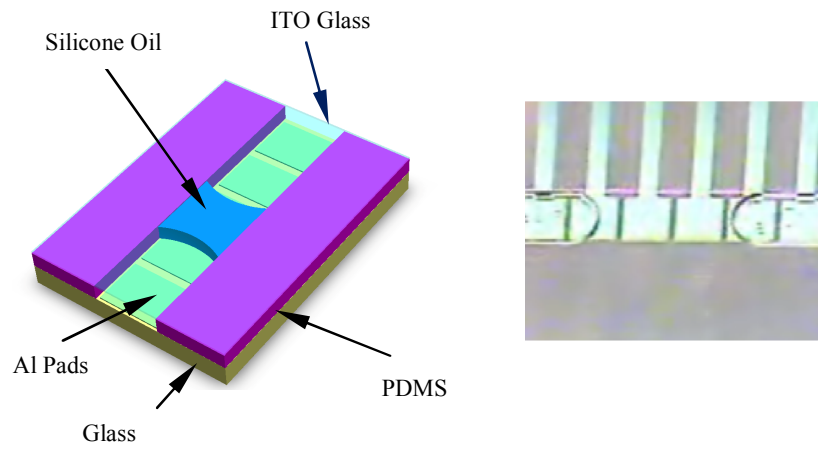

Figure 1. Schematic drawing of a single liquid piston kept in a straight microchannel with a small contact angle liquid (left) and microscope image of the fabricated device (right). 
A small silicone oil droplet was loaded from an open end of the channel with the aid of an external pneumatic source. Due to the very low surface tension of silicone oil, the contact angle on the PDMS microfluidic channel surface turned out to be far less than $90^{\circ}$. From the top view of the device as shown in Fig. 1, we can clearly see the concave edge profiles from both the sides of the silicone oil droplet, which resulted from good wettability over the inner surfaces of the microfluidic channel.

Then, the device was placed on a test platform and connected to a computer-controlled National Instruments (NI) digital I/O device, which allows us to handle a variety of driving signal patterns. The results show that the liquid piston motion closely follows the actuation signals. When the driving signal is applied to an electrode, a gradient of permittivity on the front edge line of the liquid piston is generated. It was observed that the liquid piston responded rapidly to the applied signal. When the electrode is activated sufficiently long enough for the liquid to sweep across the entire actuated area, the front edge of the moving liquid piston stops at the front edge of the leading electrode. The movement of the liquid piston is quick and highly electrically controllable. The characterization is based on the sweeping frequency and the average speed of the liquid piston. The backpressure of a single liquid piston can be improved by increasing the actuation voltage as shown in Fig. 2. The corresponding flow rate to the driving signal sweeping frequency is characterized.
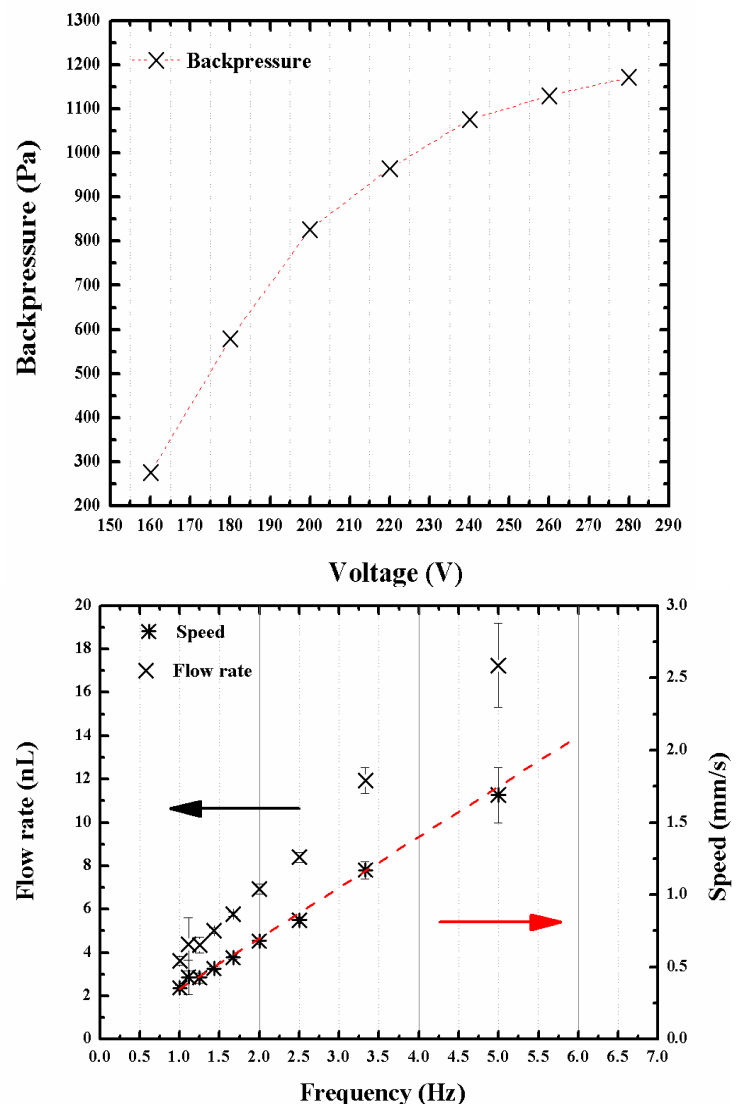

Figure 2. Characterization data of a single liquid piton. A) Backpressure vs. voltage. B) Speed and flow rate vs. driving signal sweeping frequency.

\section{Micro Gas Pump}

Based on the results of a single liquid piston experiment in a microfluidic channel, we have designed and developed a micro gas pump generating continuous flow. Different from the single liquid piston device, the micro gas pump consists of three important functional parts: 1) A closed microfluidic channel for guiding liquid pistons, 2) An array of electrodes placed along the fluidic path in the microfluidic channel for generating the actuation force on the liquid pistons, and 3) An isolation stage between inlet and outlet ports. The isolation stage in the micro gas pump, especially plays a very important role. It holds a sufficient amount of the liquid for multiple liquid pistons. In addition, within the isolation stage, high fluidic resistance is needed to avoid unwanted backflow through the short path between the inlet and outlet ports of the pump. For this, we have employed semi porous structures for increasing the fluidic resistance in the isolation stage. Low surface energy liquids can easily wet the semi porous structures providing the significantly increased surface-to-volume ratio and capillary force.

First, we fabricated, tested and characterized the isolation stage with semi porous structures to investigate its effectiveness. For this, a spin coated SU8 layer was etched by reactive ion etching (RIE) to make the structure semi porous. Fig. 3 shows lots of small semi porous SU8 features generated by oxygen RIE. After being assembled within a microfluidic channel, a silicone oil droplet was loaded in the semi porous structure.
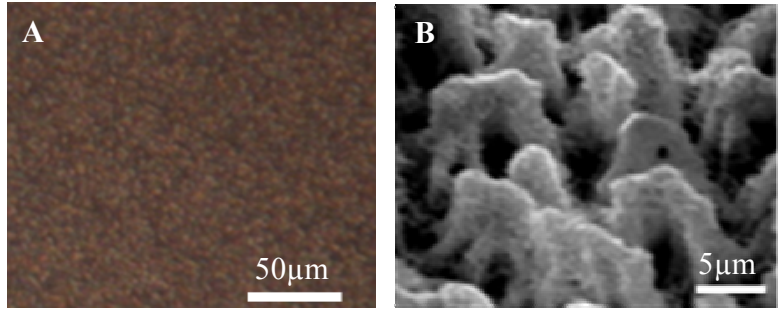

Figure 3. A) Optical and B) SEM images of SU8 semi porous structure for the isolation stage.

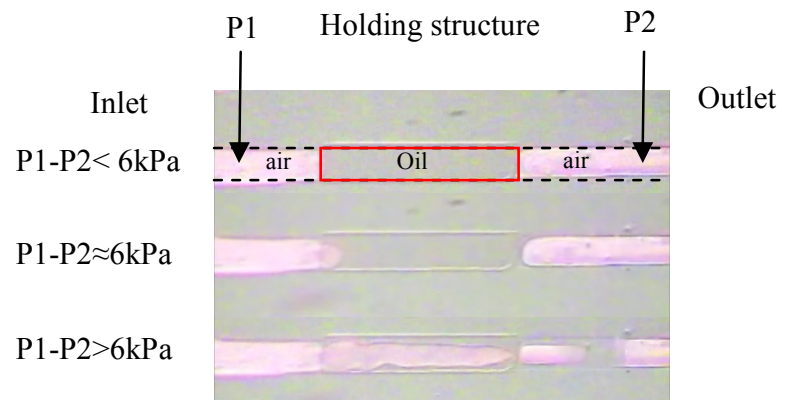

Figure 4. Snapshots of the backpressure testing for straight channel with modified semi porous SU8 (marked in red square box) structure working as isolation stage. Dash lines represent the inner surfaces of microchannel. Inlet pressure P1 kept increasing during the testing, while P2 kept constant.

An external pressure source was connected to the microchannel and an external pressure sensor through a $\mathrm{T}$ connection. The motion of liquid piston was captured through a CCD camera while monitoring the pressure recorded through a LabVIEW controlled pressure sensor. An increasing pressure was built up inside the microchannel and the liquid piston was monitored till it burst open. The threshold pressure was measured 
from this test. The liquid was seen to remain stationary until it reaches the threshold breaking pressure. Since the semi porous SU8 structures had a thickness of around $10 \mu \mathrm{m}$, addition of the structure reduces the channel height in the isolation stage. It has been observed that the semi porous SU8 structure held the silicone oil strongly and significantly increased the overall backpressure of the device up to $6 \mathrm{kPa}$. If the porosity of the structure is increased, we believe that the backpressure will also be improved.
A

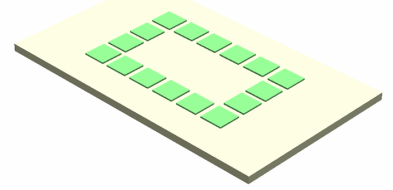

C

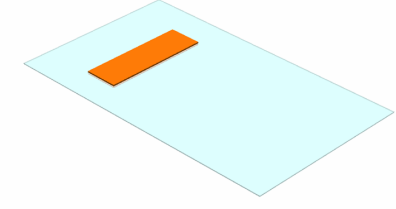

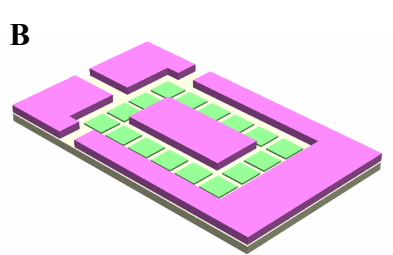

D

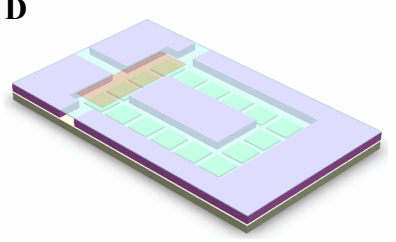

Figure 5. Brief fabrication steps, A) An array of electrode pads (green) fabricated on top of glass substrate. B) PDMS microchannel (purple) built on top of it. C) Semi porous SU8 structure (orange) was fabricated on top of cover ITO glass. D) The overview of entire device after oxygen plasma bonding.

Fabrication of the micro gas pump is very similar to that of the single liquid piston device as illustrated in Fig. 5. Like previously, an ITO coated cover glass was also utilized as a top substrate (ground) for careful monitoring of the pump. The semi porous SU8 structure was added between the inlet and outlet ports for the isolation stage. Once the fabrication was completed, the micro gas pump was mounted on the testing bench and controlled by the Labview codes. As the signal circulates, one or more liquid piston(s) can move forward and push air through the microfluidic channel. Between the liquid pistons, an air bubble can be caught and carried towards the output of the pump. Since the semi porous SU8 structure increases the liquid sorption property, it can stably hold the liquid piston at the isolation stage. Due to the delectrophoresis force acting downwards and contour force from isolation stage force acting upwards the liquid droplet can maturely split. To facilitate trapping of the gas bubble near the inlet, the duration of the applied actuation signals was intentionally increased. Moreover, the electrode pads near the inlet were designed in a triangular shape (electrode \#3, 4 in Fig. 6) to ease the transition of the gas bubble from the inlet into the circular pumping zone. During the following cycle, when the electrodes (electrode \#2-4) near the inlet port are activated to the 'ON' state again, another liquid piston is pulled from the isolation stage and fills in the microfluidic channel. The fill-in force is sufficient to bridge the gap at the inlet port. In this way, the air bubble gets trapped. In the rest of the cycling process, the liquid piston is driven electrically and moves clock-wise for carrying the air bubble towards the outlet. The liquid piston merges back into the isolation stage at the outlet, and shifts towards the inlet again.

The silicone oil imbibed semi porous structure plays the dual roles of routing the liquid back towards the inlet while maintaining sufficient backpressure. It selectively absorbs silicone oil as compared to gas; the oil gets wicked in while the gas bubble is repelled. In other words, the isolation stage acts as a gas-repelling structure, preventing the gas from back-flowing into the loading zone near the inlet.
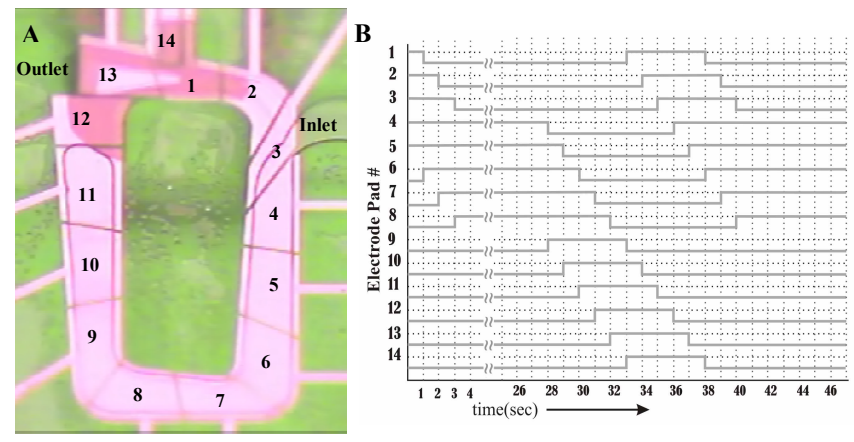

Figure 6. A) The electrode pads (pink) numbered in a clockwise sequence from the center top one. B) Actuation signals on each of the electrode pads on right.

Moreover, liquid piston movement along the circular pumping zone microchannel was tested continuously. Firstly, the speed limit of a single droplet was characterized along the clockwise path of the circular pumping zone without pressure load (zero backpressure). The average speed followed by the single droplet testing resulted in a sweeping frequency of $2.5 \mathrm{~Hz}$. We have found that the average speed of the droplet was heavily dependent on the clearing time of the outlet port and can be improved if the liquid piston can be absorbed by the isolation stage and clear the outlet port quickly enough. In other words, the silicone oil needs to be routed back to the inlet as fast as possible. A slow motion approach can be used to guarantee a successful pumping stroke in our prototype stage. A series of actuation signals were programmed using LabVIEW code. By controlling the driving signal applied to each electrode pad (Fig. 6.), a successful cyclic pumping was achieved as each step is clearly shown in Fig 7.

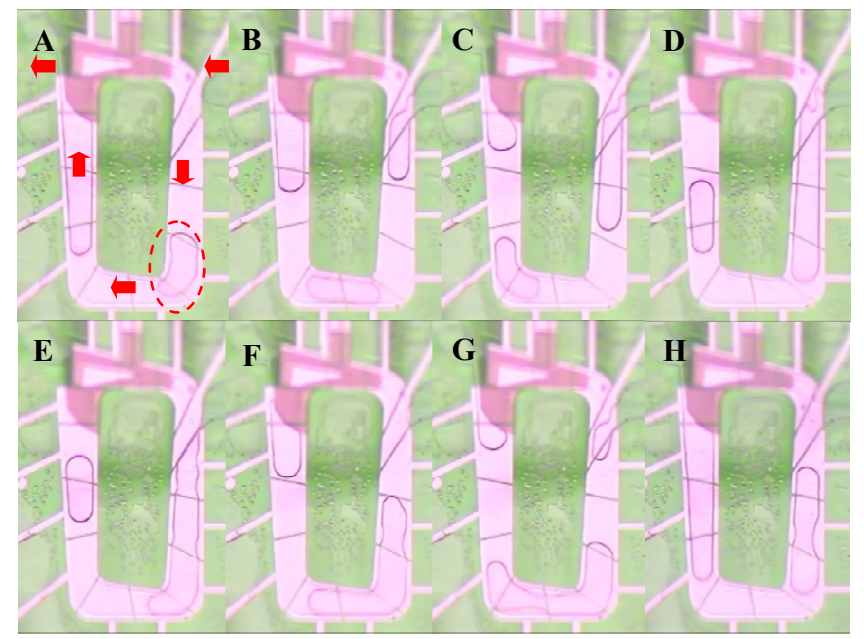

Figure 7. The continuous pumping results shown in sequenced snapshots. The pumping direction is marked in red arrows and trapped gas bubble is marked in dashed red circle.

The flow rate for our device is related to the circulating speed of the pistons, and the volume of the gas bubble captured in each 
cycle. During the cyclic pumping process, an adjustment of the time interval between the two liquid pistons at the inlet port directly affects the size of the trapped gas bubble in between. Therefore, by adjusting signals near the inlet port, the average volume of the trapped gas bubble can be determined. Post image analysis was adapted to get the volume information of the trapped bubble. The air bubble is always generated from the upper boundary of electrode \#4 as shown in Fig. 7B F and the volume of the trapped air bubble is calculated from the electrode area under the air bubble.

Based on the total volume of the trapped gas bubble per cycling time, flow rate can be further calculated. As shown in Figure 8 , the controllability of the trapped air bubble size turns out to be very good. The sizes of the trapped air bubble at the inlet are very close to what we planned to capture.

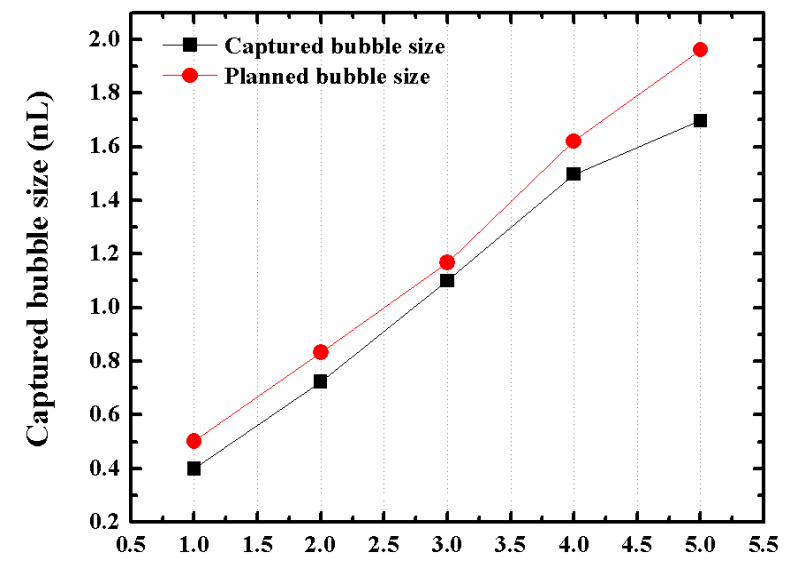

Distance from inlet (number of electrode pad)

Figure 8. The size of both planned and captured air bubble during the continuous pumping.

\section{CONCLUSION}

We have successfully demonstrated a novel micro gas pumping technique with liquid pistons. By utilizing a low surface tension liquid as working fluid (silicone oil), a complete wetting of the inner surfaces of the microfluidic channel successfully provided leak-tightness and effectively eliminated gas leakage problems including high dead-volume and backflow. The electrokinetically driven liquid piston made the pumping process highly controllable. To achieve a higher backpressure and separation between gas and liquid media, we utilized semi porous SU8 structures as an isolation stage, which has been proven to provide sufficient gas-repelling force and to increase the backpressure. This micro gas pump may bring a promising way to the efficient micro gas pumping mechanisms.

\section{REFERENCES}

[1] P. Woias, "Micropumps--past, progress and future prospects," Sensors and Actuators B: Chemical, vol. 105, pp. 28-38, 2005.

[2] M. Richter, R. Linnemann, and P. Woias, "Robust design of gas and liquid micropumps," Sensors and Actuators A: Physical, vol. 68, pp. 480-486, 1998.

[3] H. Van Lintel, F. Van de Pol, and S. Bouwstra, "A piezoelectric micropump based on micromachining of silicon," Sensors and Actuators, vol. 15, pp. 153-167, 1988.

[4] T. Bourouina, "Design and simulation of an electrostatic micropump for drug-delivery applications," Journal of micromechanics and microengineering, vol. 7, p. 186, 1997.

[5] F. Van de Pol, H. Van Lintel, M. Elwenspoek, and J. Fluitman, "A thermopneumatic micropump based on micro-engineering techniques," Sensors and Actuators A: Physical, vol. 21, pp. 198-202, 1990.

[6] C. W. Huang, S. B. Huang, and G. B. Lee, "Pneumatic micropumps with serially connected actuation chambers," Journal of micromechanics and microengineering, vol. 16, p. 2265, 2006.

[7] H. Zhou, H. Li, V. Sharma, and M. Schmidt, "A single-stage micromachined vacuum pump achieving 164 torr absolute pressure," 2011, pp. 1095-1098.

[8] C. Yamahata, C. Lotto, E. Al-Assaf, and M. Gijs, "A PMMA valveless micropump using electromagnetic actuation," Microfluidics and Nanofluidics, vol. 1, pp. 197-207, 2005.

[9] M. Doms and J. Mueller, "A micromachined vapor jet pump," Sensors and Actuators A: Physical, vol. 119, pp. 462467, 2005.

[10] A. Hatch, A. E. Kamholz, G. Holman, P. Yager, and K. F. Bohringer, "A ferrofluidic magnetic micropump," Microelectromechanical Systems, Journal of, vol. 10, pp. 215-221, 2001.

[11] J. Jiao, "Gas Pumping Effect from Dielectrophoretically Driven Liquid Droplet in a Closed Microfluidic Channel," M.S. Thesis, University of MissouriColumbia, USA, 2010.

[12] T. Jones, "Liquid dielectrophoresis on the microscale," Journal of Electrostatics, vol. 51, pp. 290-299, 2001.

[13] P. M. Young and K. Mohseni, "Calculation of DEP and EWOD forces for application in digital microfluidics," Journal of Fluids Engineering, vol. 130, p. 081603, 2008.

\section{CONTACT}

*J.Jiao, tel: +1-573-884-7867; jjw94@mail.missouri.edu 


\title{
MECHANO-FLUIDIC CHARACTERIZATION OF MICROSCALE DIELECTRIC BARRIER DISCHARGE PLASMA ACTUATORS
}

\author{
J. C. Zito ${ }^{1 *}$, R. J. Durscher ${ }^{2}$, J. Soni ${ }^{2}$, S. Roy ${ }^{2}$ and D. P. Arnold ${ }^{l}$ \\ ${ }^{1}$ Interdisciplinary Microsystems Group, University of Florida, Gainesville, Florida, USA \\ ${ }^{2}$ Applied Physics Research Group, University of Florida, Gainesville, Florida, USA
}

\section{ABSTRACT}

This article reports the fluidic and mechanical thrust characterization of microscale dielectric barrier discharge plasma actuators intended for distributed flow control applications. Particle image velocimetry and pitot measurements indicate wall jet velocities up to $2.0 \mathrm{~m} / \mathrm{s}$ while consuming an average power of $15 \mathrm{~W} / \mathrm{m}$ of electrode. Direct force measurements using a torsional balance indicate a thrust up to $3 \mathrm{mN} / \mathrm{m}$ of electrode. These microscale actuators exhibit flow characteristics similar to macroscale DBD actuators, but with an order of magnitude reduction in footprint area and mass. Furthermore, compared to similar macroscale actuators, the "thrust effectiveness" (force divided by power) is similar, and the "velocity effectiveness" (velocity divided by power) is nearly doubled.

\section{INTRODUCTION}

\section{Background \& Motivation}

Dielectric barrier discharge (DBD) plasma devices have received significant attention over recent years for applications such as active flow control, aero propulsion, biological sterilization, ozone generation, and microfluidic pumping. For active flow control applications, DBD devices offer the advantages of lack of moving parts, surface compliance, fast response and ease of construction, but generally suffer from low flow control authority (i.e. induced flow velocity, momentum transfer, and thrust).

Conventional DBD devices consist of two offset electrodes on either side of a dielectric material, as illustrated in Fig. 1. With high-voltage pulsed or AC excitation, the gas locally above the dielectric becomes weakly ionized creating a plasma discharge. This plasma imparts an electrohydrodynamic (EHD) force on the surrounding fluid, inducing a wall jet along the dielectric surface. Typical DBD actuators produce a wall jet with a velocity of $1-4$ $\mathrm{m} / \mathrm{s}$ occurring $0.5-1 \mathrm{~mm}$ above the dielectric surface. The net thrust produced ranges from a few $\mathrm{mN} / \mathrm{m}$ to over $150 \mathrm{mN} / \mathrm{m}$ (thrust per unit length electrode), and the power consumption can range up to $\sim 650 \mathrm{~W} / \mathrm{m}$, depending on both input voltage and frequency and the actuator geometry [1]. The actuator's "effectiveness" - output per consumed power-is a useful metric for comparing different designs operated at different voltage levels and frequencies. We apply this metric to define both "velocity effectiveness" (velocity divided by power) and "thrust effectiveness" (force divided by power) to compare the performance of DBD plasma actuators.

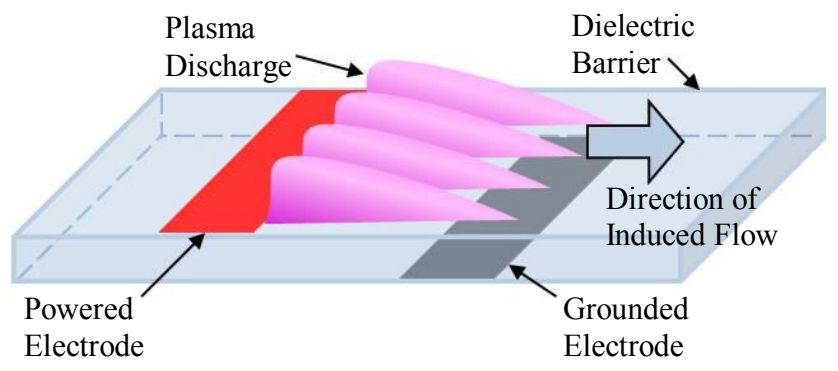

Figure 1: Schematic view of DBD plasma actuator.
For flow control applications, many efforts have been made to increase the control authority and/or effectiveness of DBD actuators by varying the input voltage amplitude and frequency, waveform shape (sinusoidal, pulsed, saw tooth, triangular, etc.), material properties, and device geometry. Extensive reviews of plasma actuators for use in flow control $[2,3]$ summarize several trends that have been validated repeatedly in experiments. For example, the electrical power consumed scales exponentially with the sinusoidal input voltage amplitude [4], $P \propto V^{\alpha}$ with $\alpha \approx 3.5$. The thrust follows a similar scaling trend with voltage up to a certain saturation point, above which increasing the input no longer increases the force. Similarly, the induced velocity also saturates; maximum velocities of $\sim 8 \mathrm{~m} / \mathrm{s}$ have been experimentally demonstrated in standard atmospheric conditions [5].

The presence of the plasma imparts a Lorentzian force density $(F)$ on the surrounding neutral fluid, which is a product of the applied electric field $(E)$ and the net separated space charge density $(q), F=q E$. The fluidic control authority of the actuator is dependent on this force density and may be improved by increasing either the applied field or the space charge density. Increasing the electric field may be accomplished by simply increasing the voltage, reducing the dielectric thickness, or through geometric manipulation of the exposed electrode. For example, it has been shown that reducing the dielectric layer thickness increases the electric field resulting in an increase in measured thrust [1] (for a given input voltage/frequency). However, reducing the dielectric thickness may lead to dielectric breakdown and device failure. Alternatively, fine-scale electrodes have been shown an effective method for increasing the thrust. For example, Abe et al. [6] found an increase in the momentum transfer (i.e., thrust) though the use of thinner copper tape electrodes. Similarly, Hoskinson et al. [7] demonstrated an exponential increase in measured thrust as the diameter of the wire electrode decreased from $0.40 \mathrm{~mm}$ to $0.11 \mathrm{~mm}$. These increases may be explained by considering the electric field around a conducting wire: for a cylindrical conductor at a given potential, the electric field is inversely proportional to the wire's radius $(E \propto 1 / r)$.

Through miniaturization of the DBD actuator geometries (both electrode size and dielectric thickness) we aim to leverage an increase in electric field in an effort to improve the fluidic control authority and actuator effectiveness. In this paper we demonstrate the successful fabrication of microscale DBD devices and experimentally analyze their power consumption, velocity distribution, and thrust production with reduced-scale geometries. We then compare our device performance with reported data from macroscale DBD plasma actuators.

\section{EXPERIMENT}

The devices are constructed using 10-mm-long thin-film titanium electrodes and a 10- m-thick polyimide dielectric on glass substrates $(500 \mathrm{~m}$ thick) using previously reported microfabrication techniques [8]. The electrodes are created using sputter deposition, and the polyimide layer is spin-coated and heatcured. The powered (top) electrodes are $1 \mathrm{~m}$ thick and range from 10 ? $\mathrm{m}$ to $500 \mathrm{~m}$ in width. The ground (bottom) electrodes are 0.1 
$\mathrm{m}$ thick and range from $10 \mathrm{~m}$ to $1 \mathrm{~mm}$ in width. All of the devices have a $100 \mathrm{~m}$ lateral gap (offset) between the electrodes. The leads are extended such that the contact probes do not interfere with the field-of-view for velocity measurements. Three devices used in these experiments are shown in Fig. 2, showing the characteristic purple glow discharge under atmospheric conditions.
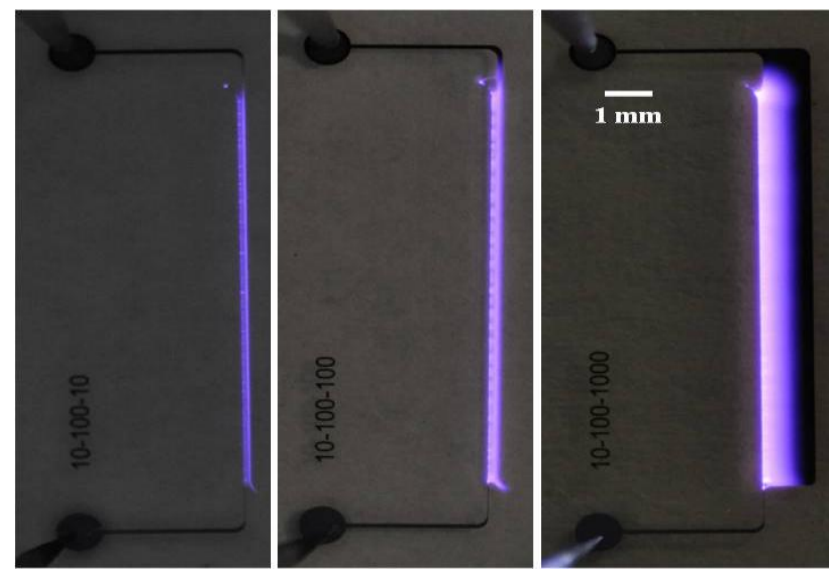

Figure 2: Some devices used for the experimental data (shown during discharge). The nomenclature corresponds to the width of the powered electrode (left electrode), electrode gap, and grounded electrode (right electrode), respectively.

For testing, a high-voltage sinusoidal input is generated using a function generator (Tektronix AFG3022B) and amplifier (Trek $30 / 20 \mathrm{~A}$ ). All of the tests are conducted at a frequency of $1 \mathrm{kHz}$. A high-voltage probe (Tektronix P6015A) measures the voltage amplitude and a current monitor (Pearson 2100) is used for current measurements. A digital oscilloscope (Tektronix DPO3014) captures both of these signals, and the average power dissipated is computed by integrating the voltage-current product over 200 periods.

Particle image velocimetry (PIV) is used to measure the instantaneous velocities induced by the plasma discharge. The PIV setup consists of a Nd:YAG laser (New Wave Research) and uses a high-speed camera (LaVision Imager Pro X 4M) with a $105 \mathrm{~mm}$ macro lens. The laser sheet is approximately $1 \mathrm{~mm}$ thick, and is positioned at the mid-point of the electrodes. The time interval $(d t)$ between image pairs is adjusted for a maximum particle displacement of $5-7$ pixels. Ondina oil is used for the PIV seed material, having a mean particle diameter of $0.8 \mathrm{~m}$ (TSI 9302 atomizer). Data is processed using LaVision's DaVis 7.2 software (mean background subtraction, multi-pass cross-correlation, outlier rejection), and the velocity field is time-averaged using 80 image pairs. The resulting vector resolution is $78.6 \mathrm{~m}$ (for both $\mathrm{x}$ and $\mathrm{y}$ directions) using a $20 \mathrm{~mm}$ wide field-of-view.

Pitot measurements are used to verify the PIV data, using a glass pipette probe with $1.5 \mathrm{~mm}$ outer diameter and $1.0 \mathrm{~mm}$ inner diameter. A differential pressure transducer (Furness Controls FCO332) with accuracy of $0.01 \mathrm{~Pa}$ is used to measure the stagnation pressure, which is converted to velocity using the formula, $p-p_{\text {atm }}=0.5 \rho v^{2}$, where the density of air $\rho=1.18 \mathrm{~kg} / \mathrm{m}^{3}$ (at $25{ }^{\circ} \mathrm{C}$ ), and $v$ is the fluid velocity $(\mathrm{m} / \mathrm{s})$. A traverse system allows precise control for incrementing the probe location. The absolute position relative to the substrate, however, is limited in accuracy by the initial manual alignment.

Direct force measurement of the time-average plasma-induced thrust is performed using a torsional balance similar to that of [9]. The balance measures the plasma actuator thrust as an angular deflection acting in opposition to torsion springs. The displacement is measured using a reflectance-based optical displacement sensor (PhilTec D100) having $40 \mathrm{~nm}$ resolution. Based on the measured displacement $x$, the angular deflection $\theta$ is calculated as $\theta=\arcsin (x / l)$ (radians), where $l$ is the moment arm length (m). From here, the force $F$ may be computed as $F=k_{\theta} \theta / l(\mathrm{~N})$, where $k_{\theta}$ is the rotational spring constant $(\mathrm{N} \cdot \mathrm{m} / \mathrm{rad})$. A viscous oil bath is used to damp out extraneous vibrations to facilitate force measurements with an estimated resolution of $35 \mathrm{nN}$.

The balance is calibrated using an electrostatic force between parallel plate electrodes, which is presumed "known" via finiteelement computation using Ansys Maxwell 2D. The rotational spring constant obtained through electrostatic calibration is validated via log decrement analysis of the transient response to a step input. Using the extracted natural frequency $\omega_{n}(\mathrm{rad} / \mathrm{s})$ from the balance and the measured mass inertia $I\left(\mathrm{~kg} \cdot \mathrm{m}^{2}\right)$, the rotational spring constant may be computed as $k_{\theta}=\omega_{n}^{2} I$ for comparison. The balance has a natural frequency of $0.37 \mathrm{~Hz}$ and a rotational spring constant of $0.292 \mathrm{~N} \cdot \mathrm{m} / \mathrm{rad}$.

\section{RESULTS}

Figure 3 shows the power consumed for microscale DBD devices with varying electrode widths operated at $1 \mathrm{kHz}$. The power consumption shows little dependency on the exposed electrode width. However, slight differences are observed for the grounded electrode width; a wider ground electrode slightly increases the power dissipation. The ability to store charge (and thus dissipate power) is related to the area of the ground electrode. The data is fit with a power-law curve to examine the dependency of power on the applied voltage. The fit lines indicate the power scales exponentially with the voltage, with the exponent $\alpha$ ranging from 3.1 to 3.6 in these data. The average power consumed (per unit length of electrode) reaches $15 \mathrm{~W} / \mathrm{m}$ at $5 \mathrm{kV}_{\mathrm{pp}}$ and $1 \mathrm{kHz}$.

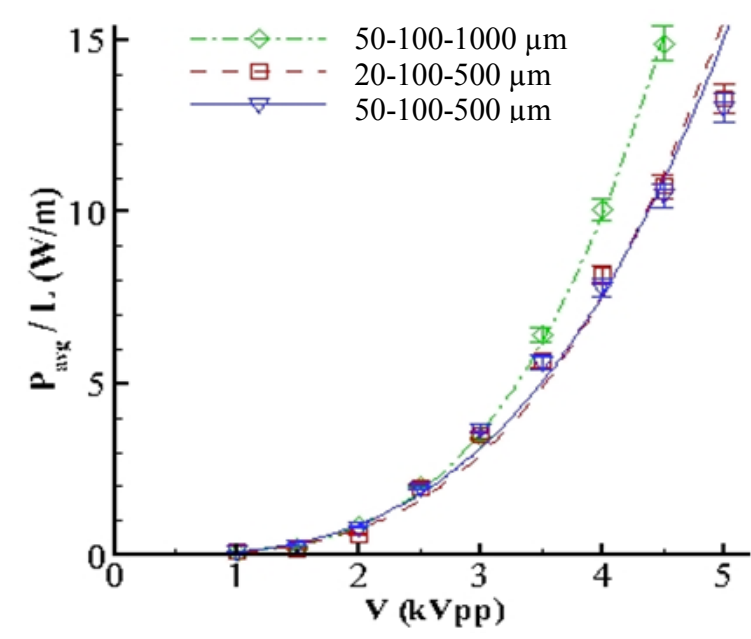

Figure 3: Power consumed for DBD devices with varying electrode geometries plotted against applied voltage, operated at $1 \mathrm{kHz}$. The nomenclature corresponds to the width of the powered electrode, electrode gap, and grounded electrode, respectively.

PIV data is shown in Fig. 4 for a microscale DBD actuator with a 10- m-wide exposed electrode and 1-mm-wide ground electrode. The actuator geometry and electrode widths are also indicated at the bottom of the velocity contour plot for reference. The velocity magnitude reaches up to $2 \mathrm{~m} / \mathrm{s}$ in the region near the electrodes, and a wall jet is established that extends over $15 \mathrm{~mm}$ 
downstream. The wall jet is similar in profile to those produced using macroscale DBD actuators.

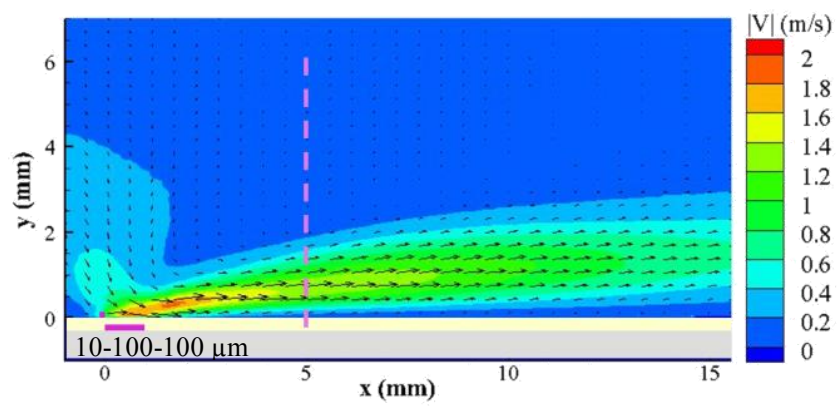

Figure 4: PIV data for devices with a 10- m-wide powered electrode and a 1-mm-wide grounded electrode. The electrode locations and geometry are indicated at the bottom of the figure; the vertical dashed line indicates the downstream location of the extracted velocity profile for comparison with pitot measurements.

To validate the PIV results, pitot measurements were made 5 $\mathrm{mm}$ downstream from the edge of the powered electrode (location indicated with dashed vertical line in Fig. 4). Figure 5 compares the results from the velocity computed from pitot measurements with an extracted profile from PIV data. The pitot measurements show good agreement with the PIV data, with slight discrepancy between the vertical location of the data points. The offset between the PIV data and pitot measurement is most likely attributed to the $1 \mathrm{~mm}$ diameter of the pitot tube over which the pressure measurement is averaged. Error in the initial manual alignment of the pitot tube may also contribute to the data offset.

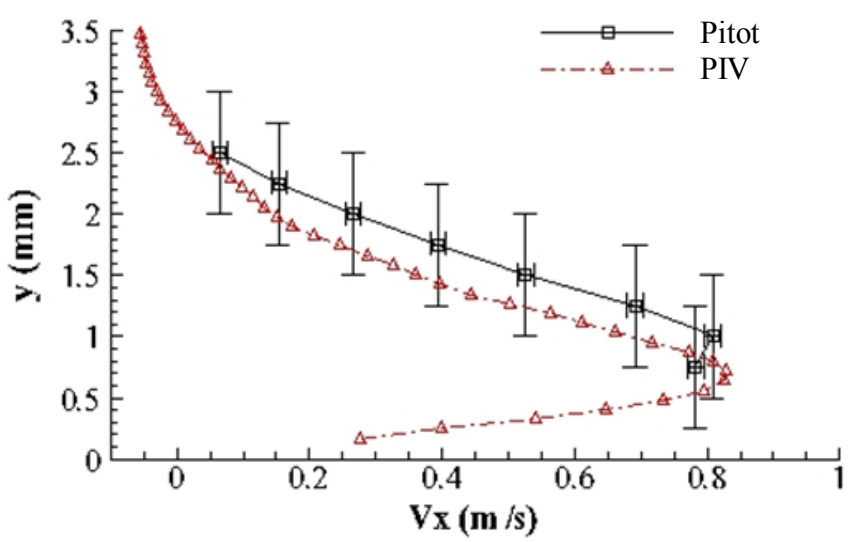

Figure 5: Velocity profile from pitot measurements at $x=5 \mathrm{~mm}$ downstream compared with the extracted PIV velocity profile for a micro DBD actuator. Vertical error bars correspond to the $1 \mathrm{~mm}$ diameter of the pitot probe; horizontal error bars represent velocity measurement error.

Measurements from the torsion balance force tests are shown in Fig. 6 for an actuator with a 10- m-wide powered electrode and 100- m-wide ground electrode. The plot shows the optically measured displacement as a function of time for various plasma excitations. Calibration measurements are made using built-in calibration electrodes for pre- and post-experiment comparison. The first two pulses correspond with a $50 \mathrm{~V}$ and $100 \mathrm{~V}$ potential applied across the calibration electrodes, providing electrostatic force and causing the balance to deflect toward the fixed electrode (away from the sensor). Following the calibration pulses, the actuator is successively stepped up in voltage from 4 to $6.5 \mathrm{kVpp}$ in $0.5 \mathrm{kVpp}$ increments. Each voltage is applied for approximately
10 seconds before turning off the actuator. Post actuator calibration is then performed for two purposes: (1) for comparison with the pre-calibration measurements for experimental consistency and (2) to provide increased resolution by using several voltages $(30-70$ $\mathrm{V}$ with $10 \mathrm{~V}$ increments) that produce displacements in the range of those measured using the actuator.

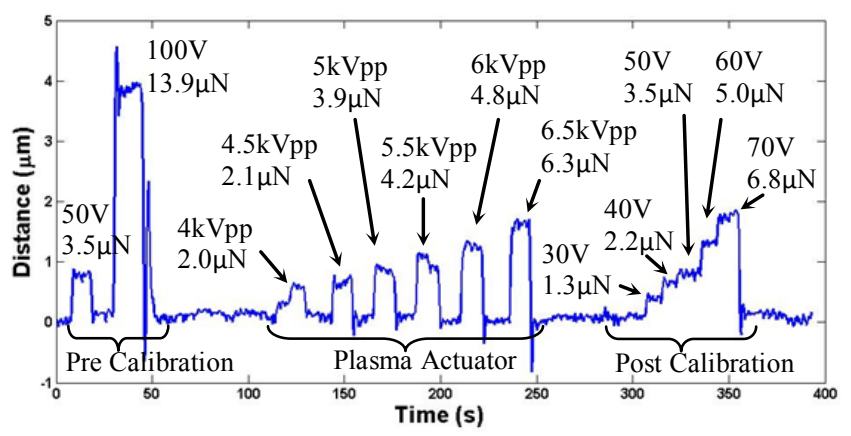

Figure 6: Displacement measurements from torsional force balance for an actuator with a powered electrode width, gap and ground electrode width of 10, 100, and $100 \mathrm{~m}$, respectively. Electrostatic calibration is performed before and after each actuator tested using parallel plate electrodes.

Using the measured deflections from the DBD actuator and comparing with the calibration measurements, the thrust is extracted for the different excitation voltages. Figure 7 plots the average force normalized by the electrode length against applied voltage for three actuators with different ground electrode widths. The device with the $1 \mathrm{~mm}$ wide ground electrode produced the greatest force, up to $3 \mathrm{mN} / \mathrm{m}$ (force normalized by electrode length). As observed in the power consumption data, devices with wider ground electrodes consumed slightly more power, which follows along with the increase in force observed here for the device with the largest ground electrode.

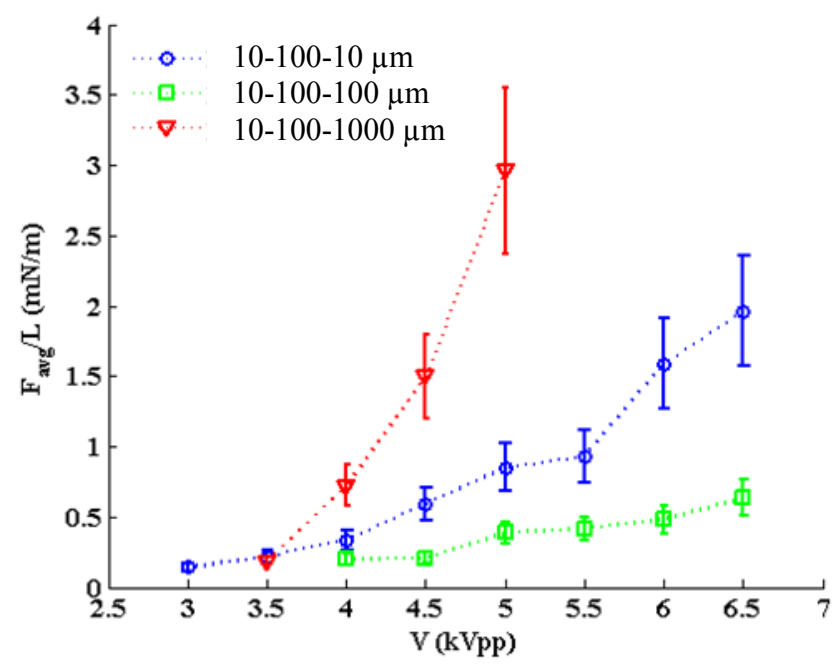

Figure 7: Average force measured for three actuators having different ground electrode widths. The devices all have 10- mwide exposed electrodes and a 100- m-wide gap. The force is normalized by the electrode length $(l=10 \mathrm{~mm})$.

The results from the microscale actuators are compared with reported macroscale DBD actuator performance [6] and summarized in Table 1. The actuator volume and mass are computed based on the DBD materials and geometries provided in [6], with electrode length normalized to $1 \mathrm{~m}$. The first row reports 
the actuator thrust effectiveness; here, the macroscale actuator indicates similar thrust and power values to the microscale actuator, and both actuators demonstrate equivalent thrust effectiveness. The fourth row of data reports the velocity effectiveness; the microscale actuator provides an $86 \%$ increase compared with [6]. On a per-volume or per-mass basis, the micro actuator out-performs the macro actuator in both thrust and velocity metrics. This is attributed to the extreme size reduction of the microscale devices, in addition to the reduction in power consumption. The thick dielectric layer (few millimeters) generally utilized for macro DBD actuators governs the voltage, and in-turn, the power required to generate a wall jet. Employment of a thinner dielectric layer $(10$ ? $\mathrm{m})$ and miniaturized electrodes allows the micro actuator to produce velocities on the order of macroscale actuators with a significant reduction in size and mass.

Table 1: Micro- and macro-DBD actuator performance metrics; comparing actuator thrust and velocity to power consumption, device geometry and material properties.

\begin{tabular}{|c|c|c|}
\hline $\begin{array}{c}\text { Performance } \\
\text { Metrics }\end{array}$ & $\begin{array}{c}\text { Micro-DBD } \\
\text { Vmax }=2 \mathrm{~m} / \mathrm{s} \\
\text { Force }=3 \mathrm{mN} / \mathrm{m} \\
\text { Pavg }=15 \mathrm{~W} / \mathrm{m} \\
(5 \mathrm{kVpp}, 1 \mathrm{kHz})\end{array}$ & $\begin{array}{c}\text { Macro-DBD [6] } \\
\text { Vmax }=1.4 \mathrm{~m} / \mathrm{s} \\
\text { Force }=3.9 \mathrm{mN} / \mathrm{m} \\
\text { Pavg }=20 \mathrm{~W} / \mathrm{m} \\
(20 \mathrm{kVpp}, 1 \mathrm{kHz})\end{array}$ \\
\hline $\begin{array}{c}\text { Thrust "Effectiveness" } \\
\text { [Force per } \\
\text { Normalized Power] } \\
(\mathrm{mN} / \mathrm{W})\end{array}$ & 0.20 & 0.20 \\
\hline $\begin{array}{c}\text { Thurst Density } \\
\text { [Force per } \\
\text { Actuator Volume] } \\
\left(\mathrm{mN} / \mathrm{m}^{3}\right)\end{array}$ & $5.29 \times 10^{6}$ & $6.84 \times 10^{4}$ \\
\hline $\begin{array}{c}\text { Thrust per } \\
\text { Actuator Mass }\end{array}$ & 0.215 & $4.10 \times 10^{-3}$ \\
\hline $\begin{array}{c}\text { Velocity "Effectiveness" } \\
\text { [Velocity per } \\
\text { Normalized Power] } \\
(\mathrm{m} / \mathrm{s}) /(\mathrm{W} / \mathrm{m}) \\
\end{array}$ & 0.13 & 0.07 \\
\hline $\begin{array}{c}\text { Velocity per } \\
\text { Actuator Volume } \\
(\mathrm{m} / \mathrm{s}) / \mathrm{m}^{3}\end{array}$ & $3.53 \times 10^{6}$ & $2.46 \times 10^{4}$ \\
\hline $\begin{array}{c}\text { Velocity per } \\
\text { Actuator Mass } \\
(\mathrm{m} / \mathrm{s}) / \mathrm{g}\end{array}$ & 1.41 & $1.44 \times 10^{-2}$ \\
\hline
\end{tabular}

To further improve the actuator thrust effectiveness and thrust density (force per actuator volume), the dielectric gap should be reduced from 100 ? $\mathrm{m}$ as reported here to few microns. The breakdown voltage for generating discharge is governed by the distance between the electrodes. Reducing the electrode gap and/or the dielectric layer thickness increases the field strength across the electrodes, leading to lower breakdown voltages and reducing the power required to operate DBD plasma actuators.

\section{CONCLUSION}

In conclusion, we have demonstrated successful operation, flow inducement, and thrust generation of DBD devices having microscale dimensions. Devices that were operated at $5 \mathrm{kV}_{\mathrm{pp}}$ and 1 $\mathrm{kHz}$ consumed $15 \mathrm{~W} / \mathrm{m}$ on average. The power consumption scales with $V^{3.1-3.6}$ indicating that the microscale devices follow similar power trends to macroscale DBD actuators [4]. The reduction in power is attributed to the reduction in the necessary breakdown voltage for discharge gained from using a thin dielectric layer. The fluid velocities reached $2.0 \mathrm{~m} / \mathrm{s}$ and the thrust was up to $3 \mathrm{mN} / \mathrm{m}$. The actuator "effectiveness"- output per consumed power-was used to compare microscale and macroscale DBD actuators. The microscale actuators demonstrate equal thrust effectiveness to macroscale devices, indicating that the DBD actuator thrust performance scales linearly with size reduction. The velocity effectiveness of microscale actuators is $86 \%$ greater than that of the macroscale DBD actuator.

Overall, the microscale DBD actuator induced velocity, thrust, and power consumption scale favorably with size reduction. The compact size, low mass, and lower voltage requirements of the microscale actuators make them implementable with minimal weight penalty. Furthermore, arrays of micro-DBD actuators could be constructed in flexible substrates (e.g. polyimide) for attachment on a curved surface, such as an airfoil.

\section{ACKNOWLEDGEMENTS}

This work was supported in part by ARO Grant W911NF-091-0511 and AFOSR Grant FA9550-09-1-0416. Travel support has been generously provided in part by the Transducer Research Foundation. The authors also gratefully acknowledge the assistance from the staff of the UF Nanoscience Research Facility.

\section{REFERENCES}

[1] F.O. Thomas, T.C. Corke, M. Iqbal, A. Kozlov, and D. Schatzman, "Optimization of Dielectric Barrier Discharge Plasma Actuators for Active Aerodynamic Flow Control", AIAA J. 47, 9 (2009).

[2] E. Moreau, "Airflow Control by Non-thermal Plasma Actuators", J. Phys. D: Appl. Phys. 40, 605 (2007).

[3] T.C. Corke, C.L. Enloe, and S.P. Wilkinson, "Dielectric Barrier Discharge Plasma Actuators for Flow Control”, Annu. Rev. Fl. Mech. 42, 505 (2010).

[4] C.L. Enloe, T.E. McLaughlin, R.D. VanDyken, K.D. Kachner, E.J. Jumper, and T.C. Corke, "Mechanisms and Responses of a Single Dielectric Barrier Plasma Actuator: Plasma Morphology", AIAA J. 42, 3 (2004).

[5] M. Forte, J. Jolibois, J. Pons, E. Moreau, G. Touchard, and M. Cazalens, "Optimization of a Dielectric Barrier Discharge Actuator By Stationary and Non-stationary Measurements of the Induced Flow Velocity: Application to Airflow Control", Exp. Fluids 43, 917 (2007).

[6] T. Abe, Y. Takizawa, S. Sato, and N. Kimura, "Experimental Study for Momentum Transfer in a Dielectric Barrier Discharge Plasma Actuator", AIAA J. 46, 9 (2008).

[7] A.R. Hoskinson, N. Hershkowitz, and D.E. Ashpis, "Force Measurements of Single and Double Barrier DBD Plasma Actuators in Quiescent Air", J. Phys. D: Appl. Phys. 41 (2008).

[8] J.C. Zito and D.P. Arnold, "Fabrication and Electrical Characterization of Microscale Dielectric Barrier Discharge Devices", Tech. Dig. Solid-State Sensors, Actuators, and Microsystems Workshop, Hilton Head Is1., SC, 6/4-7/2010.

[9] M. Gamero-Castano, "A Torsional Balance for the Characterization of MicroNewton Thrusters", Rev. Sci. Inst. 74, 10 (2003).

\section{CONTACT}

*J.C. Zito, tel: +1-352-392-1484; jcz@ufl.edu 


\section{WIRELESS IMPLANTABLE MICROSYSTEMS: Creating a Revolution in Health Care

\author{
Kensall D. Wise
} \\ Center for Wireless Integrated MicroSystems \\ Department of Electrical Engineering and Computer Science \\ The University of Michigan, Ann Arbor, MI, USA 48109-2122}

\begin{abstract}
The first integrated silicon sensors based on microelectronic technology were developed in the 1960s for medical applications. Today, they have evolved into integrated microsystems combining micropower circuits, wireless interfaces, sensors, wafer-level packaging, and energy-scavenging power sources. Catheter-tip pressure sensors have grown into smart cardiovascular stents and $1 \mathrm{~mm}^{3}$ wireless devices for treating glaucoma. Neural interfaces are launching revolutionary advances in neuroscience and in prostheses for deafness, blindness, epilepsy, Parkinson's disease, and other disorders. This paper examines the factors that limited the development of implantable microsystems in the past and continuing challenges for the future. Such devices are poised at last to provide important breakthroughs in health care.
\end{abstract}

\section{INTRODUCTION}

Since the birth of the planar process for integrated circuits in the early $1960 \mathrm{~s}$, microelectronics has seen phenomenal growth, and driven by scaling it has revolutionized many aspects of society. However, its impact on health care has been muted, and very few implantable devices have been successfully commercialized. This is surprising, because the microelectronics programs at many leading universities began by working on medical devices. It was evident very early that the small sizes facilitated by lithography would be important in developing minimally-invasive devices for diagnosing, and perhaps even treating, a variety of physiological conditions.

Some of the first work on silicon image sensors was done to develop a reading aid for the blind at Stanford University [1], and by the late 1960 s work was also underway there on implantable Doppler microsystems for arterial blood flow and for cardiac imaging [2,3]. The first projects to develop micromachined silicon sensors began at Stanford in 1966, exploiting the silicon etching work then underway for air-isolated integrated circuits at Bell Telephone Laboratories [4]. In the over forty years since those first devices were produced, a wide variety of biomedical sensors have been reported. Pacemakers have developed into sophisticated programmable microsystems whose outputs can be conditioned on levels of exertion, improving the quality of life for millions, but except for the electrodes such systems reside entirely within a welded titanium package. Progress using sensors directly exposed to the body has been slow, partly because technology had to evolve to a level suitable for commercialization and partly because the sensor was only part of the problem. To solve the entire problem, and thus permit commercialization, full systems were required. It is only the recent emergence of microsystems, combining lowpower integrated circuits, wireless connectivity, and advanced sensors that the revolution in health care originally envisioned has finally begun to occur.

\section{EARLY BEGINNINGS}

The first work on selectively-etched ("micromachined") silicon sensors was under the direction of Professor James B. Angell and had the goal of realizing multi-site microprobes for single-unit recording in the central nervous system (CNS) [5]. These devices were tested acutely in animals (Fig. 1) and by 1970 were also being used to stimulate phosphenes in human visual cortex as a basis for a visual prosthesis. The devices were successful but with nothing to shape the silicon substrate except isotropic etching, they did not yet have the yield needed to supply them in quantity to the biomedical community. Nevertheless, the etching technology demonstrated led to funding from NASA to develop catheter-tip pressure sensors for use within the chambers of the heart [6]. This led to the first silicon pressure sensors with a builtin thick rim to absorb packaging stresses (Fig. 2). By this time, anisotropic etching technology was available but in spite of using $60 \mu \mathrm{m}$-thick silicon wafers with self-terminating V-grooves for die separation, it was hard to form the $5 \mu \mathrm{m}$-thick diaphragms reproducibly with no etch-stop. The yield difficulties with the probes and pressure sensors underscored the importance of having a process consistent with high-yield and high throughput. Packaging challenges also emphasized the importance of minimizing lead count when dealing with very small implantable devices as well as the difficulties of calibration. The pressure work led to a second project with NASA-Ames to build a wafer-level gas analyzer based on chromatography [7]. Unlike the neural probes and pressure sensors, this was a system and not just a device.

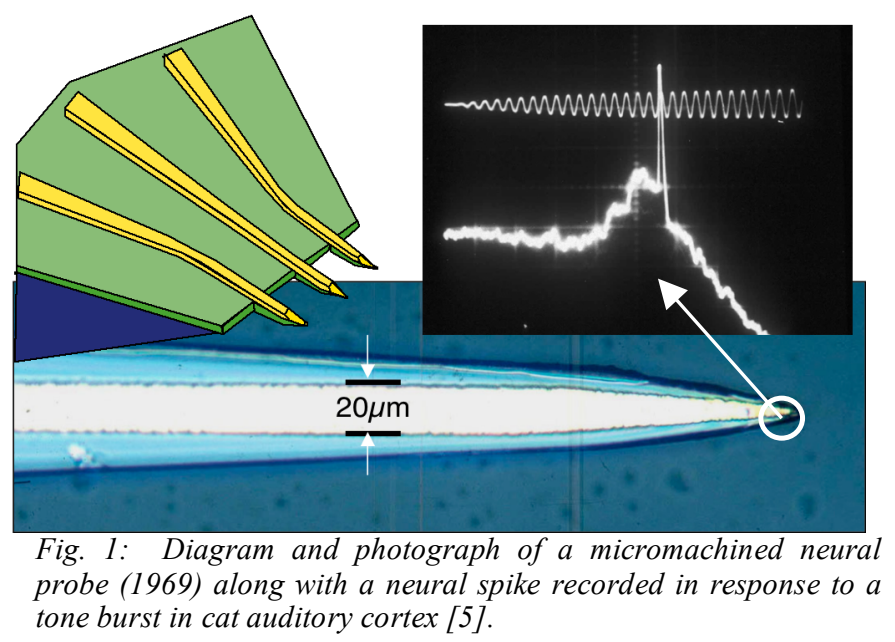

The goal of the micro gas chromatography ( $\mu \mathrm{GC})$ work was a light-weight low-power wafer-level device for the 1975 Viking Mars Lander, although applications in health care were also envisioned. The original plan for this system, shown in Fig. 3, included a gas sampling system, a spiral separation column, and a thermal-conductivity-based gas detector along with signal processing and temperature control circuitry. By 1972, 100 $\mu \mathrm{m}$-diameter column channels had been formed, had been capped using anodic silicon-glass bonding, and had yielded the first separations, but the available valve technology was not yet adequate. Work continued on the $\mu \mathrm{GC}$ through most of the 1970s at Stanford [8] and would form a basis for the re-emergence of microfluidics more than a decade later.

Solid-State Sensors, Actuators, and Microsystems Workshop Hilton Head Island, South Carolina, June 3-7, 2012 


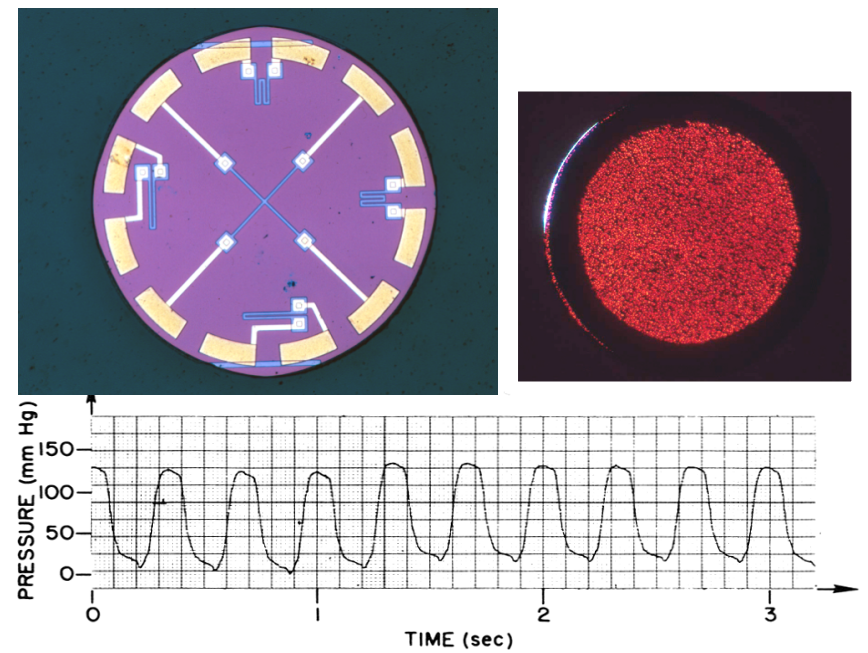

Fig. 2: A 1.6mm-diameter catheter-tip pressure sensor fabricated with a thick silicon rim using anisotropic etching (1971) together with pressure from the left ventricle of a dog [6].

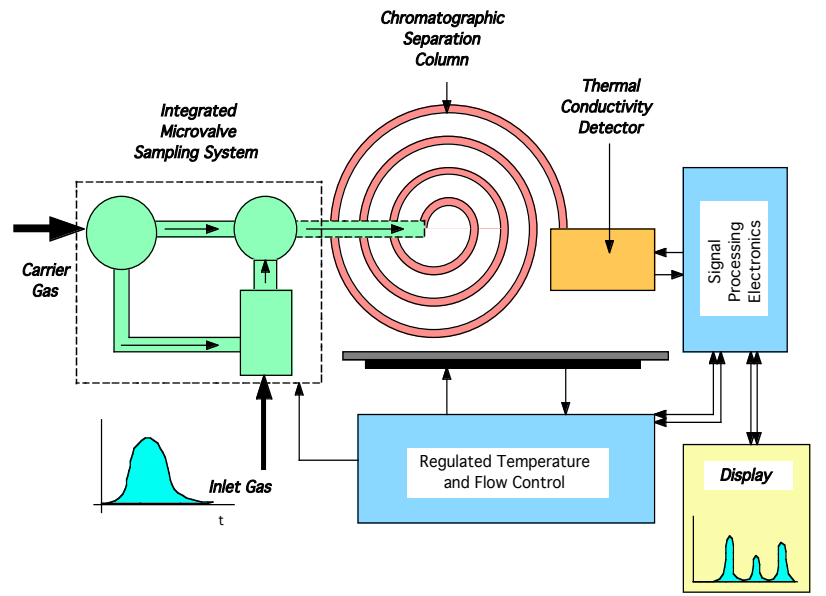

Fig. 3: Design for a wafer-scale gas chromatograph (1971).

In 1974, Fig. 4 was drawn to describe a possible wireless implantable pressure sensor. Powered by a galvanic cell formed with the body electrolyte, on-chip CMOS circuitry would charge an MOS capacitor and would form an oscillator whose frequency would be set by the sensing capacitor. Once every cycle, the oscillator would trigger a switch that would rapidly dump the stored MOS charge through an on-chip antenna, generating a current spike that could be detected externally. The technology was not ready to support for this kind of system, but the ideas were there.

These early projects defined a number of problems that silicon sensors had to overcome to be applied successfully in-vivo. First, it is important to be able to produce devices that are reproducible enough to support animal trials and can be used by physiologists and others not skilled in the art. Enough devices must be provided to establish their efficacy. Second, technology must be developed that permit high yield and high performance. Third, packaging must exist that will be well tolerated by the body and will to protect the device from it. Fourth, in any implant leads are a major concern, and the fewer the better. And fifth, it is important to solve the entire problem, not just a piece of it. It is not enough to provide a single device. An entire system must be

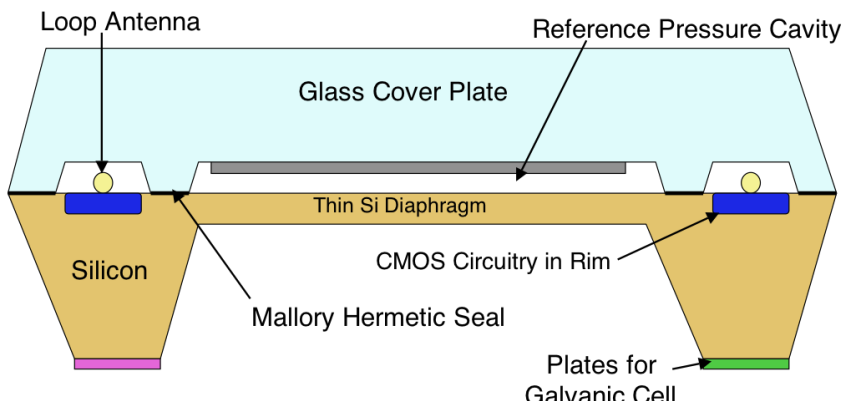

Fig. 4: Design for a self-powered wireless pressure sensor (1974). The ideas were there but the technology was not.

provided, including the transducer, the signal processing, the package, and a suitable communication link.

The 1970s focused on technology development, and a variety of other sensors emerged. The 1980s focused on devices, including flowmeters, gas sensors, and accelerometers. They saw the birth of surface micromachining [8], efforts to understand stress in deposited films, and the first chemical-vapor-deposited (CVD) sealed vacuum cavities. The first sensor conferences and journals began [9] and major funding developed for microelectromechanical systems (MEMS). In the $1990 \mathrm{~s}$, deep reactive ion etching (DRIE) entered the scene, and MEMS proliferated into subfields, including bio-MEMS. Today, wireless integrated microsystems are re-uniting portions of the field with mainstream microelectronics, driven by the needs of portable instrumentation and by a search for new frontiers that will drive the industry in the "more than Moore" era.

\section{WIRELESS IMPLANTABLE MICROSYSTEMS}

The convergence of advanced sensor technology with very low power circuits and wireless connectivity is enabling implantable devices to finally emerge. Hermetic packaging is an integral part of such systems along with a suitable power supply. The end of the scaling paradigm that has driven microelectronics for the last forty years is also driving many companies to search for interdisciplinary partnerships that can generate new high-volume highmargin products in areas such as health care, now a national priority. To assess some of the ways that wireless implantable microsystems are solving problems in health care and what the continuing challenges are, it is appropriate to look at the original devices targeted at Stanford and where they are today.

\section{Neural Interfaces}

During the past decade, neural interfaces have emerged as one of the hottest topics in bioengineering. The goal is to use multipoint recording and stimulation to better understand the CNS at the circuit/system level and serve as a basis for neural prostheses. To understand neural networks, it is important to be able to record simultaneously from dozens or even hundreds of cells, and in order to explore changes over time (learning) it is important to do so over periods from weeks to months. For use in neural prostheses, it is important to do so over years and decades.

A variety of high-density penetrating microprobes for use in CNS have been reported [10-12]. Boron etch-stops, deep dry etching (DRIE), and silicon-on-insulator (SOI) technology have made acute probe fabrication a solved problem. Such devices are now commercially available and have been used by hundreds of investigators worldwide to better understand the brain. Planar probes can be microassembled [13] to form 3D arrays (Fig. 5), permitting, in principal, every cell within a $1 \mathrm{~mm}^{3}$ block of tissue to 
be monitored. Circuitry has been integrated with such probes for site selection, signal amplification, multiplexing, self-test, and stimulus generation.

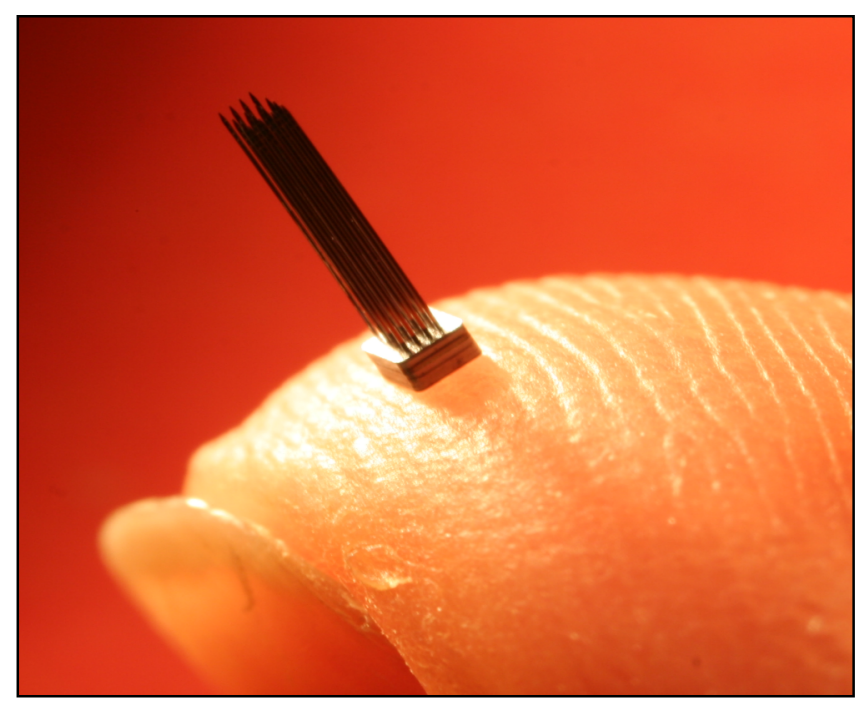

Fig. 5: A 64-site four-probe microassembled 3D electrode array with site spacings of $200 \mu \mathrm{m}$ [20].

Much of the interest in neural interfaces is due to the success of cochlear implants and deep brain stimulation (DBS). Over 150,000 cochlear prostheses have been implanted worldwide, allowing many profoundly deaf individuals to function normally in a hearing world, and deep brain stimulation has been remarkably successful in suppressing the tremor associated with Parkinson's disease with and more than 50,000 implanted to date. When the electrodes are correctly positioned in the subthalamic nucleus, tremor can be completely masked with few, if any, side effects. The biggest barrier preventing the realization of dense recording arrays, for example for capturing control signals from the motor cortex to help paralyzed individuals, is the tissue reaction around the implanted devices. Electrode arrays placed in-vivo are recognized as foreign bodies and are slowly encapsulated by astrocytes/glia. While this encapsulation is typically only a few microns thick, it can degrade recording performance over weeks or

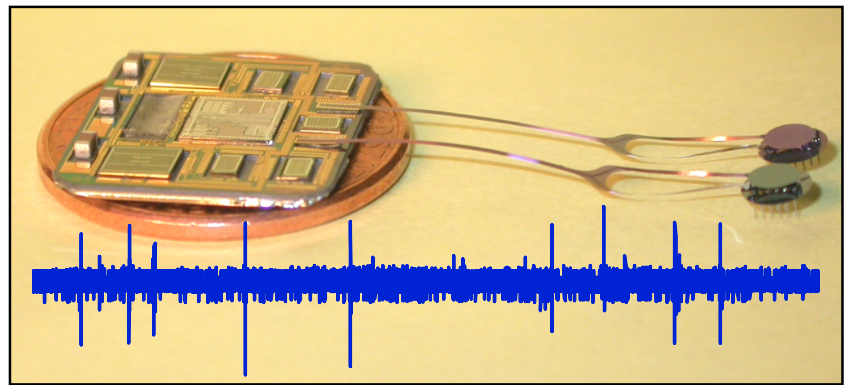

Fig. 6: A first-generation wireless neural microsystem, shown before packaging on a U.S. penny. A recording of neural activity is also shown as wirelessly recovered from the external system.

months. This may be exacerbated by probe size and/or micromotion of the probe with respect to the brain. As probe size is scaled down to cellular dimensions, dramatic reductions in tissue reaction have been observed [14]

In order to succeed in neuroscience and neural prostheses, complete neural microsystems will also be required, including embedded signal amplification, multiplexing, spike recognition, power, and a bidirectional data interface. Such systems are beginning to appear. For example, a digital spike detector chip has been reported [15] that computes per-channel means and standard deviations and allows the user to set biphasic thresholds around these baselines. The $2 \mathrm{~mm} \times 3 \mathrm{~mm}$ chip digitizes 32 multiplexed inputs at $2.56 \mathrm{Mbps}$, outputs a $5 \mathrm{~b}$ quantization of spikes above and below threshold, operates at $1.2 \mathrm{~mW}$ from $3 \mathrm{~V}$, and saves about $10 \mathrm{x}$ in typical output bandwidth. Figure 6 shows a first-generation wireless neural microsystem [16] along with an externally reconstructed spike train. Other processors are also in development. Neural microsystems will soon allow new progress in understanding the brain and in overcoming many of its disorders.

\section{Pressure Sensors}

While piezoresistive bridges have remained the approach of choice for many pressure sensing applications, in the early $80 \mathrm{~s}$ they were joined by capacitive devices, which offer easier diaphragm formation, lower temperature sensitivity, broader dynamic range, and lower power but require a vacuum-sealed reference cavity and on-chip readout circuitr0. Many pressure sensors have been developed for cardiovascular catheters [17], with some realized in widths of $350 \mu \mathrm{m}$ or less for use on guide wires within the coronary arteries of the heart. Wireless capacitive devices have also been formed on stents to allow pressure/flow sensing in the carotid arteries [18] but are not yet small enough for the coronaries. Efforts to reduce their size are being aided by the dramatic power reductions being achieved in subthreshold circuits. Figure 7 shows an intraocular pressure sensor for the improved treatment of glaucoma [19]. The system contains a microcontroller, an energyscavenging (solar) power system, an UWB wireless link, and a capacitive pressure sensor, all realized in a parylene-covered glass capsule $1 \mathrm{~mm}^{3}$ in size. The system wakes up and takes a data point every fifteen minutes, stores the data, and then returns to sleep mode. Once every day or so, it can be optically triggered to transfer its data to the external world. The system is made possible by the extremely low power of the embedded processor [20] (30pW standby, 300nW active). Pressure sensors combined with wireless interfaces and embedded electronics are forming microsystems that will significantly advance health care.
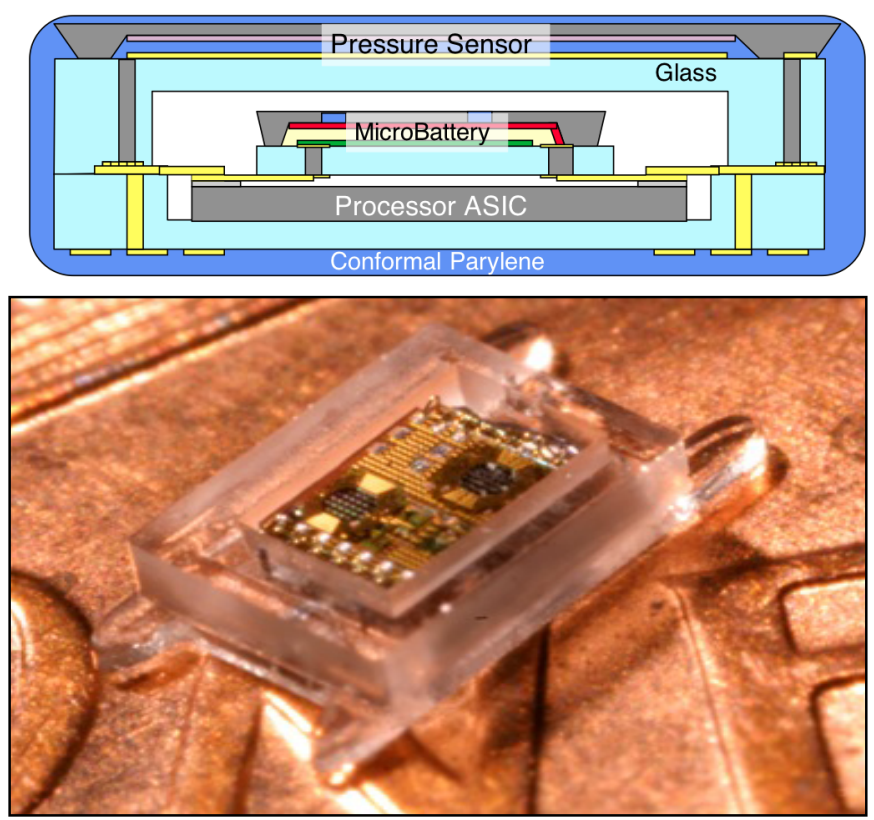

Fig. 7: Diagram of a $1 \mathrm{~mm}^{3}$ intraocular microsystem with a close up of several pressure sensors and a full system prototype on a U.S. penny. 


\section{Chromatographic Gas Analysis and Microfluidics}

Very little additional work on $\mu \mathrm{GCs}$ or microfluidics was reported until the late $90 \mathrm{~s}$; however, the last decade has seen significant efforts on $\mu \mathrm{GCs}$, including work at Sandia and at the University of Michigan [21]. This work has shown that siliconbased preconcentrators, separation columns, and detectors can separate numerous analytes in seconds to tens of seconds with detection limits less than $1 \mathrm{ppb}$. The very small size and low mass of such systems makes them very low power and able to be temperature programmed at relatively high speed to improve selectivity and avoid co-elutions. Figure 8 shows a preconcentrator, $25 \mathrm{~cm}$-long column, and chemiresistive detector on an Apple "Shuffle" MP3 player, representing the expected size of an eventual system. Micro-GC-based gas analyzers have already demonstrated the ability to detect biomarkers in breath for lung cancer and tuberculosis. Microfluidics has emerged as a field in its own right with a variety of DNA analyzers, cell capture and cancer drug screening devices, and other structures poised to significantly aid in the diagnosis and treatment of serious diseases.

\section{CONCLUSIONS}

The development of implantable devices for use in health care has been underway for at least four decades, but is only now emerging to make important impacts. Driven by spectacular successes in pacemakers, cochlear implants, and DBS, work on neural interfaces, active stents, intraocular monitors, and other microsystems is underway. There are many challenges ahead, but if past successes are any indicator, the resulting devices may work better than we even dream possible.

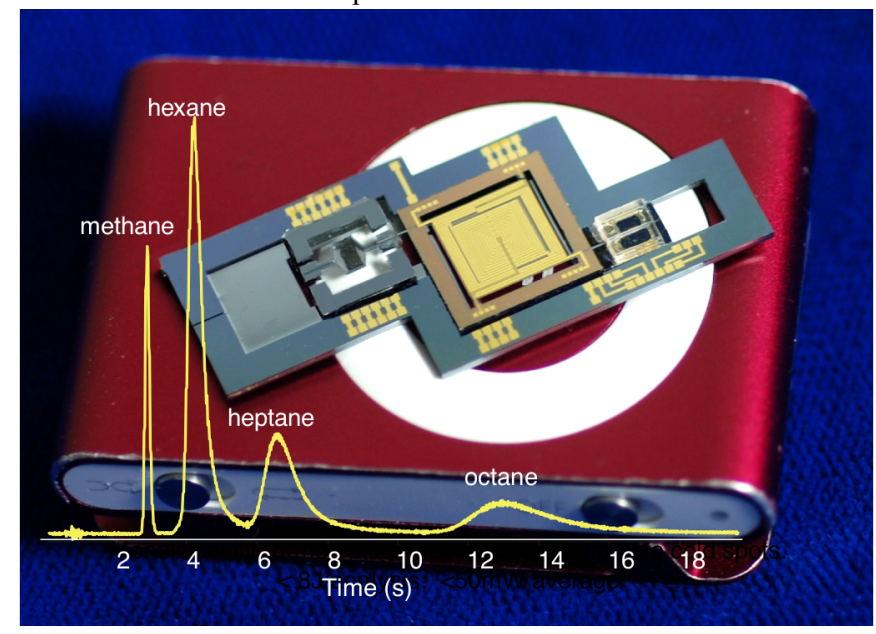

Fig. 8: A microfluidic gas analyzer on an Apple "Shuffle" MP3 player along with a column separation of alkanes $C_{5}-C_{8}$.

\section{Acknowledgments}

The work described here was supported by the Engineering Research Centers Program of the National Science Foundation under Award EEC-9986866, by the U.S. Army Research Laboratory MAST CTA under Contract W911NF, and by a gift from Ms. Polly Anderson. The many contributions from faculty, staff, and students at the Engineering Research Center for Wireless Integrated MicroSystems are gratefully acknowledged.

\section{REFERENCES}

[1] J. S. Brugler, J. D. Meindl, J. D. Plummer, P. J. Salsbury, and W. T. Young, "Integrated Electronics for a Reading Aid for the Blind," IEEE JSSC, 4, pp. 304-311, Dec 1969.
[2] R. Gill and J. D. Meindl, "Low-Power Integrated Circuits for an Implantable Pulsed Doppler Ultrasonic Blood Flowmeter," IEEE JSSC, 10, pp. 464-471, December 1975.

[3] J. D. Plummer, J. D. Meindl, and M. G. Maginness, "An Ultrasonic Imaging System for Real-time Cardiac Imaging," IEEE Int. Sol.-St. Circuits Conf.,pp.162-163, February 1974.

[4] M. P. Lepselter, "Beam-Lead Technology", Bell System Technical Journal, 45, pp. 233-254, February 1966.

[5] K. D. Wise, J. B. Angell, and A. Starr, "An Integrated Circuit Approach to Extracellular Microelectrodes," IEEE Int. Conf. on Med. and Biological Engr., Chicago, 1969.

[6] Samaun, K. D. Wise, and J. B. Angell, "An IC Piezoresistive Pressure Sensor for Biomedical Instrumentation," IEEE Trans. BME., 20, pp. 101-109, March 1973.

[7] S. C. Terry, J. H. Jerman, and J. B. Angell, "A Gas Chromatographic Air Analyzer Fabricated on a Silicon Wafer," IEEE Trans. ED, 26, pp. 1880-1886, December 1979.

[8] R. T. Howe and R. S. Muller, "Resonant-Microbridge Vapor Sensor," IEEE Trans. ED, 33, pp. 499-506, April 1986.

[9] S. Middelhoek, "The Past, Present, and Future of Transducer R\&D," Sensors and Actuators, A, pp. 2-23, 2000.

[10] K. Najafi and K. D. Wise, "An Implantable Multielectrode Array with On-Chip Signal Processing," IEEE J.SSC, pp. 1035-1045, December 1986.

[11] K. C. Cheung, K. Djupsund, Y. Dan, and L. P. Lee, "Implantable Multichannel Electrode Array Based on SOI Technology," IEEE JMEMS, 12, pp. 179-184, April 2003.

[12] R. A. Normann, "Microfabricated Electrode Arrays for Restoring Lost Sensory and Moto4r Functions," IEEE Int. Conf. on Solid-St. Sensors, Actruators, and Microsystems, pp. 959-962, June 2003.

[13] G. E. Perlin and K. D. Wise, "A Compact for ThreeDimensional Neural Microelectrode Arrays," IEEE EMBS Conf., Vancouver, BC, pp. 5806-5809, August 2008.

[14] J. L. Skousen, et al., "Reducing Surface Area while Maintaining Implant Penetrating Profile Lowers the Brain Foreign Body Response to Chronically-Implanted Planar Silicon Microelectrode Arrays," in J. Schouenborg, ed., Progress in Brain Research, vol. 194, Elsevier, 2011.

[15] R. H. Olsson, III, and K. D. Wise, "A Three-Dimensional Neural Recording Microsystem with Implantable Data Compression Circuitry," IEEE JSSC, 40, pp. 2796-2804, December 2005.

[16] A. M. Sodagar, K. D. Wise, and K. Najafi, "A FullyIntegrated Mixed-Signal Neural Processing Module for Implantable Multi-Channel Cortical Recording," IEEE Trans. BME, 54, pp. 1075-1088, July 2007.

[17] J. Ji, S. T. Cho, Y. Zhang, K. Najafi, and K. D. Wise, "An Ultraminiature CMOS Pressure Sensor for a Multiplexed Cardiovascular Catheter," IEEE Trans. ED, 39, pp. 22602267, October 1992.

[18] A. DeHennis and K. D. Wise, "A Fully-Integrated Multi-Site Pressure Sensor For Wireless Arterial Flow Characterization," IEEE JMEMS, pp. 678-685, June 2006.

[19] R. M. Haque and K. D. Wise, "An Intraocular Pressure Sensor based on a Glass Reflow Process," Solid-State Sensor, Actuator, and Microsystems Workshop, Hilton Head, S. C., June 2010.

[20] M. Seok, S. Hanson, Y.-S. Lin, Z. Foo, D. Kim, Y. Lee, N. Liu, D. Sylvester, and D. Blaauw, "The Phoenix Processor: A 30pW Platform for Sensor Applications," IEEE Symp. on VLSI Circuits, pp. 188-189, 2008.

[21] E. T. Zellers, et al., "An Integrated Micro-Analytical System for Complex Vapor Mixtures," Int. Conf. on Solid-St. Sensors, Actuators, and Microsys., Lyon, France, pp. 14911496, June 2007. 


\title{
FABRICATION OF 3D PARYLENE SHEATH PROBES FOR RELIABLE NEUROPROSTHETIC RECORDINGS
}

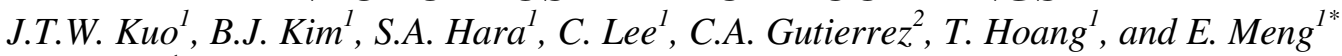 \\ ${ }^{1}$ University of Southern California, Los Angeles, California, USA \\ ${ }^{2}$ Los Angeles, California, USA
}

\begin{abstract}
3D Parylene sheath probes having Pt electrodes on the outer and inner surfaces of the sheath are introduced as a novel interface for long term intracortical neural recording. Surface micromachined Parylene channels with Pt electrodes are expanded into a 3D sheath structure by thermoforming in the presence of a custom tapered microwire that shapes the sheath. Electrochemical characterization, including electrochemical impedance spectroscopy (EIS) and cyclic voltammetry (CV), of the Pt electrodes sites was performed. EIS yielded electrode impedances ranging from $20-60 \mathrm{k} \Omega$ at $1 \mathrm{kHz}$. Electrode characterization results suggest the thermoforming process does not impact electrode performance and were in agreement with characteristics of typical neural recording electrodes. These sheath probes with their 3D structural design, soft biocompatible materials, and bioactive coatings promote integration with neural tissue enabling reliable, long term cortical recordings.
\end{abstract}

\section{INTRODUCTION}

Brain-machine interfaces for controlling motor prostheses require reliable chronic recordings from intracortical microelectrodes. Current microelectrode technologies are hampered by signal degradation over time that are attributed in part to neurotoxic factors released by microglia due to inflammatory response arising from the mechanical mismatch between the implanted rigid probe and cortical tissue; this leads to gradual retraction of neural processes from recording sites [1].

Intracortical microelectrode technologies possess short lifetime in terms of acceptable recording quality ( $<5$ years). There are two such microelectrode formats that have been used in clinical studies. The first was constructed of tapered-tip silicon pins with a single electrode at the tip [2-5]. Thermomigration was used to create trails of $\mathrm{p}^{+}$type silicon through a wafer which were exposed by removal of n-type silicon to create arrays of needles that are subsequently coated with platinum. Glass cone electrodes in which the cones are manually fabricated from pulled pipettes have achieved the most reliable long term recordings in human to date [6-12]. Microwires with de-insulated tips placed into the cone interior at different depths serve as the recording sites, with 2-4 wires per cone. Living sciatic nerve tissue or neurotrophic factors were placed within the cones prior to implantation. The ingrowth of dendritic processes into the cone toward cortical recording sites ( $\sim 3$ months) were attributed to the release of neurotrophic factors. Although, long-term recording reliability was achieved, manual fabrication of the device is labor intensive and not amenable to manufacturability nor increase in the number of recording sites.

Improvement in long term intracortical recordings is necessary to realize practical neural prostheses. Critical considerations in neural interface design include selection of appropriate biocompatible structural materials to alleviate the mechanical mismatch with tissue. Repeatability across devices and batch fabrication can be achieved by using microfabrication technologies. The used of thin film microfabricated electrodes also increases the number and density of recording sites per probe, while minimizing footprint, in order to acquire greater spatiotemporal signal information compared to microwire approaches. Finally, the use of appropriate bioactive coatings can reduce inflammatory response and promote integration into tissue.

To achieve a stable long-term intracortical interface, we introduce a novel microfabricated structure consisting of a hollow sheath in the form of a cone or cylinder that can be decorated with multiple biologically functional coatings (NGF, collagen, Matrigel, etc.) to mitigate the inflammatory response and attract and maintain growth of neural processes toward recording sites (Figure 1). Openings at the ends of the sheath further facilitate ingrowth of neural processes after recovery from implantation toward recording sites and tissue integration.
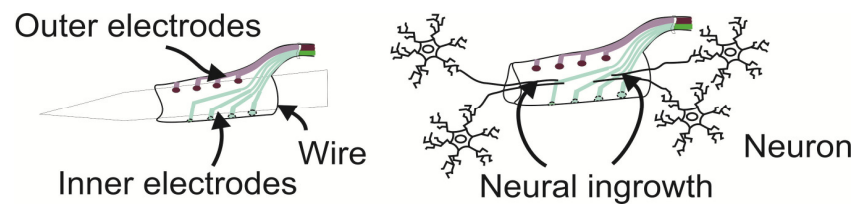

Figure 1: Conceptual drawing of Parylene sheath probe for long term intracortical recordings. A wire is inserted into the sheath and used as a surgical introducer to implant the device into the cortex (left). The wire is then removed and ingrowth of neural processes following release of bioactive coatings facilitates long term stable recordings (right).

\section{METHODS}

\section{Material Selection}

Parylene C, a Class VI biocompatible polymer that can be processed with standard micromachining technologies [13], was selected as the sheath probe structural material. The use of Parylene $\mathrm{C}$ mitigates mechanical mismatch issues between rigid probe and soft tissue present in previous technologies. Parylene $\mathrm{C}$ is flexible and much more pliable than stiff materials such as $\mathrm{Si}$ and glass possessing a low Young's modulus [14].

Only Pt was used for electrodes since no adhesion layer is needed with a Parylene substrate [15]. Pt is also highly biocompatible and widely used in bioMEMS neural interface applications [16-17].

\section{Device Design}

Sheath probes are cone or cylinder shaped with $4 \mathrm{Pt}$ electrodes (45 $\mu \mathrm{m}$ diameter) on each of the inner and outer surfaces. An integrated flexible Parylene cable $(\sim 2 \mathrm{~cm}$ long) is included. Sheath dimensions and electrode placement were selected to match the neural anatomy of the barrel cortex in rat for eventual neural recordings to capture vibrissal (whisker) movements.

The barrel cortex is organized into distinct regions that map to individual vibrissae. Individual vibrissae movements are mediated by neurons in layers $\mathrm{V}$ and VI of the barrel cortex through generation of action potentials. These layers are located at a depth of $\sim 1.5-2.0 \mathrm{~mm}$ in the rat brain and span $\sim 800 \mu \mathrm{m}$ in height [18]. Thus, the sheath structure was designed to be $800 \mu \mathrm{m}$ in length to span the targeted pyramidal layers. Electrodes were spaced equidistant from each other within the sheath length in order to effectively capture neural signals spanning the two layers. 


\section{Fabrication}

A silicon substrate was used for mechanical support during the fabrication process (Figure 2). A $5 \mu \mathrm{m}$ Parylene layer was first deposited onto the substrate to serve as the base layer of the device. Inner sheath e-beam deposited Pt electrodes $(2000 \AA)$ were then patterned by a liftoff process with negative photoresist $\mathrm{AZ}$ 5214 E-IR. These electrodes will record from dendritic processes extending into the sheath. A $1 \mu \mathrm{m}$ Parylene insulation layer was then deposited and selectively plasma etched to expose electrodes and contact pads. Sacrificial photoresist AZ 4620 was spun to a height of $8 \mu \mathrm{m}$ and patterned to form the sheath structures. A $5 \mu \mathrm{m}$ Parylene layer was then deposited over the photoresist.

Outer electrodes were patterned on top of the sheath structure using a dual layer liftoff scheme. AZ 1518 was first spun on and globally exposed. AZ 4620 was then spun on and patterned with the top electrodes. This dual photoresist AZ 1518/AZ 4620 layer scheme produced a negative sidewall profile that enhances the liftoff process for thicker photoresists and was necessary to ensure a continuous pattern from the top of the structure to the base [19]. As with inner electrodes, Pt was e-beam deposited (2000 $⿱$ A) for outer electrodes. A final $1 \mu \mathrm{m}$ Parylene insulation layer was deposited and plasma etched to create openings for outer electrodes and contact pads. Plasma etching then created sheath openings and the device cutout. Sacrificial photoresist was removed with an acetone soak and the device was released from the substrate.
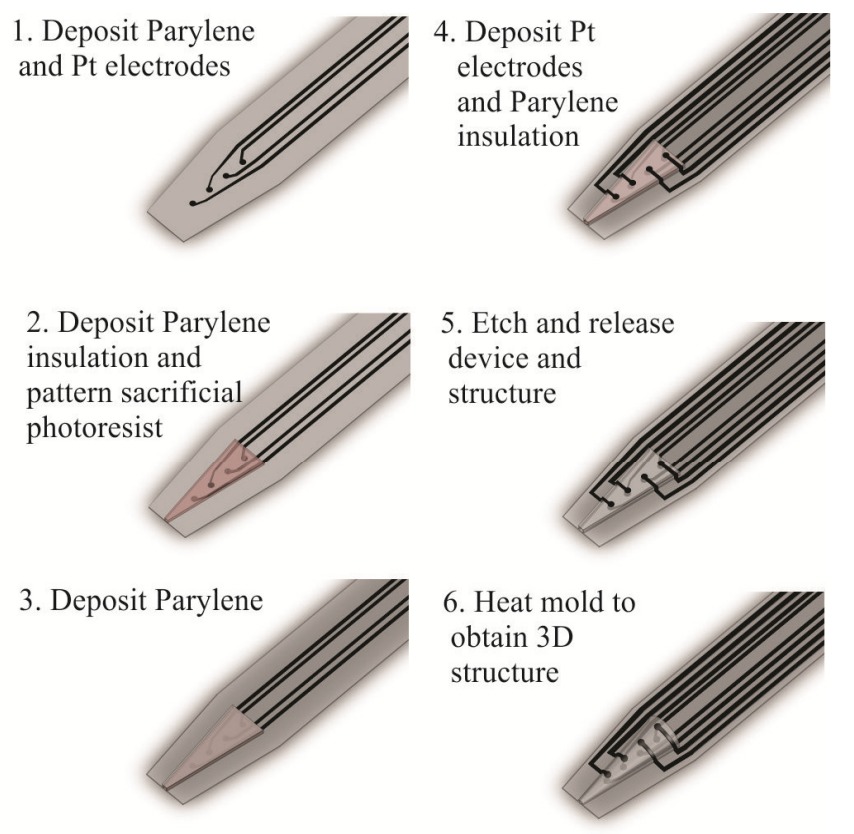

Figure 2: Abbreviated fabrication process which utilizes standard surface micromachining techniques followed by thermoforming. Final outline of device is shown throughout process for clarity.

\section{Parylene Thermoforming}

A custom tapered stainless steel or tungsten microwire was used as a mold for thermoforming of the conical or cylindrical structure (Figure 3). Microwires were etched to match the desired probe shape and supplied by MicroProbes for Life Science. The shaped microwire tip was carefully inserted into the sheath underneath a microscope. The wire and device were placed in a vacuum oven and the Parylene sheath was thermoformed by controlled temperature ramp up to $200{ }^{\circ} \mathrm{C}$ for 48 hours with nitrogen purging. After thermoforming, the wire was removed and the sheath retained its 3D shape (Figure 4).

Thermoforming not only provided the desired 3D shape but also annealed the Parylene layers [20]. This improves the adhesion between Parylene layers. Just prior to implantation, the sheaths are coated with neurotrophic factors. A zero insertion force (ZIF) connector was used to establish reversible electrical connections to the microelectrodes of the sheath through the contact pads at the end of the integrated Parylene ribbon cable (Figure 5) [21].

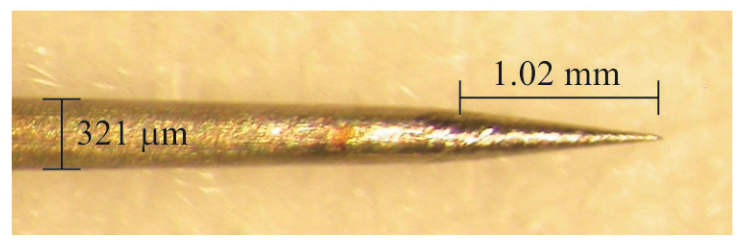

Figure 3: Custom tapered stainless steel wire for insertion into sheath for thermoforming.

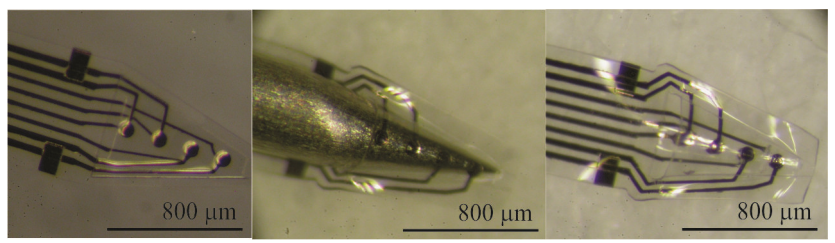

Figure 4: The sacrificial layer was first released (left).The tapered wire was inserted into the sheath (middle) and the structure was thermoformed. After thermoforming and removal of the wire, the sheath held its $3 D$ shape (right).

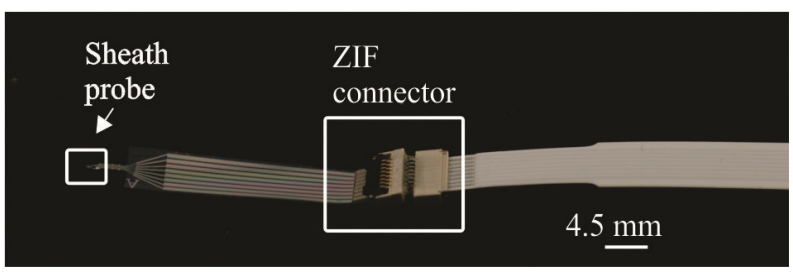

Figure 5: Sheath probe with integrated Parylene cable is attached to a ZIF connector for external electrical connection.

\section{RESULTS \\ 3D Sheath Probe Fabrication}

Sheath probes were successfully fabricated with integrated Parylene ribbon cables (Figure 6). In total, five different design variants were fabricated for eventual use in rat whisker intracortical recordings through the barrel cortex (Table 1).

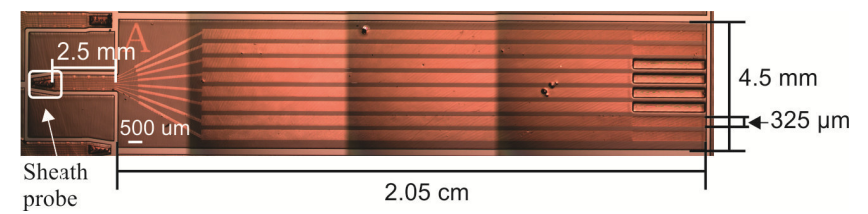

Figure 6: Photograph showing layout of unreleased probe with integrated cable still covered with photoresist. The vertical artifacts appearing along the length of the ribbon cable are due to stitching of images to obtain a view of the full length of the probe. 
Electrodes were placed to match the targeted cortical layers. 4 different cone shapes with different tapers ending at a $50 \mu \mathrm{m}$ opening were fabricated in order to test the effect of different geometries on dendritic ingrowth, if any (Figure 7). A cylinder structure was also fabricated.

Table 1: Dimensions of the different fabricated sheath structures. Electrode placement and dimensions remained constant for all device variants.

\begin{tabular}{|l|c|c|c|}
\hline $\begin{array}{l}\text { Device } \\
\text { Nomenclature }\end{array}$ & $\begin{array}{c}\text { Base Opening } \\
\text { Diameter }\end{array}$ & $\begin{array}{c}\text { Tip Opening } \\
\text { Diameter }\end{array}$ & $\begin{array}{c}\text { Sheath } \\
\text { Length }\end{array}$ \\
\hline A & $300 \mu \mathrm{m}$ & $50 \mu \mathrm{m}$ & $800 \mu \mathrm{m}$ \\
\hline $\mathrm{B}$ & $450 \mu \mathrm{m}$ & $50 \mu \mathrm{m}$ & $800 \mu \mathrm{m}$ \\
\hline $\mathrm{C}$ & $300 \mu \mathrm{m}$ & $300 \mu \mathrm{m}$ & $800 \mu \mathrm{m}$ \\
\hline A-T & $500 \mu \mathrm{m}$ & $50 \mu \mathrm{m}$ & $800 \mu \mathrm{m}$ \\
\hline B-T & $650 \mu \mathrm{m}$ & $50 \mu \mathrm{m}$ & $800 \mu \mathrm{m}$ \\
\hline
\end{tabular}
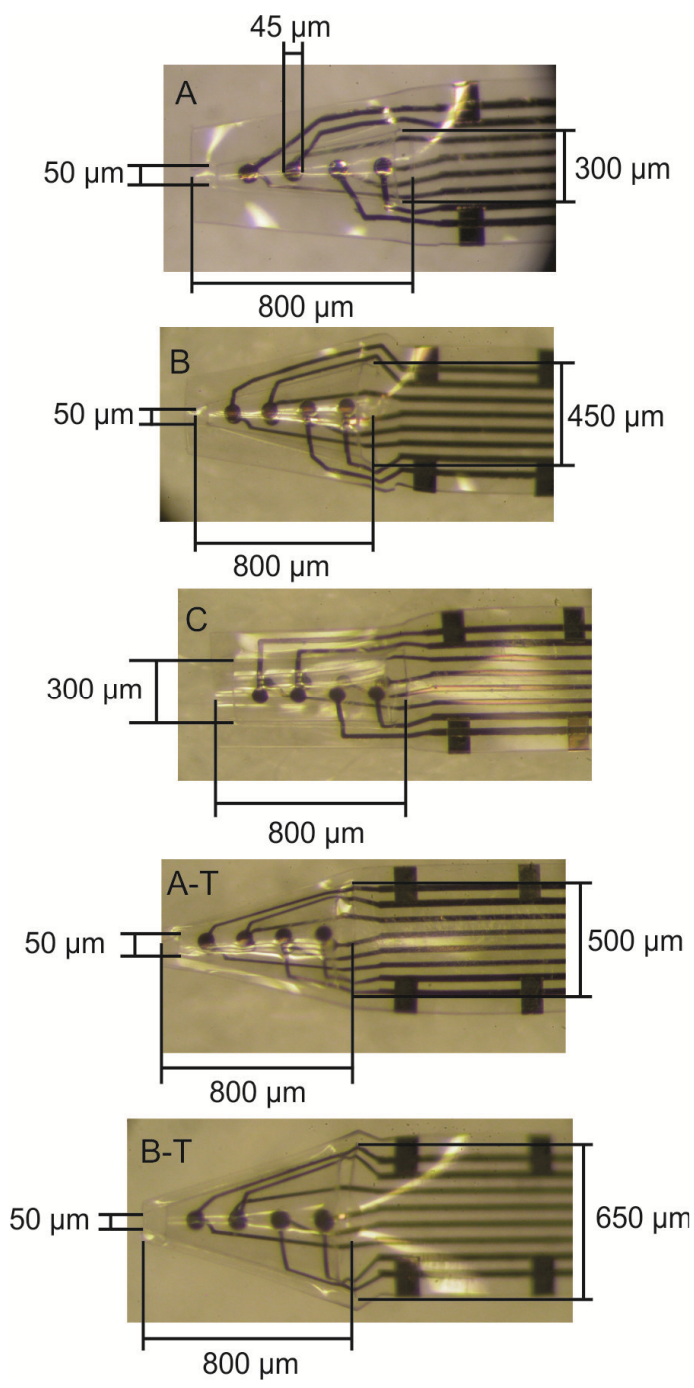

Figure 7: Photographs of the different sheath designs. Each device has been thermoformed. Electrode placement matches rat barrel cortex anatomy.

Thermoformed sheaths were mechanically robust and unlike microelectrodes supported on thin Parylene films, could be directly implanted into agarose $(0.5 \%)$ models of neural tissue without additional mechanical support.

\section{Outer Sheath Surface Electrodes}

In all designs, fabrication of the outer surface electrodes was achieved successfully with the dual photoresist layer liftoff method despite the nonplanar nature of the structure. Visual inspection through microscopy showed no discontinuity between the lead traces from the top of the structure to the bottom. Thermoformed devices exhibited no discontinuities in leads to the outer surface electrodes despite the increased strain.

A microprobe attached to a micromanipulator was carefully positioned over an electrode on the outer cone surface while another was positioned over the corresponding contact pad on the Parylene cable. A Keithley 2700 MultiMeter was used to measure the lead resistance which was observed to range from 325-389 $\Omega$ across all leads. Conductivity between the electrode and contact pad confirmed electrical continuity as leads transition from the bottom to the top of the sheath.

\section{Cyclic Voltammetry}

Cyclic voltammetry (CV) of Pt electrodes was conducted on a Gamry Reference 600 potentiostat (Figure 8). The sheath probe was placed in a $0.05 \mathrm{M}$ solution of sulfuric acid $\left(\mathrm{H}_{2} \mathrm{SO}_{4}\right)$ with one of the Pt electrodes used as the working electrode. An $\mathrm{Ag} \mathrm{Ag} / \mathrm{Cl}$ electrode in a $3 \mathrm{M} \mathrm{NaCl}$ solution was used as a reference electrode. An adjacent Pt electrode was used as the counter electrode. A scan rate of $250 \mathrm{mV} / \mathrm{sec}$ was used and cycled from -0.2 to $1.2 \mathrm{~V}$ until stable voltammograms were achieved. The resulting stable voltammogram was recorded and the procedure was repeated for the remaining Pt electrodes.

The standard voltammogram for Pt immersed in sulfuric acid was achieved for all electrodes. Oxide reduction and oxidation peaks were clearly observed as well as the hydrogen peaks indicating desorption and adsorption from the Pt surface. Thus, electrical connections from the ZIF connector to the contact pad through the Parylene cables to the recording site was verified to be electrically conductive. Micromachined thin film Pt was validated to possess the same properties as a bulk Pt microwire and so can be expected to yield similar neural recordings.

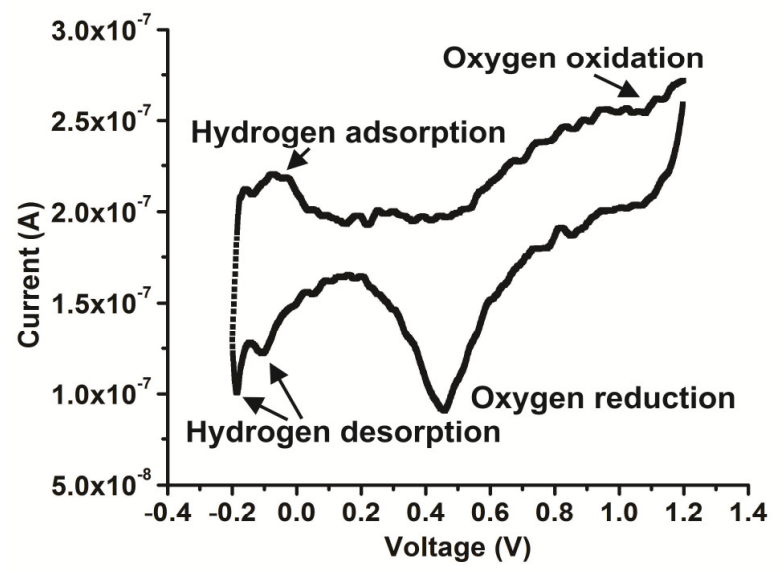

Figure 8: $\mathrm{CV}$ in $\mathrm{H}_{2} \mathrm{SO}_{4}$ of single recording site yielded standard $\mathrm{Pt}$ electrode characteristics.

Electrochemical impedance spectroscopy (EIS) was 
performed on the electrodes with the sheath probe immersed in $1 \mathrm{X}$ phosphate buffer solution (PBS). A representative measurement for a single electrode site is shown in Figure 9.

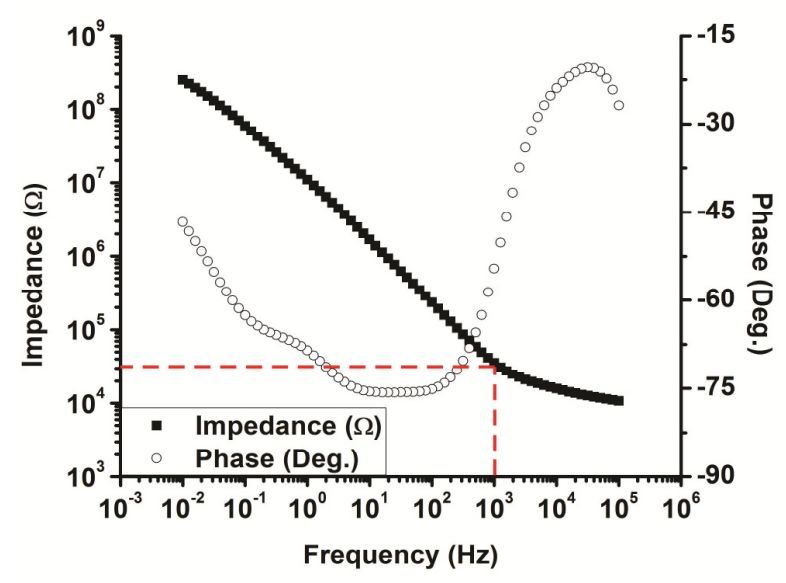

Figure 9: EIS of a single Pt recording electrode immersed in $1 X$ $P B S$. Impedance is $31.25 \mathrm{k} \Omega$ at $1 \mathrm{kHz}$.

As before, a standard phase and impedance plot were observed for all electrodes indicating that the thin film $\mathrm{Pt}$ electrodes are expected to behave in a similar fashion to $\mathrm{Pt}$ microwires for neural recordings. Recorded impedances were found to range from $20-60 \mathrm{k} \Omega$ at $1 \mathrm{kHz}$ are expected to provide adequate neural recording potential. It is desirable to limit the electrode impedance to achieve high SNR due to neural signal attenuation from the voltage divider created by electrode impedance and input impedance of recording equipment [22].

\section{CONCLUSION}

We introduced a novel 3D Parylene sheath probe that was micromachined and then thermoformed for intracortical neural recordings. Compared to earlier glass cone electrodes, this process was able to produce devices having repeatable dimensions in a batch process. Microfabrication also enabled greater yield compared to manually made cone electrodes. Parylene $\mathrm{C}$ was selected as the structural material for its biocompatibility and improved mechanical matching to tissue compared to rigid $\mathrm{Si}$ and glass.

Sheath structures were fabricated with electrodes at the outer and inner surfaces. Sheath dimensions and electrode placement were designed to match rat barrel cortex neuroanatomy. Prior to implantation in the brain, these hollow 3D structures will be coated with neurotrophic factors and immunosuppressants to promote dendritic ingrowth and reduce inflammatory response.

Electrical connectivity to probes was achieved using a ZIF connection method. Recording electrodes were characterized with $\mathrm{CV}$ and EIS. Results indicated that thermoforming did not adversely impact electrochemical behavior of the electrodes.

Future work entails evaluation of the efficacy of long term cortical recordings from the probes implanted in rat barrel cortex.

\section{ACKNOWLEDGEMENTS}

The authors wish to thank Dr. Donghai Zhu and members of the Biomedical Microsystems Lab for their assistance.

This work was sponsored by the Defense Advanced Research Projects Agency (DARPA) MTO under the auspices of Dr. Jack
Judy through the Space and Naval Warfare Systems Center, Pacific Grant/Contract No. N66001-11-1-4207.

\section{REFERENCES}

1. V. S. Polikov, P. A. Tresco, W. M. Reichert, J. Neurosci. Methods 148, 1 (2005).

2. P. K. Campbell, K. E. Jones, R. J. Huber, K. W. Horch, R. A. Normann, Biomedical Engineering, IEEE Transactions on 38, 758 (1991).

3. L. R. Hochberg et al., Nature 442, 164 (Jul 13, 2006).

4. E. M. Maynard, C. T. Nordhausen, R. A. Normann, Electroencephalogr Clin Neurophysiol 102, 228 (Mar, 1997).

5. S. Suner, M. R. Fellows, C. Vargas-Irwin, G. K. Nakata, J. P. Donoghue, IEEE Trans Neural Syst Rehabil Eng 13, 524 (Dec, 2005).

6. J. Bartels et al., Journal of Neuroscience Methods 174, 168 (Sep 30, 2008).

7. F. H. Guenther et al., Plos One 4 (Dec 9, 2009).

8. P. Kennedy et al., Journal of neural engineering 1, 72 (2004).

9. $\quad$ P. R. Kennedy, J. Neurosci. Methods 29, 181 (1989).

10. P. R. Kennedy, R. A. Bakay, Neuroreport 9, 1707 (Jun 1, 1998).

11. P. R. Kennedy, R. A. Bakay, S. M. Sharpe, Neuroreport: An International Journal for the Rapid Communication of Research in Neuroscience; Neuroreport: An International Journal for the Rapid Communication of Research in Neuroscience (1992).

12. P. R. Kennedy, S. S. Mirra, R. A. E. Bakay, Neuroscience letters 142, 89 (1992).

13. E. Meng, X. Zhang, W. Benard, in MEMS Materials and Processes Handbook R. Ghodssi, P. Li, Eds. (Springer, 2011) pp. 193-271.

14. T. A. Harder, Y. Tze-Jung, H. Qing, S. Chi-Yuan, T. YuChong, in The 15th IEEE International Conference on Micro Electro Mechanical Systems, MEMS'02. (2002) pp. 435-438.

15. D. C. Rodger et al., Sensors and Actuators B: Chemical 132, 449 (2008).

16. E. Meng, Biomedical Microsystems (CRC Press, ed. 1, 2010), pp. 408.

17. J. T. W. Kuo, L.-Y. Chang, P.-Y. Li, T. Hoang, E. Meng, Sensors and Actuators B: Chemical 152, 267 (2011).

18. M. Brecht, M. Schneider, B. Sakmann, T. W. Margrie, Nature 427, 704 (2004).

19. C. Gutierrez, E. Meng, in Transducers 2009. (IEEE, Denver, Colorado, USA, , 2009) pp. 2194-2197.

20. B. J. Kim, B. Chen, E. Meng, in Submitted to Hilton Head 2012: A Solid State Sensors, Actuators and Microsystems Workshop. (IEEE, 2012).

21. C. A. Gutierrez, C. Lee, B. Kim, E. Meng, in 16th International Solid-State Sensors, Actuators and Microsystems Conference (TRANSDUCERS). (IEEE, Beijing, China, 5-9 June 2011) pp. 2299-2302.

22. D. A. Borkholder, Stanford University (1998).

\section{CONTACT}

*E. Meng, tel: +1-213-821-3949; ellis.meng@usc.edu 


\title{
ULTRASONIC NEURAL PROBE FOR REAL TIME ELECTROMECHANICAL HISTOLOGY OF NEURAL INTERFACES
}

\author{
P. C. Chen ${ }^{1 *}$, C. J. Shen ${ }^{1,2}$, J. C. Kuo ${ }^{1}$, C. G. Clark ${ }^{2}$, B. Nijjar ${ }^{2}$, C. Schaffer ${ }^{2}$, R.F. Gilmour, Jr. ${ }^{3}$, and A. Lal ${ }^{1,2}$ \\ ${ }^{1}$ SonicMEMS Laboratory, School of Electrical and Computer Engineering \\ ${ }^{2}$ Department of Biomedical Engineering, ${ }^{3}$ Department of Biomedical Sciences, \\ Cornell University, Ithaca, NY, USA
}

\begin{abstract}
We present a neural probe with first-ever capability to measure and alter the mechanical environment of the probe. The probe is a silicon catenoidal ultrasonic horn consisting of two platinum electrode recording sites, and four polysilicon resistors for a strain gauge. The strain gauge provides transverse displacement sensitivities of $0.043 \mathrm{~V} / \mathrm{m}$ and longitudinal force sensitivities of 20 $\mathrm{mV} / \mathrm{mN}$. A miniature recording ASIC system with $50 \mathrm{~dB}$ gain neural amplifier and radio frequency module, and instrument amplifier with gain of 200 is used to transmit the neural electrical and strain signals, providing simultaneous electrical and mechanical signals from the probes. A microcontroller is used to determine and transmit the probe resonance frequencies. Using ultrasonic actuation of the probe we demonstrate in vivo measurement of insertion force reduction by a factor of 2.3, average tissue-probe stress reduction by a factor of 1.5 ,as well as breathing induced micromotion and the probe resonance frequency. These data can be useful for characterizing and improving neural probe and tissue-electrical interface reliability.
\end{abstract}

\section{INTRODUCTION}

Neural interfaces can be used to record large-scale neuronal activity and the information extracted from these recordings can, in turn, be used to control prosthetics. Neural interface technology can provide a very promising solution to treating people with paralysis or lost limbs [1]. By implanting electrodes that can record and stimulate neural activity with good spatial and temporal resolution, these technologies can help bridge a patient's cortical activity to limbs or external devices. However, the reliability of the neural interfaces is a key challenge for chronic use of neural probes for medical treatments.

Many researchers have observed that the loss of electrode function after chronic insertion is reduced to a zero signal-to-noise ratio. Studies indicate two main types of reactions resulting from electrodes. The first results from the initial insertion trauma, which can involve severing of capillaries, the extracellular matrix, and cell processes [2]. It has been observed that the initial insertion trauma causes hemorrhage and edema, in addition, to a microglia response that can fade, in some cases, after a few weeks [2]. This phenomenon of an initial insertion trauma response is supported by control studies which examine the impact of electrode stab wounds on tissue. These studies demonstrate little trace of electrode tracks several months after the stab wounds. In addition, various studies have pointed to different foreign body response reactions to electrode insertion, depending on insertion material, tip-shape, insertion velocity, and other conditions. However, despite numerous studies, in practice, the ability of maintaining a good neural interface remains a black art.

As the immune reaction to probe insertion is caused by the presence of foreign material in the body, various strategies have been proposed and attempted to reduce the immune reaction and thereby increase the lifetime of the electrodes. The effect of silicon electrode tip shape has been investigated as a potential way to reduce damage to cells. It has been demonstrated that a chisel-point silicon electrode tip shape can produce a kill zone of less than $10 \mathrm{~m}$
[3]. Additionally, electrode insertion velocity may have an effect on tissue response. Strategies have been developed to allow insertion of 100 electrodes simultaneously while producing no significant long-term damage [4]. Thinner and less stiff materials are also being investigated to determine their effect on reducing tissue immune reactions. However, one of the challenges of using very thin, flexible materials is proper insertion of the electrodes into the areas of interest. While dissolvable silk with patterned thin electronics has been used for neural recordings, these films have only been placed on the surface of the cortex [5]. Biocompatible coating techniques to decrease electrode impedance also have been investigated. Conductive polymers can decrease the initial impedance of electrodes and reduce the electrode size required to record from a given area [6]. Additionally, conductive polymer coatings, such as Poly (3,4-ethylenedioxythiophene) (PEDOT) can increase the charge injection capability of a material [7]. When inserted for long-term neural recordings, PEDOT coated electrodes exhibit lower electrode site impedance versus uncoated electrodes for a period of at least 40 days post surgery, after an initial swelling period disappears [8].

There are many challenges in terms of achieving repeatable reactions to inserted electrodes and creating viable solutions to allow decades long high quality electrical recordings from neural interfaces. However, by developing quantitative methods to assess tissue-electrode reactions in vivo and ex vivo in large numbers of animals, we can seek to better understand the problem. In this paper we use ultrasonically actuated neural probes to address reliability. We report on the first-ever multifunctional ultrasonic neural probe that can be actuated ultrasonically during insertion, greatly reducing the insertion force and reducing the average stress around the probe (Fig. 1a, b). Furthermore, the probe can measure viscoelastic properties of the probe-tissue interface and micromotion can be monitored via measuring the ultrasonic response of the probes (Fig. 1c, d). We provide measurements in mice for in situ probe histology. It is hypothesized that the reactive tissue response can be decreased by reducing the insertion force and possibly even eliminated by periodically actuating the probe with ultrasound.

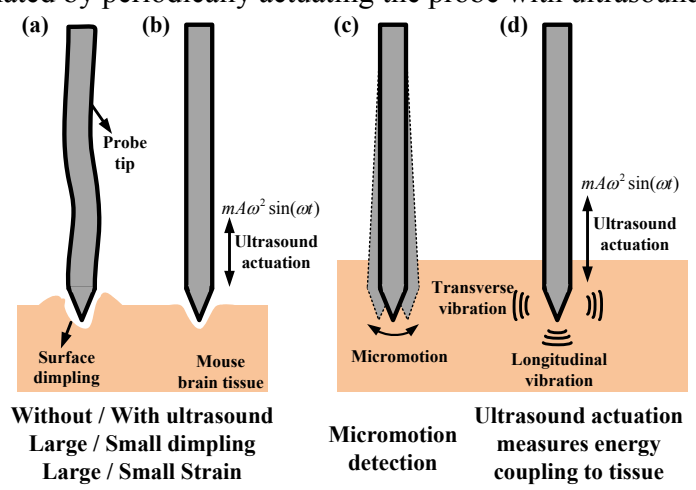

Figure 1: Probe insertion without (a) and with (b) ultrasonic actuation. Applying the ultrasonic driving voltage reduces dimpling and insertion force. (c)Micromotion detection (d) Energy coupling of the probe-tissue interface can be monitored via the ultrasonic response (longitudinal/transverse) of the probes. 


\section{PROBE PROCESS}

The silicon neural probes are designed in a catenoidal horn shape (Fig. 2) [9] for maximum tip motion, with a resonance frequency of $430 \mathrm{kHz}$. Probes are fabricated using a similar process to [9] from $<100>$ silicon wafer. Briefly, polysilicon strain gauge resistors are realized by LPCVD polysilicon implanted with boron at a dose of $2 * 10^{15}$ ions $/ \mathrm{cm}^{2}$ at $100 \mathrm{keV}$. The resistors are electrically contacted with aluminum alloy $(\mathrm{Al}+1 \%$ silicon $)$ metal lines. Insulating PECVD nitride is deposited, followed by platinum evaporation to define electrical recording sites. For back-side etching, a polymer coating Protek SR-25 is spun to protect the frontside features during DRIE probe release. This procedure avoids the large sidewall angle with $\mathrm{KOH}$ on $<100>$ wafers, and non-uniformities in $\mathrm{KOH}$ etch rates that allows $\mathrm{KOH}$ to leak to the frontside films. The new process has an improved yield near $90 \%$ versus $60 \%$ with $\mathrm{KOH}$. Lead Zirconate Titanate Oxide (PZT-4H) piezoelectric plates $\left(3.55 \times 1.25 \times 0.5 \mathrm{~mm}^{3}\right)$ are affixed to the zero-displacement nodes of the longitudinal mode shape. Based on finite element simulations, a longitudinal resonance frequency of $430 \mathrm{kHz}$ is expected, which is close to the impedance analyzer measurement on fabricated devices of $416 \mathrm{kHz}$.

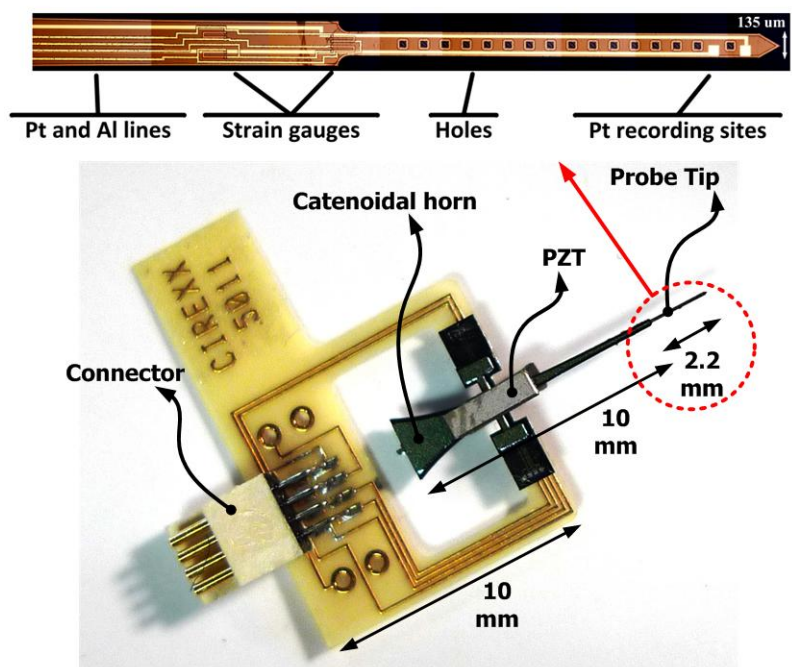

Figure 2: Miniature silicon horn with piezoelectric plates to drive the probe at its longitudinal resonance, and microphotograph of probe tip with integrated strain gauges and platinum recording sites
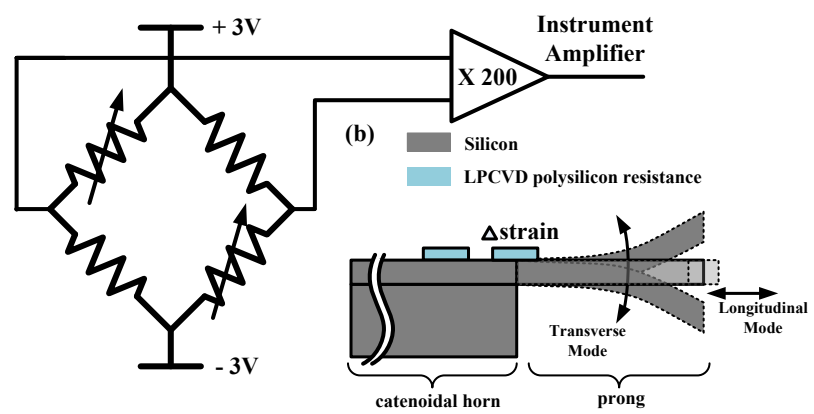

Figure 3: (a) Wheatstone bridge circuit configuration for recording insertion force and micromotion. (b) Two different modes (longitudinal/transverse) of probe tip will induce the strain signal

A prong off the main horn forms the neural probe. The prong consists of two electrical sites and polysilicon strain gauges at the interface of the horn and the prong for optimum strain measurement as shown in Fig. 3. As the probe tip bends, the resistances on the variable polyresistors change allowing monitoring of strain on the probe. We characterize this response using prong-probe dimensions of $2.2 \mathrm{~mm}$ length, $135 \mathrm{~m}$ width and $140 \mathrm{~m}$ thickness, resulting in a transverse mode displacement sensitivity of $43 \mathrm{mV} / \mathrm{m}$, and a longitudinal mode force sensitivity of $20 \mathrm{mV} / \mathrm{mN}$. Integrated holes $\left(35 \times 35 \mathrm{~m}^{2}\right)$ in the prong are fabricated to test the hypothesis that the holes may allow for better signal transduction and biocompatibility. The horn and probe is placed on a custom flexible printed circuit board (PCB) and interfaced to our custom miniature recording system.

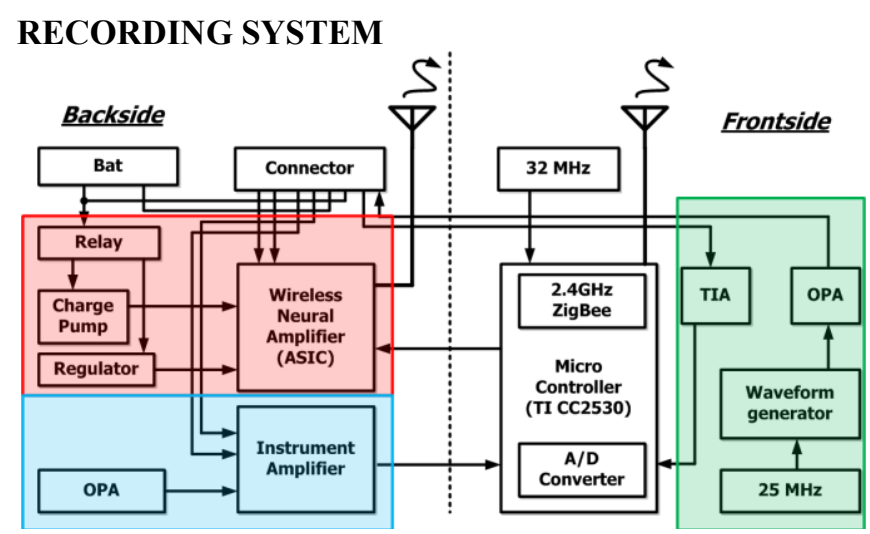

Figure 4: Control circuit block diagram. Different color correlated to the different functionalities: red- neural recording, blue insertion force and micromotion, and green - ultrasonic response.

The whole system block diagram is shown in Fig. 4. An integrated circuit was designed in Austrian Micro Systems (AMS) $0.35-\mathrm{m}$ process with four multiplexed neural amplifiers and a voltage control oscillator (VCO) as transmitter. A two-stage amplifier topology composed of differential stage followed by a common source stage resulted in a $1.25 \mathrm{~V}$ input refereed noise and $50 \mathrm{~dB}$ gain over $10 \mathrm{kHz}$ bandwidth. The VCO is designed using a differential ring oscillator topology to avoid external components. The telemetry frequency range is designed to be 82 to $84 \mathrm{MHz}$. An instrument amplifier (AD8223) with an operational amplifier (TI OPA890) is used to amplify the strain signal from the probe and send it to the analog-to-digital converter (ADC) in the microcontroller. A programmable waveform generator is controlled by the microcontroller to generate the desired frequency range of a sinusoid wave to actuate the probe and to locate the resonance frequency through a transimpedance amplifier (TIA) configured operational amplifier and send to the ADC. A microcontroller (Texas Instruments CC2530) is used to control these three different functions through a customized Lab-VIEW interface and to transmit them with a ZigBee RF interface. The system is built up on a four-layer two-sided PCB. A picture of the chronic recording system is shown in Fig. 5.

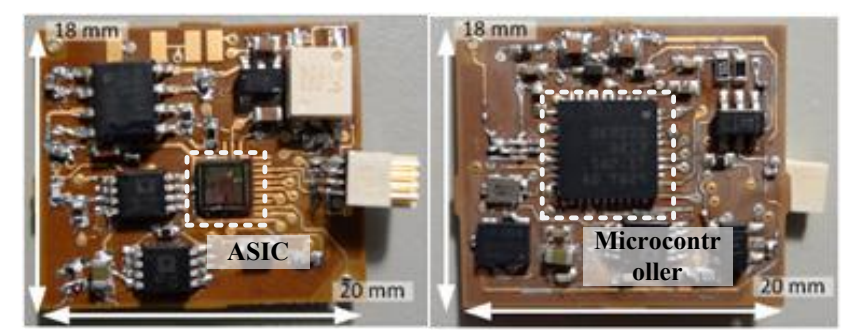

Figure 5: photograph of purposed chronic recording system 


\section{IN VIVO IMPLANTATION}

Preoperative care is administered using aseptic techniques for chronic animals. For chronic surgeries, instruments and materials are sterilized by autoclaving. Anesthesia is administered by $5 \%$ isoflurane (VetOne) and is maintained at $1.5-2 \%$ for the duration of the experiment.

At the beginning of the surgery, mice receive an injection of glycopyrrolate, an anticholinergic, intramuscularly at 0.002 $\mathrm{mg} / 100 \mathrm{~g}$ to assist in keeping the airways clear of fluid build-up and Ketoprofen $(5 \mathrm{mg} / \mathrm{kg})$ and Dexamethasone Sodium Phosphate $(0.2$ $\mathrm{mg} / \mathrm{kg}$ ) are administered subcutaneously. The mouse is secured in a stereotaxic frame by standard ear bars. After surgical preparation of the area on the head, bupivacaine $0.125 \%(\sim 0.1 \mathrm{ml})$ is injected subcutaneously above the skull. A short incision is made on top of the mouse's skull and the connective tissue is scraped away, exposing the bone.

a. Craniotomy - The dry skull is coated with a thin layer of cyanoacrylate adhesive (Vetbond). Three screws are placed in the skull to provide a framework for the dental cement to create a strong adhesion. A $5 \mathrm{~mm}$ diameter opening is drilled inside the chamber, leaving a bone flap in the center. After the bone flap is lifted off, the dura mater is kept moist with artificial cerebrospinal fluid (ACSF) and the chamber is filled with ACSF.

b. Insertion of electrode probe - The probe is secured and slowly lowered to the base of the craniotomy by a fine-precision motorized micromanipulator. The probe is gently inserted and advanced into the brain until it is approximately $200 \mu \mathrm{m}$ under the surface of the cortex. The probe is secured to the base of the skull with dental cement (Fig. 6).

c. Placement of glass coverslip - A glass coverslip is glued above the craniotomy site with cyanoacrylate and dental cement. The skin is closed around the margins of the cranial window with cyanoacrylate.

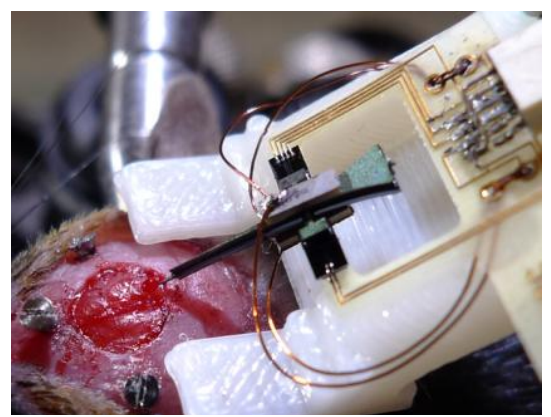

Figure 6: photograph of inserted probe in mouse brain

\section{IN VIVO EXPERIMENTAL RESULTS}

The neural probes are tested for biological efficacy by inserting them into the motor sensory cortex region of genetically modified C57BL/6 mice brains. The microglia cells were identified with green fluorescent protein (GFP). The ambulatory experiment setup is shown in Fig. 7.

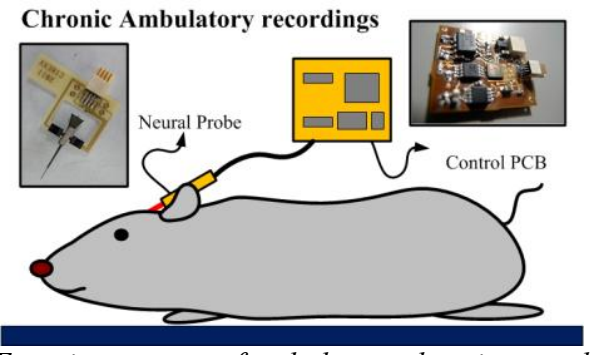

Figure 7. Experiment setup of ambulatory chronic recording.

\section{Insertion force reduction and net stress reduction}

PZT actuation is used to actuate probes during insertion while measuring the force required using a load cell. The force goes to a maximum as the probe punctures the tissue, after which the viscous drag of the tissue presents a constant force during insertion. This force can be used to monitor excessive stress caused by insertion. Different driving voltage verses the insertion forces were characterized with a $2 \%$ agar gel as brain tissue phantom (Fig. $8 \mathrm{a}$ ). The insertion force was reduced by a factor of 2.3, indicating that even at $5-\mathrm{Vpp}$ drive, the probes ultrasonically modify tissue insertion. Ultrasonic insertion causes the tissue-probe interface to have less net-stress by a factor of 1.5 , due to the ultrasonic cutting leading to less average stress and providing stress relief (Fig. 8b). The histology slices shown in Fig. 9 indicate that the ultrasonic insertion results in a smoother tissue boundary at the insertion site. The result is from the same animal with insertion into two different locations.
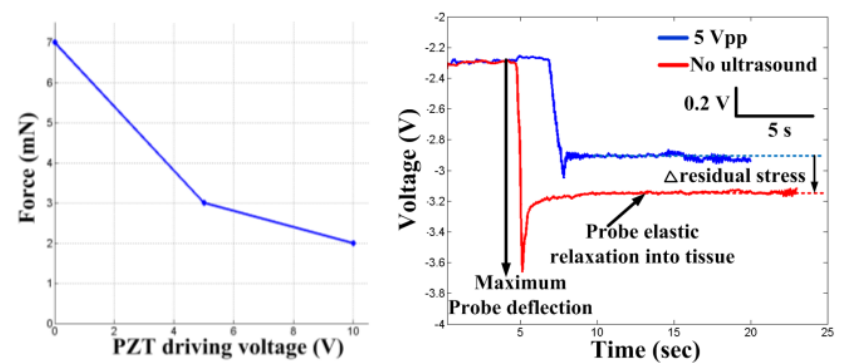

Figure 8: (a) Insertion force verse PZT driving voltage curve. (b) In vivo strain-gauge measured insertion force with no ultrasound and with 5-Vpp PZT driving voltage. Less probe deflection and residual stress by a factor of 1.5 with PZT driving was observed.
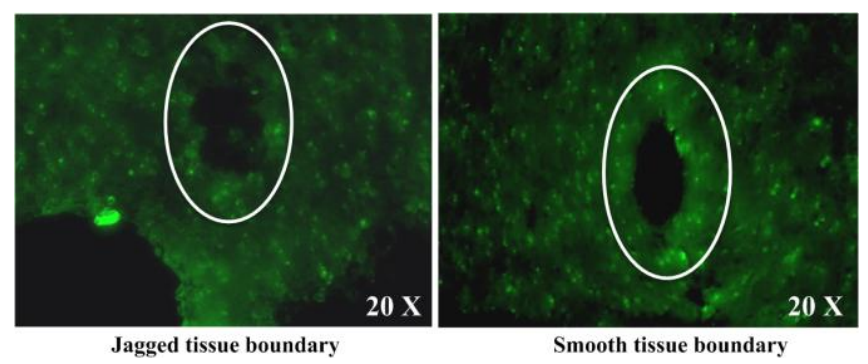

Figure 9: Histology result from the same mouse, without (left) and with (right) ultrasonic actuated insertion.

\section{Long term mechanical coupling to tissue}

The AC electro-mechanical impedance of the ultrasonic transducer at a probe longitudinal mode resonance frequency, long term mechanical coupling to tissue can be assessed. The quality factor of the resonance is indicative of the energy loss into the tissue. The measured mechanical impedance spectrum for the probe in air and after insertion into the mouse brain (Fig. 10) shows a peak broadening and a decrease in quality factor resulting from probe damping. The resonance curve shifts $3.9 \mathrm{kHz}$ with probe insertion, with peak broadening and a decreased quality factor from 34.07 to 26.72 (90\% of peak voltage) (Table 1).

Table 1: Resonance frequency, bandwidth and quality factor of the probe between air and brain tissue

\begin{tabular}{|l|c|c|}
\hline Unit & Air & Brain Tissue \\
\hline Resonance Frequency (kHz) & 415.7 & 419.6 \\
\hline Bandwidth (kHz) & 12.2 & 15.7 \\
\hline Quality Factor & 34.07 & 26.72 \\
\hline
\end{tabular}




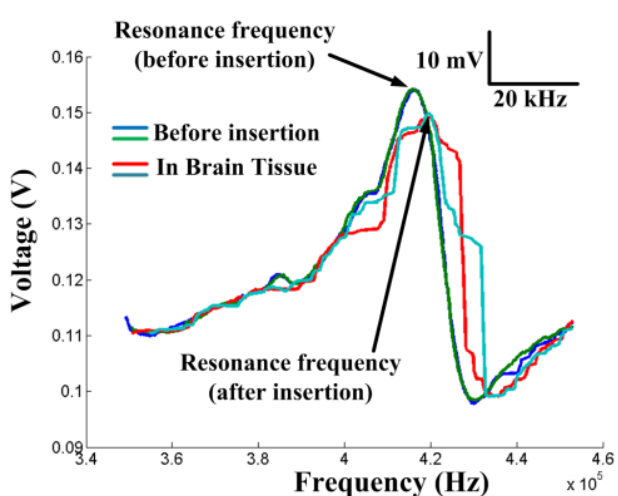

Figure 10: Measured mechanical impedance spectrum in air and in situ brain tissue

\section{Micromotion movement}

The strain-gauges can measure the strain caused by mouse motion and transmit wirelessly after insertion. We measured mouse breathing and heart rates as mechanical forces applied biologically to the mouse (Fig. 11). Studies have shown that blood flow and respiration can induce micromotion of the brain during craniotomies [10]. Our measurements indicate approximately 3- m movement induced by breathing inside the brain.

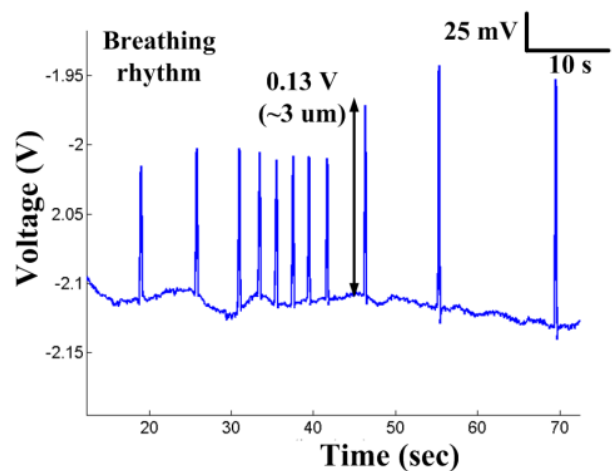

Figure 11: Strain-gauge measured breathing induced micromotion inside mouse brain.

\section{Chronic neural electrical signal measurement}

Ambulatory chronic recording after implanting the electrode for 10 days is shown in Fig. 12. The spike rates and electrode impedance from the mouse brain can be monitored over time to determine aging of the neural interface.

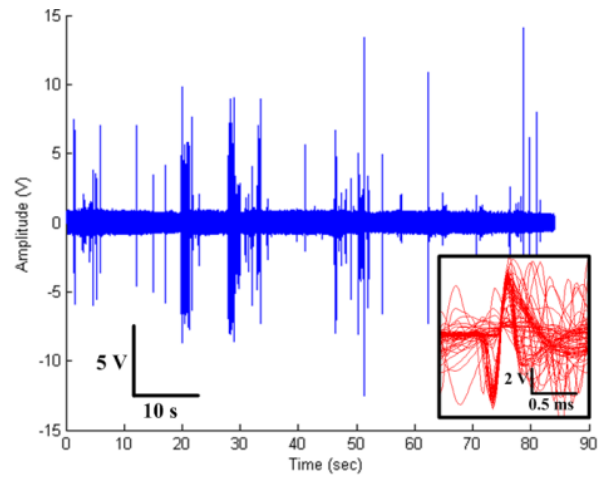

Figure 12: Chronic neural recordings from probe Pt recording sites after 10 days of implantation.

\section{CONCLUSION}

In this study, we have demonstrated a microfabricated neural probe with the capability to measure and alter the mechanical environment inside the tissue. In addition, the miniature recording system can record the signal simultaneously and chronically. We have demonstrated insertion force reduction, tissue-probe stress reduction, measurement of micromotion, and the probe resonance measurement. Future work and challenges include miniaturizing the system in an ASIC, and collecting more chronic recording data to further identify key variables that improve neural probe reliability.

\section{ACKNOWLEDGMENT}

This work was sponsored by the Defense Advanced Research Projects Agency (DARPA) MTO under the auspices of Dr. Jack Judy through the Space and Naval Warfare Systems Center, Pacific Grant/Contract No. N66001-11-1-4011. We would like to thank the Cornell Nanofabrication Facility (CNF) at Cornell University for fabrication of the probe.

\section{REFERENCES}

[1] M.A. Lebedev, J.M. Carmena, J.E. O'Doherty, M.Zacksenhouse, C.S. Henriquez, J.C. Principe, and M.A.L. Nicolelis, "Cortical ensemble adaptation to represent velocity of an artificial actuator controlled by a Brain-Machine Interface," J. Neuroscience, 25, 19 (2005), pp. 4681-93

[2] V.S. Polikov, W.M. Reichart, and P.A. Tresco, "Response of brain tissue to chronically implanted neural electrodes," J. Neurosci. Methods, 148, 1 (2005), pp. 1-18.

[3] D.J. Edell, V.V. Toi, V.M. McNeil, and L.D. Clark, "Factors influencing the biocompatibility of insertable silicon microshafts in cerebral cortex," IEEE Trans. Biomed. Eng., 39, 6 (1992), pp. 635-43.

[4] P.J. Rousche and R.A. Normann, "A system for impact insertion of a 100 electrode array into cortical tissue," in Proc. Int. IEEE EMBS Conference. (1990), pp. 494-95.

[5] D.-H. Kim, et al., "Dissolvable films of silk fibroin for ultrathin conformal bio-integrated electronics," Nat. Mater., 9, 6 (2010), pp. 511-17.

[6] K.A. Ludwig, N.B. Langhais, M.D. Joseph, S.M. Richardson-Burns, J.L. Hendricks, and D.R. Kipke, "Poly(3,4-ethylenedioxythiophene) (pedot) polymer coatings facilitate smaller neural recording electrodes," J. Neural Eng., 8, 1 (2011), pp. 014001.

[7] X.T. Cui and D.D. Zhou, "Poly(3,4-ethylenedioxythiophene) for chronic neural stimulation," IEEE Trans. Neural Syst. and Rehabil. Eng., 15, 4 (2007), pp. 502-08.

[8] K.A. Ludwig, J.D. Uram, J. Yang, D.C. Martin, and D.R. Kipke, "Chronic neural recordings using silicon microelectrode arrays electrochemically deposited with a poly(3,4-ethylenedioxythiophene) (pedot) film," J. Neural Eng., 3, 1 (2006), pp. 59-70.

[9] C.J. Shen, R.F. Gilmour, Jr. and A. Lal, "Silicon ultrasonic microprobes with collocated and concurrent strain and bio-electrical potential wireless transmission," Proc. MEMS Jan. (2011), pp.972-75.

[10] X. Liu, D.B. McCreery, R.R. Carter, L.A. Bullara, T.G. Yuen, and W.F. Agnew, "Stability of the interface between neural tissue and chronically implanted intrcortical microelectrodes," IEEE Trans. Rehabil. Eng. , 7, 3 (1999), pp. 315-326.

\section{CONTACT}

*P.C. Chen, tel: +1-607-793-3300; pc445@cornell.edu 


\title{
A MICROFABRICATED WIRELESS RF PRESSURE SENSOR MADE COMPLETELY OF BIODEGRADABLE MATERIALS
}

\author{
Mengdi Luo ${ }^{1 *}$, Chao Song ${ }^{2}$, Florian Herrault ${ }^{2}$ and Mark G. Allen ${ }^{2}$
}

${ }^{1}$ School of Materials Science and Engineering, Georgia Institute of Technology, Atlanta, Georgia, USA

${ }^{2}$ School of Electrical and Computer Engineering, Georgia Institute of Technology, Atlanta, Georgia, USA

\begin{abstract}
Implantable passive LC resonant sensors have previously been studied to wirelessly measure physiological parameters in vivo. In this work, a wireless RF pressure sensor made entirely of biodegradable materials is designed and fabricated via MEMS technologies. Such biodegradable implants may be appropriate for short-term, acute medical applications as they potentially eliminate the need for implant extraction when sensing is no longer required. Passive LC resonant sensors typically require conductors, structural materials, and dielectrics to function. Metallic zinc, known to be biocompatible, was also found to be degradable in saline and was subsequently used as the sensor conductor material, while biodegradable polymers were used as dielectrics and structural materials. Biodegradable materials are particularly challenging to process using conventional MEMS fabrication techniques because they are water-sensitive. As a result, alternative MEMS fabrication approaches using embossing and multi-layer folding were combined with traditional techniques to fabricate the pressure sensor. Experimentally, the fabricated sensor made of biodegradable materials demonstrated linear frequency response with external applied pressure. A sensitivity of $-290 \mathrm{kHz} / \mathrm{kPa}$ was measured in the $0-30 \mathrm{kPa}$ pressure range.
\end{abstract}

\section{INTRODUCTION}

Implantable resonant passive sensors were proposed more than five decades ago as a solution to wirelessly measure physical parameters within living organisms [1]. Since then, non-degradable MEMS-fabricated passive resonant sensors have been demonstrated for chronic implantable applications to monitor blood pressure and other physiological conditions [2]. Although such sensors show great promise for chronic applications, some acute or shorter-term medical applications (e.g., wound or bone healing) could also benefit from implantable monitoring sensors. Typically these sensors should be extracted when no longer needed. In these applications, if instead the sensor could be made completely biodegradable, this extraction could be avoided.

A number of researchers have proposed approaches to implantable biodegradable sensors. Wireless implantable strain sensors consisting of a micro-channel attached to a fluid reservoir were proposed in [3]. These devices utilized an external ultrasound system to assess the strain-driven filling of the channel, which could be limiting compared to RF readout. Biodegradable polymeric composite materials that could have application as components of RLC resonant sensors, prepared by emulsion polymerization of pyrrole in biodegradable polylactic acid (PLA) and polycaprolactone (PCL) polymers, are also under study [4]. Non-sensing implantable biodegradable stimulators that exploit RF coupling have also been proposed as an approach to promote bone growth. The coupling coil was fabricated using conventional micromilling of biodegradable magnesium alloys, and packaged with poly L:D lactide polymer [5].

The microfabricated pressure sensor presented in this work is also based on the passive resonant mechanism [2]. It utilizes RF interrogation while simultaneously maintaining its all-biodegradable character. The pressure sensor consists of a sensing cavity, bounded by two electrodes forming the variable capacitor and connected in series with an inductor coil (Figure 1). The inductor coil not only acts as an essential component of the resonant sensor, but also provides means for magnetically coupling the sensor to an external (e.g., outside the body) coil. When pressure is applied to the sensor, the gap between the two capacitive electrodes is reduced and the capacitor value increases. The resulting pressure-induced change in the LC resonant frequency can be measured wirelessly using the external coil.
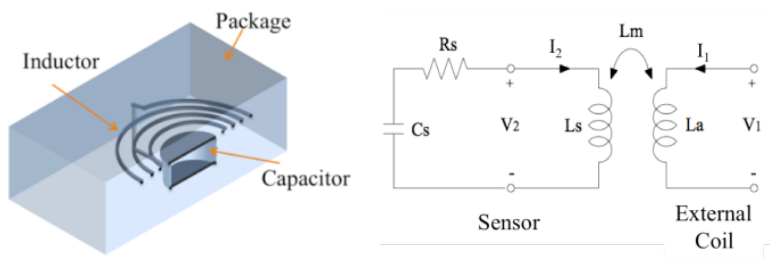

Figure 1: Schematic cross-section diagram (left) and equivalent circuit (right) of a passive $L C$ resonant sensor.

\section{DESIGN AND FABRICATION Materials}

There are several FDA-approved and commercially-available biocompatible and biodegradable polymers [6]. Some design constraints that may drive polymer choice include desired degradation time, processing/fabrication characteristics, and mechanical properties. PLA and PCL polymers have been selected in this application. PLA is used as the packaging and sensing plate material because of its mechanical properties [6], and PCL is utilized as the bonding and sealing material because of its low melting temperature (approximately $61^{\circ} \mathrm{C}$ ), which is compatible with the proposed fabrication process. Other potential biodegradable and biocompatible polymers include copolymers of PLA with polyglycolic acid (PLGA) and polyanhydrides. By varying the biodegradable polymer selection, different desired degradation times ranging from 3-24 months can be obtained [6].

The most well-known biocompatible and biodegradable metals are magnesium and iron. These metals can degrade within the body to form soluble species. Further, they have been demonstrated in biodegradable stent applications [7]. However, from a MEMS fabrication standpoint, standard approaches to create thick micro-scale conductors for high quality factor LC resonant devices, such as electrodeposition, are challenging with active elements such as magnesium. Iron is more compatible with standard electrodeposition [8], but at higher frequencies (e.g., tens of $\mathrm{MHz}$ and above), its $\mathrm{AC}$ conductivity is poor because of the relative magnetic permeability enhancement of the skin effect [9].

Zinc is considered one of the biocompatible metals and is also an essential element consumed by the human body [10]. Unlike iron, its AC electrical characteristics should enable the design and fabrication of high quality factor inductors (its bulk resistivity is only 3.5 times higher than that of copper). Zinc is also compatible with electroplating technology, making it a good candidate as a conductive material in microfabricated LC resonant biodegradable pressure sensor applications. 


\section{Device Design}

The operating frequency of passive wireless devices is typically bounded by maximum device size (at low frequencies) and signal degradation in the lossy medium of the body (at high frequencies). It has been demonstrated that operating frequencies in the $10-100 \mathrm{MHz}$ range are suitable for in vivo wireless measurements [2]. To achieve such resonant frequencies while maintaining the device area below a nominal value of $1 \mathrm{~cm}^{2}$, both the inductor and capacitor must follow specific design guidelines. Table 1 shows the design parameters of the RF pressure sensor.

Table 1: Dimensions of the conductors of the pressure sensor

\begin{tabular}{|c|c|c|c|c|c|}
\hline \multicolumn{3}{|c|}{ Zn Inductor } & \multicolumn{3}{c|}{ Zn Capacitor } \\
\hline $\begin{array}{c}\text { Number of } \\
\text { turns }\end{array}$ & $\begin{array}{c}\text { Line } \\
\text { width }\end{array}$ & $\begin{array}{c}\text { Spacing } \\
\text { width }\end{array}$ & $\begin{array}{c}\text { Gap } \\
\text { distance }\end{array}$ & $\begin{array}{c}\text { Pad } \\
\text { radius }\end{array}$ & $\begin{array}{c}\text { Pad } \\
\text { thickness }\end{array}$ \\
\hline & $(\mu \mathrm{m})$ & $(\mu \mathrm{m})$ & $(\mu \mathrm{m})$ & $(\mathrm{mm})$ & $(\mu \mathrm{m})$ \\
\hline 14 & 70 & 70 & 40 & 2.5 & 60 \\
\hline
\end{tabular}

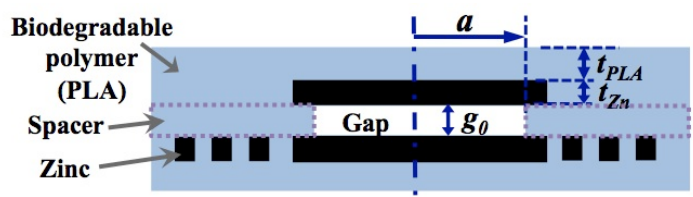

Figure 2: Schematic cross-section of the pressure sensor design.

A schematic cross-section of the pressure sensor is shown in Figure 2. The device consists of a bottom PLA layer with electroplated zinc inductor and capacitor plate, a PLA/PCL spacer layer that forms the sensor cavity, and a top PLA/Zn layer that includes the top capacitor plate. The zinc electrical interconnects between the inductor and the capacitor plates are not depicted. A first-order analytical model indicates that the center deflection of the plates varies linearly with pressure when the gap between the plates is small compared to their thicknesses. The deflection is inversely proportional to the flexural rigidity, which is related to the Young's modulus $E$ and the Poisson's ratio $v$ of the material. Finite element simulations (COMSOL 4.2) coupling electrical with mechanical analyses were performed to evaluate the suitability of the proposed sensor design. A two-dimensional axisymmetric model was used. The geometry of the simulation was simplified by neglecting the zinc coil and via structures. $E$ and $v$ of PLA as well as $\mathrm{Zn}$ were assumed to be $2 \mathrm{GPa}, 0.35$ [9] and $100 \mathrm{GPa}, 0.25$, respectively. Sensors with different thicknesses of PLA $t_{P L A}$ and radii of the deflecting diaphragm $a$ were simulated, with the dimensions shown in Table 2. The initial gap between capacitor plates was set at $40 \mu \mathrm{m}$.

Table 2: Dimensions of the sensor designs for simulation

\begin{tabular}{|c|c|c|c|}
\hline \multirow{2}{*}{ Design } & $\mathrm{a}$ & $\mathrm{t}_{\mathrm{PLA}}$ & $\mathrm{t}_{\mathrm{Zn}}$ \\
\cline { 2 - 4 } & $(\mathrm{mm})$ & $(\mu \mathrm{m})$ & $(\mu \mathrm{m})$ \\
\hline 1 & 2.4 & 200 & 60 \\
\hline 2 & 2.4 & 400 & 60 \\
\hline 3 & 4.9 & 400 & 60 \\
\hline
\end{tabular}

The simulated normalized capacitance change of the simplified sensor structure is presented in Figure 3. Applied pressures ranging from 0 to $30 \mathrm{kPa}$ were simulated. When radius of the cavity $a$ is $2.4 \mathrm{~mm}$ which is smaller than the radius of the $\mathrm{Zn}$ pad $r_{Z n}(2.5 \mathrm{~mm})$, the deflecting diaphragm consists of a Zn/PLA bi-layer plate. Although small, the capacitance change of this sensor would be detectable. Further, the thinner PLA sheet (Design 1) exhibits a higher capacitance change than the thicker PLA sheet (Design 2) for the same cavity radius because of larger deflection. The capacitance variation increases substantially for the sensor with $a=4.9 \mathrm{~mm}$ (Design 3), not only because the deflection radius is increased, but also because the deflecting diaphragm consists of a $\mathrm{Zn} / \mathrm{PLA}$ bi-layer plate embedded in a PLA ring with lower Young's modulus than the bi-layer itself. Experimentally, the deflection of the bottom plate with the zinc coils is expected to be lower than the simulated case because the coil stiffens the bottom layer.

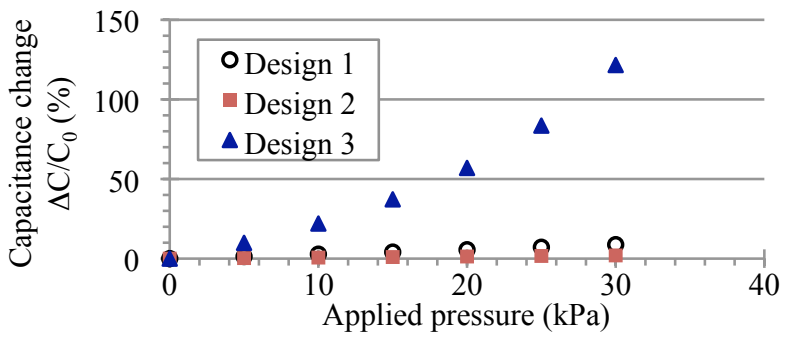

Figure 3: Normalized simulated capacitance change $\Delta C / C_{0}$ as function of external applied pressure on simplified sensor structure for different cavity dimensions and PLA thicknesses.

\section{Pressure Sensor Fabrication}

The fabrication process of the microfabricated RF pressure sensor made of biodegradable materials is schematically shown in Figure 4. Alternative fabrication approaches have been developed to augment conventional approaches since biodegradable materials may be sensitive to chemicals used in standard microfabrication techniques. The process relies on zinc electroplating followed by polymer embossing. A folding technique coupled with polymer lamination steps were utilized to create the sensor cavity and the appropriate LC resonant electrical configuration while alleviating the need for multiple electrodeposition steps.

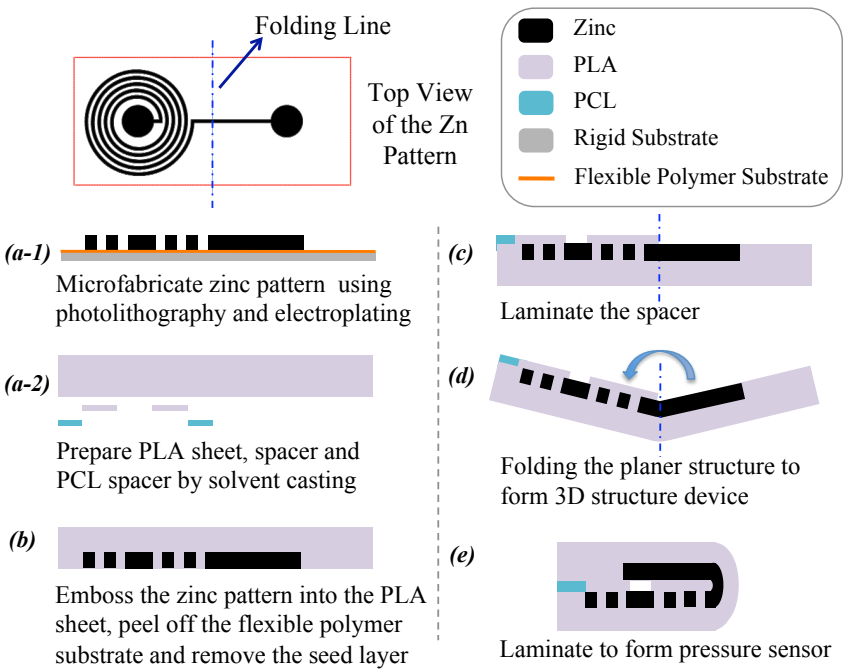

Figure 4: Fabrication process of the passive pressure sensor completely made of biodegradable materials.

The process started with the through-photoresist electrodeposition of zinc patterns (inductor and capacitor plates) onto a flexible substrate. More specifically, an $80-\mu \mathrm{m}$-thick flexible and chemically-resistant polymer film (Kapton ${ }^{\circledR}$ ) with a titanium/copper/titanium seed layer was temporarily bonded onto a rigid substrate using a thin layer of photoresist as an adhesive layer. Zinc patterns $60 \mu \mathrm{m}$ thick were electrodeposited through a photoresist mold via pulse reverse electroplating at an equivalent current density of $8 \mathrm{~mA} / \mathrm{cm}^{2}$. After the phororesist was removed, 
the metalized polymer film with microfabricated zinc patterns was separated from the rigid substrate (Figure 4(a-1)). Figure 5 shows images of the electroplated zinc inductor. PLA and PCL polymer films with designed thicknesses were prepared separately by solvent casting from dichloromethane. The PLA and PCL polymer films were cut to the appropriate designs and dimensions using a $\mathrm{CO}_{2}$ laser (Figure 4(a-2)).

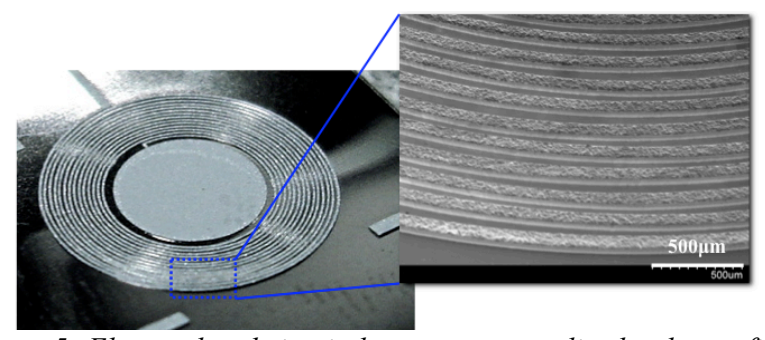

Figure 5: Electroplated zinc inductor onto metalized polymer film. The inset shows a scanning electron microscopy image of the electrodeposited zinc windings.

The flexible substrate with electroplated zinc patterns and a PLA polymer sheet fabricated as above with thicknesses ranging from 200 to $400 \mu \mathrm{m}$ were laminated under a 10-bar pressure at $160^{\circ} \mathrm{C}$ for 4 minutes. After lamination, the flexible polymer substrate was peeled off. The resulting structure consisted of the microfabricated zinc patterns embedded in a PLA polymer sheet. The remaining seed layer was removed by micro-polishing, limiting the number of wet processing steps (Figure 4(b)).

To define the sensor cavity, a biodegradable spacer consisting of a smaller PLA ring and a larger, concentrically-placed PCL ring was laminated onto the PLA sheet with embedded zinc microstructures (Figure 4(c)). A small amount of dichloromethane solvent was used as a bonding promoter. Then the PLA/Zn sheet was folded (Figure 4(d)) and laminated to form the 3-D PLA/Zn pressure sensor. The folding was performed with the assistance of selective heating along the folding line. The folding technique, adapted from [12], enables the microfabrication of a 3-D device with multiple metal layers and electrical interconnects using a single electrodeposition step. This is particularly favorable when the metallic and polymeric materials are sensitive to chemicals used in conventional microfabrication techniques. The lamination step to complete the pressure sensor fabrication was performed under pressure and at $55^{\circ} \mathrm{C}$. During the lamination, the PCL spacer softened and sealed the cavity. Finally, the device shape was defined around the inductor geometry by $\mathrm{CO}_{2}$ laser

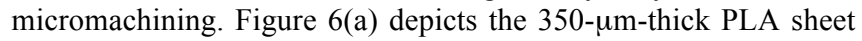
with embedded zinc patterns along with the spacers before folding and lamination. An example of a fully-fabricated and functional 10-mm-diameter device is shown in Figure 6(b). The design parameters are reported in Table 1.

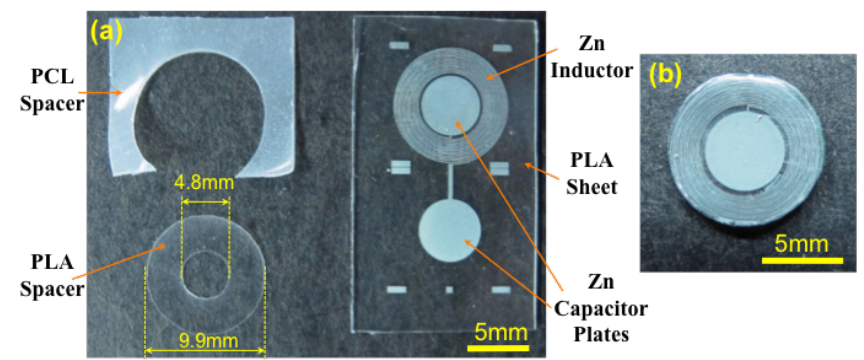

Figure 6: Microfabricated RF pressure sensor completely made of biodegradable materials, (a) before folding, and (b) after lamination and cutting.

\section{RESULTS}

Degradation Rate of Electroplated Zinc Samples

Zinc samples were electroplated for degradation tests in saline. The samples were between 1 and $2 \mathrm{~cm}^{2}$ in area and between 70 200 $\mu \mathrm{m}$ in thickness. The area and initial weight of the samples were measured after fabrication. Each specimen was immersed in $20 \mathrm{~mL}$ of Phosphate-Buffered Saline $1 \times$ (PBS buffer), and placed in an incubator at a temperature of $37 \pm 0.5^{\circ} \mathrm{C}$. Throughout a 30 -day timeframe, the weight of each specimen was measured daily. For daily weight measurements, the specimens were rinsed in deionized water and dried thoroughly. After weight measurement and before pursuing the experiment, the PBS buffer solution was refreshed by replacing half of it with fresh buffer.

The experimental results are plotted in Figure 7. The daily weight loss is normalized by the area of the $\mathrm{Zn}$ samples. The degradation rate of electroplated zinc was measured at $0.4 \mathrm{mg} /\left(\right.$ day $\left.\cdot \mathrm{cm}^{2}\right)$, which corresponds to approximately $0.6 \mu \mathrm{m} /$ day of degraded zinc material.

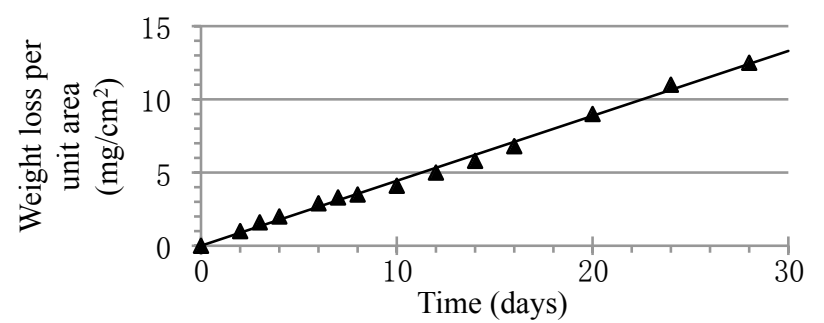

Figure 7: Biodegradability test result: daily weight loss per unit area of electroplated zinc in PBS solution.

\section{Zinc Inductor Characterization}

The inductance and quality factor of the microfabricated zinc inductor embedded in a PLA polymer layer was measured before folding using an impedance analyzer. The experimental results are presented in Figure 8. An inductance of $1.9 \mu \mathrm{H}$ was measured at frequencies below $50 \mathrm{MHz}$, with a quality factor exceeding 25 .

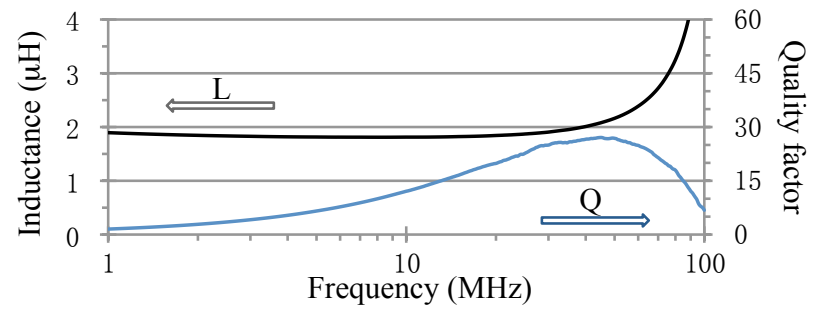

Figure 8: Measured inductance and quality factor of the electroplated zinc inductor embedded in a PLA polymer layer.

\section{Wireless Pressure Sensor Testing}

For wireless measurements, the fabricated sensor is coupled with an external coil (diameter $=1 \mathrm{~cm}, 5$ turns,). This coil has a $Q>200$ and a resonance above $100 \mathrm{MHz}$, and a coil to sensor distance of approximately $3 \mathrm{~mm}$. The impedance phase and magnitude are measured by an impedance analyzer as function of frequency. At the sensor resonance, changes in the external coil phase and magnitude are measured.

To determine the pressure sensitivity of the fabricated sensor, the sensor is placed in a sealed chamber. In these experiments, pressure was applied via an external air source. The experimental setup was shown in Figure 9. In operation, pressure is applied by turning on the air source while the valves 1 and 2 are left open. By closing valve 1 , the pressure in the chamber became stable and was measured by a pressure gauge. After electrical measurements, 
valve 2 was closed and valve 1 was re-opened as the air source pressure was modified. Valve 2 was then re-opened, and valve 1 was closed to repeat pressure and sensor electrical measurements over pressures ranging from 0 to $30 \mathrm{kPa}$. Aqueous environments could also be maintained in this apparatus by partially filling the sealed chamber containing the sensor with liquid.

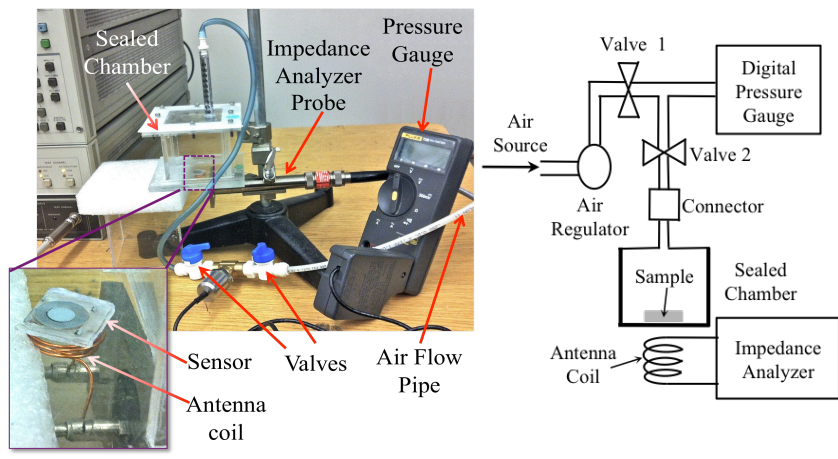

Figure 9: Pressure sensor test measurement setup (left) with schematic drawing (right).

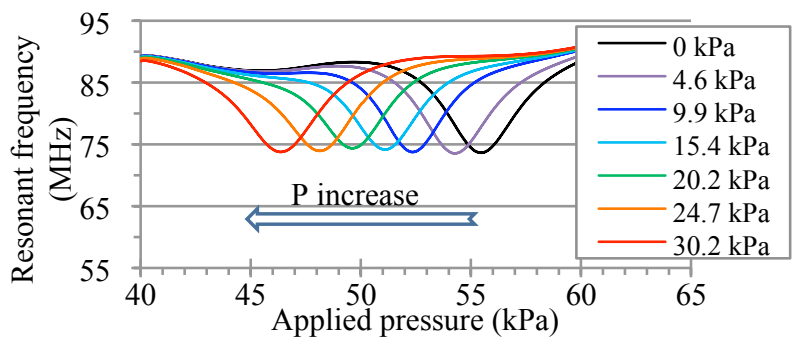

Figure 10: Impedance phase data as a function of frequency for several applied pressures.

The measured phase as a function of frequency for several applied pressures is shown in Figure 10. As the pressure increases, the minimum phase of the impedance shifts monotonically to lower frequencies because the deflection of the pressure sensitive $\mathrm{PLA} / \mathrm{Zn}$ sensor plates results in a capacitor gap decrease (i.e., a capacitance increase), and therefore a resonant frequency decrease. The impedance measurements for the microfabricated RF pressure sensor were repeated for several times under multiple pressure conditions. Figure 11 plots the resonant frequency of the sensor as a function of applied pressure in air and aqueous environments. A sensitivity of $-290 \mathrm{kHz} / \mathrm{kPa}$ was measured in air, with comparable sensitivity in water. This corresponds to a change in capacitance $\Delta \mathrm{C} / \mathrm{C}_{0}$ of $55 \%$. This sensitivity is approximately 6 times higher than that of the non-degradable implantable resonant pressure sensor in [2]. From the resonant frequency measurements, the calculated initial gap between the capacitor plates is approximately $40 \mu \mathrm{m}$, which is consistent with the expected value.

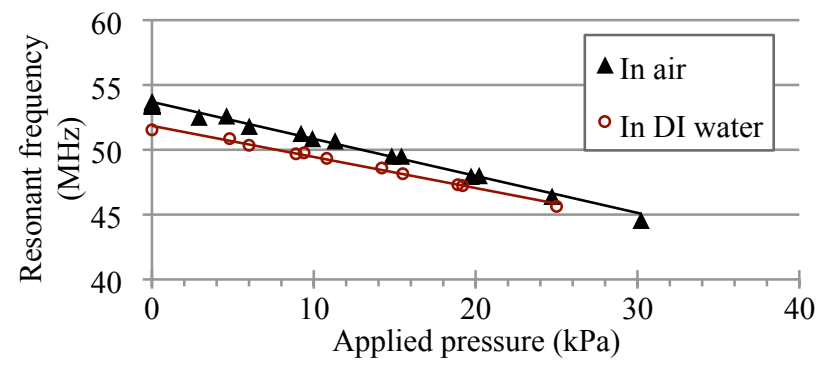

Figure 11: Resonant frequency of the sensor as a function of applied pressure in the sealed chamber.

\section{CONCLUSIONS}

A RF wireless LC resonant pressure sensor completely made of biodegradable materials was designed, microfabricated and characterized. Biocompatible zinc metal, which is compatible with MEMS fabrication techniques, was demonstrated to degrade under aqueous solutions. The zinc inductor and capacitor of the sensor were fabricated using MEMS technologies on flexible and chemically-resistant polymer and embedded in a pre-casted PLA film. The sensor was then fabricated by folding the PLA sheet with the embedded zinc microstructures. A biodegradable and adhesive spacer was utilized to form and seal the cavity during lamination.

After electrical characterization, the pressure sensor was experimentally tested in a sealed chamber with controlled pressure. The sensor response was wirelessly measured using an external coil connected to an impedance analyzer. The response time of the sensor to pressure change was instantaneous, and a sensor with sensitivity of $-290 \mathrm{kHz} / \mathrm{kPa}(-38 \mathrm{kHz} / \mathrm{mmHg})$ in air was measured experimentally. Comparable pressure-frequency behavior in an aqueous environment was observed. However, the functional lifetime of the sensors in the aqueous environment was shorter than predicted by degradation studies. These effects are currently under investigation.

\section{REFERENCES}

[1] R. S. Mackay et al., "Endoradiosonde", Nature, 197 (1957)

[2] M. A. Fonseca, J. Kroh, J. White, and M. G. Allen, "Flexible Wireless Passive Pressure Sensors for Biomedical Applications," Tech. Dig. Solid-State Sensor, Actuator, and Microsystems Workshop, Hilton Head, (2006)

[3] M. Wendlandt, F. Umbrecht, C. Hierold, F. Gattiker, J. Neuenschwander, and U. Sennhauser, "A Wireless Implantable Passive Strain Sensor (WIPSS)", European Symposium-Technical Aids for Rehabilitation, (2007)

[4] C.M. Boutry, H. Chandrahalim, and C. Hierold, "RF Conductivity of Biodegradable Conductive Polymers used for a New Generation of Partially/Fully Wireless Implantable Sensors", MEMS 2012, Paris, FRANCE, (2012)

[5] B. Finamore, G. Fedder, A. Khairi, J. Paramesh, L. Schultz, J. Burgess, P. Campbell, and L. Weiss, "Development of an Implantable Biodegradable Electrical Stimulator for Bone Repair", Biomedical Engineering Society Fall Meeting (2009)

[6] U. Edlund and A.-C. Albertsson, "Degradable Polymer Microspheres for Controlled Drug Delivery", Advances in Polymer Science, 157, (2002)

[7] R. Waksman, Biodegradable Stents: They Do Their Job and Disappear," Journal of Invasive Cardiol, 18, 2(2006)

[8] M. Schlesinger and M. Paunovic, "Modern Electroplating Fourth Edition", John Wiley \& Sons, Inc., 2000

[9] M.F. Ashby, "Engineering Materials and Processes Desk Reference", Elsevier, Inc., 2009

[10] O. I. Velikokhatnyi and P. N. Kumta, "First-principles studies on alloying and simplified thermodynamic aqueous chemical stability of calcium-, zinc-, aluminum-, yttrium- and iron-doped magnesium alloy", Acta Biomaterialia, 6, (2010)

[11] T. Kawamoto and T. Sugahara, "Examination of Properties of Bioabsorbable Osteosynthetic Material Using Finite Element Method", Journal of Hard Tissue Biology, 14, 2(2005)

[12] F. Herrault, S. Yorish, T.M. Crittenden, C.-H. Ji, and M.G. Allen, "Parylene-Insulated Ultradense Microfabricated Coils," Journal of Microelectromechanical Systems, 19, (2010)

\section{CONTACT}

*M. Luo, tel: +1-404-583-7312; mandyluo@gatech.edu 


\title{
A NOVEL MAGNETOMETER EMPLOYING MAGNETOVISCOUS EFFECT OF FERROFLUIDS
}

\author{
G. Hatipoglu ${ }^{l}$, and S. Tadigadapa ${ }^{I^{*}}$ \\ Department of Electrical Engineering, \\ Pennsylvania State University, University Park, USA
}

\begin{abstract}
In this work, we utilize ferrofluids deposited atop an ultrahigh permeability Metglas ${ }^{\circledR}\left(\mathrm{Fe}_{85} \mathrm{~B}_{5} \mathrm{Si}_{10}\right)$ amorphous thin film coated micromachined quartz resonator ( $-\mathrm{QCR}$ ) array as a unique magnetometer introduced by our group for the first time to the best of our knowledge. The magnetometer employs magnetoviscous effect of ferrofluids i.e. external magnetic field induced variations in the viscosity of a ferrofluid emulsion. The -QCR array detects these viscosity changes by virtue of its high sensitivity to viscoelastic changes in adsorbed films on its electrode. Initial measurements on the device has shown promising results, where it was possible to detect magnetic field inputs as low as $\sim 60 \mathrm{nT}$ with a SNR of 3:1.
\end{abstract}

\section{INTRODUCTION}

The scalar magnetometer introduced in this work, utilizing a highly sensitive quartz -viscometer coated with a high permeability material, tracks the viscosity changes within the ferrofluid as a sensor mechanism to detect and quantify the magnetic field strength.

Ferrofluids, which are emulsions of nano/ micro ferroparticles in a carrier liquid demonstrate highly ordered microscopic configurations under the influence of external magnetic fields [1]. The phenomenon of viscosity change of ferrofluids arising due to applied magnetic field is defined as the magnetoviscous effect [2]. The emulsion undergoes various complex structural transitions upon the application of an external magnetic field, whereby, an ensemble of Brownian magnetic nano-particles in the emulsion spontaneously organizes into columnar structures due to magnetic field induced dipole interactions [3]. The ordered chain-like columnar structures as a result of the applied magnetic field follow a hexagonal structural pattern. The length of the agglomerates and the separation distance between them are very sensitive to the small magnitude changes of the applied magnetic field as well as the frequency of the magnetic field [4].

On the other hand, the magnetization curve of an ensemble of such nano-ferroparticles is hysteresis-free. This has important consequences, as suspended superparamagnetic particles can be constantly manipulated within the fluid matrix using a magnetic field, and they do not agglomerate after the removal of the field. The particles have enough thermal energy and they are stabilized by the surfactants of about $2 \mathrm{~nm}$ thickness, which aids to recover from an aggregated state preventing further permanent aggregation. Hence, it is easy to switch on and off the magnetic interaction in the ferrofluid. The properties of the ferroluid (Ferrotec Inc, EMG 911) studied here are listed in Table 1.

Acoustic wave sensing techniques based on quartz crystal resonators $(\mathrm{QCR})$, in principle, are able to characterize not only the physical properties of the layer, but also viscoelastic properties of the layer. A typical thickness shear mode quartz crystal resonator

Table 1: Ferrofluid properties.

\begin{tabular}{|c|c|c|c|c|}
\hline $\begin{array}{c}\text { Particle } \\
\text { diameter }\end{array}$ & $\begin{array}{c}\text { Saturation } \\
\text { magnetization }\end{array}$ & $\begin{array}{c}\text { Magnetic } \\
\text { Particle Conc. }\end{array}$ & $\begin{array}{c}\text { Density } \\
\boldsymbol{a}_{\mathbf{2 5}}{ }^{\circ} \mathbf{C}\end{array}$ & $\begin{array}{c}\text { Viscosity } \\
\boldsymbol{(} \mathbf{2 7} 7^{\circ} \mathbf{C}\end{array}$ \\
\hline $10 \mathrm{~nm}$ & $\begin{array}{c}11 \mathrm{mT} \\
( \pm 10 \%)\end{array}$ & $2 \mathrm{vol} \%$ & $\begin{array}{c}890 \\
\mathrm{~kg} / \mathrm{m}^{3}\end{array}$ & $2.0 \mathrm{mPa}-\mathrm{s}$ \\
\hline
\end{tabular}

consists of a thin slice of single-crystal, piezoelectric quartz, with very large lateral dimensions in comparison to its thickness, which is sandwiched between two metal electrodes. Application of a sinusoidal electric field using the electrodes sets up a shear wave through the thickness of the quartz [5]. At resonance, the equivalent electrical circuit of the QCM is modeled as consisting of a capacitor $C_{0}$ in parallel with the motional arm of the circuit consisting of a series circuit of $L_{m}, C_{m}$, and $R_{m}$ and is commonly referred to as the Butterworth Van Dyke (BVD) model. The exceptionally low phase noise characteristic exhibited by quartz resonators allows for a precision of few parts in $10^{14}$ and is the primary reason for their widespread use in frequency control applications. The phase noise is quantitatively specified in terms of the $Q$-factor defined as the half width at the full maximum of the resonance curve or in terms of an energy dissipation factor $D$, which is inversely proportional to the decay time constant and is also equal to the $1 / Q$-factor. The resonance frequency and the $Q$ factor of the resonance are significantly affected by any surface load placed on the quartz crystal. Thus, QCRs are widely used as sensors to determine the properties of deposited layers on top such as surface mass density and recently the viscoelastic properties [6].

The QCM frequency shift resulting from the deposition of viscoelastic layer in a viscous liquid ambient can be analyzed using a continuum mechanics approach [7]. In order to model this situation, the QCM surface is considered to be in intimate contact with a layer of continuous viscoelastic slab with an infinitely thick Newtonian liquid overlayer on one of its surfaces. Under the assumption that the thickness of the bulk liquid layer is much larger than the decay length of the acoustic wave in the liquid, analytical expressions for the frequency, $Q$-factor, and dissipation factor changes with respect to liquid loading conditions can be derived [8]. Our previous work has demonstrated that the high frequency micromachined quartz resonators are especially suited for monitoring small changes in the viscoelastic loading in films adsorbed on their surface. Figure 1 compares the dependence of the frequency and $Q$-factor shift as a function of the viscosity and elastic modulus of a $35 \mathrm{~nm}$ thick soft polymer like film adsorbed on a commercial $5 \mathrm{MHz}$ and micromachined $65 \mathrm{MHz}$ resonators. It is clear that miniaturized resonators provide a greater resolution of these properties. Water-like liquid overlayer is assumed in these graphs. The miniaturized resonators provide a greater resolution for these properties. A large variation in Q-factor and frequency for small changes in the viscoelastic properties of the adsorbed films is highly desirable for accurate real-time monitoring of viscoelastic changes of the ferrofluids.

From the BVD model, the electrical admittance $Y$ of the resonator can be simply written as

$Y=G+j B=j B_{0}+Y_{m}$

where $B_{0}=\omega C_{0} ; Y_{m}=\frac{1}{R_{m}+j X_{m}} ; X_{m}=\omega L_{m}-\frac{1}{\omega C_{m}}$

$G$ and $B$ are the real and imaginary parts of admittance respectively, $B_{0}$ is the admittance of the parallel (static) arm, and $Y_{m}$ is the admittance of the motional arm consisting of series connected $R_{m}, L_{m}$ and $C_{m}$ the motional resistance, inductance and capacitance respectively. From this equation, it is possible to write 

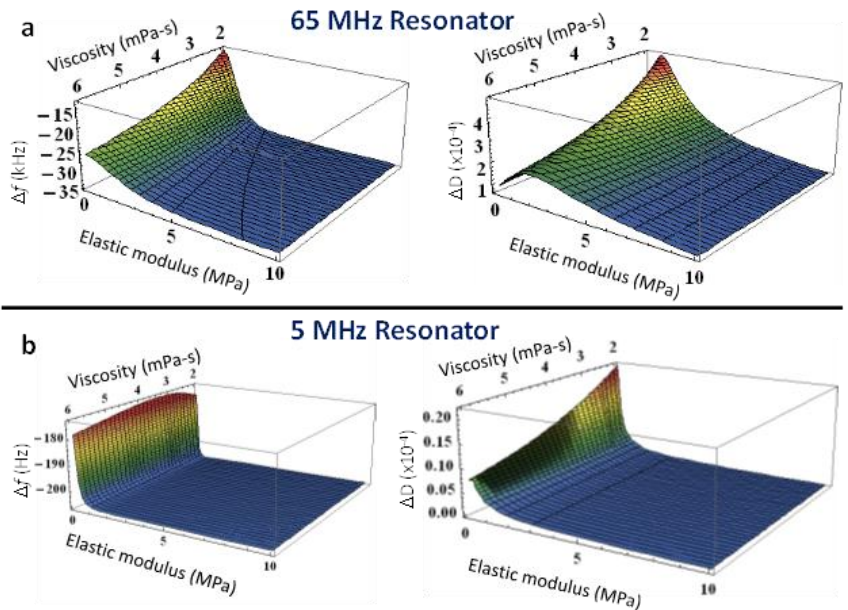

Figure 1: Comparison of the frequency and dissipation factor sensitivity of (a) $65 \mathrm{MHz}$ and (b) $5 \mathrm{MHz}$ resonators to variations in the viscosity and modulus of the adsorbed films. (Film thickness $=$ $35 \mathrm{~nm}$ and density $=1040 \mathrm{~kg} / \mathrm{m}^{3}$ have been assumed here). Clearly, the $65 \mathrm{MHz}$ resonator has a better resolution over wider range of values of these properties.

the following parametric representation is possible

$$
\left(G-\frac{1}{2 R m}\right)^{2}+\left(B-B_{0}\right)^{2}=\left(\frac{1}{2 R_{m}}\right)^{2}
$$

Thus tracking the peak of the real part of the admittance allows for a direct measurement of the motional resistance or realtime tracking of the damping effect on the quartz resonator arising due to the changing viscosity of the ferrofluid due to applied magnetic field. In this work the -QCR array electrical characteristics, affected by the magnetoviscous effect in ferrofluids, were quantitatively measured by the real-time tracking of the maximum point on the admittance curve, which in turn enables to monitor and quantify the magnetic field strength changes in real-time. In order to increase the sensitivity of the magnetometer, the -QCR is coated with an ultra-high permeability Metglas ${ }^{\circledR}\left(\mathrm{Fe}_{85} \mathrm{~B}_{5} \mathrm{Si}_{10}\right)$ amorphous thin film. Thin film of Metglas ${ }^{\circledR}$ acts as a flux concentrator [9], which is explained and discussed in the following sections in detail.

\section{THE PRINCIPLES OF MAGNETOMETER OPERATION}

\section{Device Configuration}

The -QCR array used in this work is composed of 8 resonators with each having $500 \mathrm{~m}$ diameter circular electrode. The quartz thickness in between the electrodes is $\sim 25 \mathrm{~m}$, which was etched to this final thickness from a $\sim 100 \mathrm{~m}$ thick AT-cut quartz substrate using reactive ion etching in the $\mathrm{SF}_{6}$ and Argon plasma. In this work only one pixel was utilized for the magnetometer tests, although the other pixels may be used to determine the direction of the magnetic field in future experiments. Figure 2(a) shows 3-D schematic rendering of one pixel of the array with ferrofluid loading. The device is packaged into a modified dual-in-line ceramic package with a $5 \times 5 \mathrm{~mm}$ reservoir for the ferrofluid loading. Figure 2(b) depicts the cross sectional view of the pixel with the packaging.

A cylindrical Neodymium magnet having a diameter larger than the resonator electrode area is placed in close proximity to the resonator such that the magnet provides a uniform DC magnetic bias in $+Y$-direction. This magnetic field coalesces the nanoparticles in the ferrofluid to form ordered agglomerate atop the resonator electrode, which can then be treated as a viscoleastic layer. A weak-to-moderate bias introduces some degree of columnar aggregation and orientation within the ferrofluid. It is found and experimentally shown that the low level of orientation enhances the sensitivity of the device due to enhanced magnetoviscous effect. Once a particular level of aggregation exists, the viscosity changes due to magnetic field strength are sharper and more evident [2].

The application of a periodic magnetic field in $+\mathrm{X}$ direction (in the plane of the quartz resonator electrode as shown in Fig. 2.(b) to the device, results in continuous perturbation in the ordering arrangement of the ferrofluid aggregate and therefore induces a continuous change in the viscoelastic response of the quartz resonator admittance maximum. In addition, the applied magnetic field can change the separation distance between ordered structures alter and thus the packing density could also be altered [4]. The authors postulate that the viscosity change within the fluid is mainly a function of this separation distance, which is highly sensitive to low amplitude changes in the applied magnetic field as well as its frequency [4].
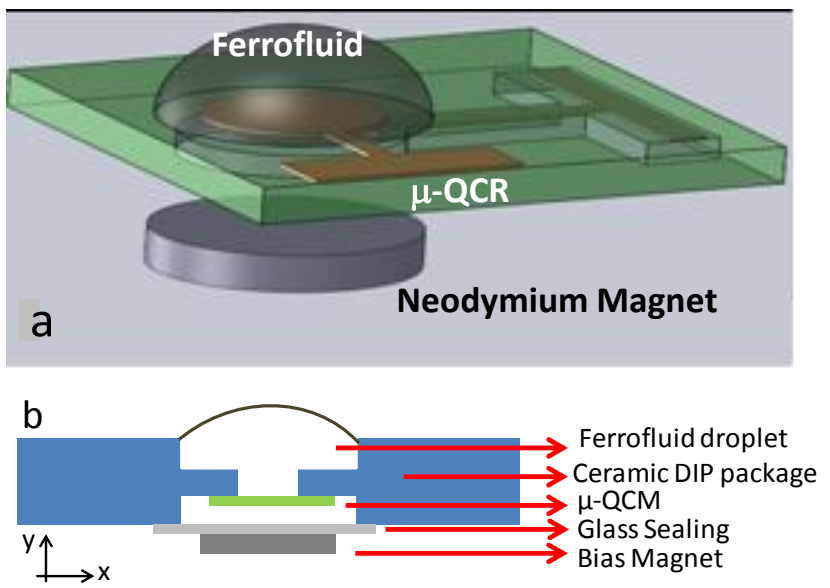

Figure 2: a) The magnetometer configuration (cross sectional view). b) $3 D$ schematic showing the resonator, magnet,and ferrofluid drop.

Figure 3 shows the experimental setup above. The Helmholtz coil has a diameter of $15 \mathrm{~cm}$ and 140 turns $(\mathrm{N}=140)$. The magnetic field is precisely controlled via the current. The $\mu$-QCR admittance characteristics were monitored in real-time using an Agilent 4294A impedance analyzer. The admittance maxima of the device was tracked and recorded as a function of the applied magnetic field.

\section{Metglas as a Flux Concentrator}

The working electrode, the top electrode in Fig. 2(a) is coated with $20 \mathrm{~nm} \mathrm{Ti} / 100 \mathrm{~nm}$ of Metglas ${ }^{\mathbb{B}}$ via ion beam deposition. The resonator area is lithographically patterned and delineated using lift-off process. Metglas ${ }^{\circledR}$ is an amorphous alloy, consisting of $\mathrm{Fe}_{85} \mathrm{~B}_{5} \mathrm{Si}_{10}$ and has a very high relative magnetic permeability $(r)$ of 45000 . This property of Metglas ${ }^{\circledR}$ is utilized to concentrate the low amplitude magnetic flux vectors into the thin film of Metglas ${ }^{\circledR}$.

Figure 4(a) shows a 2D COMSOL magnetostatics model demonstrating the flux concentration effect. A thin strip of 


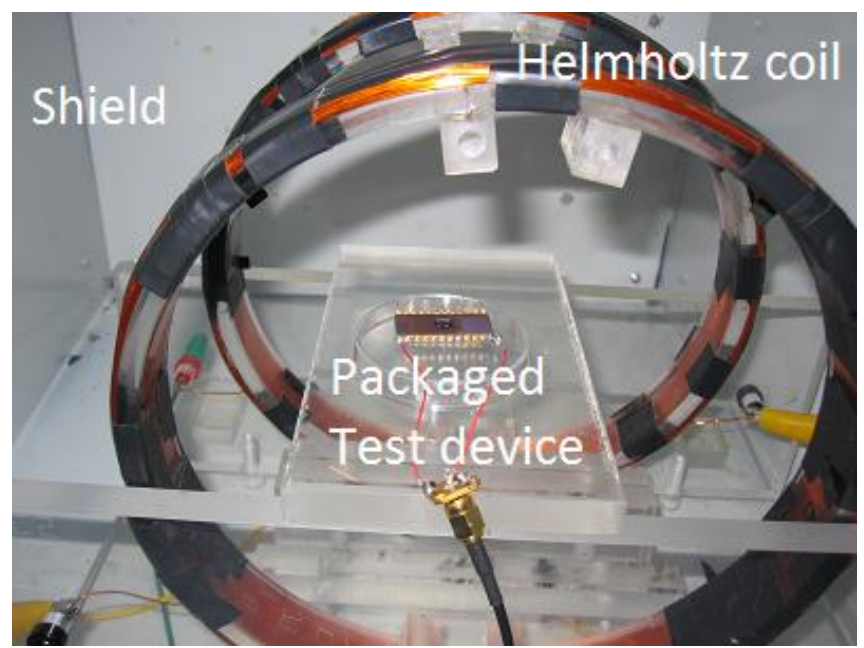

Figure 3: The Experimental Setup showing the shield, the packaged test device in DIP ceramic package and the Helmholtz coil. The -QCR array without the ferrofluid atop is unresponsive to magnetic field.

$100 \mathrm{~nm} \mathrm{Metglas}{ }^{\circledR}$ is assumed to be surrounded by air. The concentration effect is analyzed without the DC bias magnetic field from the neodymium magnet under the QCR in order to focus on the concentration effect in $+X$-direction, which is the direction of the applied magnetic field. From the left boundary of the domain to the right boundary of the domain, a uniform magnetic flux of $\sim 100 \mathrm{nT}$ is applied. As it is observed, the flux lines start to bend at the regions closer to Metglas ${ }^{\circledR}$ due to its high $r$. Moreover, the flux lines start to concentrate gradually within the length of the thin strip, reaching to a maximum in the mid section. This is shown in Fig. 4(b) where, the magnitude of the magnetic flux along the strip is plotted. The maximum peak flux that is $\sim 375 \mathrm{~T}$ corresponds to the middle point $(\mathrm{X}=1.5 \mathrm{~mm})$ of the $100 \mathrm{~nm}$ thick Metglas $^{\circledR}$ film. The flux magnitude sharply decreases to the applied uniform field that is $100 \mathrm{nT}$ at the outer regions of the Metglas ${ }^{\circledR}$ film at far ends which is $0.5 \mathrm{~mm}$ away from left and right sides of the film. Therefore, $100 \mathrm{nT}$ of the applied magnetic flux is concentrated within the Metglas ${ }^{\circledR}$ thin film to a maximum value of $\sim 375 \mathrm{~T}$, corresponding to a three orders of magnitude of enhancement. This flux concentration and enhancement aids to amplify the small magnitude changes of the applied field and therefore the sensitivity of the device. Therefore, the ferroparticles within the ferrofluid respond better to amplified magnetic field and agglomeration/deglomeration happen more effectively. Consequently, the quantification of the weak magnetic field via the

-QCR becomes more sensitive and feasible through the concentration effect.

\section{RESULTS \& DISCUSSION}

As it is emphasized above, the device is characterized by applying a square wave of magnetic field in $+\mathrm{X}$-direction (see Fig. 2(b)) through the Helmholtz coil. The characterization focuses on nano-Tesla region. The device response is tracked for randomly selected magnetic field square inputs (50\% duty cycle) of $720 \mathrm{nT}$, $360 \mathrm{nT}, 180 \mathrm{nT}, 130 \mathrm{nT}$ and $60 \mathrm{nT}$. Figure 5 shows the device response for selected magnetic fields of $720 \mathrm{nT}, 360 \mathrm{nT}$ and $60 \mathrm{nT}$ for a single on/off cycle. As the magnetic field is switched on, the admittance peak increases spontaneously to its maximum amplitude. When the field is off, the admittance peak decreases to its minimum value. A possible explanation for this admittance shift
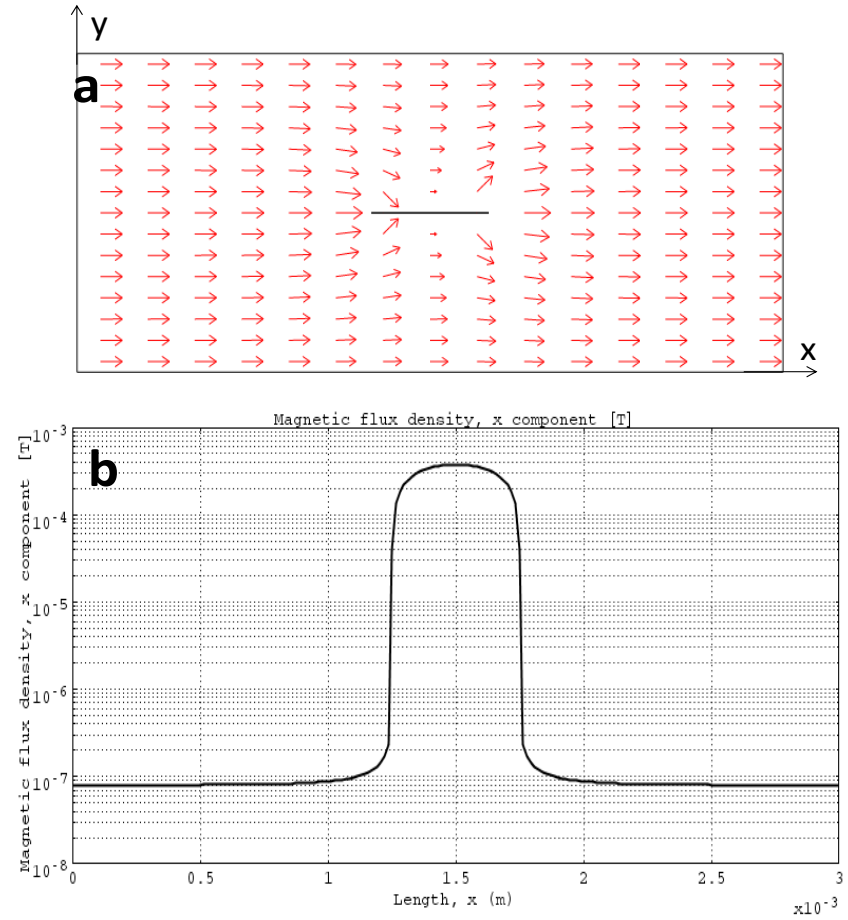

Figure 4: (a) The interaction of the magnetic flux lines with high relative permeability Metglas ${ }^{\circledR}$ thin film. A uniform field of 100 $n T$ is applied in $+x$ direction. The total length of the domain is 3 $\mathrm{mm}$ (b) The concentration effect is maximum in the middle (at $x=1.5 \mathrm{~mm}$ ) of the thin strip. The flux is concentrated about 3 orders of magnitude.

is the decreasing viscosity of the ferrofluid emulsion upon the application of the magnetic field, which perturbs the ordered state from the DC bias field. It can be thought to arise due to the increased interaction of the ferrofluid particles leading to a more tightly bound state in the presence of the magnetic field. This could arise either due to the spontaneous arrangement of the ferrofluid particles into an ordered structure or through tighter interaction leading to a more rigid network. When the magnetic field is switched off, the ferrofluid particles rapidly lose the ordered state due to their thermal energy thereby increasing the viscosity of the ferrofluid. That is why the role of the DC magnetic bias field is very important in this experiment and depending on its strength can change the response of the ferrofluid to the incident magnetic field.

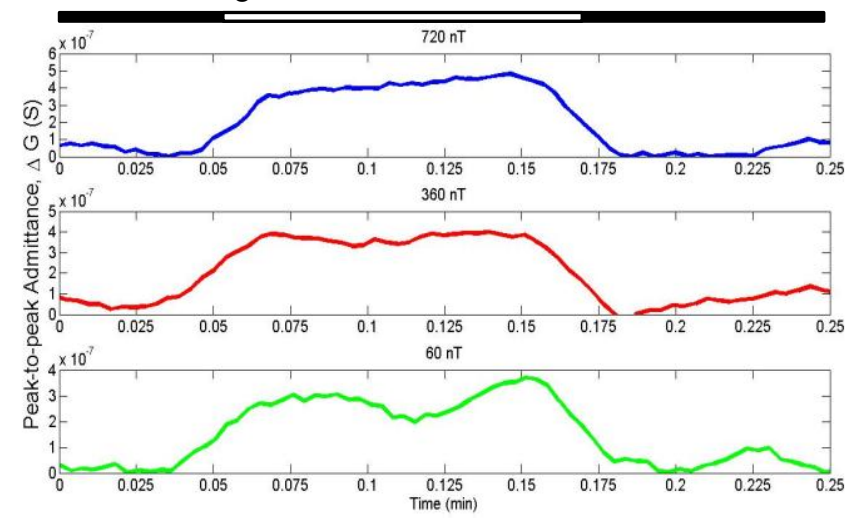

Figure 5: Maximum admittance vs time. As the magnetic field is switched on (indicated with the white bar on top), the viscosity is decreased and admittance increases. 


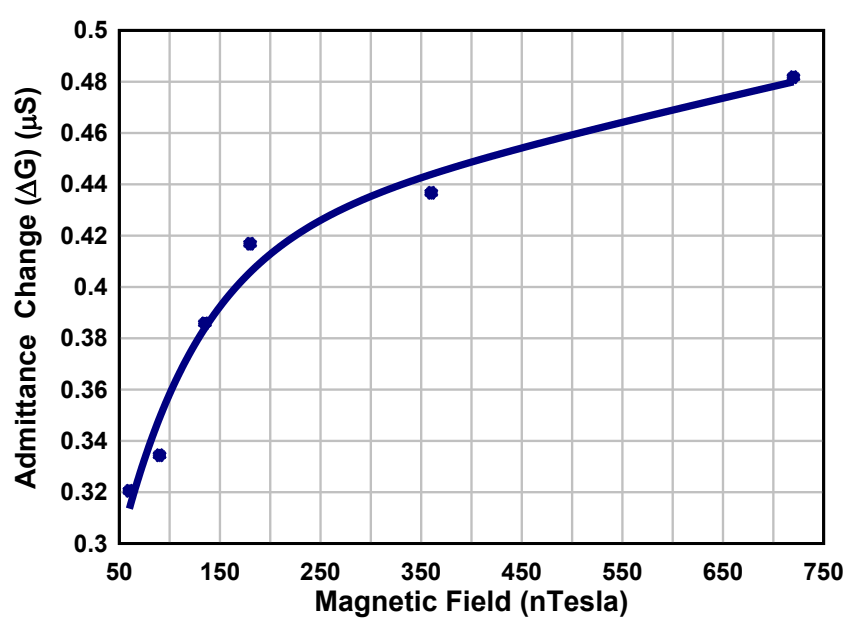

Figure 6: Peak-to-peak admittance for different square wave magnetic field amplitudes. A smooth exponential fit has been used.

The device was further characterized at the selected magnetic fields by quantifying the peak-to-peak change in resonator admittance, $\Delta G$, obtained from Fig. 5. A single value is chosen for on and off values using averaging. The peak-to-peak change in resonator admittance versus the magnetic field is plotted in figure 6 . The resonator shows a non-linear response. For the experimental conditions used, the admittance change due to magnetic field amplitude showed a sharper slope between $60 \mathrm{nT}$ and $200 \mathrm{nT}$ than at higher fields.

Despite the fact that the lowest magnetic field amplitude that has been detected is $60 \mathrm{nT}$ with a SNR of $\sim 3: 1$ with the current experimental setup and facilities, the magnetometer described here has the potential for sub-nanoTesla resolutions at room temperatures. Nevertheless, the questions relating to the linearity of response, the optimal bias magnetic field (magnitude and orientation), and the ultimate sensitivity of the sensor need to be further investigated. The other pixels can be utilized to determine the direction of the magnetic field in future studies.

\section{CONCLUSION}

In this work, a novel scalar magnetometer is proposed. The magnetometer utilizes: (i) ferrofluids and their mangetoviscous properties as the sensing mechanism, (ii) the highly sensitive QCR to detect and quantify the magnetoviscous effects, (iii) the flux concentration effect to amplify the weak magnetic field via the deposition of an ultra-high permeability thin film on working electrode, and (iv) real time magnetic field measurement by tracking the peak admittance. This study and device configuration can be also considered as a new study of the high frequency perturbation characteristics of ferrofluids.

\section{ACKNOWLEDGEMENT}

This work was supported in part by the U.S. Army Research Office under Grant W911NF-07-1-0327 and in part by the National Science Foundation (NSF) under Grant ECCS 0925438. The use of facilities at the PSU Site of the NSF National Nanotechnology Infrastructure Network (NNIN) under Agreement 0335765 is acknowledged. The authors thank to Feng Li for sputtering Metglas on the -QCR. GH acknowledges partial support from Scientific and Technical Research Council of Turkey (TUBITAK) for a 2213-International PhD. Fellowship Program.

\section{REFERENCES}

[1] V. S. Mendelev and A. O. Ivanov, "Ferrofluid aggregation in chains under the influence of a magnetic field," Physical Review E, vol. 70, p. 051502, 2004.

[2] S. Odenbach and S. Thurm, "Magnetoviscous Effects in Ferrofluids." vol. 594, S. Odenbach, Ed., ed: Springer Berlin / Heidelberg, 2003, pp. 185-201.

[3] M. Ivey, et al., "Magnetic-field-induced structural transitions in a ferrofluid emulsion," Physical Review E, vol. 63, p. 011403, 2000.

[4] C.-Y. Hong, et al., "Evidence of multiple states of ordered structures and a phase transition in magnetic fluid films under perpendicular magnetic fields," Applied Physics Letters, vol. 75, pp. 2196-2198, 1999.

[5] S. J. Martin, et al., "Characterization of a quartz crystal microbalance with simultaneous mass and liquid loading," Analytical Chemistry, vol. 63, pp. 2272-2281, 1991/10/01 1991.

[6] P. Kao, et al., "Human Serum Albumin Adsorption Study on 62-MHz Miniaturized Quartz Gravimetric Sensors," Analytical Chemistry, vol. 80, pp. 5930-5936, 2008/08/01 2008.

[7] M. V. Voinova, et al., "Viscoelastic Acoustic Response of Layered Polymer Films at Fluid-Solid Interfaces: Continuum Mechanics Approach," Physica Scripta, vol. 59, p. 391, 1999.

[8] P. Kao, et al., "Fabrication and performance characteristics of high-frequency micromachined bulk acoustic wave quartz resonator arrays," Measurement Science and Technology, vol. 20, p. 124007, 2009.

[9] Z. Fang, et al., "Enhancing the magnetoelectric response of Metglas/polyvinylidene fluoride laminates by exploiting the flux concentration effect," Applied Physics Letters, vol. 95, p. 112903, 2009.

\section{CONTACT}

*S. Tadigadapa, Tel: +1-814-865-2739; sat10@.psu.edu 


\title{
SENSITIVE UNCOOLED IR DETECTORS USING GALLIUM NITRIDE RESONATORS AND SILICON NITRIDE ABSORBERS
}

\author{
Vikrant J. Gokhale ${ }^{l}$, John Roberts ${ }^{2}$ and Mina Rais-Zadeh ${ }^{l}$ \\ ${ }^{1}$ Department of Electrical Engineering and Computer Science, \\ University of Michigan, Ann Arbor, Michigan, USA \\ ${ }^{2}$ Nitronex Corporation, Durham, North Carolina, USA
}

\begin{abstract}
This paper presents the theory and measured results of a lownoise un-cooled infrared (IR) detector, which uses a combination of piezoelectric, pyroelectric, electrostrictive, and resonant effects to achieve high sensitivity. The sensor consists of a high- $Q$ gallium nitride $(\mathrm{GaN})$ micro-mechanical resonator coated with an IR absorber layer. The IR absorber converts the IR energy into heat. The generated heat causes a shift in the frequency characteristics of the $\mathrm{GaN}$ resonators. IR detection is achieved by sensing the shift in the resonance frequency of the exposed $\mathrm{GaN}$ resonator as compared to a reference resonator. A prototype GaN-based IR sensor is implemented, showing a frequency shift of $400 \mathrm{~Hz}$ per

$\mathrm{W}$ of absorbed power. The change in the beat frequency upon IR radiation is $1830 \mathrm{ppm} / \mathrm{W}$, making it possible to sense IR radiation in nano watts range.
\end{abstract}

\section{INTRODUCTION}

The detection of infrared radiation is generally accomplished using either photonic or thermal detectors, both of which have generated a large amount of research over the last two decades [1]. The relative disadvantages of thermal detectors in terms of detectivity and response time are balanced by the fact that they do not require cooling systems, reducing the system weight, power consumption, and form factor. Furthermore, thermal detectors are better suited for radiation extending into the far wavelength IR, which is extremely useful for optical astronomy. While bolometric devices have been the most popular thermal sensors commercially, there is an increasing interest in resonant IR detectors [2]-[4]. This work presents a GaN-based resonant IR sensor that utilizes the unique material properties of $\mathrm{GaN}$ to achieve high sensitivity. A reference resonator is included in the system for self-calibration (Fig. 1). Fig. 2 shows a representative scanning electron micrograph (SEM) of a GaN detector.

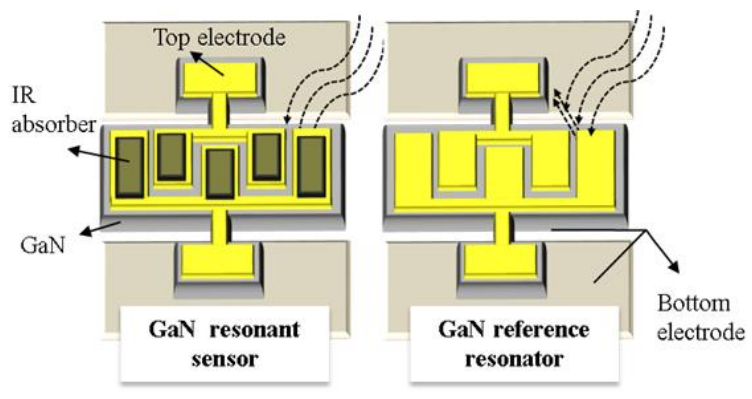

Figure 1: Schematic of a resonant sensor and a reference pair with interdigitated finger electrodes. The sensor is coated with an absorber layer. The reference resonator is either not coated with the absorber or the absorber layer on the reference resonator is blocked using the top electrode.

\section{THEORY OF OPERATION}

\section{General Resonant IR detectors}

In thermal IR detectors, radiation incident on the surface of the device increases its temperature. The conversion of incident IR

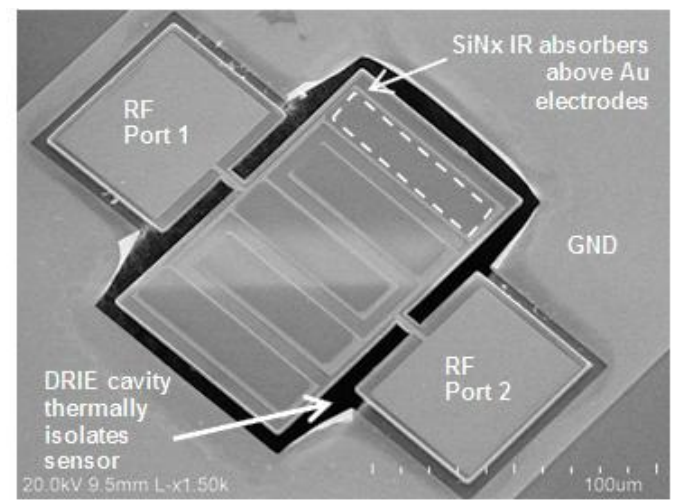

Figure 2: A SEM image of a fabricated GaN resonator with silicon nitride absorber. The dimensions of the device are 120 $\mu \mathrm{m} \times 80 \mu \mathrm{m} \times 2.15 \mu \mathrm{m}$. The tethers are $4 \mu \mathrm{m} \times 10 \mu \mathrm{m}$.

radiation power $\phi(\lambda)$ into temperature change is given by [3]

$$
\Delta T=\frac{\eta(\lambda) \phi(\lambda)}{\sqrt{G_{t h}^{2}+\omega^{2} C_{t h}^{2}}},
$$

where $G_{t h}$ is the effective thermal conductance of the device (dominated by the thermal conductance of the tethers), $C_{t h}$ is the thermal capacity of the device, $\omega$ is the rate of change of the incident signal, and $\eta(\lambda)$ is the absorption efficiency of the IR absorber layer. In resonant IR sensors, the resultant change in the resonator physical dimensions due to thermal expansion and the change in the elasticity of the resonator material are the main factors in changing the resonant frequency of the device [3]. The temperature-induced change in frequency is quantified by the wellknown temperature coefficient of frequency (TCF). Broadly speaking, the temperature dependence of the material elasticity is the dominant factor in setting the TCF and puts an upper bound on the thermal sensitivity of the resonator. By designing the device in a dually-clamped configuration and allowing it to buckle with a change in temperature, it is possible to increase the temperature sensitivity beyond TCF. However, the non-linear nature of this buckling and the fabrication challenges involved in ensuring reliable and repeatable fabrication of devices near the buckling point make this a prohibitive exercise. A second way to increase the temperature sensitivity is to use a material that has an inherently large response to heat. Pyroelectric materials are suitable candidates for this application: a pyroelectric thin film generates a spontaneous surface charge and thus a potential difference across the thickness of the open-circuit film when the temperature of the film changes (e.g., upon IR radiation). The relationship between the pyroelectric voltage and the change in temperature of a film with thickness of $t$ is given by,

$$
V=P_{V} t \Delta T
$$

Most pyroelectric materials are also piezoelectric. In a resonator made from a pyro/ piezoelectric material, this voltage $V$ can change the elastic properties of the resonator through linear 
and second-order (electrostrictive) piezoelectric interactions. In this work, we use a combination of piezo and pyroelectric effects to achieve large temperature sensitivities in resonant IR sensors.

\section{GaN Resonant IR detectors}

$\mathrm{GaN}$ is an excellent choice of material for the proposed resonant IR sensor. GaN is a single crystal wurtzite material that can be grown as a high quality thin film using metallo-organic chemical vapor deposition (MOCVD) or molecular beam epitaxy (MBE). The piezoelectric nature of $\mathrm{GaN}$ has been used to make mechanical resonators in the past by the authors [5], [6] and other researchers [7], [8]. Along with this, $\mathrm{GaN}$ is known to be a pyroelectric, with a theoretical pyroelectric voltage coefficient $\left(P_{v}\right)$ of $7 \times 10^{5} \mathrm{~V} / \mathrm{m}-\mathrm{K}$, and measured $P_{v}$ as high as $1 \times 10^{4} \mathrm{~V} / \mathrm{m}-\mathrm{K}[9]$. $\mathrm{GaN}$ is also electrostrictive [10], [11]. In $\mathrm{GaN}$, it has been found that a significant quadratic (electrostrictive) dependence of strain on electric field exists. Willatzen and Lew Yan Voon considered the case of a $\mathrm{GaN}$ thin film with $\mathrm{AC}$ and $\mathrm{DC}$ fields superimposed and derived the effect of the electrostriction on the acoustic velocity $v$ of the material [11]. Following their analysis for the acoustic velocity along the resonator length, we get,

$$
v=\sqrt{\frac{\epsilon_{31}^{\prime} c_{11}+e_{31} e_{31}^{\prime}}{\rho\left(\epsilon_{31}^{\prime}+2 M_{31} E_{3} e_{31}^{\prime}\right)}},
$$

where $\epsilon_{31}^{\prime}=\left(\epsilon_{31}-M_{31} E_{3} e_{31}\right), e_{31}^{\prime}=\left(e_{31}+M_{31} E_{3} c_{31}\right)$, and the coefficients $\epsilon, e$ and their corresponding primed counterparts represent permittivity and piezoelectric strain tensors, respectively. $M_{31}$ is the electrostrictive transverse coefficient and $E_{3}$ is the electric field across the thickness of the film. The change in the acoustic velocity is directly related to the resonant frequency. For a length-extensional resonator we have

$$
f=\frac{n}{2 L} \sqrt{\frac{C_{e f f}}{\rho}}=\frac{n v}{2 L},
$$

where $C_{e f f}$ is the effective stiffness, $\rho$ is the mass density, and $n$ is the mode of operation. Based on published values for the material properties of $\mathrm{GaN}$ and a $M_{31}$ value of $-6 \times 10^{-19} \mathrm{~m}^{2} \mathrm{~V}^{-2}$, we get the relative frequency sensitivity to voltage as large as -1000 ppm/V (Fig. 3).

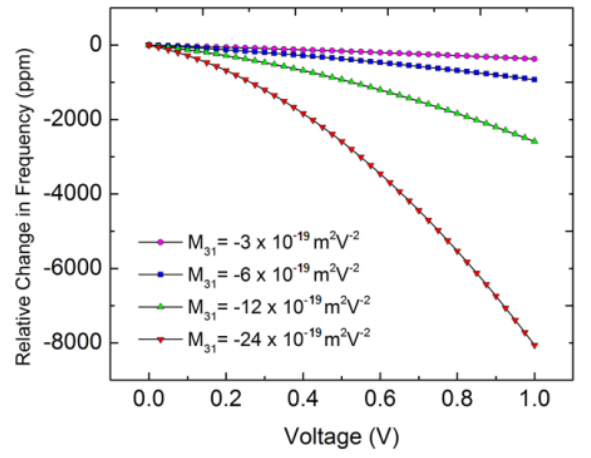

Figure 3: Predicted values of resonant frequency change due to electrostriction. For $M_{31}=-6 \times 10^{-19} \mathrm{~m}^{2} / V^{2}$ (half of $M_{33}$ ) the relative frequency change is $-1000 \mathrm{ppm} / V$ (The accurate value of $M_{31}$ is not known). The plot is derived based on the theoretical model for electrostriction in GaN [11].

The pyroelectric-electrostrictive interaction of $\mathrm{GaN}$ can be utilized as the detection mechanism for implementing resonant IR detectors. For GaN resonators presented in this work, with a thickness of $2.15 \mathrm{~m}$, and assuming a conservative value of $1 \times 10^{5}$ $\mathrm{V} / \mathrm{m}-\mathrm{K}$ for $P_{v}$, a temperature induced frequency shift of -215 $\mathrm{ppm} / \mathrm{K}$ can be achieved, which is an order of magnitude greater than the measured TCF for $\mathrm{GaN}$ resonators (i.e., $-17.7 \mathrm{ppm} / \mathrm{K}$ [5]). Thus, along with the TCF, the electrostrictive perturbation in GaN can be explored to enhance the sensitivity of thermal detectors.

\section{Sensor Scheme}

The IR sensors must be designed to work reliably at conditions that are sub-optimal for performance, in the presence of interfering signals due to shock, acceleration, and changes in ambient temperature. The best way to cancel out these interfering effects is to use a reference resonator that is similar to the sensor in all ways except one: the surface of the reference resonator does not have the absorber layer exposed. The small amount of density difference caused by the absorber and the expected variations in fabrication offset the frequency of the reference by a small amount, as compared to the sensor. This difference is enough to cause a small beat frequency which can be used as a reference to compare the frequency shift of the sensor with. In order to reduce this beat frequency, further design iterations of the reference can have the absorber layer, but coated with half the thickness of the top electrode. The frequencies of the two resonators can be described as functions of temperature by [12]

$$
\begin{aligned}
& f_{s}\left(T_{s}\right)=f_{s}\left(T_{0}\right)+A_{s} \Delta T_{s}+B_{s} \Delta T_{s}{ }^{2}+\cdots \\
& f_{r}\left(T_{r}\right)=f_{r}\left(T_{0}\right)+A_{r} \Delta T_{r}+B_{r} \Delta T_{r}{ }^{2}+\cdots
\end{aligned}
$$

Neglecting non-linear temperature dependence, we can write the beat frequency as

$$
f_{B}(T)=f_{s}\left(T_{s}\right)-f_{r}\left(T_{r}\right)=\left[A_{s} \Delta T_{s}-A_{r} \Delta T_{r}\right]+f_{B}\left(T_{0}\right)
$$

The subscripts $s, r$, and $B$ indicate the sensor, reference, and beat frequencies, respectively. $T_{0}$ is the initial temperature. The coefficients $A_{i}$ are the net coefficients of frequency change with respect to temperature, inclusive of all contributing mechanisms. Thus, the relative change in the beat frequency is a function of the incident IR radiation (Eq. 8). The beat frequency and the change thereof are easily measured using a mixer [12].

IR sensitivity $=\frac{\Delta f_{B} / f_{B}\left(T_{0}\right)}{\phi(\lambda)}=\frac{\left[A_{S} \Delta T_{S}-A_{r} \Delta T_{r}\right] / f_{B}\left(T_{0}\right)}{\phi(\lambda)} \approx \frac{A_{s} \eta(\lambda)}{G_{t h} f_{B}\left(T_{0}\right)}$

\section{EXPERIMENTAL RESULTS \\ Measurement setup}

The experimental setup for measuring the frequency response of the GaN resonators is shown in Fig. 4.

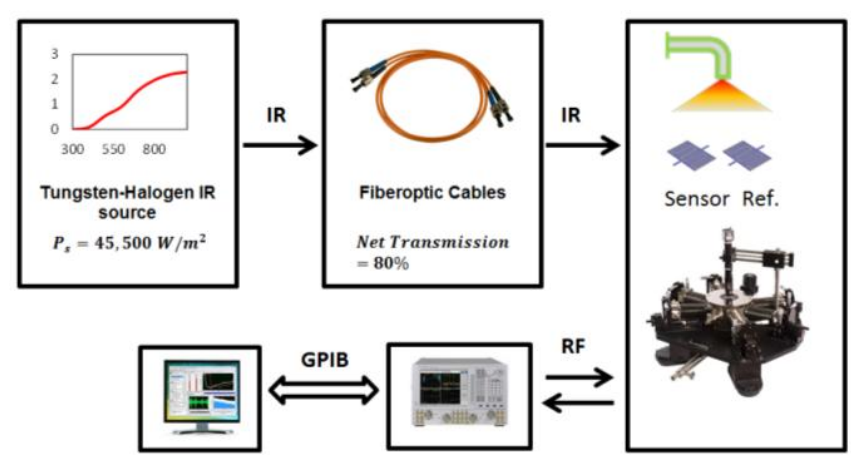

Figure 4: Schematic showing the IR measurement setup of the GaN resonators. Both sensor and reference resonators are measured using an identical procedure. 
The GaN sensor is placed in a temperature controlled probe station. IR energy is provided by a Tungsten-Halogen lamp and is coupled into the probe station using optical fibers. The RF performance of the GaN resonators is measured using an Agilent Vector Network Analyzer.

\section{Absorber Efficiency}

As seen in Equation (8), a significant factor in setting the sensitivity is the absorptivity $\eta(\lambda)$ of the IR absorber layer. GaN is only selectively absorbing at UV wavelengths. Furthermore, the metal electrodes needed for piezoelectric actuation would have highly reflecting surfaces. For example gold is almost perfectly reflecting at any thickness above $17 \mathrm{~nm}$ [13]. One solution is to coat the electrodes with a material with higher absorptivity. In this work, the GaN resonators are coated with low temperature PECVD silicon nitride $\left(\mathrm{SiN}_{\mathrm{x}}\right)$. Silicon nitride has an absorption efficiency of about 0.1 to 0.2 in the near IR spectrum (Fig. 5). To improve sensitivity, the absorber layer can be made out of carbon nanotubebased nanocomposites with an absorption coefficient of more than 0.9 over a broad spectrum [14].
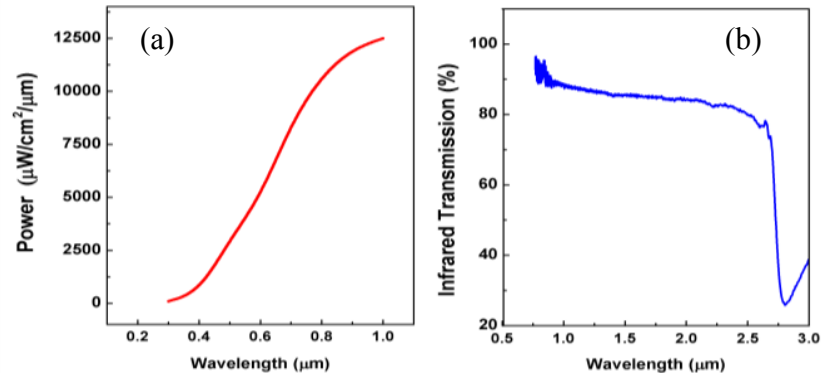

Figure 5: (a) Spectral distribution of the power supplied by the IR source. (b) Transmission spectrum for a $50 \mathrm{~nm}$ thick $S_{i} N_{x}$ film. In the relevant spectral range, $<1 \mu \mathrm{m}$, the film absorbs 10 to $20 \%$ of incident IR power. More efficient broadband absorbers can further improve the device sensitivity.

The nominal RF response of the resonator and the subsequent change when it is subject to IR radiation is given in Fig. 6. Based on the power output spectrum of the IR source, and taking into consideration fiber losses, we can calculate the power absorbed by the sensor to be 56 W. Fig 7 shows the difference in the frequency response of this sensor and a reference resonator (without the coating). From Fig. 7, the relative shift in beat frequency is $22 \mathrm{kHz}$, which gives a sensitivity of $\sim 1830 \mathrm{ppm} / \mathrm{W}$. The change in the reference frequency is only $\sim 2 \mathrm{kHz}$, uncorrelated to the IR signal, and due to random fluctuations. This gives us a minimum detectable power of $\sim 9 \mathrm{nW}$.

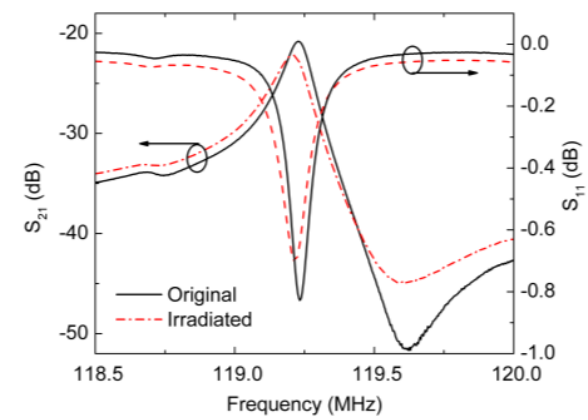

Figure 6: The change in the frequency response of a $160 \mu \mathrm{m} \times 80$ $\mu \mathrm{m} \times 2.15 \mu \mathrm{m}$ GaN resonator operated at its $5^{\text {th }}$ length-extensional mode upon radiation with $\sim 56 \mu \mathrm{W}$ IR power.
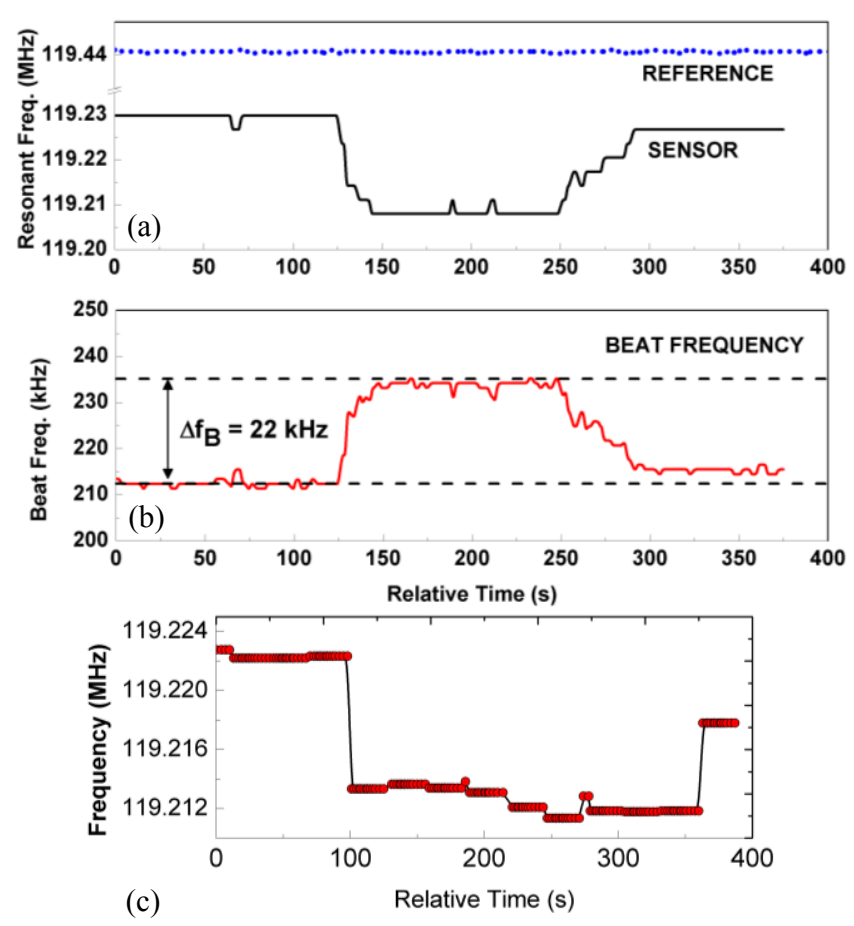

Figure 7: (a) The change in frequency of the sensor and a reference resonator on application of the IR signal. The reference resonator is identical to the sensor, except it does not have the SiNx coating. Beat frequency between the two exhibits a large change of $-21.8 \mathrm{kHz}(-183 \mathrm{ppm})$. The change in the beat frequency upon IR radiation is $10 \%$ of the original value. This can be readily detected using a mixer. (c) Faster scans reduce the overhead time due to the measurement system, but do not reduce parasitics.

The slow response seen in Fig. 7 is not the characteristic of the device, but rather of the thermal parasitics due to the probes, measurement speed, and delays inherent in the data acquisition system. We can verify this by acquiring faster (but less sensitive) scans (Fig. 7 (c)).
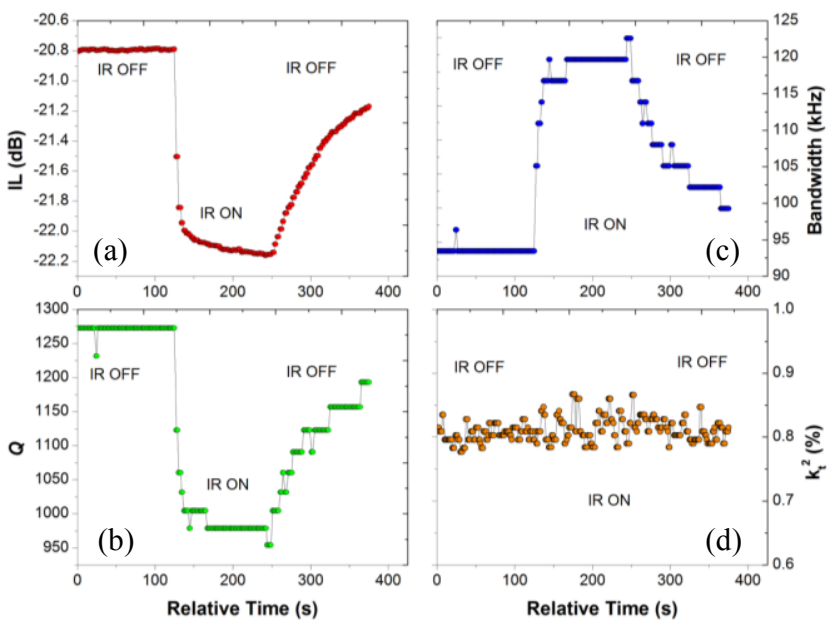

Figure 8: Figure 2: (a) Insertion loss, (b) Q, (c) bandwidth and (d) effective coupling coefficient $\left(k_{t}^{2}\right)$ of the resonator as functions of the incident IR signal. These quantities can be used in alternate measurement schemes instead of frequency sensing.

Apart from the frequency, the resonator undergoes various other changes in its performance (Fig. 8) [15], [16]. The $Q$ and the 
loss both degrade, and the bandwidth increases due to the addition of the thermal energy to the resonant system. Changes in these parameters can be used in different types of sensing schemes. Table 1 shows relevant shifts in resonator parameters for three measured sensors. The measurement conditions are the same for all devices. The important metrics for comparison of thermal detectors are the detectivity $\left(\mathbf{D}_{\mathrm{th}}^{*}\right)$, response time, and thermal fluctuation noise floor (the dominant noise mechanism for mechanical resonators [3], [17]). For the range of resonator sizes in this work, the thermal noise floor is in the range of $1 \mathrm{~K}$ to $10 \mathrm{~K}$ at room temperature. Using this design, it is possible to achieve detectivity on the order of $1 \times 10^{9} \mathrm{~cm} . \mathrm{Hz}^{0.5} . \mathrm{W}^{-1}$, with response time on the order of $1 \mathrm{~ms}$ (Fig. 9 (a)) [15]. Using better absorber layers, it is also possible to improve the sensitivity of the system (Fig. 9 (b)).

Table 1: Measured results for three different GaN IR sensors.

\begin{tabular}{|c|c|c|c|}
\hline & $\begin{array}{c}\text { Sensor 1 } \\
\text { (Fig. 6) }\end{array}$ & Sensor 2 & Sensor 3 \\
\hline \hline Dimensions & $\begin{array}{c}\mathbf{1 6 0} \boldsymbol{\mu m} \times \\
\mathbf{8 0} \boldsymbol{\mu m}\end{array}$ & $\begin{array}{c}\mathbf{1 6 0} \boldsymbol{\mu \mathbf { m } \times} \\
\mathbf{8 0} \boldsymbol{\mu \mathbf { m }}\end{array}$ & $\begin{array}{c}\mathbf{1 2 0} \boldsymbol{\mu m} \times \\
\mathbf{8 0} \boldsymbol{\mu m}\end{array}$ \\
\hline \hline Original f $\mathbf{0}(\mathbf{M H z})$ & 119.2267 & 119.4547 & 191.6475 \\
\hline$\Delta \mathbf{f}(\mathbf{k H z})$ & -21.861 & -15.615 & -10 \\
\hline \hline Original $\boldsymbol{Q}$ & 1272.566 & 813.83 & 2323 \\
\hline$\Delta \boldsymbol{Q}$ & -318.33 & -216.289 & -406.63 \\
\hline \hline Original IL (dB) & -20.804 & -24.023 & -16.522 \\
\hline$\Delta \mathbf{I L}(\mathbf{d B})$ & -1.375 & -1.309 & -1.487 \\
\hline \hline
\end{tabular}
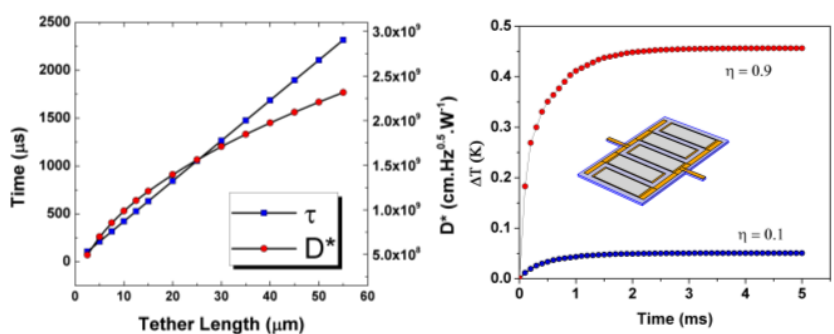

Figure 9: (a) $D^{*}$ and time constant as a function of the tether length. For the current resonator design, with an area of $160 \mu \mathrm{m} \times$ $80 \mu \mathrm{m}$, tether length of $20 \mu \mathrm{m}$ gives an optimal value for these parameters. (b) The response time of $1 \mathrm{~ms}$ is verified using COMSOL simulations for this model (inset). Improving the absorber efficiency from 0.1 to 0.9 will improve the thermal sensitivity.

\section{CONCLUSION}

Resonant IR sensors made from GaN with a sensitivity of $1830 \mathrm{ppm} / \mathrm{W}$ has been demonstrated using a sensor-reference pair. Utilizing a reference resonator enables large, perceptible changes in beat frequency due to irradiation, while cancelling out the effects of shock, acceleration, and ambient thermal change. A practical system would need further design optimization to get the best NEDT, D* and response time. The use of a broadband absorber with improved efficiency will improve the sensitivity of the detector by at least five times. Integration of the absorber and complete integration of the sensor-reference pair is ongoing work.

The interface circuitry required to measure frequency shifts can be co-fabricated on the same substrate, as $\mathrm{GaN}$ is a good material for high speed transistors. Alternate measurements schemes are also possible, using the same device. The use of the resonant detectors presented in this work is the first step to realizing IR focal plane arrays based on resonant sensors.

\section{ACKNOWLEDGENTS}

This work has been funded by NSF under Grant \#1002036, and MAST CTA under contract W911NF. The authors thank the staff at the Lurie Nanofabrication Facility for fabrication support. Travel support has been generously provided by the Transducer Research Foundation.

\section{REFERENCES}

[1] A. Rogalski, "Infrared detectors: status and trends," Progress in Quantum Electronics, 27, pp. 59-210, 2003.

[2] C. Cabuz et al., "Fabrication and packaging of a resonant infrared sensor integrated in silicon," Sensors and Actuators A, 43, pp. 92-99, 1994.

[3] J. R. Vig, R. L. Filler, and Y. Kim, "Uncooled IR imaging array based on quartz microresonators," J. Microelectromech. Sys., vol. 5, pp.131-138, 1996.

[4] P. Kao, and S. Tadigadapa, "Micromachined quartz resonator based infrared detector array," Sensors and Actuators A, vol. 149, pp. 189-192, 2009.

[5] V. J. Gokhale, J. Roberts, and M. Rais-Zadeh, "High performance bulk mode gallium nitride resonators and filters," Transducers, Beijing, PRC, pp. 926-929, 2011.

[6] A. Ansari et al., "Gallium nitride-on-silicon micromechanical overtone resonators and filters," IEEE Electron Devices Meeting, pp. 485-488, 2011.

[7] M. Faucher et al., "Amplified piezoelectric transduction of nanoscale motion in gallium nitride electromechanical resonators," Appl. Phys. Lett., vol. 94, pp. 233506, 2009.

[8] A. Muller et al., "GaN micromachined FBAR structures for microwave applications," Superlattices and Microstructures, vol. 40, pp. 426-431, 2006.

[9] M. S. Shur, A. D. Bykhovski, and R. Gaska, "Pyroelectric and piezoelectric properties of GaN-based materials," Proc. MRS, pp. G1.6, 1998.

[10] I. L. Guy, S. Muensit, and E. M. Goldys, "Electrostriction in gallium nitride," Appl. Phys. Lett, vol. 75, pp. 3641-3643 1999.

[11] M. Willatzen and L. C. Lew Yan Voon, "Static and dynamic effects due to electrostriction in GaN/AlGaN," J. Phys.: Condens. Matt., vol. 19, pp. 506202-506221, 2007.

[12] C. M. Jha et al., "High Resolution Microresonator-based digital temperature sensor," Applied Physics Letters, vol. 91, 074101-3, August 2007.

[13] W. Lang, K. Kuhl, and H. Sandmaier, "Absorbing layers for thermal infrared detectors," Sensors and Actuators A, 34, 3, pp. 243-248, 1992.

[14] Y. Sui, V. J. Gokhale, and M. Rais-Zadeh "A thin-film infrared absorber using CNT/nanodiamond nanocomposite," Proc. MRS, April 2012.

[15] M. Rais-Zadeh, "Gallium nitride micromechanical resonators for IR detection," Invited Paper, Proc. SPIE, April 2012.

[16] V. J. Gokhale, Y. Sui, and M. Rais-Zadeh, "Novel uncooled detector based on gallium nitride micromechanical resonators," Proc. SPIE, April 2012.

[17] P. W. Kruse, "A comparison of the limits to the performance of thermal and photon detector imaging arrays," Infrared Phys. Technol., 36, pp. 869-882, 1995.

\section{CONTACT}

*V. J. Gokhale, Tel: +1-734-945-5732; vikrantg@umich.edu 


\section{IN-AIR ULTRASONIC RANGEFINDING AND ANGLE ESTIMATION USING AN ARRAY OF ALN MICROMACHINED TRANSDUCERS

\author{
R.J. Przybyla ${ }^{1 *}$, S.E. Shelton ${ }^{2}$, A. Guedes ${ }^{2}$, R. Krigel ${ }^{l}$, D.A. Horsley ${ }^{2}$, and B.E. Boser ${ }^{l}$
} \\ Berkeley Sensor and Actuator Center \\ ${ }^{1}$ University of California, Berkeley, CA, USA \\ ${ }^{2}$ University of California, Davis, CA, USA}

\begin{abstract}
We describe a 2D ultrasonic depth sensor based an array of piezoelectric micromachined ultrasound transducer elements which measures range and direction to targets in air. The transducer consists of a single transmitter and seven receive elements arranged in a $6 \mathrm{~mm}$ long linear array with half wavelength spacing. The system employs pulse-echo time-of-flight and difference of arrival time to determine target range and angle and operates up to a $750 \mathrm{~mm}$ maximum range and $\pm 35^{\circ}$ angle span with $\pm 3.5 \mathrm{~mm}$ range and $\pm 3^{\circ}$ angle worst case errors, respectively.
\end{abstract}

\section{INTRODUCTION}

Optical solutions presently dominate $2 \mathrm{D}$ and $3 \mathrm{D}$ ranging systems. Although capable of relatively high resolution and frame rate, these systems suffer from high power dissipation typically in excess of several Watts [1], precluding their use in mobile applications for gesture recognition and similar applications. A further drawback of optical solutions is their sensitivity to ambient illumination and limited performance in sunlight.

Ultrasonic transducers overcome these drawbacks. Modern micromachined single element or small transmitter arrays generate sufficient ultrasonic power from a sub- $\mathrm{mW}$ electrical drive signal for target ranges up to a few meters. Compared to light, ultrasonic waves travel at roughly one million times slower velocity, translating into simpler and lower bandwidth electronics that translate into further power savings compared to optical solutions. High frequency ultrasound $(>100 \mathrm{kHz})$ is particularly attractive for chip-scale systems because several transducer elements spaced at quarter wavelength fit across a cm-sized transducer die, thus enabling $2 \mathrm{D}$ and $3 \mathrm{D}$ ranging in a very compact form factor.

Previous ultrasonic depth sensors use bulky piezoceramic arrays [2] or use large transmitters for excitation [3]. In this work, we present a $2 \mathrm{D}$ rangefinder based on a chip-scale batch-fabricated aluminum nitride (AIN) ultrasonic transducer array. We discuss the factors that affect the performance of a depth sensor, and use these to design a system which is suitable for simple gesture recognition.

\section{MICROMACHINED ULTRASOUND TRANSDUCERS}

The ultrasound transducers used in this work [4],[5] are circular piezoelectric unimorph membranes. Figure 1 shows the arrangement consisting of a $2 \mu \mathrm{m}$ thick $\mathrm{AlN} / \mathrm{Mo} / \mathrm{AlN} / \mathrm{Al}$ stack fabricated on a silicon wafer and released with a backside throughsilicon etch. Voltage applied across the electrodes results an inplane stress in the top AlN layer via the inverse piezoelectric effect. The bottom layer of AlN causes a stress gradient to form across the membrane which results in out-of-plane displacement, causing the transducer to emit a pressure wave. Similarly, an incident pressure wave causes in-plane stress in the top layer of AIN, which results in charge developing across the electrodes that can be sensed electrically.

An electrical, mechanical, and acoustical model for the device is presented in [6]. The $450 \mu \mathrm{m}$ diameter transducers are designed to have a resonant frequency $f_{o} \approx 190 \mathrm{kHz}$. At atmospheric pressure, the $\mathrm{Q}$ of the transducer is about 15 , corresponding to a bandwidth $B W=f_{d} / 2 Q \approx 6.2 \mathrm{kHz}$, and the motional impedance of each device is

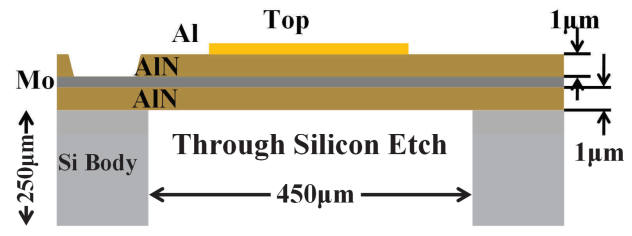

Figure 1: Schematic cross-section of a single pMUT.

Table 1: Depth Sensor Design Specifications

\begin{tabular}{l|l} 
Specification & Value \\
\hline \hline Max Range & $500 \mathrm{~mm}$ \\
Angular span & $60^{\circ}$ \\
Range accuracy & $10 \mathrm{~mm}$ \\
Range Resolution & $50 \mathrm{~mm}$ \\
Angular Accuracy & $2^{\circ}$ \\
Angular Resolution & $15^{\circ}$ \\
Update rate & $>30 \mathrm{~Hz}$ \\
\hline
\end{tabular}

approximately $2 \mathrm{M} \Omega$ at resonance. The effective surface area of each transducer is $\mathrm{SA}=0.05 \mathrm{~mm}^{2}$. The parallel plate capacitance of these devices, including bond pads and interconnect, is approximately $8 \mathrm{pF}$.

\section{SYSTEM DESIGN}

Table 1 lists the target specifications for a 2D ultrasonic depth sensor that is suitable for simple user interface tasks such as advancing a slide. Below we discuss the design of the measurement system for range and angle measurements as well as error sources that limit the range and accuracy of the system.

\section{Range Measurement}

The ranger operates by emitting an ultrasound signal and measuring the echo from a target. The transmitted signal can be either a continuous wave or a pulse. Continuous time solutions are attractive for narrow-band transmitters but suffer from multi-path fading and high receiver dynamic range requirements due to feedthrough from the transmitter to the receiver. For this reason, a pulse-echo design is used in this implementation. In this case, a short pulse is transmitted and the range $R$ estimated from the speed of sound $c \approx 340 \mathrm{~m} / \mathrm{s}$ and the delay $T=2 R / c$ of the echo. For a target at $R=1 \mathrm{~m}$, the delay is approximately $5.9 \mathrm{~ms}$.

An ideal system would transmit a narrow, sharp pulse. Short duration permits resolving closely spaced targets, while short rise time is critical to minimize timing errors due to amplitude fluctuations. Consequently, in practical, band limited systems, it is desirable to use all of the available bandwidth. In practice, this means the bandwidth of the system should be designed to be limited by the bandwidth of the ultrasound transducer, and not the interface electronics.

The ideal transmit signal is approximated in practice with a burst of a sinusoidal signal at the resonant frequency of the transmitter element. The duration $T_{p}$ of the burst involves a tradeoff between minimum and maximum target range. Since the receiver is disabled during transmission, the minimum target 
distance is $R_{\text {min }}=c T_{p} / 2$. However, bursts much shorter than $1 /(2 \mathrm{BW}) \approx 80 \mu \mathrm{s}$ corresponding to $R_{\text {min }} \approx 14 \mathrm{~mm}$ result in reduced transmit amplitude and hence smaller maximum range.

Targets spaced less than $\sim c T_{p} / 2$ in range result in overlapping echoes which are difficult to resolve as separate targets [7]. The pulse also broadens as it passes through the narrowband transducer. For a pulse that is shaped by a second order transducer response on transmission and reception, the range resolution is:

$$
\Delta_{r}=\frac{c}{2}\left(\mathrm{~T}_{\mathrm{p}}+\frac{0.27}{\mathrm{BW}}\right)
$$

For the numbers used above, the range resolution is $\Delta_{r} \approx 21 \mathrm{~mm}$.

The maximum useful range is limited by the maximum output pressure, thermal noise from the air, and noise from the electronic pickup circuit. The transmit pressure of a single element at $f_{o}$ is limited to $p_{t x} \approx 500 \mathrm{~Pa}$ by the third-order Duffing nonlinearity of the clamped-clamped membrane. Depending on the design of the transducer, the maximum output pressure will either be limited by the maximum available transmit voltage or mechanical nonlinearity.

As the ultrasonic pulse travels through the air, it is attenuated due to spreading and absorption. The roundtrip acoustic path loss is given by [8]:

$$
G_{p}=G_{a c} \frac{R_{o}}{4 R} 10^{-2 \alpha R}
$$

In this equation, $R$ is the range to the target. $\alpha$ is the attenuation constant, which is strongly dependent on frequency, and also dependent on humidity and ambient pressure [8]. At $190 \mathrm{kHz}, \alpha$ $\approx 10 \mathrm{~dB} / \mathrm{m}$. $G_{a c}$ is the acoustic gain, which depends on the size of the target, and which can also be increased by using a horn. For large targets in this design, $\mathrm{G}_{\mathrm{ac}}=20 . R_{o}=\mathrm{SA} / \lambda=27 \mu \mathrm{m}$ is the Rayleigh range, and $\lambda=c / f_{o}=1.8 \mathrm{~mm}$ is the wavelength.

The signal received by the transducer at $f_{o}$ is $i_{r x}=$ $\left(p_{r x} / \mathrm{R}_{\mathrm{m}}\right)(S A / \eta)=8 \mathrm{nA} / \mathrm{Pa}$, where $\mathrm{R}_{\mathrm{m}}$ is the motional resistance of the transducer. Random air molecule thermal vibrations are the dominant source of noise from the transducer. This noise signal is shaped by the transducer dynamics. The electronic amplifier also adds wideband thermal noise which must be filtered electronically for optimal noise performance. As mentioned above, the bandwidth of the system is critical to resolution and accuracy, so the electronic filter should not restrict the overall bandwidth of the system unless additional range is required.

For pulse detection, a threshold is generally used to distinguish faint echoes from noise. The threshold must be set high enough to detect echoes at the maximum range but to reject noise pulses which would otherwise cause large errors in the range estimate. The average time between false alarms is given in [7]:

$$
\mathrm{t}_{\mathrm{fa}}=\frac{1}{\mathrm{BW}} \mathrm{e}^{\mathrm{V}_{\mathrm{TH}}^{2} / \sigma_{\mathrm{A}}^{2}} \text {. }
$$

In practice, $\mathrm{V}_{\mathrm{TH}}^{2} / \sigma_{\mathrm{A}}^{2}=12 \mathrm{~dB}$ is a good target and corresponds to $\mathrm{t}_{\mathrm{fa}}=24$ minutes, an acceptable value in most applications. Increasing the threshold reduces the frequency of false alarms at the expense of the theoretical maximum range.

Since the dominant noise sources in the system are in the receiver, increasing the number of receive elements increases the signal power by $\mathrm{N}^{2}$ but also increases the noise power by $\mathrm{N}$. Adding transmit elements does not appreciably increase the total noise. Consequently, the on-axis SNR for a system with $\mathrm{N}_{\mathrm{tx}}$ transmitters and $\mathrm{N}_{\mathrm{rx}}$ receivers is [9]:

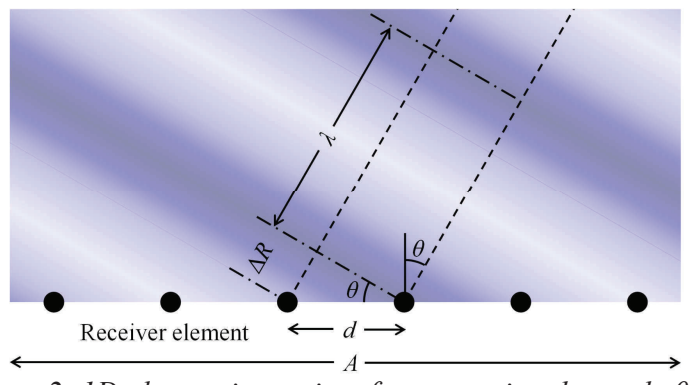

Figure 2: 1D ultrasonic receiver for measuring the angle $\theta$ of the target based on the difference in arrival time $\Delta T=\Delta R / c$ between adjacent elements.

$$
\mathrm{SNR}=\mathrm{N}_{\mathrm{tx}}^{2} \mathrm{~N}_{\mathrm{rx}} \frac{\left(\frac{\mathrm{SA}}{\eta} \frac{1}{\mathrm{R}_{\mathrm{m}}}\right)^{2} p_{t x}^{2} G_{p}^{2}}{\frac{\mathrm{kT}}{L}+\bar{v}_{a}^{2}\left(\omega_{o} C_{o}\right)^{2} \mathrm{NBW}},
$$

where $\mathrm{kT}$ is the thermal energy of the system, $L=25 \mathrm{H}$ is the effective motional inductance of the transducer, $\bar{v}_{a}^{2}$ is the spot noise variance of the front-end amplifier, $\omega_{o}=2 \pi f_{o}, C_{o}=8 \mathrm{pF}$ is the parasitic capacitance of the transducer, and NBW is the effective noise bandwidth of the electronic filter. The LHS of the denominator is mechanical noise from the transducer, and the RHS is electronic noise from the front-end amplifier. Since the noise does not depend on range, equations (2) and (4) show that the signal decreases linearly with increased range at relatively short ranges, and then begins to decrease much faster as absorption starts to become a dominant loss factor. With our system, the theoretical maximum range is $800 \mathrm{~mm}$ for a large target, and at $500 \mathrm{~mm}$ distance the SNR is $25 \mathrm{~dB}$ [9].

Amplitude noise $\sigma_{a}$ in the received signal, combined with finite burst rise time, translates into an equivalent range noise [7]:

$$
\sigma_{r}=\frac{c}{2 \mathrm{BW}} \frac{1}{2.1(\mathrm{SNR})^{1 / 2}}
$$

At the maximum range, $\mathrm{SNR}=12 \mathrm{~dB}$, which results in a worst-case $\sigma_{r}=3.3 \mathrm{~mm}$.

\section{Angle Measurement}

The goal of an angle measurement system is to measure the direction to the target from the transceiver. Figure 2 depicts a linear array of receivers used to receive a plane wave returning from a target. An incident angle $\theta$ creates a difference in the timeof-flight between neighboring elements $\Delta T=d / c \sin (\theta)$ which corresponds to a phase shift $\phi=2 \pi d / \lambda \sin (\theta)$, where $\lambda$ is the wavelength of the sound wave. Similarly, to transmit a pulse with angle $\theta$ from normal, the phase of the transmit signal should be shifted by $\phi$ between each element.

As shown in equation (4), an N-element receive array will only increase the SNR by a factor of $\mathrm{N}$ compared to a single transceiver. However, since the receive signals can be summed in the electrical domain, the signals can be shifted so that the phases align irrespective of the input phase difference. Because of this flexibility, a receive array can maintain a constant SNR across a wide span of angles.

For a linear array of $\mathrm{N}$ transducers spaced distance $d$ apart, we define the aperture of the system as $A=\mathrm{N} d / \lambda$. Aperture in the angle axis is analogous to bandwidth in the range axis, so increasing the effective aperture leads to increased angle measurement precision.

The aperture of the system determines the angular beam width of both the transmitted signal and the received signal. The 


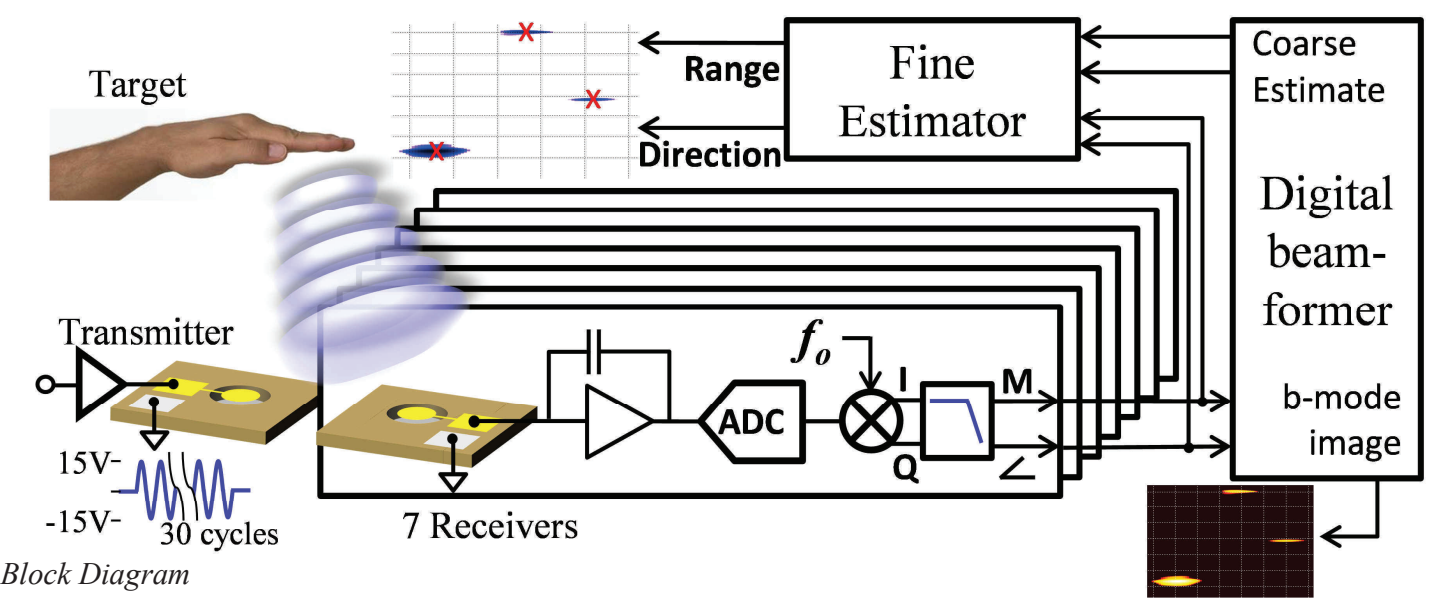

Figure 3: Block Diagram

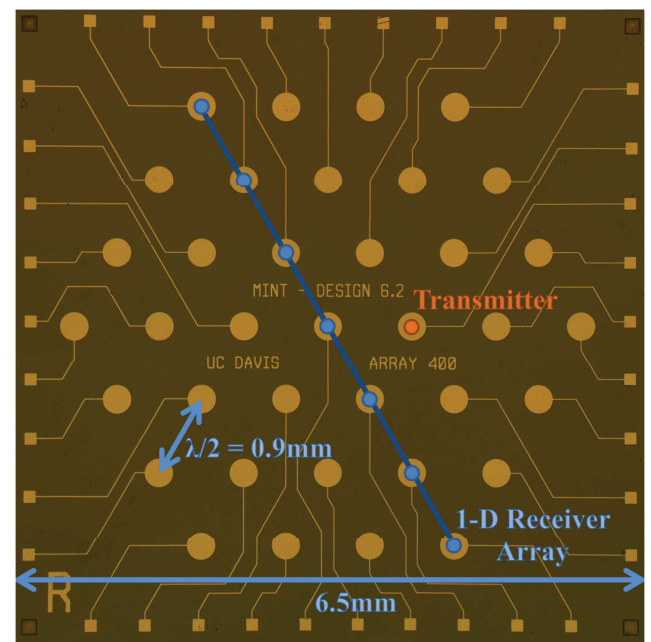

Figure 4: Optical image of a hexagonal pMUT array showing single transmit device and linear sub-array of receive devices.

directivity as a function of angle $\theta$ for a line array of isotropic sources can be shown [8] to be:

$$
D(\theta)=\frac{\sin \left(\mathrm{N} \frac{\pi d}{\lambda} \sin \theta\right)}{\mathrm{N} \sin \left(\frac{\pi d}{\lambda} \sin \theta\right)},
$$

where $d$ is the spacing between elements. To avoid large sidelobes in the directivity response, $d$ should be $\leq \lambda / 2$. We set $d=\lambda / 2$ in this design, and use a 7 element receive array, hence $A=3.5$.

For $\mathrm{N} \gg>1$, the small-angle approximation can be used to estimate the full-width half-power beam width $\theta_{b}$ as

$$
\theta_{b} \cong \frac{2.78 \lambda}{N \pi d} \cong \frac{0.886}{A} \text {. }
$$

This equation gives the beam width for a single array. If transmit and receive arrays are used, the product of the transmit and receive directivity functions must be solved for $\theta_{b}$. $\theta_{b}$ is the angular resolution of the system; for two distinct targets at the same range to be individually resolvable they must be separated by at least $\theta_{b}$ degrees. With $A=3.5, \theta_{b}=14.5^{\circ}$.

The aperture also affects the angular accuracy of the system. The rms angle error $\sigma_{\theta}$ given in [7] is

$$
\sigma_{\theta} \cong \frac{0.628 \theta_{b}}{(\mathrm{SNR})^{1 / 2}} \cong \frac{0.556}{A(\mathrm{SNR})^{1 / 2}} .
$$

At the maximum range, $\mathrm{SNR}=12 \mathrm{~dB}$, and $\sigma_{\theta}=2.3^{\circ}$, which is greater than the specification. However, at $500 \mathrm{~mm}, \mathrm{SNR}=25 \mathrm{~dB}$, which corresponds to an rms angle error $\sigma_{\theta}=0.5^{\circ}$.

Equations (7) and (8) suggest that the aperture $N d / \lambda$ should be made as large as possible to achieve the best angular performance. However, since $d \leq \lambda / 2$, achieving a smaller angular resolution requires increasing the size of the array or increasing the operating frequency. Since absorption losses in equation (2) increase at higher frequency, there is a tradeoff between angular resolution and maximum range for a given chip size.

\section{IMPLEMENTATION}

Figure 4 shows a micrograph of the seven element linear array used to receive echoes from the environment. To protect the transducer membranes from damage, the backside of the die is exposed to the air. The block diagram in Figure 3 illustrates the operation. The transmitter is excited with a $\mathrm{T}_{\mathrm{p}}=160 \mu \mathrm{sec}$ burst at $f_{o}$ and emits an omnidirectional beam. We use a transmit signal twice as long as required by the bandwidth of the transducer to allow more accurate measurement of the phase of the return echo; this increases the range resolution from $21 \mathrm{~mm}$ to $34 \mathrm{~mm}$. The transmitter power consumption, based on $\mathrm{CV}^{2} f$ losses, is $50 \mu \mathrm{W}$. The 1D receiver array captures the echoes which are amplified, digitized, and quadrature down-converted for each channel separately. Digital filtering removes wideband electronic noise. Object positions are calculated by a real-time digital postprocessor. For each angle $\theta$, the signals from each channel $k$ are shifted by a phase shift $k \phi=k 2 \pi d / \lambda \sin (\theta)$ and summed with the other channels. The angle is swept over the entire angle range, resulting in an image such as the one shown in Figure 7. Approximate target ranges and angles are extracted from this data. Subsequently, a fine estimator improves the estimate by searching each baseband signal for a pulse in temporal proximity to the coarse estimate. Refined estimates for the range and angle to the target are determined from the average time-of-flight for each channel's echo and the phase difference between adjacent receive channels.

\section{EXPERIMENTAL RESULTS}

The maximum range is limited by the signal-to-noise ratio and is dependent on the target size; the maximum range is approximately $750 \mathrm{~mm}$ for a metallic cylindrical target with $63 \mathrm{~mm}$ diameter. At $500 \mathrm{~mm}$, the $\mathrm{SNR}$ is $28 \mathrm{~dB}, 3 \mathrm{~dB}$ higher than the design value of $25 \mathrm{~dB}$. This could be due to a conservative estimate of the output pressure. Feedthrough between the transmitter and the receivers causes a dead-time during which no echo can be received, limiting the minimum range to $170 \mathrm{~mm}$ in this prototype. This is longer than the $50 \mathrm{~mm}$ expected from exponential settling of 


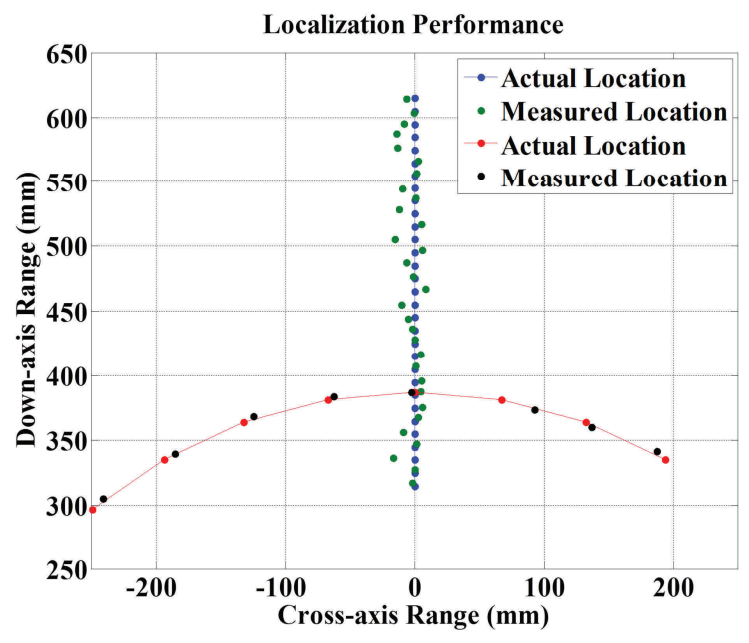

Figure 5: Measurement results showing target localization performance for distance and angle sweeps.

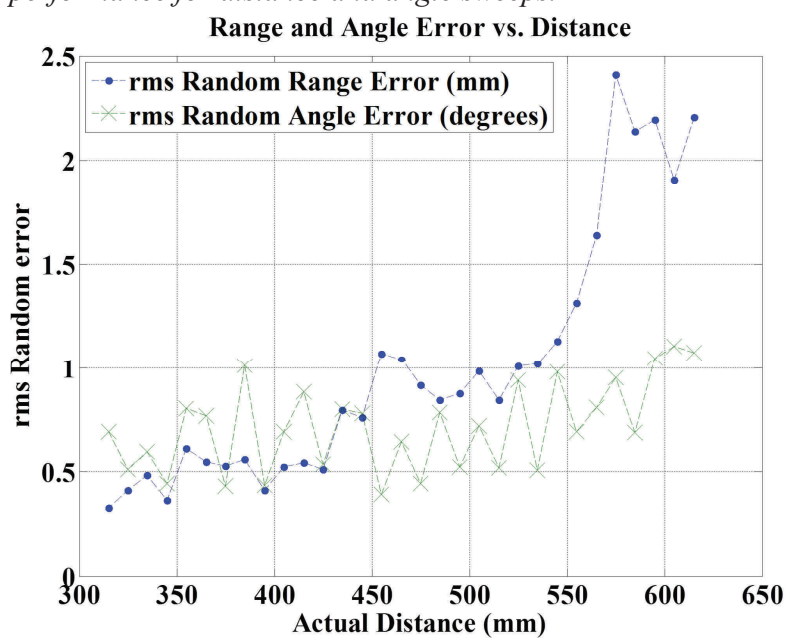

Figure 6: Random range error and random angle error vs. measured distance.

the transmit signal, and may be due to spurious short-range echoes from the test setup. The angular range of the system is $+30^{\circ}$ to $40^{\circ}$. The asymmetry is the consequence of misalignment of the transmitter with respect to the center of the receivers. The measurement time is set by the time-of-flight of the ultrasound pulse and is $4.4 \mathrm{~ms}$ at the maximum range.

Figure 5 shows the range and angle measurement performance with the cylindrical target. The target was moved both radially and on an arc with constant distance from the sensor at location 0,0 (below the plot area), and the plot shows a single representative measurement at each location; no averaging was used. The mean errors were also estimated from 100 recordings at each position and are between $\pm 1.5 \mathrm{~mm}$ and $\pm 2^{\circ}$ for the radial and $\pm 3.5 \mathrm{~mm}$ and $\pm 3^{\circ}$ for arc cases, respectively. Figure 6 shows the random range and angle error versus distance.

Figure 7 demonstrates the system's ability to create a 2D map of an author's head and hands when posing as illustrated.

\section{CONCLUSION}

We have demonstrated the first chip-scale ultrasonic depth sensor, which uses an array of thin-film aluminum nitride transducers to measure range and direction to targets in the field of view. Compared to optical solutions, ultrasonic depth sensors offer

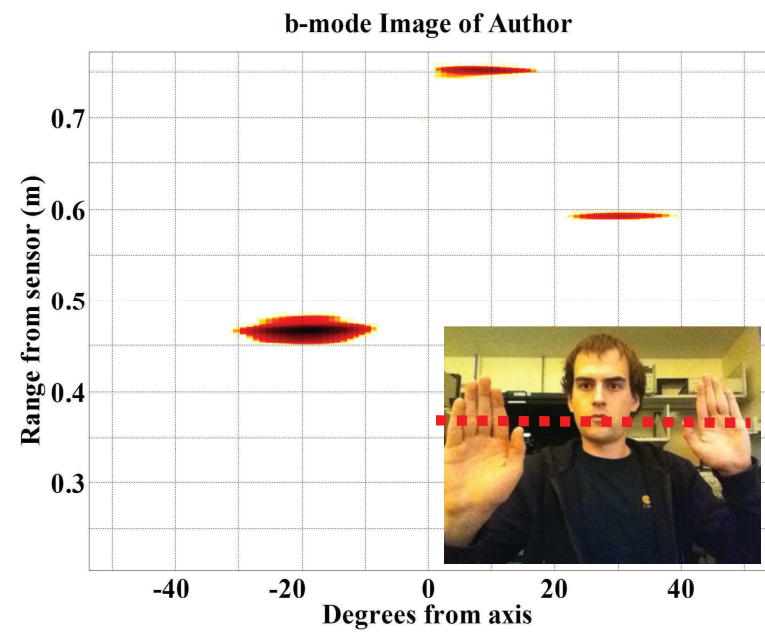

Figure 7: Ultrasound intensity plot showing echo from author's hands and head. Inset: author's pose, showing sensitive axis of system.

orders-of-magnitude reduction in power consumption, ambient light insensitivity, and chip-scale solution size. Combined with good range and angular resolution, ultrasonic solutions are ideal for simple gesture recognition and similar tasks for smart phones and other power constrained devices.

\section{REFERENCES}

[1] Microsoft Corp., Kinect Sensor Manual and Warranty, 2010.

[2] H. Furuhashi et al., "Three-dimensional imaging sensor system using an ultrasonic array sensor and a camera," in Proc. IEEE Sensors 2010, pp. 713-718, 1-4 Nov. 2010.

[3] K. Yamashita et al., "Ultrasonic micro array sensors using piezoelectric thin films and resonant frequency tuning," Sensors and Actuators A: Physical, vol. 114, no.2-3, pp. 147153, Sept. 2004.

[4] S. Shelton, et al., "CMOS-compatible AlN piezoelectric micromachined ultrasonic transducers," in Proc. IEEE Ultrasonics Symp. 2009, Oct. 2009, pp. 402-405.

[5] S. Shelton, et al., "Aluminum nitride piezoelectric micromachined ultrasound transducer arrays," in Proc. Hilton Head Solid-State Sensors, Actuators, and Microsystems Workshop 2012, June 2012.

[6] R. Przybyla, et al., "In-air rangefinding with an AIN piezoelectric micromachined ultrasound transducer," IEEE Sensors Journal, vol.11, no.11, pp.2690-2697, Nov. 2011.

[7] M. Skolnik, Introduction to Radar Systems. $3^{\text {rd }}$ edition, McGraw-Hill, 2001.

[8] D. Blackstock, Fundamentals of Physical Acoustics. John Wiley \& Sons, 2000.

[9] R. Przybyla, et al., "A Micromechanical Ultrasonic Distance Sensor With $>1$ Meter Range," in Proc. Solid-State Sensors, Actuators and Microsystems Conference (TRANSDUCERS) 2011, pp.2070-2073, Beijing, China, 5-9 June 2011.

\section{ACKNOWLEDGEMENT}

This material is based upon work supported by the Defense Advanced Research Projects Agency (DARPA) and/or the Space and Naval Warfare Center, San Diego (SPAWAR SSC-SD) under Contract No. N66001-08-C-2023.

\section{CONTACT}

*Richard J. Przybyla, rjp@berkeley.edu. 


\title{
A NOVEL, HIGH-RESOLUTION RESONANT THERMOMETER USED FOR TEMPERATURE COMPENSATION OF A COFABRICATED PRESSURE SENSOR
}

\author{
Chia-Fang Chiang ${ }^{l}$, Andrew B. Graham ${ }^{2}$,Eldwin J. $\mathrm{Ng}^{l}$, Chae Hyuck Ahn ${ }^{l}$, \\ Gary J. O'Brien', and Thomas W. Kenny ${ }^{1}$ \\ ${ }^{1}$ Stanford University, Stanford, CA, USA and ${ }^{2}$ Robert Bosch RTC, Palo Alto, CA, USA
}

\begin{abstract}
Temperature compensation of a capacitive pressure sensor with a cofabricated, high-resolution, resonant thermometer is presented in this paper. The epi-seal encapsulated resonant thermometer yields a resolution of $10 \mathrm{mK}$ due to the ultra-clean operating environment. By co-locating such a temperature sensor with a pressure sensor and fabricating them in a shared substrate, temperature measurement accuracy can be greatly improved. With this arrangement for temperature compensation, the pressure sensor shows a 100x reduction in the temperature dependence and realizes sensing accuracy within $\pm 0.1 \%$ FSO for the tested temperature range between 15 to $65^{\circ} \mathrm{C}$.
\end{abstract}

\section{INTRODUCTION}

Temperature compensation of a sensor can be made purely with clever algorithms such as using the characteristic of the slow change of temperature to distinguish a rapid signal from a false temperature induced drift [1]. Depending on the applications, software-only compensation methods may become inadequate and on-site temperature sensing or even ovenization [2] needs to be implemented to achieve certain performance. The former can be performed using a microcontroller or a compensation circuit to subtract temperature effects. In such cases, the temperature sensing capability determines the effectiveness of the compensation.

While typical on-chip temperature sensors are limited to a few tenths of a Kelvin of measurement resolution [3, 4], recent developments have shown that encapsulated resonant thermometers can offer temperature resolution of $\pm 0.35 \mathrm{mK}$ [5]. As a result of the significant increase in temperature sensing capability $\left(\sim 10^{3} \mathrm{X}\right)$, there is an opportunity to co-locate accurate thermometers with MEMS devices and provide improved temperature compensation, leading to significantly more accurate MEMS sensors.

This work uses an encapsulated resonant thermometer to compensate for temperature variations of a capacitive pressure sensor that is fabricated in the same piece of silicon as the thermometer in a single process flow. Unlike other temperature compensation methods, where sensors are typically formed separately and signal conditioned using an adjacent ASIC die, the proximity of the sensors on the shared substrate used in our approach greatly reduces inaccuracy resulting from potential temperature gradients and thermal energy propagation lag time existing between adjacent sensor and ASIC die.

\section{DEVICE DEVELOPMENT}

\section{Resonant thermometer}

The resonant thermometer used in this work is a flexural mode silicon resonator in the shape of a double-ended tuning fork (DETF), as shown in figure 1 . The structure of the DETF resonator consists of two long thin beams mechanically connected on both ends. These beams are DC biased through an anchor electrode on one side to form a clamped-free beam configuration. The beams are electrostatically driven via the outer electrodes with an AC stimulus and capacitively sensed by the inner electrode. A transimpedance amplifier (TIA) is used as an electrical oscillation feedback drive circuit to maintain vibration of the tuning fork at its fundamental mode resonant frequency [2].

The resonant frequency of the DETF resonator varies with temperature primarily due to the temperature dependency of material properties such as the Young's Modulus [6, 7]. At a constant DC bias voltage, the linear temperature coefficient of frequency $\left(T C f_{l}\right)$ of the DETF resonator can be described as

$$
T C f_{1} \approx \frac{T C E_{1}+\alpha}{2}
$$

where $T C E_{l}$ is the linear temperature coefficient of elastic modulus and $\alpha$ is the linear thermal expansion coefficient of silicon. $T C f_{l}$ defines the sensitivity of the DETF resonant thermometer. Notice that equation (1) gives an approximate relation. A complete analytic expression should also consider the influence of electrostatic actuation and axial loading and is given in [6]. Axial loading is not applicable in our case as the DETF resonator is single anchored and cannot sustain axial loading. On the other hand, for the geometry of the DETF resonant thermometer and bias conditions used in this work, electrostatic actuation induces $\sim 1.3 \%$ increase in $T C f_{l}$ compared to the solely material determined $T C f_{l}$, using $T C E_{l}:-63.75 \mathrm{ppm} /{ }^{\circ} \mathrm{C}$ and $\alpha: 2.6 \mathrm{ppm} /{ }^{\circ} \mathrm{C}$ [7].

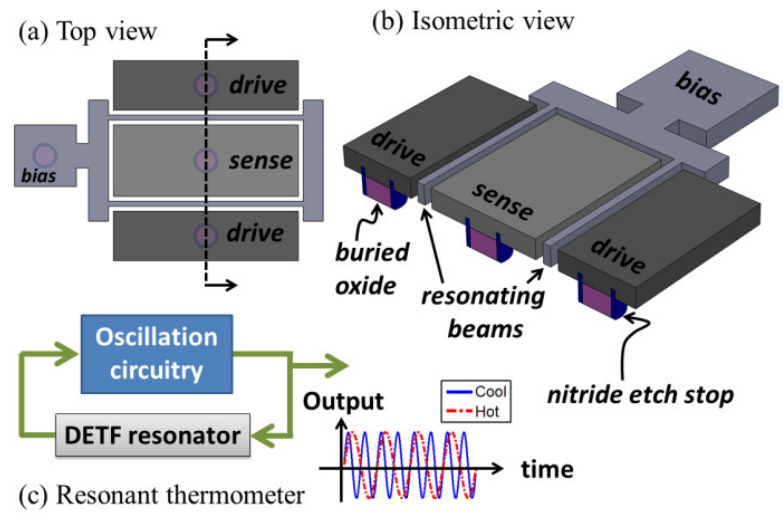

Figure 1: Schematics of a DETF resonator structure and the formation of an oscillator used as a resonant thermometer.

\section{Capacitive pressure sensor}

A capacitive absolute pressure sensor was designed to measure atmospheric pressure. Capacitive transduction was selected for its lower susceptability to temperature variations as well as the ease of fabrication integration when compared to piezoresistive transduction. Specifically, the thermal budget is not an issue for the materials used. As can be seen in figure 2, instead of using the entire diaphragm as the electrode, the device employes a reduced electrode area to increase the fractional capacitance change. This effectively improves the accuracy using our chosen measurement technique and thus can alleviate the complexity in readout circuitry design. Additionally, the temperature dependency of the sensor is reduced by sealing the capacitive cavity in low vacuum, which limits the thermal expansion effect of the trapped gas. 


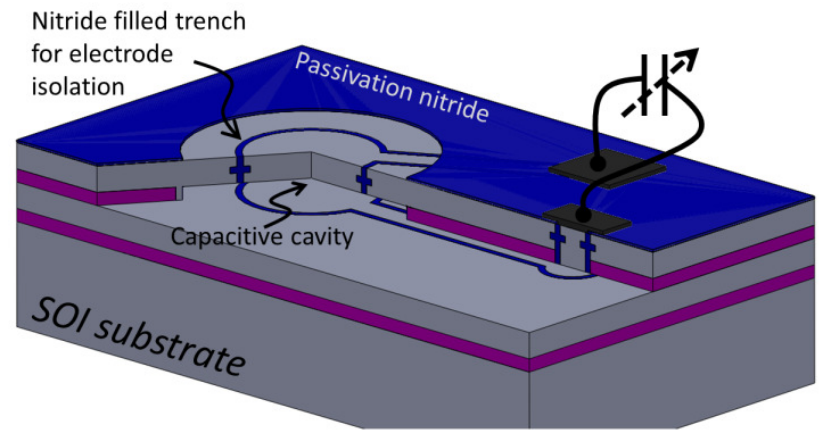

Figure 2: Structure of a capacitive pressure sensor. Electrode isolation in the diaphragm and the fixed substrate is made with a nitride filled trench.

\section{Cofabrication of the sensors}

Figure 3 shows the overall structure of the cofabricated DETF resonant thermometer and capacitive pressure sensor with their electrical functions. The integrated fabrication process is based on our previous work [8] with minor modifications. Silicon on insulator (SOI) wafers were used to construct the sensors. In the process, sacrificial silicon dioxide is used to create the cavities for the moving elements after releasing in vapor-phase hydrofluoric acid. Lateral release etch stops are created using either silicon-rich, low stress nitride or epitaxial silicon to define critical design parameters (specifically the diaphragm size of the pressure sensor), and realize a process insensitive to the release etch. After an epitaxial polysilicon deposition to seal the release etch holes (termed as "epi-seal", [9]), the deflectable diaphragm of the capacitive pressure sensor and the encapsulation of the resonant thermometer are formed simultaneously. Cross-sectional SEM images of the fabricated pressure sensor and the DETF resonant thermometer are shown in figure 4.

The epi-seal encapsulation plays a significant role in the stable performance of the DETF resonator because it provides an ultraclean, oxide-free, and hermetic environment for the resonator to operate. This frequency stability translates to high resolution of the resonant thermometer, which will be discussed in the following section.

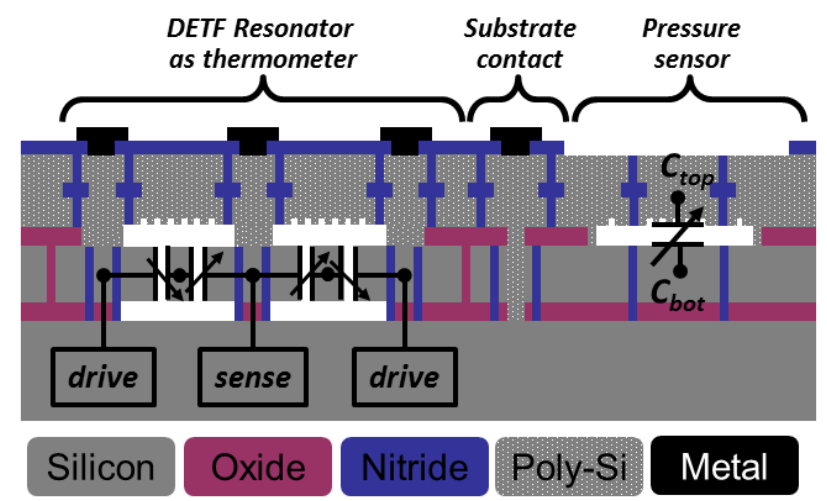

Figure 3: A cross-sectional view of the cofabricated DETF resonant thermometer and capacitive pressure sensor with their electrical functions. Structures are made with silicon on insulator wafers in a front-side only process. Electrical contacts are made with doped silicon while nitride filled trenches are used for electrical isolation.
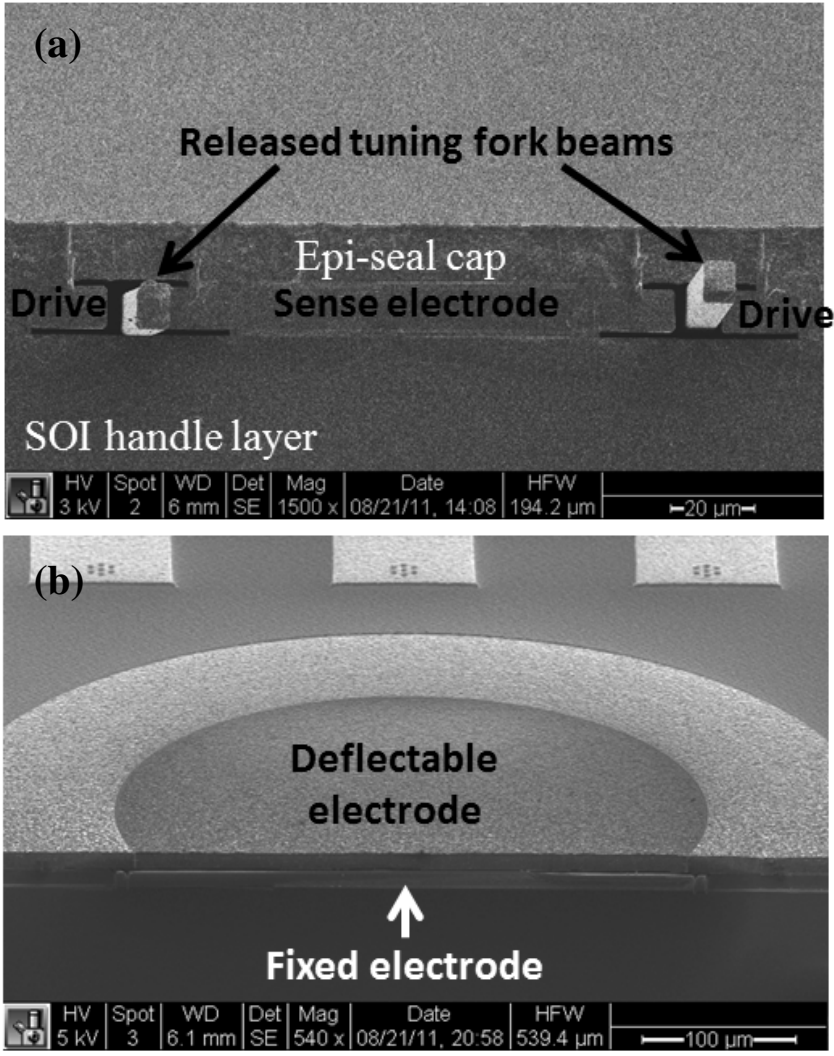

Figure 4: Cross-sectional SEM images of (a) DETF resonant thermometer and $(b)$ capacitive pressure sensor.

\section{INDIVIDUAL SENSOR PERFORMANCE}

\section{Resonant thermometer}

The sensitivity of the resonant thermometer is measured by tracking the resonant frequency change as ambient temperature varies. The test is conducted in a temperature controlled oven and the frequency is measured by using an oscillation circuit with a frequency counter. Figure 5 plots the result where the slope of $\sim$ $31 \mathrm{ppm} / \mathrm{K}$ gives the sensitivity, or $T C f_{l}$. The sensitivity could vary with silicon doping concentration and the structure orientation. Researchers have found that heavily boron doped silicon can reduce the temperature dependency and used this characteristic to demonstrate silicon resonators with highly reduced temperature sensitivity [10]. For the device fabricated in this work, the resistivity of the vibrating beams ranges from 0.22 to $0.26 \mathrm{ohm} \cdot \mathrm{cm}$. After processing, it yields sufficient conductivity for electrical access without losing temperature sensitivity.

As shown in figure 5, measurement noise causes a small error band. In order to determine the resolution of the thermometer, we used two thermometers placed side by side to track the temperature fluctuations at the same time, similar to [5]. In this test, two thermometers were placed in an oven controlled at $50 \pm 0.1{ }^{\circ} \mathrm{C}$. Thermometer 1 is the one to be calibrated and used for temperature compensation in this work. Thermometer 2 is a standard DETF resonator from a previous fabrication run, which should have very similar response to temperature, used as a reference in this case. The normalized frequency variations for the two thermometers are plotted in figures 6(a) and 6(b). By subtracting one from the other and plotting the resultant, as shown in figure 6(c), imperfections in the oven temperature control can be accounted for and thus reveal the inherent noise of the thermometer. This noise of $\sim 300 \mathrm{ppb}$ 


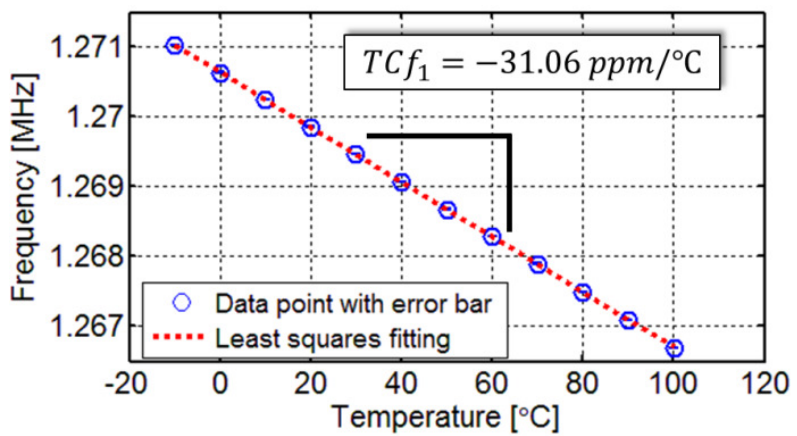

Figure 5: Measured frequency to temperature relationship of a fabricated resonant thermometer. Note the error bars are plotted and shown inside the data point circles.
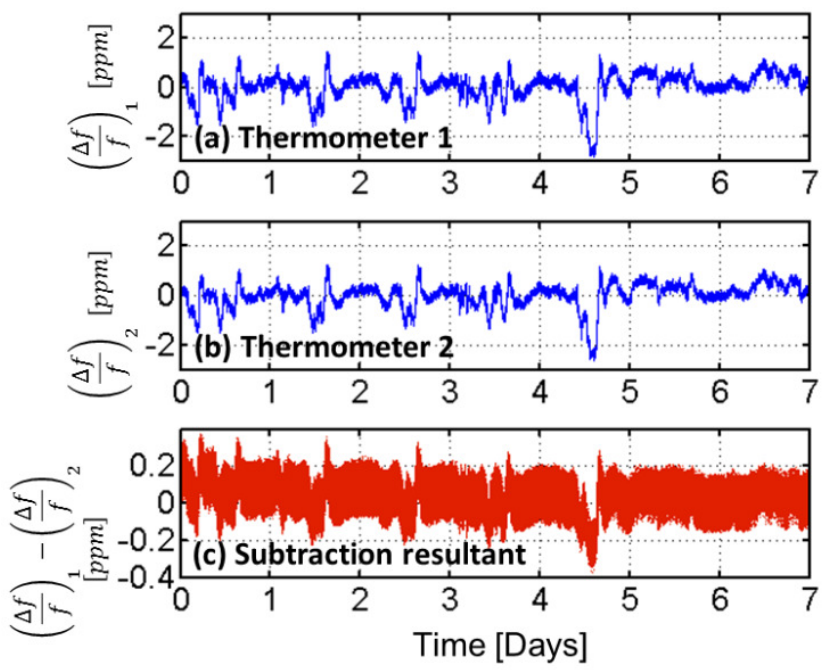

Figure 6: Stability of DETF resonant thermometers tested in a $50 \pm 0.1{ }^{\circ} \mathrm{C}$ oven. (a) and (b): frequency variations over time of two thermometers placed side by side and measured simultaneously. (c): subtraction resultant of the two resonators in order to account for the temperature control fluctuations and determine the thermometer resolution. Data is taken every second using an oscillation circuit with a frequency counter. The $\sim 300$ ppb variation corresponds to approximately $10 \mathrm{mK}$.

frequency variation is essentially the frequency stability of the DETF resonators including measurement noises from the frequency counter and oscillation circuit. Since the frequency of the resonant thermometer varies with temperature at a rate of $\sim-31$ $\mathrm{ppm} / \mathrm{K}$, the resolution of the thermometer used in the current work is approximately $10 \mathrm{mK}$. As demonstrated in [5], the measurement can be further improved by introducing the second thermometer on the same die to reduce temperature gradient effects across the two sensors.

\section{Capacitive pressure sensor}

The pressure sensor is characterized in an environmental chamber and the capacitance is readout with an Agilent 4285A LCR meter. As seen in figure 7, temperature variation causes an offset in the capacitance at a given pressure and changes the sensitivity. For the fabricated device, the capacitance offset falls in the range of 0.3 to $0.35 \mathrm{fF} /{ }^{\circ} \mathrm{C}$. This temperature dependency is undesirable and will be compensated with the co-located resonant thermometer.

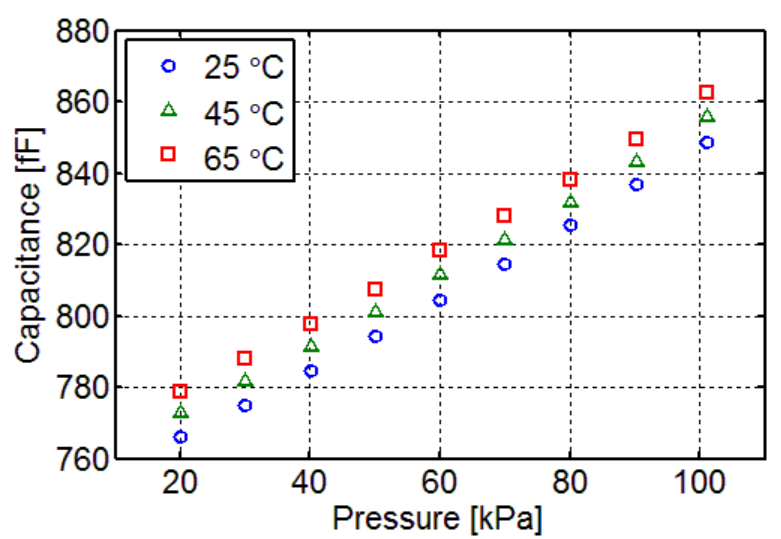

Figure 7: Capacitive response of a pressure sensor measured at various temperatures.

\section{TEMPERATURE COMPENSATION}

In order to demonstrate the feasibility of compensating for temperature induced variation of the pressure sensor output using the DETF resonant thermometer, the sensors are placed in an environmental chamber with both pressure and temperature control. The pressure is monitored by a GE Druck PACE1000 reference pressure gage with resolution of $0.0225 \mathrm{kPa}$ and adjusted by using a PFEIFFER gas dosing valve in a vacuum chamber. Temperature control is performed with a thermoelectric cooler to pump heat in and out by switching the flow direction of the actuation current.

The thermal characteristics of the pressure sensor are first captured by monitoring the capacitance at various temperatures while maintaining at constant pressure. As exemplified in figure 8, at a maintained pressure $(50 \pm 0.045 \mathrm{kPa})$, the correlation coefficient between the resonant thermometer frequency and the capacitance of the pressure sensor is found to be -0.99 over a $50 \mathrm{~K}$ temperature range. This temperature induced frequency to capacitance relationship is extracted and used for temperature compensation. A closer look at the figure, it is noticed that the capacitance exhibits a nonlinear relationship with frequency, or temperature, and a second order polynomial better fits the profile. Therefore,

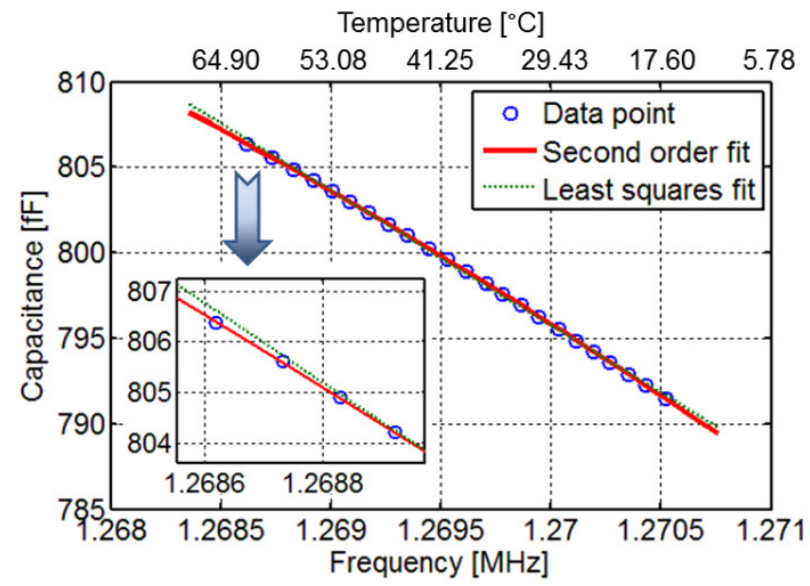

Figure 8: Correlation between the frequency of the resonant thermometer and the capacitance of the pressure sensor under a constant pressure $(50 \pm 0.045 \mathrm{kPa})$ between $15^{\circ} \mathrm{C}$ and $65^{\circ} \mathrm{C}$. A correlation coefficient of -0.99 was obtained. The inset shows a second order polynomial better fits the data. 
(a) Offset errors before and after compensation

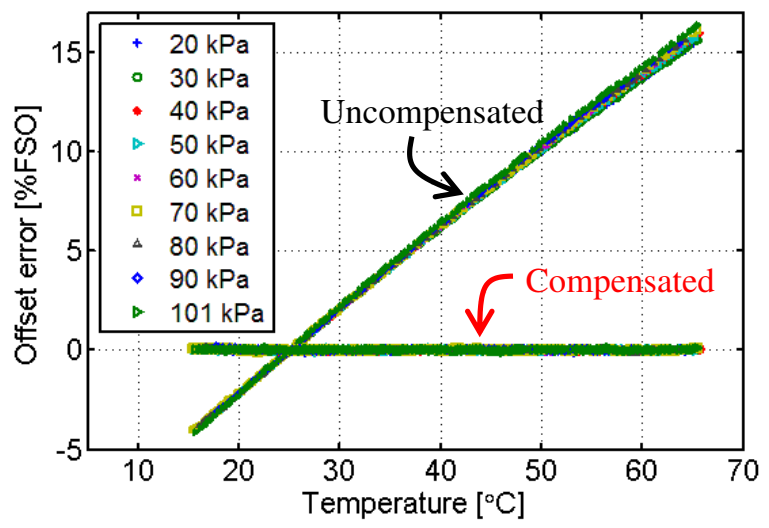

(b) Zoom in to temperature compensated data

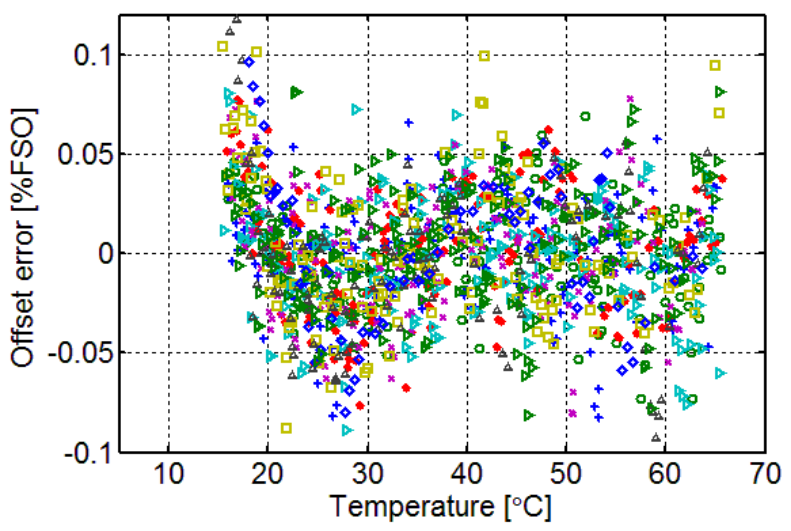

Figure 9: Offset error versus temperature ranging from 15 to 65 ${ }^{\circ} \mathrm{C}$. (a) plots data before and after temperature compensation and (b) zoom in to the offset errors of temperature compensated case. Data was compensated using the calibration result such as the one shown in figure 8 . The $\pm 0.1 \%$ FSO accuracy after compensation corresponds to $\pm 0.08 \mathrm{kPa}$.

the second order polynomial is used in temperature compensation. We performed the same characterization procedure in $10 \mathrm{kPa}$ increments from 20 to $100 \mathrm{kPa}$ to establish a complete calibration.

Figure 9 shows the resulting errors in pressure measurement as a percentage of the full scale output due to temperature variations at different pressures before and after temperature compensation. The data was normalized with respect to capacitance at $25{ }^{\circ} \mathrm{C}$ as reference. For the temperature range investigated, the offset error before compensation is $\sim 0.5 \% \mathrm{FSO} /{ }^{\circ} \mathrm{C}$. By incorporating the calibration data from the resonant thermometer, the temperature sensitivity of the pressure sensor can be significantly reduced. Figure 9(b) enlarges the compensation result. With a few exceptions, the compensated data falls within $\pm 0.1 \%$ FSO range and is concentrated within $\pm 0.05 \%$ FSO. This $\pm 0.1 \%$ FSO variation corresponds to a resolution of $\pm 0.08 \mathrm{kPa}$. Comparing the results before and after temperature compensation, a 100 fold temperature sensitivity reduction is achieved. In addition to the pressure control errors of $\pm 0.045 \mathrm{kPa}$, the random scatter of the data distribution in figure 9(b) suggests that the temperature compensation is limited by the noise associated with the capacitance measurement. As a result, it is believed the remaining challenge to further improve the accuracy lies in the measurement setup. For example, a temperature insensitive ASIC could be mounted in the same package to convert the capacitance to voltage.

\section{CONCLUSION}

A double-ended tuning fork resonant thermometer with resolution of $10 \mathrm{mK}$ and sensitivity of $-31 \mathrm{ppm} / \mathrm{K}$ was cofabricated with a capacitive pressure sensor for temperature compensation. With the proximity of the sensors, temperature gradient and thermal lag time effects can be significantly reduced, yielding accurate temperature measurement. By using the thermometer frequency versus capacitance calibration data, the temperature dependency of the pressure sensor reduces by two orders of magnitude. With this compensation, the pressure sensor achieves measurement accuracy of $\pm 0.1 \%$ FSO in the tested range of $20-100 \mathrm{kPa}$ and $15-65^{\circ} \mathrm{C}$.

\section{ACKNOWLEDGMENTS}

The authors are grateful for the support of Robert Bosch LLC, Research and Technology Center in Palo Alto, CA. The fabrication was carried out in the Stanford Nanofabrication Facility. We would also like to thank former group members Dr. Jim Salvia and Dr. Shingo Yoneoka for the oscillation circuit design and board preparation. Travel support for Chia-Fang Chiang has been generously provided by the Transducer Research Foundation.

\section{REFERENCES}

[1] H. Weinberg, "Temperature Compensation Techniques for Low g iMEMS Accelerometers," Application Note AN-598 of Analog Devices (2002).

[2] J.C. Salvia, R. Melamud, S.A. Chandorkar, S.F. Lord and T.W. Kenny, "Real-Time Temperature Compensation of MEMS Oscillators Using an Integrated Micro-Oven and a Phase-Locked Loop," JMEMS, 19, 192 (2010).

[3] M.A.P. Pertijs, K.A.A. Makinwa, and J.H. Huijsing, “A CMOS Smart Temperature Sensor with a $3 \sigma$ Inaccuracy of $\pm 0.1{ }^{\circ} \mathrm{C}$ from $-55{ }^{\circ} \mathrm{C}$ to $125{ }^{\circ} \mathrm{C}$," IEEE Journal of Solid-State Circuits, 40, 2805 (2005).

[4] Per International Electrotechnical Commission standard, class-A Pt RTD resolution: $\pm(0.15+0.002 *$ Temp. $){ }^{\circ} \mathrm{C}$

[5] E.J. Ng, H.K. Lee, C.H. Ahn, R. Melamud, and T.W. Kenny, "Stability Measurements of Silicon MEMS Resonant Thermometers," Technical Digest of the 2011 Sensors Conference, Limerick, Ireland, October 28 - 31, (2011), pp. $1257-1260$.

[6] R. Melamud, "Temperature Insensitive Micromechanical Resonators," Ph.D. dissertation, Stanford Univ., CA, (2008).

[7] M.A. Hopcroft, W.D. Nix, and T.W. Kenny, "What Is The Young's Modulus of Silicon?," JMEMS, 19, 229 (2010).

[8] C.-F. Chiang, A.B. Graham, G.J. O'Brien, and T.W. Kenny, "A Single Process for Building Capacitive Pressure Sensors and Timing References with Precise Control of Released Area Using Lateral Etch Stop," Technical Digest of the 25th International Conference on Micro Electro Mechanical Systems (MEMS '12), Paris, France, January 29 - February 2, (2012), pp. $519-522$.

[9] R.N. Candler, M.A. Hopcroft, B. Kim, W.-T. Park, R. Melamud, M. Agarwal, G. Yama, A. Partridge, M. Lutz, and T.W. Kenny, "Long-Term and Accelerated Life Testing of a Novel Single-Wafer Vacuum Encapsulation of MEMS Resonators," JMEMS, 15, 1446 (2006).

[10] A.K. Samarao and F. Ayazi, "Temperature Compensation of Silicon Resonators via Degenerate Doping," IEEE Transaction on Electron Devices, 59, 87 (2012).

\section{CONTACT}

Chia-Fang Chiang, e-mail: cfchiang@mems.stanford.edu 


\section{IT MAKES SENSE: HOW EXTREME ANALOG AND SENSING WILL CHANGE THE WORLD \\ Benedetto Vigna \\ STMicroelectronics, Geneva, Switzerland}

\begin{abstract}
Although only six years have passed since the adoption of motion sensors in the consumer market, the world of MEMS sensors has changed dramatically and is set to change even more in the future. If 2006 was the year of the Accelerometer's adoption by Nintendo ${ }^{\mathrm{TM}}$ in the Wii ${ }^{\mathrm{TM}}$ Controller, 2010 was the year of the Gyroscope's adoption by Smartphone manufacturers, like Apple ${ }^{\mathrm{TM}}$ and Fujitsu Mobile ${ }^{\mathrm{TM}}$. Moreover, combinations of these two inertial products, also known as Inertial Six DegreeOf-Freedom Motion Sensors (I6XDOF) are now starting to appear in the market, and most likely they will coexist with standalone accelerometers and gyroscopes, depending on customer needs in terms of compactness, performance, and final industrial design. This paper reports the details of the world smallest 3-axis Single Core and Dual Core silicon MEMS Coriolis Gyroscopes in production that fulfill the pressing market requirements for low power consumption, small size, slim form factor, high performance, and low cost. But it also addresses the recent trends of the Inertial Six Degree-OfFreedom, Mixed Six Degree-of-Freedom M6XDOF (3X Accelerometer plus 3X Solid State Compass), Nine Degree-ofFreedom (9XDOF) systems, realized by integrating the I6XDOF with a 3-axis magneto-resistive solid state compass, and the Ten-Degree of-Freedom (10XDOF) system, realized by adding a pressure sensor to the 9XDOF.
\end{abstract}

\section{INTRODUCTION}

Up until now, the key application driving the adoption of Motion Sensors in applications such as mobile phones and remote controllers, laptop and tablet computers, games and portable multimedia players, has been their ability to enable more intuitive user interfaces. Today, Image Stabilization, Context Awareness, Location Based Services, and Remote Monitoring are the new frontiers that will continue to drive the adoption of Motion Sensors, both in existing market segments and in many new areas, some of which are not even known today.

Linear motion detection is often required to be combined with angular velocity sensing along the pitch, yaw, and roll axes and with altitude variations in a wide range of applications where sensing three to ten degrees of freedom is required. One example is represented by Inertial Measurement Units (IMU) or Inertial Navigation Units (INU), in which accelerometers and gyroscopes are used in a complementary manner to detect linear and angular motion in many different applications: enhanced user interfaces for mobile phones, games and pointers/remote controllers, image stabilization, pedestrian and car navigation for location based services and fitness/wellness monitoring. As was the case with stand-alone accelerometers, size, performance, and price remain the driving factors for gyroscopes and for $6 \mathrm{XDOF} / 9 \mathrm{XDOF} / 10 \mathrm{XDOF}$ success in the Consumer and Industrial markets.

In Optical Image Stabilization (OIS) applications, IMUs can be used to track an object where the subject is blurred within the image due to vibrations applied to the devices. OIS systems can be implemented using pixel tracking by intensity movement or frame tracking. Typically, the IMU is embedded in video or other systems with Pan-Tilt and Zoom capabilities or mounted on moving platforms in surveillance applications [3]. Motion activation and image stabilization are features aimed to improve picture or video quality.

Inertial Navigation Unit (INU) in pedestrian/car navigation is used for Dead Reckoning (DR) to estimate the user's current location, based on a previously determined known location, when no GPS signal is available or its quality is very poor. The information from the sensor cluster is typically processed via complex Kalman filters to estimate the current position of the user. This powerful facility opens up a wide variety of applications based on the user's actual location (the so-called LBS, Location Based Services), enabling many new innovative services that will enhance everyday life. Motion Sensors are not enough on their own, but when combined with altimeters and short range radio sniffers like $\mathrm{WiFi}$, they enable an outstanding indoor navigation experience. Ten Degrees of Freedom $(10 \mathrm{XDOF})$ is mandatory to enable this application.

\section{THELMA $^{\text {TM }}$ FABRICATION PLATFORM}

All gyroscopes and accelerometers manufactured by STMicroelectronics are composed of two chips stack-assembled into one plastic Land Grid Array Package [Fig. 1]. The first chip contains the micro-machined sensing element that converts the angular rate and/or the acceleration into a capacitance variation. It is manufactured using a $1.0 \mu \mathrm{m}$ minimum lithography proprietary fabrication process, named THELMA ${ }^{\mathrm{TM}}$ which comes in two flavors:

\section{- $\mathrm{THELMA}^{\mathrm{TM}} /$ Standard}

- $\mathrm{THELMA}^{\mathrm{TM}} /$ Smeraldo

The second chip is built using an advanced CMOS process $(0.13 \mu \mathrm{m}$ node $)$ with built-in high density capacitors to amplify the small signal of the mechanical transducer and to enable an easy integration of the product in the final customer board. The motion sensor's companion CMOS chip may be analog or digital, depending on the integration of the Analog-to-DigitalConverter circuit block and on the need to have a dedicated microcontroller and memory for signal processing. The CMOS die doesn't require any additional micromachining step.

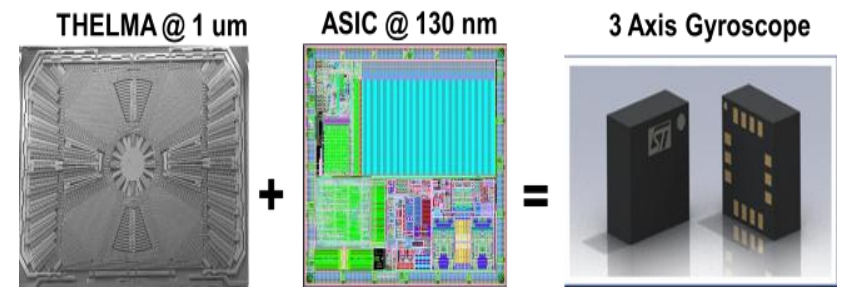

Figure 1: The micro-machined element on the left is assembled with the high performance ASIC in a single package. The package on the right measures only $3 \times 3.5 \times 1.0 \mathrm{~mm} 3$ and it's the world smallest gyro currently in high volume production. 
The THELMA ${ }^{T M}$ process requires the processing of two silicon wafers and one bonding operation. It begins with a standard silicon wafer onto which a layer of oxide $(\sim 2.5 \mu \mathrm{m})$ is first grown for electrical isolation. A thin poly-silicon layer, used for interconnections, and a second sacrificial oxide layer $(\sim 2.5 \mu \mathrm{m})$ are then deposited. Into this layer, holes are etched at the points corresponding to the supports for fixed elements and anchors for moving elements. A thicker poly-silicon epitaxial layer (from $20 \mu \mathrm{m}$ to $100 \mu \mathrm{m}$ ) is then grown on top of this, and into this third structural layer the structures for the moving and fixed elements of the device are etched with a single mask by means of a deep dry silicon etching process. Finally, the sacrificial oxide layer beneath the structures is removed by an isotropic wet etching operation to free the moving parts. The open space around the structures is then filled with a gas, usually dry nitrogen or argon, to tune the damping factor and the resonance frequency of the micro-mechanical structure. A second wafer, with mechanical stoppers, a getter (i.e. "gas eater") layer and a glass frit or metal ring is then bonded to the first wafer to protect the tiny structures during an injection molding process during which high pressures are applied. The getter is fundamental to guarantee the stability over time of the product performances since any material in the world is outgassing, and thus it lowers the quality factor of the micromachined sensing element by changing the air damping coefficient. In the Smeraldo process flavor, the sensor wafer has embedded Through Silicon Vias (TSV) [Fig. 2], enabling further die shrinkage and smarter 3-Dimensional stacking for future Wafer Level Chip Scale package solutions.
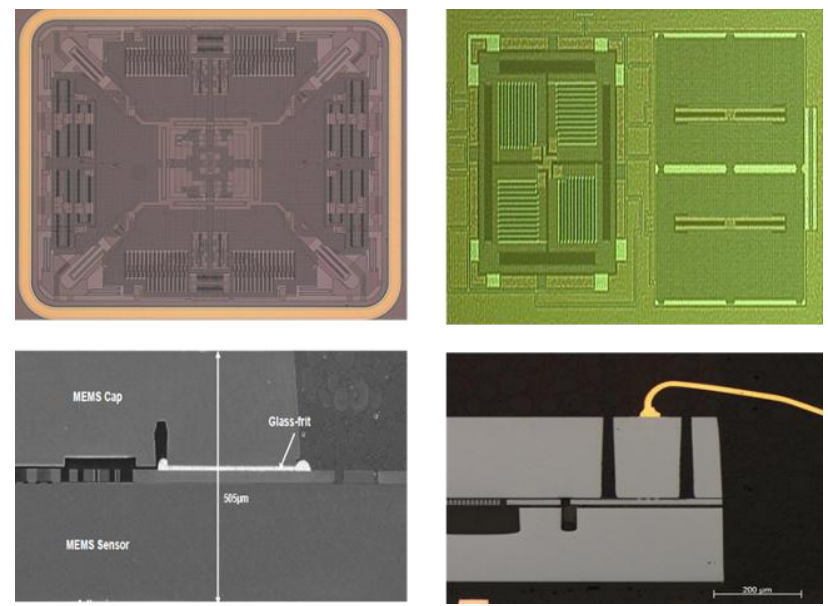

Figure 2: Accelerometer (top right picture) and Gyroscope (top left picture) realized in the THELMA ${ }^{T M}$ process. In case of the THELMA ${ }^{T M}$ Standard flavor the connection pads for wire bonding to ASIC are on the same side of the micro-machined structure, and the cap has some clearance window during the wafer bonding process (bottom left picture). In the Smeraldo flavor (bottom right picture) the connections are on the back side of the micro-machined structure, and the wafer sandwich is flat during the wafer to wafer bonding operation and at the end.

\section{THE TRI-AXIAL SINGLE CORE AND DUAL CORE CORIOLIS GYROSCOPES}

Gyroscopes enable many different applications like a more intuitive user interface, optical image stabilization, and locationbased-services. User Interface and Location Based Services require the ability to measure angular rate with relatively high full scale, from few hundred degrees/second (dps) to a few thousand dps, with a good noise density level $(0.05 \mathrm{dps} / \sqrt{\mathrm{Hz}})$, and an output data rate of up to $1 \mathrm{kHz}$; Optical Image Stabilization requires gyroscopes with a full scale lower than $100 \mathrm{dps}$, a very low noise density level $(0.01 \mathrm{dps} / \sqrt{\mathrm{Hz}})$, and an output data rate up to $10 \mathrm{kHz}$.

To address the different applications and to enable the roadmap of different players in the markets, from mobile phone and game console manufacturers to camera module and Digital Still Camera makers, STMicroelectronics developed two different types of gyroscopes assembled in the world smallest packages:

- SINGLE CORE in $3 \times 3.5 \times 1 \mathrm{~mm}^{3}$

- DUAL CORE in $4 \times 4 \times 1 \mathrm{~mm}^{3}$

DUAL CORE gyroscopes have two sensing cores [Fig 3] and thus enable two applications concurrently, while SINGLE CORE gyroscopes have only one sensing core and thus enable only one application at a time. In the first case, there are two digital outputs from which the 3 angular rates (Yaw, Pitch, and Roll) can be read simultaneously, and thus use the gyroscope to enable two applications at the same time. In the latter case instead there is only one digital output and thus only one application at a time can be enabled.

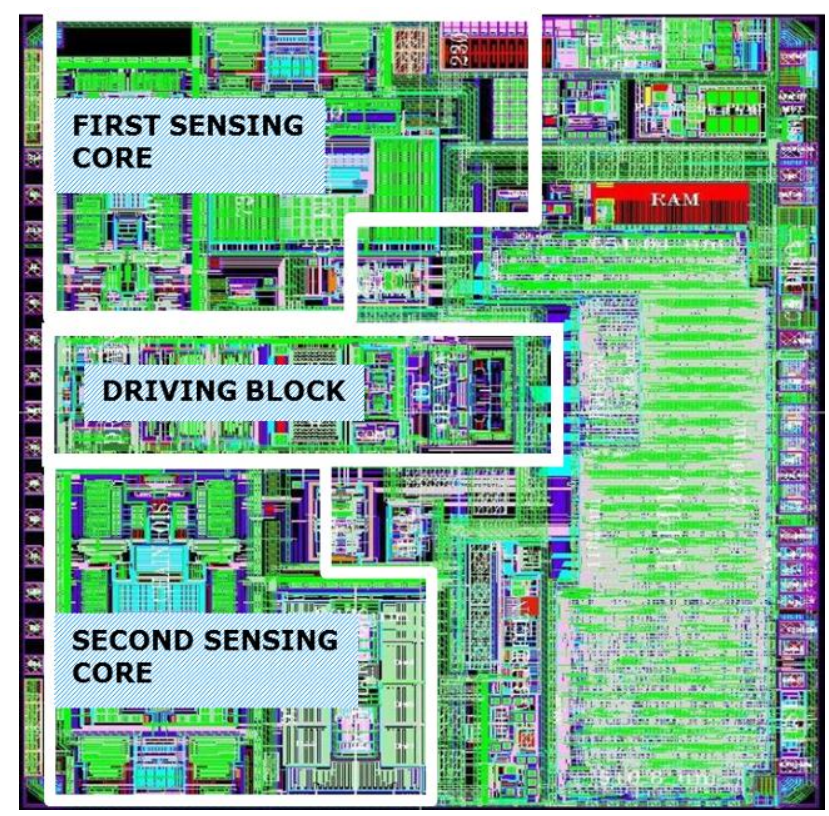

Figure 3: Each ASIC of a Gyroscope contains a driving block to resonate the tiny micro-machined mass at its resonance frequency. DUAL CORE gyroscopes have two sensing cores running in parallel and are able to detect small and high full scales with different data rates and resolutions.

The possibility of realizing these two classes of Gyroscopes by using the same chip architecture for both SINGLE and DUAL CORE gyroscopes with a single mechanical element is grounded on an innovative micro-machined transducer design that will be described in the next paragraph. It is known as "The Beating Heart".

The innovative, compact micro-mechanical transducer, which combines a triple tuning-fork structure with a single vibrating mass, is named "The Beating Heart" because of its 
working mode. The Beating Heart achieves excellent performance in terms of thermal stability, cross-axis error, and acoustic noise immunity by using a small die size.

Furthermore, the presence of a single primary vibration mode for the simultaneous excitation of the three tuning-forks, together with the ability to sense the pickoff modes in a multiplexing fashion, allows the design of a small area, low power companion chip. In fact, the presence of a single vibrating mass avoids the problems of mechanical frequency mixing and interference, extremely deleterious in gyroscopes based on multiple vibrating masses. The mechanical structure design is explained with the aid of Figure 4.
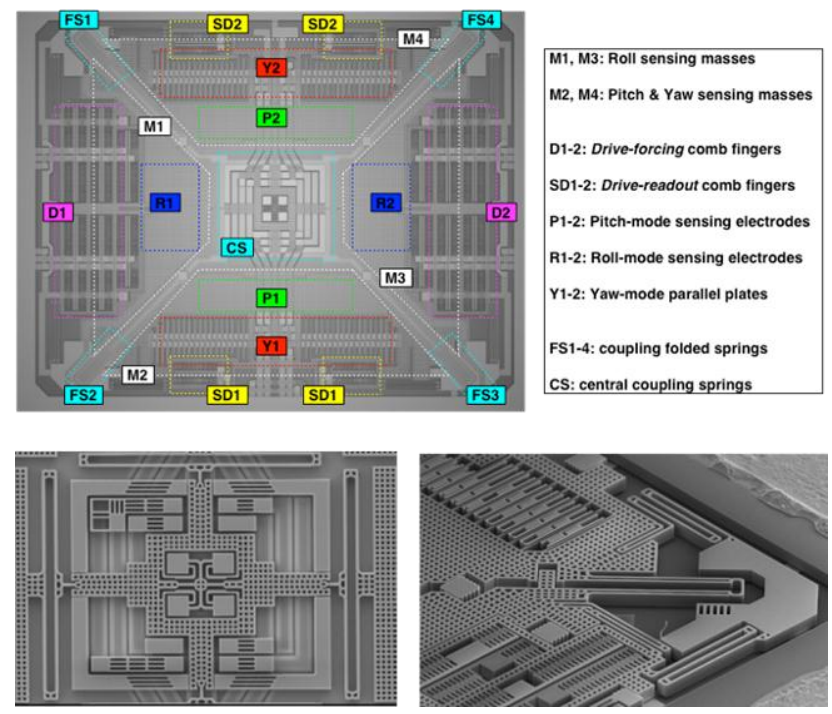

Figure 4: The poly-silicon micro-machined structure on the top is expanding and contracting at a frequency of $20 \mathrm{kHz}$ around the suspended center of mass highlighted in the bottom left picture and thanks to the spring shown on the bottom right picture.

The structure comprises four suspended plates coupled to each other by means of four folded springs connected to their outer corners, and elastically connected to a central cross-shaped hinge by an additional set of central coupling springs. The primary mode of vibration (driving mode) consists of an inplane inward/outward radial motion of the plates: on the whole, the structure cyclically expands and contracts, similarly to a beating heart (hence, the name). Primary actuation is provided with a set of comb-finger electrodes placed only on a pair of opposite plates; the mechanical motion is then propagated to the second pair by means of the coupling folded springs at the corners. The secondary modes of vibration (sensing modes) consist of an in-plane, opposite-phase motion of the second pair of plates (yaw mode), and two out-of-plane, opposite-phase motions of both pairs (pitch and roll modes).

The yaw mode is sensed by a set of parallel-plate electrodes located on the second pair of plates, whereas the pitch and roll motions are detected by sensing the capacitive variations between each plate and an electrode placed underneath; additional comb-finger electrodes are reserved for sensing the vibrating motion of the driving mode. The mechanical coupling between the two proof masses of each sensing pair allows the secondary vibrating motions to be read in differential mode, thereby improving rejection of external linear accelerations and vibrations. In addition, each secondary mode has a single resonant sensing frequency instead of two independent frequencies that require accurate matching to avoid performance degradations, which is a disadvantage of designs with uncoupled proof masses. The overall mechanical structure has frequencyunmatched primary and secondary modes, with a peak-to-peak movement of the driving mass of about $20 \mu \mathrm{m}$ and a nominal primary resonant frequency of $20 \mathrm{kHz}$. Combined with a high and time-stable quality factor (Q-Factor), guaranteed by the presence of a gettering material in the wafer-level cavity, this design choice results in an excellent level of acoustic noise isolation.

The Coriolis force exciting a secondary mode, which is proportional to both the velocity of the driving mode and the input angular rate, is directed orthogonally to both the driving axis and sensor rotation axes. The angular rate measurement is obtained from the sensed Coriolis acceleration by demodulation, once the driving mode is oscillated at constant amplitude [1].

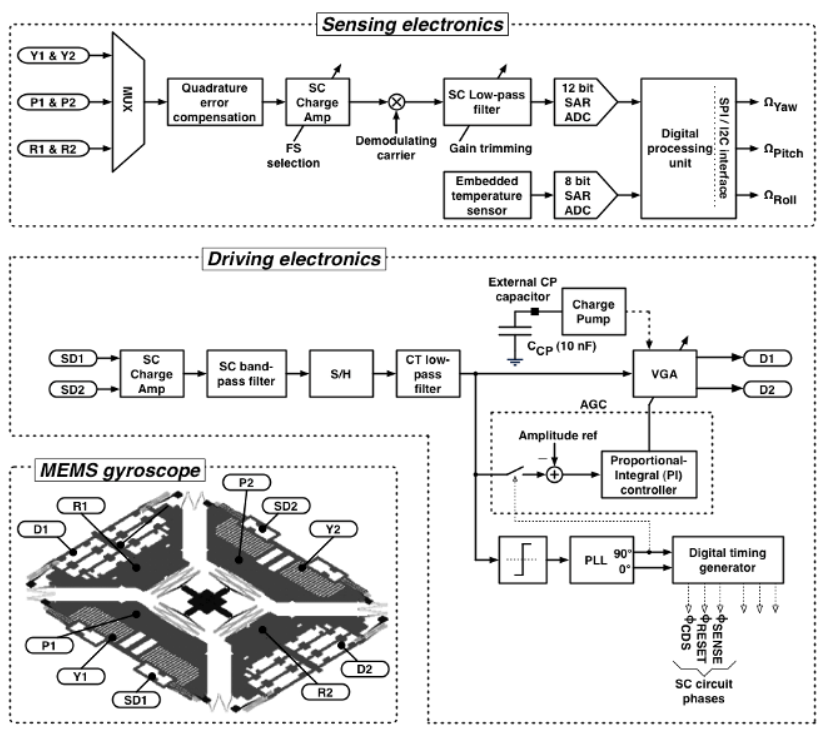

Figure 5: System Architecture Block Diagram

The primary mode is excited to oscillate at resonance by closing a feedback loop around the micro-resonator made up of the resonating masses and the drive-readout/drive-forcing combfinger electrodes. In the feedback path, the capacitive unbalancing generated by the oscillating motion of the primary mode is converted into a voltage signal by a differential charge amplifier; then, a band-pass (BP) switching-capacitor (SC) amplifier removes the residual offset and provides the necessary phase adjustment to give a total loop phase shift of $360^{\circ}$ at the resonant frequency, which is required to enforce a sustained oscillation in the electromechanical loop. The BP amplifier output is interpolated by a $2^{\text {nd }}$ order continuous-time (CT) lowpass (LP) Chebyshev filter. The resulting signal is then amplified by a variable-gain amplifier (VGA), whose gain is automatically tuned by an outer automatic gain control (AGC) loop to regulate and verify the amplitude of the sustained oscillation at the CT-LP filter output to a constant set-point value. Finally, the VGA output, boosted by a charge-pump, is fed back to the comb-drive actuating electrodes. All internal timings are generated by a PLL synchronized with the CT-LP filter output. 
A single, time-division multiplexed open-loop readout interface is used to retrieve the angular rate measurements from the Coriolis accelerations along the three sensing axes. A differential charge-amplifier front-end circuit converts the capacitive imbalance induced by the movement caused by the Coriolis forces into a voltage signal, which is then synchronously AM-demodulated using a carrier that is in-phase with the velocity of the primary mode motion. A 12-bit SAR (Successive Approximation Register) ADC performs internal $\mathrm{A} / \mathrm{D}$ conversion at a rate of $6.06 \mathrm{kHz} / \mathrm{axis}$; a $100 / 200 / 400 / 800 \mathrm{~Hz}$ output data rate (ODR) is selected by changing the decimation factor of the output sync-decimators. The final output is a 16-bit value. The compensation of the quadrature error, the most significant challenge in gyroscopes due to the non-uniform deep silicon etching profile, is performed passively, thanks to extremely good control of the spring cross section.
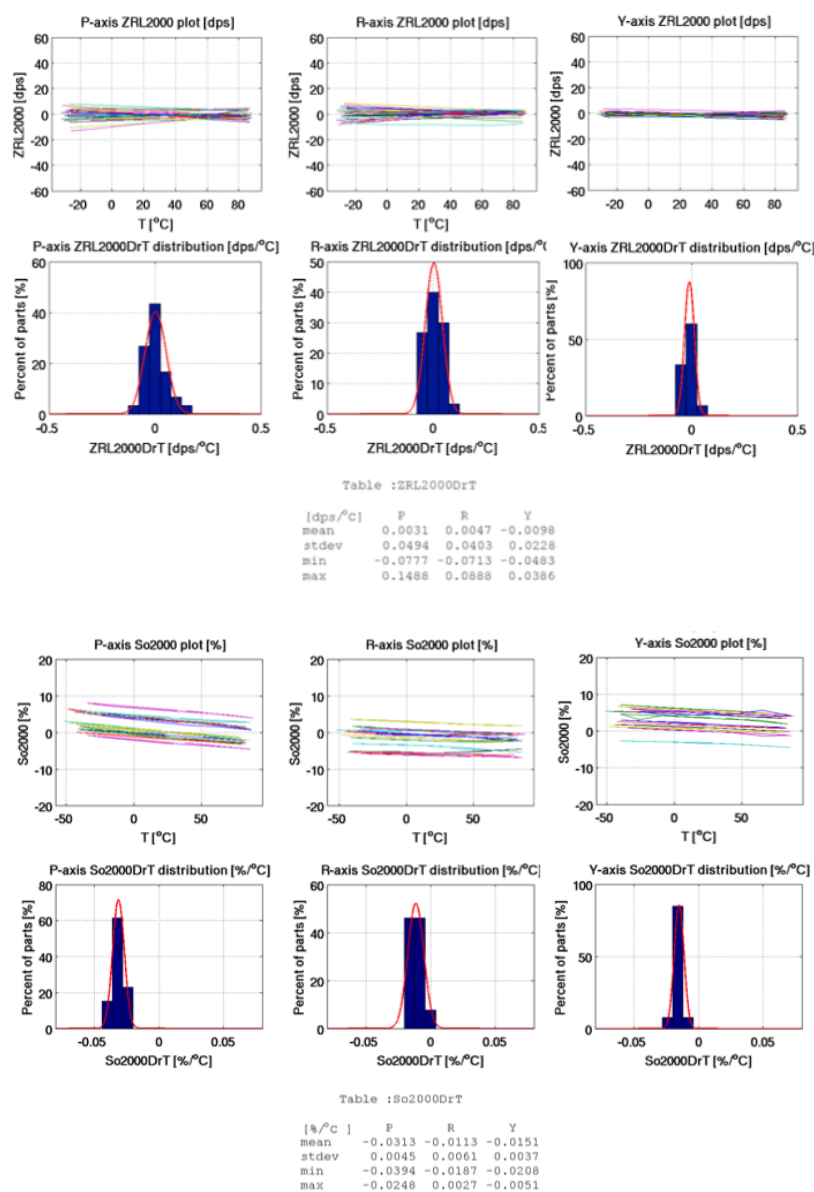
distribution of the ZRO over temperature (first 6 graphs - top). Sensitivity (So) stability over temperature and statistical distribution of So over temperature (bottom graphs).

The characterization results of 33 different samples are reported in Figure 5, with all tests performed with a Full Scale of $2,000 \mathrm{dps}$.

The overall performance results are excellent: the average noise density level is less than $0.02 \mathrm{dps} / \sqrt{\mathrm{Hz}}$ (with $\mathrm{BW}=40 \mathrm{~Hz}$ and $\mathrm{ODR}=200 \mathrm{~Hz}$. and the zero-rate output $(\mathrm{ZRO})$ and sensor scale factor (So) are very stable over temperature. With $\mathrm{FS}=2000 \mathrm{dps}$, the ZRO temperature sensitivity is less than $\pm 0.04 \mathrm{dps} /{ }^{\circ} \mathrm{C}$, while the scale factor change over the temperature range $-40^{\circ} \mathrm{C}$ to $85^{\circ} \mathrm{C}$ is within $\pm 2 \%$ of the factory trimmed value ( $\mathrm{So}=70 \mathrm{mdps} / \mathrm{LSB}$ with $\mathrm{FS}=2000 \mathrm{dps}$ ). The design robustness is assured by the tight statistical distributions of the ZRO and So temperature sensitivities. Thanks to the highly symmetric mechanical design, the cross-axis sensitivities, measured as a percentage of the nominal selected full scale, are always less than $\pm 2 \%$ and are mainly due to mounting tolerances during the packaging phase.
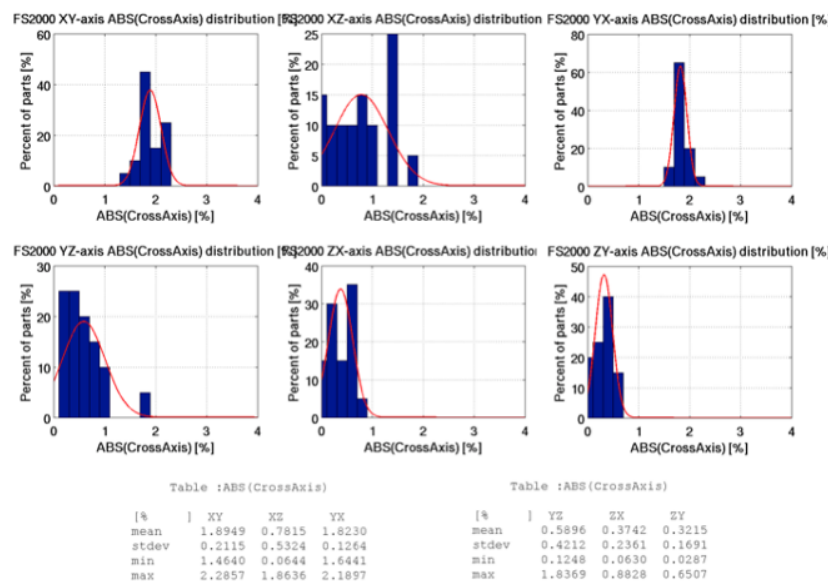

Figure 7: Statistical distributions of the cross-axis sensitivities over a set of 33 samples. Tests have been performed with $F S=2000 d p s$.

High immunity to acoustic noise is evident from data reported in Figure 7 (Test conditions FS=2000dps, ODR $=200 \mathrm{~Hz}$, output $\mathrm{BW}=50 \mathrm{~Hz}$ ).
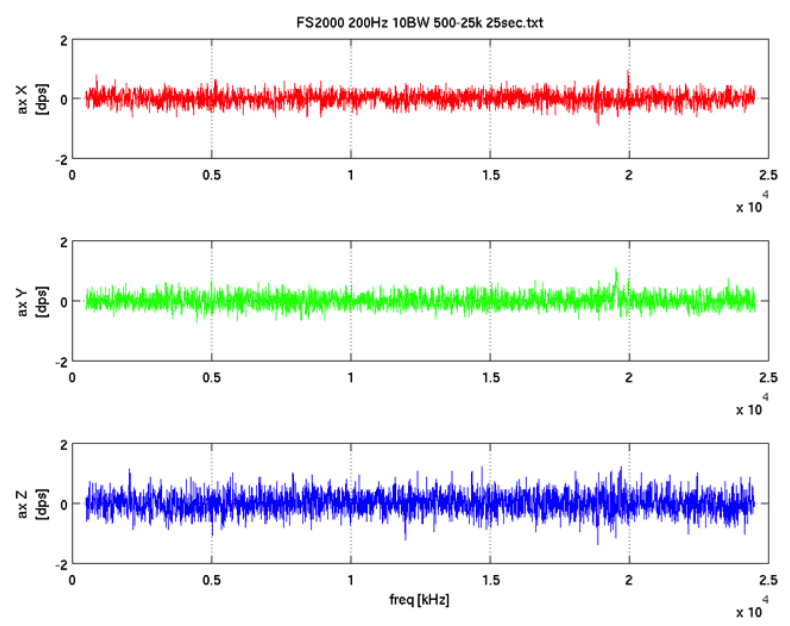

Figure 8: Gyroscope output response to an acoustical stimulus (sine noise at 90dBSPL, frequency sweeping in the range $500 \mathrm{~Hz}$ $\div 25 \mathrm{kHz}$ with steps of $5 \mathrm{~Hz}$ ). The plots show the average values of the pitch/roll/yaw angular rate outputs for each frequency of the sinusoidal acoustical stimulus.

In terms of power consumption, with a supply voltage in the range $2.16-3.6 \mathrm{~V}$, the current consumption is $5 \mathrm{~mA}$ during 
normal operation, $1.5 \mathrm{~mA}$ in sleep mode (sensing electronics switched off, but with the driving block still operative to reduce the power-on time), and $5 \mu \mathrm{A}$ in power-down mode.

Market applications will continue to require lower power consumption gyroscopes, in the range of $1 \mathrm{~mW}$, and that is where different suppliers are aiming with their product and technology roadmaps.

\section{INEMO $^{\text {TM }}$ : THE TEN ANALOG BUILDING BLOCKS OF THE FUTURE SMART DEVICES}

Depending on the application needs of the customers, on the layout constraints for the final product, and on the customer's purchasing strategy, there are cases in which Six Axis and Nine Axis Modules are preferred to discrete solutions. The ability to design and produce both tri-axis accelerometers and tri-axis gyroscopes in the same technology platform makes it easy to produce Inertial Six Degree-Of-Freedom Combos (I6XDOF). STMicroelectronics is currently in production with high performance Inertial Six Axis Motion Sensors in 3.5 x5.0 $\mathrm{x} 1.0 \mathrm{~mm} 3$ packages and is aiming to futher miniaturize the solution by exploiting the integration advantages of the THELMA ${ }^{T M}$ platform. Often customers worry about the miniaturization of the sensor itself and forget about the space required by the passives for the proper operation of the device itself. STMicroelectronics has been the first player to successfully integrate passive components, like discrete capacitors and resistors, together with micro-machined sensing elements in one package.

The Inertial Six Axis Motion Sensor is a result of the combination of volume-proven micro-mechanical structures which may be implemented on two different silicon dice or on the same die, but in either case are assembled with the companion CMOS chip in the same package by means of System-in-Package (SiP) assembly techniques [Fig. 9 and Fig. 10]. The bigger technology challenge STMicroelectronics fixed, in order to enable the integration of the Accelerometer and Gyroscope sensing element on the same chip, has been the selective realization of different vaccum levels in the two different cavities hosting the two different sets of masses.
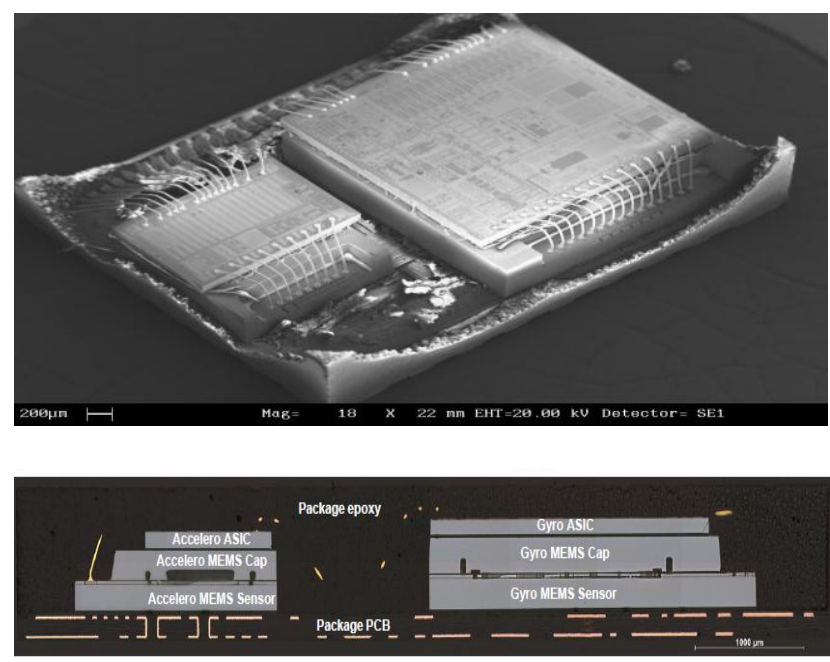

Figure 9: Six Axis Motion Sensor in 3x5.5x1.0mm ${ }^{3}$ LGA Package seen in perspective and in cross section. The Accelerometer and Gyroscope mechanical structures are located on two different silicon dice. The discrete capacitor is embedded in the same package.

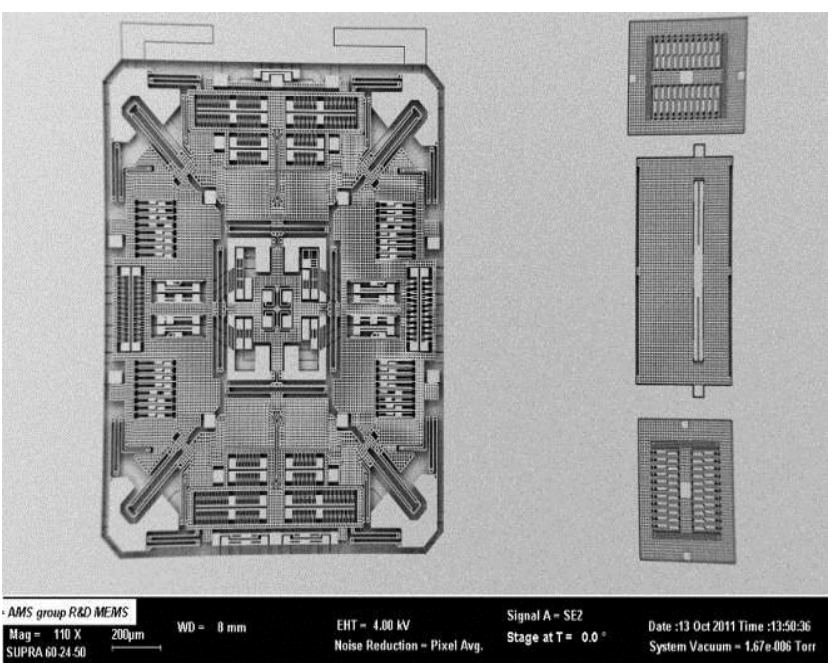

Figure 10: $3 X$ Gyroscope and $3 X$ Acceleroemeter on the same silicon die realized with the THELMA ${ }^{T M}$ micromachining platform.

Some customers, however, may not require the integration of the gyroscopes with an acceleroemter, and thus they are considering the M6XDOF, i.e. the combination of a $3 \mathrm{X}$ Accelerometer with a $3 \mathrm{X}$ Compass. As an example, there are customers that having integrated the Dual Core gyroscope in the camera module of a smartphone or a tablet, do not require another any additional gyroscope on the main PCB of the device. In this case, the best solution for the customer is the one depicted in Figure 11.

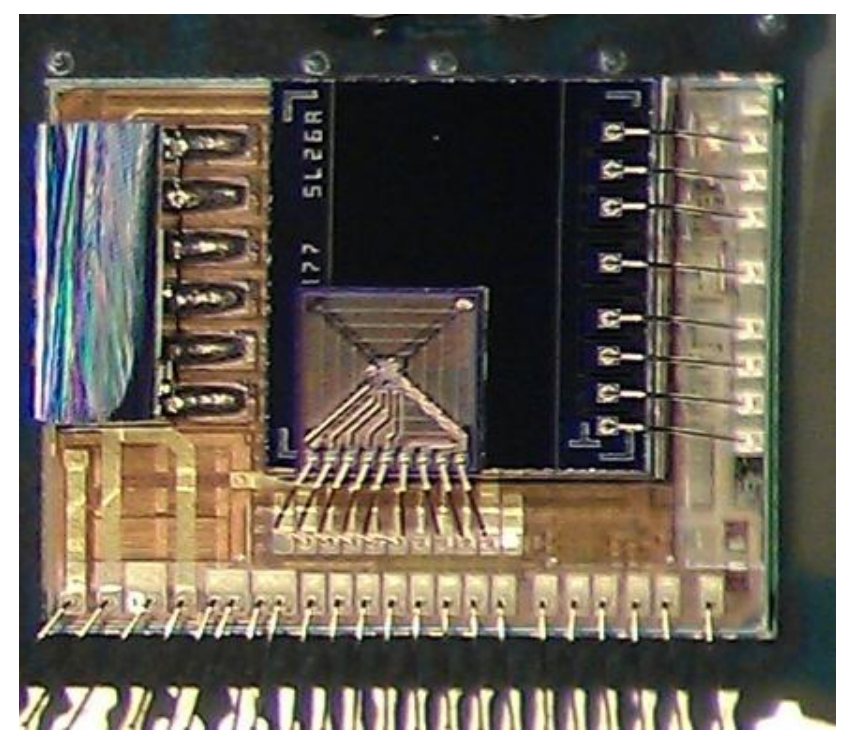

Figure 11: A $3 X$ Compass and a $3 X$ Acceleroemeter in the same package. The Z-Axis Magnetoresistive element has been assembled at $90^{\circ}$ on the ASIC for the realization of the M6XDOF.

There are many different technologies available to realize the compass, including Hall-effect devices and MagnetoImpedance-based sensors. STMicroelectronics uses the Anistotropic MagnetoResistance-based approach because of its 
very high resolution, high temperature stability, and low current consumption.

At this point, a 9XDOF sensor can be easily realized by adding all the sensors together. The 9XDOF sensor can be offered as a discrete module or as an integrated package as in Figure 12.
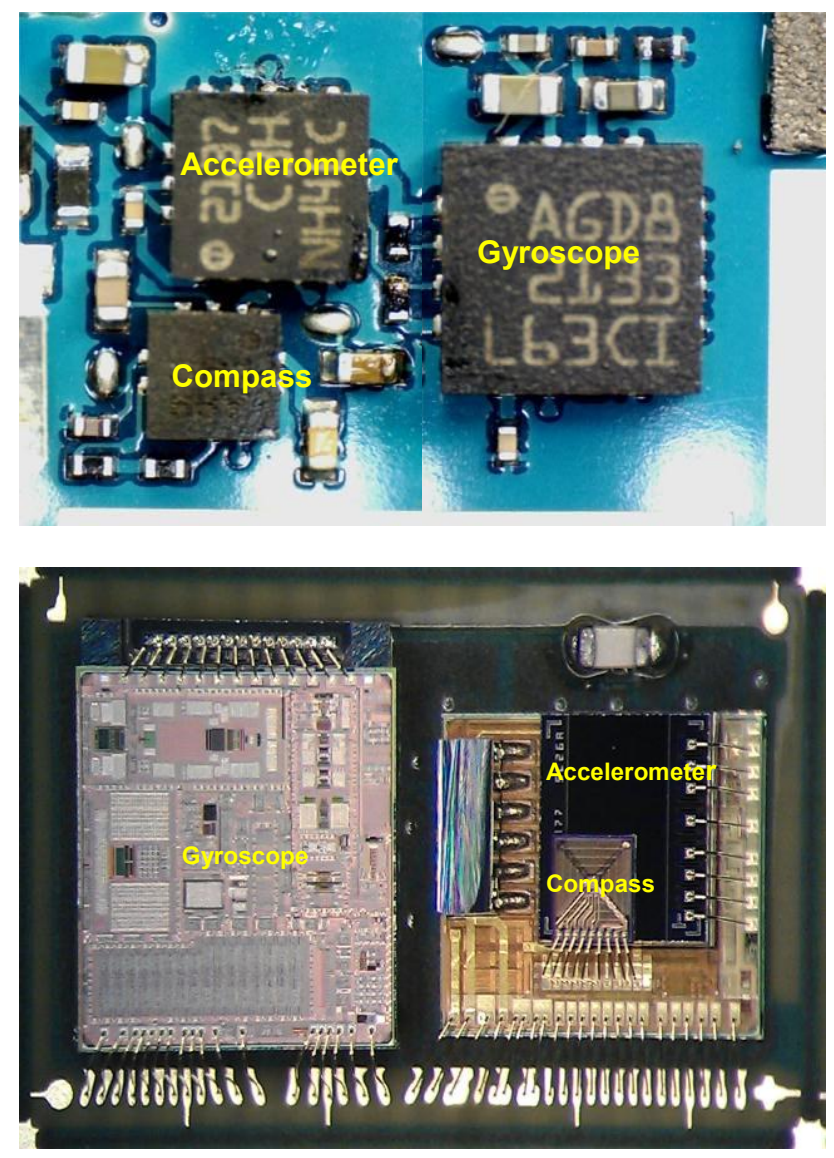

Figure 12: The three basic components of the 9XDOF are each housed in three different packages (top picture). The Accelerometer package footprint is $3 \times 3 \mathrm{~mm}^{2}$, the Gyroscope one measures $3 \times 3.5 \mathrm{~mm}^{2}$, and the Compass is $2 \times 2 \mathrm{~mm}^{2}$, totalling a footprint of $23.5 \mathrm{~mm}^{2}+$ the passive components overhead. In the bottom picture, instead, the three basic components of the 9XDOF are packaged alltogether in a single package measuring only $3.0 \times 6.5 \times 1.0 \mathrm{~mm}^{2}$ or $4 \times 4 \times 1.0 \mathrm{~mm}^{3}$

For some applications, like indoor navigation, the 9XDOF is not enough since the altitude may need to be known, and thus an altimeter is required [Fig. 13]. As a rule of thumb, the weight of atmosphere decreases $1 \mathrm{mBar}$ every eight meters of altitude, and thus by measuring the pressure, it is easy to understand at which floor one is on in a mall, for example.

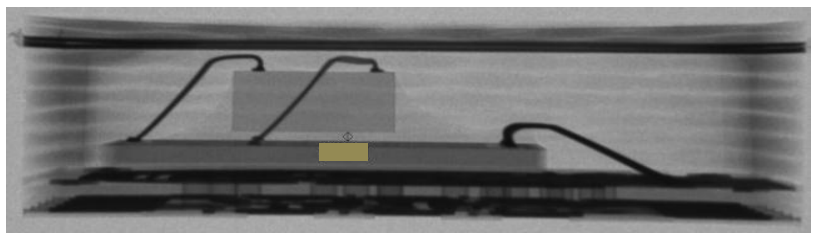

Figure 13: X-ray picture of 3x3x1mm3 packaged Pressure Sensor.

\section{THE ALTIMETER FOR THE CONSUMER MARKET}

Pressure Sensors have long been in service measuring pressure and flow in a variety of industrial, automotive, and medical applications. Micro-machined pressure sensors are fabricated in Silicon with the physical sensing mechanism being either a variable resistance or a variable capacitance. The fabrication is either "Bulk" or "Surface" micro-machining or a combination of the two. Standard silicon substrates or more expensive Silicon-On-Insulator substrates are equally used as a starting point.

The variable resistance type uses the piezo-resistive nature of silicon to convert stress in a small diaphragm into a very small resistance change. Bulk micro-machining technology is the preferred technique. The variable capacitance type uses two parallel plates. One is fixed and the other is a thin diaphragm moving in an orthogonal direction to the plane of the chip. A very small change in capacitance then occurs between these two plates. Surface micro-machining methods are preferred for this class of pressure sensors. The interface circuitry that converts resistance to voltage, or capacitance to voltage, can be integrated at chip level or at package level, as for motion sensors. But also in this case, the System-In-Package solution guarantees faster commercialization time and higher flexibility for the customer.

No ideal manufacturing process exists, but each company follows the micro-maching approach that best exploits its experience and its manufacturing assets. Nonetheless, because of the plethora of processes running in different semiconductor fabs, the high-volume consumer market players have had to accept compromises related to price, size, and performance of the pressure sensor. And this compromise has limited the wide adoption of this sensor in consumer devices, delaying the deployment of Location Based Services.

Only recently some companies have found a viable solution for the consumer market. In particular, STMicroelectronics developed a micro-machining technology called VENSENS ${ }^{\mathrm{TM}}$ in high volume production that enabled the realization of a $3 \times 3 \times 1 \mathrm{~mm} 3$ package. As Figure 14 shows, this proprietary technology enables the manufacturing of a "Full-Silicon" small footprint $\left(0.8 \times 0.8 \mathrm{~mm}^{2}\right)$, thin $(\sim 0.3 \mathrm{~mm})$ and inexpensive pressure sensor transducer that, once combined with an extreme analog ASIC, is able to detect the altitude with extreme accuracy, i.e. within $+/-1 \mathrm{mBar}$.
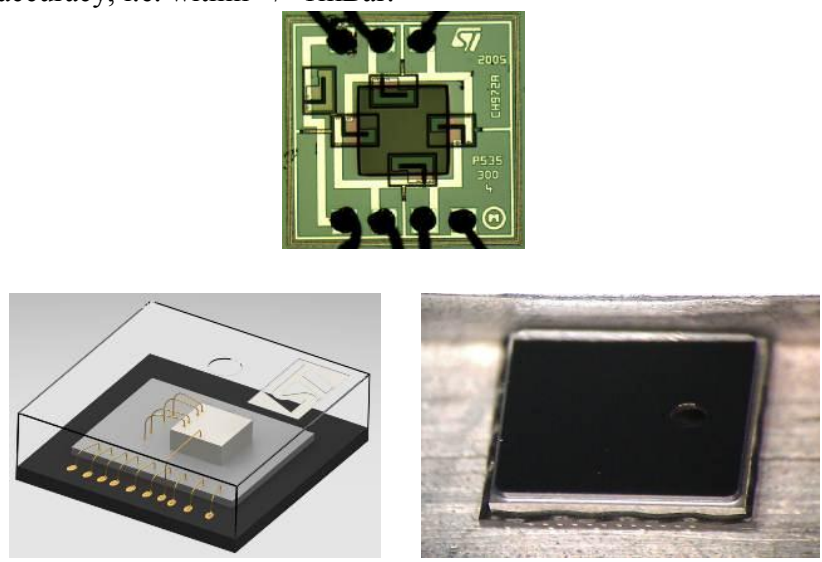

Figure 14: The VENSENS ${ }^{T M}$ piezoresistive pressure sensing element by STMicroelectronics (top picture) is packaged in a small $9 \mathrm{~mm}^{3}$ package together with its specific ASIC (bottom pictures). 


\section{VENSENS $^{\text {TM }}$ FABRICATION PLATFORM}

The VENSENS ${ }^{T M}$ process begins with a standard silicon wafer. A proprietary combination of wet and dry silicon etching steps enables the formation of a sacrificial layer on top of which a monocrystal silicon layer is grown (Figure 15) with a proprietary epitaxial process.
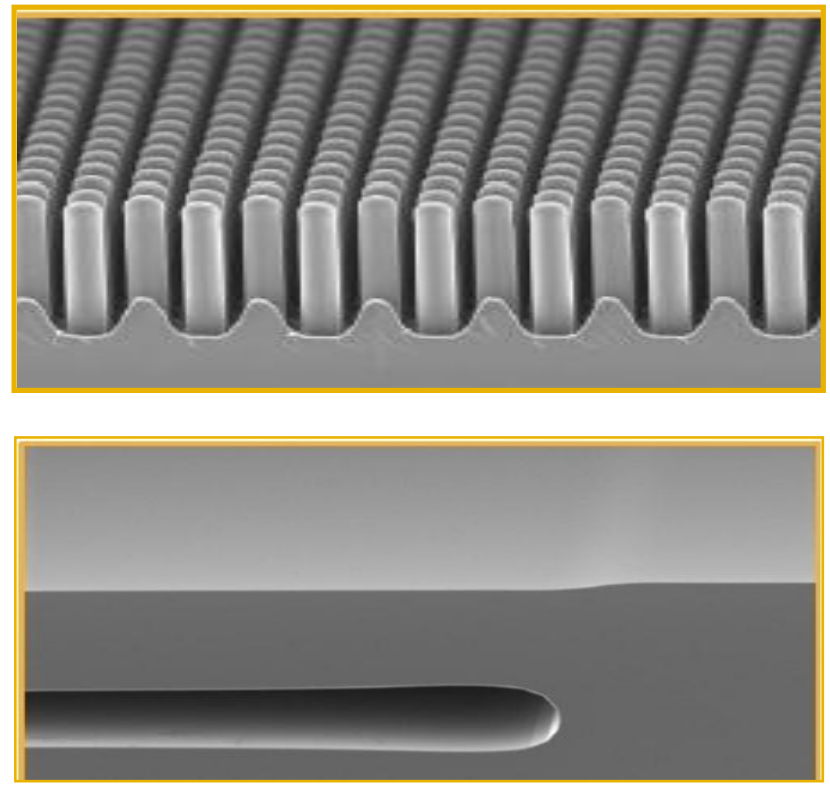

The thickness of the sacrificial layer is less than $3 \mu \mathrm{m}$ and the thickness of the structural layer can reach $20 \mu \mathrm{m}$. The end result is very similar to what is possible to get with bulk micromachining wafer-to-wafer bonding. But the big advantage is to have thinner, smaller, and mechanically more robust chips [Fig 16]. Moreover, the sealing of the cavity requires no any waferto-wafer bonding and thus the reliability of the sealing joint is definitely higher.
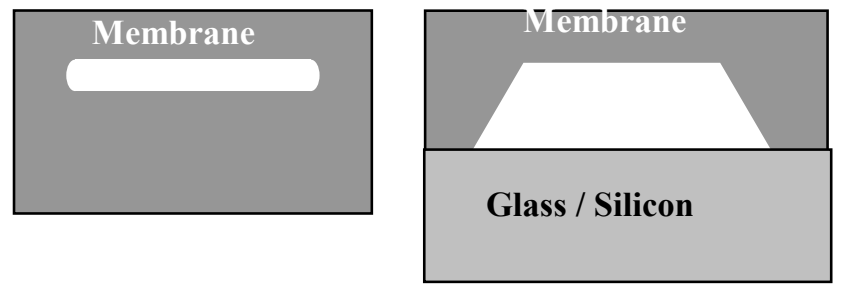

Figure 16. The two drawings are not to scale. On the left, a membrane manufactued with the VENSENS ${ }^{T M}$ process, on the right the same membrane realized with standard bulk micromachining process. The VENSENS ${ }^{T M}$ process requires no wafer-to-wafer bonding step to seal the cavity.

Because of the superior electrical properties of the monocrystal silicon, high-quality, stable resistors can be integrated in the structural layer through implantation or diffusion process. Then these resistors are connected with an aluminum metal layer to realize the four branches of a Wheatstone bridge. The bridge is sensitive to pressure changes due to the excellent piezoresitive properties of the monocrystal silicon layer.
The metal layer is then covered with a standard dielectric, like silicon-oxy-nitride, to provide the required protection against the external corrosive agents.

Because of the intrinsic vertical stopper, this solution is also highly mechanical shock compliant, and thus is able to withstand all the potential mechanical solicitations that the sensor could experience in a portable device such as a smartphone or a tablet.

\section{ALGORITHMS FOR SENSOR FUSION AND MICROCONTROLLERS}

When designing a system using multiple MEMS sensors, it is important to understand the advantages and disadvantages of accelerometers, gyroscopes, magnetometers, and pressure sensors. Sensor fusion solves key motion sensing performance issues of 6-axis modules consisting of a 3 -axis accelerometer and a 3-axis gyroscope or a 3-axis accelerometer and a 3-axis magnetic sensor.

An I6XDOF with an accelerometer and a gyroscope loses its absolute orientation as the gyro drifts over time, requiring calibration to restore an accurate heading reference. A M6XDOF with accelerometer and magnetometer is prone to data corruption in the presence of ferrous materials in the environment. A 9XDOF with an accelerometer, a gyroscope, and a magnetometer eliminates the drift that occurs with standalone sensor solutions. But these can be subject to magnetic interference. Algorithms to fuse the sensor data are required to compensate for the magnetic interference. The purpose of sensor fusion is to take each sensor measurement data as input and then apply digital filtering algorithms to compensate each other and provide accurate and responsive dynamic attitude (pitch/roll/yaw) results.

The Kalman filter is a mathematical method invented by Dr. Rudolf E. Kalman. Since its introduction in 1960, the Kalman filter has been implemented in many applications. The most well-known application is the GPS receiver itself and later, on the integration of GPS with an Inertial Navigation System (INS). This recursive digital algorithm is used to integrate or fuse GPS measurement with accelerometer and gyroscope data to achieve optimal overall system performance. The Kalman filter algorithm produces estimates of the true values of sensor measurements and their associated calculated values by predicting a value, estimating the uncertainty of the predicted value, and computing a weighted average of the predicted value and the measured value. The most weight is given to the value with the least uncertainty. The estimates produced by the algorithm tend to be closer to the true values than the original measurements because the weighted average has a better estimated uncertainty than either of the values that went into the weighted average.

The Kalman filter tries to estimate the state $\mathrm{x}$ of a discretetime controlled process that is governed by the linear difference equation,

$$
\begin{aligned}
& x_{k}=\mathbf{A} x_{k-1}+\mathbf{B} u_{k-1}+w_{k-1} \\
& z_{k}=\mathbf{H} x_{k}+v_{k}
\end{aligned}
$$

Where,

A is $\mathrm{n}$ by $\mathrm{n}$ matrix that relates the state at the previous time step $\mathrm{k}$ - 1 to the state at the current step $\mathrm{k}$, in the absence of either a driving function or process noise. 
B is $\mathrm{n}$ by 1 matrix that relates the optional control input $\mathrm{u}$ to the state $\mathrm{x}$.

$\mathbf{H}$ is $\mathrm{n}$ by m matrix that relates the state to the measurement $\mathrm{z}_{\mathrm{k}}$. $\mathrm{w}_{\mathrm{k}}$ is the process noise (random variables).

$\mathrm{v}_{\mathrm{k}}$ is the measurement noise (random variables).

$\mathrm{w}$ and $\mathrm{v}$ are assumed to be independent of each other, white, and with normal probability distributions so that,

$$
\mathrm{p}(\mathrm{w}) \sim \mathrm{N}(0, \mathrm{Q})
$$$$
\mathrm{p}(\mathrm{v}) \sim \mathrm{N}(0, \mathrm{R})
$$

where, $\mathrm{Q}$ is the process noise covariance matrix and $\mathrm{R}$ is the measurement noise covariance matrix.

The Kalman filter algorithm is based on linear dynamic system which means that the matrix $\mathbf{A}, \mathbf{B}$, and $\mathbf{H}$ are constant. To keep it simple, process and measurement covariance $\mathrm{Q}$ and $\mathrm{R}$ are also assumed as constants. Then the calculated Kalman gain $\mathbf{K}_{\mathrm{k}}$ at each time step will weigh the measurement continuously to maintain the minimum error covariance matrix $\mathbf{P}_{\mathrm{k}}$ so that the updated states will be accurate and responsive. If the process to be estimated and/or the measurement relationship to the process is non-linear, this has resulted in some of the most interesting and successful applications of Kalman filtering situations. A Kalman filter that linearizes about the current mean and covariance is referred to as an Extended Kalman Filter or EKF. In something akin to a Taylor series, we can linearize the estimation around the current estimate using the partial derivatives of the process and measurement functions to compute estimates even in the face of non-linear relationships.

When 3-axis accelerometer, 3-axis gyroscope, and 3-axis magnetometer are installed in a strap-down system such as a 9axis module for applications like human body motion tracking, 3D gaming, Unmanned Air Vehicles and air mouse, etc. quaternion method for rotation matrix calculation based on gyroscope measurements is mostly used because it doesn't have the singularity issue from the Euler angles. The EKF algorithm is implemented to fuse MEMS sensor data and then update the quaternion and gyroscope bias to output accurate attitude information for pitch, roll, and yaw. This is the so-called Attitude Heading Reference System (AHRS).

The EKF algorithm may run on a standard microcontroller external to the sensor module or on a dedicated microcontroller embedded with the sensor itself as shown in Figure 17. The speed of the microcontroller core and the memory size depend both on the application and on the accuracy the customer is looking for.

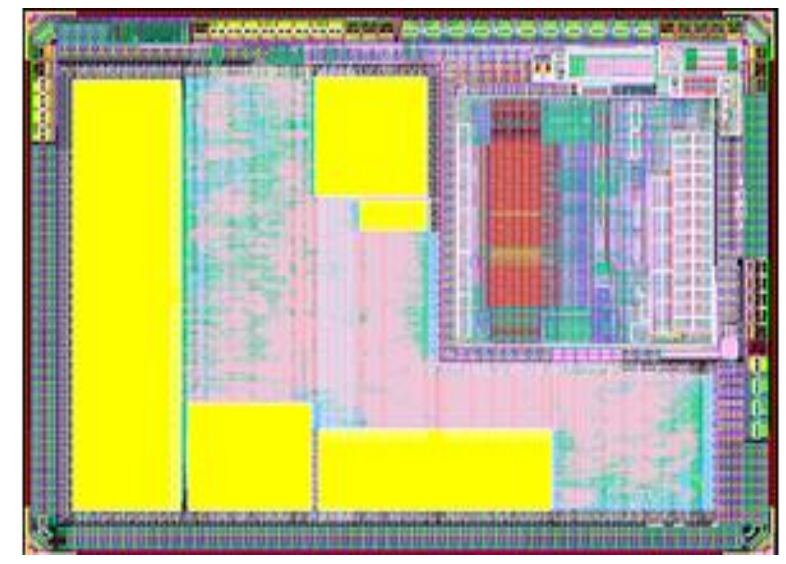

Figure 17: This picture shows a chip with the microcontroller and the memory bank required to run the EKF algorithm.

\section{CONCLUSIONS}

Accelerometers, Gyroscopes, Altimeters, Compasses and microcontrollers, whether discrete or fully integrated into single packages or single silicon dice, will continue to be an important part of all present and future smart consumer products. Enhanced User Interface, Optical Image Stabilization, Remote Monitoring, and Location Based Services have already been identified as important new applications that will benefit from these technologies...but many more will come. MEMS applications are only limited by imagination and future sensors will require more and more extreme analog chips!

\section{REFERENCES}

[1] J. Geen et al., "Single-Chip Surface Micromachined Integrated Gyroscope with $50^{\circ} \mathrm{h}$ Allan Deviation," IEEE J. Solid-State Circuits, pp. 1860-1866, Dec., 2002.

[2] R. Antonello, R. Oboe, L. Prandi, F. Biganzoli, and C. Caminada. "Open A low power 3-axis digital output MEMS gyroscope with single drive and multiplexed angular rate readout", ISSCC, Feb 2011, San Francisco

[3] B. Vigna, "STMicroelectronics Masters the Art and the Science of MEMS", Semicon Taiwan 2011, Sept.

[4] B. Vigna, "Consumer Device go SMART with MEMS", Semicon China 9/2011.

[5] C. Johnson, "Ten Technologies that will change Consumer Devices" EETimes, 1/23/12 (2012).

[6] STMicroelectronics, L3G4IS - Dual Core Gyroscope DataSheet, www.st.com/mems

[7] STMicroelectronics, L3G3200D - Single Core Gyroscope DataSheet, www.st.com/mems

[8] Massachusetts Institute of Technology, White paper - The Balance Filter, June 2007,

http://web.mit.edu/scolton/www/filter.pdf

[9] STMicroelectronics, Inc., White paper - Introduction to MEMS gyroscopes, November 2010,

http://www.electroiq.com/index/display/nanotech-articledisplay/4659348781/articles/smalltimes/nanotechmems/mems/sensors/2010/11/introduction-tomems-gyroscopes.html

[10] University of North Carolina, An Introduction to the Kalman Filter, July 2006,

http://www.cs.unc.edu/ welch/media/pdf/kalman_intro.pdf [11] IEEE transaction on biomedical engineering, Vol. 53, No. 7, July 2006, Quaternion-Based Extended Kalman Filter for Determining Orientation by Inertial and Magnetic Sensing http://ieeexplore.ieee.org/xpl/freeabs all.jsp?arnumber $=1643403$ [12] J. Esfandyari, R. De Nuccio, Gan Xu, "Solutions for MEMS Sensor Fusion", Solid State Technology, June 2011, Vol. 54, No. 7, P. 18".

[13] M. Ferraresi, "Tri Axial MEMS Gyroscope for Automotive Applications”, Smart Systems Integration, March 2012 


\section{MEMS ENABLES OSCILLATORS WITH SUB-PPM FREQUENCY STABILITY AND SUB-PS JITTER \\ R. Melamud, P.M. Hagelin, C.M. Arft, C. Grosjean, N. Arumugam, \\ P. Gupta, G. Hill, M. Lutz, A. Partridge, F. Assaderaghi \\ SiTime Corporation, Sunnyvale, California, USA}

\begin{abstract}
An integrated MEMS thermistor and resonator are at the core of SiTime's new high performance oscillator platform. These MEMS-based oscillators are designed to achieve the performance requirements of high-end communication applications. The resonator, which has an $\mathrm{f}-\mathrm{Q}$ product of $6.9 \times 10^{12}$, enables the oscillator to achieve sub- picosecond integrated phase jitter in the $12 \mathrm{kHz}$ to $20 \mathrm{MHz}$ frequency range. The MEMS thermistor, with a resistance-temperature sensitivity of $0.33 \% / \mathrm{C}$, is used to compensate for the temperature-induced frequency variations of the silicon resonator. The thermistor is key to achieving sub-ppm frequency stability in a $-40 \mathrm{C}$ to $85 \mathrm{C}$ temperature range and a sub$5 \mathrm{ppb}$ Allan deviation in 0.1 second to 10 second strides. The demonstrated system performance opens high-volume data communication markets previously unavailable to MEMS-based oscillators.
\end{abstract}

\section{INTRODUCTION}

The multi-billion dollar timing market has motivated the development of MEMS resonator-based oscillators as a replacement for quartz crystal-based oscillators. MEMS-based oscillators leverage advantages common to semiconductor products, such as miniaturization and low-cost manufacturing. This is attractive for oscillators that are aimed at high-volume consumer and industrial applications. The requirements include tight specifications on phase noise, jitter, Allan deviation, long-term stability, temperature stability, and initial frequency tolerance. Successful product adoption requires meeting these performance requirements while creating a high-yielding, robust product with reduced cost and improved lead times.

Previously commercialized MEMS-based oscillators could not provide the system performance necessary to replace temperature-compensated quartz crystal oscillators (TCXOs) and ultra-low jitter oscillators used in a wide variety of telecommunication, networking, storage, wireless, and high speed serial protocol applications. Table 1 lists some of the typical performance requirements for these applications.

Table 1: Typical performance requirements for high-end data communication applications.

\begin{tabular}{|l|c|}
\hline Specification & Requirements \\
\hline Integrated phase jitter in & $<1 \mathrm{ps}$ \\
$12 \mathrm{kHz}-20 \mathrm{MHz}$ offset from carrier & \\
\hline Allan deviation in 0.1s-10s stride & $<5 \mathrm{ppb}$ \\
\hline Temperature Stability (-40 C to $85 \mathrm{C})$ & $<5 \mathrm{ppm}$ \\
\hline Initial Frequency Tolerance & $<1 \mathrm{ppm}$ \\
\hline
\end{tabular}

We have recently demonstrated a new oscillator product platform that meets or exceeds the performance requirements described in Table $1[1,2]$. In this paper, we focus on the role of MEMS in enabling these oscillators. We present an integrated 48 $\mathrm{MHz}$ silicon resonator and silicon thermistor that are at the core of SiTime's new oscillator product platform. The resonator exhibits a systematic $\sim 3800 \mathrm{ppm}$ frequency variation over a $-40 \mathrm{C}$ to $85 \mathrm{C}$ temperature range, which is typical of uncompensated silicon

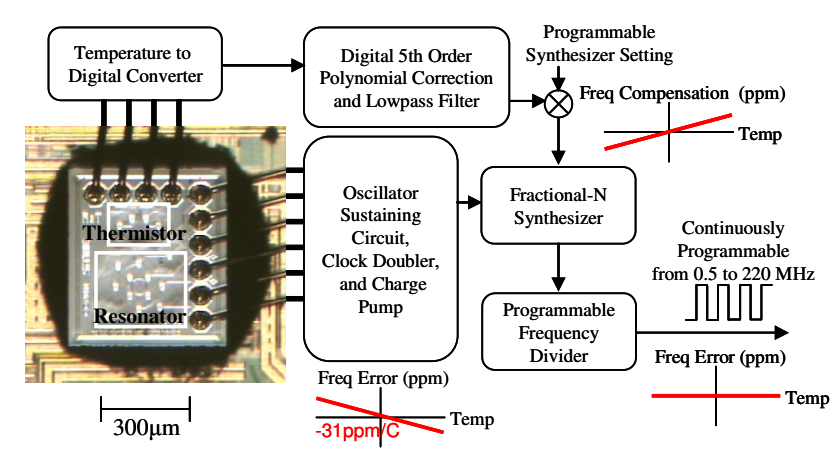

Figure 1: Block diagram of MEMS-based programmable oscillator. Micrograph shows assembled MEMS die stacked on CMOS die after dissolving plastic package. Both the resonator and thermistor are encapsulated in the MEMS die.

resonators. The output frequency of the oscillator is corrected to reduce temperature-induced variation in the resonator frequency. The compensation system uses the silicon thermistor, which is located on the same die as the resonator, and a high-resolution temperature-to-digital converter (TDC). The resulting oscillator achieves $\pm 200 \mathrm{ppb}$ frequency stability over a $-40 \mathrm{C}$ to $85 \mathrm{C}$ temperature range. This high-performance oscillator product platform is accelerating the adoption of MEMS-based oscillators in the timing market.

\section{SYSTEM OVERVIEW}

Figure 1 shows a micrograph of the MEMS die stacked on top of the $0.18 \mu \mathrm{m}$ CMOS die. The system architecture of the MEMSbased programmable oscillator achieves both temperature compensation and continuous frequency programmability. The MEMS resonator is combined with a sustaining circuit to create an electrical signal at the resonator's mechanical resonant frequency. However, due to the temperature sensitivity of the Young's Modulus of silicon, silicon resonators typically exhibit a frequency-temperature variation of $-31 \mathrm{ppm} / \mathrm{C}$ [3].

A common approach to correct for the temperature-induced frequency change is to synthesize a temperature-corrected frequency using a TDC and polynomial correction logic. The TDC consists of a temperature sensitive element, to monitor the temperature of the MEMS die, and a converter to create a digital temperature value. A fractional- $\mathrm{N}$ phase-lock loop, in conjunction with a programmable frequency divider, is used to produce the desired temperature-stable output frequency of the oscillator.

A bipolar junction transistor (BJT) temperature sensor, which is integrated on the CMOS die, has previously been used as the temperature sensor. SiTime's new oscillator platform uses a silicon MEMS thermistor as the temperature sensitive element. This improves the measurement accuracy of the resonator's temperature and enables a TDC architecture which achieves $100 \mu \mathrm{K}$ resolution in a $5 \mathrm{~Hz}$ bandwidth [1].

The MEMS resonator and thermistor are co-fabricated on the same die using the SiTime MEMS First ${ }^{\mathrm{TM}}$ process [4]. Figure 2 


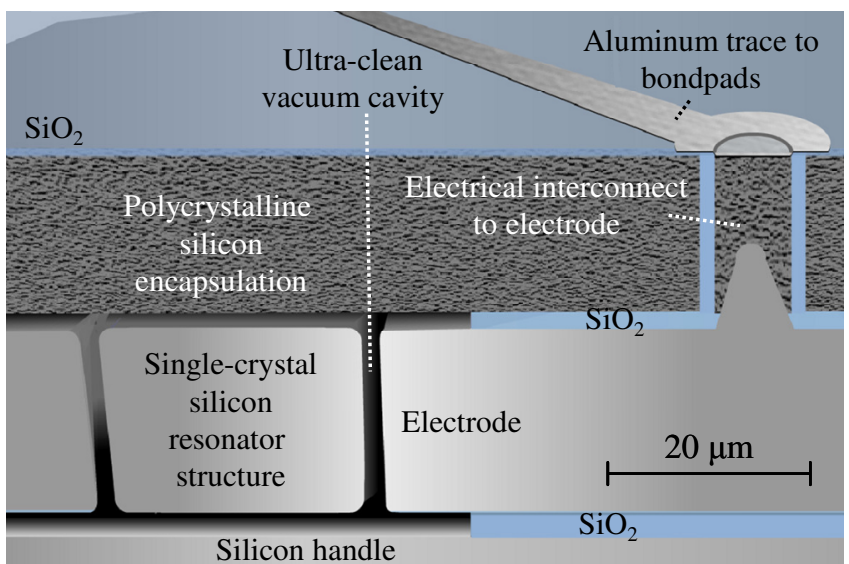

Figure 2: Cross-section diagram of SiTime's MEMS First ${ }^{T M}$ processes [4]. MEMS structures are wafer-level hermetically encapsulated using high-temperature epitaxial silicon.

shows a cross-section diagram of an encapsulated resonator. The MEMS structures are patterned in a silicon-on-insulator device layer. The cavity surrounding the structures is sealed during epitaxial deposition of the polycrystalline silicon encapsulation layer. This critical step provides a clean enclosure for the MEMS structures and protection from the external environment. The hermetic vacuum encapsulation of both devices is critical to achieving long-term frequency stability.

The packaged oscillators are produced using standard IC assembly practices. The MEMS and CMOS wafers are thinned to less than $150 \mu \mathrm{m}$ using chemical mechanical polishing (CMP). Wafer-saw dicing and automated pick-and-place are used to assemble the die onto copper lead-frames. Wirebonds connect the resonator and thermistor to the sustaining circuit and TDC on the CMOS die, respectively. The encapsulation layer on the MEMS die is mechanically robust to plastic injection molding and subsequent package singulation. Standard IC processing allows the use of low-cost, high-volume plastic packaging in contrast to the expensive and specialized ceramic or metal packaging common to quartz resonators.

\section{RESONATOR DESIGN}

The $48 \mathrm{MHz}$ resonator consists of four rings used for capacitive actuation and sensing (Figure 3). The four rings resonate in-phase in an extensional mode. The rings are coupled through frequency-matched crossed beams. A flexural-beam suspension connects the crossed beams to four mechanical anchors. The centrally-located anchors isolate the resonator from external mechanical stresses, such as those induced when the device is soldered to a circuit board. This stress isolation is an important factor in maintaining oscillator output frequency accuracy and initial frequency tolerance. Electrodes are placed on both the inside and outside of the rings for electrostatic transduction. The differential drive and sense configuration, shown in Figure 4, reduces capacitive feedthrough. High yield and tight frequency control ( $\pm 100 \mathrm{ppm})$ is achieved across a $200 \mathrm{~mm}$ wafer after CMP thinning (Figure 5).

The typical resonator quality factor, Q, of 144,000 , is a result of a parallel combination of the $\mathrm{Q}$ due to various loss mechanisms [5]. High $Q$ is critical in reducing both the far-from-carrier and close-to-carrier phase noise. Equation 1 is a simple model of phase noise, including the phase-noise floor, at a specified offset from the carrier frequency [6]:

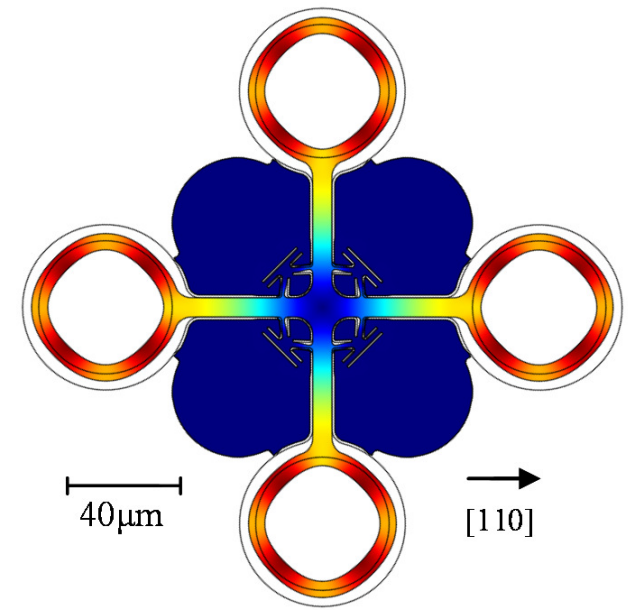

Figure 3: Finite element simulation of $48 \mathrm{MHz}$ eigenmode of resonator. Exaggerated displacement shown.

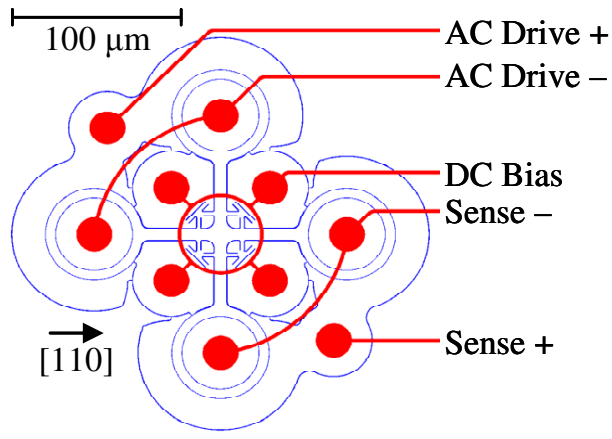

Figure 4: Drawing of silicon resonator and electrodes with metal interconnect routing in red

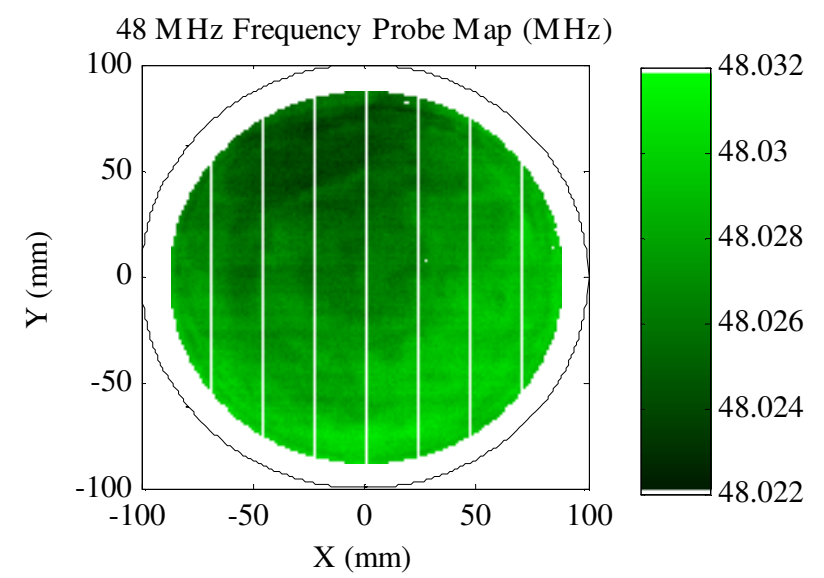

Figure 5: Resonant frequency map of $200 \mathrm{~mm}$ thinned wafer with $\sim 70,00048 \mathrm{MHz}$ resonators showing frequency variation of \pm 100 ppm across wafer.

$$
L(\Delta \omega)=10 \log \left[\frac{2 k T}{P_{s i g}} \cdot\left(\frac{\omega_{o}}{2 Q \Delta \omega}\right)^{2}+1\right]
$$

where $k$ is the Boltzmann's constant, $T$ is absolute temperature in Kelvin, $P_{\text {sig }}$ is the power output of the resonator, $\omega_{\mathrm{o}}$ is the carrier frequency, $\mathrm{Q}$ is the quality factor, and $\Delta \omega$ is the frequency offset from the carrier frequency. Increasing $Q$ helps to reduce the phase 


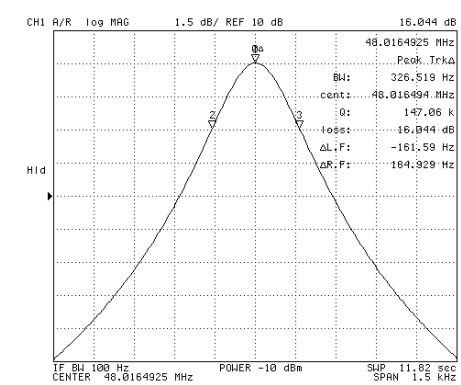

Figure 6: Network analyzer frequency response of MEMS $48 \mathrm{MHz}$ resonator at $25 \mathrm{C}$.

noise floor by reducing the motional resistance of the resonator and increasing its power output. At a given output power from the resonator, the close-to-carrier phase noise reduces with $1 / \mathrm{Q}^{2}$.

The resonator is designed to minimize the dominant energy loss mechanisms. Extensional vibration of the rings minimizes thermoelastic dissipation (TED). A $\mathrm{Q}_{\mathrm{TED}}$ of $1.3 \times 10^{6}$ was determined for this resonator using a FEA method developed by Duwel, et al. [7]. Vacuum encapsulation of the resonator reduces gas-damping losses. At a cavity pressure of less than $1 \mathrm{~Pa}$ [8], the $\mathrm{Q}$ due to gas damping far exceeds $1 \times 10^{6}$ for this resonator. Optimization of the flexural suspension to reduce displacement at the anchor minimizes anchor losses. The $\mathrm{Q}$ due to the Akhieser effect is estimated to be $8.12 \times 10^{5}$ [9]. The $\mathrm{f}-\mathrm{Q}$ product of the resonator, $6.9 \times 10^{12}$, is 5.6 times lower than the Akhieser limit of $\sim 3.9 \times 10^{13}$ for silicon resonators. We believe that the $Q$ of the resonator is limited by anchor loss mechanisms. However, the $\mathrm{f}-\mathrm{Q}$ product achieved is comparable to some of the highest $\mathrm{f}-\mathrm{Q}$ silicon resonators published (Figure 6) $[9,10]$.

\section{THERMISTOR DESIGN}

The silicon thermistor is a serpentine structure with four electrical contacts and four mechanical anchors (Figure 7). The thermistor is fabricated in the same single-crystal silicon device layer as the resonator. As such, the thermistor is hermetically vacuum encapsulated and anchored to isolate it from external mechanical stresses that adversely effect traditional BJT temperature sensors. Furthermore, the proximity of the thermistor to the resonator, less than $200 \mu \mathrm{m}$ separation, improves thermal tracking of the temperature of the resonator compared to a temperature sensor integrated onto the CMOS die.

The four-point topology of the structure allows accurate measurement of the temperature-dependent resistance independent of the resistance of the wirebonds and metal interconnects. The temperature dependence of the conductivity of silicon, $\sigma$, can be calculated from the temperature-dependent carrier density and mobility of silicon using:

$$
\sigma=q\left(n \mu_{e}+p \mu_{h}\right)
$$

where $q$ is the electron charge, $n$ is the density of electrons, $\mu_{e}$ is the electron mobility, $p$ is the density of holes, and $\mu_{h}$ is the hole mobility. The carrier density and mobility in silicon have been empirically determined and are strong functions of both temperature and dopant concentration [11]. Both factors influence the sensitivity and linearity of the thermistor resistance.

Figure 8 shows the temperature dependence of the thermistor resistance and resonator frequency. With an overall resistance change of $40 \%$ from $-40 \mathrm{C}$ to $85 \mathrm{C}$, the MEMS thermistor-based TDC signal to noise ratio is an order of magnitude better than for a BJT-based TDC [1]. While the maximum thermistor sensitivity shown is $\sim 0.33 \% / \mathrm{C}$, the temperature dependence is not linear and

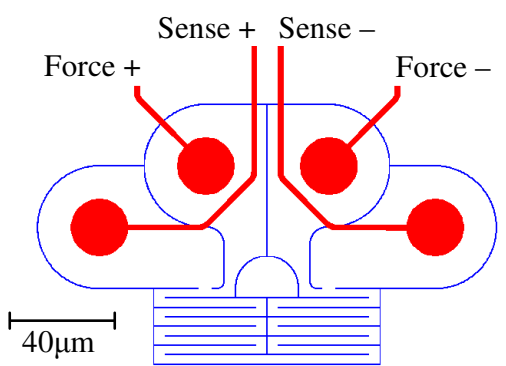

Figure 7: Drawing of silicon thermistor configured for four-point measurement with metal routing in red.

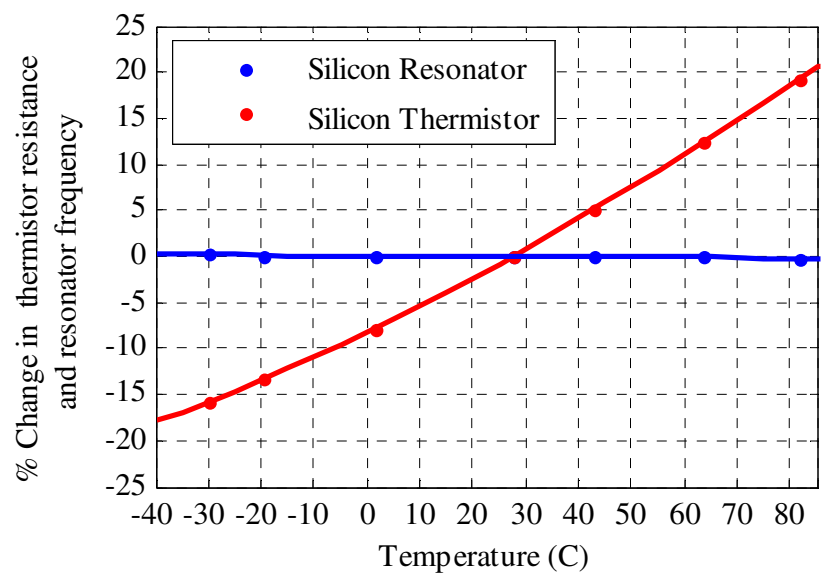

Figure 8: Temperature dependence of thermistor resistance and resonator frequency with $5^{\text {th }}$-order polynomial fit (lines) to data (points).

the thermistor sensitivity drops to $0.25 \% / \mathrm{C}$ at $-40 \mathrm{C}$. Since both the resonator and resistor have higher order temperature dependencies, a $5^{\text {th }}$-order polynomial is used to correct the frequency of the resonator relative to the TDC output.

\section{SYSTEM PERFORMANCE}

Figure 9 shows the phase noise at $25 \mathrm{C}$ of the combined 48 $\mathrm{MHz}$ resonator and sustaining circuit across extreme CMOS and MEMS manufacturing process corners. The $48 \mathrm{MHz}$ resonator combined with the low-noise sustaining circuit dominates the oscillator phase noise floor between $10 \mathrm{kHz}$ and $1 \mathrm{MHz}$. The phase noise of the $48 \mathrm{MHz}$ resonator plus sustaining circuit is below $-139 \mathrm{dBc} / \mathrm{Hz}$ at $10 \mathrm{kHz}$ offset even at the most extreme CMOS and MEMS corner. The PLL bandwidth is set to $500 \mathrm{kHz}$ to reduce noise contribution from other circuit blocks. The programmable oscillator achieves $\sim 500 \mathrm{fs}$ integrated phase jitter in the critical $12 \mathrm{kHz}$ to $20 \mathrm{MHz}$ range necessary for communication applications.

This thermistor-based TDC has been recently shown to achieve a state-of-art resolution of $<100 \mu \mathrm{K}$ in a $5 \mathrm{~Hz}$ bandwidth [1]. With a resolution of $100 \mu \mathrm{K}$, and a resonator sensitivity of $-31 \mathrm{ppm} / \mathrm{C}$, the Allan deviation has been demonstrated to be less than $5 \mathrm{ppb}$ in 0.1 second to 10 second strides.

The frequency stability of the oscillator is shown in Figure 10. The frequency error of the compensated output is dominated by the fit quality of the polynomial used to correct the resonator frequency over a particular temperature range. The compensation achieves a $\pm 200 \mathrm{ppb}$ frequency stability in the industrial range of $-40 \mathrm{C}$ to $85 \mathrm{C}$. Implementation of higher order polynomial correction could further improve the frequency stability. 


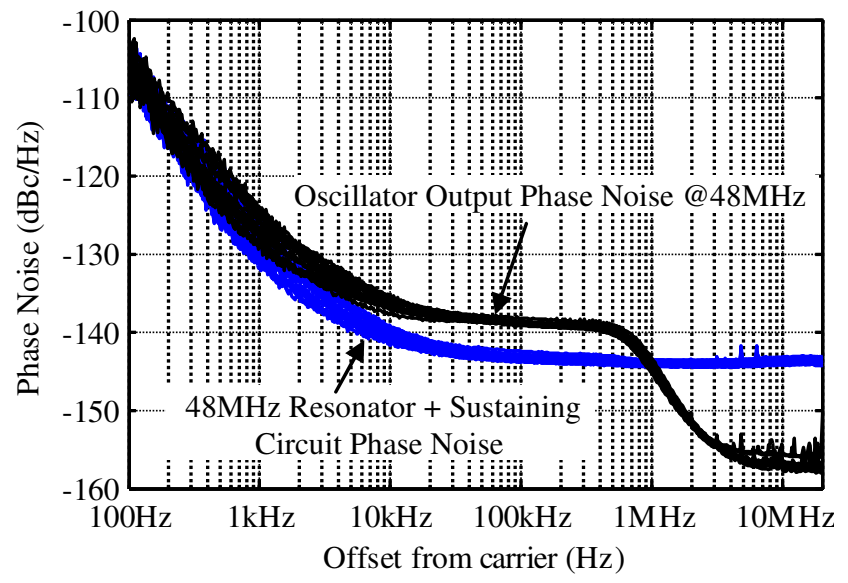

Figure 9: Phase noise at $25 \mathrm{C}$ of $48 \mathrm{MHz}$ MEMS plus sustaining circuit (blue) and compensated oscillator output (black). Nineteen devices are shown across extreme CMOS and MEMS process corners.

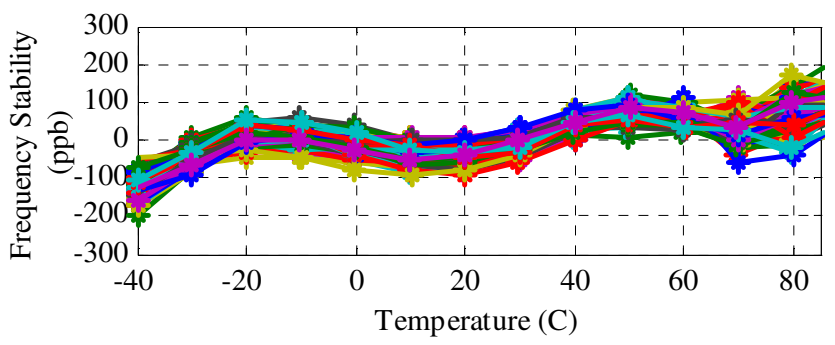

Figure 10: Frequency stability of oscillator output using 5th order polynomial compensation for 68 devices in -40 C to 85 C range.

\section{CONCLUSION}

The MEMS resonator and thermistor, described here, is instrumental in meeting the performance requirements for oscillators used in communication applications. The resonator provides the power-handling and high-Q necessary for low phase noise and low integrated phase-jitter oscillators. The thermistor is key to accurate temperature compensation of the silicon resonator. Both MEMS structures are fabricated in SiTime's MEMS First ${ }^{\mathrm{TM}}$ process that provides a robust wafer-level hermetic encapsulation for long-term stability and compatibility with standard IC packaging processes.

\section{ACKNOWLDEGEMENTS}

Michael Perrott, Jim Salvia, Fred Lee, Shouvik Mukherjee, Jin-Tae Kim, Sassan Tabatabaei, Sudhakar Pamarti, Bruno Garlepp, Cathy Lee, and Haechang Lee developed the CMOS architecture.

\section{REFERENCES}

[1] M. Perrott, J. Salvia, F. Lee, A. Partridge, S. Mukherjee, C. Arft, J-T. Kim, N. Arumugam, P. Gupta, S. Tabatabaei, S. Pamarti, H. Lee, F. Assaderaghi. "A Temperature-to-Digital Converter for a MEMS-based Programmable Oscillator With Better than \pm 0.5 ppm Frequency Stability". IEEE International Solid-State Circuits Conference, 19-23 Feb. 2012
[2] F.S. Lee, J. Salvia, C. Lee, S. Mukherjee, R. Melamud, N. Arumugam, S. Pamarti, C. Arft, P. Gupta, S. Tabatabaei, B. Garlepp, H. Lee, A. Partridge, M.H. Perrott, and F. Assaderaghi, F, "A programmable MEMS-based clock generator with sub-ps jitter performance," Symposium on VLSI Circuits (VLSIC), pp.158-159, 15-17 June 2011

[3] C. Bourgeois, E. Steinsland, N. Blanc, and N.F. de Rooij , "Design of resonators for the determination of the temperature coefficients of elastic constants of monocrystalline silicon," Proceedings of the 1997 IEEE International Frequency Control Symposium, pp.791-799, 28-30 May 1997

[4] SiTime Corporation, "SiTime's MEMS First ${ }^{\mathrm{TM}}$ Process," http://www.sitime.com/support/application-notes

[5] M. Weinberg, R. Candler, S. Chandorkar, J. Varsanik, T.W. Kenny, and A. Duwel, "Energy loss in MEMS resonators and the impact on inertial and RF devices," Solid-State Sensors, Actuators and Microsystems Conference, TRANSDUCERS 2009. pp.688-695, 21-25 June 2009

[6] Lee T.H., The Design of CMOS Radio-Frequency Integrated Circuits, 2nd Edition, Cambridge University Press, New York, 2004.

[7] A. Duwel, R.N. Candler, T.W. Kenny, and M. Varghese, "Engineering MEMS Resonators With Low Thermoelastic Damping," Journal of Microelectromechanical Systems, vol.15, no.6, pp.1437-1445, Dec. 2006.

[8] R.N. Candler, M.A. Hopcroft, B. Kim, W.-T. Park, R. Melamud, M. Agarwal, G. Yama, A. Partridge, M. Lutz, and T.W. Kenny, "Long-Term and Accelerated Life Testing of a Novel Single-Wafer Vacuum Encapsulation for MEMS Resonators," Journal of Microelectromechanical Systems, vol.15, no.6, pp.1446-1456, Dec. 2006

[9] S.A. Chandorkar, M. Agarwal, R. Melamud, R.N. Candler, K.E. Goodson, and T.W. Kenny, "Limits of quality factor in bulk-mode micromechanical resonators," IEEE International Conference on Micro Electro Mechanical Systems, MEMS 2008. pp.74-77, 13-17 Jan. 2008

[10] J.T.M van Beek and R. Puers, "A review of MEMS oscillators for frequency reference and timing applications", Journal of Micromech. Microeng. 22 (2012)

[11] S.M. Sze, Physics of Semiconductor Devices, 2nd Edition, John Wiley \& Sons, New York, 1981

\section{CONTACT}

R. Melamud,rmelamud@gmail.com 


\section{MAGNETOELECTRIC RESONANT GATE TRANSISTOR F. Li $^{l^{*}}$, R. Misra ${ }^{2}$, Z. Fang ${ }^{l}$, C. Curwen ${ }^{l}, Y$. Wu ${ }^{l}$, Q. M. Zhang ${ }^{1,3}$, P. Schiffer ${ }^{2,3}$, S. Tadigadapa ${ }^{1,3}$ and S. Datta $^{1,3}$ \\ ${ }^{1}$ Electrical Engineering, ${ }^{2}$ Physics Department, and ${ }^{3}$ Materials Research Institute, The Pennsylvania State University, University Park, Pennsylvania 16802, USA}

\begin{abstract}
Chip scale, high sensitivity magnetic sensor arrays capable of sensing below 100 picoTesla vector fields are of great interest to biomedical applications such as noninvasive medical imaging and diagnosis. Here, we present an integrated magnetoelectric resonant gate transistor (ME RGT) with nanoTesla magnetic field detection sensitivity. The device integrates Titanium (Ti)-Metglas ${ }^{\circledR}$ $\left(\mathrm{Fe}_{0.85} \mathrm{~B}_{0.05} \mathrm{Si}_{0.1}\right)$ based magnetostrictive unimorph freestanding cantilever beam coupled capacitively to the gate of an n-channel field effect transistor (FET). Using this configuration at the flexural resonance frequency of $4.9 \mathrm{kHz}$ of the cantilever, a signalto-noise ratio of $646,000 \sqrt{\mathrm{Hz}} / \mathrm{Oe}$ and a minimum detectable field of $15 \times 10^{-11} \mathrm{~T} / \sqrt{\mathrm{Hz}}$ were obtained at room temperature. This result shows a significant improvement in the thin film ME sensor integration with standard CMOS process towards on-chip biomedical imaging applications.
\end{abstract}

\section{INTRODUCTION}

Magnetoelectric (ME) effect [1] is defined as the induction of electrical polarization upon application of a magnetic field $\mathrm{H}$ and/or the magnetization upon application of an electric field E. Research has been done on both single phase materials [2] (e.g. $\mathrm{Cr}_{2} \mathrm{O}_{3}$ ) and composite laminates (e.g. PZT/ Terfenol-D, PVDF/ Metglas $^{\mathbb{B}}$ ) constructed out of a bi-layer of a magnetostrictive and piezoelectric materials. Unlike single phase materials, composite laminates have the advantage that each layer can be independently optimized to achieve a high effective ME coefficient. Recently, bulk sensors made by ME laminates were reported to exhibit large ME coupling coefficient of $21.46 \mathrm{~V} / \mathrm{cm} \cdot \mathrm{Oe}$ [3] and a high sensitivity of $2 \times 10^{-11} \mathrm{~T} / \sqrt{\mathrm{Hz}}$ [4]. However, these bulk sensors suffer from several drawbacks: (i) the size of the current prototypes of $2-10 \mathrm{~cm}$ are too large for monolithic integration with CMOS circuits and chip scale sensor arrays, (ii) the strength of coupling of the magnetic field induced strain to the piezoelectric layer is typically reduced by bonding epoxy layers [5]-[7]. In this work, we present a Metglas ${ }^{\circledR}\left(\mathrm{Fe}_{0.85} \mathrm{~B}_{0.05} \mathrm{Si}_{0.1}\right)$ thin film magnetoelectric resonant gate transistor (MERGT) by integrating a resonant magnetostrictive (MS) cantilever sensor directly atop a sensing and amplifying transistor, as shown in Fig. 1. The magnetostrictive cantilever is made from Ti/Metglas ${ }^{\circledR}$ thin film unimorph and thus can be readily integrated with the NMOS transistor readout and avoids the use of any epoxy bonding layer.

\section{THIN FILM METGLAS ${ }^{\circledR}$ AND RESONANT GATE}

The magnetoelectric resonant gate transistor combines the benefits of high-deflection sensitivity of MS cantilever sensors and FET motion sensing. A small time varying magnetic field is sensed by the resonant gate (suspended cantilever) via magnetostrictively induced flexural bending of the cantilever which, in turn, modulates the air-gap capacitance. This in turn modulates the channel charge density and therefore produces a proportional change in the drain current of the transistor amplified by the transconductance of the FET.

In order to characterize the magnetic properties of the deposited Metlgas ${ }^{\circledR}$ films, $150 \mathrm{~nm}$ thick films were first deposited on a silicon substrate and cut into $15 \mathrm{~mm}$ x $3 \mathrm{~mm}$ cantilevers. The magnetic hysteresis loop is measured by Quantum Design superconducting quantum interference device (SQUID) magnetometer. The samples are loaded in a plastic straw with the plane of the film in a direction parallel to the magnetic field. The magnetic field dependence of the magnetic moment is studied at $293 \mathrm{~K}$. The deposited films exhibit low coercive field and high saturation magnetization, as shown in Fig. 2.

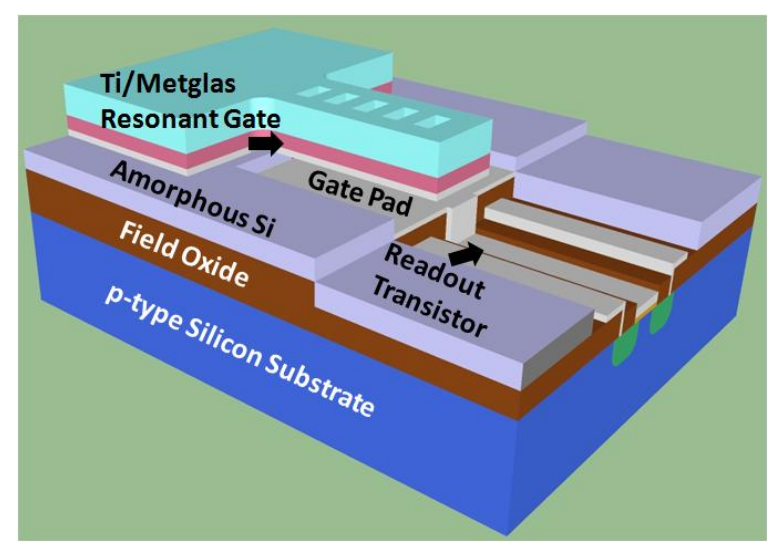

Figure 1: Schematic of the magnetoelectric resonant gate transistor (MERGT).

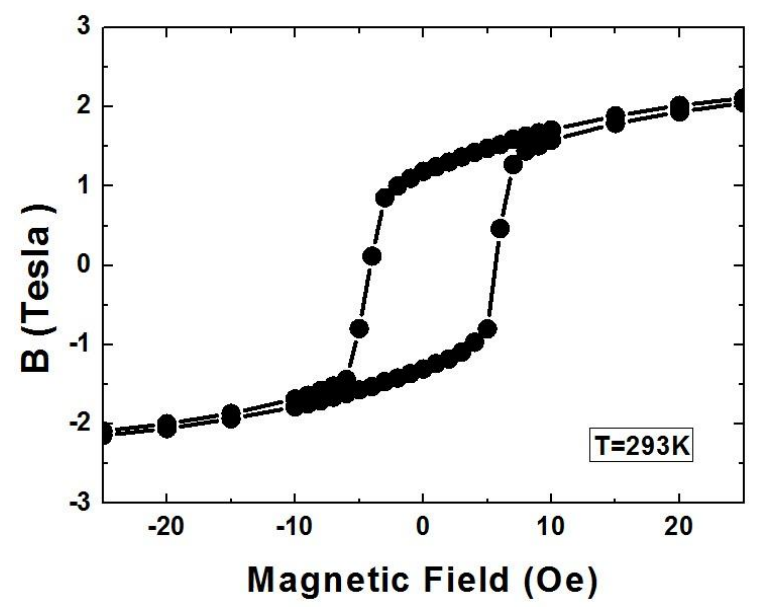

Figure 2: B-H hysteresis loop of the $150 \mathrm{~nm}$ thick ion-beam deposited Metglas ${ }^{\circledR}$ film.

Magnetostrictive coefficient of the films was characterized by using a laser vibrometer. The test cantilever was one end clamped onto the substrate holder. The entire sample was placed in a Helmholz coil and the deflection of the silicon cantilever was accurately measured as a function of the applied magnetic field as shown in the inset of Fig. 3. Figure 3 shows the measured magnetostrictive coefficient as a function of the applied magnetic field. The maximum response occurs at $4(-3)$ Oe.

The fabrication of the MERGT is realized on a NMOS transistor platform. Briefly, $5 \mathrm{~m}$ NMOS transistors with platinum gate are fabricated on p-type silicon wafers. This is followed by

the

Hilton Head Island, South Carolina, June 3-7, 2012 


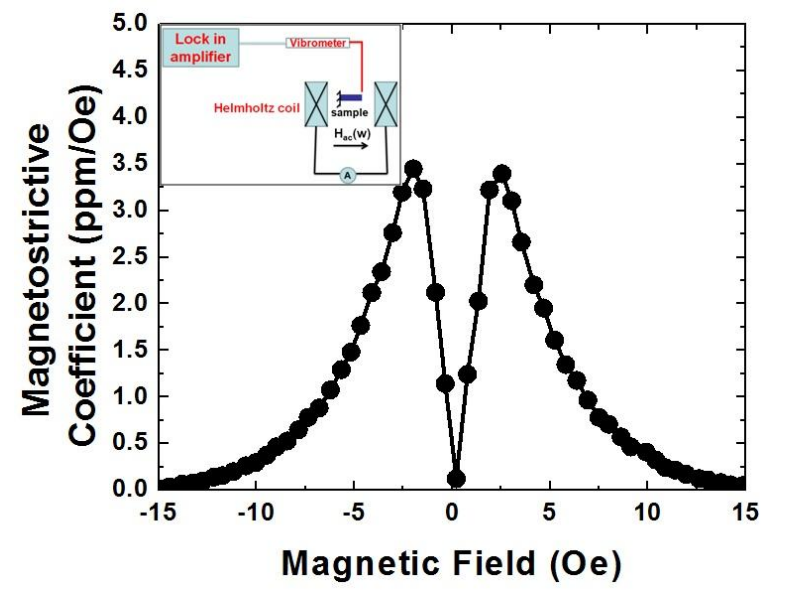

Figure 3: Magnetostrictive coefficient as a function of DC magnetic field.(Inset: laser vibrometer setup for MS coefficient characterization)

deposition of amorphous silicon sacrificial layer. The magnetostrictive cantilever consisting of $150 \mathrm{~nm}$ thick Metglas ${ }^{\circledR}$ film on $500 \mathrm{~nm}$ thick titanium films is patterned via lift-off process. Finally, the cantilevers are released using $\mathrm{XeF}_{2}$ vapor phase etching of the sacrificial amorphous silicon while protecting the anchor and other transistor regions by photoresist pattern.

Further details on the fabrication process can be found in reference [8]. Figure 4 shows the $\mathrm{ZYGO}^{\circledR}$ image of the freestanding magnetostrictive cantilever forming the gate electrode of the MERGT. An inherent problem associated with the bilayer thin film MS cantilevers is the zero field curvature of the released cantilever beam due to the residual stress mismatch between films. As can be seen from the white light interferometric image of the cantilever at zero field (inset Fig. 4), the freestanding MS cantilever is bent upwards by $5 \mu \mathrm{m}$ at the tip end due to the larger residual tensile stress in the Metglas ${ }^{\circledR}$ film in comparison to that of the Ti passive layer and therefore results in a smaller air gap capacitance.

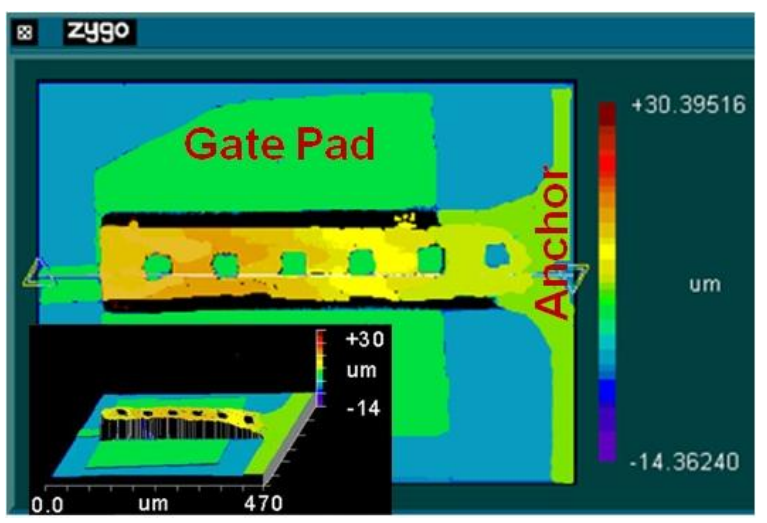

Figure 4: Top view Zygo image (SEM) of a 300 um long and 100 $\mu \mathrm{m}$ wide resonant gate. (Inset: $3 D \mathrm{ZYGO}{ }^{\circledR}$ image of the magnetostrictive top resonant gate)

We used a custom designed optical set-up to verify the functionality of the top resonant gate. The experimental set-up uses a position sensitive photo detector (PSD) to measure the deflection of the beam, as illustrated schematically in Fig. 5. A $600 \mathrm{~nm}$ wavelength laser is focused and directed onto the resonant gate tip

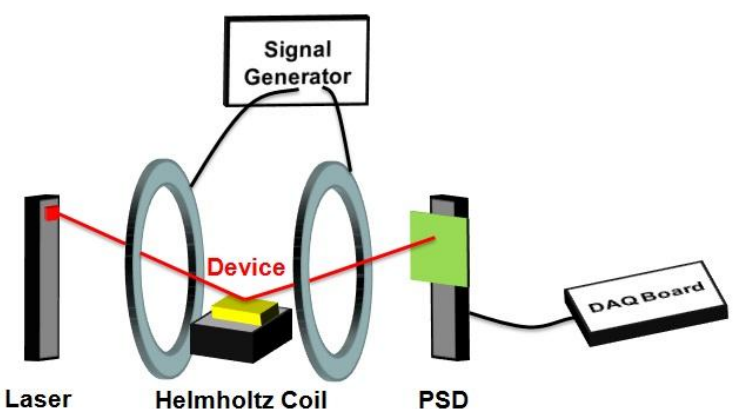

Figure 5: Schematic of optical measurement set-up using position sensitive photo detector.

and is actuated by the magnetic field generated using a Helmholtz coil. The reflected laser beam is detected by the Stiek EletroOptics 2D PSD and the output acquired using a National Instruments $^{\circledR}$ data acquisition box. HP 3314A function generator was used to drive the Helmholz coil using a triangle wave with 95\%-5\% symmetry to output a $0-1$ Oe magnetic field.

Figure 6 plots the output of PSD, which shows a linearly decreasing output as a function of the applied magnetic field using the Helmholz coil. This result confirms that the freestanding magnetostrictive cantilever responds to the incident magnetic signal via flexural bending. Calibrating the applied magnetic field the response of the PSD can be converted into an optically measured sensitivity of $4 \mathrm{mV} / \mathrm{Oe}$.

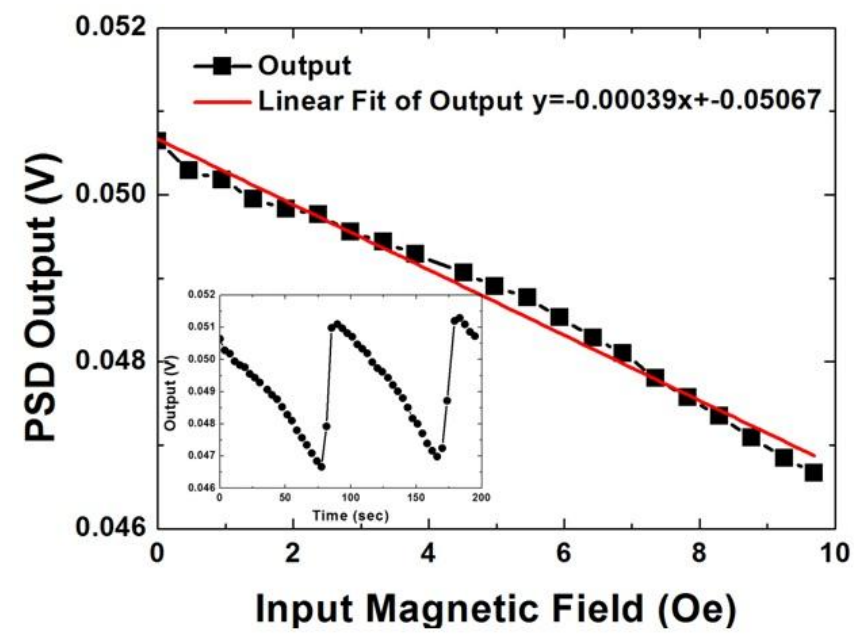

Figure 6: PSD output varies linearly with input magnetic field. (Signal generator provides a triangle wave with 95\%-5\% symmetry at a frequency of $0.01 \mathrm{~Hz}, 5.95 \mathrm{~V}_{p p}$, with an offset of 3.0 V.)

\section{MERGT CHARACTERIZATION AND DISCUSSION}

For the complete electrical characterization, the devices are placed in a ceramic dual in-line package and wire bonded to make electrical connections to the device. The performance of the MERGT was then tested in the configuration as shown in Fig. 7(a). The electromagnet controlled by the function generator provides both input ac magnetic field, $H_{i n}$, and the DC magnetic bias field, $H_{D C}$. We first characterized the top resonant gate capacitance performance. The change in capacitance was measured at $100 \mathrm{~Hz}$ with a DC magnetic bias field using an Andeen Hagerling ultra 


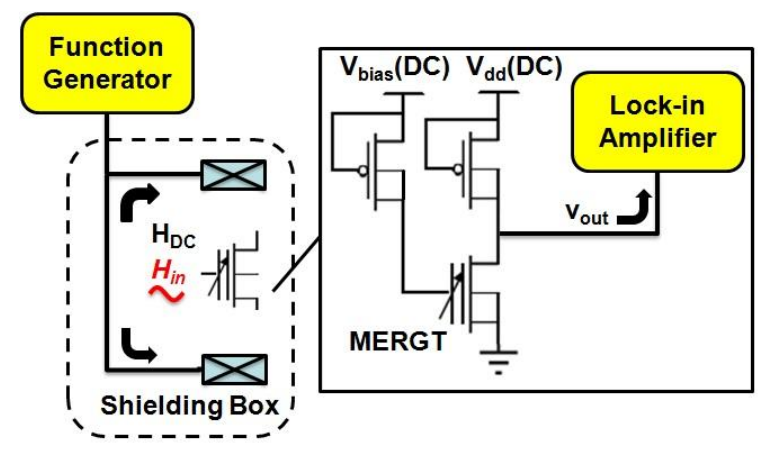

(a)

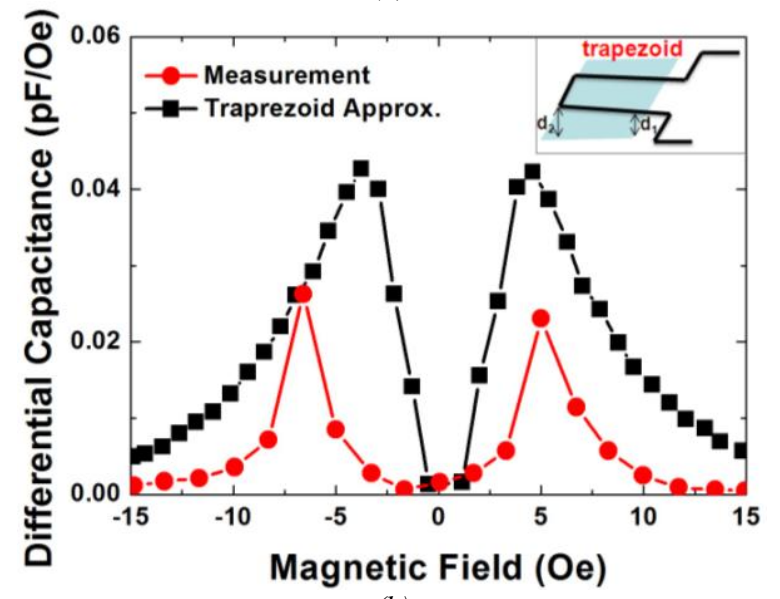

(b)

Figure 7: (a) Schematic of ac magnetic field measurement set-up. (b) Input capacitance change (measurement and calculation) as a function of DC magnetic field.

precision capacitance bridge (AH 2700A). The results, displayed in Fig. 7(b), show the comparison of differential capacitance between measurement and analytical approximation as a function of the DC magnetic bias field. The trapezoidal gap approximation for the based on a linearly bent cantilever profile overestimates the capacitance of the device and results in a shift of the peak response magnetic field. The model needs to include the $2 \mathrm{D}$ bowing of the cantilever structure to find a good agreement with the measured results [8]. The peak AC response happened at $5(-6)$ Oe.

Figure 8 shows the characterization of a completed MERGT. The output of the FET was measured using a Stanford Research SRS-830 Lock-in Amplifier. The output voltage vs. input ac magnetic field was performed in Fig. 8 (a). The measurement shows the linearity of the common source readout amplifier output following the input magnetic signal. The DC bias magnetic field was fixed at 5 Oe where the device output shows the peak response. The frequency response of the MERGT was studied in vacuum $\left(10^{-3}\right.$ Torr), as shown in Fig. 8 (b). The device was biased for the maximum ac response by a permanent magnet and then pumped down to milli-Torr pressure range using a dry pump. After the pressure had stabilized, the chamber was valved-off to maintain the chamber pressure and the chamber was physically disconnected from the pump to isolate it from the external mechanical vibration. The ac input magnetic field was then generated by a Helmholtz coil. At resonance, the output voltage increases by $\sim 20$ (in vacuum). The noise spectrum of MERGT is shown in Fig. 8 (c). The flicker noise of the input transistor limits the noise floor of the whole system [9]. Based on the results in Figs 8, we can obtain a

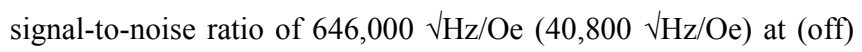
resonance, which corresponds to a minimum detectable field of $150 \mathrm{pT} / \sqrt{\mathrm{Hz}}(3 \mathrm{nT} / \sqrt{\mathrm{Hz}})$. This result represents a significant enhancement in comparison to the first integrated ME sensor demonstrated by $\mathrm{Y}$. $\mathrm{Lu}$ and A. Nathan with 40 micro Tesla detectable field [10].

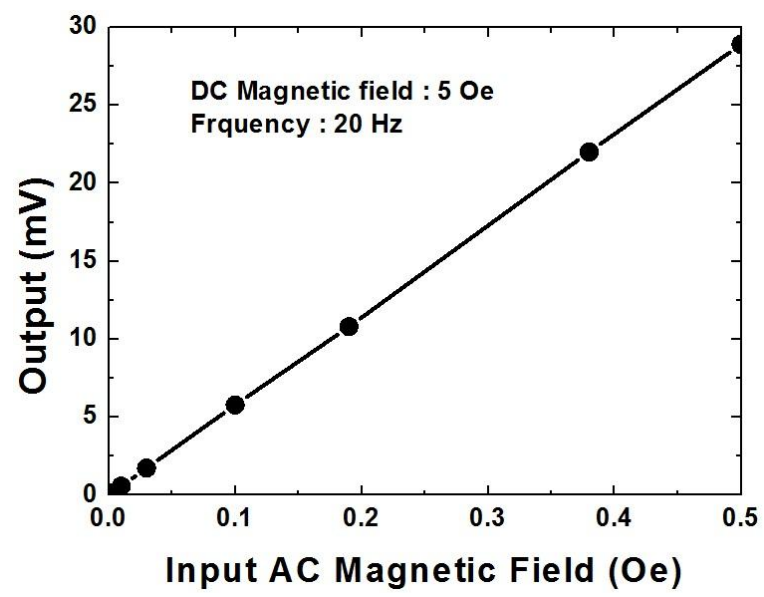

(a)

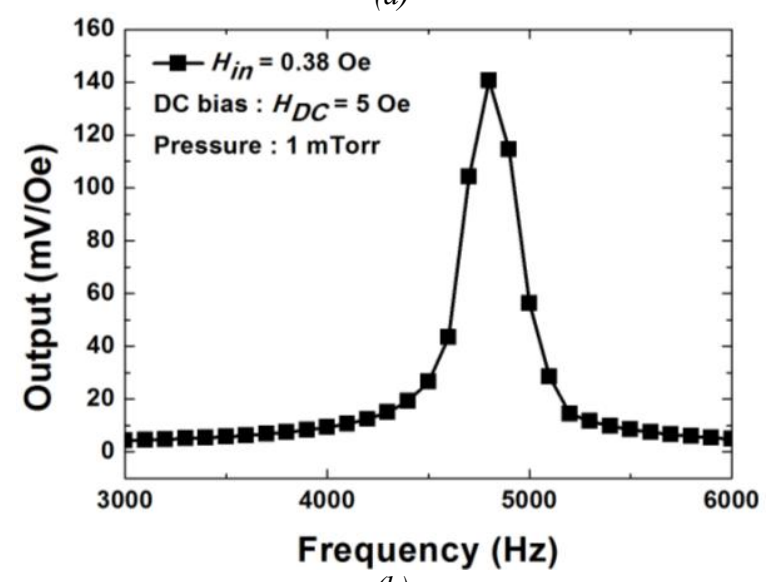

(b)

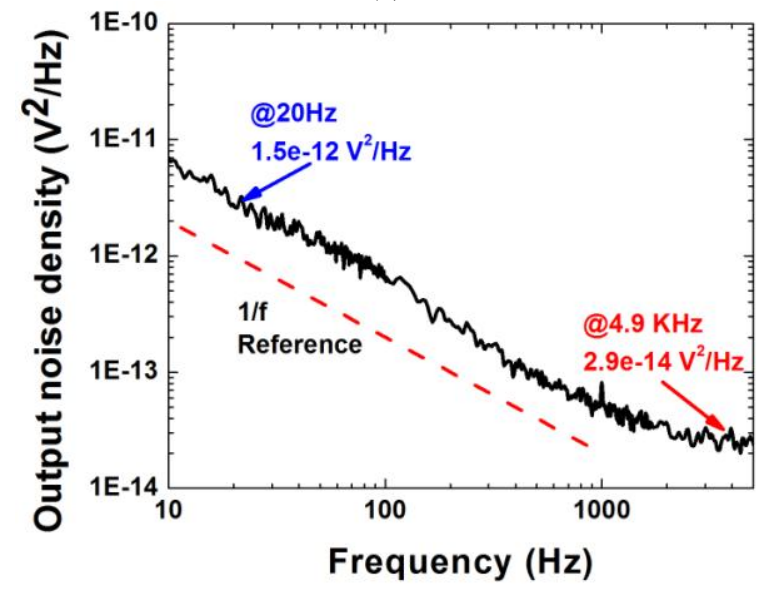

(c)

Figure 8: (a) Output voltage of common source readout amplifier circuit as a function of the input ac magnetic field. The data was measured at $20 \mathrm{~Hz}$ and $H_{D C}=5$ Oe. (b) MERGT frequency response measurement in vacuum. (c) Output noise performance of MERGT. 


\begin{tabular}{|c|c|c|c|c|c|c|c|c|}
\hline \multirow{2}{*}{$\begin{array}{l}\text { Magnetic Sensor } \\
\text { Technology }\end{array}$} & \multicolumn{8}{|c|}{ Detectable Field Range (Tesla) } \\
\hline & $10^{-14}$ & $10^{-12}$ & $10^{-10}$ & $10^{-8}$ & $10^{-6}$ & $10^{-4}$ & $10^{-2}$ & 1 \\
\hline \multicolumn{9}{|l|}{ SQUID } \\
\hline \multicolumn{9}{|l|}{ Flux gate magnetometer } \\
\hline \multicolumn{9}{|l|}{ Magnetoresistor } \\
\hline \multicolumn{9}{|l|}{ Hall-Effect Sensor } \\
\hline MERGT & & & & & & & & \\
\hline
\end{tabular}

Figure 9: MERGT sensitivity in comparison with current magnetic sensing technologies.

Finally, we have benchmarked our MERGT performance [8] in Fig. 9 by comparing the results in this work to the current magnetic sensing technologies [11] with other types of magnetic field sensors. The sensitivity of MERGT is close to the flux gate magnetometer and has the potential to achieve picoTesla detection currently achievable only using SQUID sensors. It should be noted that the noise level in this work is limited by the flicker noise of sensing transistors. By using advanced transistors such as $\mathrm{SiGe}$ Quantum well FETs with much lower flicker noise the overall noise can be reduced by at least two orders of magnitude. Furthermore, by better controlling the stress mismatch of the two constituent layers ( $\mathrm{Ti}$ and Metglas ${ }^{\circledR}$ ), the sensitivity can be further improved. These considerations suggest a strong potential of the next generation MERGT in achieving picoTesla detection at room temperature.

\section{CONCLUSION}

In summary, an integrated Metglas ${ }^{\circledR}$ MERGT with nanoTesla detection at resonance has been designed, fabricated and modeled. Such MEMS-NMOS integration using magnetostrictive cantilever and FET sensing and amplifying capability exhibits a significant enhancement in the signal-to-noise ratio and consequently smaller minimum detectable magnetic field. Also, this demonstration indicates the compatibility of the ME sensors integration with the $\mathrm{Si}$ process technology and suggests the future solutions for the ultra sensitive chip-scale magnetometers implementation.

\section{ACKNOWLEDGMENT}

The nanofabrication of the MERGT was performed at Pennsylvania State University Nanofab supported by the National Nanotechnology Infrastructure Network (NNIN). Also, the authors gratefully acknowledge the support of Jose Israel Ramirez and Thomas Jackson from Pennsylvania State University for the Metglas ${ }^{\circledR}$ ion mill sputtering.

Travel support has been generously provided by the Transducer Research Foundation.

\section{REFERENCES}

[1] L. D. Landau and E. M.Lifshitz, "Electrodynamics of Continuous Media" 1960, pp. 119-120.

[2] G. T. Rado and V. Folen, "The effective magnetoelectric coefficients of polycrystalline $\mathrm{Cr}_{2} \mathrm{O}_{3}$ annealed in perpendicular electric and magnetic fields," J. Phys. Rev. Lett., vol. 7, pp. 310, 1961.

[3] Z. Fang, S. G. Lu, F. Li, S. Datta, and Q. M. Zhang, "Enhancing the Magnetoelectric Response of Metglas/Polyvinylidene fluoride Laminates by Exploiting the Flux Concentration Effect," Appl. Phys. Lett., vol. 95, $112903,2009$.
[4] J. Zhai, Z. Xing, S. Dong, J. Li, and D. Viehland, "Detection of pico-Tesla magnetic fields using magnetoelectric sensors at room temperature," Appl. Phys. Lett., vol. 88, 062510, 2006.

[5] S. Dong, J. Zhai, J. Li, D. Viehland, "Near-ideal magnetoelectricity in high-permeability magnetostrictive piezofibere laminates with a (2-1) connectivity," Appl. Phys. Lett., vol. 89, 252904, 2006.

[6] Y. Wang, D. Gray, D. Berry, J. Gao, M. Li, F. Li, and D. Viehland, "An Extremely Low Equivalent Magnetic Noise Magnetoelectric Sensor," Adv. Mater., vol. 23, pp. 41114114, 2011.

[7] F. Li, F. Zhao, Q. M. Zhang and S. Datta, "Low-frequency voltage mode sensing of magnetoelectric sensor in package," Electronics Letters, vol. 46, no. 16, 2010.

[8] F. Li, R. Misra, Z. Fang, Y. Wu, Q. M. Zhang, P. Schiffer, S. Tadigadapa and S. Datta, "Magnetoelectric Resonant Gate Transistor with NanoTesla Sensitivity," submitted to Journal of MEMs, 2012.

[9] F. Li, Z. Fang, R. Misra, S. Tadigadapa, Q. M. Zhang, and S. Datta, "Giant Magnetoelectric Effect in Nanofabricated $\mathrm{Pb}\left(\mathrm{Zr}_{0.52} \mathrm{Ti}_{0.48}\right) \mathrm{O}_{3}-\mathrm{Fe}_{0.85} \mathrm{~B}_{0.5} \mathrm{Si}_{0.1}$ Cantilevers and Resonant Gate Transistors," Device Research Conference, vol. 37, no. 69, 2011.

[10] Y. Lu, and A. Nathan, "Thin film magnetostrictive sensor with on-chip readout and attofarad capacitance resolution," IEEE Int. Electron Devices Meet., pp. 777-780, 1996.

[11] M. J. Caruso, T. Bratland, C. H. Smith, R. Schneider, Honeywell, Inc.

\section{CONTACT}

*F. Li, Tel: +1-814-321-6158; fx1135@psu.edu 


\section{A SELF-CONTROLLED FREQUENCY TRIMMING TECHNIQUE FOR MICROMECHANICAL RESONATORS \\ A. Hajjam*, K. Dietrich, A. Rahafrooz, and S. Pourkamali \\ Department of Electrical and Computer Engineering, University of Denver, Denver, CO, USA}

\begin{abstract}
This work demonstrates the proof of concept for a selfcontrolled electronic frequency trimming technique for micromechanical single crystalline silicon resonators. Trimming is carried out via localized self-induced thermal oxidation occurring during extreme thermal actuation of the resonant structures. The cooling effect at resonance, discussed for the first time in this work, allows the localized oxidation to stop automatically as soon as the resonator frequency reaches the targeted actuation frequency applied to the structure. The viability of this concept is demonstrated by application of both off-resonance and atresonance actuation signals to fabricated resonators showing that as opposed to the off-resonance signals, the at-resonance signals with the same intensity do not lead to frequency trimming.
\end{abstract}

\section{INTRODUCTION}

As the potential emerging technology for next generation integrated resonant sensors and frequency references as well as electronic filters, micro-electro-mechanical resonators have attracted a lot of attention over the past decade. [1,2]. The resonance frequency of silicon micromechanical resonators is dependent on the physical dimensions of the resonating structure. Even very small (few nanometers) fabrication induced dimensional imprecision can lead to intolerable frequency inaccuracies for micromechanical resonators. Therefore, process variations across the substrate (e.g Non-uniformities in photolithography, etching and film thickness) leading to variations and mismatch in the mechanical resonant frequencies are a major challenge for batch fabrication of micromechanical resonators [1,3]. Achieving ppm level frequency accuracy and uniformity in batches of micromachined resonators is a daunting task necessitating postfabrication trimming [4].

Frequency trimming techniques for MEMS silicon based micromechanical resonators, range from pulsed-laserdeposition/trimming, electrostatic trimming and other process alterations. Post-fabrication frequency trimming via pulsed-laserdeposition [4,5] is unlikely to be able to provide ppm level frequency accuracy due to the difficulties in controlling the geometry and the amount of the mass deposited by the laser. Furthermore, the quality factor of the resonator is often degraded with additional materials on the resonator. An alternative method to laser enhanced material deposition for frequency trimming is based on material removal [4] which also suffers from frequency accuracy issues.

Digital trimming technique has been reported by using a high resolution fractional-N synthesizer in a phase lock loop architecture for locking a voltage-controlled oscillator to a silicon microresonator-based reference oscillator [4]. On the downside, such a digital trimming technique may not be contiguous, is power hungry, and considerably increases the overall phase noise of the oscillator and the integrated circuit die area.

Other post fabrication processes such as gold diffusion into the bulk of the resonator have also been demonstrated for frequency trimming purposes. In this method by running a current through the structure, a gold coating is diffused into the resonator silicon structure. Upon cooling, the gold diffusion slightly increases the stiffness of the resonating structure, which results in an upward shift in resonance frequency [6].

I-shaped thermally actuated micromechanical resonators are among the most suitable resonant structures offering unmatched design and transduction flexibility [7]. However, relatively narrow thermal actuators (often submicron) required to minimize power consumption of such devices, make achieving frequency accuracy even more challenging. It has previously been shown that localized thermal oxidation via joule heating can be used as a simple method for post-fabrication frequency trimming of such resonators. Using this technique, permanent frequency shifts up to $\sim 3.7 \%$ were demonstrated for a $53 \mathrm{MHz}$ resonator [8]. The approach was based on a relatively large bias current passing through the resonator structures. Joule heating caused by ohmic losses locally raises the temperature of the structure in the actuator beams (which supply the structural stiffness in the resonant mode of interest) to several hundred degrees Celsius $\left(700-1200^{\circ} \mathrm{C}\right)$. The elevated temperature causes thermal oxidation to occur on the surfaces when placed in an oxygen rich atmosphere. The change in dimensions and Young's modulus as well as internal stress, caused by oxidation, result in a permanent change in the resonant frequency of the device.

This work shows preliminary measurement results for a more advanced version of the same localized oxidation based trimming approach with the potential ability to automatically limit itself.

\section{RESONATOR DESCRIPTION}

The resonators utilized in this work are referred to as I-shaped resonators [7]. Such devices are very suitable for thermal actuation as they can easily be actuated by passing a fluctuating electrical current through the actuator beams in the middle of the structure. Their operating principle has thoroughly been explained in [7]. The resonators vibrate in the in-plane extensional resonant mode. In this mode the masses on the two ends of the beams vibrate back and forth in opposite directions. At resonance, the resistance of the pillars is modulated by the resulting alternating mechanical stress due to the piezoresistive effect. This results in a detectable alternating motional current component in the device.

Fabrication of the resonators was carried out using the standard single mask SOI-MEMS process which starts by growing a thin $(\sim 200 \mathrm{~nm})$ layer of thermal silicon dioxide on the substrate. The silicon dioxide layer is patterned to define the resonator structures. The silicon structures are then carved into the SOI device layer by deep reactive ion etching (DRIE) of silicon all the way down to the buffer oxide layer. Finally, the structures are undercut by dipping in $49 \%$ hydrofluoric acid (HF) to etch the underlying buried oxide layer (BOX) layer. At the same time the remaining oxide mask on top of the structures is also etched away. The result will be suspended single crystalline silicon resonant structures with integrated thermal actuator/piezoresistive sensors. Figure 1 shows the SEM view of a $4 \mu \mathrm{m}$ thick $18 \mathrm{MHz}$ I-shaped 
resonator which was fabricated on an N-type low resistivity SOI substrate with a BOX thickness of $5 \mu \mathrm{m}$.

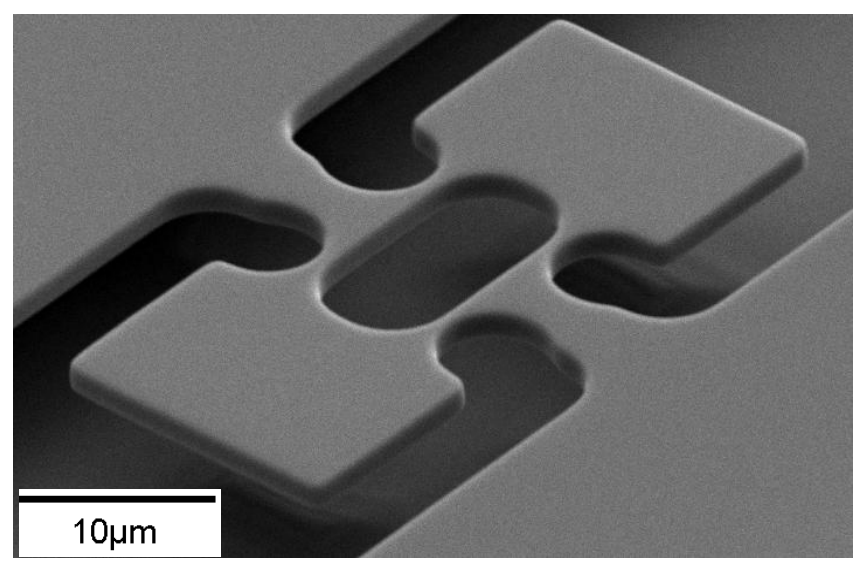

Figure 1: SEM view of an 18MHz, $4 \mu m$ thick thermalpiezoresistive I-shaped resonator. The resonator is fabricated on a low resistivity $(\sim 0.01 \Omega . \mathrm{cm}) \mathrm{N}$-type SOI substrate using a single mask process.

\section{SELF-CONTAINED TRIMMING PRINCIPLE}

As opposed to the previous work in which we applied a DC current [8], in this method localized oxidation is induced by applying an $\mathrm{AC}$ voltage $\left(V_{a c} \cos \left(2 \pi f_{a} t\right)\right)$ (Figure 2a) across the structure. The generated power has a square relationship with the applied voltage and gives birth to two frequency components at DC and twice the actuation frequency $\left(2 f_{a}\right)$ :

$$
P=\frac{v_{a c}{ }^{2} \cos ^{2}\left(2 \pi f_{a} t\right)}{R_{A}}=\frac{v_{a c}{ }^{2}}{2 R_{A}}+\frac{v_{a c}{ }^{2} \cos \left(4 \pi f_{a} t\right)}{2 R_{A}}
$$

where $R_{A}$ is the electrical resistance of the resonator and $f_{a}$ is the actuation frequency. The resulting power translates to elevated temperature of the thermal actuators. It can be shown that the $\mathrm{AC}$ temperature term is proportional but also has a $90^{\circ}$ lag in comparison to the $\mathrm{AC}$ power [9]. The resulting fluctuating temperature causes a thermal stress and therefore an alternating mechanical force inside the thermal actuators. If this applied force has the same frequency as the resonance frequency of the resonator, it actuates the device. In order for this to happen, the frequency of the actuation voltage should be half of the mechanical frequency. The resulting mechanical vibration of the resonator has a $90^{\circ}$ lag with respect to the mechanical force [9]. The mechanical vibration leads to resistance fluctuations of the resonator due to the piezoresistive effect. The AC value is proportional and in phase with the mechanical vibration. Therefore this $\mathrm{AC}$ term which adds to the electrical resistance of the device has a $180^{\circ}$ lag with respect to the applied actuation current due to thermal and mechanical delays. For N-type silicon the piezoresistive coefficient is negative which itself adds a $180^{\circ}$ lag. Thus, the resistance fluctuation is in phase with the applied voltage (blue plot in Figure $2 \mathrm{~b}$ ) and this leads to the overall resistance of : $R_{A}+r_{a c} \cos \left(2 \pi f_{0} t\right)$. As a result the ohmic power in the structure will be:

$$
P=\frac{v_{a c}{ }^{2} \cos ^{2}\left(\pi f_{0} t\right)}{R_{A}+r_{a c} \cos \left(2 \pi f_{0} t\right)}=\frac{v_{a c}{ }^{2}\left(1+\cos \left(2 \pi f_{0} t\right)\right)}{2\left(R_{A}+r_{a c} \cos \left(2 \pi f_{0} t\right)\right)}
$$

The $r_{a c}$ value itself is equal to:

$r_{a c}=\frac{R_{A}\left|\pi_{l}\right| E x_{m}}{L}$

where $\pi_{1}$ is the longitudinal piezoresistive coefficient, $E$ is the young's modules, $L$ is the length of the thermal actuator and $x_{m}$ is the vibration amplitude. The DC component of Eq. 2 can be found to be equal to:

$$
P_{D C} \cong \frac{v_{a c}^{2}}{2\left(R_{A}+r_{a c}\right)}
$$

By comparing the DC power consumptions in the two different states of off-resonance (DC part of Eq. 1) and atresonance (Eq. 4), it is obvious that at-resonance state the power consumption goes down (Figure 2c). Therefore, during resonance the static temperature decreases (Figure 2d). This difference in temperature between the two cases of at-resonance and offresonance can be utilized for self controlled trimming of the structures.

a)

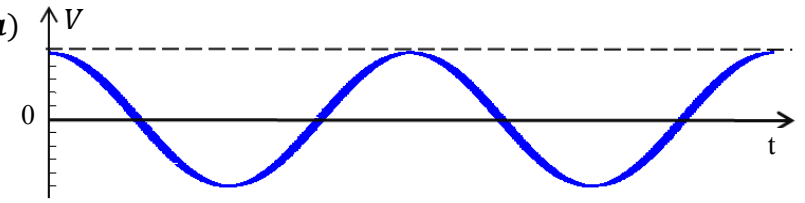

b)

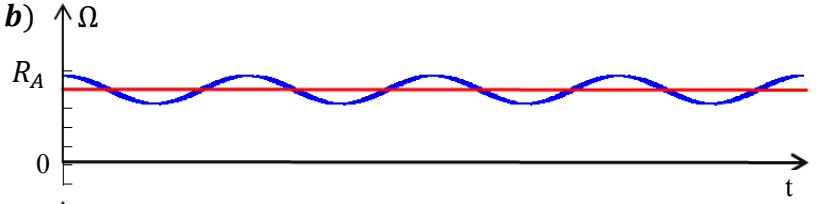

c) $\uparrow W$
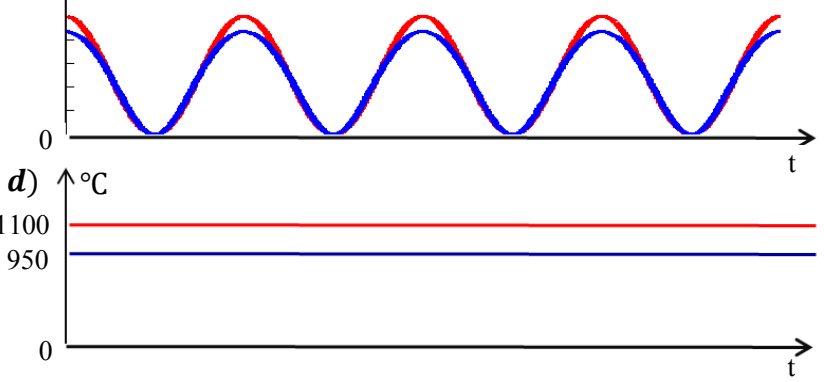

Figure 2: Waveform representation of a) Input AC voltage, b) Resistance of the resonator, c) Ohmic loss and d) Temperature in both conditions of off-resonance (red) and at-resonance (blue). It is assumed that $r_{a c}=0.15 R_{A}$. Therefore, the $D C$ power consumption drops $14 \%$ during resonance with respect to its offresonance mode. As a result assuming the initial static temperature of $1100^{\circ} \mathrm{C}$, temperature decreases to $950^{\circ} \mathrm{C}$.

Figure 3 schematically depicts the proposed self-controlled electronic frequency trimming technique for micromechanical single crystalline silicon resonators. Half the desired frequency is applied with sufficient amplitude to allow the actuators to reach the temperatures required for oxidation. As the oxidation process starts, the internal stress of the thermal actuators changes. This causes the resonance frequency of the resonator to gradually change and become closer to the desired resonance frequency. The process goes on until the resonance frequency of the resonator exactly matches the desired resonance frequency. At this point, the resonator starts vibrating and therefore the overall resonator static temperature drops significantly which could stop the local 
oxidation. In order to investigate the feasibility of this technique, assuming large vibration amplitudes and large piezoresistive coefficients, $r_{a c}$ can become comparable with $R_{D C}$. For instance, with a conservative piezoresistive coefficient of $\left(-10 \times 10^{-11} \mathrm{~Pa}^{-1}\right)$, and vibration amplitude of $100 \mathrm{x}$ smaller than the actuator length, $r_{a c}=-0.15 R_{A}$. In this case the value of $P_{D C}$ at resonance becomes $14 \%$ less than its value out of resonance. This translates to a $14 \%$ reduction of the actuator static temperature difference from the surroundings. For instance, a resonator heated up to $1100^{\circ} \mathrm{C}$ by the applied currents out of its resonance frequency, can cool down to about $950^{\circ} \mathrm{C}$ if the actuation frequency becomes equal to its mechanical resonant frequency. Therefore, the overall resonator static temperature could drop significantly if the applied actuation $\mathrm{AC}$ signal has the right frequency required for excitation of the resonant mode. Sharp dependence of thermal oxidation rate on temperature can turn small temperatures changes into major changes in the oxidation rate and completely stopping it.

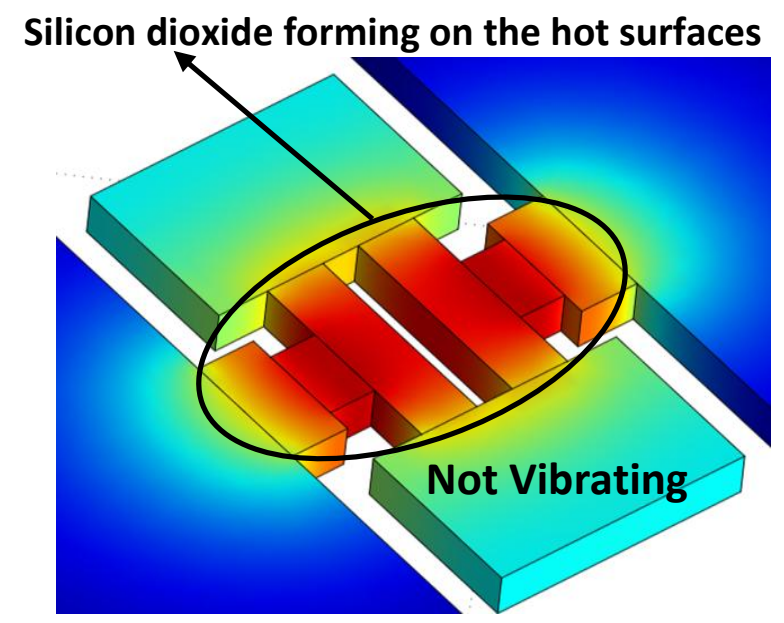

Silicon dioxide formation and freq. shift stop

Mechanical resonant frequency $\left(f_{m}\right)$ increases
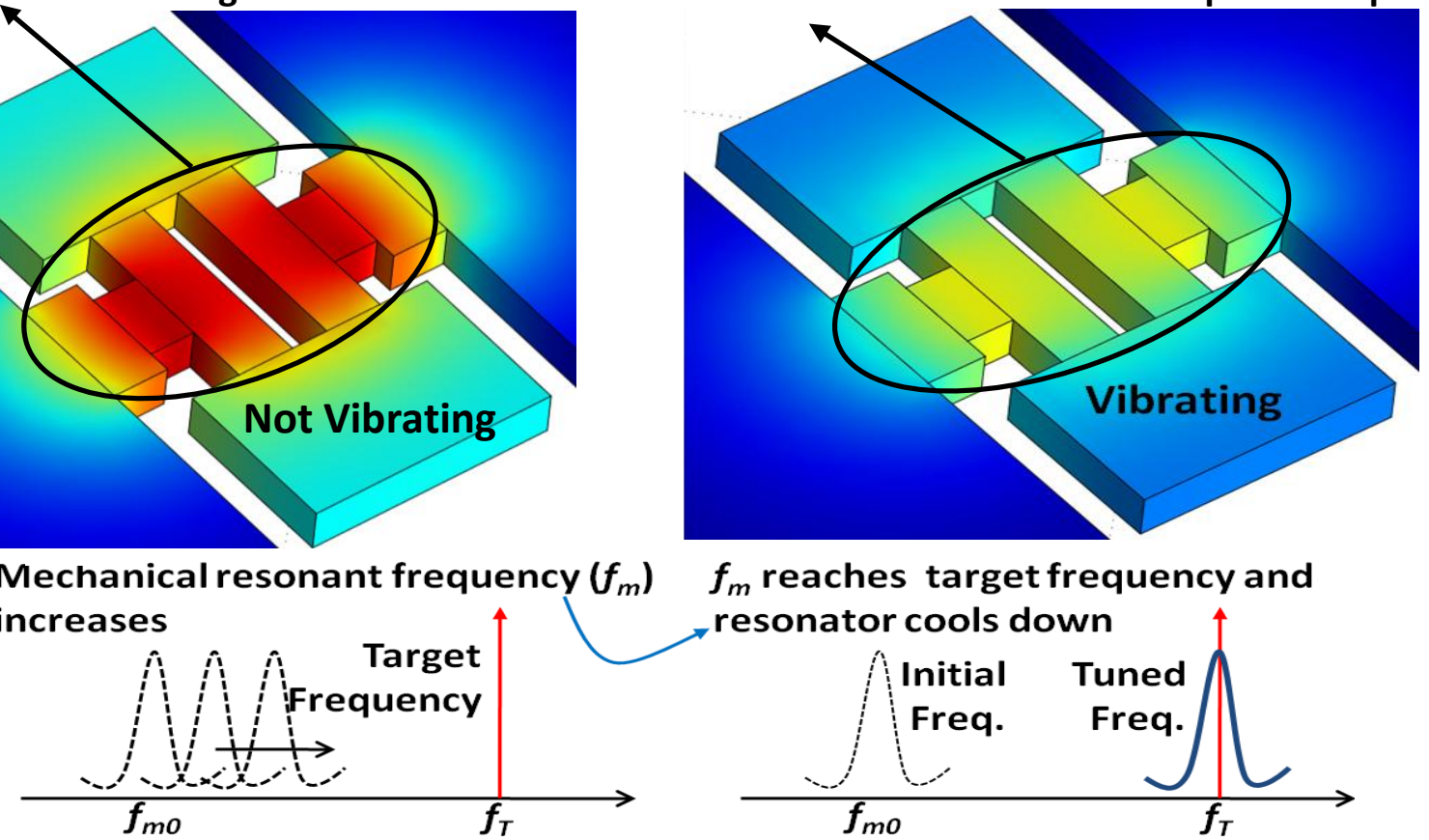

$f_{m}$ reaches target frequency and resonator cools down

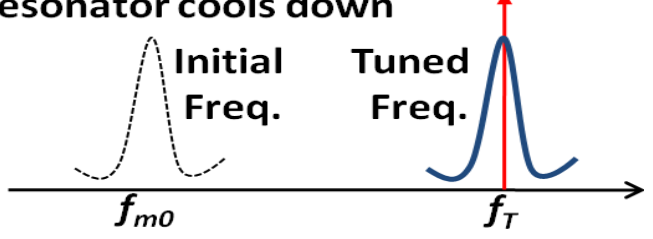

Figure 3: Schematic demo of the frequency trimming technique. When an actuation voltage with half the target resonance frequency is being applied across the actuator beams, the elevated temperature allows oxidation to take place on their surface. As a result, the structural stiffness gradually changes and the resonant frequency moves towards the target. When the mechanical resonant frequency reaches the target frequency, the resonator starts vibrating and therefore cools down. As a result, oxidation stops and the frequency will not shift further.

\section{MEASUREMENT SETUP AND RESULTS}

Figure 4 shows the schematic diagram of the test setup used for performing the localized thermal oxidation process. The

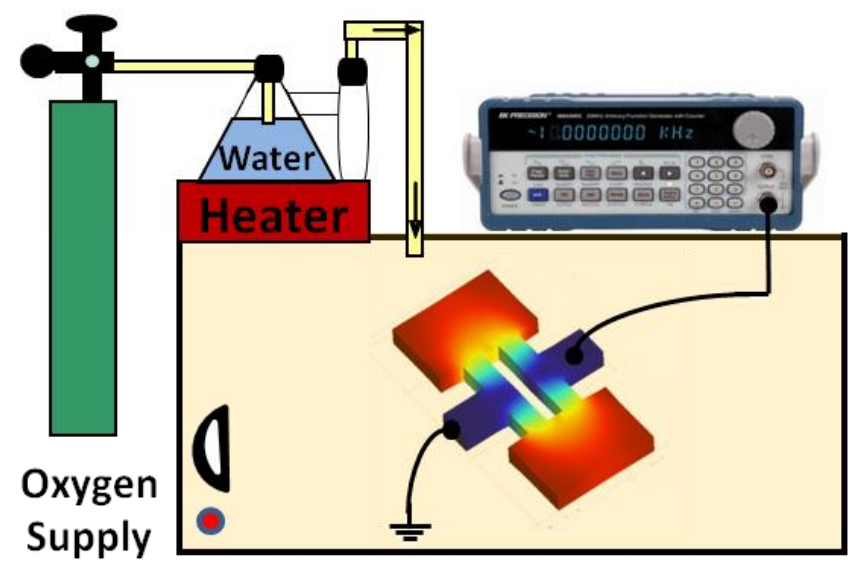

Figure 4: Schematic diagram of the setup used in this experiment. Moisture rich oxygen was driven into the chamber while a sinusoidal ac current was run through the device. resonators were placed in a chamber while connected to the test setup. Moisture rich oxygen was delivered into the chamber through an inlet tube to provide a suitable atmosphere for wet oxidation of silicon. The localized oxidation and therefore frequency trimming starts by application of a large amplitude AC voltage applied through the structure using a function generator. After this localized oxidation run, the devices were disconnected from the AC voltage source and were tested at room temperature using a network analyzer.

Figure 5 shows the change in the measured resonance frequency after different oxidation steps, for the $18 \mathrm{MHz}$ resonator of Figure 1. Different plots show data taken after localized oxidation runs via actuation by $\mathrm{AC}$ voltage with both offresonance and at-resonance frequencies. It has been shown through 4 consecutive oxidation periods with alternating off/at resonance signals applied to the device that the cooling effect is occurring. Off-resonance oxidation which has been applied in steps 1 and 3 has led to changes in the resonator frequency, whereas in steps 2 and 4 it is shown that when the excitation causes mechanical resonance, it results in almost no oxidation (no frequency trimming). 
Another set of interesting data that was collected was with regard to the resonator temperature drift. Figure 6 shows the temperature drift characteristics of the same resonator measured before and after each of the oxidation steps.

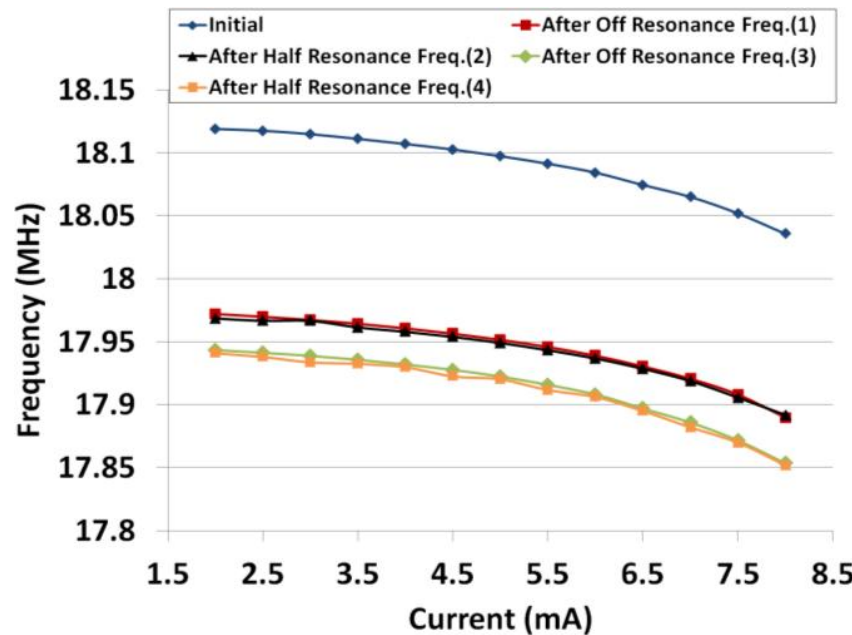

Figure 5: Measured resonance frequency of the $18 \mathrm{MHz}$ resonator of figure 1, at different bias currents. Different plots show the data taken after different localized oxidation runs at room temperature when the oxygen source was removed. As can be seen from the graph, the actuation at half the resonance frequency has had no effect on the shift in resonant frequency (steps 2 and 4), whereas each time the resonator was actuated at an off-resonance frequency, the resonance frequency has

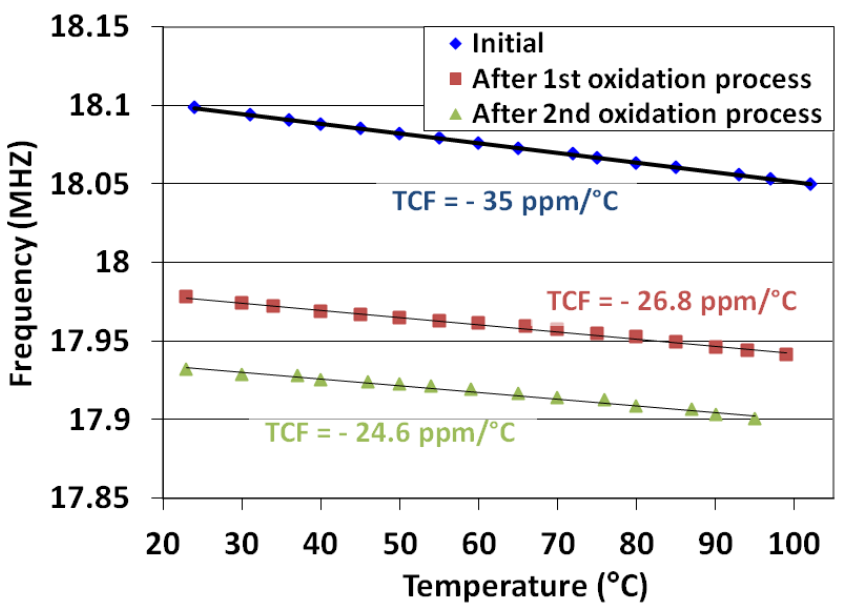

Figure 6: Temperature drift characteristics for the $18 \mathrm{MHz}$ resonator of figure 1 measured before and after each of the oxidation steps. The TCF becomes more positive (less negative) after each step due to the formation and thickening of $\mathrm{SiO}_{2}$ on the surface of the actuators.

Silicon resonators typically exhibit temperature coefficient of frequency (TCF) in the -20 to $-40 \mathrm{ppm} /{ }^{\circ} \mathrm{C}$ range [10]. Figure 6 shows that while the initial TCF for this resonator was measured to be $-35 \mathrm{ppm} /{ }^{\circ} \mathrm{C}$, its value has gradually increased (become less negative) after oxidation steps with off resonance frequency. Due to no oxidation occurring when mechanical resonance is caused, no change in TCF is expected. Increase in TCF is due to the formation and thickening of the $\mathrm{SiO}_{2}$ film (which has a positive temperature coefficient of Young's modulus) on the surface of the resonator.

\section{CONCLUSIONS}

Self-controlled post-fabrication electronic frequency trimming of individual single crystalline silicon thermal-piezoresistive resonators was demonstrated. Trimming was carried out via localized self-induced thermal oxidation while taking advantage of the cooling effect of resonators at resonance. This technique can potentially be used for automatic trimming of a whole batch of resonators after fabrication.

\section{ACKNOWLEDGEMENT}

This work was supported by National Science Foundation under grants \#0923518 and \#1056068.

\section{REFERENCES}

[1] S. Pourkamali, G. K. Ho and F. Ayazi, "Low-impedance VHF and UHF capacitive silicon bulk acoustic wave resonators," IEEE Transactions on Electron Devices, vol. 54, No. 8, 2007, pp.2017-2023.

[2] A. Hajjam, J. C. Wilson, A. Rahafrooz, and S. Pourkamali, "Fabrication and characterization of thermally actuated micromechanical resonators for airborne particle mass sensing: II. device fabrication and characterization," J. Micromech. Microeng., vol. 20, 12501, Dec. 2010.

[3] K. Sundaresan, G. K. Ho, S. Pourkamali and F. Ayazi, "Electronically temperature compensated silicon bulk acoustic resonator reference oscillators", Journal of Solid State Circuits, pp. 1425-1434, 2007.

[4] W-T. Hsu, A. R. Brown, "Frequency trimming of MEMS resonator oscillators", IEEE Intl. Freq. Control Symp. pp 1088-1091, 2007.

[5] M. A. Abdelmoneum, M. U. Demirci, S.S. Li, and C.T.-C Nguyen, "Post-Fabrication Laser Trimming of Micromechanical Filters", Technical Digest, IEEE Int. Electron Devices, pp. 39-42, 2004.

[6] A. K. Samarao and F. Ayazi, "Post-fabrication electrical trimming of silicon bulk acoustic resonators using joule heating," Proc. IEEE MEMS Conference, Italy, pp. 892-895, Jan. 2009.

[7] A Hajjam, J. C. Wilson, and S. Pourkamali, "Individual airborne particle mass measurement using high frequency micromechanical resonators," in IEEE Sensors Journal, vol. 11, pp. 2883-2890, Nov. 2011.

[8] A. Hajjam, A. Rahafrooz, J. Gonzales, R. Abdolvand and S. Pourkamali, "Localized thermal oxidation for frequency trimming and temperature compensation of micromechanical resonators," in proceedings, IEEE MEMS, Paris, France, Jan. 2012, pp. 704-707.

[9] A. Rahafrooz and S. Pourkamali, "High-Frequency Thermally Actuated Electromechanical Resonators With Piezoresistive Readout," IEEE Transactions on Electron Devices, vol.58, no.4, pp.1205-1214, April 2011.

[10] A. Hajjam, A. Rahafrooz and S. Pourkamali, "Sub-100ppb $/{ }^{\circ} \mathrm{C}$ temperature stability in thermally actuated high frequency silicon resonators via degenerate phosphorous doping and bias current optimization," Proc., IEEE IEDM, San Francisco, pp. 751-754 Dec. 2010.

\section{CONTACT}

*A.Hajjam, 2390 S.York St Denver CO, USA, Tel: +1-303-8714631; arash.hajjam@du.edu 


\section{ELECTROSTATICALLY DRIVEN ROTOR ON CONDUCTIVE LIQUID RING BEARINGS

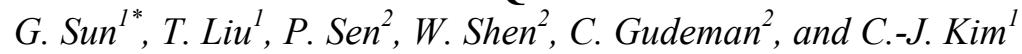 \\ ${ }^{1}$ University of California, Los Angeles (UCLA), Los Angeles, California, USA \\ ${ }^{2}$ Innovative Micro Technology (IMT), Santa Barbara, California, USA}

\begin{abstract}
This paper presents an electrostatically side-driven rotary stage featuring ionic liquid rings as both mechanical bearings and electric connections between the rotor and the substrate. An SOI-based device with multiple through-silicon vias has been fabricated and self-assembled by the liquid rings. The device has operated successfully by applying sequential voltages of $50 \mathrm{~V}_{\mathrm{DC}}$ between the rotor and the stators placed outside the rotor. Separately, the electric transmission has been verified by powering an LED on a rotating rotor $(>300 \mathrm{rpm})$ from the substrate. This is the first report of an electrostatically actuated microdevice with a liquid bearing and also the first report of a direct power transmission onto an infinitely rotating microdevice.
\end{abstract}

\section{INTRODUCTION}

Miniaturized rotating devices have been being designed over two decades by using different bearing and actuation methods. Micromotors with solid bearings, including surface dimples [1] and micro balls [2], have been actuated electrostatically [1,3], ultrasonically [4] and magnetically [5]. However, the solid-solid friction, large in microscale, requires high voltage or large current to overcome the friction and often causes serious wear [6]. Gas or air bearing [7], created through electrostatic or magnetic levitation, has no surface friction and wear but requires complicated dynamic control mechanism. Liquid bearings are considered to have a low static friction and no wear. Recently, a liquid bearing in the form of a single large droplet $[8,9]$ has been reported by actuating the rotor with electrowetting [8] or magnetically [9]. While the rotor assembly was micromachined in [9], unfortunately the device was operated with a conventional electromagnetic coil placed below the chip. More advancement is needed before one can fully utilize the advantages of liquid bearings for microdevices. In addition to the friction issue, perhaps more importantly, none of the reported micro rotating devices could transmit appreciable electrical power on to the rotor through available means (e.g., direct contacts, inductive or capacitive coupling), which has been limiting the utility of such devices.

Here, we propose to use multiple, concentric, conductive, and ring-shaped liquid bearings as both mechanical bearings and direct electric connections between the rotor and the substrate. Compared with conventional solid bearings [1,2], liquid bearings avoid dry friction, eliminate wear and enhance reliability; compared with gas bearing [7], liquid bearings can be designed to stabilize the rotation without active control using the strong surface tension in microscale; compared with a single liquid droplet [8,9], multiple conductive liquid rings allow direct electric connections; compared with multiple droplets [10], concentric liquid rings are free of contact-angle hysteresis, i.e., essentially no static friction.

By studying volume control, static and dynamic stability, payload capability, electric conductivity, and electrochemistry properties of liquid bearings, we have successfully created a new family of micro bearings: conductive liquid ring bearings. In this work, we use an ionic liquid (EMIM DCA) to form the liquid rings and incorporate them into a three-phase electrostatic side-driven rotary stage device. Direct transmission of electrical power onto the rotating rotor has also been verified. In this report, we present principle and design of the conductive liquid rings, fabrication and assembly of the electrostatically side-driven rotary stage, and testing results of the completed devices.

\section{PRINCIPLE AND DESIGN}

Figures 1 and 2 schematically show the perspective view and cross-sectional view, respectively, of electrostatically side-driven rotary stage on conductive liquid rings. Two concentric liquid rings are formed by confining the liquid inside wetting grooves and repelling it from the non-wetting superhydrophobic outer surfaces.

\section{Liquid Ring as Mechanical Bearing}

In vertical direction, the rotor is balanced by Laplace pressure, surface tension, and gravity (Fig. 2(a)). As the vertical liquid gap $h$ between rotor and substrate decreases, the supporting force resulted from Laplace pressure $\Delta P$ increases, resisting the further decrease of the gap, i.e., providing a self-regulation of the gap. Once the liquid ring is formed with rotor assembled, additional weight from the payload (i.e. static load) or acceleration shock (i.e. dynamic load) tends to decrease the liquid gap $h$ and leads to a higher Laplace pressure, maintaining the bearing until the meniscus angle $\alpha$ exceeds the advancing contact angle of the non-wetting outer surfaces.

In horizontal direction, the rotor always tends to self-center to the substrate due to the restoring force from the surface tension of the liquid (Fig. 2(b)). This restoring force can be increased by increasing the number of liquid rings. Other than vertical and horizontal stabilities, proved by our experiments, well-designed liquid rings also provide restoring force against tilting of the rotor caused by external forces.

Overall, the rotor becomes more resistant to collapsing, shifting and tilting as the liquid gap $h$ is reduced. For the device presented in this report, the gap $h$ is maintained between 100 and $200 \mu \mathrm{m}$, which is much smaller than ring width $(1 \mathrm{~mm})$ and ring diameters $(1 \mathrm{~cm}$ for inner ring and $2 \mathrm{~cm}$ for outer ring), in order to have good stability.

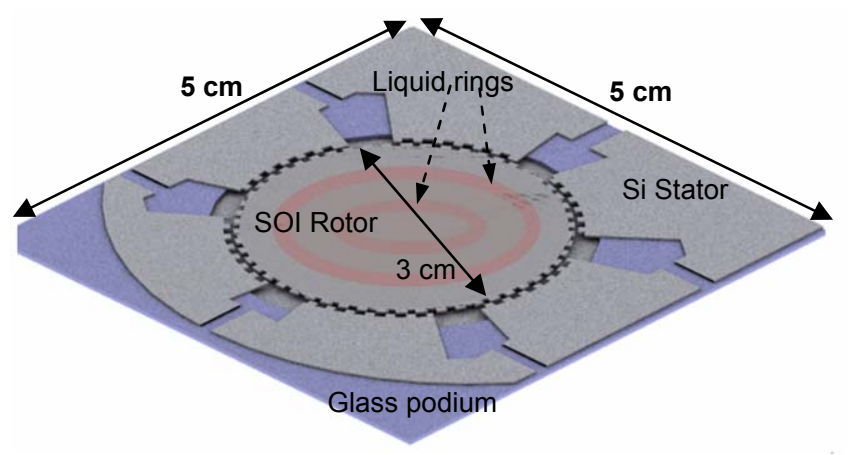

Figure 1: Perspective view of the side-driven rotary stage on conductive liquid rings. The rotor is drawn as if half-transparent to show the two rings underneath.

Solid-State Sensors, Actuators, and Microsystems Workshop Hilton Head Island, South Carolina, June 3-7, 2012 


\section{Power Transmission}

In terms of power transmission, electric current $i$ flows between the substrate and the rotor through the liquid rings and the through silicon vias (Fig. 2(c)). Each liquid ring carries an independent electrical signal. Liquid metal, e.g., mercury, has been demonstrated as an electrical signal transmission media [11]. However, most liquid metals require special operation environment or advanced packaging to prevent their oxidation, which makes the utilization of liquid metal more difficult. Instead, we have chosen ionic liquid as the conductive media as well as the mechanical bearing.

In order to deliver appreciable electrical power, ionic liquid with large electrochemical window and high electrical conductivity is preferred. Moreover, the solid-liquid interface where the electrochemical reaction happens must be taken into consideration. In the design shown in Fig. 2, each ionic liquid ring serves as an independent electrical path between rotor and substrate. Silicon on insulator (SOI)-based configuration and additional isolation trenches are used to ensure a good electrical isolation between two ionic liquid rings. In each groove, the entire bottom surface area is covered by gold (which has been confirmed to have no reaction with the ionic liquid used here) and serves as the electrodes. From this point, the ionic liquid is regarded as an electrolyte accordingly. To avoid electrolysis, the potential voltage applied on the ionic liquid should not exceed its electrochemical window.

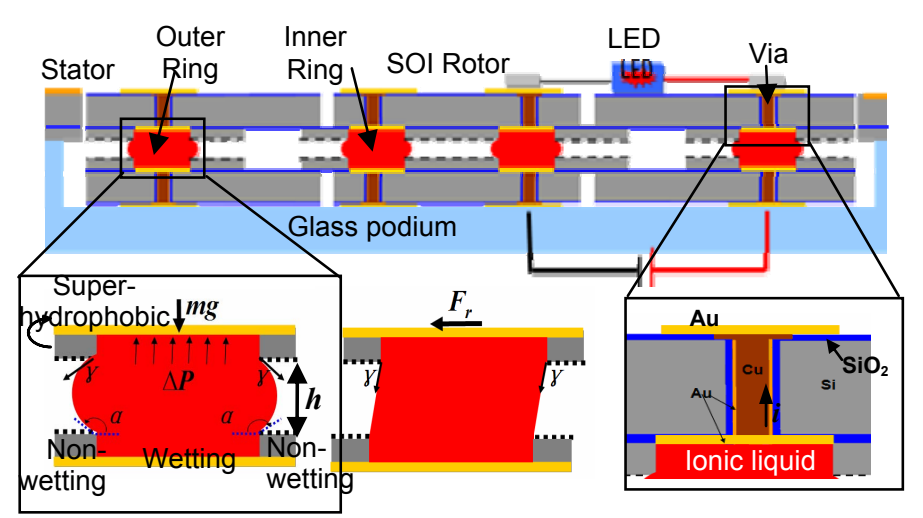

(a)

(b)

(c)

Si $\square \mathrm{SiO}_{2} \quad \mathrm{Au} \quad \mathrm{Cu} \quad$ Ionic liquid

Figure 2: Cross-sectional view of the rotary stage. (a) Vertical stability provided by Laplace pressure. (b) Horizontal stability provided by surface tension. (c) Electric transmission through liquid bearing and vias.

\section{Electrostatic Actuation}

Compared with most other actuation mechanisms, electrostatic actuation is expected to have low power consumption as no direct current flows through. A three-phase side-driven configuration has been designed for electrostatic actuation as shown in Fig. 3. The device consists of a $30 \mathrm{~mm}$-diameter rotor and 6 stators, containing multiple teeth-like electrodes (56 on rotor and 7 on each stator). The device layer (with teeth-like electrodes) of both rotor and stator is designed to be $\sim 250 \mu \mathrm{m}$ thick. The electrostatic gap between rotor and stator is $150 \mu \mathrm{m}$. With this configuration, if $50 \mathrm{~V}_{\mathrm{DC}}$ is applied, a maximum torque of $\sim 10 \mathrm{nN} \bullet \mathrm{m}$ and an open-loop control of angular accuracy of $\sim 2^{\circ}$ can be achieved. Figure 3 shows the simulation result of the electrostatic field between the rotor and a stator.

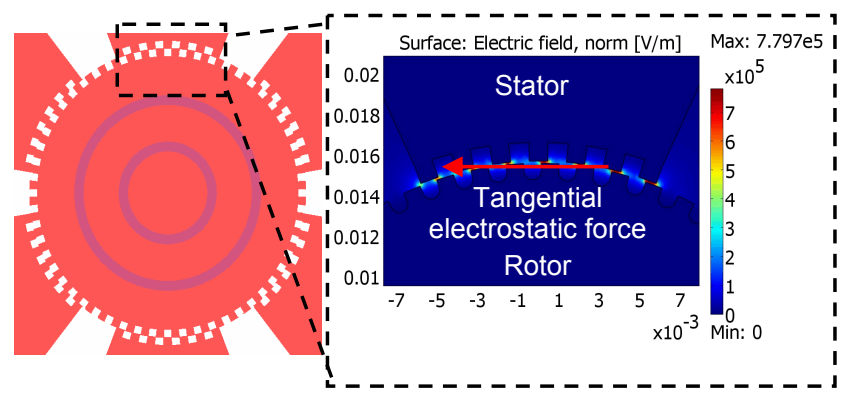

Figure 3: Layout of a three-phase side-driven rotor and simulation of its electrostatic field between the rotor and a stator that induces the electrostatic force to rotate the rotor.

\section{FABRICATION AND ASSEMBLY}

Deep reactive ion etching (DRIE) of SOI wafers, through-silicon via (TSV), black silicon, and adhesive bonding techniques were used to fabricate the rotary stage. Figure 4 shows the main fabrication steps.

An SOI wafer with highly doped layers was used. First, through-silicon vias ( $60 \mu \mathrm{m}$ in diameter; $250 \mu \mathrm{m}$ deep) were formed by DRIE and $\mathrm{Cu}$ electroplating using Innovative Micro Technology (IMT) "Super-fill" plating recipe. After polishing the surface, $\sim 2$ $\mu \mathrm{m}$ thick Au bonding pads were formed atop each $\mathrm{Cu}$ via to provide a large contact area for electrical connection. Next, electrostatic actuation electrodes and isolation groove $(250 \mu \mathrm{m}$ deep $)$ were etched in device layer by DRIE. Then, the handle layer was thinned down to $100 \mu \mathrm{m}$. A superhydrophobic surface was then formed by DRIE black silicon method, followed by another DRIE to define the wetting and non-wetting areas, which define the liquid rings. The vias were then uncovered in the wetting area followed by a $\sim 200 \mathrm{~nm}$ thick $\mathrm{Au}$ layer evaporated to ensure a direct contact with liquid rings. The stators were then bonded on a glass podium to be level with the rotor. At last, ionic liquid rings were formed and the rotor self assembled by the liquid rings.

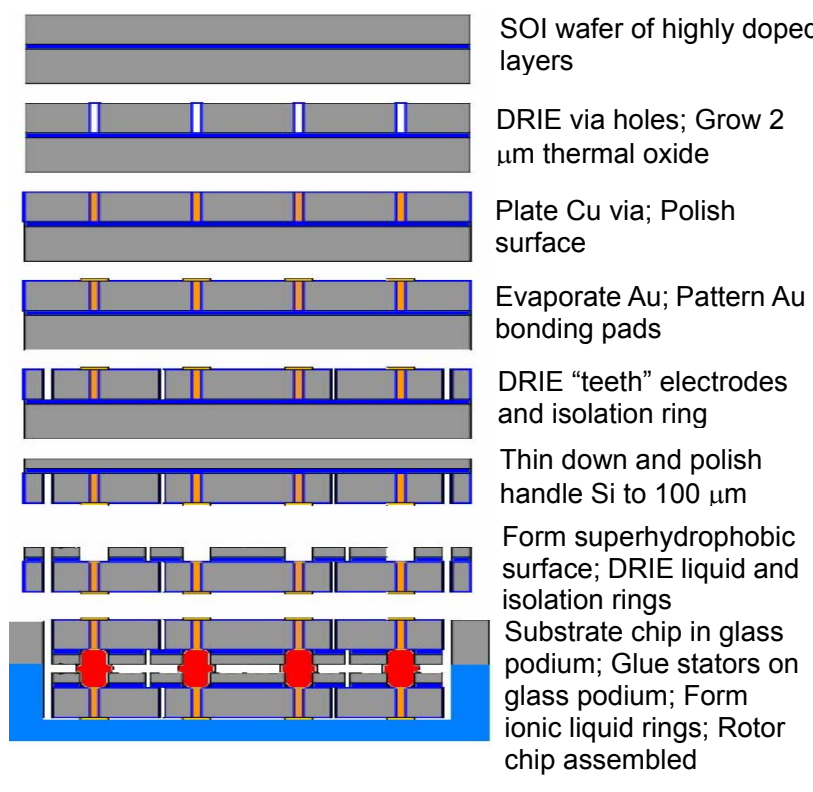

Figure 4: Fabrication process of the electrostatically side-driven rotary stage developed in this report. 
Figure 5 shows the fabrication results. On the bottom surface (i.e., the groove side) of the rotor, the black part is the non-wetting area, which is made of highly dense arrays of nanostructures. Those nanostructures have apexes of $<50 \mathrm{~nm}$ diameter, heights of $2-3 \mu \mathrm{m}$ and average pitch (i.e., period) of $\sim 400 \mathrm{~nm}$. After being treated with low surface energy coatings, e.g., Teflon ${ }^{\circledR}$ or Cytop, the nanostructured surface exhibits high superhydrophobicity (contact angle $\sim 160^{\circ}$ for ionic liquid EMIM DCA). For assembly, ionic liquid EMIM DCA-- $\mathrm{C}_{8} \mathrm{H}_{11} \mathrm{~N}_{5}$, 1-Ethyl-3-methylimidazolium dicyanamide (Sigma-Aldrich ${ }^{\circledR}$ ) was chosen for its high conductivity $(27 \mathrm{mS} / \mathrm{cm})$, low dynamic viscosity $(15-20 \mathrm{mPa} \bullet \mathrm{s})$, high surface tension $(64 \mathrm{mN} / \mathrm{m})$ [12] and negligible evaporation. After forming ionic liquid rings on both the rotor and the substrate, surface tension helped the rotor self-assemble centrally to the substrate and without tilting (Fig. 5 (c)). The liquid gap (rotor-substrate gap) is between $100 \mu \mathrm{m}$ and $200 \mu \mathrm{m}$.

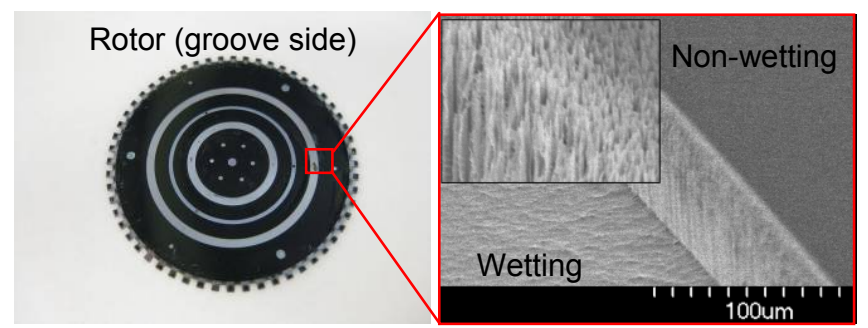

(a)

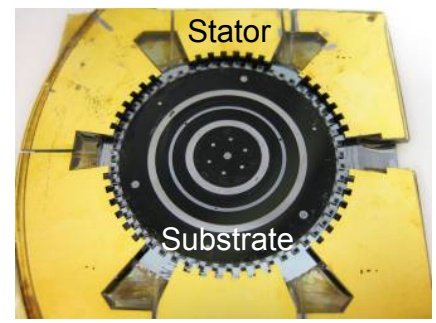

(b)

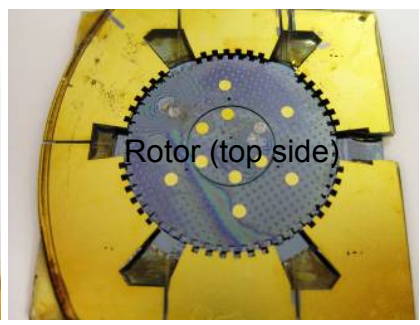

(c)
Figure 5: Fabrication result of side-driven rotary stage on conductive liquid ring bearing. (a) Rotor (bottom surface shown) and SEM picture showing the groove. (b) Substrate and six stators bonded on it. (c) Completed device after the rotor is self-assembled on the substrate by the liquid rings.

\section{RESULTS AND DISCUSSION}

\section{Electrostatic Actuation}

For the electrostatic actuation test, only the outer ionic liquid ring was formed to reduce the complexity of assembly. Figure 6 schematically shows the driving strategy. The rotor was electrically floated all the times during the operation, while the stators were connected in an alternating sequence with three distinct electrical phases. In each phase, a pair of diagonal stators was activated by applying a certain voltage. When a given phase was activated, a voltage difference $\left(50 \mathrm{~V}_{\mathrm{DC}}\right.$ in our case) between the activated stators and the rotor generated an electrostatic force. The tangential component of the electrostatic force generated a torque, which tends to realign the teeth of the rotor with the teeth of the activated stator. When the teeth of the activated stator were perfectly aligned with the teeth of the rotor, the teeth of the other two pairs of stators had a misalignment. At this point, the driving voltage switched to another phase, which activated another pair of stators. By programming the voltage phase in an appropriate sequence, a stepwise continuous rotation has been achieved in either clockwise or counterclockwise directions for more than 10 hours in a regular hotel exhibition hall without special protection.
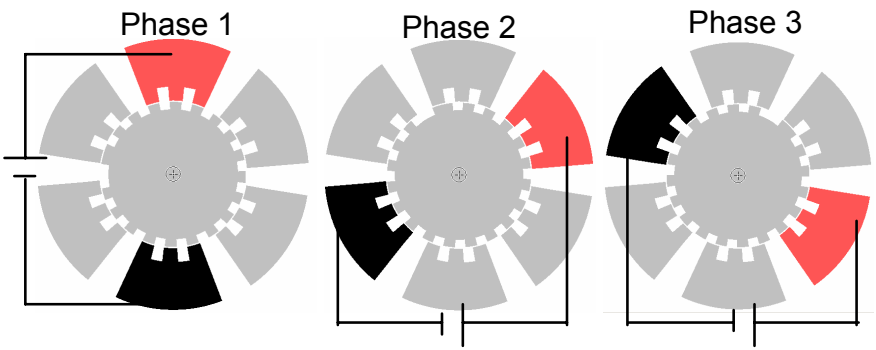

Figure 6: Three-phase electrostatic actuation.

The rotation was open-controlled and the speed was kept at a rate of $\sim 5 \%$ at all times. The wobble of rotor was measured to be less than $0.012 \mathrm{rad}$. The leakage current flow from the power supply was observed less than $10^{-3} \mu \mathrm{A}$.

\section{Power Transmission}

To verify the electrical power transmission onto a rotating rotor, we used an assembled device without outside stators, which easily facilitates the investigation of the liquid ring height, stability of rotor, and dynamic motion of high-speed rotation. An LED was placed on top of rotor and electrically connected to the Au bonding pads. Two ionic liquid rings were formed both on rotor and substrate with accurate volume control, followed by the assembly of rotor to substrate as described in the previous section. Air blowing was used to keep the rotor rotating at a rate of $\sim 300 \mathrm{rpm}$. During the rotation, a $4 \mathrm{~V}_{\mathrm{DC}}$ was applied to the substrate vias, across each of the two liquid rings, through corresponding through-rotor vias, and to the LED on the rotor. A current flow of $\sim 0.1 \mathrm{~mA}$ was observed. Figure 7 shows the side view (top) and a snap shot (top) of a rotating device with an LED. The weight of rotor, LED, and bonding wires was $\sim 700 \mathrm{mg}$, which has demonstrated a considerable payload capability of the liquid ring bearing. The LED blinking has further been confirmed on an electrostatically driven rotor, although the rotation was much slower and not continuous.

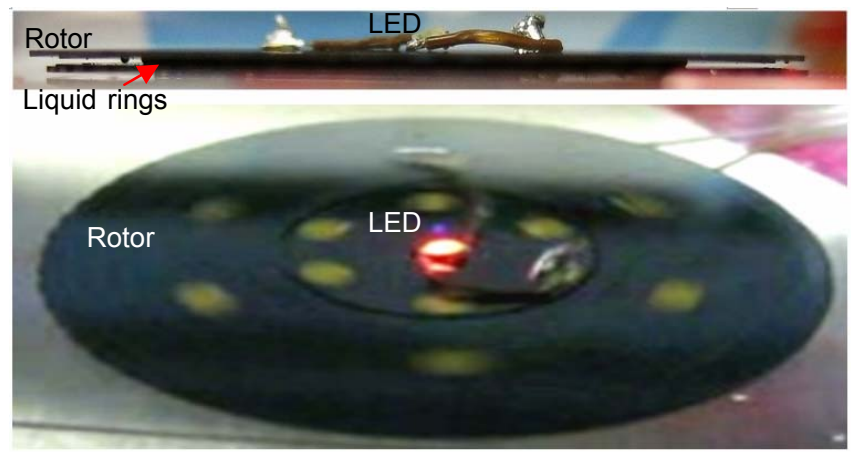

Figure 7: A red LED blinking at the center of a rotor (no stator in this device) that is being rotated by blowing air.

\section{Further Discussion}

The stability of liquid bearing has also been verified 
experimentally. The volume change of ionic liquid EMIM DCA over 30 hours at atmosphere was found less than $10 \%$, and an assembled rotary stage without protective packaging resumed even after 3 months on the shelf and remained operational for electrostatic actuation.

Although we did not discuss any application of our rotary stage, a rotating device with electrical power transmission can be potentially utilized as a secondary in-situ calibration platform for micro positioning, navigation, and timing (micro-PNT) devices, e.g., accelerometer, gyroscope, and atomic clock. In the future work, we will focus on the capacitance feedback control, optimized design of liquid rings, and further understanding of electrochemical properties of ionic liquid as a conductive element.

\section{CONCLUSIONS}

We have successfully demonstrated conductive liquid ring bearings as both mechanical bearings and electrical connection component. Fabrication of defect-free liquid rings has been the key to achieve a very small static friction - small enough to allow rotation by side-driven electrostatic actuation under a relatively low voltage $\left(50 \mathrm{~V}_{\mathrm{DC}}\right)$. The stability and reliability of liquid ring bearing have also been verified through a long-term testing. More importantly, a direct electrical power transmission to an infinitely rotating device has been demonstrated for the first time, which opens the window for meaningful applications of miniature rotating devices.

\section{ACKOWNLEDGEMENT}

This work is supported by the Defense Advanced Research Projects Agency (DARPA) under grant W31P4Q-10-1-0008. The authors would like to thank Prof. Ken Yang and Mr. Jong Jin Kim for their help on the electrostatic actuation test.

\section{REFERENCES}

[1] L.-S. Fan, Y.-C. Tai, and R.S. Muller, "IC-Processed Electrostatic Micromotors", Sensors and Actuators, 20, 41 (1989).

[2] N. Ghalichechian, A. Modafe, M.L. Beyaz, and R. Ghodssi, "Design, Fabrication, and Characterization of a Rotary Micromotor Supported on Microball Bearings", Journal of Microelectromechanical Systems, 17, 632 (2008).
[3] M. Mehregany, S.F. Bart, L.S. Tavrow, J.H. Lang, S.D. Senturia, and M.F. Schlecht, "A Study of Three Microfabricated Variable-Capacitance Motors", Sensors and Actuators, 21, 173 (1990).

[4] P. Muralt, "Ultrasonic Micromotors Based on PZT Thin Films," Journal of Electroceramics, 3, 143 (1999).

[5] C. H. Ahn, Y. J. Kim, and M. G. Allen, "A Planar Variable Reluctance Magnetic Micromotor with Fully Integrated Stator and Coils," Journal of Microelectromechanical Systems, 2, 165 (1993).

[6] M. Mehregany, S.D. Senturia, and J.H. Lang, "Measurement of Wear in Polysilicon Micromotors", IEEE Transactions on Electron Devices, 39, 1136 (1992).

[7] L.G. Frechette, S.F. Nagle, R. Ghodssi, S.D. Umans, M.A. Schmidt, J.H. Lang, "An Electrostatic Induction Micromotor Supported on Gas-lubricated Bearings", Technical Digest of the 2001 International Conference on Micro Electro Mechanical Systems, Interlaken, Switzerland, 1/21-25/01, (2001), pp. $290-293$.

[8] A. Takei, K. Matsumoto, and I. Shomoyama, "Capillary Motor Driven by Electrowetting", Lab on a Chip, 10, 1781 (2010).

[9] M. Chan, B. Yoxall, H.Park, Z. Kang, I. Izyumin, J. Chou, M.M. Megens, M.C. Wu, B.E. Boser and D.A. Horsley, "Low Friction Liquid Bearing MEMS Micromotor", Technical Digest of the 2011 International Conference on Micro Electro Mechanical Systems, Cancun, Mexico, 1/23-27/11, (2011), pp. $1237-1240$.

[10] B.E. Yoxall, M. Chan, R. S. Harake, T. Pan, and D.A. Horsley, "Rotary Liquid Droplet Microbearing", to appear in Journal of Microelectromechanical Systems.

[11] W.Shen, R.T. Edwards, and C.-J.Kim, "ElectrostaticallyActuated Metal-Droplet Microswitches Integrated on CMOS Chip", Journal of Microelectromechanical Systems, 15, 879 (2006).

[12] S.I. Fletcher, F.B. Sillars, N.E. Hudson, and P. J. Hall, "Physical Properties of Selected Ionic Liquids for Use as Electrolytes and Other Industrial Applications", Journal of Chemical \& Engineering Data, 55, 778, (2010).

\section{CONTACT}

*G. Sun, tel: +1-310-825-3977; guangyi@ucla.edu 


\title{
NANOGAP ULTRASONIC ACTUATOR FOR NON-CONTACT CONTROL OF LEVITATED INERTIAL SENSOR ROTOR
}

\author{
S. Piratla ${ }^{*}$ M. Pandey, and A. Lal
}

SonicMEMS Laboratory, School of Electrical and Computer Engineering, Cornell University, USA

\begin{abstract}
In this paper we present an ultrasonic motor with a rotor which levitates creating an air bearing with smooth rotation at 100 $600 \mathrm{rpm}$, whose motion can be controlled without contact. We demonstrate the use of a thermal actuator-driven gripper-actuator that can control the speed and direction of the rotor, without contact, while it is levitated. This same actuator can also be used to stop the rotor by direct mechanical contact. Voltages required for this actuation are in the 1-10 Volts, making the calibration platform CMOS compatible. In order to demonstrate the application of this rotor for inertial sensor calibration, we demonstrate the first integration of optically read accelerometers, read out while the rotor is spinning.
\end{abstract}

\section{KEYWORDS}

Ultrasonic, micromotor, piezoelectric, thermal actuator, levitation, accelerometer, diffraction, optical readout

\section{INTRODUCTION}

One of the most challenging problems for reaching ideal MEMS inertial sensor performance is the change in bias and scale factor over time, and over environmental variables such as shock, temperature, humidity and packaging. Several attempts have been made to eliminate bias by making individual mechanical and electrical components of the MEMS inertial sensors symmetric and higher Q. However, with limits on precision of fabrication steps such as lithography and etching, and spatial variation of material properties of thin films, bias elimination remains a hard challenge. One way to eliminate biases is to calibrate the biases actively in the sensor package itself. Micro scale stable rotating platforms with integrated inertial sensors hold the promise of performing in-situ gyroscope and accelerometer sensor calibration by applying known accelerations and rotation rates. The biases can be measured by measuring signals with sensors at known angles (Figure 1).

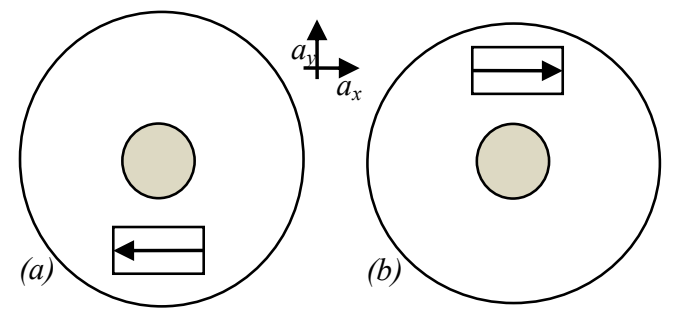

Figure 1.Calibration of $x-y$ accelerometers on a rotatable platform. In (a) the accelerometer sensitivity is negative of (b) - enabling the extraction of bias $V_{b}$ in the accelerometer measured voltage $V_{a x}=S_{a} a_{x}+V_{b}$.

However, for proper calibration of the inertial sensor scale factor the rotation of the platform needs to be free of secondary features that can complicate sensor signals. For example, a rotating platform needs to rotate smoothly without actuator artifacts such as jerks. Hence, it is critical to rotate the sensor without any physical contact. This is equivalent to suspending the rotor during calibration.

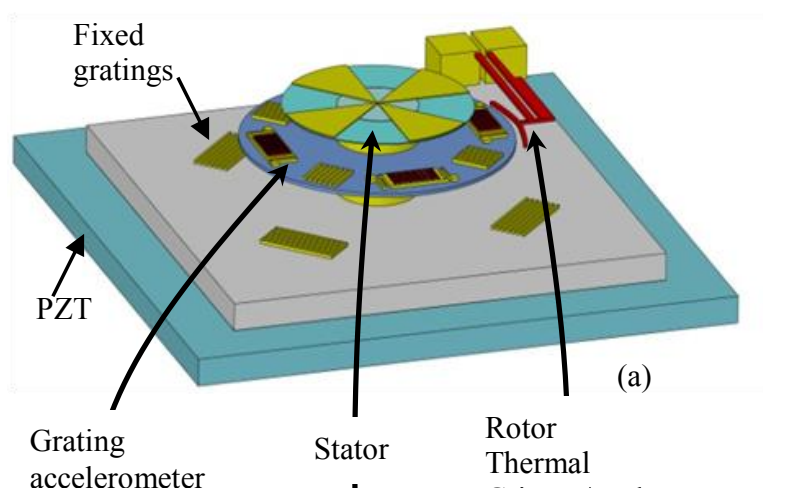

accelerometer $\quad$ Gripper/Brake

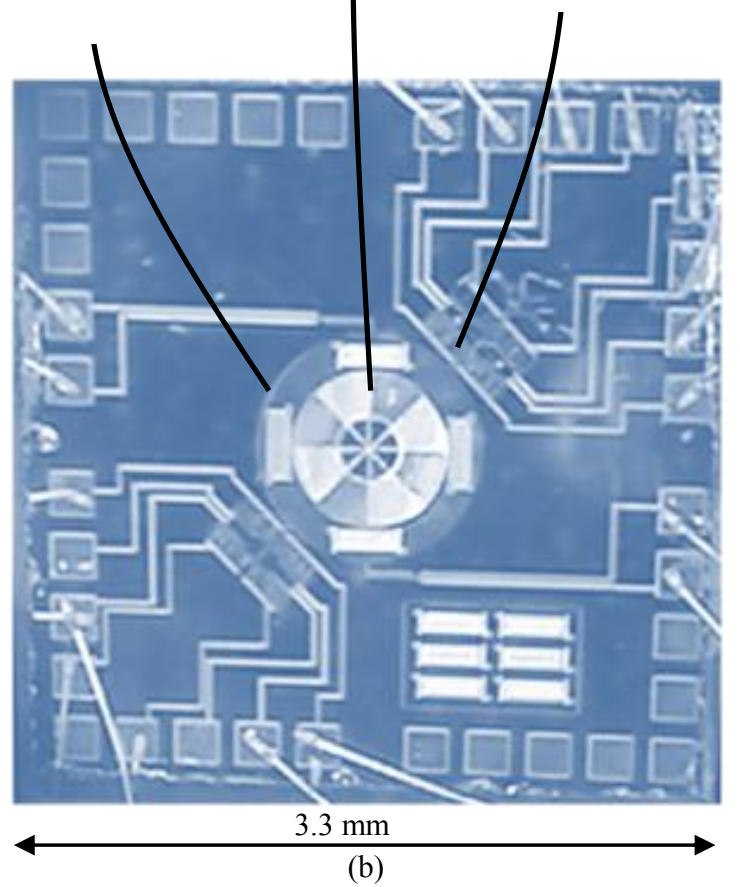

Figure 2. (a) Schematic of ultrasonically driven motor with integrated grating accelerometer. A thermal actuator is used to move a gripper close to the spinning levitated motor.

(b) Optical micrograph of wire bonded device

Many previous proof of principles exist for suspending rotating platforms. Magnetic suspension has been shown using diamagnetic rotors [1],[2]. In magnetic case, high currents are typically needed to generate sufficient magnetic field gradients for levitation. Electrostatic suspension has been achieved in implementing gyroscope itself by several investigators [3],[4]. 
In electrostatic case, relatively high voltages and vacuum are needed to achieve flotation and vacuum operation. An alternative to electromagnetic forces is to use fluidic forces to suspend rotating platforms. An alternative to suspended operation is to use viscous drag forces induced by acoustic and dynamical forces. By actuating a plate at its ultrasonic resonant modes, the resulting acoustic streaming and acoustic radiation forces can be used to levitate the rotor.

\section{PRINCIPLE OF OPERATION}

Figure 2 shows the overall concept of the inertial sensor calibration platform, implemented in MUMPS process. The rotor has thickness of $2 \mu \mathrm{m}$ and a diameter of $900 \mu \mathrm{m}$. The bulk PZT bonded to the motor is excited at amplitude of $10 \mathrm{Vpp}$ near resonant frequencies of the hub-stator, some of which are whispering gallery modes. These modes have lower air damping and are thus observed in air at high amplitudes. The rotating mode couples energy from stator to rotor through an air-fluid bearing via acoustic streaming, which is a time averaged steady flow of the fluid that usually happens due to varying acoustic intensity in the fluid medium. The resulting drag force pushes the rotor, which we have reported in our earlier work [5],[6]. Recently we optimized the acoustic coupling by programming gap as a function of theta by adding gold coverage on the stator [6],[7]. The built-in-stress in gold results in bending of the stator and rotor, giving rise to a periodic variation of stator-rotor gap, $g(\theta)=a+b^{*} \sin (n \theta)$, where a is $8.6 \mu \mathrm{m}$ and $\mathrm{b}$ is $5.8 \mu \mathrm{m}$ for $\mathrm{n}=2$. The bending of the stator allows for more space to help with the levitation.

Thermal and electrostatic actuators shown in Figure 2 can move grippers towards the spinning rotor to hold it in place during sensor operation, or control its rotation direction and rate. Accelerometers and gyros can be integrated onto the rotor, and in Fig. 2 we show an optical grating accelerometer that changes the grating spacing due to accelerations. In this paper we demonstrate non-contact operation from levitation, and also non-contact control of rotation from the grippers. We can also control the direction and rotation without contact.

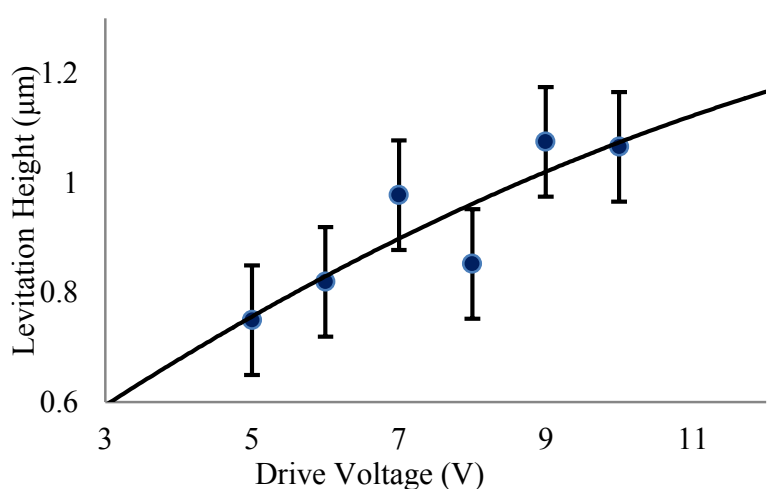

Figure 3: Variation of levitation height during rotation (measured with ZYGO) with drive voltage at $3.25 \mathrm{MHz}$. The stable levitation is at $1 / 2$ of rotor-stator gap.

\section{LEVITATION}

Levitation in fluid based systems can be achieved due to pressure built-up as a result of periodic forcing of the fluid. Here, resonant actuation of the substrate launches pressure wave in the air gap, with a wavelength $\sim 60 \mu \mathrm{m}$, which is larger than the gap between the stator and the base (about $1 \mu \mathrm{m}$ ). Hence squeeze film levitation is dominant; where periodic squeezing forces the fluid to flow out through the sides but the shear resistance prevents it.
This gives effective stiffness to the trapped air region. The resulting levitation force from a Reynolds flow based analysis is estimated by [8]:

$W=\frac{5 \varepsilon^{2}}{4 \beta}\left[\frac{\beta \cos (\beta)+\beta \cosh (\beta)-\sin (\beta)-\sinh (\beta)}{\cos (\beta)+\cosh (\beta)}\right]$

where $\beta=\sqrt{\frac{\sigma}{2}}$ with $\sigma=\frac{12 \omega \mu L^{2}}{p_{a} h_{0}^{2}}$ being the squeeze number, $\omega$ the drive frequency, the viscosity, $\mathrm{L}$ the diameter, $\mathrm{p}_{\mathrm{a}}$ the ambient pressure and $\mathrm{h}_{0}$ the initial gap.

We demonstrate that during rotation the rotor levitates and stays biased in the middle of the stator and the substrate. The larger stator deflection leads to more space for the rotor to levitate. The levitation is a function of rotation rate (rate function of drive voltage) as expected due to rotation induced lift force on the rotor (Figure 3). This compares well with the analytical results.

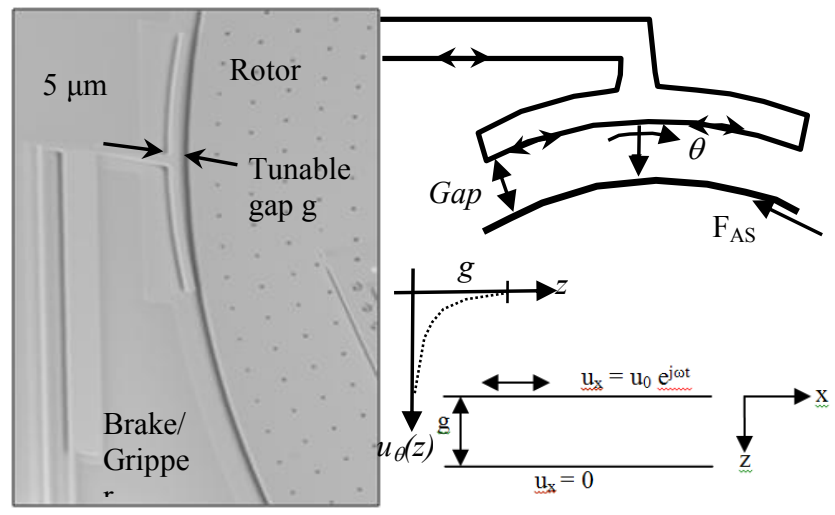

Figure 4: SEM of thermal actuator that moves the gripper towards rotor. Other structures on chip are for other testing not important to abstract. Gripper moves due to substrate motion and creates gradient in a sonic shear viscous field $\left(u_{\theta}\right)$ giving rise to acoustic streaming force $F_{A S}$.

\section{THERMAL ACTUATOR GRIPPER}

This is a thermal bimorph actuator to control the speed and direction of the rotor (Figure 4). The gripper actuator, anchored to the substrate, also moves at the PZT drive frequency.

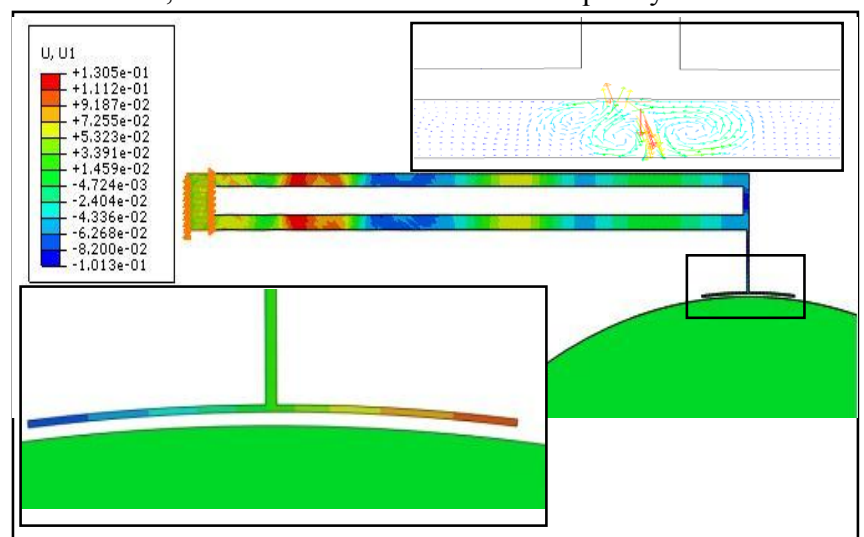

Figure 5. Ultrasonic motion of the brake pad as a function of forcing and resulting streaming.

The motion creates a gradient of velocity between gripper and rotor. This shear viscous field (shear viscous depth $0.9 \mu \mathrm{m}$ at $3.25 \mathrm{MHz}$ ) gives rise to a time averaged acoustic streaming driven force (Figure 4) [4]. Torque on the rotor is obtained from the following analysis. 
We solve the Navier-Stokes equation in the gap

$$
\rho \frac{\partial u}{\partial t}+\rho u \cdot \nabla u=f-\nabla p+\mu \nabla^{2} u
$$

As $u \cdot \nabla u$ is a second order term, it can be neglected.

No forces: $\mathrm{f}=0$

No pressure difference: $\nabla p=0$

Under these assumptions the above equation reduces to

$$
\rho \frac{\partial u}{\partial t}=\mu \frac{\partial^{2} u_{x}}{\partial z^{2}}
$$

The solution to the above equation is

$$
u_{x}=u_{0}\left(A e^{\frac{-z}{\delta}}+B\right) e^{j \omega t}
$$

Where

$\delta=\sqrt{\frac{\mu}{2 \rho \omega}}$ is the thickness of shear viscous layer.

With boundary conditions

$u_{x}=\left.u_{0}\right|_{\mathrm{z}=0}$ and $u_{x}=\left.0\right|_{\mathrm{z}=\mathrm{g}}$

we get $A=\frac{u_{0} e^{\frac{g}{2 \delta}}}{2 \sinh \left(\frac{g}{2 \delta}\right)}, B=-\frac{u_{0} e^{\frac{g}{2 \delta}}}{2 \sinh \left(\frac{g}{2 \delta}\right)}$

The resulting Reynolds stress is calculated from [8]

$$
f=\left\langle u \frac{\partial u}{\partial z}\right\rangle
$$

The total force is then obtained by integrating this term over the entire gap.

Hence the total force $=L \cdot W \cdot \int_{0}^{g}\left(u \frac{\partial u}{\partial z}\right) d z=$ W. h. $\Gamma\left(\frac{g}{\delta}\right)$

Where

$$
\Gamma\left(\frac{g}{\delta}\right)=-\frac{2 u_{0}^{2} \sin \left(\frac{g}{\delta}\right)}{4 \sinh ^{2}\left(\frac{g}{2 \delta}\right)}-\frac{u_{0}^{2}}{4 \sinh ^{2}\left(\frac{g}{2 \delta}\right)}\left(e^{-\frac{g}{\delta}}-1\right)
$$

The torque can be calculated as

$$
T=\vec{r} \times \vec{F}=R . W . h . \Gamma\left(\frac{g}{\delta}\right)
$$

The rotation rate of the rotor should be proportional to the torque. Using the expression of the torque from Eq.5 and using the values of the shear viscous depth at $3.8 \mathrm{MHz}$, we calculated the modified the rotation rate to be:

$$
\omega=\omega_{0}+A * T
$$

Where $\omega_{0}$ is the rotation rate due to the stator torque, and is the rate when the gripper-rotor gap is large with minimal effect on the rotation rate. As the gap decreases, the gripper toque increases. As can be seen in Figure 6, Eq.8 fits very well up to maximum rotation rate. After the peak in rotation rate, the equation does not fit, indicating different dynamics. Most likely, the gripper-rotor gap becomes small enough to periodically touch the rotor, resulting in periodic holding, culminating in reduced rotation rate. This part of the motion can also be modeled.

This fluidic torque from the thermal actuator can add to or counteract the acoustic streaming torque from the stator drive. If the shear viscous force due to thermal actuator gripper is in $\mathrm{CW}$ (clockwise) direction, and if the rotor is rotating in $\mathrm{CW}$ direction due to stator force, the forces add constructively and we observe an increase in rotation rate of the rotor as the gripper - rotor gap decreases (Figure 6). On the other hand, if the rotor is rotating in $\mathrm{CCW}$ (counter clockwise) direction, the rotation rate decreases as the gripper - rotor gap decreases eventually making the rotation rate zero and then reverses the direction of the rotation to $\mathrm{CW}$ (Figure 7).

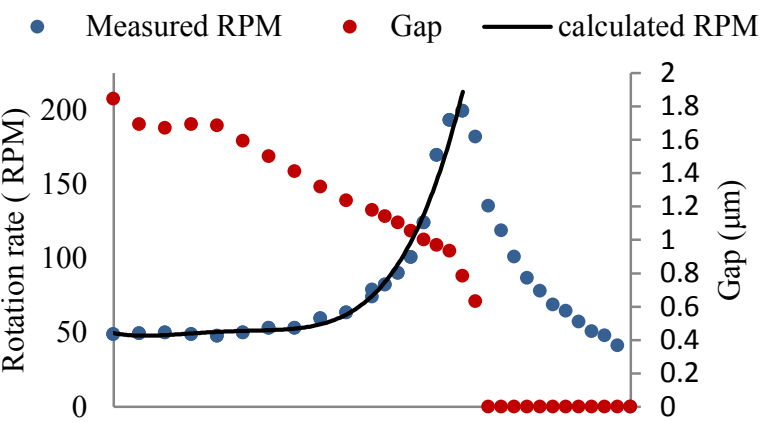

Figure 6: Rotation rate increases as gripper gap decreases with increasing actuator voltage (Figure 3 expressions predict the increase as shown). As the gripper starts touching the rotor, the rotation rate decreases.

As the drive voltage for the PZT increases $\left(12.2-12.6-13 \mathrm{~V}_{\mathrm{pp}}\right.$ in Figure 7), the stator force and the gripper force both increase since the PZT drive is the source of energy for both actuators. As the PZT drive voltage increases, the transition from $\mathrm{CW}$ to $\mathrm{CCW}$ occurs at lower gaps. This could be due to better coupling of motion to the gripper, compared to the stator. The seeming variability of direction and amplitude near the transition point (Figure 7) could be due to the unsteady contact dynamics between the rotor and the gripper. The gripper force is sufficient to drive the rotor in opposite direction, indicating that the torques generated by the stator and the gripper are comparable. At higher gripperactuator voltages, one end of the gripper touches the rotor, decreasing the rotation rate due to friction and eventually clamping it (Figure 6).

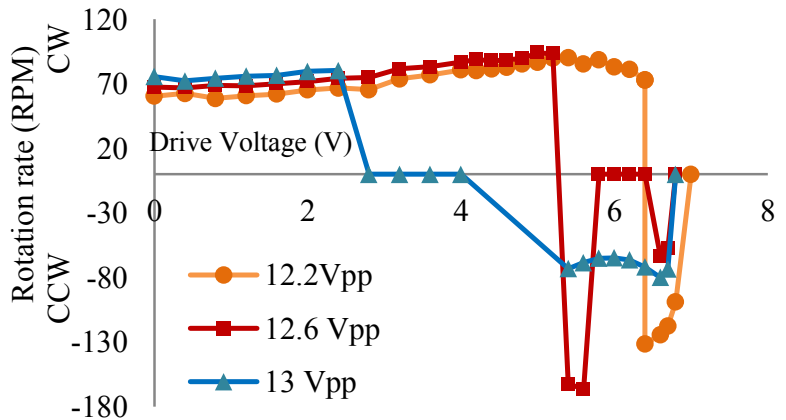

Figure 7: Rotation rate varying with thermal actuator voltage shows that shear viscous force can change direction of rotation $(C C W-$ to $C W+)$. Threshold changes with drive voltage, with increased role of shear drive.

Upon illumination from a $650 \mathrm{~nm}$ laser VCSEL diode (Thorlabs L650P007), the diffraction pattern was recorded and digitally analyzed. The diffraction pattern from G1 and G2 are orthogonal to each other and can be used to calibrate the expected and deviations of the pattern from the accelerometer. We can measure the change in the accelerometer grating response as a function of rotation rate (Figure 8). By bending of the diffraction grating, we can see that the moving grating fingers are most likely getting twisted by radial acceleration due to rotation rate. 


\section{SELF-CALIBRATING DIFFERENTIAL OPTICAL ACCELEROMETER}

A preliminary optical grating accelerometer was implemented on the rotor with built-in self-calibrating gratings. The accelerometer consists of a set of grating reflecting fingers implemented in MUMPS gold that move in circumferential direction as a result of acceleration with springs. In the middle of the moving fingers are fixed gold reflecting lines. The accelerometer provides a diffraction pattern recorded by a CMOS imager, with interference between light reflecting from the accelerometer (AG), while there are fixed gratings (G1 and G2) (Figure 8(a)).

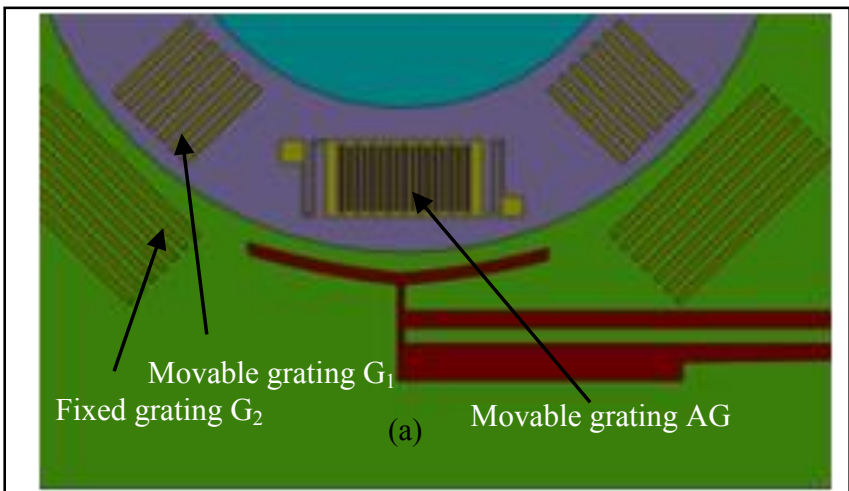

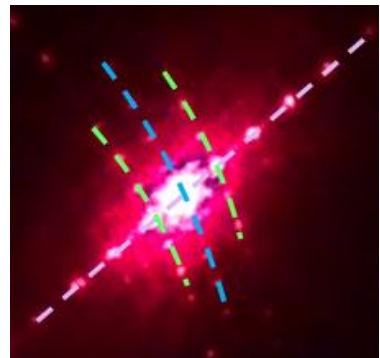

(b)

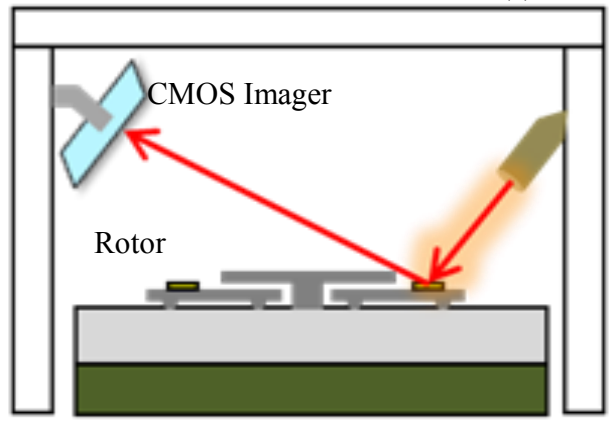

(d)

Figure 8: (a) fixed and moving gratings (b) \& (c) shows diffraction pattern changing due to rotor motion (d) Showing ultrasonic motor, VCSEL, CMOS imager for optical read out of accelerometer in a package of $10 \times 2 \times 3 \mathrm{~cm}$.

\section{CONCLUSIONS}

We have outlined a generic platform for in-situ calibration of inertial sensors, using a rotor that can be positioned at any angle and also be actuated to provide stimulus to calibrate the sensor bias and scale-factor respectively. In order to accomplish calibration, we demonstrate building blocks of a platform that do this without motion artifacts by control in levitated state. We have demonstrated that using acoustic radiation forces we can levitate spinning rotor, actuated by acoustic streaming forces. The levitation leads to rotor stabilized to middle of the gap between a stator and substrate. We have also implemented a thermal gripper that serves two purposes. With zero gap between the rotor and the gripper, the gripper acts to hold the rotor in place. By adjusting the gap to sub-micron level, we can control the rotation rate and direction of the rotation. Acoustic streaming based model of torque generation due to relative motion between the gripper and the rotor at ultrasonic frequencies is developed, which agrees with measured data. Further works needs to combine the control with integrated inertial sensors, as outlined with preliminary work on an optical grating accelerometer.

\section{ACKNOWLEDGEMENTS} support.

The authors would like to thank DARPA for financial

\section{REFERENCES}

[1] C. Shearwood, K. Y. Ho, C. B. Williams, "Development of a levitated micromotor for application as a gyroscope," Sensors and Actuators A, vol. 83, pp. 85-92, 2000.

[2] M. Kraft, M. M. Farooqui, A. G. R. Evans, "Modeling and design of an electrostaticallv levitated disk," J. Micromech Microeng, vol. 11, no. 4, pp. 423-427, 2001.

[3] M. Takao,E.Yasuo; F.Keisuke,N. Sigeru; E.Masayoshi ,'Electrostatically levitated ring-shaped rotationalgyrol/accelerometer", Japanese Journal of Applied Physics, Part 1: Regular Papers and Short Notes and Review Papers, v 42, n 4 B, p 2468-2472, April 2003.

[4] B.Dillard,V.Trent, M.Greene, E. W.Taylor, "Radiation effects on multiple DOF MEMS inertial sensors", Proceedings of SPIE - The International Society for Optical Engineering, v 8164, 2011, Nanophotonics and Macrophotonics for Space Environments $V$.

[5] V.Kaajakari, A. Lal, "Micromachined Ultrasonic Motor based on Parametric Polycrystalline Silicon Plate Excitation", Sensors and Actuators A: Physical, Vol137, No.1, pp120-128, June 2007.

[6] M. Pandey, A. Lal, "Analysis of Ultrasonic Motor in air using Fluid Structure Interaction Based Simulations", Ultrasonics 2011, Orlando Fl.

[7] S. Piratla, A. Lal, "Resonant Stator Actuation of Gear Coupled Ultrasonic Motors", Transducers 2011, Beijing, pp 2450-2453.

[8] A. Minikes and I. Buche "Levitation force induced by pressure radiation in gas squeeze films", J. Acoust. Soc. Am., Vol. 116, No. 1, July 2004.

[9] W. L. Nyborg"Acoustic Streaming near a Boundary", The Journal Of The Acoustical Society Of America, Volume 30, Number 4 April, 1958.

\section{CONTACT}

*S.Piratla, sp748@cornell.edu 


\title{
MINIATURE WIRELESS RESONANT ROTARY MOTOR ACTUATED BY LITHOGRAPHICALLY MICROMACHINED MAGNETOELASTIC FOIL
}

\author{
J. Tang, S.R. Green, Y.B. Gianchandani \\ Center for Wireless Integrated MicroSensing and Systems (WIMS ${ }^{2}$ ), University of Michigan, \\ Ann Arbor, Michigan, USA
}

\begin{abstract}
This paper presents a miniature rotary motor that is driven by wireless power transfer. This resonant motor is actuated by a $\varnothing 8$ $\mathrm{mm}$ magnetoelastic stator lithographically micromachined from Metglas $^{\mathrm{TM}} 2826 \mathrm{MB}$ foil. The prototype motor operates at a resonant frequency of $11.35 \mathrm{kHz}$ while a 3 Oe DC and a 2 Oe amplitude AC magnetic field are applied. The measured rotation speed, calculated stall torque, and output power are $44 \mathrm{rpm}, 2$ $\mathrm{nN} \cdot \mathrm{m}$ and $9 \mathrm{nW}$, respectively. The angular resolution is $\sim 23$ millidegree. Based on simulation results and the experimentally measured performance of stator prototypes, simulation parameters are derived and used in predicting the performance of a siliconmetglas stator. A significant improvement in rotation speed, resolution, and required power is predicted.
\end{abstract}

\section{INTRODUCTION}

Chip-scale rotary motors have been of interest since the 1980s. Potential applications include actuation of optical elements in microsystems for communication or microanalytical applications, or for the in-situ calibration of inertial sensors particularly rotational rate and rate-integrating sensors. Another possible application for a wirelessly-powered miniature motor is to provide propulsive force for microbots in cavities and ducts. Motor actuation methods have included electrostatic, piezoelectric, electromagnetic, and thermal [1-4]. Electrostatic motors are considered as one of the milestones in MEMS history and are simple to integrate with IC processes, but packaging requirements, output torque and payload capacity limit applicability. Piezoelectric motors have high output torque. Electromagnetic motors are attractive but present challenges for the integration of coils. In this paper, another option for chip-scale rotary motor, magnetoelastic actuation is investigated.

Magnetoelastic coupling represents the interaction between the material conditions of strain, stress and magnetization. Magnetoelastic materials exhibit strains under an external magnetic field due to the field-directed rotation and alignment of tightly-coupled, elongated structural and magnetic domains in the materials. The strain induces stress in the material which, in turn, alters the magnetization. When excited with an oscillatory field, these two simultaneously existing effects make magnetoelastic materials attractive for wireless resonant sensing. Various magnetoelastic sensing systems have been studied for pressure, temperature, liquid viscosity and density, fluid flow rate, $\mathrm{pH}$, glucose, Young's modulus, stent occlusion, etc. [5]. Utilizing magnetoelastic material for actuation of a miniature and microscale remains an open challenge. Magnetoelastic actuators can operate by mechanically resonating in response to an alternating magnetic field. The resonant motion is used to drive movement of other parts of the system. The use of magnetoelastic transduction enables wireless actuation, which simplifies device architecture and allows for remote operation. The transduction method also exhibits high payload carrying capacity as well as noise immunity due to resonant operation.

The approach followed in this effort is similar to ultrasonic rotary motors, which are typically actuated by piezoelectric or electrostatic means [6-8] and operate by generating a vibratory wave in the stator. In this case, the vibratory micro-motion is a standing flexural resonant wave and is generated magnetoelastically. The resulting vibratory shape of the stator has antinodes at which maximal out-of-plane deflection occurs. Teeth are located on the rotor such that they are slightly offset from the antinodes, resulting in a contact force with a tangential component that causes the rotor to rotate (Fig. 1). A rotor, which can contain other microsystem components, is then stacked on to the stator and rotates on the motor hub. The prototype motor presented in this paper is $8 \mathrm{~mm}$ in diameter and is able to carry loads up to $9 \mathrm{mg}$; a magnetoelastic material Metglas ${ }^{\mathrm{TM}} 2826 \mathrm{MB}$ is utilized to actuate a chip-scale rotary motor.

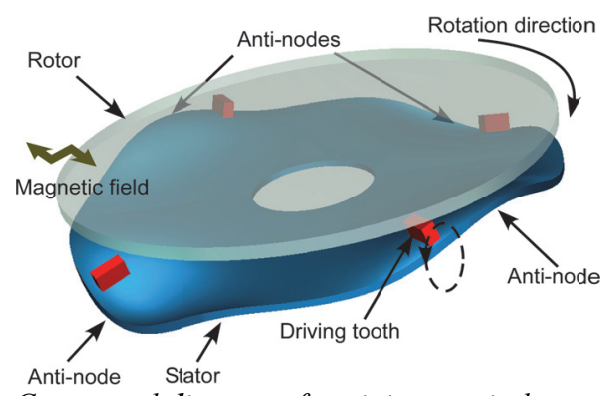

Figure 1: Conceptual diagram of a miniature wireless magnetoelastic resonant rotary motor.

\section{DESIGN AND MODELING}

A prototype megnetoelastic wireless resonant miniature motor is assembled by stacking a magnetoleastic stator and two stainless steel bases (Fig. 2). The stacked architecture is modular, which significantly simplifies the fabrication process. Each layer can be easily fabricated utilizing photochemical machining (PCM) process, as described in the fabrication section. Alignment pins ensure reasonable assembly accuracy for a stator with a diameter of $8 \mathrm{~mm}$. Two stainless steel bases provide a recess allowing stator vibration and initial positions for alignment pins and a hub. The ring-shaped stator is suspended with four crab-leg springs, which are stiff in the rotational direction but flexible in the out-ofplane direction - preventing stator rotation during rotor actuation while allowing large vertical deformation.

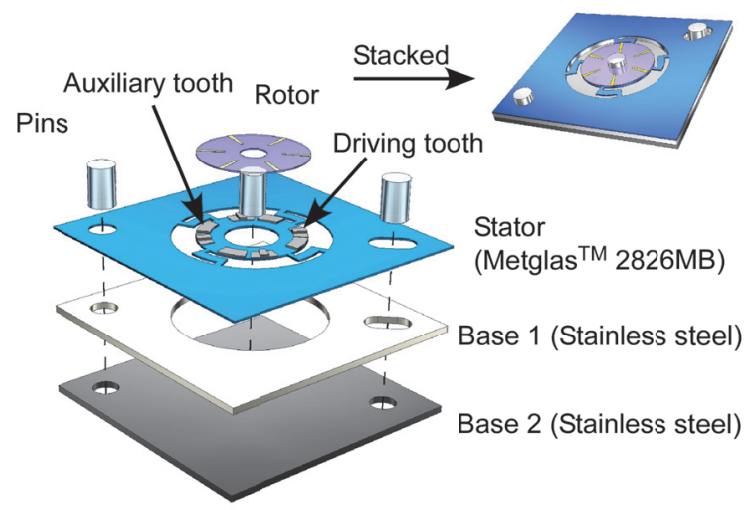

Figure 2: Schematic of a stacked prototype wireless resonant magnetoelastic miniature rotary motor.

Solid-State Sensors, Actuators, and Microsystems Workshop Hilton Head Island, South Carolina, June 3-7, 2012 
An on board coil and permanent magnets can be implemented to provide the driving $\mathrm{AC}$ and $\mathrm{DC}$ magnetic fields. For example, inductive coils for generating AC magnetic fields can be patterned on an underlying silicon substrate using standard micromachining techniques. On the same substrate, permanent magnet materials such as permalloy or samarium cobalt can be deposited and used to provide the DC fields required to bias the magnetoelastic material. However, for the prototype motor presented in this paper, external coils are used.

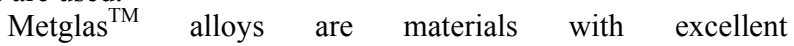
magnetostrictive properties as well as excellent mechanical properties. In this design, Metglas ${ }^{\mathrm{TM}} 2826 \mathrm{MB}$, an amorphous $\mathrm{NiFeMoB}$ alloy, is used. Its saturation magnetostriction is $12 \mathrm{ppm}$ and its DC permeability is larger than 50000 [9]. These materials are readily available in foils ( $\sim 28 \mu \mathrm{m}$ thick) and are easy to pattern utilizing PCM. Relative to other Metglas ${ }^{\mathrm{TM}}$ alloys and magnetoelastic materials, $2826 \mathrm{MB}$ also requires a small DC bias (about 2.5-3 Oe) and can resonate with a relatively small alternating field.

For a stator fabricated solely from thin Metglas ${ }^{\mathrm{TM}}$, careful consideration is required for the placement of the driving teeth. The number of driving teeth is usually the same as the number of antinodes in the desired mode shape for a resonant rotary motor [7]. The driving teeth are typically offset from the location of the antinodes in order to ensure elliptical motion of the tooth tip. However, because the Metglas ${ }^{\mathrm{TM}}$ stator is thin and light compared to any added driving teeth, the mass loading added by the driving teeth causes the antinodes to shift to the same location as the teeth. Consequently, the teeth no longer move elliptically and no rotation is generated. Therefore, as shown in Fig. 2 and 3, a design employing 8 teeth is proposed to address the issue. There are still four sets of teeth, but one set of teeth is a combination of two teeth: an auxiliary tooth and a driving tooth, each separated slightly from each other. The auxiliary teeth and driving teeth have the same mass so that the mass center and antinodes of the vibratory mode shape will be in the center between the two different teeth. However, the driving tooth is taller so only it will contact the rotor. As a result, the driving teeth can move in an elliptical manner, and drive the rotor tangentially.

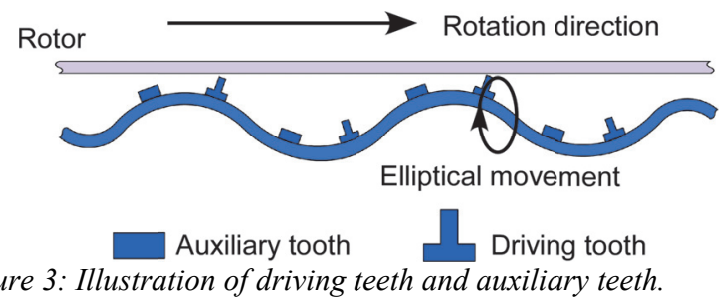

A custom magneto-mechanical harmonic finite element technique [10] is used to estimate modal displacements, shapes, and frequencies for the magnetoelastic material. Although magnetoelastic materials are generally non-linear, it is appropriate to use linearized constitutive equations describing the coupling between flux, field strength, stress, and strain in a magnetostrictive material:

$$
\begin{aligned}
& \vec{\sigma}=[C] \vec{\varepsilon}-\frac{[C][d]^{T}}{\mu_{o} \mu_{r}} \vec{B} \\
& \vec{H}=-\frac{[d][C]}{\mu_{o} \mu_{r}} \vec{\varepsilon}+\frac{1}{\mu_{0} \mu_{r}} \vec{B}
\end{aligned}
$$

where $\sigma$ is the stress vector, $C$ is the stiffness matrix, $\varepsilon$ is the strain, $d$ is the magnetostrictivity matrix, $B$ is the magnetic flux density vector, $H$ is the field strength vector, $\mu_{0}$ is the permeability of free space, and $\mu_{r}$ is the relative permeability. Equations (1) and (2) are implemented in this work utilizing COMSOL Multiphysics and coupled time-harmonic induction current and stress-strain frequency response modes. A detailed look at an FEA implementation for magnetostrictive materials is in [11]; the approach used in this work is modified for application to resonant actuators. The FEA results shown in Fig. 4 predict that a stator (inner diameter of $4 \mathrm{~mm}$ and outer diameter of $8 \mathrm{~mm}$ ) has the desired mode shape at a resonant frequency of about $11.43 \mathrm{kHz}$, with $100 \mathrm{~nm}$ out-of-plane deformation under harmonic excitation with amplitude of 2 Oe. Due to the orientation and size of the teeth and spring suspension, slightly different performance is predicted for different directions of alternating magnetic excitation. Simulation results suggest that applying magnetic field between the suspension springs gives the largest out-of-plane displacement amplitude. As desired, the vibration mode shape demonstrates that the antinodes are located in the center between a driving tooth and an auxiliary tooth.

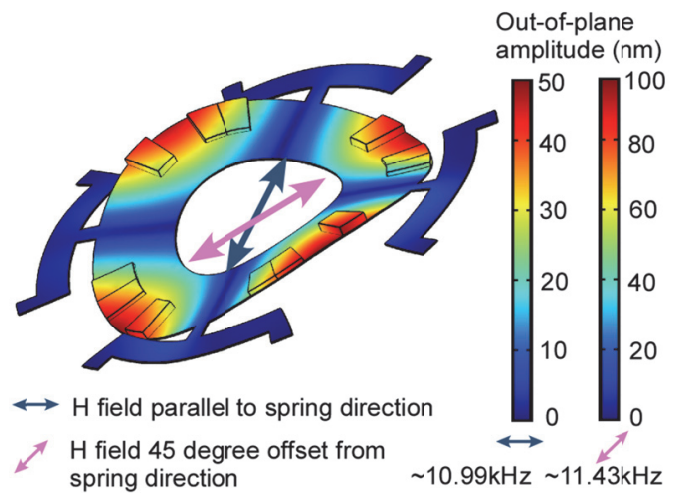

Figure 4: FEM results of out-of-plane stator vibration amplitude with different directions of AC magnetic field excitation.

\section{FABRICATION}

The fabrication process flow of a prototype motor is illustrated in Fig. 5. First, the magnetoelastic stator is batchpatterned using photochemical machining (PCM) [12] from the Metglas $^{\mathrm{TM}} 2826 \mathrm{MB}$ foil. The ring-shaped stator is patterned with inner diameter of $4 \mathrm{~mm}$ and outer diameter of $8 \mathrm{~mm}$. Other base layers are also fabricated using PCM from $0.5 \mathrm{~mm}$ thick stainless steel foils. The layers are stacked and aligned with pins, and bonded to each other with epoxy. The stainless steel auxiliary teeth and driving teeth are manually placed on the stator and fixed with epoxy. The rotor is electro-discharge-machined from magnetoelastic foil; it has a total mass of about $9 \mathrm{mg}$. A hub with diameter of about $2 \mathrm{~mm}$ is used to constrain the rotor. The overall size of the device is $2 \mathrm{~cm} \times 2 \mathrm{~cm}$ (Fig. 5).

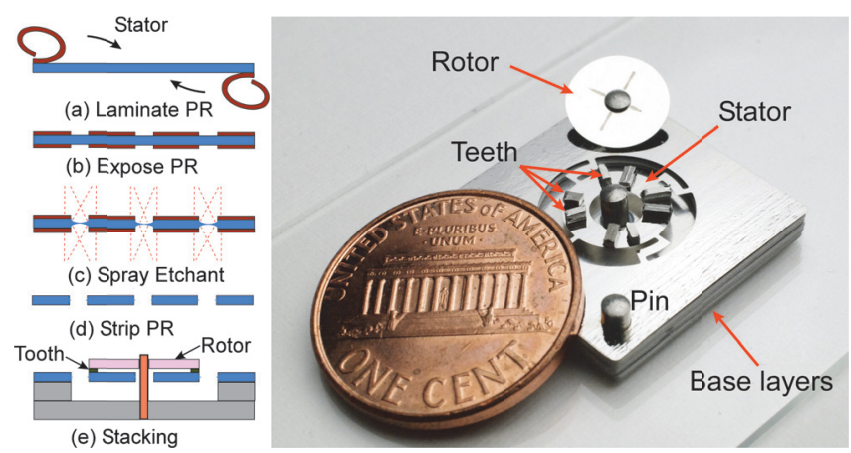

Figure 5: (Left) Fabrication process flow of a prototype magnetoelastic resonant rotary motor. (Right) Photograph of assembled prototype magnetoelastic resonant rotary motor. 


\section{EXPERIMENTAL METHODS AND RESULTS}

In the preliminary experiments reported in this paper, the motor is actuated wirelessly using two sets of external coils. Two large coils, shown in Fig. 6, are connected to a DC power supply with 2 A current, which corresponds to 3 Oe DC magnetic field. This is used to bias the magnetoelastic material into an operating region in which the strain is most sensitive to magnetic field. Another two small coils are placed inside the DC coils; these smaller coils provide an alternating magnetic field with amplitude of 2 Oe. The device under test (D.U.T.) is placed between the coils.

To measure the frequency response of the stator (before teeth have been added), a laser vibrometer and a network analyzer are used in conjunction (Fig. 6). The vibration mode shape can be determined by measuring a number of points along the rim of the stator; antinodes of a given mode shape exist at locations of strong response at a given frequency. The desired mode shape with four antinodes is confirmed to exist at $11.35 \mathrm{kHz}$, with $0.2 \mu \mathrm{m}$ unloaded out-of-plane deflection at the antinodes. As shown in Fig. 7, the frequency response is stable over at least 5 minutes of operation time.

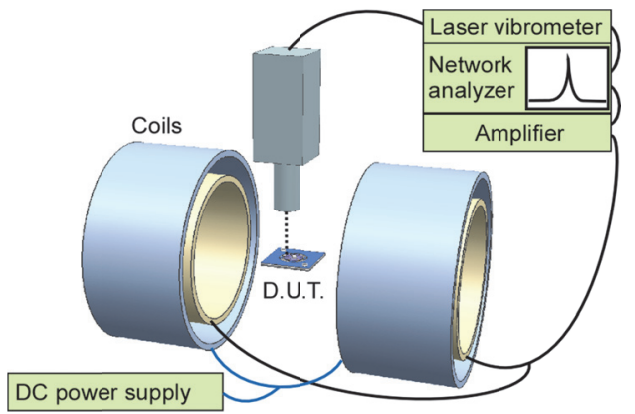

Figure 6: Experimental set up for stator vibration tests.

A video camera is used to record the rotor from above, and the video is analyzed frame-by-frame to derive the rotation speed and initial acceleration. In preliminary tests, a rotation rate of approximately $44 \mathrm{rpm}$ (4.6 radians/s) is obtained (Fig. 8). The angular resolution of about 23 milli-degree is calculated from the measured angular velocity and resonant frequency. The angular velocities from different trials are calculated from the recordings and are shown in Fig. 9. The angular acceleration, $\alpha$, before the angular velocity plateaus is derived from the data and the associated stall torque of $\sim 2 \mathrm{nN} \cdot \mathrm{m}$ is calculated using $\alpha$ and the moment of inertia of the rotor.

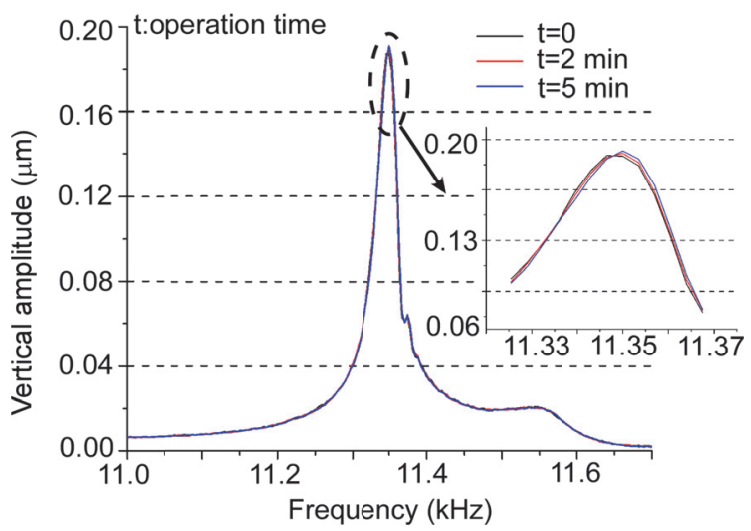

Figure 7: Frequency response of magnetoelastic stator under harmonic magnetic excitation.
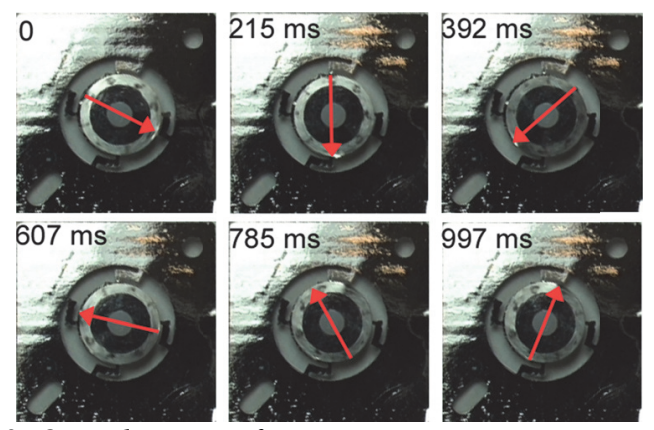

Figure 8: Optical images of rotor rotating

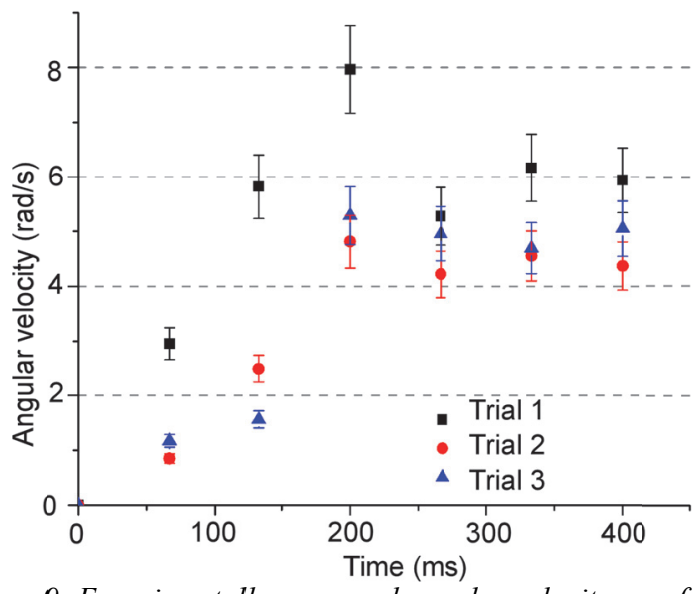

Figure 9: Experimentally measured angular velocity as a function of time under the excitation of a 3 Oe DC and a 2 Oe amplitude AC magnetic field at frequency of $11.35 \mathrm{kHz}$.

\section{DISCUSSION}

The prototype magnetoelastic wireless resonant motor demonstrates that the magnetoleastic material has significant potential for actuation in miniature and micro-scale devices. The main advantage - the wireless aspect of the actuation approach allows miniaturization and eliminates lead transfer actuator. Another obvious advantage is the ability to move large payloads; this non-optimized prototype carried a payload of about $9 \mathrm{mg}$.

A so-called silicon-Metglas ${ }^{\mathrm{TM}}$ stator is proposed and briefly analyzed. This design employs a silicon stator driven by a bonded magnetoelastic layer. Based on simulation results and the experimentally measured performance of stator prototypes, simulation parameters are derived and used in predicting performance (rotation speed and positioning resolution) for a silicon-Metglas ${ }^{\mathrm{TM}}$ architecture, as shown in Fig. 10. Simulations suggest that rotation speed is a function of the thickness of the silicon component, as shown in Fig. 11. By increasing the thickness of the silicon layer, the analyses indicate that the stepsize resolution and rotational speed can reach \pm 10 milli-degree and $1000 \%$ respectively. The predicted performance indicates improvement on rotation speed and resolution, which is crucial to many applications. The predicted performance of siliconMetglas $^{\mathrm{TM}}$ stator is compared with the measured performance of the prototype Metglas ${ }^{\mathrm{TM}}$ stator in Table I. The silicon-Metglas ${ }^{\mathrm{TM}}$ motor is expected to have a reduction in power consumption by decreasing the motor size. 


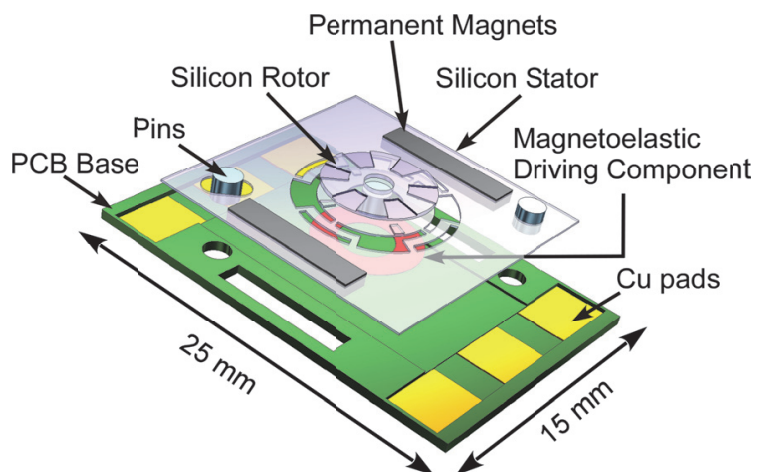

Figure 10: 3D Architecture of silicon-Metglas ${ }^{T M}$ rotary micromotor driven by $P C B$ circuits and permanent magnets.

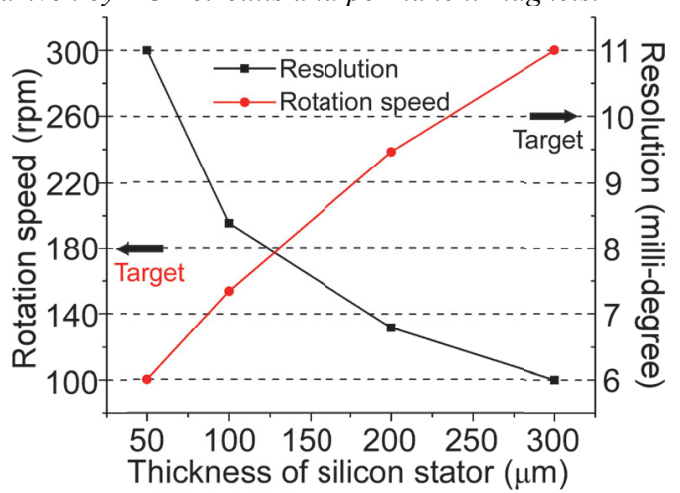

Fig. 11: Simulated dependence of step-size resolution and rotation speed on thickness of silicon stator.

Table I: Experimental performance of prototype magnetoelastic rotary motor and estimated target performance of siliconMetglas ${ }^{T M}$ stator architecture. (S) denotes simulated results while other results are measured.

\begin{tabular}{|l|c|c|}
\hline Performance & $\begin{array}{c}\text { Prototype } \\
\text { (Measured) }\end{array}$ & $\begin{array}{c}\text { Si-Metglas } \\
\text { (Predicted) }\end{array}$ \\
\hline Rotation speed $(\mathrm{rpm})$ & $\sim 44$ & $180(\mathrm{~S})$ \\
\hline Stall torque $(\mathrm{nN} \cdot \mathrm{m})$ & $\sim 2$ & - \\
\hline Step size $($ milli-degree) & $\sim 23$ & $10(\mathrm{~S})$ \\
\hline Input Power & $84 \mu \mathrm{W}(\mathrm{S})$ & $74 \mu \mathrm{W}(\mathrm{S})$ \\
\hline Output Power & $\sim 9 \mathrm{nW}$ & - \\
\hline
\end{tabular}

\section{CONCLUSION}

This work presents the analysis, fabrication, and experimental results for magnetoelastically-actuated, chip-scale rotary motors. The prototype design is actuated wirelessly by lithographically micromachined magnetoelastic foil. The Metglas ${ }^{\mathrm{TM}}$ stator has diameter of $8 \mathrm{~mm}$. A new approach that employs auxiliary teeth and driving teeth is used to customize the resonant behavior of the stator. In preliminary tests, external coils are used to provide a 3 Oe DC and a 2 Oe amplitude AC magnetic field. A rotation speed of about $44 \mathrm{rpm}$ is achieved. Associated stall torque and output power are calculated to be $\sim 2 \mathrm{nN} \cdot \mathrm{m}$ and $\sim 9 \mathrm{nW}$, respectively. The angular step size is about 23 milli-degree.

As the next step, a silicon-Metglas ${ }^{\mathrm{TM}}$ motor is proposed and analyzed using parameters derived from simulation results and experimental prototype results. Predicted results suggest significant performance improvement in rotational speed, angular resolution and power consumption. The results and analysis in this work indicate that bulk magnetoelastic material is a viable candidate for wireless actuation in MEMS devices. Future work will focus on the proposed silicon-Metglas ${ }^{\mathrm{TM}}$ motor.

\section{ACKNOWLEDGEMENTS}

The authors acknowledge Metglas, Inc. for the samples provided for this project. This work was supported in part by a DARPA project (PASCAL), and the University of Michigan.

\section{REFERENCES}

[1] L.S. Fan, Y.C. Tai, R.S. Muller, "IC-processed Electrostatic Micromotors," Sensors and Actuators, 20, (1989), pp 41-47.

[2] V. Kaajakari, A. Lal, "Micromachined ultrasonic motor based on parametric polycrystalline silicon plate excitation," Sensors and Actuators A, 137, (2007), pp. 120-128.

[3] C.H. Ahn, Y.J. Kim, M.G. Allen, "A Planar Variable Reluctance Magnetic Micromotor with Fully Integrated Stator and Coils," Journal of Microelectromechanical Systems, 2 (4), (1993), pp. 165-173.

[4] J.S. Park, L.L. Chu, A.D. Oliver, Y.B. Gianchandani, "BentBeam Electrothermal Actuators - Part II: Linear and Rotary Microengines," Journal of Microelectromechanical Systems, 10 (2), (2001), pp. 255-262.

[5] C.A. Grimes, S.C. Roy, S. Rani, and Q.Y. Cai, "Theory, Instrumentation and Applications of Magnetoelastic Resonance Sensors: A Review," Sensors, 11, (2011), pp. 2809-2844.

[6] M. Suzuki, K. Tani, T. Sakahura, "Development of a new type piezoelectric micromotor," Sensors and Actuators A, 83, (2000), pp. 244-248.

[7] A. Iino, K. Suzuki, M. Kasuga, M. Suzuki, T. Yamanaka, "Development of a self-oscillating ultrasonic micro-motor and its application to a watch," Ultrasonics, 38, (2000), pp. 54-59.

[8] Ph. Robert, J.S. Danel, P. Villard, "The electrostatic ultrasonic micromotor," Journal of Micromechanics and Microengineering, 7, (1997), pp. 170-172.

[9] Metglas, Inc. "Magnetic Alloy 2826MB (nickel-based) Technical Bulletin". [Online]. Available: http://www.metglas.com

[10] S.R. Green, Y.B. Gianchandani, "Wireless Magnetoelastic Monitoring of Biliary Stents," Journal of Microelectromechanical Systems, 18 (1), (2009), pp. 64-78.

[11] J. Benatar, "FEM Implementations of Magnetostrictive-Based Applications", MS thesis, Univ. of Maryland, 2005.

[12] ASM Handbook, 16, ASM International, 1989.

\section{CONTACT}

*J. Tang tel: +1-765-714-5838; juntang@umich.edu 


\title{
A VIRTUAL MICROFLUIDIC CHANNEL WITH OIL-REPELLANT SURFACES FOR GUIDING LOW SURFACE TENSION LIQUIDS
}

\author{
Riberet Almeida and Jae Wan Kwon
}

University of Missouri-Columbia, USA

\begin{abstract}
This paper reports for the first time a novel technique to form a 'virtual' microfluidic channel for guiding low surface tension liquids. Confining and manipulating non-aqueous low surface tension liquids within a desired area are not trivial but pose great challenges in microfluidic devices. Newly developed oil-repellant surfaces formed by using a plasma assisted surface micromachining process have been employed to control the surface wettability for low surface tension liquids. Such oleophophobic surfaces have been utilized to form microfluidic channels without sidewalls. Various low surface tension liquids have been electrically actuated and successfully guided along the 'virtual' microfluidic channel.
\end{abstract}

\section{INTRODUCTION}

Control of surface wettability for manipulating liquids has been widely adapted in many practical applications [1]. Although manipulating and controlling aqueous solutions are relatively easy, it is very difficult to prevent spreading of non-aqueous low surface tension liquids (oils including decane, octane, and hexadecane) onto non-desired regions. In addition, superoleophobic surfaces inside microfluidic channels have not been broadly explored yet. Moreover, inherently oleophobic $\left(\theta>90^{\circ}\right.$ on a smooth surface) surface chemistries are naturally unavailable. This means that the Wenzel state lowers the contact angle further, while the Cassie state is inherently unstable on most geometries [2]. Unlike superhydrophobic surfaces, superoleophobic surfaces cannot be easily fabricated with nano-structures [3]. And the use of micro/nano-structured features to prevent lateral spreading of low surface tension liquids has not been very effective. The relationship between oleophilic surfaces and superoleophobic surfaces is more complex than the relationship between hydrophilic and superhydrophobic surfaces [2]. In fact, the surface chemistry of the previously reported superoleophobic surfaces using various fluorinated coatings over re-entrant geometries is actually slightly oleophilic [2]. When the oil drop falls into the Wenzel state due to perturbation, the oil drop can simply be pinned or spread laterally. Thus, it is not clear how oleophobically stable the surface is to lateral flows. We have developed an oil-repellent surface which can resolve the concerns described above.

\section{OIL REPELLANT SURFACE}

Since the oleophobicity is governed by the surface geometry, we designed an oil-repellant surface by combining laterally and vertically oriented re-entrant geometries to liquids.

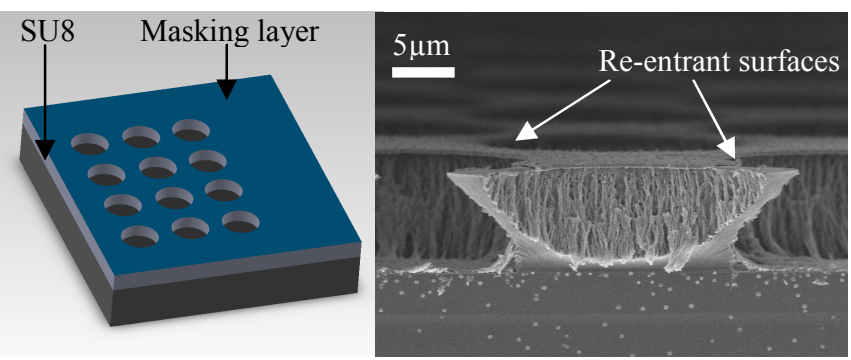

Figure 1: Schematic view and SEM image of micromachined features comprising the oil-repellant surface.
Thus, such surfaces can prohibit laterally approaching low surface tension liquids. In order to minimize $\phi_{\mathrm{s}}$, which is the areal fraction of the liquid-air interface, occluded by the texture in the CassieBaxter model, we utilized surface micromachined features as shown in Fig. 1. The geometry of the features can act as oilrepelling features to the lateral flow of low surface tension liquids. The re-entrant features in the textured area formed by reactive ion etching can clearly be seen in the SEM image. By application of the very uniquely micromachined features with a hydrophobic coating, selective wetting by a non-aqueous low surface tension liquid has been successfully achieved as illustrated in Fig. 2.
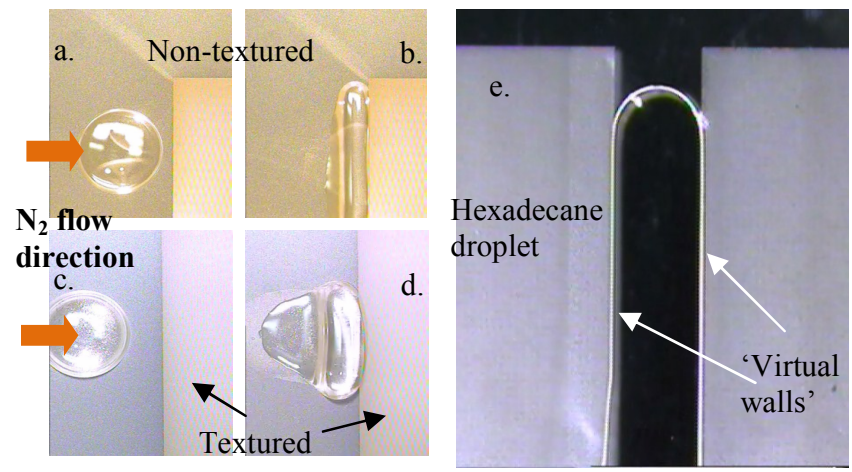

Figure 2: Silicone oil droplet (a, b) and castor oil droplet $(c, d)$ pneumatically actuated $(a \rightarrow b, c \rightarrow d)$ from non-textured towards the textured oil-repellant region. e. Hexadecane moves along an un-textured path due to the presence of 'virtual' walls.

As can be seen in the above image, a drop of hexadecane selectively wets the non-textured region. The textured regions turn out to be too oil-repellant to allow the hexadecane droplet to wet the surface. Even when pushed by a strong pneumatic force, the oil droplet cannot move over the textured region. Various low surface tension liquids introduced between the textured areas have been well confined within the oleophilic pathways as shown in Fig. 2e. The gas-liquid interface is pinned precisely at the boundary between the textured and non-textured regions. Because there are no physical walls on the sides of the liquid stream, it can be looked upon as confinement by the presence of 'virtual' walls.

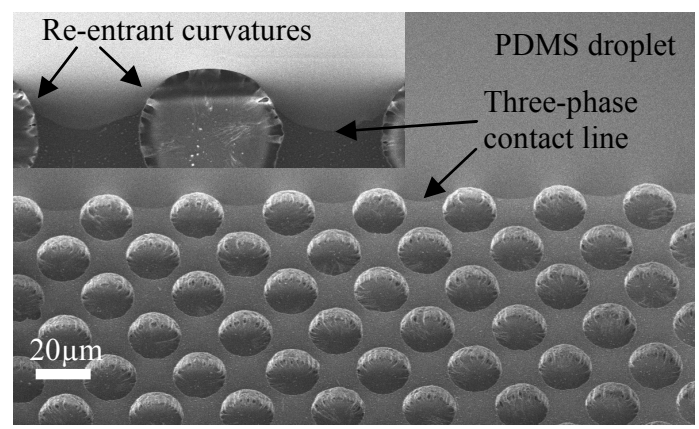

Figure 3: PDMS droplet at the edge of an oil-repellant surface for mimicking the behavior of the oil droplet three-phase contact line at these features.
Solid-State Sensors, Actuators, and Microsystems Workshop Hilton Head Island, South Carolina, June 3-7, 2012 
The oil-repellant behavior arises most importantly from the interaction of the three-phase contact line with the re-entrant geometries of the micromachined features on the surface. A further study about the interaction of the three-phase contact line with the boundary between the textured and non-textured areas was carried out by using scanning electron microscopy (SEM). For this, a PDMS droplet, which has a similar surface tension to that of silicone oil, was placed on the non textured surface and pneumatically pushed towards the micro textured area and then cured. Clear images of the representation of an oil droplet by PDMS at the boundary were obtained as shown in Fig. 3. The fluidic resistance faced by the three-phase contact line at the boundary prevents its further advancement.

\section{VIRTUAL MICROFLUIDIC CHANNEL WITH BUILT-IN LIQUID DRIVERS}

This phenomenon has been further exploited to form a 'virtual' microfluidic channel for guiding low surface tension liquids. In order to drive the low surface tension liquid confined between two 'virtual' walls, we have implemented electrodes only under the non-textured regions to provide the necessary dielectrophoretic forces. The brief fabrication steps are shown in Fig. 4.

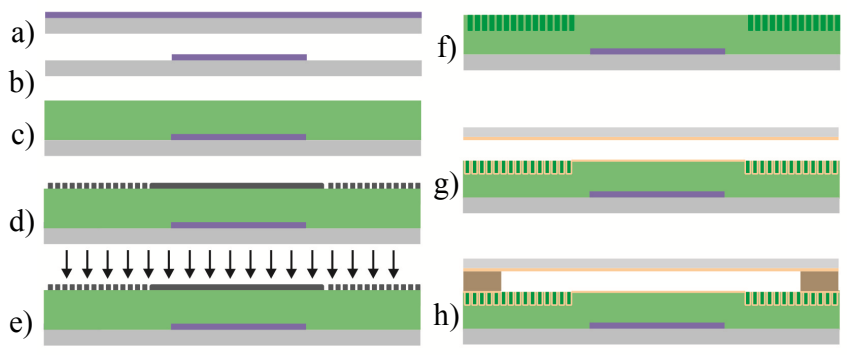

Figure 4: Fabrication steps outlined a) Metallization b) Patterning of metal layer to define electrodes c) SU8 layer d) Metallization and patterning defining textures e) $\mathrm{O}_{2}$ RIE etch $\mathrm{f}$ ) Stripping of metal layer g) Coating substrate surface and coverslip with hydrophobic layer h) Final device assembly via spacers.

Fabrication starts with the patterning of a metalized glass substrate which defines the underlying dielectrophoretic (DEP) electrodes. On top of these electrodes, a $10 \mu \mathrm{m}$ thick SU8 layer was deposited and cured followed by a metallization and patterning step. The patterned metalized layer acts as a mask to the subsequent surface micromachining step which defines the oilrepellant features. Surface micromachining of the cured SU8 was carried out by etching in a reactive ion etcher (RIE) with oxygen. The micromachined surface as well as the top cover glass substrate was then made hydrophobic with the application of a very thin Cytop layer (100nm). The top cover substrate was then assembled over a $25 \mu \mathrm{m}$ thick spacer and bonded. Since the electrodes are not in direct contact with the liquids, we believe that dielectric as well as conducting liquids can be driven along the virtual microfluidic channel. As soon as a test liquid was loaded into the device, the liquid remained confined between the 'virtual' walls. Compared to viscous fluids such as silicone and castor oil, less viscous fluids such as hexadecane and Fluorinert FC-70 flow more easily along the paths confined by the textured oil-repellant surfaces. Activation of the electrodes with a DC voltage source, thereby generating dielectrophoretic forces in the oil droplet cause it to move along the 'virtual' microfluidic channel (non-textured area) as shown in
Fig. 5. The liquid-air interfaces along the 'virtual' walls still look clearly straight. In addition, the actuation voltages for various test liquids with different properties are summarized in Table 1.

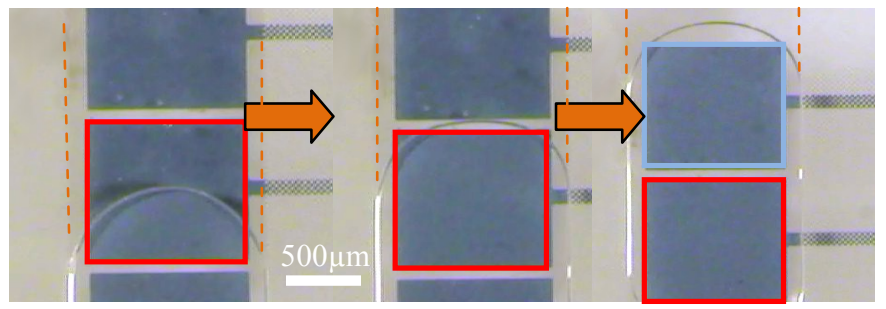

Figure 5: Droplet (Silicone oil) actuated across the desired path using liquid Dielectrophoresis (L-DEP) by actuating one electrode at a time.

\section{CONCLUSION}

A virtual microfluidic channel for low surface tension liquids has been successfully demonstrated. An oil-repellant surface was first designed and tested. Implementation of 'virtual' walls generated by the oil-repellant surfaces was carried out to confine low surface tension liquids. Electrical actuation of various test liquids between the 'virtual' walls has shown successful outcomes. This technique can be applied to many microfluidic devices dealing with various low surface tension liquids.

Table 1: Actuation voltages with and without the use of micro textured guiding of various low surface tension liquids

\begin{tabular}{|l|c|c|c|c|}
\hline Test liquids & $\begin{array}{c}\text { Actuation } \\
\text { voltage } \\
\text { without } \\
\text { micro } \\
\text { textures }\end{array}$ & $\begin{array}{c}\text { Actuation } \\
\text { voltage } \\
\text { with } \\
\text { micro } \\
\text { textures }\end{array}$ & $\begin{array}{c}\text { Di- } \\
\text { electric } \\
\text { constant }\end{array}$ & $\begin{array}{c}\text { Surface } \\
\text { tension } \\
(\mathbf{m J} / \\
\left.\text { cm }^{2}\right)\end{array}$ \\
\hline $\begin{array}{l}\text { Silicone oil } \\
(50 c S t)\end{array}$ & $310 \mathrm{~V}$ & $325 \mathrm{~V}$ & 2.75 & 20.3 \\
\hline $\begin{array}{l}\text { Castor oil } \\
(985 \mathrm{cSt})\end{array}$ & $316 \mathrm{~V}$ & $450 \mathrm{~V}$ & 4.7 & 39 \\
\hline $\begin{array}{l}\text { Hexa- } \\
\text { decane } \\
(3.02 \mathrm{cSt})\end{array}$ & $\sim 400 \mathrm{~V}$ & $420 \mathrm{~V}$ & 2.05 & 27.4 \\
\hline $\begin{array}{l}\text { FC-70 } \\
(24 \mathrm{cSt})\end{array}$ & $188 \mathrm{~V}$ & $210 \mathrm{~V}$ & 1.98 & 18 \\
\hline
\end{tabular}

\section{ACKNOWLEDGEMENT}

This research was supported by the National Science Foundation under Grant No. ECCS 0901618.

\section{REFERENCES}

[1] N. Verplanck, et al., "Wettability switching techniques on superhydrophobic surfaces," Nanoscale Research Letters, vol. 2, pp. 577-596, 2007.

[2] V. Jokinen, et al., "Controlled Lateral Spreading and Pinning of Oil Droplets Based on Topography and Chemical Patterning," Langmuir, 2011.

[3] A. Tuteja, et al., "Robust omniphobic surfaces, "Proceedings of the National Academy of Sciences, vol. 105, p. 18200 , 2008 .

CONTACT R. Almeida;+1-573-884-7867; rab5b@mail.missouri.edu 


\title{
ACOUSTO-OPTIC FREQUENCY MODULATOR
}

\author{
S. Tallur ${ }^{*}$, and S.A. Bhave
}

OxideMEMS Lab, Cornell University, Ithaca, New York, USA

\begin{abstract}
Frequency modulation of a continuous wave laser source enables generation of photons at multiple frequencies from a single pump laser. Such multiple closely spaced laser lines are important for high data rate QAM in dense-WDM networks. In this paper we present a monolithic silicon acousto-optic frequency modulator (AOFM) operating at $1.09 \mathrm{GHz}$. We demonstrate frequency modulation of a $1564 \mathrm{~nm}$ wavelength pump laser, resulting in generation of sideband laser lines spaced by $0.009 \mathrm{~nm}$.
\end{abstract}

\section{INTRODUCTION}

On-chip optical frequency modulation has been demonstrated by coupling surface acoustic waves (SAW) to InAs/GaAs quantum dots $[1,2]$. This phenomenon has not been demonstrated in silicon to date. The silicon acousto-optic modulator (AOM) presented in [3] has a mechanical resonance frequency of $1.1 \mathrm{GHz}$ but an optical cavity $3 \mathrm{~dB}$-linewidth $\approx 5.88 \mathrm{GHz}$. The mechanical response of the resonator is hence slower than the lifetime of the photons inside the cavity and thus results in predominant amplitude modulation (AM) of the laser light. Here we present a modulator with an optical cavity linewidth $\approx 1.27 \mathrm{GHz}$. The rate of the mechanical vibrations is comparable to the photon lifetime, which results in Doppler-shift of the circulating intra-cavity optical field, thus causing frequency modulation (FM). Such an Acousto-Optic Frequency Modulator (AOFM) converts photons at the pump laser frequency to other frequencies on account of mechanical motion.

When the mechanical vibration frequency is greater than half of the optical cavity $3 \mathrm{~dB}$-linewidth, the resonator is termed to lie in the "resolved sideband regime" [4]. In this case, the motion of the optomechanical resonator results in asymmetric higher and lower frequency optical sidebands spaced by the mechanical resonance frequency. The asymmetry can be explained solely by coincident $\mathrm{AM}$ and FM [5]. For the modulator presented here, the lower frequency sideband has $>12 \mathrm{X}$ higher intensity than the higher frequency sideband at $1.09 \mathrm{GHz}$, indicating strong $\mathrm{FM}$ plus some AM.

\section{DEVICE DESIGN}

When the mechanical resonance frequency for the modulator, $\Omega_{\mathrm{m}}$, is greater than half of the optical cavity linewidth $\kappa / 2=$ $\omega_{\text {opt }} / 2 Q_{\text {opt }}$, the dependence of the intra-cavity field amplitude on the mechanical frequency is complex [6] and one can write the exact solution [4]:

$$
a_{p}(t)=s \sqrt{\kappa} \sum_{n=-\infty}^{n=+\infty} \frac{(-i)^{n} J_{n}(\beta)}{\frac{\kappa}{2}+i\left(\Delta+n \Omega_{m}\right)} e^{i\left(\omega+n \Omega_{m}\right) t+i \beta \cos \left(\Omega_{m} t\right)}
$$

The modulation index, $\beta$, depends linearly on the amplitude of mechanical displacement of the micro-ring. The offset of the pump laser frequency from the cavity resonance frequency is denoted by the detuning, $\Delta=\omega_{\text {laser }}-\omega_{\text {opt }}$. The electro-acoustically driven motion of the cavity leads to a combination of AM and FM of the laser light as seen in equation 1.

The modulator presented in [3] is extended to an array of mechanically coupled ring resonators for large electrostatic driving force [7]. Figure 1 shows a scanning electron micrograph (SEM) of the device. Mechanical motion of the ring resonators is actuated through capacitive air gap electrostatic transduction. The lithographically defined air gap is $130 \mathrm{~nm}$. Input laser light is coupled into the optomechanical resonator using an integrated waveguide.

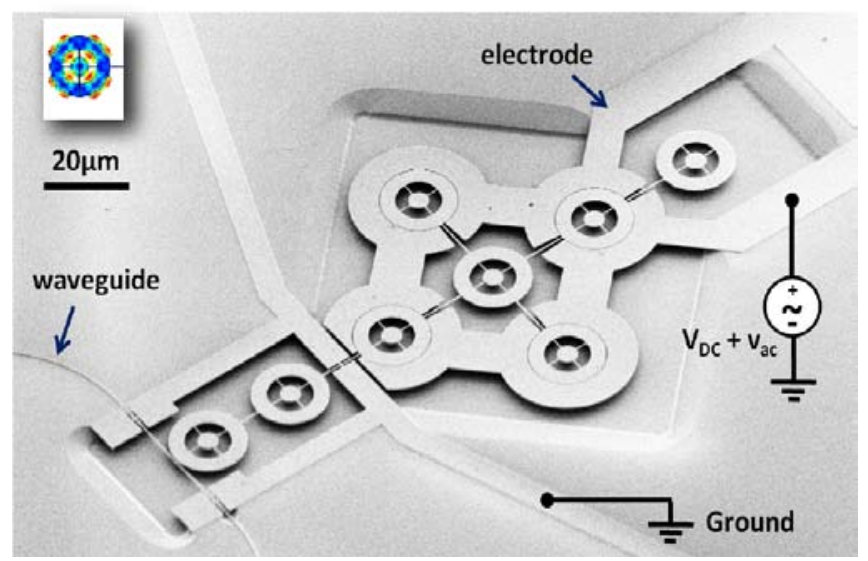

Figure 1: SEM of the acousto-optic frequency modulator. (Top-left inset: COMSOL mode shape of the compound radial expansion mode of the ring resonator at $1.09 \mathrm{GHz}$ )

\section{EXPERIMENTAL RESULTS AND DISCUSSION}

To probe the optical response of the modulator, light from a Santec TSL-510 tunable laser is coupled into the waveguide using grating couplers. We choose an optical resonant mode at a wavelength of $1564 \mathrm{~nm}$ with an optical quality factor of 150,000 , which corresponds to a cavity linewidth $\approx 1.27 \mathrm{GHz}$. The laser wavelength is blue detuned to the half maximum point of this optical cavity mode and the transmission of the modulator is obtained by performing a 2-port measurement using an Agilent N5230A network analyzer. A mechanical quality factor of 1,800 was measured for the resonant mode at $1.09 \mathrm{GHz}$ as shown in Figure 2.

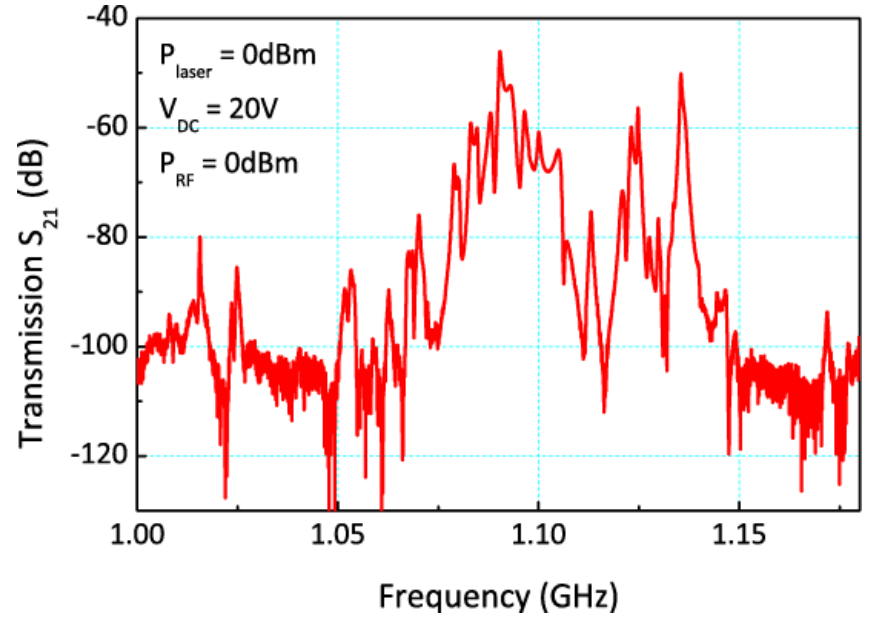

Figure 2: RF transmission of the AOFM. Multiple peaks corresponding to different modes of vibration of the device are seen. The mechanical resonant mode at $1.09 \mathrm{GHz}$ that corresponds to in phase motion of all the rings in the array has an insertion loss of $46 \mathrm{~dB}$. 
For observing the optical sidebands for the modulator, we use a Thorlabs SA210-12B scanning Fabry-Perot (FP) interferometer. A bias-tee is used to apply a combination of DC bias voltage and AC voltage at $1.09 \mathrm{GHz}$ using an Agilent E8257D PSG Analog Signal Generator. The wavelength of the FP cavity is scanned across its entire range by sweeping the voltage on the piezocontroller in the FP cavity control box. The transmitted light intensity is measured using an internal photodiode, amplified by a transimpedance amplifier inside the control box, and displayed on an oscilloscope. An illustration of the setup is shown in Figure 3.

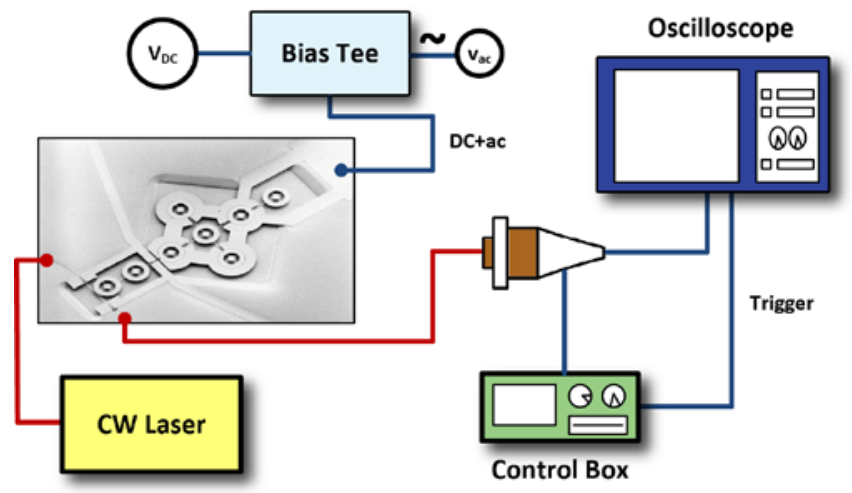

Figure 3: Experimental setup for characterization of the optical sidebands of the AOFM using a scanning FP interferometer. As the FP cavity scans across wavelengths, its output shows a peak on the oscilloscope whenever it passes across a sideband.

The optical sidebands are analyzed for relative detuning $(2 \Delta / \kappa)$ values of 0.5 and 1.5 respectively. Figure 4 shows the measured intensity values at the sidebands, normalized to the intensity at the input laser frequency incident on the FP interferometer. A combination of $20 \mathrm{~V} \mathrm{DC}$ and $8 \mathrm{dBm}$ RF power was applied at the input electrode of the modulator. The observed asymmetry in the sideband intensities results from coincidental AM and FM [5]. This is in sharp contrast to perfectly symmetric sideband generation on account of pure AM or pure FM.

The lower frequency sideband is enhanced by the optical cavity, while the higher frequency sideband is filtered out. This effect is maximized for $\Delta=\Omega_{m}$, which corresponds to a relative detuning of 1.5 (Figure 4(b)). For this case, the ratio of the sideband heights is $12.67: 1$, which compares well to the calculated value of 10.8 from simulation following equation 1 . In comparison, we measure a ratio of $2.2 \mathrm{X}$ for relative detuning of 0.5 (Figure 4(a)). The modulation index, $\beta$, which appears in equation 1 , was calculated by calculating the amplitude of mechanical displacement of the micro-ring by following the derivation in [8]. Although our final goal is pure FM of laser light, the asymmetry due to presence of some AM serves as a proof of concept.

\section{CONCLUSION}

We demonstrate frequency modulation of a continuous wave laser of wavelength $1564 \mathrm{~nm}$ using a silicon acousto-optic modulator operating at $1.09 \mathrm{GHz}$, thus resulting in generation of sideband laser lines spaced apart by $9.23 \mathrm{pm}$. This constitutes the first demonstration of electro-acoustically generating photons of different colors from a single pump laser in silicon.

\section{REFERENCES}

[1] M. Metcalfe, et al., "Resolved sideband emission of InAsGaAs quantum dots strained by surface acoustic waves", Phys. Rev. Lett. 105, 037401 (2010). (a)

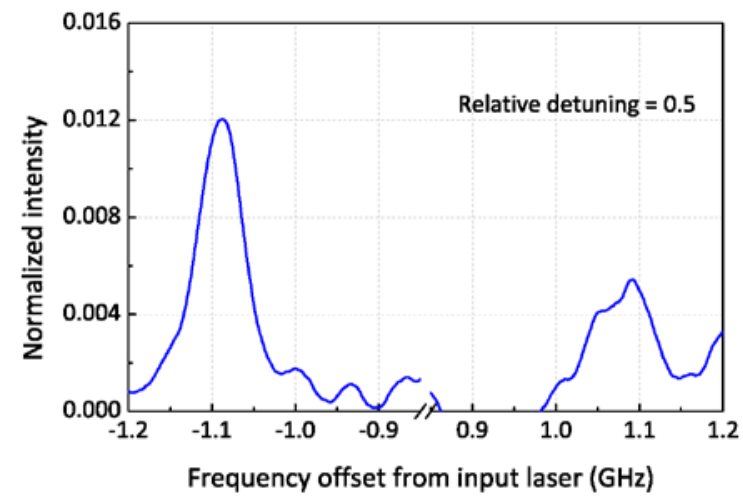

(b)

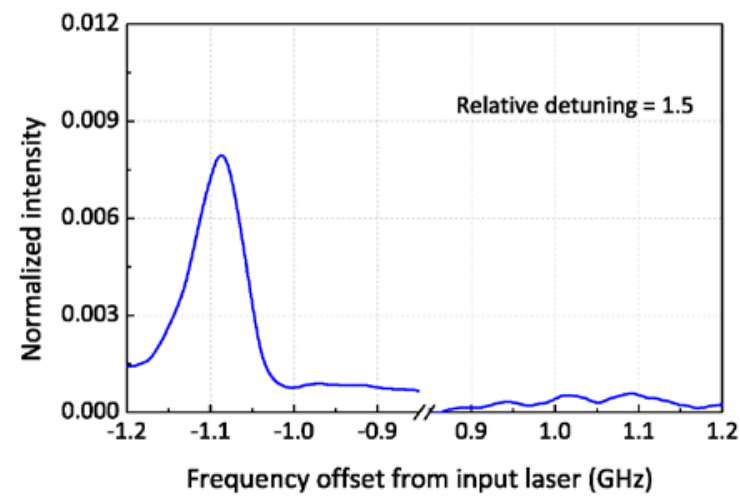

Figure 4: Motional sidebands at $\pm 1.09 \mathrm{GHz}$ offsets from laser frequency measured for relative detuning value of (a) 0.5, and (b) 1.5. In panel (b), the peak intensities at the sidebands have a ratio of 12.67:1. The asymmetry of the sidebands confirms simultaneous $A M$ and $F M$.

[2] D.A. Fuhrmann, et al., "Dynamic modulation of photonic crystal nanocavities using gigahertz acoustic phonons", Nature Photonics, 5, pp. 605-609 (2011).

[3] S. Sridaran and S. A. Bhave, "1.12 GHz opto-acoustic oscillator", 25th IEEE International Conference on Micro Electro Mechanical Systems, Paris, France (2012) pp 644647.

[4] A. Schliesser, et al., "Resolved-sideband cooling of a micromechanical oscillator", Nature Physics 4, 415-419 (2008).

[5] H. Roder, "Amplitude, Phase, and Frequency Modulation", Proceedings of the Institute of Radio Engineers, 19(12), pp. 2145-2176 (1931).

[6] T. Carmon, et al., "Temporal Behavior of Radiation-PressureInduced Vibrations of an Optical Microcavity Phonon Mode", Phys. Rev. Lett. 94, 223902 (2005).

[7] S.-S. Li, Y.-W. Lin, Z. Ren, and C. T.-C. Nguyen, "An MSI micromechanical differential disk-array filter", $14^{\text {th }}$ Int. Conf. on Solid-State Sensors \& Actuators (Transducers'07), Lyon, France (2007) pp. 307-311.

[8] S. Tallur, T. J. Cheng, S. Sridaran and S. A. Bhave, "Motional impedance analysis: bridging the 'gap' in dielectric transduction", $65^{\text {th }}$ IEEE Frequency Control Symposium, San Francisco, California (2011) pp. 135-138.

\section{CONTACT}

*S. Tallur, tel: +1-607-279-7818; sgt28@cornell.edu 


\title{
AUTONOMOUS MICROSYSTEMS FOR ASTROBIOLOGY: DEVELOPMENT OF, AND SPACEFLIGHT RESULTS FROM, THE O/OREOS NANOSATELLITE
}

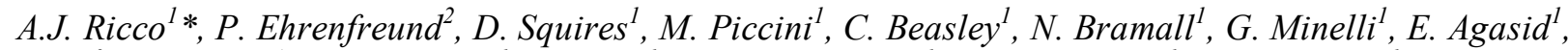

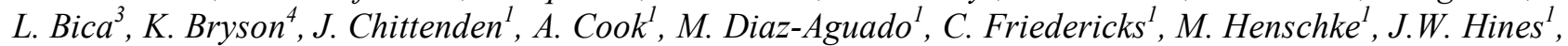

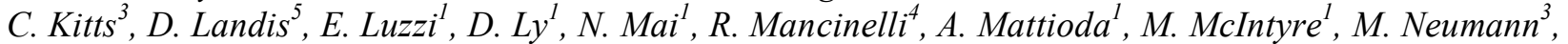

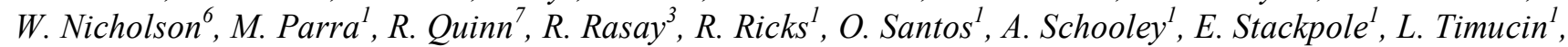 \\ B. Yost ${ }^{1}$, and A. Young ${ }^{3}$ \\ ${ }^{1}$ NASA Ames Research Center, Moffett Field, California, USA \\ ${ }^{2}$ Space Policy Institute, Washington, DC, USA \\ ${ }^{3}$ Robotic Systems Laboratory, Santa Clara University, Santa Clara, California, USA \\ ${ }^{4}$ Bay Area Environmental Research Institute, Sonoma, California, USA \\ ${ }^{5}$ Draper Laboratory, Cambridge, Massachusetts, USA \\ ${ }^{6}$ University of Florida, Kennedy Space Center, Florida, USA \\ ${ }^{7}$ SETI Institute, Mountain View, California, USA
}

\begin{abstract}
We designed, developed, constructed, tested, launched, and are now analyzing data telemetered from orbit by the O/OREOS (Organism/ Organic Exposure to Orbital Stresses) nanosatellite. Measuring $\sim 10 \times 10 \times 34 \mathrm{~cm}$ and weighing $5.5 \mathrm{~kg}$, O/OREOS (Figure 1) launched in November 2010 into 650-km Earth orbit as a secondary payload from Kodiak, Alaska. O/OREOS comprises three conjoined $10-\mathrm{cm}$-cubes, each a fully integrated microsystem: a "bus" including communications, power, and control functions; the Space Environment Survivability of Living Organisms (SESLO) experiment, and the Space Environment Viability of Organics (SEVO) experiment. We will present results from SEVO's real-time analysis of the photostability of organics and biomarkers over space exposure times of one year, and SESLO's data on the survival and metabolic activity of microorganisms at 3 timepoints spanning 6 months.
\end{abstract}

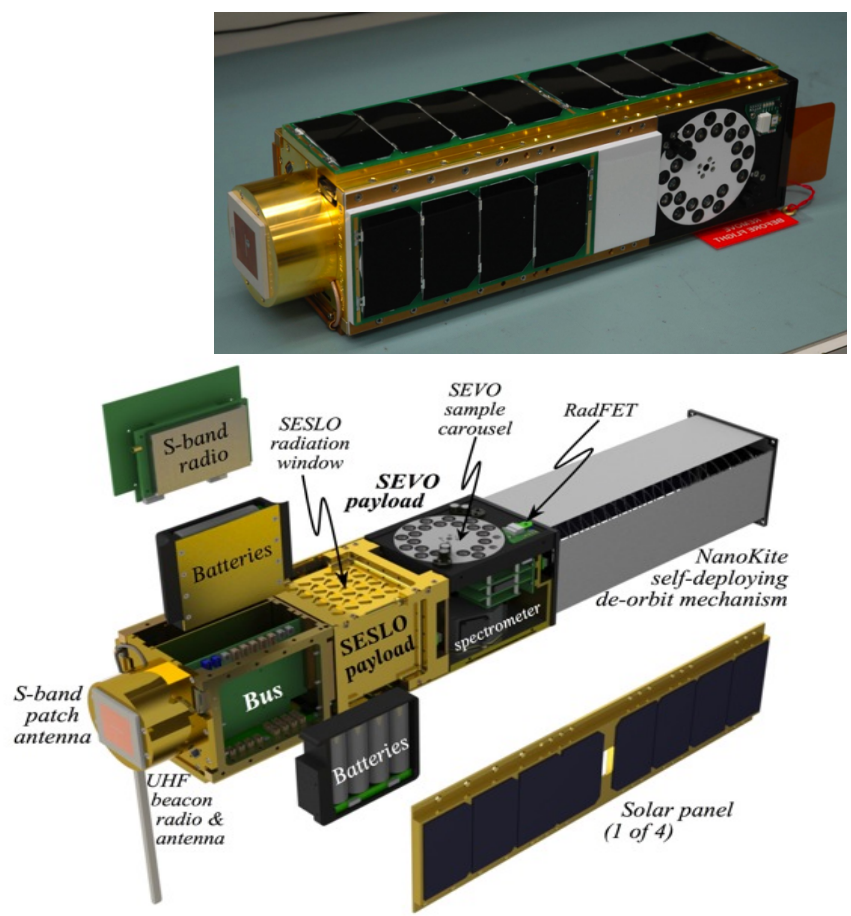

Figure 1: Photograph (top) and exploded solid model (bottom) of O/OREOS nanosatellite (10 × $10 \times 34 \mathrm{~cm}$, excluding nanokite).

9780964002494/HH2012/\$25@2012TRF

DOI 10.31438/trf.hh2012.25

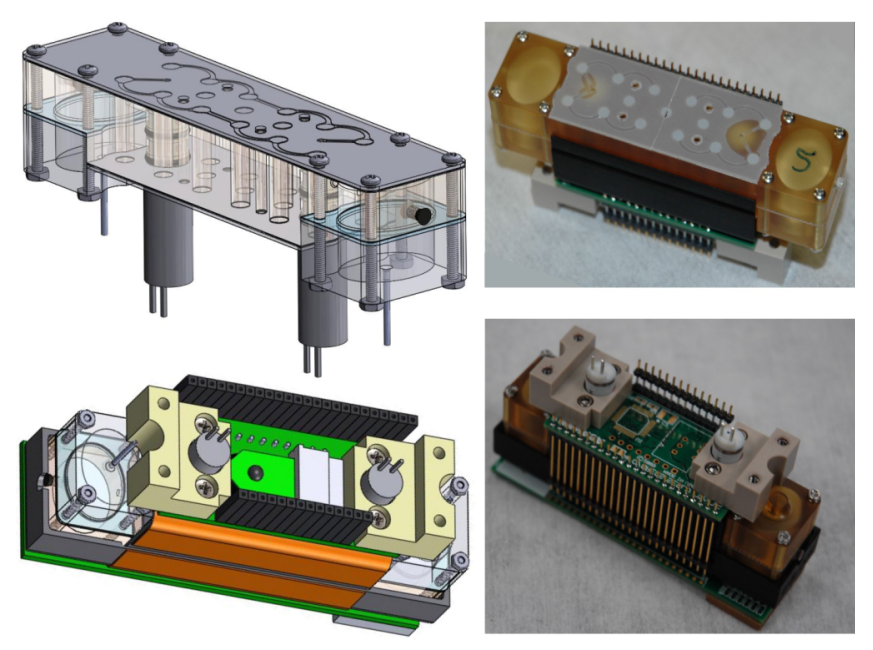

Figure 2: Four different views (solid model at left, photographs at right) of one of three bioblocks included in the SESLO payload. The two solenoid valves protrude down from the block (upper left); reservoirs are the elliptical structures at both ends of the block; heater is orange; radiation-sensitive FET (RadFET), which measures integrated radiation dose at two locations in each of the two payload cubes, is a black dot near the center of the bottom left.

\section{INTRODUCTION}

Small autonomous satellites under $10 \mathrm{~kg}$, referred to as nanosatellites or cubesats, can simultaneously reduce the cost and increase the accessibility of space science experiments [1]. Leveraging and integrating recent advances in nano-, micro-, and miniature technologies in fields from biotechnology to materials to sensors to telecommunications, cube/nanosatellites are under development by nearly 100 universities, numerous small commercial ventures, large aerospace companies, a dozen developing nations, and the world's major space agencies. Despite their small size, such spacecraft can support complex science, as in our drug-dosedependence experiments aboard PharmaSat [2]. The coupling of autonomy and telemetry in these orbital "free flyers" can provide near-real-time kinetic data as space science experiments progress.

Astrobiology seeks understanding of the origins, evolution, development, and future of life in the universe. O/OREOS addresses this by characterizing the space environment degradation of organic starting materials for life and biomarkers, and by probHilton Head Island, South Carolina, June 3-7, 2012 
ing the details of microorganism survival under the influence of microgravity and ionizing radiation. It also supports NASA's scientific exploration program by investigating Earth's local space environment and space biological effects relevant to future lunar and Mars missions.
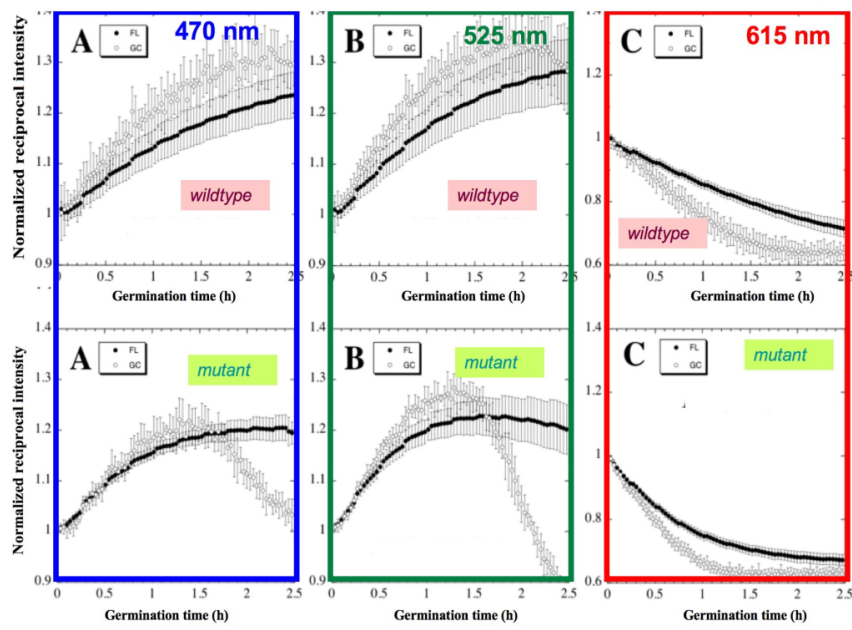

Figure 3: Growth curves (as normalized reciprocal intensity) after 14 days in space (solid dots) and an identical ground control (open circles) for B. subtilis wildtype and mutant strains at three wavelengths. Error bars are for $4-6$ wells of each strain of the microbe. The data show that growth is somewhat slower in the microgravity environment of space due to differences in mass transport of nutrients and waste products.

\section{DETAILS, RESULTS, AND DISCUSSION}

Soon after its orbital deployment and then after 3 and 6 months in space, SESLO characterized the growth, activity, health and adaptation of microorganisms to the stresses of outer space. SESLO consists of three miniature "bioblock" modules, each made by a combination of miniature machining and thin-film processing to include twelve $75-\mu \mathrm{L}$ sample wells plus a pair of valves and reservoirs (Figure 2). Groups of 6 wells are microfluidically connected via a solenoid valve to a reservoir containing growth medium the microbes.

Absorbance through each $2.8 \times 12 \mathrm{~mm}$ well was monitored using 3-color (RGB) LED illumination and an intensity-tofrequency detector. Prior to spaceflight, microbes were dried onto microwell walls and bioblocks sealed with gas-permeable mem-
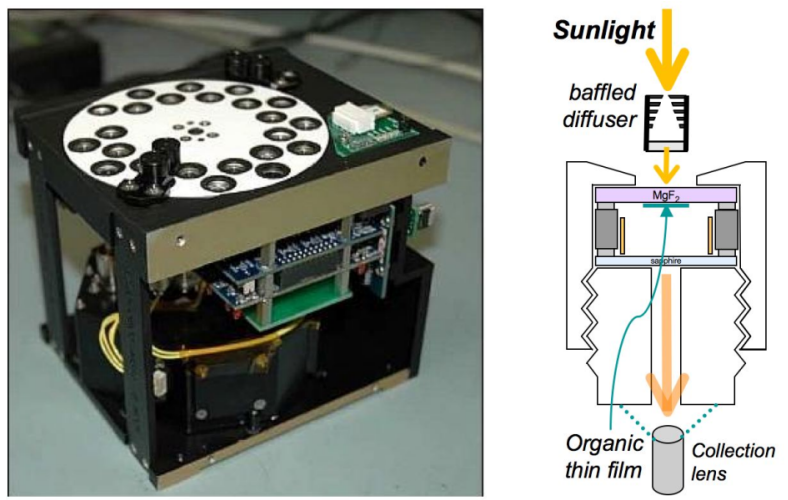

Figure 4: SEVO spectrometer payload cube (left) and cross sectional diagram of one of the 24 SEVO sample cells (right), $1 \mathrm{~cm}$ in diameter. $\mathrm{MgF}_{2}$ optical flat (at top of cell) transmits solar radiation to vacuum UV wavelengths, including the Lyman alpha hydrogen emission at $124 \mathrm{~nm}$. branes. The growth medium included the viability dye Alamar blue, which changes color due to cellular metabolic activity. Figure 3 shows initial growth curves telemetered from orbit [3].

SEVO tracked the outer space photostability of organics; solar and space radiation are attenuated only by $1.5-\mathrm{mm} \mathrm{MgF}_{2}$ windows that support thin-film samples. Four molecular classespolycyclic aromatic hydrocarbon, amino acid, quinone, metalloporphyrin-represent "bio-building-blocks" and biomarkers. SEVO comprises a miniaturized UV-visible-NIR spectrometer and a 24-sample carousel that holds hermetically sealed microcells (Figure 4). Integral optics enable the use of the Sun as the light source. After transiting a given organic film, light travels via optical fiber to the spectrometer $(<2 \mathrm{~nm}$ spectral resolution; $200-$ $1000 \mathrm{~nm}) .16$ spectral acquisitions are averaged and stored biweekly for each cell, then telemetered. Figure 5 shows a SEVOacquired solar reference spectrum. Details of the spectroscopically-measured chemical changes of organic thin films resulting from over one year of full solar exposure in orbit will be reported in a forthcoming publication [4].

\section{CONCLUSIONS}

The success of the O/OREOS mission demonstrates the utility of low-cost nanosats as space science research and technology development platforms that can capitalize on the latest technologies to fly instruments that are truly state of the art. The O/OREOS Mission was supported by the NASA Astrobiology Small Payloads Program and Planetary Protection Office.

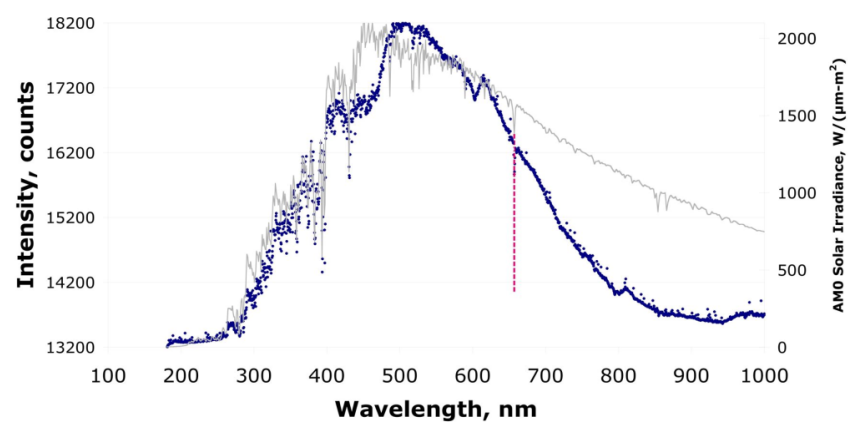

Figure 5: Solar spectrum measured at an orbital altitude of 650 $\mathrm{km}$ by the SEVO spectrometer (dots, left axis) compared to a reference solar spectrum (gray line, right axis) showing excellent alignment of spectral features. Organic thin film absorbances are measured using the sun as the light source for the spectrometer.

\section{REFERENCES}

[1] K. Woellert, P. Ehrenfreund, et al., "Cubesats: Cost-Effective Science and Technology Platforms for Developing Nations," Adv. Space Res., 47, 663 (2011).

[2] A.J. Ricco, M. Parra et al., "PharmaSat: Drug Dose Dependence Results from An Autonomous Microsystem-Based Small Satellite," Tech. Digest 2010 Solid-State Sensors, Actuators, and Microsystems Workshop, Transducer Research Foundation: San Diego (2010); pp. 110-113.

[3] W.L. Nicholson, A.J. Ricco, et al., "The O/OREOS Mission: First Science Data from the SESLO Payload," Astrobiology, 11, 951-958 (2011).

[4] A. Mattioda, A. Cook, et al., "The O/OREOS Mission: First Science Data from the Space Environment Viability of Organics (SEVO) Payload," Astrobiology, in preparation (2012).

\section{CONTACT}

*A.J. Ricco, tel: +1-650-604-4276; ajricco@stanford.edu 


\title{
MICROFLUIDIC OPTOMECHANICAL OSCILLATORS
}

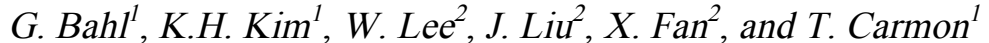

${ }^{1}$ EECS and ${ }^{2}$ BME departments, University of Michigan, Ann Arbor, Michigan, USA

\section{ABSTRACT}

We demonstrate a new class of fused silica MEMS that bridge between optomechanical and fluidic microsystems. Since fused silica is non-conductive and optically transparent, we employ "touch-less" excitation by means of optical electrostriction and radiation pressure to excite surface-acoustic-wave whispering gallery modes and wineglass modes in these devices. This is the first demonstration of mechanical vibration that interacts with fluids, excited in a microstructure via optical forces. These mechanical oscillators operate over several orders-of-magnitude in frequency from $8.5 \mathrm{MHz}-11 \mathrm{GHz}$.

\section{INTRODUCTION}

Resonant MEMS cantilevers with embedded microfluidic channels provide sub-femtogram resolution for mass sensing of analytes in fluidic media [1] using electrostatic transduction. Meanwhile, optical microresonators have proven their use in nanoparticle detection experiments in both wet [2] and dry environments $[3,4]$. Additionally, it is known that light can also be used to excite [5-7] and attenuate [8] mechanical vibration in microdevices by exploiting radiation pressure [5,7], gradient force [9], and electrostriction [6,8]. The excitation of mechanical vibration using optical forces in fluidic media, however, has not been achieved due to the large viscous damping experienced. In this work, we enable this capability by confining the test liquid (distilled water) inside a hollow microfluidic optomechanical $(\mu \mathrm{FOM})$ resonator. We show, for the first time, a device that in the presence of a liquid preserves ultra-high optical quality factors, and can be used to optically drive high-Q mechanical modes of the liquid-containing resonator. The methods employed here are directly applicable towards actuating optically transparent shelltype MEMS resonators for gyroscope applications. Hybrid

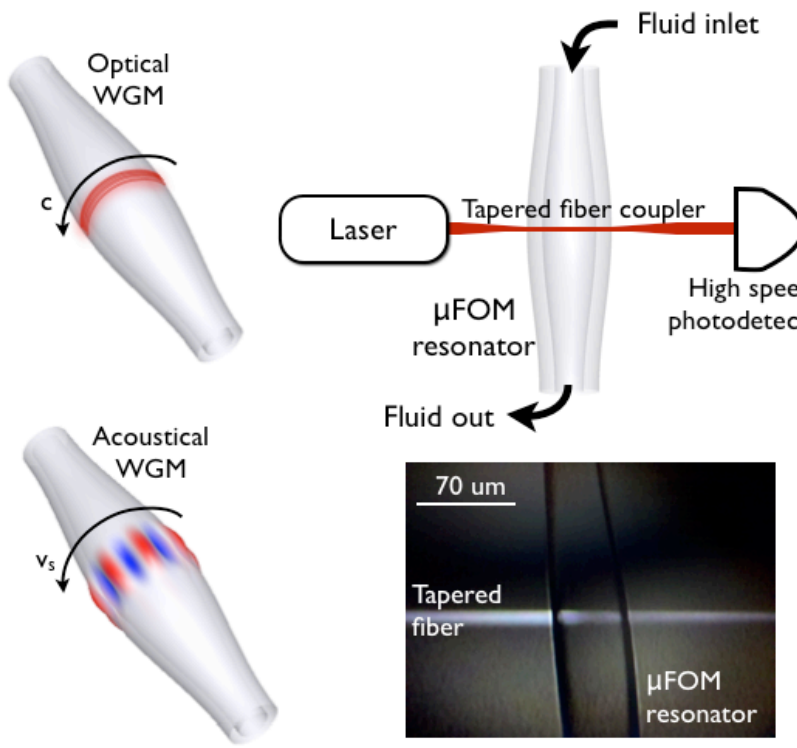

Figure 1. The optical modes and the mechanical modes of a fused silica glass $\mu F O M$ device are both whispering-gallery modes (WGM). Laser light at $1.5 \mu \mathrm{m}$ wavelength is coupled into the optical modes via a tapered optical fiber. Light then excites mechanical vibration by means of optical electrostriction. optomechanical bio/chemical sensors are also enabled.

\section{FABRICATION AND TESTING}

The $\mu \mathrm{FOM}$ device is fabricated with a commercial fused silica glass capillary that is pulled lengthwise while being heated under IR-laser illumination. The radius of the capillary is modulated in this process by varying laser power (Fig 1). The glass wall thickness is easily controlled between $5 \mu \mathrm{m}-45 \mu \mathrm{m}$ with a choice of an appropriate starting capillary. Combined with a glassblowing technique [10] the capillary wall can be as thin as $560 \mathrm{~nm}$. Such glass-based capillary devices are naturally integrated for rapid delivery of small volumes of analyte for sensing applications. We perform all the described experiments with distilled water present inside the $\mu \mathrm{FOM}$ device.

\section{EXPERIMENT}

At its widest the fused silica device forms an optical microresonator (Fig 1) that allows the 3D confinement of light due to total internal reflection. The optical modes are whispering gallery modes (WGMs) residing at the outer surface of the device. Light is coupled into these optical WGMs by means of a tapered optical fiber. Measurements indicate optical quality factors in excess of $\mathrm{Q}_{0}=10^{8}$. Mechanical oscillations in the device modulate or scatter the light in the optical WGM and are therefore observable. The minimum power observed to excite these oscillations is in approximately $160 \mu \mathrm{W}$.

The mechanical modes that we probe in this study are acoustical whispering-gallery modes [11]. Previously inaccessible using MEMS based techniques (electrostatic, thermal, piezoelectric actuation), these oscillations are excited by means of optical electrostriction as described in [6]. Mechanical modes ranging over three orders-of-magnitude in frequency are obtained (Fig 2). In addition, low frequency wineglass modes (Fig 2a) can be actuated by means of centrifugal radiation pressure. The wineglass mode and the $99 \mathrm{MHz}$ acoustical WGM (Fig 2b) are of interest as they causes significant deformation of the inner surface (Fig 3a,b), allowing strong interaction with fluids or analytes confined inside.

The mechanical quality factor $\left(\mathrm{Q}_{\mathrm{m}}\right)$ for the $99 \mathrm{MHz}$ mode is measured to be 4700 with distilled water present inside the capillary. This value compares extremely well against the previously measured $\mathrm{Q}_{\mathrm{m}}=12,300$ in a solid silica sphere (dry) for a $95 \mathrm{MHz}$ mode [8]. The displacement of the inner surface in the case of the higher frequency modes (Fig $\mathbf{2 c , d}$ ) can be improved by using thinner-walled devices [10]. Finally, the vibration can be kept stable for long periods of time, as required for sensor applications (Fig 3c).

\section{DISCUSSION}

Mechanical WGMs have a distinct advantage for sensor applications as the absence of any stationary nodes provides some level of insensitivity to position of the analyte. This is different from using standing-wave resonators or cantilever resonators [1] where sensitivity has a strong dependence on position. In addition to high- $\mathrm{Q}_{\mathrm{m}}$ mechanical WGMs, we have also observed breathing mode oscillations (typically $30 \mathrm{MHz}$ ) in these $\mu \mathrm{FOM}$ devices. 
a
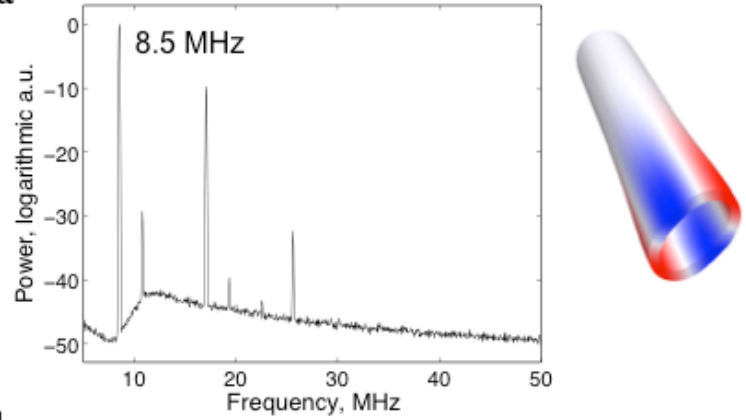

b

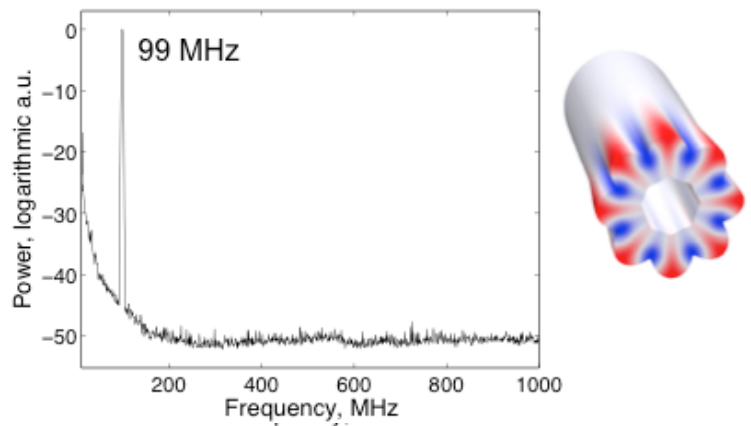

C
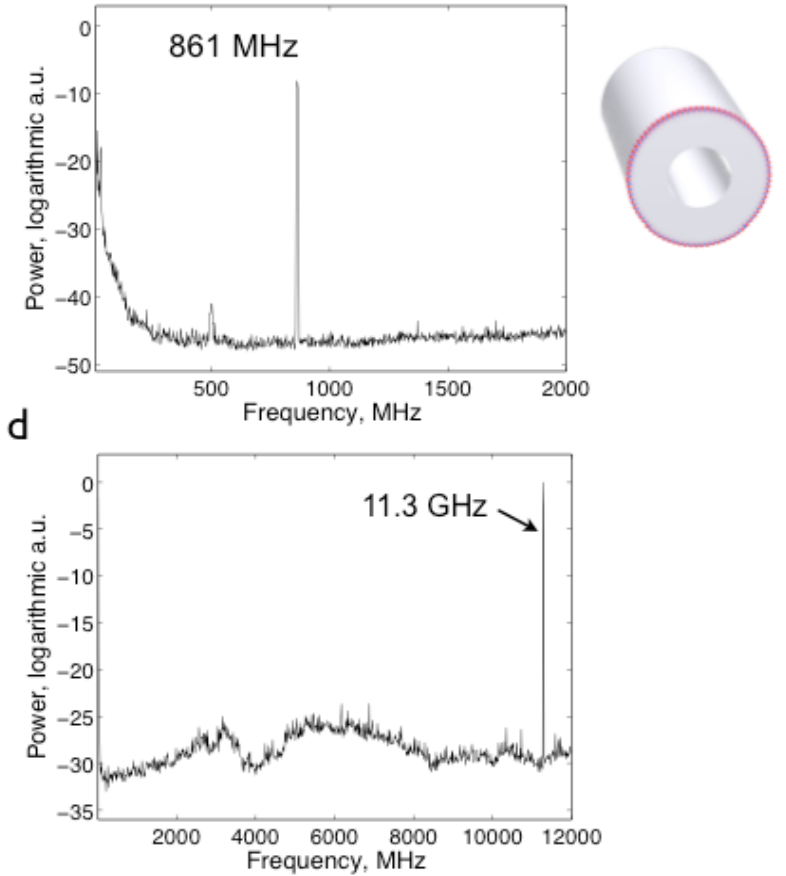

Figure 2. Mechanical vibrations ranging from $8.5 \mathrm{MHz}-11 \mathrm{GHz}$ can be excited on $\mu F O M$ devices with liquid present inside the device. The mechanical whispering gallery modes $(b, c, d)$ are traveling modes. The wineglass mode (a) is a standing wave mechanical resonance as evidenced by the harmonics present in the electrical spectrum. All these modes can interact with fluids in the device, provided the device wall thickness is comparable to the acoustical wavelength. This is easily seen for the $99 \mathrm{MHz}$ mode.

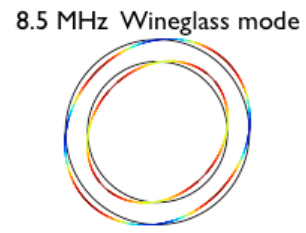

b

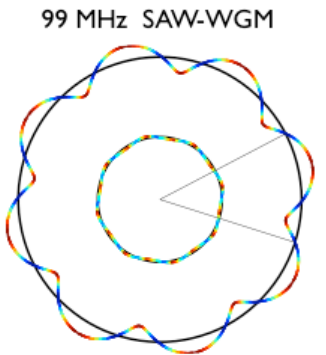

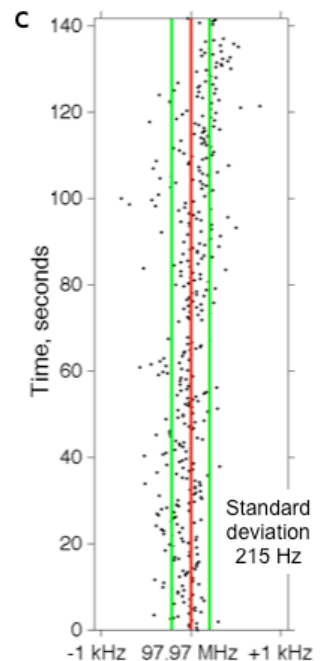

Figure 3. (a) The wineglass mode causes significant surface displacement of the inner wall of the capillary. (b) For the $99 \mathrm{MHz}$ mode, the ratio of inner surface deformation to outer surface deformation is approximately $16 \%$. (c) This oscillation exhibits good stability under constant input laser power.

\section{CONTACT}

Gaurav Bahl, bahlg@umich.edu

Tal Carmon, tcarmon@umich.edu

\section{REFERENCES}

[1] Burg, T.P., et al., "Weighing of biomolecules, single cells and single nanoparticles in fluid." Nature, 2007. 446(7139): p. 1066-1069.

[2] Vollmer, F and Arnold, S, "Whispering-gallery-mode biosensing: label-free detection down to single molecules," Nature Methods 5, 7 (2008).

[3] He, L., et al., "Detecting single viruses and nanoparticles using whispering gallery microlasers", Nature Nanotechnology, 2011. 6: p. 428-432.

[4] Eichenfield, M., et al, "A picogram- and nanometer-scale photonic-crystal optomechanical cavity," Nature 459, p. 550 (2009).

[5] Carmon, T., et al, "Temporal behavior of radiation-pressureinduced vibrations of an optical microcavity phonon mode" Physical Review Letters 94, 223902 (2005).

[6] Bahl, G., et al, "Stimulated optomechanical excitation of surface acoustic waves in a microdevice". Nature Communications 2, 403 (2011).

[7] Rokhsari, H., et al, "Radiation-pressure-driven micromechanical oscillator" Optics Express 13, 5293 (2005).

[8] Bahl, G., et al, "Observation of Spontaneous Brillouin Cooling" Nature Physics 8, 3 (2012) doi:10.1038/nphys2206.

[9] Povinelli, M. L. et al. "Evanescent-wave bonding between optical waveguides." Optics Letters 30, 3042-3044 (2005).

[10] Lee, W., et al, "A quasi-droplet optofluidic ring resonator laser using a micro-bubble.” Applied Physics Letters 99, 091102 (2011)

[11] John Zehnpfennig, et al., "Surface optomechanics: Calculating optically excited acoustical whispering gallery modes in microspheres." Optics Express, Vol. 19, pp. 14240-8 (2011). 


\title{
RECONFIGURABLE SIBAR FILTERS WITH SIDEWALL ALUMINUM NITRIDE SIGNAL TRANSDUCTION
}

\author{
R. Tabrizian ${ }^{*}$ and F. Ayazi
}

School of Electrical and Computer Engineering, Georgia Institute of Technology, Atlanta, Georgia, USA

\section{ABSTRACT}

This paper reports, for the first time, on a versatile low-loss filter technology that can cover a wide frequency range $(30 \mathrm{MHz}$ to $3 \mathrm{GHz}$ ) and has electronic bandwidth and frequency tunability. The reconfigurable MEMS filters implemented using this technology have lithographically-defined frequencies, and use high- $Q$, low-motional-resistance silicon bulk acoustic resonators (SiBARs) with sidewall aluminum nitride (AlN) signal transduction. A proof-of-concept one-stage ladder filter at $\sim 100$ $\mathrm{MHz}$ implemented using this technology shows an insertion loss (IL) of $\sim 5 \mathrm{~dB}$ (with $50 \Omega$ termination) and a tunable bandwidth ranging between 110-360 kHz. An array of two adjacent channelselect filters with different bandwidths is also demonstrated.

\section{INTRODUCTION}

The fast growth of multiband and multifunction wireless communication systems has resulted in an increasing demand for a single-chip reconfigurable filter array. A few filter technologies with different levels of reconfigurability have been demonstrated to fulfill this demand, such as integrated tunable LCs [1] and evanescent mode cavity filters [2]. In these technologies, which are typically large in size, filter reconfiguration is mainly addressed by tuning the center frequency of a wideband filter using voltage tunable capacitors. Since filters implemented using these technologies are limited in their maximum achievable $Q$, they cannot be utilized to implement direct narrowband channel selection at RF to obviate subsequent down-conversion to baseband. Bulk acoustic wave (BAW) filters have been proposed to realize narrow bandwidth channel-select filters at RF [3-6]. However, since the resonance frequency of thickness-mode BAW devices (such as FBAR and SMR) is determined by thin films and substrate thicknesses and hence is nearly constant across the wafer [3-5], array of channel-select filters with frequencies covering the entire RF band cannot be realized without multiple film depositions, which makes their fabrication excessively complex and expensive. Solutions based on lateral-extensional resonators have the advantage of lithographically-defined frequencies; however, their application has been severely limited so far due to the low efficiency of electrostatic [6] and transverse piezoelectric $\left(d_{31}\right.$-based) transduction [7], which results in large motional impedances not suitable for construction of low loss filters.

\section{CONCEPT}

A truly reconfigurable filter technology should offer center frequency, bandwidth, and order reconfiguration in a wide frequency range. This requires a low-impedance, high- $Q$ resonator technology that can cover a useful spectrum via tuning and/or arraying (e.g., $30 \mathrm{MHz}$ to $3 \mathrm{GHz}$ ) and can lend itself well to electrical coupling to create arbitrary filter responses. This has been difficult to achieve using acoustic resonators due to various issues related to physical size, impedance, $Q$ factor and tuning. In our earlier work, we had reported on sidewall AIN transduction of high- $Q$ silicon bulk acoustic resonators (SiBAR) with rectangular bar geometry [8]. These are one-port width-extensional resonators with lithographically-defined frequencies. By engineering the dimensions, especially the thickness of the bar structures, lateral BAW resonators with very low motional resistances can be realized, enabled by the very efficient longitudinal piezoelectric coefficient $\left(d_{33}\right)$ of an AIN layer covering the large sidewalls of the device. Furthermore, this transduction configuration results in inherently negligible charge cancellation at the transduction port, since the metallic electrodes sandwiching the AlN layer are extended over uni-stress surfaces with uniformly-excited charge distribution (Fig. 1(a)). These resonators can essentially be interpreted as lateral FBARs sandwiching a layer of silicon with lithographically-defined dimensions. Following this interpretation, these devices not only inherit AIN FBAR advantages, but also surpass them in several aspects: since the resonance frequency of AlN-on-sidewall SiBARs is mainly defined by the lateral dimensions of their central silicon structure, bandpass filters with any desirable frequency can be implemented on a common substrate, while a single deposition of a piezoelectric AlN layer suffices for providing efficient electromechanical transduction to all the resonators. Furthermore, since these resonators have two dimensions with frequency dependencies extended at the two extremes (i.e., small frequency dependency on the length as opposed to high frequency sensitivity to the width of the resonator), a large range of frequencies can be realized, comfortably extending from $30 \mathrm{MHz}$ to $3 \mathrm{GHz}$ to cover the entire VHF and UHF bands. Last, but not least, real-time bandwidth and frequency tuning can be realized by running small currents through the silicon body of individual resonators. In this paper, we use these resonators in ladder-type topology to implement reconfigurable narrowband filters with extremely sharp skirts and low pass-band insertion loss.

\section{RECONFIGURABLE BANDPASS FILTER DESIGN}

A one-stage ladder-type filter consist of two AlN-on-sidewall SiBARs with high $Q(\sim 7,800$ in air $)$ and low motional resistance, one in series and one in parallel with offset frequency. Figure 1((b) and (c)) schematically shows the filter construction as well as the principle of operation.
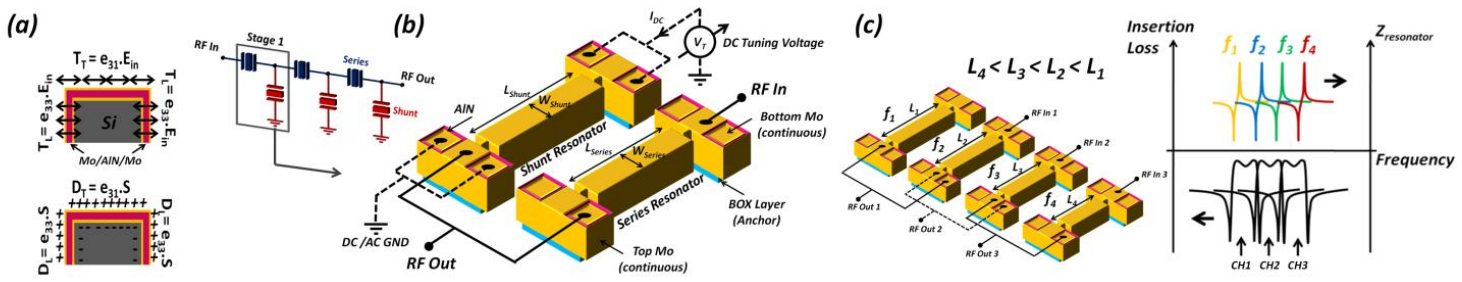

Figure 1: (a) The cross section of the resonator showing the actuation/sense mechanisms; (b) reconfigurable SiBAR filter with sidewall AlN transduction and thermal bandwidth and frequency tuning: one-stage ladder-type filter; (c) an array of adjacent channel-select ladder-type filters; each resonator serve as series and shunt element for two adjacent filters resulting in intrinsically-adjacent filter array. 
The small frequency offset and hence filter bandwidth is achieved by modifying the length of the SiBARs. Larger offsets can be obtained by adjusting the width of the resonator (primary frequency determining dimension). Furthermore, having a large negative TCF $\left(-32 \mathrm{ppm} /{ }^{\circ} \mathrm{C}\right)$, considerable frequency drift can be achieved by thermal tuning of each resonator of the filter, independently. This is done by passing a DC current via the body of the resonator resulting in the Joule heating. Large tuning ranges can be achieved using this technique by applying small DC voltages across the resonator.

\section{MEASUREMENT}

Figure 2 shows the SEM picture of the fabricated AlN-onsidewall SiBAR. The AC ground ports of this resonator can be used to apply tuning voltage across the resonator. Figure 3 shows the frequency response of two filters built using this technology as well as the configuration of the filters. Since the same resonator defines the pass-band transition of both filters, intrinsically adjacent bandpass channel-select filters can be implemented using this configuration. Figure 4 shows the thermal tuning characteristic of a single resonator. Large tuning ranges have been achieved with parabolic voltage dependency of resonance frequency. By applying this tuning technique to the shunt resonator of the ladder-type filter, significant bandwidth tuning is achieved. Figure 5 shows the frequency response of a one-stage ladder filter at $\sim 100 \mathrm{MHz}$ with IL $<5 \mathrm{~dB}$ (with $50 \Omega$ termination) and a tunable bandwidth ranged between 110-360 kHz. $227 \%$ of bandwidth tuning has been achieved with application of less than 2 volts.

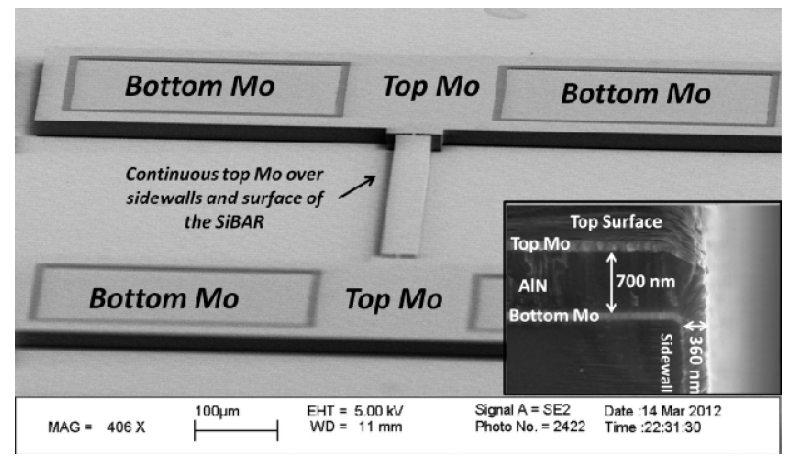

Figure 2: SEM picture of a SiBAR with sidewall AlN; the inset demonstrates the cross-section of these resonators showing the continuity of AlN and Mo films across the top surface and sidewalls of the SiBAR.

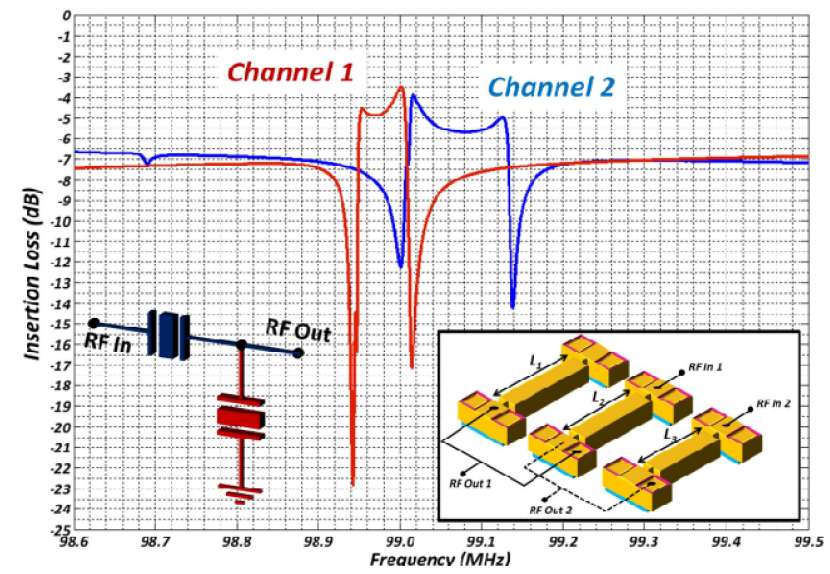

Figure 3: Frequency response of two adjacent filters; inset shows the configuration of these filters: the same resonator operates as the series and shunt resonator of channel 1 and 2 respectively.

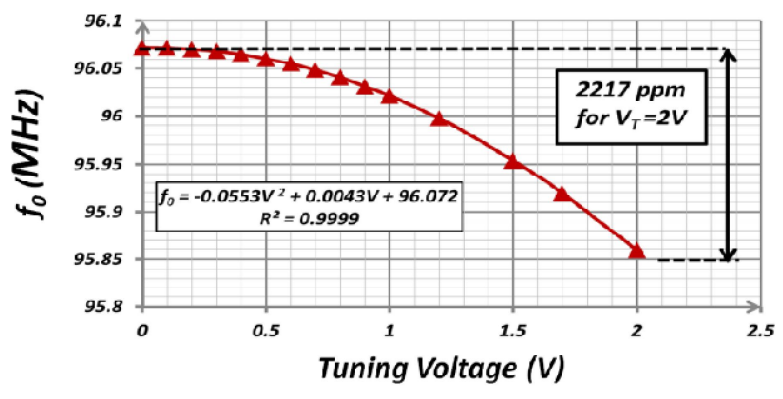

Figure 4: Frequency tuning characteristic of AlN-on-sidewall SiBAR showing large tuning achievable with small voltages and parabolic voltage dependency of the resonance frequency.

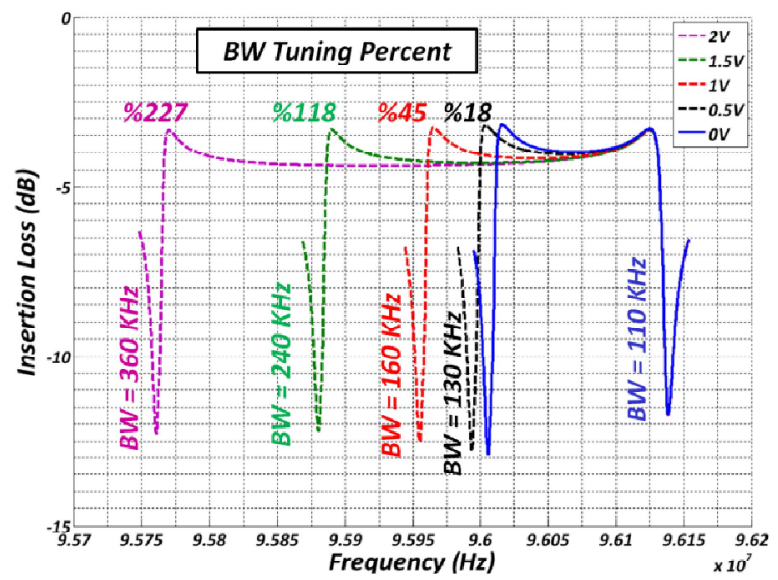

Figure 5: Bandwidth tunability for different voltages; the tuning will be done by passing current through the shunt resonator which results in its frequency decrease due to the SiBAR's negative TCF.

\section{ACKNOWLEDEMENT}

This work has been supported by National Science Foundation. Travel support has been generously provided by the Transducer Research Foundation.

\section{REFERENCES}

[1] M. Rais-Zadeh, et al., IEEE Journal of Microelectromechanical Systems, Volume 18, Issue 3, August 2009, pp. 942-949.

[2] H. Joshi, et al., IEEE MTT-S International Microwave Symposium Digest, June 2009, pp. 629-632.

[3] W. Pan and F. Ayazi, Tech. Digest 2008 IEEE International Frequency Control Symposium (IFCS 2008), Honolulu, HI, May 2008.

[4] K. M. Lakin, "Thin Film Resonators and High Frequency Filters," TFR Technologies, www.triquint.com.

[5] Lih Feng Cheow, et al., Solid State Sensor, Actuator and Microsystems Workshop (Hilton Head 2006), Hilton Head Island, South Carolina, June 4-8, 2006, pp. 304-307.

[6] S.-S. Li, et al., Int. Conf. on Solid-State Sensors, Actuators and Microsystems (Transducers), Lyon, France, June 2007. pp. 307-311.

[7] G. Piazza, et al., International Conference on Solid-State Sensors, Actuators and Microsystems (Transducers), Seoul, South Korea, June 2005. pp. 2065-2068.

[8] R. Tabrizian and F. Ayazi, Int. Conf. on Solid-State Sensors, Actuators and Microsystems (Transducers), Beijing, China, June 2011. pp. 1520-1523.

*R. Tabrizian, tel: +1-404-259-7322; roozbeh@gatech.edu 


\title{
THREE-DIMENSIONAL HIERARCHICAL MICROBATTERY ELECTRODES FOR TUNABLE HIGH ENERGY AND POWER DENSITY BATTERIES
}

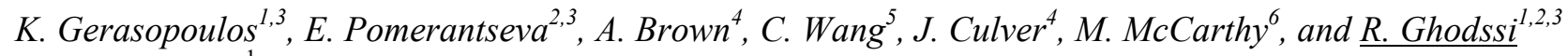 \\ ${ }^{1}$ MEMS Sensors and Actuators Laboratory, Institute for Systems Research; \\ ${ }^{2}$ Department of Electrical and Computer Engineering; \\ ${ }^{3}$ Department of Materials Science and Engineering; \\ ${ }^{4}$ Department of Plant Sciences and Landscape Architecture; \\ ${ }^{5}$ Department of Chemical and Biomolecular Engineering \\ University of Maryland, College Park, MD 20742, USA \\ ${ }^{6}$ Department of Mechanical Engineering and Mechanics, Drexel University, Philadelphia, PA 19104, USA
}

\begin{abstract}
We present a novel approach for the fabrication of hierarchical electrodes that integrates bottom-up nanostructure self-assembly and top-down microfabrication processes. The electrodes consist of virus-templated nanostructures self-assembled on three-dimensional micropillars. Active battery material $\left(\mathrm{V}_{2} \mathrm{O}_{5}\right)$ is conformally deposited using atomic layer deposition (ALD) on the hierarchical micro/nano network. Electrochemical testing of these electrodes indicates a 3 -fold increase in energy density compared to nanostructures alone, while maintaining the high power characteristics. Investigation of capacity scaling for varying active material thickness reveals underlying limitations and highlights the importance of our method in controlling both energy and power density with structural hierarchy.
\end{abstract}

\section{INTRODUCTION}

Three-dimensional micro/nano electrodes have been extensively researched as components for next-generation microbatteries [1]. Microstructures can increase material loading for the same device footprint, but lack in power density due to micrometer-size diffusion lengths. Nanostructures enable faster reaction kinetics but the electrode porosity reduces active material mass, thus limiting the available energy density. While the advantages of micro/nano structures have been independently demonstrated, no architecture integrating both components has been presented. In this work, the benefits of both length scales are combined for the first time, through the integration of virusstructured nanomaterials and 3-D micropillars. The experimental results show tunable energy density scaling (due to microstructures) and retention of high rate performance/power density (due to the nanostructures).

The key enabling technology in our approach is the use of the Tobacco mosaic virus (TMV) as a template for the synthesis of nanostructured materials. The TMV is a cylindrical plant virus which can be genetically modified to facilitate electroless metal coating and self-assembly onto various surfaces. The metallic thin film encasing the virus is uniform, highly conductive, and enables the rigidity of the resulting 3D nano-network. Previously we have demonstrated core/shell virus-templated Li-ion battery electrodes as well as photolithographic patterning and 3-D assembly of the particles [2-5]. These capabilities enable fabrication on substrates compatible with standard semiconductor processes, which is important factor in our approach. In this work, hierarchical electrodes are fabricated and their energy and power density are for the first time characterized.

\section{FABRICATION}

The electrodes were fabricated on silicon wafers with an evaporated gold seed layer. A three-spin process in AZ9260 photoresist was employed to form a $65 \mu \mathrm{m}$ thick polymer mold.
Gold pillars with $20 \mu \mathrm{m}$ diameter were electroplated from a commercial sulfite-based solution. Based on the dimensions in this design, a three-fold increase in area can be achieved. After electrodeposition, the photoresist was removed in acetone and the micropillars were coated with the metalized TMV template as described previously [2-5]. Finally, $\mathrm{V}_{2} \mathrm{O}_{5}$ layer was deposited using ALD at $170^{\circ} \mathrm{C}$. The electrodes fabrication process is schematically illustrated in Figure 1. Electrodes with and without micropillars were tested to investigate the capacity increase in the hierarchical design at various current densities and rates as well as different thicknesses of active materials.

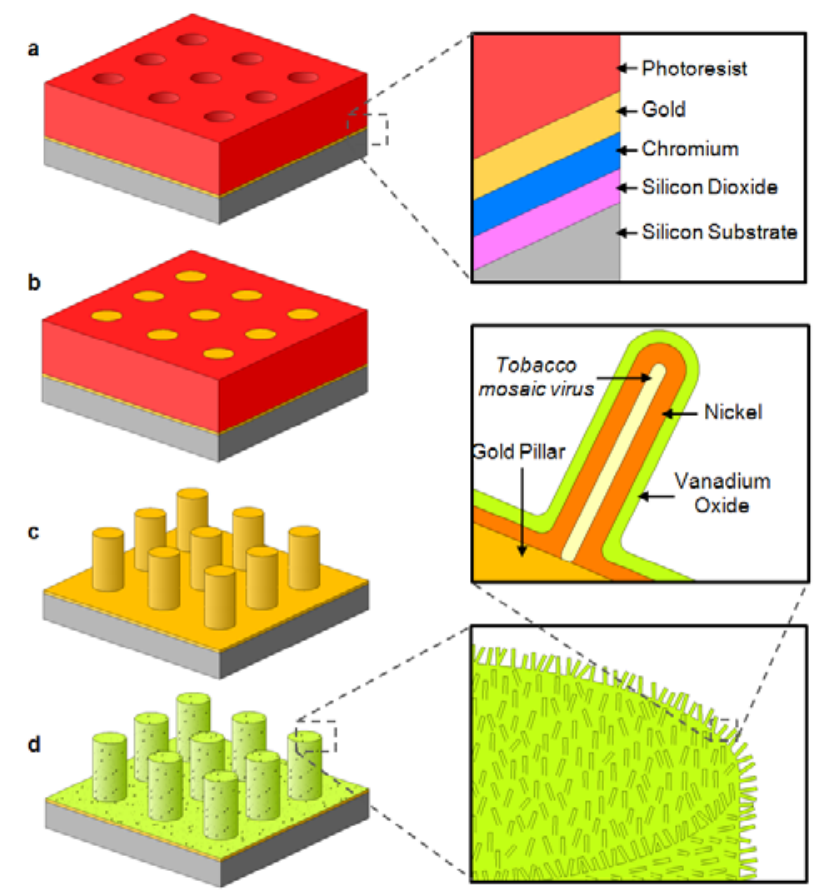

Figure 1: Schematic representation of the hierarchical electrode fabrication: (a) the photoresist mold is patterned on the silicon wafer (the inset shows the full layer structures); (b) gold is electroplated through the mold; (c) the mold is dissolved in an acetone bath; (d) TMV self-assembles onto the chip surface and then coated with $\mathrm{Ni}$ and $\mathrm{V}_{2} \mathrm{O}_{5}$; insets show the textured micro/nano structure of the electrode and an individual viral nanorod.

\section{RESULTS}

Figure 2 shows SEM and TEM images of the hierarchical electrodes with very uniform TMV coating of gold micropillars. The highly porous 3D nanonetwork is formed by virus particles (Fig. 2c). Figure 2d shows a cross-section TEM image taken from the sidewall of the pillar demonstrating that the $\mathrm{V}_{2} \mathrm{O}_{5}$ coating is 
uniform regardless of the orientation of the particle. This is a significant attribute of ALD, which is ideally suited to create uniform, conformal coatings of complex 3D geometries with precise control over film thickness.

Figure 3a shows discharge/charge curves for electrodes with and without micropillars. The thickness of $\mathrm{V}_{2} \mathrm{O}_{5}$ in these experiments was $30 \mathrm{~nm}$ (1000 ALD cycles). The capacity for the hierarchical electrode is $38 \mu \mathrm{Ah} \mathrm{cm}^{-2}$, while the nanostructured only electrode exhibits a capacity of $13 \mu \mathrm{Ah} \mathrm{cm}^{-2}$. This is in excellent agreement with the increase in mass and surface area. As indicated in Figure 3b, where the capacity versus cycle number for the first 35 cycles of operation is plotted. This 3-fold increase in energy density is demonstrated throughout the course of testing without batteries failure.

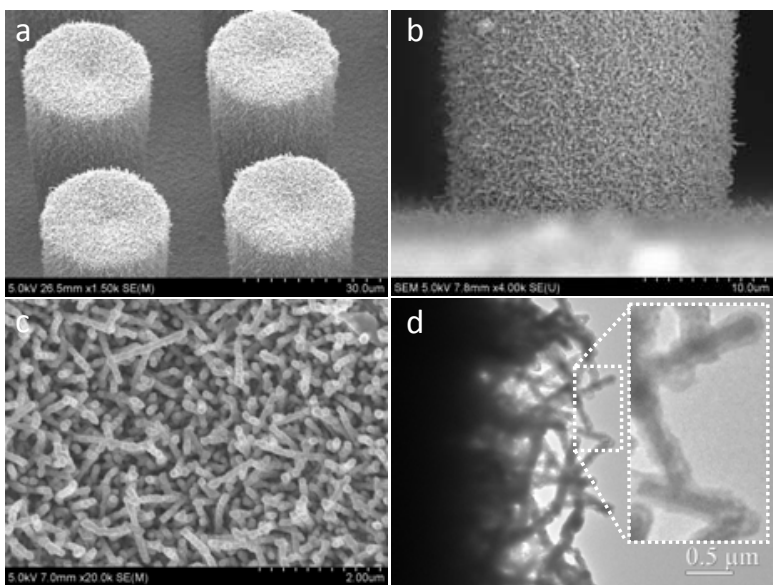

Figure 2: SEM and TEM images of the hierarchical electrodes: (ab) micropillars coated with the $\mathrm{TMV} / \mathrm{Ni} / \mathrm{V}_{2} \mathrm{O}_{5}$ layers $\left(\mathrm{V}_{2} \mathrm{O}_{5}\right.$ was deposited for 1000 ALD cycles); (c) close-up image showing the top of micropillar; (d) the sidewall of a micropillar, showing uniform $\mathrm{V}_{2} \mathrm{O}_{5}$ coverage regardless TMV particles orientation.
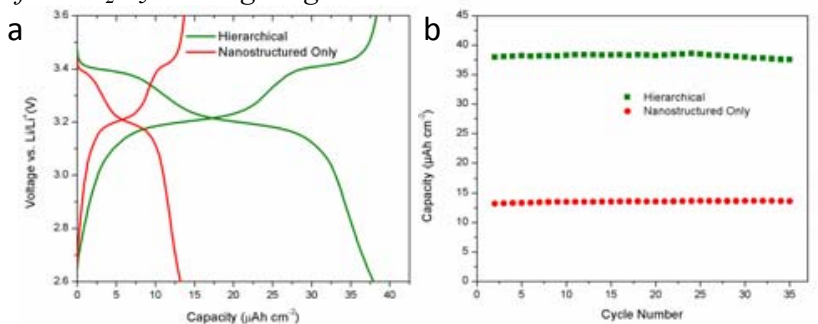

Figure 3: Electrochemical characterization of virus-structured electrodes with and without micropillars (a) galvanostatic charge/discharge curves at a current of $10 \mu \mathrm{A}$ (electrode footprint area was $1.1 \mathrm{~cm}^{2}$ ); (b) capacity vs. cycle number for the samples used in a for the first 35 cycles of operation.
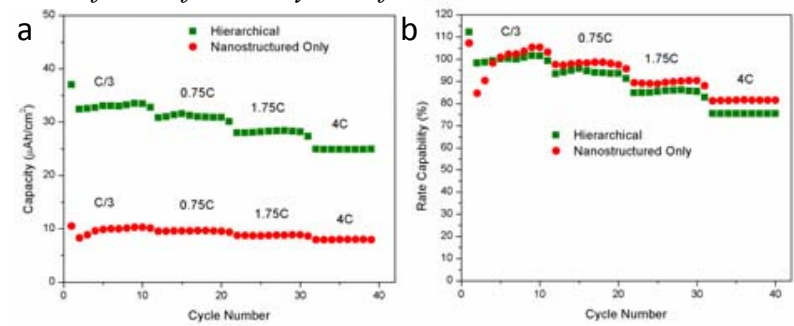

Figure 4: Rate capability data at different $C$-rates for hierarchical and nanostructured only electrodes: (a) capacity vs. cycle number; (b) capacity retention vs. cycle number (the data is presented as the ratio of $C_{i} / C_{\text {inav }}$, where $C_{i}$ is the capacity for each cycle, and $C_{\text {inav }}$ is the average initial capacity for the lower C-rate).
The rate performance results are shown in Figure 4. In these tests, electrodes were cycled in increments of 10 cycles for progressively increasing current values. The capacity remains 3 times higher for the hierarchical electrodes for all C-rates (Fig. 4a), while both types of electrodes demonstrate similar rate capabilities (Fig. 4b). This result indicates that the addition of micropillars increases the energy density of the electrodes without affecting the high rate performance. The morphology and discharge/charge curves for hierarchical electrodes with different $\mathrm{V}_{2} \mathrm{O}_{5}$ thicknesses are shown in Figure 5. Interestingly, the capacity increase exhibits a non-linearity as the thickness of the active material increases from $\sim 60 \mathrm{~nm}$ to $\sim 120 \mathrm{~nm}$ (Fig. $5 \mathrm{~d}$ ). This behavior is caused by progressive blocking of open spaces between closely-packed adjacent TMV nanorods with increasing deposition thickness (Fig. 5e). This identifies inherent limitations in increasing nanostructure thickness and highlights the importance of our technology for independently controlling energy and power density.
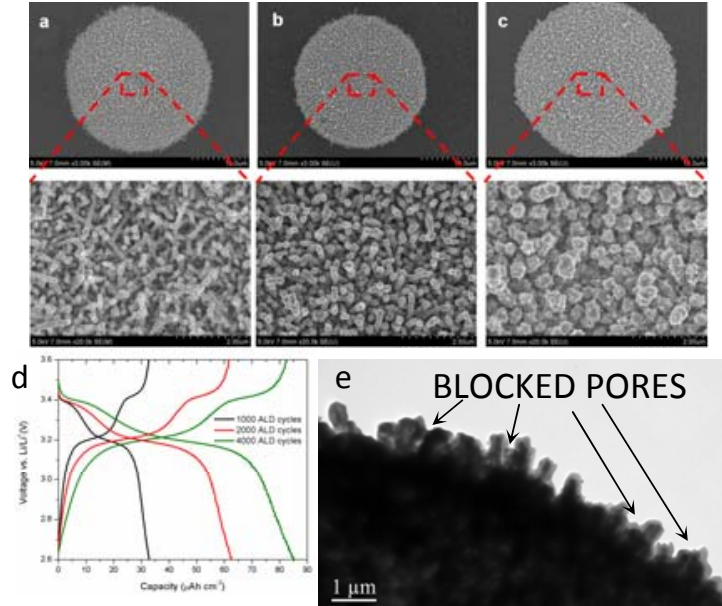

Figure 5: Hierarchical electrode morphology and electrochemical performance for different $\mathrm{V}_{2} \mathrm{O}_{5}$ thickness: (a-c) SEM images showing the top of micropillars for 1000, 2000 and 4000 ALD cycles of $\mathrm{V}_{2} \mathrm{O}_{5}$, respectively; (d) galvanostatic discharge/charge curves for hierarchical electrodes at a current of $12 \mu \mathrm{A} ; V_{2} \mathrm{O}_{5}$ was deposited for 1000, 2000, and 4000 ALD cycles, aiming at thicknesses of $30 \mathrm{~nm}, 60 \mathrm{~nm}$, and $120 \mathrm{~nm}$, respectively; (e) cross section TEM image taken from the sidewall of micropillar for the sample with $4000 \mathrm{ALD}$ cycles of $\mathrm{V}_{2} \mathrm{O}_{5}$.

\section{REFERENCES}

[1] T.S. Arthur, D.J. Bates, N. Cirigliano, D.C. Johnson, P. Malati, J.M. Mosby, E. Perre, M.T. Rawls, A.L. Prieto, B. Dunn, "Three-dimensional electrodes and battery architectures", MRS Bulletin, 36, 523 (2011).

[2] K. Gerasopoulos, M. McCarthy, P. Banerjee, X. Fan, J.N. Culver, and R. Ghodssi, "Biofabrication methods for the patterned assembly and synthesis of viral nanotemplates", Nanotechnology 21, 055304 (2010).

[3] K. Gerasopoulos, X. Chen, J. Culver, C. Wang, and R. Ghodssi, "Self-assembled $\mathrm{Ni} / \mathrm{TiO}_{2}$ nanocomposite anodes synthesized via electroless plating and atomic layer deposition on biological scaffolds", Chem. Comm. 46, 7349 (2010).

[4] E. Pomerantseva, K. Gerasopoulos, X. Chen, G. Rubloff, and R. Ghodssi, "Electrochemical performance of the nanostructured biotemplated $\mathrm{V}_{2} \mathrm{O}_{5}$ cathode for lithium-ion batteries", J. Power Sources 206, 282 (2012).

\section{CONTACT}

Professor Reza Ghodssi: Tel.: +1 301405 8158; E-mail: ghodssi@umd.edu 


\section{HOW WIRELESS HEALTH WILL CHANGE HEALTH CARE DELIVERY M.J. Zdeblick, PhD. ${ }^{{ }^{*}}$ \\ ${ }^{1}$ Proteus Biomedical, Inc., Redwood City, California, USA}

\begin{abstract}
The miniature supercomputer with high resolution screen we carry in our pocket to make phone calls will help bring modern health care to all corners of the world. Instead of building expensive hospitals throughout the developing world, low-cost, high-volume devices wirelessly networked together will help deliver health care to the billions of people who have none today. This application space is rich in opportunities for those who wish to explore new ways of caring for people who today receive little if any medical services. Examples will illustrate how wireless technology and resultant enabled systems will change the way we educate, diagnose, and treat patients throughout the world.
\end{abstract}

\section{INTRODUCTION}

"So many people use apps every day to keep up with their friends, with the news, find out when the next bus will turn up or which train to catch. I want to make using apps to track blood pressure, to find the nearest source of support when you need it and to get practical help in staying healthy the norm"

U.K. Health Secretary Andrew Lansley in Marie Claire magazine.

A typical hospital - complete with power plant, parking, and all expected amenities - in an urban United States (US) location costs approximately US $\$ 1 \mathrm{~B}$ to build [1]. While this is an affordable investment for a large city in the US, the sum exceeds the 2010 GDP of 22 countries, represents at least $10 \%$ of the GDP of 80 countries, and at least $1 \%$ of the GDP of 134 countries [2]. Even one such hospital represents an unaffordable luxury for the majority of countries worldwide. The US model of health care delivery is simply not scalable to much of the rest of the world.

For many regions, such a hospital-centric model fails due to the lack of infrastructure needed by patients to travel to these centers [3]. As a result, billions on this planet receive little or no health care. In some cases, a health care delivery platform based on cellular technologies competes with nothing but local tribal knowledge. Technology that brings health care to people where they live creates opportunity at many levels, from the individual, through the community, up to an entire country.

At the other end of the economic spectrum, Western countries are bankrupting themselves by delivering health care using models designed when there were few medicines, no sophisticated medical devices, and most people died before they turned 60 years old. Indeed, Western hospitals provide a level of care absolutely unimaginable only a century ago. Advanced surgical tools and procedures, combined with mature imaging modalities including Magnetic Resonance Imaging (MRI), Computerized Tomography (CT), and Positron Emission Tomography (PET) offer to a patient in critical need of care the very highest chances of restoring health.

On the other hand, an increasing portion of Western health care budgets are dedicated to treating the consequences of "affluenza," such as obesity, diabetes, stroke, and heart failure. These diseases persist for years, and the pathways lead to very serious - and expensive - complications. Based on data from the Center for Medicare and Medicaid Studies, it has been estimated that $76 \%$ of Medicare spending is on patients with five or more chronic diseases [4]. Unless treated in a nursing home, most patients or their caregivers treat themselves between visits to the physician. It is here where there is an opportunity to improve health, provide quality care and reduce overall health care costs. For example, a diabetic who changes diet, regularly exercises and takes medicines as prescribed, is more likely to avoid dependency on insulin, become healthier and happier, and consume fewer health care dollars. By providing feedback to patients and their caregivers between physician visits, mobile technology can make an important contribution to Western medicine.

Engineers have been developing sensors to provide such feedback for many years. Blood glucose sensors for diabetes are an essential element in the treatment of that disease. Could other sensor systems make similar contributions to other diseases? Sensing and communication systems based on mobile technology may fundamentally change how individuals interact with the collective knowledge and skills of the modern world when confronted with a medical challenge.

Mobile (wireless) technologies may enable many new models for delivering health care, both in the developed and developing communities around the world. This article discusses how mobile technology will affect three of the most important aspects of health care. The first is education, as sophisticated medical equipment is useless in poorly trained hands and minds. The second is measurement systems, as modern health care often relies upon technology to manage disease. The third is effective and secure use of medicines, as diagnosis is ineffective without valid medicines that are used as prescribed. Furthermore, this article will provide existing and future examples of the use of mobile technology for each of these three aspects or health care, and how they might be used to integrate the developed and developing worlds.

\section{EDUCATION}

"Knowledge, therefore, becomes more expensive per unit over time, and the number of units increases exponentially."

\section{"Max Planck pointed out that each unit of new knowledge costs more than the last, because the easier answers come first and give us new techniques to apply to the next" \\ Donald Kennedy, former President of Stanford University}

\section{The Western University Model}

Since 1088, when the University of Bologna began a long tradition of education at Western-style Universities, the education system has been comprised of three fundamental elements: teachers who teach, students who learn, and buildings that house them. Of course, there were centers of learning in Ancient Greece, China, Persia, and Pakistan that came earlier in time, but they were organized differently. A European University was comprised of a community of teachers and taught; was granted administrative autonomy from their rulers; evolved curricula; set and reached research objectives; and, perhaps most importantly, granted credentials to those deemed worthy. These centers of learning attracted students and teachers from great distances to a single location. This concentration of people facilitated both the acquisition of "each unit of new knowledge" and the distribution of already-obtained knowledge to the next generation. The "Western University Model" proved to be both productive and effective, and rapidly spread throughout $\mathrm{W}$. Europe in the $11^{\text {th }}$ and $12^{\text {th }}$ Century, E. Europe in the $14^{\text {th }}$ and $15^{\text {th }} \mathrm{C}$, the Americas starting in the $16^{\text {th }}$ $\mathrm{C}$, Australia since the $19^{\text {th }} \mathrm{C}$, and Africa and Asia since the $19^{\text {th }} \&$ $20^{\text {th }} \mathrm{C}$. 
A strong correlation exists between the date of the first arrival of Western-style Universities and economic progress. While this is not the sole determinant of economic or other success, according to Niall Ferguson [5], the Western Education model is one of the 8 "killer apps" that lead to the success of the "West over the Rest" over the last 500 years. Notably, in Zimbabwe, one of the poorest parts of Africa, the first University was only established in 1952.

So, while the Western Model of Education has served the West and now the Rest well in the past, will it evolve and adapt to the needs and conditions of the modern societies, or will it be replaced by a disruptive solution based on technology? Is the University of Bologna model still the most efficient way of teaching the next generations of students? If one separates the Research function of a University from the Distribution of Knowledge role, one sees that mobile communication systems may replace - or perhaps complement - the campus of buildings. At what point do the taught teach themselves?

\section{African health care by the numbers}

The challenge of providing modern health care in Africa is immediately evident upon review of some simple statistics [6][7][8]. While the continent of Africa and its fifty-four countries has the same number of medical schools as the United States, Africa is three times larger in terms of surface area and population. More importantly, GDP per person in Africa $(\$ 1,600)$ is less than a tenth of the poverty rate in the US $(\$ 22,350)$.

\begin{tabular}{lccc} 
& United States & & Africa \\
\cline { 2 - 2 } Size & $3.79 \mathrm{M} \mathrm{miles} \mathrm{m}^{2}$ & & $11.7 \mathrm{M} \mathrm{miles}{ }^{2}$ \\
Population & $312 \mathrm{M}$ & & $1000 \mathrm{M}$ \\
Per capita GDP & $\$ 47,000$ & & $\$ 1,600$ \\
\# of medical schools & 134 & 137 \\
Doctors / 1000 & 2.3 & 0.27
\end{tabular}

Table 1: Africa continent of 54 countries compared to US.

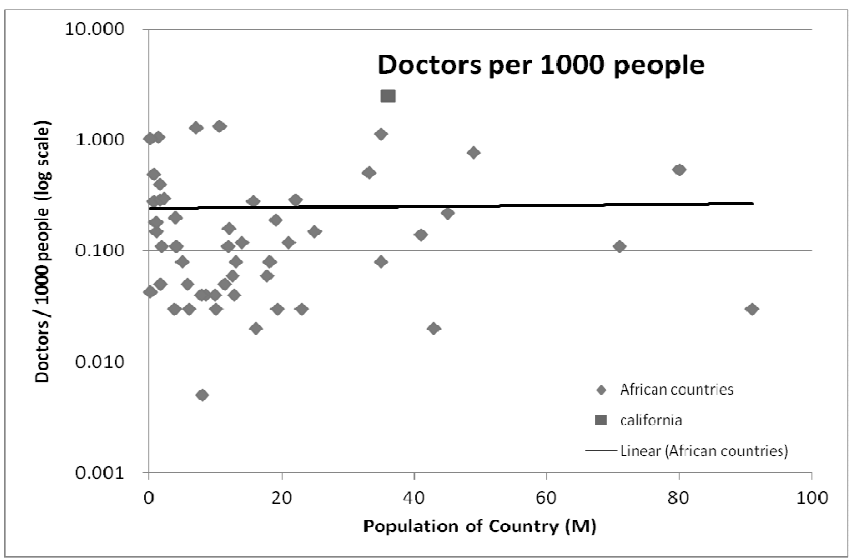

Figure 1: Doctors per 1000 people for various African countries.

Many African medical school (and other college) graduates migrate to wealthier countries around the world, leaving a doctor / patient ratio only a tenth that of the US. Think of this as an engineering problem: "How can technology increase the effective number of doctors in Africa by a factor of ten, in less than ten years?"

One approach is to use mobile health to train a much larger number of local health care providers than are currently being educated at the existing African medical schools. Another would be to find a way to encourage those alumni to stay in Africa. A third avenue would be to create a system where doctors in other parts of the world can have a virtual presence in even the most remote parts of Africa. Mobile Health can play a significant role in all three of these non-exclusive threads.

\section{Topol-Khan Academy}

Recently, Eric Topol MD, mused that a medical version of the Khan Academy could be used to help train medical care providers [9]. The Khan Academy (www.khanacademy.org) is a structured on-line education system comprised of over 3000 video lectures covering topics ranging "from arithmetic to physics, finance, and history and 315 practice exercises." The system has served individuals interested in learning specific skills as well as assisting classrooms achieve their academic goals while allowing each student to advance at an individual pace. For this latter case, higher level summaries of student performance help teachers focus their class time on specific topics that are giving individuals particular difficulty. The Khan Academy is currently being used on comparative basis in a Los Altos, CA, school district to measure its relative performance with the pre-existing teaching methods.

Could a medical version of the Khan Academy be used to train medical practitioners in the developing world using wireless systems that convert a village hut into a medical school? While this might seem far-fetched, an on-line search reveals this telling note posted on The Student Doctor Network:

\section{"Khan Academy \\ So $i$ noticed that these are great videos for physics chem and bio...does anybody know of any good sites for OCHEM.... i have to take this mcat June 17... and I just want to clarify a couple of things in orgo..."}

In fact, medical students planning to take the MCAT's are already using the Khan Academy. What is to stop such a network from teaching an entire generation of medical students in, for example, Africa? Skeptics might argue that while the Khan Academy approach could teach Organic Chemistry, it couldn't possibly teach surgery, or other skills of the profession that are best learned as an apprentice. This may be true for many branches of medicine - for now. On the other hand, could something like Topol-Khan significantly increase the effectiveness of existing medical school programs in Africa? Could it teach pharmacists how to diagnose malaria or worms and sell a prescription? Could one be taught how to use the mobile technologies described herein and elsewhere to provide health care where there is none?

Mobile phones and tablets are being adopted in even the most remote corners of the world. The distribution of knowledge is extremely valuable to everyone, closely following food and water in the pyramid of fundamental needs and wants. The increasing affordability of cell phones and tablets connected to the internet has enabled these technologies to become one of the most popular means of distributing knowledge. Clearly, something like a TopolKhan Academy could make an important complement to any mobile sensing instrument by giving local people the knowledge and skills to use such tools effectively.

\section{International Medical Schools}

The US graduates about 20,000 doctors per year. If all of these doctors left the US and went to Africa, in one-hundred years Africa would have the same number of doctors per 1000 people as the US today. Of course, relatively few American-trained doctors practice medicine in Africa; a much higher percentage of Africantrained doctors make the opposite journey. One way of retaining and attracting additional people to Africa is to provide 
complementary sources of income for trained people residing in Africa. One way of doing this would be for trained staff in Africa to perform and get paid for some of the routine analyses needed in high-cost regions of the world.

Training African doctors to analyze the diseases of Western patients may seem counter-intuitive - even nonsensical, as many people default to the opposite mental model of how the West will help Africa: have local, untrained, unskilled people take pictures of skin rashes or collect information of the symptoms of disease, and send them through e-mail or text messages to a trained professional in America, who will diagnose the underlying condition. While such model would certainly attract a number of highly altruistic doctors in the West, it would be hardly sustainable: who would reimburse our doctors to perform such services for poor people in Africa? Even wealthy Americans can hardly afford the services of their own physicians, let alone patients in the developing world. Instead, more practical and scalable might be the model where an African doctor with one electronic eye and ear in America and both feet in Africa, multiplexes between diagnosing and treating local people while earning a reasonable fee for performing analytical services for high-cost Western hospitals. Even if the number of remote analyses an African doctor made per week is very low, being part of a Western medical system would bring tremendous advantages to that doctor's local practice: knowledge, prestige, and income. Today, it is routine for many American hospitals to send radiographs to qualified Radiologists in India for reading and interpretation. Could South Africa become the site where such a model goes viral in Africa?

The cost of an iPad is about $\$ 500$ - about one-third of the per capita GDP of Africa. In the right hands and as a component of a formal training process, it could become a critical tool in educating a continent full of doctors. Yet by itself it would be inadequate: the iPad cannot teach surgery or anesthesiology, or can it?

Perhaps a hybrid model of medical school education and practice may arise in the developing world. Those disciplines that can be taught and practiced using mobile technology will blossom, while centers with "big iron" capital equipment will be required for those systems that cannot be "mobilized."

A hybrid solution such as this provides a "St. James Bible" opportunity for those in the business of educating the next generation of medical students: the teaching materials created over the next few years may be used to educate millions of doctors who treat billions of patients. Similarly, medical devices developed as accessories and add-ons to a cell phone or tablet could both enable this process and service entire continents of people.

Imagine a consortium of the best medical schools each "adopting" an African medical school. Each paired with a bioengineering department that designs mobile medical systems defined through these interactions. If such noble efforts were coordinated and became part of formal training programs based on a Topol-Khan Academy model, could they produce - within one person's lifetime - a cadre of self-sustainable doctors in Africa capable of providing care to all of its citizens?

\section{MOBILE MEASUREMENT SYSTEMS}

"Engineers who are great in physics and calculus but can't think in new ways about old objects are doomed to think in old ways about new objects"

John Ratzenburger, paraphrasing a Stanford study

In this section, we will look at some examples of how various organizations are beginning to use wireless technology to deliver health care outside of the clinic or hospital. With thousands of health-related apps available on smart phones, where does one start? A cursory review finds many examples of information tools, but fewer, though much more interesting, apps that make the smart phone an essential element of a medical device. A sampling follows of mobile health innovations that promise improvement in quality, accessibility, and affordability of care in an era of increasing need and increasingly constrained resources. Mobile health care technology is just beginning to become a common adjunct to diagnosis, therapy, and management of care.

\section{mHealth Alliance}

The mHealth Alliance was launched by the UN Foundation, the Rockefeller Foundation, and the Vodaphone Foundation in 2009 to promote the use of wireless technologies in the delivery of health care. Each year, mHealth Alliance sponsors an Innovator's Challenge. The 2011 winners represent their sampling of who is innovating in this space (from www.mHealthAlliance.org):

Mobile system that facilitates pregnancy surveillance, registration and emergency response (Alain Labrique for mCare)

Mobile technology that reduces child mortality (Ann RoosWeil for Pesinet; Mali).

A personalized voice-based information retrieval and transaction system (Ashok Jhunjhunwala for Voice Net; India).

Field-portable microscope and diagnostics tools (Aydogan Ozcan; Africa, South America, and South Asia).

Instant drug quality verification system via text messaging (Bright Simmons for mPedigree; Africa and South Asia).

A localized multimedia (Derek Treatman for CommCare Sense; India).

Doctor directories and bulk SMS system (Eric Woods for MDNet; Ghana and Liberia).

SIM card applications the least expensive phones (Isaac Holeman; Malawi and Cameroon).

Automated phone calls and SIM message system ( Joaquin Blaya for MiDoctor). India).

SMS-based mother and child tracking system (K.S. Shankar ;

Clinical decision-support system (Martin Were for AMPATH; Kenya).

\section{Medic Mobile}

MedicMobile creates technology that converts $\$ 15$ cell phones into extensions of existing open-source platforms, including FrontlineSMS, OpenMRS, Ushahidi, Google Apps, and HealthMap. These SIM apps enable a broad range of sophisticated tools to manage a wide range of health care delivery. MedicMobile.org lists: "community health worker coordination and management, community mobilization for vaccination and satellite clinics, logistics and supply chain management, referrals, routine data collection, and mapping of health services."

A six-month pilot program in Malawi saved the clinical staff 1,200 hours and over $\$ 3,000$ in motorbike fuel. 100 patients received treatment for tuberculosis after their symptoms were communicated using text messaging.

In the developing world, lack of infrastructure prevents health workers from delivering efficient healthcare to rural areas. Networking together very inexpensive phones using easy-to-use, meaningful software is establishing mobile technologies as the platform upon which other services will be built.

\section{Sense A/S blood pressure measuring patch}

The Danish company Sense A/S develops ContiPress ${ }^{\mathrm{TM}}$, an adhesive patch worn on the arm that continuously measures the patient's blood pressure. The ContiPress ${ }^{\mathrm{TM}}$ patch includes electrodes, electronics, and a wireless communication system. The 
unit monitors and stores information about the patient's blood pressure over 24 hours, which can be used to aid in the diagnosis of related conditions.
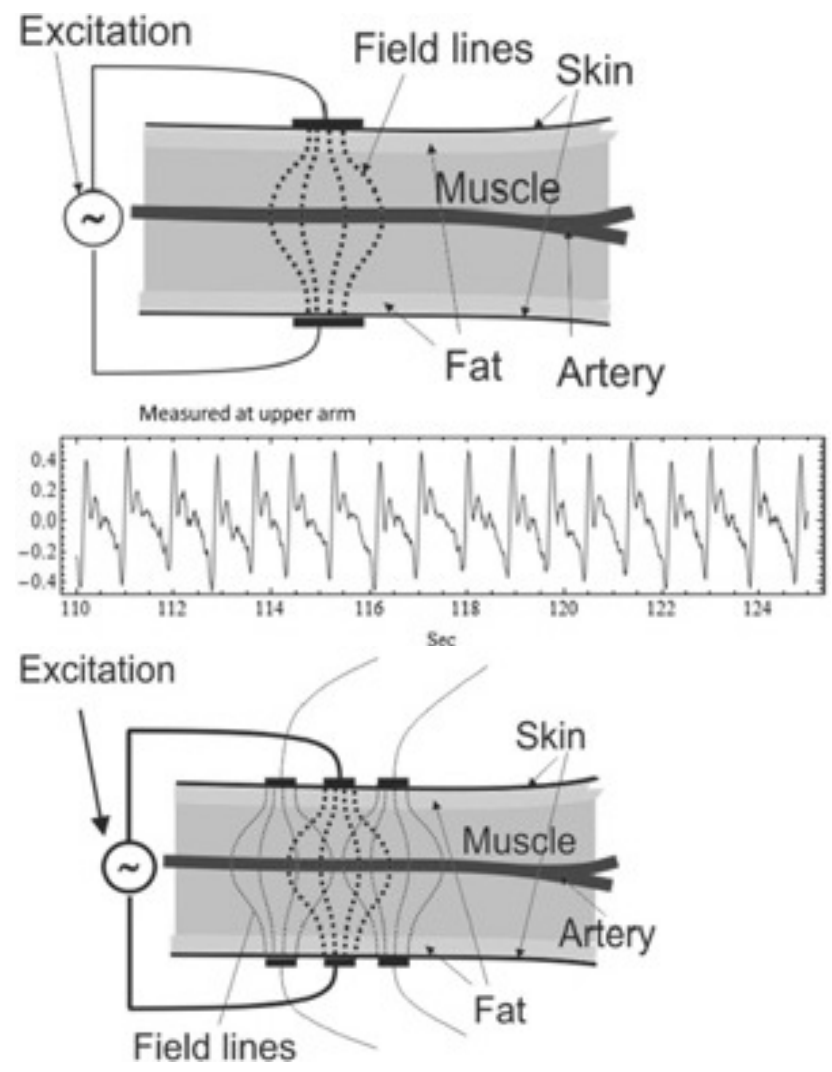

Figure 2: Estimating Blood pressure by measuring impedance.

\section{Head Ballistocardiogram and Head ECG}

Under the direction of Prof. Sodini, researchers at MIT have been developing portable sensors that measure both the ECG and Ballistocardiogram. Such novel metrics delivered in a wearable form-factor may provide new approaches to managing chronic disease [10].

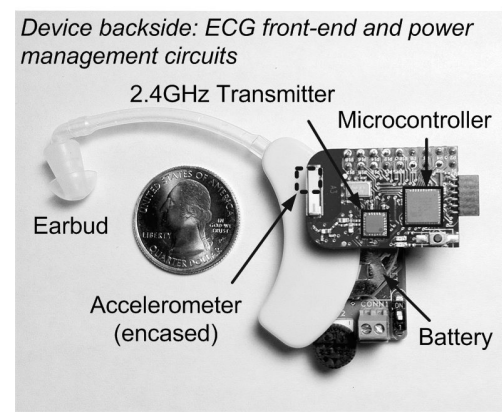

Wearable Heart Monitor

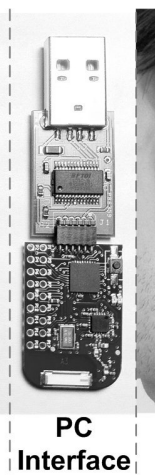

Interface
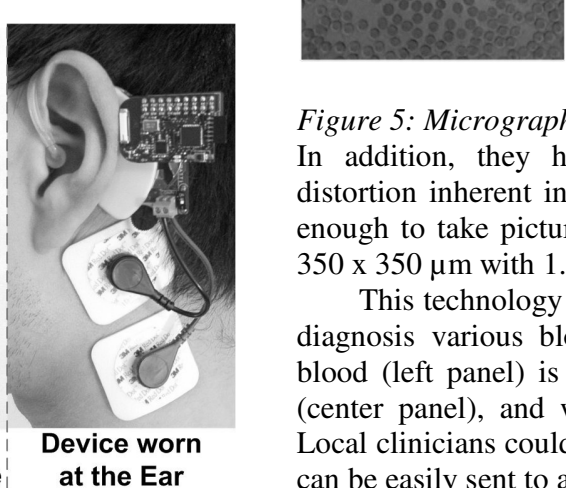

Figure 3: Head-mounted ECG / BCG system

Low-cost, cell-phone powered stethoscope ideal for remote care

Originally intended as a way of diagnosing tuberculous pericarditis, this mobile stethoscope kit created by Oxford University and University of Cape Town allows a remote physician access to the acoustic information detectable by a stethoscope using a low-cost cell phone [11].

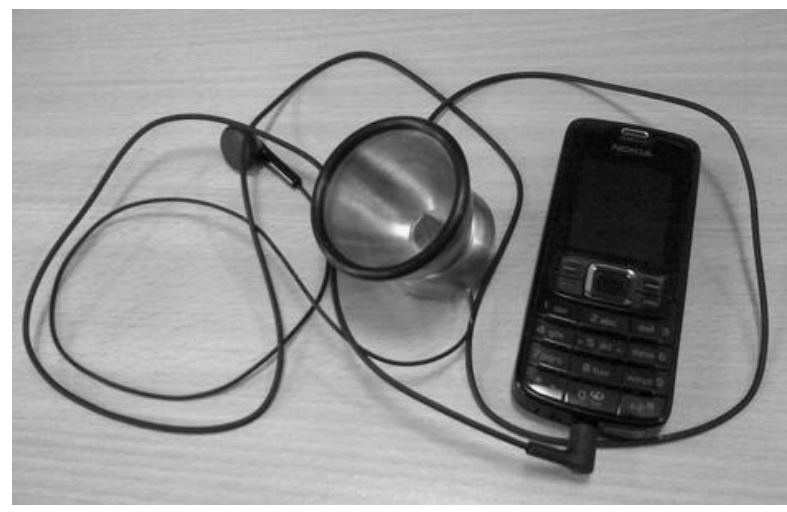

Figure 4: Recordable stethoscope networked with low-cost phone

The device could be used not only in the direct diagnosis of diseases, but also as a training tool. In-training clinicians could diagnose with a traditional stethoscope and use this device to confirm; recordings could be used as part of a Topol-Khan Academy program to rapidly teach the proper use of a stethoscope.

\section{Smart phone microscope}

Researchers at the University of California at Davis have demonstrated a low-cost add-on to a common smart phone that converts the camera into a microscope [12]. This is accomplished through the wise placement of either a $1 \mathrm{~mm}$ diameter sapphire ball lens or a GRIN lens placed over the imaging lens of the phone.
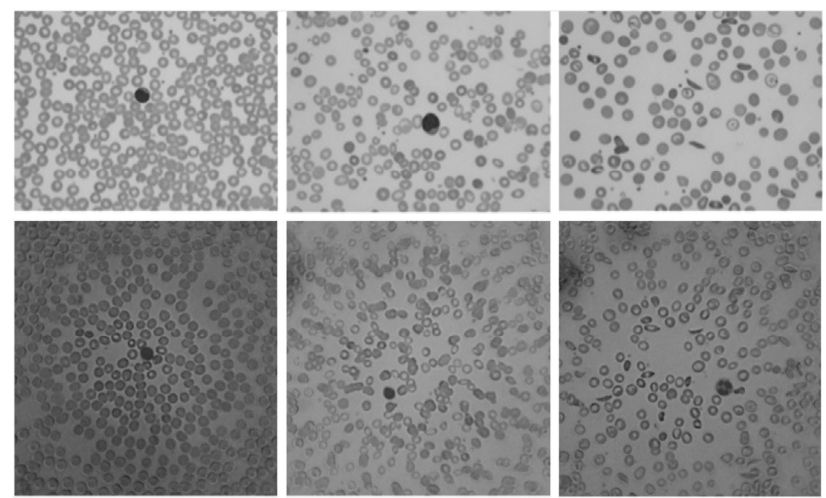

Figure 5: Micrographs of blood smears taken with smartphone In addition, they have developed software that corrects the distortion inherent in this optical system to provide images good enough to take pictures of with a field of view of approximately $350 \times 350 \mu \mathrm{m}$ with $1.5 \mu \mathrm{m}$ resolution.

This technology could be rapidly distributed in rural places to diagnosis various blood diseases. In the above images, normal blood (left panel) is compared with iron deficient anemic blood (center panel), and with sickle cell anemic blood (right panel). Local clinicians could be trained to interpret these images and they can be easily sent to a medical facility for confirmation.

\section{Portable ECG using cell phone}

A number of organizations are developing or introducing cellphone-based devices that use dry electrodes on the phone or its sleeve to detect and record an ECG. Examples include the EPI Life by Ephone International. 


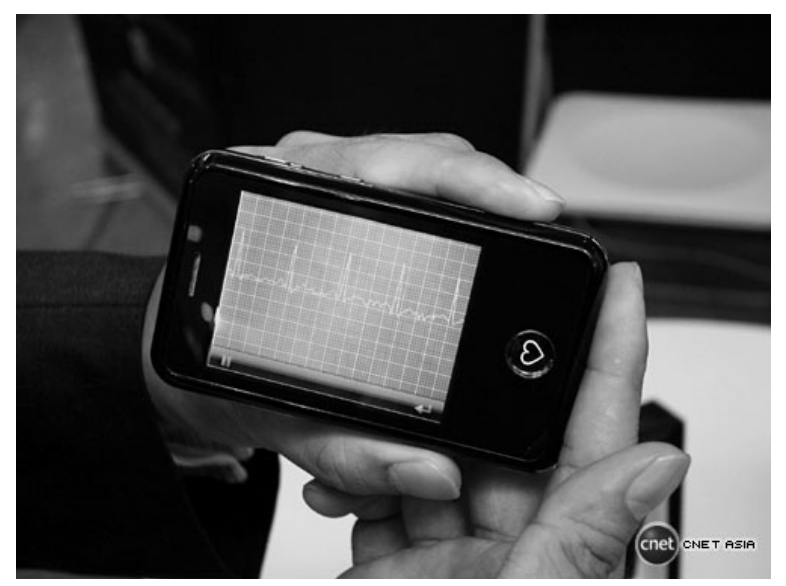

Figure 6: ECG using the EPI Life cell phone.

\section{Portable Ultrasound using cell phone}

GE and Mobisante both recently announced portable, lowcost ultrasound devices that may replace the stethoscope in many clinical settings. One barrier in the West is reimbursement: would each use of a $\$ 5,000$ device deserve the same fee derived from much more expensive systems? In Africa, this lower cost and portability could lead to Médecins Sans Hôpitals.

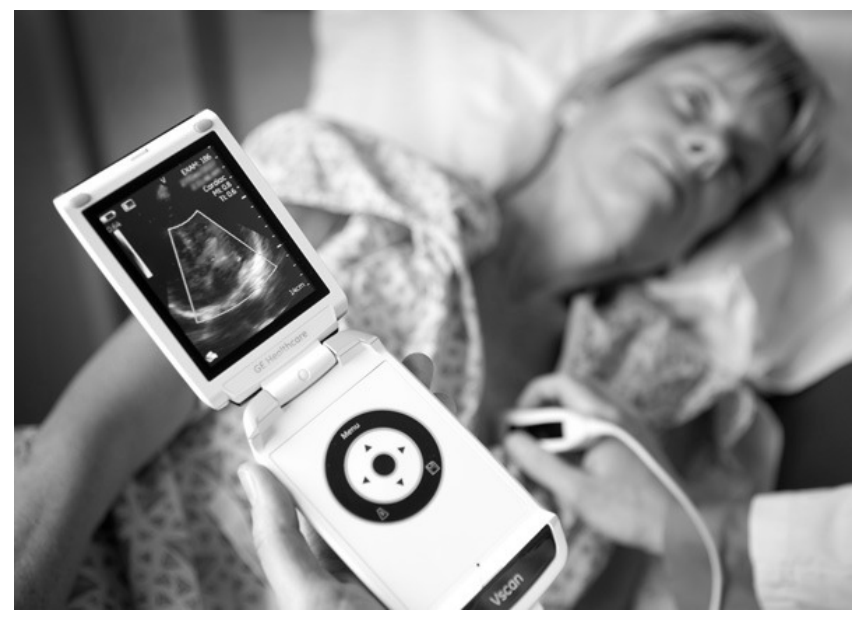

Figure 7: Portable ultrasound.

\section{Apple Computer}

Apple Computer recently announced a security configuration that would make the now-ubiquitous iPad HIPAA-compliant [13]. Healthcare providers now can use the FaceTime video chat function to securely talk to patients and other clinicians. Regulatory compliance provides entree to Western health care, but the marginal utility of such a system is much higher in Africa.

\section{SECURE AND EFFECTIVE USE OF MEDICINES \\ "Drugs don't work in patients that don't take them" \\ C. Everett Koop}

\section{Secure packaging counters counterfeit drugs}

Drug counterfeiting is an estimated $\$ 75$ billion business in 2010, and fake medications are responsible for at least 700,000 deaths every year [14][15]. Malaria medication is especially at risk; according to the World Trade Organization, fake malaria drugs kill 100,000 Southeast Asians a year [14].

A new system from HP and mPedigree assigns a code that is revealed by scratching off a coating on the packaging of participating drugs. This code can be text messaged by the consumer at the point of purchase to a free SMS number to verify the authenticity of the drug package. If the drug package is real, the patient receives a reply of "OK," and other information like the expiration date. If the drug packaging contains a counterfeit code, the consumer receives a message alerting them that the pack may be a fake, as well as a phone number to report the incident [15].

Unfortunately, this system validates the package of the medicines, and not the medicines themselves. The Healthstore Foundation, a non-profit founded in 1997 to create self-sustaining pharmacies in developing nations and with which the author was once affiliated, provides a stable, sustainable supply of genuine medicines to rural, under-developed regions. (76 pharmacies were established in Kenya by the end of 2010, serving about 500,000 patients.) One common counterfeiting method was documented by a sting operation performed by Glaxo-Smith-Kline: good medicines are first emptied from authenticated packages, which are then refilled with counterfeit medicines and resealed to look like genuine, authentic packages [16]. Thus, authenticating drug packages and not the medicines themselves may provide a false sense of security. Nevertheless, this appears to be the best available system to counter the sellers of counterfeit medicines.

\section{Digital Medicines}

The author and other researchers at Proteus Biomedical have developed a digital health feedback system comprised of a wearable sensor, an ingestion sensor and a viewing application [17]. The "MEMS" contribution to this system is a silicon chip coated with $\mathrm{CuCl}$ and $\mathrm{Mg}$ on opposite surfaces that is embedded in each tablet or capsule. Upon digestion in the stomach, the two dissimilar materials combine with the stomach juices to power up the CMOS circuit. The circuit, in turn, modulates the current between those two materials and thus the rate of reaction of $\mathrm{Mg} \rightarrow$ $\mathrm{Mg}^{+}$and $\mathrm{Cu}^{+} \rightarrow \mathrm{Cu}$. This modulation of chemical reactions allows information to be communicated from the ingestion sensor to the wearable sensor.

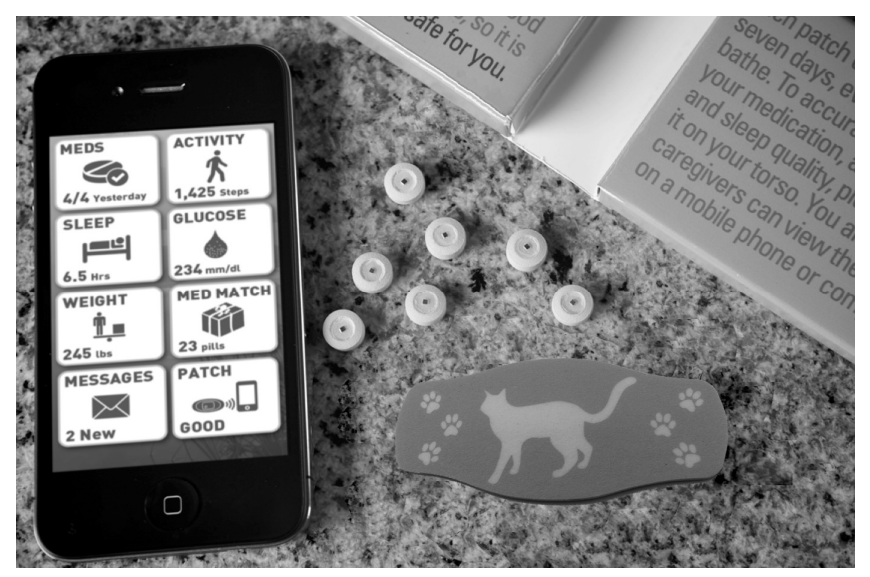

Figure 8: Digital medicine system used to manage chronic disease

The initial use will be a digital health feedback system that helps a patient manage chronic disease by providing feedback of their medicine ingestion, activity rate, heart rate and similar indicators of their health. Many medicines do not achieve their full therapeutic value when used outside of a hospital simply because 
they are not ingested as prescribed. The entire disease category of XDR TB (Extremely Drug Resistant Tuberculosis) is caused simply because patients do not follow a full course of inexpensive antibiotics, allowing resistant strains to evolve and multiply. Examples of effects of poor adherence in the treatment of heart failure, mental illness, diabetes, oncology, and organ transplant are found throughout the medical literature [18][19][20].

An illustrative example of how the Proteus system may be used is the treatment of diabetes. There are three therapeutic pathways to treat a pre-insulin-dependent diabetic: diet, exercise and medicine. By far the most common prescribed medicine for diabetes is generic metformin. Proteus' health management system will directly measure two of these three categories: exercise and digestion of "digital" metformin.

But tracking data is only the beginning. Closing the feedback loop is crucial and can be achieved by sharing of the data with friends, family members, physicians, pharmacists (more common in small towns and in the UK than in urban US) or other diabetics.

Games will be set up allowing individuals or teams to get fun feedback for healthy behavior. For example, a patient might sign up for "MedMatch" medicine, a pill is donated to an individual in Nairobi. A program might be geared toward Breast Cancer survivors who today walk or run in fundraising "marathons." This "game" would track their heart rate and step count during the event and their taking of prescribed Tomoxifen between fund-raisers; the "teams" with the highest adherence between fundraisers might win awards such as having their photos taken with athletes like Lance Armstrong, himself a cancer survivor, at the next fundraiser. This way, the fundraisers are helping themselves while they are raising funds to finance research that helps others.

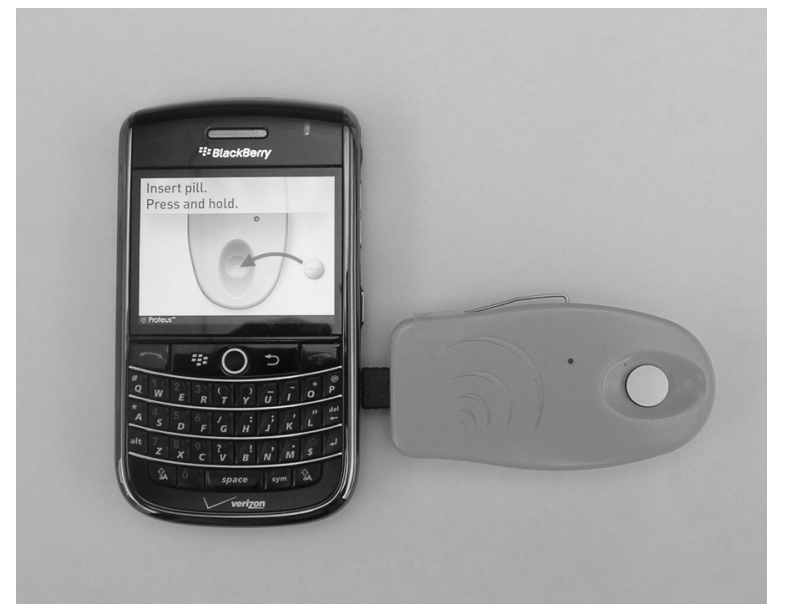

Figure 9: Digital medicine system used to fight counterfeit drugs

Another important use of Proteus' digital medicines will be to give pharmacists the ability to validate the integrity of their medicines - before selling them. The prototype shown in Figure 9 is targeted for use in the developing world, where the dongle detects the chip inside the pill and communicates its ID to a central database using the phone. The approach would be robust against even the most committed counterfeiter, who today re-packages useless medicines into valid packages.

\section{SUMMARY}

The application of mobile technology to health is still in its infancy. Cell phones that record ECG's, listen to heart-beats, take micrographs of blood smears, measure blood pressure and blood glucose levels, are only the tip of the spear that will lance disease in places that today have little access to modern medicine. iPads may open a new window on how the next generation of doctors will be trained and serve in both the developed and developing regions. It is a realm full of opportunity for those who build systems that measure, detect, sample, or otherwise sense. The collision between miniaturization and health care creates an opportunity to help build electronic bridges between continents of people, and make lasting impact on the upward march of humanity.

\section{REFERENCES}

[1] Personal conversation with Michael Zdeblick, VP in charge of new building at Rush-Presbyterian Hospital, Chicago, 2012.

[2] World Bank, accessed by Wikipedia December 20, 2011.

[3] Personal conversation with Scott Hillstrom, Chairman of The Healthstore Foundation, and Gunther Faber, former VP of Africa for GSK.

[4] Kimberly Swartz, Projected Costs of Chronic Diseases, The Hastings Center

[5] N. Ferguson, Civilization, The Penguin Press, 2011.

[6] World Bank, op cit.

[7] www.nationmaster.com

[8] en.wikipedia.org/wiki/List_of_medical_schools_in_Africa

[9] Eric Topol, MD., Oral presentation at Proteus, Feb 2012.

[10] A Continuous, Wearable, and Wireless Heart Monitor using Head Ballistocardiogram (BCG) and Head Electrocardiogram (ECG), Sodini, et al, EMBC 2011, p 491.

[11] Dr. Gari Clifford, Oxford's Institute for Biomedical Engineering, Press Release 16 sep 2011.

[12] Cell-Phone-Based Platform for Biomedical Device Development and Education Applications, Smith ZJ, Chu K, Espenson AR, Rahimzadeh M, Gryshuk A, et al. (2011) CellPhone-Based Platform for Biomedical Device Development and Education Applications. PLoS ONE 6(3): e17150. doi:10.1371/journal.pone.0017150

[13] http://www.zdnet.com/blog/apple/facetime-calls-areencrypted-and-hipaa-compliant-when-using-properencryption $/ 11166$

[14] Nilesh K. Patel, The manufacturing of fake drugs is a vast and underreported problem, particularly affecting poorer countries, pharmainfo.net

[15] www.HP.com

[16] Scott Hillstrom, op cit.

[17] Au-Yeung KY, Moon GD, Robertson TL, DiCarlo LA, Epstein MS, Weis SE, Reves RR, Engel G. Early clinical experience with networked system for promoting patient selfmanagement. Am J Manag Care. 2011; 17: e277-87.

[18] Nonadherence to adjuvant tamoxifen therapy in women with primary breast cancer, Ann H Partridge, Philip S Wang, Eric P Winer, Jerry Avorn, J Clin Oncol, Vol. 21 No. 4 pp 602-6

[19] Fischl M, Castro J, Monroig R, et al. Impact of directly observed therapy on long-term outcomes in HIV clinical trials. In: Program and abstracts of the 8th Conference on Retroviruses and Opportunistic Infections; February 4-8, 2001; Chicago. Abstract 528.

[20] Frangou, S.; Sachpazidis, Ilias; Stasinakis, A.; Sakas, Georgios. Telemonitoring of medication adherence in patients with schizophrenia. Telemedicine Journal and EHealth 2005; 11: 675-683.

\section{CONTACT}

*M.J. Zdeblick, tel: +1-650-632-4031 x105, 2600 Bridge Parkway, Redwood City CA, 94065, Mzdeblick@ProteusBiomed.com 


\title{
RF MEMS CAPACITOR INTEGRATION
}

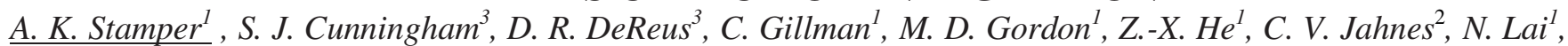 \\ S. L. Luce ${ }^{I}$, J. Maling ${ }^{1}$, A. S. Morris ${ }^{3}$, W. J. Murphy ${ }^{I}$, I. Vitomirov ${ }^{3}$, E. J. White ${ }^{I}$, and B. K. Wong ${ }^{I}$ \\ ${ }^{1}$ IBM, Inc., Essex Junction, VT USA, ${ }^{2}$ IBM, Inc. Yorktown Heights, NY USA \\ ${ }^{3}$ Wispry Inc., Irvine, CA USA
}

\begin{abstract}
MEMS capacitor switches have been integrated with $50 \mathrm{~V}$ LDMOS/5V-CMOS $0.18 \mu \mathrm{m}$ generation ICs. The MEMS were formed with the final three Ti-cladded $\mathrm{AlCu}$ wiring levels in $\mathrm{SiO}_{2}$ using a novel planar sacrificial silicon cavity process [1-3]. The MEMS cavities were hermetically sealed at less than atmospheric pressure at wafer level with a CVD dielectric lid. After Pb-free solder bumping, the MEMS chips are packaged in organic laminate packages. The capacitor portion of the MEMS beam has a capacitance density of $\sim 0.12 \mathrm{fF} / \mu^{2}$ and the pull-in, restoring, selfactuation, and break down voltages are on the order of $30 \mathrm{~V}, 10 \mathrm{~V}$, $>45 \mathrm{~V}$, and $>150 \mathrm{~V}$ for $0.2 \mathrm{pF}$ devices, respectively. MEMS cycling lifetime of 500 million cycles has been demonstrated. This paper summarizes the critical integration, yield, and reliability issues associated with the L1 qualification of this HV CMOS [4], RF MEMS technology.
\end{abstract}

\section{INTRODUCTION}

This is the first report of the L1 manufacturing qualification and early production ramp of a monolithically integrated RF CMOS-MEMS capacitor technology. The technology is in manufacturing production to provide chips for antenna tuning in cell phones. The MEMS capacitors are integrated in large preprogrammed tunable arrays with CMOS controllers and on-chip $40 \mathrm{~V}$ operation. Figure 1 shows an image of a 64 MEMS tunable capacitor chip. This paper summarizes the critical RF MEMS technology integration, yield, and reliability issues.

\section{MEMS TECHNOLOGY OVERVIEW \\ MEMS Capacitor Design and Fabrication}

The metal/oxide/metal MEMS beam is formed in a planar silicon cavity and the beam is released by removing the sacrificial silicon through holes in the oxide lid using $\mathrm{XeF}_{2}$ gas. The cavity is hermetically sealed by depositing CVD oxide and nitride to form a dielectric lid. The $\sim 70 \mu \mathrm{m}$ by $400 \mu \mathrm{m}$ asymmetric bridge MEMS beams are formed with two beams per cavity with nominal capacitances of $0.1,0.2$ or $0.4 \mathrm{pF}$ per beam. The MEMS capacitor electrodes are formed between the lower $\mathrm{AlCu}$ wire in the beam and the $\mathrm{AlCu}$ wire under the beam, both of which are coated with a thin MEMS capacitor dielectric to prevent aluminum-silicon reaction during fabrication and capacitor electrode shorting during actuation (Figures 2-3). 50nm tall oxide bumpers are formed inside patterned openings in the lower MEMS beam electrode (Figures 2-4) to prevent actuator-actuator contact, resulting in cycling lifetimes of $>500$ million cycles. The patterned openings in the lower beam electrode also act to reduce AlCu hillock density and height. The asymmetric actuators (Act) and RF capacitor (Cap) combined with the use of a longitudinal spring (Figure 5) act to minimize capacitance variability.

\section{MEMS Capacitor Electrode Topography}

We optimized the Ti-cladded AlCu metallurgy to reduce hillock density and height such that the average AFM-measured, random RMS roughness under the lower MEMS beam electrode and over the fixed electrode under the MEMS cavity are $2.3 \mathrm{~nm}$ and $4.3 \mathrm{~nm}$, respectively (Figure 6). We sporadically observe metal hillocks under the lower MEMS beam electrode, due to random process variability (Figures 7-8), and these hillocks can reduce capacitance density and act as cycling stiction nucleation points.

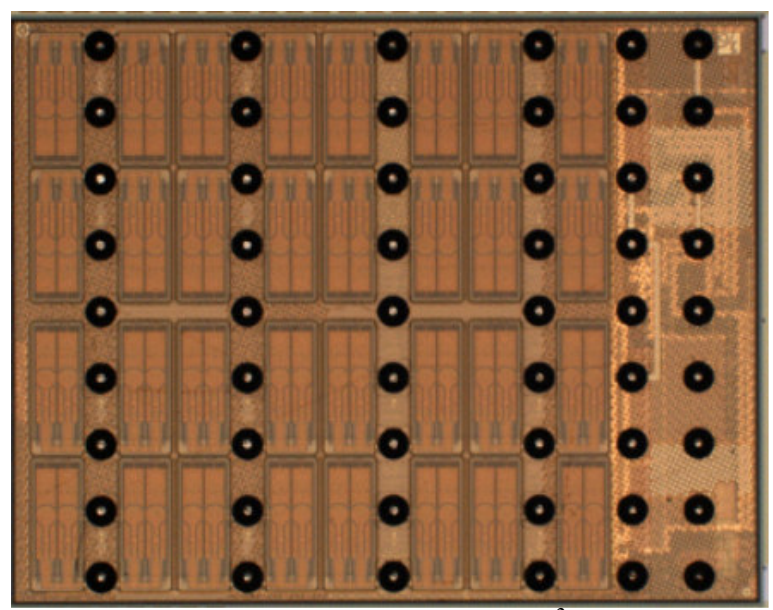

Figure 1: Optical image of $\sim 2.2 \times 2.8 \mathrm{~mm}^{2} 64$ MEMS capacitor Wispry tunable impedance matching chip post solder bumping.

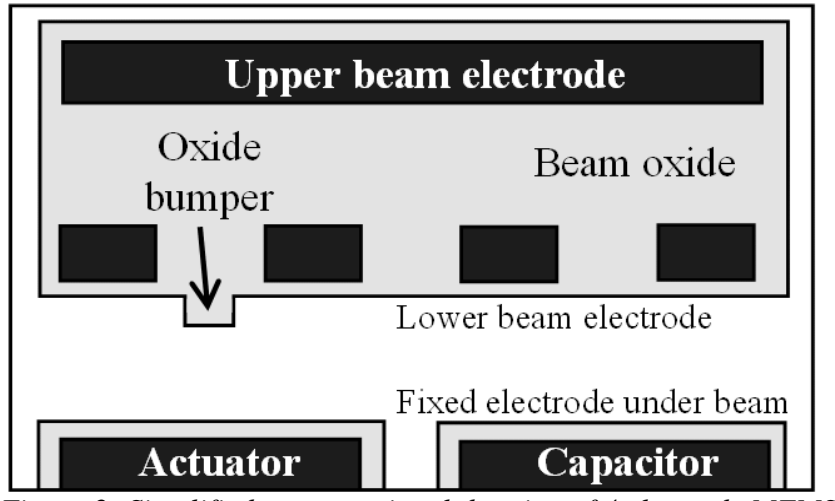

Figure 2: Simplified cross-sectional drawing of 4 electrode MEMS capacitor beam showing actuator and capacitor electrodes.

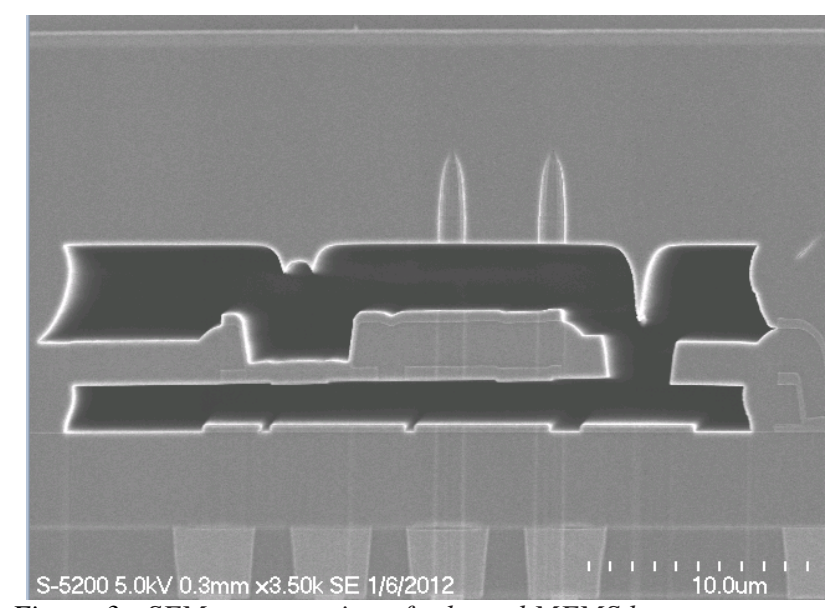

Figure 3: SEM cross-section of released MEMS beam test structure showing cavity, beam, and 10 1 m thick lid. Hilton Head Island, South Carolina, June 3-7, 2012 


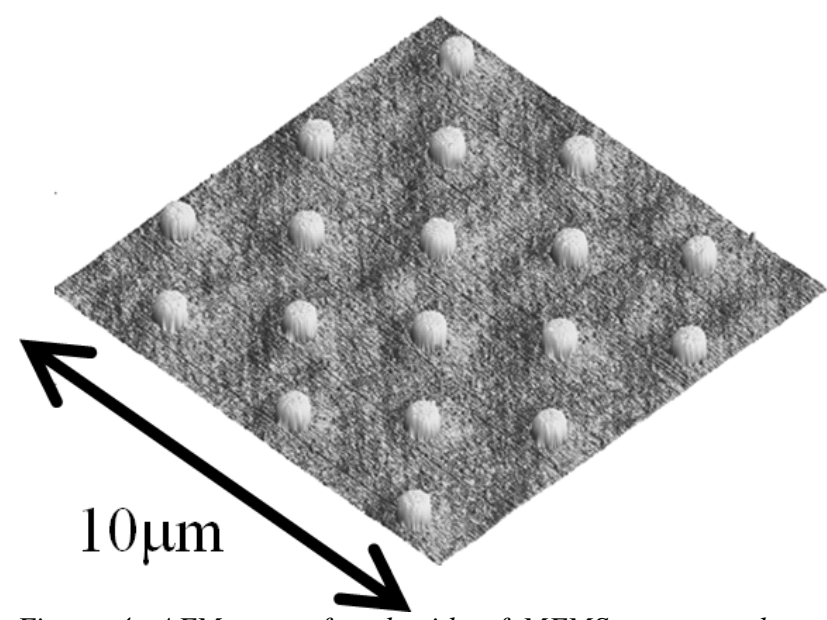

Figure 4: AFM map of underside of MEMS actuator electrode showing 50nm deep oxide bumper array (70nm min-max height).

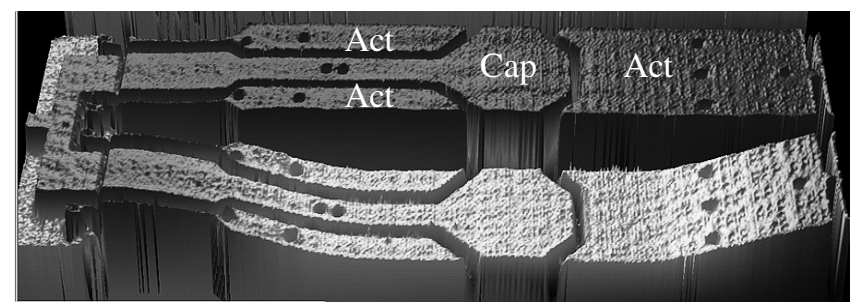

Figure 5: Optical profiler image of non-actuated (top) and actuated (bottom) MEMS capacitor beam including labels for the RF capacitor head (Cap) and actuators (Act).
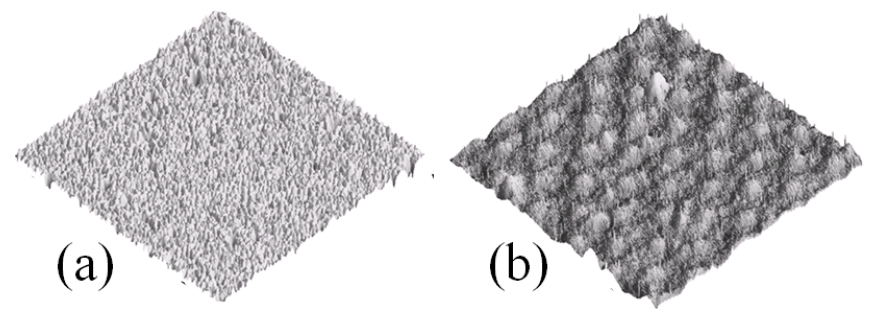

Figure 6: Typical AFM images for $400 \mu^{2}$ areas (a) above the fixed electrode surface under the MEMS beam (63nm min-max) and (b) under the lower MEMS electrode (27nm min-max).

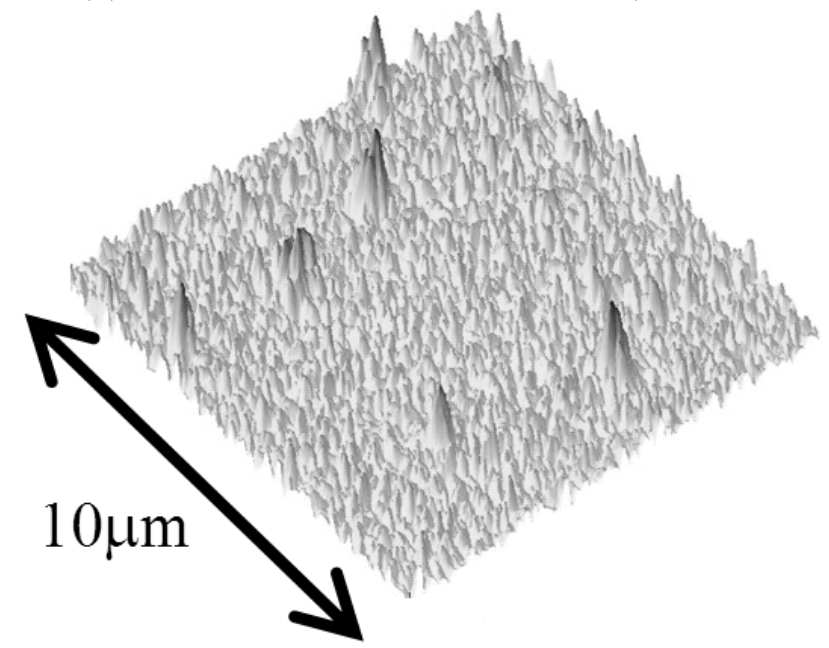

Figure 7: AFM roughness map of lower fixed MEMS capacitor electrode showing severe hillocks (64nm min-max height).

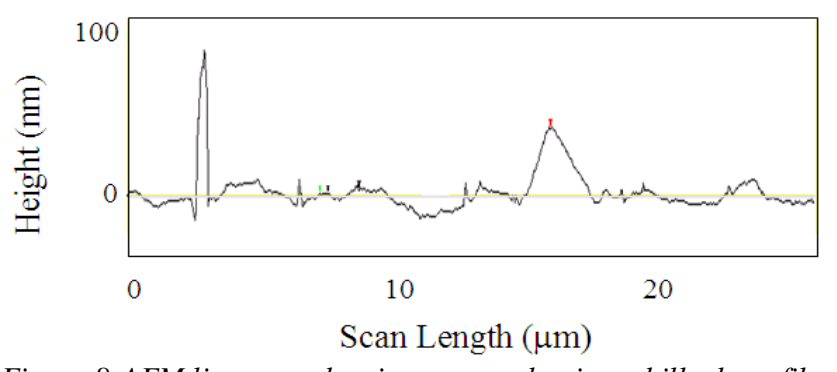

Figure 8:AFM line scan showing severe aluminum hillock profiles.

\section{MEMS CAPACITOR ELECTRICAL TEST}

The MEMS capacitor is operated by applying $40 \mathrm{~V}$ between the moving actuator electrodes and the fixed actuator electrodes under the MEMS beam (Figures 2 and 5). The operating voltage of $40 \mathrm{~V}$ is higher than the pull-in voltage. The MEMS beam consists of a metal/oxide/metal sandwich with oxide composing $\sim 2 / 3$ of the volume. The low CTE of oxide combined with both the longitudinal spring at the end of the large actuators in the MEMS beam and the volume matching of the high CTE upper and lower MEMS beam metal act to minimize process- and temperatureinduced variability in beam shape. We measure $<2 \%$ capacitance and $<2 \mathrm{~V}$ pull-in voltage $-25^{\circ} \mathrm{C}$ to $100^{\circ} \mathrm{C}$ variability.

MEMS capacitance is defined as the $0-40 \mathrm{~V}$ delta capacitance difference (Figure 9). MEMS capacitor functional yields are determined by the delta capacitance and pull-in voltage (VPI). Figure 10 shows measured MEMS delta-capacitance and VPI data for $\sim 1 / 2$ year of early manufacturing production. The MEMS deltacapacitance has significantly increased in the past $1 / 2$ year, due to reduction of MEMS cavity residuals and metal hillocks on the MEMS capacitor electrodes. The primary functional yield loss mechanisms are delta-capacitance and VPI variability. VPI is proportional to (beam thickness) ${ }^{1.5}$ and (actuation gap) ${ }^{1.5}$ [5]. In addition, stress gradient changes in the MEMS beam induce positive or negative beam curvature which changes the actuation gap and resultant VPI. For example, a tensile beam stress gradient, i.e. having a more tensile film on the top of the beam, causes the beam center to bend downwards in a concave up fashion, resulting in reduced VPI due to a smaller actuation gap. Table 1 summarizes measured MEMS beam thickness and stress gradient variability and their effect on VPI variability for a MEMS capacitor centered with VPI $=30 \mathrm{~V}$. We actively modulate the expected released MEMS beam shape to center it at target by changing the thickness of the nominally $80 \mathrm{~nm}$ oxide thickness over the beam, which changes the MEMS beam stress gradient and resultant beam curvature. Figure 11 shows a 5V VPI shift per $10 \mathrm{~nm}$ of oxide thickness over the beam, primarily because of increased beam curvature and resultant air gap change.

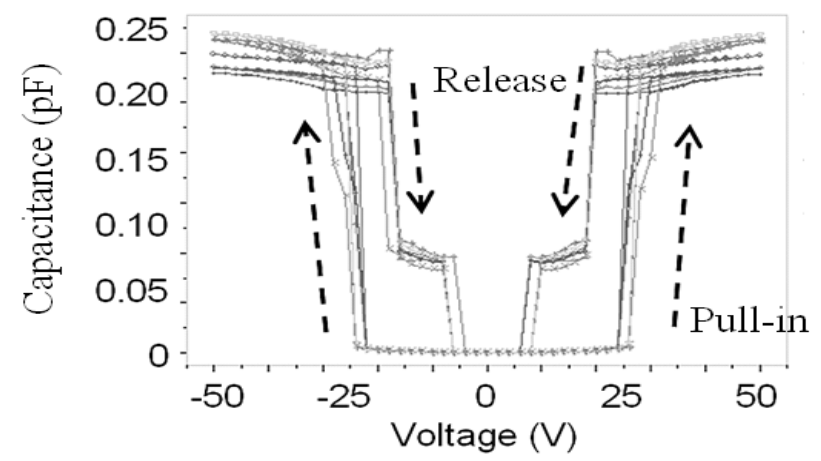

Figure 9: $M E M S$ bipolar $C$ - $V$ data for seven individual $0.2 p F$ wafer center, middle, and edge radius capacitors. 
In addition to the obvious problem of no MEMS capacitor actuation when VPI is greater than the $40 \mathrm{~V}$ operation voltage, a low VPI causes yield loss for self-actuation. Self-actuation occurs because the actuation force is proportional to (voltage) ${ }^{2}$ and the RF voltage across an unactuated MEMS capacitor causes it to actuate. For the MEMS design and test data shown in this paper, selfactuation falls below specification (40V) when VPI is below $\sim 26 \mathrm{~V}$.
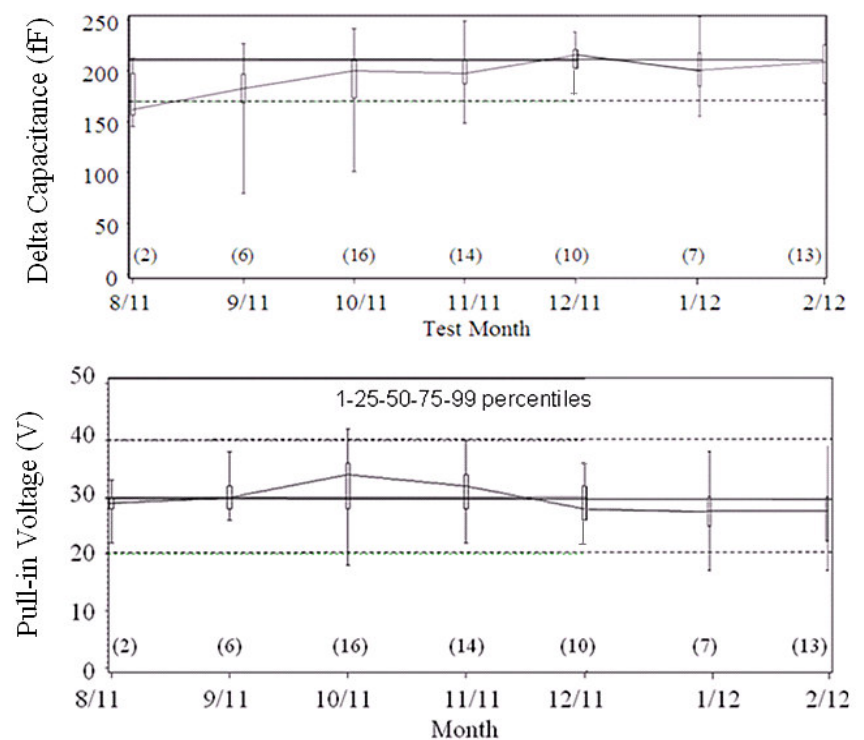

Figure 10: 200fF MEMS capacitor delta capacitance and pull-in voltage data for $\sim 70$ production lots with 6-25 wafers/lot and 10 chips/wafer (lots/month shown in parenthesis).

Table 1: MEMS beam process effect on pull-in voltage variability.

\begin{tabular}{|c|c|c|}
\hline Parameter & $\begin{array}{c}\text { Measured } \\
\text { Variability }\end{array}$ & $\begin{array}{c}\text { Predicted VPI } \\
\text { variability }\end{array}$ \\
\hline Beam oxide stress gradient & $28+/-6 \mathrm{MPa} / \mu \mathrm{m}$ & $+/-2.7$ \\
Beam oxide thickness & $2+/-0.1 \mu \mathrm{m}$ & $+/-2.3$ \\
\hline Beam metal thickness & $+/-20 \mathrm{~nm}$ & $+/-3.0$ \\
\hline Under/over beam ox thick & $80+/-5 \mathrm{~nm}$ & $+/-2.5$ \\
Under/over beam ox stress & $280+/-14 \mathrm{MPa}$ & $+/-1.0$ \\
\hline Lower cavity height & $1.9+/-0.2 \mu \mathrm{m}$ & $+/-4.4$ \\
\hline Total VPI variability & $+/-7 \mathrm{~V}$ & $+/-6.9$ \\
\hline
\end{tabular}

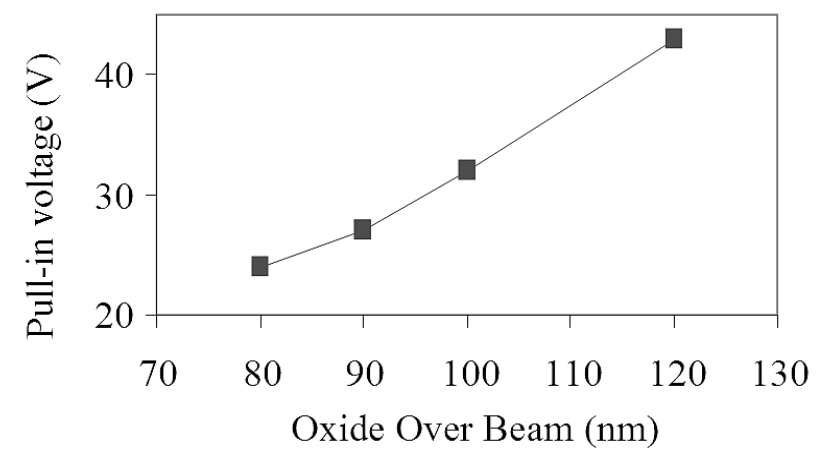

Figure 11: VPI vs. oxide thickness over the MEMS beam.

\section{MEMS Cavity Integrity}

Upon exiting the fab, wafers are processed through standard $\mathrm{Pb}$-free bump flip-chip assembly processes that include passivation, bumping, test, back-side grind, back-side coating, and dicing. The chips are then flip-chip mounted to an organic laminate carrier substrate with under fill and finally the substrate is mounted to a printed circuit board. Figure 12 shows MEMS cavity cracks that occurred on $\sim 6 \mathrm{~mm}^{2}$ packaged chips with 32 MEMS cavities per chip. We determined that these lid cracks became visible during back side grind and chip dicing and were exacerbated by the acute angle in the corner of the upper MEMS cavity (Figure 12). Lid cracks in packaged chips destroy the hermetic seal, which results in immediate stiction of the MEMS beam during electrical test. Lid cracking, which was modeled using finite element analysis, was eliminated by increasing the lid thickness from $6 \mu \mathrm{m}$ to $10 \mu \mathrm{m}$, which reduced the modeled maximum principle stress by a factor of $\sim 2.8$ and also reduced the $130^{\circ} \mathrm{C}, 91 \%$ R.H. pressure cooker stress fail rate of packaged chips from $\sim 20 \%$ to $0 \%$.

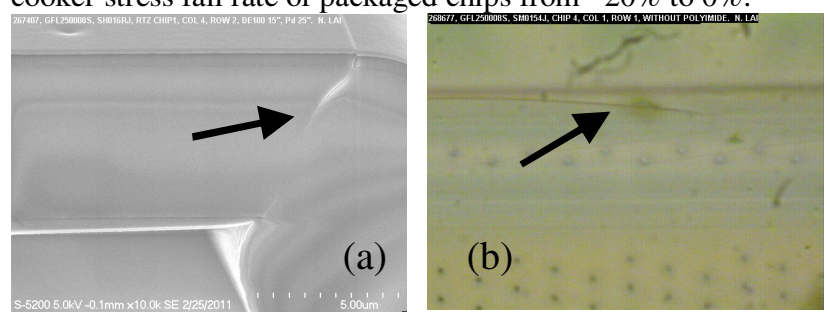

Figure 12: (a) SEM cross-section and (b) optical top view micrograph showing depackaged MEMS chip cavity cracks originating in cavity corner with $6 \mu$ m thick lid.

\section{MEMS CAPACITOR RELIABILTY Cycling}

MEMS capacitor cycling is a challenging reliability stress. End user specifications are variable but typically are 0.1 to 1 billion cycles over the operating temperature. Historically, we observed two primary cycling fail modes: 0V stiction-induced capacitance increase and $40 \mathrm{~V}$ defect- or wear-in-induced capacitance change [1]. Figure 13 shows representative cycling data for a single MEMS capacitor driver which connects 7 MEMS capacitors in parallel on a chip with 64 MEMS capacitors. Most drivers do not fail during our cycling tests (Chip 1) and, when they do, they fail for actuator stiction on one or more of the seven MEMS capacitors (Chip 2), which increases the $0 \mathrm{~V}$ capacitance. 2 of the 7 MEMS capacitors in Chip 2 exhibited actuator stiction fails, the first at 290 and a second at 350 million cycles. We sometimes observe a simultaneous $0 \mathrm{~V}$ and $40 \mathrm{~V}$ increase in capacitance (Chip 3), which may mean that an actuator stiction fail caused the actuated beam shape to change, resulting in a $40 \mathrm{~V}$ capacitance change. Cycling fails are rarely accompanied by dielectric breakdown, which means that the MEMS capacitor drivers continue to operate after the initial cycling fail. By cycling large numbers of chips and drivers, we calculated a fail rate of 1000 ppm at 500 million cycles.

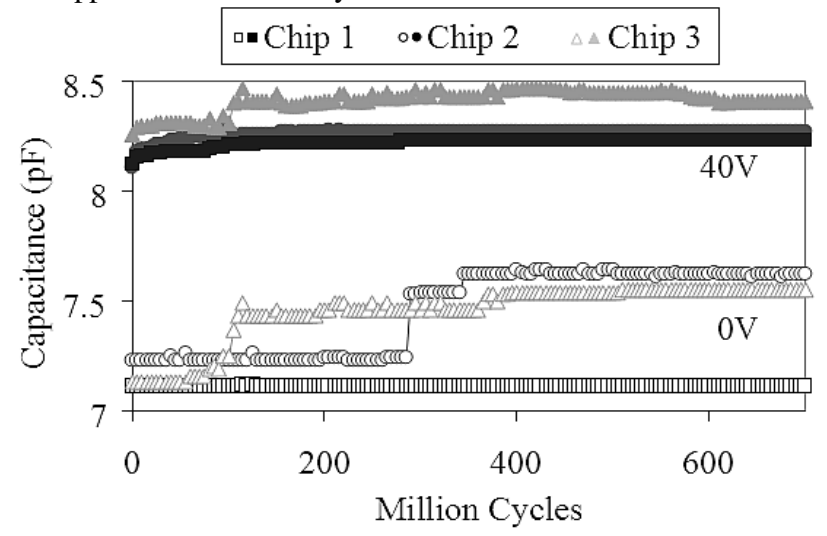

Figure 13: Cycling data for a 7 MEMS capacitor driver showing OV (open symbol) and 4OV (closed symbol) capacitance. 
Failure analysis of cycling fails (Figure 14) consistently shows actuator stiction in areas between the oxide bumpers. We believe that this stiction is due to the random presence of metal hillocks under the MEMS beam, which leads to electric field enhancement and accelerated stiction fails. These metal hillocks provide a high electric field actuator stiction point during cycling and the likelihood of their causing a stiction fail increases over time as the oxide bumpers are eroded. Figure 15 shows a severe case of hillocks under the MEMS beam.
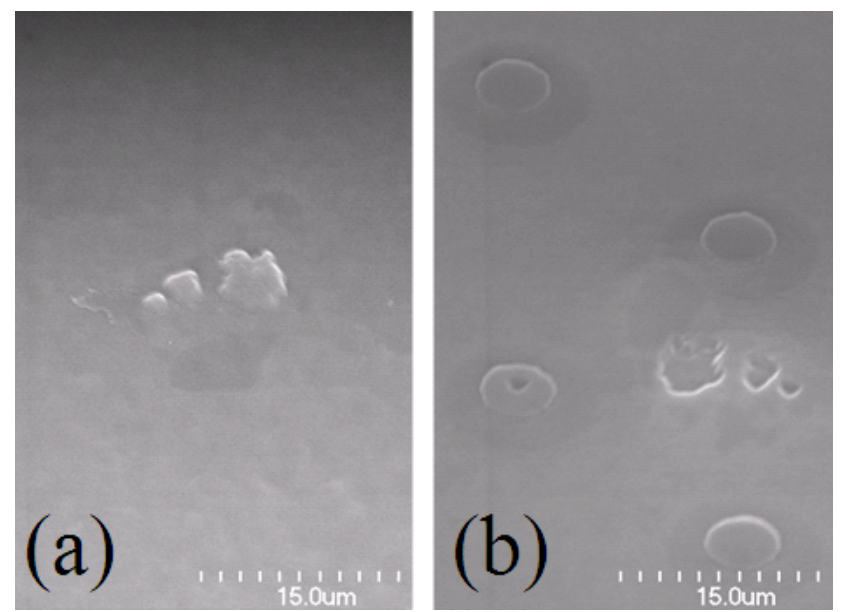

Figure 14: Typical MEMS cycling stiction fail showing (1) top side of fixed electrode and $(b)$ underside of movable electrode.

\section{MEMS Capacitor breakdown voltage}

The MEMS capacitor dielectric needs to be reliable up to $40 \mathrm{~V}$ DC actuation voltage and $80 \mathrm{~V}$ peak-to-peak RF voltage. Figure 16 shows measured actuated MEMS capacitor voltage breakdown (VBD) for the MEMS bridge beam used in the tunable capacitor chip (Figure 5). VBD is centered at about $150 \mathrm{~V}$ with little waferto- wafer and lot-to-lot variability and exceeds the $80 \mathrm{~V}$ specification. We measure both capacitor to capacitor and ganged actuator-capacitor to actuator-capacitor electrode VBD and observe slightly lower values for the ganged actuator-capacitor measurement, as expected from the larger area.

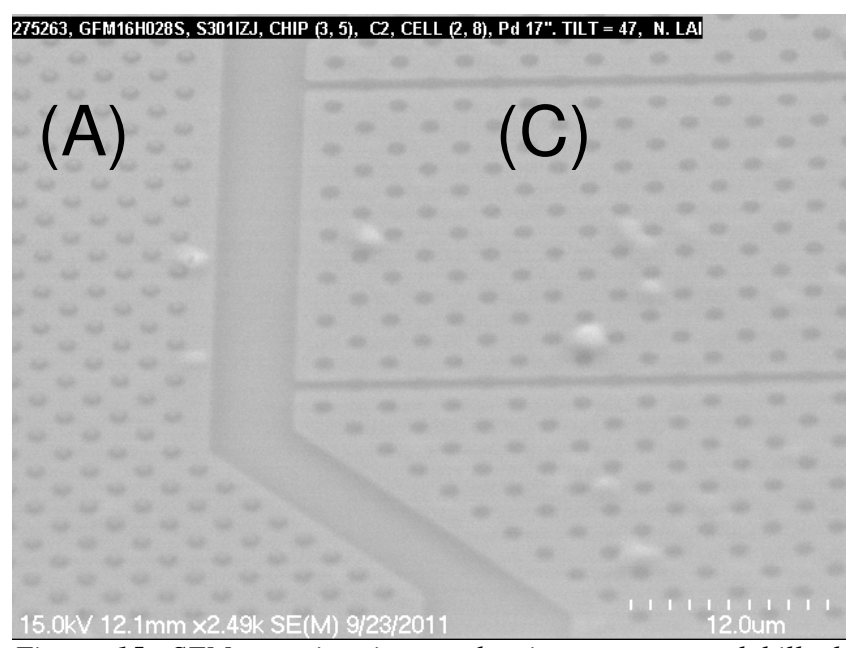

Figure 15: SEM top view image showing severe metal hillocks under the MEMS beam electrode on the actuator $(A)$ and capacitor (C) electrodes.

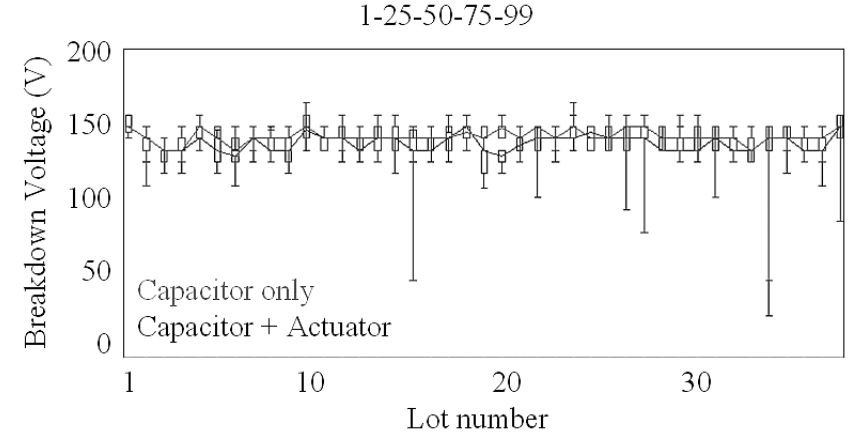

Figure 16: MEMS capacitor breakdown voltage.

\section{SUMMARY}

We have successfully L1 production qualified RF-MEMS capacitor switches into a $0.18 \mu \mathrm{m}$ high voltage CMOS process flow and begun the early manufacturing ramp. The MEMS capacitors were formed using a sacrificial silicon cavity process are hermetically sealed at wafer level, and flip chip packaged in organic laminate packages. No fails occurred during $130^{\circ} \mathrm{C}, 91 \%$ R.H. pressure cooker stressing of packaged chips. MEMS cycling lifetime of 500 million cycles has been demonstrated. The primary functional yield limiters are due to pull-in voltage and MEMS capacitance variability. This RF-MEMS technology has demonstrated preliminary yield and reliability sufficient for the initial application of cell phone antenna tuning.

\section{REFERENCES}

[1] A. K. Stamper, C. V. Jahnes, S. R. Dupuis, A. Gupta, Z.-X. He, R. T. Herrin, S. L. Luce, J. Maling, D. R. Miga, W. J. Murphy, E. J. White, S. Cunningham, D. R. DeReus, I. Vitomirov, and A. S. Morris, "Planar MEMS RF Capacitor Integration" in Proc. $16^{\text {th }}$ Transducers, Beijing, China, June 59, 2011, pp.1803-6.

[2] C. V. Jahnes, N. Hoivik, J. M. Cotte, M. Lu, and J. H. Magerlein, "Evaluation of O2 Plasma and XeF2 Vapor Etch Release Process for RF MEMS, Switches Fabricated using CMOS Interconnect Processes" in Proc. Solid State Sensors, Actuators, and Microsystems Workshop, Hilton Head Island, SC, United States, June 4-8, 2006 pp. 360-3.

[3] S. Natarajan, S. Cunningham, A. Morris, \& D. DeReus, "CMOS Integrated Digital RF MEMS Capacitors", $11^{\text {th }}$ Meeting on Silicon Monolithic Integrated Circuits in RF Systems, Phoenix, AZ, Jan. 17-19, 2011 pp. 173-176.

[4] R. Minixhofer, N. Feilchenfeld, M. Knaipp, G. Röhrer, J. M. Park, M. Zierak, H. Enichlmair, M. Levy, B. Löffler, D. Hershberger, F. Unterleitner, M. Gautsch, K. Chatty, Y. Shi, W. Posch, E. Seebacher, M. Schrems, J. Dunn, and D. Harame, "A 120V 180nm High Voltage CMOS smart power technology for System-on-chip integration", Proc. 22nd Int. Symp. Power Semicond. Dev. \& IC's, Hiroshima, Japan, June 7-10, 2010 pp. 75-78.

[5] Peter M. Osterberg and Stephen D. Senturia in J. Micromechanical Systems, Vol. 6, No. 2, June 1997.

\section{CONTACT}

*A. K. Stamper, tel: +1-802-769-8466; astamper@us.ibm.com 


\section{TUNABLE MOBILE HANDSET ANTENNA IMPEDANCE MATCH BASED ON RF MEMS BI-STATE CAPACITORS \\ X. Zhu ${ }^{l^{*}}$, A. Morris ${ }^{3}$, G. Hutcheson ${ }^{l}$, M. Brobston ${ }^{1}$, Y. J. Shin ${ }^{2}$ \\ S. Cunningham ${ }^{3}$, J. H. Lee ${ }^{2}$ and J. Hilbert ${ }^{3}$ \\ ${ }^{1}$ Samsung Telecommunications America, Richardson, Texas, USA \\ ${ }^{2}$ Samsung Electronics, Suwon, South Korea \\ ${ }^{3}$ WiSpry, Inc., Irvine, California, USA}

\begin{abstract}
This paper presents the design, implementation and test result of a Tunable Matching Network (TMN) based on RF MEMS bistate capacitor technology. The targeted application is to improve multi-mode multi-band (MMMB) mobile handset RF performance by providing optimum matching at the antenna RF feed point. The need for such a device is described followed by application requirements. The methodology for selecting topology is discussed, the design flow is illustrated, and the design result is summarized. The RF performance improvement provided by the TMN is demonstrated under both the lab bench, and field test environments.
\end{abstract}

\section{INTRODUCTION}

As mobile communications evolves from $2 \mathrm{G}, 3 \mathrm{G}$ to LTE/LTE-Advanced, and with the booming of the smart phone market, the MMMB RF front end that was previously only available in high-end smart phones has migrated to mid-range even low-end mobile devices. Multi-mode indicates that the handset supports a wide range of communication standards, e.g. GSM, GPRS, EDGE, CDMA, WCDMA, and LTE in latest products. Multi-band denotes that the device is able to operate at a multiple frequency bands, e.g. 700, 800, 900, 1700, 1800, 1900, 2100 and even $2600 \mathrm{MHz}$. The most popular type of antenna in handsets is the PIFA(Planar Inverted F Antenna) type which typically has only 2 resonant frequencies, one at $1900 \mathrm{MHz}$ and the other one at 800 or $900 \mathrm{MHz}$. When a handset operates at any frequency band away from these two resonant bands, the transmit efficiency and receive sensitivity are reduced compared to those at the resonances. Moreover, to obtain maximum radiation/sensitivity to meet stringent carrier RF performance specifications, $\lambda / 4$ structure length is desired leading to a large antenna volume. However, the consumer's want for a slim form factor together with the complexity of hardware due to ever increasing functionality, the large size of display and the large capacity battery has squeezed the available space for the phone antenna. Furthermore, the phone must meet specifications under a range of typical operating conditions, e.g. phone held by hand, phone held by hand and close to head, and phone in flip/slide open position. To make the situation challenging, every phone design has a different physical geometry requirement for the antenna. Therefore, the electrical characteristic of the antenna in each phone is dramatically different. Fig. 1 represents measurement antenna feed point impedance for 5 handsets in different operational conditions for GSM850 band (from 824 to $894 \mathrm{MHz}$ ) which is the lowest frequency band in our design. Some similarity can be observed among these traces. As frequency goes up, the impedance changes more dramatically and the antennas show less resemblance to each other. Usually, a mobile handset antenna is well designed for one operational condition while it will be easily de-tuned under other operational environments, as shown by traces in Fig. 1 swinging from the center to the edge of Smith chart. Meeting all of these requirements within a short product development cycle is difficult and it may not be possible for the engineers to find an acceptable antenna solution. Therefore a general tunable antenna impedance match solution able to be dropped into different product designs to facilitate the product development is preferred.

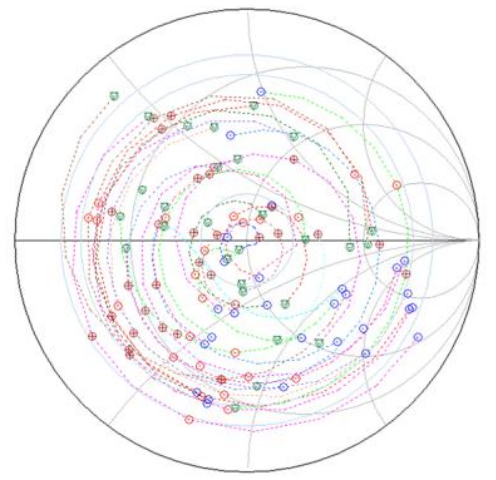

Figure 1: Measured antenna impedance data of 5 different phones under different operation condition at GSM850 band. The blue circles mark the start of TX band, and the red ones label the end of $T X$ band. The red circles with cross mark the start of $R X$ and the green circles with triangle mark the end of $R X$ band.

After decades of development effort, RF MEMS technology finally has matured to provide a viable tunable RF front end solution[1]. Several approaches have explored, e.g. antenna resonant element tuning[2][3] and power amplifier output impedance tuning[4]. However, these solutions are either targeted for a single band or impedance at a single frequency. In this paper, a solution for matching arbitrary impedance simultaneously of transmit and receive is presented.

\section{DESIGN TARGET AND METHODOLOGY}

As aforementioned, the desired TMN device should have the capability to cover the entire Smith chart, but in practice, the impedance within VSWR $\leq 10: 1$ region is specified because it represents majority of measured impedances that need to be matched. One important aspect in TMN design for antenna impedance match is simultaneously matching of TX and RX band impedances. The reason is that CDMA, WCDMA, and LTE FDD all operate using frequency division duplexing(FDD) in which transmit(TX) and receive(RX) signals simultaneously at different frequencies and thus a compromise between of the match for TX and RX has to be made. Whereas for the case of TDD system (such as GSM or LTE TDD) or of PA impedance tuning, only a single frequency at the specified time slot needs to be considered therefore a perfect match can be always achieved theoretically. This simultaneous match of TX and RX impedance makes it impossible to derive a closed-form solution for an arbitrary antenna because there is no general relationship between TX and RX frequency impedance across antenna designs.

To evaluate matching performance, the term transducer 
gain(TG)[5] or transducer power gain[6], which is widely used in the power amplifier design, is used as the most important criterion to determine the quality of matching,

$$
T G=\frac{\left|S_{21}\right|^{2}\left(1-\left|\Gamma_{S}\right|^{2}\right)\left(1-\left|\Gamma_{L}\right|^{2}\right)}{\left|1-\Gamma_{S} \Gamma_{i n}\right|^{2}\left|1-S_{22} \Gamma_{L}\right|^{2}}
$$

where $\Gamma_{L}$ is the reflection coefficient of the antenna to be matched, $\Gamma_{S}$ is the source reflection coefficient which is zero for perfect $50 \Omega$ source, $\Gamma_{i n}$ is the reflection coefficient of TMN device with the other port connected to the antenna, and $S_{21}$ and $S_{22}$ are TMN Sparameters. The return loss is only used as a monitoring parameter. The rationale is that maximizing the total amount of power radiating out of the antenna, versus minimizing the power reflected by the antenna, determines the performance of the RF front-end; the non ideal reactive component in the network and unwanted resonances can yield a good return loss but inferior transducer gain. To benchmark the antenna performance improvement provided by the TMN device, relative transducer gain is used, which is defined as the ratio between transducer gain with and without the TMN is defined as

$$
R T G=\frac{\left|S_{21}\right|^{2}}{\left|1-S_{22} \Gamma_{L}\right|^{2}}
$$

The selected circuit topology can be explained by a Yin-Yang theory described by Dr. Randy Rhea [7][8] which is shown in Fig 2. A single ' $\mathrm{L}$ ' section of $\mathrm{L}-\mathrm{C}$ network is capable to provide impedances over half of the Smith chart, which implies the conjugate of any impedance in such area can be well matched. Therefore, by cascading Yin, (e.g. Type 1) and Yang (e.g. Type 3), any impedance across the entire Smith chart can theoretically be matched if not considering matching bandwidth (for simultaneous TX and RX impedance match).

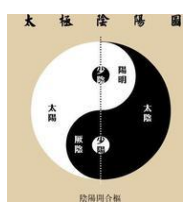

(a)

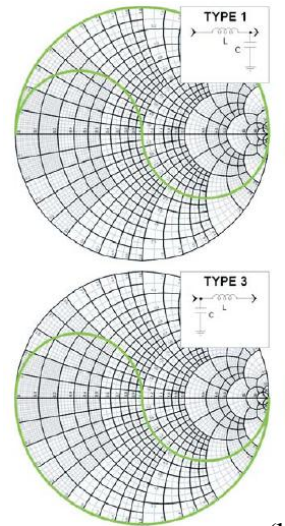

(b)

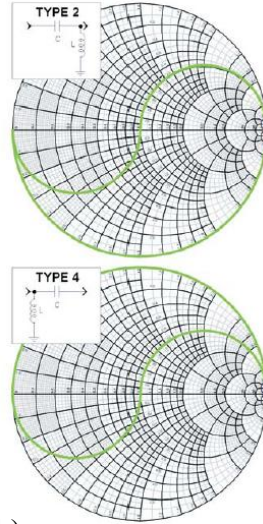

Figure 2: (a) A Chinese Yin-Yang Symbol, (b) The basic concept of Yin-Yang of matching[7].

To implement with available non-ideal reactive components, only 2 sections of L-C are used to minimize the insertion loss. A tunable capacitor is required in parallel with series inductors to achieve a pseudo-tunable inductor. Because any physical capacitor has parasitic inductance and capacitance associated with its RF leads and any tuning element has tuning ratios, the maximum total required capacitance is chosen empirically depending on the coverage and TG requirement. The Type 1 and Type 3 combination is selected following these criteria. A shunt tunable capacitor is added at the common node of two L-C network translating it into the bridged double- $\pi$ circuit topology as shown in Fig. 3(a). One purpose for the added shunt capacitor is to make the network more like a transmission line to mitigate the degradation of impedance match when load impedance falls in VSWR $<2: 1$ circle, especially when operating at high frequency band. The pair of tunable capacitors associated with each inductor is converted into a single capacitor in connecting the two distal series inductor terminals to reduce parasitic at the common node between two inductors and to minimize the total amount of tunable capacitance required. Each capacitor in the schematic is made of a bank of capacitors which is shown in Fig 3(b). Each capacitor in the capacitor bank has only two states, UP state (min. capacitance) and DOWN state (max. capacitance). The Least Significant Bit (LSB) capacitor in the bank determines the tuning resolution of each capacitor bank.

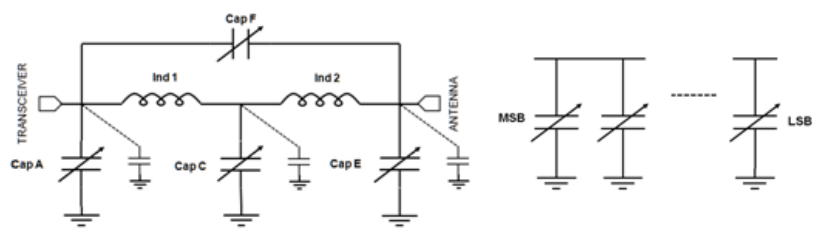

(a)

(b)

Figure 3: (a) Proposed double $\pi$ tunable match network for generic antenna impedance match. (b) Illustration of each tunable capacitor which is made of a bank of capacitors.

An optimization process has been used to determine the value of two inductors and max capacitance for each of five tunable capacitors to maximize impedance coverage over the frequency bands of interest. The minimum capacitance is set by MEMS device inherent tuning ratio. At the initial design stage, the parasitic capacitance was assumed to be a percentage value of the total capacitance at each circuit node and is later replaced with precise parameters derived from HFSS simulation and measurement.

High tuning resolution provides fine tuning steps of impedance. This is primarily required for tuning at high frequency, e.g. $\sim 2 \mathrm{GHz} v s .<1 \mathrm{GHz}$, since tunable component impedance changes more significantly due to the frequency multiplication factor. However, a finer resolution means more physical capacitors are required in the capacitor bank, therefore more parasitic from MEMS capacitors themselves and their interconnections will add to each circuit node which reduces the effective capacitor tuning ratio. A compromise between the best circuitry topology, available resolution and parasitic is made leading to a nominal step size of $1 / 8 \mathrm{pF}$.

\section{SIMULATION RESULTS}

Detailed RF analysis of the RF MEMS capacitor and TMN module substrate is implemented in Ansoft's HFSS, and the entire integrated TMN module simulation is performed in Agilent's ADS. Antenna impedances of multiple handsets under different operation bands and operation conditions have been used to determine the optimum capacitance settings and highest achievable performance. Results are shown in Fig. 4. After the RF MEMS tunable capacitor bank partition and layout are determined, an independent sweep of two inductors within acceptable range is performed to squeeze out more performance, and to balance the high frequency band and low frequency band performance. The result is represented in the contour plot format of RTG as example shown in Fig 5. The green area is preferred region for inductor value selection. 


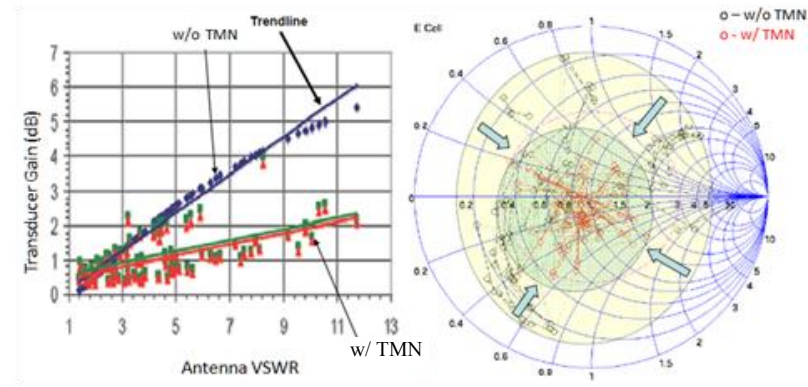

Figure 4: Simulation of antenna performance improvement by $T M N$.

(a) Improvement of TG over wide range of VSWR;

(b) Improvement of $\Gamma$ (dashed line connects $T X$ and $R X$ pairs, green dots connect without TMN and red dots connect with TMN).

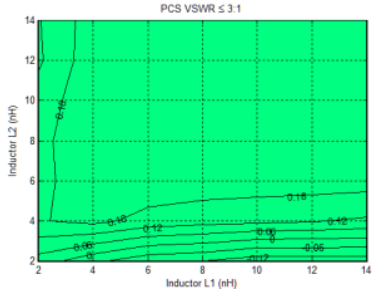

(a)

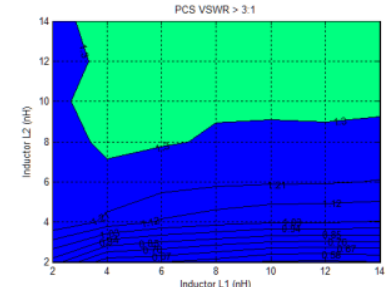

(b)
Figure 5: RTG contour plot by sweeping values of two inductors independently. The green area is preferred region for inductor value selection.

The final simulation result of TMN devices with five different operation band and different operational environments across multiple handset antennas is tabulated in Table 1. The average transducer power gain of $\mathrm{TX}$ and $\mathrm{RX}$ is used to evaluate improvement of the transmit efficiency/receive sensitivity. The data is divided into two regions: a low VSWR region where the antenna is well tuned by design and a high VSWR region where the antenna is de-tuned. In general, the TMN significantly improves the antenna performance in the high VSWR regions while having minimal impact on the RF performance loss in the low VSWR regions. The lower bands show much better improvement than high bands due to two factors: the network is optimized toward low band application which is the greatest challenge for small volume antenna design and the parasitic in the network have less impact at low band than at high band.

Table 1. Simulated average antenna transducer gain improvement with the insertion of TMN RF MEMS device

\begin{tabular}{|l|c|c|}
\hline \multicolumn{1}{|c|}{ Band } & Low VSWR Region & High VSWR Region \\
\hline $800 \mathrm{MHz}$ & -0.09 & 1.33 \\
\hline $900 \mathrm{MHz}$ & 0.11 & 1.63 \\
\hline $1800 \mathrm{MHz}$ & -0.01 & 1.23 \\
\hline $1900 \mathrm{MHz}$ & 0.05 & 0.99 \\
\hline $2100 \mathrm{MHz}$ & -0.23 & 0.78 \\
\hline
\end{tabular}

\section{IMPLEMENTATION}

The high level system design is shown in Fig. 6. As explained above, a bridged double- $\pi$ topology is chosen to provide a general purpose impedance match, although this may not be the best solution for all handset antennas. The motivation is that a stationary hardware platform with programmable control firmware capability will reduce number of handset product development cycles, easing the adoption across other product lines.

Due to limited mobile handset PCB board space and to simplify the control of the MEMS array, a vertically integrated solution RF MEMS above IC[9][10], is chosen. A complete device layout and die photo is shown in Fig. 7. While CMOS control circuitry extends over the entire die, the noisy portion of the digital control and high voltage charge pump is placed to one side of MEMS capacitor banks. This minimizes the electrical interference between CMOS and MEMS portions. The total die size is about $2.2 \times 2.8 \mathrm{~mm}$
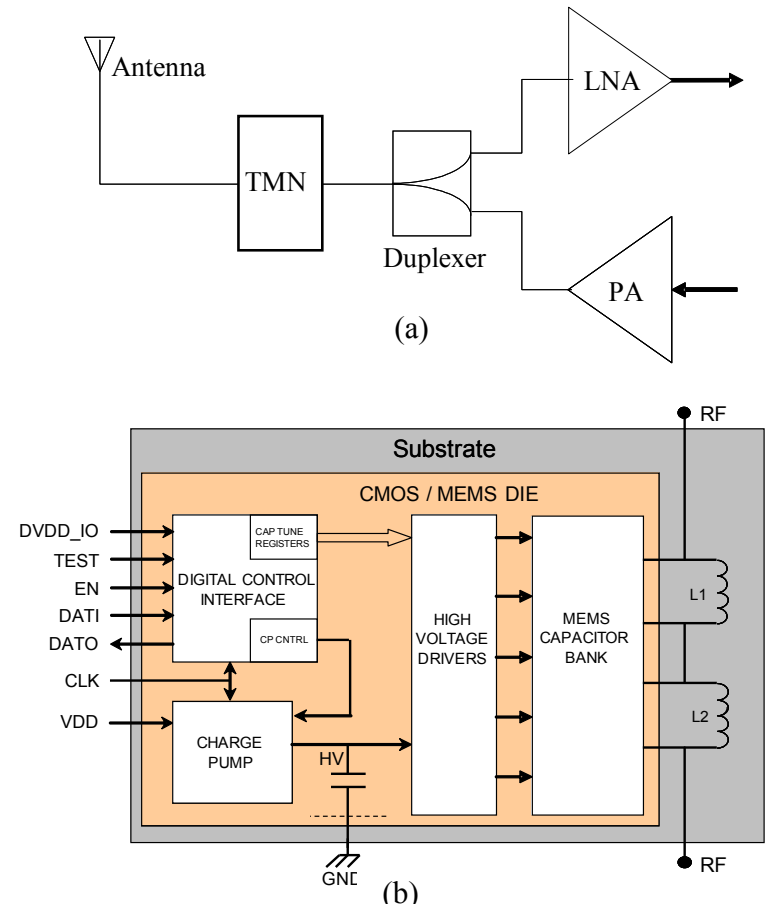

Figure 6: System architecture.

(a) TMN insertion point in the RF front end of mobile device.

(b) High level system schematic of TMN devices.

The bi-state capacitors are fabricated in layers above the CMOS for further isolation from the substrate. The fixed capacitor plate and the fixed actuator plate are fabricated immediately above the CMOS and are covered by a thin, $80 \mathrm{~nm}$, oxide dielectric and ALD $\mathrm{Al}_{2} \mathrm{O}_{3}$. A lower silicon sacrificial layer is deposited and planarized above the fixed capacitor plates. The metal-oxide-metal composite beam is deposited on the lower sacrificial layer. The metal for the fixed capacitor plate and the composite beam is $\mathrm{AlCu}$ with a TiN capping layer. Under the lower beam metal and on top of the silicon sacrificial layer is a $2^{\text {nd }} 80 \mathrm{~nm}$ oxide layer. When the bi-state capacitor is closed, the oxide- $\mathrm{Al}_{2} \mathrm{O}_{3}$-oxide defines the capacitance density, except for a residual air gap. To maintain symmetry of the MEMS beam, an 80nm oxide is deposited on the upper metal. The symmetric metal-oxide-metal composite provides excellent stress and thermal matching to minimize the deformations induced by process stresses or thermal mismatch. 


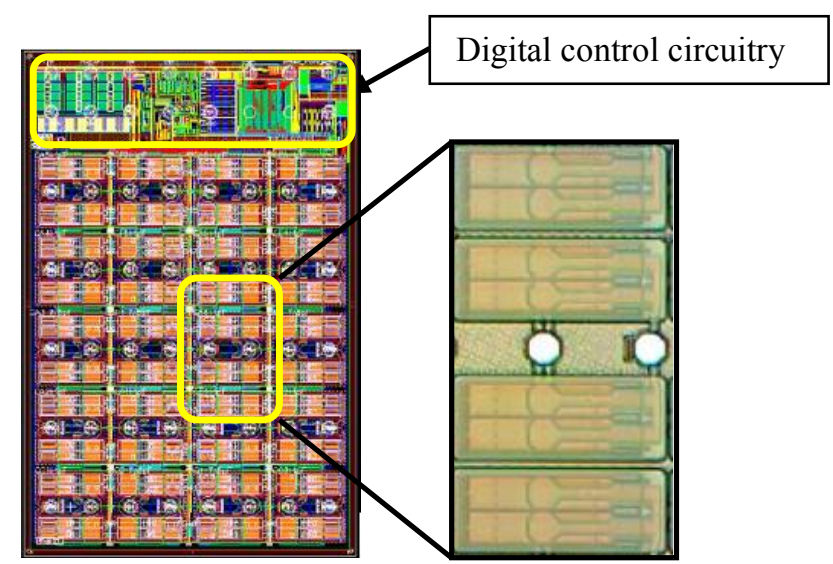

Figure 7: Chip layout and unit cell photo.

Above the MEMS bi-state capacitor, a $2^{\text {nd }}$ silicon sacrificial layer is deposited to form a cavity above the beam. The $2^{\text {nd }}$ silicon sacrificial layer is encapsulated by an oxide layer that is perforated to allow the $1^{\text {st }}$ and $2^{\text {nd }}$ silicon sacrificial layers to be vented by a $\mathrm{XeF}_{2}$ process. Once the silicon is vented, the cavity is sealed by a sub-atmospheric oxide CVD process and passivated with a silicon nitride layer. The wafer level encapsulation process provides a hermetic environment for the operation of the bi-stable capacitor. A cross-section of the MEMS bi-state capacitor is shown in Fig. 8, which shows the capacitor integrated above the HV RF CMOS and shows the hermetic encapsulation above the capacitor.

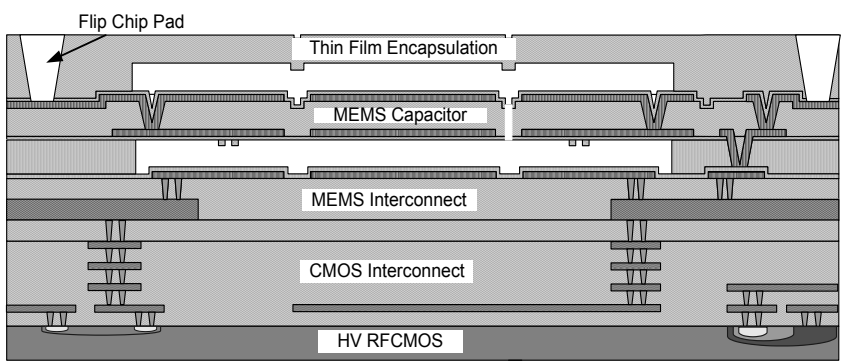

Figure 8: Cross-section of MEMS bi-state capacitor integrated with HV RF CMOS and hermetic encapsulation.

Fig. 7 shows a photograph of a unit cell that is comprised of four bi-state capacitors and two RF flip-chip pads. Each unit cell is defined by its total capacitance, which are comprised of combinations of $0.4 \mathrm{pF}, 0.2 \mathrm{pF}$, and $0.1 \mathrm{pF}$ sized bi-state capacitors. A TMN device is comprised of 20 cells, which are divided among four tunable capacitor banks (Cap A, C, E, and F). The unit cells have 4 capacitor elements that change states simultaneously. The fractional cells are 3-bit binary eight-state cells.

The TMN die includes the integrated CMOS and MEMS bistate capacitors necessary to form the four banks of the bridged double $\pi$ tunable match network. TMN die is flip chip mounted to a laminate substrate, which will include interconnects to connect cells together in the double $\pi$ network configuration. This laminated substrate can be either the phone PCB board or interpose board. The two match network inductors can be designed into the substrate metal layers, which will results in a complete module, or can be designed into the phone PCB with discrete components. The TMN die is shown flip-chip mounted to a laminate substrate in Fig. 9.

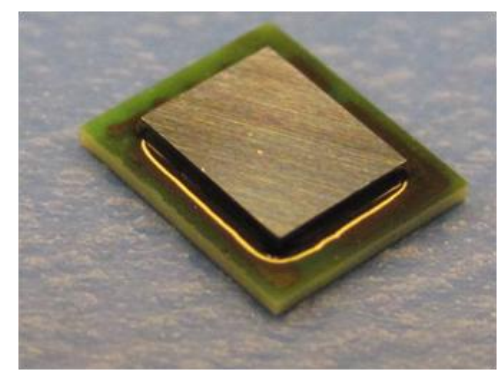

Figure 9: TMN die flip chip mounted to laminate substrate with die underfill.

\section{TEST RESULTS}

The TMN device RF performance is assessed on an evaluation board by two port measurements. The settings of the 5 capacitors on the TMN device is derived by ADS simulation under specified operation frequency band and impedance to be matched. The $50 \Omega$ VNA measured results are converted to specified antenna loading condition to compare with simulation results. Very good correlation between simulation and measurement, both in magnitude and phase, has been demonstrated in Fig. 10.

The device has been integrated into the product devices with one example shown in Fig. 11. This handset has gone through standard TRP/TIS measurement and field tests. Improvement across all band have been observed. For antenna operating at its non-optimized mode, several $\mathrm{dB}$ enhancements has been recorded.
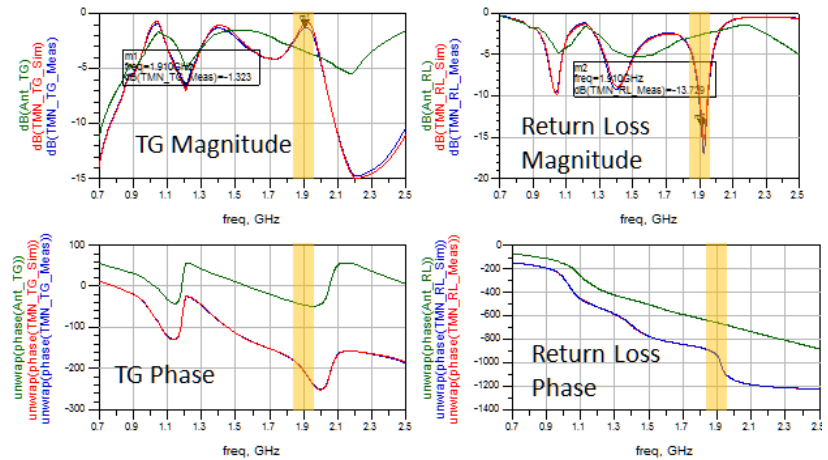

Figure 10: Lab measurement vs. simulation. Green traces are data without TMN device, red traces are simulation data with $T M N$ device and blue traces are measurement data. The vertical yellow stripes mark the operating frequency band where antenna impedance is supposed to be tuned.

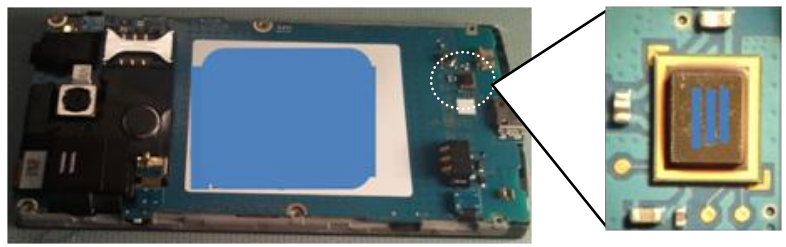

Figure 11: Photos of TMN device and its presence in a mobile handset product.

\section{CONCLUSION}

An RF MEMS tunable capacitor enabled tunable mobile handset antenna impedance match is presented. The device performance can be very well predicted with careful and 
comprehensive design. The results from a general purpose design reveal the capability of RF MEMS tunable devices to improve RF performance for a wide range of antenna product designs. This demonstrates that the RF-MEMS TMN is a viable technology solution to meet MMMB mobile handset challenges. With circuitry topology tailored toward a particular product, even more RF performance improvement is expected but at the cost of longer product development cycle.

\section{ACKNOWLEGEMENT}

We greatly appreciate all of the support and constructive collaboration from various Samsung engineering teams, WiSpry engineering team, Jazz and IBM process development teams.

\section{REFERENCES}

[1] G. M. Rebeiz, "RF MEMS: Theory, Design and Technology," Wiley, New Jersey, 2003.

[2] J. Park, etc., "Tunable planar inverted-F antenna using rf MEMS switch for the reduction of human hand effect," IEEE MEMS Conference, pp. 163-166 (2007).

[3] P. Steeneken, "A Five-Band Reconfigurable PIFA for Mobile Phones," K. Boyle, IEEE Trans. on Antenna and Propagation, Vol. 55, No. 11, pp. 3300-3309, Nov. 2007.

[4] A. van Bezooijen, "Mobile phone performance improvements using an adaptively controlled antenna tuner", 2011 IEEE MTT-S, 5-10 June 2011.

[5] R. Collin, "Foundations of Microwave Engineering," 2nd edition, IEEE press, 2001.

[6] D. Pozar, "Microwave Engineering," 2nd edition, John Wiley and Sons, 1998.

[7] R. Rhea, "The Yin-Yang of Matching: Part 1-Basic Matching Concepts Ying-Yang match", High Frequency Electronics, March 2006.

[8] R. Rhea, "The Yin-Yang of Matching: Part 2-Practical Matching Techniques", High Frequency Electronics, April 2006.

[9] A. K. Stamper, etc., "Planar MEMS RF Capacitor Integration," pp. 1803-1806, Transducers' 11.

[10] S. Natarajan, S. Cunningham, A. Morris, \& D. DeReus, "CMOS Integrated Digital RF MEMS Capacitors", 11th Meeting on Silicon Monolithic Integrated Circuits in RF Systems, Phoenix, AZ, Jan. 17-19, 2011 pp. 173-176.

\section{CONTACT}

*X. Zhu, tel: +1-972-795-9383; xzhu@sta.samsungtelecom.com 


\section{ULTRA-MINIATURIZED POWER CONVERTER MODULES USING MICROMACHINED COPPER SCAFFOLDS

\author{
C.D. Meyer ${ }^{1,2 *}$, S.S. Bedair ${ }^{1}$, B.C. Morgan ${ }^{1}$ and D.P. Arnold ${ }^{2}$ \\ ${ }^{1}$ U.S. Army Research Laboratory, Adelphi, Maryland, USA \\ ${ }^{2}$ University of Florida, Gainesville, Florida, USA
}

\begin{abstract}
This paper presents ultra-miniature $\left(<2 \mathrm{~mm}^{3}\right)$ power converter modules with high power density $\left(77 \mathrm{~W} / \mathrm{cm}^{3}\right)$ enabled by micromachined 3D copper scaffolds containing high-inductancedensity $\left(130 \mathrm{nH} / \mathrm{mm}^{2}\right)$ air-core power inductors. The scaffolds are formed by electroplating multiple, $30-\mu \mathrm{m}$-thick layers of copper within patterned photoresist molds, yielding freestanding copper traces with $>10: 1$ aspect ratios. After backfilling with a chemically-resistant polymer, the scaffolds are then fully detached from the silicon fabrication wafer by wet-etching an underlying oxide layer. Surface-mount switch/controller chips and capacitors are attached by solder reflow to the copper scaffolds, forming selfcontained converter modules.
\end{abstract}

\section{INTRODUCTION}

While modern power converters for portable electronics are typically realized with a switch/controller IC that is connected at the PCB level to a handful of off-chip passives, self-contained "single-chip" power converter modules are highly desired for size, performance, cost, and system integration. Reduced-size-scale dcdc converters could replace series regulators in mobile communications devices, providing greater efficiency in downconverting battery voltage levels to the continually decreasing supply voltages required by LSI circuits [1]. Diminutive size and weight will also be prerequisite to the mobility of future envisioned autonomous robotic microsystems [2], which will further require a sort of mobile "pico-grid" for efficient energy transfer to a system of sensors, actuators, and processors having vastly divergent power needs [3].

The power inductor remains one of the greatest impediments to the miniaturization of power converters, and it increasingly dominates the overall size as converter volume decreases. Inductors by their nature require large areas or high-magneticpermeability materials to increase induction. Emerging highfrequency converter circuits switching at 10's-100's of MHz have reduced inductance requirements down to 10's-100's of $\mathrm{nH}$. However, magnetic materials exhibit greatly increased core losses at these frequencies due to eddy currents and domain dynamics [4], [5]. On the other hand, air-core microinductors, common in RF applications, typically have inductances that are too low for most power converters and yield low quality factors at sub-GHz frequencies.

At Hilton Head 2010 [6] we presented a process for realizing $3 \mathrm{D}$ air-core microinductors and transformers formed in $10-\mu \mathrm{m}$ thick copper layers. Leveraging strong magnetic coupling between vertically stacked, tightly wound planar spirals, the devices yielded high inductance densities $>100 \mathrm{nH} / \mathrm{mm}^{2}$ [7]. However, the process required sequential removal of the molding and seed metal at each layer, so that photoresist posts would remain between windings to prevent electrical shorting. Although the inductors provided high quality factors $>20$ on Pyrex substrates, they would need to be fabricated monolithically on an IC converter substrate in order to form a compact power converter. However, this approach would increase high-frequency losses due to coupling with the conductive substrate and would require extra processing to protect the freestanding structures during packaging.
In this article, we now report an extension of our 3D copper process with $3 \mathrm{x}$ thicker layers to realize inductors that not only have lower dc resistance (DCR) but also are sufficiently robust to be detached from the fabrication wafer. With aspect ratios up to 10:1 per layer, the process results in tighter coil turns than have been achieved in other 3D metal efforts [8], [9], [10]. Furthermore, the multilayer copper process is generalized into a flexible microfabrication platform that enables the formation of air-core microinductors within other copper scaffolding structures. When detached from the silicon fabrication wafer, the copper scaffolding functions as an interposer for interconnecting power IC and passives all within a highly compact, integrated module, as depicted in Fig. 1. The scaffolds are constructed of three independently molded layers of $30-\mu \mathrm{m}$-thick electroplated copper. For the purpose of demonstration, scaffolds containing $130 \mathrm{nH}$ microinductors were microfabricated and tested with commercially available boost converter chips and surface-mount capacitors that were attached by solder reflow.

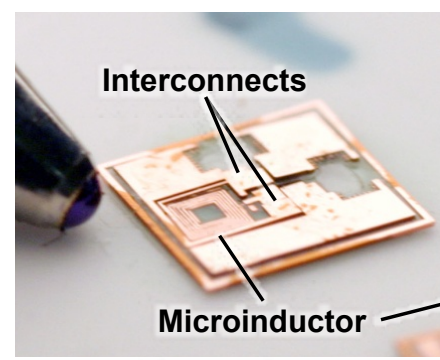

(a)

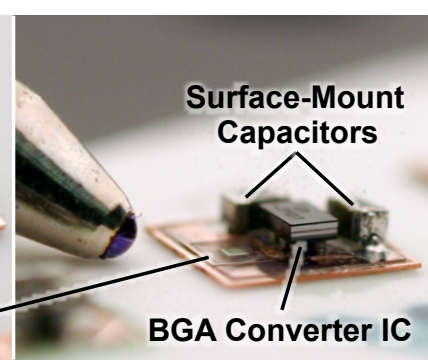

(b)
Figure 1: Photographs of converter module. (a) Multilevel copper scaffolding detached from Si. (b) Surface-mount capacitors and $B G A$ converter chip soldered to copper pads. Tip of ballpoint pen shown (left) for size reference.

\section{BACKGROUND AND DESIGN}

Chip-scale power modules have previously been demonstrated by integrating the inductor into an interposer that sits between the power IC and the circuit board that is to be powered. Hayashi, et al. [1] fabricated solenoidal-winding inductors within a $525-\mu \mathrm{m}$-thick ferrite wafer to which IC chips were flip-chip ultrasonic bonded using gold stud bumps. Wang, et al. [11] fabricated spiral-winding inductors using a silicon wafer as a mold, which was subsequently etched away and back-filled with a ferritepolymer composite; an IC chip and capacitors were mounted onto the $0.6-\mathrm{mm}$-thick inductor by solder reflow.

In contrast, the use of detached copper scaffolds in this work results in an inductor interposer platform that, at a total thickness of $90 \mu \mathrm{m}$, is several times thinner than these previous results. The use of stacked, planar spiral windings within an interposer is also unique and results in exceptionally high volumetric inductance density. As depicted in Fig. 2, the inductor design consists of six turns in each of the two $30-\mu \mathrm{m}$-thick winding layers, which are arranged in a square-shaped spiral layout with a $1 \mathrm{~mm}$ outer diameter, $32 \mu \mathrm{m}$ trace width, and $16 \mu \mathrm{m}$ lateral spacing between 


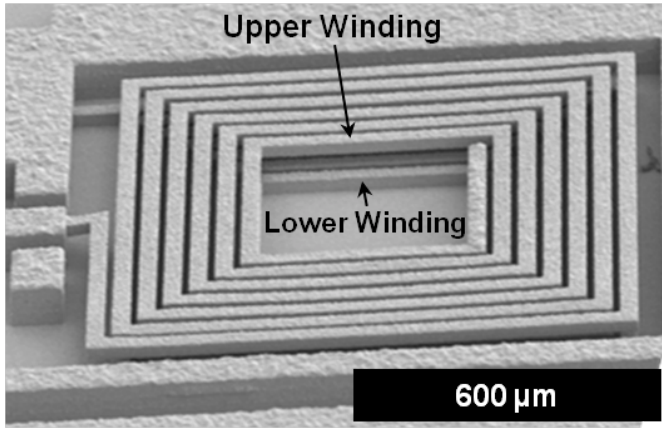

Figure 2: SEM of air-core microinductor realized in three layers of $30 \mu \mathrm{m}$ thick electroplated copper.

adjacent turns. Because all three copper layers were deposited with equal thicknesses, the vertical gap between the upper and lower winding layers was similarly $30 \mu \mathrm{m}$.

\section{FABRICATION}

The copper scaffolds are fabricated by the process illustrated in Fig. 3. The scaffolds are constructed on standard, 500- $\mu$ m-thick, 100-mm-diameter silicon wafers. First, a $2-\mu \mathrm{m}$-thick insulating layer of silicon dioxide is deposited via PECVD, which forms the sacrificial release layer for later detachment. Next, a $20 \mathrm{~nm}$ layer of Ti is sputter deposited over the oxide to improve the adhesion with copper. The copper scaffolds are then sequentially constructed layer-by-layer by electroplating copper onto thin sputter-deposited copper seeds through photoresist molds. The details are explained next.

(a) Sequential plating through molds.

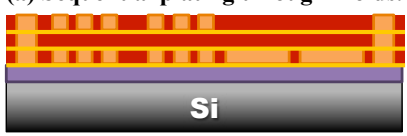

(b) Removal of molds and seeds.

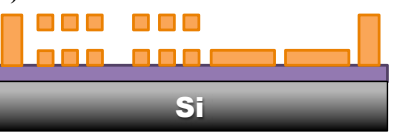

(c) Backfill with polymer.

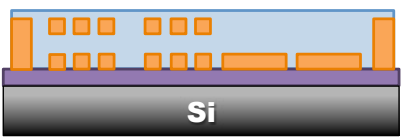

(d) Oxide etch to release.

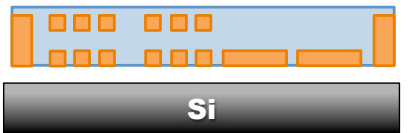

(e) Attachment to target substrate.

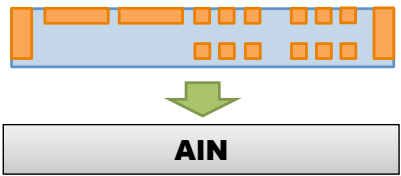

(f) Reflow of surface mount parts.

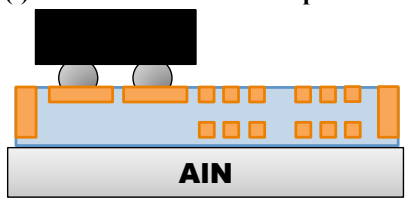

Figure 3: Illustration of process sequence to realize integrated converter module by detaching multilevel copper from silicon wafer and attaching surface-mount parts by solder reflow.

At the start of each layer, the surface is cleaned by RF sputter etching to remove any oxide from the surface of the preceding electroplated copper. Then a $200 \mathrm{~nm}$ seed of copper is sputter deposited over the entire wafer surface. Higher power dc sputtering (1.2 kW, 12-inch-diameter target) yields improved adhesion of the copper seed to underlying photoresist as compared to lower power. Positive-tone AZ9245 photoresist is spun to a thickness of $30 \mu \mathrm{m}$ and cured. Contact lithography is used to expose the regions where copper is to be plated, and the photoresist is developed by immersion in AZ400K developer. Ashing in $\mathrm{O}_{2}$ plasma is then used to clean the copper seed of any residual photoresist scum in the developed regions.

The thick copper structures of each layer are electroplated in an acid copper sulfate electrolyte bath composed of $15 \mathrm{~g} / \mathrm{L} \mathrm{Cu}$ and $210 \mathrm{~g} / \mathrm{L}$ sulfuric acid [12]. The electroplating is timed such that the copper fills just to the top of the photoresist mold, and no further planarization steps are taken. The sequence of sputter deposition, photolithography, and electroplating is repeated for each layer. Three layers are used to form the scaffolds, as shown in Fig. 3a.

Finally after all layers have been deposited, the photoresist molds and copper seeds are all removed simultaneously by ultrasonic agitation Baker PRS-3000 N-methyl-2-pyrrolidone (NMP) photoresist stripper. Subsequent etching in a solution of ceric ammonium nitrate removes the bottommost copper seed. After the wet processing steps, the wafer is rinsed in water and dried on a hotplate (Fig. 3b). The process has been tested with up to four $30-\mu \mathrm{m}$-thick layers of copper and yields critical dimensions $\sim 5 \mu \mathrm{m}$ for aspect ratios up to 20:1, as demonstrated in Fig. 4.

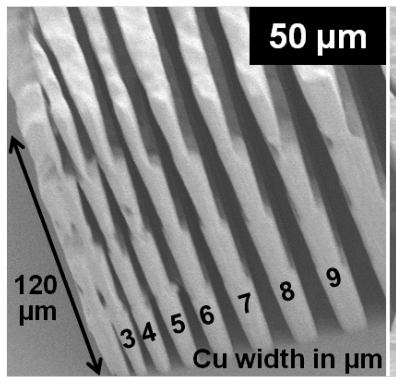

(a)

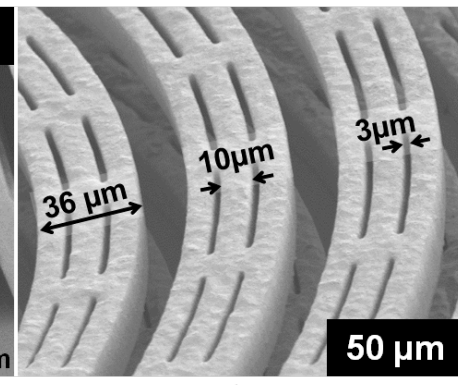

(b)
Figure 4: (a) Fins implemented in four layers of 30um thick copper illustrate fine resolution capability, (b) as used, for example, to form slits in an inductor winding.

The 3D freestanding copper scaffolds are then backfilled with Brewer ProTEK A2-22 protective polymer, which is specifically formulated for resistance to hydrofluoric acid [13] (Fig. 3c). After curing on a hotplate, the backfilled copper scaffolds are released from the wafer by sacrificial oxide etching in hydrofluoric acid (Fig. 3d). For testing purposes, the individual converter modules are affixed to an aluminum nitride substrate (Fig. 3e), though in practice the scaffolds would likely be connected by solder reflow to a PCB containing the broader circuit of the desired application. A BGA-style converter chip and surface-mount capacitors are mounted onto landing pads on the scaffolds by solder reflow (Fig. 3f).

\section{RESULTS}

\section{Inductor Impedance Characterization}

Prior to attaching the converter chip and capacitors, the impedance of the inductor was measured in order to verify functionality after encapsulation and wafer detachment. Impedance was measured using an Agilent E8361A network analyzer with excitation frequency swept from $10 \mathrm{MHz}-1 \mathrm{GHz}$ and RF probes landed at one-port ground-signal-ground pads formed in the copper scaffolding at the input of the inductor. As plotted in Fig. 5, the inductor yielded $130 \mathrm{nH}$ inductance with a self-resonant frequency of $370 \mathrm{MHz}$. A peak quality factor (ratio of imaginary to real part of impedance) of 15 was obtained at $160 \mathrm{MHz}$. The inductor dc resistance was measured at $0.695 \Omega$ using a Keithley 2400 SourceMeter with four-point probing and $20 \mathrm{~mA}$ source current.

The performance parameters of the inductor are summarized 


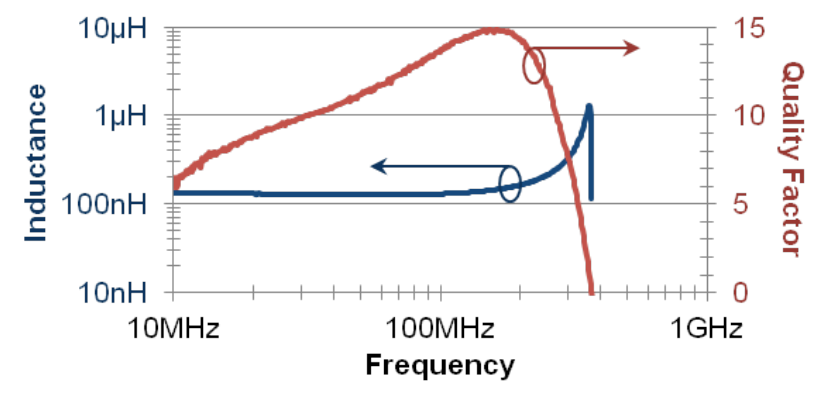

Figure 5: Plots of frequency-dependent inductance and quality factor of micromachined inductor after encapsulation and detachment from Si wafer.

in Table 1 for comparison with several example inductors from the literature that have been tested within chip-scale power converter circuits. Compared to the air-core microinductor reported in [14], the inductor presented here features $>10 \mathrm{x}$ the inductance while occupying the same lateral area at $1 \mathrm{~mm}^{2}$. Similar levels of inductance and peak quality factor are found in the ferrite-core surface-mount 0402-sized inductor tested in [15], but at roughly $5 \mathrm{x}$ the volume. The magnetic-film-core microinductors were designed for low DCR and are larger than even the 0402 surface-mount part.

Table 1: 3D copper microinductor of this work compared to those demonstrated in $\mathrm{mm}^{3}$-scale converters in other works. Parameters are inductance $(L)$, dc resistance (DCR), peak quality factor $\left(Q_{\max }\right)$ and inductor volume.

\begin{tabular}{|l|l|r|l|r|l|}
\hline \multicolumn{1}{|c|}{ Example } & Core & \multicolumn{1}{c|}{ L } & \multicolumn{1}{c|}{ DCR } & Q $\max$ & Volume \\
\hline This work & Air & $\mathbf{1 3 0} \mathbf{n H}$ & $\mathbf{0 . 7} \boldsymbol{\Omega}$ & $\mathbf{1 5}$ & $\mathbf{0 . 0 9} \mathbf{m m}^{\mathbf{3}}$ \\
\hline Micro. on Si [14] & Air & $11 \mathrm{nH}$ & $0.4 \Omega$ & 30 & $0.31 \mathrm{~mm}^{3}$ \\
\hline Surface-mount[15] & Ferrite & $140 \mathrm{nH}$ & $0.3 \Omega$ & 12 & $0.49 \mathrm{~mm}^{3}$ \\
\hline Micro. on Si [15] & $\mathrm{NiFe}$ & $150 \mathrm{nH}$ & $0.2 \Omega$ & 4 & $1.26 \mathrm{~mm}^{3}$ \\
\hline Micro. in Si [11] & $\mathrm{NiZn}$ & $350 \mathrm{nH}$ & $0.1 \Omega$ & 10 & $5.40 \mathrm{~mm}^{3}$ \\
\hline Micro. in NiZn [1] & NiZn & $1650 \mathrm{nH}$ & $0.02 \Omega$ & 20 & $6.43 \mathrm{~mm}^{3}$ \\
\hline
\end{tabular}

\section{Converter Testing}

The copper scaffolding platform was tested in conjunction with a Texas Instruments TPS61240 boost converter chip, which is designed to output a fixed voltage of approximately $5 \mathrm{~V}$. The TPS61240 was selected for having one of the highest switching frequencies amongst commercially available boost converters at up to $4.5 \mathrm{MHz}$ and, as such, called for one of the lowest values of inductance at $1 \mu \mathrm{H}$. Because the microinductor was significantly smaller-valued at $130 \mathrm{nH}$, the converter was only able to effectively regulate the output voltage at light load currents in the pulse frequency modulation (PFM) mode of operation. Two surface-mount $4.7 \mu \mathrm{F}$ capacitors with $1.0 \mathrm{~mm} \times 0.5 \mathrm{~mm}$ footprints were soldered onto the converter module for source and load filtering.

Two Keithley 2400 SourceMeters were used in the test setup and were connected by needle probes to the converter module: one in source mode at the input regulated the input voltage $V_{\text {in }}$ and measured the input current $I_{i n}$; the other in electronic load mode at the output regulated the output current $I_{\text {out }}$ and measured the output voltage $V_{\text {out }}$. Fig. 6 indicates these quantities on a simplified boost circuit diagram. Input and output powers were calculated from these measurements as the product of voltage and current, and efficiencies were calculated as the ratio of output to input power. The output current $I_{\text {out }}$ was swept from $1 \mathrm{~mA}$ to $30+\mathrm{mA}$ for several values of $V_{i n}$, with $V_{\text {out }}$ fixed at $5 \mathrm{~V}$ by the converter circuit.

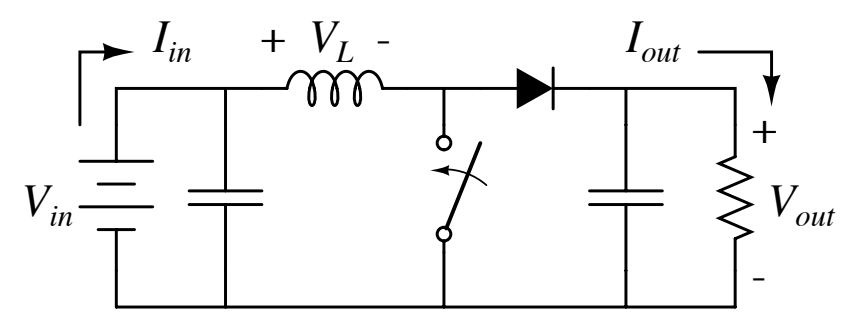

Figure 6: Simplified circuit diagram of boost converter circuit. Diagram indicates measurement points of input voltage $\left(V_{i n}\right)$ and current $\left(I_{\text {in }}\right)$, output voltage $\left(V_{\text {out }}\right)$ and current $\left(I_{\text {out }}\right)$, and inductor voltage $\left(V_{L}\right)$.

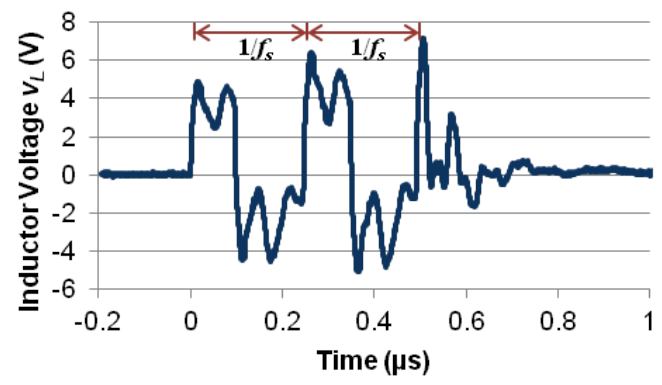

Figure 7: Inductor voltage $v_{L}(t)$ measured as a function of time for one series of high frequency pulses.

The inductor voltage $v_{L}(t)$ was monitored using a LeCroy oscilloscope with high impedance probes. As shown in the waveform in Fig. 7, the inductor was intermittently switched with a high frequency pulse sequence - the amplitude, duty cycle $(D)$, and frequency $\left(f_{s}\right)$ of which were dependent on the input voltage. These pulse sequences were repeated at lower frequencies as necessary to maintain an output voltage with $<100 \mathrm{mV}_{\mathrm{p} \text {-p }}$ ripple (i.e. as a function of output current).

As plotted in Fig. 8, the converter efficiency was relatively insensitive to output current but fell off dramatically with decreasing input voltage (increasing conversion ratio). For input voltages of $3.0 \mathrm{~V}, 3.5 \mathrm{~V}$, and $4.0 \mathrm{~V}$, the switching frequencies $\left(f_{s}\right)$ within each pulse sequence were measured to be $3.9 \mathrm{MHz}$, $4.1 \mathrm{MHz}$, and $4.2 \mathrm{MHz}$ with duty cycles $D$ of $0.5,0.4$, and 0.3 , respectively. Greater duty cycles were associated with lower efficiencies, since the longer pulse width $\left(v_{L}>0 \mathrm{~V}\right)$ more nearly approached the $L / R$ time constant of the inductor. A peak

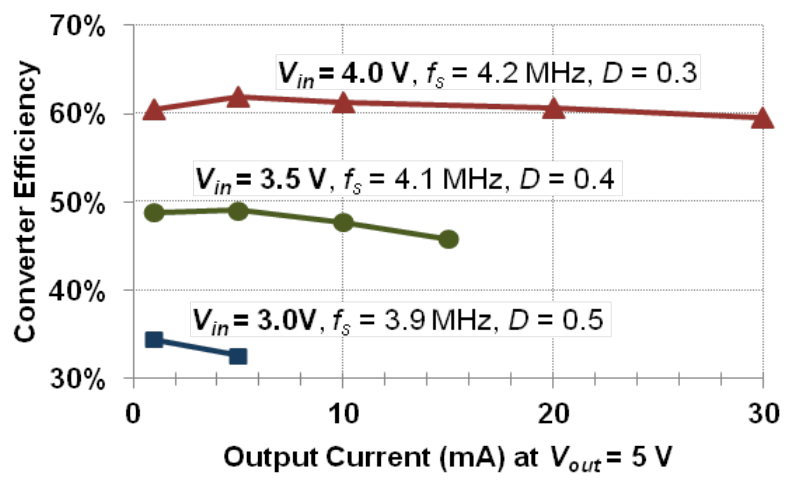

Figure 8: Plot of the converter module efficiency measured as a function of output current with $5 \mathrm{~V}$ output and several different input voltages. 
efficiency of $62 \%$ was measured with $V_{\text {in }}=4 \mathrm{~V}$ and $I_{\text {out }}=5 \mathrm{~mA}$, while a maximum power output of $153 \mathrm{~mW}$ was recorded at $60 \%$ efficiency with $V_{\text {in }}=4.0 \mathrm{~V}$ and $I_{\text {out }}=30 \mathrm{~mA}$. At output currents greater than those reported in Fig. 8, the converter exhibited control loop instability as it switched to the pulse-width modulation (PWM) mode of operation.

\section{CONCLUSION AND FUTURE WORK}

The process of 3D micromachining detachable copper scaffolds represents a powerful new capability for realizing ultraminiature power systems, enabling high performance passives to be built into self-contained modules with minimal volume. With its low profile and wafer detachment, the microfabricated inductor presented in this work provided exceedingly high volumetric inductance density $\left(1.4 \mu \mathrm{H} / \mathrm{mm}^{3}\right)$. As a result, high power density $\left(77 \mathrm{~W} / \mathrm{cm}^{3}\right)$ was obtained from testing the $130 \mathrm{nH}$ inductor in combination with a commercially available $\sim 4 \mathrm{MHz}$ boost converter chip, despite the converter being designed for a much larger inductor $(1 \mu \mathrm{H})$.

The 3D copper scaffolding is particularly complementary for use with emerging power converters of higher switching frequency (tens to hundreds of $\mathrm{MHz}$ ), which require even smaller passive components. The process may additionally be used to form high dielectric strength capacitors through interleaved plates of high aspect ratio copper. Because the scaffolds are fully exposed and freestanding, it may be possible to selectively backfill inductor and capacitor structures with magnetic and dielectric composites, respectively, prior to encapsulating the full scaffold package.

Deformable sockets formed in the copper scaffolding (Fig. 9) could ease the integration of the smallest-sized surface-mount components (with $400 \mu \mathrm{m} \times 200 \mu \mathrm{m}$ footprint), which are problematic to attach by solder reflow due to tomb-stoning and misalignment [16], [17]. The converter IC and capacitors could then be integrated with the scaffolding before embedding to enable wafer-level packaging of fully integrated converter modules.

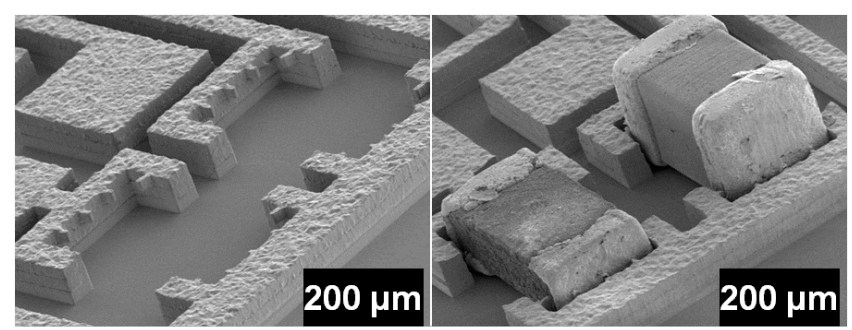

(a)

(b)

Figure 9: Deformable copper sockets (a) before and (b) after fitting with a 01005-sized resistor and capacitor.

\section{ACKNOWLEDGMENTS} 08-2-0035.

This work was supported in part by ARO Grant \#W911NF-

\section{REFERENCES}

[1] Z. Hayashi, Y. Katayama, M. Edo, and H. Nishio, "HighEfficiency DC-DC Converter Chip Size Module With Integrated Soft Ferrite," IEEE Trans. Magn., 39, 3068 (2003).

[2] B. Morgan, et al., "Power for autonomous Microsystems," Proc. $27^{\text {th }}$ Army Science Conference 2010, Orlando, FL, $11 / 29-12 / 2 / 10,5$ pages.

[3] B. Morgan, et al., "Power Considerations for MAST
Platforms," Proc. SPIE Defense Security \& Sensing Conf. 2010, Orlando, FL, 4/5-9/2010, 12 pages.

[4] G. Bertotti, "General properties of power losses in soft ferromagnetic materials," IEEE Trans. Magn., 24(1), 621 (1998).

[5] R. Meere, et al., "Magnetic-Core and Air-Core Inductors on Silicon: A Performance Comparison up to $100 \mathrm{MHz}$," IEEE Trans. Magn., 47, 4429 (2011).

[6] C.D. Meyer, S.S. Bedair, B.C. Morgan, and D.P. Arnold, "Micromachined Thick-Film Copper Power Inductors and Transformers for Integrated Power Converters," Technical Digest of the 2010 Solid-State Sensors, Actuators, and Microsystems Workshop, Hilton Head Isl., SC, 6/6-10/10, Transducer Research Foundation, Cleveland (2010), pp. 455458.

[7] C.D. Meyer, S.S. Bedair, B.C. Morgan, and D.P. Arnold, "High-Inductance-Density, Air-Core Power Inductors and Transformers Designed for Operation at 100-500 MHz," IEEE Trans. Magn., 46, 2236 (2010).

[8] J.Y. Park and M.G. Allen, "Packaging-Compatible High Q Microinductors and Microfilters for Wireless Applications," IEEE Trans. Advanced Packaging 22 (2), 207 (1999).

[9] J.-B. Yoon, B.-I. Kim Y.-S. Choi, and E. Yoon, "3-D Construction of Monolithic Passive Components for RF and Microwave ICs Using Thick-Metal Surface Micromachining Technology," IEEE Trans. Microwave Theory and Techniques, 51(1), 279 (2003).

[10] Z. Popovic, et al., "Micro-fabricated Micro-coaxial Millimeter-wave Components," Proc. Intl. Conf. on Infrared, Millimeter and Terahertz Waves, Pasadena, CA, 9/15-19/08, IEEE, pp. 1-3 (2008).

[11] M. Wang, J. Li, K.D.T. Ngo, and H. Xie, "A SurfaceMountable Microfabricated Power Inductor in Silicon for Ultracompact Power Supplies," IEEE Trans. Power Electron., 26, 1310 (2011).

[12] M. Schlesinger and M. Paunovic, Eds., Modern Electroplating, John Wiley \& Sons, New York, 2000.

[13] "ProTEK A2 Etch Protective Coating Technical Data Sheet," Brewer Science, Rolla, Missouri (2007).

[14] H.J. Bergveld, et al., "A 65-nm-CMOS 100-MHz 87\%efficient DC-DC down converter based on dual-die Systemin-Package integration," Proc. IEEE Energy Conversion Congress \& Exposition 2009, San Jose, CA, 9/20-24/09, pp. 3698-3705.

[15] R. Meere, T. O’Donnell, H.J. Bergveld, N. Wang, and S.C. O'Mathuna, "Analysis of Microinductor Performance in a 20$100 \mathrm{MHz}$ DC/DC Converter," IEEE Trans. Power Electron., 24, 2212 (2009).

[16] H.T. Ming, T.K. Ming, and L. Khor, "Tombstone reduction by reflow profile optimization, SMT stencil design and pad design," Proc. IEEE/CPMT Intl. Electronic Manufacturing Tech. Symposium, Melaka, MY, 11/30-12/2/10, IEEE pp. 15 (2010).

[17] J. Hoo, A. Lingley, R. Baskaran, X. Xiong, and K.F. Bohringer, "Parallel Assembly of 01005 Surface Mount Technology Components with 100\% Yield," Proc. IEEE Intl. Conf. on Micro Electro Mechanical Systems, Wanchai, HK, 1/24-28/10, IEEE, pp. 532-535 (2010).

\section{CONTACT}

*C.D. Meyer, tel: +1-301-394-4286; christopher.d.meyer1.civ@mail.mil 


\section{CHAOTIC BEHAVIOUR OF A MEMBRANE RESONATOR WITH NANOWIRE ARRAY \\ Yuerui Lu*, Manoj Pandey, and Amit Lal \\ SonicMEMS Laboratory, School of Electrical and Computer Engineering \\ Cornell University, Ithaca, NY, USA}

\begin{abstract}
We report the first chaotic behavior of a membrane mechanical resonator with a nanowire (NW) array structured surface. Compared with the control sample without NW array, the membrane resonator with NW array structured surface, can easily transition from linear operation to a nonlinear motion at resonance, and further to a chaotic state, through an increase in its dynamic operating amplitude. The high nonlinearity of the device spreads the concentrated excitation energy from a single frequency to a broad-band frequency range, which could be useful for line-spectrum vibration isolation. We develop a set of possible reasons for the chaotic behavior, which aid in future investigation of controlled chaotic structures.
\end{abstract}

\section{INTRODUCTION}

Nano-scale resonance systems can transition from a linear resonance operation to a nonlinear one through an increase in its dynamic operating amplitude. In the nonlinear regime of nano-electro-mechanical system (NEMS), phenomena, such as hysteresis [1], spring hardening [2], and pull-in [3], can occur. The nonlinear dynamical properties of the NEMS have been exploited for many applications. For instance, noise-enabled transitions in a nonlinear resonator were analyzed to improve the precision in measuring the linear resonance frequency [4], and a homodyne measurement scheme for a nonlinear resonator was proposed for increasing the mass sensitivity and reducing the response time [5].

The nonlinear dynamics of NEMS system might lead to the appearance of chaos. Chaotic behavior of a dynamical system is highly sensitive to initial conditions. Chaos can be observed in many natural systems, such as weather, where infinitesimally small perturbations can cause large effects. For chaotic systems, small differences in initial conditions will result in widely diverging outcomes, leading to system outcome prediction impossible [6]. Normally, such behavior may be investigated or explained through analysis of a chaotic mathematical model, or through analytical techniques such as recurrence plots and Poincaré maps. Nonlinearity, especially chaos, in NEMS systems has mostly been seen as a problem, but here we might want to ask whether one can explore chaos to enhance its applicability.

Here, we report the first chaotic behavior of a membrane mechanical resonator with nanowire $(\mathrm{NW})$ array structured surface. Compared with the control sample without NW array, the membrane resonator with NW array structured surface (Fig.1), can easily transit from linear resonance operation to a nonlinear one, and further to a chaotic state, through an increase in its dynamic operating amplitude. The high nonlinearity of our device will help to spread the concentrated excitation energy from line-spectrum frequency to a broad-band frequency range (Fig.2 \& 3), which could be used for line-spectrum vibration isolation [7]. Vibration isolation is needed for MEMS device [8]; for example, inertial sensors, especially MEMS gyroscopes, can be interfered or even damaged by the undesired modes of vibration present in mechanically harsh environments. For a linear vibration isolation system, frequency conservation is the main characteristics. For nonlinear oscillations, external harmonic excitation from noise can exhibit a great variety of harmonic responses, sometimes countless when chaos appears
The concentrated energy therefore spreads from the frequency of excitation to a broad-band frequency range (Fig.2). For the given input energy, the response energy at the excitation frequency for the chaotic vibration system is much less than that for the linear vibration system [7]. Hence, a chaotic energy dissipater could be used to isolate sensors from external vibrations.

\section{DEVICE FABRICATION}

(a)

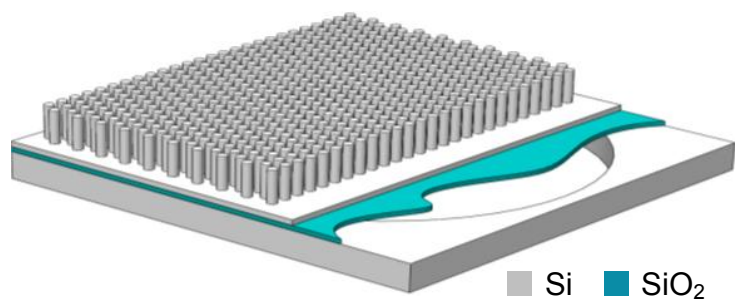

(b)
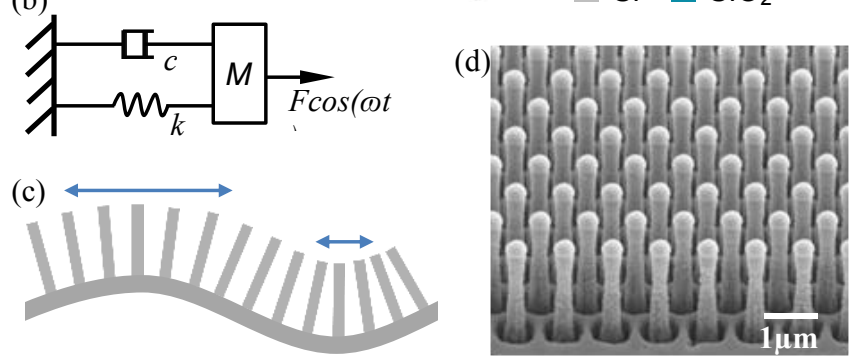

Figure 1: (a) Schematic for device structure. Vertical Si nanowire $(\mathrm{NW})$ array were patterned on $\mathrm{Si} / \mathrm{SiO}_{2}(1 \mu \mathrm{m} / 2 \mu \mathrm{m}$ in thickness) bilayer circular membrane (diameter $800 \mu \mathrm{m}$ ). (b) Schematic for the single degree of freedom model with damping. (c) Schematic for the space modulation of the NWs by the standing wave of the membrane. (d) SEM image of the $N W$ array.

For device fabrication, we used electron beam lithography to pattern the vertical silicon NW arrays, on a SOI wafer, by silicon reactive ion etching (RIE) using $\mathrm{SiO}_{2}$ nano-posts as the etching mask. The vertical silicon NW array has $300 \mathrm{~nm}$ of wire diameter, 1 $\mu \mathrm{m}$ of pitch and $3.5 \mu \mathrm{m}$ of height (Fig.1b). These NW arrays show uniform wire diameter, controllable wire orientation and wire pitch spacing. Optical lithography with backside alignment was used to pattern the backside circular cavity $(800 \mu \mathrm{m}$ diameter $)$, followed by a silicon DRIE to etch through the silicon substrate using the SOI buried oxide as the stopping layer. A PZT plate was adhesively bonded to the silicon backside to actuate the membrane.

\section{EXPERIMENTAL OBSERVATIONS}

The vibration response of the membrane was measured by an optical interferometer $\left(\right.$ PolyTec $\left.^{\mathrm{TM}}\right)$. Power-spectrum density characteristics (Fig.2) of the devices were measured by using a power spectrometer to analyze the output signal from the interferometer. The vibration amplitude versus time was measured using an oscilloscope and a spectrum analyzer. For the membrane with NWs, we observed the fundamental resonance mode $\left(f_{l}\right)$ at $163 \mathrm{kHz}$. When the system operates in linear range with low driving voltage $20 \mathrm{mV}$, the output signal shows monotonic spectrum (Fig. 
2a top). The device can easily transition from linear resonance operation to nonlinear one, by increasing the PZT driving voltage. Initially, super harmonic nonlinear modes, such as $2 f_{l}$ and $3 f_{l}$, showed up at higher driving voltage $(100 \mathrm{mV})$. Since the samples with and without NW are on the same chip and share the same PZT for actuation, the coupling from the PZT was the very similar in two samples. In the non-membrane area, the amplitudes of the NW versus non-NW samples were in the ratio of around 1:3, since the sample without NW has higher Q value (Table 1). When we increased the driving voltage further, the resonance modes and super harmonic modes will start to split into symmetric sub-bands, through bifurcation process. Finally, chaotic behavior of the device was observed (Fig.2a bottom), at $300 \mathrm{mV}$ driving voltage. In the chaotic state of the device, the concentrated excitation energy was spread from line-spectrum frequency to many peaks over broad frequency range. The measurement of upper frequency range was limited by the range of the spectrum analyzer. The nonlinearity
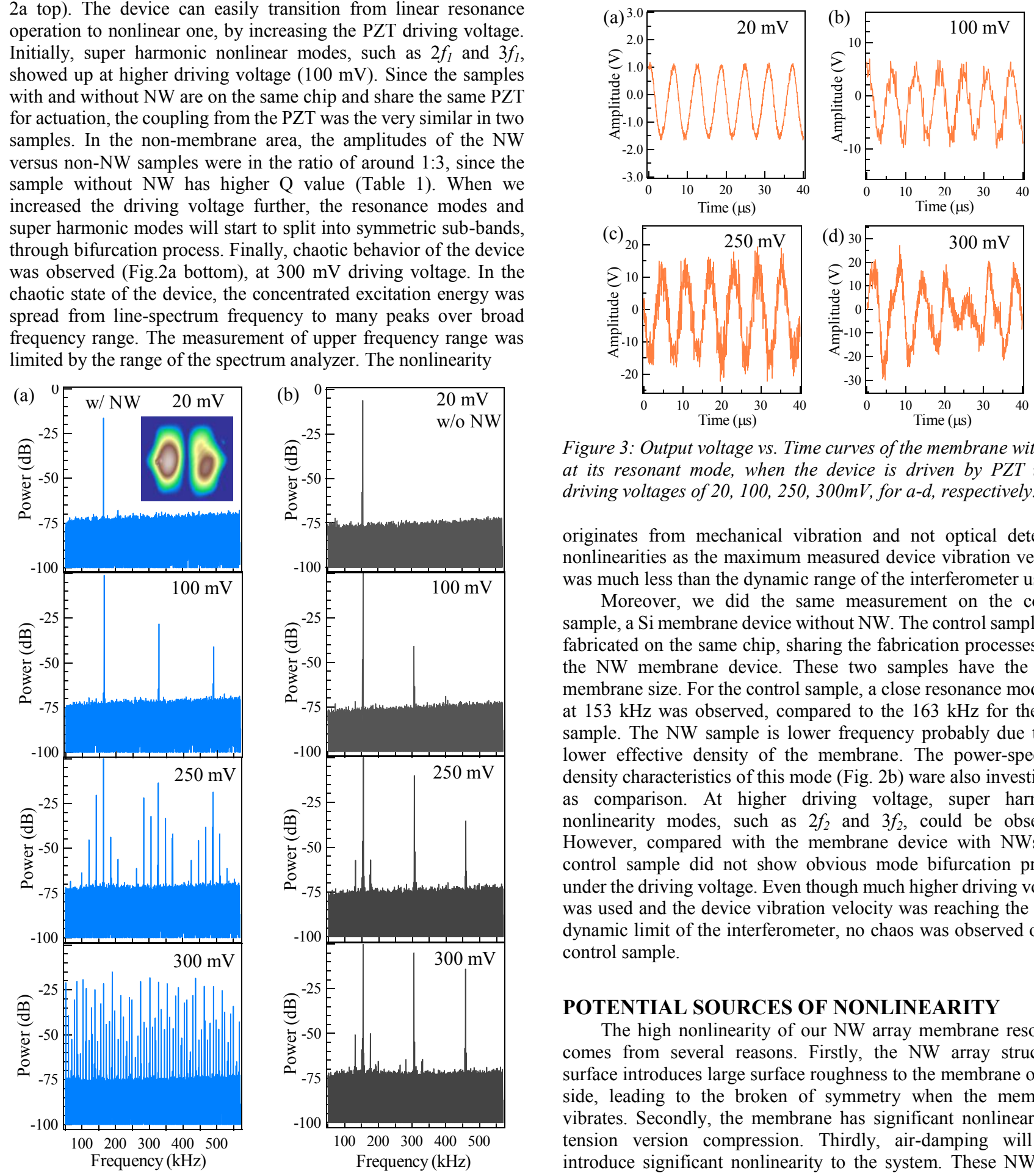

Figure 3: Output voltage vs. Time curves of the membrane with $\mathrm{NW}$ at its resonant mode, when the device is driven by PZT under driving voltages of $20,100,250,300 \mathrm{mV}$, for a-d, respectively.

originates from mechanical vibration and not optical detection nonlinearities as the maximum measured device vibration velocity was much less than the dynamic range of the interferometer used.

Moreover, we did the same measurement on the control sample, a Si membrane device without NW. The control sample was fabricated on the same chip, sharing the fabrication processes with the NW membrane device. These two samples have the same membrane size. For the control sample, a close resonance mode $\left(f_{2}\right)$ at $153 \mathrm{kHz}$ was observed, compared to the $163 \mathrm{kHz}$ for the NW sample. The NW sample is lower frequency probably due to the lower effective density of the membrane. The power-spectrum density characteristics of this mode (Fig. 2b) ware also investigated as comparison. At higher driving voltage, super harmonic nonlinearity modes, such as $2 f_{2}$ and $3 f_{2}$, could be observed. However, compared with the membrane device with NWs, the control sample did not show obvious mode bifurcation process under the driving voltage. Even though much higher driving voltage was used and the device vibration velocity was reaching the upper dynamic limit of the interferometer, no chaos was observed on the control sample.

\section{POTENTIAL SOURCES OF NONLINEARITY}

The high nonlinearity of our NW array membrane resonator comes from several reasons. Firstly, the NW array structured surface introduces large surface roughness to the membrane on one side, leading to the broken of symmetry when the membrane vibrates. Secondly, the membrane has significant nonlinearity in tension version compression. Thirdly, air-damping will also introduce significant nonlinearity to the system. These NWs can enhance the total surface area by a factor of 10 , leading to larger the air-damping force. In following section, we would like to focus on the modeling of nonlinearity from air-damping.

2. (a) Power-spectrum density characteristics of membrane with $N W$ at its resonant mode, when the device is driven by PZT under driving voltages of 20,100, 250, $300 \mathrm{mV}$. Inset shows the measured standing wave vibration amplitude $2 D$ mapping pattern of the mode $163 \mathrm{kHz}$. During mapping, the actuation frequency was fixed at the resonance mode and the interferometer laser gun was controlled by a stage controller for lateral movement with sub-0.5 $\mu \mathrm{m}$ lateral resolution. (b) power-spectrum density of the control membrane without $N W$, when it is driven at the same driving voltages correspondingly.

\section{MODELING AND ANALYSIS}

By considering damping, the nonlinear partial differential equation governing the membrane can be obtained as equation [9]:

$$
\rho h \frac{\partial^{2} Z}{\partial t^{2}}+D \nabla^{4} Z+\zeta \frac{\partial Z}{\partial t}=f(r, \theta, t)
$$


where $Z(r, \theta, t)$ is the transverse displacement, $\zeta$ is the damping coefficient, $h$ is the effective membrane thickness, $\rho$ is the effective mass density, and $D$ is the effective flexural rigidity of the membrane, and $\nabla^{2}$ is the Laplacian.

By solving this equation for a circular membrane with radius $r_{0}$, the resonant frequencies and their corresponding transverse displacement profiles at different mode shapes are obtained as follows [10]:

$$
\begin{aligned}
& f_{m n}=\frac{1}{2 \pi} \sqrt{\frac{\gamma_{m n}^{4} D}{\rho h}} \\
& Z_{m n}(r, \theta)=\left[J_{m}\left(\gamma_{m n} r\right)-\frac{J_{m}\left(\gamma_{m n} r_{0}\right)}{I_{m}\left(\gamma_{m n} r_{0}\right)} I_{m}\left(\gamma_{m n} r\right)\right] \\
& \cdot\left[A_{1 m} \sin (m \theta)+A_{2 m} \cos (m \theta)\right]
\end{aligned}
$$

where the functions $J_{m}\left(\gamma_{m n} r\right)$ and $I_{m}\left(\gamma_{m n} r\right)$ are the Bessel function and the modified Bessel function of the first kind, $\gamma_{m n}$ are the eigenvalues and the subscripts $m$ and $n$ represent the number of nodal diameters and nodal circles for the vibration mode, respectively.

Symmetric circular discs under high-amplitude excitation have been shown to undergo bifurcation, leading to chaotic motion [11]. This happens due to the presence of cubic nonlinearity in the system, which becomes prominent at large amplitude vibrations, where energy is spread into different modes of the disc. The mode mixing results in the excitation of other energy modes, from what is being directly forced. This results in the quasi-periodic response of the directly excited mode which may become chaotic. It has also been shown that if structural symmetry in such systems is broken, the chaotic transition becomes possible at a much lower forcing [11]. Additionally the route to chaos is through period doubling, which is what is observed in the current system. Hence the presence of NW brings down the forcing threshold for the chaos, which can now be observed at relatively lower amplitude forcing. In order to handle the complexity of the analysis we obtain a reduced ordered set of equations for the system at hand by projecting the governing partial differential equation of the system motion, on to the dominant linear modes and hence obtain a set of ordinary differential equations, which are integrated using a $4^{\text {th }}$ order Runge-Kutta based ODE solver. The governing equations used for the simulations are [12]:

$$
\ddot{x}+\alpha \dot{x}+\gamma \dot{x}^{2}-\omega_{0}^{2} x+\beta x^{3}=F \cos (\omega t)
$$

where $x$ is the position displacement, $\alpha$ is the linear damping coefficient, $\gamma$ is the quadratic damping coefficient, $\omega_{0}$ is the resonance frequency of the system, $\beta$ is the Duffing's cubic nonlinearity coefficient, $F$ is the external excitation force and $\omega$ is the frequency of the external force.

Previous theoretical reports [12] showed that damping force plays an important role in the nonlinear dynamics of a Duffing's oscillation system, which is also confirmed by our simulation results. We simulated the Poincare maps (Fig. 4) for two different cubic nonlinear systems with: 1) linear damping, and 2) quadratic damping. Poincare map for the system is obtained by plotting the solution at every forcing time period, which shows the periodic vibrations at the forcing frequency, with one point, while period doubling is shown with twice the number of points. Figure $4 \mathrm{~b}$ shows that quadratic damping has lower forcing threshold value for the chaos, among these three systems. For the system without damping, we did not get chaotic behavior in the force range simulated, which indicates that it has the highest forcing threshold value for the chaos. Therefore, the addition of damping or quadratic damping term of same order significantly reduces the forcing threshold value for chaos.

From our experiments, we observed that the membrane device with NWs did show higher damping, compared with the membrane without NWs. We measured three resonance modes on each device (Table 1). The modes for the device with NWs showed much lower quality factor values $(40 \sim 75)$ than that of the modes from the device without NWs (250 350). The enhanced air damping might be due to the enhanced total surface area, by a factor of 10 , from these high aspect ratio NW structures. The squeeze film damping between the nanowires is also expected to increase the damping

Based on fluid dynamics theory $[13,14]$, the drag force on an object is generally dependent on its velocity, $v$, and there exist two
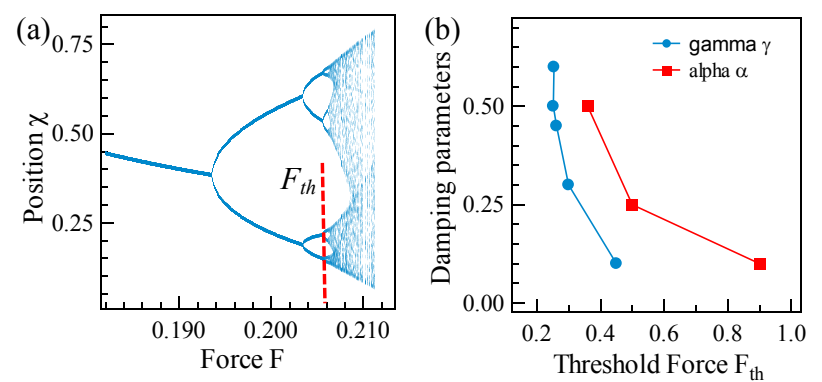

Figure 4: (a) Simulated Poincaré map of Position x vs. Force for a forced Duffing's oscillator with quadratic damping. Red dashed line indicates the threshold force $\left(F_{t h}\right)$ value for chaos. b) Simulated threshold force values as a function of the parameters for linear damping (alpha) and quadratic damping (gamma).
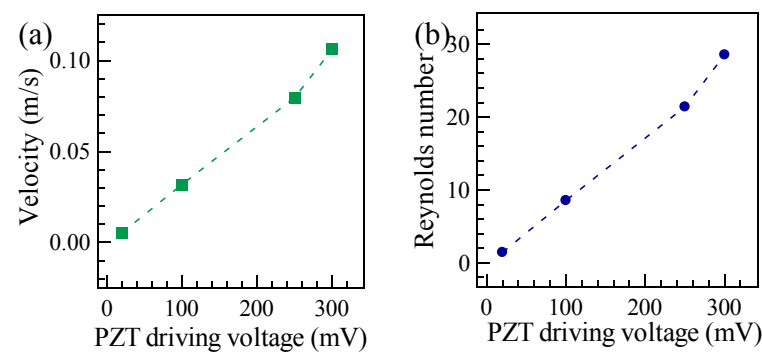

Figure 5: (a) Measured velocity as a function of the PZT driving voltage. (b) Calculated Reynolds number of our device based on the measured velocity and equation 5.

regimes where this dependence is either linear or quadratic. The parameter that differentiates these two regimes is the Reynolds number, $R_{e}$, which is the ratio of inertial to viscous forces,

$$
R_{e}=\rho l v / \eta
$$

where $\rho$ is the fluid density, $l$ is the characteristic cross-sectional length, $v$ is the velocity and $\eta$ is the dynamic fluid viscosity. At high Reynolds number $\left(R_{e}>10^{3}\right)$, drag is approximately dependent on the square of the velocity [14]:

$$
f_{d}(v)=\frac{1}{2} \rho C_{d} A v^{2}
$$

where $f_{d}(v)$ is the velocity-dependent drag force where $C_{d}$ is the drag coefficient, $A$ is the cross-sectional area and. The drag coefficient, $C_{d}$, depends on the Reynolds number. At low Reynolds 
number $\left(R_{e}<1\right)$, drag is linear with velocity [14]:

$$
f_{d}(v)=12 \eta A v / l
$$

Table 1: Comparison of the measured quality factor (in air) for the modes of the membrane sample with $N W$ and without $N W$.

\begin{tabular}{|c|c|c|c|}
\hline \multicolumn{2}{|c|}{ Membrane with NW } & \multicolumn{2}{c|}{ Membrane without NW } \\
\hline$f(\mathrm{kHz})$ & $Q$ & $f(\mathrm{kHz})$ & $Q$ \\
\hline 163 & 42 & 153 & 337 \\
\hline 107 & 38 & 101 & 265 \\
\hline 234 & 73 & 218 & 352 \\
\hline
\end{tabular}

Based on our measured velocity (Fig. 6a), the Reynolds number of our device is in the range of 1-50 (Fig. 6b). Therefore, the damping force for our system should be a combination of linear and quadratic damping. Moreover, based on equation 3 and 4, both linear and quadratic damping forces could be enhanced by the NW structure, due to the enhanced surface area.
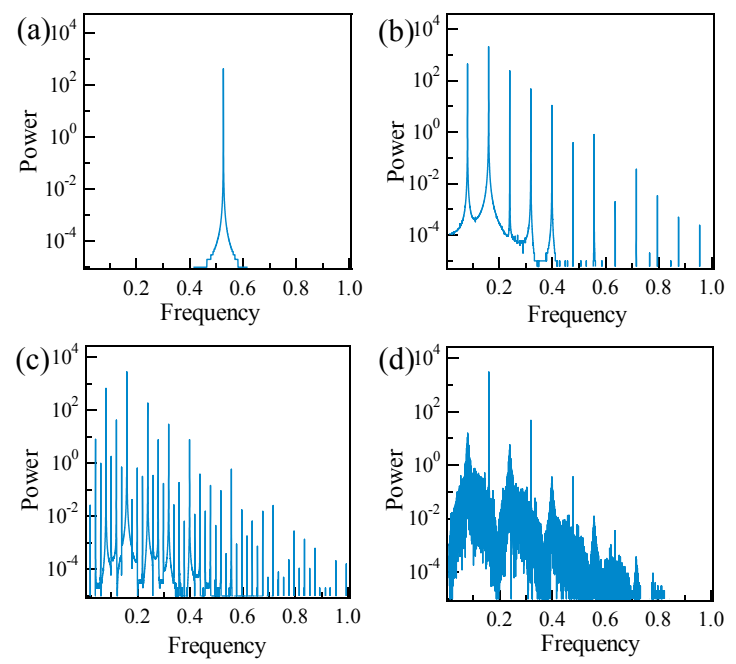

Figure 6: Simulated FFT of the motion at four different forcing levels: a) $F=0.2$ Single frequency responses, b) $F=0.34$ Multiple harmonics, c) $F=0.206$ Side-band bifurcation formation, d) $F=0.38$ Chaotic response.

Moreover, it has been previously shown [15] that in beam vibration problems, combination resonance can occur when the sum of the natural frequency of certain modes of the system is close to the sum of other modes of the system. In this case energy can transfer from higher modes of the system to the lower ones but no other way. Excitation close to the higher modes can lead to vibration of lower modes. This also leads to periodic modulation of primary mode being excited. However for this combination of the frequencies to be in an internal resonance, it is necessary that the governing equation have a quadratic nonlinearity term. This term seems to originate from the quadratic damping term that can be used to model the air drag. Here, the enhanced quadratic damping term from NWs might also trigger internal resonances, leading to lower threshold forcing value for chaos.

Of course, chaotic motion is a very complicated dynamic process, which is related to many factors [15]. In order to have a better understanding on the chaotic behavior of our device, more theoretical and experimental work is needed. Although hard to model, our system will be useful where energy dispersion is required. Also, our device provides a good platform to investigate nonlinearity dynamics and potential chaos engineering.

\section{CONCLUSION}

Chaotic behavior of a membrane mechanical resonator with NW array structured surface has been presented for the first time. Compared with the control sample without NW array, the membrane resonator with NW array structured surface, can easily transit from linear resonance operation to a nonlinear one, and further to a chaotic state, through an increase in its dynamic operating amplitude. The high nonlinearity of our device will help to spread the concentrated excitation energy from line-spectrum frequency to a broad frequency range. Our system will be useful where energy dispersion is required, such as for line-spectrum vibration isolation. Moreover, simulations on the dynamics of nonlinear systems with different damping have been investigated and possible reasons for the chaotic behavior are discussed.

\section{REFERENCES}

[1] I. Kozinsky, H. W. C. Postma, O. Kogan, A. Husain, M. L. Roukes, "Basins of Attraction of a Nonlinear Nanomechanical Resonator”, Physical Review Letters 99, 207201 (2007).

[2] I. Kozinsky, H. W. C. Postma, I. Bargatin, M. L. Roukes, "Tuning nonlinearity, dynamic range, and frequency of nanomechanical resonators", Applied Physics Letters 88, 253101 (2006).

[3] S. N. Cha et al., "Fabrication of a nanoelectromechanical switch using a suspended carbon nanotube", Applied Physics Letters 86, 083105 (2005).

[4] J. S. Aldridge, A. N. Cleland, "Noise-Enabled Precision Measurements of a Duffing Nanomechanical Resonator", Physical Review Letters 94, 156403 (2005).

[5] E. Buks, B. Yurke, "Mass detection with a nonlinear nanomechanical resonator", Physical Review E 74, 046619 (2006).

[6] S. H. Kellert, In the Wake of Chaos: Unpredictable Order in Dynamical Systems. University of Chicago Press. p. 32, 1993.

[7] J. Lou, et al. "Application of chaos method to line spectra reduction" Journal of Sound and Vibration. 286, 645 (2005).

[8] S. Yoon, et al. "Analysis and wafer-level design of a high-order silicon vibration isolator for resonating MEMS devices", J. Micromech. Microeng. 21, 015017 (2011).

[9] S. P. Timoshenko, S. Woinowsky-Kringer, Theory of Plates and Shells (New York: McGraw-Hill), 1959.

[10] M. Olfatnia, V. R. Singh, T. Xu, J. M. Miao, L. S. Ong, "Analysis of the vibration modes of piezoelectric circular microdiaphragms", J. Micromech. Microeng, Vol. 20, pp. 085013, 2010.

[11] C. Touze, O.Thomas, M.Amabili, "Transition to chaotic vibrations for harmonically forced perfect and imperfect circular plates", International Journal of Non-Linear Mechanics ,Vol. 46, Issue 1, pp. 234-246, (2011).

[12] A. Sharma, V. Patidar, G. Purohit, K.K. Sud, "Effects on the bifurcation and chaos in forced Duffing oscillator due to nonlinear damping", Communications in Nonlinear Science and Numerical Simulation, Vol.17, Iss.6, pp.2254-69, (2012).

[13] E. M. Purcell, Am. J. Phys. 453, (1977).

[14] J. P. Owen, W. S. Ryu, "The effects of linear and quadratic drag on falling spheres: an undergraduate laboratory", European Journal of Physics 26, 1085 (2005).

[15] C. Touzé, S. Bilbao, O. Cadot, "Transition scenario to turbulence in thin vibrating plates", Journal of Sound and Vibration 331, 412 (2012).

\section{CONTACT}

*Yuerui Lu, Email: yl676@cornell.edu 


\title{
LARGE AREA SEMI-PERMEABLE ENCAPSULATION MEMBRANES USING CARBON NANOTUBE COMPOSITES
}

\author{
Armon Mahajerin and Liwei Lin \\ Berkeley Sensor and Actuator Center \\ University of California, Berkeley, USA
}

\begin{abstract}
A batch-fabrication methodology to create large area vacuumsealed cavities on top of silicon substrates is reported. This process uses a semi-permeable membrane made of carbon nanotubes (CNTs) and polysilicon. The dense forest of CNTs, conformally coated with polysilicon by low pressure chemical vapor deposition (LPCVD), functions as the semi-permeable membrane. When the top surface is opened via plasma etching of polysilicon, the membrane is penetrable to liquid and vapor. Hydrofluoric acid (HF) vapor is used to etch the underlying oxide before sealing the film with another LPCVD of polysilicon, rendering it impermeable. Sealed areas of more than $1 \times 1 \mathrm{~mm}^{2}$ have been fabricated.
\end{abstract}

\section{INTRODUCTION}

Microelectromechanical systems (MEMS), which function as sensors or actuators, have undergone considerable growth in the past two decades. One of the key developments has been the miniaturization of MEMS, making it possible to integrate their processing with that of integrated circuit (IC) processing to reduce the costs of manufacturing and packaging [1]. However, a key difference between MEMS and IC devices is that MEMS require some form of interaction with their environments for signal processing. This can be problematic when considering process flow compatibility. The key issue for MEMS process development is the packaging of the devices, which is often the primary barrier to the commercialization of MEMS devices. It also tends to contribute to up to $70 \%$ of the total cost for a MEMS device development cycle [2]. Due to the widely varying types of MEMS device materials and applications, a universally adopted industry standard remains elusive. Pressure, chemical, or fluidic sensors should to be open to the environment in order to function. On the other hand, accelerometers, gyroscopes, or oscillators should be hermetically sealed, sometimes with an anti-stiction agent and/or buffer gas [3]. Moisture penetration may also lead to corrosion within the package.

One trend in industry packaging methodologies is the shift from die level packaging to wafer level packaging. Die level encapsulation involves dicing the chips on a wafer then packaging them individually with separate substrates. The drawbacks to this approach include special handling requirements so as to not damage the MEMS devices and reduced throughput since individual dies must first be packaged then tested for meeting performance specifications.

Wafer level packaging mitigates a number of issues in die level packaging [4]. Importantly, incorporating encapsulation into the device fabrication steps prior to dicing provides an inherent protective lid for the MEMS structures during fabrication and back-end assembly. The wafer level encapsulation may be formed by interfacial bonding between the device substrate and a separate sealing wafer or by thin film deposition with the latter approach offering savings in material costs. Thin film encapsulation also eliminates the need for aligning separate wafers as well as the seal ring around each device, thereby increasing available space to enhance yield and throughput. Lastly, the lower topography of the packaged devices permits post encapsulation processing.
The nature of thin films can limiting compared to bonded caps when considering the strength and size of the membrane, since these films may collapse or break under vacuum loads. Some thin film processes use the permeability of that membrane to provide a pathway for etchants to sacrificial layers, and they must be sealed afterward. Other thin films may simply be patterned with a small channel near the base in lieu of permeability [5]. In either case, for larger areas it's often necessary to bolster the membrane with a hard film like silicon nitride, which may be problematic for processing due to slow deposition.

Polysilicon is one such material that can be made permeable for thicknesses around less than $1 \mu \mathrm{m}$ [6,7]. However, incorporating this into a fabrication process requires numerous steps of doping, annealing, hard mask under layers, etc. that can be tedious. Thickness and area limitations are also an issue. However, combining polysilicon with another material such as CNTs opens possibilities to use thicker films for larger areas. The results presented here aim to improve the flexibility in designing the thickness and strength of encapsulation membranes compared to other thin film approaches. The concept of using a composite film of polysilicon and CNTs is discussed in the following section.

\section{CONCEPT}

CNTs are attractive materials for a number of reasons. They may be grown with very large aspect ratios and possess semiconducting properties along with good mechanical strength and self-alignment when grown in a forest. In this paper's process CNT height may be set anywhere from $5 \mu \mathrm{m}$ to $50 \mu \mathrm{m}$ with short processing times, with CNTs serving as a skeletal framework for deposited encapsulation films. Moreover, the inclusion of CNTs into a composite film generates a natural porosity in the film due to the spacing between individual CNTs. Thus, a semi-permeable membrane forms by coating the CNT forest with a material like polysilicon. Fig. 1 below shows a schematic of the composite film.

\section{CNT/Polysilicon Encapsulation Membrane}

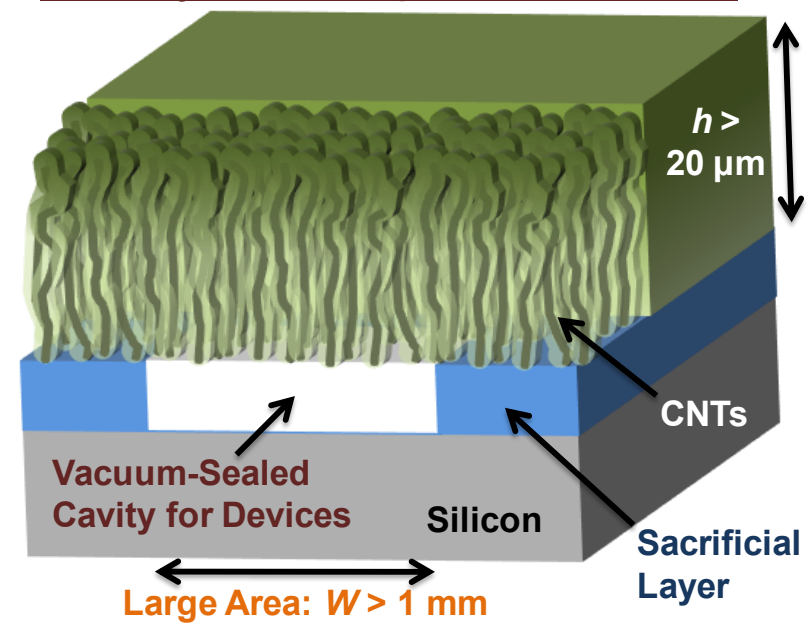

Figure 1: Conceptual illustration of encapsulation using a semipermeable CNT/polysilicon membrane. 


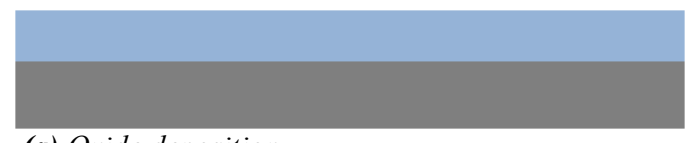

(a) Oxide deposition

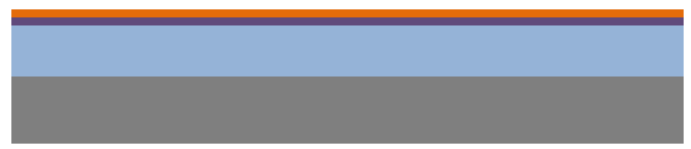

(b) Evaporation of Al and Fe films

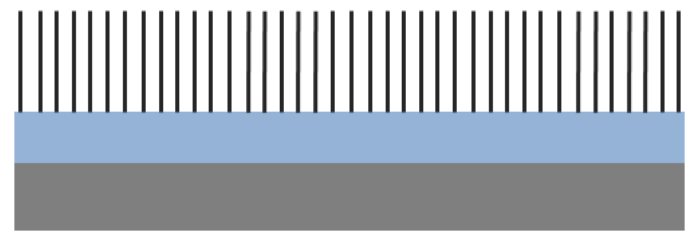

(c) CNT growth

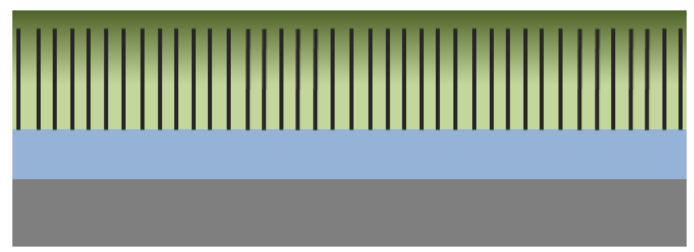

(d) First polysilicon LPCVD

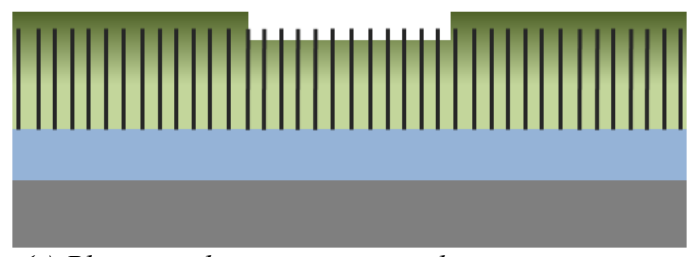

(e) Plasma etching to create window

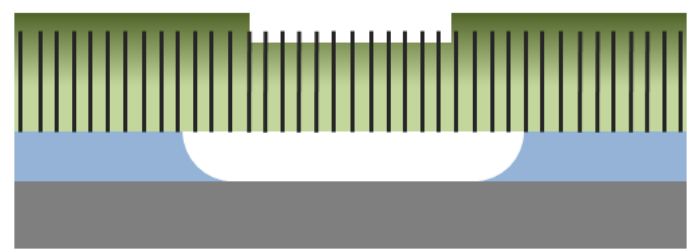

(f) $H F$ vapor etching of oxide

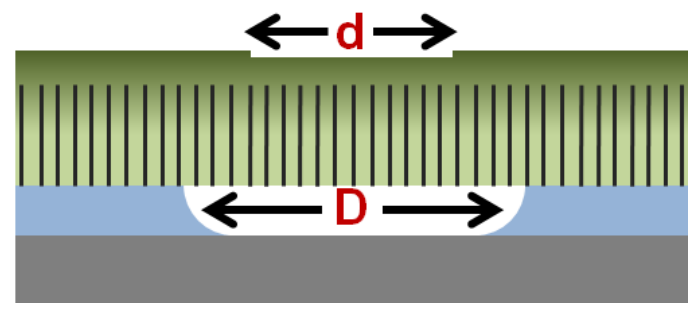

(g) Second polysilicon LPCVD (sealing)

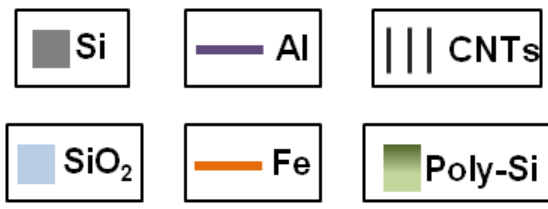

Figure 2: Fabrication process for developing the CNT/polysilicon composite and forming the sealed cavity.

\section{FABRICATION}

The process flow for testing the $\mathrm{CNT} /$ polysilicon composite, performing oxide etching, and sealing the cavity is shown in Fig. 2. The fabrication begins with a bare silicon wafer. Low-temperature oxide is deposited as a sacrificial layer onto the substrate via LPCVD in a furnace at a process temperature of $450^{\circ} \mathrm{C}$. The deposition rate is roughly $700 \mathrm{~nm} /$ hour. In a fully realized MEMS process the device should be embedded within the oxide. If much thicker films are needed, oxide from a liquid source of tetraethyl orthosilicate (TEOS) may be deposited with plasma enhanced chemical vapor deposition (PECVD) to drastically reduce the deposition time compared to a furnace process.

Next, two metal films are deposited using electron-beam evaporation at a pressure of $10^{-6}$ torr or less (Fig. 2b). First, an aluminum film of $10 \mathrm{~nm}$ is evaporated, followed by $5 \mathrm{~nm}$ of iron. The Al layer serves as a barrier against interaction between the iron and the substrate underneath. In addition, Al also supports proper nucleation of $\mathrm{Fe}$, which serves as the catalyst for the CNT growth process.

After metal deposition the CNTs may be grown in a simple furnace at a temperature of $720^{\circ} \mathrm{C}$ with hydrogen $\left(\mathrm{H}_{2}\right)$ and ethylene $\left(\mathrm{C}_{2} \mathrm{H}_{4}\right)$ gas flow (Fig. 2c). After approximately two minutes CNTs begin to grow at a rate of roughly $5 \mu \mathrm{m} / \mathrm{min}$. One should note that this growth rate is sensitive to the thickness of the iron film, but a $30 \mu \mathrm{m}$ CNT height may typically be grown in less than $8 \mathrm{~min}$. Fig. 3 shows two different heights for CNT forests, both of which are vertically aligned and dense. CNT forests as tall as $50 \mu \mathrm{m}$ have been successfully grown as well. Depending on the required area for a particular application, modulating the CNT height is quite easy, compared to various thin film approaches which may require different materials or steps in order to thicken the film and make it more robust.

Once the CNT forest is in place, the first LPCVD of polysilicon occurs with a deposition temperature is $615^{\circ} \mathrm{C}$ (Fig. $2 \mathrm{~d}$ ). The minimum time for this step was found to be $45 \mathrm{~min}$ in order to fill the top portion of the CNT forest fully with polysilicon. Fig. 4 shows the coating behavior of the polysilicon throughout the height of the CNTs. The thickness of deposited polysilicon is lower near the bottom of the forest primarily because the top $5 \mu \mathrm{m}$ fill more quickly, thereby constricting gas diffusion through the forest. This is favorable in that the polysilicon forms a natural seal at the top of the composite film.
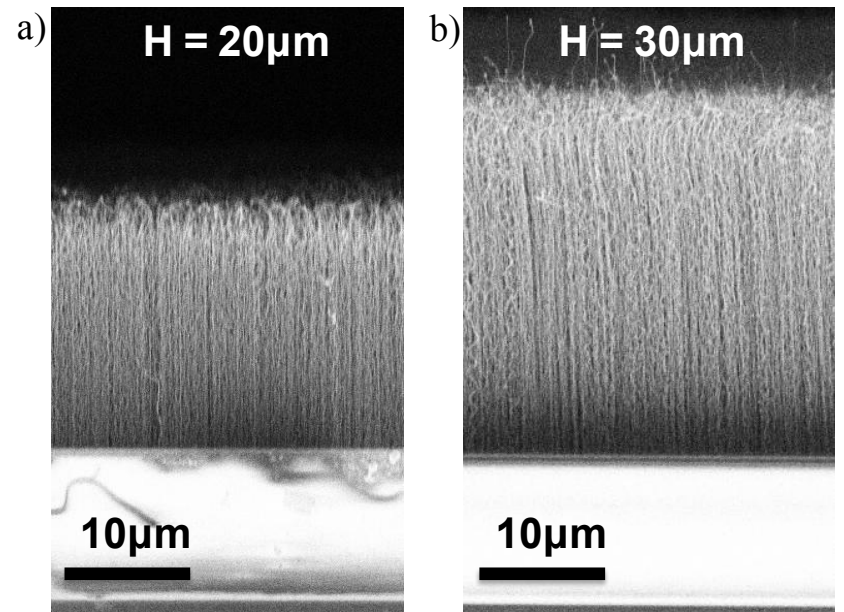

\section{$10 \mu \mathrm{m}$}

Figure 3: SEM photographs showing CNT forest (a) after 4 min growth, resulting in a $20 \mu \mathrm{m}$ tall forest; and (b) after 5 min growth, resulting in a $30 \mu \mathrm{m}$ tall forest. 


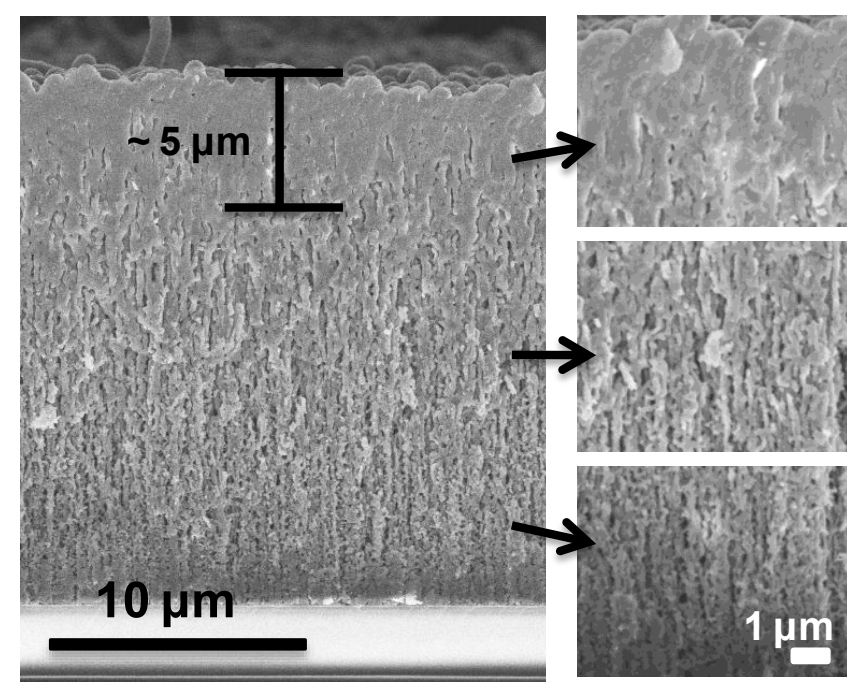

Figure 4: SEM photograph showing varying thickness of deposited polysilicon on CNTs from top to bottom as well as naturally occurring pores that result from the polysilicon coating.

Next, a select area on top of the composite must be etched to remove the polysilicon (Fig. 2e). This opens the vertical pathway for liquids and gas to travel through the membrane to the underlying material. A gas mixture of $90 \%$ sulfur hexafluoride $\left(\mathrm{SF}_{6}\right)$ and $10 \%$ oxygen $\left(\mathrm{O}_{2}\right)$ flowing at $66 \mathrm{sccm}$ will etch polysilicon at roughly $0.45 \mu \mathrm{m} / \mathrm{min}$ under $50 \mathrm{~W}$ power. The top surface of the film darkens as polysilicon is removed, indicating the CNTs have been exposed. The profile of the opened etchant access window may be seen in Fig. 5. Rectangular cavity windows as wide as $1250 \mu \mathrm{m}$ along the shorter direction were used.

Once the pores in the composite film are exposed, sacrificial oxide is removed with HF vapor penetration (Fig. 2f). The chip or wafer is set onto an electrostatic chuck, held at $45^{\circ} \mathrm{C}$, and placed over the $\mathrm{HF}$ vapor bath, which is kept at $35^{\circ} \mathrm{C}$, in order to prevent condensation of HF or reaction byproducts within the cavity. Lastly, another polysilicon LPCVD of $45 \mathrm{~min}$ seals the film and the newly created cavity (Fig. 2g).

\section{EXPERIMENTAL RESULTS}

\section{HF Vapor Etching of Underlying Oxide}

HF vapor penetration occurred almost instantly during experiments and cleared out buried oxide at a rate of nearly 1 $\mu \mathrm{m} / \mathrm{min}$. Cavities wider than $1 \mathrm{~mm}$ may be generated in less than $6 \mathrm{~min}$. However, there is a trade-off between the thicknesses of the oxide layer and the composite film. Specifically, as the oxide thickness increases to $10 \mu \mathrm{m}$ and beyond, lateral undercut or overetch occurs. Over-etch is defined based on the width of the window, $d$, and the width of the resultant cavity, $D$ as indicated in Fig. 2g. Then,

$$
\% O E=\frac{D-d}{d}
$$

Two sets of composite films with differing CNT heights of 20 $\mu \mathrm{m}$ tall and $30 \mu \mathrm{m}$ were tested against over-etching. The thinner membrane exhibited far less over-etch, up to $12 \%$, as a function of increasing exposure to $\mathrm{HF}$ vapor. On the other hand, the thicker membrane reached over-etch values of almost $100 \%$, or twice the intended cavity width as defined by the window on the composite membrane. The primary reason for this is that the permeability of the $\mathrm{CNT}$ /polysilicon composite comes from the pores in the

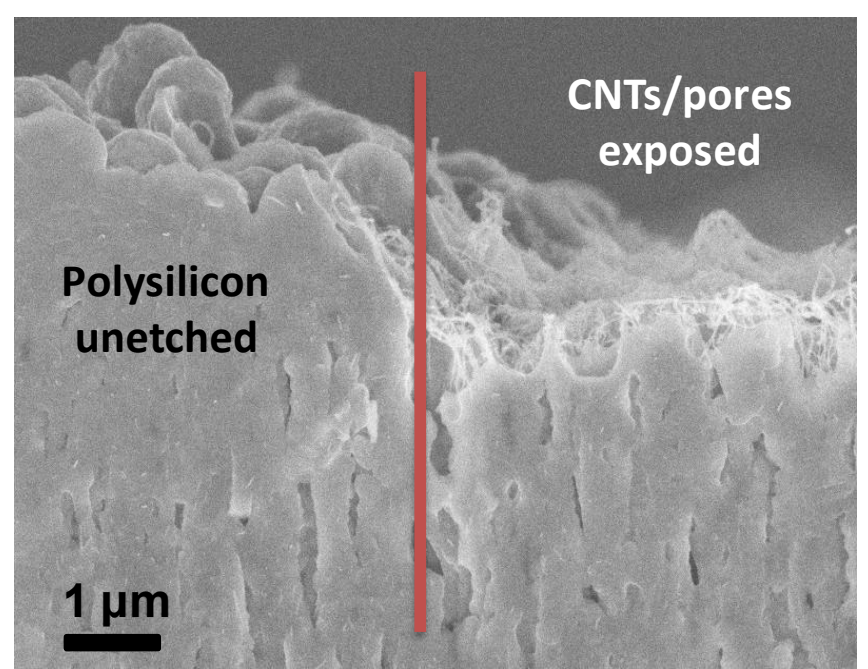

Figure 5: SEM photograph of composite film's top surface profile after plasma etching, which exposes CNTs and opens the underlying pores to liquid and vapor.

membrane and not the CNTs themselves. Therefore, there is a degree of lateral permeability in the membrane, evident even when HF vapor time is minimal ( $2 \mathrm{~min})$. The extent of over-etch may be modulated by reducing the thickness, essentially the CNT forest height. However, reducing thickness also reduces strength and limits how large an area may be safely sealed without membrane collapse. Nevertheless, the fabrication of the composite film is versatile enough such that changing the CNT height for different applications is seamless.

\section{Sealing the Membrane}

The second polysilicon only deposited onto the top of the membrane, as the top section is nearly filled from the first polysilicon deposition even after plasma etching. This is important for ensuring that polysilicon does not deposit onto the surface of the cleared cavity substrate or a device contained within. In addition, it's noted that defining the etch window creates a step height of roughly $2-3 \mu \mathrm{m}$ on top of the composite membrane. This is not an issue for the sealing step after oxide removal, but for subsequent post-encapsulation processing a planar surface may be required. In this case the top surface of the membrane may be smoothened with self-planarizing TEOS deposition step. This permits post-seal processing steps such as opening electrical contacts.

A surface profilometer was used to examine the membranes for possible deflection after sealing with LPCVD due to the pressure difference between inside the cavity and outside atmosphere. This deflection is a function of the membrane thickness and cavity width. For example, in the case of $1000 \mu \mathrm{m}$ wide etch windows, the membrane built with $25 \mu \mathrm{m}$ tall CNTs showed a concave up membrane shape with maximum deflection of $7 \mu \mathrm{m}$ in the center of the cavity. Increasing the CNT height to $45 \mu \mathrm{m}$ for the same cavity size improved the strength of the film against pressure loading with no measurable curvature of deflection. These results take into account the step height between the window and unetched areas as discussed above.

\section{CONCLUSIONS}

Wafer level encapsulation using a CNT/polysilicon composite film improves upon the flexibility in designing the thickness and area of an encapsulation membrane compared to other thin film 
approaches. The membrane contains a natural porosity that facilities removal of sacrificial oxide via $\mathrm{HF}$ vapor. The fabrication of the CNT/polysilicon composite is highly tunable with regard to the CNT forest height that supports the membrane. Etching behavior of buried oxide using HF vapor was characterized for different membrane thicknesses, based on the CNT heights, confirming that lateral over-etch may be reduced by using shorter CNTs. For large area applications (e.g. $>1 \mathrm{~mm}^{2}$ ) in which thicker CNTs are needed to withstand against deflection, the etch window may be designed smaller than the intended cavity size to compensate for lateral undercut. Overall the process technology shows promise and versatility for a simplified approach to waferlevel encapsulation of MEMS devices.

\section{ACKNOWLEDGEMENTS}

Travel support has been generously provided by the Transducer Research Foundation.

\section{REFERENCES}

[1] R.N. Candler, W.-T. Park, H. Li, G. Yama, A. Partridge, M. Lutz, and T.W. Kenny, "Single Wafer Encapsulation of MEMS Devices", IEEE Transactions on Advanced Packaging, 26, 3 (2003).
[2] H.M. Clearfield, J.L. Young, S.D. Wijeyesekera, and E.A. Logan, "Wafer-Level Chip Scale Packaging: Benefits for Integrated Passive Devices", IEEE Transactions on Advanced Packaging, 23, 2 (2000).

[3] S.-H. Choa, "Reliability of MEMS packaging: vacuum maintenance and packaging induced stress", Microsystem Technologies, 11 (2005).

[4] M. Esashi, "Wafer level packaging of MEMS", Journal of Micromechanics and Microengineering, 18 (2008).

[5] C. O'Mahony, M. Hill, Z. Olszewski, and A. Blake, "Waferlevel thin-film encapsulation for MEMS", Microelectronic Engineering, 86 (2000).

[6] R. He and C.-J. Kim, "On-Wafer Monolithic Encapsulation by Surface Micromachining With Porous Polysilicon Shell", Journal of Microelectromechanical Systems, 16, 2 (2007).

[7] K.S. Lebouitz, A. Mazaheri, R.T. Howe, and A.P. Pisano, "Vacuum Encapsulation of Resonant Devices Using Permeable Polysilicon", Technical Digest of the $12^{\text {th }}$ Annual IEEE International Conference on MEMS (1999), pp. 470475 .

\section{CONTACT}

*A. Mahajerin, tel: 1-510-642-8983; armonmah@me.berkeley.edu 


\title{
DIRECT-WRITE SELF-ASSEMBLY OF 3D COLLOIDAL MICROSTRUCTURES
}

\author{
Justin Beroz, Mostafa Bedewy, and A. John Hart \\ Mechanosynthesis Group, Department of Mechanical Engineering \\ University of Michigan, Ann Arbor, Michigan, USA
}

\begin{abstract}
We present a direct-write technique for assembly of microscale 3D colloidal crystals on substrates. We use a custombuilt high-resolution liquid manipulation system to dispense colloidal suspensions through a capillary tip. Using this system, we establish a liquid bridge between the capillary tip and a temperature-controlled substrate, initiating crystal growth upward from the substrate. We demonstrate construction of cone-shaped and tower structures by controlling the dynamic shape of the liquid meniscus during crystal precipitation. Interplay between lateral capillary forces and granular cohesion governs assembly. Finally, we show that confinement of the meniscus on microfabricated template features enables assembly of discrete particle clusters.
\end{abstract}

\section{INTRODUCTION}

Spanning from the nanometer to millimeter scales, discrete components can serve as building blocks for optical components, filters, biosensors, micromechanisms, electronics, and many other novel materials and devices. New techniques are needed to assemble a growing library of these building blocks with high precision and throughput, ranging from semiconductor nanoparticles to polymer microspheres and miniature circuit elements. In particular, the proportional relationship between liquid meniscus curvature and capillary pressure is a versatile means for ordering ensembles of particles at liquid-air interfaces, and enables fabrication of closely packed layers and discrete clusters of micro and nanoparticles on substrates. Collectively, these techniques are known as evaporative self-assembly, and are, for example, implemented by dispensing droplets onto the target substrate by inkjet printing [1], or by drawing a meniscus across the substrate [2]. However, it is highly challenging to arrange particles into well-organized 3D shapes by current methods. Here, we demonstrate that $3 \mathrm{D}$ colloidal assemblies can be directly fabricated on flat and microstructured surfaces by dynamic mechanical control of a liquid meniscus during evaporation.
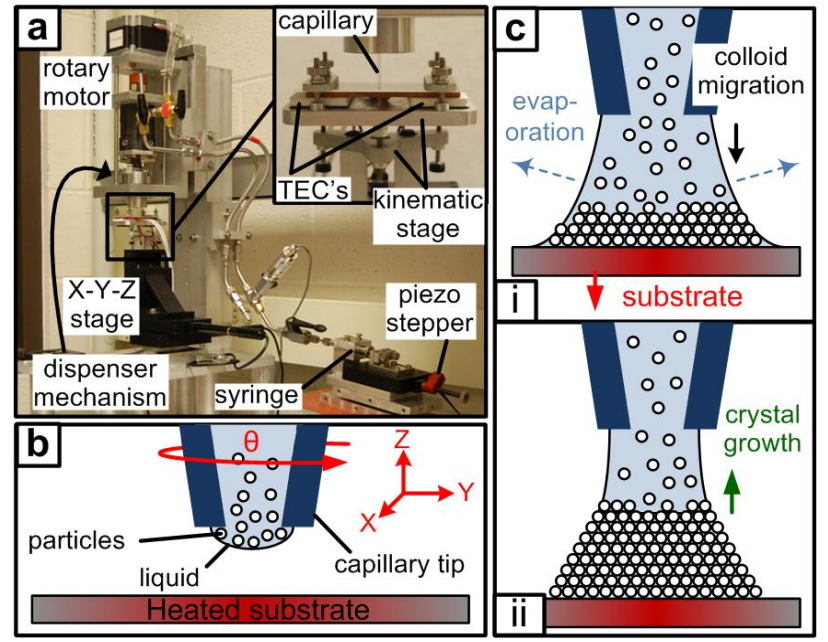

Figure 1: (a) Liquid dispensing system (adapted from [3]); (b) schematic of 4-DOF motion control, and (c) schematic of directwrite assembly process.
To facilitate this study, we designed and built a precision instrument (Fig. 1a, [3]), which can simultaneously deposit and manipulate nano- and microliter liquid droplets on a temperature controlled substrate, in four degrees of freedom (Fig. 1b). In this paper, we introduce a technique for fabricating 3D colloidal microstructures using vertical stage motion. We contact the heated substrate with a liquid that is displaced slightly from the end of the capillary tip, thereby creating a liquid bridge between the capillary tip and substrate (Fig. 1c). Particles in the liquid migrate towards the substrate due to sedimentation and/or evaporation-driven liquid flow. When the evaporation rate locally exceeds the rate of liquid replenishment, the meniscus recedes upward from the substrate, building a colloidal crystal whose geometry is determined by the dynamic shape of the receding meniscus.

Via concerted control of vertical substrate motion and liquid dispensing rate, we can control the liquid bridge profile between the tip and the heated substrate. As shown in Figure 2, this control allows us to fabricate colloid crystals, consisting of $10 \mathrm{~m}$ polystyrene particles, in the shape of cones and towers on flat substrates.

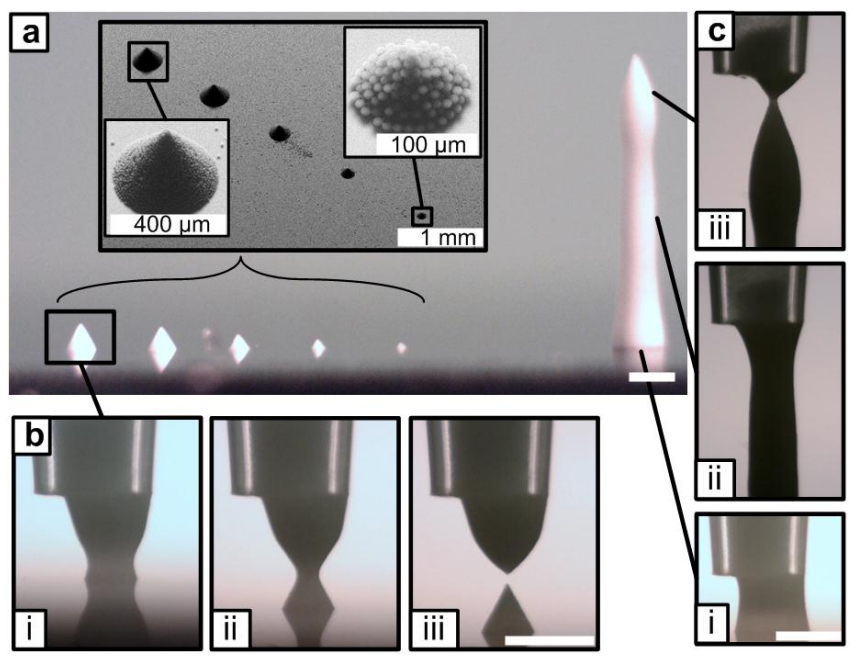

Figure 2: Extrusion of crystals of $10 \mathrm{~m}$ polystyrene spheres into conical and tower structures by drawing from a capillary tip (all scale bars $=500 \mathrm{~m}$ unless noted)

\section{PROCESS FUNDAMENTALS}

In order to understand how these structures precipitate from the liquid bridge, we present a conceptual description of the particle assembly mechanisms observed during crystal growth, which arise due to capillary forces. Specifically, the local curvature of a liquid meniscus is proportional to the pressure difference across the interface, as described by the Laplace-Young equation. This capillary pressure, in concert with the effects of surface tension, produce two distinct phenomena which we call surface assembly and bulk cohesion. Formation of these crystal structures may be understood by considering the interaction between colloid organization at the liquid-air interface and coherence in the bulk material. 


\section{Assembly at the Three-Phase Contact Line}

Suspended colloid particles that are at the liquid-air interface locally deform the liquid meniscus, which gives rise to lateral capillary attraction between particles due to interface tension and Laplace capillary pressure. This drives the colloids to assemble into closed-packed domains along the liquid-air interface. This has been explored thoroughly in the context of self-assembly of closed-packed monolayers and multilayers of particles on surfaces, using a variety of techniques, including [4] drop-casting, bladecasting, and Langmuir-Blodgett drawing. After evaporation, particles remain arranged in the crystal lattice due to Van der Waals attraction to neighboring particles and to the surface.

Our process may be regarded as a 3-dimensional (3D) analog to these well established planar self-assembly techniques. However, here are important differences with regard to our process due to the 3D contour of the liquid bridge. As shown in Fig. 3, evaporation-induced flows pull particles towards the liquidsubstrate contact line, where the particles pack due to lateral capillary forces at the interface, thus causing a colloid crystal nucleate and to grow. In this respect, the colloid microstructure may be thought of as precipitating from the meniscus-crystal contact line in the direction of meniscus recession.

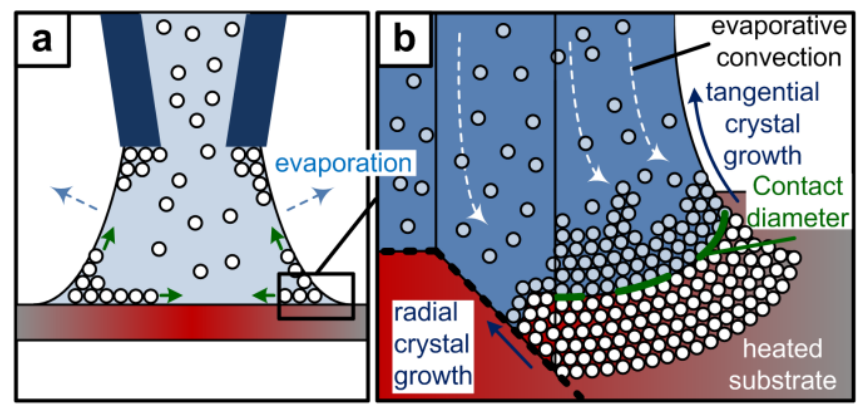

Figure 3: (a) Schematic illustrating the surface assembly process within the liquid bridge; (b) isometric view with wedge cut illustrates crystal growth tangent to the meniscus, and inwards radial crystal growth, which adds thickness to the crystal.

Meniscus recession may occur tangent to the meniscus surface, thereby precipitating a closed-packed outer surface; and radially inwards, thereby thickening the closed packed surface to form a multi-layered 'crust' (Fig. 3). Note that evaporationinduced convection can transport particles to the meniscus surface for tangential crystal growth, and to the underside of the hexagonal-packed surface for radial crystal growth.

Several factors influence the integrity of the closed packed crystal. One obvious culprit is monodispersity in colloid diameter. Larger-than-average particles in the crystal lattice create defects that can propagate through the crystal. This may be minimized by using highly monodisperse particles. Additionally, crystal growth may nucleate at multiple locations, which leads to grain boundaries at the intersection of crystals with different lattice orientations. These defect mechanisms are well documented in the literature for 2D capillary self-assembly [4]. Geometry provides a unique constraint for our process. The crystallization process is confined by the curvature of the meniscus, and defects may be created to accommodate this geometric confinement. One can imagine that the defect density will increase radially inwards from the outer surface, ultimately resulting in jammed, amorphously packed colloids at the center.

\section{Bulk Cohesion}

In contrast to the surface assembly just described, capillary forces can effectuate bulk cohesion between particles. Here, the colloid particles behave as an amorphously packed wet granular material, which may exist in four distinct phases according to the volume fraction of liquid in the void spaces between the particles [5]. At very low saturation, small liquid bridges form between particles near contact points (Fig 4a-i). Surface tension and capillary pressure within the liquid bridges constitute a normal capillary force attraction, which causes high cohesion amongst the particles.

Increase in liquid content fills more of the void space between the particles, which leads to coalescence of capillary bridges (Fig. 4a-ii) and a decrease in particle cohesion. At the limit where all void space is filled with liquid (Fig. 4a-iii), the particles experience weak cohesion due to lateral capillary forces at the peripheral liquid air interface. Further increase in liquid content dilutes particles into a slurry (Fig. 4a-iv), and cohesion is lost.

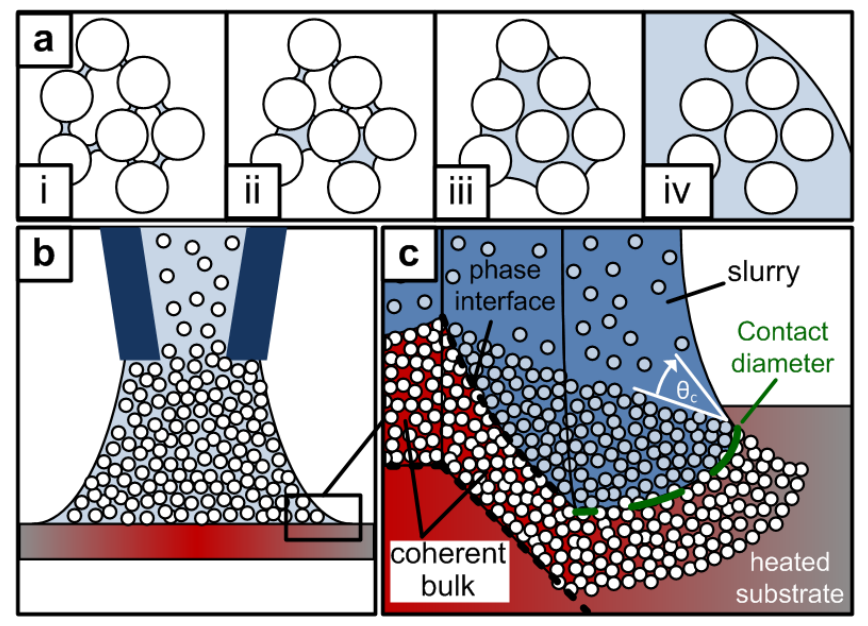

Figure 4: (a) Schematic illustrating the phases of granular bulk cohesion [5]; (b) illustration of particles sedimenting within the liquid bridge; (c) isometric view with wedge cut illustrates the contact angle, $\theta_{c}$, created at the contact diameter at the interface between the cohesive bulk phase and liquid bridge slurry.

During extrusion of a crystal structure by our method, particles may sediment onto the substrate inside the liquid bridge (Fig. 4b). Substrate heating accelerates liquid evaporation, thereby introducing air voids in the interstitial spaces and effectuating cohesion amongst the sedimented particles (i.e., phases $i$ and ii, Fig. 4a). Collectively, the particles behave as a distinct bulk wet granular material that, we believe, develops a discernable interface with respect to the liquid bridge slurry above (Fig. 4c). The meniscus of the liquid bridge slurry pins to the cohesive bulk at the peripheral contact diameter with contact angle $\theta_{c}$, as illustrated. An amorphous crystal structure may be grown by simply pulling on the meniscus so that this contact diameter recedes by slipping over the cohesive bulk material.

\section{RESULTS}

Based on this understanding, we demonstrate control of the process parameters to direct the crystal structure contour, and realize $3 \mathrm{D}$ structures, ranging from cones to towers. Utilizing the capabilities of our liquid dispenser system, we are able to control the following parameters for fabricating colloid microstructures: profile of the liquid bridge between the capillary tip and 
precipitating crystal structure, initial particle concentration, and substrate patterning. In the following section, we demonstrate, experimentally, the possibilities within this design space and enumerate the interplay between surface assembly and bulk cohesion in guiding 3D colloid assembly.

\section{Contact Diameter (i.e., slope) Control}

During the assembly process, the meniscus of the liquid bridge extending from the capillary tip is locally pinned to the crystal structure at the contact diameter, as illustrated in Fig. 3 and Fig. 4. Due to contact angle hysteresis, this angle may vary between characteristic advancing and receding contact angles.

By controlling the rate of liquid dispensing and the downward speed of the substrate, we can control this contact angle (within the advancing-receding range). This, in turn, enables us to control the evolution of the contact diameter with time, and this determines the slope of the particle assembly. Qualitatively speaking, larger contact angles (i.e., near the characteristic advancing contact angle) increase the contact diameter over the course of crystal growth, and smaller contact angles (i.e., near the characteristic receding contact angle) decrease the contact diameter. Two limits of this technique are conical structures (Fig. 2a,b), where we constrict this precipitation diameter at a constant rate during crystal growth; and towers (Fig. 2a,c), where we maintain a constant contact diameter and can extrude a vertical column of indefinite length.

To better understand the mechanics of assembly, we fabricated an array of conical crystals, where we systematically varied the initial contact diameter, substrate motion rate, and liquid volume fraction in the tip. In all experiments, we held the substrate temperature at $70^{\circ} \mathrm{C}$, and did not dispense additional liquid once the bridge was formed. Under these control parameters, we fabricated cones ranging in height from $50 \mathrm{~m}$ to $550 \mathrm{~m}$, and having a wide range of height-to-base diameter aspect ratios (0.41.2). Notably, we observed various slope contours, such as those depicted in Fig. 5a,b.

In order to interpret these results, we studied the evolution of the liquid meniscus over the course of crystal growth. Fig. 5a,b illustrates the evolution of the liquid bridge meniscus profile over time by overlaying images captured at periodic time intervals (Fig. $5 \mathrm{a}=10 \mathrm{sec}$, Fig. $5 \mathrm{~b}=5 \mathrm{sec}$ ). For each instant, we measured the contact angle of the liquid meniscus with respect to slope of the final structure, and identified three distinct stages, which are labeled (i), (ii), and (iii) in Fig. 5a,b.

(i) Upon establishing a liquid bridge, the meniscus is initially pinned to the substrate at a relatively large contact angle. Withdrawing the substrate stretches the meniscus, thereby decreasing the contact angle until it reaches the characteristic receding angle with respect to the structure slope, which we measure to be $\sim 11-15^{\circ}$.

(ii) As crystal growth proceeds, the meniscus contact line recedes at this constant angle. Notably, this contact angle is the same in Fig. 5a and Fig. 5b (within measurement error), even though the structure contours are significantly different. We believe this indicates that bulk cohesion is present within the volume, and the meniscus is slipping over the cohesive material interface at a characteristic receding contact angle, which we would expect to be independent of the interface contour. Our hypothesis is supported by observation of amorphous packing at the crystal surface in this region, as shown by the SEM images (Fig. 5c,d).

(iii) Towards the apex of the cone, we observe an increase in measured contact angle accompanied by a change in slope. Here, we propose that the liquid content in the bridge has lowered to the point where the entire liquid bridge becomes a coherent granular material. At this point, although we state a meniscus contact angle in the contour plots (Fig. 5a,b), formally calling this a contact angle is no longer appropriate. A more accurate interpretation is that we have a coherent wet granular column, which necks under the applied tension until rupture occurs.

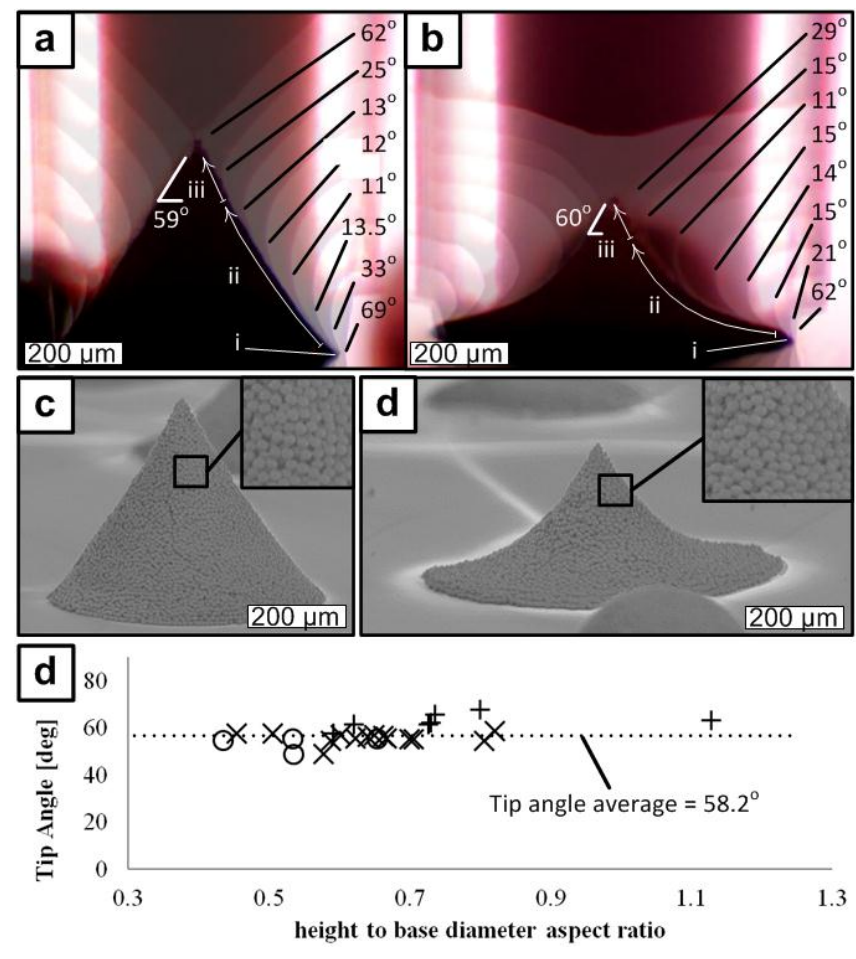

Figure 5: $(a, b)$ contour plots demonstrating the evolution of the meniscus during crystal growth; (c, d) SEM images of these structures reveal amorphous crystal packing; (d) the slopes for all cones converge to $\sim 60^{\circ}$ near the apex.

A further interesting observation is that the apex slope is approximately the same for all the cones we fabricated in this study, even though the initial slopes vary. The plot of cone apex angle vs. aspect ratio (Fig. 5d) shows that this angle appears to be independent of aspect ratio and draw speed. This seems to indicate a characteristic yielding behavior for this cohesive wet granular material.

\section{Crystallinity Control}

For surface assembly to occur, the suspended particles must have enough mobility to travel to, and arrange at, the liquid air interface. Particle cohesion and physical obstruction can terminate surface assembly by arresting free particles from maneuvering to the liquid-air interface, or limiting the space available to arrange in a closed-packed lattice, respectively.

In our technique, sedimentation of particles is substantial, and thus growth of the bulk cohesive phase through these sedimented particles is responsible for terminating the radial and tangential growth of surface assembled crystals. By controlling the initial concentration of particles at bridge formation, we can prescribe how much time free particles have to assemble at the surface before termination by the growing coherent bulk phase.

Figure 6 shows three different broken particle towers fabricated with different initial particle concentrations at liquid bridge formation. Note that these 'initial concentrations' are 
qualitative. We controlled this by simply allowing different amounts of time for particle sedimentation in the capillary tip before creating the liquid bridge with the substrate. The tower with the highest initial particle concentration (Fig. 6a) exhibits an entirely amorphous outer surface and inner volume. Here, the initial particle concentration is high enough that there is already coherence among the particles, so surface assembly is completely arrested. At lower initial particle concentration (Fig. 6b), we achieved a polycrystalline surface approximately 1 particle thick. At our lowest prepared particle concentration (Fig. 6c), we achieved a hexagonal closed packed outer surface on the tower. This closed packed layer was able to grow radially inwards a thickness of several particle layers before becoming jammed by the amorphous bulk phase.

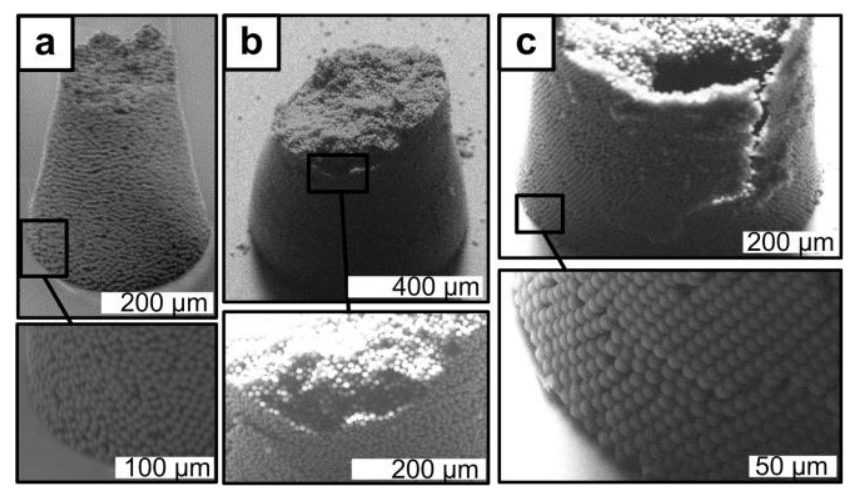

Figure 6: Three broken towers, fabricated from liquid bridges having different initial particle concentration (decreasing left to right) each reveal differences in crystal packing at the surface.

\section{Discrete Particle Assemblies}

In the results presented so far, the base diameter of the crystal structure is limited by the size of the capillary tip and the geometry of the liquid bridge. Here, we show that pinning the meniscus to a substrate microfeature allows fabrication of highly ordered assemblies comprising discrete numbers of particles. Notably, these structures are substantially smaller than the tip diameter, and their placement is determined by registry with the guide feature. This enables precision deterministic fabrication of colloid crystal structures with dimensions and placement precision finer than the capillary tip dimensions and substrate motion resolution, respectively.

To demonstrate this principle, we chose to assemble particles onto vertically aligned carbon nanotube (CNT) microstructures. As shown in Fig. 7a, we assembled a small crystal arrangement of particles across the opening of a CNT hollow micro cylinder (50 $\mathrm{m} \mathrm{ID}, 80 \mathrm{~m}$ OD). Notice that the meniscus can easily adapt to misalignment between the capillary tube axis and CNT microcylinder without adversely affecting particle placement accuracy. Similarly, we deposited clusters of two, three, and four particles on top of densified CNT micropillars (Fig. 7b,c,d respectively). For these results, the liquid (water) wicked through the CNT pillar, causing it to densify due to capillary forces. Thus, we are demonstrating the potential for using the suspension liquid to affect the organization on a surface by other capillary-driven mechanisms in concert with particle crystal growth.

This result also demonstrates the potential of this technique to deterministically place individual, and discrete arrangements of, microparticles by engineering microfeatures having suitable geometry and surface energy. Our technique can potentially be scaled down to smaller sizes. One can imagine, for instance, depositing a single $500 \mathrm{~nm}$ particle on a $1 \mathrm{~m}$ diameter post.

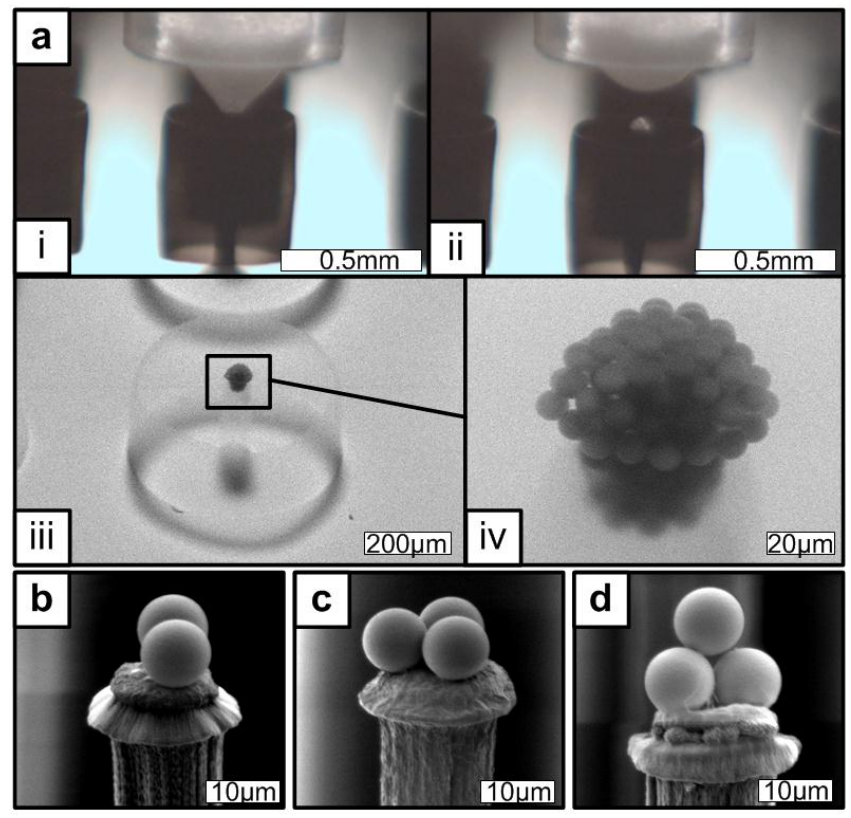

Figure 7: The colloid suspension meniscus can be pinned to diameters smaller than the capillary tip diameter by substrate microfeatures, such as (a) concentric carbon nanotube (CNT) microcylinders and solid CNT micropillars in order to deposit discrete arrangements of particles such as a (b) dimer, (c) trimer, and (d) tetramer.

\section{CONCLUSION}

Our approach to direct-write evaporative self-assembly contrasts conventional approaches to inkjet printing [6] and electrohydrodynamic printing $[7,8]$, which are limited by the size, ejection rate, and spread of droplets on the substrate. We showed that, by maintaining direct mechanical control of the meniscus, it is possible to directly build a complex 3D shape or to direct individual particles towards guide features on the substrate. This is potentially advantageous for heterogeneous assembly and integration of pre-made components such as microelectronic elements on circuit boards or MEMS devices. Deterministic colloid assemblies may be useful as miniature filters or photonic crystals within lab-on-a-chip systems, or model granular materials having engineerable thermal, electrical, or mechanical properties. Additionally, this technique can potentially be scaled down to assemble colloidal nanoparticles by using smaller diameter tips. This may be interesting for direct fabrication of novel photonic crystal arrays and other metamaterials.

\section{REFERENCES}

[1] Calvert, P., Chem. Mat., 2001. 13(10): p. 3299-3305.

[2] Bodnarchuk, M., et. al., ACS Nano, 2010. 4(1): p. 423-431.

[3] Beroz, J., et al., Rev. Sci. Instrum., 2012. 83: 015104.

[4] Galisteo-Lopez, J.F., et al., Adv. Mater., 2011. 23: p. 30-69.

[5] Mitarai, N., Nori, F., Adv. in Physics, 2006. 55(1): p. 1-45.

[6] Derby, B., Ann. Rev. Mater. Res., 2010. 40: p. 395-414.

[7] Mishra, S., et al., J. Micromech. Microeng., 2010. 20(9).

[8] Park, J.U., et al., Nano Letters, 2010. 10(2): p. 584-591.

\section{CONTACT}

*A.J. Hart, tel: +1-734-615-6146; ajohnh@umich.edu 


\title{
A FERROFLUID-BASED PRESSURE SENSOR FOR BIOMEDICAL APPLICATIONS
}

\author{
Girish Chitnis ${ }^{* 1,3}$ and Babak Ziaie ${ }^{2,3}$
}

\author{
${ }^{1}$ School of Mechanical Engineering, Purdue University, West Lafayette, Indiana, USA \\ ${ }^{2}$ School of Electrical and Computer Engineering, Purdue University, West Lafayette, Indiana, USA \\ ${ }^{3}$ Birck Nanotechnology Center, Purdue University, USA
}

\begin{abstract}
This paper presents a new pressure sensor design based on magnetic liquid displacement and its corresponding inductance change. The design of pressure sensor is presented followed by its fabrication and in vitro test results. Experimental results show a nonlinear relationship between applied pressure and change in resonant frequency. The initial linear region $(0-20 \mathrm{mmHg})$ shows a sensitivity of $6 \mathrm{kHz} / \mathrm{mmHg}$. Simple design and easy fabrication process make this pressure sensor an attractive option for many biomedical applications.
\end{abstract}

\section{INTRODUCTION}

Wireless measurement of physiological pressures has been pursued for several decades following the pioneering work of Farrar et al. in 1957 to measure gastrointestinal pressure [1]. A decade later, further miniaturization of implantable pressure sensors was utilized by Collins [2] to measure intraocular pressure using a passive LC transponder. Since the rise of MEMS and microfabrication technology over the past couple of decades, many pressure sensors have been reported that are designed to measure pressure at various anatomical locations such as blood vessels [3], [4], eye [5-8], bladder [9], cranium [10], etc. Efforts in this area have been focused on passive (LC) and active (pressure sensor plus interface and RF electronics, powered through inductive methods or batteries) transponders. Passive devices have an inherent advantage over active ones since they do not need any external power source. Most passive transponders rely on changes in capacitance or inductance for pressure measurement. Although conceptually simple, their fabrication requires complex techniques. Here we present a much simpler passive pressure sensing transponder based on the novel concept of ferrofluid movement in and around an integrated coil. Inductance of the coil varies with applied pressure due to transfer of ferrofluid trapped in the sensor. Since the coil itself has some built-in stray capacitances, no external capacitor is needed to create the LC tank (inductor selfresonant frequency is therefore a function of applied pressure). Although there have been reports on ferrofluid-based gas flow sensor [11], inclinometer [12], and LVDT sensor [13]; the transducer presented here is the first implementation of a wireless passive pressure sensor using ferrofluid.

\section{DESIGN}

The working mechanism of the ferrofluid-based pressure sensor relies on high magnetic permeability of dispersed ferrite nanoparticles and fluidity of the dispersion medium. Figure 1 depicts the concept of the ferrofluid-based pressure sensor. It consists of four major components: 1) a needle, 2) ferrofluid, 3) a planar coil, and 4) a polymeric membrane. The needle penetrates a body cavity by piercing through a membrane such as sclera, blood vessel wall, or bladder wall to establish a direct hydraulic contact between ferrofluid and the biological inner fluid (aqueous humor, blood, urine, etc.) (Fig.1a). When internal pressure is higher than the atmospheric pressure, ferrofluid flows through the needle and the hole at the center of the coil, deflecting the polymeric (a)

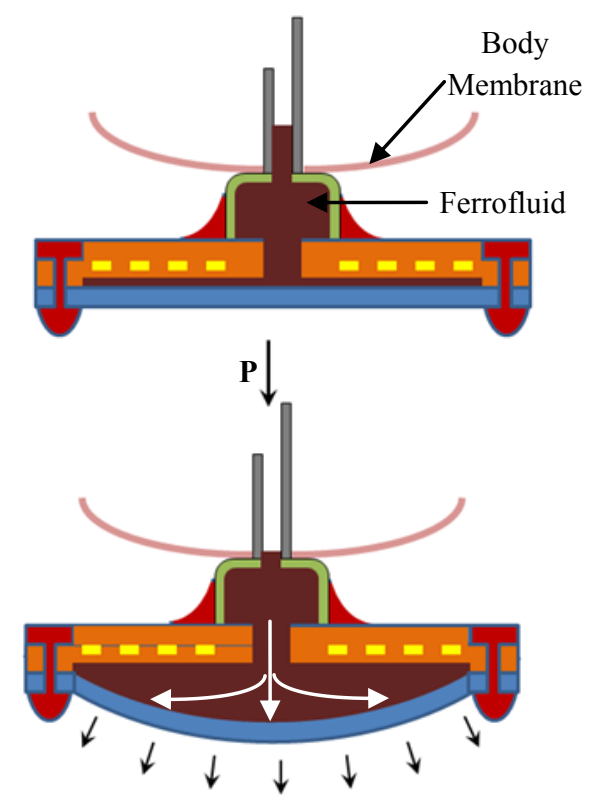

Figure 1: Schematic diagram of ferrofluid pressure sensor (dimensions not to scale)

membrane away from the coil (Fig. 1b). As a result, ferrofluid occupies more volume on top of the coil. Since inductance is directly proportional to the volume of the magnetic material in proximity to the coil, the pressure increase leads to an increase in the inductance (assuming the density of nanoparticles in the ferrofluid is constant). In our structure, stray capacitance is fixed and is not affected by change in the pressure; leading to a decrease in the self-resonant frequency of the coil as external pressure increases. For a given pressure, the change in resonant frequency of the coil depends on deflection of the polymeric membrane as well as magnetic permeability of ferrofluid. The geometry (radius and thickness) and material of the membrane can be altered to achieve desired sensitivity and range. Magnetic permeability of the ferrofluid can be altered by changing concentration of ferrite nanoparticle in the colloid. In the present design, we use $100 \mathrm{~m}$ thick Polyethylene terephthalate (PET) sheet with deflectable radius of $10 \mathrm{~mm}$ as polymeric membrane. The ferrofluid (APG 311 by Ferrotec, Inc.) has ferrite volume concentration of $2 \%$ (particle size $10 \mathrm{~nm}$ ) dispersed in synthetic hydrocarbon with overall magnetic permeability of 1.1 (measured at $\mathrm{f}=10 \mathrm{MHz}$ ). A polyimide based spiral coil with 20 turns, $18 \mathrm{~m}$ thick copper (2 layers) and $25 \mathrm{~m}$ polyimide (3 layers) is used for its high quality factor.

\section{FABRICATION}

Figure 2 shows the fabrication process flow of the ferrofluidbased pressure sensor. Polyimide flexcoil $(L \approx 10 \quad H)$ was used for fabrication of this prototype (Fig. 2a). The coil was designed in- 
(a)

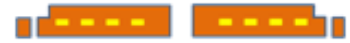

(c)

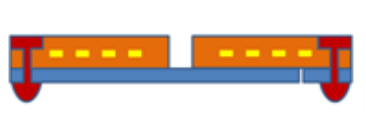

(e)

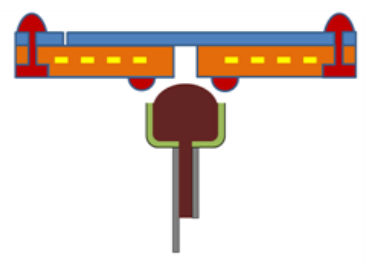

(g)

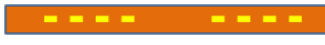

(b)

(d)

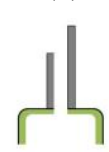

(f)

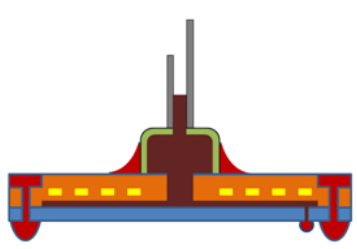

(h)
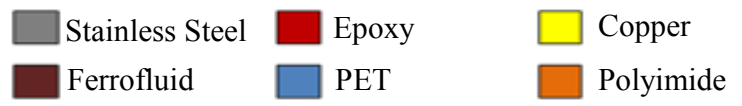

Figure 2: Fabrication process flow.

house but fabrication was outsourced to Parlex USA, Inc. A 100 $m$ thick PET sheet (transparency) was used as the polymeric membrane (Fig. 2b). Both flex-coil and transparency were machined to desired shape using a $\mathrm{CO}_{2}$ laser (Universal® Laser Systems), (Fig. 2c-d). The coil and membrane were bonded together using standard five minute epoxy (Fig. 2e). High viscosity epoxy was used to ensure that the membrane only bonds at the edges. Holes were laser-drilled near the edges to ensure a leakproof anchor. Note that an additional space around the coil needs to be provided for the anchors which lead to the physical size of the coil being different from its electrical size. Similarly, movable diameter of the PET membrane is smaller than its actual physical size. Commercially available hypodermic needle (26 gauge) was

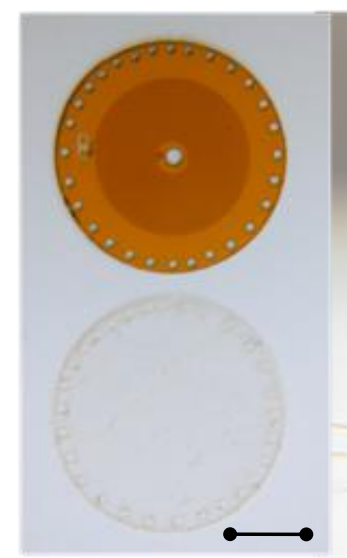

(a)

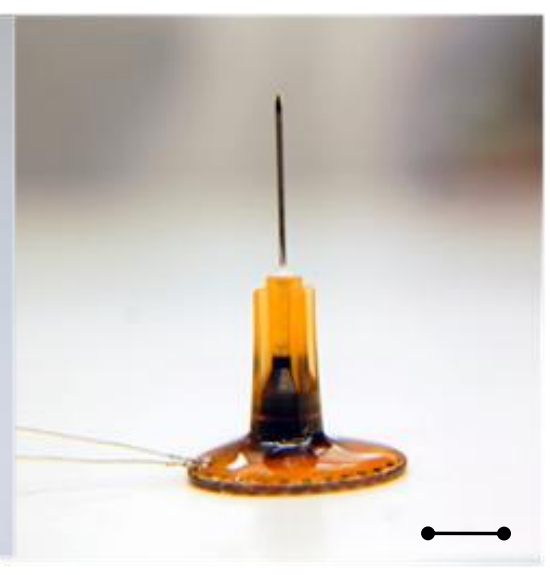

(b)
Figure 3: (a) Laser machined flex-coil and transparency (b) assembled ferrofluid pressure sensor pressure sensor (Scale bar: $5 \mathrm{~mm}$ ). mechanically machined to create the ferrofluid chamber (Fig. 2f). Chamber and needle prefilled with ferrofluid, was then bonded to the coil/membrane assembly using epoxy glue (Fig. 2 g). An escape hole was provided in the transparency to allow air to purge and allow the ferrofluid to completely fill the gaps. This escape hole was sealed later using epoxy glue (Fig. 2h). Figure 3 shows photographs of the laser-machined coil and transparency (Fig. 3a) along with a completely assembled ferrofluid based pressure sensor (Fig. 3b). Table 1 lists all the final parameters of the pressure sensor.

Table 1: Ferrofluid pressure sensor parameters

\begin{tabular}{lc}
\hline Polymeric membrane \\
\hline Thickness (mm) & 0.100 \\
Diameter & 10 \\
Movable (mm) & 15 \\
Total (mm) & \\
Coil & 0.250 \\
Thickness (mm) & 10 \\
Diameter & 15 \\
Electrical (mm) & \\
Physical (mm) & Gauge 26 \\
Hypodermic Needle & 0.260 \\
I.D. $($ mm) & 0.464 \\
O.D. $(\mathrm{mm})$ & 10 \\
Length (mm) &
\end{tabular}

\section{RESULTS AND DISCUSSION}

The pressure sensor was characterized against a water column pressure as shown in Figure 4a. The needle was inserted in a water-filled rubber balloon to mimic its insertion through a body cavity (Fig. 4b). Self-resonant frequency of the sensor was measured using an impedance analyzer. It is important to note that although here the frequency is measured through direct wired connection to the analyzer, similar measurement can be performed wirelessly by using phase-dip or other techniques. Resonant frequency was measured for pressures from 0 to $50 \mathrm{mmHg}$ while increasing and decreasing the pressure in steps of $5 \mathrm{mmHg}$. Figure 5a shows the measured impedance of the device at various pressures (not all the measurements are shown to maintain clarity of the diagram). The resonant frequency plotted against applied pressure is shown in Figure 5b.

It is safe to assume that if the nanoparticle concentration is kept constant, the change in inductance is proportional to volume of the ferrofluid surrounding the coil. Considering that the circular membrane would deflect to a spherical segment, change in ferrofluid volume will be proportional to the cube of deflection. Hence if we assume linear deflection region for the polymer membrane, change in inductance is expected to be proportional to the cube of applied pressure. As expected, figure 5b shows a very good fit for such cubic trend. One can consider the response to be almost linear in the range $(0-20 \mathrm{mmHg})$ where it shows a sensitivity of $\sim 6 \mathrm{kHz} / \mathrm{mmHg}$. Sensitivity and linear range of the sensor can be easily improved by simply adjusting geometry of the deflecting membrane. Considering the current thickness of $100 \mu \mathrm{m}$ there is considerable room to improve sensitivity. 


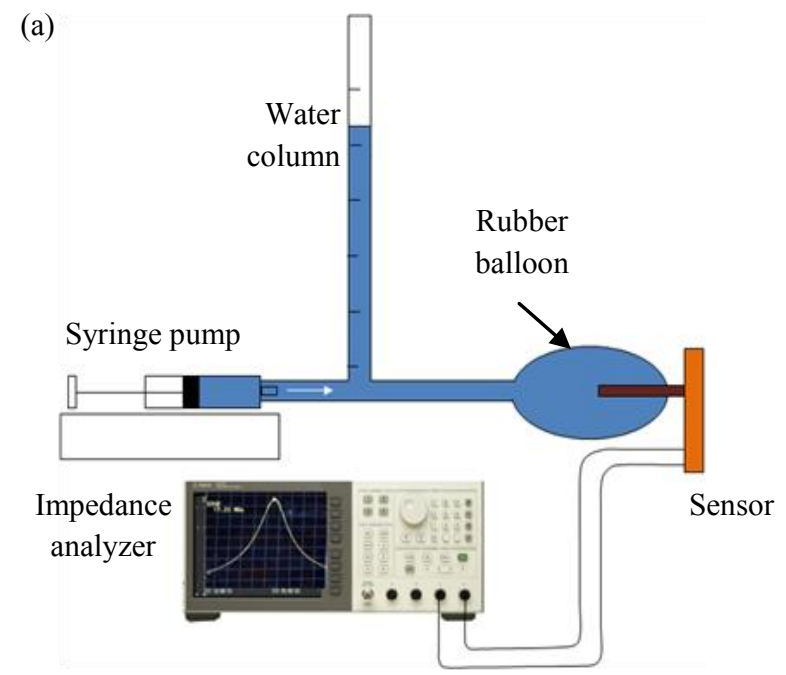

(b)

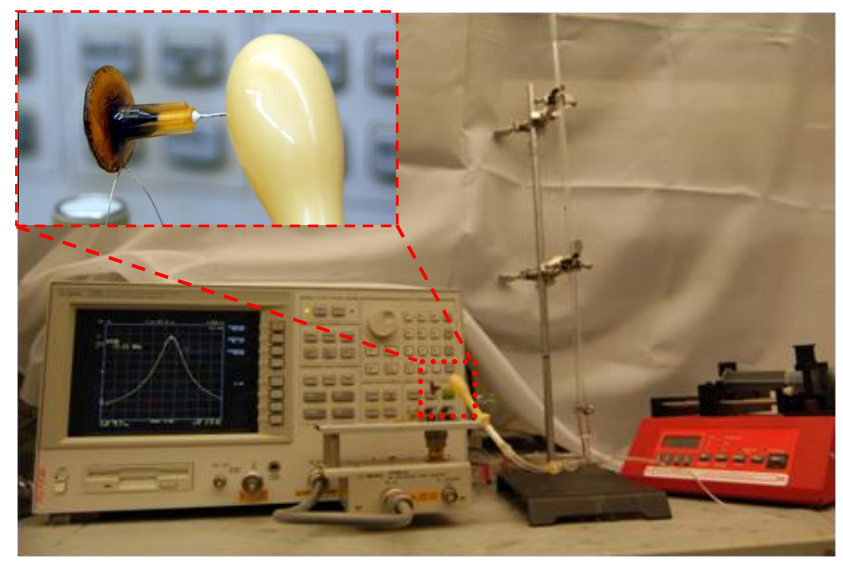

Figure 4: (a) Schematic diagram of the experimental set up (b) Photograph of the experimental set up (inset shows pressure sensor inserted in a rubber balloon)

It is important to note that the sensor uses only one coil in open configuration. Since there is no need to bring the contact out one can fabricate such coil in a single layer of metallization. Also, since this sensor does not need an additional capacitor, any established coil fabrication technique can be used to achieve low resistance and high quality factor.

Apart from the advantages, there are some concerns that need to be addressed. In the current design ferrofluid is directly exposed to exteriors through needle opening. This can lead to leakage of ferrofluid leading to a dysfunctional sensor. Further if the ferrofluid level drops below the needle while pressurizing, it is easy to trap a bubble inside the chamber which would not only obstruct ferrofluid movement but also affect effective magnetic permeability of the system. Use of a high gauge (i.e. small inner diameter) needle resolves these issues to some extent since surface tension helps confining the ferrofluid. However a physical barrier separating the exterior from ferrofluid can improve reliability significantly. Although biocompatibility of the device in its current form is not validated, most of the structural components can be replaced if needed. The only irreplaceable component, ferrite nanoparticles, is shown to be biocompatible [14, 15], and it has

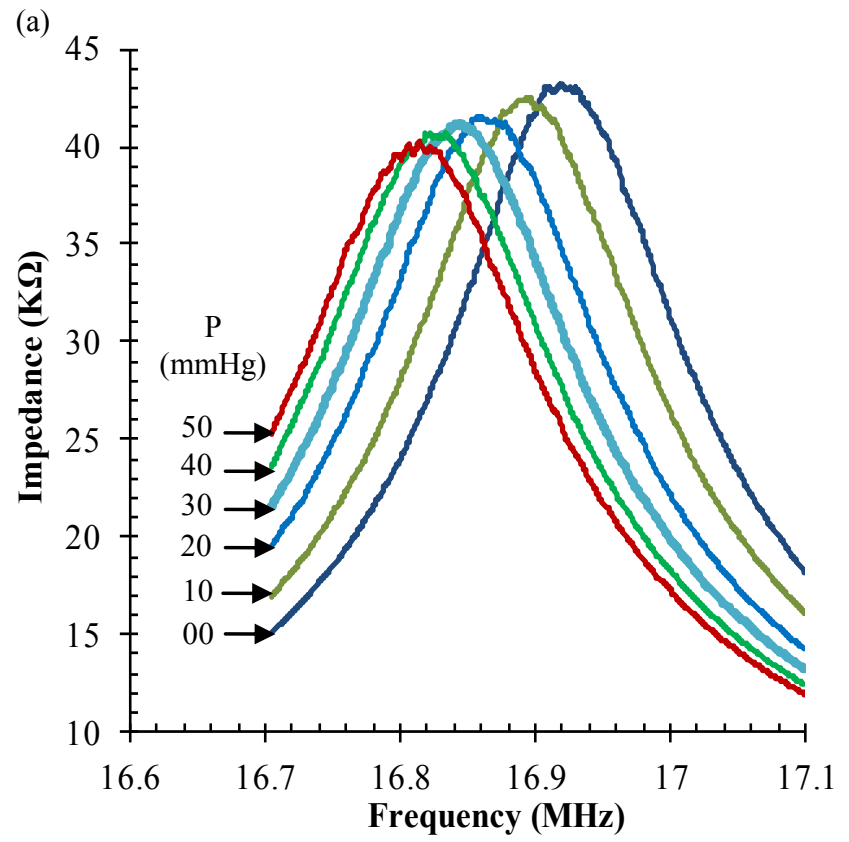

(b)

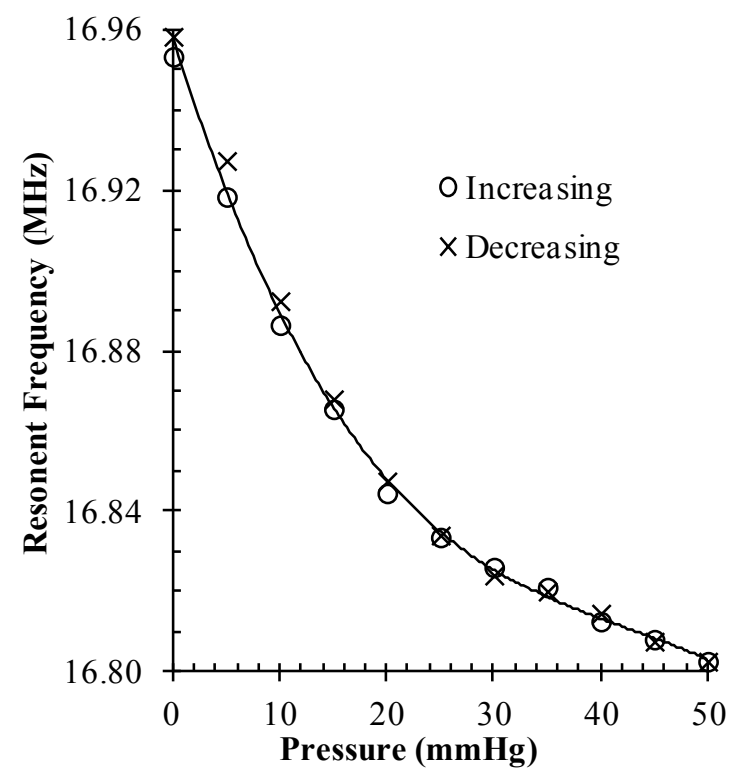

Figure 5: Measurements (a) Frequency sweep of impedance measurement at various applied pressures (b) Self-resonant frequency $(\mathrm{MHz})$ plotted against applied pressure $(\mathrm{mmHg})$

been proposed as an implant for the treatment of retinal detachment [16]. Finally, for some implant sites such as urinary bladder, which is not exposed to atmospheric air, an absolute pressure sensor is preferable. Ferrofluid pressure sensor can be easily converted to absolute pressure sensor by simply including a reference chamber across the polymeric membrane as shown in Fig. 6.

\section{CONCLUSION}

In this paper, we discussed a new pressure sensing technique based on magnetic liquid displacement and corresponding 


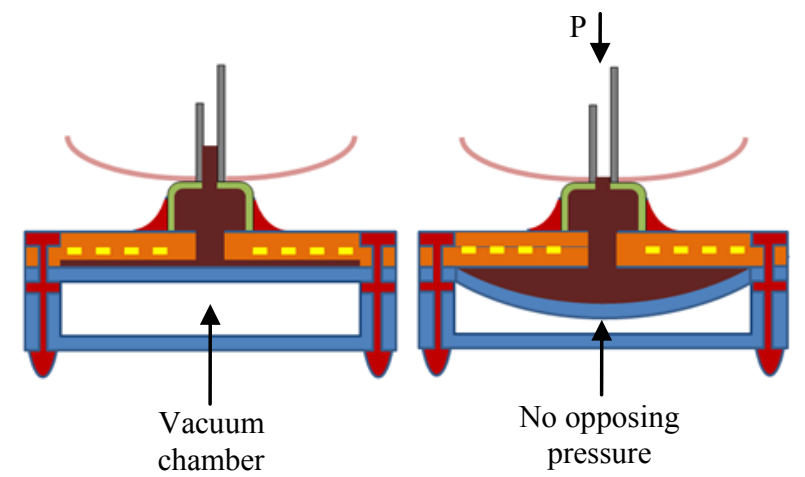

Figure 6: Proposed schematic diagram of an absolute pressure sensor based on ferrofluid movement.

inductance change. A pressure sensor was designed, fabricated, and tested in vitro. Measurements show a cubic relation between applied pressure and inductance change. The initial linear region $(0-20 \mathrm{mmHg})$ shows a sensitivity of $6 \mathrm{kHz} / \mathrm{mmHg}$. Finally, a design variation was suggested to fabricate an absolute pressure sensor based on ferrofluid movement. Simple design and easy fabrication process make this pressure sensor an attractive option for many biomedical applications.

\section{ACKNOWLEDGEMENTS}

The authors wish to thank staff of the Birck Nanotechnology Center at Purdue University for their assistance in fabrication of the transponder

\section{REFERENCES}

[1] J. T. Farrar, V. K. Zworykin, and J. Baum, "PressureSensitive Telemetering Capsule for Study of Gastrointestinal Motility," Science, vol. 126, no. 3280, pp. 975-976, Nov. 1957.

[2] C. C. Collins, "Miniature passive pressure transensor for implanting in the eye," IEEE transactions on bio-medical engineering, vol. 14, no. 2, pp. 74-83, Apr. 1967.

[3] M. G. Allen, "Micromachined endovascularly-implantable wireless aneurysm pressure sensors: from concept to clinic," in The 13th International Conference on Solid-State Sensors, Actuators and Microsystems, 2005. Digest of Technical Papers. TRANSDUCERS '05, 2005, vol. 1, pp. 275-278.

[4] B. Ziaie and K. Najafi, "An Implantable Microsystem for Tonometric Blood Pressure Measurement," Biomedical Microdevices, vol. 3, no. 4, pp. 285-292, 2001.

[5] T. Eggers et al., "Wireless intra-ocular pressure monitoring system integrated into an artificial lens," in Microtechnologies in Medicine and Biology, 1st Annual International, Conference On. 2000, 2000, no. 1, pp. 466-469.

[6] K. Stangel, S. Kolnsberg, D. Hammerschmidt, B. Hosticka, H. Trieu, and W. Mokwa, "A programmable intraocular CMOS pressure sensor system implant," Solid-State Circuits, IEEE Journal of, vol. 36, no. 7, pp. 1094-1100, 2001.
[7] D. C. Rodger, S. Saati, and M. S. Humayun, "Microfabricated Implantable Parylene-Based Wireless Passive Intraocular Pressure Sensors," Journal of Microelectromechanical Systems, vol. 17, no. 6, pp. 1342-1351, Dec. 2008.

[8] G. D. Chitnis, T. Maleki, B. Samuels, L. B. Cantor, and B. Ziaie, "An ocular tack for minimally invasive continuous wireless monitoring of intraocular pressure," in 2012 IEEE 25th International Conference on Micro Electro Mechanical Systems (MEMS), 2012, no. February, pp. 922-925.

[9] J. Melgaard and N. J. M. Rijkhoff, "Detecting the onset of urinary bladder contractions using an implantable pressure sensor.," IEEE transactions on neural systems and rehabilitation engineering: a publication of the IEEE Engineering in Medicine and Biology Society, vol. 19, no. 6, pp. 700-8, Dec. 2011.

[10] M. Ghannad-Rezaie, L. J.-S. Yang, H. J. L. Garton, and N. Chronis, "A Near-Infrared Optomechanical Intracranial Pressure Microsensor," Journal of Microelectromechanical Systems, vol. 21, no. 1, pp. 23-33, Feb. 2012.

[11] H. Oka and J. Iwata, "Fundamental Behavior of Micro Gas Flow Sensor Using Magnetic Fluid," IEEE Translation Journal on Magnetics in Japan, vol. 2, no. 6, pp. 563-565, Jun. 1987.

[12] B. Ando, A. Ascia, and S. Baglio, "A Ferrofluidic Inclinometer in the Resonant Configuration," IEEE Transactions on Instrumentation and Measurement, vol. 59, no. 3, pp. 558-564, Mar. 2010.

[13] M. Felix, A. Lizarraga, A. Islas, and A. Gonzalez, "Analysis of a ferrofluid core LVDT displacement sensor," in IECON 2010 - 36th Annual Conference on IEEE Industrial Electronics Society, 2010, pp. 1769-1772.

[14] A. Tomitaka, A. Hirukawa, T. Yamada, S. Morishita, and Y. Takemura, "Biocompatibility of various ferrite nanoparticles evaluated by in vitro cytotoxicity assays using HeLa cells," Journal of Magnetism and Magnetic Materials, vol. 321, no. 10, pp. 1482-1484, May 2009.

[15] D. H. Kim, S.-H. Lee, K.-N. Kim, K.-M. Kim, I.-B. Shim, and Y.-K. Lee, "Cytotoxicity of ferrite particles by MTT and agar diffusion methods for hyperthermic application," Journal of Magnetism and Magnetic Materials, vol. 293, no. 1, pp. 287292, May 2005.

[16] O. T. Mefford et al., "Field-induced motion of ferrofluids through immiscible viscous media: Testbed for restorative treatment of retinal detachment," Journal of Magnetism and Magnetic Materials, vol. 311, no. 1, pp. 347-353, Apr. 2007.

\section{CONTACT}

*G. D. Chitnis, tel: +1-413-561-5226; gchitnis@purdue.edu 


\title{
HYDROGEL CHECK VALVE WITH NON-ZERO CRACKING PRESSURE FOR USE AS A POTENTIAL ALTERNATIVE HYDROCEPHALUS TREATMENT METHOD
}

\author{
H.N. Schwerdt ${ }^{*}$, R. Bristol ${ }^{2}$, and J. Chae ${ }^{l}$ \\ ${ }^{1}$ Arizona State University, Tempe, Arizona, USA \\ ${ }^{2}$ Phoenix Children's Hospital, Phoenix, Arizona, USA
}

\begin{abstract}
An alternative method of treating hydrocephalus by means of cranially confining and miniaturizing draining mechanisms may offer greater safety and reliability than standard cerebrospinal fluid (CSF) shunts that suffer from high failure rates partly related to their physically large form (2 long catheters and 1 valve) and implant coverage (brain to distal organs). This passive check valve targets restoration of near natural CSF draining operations and attempts to mitigate common valve complications such as back flow and steady state leakage. A simple perforated hydrogel membrane forms the basic check valve structure and its swelling characteristics provide the sealing to diminish leakage. Preliminary measurements demonstrate its ability to operate within an appropriate range of $-800<\Delta P<600 \mathrm{mmH}_{2} \mathrm{O}$, establish a targeted $P_{T} \approx 20-110 \mathrm{mmH}_{2} \mathrm{O}$, and substantially lower reverse flow leakage for $\triangle P<P_{T}$.
\end{abstract}

\section{INTRODUCTION Background}

Hydrocephalus, a chronic neurological and, in most cases, congenital disorder, occurs due to excessive accumulation of CSF within the ventricles of the brain causing fatally high increases in intracranial pressures (ICP) [1-3]. The disorder may lead to abnormal enlargement of the head, convulsions, mental disabilities, and even death. Most forms of this condition are thought to originate due to faulty arachnoid granulations that serve as natural valve formations protruding from the meningeal membrane, arachnoid mater, surrounding the brain and that normally direct CSF from the subarachnoid space (SAS) of the brain to the sagittal sinus immediately exterior to the meninges and enclosed within the cranium [2]. Current methods to treat hydrocephalus involve, primarily, implanting a macroscopic valve (CSF shunt) into the brain to divert the accumulating CSF through an intracranial and subcutaneous long tube (catheter) outside of the cranium and to the abdominal cavity or other distal organs $[1,3,4]$ (Fig. 1). However, more than $50 \%$ of these shunts fail within the first two years of implantation, instigating additional invasive surgeries [3]. Some of these complications may be attributed to the shunts' physically large and lengthy form making them susceptible to external forces and/or siphoning effects. Additionally, intracranial catheters potentially expose the brain and increase risks of infection.

\section{Current Technology}

Normally closed MEMS check valves have been studied for over 20 years, and target mainly 3 operating parameters [5-9]: 1) high diodicity or unidirectionality of flow $\left.\left(Q_{O} \geq 0\right), 2\right)$ zero leakage when applied differential pressure $(\triangle P)$ is less than the cracking pressure $\left(P_{T}\right)$, and 3$)$ a designated non-zero or zero $P_{T}$ (the former is targeted for CSF draining). However, existing work usually reveals deficiencies in one or more of these targeted properties, and especially so in avoiding reverse flow leakage that occurs due to inadequate sealing of the valve when $\Delta P<P_{T}[5,8,9]$. Other limitations may involve reverse flow leakage at higher back pressures $(\triangle P<<0)$ [5], permanent deformation that directly diminishes repeatability [8], stiction of the valve seat that affects reproducibility and yield, and/or finite leakage observed after longer or multiple periods of operation undermining its utility in permanent chronic implants. To reduce many of these failure mechanisms, more complex valve designs and fabrication processes have been developed to realize adequate sealing with low or virtually zero static and reverse flow leakage and higher reproducibility and durability [10-11]. These more complex valves were formed on PDMS and were constructed for application in drug delivery [10] or regulating intraocular pressure (IOP) [11] for glaucoma treatment and demonstrated effective operation as implemented in animal models. Unlike drainage devices used in treating glaucoma where $Q_{O} \approx 1-5 \mu \mathrm{L} / \mathrm{min}$ and $P_{T} \approx 68-203 \mathrm{mmH}_{2} \mathrm{O}$, CSF draining for hydrocephalus involves substantially higher flow rates $\left(Q_{O} \approx 0.1-3.0 \mathrm{~mL} / \mathrm{min}\right)$ and may target more variable cracking pressures $\left(P_{T} \approx 10-230 \mathrm{mmH}_{2} \mathrm{O}\right)$ [4]. "Arachnoid mimicking" microvalve arrays using parylene/PDMS composite flap valves were recently developed for application in hydrocephalus, but retained challenges in leakage and diodicity [5].

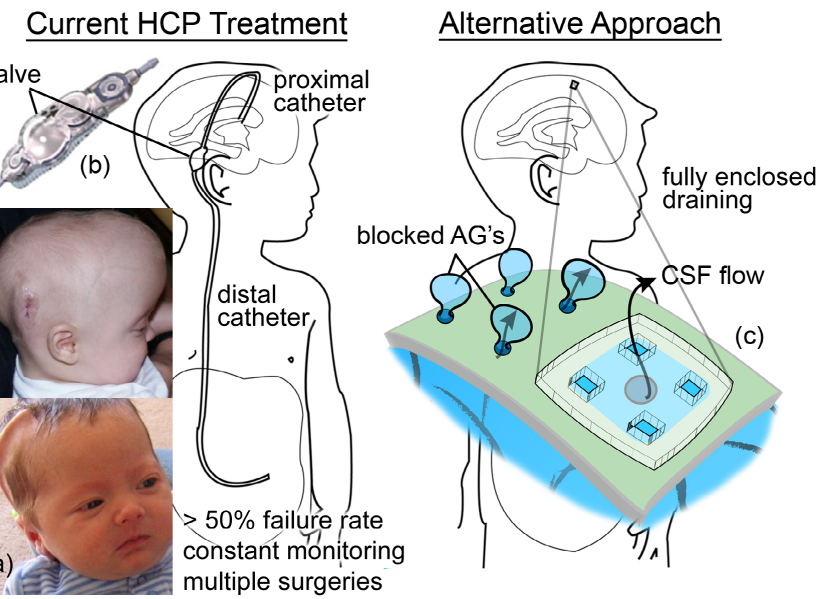

Figure 1: Depiction of current hydrocephalus (HCP) treatment methods versus a fully enclosed approach that would eliminate the need for relatively large subcutaneous catheters and valves. (a) Photographs of infants with HCP demonstrating noticeable shunt protruding the skin as well as enlargement of the head. (b) Photograph of commercial macroscopic shunt valve used to regulate CSF flow through connected catheters (Medtronic, Inc.). (c) Illustration of how HCP affects normal CSF draining through malfunctioning blocked arachnoid granulations (AGs) and how a microvalve may be appended to direct CSF flow from the brain at the associated meningeal membrane without requiring excess through-cranium catheters or distal tubing.

\section{Novelty and Impact}

The check valve presented herein aims to alleviate challenges in valve sealing and finite leakage by utilizing the intrinsic swelling behavior of hydrogels. Hydrogel polymers swell and expand when immersed in liquids that diffuse through microscopic pores in the gel matrix. This swelling phenomenon forms the basic passive check valve functionality and may effectively provide secure sealing between the valve seat and inlet to minimize static and reverse flow leakage issues. Hydrogel materials have been 
employed in valves to create environmentally (ie. $\mathrm{pH}$ or temperature) responsive valves for microfluidic lab-on-a-chip applications for in-plane operation [9]. Unlike previously reported hydrogel valves, the valve of this work is composed of only silicon and hydrogel and is fabricated using planar micromachining techniques for out-of-plane operation. As a potential hydrocephalus treatment method, this valve may be concealed subcranially and effectively substitute CSF draining operations of the obstructed arachnoid granulations (Fig. 2). Subsequent sections of this paper will delineate the passive check valve's design and fundamental operation, fabrication process, and measurement results.

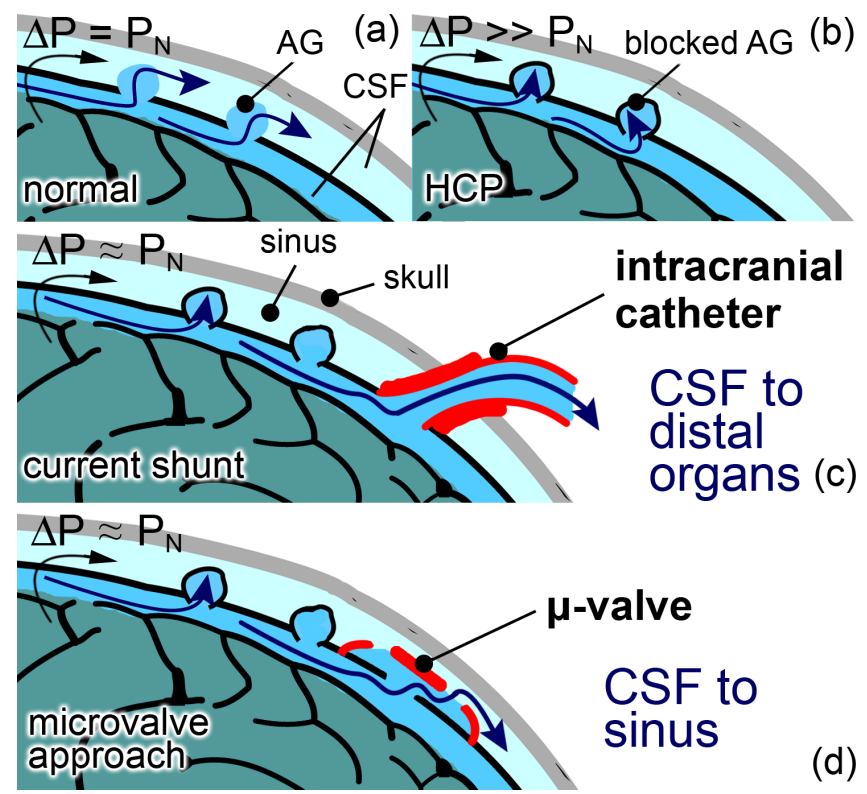

Figure 2: Illustration of arachnoid granulation function as a oneway valve (protruding structures from the dura mater) to direct CSF flow from the subarachnoid space (SAS) enclosing the brain to the sagittal sinus (top right). (a) Under normal conditions the intracranial differential pressure (ICP) is around $-100<\Delta P<350$ $\mathrm{mmH}_{2} \mathrm{O}$ ( $P_{\mathrm{N}}$, in figure, indicates this normal ICP range). (b) With hydrocephalus $\triangle P$ may reach fatally higher levels due to buildup of CSF within the brain. (c) Current shunts direct CSF from the brain to the stomach or other distal organs, requiring throughcranium catheters. (d) The hydrogel microvalve attempts to alleviate this perilous condition to substitute CSF draining operations of blocked arachnoid granulations (AGs) and diverting CSF directly to the sinus, as in normal conditions.

\section{MATERIALS AND METHODS}

\section{Operation}

The check valve essentially functions to provide a normally closed one way conduit for fluid flow and is in the form of a single suspended perforated square diaphragm made of hydrogel anchored to a silicon substrate (Fig. 3). Both valve structures, hydrogel and silicon, are approved biocompatible materials $[12,13]$. In its closed state $\left(\triangle P<P_{T}\right)$, the water induced expansion of the hydrogel seals the inlet port and prevents fluid outflow. At $\triangle P \geq P_{T}$, sufficient inlet pressure overwhelms hydrogel swelling forces allowing the valve to open, at which point the opened valve's fluid flow rate $\left(Q_{O}\right)$ is governed by the level of displacement of the hydrogel membrane. The valve's hydrogel material properties and dimensions determine the operating range of $Q_{O}$ along with $\Delta P$, as well as $P_{T}$.
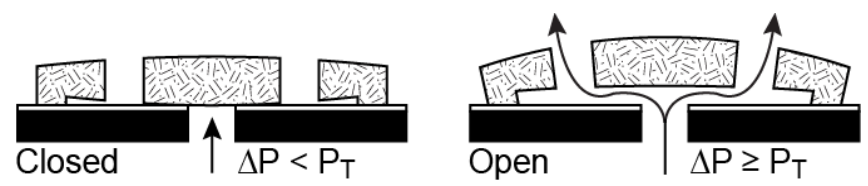

Figure 3: Designated closed and open states of the hydrogel microvalve. In the closed state (left) the hydrogel's swelling force allows the perforated membrane to seal the inlet channel and block flow (when $\triangle P<P_{T}$, cracking pressure). In the open state, when ICP reaches $P_{T}\left(\triangle P \geq P_{T}\right)$, the membrane deflects upwards to allow fluid to flow through the perforations through the membrane.

\section{Design}

The check valve targets operation at $Q_{O} \approx 0.1-3.0 \mathrm{~mL} / \mathrm{min}$, $200<\Delta P<600 \mathrm{mmH}_{2} \mathrm{O}$, and $P_{T} \approx 10-230 \mathrm{mmH}_{2} \mathrm{O}$ as pertains to observed CSF draining in current hydrocephalus shunts and normal and increased ICP levels found in hydrocephalus patients $[1,3,4]$. A perforated square diaphragm performs basic valve operations by deflecting and allowing fluid to flow through exposed perforations in response to the pressure $(\Delta P)$ across the valve. The deflection of a suspended square membrane in response to pressure may be estimated by [14],

$$
w_{0}=0.802 b \cdot\left(\frac{P b}{E t}\right)^{1 / 3}
$$

where $w_{0}$ is maximum center displacement, $b$ is membrane side length halved, $P$ is pressure, $E$ is Young's modulus $(E \approx 100-300$ $\mathrm{kPa}$ for hydrogels) [15], and $t$ is membrane thickness. Volumetric fluid flow rate $\left(Q_{O}\right)$ through the valve may then be modeled by a linear expression, $Q_{O}=\Delta P / R_{h}$, where $R_{h}$ is hydraulic resistance, which for a rectangular orifice may be expressed as,

$$
R_{h} \approx \frac{12 \mu L}{w h^{3}(1-0.603 h / w)}
$$

where $\mu$ is dynamic viscosity of the fluid ( $\mu($ water $) \approx 0.68$ at $38^{\circ} \mathrm{C}$ ), $L$ is channel length, $w$ is channel width, and $h$ is channel height. $R_{h}$ may be modeled as a series configuration of resistors correlated with the inlet channel of the silicon substrate, the variable gap formed by deflection of the hydrogel membrane, and the perforation outlet vias of the membrane. Based on this simplified model and the intended pressure range $(\Delta P)$ and targeted flow rates $\left(Q_{O}\right)$, various dimensions for the check valve are selected in the range of $b=600-1150 \mu \mathrm{m}, t \approx 100-260 \mu \mathrm{m}$, inlet channel diameter of 400-700 $\mu \mathrm{m}$, and perforation outlet diameters of 200-400 $\mu \mathrm{m}$.

Along with its physical dimensions ( $b$ and $t$ ), flow characteristics of the passive membrane valve are predominantly governed by the hydrogel material properties that will determine $E$ in (1) as well as $P_{T}$. According to Flory theory and its extensions [16], the volumetric swelling ratio for a highly swollen gel network may be estimated by

$$
\left(\frac{V}{V_{0}}\right)^{-2 / 3}=\frac{k}{\zeta Z}
$$

where $V$ and $V_{0}$ are swollen and dry gel volumes, respectively, $k$ is gel's elasticity, $\zeta$ is degree of gel ionization, and $Z$ is potential ions per dry gel volume. Flory theory also suggests a linear relationship between $k$ and the ratio of crosslinker, which may further indicate that the hydrogel volume is to the $(-2 / 3)$ power proportional to crosslinker ratio. This swelling dependence may be used to predict $P_{T}$ of the valve. The hydrogel membrane remains swollen as it is immersed in liquid at all times. 


\section{Fabrication}

The fabrication process is outlined in Fig. 4 and involves i) patterning and hardbaking $4 \mu \mathrm{m}$ thick photoresist (AZ P4330-RS, AZ Electronic Materials) sacrificial layers on $0.2 \mu \mathrm{m}$ plasma enhanced chemical vapor deposited (PECVD) $\mathrm{SiO}_{2} 500 \mu \mathrm{m}$ thick silicon $<100>$ substrates followed by adhesion promotion, ii) application and UV exposure patterning of hydrogel solution confined by $150 \mu \mathrm{m}$ rubber spacers between mask and substrate, iii) deep reactive ion etching (DRIE) of backside inlet channels through silicon, followed by wet etching of topside exposed $\mathrm{SiO}_{2}$ and photoresist, and iv) immersion in water to swell hydrogels. Completed valves are shown in Fig. 5. The chemical composition of the hydrogel solution comprises the monomer, 2-hydroxyethyl methacrylate (HEMA), crosslinker, ethylene glycol dimethacrylate (EGDA), and photoinitiator, 2,2-dimethoxy-2-phenylacetophenone (DMPA), at a volumetric ratio of 1.0/0.04/0.1.

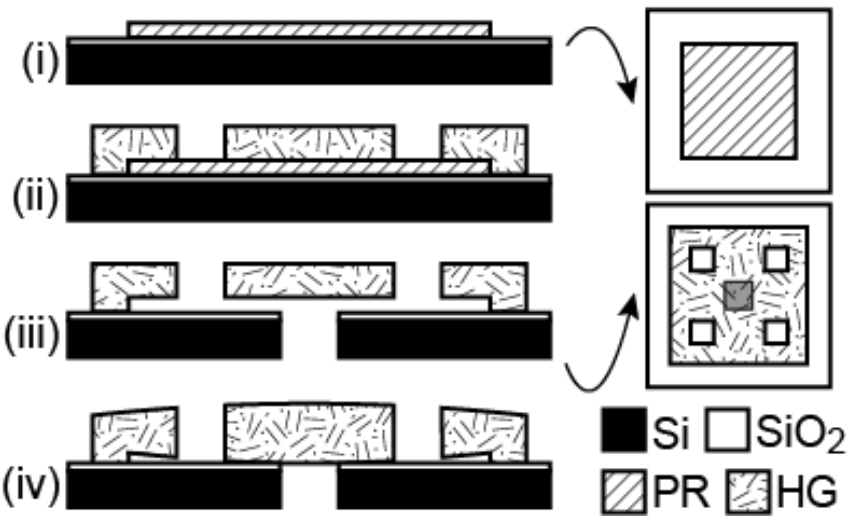

Figure 4: Cross-sectional (left) and top-view (right) fabrication process flow diagrams. (i) $4 \mu \mathrm{m}$ thick sacrificial photoresist (PR) is spun and patterned on a silicon wafer, coated with $0.2 \mu \mathrm{m}$ thick PECVD SiO ${ }_{2}$, followed by overnight treatment in an adhesion promoter. (ii) $150 \mu \mathrm{m}$ thick rubber spacers are fixed to the perimeter of the wafer and hydrogel (HG) solution is poured into the enclosed area, and patterned by UV. Uncured HG is rinsed away with isopropyl alcohol. (iii) Backside channels through silicon are etched using DRIE to expose topside $\mathrm{SiO}_{2}$. Remaining $\mathrm{SiO}_{2}$ and sacrificial $\mathrm{PR}$ are etched away using buffered hydrofluoric acid and acetone/isopropyl alcohol, respectively. (iv) Fabricated hydrogel microvalves are placed in water to acquire their swollen state and seal the channels in their initial closed state.

\section{RESULTS}

\section{Measurements}

Benchtop testing of the hydrogel check valve was performed using continuous syringe pump injection of water to generate $\Delta P$ across the valve and recording output steady state $Q_{O}$ (Fig. 6). Hydrogel membrane thicknesses $(t)$ ranged from 110 to $260 \mu \mathrm{m}$ and $b$ from 0.6 to $1.15 \mathrm{~mm}$. The intended operating fluid, CSF, has a density of $1.003-1.008 \mathrm{~g} / \mathrm{cm}^{3}$ and $\mathrm{pH}$ of 7.4 [2], which closely match those of water and may validate its use as the working fluid for benchtop testing. $\Delta P$ across the valve was acquired by converting voltage output from a differential pressure transducer (PX26-001DV, Omega). $Q_{O}$ was monitored by measuring float height through a rotameter (FL-1441-G, Omega) with 5\% accuracy and $\sim 3.5 \mu \mathrm{L} / \mathrm{min}$ resolution at discrete intervals (15 to 45 minutes) upon stabilization of $\Delta P$.

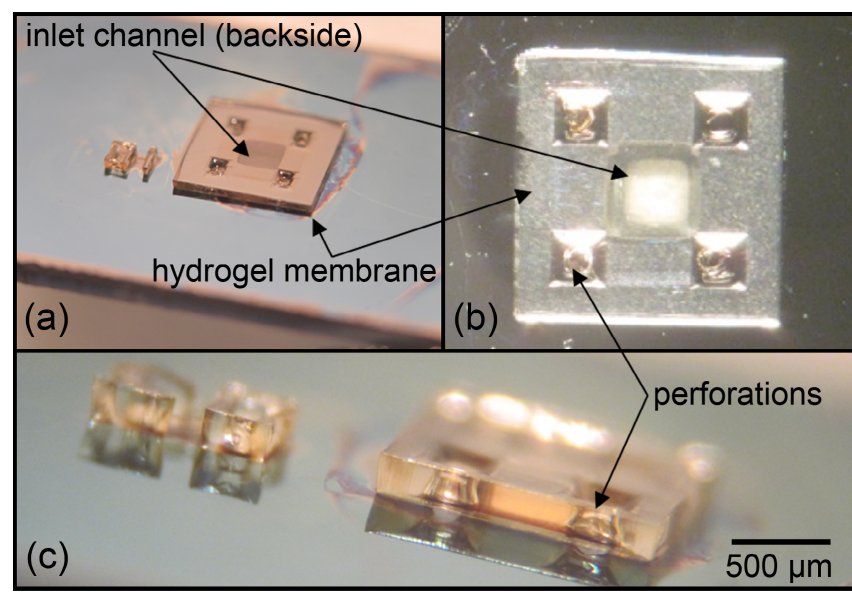

Figure 5: Photographs of fabricated hydrogel microvalves. (a) Angled die level view. (b) Top view of hydrogel microvalve. (c) Cross-sectional view of microvalve with scale bar.

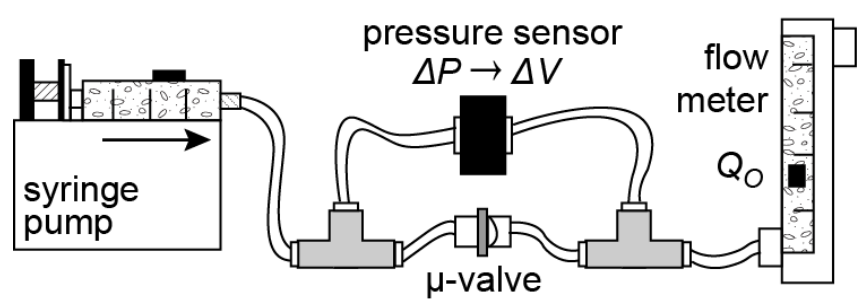

Figure 6: Experimental test setup. A syringe pump (left) manually generates pressure across the $\mu$-valve (bottom center). A pressure sensor (top center) measures differential pressure $(\Delta P)$ across the valve through its voltage output ( $\Delta V)$. A flow meter (right) measures the output flow rate of the microvalve $\left(Q_{O}\right)$.

\section{Discussion}

Fluid flow response for several different hydrogel membrane geometries are shown in Fig. 7 and demonstrate high diodicity with no observable static or reverse flow leakage and targeted $P_{T} \approx 20-110 \mathrm{mmH}_{2} \mathrm{O}$. The valves are capable of tolerating high back pressures $\left(\triangle P<-800 \mathrm{mmH}_{2} \mathrm{O}\right)$ without noticeable deformation or degradation while maintaining desired $P_{T}$. Fig. 8 shows preliminary over time measurements demonstrating the valve's ability to retain targeted $P_{T}$ with a variability of $\pm 7.5 \mathrm{mmH}_{2} \mathrm{O}$. Table 1 summarizes the measured results of the valves along with comparison to targeted specifications.

The maximum $Q_{O}$ was slightly under $0.20 \mathrm{~mL} / \mathrm{min}$ at the maximum $\triangle P=600 \mathrm{mmH}_{2} \mathrm{O}$. Depending on the treatment requirements for hydrocephalus, higher flow rates may be needed signifying lowering the hydraulic resistance, $R_{h}$, of the microvalve. This may be implemented indirectly by simply utilizing a parallel array of multiple valves. Changes in hydrogel membrane geometry and dimensions may also be made to decrease $R_{h}$ as well as optimize other flow parameters as required for the specific treatment protocol.

The fabrication process revealed several challenges in yield and reproducibility mainly owing to stiction and photoresist release. Nonetheless, slight optimizations by increasing the thickness of the sacrificial photoresist layer and/or treating or coating hydrogel or $\mathrm{SiO}_{2}$ surfaces may alleviate these challenges. 


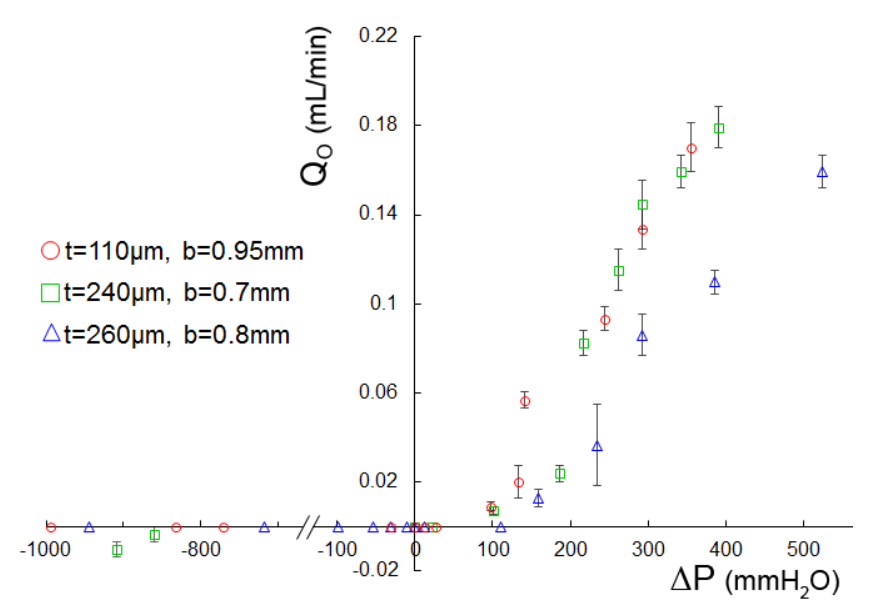

Figure 7: Measured flow response of hydrogel microvalves demonstrating $P_{T} \approx 20-110 \mathrm{mmH}_{2} \mathrm{O}$ that is within targeted $P_{T} \approx 10$ $230 \mathrm{mmH}_{2} \mathrm{O}$ with static and reverse flow sealing and high diodicity where $Q_{O}=0$ at steady state $\left(\triangle P=0 \mathrm{mmH}_{2} \mathrm{O}\right)$ and at high back pressures $\left(\triangle \mathrm{P}<-800 \mathrm{mmH}_{2} \mathrm{O}\right)$.

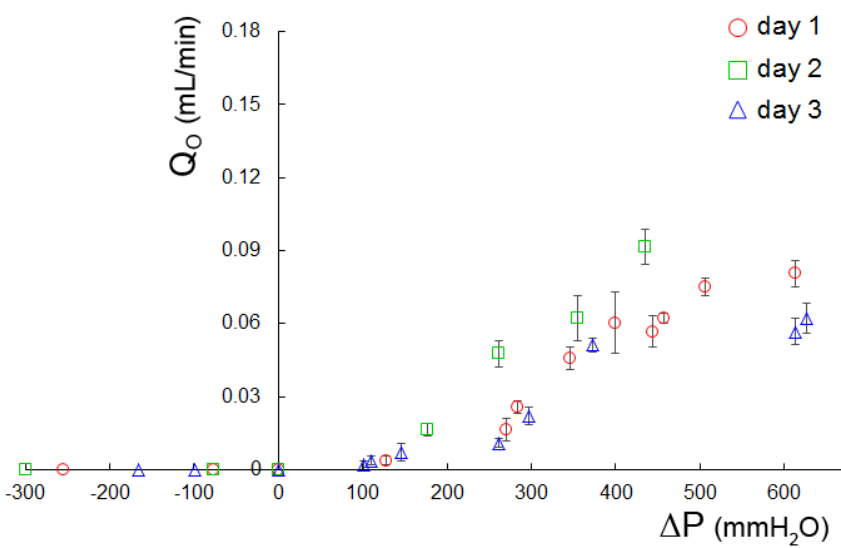

Figure 8: Preliminary over time measurements of a single hydrogel microvalve device depicting low variability of $P_{T}$.

\section{CONCLUSION}

A hydrogel microvalve operating at targeted $\triangle P$ and $Q_{O}$ levels suitable for CSF draining with negligible or zero observable leakage may strengthen its potential utility as an alternative hydrocephalus treatment method. The valve is relatively simple in design and only comprises two materials, silicon and hydrogel, both of which are biocompatible. Benchtop testing to characterize its long term performance and durability are ongoing. Furthermore, additional tests may be performed to characterize valve fluid flow properties as relates to its geometry and hydrogel material composition to confirm manual controllability of $P_{T}$ as well as other flow parameters (ie. hydraulic resistance, $R_{h}$ ). Future work to ensure its reliability and durability as a permanent medical implant will entail long term and in vivo testing.

Table 1: Targeted and measured specifications of hydrogel check valve.

\begin{tabular}{lcc}
\hline \hline & Targeted & Measured \\
\hline$\Delta P$ & $-200-600 \mathrm{mmH}_{2} \mathrm{O}$ & $-800-600 \mathrm{mmH}_{2} \mathrm{O}$ \\
$Q_{O}$ & $0.1-3.0 \mathrm{~mL} / \mathrm{min}$ & $0.01-0.20 \mathrm{~mL} / \mathrm{min}$ \\
$P_{T}$ & $10-230 \mathrm{mmH}_{2} \mathrm{O}$ & $20-110 \mathrm{mmH}_{2} \mathrm{O}$ \\
\hline \hline
\end{tabular}

\section{ACKNOWLEDGMENT}

This project was partially supported by the Arizona State University Graduate and Professional Student Association's JumpStart Grant. Travel support has been generously provided by the Transducer Research Foundation.

\section{REFERENCES}

[1] A. Chabrerie and P.M. Black, "Ventricular Shunts," Journal of Intensive Care Medicine, 15, 218 (2002).

[2] A. Brodbelt and M. Stoodley, "CSF Pathways: A Review," British Journal of Neurosurgery, 21, 510 (2007).

[3] J.M. Drake, J.R.W. Kestle, and S. Tuli, "CSF Shunts 50 Years On-Past, Present, and Future," Children's Nervous System, 16,800 (2000).

[4] M. Czosnyka, Z. Czosnyka, H. Whitehouse, and J.D. Pickard, "Hydrodynamic Properties of Hydrocephalus Shunts: United Kingdom Shunt Evaluation Laboratory," Journal of Neurology, Neurosurgery, and Psychiatry 62, 43 (1997).

[5] J. Oh, G. Kim, F. Kralick, and H. Noh, "Design and Fabrication of a PDMS/Parylene Microvalve for the Treatment of Hydrocephalus," Journal of Microelectromechanical Systems, 20, 811 (2011).

[6] K. Oh and C. Ahn, "A Review of Microvalves," Journal of Micromechanics and Microengineering, 16, R13 (2006).

[7] P. Chen, D. Rodger, E. Meng, M. Humayun, and Y. Tai, "Surface-Micromachined Parylene Dual Valves for On-Chip Unpowered Microflow Regulation," Journal of Microelectromechanical Systems, 16, 223 (2007).

[8] J. Tirén, L. Tenerz, and B. Hok, "A Batch-Fabricated NonReverse Valve with Cantilever Beam Manufactured by Micromachining of Silicon," Sensors and Actuators, 18, 389 (1989).

[9] D. Kim and D. Beebe, "A Bi-Polymer Micro One-Way Valve," Sensors and Actuators, 136, 426 (2007).

[10] R. Lo, P. Li, S. Saati, R.N. Agrawal, M.S. Humayan, and E. Meng, "A Passive MEMS Drug Delivery Pump for Treatment of Ocular Diseases," Biomedical Microdevices, 11, 959 (2009).

[11] S. Moon, S. Im, J. An, C.J. Park, H.G. Kim, S.W. Park, H.I. Kim, and J. Lee, "Selectively Bonded Polymeric Glaucoma Drainage Device for Reliable Regulation of Intraocular Pressure," Biomedical Microdevices, 10.1007/s10544-0119609-4 (2011).

[12] J. Montheard, M. Chatzopoulos, and D. Chappard, "2Hydroxyethyl Methacrylate (HEMA): Chemical Properties and Applications in Biomedical Fields," Journal of Macromolecular Science Part C, 32, 1 (1992).

[13] G. Kotzar, M. Freas, P. Abel, A. Fleischman, S. Roy, C. Zorman, J.M. Moran, and J. Melzak, "Evaluation of MEMS Materials of Construction for Implantable Medical Devices," Biomaterials, 23, 2737 (2002).

[14] S. Timoschenko, Theory of Plates and Shells, McGraw-Hill, New York, 1940.

[15] H. Li, T.Y. Ng, Y.K. Yew, and K.Y. Lam, "Modeling and Simulation of the Swelling Behavior of $\mathrm{pH}$-stimulusResponsive Hydrogels," Biomacromolecules, 6, 109 (2005).

[16] S.H. Gehrke, G.P. Andrews, and E.L. Cussler, "Chemical Aspects of Gel Extraction," Chemical Engineering Science, 41, 2153 (1986).

\section{CONTACT}

*H.N. Schwerdt; hschwerd@asu.edu 


\section{A LARGE PISTON DISPLACEMENT MEMS MIRROR WITH ELECTROTHERMAL LADDER ACTUATOR ARRAYS FOR FOURIER TRANSFORM SPECTROSCOPY APPLICATIONS

\author{
S.R. Samuelson ${ }^{*}$ and H. Xie
}

Department of Electrical and Computer Engineering, University of Florida, Gainesville, Florida, USA

\begin{abstract}
A large displacement piston motion micromirror is designed, fabricated and tested with device features tuned to application in a hand-portable Fourier Transform Spectroscopy (FTS) system. The fabricated MEMS mirror is based on electrothermal actuation and has a footprint of $1.9 \times 1.9 \times 0.5 \mathrm{~mm}^{3}$ with a mirror aperture of $1.02 \mathrm{~mm}$. The application optimized device holds key features of ultralow maximum tilt of $0.25^{\circ}$, a large, strongly linear motion of $90 \mu \mathrm{m}$ achievable at only $1.2 \mathrm{~V} \mathrm{dc}$ and a maximum theoretical spectral resolution of $55.6 \mathrm{~cm}^{-1}$. This device is further characterized with pertinent characteristics for applicability to rapid scan FTS system configurations.
\end{abstract}

\section{INTRODUCTION}

MEMS-based FTS systems based on amplitude division interferometry possess the same benefits as their macro-scale counterparts, namely the multiplex and throughput advantages, which result in fast measurement and high sensitivity [1]. These systems are constrained by spectral resolution, which is limited by the achievable optical path difference (OPD). These systems in the micro-scale have been investigated and demonstrated both based on fabrication via LIGA and surface/bulk micromachining [2]. The primary actuation methodologies employed include electrostatic, electrothermal, and electromagnetic. An electrostatic device that includes a fully integrated system can achieve a maximum displacement of $25 \mu \mathrm{m}$ [3] while a fabricated electrostatic mirror as a standalone device has been reported to achieve up to $38.5 \mu \mathrm{m}$ displacement via in plane actuation [4]. Another device is also a discrete mirror that stacks comb drives to achieve an out of plane displacement of $200 \mu \mathrm{m}$ at resonance under vacuum at $100 \mathrm{~Pa}$ [5]. Electromagnetic devices have been constructed via LIGA technology and are able to achieve $54 \mu \mathrm{m}$ effective displacement [6].

Prior work has been accomplished with electrothermal devices that have yielded a device with $308 \mu \mathrm{m}$ of effective displacement [7]. The work employed an LSF-LVD electrothermal micromirror that used both sides of the mirror surface in a tilt insensitive FTS system to combat the high tilt inherent in the mirror design. This system allowed four times the OPD via the path length varying through both arms of the FTS system. This contrasts with the usual doubling of path length in traditional Michelson interferometer systems and thus allowed a spectral resolution of $8.1 \mathrm{~cm}^{-1}$. The shortcoming of this system stems from the all-purpose design orientation of the micromirror that allowed large displacement but additionally generated large tilt that necessitated the tilt insensitive system, which added additional optical components and thus increased system size. Additionally, tilt compensation only removed beam misalignment from the actuator axis but did not restrict travel of the matched beams in tandem or remove tilt for the off-actuator axis.

This work carries over the goals of the prior work to create a system that is compact, portable and of low cost such that it may be employed in areas such as chemical analysis and biohazard detection [7]. To add to these goals, it is of paramount importance to reduce tilt while not excessively sacrificing displacement as well as increasing robustness of the system for portable use.

\section{MEMS MIRROR DESIGN}

Actuator Structure and Function

With these goals firmly in mind, the basic conceptualization of the electrothermal actuator was chosen to be based on a foldeddual-S-shaped-bimorph (FDSB) electrothermal actuator design to achieve large displacement at low drive voltage [8]. The fundamental principle of electrothermal actuation is to induce a temperature change through Joule heating which is generated by applying an electrical current to a resistor built in the bimorph; as a consequence of this heating, the difference of thermal stresses due to different coefficients of thermal expansion (CTEs) between the two primary layers of the bimorph results in bending of the cantilevered structure. The core of this actuator design is the Sshaped bimorph (Fig. 1(a)), which consists of three sections $\mathrm{L}_{1}, \mathrm{~L}_{2}$ and $\mathrm{L}_{3}$ which contain the following thin film layers of $\mathrm{Al} / \mathrm{SiO}_{2}$, $\mathrm{SiO}_{2} / \mathrm{Al} / \mathrm{SiO}_{2}$ and $\mathrm{SiO}_{2} / \mathrm{Al}$ respectively. The initial position of this beam, after release from the silicon substrate on which it is fabricated, will demonstrate the S-shaped structure as shown in Fig. 1(b) which is given via FEM simulation in COMSOL [9]. Section $\mathrm{L}_{1}$ generates initial upward curvature while $\mathrm{L}_{3}$ generates downward curvature since $\mathrm{Al}$ has much larger CTE than $\mathrm{SiO}_{2}$. Additionally, section $\mathrm{L}_{2}$ provides an overlap that protects the junction between the two active sections and provides approximate self-compensation of thermal strain for an effectively straight section.

(a)

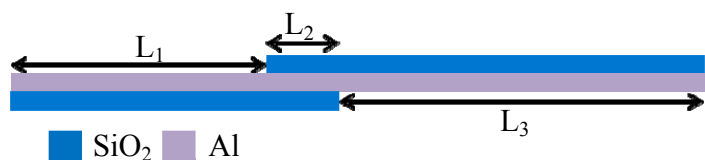

(b)

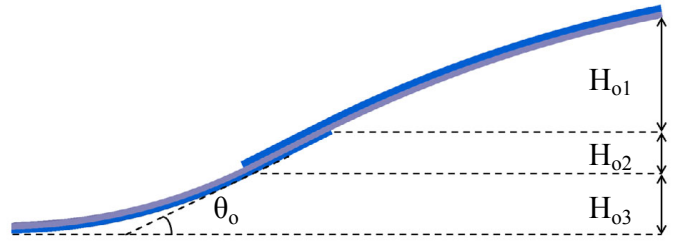

Figure 1: (a) Three constituent sections of S-shaped bimorph, (b) Initial vertical displacement on release.

A hinge is implemented that is of the same approximately self-compensated structure as $\mathrm{L}_{2}$ which joins two S-curves to form a FDSB actuator. This actuator structure results in a pure vertical displacement. In order to further increase the vertical displacement, two FDSB actuators are connected in series to form a ladder actuator (Fig. 2). With $\mathrm{R}_{\mathrm{o} 1}$ and $\mathrm{R}_{\mathrm{o} 3}$ signifying the initial radius of curvature of $\mathrm{L}_{1}$ and $\mathrm{L}_{3}$ respectively, the initial displacement of the FDSB ladder actuator can be expressed as follows (Eq. 1-3):

$$
\begin{aligned}
& H_{o}=4\left(H_{o 1}+H_{o 2}+H_{o 3}\right) \\
& H_{o}=4\left[R_{o 1}\left(1-\cos \theta_{o}\right)+L_{2} \sin \theta_{o}+R_{o 3}\left(1-\cos \theta_{o}\right)\right]
\end{aligned}
$$




$$
H_{o}=4\left[R_{o 1}\left(1-\cos \left(\frac{L_{1}}{R_{o 1}}\right)\right)+L_{2} \sin \left(\frac{L_{1}}{R_{o 1}}\right)+R_{o 3}\left(1-\cos \left(\frac{L_{1}}{R_{o 1}}\right)\right)\right](3)
$$

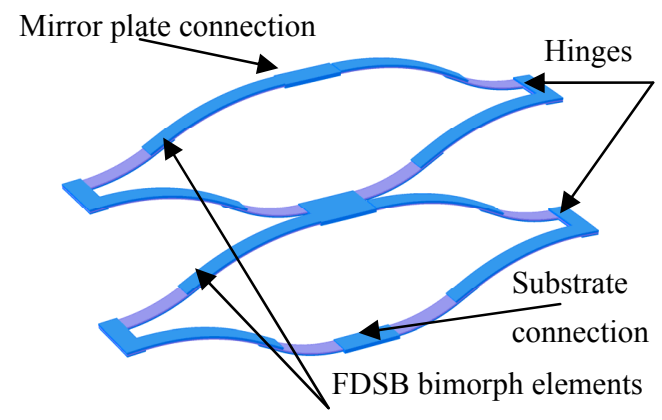

Figure 2: Full ladder actuator FEM simulation with stacked FDSB bimorph elements.

In addition to these equations which determine initial displacement of the actuator structure, an analytical model for the induced-strain actuator structure can determine the radii of curvature of the separate sections where thermal stress is the only contribution to the induced strain [10]. The primary components of the actuator are the bimorph layers of $\mathrm{SiO}_{2}$ and $\mathrm{Al}$. However, there are additional layers in the actuator that qualifies as a multimorph. These different layers include thin $\mathrm{SiO}_{2}$ and $\mathrm{Cr}$ layers that function as insulation and adhesion layers. Finally, there is a Pt layer that functions as a heater to allow greater Joule heating uniformity. Although these layers are contributory to the actuator structure and classify it as a multimorph as shown in accurate detail in Fig 3, the primary functional layers remain that of the bimorph and consideration of the full multimorph stack can be made in FEM simulation and experimental results to show this as a valid simplification [8].

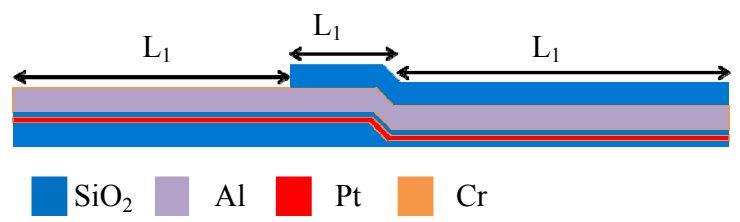

Figure 3: Cross section of S-shaped bimorph with all layers.

\section{Actuator Optimization}

Optimization of this design begins with addressing the need to reduce tilt. To address this, the following equation shows angular responsivity $\gamma$ in a single bimorph, which can be utilized as an approximate guideline for design constraints [11].

$$
\gamma=\frac{\theta_{T}}{\Delta T}=L_{b}\left(\alpha_{1}-\alpha_{2}\right) \beta_{r}
$$

In Eq. (4) the variables are as follows: thermal response angle from Joule heating $\theta_{\mathrm{T}}$; average temperate rise as a result of Joule heating $\Delta \mathrm{T}$; curvature coefficient of the bimorph combination $\beta_{\mathrm{r}}$; coefficient of thermal expansion from the top layer $\alpha_{1}$ and $\alpha_{2}$; and bimorph length $\mathrm{L}_{\mathrm{b}}$. From this equation it is found that the angular responsivity is directly proportional to the length of the bimorph in question. Reducing the bimorph lengths for each section by approximately $60 \%$ over the previous designs allows a significant and proportional reduction in angular responsivity, which strongly reduces sensitivity to signal nonlinearities and allows piston motion, when properly driven, to be the pure system output. This equation offers a guideline that does not consider the complex interactions between bimorph segments and compensated sections but presents a simple rule of thumb that may be validated most directly and clearly through experimental results.

In addition to angular responsivity, actuator stiffness is a critical aspect of mirror design as it informs robustness. It has been demonstrated that the compensating section of the actuator structure increases robustness of the actuator [8]. From this understanding, the overlap has been increased as a proportion of the S-shaped structure. This has yielded an approximately $30 \%$ increase in overlap to strengthen the actuator structure, which leads to an increase in device rigidity and vertical stiffness, all facets of a robust micromirror.

These improvements lower susceptibility of the mirror to rotation or breakage, which yields significant benefit for an intentionally mobile, piston actuating mirror. The tradeoff of such gains is requisite reduction in multimorph length, and in turn reduced actuation range. This has been handily addressed by stacking the FDSB actuators as illustrated in Fig. 4. This renders a mirror design with three stacked pairs of ladder actuators evenly spaced on a mirror edge and symmetric with the opposing edge (Fig. 5). The full mirror design is simulated via FEM simulation in COMSOL and shows the results of the optimizations of reducing angular responsivity and increasing rotational actuator stiffness [9].

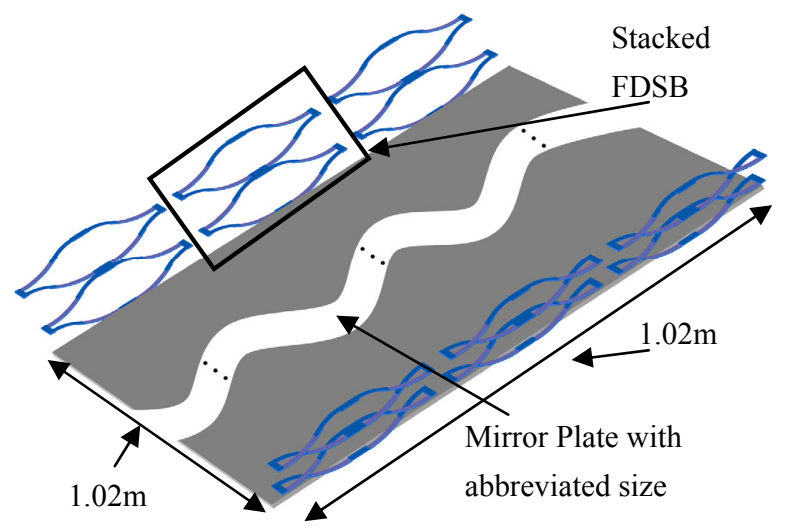

Figure 4: Full FEM of ladder actuator device with partially omitted mirror plate size.

\section{FABRICATION AND CHARACTERIZATION Device fabrication}

The fabrication process (Fig. 5) is a combined surface and bulk micromachining process that follows generally, with some variations, the process previously reported in [12]. The process utilizes silicon on insulator (SOI) wafer with the following features: a $40 \mu \mathrm{m}$-thick device layer, a $2 \mu \mathrm{m}$ buried oxide (BOX) layer and a $400 \mu \mathrm{m}$ handling layer. To begin, $1 \mu \mathrm{m}$ of PECVD $\mathrm{SiO}_{2}$ is deposited and then patterned through a buffered oxide etch primarily for the actuator (Fig. 5(a)). A $0.05 \mu \mathrm{m}$ PECVD $\mathrm{SiO}_{2}$ adhesion layer is then added to the wafer. Platinum for Joule heating is then sputtered and lifted off (Fig. 5(b)). PECVD $\mathrm{SiO}_{2}$ is again deposited for $0.2 \mu \mathrm{m}$ and patterned via dry etch to function as thermal isolation (Fig. 5(c)). Aluminum is evaporated for $1.0 \mu \mathrm{m}$ and lifted off for the actuator as well as traces (Fig. 5(d)). The final $\mathrm{SiO}_{2}$ layer is deposited via PECVD for $1.4 \mu \mathrm{m}$ and patterned via RIE dry etch primarily to complete the actuator (Fig. 5(e)). Next, photoresist is spun on a carrier wafer and applied to the frontside of the process wafer and oven-baked (Fig. 5(f)). After, backside bulk silicon anisotropic etch is performed until reaching the BOX. Thereafter, RIE etch removes the BOX (Fig. 5(g)). The carrier wafer is separated from the process wafer and the devices 
are split apart as chunks of devices aided by the previous backside anisotropic bulk silicon etch (Fig. 5(h)). Finally, the individual devices are released through a combination of anisotropic and isotropic etching in DRIE to release the devices and generate a final device configuration (Fig. 5(i)).

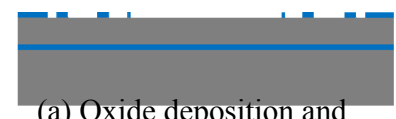

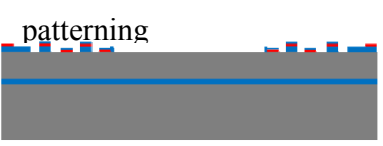

(c) Oxide deposition and contact opening

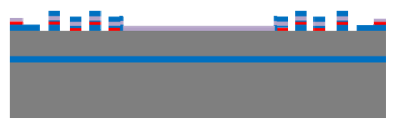

(e) Oxide deposition and

patterning

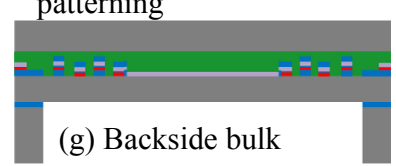

silicon and BOX etch

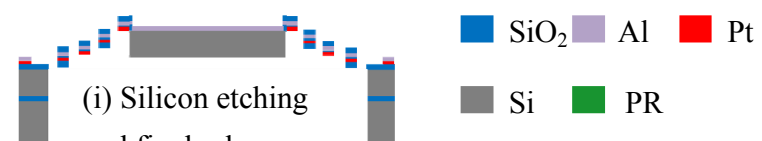

and final release

Figure 5: Fabrication process for device.

\section{Device characterization}

The final state of the device after fabrication and release is seen in Fig. 6(a). The mirror plate in Fig. 6(a) consists of the $40 \mu \mathrm{m}$-thick silicon device layer for the purposes of optical flatness. It can be seen in Fig. 6(c) that some sections of the bimorphs are exposed with $\mathrm{SiO}_{2}$ and the rest with $\mathrm{Al}$ while the embedded platinum for Joule heating is buried. The footprint of the device is $1.9 \mathrm{~mm} \times 1.9 \mathrm{~mm}$, and the mirror plate is $1.02 \mathrm{~mm} \times 1.02 \mathrm{~mm}$ which yields a fill factor of $28 \%$. From this design the individual ladder actuators have an average resistance of $15 \mathrm{ohms}$ with each individual ladder actuator capable of being driven through a distinct driving signal. The variance of these resistors is $+7 \mathrm{ohms}$ and $-2 \mathrm{ohms}$. The overall reduction of inactuator resistance is only $20 \%$. However, when factoring in the resistance from electrical paths over previous designs, the trace length is reduced by $\sim 70 \%$ [12]. Considering these two reductions, resistance, is reduced from $120 \mathrm{ohms}$ to $\sim 25 \mathrm{ohms}$. The remaining difference from this can be accounted for in fabrication variation.

The dc response of the device is measured by an Olympus BX51 microscope and a Quadra-Chek 200 geometry measuring system. From this setup, the mirror can step in piston motion through $90 \mu \mathrm{m}$ with a signal ranging from 0 to $1.2 \mathrm{~V}$ dc (Fig. 7). The observed linearity results from the reduced angular responsivity and the strong mechanical coupling of the ladder actuators. The maximum current draw of the complete device is $242 \mathrm{~mA}$. In addition to the displacement measurements, the tilt through the entire dc piston actuation range is measured. The maximum recorded tilt angle is $0.25^{\circ}$ during the entire dc piston actuation range on the actuator axis (Fig. 7). Additionally, there was no measureable off actuator tilt with this experimental setup.
This results in a tilt reduction of $85 \%$ over the previous electrothermal piston, low tilt micromirrors used in FTS applications [7].

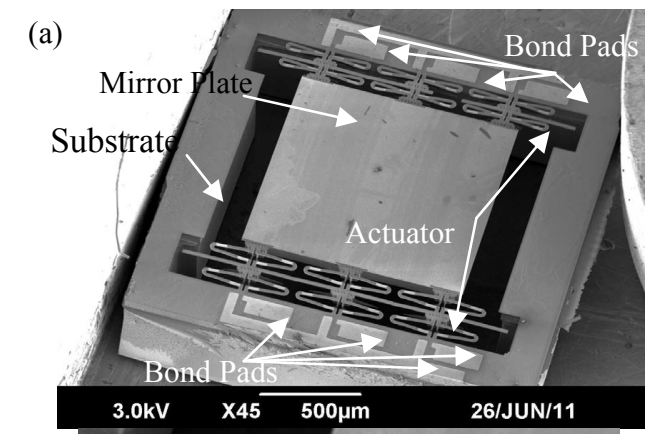

(b)

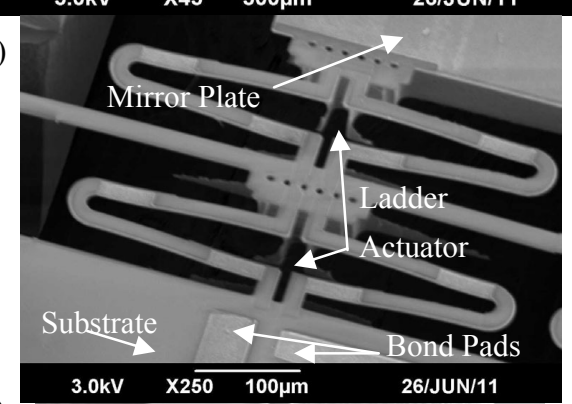

(c)

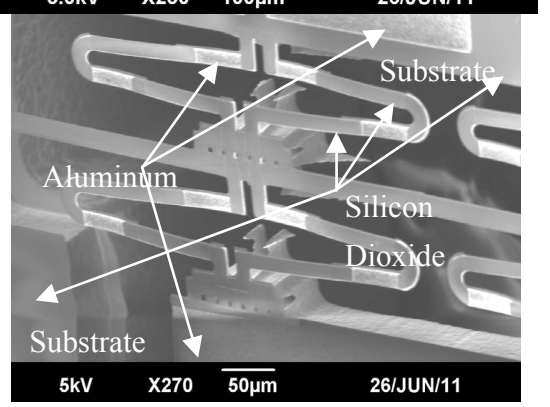

Figure 6: (a) SEM of the whole micromirror device, (b) SEM of the two-level ladder actuator, (c) SEM of the two-level ladder actuator at a different imaging angle with materials labeled (Platinum embedded and not shown).

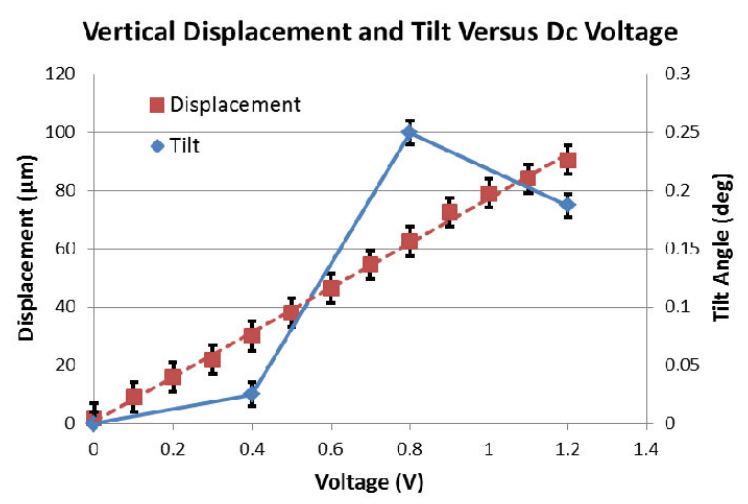

Figure 7: Recorded data points for displacement vs. given voltage at increments of $0.1 \mathrm{Vdc}$ and maximum tilt variation of only $0.25^{\circ}$.

For ac measurements, the micromirror is mounted on an optical breadboard for precise spacing from a 2D position sensitive detector (PSD). The driving signal ranges from 0.7 to $1.1 \mathrm{~V}$ at 
$0.33 \mathrm{~Hz}$. The signal is un-tuned and so does not adjust for layer thickness mismatch in the actuators from fabrication or for release step undercut of the sidewall and mirror edges. For a practical, unoptimized ac driving signal, the mirror produces a maximum of $0.22^{\circ}$ tilt on the actuator axis and $0.097^{\circ}$ on the off-actuator axis as measured via the PSD (Fig. 8).

Frequency characterization for the device also shows that the first resonant frequency or piston mode is found at $438 \mathrm{~Hz}$ with the second or torsional mode at $702 \mathrm{~Hz}$. This indicates that the design constraints have surpassed the frequency response over previous designs where $406 \mathrm{~Hz}$ was the torsional mode peak [12]. The results indicate a bandwidth amply wide to surpass the baseline definition of rapid-scan interferometers wherein the optical velocity is greater than $0.1 \mathrm{~cm}^{-1}[1]$.

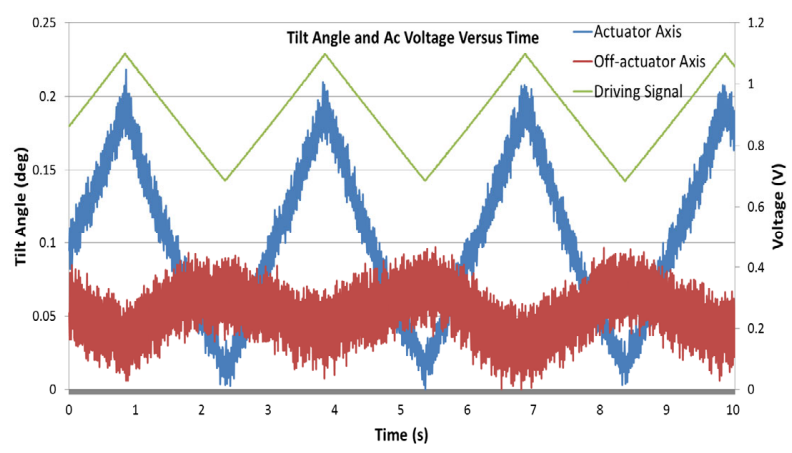

Figure 8: Tilt angle and recorded ac voltage applied to all actuators for piston motion yields a maximum tilt variation of only $0.22^{\circ}$ on the actuator axis and $0.097^{\circ}$ on the off-actuator axis.

\section{Characterization Results Interpretation}

The tilt of the mirror can be reduced in the ac and dc cases by tuning the driving voltage amplitudes to match the resistor variance of the actuators; however, due to resistor drift of several ohms during the heating and cooling cycles, such a static compensation is valid for only short intervals and requires regulation through feedback to remain beneficial.

As the tilt in the system ranges from minimum tilt to maximum tilt, it will be possible to utilize previously developed techniques of waveform shaping to correctly adjust the shift in mirror tilt while taking into account the thermal response time of the electrothermal micromirror [13]. The feedback component providing the information from this method will employ a heliumneon laser that will provide tilt information and real-time position information in accordance with established methods for reference sources [1][14]. This will permit effective integration into a practical system that will fulfill the requirements of a rapid-scan FTS system.

\section{CONCLUSION}

A unique ladder actuator that can provide ultralow tilt with large piston motion has been demonstrated. A micromirror based on such ladder actuators shows $25^{\circ}$ tilt at $90 \mu \mathrm{m}$ piston displacement. The maximum tilt is reduced by $85 \%$ compared to the previous design. This micromirror can produce a maximum wavenumber resolution of $55.6 \mathrm{~cm}^{-1}$. Prior large displacement MEMS mirrors have sacrificed stability in pure vertical displacement for larger range. In the pursuit of miniaturization, eliminating the need for physical tilt compensation streamlines and explains a practical path to a completely miniaturized system with concrete uses.

\section{ACKNOWLEDGEMENTS}

This work is supported by the National Science Foundation under the award \#0901711.

\section{REFERENCES}

[1] P. R. Griffiths and J.A. de Haseth, Fourier Transform Infrared Spectrometry, 2nd ed. Hoboken, N.J.: WileyInterscience, 2007.

[2] H. Xie and Z. M. Qi, "MEMS-Based Optical Chemical Sensors" in Semiconductor Device-Based Sensors for Gas, Chemical, and Biomedical Applications, F. Ren, Ed., CRC Press, 2011, ch. 7, pp. 267-302.

[3] K. Yu, D. Lee, U. Krishnamoorthy, N. Park, and O. Solgaard, "Micromachined Fourier transform spectrometer on silicon optical bench platform," Sensors and Actuators A: Physical, vol. 130-131, no. 0, pp. 523-530, Aug. 2006.

[4] O. Manzardo, H. P. Herzig, C. R. Marxer, and N. F. de Rooij, "Miniaturized time-scanning Fourier transform spectrometer based on silicon technology," Optics Letters, vol. 24, no. 23, pp. 1705-1707, Dec. 1999.

[5] T. Sandner, A. Kenda, C. Drabe, H. Schenk, and W. Scherf, "Miniaturized FTIR-spectrometer based on optical MEMS translatory actuator," Proceedings MOEMS and Miniaturized Systems VI, vol. 6466, pp. 646602-64660212, Jan. 2007.

[6] C. Solf, J. Mohr, and U. Wallrabe, "Miniaturized LIGA Fourier transformation spectrometer," Proceedings IEEE Sensors, 2003, vol. 2, pp. 773- 776, 22-24 Oct. 2003.

[7] L. Wu, A. Pais, S. R. Samuelson, S. Guo, and H. Xie, “A mirror-tilt-insensitive Fourier transform spectrometer based on a large vertical displacement micromirror with dual reflective surface," TRANSDUCERS 2009, pp. 2090-2093, 21-25 June 2009.

[8] K. Jia, S. Pal, and H. Xie, "An Electrothermal Tip-TiltPiston Micromirror Based on Folded Dual S-Shaped Bimorphs," Journal of Microelectromechanical Systems, vol. 18, no. 5, pp. 1004-1015, Oct. 2009.

[9] COMSOL, COMSOL Multiphysics 4.2, 2011.

[10] M. S. Weinberg, "Working equations for piezoelectric actuators and sensors," Journal of Microelectromechanical Systems, vol. 8, no. 4, pp. 529-533, Dec. 1999.

[11] H. Xie, S. Todd, A. Jain, and G. K. Fedder, "Single-Crystal Silicon Based Electrothermal MEMS Mirrors for Biomedical Imaging Applications" in MEMS/NEMS Handbook Volume 5: Medical Applications and MOEMS, C.T. Leondes, Ed., New York, N.Y.: Springer US, 2006, pp. 101-143.

[12] L. Wu, S. R. Samuelson, J. Sun, W. Lau, S. Choe, B. S. Sorg, K. Jia, and H. Xie, "A 2.8-MM imaging probe based on a high-fill-factor MEMS mirror and wire-bonding-free packaging for endoscopic optical coherence tomography," IEEE 24th International Conference on Micro Electro Mechanical Systems (MEMS), Cancun, Mexico, 2011, pp. 33-36.

[13] S. Pal and Huikai Xie, "Pre-Shaped Open Loop Drive of Electrothermal Micromirror by Continuous and Pulse Width Modulated Waveforms," IEEE Journal of Quantum Electronics, vol. 46, no. 9, pp. 1254-1260, Sep. 2010.

[14] R. J. Bell, Introductory Fourier Transform Spectroscopy. New York, N.Y.: Academic Press, 1972.

\section{CONTACT}

*S.R. Samuelson, tel: +1-352-846-0441; cap315@ufl.edu 


\title{
GENERATION OF PRESSURE BY EWOD-ACTUATED DROPLETS
}

\author{
J. Jenkins ${ }^{*}$ and C.-J. Kim
}

\author{
Mechanical and Aerospace Engineering Department, University of California, Los Angeles (UCLA) \\ Los Angeles, California, U.S.A.
}

\begin{abstract}
We demonstrate the accumulation of pumping pressure developed by multiple droplets in series actuated simultaneously by electrowetting-on-dielectric (EWOD). To measure pressure generated by EWOD, water droplets in a silicone oil medium are confined to a microchannel and driven together against a known pneumatic pressure until they can no longer advance forward. The total pressure withstood is found to scale approximately linearly with the number of droplets. Using $80 \mathrm{~V}_{\mathrm{AC}}$ at $1 \mathrm{kHz}$, while a single droplet was measured to develop $1.4 \mathrm{kPa}$ of pressure, up to 6 droplets together generated $7.6 \mathrm{kPa}$, opening the door for EWOD to be used as a pumping mechanism in high pressure applications.
\end{abstract}

\section{INTRODUCTION}

Electrowetting-on-dielectric (EWOD) [1-2] is a proven scheme for microfluidic actuation, especially popular for dropletbased digital microfluidics [3-7] in lab-on-a-chip [5-10] as well as other [11-14] applications. It carries the advantages of low power consumption and small liquid volume requirements, as it directly manipulates discrete individual droplets. The ability to move droplets around a device area atop patterned electrodes has evolved the essential droplet functions of dispensing, translation, splitting and mixing [3-5]. Most applications derive their functionality by combining these functions to perform higher order tasks. At the core of EWOD actuation, however, is a pressure gradient, internal to the droplet, which gives rise to its motion. In our experiments, we harness this pressure to work against an external pressure, demonstrating EWOD's value not only as a means of displacing a droplet of interest from one place to another, but also as a viable mechanism for pumping other media at elevated pressures.

In a typical EWOD device configuration (Figure 1), voltage is applied between electrodes above and beneath a liquid droplet, at least one of which is covered by a thin dielectric layer. The presence of the electric field tightens (increases) the curvature of the liquid meniscus at the liquid-solid contact line, where the electric field is highly concentrated, and the apparent contact angle of the liquid on the solid surface decreases [15, 16], which is equivalent to the surface becoming more wetting to the liquid. Actuating an electrode that sits beneath only a portion of the contact line results in a disparity in meniscus curvature from one end of the droplet to the other, and hence, an internal pressure gradient. Calculations have predicted this pressure to be as high as $9 \mathrm{kPa}$ at $100 \mathrm{~V}$ for a typical device configuration under ideal conditions [1] (that is, without taking contact angle hysteresis into account), but have not been rigorously confirmed by experimental measurements on devices of this kind. Furthermore, early work on continuous electrowetting suggested that the pressure drops across individual droplets placed in series could theoretically be accumulated [17], transmitting the effect of each through a surrounding medium to its neighbor, but this effect had yet to be proven or explored in experiments. In order to use EWOD actuation for pumping with wide applicability, including devices with large resistance, back pressure, or perhaps to pump ancillary media by driving against a deflecting membrane [18], we need to first verify that the total pumping pressure available indeed increases with the number of droplets actuated and then test whether the correlation between pressure and number of droplets is one of strict summation. Hilton Head Island, South Carolina, June 3-7, 2012

Figure 1: Cross-section schematic of typical EWOD device configuration.

Although EWOD is usually used to move a liquid volume of interest itself, some have shown that it is suitable for use more generally as pump components analogous to pistons and valves [19]. Microchannels filled with dodecane oil contained water droplets strategically positioned and held in place by constrictions in channel width and height. Under actuation of $65 \mathrm{~V}_{\mathrm{DC}}$ to $80 \mathrm{~V}_{\mathrm{DC}}$, the droplet menisci were able to advance into the narrower segments. This mechanism was employed with an appropriate actuation sequence such that some droplets acted as inlet and outlet valves, opening or blocking the channel depending on actuation state. Another larger droplet acted as a piston, advancing into the flow channel to displace oil through the open valves. In this way, significant flow rates of the oil medium were achieved.

Yet other work showed the development of a micropump based on oscillating continuous electrowetting actuation of a mercury droplet within a microchannel [18]. The device consisted of components fabricated from three substrates stacked together, forming a microchannel for the mercury droplet, silicone rubber membranes deflected by the droplet motion, and inlet and outlet chambers with metallic check valves, into which water was drawn and later expelled by the deflecting membrane.

Since driving pressure increases with actuation voltage, higher pressures could be achieved using one droplet alone and increasing the voltage applied, however, such an approach is significantly limited. High actuation voltage leads to the wellknown contact angle saturation, electrolysis and eventually dielectric breakdown. Adding droplets rather than increasing the actuation voltage avoids these problems while maintaining relatively low power consumption. Our work presents a micropumping scheme that offers a simple device with versatility, convenience, and a capability of vastly higher output pressure.

\section{EXPERIMENTAL PROCEDURE \\ Device Fabrication}

To direct accumulated pressure, a microchannel is fabricated atop an otherwise common-configuration EWOD device. As shown in Figure 2(a), $\mathrm{Cr} / \mathrm{Au}$ electrodes and contact pads are patterned on a glass substrate, laid out in a long row. This is followed by deposition of a $1.2 \mu \mathrm{m}$ PECVD $\mathrm{Si}_{\mathrm{x}} \mathrm{N}_{\mathrm{y}}$ dielectric layer. A $50 \mu \mathrm{m}$ thick $\mathrm{KMPR}^{\circledR} 1050$ (MicroChem) layer is then spincoated and patterned to form channel walls. A glass slide coated with indium tin oxide (ITO) to act as the grounding electrode serves as a cover plate, shown in Figure 2(b). A $100 \mathrm{~nm}$ thick 


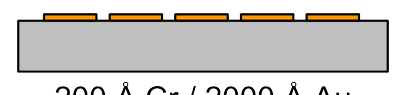

$200 \AA \mathrm{A} \mathrm{Cr} / 2000 \AA \mathrm{Au}$ deposited and patterned

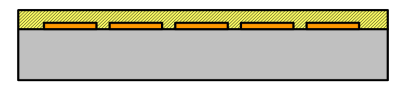

$1.2 \mu \mathrm{m}$ silicon nitride deposited by PECVD

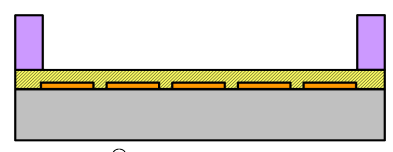

KMPR $^{\circledR} 1050$ spin-coated and patterned

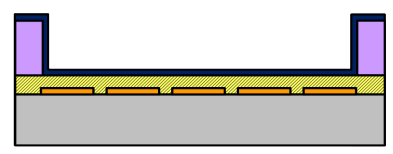

Cytop hydrophobic coating spin-coated

(a)

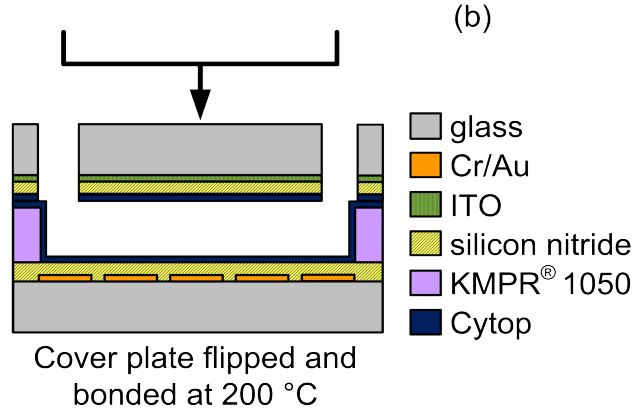

(c)

Figure 2: Process flow for (a) device substrate, (b) cover plate, and (c) substrate-cover assembly. Note the sidewall, not generating EWOD force, only retards the motion.

$\mathrm{Si}_{\mathrm{x}} \mathrm{N}_{\mathrm{y}}$ layer is deposited on the cover plate for isolation. Next, access holes $1 \mathrm{~mm}$ in diameter are drilled in the cover plate to allow the later addition of water droplets and oil medium. A hydrophobic coating of $2 \%$ Cytop solution is spin-coated on both the device substrate and cover plate. The cover plate is flipped, aligned to the substrate as in Figure 2(c), and clamping force is applied to the assembly on a hot plate at $200{ }^{\circ} \mathrm{C}$, allowing the Cytop to reflow and bond the cover plate to the substrate. Finally, tubing is epoxied over the drilled access holes for liquid filling.

\section{Pressure Measurement}

The pressure generated is determined by equilibrating actuated droplets against a known external source. We use a tank of compressed nitrogen with a finely controlled regulator to adjust this backpressure supplied to the outlet of the device microchannel. The channel is first filled with silicone oil to act as an effective medium for pressure transmission between the droplets. Before applying any pressure from the nitrogen tank, deionized water droplets are dispensed from a reservoir by EWOD and moved into position in the microchannel individually, arranged in series and with at least one grounded electrode space between them to avoid unintentional actuation in the reverse direction.
With the inlet behind the train of droplets left open to atmosphere throughout the experiments, the pressure from the tank is steadily increased through the regulator. When the supplied backpressure is sufficient to overcome the droplet's friction force (i.e., static resistance) at the channel walls, they slowly begin to move backward. The droplets are then driven against the tank pressure by EWOD with $80 \mathrm{~V}_{\mathrm{AC}}$ at $1 \mathrm{kHz}$. In our device, the sidewalls of the channel are passive, and do not contribute to the droplet's pressure development. On the contrary, due to friction, the presence of the sidewalls retards the motion as compared to an open EWOD configuration, requiring higher voltage to overcome. Recent work has shown that electrodes may be incorporated into the channel sidewalls [20], aiding in the forward driving force, and thus reducing the applied voltage necessary to move droplets. However, such a configuration requires a significantly more complex fabrication process including the production of a shadow mask with microscale resolution by front- and backside throughwafer etching.

We confirmed experimentally that water, with a contact angle on Cytop of approximately $110^{\circ}$, completely fills the channel corners when in an air medium (Figure 3(a)). A droplet was placed in a passive microchannel and pressure was applied to one end by way of the nitrogen tank. A digital pressure gauge was connected to the outlet of the channel and the pressure on this side of the droplet was monitored over time. With $3 \mathrm{kPa}$ of applied pressure to the inlet, no pressure change aft of the droplet was detected for several hours, implying that the droplet completely filled the corners of the channel, letting none of the applied pressure leak past it.

(a)
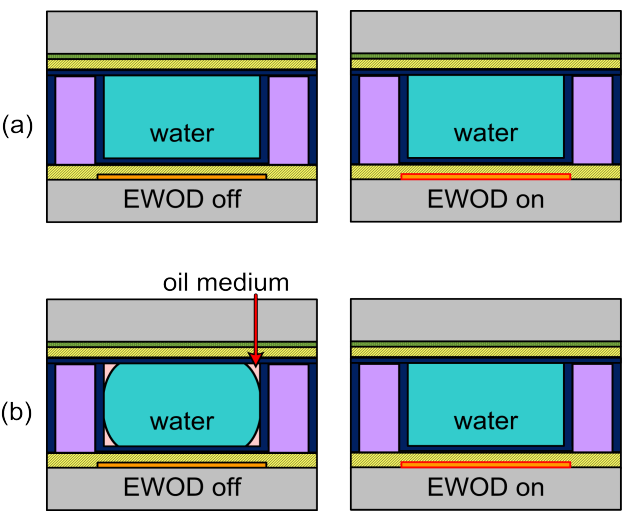

Figure 3: Cross-section schematic (showing a "front view" of the droplet, orthogonal to the cross-section plane of Figure 2) of water droplet (a) in air medium over unactuated (left) and actuated (right) electrodes, (b) in oil medium over unactuated (left) and actuated (right) electrodes. In air medium (a), water (contact angle $\sim 110^{\circ}$ ) fills the corners naturally with or without EWOD actuation, which was confirmed by experiments. In oil medium (b), on the other hand, water (contact angle $\sim 160^{\circ}$ ) does not fill the corners unless EWOD is on.

However, during our cumulative pressure generation experiments, we found that actuating series of droplets primarily compressed the volume of air trapped between them in the channel, rather than contributing additional total pumping pressure. For this reason, we performed our experiments in a medium of silicone oil, whose incompressibility allowed for effective transmission of pressure from one droplet to the next. In trade-off, however, the water makes a much higher contact angle with the channel walls, $\sim 160^{\circ}$, which fails to fill the corners (Figure 3(b)) 
when no actuation voltage is applied. To overcome the potential problem of pressure leak through the corners, we have modified the electrode actuation scheme as compared to typical EWOD devices: at least three electrodes are allotted per droplet as shown in Figure 4. The electrode under each droplet's leading meniscus is actuated as well as the one beneath its midsection. The former induces the droplet to wet and advance along the leading electrode. The latter does not provide any driving pressure, but reduces the effective contact angle at the channel surfaces to help spread the droplet to the full channel width, aiding in preventing leakage of the surrounding medium past the droplet (Figure 3(b)). The electrode under the droplet's trailing meniscus is grounded so as to remain in a hydrophobic state, establishing the droplet driving pressure.

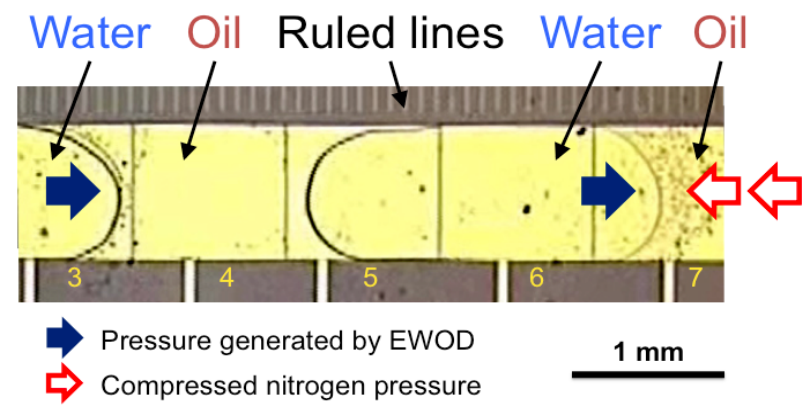

Figure 4: Top view photo of two droplets actuated simultaneously by EWOD to oppose a pressure from right. Electrodes 2 (not shown), 3, 6 and 7 are actuated with $80 V_{A C}$ at $1 \mathrm{kHz}$, while electrodes 1 (not shown), 4 and 5, as well as the transparent top cover plate are grounded. Ruled lines help to identify point of droplet stalling.

Actuation of multiple droplets together is observed to counteract higher tank pressures, which is adjusted until the droplets stall (Figure 4). Ruled lines patterned on the surface of the device help to identify the moment at which the droplets are no longer able to move forward against the compressed nitrogen, providing a visual stationary reference point. At the stalling pressure, removing voltage from any one of the droplets allows the entire group to move backward down the channel under the backpressure. Reinstating voltage prevents the loss of further ground. The balanced pressure is read from a digital gauge connected to a parallel on-chip channel, crosschecked with the reading of the inlet regulator, and recorded. This pressure is reported as that generated by the procession of droplets. A side view schematic of the experimental setup is shown in Figure 5.

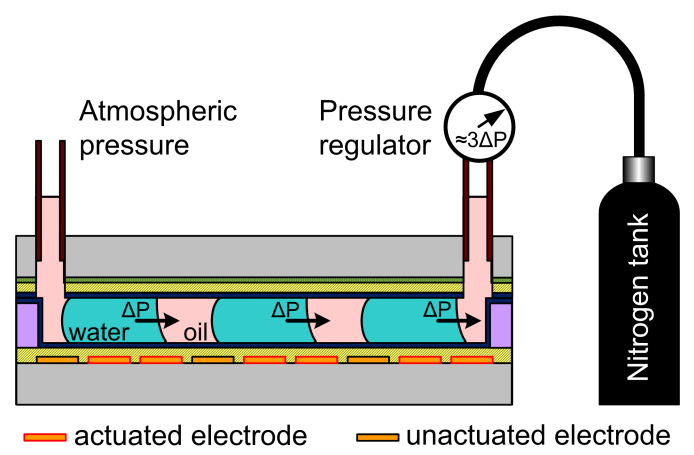

Figure 5: Side view schematic of experimental setup showing multiple droplets (3 shown) in series pushing against the regulated pressure.

\section{RESULTS AND DISCUSSION}

Measured pressure results are plotted versus the number of droplets actuated in series in Figure 6, along with a linear curve fit. Each point represents an average of four experimental measurements for each series of droplets. The data show that with each additional droplet, the total pressure withstood increases nearly proportionally. In these experiments, each droplet contributes approximately $1.4 \mathrm{kPa}$ of pressure to the total. The frictional hindrance to droplet motion due to the channel sidewalls appears to be constant for each droplet; the pressure increase by each additional actuated droplet does not appear to diminish at higher numbers of droplets. With up to six droplets, the output pressure of $7.6 \mathrm{kPa}$ achieved is higher than the $2.0-2.5 \mathrm{kPa}$ seen by the aforementioned single piston EWOD-based pumping system under similar voltage [19], on account of our multiple droplets working together. Extrapolation of the plot implies that even higher pressures for more demanding applications can be achieved simply through the actuation of a greater number of droplets.

\section{Total pressure generated vs. number of droplets}

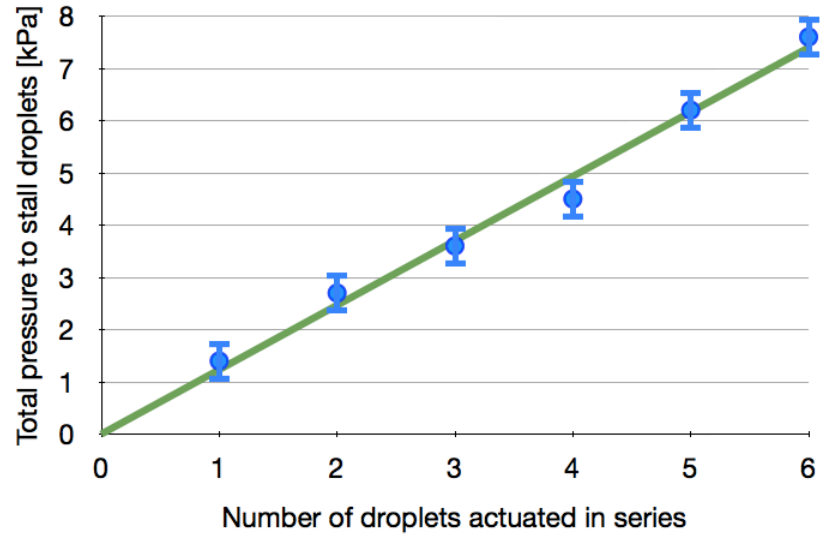

Figure 6: Plot of mean total accumulated pressure versus number of droplets in series actuated by EWOD simultaneously. Error bars represent variation in gauge reading across 4 experiments for each point.

Expected performance was calculated from the LippmannYoung and Young-Laplace equations that are often used to describe EWOD actuation [1,2]. For the electrode configuration used in our experiments, the resulting equation for internal droplet pressure gradient $\Delta P$ is given as:

$$
\Delta P=\frac{1}{2} \frac{\varepsilon \varepsilon_{o} V^{2}}{t d}
$$

where $\varepsilon$ is the dielectric constant of the dielectric layer, $\varepsilon_{\mathrm{o}}$ is the permittivity of free space, $V$ is applied voltage, $t$ is the thickness of the dielectric layer and $d$ is the microchannel height.

Frictional resistance forces resulting from contact with the substrate, cover plate and channel sidewalls were taken into account, approximated for each surface using contact angle hysteresis force [21], $F_{\text {hyst }}$, which can be written as:

$$
F_{\text {hyst }}=w \sigma\left(\cos \theta_{r}-\cos \theta_{a}\right)
$$

where $w$ is the width of the contact area between the liquid and solid surface, $\sigma$ is the interfacial tension between the liquid and the surrounding medium, and $\theta_{r}$ and $\theta_{a}$ are receding and advancing 
contact angles, respectively.

With $50 \mu \mathrm{m}$ channel height, $1.4 \mathrm{~mm}$ channel width, $80 \mathrm{~V}_{\mathrm{AC}}$ actuation voltage, $1.2 \mu \mathrm{m}$ thick dielectric layer, material properties for silicon nitride and the approximate receding and advancing contact angles of $158^{\circ}$ and $162^{\circ}$, respectively, for Equations 1 and 2 , each droplet is calculated to withstand a pressure of $3.9 \mathrm{kPa}$. The measured value of approximately $1.4 \mathrm{kPa}$ per droplet represents $36 \%$ of this expected value. We anticipate that improvements in the quality of fabrication, especially in the smoothness of the surfaces will bring experimental results closer in line with their theoretical values. Spin-coating of the Cytop hydrophobic layer on features as tall as the channel sidewalls may result in non-uniform coverage. Furthermore, Cytop reflow during the bonding of the cover plate may have caused pooling in the corners of the channel.

\section{CONCLUSIONS}

We have shown that high pressure pumping can be achieved using EWOD actuation of droplets. While each droplet exerts a pressure as a function of the EWOD voltage applied, the total output pressure of such a micropumping device is a function of the number of droplets simultaneously actuated. We observed that pressure sums from one droplet to the next in series, measuring a total pressure for up to six droplets that was approximately six times that measured for a single droplet. As such, voltage need not be increased to develop high pumping pressures, but may be fixed to that which is allowed or available by the device in hand or required by the application. Devices may be designed with a large number of droplets to meet pressure needs, with the ability to increase or decrease pressure as necessary by simply controlling the signal to the electrodes, turning the contributions of certain individual droplets on or off. Seemingly limited only by fabrication capabilities, current values suggest even a full atmosphere of pumping pressure could be realized with 70 droplets pumping together.

\section{ACKNOWLEDGEMENTS}

The authors would like to thank Dr. Wyatt Nelson, Dr. Prosenjit Sen and all the members of the UCLA Micro and Nano Manufacturing Laboratory for their helpful advice and discussion.

\section{REFERENCES}

[1] J. Lee, H. Moon, J. Fowler, T. Schoellhammer and C.-J. Kim, "Electrowetting and electrowetting-on-dielectric for microscale liquid handling", Sensor. Actuat. A-Phys., 95, 259 (2002).

[2] H. Moon, S.K. Cho, R.L. Garrell, and C.-J. Kim, "Low voltage electrowetting-on-dielectric”, J. Appl. Phys., 92, 4080 (2002).

[3] S.K. Cho, H. Moon, and C.-J. Kim, "Creating, Transporting, Cutting, and Merging Liquid Droplets by ElectrowettingBased Actuation for Digital Microfluidic Circuits", J. Microelectromech. Syst., 12, 70 (2003).

[4] M.G. Pollack, A.D. Shenderov, and R.B. Fair, "Electrowetting-Based Actuation of Droplets for Integrated Microfluidics", Lab Chip, 2, 96 (2002).

[5] P. Paik, V.K. Pamula, and R.B. Fair, "Rapid droplet mixers for digital microfluidic systems", Lab Chip, 3, 253 (2003).

[6] H. Moon, A.R. Wheeler, R.L. Garrell, J.A. Loo, and C.-J. Kim, "An integrated digital microfluidic chip for multiplexed proteomic sample preparation and analysis by MALDI-MS", Lab Chip, 6, 1213 (2006).
[7] V. Srinivasan, V.K. Pamula, and R.B. Fair, "An integrated digital microfluidic lab-on-a-chip for clinical diagnostics on human physiological fluids", Lab Chip, 4, 310 (2004).

[8] P.Y. Keng, S. Chen, H. Ding, S. Sadeghi, G.J. Shah, A. Dooraghi, M.E. Phelps, N. Satyamurthy, A.F. Chatziioannou, C.-J. Kim, and R.M. van Dam, "Micro-chemical synthesis of molecular probes on an electronic microfluidic device", Proc. Natl. Acad. Sci. USA, 109, 690 (2012).

[9] W.C. Nelson, I. Peng, G.-A. Lee, J.A. Loo, R.L. Garrell, and C.-J. Kim, "Incubated Protein Reduction and Digestion on an Electrowetting-on-Dielectric Digital Microfluidic Chip for MALDI-MS", Anal. Chem., 82, 9932 (2010).

[10] N. Vergauwe, D. Witters, F. Ceyssens, S. Vermeir, B. Verbruggen, R. Puers, and J. Lammertyn, "A Versatile Electrowetting-Based Digital Microfluidic Platform for Quantitative Homogeneous and Heterogeneous Bio-Assays", J. Micromech. Microeng., 21, 054026 (2011).

[11] P. Sen and C.-J. Kim, "A Fast Liquid-Metal Droplet Microswitch Using EWOD-Driven Contact-Line Sliding”, J. Microelectromech. Syst., 18, 174 (2009).

[12] W.C. Nelson, H.P. Kavehpour, and C.-J. Kim, “A miniature capillary breakup extensional rheometer by electrostatically assisted generation of liquid filaments", Lab Chip, 11, 2424 (2011).

[13] Y.-Y. Lin, C.-W. Lin, L.-J. Yang, and A.-B. Wang, "Microviscometer based on electrowetting on dielectric", Electrochim. Acta, 52, 2876 (2007).

[14] P. Mach, T. Krupenkin, S. Yang, and J. Rogers, "Dynamic tuning of optical waveguides with electrowetting pumps and recirculating fluid channels", Appl. Phys. Lett., 81, 202 (2002).

[15] F. Mugele and J. Buehrle, "Equilibrium drop surface profiles in electric fields", J. Phys.: Condens. Matter, 19, 375112 (2007).

[16] W.C. Nelson and C.-J. Kim, "Droplet Actuation by Electrowetting-on-Dielectric: A Review", J. Adhes. Sci. Technol., In press.

[17] E. Colgate, H. Matsumoto, "An investigation of electrowetting-based actuation", J. Vac. Sci. Technol. A, 8, 3625 (1990).

[18] K.-S. Yun, I.-J. Cho, J.-U. Bu, C.-J. Kim, and E. Yoon, "A surface-tension driven micropump for low-voltage and lowpower operations", J. Microelectromech. Syst., 11, 454 (2002).

[19] J. Kedzierski, S. Berry, and B. Abedian, "New Generation of Digital Microfluidic Devices", J. Microelectromech. Syst., 18, 845 (2009).

[20] S. Morishita, M. Kubota, and Y. Mita, "Integration of EWOD pumping device in deep microfluidic channels using a threedimensional shadowmask", Proc Int. Conf. MEMS, Paris, France, 1/29-2/2, IEEE (2012), pp. 1045-1048.

[21] E.B. Dussan V. and R.T.-P. Chow, "On the ability of drops or bubbles to stick to non-horizontal surfaces of solids", J. Fluid Mech., 137, 1 (1983).

\section{CONTACT}

*J. Jenkins, tel: +1-310-825-3977; jamesrj@UCLA.edu 


\section{A HIGHLY DENSE NANONEEDLE ARRAY FOR INTRACELLULAR GENE DELIVERY \\ Seung-Joon Paik ${ }^{1 *}$, Seonhee Park ${ }^{2}$, Vladimir Zarnitsyn ${ }^{2}$, Seungkeun Choi ${ }^{1}$, Xin Dong Guo ${ }^{2}$, Mark R. Prausnitz ${ }^{2}$, and Mark G. Allen ${ }^{1,2}$ \\ ${ }^{1}$ School of Electrical and Computer Engineering \\ ${ }^{2}$ School of Chemical and Biomolecular Engineering \\ Georgia Institute of Technology, Atlanta, Georgia, USA}

\begin{abstract}
We present a dense needle array with sharp nano-tips for intracellular gene and drug delivery, with the ultimate goal of gene correction in a high throughput manner. The nanoneedles are fabricated using isotropic dry etching of silicon, followed by anisotropic dry etching and thermal-oxidation-based tip sharpening. The nanoneedle density is as large as $10^{6}$ needles per square centimeter. In-vitro intracellular delivery tests demonstrated that the nanoneedle array can effectively deliver molecular probes into cells without causing significant cell death from cell membrane penetration. Uptake of molecular probes by up to $34 \%$ of cells after nanoneedle treatment was observed.
\end{abstract}

\section{INTRODUCTION}

Investigation into cellular functions requires the ability to apply specific and controlled treatment to cells. Such treatment includes the delivery of biological effectors across cell membranes using various approaches such as chemical, mechanical or electrical perturbations to cells [1]. In addition to investigation, gene correction represents an active treatment in which genetic diseases and cancer may be treated by correcting the causative mutations in the genetic code. Sickle cell disease is the most common inherited blood disorder caused by a mutation of a single nucleotide of a gene. The disease causes hemoglobin-containing red blood cells to tend to deform, clump and break apart, resulting in clogged blood vessels and causing severe pain, serious infection and organ damage. Bone marrow transplantation, the only permanent cure for the disease, has the undesirable limitation of donor compatibility. Research has shown that transforming defective blood-forming cells into normal ones by inserting corrective genes can alleviate the disease [2].

Several techniques currently exist for gene correction, including biological methods or physical injection of therapeutic agents into cells. Physical methods such as conventional microinjection typically use glass microcapillaries; however, these

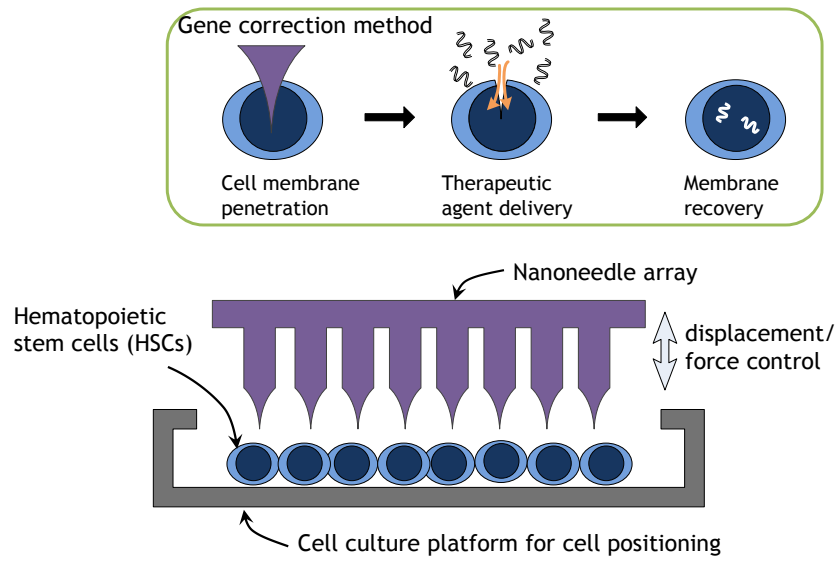

Figure 1: Schematic diagram of intracellular delivery system for insertion of therapeutic genes.

9780964002494/HH2012/\$25C2012TRF

DOI 10.31438/trf.hh2012.40 techniques have relatively low throughput due to the time-consuming operation of targeting individual cell nuclei [1]. A micron-scale microcapillary array, made of $\mathrm{SiO}_{2}$, was previously demonstrated for fluorescent dye injection [3] and DNA injection [4] to plant cells. In this paper we propose a silicon nanoneedle array with needle tip diameters in tens of nanometers, enabling pinpoint injection of individual cells in a high throughput manner without causing cell death by puncture (Fig. 1).

\section{FABRICATION}

Fabrication proceeds by patterning a positive photoresist mask (SC1813, Shipley) on a silicon wafer bearing 0.7 micron of thermal oxide. After oxide etching, isotropic dry etching of $\mathrm{Si}$ with an $\mathrm{SF}_{6}$ plasma is performed in order to achieve sharp tips utilizing under-etching of silicon under the etch mask (Figs 2a and 2b). Arrays of circular patterns of $7 \mu \mathrm{m}$-diameter remain attached to the silicon substrate after the isotropic etching, so that the circular pattern can act as an etch mask during the subsequent anisotropic etching process (Fig. 2c). Due to the directivity from a DC bias during the isotropic etch step [5], a silicon neck region is formed several microns below the mask. After removing residual masks and cleaning, nano-scale silicon tips are formed using an oxidation sharpening process [6] (Fig. 2e). The nanoneedle array is separated into individual $5 \mathrm{~mm} \times 5 \mathrm{~mm}$ die and the remaining $\mathrm{SiO}_{2}$ is removed using HF (Fig. 2f). Figure 3 shows formation of silicon nano-tips after growth of $0.8 \mu \mathrm{m}$-thick thermal oxide. Figures 3(a) and 3(b) show SEM micrographs of structures during the fabrication process corresponding to the fabrication steps shown in Figures 2(d) and 2(f), respectively.

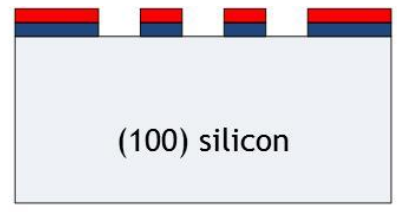

(a) Etch mask patterning

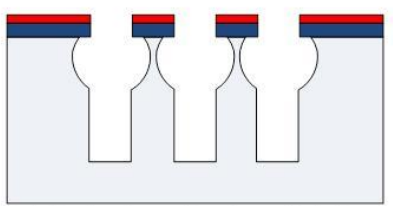

(c) Anisotropic dry etching

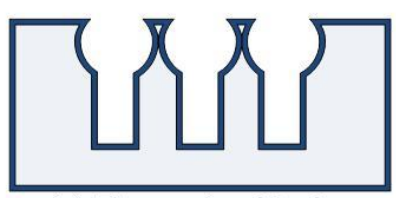

(e) Thermal oxidation

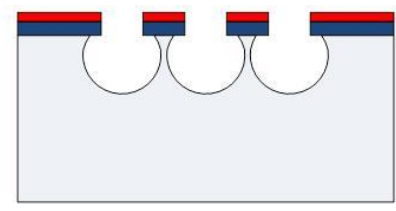

(b) Isotropic dry etching

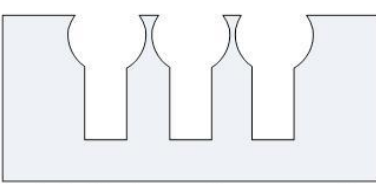

(d) Etch mask removal

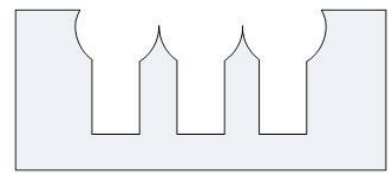

(f) Thermal oxide removal
Figure 2: Fabrication process for highly dense silicon nanoneedles (photoresist in red and $\mathrm{SiO}_{2}$ in blue).

Solid-State Sensors, Actuators, and Microsystems Workshop Hilton Head Island, South Carolina, June 3-7, 2012 
In addition to circular patterns, arrays of regular hexagons and squares were also investigated as etch mask patterns. The sizes of hexagons and squares are determined to inscribe $7 \mu \mathrm{m}$-diameter circles. The pitch of the patterns is designed to be $10 \mu \mathrm{m}$, which is close to the typical diameter of cells of interest. In order to achieve regular feature pitch, the circular and hexagonal patterns are arranged in a honeycomb shape as shown in the left side of Figure 4. The square patterns are arranged in a waffle pattern due to the geometric limit of the diagonal length of square patterns.

The profile dependence of nano-tips on the initial shapes of the patterns is shown in Figure 4. The nano-tips were fabricated by
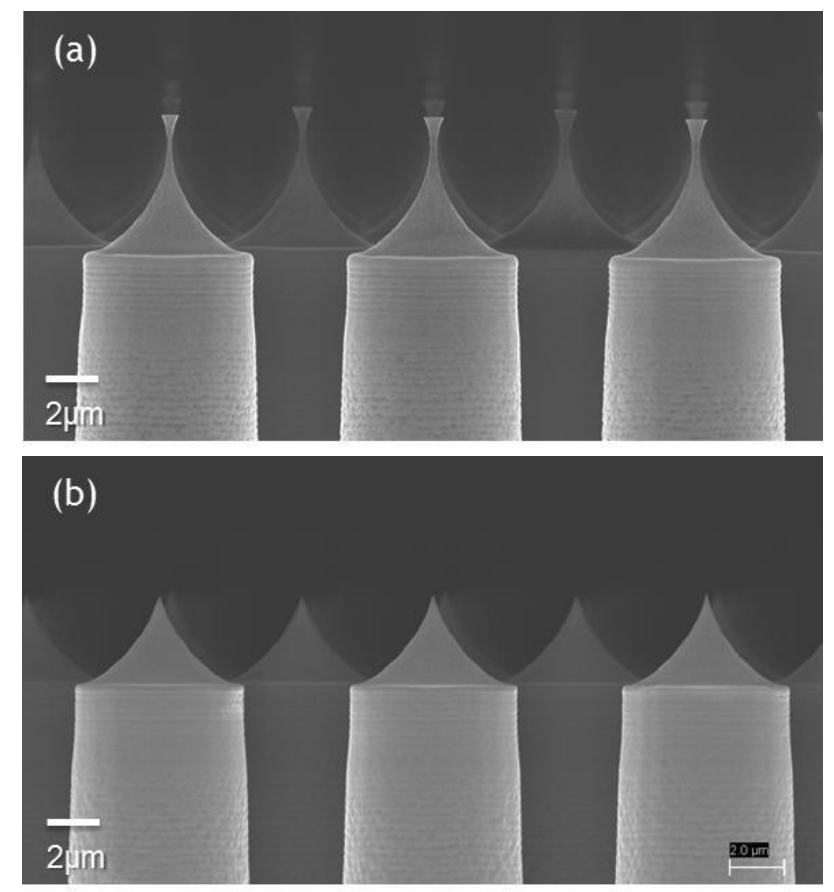

Figure 3: Formation of sharp tips using thermal oxidation. (a) Before oxidation and (b) after oxidation and removal of oxide layer.

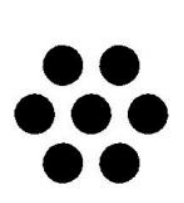

(a) Circles

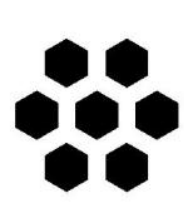

(b) Hexagons

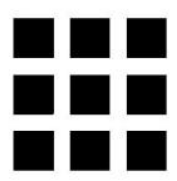

(c) Squares
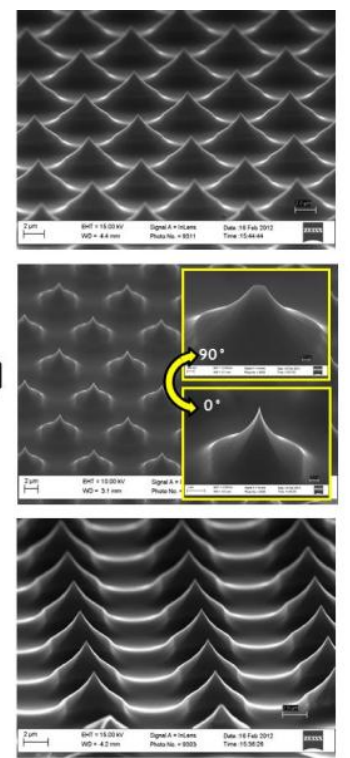
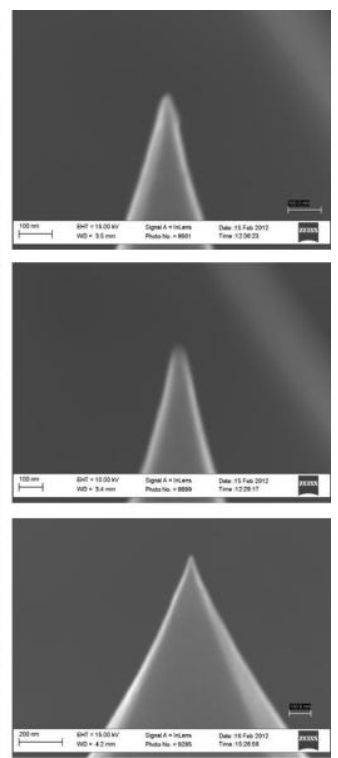

Figure 4: Fabricated nano-tips based on three shapes of mask patterns. using isotropic etching and thermal-oxidation-sharpening processes. The center pictures show an overall view of the tips of circular, hexagonal and square patterns, respectively. The pictures on the right side show a detailed view of the tips, showing tens of nanometers of tip diameter independent of initial shape. Since the micro loading effect during isotropic etching affects the tip profile, gaps between patterns are supposed to be uniform; however, because of a photomask irregularity in the hexagonal patterns, the gaps between vertical edges are wider than those of diagonal edges by a few hundred nanometers. This sub-micron error creates a different etch depth under the mask, resulting in the formation of blades instead of tips. The two insets of Figure 4(b) show orthogonal views of the fabricated nanoneedles, illustrating the blades.

Figure 5 shows individualized nanoneedle arrays separated into $5 \mathrm{~mm} \times 5 \mathrm{~mm}$ die, and corresponding SEM micrographs. The height, width and pitch of the nanoneedles are $28 \mu \mathrm{m}, 7 \mu \mathrm{m}$, and $10 \mu \mathrm{m}$, respectively. The radius of a nanoneedle tip is approximately $16 \mathrm{~nm}$. In the experiments described below, nanoneedles fabricated from circular patterns were utilized.

\section{INTRACELLULAR DELIVERY}

Materials and methods

As a model cell line, confluent monolayers of human prostate cancer cells (DU145) are cultured in dishes containing a culture medium solution. A calcein fluorescent dye and a fluorescein isothiocyanate labeled bovine serum albumin (FITC-BSA) are used as model molecules that normally do not cross intact cell membranes. The molecular weight and radius of calcein are $623 \mathrm{Da}$ and $0.6 \mathrm{~nm}$, and those of BSA are $66 \mathrm{kDa}$ and $3.6 \mathrm{~nm}$, respectively[7].

The backside of a nanoneedle array die is attached to a customized poly(methyl methacrylate) (PMMA) column with base dimension comparable to the die. A substrate bearing cancer cells is immersed in culture medium containing calcein fluorescent dye. The PMMA column bearing the nanoneedle array is placed into contact with the cell-bearing substrate and manually pressed into the cells for $10 \mathrm{~s}$. After removal of the nanoneedles, the cells are left quiescent for $5 \mathrm{~min}$ to recover from penetration, and are washed in saline. A propidium iodide (PI) dye is applied onto the cells after the nanoneedle treatment to stain cells killed possibly by needle

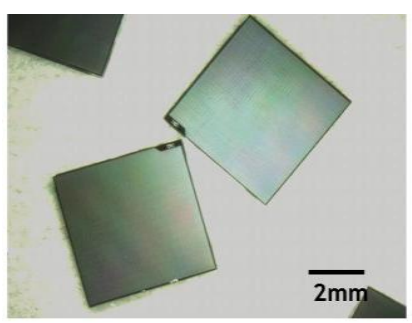

(a) Nanoneedle array dies

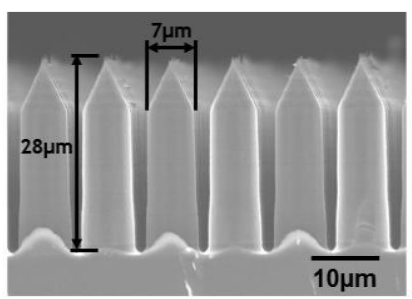

(c) Side profile of nanoneedles

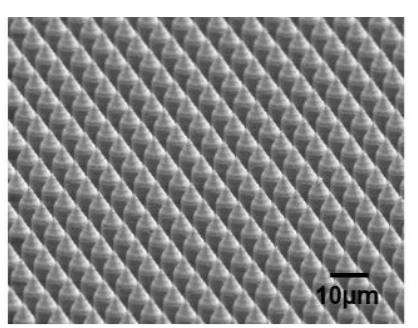

(b) SEM micrograph of nanoneedles

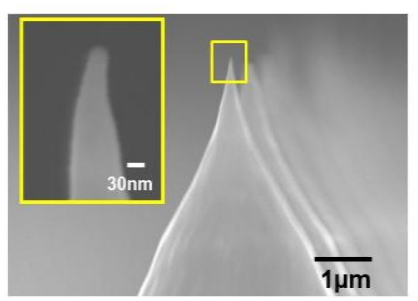

(d) Sharp tip profile
Figure 5: Fabricated $5 \mathrm{~mm} \times 5 \mathrm{~mm}$ nanoneedle array with the tip density of $10^{6} / \mathrm{cm}^{2}$. The radius of a tip is approximately $16 \mathrm{~nm}$. 
penetration and then washed with saline. This protocol of cell treatment results in the nanoneedle treated cells showing better uptake of calcein than the control group with no significant increase of cell death as shown in Figure 6. Nonetheless, leveling between the attached nanoneedle array and mono-layered cells and uniform application of force were challenging, causing significant variability.

Several protocol changes were implemented to improve the uniformity and reproducibility of the experiment. It was observed that the bare silicon surface is relatively hydrophobic and the nano-patterned silicon is even more so. Direct placement of a nanoneedle array onto cells in solution may trap air, preventing good contact between the nanoneedles and cells. Wetting the surface of the arrays prior to cell contact could alleviate this issue. A droplet of the culture medium solution containing calcein is therefore introduced to the surface of the nanoneedle array, covering the array without overflowing, prior to insertion. The wetted array is then placed in contact with a substrate bearing cells as above. To improve reproducibility of the needle-cell interaction force, a known constant force $(1.24 \mathrm{~g} \cdot \mathrm{f}$, approximately $12 \mathrm{mN})$ was placed on top of the needle array instead of the previously-used manual pressure technique to generate a uniform downward needle-to-cell force. The nanoneedle array was kept in place for $1 \mathrm{~min}$. To reproducibly remove the array, a previously-attached double-sided adhesive tape placed under the weight was utilized as a convenient lifting point. The freestanding weight combined with pre-application of calcein-bearing solution to the array assists self-leveling of the nanoneedle array and enables the application of uniformly-distributed force to the cells. After removing the nanoneedles, the cells are left quiescent for $10 \mathrm{~min}$ to allow diffusion of probe molecules and to recover from penetration, followed by a saline wash as described above.

Numbers of cells from control, nanoneedle and bare silicon groups respectively are counted by adopting the ruler method of a hemocytometer. Since a confluent monolayer of cells is grown on the substrate and the field of view of a fluorescent microscope is limited to $3.5 \mathrm{~mm}^{2}$, the center grid of the hemocytometer is sufficient for counting. Therefore cells in five regions of $0.2 \mathrm{~mm} \times 0.2 \mathrm{~mm}$ area within a $1 \mathrm{~mm} \times 1 \mathrm{~mm}$ square are counted.

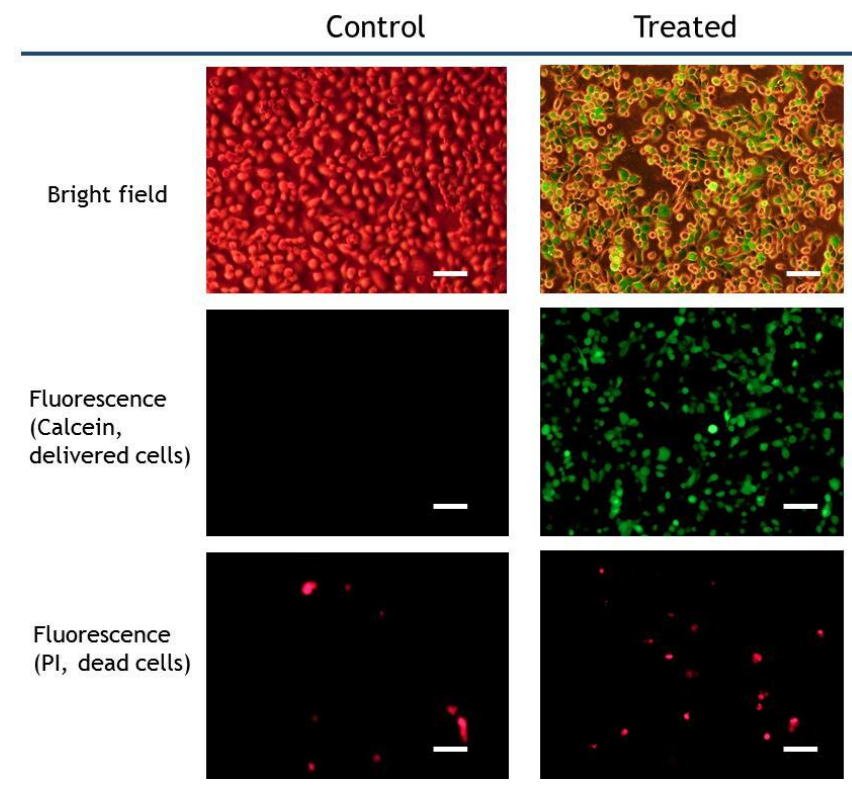

Figure 6: Intracellular delivery test and cell death assay with nanoneedles to DU145 cells (scale bar; $100 \mathrm{~m}$ ).

\section{Results and discussion}

As a preliminary result, nanoneedle-treated cells show intracellular uptake of a small model probe (calcein dye) as shown in Figure 6. The number of treated cells that expresses green color in this field of view is over $70 \%$ while the control shows no uptake. However, as mentioned before, leveling issue and non-uniformity of applied force result in a lack of repeatability.

Given a precise control of force application and uniformity, three groups of cells are treated with no die (negative control), nanoneedle die (experiment) and bare silicon die (negative control), respectively. Figure 7 shows representative pictures of a silicon die group and a nanoneedle die group after treatment. In the silicon die case, most of the cells originally on the cell culture substrate were transferred onto the silicon die, leaving a few cells with delivered dye on the original substrate. Most of the cells in the nanoneedle die case, however, remain on the original substrate, resulting in an approximate $30 \%$ delivery ratio. Note that the narrow "L" shaped cell-empty region in the bottom center picture is caused by an extruded frame surrounding the nanoneedle die, which is higher than nanoneedles by a few microns due to the nature of the oxidation sharpening process.

Figure 8 summarizes the results of three groups. After each treatment, most of the cells in the no-die control group and the nanoneedle die group remain attached on their original substrates, whereas approximately half of the cells in the silicon die control group are detached from their original substrates. In the silicon die control group, among those cells still adhering to their original substrate, an almost negligible dye uptake as low as $4 \%$ of cells was observed, along with $1.5 \%$ of cell death. The nanoneedle group shows the highest uptake of the probe molecule up to $34 \%$, while the no-die control group shows no uptake. According to the PI staining, there was no significant difference in cell death between control and nanoneedle application.

To investigate intracellular introduction of larger molecules, a nanoneedle array which has tips fabricated from square patterns without trenches, with height of $3 \mu \mathrm{m}$, was applied. The techniques of the wetting of the nanoneedle array with the culture medium solution and the application of the constant force containing BSA was applied as above. Since calcein is delivered into cells more easily than macromolecules (i.e. BSA) [7], however, the waiting time for diffusion after the treatment was increased to $40 \mathrm{~min}$. The intracellular uptake of the FITC-BSA by nanoneedles is shown in Figure 9. The nanoneedle technique enables BSA macromolecules to cross the cell membrane and enter the intracellular space.
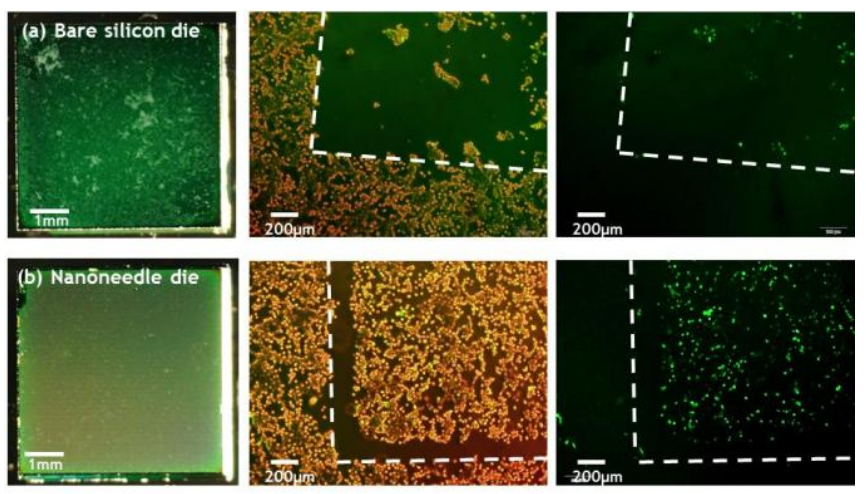

Figure 7: Photographs of dies and treated cells after intracellular delivery tests with (a) a bare silicon die and (b) a nanoneedle array die. (Left) Top surface of the dies, (center) bright filed pictures of the cell-bearing substrate, and (right) fluorescent pictures. White dashed lines mean boundaries of treated area by each die. 


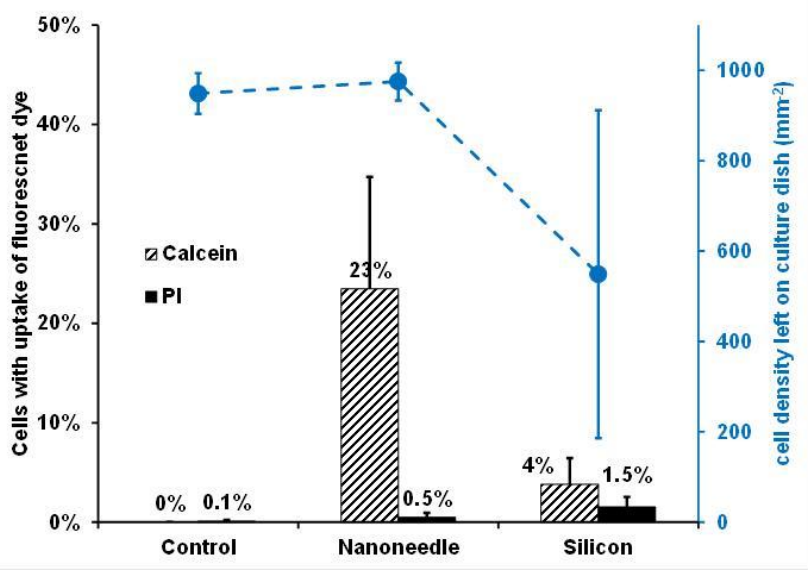

Figure 8: Percent of cell numbers expressing fluorescent light on the substrate after nanoneedle treatment. Cell density left on culture substrates drops significantly after bare silicon treatment. (error bars: standard deviation)

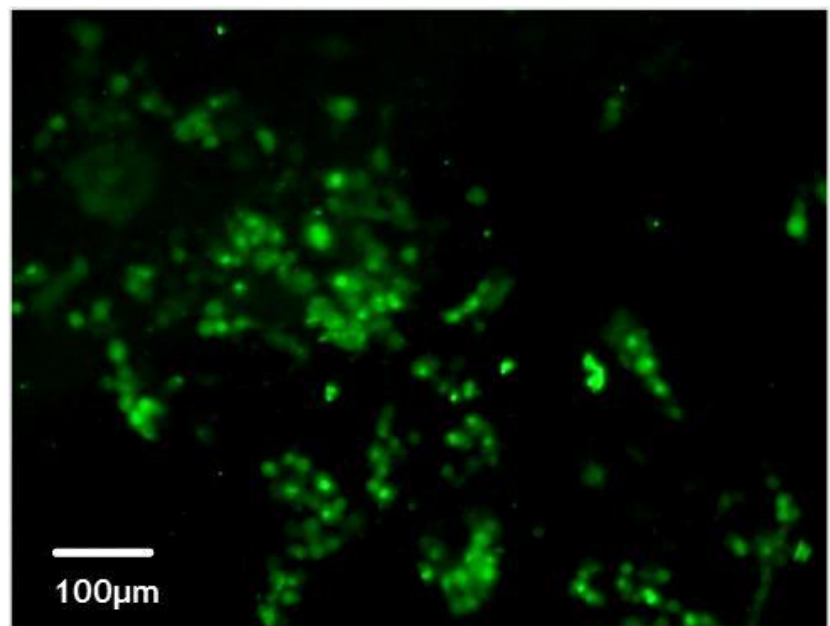

Figure 9: Fluorescent image of cells after BSA delivery with a nanoneedle array

\section{CONCLUSION}

By exploiting conventional isotropic etching and a thermal-oxidation-based sharpening process, high-density nanoneedle arrays were produced. The nanoneedles possessed sharp tips as small as $16 \mathrm{~nm}$ in radius and a high density as large as $10^{6}$ per square centimeters regardless of shapes of mask patterns as long as opening gaps between array elements were consistent. The silicon-micromachined nanoneedles were able to deliver small molecules as well as macromolecules into cells by mechanically penetrating cell membranes. The punctured cells showed uptake of calcein and BSA, respectively, with no significant cell death caused by membrane penetration. The demonstration of intracellular delivery with nanoneedles shows promise for high throughput gene transfer. Additional study on correlation of intracellular uptake of macromolecules with mechanical parameters of nanoneedle treatment for cell membrane penetration, and subsequent demonstration of gene incorporation and expression, is required to further validate this technique.

\section{ACKNOWLEDGMENTS}

This work was supported by National Institutes of Health under contract No. 1 PN2 EY018244-01.

\section{REFERENCES}

[1] A.K. Shalek, J.T. Robinson, E.S. karp, J.S. Lee, D. Ahn, M. Yoon, A. Sutton, M. Jorgolli, R.S. Gertner, T.S. Gujral, G. MacBeath, E.G.Yang, and H. Park, "Vertical Silicon Nanowires As A Universal Platform For Delivering Biomolecules Into Living Cells", Proceedings of the National Academy of Science, 107, 1870 (2010).

[2] T.I. Pestina, P.W. Hargrove, D. Jay, J.T. Gray, K.M. Boyd, and D. A. Persons, "Correction Of Murine Sickle Cell Disease Using $\gamma$-Globin Lentiviral Vectors To Mediate High-Level Expression Of Fetal Hemoglobin", Molecular Therapy, 17, 245 (2009).

[3] K. Chun, G. Hashiguchi, H. Toshiyoshi, H. Fujita, Y. Kikuchi, J. Ishikawa, Y. Murakami, and E. Tamiya, "An Array Of Hollow Microcapillaries For The Controlled Injection Of Genetic Materials Into Animal/Plant Cells", Technical Digest of the $12^{\text {th }}$ IEEE International Conference on Micro Electro Mechanical Systems, Orlando, FL, 1/17-21/99 (1999), pp. 406-411.

[4] K. Chun, G. Hashiguchi, H. Toshioyoshi, B.L. Pioufle, J. Ishikawa, Y. Murakami, E. Tamiya, Y. Kikuchi, and H. Fujita, "DNA Injection Into Plant Cell Conglometrates By Micromachined Hollow Microcapillary Arrays", Technical Digest of the $10^{\text {th }}$ International Conference on Solid-State Sensors and Actuators, Sendai, Japan, 6/7-10/99 (1999), 1B3.2

[5] S.-J. Paik, S. Byun, J.-M. Lim, Y. Park, A. Lee, S. Chung, J. Chang, K. Chun, and D. Cho, "In-Plane Single-Crystal-Silicon Microneedles For Minimally Invasive Microfluid Systems", Sensors and Actuators A: Physical, 114, 276 (2004).

[6] C. Hong and A.I. Akinwande, "Oxidation Sharpening Mechanism For Silicon Tip Formation", Electrochemical and Solid-State Letters, 8, F13 (2005).

[7] H.R. Guzman, D.X. Nguyen, A.J. McNamara, and M.R. Prausnitz, "Equilibrium Loading Of Cells With Macromolecules By Ultrasound: Effects Of Molecular Size And Acoustic Energy", Journal of Pharmaceutical Sciences, 91, 1693 (2002).

\section{CONTACT}

*S.-J. Paik, tel: +1-404-894-8807; spaik8@gatech.edu 


\section{CHRONICALLY IMPLANTABLE, 121-CHANNEL, POLYMER MICROELECTRODE ARRAY WITH HERMETICALLY-SEALED WIRELESS INTERFACE

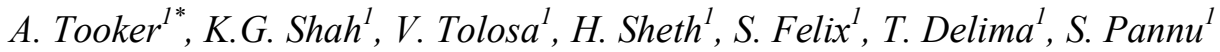 \\ ${ }^{1}$ Lawrence Livermore National Laboratory, Livermore, California, USA
}

\begin{abstract}
We present here the first demonstration of a 121-channel, chronically-implantable, wireless neural interface. This fullyimplantable device enables animals to be freely-behaving in their natural environment, unconstrained by external wires. This neural interface includes a polymer microelectrode array, capable of both stimulating and recording from neural tissue, integrated with a fully-biocompatible, hermetically-sealed, microelectronics package. The $121 \mathrm{recording} /$ stimulating electrodes demonstrate a high degree of reproducibility and repeatability.
\end{abstract}

\section{INTRODUCTION}

There is an ever growing need for reliable, biocompatible neural interfaces for chronic recording and stimulation of neural tissue. This capability is required for identifying and understanding the underlying mechanisms of the complex nervous system and developing neuroprosthetics to treat debilitating chronic neurological disorders such as deafness, blindness, spinal cord injury, depression, and several brain-related diseases. Most of the research used to investigate these conditions requires animals to be in their natural environment and not perturbed by the presence of personnel and the physical wires tethering them to measurement equipment. Thus, it is clear that a reliable penetrating neural interface with high electrode density and wireless telemetry is a critical technology component for scientific research and clinical applications. This work presents a fullyimplantable, wireless, neural interface for recording and stimulation of neural tissue. This neural interface consists of a polymer microelectrode array integrated with a microelectronics packaged that is hermetically sealed in a biocompatible package.

\section{Wireless Neural Interfaces}

Wireless neural interfaces have been developed by several groups [1-9] in recent years. There are four major elements that distinguish this work from previously published work.

1. The use of a polymer microelectrode array.

2. The microelectronics package is fully-implantable.

3. The microelectronics package is hermetically sealed.

4. There are 121 channels, all capable of stimulating and recording from neural tissue.

While each of these elements has been previously published [115], this is the first demonstration of the combination of all 4 of these elements. Similar demonstrations of these elements have been developed [8-9] primarily for use in retinal implants to restore site. These implants, however, have only 16-64 channels and are typically only capable of neural stimulation, not recording.

\section{Polymer Microelectrode Array}

There has been significant work done in the fabrication of both polymer [7-15] and silicon [1-6] microelectrode arrays for stimulation of and recording from neural tissue. These microelectrode arrays consist of discrete electrodes that are connected, via metal traces, to output leads and/or other signal processing circuitry. Neuronal stimulation and recording is conducted at these electrode sites.

Silicon microelectrode arrays [1-6] can be easily and efficiently fabricated, primarily due to the prevalence of silicon in the microelectronics industry and the resulting wealth of established fabrication techniques. Unfortunately, there are significant concerns regarding the suitability for long-term (i.e. chronic) in vivo studies, as silicon is not a biocompatible material [16]. Further, strain can be induced on the neural tissue due to the mismatch between the mechanical properties of silicon and neural tissue and the continuous micro-motion of the tissue. This can cause chronic injury and glial scarring at the implantation site.

Polymer microelectrode arrays [7-15] are an attractive alternative. The rapid development of MEMS fabrication techniques and the increasing presence of these polymers ensures that polymer-based microelectrode arrays can be fabricated with the same ease and efficiency as silicon microelectrode arrays. The mechanical properties of these polymer microelectrode arrays are more closely matched to that of neural tissue, thus imparting less strain and minimizing injury and glial scarring at the implantation site [17]. In addition, these polymers, specifically polyimide and parylene, are both biocompatible materials; thus, they are more suitable for chronic implantation than silicon [18].

Several wireless neural interfaces have been developed using silicon [1-6] and polymer [7-9] microelectrode arrays. The microelectrode array presented here is a polyimide microelectrode array.

\section{Fully-Implantable Microelectronics Package}

The microelectronics package presented here is fullyimplantable. Previous work (mostly retinal implants) has demonstrated the use of a fully-implantable microelectronics package [1,4,7-9]. Several groups, however, have used percutaneous or head-mounted microelectronics package [2-3,5-6]. There are two significant advantages for a fully-implantable package, especially for chronic in vivo studies. First, as the packages are fully-implantable they are less prone to breakage or damage. Second, fully-implantable packages are less prone to infection, as there are no open wound/surgical insertion sites.

\section{Hermetically-Sealed Microelectronics Package}

In addition to being fully-implantable, the microelectronics package is hermetically-sealed. Some previous work has demonstrated the use of a hermetically-sealed microelectronics package, although this has been limited to retinal implants [7-9]. The alternative to hermetic sealing is a polymer coating [1,4]. Polymer-coated microelectronics packages, however, are susceptible to sealing problems due to both potential nonuniformities in polymer coatings and adhesion issues. Hermetically-sealed microelectronics packages do not suffer from either coating non-uniformity or material adhesion problems; thus, they are more suitable for chronic, long-term in vivo studies.

\section{1-Channels for Neural Stimulation and Recording}

In general, more channels (i.e. electrodes) correlates to an increased number of neurons as potential targets for either stimulation or recording. Most wireless neural interfaces are limited to 15-16 channels [2-3,5-8]. Recently, several groups have demonstrated larger channel numbers, generally 60-100 channels $[1,4,9]$. We present here the first demonstration of a 121-channel wireless neural interface. 


\section{WIRELESS NEURAL INTERFACE - FABRICATION AND ASSEMBLY}

There are three main components to this wireless neural interface: 1) polymer microelectrode array, 2) hermetically-sealed microelectronics package, and 3) inductive coils for power and data transfer.

\section{Polymer Microelectrode Array}

Although the use of flexible polyimide minimizes the injury and glial scarring caused by the implantation of the microelectrode array, it does not eliminate it. The amount of injury and scarring can be further reduced by minimizing the size of the implanted microelectrode array. Unfortunately, typical efforts to minimize the size also limit the number of stimulation and recording sites (electrodes). In order to effectively decrease the size of the probe, while maintaining the electrode count, additional trace metal layers are required. Generally, the width of the microelectrode arrays is $20-30$ times the thickness. Thus, although the additional metal layers necessitate additional polyimide insulating layers, a significant decrease in microelectrode array width can be achieved with only a marginal increase in thickness. The resulting reduction in cross-sectional area will cause less injury and scarring while maintaining the same number of electrodes.

There are several examples of polyimide microelectrode arrays with one layer of trace metal [7-11]. In addition, some work has demonstrated the use of two layers of trace metal [12-15]. We present here a fabrication process for microelectrode arrays utilizing three layers of trace metal.

The general fabrication process for these 3-layer metal microelectrode arrays is summarized in Figure 1. The processing steps are summarized below:

1. Polyimide 1 Deposition $(5 \mathrm{~m})$

2. Deposition/Patterning of Trace Metal $1(\mathrm{Ti} / \mathrm{Au} / \mathrm{Ti})$

3. Polyimide 2 Deposition $(2 \mathrm{~m})$ and Inter-layer Via Etching $\left(\mathrm{O}_{2}\right.$ Plasma $)$

4. Deposition/Patterning of Trace Metal $2(\mathrm{Ti} / \mathrm{Au} / \mathrm{Ti})$

5. Polyimide 3 Deposition $(2 \mathrm{~m})$ and Inter-layer Via Etching $\left(\mathrm{O}_{2}\right.$ Plasma $)$

6. Deposition/Patterning of Trace Metal $3(\mathrm{Ti} / \mathrm{Au} / \mathrm{Ti})$

7. Deposition/Patterning of Electrode Metal (Ir)

8. Polyimide 4 Deposition $(5 \mathrm{~m})$ and Electrode Via Etching $\left(\mathrm{O}_{2}\right.$ Plasma)

9. Device Outline Etching $\left(\mathrm{O}_{2}\right.$ Plasma $)$

We have demonstrated this process using polyimide. The same fabrication process can be used with other polymers, such as parylene and silicones.

Photographs of the three-layer metal, polyimide microelectrode array are shown in Figure 2. The polymer microelectrode array consists of 5 distinct probes, all routed together and joined at a single connector. Each probe has 4 shanks; each shank has 6 recording/stimulating electrodes plus 1 reference electrode. This creates a single polyimide microelectrode array with 121 electrodes (all of the reference electrodes are shorted together). The flexibility of the individual probes ensures the final device can be easily assembled into a three-dimensional superstructure ( 4 x 5 array of shanks).

\section{Hermetically-Sealed Microelectronics Package and Inductive Coils}

The microelectronic components need to be hermetically sealed and separated from the neural tissue for two basic reasons. First, the electronics are made of non-biocompatible materials that may elicit a cytotoxic response from the neural tissue. Second, the electronics would corrode (and be shorted out) due to moisture and the harsh, biological environment of the body. Therefore, the microelectronics package consists of a biocompatible metal can attached to a ceramic substrate. The electronics are then sealed inside this can (Figure 3), effectively preventing physical interaction with the neural tissue.
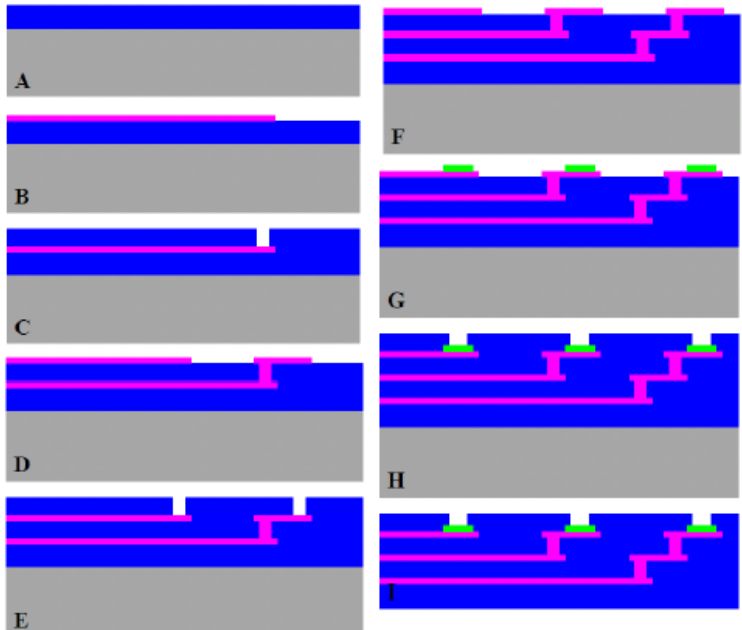

Silicon Polyimide

$\mathbf{T i} / \mathbf{A u} / \mathbf{T i}$

Ir

Figure 1: Cross-sectional view of the general fabrication process for three-layer trace metal, polyimide microelectrode arrays. (Images are not drawn to scale.)

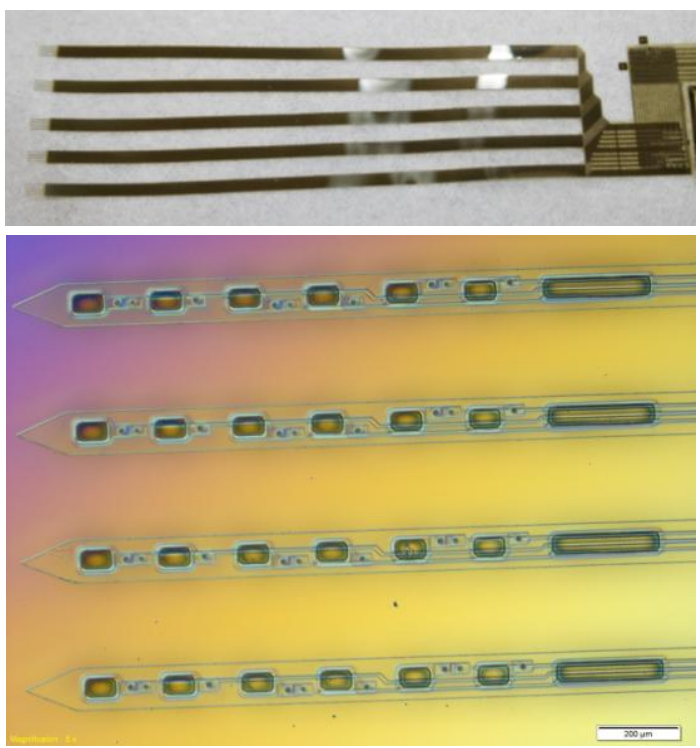

Figure 2: The entire polyimide microelectrode array, with 5 probes, is shown at the top. All of the probes are joined at a single connector (right). An enlargement of one of the probe tips (bottom) shows the 4 shanks, each with 6 electrodes for recording/stimulating and 1 reference electrode.

A variety of electronic components are used to convert the wireless data and power signals into the 121 individual electrical impulses that are delivered to the neural tissue. The electronics contained in this wireless neural interface are:

1. A power telemetry chip that converts the incoming power telemetry signal into the necessary voltages that operate the stimulator chip.

2. A stimulator chip that uses the incoming data signal 
and generates the independent electrical impulses. (This chip is scalable to 256 channels.)

3. Associated surface mount circuitry (both active and passive), such as an oscillator (for the chip clock), resistors, capacitors, diodes, and an inverter.

This wireless neural interface does not contain a battery, rather, the wireless data and power signals are transferred to it using inductively coupled coils. The inductive coils for wireless telemetry (power and data transfer) are fabricated using gold wire with polyimide coating for insulation.
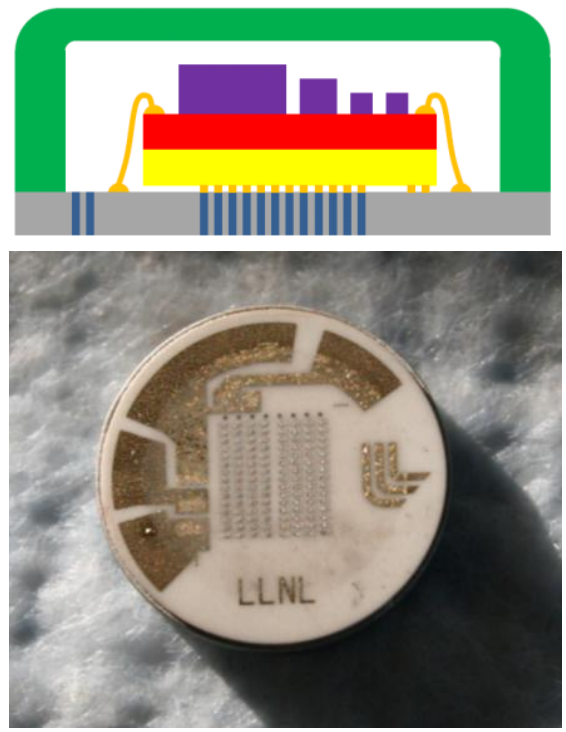

Figure 3: Cross-sectional schematic of the microelectronics package (top). The electronics are hermetically-sealed inside the metal can (green). The stimulator and power telemetry chips (yellow), silicon interconnect board (red), and surface mount circuitry (purple) are shown. The metal can (13 mm diameter, 3.8 $\mathrm{mm}$ tall) is brazed on a ceramic disc (gray) with high-density vias (blue) for connections between the electronics and the microelectrode array.

The electronic components are assembled using several microelectronics packaging/assembly methods in a manner that minimizes the total volume of the device. These components are assembled inside a hermetically-sealed package. The package consists of a ceramic substrate that contains an array of hermetic electrical feedthroughs which transmits electrical signals to and from the electronics and microelectrode array. The ceramic substrate is uniformly coated with a biocompatible metal stack. The metal is then lithographically-patterned on one side for the attachment of the electronics components and on the other side for the attachment of the polymer microelectrode array and the inductive coils. The stimulator chip is assembled directly on the ceramic substrate with flip-chip bonding. The remainder of the electronics components (power telemetry chip and other circuitry) are assembled onto a silicon interconnect board. High-aspect-ratio wire-bonds serve as interconnects between the power telemetry chip, the interconnect board, and the stimulator chip. The ceramic substrate is brazed to a biocompatible niobium ring. Once all of the electronics components are assembled onto the ceramic substrate, the microelectronics package is then hermetically-sealed by laser welding a niobium cap to the ring. Finally, the microelectrode array and inductive coils are connected to the ceramic substrate with gold rivet bonds [19] (Figure 4).

\section{NEURAL INTERFACE CHARACTERIZATION Electrochemical Testing}

The iridium activation was performed using biphasic potential pulsing in phosphate-buffered saline to form an activated iridium oxide film (AIROF). Individual iridium electrodes were characterized to determine charge storage capacity (CSC) and impedance both before and after activation. Cyclic voltammetry (CV) and electrochemical impedance measurements were made with a Princeton Applied Research (PAR) potentiostat using vendor-supplied software. All measurements were made in a three-electrode cell using a $\mathrm{Pt}$ counter electrode, an $\mathrm{Ag} / \mathrm{AgCl}$ reference electrode, and phosphate-buffered saline $(\mathrm{pH} 7.4)$ as the electrolyte. Potential cycling for the CVs was performed between $-600 \mathrm{mV}$ and $+800 \mathrm{mV}$ at a scan rate of $100 \mathrm{mV} / \mathrm{s}$.

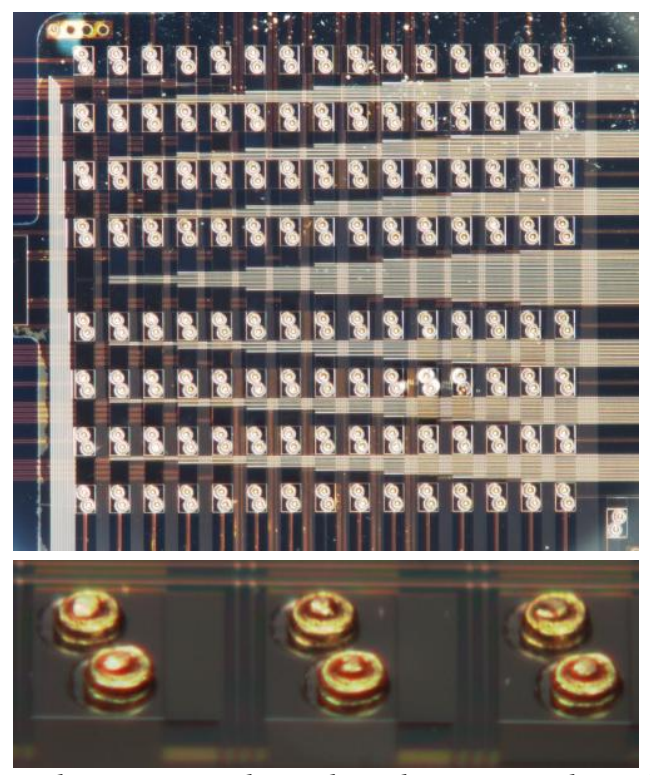

Figure 4: The top image shows the polymer microelectrode array with gold rivet bonds to the ceramic substrate. The bottom image shows an enlarged view of three rivet bond connections. Each electrode has two rivet bonds (for redundancy). Each pair of rivet bonds has a pitch of $305 \mu \mathrm{m}$. The rivet bonds are each $\sim 80 \mu \mathrm{m}$ in diameter.

Typical CV curves for 12 of the 121 electrodes are shown in Figure 5. The recording/stimulating electrodes are $40 \mathrm{~m}$ by 65 $\mathrm{m}$. As can be seen, the CVs for the different recording/stimulating electrodes overlap quite well and the characteristic iridium oxide peaks are clearly visible after activation. Post-activation, the average CSC for the recording/stimulating electrodes on a single device is 30.81 $\mathrm{mC} / \mathrm{cm}^{2}$, with a standard deviation of $0.70 \mathrm{mC} / \mathrm{cm}^{2}$. Postactivation, the average impedance for the recording/stimulating electrodes on a single device is $46.00 \mathrm{k} \Omega$, with a standard deviation of $4.52 \mathrm{k} \Omega$ (at a frequency of $948.7 \mathrm{kHz}$ ). Thus, for a single device, there is no significant difference in electrode characteristics.

\section{Biocompatibility Testing}

The selection and evaluation of materials and devices intended for use in humans requires a structured program of assessment to establish biocompatibility and safety. These wireless interfaces have passed the ISO-10993 array of biocompatibility testing for chronically implanted devices, in accordance with the FDA regulations. This includes assessing the 
biological reactivity, allergenic potential, sensitizing capacity, systemic toxic effects, immune response, and genetic mutations in animal models. The completely assembled interfaces (although with platinum electrodes), including microelectronics package, have successfully passed this biocompatibility testing. The neural interfaces with iridium electrodes have not been subjected to the complete array of testing, but have successfully passed basic cytotoxicity screening. We expect the neural interfaces with iridium electrodes to also successfully pass biocompatibility testing, due to the inherent similarities in material selection and fabrication process.

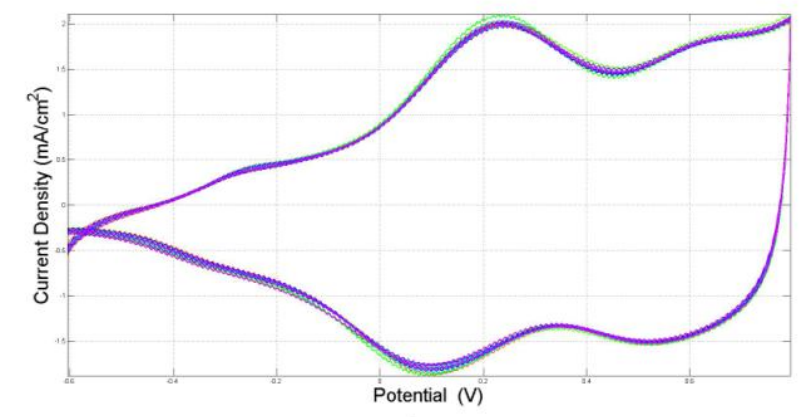

Figure 5: Typical CV curves of 12 representative recording/stimulating electrodes after electrochemical activation. The CVs overlap extremely well and the characteristic iridium peaks are clearly visible.

\section{CONCLUSION}

We have presented here the first demonstration of a 121channel, fully-implantable, wireless, neural interface for recording and stimulation of neural tissue. The neural interface consists of a polymer microelectrode array integrated with a hermeticallysealed, biocompatible, microelectronics package. The fully assembled neural interfaces are currently undergoing further functional and lifetime testing in preparation for in vivo studies.

\section{ACKNOWLEDGEMENTS}

We would like to thank Professor Wentai Liu's group at the University of California, Santa Cruz, for the microelectronics.

This work performed under the auspices of the U.S. Department of Energy by Lawrence Livermore National Laboratory under contract DE-AC52-07NA27344. LLNL-CONF544073

\section{REFERENCES}

[1] A. Sharma, L. Rieth, P. Tathireddy, R. Harrison, H. Oppermann, M. Klein, M. Topper, E. Jung, R. Normann, G. Clark, and F. Solzbacher, "Long term in vitro functional stability and recording longevity of fully integrated wireless neural interfaces based on the Utah Slant Electrode Array," J. Neural Eng., 8 (4), (2011).

[2] E. Greenwald, M. Mollazadeh, C. Hu, W. Tang, E. Culurciello, and N. Thakor, "A VLSI Neural Monitoring System With Ultra-Wideband Telemetry for Awake Behaving Subjects," IEEE Trans. Biomed. Cir. Sys., 5 (2), pp. 112 118, 2011.

[3] D. Fan, D. Rich, T. Holtzman, P. Ruther, J. Dalley, A. Lopez, M. Rossi, J. Barter, D. Salas-Meza, S. Herwik, T. Holzhammer, J. Morizio, and H. Yin, "A Wireless MultiChannel Recording System for Freely Behaving Mice and Rats," PLoS ONE, 6 (7), e22033 (2011).
[4] C. Chestek, V. Gilja, P. Nuyujukian, J. Foster, J. Fan, M. Kaufman, M. Churchland, Z. Rivera-Alvidrez, J. Cunningham, S. Ryu, and K. Shenoy, "Long-term stability of neural prosthetic control signals from silicon cortical arrays in rhesus macaque motor cortex," J. Neural Eng., 8 (4), (2011).

[5] A. Bonfanti, G. Zambra, G. Baranauskas, G. Angotzi, E. Maggiolini, M. Semprini, A. Vato, L. Fadiga, A. Spinelli, and A. Lacaita, "A wireless microsystem with digital data compression for neural spike recording," Microelec. Eng., 88, pp. $1672-1675$ (2011).

[6] M. Azin, D. Guggenmos, S. Barbay, R. Nudo, and P. Mohseni, "A Miniaturized System for Spike-Triggered Intracortical Microstimulation in an Ambulatory Rat," IEEE. Trans. Biomed. Eng., 58 (9), pp. 2589 - 2597, (2011).

[7] A. Sodogar, G. Perlin, Y. Yao, K. Najafi, and K. Wise, "An Implantable 64-Channel Wireless Microsystem for SingleUnit Neural Recording," IEEE J. Solid-State Cir., 44 (9), pp. $2591-2604$ (2009).

[8] S. Kelly, D. Shire, J. Chen, P. Doyle, M. Gingerich, W. Drohan, L. Theogarajan, S. Cogan, J. Wyatt, and J. Rizzo, "Realization of a 15-Channel, Hermetically-Encased Wireless Subretinal Prosthesis for the Blind," IEEE-EMBS Conf., pp. 200-203 (2009).

[9] http://www.artificialretina.energy.gov

[10] S. Lee, J. Jung, Y. Chae, J.-K. Suh, and J. Kang, "Fabrication and characterization of implantable flexible nerve cuff electrodes with Pt., Ir, and IrOx films deposited by RF sputtering," J. Micromech. Microeng., 20 (3), (2010).

[11] B. Rebehn, C. Bosman, R. Oostenveld, P. Fries, and T. Stieglitz, "A MEMS-based flexible multi-channel ECoGelectrode array," J. Neural Eng., 6 (3), (2009).

[12] A. Mercanzini, K. Cheung, D. Buhl, M. Boers, A. Maillard, P. Colin, J.-C. Bensadoun, A. Bertsch, and P. Renaud, "Demonstration of cortical recording using novel flexible polymer neural probes," Sens. Actuators A, 143, pp. 90-96 (2008).

[13] J. Seymour, N. Langhals, D. Anderson, and D. Kipke, "Novel multi-side, microelectrode arrays for implantable neural applications," Biomed. Microdevices, 13, pp. $441-452$ (2011).

[14] T. Doerge, S. Kammer, M. Hanauer, A. Sossalla, S. Steltenkamp, "Novel method for flexible double sided microelectrode fabrication process," Bioengineered and Bioinspired Systems IV Book Series: Proc. Of SPIE, 7365 (2009).

[15] T. Stieglitz, "Flexible biomedical microdevices with doublsided electrode arrangements for neural applications," Sens. Actuators A, 90 pp. 203-211 (2001).

[16] R. Biran, D. Martin, and P. Tresco, "Neuronal cell loss accompanies the brain tissue response to chronically implanted silicon microelectrode arrays," Exp. Neurology, 195, pp. 115-126 (2005).

[17] V. Polikov, P. Tresco, and W. Reichert, "Response of brain tissue to chronically implanted neural electrodes," J. Neurosci. Meth., 148, pp. 1-18 (2005).

[18] Y. Sun, S. Lacour, R. Brooks, R. Rushton, J. Fawcett, and R. Cameron, "Assessment of the biocompatibility of photosensitive polyimide for implantable medical device use," J. Biomed. Mat. Res. A, 90A (3), pp. 648-655 (2009).

[19] T. Stieglitz, H. Beutel, and J.-U. Meyer, "Microflex-A New Assembling Technique for Interconnects," J. Intelligent Mat. Sys. And Struc., 11 (6), pp. 417-25 (2000).

\section{CONTACT}

*A. Tooker, tel: +1-925-422-2326; tooker1@1lnl.gov 


\title{
FLIP-CHIP BASED PACKAGING FOR LINEAR RATCHETING MICROACTUATORS ENABLES 3D STACKS OF MOVEBLE MICROELECTRODES FOR THE BRAIN
}

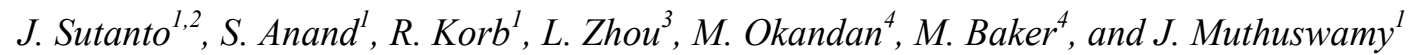 \\ ${ }^{1}$ School of Biological and Health System Engineering, Arizona State University, Tempe, AZ, USA \\ ${ }^{2}$ Amkor Technology, Platform Technology Development, Chandler, AZ, USA \\ ${ }^{3}$ School of Electrical, Computer and Energy Engineering, Arizona State University, Tempe, AZ, USA \\ ${ }^{4}$ Sandia National Laboratories, Albuquerque, NM, USA
}

\begin{abstract}
MEMS based movable microelectrodes now offer the exciting possibility of developing a fully autonomous implantable neural prosthetic system. However, critical interconnect and packaging challenges remain. We report here a novel flip-chip based interconnect and packaging approach for the implantable MEMS microelectrodes with complex moving structures on the die. The proposed approach is chip-scale, versatile (applicable to a variety of substrates and dies), scalable (allowing the realization of 3D stacks), and low-cost. We report here successful in vivo testing of this non-hermetically encapsulated flip-chip based package in long-term rodent experiments.
\end{abstract}

\section{INTRODUCTION}

Emerging prosthetic devices for the brain will enable amputees or patients with peripheral neuropathy, or spinal-cord injuries or stroke to control external robotic devices using their brain signals. The success of brain prosthetic devices is critically dependent on the reliability of implants used to monitor the single neuronal electrical activities in the brain. Several studies have successfully demonstrated the potential of moveable microelectrodes in improving reliability of neuronal recordings by repositioning and seeking new neurons in the event of a failure of the neuron-electrode interface [1-4]. However, the size and weight of the movable microelectrodes are often large, interfering with animal or patient movement/behavior. Therefore, there is a need for a MEMS based movable microelectrode device that can be eventually integrated with advanced signal conditioning and control circuitry towards a fully autonomous neural prosthetic system for the brain. The development of chevron-latch microactuators for movable microelectrodes was reported earlier, where the MEMS chip was packaged on an Au-plated wire bonding package mounted on a $15 \mathrm{~mm}$ x $20 \mathrm{~mm}$ PCB for longterm rodent experiments [5-6]. However, such wire-bonded package and interconnects are bulky, not scalable and cumbersome for implantable applications.

Conventional flip-chip packaging approaches have serious technical problems when applied to MEMS devices with complex moving structures. Additional challenges arise in the case of implantable applications. First, there is a need for high-aspect ratio first-level interconnects (FLI) that will allow sufficient room for the MEMS structures on the die to move and function. Second, flux dissipation in the reflow process is highly uncontrolled resulting in catastrophic contamination of the MEMS structures. Third, even without the underfill, leaks during the encapsulation process can also jam the MEMS structures. Due to the risk of such contamination, the FLI are often moved hundreds of microns away from active MEMS structures increasing the form-factor of the device. We report here a novel low-temperature flip-chip based approach [7-8] using Ag epoxy or solder for FLI. FLI bumps with high aspect ratio $(>2)$ can be made within $70 \mu \mathrm{m}$ from active MEMS structures without any hindrance to MEMS function. The

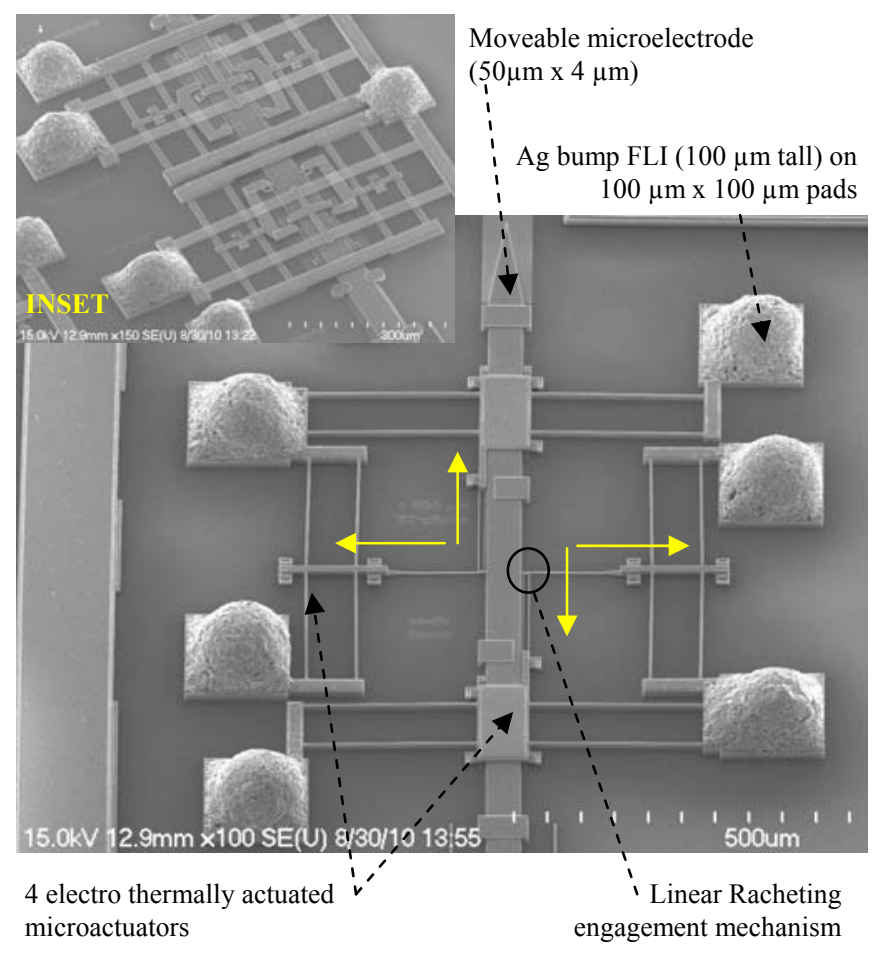

Figure 1. Electro-thermally actuated neural microelectrodes w/ linear ratcheting mechanism. INSET: Previously reported chevron-actuators

flip-chip approach is versatile in that it can be applied to a variety of substrates such as silicon, glass or plastics such as parylene or polyimide.

\section{THEORY}

A. Arrays of MEMS microelectrodes

Arrays of three electrothermally actuated microelectrodes on a 3 $\mathrm{mm} \times 7 \mathrm{~mm}$ chip are microfabricated using SUMMITV ${ }^{\mathrm{TM}}$ process (Sandia Ultraplanar Multi-level Micromachine Technology). In

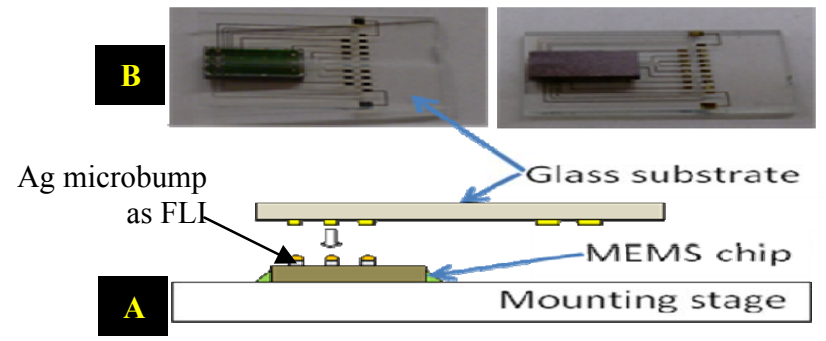

Figure 2. A: Showing the schematic of the flip-chip process where Ag microbumps are used as the FLI. B: MEMS die and glass substrate after flip-chip process

Solid-State Sensors, Actuators, and Microsystems Workshop Hilton Head Island, South Carolina, June 3-7, 2012 
this report, a new generation of MEMS microactuator called the linear ratcheting microactuator is presented as shown in Fig. 1. Compared with the previously reported Chevron-design, the linear ratcheting technique provide a number of advantages, such as higher actuation force $(480 \mu \mathrm{N}$ compared to $220 \mu \mathrm{N})$, smaller actuation step $(6.5 \mu \mathrm{m}$ compared to $8.8 \mu \mathrm{m})$, and more reliable microelectrode movement (both forward and backward).

The current generation of MEMS microelectrode array attempts to address some of the shortcomings of the earlier Chevron-type actuators identified earlier by long-term in vivo testing. The microelectrode has a linear array of teeth spaced 6.5 $\mu \mathrm{m}$ apart at the edges. The 'pawl', a peg-like structure engages the teeth of the electrode and locks it in position, preventing displacement in rest conditions. The new actuators have a simplified movement mechanism with only one 'pawl' that engages with the microelectrode teeth as shown in Fig.1. It has two pairs of electrothermal Chevron actuators that perform identical function. 'Forward drive' actuator and 'disengage forward drive' actuator control the forward movement of the microelectrode and 'reverse drive' and 'disengage reverse drive' actuator control the backward movement of the electrode. The central shuttle of the drive and disengage actuators are both connected in an L-shape arrangement to a 'pawl' that engages the microelectrode. A set of timed pulse waveforms were applied to these actuators to operate the pawl and move the linear ratchet/microelectrode in a given direction.

\section{B. Flip-Chip Packaging technology}

The MEMS die is flip-chipped to the glass substrate via a novel Ag bump FLI as shown in Fig. 2. For more detail on this process, the reader is referred to our earlier report [7-8]. The key steps involved in the current flip-chip process are:

1. Kitting process to prepare the three main components: the MEMS chip, glass substrate, and Omnetics ${ }^{\mathrm{TM}}$ (Omnetics connector corporation) TLI (Third-level interconnects);

2. Bumping process involved dispensing Ag epoxy on each $\mathrm{Al}$ pad to create microscale bumps to build the FLI (First level Interconnects) with a height of about $100 \mu \mathrm{m}$ (total of 18 bumps per testing sample).

3. Flip chip process to a) align the die with respect to the transparent glass substrate, and $b$ ) bond the die and substrate

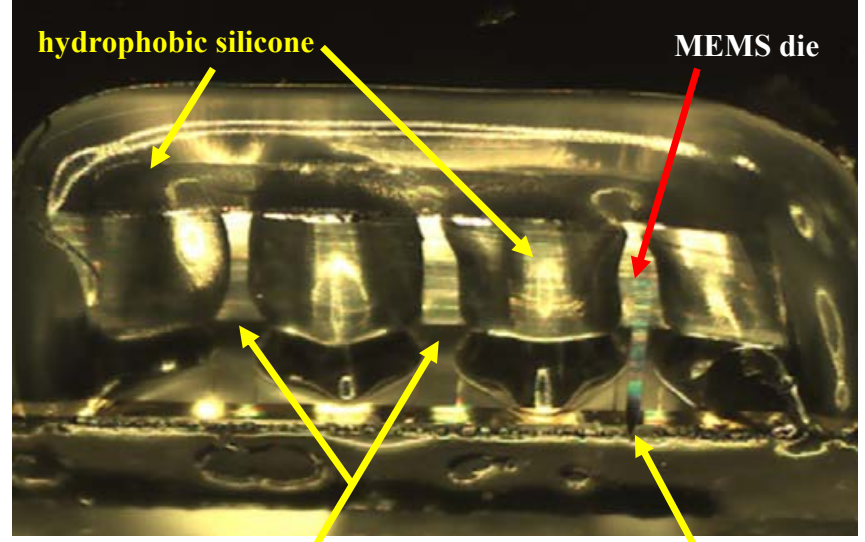

3 of $150 \mu \mathrm{m} \times 100 \mu \mathrm{m}$ hydrophobic micro-channnels

One MEMS microelectrode

$(50 \mu \mathrm{m} \times 4 \mu \mathrm{m})$

Figure 3: Micrograph of the non-hermetic encapsulation (NHE) - with the MEMS die facing down. 3 microchannels each $150 \mu \mathrm{m}(\mathrm{W})$ by $100 \mu \mathrm{m}(\mathrm{H})$ were patterned in hydrophobic silicone to allow egress of microelectrodes. together through the FLI Ag bumps. The sub-steps involved in this process were as follows: (a) the glass substrate was picked up by a suction piston; (b) the MEMS chip located on the chuck was positioned to align it with the glass substrate. To properly bond the FLI bumps the glass substrate was positioned on top of the die with a joint pressure of $20-30 \mathrm{MPa} / \mathrm{bump}$; While the Ag epoxy is still wet (uncured), the chuck was then heated up to $120^{\circ} \mathrm{C}$ for $1 \mathrm{~min}$ to fully cure the Ag epoxy bumps.

4. Connect the TLI to bond the Omnetics ${ }^{\mathrm{TM}}$ connector to the glass substrate by using Ag epoxy. The process was similar to step no 3 .

5. Create non-hermetic encapsulation; In order to create nonhermetic encapsulation on the front side of the die, hydrophobic silicone microstructures were patterned using the dispensing system as shown in the SEM of Fig. 3. A non-hermetic encapsulation (NHE) is important to allow the microelectrodes to move freely inside the brain after implantation and while simultaneously preventing the cerebro-spinal fluids in the brain from entering or contaminating the active MEMS structures. Our previous work achieved a non-hermetic encapsulation by using a screen-mesh with the opening of $60 \mu \mathrm{m}$ coated with silicone gel. In this paper, we report the results of a novel NHE technique compatible with flip-chip process using a non-flow silicone underfill to non-hermetically encapsulate the front edge (from where the microelectrodes emerge before entering the brain) of the integrated package as shown in Fig. 4. Patterns of silicone microstructures were created in the NHE. The $100 \%$ silicone was an auto/marine sealant, clear and commercially available in the hardware store. Pneumatic dispensing technique (EFD Inc.) was used to pattern the silicone microstructures in the NHE on the front edge of the die. The other three edges of the die are nearhermetically sealed using epoxy.

6. Hard protection was a process to apply hard epoxy over the die to protect and introduce a near hermetic seal.

\section{RESULTS}

Compared with the wire-bonding approach reported earlier, the flip-chip technology offered significant reduction in a) the total

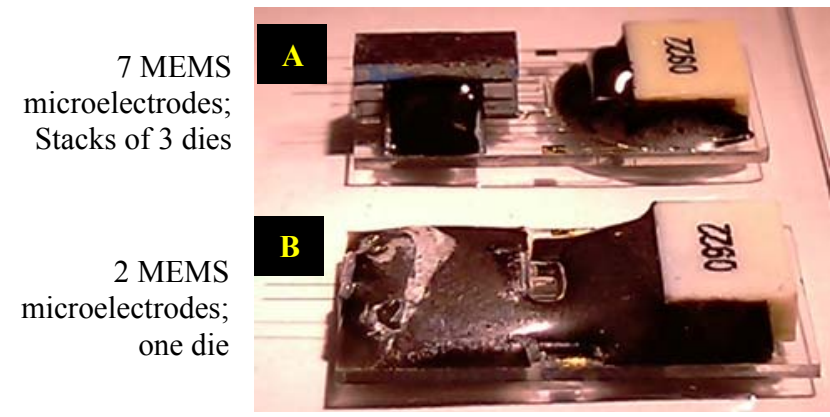

Figure 4. A: Stack of 3 dies, where 7 out of 9 MEMS moveable microelectrodes are actuated B: One die design, where 2 out of 3 MEMS moveable microelectrodes are actuated outside the package boundary

weight of package from 5 grams to 0.5 grams and b) the package thickness from $5 \mathrm{~mm}$ to less than $1 \mathrm{~mm}$. One of the advantages of flip-chip packaging is the ability to scale up the number of chips without significantly increasing the X-Y form factor by facilitating 3D stacks. The flip-chip technology is extended to realize a chipscale 3D stack using flip-chip technology and packaging processes. A 3D flip-chip stack of 3 MEMS microactuator dies is shown in Fig. 4A. Such 3D stacks enable the development of high-density movable microelectrodes that enable the monitoring of ensemble of neurons. For prosthetic applications, higher degree of freedom 


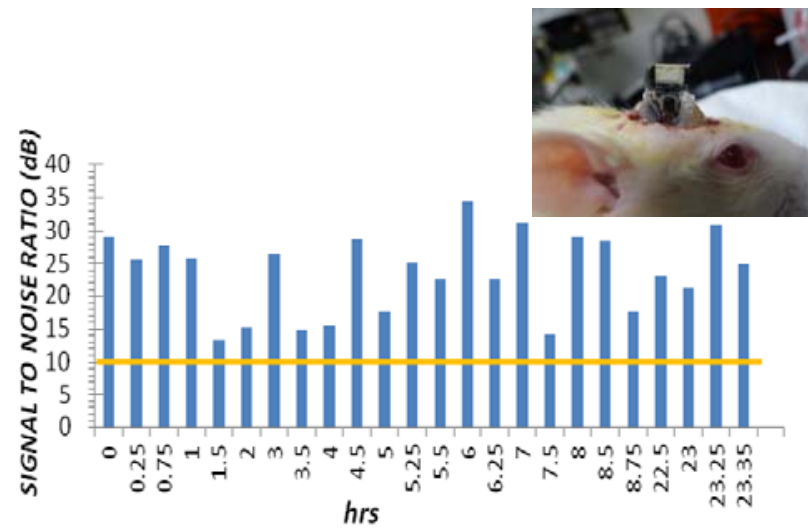

Figure 5: SNR (signal-to-noise ratio) over $24 \mathrm{hrs}$ of continuous neural recordings from rodents. SNR is always above the 10 $\mathrm{dB}$ threshold that corresponds to units that are barely discernible.

and precision can arguably be achieved by sampling larger numbers of neurons.

The packaged MEMS moveable electrodes were surgically implanted in the rodent brain. The packaged device is mounted on the rodent skull. Four mounting screws are initially attached to the bone/skull surrounding the perimeter of the craniotomy, and the microelectrodes are implanted in the motor cortex. After opening the skull, the dura is carefully resected; followed by introducing gel foam on the perimeter of the craniotomy. Silicon microchannels were patterned inside the mould cavity with three holes at the same spacing as the ones for MEMS microelectrodes. A small spacer (made of silicone) was then made between the skull and the MEMS package to accommodate the curvature of the skull. By using stereotactic equipment, the front side of the MEMS package is brought in contact with the spacer, followed by placing dental cement surrounding the package. The dental cement is anchored to the screws. A test under pressurized liquid immersion was done on the MEMS package after SHS was applied to determine its break-down pressure, which was found to be $80 \mathrm{~cm}$ of water. The pilot in vivo test of NHE implanted on the rodents' skull shows no liquid infiltration after implant for more than 77 days. The test eventually failed due to cracks in the glass substrate

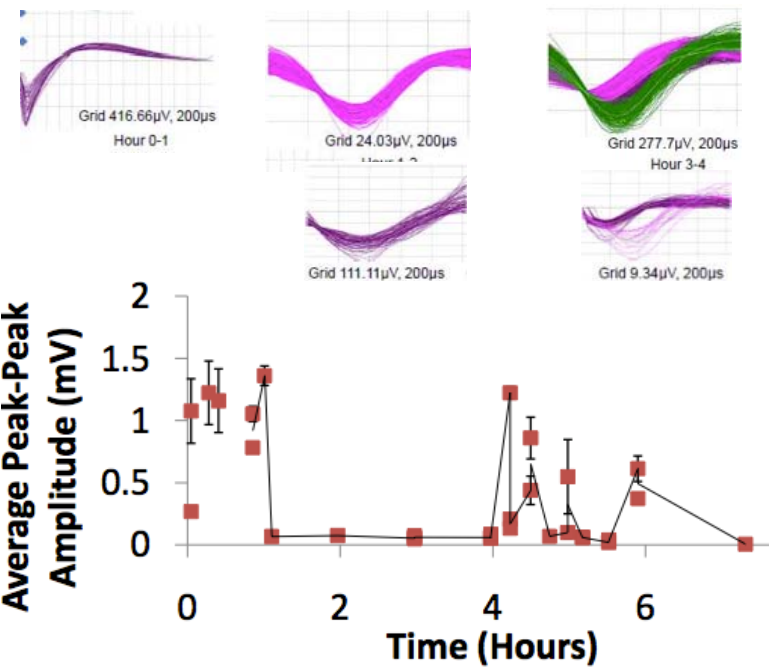

Figure 6: Trend in average peak-to-peak amplitudes in the neural action potentials over the first 7 hours are plotted with typical action potentials observed at different time points shown above. while no damage was observed in the NHE. Figure 5 shows the signal-to-noise ratio (SNR) of neural recording from one MEMS microelectrodes over 24 hours of nearly continuous recording immediately after surgery. SNR is over $10 \mathrm{~dB}$ - which corresponds to the threshold where the action potential waveforms are barely discernible. The peak-to-peak amplitudes of single unit activities over 7 hours on a free behaving rodent are shown in Fig. 6.

\section{CONCLUSIONS}

A novel flip-chip based packaging approach for MEMS devices was developed and was successful tested for monitoring single neuronal activity in long-term rodent experiments. The proposed approach is a low-temperature process that is applicable to silicon, glass or plastic substrates and also a variety of MEMS devices that involve complex moving structures on their die. The non-hermetic encapsulation was found to be adequate in keeping the brain fluids out of the MEMS device over a period of 77 days The interconnects were successfully tested by obtaining continuous neural recording over a period of $24 \mathrm{hrs}$ immediately after implantation. The flip-chip interconnects through Ag bumps provided chip-scale interconnects that allowed for the development of 3D stacks of MEMS dies. A stack of 3 MEMS dies was developed that was mechanically stable. The electrical interconnects for the stack is a work in progress.

\section{ACKNOWLEDGEMENTS}

We would like to thank NIH (NS055312-S1) for funding this research and the Center for Solid State Electronic Research (CSSER) at Arizona State University for use of their clean room facilities.

\section{REFERENCES}

[1] S. Chakrabarti, P. Hebert, M. T. Wolf, M. Campos, J. W. Burdick, and A. Gail, "Expert-like performance of an autonomous spike tracking algorithm in isolating and maintaining single units in the macaque cortex," $J$ Neurosci Methods, vol. 205, pp. 72-85, 2012.

[2] J. G. Cham, E. A. Branchaud, Z. Nenadic, B. Greger, R. A. Andersen, and J. W. Burdick, "Semi-chronic motorized microdrive and control algorithm for autonomously isolating and maintaining optimal extracellular action potentials," J Neurophysiol, vol. 93, pp. 570-9, 2005.

[3] M. S. Fee and A. Leonardo, "Miniature motorized microdrive and commutator system for chronic neural recording in small animals," J Neurosci Meth, vol. 112, pp. 83-94, Dec 152001.

[4] A. Jackson and E. E. Fetz, "Compact movable microwire array for long-term chronic unit recording in cerebral cortex of primates," J Neurophysiol, vol. 98, pp. 31093118, 2007.

[5] N. Jackson, A. Sridharan, S. Anand, M. Baker, M. Okandan, and J. Muthuswamy, "Long-Term Neural Recordings Using MEMS Based Movable Microelectrodes in the Brain," Front Neuroeng, vol. 3, p. 10, 2010.

[6] J. Muthuswamy, M. Okandan, A. Gilletti, M. S. Baker, and T. Jain, "An array of microactuated microelectrodes for monitoring single-neuronal activity in rodents," IEEE T Bio-med Eng, vol. 52, pp. 1470-7, Aug 2005.

[7] J. Sutanto, S. Anand, C. Patel, and J. Muthuswamy, "Novel First-Level Interconnect Techniques for Flip-chip on MEMS devices " J Microelectromech S, vol. 21, pp. 132144,2012 
[8] J. Sutanto, S. Anand, A. Sridharan, R. Korb, L. Zhou, M. Baker, M. Okandan, and J. Muthuswamy, "Packaging and Non-Hermetic Encapsulation Technology for Flipchip on Implantable MEMS Devices," $J$ Microelectromech $S$, vol. in press, 2012.

\section{CONTACT}

Jit Muthuswamy

Associate Professor, Biomedical Engineering

ECG 334, P.O. Box 879709

School of Biological and Health Systems Engineering

Arizona State University

Tempe, AZ 85287-9709

Ph: (480) 9651599

jit@asu.edu 


\title{
HIGH STRAIN AND BIOCOMPATIBLE SCREEN PRINTED NANOCOMPOSITE BASED CONDUCTIVE PDMS STRAIN SENSORS
}

\author{
C. Lee, L. Jug and E. Meng \\ University of Southern California, Los Angeles, California, USC
}

\begin{abstract}
Strain sensors capable of operating in high strain conditions remain a technical challenge. In particular, biocompatible strain sensor technology is needed that satisfies the following requirements: (1) construction from low modulus materials that approach values of soft biological tissues and (2) high strain operation ( $\geq 20 \%)$. We present a screen printed, nanocomposite-based conductive polydimethylsiloxane (CPDMS) strain sensor capable of $40 \%$ strain operation with a gauge factor (GF) $>100$. Strain sensing using CPDMS sensors containing multi-walled carbon nanotubes (MWNT), graphene nanoplatelets (GNP), or a mixture of both nanocarbon filler materials was demonstrated. The combination of high strain operation, high GF, and biocompatible construction pave the way for minimally invasive in vivo strain measurements. Strain sensors were characterized according to their conductivity, zero current resistance (ZCR), thermal coefficients of resistance (TCR), and gauge factor.
\end{abstract}

\section{INTRODUCTION}

Typical strain sensors are made from silicon, metal, or other hard materials using microelectromechanical systems (MEMS) technology [1-2]. These devices are efficient strain sensors; however, they are limited to low strain applications, and their modulus of elasticity does not match that of soft tissues [3], thus limiting their ability to be used in vivo for measuring the strain of soft tissues. Recent research has shown that polydimethylsiloxane mixed with nanocarbon filler (typically multi-walled carbon nanotubes) has potential as a piezoresistive material for strain gauge applications [4-8] but only low gauge factors $(\sim 12)$ were achieved [9]. Such composites become conductive once the filler concentration reaches the percolation threshold. The percolation threshold of CNTs is dependent on aspect ratio, diameter, degree of conglomeration and alignment and therefore ranges widely from $0.005 \mathrm{vol} \%$ to several vol $\%$ [10].

We investigated a GNP/MWNT blend mixed with PDMS to achieve a low percolation threshold with MWNTs while conductive networks above the percolation threshold were created using GNPs. GNPs are also lower in cost and improve the consistency of the prepolymer for screenprinting. The GNP/MWNT and PDMS composite form CPDMS that is sandwiched between and supported by two layers of transparent, medical grade PDMS.

A medical grade PDMS (USP Class VI and ISO 10993-1) was selected for the polymer matrix to improve elongation properties of the resulting CPDMS for high strain operation, which, in our past work, was limited by

9780964002494/HH2012/\$25C2012TRF

DOI 10.31438/trf.hh2012.43 fracture of the piezoresistive material under strain (failed at $1.5 \%$ strain [11]). PDMS-based strain sensors are low cost, simple to manufacture, and well suited for in vivo use because of their low Young's modulus (closer match to that of organs and tissues than silicon) and biocompatibility. Our intended application is in vivo measurement of urinary bladder fullness which requires reliable operation up to $\sim 20 \%$ strain.

\section{THEORY}

Gauge factor is a useful measure of merit for strain sensors and is equal to the normalized change in resistance divided by the strain:

$$
G F=\frac{\Delta R / R}{\Delta L / L}=\frac{\Delta R / R}{\varepsilon}
$$

where $R$ is the nominal as-fabricated, undeformed strain gauge resistance, $\Delta R$ is the total change in resistance, $L$ is the original unstrained strain gauge length, $\Delta L$ is the change in length following application of strain, and $\varepsilon$ is the applied strain. Metal and semiconductors are typical materials used for strain gauges. However, metal strain gauges typically have relatively low gauge factors $(\mathrm{GF} \sim 2)$ and although semiconductors have much better gauge factors (GF 100), they can only be used for low strain (< $0.1 \%$ ) applications. Thick film materials for strain sensing have reported GFs in the range of 2-10 [12-14] which are promising for high strain operation applications such as measurement of strain/fullness of the bladder as part of a neuroprosthetic approach to management of lower urinary tract dysfunction [11].

\section{METHODS \\ Materials}

Graphene nanoplatelets and multiwalled carbon nanotubes were obtained from cheaptubes.com (Brattleboro, VT), PDMS from Factor II (A-103, MDX44210, Lakeside, AZ), Stoddard solvent and isopropyl alcohol (IPA) from VWR (Brisbane, CA), and 34 gauge wire from EIS/Fay Wire (Elmhurst, IL).

\section{Sensor Fabrication}

To prepare the CPDMS prepolymer mixture, a precision scale was used to measure out a predetermined amount of carbon filler material, either graphene nanoplatelets (GNP) or multiwalled carbon nanotubes (MWNT), and PDMS base into a glass beaker. To this was added $10-15 \mathrm{~mL}$ of a solvent mixture consisting of a 30:70 ratio of IPA and Stoddard solvent (by volume).

Solid-State Sensors, Actuators, and Microsystems Workshop Hilton Head Island, South Carolina, June 3-7, 2012 
The mixture was then placed in an ultrasonic bath (Bransonic 3510, Branson Ultrasonic Corp., Danbury, CT) for 15-18 hours to achieve thorough mixing of the filler material with the PDMS base and evaporate organic solvent. Using this method, CPDMS base with a range of concentrations of MWNT and GNP were prepared (Figure 1).

a

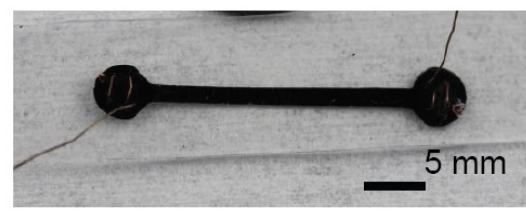

b

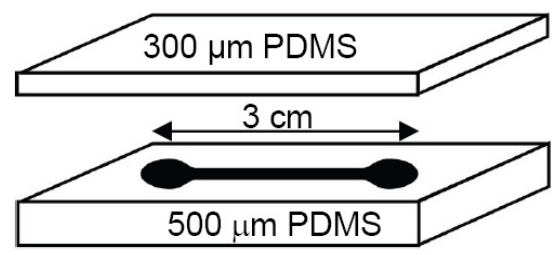

C

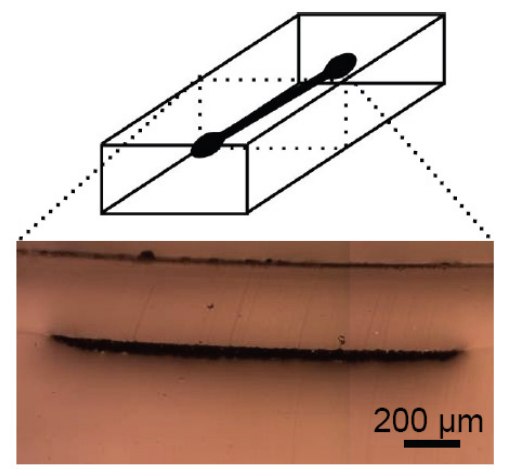

Figure 1: (a) Optical micrograph, (b) three dimensional exploded illustration showing sensor construction, and (c) illustration and optical micrograph showing cross section of strain sensor. (Dimensions of top surface: $9.5 \mathrm{~mm} \times 40 \mathrm{~mm}$.)

Crosslinker (in a ratio of 1:10 to the base) was added to the nanocomposite conductive filler-PDMS base mixture using a planetary mixer (Thinky Corp., Laguna Hills, CA) and the obtained conductive prepolymer was screen printed onto a $500 \mu \mathrm{m}$ thick layer of nonconductive medical grade PDMS using a lithographically defined brass screen using a previously developed method [11]. Briefly, CPDMS prepolymer was spread across a custom stencil using a plastic squeegee. The stencil was made by etching away a lithographically defined pattern in a $76 \mu \mathrm{m}$ thick brass shim $(4.5 " \times 6 ")$ sandwiched between two sheets of a negative, dry-film photoresist. The photoresist was exposed using a high resolution transparency mask (Mikacolor, Los Angeles, CA) and UV light source $\left(45 \mathrm{~mJ} / \mathrm{cm}^{2}\right)$ and developed in a dilute sodium hydroxide bath. The exposed brass regions were etched away using ferric chloride. The parts for this etching kit were purchased from MicroMark (Berkeley Heights, NJ).
The screen-printed CPDMS was then placed under a vacuum for 3-4 hours to remove any remaining solvent and then cured at $80^{\circ} \mathrm{C}$. A second layer of nonconductive medical grade PDMS was added to fully encapsulate the CPDMS. Robust electrical connections were made by threading fine wires through the contact pads several times. For the purposes of saline soak testing, an additional layer of PDMS was added to insulate the threaded wires.

\section{Electrical Properties}

Zero current resistance (ZCR) was measured by recording the resistances of the devices from $1-10 \mu \mathrm{A}$ and extrapolating the resistances back to zero current.

Temperature coefficient of resistance (TCR) was calculated by measuring the device resistance at different temperatures from room temperature to $90^{\circ} \mathrm{C}$ and taking the slope of the resulting curve.

Gauge factor was obtained from data acquired using a motorized stage (Thorlabs, Z812) that allowed for user defined strain patterns and a precision multimeter (Keithley 2400, Keithley Instruments, Cleveland, OH). The sensors were mounted to the motorized stage using custom acrylic clamps with a known separation distance (Figure 2). Clamp separation was increased by discrete steps via computer control of the stage to achieve known levels of unaxial strain. Sensor performance under various strain regimens was evaluated.

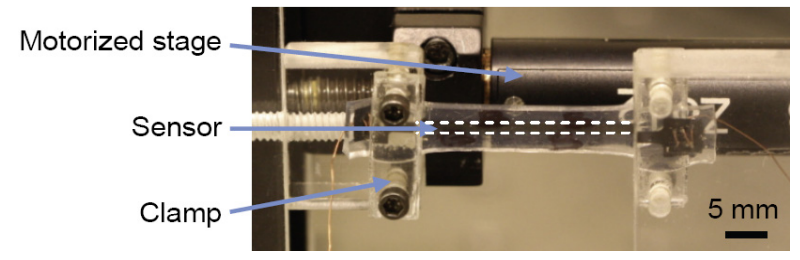

Figure 2: Gauge factor test set up.

\section{RESULTS AND DISCUSSION}

CPDMS mixtures were made with concentrations of MWNT from $2.5 \mathrm{wt} \%$ to $15 \%$. Under $5 \%$, the devices were not conductive and over $10 \%$, the mixture was too viscous to be screen-printed. GNP concentrations were varied from $5 \%$ to $15 \%$. Under $10 \%$, the GNP devices were not conductive and over $12.5 \%$, the CPDMS would not cure. Based on these observations, mixtures of GNP and MWNT were selected ranging from 2.5 to $7.5 \%$ MWNT and 1.5 to $15 \%$ GNP (Table 1).

\section{Electrical Characteristics}

For electrical characterization of the CPDMS material, $35 \times 45 \mathrm{~mm}$ rectangular patterns were printed onto a non-conductive PDMS substrate to ensure that the edges effects were minimized. However, subsequent 4 point probe measurements performed directly on unencapsulated sensors $(1.8 \times 30 \mathrm{~mm})$ yielded identical resistance values, therefore 4 point measurements were 
made directly on exposed, unencapsulated sensors. The thickness of the film was measured by taking sections through a screen-printed sensor element and measuring the average thickness using NIH ImageJ software (Figure 1c).

Table 1. Gauge factor and other manufacturing notes describing different compositions $(N C=$ not conductive. Chloroform was used as a non-polar solvent until it was determined that it is not a suitable solvent for MDX-4 4210. All devices, therefore, were produced using Stoddard solvent and IPA mixture unless otherwise noted.).

\begin{tabular}{|c|c|c|l|}
\hline \multicolumn{2}{|c|}{ Composition } & \multirow{2}{*}{ GF } & \multicolumn{2}{|}{ Notes } \\
\cline { 1 - 2 } MWNT (\%) & GNP (\%) & & \\
\hline 3.5 & 0 & NA & NC \\
\hline 5 & 0 & 99.8 & \\
\hline 10 & 0 & 10.24 & \\
\hline 15 & 0 & NA & Not printable \\
\hline 0 & 5 & NA & NC \\
\hline 0 & 10 & NA & NC \\
\hline 0 & 12.5 & 17.25 & \\
\hline 3.5 & 1.5 & 88.75 & \\
\hline 5 & 10 & 1.18 & Did not cure, chloroform \\
\hline 7.5 & 5 & 2.7 & Chloroform \\
\hline 2.5 & 15 & 2.6 & Did not cure, chloroform \\
\hline 2.5 & 15 & 20.5 & $\begin{array}{l}\text { Did not cure, chloroform, } \\
\text { Parylene coated }\end{array}$ \\
\hline
\end{tabular}

The measured conductivity of the CPDMS was similar to the results of other studies [5-6] (Figure 3), with MWNT devices becoming conductive at $5 \mathrm{wt} \%$. An upper limit to conductivity was found with GNP at $12.5 \%$, which was as conductive as the MWNT samples reported in [5]. Therefore, although conductivity could not be achieved at low concentrations of GNP below $12.5 \%$, once GNP loaded samples became conductive (reached the percolation threshold), they were as conductive of their MWNT counterparts. This trend also appeared when measuring zero current resistance. Although the resistance of the 10\% GNP sample was much higher than the $10 \%$ MWNT sample, when the concentration of GNP was increased to $12.5 \%$, the resistance dropped down to below the $10 \%$ MWNT resistance (Figure 4).

Pure MWNT devices exhibited negative TCR, whereas pure GNP devices had slightly positive TCR. GNP/MWNT composites had negative TCR (Figure 5). GNP/MWNT CPDMS that were first annealed $\left(90^{\circ} \mathrm{C}\right)$ exhibited increased TCR (data not shown).

The gauge factors of the devices were calculated from resistance data obtained under known applied strains up to $40 \%$ strain (Figure 6). Gauge factor (along with variability of gauge factor) was observed to increase as the amount of filler material was reduced (Table 1, Figure 7). This is consistent with results reported elsewhere (Table 2) [5-6, 8]. Gauge factor was inversely related to conductivity. Interestingly, gauge factor for sensors with filler content $>12.5 \mathrm{wt} \%$ was increased for CPDMS sesors with Parylene coating. $\quad 1.5 \% \quad$ GNP/3.5 \%MWNT composite CPDMS sensors had similar TCR and gauge factor as the pure 5\% MWNT device but had greater conductivity.

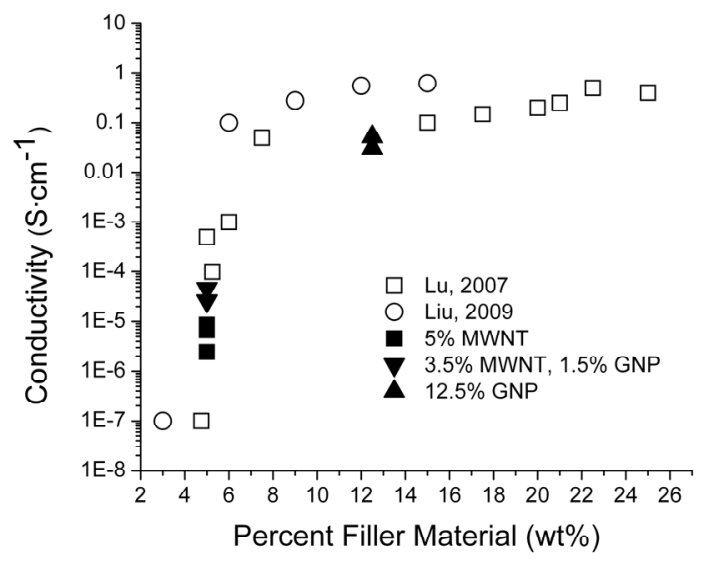

Figure 3: Conductivity measurements for CPDMS samples compared to previously reported data.

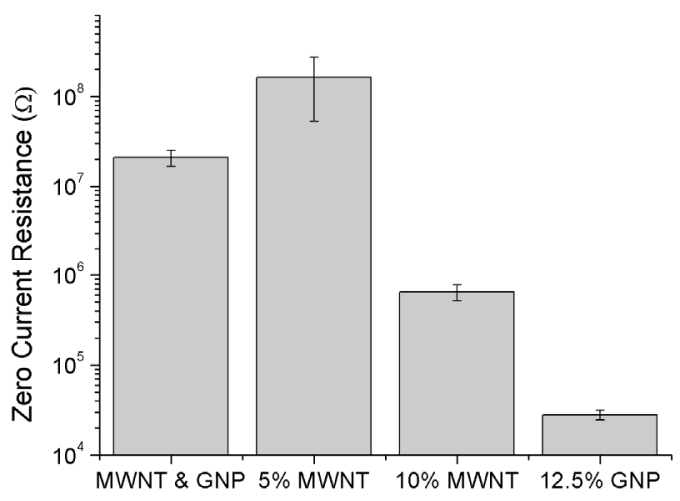

Figure 4: Zero current resistance data (In the composite sample, MWNT \& GNP concentrations are $3.5 \%$ and $1.5 \%$ respectively.). Data are mean $\pm S E(n=6-12)$.

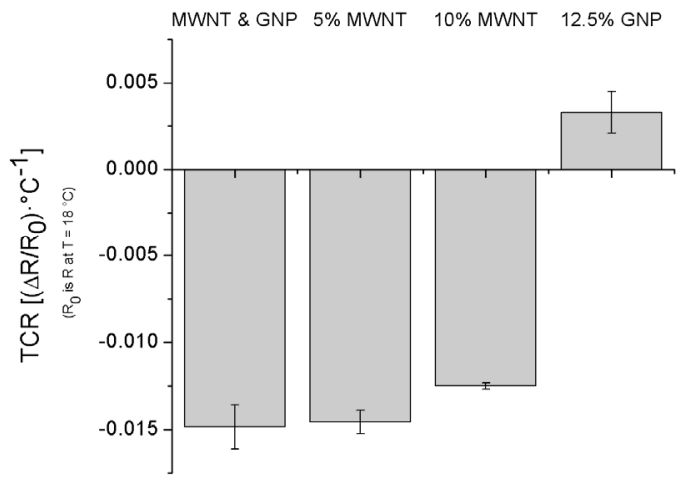

Figure 5: Temperature coefficient of resistance (TCR) data (In the composite sample, MWNT \& GNP concentrations are $3.5 \%$ and $1.5 \%$ respectively.). Data are mean $\pm S E(n=3-5)$. 


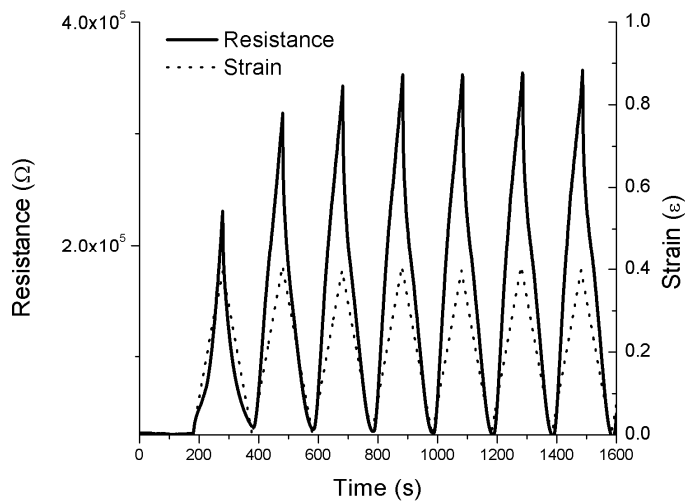

Figure 6: Representative raw gauge factor data. In this case, $40 \%$ strain was applied.

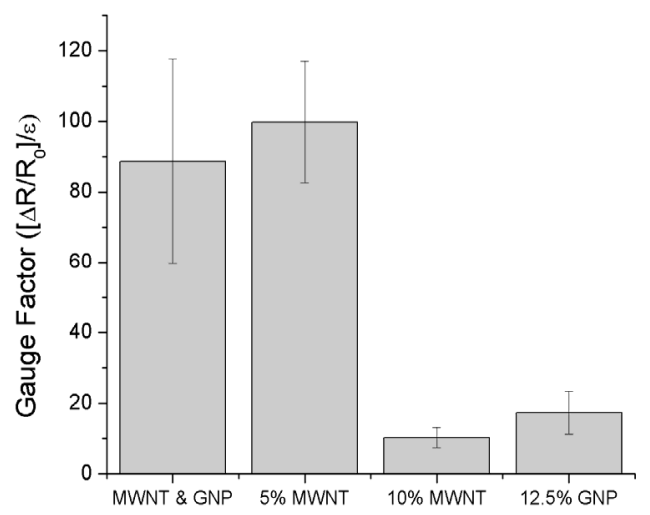

Figure 7: Calculated gauge factor for CDPMS strain sensors having various concentrations and compositions of filler material. Data are mean $\pm S E(n=4-5)$.

Table 2: Gauge factors and achieved strain for CPDMS strain sensors reported in literature.

\begin{tabular}{|c|c|c|}
\hline Reference & Max GF & Max Applied Strain \\
\hline Lu, 2007 [5] & 12.3 & $1.2 \%$ \\
\hline Liu, 2009 [6] & 2.75 & $40 \%$ \\
\hline Giannone, 2009 [8] & 5.6 & $8.5 \%$ \\
\hline
\end{tabular}

Soak testing of strain sensors was performed in $1 \mathrm{X}$ PBS $\left(37^{\circ} \mathrm{C}\right.$ for 21 days) and is ongoing. A decrease in resistance of $42 \%$ over this period was observed, pointing to penetration of conductive ions into the polymer, which can be mitigated by Parylene coating.

\section{CONCLUSION}

Strain sensors made from MWNT and GNP filled PDMS screen-printed onto PDMS were manufactured and characterized according to their zero current resistance, TCR and gauge factor. Due to their high gauge factor, large strain capability, and construction from medical grade materials, these devices are promising for in vivo applications. Coupled with a micro-hydrostatic pressure monitor, these devices could be used to measure bladder fullness for the thousands of people suffering from loss of control of micturition from spinal cord injuries and neurogenic bladder.

\section{ACKNOWLEDGEMENTS}

This work was funded in part by an NSF CAREER Award (ECS-0547544). The authors would like to thank Dr. Donghai Zhu, Mr. Eric Welder, and the members of the USC Biomedical Microsystems Laboratory for their assistance.

\section{REFERENCES}

[1] G. T. A. Kovacs, Micromachined Transducers Sourcebook. McGaw-Hill Companies, Inc. (1998).

[2] M. Madou, Fundamentals of Microfabrication. CRC Press (1997).

[3] G. R. Higson, "Recent advances in strain gauges", Journal of Scientific Instrumentation, 41, 405-414 (1964).

[4] J. Engel, J. Chen, N. Chen, S. Pandya, and C. Liu, "Multiwalled carbon nanotube filled conductive elastomers: materials and application to micro transducers", 19th IEEE International Conference on MEMS, Istanbul, Turkey, 1/22-26/2006, IEEE (2006), pp. 246-249

[5] J. Lu, M. Lu, A. Bermak, Y. Lee, "Study of piezoresistance effect of carbon nanotube-PDMS composite materials for nanosensors", 7th IEEE Conference on Nanotechnology, Hong Kong, China, 8/25/2007, IEEE-NANO (2007) pp. 1240-1243.

[6] C.-X. Liu and J.-W. Choi, "Patterning conductive PDMS nanocomposite in an elastomer using microcontact printing", J. Micromech. and Microeng., 19, 085019 (2009).

[7] I. Kang, et al., "A carbon nanotube strain sensor for structural health monitoring", Smart Materials and Structures, 15, 737-748 (2006).

[8] P. Giannone and S. Graziani, "Flexible tailored sensors for large deformation monitoring", Instrumentation and Measurement Technology Conference, Singapore, 5/57/09, IEEE (2009) pp. 593-596.

[9] R. Rizvi, B.Cochrane, E. Biddiss and H. Naguib "Piezoresistance characterization of poly(dimethylsiloxane) and poly(ethylene) carbon nanotube composites", Smart Mater. Struct., 20, 094003, (2011).

[10] M. Moniruzzaman and K. I. Winey, "Polymer nanocomposites containing carbon nanotubes", Macromolecules, 39, 5194-5205 (2006).

[11] C. A. Gutierrez and E. Meng, "Low-cost carbon thick-film strain sensors for implantable applications", J. Micromech. Microeng., 20, 095028 (2010).

[12] J. E. Brignell, N. M. White, and A. W. J. Cranny, "Sensor applications of thick-film technology", IEE Proc. 135, 7784 (1988)

[13] J. S. Shah, "Strain sensitivity in thick-film resistors", IEEE Trans. Compon., Hybrids, Manuf. Technol. 3, 554-64 (1980).

[14] M. Prudenziati and B. Morten, "Piezoresistive properties of thick-film resistors: an overview", Microelectronics International, 3, 20-37 (1986).

\section{CONTACT}

*E. Meng., tel: (213) 821 3949; ellis.meng@usc.edu 


\title{
MEMS FORCE PROBES FOR CELL MECHANOBIOLOGY AT THE MICROSECOND SCALE
}

\author{
J.C. Doll and B.L. Pruitt* \\ Stanford University, Stanford, California, USA
}

\begin{abstract}
We present high-speed force probes with on-chip actuation and sensing for the measurement of $\mathrm{pN}$-scale forces at the microsecond time scale. We achieve a high resonant frequency in water (1-200 kHz) with requisite low spring constants $(0.3-40$ $\mathrm{pN} / \mathrm{nm})$ and low integrated force noise $(1-100 \mathrm{pN})$ by targeting probe dimensions on the order of $300 \mathrm{~nm}$ thick, 1-2 $\mu \mathrm{m}$ wide and 30-200 $\mu \mathrm{m}$ long. Forces are measured using silicon piezoresistors while the probe is actuated thermally.
\end{abstract}

\section{INTRODUCTION}

A variety of instruments have been developed for the precise measurement of forces and displacements in the past 25 years. The most commonly used techniques are atomic force microscopy, magnetic tweezers, and optical tweezers. These tools are capable of measuring force $\left(10^{-14}-10^{-8} \mathrm{~N}\right)$, displacement $\left(10^{-10}-10^{-4} \mathrm{~m}\right)$ and time $\left(10^{-4}-10^{2} \mathrm{sec}\right)$ over many orders of magnitude [1]. However, none are capable of simultaneously measuring and delivering $\mathrm{pN}$ scale forces with microsecond time resolution.

The ideal force probe needs to have comparable stiffness to the system of interest (e.g. $0.1-10 \mathrm{pN} / \mathrm{nm}$ for cells and molecules) while maintaining a high resonant frequency during operation in liquid (e.g. $100 \mathrm{kHz}$ ). Optical and magnetic tweezers can be built with vanishingly small spring constants (e.g. $10^{-3} \mathrm{pN} / \mathrm{nm}$ ), but the relatively large mass of the bead limits their bandwidth to 1-10 $\mathrm{kHz}$. Microcantilevers have been fabricated with measurement bandwidths of over $5 \mathrm{MHz}$ in water, but their spring constants have been correspondingly large, again due to their relatively large size.

In order to address this technological gap, we have developed force probes with on-chip piezoresistive force readout and thermal actuation. A cantilever beam structure minimizes the probe mass while allowing simple spring constant tuning via the length of the probe. Microcantilevers with integrated sensing and actuation have been reported previously [2-4]. However, they were designed for high-speed scanning probe microscopy, so were relatively large (e.g. $100 \mu \mathrm{m}$ wide x $3 \mu \mathrm{m}$ thick) and optimized for displacement resolution at the expense of force resolution. In contrast, our devices are optimized for single point force measurements and are 10-100x smaller than prior integrated devices.

\section{DESIGN}

The force probe topology is presented in Figure 1. The probe consists of a narrow, soft sensor at the tip and a wide, stiff actuator at the base. We have designed and fabricated two different probe varieties: sensor-only devices (PR) and thermally actuated devices (PRT). The PR devices do not include an actuator but retain the stiffened base in order to define the force probe length from the front of the wafer rather than during the imprecise backside release etch. Placement of the stiff, heavy actuator at the base of the probe maximizes the tip deflection range, maximizes the overall resonant frequency of the probe, minimizes the thermal actuator time constant and decouples the mechanical design of the sensor and actuator portions.

The piezoresistor is designed using numerical optimization [5]. Although the piezoresistor design problem is not convex, seeding the numerical optimizer with several random initial guesses leads to a globally optimal design. Our design optimization code now captures effects such as self-heating in piezoresistive cantilevers [6] and integrates thermal modeling and temperature constraints. The piezoresistors are designed to minimize the RMS force noise in the intended measurement bandwidth while constraining the increases in the maximum and tip temperatures to approximately 5 and $1 \mathrm{~K}$ during operation in water, respectively.

The tradeoff between spring constant and resonant frequency improves as the size of the force probe decreases. A cantilever beam thickness of $300 \mathrm{~nm}$ was chosen due to the rapid reduction in thermal conductivity and uncertainty in the effective elastic modulus with any further reduction in thickness. But a thin device requires a shallow piezoresistor. The open source modeling and optimization code that we developed handles diffused, epitaxial and ion implanted piezoresistors (via lookup tables). We opted to fabricate the piezoresistors using $\mathrm{POCl}_{3}$ predeposition due to the relatively simple fabrication process and higher performance of $n$ type compared with p-type piezoresistors for this application [5]. Metal interconnects connect the piezoresistor to the bondpads, minimizing both the excess interconnect resistance and mechanical crosstalk during simultaneous actuation and sensing [3].

The actuator consists of a silicon heater, $\mathrm{SiO}_{2}$ etch stop and a layer of $\mathrm{Al}$. The heater increases the temperature of the actuator region, deflecting the probe tip due to the mismatch in thermal expansion coefficients between $\mathrm{Si}(2.6 \mathrm{ppm} / \mathrm{K})$ and $\mathrm{Al}(23 \mathrm{ppm} / \mathrm{K})$. The $\mathrm{SiO}_{2}$ thickness $(100 \mathrm{~nm})$ is chosen to minimize the thermal resistance between the heater and $\mathrm{Al}$ while providing an adequate etch stop. The thickness of the Al layer $(1000 \mathrm{~nm})$ is chosen to place the neutral axis at the $\mathrm{SiO}_{2}-\mathrm{Al}$ interface. The length of the actuator portion is a tradeoff between the heater time constant,

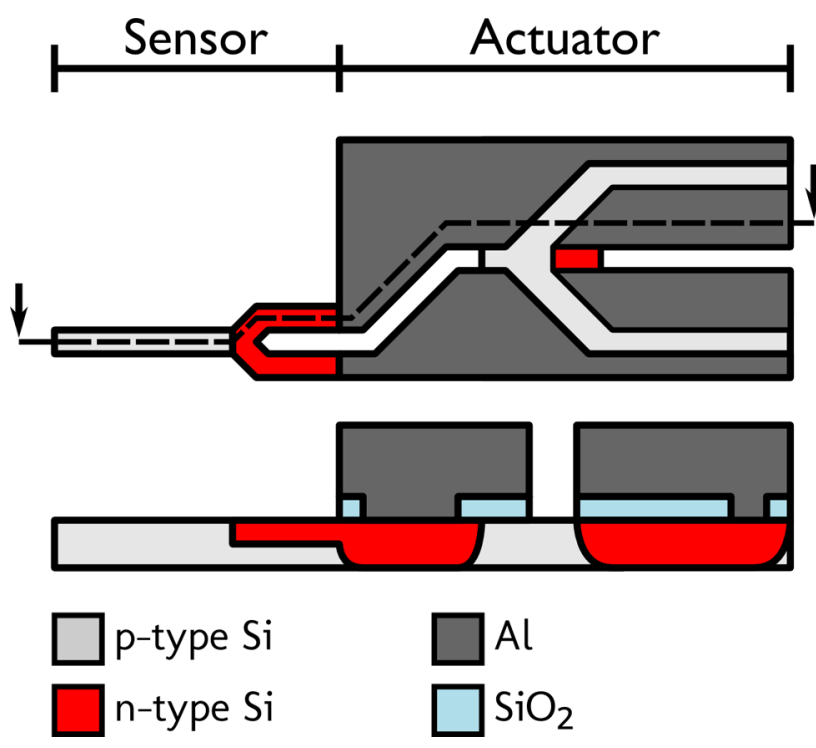

Figure 1: Layout of a force probe with integrated piezoresistive sensing and thermal actuation (PRT). The probe consists of a narrow, soft sensor attached to the end of a wide, stiff actuator. Placement of the actuator at the base rather than the tip leads to numerous performance benefits and decouples the mechanical and thermal design of the actuator and sensor.

Solid-State Sensors, Actuators, and Microsystems Workshop Hilton Head Island, South Carolina, June 3-7, 2012 
overall resonant frequency of the probe, and the maximum tip deflection. The overall resonant frequency of the probe is modeled using both finite element analysis and numerically using the Rayleigh-Ritz method. The target maximum tip deflection for the devices is $500 \mathrm{~nm}$ for the study of mammalian cochlear hair cells.

\section{FABRICATION}

The seven mask fabrication process is illustrated in Figure 2. The PR and PRT probes are fabricated using the same process and use identical film thicknesses.

The process starts with a (100) oriented $100 \mathrm{~mm}$ diameter silicon-on-insulator (SOI) wafer with a $340 \mathrm{~nm}$ thick device layer, $400 \mathrm{~nm}$ thick buried oxide (BOX) and $400 \mu \mathrm{m}$ thick handle wafer. The device layer is p-type silicon with a resistivity of 10-20 $\Omega$-cm. Poly-on-insulator (POI) wafers with comparable device and BOX layer thicknesses were fabricated and processed alongside the SOI wafers for process development. All lithography steps are performed using $0.7 \mu \mathrm{m}$ thick SPR955 (unless otherwise noted) on an ASML PAS 5500/60 stepper with backside alignment capability. The critical dimensions and overlay tolerances of the process are $0.5 \mu \mathrm{m}$ and $0.25 \mu \mathrm{m}$, respectively.

First, alignment marks are patterned and etched $120 \mathrm{~nm}$ into the device layer. The wafer is rotated by 45 degrees during the alignment mark exposure so that the piezoresistors will be oriented in the $<100>$ direction $( \pm 0.5$ degrees as set by the device layer orientation tolerance).

The oxide diffusion mask is formed next. The wafers are diffusion cleaned and a $90 \mathrm{~nm}$ thick thermal oxide layer is grown by oxidizing them in steam at $900 \mathrm{C}$. The thermal oxidation decreases the thickness of the device layer from 340 to the target thickness of $300 \mathrm{~nm}$. An additional $320 \mathrm{~nm}$ thick layer of low temperature oxide (LTO) is deposited on the wafers at $400 \mathrm{C}$ in order to increase the thickness of the oxide mask and prevent the diffusion of phosphorus through the oxide mask during the high temperature contact diffusion. After the LTO deposition the wafers are annealed in $\mathrm{N}_{2}$ at $1100 \mathrm{C}$ for 20 hours in order to densify the LTO and noticeably reduces strain gradients in the device layer.

The piezoresistive sensor is formed using two separate diffusions. First, the piezoresistor contacts are $\mathrm{POCl}_{3}$ doped at $900 \mathrm{C}$ for 20 minutes. Afterwards the thin layer of phosphosilicate glass (PSG) deposited on the surface is stripped in a dilute 50:1 HF solution, preventing undercut during the next step while leaving the underlying oxide mask intact. In the second diffusion the window in the oxide diffusion mask is opened further to dope the entire piezoresistor region at $825 \mathrm{C}$ for 30 minutes. The oxide windows are wet etched in 20:1 buffered oxide etchant (BOE) after the photoresist has been rendered hydrophilic in a $60 \mathrm{sec}$ low temperature $\mathrm{O}_{2}$ plasma pretreatment step. The high surface phosphorus concentration leads to slight upward device curvature. Minimizing the piezoresistor length reduces both the static tip deflection and the tip temperature increase during operation.

All of the remaining oxide is stripped and the device layer is patterned and etched via reactive ion etching (RIE). Afterwards the wafer is cleaned, $100 \mathrm{~nm}$ of LTO is deposited and oxide vias are patterned and wet etched. As in the rest of the oxide etch steps the wafer is pretreated with $\mathrm{O}_{2}$ plasma in order to render the photoresist hydrophilic and the vias are etched using 20:1 BOE. The etch is timed by visually monitoring the backside of the wafer and over etching by $20 \%$. After the wafers are cleaned in PRS1000 , the native oxide is removed with a brief 50:1 HF dip before rushing the wafers to the sputterer load lock for metal deposition.

In the sputterer load lock the wafers are heated to $200 \mathrm{C}$ for 3 minutes using quartz lamps to drive off any moisture before depositing $50 \mathrm{~nm}$ thick Ti and $1000 \mathrm{~nm}$ thick Al layer. The Ti layer provides a lower barrier height than Al to n-type silicon ( 0.5 vs 0.7 $\mathrm{eV}$ ) and serves as a contact diffusion barrier. The metal is lithographically patterned and etched back via RIE, stopping on the LTO layer. Afterwards the wafers are rinsed in water to eliminate residual $\mathrm{HCl}$ on the wafer before stripping the photoresist mask. Next a $400 \mathrm{~nm}$ thick LTO layer is deposited on the wafer and a trench is patterned and etched via RIE into the oxide around the force probe. The trench prevents cracks in the BOX from snapping the force probes, and increases probe yield from about $35 \%$ to $70 \%$ (and up to $90 \%$ ) [7].

The backside of the wafer is patterned with a $7 \mu \mathrm{m}$ thick layer of SPR220 and baked for two hours at 90C to harden it. The frontside of the wafer is left unprotected in order to maximize heat transfer during the backside DRIE. The wafers are shipped to the Lurie Nanofabrication Facility (LNF) at the University of Michigan for the backside etch. A staff member at the LNF bonds the device wafer to a carrier wafer using CrystalBond 555 (CB) using a custom bonding tool that eliminates air pockets in the $\mathrm{CB}$. After the wafers are etched and shipped back, the backside photoresist is stripped and the wafer is debonded from the carrier by soaking in $70 \mathrm{C}$ water for 30 minutes. The LTO coating deposited earlier prevents metal corrosion during this step. Afterwards the wafer is cleaned in fresh DI water and air dried.

The wafers are cleaned in a downstream $\mathrm{O}_{2}$ plasma asher to remove residual DRIE passivation polymer. However a thin layer of fluorocarbon polymer remains on the wafer because it is not volatile in pure $\mathrm{O}_{2}$ plasma. Without further cleaning a thin web of polymer remains on the wafer, rendering most of the devices unusable. A second polymer cleanup step is performed in a $\mathrm{CF}_{4} / \mathrm{O}_{2}$ plasma in order to remove the remaining fluorocarbon polymer. (a)

(b)

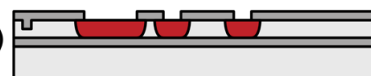

(c)

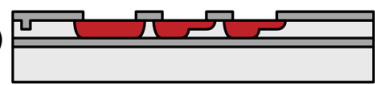

(d)

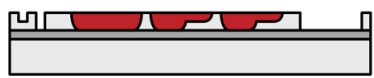

(e)

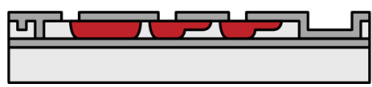

(f)

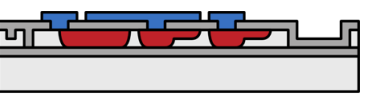

(g)

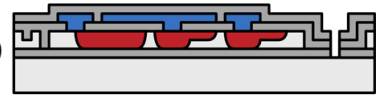

(h)

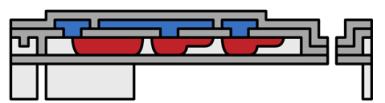

(i)

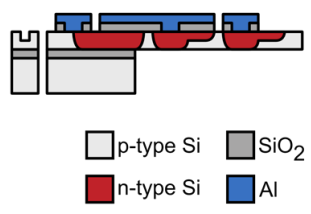

Figure 2: Fabrication process for force probes with integrated piezoresistive sensing and thermal actuation (PRT). (a) Alignment marks are patterned and etched. (b) The wafers are oxidized, coated with additional low temperature oxide (LTO) and annealed. Windows are opened in the oxide mask and the contacts are $\mathrm{POCl}_{3}$ doped at 900C. (c) The oxide windows are opened further and the piezoresistors are $\mathrm{POCl}_{3}$ doped at $825 \mathrm{C}$. (d) The oxide is stripped and the device layer is patterned and etched via reactive ion etching (RIE). (e) A layer of LTO is deposited and vias are opened before (f) the Ti/Al metal stack is deposited and patterned via RIE. (g) An additional layer of LTO is deposited and cracks are patterned into the oxide stack in order to increase device yield. (h) The backside of the wafer is patterned, bonded to a carrier wafer, etched via DRIE and debonded. (i) The BOX and LTO layers are etched via vapor HF in order to release the probes. 
Finally, the BOX and LTO layers are etched in vapor HF in order to release the force probes. The wafers are etched from the frontside, but cracks in the BOX allow the vapor HF to attack the BOX and LTO layers from both sides at once. The top LTO coating is approximately the same thickness as the BOX and minimizes undercut of the LTO layer underneath the metal interconnects. No forming gas anneal (FGA) is used because we found that it has a detrimental effect on the contact resistivity, even with the Ti diffusion barrier, due to the shallow piezoresistors.

Scanning electron micrographs (SEMs) of finished PR and PRT devices are presented in Figure 3. After cleanroom fabrication is complete, the devices are snapped out of the wafer using snap tabs defined during the backside DRIE step. The devices are epoxied to custom printed circuit boards (PCBs) and ultrasonically wirebonded with Al wire. Afterwards the wirebonds are coated with epoxy and the entire assembly is coated with a 200-300 nm thick layer of parylene N. Passivation is required for operation in water but is not necessary for experiments in air or vacuum.
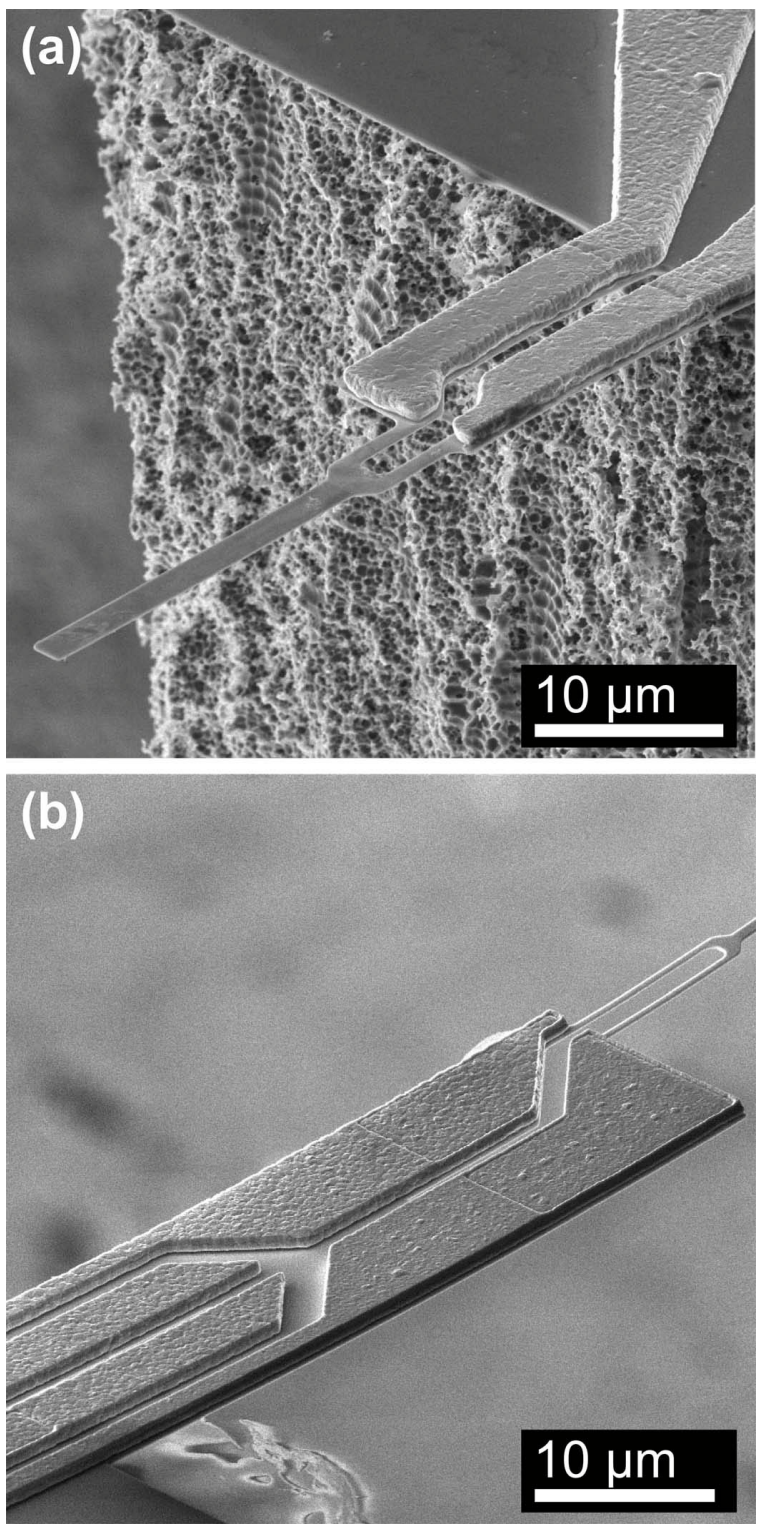

Figure 3: Scanning electron micrographs (SEMs) of finished (a) PR and (b) PRT force probes.
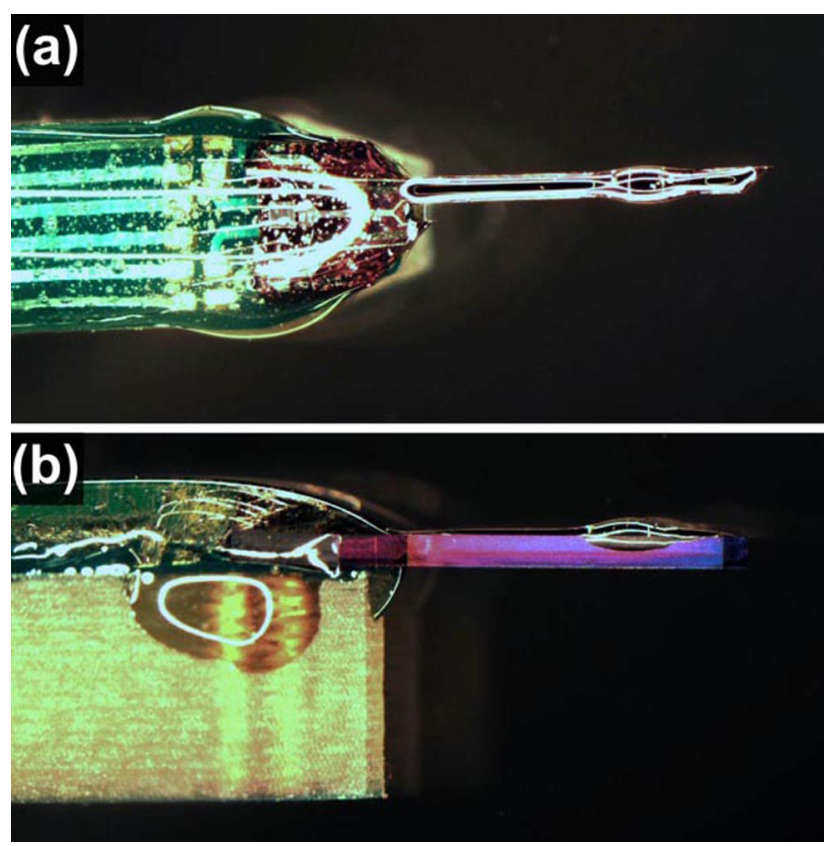

Figure 4: (a) Top and (b) side views of a fully assembled and passivated device. First, the silicon die is epoxied and wirebonded to the PCB. Next, the wirebonds and long silicon extension are coated with epoxy. Finally, the entire assembly is coated with a 200-300 nm thick layer of parylene $N$. The long extension enables compatibility with both upright and inverted microscopes.

Optical micrographs of a finished, assembled device are presented in Figure 4. Note the long silicon extension, which enables compatibility with upright microscopes with a working distance as small as $1 \mathrm{~mm}$. The device PCB includes a pair of through holes for attachment to a three-axis micromanipulator.

\section{PROBE CHARACTERIZATION}

The high dopant concentration at the contact surface ( $\sim \mathrm{e} 20 / \mathrm{cc})$ yields Ohmic contacts. The sheet resistance $(110 \Omega / \square)$ and contact resistivity $\left(10-20 \mu \Omega-\mathrm{cm}^{2}\right)$ were calculated from Van der Pauw and Kelvin Bridge test structures and confirmed using the transfer length method on finished devices. The high contact doping both minimizes the contact resistivity and overall contact resistance of the one-dimensional transmission line. Overall, the contacts and metal interconnects contribute about $340 \Omega$ of excess resistance. In contrast, devices without the two-step diffusion and optimized metallization processes had excess resistances of 1-2 $\mathrm{k} \Omega$, severely degrading the piezoresistor force resolution.

Each silicon die contains two identical force probes. The compensation probe, set far back from the tip of the device, provides both temperature and crosstalk compensation. A Wheatstone bridge circuit transduces the resistance change of the piezoresistors into a voltage output. The main and compensation probes are placed in the bottom legs of the bridge while two potentiometers balance the top legs. It is essential to place the main and compensation probes in opposite sides of the bridge in order to compensate the actuator-sensor crosstalk during simultaneous actuation and sensing.

The Wheatstone bridge bias is generated by a $5 \mathrm{~V}$ voltage reference (ADR445), taken through a potentiometer controlled voltage divider to set the desired bias and voltage buffered. The Wheatstone bridge signal is amplified in two stages in order to maintain sufficient bandwidth. First, a low noise instrumentation 


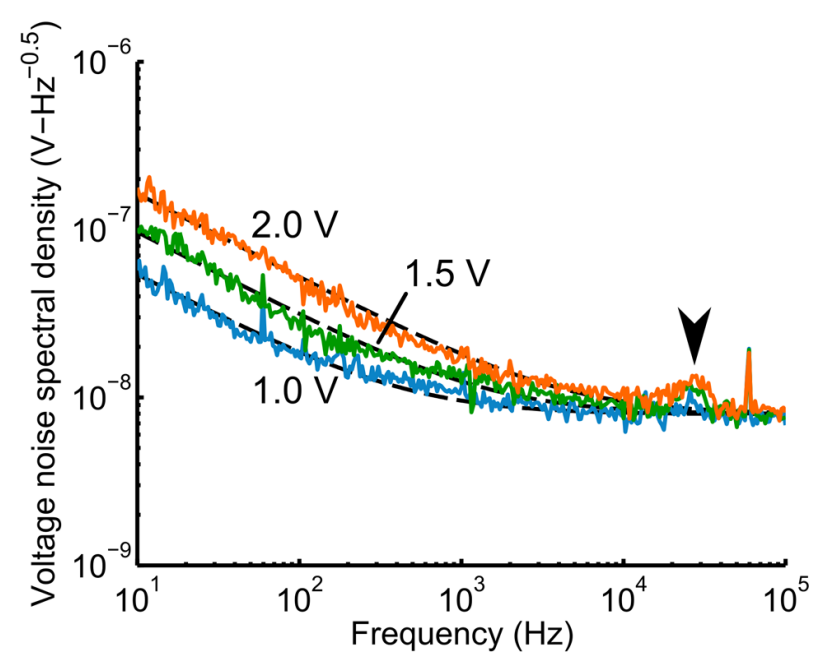

Figure 5: Example piezoresistor noise spectra in air for Wheatstone bridge bias voltages of $1,1.5$ and $2 \mathrm{~V}$. The force probe is capable of self-detecting its thermomechanical resonance in air ( $f_{0}=30 \mathrm{kHz}, Q=3.6$ ). The Hooge factor is calculated by fitting a noise model (dashed lines) to the measured spectra. For the device presented here, $\alpha=1.1 \pm 0.4 \times 10^{-5}$.

amplifier (INA103) amplifies the signal 100x $\left(\mathrm{f}_{-3 \mathrm{~dB}}=800 \mathrm{kHz}\right)$. A second-stage variable gain amplifier (THS4031) provides further gain without compromising the measurement bandwidth.

Example piezoresistor noise spectra are presented in Figure 5. The piezoresistor exhibits $1 / \mathrm{f}$ noise due to bulk mobility fluctuations. The Hooge factor $(\alpha)$ is calculated by fitting a noise model to the measured spectra. The electrically active dopant concentration profile is measured via spreading resistance analysis (Solecon Laboratories) and used to calculate the number of carriers in the piezoresistor. The effective number of carriers, accounting for the variation in current density through the depth of the piezoresistor, is about $20 \%$ smaller than the total number of carriers and is used to model the $1 / \mathrm{f}$ noise.

The piezoresistor displacement sensitivity is calibrated by applying a known deflection to the tip of the force probe. We mount the probe on a piezoelectric stage with capacitive position readout and deflect the probe with a sub-micron $\mathrm{AC}$ deflection using a stationary, stiff AFM cantilever. Example calibration data is shown in Figure 6. The measured and predicted displacement sensitivities match to within about $10 \%$. The model prediction is based upon experimentally inferred sensitivity and resistance factors $\left(\beta^{*}=0.23 \pm 5 \%, \gamma=0.83\right.$ to 0.96$)$.

Our piezoresistive sensors operate near the thermodynamic force noise limit. For example a $98 \mu \mathrm{m}$ long, $2 \mu \mathrm{m}$ wide probe has a resonant frequency in air of $28 \mathrm{kHz}$, quality factor of 3.8 and spring constant of $1.63 \pm 0.13 \mathrm{pN} / \mathrm{nm}$ (measured via thermomechanical noise). The overall force noise from $1 \mathrm{~Hz}$ to 28 $\mathrm{kHz}$ is $4.4 \pm 0.4 \mathrm{pN}$ for a $2 \mathrm{~V}$ bridge bias (to limit self-heating), compared with the thermomechanical force noise limit of $1.0 \mathrm{pN}$. Similarly results (i.e. operation at about $4 \mathrm{x}$ the thermomechanical noise floor) are obtained when the devices are operated in water.

\section{CONCLUSIONS}

We have summarized the fabrication and sensing performance of our force probes. Their force resolution, actuation speed and actuator-sensor crosstalk each represent a 10-20 fold improvement over prior devices and pave the way for $\mathrm{pN}$-scale force measurements at the microsecond time scale in water.
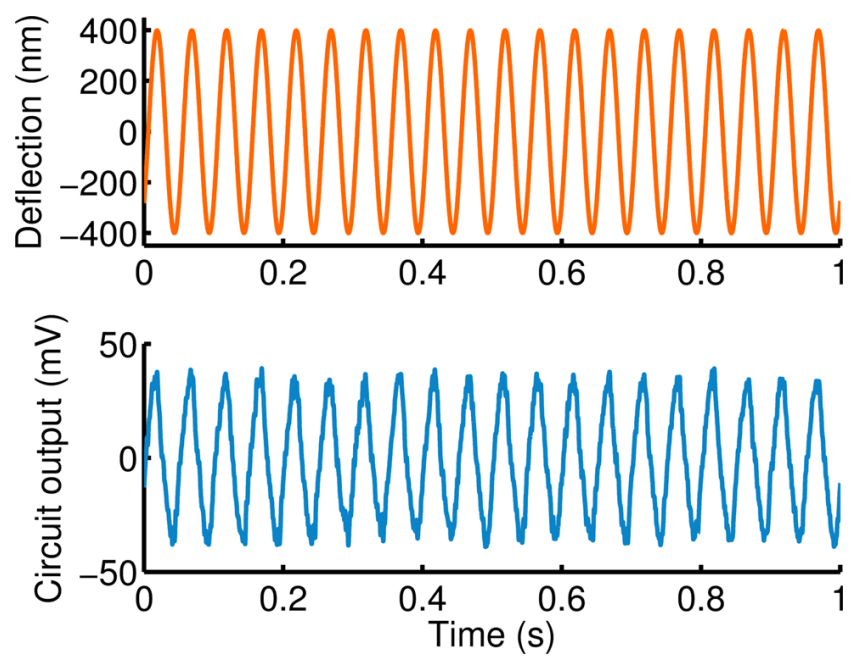

Figure 6: Example of displacement sensitivity calibration. The force probe is deflected $400 \mathrm{~nm}$ at $20 \mathrm{~Hz}$ and the output of the measurement circuit is recorded. The average displacement sensitivity for this probe was $73.5 \pm 10.3 \mathrm{~V} / \mathrm{m}$-V compared with a model prediction of $80.8 \mathrm{~V} / \mathrm{m}-\mathrm{V}$.

\section{ACKNOWLEDGEMENTS}

Fabrication work was performed at the Stanford and Lurie Nanofabrication Facilities (members of the NNIN) supported by the NSF under Grant ECS-9731293. This work was supported by the NIH under grant EB006745, the NSF under CAREER Award ECS-0449400, COINS NSF-NSEC ECS-0425914, and CPN NSFNSEC PHY-0425897, and by DARPA under YFA N66001-09-12089. JCD was supported in part by NDSEG and NSF Graduate Research fellowships. The authors are grateful to A.J. Ricci and A.W. Peng of Stanford University for their helpful input on the probe design and characterization, and S. Martin and R. Hower of the University of Michigan for performing the backside DRIE.

\section{REFERENCES}

[1] K.C. Neuman and A. Nagy, "Single-molecule force spectroscopy: optical tweezers, magnetic tweezers and atomic force microscopy", Nature Methods (2008).

[2] S.C. Minne, S.R. Manalis and C.F. Quate, "Parallel atomic force microscopy using cantilevers with integrated piezoresistive sensors and integrated piezoelectric actuators", Applied Physics Letters (1995).

[3] Y.-S. Kim et al., "PZT cantilever array integrated with piezoresistor sensor for high speed parallel operation of AFM", Sensors and Actuators A (2003).

[4] G.E. Fantner et al., "Use of self-actuating and self-sensing cantilevers for imaging biological samples in fluid", Nanotechnology (2009).

[5] J.C. Doll, S.-J. Park and B.L. Pruitt, "Design optimization of piezoresistive cantilevers for force sensing in air and water", Journal of Applied Physics (2009).

[6] J.C. Doll et al, E.A. Corbin, W.P. King and B.L. Pruitt, "Selfheating in piezoresistive cantilevers", App. Phys. Lett. (2011).

[7] G.C. Hill et al, "Patterned cracks improve yield in the release of compliant microdevices from silicon-on-insulator wafers", J. Micromech. Microeng. (2011).

\section{CONTACT}

*B.L. Pruitt; pruitt@stanford.edu 


\title{
A 1.5 MICRO-LITER MICROBIAL FUEL CELL ARRAY FOR RAPID SCREENING OF EXOELECTROGENIC BACTERIA
}

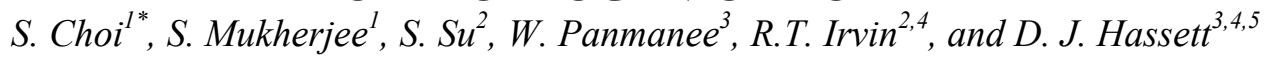 \\ ${ }^{1}$ School of Electronic and Computing Systems, University of Cincinnati, Ohio, USA \\ ${ }^{2}$ Depaertment of Microbiology and Immunology, University of Alberta, CA, \\ ${ }^{3}$ Department of Molecular Genetics, Biochemistry \& Microbiology, University of Cincinnati, Ohio, USA, \\ ${ }^{4}$ Pilus Energy LLC, Cincinnati, Ohio, USA, ${ }^{5}$ Cincinnati VA Hospital, Cincinnati, OH USA
}

\begin{abstract}
We report an array of six MEMS microbial fuel cells (MFCs), a compact and reliable platform for rapid screening of exoelectrogenic bacteria. The presented device contains vertically stacked $1.5 \mu \mathrm{L}$ anode/cathode chambers separated by a proton exchange membrane (PEM), and represents the smallest MEMS MFC array. Within just five hours, we successfully determined the electricity generation capacity of two known bacterial electrogens and another metabolically more voracious organism with 4 isogenic mutants of this strain constructed with the hypothesis that such mutations could alter their electrogenic properties. In addition, the percent deviation of all six MFC units was less than $1.4 \%$ from their open circuit voltages recorded, which is far less than that of mL-sized MFC arrays and even MEMS MFC arrays.
\end{abstract}

\section{INTRODUCTION}

Driven by increasing concerns over the energy-climate crisis and environment pollution, microbial fuel cells (MFCs) have been a major focus for renewable energy production [1]. MFCs are powered by live microorganisms to efficiently catalyze degradation of a broad range of organic substrates under natural conditions, and, as such, offer clean and sustainable features [2]. Thus far, only a limited number of bacteria species and their optimal growth conditions have been studied for use in various MFCs. This reveals a significant deficiency of the essential knowledge needed to ascertain which bacterial species or consortia may be best suited for generating optimal power density in MFCs [3]. This deficiency is largely caused by limitations in current screening methods based on larger scale two-bottle MFCs that require longer start-up times (from days to weeks), significant space and materials (hundreds of milliliters to liters), and labor-intensive control for MFC experiments, either in series or in parallel circuits [4]. This limitation has motivated efforts to miniaturize MFC arrays, such that the effective chamber volumes are reduced to the microliter scale in a well-controlled manner. However, recently proposed 9-well pipette tip-based miniature MFC array [3] and 24-well MEMS MFC array [5, 6] still has several limitations; (1) large sized anodic chamber volume $(>400 \mu \mathrm{L})$, and (2) high percent deviation in each MFC unit $(>8 \%)$. Here, we developed an ultra-small $(1.5$ $\mu \mathrm{L})$, reproducible, and practical parallel analyses platform for rapid screening of exoelectrogenic bacteria. Normally, miniaturizing MFCs into a few micro-scale levels is exceptionally challenging because the devices become vulnerable to the $\mathrm{O}_{2}$ invasion into the anode chamber, leading to a significant increase in the start-up time and decrease in current generation [7]. Typically, microorganisms consume $\mathrm{O}_{2}$ immediately and mitigate abiotic $\mathrm{O}_{2}$ reduction on the anode; this $\mathrm{O}_{2}$-scavenging reaction occurs in macro-sized MFCs having high biomass concentration in an anode chamber. However, the micro-sized MFC has a small anode chamber volume, and the small-area anode had a small number of $\mathrm{O}_{2}$-utilizing microorganisms [7]. Therefore, in order to minimize $\mathrm{O}_{2}$ intrusion in such a small MFC array, we used $254 \mu$ m-thick PTEE (polytetrafluoroethylene) gaskets (five orders of oxygen permeability

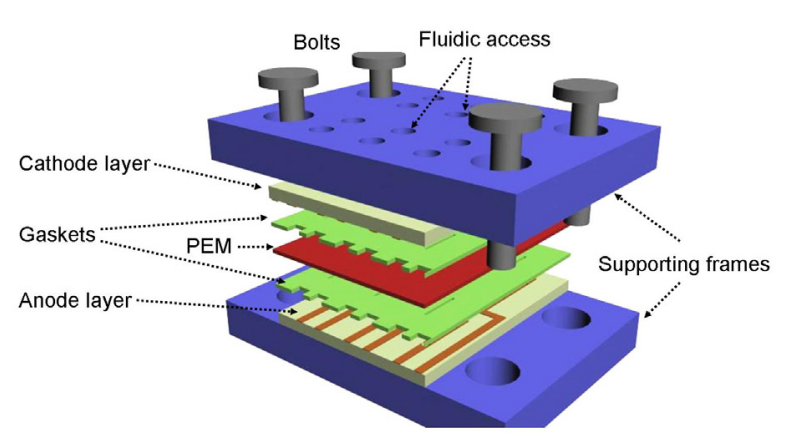

Figure 1: Schematic of the 6-well MEMS MFC array system.
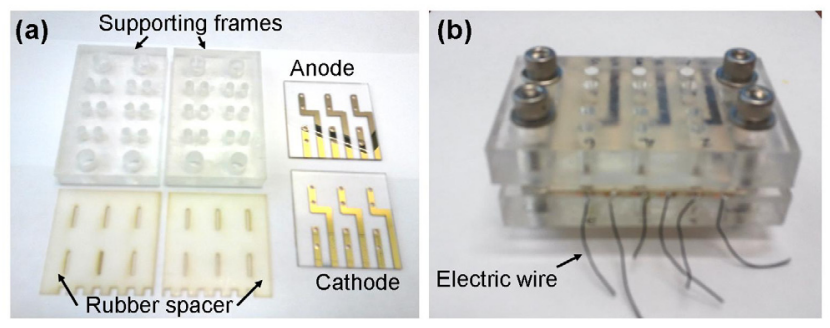

Figure 2: (a) Individual layers of the MFC array and (b) fully assembled MFC array.

lower than that of other polymer gaskets) and PEEK (Polyetheretherketone) polymer tubings (fifty times lower magnitude than that of conventional fluorinated ethylene propylene tubings) [8]. Finally, we applied this technique for studies of selected hypothesis-driven genes that could alter power density in $P$. aeruginosa isogenic mutants. These efforts demonstrated that spatially distinct wells of the MFC array displayed highly comparable performance characteristics and identified which of the hypothesis-driven, selected genes in $P$. aeruginosa triggered higher power density. The hyperpiliated pilT mutant of $P$. aeruginosa displayed the highest current generation, 2-fold higher than the well known electrogenic bacterium, Shewanella oneidensis.

\section{MATERIALS AND METHODS}

\section{Device fabrication and assembly}

A schematic of the MFC array is shown in Fig. 1. The MFC array consisted of five functional layers; an anode electrode layer (Cr/Au on PMMA (polymethyl methacrylate)), an anode chamber layer (gasket), a PEM (proton exchange membrane), a cathode chamber layer (gasket), and a cathode electrode layer $(\mathrm{Cr} / \mathrm{Au}$ on PMMA). The $1.5 \mu \mathrm{L}$ anode/cathode chamber was defined by a 254 $\mu \mathrm{m}$-thick gasket; the exposed electrode area per cell is $6 \mathrm{~mm}^{2}$ (Fig. 2a). The array was assembled by the two supporting frames with 
bolts and nuts (Fig. 2b). Each layer except for the PEM was micro-patterned by using laser micromachining. First, we cut two 5 x $5 \mathrm{~cm}$ PMMA chips with the laser and drilled 12 holes on each chip for inlets/outlets. The chips were then coated with $\mathrm{Cr} / \mathrm{Au}(20$ $\mathrm{nm} / 200 \mathrm{~nm}$ ) by an electron-beam evaporator and patterned to define the electrodes using a lift-off process. The $254 \mu \mathrm{m}$-thick PTEE rubber gaskets were micro-patterned to define the anode/cathode chambers. All the layers were manually stacked in sequence while carefully aligning the tubing holes for the microfluidic channels. For fluidic access, 24 sterile PEEK polymer tubings were plugged into the holes to form twelve independent routes for anolyte/catholyte access. The individual microfluidic access path is very important to provide independent and reliable results for each MFC unit.

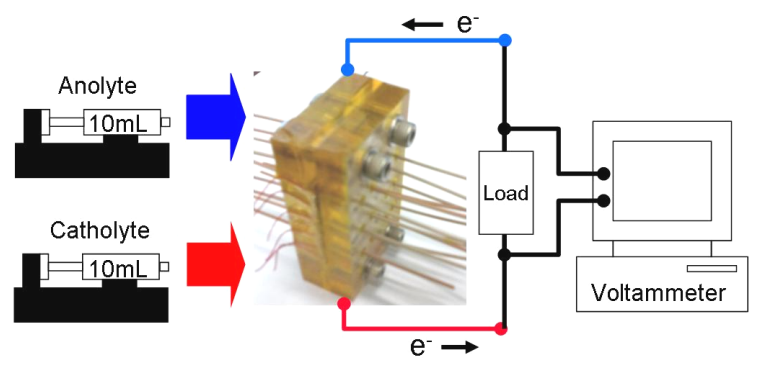

[ Solution Injection ] [ [ LL-MFC ] [ Data Acquisition ]

Figure 3: Schematic of test setup for monitoring the MEMS MFC array. The array has 6 microfluidically-controlled micro-sized MFCs integrated on a single chip. All six MFCs are connected to 150- $\Omega$ external loads and output voltages are recorded every 10 $\min$.

\section{Inoculum}

For the selection of hyper-electrogenic strains, we used Luria broth in the anodic chamber, and, for the sake of convenience, aerobic $50 \mathrm{mM}$ potassium ferricyanide in the cathode buffered with $100 \mathrm{mM}$ potassium phosphate, $\mathrm{pH}$ 7.0. Six microorganisms were tested; Wild-type $S$. oneidensis MR1, Wild-type $P$. aeruginosa $P A O 1$ and isogenic nirS, lasl, bdlA and pilT mutants, respectively. $P$. aeruginosa $P A O 1$ mutants were generated using classical allelic replacement techniques with gentamicin or tetracycline cassettes inserted with selected genes followed by sucrose counter-selection to resolves merodiploids as described by Hoang et al. [9].

\section{Measurement setup}

We measured the potentials between the anodes and cathodes using a data acquisition system (CompactRIO System, National Instrument) and recorded them every $10 \mathrm{~min}$. via LabVIEW. An external resistor $(150 \Omega)$, connected between the electrodes of each MFC unit, closed the circuit. We calculated current through the resistor via Ohm's law. Current density was normalized to the anode area $\left(6 \mathrm{~mm}^{2}\right)$ or anode chamber volume $(1.5 \mu \mathrm{L})$. We droved anolyte and catholyte to fill the anode/cathode chambers with syringe pumps, and supplied the solutions continuously at a very low flow rate, under $20 \mu \mathrm{L} /$ hour.

\section{RESULTS AND DISCUSSION}

Open circuit voltages

We first compared the Open Circuit Voltages (OCV; cathode potential minus anode potential with an infinite load) in the

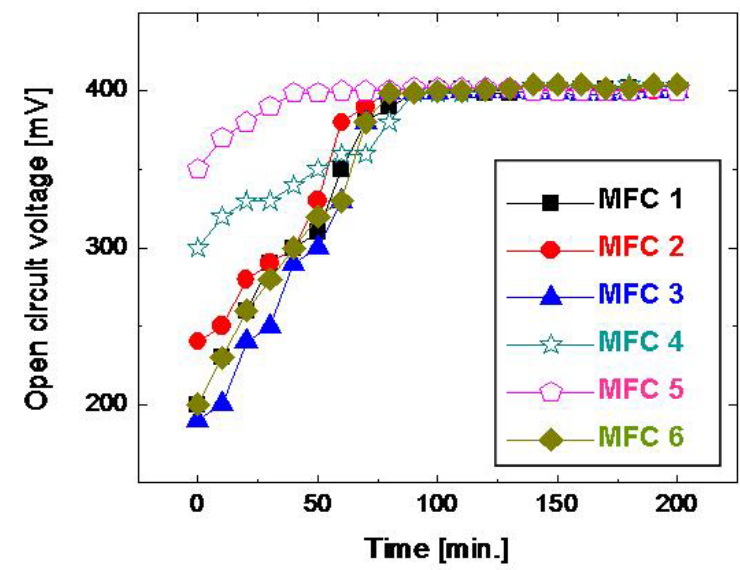

Figure 4: Open circuit voltages (OCV) of the MFC units in the array.

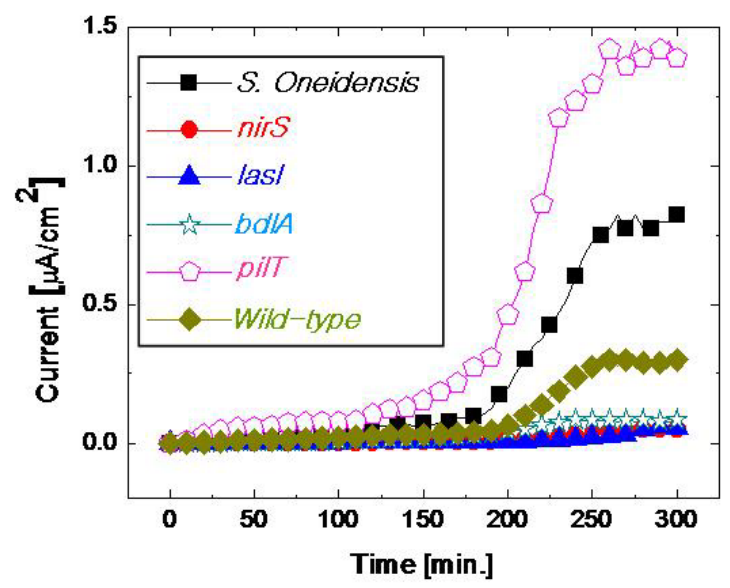

Figure 5: Currents produced from six MFC units, in which different microorganisms are inoculated, respectively. pilT mutant of $P$. aeruginosa (pentagons) produced the highest current.

continuous-mode MFC units. No bacteria were inoculated in the anode chambers for these experiments, in which the cathode potentials were controlled by ferricyanide at about $300 \mathrm{mV}$. The MFC array was built airtight and all solutions were sparged with $\mathrm{N}_{2}$ before injection. As shown in Fig. 4, the OCVs of the six MFCs increased and reached a value of approximately $402 \mathrm{mV}$ with less than $1.4 \%$ variation, which is far less than that of $\mathrm{mL}$-sized MFC array $(25 \%)$ and even other MEMS MFC arrays ( $>8 \%)$ [2, 10, 11]. We believe that this low percent deviation is attributed to the (1) significantly reduced chamber volume in a well-controlled manner and (2) more effective oxygen-tight interface. As the size of the MFCs becomes smaller, it has been observed that the background current decreased drastically in the $\mathrm{nA}$ range [12]. Since we used a $\mathrm{mm}^{2}$ scale anode area, the background current was almost negligible and became stable. Also, we recently reported that $\mathrm{O}_{2}$ penetrating into the anode chamber abiotically reacted with the anode since the anode potential was negative enough $(-0.3$ to $-0.1 \mathrm{~V}$ versus $S H E)$ to reduce $\mathrm{O}_{2}$ into $\mathrm{H}_{2} \mathrm{O}$ or low $\mu \mathrm{M}$ levels of $\mathrm{H}_{2} \mathrm{O}_{2}$. This reduction will create a negative current that competes with the positive current produced by the microorganisms. To date, prior micro-sized MFC arrays were not able to minimize the $\mathrm{O}_{2}$ invasion into the anode 
Table 1: Micro-sized MFC arrays

\begin{tabular}{|c|c|c|c|c|c|}
\hline & (1) Biffinger et al. 2009 [3] & (2) Hou et al. 2009 [11] & (3) Hou et al. 2011a [5] & (4) Hou et al. 2011b [6] & (5) This work \\
\hline \# of MFC units & 9 & 24 & 24 & 24 & 6 \\
\hline $\begin{array}{c}\text { \# of actual bacterial species to be } \\
\text { screened }\end{array}$ & 9 & 24 & 24 & 6 & 6 \\
\hline Bacteria & Shewanella sp. & Shewanella $s p$. & $\begin{array}{l}\text { Shewanella \& } \\
\text { Arthrobacter sp. }\end{array}$ & Shewanella sp. & $\begin{array}{c}\text { Shewanella \& } \\
\text { Pseudomonas sp. }\end{array}$ \\
\hline Operation/fluidic access & Batch mode / no access & Batch mode / no access & Batch mode / no access & $\begin{array}{l}\text { Continuous mode / } \\
4 \text { common access }\end{array}$ & $\begin{array}{l}\text { Continuous mode / } \\
6 \text { independent access }\end{array}$ \\
\hline Sustainability & $<15$ hours & $<17$ hours & NA & $>$ Several days & > Several days \\
\hline Anode/cathode material & Graphite felt/Carbon paper & Gold/Carbon Cloth & Carbon Cloth /Carbon Cloth & Gold/Carbon paper & Gold/Gold \\
\hline Anode area & $1.1 \mathrm{~cm}^{2}$ & $0.38 \mathrm{~cm}^{2}$ & $0.38 \mathrm{~cm}^{2}$ & $0.38 \mathrm{~cm}^{2}$ & $0.06 \mathrm{~cm}^{2}$ \\
\hline Anode volume & $500 \mu \mathrm{L}$ & $600 \mu \mathrm{L}$ & $600 \mu \mathrm{L}$ & $400 \mu \mathrm{L}$ & $1.5 \mu \mathrm{L}$ \\
\hline Membrane & Nafion 117 & Nafion 117 & Nafion 117 & Nafion 117 & Nafion 117 \\
\hline Catholyte & Potassium ferricyanide & Potassium ferricyanide & Air & Potassium ferricyanide & Potassium ferricyanide \\
\hline Start-up & $<2$ hours & $<2$ hours & $<20$ hours & NA & $<2$ hours \\
\hline Deviation between array cells & $8 \%$ & $6 \sim 14 \%$ & $16.4 \sim 22 \%$ & NA & $1.4 \%$ \\
\hline Current density & $\sim 2.5 \mu \mathrm{A} / \mathrm{cm}^{2}$ & $\sim 0.5 \mu \mathrm{A} / \mathrm{cm}^{2}$ & $\sim 0.2 \mu \mathrm{A} / \mathrm{cm}^{2}$ & NA & $\sim 1 \mu \mathrm{A} / \mathrm{cm}^{2}$ \\
\hline
\end{tabular}

chamber since they used PDMS or adhesive as a gasket, both of which have high $\mathrm{O}_{2}$ permeability [7]. Eventually, they could have different amounts of $\mathrm{O}_{2}$ dissolved in the chambers, leading to significant deviations in each MFC unit. Therefore, $\mathrm{O}_{2}$ leakage in the micro-scale MFC array was the dominant factor for their unreliable and irreproducible properties while high percent deviation in the conventional macro-scale MFCs was mainly due to the large background noise and the large variations in the growth, evolution, and metabolism of the consortium of microbes in the uncontrollable macro-scale environmental surroundings. Here, in order to have low percent deviation between MFC units in the array, we reduced the chamber volume to $1.5 \mu \mathrm{L}$, which is the smallest chamber among all the reported MFC arrays. In addition, we minimized $\mathrm{O}_{2}$ intrusion by using $254 \mu \mathrm{m}$-thick PTEE gaskets and PEEK polymer tubings [8].

\section{Current generation and start-up time}

Six bacterial cultures (one $S$. oneidensis and five different $P$. aeruginosa strains) and potassium ferricyanide were continuously supplied to the anode and cathode chambers, respectively, with flow driven by rate-controlled syringe pumps. Normally, the MFCs are operated under batch mode for better accumulation and acclimation of bacteria for optimal biofilm formation on their anode surfaces [7, 12], a trait necessary for mediator-less current generation. Once current generations are observed after bacterial inoculation, solutions are supplied under continuous mode. However, as the size of the MFC is scaled towards the micro regime, it is difficult to notice the biofilm formation by monitoring its current generation because it becomes extremely sensitive to even minute $\mathrm{O}_{2}$ leakage and interrupt bacteria from attaching the surface for anode-respiration, dramatically decreasing current generation and increasing start-up time [7, 8]. That is why we continuously flowed all culture solutions even for biofilm formation on the anode but at a very low flow rate $(<20 \mu \mathrm{L} /$ hour $)$. This continuous operation helped keep a high flow by flushing out $\mathrm{O}_{2}$ that had diffused into the anode and support better anode-electron transfer for the bacteria, ultimately decreasing start-up phase and increasing current density. Reducing oxygen diffusion is the key factor to increasing the performance of micro-sized MFCs because $\mathrm{O}_{2}$ diffusion into the anode chamber could compete with extracellular electron transfer to the anode and, therefore, decrease coulombic efficiency and current generation. For example, it has been reported that Geobacter $s p$. can grow with $\mathrm{O}_{2}$ as a terminal electron acceptor, and this diverts electrons away from the anode, resulting in lower current density [14]. Maximizing the performance of an individual MFC unit on the array is critical to the successful development of a sensitive MFC array. All six MFCs started to generate current about $100 \mathrm{~min}$. after inoculum injection (Fig. 5). Then, the current gradually increased and reached a peak value in 5 hours. This demonstrates the capability of our MEMS MFC array for a fast analysis of exoelectrogenic bacteria compared to that of most mL-scale MFCs (several days) and it is comparable to that of other MEMS MFC arrays. We successfully determined the electrogenic capacity from 4 mutants of $P$. aeruginosa with high sensitivity, which has never been investigated. The hyperpiliated pilT mutant of $P$. aeruginosa displayed the highest current generation, 2-fold higher than that of wild-type $S$. oneidensis. This finding is quite interesting because reducing twitching motility could increase the current generation. This work validated the utility of our MFC array by studying how strategic genetic modifications impact the electrochemical activity of $P$. aeruginosa strains.

\section{Comparison to other micro-sized MFC arrays}

Table 1 summarizes specifications of prior micro-sized MFC array work and compares our MEMS MFC array with them. Initial MFC arrays were operated under batch mode without fluidic access. Even the first MFC array [3] was completely exposed to air and was not able to remain active in anaerobic environments, eliminating its ability to study many other bacteria strains that expire when exposed to aerobic environments for extended periods of time (Geobacter sp., Clostridium sp., etc.). The second and third MFC arrays [5,6,11] were also exposed to some air since the device required solution loading into each chamber between manual assembly steps for the array, leading to unreliable/irreproducible results. Another common concern with these three MFC arrays can cause contamination from unit to unit during operation or fabrication. Moreover, lack of fluidic access to each MFC unit hampers long-term analysis abilities ( $<17$ hours). In this sense, continuous mode is ideal for the MFC array. Very recently, Hou et al., for the first time, reported a microfluidically-controlled MFC array [6]. To 
simplify the intricate microfluidic accesses, they used 4 common fluidic access to the anode chamber and one common access to the cathode chamber, demonstrating long term screening studies of electrogens. However, they have not presented enough valuable data we can compare to with other MFC arrays. Moreover, the device does not provide independent access of the 24 spatially distinct MFC units and thus, in actuality, only 6 different microbial species can be screened. Our MFC array significantly reduced the chamber volume and anode area, leading to (1) low background current, (2) very low deviation between MFC units, and (3) short start-up time despite the fact that the reduced chamber becomes more sensitive to oxygen invasion and diverts electrons away from the anode.

Gold has been identified as a potential material for micro-sized MFC anode development. Gold is biocompatible, highly conductive, and is compatible with conventional microfabrication modalities for the development of a micro-sized MFC platform. However, our preliminary results with Geobacter $s p$. and other previous studies with Shewanella $s p$. suggested that bare gold is a poor electrode material for the anode of MFCs because gold does not contain functional groups, such as redox-capable quinones, a natural electron acceptor for anaerobic respiration [7, 15]. As shown in Table 1, the MFC array using graphite felt as an anode generated more than 5 times larger current density than that using gold. We believe that the most effective approach for developing efficient micro-sized MFC anodes will be to chemically modify the gold surface for better electronic coupling between gold electrodes and bacteria along with maintaining compatibility to MEMS techniques. For example, current production with $S$. putrefaciens increased 100 -fold when the gold surface was coated with a SAM of 11-mercapto-undecanoic acid [16]. This was due to bacterial cytochromes conducting the final electron transfer from the bacteria and the carboxylic acid termini of SAM-modified electrodes can accommodate cytochromes on an electrode surface through strong hydrogen bonding with the peptide bonds within the protein backbone. Another option for the micro-sized MFC anode might be alternative electrode materials or non-conventional nanoscale electrodes such as nanowires, nanotubes, or graphenes, meeting micro-/nano-fabrication process requirements.

\section{CONCLUSION}

In this work, we developed a $1.5 \mu \mathrm{L}$ MFC array that allows for reliable and sensitive bacterial screening within just five hours. The OCVs of 6 spatially distinct MFC units on the array differed by less than $1.4 \%$. Ultimately, we were able to validate the utility of our MFC array by studying selected hypothesis-driven genes in $P$. aeruginosa. We expect that this work highlights the potential realization of a practical tool for efficient high-throughput bacterial screening and fundamental MFC understanding, which may further improve power extraction in MFCs. Our future work is directed toward the development of much higher throughput micro-sized MFC arrays with independent fluidic accesses.

\section{ACKNOWLEDGEMENT}

We thank Prof. Jason Heikenfeld for stimulating discussions and offering part of the fabrication facilities. We would also like to thank Ohio Center for Microfluidic Innovation (OCMI) at the University of Cincinnati for supporting this work.

\section{REFERENCES}

[1] Y. Yang, G. Sun, and M. Xu, "Microbial fuel cells come of age," J. Chem. Technol. Biotechnol. 86, 625 (2010)

[2] L. Logan, and J.M. Regan, "Microbial fuel cells - challenges and applications," Environ. Sci. Tehcnol., 40, 5172 (2006)
[3] J. Biffinger, M. Ribbens, B. Ringeisen, J. Pietron, S. Finkel, and K. Nealson, "Characterization of electrochemically active bacteria utilizing a high-throughput voltage-based screening assay," Biotechnology and Bioengineering, 102, 436 (2009)

[4] X. Cao, X. Huang, X. Zhang, P. Liang, and M. Fan, "A mini-microbial fuel cell for voltage testing of exoelectrogenic bacteria," Front. Environ. Sci. Engin. China. 3, 307 (2009)

[5] H. Hou, L. Li, P. de Figueiredo, and A. Han, "Air-cathode microbial fuel cell array: A device for identifying and characterizing electrochemically active microbes," Biosensors and Bioeletronics, 26, 2680 (2011)

[6] H. Hou, L. Li, P. de Figueiredo, and A. Han, "Microfluidic microbial fuel cell array for multiplexed long-term parallel analysis of microbial activities, "Proceedings of 15 th International Conference on Miniaturized Systems for Chemistry and Life Sciences ( $\mu$-TAS) 2011, Seattle, USA, (2011), pp. 1424-1426.

[7] S. Choi, H.-S. Lee, Y. Yang, P. Parameswaran, C.I. Torres, B.E. Rittmann and J. Chae, "A $\mu \mathrm{L}$-scale Micromachined Microbial Fuel Cell Having High Power Density," Lab on a Chip, 11, 1110 (2011)

[8] S. Choi, J. Chae, " $\mu L$-Scale microbial fuel cell with optimal power generation and biofilm formation," Proceedings of $25^{\text {nd }}$ IEEE International Conference on Micro Electro Mechanical Systems (MEMS) 2012, Paris, France, (2012), pp.43- 46.

[9] T.T. Hoang, A.J. Kutchma, A. Becher, and H.P. Schweizer, "Integration-proficient plasmids for Pseudomonas aeruginosa: site-specific integration and use for engineering of reporter and expression strains," Plasmid, 43, 59 (2000)

[10] P. Aelterman, K. Rabaey, H.T. Pham, N. Boon, and W. Verstraete, "Continuous electricity generation at high voltages and currents using stacked microbial fuel cells," Environ. Sci. Technol., 40, 3388, (2006)

[11] H. Hou, L. Li, Y. Cho, P. de Figueiredo, and A. Han, "Microfabricated Microbial Fuel Cell Arrays Reveal Electrochemically Active Microbes," PLOS one, 4, e6570, (2009)

[12] F. Qian, M. Baum, Q. Gu, and D. E. Morse, "A $1.5 \mu l$ microbial fuel cell for on-chip bioelectricity generation," Lab on a Chip, 9 , 3076 (2009)

[13] Z. He, J. Kan, Y. Wang, Y. Huang, F. Mansfeld, and K.H. Nealson, "Electricity production coupled to ammonium in a microbial fuel cell," Environ. Sci. Technol. 43, 3391 (2009)

[14] W.C. Lin, M.V. Coppi, D.R. Lovley, "Geobacter sulfurreducens Can Grow with Oxygen as a Terminal Electron Acceptor," Appl. Environ. Microbiol. 70, 2525 (2004)

[15] H. Richter, K. McCarthy, K.P. Nevin, J.P. Johnson, V.M. Rotello, and D.R. Lovley, "Electricity generation by Geobacter Sulfurreducens attached to gold electrodes," Langmuir, 24, 4376 (2008)

[16] S.R. Crittenden, C.J. Sund, and J.J. Sumner, "Mediating electron transfer from bacteria to a gold electrode via a self-assembled monolayer," Langmuir, 22, 9473 (2006)

\section{CONTACT}

*S. Choi; seokheun.choi@uc.edu 


\section{A MICROMACHINED CHEMICAL SENSOR FOR SEA FLOOR ENVIRONMENTS: INITIAL RESULTS}

Tao $\mathrm{Li}^{l^{*}}$, Kang Ding ${ }^{2}$, William E. Seyfried, $\mathrm{Jr}^{2}{ }^{2}$, and Yogesh B. Gianchandani ${ }^{1}$

${ }^{1}$ Center for Wireless Integrated MicroSensing and Systems (WIMS ${ }^{2}$ ), University of Michigan, Ann Arbor, MI

${ }^{2}$ Department of Geology and Geophysics, University of Minnesota, Minneapolis, MN

\section{ABSTRACT}

This paper reports a micromachined chemical sensor intended for harsh environments such as those near hydrothermal vents at the sea floor. The sensor is intended to measure the $\mathrm{pH}$, dissolved $\mathrm{H}_{2}$ and $\mathrm{H}_{2} \mathrm{~S}$, and temperature. The device is $12 \mathrm{~mm}$ in diameter and $6 \mathrm{~mm}$ in effective length, including packaging. A $\mathrm{YSZ}|\mathrm{HgO}| \mathrm{Hg}$ solid electrode is used as a common electrode for measurement of the three chemical quantities. The YSZ diaphragm used for this electrode is $<500 \mu \mathrm{m}$ thick, allowing sufficient ion conductance at temperatures $>35^{\circ} \mathrm{C}$. The other electrodes are thin-film metal electrodes fabricated on the same YSZ diaphragm: an $\mathrm{Ag} \mid \mathrm{AgCl}$ reference electrode for $\mathrm{pH}$ sensing, an $\mathrm{Ag} \mid \mathrm{Ag}_{2} \mathrm{~S}$ electrode for $\mathrm{H}_{2} \mathrm{~S}$ sensing, and a Pt electrode for $\mathrm{H}_{2}$ sensing. The two temperature sensors are thin film Pt resistors. Initial tests have been performed to test the functionality of the $\mathrm{pH}$ and $\mathrm{H}_{2} \mathrm{~S}$ sensors; measurements are in line with expectations.

\section{INTRODUCTION}

In situ chemical sensing in harsh geological environments is important for many applications. One example is the exploration of hydrothermal vents located at mid-ocean ridges. On the adjacent sea floor, these vents not only create rich mineral deposits that drive mining interests, but also nurture more than 1300 previously-unknown biological species that do not depend on the sun [1]. Knowledge of chemical components and quantities, especially $\mathrm{pH}$ and redox (e.g. dissolved $\mathrm{H}_{2}$ and $\mathrm{H}_{2} \mathrm{~S}$ ), can provide invaluable insight into such biological communities and lead to better understanding of the bio-geochemical environments at these locations [2-3]. Rapid and direct in situ measurement of these quantities is essential to obtain accurate and meaningful readings. However, these measurements also present significant challenges due to high temperature and high pressure that are typical in these and other geochemical environments.

Chemical sensors designed to meet the needs in these extreme geochemical conditions are few [2-5]. A sensor based on yttriastabilized-zirconia (YSZ) ion-conductive ceramic was reported for sensing of $\mathrm{pH}$, dissolved $\mathrm{H}_{2}$ and dissolved $\mathrm{H}_{2} \mathrm{~S}$, and deployed at deep-sea hydrothermal vents at temperatures up to $400^{\circ} \mathrm{C}$ and pressure levels up to $40 \mathrm{MPa}$ [2-3]. This conventional sensor had $2.5 \mathrm{~cm}$ diameter and $>30 \mathrm{~cm}$ length. Reduced sensor size is desirable to allow more precise measurement of high-gradient conditions. It can also enable spatial distribution of sensor clusters to allow simultaneous characterization at multiple sites in a biological community near the vents.

This paper reports the design and initial results of a micromachined chemical sensor based on a thin YSZ diaphragm for measurement of $\mathrm{pH}$, dissolved $\mathrm{H}_{2}$, dissolved $\mathrm{H}_{2} \mathrm{~S}$ and temperature (Fig. 1). The sensor design is intended to provide compatibility with environments that have high pressure $(>10$ $\mathrm{MPa})$ and relatively high temperature $\left(\leq 150^{\circ} \mathrm{C}\right)$, such as that in a typical biological community near the vents (Fig. 2). The sensor has $12 \mathrm{~mm}$ diameter and $\approx 6 \mathrm{~mm}$ effective length with packaging. The design details are described, along with the fabrication process. Preliminary tests of the sensor have been performed in regular laboratory settings to verify the validity of the design and the functionalities of the sensor. The measurement results are

reported.

\section{DEVICE CONCEPT}

The micromachined sensor is intended to sense $\mathrm{pH}$, dissolved $\mathrm{H}_{2}$, dissolved $\mathrm{H}_{2} \mathrm{~S}$, and temperature. To measure the three chemical quantities, three electrochemical cells are included (Table I). The YSZ $|\mathrm{HgO}| \mathrm{Hg}$ solid electrode is a common electrode used in all three electrochemical cells. It is used for $\mathrm{pH}$ sensing with a $\mathrm{Ag} \mid \mathrm{AgCl}$ reference electrode. The ion conductance of the YSZ ceramic in the electrode allows measurement of the activity of $\mathrm{H}^{+}$ ions in fluid with presence of other chemical components [6]. This electrode is also used as a reference electrode to eliminate the effect of $\mathrm{pH}$ on output voltage potentials, both in the measurement of dissolved $\mathrm{H}_{2}$ with a $\mathrm{Pt}$ sensing electrode, and in the measurement of dissolved $\mathrm{H}_{2} \mathrm{~S}$ with a $\mathrm{Ag} \mid \mathrm{Ag}_{2} \mathrm{~S}$ sensing electrode. The voltage potential between the electrodes in each cell is related
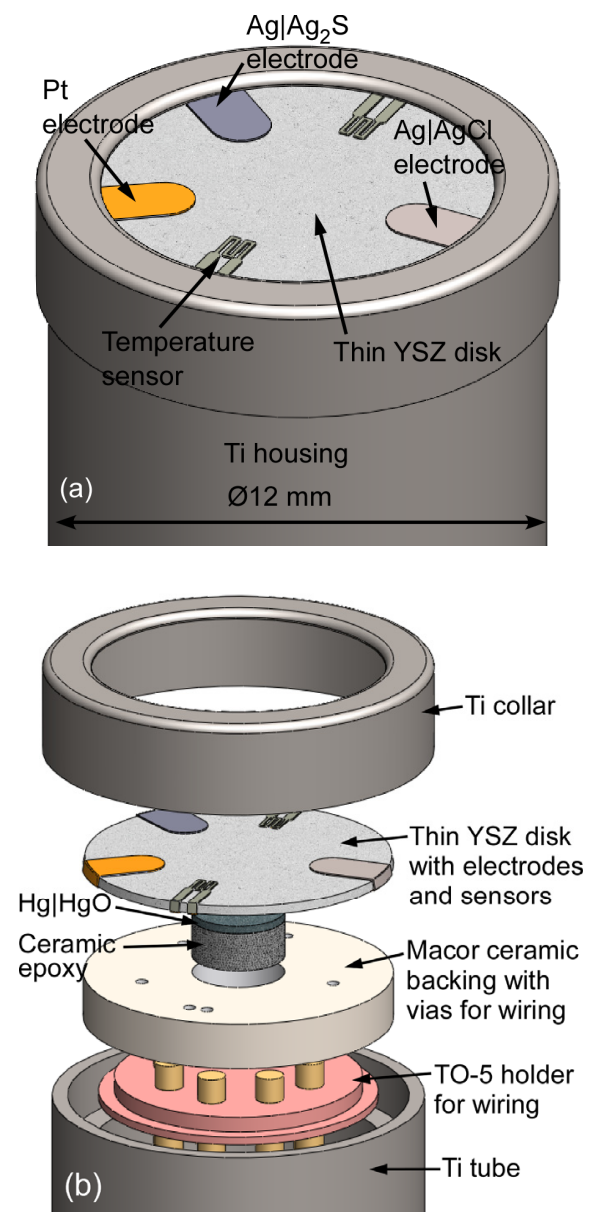

Figure 1: Schematics of the micromachined sensor proposed for analysis of geofluids in harsh environments. (a) Perspective view of the full device, showing sensing electrodes and temperature sensors (RTDs) on the thin YSZ disk and the $\$ 12 \mathrm{~mm}$ titanium housing. (b) Exploded view showing individual components inside the Ti housing.

Solid-State Sensors, Actuators, and Microsystems Workshop Hilton Head Island, South Carolina, June 3-7, 2012 


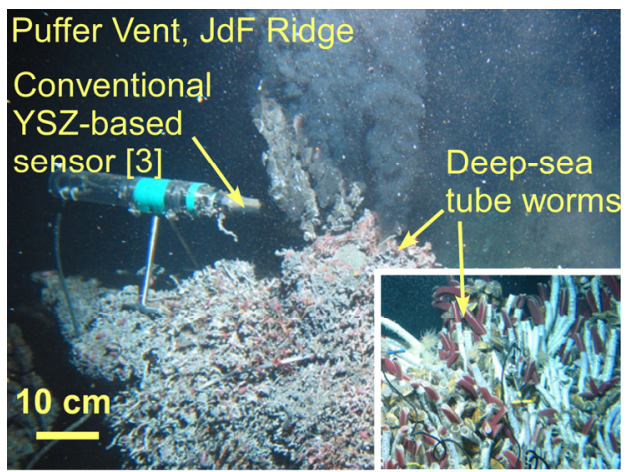

Figure 2: Exploration of deep sea hydro-thermal vents using manned submersible assets [3]. Photo shows deployment of a conventional YSZ-based sensor unit at Puffer Vent of Juan de Fuca Ridge.

Table I: Electrochemical cells and their corresponding electrodes in the micromachined chemical sensor.

\begin{tabular}{|l|l|}
\hline Sensing target & \multicolumn{1}{|c|}{ Electrochemical cell } \\
\hline $\mathrm{pH}$ & $\mathrm{Ag}|\mathrm{AgCl}|$ Target solution $|\mathrm{YSZ}| \mathrm{HgO} \mid \mathrm{Hg}$ \\
\hline Dissolved $\mathrm{H}_{2}$ & $\mathrm{Pt} \mid \mathrm{Target}$ solution $|\mathrm{YSZ}| \mathrm{HgO} \mid \mathrm{Hg}$ \\
\hline Dissolved $\mathrm{H}_{2} \mathrm{~S}$ & $\mathrm{Ag}\left|\mathrm{Ag}_{2} \mathrm{~S}\right|$ Target solution $|\mathrm{YSZ}| \mathrm{HgO} \mid \mathrm{Hg}$ \\
\hline
\end{tabular}

to the corresponding target chemical quantity by $[3,7]$ :

$$
\begin{aligned}
& V_{p H, T, P}=V_{p H}^{0}-\frac{2.303 R T}{F}\left(\log a_{\mathrm{Cl}^{-}}-\frac{1}{2} \log a_{\mathrm{H}_{2} \mathrm{O}}-\mathrm{pH}_{\mathrm{t}}\right) \\
& V_{\mathrm{H}_{2}, T, P}=V_{\mathrm{Hg} \mid \mathrm{HgO}}^{0}+\frac{2.303 R T}{2 F} \log \left(\frac{K_{H} \cdot \gamma_{\mathrm{H}_{2}} \cdot m_{\mathrm{H}_{2}}}{a_{\mathrm{H}_{2} \mathrm{O}}}\right) \\
& V_{\mathrm{H}_{2} S, T, P}=V_{\mathrm{H}_{2} \mathrm{~S}}^{0}+\frac{2.303 R T}{2 F} \log \left(\frac{K_{H} \cdot \gamma_{\mathrm{H}_{2} \mathrm{~S}} \cdot m_{\mathrm{H}_{2} \mathrm{~S}}}{a_{\mathrm{H}_{2} \mathrm{O}}}\right)
\end{aligned}
$$

where $V_{p H, T, P}, V_{H 2, T, P}, V_{H 2 S, T, P}$ are measured voltage potentials in corresponding cells, $V_{p H}^{0}$ and $V_{H 2 S}^{0}$ are the standard cell potentials at the appropriate temperatures and pressure and the values can be determined from the standard state potentials of $\mathrm{Ag}|\mathrm{AgCl}, \mathrm{Ag}| \mathrm{Ag}_{2} \mathrm{~S}$ and $\mathrm{Hg} \mid \mathrm{HgO}$ electrodes (i.e. $V_{A g \mid A g C l}^{0}, V_{A g \mid A g 2 S}^{0}$ and $V_{H g \mid H g O}^{0}$, respectively), $a$ is the activity of the chemical component, $R$ is the gas constant, $F$ is the Faraday constant, $T$ is temperature in $\mathrm{K}, p H_{t}$ is the $\mathrm{pH}$ to be measured, $K_{H}$ is the Henry's law constant, $\gamma_{H 2}$ and $\gamma_{H 2 S}$ are the activity coefficients of $\mathrm{H}_{2}$ and $\mathrm{H}_{2} \mathrm{~S}$, and $m_{H 2}$ and $m_{H 2 S}$ are the dissolved $\mathrm{H}_{2}$ and $\mathrm{H}_{2} \mathrm{~S}$ concentrations, respectively. With a DC measurement of the corresponding cell potential at the target temperature and pressure, direct sensing of each chemical quantity can be obtained. Temperature sensing is performed by two Pt-film resistance-temperature-detectors (RTD) through a measurement in resistance change of the Pt films.

The schematics of the sensor design are shown in Fig. 1 and Fig. 3. All sensor components are integrated at the end of a titanium tube. A thin YSZ disk/diaphragm is used for ion conduction to the underneath $\mathrm{Hg} \mid \mathrm{HgO}$ paste electrode. The thickness of the YSZ disk should be small enough $(<<1 \mathrm{~mm})$ to allow sufficient ion conduction for the sensing purpose at temperatures below $100^{\circ} \mathrm{C}$. The disk is also a common substrate for other thin metal-film electrodes $\left(\mathrm{Ag} \mid \mathrm{AgCl}, \mathrm{Pt}\right.$ and $\mathrm{Ag} \mid \mathrm{Ag}_{2} \mathrm{~S}$ ) and RTDs. Electrical connections from these electrodes/RTDs run over a $10^{\circ}$-tapered sidewall of the YSZ disk to the backside. No through-hole or cavity is present on the YSZ surface to avoid leakage under pressure. The YSZ disk is supported by a Macor ${ }^{\mathbb{B}}$ ceramic disk, which has feedthroughs for electrical connection

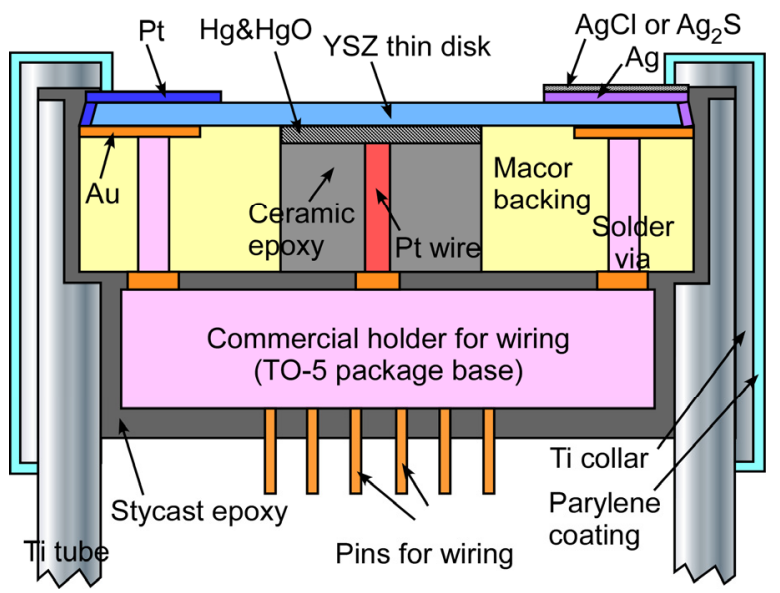

Figure 3: Schematic of the micromachined sensor in $2 D$ crosssectional view, showing integration of individual components. Note that parylene is selectively coated on the sensor external surface, covering RTDs but not other electrodes.

from the backside of the YSZ disk to a commercial TO-5 holder for cable wiring. The $\mathrm{Hg} \mid \mathrm{HgO}$ paste is sealed inside a cavity in the Macor disk by ceramic epoxy, with a Pt wire going out for electrical connection (Fig. 3). The Ti tube covers all sidewalls of internal components, and a Ti collar wraps around the edge of the YSZ front surface, minimizing potential leak path. High-strength vacuum-sealing Stycast epoxy encapsulates all internal components and seals the Ti housing. Selective coating of parylene or other polymers provides additional device sealing and electrical insulation for RTDs.

\section{DEVICE FABRICATION}

The YSZ disks were custom-made using Type ZDY-4 material (CoorsTek, Inc., CO, USA). In order to deposit thin-film metal electrodes/RTDs on the surface of the YSZ disk, a shadow mask was used. The metal films, including Pt and Ag on the front side and $\mathrm{Au}$ on the backside of the disk, were sequentially deposited by sputtering or evaporation through the corresponding patterns on the shadow mask. The thicknesses of the metal films are $200 / 1000 \AA$ for $\mathrm{Ti} / \mathrm{Pt}$, and $500 / 20000 \AA$ for $\mathrm{Ti} / \mathrm{Ag}$, and $500 / 5000 \AA$ for $\mathrm{Ti} / \mathrm{Au}$. Features were included on the shadow mask to allow alignment between different metal films. The thin-

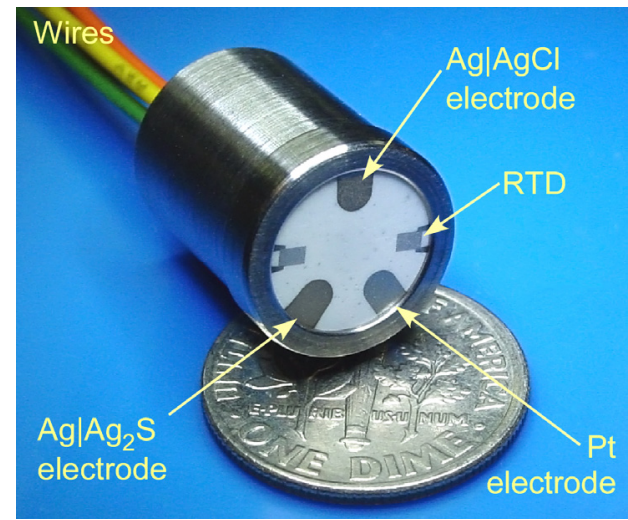

Figure 4: Photo of an integrated device on a US dime, showing $\mathrm{Ag}|\mathrm{AgCl}, \mathrm{Ag}| \mathrm{Ag}_{2} \mathrm{~S}$ and Pt electrodes and $30 \mu \mathrm{m}$-wide Pt lines for RTDs, before selective parylene coating. The $15 \mathrm{~mm}$ length of the Ti tube is for demonstration only. Effective sensor length from YSZ disk to TO-5 holder: $\approx 6 \mathrm{~mm}$. 


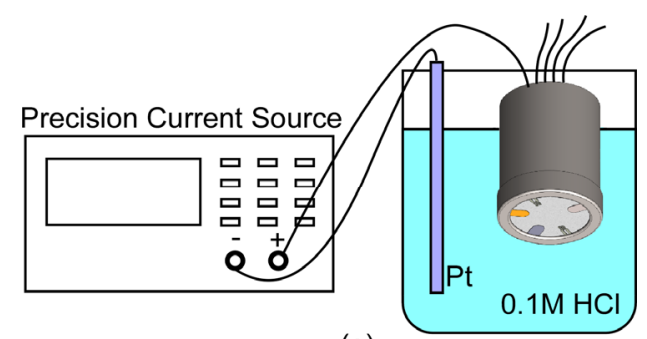

(a)

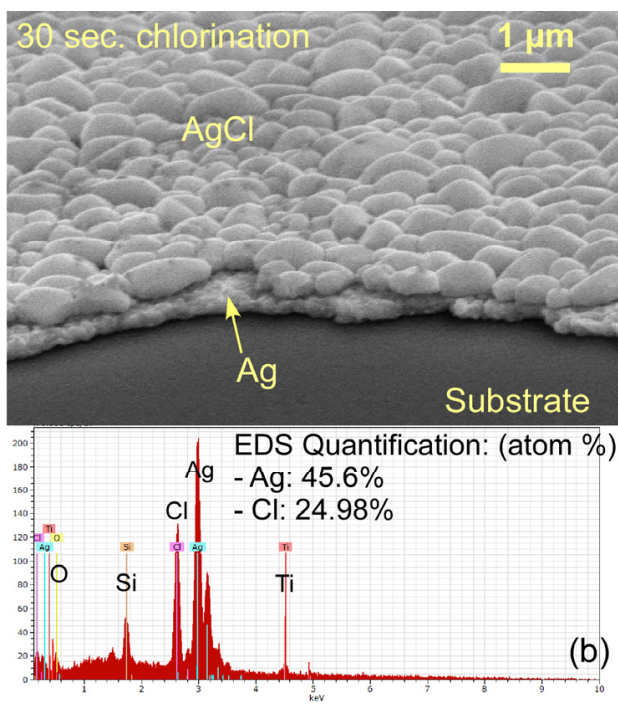

Figure 5: (a) Setup for silver chlorination. The counter electrode is a silicon wafer evaporation-coated with $5000 \AA$ platinum. Current density: $2 \mathrm{~mA} / \mathrm{cm}^{2}$. (b) SEM image and EDS analysis result of the chlorinated Ag film on a glass test substrate.

film RTDs consist of $30 \mu \mathrm{m}$-wide folded Pt lines and have a resistance of $\approx 200 \Omega$ each. The $10^{\circ}$-tapered edge of the YSZ disk allowed metal to be deposited on the slope to make the electrical connection to the backside of the disk.

The $\mathrm{Hg} \mid \mathrm{HgO}$ paste was formed by mixing $\mathrm{Hg}(99.9998 \%$ purity) and $\mathrm{HgO}$ (99.998\% purity) (Alfa Aesar, MA, USA). The Macor ceramic disk was custom-made by micromilling using material obtained from McMaster, NJ, USA. The disk has a thickness of $1.588 \mathrm{~mm}$. The center cavity on the disk for $\mathrm{Hg} \mid \mathrm{HgO}$ paste and ceramic epoxy has a diameter of $3 \mathrm{~mm}$. The electrical feedthroughs/vias have a diameter of $500 \mu \mathrm{m}$. The Ti tube and collar were custom-machined from Grade $5 \mathrm{Ti}$ alloy (Ti-6Al-4V, McMaster, NJ, USA), which has excellent mechanical strength and corrosion resistance. A photo of an integrated device is shown in Fig. 4. The effective length of the sensor from the YSZ disk to the TO-5 holder is $\approx 6 \mathrm{~mm}$.

After the sensor components were integrated, the $2 \mu \mathrm{m}$-thick $\mathrm{Ag}$ films on the YSZ disk were activated by chlorination and sulfurization, turning the $\mathrm{Ag}$ films into $\mathrm{Ag} \mid \mathrm{AgCl}$ and $\mathrm{Ag} \mid \mathrm{Ag}_{2} \mathrm{~S}$ electrodes, respectively. For silver chlorination, the sensor was dipped into an $\mathrm{HCl}$ solution with a concentration of $0.1 \mathrm{M}$ (Fig. 5a). The counter electrode was a silicon wafer with an evaporated $5000 \AA$-thick Pt film. The current density used for chlorination was $2 \mathrm{~mA} / \mathrm{cm}^{2}$. This current level has been reported to provide a uniform coating of $\mathrm{AgCl}$ with a grain size around $1 \mu \mathrm{m}$ [8]. For silver sulfurization, an electrochemical cell of $\mathrm{Ag}|\mathrm{AgCl}| \mathrm{S}^{2-}$ $\left|\mathrm{Ag}_{2} \mathrm{~S}\right| \mathrm{Ag}$ was formed. The electrodes in the cell were a separately-built $\mathrm{Ag} \mid \mathrm{AgCl}$ electrode and the target $\mathrm{Ag}$ film on the sensor; a $\mathrm{Na}_{2} \mathrm{~S}$ solution was used as the electrolyte. The $\mathrm{Ag} \mid \mathrm{AgCl}$ electrode that was formed on the sensor was protected by a photoresist coating. The target $\mathrm{Ag}$ film on the sensor was sulfurized by externally shorting the cell.

\section{EXPERIMENTAL RESULTS}

The chlorination procedure was characterized using a Ag film coated on a glass substrate. This Ag film was deposited by sputtering in the same session when the Ag films on the YSZ disks were deposited. The obtained $\mathrm{Ag} \mid \mathrm{AgCl}$ film is shown in the upper portion of Fig. 5(b). The $\mathrm{AgCl}$ layer has a grain size of $\approx 1 \mu \mathrm{m}$ as expected and is uniformly coated on the remaining Ag layer. Energy dispersive spectroscopy (EDS) was done to characterize the electrode and the obtained spectrum is shown in the lower portion of Fig. 5(b). According to the EDS quantification result, the electrode has $45.6 \%$ atom percentage for $\mathrm{Ag}$ and $24.98 \%$ for $\mathrm{Cl}$, suggesting a ratio of $\approx 1$ between $\mathrm{Ag}$ and $\mathrm{AgCl}$.

As a common electrode for all three chemical sensing functions, the $\mathrm{YSZ}|\mathrm{HgO}| \mathrm{Hg}$ solid electrode is the core component of the sensor. It also presented the most challenge in the fabrication of all four electrodes. $\mathrm{pH}$ measurements in a regular laboratory setting using this electrode can provide direct verification of its integrity and functionality. In these measurements, hydrochloric acid solutions with $\mathrm{pH}$ ranging in 25.5 were used. The $\mathrm{pH}$ values were verified with a double-junction pH meter (Oakton ${ }^{\circledR}$ pHTestr $3+$ ), which has an accuracy of 0.1 . Before it was used, the meter was calibrated with certified $\mathrm{pH}$ buffer solutions from Fisher Scientific. The $\mathrm{HCl}$ solutions were heated on a hotplate to $35^{\circ} \mathrm{C}$, and a thermocouple was used to monitor the solution temperature. The voltage potential between the $\mathrm{Ag} \mid \mathrm{AgCl}$ and $\mathrm{YSZ}|\mathrm{HgO}| \mathrm{Hg}$ electrodes on the sensor was read out by an Agilent 34401A multimeter with its input resistance set at $>10 \mathrm{G} \Omega$.

As shown in Fig. 6, the plot of measured $V_{p H, T, P}$ vs. pH has a slope of $123.2 \mathrm{mV} / \mathrm{pH}$. This slope is about double the Nernst slope of $61.14 \mathrm{mV} / \mathrm{pH}$ at $35^{\circ} \mathrm{C}$. This is due to the presence of both $\mathrm{H}^{+}$ and $\mathrm{Cl}^{-}$ions in the solutions. The $\mathrm{YSZ}|\mathrm{HgO}| \mathrm{Hg}$ electrode is sensitive to $\mathrm{H}^{+}$ions while the $\mathrm{Ag} \mid \mathrm{AgCl}$ electrode is sensitive to $\mathrm{Cl}^{-}$ ions. In a regular laboratory environment, the strong $\mathrm{HCl}$ acid can generate equal concentrations of $\mathrm{H}^{+}$and $\mathrm{Cl}^{-}$in the solution

$$
\log a_{\mathrm{Cl}^{-}}=\log a_{\mathrm{H}^{+}}=-\mathrm{pH}_{\mathrm{t}}
$$

Assume the activity of $\mathrm{H}_{2} \mathrm{O}$ in the regular lab environment is 1 ,

$$
\log a_{\mathrm{H}_{2} \mathrm{O}}=0
$$

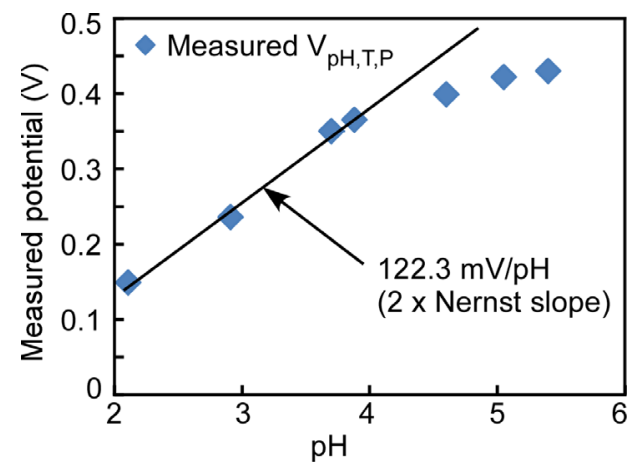

Figure 6: Measured potential between the $\mathrm{Ag} \mid \mathrm{AgCl}$ and $Y \mathrm{SZ}|\mathrm{HgO}| \mathrm{Hg}$ electrodes, vs. $\mathrm{pH}$ of $\mathrm{HCl}$ solutions at $35^{\circ} \mathrm{C}$. The straight line plots double the Nernst slope of $61.14 \mathrm{mV} / \mathrm{pH}$ at $35^{\circ} \mathrm{C}$ for comparison. This slope is due to the effect of both $\mathrm{H}^{+}$and $\mathrm{Cl}^{-}$. For $\mathrm{pH}>4, V_{p H, T, P}$ saturates. This may be due to activity of $\mathrm{H}^{+}$and $\mathrm{OH}^{-}$from $\mathrm{H}_{2} \mathrm{O}$ at higher $\mathrm{pH}$, and also of low $\mathrm{Cl}^{-}$for $\mathrm{Ag} \mid \mathrm{AgCl}$ electrode. 
Sensor Test with 2-12 $\mathrm{mMol} / \mathrm{kg} \mathrm{H}_{2} \mathrm{~S}$ in Water

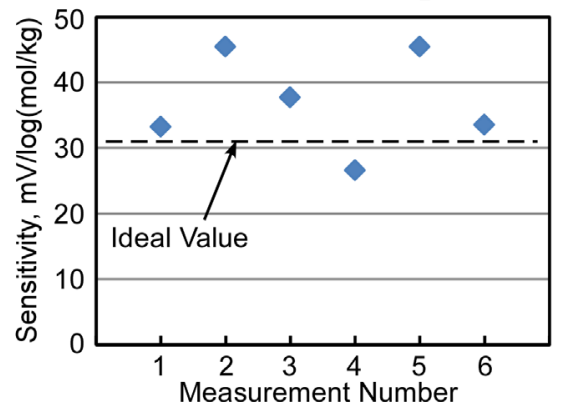

Figure 7: Sensitivity of measured sensor responses to the $\mathrm{H}_{2} \mathrm{~S}$ concentration in water, and comparison to the ideal value of 30.6 $\mathrm{mV} / \log (\mathrm{mol} / \mathrm{kg})$. The sensor output potential was taken between the $\mathrm{YSZ}|\mathrm{HgO}| \mathrm{Hg}$ and $\mathrm{Ag} \mid \mathrm{Ag}_{2} \mathrm{~S}$ electrodes at $35^{\circ} \mathrm{C}$. Differences from the ideal value are related to uncertainty in the $\mathrm{H}_{2} \mathrm{~S}$ concentration.

Equation (1), which provides the relationship between the measurement voltage potential and $\mathrm{pH}$, can be simplified as

$$
V_{p H, T, P}=V_{p H}^{0}+2 \cdot \frac{2.303 R T}{F} \mathrm{pH}_{\mathrm{t}}
$$

This indicates that the expected slope of $V_{p H, T, P}$ vs. $\mathrm{pH}$ is double the Nernst slope. At $35^{\circ} \mathrm{C}$, the expected slope is $122.3 \mathrm{mV} / \mathrm{pH}$, and the measured value is comparable to it. This indicates the validity of the $\mathrm{pH}$ sensor design and the integrity of the YSZ $|\mathrm{HgO}| \mathrm{Hg}$ core electrode.

Also shown in Fig. 6, $V_{p H, T, P}$ saturates at $\mathrm{pH}$ values $>4$. This may be due to activity of $\mathrm{H}^{+}$and $\mathrm{OH}^{-}$from $\mathrm{H}_{2} \mathrm{O}$ at higher $\mathrm{pH}$, and also low $\mathrm{Cl}^{-}$activity at low $\mathrm{HCl}$ concentration for the $\mathrm{Ag} \mid \mathrm{AgCl}$ electrode.

$\mathrm{H}_{2} \mathrm{~S}$ sensing was tested using hydrogen sulfide water (Grainger, Inc., USA) with varying concentration estimated in the range of $2-12 \mathrm{mMol} / \mathrm{kg}$. The sensor output potential was taken between the $\mathrm{YSZ}|\mathrm{HgO}| \mathrm{Hg}$ and $\mathrm{Ag} \mid \mathrm{Ag}_{2} \mathrm{~S}$ electrodes at $35^{\circ} \mathrm{C}$. The sensitivity of the sensor - i.e. the change in output potential per unit change in the $\mathrm{H}_{2} \mathrm{~S}$ concentration - was typically 26-45 $\mathrm{mV} / \log (\mathrm{mol} / \mathrm{kg})$ (Fig.7). The ideal value is $30.6 \mathrm{mV} / \log (\mathrm{mol} / \mathrm{kg})$ according to Equation 3. Differences from the ideal value are related to uncertainty in the $\mathrm{H}_{2} \mathrm{~S}$ concentration, which can vary from the estimated value due to reasons such as oxidation of dissolved sulfide into sulfate.

\section{DISCUSSION}

As described in the experimental results, the sensor responds to both $\mathrm{H}^{+}$and $\mathrm{Cl}^{-}$concentrations in the $\mathrm{pH}$ measurement without a buffer solution that provides a constant $\mathrm{Cl}^{-}$concentration for the $\mathrm{Ag} \mid \mathrm{AgCl}$ electrode. This makes it difficult to use the sensor in regular applications, though approaches such as a coating of $\mathrm{KCl}$ gel on the $\mathrm{Ag} \mid \mathrm{AgCl}$ electrode can provide simple solutions [8]. However, in the target environments at hydrothermal vents, the sea water presents a saturated $\mathrm{Cl}^{-}$concentration that is mostly constant, rendering these additional techniques unnecessary.

The sensor has been tested for $\mathrm{pH}$ and $\mathrm{H}_{2} \mathrm{~S}$ measurement in a laboratory environment. Although the sensor is designed for high pressure and relatively high temperature applications, its performance in harsh environments remains to be evaluated. This, together with the sensing function for dissolved $\mathrm{H}_{2}$ and temperature, will be verified in future efforts.

\section{CONCLUSIONS}

A micromachined chemical sensor intended for applications in harsh environments has been design and implemented. Sensing functions for $\mathrm{pH}$, dissolved $\mathrm{H}_{2}$ and $\mathrm{H}_{2} \mathrm{~S}$, and temperature are included. Techniques for silver chlorination and sulfurization have been developed and characterized to activate the $\mathrm{Ag} \mid \mathrm{AgCl}$ reference electrode for $\mathrm{pH}$ sensing and the $\mathrm{Ag} \mid \mathrm{Ag}_{2} \mathrm{~S}$ sensing electrode for $\mathrm{H}_{2} \mathrm{~S}$ sensing. Initial measurement results for $\mathrm{pH}$ and $\mathrm{H}_{2} \mathrm{~S}$ sensing in a regular laboratory environment indicate validity of the sensor design and the integrity of the $\mathrm{YSZ}|\mathrm{HgO}| \mathrm{Hg}$ core component. Further characterization of sensor functions as well as device testing under harsh environments will be pursued in future effort.

\section{ACKNOWLEDGEMENT}

The research effort was supported in part by the National Science Foundation. Facilities used for this research include the Lurie Nanofabrication Facility (LNF) operated by the Solid-State Electronics Laboratory (SSEL) at the University of Michigan.

\section{REFERENCES}

[1] S. Begley, "Mining's Final Frontier", Newsweek, pp. 50-52, Sep. 20, 2010

[2] K. Ding and W.E. Seyfried, Jr., "Direct $\mathrm{pH}$ measurement of $\mathrm{NaCl}$-bearing fluid with an in situ sensor at $400^{\circ} \mathrm{C}$ and 40 megapascals," Science, 272, pp. 1634-1636, 1996

[3] K. Ding and W.E. Seyfried, Jr., "In situ measurement of $\mathrm{pH}$ and dissolved $\mathrm{H} 2$ in mid-ocean ridge hydrothermal fluids at elevated temperatures and pressures," Chem. Rev., 107, pp. 601-622, 2007

[4] Y. Pan and W.E. Seyfried, Jr., "Experimental and theoretical constraints on $\mathrm{pH}$ measurements with an Iridium oxide electrode in aqueous fluids from 25 to $175^{\circ} \mathrm{C}$ and $25 \mathrm{MPa}$.," J. Solution Chem., 37, pp. 1051-1062, 2008

[5] L.W. Niedrach, "A new membrane-type $\mathrm{pH}$ sensor for use in high temperature-high pressure water," J. Electrochem. Soc., 127 , pp. 2122-30, 1980

[6] D.D. Macdonald, S. Hettiarachchi, and S.J. Lenhart, "The thermodynamic viability of yttria-stabilized zirconia $\mathrm{pH}$ sensors for high temperature aqueous solutions," J. Solution Chem., 17(8), pp. 719-732, 1988

[7] K. Ding, W.E. Seyfried, Jr., M.K. Tivey, and A.M. Bradley, "In situ measurement of dissolved $\mathrm{H}_{2}$ and $\mathrm{H}_{2} \mathrm{~S}$ in hightemperature hydrothermal vent fluids at the Main Endeavour Field, Juan de Fuca Ridge," Earth and Planetary Science Letters, 186(3-4), pp. 417-25, Apr. 2001

[8] I-Y. Huang, R.-S. Huang, and L.-H. Lo, "Improvement of integrated $\mathrm{Ag} / \mathrm{AgCl}$ thin-film electrodes by $\mathrm{KCl}$-gel coating for ISFET applications," Sensors and Actuators B: Chemical, 94(1), pp. 53-64, 2003

\section{CONTACT}

*Tao Li, 1301 Beal Ave., Ann Arbor, MI, 48109, USA; tel: 1-734615-7983; fax: 1-734-763-9324; email: litz@umich.edu 


\title{
A SMART BIOCHIP WITH HAND-HELD ANALYZER FOR MONITORING SPECIFIC ENVIRONMENTAL BIOMARKERS
}

\author{
Jungyoup (Jay) Han ${ }^{1 *}$, Junhai Kai ${ }^{l}$, Se Hwan Lee ${ }^{l}$, Aniruddha Puntambekar ${ }^{1}$, \\ Ji-Youn Lim ${ }^{2}$, Donggeun Sul ${ }^{2}$, and Chong H. Ahn ${ }^{1,3}$ \\ ${ }^{1}$ Siloam Biosciences, Inc., Cincinnati, USA \\ ${ }^{2}$ Graduate School of Medicine, Korea University, Seoul, Republic of Korea \\ ${ }^{3}$ School of Electronics \& Computing Systems, University of Cincinnati, USA
}

\begin{abstract}
A new smart disposable biochip with a hand-held analyzer for monitoring specific environmental proteomic biomarkers has been designed, developed and fully characterized using polymer microfabrication technology and chemiluminescence immunoassay detection method. High sensitivity, small size, and low cost of the developed system are very suitable for exposure monitoring as a point-of-care (POC) platform. In this paper, Rho GDP inhibitor and triose phosphate isomerase as potential environmental biomarkers of di(2-ethylhexl) phthalate (DEHP) and human disease associate with DEHP were used for the developed biochip with the analyzer, demonstrating a POC platform. The smart biochip based analysis only requires $<\sim 30$ minutes, comparing to $4 \sim 5$ hours of a conventional ELISA.
\end{abstract}

\section{INTRODUCTION}

As the most commonly used phthalate ester and well-known endocrine-disruptors, di(2-ethylhexyl) phthalate (DEHP) is widely used in variety of consumer products, such as building products, car products, clothing, food packaging, children's products and some medical devices made of polyvinyl chloride. DEHP, an environmentally toxic substance has been reported to have cytotoxic, immunotoxic, genotoxic and reproductive toxic properties, and in long term feed toxicological studies it has been shown to be carcinogenic in mice and rats [1].

The development of biochip has evolved through rapid growth over the last decade and has shown numerous applications in a number of biochemical analysis operations such as DNA analysis, proteomics analysis and clinical analysis, [2,3]. A significant application area for this technology is clinical diagnostics. The detection of specific diseases is possible by incorporating biomarkers specific to such agents. The early, rapid, and sensitive point-of-care testing (POCT) of the disease state becomes a vital goal for clinical diagnoses. So the microfluidic chips based on MEMS (Micro Electro Mechanical Systems) has been introduced for biomarker measurements for disease analysis and prevention. Such devices have many preferable advantages such as low sample/reagent volume, short analysis time, less sample waste, cost effectiveness, compatible manufacture processes, and potential for making disposable devices.

The development of biochip for clinical analysis has rapidly evolved over the last decade. Initial biochip research has also been followed by the development of $\mu$ TAS (Micro total analysis system), which has shown numerous applications in biochemical analysis. The explosive development of biochip for $\mu$ TAS has been motivated by its capabilities in miniaturizing size, reducing detection time, improving sensitivity, and providing ease-in-operation. Specifically, there has been a large demand for the development of fully integrated plastic microfluidic immunoassay chips, which are ideal solutions for point-of-care testing for monitoring human biomarkers level in disease analysis and prevention. Such devices have many favorable advantages such as low sample/reagent volume, short analysis time, less sample wastage, cost effectiveness, compatible manufacturing processes, and potential for making disposable devices.

The paper is organized as follows: first we introduce a protocol of the chemiluminescence-based sandwich immunoassay sequence in the biochip and design scheme of the developed biochip with solid propellant nitrogen gas generator. Next configuration of the developed hand-held analyzer and biochip are demonstrated. Then, result of the testing detection of biomarkers and operation of the developed hand-held analyzer with biochip is discussed.

\section{DESIGN AND FABIRCATION}

\section{Solid Phase Immunoassay on biochip}

Sandwich immunoassay is a kind of solid phase immunoassay applied for the determination of macromolecular antigens, e.g., protein and peptide, where simultaneous binding of two antibodies to the antigen is allowed without steric hindrance. The sample is incubated onto a solid phase, which is coated with an excess of affinity-purified anti-analyte (capture antibody) and blocked by high concentration dummy protein solution.

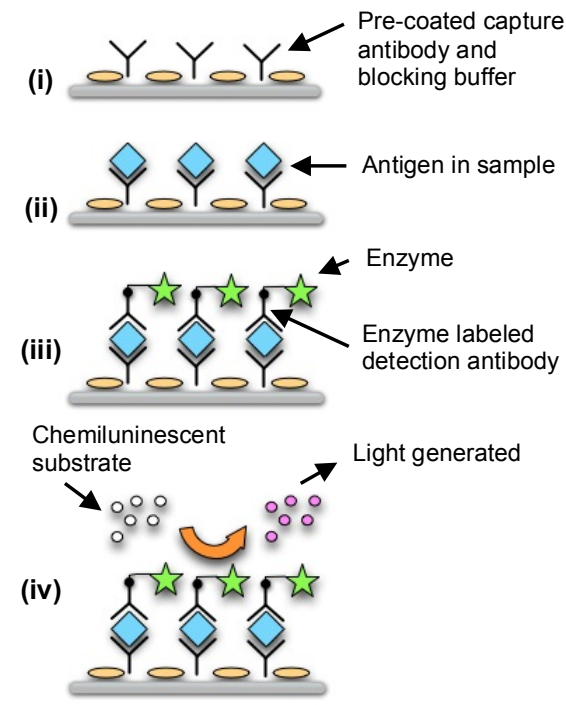

Figure 1: Protocol of the chemiluminescence-based sandwich immunoassay sequence in the biochip: (i) incubating capture anti-body and blocking; (ii) incubating target analyte; (iii) incubating enzyme labeled detection antibody; and (iv) catalyzing chemiluminescent substrate.

During the subsequent incubation, the capture antibody binds the analyte specifically. All other sample constituents are washed out and then bound analyte is quantified in a second step by adding an excess of labeled anti-analyte antibody (detection antibody). After incubation the unbound antibody is washed out and the signal from the solid-phase-bound detection antibody is directly related to the analyte concentration in the sample. For 
chemiluminescence immunoassay, the detection antibody is labeled with chemiluminescence enzyme that catalyzes chemiluminescence substrate to generate the light signal. Figure 1 shows the protocol of chemiluminescence based sandwich immunoassay.

Passive adsorption of protein follows typical saturation principles. In addition, the saturation will occur in molecular density in range of $10^{-9} \sim 10^{-8} \mathrm{~mol} / \mathrm{m}^{2}$ in most hydrophobic polymer surface depending on the protein molecular weight, solution condition, and surface condition. Increasing surface-volume ratio and shortening diffusion distance should decrease the incubation time of protein adsorption on the solid surface. That means the assay time for solid phase immunoassay will be significantly reduced by using microfluidic biochips.

\section{Microfluidic "Smart" Biochip}

Figure 2 illustrates the developed biochip for simplicity. The biochip is composed of six (6) microfluidic chambers for assay reagents and sample. The capture antibody is pre-conjugated onto the detection chamber. Four chambers are preloaded with reagent solution: detection antibody, $1^{\text {st }}$ washing buffer, $2^{\text {nd }}$ washing buffer and chemiluminescence substrate. A two-step buffer wash ensures that any residue of the HRP-labeled secondary antibody does not interact with the chemiluminescence substrate to produce a false signal. The empty "sample" chamber will be loaded with target sample before testing.

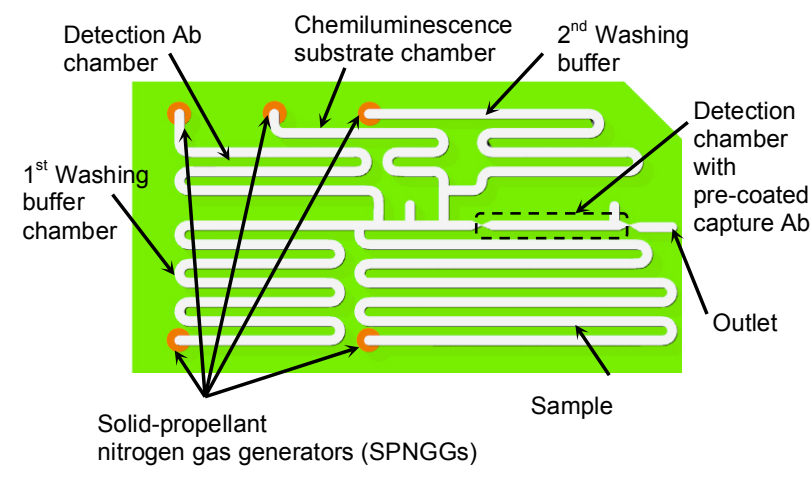

Figure 2: Schematic diagram of the developed "smart" biochip $(60 \mathrm{~mm} \times 30 \mathrm{~mm})$, which has microchannels for detection and pre-coated capture antibody, sample solution, washing buffers and substrate solution.

As shown in Figure 2, the biochip contains a detection chamber where the capture antibody was coated prior to assembly. After assembly, all the microfluidic channels in the biochip were coated with a blocking agent to minimize non-specific adsorption. Following this, all the reagents and buffer solutions were loaded using capillary sampling. Using selective surface modification techniques we can ensure that only the reagent and buffer chambers are hydrophilic whereas the remaining portions are strongly hydrophobic. The passive valves at the end of each chamber, and the air gap channels further ensure that the liquids only fill up the relevant chambers. Following this step, each of the reagent/buffer inlets in sealed to ensure that no liquid flows out when pressure is applied.

A key advantage of the developed biochip is the solid-propellant based microfluidic pump scheme [4]. This allows us to develop a fully integrated biochip that includes all the fluidic components equipped on-chip. Eliminating bulky, power-hungry components such as pumps affords size and cost benefits whereas eliminating fluidic interconnects to such components improves the chip reliability.
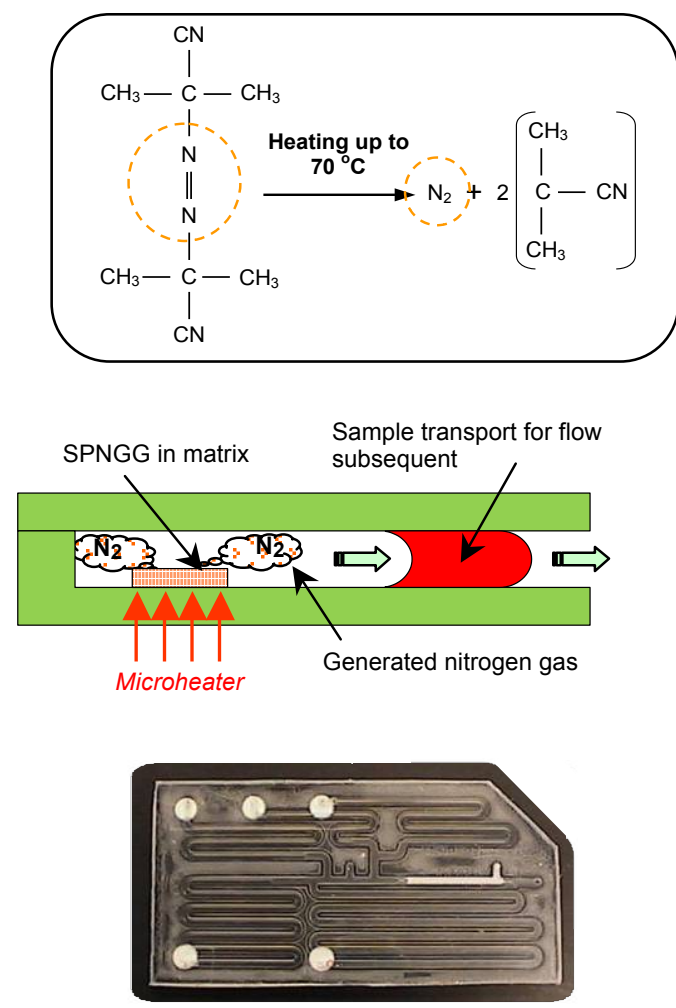

Figure 3: (a) Decomposition mechanism of the solid-propellant nitrogen gas generator (SPNGG); (b) schematic diagram of solid-propellant nitrogen gas generator (SPNGG) operation [4] and (c) photograph after assembly of biochip with SPNGGs (5 circular spots at the end of the chambers shown)

The functional pressure generator consists of a solid chemical propellant positioned on a resistive microheater. The solid propellant (AIBN: azobis-isobutyronitrile) decomposes at $70^{\circ} \mathrm{C}$ and releases non-toxic Nitrogen gas a by-product as shown in Figure 3(a). This device can be easily integrated with the biochip using standard lithography/screen-printing techniques. The amount of Nitrogen generated is governed by the applied thermal power and can be easily regulated. Due to its compact size, easy fabrication, easy integration, high reliability, biologically inert gas output, and functionality of gas generation, this pressure generator serves as an excellent driving mechanism for the biochip application

\section{Hand-held Analyzer}

We have developed a chemiluminescence based electronic analyzer which is capable of analyzing low-level light signal output from a dedicated biochip and controls the flow sequence of the biochip and captures the light signal from the biochip using an in-built photo detection circuitry and displays the captured data as a corresponding concentration value, shown in Figure 4 . The analyzer can be configured for use of a biochip towards both enzyme activity detection and protein biomarker concentration measurement using sandwich immunoassay protocol.

The analyzer is comprised of two major user interface controls: a) keypad and b) 16 character LCD display. In addition to the interface, the analyzer also incorporates a PS/2 data port 
with USB communication protocol. The analyzer can be connected to a personal computer (PC) and programmed using dedicated software installed on the computer.

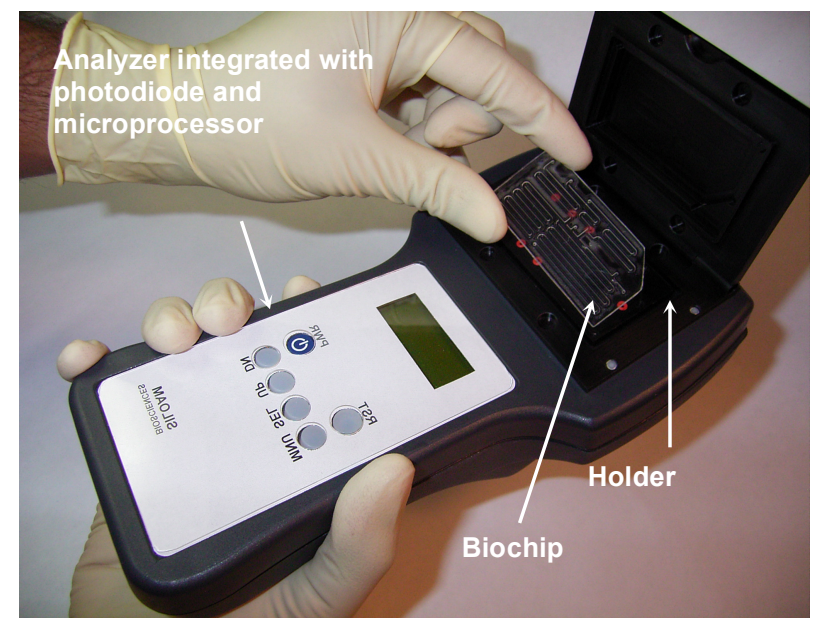

Figure 4: Developed hand-held analyzer integrated with photodiode and microprocessor

The microprocessor unit is built on the commercially available microprocessor PIC16F877A from Microchip Inc. The microprocessor is integrated with digital $\mathrm{I} / \mathrm{O}$ ports and 8 analog input channels. The system has two $5 \mathrm{~V}$ regulated power supplies: one supply is dedicated for microprocessor alone and the second $5 \mathrm{~V}$ regulated power supply is utilized for all the peripheral devices used along with the system. The microprocessor configured on the mother board controls the heater board which drives microheaters for operation of SPNGGs with pre-programmed software and receives light signal thru the optical detection module.

The holder was specially designed for tight light sealing and integration of five (5) microheaters underneath of SPNGGs on a biochip well aligned. Optical signal detection module is attached to the bottom of the holder with a hollow hole aligned with the detection chamber of the biochip.

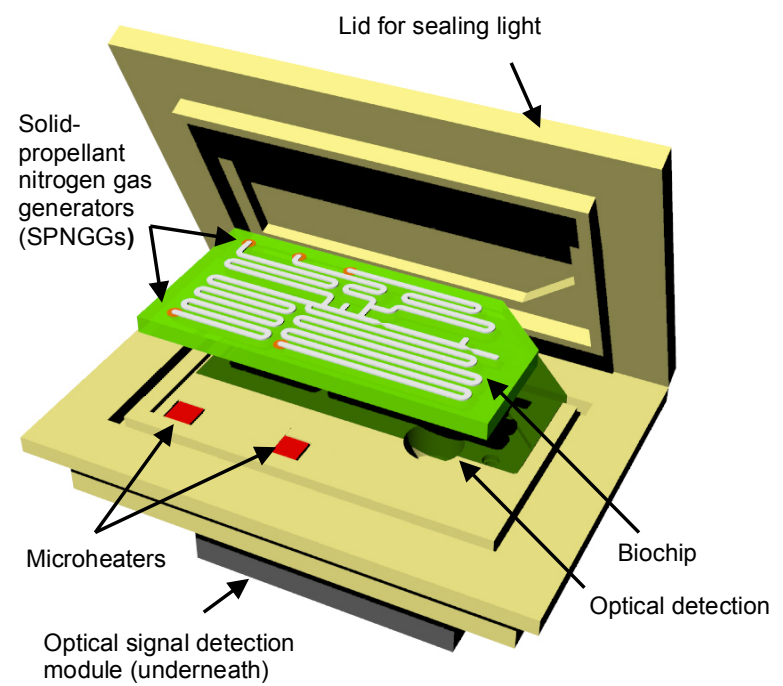

Figure 5. Schematic diagram of developed biochip, holder and optical signal detection module. Five (5) microheaters are deposited on the holder, which are aligned with solid-propellant nitrogen gas generators (SPNGGs) on the biochip.

\section{RESULT AND DISCUSSION}

We have developed the fully integrated biochip including solid-propellant nitrogen gas generators for evaluating the fluidic sequencing of the biochip with sandwich immunoassay. Microphotographs of the biochip are shown is Figure 6. In the paper we used five SPNGGs. After loading target analyte into the sample chamber, the first SPNGG fired to deliver target analyte to the detection chamber pre-coated with capture antibody. Then the second SPNGG to deliver the detection antibody into the detection chamber, pushing the target analyte incubated at the detection chamber for the previous step. With completion of second flow, the third SPNGG were used for delivery of the $1^{\text {st }}$ and $2^{\text {nd }}$ washing buffer, sequentially. After ignition of the fifth SPNGG for substrate delivery the analyzer read the optical signal developed from the detection chamber. Note that this image sequence uses color dyes for visualization.

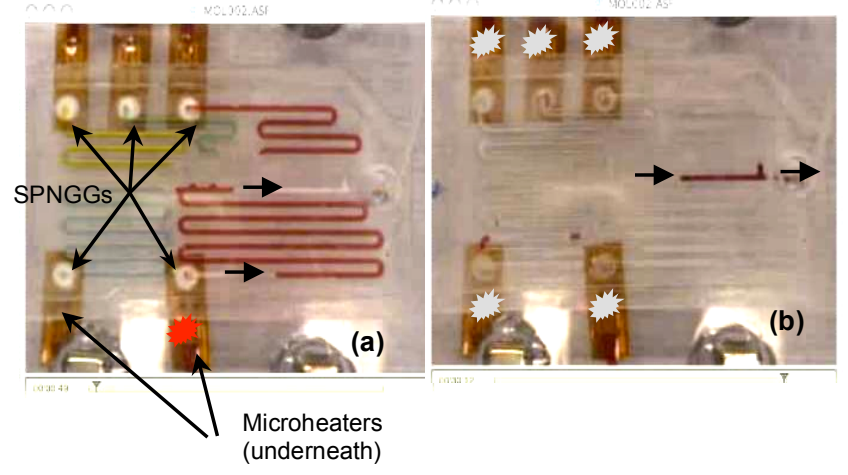

Figure 6: Operation of the smart biochip with five SPNGGs, microheaters underneath each SPNGG, and chambers: (a) the sample is delivered to detection chamber by the first SPNGG and (b) after completing all immunoassay sequences, the biochip is ready for detection of optical signal using the analyzer.

The SPNGG successfully delivered the reagents at controlled rates ( 22 minute) for completing on-chip assay. By modulating the nitrogen gas generation rate of SPNGG, we can optimize the flow rates and delivery times to ensure that the immunoassay sequence is completed satisfactorily. During optimization of the flow rate, there were significant "backflow" where the assay reagent entered a channel adjoining the detection chamber. For example, the sample partially entered the delivery channel for detection antibody and buffer/substrate. However, using the two-step wash sequence, any residue due to backflow can be pumped out before unwanted interactions.

Previously we have reported identification of toxicological biomarkers of DEHP [5]. Through the western blotting analysis for HepG2 secreted proteins, we have clearly shown three (3) up-regulated expressions of proteins, Rho-GDP initiator (Rho-GDI), gelsolin, and triose phosphate isomerase (TIM). A low concentration of DEHP led to up-regulation of Raf kinase inhibitory protein (RKIP) in HepG2 cells, while a high concentration of DEHP reduced the expression of RKIP. From this result we selected Rho GDP initiator and TIM as potential phthalate biomarkers.

As seen in Figure 7, the chemiluminescence -based sandwich immunoassay of selected proteins on the developed smart biochip 
with the hand-held analyzer was successfully evaluated $\left(>R^{2}=0.99\right.$ for both Rho-GDI and TIM).
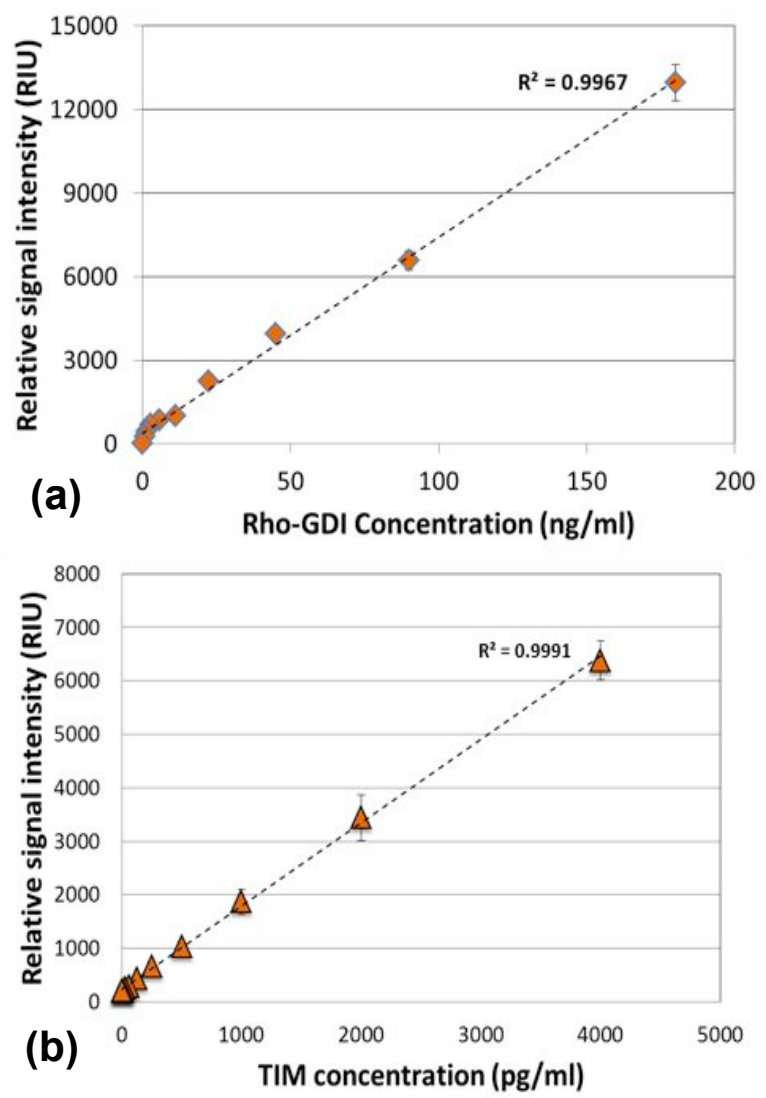

Figure 7: Immunoassay test results for concentrations of (a) Rho-GDI and (b) Triose phosphate isomerage (TIM) using developed smart biochip with hand-held analyzer. This clearly demonstrates the ability of the developed biochip and analyzer for high sensitivity detection of specific phthalate biomarkers.

In case of POCT application, a battery powered hand-held photodiode based optical analyzer was developed and characterized. By combining with the hand-help analyzer, the biochip exhibits powerful capabilities as the key sensing unit in terms of rapid and accurate point-of-care testing for clinical diagnostics. The features of high sensitivity and rapid analysis from the proposed system makes it uniquely well suited for POCT of specific environmental biomarkers for early warning of disease.

\section{ACKNOWLEDGEMENT}

This work was partially supported by the Ministry of Environment of South Korean Government as "The Eco-Technopia 21 project" (2009-09001-0075-0).

\section{REFERENCES}

[1] R Kavlock, D Barr, K Boekelheide, W Breslin, "NTP-CERHR expert panel update on the reproductive and developmental toxicity of di(2-ethylhexyl) phthalate," Reproductive Toxicology. 22, 291-399 (2006)

[2] C Malines, M Niggemann, and BD MacCraith, "Multi-analyte optical chemical sensor employing a plastic substrate," Measurement Science and Technology, 11(8) 1105-1110, (2000)
[3] R Ehrnstorm, "Miniaturization and integration: challenges and breakthrough in microfluidics," Lab on a Chip, 2, $26 \mathrm{~N}-30 \mathrm{~N}(2002)$

[4] J Han, S Lee, and C Ahn, "A disposable on-chip pressure actuator using IR-induced thermolysis for sample transport," MicroTAS 2006, Kyoto, Japan 521-523 (2006)

[5] S Choi, SY Park, J Jeong, E Cho, S Phark, M Lee, D Kwak, JY Lim, D Sul, "Identification of toxicological biomarkers of di(2-ethylhexyl) phthalate in proteins secreted by HepG2 cells using proteomic analysis," Proteomics, 10(9), 1831-46 (2010)

\section{CONTACT}

*J. Han, tel: +1-513-429-2976; jhan@siloambio.com 


\title{
ADVANCING NEXT-GENERATION PROTEOMICS: A POLYMER-PATTERNED MICROCHAMBER ENABLES INTEGRATION OF THE DISTINCT PROTEIN SEPARATIONS COMPRISING TWO-DIMENSIONAL ELECTROPHORESIS
}

\author{
Augusto M. Tentori, Alex J. Hughes, and Amy E. Herr \\ UC Berkeley/UCSF Joint Graduate Group in Bioengineering, Berkeley, CA, USA
}

\begin{abstract}
We introduce a new microfluidic approach for rapid, integrated two-dimensional electrophoresis (2DE), which uses a fundamentally different design strategy for integrating two the distinct assay stages of isoelectric focusing (IEF) and size-based electrophoretic separations. A novel device microchamber architecture coupled with spatially heterogeneous polymers enables the separated species from the $1^{\text {st }}$ dimension to be transferred for sizing separations without discretization into individual side channels. The carrier ampholyte based $\mathrm{pH}$ gradient is confined to the microchamber by incorporating immobilines into the polyacrylamide (PA) gel regions flanking the microchamber. The resulting $\mathrm{pH}$ is both linear and stable, with cathodic drifts $<3 \mu \mathrm{m} / \mathrm{min}$. Sample loading and focusing is rapidly achieved in $<30 \mathrm{~min}$. Species with isoelectric points ( $\mathrm{pI}$ ) of $<0.1 \mathrm{pH}$ units can be resolved and we estimate peak capacities of $\sim 100$ in the 1 st dimension. Protein transfer (enabled by ionic mobilization) and separation are rapid $(<10 \mathrm{~min})$ and preserve $1^{\text {st }}$ dimension separation information with band broadening factors $<1.3 \mathrm{x}$ and position drifts of $<50 \mu \mathrm{m}$.
\end{abstract}

\section{INTRODUCTION}

In biological processes, proteins are dynamic, ubiquitous conduits for transfer of information into action. Proteomic assays are central to the study of protein-mediated signaling. In particular, multidimensional protein separations enable the specific identification of protein species even amidst complex sample backgrounds.

Conventional 2DE integrates two serial assays to separate proteins by isoelectric point $(\mathrm{pI})$ and size [1]. Separating complex protein samples by IEF and protein sizing yields two physicochemical properties for each protein and enables the identification of species with a higher degree of precision then available from a single stage assay. For example, protein size separations are used for the identification of specific proteins; combined with charge separations, specific isoforms can also be identified [2]. Additionally, 2D separations provide better separation resolution and higher peak capacities compared to $1 \mathrm{D}$ assays, making 2D assays more suitable for the analysis of complex biological samples [3].

While powerful, conventional benchtop multidimensional separations are low throughput, semi-quantitative, and require manual intervention by a trained operator. Progress in proteomics is hindered by limited innovation in analytical technology.

Consequently, we introduce the development of a new microfluidic 2DE technology, as is critical to advancing the much needed 'proteomics revolution'. Unfortunately, conventional 2DE is labor and time intensive, making the assay a bottleneck for protein studies. Prior efforts to develop microfluidic 2DE have attempted to overcome these limitations, but suffer from information losses during transfer to the second stage. The as-ofyet unreported work we detail here introduces a fundamentally different design strategy for integrating two distinct assay stages. A novel device architecture (microchamber) coupled with spatially heterogeneous polymers overcomes key technical gaps that have plagued advances in on-chip proteomics.

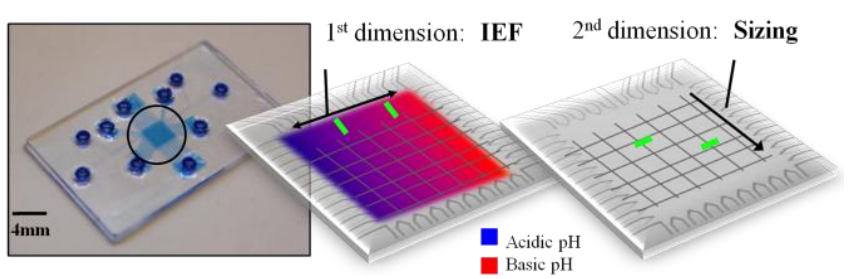

Figure 1: Polymer photopatterning in microchamber enables $2 D E$. Etched glass microdevice device features a $4 x 4 \mathrm{~mm}$ microchamber spanned by side channels for sample loading and electric field control. A multi-step PA photopolymerization protocol allows definition of spatially heterogeneous sieving media within the microchamber for IEF and subsequent size separations.

Established microfluidic 2DE employs intersecting microchannel networks supporting IEF and sizing. These integration efforts to unify $2 \mathrm{DE}$ in a microdevice have been severely limited owing to reliance on intersecting microchannel networks. In one implementation, species focused in a single channel are mobilized to a channel intersection using a low dispersion electroosmotic flow. When a band of interest reaches the intersection, the electric field direction is reversed to transfer individual bands to the second dimension [4]. In another incarnation species are focused in a channel flanked by multiple channels. The bands are then transferred to the array of channels for the second separation stage $[5,6]$.

In both cases, the continuous first stage assay is discretized in sampling to the second stage. Total peak capacity and $1^{\text {st }}$ dimension separation resolution depend on this sampling frequency in addition to the $1^{\text {st }}$ dimension separation performance. This general strategy for microfluidic 2DE leads to significant information losses that are not experienced with conventional benchtop approaches [7-9]. Thus, there is a critical need to introduce technology that combines the benefits microfluidic (speed, automation, low sample needs) and macroscale (lossless transfer) approaches.

Three major advances underpin our unique approach. Firstly, we introduce a new design utilizing microchamber and channel networks housing functional hydrogels (Figure 1). In contrast to previous studies, we use this approach to integrate multiple electrophoresis separations. Secondly, we utilize a novel photopatterning approach that allows spatial definition of physicochemical properties of hydrogels within this geometry. This marks a significant advance on state-of-the-art, as spatial definition of chemical properties has not been previously demonstrated. Thirdly, we demonstrate a novel integration approach that harnesses chemical mobilization of the first stage into distinct chamber regions, thus ensuring a transfer process with minimal losses between the two separation modes.

\section{METHODS AND RESULTS \\ Device Fabrication}

Etched glass chips were designed in-house using commercial computer-aided design (CAD) software. Designs were then sent to Hilton Head Island, South Carolina, June 3-7, 2012 
a commercial vendor (Caliper Life Sciences) for fabrication using well established glass wet-etching protocols. Subsequent customizations of the glass devices were all done in-house. PA gels within the microchannels of the device were photopolymerized using conventional UV photoinitiator (VA-086) and PA precursor and cross-linker reagents. Photopatterning was achieved using chrome photomasks in conjunction with an exposure system consisting of an inverted epifluorescence microscope coupled to a mercury lamp. Photopatterning protocols in conjunction with precursor solution wash steps enable the localization of the chemical and physical properties of the PA gel matrix with a high degree of spatial precision.

\section{pH Gradient Confinement}

A novel PA photopolymerization protocol allows us to confine the chemical conditions required for IEF within the microchamber (Figure 2). While achievable in a single channel, spatially confined IEF in a 2D geometry is a key component of our integration strategy and has never previously been reported. Chip designs that allow precise electric field control in 2D geometries have not allowed control over the conditions for the formation of the $\mathrm{pH}$ gradient in specific regions. Specifically, to achieve voltage shaping and therefore precise electrophoretic transport in a 2D geometry, side channel arrays flanking the main chamber are required $[10,11]$. These long control channels complicate the control of chemical conditions within the chamber by conventional approaches. This challenge was overcome by our novel fabrication approach. We achieve $\mathrm{pH}$ gradient confinement by using immobilized buffers to establish the basic and acidic boundary conditions flanking the microchamber. Immobilines are acrylamido weak acids and bases that can be incorporated into PA gels. Combinations of immobilines are used to achieve gels buffered at basic ( $\mathrm{pH}$ 9.3) and acid ( $\mathrm{pH} 3.6)$ conditions.

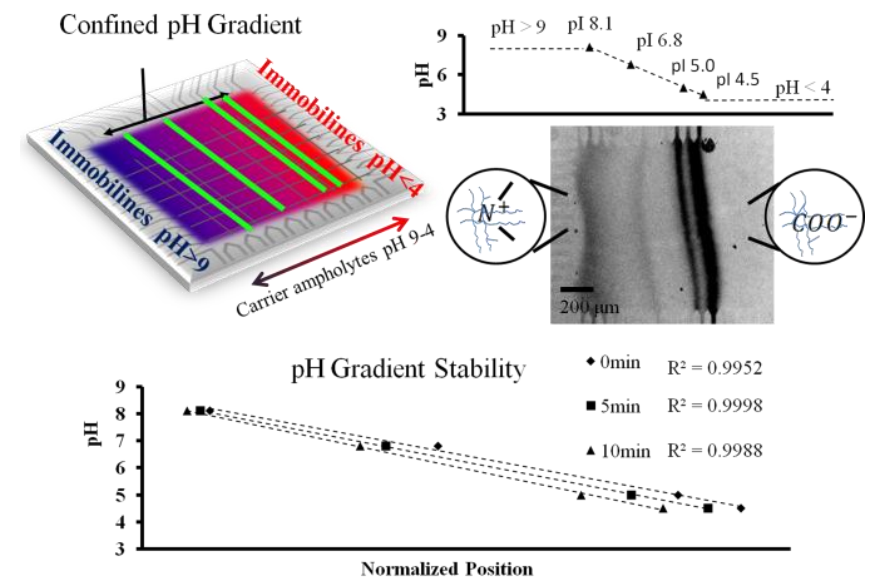

Figure 2: pH gradient confinement. Immobilines (acrylamido weak acid and bases) are incorporated into the gel flanking the microchamber. The presence of the stationary $\mathrm{pH}$ boundaries constrains the $p H$ gradient created by the (non-immobilized) carrier ampholytes within the chamber, demonstrated by the inverted micrograph of fluorescent UV pI markers performed in the $2 \mathrm{D}$ geometry. This $\mathrm{pH}$ gradient is stable with cathodic drifts $<3 \mu \mathrm{m} / \mathrm{min}$.

Gels containing (non-immobilized) polybuffer broad range (pH 4-9) carrier ampholytes are then patterned in the chamber. Upon application of an electric field, the carrier ampholytes migrate to their $\mathrm{pI}$. Ampholytes buffer at their $\mathrm{pI}$ and therefore using mixtures of several ampholytes with a large $\mathrm{pI}$ range, the $\mathrm{pH}$ gradient is established. Typically, the $\mathrm{pH}$ gradient for IEF using carrier ampholytes is formed by having a basic buffer (catholyte) and an acidic buffer (anolyte) at the terminals. In our approach, the stationary $\mathrm{pH}$ boundary conditions flanking the chamber constrain the $\mathrm{pH}$ gradient created by the carrier ampholytes within the chamber. $\mathrm{pH}$ gradients formed using our unique approach are stable with minimal cathodic drifts of $<3 \mu \mathrm{m} / \mathrm{min}$. Importantly, this approach enables highly flexible design, as the $\mathrm{pH}$ gradient range can be easily tuned for the desired application.

\section{Spatially Constrained IEF in 2D Geometry}

In order to integrate the chamber-based IEF with subsequent separations, a 1D "lane" for IEF in the chamber must be defined under the constrained $\mathrm{pH}$ gradient approach. Proper loading and precise voltage protocols are necessary to achieve focusing on a $1 \mathrm{D}$ lane in the chamber.

\section{A Confined GFP focusing in microchamber}

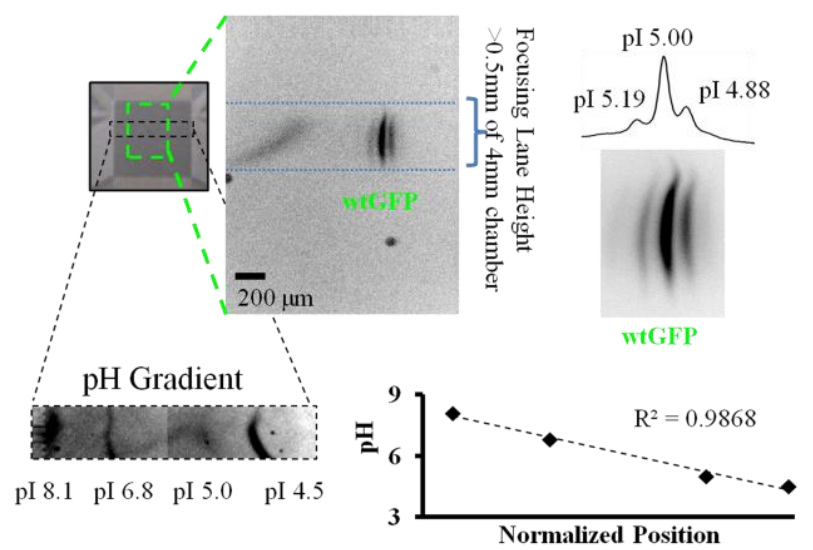

B Comparative GFP focusing in single channel
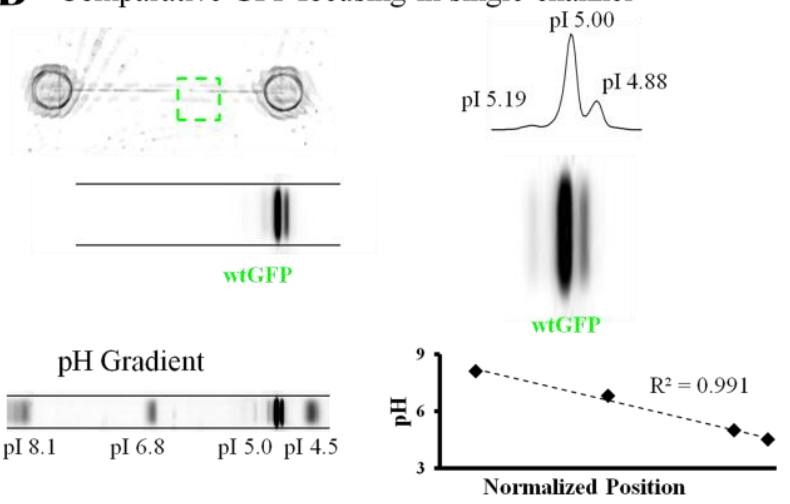

Figure 3: Confined high resolution and rapid IEF in microchamber. (A) Inverted fluorescence micrograph of wtGFP focusing in a confined ' $1 D$ lane' in the microchamber geometry. Sample loading is performed through a single side channel and electric field control minimizes dispersion. Stable, focused 3 isoform pattern of wtGFP (pIs 5.19, 5.00, 4.88) is visible <7min after loading into chamber. The $\mathrm{pH}$ gradient from 4-9 is linear and constrained within the microchamber. (B) For comparison, wtGFP focusing in a single $10.4 \mathrm{~mm}$ channel.

Confinement is achieved by loading the protein sample through a single channel and precise control of the electric field 
within the microchamber to prevent dispersion $[12,13]$. In the current design, samples are electrophoretically injected from a single channel in the cathode side. After the desired amount of material has been loaded, the loading well is washed with catholyte buffer. An additional benefit of our $\mathrm{pH}$ gradient confinement approach using immobilines is that ampholytes in entire chamber are uniformly focused. Without these uniform conditions, IEF on a 1D lane is not possible, as conductivity variations in focused and unfocused regions leads to substantial dispersion due to nonuniform electric fields.

Sample loading and focusing is rapid, taking less than $30 \mathrm{~min}$ to complete. Focusing occurs in a $<0.5 \mathrm{~mm}$ lane, thus enabling seamless integration with other regions of the microchamber. IEF performed in this configuration enables the separation of GFP isoforms resulting in resolution $<0.1 \mathrm{pH}$ units and peak capacities of $\sim 100$ in the first dimension (Figure 3). Further design improvements would include distinct wells for sample loading and catholyte reservoirs, thereby ensuring completely hands-free protocols. Resolving power on IEF systems depends on the steepness of the $\mathrm{pH}$ gradient and the separation axis length. The separation performance of this system can therefore be further enhanced by constraining narrower $\mathrm{pH}$ ranges and increasing the size of the microchamber.

\section{Transfer and Separation}

Low dispersion integration of the separate assay stages has been a hurdle to realizing high total assay performance. A major challenge is inherent to IEF, as proteins focused at their $\mathrm{pI}$ have no net charge and thus will not be mobilized unless the local $\mathrm{pH}$ is disrupted [14].

We initiate mobilization from the IEF stage to protein sizing by disrupting the $\mathrm{pH}$ gradient using electrophoretic introduction of a salt in the perpendicular direction to focusing (Figure 4). The introduction of an ionic species will cause the local hydronium ion and hydroxide ion levels to change in order to maintain the electroneutrality condition. Consequently, the local $\mathrm{pH}$ is altered allowing proteins to be mobilized given that they are no longer at their pI.

The mobilized proteins separate based on their relative mobilities. Protein transfer and separation is rapid, taking $<10 \mathrm{~min}$ to complete and preserves $1^{\text {st }}$ dimension separation information with band broadening of only $<1.3 x$ (competing microfluidic approaches result in effective band broadening of $\sim 10 \mathrm{x}$ due to undersampling). The bands drift $<50 \mu \mathrm{m}$ towards the acidic end during transfer, but retain their relative positions. Total assay time is $<1 \mathrm{hr}$.

The two key characteristics the ideal transfer mechanism would provide would be speed and transfer uniformity. A fast transfer will improve total assay performance as diffusion resulting from the stopping of the focusing electric field will be kept to a minimum. A uniform transfer will retain $1^{\text {st }}$ dimension separation information intact as well as enable size and electrophoretic mobility information to be extracted from the $2^{\text {nd }}$ separation. We are currently testing the uniformity of the transfer for broad $\mathrm{pH}$ ranges.

Another approach for mobilizing focused species is the introduction of an ionic detergent such as Sodium dodecyl sulfate (SDS) to impart a net charge on the proteins. We are currently testing how transfer performance will be affected with the introduction of ionic detergents compared to the demonstrated detergent-free transfer.

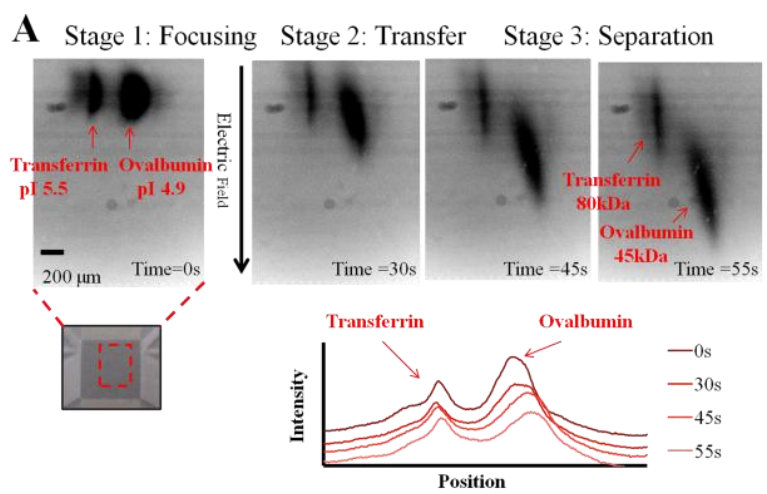

B Comparative single channel focusing

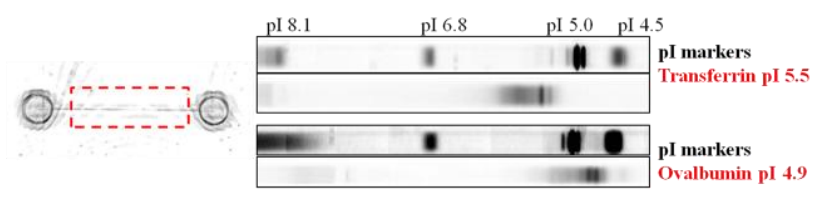

Figure 4: Microchamber integration enables lossless transfer for subsequent size separation. (A) Inverted fluorescence micrographs of CE540 labeled transferrin and ovalbumin focusing in confined '1D lane' in microchamber and subsequent transfer. Upon switching electric field direction and electrophoretic loading of a salt, the $\mathrm{pH}$ gradient is disrupted and the proteins migrate through the sieving matrix with mobilities proportional to their molecular weights. Offset intensity profile plots of transferrin and ovalbumin as they migrate through the chamber. Band broadening factors of $<1.3 x$ and position drifts $<50 \mu m$ are observed. (B) For comparison, transferrin and ovalbumin focusing in single channels.

\section{CONCLUSIONS}

Our approach combines the benefits of microfluidic (speed, automation, small volumes) and macroscale (lossless transfer) platforms. We are working towards developing capabilities for extracting both $\mathrm{pI}$ and molecular weight information from unlabeled biological samples. In addition, we hope to continue to improve total assay performance by further optimizing chip designs and experimental protocols. We see these advances as forming a new design paradigm for automated multi-stage assays with relevance spanning from fundamental biological inquiry to study of protein diagnostic biomarkers.

\section{ACKNOWLEDGEMENTS}

The authors gratefully acknowledge members and alumni of the Herr Laboratory at UC Berkeley for assistance and helpful discussions. The authors gratefully acknowledge financial support from the U.S. Department of Homeland Security's ORISE Fellowship (AMT) and the U.S. Department of Defense's NDSEG Fellowship (AJH). Partial conference travel support was generously provided by the Transducer Research Foundation (AMT). This work was funded in part by the National Science Foundation through CAREER Award (AEH, grant \# 1056035). $\mathrm{AEH}$ is an Alfred P. Sloan Foundation Research Fellow in chemistry.

This research was performed under an appointment to the Department of Homeland Security (DHS) Scholarship and Fellowship Program, administered by the Oak Ridge Institute for Science and Education (ORISE) through an interagency agreement between the US Department of Energy (DOE) and DHS. ORISE is 
managed by Oak Ridge Associated Universities (ORAU) under DOE contract number DE-AC05 06OR23100. All opinions expressed in this paper are the authors' and do not necessarily reflect the policies and views of DHS, DOE, or ORAU/ORISE.

\section{REFERENCES}

[1] Jung K., J. Reiche, A. Boehme, C. Stephan, S. A. Loening, D. Schnorr, W. Hoesel and P. Sinha (2004). "Analysis of subforms of free prostate-specific antigen in serum by twodimensional gel electrophoresis: potential to improve diagnosis of prostate cancer." Clin Chem 50(12): 2292-2301.

[2] O'Farrell, P. H. (1975). "High resolution two-dimensional electrophoresis of proteins." J Biol Chem 250(10): 40074021.

[3] Giddings, J. C. (1984). "Two-dimensional separations: concept and promise." Anal Chem 56(12): 1258A-1260A, 1262A, 1264A passim.

[4] Herr A. E., J. I. Molho, K. A. Drouvalakis, J. C. Mikkelsen, P. J. Utz, J. G. Santiago and T. W. Kenny (2003). "On-chip coupling of isoelectric focusing and free solution electrophoresis for multidimensional separations." Anal Chem 75(5): 1180-1187.

[5] Yang S., J. Liu, C. S. Lee and D. L. Devoe (2009). "Microfluidic 2-D PAGE using multifunctional in situ polyacrylamide gels and discontinuous buffers." Lab Chip 9(4): 592-599.

[6] Liu, J., C. F. Chen, S. Yang, C. C. Chang and D. L. Devoe (2010). "Mixed-mode electrokinetic and chromatographic peptide separations in a microvalve-integrated polymer chip." Lab Chip 10(16): 2122-2129.
[7] Li, X., D. R. Stoll and P. W. Carr (2009). "Equation for peak capacity estimation in two-dimensional liquid chromatography." Anal Chem 81(2): 845-850.

[8] Seeley, J. V. (2002). "Theoretical study of incomplete sampling of the first dimension in comprehensive twodimensional chromatography." J Chromatogr A 962(1-2): 2127.

[9] Davis, J. M., D. R. Stoll and P. W. Carr (2008). "Effect of first-dimension undersampling on effective peak capacity in comprehensive two-dimensional separations." Anal Chem 80(2): 461-473.

[10] Lerch, M. A. and S. C. Jacobson (2007). "Electrokinetic fluid control in two-dimensional planar microfluidic devices." Anal Chem 79(19): 7485-7491.

[11] Lerch, M. A., M. D. Hoffman and S. C. Jacobson (2008). "Influence of channel position on sample confinement in twodimensional planar microfluidic devices." Lab Chip 8(2): 316-322.

[12] He, M. and A. E. Herr (2009). "Microfluidic polyacrylamide gel electrophoresis with in situ immunoblotting for native protein analysis." Anal Chem 81(19): 8177-8184

[13] He, M. and A. E. Herr (2010). "Polyacrylamide gel photopatterning enables automated protein immunoblotting in a two-dimensional microdevice." J Am Chem Soc 132(8): 2512-2513.

[14] R. Rodriguez-Diaz, M. Zhu, T. Wehr (1997) "Strategies to improve performance of capillary isoelectric focusing." Journal of Chromatography A, 772 145-160

\section{CONTACT}

atentori@berkeley.edu, aeh@berkeley.edu

http://herrlab.berkeley.edu 


\section{MULTICOLOR QUANTUM DOT-BASED FLUORESCENCE EXCITATION FOR CELLULAR MICRO-ARRAY SCREENING

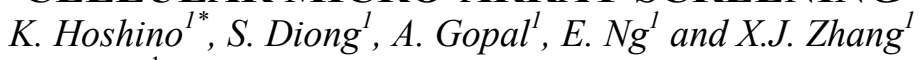 \\ ${ }^{1}$ Department of Biomedical Engineering, The University of Texas at Austin, Austin, Texas, USA}

\begin{abstract}
We have developed a colloidal quantum dot (QD) - based multicolor excitation light source array designed for high throughput lab-on-a-chip cell screening and imaging. We have demonstrated preliminary measurements of fluorescence excitation that is suitable for the arrayed fluorescence immunoassay. Ex vivo transmission microscopy and fluorescence imaging of cultured cancer cells (MDA-MB 231 and SKBr3) were performed to demonstrate the efficacy of the QD-based light source. Our lowcost light source array is highly compatible with commercially available fluorescence microscopes and fluorescent markers commonly used for biomedical imaging and screening.
\end{abstract}

\section{INTRODUCTION}

Colloidal quantum dots (QDs) have demonstrated significant potential as fluorescent markers for bioimaging and sensing [1-3]. Emission wavelengths can be easily tailored by proper choice of materials and the size of the QDs. Semiconductor QDs are much more stable and slow to photobleaching compared with commonly used organic fluorescent dyes.

Here we demonstrate another important advantage of colloidal QDs, which is the compatibility with the advanced microfabrication technologies. In previous studies, we have shown patterning of colloidal QDs on silicon substrates [4-5]. The feature size of patterned QDs can be arbitrary chosen from millimeter scale down to single molecular order. Those patterned QDs can be also used as lumophores of light emitting diodes (LEDs) directly integrated onto silicon microdevices. We constructed QDLEDs onto several types of silicon MEMS structures [6].

One strong potential application for integrated QD light source is lab-on-a-chip bioimaging systems [6-7]. Hyperspectral analysis of cells using multiple fluorescent markers demonstrated efficacy in identification of cancer cell types [8]. In such immunofluorescence bioimaging systems, multiple excitation light source is crucial to efficiently excite different types of fluorescent markers that have different absorption wavelength bands. Colloidal QDs easily enable integration of multicolor sources on a single substrate. Furthermore, QD based illumination systems are highly compatible with solid state excitation configuration. colloidal QDs can be either electrically excited or excited by an UVLED. Solid state light sources have been drawing attention for imaging and spectroscopy [9-10], because it can construct a highly integrated, low-cost, energy efficient illumination setup. It also enables time controlled fluorescence measurements such as fluorescence lifetime measurement [9].

In this paper, we propose a colloidal QD based multicolor light source designed for fluorescence biosample imaging. Excited by a high power UVLED, patterned Colloidal QDs work as an excitation source for transmission microscopy and immunofluorescent cell imaging.

\section{EXPERIMENTAL SETUP}

Patterned QD light source

The two key techniques we introduced here are patterning of colloidal QDs on a glass substrate and UV excitation utilizing total internal reflection in the glass substrate. Figure 1 shows an illustration of the fabrication procedure and the experimental setup. Array of colloidal QD patterns were fabricated onto a glass slide by the micro contact printing technique. The colloidal QDs are $\mathrm{CdSe} / \mathrm{ZnS}$ core-shell nanoparticles. PDMS stamps formed by SU-8 patterned molds were used to transfer films of quantum dots onto the substrate. The details of the stamping techniques are described in [5], where we have previously demonstrated patterning of QDs as fine as $500 \mathrm{~nm}$. An UV light from a high-power LED (LedEngin, $200 \mathrm{~mW}$ ) with the emission wavelength peaked at $405 \mathrm{~nm}$ or 365 $\mathrm{nm}$ is focused and coupled to the substrate glass slide to induce total internal reflection. The QD films are excited by the UV evanescent field on the surface of the glass slide.

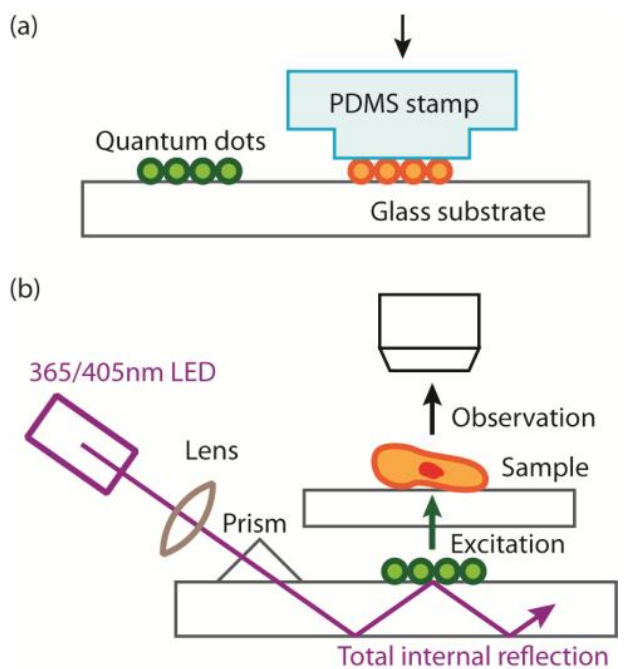

Figure 1: (a) Fabrication procedure and (b) experimental setup of the arrayed multicolor light source

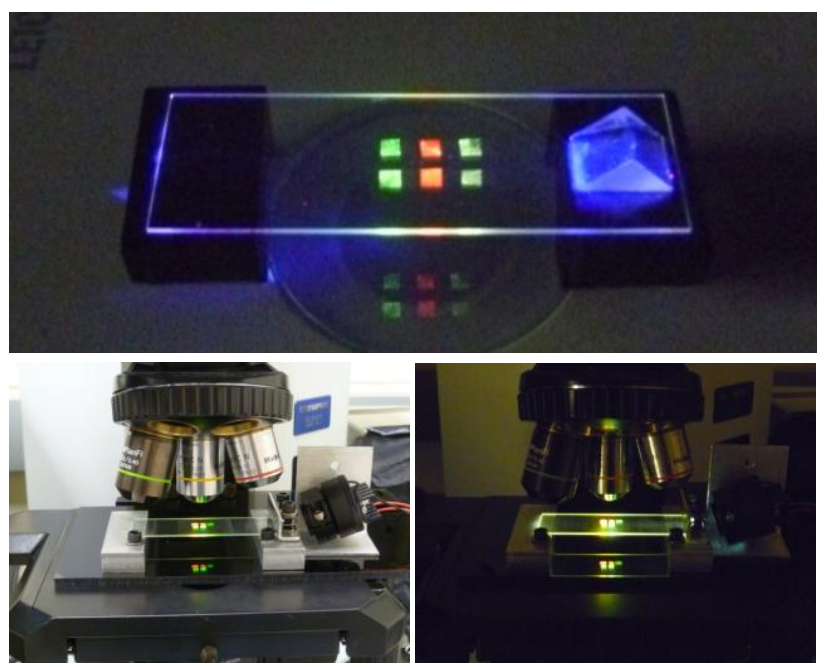

Figure 2: Emission from the $Q D$ array and experimental setup with a standard fluorescence microscope

Solid-State Sensors, Actuators, and Microsystems Workshop Hilton Head Island, South Carolina, June 3-7, 2012 
Figure 2 shows photographs of the emission from the QD arrays and the experimental setup built in a standard fluorescence microscope (Olympus BX51). Because the evanescent field decays quickly in the near-field and does not transmit energy in the farfield, intensities of the UV light observed with the microscope is almost negligible compared with those of the QDs that are excited by the evanescent field. Figure 3 shows the emission spectra of the QD array. Peak emission wavelengths can be easily chosen by using QDs with a proper average diameter.

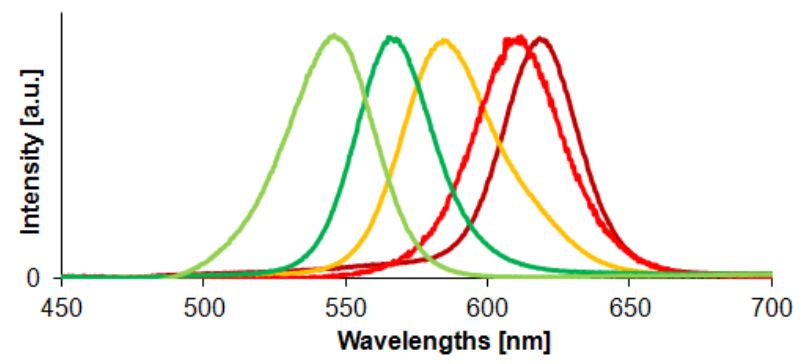

Figure 3. Emission spectra of the $Q D$ array. Emission peaks ranging from $540 \mathrm{~nm}$ to $620 \mathrm{~nm}$ were used in the measurement.

\section{Fluorescence excitation measurement}

Figure 4 shows the setup the fluorescence excitation experiment. QDs with an emission peak at $540 \mathrm{~nm}$ and $655 \mathrm{~nm}$ were used as the excitation source (light source QDs) and the fluorescent marker (sample QDs), respectively. The sample QD is located on a $1 \mathrm{~mm}$-thick glass slide, which roughly defines the distance between the excitation QD light source and the sample QD. A UV-cut plastic thin film and a green color film filter are inserted between the light source QD and the sample QDs. The purposes of these filters are: (1) To avoid cross direct excitation of sample QDs by stray lights from the UV LED and (2) To remove crosstalk from the light source QD to the fluorescence from the sample QD. A microscopic spectrometer consisting of a monochromator (Princeton Instruments Acton SP2150) and a cooled CCD (Princeton Instruments Pixis 400) measured the fluorescence from the sample QDs along with the excitation from the light source QDs.

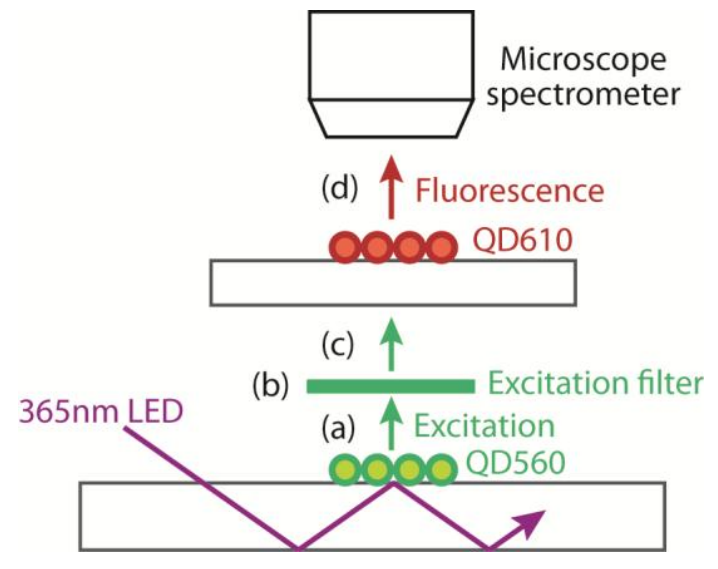

Figure 4: Fluorescence excitation setup with the light source $Q D$ (emission peaked at $560 \mathrm{~nm}$ ) and sample QD (emission peaked at $610 \mathrm{~nm})$.
Figure 5(a) shows the spectrum measured for the emission of the light source QDs (QD540) without any filter attached (see also figure 4(a) for the setup). One common issue of using two-step excitation as introduced here is cross excitation from the primary excitation source. As one can see in figure 2 top, some UV emission is visible at the edges of the slide glass and the prism. These stray lights may cause unwanted cross excitation of the sample QDs. In the actual experimental setup, a black shield box was attached to cover the observation area to block stray lights from the UVLED. In the area around the light source QD, scattering of UV light is small enough and not visible. Direct emission from UVLED cannot be found in the spectrum in figure 5(a). Another issue is the crosstalk between the light source QD and the fluorescence of the sample QD. In figure 5(a), detectable emission is found in the range of $>600 \mathrm{~nm}$, which will interfere with the observation of fluorescence from the sample. A green filter is used to trim the portion of $>600 \mathrm{~nm}$ emission. Transmission spectrum of the green filter is shown in the figure 5(b). Figure 5(c) is the spectrum of the light source QD with the filters attached. The half width of excitation in figure $5(\mathrm{~d})$ was $25 \mathrm{~nm}$, and is narrower than $29 \mathrm{~nm}$ measured for the emission from the light source QD. Figure 5(d) is the spectrum measured for the sample QD, along with the excitation light from the QD through the green and UVcut filters. Fluorescence from the sample QDs (QD620) is clearly visible in the spectrum.

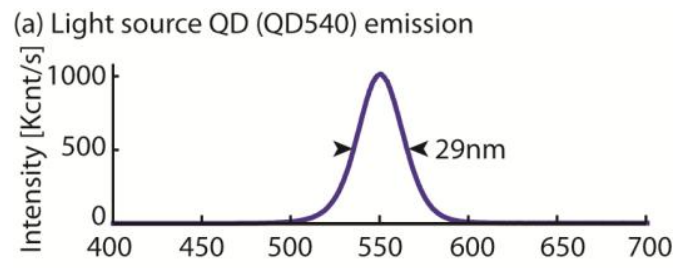

(b) Filter transmission
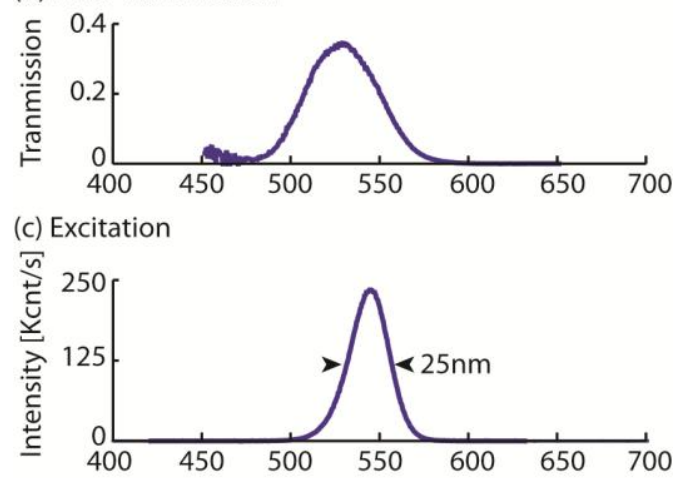

(d) Excitation and fluorescence

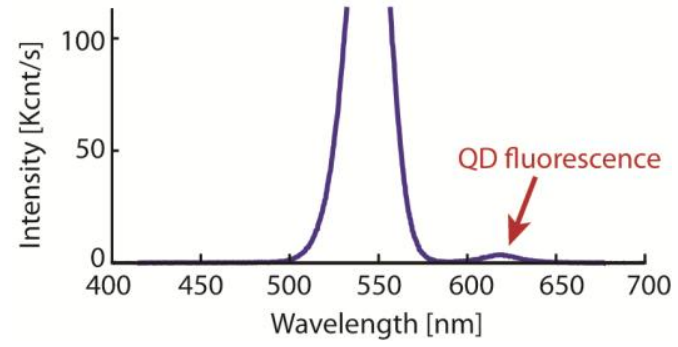

Figure 5: Spectrum showing the QD excitation (peaked at 540nm) and the fluorescence (peaked at610nm) 


\section{Power density of the QD light source}

The power density of the QD light source can be evaluated by the photon count measured by the spectrometer. The emission power $p_{i}$ of a single photon with a wavelength of $\lambda_{i}$ is:

$$
p_{i}=h v=h \cdot \frac{c}{\lambda_{i}}
$$

where $h$ is the Planck constant, $c$ is the speed of light. The emission energy measured by the CCD can be obtained by adding up energies measured for all the wavelengths of $\lambda_{i}(i=1 \sim N)$

$$
p=\sum_{i=1}^{N} p_{i} \cdot \text { count }_{i},
$$

Where count is the value of photon count per second for a wavelength $\lambda_{i}$.

The microscope objective collects only a part of the entire QD emission. We use an objective with a numerical aperture (NA) of $\sin \theta=0.65$. The emission $P_{\mathrm{m}}[\mathrm{W}]$ from the measured area is:

$$
P_{\mathrm{m}}=p \cdot(4 \pi) / 2 \pi \int_{0}^{\theta} \sin \varphi \cdot \mathrm{d} \varphi .
$$

The microscope spectrometer measures the intensity of lights through an opening slit that corresponds to $15 \mu \mathrm{m} \times 170 \mu \mathrm{m}$ on the sample plane. The power density $\left[\mathrm{W} / \mathrm{cm}^{2}\right]$ is thus given as

$$
P=P_{\mathrm{m}} \cdot 10^{-2} \times 10^{-2} /\left(15 \cdot 10^{-6} \times 170 \cdot 10^{-6}\right)
$$

Applying (1)-(4) to the measurement in figure 5(c), the power density of the QD at a point (c) becomes:

$$
P=2.1 \times 10^{-6}\left[\mathrm{~W} / \mathrm{cm}^{2}\right]
$$

\section{CANCER CELL IMAGING}

\section{Transmission-mode illumination}

The QD light source was first used as a transmission-mode illumination source to perform observation of cancer cells. A $150 \mathrm{~m}$-thick cover glass slip, on which cancer cells are fixed, is placed on a QD light source. The cells are stained with HEMA 3 (Fisher Scientific), which is commonly used to visualize cell nucleus. HEMA 3 contains eosin Y that stains the cytoplasm and methylene blue that stains the nucleus of the cell. The dye absorbs visible light and makes the stained area visible under transmissionmode illumination. The transmission spectrum of the HEMA3 dye is shown in figure 6 .

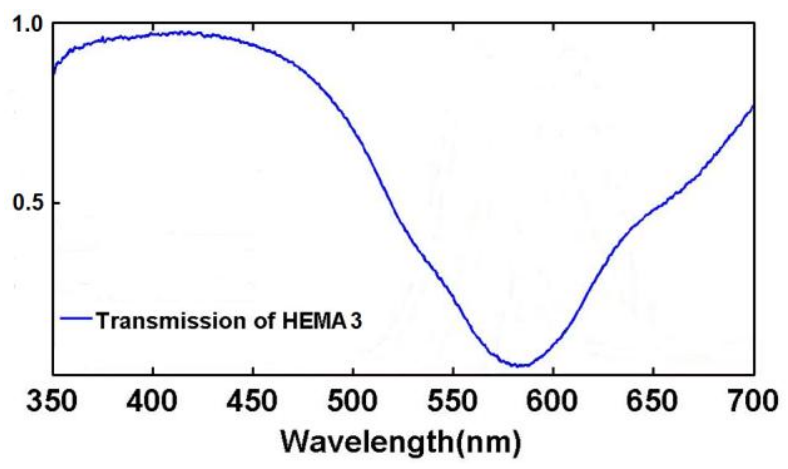

Figure 6: Absorption spectrum of the HEMA3 dye. It absorbs lights in visible range of roughly 540-620nm.

We can use a light source with emission wavelengths complementarily peaked in the absorption peak of HEMA 3 dye to clearly observe the stained area. Absorption peak of HEMA3 ranges roughly in the visible region of 540-620nm. Figure 7 shows an example of cells imaged with the multicolor light sources. MDA-MB231 breast cancer cells were used as the sample. We were able to clearly observe and determine the nucleus to cytoplasm ratio, which is an important factor to distinguish cancer cells from normal cells.
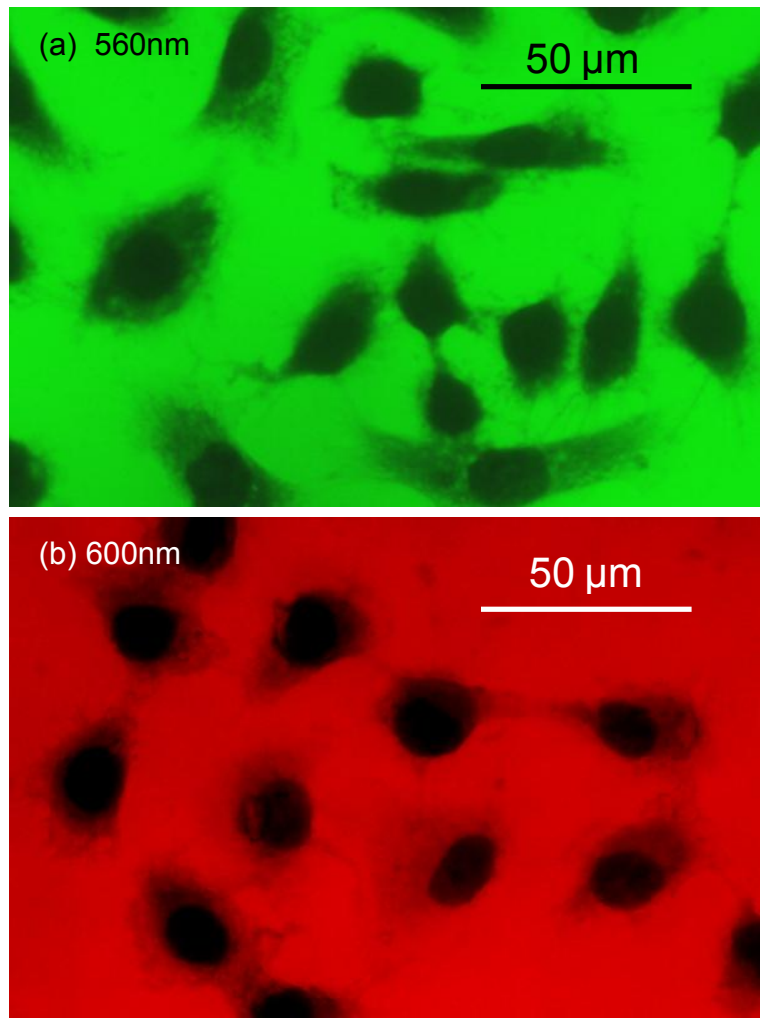

Figure 7: MDA-MB232 cells imaged with transmission mode illumination with (a) QD560 and (b) QD600. The cells are stained with the HEMA3 dye.

\section{Fluorescence excitation}

We performed fluorescence imaging of cultured cancer cells. A breast cancer cell line $\mathrm{SKBr} 3$ was used. SKBr3 is known to overexpress the gene product of Human Epidermal Growth Factor Receptor 2 (HER2).
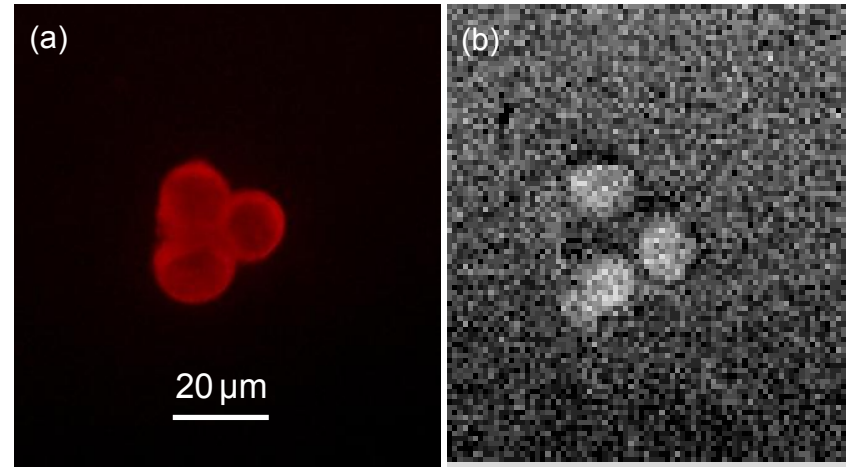

Figure 8. Fluorescence images of SKBr3 cancer cells. (a) Imaged with a standard fluorescence excitation setup. (b) Imaged with QD540 excitation source. 
Her2 is often used as a biomarker for cancer cell identification or targeted therapy, since Her2 overexpression is found in a certain aggressive type of breast cancer. Her2 is thus clinically used to characterize the type of breast cancer. Figure 8 (a) is a fluorescence image of $\mathrm{SKBr} 3$ cells taken with a standard fluorescence microscope (Olympus BX51). After the cultured cells are trypsinized from a culture dish, they were fixed on a glass slide and stained with AlexaFluor 594 dye conjugated with Her2 antibodies. A color CCD camera (Olympus DP71) and a filter cube (excitation peak wavelength $535 \mathrm{~nm}$, bandwidth $50 \mathrm{~nm}$, dichroic mirror $565 \mathrm{~nm}$ long-pass and emission peak wavelength $610 \mathrm{~nm}$, bandwidth $75 \mathrm{~nm}$ ) were used for Figure $8(\mathrm{a})$. Figure 8 (b) is a fluorescence image taken with the QD540 excitation light source. The same filter cube as figure 8(a) was used for fluorescence observation. A cooled monochromatic CCD (Princeton Instruments Pixis 400) was used for imaging. Although the image is dimmer compared with the standard excitation setup, the same cells are fluorescently observed with our integrated QD light source.

\section{CONCLUSION}

We have developed an arrayed colloidal QD excitation light source that can be easily used in commercially available microscopic observation setup. We characterized the QD light source by fluorescence excitation measurements of test QD samples. The setup is then successfully used for imaging of ex-vivo cancer cell transmission-mode illumination microscopy and immunofluorescence imaging.

Our arrayed excitation is especially suitable for patterned immuno-fluorescent assay [11-12] or cellular microarray imaging [13], where multiple biomarkers are tested in a microarrayed configuration. The QDs used here can be electrically excited in an LED configuration, which will further extend the possibility and versatility of our illumination setup.

\section{REFERENCES}

[1] X. Wu, H. Liu, J. Liu, K.N. Haley, J.A. Treadway, J.P. Larson, N. Ge, F. Peale, and M.P. Bruchez, "Immunofluorescent labeling of cancer marker Her2 and other cellular targets with semiconductor quantum dots," Nature Biotechnology 21, 41-46, 2003.

[2] X. Michalet, F. F. Pinaud, L.A. Bentolila, J.M. Tsay, S. Doose, J.J. Li, G. Sundaresan, A.M. Wu, S. S. Gambhir, S. Weiss, "Quantum Dots for Live Cells, in Vivo Imaging, and Diagnostics," Science 307, 538-544, 2005.

[3] I.L. Medintz, H.T. Uyeda, E.R. Goldman and H. Mattoussi, "Quantum dot bioconjugates for imaging, labelling and sensing," Nature Materials 4, 435-446, 2005.

[4] A. Gopal, K. Hoshino \& X.J. Zhang, "Photolithographic patterning of sub-wavelength colloidal quantum dot based inorganic light emitting diodes on silicon," Applied Physics Letters, 96, 13, 131109, 2010.

[5] K. Hoshino, T.C. Turner, S. Kim, A. Gopal and X.J. Zhang, "Single molecular stamping of self-assembled sub-10nm colloidal quantum dots," Langmuir 2008, 24, 13804-13808.

[6] A. Gopal, Z. Wang, K. Hoshino and X.J. Zhang, "Multispectral Analysis of Cancer Cells using Quantum Dot LEDs patterned on chip", The 16th International Conference on Solid-State Sensors, Actuators and Microsystems (Transducers 2011), pp.1817-1820.

[7] B. Yao, G. Luo, L. Wang, Y. Gao, G. Lei, K. Ren, L. Chen, Y. Wang, Y. Hub and Y. Qiu, "A microfluidic device using a green organic light emitting diode as an integrated excitation source," Lab on a Chip 5, 1041-1047, 2005.

[8] J.W. Uhr, M.L. Huebschman, E.P. Frenkel, N.L. Lane, R. Ashfaq, Huaying Liu, D.R. Rana, L. Cheng, A.T. Lin, G.A. Hughes, X.J. Zhang, H.R. Garner, "Molecular profiling of individual tumor cells by hyperspectral microscopic imaging," Translational Research 2011 (in press).

[9] T. Araki, H. Misawa, "Light emitting diodebased nanosecond ultraviolet light source for fluorescence lifetime measurements," Review of Scientific Instruments 66, 5469, 1995.

[10] E. Entcheva, Y. Kostov, E. Tchernev, L. Tung, "Fluorescence imaging of electrical activity in cardiac cells using an allsolid-state system," 51, $333-341,2004$

[11] E. Ng, A. Gopal, K. Hoshino, \& X.J. Zhang, "Multicolor Microcontact Printing of Proteins on Nanoporous Surface for Patterned Immunoassay," Applied Nanoscience, 1, 79-85, 2011.

[12] E. Blinka, K. Loeffler, Y. Hu, A. Gopal, K. Hoshino, K. Lin, X. Liu, M. Ferrari \& X.J. Zhang, "Enhanced Microcontact Printing of Proteins on Nanoporous Silica Surface," IOP Nanotechnology, 21, 415302, 2010.

[13] H.C. Moeller, M.K. Mian, S. Shrivastava, B.G. Chung \& A. Khademhosseini, "A microwell array system for stem cell culture," Biomaterials 29, 752-763, 2008.

\section{CONTACT}

*K. Hoshino, tel: +1-512-475-6872; hoshino@mail.utexas.edu 


\title{
SYNTHESIS OF DIVERSE TRACERS ON EWOD MICRODEVICE FOR POSITRON EMISSION TOMOGRAPHY (PET)
}

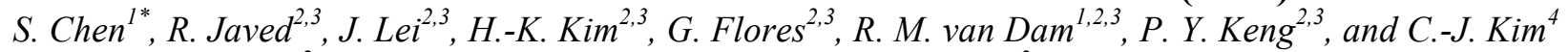 \\ ${ }^{1}$ Bioengineering Dept., ${ }^{2}$ Dept. of Molecular and Medical Pharmacology, ${ }^{3}$ Crump Institute for Molecular Imaging, \\ ${ }^{4}$ Mechanical and Aerospace Engineering Dept., University of California, Los Angeles (UCLA), California, U.S.A.
}

\section{ABSTRACT}

We demonstrate the synthesis of diverse tracers (sugar, DNA nucleoside, protein labeling compound, and neurotransmitter) using a single electrowetting-on-dielectric (EWOD) chip design with multifunctional electrodes for heating, temperature sensing, and EWOD driving. Furthermore, we present a key advantage of microfluidic chemical synthesis in increasing specific activity (radioactivity per mass of tracer) compared to macroscale synthesis. These are important steps toward more affordable, specific, and universal tracer production for use in positron emission tomography (PET).

\section{INTRODUCTION}

Positron emission tomography (PET) is a sensitive type of functional medical in vivo imaging for specific, quantitative, spatial, and temporal measurement of biochemical processes. Applications of PET include studying disease mechanisms, detecting early stage diseases, monitoring response to therapy, and developing therapeutics [1].

Since PET requires administration of a positron-emitting tracer with biological activity, availability of such tracers dictates ability to perform imaging. Over 1,600 PET tracers have been synthesized to date, but only several tracers are routinely available to imaging centers by commercial radiopharmacies [2]. Leveraging the radiopharmacies as a supply of radioisotope ( $\left[{ }^{18} \mathrm{~F}\right]$ fluoride) rather than tracers, and moving tracer production into the enduser's facility has potential to significantly increase diversity of tracers for the end user. Microfluidic tracer production could serve as the missing technological link by eliminating the need of the large capital investment involved in current PET probe production, (i.e. bulky synthesizers and expensive hot cells for radiation shielding).

After initial reports of synthesizing ${ }^{18} \mathrm{~F}$-labeled PET tracers with electrowetting-on-dielectric (EWOD) technology [3],[4], the digital microfluidic device has been used in conjunction with onchip radioactivity detection for optimizing microscale radiosynthesis conditions, and it has been demonstrated that its produced tracers have passed the numerous quality control requirements for human use [5]. While our earlier work demonstrated proof-of-concept radiosynthesis of $\left[{ }^{18} \mathrm{~F}\right]$ fluoro-2deoxy-D-glucose $\left(\left[{ }^{18} \mathrm{~F}\right] \mathrm{FDG}\right)[4]$ and $1-\left[{ }^{18} \mathrm{~F}\right]$ fluoro-4-nitrobenzene $\left(\left[{ }^{18} \mathrm{~F}\right] \mathrm{FNB}\right)[3]$, in this report we use one EWOD chip design and diversify synthesis to other PET tracers currently used in clinical and preclinical research: $\left[{ }^{18} \mathrm{~F}\right] \mathrm{FDG}$ (sugar), 3'-deoxy-3'$\left[{ }^{18} \mathrm{~F}\right]$ fluorothymidine $\left(\left[{ }^{18} \mathrm{~F}\right] \mathrm{FLT}\right.$, DNA nucleoside), tert-butyl 4$\left[{ }^{18} \mathrm{~F}\right]$ fluorobenzoate (the intermediate of $\left[{ }^{18} \mathrm{~F}\right] \mathrm{FSB}$, a prosthetic group for protein labeling), and $\left[{ }^{18} \mathrm{~F}\right]$ fallypride (neurotransmitter).

General aspects of microfluidics are attractive for tracer synthesis, because shorter reaction times result in less radioisotope decay and the small volumes match the nanogram amounts of tracer sufficient for PET imaging [6]. EWOD-based digital microfluidics has additional advantages in electronic control of droplet movement on a generic chip for easy reconfiguration to synthesize a variety of tracers. Our chip is also open to operate in air (i.e., no oil medium) without sidewalls, allowing rapid drying, evaporation, and solvent exchange; these are critical steps for many radiosynthesis processes that are water-sensitive but begin with cyclotron-produced $\left[{ }^{18} \mathrm{~F}\right]$ fluoride in $\left[{ }^{18} \mathrm{O}\right] \mathrm{H}_{2} \mathrm{O}$.

Furthermore, we present an important advantage of microfluidic radiosynthesis in increasing specific activity (radioactivity per mass of tracer). High specific activity is important in neurotransmitter tracers like $\left[{ }^{18} \mathrm{~F}\right]$ fallypride, where natural in vivo receptor quantities are low and easily saturated. Microfluidic synthesis should produce tracers with increased specific activity by reducing isotopic dilution and contamination from solvents, reagents, and transport lines [7]. We present the first experimental results comparing microfluidic synthesis with automated macroscale synthesis, demonstrating over 30 fold increase in specific activity when using the same reagents, concentrations, and heating times.

\section{DEVICE AND MATERIALS \\ EWOD Device Fabrication}

Our EWOD device has six inlets and droplet pathways that meet at a circular reaction site (Figure 1). The reaction site consists of 4 concentric multifunctional electrodes, each a resistive element that can be used for EWOD actuation of droplets or feedbackcontrolled heating. This multi-element reaction site can center the droplet and maintain temperature more uniformly than a singleelement heater as the droplet shrinks during evaporations [4].

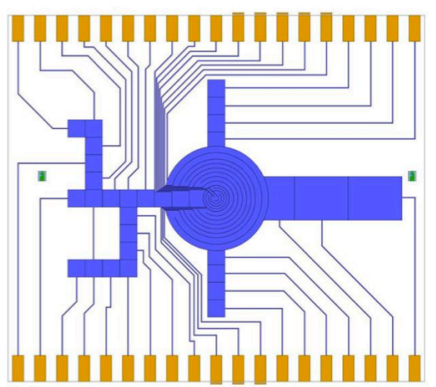

(a)

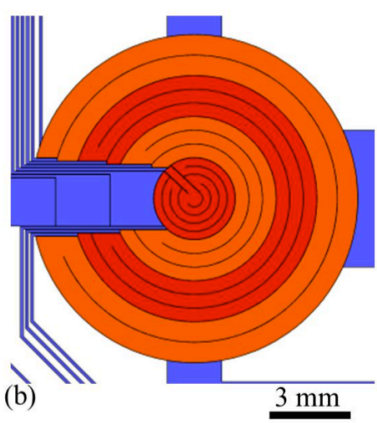

Figure 1: EWOD chip design for tracer synthesis. (a) device layout with multifunctional ITO electrodes (blue) and gold contacts (yellow), (b) the reaction site zoomed to show the individually controlled 4 concentric resistive heating rings.

EWOD chips and cover plates were fabricated from $700 \mu \mathrm{m}$ thick glass wafers coated with $140 \mathrm{~nm}$ ITO (Semiconductor Solutions). $\mathrm{Cr}(20 \mathrm{~nm})$ and $\mathrm{Au}(200 \mathrm{~nm})$ were evaporated onto the wafers. $\mathrm{Au}, \mathrm{Cr}$, and ITO layers were etched to form EWOD electrodes, heaters, connection lines, and contact pads. PECVD silicon nitride $(2 \mu \mathrm{m})$ was deposited as a dielectric on the device plate, and a thinner layer $(100 \mathrm{~nm})$ was deposited on the cover plate. Teflon ${ }^{\circledR}(250 \mathrm{~nm})$ was spin-coated and annealed at $340^{\circ} \mathrm{C}$ under vacuum to make the surfaces hydrophobic.

Test slides for testing reaction conditions were made from 25 x $25 \times 1 \mathrm{~mm}$ glass microscope slides (Corning), on to which 250 $\mathrm{nm}$ Teflon ${ }^{\circledR}$ was spin-coated and annealed at $340^{\circ} \mathrm{C}$.

\section{Device Actuation}

EWOD actuation voltage for droplet movement was generated from a $10 \mathrm{kHz}$ signal (33220A waveform generator, Hilton Head Island, South Carolina, June 3-7, 2012 
Agilent Technologies) amplified to 100 Vrms (Model 601C, Trek). The voltage was applied selectively to desired electrodes for droplet movement by individually addressable relays (AQW610EH PhotoMOS relay, Panasonic) that were controlled by a LabVIEW program using a digital I/O device (NI USB-6509, National Instruments).

A second digital I/O device (NI USB-6250, National Instruments) was used to control a multichannel heater driver that was designed and built in house to measure and maintain feedbackcontrolled temperatures over the chip's four individual resistive heaters. Each chip's multifunctional electrodes were individually connected to a switch to alternate between EWOD actuation voltage or temperature measurement and heating.

To test reaction conditions with the Teflon ${ }^{\circledR}$ coated glass slides, a Peltier module (Model 5R7-001, McShane) was used for temperature control.

\section{Macroscale Radiosynthesizer}

Automated Radiochemistry Platform (ARC-P) was used for conventional synthesis of $\left[{ }^{18} \mathrm{~F}\right] \mathrm{FDG}$ to compare specific activity with microfluidic synthesis [8]. ARC-P is a semi-automated inhouse macroscale synthesizer with high temperature, high pressure, and multi-pot capabilities.

\section{Solution Preparation}

Anhydrous acetonitrile ( $\mathrm{MeCN}, 99.8 \%)$, anhydrous dimethyl sulfoxide (DMSO, 99.9\%), anhydrous methanol (MeOH, 99.8\%), $\mathrm{O}$-( $N$-succinimidyl)- $N, N, N^{\prime}, N^{\prime}$-tetramethyluronium tetrafluoroborate (TSTU, 98\%), 2,3-dimethyl-2-butanol (thexyl alcohol), potassium carbonate $\left(\mathrm{K}_{2} \mathrm{CO}_{3}, 99 \%\right), 4,7,13,16,21,24$-hexaoxa1,10-diazobicyclo (8.8.8)-hexacosane ( $\mathrm{K} \quad 2.2 .2, \quad 98 \%)$, and mannose triflate (FDG precursor) were purchased from SigmaAldrich. $1 \mathrm{~N} \mathrm{HCl}$ (certified, Fisher Chemicals) was purchased from Fisher Scientific. Tetrabutylammonium bicarbonate $\left(\mathrm{TBAHCO}_{3}\right)$, 3-N-Boc-5'-O-dimethoxytrityl-3'-O-nosyl-thymidinie

(FLT precursor), 4-(tert-butoxycarbonyl)-trimethylbenzeneammonium triflate (tert-butyl 4-fluorobenzoate precursor), and tosyl-fallypride (fallypride precursor) were purchased from ABX Advanced Biochemical Compounds (Radeberg, Germany) and used as received.

No-carrier-added $\left[{ }^{18} \mathrm{~F}\right]$ fluoride ion was obtained from the UCLA Crump Institute for Molecular Imaging Cyclotron and Radiochemistry Technology Center by irradiation of $97 \%{ }^{18} \mathrm{O}$ enriched water with an $11 \mathrm{MeV}$ proton beam using an RDS-112 cyclotron (Siemens).

\section{RESULTS AND DISCUSSION}

Macroscale and microscale specific activity comparison

Specific activity is the radioactivity per total mass of tracer compound $\left({ }^{18} \mathrm{~F}\right.$-labeled and ${ }^{19} \mathrm{~F}$-labeled $)$. Nonradioactive $\left({ }^{19} \mathrm{~F}\right.$ labeled) molecules compete with radioactive $\left({ }^{18} \mathrm{~F}\right.$-labeled) ones for binding or uptake, resulting in lower imaging signal strength and lower contrast. High specific activity has added importance in neural imaging where receptors quantities are low and easily saturated. This is especially significant where the numbers of receptors is extremely small in the brain of small animals [9].

Microfluidic radiosynthesis can be beneficial to increase specific activity because greater mass and heat transfer result in shorter reaction times and allow less radioisotope decay. Also, reduced volumes are hypothesized to reduce contamination by nonradioactive fluorine-19 from solvents and reagents [7].

We compared specific activity of microfluidic synthesis with that of conventional synthesis by producing $\left[{ }^{18} \mathrm{~F}\right] \mathrm{FDG}$ simultaneously with an EWOD device and also an ARC-P macroscale radiosynthesier from the same batch of $\left[{ }^{18} \mathrm{~F}\right]$ fluoride. The synthesis process on EWOD device has been described by Keng et al. [5]. The same reagent sources were used for both synthesizers, but the macroscale synthesis used 80 times more reagents.

Product from each synthesizer was extracted and passed through a purification cartridge. After the final $\left[{ }^{18} \mathrm{~F}\right] \mathrm{FDG}$ product was eluted, its radioactivity was measured with a dose calibrator (CRC-25 PET, Capintec) and its radiochemical purity was evaluated using radio-TLC (MiniGITA star, Raytest). After overnight radiodecay, concentration and mass of the tracer was measured by UV absorbance and an analytical balance.

EWOD synthesis had over 30 fold increase in specific activity compared to automated macroscale synthesis, when using the same reagents, concentrations, and heating times (Table 1).

Table 1: $\left.{ }^{18} F\right] F D G$ was produced in parallel by an EWOD device and automated macroscale system using the same reagent sources and procedure (except for 80x increase in macroscale synthesis reagent volumes). Specific activity of radiotracers produced by EWOD showed 34.5 fold average increase.

\begin{tabular}{|c|c|c|c|}
\hline & \multicolumn{2}{|c|}{ 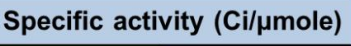 } & \multirow{2}{*}{ Improvement Ratio } \\
\hline & EWOD & Macroscale & \\
\hline Exp 1 & 0.74 & 0.014 & 55.0 \\
\hline Exp 2 & 2.86 & 0.091 & 31.4 \\
\hline Average & 1.80 & 0.052 & 34.5 \\
\hline
\end{tabular}

\section{Reaction condition optimization}

Different volumes, concentrations, and temperatures were used for each tracer synthesis after conditions had been optimized for microscale reactions on a Teflon-coated glass substrate, which mimics an EWOD chip (Figure 2). Herein we adapted the bulky alcohol method to $\left[{ }^{18} \mathrm{~F}\right] \mathrm{FLT}$ and $\left[{ }^{18} \mathrm{~F}\right]$ fallypride synthesis on EWOD to assist the nucleophilic fluorination reactions [10]. The protic solvent enabled high radiofluorination efficiencies $(80-85 \%)$ at lower temperature $\left(100{ }^{\circ} \mathrm{C}\right)$; in comparison, the conventional method requires higher temperatures $\left(130-180{ }^{\circ} \mathrm{C}\right)$ to achieve comparable fluorination efficiencies.

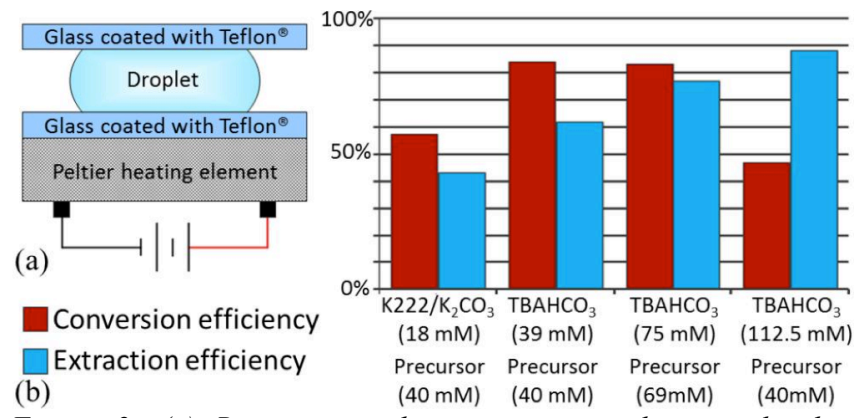

Figure 2: (a) Reaction conditions were tested using droplets sandwiched between glass coated with Teflon ${ }^{\circledR}$ and heated by a Peltier element. This can be done more intensively with the EWOD chip. (b) Data showing improved $\left[{ }^{18} F\right] F L T$ fluorination efficiency with different concentrations of tetrabutylammonium bicarbonate ( $\mathrm{TBAHCO}_{3}$ ) and the FLT precursor.

\section{Synthesis of 3'-deoxy-3' $-\left[{ }^{18}\right.$ F $]$ fluorothymidine $\left(\left[{ }^{18}\right.\right.$ F $]$ FLT $)$}

$\left[{ }^{18} \mathrm{~F}\right] \mathrm{FDG}$ is the most widely used PET tracer with applications in oncologic, neurological, and cardiac imaging; but it is not target-specific, which can lead to false positives due to unrelated natural causes of increased glucose uptake and glycolysis like inflammation and muscle activity, and has high background in 
areas such as the heart, brain, and the vicinity of the bladder. $\left[{ }^{18} \mathrm{~F}\right] \mathrm{FLT}$, a DNA nucleoside analog of thymidine, is used to estimate cell proliferation, in many cases a more specific hallmark of malignant tumors.

The two-step reaction is illustrated in Figure 3. The first step is the fluorination reaction in which FLT precursor, 3-N-Boc-5'-Odimethoxytrityl-3'-O-nosyl-thymidinie, is labeled with activated $\left[{ }^{18} \mathrm{~F}\right]$ fluoride to produce the fluorinated intermediate, 3-N-Boc-5'O-dimethyltrityl-3'-deoxy-3'fluorothymidine. The second step is the hydrolysis reaction using $\mathrm{HCl}$ to remove the dimethoxytrityl and the tert-butoxycarbonyl protecting groups.
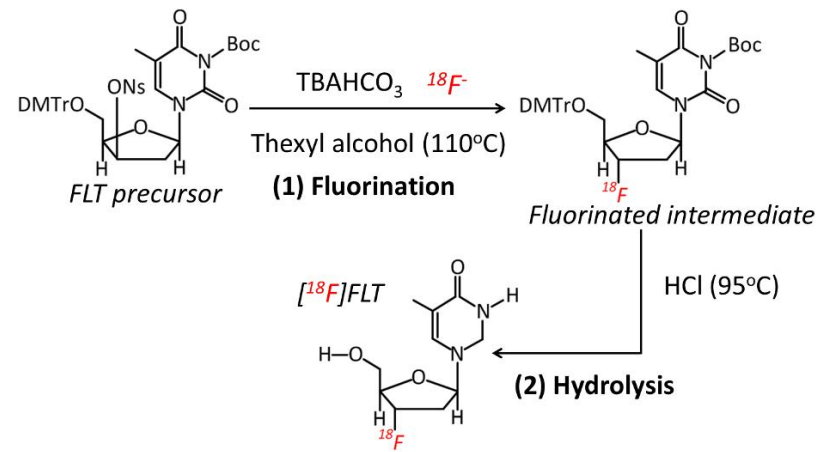

Figure 3: Two-step synthesis of $\Gamma^{18}$ FlFLT.

The multistep $\left[{ }^{18} \mathrm{~F}\right] \mathrm{FLT}$ synthesis was performed on EWOD: first, $3 \mu \mathrm{L}$ of the $\left[{ }^{18} \mathrm{~F}\right]$ fluoride mixture is pipetted onto the EWOD chip through a dedicated loading site on the cover plate edge and transported to the heater by EWOD actuation. This loading process is repeated an additional time. The $6 \mu \mathrm{L}\left[{ }^{18} \mathrm{~F}\right]$ fluoride mixture is heated to $105{ }^{\circ} \mathrm{C}$ for 1 minutes to evaporate the solvent. The $\left[{ }^{18} \mathrm{~F}\right]$ fluoride mixture is dried via azeotropic distillation by transporting three $\mathrm{MeCN}$ droplets $(3 \mu \mathrm{L}$ each) through the first loading edge into the heater site and heating at $105{ }^{\circ} \mathrm{C}$ for 1 minute. This drying step is repeated one additional time.

For the fluorination reaction, $4 \mu \mathrm{L}$ of FLT precursor in thexyl alcohol $(2 \mu \mathrm{L} ; 45 \mathrm{mM})$ and $4 \mu \mathrm{L}$ of $\mathrm{MeCN}$ are pipetted onto the EWOD chip through the second loading edge to avoid crosscontamination. The droplets are moved to the dried $\left[{ }^{18} \mathrm{~F}\right]$ fluoride residue on the heating site by electrowetting, and the reaction droplet is heated at $110^{\circ} \mathrm{C}$ for 5 minutes.

For the deprotection step, $2.5 \mu \mathrm{L}$ of $\mathrm{HCl}(1 \mathrm{~N})$ and $2.5 \mu \mathrm{L}$ of $\mathrm{MeCN}$ are added and transported to the heater region to perform the hydrolysis reaction. The reaction droplet is heated at $95{ }^{\circ} \mathrm{C}$ for 5 mins. After the synthesis, the cover plate is removed and the crude $\left[{ }^{18} \mathrm{~F}\right] \mathrm{FDG}$ product is extracted by pipette using $25 \mu \mathrm{L}$ of $\mathrm{MeCN} \mathrm{H}_{2} \mathrm{O}$ and $100 \mu \mathrm{L}$ of $\mathrm{H}_{2} \mathrm{O}$.

The product is then confirmed with high performance liquid

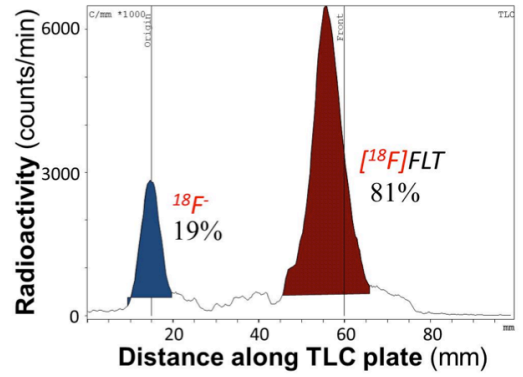

Figure 4: Radio-TLC analysis to determine radiofluorination efficiency of $\left[^{18} \mathrm{~F}\right] F L T$. chromatography (HPLC) and analyzed with radio-thin layer chromatography (TLC) (Figure 4). Fluorination yield on EWOD was measured to be $81 \% \pm 1 \%(n=4)$.

\section{Synthesis of tert-butyl $4-\left[{ }^{18}\right.$ F]fluorobenzoate}

Tert-butyl 4- $\left[{ }^{18} \mathrm{~F}\right]$ fluorobenzoate is the fluorinated intermediate of N-succinimidyl-4- $\left[{ }^{18} \mathrm{~F}\right]$ fluorobenzate ( $\left.\left[{ }^{18} \mathrm{~F}\right] \mathrm{SFB}\right)$, a prosthetic agent for radiolabeling antibodies and other biomolecules that are sensitive to harsh reaction conditions (temperature, solvents, $\mathrm{pH}$ ). The three-step synthesis of $\left[{ }^{18} \mathrm{~F}\right] \mathrm{SFB}$ is illustrated in Figure 5. The first step is the aromatic nucleophilic fluorination of the 4-(tert-butoxycarbonyl)-trimethylbenzene ammonium triflate (SFB precursor), which involved harsher reaction conditions in comparison to aliphatic fluorination reactions. The second step is a hydrolysis reaction using trifluoroacetic acid to hydrolyze the t-butyl ester. The third step for final product conversion is TSTU activation.

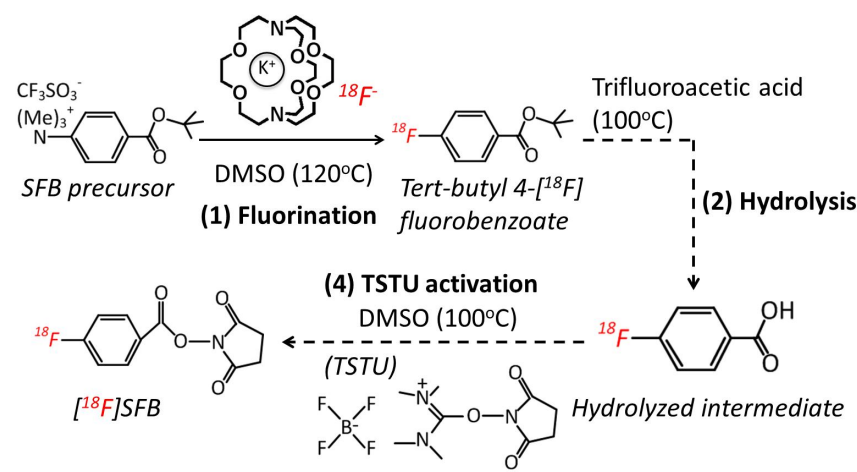

Figure 5: Three-step synthesis of $\left[{ }^{18} \mathrm{~F}\right] \mathrm{SFB}$.

For tert-butyl $4-\left[{ }^{18} \mathrm{~F}\right]$ fluorobenzoate synthesis on EWOD, 3 $\mu \mathrm{L}$ of $\left[{ }^{18} \mathrm{~F}\right]$ fluoride mixture pipetted onto the EWOD chip and moved by electrowetting to the heater. The solvent is evaporated at $105{ }^{\circ} \mathrm{C}$ for 1 minute. Two azeotropic drying steps are then performed in which $9 \mu \mathrm{L}$ of $\mathrm{MeCN}$ are added to the residue site and heated at $105{ }^{\circ} \mathrm{C}$ for 1 minute. For fluorination, $6 \mu \mathrm{L}$ of the SFB precursor dissolved in DMSO was loaded to the chip and heated at $120{ }^{\circ} \mathrm{C}$ for 12 minutes.

The reaction droplet was extracted and analyzed by HPLC and radio-TLC. Fluorination yield on EWOD was measured to be $83 \%+11 \%(n=4)$.

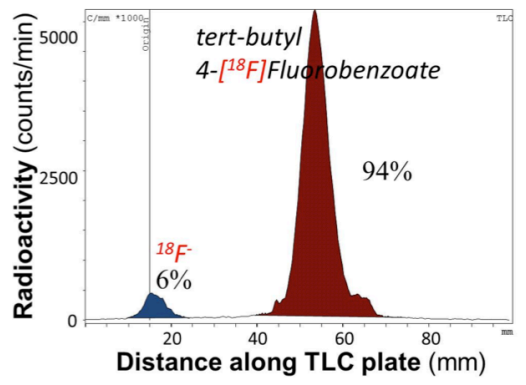

Figure 6: Radio-TLC analysis to determine radiofluorination efficiency of $\left[{ }^{18} \mathrm{~F}\right]$ tert-butyl $4-\left[{ }^{18} \mathrm{~F}\right]$ fluorobenzoate.

Synthesis of $\left[{ }^{18} \mathrm{~F}\right]$ fallypride

$\left[{ }^{18} \mathrm{~F}\right]$ fallypride is a dopamine receptor antagonist, useful for studying Alzheimer's and Parkinson's disease. $\left[{ }^{18} \mathrm{~F}\right] \mathrm{SFB}$ and $\left[{ }^{18} \mathrm{~F}\right]$ fallypride are not commercially available despite their proven effectiveness. $\left[{ }^{18} \mathrm{~F}\right]$ fallypride is synthesized by a single-step fluorination reaction as shown in Figure 7.

For this synthesis on EWOD, $7 \mu \mathrm{L}$ of $\left[{ }^{18} \mathrm{~F}\right]$ fluoride mixture 


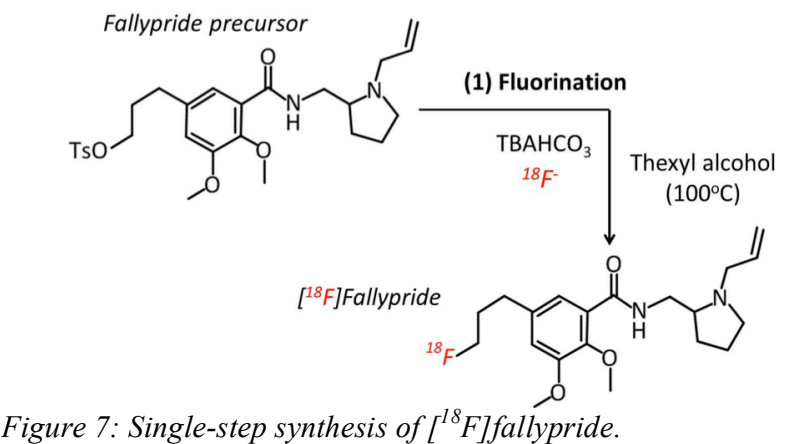

was pipetted onto the chip, moved to the heater by EWOD actuation, and heated at $105{ }^{\circ} \mathrm{C}$ for $1 \mathrm{~min}$. Azeotropic drying was performed twice by adding $12 \mu \mathrm{L}$ of $\mathrm{MeCN}$ and heating at $105^{\circ} \mathrm{C}$ for $1 \mathrm{~min}$. For fluorination, $2 \mu \mathrm{L}$ of FLT precursor and $6 \mu \mathrm{L}$ of $\mathrm{MeCN}$ were added and heated at $100{ }^{\circ} \mathrm{C}$ for 7 minutes. After extraction and analysis, $\left[{ }^{18} \mathrm{~F}\right]$ fallypride fluorination yield was measured to be $82 \% \pm 9 \%(n=3)$.

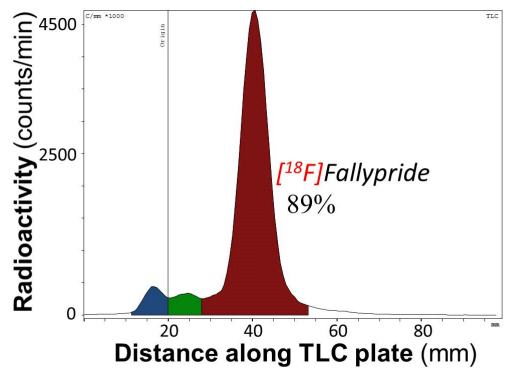

Figure 9: Radio-TLC analysis to determine radiofluorination efficiency of $\left[{ }^{18} \mathrm{~F}\right]$ fallypride.

\section{CONCLUSION}

We have demonstrated the synthesis of diverse tracers from cyclotron-produced $\left[{ }^{18} \mathrm{~F}\right]$ fluoride solutions using one design of an EWOD device with multifunctional electrodes for heating. All 4 radiolabeled compounds have been reliably synthesized on EWOD chip with conversion efficiencies between $70-90 \%$, comparable to or greater than those of commercially available macroscale commercial synthesizers (Table 2). In addition to aliphatic nucleophilic fluorination reactions, we have also demonstrated aromatic nucleophilic fluorination, which generally requires harsher reaction conditions. This success indicates the final goal

Table 2: Four diverse radiolabeled molecules synthesized by EWOD with the same chip design.

\begin{tabular}{|c|c|c|c|c|c|}
\hline Tracer & Molecule & Application & $\begin{array}{c}\text { Radiopharmacy } \\
\text { availability }\end{array}$ & \begin{tabular}{|c|}
$\begin{array}{c}\text { Fluorination } \\
\text { yield } \\
\text { (on EWOD) }\end{array}$ \\
\end{tabular} & $\begin{array}{l}\text { Hydrolysis } \\
\text { yield } \\
\text { (on EWOD) } \\
\end{array}$ \\
\hline $\begin{array}{c}\Gamma^{18} \text { Flfluoro-2- } \\
\text { deoxy-D-glucose } \\
\left(I^{18} F \mid F D G\right)\end{array}$ & HOS & $\begin{array}{c}\text { Sugar } \\
\text { metabolism }\end{array}$ & Yes & $\begin{array}{c}89 \% \pm 9 \% \\
(n=3)\end{array}$ & $\begin{array}{c}97 \% \pm 3 \% \\
(n=3)\end{array}$ \\
\hline $\begin{array}{c}3^{\prime} \text {-deoxy- } 3^{\prime}-I^{8} \mathrm{FJ} \\
\text { fluorothymidine } \\
\left(I^{18} \mathrm{~F} / \mathrm{FLT}\right)\end{array}$ & & $\begin{array}{c}\text { DNA } \\
\text { proliferation }\end{array}$ & Limited & $\begin{array}{c}81 \% \pm 1 \% \\
(n=4)\end{array}$ & $\begin{array}{c}100 \% \pm 0 \% \\
(\mathrm{n}=4)\end{array}$ \\
\hline $\begin{array}{l}\left.\text { tert-butyl } 4-\Gamma^{18} \mathrm{~F}\right] \\
\text { fluorobenzoate }\end{array}$ & & $\begin{array}{l}\text { Protein } \\
\text { labeling }\end{array}$ & No & $\begin{array}{c}83 \% \pm 11 \% \\
(n=4)\end{array}$ & (not applicable) \\
\hline${ }^{18}$ Flfallypride & & $\begin{array}{c}\text { Neural } \\
\text { transmitter } \\
\text { binding }\end{array}$ & No & $\begin{array}{c}82 \% \pm 9 \% \\
(n=3)\end{array}$ & (not applicable) \\
\hline & & & & & \\
\hline
\end{tabular}

of an affordable, reliable, and flexible synthesizer, which would empower final users to produce tracers of their choice locally, is reachable in the foreseeable future.

We have shown that EWOD-based digital microfluidic reactions can carry out high-yield chemical reactions, and provided evidence of an additional advantage in increased specific activity of the tracers. We will investigate EWOD device geometry further to understand the factors that influence specific activity and develop a more generic synthesizer EWOD chip for increased specific activity. The efficacy of the synthesized PET tracers will be demonstrated by use in small animal preclinical imaging.

\section{ACKNOWLEDGEMENT}

This work was supported in part by the UCLA Foundation from a donation made by Ralph \& Marjorie Crump for the UCLA Crump Institute for Molecular Imaging and the Department of Energy [DE-SC0005056]. We thank Prof. David Stout for use of radiolabeling facilities, Dr. Gaurav Shah for initial demonstration of the EWOD compatibility with typical radiochemistry solvents, Dr. Wyatt Nelson for developing the multi-functional EWOD electrodes, and Dr. Saman Sadeghi and Bob Silverman for developing the multi-channel temperature controller and software.

\section{REFERENCES}

[1] M.E. Phelps, "Positron emission tomography provides molecular imaging of biological processes", Proc. National Academy of Science, 97, 9226-9233, (2000).

[2] R. Iwata, Reference book for PET radiopharmaceuticals, CYRIC, Tohoku University, Sendai, Japan, 2004.

[3] S. Chen, H. Ding, G.J. Shah, R.M. van Dam, and C.-J. Kim, "EWOD microdevices for synthesis of ${ }^{18} \mathrm{~F}$-labeled tracers for positron emission tomography (PET)", Tech. Dig. Solid-State Sensor and Actuator Workshop, Hilton Head Island, SC, 6/66/10, Transducer Research Foundation, (2010), pp. 37-40.

[4] S. Chen, P.Y. Keng, G.J. Shah, R.M. van Dam, and C.-J. Kim, "Synthesis of ${ }^{18} \mathrm{~F}$-labeled probes on EWOD platform for positron emission tomography (PET) preclinical imaging", Proc' Int. Conf. MEMS, Cancun, Mexico, 1/23-1/27, IEEE, (2011), pp. 980-983.

[5] P.Y. Keng, S. Chen, H. Ding, S. Sadeghi, G.J. Shah, A. Dooraghi, M.E. Phelps, N. Satyamurthy, A.F. Chatziioannou, C.J. Kim, and R.M. van Dam, "Micro-chemical synthesis of molecular probes on an electronic microfluidic device", Proc. National Academy of Science, 109, 3, 690-695, (2012).

[6] A.M. Elizarov, "Microreactors for radiopharmaceutical synthesis", Lab Chip, 9, 10, 1326-1333 (2009).

[7] R. Bejot, A.M. Elizarov, E. Ball, J. Zhang, R. Miraghaie, H.C. Kolb, and V. Gouverneur, "Batch-mode microfluidic radiosynthesis of $\mathrm{N}$-succinimidyl-4-[ $\left.{ }^{18} \mathrm{~F}\right]$ fluorobenzoate for protein labeling", J Label Compd. Radiopharm, 54, 3, 117-122 (2011).

[8] M.A. Eddings, S. Olma, M. Wang, Y. Deng, H. Ding, N. Satyamurthy, K. Shen, and R.M. van Dam, "Automated Radiochemistry Platform (ARC-P): plug-and-play radiochemistry modules for reconfigurable radiosynthesis," Int. Symp. Radiopharmaceutical Sciences, Edmonton, Canada, 7/12, Society of Radiopharmaceutical Sciences (2009), poster.

[9] M.P. Kung and H.F. Kung, "Mass effect of injected dose in small rodent imaging by SPECT and PET," Nuclear Medicine and Biology, 32, 7, 673-678, (2005).

[10] B.S. Moon, J.H. Park, H.J. Lee, J.S. Kim, H.S. Kil, B.S. Lee, D.Y. Chi, B.C. Lee, Y.K. Kim, and S.E. Kim, "Highly efficient production of $\left[{ }^{18} \mathrm{~F}\right]$ fallypride using small amounts of base concentration," Applied Radiation and Isotopes, 68, 12, 22792284 (2010).

\section{CONTACT:}

*Supin Chen, tel: +1-310-825-3977; supin.chen@engineering.ucla.edu 


\title{
TOBACCO MOSAIC VIRUS BIOTEMPLATED ELECTROCHEMICAL BIOSENSOR
}

\author{
Hadar Ben-Yoav ${ }^{l^{*}}$, Adam D. Brown ${ }^{2}$,Ekaterina Pomerantseval ${ }^{1}$, Deanna L. Kelly, James N. Culver ${ }^{2}$,
} and Reza Ghodssi $i^{*}$

${ }^{1}$ MEMS Sensors and Actuators Laboratory, Department of Electrical and Computer Engineering, Institute for

Systems Research,

\author{
${ }^{2}$ Institute for Bioscience and Biotechnology Research, University of Maryland, College Park, Maryland, USA
}

${ }^{3}$ Maryland Psychiatric Research Center, University of Maryland School of Medicine, Baltimore, Maryland, USA

\section{ABSTRACT}

This work demonstrates the first utilization of virus molecules as nano-scale biotemplates assembled on an electrochemical biosensor, allowing for an 8-times increased signal and an improved biosensing performance of 9.5 -fold. The versatile and inexpensive biological Tobacco mosaic virus was integrated as a high aspect ratio, low footprint, low-cost, easy to genetically functionalize, nanostructured three-dimensional scaffold for the synthesis of novel multifunctional electrodes. The biotemplated scaffold allows for an increased surface area resulting in higher electrochemical currents, better signal-to-noise ratio and improved sensitivity when incorporated into miniaturized biosensors.

\section{INTRODUCTION}

\section{Electrochemical Biosensors}

Electrochemical biosensors are based on a bioelectrochemical interaction process, where electrochemical species are consumed or generated. Most electrochemical measurements detect oxidation/reduction of the product generated by biological conversion of the analyte undergoing redox reactions on the sensor's surface and solely respond to electro-active species. Electrochemical measurements are classified according to the variable being measured: amperometry, potentiometry and conductometry $[1,2]$.

In recent decades, nanostructured materials have received much attention due to unique properties they offer as robust platforms for electronic and optical signal transduction. One of its major contributions is the design of a new generation of miniaturized biosensing devices. Furthermore, biofunctional nanoparticles can produce a synergistic effect between catalytic activity, conductivity, and biocompatibility to accelerate the signal transduction, leading to the quick development of stable, specific, selective and sensitive biosensors in different fields. An important point besides the biosensing applicability of nanomaterials is the use of manipulation techniques for their integration in fabrication techniques [3]. By integrating with electrochemical sensor fabrication, these materials provide interesting properties such as increased surface area. The augmented surface area leads to higher signal-to-noise ratio, increased sensitivity and dynamic range, short distances for mass transport and charge transfer as well as their ability to create complex nano-bio-architectures that allow volume change and unique selective biological functionality. Nanomaterials allow the development of new architectures applied in electrochemical sensing and biosensing devices [4, 5]. For example, $\mathrm{Ye}$ and colleagues presented a multi-walled carbon nanotube (MWNT)-based electrochemical biosensor for glucose detection [5]. The higher surface area of the well-aligned MWNT generated higher electrochemical currents that were correlated to a highly sensitive sensor.

\section{Tobacco mosaic virus}

Biological nanomaterials provide a versatile and cost effective solution for the fabrication of high surface area nanoarchitectures. One category of these biological nanostructures is plant and bacteria virus particles consisting of macromolecular assemblies of nucleic acid packaged by many copies of coat proteins. These molecules display some unique advantages as they show exceptional stability in a wide range of temperatures and $\mathrm{pH}$ values, in addition to their surface-exposed functional groups, selfassembly and tunability [6].

Among the available plant viruses [7], Tobacco mosaic virus (TMV) is the most extensively studied filamentous plant structure for nanoscale applications. The TMV virion is a rigid rod consisting of about 2,130 identical coat protein subunits stacked in a helix around a single strand of plus sense RNA, forming a $4 \mathrm{~nm}$ diameter channel through the $300 \mathrm{~nm}$ long virion axis. Properties of the TMV system that make it particularly useful as a selfassembling macromolecular template for nanomaterials include: 1) its known three-dimensional structure [8]; 2) a wealth of biophysical information on its self-assembly characteristics [9]; 3) the availability of creating novel virus structures and surfaces via established molecular techniques [10]; 4) a wide range of existing coat protein variants with diverse assembly properties [11], and 5) the ability to easily purify large quantities of virus and coat protein from infected plants. TMV-structured metal and metal-oxide nanowires have been synthesized using several techniques [12, 13], transport properties have been studied [14], and potential applications in nano-scale devices have been investigated through proof-of-concept demonstrations [15].

Previous work with engineered mutations of the TMV has resulted in enhanced particle coatings and templates that can be readily integrated into microfabricated devices and has established a novel patterning process. Efficient templates for metallic coatings have been achieved through the introduction of one (TMV-1cys) or two (TMV-2cys) cysteine residues within the coat protein open reading frame. Cysteines are amino acids with thiol groups that show enhanced metal binding properties based on strong, covalent-like interactions. One and two-step electroless plating methods have been used for the fabrication of TMV-2cysbased wires coated with gold, silver and palladium clusters that show more uniform coating compared to the wild-type virus [16]. Additionally, the rod-shaped viruses can be directionally attached to various surfaces and coated to create high aspect ratio nickel, cobalt and platinum materials (TMV-1cys). Alternative pathways were explored for patterning the viral molecules in microfabricated electrodes as well as controlled environments, utilizing nucleic acid hybridization [17]. Recent work in our team has focused upon the development of novel inorganic structures using the TMV and their application in energy storage devices. A simple and versatile approach for the selective patterning of both metal-coated and uncoated TMV using lift-off processing has been developed [18]. The high aspect ratio of the coated TMV in addition to its robustness was utilized in the development of high surface area nickel-zinc [19] and Li-ion microbatteries [20, 21]. By the integration of high aspect ratio biotemplated TMV scaffold in electrode fabrication process, high surface area electrochemical sensors can be realized improving the overall biosensing performance. 


\section{Mental Health Applications}

The medical management of mental health care is one of the most debilitating and costly of all disorders. A major unmet need is the ability to have an objective, real time analysis of the disorder at the point-of-care. Schizophrenia is a common mental health disorder, has high social and economic impact, and manifests early - mostly during adolescence and early adulthood. It is frequently preceded by premorbid pattern of rather unspecific but nevertheless handicapping symptoms that may be present for several years before clinical manifestation. Schizophrenia is a complex disorder involving difficulties with reality distortion, cognitive impairments, and work and social dysfunction. Often, it is also associated with symptoms of moodiness and anxiety. Once diagnosed, patients need to remain on antipsychotic medications lifelong. One of the biggest challenges of treatment is that many people discontinue their treatment which can lead to relapse and rehospitalizations, having a major impact on patient and society healthcare costs [22, 23]. Finding a method for continually monitoring patient status would allow for psychiatrists to more effectively treat these mental disorders.

Over the past few years, genetic analysis has found a strong relation between the gene encoding for Neuregulin-1 (NRG1) and schizophrenia [24]. Studies have obtained supporting evidence for NRG1 as a candidate gene for the disorder [25]. Moreover, the identification of NRG1 receptor ErbB4 as an additional candidate risk gene for schizophrenia strongly suggests that this signaling pathway participates in the pathophysiology of the disorder [26]. By monitoring NRG1 concentration during various stages of schizophrenia, mental health care management can be studied and improved.

Microfluidic lab-on-a-chip (LOC) microsystems provide numerous advantages in clinical diagnostics, environmental monitoring and biomedical research fields. These translational technologies hold potential to improve upon the resolution, regulation, sensitivity, flexibility, and cost-savings over more traditional approaches, bringing bench top methods into the pointof-care. By the integration of MEMS sensing devices with mental health analysis, pre-clinical assessments and the overall chance of success of personalized medical care at the point-of-care can be significantly improved.

\section{RESULTS}

One of the challenges with miniaturized biosensing devices is the high background signal and low signal-to-noise ratio that decrease the performance of biosensors. By increasing the signal, electrochemical biosensors can improve their bio-detection efficiency resulting in higher sensitivity. Here, we used gold planar square electrodes $(20 \mathrm{~nm} \mathrm{Cr} / 180 \mathrm{~nm} \mathrm{Au}$, surface area $=0.49$ $\mathrm{cm}^{2}$ ) that were patterned onto a silicon dioxide substrate via DC sputtering and were realized using both wet etching and lift-off techniques. Virus modification of the electrode surface was performed to enhance the total surface area. Fabrication was carried out by submerging the electrodes in a solution of rodshaped $300 \times 18 \mathrm{~nm}$ TMV vertical viruses in $0.1 \mathrm{M}$ phosphate buffer ( $\mathrm{pH} 7$ ) for 18 hours, allowing for self-assembly of the virus particles on the microfabricated planar gold electrodes via the previously mentioned cysteine residues. Following TMV selfassembly, nickel was electroless deposited for 5 minutes [19]. Sequentially, gold was electroless plated in a gold plating bath $\left(0.007 \mathrm{M} \mathrm{KAu}(\mathrm{CN})_{2}, 1.4 \mathrm{M} \mathrm{NH} \mathrm{Nl}_{4}, 0.2 \mathrm{M}\right.$ Sodium citrate, and $1 \mathrm{M}$ $\mathrm{NaH}_{2} \mathrm{PO}_{2}$. $\mathrm{pH}$ 7.0-7.5) for 45 minutes at $90^{\circ} \mathrm{C}$ to form a conformal gold coating on the virus particles. Fig. 1 shows SEM images of the surface of the electrode modified with nickel-coated TMV-1cys after gold deposition (TMV/Ni/Au modified electrode). TMV- 1cys were observed to attach vertically on the electrode surface. A TEM image of the nanocomposite virus particle (Fig. 2) shows approximately $67 \mathrm{~nm}$ nickel and $46 \mathrm{~nm}$ gold coatings. The thin films of the nickel and the gold maintain the high aspect ratio of the TMV scaffold after plating, increasing the electrochemically active surface area of the electrode.
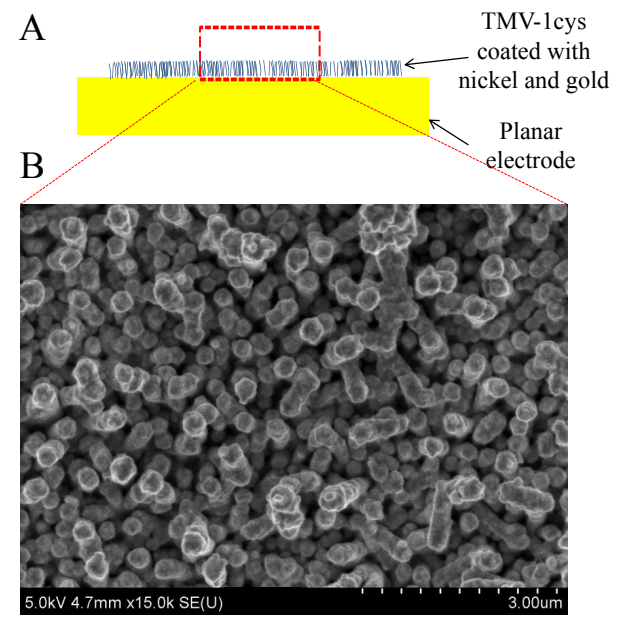

Figure 1: Self-assembled TMV-1cys molecules coated with nickel and gold layers on the surface of planar electrode. (A) Cross section schematic. (B) Scanning electron micrograph.

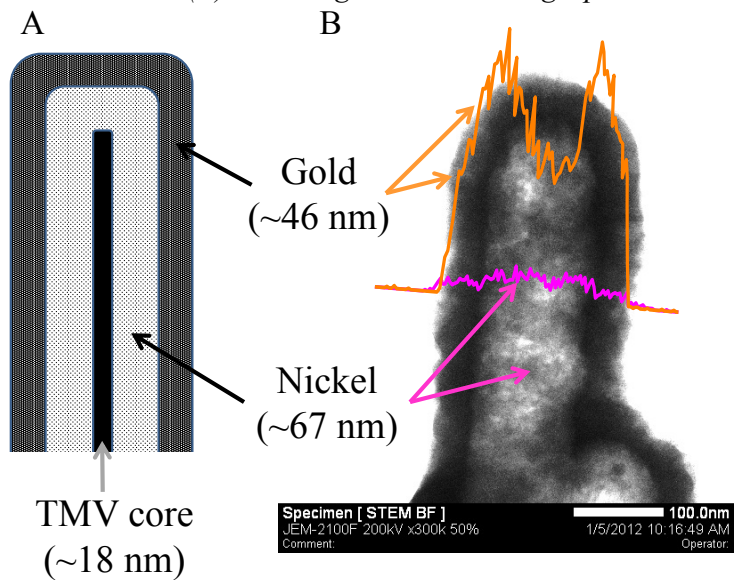

Figure 2: (A) Schematic of the TMV coatings. (B) Transmission electron microscopy analysis of the TMV-1cys coated with nickel and gold layers with EDS profiles of nickel (pink / dark gray) and gold (orange / light gray).

The modified electrodes were electrochemically characterized using cyclic voltammetry (CHI660D single channel potentiostat from $\mathrm{CH}$ Instruments, Austin, TX. Commercial Ag/AgCl reference electrode. Pt wire counter electrode) in the presence of a redox couple $5 \mathrm{mM}$ ferrocyanide, $5 \mathrm{mM}$ ferricyanide, $10 \mathrm{mM}$ phosphate buffers saline (PBS) solution. Fig. 3 illustrates the increased signal from the modified electrodes compared to unmodified electrode with cyclic voltammograms of the generated electrochemical reduction and oxidation reactions. The electrodes produced reversible nernstian characteristics where the $\mathrm{TMV} / \mathrm{Ni} / \mathrm{Au}$ modified electrode generated the highest electrochemical current (8-fold higher oxidation peak current in comparison with the unmodified planar electrode) due to the high active surface area. The oxidation and the reduction peaks generated by the $\mathrm{TMV} / \mathrm{Ni} / \mathrm{Au}$ modified electrodes varied in compare to the other types of electrodes. This variation is may be due to increase with the uncompensated resistance in the new high aspect ratio electrochemical interface. Furthermore, the potential presence of 
nickel uncoated with gold on the TMV scaffold may result in changes with the standard reduction potential of the redox couple. The increased electrochemical activity of the $\mathrm{Ni} / \mathrm{Au}$ modified electrode compared to unmodified electrode may be due high surface roughness resulted by the nickel and the gold electroless plating. Furthermore, the TMV modified electrode impeded the generated electrochemical current that was attributed to the nonconductive virus coating on the electrode surface.

The effective surface area of the unmodified and the modified electrodes were calculated from Bard and Faulkner [27]:

$$
I_{\text {peak }}=0.4463\left(F^{3} / R T\right) n^{3 / 2} A D^{1 / 2} C^{*} v^{I / 2}
$$

Where $F\left[\mathrm{C} \mathrm{mol}^{-1}\right]$ is the Faraday constant, $R\left[\mathrm{~J} \mathrm{~mol}^{-1} \mathrm{~K}^{-1}\right]$ is the gas constant, $T[\mathrm{~K}]$ is the temperature, $n$ is the stoichiometric number of electrons involved in an electrode reaction, $A\left[\mathrm{~cm}^{2}\right]$ is the effective surface area of the electrode, $D\left[\mathrm{~cm}^{2} \mathrm{~s}^{-1}\right]$ is the diffusion coefficient of the electro-active species, $C^{*}\left[\mathrm{~mol} \mathrm{~cm}^{-3}\right]$ is the bulk concentration of the electro-active species, $v\left[\mathrm{~V} \mathrm{~s}^{-1}\right]$ is the linear potential scan rate, and $I_{\text {peak }}[\mathrm{A}]$ is the peak current. Effective surface area calculations demonstrated that TMV/Ni/Au resulted in the highest area (Table 1), a characteristic important for the high performance of electrochemical sensors due to the surface area limited reaction kinetics.

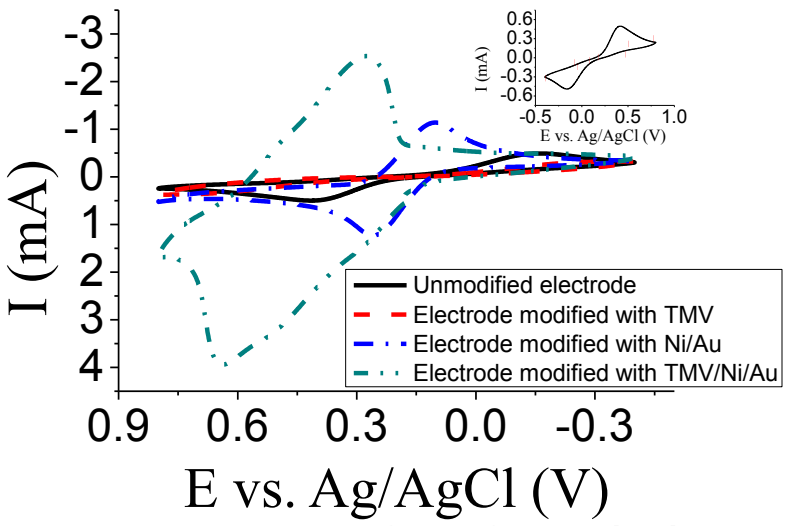

Figure 3: Cyclic voltammograms of the planar electrode modified with either the TMV, the Ni/Au electroless deposition, or both. Inset shows only the unmodified and the TMV modified electrodes.

Table 1: Measured effective surface area and bio-detection efficiency analysis for the unmodified and the modified electrodes.

\begin{tabular}{|c|c|c|}
\hline $\begin{array}{c}\text { Electrode } \\
\text { modification }\end{array}$ & $\begin{array}{c}\text { Effective } \\
\text { surface area } \\
{\left[\mathbf{c m}^{2}\right]}\end{array}$ & $\begin{array}{c}\text { Bio-detection } \\
\text { efficiency [\%] }\end{array}$ \\
\hline Unmodified & 0.42 & 1.7 \\
\hline $\mathrm{TMV}$ & 0.32 & 2.1 \\
\hline $\mathrm{Ni} / \mathrm{Au}$ & 1.05 & $\begin{array}{c}-1.1 \text { (poor } \\
\text { efficiency) }\end{array}$ \\
\hline $\mathrm{TMV} / \mathrm{Ni} / \mathrm{Au}$ & 3.34 & $\mathbf{1 6 . 1}$ \\
\hline
\end{tabular}

In this study the target NRG1 polymorphism ssDNA (SNP8NRG243177) bio-detection efficiency was electrochemically analyzed using thiolated ssDNA probe to NRG1 assembled on the high surface area $\mathrm{TMV} / \mathrm{Ni} / \mathrm{Au}$ modified electrode. In short, electrodes were incubated in a solution containing $10 \mathrm{mM}$ PBS, $100 \mathrm{mM} \mathrm{NaCl}, 10 \mu \mathrm{M}$ Tris (2carboxyethyl) phosphine (TCEP) and $1 \mu \mathrm{M}$ probe ssDNA for 3 hour followed by rinsing with PBS. Afterwards, the electrodes were incubated for 24 hour in PBS solution containing $1 \mathrm{mM}$ of 6mercapto-1-hexanol (MCH). $\mathrm{MCH}$ is used to passivate any exposed regions on the surface to reduce non-specific binding during DNA hybridization. Incubation with either the noncomplementary or the target sequences was performed in a $4 x$ saline sodium citrate (SSC) buffer containing $1 \mu \mathrm{M}$ of the target DNA for 1 hour.

Biosensing performance was studied with cyclic voltammetry in the presence of the previously described redox ferrocyanide/ferricyanide solution (Fig. 4). All electrodes demonstrated reversible nernstian characteristics in the presence of complementary NRG1 target and non-complementary ssDNA. Moreover, the TMV/Ni/Au modified electrode demonstrated the largest current differentiation upon DNA hybridization. DNA hybridization causes stronger electrostatic repulsion forces with the negatively charged electro-active species in the electrolyte, hence impeding the electrochemical reaction at the electrode [28]. Biodetection efficiency (ratio of oxidation peak current decrease between non-complementary and complementary ssDNA) analysis (Table 1) demonstrated 9.5-fold improved bio-detection performance for the 3D TMV/Ni/Au modified electrode compared to the unmodified electrode. This increase is not only due to the higher effective surface area (factor of 8), but also due to the inherent properties of nanomaterials improving biosensing performance, e.g. augmented amount of probes functionalizing the surface of the electrode and heightened target concentration near the electrochemically reactive surface.

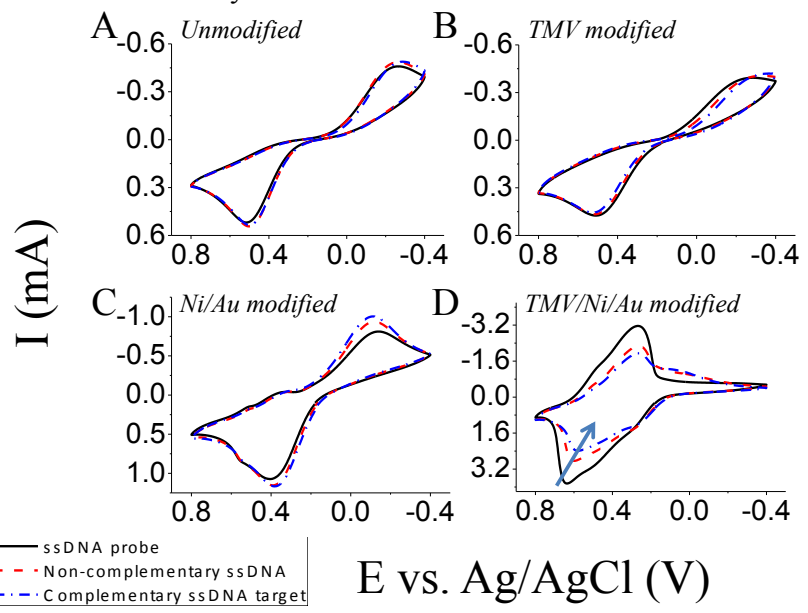

Figure 4: Cyclic voltammograms of the (A) planar unmodified electrode, (B) TMV modified electrode, (C) Ni/Au modified electrode, and (D) TMV/Ni/Au modified electrode following introduction of ssDNA NRG1 probe (black solid), noncomplementary sSDNA (red dashed), and complementary NRG1 target SSDNA (blue dashed-dot). Arrow indicates current reduction upon DNA hybridization.

\section{CONCLUSIONS}

This work demonstrated the first utilization of TMV molecules coated with nickel and gold as nano-scale biotemplates assembled on an electrochemical biosensor for schizophrenia analysis. The biotemplated TMV modified sensor generated heightened electrochemical activity that improved the biosensing performance. Given the large set of biological recognition events that can be detected electrochemically and the innately high compatibility of TMV-biotemplate integration with conventional microfabrication technology, we believe this work has broad applications in biological electrochemical sensing and transduction. Specifically, we envision the utilization of TMV as a high aspect ratio nano-scale 3D scaffold for high surface area microelectrodes integrated in MEMS sensing devices improving 
dramatically their performance. We believe TMV-biotemplated LOC devices leverage electrochemical biosensing reactions that will lead to the creation of new families of low-cost, rapid and sensitive miniaturized biosensors for point-of-care analysis.

\section{ACKNOWLEDGMENTS}

The authors acknowledge the Robert W. Deutsch Foundation and National Science Foundation Emerging Frontiers in Research and Innovation (EFRI) for financial support. The authors also thank the Maryland Nanocenter and its Fablab for cleanroom facility support. The authors grateful to Prof. Yosi ShachamDiamand and Prof. Alexandra Inberg from the Tel Aviv University for the fruitful discussion on gold electroless deposition.

\section{REFERENCES}

[1] A. Le Goff, M. Holzinger, and S. Cosnier, "Enzymatic Biosensors based on SWCNT-Conducting Polymer Electrodes", Analyst, 136, 1279 (2011).

[2] W. Siangproh, W. Dungchai, P. Rattanarat, and O. Chailapakul, "Nanoparticle-based Electrochemical Detection in Conventional and Miniaturized Systems and Their Bioanalytical Applications: A Review", Analytica Chimica Acta, 690, 10, (2011).

[3] R.H. Baughman, A.A. Zakhidov, and W.A. de Heer, "Carbon Nanotubes - The Route Toward Applications”, Science, 297, 787 (2002).

[4] J. Wang, "Carbon-Nanotube based Electrochemical Biosensors: A Review”, Electroanalysis, 17, 7 (2005).

[5] J.-S. Ye, Y. Wen, W. De Zhang, L. Ming Gan, G.Q. Xu, and F.-S. Sheu, "Nonenzymatic Glucose Detection using Multiwalled Carbon Nanotube Electrodes", Electrochemistry Communications, 6, 66 (2004).

[6] D.J. Evans, "The Bionanoscience of Plant Viruses: Templates and Synthons for New Materials", Journal of Materials Chemistry, 18, 3746 (2008).

[7] J.C. Smith, K.-B. Lee, Q. Wang, M.G. Finn, J.E. Johnson, M. Mrksich, and C.A. Mirkin, "Nanopatterning the Chemospecific Immobilization of Cowpea Mosaic Virus Capsid", Nano Letters, 3, 883 (2003).

[8] K. Namba, R. Pattanayek, and G. Stubbs, "Visualization of Protein-Nucleic Acid Interactions in a Virus: Refined Structure of Intact Tobacco Mosaic Virus at $2.9 \AA$ Resolution by X-ray Fiber Diffraction", Journal of Molecular Biology, 208, 307 (1989).

[9] A.C. Durham, J.T. Finch, and A. Klug, "States of Aggregation of Tobacco Mosaic Virus Protein", Nature New Biology, 229, 37 (1971).

[10] J.N. Culver, W.O. Dawson, K. Plonk, and G. Stubbs, "SiteDirected Mutagenesis Confirms the Involvement of Carboxylate Groups in the Disassembly of Tobacco Mosaic Virus", Virology, 206, 724 (1995).

[11] J.N. Culver, "Tobacco Mosaic Virus Assembly and Disassembly: Determinants in Pathogenicity and Resistance", Annual Review of Phytopathology, 40, 287 (2002).

[12] M. Knez, M. Sumser, A.M. Bittner, C. Wege, H. Jeske, T.P. Martin, and K. Kern, "Spatially Selective Nucleation of Metal Clusters on the Tobacco Mosaic Virus", Advanced Functional Materials, 14, 116 (2004).

[13] M. Knez, A. Kadri, C. Wege, U. Gösele, H. Jeske, and K. Nielsch, "Atomic Layer Deposition on Biological Macromolecules: Metal Oxide Coating of Tobacco Mosaic Virus and Ferritin", Nano Letters, 6, 1172 (2006).

[14] M. Knez, M. Sumser, A.M. Bittner, C. Wege, H. Jeske, S.
Kooi, M. Burghard, and K. Kern, "Electrochemical Modification of Individual Nano-Objects", Journal of Electroanalytical Chemistry, 522, 70 (2002).

[15] R.J. Tseng, C. Tsai, L. Ma, J. Ouyang, C.S. Ozkan, and Y. Yang, "Digital Memory Device based on Tobacco Mosaic Virus Conjugated with Nanoparticles", Nature Nanotechnology, 1, 72 (2006).

[16] S.-Y. Lee, E. Royston, J.N. Culver, and M.T. Harris, "Improved Metal Cluster Deposition on a Genetically Engineered Tobacco Mosaic Virus Template", Nanotechnology, 16, S435 (2005).

[17] H. Yi, S. Nisar, S.-Y. Lee, M.A. Powers, W.E. Bentley, G.F. Payne, R. Ghodssi, G.W. Rubloff, M.T. Harris, and J.N. Culver, "Patterned Assembly of Genetically Modified Viral Nanotemplates via Nucleic Acid Hybridization", Nano Letters, 5, 1931 (2005).

[18] K. Gerasopoulos, M. McCarthy, P. Banerjee, X. Fan, J.N. Culver, and R. Ghodssi, "Biofabrication Methods for the Patterned Assembly and Synthesis of Viral Nanotemplates", Nanotechnology, 21, 055304 (2010).

[19] K. Gerasopoulos, M. McCarthy, E. Royston, J.N. Culver, and R. Ghodssi, "Nanostructured Nickel Electrodes using the Tobacco mosaic virus for Microbattery Applications", Journal of Micromechanics and Microengineering, 18, 104003 (2008).

[20] E. Pomerantseva, K. Gerasopoulos, X. Chen, G. Rubloff, and R. Ghodssi, "Electrochemical Performance of the Nanostructured Biotemplated V2O5 Cathode for Lithium-ion Batteries”, Journal of Power Sources, 206, 282 (2012).

[21] K. Gerasopoulos, X. Chen, J. Culver, C. Wang, and R. Ghodssi, "Self-assembled Ni/TiO2 Nanocomposite Anodes Synthesized via Electroless Plating and Atomic Layer Deposition on Biological Scaffolds", Chemical Communications, 46, 7349 (2010).

[22] R.A. Carlstedt, Handbook of Integrative Clinical Psychology, Psychiatry, and Behavioral Medicine: Perspectives, Practices, and Research, Springer Publishing Company, New York, 2010.

[23] B.J. Sadock, H.I. Kaplan, and V.A. Sadock, Kaplan \& Sadock's Synopsis of Psychiatry: Behavioral Sciences/Clinical Psychiatry, $10^{\text {th }}$ ed., Lippincott Williams \& Wilkins, Philadelphia, 2007.

[24] B. Rico and O. Marín, "Neuregulin Signaling, Cortical Circuitry Development and Schizophrenia", Current Opinion in Genetics \& Development, 21, 262 (2011).

[25] P.J. Harrison and A.J. Law, "Neuregulin 1 and Schizophrenia: Genetics, Gene Expression, and Neurobiology", Biological Psychiatry, 60, 132 (2006).

[26] T. Walsh, J.M. McClellan, S.E McCarthy, A.M. Addington, S.B. Pierce, G.M. Cooper, A.S. Nord, M. Kusenda, D. Malhotra, A. Bhandari et al., "Rare Structural Variants Disrupt Multiple Genes in Neurodevelopmental Pathways in Schizophrenia", Science, 320, 539 (2008).

[27] A.J. Bard and L.R. Faulkner, Electrochemical Methods: Fundamentals and Applications, John Wiley \& Sons, New York, 2001.

[28] E. Katz and I. Willner, "Probing Biomolecular Interactions at Conductive and Semiconductive Surfaces by Impedance Spectroscopy: Routes to Impedimetric Immunosensors, DNASensors, and Enzyme Biosensors", Electroanalysis, 15, 913 (2003).

\section{CONTACT}

*Reza Ghodssi, tel: +1-301-405-8158; GHODSSI@UMD.edu Hadar Ben-Yoav, tel: +1-301-405-2168; BENYOAV@,UMD.edu 


\section{HIGH-DENSITY, BIO-COMPATIBLE, AND HERMETIC ELECTRICAL FEEDTHROUGHS USING EXTRUDED METAL VIAS

\author{
K. G. Shah ${ }^{1 *}$, T. DeLima ${ }^{l}$, S. Felix ${ }^{l}$, H. Sheth ${ }^{l}$, V. Tolosa ${ }^{l}$, A. Tooker ${ }^{l}$, and S. Pannu ${ }^{l}$
} \\ ${ }^{1}$ Lawrence Livermore National Laboratory, Livermore, California, USA}

\begin{abstract}
Implanted medical devices such as pacemakers and neural prosthetics require that the electronic components that power these devices are protected from the harsh chemical and biological environment of the body. Typically, the electronics are hermetically sealed inside a bio-compatible package containing feedthroughs that transmit electrical signals, while being impermeable to particles or moisture. We present a novel approach for fabricating one of the highest densities of biocompatible hermetic feedthroughs in alumina $\left(\mathrm{Al}_{2} \mathrm{O}_{3}\right)$. Alumina substrates with laser machined vias of $200 \mu \mathrm{m}$ pitch were conformally metallized and lithographically patterned. Hermetic electrical feedthroughs were formed by extruding metal studbumps partially through the vias. Hermeticity testing showed leak rates better than $9 \times 10^{-10}$ torr-1/s. Based on our preliminary results and process optimization, this extruded metal via approach is a high-density, low temperature, cost-effective, and robust method of miniaturizing electrical feedthroughs for a wide range of implantable bio-medical device applications.
\end{abstract}

\section{INTRODUCTION}

\section{Background and Context}

Electrically active bio-medical devices (such as pacemakers and neural prosthetics) have the ability to diagnose, monitor, and treat a wide range of diseases [1-2]. In many of these applications, it is necessary to chronically implant the electronics that enable interactions with the tissue using electrical signals.

In the example of cochlear implants, it is desired that the implant continue to function over the patient's entire lifetime without causing any adverse cytotoxic reaction from the tissue [3]. Thus, the non-bio-compatible components (integrated circuits, passive components, batteries, etc) are hermetically sealed in order to separate them from the body. At the same time, the electronic circuits need to be protected from the harsh biological environment of the body which can cause electrical shorts or corrosion, and ultimately lead to open circuits. Typically, these issues are overcome by encapsulating or protecting the electronics inside a biocompatible and hermetically sealed electronics package. The electronics package often incorporates an array of electrical feedthroughs that allow the transmission of electrical signals between the interior and exterior of the package, while maintaining a seal that prevents transfer of particles or fluids [4-5]. It is desirable that the feedthroughs be fabricated from chronically biocompatible materials, while meeting stringent specifications for hermeticity.

In the case of retinal prosthetics, the number of electrical feedthroughs or channels may directly affect the image quality that can be restored to the patient. Simply increasing the number of feedthroughs is typically not feasible because it increases the size of implant, which may make it impractical for implantation. It is therefore necessary to increase the density of electrical feedthroughs so that the channel count can be increased without significantly affecting device size [6-7].

\section{State-of-Art}

A common method of making hermetic feedthroughs is by brazing metal pins inside the vias of an insulating substrate. While this method can consistently result in hermetic feedthroughs, the pitch is limited, in many cases to as high as $400-500 \mu \mathrm{m}$.

Another common approach is to laser machine vias into an insulating substrate, and to fill the vias by stencil printing a metal paste and co-firing at high temperature. Since the metal paste contains organic binders or thinners that are driven out during the firing process, it is common for voids to form, which may negatively impact the hermeticity of the feedthroughs. Commercially available feedthroughs using this technology for bio-medical applications have a pitch in the range of 400-600 $\mu \mathrm{m}$.

Methods such as LTCC (Low Temperature Co-Fired Ceramics) use thin ceramic-glass sheets, into which vias are punched or laser machined, and filled with metal paste. Multiple ceramic layers are aligned and sintered at temperatures up to about $1000{ }^{\circ} \mathrm{C}$. A major limitation with this technology in addition to lack of scalability, is the misalignment of individual feedthroughs due to uneven shrinkage of the ceramic during the sintering process. In contrast, the electronic components that are attached to these feedthrough arrays are lithographically patterned, and have a high repeatability and accuracy of both size and location of bond pads. Due to the mismatch in pad versus feedthrough locations, it becomes necessary to use more compliant attachment processes, further limiting scalability of this technology [8].

State-of-art electrical feedthrough technologies including those mentioned above are high-cost, lack scalability, and have inherent material incompatibilities [4]. Importantly, limited density (or pitch) can directly affect the performance of a biomedical device, for example, as cochlear implants with limited fidelity, or retinal prostheses with limited image resolution. Thus, there is currently an unmet need for high-density, bio-compatible electrical feedthrough arrays.

\section{METHODS}

\section{Extruded Via Concept Overview}

We present a novel approach for fabricating high-density hermetic feedthroughs from chronically bio-compatible materials using an extruded metal via process (Figure 1).

First, a non-conductive alumina substrate $\left(\mathrm{Al}_{2} \mathrm{O}_{3}\right)$ is laser machined to form an array of densely-packed vias. Next, the ceramic is conformally metallized $(\mathrm{Ti} / \mathrm{Au})$ and lithographically patterned on both sides of the ceramic substrate. The novel step involves forming metal stud bumps centered over the vias. Since the stud bump diameter is greater than the via size, the metal partially extrudes through the via while bonding to the metallized ceramic. The combination of extrusion, ultrasonic energy, elevated temperature, and force is expected to form a hermetic seal. Simultaneously, the diffusion of the stud bump into the conformal metallization provides an electrically conductive path between the two surfaces of the alumina substrate. Optionally, after stud bumping, the bumps are coined by thermo-compression to drive the metal deeper, and to provide a flat ceramic surface onto which electronic components may be assembled. 


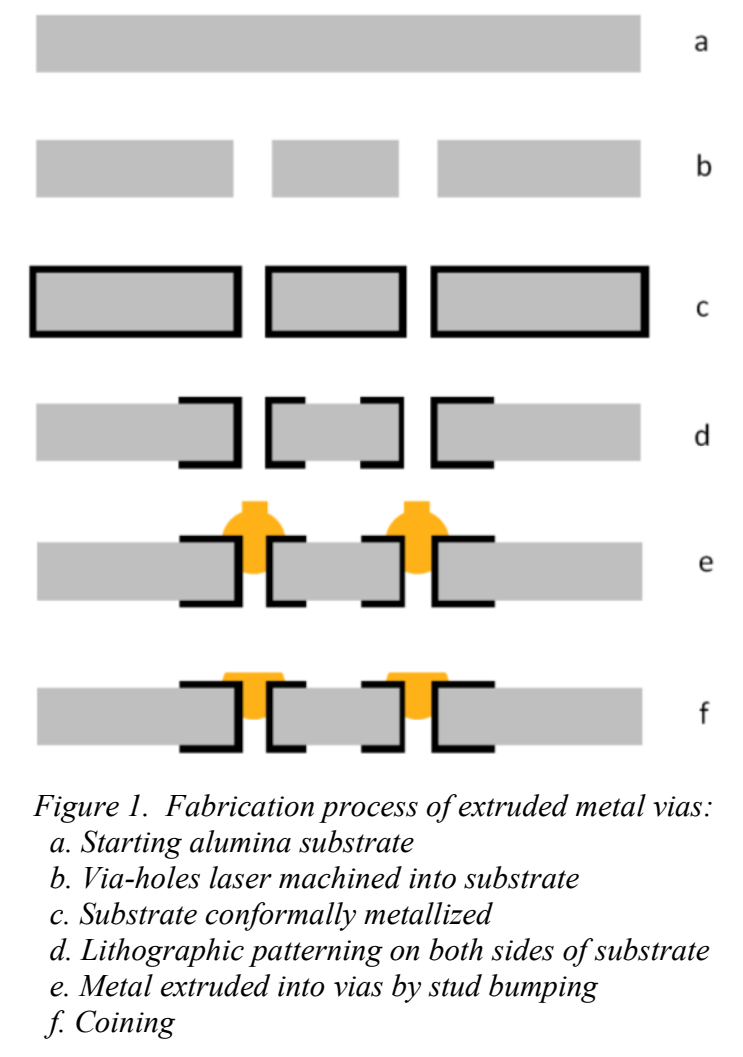

\section{Fabrication Process}

Alumina $\left(\mathrm{Al}_{2} \mathrm{O}_{3}\right)$ was chosen as the insulating material because of its demonstrated bio-compatibility, chemical inertness, and use in bio-medical devices, such as retinal prostheses, cochlear implants, and neural stimulators [9]. As the starting substrate, this study used $99.6 \%$ purity alumina substrates with a thickness of 250 $\mu \mathrm{m}$ and a surface roughness of $25-50 \mathrm{~nm} \mathrm{Ra}$. An array of vias was laser machined using commercial $\mathrm{CO}_{2}$ laser processing technology at a pitch of $200 \mu \mathrm{m}$ (equivalent to a density of $\sim 2500 \mathrm{vias} / \mathrm{cm}^{2}$ ). It is important to note that the laser machined vias have an inherent taper that results in the via openings on the laser entry-side to be larger in diameter than the exit-side. The average exit-side via diameter for our samples was $53 \mu \mathrm{m}$ (standard deviation 2.7).

The laser machined substrates were conformally metallized on both sides with a sputtered $\mathrm{Ti} / \mathrm{Au}$ thin film. The metal is lithographically patterned on one side for the attachment of the electronics, and patterned on the opposite side for attachment to an implantable microelectrode array [10].

The critical aspect of the extruded via process is the optimization of process parameters for stud-bumping such that a consistent hermetic seal can be formed. It is expected that the seal is formed along the wall of the via when a stud bump of larger diameter than the via is extruded through it. A five-factor, half fractional factorial design of experiments containing 16 unique stud bumping parameter sets was performed to find the optimal combination of process conditions that result in hermetic feedthroughs. The critical bumping parameters selected for optimization were ultrasonic bonding power, time that ultrasonic energy is applied (or ultrasonic time), bond force, substrate temperature, and wire hardness. Commercially available $25 \mu \mathrm{m}$ diameter gold bonding wire of high and low hardness was utilized. Stud bumps were formed with an F\&K Delvotec 5610 bonder. A variety of bonding parameters were utilized to determine the appropriate upper and lower bounds of bonding parameters for the design of experiments.

Optionally, the stud bumps on the alumina substrate are coined by thermo-compression on a flip-chip bonder. The coining process was designed to make the substrates planar, but as will be discussed later, it improved the hermeticity of feedthroughs.

\section{Testing methods}

The performance of the extruded metal vias was quantified by measuring their hermeticity and electrical properties.

Electrical testing consisted of verifying electrical continuity of the feedthrough and measurement of the electrical resistance with a multimeter. An acceptable electrical resistance of the via is highly dependent on application, and will be discussed in the later section.

Hermeticity of the feedthroughs was measured using a helium leak test. An Adixen ASM 182 TD + helium leak detector (detection limit $\sim 5 \times 10^{-12}$ torr-1/s), was fitted with a custom sealing fixture that allowed a vacuum (of $1 \times 10^{-3}$ torr) to be applied between the ceramic substrate and the helium mass spectrometer. Once vacuum was achieved, helium was flooded on the outer surface of the ceramic while recording any fluctuations in the leak rate. We considered a hermetic feedthrough if the leak rate was better than $9 \times 10^{-10}$ torr- $1 / \mathrm{s}$, which exceeds the MIL-STD-883 specification for the expected internal volume of our package.

\section{RESULTS}

The extruded via process was successfully optimized to create hermetic electrical feedthroughs in alumina substrates. In the design of experiments, each test of bonding parameters was performed on alumina substrates containing single laser machined vias.

Figure 2 shows a top-view and cross-sectional view of a single hermetic electrical feedthrough, with and without the optional coining step. The coining step planarized the extruded vias to the alumina substrate, which is preferred for subsequent processing and assembly steps.
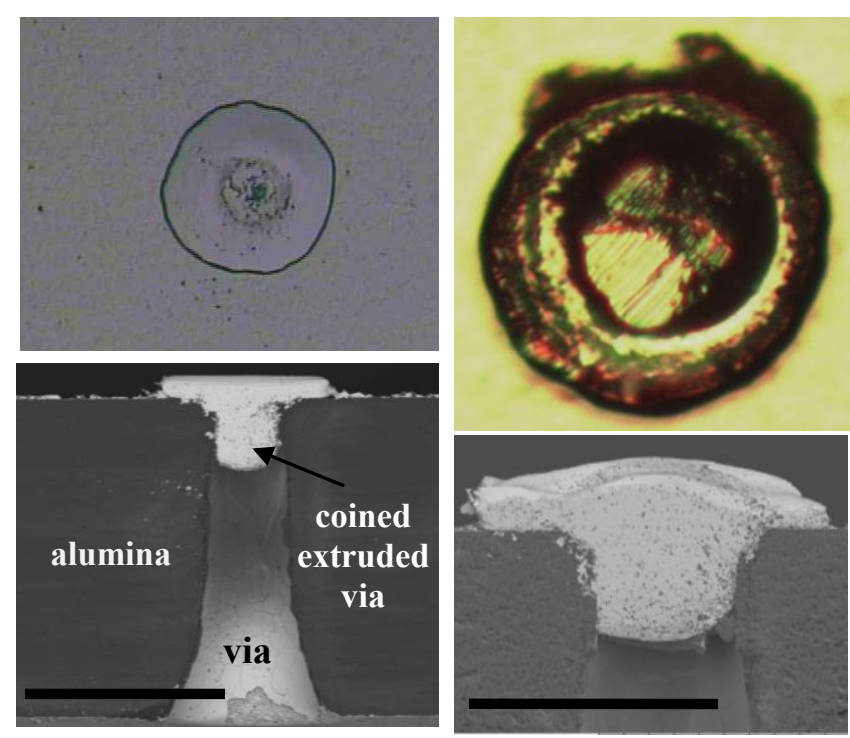

Figure 2. Left: Top-view and cross-sectional view of extruded via after coining; Right: Top-view and cross-sectional closeup of extruded via, no coining. (scale bars $=100 \mu \mathrm{m}$ ) 
A preliminary analysis of the settings that resulted in hermetic feedthroughs shows a wide process window for the parameters chosen for the design of experiments (Table 1). Six of the sixteen tests yielded hermetic feedthroughs with leak rates better than MIL-STD-883 specification.

However, a closer look at the electrical testing results showed that only two of these six substrates have acceptable electrical performance (highlighted in Table 1). Test \#4 had an electrical resistance of $50 \Omega$, while Test $\# 1$ had a resistance of $250 \Omega$. The remaining tests had resistances over $1000 \Omega$, which may not be acceptable for some device applications.

Table 1. Process parameters that resulted in hermetic feedthroughs. Highlighted rows indicate tests with acceptable electrical performance

\begin{tabular}{|c|l|l|l|l|l|}
\hline$\#$ & $\begin{array}{c}\text { Wire } \\
\text { Hardness }\end{array}$ & Force & $\begin{array}{c}\text { Ultrasonic } \\
\text { power }\end{array}$ & $\begin{array}{c}\text { Ultrasonic } \\
\text { time }\end{array}$ & $\begin{array}{c}\text { Bonding } \\
\text { temperature }\end{array}$ \\
\hline 1 & Hard & Low & Low & Low & Low \\
\hline 2 & Soft & Low & Medium & High & Low \\
\hline 3 & Hard & High & Medium & High & Low \\
\hline 4 & Hard & High & High & Low & Low \\
\hline 5 & Soft & Low & Low & Low & High \\
\hline 6 & Hard & Low & Medium & High & High \\
\hline
\end{tabular}

An unexpected outcome of the optional coining process was an improvement in hermeticity. Five extruded metal vias that initially failed hermeticity testing were thermo-compressively coined using a flip-chip bonder. Two of these five samples were hermetic when subsequently leak tested.

Figure 3 shows an array of 12 vias $(200 \mu \mathrm{m}$ pitch $)$, before and after extruding vias with gold stud bumps.
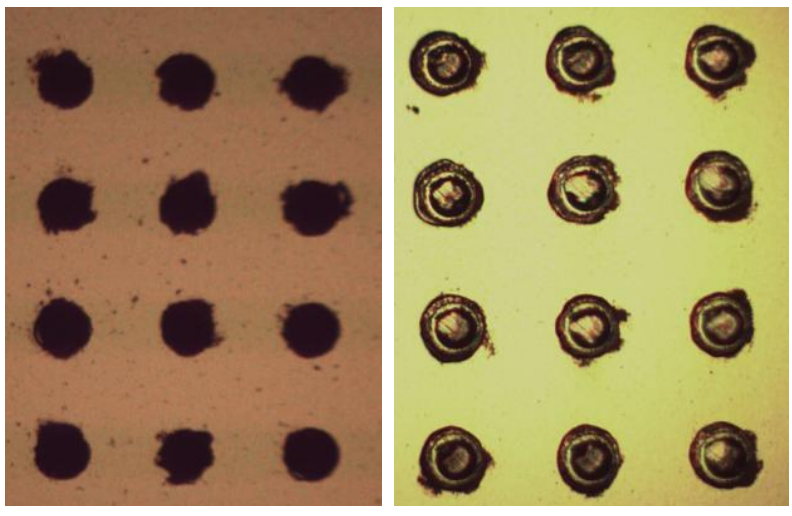

Figure 3. Left: metallized bare ceramic substrate with laser machined vias of $200 \mu \mathrm{m}$ pitch (Schematic in Figure 1c). Right: gold vias extruded through ceramic vias (schematic in Figure 1e)

\section{DISCUSSION AND CONCLUSIONS}

We have demonstrated a feedthrough pitch of $200 \mu \mathrm{m}$, which is one of the lowest shown in literature, especially when compared with conventional bio-medical feedthrough technologies that achieve pitches in the $350-600 \mu \mathrm{m}$ range.

In addition, the extruded feedthrough process minimized the required thickness of the ceramic substrate $(250 \mu \mathrm{m})$. Conventional processes that use metal pastes often create voids in the feedthroughs because the organic binders or thinners outgas during sintering or thermal processing. The presence of voids necessitates a larger ceramic thickness (greater than $400 \mu \mathrm{m}$ ) to reduce the likelihood that a series of voids will form a leakage path through the ceramic, and render it non-hermetic. The extruded via process can achieve hermetic feedthroughs at roughly half the thickness of conventional feedthrough technologies because it uses bulk metal wire to seal the via openings.

In addition to the high density, this approach can be used to form hermetic feedthroughs at extremely low processing temperature $\left(150{ }^{\circ} \mathrm{C}\right)$, and has the ability to rapidly create feedthroughs without complicated processing steps. In contrast, existing technologies often require high processing temperatures, which amplifies problems with feedthrough failure due to mismatch in thermal coefficient of expansion between the ceramic substrate and the metal feedthrough.

Another advantage of the low-temperature processing is the flexibility that our method provides for bio-medical device assembly. Currently, many devices are assembled by first fabricating the feedthrough array (at high temperature), followed by a high-temperature brazing process to attach the ceramic to a bio-compatible metal ring. The braze process often results in hermeticity failure due to strain induced from a mismatch in thermal expansion between the ceramic and metal. The extruded via process would enable a "via-last" assembly, in which all electronic components can be assembled first. This would reduce the chance of failure of the feedthrough array, and also make it easier to perform electrical testing of the assembled components before they are hermetically sealed.

Currently, a disadvantage of the extruded via process is the relatively high electrical resistances. However, this may be acceptable for many neural prostheses applications that use microelectrode arrays. Microelectrode arrays attached to feedthroughs often have trace resistances on the order of $1-10 \mathrm{k} \Omega$. Moving forward, we propose to reduce the electrical resistances either by electroplating a thicker metal layer, or by using atomic layer deposition processes to achieve a more conformal metal coating in the vias.

Table 2 qualitatively compares the extruded via process with conventional feedthrough technologies.

Table 2. Comparison of extruded metal via process with state-ofart bio-medical feedthrough technologies.

\begin{tabular}{|l|l|l|l|l|}
\hline Method & Hermeticity & Density & $\begin{array}{l}\text { Electrical } \\
\text { resistance }\end{array}$ & $\begin{array}{l}\text { Processing } \\
\text { temperature }\end{array}$ \\
\hline Brazed pins & High & Poor & Excellent & High \\
\hline LTCC & Moderate & Moderate & Excellent & Moderate \\
\hline $\begin{array}{l}\text { Stencil- } \\
\text { printed } \\
\text { metal pastes }\end{array}$ & Moderate & Moderate & Excellent & High \\
\hline $\begin{array}{l}\text { Extruded } \\
\text { metal vias }\end{array}$ & High & Excellent & Moderate & Low \\
\hline
\end{tabular}

\section{FUTURE WORK}

Moving forward, we hope to improve and expand the extruded metal via process. First, further optimization of the design of experiments will be performed to find the reliable process window for stud bumping. Second, improvements will be made to the metallization process to reduce resistances so that this technology can have broader applications, especially for semiconductor devices. Third, platinum bonding wire will be adopted due its superior bio-compatibility in the presence of a corrosive environment. Finally, we hope to extend this process to insulating substrates that can be lithographically etched, which will enable further miniaturization of feedthrough densities, and ultimately lead us to realize neural prostheses with ultra-high channel counts. 


\section{ACKNOWLEDGEMENTS:}

This work was performed under the auspices of the U.S. Department of Energy by the Lawrence Livermore National Laboratory, Contract number DE-AC52-07NA27344 and funded by the National Institute on Deafness and Other Communication Disorders, contract Y1-DC-8002-01.

\section{REFERENCES}

[1] T. Stieglitz, M. Schuettler, and K. P. Koch, "Implantable biomedical microsystems for neural prostheses.," IEEE engineering in medicine and biology magazine : the quarterly magazine of the Engineering in Medicine \& Biology Society, vol. 24, no. 5, pp. 58-65 (2005)

[2] J. H. Prescott, S. Lipka, S. Baldwin, N. F. Sheppard, Jr, J. M. Maloney, J. Coppeta, B. Yomtov, M. A. Staples, J. T. Santini, Jr, "Chronic, programmed polypeptide delivery from an implanted, multireservoir microchip device." Nature biotechnology, vol. 24, no. 4, pp. 437-8, (2006)

[3] T. J. Balkany, A. V. Hodges, O. Gómez-Marín, P. A. Bird, S. Dolan-Ash, S. Butts, F. F. Telischi Mee. "Cochlear reimplantation", The Laryngoscope, 109: 351-355 (1999).

[4] G. Jiang and D. D. Zhou, Technology Advances and Challenges in Hermetic Packaging for Implantable Medical Devices. Springer New York, New York, NY 2010.

[5] J. Coppeta et al., "Low Temperature Methods for Hermetically Sealing Reservoir Devices," U.S. Patent 7,114,312 B22006.

[6] J. Winter, S. Cogan, J. Rizzo. "Retinal Prostheses: current challenges and future outlook". Journal of Biomaterials Science, Polymer Edition, Volume 18, Number 8, pp. 10311055(25), (2007).

[7] J. Weiland, W. Fink, M. Humayun, W. Liu, D. Rodger, Y.C. Tai, M. Tarbell. "Progress towards a high-resolution retinal prosthesis". IEEE EMBS conference, pp. 7373 - 7375, (2005).

[8] T. Guenther, C.W.D. Dodds, N. H. Lovell, G. J. Suaning. "Chip-scale hermetic feedthroughs for implantable bionics", IEEE EMBS conference pp. 6717 - 6720, (2011).

[9] T. Stieglitz, "Manufacturing, assembling and packaging of miniaturized neural implants". Microsystem technologies, vol 16, no. 5, 723-734, (2010).

[10] A. Tooker, K. Shah, V. Tolosa, H. Sheth, S. Felix, T. Delima, S. Pannu. "Chronically Implantable, 121-Channel, Polymer Microelectrode Array with Hermetically-Sealed Wireless Interface". Accepted, Solid-State Sensors, Actuators, and Microsystems Workshop, Hilton Head, (2012).

\section{CONTACT}

* Kedar G. Shah, Tel: +1-925-422-7296; kshah@1lnl.gov

LLNL-CONF-544091 


\section{HIGHLY STABLE SURFACE FUNCTIONALIZATION OF HIGH-ASPECT-RATIO MICROGAS CHROMATOGRAPHY COLUMNS USING LAYER-BY-LAYER SELF-ASSEMBLY OF $\mathrm{SiO}_{2}$ NANOPARTICLES Dong Wang $^{l+}$, Hamza Shakeel ${ }^{2 \dagger}$, Reza Montazami ${ }^{3}$, Gary Rice ${ }^{4}$, James R. Heflin ${ }^{l}$, and Masoud Agah ${ }^{2 *}$ ${ }^{1}$ Department of Physics, Virginia Tech, USA \\ ${ }^{2}$ VT MEMS Lab, Bradley Department of Electrical \& Computer Engineering, Virginia Tech, USA \\ ${ }^{3}$ Department of Mechanical Engineering, Iowa State University, USA \\ ${ }^{4}$ Department of Chemistry, College of William \& Mary, USA \\ ${ }^{\dagger}$ These authors contributed equally to this work}

\begin{abstract}
A novel selective surface functionalization of 3D micro-silicon channels using a $\mathrm{SiO}_{2}$ nanoparticles (NPs) coating deposited using Layer-by-Layer (LbL) self-assembly technique is reported here. Additionally, application of $\mathrm{SiO}_{2} \mathrm{NPs}$ coating as a stationary phase for chromatographic separation is also presented for the first time. Compared to previously reported techniques, this method enables a simple, low-cost and parallel processing scheme in obtaining homogenous and stable NPs coatings inside the microfluidic channel. Moreover, the reported scheme also provides good repeatability and ease of control over the coating thickness. The $\mathrm{SiO}_{2}$ NPs functionalized microfabricated gas chromatography columns with wide single capillary channels (1 m-long, 150 m-wide, 240 m-deep) and very narrow multicapillary channels (25 cm-long, $30 \mathrm{~m}$-wide, $240 \mathrm{~m}$-deep, 16 parallel channels) successfully separated a multicomponent gas mixture with a wide range of boiling points.
\end{abstract}

\section{INTRODUCTION}

\section{Microgas Chromatography ( $\mu \mathrm{GC})$}

Gas chromatography (GC) is a reliable chemical analysis technique that is used to separate and identify the constituents of complex gas mixtures. GC has applications in vast areas such as environment monitoring, food processing, pharmaceutical industry, biomedical science, forensic and trace analysis. A chromatographic process (schematically shown in Fig. 1) starts with the injection and vaporization of an unknown sample mixture (analytes); after which the analytes traverse through the separation column via a carrier gas (mobile phase). Columns are typically coated with a polymer (stationary phase) to physically separate the constituents of a mixture into separate compounds in a time domain. Afterwards, the separated components are eluted into a detector that generates a chromatogram based on the retention time of each compound. Each

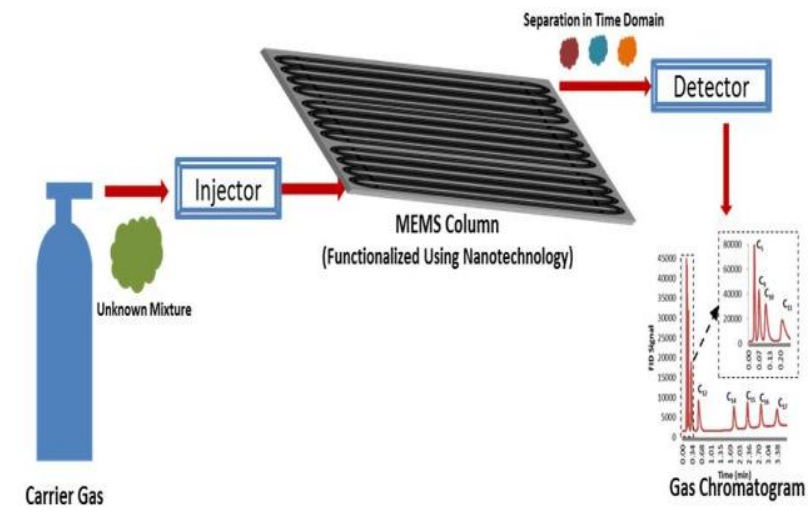

Figure 1. Schematic of a GC system peak in a given chromatogram represents a particular compound.

Traditional bench-top GC instruments, though widely used for over 50 years, are bulky, expensive, time and energy consuming. Due to recent advancement and emergence of microelectromechanical systems (MEMS), energy-efficient integrated $\mu \mathrm{GC}$ systems have attracted considerable attention because of its portability, fast analysis time, and low cost [1-3]. This system, upon complete realization, could enormously expand the range of GC applications in in-situ air quality monitoring, biomedical analysis, and homeland security. As a key component in a $\mu \mathrm{GC}$ system, conventional meters-long separation columns are replaced with coin-sized microfabricated silicon columns. The separation ability and efficiency of the column is directly related to the quality of the stationary phase. Using conventional coating techniques for rectangular-shaped microfabricated columns present major challenges towards getting reproducible and uniform coating. Conventional coating techniques also suffer from limitation in high temperature applications and poor stability (organic coatings) or requirements of expensive instruments and elaborate operation (inorganic coatings) [4-7]. The high thermal stability and high surface area of $\mathrm{SiO}_{2} \mathrm{NPs}$ present an excellent candidate for the stationary phase if employed using simple and low cost methods.

\section{Layer-by-Layer (LbL) Self-Assembly Technique}

Developed first in 1991[8], the LbL self-assembly technique was utilized to develop Ionic Self-Assembly Multilayers (ISAM), which have been widely used in electromechanical actuators [9] as well as in electro-optical and electrochromic devices $[10,11]$. In short, by alternatively dipping the substrate into two aqueous solutions of materials with opposite charges (positive and negative), LbL thin films of the two materials, bonded together by electrostatic force, will build up on each other (two adjacent layers of those two materials are called one bilayer). Thorough rinsing in between each deposition step washes away excess coating materials from the previous stage while leaving enough surface charge for the material to be electrostatically deposited in the next step. Thus, uniform, dense, and sturdy stacks of layers can be deposited on the surface of the substrate with a wide variety of configurations. Additionally, a desired film thickness can be achieved by simply terminating the procedure at a certain number of bilayers. Therefore, with a relatively simple concepts and straightforward operations, the LbL self-assembly technique could be conveniently incorporated into the manufacturing of silicon-based GC devices. In this article, by utilizing the $\mathrm{LbL}$ technique for the first time, high-aspect-ratio (HAR) $\mu \mathrm{GC}$ columns with different configurations are functionalized with $\mathrm{SiO}_{2}$ NPs coatings used as a stationary phase.

\section{FABRICATION}

The fabrication scheme incorporating LbL self-assembled $\mathrm{SiO}_{2}$ NPs coating, schematically shown in Fig. 2, can be divided into three major steps, which include column fabrication, LBL 
deposition of NPs, and anodic bonding of the silicon substrate with Pyrex followed by deactivation of the silica surface.

\section{Column Fabrication}

The fabrication of HAR $1 \mathrm{~m}$-long, 150 m-wide, 240 m-deep single capillary columns ( SCC) and $25 \mathrm{~cm}$-long, $30 \mathrm{~m}$-wide, 240 m-deep multicapillary columns ( MCC) with an array of 16 channels (Fig. 3H) working in parallel starts with anisotropic etching of plain silicon wafers. In the first step, wafers are spin coated with AZ9260 photoresist at $3000 \mathrm{rpm}$, soft-baked at $110^{\circ} \mathrm{C}$ for 1 minute and exposed for 45 seconds using a mask aligner (MA-6). After development using AZ400K, wafers are hard-baked at $110^{\circ} \mathrm{C}$ for 2 minutes. Deep reactive ion etching (DRIE) with photoresist as an etch mask is used to etch the wafers in order to get the desired channel dimensions. Afterwards, the wafer is diced into individual devices. Selective deposition of NPs inside the channels is achieved by using a liftoff procedure explained in the next section (Step 1, Fig. 2).

\section{LbL Deposition of $\mathrm{SiO}_{2} \mathrm{NPs}$}

Keeping the photoresist intact, the $\mathrm{LbL}$ deposition of $\mathrm{SiO}_{2} \mathrm{NPs}$ inside HAR microfluidic channels starts with alternatively dipping each device into a positively charged long-chain inert polymeric aqueous solution (10 mM polyallylamine hydrochloride, $\mathrm{PAH}$, from Sigma-Aldrich), and negatively charged $\mathrm{SiO}_{2} \mathrm{NPs}$ suspension (Nissan Chemical, $45 \mathrm{~nm}$ average particle size). Because of the much narrower channels, the MCC column deposition uses the $\mathrm{SiO}_{2}$ NPs suspension at $1 / 3$ of its original concentration. This prevents the formation of bulky NPs structures between the channel walls. Following our earlier reported findings [11] to guarantee excellent $\mathrm{PAH} / \mathrm{SiO}_{2} \mathrm{NPs}$ LbL coating uniformity, the $\mathrm{pH}$ value of the PAH solution and $\mathrm{SiO}_{2} \mathrm{NPs}$ suspension are adjusted to the values of $7.0( \pm 0.1)$ and $9.0( \pm 0.1)$, respectively, by adding $\mathrm{HCl}$ and $\mathrm{NaOH}$ solutions. The LbL coating is then performed in an automatic dipping system (StratoSequence VI Robot, nanoStrata Inc.) in which every 2.5 minutes of $\mathrm{PAH}$ and $\mathrm{SiO}_{2} \mathrm{NPs}$ coating step is followed by three 1 minute DI water rinsing steps (Step 2, Fig. 2). The LbL deposition scheme presented here affords a homogenous $\mathrm{SiO}_{2}$ NPs coverage both on the interior microchannel surfaces and on the photoresist at the top of chip. After coating, the samples are rinsed thoroughly with deionized (DI) water and dried with low flux nitrogen. NPs coated devices are then dipped in acetone and sonicated for 12 minutes. This liftoff procedure removes the NPs deposited on photoresist without removing the coating inside the trenches. The yield of lift-off process is important especially for the microfluidic applications in which the NPs coated silicon channels are to be sealed with a glass substrate (Step 3, Fig. 2).

\section{Anodic Bonding and Deactivation of the Silica Surface}

Before anodic bonding, the devices are first calcinated in a furnace at a high temperature $\left(500^{\circ} \mathrm{C}\right)$ for 4 hours to fuse the $\mathrm{SiO}_{2}$ NPs together and to ensure the stability of the coating during temperature programming of the GC columns. Afterwards, the silicon trenches are sealed to a Pyrex wafer using anodic bonding at $400^{\circ} \mathrm{C}$ and $1250 \mathrm{~V}$ (Step 4, Fig. 2). Next, deactivated capillary tubes (220 m outer diameter, $100 \mathrm{~m}$ internal diameter, $25 \mathrm{~cm}$ length) are installed at the inlet and outlet ports using epoxy. Then, $10 \mathrm{mM}$ chlorodimethyl octadecyl silane (CDOS) diluted in toluene is pumped into the chip by nitrogen (Step 5, Fig. 2). This overnight process deactivates NPs and the Pyrex cover surface [12]. Finally, the solvent is blown out by nitrogen and the device is ready for chromatographic analysis.

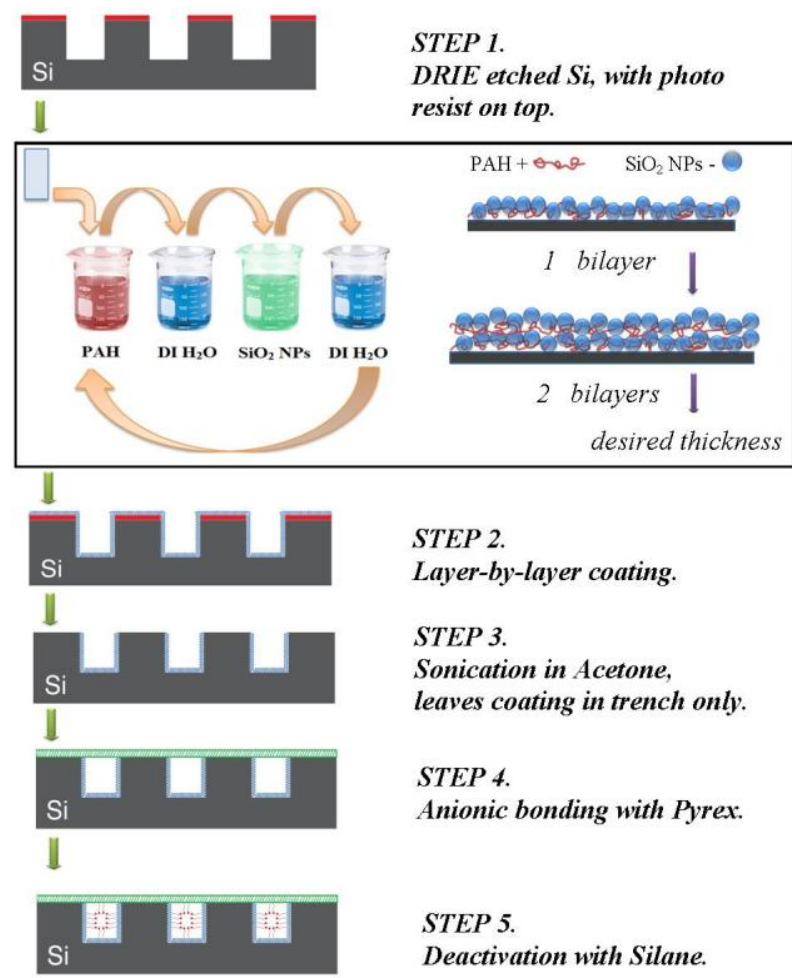

Figure 2. Schematic process flow of $\mathrm{SiO}_{2} \mathrm{NPs}$ coating using Layer-by-Layer self-assembly technique in the Si trench.

\section{CHARACTERIZATION OF $\mathrm{SiO}_{2}$ NPS COATING}

As displayed in Fig. 3 (A-D), the bottom and sidewalls of the trenches are completely covered by $\mathrm{SiO}_{2} \mathrm{NPs}$, while at the same time NPs are neatly removed by the lift-off process from the top surface. This is the first successful demonstration of combining microlithography and LbL self-assembly to selectively deposit NPs on the interior surfaces of silicon microchannels. Fig. 3E and Fig. $3 \mathrm{~F}$ show the SEM images of 5 and 10 bilayers of $\mathrm{SiO}_{2} \mathrm{NPs}$ deposited inside silicon trenches, respectively. The thickness measured directly from the SEM images are $261 \mathrm{~nm}$ to $316 \mathrm{~nm}, 399$ $\mathrm{nm}$ to $486 \mathrm{~nm}$, and $554 \mathrm{~nm}$ to $667 \mathrm{~nm}$ for 5, 10, and 15 bilayers, respectively. Although a lower concentration of $\mathrm{SiO}_{2} \mathrm{NPs}$ suspension is used for MCC, the 10 bilayers coating inside its channel (Fig. 2G) has approximately the same thickness (396 nm) as the 10 bilayers coating inside the SCC (Fig. $2 \mathrm{H})$. This means the coating for the $\mathrm{SiO}_{2}$ NPs suspension at $1 / 3$ of its original concentration is sufficient to deposit a layer of $\mathrm{SiO}_{2} \mathrm{NPs}$ over the previous $\mathrm{PAH}$ layer to neutralize and reverse the surface charge, while the excess $\mathrm{SiO}_{2}$ NPs during the SCC coating with higher concentration suspension is washed off during the rinsing steps. 

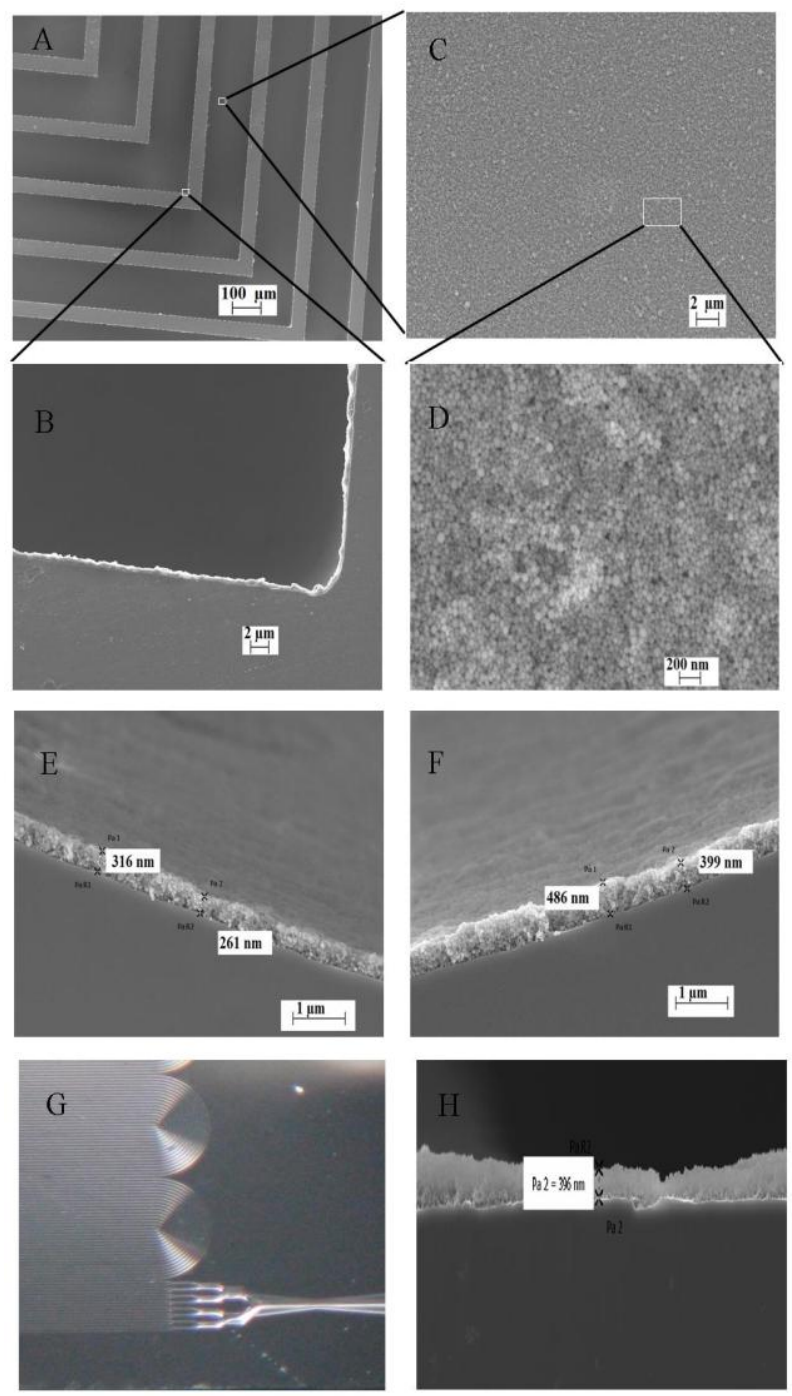

Figure 3. SEM images of the $10 \mathrm{BLs} \mathrm{SiO}_{2} \mathrm{NPs}$ coated single capillary column $(A-D)$, on the side wall $(B)$, bottom of the channel (C-D); cross-section view of silicon trench with $5 \mathrm{BLS}$ (E) and $10 \mathrm{BLs}$ of $\mathrm{SiO}_{2} \mathrm{NPs}$ coating $(F)$. Optical image of multicapillary column $(G)$ and the SEM image of its cross-section with $10 \mathrm{BLs} \mathrm{SiO}_{2} \mathrm{NPs}$ coating on the side wall of Si trench $(H)$.

\section{SEPERATION TEST AND RESULTS}

\section{Experimental Setup}

A commercial GC system (HP 5890 Series II), equipped with an autosampler, electronic pressure controller, and flame ionization detector (FID), was used for characterizing the separation ability of the GC columns with the $\mathrm{SiO}_{2}$ NPs stationary phase. Highly purified nitrogen (from Airgas Inc.) was used as carrier gas. Inlet and detector temperatures are set to $280^{\circ} \mathrm{C}$ and the split ratio is set to 100:1. Standard chemicals were purchased from Sigma-Aldrich and used for all experiments.

\section{Separation Results}

In order to evaluate the chromatographic performance, the functionalized GC columns were placed in a GC oven. All columns were first purged with dry nitrogen to remove any trapped
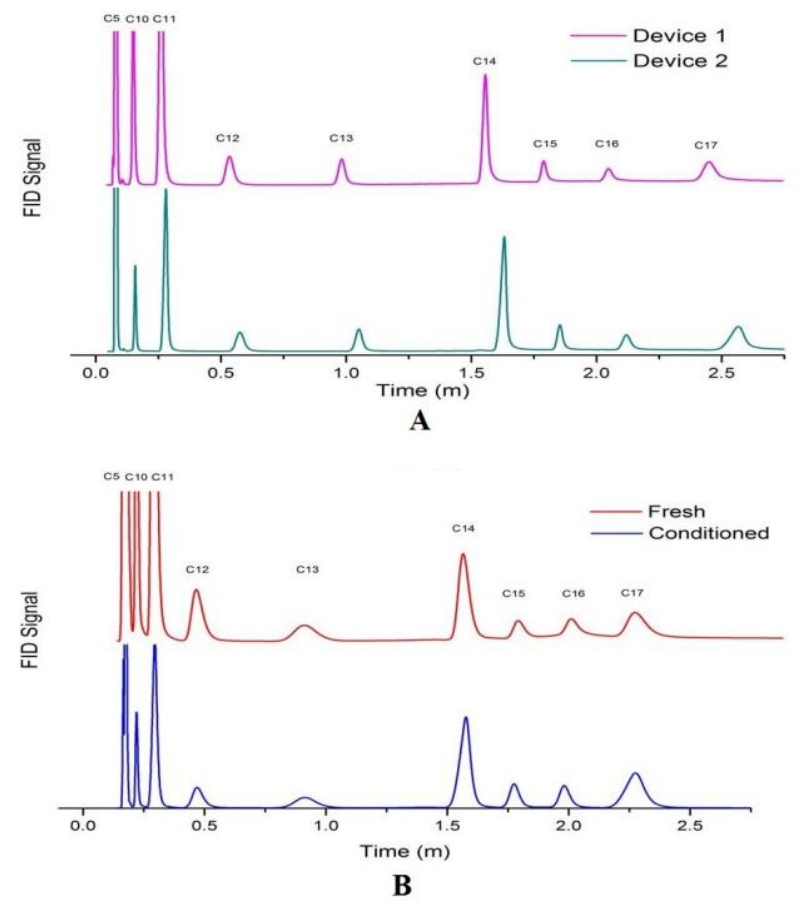

Figure 4. Example of 9 alkane compounds separation by two $10 \mathrm{BLs} \mathrm{SiO}_{2} \mathrm{NPs}$ coated MEMS GC columns (A) and one column before and after 24 hours conditioning under 7.5 psi at $160{ }^{\circ} \mathrm{C}(B)$.

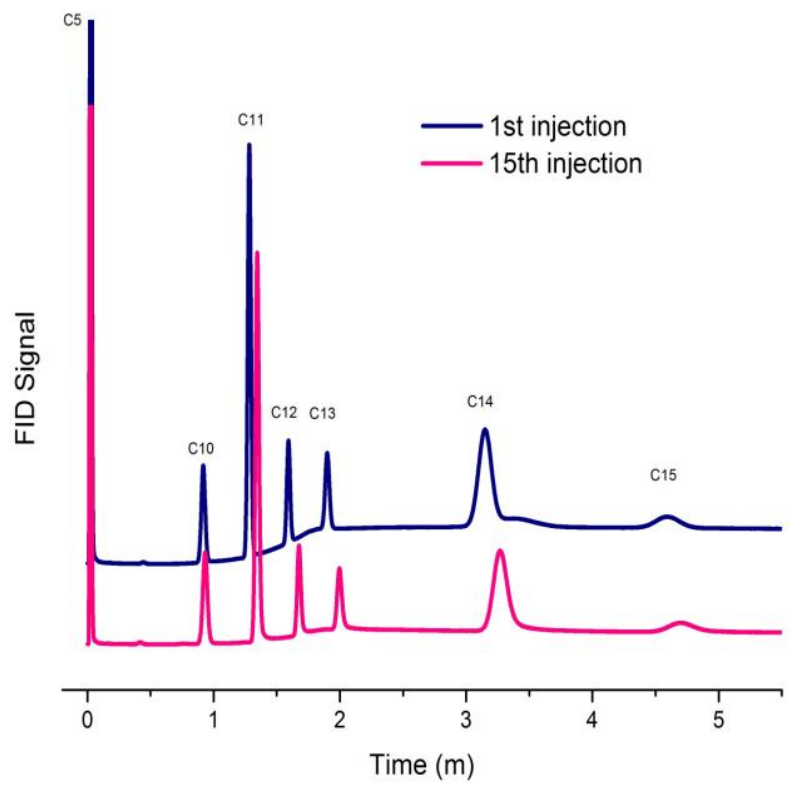

Figure 5. Chromatographic separation result by a $10 \mathrm{BLs} \mathrm{SiO}_{2}$ NPs coated MEMS GC multicapillary column for the 1st and 15 th injection.

oxygen in the system. The columns were then conditioned gradually from ambient temperature to slightly above the maximum 
operating temperature $\left(120^{\circ} \mathrm{C}\right)$ at a temperature ramp rate of $1^{\circ} \mathrm{C} / \mathrm{min}$ and a constant carrier gas flow of 7.5 psi until a constant baseline was observed.

The effectiveness of the $\mathrm{SiO}_{2} \mathrm{NPs}$ coating as a stationary phase for different channel geometries was tested on both SCCs and MCCs. All the chromatograms in Fig. 4 ( SCC) and Fig. 5 ( MCC) exhibited successful separations of alkanes (n-pentane, n-decane, n-undecane, n-dodecane, n-tridecane, n-tetradecane, n-pentadecane, $n$-hexadecane and n-heptadecane) spanning a wide range of boiling points $\left(32^{\circ} \mathrm{C} \sim 202^{\circ} \mathrm{C}\right)$ with high stability and repeatability (less than $4 \%$ deviation in retention time). Fig. 4B shows the long-term stability of this novel stationary phase after $\mathrm{SiO}_{2}$ NPs coated GC columns were subjected to the 7.5 psi flow and $160^{\circ} \mathrm{C}$ temperature for 24 hours. Similarly, Fig. 5 demonstrates the time stability of NPs-based stationary phase after 15 injections on the MCC.

\section{CONCLUSION}

$\mathrm{SiO}_{2}$ NPs coating fabricated by the LbL technique has been incorporated into GC separation columns and utilized as a new stationary phase for the first time. With simple and parallel fabrication procedures, $\mathrm{LbL}$ technique has shown the ability to generate homogenous conformal $\mathrm{SiO}_{2} \mathrm{NPs}$ coating with easy nanoscale thickness control inside the GC columns with different configurations. GC columns (both single and multi- capillary) with this stationary phase provided good separations of an alkane mixture with a wide range of boiling points.

\section{ACKNOWLEDGEMENT}

This work is supported primarily by the National Science Foundation under Award No ECCS-1002279. The authors also want to thank Transducer Research Foundation for generously providing travel support.

\section{REFERENCES}

[1] M. A. Zareian-Jahromi, M. Ashraf-Khorassani, L. T. Taylor, and M. Agah, "Design, Modeling, and Fabrication of MEMS-Based Multicapillary Gas Chromatographic Columns," Journal of Microelectromechanical Systems, 18, 28 (2009).

[2] H. Shakeel, G. Rice, and M. Agah, "First Reconfigurable MEMS Separation Columns for Micro Gas Chromatography," 2012 IEEE 25th International Conference on Micro Electro Mechanical Systems (MEMS), Paris, France, 2012, pp. 823-826.

[3] H. Shakeel and M. Agah, "High-Performance Multicapillary Gas Separation Columns with MPG Stationary Phases," IEEE Sensors, Limerick, Irland, 2011, pp. 1909-1912.
[4] S. Reidy, G. Lambertus, J. Reece, and R. Sacks, "High-Performance, Static-Coated Silicon Microfabricated Columns for Gas Chromatography," Analytical Chemistry, 78, 2623 (2006).

[5] A. Malainou, "The Fabrication of a Microcolumn for Gas Separation Using Poly(dimethylsiloxane) as the Structural and Functional Material," Journal of Micromechanics and Microengineering, 18, 105007 (2008).

[6] L.-M. Yuan, C.-X. Ren, LiLi, P. Ai, Z.-H. Yan, M. Zi, and Z.-Y. Li, "Single-Walled Carbon Nanotubes Used as Stationary Phase in GC," Analytical Chemistry, 78, 6384 (2006).

[7] J. Vial, D. Thiébaut, F. Marty, P. Guibal, R. Haudebourg, K. Nachef, K. Danaie, and B. Bourlon, "Silica Sputtering as a Novel Collective Stationary Phase Deposition for Microelectromechanical System Gas Chromatography column: Feasibility and First Separations," Journal of Chromatography A, 1218, 3262 (2011).

[8] G. Decher, J. D. Hong, and J. Schmitt, "Buildup of Ultrathin Multilayer Films by a Self-Assembly Process, 1 Consecutively Adsorption of Anionic and Cationic Bipolar Amphiphiles on Charged Surfaces," Makromolekulare Chemie, Macromolecular Symposia, 46, 321 (1991).

[9] R. Montazami, S. Liu, Y. Liu, D. Wang, Q. Zhang, and J. R. Heflin, "Thickness Dependence of Curvature, Strain, and Response Time in Ionic Electroactive Polymer Actuators Fabricated via Layer-by-Layer Assembly," Journal of Applied Physics, 109, 104301 (2011).

[10] R. Montazami, V. Jain, and J. R. Heflin, "High Contrast Asymmetric Solid State Electrochromic Devices Based on Layer-by-Layer Deposition of Polyaniline and Poly(aniline sulfonic Acid)," Electrochimica Acta, 56, 990 (2010).

[11] S. E. Yancey, W. Zhong, J. R. Heflin, and A. L. Ritter, "The Influence of Void Space on Antireflection Coatings of Silica Nanoparticle Self-Assembled Films," Journal of Applied Physics, 99, 034313 (2006).

[12] A. D. Radadia, R. I. Masel, M. A. Shannon, J. P. Jerrell, and K. R. Cadwallader, "Micromachined GC Columns for Fast Separation of Organophosphonate and Organosulfur Compounds," Analytical Chemistry, 80, 4087 (2008).

CONTACT

*Dr. M. Agah, tel: +1-540-231-2653; Agah@VT.edu 


\section{ION-SENSITIVE FIELD EFFECT TRANSISTOR SENSORS USING SUSPENDED GRAPHENE NANORIBBON PATTERNED BY SHRINK LITHOGRAPHY \\ B. Zhang and T. Cui* \\ University of Minnesota, Minneapolis, USA}

\begin{abstract}
Low-cost and facile shrink lithography derived graphene nanoribbon (GNR) was suspended on the channel to form an ionsensitive field effect transistor (ISFET), and its $\mathrm{pH}$ sensing application is presented. By combining a thermoplastic film with a molding process, GNR patterns $50 \mathrm{~nm}$ wide were achieved in a low-cost and simple way. Ambipolar characteristics of annealed suspended GNR ISFET present an enhanced ambipolar effect. In comparison, unsuspended GNR and microscale graphene sheets based ISFETs were characterized under the same design, fabrication, and measurement conditions, showing that the suspended GNR ISFET is superior in sensitivity and detection limits.
\end{abstract}

\section{INTRODUCTION}

Graphene nanoribbons (GNRs) become an attractive nanostructure with superior electrical, chemical, and mechanical properties [1-3]. Several GNR synthesis approaches were developed, including lithographic patterning [4] and unzipping of carbon nanotubes [5]. More recently, GNR has been employed as a sensing structure for the detection of various ions, providing unique advantages due to its tunable ambipolar field-effect characteristics [3], relatively low $1 / \mathrm{f}$ noise, and biocompatibility [6]. However, the potential applications of GNR are hampered by its limited availability due to the complexity and high-cost fabrication methods. In the meanwhile, current study has been focused on graphene attached on silicon oxide substrates [7], and charge traps at the interface and in the oxide have been reported as external scattering centers and degrade electrical properties in graphene [8].

Therefore, in this paper, an ion-sensitive field effect transistor (ISFET) using a suspended GNR formed by low-cost and facile shrink lithographic patterning was demonstrated. Herein, shrink lithography combines a thermoplastic film with a molding process to successfully achieve GNR patterns in a low-cost and simple way. The ambipolar characteristics of the suspended GNR ISFET were measured in different $\mathrm{pH}$ solutions. Moreover, its low electrical noise due to the suspension structure and annealing effect gives a very promising way to achieve relatively high sensitivity. In comparison, an unsuspended GNR and microscale graphene sheets based biosensors were characterized under the same design, fabrication, and measurement conditions, demonstrating that the suspended GNR ISFET is superior in its sensing performance.

\section{DESIGN AND FABRICATION}

The fabrication process of the suspended GNR ISFET is illustrated in Fig. 1. We started with a graphene plate genearated by mechanical exfoliation method. A photoresist layer (Shipley S1818) was used as a transfer stamp to exfoliate graphene patterns from highly oriented pyrolytic graphite (HOPG), and transferred patterns onto a $\mathrm{SiO}_{2} / \mathrm{Si}$ substrate. First, patterns in microscale were formed on the surface of HOPG by photolithography followed by an oxygen plasma etching with STS etcher (Model 320, 30 seconds). A layer of S1818 was spun on the pre-treated HOPG, and a thermal releasing tape was used to gently peel graphene flakes off, and transferred them onto the $\mathrm{SiO}_{2} / \mathrm{Si}$ substrate. The substrate was pre-patterned with marks for alignment and location.
After stripping photoresist off, a microscale graphene plate was generated on the $\mathrm{SiO}_{2} / \mathrm{Si}$ substrate.

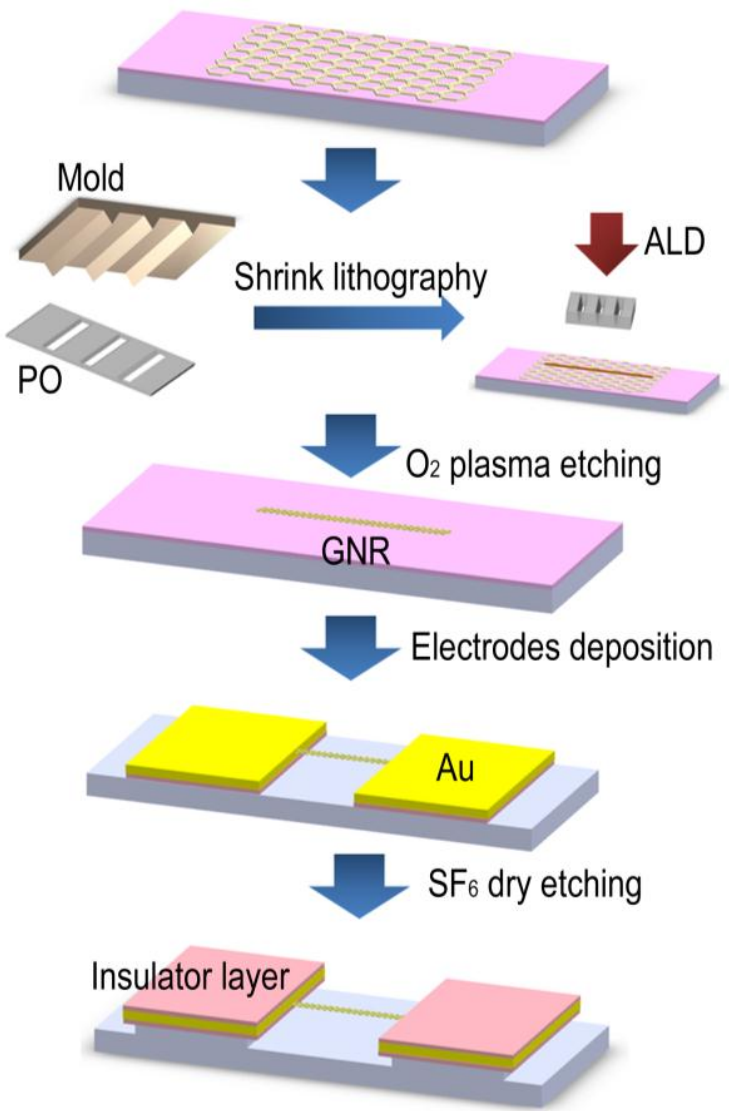

Figure 1: Schematic of fabrication process of a suspended GNR ISFET by shrink lithography.

Next, a typical mold is fabricated by conventional photolithography, $\mathrm{KOH}$ etching, and subsequent electroplating. A cleaned silicon wafer with $\mathrm{SiO}_{2} 300 \mathrm{~nm}$ thick was patterned by photolithography with photoresist of Shipley S1813 about $1 \mathrm{~m}$ thick spin-coated at $3,000 \mathrm{rpm}$ for $30 \mathrm{~s}$ and exposed for $6 \mathrm{~s}$, followed by buffered HF (BOE 10:1) etching for 7 minutes. The patterned wafer was etched by $20 \% \mathrm{KOH}$ at $80^{\circ} \mathrm{C}$ for 10 minutes, and the $\mathrm{SiO}_{2}$ was the passivation layer. To impenetrate the shrink film, an additional KMPR (MicroChem Inc.) layer around the patterns was deposited. Chromium/gold layers 50/50 nm thick were deposited on the substrate by an AJA sputter system (Model ATC 2000). Nickel electroforming was conducted on a nickel plating station (SE-101, Digital Matrix Co., U.S.) using the patterned wafer as mandrel. A nickel sulfamate bath and a pulse reverse power were used to obtain uniform and stress free nickel mold. After electroforming, the silicon wafer was completely removed by $20 \% \mathrm{KOH}$ at $80^{\circ} \mathrm{C}$. The fabricated nickel mold was cleaned by acetone and de-ionized water.

The mold is embossed against a polyolefin (PO) thermoplastic film to obtain impenetrated patterns. After preparation of the 
mold, it was embossed against the shrink film and held for 2 minutes by a Manual Presser (Model Grimco 12-1-HT). The shrink film (Sealed Air Nexcel multilayer shrink film 955D) formed by 5 layers of co-extruded polyolefin (PO) was purchased from Sealed Air. The mold was released, and the PO shrink film with impenetrated patterns was made. Sequentially, the patterned PO shrink film was placed between two silicon wafers for uniform heating to desired temperatures in a slowly rising process for approximately 5 minutes and held for 10 minutes for shrinkage in a convection oven (Model 280A). Due to the adhesion of shrink polymer film at high shrink temperature, the PO shrink film bonded with silicon very well, as the shadow mask for $\mathrm{Al}_{2} \mathrm{O}_{3}$ deposition. After that, oxygen plasma was applied to pattern the graphene nanoribbon $50 \mathrm{~nm}$ wide. By using shrink lithography, extreme narrow patterns were obtained on the PO shrink film. A layer of $\mathrm{Al}_{2} \mathrm{O}_{3} 8 \mathrm{~nm}$ thick was deposited, and the shrunk PO film was acting as a shadow mask. After the PO film was stripped, an $\mathrm{Al}_{2} \mathrm{O}_{3}$ nanoribbon was left on the graphene as the mask. The substrate was followed by oxygen plasma etching with STS etcher (Model 320, 10 seconds). The masks on the substrate assisted the alignment and location. The substrate was immersed into a $\mathrm{KOH}$ solution to remove the $\mathrm{Al}_{2} \mathrm{O}_{3}$ mask. Chromium/gold electrodes $50 / 200 \mathrm{~nm}$ thick were deposited and patterned on the two ends of grapheme nanoribbon by an electron-beam evaporation (Model SEC 600) and metal lift-off. The $\mathrm{SiO}_{2}$ layer $300 \mathrm{~nm}$ thick underneath graphene ribbons was etched away by buffered HF (BOE 10:1) for 7 minutes. The suspended structure was obtained by $\mathrm{SF}_{6}$ etching followed by Super Critical Dryer (Model 915 B).
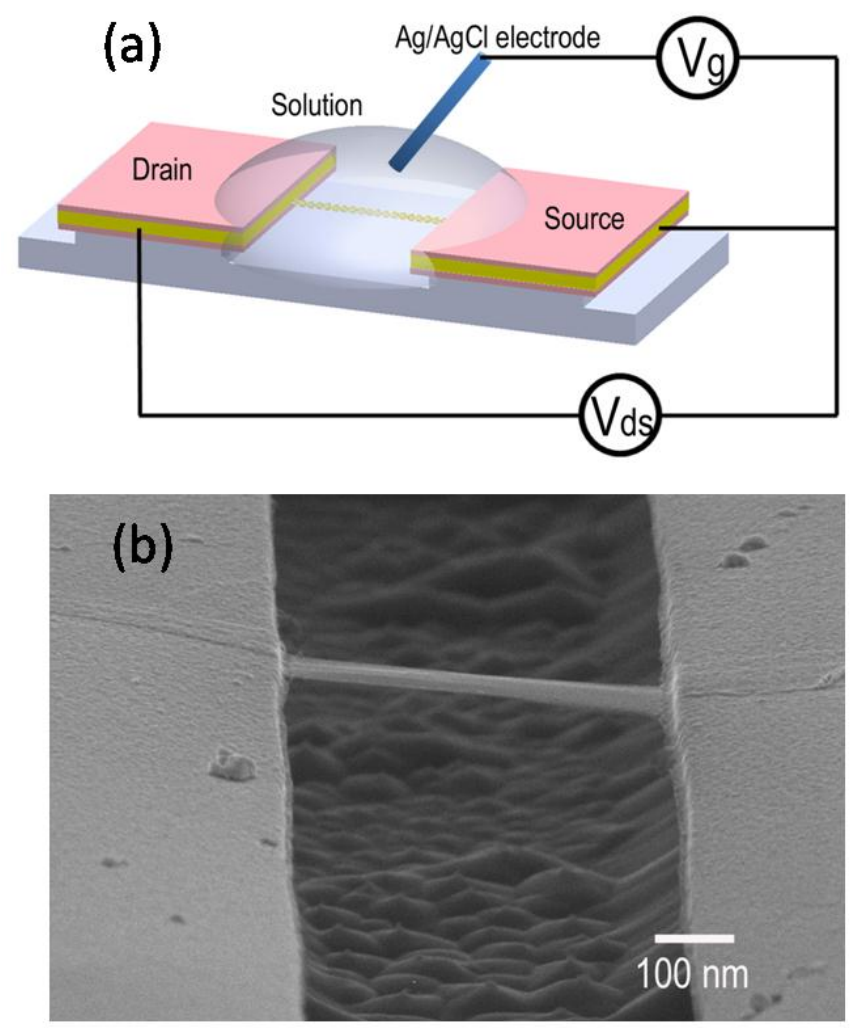

Figure 2: (a) Schematic of a suspended GNR ISFET. (b) SEM image of the suspended GNR structure. The width of the GNR is about $50 \mathrm{~nm}$.

Fig. 2a shows the structure of the suspended GNR ISFET. Target solutions were introduced into the recording chamber, and an $\mathrm{Ag} / \mathrm{AgCl}$ reference electrode was immersed to apply a desired gate voltage. Electrical measurements were carried out using a semiconductor analyzer (HP 4145B). The suspended GNR was inspected by scanning electron microscope (SEM). As shown in Fig. 2(b), the GNR about $50 \mathrm{~nm}$ wide is successfully suspended by the dry etching of silicon.

\section{RESULTS AND DISCUSSIONS}

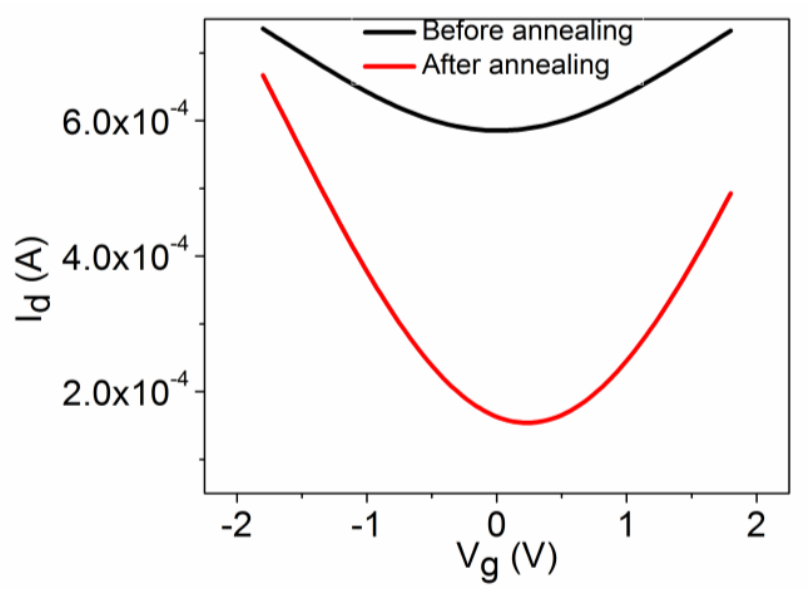

Figure 3: Ambipolar characteristics of GNR ISFET are measured in a PBS buffer solution at room temperature. Annealing bias voltage at a set point of $3.3 \mathrm{~V}$ is applied by $V_{d s}$ without a gate voltage. The annealing process contributes the enhanced ambipolar behavior.

Drain-to-source current versus solution gate voltage was recorded to investigate the electrical properties of the suspended GNR ISFET. Fig. 3 presents ambipolar characteristics of GNR ISFET measured in a PBS buffer solution at room temperature, showing the transition from p-type region to n-type region at the Dirac point of about $0.2 \mathrm{~V}$. After applying the annealing bias voltage to a predefined set point of $3.3 \mathrm{~V}$, the enhanced ambipolar behavior demonstrates larger intrinsic bandgap of GNR due to the annealing process, compared with the device before annealing. The enhanced ambipolar behavior after annealing is contributed by the thermal energy [9] and the suspended structure change [10] introduced by annealing. The annealing effect is responsible for the larger energy band gap, comparable to those of possibly much narrower ribbons [10].

The $\mathrm{pH}$ electrical characteristics were also investigated. First, the annealed suspended GNR ISFET was used to detect different $\mathrm{pH}$ solutions, and compared with unsuspended GNR prior to annealing and a normal graphene sheet in microscale under the same design, fabrication and measurement conditions. As shown in Fig. 4(a), solutions from $\mathrm{pH} 5$ to $\mathrm{pH} 9$ were delivered to the suspended GNR ISFET sequentially. The electrolyte gate response of the device drain-to-source current was measured for each solution. While the ISFET was biased at $\mathrm{V}_{\mathrm{ds}}$ of $1 \mathrm{~V}$, and the gate voltage was applied from -1.8 to $1.8 \mathrm{~V}$. It is obviously observed that the Dirac point of GNR shifts positively from $\mathrm{pH} 5$ to $\mathrm{pH}$, suggesting that $\mathrm{H}^{+}$ions act as n-type dopants to the GNR. Adsorption of a positively charged $\mathrm{H}^{+}$induces additional negative charge in the GNR, thus the n-type doping of the GNR shifts the Dirac point to more negative gate voltages. The suspended GNR behaves as a p-type material when negative gate potential is applied, and the $I_{d}$ of the ISFET increases with the increase of $p H$ values. On the contrary, when the gate potential is switched to be 
positive, transition from p-type region to n-type region occurs, and the $I_{d}$ of the ISFET decreases with the increased $\mathrm{pH}$ values.
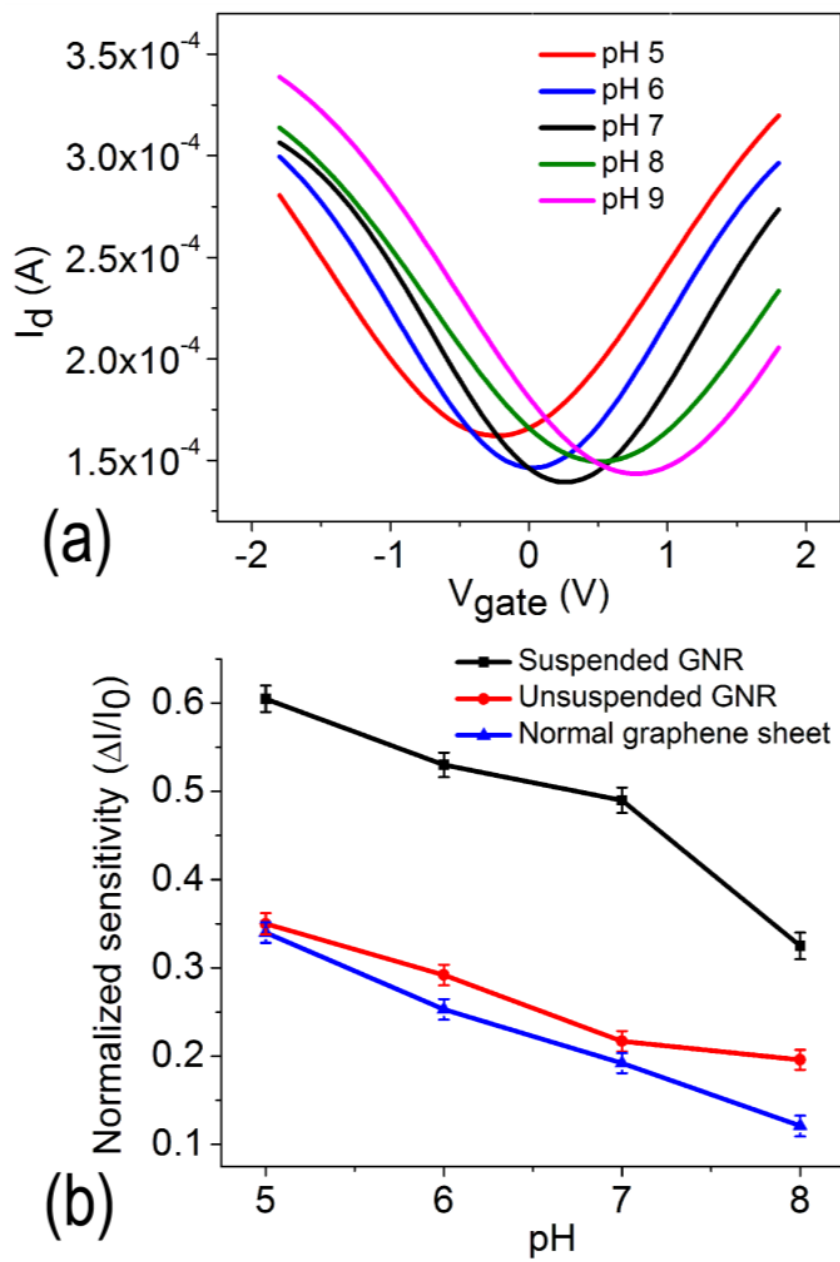

Figure 4: (a) Ambipolar characteristics of suspended GNR ISFET with different $p H$ solutions. The Dirac point of suspended GNR shifts significantly from $\mathrm{pH} 5$ to $\mathrm{pH}$ 9. (b) Normalized sensitivity of different types of ISFET is measured. In order to get a clear readout, a normalized sensitivity is deduced. Id for $\mathrm{pH} 9$ solution is used as an initial current $I_{0}$, and other Id tested under different $\mathrm{pH}$ subtracted $I_{0}$ to get $\Delta I$. Normalized sensitivity is represented as $\triangle I / I_{0}$. Suspended GNR presents better sensitivity than the unsuspended GNR and normal graphene.

The sensitivity of different types of ISFET was also investigated. The ISFET was biased at $\mathrm{V}_{\mathrm{ds}}$ of $1 \mathrm{~V}$, and the gate voltage was fixed at $1.8 \mathrm{~V}$. Different solutions from $\mathrm{pH} 5$ to $\mathrm{pH} 9$ were introduced to the suspended GNR ISFET, unsuspended GNR ISFET and normal graphene ISFET, respectively. The width of the suspended GNR and unsuspended GNR is about $50 \mathrm{~nm}$, and the normal graphene sheet is in micrometer scale. For a clear readout, a normalized sensitivity was deduced. $\mathrm{I}_{\mathrm{d}}$ for a $\mathrm{pH} 9$ solution was used as an initial source-drain current, and other $I_{d}$ values tested under different $\mathrm{pH}$ values subtract, and then divided by the initial $I_{d}$ to get the normalized sensitivity. As shown in Fig. 4(b), the normalized sensitivity was compared among the three types of ISFET, demonstrating that suspended GNR ISFET was superior in $\mathrm{pH}$ sensing performance. Two factors play important roles for the better sensitivity of suspended GNR. One factor is due to the suspended gap under the GNR. The $\mathrm{H}^{+}$ion concentration becomes non-uniform due to the field presented in the suspended gap [11]. The other one is that the charge traps at the interface and in the oxide, which act as external scattering centers and degrade transport properties $[12,13]$ greatly by suspension.

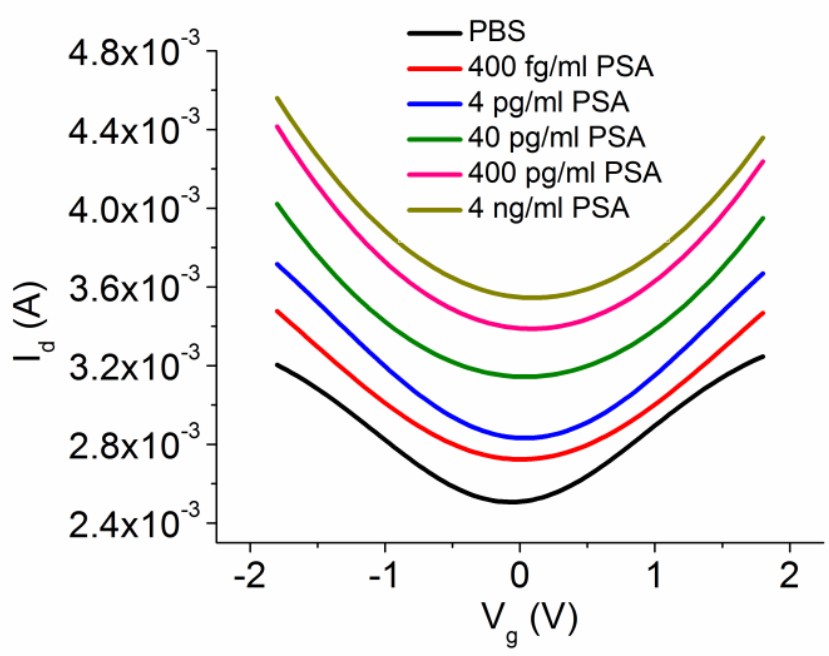

Figure 5: Ambipolar characteristics of suspended GNR ISFET with different PSA solutions induced. The Dirac point of suspended GNR does not shift as significantly as pH detection.

Furthermore, we also investigated the cancer bio-marker detection by modifying the surface of GNR with receptor antibodies. Cancer bio-markers are molecules in blood or tissue, associated with cancer and whose measurement or identification is elucidated very critical and efficient in disease prediction, diagnosis, and monitoring [14]. In the meanwhile, the clinical utility of PSA to discriminate health and disease requires the capability to detect extremely low concentration [15], which is also especially important to understand cellular processes and to search for new protein biomarkers [16]. Herein, after the surface modification of suspended GNR, the anti-PSA was immobilized on the surface of a suspended GNR, and the ISFET was blocked with bovine serum albumin. Given that the conductance of graphene is determined by the charge carrier density and mobility, it is evident that changes in density and/or mobility of charge carriers must be responsive when molecules or ions are absorbed [17]. Therefore, the conductance of the suspended GNR modified with the PSA capture antibody shifts as the concentration change of PSA solutions.

As shown in Fig. 5, different concentrations of PSA solutions from 0 to $4 \mathrm{ng} / \mathrm{ml}$ were introduced to the surface modified suspended GNR ISFET sequentially. The ambipolar characteristics of suspended GNR ISFET were measured at different PSA solutions. The ISFET was biased at $\mathrm{V}_{\mathrm{ds}}$ of $1 \mathrm{~V}$, and the gate voltage was applied from -1.8 to $1.8 \mathrm{~V}$. There is a small right shift of the Dirac point of suspended GNR from 0 to $4 \mathrm{ng} / \mathrm{ml}$ in the PSA solutions with a much less shift compared with a $\mathrm{pH}$ detection. The shift of Dirac point is due to the electrostatic gating effect from the adsorbed charge species adopted to explain the shift of $\mathrm{I}_{\mathrm{d}^{-}}$ $\mathrm{V}_{\mathrm{g}}$ curve of carbon nanotube transistors. The positive shift of Dirac point is due to the negative charges of the adsorbed PSA which can only be balanced by a positive gate voltage. However, the interaction between GNR and PSA immunoreaction bonding is relative weak compared to the $\mathrm{H}^{+}$. Therefore, the positive shift of Dirac point is not obvious, and the conductance change becomes dominant. 


\section{CONCLUSIONS}

In summary, we have investigated the fabrication of suspended GNR ISFET using shrink lithography, which is very cost effective due to the inexpensive thermoplastics and the molding process. The $\mathrm{pH}$ value was characterized based on the suspended GNR ISFET to show positively shift of the Dirac point with the increase of $\mathrm{pH}$ values. Annealing effect on the ambipolar characteristics of a suspended GNR was also investigated. In comparison, the ISFETs based on unsuspended GNR and microscale graphene sheets were characterized under the same design, fabrication, and measurement conditions, showing that suspended GNR ISFET was superior in sensitivity. Moreover, this low-cost detection platform can be extended to the recognition of other antigens, which may open a new way to diagnose cancer and other complex diseases.

\section{ACKNOWLEDGMENT}

The authors acknowledge the assistance of fabrication and characterization from the Nanofabrication Center and the Characterization Facility at the University of Minnesota.

\section{REFERENCES}

[1] X. Wang, H. Dai, "Etching and Narrowing of Graphene from the Edges", Nat. Chem. 2, 661, (2010).

[2] X. Li, X. Wang, L. Zhang, S. Lee, H. Dai, "Chemically Derived, Ultrasmooth Graphene Nanoribbon Semiconductors", Science. 319, 1229, (2008).

[3] D. V. Kosynkin, A. L. Higginbotham, A. Sinitskii, J. R. Lomeda, A. Dimiev, B. K. Price, J. M. Tour, "Longitudinal Unzipping of Carbon Nanotubes to Form Graphene Nanoribbons", Nature. 458, 872, (2009).

[4] L. Tapaszto', G. Dobrik, P. Lambin, P. Biro', la' szlo' "Tailoring the Atomic Structure of Graphene Nanoribbons by Scanning Tunnelling Microscope Lithography", Nat. Nanotech. 3, 397, (2008)

[5] L. Jiao, L. Zhang, L. Ding, J. Liu, H. Dai, “Aligned Graphene Nanoribbons and Crossbars from Unzipped Carbon Nanotubes", Nano Res. 3, 387, (2010).

[6] K. R. Ratinac, W. Yang, S. P. Ringer, F. Braet, "Toward Ubiquitous Environmental Gas SensorssCapitalizing on the Promise of Graphene", Environ. Sci. Technol. 44, 1167, (2010).

[7] Y. Huang, X. Dong, Y. Shi, C. M. Li, L. Li, P. Chen, "Nanoelectronic Biosensors Based on CVD Grown Graphene", Nanoscale, 2, 1485, (2010).
[8] Z. Cheng, Q. Li, Z. Li, Q. Zhou, Y. Fang, "Suspended Graphene Sensors with Improved Signal and Reduced Noise", Nano. Lett. 10, 1864, (2010).

[9] T. Shimizu, J. Haruyama, D. C. Marcano, D. V. Kosinkin, J. M. Tour, K. Hirose, K. Suenaga, "Large Intrinsic Energy Bandgaps in Annealed Nanotube-Derived Graphene Nanoribbons", Nat. Nanotech. 6, 45, (2010).

[10] M. Lin, C. Ling, Y. Zhang, H. J. Yoon, M. M. Cheng, L. A. Agapito, N. Kioussis, N. Widjaja, Z. Zhou, "RoomTemperature High On/Off Ratio in Suspended Graphene Nanoribbon Field-Effect Transistors", Nanotechnol. 22, 265201, (2011).

[11] F. Bendriaa, F. Le Bihan, A.C. Salaün, T. MohammedBrahim, O. Bonnaud, "Highly Sensitive Suspended-Gate Ion Sensitive Transistor for the Detection of $\mathrm{pH}^{\text {", Proceedings of }}$ SPIE, 5866, 433, (2005).

[12] Y. Lin, P Avouris, "Strong Suppression of Electrical Noise in Bilayer Graphene Nanodevices”, Nano. Lett. 8, 2119, (2008).

[13] G. Liu, W. Stillman, S. Rumyantsev, Q. Shao, M. Shur, A. A. Balandin, "Low-Frequency Electronic Noise in the DoubleGate Single-Layer Graphene Transistors", Appl. Phys. Lett. 95, 033103, (2009).

[14] R. Etzioni, N. Urban, S. Ramsey, M. McIntosh, S. Schwartz, B. Reid, J. Radich, G. Anderson, L. Hartwell, "The Case for Early Detection", Nat. Rev. 3, 1, (2003).

[15] H. Lilja, D. Ulmert, A. J. Vickers, "Prostate-Specific Antigen and Prostate Cancer: Prediction, Detection and Monitoring", Nat. Rev. 8, 268, (2008).

[16] L. A. Tessler, J. G. Reifenberger, R. D. Mitra, "Protein Quantification in Complex Mixtures by Solid Phase SingleMolecule Counting", Anal. Chem. 81, 7141, (2009).

[17] E. H. Hwang, S. Adam, S. Das. Sarma, "Transport in Chemically Doped Graphene in the Presence of Adsorbed Molecules", Phys. Rev. B. 76, 195421, (2007).

\section{CONTACT}

*T. Cui, tel: +1- 612-626-1636; tcui@me.umn.edu 


\title{
NANOPOROUS-BASED HYDROPHILIC POLYMER MICROCHANNEL USING A SPRAY LAYER-BY-LAYER ELECTROSTATIC NANO-ASSEMBLY
}

\author{
K.K. Lee * and C.H. Ahn \\ Microsystems and BioMEMS Laboratory, \\ School of Electronics and Computing Systems, University of Cincinnati, Cincinnati, Ohio, USA
}

\begin{abstract}
A simple, versatile, cost-effective, and high-throughput spray layer-by-layer (LbL) electrostatic nano-assembly technology has been developed, characterized and applied to polymer microchannels with nanoporous surfaces sprayed on cyclic olefin copolymer (COC) for a capillary micropump or separator. The pumping capabilities achieved from the sprayed nanoporous surfaces were mainly due to the strong hydrophilic properties of the multi-coated bilayers of hydrophilic silica nanoparticles. The hydrophilic nature of nanoporous surface of the COC microchannel was characterized by measuring the length of a test liquid driven by the capillary forces produced through the microchannel. The spray LbL method developed in this work can be an innovative approach for the modification of various surfaces of polymer microchannels.
\end{abstract}

\section{INTRODUCTION}

Flow control of biological samples or reagents using capillary force through a microchannel is very attractive for the on-chip microfluidic devices of biological sensing or diagnostic systems [1, 2]. Specifically, superhydrophilic wetting properties have significant effects on liquid behavior in the surface control applications such as capillary pumping or separator [3]. So, the control of surface energy over the microchannel walls is desirable for enhancing the wetting properties of microchannel surfaces.

Polymer-based microfluidic devices are alternative to those fabricated from glass and silicon substrates along with the standard lithographic microfabrication technology [4]. Polymers are suitable for single-use disposable devices since they offer low cost, good biocompatibility and mass manufacture using high-throughput microfabrication techniques such as hot embossing or injection molding. Various polymer materials such as COC, polyimide, polymethylmethacrylate (PMMA), polydimethylsiloxane (PDMS) and polycarbonate (PC) have been used for the microfluidic devices. Among them, COC is of particular interest due to its combination of excellent UV transparency, low autofluorescence, low oxygen permeability, high mechanical strength, and compatibility with a broad range of chemicals and solvents [5].

However, like most other polymers, COC has a hydrophobic nature, which causes many problems in the applications of biochip or lab chip such as sample loss, degradation of resolution in separations, and difficulties with accurate quantitative analyses. In order to minimize these problems, it is necessary to chemically modify or fuctionalize the walls of the COC microchannels. Various techniques of polymer surface modification have been developed [6-10]. However, most of these procedures are not suitable for microfluidic devices since they rarely allow selective patterning on the surface of microchannel. Furthermore, they require additional special instruments, harsh processing conditions, and high cost. So, there is still a large demand for the development of a new technique for attaining highly hydrophilic surfaces on the patterned COC microchannels.

In this work, a new simple, fast, versatile, effective and functional coating method using a spray layer-by-layer (LbL) nano-assembly has been developed to selectively modify the surface of COC polymer microchannels for various microfluidic devices. The major advantage of this spray LbL is a dramatically reduced processing time needed for the multilayer formation at room temperature. Surface functionality can be easily controlled by choosing appropriate polyelectrolytes.

\section{NANO ASSEMBLY}

An aluminum master mold was fabricated first using a micromilling machine (Microlution 5100S) for the replica of COC microchannels. Then, COC substrates were patterned with microfluidic channels using hot embossing technique for the spray $\mathrm{LbL}$ process. The dimension of fabricated microchannel was 400 $\mu \mathrm{m} \times 200 \mu \mathrm{m} \times 47 \mathrm{~mm}$. Then, the spray LbL nano-assembly process with silica nanoparticles was followed for the surface modification of the patterned COC microchannel.

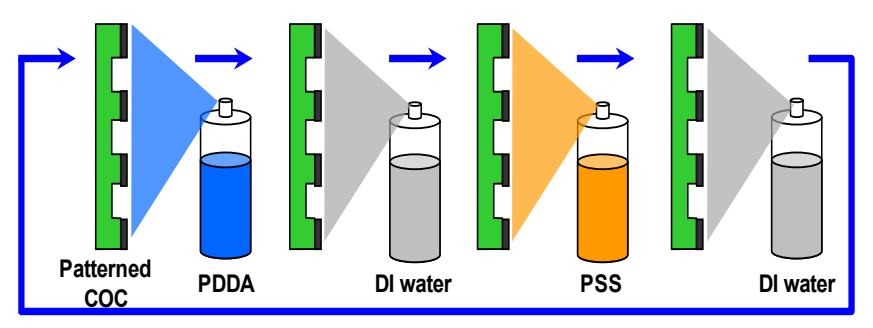

Figure 1: Schematic illustration of spray-LbL nanoassembly of multilayer films on patterned COC microchannels.

Figure 1 shows the conceptual diagram of the spray LbL technology. The surface was modified by successive spraying of poly (dimethyldiallyl-ammonium chloride) (PDDA) and poly (sodium 4-styrenesulfonate) (PSS) (Sigma-Aldrich, St. Louis, MO) as an adhesion promoter on the COC microchannel surfaces, which were already activated by oxygen plasma. Rinsing step was needed after a complete layer was formed. Then, the layer of oppositely charged polyion was sprayed. The polyion solutions and rinsing solutions were supplied by enforced spraying in this process. As hydrophilic nanoparticles with different sizes, SM-30 colloidal silica $30 \mathrm{wt} \%$ (7 $\mathrm{nm}$ diameter silica particles, Sigma-Aldrich), Ludox HS-40 colloidal silica 40 wt \% (12 nm diameter silica particles, Sigma-Aldrich), Ludox TM-40 colloidal silica 40 wt \% (22 nm diameter silica particles, Sigma-Aldrich), and silica nanospheres (5\% aqueous dispersion, $50 \mathrm{~nm} \& 100 \mathrm{~nm}$ diameter silica nanoparticles, Polysciences Inc.) were used for the nanoassembly. A typical multilayer build-up process by spray LbL is schematically described in Figure 2. In Figure 2, 3s, 27s, 20s, and $10 \mathrm{~s}$ indicate the desired processing time in seconds for each process. A polyelectrolyte (PDDA or PSS or silica) was applied by spraying. Spraying is suspended but drainage and evaporation of water continue. The film was spray-rinsed with deionized (DI) water. Then, spray rinsing was suspended but drainage and evaporation of water continue. The total deposition time per layer was 60 seconds. Negatively charged colloidal silica nanoparticles (with average particle sizes of 7, 12, 22, 50, and $100 \mathrm{~nm}$ ) were diluted to 0.3 wt $\%$ in DI water. Polymer solutions (PDDA, PSS) 
were diluted to $0.01 \mathrm{M}$ in DI water.

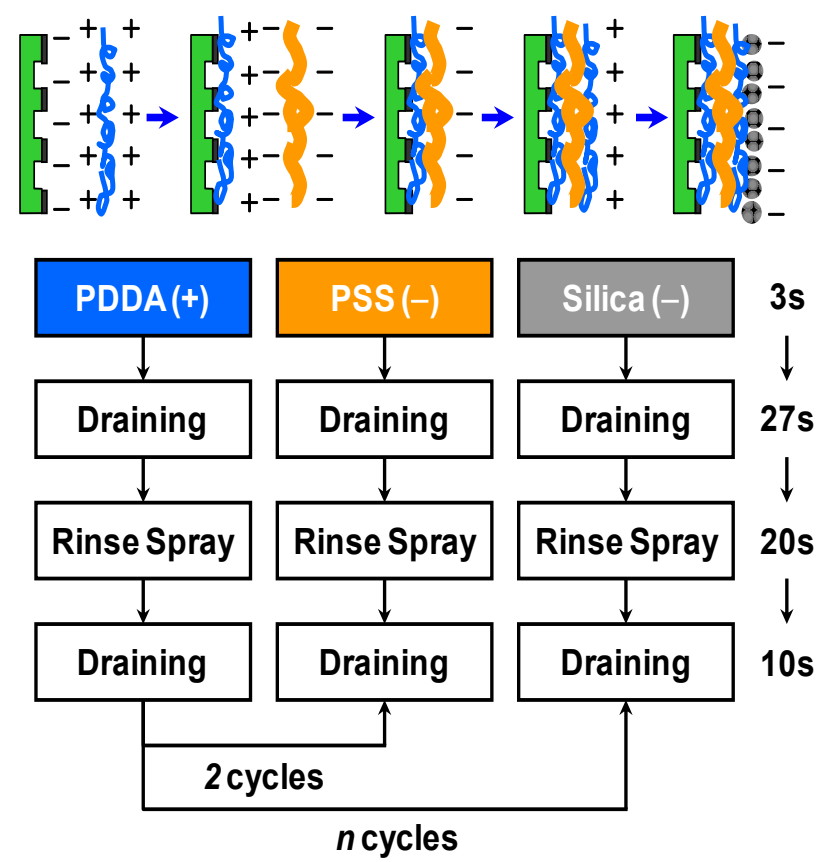

Figure 2: Nano-assembly protocol for the deposition of polyelectrolyte multilayer films on patterned COC microchannels by spray LbL, where $n$ is the number of bilayers. Spraying times and polyelectrolyte concentrations were based on the previous work [11], and no drying step was performed between layer depositions.

\section{EXPERIMENT AND RESULTS}

\section{Contact Angle Measurement}

The wettability of the spray coated surfaces of COC was characterized in ambient air at room temperature using a contact angle analyzer based on the sessile drop method. The mean contact angles are determined by averaging values measured at five different points on the sample surface. In Figure 3, contact angles on the modified COC surfaces were measured as a function of the nanoparticle sizes. The lowest contact angle with smallest standard deviation was obtained in the COC surface coated with 12 $\mathrm{nm}$ silica nanoparticles. So, the $12 \mathrm{~nm}$ silica nanoparticle was chosen for the spray LbL nano-assembly on the COC microchannels as the surface control applications such as capillary pumping or separator.

\section{Surface Characterization with SEM and EDS}

The COC microchannel and the nano silica film formed by silica nanoparticles were characterized by SEM (XL-30, ESEM, Philips) as shown in Figure 4. Silica nanoparticles can be seen in the microchannels and the inset SEM image is a high resolution image of the nanoparticles. These close and uniform microstructures, which contribute to absorb liquid and enhance the nanoporous capillary action, can be observed in the SEM image.

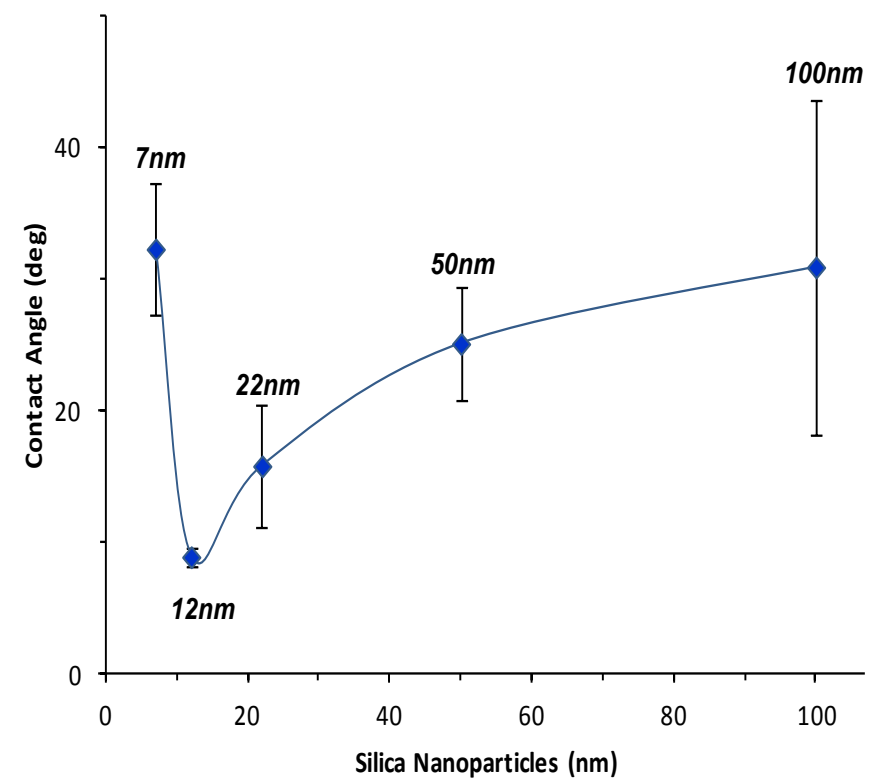

Figure 3: Variation of contact angle on silica nanoparticle sizes with DI water droplets.

In order to confirm the silica nano-assembly, energy dispersive X-ray spectroscopy (EDS) analysis was performed over the microchannel area with and without the silica nanoparticles as shown in Figure 5. The signature of silicon and oxygen peaks in EDS spectra were shown at the microchannel area with the spray silica nanoparticles. This clearly showed that the silica film was successfully constructed inside the COC microchannel by the spray LbL nano-assembly of the silica nanoparticles.

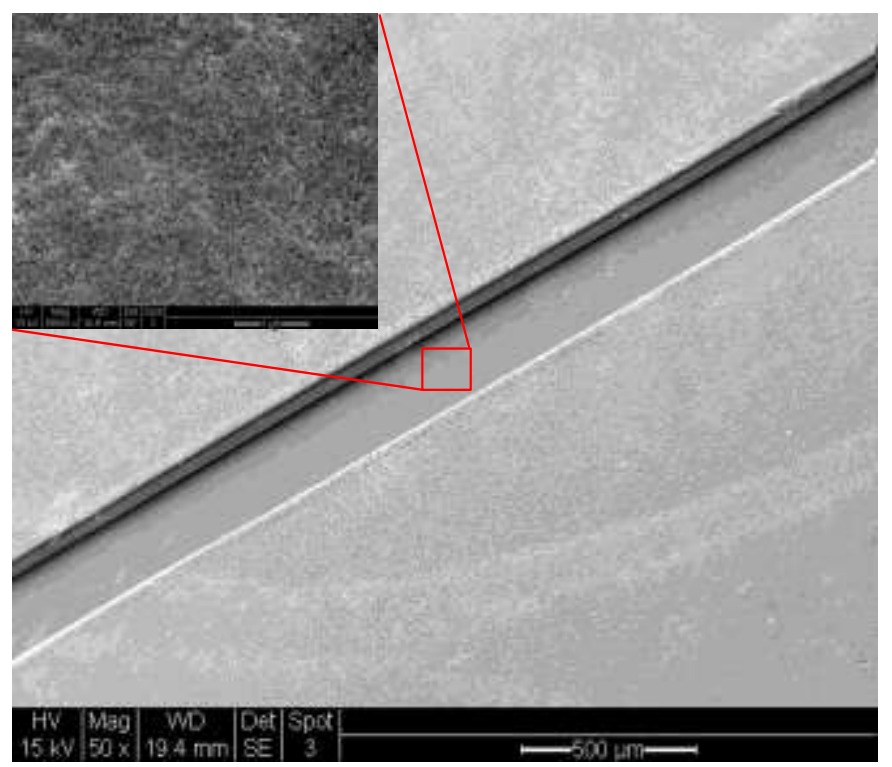

Figure 4: SEM images of COC microchannels after the spray $L b L$ nano-assembly of silica nanoparticles. 


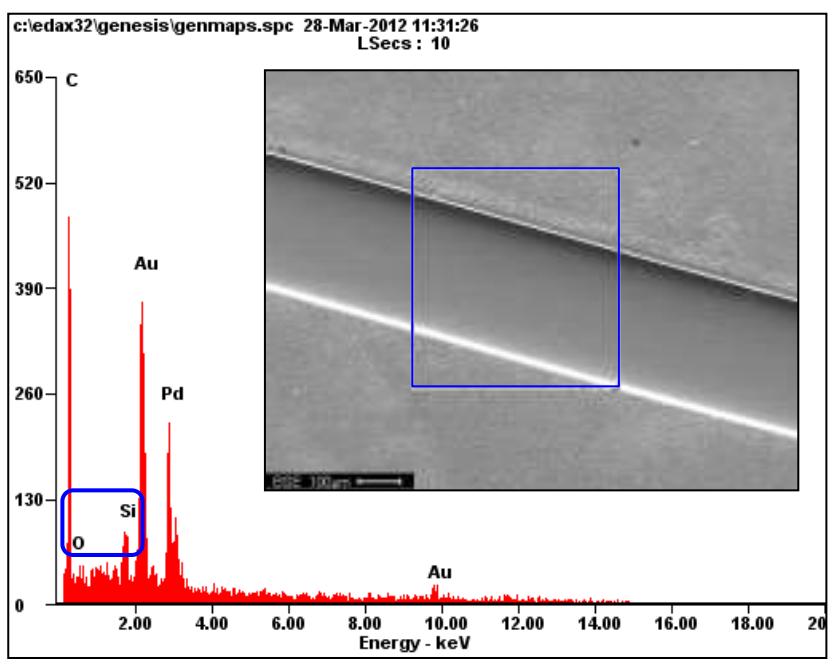

(a)

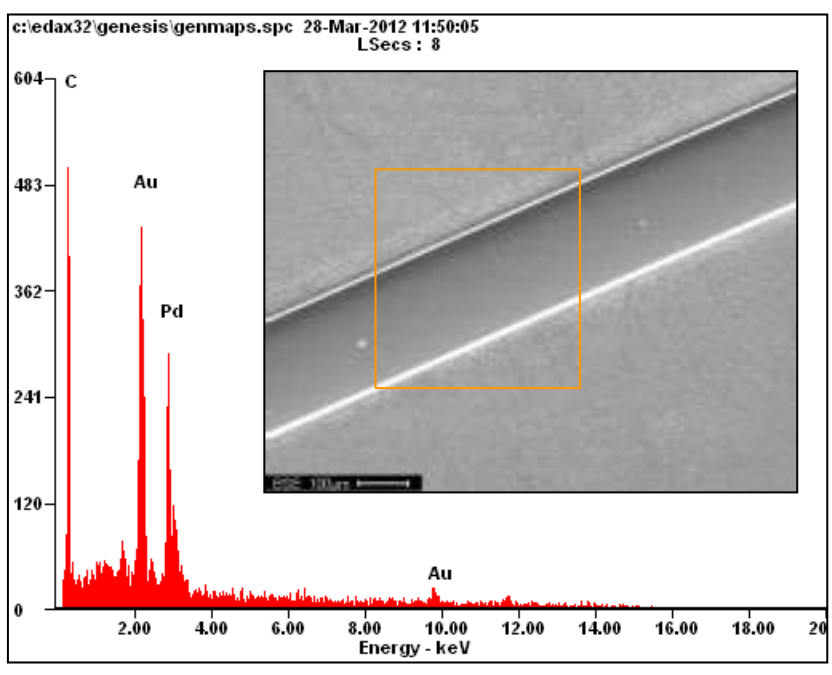

(b)

Figure 5: Energy dispersive X-ray spectroscopy (EDS) spectra of the (a) silica nanoparticle coated COC and (b) bare COC.

\section{Time-dependent Changes in Contact Angle}

Figure 6 shows the changes in contact angle for a first water drop $(3 \mu \mathrm{L})$ as a function of time. The nanoporous multilayer thin films were deposited from the spray LbL assembled silica nanoparticles and a polycation (PDDA) solution. All processing conditions such as spraying time and polyelectrolyte concentration were based on the previous work [11]. The contact angles of all surfaces were decreased over the time. Highly wettable surfaces were obtained with 5,10 and 15 coatings of bilayers, but superhydrophilic behavior was only achieved in 5 coatings of bilayers for the film that completely wets (contact angle below $5^{\circ}$ ) with DI water after 350 seconds. This dramatic drop in contact angles indicates that the COC surface can be super-hydrophilic with silica nanoparticles due to the intrinsically high level of wettability of the silica nanoparticles coupled with the nanoporous nature of the multilayer surface. Thus, the 5 coatings of [PDDA/silica] bilayers were chosen as optimum coatings of bilayers to obtain the maximum nanoporous capillary pumping effect in this process.

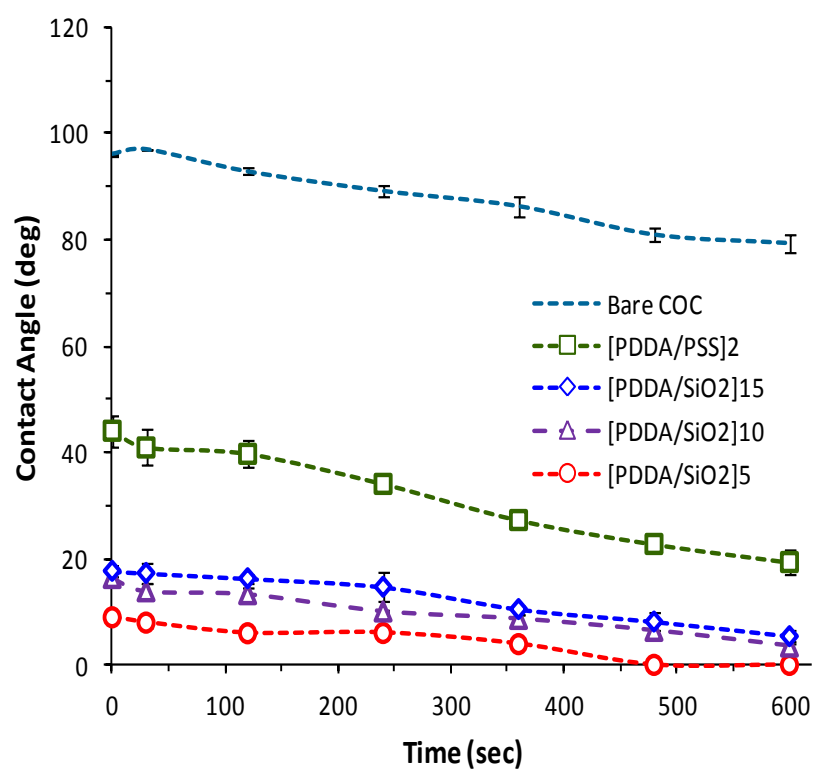

Figure 6: Time-dependent changes in contact angle with the different number of silica layers.

\section{Measurement of Capillary Flow}

Two types of microchannels 200 and $400 \mu \mathrm{m}$ wide in horizontal platform were prepared to characterize the nanoporous capillary pumping effect. All microchannel surfaces were coated with 5 number of [PDDA/silica] bilayers. A droplet of $3 \mu \mathrm{l}$ of red food dye was placed on the inlet of the microchannel using a micropipette. A flow was produced through the microchannel to reach the outlet reservoir by autonomous capillary. As shown in Figure 7, the liquid front of the food dye can move up to $47 \mathrm{~mm}$ without any additional forces within approximately 12 seconds. It is found that the liquid moves quickly at the beginning time, and the speed of the movement slightly decreases at the succeeding time until the liquid reached the outlet reservoir.

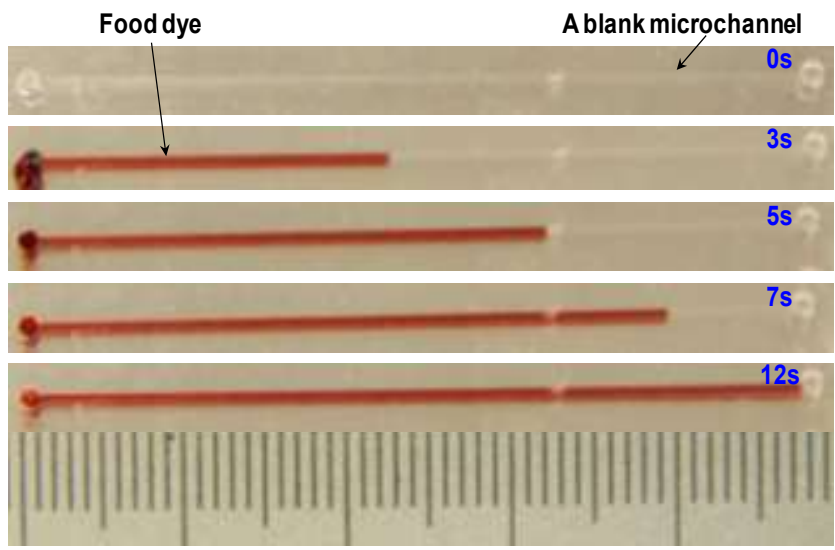

Figure 7: Flow tests for the COC microchannels with a dimension of $400 \mu \mathrm{m} \times 100 \mu \mathrm{m} \times 47 \mathrm{~mm}$. 
Figure 8 shows the relationship between the relative distance of liquid-gas interface locations from inlet to outlet reservoirs and the time. The liquid front location of the food dye moved approximately $42 \mathrm{~mm}$ for the initial seven seconds, and move 5 $\mathrm{mm}$ during the succeeding three seconds when the width of microchannel is $200 \mu \mathrm{m}$. As the width is $400 \mu \mathrm{m}$, the liquid-gas interface location could only move $37 \mathrm{~mm}$ for the first seven seconds, but moves $6 \mathrm{~mm}$ during the succeeding three seconds. Thus, the capillary pumping effect was decreasing with the increasing width of microchannels.

\section{CONCLUSION}

In conclusion, the spray LbL nano-assembly technique creates the nanoporous infiltration networks with silica nanoparticles on the patterned COC microchannels. The nanocapillary effect of the networks, which was confirmed from the measured contact angles, ensures the superhydrophilic surfaces developed on the hydrophobic COC microchannels. The effects were successfully characterized in a horizontal microchannel on COC testing platform. The result shows that this capillary pump can drive the food dye $47 \mathrm{~mm}$ long without additional power within 12 seconds. The nanocapillary driven coating networks can be a general platform for the highly desirable modification of various surfaces of polymer microchannels.

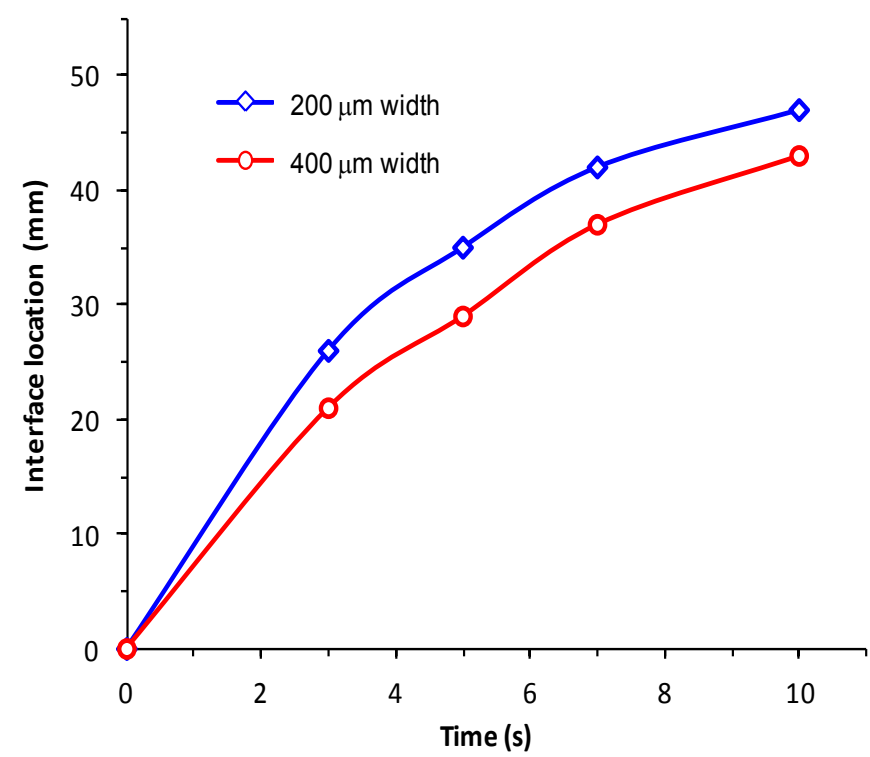

Figure 8: Capillary pumping rate versus time with two types of microchannels 200 and $400 \mu \mathrm{m}$ wide in horizontal platform.

\section{REFERENCES}

[1] C.F. Kung, C.F. Chiu, C.F. Chen, C.C. Chu, "Blood flow driven by surface tension in a microchannel," Microfluidics and Nanofluidics, 6, 693 (2009).

[2] A.C. Henry, T.J. Tutt, M. Galloway, Y.Y. Davidson, C.S. McWhorter, S.A. Soper, R.L. McCarley, "Surface modifcation of poly(methyl methacrylate) used in the fabrication of microanalytical devices," Analytical Chemistry, 72, 5331 (2000).

[3] V. Bulmus, H. Ayhan, E. Piskin, "Modified PMMA monosize microbeads for glucose oxidase immobilization," Chemical
Engineering Journal, 65, 71 (1997).

[4] M.A. Burns, B.N. Johnson, S.N. Brahmasandra, K. Handique, J.R. Webster, M. Krishnan, T.S. Sammarco, P.M. Man, D. Jones, D. Heldsinger, C.H. Mastrangelo, D.T. Burke, "An Integrated nanoliter DNA analysis Device," Science, 282, 484 (1998).

[5] R.R. Lamonte, D. McNally, "Uses and processing of cyclic olefin copolymers," Plastics Engineering, 56, 51 (2000).

[6] J.K. Liu, M.L. Lee, "Permanent surface modification of polymeric capillary electrophoresis microchips for protein and peptide analysis," Electrophoresis, 27, 3533 (2006).

[7] V. Dolnik, "Wall coating for capillary electrophoresis on microchips," Electrophoresis, 25, 3589 (2004).

[8] D. Belder, M. Ludwig, "Surface modification in microchip electrophoresis," Electrophoresis, 24, 3595 (2003).

[9] H. Makamba, J. H. Kim, K. Lim, N. Park, J. H. Hahn, "Surface modification of poly(dimethylsiloxane) microchannels," Electrophoresis, 24, 3607 (2003).

[10] C.H. Ahn, J.W. Choi, G. Beaucage, J.H. Nevin, J.B. Lee, A. Puntambekar, J.Y. Lee, "Disposable Smart Lab on a Chip for Point-of-Care Clinical Diagnostics," Proceedings of the IEEE, 92(1), 54 (2004)

[11] A. Izquierdo, S.S. Ono, J.C. Voegel, P. Schaaf, G. Decher, Langmuir, "Dipping versus Spraying: Exploring the Deposition Conditions for Speeding Up Layer-by-Layer Assembly," Langmuir, 21, 7558 (2005).

\section{CONTACT}

*K.K. Lee, tel: +1-513-535-9030; Leek8@mail.uc.edu 


\title{
RAPID AND FACILE FABRICATION OF 3D-NETWORKED CIRCULAR MICROFLUIDIC CHANNELS IN PDMS
}

\author{
Jiwon Lee, Jungwook Paek, and Jaeyoun Kim* \\ Department of Electrical and Computer Engineering, \\ Iowa State University, Ames, Iowa, USA
}

\begin{abstract}
In this paper, we propose a new fabrication method which establishes a simple, rapid, and cost-effective way of generating 3D-networked circular microfluidic (MF) channels. Due to the growing interests to mimic biological vasculatures [13], recently techniques to develop circular MF channels with 3D networks are on demand. By adapting sucrose fibers as eco-friendly sacrificial templates, we established rapidly prototype pre-designed scheme which does not require cumbersome fume-hoods or cleanroom facilities. Moreover, easy control of thicknesses and easy modification of the structure coming from the water-dissolvable nature of sucrose fiber makes various structures feasible in 3Dnetworked circular MF applications.
\end{abstract}

\section{INTRODUCTION}

2D-networked rectangular microfluidic (MF) channels have been the workhorse of lab-on-a-chip (LoC) devices. Recent expansion in the LoC's scope of application, however, introduced an increasingly diverse set of requirements on the MF channel's cross-sectional shape and topology. Of special interest among them include the demands for MF channels with circular crosssection [1-2] and their non-planar 3D deployment [3-4]. While these new architectures will certainly benefit the on-going efforts to mimic biological vasculatures, the fabrication schemes have been complicated and limited as well in their ability to realize MF channel junctions that are physiologically realistic [5].

Circular MF channels have been fabricated through poly (dimethylsiloxane) (PDMS) molding of cylindrical templates predominantly with a variety of templates. Templates with sufficient strength, such as metal wires and nylon threads were physically pulled out, but the scheme cannot produce internally starting and ending MF channels within the PDMS blocks [6]. The use of chemically etchable templates allowed more complex geometries but inevitably brought complicated chemical processes which in general are time-consuming and high-temperature processes. As an alternative, rectangular MF channels were circularized by filling them with curable liquid and applying a strong air stream which bores cylindrical openings. Such a scheme is advantageous for its applicability to conventionally prepared 2D MF networks, whereas it required additional steps involving special solvents.

The realization of non-planar MF networks to study biological systems has also been pursued actively. Layer-by-layer assembly of planar structures [6-7], with vertical via connecting MF channels in different layers, has been the most commonly adopted whereas it was not true 3D MF channels trajectories. Instead, in the plug-in-and-mold scheme, PDMS MF "pipes" were cut out from a 2D MF network prepared in advance and then manually shaped into 3D topologies before getting immersed in liquid-phase PDMS to be cured. Another more recent scheme, omni-directional printing, fabricated 3D MF channel networks by depositing filaments of fugitive ink that can be liquefied and drained after the embedding material got solidified [8].

Thus, a new fabrication scheme should accomplish both circular MF channels and 3D trajectories simultaneously. In particular, it is highly desired to establish a simple and accessible, yet flexible, rapid prototyping technique for 3D-networed circular MF channels. Some of the previously mentioned schemes actually meet the dual requirements. The omni-directionally printed 3D MF channels [8] exhibit circular cross-section thanks to the filamentary morphology of the nozzle-injected ink. The schemes in Refs [8], [9], and [10] also accomplished the dual goal using 3D-shapable cylindrical templates as well as complex instruments and materials. These schemes, however, are not suitable for readily accessible rapid prototyping or compatible with PDMS. Another attempt, the simple overlapping method of two cylindrical templates would result in a physiologically un-realistic junction shape with little contact area. The formation of a proper junction requires attaching one end of the template to the side of another. With the template materials not providing effective ways for the task, the authors of the previous work had to fuse the overlapping areas mechanically or thermally, a cumbersome process that may further deform the junction shape.

In this work, we demonstrate a new scheme for rapid prototyping of MF channels with circular cross-sections and 3D trajectories, with an emphasis on realizing physiologically realistic channel branches. Our work differs from the previous ones in providing a scheme to rapidly prototype pre-designed, rather than randomly shaped, 3D networks of circular MF channels. We also focus on achieving the dual goal using low-temperature, waterbased processes so that the need for fume-hoods or cleanroom facilities can be eliminated. In this regard, we established techniques to make junctions between the sucrose fiber templates and devised methods to efficiently control the trajectories of the templates. The technique is also compatible with a rapid prototyping method, the conventional PDMS molding process. In addition, we exploited the wide variety in the achievable shape of the sucrose template to implement structures other than MF channels as well.
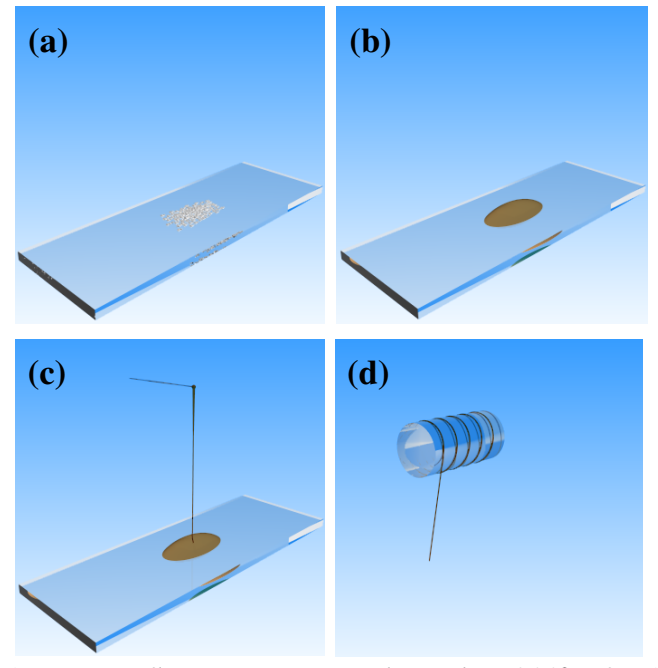

Figure 1: Process flow (a) sucrose is heated on $200^{\circ} \mathrm{C}$ for $15 \mathrm{~min}$, (b) at light brown color, ramp down to $125^{\circ} \mathrm{C}$ for 10min, (c) pull sucrose fibers at $0.1 \sim 0.5 \mathrm{~m} / \mathrm{s},(d)$ coil it around a cylindrical PDMS base template 


\section{MATERIAL SELECTION}

The key enabling factor is the use of sucrose as the sacrificial material. It is highly water-soluble and can be pulled into cylindrical fibers at $<200^{\circ} \mathrm{C}$ for various thicknesses. The resulting fibers also exhibit mechanical strength and surface smoothness adequate for handling/shaping and formation of transparent MF channels, respectively. These advantageous features of sugarbased sacrificial template materials have been exploited formerly. Bellan et al [11] fabricated 3D artificial vascular structures by melt-spinning random sugar fiber networks with a cotton-candy machine and then replicating them with PDMS, epoxy, and poly (caprolactone). Li et al [12] also fabricated bundles of poly-(Llactic acid) (PLLA) tubules for nerve scaffolds by coating sucrose fibers with PLLA and then dissolving the fibers. Former studies, however, are targeted random or simple tubular structures only. We fully exploit the mechanical and thermal properties of sucrose fiber to realize pre-designed templates in a more controlled fashion.

PDMS molding, which is popularly used for its easy curing mechanism, transparency, and flexibility, is selected as to generate MF structures. Due to the temperature budget and chemical-free dissolving mechanism of sucrose, it is suitable to be used with PDMS as well as it enables the fabrication of complex MF channels in bench-top settings. Figure 2 offers an example of the structure created with sucrose as a sacrificial 3D template for the PDMS based MF structure.
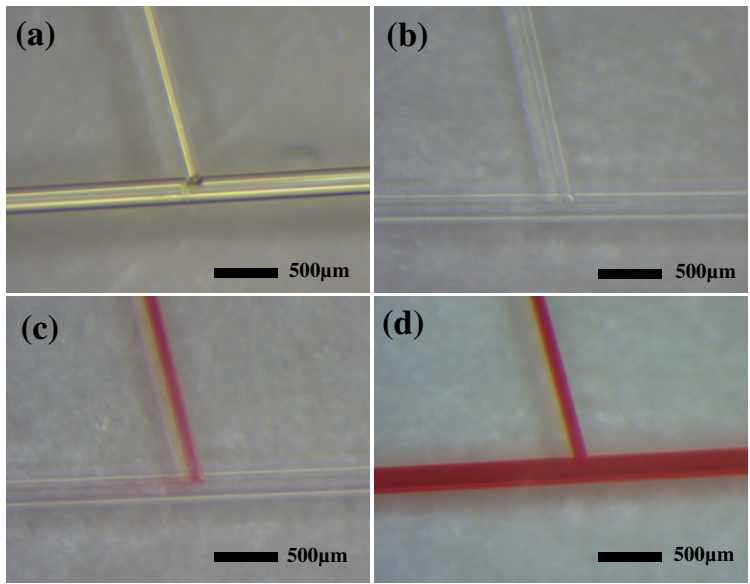

Figure 2: Microscopic images of a PDMS MF junction made with two sucrose fibers: (a) original sucrose fiber assembly, (b) after dissolving the sucrose, $(c-d)$ red food dye was injected to visualize the junction

\section{FABRICATION METHOD}

\section{Preparation of sucrose fibers}

Steps for sucrose fiber pulling are described in Figs. 1(a)-(c). First, crystalline sucrose was melted at $200^{\circ} \mathrm{C}$. Upon turning into light-brown color after 10-15 min, the temperature was ramped down to $125^{\circ} \mathrm{C}$ and maintained for additional $10 \mathrm{~min}$. This particular caramelization temperature extends the duration of the caramelized state up to 3 hours. Then sucrose fibers were pulled at $0.1-0.5 \mathrm{~m} / \mathrm{s}$ with an optical fiber. The diameter of the pulled sucrose fiber was varied between few tens to several hundred micrometers rely on the speed. Both manual pulling and micromanipulator automated pulling is possible depending on the length and the thickness of fiber required.

\section{Assembly of sucrose fibers}

The water-soluble nature and high tensile strength of the sucrose fibers facilitates their manual handling and assembly. Sucrose fibers pulled out of caramelized sucrose instantly solidify. Subsequently, pulled fibers were assembled into a 3D network on or around the base PDMS template. First, the PDMS base plate was made with standard PDMS process. Sylgard184 base and curing agent were mixed at 10:1 ratio and cured for 3 hours at $60^{\circ} \mathrm{C}$. Then, two or more sucrose fibers are located on top of the base plate, used as substrate of final product. Subsequently, one end of a sucrose fiber was closely applied to the cylindrical body of another sucrose fiber to create junctions. Then, two sucrose fibers were easily attached to each other by applying small amount of water. Figure 2(a) shows original sucrose fibers made with above method. To secure the bonding, adhesives made with additional sucrose and water as $2: 3$ weight-ratios and heated on $90^{\circ} \mathrm{C}$ hot plate temperature could also be used. In addition, sucrose-water adhesives make different junction angles feasible, but large quantity of them can cause residues while water should be continuously supplied to maintain the optimum weight-ratio. Not only multiple branches were made by connecting sucrose fibers, but also with a lengthy single fiber we could make different types of patterns. In general, we can easily withdraw a couple meters of a thin sucrose fiber. Especially when the diameter of sucrose fiber is less than $50 \mu \mathrm{m}$, it is flexible enough to be coiled or located around any shapes depending on the PDMS template. For instance, a sucrose fiber can be coiled around a PDMS cylinder to make a solenoidal MF structure as illustrated in Fig. 1(d).

\section{Dissolving of Sucrose fibers}

After being assembled on or around the PDMS base template, sucrose template structure was placed inside a container and subsequently filled with another batch of PDMS. Once PDMS is completely cured, the sucrose fiber assembly was dissolved in warm $\left(\sim 70^{\circ} \mathrm{C}\right)$ water. This low temperature, water-based template removal makes our scheme much simpler than the other methods relying on chemical etching. To aid the complete removal of sucrose, flow of warm water was injected with a syringe.

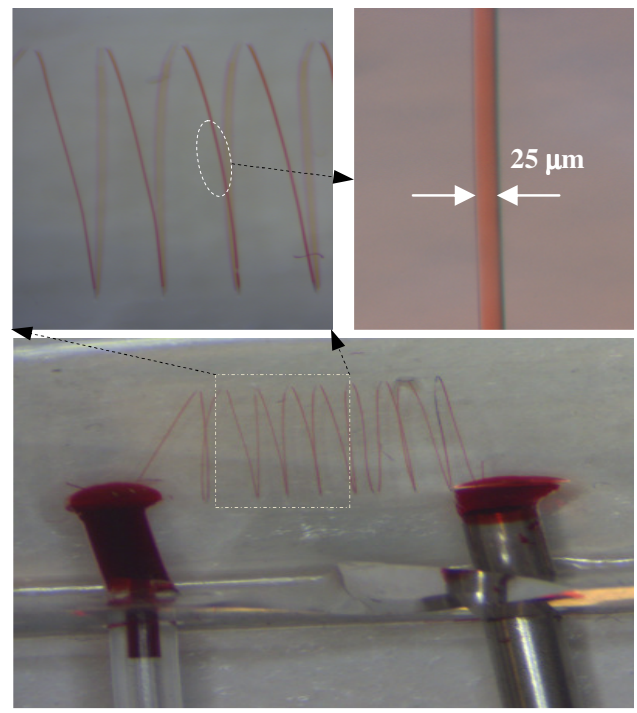

Figure 3: Microscopic images of a $25 \mu$ m-diameter MF channel forming a 3D solenoidal network with a $5 \mathrm{~mm}$-diameter size core 


\section{RESULT AND DISCUSSION \\ Microfluidic channels}

Figure 2 (a-d) shows a MF channel smoothly branching from a bigger one. From the initial sucrose fiber template (Fig. 2(a)) to the water-filled MF channel in PDMS (Fig. 2(b)), we can observe smooth surface. Surface quality and the circular shape of sucrose fiber are characterized further in the following sections. Especially, the pattern of gradual spreading red food dye (Fig. 2(c)) affirms that the junction was formed at the side of the bigger channel, a proper form for vaso-mimetic applications.

Not only simple junctions but we also created a MF channel with solenoidal trajectory as shown in Fig. 3. The diameter of the MF channel and the solenoid is $25 \mu \mathrm{m}$ and $5 \mathrm{~mm}$, respectively. Like the presented solenoidal structure, we can generate various structures with long yet compact vessel-like structures according to the base template trajectory and how we embed sucrose fibers.
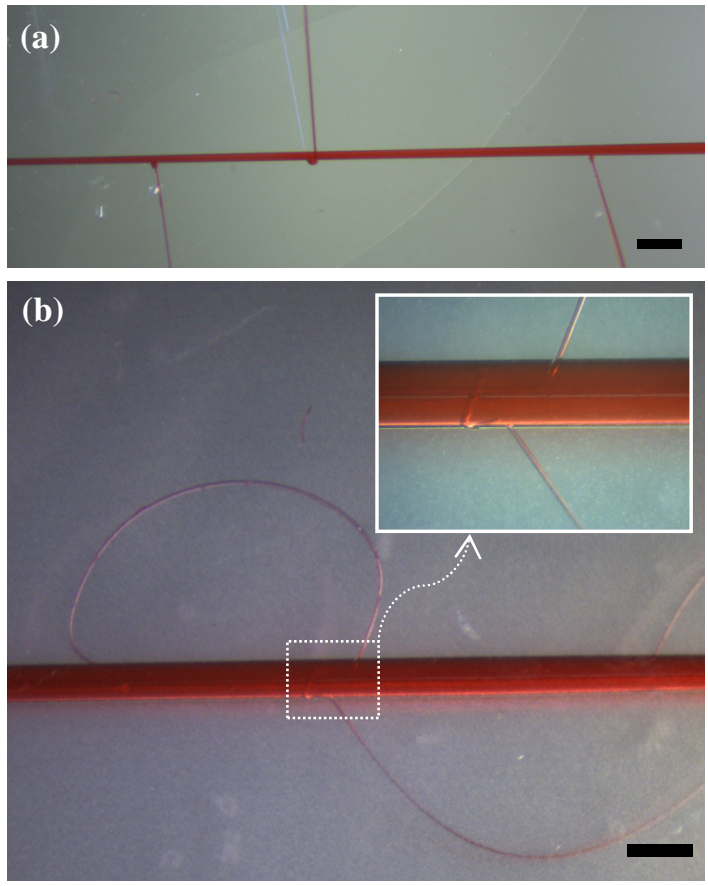

Figure 4: Microscopic images of PDMS MF structures filled with red dyes (a) branches made with assembly of straight fibers (b) A $3 D$ assembly of curved fibers attached at different angles which start and end internally. And the inlet shows magnified image of its junctions. Scale bars: $1 \mathrm{~mm}$

\section{D networked trajectories}

Various structures can be realized due to the waterdissolvable nature of sucrose as introduced in the former fabrication sections. Especially the power of this technique reveals when we make angled 3D structures. Not only angles in the azimuth direction but also in the elevation direction are feasible by simply applying of trapezoidal pyramid PDMS block template with different angles underneath each sucrose fiber. When higher quantity of water-sucrose adhesives is applied, it is also possible to make the same angled branch structure in both directions without having supporting blocks. For example, the structure shown in the Fig 4(a) provides the three junctions made only with adhesive strength. Due to the tapering, the thickness of the center channel varies that the average thickness is $220 \mu \mathrm{m}$. The thicknesses of branches fall into the range of $60 \pm 5 \mu \mathrm{m}$.
Figure 4(b) illustrates internally looped MF channels embedded in the PDMS molding. There are three internally starting and ending fibers in the figure, but two of them are cropped to enhance the visibility. All three curved structures are shaped by applying steam prior to attachment. Also, the inlet of Fig. 4(b) shows magnified image of junctions to illustrate their smooth and angled transition of the structure. The thickness of the center channel is $586 \mu \mathrm{m}$ while branches are measured as $53 \mu \mathrm{m}$ for the top one and $45 \mu \mathrm{m}$ for the bottom one accordingly. Particularly, this structure demonstrates the great potentials in obtaining various shapes and size structures. The location and the angle of junctions can be further controlled by micro-manipulator. Especially, the images prove its physiologically realistic bifurcation of MF channels.

\section{D shaped and tapered structure}

Figure 5(a) illustrates a tapered and 3D shaped MF channel. By starting with the thicker pulling rod and varying the instant pulling speed, we could manually make a tapered structure. Then, a point of the pulled sucrose fiber was exposed to steam for a few seconds while forming a curve. Instant removal of steam is required to prevent breaking of the fiber. This effortless tapering and shaping offer great potentials of generating more complicated 3D networks. In particular, with the right equipment set-up, such as a computer-controlled manipulator, more complicated and compact drawing to realize vaso-mimetic structures are possible.

Figure 5(b-c) shows microscopic images of an original sucrose fiber. This particular sucrose fiber is obtained by manually withdrawn for $51 \mathrm{~cm}$ and timed as $3.4 \mathrm{sec}$. Then, to avoid the abrupt change of thickness, only the mid-point of the fiber is used to be measured and averaged. The diameter of fiber is measured as $123 \mu \mathrm{m}$. As we observe in images, the both cylindrical body and the cut edge surfaces are smooth at the micron scale. Also, cross sectional view in (c) proves the circular shape of MF channel to contribute to the realization of the vaso-mimetic LoCs.

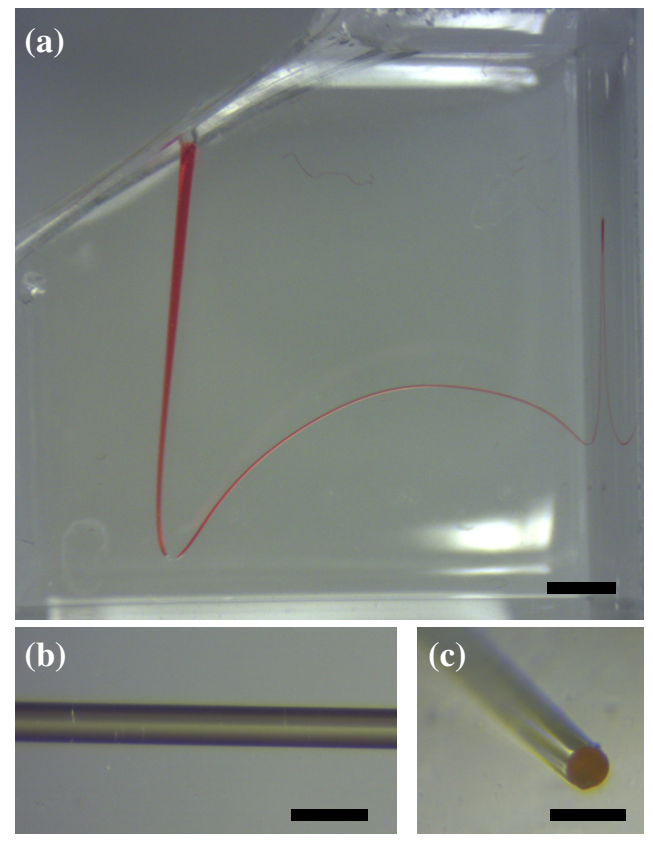

Figure 5: Microscopic images: (a) 3D MF channel made with a tapered sucrose fiber and shaped with steam $(b)$ the top view and (c) the angled-cross sectional view of the original sucrose fiber pulled at $3.4 \mathrm{~cm} / \mathrm{s}$. Scale bars: $250 \mu \mathrm{m}$ 

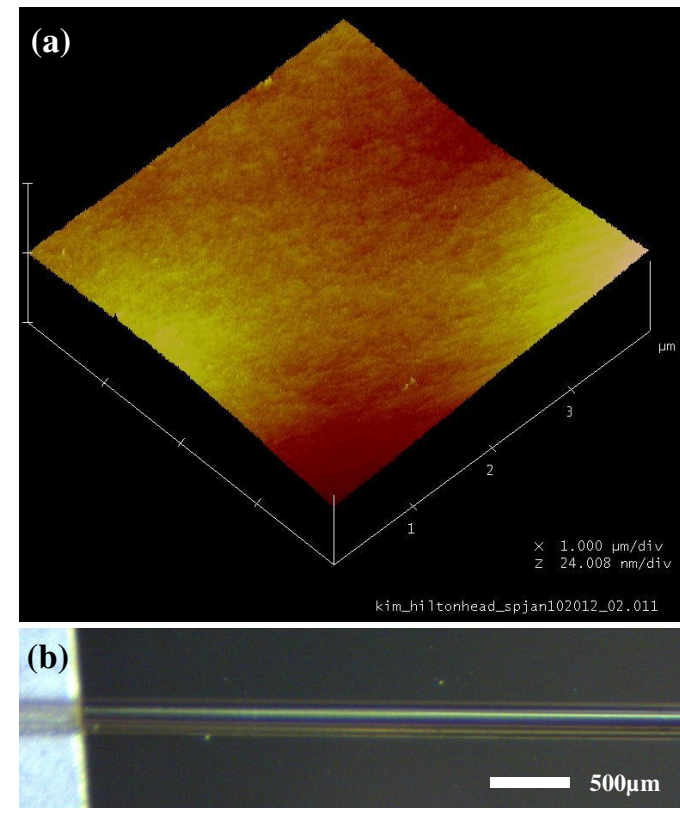

Figure 6: (a) 3D AFM scan of $16 \mu^{2}$ area of the outer surface of sucrose fiber, (b) a microscopic image of its original fiber

We further investigated surface roughness of the sucrose fiber by using AFM (atomic force microscopy) scan. Figure 6 shows the 3D AFM scans of $16 \mu \mathrm{m}^{2}$ area. The measurement scan was carried out over ten randomly selected locations on a single fiber. Then, Nanoscope program is used to characterize the surface. The root mean square roughness value was found to be $1.1 \pm 0.4 \mathrm{~nm}$, confirming its excellent surface smoothness.

\section{CONCLUSION}

In summary, we established a simple, rapid, and cost-effective fabrication scheme to realize 3D networks of circular MF channels by adopting sucrose as the sacrificial material. Various MF structures shown in the manuscript prove the great potential of our fabrication method. The excellent chemical, mechanical, and thermal properties of sucrose allowed a high level of flexibility in the attainable network topologies. Especially, the easy tapering and curving modification of the sucrose template can be greatly used for the formation of physiologically realistic junctions.

\section{ACKNOWLEDGEMENT}

The authors were supported by NSF CAREER award (0954845).

\section{REFERENCES}

[1] M. Abdelgawad, C. Wu, W.-Y. Chien, W. R. Geddie, M. A. S. Jewett and Y. Sun, "A fast and simple method to fabricate circular microchannels in polydimethylsiloxane (PDMS)," Lab on a Chip, 11, 545 (2011).

[2] S.-H. Song, C.-K. Lee, T.-J. Kim, I.-C. Shin, S.-C. Jun and H.-I. Jung, "A rapid and simple fabrication method for 3dimensional circular microfluidic channel using metal wire removal process," Microfluid Nanofluid, 9, 533 (2010).

[3] H. Wu, T. W. Odom, D. T. Chiu, and G. M. Whitesides, "Fabrication of Complex Three-Dimensional Microchannel Systems in PDMS," J. Am. Chem. Soc., 125, 554-559 (2012).

[4] Y. Luo and R. N. Zare, "Perforated membrane method for fabricating three-dimensional polydimethylsiloxane microfluidic devices," Lab Chip, 8, 1688 (2008).

[5] J. M. Rosano, N. Tousi, R. C. Scott, B. Krynska, V. Rizzo, B. Prabhakarpandian, K. Pant, S. Sundaram, and M. F. Kiani, "A physiologically realistic in vitro model of microvascular networks," Biomed Microdevices, 11, 5 (2009).

[6] M. K. S. Verma, A. Majumder, and A. Ghatak, "Embedded Template-Assisted Fabrication of Complex Microchannels in PDMS and Design of a Microfluidic Adhesive," Langmuir, 22, 10291-10295 (2006).

[7] Y. Jia, J. Jiang, X. Ma, Y. Li, H. Huang, K. Cai, S. Cai, and $\mathrm{Y}$. Wu, "PDMS microchannel fabrication technique based on microwire-molding," Chinese Science Bulletin, 53, 39283936 (2008).

[8] W. Wu, A. DeConinck, and J. A. Lewis, "Omnidirectional Printing of 3D Microvascular Networks," Advanced Materials, 23, H178-H183 (2011).

[9] Y. Du, M. Ghodousi, H. Qi, N. Haas, W. Xiao, and A. Khademhosseini, "Sequential assembly of cell-laden hydrogel constructs to engineer vascular-like microchannels," Biotechnology and Bioengineering, 108, 1693-1703 (2011).

[10] C. Xia and N. X. Fang, "3D microfabricated bioreactor with capillaries," Biomedical Microdevices 11, 1309-1315 (2009).

[11] L. M. Bellan, S. P. Singh, P. W. Henderson, T. J. Porri, H. G. Graighead and J. A. Spector, "Fabrication of an artificial 3dimensional vascular network using sacrificial sugar structures," Soft Matter, 5, 1354 (2009)

[12] J. Li, T. A. Rickett, and R. Shi, "Biomimetic Nerve Scaffolds with aligned Intraluminal Microchannels: A "Sweet" Approach to Tissue Engineering," Langmuir, 25, 1813 (2009)

[13] L. K. Fiddes, N. Raz, S. Srigunapalan, E. Tumarkan, C. A. Simmons, A. R. Wheeler, E. Kumacheva, "A circular crosssection PDMS microfluidics system for replication of cardiovascular flow conditions," Biomaterials, 31, 3459 (2010).

\section{CONTACT}

*Jaeyoun Kim, tel: +1-515-294-4214; plasmon@istate.edu 


\section{THREE DIMENSIONALLY-PRINTED (3DP) MICROFLUIDIC DEVICES \\ O.H. Paydar ${ }^{1}$, C.N. Paredes ${ }^{2}$, and R.N. Candler ${ }^{2,3}$ \\ ${ }^{1}$ Biomedical Engineering Interdepartmental Program, ${ }^{2}$ Department of Electrical Engineering, \\ ${ }^{3}$ California NanoSystems Institute \\ University of California, Los Angeles, USA}

\begin{abstract}
This paper introduces the use of three-dimensionally printed (3DP) molds for rapid fabrication of complex and arbitrary microchannel geometries unattainable through existing soft lithography techniques. These molds are printed directly from computer-aided design (CAD) files, making rapid prototyping of microfluidic devices possible in hours. These 3D-printed structures enable precise control of various device geometries, such as the profile of the channel cross-section and variable channel heights in a single device. We report fabrication of complex $3 \mathrm{D}$ channels in a polydimethylsiloxane (PDMS) polymer. Device characterization, including surface roughness and resolution, are assessed.
\end{abstract}

\section{INTRODUCTION}

Soft Lithography

Polymer-based microfluidic devices have revolutionized biomedical research and diagnostics due to the many advantages that elastomers have over rigid materials, such as silicon or glass [1-3], with polydimethylsiloxane (PDMS) being the most common elastomer used in microfluidic devices. PDMS is well suited to microfluidics due to its potential for fast prototyping, low cost, and gas-permeability. Additionally, PDMS is optically transparent (240 $\mathrm{nm}-1100 \mathrm{~nm}$ ), nontoxic, electrically insulating, impermeable to liquids, and the PDMS prepolymer conforms to the contours of the master-mold with high fidelity ( 10 's $\mathrm{nm})$.

Presently, the fabrication of a mold requires silicon wafers with positive surface relief structures $[4,5]$, typically a photoresist, to shape the PDMS prepolymer. Defining these microstructures requires cleanroom access, microfabrication tools, extensive training, and expensive reagents. Moreover, this method, based on integrated circuit (IC) fabrication processes, is constrained to planar structures. The simplest 3D structures require multiple lithography steps, precise layer alignment, and are typically limited to extrusions of 2-D geometries. Consequently, complex threedimensional (3D) structures, including vias $[6,7]$ and multi-tier devices [8], remain a fabrication challenge.

Innovative alternatives to cost-prohibitive microfluidic device fabrication include a high-resolution shrink film device with high aspect ratio (HAR) channels [9] and inkjet-printed devices [10-12]. The shrink film devices suffer from similar design limitations to those made by IC processes, including single channel heights and lack of 3D shape control. The inkjet-printed microfluidic devices provide another inexpensive alternative, but are still limited to planar channels defined on the surface of a glass slide. While these solutions do provide a cost-effective approach to microfluidics, they do not fully address the needs of $3 \mathrm{D}$ microfluidics. A threedimensional fabrication method would allow for the possibility of increased port density and device complexity, improved throughchannel (via) formation, and the possibility of more physiologically relevant geometries, such as channels with round cross sections.

\section{Rapid Prototyping - Three Dimensional (3D) Printing}

Commercially available rapid prototyping technologies, specifically 3D printing, have been available for nearly a decade. However, recent advances (e.g., biocompatibility, multi-material printing) and improved resolution enable, for the first time, rapid prototyping of structures that can be used to mold PDMS microchannels with tens of microns resolution and nearly arbitrary topography (e.g., variable channel height and cross section profile). These molds are printed directly from computer-aided design (CAD) files, making rapid prototyping of microfluidic devices possible in hours. Three-dimensional printed structures enable precise control of various device geometries, such as the profile of the channel cross-section and varying channel heights in a single device. Three-dimensional printed microfluidic devices will give researchers unprecedented design flexibility to quickly and inexpensively create more complex channels.

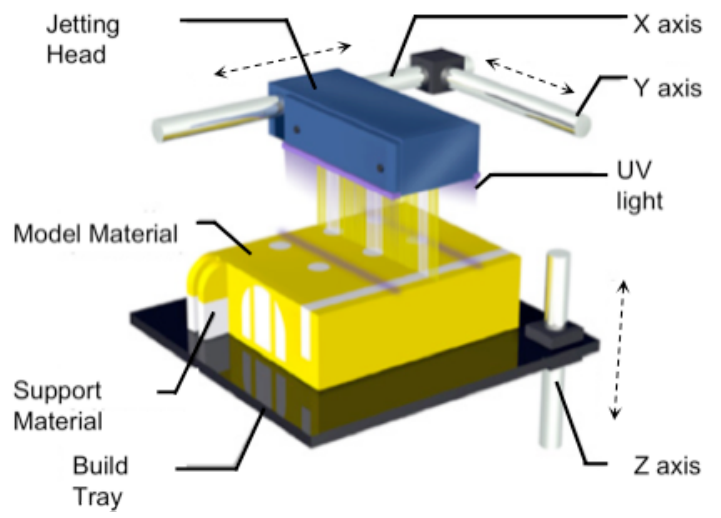

Figure 1. Three-dimensional (3D) printing process using the Objet24 printer. Model (yellow) and support (white) photopolymers are dispensed and immediately UV-cured in 28micron layers. The gel-like support material is removed with pressurized water, revealing the final device. The resolution limit of this technology is 600x600x900 dpi-capable of $\sim 45 \mu \mathrm{m}$ line widths. Reprinted with permission from Objet Geometries Ltd.

Similar rapid-prototyping technologies including stereolithography are capable of milli- and micron-scale manufacturing. As a serial writing process, this technology has encountered throughput issues in the past, although recent advances may help overcome this limitation [13].

\section{MATERIALS AND METHODS}

\section{Design, Print, and Pour}

Microchannel molds are printed directly from computer-aided design (CAD) files (Autodesk Inventor, Autodesk, Inc., San Rafael, CA, USA), making design and rapid prototyping of devices possible in hours. The fully automated printing process simultaneously jets a photocurable polymer and a support material in 28- $\mu \mathrm{m}$ layers (Figure 1). Each layer of the model and support material is immediately UV-cured. Upon completion of the printing process, a high-pressure water jet exposes the final device design by dissolving the gel-like support material. The 3D printed device becomes the replica mold master and replaces time consuming, expensive microfabrication (Figure 2). 


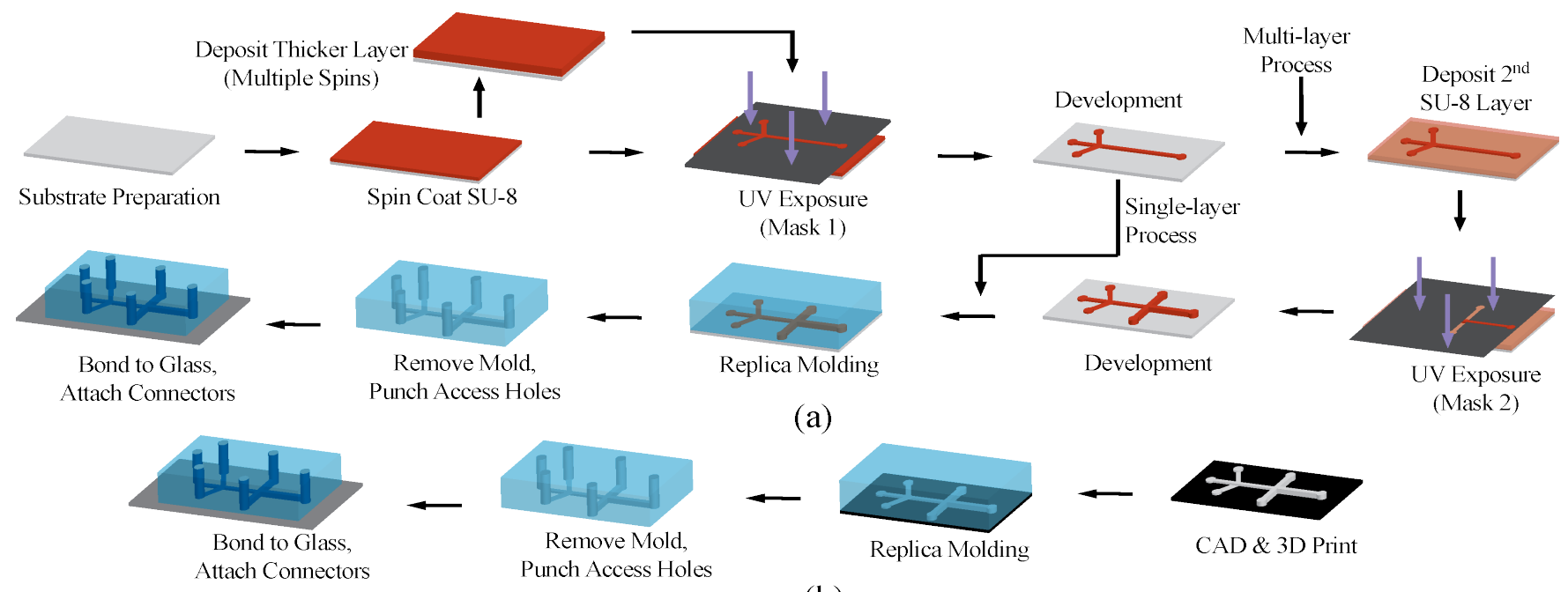

(b)

Figure 2. Conventional and $3 D$ printed mold fabrication processes. (a) Conventional master-mold fabrication requires cleanroom access, and multi-layer devices require several iterations of photolithography. (b) For 3D printed molds, a CAD file is directly exported to the $3 D$ printer for mold fabrication.

A base-to-catalyst mixture $(9.1 \% \mathrm{w} / \mathrm{w})$ of silicone elastomer (Sylgard 184, Dow Corning, Midland, MI, USA) was poured onto the master, desiccated, and cured at $75^{\circ} \mathrm{C}$. The device was removed from the mold and access holes were punched into the device to define the inlet(s) and outlet(s). To complete the process, the device was treated with $\mathrm{O}_{2}$ plasma and bonded to a glass substrate.

\section{Characterization-Surface Roughness}

Because PDMS conforms to the contours of the mold with high fidelity, it was imperative to characterize the surface topology. Limitations of printer resolution due to droplet size or droplet spreading during curing can lead to leakage or lack of faithful reproduction of design features. To investigate these limitations, surface roughness of 3D printed samples $\left(2.5-\mathrm{mm}^{2}\right)$ was quantified and compared to surface roughness measurements of conventional replica-mold materials, silicon and photoresist (SU-8). The printer provides two options for surface finish, glossy and matte, both of which were characterized.

The surface roughness measurements were taken with an optical profiler (Wyko NT3300, Veeco, Fullerton, CA, USA). Vertical scanning interferometry (VSI) resolves the surface topography by detecting the interference between emitted light and the reflected beam. Because of poor reflectivity, the 3D printed samples were sputter coated with a 100-Angstrom layer of chromium/gold. The average of the absolute value of deviation from the mean surface height is used as a measure of the surface roughness, $R_{a}$.

\section{Design of Microfluidic Devices}

The 3D printing process was used to fabricate three types of devices molds - gradient generators, size-exclusion filters, and double helices. The molds were used to fabricate PDMS devices to demonstrate the ability to fabricate functional devices with control of $3 \mathrm{D}$ geometry, as discussed in the following section.

\section{RESULTS AND DISCUSSION}

Three-dimensionally printed devices, regardless of finish, have predictably rougher surface topology than traditional microfluidic molds (Figure 3). Because microfluidic channels are molded from photoresist relief structures, we compared the roughness of the SU-8 sample with the $3 \mathrm{D}$ printed material. The surface roughness of both matte and glossy samples were an order of magnitude larger than conventional microfabricated templates.

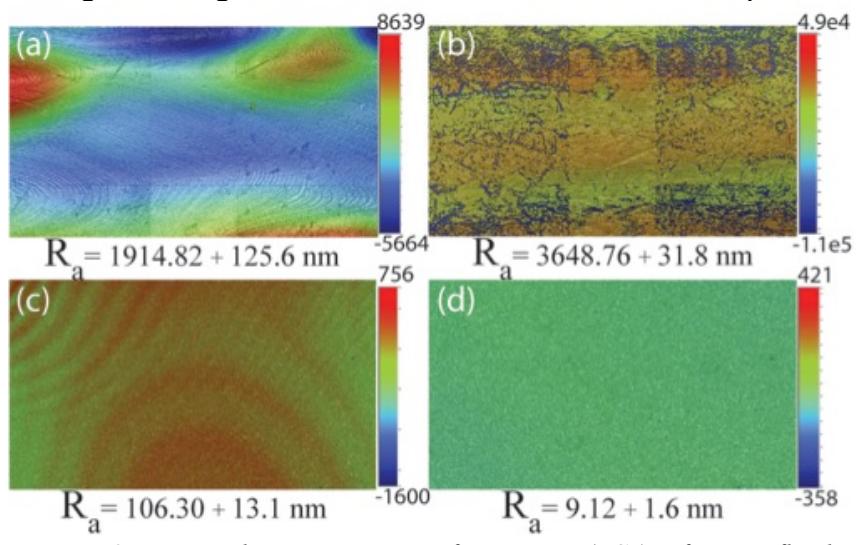

Figure 3. Vertical scanning interferometry (VSI) of microfluidic master materials. The glossy (a) and matte (b) $3 D$ printed surface roughness, $R_{a}$, is an order of magnitude larger than the channeldefining SU-8 feature (c). Silicon (d) is also represented. All dimensions are given in nanometers.

Fortunately, the periodic minima and maxima (figure 3a)-a consequence of the jetting process - that define the surface topology do not affect the ability of the device to bond to glass, likely due to the low elastic modulus of PDMS.

\section{Characterization of 3D-Printed Devices}

Characterization of a replica-molded, 3D-printed gradient generator designed with $150-\mu \mathrm{m}$-wide square channels highlighted important design considerations. A scanning electron microscope (SEM) image of the gradient generator outlet (Figure 4) clearly illustrates enlarged channels. As can be seen, spreading occured during the $3 \mathrm{D}$ printing process, leading to a $133 \%$ increase in channel diameter. To avoid channels reflowing into one another, the CAD was modified to account for the droplet spread and the separation between channels was increased. 


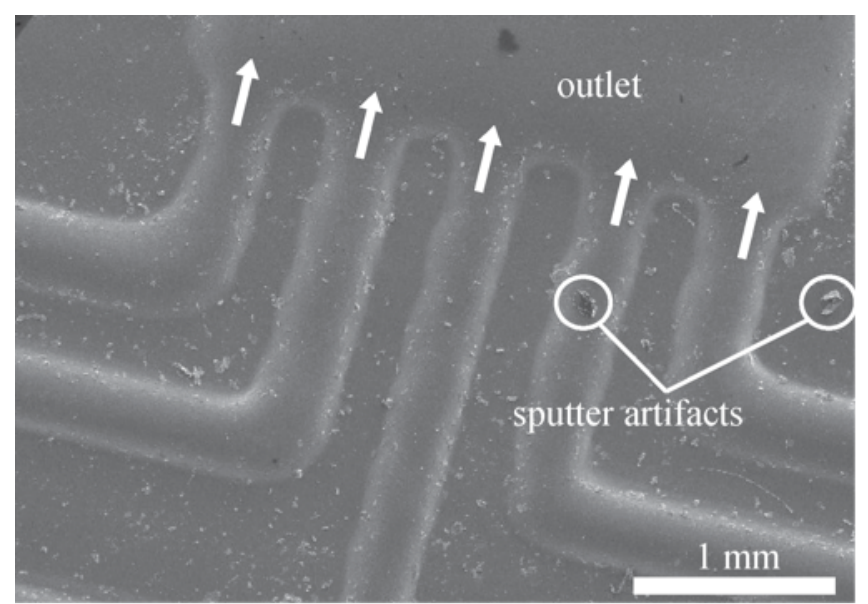

Figure 4. Scanning electron micrograph (SEM) at the outlet of a $3 D$-printed mold of the gradient generator. Channels designed to be 150-um-wide have spread to 350 micrometers. This $133 \%$ increase was a result of the photocurable polymer spreading prior to the UV cure. Arrows represent the direction of fluid flow after the PDMS was cast and the device was completed.

After removing the PDMS from the 3D printed master and attaching connectors to the inlets and outlet of the device, the three input streams were filled with red, yellow, and blue dyes. Flow rates below $100 \mu \mathrm{L} / \mathrm{min}$ generated a gradient at the device outlet, but because laminar flow dominates in this regime, larger flow rates resulted in the same three-color streams at the outlet.

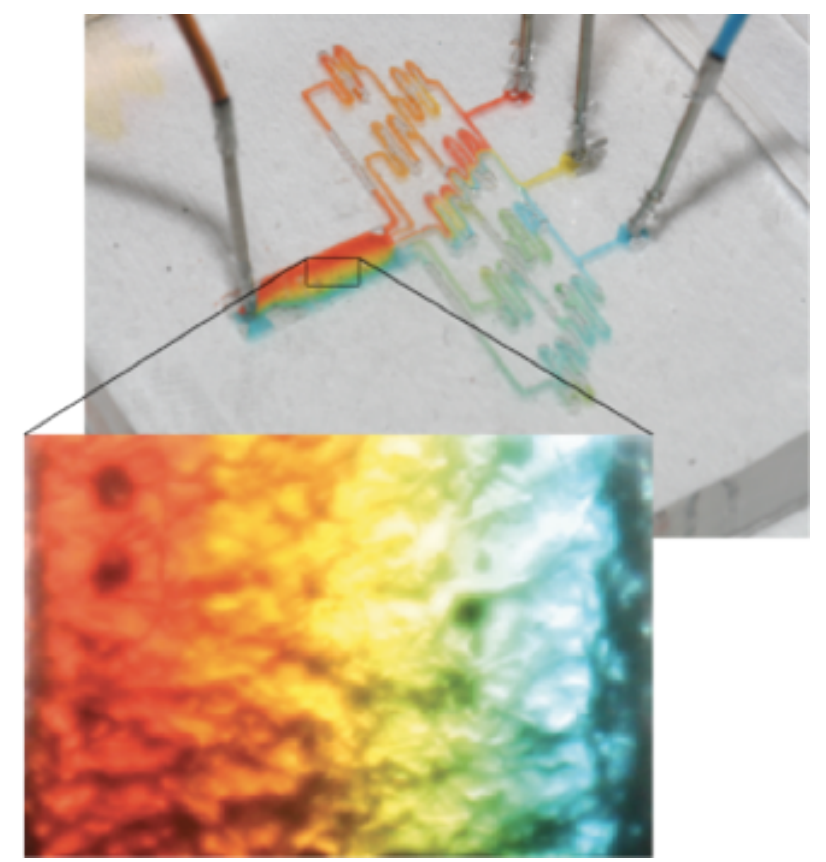

Figure 5. Three-dimensional printed (3DP) gradient generator. The device was designed with $150 \mu$ m-wide channels. Inset shows the color gradient generated between the red, yellow, and blue input streams.

Unlike conventional microfluidic device fabrication, the manufacturing of 3D printed master-molds for multi-tier devices is no more difficult than the fabrication of simple 2D geometries.

A relatively simple multi-level structure, which would require four separate exposures and precise alignment with conventional microfabrication was designed and printed in less than one hour. This step-structure (Figure 6) was designed with an initial $400-\mu \mathrm{m}-$ tall inlet and each successive step reduces the channel height by $150 \mu \mathrm{m}$. This type of device is representative of size-exclusion filters where changes in the channel height can funnel different sized particles into separate collection reservoirs.

The ability to design arbitrary channel heights could open up new design spaces for microfluidic devices, such as size-based particle filters or biomimetic channels that reproduce the exact shape of blood vessels.

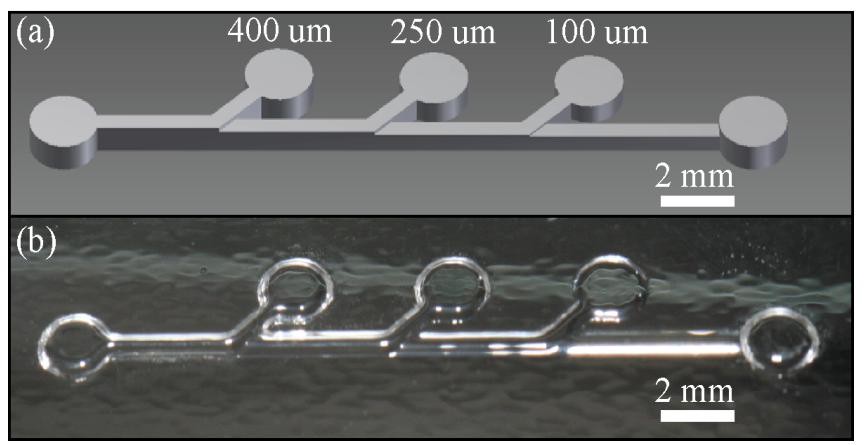

Figure 6. (a) Computer-aided design (CAD) schematic of multitier device (i.e. size-exclusion filter). Steps were integrated into the design and printed. Each step is 150 um shorter than the previous. (b) The molded PDMS device with three different channel heights ranging from 100 to $400 \mu \mathrm{m}$.

Figure 7 shows the full capability of the 3D printing technique, where double helices have been fabricated in PDMS. Each strand of the double helix structure was designed in CAD as a 1-mm diameter circular channel with a 5-mm pitch (Figure 7a). The strands were anchored onto the same base and their inner surfaces were separated by 2 millimeters. The PDMS prepolymer was cast around the device shaping the helical channels. The mold was removed following the curing process. Removal of the mold was not difficult because the mold material was observed to impede full curing of the PDMS. The PDMS would fully cure after contact with the mold was removed. For illustration purposes, a single channel was filled with dye (Figure 7b). The channels were then filled with different colored dyes to emphasize the ability to create over- and underpasses (Figure 7c).

Of particular importance, the channels were fully defined in the PDMS polymer. This technique allows the user to bypass $\mathrm{O}_{2}$ plasma treatment and avoid bonding the device to glass, which further simplifies the device manufacturing process. It also allows for the channel to be fabricated from the same material, as opposed to bonding PDMS onto a substrate. However, without oxygen plasma treatment, the untreated PDMS remains hydrophobic $\left(109^{\circ}\right)$ and obstructs flow. To overcome this potential challenge the device can be submerged in $1 \mathrm{M} \mathrm{NaOH}$ for 24 hours. The resulting surface treatment is more stable than radio-frequency plasma or corona discharge and relieves the flow obstruction of native PDMS [14].

\section{The Advantage over 2D Fabrication Technology}

Three-dimensional printing facilitates increased microchannel complexity and simplifies fabrication at a low cost $(<\$ 1 /$ mold of material costs). In addition to the technical advantages, this economical alternative can help accelerate research progress by increasing access to a once-limited field. 


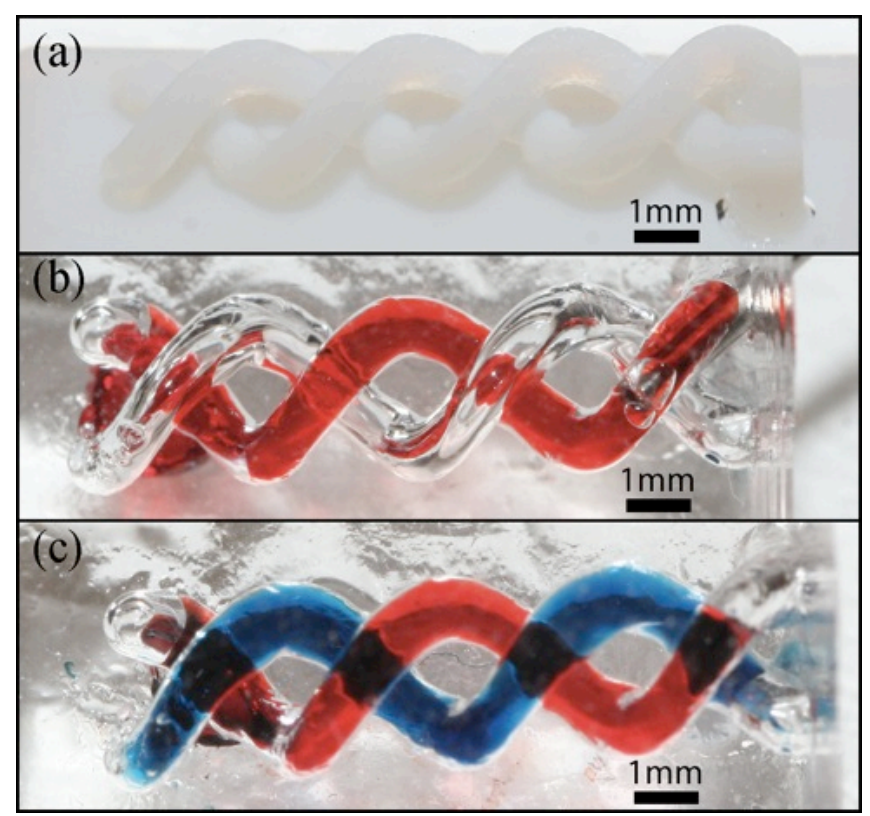

Figure 7. Truly $3 D$ microchannels developed from $3 D$ printed molds. (a) PDMS surrounding the mold. Each strand of the double helix was designed to have a $1 \mathrm{~mm}$ diameter and a $5 \mathrm{~mm}$ pitch. (b) A single channel filled with colored water. (c) Both channels filled with solutions, emphasizing the ability to create over- and underpasses.

\section{CONCLUSION}

Microfluidic structures fabricated through traditional soft lithography processes are limited to rectangular extrusions of highresolution $2 \mathrm{D}$ structures. While $3 \mathrm{D}$ printed structures do not currently possess the resolution of photolithographically defined structures, they do enable enhanced control of device geometries, such as the profile of the channel cross-section and varying channel heights in a single device. Because of this design flexibility, 3D printed microfluidic technology will give researchers the ability to quickly and inexpensively create channels with unprecedented shape and complexity.

\section{FUTURE WORK}

Future efforts will focus on the creation of high-density embedded channel networks. The process requires selectively dissolving the $3 \mathrm{D}$ printed material without damaging the surrounding PDMS structure. Also, we will continue investigating resolution limits of the 3D-printed mold technology and methods to improve this resolution.

\section{ACKNOWLEDGEMENTS}

The authors would like to thank the Nanoelectronics Research Facility (NRF) at UCLA for making device characterization possible. We would also like to express our gratitude to Joe Zendejas, without whom this work would not be possible. Student travel support has been generously provided by the Transducer Research Foundation.

\section{REFERENCES}

[1] A. Mata, A. J. Fleischman, and S. Roy, "Characterization of polydimethylsiloxane (PDMS) properties for biomedical micro/nanosystems," Biomedical Microdevices, vol. 7, pp. 281-293, May 012005.

[2] J. C. McDonald, D. C. Duffy, J. R. Anderson, D. T. Chiu, H. Wu, O. J. A. Schueller, and G. M. Whitesides, "Fabrication of microfluidic systems in poly (dimethylsiloxane)," Electrophoresis, vol. 21, pp. 27-40, 2000.

[3] J. C. McDonald and G. M. Whitesides, "Poly(dimethylsiloxane) as a Material for Fabricating Microfluidic Devices," Accounts of Chemical Research, vol. 35, pp. 491-499, Jul 2002.

[4] C. S. Effenhauser, G. J. M. Bruin, A. Paulus, and M. Ehrat, "Integrated capillary electrophoresis on flexible silicone microdevices: analysis of DNA restriction fragments and detection of single DNA molecules on microchips," Anal. Chem., pp. 3451-3457, Feb 011997.

[5] Y. Xia and G. M. Whitesides, "Soft lithography," Angew. Chem. Int. Ed., pp. 550-575, May 011998.

[6] C. Carlborg, T. Haraldsson, M. Cornaglia, G. Stemme, and W. van der Wijngaart, "A High-Yield Process for 3-D LargeScale Integrated Microfluidic Networks in PDMS," Microelectromechanical Systems, Journal of, vol. 19, pp. 1050-1057, 2010.

[7] J. R. Anderson, D. T. Chiu, R. J. Jackman, O. Cherniavskaya, J. C. McDonald, H. Wu, S. H. Whitesides, and G. M. Whitesides, "Fabrication of Topologically Complex ThreeDimensional Microfluidic Systems in PDMS by Rapid Prototyping," Analytical Chemistry, vol. 72, pp. 3158-3164, Jul 2000.

[8] M. A. Unger, H.-P. Chou, T. Thorsen, A. Scherer, and S. R. Quake, "Monolithic microfabricated valves and pumps by multilayer soft lithography," Science, vol. 288, pp. 113-116, May 012000.

[9] D. Taylor, D. Dyer, V. Lew, and M. Khine, "Shrink film patterning by craft cutter: complete plastic chips with high resolution/high-aspect ratio channel," Lab on a Chip, vol. 10, p. 2472, 2010.

[10] M. Watanabe, "Refreshable microfluidic channels constructed using an inkjet printer," Sensors and Actuators B: Chemical, vol. 122, pp. 141-147, Apr 2007.

[11] M. Watanabe, "Microfluidic devices easily created using an office inkjet printer," Microfluidics and Nanofluidics, vol. 8, pp. 403-408, Nov 042009.

[12] M. Watanabe, "An inkjet-printed microfluidic device for liquid-liquid extraction," The Analyst, vol. 136, p. 1420, 2011.

[13] F. P. W. Melchels, J. Feijen, and D. W. Grijpma, "A review on stereolithography and its applications in biomedical engineering," Biomaterials, vol. 31, pp. 6121-6130, Aug 01 2010.

[14] I. Hoek, F. Tho, and W. M. Arnold, "Sodium hydroxide treatment of PDMS based microfluidic devices," Lab on a Chip, vol. 10, pp. 2283-2285, 2010.

\section{CONTACT}

*O.H. Paydar, tel: +1-317-332-3302; omeed.paydar@ucla.edu 


\title{
A MICRO GAS CHROMATOGRAPHY COLUMN WITH AN EMBEDDED OUT-OF-PLANE THERMAL CONDUCTIVITY DETECTOR
}

\author{
Shree Narayanan and Masoud Agah
}

VT MEMS Laboratory, Bradley Department of Electrical and Computer Engineering, Virginia Tech, USA

\begin{abstract}
We present the design, fabrication and experimental evaluation of a unique micro gas chromatography ( $\mu \mathrm{GC})$ column with an integrated micro thermal conductivity detector ( $\mu \mathrm{TCD})$. The resistors are dry released to form $170 \mu \mathrm{m}$ diameter coils. The high isolation of the sensing element provides for an improved sensitivity by an order of magnitude over our previously reported designs. The chip incorporates a 2-port design approach and a device size of $2 \mathrm{~cm} \times 4 \mathrm{~cm}$. Fabricated using a four-mask process, the resistors and microfluidics are independently processed on borofloat and silicon wafers, respectively, and subsequently anodic bonded. The separation column is static coated without damage to the resistors. The coated column-detector chip has successfully separated a representative hydrocarbon mixture in less than 2 minutes while consuming only $50 \mathrm{~mW}$ of power for TCD operation.
\end{abstract}

\section{INTRODUCTION}

Gas chromatography (GC), the technique of separating a mixture of gases into its components, has applications ranging across space exploration, homeland security, dissolved gas analysis and food quality testing [1]. Conventional gas chromatography is an established field that is more suited to lab testing owing to large bulky instruments that consume large power. Micro gas chromatography $(\mu \mathrm{GC})$ refers to the miniaturization of a $\mathrm{GC}$ system using microfabrication techniques. The resulting advantages include lower power consumption and batch fabrication of components that reduces cost. In addition, their light weight enables field-use. Microfabrication allows the development of innovative structures and geometries to test the theoretical extremes of optimized performance that might otherwise be difficult in conventional GC $[2,3]$.

Multiple efforts have been invested into the development of the individual components of a $\mu \mathrm{GC}$ system and their integration. Some noteworthy attempts include the hybrid integration of detectors reported in $[4,5]$. However, the effort towards monolithic integration of detectors with separation column has been minimal and needs further investigation [6, 7]. Previously, we have reported the integration of a TCD with a separation column, in a unique configuration that saved space, reduced the number of fluidic interconnections, and at the same time provided for the thermostatted operation of the separation column [8].

In this paper, we present an improved version of the thermal conductivity detector wherein the resistors are high aspect ratio (HAR) fabricated with a modified silicon sacrificial layer dry etch (SSLDE) process. These released structures were anodic bonded with the separation column and resulted in an order of magnitude increased sensitivity while requiring only $50 \mathrm{~mW}$ for operation.

\section{THEORY \\ GC System}

A GC system comprises of three main components viz., preconcentrator, separation column, and detector. The preconcentrator is responsible for amplifying the compounds of interest to a level that will exceed the limit of detection of the detector $[9,10]$. The concentrated sample from the preconcentrator is injected into a column whose task is to separate the gases. In its conventional form, a separation column is a capillary whose internal surface is coated with a compound referred to as the stationary phase. As the mixture is carried along by a carrier gas through the capillary, different compounds interact with the stationary phase to a different extent resulting in separation. In the microfabrication world, these capillaries are typically channels etched in silicon. The eluted compounds then pass into a detector such as a flame ionization detector (FID), chemiresistors, a thermal conductivity detector (TCD), or a surface acoustic wave (SAW) sensor.

\section{Monolithic Integration of Detector}

We have opted to integrate a TCD, owing to its universal detection capability, simplicity and robustness. It consists of a heated resistor placed in a carrier gas which is often helium due to its high thermal conductivity $(0.1513 \mathrm{~W} / \mathrm{m} . \mathrm{K})$. When a component of differing thermal conductivity comes in contact with the resistor, the heat flux gradient is altered and hence, the temperature of the resistor changes. By measuring this change as a differential measurement against a reference resistor, which does not come in contact with the eluted compound, a signal corresponding to the eluted compound is obtained. We have previously shown a technique to place the reference resistor close to the inlet of the separation column and detect the compound, though with limited sensitivity [11]. By suspending the resistors into the gas stream, the sensitivity of detection can be increased [12].

Prior reports in literature to suspend the resistor have employed under-etching thin metal films on silicon to simultaneously form the fluidic channel [7, 13]. This limits our ability to manipulate the silicon substrate. Further, the Borofloat lid remains unused. In this paper, we have released a resistor which is deposited on Borofloat, and yet released, leaving the silicon free to be etched to our needs. In particular we have used a modified form of the sacrificial silicon layer dry etch (SSLDE) technique to ensure that in addition to the release, the Borofloat is still suitable to be bonded to the silicon separation column [14].

\section{FABRICATION}

The device consists of a silicon substrate hosting the separation microfluidic channel and a Borofloat wafer hosting the TCD which are separately processed and finally bonded together. The process flow is summarized in Fig. 1.

A $700 \mu \mathrm{m}$ thick Borofloat wafer is blanket deposited with a 650nm thick sacrificial PECVD oxide. AZ5214 was spun coated and patterned in image reversal mode to define the anchor. A 200nm thick sacrificial silicon was e-beam evaporated and lifted off on the patterned AZ5214. Another $1.5 \mu \mathrm{m}$ thick "structural" PECVD oxide was then blanket deposited. Following this, a $60 \mathrm{~nm} / 70 \mathrm{~nm}$ thick $\mathrm{Cr} / \mathrm{Ni}$ metal stack was evaporated and lifted off using the mask of the resistor. A $1.2 \mu$ m thick AZ5214, patterned in image reversal mode, on the metallic resistor served as the mask for further processing. The wafer was diced prior to the releasing step. The releasing step consisted of an initial 5 minute wet etch of the structural oxide in Buffered Oxide Etch (BOE), revealing the sacrificial silicon. The device was then mounted on a handle wafer and the sacrificial silicon etched away using the SSLDE process 
[14]. This was performed in an Alcatel AMS100 i-Speeder DRIE, with $0.1 \mathrm{mbar}$ chamber pressure, $1500 \mathrm{~W}$ coil power, no substrate bias at $20^{\circ} \mathrm{C}$, platen height of $200 \mathrm{~mm}$, and a $\mathrm{SF}_{6}$ flow rate of $300 \mathrm{sccm}$. The chemistry of $\mathrm{SF}_{6}$ enables high lateral etch rates of up to $15 \mu \mathrm{m} / \mathrm{min}$. In the absence of the sacrificial PECVD oxide, the Borofloat surface is exposed to the aggressive $\mathrm{SF}_{6}$ plasma, while the resistors are being under etched. The surface is then rendered unsuitable for anodic bonding. Upon release, the device is raised to $400^{\circ} \mathrm{C}$ on a hot plate in atmosphere. This step anneals the thin metal film and causes further curling, giving the active element of the resistors a coil shape as shown in Fig. 2. The coil has a cross sectional diameter of $168 \mu \mathrm{m}$, giving it sufficient coverage over the entire fluidic channel cross-section. which is $400 \mu \mathrm{m}$ wide and $250 \mu \mathrm{m}$ deep. The interconnects and bond pads are anchored to the substrate. The photoresist was then ashed in an oxygen plasma.
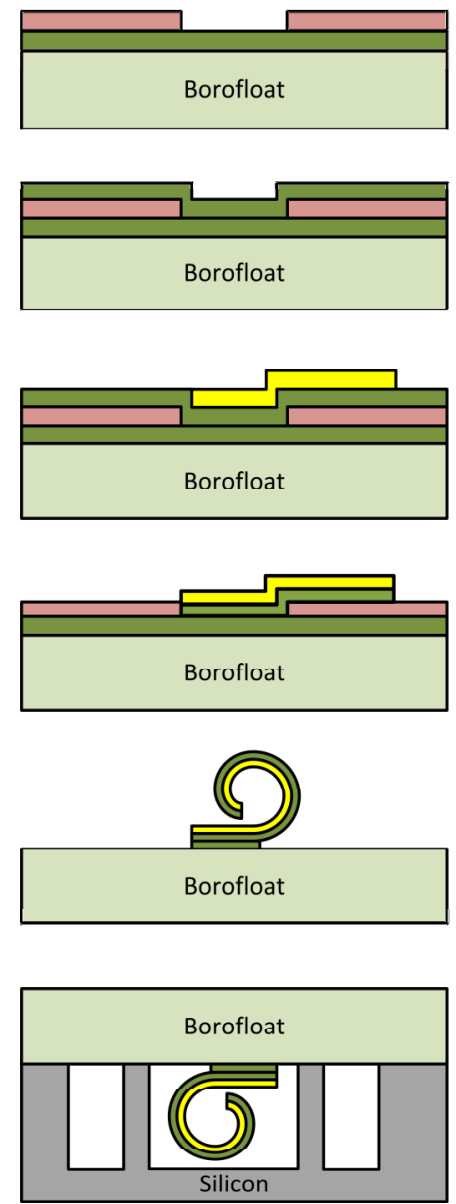

Figure 1: Fabrication of released coiled metal resistors by sacrificial etch of silicon. The photoresist used as a mask to protect the metal while under-etching has been avoided in the diagram, for simplicity

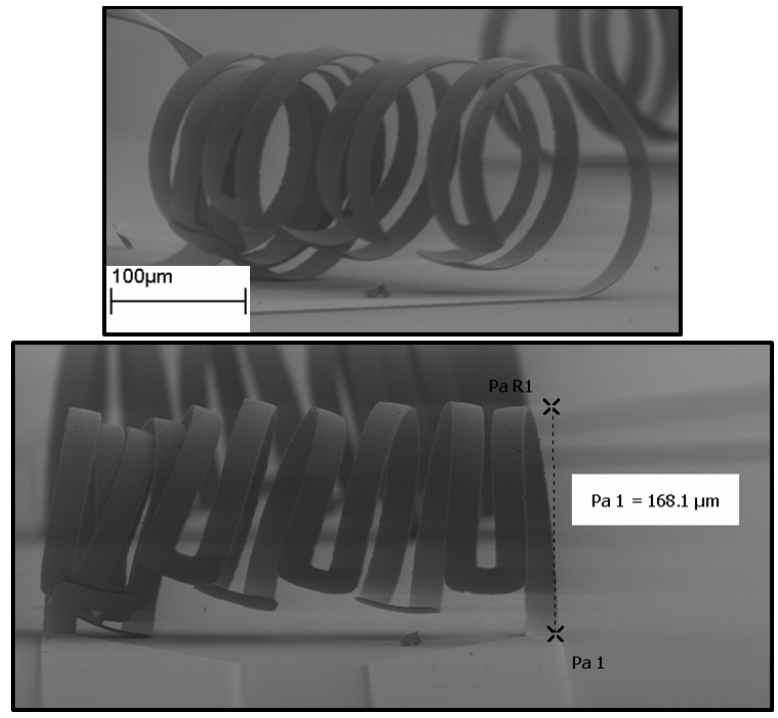

Figure 2: SEM image showing a side view (on top) and the front view (bottom) of the released coils.

The separation column was fabricated on a $500 \mu \mathrm{m}$ thick single-side polished silicon wafer. First, the wafer was spun coated with S1813. The photoresist was then patterned and etched to a shallow depth of $2-3 \mu \mathrm{m}$. This shallow etch prevents contact between the metal interconnects on the Borofloat wafer and the walls of the separation column in silicon, upon bonding. The photoresist was stripped and the wafer spun coated with AZ9260. The $8 \mu \mathrm{m}$-thick photoresist was patterned with a mask for subsequent deep etching of the channels. The Alcatel DRIE was employed in an anisotropic process, resulting in $250 \mu \mathrm{m}$-deep channels for the separation column. Finally, the photoresist was stripped off, and the silicon wafer also diced into individual devices.

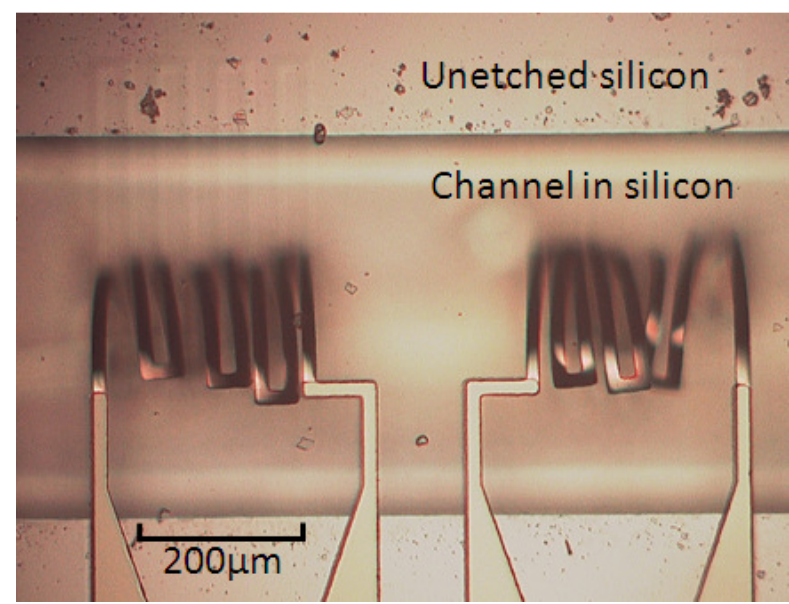

Figure 3: View of the released structures within the channel in a bonded device.

The diced detector on Borofloat and the diced separation column on silicon were aligned and brought into contact using a pick and place bonder followed by anodic bonding. Fig 3 shows the alignment of the resistors in the fluidic channel, while seeing through the Borofloat wafer. The coiled structures are mostly outof-focus due the out-of-plane bending. They align into the fluidic channel etched in silicon, which acts as a recess. 
Capillary tubes were fixed to the inlet and outlet ports of the device. The device was then static coated with polydimethylsiloxane [15]. This was achieved by filling the channel with a solution of polydimethylsiloxane (OV1) in pentane at 40psi followed by drawing vacuum at one end with the other end sealed with wax. Vaporization of pentane leaves behind a thin film of polydimethylsiloxane which serves as the stationary phase. It should be noted that the flow of the viscous solution through the resistors did not cause the released structures to detach, at the point of anchor. This is a current point of investigation to understand the limit of fluidic flow, which will cause the resistors to detach. An optical image of the packaged device is shown in Fig 4.

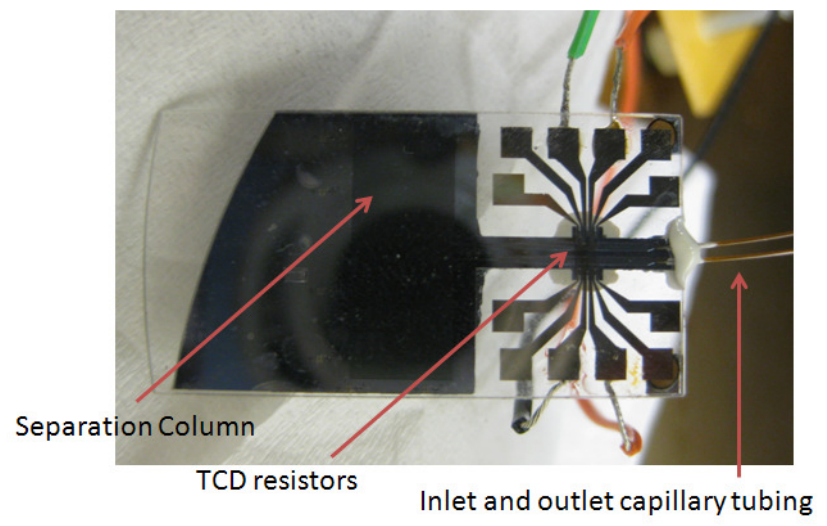

Figure 4: Optical image of the packaged device.

\section{RESULTS AND DISCUSSSION}

A $1 \mathrm{~m}$-long, 100 $\mu \mathrm{m}$-wide separation column was fabricated with the released resistors and packaged as show in Fig 5. A current of $10 \mathrm{~mA}$ was sourced into a Wheatstone bridge with the two resistors in each of its arms. The differential voltage was fed into an Agilent 34401A and recorded through LabVIEW. The device was installed in an HP5890. The injector inlet of the HP5890 was maintained at 11 psi of Helium@ $280^{\circ} \mathrm{C}$ while the oven itself was held at $65^{\circ} \mathrm{C}$. The device was tested by injecting a $0.2 \mu \mathrm{l}$ sample from a mixture of $20 \mu \mathrm{l}$ each of n-nonane, $\mathrm{n}$-decane, $n$-undecane and n-dodecane diluted in $200 \mu \mathrm{l}$ of n-octane. The response from the TCD is shown in Fig. 5. The suspended nature of the resistors resulted in a reduction in power by a factor of four. Additionally, our non-suspended TCD could barely detect $5000 \mathrm{ppm}$ of pentane in octane while we could readily achieve $500 \mathrm{ppm}$ sensitivity with our new released resistors.

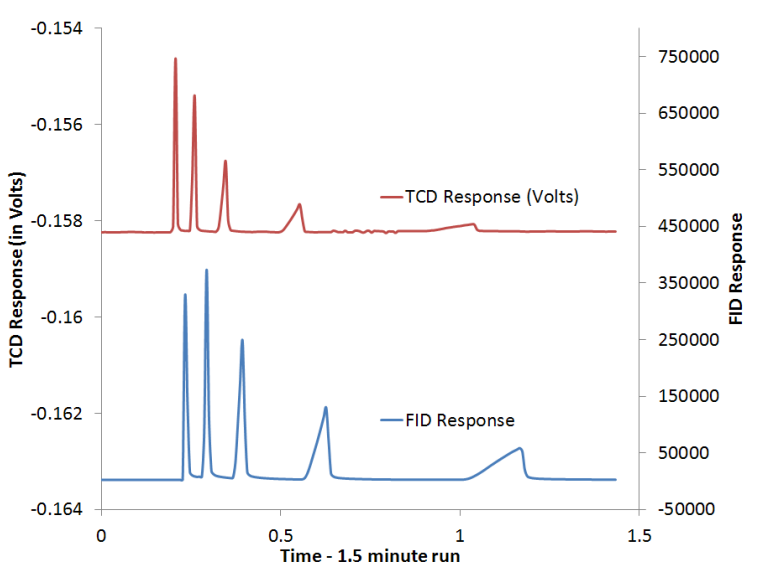

Figure 5: Comparison of the TCD and FID response to a mixture of n-hexane, $n$-octane, $n$-nonane, $n$-decane and nundecane.

\section{ACKNOWLEDGEMENTS}

This work has been supported primarily by the National Science Foundation CAREER award (ECCS-0747600). Travel support has ben generously provided by the Transducer Research Foundation. The authors would like to thank Mr. Don Leber with the Virginia Tech MicrOn cleanroom for his technical assistance and the staff at Nanoscale Characterization and Fabrication Laboratory (NCFL).

\section{REFERENCES}

[1] G. Monti, et al., "Monitoring Food Quality by Microfluidic Electrophoresis, Gas Chromatography, and Mass Spectrometry Techniques: Effects of Aquaculture on the Sea Bass (Dicentrarchus labrax)," Analytical Chemistry, vol. 77, pp. 2587-2594, 2005/04/01 2005.

[2] H. Shakeel, et al., "First reconfigurable MEMS separation columns for micro gas chromatography," 2012, pp. 823-826.

[3] B. Alfeeli, et al., "Low pressure drop micro preconcentrators with cobweb Tenax-TA film for analysis of human breath," 2011, pp. 916-919.

[4] S. K. Kim, et al., "Microfabricated Gas Chromatograph for the Selective Determination of Trichloroethylene Vapor at Sub-Parts-Per-Billion Concentrations in Complex Mixtures," Analytical Chemistry, vol. 83, pp. 7198-7206, 2011/09/15 2011.

[5] P. R. Lewis, et al., "Recent advancements in the gasphase MicroChemLab," Sensors Journal, IEEE, vol. 6, pp. 784-795, 2006.

[6] R. Manginell, et al., "A Monolithically-Integrated $\mu \mathrm{GC}$ Chemical Sensor System," Sensors, vol. 11, pp. 65176532, 2011.

[7] B. C. Kaanta, et al., "A monolithically fabricated gas chromatography separation column with an integrated high sensitivity thermal conductivity detector," Journal of Micromechanics and Microengineering, vol. 20, p. 055016, 2010.

[8] S. Sreedharan Nair, et al., "A 2-Port Static Coated Micro Gas Chromatography Column with an Embedded Thermal Conductivity Detector," Sensors Journal, IEEE, pp. 1-1, 2011.

[9] B. Alfeeli and M. Agah, "Toward Handheld Diagnostics 
of Cancer Biomarkers in Breath: Micro Preconcentration of Trace Levels of Volatiles in Human Breath," Sensors Journal, IEEE, pp. 1-1, 2011.

[10] B. Bae, et al., "A fully-integrated MEMS preconcentrator for rapid gas sampling," 2007, pp. 14971500.

[11] S. Narayanan, et al., "A micro gas chromatography chip with an embedded non-cascaded thermal conductivity detector," Procedia Engineering, vol. 5, pp. 29-32, 2010.

[12] C. Lyons, et al., "A high-speed mass flow sensor with heated silicon carbide bridges," 1998, pp. 356-360.

[13] D. Cruz, et al., "Microfabricated thermal conductivity detector for the micro-ChemLab ${ }^{\mathrm{TM}}, "$ Sensors and Actuators B: Chemical, vol. 121, pp. 414-422, 2007.

[14] S. Frederico, et al., "Silicon sacrificial layer dry etching (SSLDE) for free-standing RF MEMS architectures," 2003, pp. 570-573.

[15] S. Reidy, et al., "High-performance, static-coated silicon microfabricated columns for gas chromatography," Analytical Chemistry, vol. 78, pp. 2623-2630, 2006.

\section{CONTACT}

*Dr. Masoud Agah, tel: +1-540-231-2653; agah@ vt.edu 


\title{
3D FOCUSING IN PARALLEL MICROCHANNELS FOR HIGH-THROUGHPUT SYNTHESIS OF POLYMERIC NANOPARTICLES
}

\author{
Jong-Min Lim ${ }^{1,2,3}$, Pedro M. Valencia , Minsoung Rhee ${ }^{1,2,3}$, \\ Robert S. Langer ${ }^{3,4}$, Omid C. Farokhzad ${ }^{2},{ }^{*}$, and Rohit Karnik ${ }^{1,}{ }^{*}$
}

${ }^{1}$ Department of Mechanical Engineering, Massachusetts Institute of Technology, Cambridge, MA, USA

${ }^{2}$ Department of Anesthesiology, Brigham and Women's Hospital - Harvard Medical School, Boston, MA, USA

${ }^{3}$ Koch Institute for Integrative Cancer Research, Massachusetts Institute of Technology, Cambridge, MA, USA

${ }^{4}$ Department of Chemical Engineering, Massachusetts Institute of Technology, Cambridge, MA, USA

\begin{abstract}
We have developed a multilayer microfluidic device for the parallelization of nanoparticles synthesis by microfluidic rapid nanoprecipitation in 3D hydrodynamic flow focusing geometry. Since the shape and position of interconnecting holes between upper and lower poly(dimethylsiloxane) (PDMS) channels could be optimized, stable vertical focusing could be achieved. The poly(lactide-co-glycolide)- $b$-polyethyleneglycol (PLGA-PEG) nanoparticles with various sizes could be synthesized with polymers of different molecular weights and concentrations. In addition, the size of nanoparticles could be precisely tuned simply by changing the flow ratio of organic stream to the total flow rate of water when the molecular weights and concentrations of polymer were fixed.
\end{abstract}

\section{INTRODUCTION}

Use of microfluidics for synthesis of nanoparticles has been of great interest due to controllability in their physicochemical properties and continuous flow process ${ }^{1-3}$. Recently, our group demonstrated microfluidic synthesis of poly(lactide-co-glycolide)- $b$-polyethyleneglycol (PLGA-PEG) nanoparticles for drug delivery systems using rapid nanoprecipitation by mixing polymer solution in acetonitrile with water in hydrodynamic flow focusing geometry ${ }^{4}$. More recently, we reported a single channel microfluidic device with three sequential holes to achieve the 3D hydrodynamic flow focusing 5 . We could enhance the robustness of operation by adopting the $3 \mathrm{D}$ hydrodynamic flow focusing geometry that isolates the precipitating polymers from poly(dimethylsiloxane) (PDMS) channel walls, eliminating microchannel fouling. However, applications of the microfluidic synthesis were limited to in vitro studies due to low throughput. In addition, the precision of manual drilling was critical to achieve stable 3D hydrodynamic flow focusing.

Herein, we reported the parallelization of nanoparticles synthesis in microfluidic system by using multilayer microfluidic device that enable sufficient throughput for in-vivo studies. Since the interconnecting holes between upper and lower PDMS channels were originated from the patterned SU-8 posts, the shape and position of interconnecting holes could be optimized. In addition, the precision of the interconnecting holes position could be significantly enhanced by using conventional multilayer photolithography. As a result, more stable vertical focusing could be achieved. Moreover, only four external tubing connections were required due to the 3D fluidic architecture design.

Smaller nanoparticles could be synthesized reproducibly from the microfluidic rapid nanoprecipitation method since the mixing time scale was shorter than that of conventional bulk synthesis method. In addition, the size of nanoparticles could be precisely controlled simply by changing the flow rates of fluids when the molecular weights and concentrations of polymer were fixed. Because the threshold of renal clearance of nanoparticles is about $5.5 \mathrm{~nm}$ and the minimum size from conventional bulk synthesis method is $30 \mathrm{~nm}^{6,7}$, the sub-30 nm PLGA-PEG nanoparticles synthesized from 3D hydrodynamic flow focusing can be used for optimizing the size of nanomedicine ${ }^{8}$.

\section{EXPERIMENTAL}

\section{Materials}

As the polymeric precursor, PLGA-PEG (Boehringer Ingelheim $\mathrm{GmbH}$, Germany) at molecular weights of $\mathrm{PLGA}_{10 \mathrm{~K}}-\mathrm{PEG}_{5 \mathrm{~K}}$, $\mathrm{PLGA}_{27 \mathrm{~K}}-\mathrm{PEG}_{5 \mathrm{~K}}, \mathrm{PLGA}_{45 \mathrm{~K}}-\mathrm{PEG}_{5 \mathrm{~K}}$, and $\mathrm{PLGA}_{95 \mathrm{~K}}-\mathrm{PEG}_{5 \mathrm{~K}}$ were dissolved in acetonitrile (Aldrich) at concentrations of $10-50$ $\mathrm{mg} / \mathrm{mL}$. Pure acetonitrile and deionized water were employed as vertical and lateral sheath stream, respectively. To obtain the confocal microscope images, aqueous solution of Rh6G (Aldrich) and FITC (Aldrich) were used as core and vertical sheath fluids, respectively.

\section{Preparation of Multilayer PDMS Microfluidic device}

Multilayer 3D hydrodynamic flow focusing microfluidic device was prepared using multilayer photolithography and multilayer soft lithography 9 . The multilayer device preparation procedure was illustrated in Figure 1. Master molds for the bottom and upper layers were patterned in SU-8 photoresist via conventional photolithography. Post arrays for interconnecting holes were patterned in SU-8 on top of the bottom layer with precise alignment using an UV aligner.

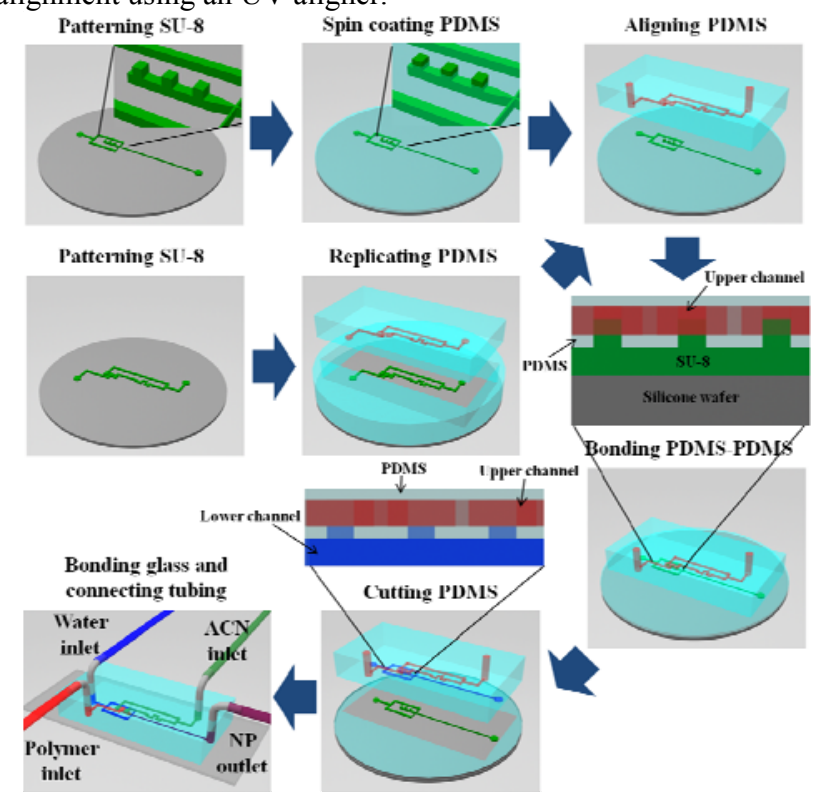

Figure 1: Schematic illustration of fabrication procedure for the multilayer $3 D$ hydrodynamic flow focusing device. 
A thin PDMS membrane was spin-coated on the master mold for bottom layer with post arrays. The spin speed was tuned to precisely control the membrane thickness to be slightly lower than the interconnect post height. The upper PDMS microchannel was prepared using conventional replica molding of PDMS from the SU-8 master for upper layer. The upper PDMS channel was aligned under a microscope and bonded on top of bottom PDMS membrane using oxygen plasma treatment. To assemble the final device, the multilayer PDMS channel was bonded with a slide glass via oxygen plasma and connected to tubing.

\section{Preparation of PLGA-PEG Nanoparticles}

Monodisperse PLGA-PEG nanoparticles were synthesized using the multilyer PDMS microfluidic device. The PLGA-PEG precursor in acetonitrile was introduced to the microchannel connected with the middle interconnecting holes. Pure acetonitrile was introduced to the microchannel connected with the first and the third interconnecting holes. The water flow rate were maintained at $50 \mu \mathrm{L} / \mathrm{min}$ and $400 \mu \mathrm{L} / \mathrm{min}$ for single $3 \mathrm{D}$ hydrodynamic flow focusing and 8 parallel 3D hydrodynamic flow focusing, respectively. During the nanoparticle synthesis, the flow rates were controlled by syringe pumps (Harvard Apparatus) and the flow motion was observed using an inverted microscope (Nikon, Eclipse TS 100). To confirm the vertical focusing, laser scanning confocal microspcope (Carl Zeiss MicroImaging, LSM 510) was used. In case of the bulk synthesis, we mixed $100 \mu \mathrm{L}$ of polymeric precursor solution drop-wise with $1 \mathrm{~mL}$ of water for about $2 \mathrm{hrs}^{10,11}$.

\section{PLGA-PEG Nanoparticles Characterization}

The size distribution by volume fraction of PLGA-PEG nanoparticles were measured using dynamic light scattering with Zetasizer Nano ZS (Malvern Instruments Ltd., U.K.). It is noteworthy that the nanoparticles size did not vary significantly after washing with deionized water several times. The synthesized PLGA-PEG nanoparticles were imaged by TEM (JEOL 200CX) with negative staining by uranyl acetate (Electron Microscopy Sciences).

\section{RESULTS AND DISCUSSION}

Multilayer PDMS microfluidic device for parallel 3D hydrodynamic flow focusing could be prepared using multilayer photolithography and multilayer soft lithography ${ }^{9}$. Figure 2 shows the digital camera image of the 8 parallel 3D hydrodynamic flow focusing device. To clearly demonstrate the upper and lower microchannels, the interconnecting holes were closed by spincoating the PDMS thicker than the height of post arrays. In addition, the red and blue food coloring dyes were filled in the upper and lower microchannels, respectively.

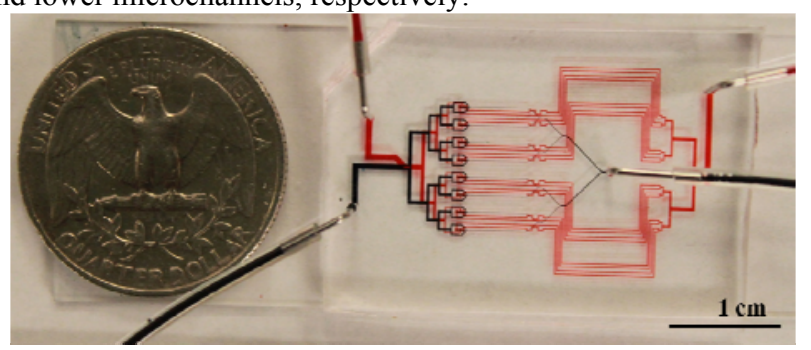

Figure 2: Digital camera images of 8 parallel 3D hydrodynamic flow focusing device.

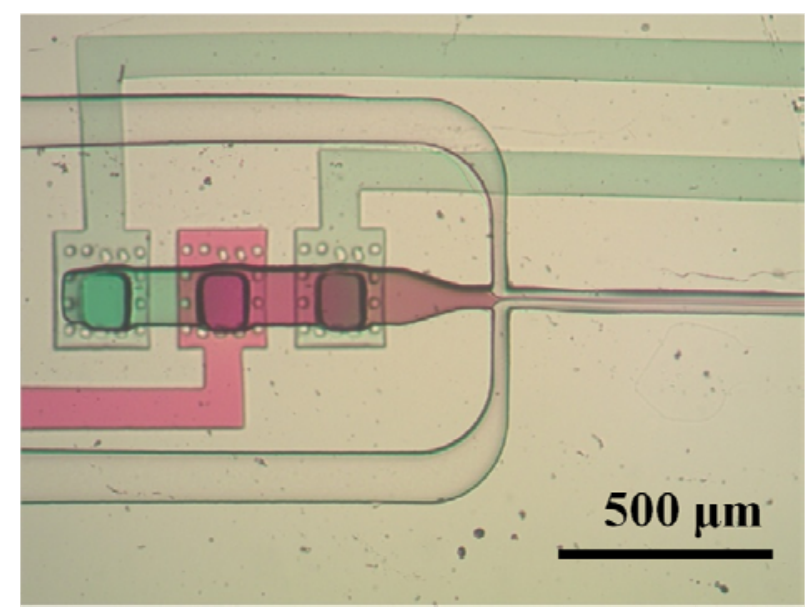

Figure 3: Optical microscope image of the 3D hydrodynamic flow focusing device.

It is noteworthy that the microchannels for the upper sheath stream were meandered to match the resistances of the microchannel lengths between upper and bottom streams.

Figure 3 shows optical microscope image of the device for nanoparticles synthesis. In case of the device used for nanoparticle synthesis, the thickness of spincoated PDMS was slightly lower than the height of post arrays (see Figure 1). As a result, the organic stream could flow from the upper microchannel to the lower microchannel. To confirm the interlayer flow, polymeric precursor and pure acetonitrile were denoted using red and green food coloring dyes, respectively (see Figure 3). Since the interlayer flows occurred only at the interconnecting holes, tubing of the device could be significantly simplified by using the multilayer microfluidic device. As a result, only 3 inlets and 1 outlet are required for a parallel 3D hydrodynamic flow focusing device.

The lateral position of inlet holes significantly affects the vertical focusing in 3D hydrodynamic flow focusing with three sequential inlets. The precision of manual drilling was critical in our previous single layer $3 \mathrm{D}$ hydrodynamic flow focusing device ${ }^{5}$. In case of the multilayer 3D hydrodynamic flow focusing device, precise alignment of interconnecting holes could be achieved by using conventional multilayer photolithography with UV aligner. In addition, the shape of interconnecting holes could be optimized. The effect of interconnecting holes geometry could be examined by using 3D finite element simulations using COMSOL (COMSOL Inc., Burlington, MA). Because deep microchannel with inlet holes having slightly larger diameter than the width of microchannel could achieved the most uniform distribution of vertically focused stream $^{5}$, the width and height of lower microchannel were fixed to $100 \mu \mathrm{m}$ and the width of interconnecting holes were fixed to $120 \mu \mathrm{m}$ during the 3D finite element simulations. Out of three sequential interconnecting holes, the first interconnecting hole for the bottom sheath fluid was excluded during the simulation because its influence to the vertical focusing is negligible. Round interconnecting holes resulted in the non-uniform distribution of concentration profile (see Figure 4a). Likewise, rectangular interconnecting holes smaller than $100 \mu \mathrm{m}$ in the lengthwise direction resulted in arched vertically focused stream (see Figure 4b). When rectangular interconnecting holes with larger than 100 $\mu \mathrm{m}$ in the lengthwise direction were used, the most uniform concentration profile of the vertically focused stream could be achieved (see Figure 4c and 4d). 


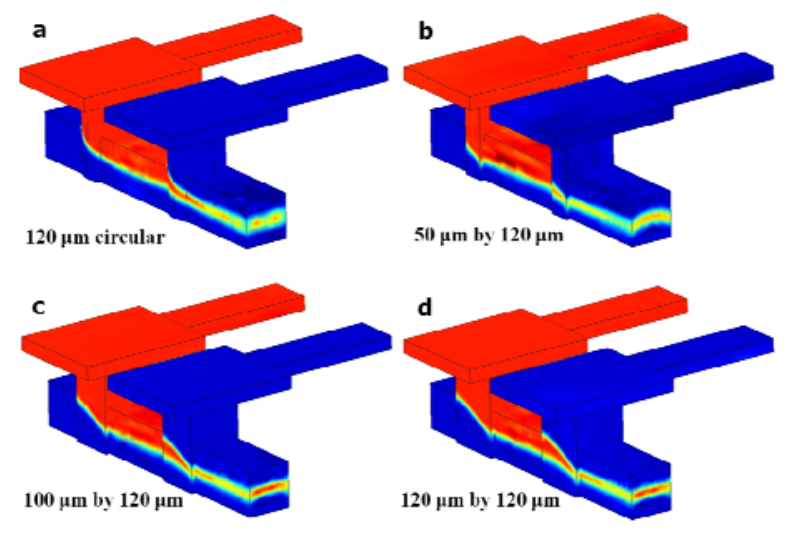

Figure 4: Perspective views of $3 D$ finite element simulations at the vertical focusing region with (a) round (120 $\mu \mathrm{m}$ in diameter), (b) rectangular $(50 \mu \mathrm{m} \times 120 \mu \mathrm{m})$, (c) rectangular $(100 \mu \mathrm{m} \times 120 \mu \mathrm{m})$, and $(d)$ square $(120 \mu \mathrm{m} \times 120 \mu \mathrm{m})$ shapes of interconnecting holes. Here, the fraction of polymer flow in organic flow $(f)$ is 0.3 . Both width and height of lower microchannel are $100 \mu \mathrm{m}$.

Since some residual PDMS may remain on top of the post arrays during the spincoating of PDMS if the length of post arrays were too long, the length of interconnecting holes was fixed at $100 \mu \mathrm{m}$ for the device fabrication.

The flow within the multilayer 3D hydrodynamic flow focusing device was examined using confocal microscopy. We used aqueous Rh6G (red) and FITC (green) fluorescent dye solutions as the vertically focused stream and the vertical sheath streams, respectively. As predicted in 3D finite element simulations, stable and uniform vertical focusing could be achieved when the rectangular interconnecting holes $(100 \mu \mathrm{m} \times 120 \mu \mathrm{m})$ were used (see Figure 5a and 5b).
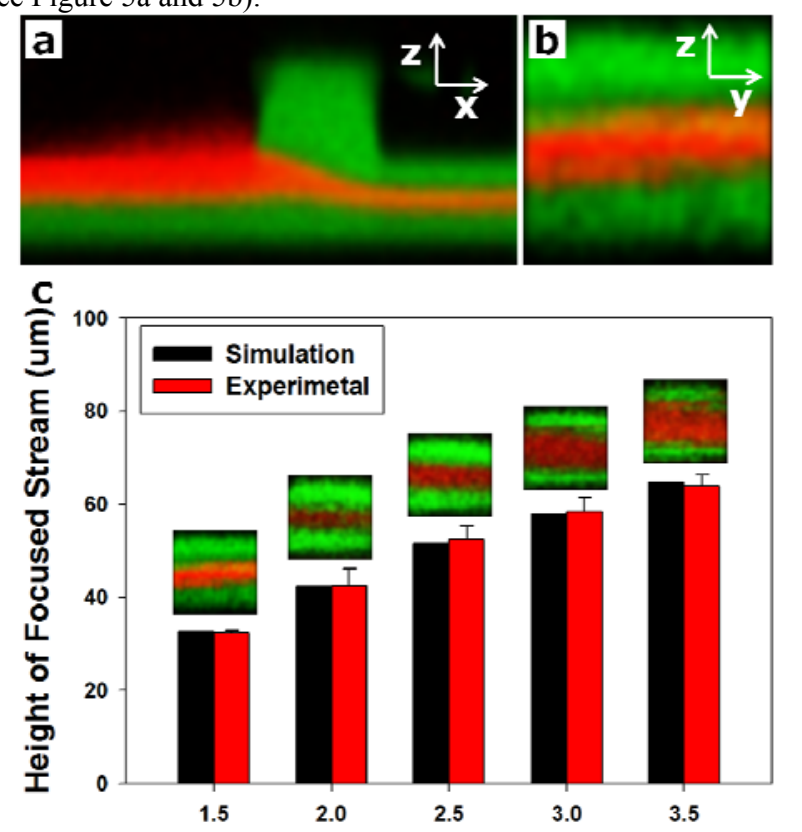

Fraction of Polymer Flow in Organic Flow

Figure 5: (a) cross-sectional front view and (b) cross-sectional side view from confocal microscope data at the vertical focusing region (c) Height of vertically focused polymeric stream as a function of the fraction of polymer flow in organic flow $(f)$.
In addition, the heights and shapes of vertical focusing streams obtained by confocal microscopy are collectively in good agreement with the 3D finite element simulations at various fraction of polymer flow in organic flow $(f)$ (see Figure $5 \mathrm{c}$ ). Since the resistances of microchannels for the vertical sheath streams were matched by meandering the microchannel for the upper sheath stream, the core stream could be located at the middle of the bottom microchannel. In addition, uniform concentration profile of the vertically focused stream could be achieved due to the optimal shapes of interconnecting holes. As a result, the vertically focused stream did not touch the PDMS microchannels even at high $f$ (see Figure 5c).

To enhance the production yield of nanoparticles, we performed parallelization of nanoparticles synthesis by using the multilayer microfluidic 3D hydrodynamic flow focusing. By using the multilayer PDMS microfluidic device, PLGA-PEG nanoparticles with various sizes could be synthesized with polymers of different molecular weights and concentrations (see Figure 6a). The nanoparticles synthesized from the microfluidic 3D hydrodynamic flow focusing were smaller compared to the nanoparticles synthesized from the bulk synthesis method because the mixing time $\left(\tau_{\text {mix }}\right)$ is shorter than the characteristic aggregation time scale $\left(\tau_{\text {agg }}\right)$ in the microfluidic rapid mixing ${ }^{12}$.
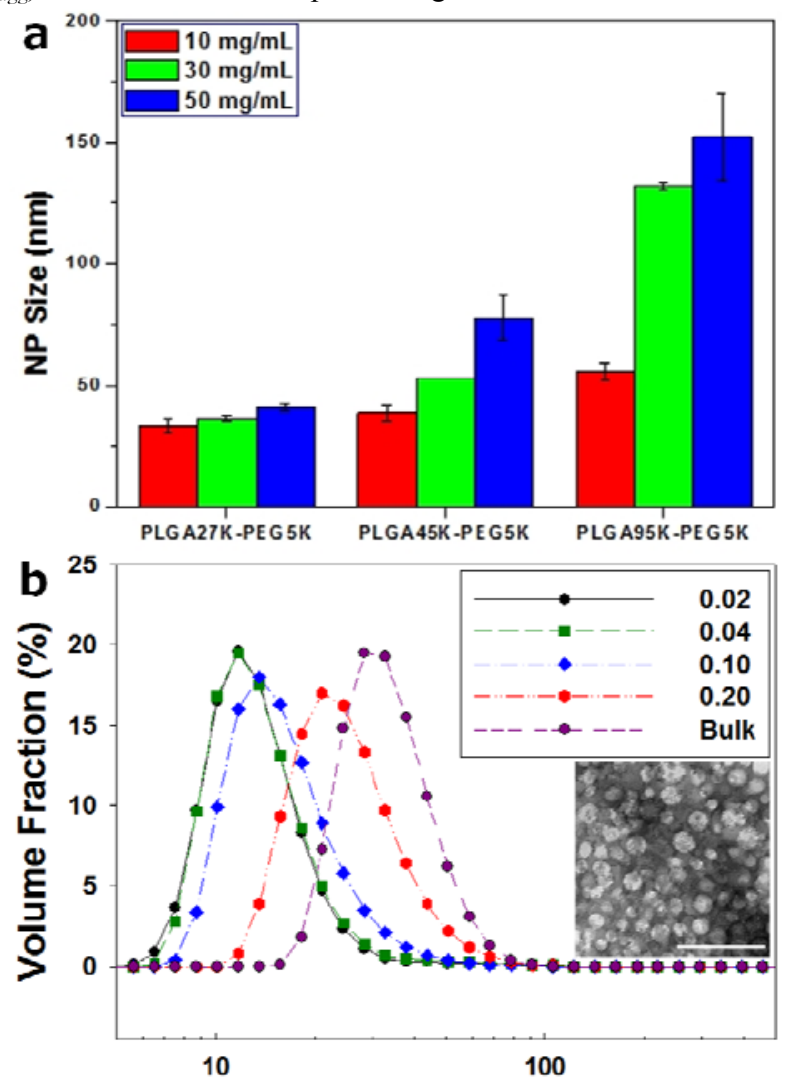

Nanoparticle Diameter (nm)

Figure 6: (a) Effect of PLGA-PEG precursor concentrations and molecular weights on the size of synthesized PLGA-PEG nanoparticles. (b) Effect of flow ratio on nanoparticles size. The inset shows TEM image of the nanoparticles prepared by microfluidic $3 D$ hydrodynamic flow focusing when the flow ratio of organic stream to the total flow rate of water $(R)$ is 0.04 . Scale bar is $100 \mathrm{~nm}$. 
When molecular weight and concentration of polymeric precursor are same, the size of nanoparticles depends on the mixing time $\left(\tau_{\text {mix }}\right)$ for hydrodynamic flow focusing ${ }^{4}$. The mixing time $\left(\tau_{\text {mix }}\right)$ for hydrodynamic flow focusing can be estimated as follows:

$$
\tau_{\text {mix }} \sim \frac{w_{f}^{2}}{4 D} \approx \frac{w^{2}}{9 D} \frac{1}{(1+1 / R)^{2}}
$$

Where $D$ is diffusivity of the solvent, $w_{f}$ is width of the focused stream, $w$ is channel width, and $R$ is the ratio of flow rate of the polymeric stream to the total flow rate of water. Since $\tau_{\text {mix }}$ for hydrodynamic flow focusing is function of flow ratio of organic stream to the total flow rate of water $(R)$, the size of nanoparticles could be controlled in 3D hydrodynamic flow focusing simply by changing the flow ratio of organic stream to the total flow rate of water $(R)$. Eq. 1 predicts mixing time $\left(\tau_{\operatorname{mix}}\right)$ in the range of 0.017 $1.2 \mathrm{~ms}$ for typical flow ratios $(\mathrm{R}=0.02-0.2)$ in our device. The size of nanoparticles decreased from about $24 \mathrm{~nm}$ to $14 \mathrm{~nm}$ as the mixing time $\left(\tau_{\text {mix }}\right)$ decreased when $\mathrm{PLGA}_{10 \mathrm{~K}}-\mathrm{PEG}_{5 \mathrm{~K}}$ with low concentration (i.e. $10 \mathrm{mg} / \mathrm{mL}$ ) was used as the polymeric precursor (see Figure $6 \mathrm{~b}$ ). It is noteworthy that the decrease in the nanoparticles size saturated when mixing time $\left(\tau_{\text {mix }}\right)$ was shorter than $0.066 \mathrm{~ms}$ (i.e. $\mathrm{R}=0.04$ ).

\section{CONCLUSION}

We could demonstrate the parallelization of PLGA-PEG nanoparticle synthesis in a microfluidic system by using multilayer microfluidic device. Since the shape and position of interconnecting holes between upper and lower PDMS channels could be optimized, stable vertical focusing could be achieved even at high fraction of polymer flow in organic flow $(f)$. The PLGA-PEG nanoparticles with various sizes could be synthesized with polymers of different molecular weights and concentrations. The nanoparticles synthesized from the microfluidic 3D hydrodynamic flow focusing were smaller compared to the nanoparticles synthesized from the bulk synthesis method because the mixing time $\left(\tau_{\text {mix }}\right)$ is shorter than the characteristic aggregation time scale $\left(\tau_{\text {agg }}\right)$ in the microfluidic rapid mixing. In addition, the size of nanoparticles could be precisely tuned simply by changing the flow ratio of organic stream to the total flow rate of water $(R)$ when the molecular weights and concentrations of polymer were fixed.

\section{ACKNOWLEDGMENTS}

This research was supported by the Koch-Prostate Cancer Foundation Award in Nanotherapeutics (R.L. and O.C.F.), by the Concept Development Grant 5P50CA090381-09 from the Dana Farber Cancer Institute Prostate SPORE (O.C.F.), and by NIH Grants CA119349 (R.L. and O.C.F.) and EB003647 (O.C.F.). P.M.V. is supported by NSF Graduate Research Fellowship.

\section{REFERENCES}

[1] Y. J. Song, J. Hormes, and C. Kumar, "Microfluidic synthesis of nanomaterials," Small, 4, 698 (2008).

[2] A. Jahn, J. E. Reiner, W. N. Vreeland, D. L. DeVoe, L. E. Locascio, and M. Gaitan, "Preparation of nanoparticles by continuous-flow microfluidics," Journal of Nanoparticle Research, 10, 925 (2008).

[3] S. Marre and K. F. Jensen, "Synthesis of micro and nanostructures in microfluidic systems," Chemical Society Reviews, 39, 1183 (2010).

[4] R. Karnik, F. Gu, P. Basto, C. Cannizzaro, L. Dean, W. Kyei-Manu, R. Langer, and O. C. Farokhzad, "Microfluidic platform for controlled synthesis of polymeric nanoparticles," Nano Letters, 8, 2906 (2008).

[5] M. Rhee, P. M. Valencia, M. I. Rodriguez, R. Langer, O. C. Farokhzad, and R. Karnik, "Synthesis of Size-Tunable Polymeric Nanoparticles Enabled by 3D Hydrodynamic Flow Focusing in Single-Layer Microchannels," Advanced Materials, 23, H79 (2011).

[6] H. S. Choi, W. Liu, P. Misra, E. Tanaka, J. P. Zimmer, B. I. Ipe, M. G. Bawendi, and J. V. Frangioni, "Renal clearance of quantum dots," Nature Biotechnology, 25, 1165 (2007).

[7] F. Alexis, E. Pridgen, L. K. Molnar, and O. C. Farokhzad, "Factors affecting the clearance and biodistribution of polymeric nanoparticles," Molecular Pharmaceutics, 5, 505 (2008).

[8] H. Cabral, Y. Matsumoto, K. Mizuno, Q. Chen, M. Murakami, M. Kimura, Y. Terada, M. R. Kano, K. Miyazono, M. Uesaka, N. Nishiyama, and K. Kataoka, "Accumulation of sub-100 nm polymeric micelles in poorly permeable tumours depends on size," Nature Nanotechnology, 6, 815 (2011).

[9] E. P. Kartalov, C. Walker, C. R. Taylor, W. F. Anderson, and A. Scherer, "Microfluidic vias enable nested bioarrays and autoregulatory devices in Newtonian fluids," Proceedings of the National Academy of Sciences of the United States of America, 103, 12280 (2006).

[10] J. Cheng, B. A. Teply, I. Sherifi, J. Sung, G. Luther, F. X. Gu, E. Levy-Nissenbaum, A. F. Radovic-Moreno, R. Langer, and O. C. Farokhzad, "Formulation of functionalized PLGA-PEG nanoparticles for in vivo targeted drug delivery," Biomaterials, 28, 869 (2007).

[11] J. M. Chan, P. M. Valencia, L. F. Zhang, R. Langer, and O. C. Farokhzad, "Polymeric Nanoparticles for Drug Delivery," in Cancer Nanotechnology: Methods and Protocols. vol. 624, ed Totowa: Humana Press Inc, 2010, pp. 163-175.

[12] B. K. Johnson and R. K. Prud'homme, "Mechanism for rapid self-assembly of block copolymer nanoparticles," Physical Review Letters, 91, 118302 (2003).

\section{CONTACT}

* R. Karnik, tel: +1-617-324-1155; karnik@mit.edu

* O. C. Farokhzad, tel: +1-617-732-6093; ofarokhzad@partners.org 


\title{
AUTONOMOUS RAILING AND TRAPPING OF MICROBEADS FOR CONTINUOUS FLOW MULTI-STAGE MICROFLUIDIC PROCESSES
}

\author{
Ryan D. Sochol ${ }^{l}$, William E. R. Krieger ${ }^{l}$, Luke P. Lee ${ }^{2}$ and Liwei Lin ${ }^{l}$ \\ ${ }^{1}$ Department of Mechanical Engineering, ${ }^{2}$ Department of Bioengineering \\ Berkeley Sensor and Actuator Center, Biomolecular Nanotechnology Center \\ University of California, Berkeley, USA
}

\begin{abstract}
"Multi-stage" fluidic mixing processes are critical to a wide range of chemical and biological assays (e.g., immunoassays). Unfortunately, the majority of biochemical assays suffer from laborious and time-intensive fluidic mixing procedures in which distinct reagents and/or washes are loaded sequentially and separately (i.e., one-at-a-time). Previously, we introduced the first microfluidic railing system capable of hydrodynamically guiding microbeads into discrete, adjacent flow streams in order to autonomously accomplish multi-stage fluidic mixing reactions on-chip. However, microbead immobilization and signal detection were only possible after reaction completion, which poses a significant problem for bead-based assays that require microbead visualization during intermediate phases of multi-stage processes. To overcome this limitation, here we present a single-layer, "continuous flow" microfluidic system that utilizes microposts arrayed in sections at angles of $1^{\circ}, 15^{\circ}$, and $1^{\circ}$ (with respect to the flow direction) to: ( $i$ ) rail suspended microbeads into a trapping area, (ii) trap select numbers of microbeads, and then (iii) transport subsequent microbeads into adjacent flow streams, respectively. By enabling both autonomous bead-based mixing reactions as well as microbead immobilization during each fluidic mixing stage, the presented continuous flow microfluidic "rail-and-trap" system offers a simple, yet powerful methodology for applications in chemical and biological fields, such as molecular diagnostics.
\end{abstract}

\section{KEYWORDS}

Microfluidic Railing, Microbeads, Trapping, Continuous Flow

\section{INTRODUCTION}

Microbead Functionalization for Biochemical Assays

For multi-stage chemical and biological reaction processes that require fluidic mixing with numerous reagents and/or washes, microfluidic systems offer a variety of benefits, such as low reagent volumes and enhanced reaction kinetics [1-3]. Bead-based microfluidic technologies are especially advantageous for accomplishing biochemical assays (i.e., by using microbeads as substrates) due to high surface-to-volume ratios and the ability to mix-and-match microbeads corresponding to specific screenings [4-9]. In particular, microbeads can be functionalized with diverse surface modifications, such as molecular probes capable of detecting DNA analytes and inflammatory cytokines [9-12]. Unfortunately, bead-based microfluidic systems that are employed to perform multi-stage biochemical assays near-universally require external observation and/or regulation during device operation.

\section{Microfluidic Railing for Continuous Flow Mixing Processes}

Recently, microfluidic research has shifted toward systems with autonomous on-chip functionalities [13-15], and microfluidic railing techniques offer powerful means for autonomously transporting microparticulates into discrete, parallel flow streams (i.e., under continuous input flow conditions). Previously, researchers have achieved microfluidic railing for both photopatterned microstructures $[16,17]$ and microdroplets [18-20]. For example, multi-layer photolithographic processes have been used to etch grooves into microchannels that act as tracks in order to rail (i.e., guide) photopolymerized microstructures $[16,17]$ and microdroplets [18]. To bypass the limitations associated with multi-layer fabrication processes (e.g., increased costs, time, and labor), researchers have also developed single-layer microfluidic systems for railing microdroplets using comparatively smaller microposts (alternatively referred to as micropillars) $[19,20]$. Previously, we presented the first microfluidic railing system capable of handling suspended microbeads, which we employed to passively accomplish a 10-stage molecular synthesis process on microbead substrates [21]. Here, we adapt this bead-based railing system to enable microbead immobilization and visualization during each step of multi-stage fluidic mixing processes (Fig. 1).

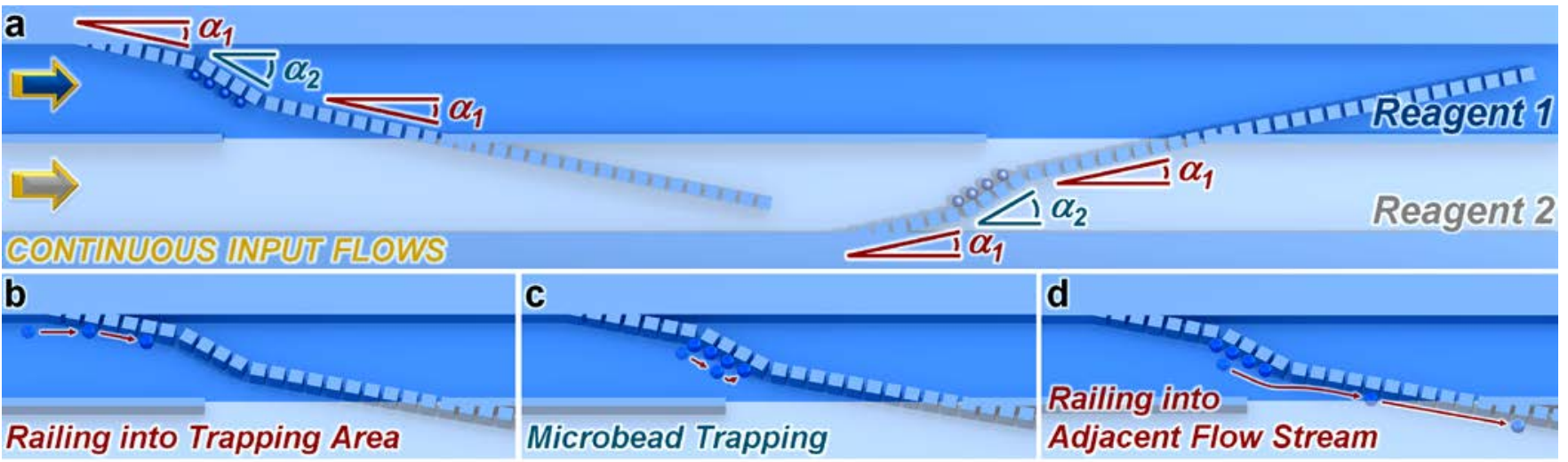

Figure 1: The microfluidic "rail-and-trap" concept. (a) Overview of an example system with two reagents inputted under continuous flow conditions. Microposts are arrayed in sections at either a low angle, " $\alpha_{1}$," or a higher angle, " $\alpha_{2}$," (with respect to the flow direction) in order to prevent or promote microbead immobilization, respectively. (b) Initially, suspended microbeads are railed into the trapping area due to the low $\alpha_{1}$. (c) The higher $\alpha_{2}$ promotes microbead trapping in the gaps between microposts. (d) After select numbers of microbeads are trapped, subsequent beads are railed into discrete, adjacent fluidic streams. This process can be repeated as desired (and additional reagents and/or washes can be loaded in parallel) in order to autonomously rail-and-trap microbeads during multi-stage mixing reactions. 

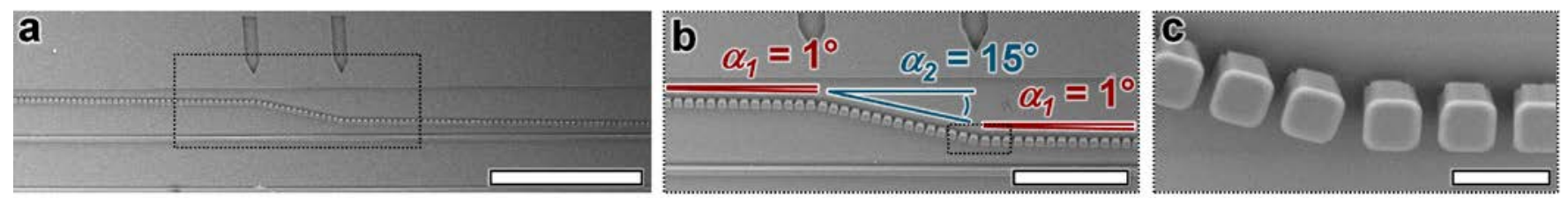

Figure 2: Microfabrication results for the microfluidic rail-and-trap system. Microposts are arrayed in sections at $\alpha_{1}=1{ }^{\circ}, \alpha_{2}=15^{\circ}$, and

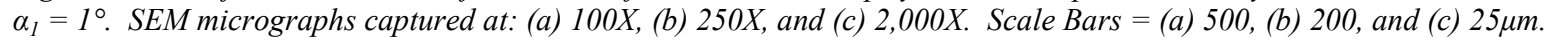

\section{CONCEPT}

The ability to visualize microbeads during specified points of multi-stage reaction processes is a critical requirement for a variety of biochemical assays. For example, we previously achieved beadbased cytokine detection via a multi-stage aptamer-based sandwich assay that consisted of three fundamental steps [22]. First, a fluorescent aptamer was conjugated to the microbead substrates, which resulted in the highest bead-based fluorescent intensities (i.e., for the positive control). Then, a complementary quencher was bound to the fluorescent aptamer, which quenched the fluorescent signal (i.e., for the negative control). Lastly, an inflammatory cytokine, IFN- $\gamma$, displaced the quencher by binding to the fluorescent aptamer, which resulted in a detectable increase in the fluorescent intensity on the microbead surfaces [22]. For such assays, the utility of our prior microfluidic railing system remains limited, despite the broad advantages associated with autonomous bead-based fluidic mixing on-chip [21].

To overcome this drawback, here we present a microfluidic "rail-and-trap" system (Fig. 1). Previously, we observed that square microposts arrayed at an angle of $1^{\circ}$ successfully railed microbeads by preventing microbead immobilization in the system; however, higher angles (e.g., 15 ${ }^{\circ}$ ) were found to promote microbead immobilization in the gaps between microposts [21]. In this work, we exploit these phenomena in order to passively: (i) transport suspended microbeads into distinct, parallel flow streams, and (ii) immobilize select numbers of microbeads during each phase of multi-stage fluidic processes. Figure 1a shows illustrations of the microfluidic rail-and-trap concept, which consists of square-shaped microposts (i.e., of approximately the same size as the microbeads) arrayed in sections at either a low angle, $\alpha_{1}$, or a higher angle, $\alpha_{2}$, in order to prevent or promote microbead immobilization, respectively. Under continuous flow conditions, microbeads are initially guided toward the trapping area due to the low $\alpha_{1}$ (Fig. 1b). The higher $\alpha_{2}$ promotes microbead immobilization in the gaps between microposts (Fig. 1c). After select numbers of microbeads are immobilized, subsequent microbeads are transported into the distinct, adjacent fluidic stream (Fig. 1d). This process can be repeated continuously as desired. Furthermore, additional fluidic reagents and/or washes can be inputted in parallel to tailor the microfluidic rail-and-trap system for a variety of multi-stage fluidic reaction processes.

\section{MICROFABRICATION}

The prototype rail-and-trap system was fabricated via standard and universally employed single-layer soft-lithography processes using polydimethylsiloxane (PDMS). Briefly, an $18 \mu \mathrm{m}-$ high layer of SU-8 negative photoresist was spin-coated onto a clean $\mathrm{Si}$ wafer. Using a photomask, the device patterns were UV exposed onto the layer of photoresist via contact photolithography. The wafer was then developed to become a positive master for the micromolding process. Next, the silicone elastomer, PDMS, was mixed at a 10:1 (base : curing agent) ratio and poured onto the master. After curing of the PDMS at $55{ }^{\circ} \mathrm{C}$, the elastomer was removed from the master. Individual devices were cut and then punched with holes at inlet and outlet locations. Glass slides were washed in successive dishes of Acetone, Isopropanol, and DI water, while the PDMS devices were washed in successive dishes of Isopropanol and DI water. The glass slides and PDMS devices were dried with a $\mathrm{N}_{2}$ gun, and then exposed to UV ozone for 5 minutes. Lastly, the PDMS devices were thermally bonded to the glass slides at $55{ }^{\circ} \mathrm{C}$. The final devices included arrays of square-shaped microposts $\left(15 \times 15 \mu \mathrm{m}^{2}\right)$, with an interpost spacing of $5 \mu \mathrm{m}$. The microchannels included a width of $200 \mu \mathrm{m}$. Microfabrication results are shown in Figure 2.

\section{RESULTS \\ COMSOL Multiphysics Fluid Dynamics Simulations}

Three-dimensional COMSOL Multiphysics fluid dynamics simulations were performed to provide insight into the microfluidic rail-and-trap system (Fig. 3). The model included spherical microbeads (15 $\mu \mathrm{m}$ in diameter) placed in contact with arrayed square microposts $\left(15 \times 15 \mu \mathrm{m}^{2}\right)$ in each section of the system (i.e., $\alpha_{1}=1^{\circ}, \alpha_{2}=15^{\circ}$, and $\alpha_{1}=1^{\circ}$ ). The pressure field simulation results showed higher pressure drops across the arrayed microposts in the $\alpha_{2}=15^{\circ}$ section compared to the $\alpha_{1}=1^{\circ}$ sections. Additionally, the fluid velocity field vectors (overlaid red arrows) located at the top right of the microbeads had opposite directionalities for the $\alpha_{1}=1^{\circ}$ sections versus the $\alpha_{2}=15^{\circ}$ section, with the velocity field vectors directed into the gap for the $\alpha_{2}=15^{\circ}$ case. These simulation results suggest that the $\alpha_{1}=1^{\circ}$ sections would promote railing of suspended microbeads along the arrayed microposts, while the $\alpha_{2}=15^{\circ}$ section would be more likely to promote microbead trapping in the gaps between microposts.

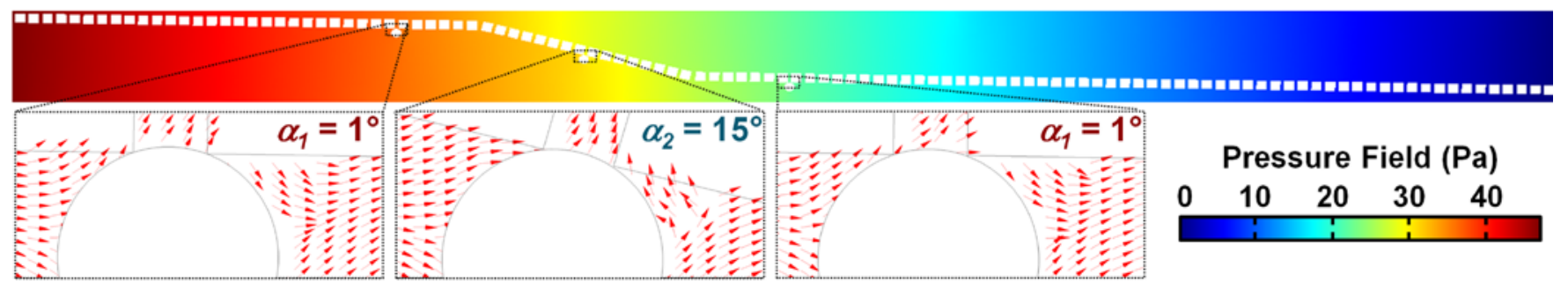

Figure 3: Three-dimensional COMSOL Multiphysics fluid dynamics simulation results for the microfluidic rail-and-trap system. The model consists of spherical microbeads $\left(15 \mu \mathrm{m}\right.$ in diameter) in contact with arrayed microposts $\left(15 \times 15 \mu \mathrm{m}^{2}\right)$ in each section of the system (i.e., $\alpha_{1}=1^{\circ}, \alpha_{2}=15^{\circ}$, and $\alpha_{1}=1^{\circ}$ ). (Top) Pressure field across the rail-and-trap system. (Bottom) Expanded views of each of the microbeads. Overlaid 'red arrows' denote the direction of the fluid velocity field vectors. 

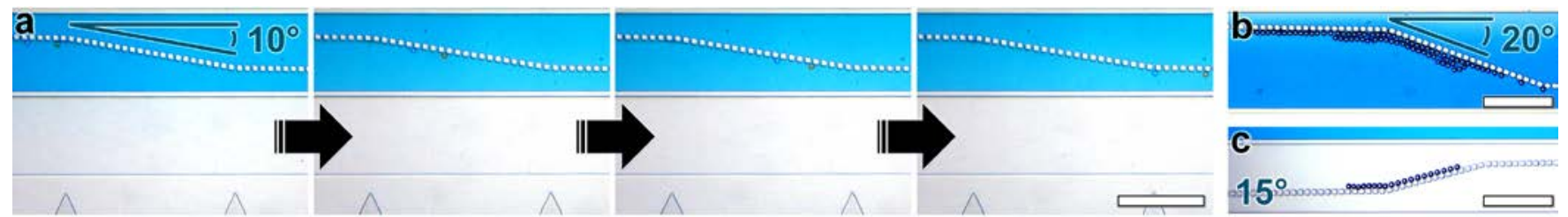

Figure 4: Experimental results for microbead handling in various microfluidic rail-and-trap testing systems. (a) Sequential micrographs of a suspended microbead bypassing the trapping area of a system with $\alpha_{1}=1^{\circ}$ and $\alpha_{2}=10^{\circ}$. (b) A micrograph of the trapping area of a system with $\alpha_{1}=1^{\circ}$ and $\alpha_{2}=20^{\circ}$ after the microbead loading process. (c) A micrograph of the trapping area of a system with $\alpha_{1}=1^{\circ}$ and $\alpha_{2}=15^{\circ}$ after the microbead loading process. Suspended streptavidin-coated polystyrene microbeads are $15 \mu \mathrm{m}$ in diameter; 'Orange' and 'blue' microbeads show a singular mobile microbead at two time-points within one second; Scale Bars $=200 \mu \mathrm{m}$.

\section{Experimental Characterization of Rail-and-Trap Performance}

Experiments with suspended streptavidin-coated polystyrene microbeads $(15 \mu \mathrm{m}$ in diameter) were performed using testing systems with varying $\alpha_{2}$ (i.e., while $\alpha_{1}$ was held constant at $1^{\circ}$ ) in order to investigate the effects of $\alpha_{2}$ on rail-and-trap performance. Previously, we found that values of $\alpha$ as low as $2.5^{\circ}$ resulted in microbead immobilization with the railing system, with higher proportions of microbead trapping observed as the value of $\alpha$ increased [21]. In this study, a variety of testing devices were fabricated with values of $\alpha_{2}$ ranging from $5^{\circ}$ to $25^{\circ}$ (with $\alpha_{1}=1^{\circ}$ ). A blue-dyed microbead suspension and a clear wash solution of phosphate buffered saline (PBS) were simultaneously loaded via separate inlets under continuous, constant input flow conditions. Specifically, two syringe pumps - each set at a constant flow rate of $1.5 \mu \mathrm{l} / \mathrm{min}$ - were used to control the input flow rates for each solution/suspension in parallel.

For testing systems in which $\alpha_{2}$ was too low (i.e., $\alpha_{2} \leq 10^{\circ}$ ), microbeads were observed to bypass the trapping areas without being immobilized. For example, Figure 4a shows sequential micrographs of a suspended microbead railing along the $\alpha_{2}=10^{\circ}$ trapping area, instead of being immobilized. Conversely, for systems where $\alpha_{2}$ was too high (i.e., $\alpha_{2} \geq 20^{\circ}$ ), microbead immobilization in the trapping area was promoted too strongly, which caused device failure because subsequent microbeads were prevented from bypassing fully occupied trapping areas. For example, Figure $4 \mathrm{~b}$ shows an $\alpha_{2}=20^{\circ}$ testing system after the bead loading process, with high numbers of microbeads immobilized on top of previously trapped microbeads. Experimental device runs revealed that the prototype systems with $\alpha_{2}=15^{\circ}$ were the most effective systems tested for trapping select numbers of microbeads without preventing subsequent microbeads from railing into the distinct, parallel flow streams (Fig. 4c).
Figure 5 shows experimental results for the microfluidic railand-trap system with $\alpha_{1}=1^{\circ}$ and $\alpha_{2}=15^{\circ}$. Under continuous input flow conditions, suspended microbeads were successfully: (a) railed into the $\alpha_{2}$ trapping area for immobilization, (b) transported along the micropost array rails from the top solution (blue) into the discrete, adjacent bottom solution (clear), (c) directed to bypass previously immobilized microbeads in the $\alpha_{2}$ trapping area, and $(d)$ transported along the micropost array rails from the bottom solution (clear) back into the parallel top solution (blue). Multiple experimental device runs confirmed that this process was repeatable. One caveat to this initial demonstration, however, was that select numbers of microbeads were found to immobilize in the $\alpha_{1}$ section directly preceding the $\alpha_{2}$ trapping areas (Fig. 4c; Fig. 5a, c). This phenomenon necessitated that higher numbers of microbeads be loaded initially in order to account for the expected loss of microbeads. Thus, one focus area for future research would be to adapt the current design to eliminate this issue. Despite this limitation, the overall device performance for the prototype system was not impacted significantly (Fig. 5). Rather, the majority of microbeads were still found to rail-and-trap as designed.

\section{CONCLUSIONS}

Microfluidic processors that are capable of autonomous onchip functionalities are critical to the advancement of lab-on-a-chip applications, such as point-of-care (POC) molecular diagnostics and biomarker screening. This work represents the first demonstration of a microfluidic system that functions autonomously under continuous input flow conditions to both transport suspended microbeads into distinct, parallel flow streams for fluidic mixing, while immobilizing select numbers of microbeads at each step of multi-stage fluidic processes as desired.

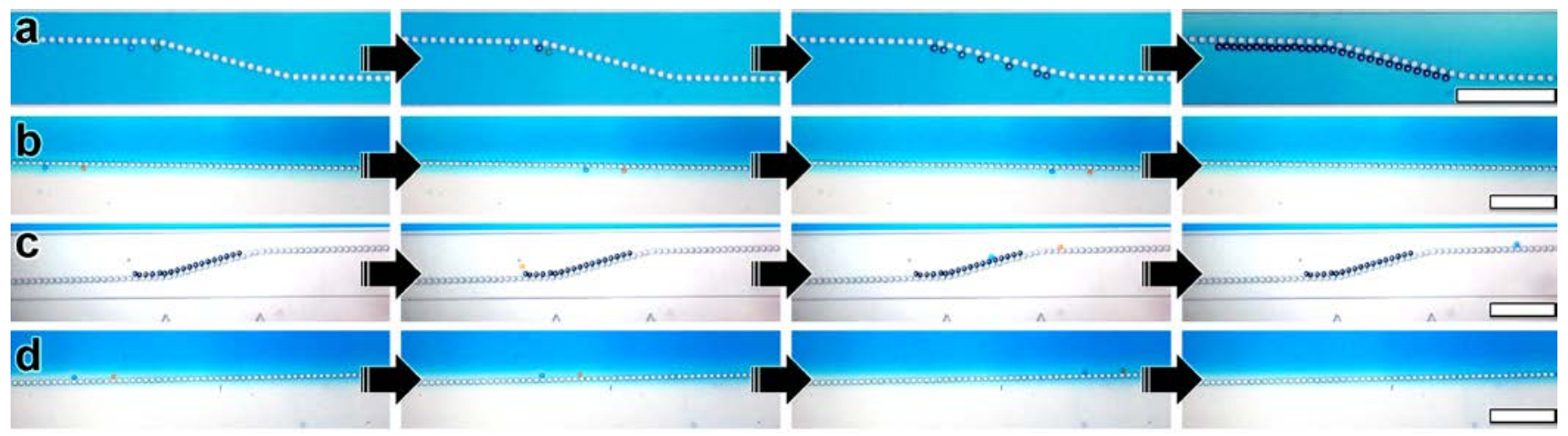

Figure 5: Sequential micrographs of experimental results for microbead dynamics in the microfluidic rail-and-trap system with $\alpha_{1}=1^{\circ}$ and $\alpha_{2}=15^{\circ}$. (a) Microbeads are railed into the trapping area and then immobilized. (b) A microbead is railed from the top solution (blue) into the bottom wash solution (clear). (c) A microbead bypasses previously immobilized microbeads. (d) A microbead is railed from the bottom wash solution (clear) back to the top solution (blue). Suspended streptavidin-coated polystyrene microbeads are 15 um in diameter; 'Orange' and 'blue' microbeads show a singular mobile microbead at two time-points within one second; Scale Bars $=200 \mu$ m. 
Specifically, microposts arrayed in sections at angles of $1^{\circ}, 15^{\circ}$, and $1^{\circ}$ (with respect to the flow direction) were utilized to: (i) guide suspended microbeads into a designated trapping area, (ii) immobilize select numbers of microbeads in the trapping area, and then (iii) rail subsequent microbeads into discrete, adjacent flow streams, respectively. Both theoretical simulations and experimental methods were employed to characterize the presented microfluidic rail-and-trap system. Experimental results revealed that trapping areas with microposts arrayed at lower angles (i.e., $\alpha_{2} \leq 10^{\circ}$ ) were unable to immobilize suspended microbeads within the device, while microposts arrayed at higher angles (i.e., $\alpha_{2} \geq 20^{\circ}$ ) resulted in device failure because subsequent microbeads immobilized on top of previously arrayed beads. In contrast, systems that included trapping areas with microposts arrayed at an angle of $15^{\circ}$ were found to rail-and-trap effectively. One limitation of the presented system was that a select number of microbeads were found to unintentionally immobilize in the railing section directly preceding fully occupied trapping areas. Although future work should focus on modifying the current system to overcome this issue, the overall device performance was not impacted significantly. Thus, the presented microfluidic rail-andtrap system provides a robust single-layer, continuous flow technique for autonomously accomplishing multiple fluidic mixing processes with suspended microbeads (e.g., for chemical and biological assays), with the unique capability of enabling microbead visualization and signal detection during each phase of multi-stage reactions.

\section{ACKNOWLEDGEMENTS}

The authors greatly appreciate the help and support of Kosuke Iwai, Adrienne Higa, and Paul Lum. The authors also thank the members of the Liwei Lin Laboratory, the Biologically-inspired Photonics-Optofluidics-Electronics Technology and Science (BioPOETS) Laboratory, and the Micro Mechanical Methods for Biology $\left(\mathrm{M}^{3} \mathrm{~B}\right)$ Laboratory. This work is supported in part by the DARPA N/MEMS program under the Micro/Nano Fluidics Fundamentals Focus (MF3) center.

\section{REFERENCES}

[1] P. Yager, T. Edwards, E. Fu, K. Helton, K. Nelson, M. R. Tam, and B. H. Weigl, "Microfluidic diagnostic technologies for global public health," Nature, 442, pp. 412-418, (2006).

[2] T. Thorsen, S. J. Maerkl, and S. R. Quake, "Microfluidic large-scale integration," Science, 298, pp. 580-584, (2002).

[3] A. D. Tadmor, E. A. Ottesen, J. R. Leadbetter, and R. Phillips, "Probing Individual Environmental Bacteria for Viruses by Using Microfluidic Digital PCR,” Science, 333, pp. 58-62, (2011).

[4] K. Iwai, W.-H. Tan, H. Ishihara, and S. Takeuchi, "A resettable dynamic microarray device," Biomedical Microdevices, (2011).

[5] W. H. Tan and S. Takeuchi, "A trap-and-release integrated microfluidic system for dynamic microarray applications," Proceedings of the National Academy of Sciences of the United States of America, 104, pp. 1146-1151, (2007).

[6] W. H. Tan and S. Takeuchi, "Dynamic microarray system with gentle retrieval mechanism for cell-encapsulating hydrogel beads," Lab on a Chip, 8, pp. 259-266, (2008).

[7] J. H. Wang, W. Wang, Y. H. Liu, L. B. Duo, L. J. Huang, and X. F. Jiang, "The method of single-nucleotide variations detection using capillary electrophoresis and molecular beacons," Molecular Biology Reports, 36, pp. 1903-1908, (2009).

[8] E. Verpoorte, "Beads and chips: new recipes for analysis,"
Lab on a Chip, 3, pp. 60N-68N, (2003).

[9] R. D. Sochol, B. P. Casavant, M. E. Dueck, L. P. Lee, and L. Lin, "A dynamic bead-based microarray for parallel DNA detection," Journal of Micromechanics and Microengineering, 21, p. 054019, (2011).

[10] N. Tuleuova, C. N. Jones, J. Yan, E. Ramanculov, Y. Yokobayashi, and A. Revzin, "Development of an Aptamer Beacon for Detection of Interferon-Gamma," Analytical Chemistry, 82, pp. 1851-1857, (2010).

[11] S. A. Dunbar, "Applications of Luminex (R) xMAP (TM) technology for rapid, high-throughput multiplexed nucleic acid detection," Clinica Chimica Acta, 363, pp. 71-82, (2006).

[12] F. J. Steemers and K. L. Gunderson, "Whole genome genotyping technologies on the BeadArray (TM) platform," Biotechnology Journal, 2, pp. 41-49, (2007).

[13] D. C. Leslie, C. J. Easley, E. Seker, J. M. Karlinsey, M. Utz, M. R. Begley, and J. P. Landers, "Frequency-specific flow control in microfluidic circuits with passive elastomeric features," Nature Physics, 5, pp. 231-235, (2009).

[14] B. Mosadegh, C.-H. Kuo, Y.-C. Tung, Y.-s. Torisawa, T. Bersano-Begey, H. Tavana, and S. Takayama, "Integrated elastomeric components for autonomous regulation of sequential and oscillatory flow switching in microfluidic devices," Nature Physics, 6, pp. 433-437, (2010).

[15] J. A. Weaver, J. Melin, D. Stark, S. R. Quake, and M. A. Horowitz, "Static control logic for microfluidic devices using pressure-gain valves," Nature Physics, 6, pp. 218-223, (2010).

[16] S. E. Chung, S. A. Lee, J. Kim, and S. Kwon, "Optofluidic encapsulation and manipulation of silicon microchips using image processing based optofluidic maskless lithography and railed microfluidics," Lab on a Chip, 9, pp. 2845-2850, (2009).

[17] S. E. Chung, W. Park, S. Shin, S. A. Lee, and S. Kwon, "Guided and fluidic self-assembly of microstructures using railed microfluidic channels," Nature Materials, 7, pp. 581587, (2008).

[18] P. Abbyad, R. Dangla, A. Alexandrou, and C. N. Baroud, "Rails and anchors: guiding and trapping droplet microreactors in two dimensions," Lab on a Chip, 11, pp. 813-821, (2011).

[19] C. Kantak, S. Beyer, L. Yobas, T. Bansal, and D. Trau, “A 'microfluidic pinball' for on-chip generation of Layer-byLayer polyelectrolyte microcapsules," Lab on a Chip, 11, pp. 1030-1035, (2011).

[20] S. Zhang, L. Yobas, and D. Trau, "A Microfluidic Device for Continuous Flow Layer-by-Layer Encapsulation of Droplets with Polyelectrolytes," presented at The 12th International Conference on Miniaturized Systems for Chemistry and Life Sciences (MicroTAS 2008), San Diego, CA, USA, (2008), pp. 1402-1404.

[21] R. D. Sochol, R. Ruelos, V. Chang, M. E. Dueck, L. P. Lee, and L. Liwei, "Continuous flow layer-by-layer microbead functionalization via a micropost array railing system," presented at The 16th International Solid-State Sensors, Actuators and Microsystems Conference (Transducers 2011), (2011).

[22] R. D. Sochol, L. Lo, R. Ruelos, V. Chang, D. Bahri, K. Iwai, J. C. Lo, M. E. Dueck, L. P. Lee, and L. Lin, "Microfluidic Ping Pong for Cytokine Detection," presented at The IEEE 24th International Conference on Micro Electro Mechanical Systems (MEMS 2011), Cancun, Mexico, (2011).

\section{CONTACT}

*Ryan D. Sochol, tel: +1-410-935-8971; rsochol@gmail.com 


\title{
MICROFLUIDIC BIOFILM OBSERVATION, ANALYSIS AND TREATMENT (MICRO-BOAT) PLATFORM
}

\author{
Y.W. Kim ${ }^{* 1,2}$, M.P. Mosteller ${ }^{* 1}$, M.T. Meyer ${ }^{1,3}$, H. Ben-Yoav ${ }^{1,2}$,W.E. Bentley ${ }^{3}$ and R. Ghodssi, ${ }^{1,2,3}$ \\ ${ }^{1}$ MEMS Sensors and Actuators Laboratory, ${ }^{1}$ Institute for Systems Research \\ ${ }^{2}$ Department of Electrical and Computer Engineering, ${ }^{3}$ Fischell Department of Bioengineering \\ University of Maryland, College Park, MD, USA
}

\begin{abstract}
We present the first demonstration of a novel bacterial biofilm treatment technique showing a $56 \%$ average decrease in bacterial cell viability compared to traditional antibiotic treatments in a Micro-BOAT platform. Integrated linear array charge-coupled devices achieve spatially realized optical density monitoring, correlating to both average biomass and localized biofilm morphology. For on-chip demonstration of biofilm treatment, a unique bioelectric effect using a superpositioned direct and alternating current electric field is applied in the presence of antibiotics. Use of the platform demonstrated successful real-time monitoring of biofilm treatment and validated an on-chip bioelectric effect showing a decrease in both bacterial cell viability and overall biomass.
\end{abstract}

\section{INTRODUCTION}

Bacterial biofilms are complex communities composed of a group of communicating bacteria and an extracellular matrix (ECM) [1]. The presence of the ECM limits molecular diffusion within the biofilm, therefore requiring higher doses of antibiotics for effective treatment [1]. In addition, since bacteria in close proximity can readily exchange genes such as those that promote antibiotic resistance, biofilms typically develop higher resistivity to antibiotics compared to planktonic bacteria [2]. Hence, once biofilms are established, their harmful toxin secretion can lead to severe infections that are difficult to treat, often requiring between 500-5000 times greater concentration of antibiotics compared to bacterial suspensions [3]. High doses of antibiotics are ill-advised, as they increase the risk of harmful side effects and the proliferation of multidrug resistant bacteria. Due to the high doses of antibiotics needed for biofilm treatment and the consequent emergence of multidrug resistant bacteria, a highly efficient biofilm treatment method utilizing low concentrations of antibacterial agents is of great interest.

When biofilms are treated by simultaneously combining antibiotics with electric fields, the synergistic effect results in dramatically improved biofilm treatment efficacy and is known as the bioelectric effect [3-5]. While this method has previously been shown utilizing electric fields exceeding biocompatible limits, recent work in our group has developed a new bioelectric effect capable of efficient biofilm treatment with parameters well suited for clinical applications [4, 7]. The new bioelectric effect utilizes a superpositioned DC and AC electric field based on the potential interactions of both field types in conjunction with low doses of gentamicin below the minimal inhibitory concentration (MIC) [8]. Demonstration of this method in a microsystem utilizing planar electrodes is critical to enabling future in vivo biofilm treatments in infected prosthetics and implants due to the necessary miniaturization of the system. The integration of this technique with microfluidics and optoelectronics enables the demonstration of the bioelectric effect at this scale.

In this work, we have developed a microfluidic biofilm observation, analysis and treatment (Micro-BOAT) platform for real-time spatiotemporal biofilm monitoring and treatment, allowing us to achieve the first demonstration of the enhanced

* Indicates equally contributing first authors bioelectric effect in a micro-scale system. Detection of biofilm growth is achieved via the optical density (OD) properties of the biofilm as its density increases and has been demonstrated previously as a viable metric of biofilm development correlating to bacterial biomass [10]. The spatial resolution of the integrated charge-coupled devices (CCD) enables real-time tracking of localized biofilm morphology in the channel. End-point cell viability studies utilizing fluorescence microscopy further verify efficacy of the biofilm treatment. The use of the Micro-BOAT platform enables characterization of the bioelectric effect in environments representative of in vivo applications using a noninvasive monitoring technique, while simultaneously providing a low cost, high throughput means of treatment evaluation.

\section{METHODS}

\section{Micro-BOAT Platform}

Optical density monitoring of biofilm growth in a microfluidic channel has been previously validated in our group [10]. This device utilized individual commericial-off-the-shelf (COTS) photodiodes to detect biofilm OD at two locations within the microfluidic chamber. Based on this work, the Micro-BOAT platform is an integrated microsystem consisting of both COTS components as well as microfabricated elements to achieve fullchannel analysis of biofilm optical density. CCD devices and supporting electronics are featured in parallel on a $9.5 \mathrm{~cm} \times 8.1 \mathrm{~cm}$ printed circuit board (PCB) to enable six parallel experiments on a single chip. Microfluidic chambers for biofilm growth and electrode structures for applying the enhanced bioelectric effect are constructed using microfabrication techniques on a separate module of the platform. A schematic of the microsystem is provided in figure 1 . The CCDs (TAOS TSL202R) feature $128 \times 1$ linear pixel arrays with individual photodiodes measuring $120 \mu \mathrm{m}$ (H) by $70 \mu \mathrm{m}(\mathrm{W})$ spaced $55 \mu \mathrm{m}$ apart, thereby spanning an overall array length of $1.6 \mathrm{~cm}$. The microfluidic module is comprised of molded polydimethylsiloxane (PDMS) channels bonded to a transparent Pyrex ${ }^{\mathrm{TM}}$ substrate to allow for light transmission to the CCD photopixels.

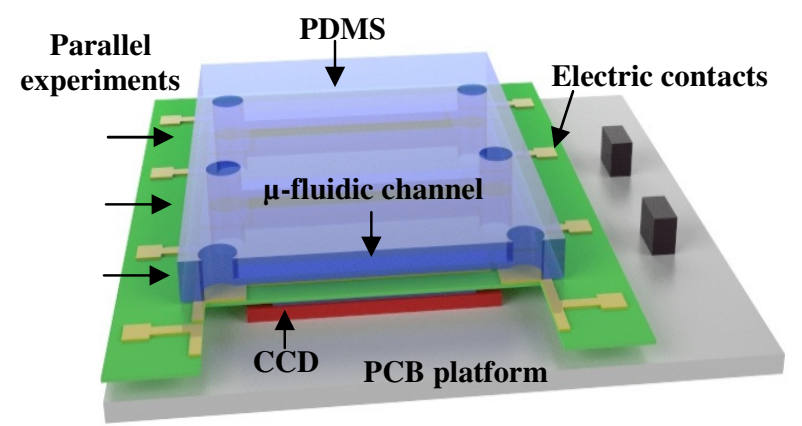

Figure 1: Cross-sectional schematic of the integrated Micro-BOAT platform with CCD components. Electric contacts introduce the enhanced bioelectric effect to biofilms within the microfluidic channel. Hilton Head Island, South Carolina, June 3-7, 2012 
Gold electrodes patterned on the Pyrex $^{\mathrm{TM}}$ substrate provide an electric field for the bioelectric effect while simultaneously limiting peripheral light from disrupting optical density measurements. The electrodes are spaced $2 \mathrm{~mm}$ apart along the full length of the microfluidic channel. The generated electric field is therefore perpendicularly in-plane to the direction of fluid flow in the channel, with the resultant electrophoretic force being orthogonally out-of-plane with respect to the flow direction. The linear photopixel arrays of the CCD/PCB module are aligned to the channels of the microfluidic/electrode module to enable optical density measurement. Uniform illumination of the Micro-BOAT sensors, necessary for proper device calibration, is achieved through a diffused LED light panel tuned to $630 \mathrm{~nm}$ by a polycarbonate lighting gel film in order to match the peak sensitivity of the CCD devices.

The microfluidic device module of the Micro-BOAT platform is fabricated via the process shown in figure 2 .

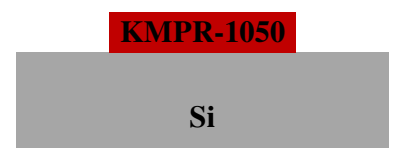

(a) KMPR-1050 patterning (100 $\mu \mathrm{m}$ thick) and silanization

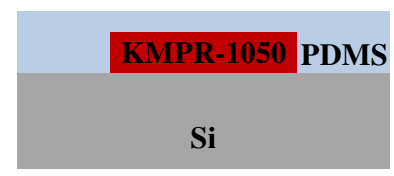

(b) PDMS molding and curing

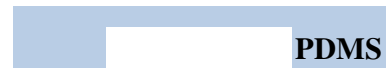

(c) PDMS removal from mold

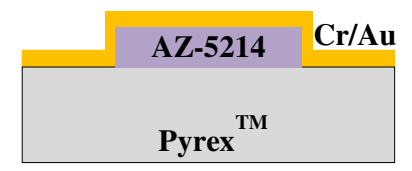

(d) AZ-5214 patterning and $\mathrm{Cr} / \mathrm{Au}$ deposition (Cr 15nm / Au 200nm)

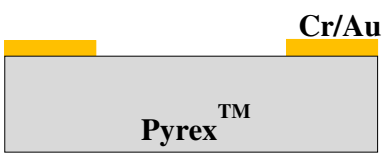

(e) $\mathrm{Cr} / \mathrm{Au}$ liftoff

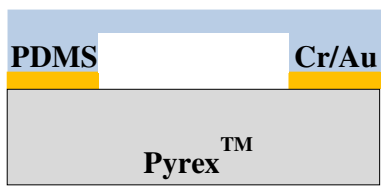

(f) Bonding of PDMS channel
Figure 2: Fabrication process flow for the microfluidic and electrode module of the Micro-BOAT platform. The direction of fluid flow within the micro-channels is shown perpendicular to the page.

A mold of the microfluidic structures is fabricated by patterning $100 \mu \mathrm{m}$-thick KMPR-1050 on a silicon substrate using contact photolithography. To avoid adhesion of PDMS to the channel mold during curing, vapor phase silanization in a vacuum dessicator is performed for one hour using trimethylchlorosilane (Silane M3). Using this technique, a single mold can be reused multiple times without quality reduction in the PDMS channels. PDMS (Sylgard 184, Dow Corning) in a 10:1 ratio is then poured over the mold and cured in a furnace for 20 minutes at $80^{\circ} \mathrm{C}$. After cooling, the PDMS is removed from the mold and cut to fit the Pyrex ${ }^{\text {TM }}$ substrate. To fabricate the gold electrodes for the bioelectric effect, AZ-5214 photoresist is initially patterned on a Pyrex ${ }^{\mathrm{TM}}$ substrate using contact photolithography to achieve a $1.6 \mu \mathrm{m}$ thickness profile. E-beam evaporation of $\mathrm{Cr} / \mathrm{Au}(15 \mathrm{~nm} / 200 \mathrm{~nm})$ and liftoff is then performed to complete the electrode structures. Finally, the PDMS is reversibly bonded to the gold-patterned substrate by applying methanol to the PDMS, then aligning and placing it onto the Pyrex $^{\mathrm{TM}}$ chip. Here, reversible bonding is preferred to allow for disassembly, cleaning, and reuse of the patterned substrate for multiple experiments. The resulting microfluidic chambers measure $100 \mu \mathrm{m}$ deep, $2000 \mu \mathrm{m}$ wide, and
$1.75 \mathrm{~cm}$ long. The microfluidic chamber is integrated with external Tygon tubing via interface ports that are drilled in the PDMS using a $2 \mathrm{~mm}$ diameter dermatological punch.

\section{Bioelectric Effect}

A combinatorial biofilm treatment using electric fields and antibiotics, known as the bioelectric effect, has been demonstrated to improve efficacy of antimicrobial drugs [3-6]. Since the treatment was first demonstrated in 1994 [3], studies have utilized either a direct or alternating current (DC or AC) electric field to achieve the bioelectric effect [3-6]. As electric fields are applied along with antibiotics, the fields can induce a $\mathrm{pH}$ gradient and increase antibiotic permeability into the biofilm [4]. The bioelectric effect has also demonstrated biocidal characteristics with different antibiotics [6]. However, a successful clinical application has not yet been realized due to remaining challenges, including electrolysis of the media as a result of the high electric fields applied for the effect. When the intensity of the electric potential is greater than the limit voltage $(0.8 \mathrm{~V})$, electrolysis of the surrounding media results in the generation of toxic molecules such as hydrogen ions, thus interrupting cell metabolism and resulting in the death of surrounding healthy cells [9].

Recent work in our group has developed an enhanced bioelectric effect capable of efficient biofilm treatment with parameters well-suited for clinical applications [7]. The intensity of the electric field is characterized by using an electric field potential below the electrolysis threshold of the media $(0.8 \mathrm{~V})$, which corresponds to $2 \mathrm{~V} / \mathrm{cm}$ for this platform. By avoiding electrolysis induction, this treatment method can be directly applied to clinical applications. In the experiments, we introduced a superpositioned DC and AC field with gentamicin based on the potential synergistic effects of different electric fields [7]. The DC component of the superpositioned field was characterized to avoid electrolysis induction [7]. The frequency of the AC component was selected based on literature $[4,5]$ and the magnitude of the field matched to the DC component. Thus, the superpositioned electric field was composed of a $1.25 \mathrm{~V} / \mathrm{cm}$ sinusoidal signal at $10 \mathrm{MHz}$ (AC component) with a $1.25 \mathrm{~V} / \mathrm{cm}$ DC offset (DC component). The concentration of gentamicin $(10 \mu \mathrm{g} / \mathrm{mL})$ was selected to be lower than the minimal inhibitory concentration (MIC) $(\approx$ $32 \mu \mathrm{g} / \mathrm{mL}$ ) [8] to minimize the concentration of antibiotics. In doing so, the bioelectric effect has a critical advantage, by deterring the development of antibiotic resistive bacterial strains.

\section{Experimental Procedures}

The demonstration of the superpositioned bioelectric effect in a micro-scale device has been achieved using the Micro-BOAT platform. Bacterial cultures of Escherichia coli BL21 pGFP are grown in Lysogeny Broth (LB) media to an $\mathrm{OD}_{600}$ of $0.25 \mathrm{AU}$. Testing is performed by initially inoculating a microfluidic chamber with bacterial suspension for 2 hours at $37^{\circ} \mathrm{C}$ with no flow to allow microbial attachment to occur on the channel substrate. LB media is then flowed continuously through the channel for 24 hours at $20 \mu \mathrm{L} / \mathrm{h}$ by a syringe pump (Cole-Parmer Instrument Company, USA) to replenish nutrients and foster biofilm growth. For the channel dimensions presented here, this equates to an effective flow velocity of $30 \mu \mathrm{m} / \mathrm{s}$ and has been optimized to provide an environment conducive to biofilm growth. Following the 24 hour growth period, four treatments are performed on the mature biofilms: (1) control (no antibiotic or electric field), (2) only superpositioned electric field, (3) only gentamicin, and (4) enhanced bioelectric effect (superpositioned electric field with gentamicin). Control biofilms are continuously provided LB media at the same flow rate in order to determine steady-state biofilm growth. Biofilms treated with gentamicin, both 
in antibiotic-only experiments and those featuring the enhanced bioelectric effect, are done so with a $10 \mu \mathrm{g} / \mathrm{mL}$ concentration of the drug [7]. The electric field featured in the biofilm experiments was introduced by a function generator (Agilent Inc., USA) [7]. Each of the four biofilm treatments is conducted with 3 experiment samples to demonstrate repeatability of the various assays.

Optical density measurements are taken non-invasively in real-time with respect to both average OD change as well as localized morphology. Measurements are obtained every 8 minutes from the CCD devices to monitor the spatiotemporal development of the bacterial biofilms beginning after the initial two-hour inoculation period. Additionally, bacterial cell viability staining and fluorescence microscopy is used to provide end-point analyses of cell viability within the biofilms to verify treatment efficacy. Since the strain of bacteria used (BL21 pGFP) fluoresces green when the bacteria are metabolically active, only propidium iodide (PI) (Invitrogen Inc., USA) is needed to stain dead bacteria allowing them to fluoresce red. Following biofilm treatments, the microfluidic chamber is initially rinsed with phosphate buffered saline (PBS) solution at a rate of $200 \mu \mathrm{L} / \mathrm{h}$ for one hour to quantify only adherent bacteria. The stain is then supplied at a concentration of $1.5 \mu \mathrm{L}$ PI per $1000 \mu \mathrm{L}$ PBS at a flow rate of $200 \mu \mathrm{L} / \mathrm{h}$ for an additional 2 hours and unabsorbed stain rinsed from the microchannel using PBS at the same flow rate for an additional one hour. The ratios of live bacteria surface coverage with respect to the total biomass surface coverage for different treatments are analyzed using the software package ImageJ (Image J 1.44, USA). For each experiment, both optical density and viability analyses are performed to confirm the OD method for biofilm monitoring

\section{RESULTS AND DISCUSSION \\ Optical Density Monitoring}

Figure 3 presents the average change in biofilm OD along the entire microfluidic channel for each of the four treatments, normalized to their initial optical densities. Before applying treatment, mature E. coli biofilms are formed in the microfludic channels for 24 hours, as described previously. Each treatment is then performed continuously for an additional 24 hours. As shown in figure 3, the control and electric field treatments resulted in overall increases in biofilm optical density, corresponding to bacterial biomass growth. In contrast, those biofilms treated with gentamicin and the enhanced bioelectric effect showed decreases in biomass over the course of the treatments. After treatment, biofilms subject to the bioelectric effect experienced a net $40 \%$ decrease in OD which implied substantial biomass decrease, while the control case demonstrated a $260 \%$ increase in OD as shown in figure 4(a). The opposing trends in OD change between the control and bioelectric effect demonstrate that the combination of a superpositioned electric field in the presence of antibiotics significantly improves biofilm treatment. Additionally, figure 4(b) illustrates the capabilities of the microsystem to monitor localized biofilm morphology in real-time without conventional instruments to get this image such as confocal microscope, a critical advantage of this platform. Spatial understanding of the biological system aids in filtering of signal disturbances due to free-flowing particulates in the microchannel, thus enabling more accurate biofilm analyses.

\section{Biofilm Bacteria Viability}

In addition to the biomass change monitoring by OD measurements, efficacy of each of the biofilm treatments was evaluated using a live/dead cell staining method to verify the treatment efficacies. Dead cells are stained red with propidium iodide, and viable cells appear green due to the expression of GFP.

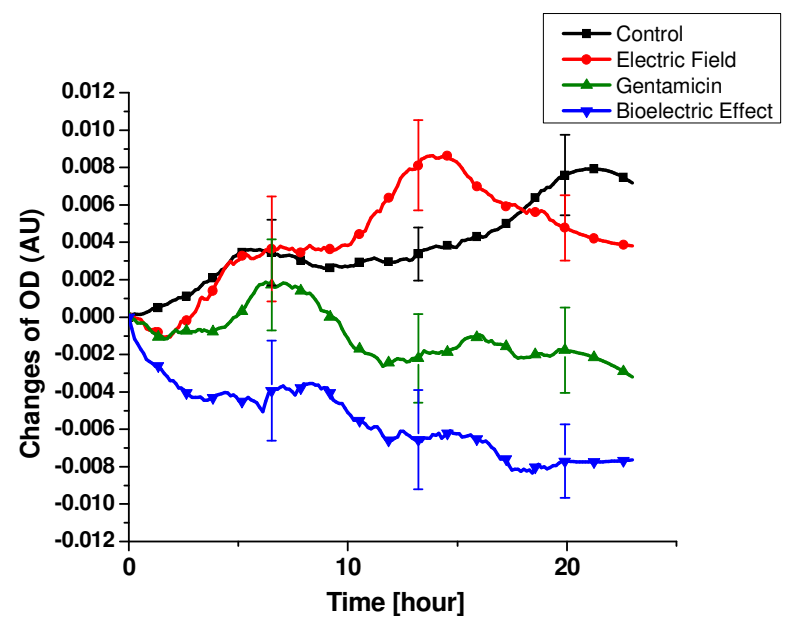

Figure 3: Measured changes in OD during biofilm treatment using the Micro-BOAT platform. Each curve represents the average OD change for three samples with standard deviations shown at representative time points. (a)

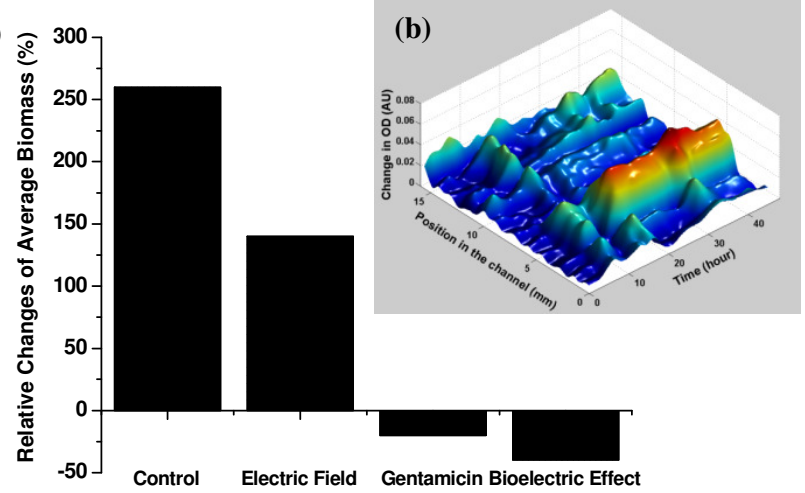

Figure 4: (a) Relative average changes of biomass after treatments in the microfluidic channel. Biofilms treated with the bioelectric effect had a decrease of approximately $40 \%$ in overall biomass while control and electric field treatments resulted in biomass increases of $260 \%$ and $140 \%$ respectively. (b) Surface reconstruction of biofilm morphology changes in the channel over time. Waterfall images are reconstructed using MATLAB based on the $O D$ from the CCDs. The result illustrates the capability to investigate localized biofilm morphology differences.

Figure 5 shows bright field total fluorescence (left column) and only green fluorescence (right column) for each of the four treatments. The total fluorescence images represented the overall biomass in the microfluidic channel. As shown in figure $5(\mathrm{~g})$, biofilms treated with the superpositioned bioelectric effect showed the least amount of bacterial biomass, demonstrating the enhanced effect of this biofilm treatment compared to the other methods. Viable bacteria within the biofilms were inspected using a green fluorescence filter with results shown in the right column of figure 6. Biofilms treated with the bioelectric effect show a lower density of live cells compared to other treatments, thus demonstrating the capability of this method to reduce both overall bacterial biomass as well as cell viability. A quantitative analysis of viable biofilm bacteria was performed using the image processing program ImageJ. The percentage of viable bacteria in each treatment was calculated based on the surface coverage of the green fluorescence images with respect to the coverage of total fluorescent biomass (figure 5). Thus, this approach represents the percentage of viable bacteria within a given biomass, which is clinically important. 

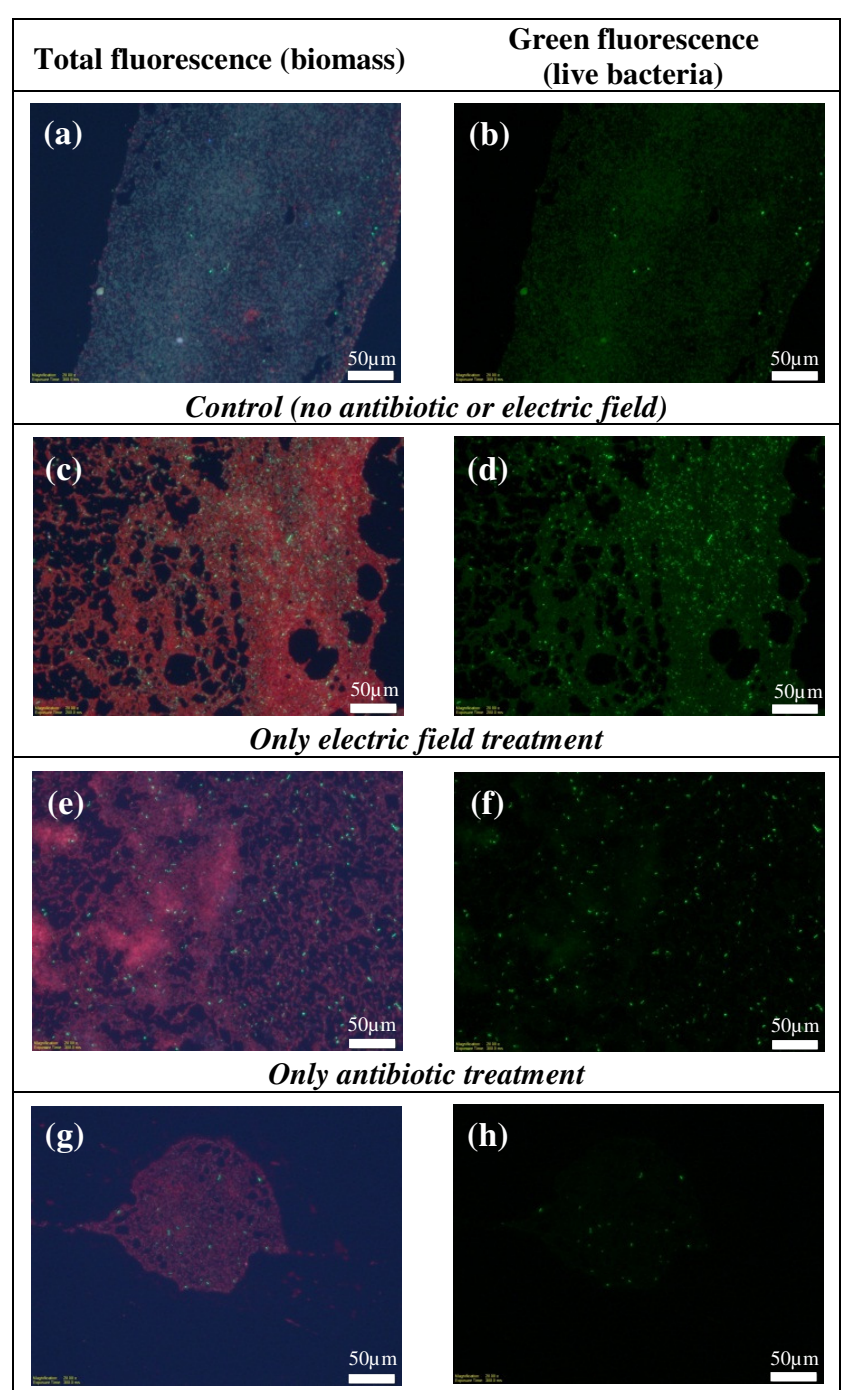

Bioelectric Effect (Electric field with the antibiotic)

Figure 5: Representative fluorescence microscopy images of biofilms in the channel after treatment. Biofilms treated with the bioelectric effect showed less biomass (left column) as well as low live bacterial density (right column).

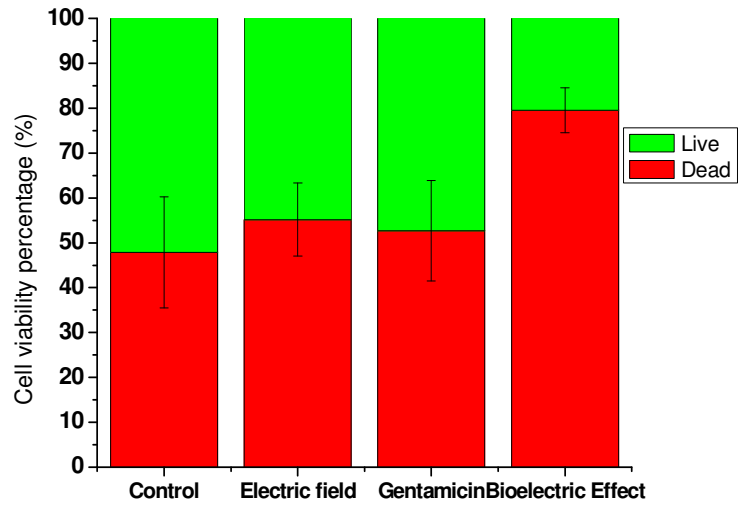

Figure 6: Results of cell viability studies. Using the bioelectric effect treatment, the density of dead cells was increased an average of $56 \%$ compared to the antibiotic treatment.

As shown in figure 6, the bioelectric effect shows $56 \%$ improved treatment efficacy compared to only antibiotic treated biofilms.
Based on the results in figures 3-6, the on-chip bioelectric effect demonstrates not only decreased biomass growth, but also an increased biocidal effect to biofilm bacteria. The effective use of the enhanced biofilm treatment using planar electrodes facilitates the development of a micro-scale biofilm treatment system, allowing this concept to be applied to clinical applications.

\section{CONCLUSIONS}

The successful treatment of biofilms using the bioelectric effect for improved antibiotic efficacy is demonstrated in the Micro-BOAT platform. Using the platform, parallel biofilm treatment experiments were conducted to determine the relative efficacy of each treatment. The demonstration of the bioelectric effect at the micro-scale lays the foundation for future in-vivo bacterial treatment concepts. Furthermore, the unique capability of the CCD-enhanced Micro-BOAT platform to perform temporally and spatially resolved monitoring makes it useful for future drug discovery studies.

\section{ACKNOWLEDGEMENT}

The authors would like to acknowledge the Robert W. Deutsch Foundation and the National Science Foundation Emerging Frontiers in Research and Innovation (EFRI) for financial support. The authors would also like to thank the Maryland Nanocenter and its Fablab for facility support.

\section{REFERENCES}

[1] J.W. Costerton, P.S. Stewart, and E.P. Greenberg, "Bacterial Biofilms: A Common Cause of Persistent Infections," Science, 21, 284 (1999).

[2] R.M. Donlan, "Biofilms and Device-Associated Infections," Emerging Infectious Diseases, 7, 2 (2001).

[3] J.W. Costerton, B. Ellis, K. Lim, F. Johnson, and A.E. Khoury, "Mechanism of Electrical Enhancement of Efficacy of Antibiotics in Killing Biofilm Bacteria," Antimicrob. Agents Chemother, 38 (1994).

[4] J.L. Pozo, M.S. Rouse, and R. Patel, "Bioelectric Effect and Bacterial Biofilms. A Systematic Review", Int. J. Artif. Organs, 30 (2008).

[5] F. Pedarros-Caubet et al., "A Radio Frequency Electric Current Enhances Antibiotic Efficacy against Bacterial Biofilms," Antimicrob. Agents Chemother, 48 (2004).

[6] J.L. del Pozo, M.S. Rouse, J.N. Mandrekar, M.F. Sampedro, J.M. Steckelberg, and R. Patel, "Effect of Electrical Current on the Activities of Antimicrobial Agents against Psuedomonas Aeruginosa, Staphylococcus Aureus, and Staphylococcus Epidermidis Biofilms," Antimicrob. Agents Chemother, 53 (2009).

[7] Y.W. Kim, H. Ben-Yoav, H.C. Wu, W.E. Bentley, and R. Ghodssi, "An Enhanced Superpositioned Bioelectric Effects for Biofilm Treatment," US Provisional Patent Application, September 2011.

[8] S. A. Salmon, J. L. Watts, "Minimum Inhibitory Concentration Determinations for Various Antimicrobial Agents against 1570 Bacterial Isolates from Turkey Poults", Avian Diseases, 44, (2000).

[9] J.O. Bockris and K.N. Reddy, Modern Electrochemistry, Kluwer Academic / Plenum Publishers, New York, 2000.

[10] M.T. Meyer, V. Roy, W.E. Bentley, and R. Ghodssi, "Development and validation of a microfluidic reactor for biofilm monitoring via optical methods," J. Micromech. Microeng., 21, 054023 (2011).

\section{CONTACT}

*R. Ghodssi, tel: +1-301-405-8158; ghodssi@umd.edu 


\section{MICROFLUIDIC DEVICES FOR RAPID LABEL-FREE SEPARATION OF CELLS AND POINT-OF-CARE DIAGNOSTICS

\author{
S. Bose ${ }^{l}$, M.-H. Hollatz ${ }^{l}$, C.-H. Lee ${ }^{l}$, J. M. Karp ${ }^{1,2}$ and R. Karnik ${ }^{l}$ \\ ${ }^{1}$ Massachusetts Institute of Technology, Cambridge, Massachusetts, USA \\ ${ }^{2}$ Harvard Medical School, Boston, Massachusetts, USA
}

\section{ABSTRACT}

Cell sorting serves an important role in clinical diagnosis and biological research. Most of the existing microscale sorting techniques are either non-specific to antigen type or rely on capturing cells making sample recovery difficult. We demonstrate a simple; yet effective technique for isolating cells in an antigen specific manner by using transient interactions of the cell surface antigens with asymmetric receptor patterned surface. Using microfluidic devices incorporating P-selectin patterns we demonstrate separation of HL60 cells from K562 cells. We achieved a sorting purity above $90 \%$ and efficiency greater than $85 \%$ with this system. We further demonstrate the clinical significance of the method by demonstrating single step separation of neutrophils from whole blood. When whole blood is introduced in the device, the granulocyte population gets separated exclusively yielding neutrophils of high purity $(<10 \%$ contamination). To our knowledge, this is the first ever demonstration of continuous label free sorting of neutrophils from whole blood.

\section{INTRODUCTION}

Separation and analysis of cells based on surface markers plays an important role in biological research and clinical diagnosis. Existing microscale cell sorting techniques are either non-specific to antigen type, require expensive accessory equipment for their operation, or capture cells on adhesive surfaces and necessitate special elution procedures for recovering the cells. We have recently demonstrated that transient receptor-ligand interactions that result in cell rolling [1] on a surface under fluid flow can be used to control the flow of cells on a receptorpatterned substrate [2], which paves the way for a new technique for label-free cell separation. Specifically, we found that when a rolling cell encounters a patterned P-selectin receptor edge, it is deflected from its direction of flow and follows the edge [2]. We envision a microfluidic device that would perform label-free separation of cells by rolling on receptor patterned surfaces (Figure $1)$.

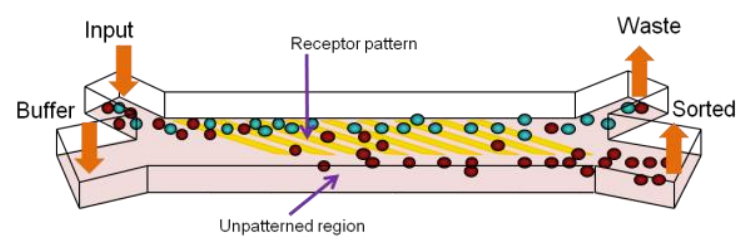

Figure 1: Schematic of the cell separation device. Cells are introduced from one side in a buffer flow. Rolling of cells on patterned receptors causes them to separate laterally from where they can be collected in different channels.

The technique relies on cell rolling - a physiological phenomenon exhibited by several types of cells including leukocytes, hematopoietic stem cells and cancer cells, which is mediated by glycoproteins known as selectins. In our earlier work we studied the dynamics of the rolling of HL60 cells on P-selectin pattern edges and described the optimal condition under which the deflection of cells on these asymmetric patterns can be maximized
[3]. In this work we report design of microfluidic devices for continuous-flow separation of i) HL60 cells from K562 cells and ii) granulocytes from whole blood, based on transient adhesive interactions between PSGL-1 expressed on the surface of HL60 cells and granulocytes with P-selectin receptors patterned in the device. P-selectin was patterned by selective surface chemistry on gold-patterned glass slides. The design allows target cells to settle gravitationally along the length of the channel, after which the cells get displaced laterally into a non-patterned region for quick elution.

\section{EXPERIMENTAL}

Gold coated glass slides (EMF corp.) were patterned using a positive photoresist as an etching mask. The substrates were cleaned with piranha $(3: 1, \mathrm{H} 2 \mathrm{SO} 4: \mathrm{H} 2 \mathrm{O} 2)$ and immersed in $1 \%$ PEG-trimethoxysilane (Gelest) solution in toluene overnight followed by treatment with $1 \mathrm{mM}$ dithio-bis-succinimidyl propionate (DSP, Pierce Biotech.) in DMF for $2 \mathrm{~h}$, after which they were washed in ethanol. Finally the substrates were incubated with P-selectin (R\&D Systems) and stored in 1\% BSA solution until used in the experiments (Figure 2).

The flow cell was molded in PDMS using standard lithography techniques, and consisted of a serpentine channel of rectangular cross-section (100 $\mathrm{m} \mathrm{x} 1 \mathrm{~mm})$ with total length of 20 $\mathrm{cm}$. The device was attached to the substrate after alignment by applying vacuum, creating a reversible bond.

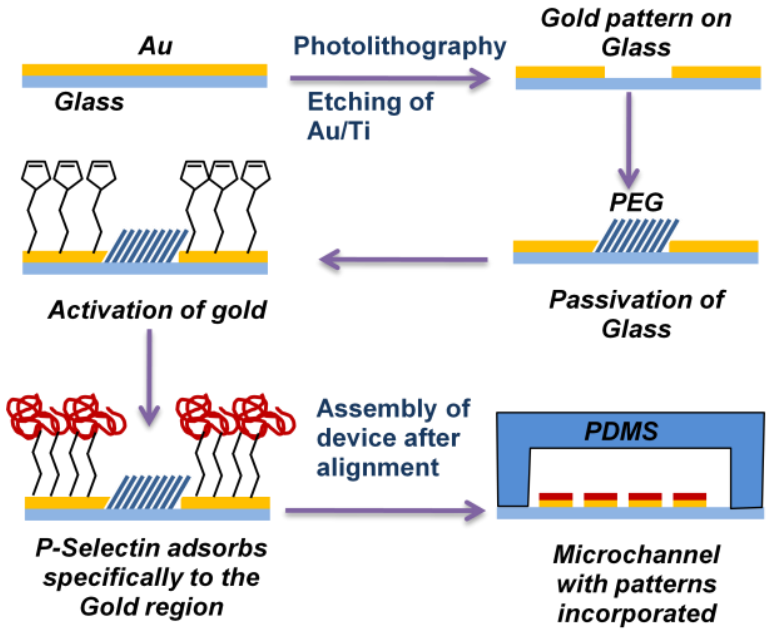

Figure 2: Fabrication process to create receptor pattered substrate through selective chemistry followed by alignment of device.

HL60 cells and K562 cells (ATCC) stained with CellTracker Red and Green respectively, were mixed in 1:1 ratio with a total concentration of 1 million cells per $\mathrm{mL}$, and injected into the device assembly alongside a buffer stream. Flux of the flowing cells was recorded at different locations along the channel length using an epifluorescence microscope. For the separation of granulocytes, anticoagulated human whole blood was used instead of the cell mixture. The samples were analyzed via flow cytometry. 


\section{RESULTS AND DISCUSSION}

When a stream of HL60 and K562 cell mixture was introduced parallel to a buffer stream in 1:9 ratio at a wall shear stress of $0.5 \mathrm{dyn} / \mathrm{cm}^{2}$, we observed that HL60 cells specifically interacted with the patterns, followed the edges of the patterns, and were displaced laterally in a device length-dependent manner (Figure 3a). In contrast, the K562 cells showed a passive spreading across the width of the channel, possibly due to cell-cell hydrodynamic interactions. As evident from Figure $3 \mathrm{a}$, the efficiency and purity of the separation can be tailored depending on the fraction of the flow isolated at the channel exit. Collecting $25 \%$ of the flow from the purified end resulted in a purity of $94 \%$ of the separated HL60 population and a recovery efficiency of $\sim 85 \%$ (Figure $3 b$ ).
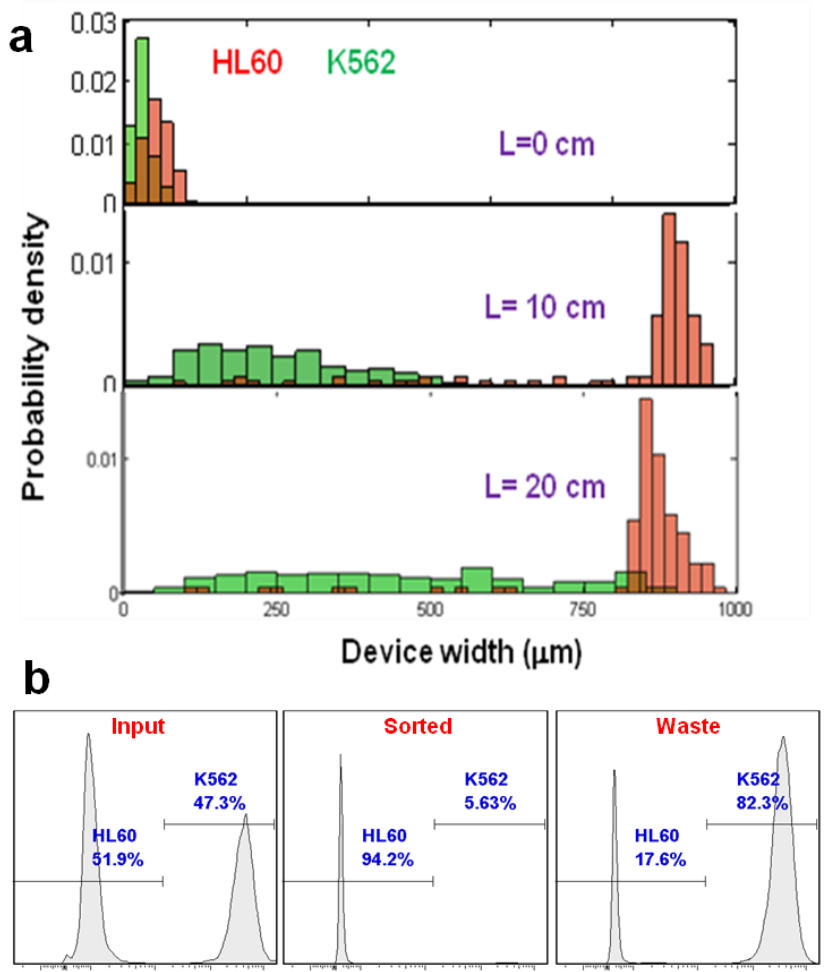

Figure 3: Separation of HL60 cells from K562 cells. (a) Distribution of the flux of HL60 and K562 cells evolving along the length of device. (b) Analysis of the input, sorted and waste samples via flow cytometry.

Next, we infused anticoagulated whole blood into the device and observed that similar to HL60 cells, neutrophils specifically attached and rolled on the P-selectin patterns getting displaced. The composition of the input and sorted sample was found by staining the samples using a pan leukocyte marker (CD45) and granulocyte specific marker (CD66). Amazingly, we found that the sorted sample was highly enriched in granulocytes $(>90 \%)$ as compared to $\sim 47 \%$ in the input (Figure 4 ), and significantly depleted of RBCs. We operated these devices for more than $3 \mathrm{~h}$ and did not find appreciable difference in performance indicating that the surfaces are resistant to bio-fouling.
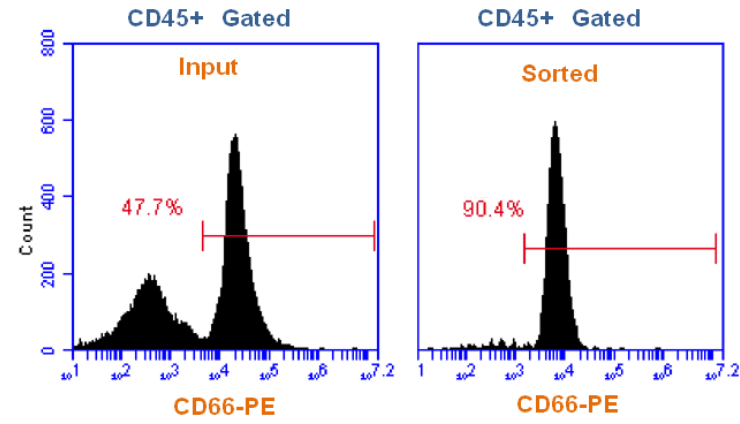

Figure 4: Separation of granulocytes from whole blood. The granulocyte composition of the input and sorted samples were analyzed via flow cytometry after staining by CD45-FITC (pan leukocyte marker) and CD66-PE (granulocyte marker) and are shown in the figure.

\section{CONCLUSION}

In this paper we demonstrated high efficiency, high purity separation of HL60 cells from K562 cells and granulocytes from whole blood by rolling on surfaces patterned with P-selectin in a continuous flow. Further development of this technology could lead to separation and detection of different cell types based on their differential rolling behavior on receptor edges. Although Pselectin was used in this study, other molecules that enable transient cell-surface interactions can potentially be used to target cells based on surface markers. This approach to continuous-flow separation of cells based on surface markers is promising for diagnostic applications and also as a general cell sorting technique.

\section{ACKNOWLEDGEMENTS}

The authors acknowledge the funding from the Deshpande Center for Technological Innovation at MIT (R.K. and J.M.K.), NIH grants HL-095722 and HL-097172 (J.M.K.) and NSF CAREER award 0952493 through the Chemical and Biological Separations program (R.K.) . The devices were fabricated at the Microsystems Technology Laboratory at MIT.

\section{REFERENCES}

[1] S. Q. Chen, R. Alon, R. C. Fuhlbrigge, and T. A. Springer, “ Rolling and transient tethering of leukocytes on antibodies reveal specializations of selectins “, PNAS, pp. 3172, vol 98, 1997.

[2] R. Karnik, S. Hong, H, Zang, Y. Mei, D. Anderson, J. Karp and R. Langer, "Nanomechanical control of cell rolling in two dimensions through surface patterning of receptors", Nano Letters., pp. 1153, vol 8, 2008.

[3] C-H Lee et.al., "Examining the lateral displacement of HL60 cells rolling on asymmetric P-selectin patterns", Langmuir, pp. 240, vol 27(1), 2011.

\section{CONTACT}

*R. Karnik, tel: +1-617-324-1155; karnik@mit.edu 


\title{
MICROSCALE SURFACE ENERGY PROPERTIES FOR ENHANCED SURFACE CONDENSATION AND SAMPLING OF EXHALED BREATH METABOLITES
}

\author{
Hamzeh K. Bardaweel ${ }^{l}$, Konstantin Zamuruyev ${ }^{l}$, Jean-Pierre Delplanque ${ }^{l}$, Nicholas J. Kenyon ${ }^{l}$, \\ Christopher Carron ${ }^{2}$, Oliver Brand ${ }^{2}$, Cristina E. Davis ${ }^{1 *}$ \\ ${ }^{1}$ University of California, Davis, CA, USA \\ ${ }^{2}$ Georgia Institute of Technology, Atlanta, GA, USA
}

\begin{abstract}
A MEMS-based exhaled breath sampler for the capture of both volatile and non-volatile biomarker metabolites is presented. The surface of the sampler is made superhydrophobic to enhance dropwise condensation and patterned with a radially-distributed wettability gradient that routes exhaled breath condensate droplets toward a central collection point. Results show that the surface of the sampler promotes dropwise condensation, and enables a freeenergy-driven mechanism to collect exhaled breath condensate from the surface. Contact angles, measured from the most hydrophobic to the least hydrophobic region, range from 157.0 to 126.8 degrees. Maximum contact angle hysteresis is approximately 1.8 degrees. Droplets deposited on the surface move at a rate of $6 \mathrm{~mm} / \mathrm{sec}$ towards the central collection point.
\end{abstract}

\section{INTRODUCTION}

Exhaled human breath analysis has become attractive as a diagnostic tool for various diseases including cancer, asthma, and respiratory infections [1-3]. One of the technological challenges for collecting exhaled breath samples from subjects is the design of an efficient and reliable breath sampler [4, 5]. Exhaled breath is usually collected with commercially available collection devices, such as the EcoScreen [6] and Rtube ${ }^{\mathrm{TM}}$ [7]. The available breath sampling technologies are power hungry, bulky, and show variability in performance [5]. There is a need for portable, efficient, and robust point-of-care breath samplers. Here we report our efforts toward fabricating a MEMS-based breath sampler for both volatile and non-volatile biomarker metabolite capture.

\section{DESIGN OF SAMPLER SURFACE}

Compared to currently used breath samplers, our MEMSbased sampler enhances the condensation process. It enables a free-energy-driven mechanism to collect exhaled breath condensate from a central point of the surface. The design of the MEMS-based sampler utilizes specifically-tailored superhydrophobic surfaces and a wettability gradient. The surface of the sampler is made superhydrophobic to promote dropwise condensation over filmwise condensation. The dropwise mode is preferable because it allows one order of magnitude larger coefficients of heat transfer between the surface and the exhaled breath gas [8]. The surface of the sampler is also patterned with a spatially-distributed wettability gradient that provides a freeenergy driven mechanism to route exhaled breath condensate droplets toward the collection point. In addition, the wettability gradient contributes to maintaining dropwise condensation on the sampler surface by continuously removing droplets from the surface, and thus freeing prior nucleation sites for new droplets to nucleate.

The wettability of a flat surface is described by Young's equation

$$
\cos \theta_{e}=\frac{\gamma_{S V}-\gamma_{S L}}{\gamma_{L V}}
$$

where $\theta_{e}$ is the equilibrium contact angle and $\gamma_{S V}, \gamma_{S L}$, and $\gamma_{L V}$ are the solid-vapor, solid-liquid, and the liquid-vapor interfacial free energies per unit area, respectively. Wenzel [9] proposed a mathematical model to predict the contact angle of a droplet on a rough surface, given by

$$
\cos \theta_{r}^{W}=r \cos \theta_{e}
$$

where $r$ is a roughness factor defined as the ratio of the actual area of the rough surface to the projected area. Wenzel's model assumes that the liquid completely fills the grooves of the rough surface. Cassie [10] proposed another mathematical model originally developed to describe the contact angle of a droplet on a chemically non-uniform surface, that can also be used to describe the contact angle of a droplet on a rough surface [11]

$$
\cos \theta_{r}^{C}=f_{s}\left(1+\cos \theta_{e}\right)-1
$$

where $f_{s}$ is the ratio of the area of the rough surface contacting the droplet to the projected area. The Cassie model assumes that the liquid is completely suspended by the micro-patterns.

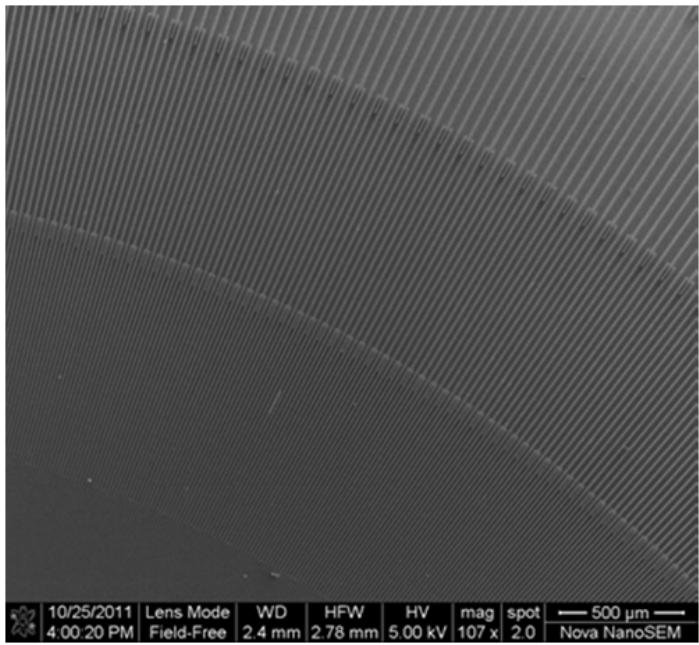

Figure 1. SEM image of the micropatterned sample surface.

Transport of droplets on graded surfaces has been investigated as a method of free-energy-driven droplet movement [12, 13]. Here, the presence of a surface wettability gradient induces the net mass transport of droplets. A droplet tends to move towards the more wettable side if it experiences an imbalance in surface tension forces across its edges, i.e. across the two opposite sides of the liquid-solid contact lines. Wettability gradients can be used to remove droplets from the surface by creating a spatial variation in the physical or chemical properties of the surface.

As the droplet moves along the surface, a resistance force is developed typically attributed to the presence of local defects [11]. The resistance force is measured by contact angle hysteresis 


$$
\left(\cos \theta_{R}-\cos \theta_{A}\right)=\frac{m g(\sin \alpha)}{\omega \gamma_{L V}}
$$

where contact angle hysteresis $\left(\theta_{R}-\theta_{A}\right)$ is defined as the difference between receding $\theta_{R}$ and advancing $\theta_{A}$ contact angles, $g$ is the acceleration of gravity, $\alpha$ is the minimum angle of tilt at which a droplet will spontaneously move, and $m$ and $\omega$ are the mass and width of the droplet base, respectively [14]. Thus a successful, free-energy driven droplet transport on roughened surface requires creating an imbalance in capillary forces across the edges of the droplet, and minimizing contact angle hysteresis.

Dropwise condensation on superhydrophobic surfaces has been investigated thoroughly by many research groups [15-18]. Dropwise condensation is the preferred regime of condensation due to its higher rate of heat transfer, compared to the filmwise condensation regime [8]. However, initiating and maintaining dropwise condensation has proven challenging $[15,18,19]$. While initiating dropwise condensation requires non-wettable surfaces, maintaining dropwise condensation requires continuous removal of small droplets from the surface. During filmwise condensation the condensed liquid tends to wet the surface, and a film of liquid is formed on the surface by droplets coalescence. The presence of the liquid film significantly reduces heat transfer across the surface and, consequently, the rate of condensation decreases as well. In configurations where wettability is poor, the formation of a liquid film is impeded, and the surface is covered with a distribution of droplets with various sizes; this is dropwise condensation. In this regime, heat transfer between the surface and the humid air is only affected where droplets are present. The larger droplets with sizes greater than $10 \mu \mathrm{m}$ [20] have a large thermal resistance and thus behave locally like a liquid film, significantly hindering heat transfer. Very often dropwise condensation turns quickly into filmwise condensation, reducing the effectiveness of heat transfer and thus the condensation process.

\section{SAMPLER SURFACE FABRICATION}

Both chemical composition and physical roughness of the surface contribute to its wettability. In this work, the surface is patterned with micro-fabricated features, namely etched grooves, to increase its hydrophobicity. The wettability gradient is obtained by gradually varying the roughness of the micro-patterned surface. The surface is subsequently coated with a low energy interface material to make it superhydrophobic. Silicon is chosen as the material of the sampler surface because of its high thermal conductivity and its adaptability with microfabrication techniques. The surface is patterned using contact photolithography and deep reactive ion etching is used to etch the characteristic groove/ridge structure. The widths of the ridges and grooves modulate the surface wettability and, thus, are changed radially to establish a wettability gradient in the radial direction of the circular sampler geometry. Polybutadiene (Aldrich, MW= $420000,36 \%$ cis 1,4 addition, 55\% trans 1,4 addition, 9\% 1,2 addition) is then dissolved in toluene $(99.5 \%$ purity) at a concentration of $5 \%(\mathrm{w} / \mathrm{w})$ [21]. The surface of the sampler is spin-coated with the prepared solution and annealed in a vacuum oven to remove entrapped solvent. The coated surface is plasma treated in a vacuum chamber. Lastly the plasma fluorinated polybutadiene film is treated in a vacuum chamber. Further details on plasma fluorination are provided by Woodward et al. [21, 22]. Figure 1 shows a SEM picture of the micro-patterned sampler surface. The surface consists of micropatterned concentric regions. Each region consists of a periodic arrangement of radially patterned ridges and grooves with the and grooves decreasing) toward the center of the surface.

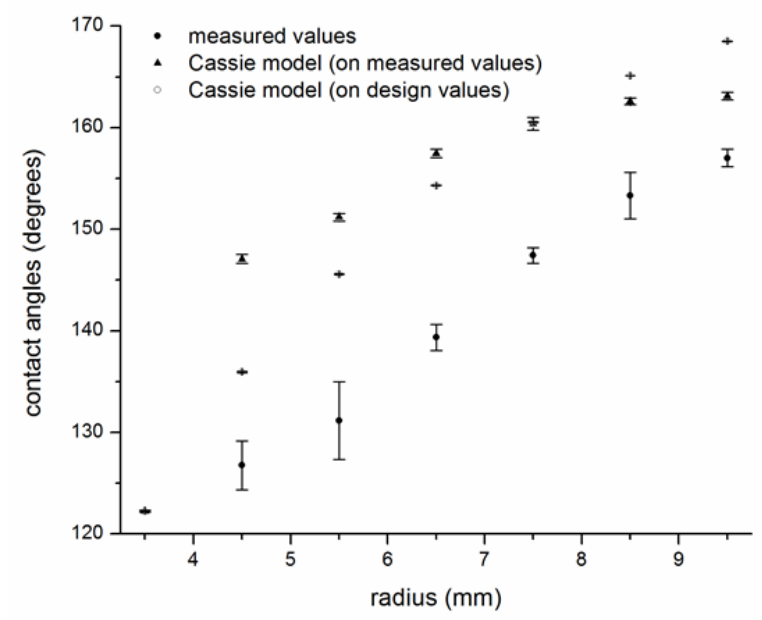

Figure 2. Contact angle distribution on the surface of the sampler.

Thus, the surface roughness is tuned by increasing the number of walls and grooves toward the center. The collection point is an un-patterned circular area at the center, $8 \mathrm{~mm}$ in diameter. The grooves are approximately $60 \mu \mathrm{m}$ deep. The sampler is $20 \mathrm{~mm}$ in diameter and fabricated on $500 \mu \mathrm{m}$ thick silicon wafers.

\section{RESULTS AND DISCUSSION}

In characterizing the surface of the sampler we designed two sets of experiments. The first set of experiments investigated the movement of droplets on the surface of the sampler. In Figure 2, the contact angles of water droplets deposited on the surface of the sampler were measured with a goniometer (Ramé-hart Model 250). Both measured and Cassie-modeled contact angles are shown. Here, contact angles predicted by the Wenzel model (not shown) deviate sharply from measured data. This can be explained if the droplets deposited on the surface of the sampler are lifted by the micro-patterns (Cassie model). The plot shows that contact angles predicted by the Cassie model show similar trends to measured contact angles. In calculating the contact angles predicted by the Cassie model, the actual dimensions of the fabricated grooves/ridges were measured (with an SEM Nova ${ }^{\mathrm{TM}}$ NanoSEM 230) and substituted in the model. The wettability gradient is distributed between the outer edge of the sampler surface, which is the most hydrophobic region, and the center collection point of the sampler, which is the least hydrophobic region. The measured contact angles decreased gradually from 157.0 to 126.7 degrees toward the center of the sampler surface. The maximum measured contact angle hysteresis $\left(\theta_{R}-\theta_{A}\right)$ is approximately 1.8 degrees. Thus, resistance for movement of droplets deposited on the surface is minimized.

Figure 3 shows an example of a video capture sequence of a series of purified water droplets deposited with a syringe on the surface of the sampler. The droplets size ranged between 2-10 $\mu \mathrm{L}$. As the droplet was deposited on the surface it moved along the wettability gradient toward the center of the surface driven by capillarity. To successfully maintain the mobility of the droplet on the surface, the difference in capillary forces must overcome the induced resistance force, described by equation 4 .

In the second set of experiments the behavior of the sampler surface under condensation conditions was investigated. In condensation tests, the surface of the sampler was actively cooled with a thermoelectric element (Custom Thermoelectric, model number: 19811-9L31-02CN1). The average surface temperature was $16{ }^{\circ} \mathrm{C}$. 

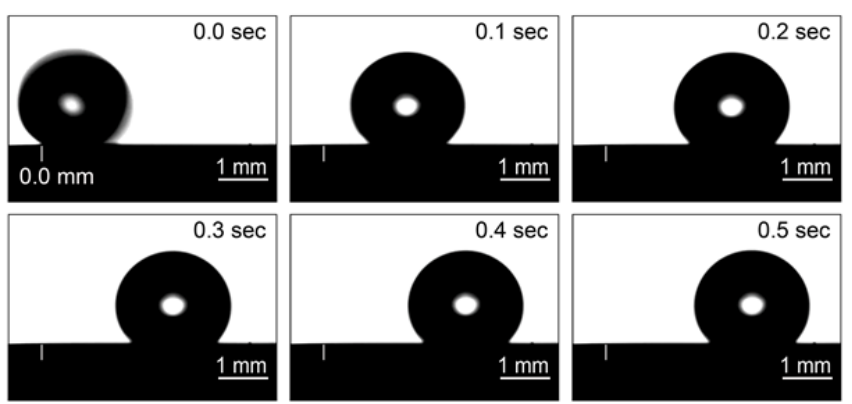

Figure 3. Radial movement of the droplet.

The test was done at atmospheric pressure and ambient temperature $\left(22.2{ }^{\circ} \mathrm{C}\right)$. The relative humidity of the room air was measured at $\phi=48 \%$. Video imaging of the condensation process was captured with a camera (SENTECH) attached to an optical microscope (CARL ZEISS, model number: 452825). The observed condensation process is shown Figure 5. Initially, droplets nucleate on the surface without significant interactions between them. As droplets grow, some of them coalesce and continue to grow in size to form larger droplets. After nucleation and initial growth, droplets merge at their contact lines with other droplets. As droplets coalesce, new nucleation sites are available - allowing for new nuclei to grow. As condensation progresses, droplets continue growing in size. Once they reach a critical size (i.e. the droplets "feel" the wettability gradient across their edges), they move in a directional manner along the wettability gradient.

The ability of the super-hydrophobic micro-patterned surface to promote dropwise condensation is demonstrated in Figure 5. This shows the sampler surface at the end of a condensation test. Two forms of sweeping actions took place during the condensation process. First, we observe "local sweeping" when nucleated droplets sweep the surface as they grow in size and coalesce. Secondly, "radial sweeping" is observed as larger droplets sweep the surface and swallow smaller droplets as they move along the wettability gradient toward the collection point. Radial sweeping clears the smaller droplets from the surface of the sampler, and therefore creates new sites for droplet nucleation and growth.

\section{CONCLUSIONS}

In this article, we have performed initial characterization of a MEMS-based exhaled breath sampler. The surface of the sampler is made superhydrophobic and promotes dropwise condensation over filmwise condensation. The surface of the sampler is also patterned with a spatially-distributed wettability gradient that provides a free-energy driven mechanism to route exhaled breath condensate droplets toward the collection point at the center of the condenser. Maximum measured contact angle hysteresis is approximately 1.8 degrees. Droplets deposited on the surface move in directional manner, toward the center of the sampler. Upon condensation, droplets nucleate and coalesce. As they grow, they move and sweep the surface, swallowing smaller droplets. Large droplets move along the pre-designed wettability gradient, toward the center of the sampler surface. New nucleation sites become continuously available as a result of droplets removal, allowing for new nuclei to grow. Since heat transfer is mostly suppressed at locations where droplets have diameters larger than $10 \mu \mathrm{m}$, this method of removing droplets from the surface during dropwise condensation is effective.
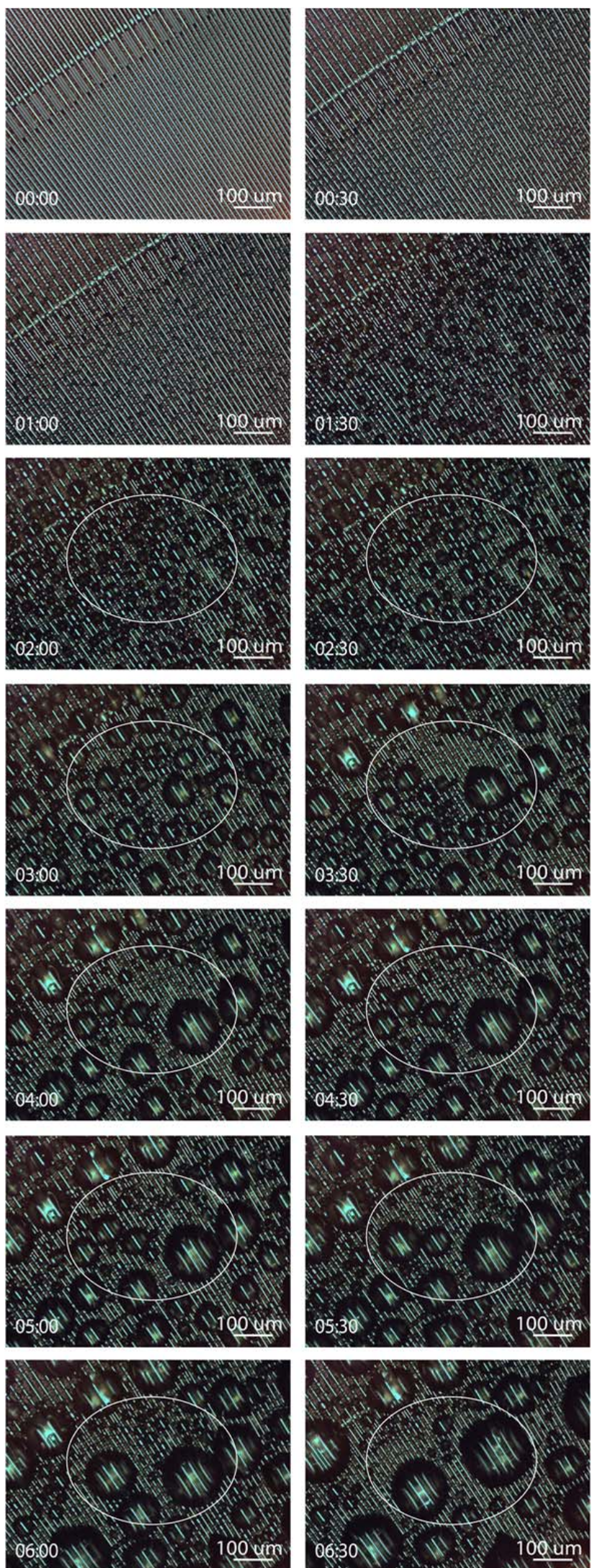

Figure 4. Condensation cycle: droplet nucleation, growth, local sweeping, radial sweeping, new sites are available for nucleation, and a new condensation cycle starts. 


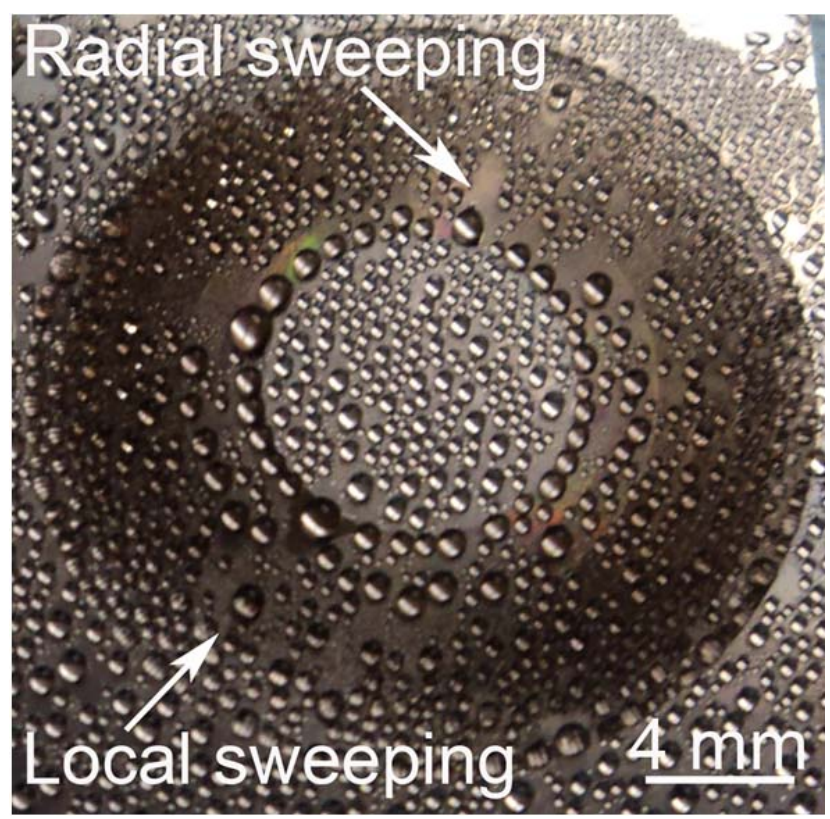

Figure 5. Sampler surface at the end of the condensation test.

\section{REFERENCES:}

[1] Manolis, A., "The diagnostic potential of breath analysis." Clinical Chemistry, 1983. 29(1): p. 5-15.

[2] Kharitonov, S.A. and P.J. Barnes, "Biomarkers of some pulmonary diseases in exhaled breath." Biomarkers, 2002. 7(1): p. 1-32.

[3] Horvath, I., Hunt, J., Barnes, P. J., "Exhaled breath condensate: methodological recommendations and unresolved questions." European Respiratory Journal, 2005. 26(3): p. 523-548.

[4] Liu, J., Conrad, D. H., Chow, S., Tran, V. H., Yates, D. H., Thomas, P. S., "Collection devices influence the constituents of exhaled breath condensate." European Respiratory Journal, 2007. 30(4): p. 807-808.

[5] Rosias, P. P., Robroeks, C. M., Kester, A., den Hartog, G. J., Wodzig, W. K., Rilkers, G. T., Zimmermann, L. J., van Schayck, C. P., Joebsis, Q., Dompeling, E., "Biomarker reproducibility in exhaled breath condensate collected with different condensers." European Respiratory Journal, 2008. 31(5): p. 934-942.

[6] Carpagnano, G. E., Kharitonov, S. A., Foschino-Barbaro, M. P.Resta, O., Gramiccioni, E., Barnes, P. J., "Increased inflammatory markers in the exhaled breath condensate of cigarette smokers." European Respiratory Journal, 2003. 21(4): p. 589-593.

[7] Esther, C. R., Jasin, H. M., Collins, L. B., Swenberg, J. A., Boysen, G., "A mass spectrometric method to simultaneously measure a biomarker and dilution marker in exhaled breath condensate." Rapid Communications in Mass Spectrometry, 2008. 22(5): p. 701-705.

[8] Rose, J.W., "Dropwise condensation theory and experiment: a review." Proceedings of the Institution of Mechanical Engineers Part A-Journal of Power and Energy, 2002. 216(A2): p. 115-128.

[9] Wenzel, R.N., "Surface Roughness and Contact Angle." Journal of Physical and Colloid Chemistry, 1949. 53(9): p. 1466-1467.

[10] Cassie, A.B.D., "Contact Angles." Discussions of the Faraday Society, 1948. 3: p. 11-16.
[11] Berthier, J., "Microdrops and digital microfluidics." Micro \& nano technologies 2008, Norwich, NY: William Andrew Pub. xviii, $441 \mathrm{p}$.

[12] Chaudhury, M.K. and G.M. Whitesides, "How to Make Water Run Uphill." Science, 1992. 256(5063): p. 1539-1541.

[13] Callies, M. and D. Quere, "On water repellency." Soft Matter, 2005. 1(1): p. 55-61.

[14] Furmidge, C.G., "Studies at Phase Interfaces .1. Sliding of Liquid Drops on Solid Surfaces and a Theory for Spray Retention." Journal of Colloid Science, 1962. 17(4): p. 309-\&.

[15] Wier, K.A. and T.J. McCarthy, "Condensation on ultrahydrophobic surfaces and its effect on droplet mobility: Ultrahydrophobic surfaces are not always water repellant." Langmuir, 2006. 22(6): p. 2433-2436.

[16] Dorrer, C. and J. Ruhe, "Condensation and wetting transitions on microstructured ultrahydrophobic surfaces." Langmuir, 2007. 23(7): p. 3820-3824.

[17] Narhe, R.D. and D.A. Beysens, "Nucleation and growth on a superhydrophobic grooved surface." Physical Review Letters, 2004. 93(7).

[18] Boreyko, J.B. and C.-H. Chen, "Self-Propelled Dropwise Condensate on Superhydrophobic Surfaces." Physical Review Letters, 2009. 103(18): p. 184501.

[19] Varanasi, K. K., Hsu, M., Bhate, N., Yang, W. S., Deng, T., "Spatial control in the heterogeneous nucleation of water." Applied Physics Letters, 2009. 95(9).

[20] Graham, C. and P. Griffith, "Drop Size Distributions and Heat-Transfer in Dropwise Condensation." International Journal of Heat and Mass Transfer, 1973. 16(2): p. 337-346.

[21] Woodward, I. S., Schofield, W. C. E., Roucoules, V., Bradley, T. J., Badyal, J. P. S., "Micropatterning of plasma fluorinated super-hydrophobic surfaces." Plasma Chemistry and Plasma Processing, 2006. 26(5): p. 507-516.

[22] Woodward, I., Schofield, W. C. E., Roucoules, V., Badyal, J. P. S., "Super-hydrophobic surfaces produced by plasma fluorination of polybutadiene films." Langmuir, 2003. 19(8): p. $3432-3438$.

\section{CONTACT}

* C.E. Davis, tel: +1 530754 9004; cedavis@ucdavis.edu

\section{ACKNOLWLEDGEMENTS}

This work was generously supported by The Hartwell Foundation [CD, JPD]. Partial support was also provided by the United States Air Force Research Laboratory through contract FA8650-08-C-6832 and UES, Inc. [CD, JPD, OB]. This work was also supported in part by the National Center for Research Resources (NCRR) under Grant UL1RR024146-06 [CD], a component of the National Institutes of Health (NIH). Graduate fellowship support [KZ] was provided by award number P42ES004699 from the National Institute of Environmental Health Sciences (NIEHS).

The contents of this manuscript are solely the responsibility of the authors and do not necessarily represent the official view of the funding agencies. 


\title{
SELF-FORMED, NATURALLY-OPTIMIZED MICROFLUIDIC CHANNELS IN POLYDIMETHYLSILOXANE (PDMS)
}

\author{
Abigail Vinson and Joseph A. Potkay"
}

Advanced Platform Technology Center, Department of Veterans Affairs Medical Center, Cleveland, Ohio, USA

Case Western Reserve University, Cleveland, Ohio, USA

\begin{abstract}
This paper presents initial results for a method to naturally and automatically form microfluidic channels and interconnects through the displacement of a viscous fluid by a gas stream. This new procedure provides an easy means to construct microfluidic networks that automatically and efficiently connect microfluidic channels with low pressure drop and minimal stasis. It is a direct supplement to current microfabrication methods and is in contrast to procedures that utilize complicated construction techniques and complex channel designs. This work demonstrates the concept using PMDS. Fully-formed microfluidic channels created using this approach exhibit round cross-sections, smooth transitions between channels, and achieve the same fluidic resistance as their unmodified counterparts while utilizing $46 \%$ less total channel volume.
\end{abstract}

\section{INTRODUCTION}

Efficient microfluidic channels and routing are critical, and yet remain a challenge, for many lab-on-a-chip systems. In these systems, microfabrication is employed to create microfluidic systems that harness advantageous properties at the micro-scale, such as increased surface-to-volume ratio, reduced sample size, increased transport, or increased surface interactions. However, these systems are often implemented with traditional microfabrication techniques resulting in rectangular cross sections, sharp corners, and abrupt transitions between channels of different dimensions. These properties can be a source of unintended problems. In separation systems such as chromatography columns [1], for example, square cross sections provide dead volume causing peak widening and decreased resolution. Round cross sections are generally preferable. In artificial organ systems [2], non-uniform shear stresses and flow stasis instigate blood coagulation and initiate device failure. For many applications, it is thus advantageous to have channels with round cross sections, rounded corners, smooth transitions between channels of different dimensions, uniform flow distribution and shear stress, minimal stasis, and no turbulence. However, these properties have traditionally been difficult to achieve due to the limitations of lithography-based microfabrication techniques. A method to simply overcome these drawbacks would be highly advantageous, improving microfluidic systems and simplifying the design process.

Routing of fluids to the microfluidic channels is another serious concern. Inefficient fluid distribution networks can use excessive area, create regions of stasis/stagnation, and exhibit flow disturbances, increasing pressure drops. For systems with a large number of microchannels, a branching network is required which efficiently routes fluid from large input and output channels to microchannels where sensing, analysis or modification takes place. In order to address this issue, researchers have attempted to design efficient channel networks or endeavored to mimic biological principles [3] or natural scaling rules, such as Murray's Law [4]. In this paper, instead of attempting to design microfluidic interconnects within the limits of current construction methods, we instead allow nature to automatically optimize their structure.

It has been shown previously [5-7] that when a viscous liquid filling a channel is displaced by a stream of gas, a portion of the liquid remains deposited on the channel walls due to its viscosity. This method can also convert rectangular PDMS cross sections into round ones [7]. In this paper, we extend this technique to produce automatically-optimized, self-formed microfluidic channels and interconnects.

\section{THEORY}

In 1926, Murray investigated the branching of arteries in the human vascular system. He discovered that, in order to minimize biological work, its vessels are connected so that the total flow in the system is carried by a set of vessels whose radii cubed sum to a constant value [4].

$$
\Sigma \mathrm{r}^{3}=\text { constant }
$$

In other words, at each stage of branching, the cube of the radius of a parent vessel equals the sum of the cubes of the daughter vessels [4]. This relationship has additionally been found to apply to other natural branching systems $[8,9]$.

More recently, Murray's law has been generalized to apply to fluidic branching systems with non-Newtonian fluids [10] or those that have other optimality principles [11]. This generalized version of Murray's law replaces the exponent with a constant, C, that is dependent on the properties of the specific system.

$$
\Sigma r^{c}=\text { constant }
$$

In branching systems, it has been generally found that the value of the constant $\mathrm{c}$ has been naturally selected to minimize required work.

\section{DESIGN}

The structure exploited to test this concept is shown in Fig. 1 and is modeled after a generic microfluidic system. That is, in a typical microfluidic system, the fluid is first routed into a large channel with low pressure drop that is large enough to interface

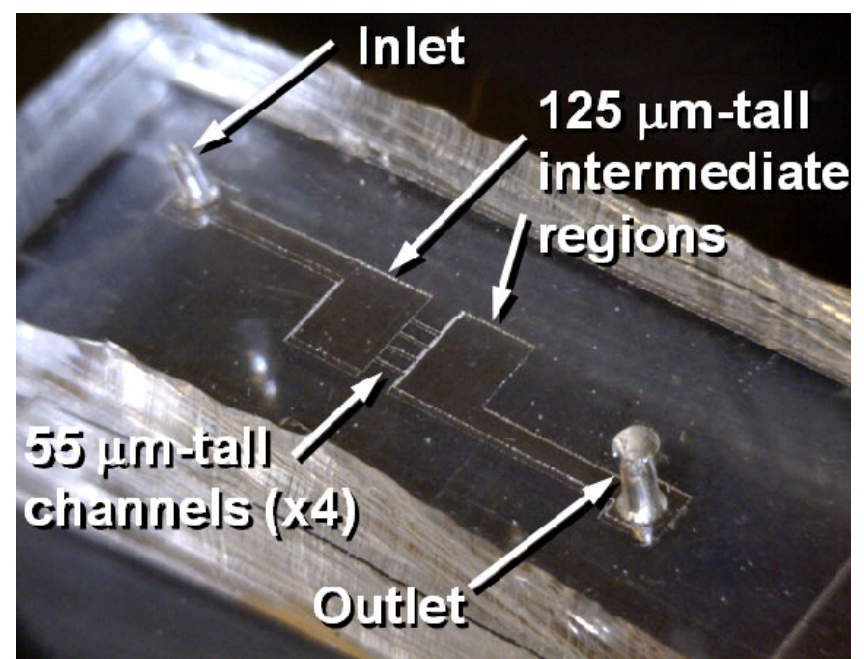

Figure 1: Completed microfluidic test structure. This device is referred to as the "unmodified structure" throughout this paper. Hilton Head Island, South Carolina, June 3-7, 2012 
with the macroscopic world. This large channel then distributes the fluid to anywhere from one to many smaller microfluidic channels that take advantage of properties at the microscale to achieve sensing, analysis, treatment, or separation of components in the fluid, depending on the application. After analysis or modification in the microchannels, the fluid is then routed to a larger channel (with low pressure drop) and can then exit the structure via the device outlet.

This test structure in Fig. 1 was designed to mimic a generic microfluidic system as described above and consists of two 125 $\mu \mathrm{m}$-tall channels connected via four $55 \mu \mathrm{m}$-high center channels. The input and output are separated from the $55 \mu \mathrm{m}$-high center channels through a loosely-bound intermediate region where the auto-optimization occurs. Finally, this test structure was utilized because it exhibits all of the problems associated with standard microfluidic systems constructed using conventional lithographybased techniques: sharp corners, regions of low and high flow, abrupt changes in channel dimension and rectangular cross sections.

\section{FABRICATION}

Microfabrication of the device followed standard PDMS micromolding techniques [12] and proceeded as follows. Masks were created using an image editing program and printed onto a transparency using an Epson Stylus C88+ Inkjet Printer. Microchem SU-8 (2000 series) was utilized to create a mold. Two lithography steps were performed on a 4" silicon wafer to produce both 55 and $125 \mu \mathrm{m}$-tall features on the same mold. A Kinsten KVB-30D UV exposure system was used to cross-link the SU-8. After development in SU-8 Developer, the mold was employed to create PDMS devices. Dow Corning Sylgard 184 was mixed, degassed and poured onto the photoresist mold in a petri dish and cured. After curing, inlet and outlet holes were punched into each device using a $2 \mathrm{~mm}$ diameter needle. The molded PDMS was then bonded to a flat PDMS substrate using oxygen plasma bonding [13] forming an enclosed device. For fluidic access, custom connectors were bonded over the inlet and outlet of the device using a combination of oxygen plasma bonding and PDMS epoxy. A completed device, prior to the attachment of connectors, is shown in Fig. 1.

\section{EXPERIMENTAL}

To begin, the fluidic channels in the structure were completely filled with PDMS using a $10 \mathrm{ml}$ syringe and $5 \mu \mathrm{m}$ syringe filter. The filter removes all large debris and particles which can clog the microchannels during the experiment. Silicone oil (Gelest DMS-T01) can be used to dilute the PDMS and decrease its viscosity [7]. In this work, a 3:1 ratio of PDMS to silicone oil has been employed. A stream of nitrogen gas was then forced through the device at a fixed flow rate using an Omega FMA5508 mass flow controller. At the micro-scale, where gravity and inertial effects are negligible compared to viscous and surface effects, a stable cylindrical gas-liquid interface is formed which minimizes the amount of work required to displace the viscous PDMS inside the device. After steady state was achieved and the gas/liquid interface was stable, channel formation was visually recorded using a BigC Dino-Lite Digital Microscope Camera. The flow rate was then either ramped to investigate higher flow rates or the device was heated to cure the PDMS and fix the microfluidic network. Pressure across the device was monitored using a Honeywell 26PC series pressure sensor and Keithley 2000 digital multimeter. Pressure and flow were converted to fluidic resistance for easier comparison of channel properties at various flow rates.

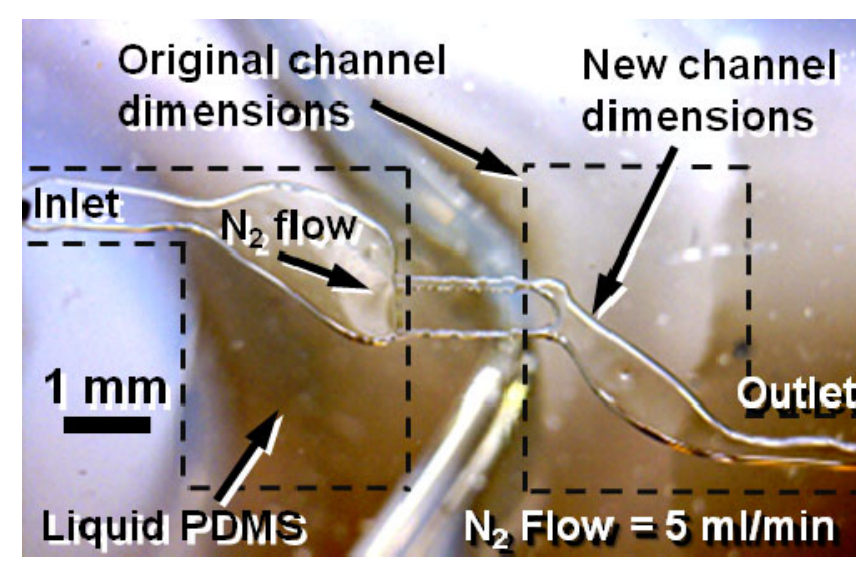

(a)

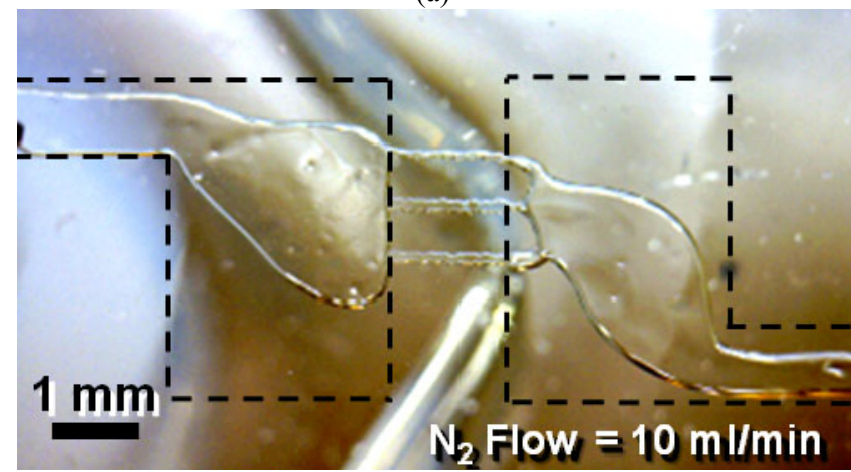

(b)

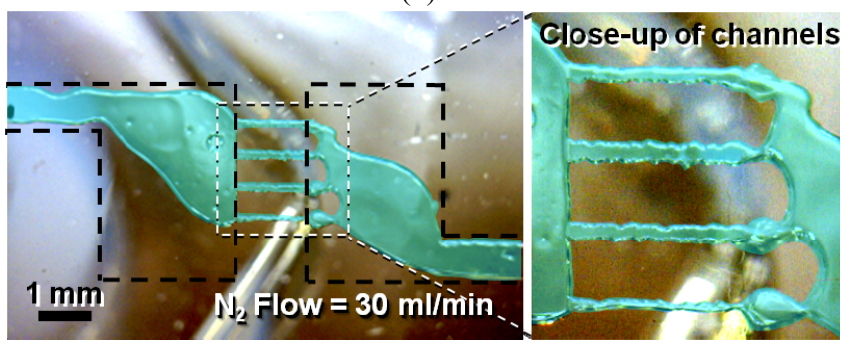

(c)

Figure 2: The structure of the self-formed channels as a function of flow rate. Each of the displayed images has four $55 \mu$-tall channels in the center of the image. However, two of the $55 \mu \mathrm{m}$ channels are not cleared of PDMS until higher flow rates, presumably due to their thinner width (see close-up). In (c), the channel was colored for improved visibility. The unmodified, original channel shape is displayed in Fig. 1.

\section{RESULTS \& DISCUSSION}

Figure 2 shows channel structures formed using this method at three nitrogen flow rates. As can been seen, the process significantly modifies the overall channel structure when comparing unmodified (dotted lines in Fig. 2) to treated channels. In addition, flow channel shapes are interesting and vary considerably from those designed by human researchers. The width of the $125 \mu \mathrm{m}$ inlet and outlet regions and the number of 55 $\mu \mathrm{m}$ channels cleared of PDMS was a function of the $\mathrm{N}_{2}$ flow rate as well as the time exposed to flowing $\mathrm{N}_{2}$ gas. In general, higher flow rates and longer $\mathrm{N}_{2}$ exposure times resulted in larger channel widths and a larger number of clear $55 \mu \mathrm{m}$ channels. All angles within the channel are reduced. Total channel volume for a $\mathrm{N}_{2}$ flow rate of $30 \mathrm{ml} / \mathrm{min}$ decreased by $46 \%$. The $125 \mu \mathrm{m}$-tall inlet region was generally wider than the outlet region suggesting that pressure 

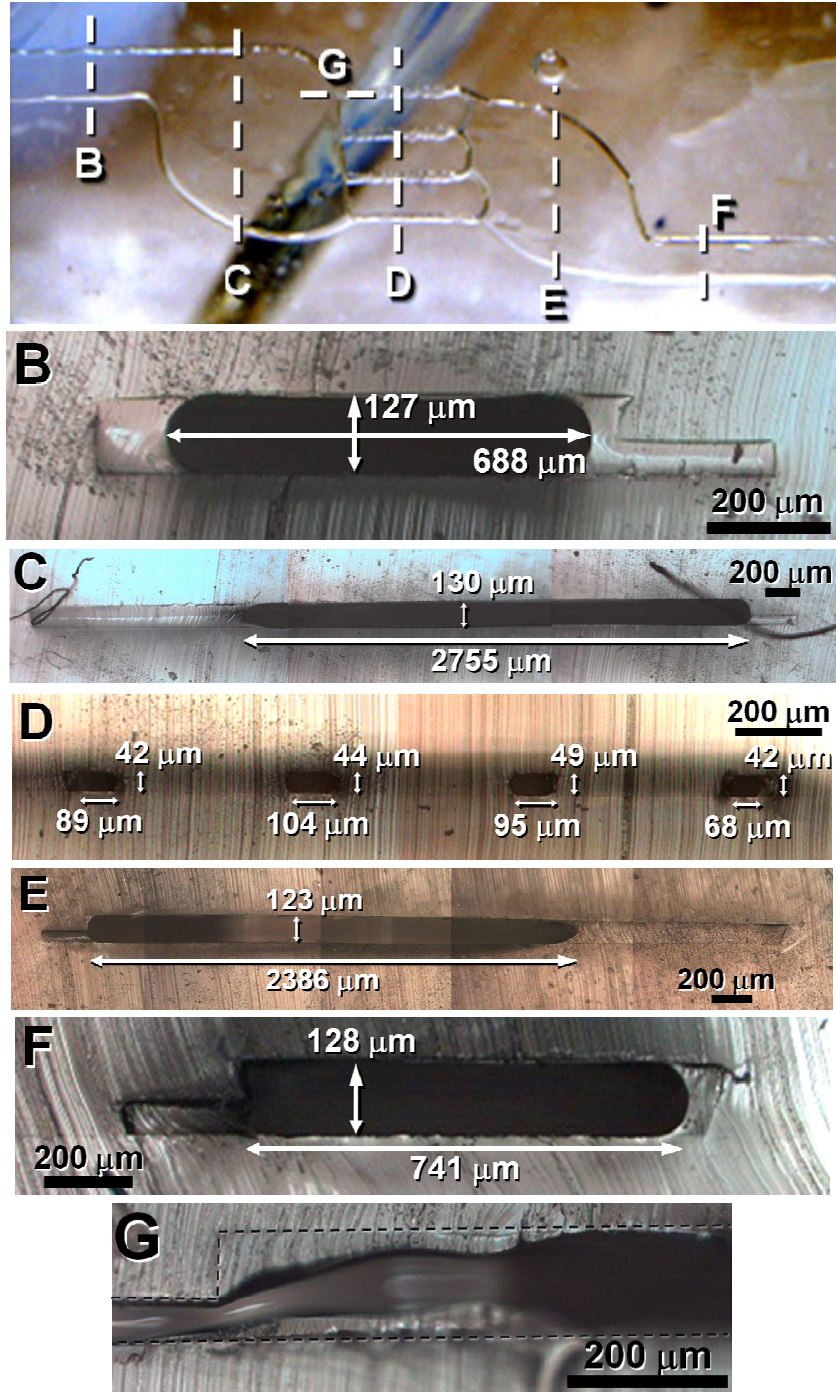

Figure 3: Cross sections of self-formed channels. Letters in bottom images correspond to locations annotated in top image. $G$ shows the transition between the 55 and $125 \mu$ m-high channels. Areas of low flow in the original channel are filled in by PDMS.

has an impact on channel formation. The roughness and differing size of the $55 \mu \mathrm{m}$ channels shows the effect of utilizing inkjet printed masks.

Figure 3 shows cross-sections of the device at various points along the newly-formed channel structure for an $\mathrm{N}_{2}$ flow rate of 30 $\mathrm{ml} / \mathrm{min}$. The process converted all channels from rectangular to round or oval cross sections. Fig. 3a displays another advantage of this method. The smaller rectangular portion of the flow channel on the bottom right is due to mask misalignment and is inefficient for fluid flow due to its smaller height. After processing, this area is completely filled in, eliminating its impact on device performance. Figure $3 \mathrm{f}$ displays a cross section along where the channel transitions from 125 to $55 \mu \mathrm{m}$. Prior to processing, the transition is abrupt and displayed as a dotted line in the figure. After processing, the transition is smooth, resulting in less flow stagnation in the final device.

Figure 4 displays the pressure (Fig. 4a) and fluidic resistance (Fig. 4b) measured across a device during channel formation. Pressure initially increases until nitrogen gas enters the channel structure. It then decreases rapidly and stabilizes as the self-formed

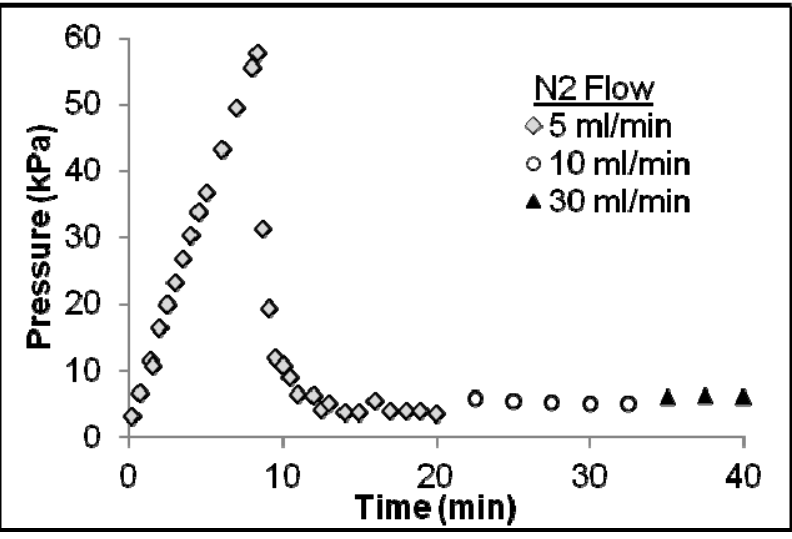

(a)

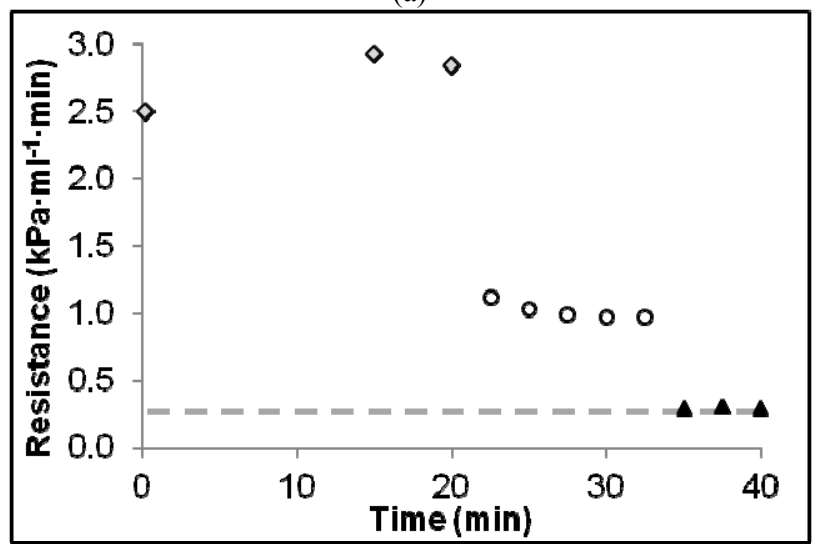

(b)

Figure 4: Pressure (a) and fluidic resistance (b) for a device during the channel formation process. Initial $N_{2}$ flow was set to 5 $\mathrm{ml} / \mathrm{min}$ (open diamonds) until steady state was achieved. It was then raised to $10 \mathrm{ml} / \mathrm{min}$ (open circles) and allowed to settle and then $30 \mathrm{ml} / \mathrm{min}$ (filled triangles). In (b), the y-axis is zoomed-in to show smaller values of fluidic resistance in more detail. At an $\mathrm{N}_{2}$ flow rate of $30 \mathrm{ml} / \mathrm{min}$, the fluidic resistance of the naturally formed channels (filled triangles) is equivalent to that of the unmodified structure (dotted line). The unmodified structure is shown in Fig. 1.

channel structure for that flow rate becomes established. For an $\mathrm{N}_{2}$ flow rate of $30 \mathrm{ml} / \mathrm{min}$, the self-formed fluidic network has the same final fluidic resistance as the initial, uncoated structure. The initial resistance is shown as a dotted line in Fig. 4b.

Table 1 compares channel properties at various points along the microfluidic network as labeled in Fig. 3 and attempts to determine the relationship between flow channels at each level within self-formed channel network. According to Eqn. 2 which describes branching systems that minimize work, the sum of all radii raised to the power $\mathrm{c}$ at each level should be constant. Table 1 thus compares the sum of the hydraulic radii, hydraulic radii squared, hydraulic radii cubed and a term proportional to fluidic conductance to determine the constant c. Since the formed channels are oval in shape and a radius is thus not defined, the channels' hydraulic radii were used in calculations. Only the sum of the hydraulic radii is roughly constant, indicating that the optimality principles for this method differ from those in the natural vasculature $(\mathrm{c}=3)$ [4]. The exact nature of channel structure optimization is still under investigation. 
Table 1: Comparison of self-formed channel properties at various locations annotated in Fig. 3. $\Sigma R_{H}^{3}$ is the sum of all hydraulic radii cubed for all channels at each location in Fig. 3. $W$ and $H$ are the channel width and height. $W H^{3}$ is proportional to hydraulic conductance.

\begin{tabular}{|l|c|c|c|c|c|}
\hline Location & $\boldsymbol{B}$ & $\boldsymbol{C}$ & $\boldsymbol{D}$ & $\boldsymbol{E}$ & $\boldsymbol{F}$ \\
\hline \hline$\Sigma \mathbf{R}_{\mathbf{H}}{ }^{\mathbf{3}}$ & $1.0 \times 10^{6}$ & $1.9 \times 10^{6}$ & $1.0 \times 10^{5}$ & $1.6 \times 10^{6}$ & $1.6 \times 10^{6}$ \\
\hline$\Sigma \mathbf{R}_{\mathbf{H}}{ }^{\mathbf{2}}$ & $1.0 \times 10^{4}$ & $1.5 \times 10^{4}$ & $3.5 \times 10^{3}$ & $1.4 \times 10^{4}$ & $1.4 \times 10^{4}$ \\
\hline $\boldsymbol{\Sigma} \mathbf{R}_{\mathbf{H}}$ & $\mathbf{1 . 1 \times 1 0 ^ { 2 }}$ & $\mathbf{1 . 2 \times 1 0 ^ { 2 }}$ & $\mathbf{1 . 2 \times 1 0 ^ { 2 }}$ & $\mathbf{1 . 2 \times 1 0 ^ { 2 }}$ & $\mathbf{1 . 2 \times 1 0 ^ { 2 }}$ \\
\hline $\boldsymbol{\Sigma} \mathbf{W H}^{\mathbf{3}}$ & $1.2 \times 10^{9}$ & $5.9 \times 10^{9}$ & $2.2 \times 10^{7}$ & $4.3 \times 10^{9}$ & $1.4 \times 10^{9}$ \\
\hline
\end{tabular}

\section{CONCLUSIONS}

In summary, this new method can be used to form naturally optimized microfluidic networks. Furthermore, it converts rectangular cross sections into round ones, eliminates sharp corners, provides smooth transitions between channels of different dimensions, and eliminates channel dead volume. Channel volume decreased by $46 \%$ (compared to the unmodified structure); pressure drop did not increase significantly (within the accuracy of the sensor). Data did not follow Murray's law for natural branching systems. Instead, the sum of hydraulic radii $\left(\mathrm{R}_{\mathrm{H}}\right)$ for all channels at each level was constant. Overall, the process appears to vastly improve the properties of microfluidic channels and routing formed using standard microfabrication techniques.

\section{ACKNOWLEDGEMENTS}

We thank Dr. Ronald Triolo, Dr. Christian Zorman and Mr. Robert (Chris) Roberts for facilities used to complete this work. We additionally thank Mr. Robert (Chris) Roberts for proofreading. This work was supported by Department of Veterans Affairs Rehabilitation Research and Development (VA RR\&D) Grant F7404-R and VA RR\&D Grant C3819C, The Advanced Platform Technology Research Center of Excellence.

\section{REFERENCES}

[1] J.A. Potkay, G.R. Lambertus, R.D. Sacks, and K D. Wise, "A Low Power Pressure- and Temperature-Programmable Micro Gas Chromatography Column," Journal of Microelectromechanical Systems (JMEMS), 16, 5 (2007), pp. 1071-1079.
[2] J.A. Potkay, M. Magnetta, A. Vinson, and B. Cmolik, "Bioinspired, efficient, artificial lung employing air as the ventilating gas," Lab Chip, 11, 17, (2011), pp. 2901 - 2909.

[3] RW. Barber and D.R. Emerson, "Optimal design of microfluidic networks using biologically inspired principles," Microfluidics and Nanofluidics, 4, 3 (2008), pp. 179-191.

[4] C.D. Murray, "The Physiological Principle of Minimum Work: I. The Vascular System and the Cost of Blood Volume", PNAS, 12, 3 (1925), pp. 207-214.

[5] G.I. Taylor, "Deposition of a viscous fluid on the wall of a tube", Journal of Fluid Mechanics, 10, 2 (1961), pp. 161-165.

[6] A. Polynkin, J.F.T. Pittman, and J. Sienz, "Gas displacing liquids from non-circular tubes: high capillary number flow of a shear-thinning liquid", Chemical Engineering Science, 60, 6 (2005), pp. 1591-1602.

[7] M. Abdelgawad, C. Wu, W. Chien, W.R. Geddie, M.A.S. Jewett, and Y. Sun, "A fast and simple method to fabricate circular microchannels in polydimethylsiloxane (PDMS)", Lab on a Chip, 11, 3 (2005), pp. 545-551.

[8] K.A. McCulloh, J.S. Sperry, and F.R. Adler, "Water transport in plants obeys Murray's law," Nature, 421 (2003), pp. 939942.

[9] T.F. Sherman, "On connecting large vessels to small. The meaning of Murray's law." The Journal of General Physiology, 78, 4 (1981), pp. 431-453.

[10] R. Revellin, F. Rousset, D. Baud, and J. Bonjour, "Extension of Murray's law using a non-Newtonian model of blood flow," Theoretical Biology and Medical Modeling, 6, 7 (2009).

[11] G.S. Kassab, "Scaling laws of vascular trees: of form and function," American Journal of Physiology, 290, 2 (2006).

[12] D.C. Duffy, J.C. McDonald, O.J.A. Schueller and G.M. Whitesides, "Rapid prototyping of microfluidic systems in poly(dimethylsiloxane)", Anal. Chem., 70, 23 (1998), pp. 4974-4984.

[13] S. Bhattacharya, A. Datta, J.M. Berg, and S. Gangopadhyay, "Studies on surface wettability of poly(dimethyl) siloxane (PDMS) and glass under oxygen-plasma treatment and correlation with bond strength," JMEMS, 14, 3 (2005), pp. 590-597.

\section{CONTACT}

*J.A. Potkay, joseph.potkay@case.edu; Fax: +1-216-707-6420; ResearcherID: A-1977-2011 


\title{
DELAYED LEIDENFROST PHENOMENA ON BIOTEMPLATED NANOSTRUCTURED SURFACES
}

\author{
S. King, M. Rahman, E. Olceroglu, and M. McCarthy ${ }^{*}$
}

Drexel University, Philadelphia, Pennsylvania, USA

\section{ABSTRACT}

Using the Tobacco mosaic virus (TMV) as a nanoscale template, superhydrophilic surfaces have been fabricated and characterized showing a delay of the Leidenfrost phenomena during droplet cooling. In this work, we show that the Leidenfrost point (LFP) can be delayed by over $100^{\circ} \mathrm{C}$ with the addition of TMV nanostructures, and up to $250^{\circ} \mathrm{C}$ by using dual-scale hierarchical structures. Additionally, increases in heat transfer by nearly three orders of magnitude have been achieved as compared to flat samples at similar temperatures.

\section{INTRODUCTION}

The Leidenfrost point is the temperature at which a liquid will begin film boiling and is commonly seen when a droplet of water is placed in a hot frying pan. The droplet slides around, hovering on a cushion of vapor without wetting the surface. Eliminating this phenomenon is important in high-temperature processes where liquid-to-surface contact is critical, such as replacing nuclear reactor coolant after loss-of-coolant incidents [1]. This Late News Paper reports the first demonstration of delayed Leidenfrost effect using nanostructures that can be applied to a variety of surfaces using a simple yet powerful biotemplating technique.
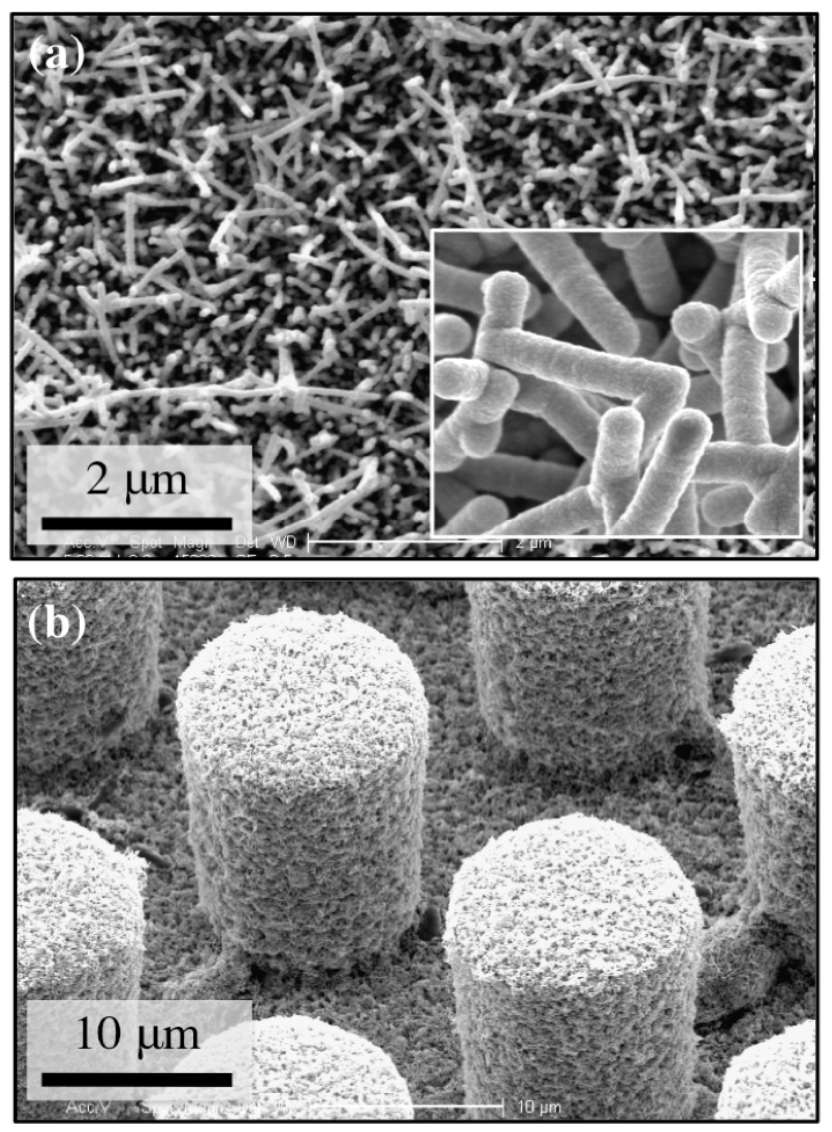

Figure 1: SEM images of (a) nickel-coated TMV nanostructures at two magnifications and (b) hierarchical structures comprised of SU-8 posts coated with TMV nanostructures.

\section{FABRICATION}

By manipulating interfaces with nanostructures, substantial increases in heat transfer can be achieved. However, traditional nanofabrication methods are difficult to integrate with real-world cooling schemes. The current work uses a simple room temperature nanofabrication process, using the self-assembly and mineralization of the TMV to create superhydrophilic surfaces for enhanced heat transfer. By sequentially submerging a surface in three room temperature solutions (TMV suspension, catalyst, and electroless nickel solution) conformal virus-templated nickel coatings are created. A similar fabrication method has been previously reported at Hilton Head 2010 and Transducers 2011 for superhydrophobic water-repellent surfaces [2] and 3D microbattery electrodes [3]. This work reports superhydrophilic surfaces for increased wetting and phase-change heat transfer. This process requires no special equipment and can be easily extended to both micro and macroscale manufacturing. Figure 1 shows SEM images of virus-templated nanostructures, and a hierarchical surface with SU-8 pillars coated with TMV. In this work, flat nickel surfaces (contact angle of $80^{\circ}$ ), TMV nanostructured surfaces $\left(<10^{\circ}\right)$, and hierarchical surfaces with post heights of $20 \mu \mathrm{m}$ and $40 \mu \mathrm{m}\left(\sim 0^{\circ}\right)$ were fabricated and tested.

\section{EXPERIMENTAL RESULTS}

The evaporation of $6 \mu \mathrm{L}$ water droplets on heated surfaces was measured using high-speed imaging to determine the LFP, the temperature corresponding to the longest evaporation time. Figure 2 shows the evaporation time versus surface temperature for each sample. As the surface temperature is increased the evaporation time decreases until the onset of film boiling, at which point the time to evaporate begins to increase for each sample. As compared to the flat sample $\left(\mathrm{LFP}=200^{\circ} \mathrm{C}\right)$, the nanostructures offset LFP by $100^{\circ} \mathrm{C}$, the $20 \mu \mathrm{m}$ hierarchical samples offset LFP by $175^{\circ} \mathrm{C}$, and the $40 \mu \mathrm{m}$ hierarchical samples offset LFP by $250^{\circ} \mathrm{C}$. We believe the posts bridge the vapor film and capillary pressure maintains the wetted surface. Figure 3 shows high-speed imaging of droplet-

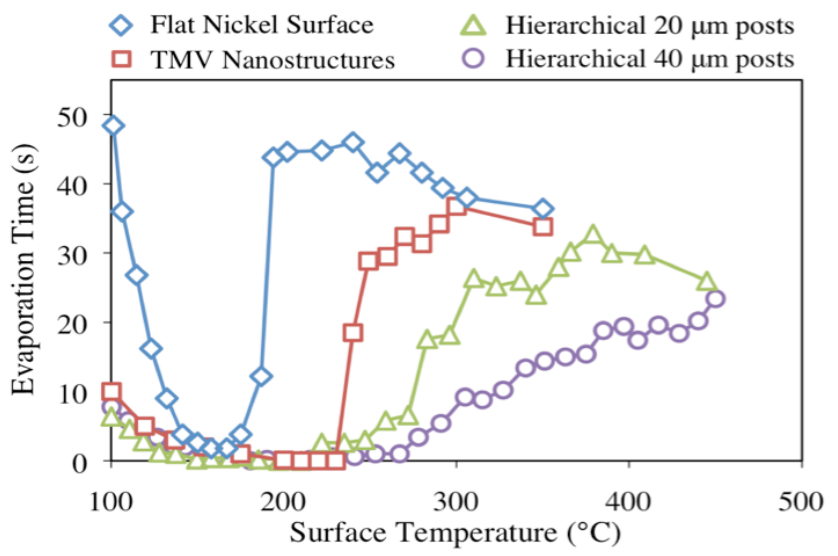

Figure 2: Experimental results of droplet evaporation time as a function of surface temperature for each sample, showing an offset in LFP with the addition of nano and microstructures. The LFP is defined as the surface temperature at the largest evaporation time. 
(a)
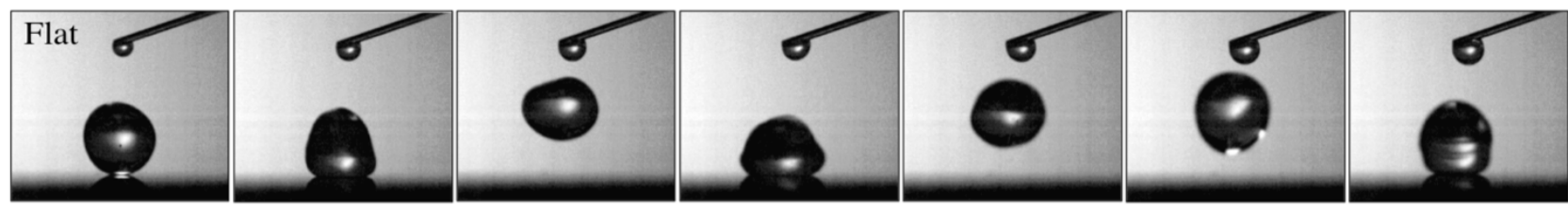

(b)
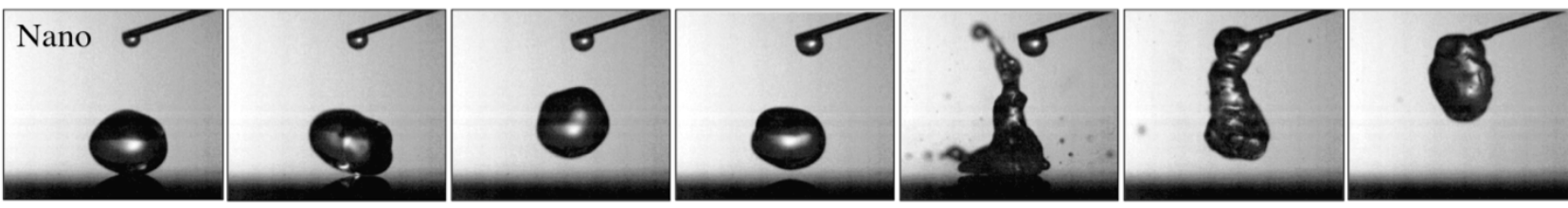

(c)

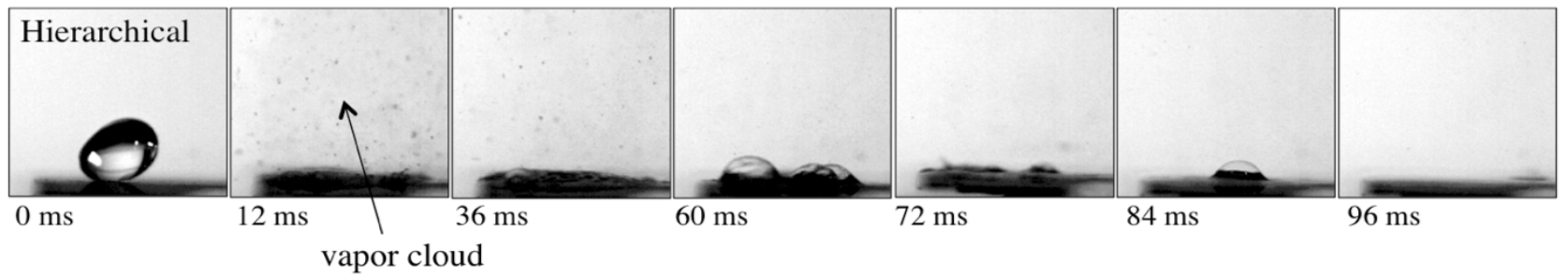

Figure 3: High-speed imaging of droplet-surface interactions at $T_{\text {Surface }}=200^{\circ} \mathrm{C}$ for (a) flat nickel surface, (b) nanostructured TMV surface, and (c) hierarchical surface with $40 \mu \mathrm{m}$ tall posts. It can be seen that the flat surface is undergoing the Leidenfrost phenomena, where the droplet does not wet the hydrophilic surface because of a thin vapor layer generated by the slow evaporation of the droplet. The nanostructured surface shows similar behavior but periodically bridges this vapor layer, leading to faster evaporation and droplet deformation. The hierarchical sample immediately wets and vaporizes the droplet quickly.

interactions at $\mathrm{T}_{\text {Surface }}=200^{\circ} \mathrm{C}$. The flat surface (Fig.3a) undergoes classical Leidenfrost behavior, bouncing on the surface and not wetting. Droplets initially bounce on the nanostructured surface (Fig. $3 \mathrm{~b}$ ), but eventually (at $\mathrm{t}=72 \mathrm{~ms}$ ) bridge the vapor layer causing a violent rupture. This process continues until the droplet has evaporated. Figure $3 \mathrm{c}$ shows the droplet immediately wetting the hierarchical sample and evaporating in under $100 \mathrm{~ms}$.

Knowing the time to evaporate a fixed volume of water, the heat transfer rate per droplet is calculated. Figure 4 shows heat transfer rates above and below the LFP for flat surfaces. It can be seen that at $\mathrm{T}_{\text {Surface }}=100^{\circ} \mathrm{C}$, the structured samples increase heat transfer by a factor of five-to-eight. At $\mathrm{T}_{\text {Surface }}=250^{\circ} \mathrm{C}$, the structured surfaces increase heat transfer by almost three orders of magnitude.

\section{CONCLUSION}

This work demonstrates the feasibility of using biotemplated nanostructures based on the TMV for delaying LFP. Offsets of $250^{\circ} \mathrm{C}$ and a nearly three order of magnitude increase in heat transfer have been demonstrated.

\section{ACKNOWLEDGEMENTS}

Travel support has been generously provided by the Transducer Research Foundation.

\section{REFERENCES}

[1] H. Kim, et al, "On the effect of surface roughness height, wettability, and nanoporosity on Leidenfrost phenomena", Appl. Phys. Lett. 98, 083121 (2011)

[2] M. McCarthy, et al, "Biomimetic Superhydrophobic Surfaces using Viral Nanotemplates for Self-Cleaning and Dropwise Condensation”, Hilton Head '10, June 6-10, Hilton Head, SC.

[3] K. Gerasopoulos, et al, "A Hierarchical Approach for the Fabrication of Three-dimensional Microbattery Electrodes," Transducers 2011, June 5-9, 2011, Beijing, China.

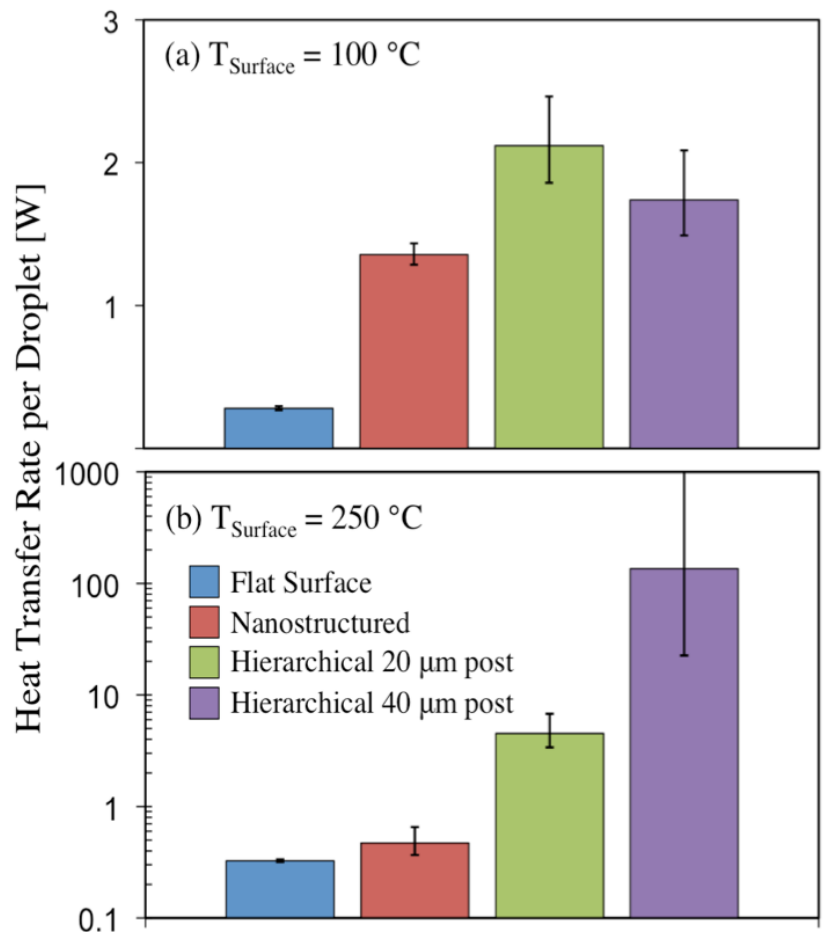

Figure 4: Heat transfer rate per droplet for each sample for (a) $T_{\text {Surface }}=100^{\circ} \mathrm{C}$ and (b) $T_{\text {Surface }}=250^{\circ} \mathrm{C}$ (on a log scale), showing substantial increases in heat transfer with nano/microstructures.

\section{CONTACT}

*Matthew McCarthy, Drexel University, 115 Randell, 3141 Chestnut St., Philadelphia, PA, 19104, mccarthy@coe.drexel.edu, 215-895-2841. 


\title{
HIGH PERFORMANCE CARBON NANOTUBE/SILICON HETEROJUNCTION SOLAR CELLS
}

\author{
Xiaokai Li ${ }^{l}$, Yeonwoong Jung ${ }^{2}$, Yong Sun ${ }^{2}$, Mark A. Reed ${ }^{2}$ and André D. Taylor ${ }^{1 *}$ \\ ${ }^{1}$ Chemical \& Environmental Engineering Department \\ ${ }^{2}$ Electrical Engineering Department \\ Yale University, New Haven, CT 06511 USA
}

\begin{abstract}
A key obstacle for Terra-watt deployment of solar cells is the high cost of materials and fabrication processes. Here, we report the development of an advanced spraying technique for the fabrication of carbon nanotube (CNT) and crystalline silicon ( $\mathrm{Si}$ ) heterojunction solar cells for both planar and dual radial geometries. The nanostructured dual radial p-n junction array solar cells offer a great opportunity to produce efficient solar cells with low cost materials. We have optimized the spray technique and engineered the $\mathrm{CNT} / \mathrm{Si}$ interface for $\mathrm{CNT} / \mathrm{Si}$ solar cell of planar geometry and achieved power conversion efficiency (PCE) of $8.5 \%$ at $\mathrm{AM} 1.5 \mathrm{G}$. For $\mathrm{CNT} / \mathrm{Si}$ solar cell of dual radial geometry, initial test revealed an ideality factor of 3.85 , which suggests that these devices can be further optimized beyond this initial proof of concept.
\end{abstract}

\section{INTRODUCTION}

Photovoltaic (PV) solar cells are attractive candidates for clean and renewable power. Although significant advancements have been made in the energy conversion efficiency, the normalized energy costs of PVs still remain higher than using traditional fossil fuels [1]. Nanostructured radial p-n junction arrays offer a great opportunity to produce efficient solar cells, by decoupling light absorption and charge separation. This unique architecture facilitates improved light scattering and trapping using low cost materials. Previously both Si nanowire (SiNW) (Fig. 1a) [2] and nanopore (Fig. 1b) [3] radial p-n junction architectures have been explored. The cells showed performance in SiNWs and nanopore structures superior to that in planar ones, demonstrating a promising power conversion efficiency (PCE) of 11\% [4]. However, these geometry cells required a series of complicated fabrication processes with some steps at elevated $\left(1000{ }^{\circ} \mathrm{C}\right)$ temperature.

Recent research efforts toward high-efficiency solar cells have been driven by the use of novel materials with superior materials properties coupled with low cost fabrication processes. In this regard, $\mathrm{CNT} / \mathrm{Si}$ heterojunction solar cells satisfy both these criteria with the following advantages: (1) CNTs possess excellent electronic (e.g., high carrier mobility and reduced scattering), structural (e.g., low density and mechanical flexibility), and optical (e.g., wide range of direct band gaps and strong photo-absorption) properties, suitable for photovoltaic components. (2) CNTs can be processed by wet chemical methods at low temperature and are easy to extrinsically $p$-dope (e.g., acid treatments) for controlling photovoltaic properties. (3) heterojunction is formed via a simple/inexpensive solution casting or mechanical transfer of CNTs onto planar Si substrate, which is also compatible with well-established Si-based photovoltaics, presently the dominant technology of the market. (4) CNT/Si solar cells are less susceptible to air/environment-sensitive degradation, which CNT-organic solar cells generally suffer from.

Here we show a new approach that exploits the radial junction of $\mathrm{Si}$ hollow pillar arrays with doped single walled carbon nanotubes. Remarkably, no radial heterojunction devices with

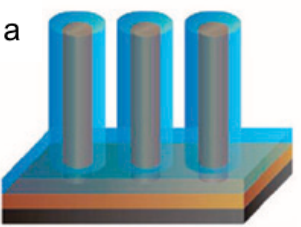

CNT and Si have been reported to date due to technological challenges of conformal wrapping $\mathrm{Si}$ pillars or pores with nanometer scale thin $\mathrm{CNT}$ films. Previously reported $\mathrm{CNT} / \mathrm{Si}$ devices only have the CNT sitting on the top of the pillars leading to low efficiency of $1.29 \%$ [5]. Our results reveal that this new architecture is viable and can be further improved using several processing steps that we previously developed for multifunctional composite materials [6].

Figure 1. (a) Schematic cell design with the single crystalline $n-S i$ $\mathrm{NW}$ core in brown, the polycrystalline $\mathrm{p}$-Si shell in blue. (b) $\mathrm{Si}$ nanohole solar cell with radial $p$-n junctions via thermal phosphorus dopant diffusion. The $n+$ layer is shown in purple bronze, the p-Si substrate in gray. (Figure 1 a and $b$ are adapted from reference [1] and [2] respectively.)

\section{RESULTS AND DISCUSSION}

We developed a low temperature spraying technique that allows the CNTs to wrap around and inside the hollow Si pillars (Fig. 2) forming a CNT/Si dual radial heterojunction (Fig. 3). a
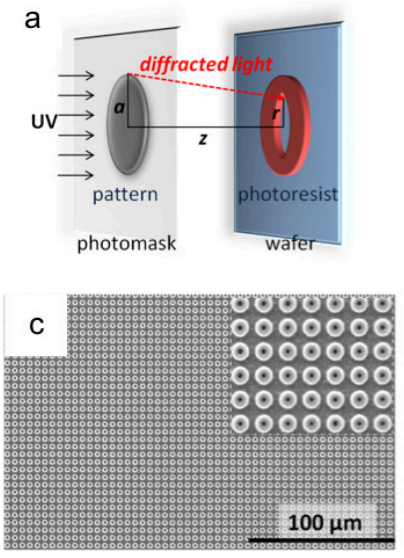

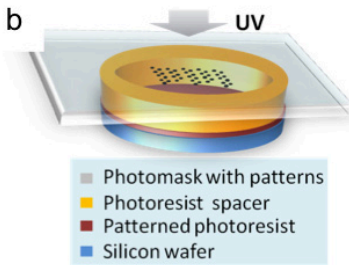

a Silicon wafer

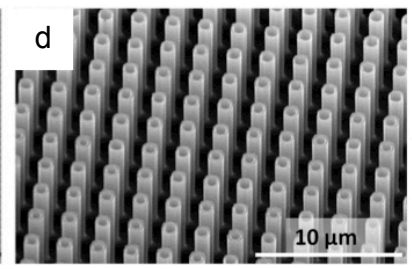

Figure 2. Hollow Si pillar fabrication procedure. (a) Schematic to illustrate the principle of diffraction lithography. (b) Schematic to illustrate the experimental set-up. (c) SEM image to show a large-area uniformity of photoresist rings. (d) SEM image to show an array of vertically aligned hollow Si pillars.

Previously, CNTs have been sprayed on flat $\mathrm{Si}$ wafers to make CNT/Si solar cells of flat geometry with a 4\% PCE [7]. Using our advanced spraying techniques, we demonstrate an $8.5 \%$ Hilton Head Island, South Carolina, June 3-7, 2012 
PCE at AM1.5 (Fig. 4a). Based on this success, we have optimized the hollow pillar structures and increased the interfacial area $(10 \mathrm{X})$ over the flat geometry $(10 \mu \mathrm{m}$ tall, $0.3 \mu \mathrm{m}$ and $1 \mu \mathrm{m}$ inner and outer diameter respectively with $1 \mu \mathrm{m}$ spacing). Initial results show an ideality factor of 3.68 (Fig. 4b).

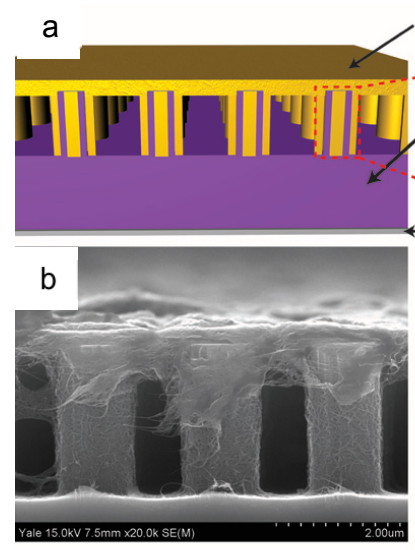

Side View
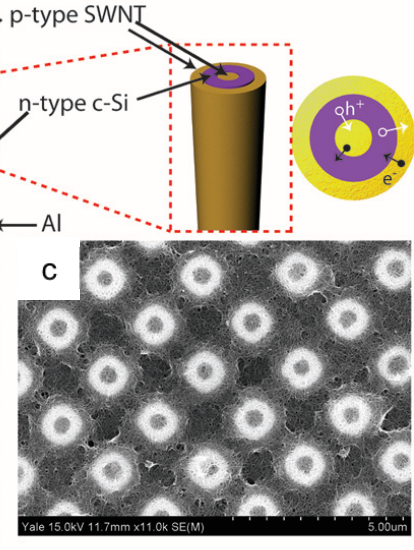

Top View
Figure 3. CNT/ Si dual radial heterojunction solar cell. (a) illustration of the heterojuntion and SEM picture of (b) side view and (c) top view.

At present, the ideality factor (3.68 in Fig. 4b) of the dual radial CNT/Si solar cell is not as high as that (1.95 in Fig. 4a) of the planar CNT/Si solar cell. This high ideality factor suggests that the interface states of heterojunctions need to be further improved since the bulk properties of used CNT and Si are identical. The $\mathrm{CNT}$ in the dual radial geometry cell is interfaced with top-down dry-etched $\mathrm{Si}$, which inherently possesses large defects on the surface, while the planar cell is made of CNT interfaced with the pristine, single-crystalline Si surface. Un-optimized interfaces lead to large carrier recombination, short carrier lifetime, and thus a high ideality factor. Efforts are presently being made to optimize the interfacial properties toward suppressed carrier recombination, e.g., better adhesion between $\mathrm{CNT}$ and $\mathrm{Si}$, improving the surface crystallinities of $\mathrm{Si}$ tubes by utilizing various surface passivation/etching techniques. Nevertheless, one noticeable advantage of this dual radial heterojunction solar cell is its large optical absorption due to greatly increased interfacial areas over either conventional planar cells or dual radial heterojunction cells made of solid pillars without inner holes. This benefit is believed to be significant enough to compensate for the high ideality factor, thus leading to a higher overall efficiency.

We can control the feature sizes of vertically aligned hollow Si pillars using our recently developed single step diffraction optical lithography [8]. (Fig. 2) This simple and well-controlled method relies on the diffraction of UV light from opaque patterns on a photomask, and utilizes the central diffraction maximum, which forms behind the patterns. This single-step lithographic approach doesn't require any extra cost over conventional optical lithographies since the hollowness of the photoresist is formed through a spontaneous diffraction effect, which is also highly controllable without any need for extra experimental apparatus. Moreover, this method can fabricate sub-wavelength features on a large-scale, e.g., the radial thickness of our Si tubes are down below $\sim 100 \mathrm{~nm}$ which are $<\sim 1 / 4$ of the used wavelength, which conventional optical lithographies can hardly achieve. Other non-optical (e.g., e-beam lithography) or alternative optical (e.g., interference) lithographies capable of sub-wavelength feature fabrication are generally very costly and require a series of fabrication process and experimental apparatus, unlike ours.
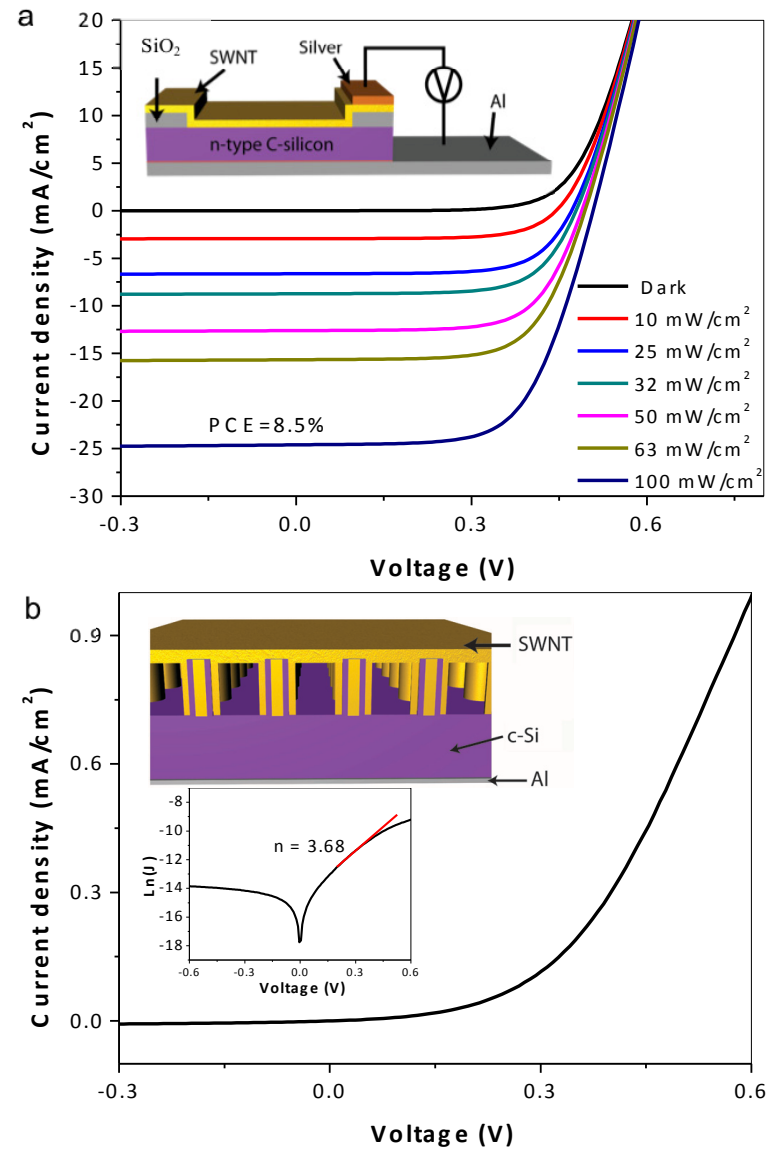

Figure 4. CNT/Si heterojunction solar cell. (a) J-V curve of flat heterojunction. The inset illustrates the flat heterojuntion. The ideality factor of the device is 1.95. (b) Dark J-V for CNT/Si dual radial heterojuntion. The ideality factor of the device is 3.68 .

Fig. 2a illustrates the experimental arrangement for the case of diffraction from the circular disk on a transparent photomask with an accurately controlled separation $\mathrm{z}$ introduced between the photomask and a photoresist-coated wafer. Consequently, light diffracted behind the disk $\mathrm{z}$ is selectively chosen to expose the photoresist, forming a bright spot in the center of the shadow of the disk. Fig. 2b shows the schematic experimental set-up where the separation $\mathrm{z}$ was accomplished by an intentionally created photoresist spacer attached to the photomask. Fig. 2c illustrates an SEM image of a large-area array of photoresist rings. Fig. 2d shows the resultant hollow Si pillar array after reactive ion etching process.

\section{CONCLUSIONS}

In summary, we have described an ordered dual radial heterojunction structure from a low temperature solution process, which promises higher light absorption, better carrier separation and collection, and higher solar cell efficiency.

\section{ACKNOWLEDGEMENTS}

X.L. and A.D.T. acknowledge financial support by the SOLAR program of the National Science Foundation under DMR-0934520. 


\section{REFERENCES}

[1] N.S. Lewis, "Toward Cost-Effective Solar Energy Use", Science, 798, 315 (2007).

[2] E.C. Garnett, P. Yang, "Silicon Nanowire Radial P-N Junction Solar Cells", Journal of the American Chemical Society, 9224, 130 (2008).

[3] K.Q. Peng, X. Wang, L. Li, X.L. Wu, S.T. Lee, "High-Performance Silicon Nanohole Solar Cells", Journal of the American Chemical Society, 6872, 132 (2010).

[4] D.R. Kim, C.H. Lee, P.M. Rao, et al., "Hybrid Si Microwire and Planar Solar Cells: Passivation and Characterization", Nano Letters, 2704, 11 (2011).

[5] Q. Shu, J. Wei, K. Wang, et al., "Hybrid Heterojunction and Photoelectrochemistry Solar Cell Based on Silicon Nanowires and Double-Walled Carbon Nanotubes", Nano Letters, 4338, 9 (2009).
[6] X. Li, F. Gittleson, M. Carmo, R.C. Sekol, A.D. Taylor, "Scalable Fabrication of Multifunctional Freestanding Carbon Nanotube/Polymer Composite Thin Films for Energy Conversion", ACS Nano, 1347, 6 (2012).

[7] Z. Li, V.P. Kunets, V. Saini, et al., "Light-Harvesting Using High Density p-type Single Wall Carbon Nanotube/n-type Silicon Heterojunctions", Acs Nano, 1407, 3 (2009).

[8] Y. Jung, A. Vacic, Y. Sun, E. Hadjimichael, M. Reed,"Mapping of Near Field Light and Fabrication of Complex Nanopatterns by Diffraction Lithography", Nanotechnology, 045301, 23 (2012).

\section{CONTACT}

*André D. Taylor, tel: (203) 432-2217; andre.taylor@yale.edu 


\title{
LOW COST LOCALIZED BONDING OF PMMA MICROFLUIDICS USING MICROWAVE SUSCEPTORS
}

\author{
A. Toossi ${ }^{1}$, D. Sameoto ${ }^{2}$, and M. Daneshmand ${ }^{1}$
}

${ }^{1}$ Electrical and Computer Engineering Department, University of Alberta, Edmonton, Alberta, Canada

${ }^{2}$ Mechanical Engineering Department, University of Alberta, Edmonton, Alberta, Canada

\begin{abstract}
A novel, low cost localized heating technique is demonstrated for bonding of thermoplastic microfluidic devices. In this technique, low-cost widely accessible commercial microwave ovens are used as the primary energy source. Microwave susceptors made from $15 \mathrm{~nm}$ thick gold are designed using our recently developed models and used as localized heating elements. Using this technique, polymethylmethacrylate (PMMA) microfluidic channels are bonded and sealed in less than 45 seconds of applied microwave power.
\end{abstract}

\section{INTRODUCTION}

PMMA is one of the most common thermoplastics used in fabrication of microfluidics due to its optical transparency, chemical compatibility, relatively low price and wide accessibility [1]. One of the biggest challenges in fabrication of microfluidics is achieving high yield bonding processes with minimum damage to the channels. For bonding thermoplastics, it is preferable to have the interface heated selectively and efficiently so as to minimize time and energy required.

Microwave bonding concept is based on having thin (smaller than the skin depth) electrically conductive intermediate layers at the bonding interface which accommodates the current induced by microwave radiations. The surface currents will then generate localized heat required for bonding. Other groups [2]-[5] have applied this technique to polymers but used either customized microwave cavities [2] or needed solvents or conductive polymers to achieve high strengths [4]-[5]. Here, we propose to utilize metallic intermediate susceptor layer to provide fast, inexpensive and efficient bonding. To our knowledge this is the first study on efficient design of metallic microwave susceptors for bonding microfluidics in commercial microwave ovens. However, none of the previously published results provide rapid localized heating in inexpensive fashion.

\section{MICROWAVE HEATING SYSTEM}

The microwave heating system used in this study consisted of a commercial microwave oven (Panasonic NNSA630W), PMMA substrates (1x1x0.06"Plaskolite C OPTIX Acrylic), and thin metallic microwave susceptors (gold).

Metal parts much smaller than the microwave wavelength do not heat efficiently inside the microwave oven. On the other hand, metallic patterns that are too large or contain acute angles are prone to micro-sized explosions. Therefore, susceptor design is vital in achieving controllable, efficient heating at the bonding interface. In our previous study [6], we have presented microwave susceptor design details based on electromagnetic simulations and this work is the first successful bonding of thermoplastics using these designed susceptors. Designed microwave susceptor patterns were produced by sputtering $15 \mathrm{~nm}$ of gold on acrylic substrates through a stencil cut from 0.035 inch thick acrylic sheets using a Versa Laser VLS 3.5 $\mathrm{CO}_{2}$ laser cutter as shown in Figure 1 (a). The heating curve of the fabricated susceptors is shown in Figure 2.

To avoid excessive field concentration, an area of intermediate electric field intensity is chosen as our test location

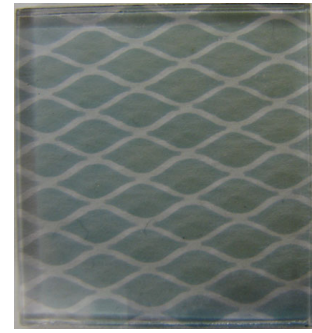

(a)

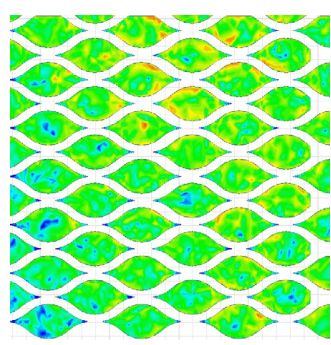

(c)

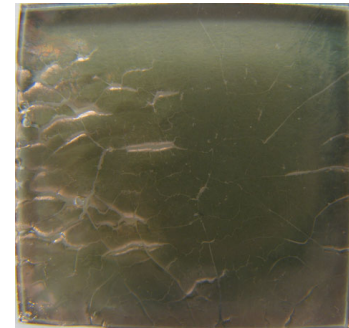

(b)
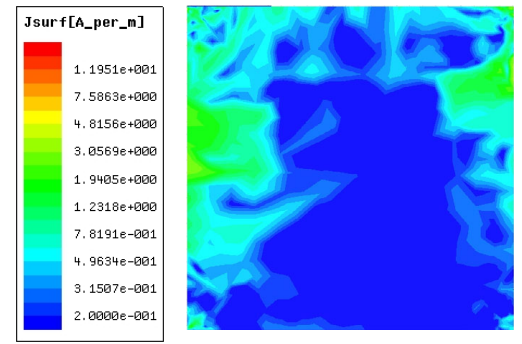

(d)
Figure 1: (a) Picture of the fabricated microwave susceptor. (b) Picture of the non-patterned gold susceptor after the test, explosions are caused by large stress generations along the surface as a result of non-uniform heating. (c) Simulation results showing "Magnitude of Surface Current Density" (MSCD) of the susceptor in the selected test location. (d) Simulation results showing MSCD of the non-patterned susceptor in the test location. Simulation results are based on normalized microwave input power of $1 \mathrm{~W}$ to the microwave cavity. ANSYS HFSS software was used for electromagnetic simulations.

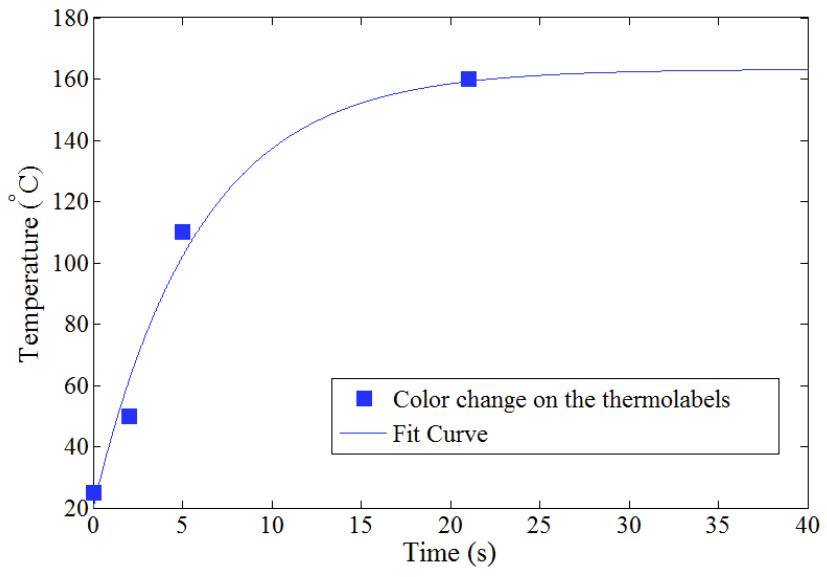

Figure 2: Temperature. vs. time of the designed microwave susceptor pattern. Temperature data was taken using Nichiyu Giken Kogyo co. thermolabels attached to the surface of the susceptors. Time axis origin is set to microwave source activation time. Hilton Head Island, South Carolina, June 3-7, 2012 

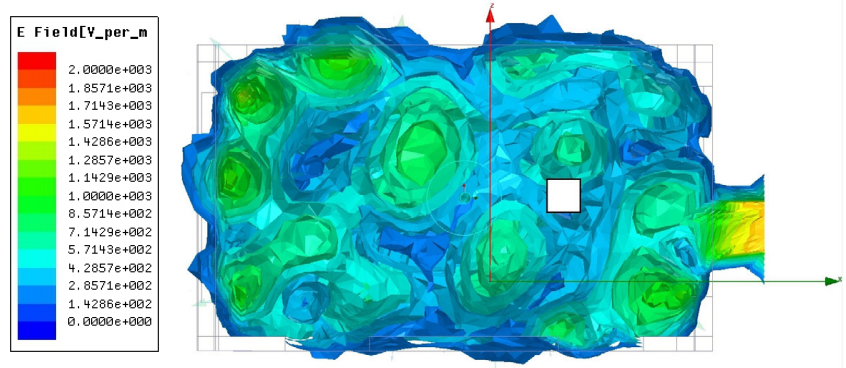

Figure 3: Selected test location (white square) shown in the crosssection of the electric field distribution of the microwave cavity

-(Figure 3). For bonding tests, microfluidic channels and reservoirs were cut into acrylic substrates using a $\mathrm{CO}_{2}$ laser cutter.

\section{RESULTS}

Two PMMA substrates, one with microfluidic channels and the other coated with gold susceptors were held together by elastic bands to exert moderate pressure and were then placed at the test location inside the microwave oven cavity. After running the microwave at $100 \%$ power $(1200 \mathrm{~W})$ for 45 seconds the substrates were bonded and channels were sealed without leaks (Fig. 4).

Bonding is very consistent over the $1 \times 1$ " size of our substrates due to relative field uniformity. Heating uniformity is related to the uniformity of the MSCD and shows marked improvements in discrete metallic susceptors over a solid metal film of the same size.

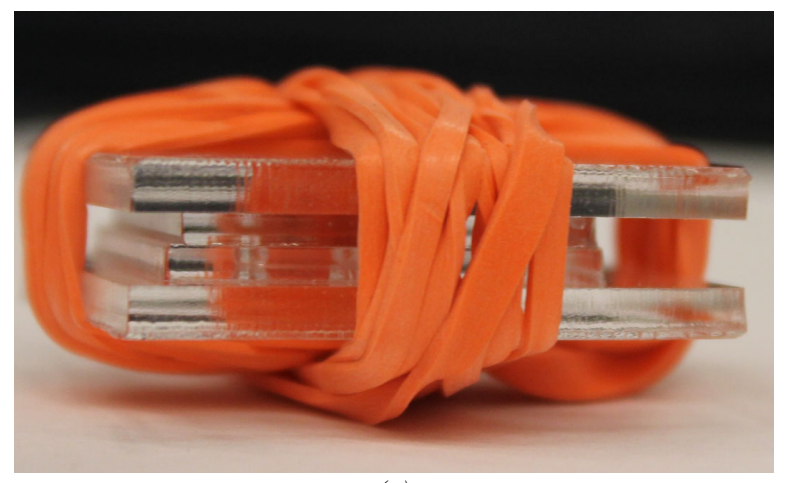

(a)

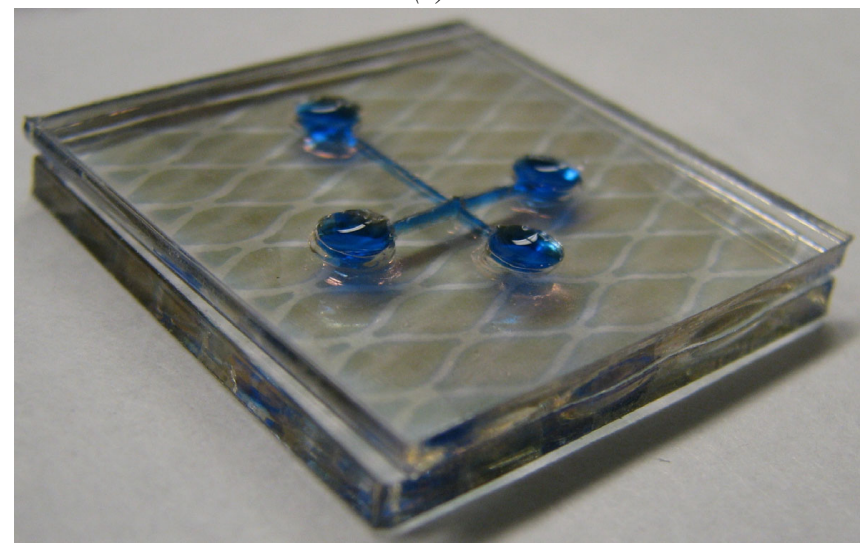

(b)

Figure 4: (a) Bonding samples and their attachment before the bonding test (b) bonded microfluidic channels filled with blue ink for leakage test

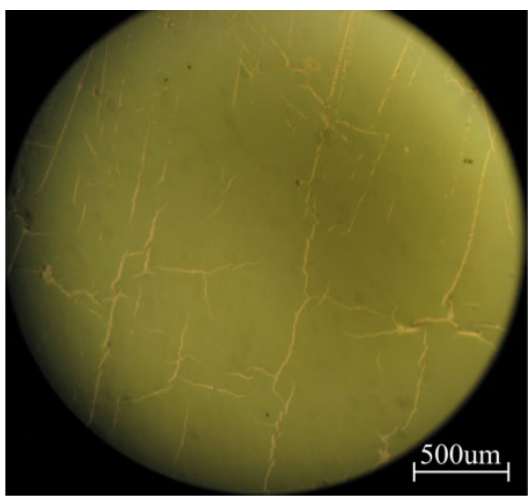

Figure 5: Microscopic images of cracks produced in the gold layer due thermal mismatch after 15 seconds of microwave radiation at $100 \%$ power.

\section{CONCLUSIONS}

PMMA bonding using microwave susceptors and commercial microwave ovens provides inexpensive, rapid and easy microfluidic device fabrication. This is the first demonstration of how specific metal susceptor designs can produce improved heating uniformity and polymer bonding. Future studies will focus on characterizing the bond strength for different power levels and heating times and the use of other lower-melting point materials as an intermediate layer for bonding in less time.

\section{ACKNOWLEDGEMENTS}

The authors would like to thank Dr. Subir Bhattacharjee for providing access to his $\mathrm{CO}_{2}$ Laser Cutter. This research was funded by NSERC and CMC Microsystems.

\section{REFERENCES}

[1] C. Tsao and D. L. DeVoe, "Bonding of thermoplastic polymer microfluidics", Microfluidics and Nanofluidics, vol. 6, no. 1, pp. 1-16, November, (2008).

[2] Kin Fong Lei, W. J. Li, N. Budraa, and J. D. Mai, "Microwave bonding of polymer-based substrates for micronano fluidic applications", in TRANSDUCERS, Solid-State Sensors, Actuators and Microsystems, 12th International Conference on, vol. 2, pp. 1335-1338, (2003).

[3] N. K. Budraa, H. W. Jackson, M. Barmatz, W. T. Pike, and J. D. Mai, "Low pressure and low temperature hermetic wafer bonding using microwave heating", in Twelfth IEEE International Conference on Micro Electro Mechanical Systems (MEMS '99), pp. 490-492, (1999).

[4] M. Rahbar, S. Chhina, D. Sameoto, and M. Parameswaran, "Microwave-induced, thermally assisted solvent bonding for low-cost PMMA microfluidic devices", Journal of Micromechanics and Microengineering, vol. 20, no. 1, p. 015026, January, (2010).

[5] R. J. Holmes, C. McDonagh, J. A. D. McLaughlin, S. Mohr, N. J. Goddard, and P. R. Fielden, "Microwave bonding of poly(methylmethacrylate) microfluidic devices using a conductive polymer", Journal of Physics and Chemistry of Solids, vol. 72, no. 6, pp. 626-629, Jun., (2011).

[6] A. Toossi, M. Daneshmand, D. Sameoto, "Microwave Susceptor Design for Wafer Bonding Applications", Accepted in IEEE MTT-S Int. Microwave Symp. Dig., July, (2012). 


\title{
PHOTORESIST-DERIVED POROUS CARBON FOR INTEGRATED ON-CHIP ENERGY STORAGE
}

\author{
B.I. Hsia, M. Vincent, M.S. Kim, C. Carraro, and R. Maboudian* \\ Department of Chemical and Biomolecular Engineering, University of California, Berkeley, Berkeley, CA
}

\begin{abstract}
We have developed a facile, and scalable fabrication process for high surface area porous carbon for microsupercapacitor applications. A porous carbon film is synthesized by pyrolysis of commercial SPR-220 photoresist in an $\mathrm{H}_{2} / \mathrm{Ar}$ mixture and tested for specific capacitance. A capacitance of about $2 \mathrm{mF} / \mathrm{cm}^{2}$ was achieved, matching or exceeding numerous other proposed microsupercapacitor technologies. The material was also demonstrated to be robust under cycling in aqueous electrolyte, retaining $>92 \%$ of its original capacitance after $10^{6}$ cycles.
\end{abstract}

\section{INTRODUCTION}

The increasing technological demand for autonomous sensing platforms necessitates the development of integrated energy storage for powering sensors, actuators, and communications microsystems. Supercapacitors, one potential candidate for on-chip energy storage, store charge electrostatically at the electrode/electrolyte interface, in contrast batteries, which rely on electrochemical energy storage in the bulk electrode. Because of this interfacial charge storage mechanism, supercapacitors charge and discharge much more quickly (in seconds vs. in minutes or hours for batteries) and have much longer cycle lifetime $\left(10^{6} \mathrm{vs}\right.$. $10^{3}$ cycles for batteries), but they require high surface area electrodes to achieve good energy density [1]. Because of this high surface area requirement, activated carbon is a popular material choice for supercapacitor electrodes [2]. However, for integrated, planar, on-chip supercapacitors, activated carbon presents many fabrication challenges including patterning and placement of electrodes [3].

For on-chip supercapacitor applications, an effective approach is utilization of photoresist-derived carbon since photoresist processing is well-established in microfabrication methodologies. However, prior reports on photoresist-derived carbon from AZ4300 and microstructured SU-8 resists show relatively low capacitance values unless the electrode material is chemically activated or decorated with electroactive materials [4] [5] [6] [7]. In this manuscript, we demonstrate that the pyrolysis of another readily available photoresist, SPR-220, yields a highly capacitive film without any additional treatment. The resultant capacitance from our procedure is also competitive with other proposed carbon-based microsupercapacitor electrode materials including inkjet-printed carbon [3], carbide-derived carbon [8], and carbon nanotubes [9].

\section{METHODS}

The fabrication procedure, inspired by Ref. [4], is illustrated in Fig. 1. A commercially available photoresist, SPR-220, is spin coated onto a substrate, and soft baked at $115^{\circ} \mathrm{C}$ for 3 minutes. Standard lithographic patterning can then be used to pattern the photoresist if desired. The photoresist is then annealed at $300^{\circ} \mathrm{C}$ for 30 minutes in 1 Torr of Ar to drive off residual solvent. This preanneal is followed by a $900^{\circ} \mathrm{C}$ pyrolysis in 1 Torr of a $10 \% \mathrm{H}_{2} / 90 \%$ Ar mixture. After 1 hour, the sample is allowed to cool to room temperature in the mixed gas environment.

\section{RESULTS \& DISCUSSION}

Figure 2 shows an optical image of a film patterned into an interdigitated comb electrode configuration on a $\mathrm{Si}$ substrate, before and after pyrolysis. While the lateral dimensions are approximately maintained after annealing, the film thickness is reduced by about a factor of 5 after annealing, from $10 \mu \mathrm{m}$ to 2 $\mu \mathrm{m}$, as determined by profilometry.

Figure 3 shows the Raman spectra of unannealed and annealed photoresist in comparison to commercial activated carbon. Both the activated carbon and annealed photoresist show the characteristic peaks of graphitic carbon, the D- and G- peaks, at about $1350 \mathrm{~cm}^{-1}$ and $1600 \mathrm{~cm}^{-1}$ respectively, confirming the carbonaceous nature of the fabricated carbon film.

The capacitance of a $2 \mu \mathrm{m}$ thick porous carbon film was measured via cyclic voltammetry $(\mathrm{CV})$ in a $3.5 \mathrm{M} \mathrm{KCl}$ solution in a three-probe configuration with a $\mathrm{Pt}$ counter electrode and an $\mathrm{Ag} / \mathrm{AgCl}$ reference electrode, and the result is presented in Fig. 4. The CV curve shape is semi-rectangular for biases between 0 and $+0.7 \mathrm{~V}$ indicating ideal capacitive behavior. The sharp current increase for bias $<+0.8 \mathrm{~V}$ and $<-0.2 \mathrm{~V}$ likely stem from irreversible processes at the electrode surface. The other small irregularities likely stem from pseudocapacitive reactions at interfacial functional groups [10]. Specific capacitance is calculated via Eq. 1, where $I$ is the current taken at a midpoint voltage, $+0.25 \mathrm{~V}, d V / d t$ is the voltage sweep rate, and $A$ is the projected area of the film.

$$
C=\frac{I}{d V / d t} \frac{1}{A}
$$

The measured capacitance is measured to be about $2 \mathrm{mF} / \mathrm{cm}^{2}$ for all scan rates, matching or exceeding the capacitance for inkjetprinted activated carbon [3] and carbon nanotubes [9]. Carbidederived carbon, with an areal capacitance of $30 \mathrm{mF} / \mathrm{cm}^{2}$ for $2 \mu \mathrm{m}$ films, has demonstrated greater capacitance values, but requires a lengthier fabrication including high temperature annealing in a chlorine environment and carbon etching [8]. Additionally, we predict that thicker photoresist films may give increased areal capacitance, and that chemical treatments may improve porosity and pseudocapacitance [6] [10]. Previous tests on annealed AZ4300 and microstructured SU-8 photoresists gave capacitances of $8 \mu \mathrm{F} / \mathrm{cm}^{2}$ [4] and $<60 \mu \mathrm{F} / \mathrm{cm}^{2}$ [6] respectively, indicating that our process likely yields a much more porous, high surface area film, resulting in a capacitance improvement of two orders of magnitude. To demonstrate the robustness of the film, a cycle lifetime test was also performed that showed $<8 \%$ drop in capacitance over one million charge/discharge cycles, as shown in Fig. 5. This compares favorably with Ref. [6], where a chemically activated pyrolyzed carbon electrode showed a $>12 \%$ drop in capacitance over one thousand cycles.

In summary, a simple, easily integratable process has been developed for fabrication of porous carbon electrodes via a photoresist annealing process. These electrodes exhibit a high specific capacitance and a long cycle lifetime in aqueous electrolyte. A planar, integrated, two-electrode device is easily fabricated via standard photolithographic techniques. Future work will focus on chemical pretreatments to increase specific surface area and pseudocapacitance, and device fabrication and characterization in a variety of electrolytes. 


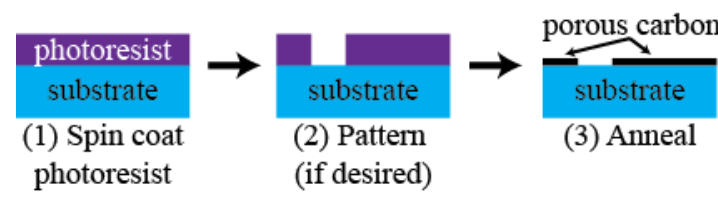

Figure 1: Fabrication schematic

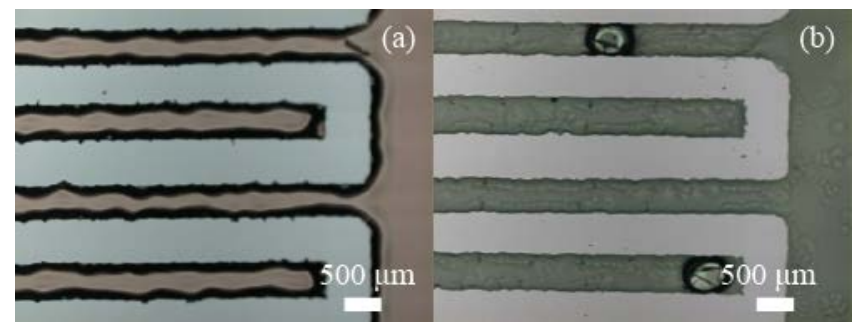

Figure 2: Optical image of comb electrode pattern (a) before (photoresist) and (b) after annealing (porous carbon). Lateral size of patterned structures is not significantly altered by annealing.

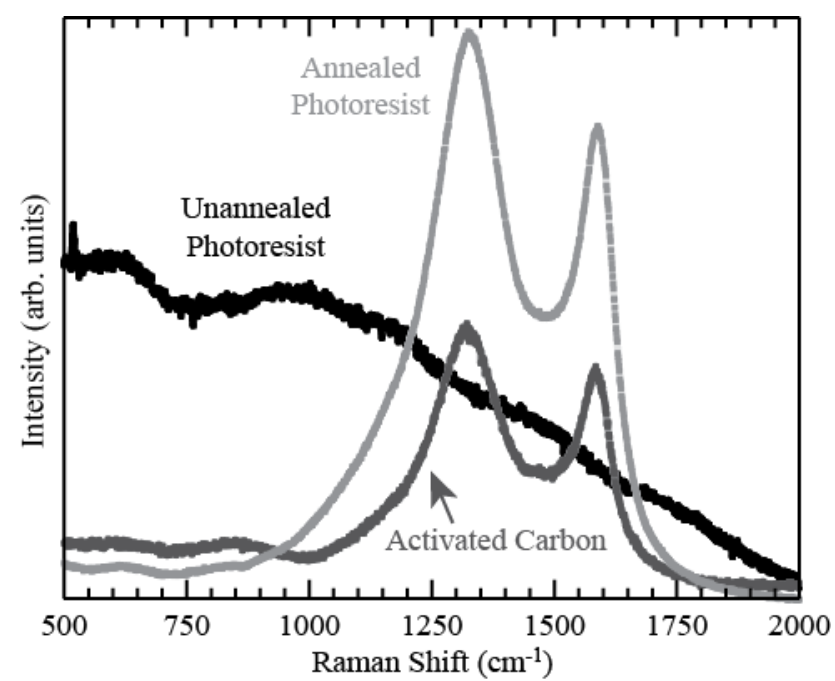

Figure 3: Raman spectra of unannealed photoresist, annealed photoresist, and activated carbon.

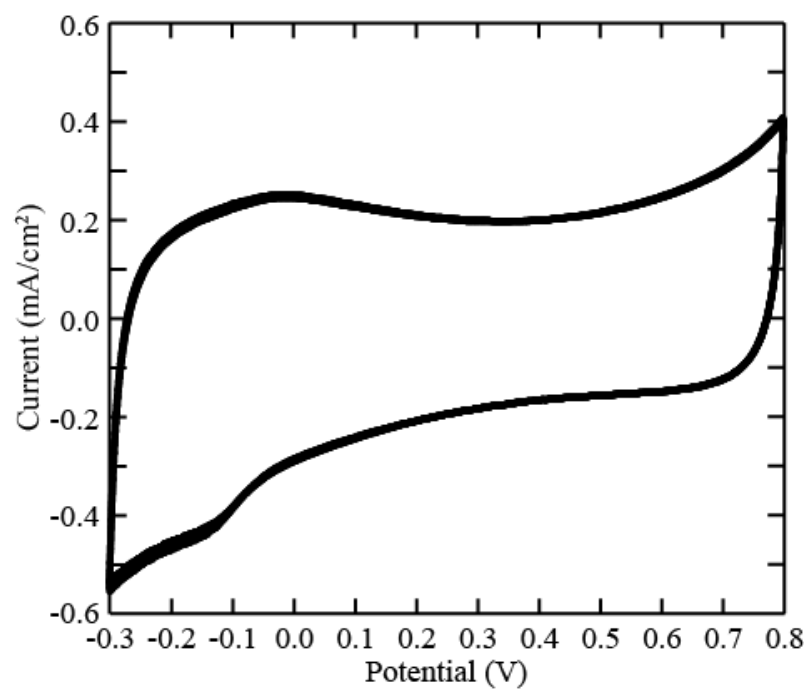

Figure 4: Cyclic voltammogram of $2 \mu \mathrm{m}$ thick annealed photoresist film in $3.5 \mathrm{M} \mathrm{KCl}$. Scan rate is $100 \mathrm{mV} / \mathrm{s}$.

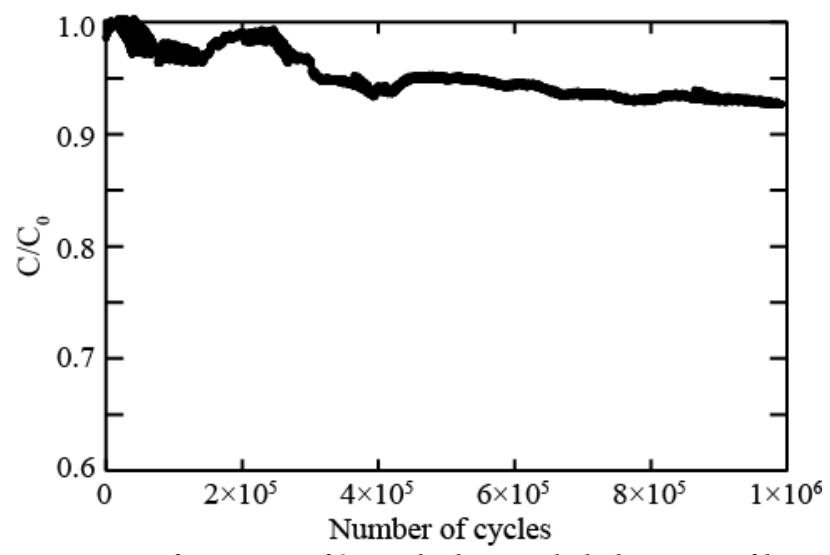

Figure 5: Lifetime test of $2 \mu \mathrm{m}$ thick annealed photoresist film in $3.5 \mathrm{M} \mathrm{KCl}$. Scan rate is $5 \mathrm{~V} / \mathrm{s}$ and potential window is $-150 \mathrm{mV}$ to $150 \mathrm{mV}$.

\section{REFERENCES}

[1] P. Simon and Y. Gogotsi, "Materials for Electrochemical Capacitors," Nature Mat., vol. 7, pp. 845-854, 2008.

[2] E. Frackowiak, "Carbon materials for supercapacitor application," Physical Chemistry Chemical Physics, vol. 9, pp. 1774-1785, 2007.

[3] D. Pech, M. Brunet, P.-L. Taberna, P. Simon, N. Fabre, F. Mesnilgrente, V. Conedera and H. Durou, "Elaboration of a microstructured inkjet-printed carbon electrochemical capacitor," J. Power Sources, vol. 195, no. 4, p. 1266-1269, 2010.

[4] S. Ranganathan, R. McCreery, S. M. Majji and M. Madou, "Photoresist-Derived Carbon for Microelectromechanical Systems and Electrochemical Applications," J. Electrochem. Soc., vol. 147, no. 1, pp. 277-282, 2000.

[5] M. Beidaghi and C. Wang, "Micro-supercapacitors bsaed on three dimensional interdigital polypyrrole/C-MEMS electrodes," Electrochimica Acta, vol. 56, pp. 9508-9514, 2011.

[6] M. Beidaghi, W. Chen and C. Wang, "Electrochemically activated carbon micro-electrode arrays for electrochemical micro-supercapacitors," Journal of Power Sources, vol. 196, pp. 2403-2409, 2011.

[7] W. Chen, M. Beidaghi, V. Penmatsa, K. Bechtold, L. Kumari, W. Z. Li and C. Wang, "Integration of Carbon Nanotubes to C-MEMS for On-chip Supercapacitors," IEEE Transactions on Nanotechnology, vol. 9, pp. 734-740, 2010.

[8] J. Chmiola, C. Largeot, P.-L. Taberna, P. Simon and Y. Gogotsi, "Monolithic Carbide-Derived Carbon Films for Micro-Supercapacitors," Science, vol. 328, no. 5977, pp. 480483, 2010.

[9] Y. Q. Jiang, Q. Zhou and L. Lin, "Planar MEMS Supercapacitor using Carbon Nanotube Forests," Proc. IEEE Micr. Elect., pp. 587-590, 2009.

[10] E. Frackowiak and F. Beguin, "Carbon materials for the electrochemical storage of energy in capacitors," Carbon, vol. 39, pp. 937-950, 2001

\section{CONTACT}

*R. Maboudian, tel: +1-510-643-7957; maboudia@berkeley.edu 


\title{
WET ETCHING \& UNIFORM WAFER-LEVEL THINNING OF BULK PIEZOELETRIC CERAMICS ON SILICON
}

\author{
Ethem Erkan Aktakka ${ }^{*}$ Rebecca L. Peterson, and Khalil Najafi \\ Center for Wireless Integrated MicroSensing and Systems (WIMS ${ }^{2}$ ) \\ University of Michigan, Ann Arbor, MI, USA
}

\section{ABSTRACT}

This paper presents new micro-fabrication tools and material/process characterization for wafer-level lapping and wetetch patterning of bulk PZT on silicon. The process enables precise control over final film thickness $(5-100 \mu \mathrm{m})$ with high wafer-level uniformity $( \pm 0.5 \mu \mathrm{m})$, and the lapping-rate $(10-30 \mu \mathrm{m} / \mathrm{min})$ and surface roughness $(43 \mathrm{~nm})$ are characterized. Additionally, surface micro-machining of suspended PZT structures is demonstrated by bonding and thinning over pre-patterned silicon features. Finally, a new wet-etching process is developed for low-undercut $(0.6: 1)$ patterning of $>5 \mu \mathrm{m}$ thick PZT films, and $\mathrm{d}_{33 \text {-EFF }}$ piezoelectric strain coefficients on thinned and patterned bulk PZT-5A and $5 \mathrm{H}$ films are measured as 140 and $311 \mathrm{pm} / \mathrm{V}$, respectively.

\section{INTRODUCTION}

Recently, we introduced a micro-fabrication technology for integration of bulk PZT-5A substrates on silicon by solder bonding and thinning, to realize mm-scale diaphragm actuators [1] and energy harvesters [2]. In this paper, we report new microfabrication methods to achieve precise PZT film thickness with excellent wafer-level uniformity and reduced minimum film thickness $(\sim 5 \mu \mathrm{m})$ in a reliable process. We also report here surface micro-machining of PZT diaphragms, wet-etching of thick PZT films, and integration of PZT-5H for higher piezoelectric response than PZT-5A (Fig. 1). These processes are critical to enable batchmode fabrication of miniaturized and high-performance microspeakers, microphones, microfluidic devices, and complex-shape actuators. The microfabrication techniques introduced here offer increased reliability, fabrication flexibility, and higher material quality over existing piezoelectric film deposition methods.

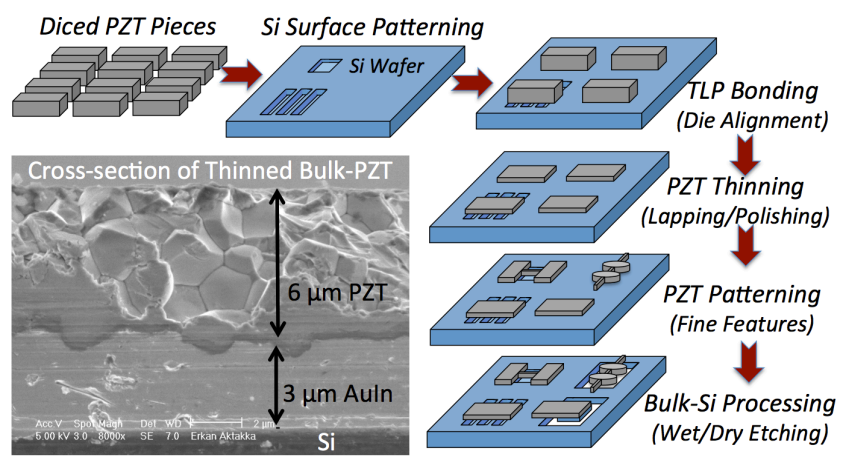

Figure 1: Bulk-PZT on Si process and SEM image of final PZT film.

\section{UNIFORM WAFER-LEVEL THINNING}

Minimizing wafer-level variation of thinned-PZT thickness is critical for batch-mode MEMS fabrication. It can be as high as $\pm 18 \mu \mathrm{m}$ on a typical wafer, often due to increased lapping rates near the wafer edge. A new method is developed to improve waferscale thinning uniformity and to precisely define the final PZT thickness, by leveraging the silicon-wafer surface as a thinningstop layer (Fig. 2). A $0.5 \mu \mathrm{m}$ thermal-oxide layer enhances surface hardness, and the lapping rate decreases very effectively $(\sim 100 \times)$ upon leveling of PZT with the $\mathrm{Si} / \mathrm{SiO}_{2}$ surface (Fig. 3). Highly uniform film thickness $( \pm 0.5 \mu \mathrm{m})$ is obtained across 4 -inch wafers.
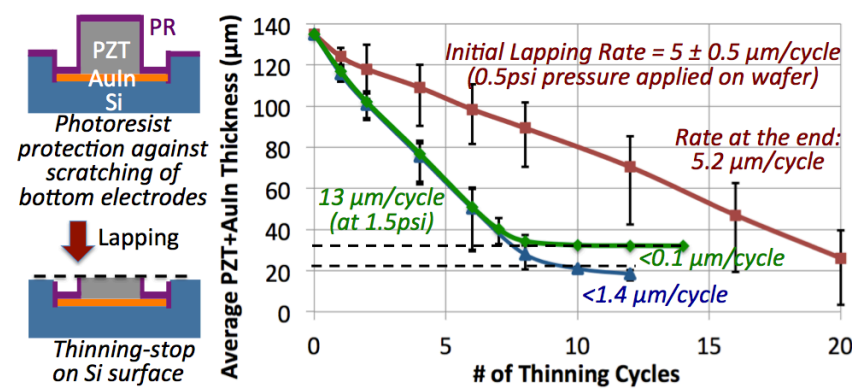

Test Stop Height of Decrease in lapping |Wafer-level thickness uniformity Wafer layer stop layer rate at the stop layer Max Deviation Standard Dev.

\begin{tabular}{c|c|c|c|c|c}
\hline$\square 1$ & $\mathrm{~N} / \mathrm{A}$ & - & - & $+/-17.2 \mu \mathrm{m}$ & $11.8 \mu \mathrm{m}$ \\
\hline $\mathbf{\Delta}$ & $\mathrm{Si}$ & $22.5 \mu \mathrm{m}$ & $91.3 \%$ & $+/-1.8 \mu \mathrm{m}$ & $1.1 \mu \mathrm{m}$ \\
\hline 3 & $\mathrm{Si} / \mathrm{SiO}_{2}$ & $32.5 \mu \mathrm{m}$ & $99.2 \%$ & $+/-0.55 \mu \mathrm{m}$ & $0.28 \mu \mathrm{m}$
\end{tabular}

Figure 2: Change in the lapping rates when stop-layer is reached.

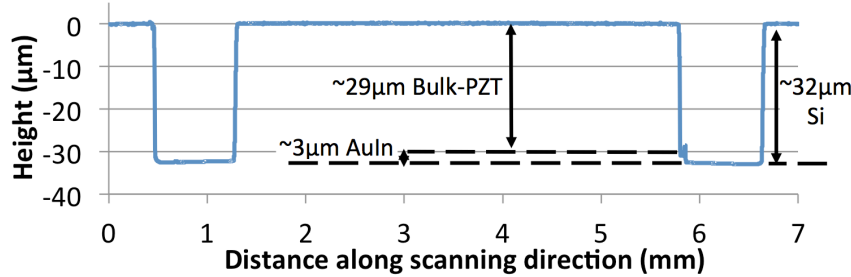

Figure 3: Flatness and thickness profile of a thinned-PZT die on Si.

For many applications, surface roughness of the final film is also a critical parameter. The average roughness on $20-\mu \mathrm{m}$ thick lapped/polished PZT films is characterized to be $43 \mathrm{~nm}$, which is acceptable for most MEMS and can be improved further by extending the polishing period (Fig. 4).
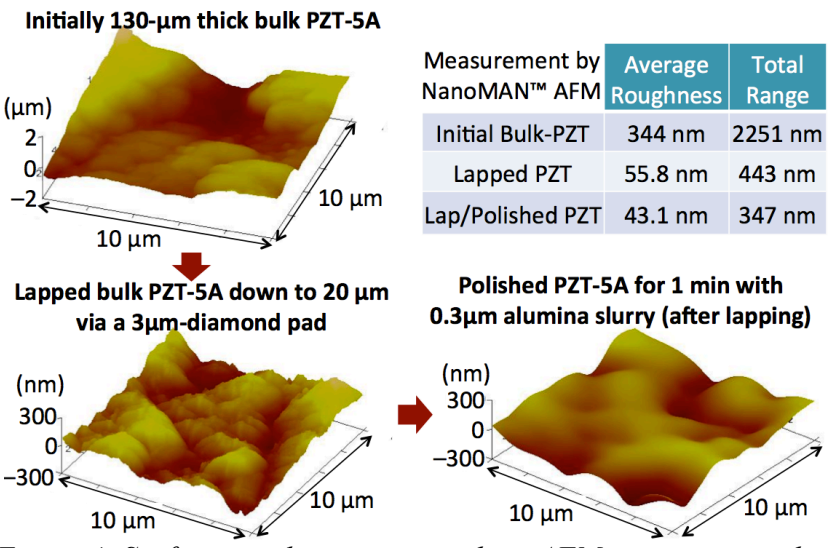

Polished PZT-5A for 1 min with $0.3 \mu \mathrm{m}$ alumina slurry (after lapping)

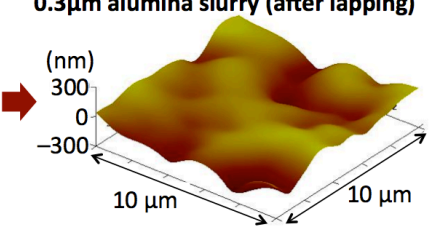

For device-release purposes, PZT bonding/thinning over prepatterned silicon features is demonstrated as an alternative to expensive and time-consuming backside through-wafer etching. Suspended structures with up to 1:40 thickness:diameter ratio are fabricated, which can be used for diaphragm actuation in acoustic, ultrasonic and microfluidic applications (Fig. 5-6). This unique surface micro-machining capability also facilitates easier packaging, and is not attainable with other piezoelectric-film deposition methods, where a flat deposition surface is required. Hilton Head Island, South Carolina, June 3-7, 2012 

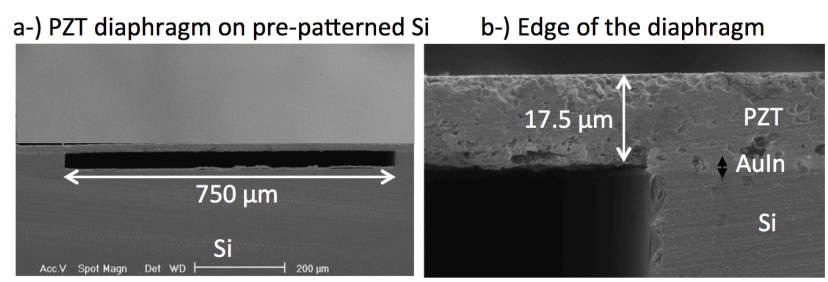

Figure 5: Suspended PZT layer bonded and thinned over Si cavity. a-) Bonding and lapping of PZT on pre-patterned Si features
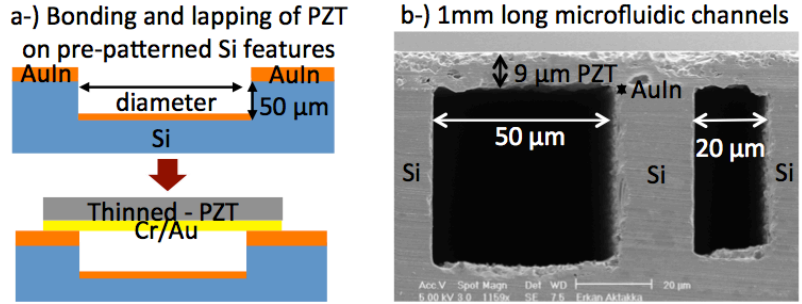

c-) Measured min. PZT thickness obtained for different membrane sizes

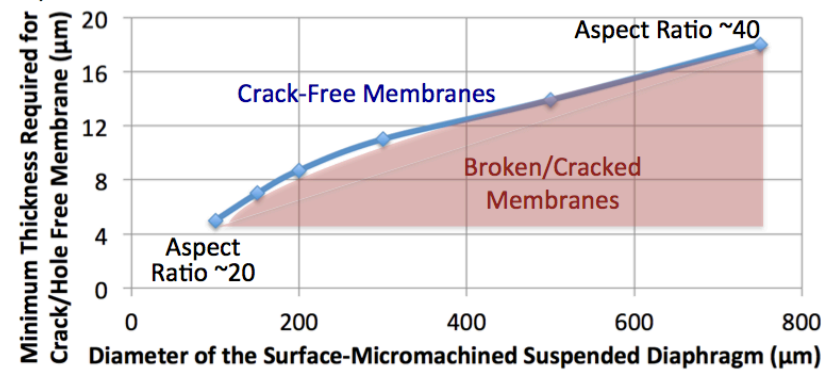

Figure 6: Minimum PZT thickness obtained versus diaphragm size.

\section{LOW-UNDERCUT WET-ETCHING}

Wet-etching of PZT structures is highly desirable, since it provides a cost-effective, high-throughput process, and enables lithographically-defined features. However, previous studies mostly focused on sol-gel spin-coated 1-2 $\mu \mathrm{m}$ thick films, with high-undercut ratios of $1.5: 1$ to $5: 1$ [3-6]. For etching through the complex composition of $>5 \mu \mathrm{m}$ thick PZT films, using the right mix of chemical reagents is critical, since residues due to reaction by-products cause unreliable etch rates and increased undercut. Here, a new mixture is utilized, $\mathrm{BHF}: \mathrm{HNO}_{3}(67 \%): \mathrm{HCl}(38 \%)$ with 2:2:1 ratio, which is $14 \times$ diluted by $\mathrm{DI}-\mathrm{H}_{2} \mathrm{O}$ to achieve a controllable etch rate and prevent photoresist-mask delamination. The solution is heated to $40^{\circ} \mathrm{C}$ in order to increase etching efficiency and obtain a better undercut profile. The etch process is expected to follow the below equations, where $\mathrm{PbClF}$ residue is converted into $\mathrm{PbCl}_{2}$, which has a higher solubility in water [7].

$$
\begin{aligned}
\mathrm{Pb}(\mathrm{Ti}, \mathrm{Zr}) \mathrm{O}_{3}(\mathrm{~s})+\mathrm{HCl}(\mathrm{aq}) & +\mathrm{HF}(\mathrm{aq}) \rightarrow\left[\mathrm{TiF}_{6}\right]^{2-}(a q)+\left[\mathrm{ZrF}_{6}\right]^{2-}(a q) \\
+\left[\mathrm{PbCl}_{4}\right]^{2-}(a q)+\mathrm{PbClF}(\mathrm{s})+\mathrm{H}_{2} \mathrm{O}(\mathrm{l}) & (1) \\
\mathrm{PbClF}(\mathrm{s})+\mathrm{HNO}_{3}(a q) \rightarrow \mathrm{PbCl}_{2}(\mathrm{~s}) & +\mathrm{Pb}^{2+}(a q) \\
& +\mathrm{NO}_{3}^{-}(a q)+\mathrm{HF}(a q)
\end{aligned}
$$

To minimize undercut, multiple cycles of lithography followed by wet-etching are used (Fig. 7). Only a portion of the total thickness is etched in each cycle (etch rate $2-3 \mu \mathrm{m} / \mathrm{min}$ ), followed by ultrasonic cleaning. Then the photoresist mask is removed and a new photoresist layer is added to coat the previously-created undercut region. Patterning of $18 \mu \mathrm{m}$ thick PZT in two cycles yields an undercut ratio of 0.6:1 (Fig. 8).

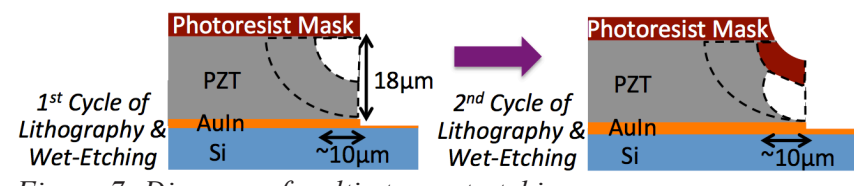

Figure 7: Diagram of multi-step wet-etching process.

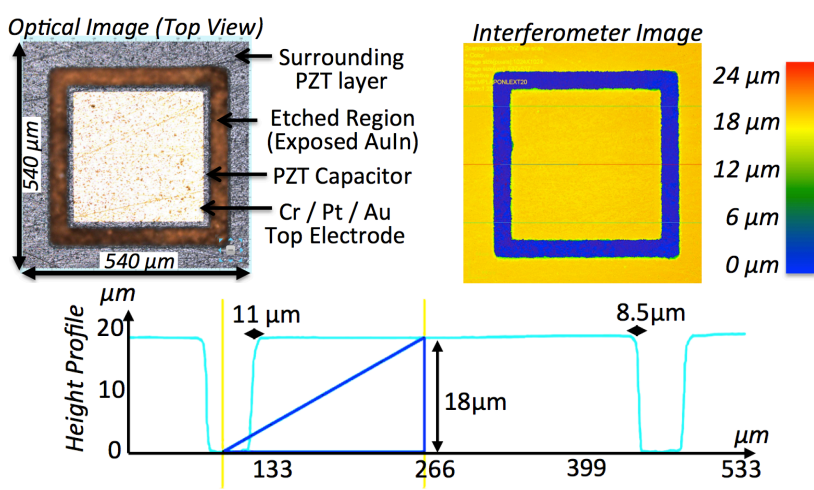

Figure 8: Wet-etched $18 \mu \mathrm{m}$ thick PZT layer with 0.6:1 undercut. MEASURED PIEZOELECTRIC PROPERTIES

Preservation of bulk piezoelectric properties in the final bonded/thinned/patterned $\mathrm{PZT}-5 \mathrm{~A} / 5 \mathrm{H}$ films is confirmed via piezo-response force microscopy (Fig. 9). The $d_{33}$ values calculated from measured $\mathrm{d}_{33 \text { eff }}$ [8] are close to the values in vendor datasheets for the bulk materials (Table 1), and are notably higher than values of existing thin-film-deposited piezoelectrics.

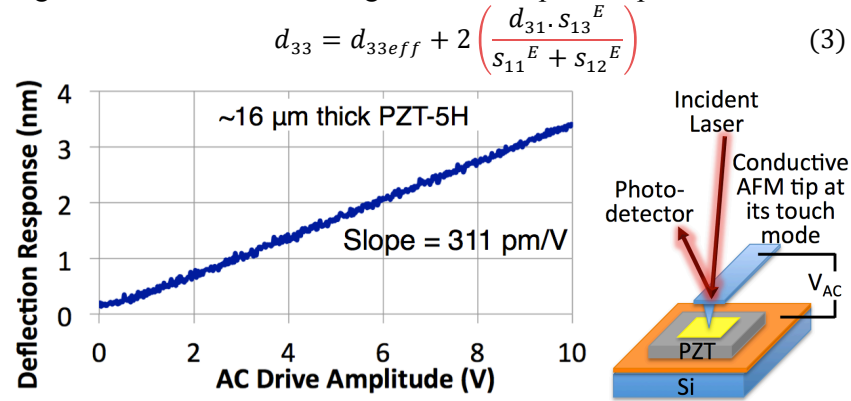

Figure 9: Piezoelectric response measured on $300 \mu \mathrm{m} \times 300 \mu \mathrm{m}$, $16 \mu \mathrm{m}$ thick PZT5A/5H capacitors via piezo-force-microscopy.

Table 1: Measured longitudinal piezoelectric strain coefficients on $16 \mu \mathrm{m}$ thick PZT-5A/5H films on Si without any repolarization.

\begin{tabular}{c|c|c|c} 
& $\begin{array}{c}\mathrm{d}_{33-E F F} \\
\text { (Measured) }\end{array}$ & $\begin{array}{c}\mathrm{d}_{33} \\
\text { (Calculated) }\end{array}$ & $\begin{array}{c}\mathrm{d}_{33} \\
\text { (Vendor Datasheet) }\end{array}$ \\
\hline PZT-5A & $140 \mathrm{pm} / \mathrm{V}$ & $398 \mathrm{pm} / \mathrm{V}$ & $390 \mathrm{pm} / \mathrm{V}$ \\
\hline PZT-5H & $311 \mathrm{pm} / \mathrm{V}$ & $772 \mathrm{pm} / \mathrm{V}$ & $650 \mathrm{pm} / \mathrm{V}$
\end{tabular}

\section{ACKNOWLEDGEMENT}

This work is partially supported by DARPA awards, Hybrid Insect MEMS \#N66001-07-1-2006 and PASCAL \#W31P4Q-12-1-0002.

\section{REFERENCES}

[1] E.E. Aktakka, H. Kim, K. Najafi, Transducers'09, (2009), pp. 849-852.

[2] E.E. Aktakka, R.L. Peterson, K. Najafi, Transducers'11, (2011), pp. 1649-1652.

[3] L.-P. Wang, R. Wolf, Q. Zhou, S. Trolier-McKinstry, R. J. Davis, MRS Symp., Vol. 657 (2001), pp. EE5.39.1- EE5.39.6.

[4] Y.B. Jeon, C.W. Wong, S.-G. Kim, Journal of Electroceramics, 13, 509 (2004).

[5] C. Cai, J. Huang, Y. Zhai, W. Ma, W. Liu, Chinese Optics Letters, 8, 210 (2010).

[6] L. Che, E. Halvorsen, X. Chen, Journal of Micromechmanics and Microengineering, 21, 105008 (2011).

[7] K. Zheng, J. Lu, J. Chu, Jpn. J. Appl. Phys., 43, 3934 (2004).

[8] K. Prume, P. Muralt, F. Calame, T. Schmitz-Kempen, S. Tiedke, IEEE Trans. UFFC, 54, 8 (2007).

\section{CONTACT}

* E. E. Aktakka, tel: +1-734-272-3170; aktakka@umich.edu K. Najafi, tel: +1-734-763-6650; najafi@umich.edu 


\title{
HIGH PERFORMANCE SPUTTERED THERMOELECTRIC JUNCTIONS FOR MICROMECHANICAL DEVICES
}

\author{
R.P. Shea ${ }^{l}$, A.S. Gawarikar ${ }^{1}$, and J.J. Talghader ${ }^{{ }^{*}}$ \\ ${ }^{1}$ University of Minnesota, Minneapolis, Minnesota, USA
}

\begin{abstract}
The deposition and characterization of thin co-sputtered $\mathrm{Bi}_{2} \mathrm{Te}_{3}$ and $\mathrm{Sb}_{2} \mathrm{Te}_{3}$ thermoelectric films of thickness comparable to the carrier mean free path is presented. Measurements of the Seebeck coefficient, resistivity, and thermal conductivity are described. The test microstructures are highly thermally isolated and incorporate a micro-heater and thermoelectric junction. Two compositions of each material have been studied. The $\mathrm{Sb}_{2} \mathrm{Te}_{3}$ shows resistivities of $29.72 \mu \Omega-\mathrm{m}$ and $26.22 \mu \Omega-\mathrm{m}$, Seebeck coefficients of $118 \mu \mathrm{V} / \mathrm{K}$ and $131 \mu \mathrm{V} / \mathrm{K}$, and a thermal conductivity of $1.02 \mathrm{Wm}^{-1} \mathrm{~K}^{-1}$, while the $\mathrm{Bi}_{2} \mathrm{Te}_{3}$ films show resistivities of $5.22 \mu \Omega-\mathrm{m}$ and $6.15 \mu \Omega-\mathrm{m}$, Seebeck coefficients of $-51 \mu \mathrm{V} / \mathrm{K}$ and $50 \mu \mathrm{V} / \mathrm{K}$, and a thermal conductivity of $.88 \mathrm{Wm}^{-1} \mathrm{~K}^{-1}$. The materials systems exhibit thermoelectric figures of merit of $0.93 \times 10^{-3}$ and $1.23 \times 10^{-3}$.
\end{abstract}

\section{INTRODUCTION}

$\mathrm{Bi}_{2} \mathrm{Te}_{3} / \mathrm{Sb}_{2} \mathrm{Te}_{3}$ junctions are among the best thermoelectric devices in existence; however, thin films of these materials are difficult to deposit and utilize in micromachined devices. Boyer and Cisse have shown that the thermoelectric properties degrade at thicknesses below 500nm in epitaxial MJCVD films due to limited mean-free path of carriers [1]. Unfortunately, micromechanical and nanosystems require films that are thinner than this threshold. They also require films that are compatible with common and accessible deposition techniques, such as sputtering or evaporation.

The deposition of thin films of $\mathrm{Bi}_{2} \mathrm{Te}_{3}$ and $\mathrm{Sb}_{2} \mathrm{Te}_{3}$ using methods such as MJCVD [1], flash evaporation [2], and coevaporation $[3,4]$ has been widely studied but limited work has been done on sputtered films of these materials, in particular $\mathrm{Sb}_{2} \mathrm{Te}_{3}$. Various groups have studied co-sputtering of $\mathrm{Bi}_{2} \mathrm{Te}_{3}[5,6]$ as well as reacting alternating films of sputtered $\mathrm{Bi}$ and $\mathrm{Te}$ [7], but to our knowledge, no study exists of thermoelectric junctions of sputtered $\mathrm{Bi}_{2} \mathrm{Te}_{3}$ and $\mathrm{Sb}_{2} \mathrm{Te}_{3}$ thin films incorporated into a micromechanical device.

The fabricated device consists of a highly thermally isolated micro-heater connected to a thermoelectric junction. A diagram of

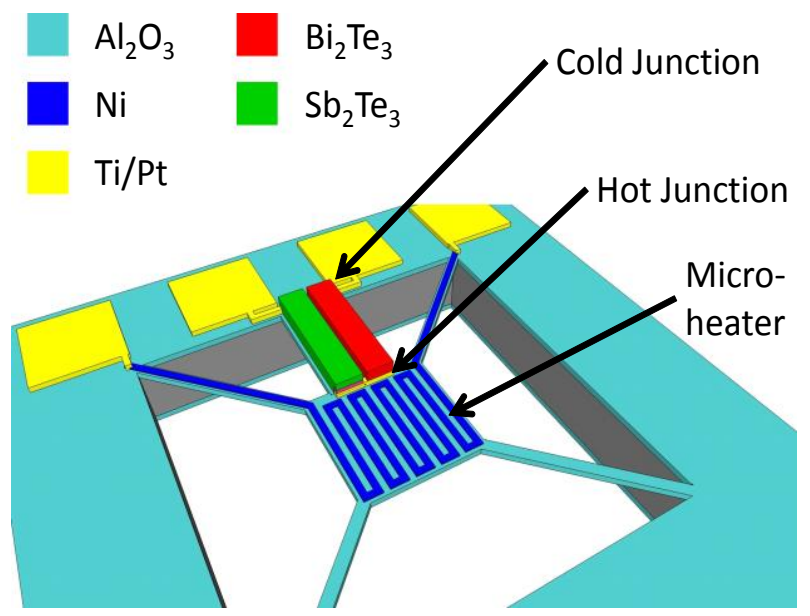

Figure 1: Conceptual image of the fabricated devices showing layer structure and device layout. this design can be seen in Figure 1. The hot side of the thermoelectric element lies on the same thermally isolated structure as the micro-heater while the cold side lies on the substrate. This allows us to accurately control the temperature difference between the two sides of the junction and therefore characterize its thermoelectric properties. Our thermoelectric films range in thickness from $225 \mathrm{~nm}$ to $272 \mathrm{~nm}$ and the thermal isolation of the micro-heater is on the order of $5 \times 10^{-7} \mathrm{~W} / \mathrm{K}$.

\section{FABRICATION}

The thermoelectric junctions were fabricated using a series of surface micromachining processes followed by a through-wafer etch release using the Bosch process. Fabrication began with an LPCVD low-stress nitride layer which was deposited on both sides of the wafer, but removed from the back side with a $\mathrm{CF}_{4} / \mathrm{O}_{2}$ plasma etch. This layer is intended to provide electrical insulation between the substrate and bond pads. Next, an atomic layer deposition (ALD) film of $\mathrm{Al}_{2} \mathrm{O}_{3}$ was deposited on both sides of the wafer which serves to electrically isolate ensuing layers from the substrate as well as provide structural support and act as an etch stop layer for the final release. Next a Ni layer was deposited to form the micro-heater used to characterize the device. This layer and the following metal and thermoelectric layers were patterned using a lift-off technique. Ti/Pt was then deposited both to form pads for wire bonding and to connect the thermoelectric materials and the micro-heater to these pads. A $\mathrm{Cr} / \mathrm{Au}$ layer was then deposited on top of the $\mathrm{Pt}$ of the bond pads to thicken them for wire bonding.

Thermoelectric films were deposited by RF magnetron cosputtering the base elements. The elemental composition of cosputtered films has been measured on test samples using Rutherford Back Scattering (RBS) for $\mathrm{Bi}_{2} \mathrm{Te}_{3}$ and Energy Dispersion X-ray Spectrometry (EDX) for $\mathrm{Sb}_{2} \mathrm{Te}_{3}$. In this study, the sputtering power of Te was held constant at $27 \mathrm{~W}$ while the sputtering powers of $\mathrm{Sb}$ and $\mathrm{Bi}$ were varied. The films are deposited at a substrate temperature of $260^{\circ} \mathrm{C}$ for in $\mathrm{Bi}_{2} \mathrm{Te}_{3}$ and $230^{\circ} \mathrm{C}$ in $\mathrm{Sb}_{2} \mathrm{Te}_{3}$ as suggested in [8]. The measured $\mathrm{Te}$

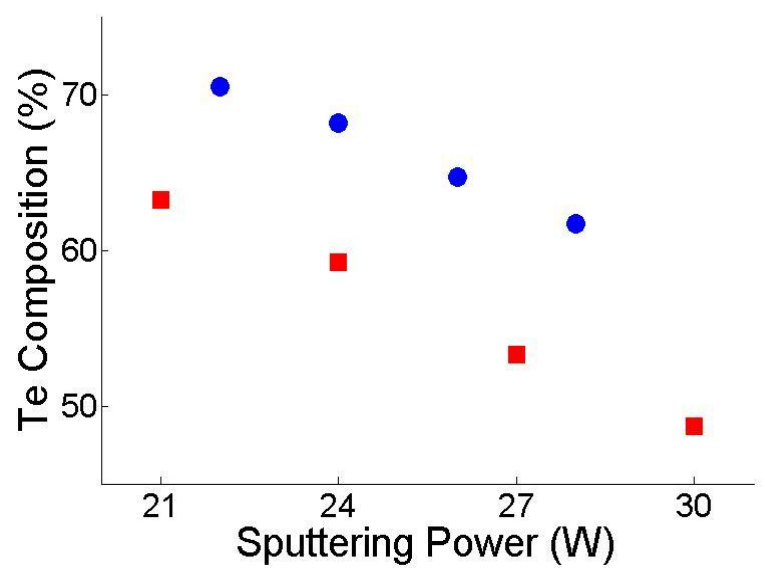

Figure 2: Tellurium composition vs Bi/Sb sputtering power for cosputtered $\mathrm{Bi}_{2} \mathrm{Te}_{3}(\square)$ and $\mathrm{Sb}_{2} \mathrm{Te}_{3}(\mathrm{O})$ with Te sputtering power held constant at $27 \mathrm{~W}$. 
composition of films for each compound can be seen in Figure 2. For the Seebeck coefficient, resistivity, and thermal conductivity data presented here, two recipes were used for each material. Batch A used sputtering powers of $22 \mathrm{~W}$ for $\mathrm{Bi}$ and $26 \mathrm{~W}$ for $\mathrm{Sb}$ while Batch B used sputtering powers of $23 \mathrm{~W}$ for $\mathrm{Bi}$ and $27 \mathrm{~W}$ for $\mathrm{Sb}$. In all cases, the sputtering power of Te was $27 \mathrm{~W}$. These values correspond to a sputtering power density of $3.82 \mathrm{~W} / \mathrm{in}^{2}$ at $27 \mathrm{~W}$ for the 3 " targets used. Film thickness was measured using standard profilometry and measured sputtering rates range from 5$6 \mathrm{~nm}$ per minute.

After the thermoelectric films are deposited, a second layer of ALD $\mathrm{Al}_{2} \mathrm{O}_{3}$ was deposited to encapsulate the films during the ensuing etch steps. This layer was then patterned using a $\mathrm{BCl}_{3}$ plasma etch to both define the support structure and plates of the devices and to open vias to enable electrical contact to the bond pads. The back-side $\mathrm{Al}_{2} \mathrm{O}_{3}$ was pattered in the same way to define the etch pits used for release. Finally the devices were released using a through wafer Bosch etch leaving free-standing thermal isolation structures containing micro-heaters and the hot side of thermoelectric junction. The nitride layer is mostly removed during the Bosch etch, but often requires a secondary plasma etch to fully remove. The material, deposition method, and thickness of each layer are seen in Table 1 and a diagram of the device layout can be seen in Figure 1.

Table 1: Material, layer thickness, and deposition technique for sequential fabrication steps.

\begin{tabular}{|c|c|c|}
\hline Material & Thickness $(\mathrm{nm})$ & Technique \\
\hline $\mathrm{LSN}$ & 200 & LPCVD \\
\hline $\mathrm{Al}_{2} \mathrm{O}_{3}$ & 25 & ALD \\
\hline $\mathrm{Ni}$ & 15 & E-Beam Evap. \\
\hline $\mathrm{Ti} / \mathrm{Pt}$ & $5 / 10$ & E-Beam Evap. \\
\hline $\mathrm{Cr} / \mathrm{Au}$ & $15 / 185$ & E-Beam Evap. \\
\hline $\mathrm{Bi}_{2} \mathrm{Te}_{3}$ & $253(\mathrm{~A}) 272(\mathrm{~B})$ & RF Sputter \\
\hline $\mathrm{Sb}_{2} \mathrm{Te}_{3}$ & $229(\mathrm{~A}) 225(\mathrm{~B})$ & RF Sputter \\
\hline $\mathrm{Al}_{2} \mathrm{O}_{3}$ & 25 & ALD \\
\hline
\end{tabular}

\section{CHARACTERIZATION}

Temperature Coefficient of Resistivity

An important factor in the characterization of the thermoelectric films is the temperature coefficient of resistance (TCR) of the micro-heater. The TCR, $\alpha$, is given by

$$
R(T)=R\left(T_{0}\right)(1+\alpha \Delta T)
$$

In our case this was measured by placing the device die on a standard Peltier cooler/heater and measuring the device resistance compared to the temperature measured by a reference thermistor. The TCR measured was $.31 \% / \mathrm{K}$ for the $\mathrm{Ni}$ micro-heaters and $.12 \% / \mathrm{K}$ for $\mathrm{Ni} / \mathrm{Fe}$.

\section{Seebeck Coefficient}

The Seebeck coefficient of the released devices was measured by varying the temperature of the hot junction using the microheater and monitoring this temperature change using the resistance of the heater. The Seebeck coefficient, $\sigma$, is given by

$$
\sigma=\frac{\Delta V}{\Delta T}
$$

where $\Delta \mathrm{V}$ is measured and $\Delta \mathrm{T}$ was calculated from the change in resistance measured as the current varies.

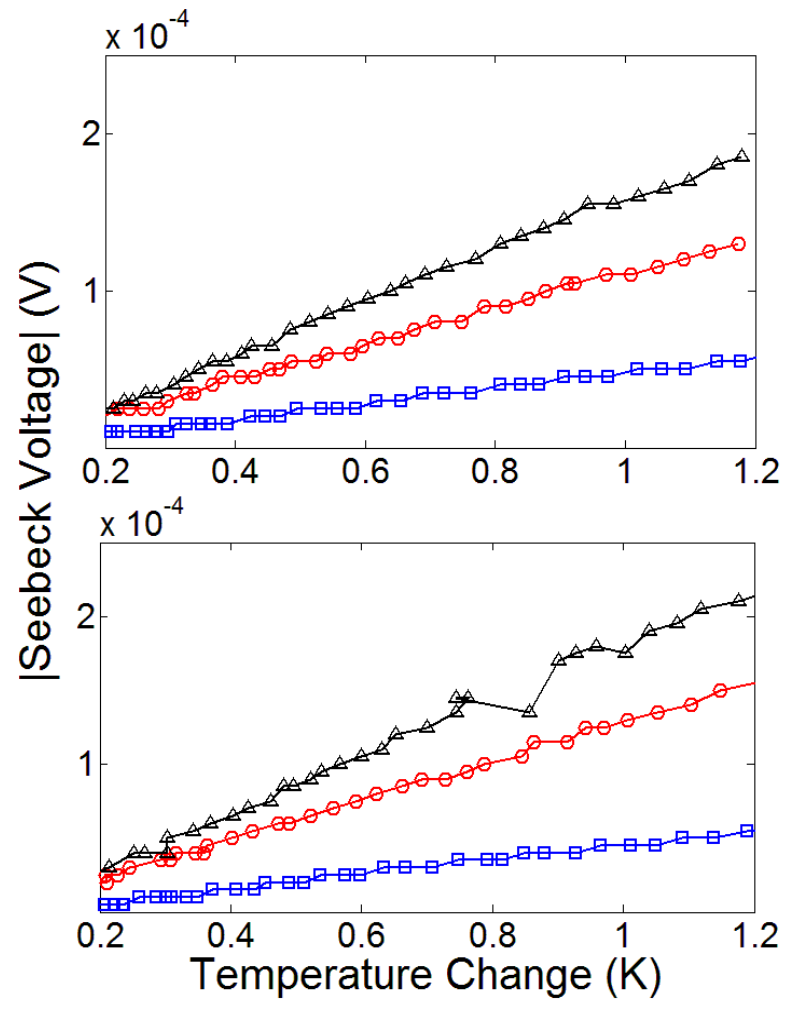

Figure 3: Seebeck voltage vs. temperature change for released Batch A (bottom) and Batch B (top) thermoelectric junctions (4), $\mathrm{Sb}_{2} \mathrm{Te}_{3}$ test structures (O), and $\mathrm{Bi}_{2} \mathrm{Te}_{3}$ test structures ( $\square$ ).

To measure the Seebeck coefficient of devices we contacted the bond pads of released structures using four probe tips. The micro-heater was connected to a source meter which allows us to source a variable current to the heater while measuring its resistance. The thermoelectric element was connected to a voltage meter via a low noise pre-amplifier to measure the resulting change in Seebeck voltage. The temperature change, $\Delta \mathrm{T}$, of the microheater, and therefore the hot side of the junction can then be calculated using Equation 1. From Equation 2 we can see that the Seebeck coefficient is directly related to the slope of the Seebeck voltage vs. temperature change plot seen in Figure 3. We have tested both thermoelectric junctions, like those seen in Figure 4, as well as test structures comprised of single materials connected to Pt lines. In Figure 3, the absolute value of the Seebeck voltage is plotted for comparison and the calculated Seebeck coefficient values for both batches are shown in Table 2 .

Table 2: Seebeck coefficents measured for test structures and junctions for each recipe.

\begin{tabular}{|c|c|c|c|}
\hline Material & Batch & $\begin{array}{c}\text { Power Ratio } \\
(\mathrm{Sb}, \mathrm{Bi}) / \mathrm{Te}(\mathrm{W})\end{array}$ & $\begin{array}{c}\text { Seebeck } \\
\text { Coef. }\end{array}$ \\
\hline $\mathrm{Sb}_{2} \mathrm{Te}_{3}$ & A & $26 / 27$ & $118 \mu \mathrm{V} / \mathrm{K}$ \\
\hline $\mathrm{Bi}_{2} \mathrm{Te}_{3}$ & $\mathrm{~A}$ & $22 / 27$ & $-51 \mu \mathrm{V} / \mathrm{K}$ \\
\hline $\mathrm{Junction}_{\mathrm{Sb}} \mathrm{Te}_{3}$ & A & $26 / 27 \& 22 / 27$ & $168 \mu \mathrm{V} / \mathrm{K}$ \\
\hline $\mathrm{Bi}_{2} \mathrm{Te}_{3}$ & $\mathrm{~B}$ & $27 / 27$ & $133 \mu \mathrm{V} / \mathrm{K}$ \\
\hline $\mathrm{Junction}^{\mid}$ & B & $23 / 27$ & $-50 \mu \mathrm{V} / \mathrm{K}$ \\
\hline
\end{tabular}




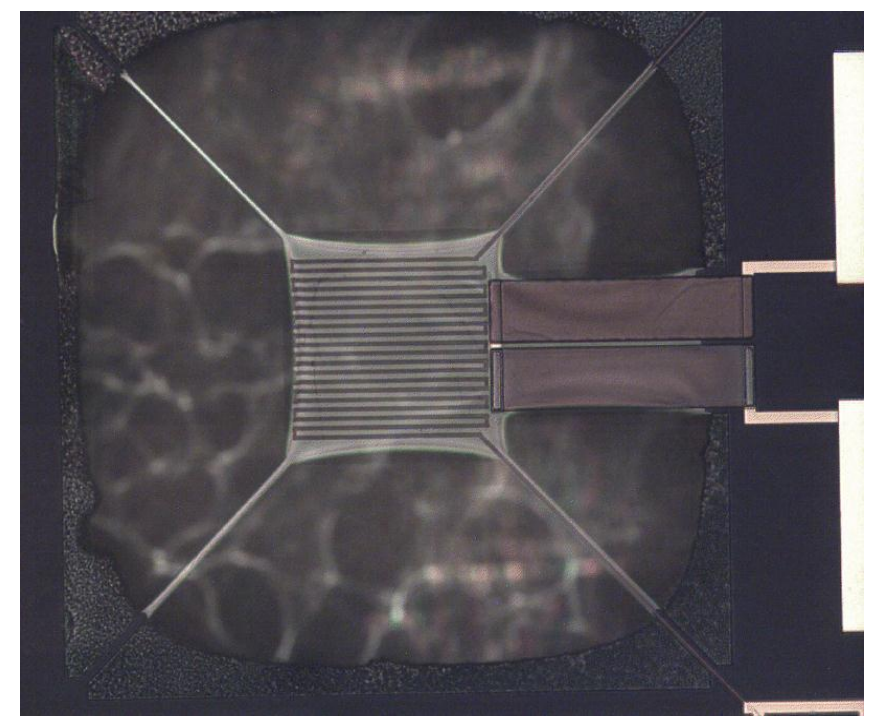

Figure 4: Optical image of the released device with the microheater seen on the center plate and the thermoelectric junction to the right of the plate.

\section{Thermal Conductivity}

The thermal conductivity $(\kappa)$ of our materials was characterized using test structures like that seen in Figure 5. This structure consists of a thermally isolated micro-heater with wide legs composed of mostly the thermoelectric material being studied. With this geometry, the dominant heat transfer mechanism is thermal conduction through the material of interest. When placed in vacuum, conduction through air and convection can be ignored and the heat balance equation of the structure simplifies to

$$
\mathbf{I}^{2} \mathbf{R}=\mathbf{G} \Delta \mathbf{T}
$$

Testing was done on samples using the Batch A recipe and using a $\mathrm{NiFe}$ micro-heater having a TCR of $.12 \% / \mathrm{K}$. Again, we sourced a varying current while measuring the resistance of the micro-heater and the total thermal conductance of the structure can be calculated

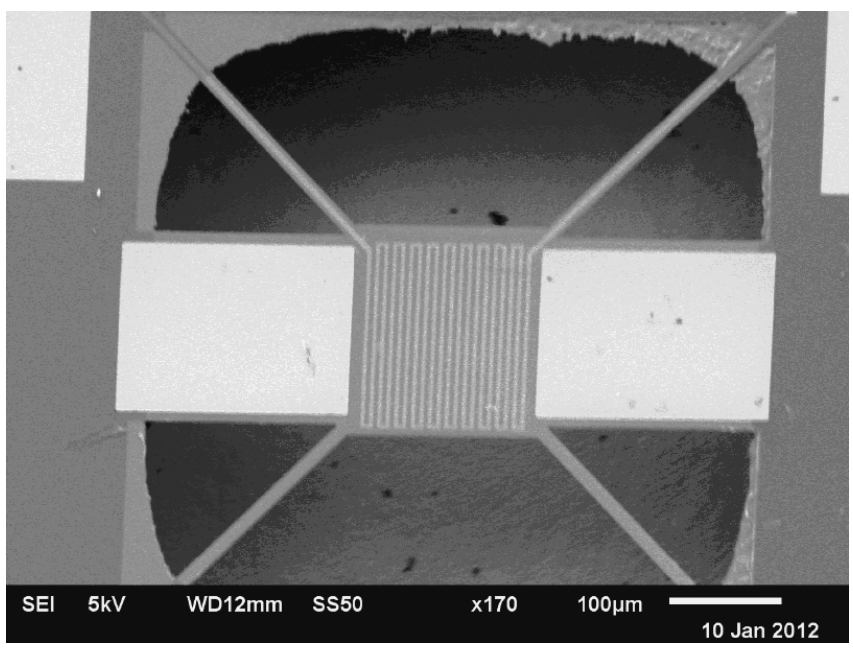

Figure 5: SEM micrograph of the test structures used to characterize the thermal conductivity of the thermoelectric materials. A micro heater supported by large films of the material being studied.

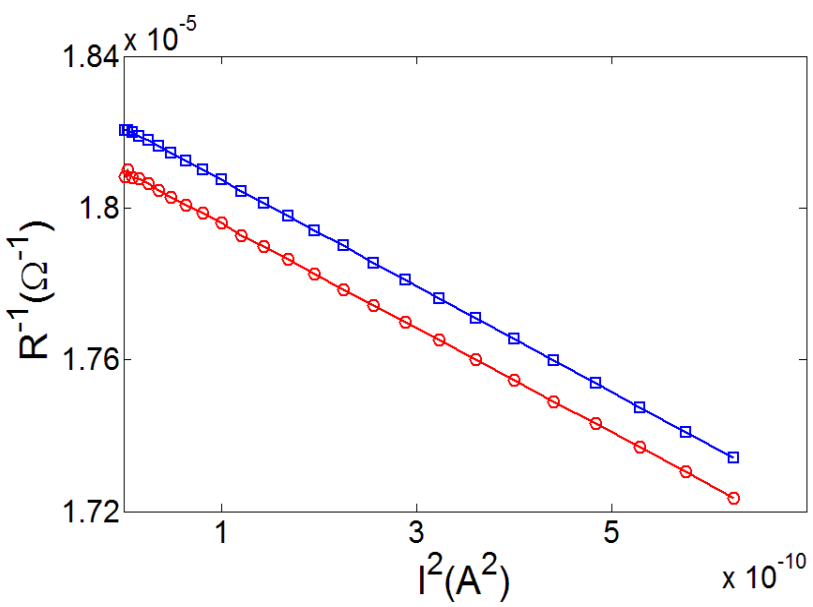

Figure 6: Thermal conductivity data $\left(I^{2}\right.$ vs. $\left.R^{-1}\right)$ for $\mathrm{Sb}_{2} \mathrm{Te}_{3}$ (०) and $\mathrm{Bi}_{2} \mathrm{Te}_{3}$ ( $\square$ ) test structures for films using the Batch $\mathrm{A}$ recipe.

as the micro-heater TCR divided by the slope of the $\mathrm{I}^{2}$ vs. $\mathrm{R}^{-1}$ plot seen in Figure 6. In order to extract the $\kappa$ values of our materials from the overall thermal conductance of the structure, we made a few assumptions about the other materials involved. We have assumed that $\kappa=2.6 \mathrm{Wm}^{-1} \mathrm{~K}^{-1}$ for $\mathrm{ALD} \mathrm{Al}_{2} \mathrm{O}_{3}$, a value that has been measured previously by our group [9], and that $\kappa=25 \mathrm{Wm}^{-1} \mathrm{~K}^{-1}$ for $\mathrm{Ni} / \mathrm{Fe}$. These assumptions are conservative, but have little impact on the final calculation due to the relatively small amount of $\mathrm{Ni} / \mathrm{Fe}$ in the support structure. The thermal conductance due to convection, conduction through air, and radiation were assumed to be negligible. Our final calculated values for these materials are $\kappa=.88 \mathrm{Wm}^{-1} \mathrm{~K}^{-1}$ for $\mathrm{Bi}_{2} \mathrm{Te}_{3}$ and $\kappa=1.02 \mathrm{Wm}^{-1} \mathrm{~K}^{-1}$ for $\mathrm{Sb}_{2} \mathrm{Te}_{3}$.

The thermal conductivity of devices such as those seen in Figure 4 was measured at around $5 \times 10^{-7} \mathrm{~W} / \mathrm{K}$ for both batches. This is a higher value than expected given the measured $\kappa$ values for the thermoelectric materials. We suspect that this is due to significant thermal conductivity due to radiation in our devices which becomes more apparent for lower G structures. The infrared absorption of Ni micro-heater devices was measured using an FTIR microscope and revealed an average absorption of $27 \%$ in the $8-12 \mu \mathrm{m}$ peak room-temperature thermal emission range. Using the Stephan Boltzman Law we can estimate the thermal conductance due to radiation $1.32 \times 10^{-7} \mathrm{~W} / \mathrm{K}$ which is approximately $15 \%$ of the total thermal conductance of our test structures. This means that our calculated $\kappa$ values may have been somewhat conservative, as some of the thermal conductance attributed to the films was actually due to radiation. Unfortunately, we were unable to measure $\kappa$ values for the Batch B devices, but given the relatively small deviation in $\kappa$ with small changes in composition for this material system [10], we assume the same $\kappa$ values for both batches when calculating the figure of merit. This is a non-ideal assumption but given the conservative nature of our calculation, we felt it was reasonable.

\section{Resistivity}

Film resistivity $(\rho)$ is defined as

$$
\rho=\frac{\mathbf{R A}}{\mathbf{L}}
$$

Where $\mathrm{R}$ is the resistance of the film in Ohms, $\mathrm{L}$ is the length, and A is the cross sectional area of the film. Since it is impossible to isolate the resistivity of the individual materials while testing 
junctions, electrical testing included both thermoelectric junctions and test structures which were simply long resistors of single materials. The resistance test structures consisted of $1730 \mu \mathrm{m}$ long, $3 \mu \mathrm{m}$ wide resistors contacted in a four point resistance measurement test structure. Due to lift-off issues with $3 \mu \mathrm{m}$ widths, the Batch $\mathrm{A} \mathrm{Sb}_{2} \mathrm{Te}_{3}$ resistivity was tested on $5 \mu \mathrm{m}$ wide resistors of the same length. Film thicknesses are given in Table 1. Resistance was measured using a current of $10 \mu \mathrm{A}$. The resistivities calculated for Batch A are 5.22 $\mu \Omega-\mathrm{m}$ for $\mathrm{Bi}_{2} \mathrm{Te}_{3}$ and $29.72 \mu \Omega-\mathrm{m}$ for $\mathrm{Sb}_{2} \mathrm{Te}_{3}$. For Batch B we calculated $6.15 \mu \Omega-\mathrm{m}$ for $\mathrm{Bi}_{2} \mathrm{Te}_{3}$ and $26.22 \mu \Omega-\mathrm{m}$ for $\mathrm{Sb}_{2} \mathrm{Te}_{3}$.

The resistance of unreleased thermoelectric junctions like those shown in Figure 4 was tested over a variety of film widths. The measured values were significantly higher than expected given the resistivity values previously measured on test structures. We suspect this is due to a contact resistance issue since the resistance values tend to converge to the expected value in the narrower junctions where the film geometry is closer to that of the test structures. Also, for reasons we do not yet understand, the resistance of the junctions drops significantly after etch release, bringing them to within $15 \%$ of the expected value. An example are the $54 \mu \mathrm{m}$ wide Batch A junctions which have an expected resistance of $1164 \Omega$ given their geometry and measured resistivity. Before release the average resistance measured is $1727 \Omega$ while this drops to $1304 \Omega$ after release.

Figure of Merit

The thermoelectric figure of merit $(\mathrm{Z})$ is defined as

$$
Z=\frac{\left(\sigma_{p}-\sigma_{n}\right)^{2}}{\left[\left(\kappa_{\rho} \rho_{\rho}\right)^{2}+\left(\kappa_{n} \rho_{n}\right)^{2}\right]^{\frac{1}{2}}}
$$

which simplifies to $z=\sigma^{2} \kappa^{-1} \rho^{-1}$ when dealing with a single material [10]. Table 3 shows the measured parameters for each film and the calculated $\mathrm{Z}$ value. Combining the properties of the two materials using Equation 3 we calculate an overall $\mathrm{Z}$ value of $0.93 \times 10^{-3}$ for the Batch A material system $1.23 \times 10^{-3}$ for the batch B material system.

Table 3: Measured resistivity ( $\rho)$, thermal conductivity $(\kappa)$, Seebeck coefficient $(\sigma)$, and calculated figure of merit (Z) for the films presented. The * on the Batch $B$ values indicates that the values were assumed equal to Batch $A$ as explained in the text.

\begin{tabular}{|l|l|l|l|l|}
\hline Recipe & $\rho(\mu \Omega-\mathrm{m})$ & $\kappa(\mathrm{W} / \mathrm{mK})$ & $\sigma(\mu \mathrm{V} / \mathrm{K})$ & $\mathrm{Z}^{*} 10^{4}$ \\
\hline $\mathrm{Sb}_{2} \mathrm{Te}_{3} \mathrm{~A}$ & 29.72 & 1.02 & 118 & 4.59 \\
\hline $\mathrm{Sb}_{2} \mathrm{Te}_{3} \mathrm{~B}$ & 26.22 & $1.02^{*}$ & 133 & 6.61 \\
\hline $\mathrm{Bi}_{2} \mathrm{Te}_{3} \mathrm{~A}$ & 5.22 & .88 & -51 & 5.66 \\
\hline $\mathrm{Bi}_{2} \mathrm{Te}_{3} \mathrm{~B}$ & 6.15 & $.88^{*}$ & -50 & 4.62 \\
\hline
\end{tabular}

\section{CONCLUSIONS}

Microstructures have been fabricated with a high degree of thermal isolation, $\mathrm{G} \sim 5 \times 10^{-7}$, which contain a micro-heater and $\mathrm{Sb}_{2} \mathrm{Te}_{3} / \mathrm{Bi}_{2} \mathrm{Te}_{3}$ thermoelectric junction. The thermoelectric properties, Seebeck coefficients, resistivities, and thermal conductivities of these materials have been measured for two different compositions of each material. The resulting figures of merit for these materials systems are $0.93 \times 10^{-3}$ and $1.23 \times 10^{-3}$.

These values are sufficient for applications such as micro-coolers, infrared detector readouts, and temperature controllers using films thin enough for MEMS-based devices.

\section{ACKNOWLEDGEMENTS}

The authors would like to thank DARPA MTO for their support under Army Research Office contract W911QX-12-C0002. The views expressed are those of the authors and do not reflect the official policy or position of the Department of Defense or the U.S. Government. Approved for public release, distribution unlimited.

\section{REFERENCES}

[1] A. Boyer and E. Cissé, "Properties of thin film thermoelectric materials: application to sensors using the Seebeck effect," Materials Science and Engineering: B, 13, 2 (1992).

[2] N.G. Patel and P.G. Patel, "Thermoelectric cooling efficiency in a $\mathrm{p}-\mathrm{Sb}_{2} \mathrm{Te}_{3}-\mathrm{n}-\mathrm{Bi}_{2} \mathrm{Te}_{3}$ thin film thermocouple," Solid-State Electronics, 35, 9 (1992).

[3] H. Zou, D.M. Rowe, and S.G.K. Williams, "Peltier effect in a co-evaporated $\mathrm{Sb}_{2} \mathrm{Te}_{3}(\mathrm{P})-\mathrm{Bi}_{2} \mathrm{Te}_{3}(\mathrm{~N})$ thin film thermocouple," Thin Solid Films, 408, 1-2 (2002).

[4] L.M. Goncalves, P. Alpium, G. Min, D.M. Rowe, C. Couto, and J.H. Correia, "Optimization of $\mathrm{Bi}_{2} \mathrm{Te}_{3}$ and $\mathrm{Sb}_{2} \mathrm{Te}_{3}$ thin films deposited by co-evaporation on polyimide for thermoelectric applications," Vacuum, 82, 12 (2008).

[5] D. Kim and G. Lee, "Effect of rapid thermal annealing on thermoelectric properties of bismuth telluride films grown by co-sputtering," Materials Science and Engineering: B, 131, 13 (2006).

[6] H. Bottner, J. Nurnus, A. Gavrikov, G. Kuhner, M. Jagle, C. Kunzel, D. Eberhard, G. Plescher, A. Schubert, K.-H. Schlereth, "New thermoelectric components using microsystem technologies," Journal of Microelectromechanical Systems, 13, 3, (2004).

[7] C. Liao and T. She, "Preparation of bismuth telluride thin films through interfacial reacting," Thin Solid Films, 515, 2021 (2007).

[8] A. Gross, G. Hwang, B. Huang, H. Yang, N. Ghafouri, H. Kim, C. Uher, M. Kaviany, K. Najafi, "High-performance micro scale thermoelectric cooler: An optimized 6-stage cooler," Solid-State Sensors, Actuators and Microsystems Conference, Denver, CO, 6/21-25/09, IEEE, Piscataway (2009), pp. 2413-2416.

[9] N. T. Gabriel and J. J. Talghader, "Thermal conductivity and refractive index of hafnia-alumina nanolaminates," Journal of Applied Physics, 110, 4, (2011).

[10] D.M. Rowe, CRC Handbook of Thermoelectrics, CRC Press, Boca Raton, 1995.

\section{CONTACT}

*J.J. Talghader, tel: +1-612-625-4524; joey@umn.edu 


\title{
CHARACTERIZATION AND PRODUCTION CONTROL FOR DRIE OF MEMS ACCELEROMETERS USING OPTICAL DISPLACEMENT TRANSDUCER MEASUREMENTS
}

\author{
B. Keese ${ }^{I^{*}}$, B. Homeijer ${ }^{1}$, J.L. Williams ${ }^{1}$ and P. Knutrud ${ }^{2}$ \\ ${ }^{1}$ Hewlett-Packard Co., Technology Development Operations, Corvallis, OR , USA \\ ${ }^{2}$ Inspectrology, Sudbury, MA, USA
}

\begin{abstract}
A Fiber Optic Displacement Transducer is used to measure MEMS device motion after the active elements are released by etching. This paper discusses the use of such measurements to determine the resonant frequency of MEMS devices, the use of this data to characterize and improve Silicon DRIE processing and also describes incorporating this technique as an off-axis measurement system within an existing automated metrology tool. These measurements can be conducted quickly and non-destructively immediately following release etch, and this data has proven more informative than previous methods using the dimensions of etched features to predict the resonant frequency of MEMS devices.
\end{abstract}

\section{INTRODUCTION}

The invention and widespread use of Bosch DRIE processing has facilitated a dramatic increase in the number, type and success of MEMS devices being fabricated [1]. The very deep, high aspect ratio features created using this etch process allow the manufacture of the various sensors, actuators and resonators critical to the MEMS industry. Yet these same dimensional considerations create significant challenges for in-process dimensional measurement. The top-down optical or SEM dimensional measurement traditionally used in microelectronics is often inadequate to characterize and control MEMS processing, and mechanical or beam probes of various sorts are excluded by the geometry of the structures being produced. Thus, an alternate and ultimately more informative measurement has been developed, using a fiber optic lever transducer. These measurements can be conducted quickly and non-destructively immediately following release etch, and the data closely predicts the final performance of the assembled MEMS. This technique has proven more reliable than previous methods which used the dimensions of etched features as a proxy to predict the resonant frequency of the devices.

The seismic grade MEMS accelerometers recently announced by HP [2-4] consist of a hermetically sealed stack of three silicon wafers. The first wafer has a cavity etched into the bulk silicon. The second wafer is plasma bonded to this cavity wafer, and receives a Bosch etch to release the proof mass and form the flexure springs on which the mass is suspended (Figure 1a,b). The third "cap" wafer is bonded to the above two-wafer assembly, providing sensing electronics and hermetically sealing the device.

The final etched dimensions of the flexure springs determine their stiffness and strongly impact the performance of the MEMS accelerometers. However, due to the depth and the high aspect ratio of the etched flexures, meaningful three-dimensional measurements are very difficult to obtain, while the behavior of the etch is more complex than can be characterized using top-down width measurements alone (Figure 1c). Although cross-section SEM micrographs were essential for our initial work, the timeintensive and destructive nature of the technique limited its usefulness for controlling manufacture of the accelerometers in commercial volumes.
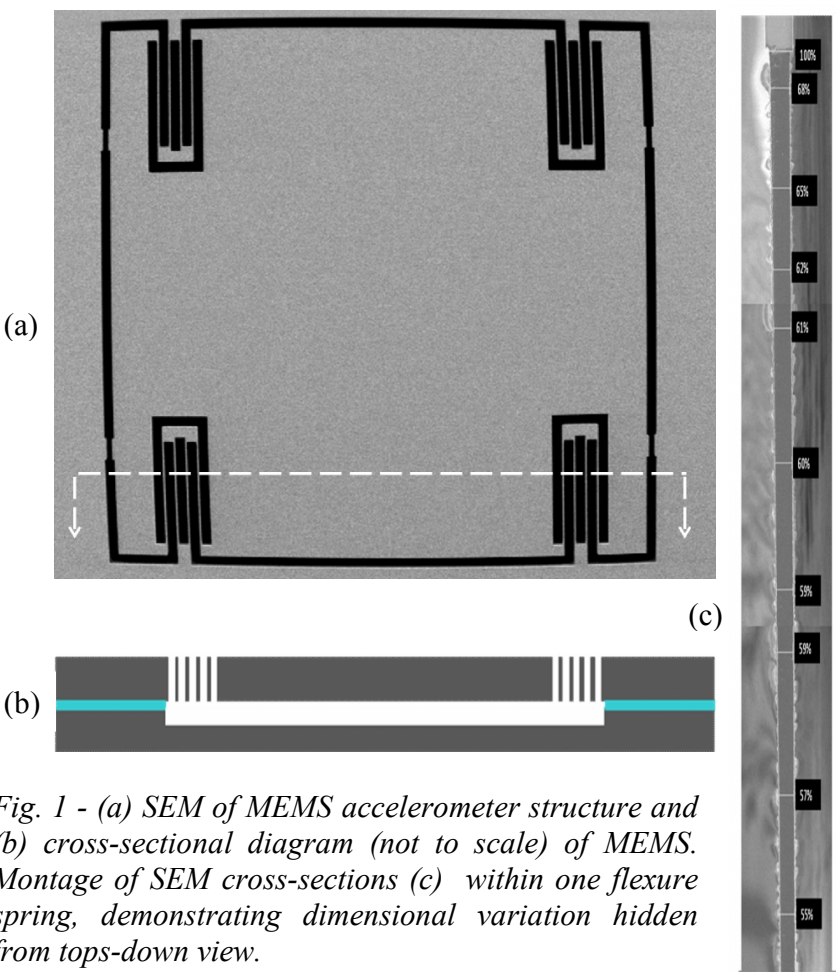

(b)

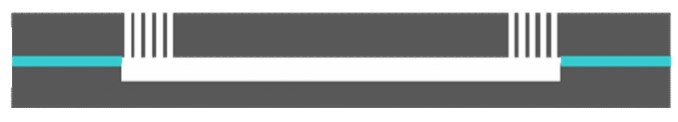

Fig. 1 - (a) SEM of MEMS accelerometer structure and (b) cross-sectional diagram (not to scale) of MEMS. Montage of SEM cross-sections (c) within one flexure spring, demonstrating dimensional variation hidden from tops-down view.

It was observed that the active MEMS elements will oscillate once etching has released the movable portion of the MEMS device (Figure 2). This movement can be recorded and analyzed to determine the resonant frequency of the MEMS structure, which is the critical parameter of interest. A simple technique to obtain this signal uses an intensity modulated fiber optic lever transducer.

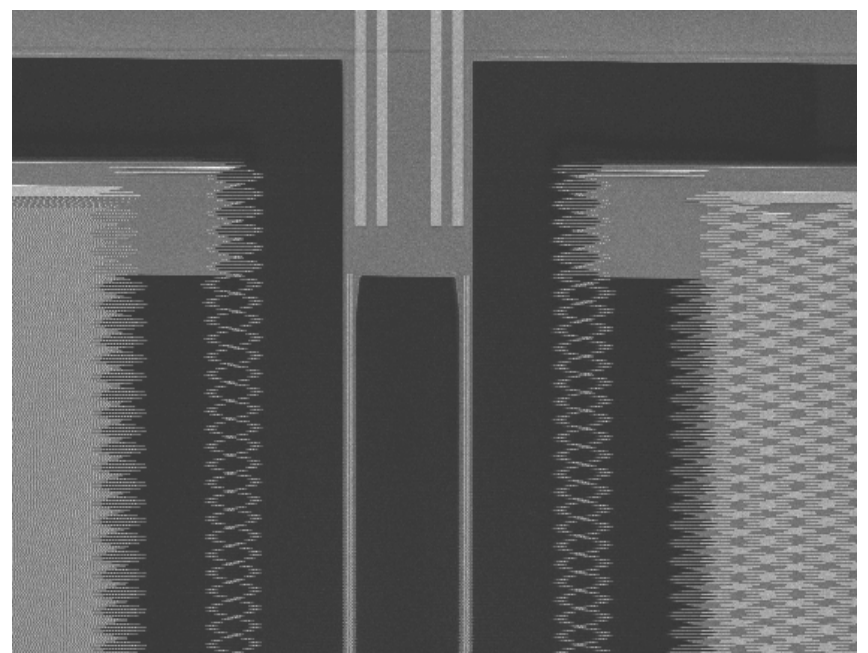

Figure 2-Observed oscillation of released accelerometer in SEM 
A fiber optic bundle in which adjacent fibers act as either illumination transmitting fibers or as reflected intensity receiving fibers will form an intensity-modulated optic lever displacement transducer [5], a transducer sensitive to displacement of a sample illuminated by the bundle. The intensity of the reflected light signal changes as a function of the distance to the illuminated sample, illustrated in Figures 3.
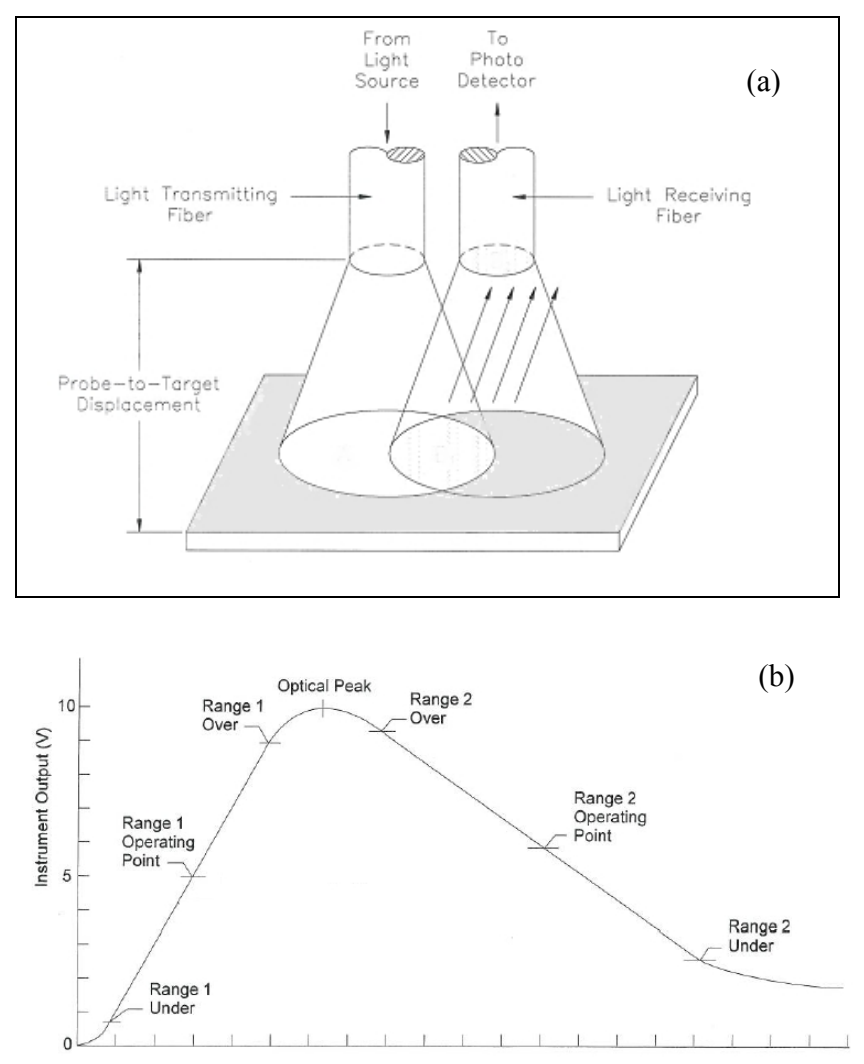

Probe to Target Gap, Displacement (uin or um)

Figure 3 - (a) Intensity modulated optic lever principle [6] and (b) Probe intensity calibration curve [6].

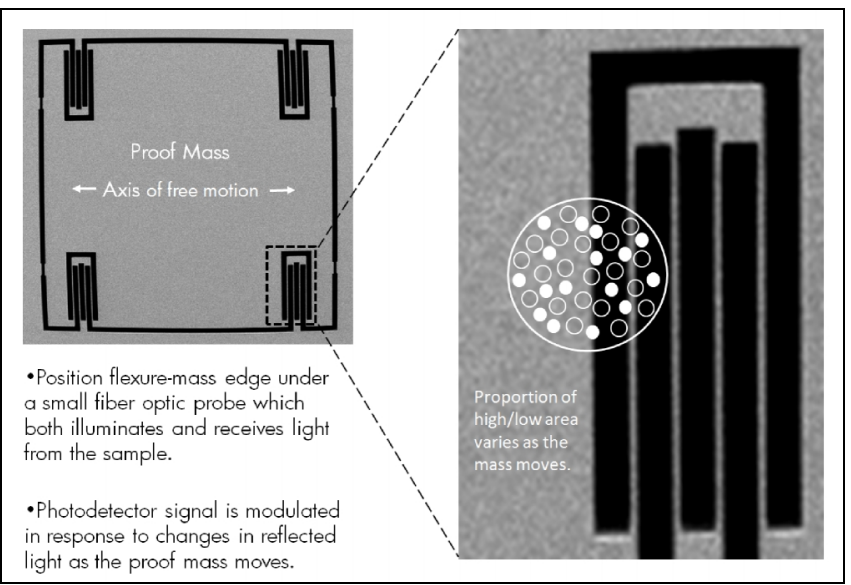

Figure 4 - Schematic of signal generation principal

By positioning the free moving portion of a MEMS device partially under a fiber optic transducer, the reflected signal produced is an area average of the signal from the unetched (high) and etched (low) portions of the device. By then stimulating its motion mechanically, the reflected light signal is modulated as the proportion of high and low topography under the beam changes (Figure 4). The signal data is fit to a damped sinusoidal model, from which the resonant frequency of the MEMS device, the initial amplitude and quality factor of the signal can be calculated.

\section{MANUAL SYSTEM \\ IMPLENTATION}

A manually operated measurement system was built, utilizing the fiber optic transducer principle. An MTI 2020R fiber optic probe was mounted to a microscope frame and wafer stage, allowing $\mathrm{X}$-, $\mathrm{Y}$ - and $\mathrm{Z}$-axis positioning of the individual die on sample wafers to be brought under the probe, shown in Figure 5. The optical probe is controlled by an MTI-2100 Fotonic Sensor, which converts the reflected light signal to a $0-10$ Volt electrical output signal and provides various options for filtering the output signal. The output voltage signal is routed to a DAQ board (Natl. Instr. PCIe-6321) in a PC, which samples and stores the signal. The stored signal can be analyzed with various techniques. In this case, the data are compared to a damped sinusoid model using a MATLAB program, and frequency, initial amplitude, quality factor and $\mathrm{R}^{2}$ are extracted from each set of fit data.

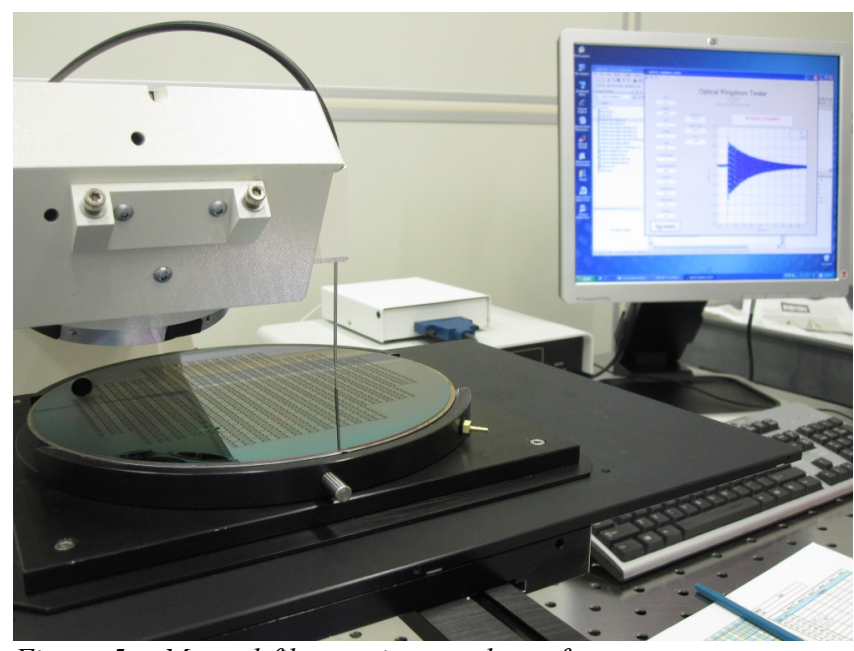

Figure 5 - Manual fiber optic transducer frequency measurement system

\section{OPERATION} follows:

Using the above apparatus, measurements are taken as

A sample wafer is loaded by hand onto the wafer stage, and the stage is moved to bring the unetched upper surface of the wafer assembly into the plane of focus of the optical probe. The probe is operated at the left (high) end of 'Range 2' as shown in Figure 3b, with the upper surface of the wafer assembly approximately $300 \mu \mathrm{m}$ from the probe tip. This returns a voltage signal of approximately 8 Volts with good contrast to the lower, etched regions, which provide a signal of roughly 2.5 Volts. The probe illumination intensity can be adjusted through the MTI-2100 Fotonic Sensor as needed.

Using the wafer stage the sample is moved, centering the edge of an etched flexure spring in the illuminated area under the fiber optic probe, causing the return signal voltage to fall to about half the value given by the unetched upper surface. The edge of the wafer assembly is then tapped gently with a metal rod to excite the accelerometers into motion, modulating the return signal, which is captured by the DAQ board and stored on the PC. The MATLAB program then analyzes the stored data, and logs the parameters to a 
text file. Using this manual system, the time to move, focus and measure each new sample on a wafer is about 30 seconds.

\section{RESULTS}

Due to the large volume of data and many cycles over which the signal can be obtained, measurements taken in this manner can be fit to the damped sinusoid model with correlation coefficients above 0.9 for typical measurements (Figure 6).

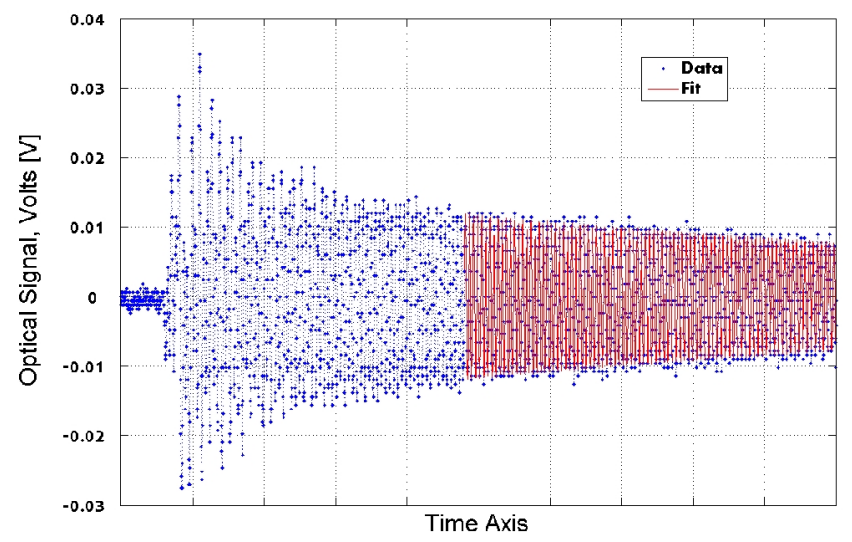

Figure 6-Typical optical signal fit to damped harmonic model.
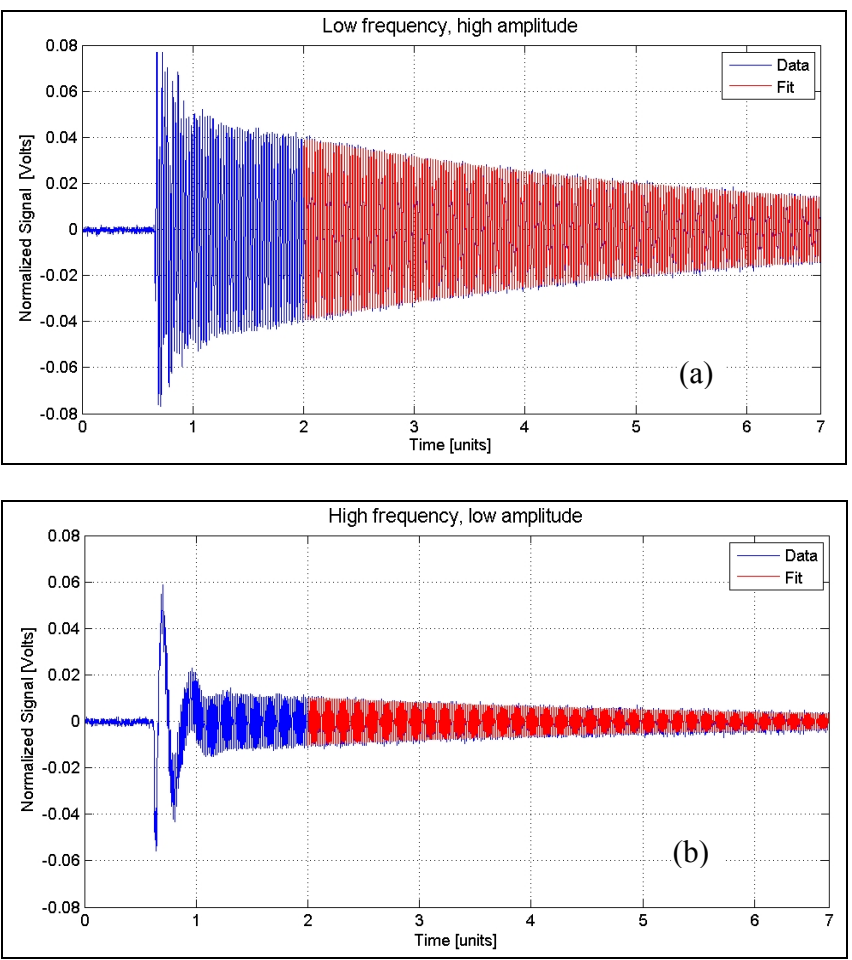

Figure 7 - Signals typical of (a) weak and (b) stiff flexure springs.

This data has proven extremely valuable when optimizing the BOSCH DRIE recipe parameters, making immediate feedback available as recipe parameter experiments were designed and etched. Over-etching and/or poor passivation were known to produce flexure springs with narrow cross-sectional shape, and low resonant frequencies and high amplitudes consistent with weak springs were measured for parts etched under those conditions (Figure 7a). Conversely, flexures which were under-etched or over-passivated during etch gave high resonant frequencies and lower amplitudes (Figure 7b) consistent with stiffer, wider springs. Improvements in across-the-wafer frequency response uniformity (Figure 8) were thus quickly obtained.

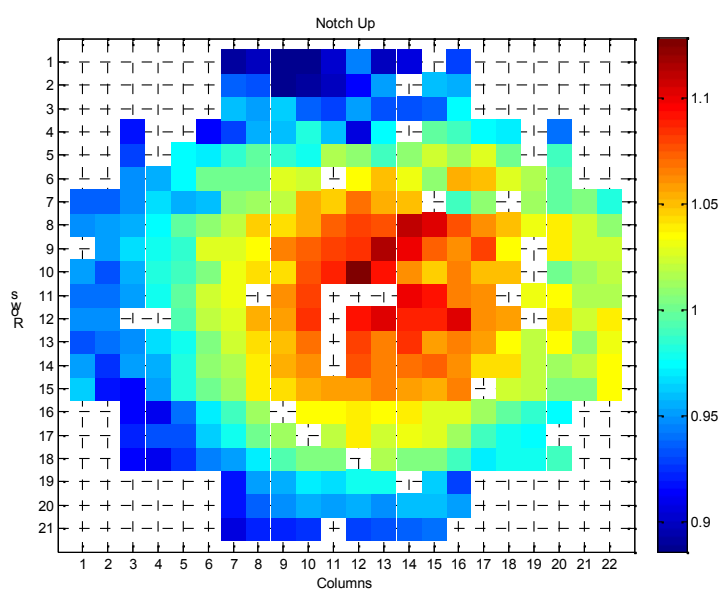

Figure 8-Typical normalized cross-wafer frequency uniformity.

The data obtained from this measurement system has also proven quite useful in diagnosing and resolving manufacturing process excursions. Whereas top-down width measurements failed to detect that a photolithography process shift had occurred, these optical measurements detected the shift in device frequency and allowed quick response. Additionally, brken or fractured flexure springs which are difficult to detect using automated top-down inspection techniques are readily identifiable within the frequency response data.

Most importantly, the optical data proved to be a reliable indicator of the frequency which can be expected from the finished devices when tested electrically, over a wide range of designs, frequencies and etch conditions (Figure 9).

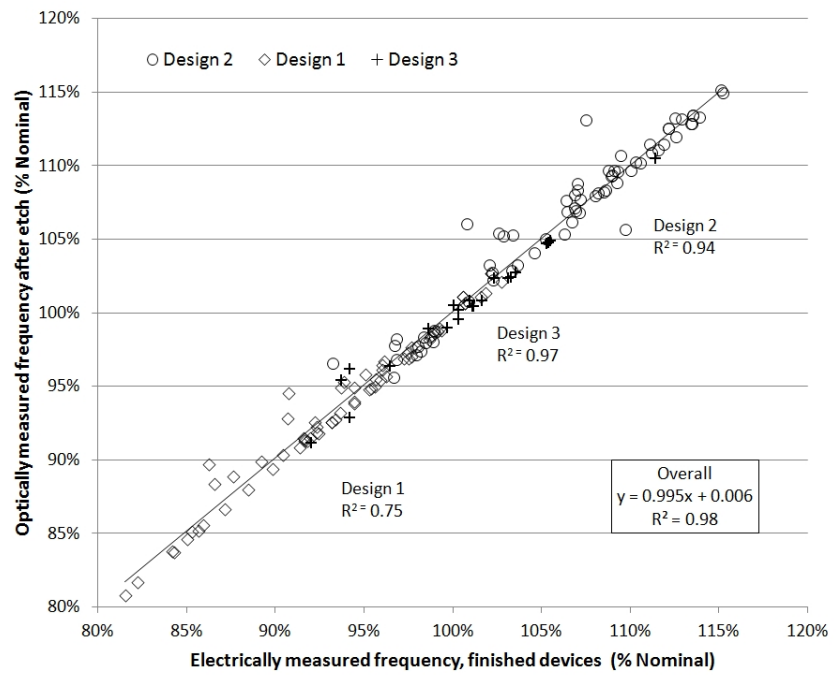

Figure 9 - Correlation of optical frequency measurements done in-process to electrical frequency measurements taken on finished devices. 


\section{AUTOMATED SYSTEM}

To provide the extensive data required to produce the HP seismic grade MEMS accelerometers in commercial volumes with demonstrated process control, a high degree of automation is required of the measurement system. As such, fiber optic transducers have been integrated into existing measurement equipment as an off-axis metrology system, allowing each device to be measured following release etch.

\section{IMPLENTATION}

An MTI 2020R fiber optic probe was fixed to each side of the microscope frame of an existing IVS-130, allowing access to the entire surface of the $200 \mathrm{~mm}$ wafer assemblies (Figure 10). To provide adequate working distance for wafer handling, each probe is fitted with a KD-LS-1A Fiber Optic Probe Extender (MTI), which increases the working distance to approximately $12 \mathrm{~mm}$. As on the manual system, the probes are controlled by an MTI-2100 Fotonic Sensor, converting the reflected light to a $0-10$ Volt electrical output signal and providing various options for filtering the output signal.

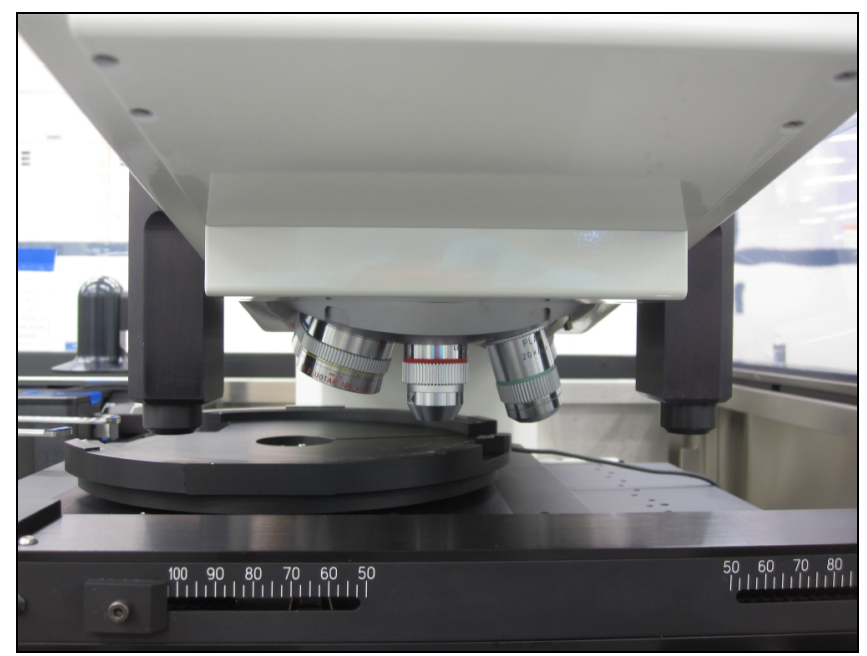

Figure 10: Fiber optic probes and extenders mounted to either side of IVS-130 microscope frame.

Following recipe selection via SECS and automated wafer loading, a modified control program on the IVS-130 routes the samples selected for measurement to either the left or right probe, based on the location of the sample on the wafer assembly. On the automated system, the output voltage signals are again routed to a DAQ board (Natl. Instr. PCIe-6321) housed in a separate PC, which samples, stores and analyzes the signal using proprietary software, and then provides the extracted measurement parameters to the computer controlling the IVS-130 (Figure 11). The IVS-130 control computer reports these parameters as it would any other dimension or overlay measurement.

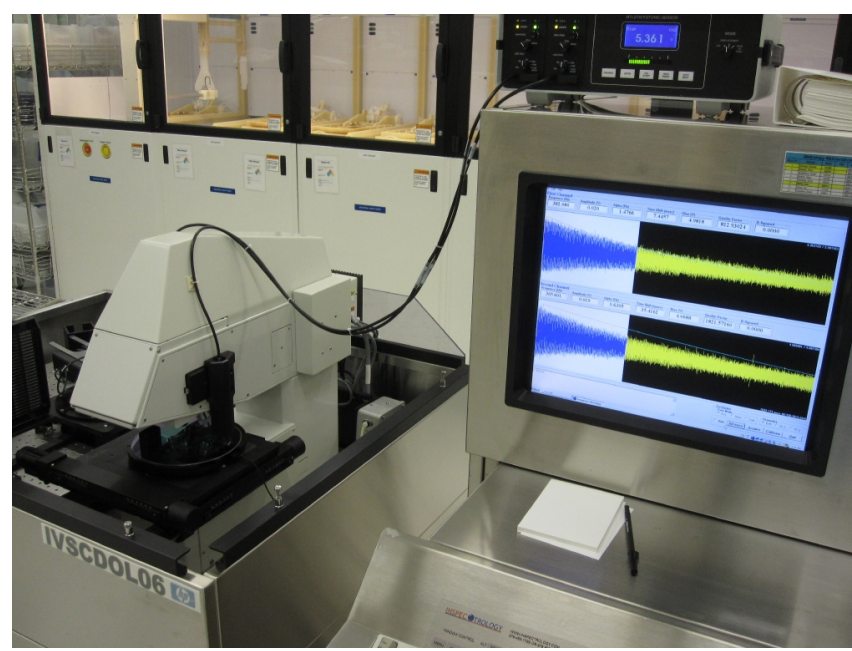

Figure 11: Automated system displaying signal analysis screen.

Rather than striking the samples, the automated system uses the motion of the wafer assembly on the stage to stimulate the accelerometers into motion. Driving the sample to a stop under the fiber optic probe results in a gentle acceleration to which the devices respond.

\section{RESULTS}

Although gentle, the wafer stage move and stop under the fiber optic probes provides sufficient stimulus to cause the accelerometers to resonate detectably (Figure 12). Again, due to the large volume of data and many cycles over which the signal is obtained, measurements taken in this manner can be fit to a damped sinusoid model with high confidence. Operating the optical probes in the manner described results in a sensitivity to the lateral motion of an edge of approximately $20 \mathrm{mV} / \mu \mathrm{m}$.

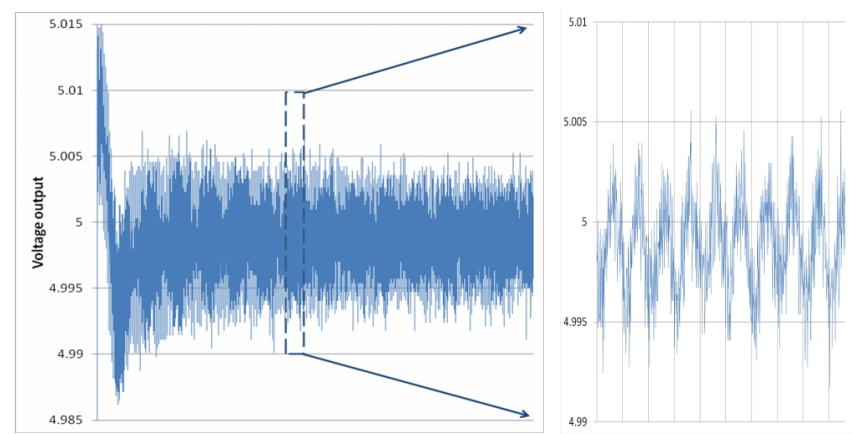

Figure 12: Initial 0.8secs of signal output (a) and section of same signal on an expanded time axis (b).

Data taken on the automated system matches data taken from the manual system to within a fraction of one Hertz in all instances (Figure 13). Both of the probes on the automated system are shown to match the single-probe manual system, thus they also match one another. 


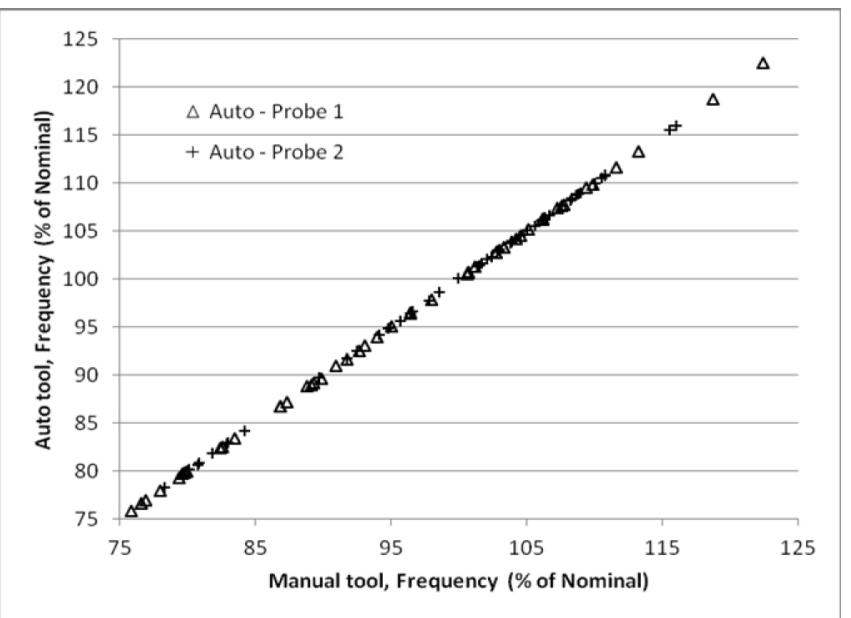

Figure 13: Correlation of frequency measurements taken on Automatic and Manual systems.

All of the original capabilities of the IVS measurement tool remain intact, and the tool still performs dimensional and overlay measurements. Frequency measurement data can be taken in the same program as, and interspersed with, dimensional or overlay data. For frequency measurement, move-acquire-measure (MAM) time is 11 seconds, allowing extensive datasets to be taken. The tool is SECS compliant and is interfaced to an automated factory control system for recipe selection and data transfer.

\section{CONCLUSION}

The resonant frequency of MEMS devices can be obtained by using a fiber optic transducer to detect the motion of the device. This relatively simple device - no lasers, no moving parts - was used to build a manual measurement system and has since been incorporated into an available automated tool as an off-axis measurement system, offering programmable sample selection, cassette-to-cassette wafer handling and recipe selection via SECS interface. Data obtained using this tooling has a high signal to noise ratio, is extremely repeatable, with excellent fit to the modeled damped sinusoidal behavior. These measurements can be conducted quickly and non-destructively immediately following the release etch and have proven quite useful in characterizing and controlling the fabrication processes used to form the flexure springs of the devices. The measurements also correlate very well to the resonant frequency as determined electrically in the completed devices.

\section{ACKNOWLEDGEMENT}

The authors would like to thank Mr. Paul Slazas of MTI for his advice and assistance in selecting and using the fiber optics and instruments.

\section{REFERENCES}

[1] F. Laermer and A. Urban, "BOSCH-DRIE Shaping MEMS History, Applications and Future Directions", Technical Digest of the 2010 Solid-State Sensors, Actuators, and Microsystems Workshop, Hilton Head Isl.,SC 6/6-10/10, Transducer Research Foundation, Cleveland, OH (2010), pp. 1-6.

[2] HP News Release (2010, Feb. 15), "Shell and HP to Develop Ultrahigh-Resolution Seismic Sensing Solution” [Online]. Available: http://www.hp.com/hpinfo/newsroom/press/2010/100215xa.ht $\underline{\mathrm{ml}}$

[3] B.D. Homeijer, et.al., "Hewlett-Packard's Seismic Grade MEMS Accelerometer", Proc.24th IEEE Conf. on MEMS, Cancun,MX, Jan. 2011

[4] D.J. Milligan, B.D. Homeijer and R.G. Walmsley, "An UltraLow Noise MEMS Accelerometer for Seismic Imaging", Proc.10th IEEE Conf. on Sensors, Limerick,IE, Nov. 2011

[5] R.O. Cook and C.W. Hamm, "Fiber optic lever displacement transducer”, Applied Optics, Vol.18, No. 19, pp. 3230-3241, Oct. 1, 1979.

[6] MTI Instruments, "An Introduction to the Fotonic Sensor", Application notes [On-line] Available: http://www.mtiinstruments.com/products/fiberopticmeasurem ent.aspx

\section{CONTACT}

*B. Keese, tel: +1-541-715-2497; william.keese@hp.com 


\title{
TITANIA SILICATE / FUSED QUARTZ GLASSBLOWING FOR 3-D FABRICATION OF LOW INTERNAL LOSS WINEGLASS MICRO-STRUCTURES
}

\author{
D. Senkal, C.R. Raum, A.A. Trusov, and A.M. Shkel \\ MicroSystems Laboratory, University of California, Irvine, CA, USA
}

\begin{abstract}
We report, for the first time, a fabrication process for building highly smooth, symmetric 3-D dynamic wineglass structures using low internal loss materials, namely fused quartz and ultra low expansion titania silicate glass (ULE TSG). The approach consists of a high temperature (at $1650{ }^{\circ} \mathrm{C}$ ) micro-glassblowing process and a novel inverted-wineglass architecture that provides self-aligned stem structures. An in-house process capability of $1800{ }^{\circ} \mathrm{C}$ glassblowing with a rapid cooling rate of $500{ }^{\circ} \mathrm{C} / \mathrm{min}$ was developed. Feasibility of the process has been demonstrated by fabrication of TSG wineglass micro-structures. The method may enable new classes of TSG and fused quartz MEMS devices with extremely low surface roughness, $0.23 \mathrm{~nm}$ surface average $(\mathrm{Sa})$, intrinsically low internal losses $\left(\mathrm{Q}_{\mathrm{TED}}>5 \mathrm{E}+10\right)$ and dynamically balanced structures.
\end{abstract}

\section{INTRODUCTION}

Maximization of the quality (Q) factor is key to enhancing performance of vibratory MEMS devices in demanding signal processing, timing and inertial applications [1]. The macro-scale hemispherical resonator gyroscope (HRG) with Q-factors over $25 \mathrm{E}+6$ [2] motivates the investigation of 3-D fused quartz microwineglass structures for use as vibratory elements.

With the emergence of novel fabrication techniques batch fabrication of 3-D wineglass structures are becoming possible. For instance, hemispherical shells fabricated by deposition of polysilicon [3] or silicon nitride [4] thin films into isotropically etched cavities have recently been demonstrated. Alternative fabrication techniques include "3-D SOULE" process for fabrication of mushroom and concave shaped spherical structures [5] as well as blow molding of bulk metallic glasses into preetched cavities [6]. However, MEMS wineglass resonators with sufficient smoothness and low thermoelastic dissipation (TED) have not yet been demonstrated in the literature. To take full advantage of the 3-D wineglass architecture, fabrication techniques with low surface roughness as well as materials with high isotropy and low TED are desired.

It has been demonstrated that MEMS devices can reach the fundamental $\mathrm{Q}_{\mathrm{TED}}$ limit by using a combination of balanced mechanical design and vacuum packaging with getters [7]. TED is caused by local temperature fluctuations due to vibration and the associated irreversible heat flow, which results in entropic dissipation. TED can be reduced either by decoupling the mechanical vibrations from the thermal fluctuations or by using materials with low coefficient of thermal expansion (CTE). This paper focuses on materials with low CTE, such as fused quartz $\left(0.5 \mathrm{ppm} /{ }^{\circ} \mathrm{C}\right)$ or ultra low expansion titania silicate glass $\left(0.03 \mathrm{ppm} /{ }^{\circ} \mathrm{C}\right)$, which can provide a dramatic increase in fundamental $\mathrm{Q}_{\mathrm{TED}}$ limit $\left(\mathrm{Q}_{\mathrm{TED}}>7 \mathrm{E}+10\right.$ for a $\mathrm{TSG}$ wineglass). However, when compared to silicon, titania silicate glass and fused quartz dry etching suffers from order of magnitude higher surface roughness, lower mask selectivity and aspect ratios [8].

Pyrex glassblowing at $850^{\circ} \mathrm{C}$ on a silicon substrate was previously demonstrated for fabrication of smooth, symmetric 3-D structures [9-11]. However, TSG glassblowing requires upwards of $1600{ }^{\circ} \mathrm{C}$ glassblowing temperature due to its higher softening point, which prevents the use of fabrication processes that rely on a a) Fused quartz is etched and TSG device layer is bonded on top.
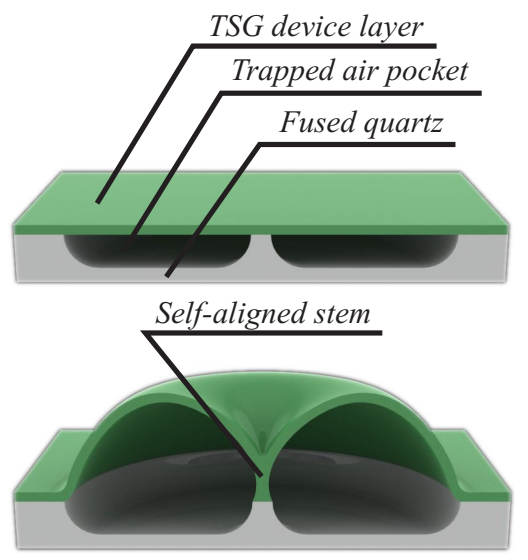

b) TSG/fused quartz stack is glassblown at $1650^{\circ} \mathrm{C}$, creating a selfaligned stem.

c) Inverted-wineglass structure is released along the perimeter (e.g. laserablation).

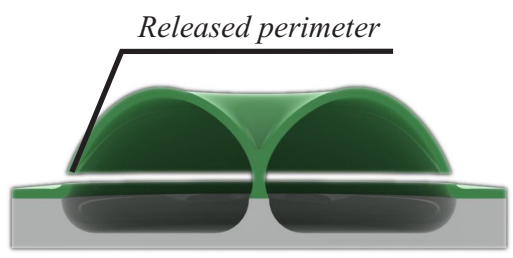

Figure 1: High temperature glassblowing process for fabrication of ULE TSG / fused quartz inverted-wineglass structures.

silicon substrate. This paper explores the hypothesis that high temperature glassblowing $\left(1650^{\circ} \mathrm{C}\right)$, may serve as an enabling mechanism for wafer-scale fabrication of TSG / fused quartz 3-D wineglass structures. The approach consists of a high temperature micro-glassblowing process and a novel inverted-wineglass architecture that provides self-aligned stem structures, Fig. 1. An in-house process capability of $1800{ }^{\circ} \mathrm{C}$ glassblowing with a rapid cooling rate of $500{ }^{\circ} \mathrm{C} / \mathrm{min}$ was developed. Feasibility of the process has been demonstrated by fabrication of TSG / fused quartz micro-wineglass structures.

\section{DESIGN TRADE-OFFS}

To analyze the effects of geometric design parameters as well as the material choice on fundamental Q-factor limits, parametric finite element analysis (FEA) was conducted using Comsol Multiphysics Package.

\section{Anchor Loss}

The focus of the analysis was to compare the invertedwineglass architecture proposed in this paper vs. previously fabricated glassblown structures with large attachment diameter, Fig. 2 [11]. The structure in Fig. 2(a) was fabricated by first glassblowing a spherical structure through a stencil layer and then laser micromachining the cap to create a wineglass structure.

The structure in Fig. 2(a) had a shell diameter of $1142 \mu \mathrm{m}$, anchor diameter of $600 \mu \mathrm{m}$ and average thickness of $4 \mu \mathrm{m}$, which gives roughly 1:2 attachment to shell diameter ratio. In contrast, the inverted-wineglass structure with the integrated stem, Fig. 2(b), had a shell diameter of $4200 \mu \mathrm{m}, 300 \mu \mathrm{m}$ anchor diameter and an average thickness of $80 \mu \mathrm{m}$, giving a 1:14 anchor to shell diameter ratio. 


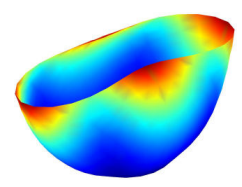

(a) without stem

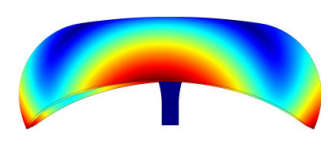

(b) with stem

\begin{tabular}{lccccc}
\hline & \multicolumn{4}{c}{$\mathbf{Q}_{\text {TED }}$} & Q $_{\text {anchor }}$ \\
\cline { 2 - 5 } & Silicon & Pyrex & FQ & TSG & \\
\hline (a) w/o stem & $1 \mathrm{E}+05$ & $1 \mathrm{E}+06$ & $4 \mathrm{E}+07$ & $1 \mathrm{E}+10$ & $3 \mathrm{E}+03$ \\
(b) with stem & $6 \mathrm{E}+04$ & $7 \mathrm{E}+06$ & $2 \mathrm{E}+07$ & $\mathbf{7 E}+\mathbf{1 0}$ & $\mathbf{5 E}+\mathbf{1 0}$ \\
\hline
\end{tabular}

Figure 2: Fundamental $Q_{T E D}$ and $Q_{\text {anchor limits for (a) without }}$ stem, (b) with stem, obtained using finite element analysis.

To simulate the accoustic loss in an infinite medium, a perfectly matched layer (PML) was used for the substrate domain. PML works by absorbing accoustic waves over a large frequency range at any non-zero angle of incidence. The simulation was run for perfectly symmetric structures, neglecting the contribution of mass imbalance to the anchor loss. For this reason, the values obtained from FEA represent the fundamental anchor loss limit of the structures. The wineglass structure with 1:2 anchor to shell diameter ratio Fig. 2(a) had a fundamental $Q_{\text {anchor }}$ limit of 3000, which is in close agreement with the experimentally obtained quality factor of 1256 [11]. In contrast, the analysis of the wineglass structure with the integrated stem (1:14 ratio), Fig. 2(b), showed virtually zero anchor loss $\left(\mathrm{Q}_{\text {anchor }}>5 \mathrm{E}+10\right)$.

\section{Thermoelastic Dissipation}

The goal of the analysis was to understand the effect of CTE on $\mathrm{Q}_{\mathrm{TED}}$. Four different materials: Silicon, pyrex, fused quartz and TSG were investigated, Fig. 2. Energy loss caused by TED was analyzed using a coupled thermo-mechanical model. The model was solved for the $n=2$ wineglass modes, and $Q_{T E D}$ values were extracted from the ratio of the real and imaginary parts of the eigenfrequencies. The difference in $\mathrm{Q}_{\mathrm{TED}}$ between the two geometries was limited to within one order of magnitude, whereas the material choice had a huge impact on the $\mathrm{Q}_{\mathrm{TED}}$. TSG (with the lowest CTE among the materials investigated) had the highest fundamental $\mathrm{Q}_{\mathrm{TED}}$ value at $7 \mathrm{E}+10$, which was followed by fused quartz at $\mathrm{Q}_{\mathrm{TED}}>2 \mathrm{E}+7$.

\section{FABRICATION PROCESS}

Fabrication process for TSG wineglass structures consists of four main steps, namely: (1) Etching of the fused quartz (or fused silica) substrate, (2) bonding of TSG device layer to fused quartz, (3) glassblowing, and finally (4) releasing the wineglass structure by etching around the perimeter, Fig. 1. The process starts by LPCVD deposition of a $2 \mu \mathrm{m}$ PolySi hard mask onto the fused quartz substrate. After which, the cavity openings were defined on the PolySi hard-mask using RIE. Then, $\sim 150 \mu \mathrm{m}$ deep toroidal cavities were wet etched into the substrate wafer using concentrated HF (49\%). In order to establish the etch rate of HF on fused quartz and TSG, 75 minute test runs were performed at room temperature. Etch depth was measured every 15 minutes by stopping the etch and measuring using a DEKTAK 3 profilometer. Linear regression fits showed an etch rate of $1.07 \mu \mathrm{m} / \mathrm{min}$ for fused quartz and $2.86 \mu \mathrm{m} / \mathrm{min}$ for TSG, with a linearity of $\mathrm{R}^{2}=0.996$ and $\mathrm{R}^{2}=0.997$ respectively. Once the etch was complete, PolySi layer was stripped and the wafers were thoroughly cleaned using RCA clean.

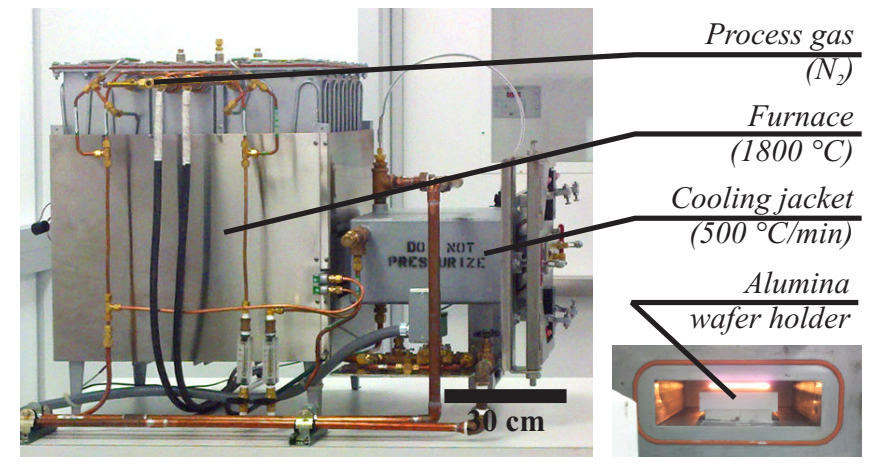

Figure 3: Process capability of $1800^{\circ} \mathrm{C}$ glassblowing with a rapid cooling rate of $500^{\circ} \mathrm{C} / \mathrm{min}$ was developed.

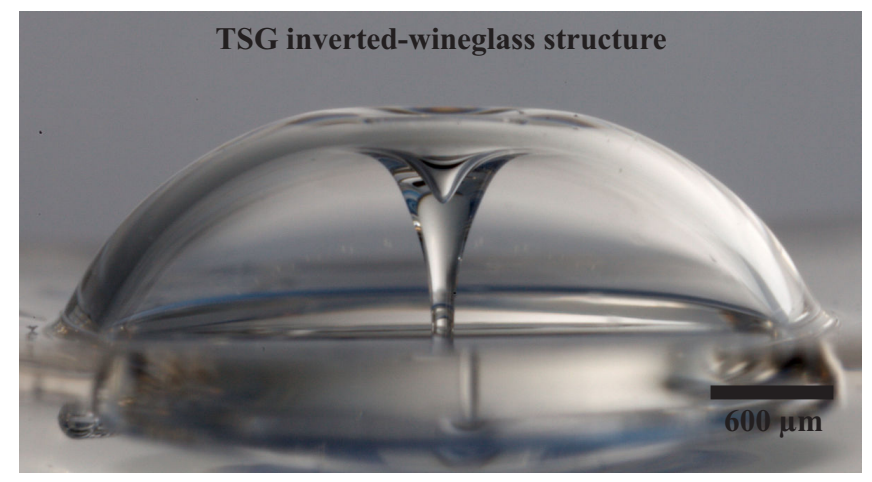

Figure 4: Optical photograph of glassblown TSG invertedwineglass structure. Outer diameter is $4200 \mu \mathrm{m}$.

The next step of the fabrication process is bonding of the TSG device layer onto the etched fused quartz wafer. Due to the subsequent high temperature glassblowing process the bond needs to survive up to $1650{ }^{\circ} \mathrm{C}$, which prevents the use of intermediate materials. For this reason, a plasma activated fusion bonding process was developed, Fig. 1(a). The bond is performed by plasma activating the TSG and fused quartz wafers and then bringing them into optical contact. Since the process relies on hydrogen bonds to keep the two wafers together, highly polished and clean surfaces $(<1 \mathrm{~nm} \mathrm{Sa})$ are required. Once cured, the bond creates a seamless hermetic seal around the etched cavities without using any intermediate material.

The TSG / fused quartz wafer stack is then glassblown at $1650{ }^{\circ} \mathrm{C}$ in a custom-built high temperature furnace with a rapid cooling rate of $500{ }^{\circ} \mathrm{C} / \mathrm{min}$, Fig. 3. The furnace consists of two main chambers that are connected to each other through a third vestibule chamber. The first chamber is used for heating and can go up to $1800{ }^{\circ} \mathrm{C}$, the second chamber is enveloped by a water cooled jacket, that maintains $<200{ }^{\circ} \mathrm{C}$ temperature. The samples are transported between the heating and cooling chambers by using a sliding alumina wafer holder. A typical glassblowing run involves keeping the wafer stack at glassblowing temperature for one minute and then extracting the wafer stack into the water cooled jacket for solidification.

During glassblowing, two phenomenon occur simultaneously: TSG layer becomes viscous due to the elevated temperature, the air inside the etched cavity expands, creating the 3-D glassblown structure. Because the TSG wafer is bonded both around the circular cavity and the cylindrical post in the middle, the glassblown structure creates a self-aligned TSG stem, Fig. 4. 


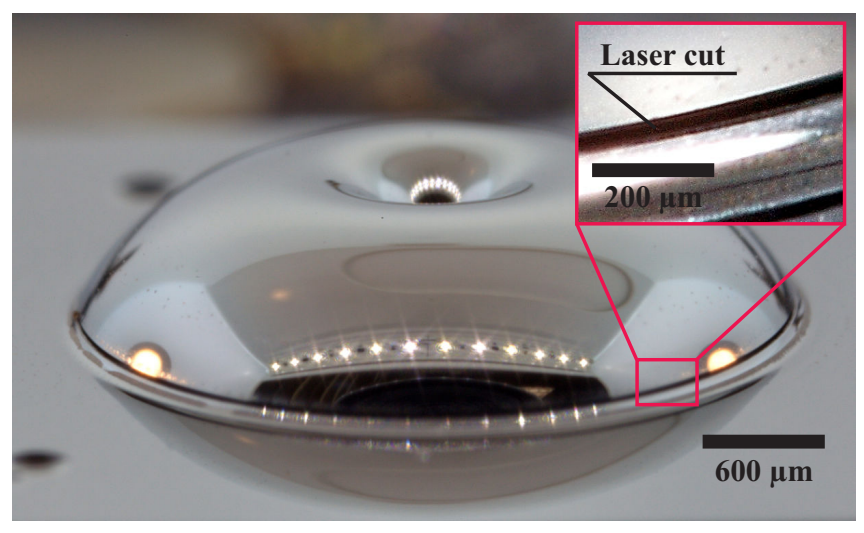

Figure 5: Optical photograph of inverted-wineglass, released along the perimeter using laser ablation and coated with iridium.

The final step of the fabrication process is to release the wineglass around its perimeter, which can be accomplished by laser ablation or dry etching of the device layer. The wineglass structure in Fig. 5 was released with laser ablation, using a 2-axis laser micromachining system, Resonetics RapidX 250. The system was upgraded to 3-axis by installing a custom built rotary stage assembly from National Aperture, Inc. The wineglass structure was mounted onto the rotary stage and its axis of symmetry was aligned with the rotation axis with the help of an $x-y$ stage. Laser ablation was performed by focusing the laser onto the perimeter of the wineglass at a perpendicular angle and rotating the wineglass structure at constant angular velocity. For the laser source, an ArF (193 nm) excimer laser (Coherent COMPexPRO 110) was used with $20 \mathrm{~ns}$ pulse duration, $50 \mathrm{~Hz}$ repetition rate and a laser spot size of $40 \mu \mathrm{m}$.

\section{METROLOGY}

In this section we analyze the surface roughness and the material composition of TSG device layer before and after glassblowing.

\section{Surface Roughness}

Surface roughness measurements of TSG glassblown samples were performed using an atomic force microscope (AFM) from Pacific Nanotechnology (Nano-R). With a sensor noise level of $<0.13 \mathrm{~nm}$ in the z-direction, Nano-R can resolve sub-nanometer features. Samples were cleaned using standard solvent clean (Acetone, IPA, methanol) before each scan. No additional treatment was performed on the samples. The AFM was run in tapping mode, using a $10 \mathrm{~nm}$ radius probe tip (Agilent U3120A).

Surface roughness of the samples before and after glassblowing were analyzed, with the hypothesis that glassblowing can improve the surface roughness. Highly polished TSG wafers were used for the device layer, which was verified by AFM scans, showing a surface roughness of $0.40 \mathrm{~nm} \mathrm{Sa,} \mathrm{Fig.} \mathrm{6(b).}$ Characterization of the glassblown samples showed a two-fold improvement in surface roughness, down to $0.23 \mathrm{~nm} \mathrm{Sa}$, Fig 6(a). We also observed that the angstrom level scratches in Fig 6(b), associated with the lapping operation, disappeared after glassblowing, Fig 6(a), confirming the hypothesis.

Two-fold improvement in surface roughness is attributed to viscous flow of the glass layer and the associated surface tension forces. As the glassblowing is performed above the glass softening temperature, TSG device layer becomes viscous and the surface tension forces become active, working towards minimizing the

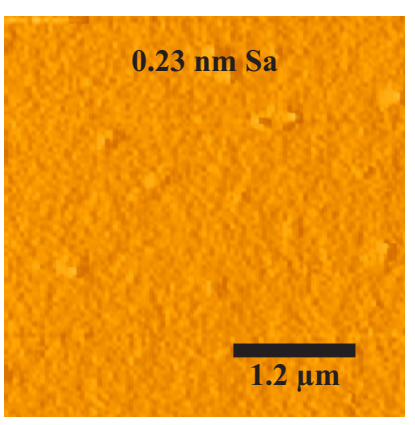

(a) After glassblowing

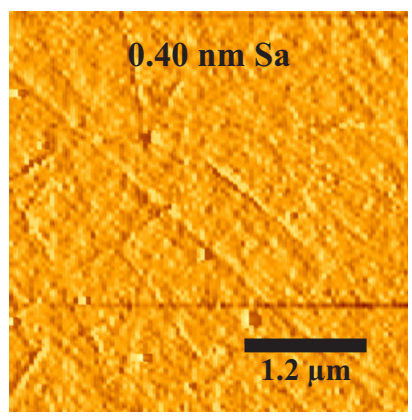

(b) Before glassblowing

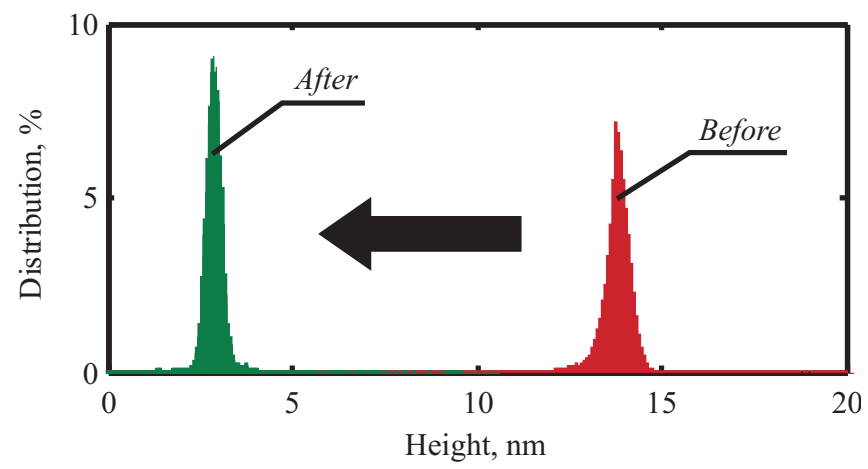

Figure 6: AFM surface profiles of TSG, (a) before and (b) after glassblowing. Glassblowing creates extremely smooth $(0.2 \mathrm{ppm}$ relative roughness) TSG structures.

surface area of the glass structure. This creates an effect analogous to "stretching out" the wrinkles on the surface, lowering the surface roughness.

\section{Material Composition}

For resonant and optical applications, it is critical that TSG retains its original material composition and properties after glassblowing, which are structural integrity, material uniformity and optical transparency. We found that glassblowing temperature and the rate of cooling are the most important parameters that affect the quality of the TSG layer after glassblowing.

The structure in Fig. 7 was glassblown using a conventional high temperature furnace at $1600^{\circ} \mathrm{C}$, which does not allow removal of the samples at elevated temperatures. For this reason, the structure was left to cool-down to room temperature over an 8 hour period. Recrystallization as well as micro-cracks were observed on the surface. In order to establish the nature of the recrystallization electron dispersive spectroscopy (EDS) was employed. Philips XL-30 FEG SEM with a Thermo Scientific UltraDry silicon drift X-ray detector was used for EDS characterization. An acceleration voltage of $10 \mathrm{kV}$ was used at $10 \mathrm{~mm}$ working distance, and samples were coated with $5 \mathrm{~nm}$ of sputtered iridium to prevent charging.

EDS analysis of the crystals in Fig. 7 revealed higher concentrations of titanium, implying that $\mathrm{TiO}_{2}$ is exsolving from the $\mathrm{SiO}_{2} / \mathrm{TiO}_{2}$ matrix. In contrast, the structure in Fig. 8 was glassblown using rapid cooling by bringing the temperature of the sample from $1650{ }^{\circ} \mathrm{C}$ to $\sim 200{ }^{\circ} \mathrm{C}$ within a minute. No microcracks or recrystallization were observed, as can be validated by the optical transparency. EDS spectral plots showed homogeneous $\mathrm{SiO}_{2}$ and $\mathrm{TiO}_{2}$ distribution in Fig. 8 as opposed to heterogeneous distribution in Fig. 7. 

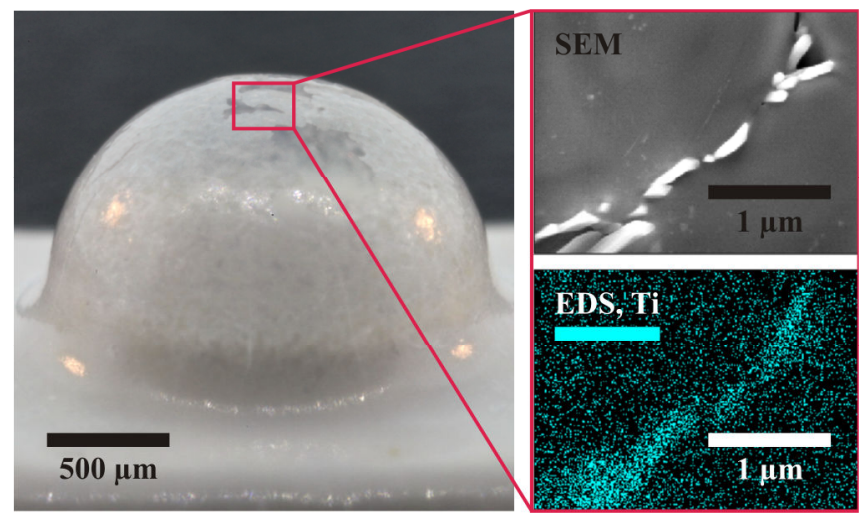

Figure 7: Slow cooling of TSG (> $8 \mathrm{hr}$.) causes recrystallization.
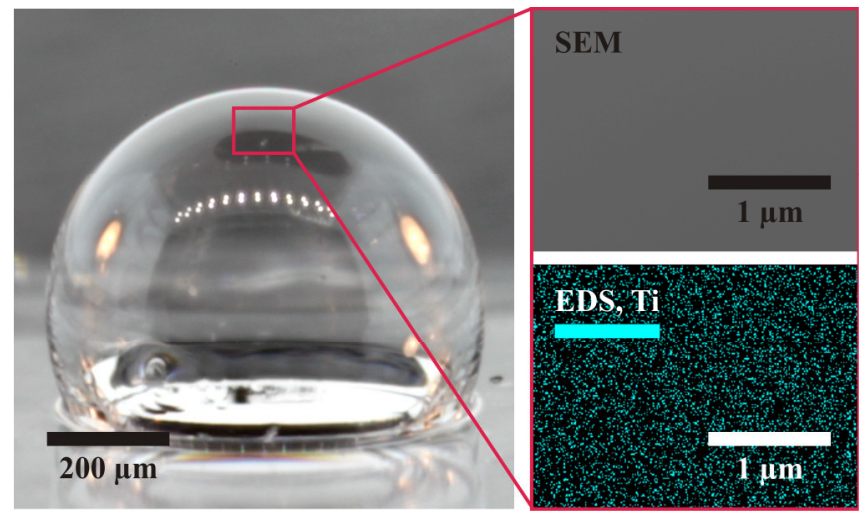

Figure 8: Glassblowing with rapid cooling of TSG $(<1$ min.) prevents recrystallization.

EDS was used to obtain the spectral signatures of TSG before and after glassblowing. No change in the composition of TSG was observed after glassblowing, Fig. 9. EDS spectrums also revealed 7 to 8 weight percent of $\mathrm{TiO}_{2}$ in TSG, which is in agreement with the nominal $\mathrm{TiO}_{2}$ concentration of Corning ULE TSG.

\section{CONCLUSIONS}

A new high temperature micro-glassblowing process for fabrication of 3-D low internal loss ULE TSG wineglass structures was developed and experimentally demonstrated. The process was enabled by the development of an in-house high temperature glassblowing capability of $1800^{\circ} \mathrm{C}$ with a cooling rate of $>500{ }^{\circ} \mathrm{C} / \mathrm{min}$ and plasma activated fusion bonding of TSG to fused quartz. EDS spectral analysis of TSG before and after glassblowing revealed that the material retained its properties after glassblowing with no recrystallization or change in glass composition. AFM surface scans of the glassblown structures showed extremely low surface roughness $(0.23 \mathrm{~nm} \mathrm{Sa})$. A novel inverted-wineglass architecture was also implemented, providing self-aligned stem structures with a fundamental anchor loss limit of $\mathrm{Q}_{\text {anchor }}>7 \mathrm{E}+10$. The method may enable new classes of TSG / fused quartz MEMS devices with extremely low surface roughness, intrinsically low internal losses $\left(\mathrm{Q}_{\mathrm{TED}}>5 \mathrm{E}+10\right)$ and dynamically balanced structures.

\section{ACKNOWLEDGEMENTS}

This material is based upon work supported by DARPA/SPAWAR under Grant N66001-10-1-4074 (Program Manager Tayo Akinwande) \& DARPA grant W31P4Q-11-1-0006.

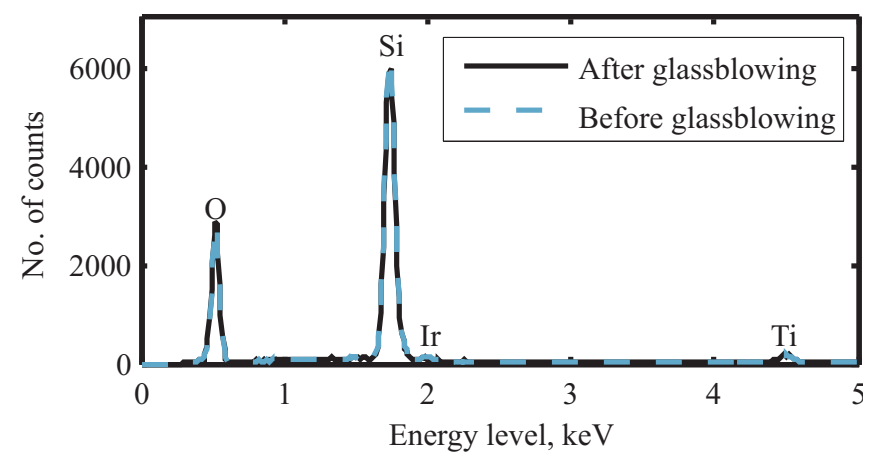

Figure 9: EDS spectral analysis of TSG reveals that composition of TSG does not change after glassblowing.

\section{REFERENCES}

[1] M. Weinberg, R. Candler, S. Chandorkar, J. Varsanik, T. Kenny, A. Duwel, "Energy Loss in MEMS Resonators and the Impact on Inertial and RF Devices," Proc. Transducers'09, Denver, Colorado, USA, June 21-25, 2009, pp. 688-695.

[2] D.M. Rozelle, "The hemispherical resonator gyro: From wineglass to the planets", in Proc. AAS/AIAA Space Flight Mechanics Meeting, Feb. 2009, pp. 1157-1178.

[3] L. D. Sorenson, X. Gao, F. Ayazi, "3-D Micromachined Hemispherical Shell Resonators With Integrated Capacitive Transducers," Proc. IEEE MEMS'12, Paris, France, Jan. 29 Feb. 2, 2012, pp. 168-171.

[4] C.L. Fegely, D.N. Hutchison, S.A. Bhave, "Isotropic etching of 111 SCS for wafer-scale manufacturing of perfectly hemispherical silicon molds," Proc. Transducers'11, Beijing, China, June 5-9, 2011, pp 2595-2598.

[5] K. Visvanathan, Li Tao, Y. B. Gianchandani, "3-D-soule: A fabrication process for large scale integration and micromachining of spherical structures", Proc. IEEE MEMS'11, Jan. 21 - 27, 2011, pp. 45-48.

[6] B. Sarac, G. Kumar, T. Hodges, S. Ding, A. Desai, J. Schroers, "Three-Dimensional Shell Fabrication Using Blow Molding of Bulk Metallic Glass," IEEE J. Microelectromech. Syst., vol. 20, no. 1, pp. 28-36, 2011.

[7] I.P. Prikhodko, S.A. Zotov, A.A. Trusov, A.M. Shkel, "Subdegree-per-hour silicon MEMS rate sensor with 1 million Qfactor," Proc. Transducers'11, Beijing, China, June 5-9, 2011, pp. 2809-2812.

[8] Chen, W., Sugita, K., Morikawa, Y., Yasunami, S., Hayashi, $\mathrm{T}$., \& Uchida, $\mathrm{T}$, "Application of magnetic neutral loop discharge plasma in deep silica etching," J. Vacuum Science \& Technology, vol. 19, no.6, pp. 2936-2940, 2001.

[9] E.J. Eklund, A.M. Shkel, "Glass Blowing on a Wafer Level," IEEE J. Microelectromech. Syst., 16(2), pp. 232-239, 2007.

[10] I.P Prikhodko, S.A. Zotov, A.A. Trusov A.M. Shkel, "Microscale Glass-Blown Three-Dimensional Spherical Shell Resonators," IEEE J. Microelectromech. Syst., vol. 20, no. 3, pp.691-701, 2011.

[11] D. Senkal, I. P. Prikhodko, A. A. Trusov, A. M. Shkel, "Micromachined 3-D Glass-Blown Wineglass Structures for Vibratory MEMS Applications," Technologies or Future Micro-Nano Manufacturing, Napa, California, USA, August 8 - 10, 2011, pp. 166-169.

\section{CONTACT}

* A.A. Trusov, tel: +1-949-824-6314; atrusov@uci.edu. 


\section{ULTRA-STABLE EPITAXIAL POLYSILICON RESONATORS

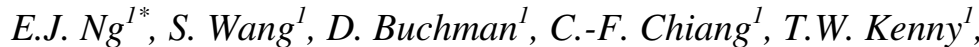

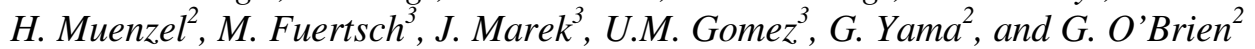 \\ ${ }^{1}$ Stanford University, Stanford, California, USA \\ ${ }^{2}$ Robert Bosch RTC, Palo Alto, California, USA \\ ${ }^{3}$ Robert Bosch GmbH, Reutlingen, Germany}

\begin{abstract}
Epitaxially grown polycrystalline silicon (epi-poly) has shown great promise as a MEMS material, offering isotropic material properties with minimal residual stress and stress gradients. While epi-poly has been used previously for MEMS inertial systems or sensors, its use in high precision resonator applications has been restricted to an encapsulation layer for resonators fabricated in single crystal silicon. In this work, encapsulated resonators fabricated with epi-poly as the functional layer were tested alongside single crystal silicon devices with regard to resonant frequency, quality factor, temperature dependence, and stability. Test results indicate that epi-poly resonators have very similar mechanical properties and stability to single crystal silicon resonators when operated in a clean package.
\end{abstract}

\section{INTRODUCTION}

Polycrystalline silicon was introduced in the early 1980s for surface micromachining [1] and has since become one of the most common MEMS materials. However, conventional low-pressure chemical vapor deposited (LPCVD) polysilicon has residual stress and stress gradients that are difficult to control, making the deposition of thick functional layers challenging [1,2]. In the 1990s, epitaxially grown polycrystalline silicon (epi-poly) was developed [3-8], allowing for a high deposition rate with minimal residual stress and stress gradients [7,8]. This enabled thick depositions $(>10 \mu \mathrm{m})$, necessary for many surface micromachined MEMS devices. Since then, it has been used as functional material in MEMS accelerometers, gyroscopes and other products [4,5]. Particularly in gyroscopes, the stability of mechanical characteristics of the material is critical to performance. Epi-poly gyroscopes made by Bosch have an outstanding reputation for long-term stability in harsh environments, such as automobiles.

Despite this excellent reputation for epi-poly, engineers building precision time references, such as at SiTime [9], have relied exclusively on single crystal silicon as a functional material. Our group has shown that single crystal silicon resonators offer excellent long-term stability [10,11], and SiTime is currently marketing timing products with sub-ppm frequency stability over time and temperature based on single crystal silicon resonators. An important ingredient in the stability of these resonators has been the use of high-temperature encapsulation with epi-poly as the sealing layer (epi-seal) [12]. This encapsulation process has emerged as a basis for the characterization of single crystal silicon, with resonators demonstrating many better-than-reported properties when packaged as such $[10,11,13]$. Single crystal silicon, however, is highly anisotropic [14] and is challenging to implement in devices for which isotropic material properties are desired, such as bulk-mode resonators and wine-glass resonators.

Epi-poly, on the other hand, has a polycrystalline structure with isotropic material properties. Because of its low stress and stress gradients, thick layers are possible for MEMS applications. However, its performance as a material for high precision MEMS still remains unknown. Material stability is crucial for these devices - drift over time could severely limit the performance of many devices. Thus, the question we seek to answer is: Just how stable are epi-poly resonators, when properly packaged?

To answer this question, double-ended tuning fork (DETF) resonators (Fig. 1) were fabricated in single crystal silicon as well as epi-poly. DETF resonators were chosen because they have been well-characterized by our group previously in epi-seal packages [15]. Of interest here are the mechanical properties of epi-poly compared to those of single crystal silicon for MEMS devices - for resonators, these are the resonant frequency, quality factor, temperature dependence, and stability. To measure the resonant frequency, quality factor, and temperature dependence, frequency sweeps with a network analyzer was conducted on the resonators.

A precise measure of resonator frequency stability is more difficult to obtain. Silicon resonators are known to have approximately $-31 \mathrm{ppm} /{ }^{\circ} \mathrm{C}$ frequency-temperature dependence [16] - even a slight variation in the environmental temperature causes a significant change in frequency, limiting stability measurements. To filter out the temperature dependence, multiple identical single anchored DETF resonators were fabricated in proximity with a common anchor (Fig. 1). Measuring the resonant frequency over time and subtracting the relative frequency changes greatly reduces temperature variation effects, yielding a measure of stability [10].

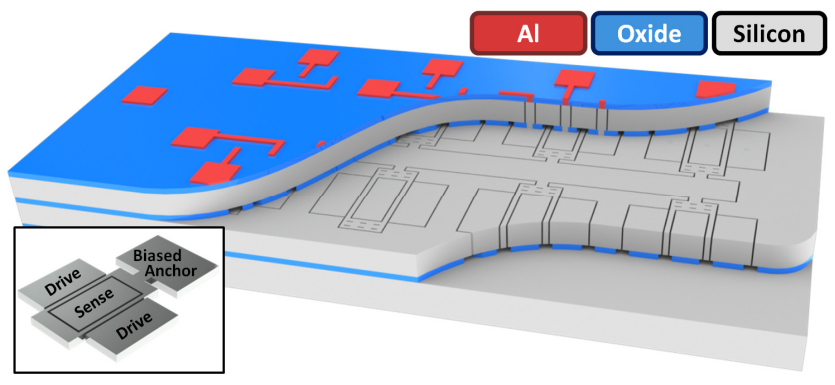

Figure 1: Multiple single-anchored double-ended tuning fork (DETF) resonators in proximity with a common anchor in an episeal process. (Inset) Single-anchored DETF resonator with resonating beams in-between sense and drive electrodes.

\section{FABRICATION}

To elucidate the difference between single crystal silicon and epi-poly for MEMS, resonators were fabricated in an epi-seal process in both single crystal silicon as well as epi-poly (Fig. 2) [12]. For single crystal silicon devices, the process started with an n-type phosphorus-doped silicon-on-insulator (SOI) wafer with a 2 $\mu \mathrm{m}$ buried oxide layer and a $20 \mu \mathrm{m}$ (100) device layer with a resistivity of $16 \mathrm{~m} \Omega-\mathrm{cm}$. A similar epi-poly silicon-on-insulator (PSOI) wafer was fabricated by first growing a $2 \mu \mathrm{m}$ silicon dioxide layer on a wafer (Fig. 2a) and then depositing an n-type phosphorus-doped $20 \mu \mathrm{m}$ layer of epi-poly with a resistivity of 8 $\mathrm{m} \Omega-\mathrm{cm}$. The epi-poly deposition process has previously been used in our group for encapsulation, but is used in this work as the functional layer. 


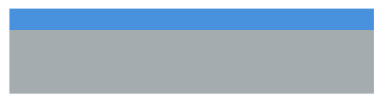

(a)

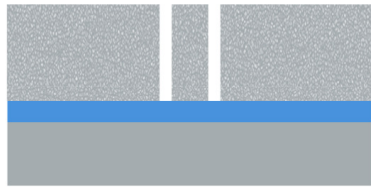

(b)

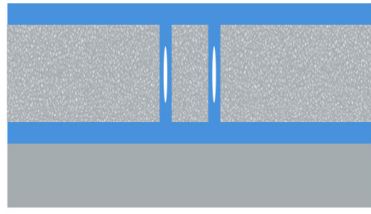

(c)

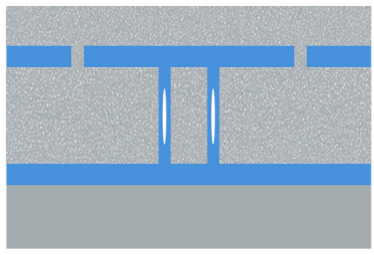

(d)

Al

Oxide Silicon

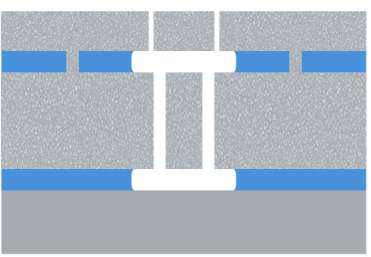

(e)

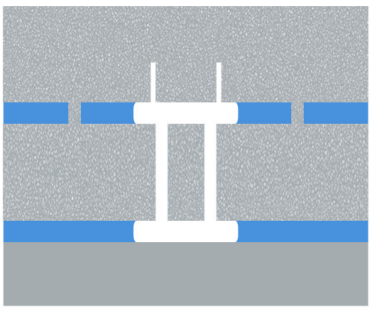

(f)

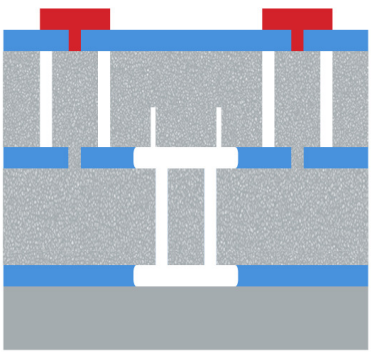

(g)
Figure 2: Cross-section view of the epi-seal process using epi-poly as the device layer. (a) Oxide is grown on a handle wafer; (b) Epipoly is deposited to form a PSOI (polysilicon on insulator) and resonator designs are etched in epi-poly; (c) trenches are filled with oxide; (d) electrical contacts are etched in the oxide and a first epi-poly encapsulation layer is deposited; (e) vents are etched in the cap and the oxide around the device is released with HF vapor; (f) the device is sealed with a second layer of epi-poly; ( $g$ ) Electrical isolation and contacts are defined, completing the process. Single crystal silicon devices were also fabricated in the same manner starting from an SOI (silicon on insulator) in (b).

Epi-poly deposition was performed in an Applied Materials Centura EPI reduced-pressure epitaxial reactor. $A$ thin (approximately $100 \mathrm{~nm}$ ) seed layer of polysilicon was first deposited using silane $\left(\mathrm{SiH}_{4}\right)$ in a $\mathrm{H}_{2}$ carrier at $800^{\circ} \mathrm{C}$ and 600 Torr with a $\mathrm{SiH}_{4}$ gas flow rate of $60 \mathrm{sccm}$ for $90 \mathrm{~s}$. This acts as a nucleation layer for polysilicon deposition on oxide. Dichlorosilane (DCS, $\mathrm{SiH}_{2} \mathrm{Cl}_{2}$ ) at $1080^{\circ} \mathrm{C}$ and 30 Torr was used to grow $20 \mu \mathrm{m}$ of polysilicon epitaxially with a flow rate of $400 \mathrm{sccm}$ (DCS) in a $\mathrm{H}_{2}$ carrier. The polysilicon was doped in-situ with 100 sccm of phosphine $\left(\mathrm{PH}_{3}\right)$. Growth rates were approximately 1 $\mu \mathrm{m} / \mathrm{min}$. Due to chamber limitations, $3 \mu \mathrm{m}$ of polysilicon was grown at a time, with the wafer kept under load-lock and $\mathrm{H}_{2}$ baked at $1130^{\circ} \mathrm{C}$ for $300 \mathrm{~s}$ between deposition steps to ensure a high quality epitaxial deposition. Chemical Mechanical Polishing (CMP) was then used to planarize the polysilicon wafer surface before further processing.

Following the epi-seal fabrication process as previously detailed [12], identical resonator designs were etched into the device layer of the SOI and the PSOI wafers using Deep Reactive Ion Etching (DRIE) for a capacitive transduction gap with a trench width of $1.5 \mu \mathrm{m}$ (Fig. 2b). Tetraethyl orthosilicate (TEOS) oxide was used to refill the trenches and create the oxide spacer between the device layer and the encapsulation (Fig. 2c). Vias were plasma etched in the oxide to allow for electrical access to the electrodes. Thereafter, a first layer of epi-poly $(\sim 6 \mu \mathrm{m})$ was deposited (Fig. $2 \mathrm{~d}$ ) using the same epi-poly process as described above. Vent holes were etched in this layer using DRIE and the oxide around the device was released with $\mathrm{HF}$ vapor (Fig. 2e). A second epi-poly sealing layer $(\sim 20 \mu \mathrm{m})$ was deposited over the vents to seal the device and to provide mechanical stability (Fig. 2f), with a high temperature $\left(1130^{\circ} \mathrm{C}\right) \mathrm{H}_{2}$ bake performed prior to deposition to remove the native oxide around the device. The epitaxial deposition also seals the device cavity under low pressure with $\mathrm{H}_{2}$ remaining in the cavity. For electrical access through the cap to the electrodes, isolation trenches were etched with DRIE. Another layer of oxide was then deposited over the trenches. Finally, aluminum electrical contact pads were patterned (Fig. 2g). To reduce the pressure in the cavity even further, $\mathrm{H}_{2}$ was diffused out by annealing in a nitrogen flow at $400^{\circ} \mathrm{C}$ for 90 hours.

Single crystal silicon and epi-poly cross-sections are compared in Fig. 3 (a) and (b), with the granular structure of epipoly clearly noticeable. From another view (Fig. 3c), the epi-poly grains are seen to be columnar and increasing in size from the base $(\sim 200 \mathrm{~nm})$ to the top $(\sim 5 \mu \mathrm{m})$, as also reported in [8]. This occurs as the silicon deposition on top of the nucleation layer continues epitaxially, during which the deposited silicon conforms to the underlying lattice, adding to the existing polycrystalline structure, producing grains that are columnar and growing larger in plane.

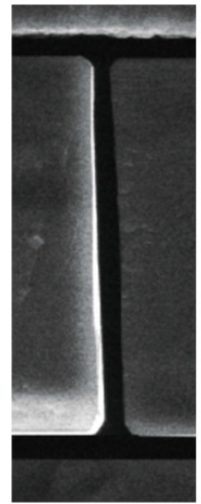

(a)

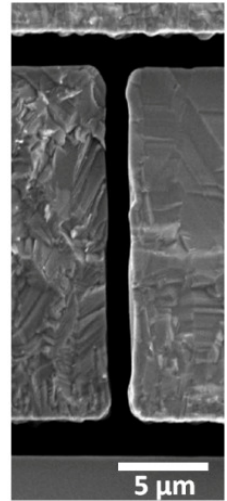

(b)

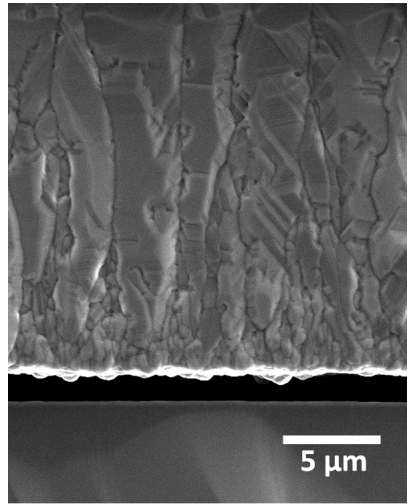

(c)
Figure 3: (a) Transduction region for single crystal silicon; (b) Transduction region for epi-poly; (c) Grain structure of epi-poly.

Since the wafers are baked in $\mathrm{H}_{2}$ at high temperatures $\left(1130^{\circ} \mathrm{C}\right)$ and low pressures $(30$ Torr) during the epitaxial sealing step, silicon migration is expected to occur to minimize surface energy - in single crystal silicon, this has the desirable effect of smoothing out the DRIE scallops. For epi-poly, however, the different crystal orientations result in grain growth along the randomly oriented grains and cause a rough surface with numerous bumps. From SEM images, the surface roughness of the epi-poly is apparent and is about $\sim 0.3 \mu \mathrm{m}$. For the resonators tested in this work, the transduction gap size is $1.5 \mu \mathrm{m}$ and thus no adverse effects from the roughness were observed during resonator testing.

On the completed resonator die (Fig. 4), only the final aluminum electrical traces are visible. The resonators are buried underneath in a vacuum, protected from dust, oxygen, moisture, and other contaminants in the environment. 


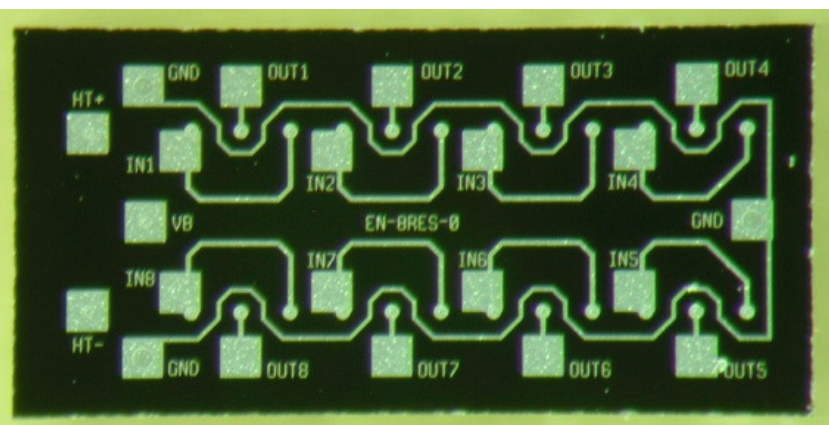

Figure 4: Completed resonator die $(2 \mathrm{~mm} \times 1 \mathrm{~mm})$ with multiple DETF resonators. The resonators are buried in a vacuum under a thick polysilicon seal, protecting them from dust, oxygen and moisture. Only the final aluminum electrical traces are visible.

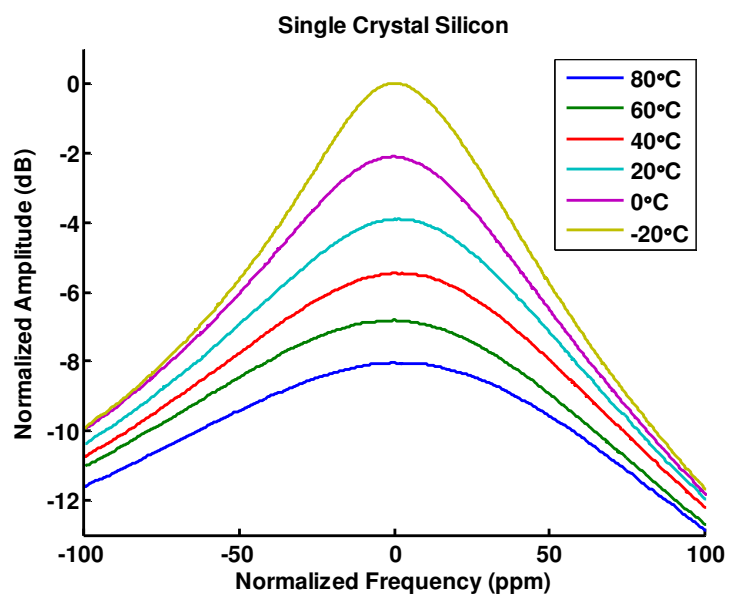

(a)

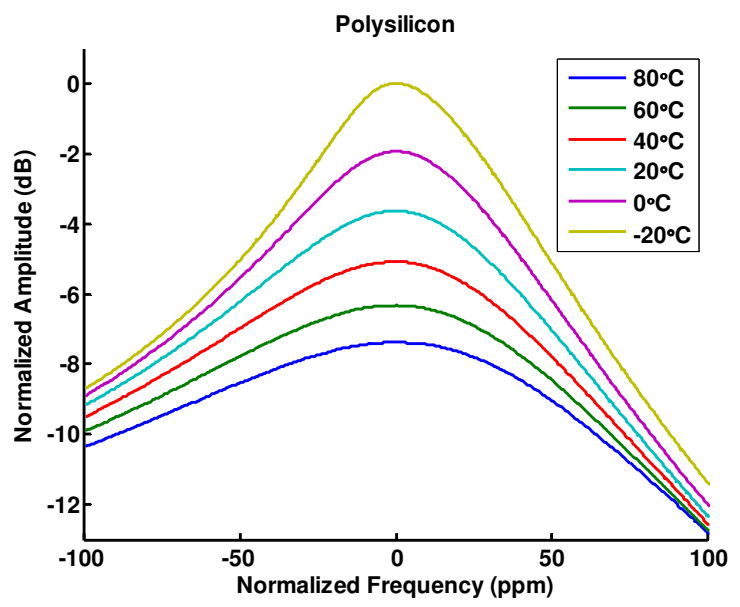

(b)

Figure 5: Experimentally measured resonant peaks normalized at peak frequency for (a) single crystal silicon, and (b) epi-poly.

\section{TESTING METHODOLOGY AND RESULTS}

Single-anchored, double-ended tuning fork (DETF) resonators (Fig. 1, 4) were used for material characterization. These resonators had nominal beam dimensions of $200 \mu \mathrm{m} \times 6 \mu \mathrm{m}$, giving a theoretical resonant frequency of about $1.3 \mathrm{MHz}$. Measured resonant frequencies were approximately $1.27 \mathrm{MHz}$ with a bias voltage of $8 \mathrm{~V}$ for single crystal silicon resonators oriented in the $\langle 110\rangle$ direction as well as epi-poly resonators. Frequency sweeps revealed very similar response for single crystal silicon and epi-poly resonators at various temperatures (Fig. 5). Plotting resonant frequency (Fig. 6a) and quality factor (Fig. 6b) against temperature also indicate that epi-poly resonators are very similar to single crystal silicon resonators, owing to the similarity in the material properties that affect dynamic MEMS devices. Temperature coefficient of frequency (TCf) differences observed between single crystal silicon and epi-poly is likely to have been due to the doping concentration difference $\left(\sim 2 \times 10^{18} \mathrm{~cm}^{-3}\right.$ for single crystal vs. $\sim 6 \times 10^{18} \mathrm{~cm}^{-3}$ for epi-poly), which is expected to affect the TCf [17].

For stability measurements, the frequencies of two identical DETF resonators on the same die were measured simultaneously with oscillation circuitry and frequency counters in a temperature chamber maintained at $70^{\circ} \mathrm{C}$ as in [10]. The resonant frequencies of the resonator pair exhibit near-identical time variations, indicating that both are responding to changes in the temperature of the test environment. To cancel the temperature dependence effect and to obtain a measure of the stability apart from temperature fluctuations, the difference in frequency (in parts-perbillion) between the pair of identical resonators in proximity is measured simultaneously (Fig. 7). The drift for both single crystal silicon and epi-poly resonators is well below $100 \mathrm{ppb}$ over 4 days. Most importantly, very similar residual drift is seen for both single crystal and epi-poly resonators. In fact, there are essentially no detectable differences between any of the measured characteristics of the single crystal silicon resonators and the epi-poly resonators, when operated in ultra-clean packages.

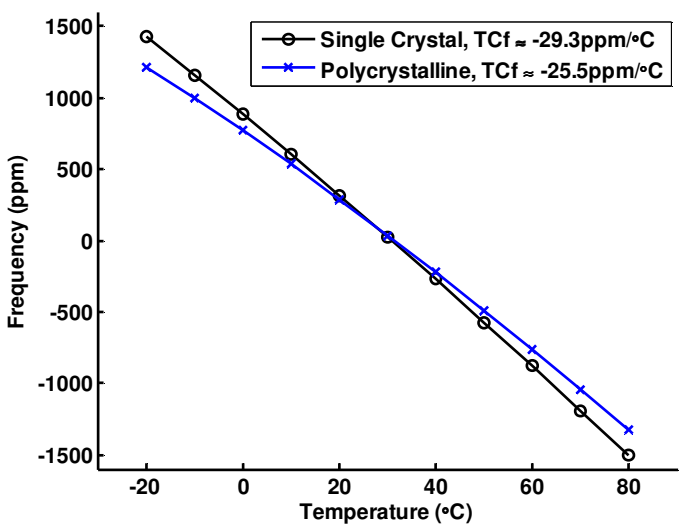

(a)

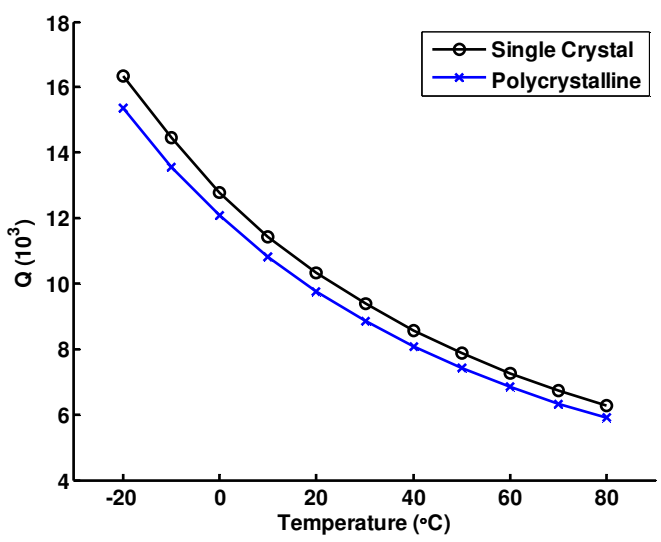

(b)

Figure 6: (a) Frequency vs. temperature and (b) quality factor vs. temperature plots for single crystal silicon and epi-poly. 

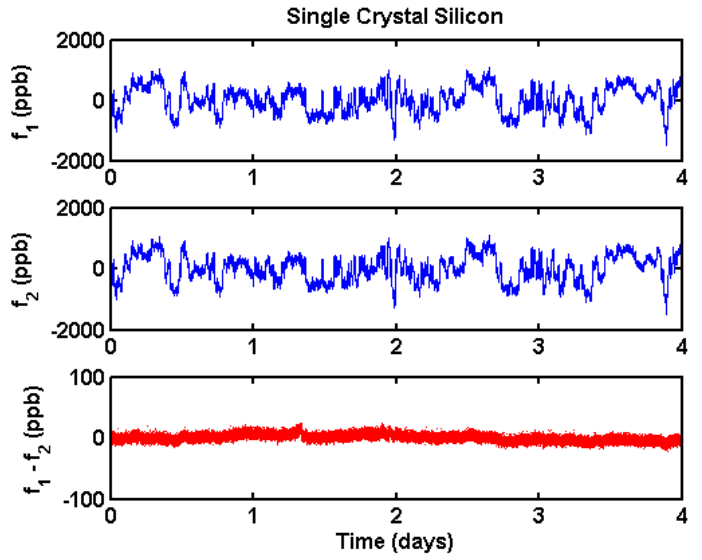

(a)
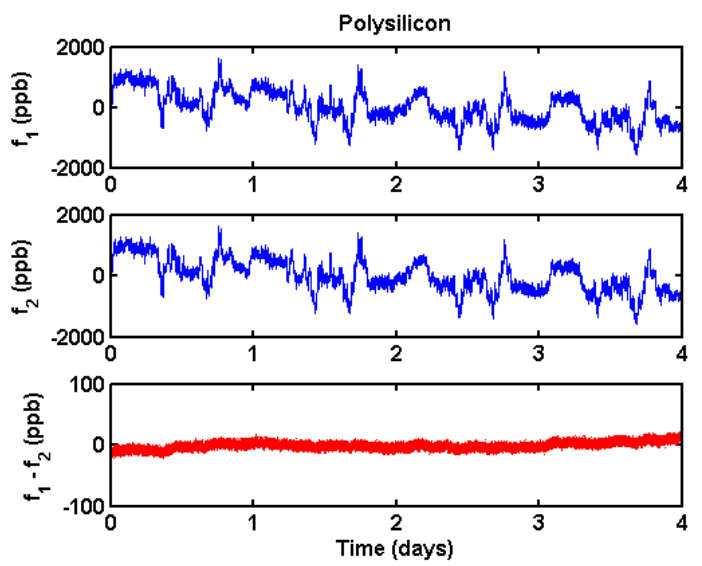

(b)

Figure 7: Stability measurements over 4 days showing the resonant frequencies of two resonators and the frequency difference in parts-per-billion for $(a)$ single crystal silicon and $(b)$ epi-poly devices.

\section{CONCLUSION}

Double-ended tuning fork resonators were fabricated in epitaxially grown polycrystalline silicon with a clean, high temperature encapsulation process epi-seal. These epi-poly resonators demonstrate very similar stability and mechanical properties (resonant frequency, quality factor, and temperature dependence) to their single crystal silicon counterparts, indicating that epi-poly silicon-on-insulator (PSOI) wafers may be used for many high performance MEMS applications where the anisotropy of single crystal silicon may be undesirable.

\section{ACKNOWLEDGEMENTS}

This work was supported by DARPA grant N66001-10-14094, "Microscale Resonant Thermometers", managed by Dr. Andrei Shkel. Fabrication work was performed at the Stanford Nanofabrication Facility (a member of the National Nanotechnology Infrastructure Network). The authors would like to thank the staff at the Stanford Nanofabrication Facility, in particular M. M. Stevens for the assistance with the epitaxial reactor. The authors would also like to thank J. S. Salvia for the oscillator circuit design.

\section{REFERENCES}

[1] R.T. Howe and R.S. Muller, "Polycrystalline and amorphous silicon micromechanical beams: annealing and mechanical properties," Sensors Actuators, vol. 4, p. 447-454, 1983.

[2] H. Guckel, J. J. Sniegowski, T. R. Christenson, and F. Raissi, "The application of fine-grained tensile polysilicon to mechanically resonant transducers," Sensors Actuators A, vol. 21, pp. 346-351, 1990.

[3] K.Funk, A.Schlip, M.Offenberg, B.Eisner, and F. Larmer, "Surface Micromachining of Resonant Silicon Structures," IEEE Transducers 1995.

[4] J.Munchow, H.Muenzel, M.Offenberg, and W.Waldvogel, US Patent 5,616,514, "Method of Fabricating a Micromechanical Sensor," 1997.

[5] M.Lutz, W.Golderer, J.Gerstenmeier, J.Marek, B.Maihofer, S.Mahler, H.Muenzel, and U.Bischof, "A precision yaw rate sensor in silicon micromachining," IEEE Transducers 1997, pp. 847 - 850 .

[6] M.Furtsch, "Mechanical Properties of Thick Polycrystalline Silicon Films suitable for Micromachining," PhD Thesis, 1999.

[7] P.T.J. Gennissen, M. Bartek, P.J. French, and P.M. Sarro, "Bipolar-compatible epitaxial poly for smart sensors: stress minimization and applications," Sensors Actuators A, vol. 62, pp. 636-645, 1997.

[8] P. Langea, M. Kirstena, W. Riethmüllera, B. Wenka, G. Zwickera, J.R. Moranteb, F. Ericsonc, and J.Å. Schweitzc, "Thick polycrystalline silicon for surface-micromechanical applications: deposition, structuring and mechanical characterization," Sensors Actuators A, vol. 54, pp. 674-678, 1996.

[9] SiTime Corporation [Online]. Available: www.sitime.com

[10] E.J. Ng, H.K. Lee, C.H. Ahn, R.Melamud, and T. W. Kenny, "Stability measurements of silicon MEMS resonant thermometers," IEEE Sensors 2011, pp. 1257-1260.

[11] B. Kim, R. N. Candler, M. Hopcroft, M. Agarwal, W.-T Park, and T. W. Kenny, "Frequency Stability of Encapsulated MEMS Resonator," Sensors and Actuators A: Physical, vol. 136, pp. 125-131, 2007.

[12] R.N. Candler, W.T. Park, H.M. Li, G.Yama, A.Partridge, M. Lutz, and T.W. Kenny, "Single-wafer encapsulation of MEMS Devices,” IEEE Trans. Adv. Packaging, vol. 26, no. 3, pp. 227, 2003.

[13] S. Yoneoka, Y. Q. Qu, S. Wang, M. W. Messana, A. B. Graham, J. Salvia, B. Kim, R. Melamud, G. Bahl, and T. W. Kenny, "High-cyclic fatigue experiments of single crystal silicon in an oxgen-free environment," IEEE MEMS 2010, pp. 224-227.

[14] M.A. Hopcroft, W.D. Nix, and T.W. Kenny, "What is the Young's Modulus of Silicon?” JMEMS, vol. 19, pp. 229-238, 2010.

[15] M. Agarwal, S. A. Chandorkar, H. Mehta, R. N. Candler, B. Kim, et al., "A study of electrostatic force nonlinearities in resonant microstructures," Appl. Phys. Lett. 92, 104106, 2008.

[16] R. Melamud, S. A. Chandorkar, B. Kim, H. K. Lee, J. Salvia, et al., "Temperature Insensitive Composite Micromechanical Resonators," JMEMS, vol. 18, no. 6, pp. 1409-1419, 2009.

[17] A.K. Samarao and F. Ayazi, "Temperature Compensation of Silicon Resonators via Degenerate Doping," IEEE Trans. Elect. Dev., vol. 59, pp. 87-93, 2012.

\section{CONTACT}

Eldwin Ng, email: eldwin@stanford.edu 


\author{
WINEGLASS-ON-A-CHIP \\ P. Shao, L.D. Sorenson, X. Gao, and F. Ayazi \\ Georgia Institute of Technology, Atlanta, GA, USA
}

\begin{abstract}
This paper introduces free-standing, stem-supported silicon dioxide hemispherical shells ( -wineglasses) that are thermallygrown and have high-quality-factor resonances due to unrestricted support stem diameter and low internal thermoelastic damping. The fabrication process offers a direct path to batch fabrication of hemispherical wineglass microstructures with ultra-thin conformal conductive coatings using atomic layer deposition (ALD). A novel assembly method forms capacitive electrodes for rapid electrical characterization of the silicon dioxide -wineglass resonators. A quality factor of 5,600 is measured for the $m=4$ resonance of a 740 $\mu \mathrm{m}$ diameter, $2 \mu \mathrm{m}$ thick thermal oxide shell with $30 \mathrm{~nm}$ ALD TiN coating and $77 \mu \mathrm{m}$ diameter silicon support stem.
\end{abstract}

\section{INTRODUCTION}

Three-dimensional microstructures hold great potential for a multitude of applications. An example of a desirable and truly 3D structure is a hemispherical wineglass (Figure 1). Such $\mu$ wineglasses can yield very high mechanical quality factors at low frequencies (low $\mathrm{kHz}$ ) due to their thin flexural shell and highlybalanced symmetry, all within a small die area. However, difficulty in fabricating free-standing, stem-supported wineglass hemispherical shells with capacitive transduction electrodes has prevented thorough mechanical characterization of these structures. This paper introduces thermally-grown silicon dioxide hemispherical shells released from microfabricated single-crystal silicon molds, which are subsequently blanketed with an ultra-thin atomic layer deposition (ALD) conductive coating for electrical actuation and sensing. The nearly ideal symmetry of the shells confines the vibration energy near the shell rim, minimizing acoustic radiation through the support into the substrate. Furthermore, the low coefficient of thermal expansion of thermally-grown oxide results in reduced internal thermoelastic damping [1]. The oxide wineglass-on-a-chip resonators share these advantageous features with ultra-high-performance macroscale hemispherical resonator gyroscopes (HRGs) [2].

Several reports have been made in literature on different approaches for fabricating microscale hemispherical shells. PMMA and boron-doped silicon shells were first fabricated in 1979 for thermonuclear fusion research [3]. Two years later, shells made from gold or oxide were fabricated by a similar technique [4]. However, these shells were fully detached from the substrate, and the resonance characteristics of these shells were not measured. A blow-molding method based on thermoplastic forming of bulk metallic glass has been used to fabricate 3D micro shells [5]. UCI has recently reported 'inverted' Pyrex wineglass structures fabricated by wafer-level glassblowing. The inverted Pyrex hemispherical structures are mechanically supported at the rim, limiting their use as high- $Q$ resonators [6]. The same group has demonstrated stem-supported hemispherical shells through laser-cutting of individual glass spheres [7]. However, laser-cutting is a serial process that is difficult to implement and control at wafer-level. Furthermore, the stem support diameter is determined by the opening size in the silicon stencil wafer, which must be large to create large diameter shells.

Our group recently reported polysilicon hemispherical shell resonators with integrated capacitive transducers for electrical operation [1]. The isolated electrodes are created by boron-doping of n-type silicon wafers, forming PN junctions for isolation. The

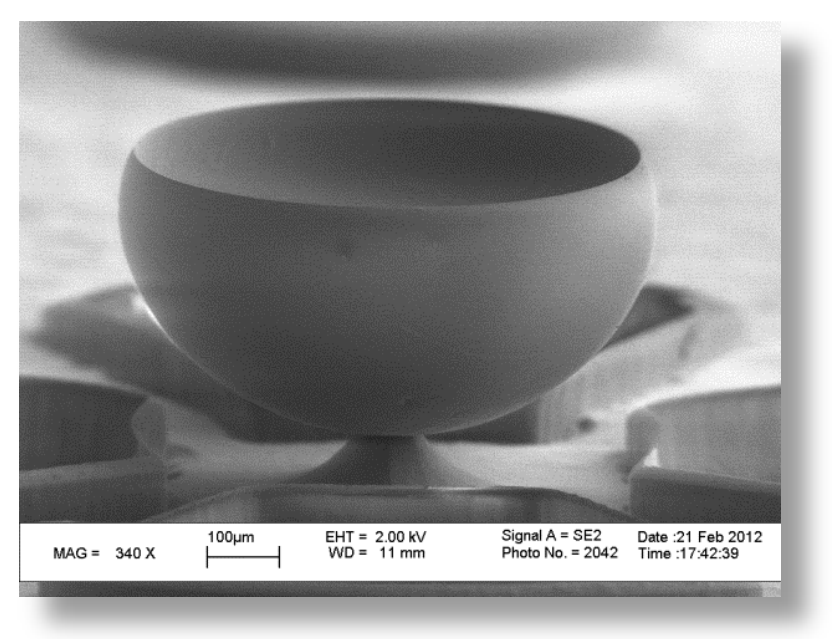

Figure 1: Perspective view of a fully-released $2 \mu \mathrm{m}$ thick, $740 \mu \mathrm{m}$ diameter, TiN-coated thermally-grown silicon dioxide -wineglass with small support (77 $m$ diameter).

capacitive gap is defined by the sacrificial oxide layer, whose thickness is limited to a few microns. In this work, a novel assembly method forms electrodes with large capacitive gaps surrounding the shell for rapid characterization of the oxide shell resonances, enabling full electrical characterization of microscale stem-supported hemispherical shells.

\section{MATERIAL SELECTION}

Various energy dissipation mechanisms limit resonator quality factor. Low frequency resonators operate in the Akhieser regime, so the limit of $Q$ is inversely proportional to frequency [8]. However, factors such as surface roughness, support loss, and thermoelastic damping (TED) may dominate the energy dissipation, leading to lower $Q$ than the Akhieser limit predicts [1]. Surface roughness is ultimately determined by the smoothness of the mold and support loss can be suppressed by anchor design. TED can be alleviated through material selection.

To minimize TED, which originates from the coupling of the stress and thermal fields, a material with a low coefficient of thermal expansion (CTE) over the operating temperature range is desired. For example, quality factors greater than 25 million have been achieved, at the macroscale, using fused quartz as the structural material for the HRG [2]. Table 1 compares fused quartz, thermally-grown oxide, and titania silicate (ultra-low expansionULE) glass. Thermal oxide has a similar value of CTE to fused quartz and is very stable over a large temperature range. It can be noted that titania silicate glass show a very low $\mathrm{CTE}\left(\sim 60 \mathrm{ppb} /{ }^{\circ} \mathrm{C}\right)$ near room temperature. However, this ramps up rather quickly at $40^{\circ} \mathrm{C}$ and will reach $7 \mathrm{ppm} /{ }^{\circ} \mathrm{C}$ at $95^{\circ} \mathrm{C}$ [9]. Therefore, thermal oxide was selected as the -wineglass structural material for low TED.

Table 1.CTEs of different materials.

\begin{tabular}{|c|c|c|}
\hline Material & $\begin{array}{c}\mathrm{CTE} @ 25^{\circ} \mathrm{C} \\
\left(\mathrm{ppm} /{ }^{\circ} \mathrm{C}\right)\end{array}$ & $\begin{array}{c}\mathrm{CTE} @ 95^{\circ} \mathrm{C} \\
\left(\mathrm{ppm} /{ }^{\circ} \mathrm{C}\right)\end{array}$ \\
\hline Fused quartz [10] & 0.45 & 0.58 \\
\hline Silicon dioxide (thermal) [11] & 0.5 & 0.8 \\
\hline $\begin{array}{c}\text { Titania silicate glass [9] } \\
\text { (Ultra low expansion glass) }\end{array}$ & 0.06 & 7 \\
\hline
\end{tabular}

Solid-State Sensors, Actuators, and Microsystems Workshop Hilton Head Island, South Carolina, June 3-7, 2012 


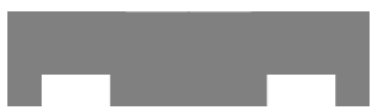

(a)

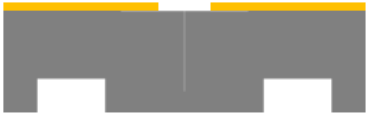

(b)

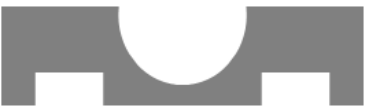

(c)

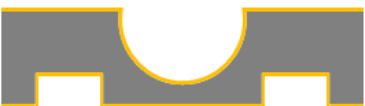

(d)

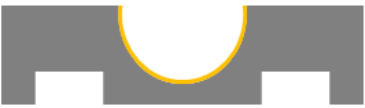

(e)

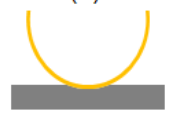

(f)

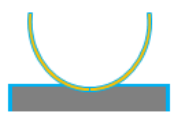

(g)

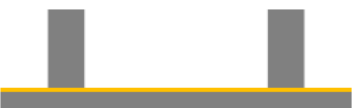

(h)

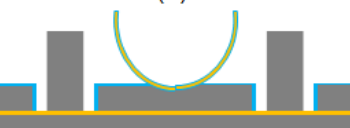

(i)

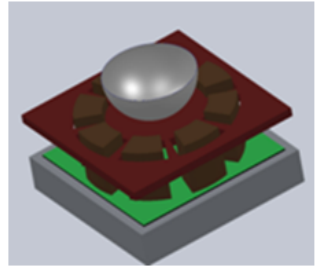

(j)

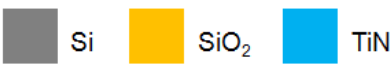

Figure 2 (color online): Fabrication and assembly process flow of -wineglass: (a) etch back side blind holes for electrode assembly; (b) pattern front side PECVD oxide mask; (c) isotropically etch silicon mold in $\mathrm{SF}_{6}$ plasma and remove oxide mask; (d) grow thermal oxide; (e) remove oxide on top side and back side while photoresist is protecting oxide in mold; (f) release -wineglass by $\mathrm{XeF}_{2} ;$ (g) atomic layer deposition (ALD) conformal coating of titanium nitride (TiN) layer; (h) etch electrode pillars on siliconon-insulator (SOI) wafer; (i) assembly of two dies with adhesive in between (not shown); (j) three-dimensional view of assembled wineglass.

\section{FABRICATION AND ASSEMBLY PROCESS}

The silicon hemispherical molding technique introduced in [1] has been tailored for thermal growth of silicon dioxide shells. Figure 2 shows the wafer-level batch-fabrication process for oxide shells and the electrode assembly. Blind holes around the shell are first etched from back side of the wafer using the Bosch process. Later in the process, these blind holes will become through holes for electrode assembly. A $7 \mathrm{~m}$ thick PECVD oxide layer is deposited on the front side at $300^{\circ} \mathrm{C}$. A circular opening is then etched into the PECVD oxide mask by a front side to back side alignment. Silicon is isotropically etched by $\mathrm{SF}_{6}$ plasma to create a symmetric hemispherical mold. After the PECVD oxide mask is removed in hydrofluoric acid (HF), oxide is thermally grown at $1100^{\circ} \mathrm{C}$ using a wet oxidation method and reaches a final thickness of $3.3 \mathrm{~m}$. Due to the diffusion limited nature of thermal oxidation, oxide is very conformal to the shape of the silicon hemispherical mold. The thermally-grown oxide on the wafer surface is removed in $\mathrm{C}_{4} \mathrm{~F}_{8}$ plasma while the hemispherical mold is protected by photoresist. The remaining photoresist is removed in Piranha, leaving only oxide in the hemispherical mold. The oxide shell is released by etching the surrounding silicon in $\mathrm{XeF}_{2}$. Figure 3 shows an optical image of an array of released oxide shells.

To enable electrical testing, the shells must be coated with a conformal conductive layer that electrically connects the shell and the supporting substrate, providing a path for polarization voltage.
Compared to evaporation and sputtering, atomic layer deposition (ALD) provides a higher quality film and a more uniform conformal coating. $30 \mathrm{~nm}$ of titanium nitride (TiN) is deposited at $250^{\circ} \mathrm{C}$, conformally coating the sharp transition between the oxide shell and the silicon stem. A resistance of $800 \Omega$ was measured between the TiN-coated shell and substrate, confirming that this layer provides sufficient electrical connection.

Electrode pillars are etched by high aspect ratio silicon etcher on silicon-on-insulator (SOI) wafers using the Bosch process to ensure electrical isolation between electrodes. Finally, the shells and electrodes are assembled under microscope. Adhesive is dispensed and hard-baked between the two wafers to secure them together. Figure 4 shows a bird's eye view of an assembled device with electrodes for testing.

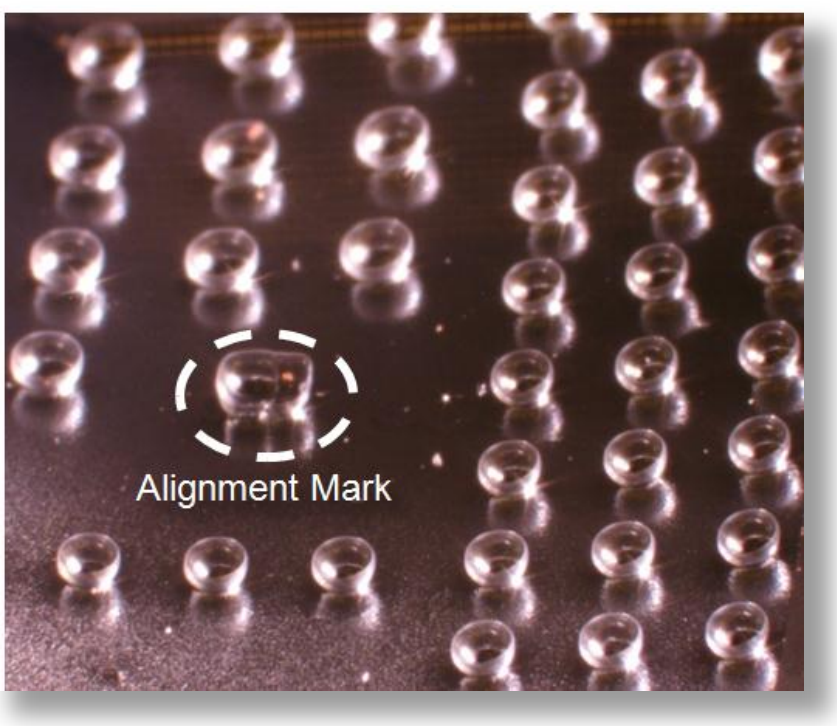

Figure 3: Wafer-level fabrication of -wineglass (field of view: $2 \mathrm{~cm}$ by $2 \mathrm{~cm}$ ).

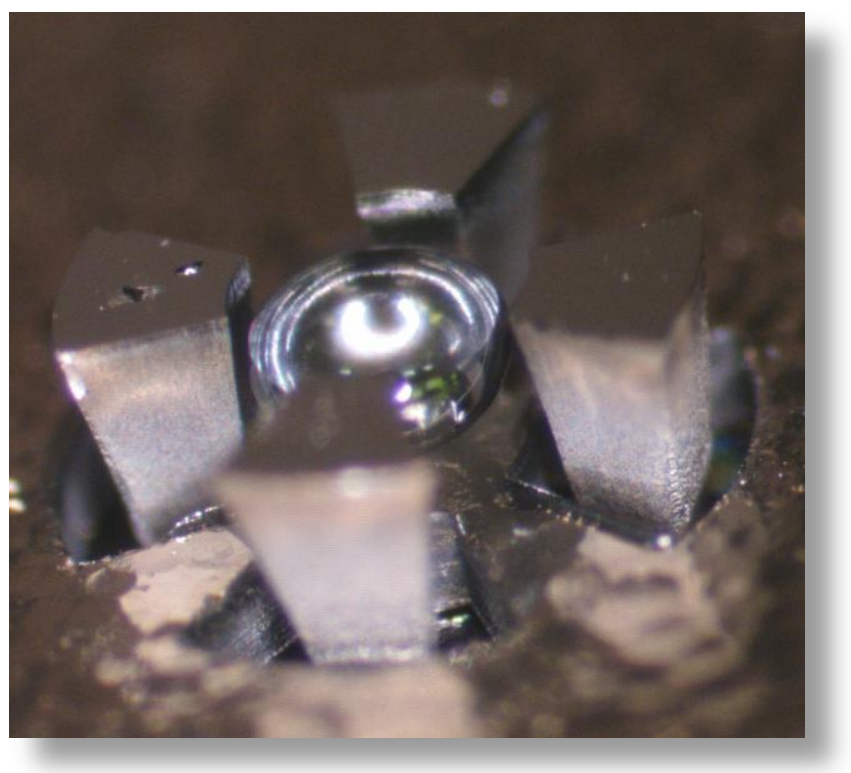

Figure 4: Optical image of assembled oxide -wineglass with electrode pillars for electrical testing. The -wineglass is coated with $30 \mathrm{~nm}$ TiN by atomic layer deposition (ALD). 


\section{PROCESS CHARACTERIZATION}

Axial symmetry is an important geometric requirement of wineglass structures. A software tool was developed for SEM image analysis to characterize the circular symmetry of the fabricated shells [1]. For the example shell with equatorial radius of $552.5 \mathrm{~m}$ shown in Figure $5 \mathrm{a}$, a radial standard deviation smaller than $0.61 \%$ is reported (Fig. $5 b$ ), demonstrating that the shells are highly balanced. One uncertainty in this analysis is tilting of SEM stage and the mounting of the sample. If the sample is not mounted perpendicular to the electron gun, the rim of the shell will appear elliptical, resulting in systematic measurement error.

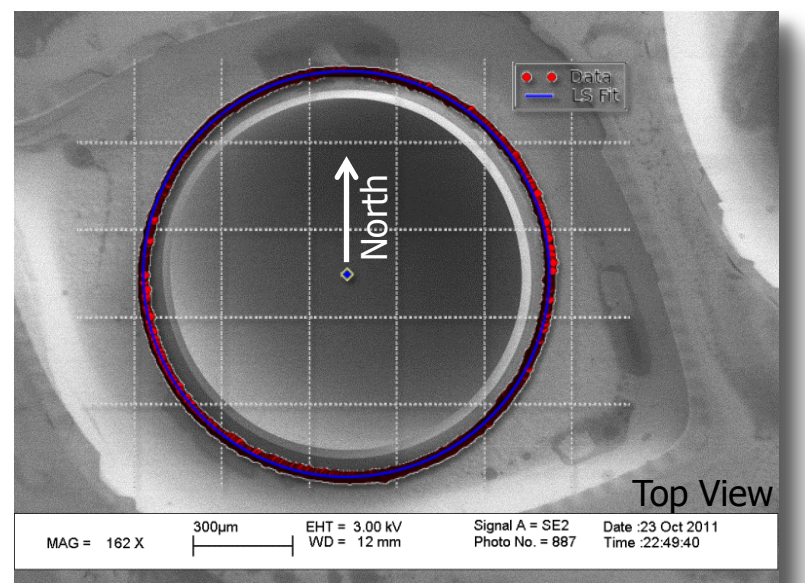

(a)

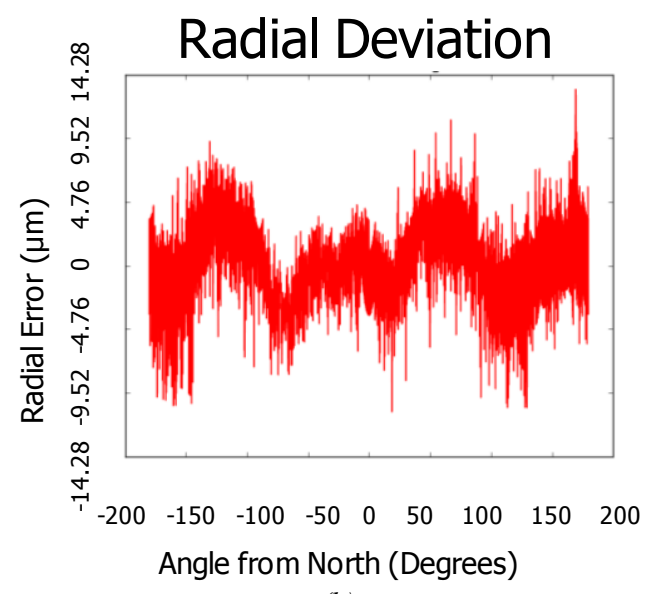

(b)

Figure 5: (a) least squares fit to the top view SEM image of wineglass; (b) radial error of -wineglass results with a standard deviation of $0.61 \%(3.37 \mathrm{~m}$ in diameter of $1105 \mathrm{~m})$.

Figure 6 presents additional details of the assembled wineglass with electrodes. Although $3.3 \mathrm{~m}$ oxide was initially grown in the silicon mold, only $1.6 \mathrm{~m}$ is left at the rim after the $\mathrm{XeF}_{2}$ release (Fig. 6a). The etching of oxide in $\mathrm{XeF}_{2}$, which is known as a high selectivity silicon etchant, is not negligible during silicon etching to this depth. The shell thickness is also found to vary along the depth of shell. During $\mathrm{XeF}_{2}$ release, the top part of the shell is released first and exposed to $\mathrm{XeF}_{2}$ from two sides. Thus, more oxide is consumed at the rim than at the unexposed base. By cleaving the released shell, thickness variation along the depth of shell could be measured under SEM (Fig. 6b). The thickness smoothly tapers from $1.6 \mathrm{~m}$ at the rim to $2.2 \mathrm{~m}$ close to the base. Thickness variation in the depth direction is tolerable since it has no impact on the axial symmetry of the shell. The shell rim is also found to have a reentrant extension with projected dimension of $23 \mathrm{~m}$ near its rim (Fig. 6a). This is due to the nature of the isotropic mold formation, since $\mathrm{SF}_{6}$ cannot etch laterally to the oxide mask opening.

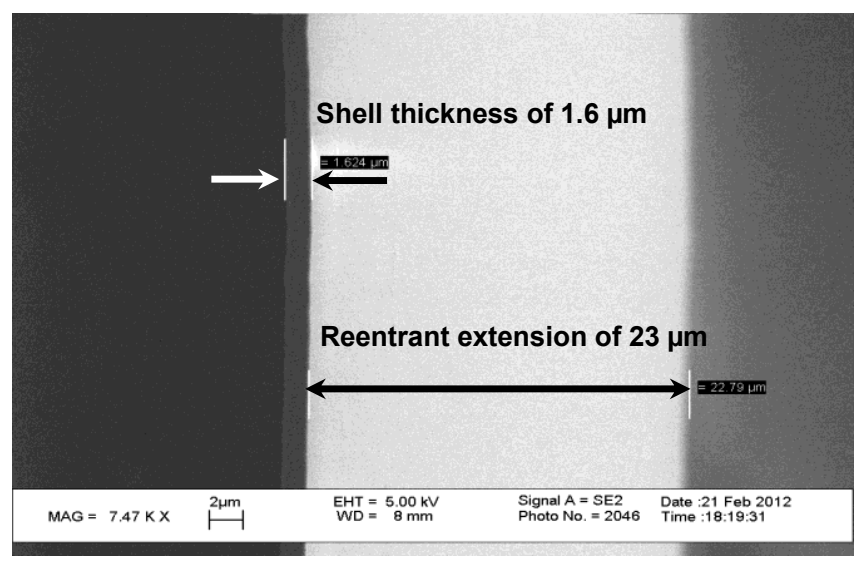

(a)

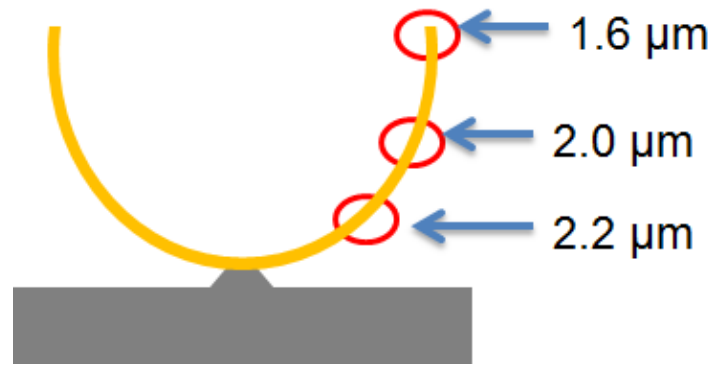

(b)

Figure 6: Detailed geometric characterization of assembled wineglass: (a) (top view) shell thickness of $1.6 \mathrm{~m}$ and reentrant projection of $23 \mathrm{~m}$; (b) thickness variation along the depth of the shell (measured by SEM), showing measured shell thickness at different locations schematically.

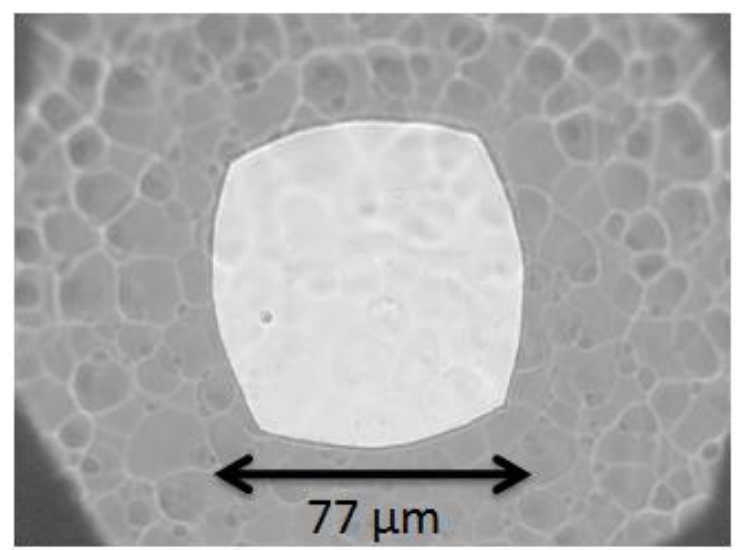

Figure 7: Top-view optical microscope image of octagonal-shaped support (77 m support diameter). Substrate roughness created by $\mathrm{XeF}_{2}$ release is visible through the transparent shell. 


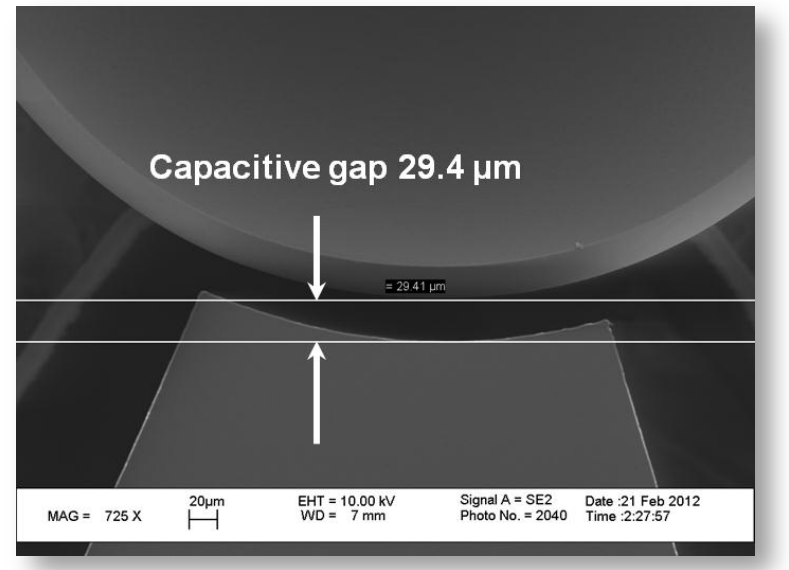

Figure 8: Capacitive gap of $29.4 m$ between -wineglass and excitation electrode, giving efficient electrostatic transduction.

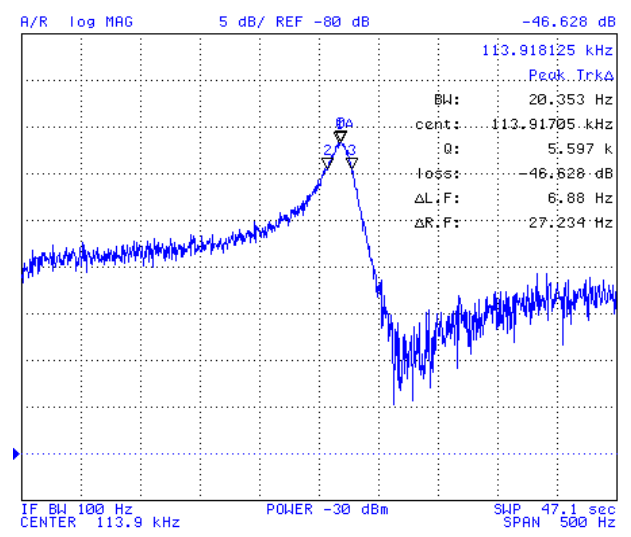

(a)

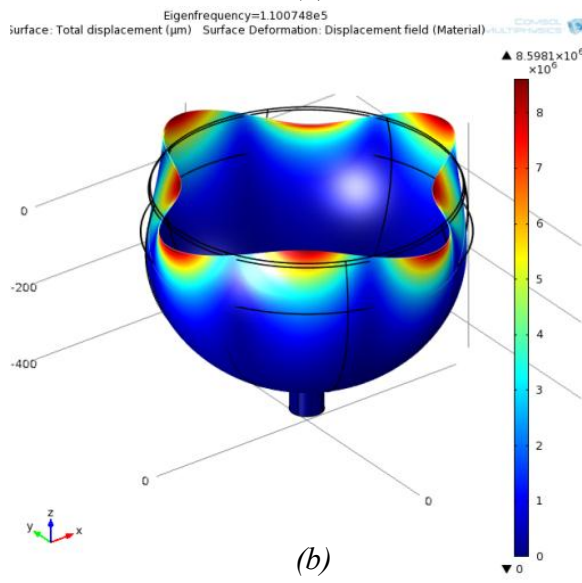

Figure 9: Electrical testing result of -wineglass (a) resonance peak of wineglass mode $(m=4)$ at $113.9 \mathrm{kHz}$ with quality factor of 5,600; (b) COMSOL FEA modal analysis showing simulated resonance frequency of $110.1 \mathrm{kHz}$.

The support diameter is an important geometric parameter of the -wineglass. It has a strong effect on the resonance frequency and may allow support loss to limit the quality factor. Figure 7 shows an optical microscope image of the support structure. Interestingly, although $\mathrm{XeF}_{2}$ is well-known for etching silicon isotropically, the etching does show some crystalline preference evidenced by an octagonal-shaped support with $77 \mathrm{~m}$ diameter. Due to the transparency of the oxide shell, the substrate surface roughness that is formed during $\mathrm{XeF}_{2}$ release is visible in Figure 7.
Figure 8 shows the $29.4 \mathrm{~m}$ capacitive gap between the wineglass structure and electrode pillar. A smaller gap will give better electrical transduction, thus lowering the motional impedance, while a larger gap can allow large drive amplitude.

\section{TESTING RESULTS}

The $740 \mathrm{~m}$ device was tested in vacuum as a one-portresonator. An Agilent 4395A network analyzer supplies AC drive voltage to one electrode pillar and a sense current is generated by the change in capacitance across the polarized gaps due to vibration of the shell. A trans-impedance amplifier (TIA) is used to amplify the signal from -wineglass. Using the electrical testing platform, we were able to excite the $\mathrm{m}=4$ resonance mode at 113.9 $\mathrm{kHz}$ with a quality factor of 5,600 (Fig. 9a). Eigenfrequency analysis by COMSOL FEA software predicts a resonance frequency of $110.1 \mathrm{kHz}$ (Fig. 9b), which is in good agreement with the measured frequency.

\section{CONCLUSION}

A wafer-level fabrication method was developed for highlysymmetric thermally-grown oxide -wineglasses. An ALD TiN conductive layer was able to establish sufficient electrical connection between the -wineglass and the substrate. A novel assembly process was developed for the electrical testing platform. The $\mathrm{m}=4$ resonance of assembled -wineglass was measured in vacuum, with a $Q$ of 5,600 at $113.9 \mathrm{kHz}$, demonstrating the strong potential of these devices for resonator applications.

\section{ACKNOWLEDGMENTS}

This work was supported by the DARPA Microsystem Technology Office, Microscale Rate Integrating Gyroscope (MRIG) program under contract \#HR0011-00-C-0032 led by Northrop Grumman. The authors would like to thank the cleanroom staff at Georgia Tech's Institute for Electronics and Nanotechnology (IEN) for fabrication support.

\section{REFERENCES}

[1] L. Sorenson, in IEEE International Conference on Micro Electro Mechanical Systems (MEMS 2012), Paris, France, 2012, pp. 168-171.

[2] D. Rozelle, in Proc. 19th AAS/AIAA Space Flight Mechanics Meeting, 2009, pp. 1157-1178.

[3] K. D. Wise, Journal of Vacuum Science and Technology, vol. 16, no. 3, pp. 936-939, 1979.

[4] K. D. Wise, Journal of Vacuum Science and Technology, vol. 18, no. 3, pp. 1179-1182, 1981.

[5] B. Sarac, Journal of Microelectromechanical Systems, vol. 20, no. 1, pp. 28-36, Jan. 2011.

[6] I. P. Prikhodko, Journal of Microelectromechanical Systems, vol. 20, no. 3, pp. 691-701, May 2011.

[7] D. Senkal, in Technologies for Future Micro-Nano Manufacturing, Napa, California, USA, 2011.

[8] R. Tabrizian, presented at the Solid-State Sensors, Actuators and Microsystems Conference, May 2009, pp. 2131-2134.

[9] http://www.corning.com/assets/0/965/989/1081/D20FD2EA7264-43DD-B544-E1CA042B486A.pdf.

[10] J. A. Beattie, Proceedings of the American Academy of Arts and Sciences, vol. 74, no. 11, pp. 371-388, Dec. 1941.

[11] I. Blech, Journal of Applied Physics, vol. 53, no. 6, pp. 42024207, May 1982.

\section{CONTACT}

*P. Shao, tel: +1-404-988-5782; pengshao@gatech.edu 


\title{
A MEMS CONTROLLED CAVITY OPTOMECHANICAL SENSING SYSTEM
}

\author{
H. Miao ${ }^{1,2}$, K. Srinivasan ${ }^{l}$, and V.A. Aksyuk ${ }^{l}$ \\ ${ }^{1}$ Center for Nanoscale Science and Technology, National Institute of Standards and Technology, Gaithersburg, \\ Maryland, USA \\ ${ }^{2}$ Maryland Nanocenter, University of Maryland, College Park, Maryland, USA
}

\begin{abstract}
A novel high optical Q Si microdisk photonic cavity sensor is integrated to read out mechanical motion of an electrostatically actuated cantilever achieving $4.6 \mathrm{fm} / \mathrm{Hz}^{1 / 2}$ displacement sensitivity for sub- $\mathrm{W}$ input power. The readout gain is electrically-tunable by a factor of 200 . Using cold-damping feedback we reduce the thermal mechanical vibration by a factor of $>1000$, flattening the mechanical transfer function, and demonstrate broadband sensing with $100 \mathrm{kHz}$ bandwidth, $>2.5$ times the fundamental mechanical frequency. With $<20 \mu \mathrm{m}$ footprint and intrinsic readout bandwidth of $>500 \mathrm{MHz}$, cavity optomechanics enables a practical and flexible MEMS sensing platform with a broad application range.
\end{abstract}

\section{INTRODUCTION Motivation}

Sensors based on micro and, more recently, nano-mechanical systems continue to develop rapidly. They enable a wide variety of applications, from ubiquitous, mature technology of MEMS accelerometers, to gyroscopes [1], rapidly advancing in performance, from biomedical sensing [2] and atomic force microscopy (AFM) $[3,4]$ with mechanically simple cantilevers, to measurement of torque from single flux quanta in superconductors [5] and spin-polarized electron currents [6], with specially designed, complicated micro- and nano-devices. Generally, shrinking the sensor size allows increasing the mechanical responsivity by making it more compliant, while simultaneously increasing the bandwidth by lowering the inertia, thus reaching higher mechanical gain-bandwidth products. The sensor precision is fundamentally limited by the thermal Langevin force noise, which is related to the sensor dissipation via the fluctuation dissipation theorem. This noise can be minimized by making the appropriate materials, design and processing choices to achieve very low dissipation rates, with mechanical quality factors $(\mathrm{Q})$ in the $10^{5}$ to $10^{6}$ range [7]. In addition to lowering the sensor input noise floor, high Q increases the sensor responsivity (mechanical gain) in a narrow region of frequencies near resonance. While the gain is increased proportional to the $\mathrm{Q}$, the bandwidth, over which this high gain is available, drops as $1 / \mathrm{Q}$.

In a typical mechanical sensor the change in the mechanical position, in response to the driving forces and the environment, is detected by some linear readout means, such as electrostatic, piezoresistive, piezoelectric or optical. To operate the sensor at the fundamental limit of precision imposed by the thermal mechanical noise, it is necessary that the noise introduced by the readout be below the mechanically amplified Langevin force noise, over the whole sensor bandwidth. While this requirement is easier to achieve for near-resonant operation due to very high mechanical gain in this narrow band frequency region, it is a much more challenging task for broadband sensing, where the readout precision is often the dominant limitation of the sensor performance.

The main motivation of this work is to develop and demonstrate a technique that simultaneously advances the on-chip MEMS position readout precision and bandwidth by several orders of magnitude. Such technology should be fully integrated, stable, compatible with many types of mechanical sensors, have small footprint, low power dissipation and require minimal external infrastructure. It should also allow tailoring the readout gain and dynamic range to specific application requirements. We realize such a technique based on integrated interferometric optical readout or cavity optomechanics, achieving $4.6 \mathrm{fm} / \mathrm{Hz}^{1 / 2}$ displacement sensitivity for sub- W input optical power.

Taking advantage of this high sensitivity, we use feedback and electrostatic actuation to reduce the thermal mechanical vibration of the sensing element by a factor of 1000, tune the mechanical transfer function to remove the resonant peak at the fundamental frequency of $\approx 39 \mathrm{kHz}$ and extend the measurement bandwidth to $100 \mathrm{kHz}$ ( $>2.5$ times the fundamental frequency). The input-referred sensor readout noise remains below the thermal mechanical noise over this whole frequency range.

\section{Approach}

The fundamental benefits of optical readout are small footprint and high sensitivity, both related to the wavelength of light, low power dissipation, absence of intrinsic thermal noise due to photon shot noise limited operation, and very high intrinsic bandwidth, related to the optical frequency. Optical cavities afford even higher sensitivity at the expense of slight reduction in bandwidth by capturing the photons and making them interact with the mechanical degree of freedom over the cavity lifetime.

Silicon nanophotonics provides a path to integrating such cavities on chip, achieving high optical quality factors up to $10^{6}$ in a system compatible with MEMS and CMOS processes and materials. Such fiber pigtailed and connectorized Si devices operate at telecommunication wavelength, where highly stable, low noise, compact and economical diode laser sources, as well as low noise detectors, are readily available.

We have chosen a relatively simple $\mathrm{Si}$ microdisk cavity design, where the light circulates along the microdisk edge in a whispering gallery mode. The optical connection to the cavity is provided through evanescent coupling from a fixed on-chip $\mathrm{Si}$ waveguide, which is end-fire coupled to conventional optical fibers glued into v-groves. However, in contrast to the previous cavity optomechanical work $[8,9]$, we are not sensing the mechanical breathing mode of the microdisk, because such mode is very stiff, high frequency and is not easily coupled to for sensing external mechanical forces without degrading the optical performance.

In a novel design, illustrated in Figure 1d, we separate the optical and the mechanical parts of the system. The microdisk itself does not move. Instead we introduce a flat $\mathrm{SiN}_{\mathrm{x}}$ dielectric ring suspended a short distance above the microdisk top surface and movable vertically toward and away from it. The ring is mechanically attached to a soft cantilever actuator, comprising the mechanically active part of the sensor. Because the microdisk is thin, a significant fraction of the optical mode power density is located outside the microdisk, in the evanescent tails below and above it, where it is affected by the ring. As the ring moves closer to the disk, a larger fraction of the optical power travels inside the ring dielectric, increasing the effective index and shifting the frequency of the optical resonance lower. With the ring a few hundred nanometers above the disk, the strong coupling between the cavity optical frequency and the ring position forms the basis for our sensitive optical readout. 

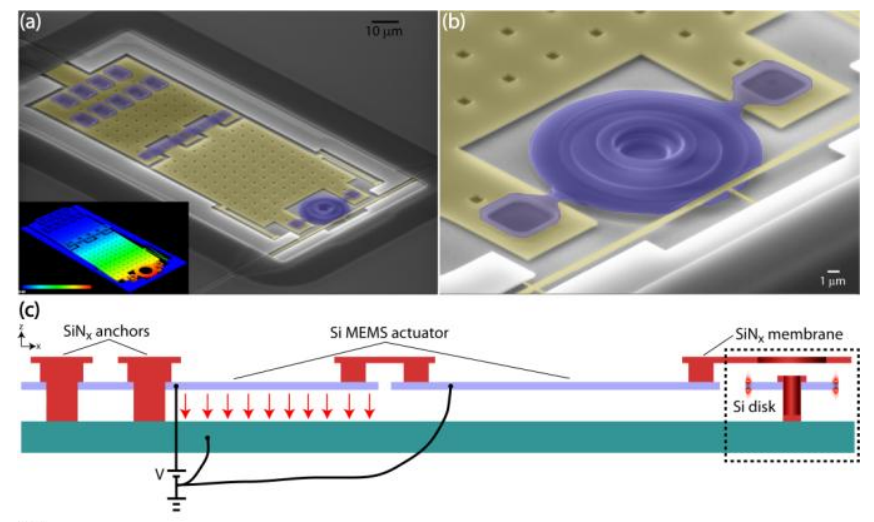

(d)

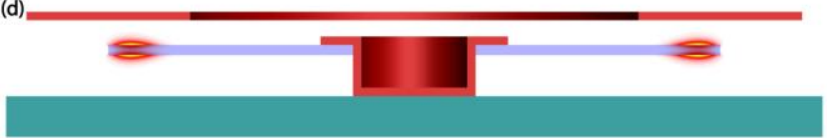

Figure 1: Device geometry. (a) SEM of the fabricated device. (inset) Finite element simulation of the fundamental mechanical mode of the mechanical structure. (b) Zoomed-in view of the sensor area. (c)-(d) $2 D$ cross-sectional illustrations of the device. The illustration shown in (c) is a cross section taken through the key elements of the mechanical sensor. In these cross-section illustrations, the $Z$ axis scale has been adjusted for clarity.

\section{EXPERIMENTAL}

\section{Design and fabrication}

Figure 1 shows the SEM and the illustrated cross sections of the sensing system. A 19 m outer diameter, $200 \mathrm{~nm}$ thick silicon nitride ring is placed within the evanescent field of a $15 \mathrm{~m}$ diameter, $240 \mathrm{~nm}$ thick silicon microdisk optical resonator, with a variable distance $\mathrm{Z}$ between them. The microdisk is fixed to the substrate via a silicon nitride anchor, while the ring is attached to a MEMS actuator. The actuator is a silicon cantilever consisting of two electrically separate parts that are mechanically joined by a dielectric silicon nitride bridge, with one side fixed to the substrate via silicon nitride anchors. Voltage is applied to the first part of the cantilever, while the substrate, $1 \mathrm{~m}$ below, is kept at the ground potential. The attractive electrostatic force bends the first part toward the substrate. The second part of the cantilever, maintained at the ground potential, serves as a mechanical lever arm to achieve a larger range of motion at the ring location. The resulting soft cantilever with the sensor ring has a fundamental mechanical bending mode (Figure 1a, inset) with a measured frequency of $\approx 38.5 \mathrm{kHz}$ and the stiffness at the sensor attachment point of $\approx 0.04$ $\mathrm{N} / \mathrm{m}$ from the FEM model.

The fabrication process was started with a silicon-on-insulator (SOI) wafer with $240 \mathrm{~nm}$ top silicon layer and $1 \mathrm{~m}$ buried oxide (BOX) layer. The device layer is patterned via electron beam lithography and dry etched down to the BOX layer to produce silicon microdisks, access waveguides and the actuators. The waveguide is linearly tapered down to a width of $125 \mathrm{~nm}$ at its end for low loss coupling with optical fibers. A sacrificial silicon dioxide layer $\quad(\approx 600 \mathrm{~nm}$ thick $)$ and a low stress silicon nitride layer $(\approx 200 \mathrm{~nm})$ are sequentially deposited in a low temperature oxide (LTO) and a silicon nitride low pressure chemical vapor deposition (LPCVD) furnace, patterned via optical lithography, and dry etched to form the nitride ring, nitride anchors and bridge and an electrical pad to ground the substrate. A photolithography, buffered oxide etch, and ion implantation (Boron) process was used to dope the first part of the cantilever and the pad to a doping level of $>10^{19} \mathrm{~cm}^{-3}$ for low contact resistivity. Another $1 \mathrm{~m}$ oxide layer was deposited. The wafer was annealed for $1 \mathrm{~h}$ at $1000{ }^{\circ} \mathrm{C}$ in an ambient $\mathrm{N}_{2}$ environment. Another photolithography, metal deposition, liftoff, dry etching and TMAH wet etching process was used to define the $\mathrm{V}$-grooves for fiber pigtailing. The device was finally placed in $49 \% \mathrm{HF}$ to undercut and release the movable structures, and critical point dried. Fibers are placed in the Vgrooves, aligned and cured into place with ultraviolet (UV) curable epoxy, with a fiber to fiber loss of $16 \mathrm{~dB}$.

\section{Transducer characteristics: static}

Figure $2 \mathrm{a}$ shows the electrostatic tuning of $Z$ and of the optical resonant wavelength as a function of the DC voltage applied to the actuator. The tuning of $Z$ is measured using a white light interferometric profiler. The resonant wavelength at various DC voltage values is measured via optical spectroscopy using a tunable wavelength laser. The inset of Fig. $2 b$ shows the transmission spectrum of one of the optical modes that is used for subsequent experiments. The linewidth of the optical mode is 4.1 $\mathrm{pm}$, corresponding to an optical Q of $3.8 \times 10^{5}$. To detect small motions of the cantilever we tune the laser to a set point on the linear slope of the optical resonance line (Figure $2 \mathrm{~b}$, inset). The change of the optical resonance frequency due to the mechanical motion causes a proportional change in the detected optical intensity. The sensor readout gain is proportional to the line slope given by the optical $\mathrm{Q}$ and the optomechanical coupling $g_{O M}=d \omega_{c} / d Z$, where $\omega_{c}$ is the optical resonance frequency. Figure $2 \mathrm{~b}$ shows the optomechanical coupling, calculated from Fig. 2a, which is increased by a factor of more than 200 (from $g_{O M} / 2 \pi=65$ $\mathrm{MHz} / \mathrm{nm}$ to $g_{O M} / 2 \pi=13.4 \mathrm{GHz} / \mathrm{nm}$ ) when the applied voltage is increased from $2.5 \mathrm{~V}$ to $8 \mathrm{~V}$, thus broadly adjusting the gain of the readout.
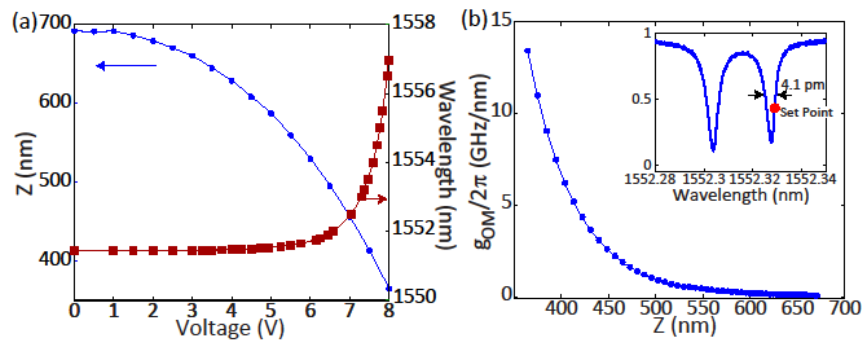

Figure 2: Static properties of the system. (a) Displacement and resonant wavelength as a function of bias voltage. (b) Optomechanical coupling as a function of displacement. Inset shows the transmission spectrum of the optical mode. In (a) and (b) the dots are the positions where the measurements were made.

\section{Transducer performance: dynamics and feedback}

We test the cavity optomechanical sensor by measuring the dynamics of the fundamental cantilever bending mode of the actuator. A CW light from a tunable wavelength laser is launched into the device, with an estimated optical power of $\approx 250 \mathrm{nW}$ in the waveguide, and $\approx 40 \mathrm{nW}$ out-coupled to a photodetector with a gain of $\approx 1.9 \times 10^{6} \mathrm{~V} / \mathrm{W}$ and a $3 \mathrm{~dB}$ bandwidth of $200 \mathrm{kHz}$. The wavelength is tuned to a pre-specified transmitted power set point as shown in the inset of Fig. 2b. A small motion of the cantilever results in a linear modulation of the transmitted optical power. The resulting photodetector voltage proportional to the mechanical displacement is recorded with an electrical signal analyzer.

We have measured two characteristics of our device (placed in a vacuum chamber with a pressure of $0.3 \mathrm{~Pa}$ ) under various 
conditions: the mechanical displacement noise spectral density and the sensor transfer function, shown in Fig. $3 a$ and $3 b$, respectively. The blue curves on Fig. $3 a$ and $3 b$ are taken under a fixed actuator DC bias voltage of $3 \mathrm{~V}$. To measure the transfer function, a small $\mathrm{AC}$ voltage with varying frequency is added to the DC bias, and the cantilever response is recorded in a lock-in measurement, normalized to the AC voltage. The black curve in Fig. 3a shows the noise background of the measurement.
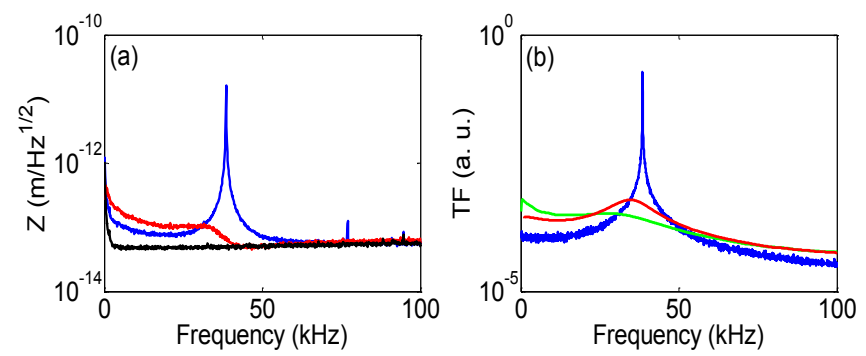

Figure 3: Device dynamics with and without cold damping. (a) Displacement noise spectra at 3 V DC bias with (red) and without (blue) feedback cooling. (b) Transfer functions (TF) corresponding to (a) (blue and red), and with higher feedback gain (green). Black curve in (a) shows the photodetector limited measurement sensitivity for both the undamped case and the damped case which are not distinguishable.

Although the high mechanical Q is desirable to reduce the thermal mechanical noise, the resulting highly non-uniform transfer function severely limits the sensor dynamic range if a broadband mechanical measurement is considered. We overcome this limitation by introducing a cold damping electrical feedback loop that modifies the sensor mechanical transfer function, reducing its variation across the frequency range. The red curves in Fig. $3 \mathrm{a}$ and $3 \mathrm{~b}$ show the noise spectrum and the corresponding transfer function at the same bias of $3 \mathrm{~V}$ with feedback cooling. The green curve in Fig. $3 \mathrm{~b}$ illustrates the transfer function with a higher damping gain setting, where the mechanical $Q$ is approaching 1. Considering the measured intrinsic $Q$ of $\approx 1400$, the root mean square thermal vibration amplitude of the mechanical mode is reduced by a factor of $>1000$. While the cold damping in itself does not reduce the input thermal force noise of the sensor, it does reduce any back-action that the sensor may exert on the physical system being measured by reducing the effective temperature of the sensor mechanical mode.

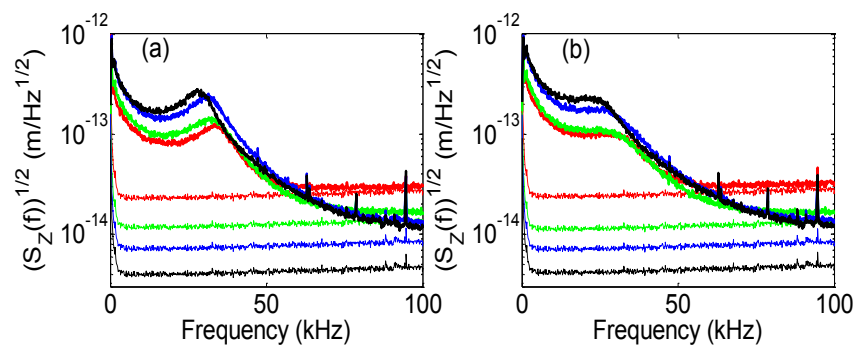

Figure 4: Displacement spectra at various bias voltages. (a) Under-damped case. (b) Critically damped case. The bias voltages for the red, green, blue and black curves are $4.5 \mathrm{~V}, 5.5 \mathrm{~V}, 6.25 \mathrm{~V}$ and $6.75 \mathrm{~V}$, respectively. The thicker lines show the measured ring displacement noise, the thinner lines are the corresponding photodetector limited background curves. The highest achieved readout sensitivity corresponds to the thin black lines.
The large reduction of the mechanical vibration of the sensor by the feedback allows working at increased DC bias, reaching higher readout gain and lower noise. Figure 4 shows the displacement noise spectra and the measurement noise backgrounds at increasing DC bias voltages. The degree of damping can be adjusted independently of the bias voltage, as illustrated by the two sets of curves in Fig. $4 \mathrm{a}$ and $4 \mathrm{~b}$. In addition to damping, our simple feedback choice also results in the reduction of the closed-loop mechanical stiffness. The displacement sensitivity increases by more than a factor of 15 when the bias voltage is increased from $4.5 \mathrm{~V}$ to $6.75 \mathrm{~V}$, and is limited by the photo detector noise to $4.6 \pm 0.6 \mathrm{fm} / \mathrm{Hz}^{1 / 2}$ at a bias voltage lever of $6.75 \mathrm{~V}$, which is only 2.3 times the standard quantum limit of the system.

\section{CONCLUSION \\ Summary}

We have realized a novel, fully-integrated and fiber-pigtailed high-precision optical readout of MEMS mechanical motion based on cavity optomechanics. The optical interferometric sensing of mechanical motion is achieved by evanescent coupling of a high $\mathrm{Q}=3.8 \times 10^{5}$ optical mode in a stationary Si microdisk optical cavity with a movable $\mathrm{SiN}_{\mathrm{x}}$ dielectric ring structure in close proximity above the microdisk. This design achieves large optomechanical coupling for high readout gain, while separating mechanical and optical parts of the system for independent optimization.

We demonstrate readout sensitivity below $5 \mathrm{fm} / \mathrm{Hz}^{1 / 2}$ limited by photodetector noise, with less than $1 \mathrm{~W}$ input optical power. The intrinsic bandwidth of our readout is above $500 \mathrm{MHz}$ limited by the photon lifetime in the optical cavity, while in this experiment it was limited by the detector bandwidth of $200 \mathrm{kHz}$. Our nanophotonic readout is highly stable and requires no optical alignment. The integrated actuator can tune the optomechanical readout gain by a factor of 200 .

While the total noise in our system is dominated by the thermal mechanical noise, it is noteworthy that the readout sensitivity approaches the standard quantum limit near resonance. From the practical perspective, the high sensitivity allows broadband mechanical sensing at frequencies much larger than the fundamental mechanical resonance. We have used the readout and the actuator in a feedback loop to flatten the mechanical transfer function to enable broadband measurements, dampen thermal mechanical motion of our sensor by a factor of $>1000$ and measure its vibration noise power spectrum over a broad mechanical bandwidth of $0 \mathrm{kHz}$ to $100 \mathrm{kHz}$, more than 2.5 times the fundamental mechanical resonance frequency of $\approx 39 \mathrm{kHz}$.

\section{Outlook}

We believe our integrated, stable MEMS sensing platform can enable a variety of scientific and technical sensing applications due to a combination of very high sensitivity, very high bandwidth and decoupling of mechanical and optical design parameters. The platform uses conventional MEMS materials and processes. The readout approach is suitable for high and low mechanical frequency systems with a broad range of stiffness and mass. The optomechanical gain can be fixed or widely tunable. For fixed optical gain operation the actuator may be unnecessary, enabling all-optical sensing. The ability to electrically tune the operating wavelength by up to several nanometers makes possible operation with practical and economical telecom fixed wavelength laser diodes. The small footprint, fiber readout and miniscule power consumption may enable novel sensor array applications. 


\section{REFERENCES}

[1] S. A. Zotov, M. C. Rivers, A. A. Trusov and A. M. Shkel, "Folded MEMS pyramid inertial measurement unit", IEEE Sensors Journal, 11, 2780-2789 (2011).

[2] O. Sahin, S. Magonov, C. Su, C. F. Quate and O. Solgaard, "An atomic force microscope tip designed to measure timevarying nanomechanical forces", Nature Nanotechnoloty. 2, 507-514 (2007).

[3] J.L. Arlett, E.B. Myers and M. L. Roukes, "Comparative advantages of mechanical biosensors", Nature Nanotechnology, 6, 203-215 (2011).

[4] K. Srinivasan, H. Miao, M. T. Rakher, M. Davanco and V. Aksyuk, "Optomechanical transduction of an integrated silicon cantilever probe using a microdisk resonator", Nano Letter, 11, 791-797 (2011).

[5] C. A. Bolle, V. Aksyuk, F. Pardo, P. L. Gammel, E. Zeldov, E. Bucher, R. Boie, D. J. Bishop and D. R. Nelson, "Observation of mesoscopic vortex physics using micromechanical oscillators", Nature 399 43-46 (1999).

[6] G. Zolfagharkhani, A. Gaidarzhy, P. Degiovanni, S. Kettemann, P. Fulde and P. Mohanty "Nanomechanical detection of itinerant electron spin flip", Nature Nanotechnology, 3 720-723 (2008).

[7] J. Yang, T. Ono and M. Esashi, "Surface effects and high quality factors in ultrathin single-crystal silicon cantilevers", Applied Physics Letters, 77, 3860 (2000).

[8] T. J. Kippenberg and K. J. Vahala, "Cavity opto-mechanics", Optics Express 15, 17172-17205 (2007).

[9] M. Eichenfield, J. Chan, R. M. Camacho, K. J. Vahala and O. Painter, "Optomechanical crystals", Nature, 462, 462, 78-82 (2009).

\section{CONTACT}

*V. A. Aksyuk, tel: +1-301-975-2867; vladimir.aksyuk@nist.gov 


\title{
A SINGLE-CHIP CMOS-MEMS MICROGRAVITY ACCELEROMETER
}

\author{
Y. Zhang ${ }^{*}$, G. Meng, and N. Yazdi \\ Evigia Systems, Inc., Ann Arbor, Michigan, USA
}

\begin{abstract}
This paper reports a single-chip CMOS-MEMS microgravity accelerometer for precision acceleration measurements at micro-g levels. This sensor employs a novel four-quadrant symmetric device structure with full-bridge capacitive output, and a robust fabrication process to form thick single-crystalline silicon sensors atop of CMOS wafers providing high-sensitivity, low-noise and high-stability. The accelerometer is sealed at wafer-level using silicon-caps to lower drift and shield EMI. It has a sensitivity of $1.8 \mathrm{~V} / \mathrm{g}$ and noise floor of $1.2 \mu \mathrm{g} / \mathrm{rt}-\mathrm{Hz}$.
\end{abstract}

\section{INTRODUCTION}

Capacitive micro-accelerometers [1] have advantages of high sensitivity, good dc response, low noise, low drift, low temperature sensitivity, low-power dissipation, and large readout bandwidth. High-sensitivity in the capacitive accelerometer designs, including both combined surface-bulk micro-machined accelerometers [2] and SOI devices [3], is typically attained by using larger lateral dimensions and thick proof-masses in sensor structures. These devices are hybrid packaged with the interface circuitry resulting in larger parasitic, lower electrical signal-to-noise ratio, and EMI susceptibility through the sensor/circuit interconnects. Surface micro-machined accelerometers [4] have the advantages of monolithic integration with the interface circuit. The proof-mass is however small, resulting in a lower mechanical sensitivity and typically 10 's-100's of micro-g mechanical noise floor.

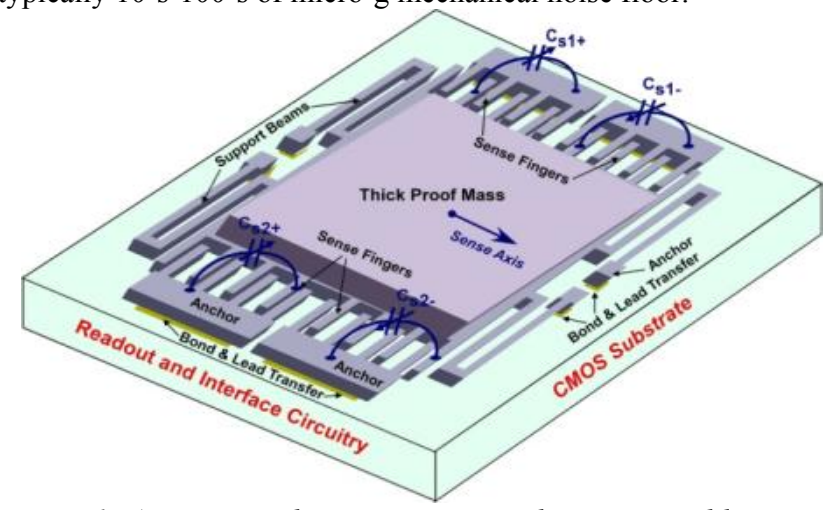

Figure 1: An integrated microgravity accelerometer and low-noise CMOS interface circuitry. The sensor fabrication is completed after integration with the CMOS wafer.

In this paper, we present a novel fully-symmetric highsensitivity silicon accelerometer integrated with the interface CMOS circuitry. Figure 1 shows the overall CMOS-MEMS accelerometer device structure. It is a lateral accelerometer with $50-100 \mu \mathrm{m}$ thick interdigitated fingers and $3-5 \mu \mathrm{m}$ air gap. The sensor achieves high-sensitivity and low-noise via use of thick single-crystalline structure and low-parasitic CMOS integration. Mechanical and temperature stability is improved by using a fullydifferential four-quadrant symmetric sense and readout. The sensor structure is fabricated on a SOI wafer. It uniquely forms a four-quadrant symmetric capacitive full-bridge with isolated sense capacitor leads, which enables the formation of a full-bridge electronic readout without any reference capacitors. The sensing fingers are formed by front-side high aspect ratio etching of the device layer. The handle layer of the SOI wafer is used to define a thick proof mass. The sensor wafer is directly integrated with a CMOS wafer that makes multiple low-parasitic local leads between sensors and circuits possible. This wafer-level integration also simplifies the final sensor package, thus further reduces the manufacturing cost. The sensors and the IC circuitry are hermetically packaged at the wafer level. This level of sensor package protects the sensors from ambient and also serves as EMI shield.

\section{SENSOR DESIGN}

The proper design of MEMS sensors is primarily important in accomplishing good sensor performance. The major design goal is to achieve high sensitivity, low mechanical noise-floor, low crossaxis sensitivity and low temperature sensitivity while minimizing the overall sensor size and maintaining a high-yield fabrication process. This microgravity accelerometer uses capacitive sensing scheme due to its good dc-response, high-sensitivity, low-noise and low-temperature drift. The static open-loop sensitivity of a capacitive accelerometer is:

$$
\frac{\Delta C_{\text {static }}}{a}=N \frac{\varepsilon_{0} A m}{g_{s}^{2} k}=N \frac{\varepsilon_{0} A}{g_{s}^{2} \omega_{r}^{2}}
$$

where $\Delta C_{\text {static }}$ is the sensor capacitance change, $a$ is the input acceleration, $m$ is the proof mass, $k$ is the suspension spring constant, $A$ is the sense area per electrode and $N$ is the number of electrodes, $g_{s}$ is the air gap, $\omega_{r}$ is the mechanical self-resonance frequency, and $\varepsilon_{0}$ is the air permittivity. Mechanical noise is caused by the Brownian motion of the gas molecules surrounding the proof mass and the Brownian motion of the proof mass suspension or anchors. The Total Noise Equivalent Acceleration (TNEA rms- $\left[\mathrm{m} / \mathrm{sec}^{2} / \sqrt{\mathrm{Hz}}\right]$ ) can be calculated by

$$
T N E A=\frac{\sqrt{4 K_{B} T D}}{m}=\sqrt{\frac{4 K_{B} T \omega_{r}}{Q m}}
$$

where $K_{B}$ is the Boltzmann constant and $T$ is the temperature in Kelvin, $D$ is the damping factor, and $Q$ is the quality factor. For the parallel plates that are not at low-pressure ambient and their width and length are significantly larger than the air gap, squeeze film damping is the dominant regime. The squeeze film damping can be calculated based on the geometries and the surrounding gas viscosity $(\mu)$. For instance, the damping factor $D$ for $N$ interdigitated fingers with length of $L$ and thickness of $T$, separated by gap of $g_{s}$ is given by:

$$
D=0.427 N \mu \frac{T^{3} L}{g_{s}^{3}}=0.427 N \mu \frac{A T^{2}}{g_{s}^{3}}
$$

Equations (1)-(3) indicate that the target high-performance of the microgravity accelerometer requires formation of thick large mass, soft suspension, narrow air gap over a large area, and small damping simultaneously.

\section{Sensor Device Structure}

The sensor structure is designed to be fabricated on a SOI wafer and integrated with the CMOS interface circuit at wafer 
level. As shown in Fig. 2, each accelerometer has four sets of interdigitated sensing finger pairs and each finger pair has one moving finger and one fixed finger to form a parallel-plate capacitor. The moving fingers are attached to the proof mass while the fixed fingers are anchored to substrate. The sensing fingers are formed by front-side high aspect ratio etching in the device layer, which will allow formation of $2-5 \mu \mathrm{m}$ air gap with $50-100 \mu \mathrm{m}$ finger thickness. The finger gaps are asymmetrical $\left(\mathrm{g}_{0}>\mathrm{g}_{\mathrm{s}}\right)$ to provide a net capacitance change when the proof mass displaces with respect to the fixed fingers under presence of acceleration. For $\mathrm{g}_{0}>4 \mathrm{~g}_{\mathrm{s}}$ the capacitance sensitivity is reduced only $\sim 6 \%$ compared with the case where the sense element is formed by two parallel plates separated by a gap of $g_{s}$. The thick handle layer of the SOI wafer is used to define a thick proof mass $(200-400 \mu \mathrm{m}$ thick), and the proof mass is supported by four folded and suspended support beams. Because of the usage of the thick handle layer as the proof mass and the design of the folded support beams as the soft suspension, a high sensitivity is obtained while the overall sensor size is much reduced.
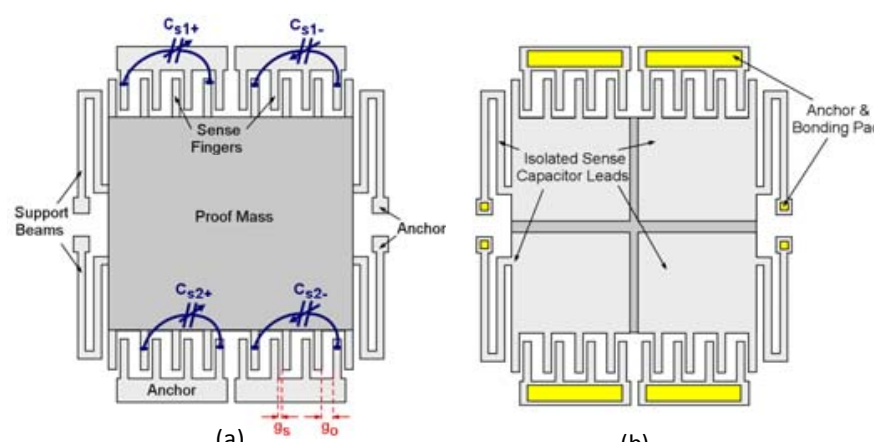

(a)

(b)

Figure 2: A microgravity accelerometer sensing element: (a) a top view and (b) a bottom view.

The high capacitive sensitivity and low mechanical noise are achieved by a symmetric capacitive full-bridge structure to directly interface with a fully-differential readout front-end without any need for reference capacitors. The full-bridge structure improves the overall sensor module sensitivity and lowers drift and temperature sensitivity. In general, this sensor-readout approach is not possible with many commonly used MEMS accelerometer structures in which the proof mass forms a common sensecapacitance node. In this design, however, the device layer and the handle layer are electrically isolated from each other, and its four electrodes of the sense capacitive bridge are isolated at the same step as the sensing finger definition, as shown in Fig. 2(b), such that it makes a capacitive full-bridge possible.

\section{Sensor Geometrical Design and Noise Performance}

The overall sensor design is driven by minimizing the combined electrical and mechanical noise in the smallest die size. Figure 3 illustrates a simulated noise performance with respect to the sensing air gap in the designed accelerometer with two different proof mass thicknesses of $250 \mu \mathrm{m}$ and $350 \mu \mathrm{m}$. The length, thickness, and separation of the interdigitated fingers are $180 \mu \mathrm{m}, 80 \mu \mathrm{m}$, and $7 \mu \mathrm{m}$, respectively, in the simulation. Based on the simulation results, an air gap of $4 \mu \mathrm{m}$ is selected, which results in a lower noise-floor of $0.32 \mu \mathrm{g}-\mathrm{rms} / \sqrt{\mathrm{Hz}}$ with $350 \mu \mathrm{m}$ proof mass, and $0.45 \mu \mathrm{g}-\mathrm{rms} / \sqrt{\mathrm{Hz}}$ with $250 \mu \mathrm{m}$ proof mass at 1 atmosphere ambient pressure.

Table 1 presents the summary of the sensor design parameters. The cross axis sensitivity was analyzed by means of

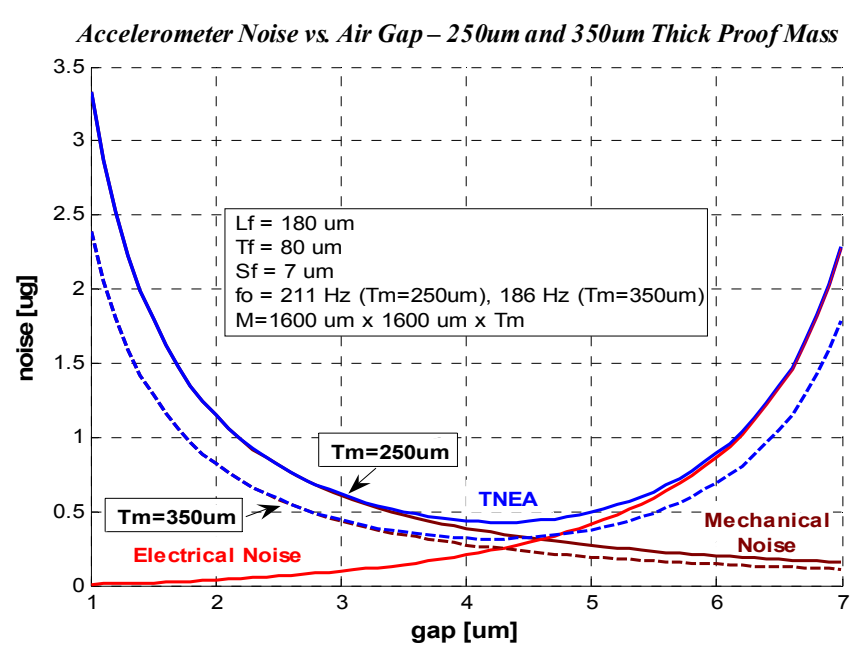

Figure 3: The rms-noise of the designed accelerometer with a lateral dimension of $1600 \mu \mathrm{m} \times 1600 \mu \mathrm{m}$ and thickness of $250 \mu \mathrm{m}$ and $350 \mu \mathrm{m}$ of proof mass. The air gap of $4 \mu \mathrm{m}$ is selected.

FEM simulations and comparisons of sensor structure frequency modes. The $\mathrm{z}$-axis mode is the $2^{\text {nd }}$ mode and mostly impacting the cross-axis sensitivity. The cross-axis sensitivity is minimized by the proper geometrical design of the support beams, by reducing the distance of proof mass gravity center to the support beam plane, and by optimizing the ratio of the proof mass thickness to the beam height. In addition, the four-quadrant symmetrical device structure helps with reducing the cross axis sensitivity. The sensor $\mathrm{z}$-axis cross axis sensitivity is less than $0.3 \%$, and the $y$-axis cross axis is about an order of magnitude smaller.

Table 1: Summary of the accelerometer design parameters.

\begin{tabular}{|l|l|}
\hline Design Parameter & Value \\
\hline Sense fingers & \\
Sense gap & $4 \mu \mathrm{m}$ \\
Length & $180 \mu \mathrm{m}$ \\
Thickness & $80 \mu \mathrm{m}$ \\
Width & $5 \mu \mathrm{m}$ \\
Separation gap & $8 \mu \mathrm{m}$ \\
Number & $4 \times 36$ \\
\hline Proof mass & $1600 \mu \mathrm{m} \times 1600 \mu \mathrm{m} \times 350 \mu \mathrm{m}$ \\
\hline Resonance frequency & $186 \mathrm{~Hz}$ \\
\hline Cross-axis sensitivity & better than $0.3 \%$ \\
\hline Rest capacitance & $4 \times 1.2 \mathrm{pF}$ \\
\hline Capacitive sensitivity & $4 \times 1.7 \mathrm{pF} / \mathrm{g}$ \\
\hline Simulated TNEA & $<0.4 \mu \mathrm{g}-\mathrm{rms} / \mathrm{rt}-\mathrm{Hz}$ \\
\hline
\end{tabular}

\section{Sensor Stability}

The mechanical and temperature stability is obtained by constructing sensor structures with all single-crystal silicon which has excellent stable mechanical characteristics resulting in higher sensor mechanical stability and minimum temperature related stress variations. The bonding pads at the anchors are formed by metal bond. However, these metal pads will not impact the device performance adversely because they are connected to the substrate rigidly. Furthermore the symmetrical full-bridge structure also helps to lower any potential residual effects and to cancel temperature effect as well. In addition, the high stability over wide temperature range will be achieved by employing folded-beam suspensions with which any residual thermo-mechanical stresses induced by the CMOS dielectric and metal layers can be absorbed. 


\section{SENSOR FABRICATION AND INTEGRATION}

The sensor fabrication process is fully compatible with postCMOS processing, and enables formation of the designed sensor structure and its CMOS integration. All fabrication steps are processed in batch and performed at wafer-level. The combination of the steps is a high-yield process, which can be performed in a standard MEMS fabrication facility. All these features provide a clear competitive advantage for accelerometer manufacturing. The fabrication, integration and packaging consist of four parts as shown in Fig. 4.
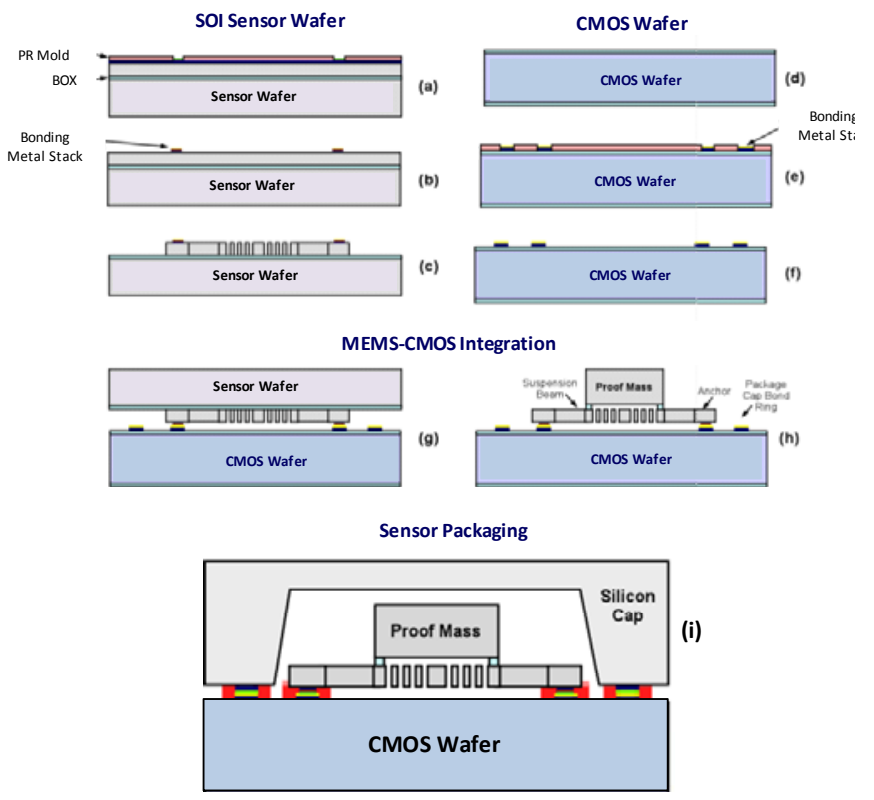

Figure 4: CMOS-MEMS microgravity accelerometer fabrication, integration and packaging process flow.

The first part includes two masking steps performed on a SOI wafer with $50-100 \mu \mathrm{m}$ device layer thickness and $200-400 \mu \mathrm{m}$ thick handle layer. The process starts with electroplating the bonding metal stack on the device layer using a photo resist mold (Fig. 4(a)). The plating seed layer and photo resist mold are removed after electroplating (Fig. 4(b)). It is followed by patterning and DRIE etching on device layer to define sensing fingers, anchors, and suspension support beams. The etch mask is removed to complete the first part of the process (Fig. 4(c)).

The second part of the process has one masking steps and is performed on the CMOS wafers (Fig. 4(d)) containing the interface circuitry. $\mathrm{Cr} / \mathrm{Au}$ metal layers are deposited and patterned for bonding pads (Fig. 4(e)). These pads are in contact the CMOS pads and transfer the leads from the CMOS circuits to the sensors. Metal bonding ring for sensor cap package can also be defined at this step.

The third part of the process includes a wafer bonding and a single masking step for proof mass etching after wafer bonding. The fabricated sensor wafer is flipped, aligned to the bonding pads on the CMOS wafer. Then slight pressure is applied and the sensor wafer and the CMOS wafer are bonded at temperature $<380^{\circ} \mathrm{C}$ (Fig. $4(\mathrm{~g})$ ). The thick proof-mass is etched on the SOI handle layer by DRIE after wafer bonding. The sensor structure is released by a dry etching the SOI buried oxide layer (Fig. 4(h)).

The fourth part of the process is the sensor micro-package step. The bonded and release etched CMOS-MEMS wafer is encapsulated at wafer-level by a cavity etched silicon cap wafer to protect it from the environment prior to die singulation. The sealed cap also isolates the sensor from the environment as well as the ambient humidity and pressure variations. Finally the capped sensor-circuitry wafer can be singulated into individual sensor die (Fig. 4(i)). Figure 5 is a picture taken from a fully fabricated sensor wafer prior to its final micro-package. An enlarged view of multiple sensor dies is shown, as well.

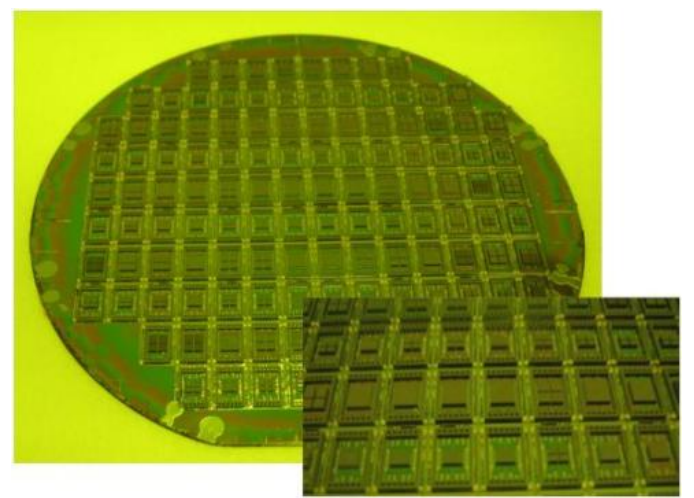

Figure 5: Pictures of a fabricated sensor wafer and a close-up multiple dies prior to the sensor package.

Figure 6 shows the SEM of a single accelerometer after DRIE etching of proof mass from its handle layer. Four sets of sensing fingers can be seen from the figure. The proof mass quadrants are electrically isolated from each other from the device layer side (facing down). They are mechanically connected rigidly through the handle layer as shown in the figure. Figure 7 is a close-up view for a portion of the accelerometer. The sensing fingers, the support beam, one of the proof mass quadrant, and the lead transfer metal pad from fingers attached to the proof mass are all shown in the figure.

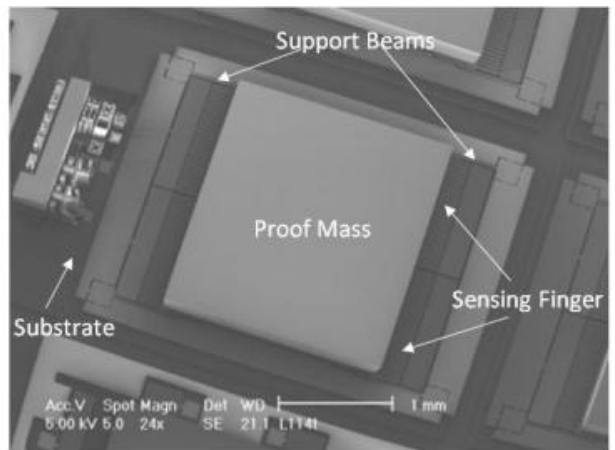

Figure 6: The accelerometer after wafer bonding and proof mass definition.

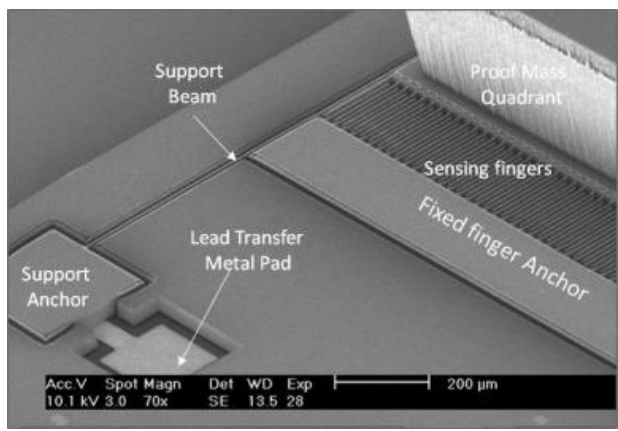

Figure 7: A close-up view of a portion of the fabricated accelerometer. 
The wafer-level micro-packaged CMOS-MEMS accelerometers are shown in Fig. 8. At this step the completed wafer can be diced to individual sensor chip and package it in its housing as needed. Figure 9 presents a single CMOS-MEMS sensor chip that is packaged in standard 64-pin DIP IC housing for testing.

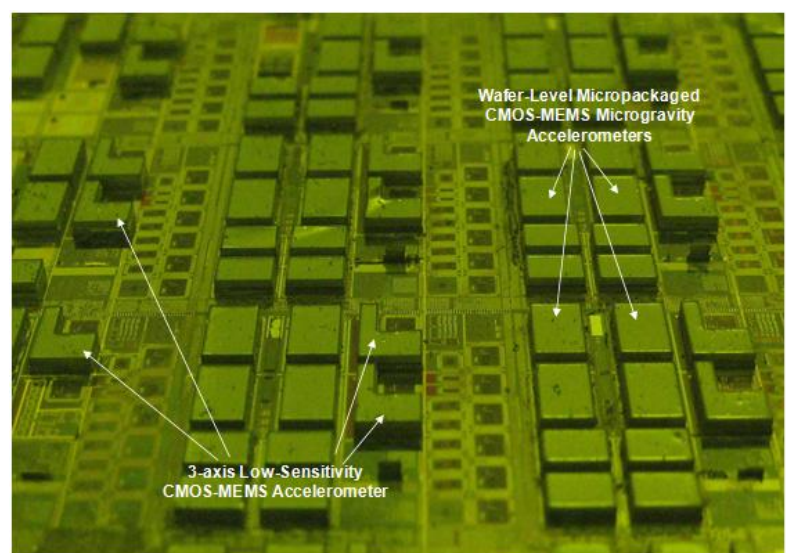

Figure 8: A picture taken from a fully fabricated, integrated and wafer-level micro-packaged CMOS-MEMS accelerometer wafer.

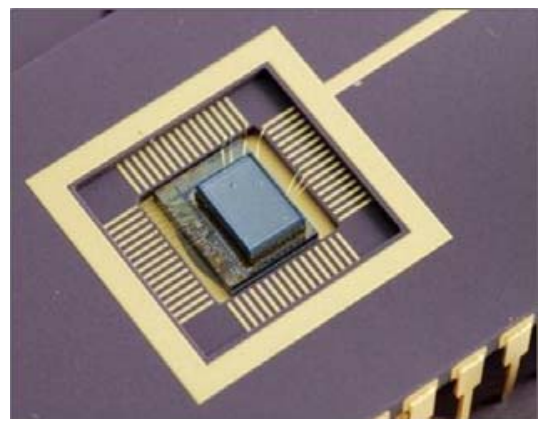

Figure 9: A singulated CMOS-MEMS microgravity accelerometer chip packaged in a standard 64-pin DIP IC housing and wire bonded for testing.

\section{SENSOR MEASUREMENTS}

The fabricated microgravity accelerometers were tested both at the wafer-level by probing and at device level with CMOS interface circuitry. Figure 10(a) presents the measured capacitance response versus a sweeping dc voltage for one set of the sense capacitors. This is a static open-loop test. The measured sensitivity under this condition is $1.7 \mathrm{pF} / \mathrm{g}$ for a single set of sense fingers corresponding to a total sensitivity of $4 \times 1.7 \mathrm{pF} / \mathrm{g}$ from all four sets of interdigitated sense fingers. The noise floor for this accelerometer is $1.2 \mu \mathrm{g} / \mathrm{rt}-\mathrm{Hz}$.

The fabricated and micro-packaged accelerometer was also tested together with its interface CMOS read-out circuit. The sensor chips were mounted and wire bonded in standard 64-pin DIP IC housing as shown in Fig 9. Figure 10(b) is a screen shot showing the measurement result for a single packaged CMOSMEMS accelerometer in turn-over tests. The sensitivity is $1.8 \mathrm{~V} / \mathrm{g}$ with power dissipation of $6 \mathrm{~mW}$ at $3 \mathrm{~V}$.

\section{CONCLUSION}

In conclusion, a single-chip CMOS-MEMS microgravity accelerometer has been designed, fabricated, and tested. This sensor has a fully-symmetrical full-bridge sense capacitor enabling precision readout without any need for reference capacitors. Its single-crystal silicon sensor structure improves long term-stability.
Test results indicate that this MEMS-CMOS microgravity accelerometer has high sensitivity and low thermo-mechanical noise. Because of its high performance and CMOS manufacturing compatibility, this microgravity accelerometer can be readily used in applications demanding precision, including inertial navigation.

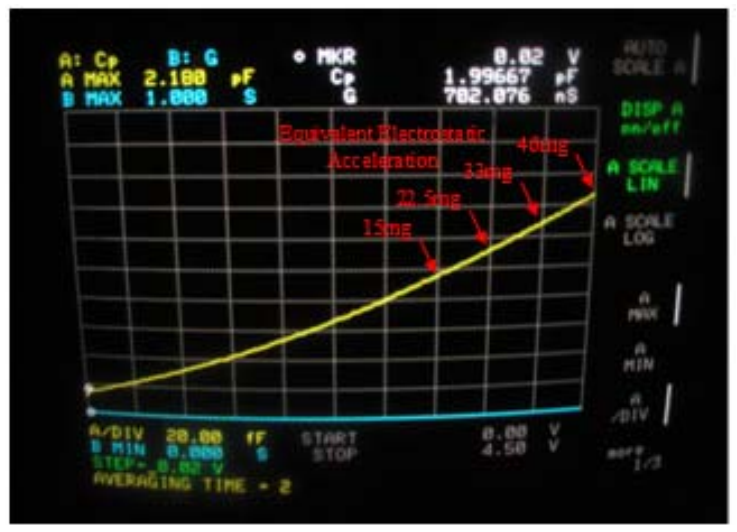

(a)

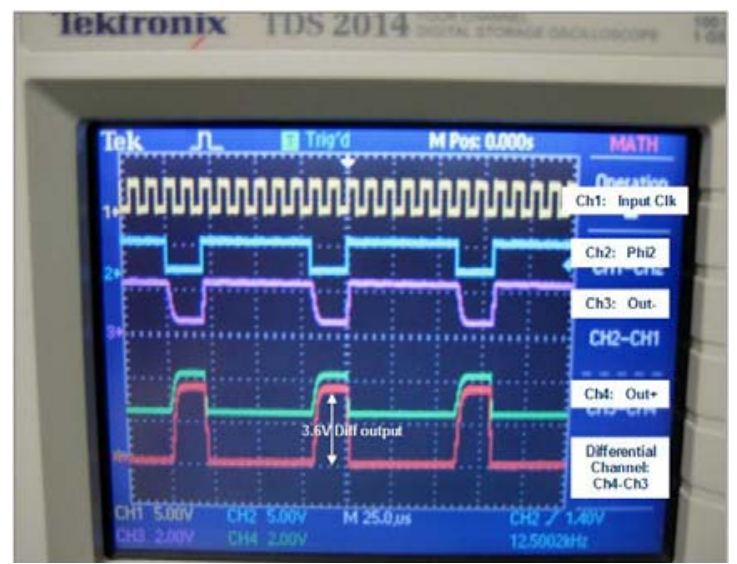

(b)

Figure 10:Measurement results: (a) measured sensitivity of one group sense fingers $4 \times 1.7 \mathrm{pF} / \mathrm{g}$ by sweeping dc voltage and (b) measured sensitivity of $1.8 \mathrm{~V} / \mathrm{g}$ for an integrated CMOS-MEMS sensor using turn-over test.

\section{REFERENCES}

[1] N. Yazdi, A. Ayazi, and K. Najafi, "Micromachined Inertial Sensors", Proceedings of the IEEE, vol. 86, no. 8, pp. 16401659, August 1998.

[2] N. Yazdi, K. Najafi, "An All-Silicon Single-Wafer Micro-g Accelerometer with a Combined Surface and Bulk Micromachining Process," IEEE/ASME Journal of Microelectromechanical Systems (JMEMS), vol. 9, no. 4, December 2000, pp. 544-550.

[3] B. Amini, F. Ayazi, "A 2.5-V 14-bit $\Sigma \Delta$ CMOS SOI capacitive accelerometer", IEEE Journal of Solid-State Circuits (JSSC), v. 39, no. 12, Dec. 2004, pp.2467-2476.

[4] B. E. Boser, R.T. Howe, "Surface Micromachined Accelerometers", IEEE Journal of Solid-State Circuits (JSSC), v. 31, no. 3, Mar. 1996, pp.366-375.

\section{CONTACT}

*Y. Zhang, tel: +1-734-302-1140; yzhang@evigia.com 


\section{ACHIEVING LONG-TERM BIAS STABILITY IN HIGH-Q INERTIAL MEMS BY TEMPERATURE SELF-SENSING WITH A 0.5 MILLICELCIUS PRECISION

\author{
I.P. Prikhodko, A.A. Trusov, and A.M. Shkel
} \\ MicroSystems Laboratory, University of California, Irvine, CA, USA}

\begin{abstract}
We present long-term bias drift compensation in high-quality $(Q)$ factor MEMS gyroscopes using real-time temperature selfsensing. The approach takes advantage of linear temperature dependence of the drive-mode resonant frequency for selfcompensation of temperature-induced sense-mode drifts. The approach was validated by a vacuum packaged silicon quadruple mass gyroscope, with signal-to-noise ratio (SNR) enhanced by isotopic $Q$-factors of 1.2 million. Owing to high $Q$-factors, a measured frequency stability of $0.01 \mathrm{ppm}$ provided a temperature self-sensing precision of $0.0004{ }^{\circ} \mathrm{C}$, on par with the state-of-the-art MEMS resonant thermometers. Real-time self-compensation yielded a total bias error of $0.5 \% \mathrm{hr}$ and total scale-factor error of $700 \mathrm{ppm}$ over temperature variations. This enabled repeatable long-term rate measurements required for MEMS gyrocompassing with a milliradian azimuth precision.
\end{abstract}

\section{INTRODUCTION}

In recent years several groups have reported silicon MEMS gyroscopes with sub-degree per hour Allan deviation of bias [1-3]. However, long-term bias and scale-factor drifts limit their potential in real-world missions. The drift source for most MEMS is their inherent sensitivity to temperature variations. An uncompensated bias sensitivity of $500(\% / \mathrm{hr}) /{ }^{\circ} \mathrm{C}$ is typical for MEMS gyroscopes [4]. Whereas, quartz and silicon MEMS resonators used in timing applications exhibit orders of magnitude higher long-term stability, due to more advanced compensation techniques. For instance, high-stability dual-mode oscillators use the secondary mode as a thermometer for the compensation of primary mode drifts [5]. In contrast, conventional approaches for gyroscope's calibration rely on third-order thermal models and external temperature sensors, which suffer from such effects as thermal lag and temperatureinduced hysteresis. These limitations motivate the development of new real-time self-calibration methods for inertial MEMS.

Fused quartz hemispherical resonator gyroscope utilizes a temperature compensation technique which uses the resonant frequency as a measure of gyroscope temperature [6]. Successful implementation of this self-sensing technique relies on two main factors. The first is linearity of the temperature-frequency dependence of the resonator material, and the second is high frequency stability, brought forth by a high $Q$-factor. Recently, we introduced a MEMS quadruple mass gyroscope (QMG) [2,3,7], which satisfies these requirements with $Q$-factor above 1 million, and linear temperature coefficient of frequency (TCF), thanks to the single crystalline silicon resonator body, Fig. 1 inset.

Frequency-based measurements of temperature also provides inherently better stability then amplitude-based (voltage) readings commonly employed in temperature sensors (1 ppm stability and repeatability is easy in frequency domain, but almost impossible in analog signal domain). These make high-resolution self-sensing potentially possible in silicon MEMS technology. In this paper, we demonstrate that the resonant temperature self-sensing can be used in real-time for high- $Q$ MEMS gyroscopes (Fig. 2) to yield a subdegree per hour total bias error over temperature variations. We also demonstrate that the long-term stability provided by the selfcompensation approach allows for repeatable measurements of small angular rates, required for gyrocompassing applications.

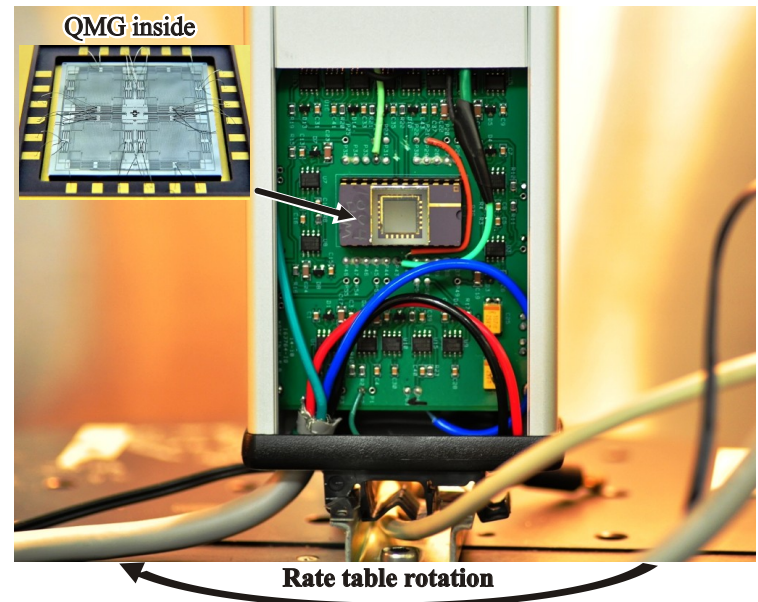

Figure 1: Photo of the experimental setup, showing the vacuum packaged $Q M G$ rate sensor, $P C B$ electronics, and a rate table.

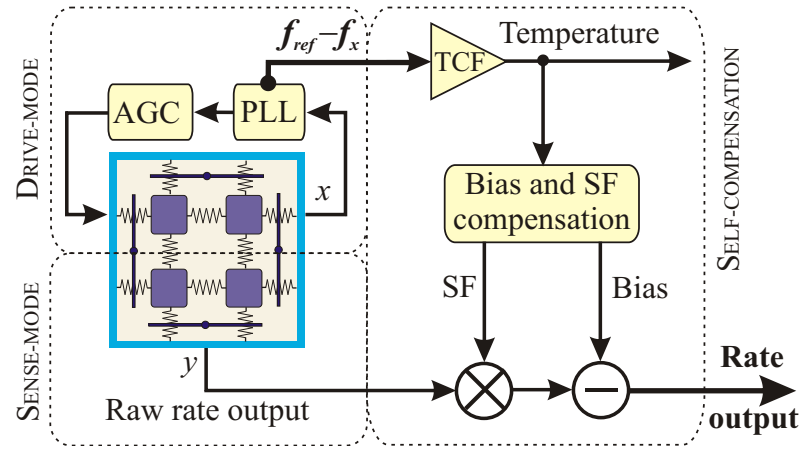

Figure 2: Signal processing using drive-mode frequency for selfcompensation of temperature-induced bias and scale-factor drifts.

\section{TEMPERATURE SENSITIVITY ANALYSIS}

Here we analyze temperature-induced drift sources in high- $Q$ gyroscopes and show the importance of temperature compensation.

\section{Temperature-Induced Drifts}

Operation of vibratory $z$-axis angular rate gyroscopes is based on energy transfer between two vibratory modes, Fig. 2 . The drivemode is continuously excited at resonance, and the sense-mode is used for the rate detection. The amplitude $(y)$ of the sense-mode is proportional to the rate $\left(\Omega_{z}\right)$, with scale-factor $(S F)$ and bias $(B)$ :

$$
y=S F \times\left(\Omega_{z}+B\right) .
$$

Assuming a worst-case scenario, the sense-mode is openloop, and thus more susceptible to temperature variations. Scalefactor and bias are functions of the angular gain $(k \leq 1)$, the sensemode natural frequency $\omega_{y}$, and the drive amplitude $(x)$ [8]:

$$
S F=2 k Q_{\text {eff }} x / \omega_{y}, \quad B=\Delta(1 / \tau) \sin \left(2 \theta_{\tau}\right) / 2 .
$$

Here, $\Delta(1 / \tau)$ is the damping mismatch between vibratory modes, $\theta_{\tau}$ is the principal axis of damping, and $Q_{e f f}$ is the effective $Q$-factor:

$$
Q_{\text {eff }}=Q_{y} / \sqrt{1+4 Q_{y}^{2}\left(\Delta \omega / \omega_{y}\right)^{2}},
$$

which reaches maximum $Q_{y}$ at zero frequency mismatch $(\Delta \omega=0)$. 


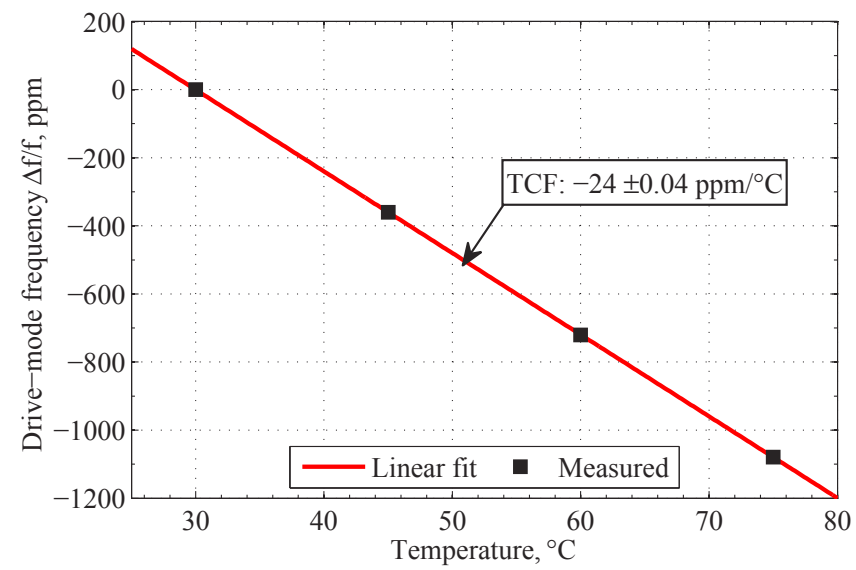

Figure 3: Linear frequency-temperature dependence, revealing a $-24 \pm 0.04 \mathrm{ppm} /{ }^{\circ} \mathrm{C} \mathrm{TCF}$ over temperature range of $30^{\circ} \mathrm{C}$ to $75^{\circ} \mathrm{C}$.

It follows from (2) and (3) that the mode-matched condition over wide temperature range, i.e. $\Delta \omega=0$ and $\Delta(1 / \tau)=0$, is desired to ensure stability of scale-factor and bias. While these requirements can be satisfied by a symmetric transducer design, the temperature dependence of the rest of the parameters in (1)-(3) still reduce the overall accuracy and repeatability of rate measurements. The only parameters with negligible sensitivities are the drive amplitude $x$, stabilized by an automatic gain control (AGC), and the angulargain factor, $k$, with a sub-ppm $/{ }^{\circ} \mathrm{C}$ stability over temperature [6].

Thus, the parameter with the highest sensitivity is either the sense-mode frequency, $\omega_{y}$, or the sense-mode quality factor, $Q_{y}$. Typical temperature sensitivity of a resonant frequency for silicon MEMS is only $-31 \mathrm{ppm} /{ }^{\circ} \mathrm{C}$ near room temperature. Whereas, the sensitivity of $Q$-factor depends on the dominant energy loss mechanism. For the thermo-elastic dissipation, with the associated strong $1 / T^{3}$ temperature dependence [2], the $Q$-factor sensitivity is the primary factor that contributes to the scale-factor fluctuations.

\section{Scale-Factor and Bias Sensitivities}

The relationship between the scale-factor (as well as bias) and temperature is non-linear; however, for limited temperature range, we can consider the change to be linear. Quantitatively, we can estimate the effect of the $Q$-factor on scale-factor by taking the derivative of the expression (2) with respect to temperature. The result is divided by the nominal scale-factor to find the relative change (assuming $\Delta \omega=0$ ):

$$
\frac{1}{S F} \frac{d S F}{d T}=\frac{1}{Q_{y}} \frac{d Q_{y}}{d T} .
$$

Previously we reported experimental temperature characterization of the $Q$-factor for the vacuum sealed QMG, which confirmed that the dominant energy loss mechanism is thermoelastic dissipation [2]. Linear fit to the $Q(T)$ data [2] reveals $d Q_{y} / d T$ of $12,000^{\circ} \mathrm{C}^{-1}$, yielding a scale-factor sensitivity of $10,000 \mathrm{ppm} /{ }^{\circ} \mathrm{C}$ near room temperature. Calculation of the derivatives $d Q_{y} / d T$ at different temperature shows that the sensitivity varies from $5,000 \mathrm{ppm} /{ }^{\circ} \mathrm{C}$ to $20,000 \mathrm{ppm} /{ }^{\circ} \mathrm{C}$ over wider temperature range of $-40{ }^{\circ} \mathrm{C}$ to $120{ }^{\circ} \mathrm{C}$.

In a similar manner, analysis of the bias sensitivity shows that even for a symmetric design with constant $\Delta(1 / \tau)$ over temperature, drifts can be caused by variations of the principal axis of damping, $\theta_{\tau}$. In principle, the effect can be minimized by applying the drive force at the angle $\theta=\theta_{\tau}$, but temperature variations can still shift the angle $\theta_{\tau}$. This analysis suggests that high- $Q$ gyroscopes require temperature compensation for repeatable rate measurements.

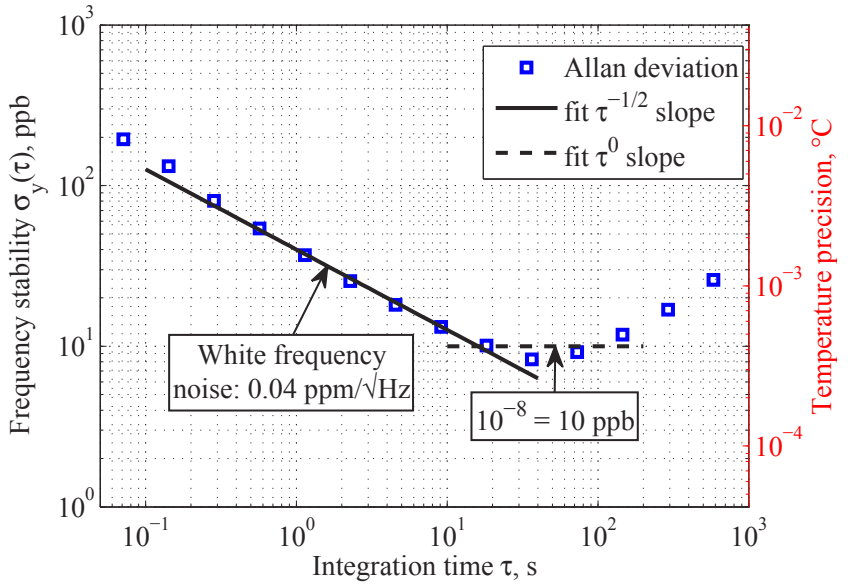

Figure 4: Experimental demonstration of $10^{-8}$ frequency stability, which translates to $4 \cdot 10^{-4}{ }^{\circ} \mathrm{C}$ precision of temperature self-sensing.

\section{TEMPERATURE SELF-SENSING}

In this section we describe the temperature compensation method and demonstrate its accuracy and precision using QMG.

\section{Frequency-Based Temperature Sensing}

While the gyroscope drive-mode is controlled by a phaselocked loop (PLL) and AGC loop, Fig. 2, the open-loop sensemode mode is susceptible to temperature variations, as shown in the previous section. To compensate for temperature-induced scale-factor and bias drifts, we propose to use the drive-mode frequency as a built-in thermometer, which is free from any spatial or temporal thermal lag. The silicon resonator frequency changes linearly with the temperature, so by monitoring this change, onchip gyroscope temperature can be measured directly.

Scale-factor and bias drifts are obtained using the temperature sensitivity coefficients, which are measured experimentally in a calibration run. Fig. 2 shows the real-time self-compensation signal processing. The instantaneous frequency change is first converted to the temperature using the measured TCF. Once the temperature is obtained, it is converted to the scale-factor and bias drifts. Finally, the raw gyroscope output is corrected by multiplying it with the scale-factor change, followed by subtraction of bias drifts.

\section{Self-Sensing Accuracy and Precision}

The QMG transducer [2,3,7] was chosen for the evaluation of the approach due to its symmetric high- $Q$ design and isotropy of both frequency and damping. Stand-alone QMGs were fabricated using an SOI process with a $100 \mu \mathrm{m}$ thick device layer. Singulated devices were vacuum sealed using getters inside a ceramic package, providing sub-mTorr vacuum sustainable over many years. Characterization of the packaged QMGs showed drive- and sense-mode $Q$-factors of 1.17 million at $25^{\circ} \mathrm{C}$ and 0.7 million at $100^{\circ} \mathrm{C}$, with $\Delta Q / Q$ symmetry of $1 \%$, or $\Delta(1 / \tau)$ of $10^{\circ} / \mathrm{hr}$ [2]. Frequency symmetry $\Delta \omega$ over temperature range of $30{ }^{\circ} \mathrm{C}$ to $75^{\circ} \mathrm{C}$ was also confirmed with a $0.2 \mathrm{ppm} /{ }^{\circ} \mathrm{C}$ uncertainty. High $Q$-factor is expected to provide a sub-ppm frequency stability, required for high-precision frequency-based temperature sensing.

The signal processing for temperature self-sensing takes advantage of high $Q$-factors and linear TCF of the silicon QMG. As shown in Fig. 2, a PLL monitors the resonant frequency change relative to a high-stability frequency reference, which is then converted to temperature. Accuracy of the frequency-to-temperature conversion thus relies on accuracy and linearity of the TCF value. The TCF was measured over a temperature range of $30^{\circ} \mathrm{C}$ to $75^{\circ} \mathrm{C}$ 


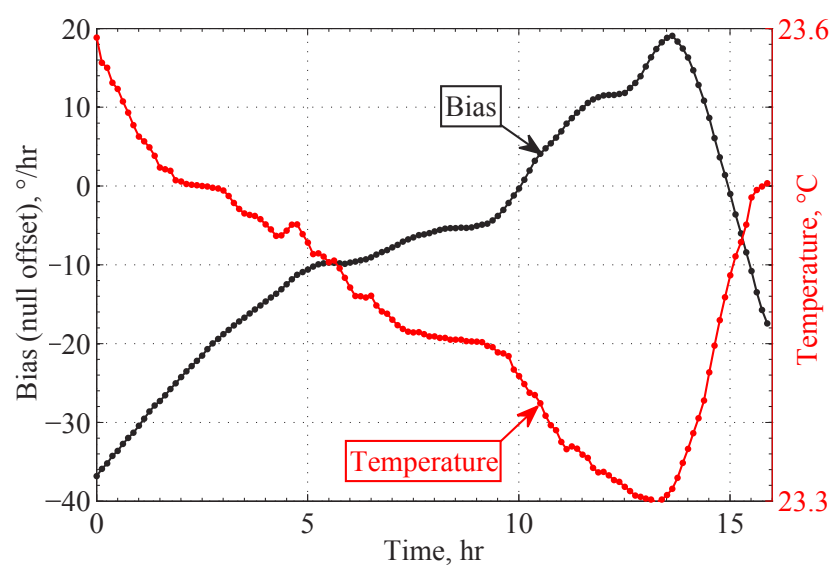

Figure 5: Experimentally measured $99 \%$ temperature correlation of a gyroscope bias during a $16 \mathrm{hr}$ run (uncompensated data).

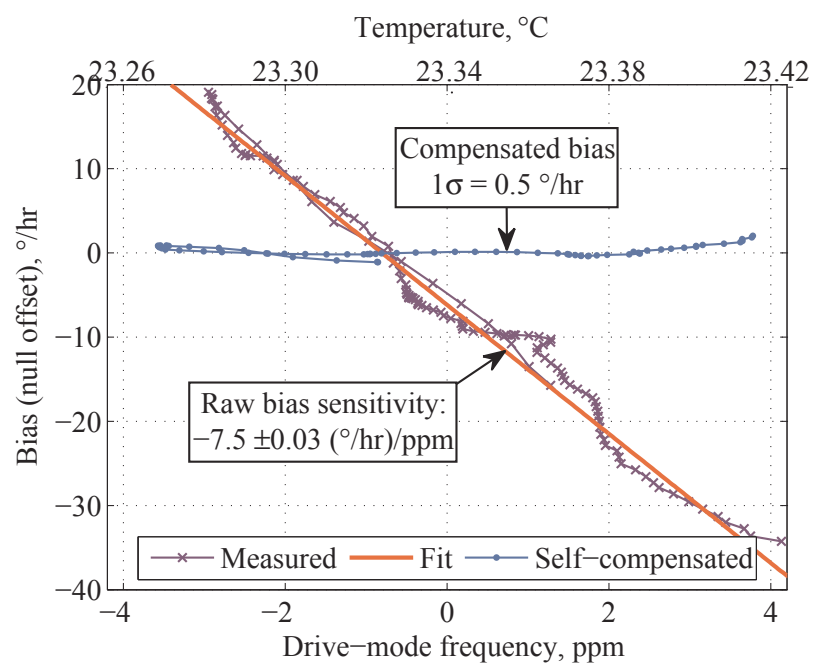

Figure 6: Self-compensated gyroscope bias, revealing a 1- $\sigma$ bias total error of $0.5 \%$ hr over temperature during 16 hour run.

using a thermistor from GEC Instruments with accuracy and precision of $10^{-4}{ }^{\circ} \mathrm{C}$. The TCF of $-24 \mathrm{ppm} /{ }^{\circ} \mathrm{C}$ was calculated from a linear fit to the frequency-temperature data with an accuracy of 0.04 ppm $/{ }^{\circ}$ C, Fig. 3 .

The precision of the frequency-based temperature sensing depends on the resonant frequency stability of the anti-phase drivemode of the gyroscope. Owing to high- $Q$ factor, we demonstrated a $40 \mathrm{ppb} / \sqrt{\mathrm{Hz}}$ white frequency noise, resulting in $10 \mathrm{ppb}$ frequency stability at $30 \mathrm{~s}$ for the QMG (near room temperature) [7], Fig. 4. Using the TCF of $-24 \mathrm{ppm} /{ }^{\circ} \mathrm{C}$, the measured frequency stability translates to temperature self-sensing precision of $0.002{ }^{\circ} \mathrm{C}$ at $1 \mathrm{~s}$ averaging time $0.0004^{\circ} \mathrm{C}$ at $30 \mathrm{~s}$ averaging time (with an accuracy of $0.002{ }^{\circ} \mathrm{C}$ ), on par with the state-of-the-art MEMS resonant thermometers [9]. Next, we demonstrate the self-compensation of QMG drifts using the high-resolution frequency thermometer.

\section{DEMONSTRATION OF SELF-COMPENSATION}

Here we demonstrate self-compensation of QMG output drifts. As discussed earlier, the frequency and damping symmetry of the QMG significanlty reduce scale-factor and bias temperature sensitivities. Nevertheless, imperfections in fabrication, packaging, and electronic component drifts contribute to sensor's temperature sensitivity. To experimentally measure output drifts, we mounted

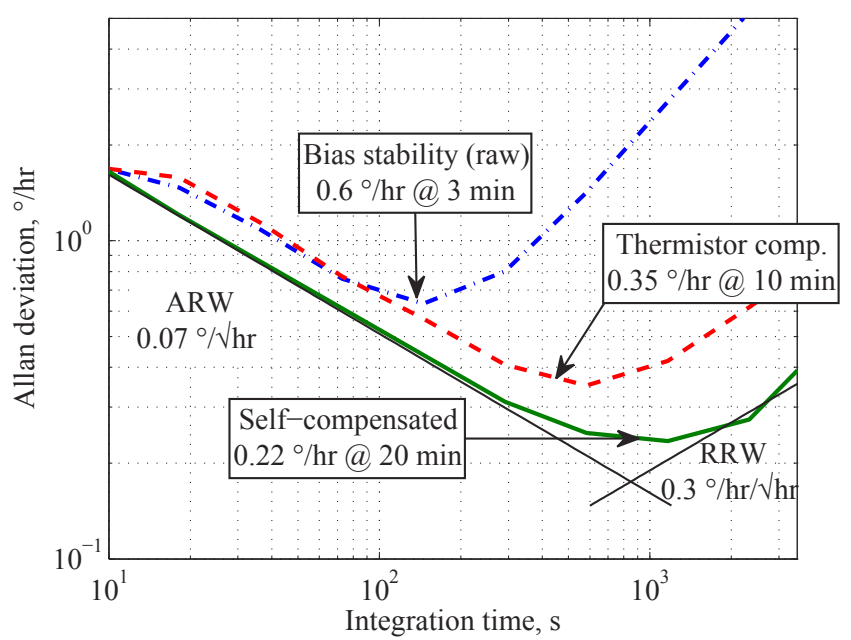

Figure 7: Allan deviation of gyroscope outputs over temperature variations, showing a $0.2 \%$ r self-compensated bias instability.

the QMG with its input axis horizontal on a rotary platform and separated scale-factor from sensor's bias by virtue of continuous rotation in a horizontal plane. The method is similar to carouseling technique used for continuous modulation of constant rotation rate of the Earth [3]. The gyroscope control (Fig. 2) was implemented in HF2 Zurich Instruments (ZI) hardware. From the measurements a strong correlation between the gyroscope bias and temperature was observed during a $16 \mathrm{hr}$ run, Fig. 5 . The measured $99 \%$ correlation confirmed environmental changes to be the primary drift source.

\section{Self-Compensation of Bias and Scale-Factor Drifts}

As expected, a linear relationship is observed for bias (null offset) as a function of the on-chip gyroscope temperature, Fig. 6. The direct gyroscope temperature was self-sensed by monitoring its resonant frequency. The temperature sensitivity coefficient of $-7.5 \pm 0.03(\% / \mathrm{hr}) / \mathrm{ppm}$ was found by linear least squares fitting, which translates to the bias coefficient of $-180 \pm 0.8\left({ }^{\circ} / \mathrm{hr}\right) /{ }^{\circ} \mathrm{C}$. This sensitivity coefficient was used to perform self-compensation of temperature-induced bias drifts near the room temperature. Selfcompensation removed the linear trend and enabled a total $1-\sigma$ bias error of $0.5^{\circ} / \mathrm{hr}$ over day/night temperature variations, Fig. 6 .

The scale-factor sensitivity was characterized over a wider temperature range of $24{ }^{\circ} \mathrm{C}$ to $60{ }^{\circ} \mathrm{C}$ using a custom-built packagelevel heater for separation of transducer drifts from interface electronics drifts. Temperature change of $36^{\circ} \mathrm{C}$ resulted in $30 \%$ drop of the scale factor, yielding a temperature coefficient of $9,460{ }^{\circ} \mathrm{C} \mathrm{ppm} /{ }^{\circ} \mathrm{C}$, in a fairly good agreement with the theoretical value of $10,000 \mathrm{ppm} /{ }^{\circ} \mathrm{C}$. The measured sensitivity coefficient was used for self-compensation of scale-factor changes, yielding a total $1-\sigma$ scale-factor error of $700 \mathrm{ppm}$ over temperature variations, confirming the feasibility of the approach.

\section{Long-Term Stability Analysis}

Allan deviation analysis allows determination of noise processes present in a gyroscope output. As discussed earlier, the advantage of the proposed method is absence of thermal lag. Fig. 7 compares the compensation accomplished by using self-sensing vs. using a high-precision external thermistor from GEC Instruments. For long-term stability assessment we compared rate random walk (RRW) values, Fig. 7. Allan deviation of the raw data (from Fig. 5) revealed the bias instability of $0.6 \% \mathrm{hr}$ after 3 min integration time. For longer integration times the output was dominated by the temperature ramp with a $\tau^{+1}$ slope. 


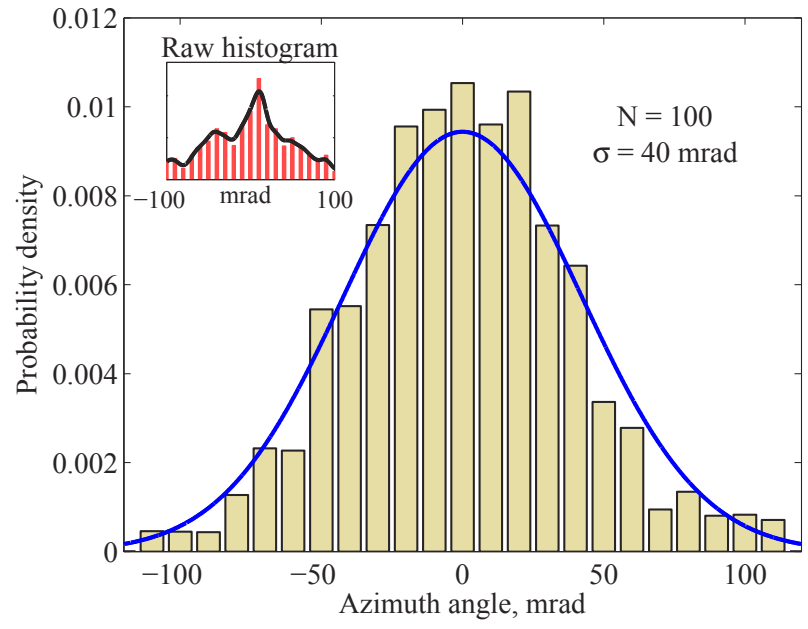

Figure 8: Distribution of gyrocompassing errors, showing normal distribution after self-compensation. Inset: distribution of raw data

Bias drift compensation using the external thermistor resolved RRW process of $1 \% / \mathrm{hr} / \sqrt{\mathrm{hr}}$, showing that the output was no longer affected by temperature variations. Despite an excellent thermistor accuracy, conventional compensation did not significantly improve the bias instability due to apparent thermal lag. In contrast, selfcompensation resulted in a threefold improvement of the bias instability, providing a $0.2 \% \mathrm{hr}$ value at $20 \mathrm{~min}$. Most importantly, the RRW improved down to $0.3 \% / \mathrm{hr} / \sqrt{\mathrm{hr}}$, indicating long-term stability. Next, we use gyrocompassing application to illustrate the benefits of self-compensation for improving repeatability.

\section{Self-Compensation for Gyrocompassing}

Frequency-based temperature self-sensing can be applied to various applications. One of the demanding inertial applications that require long-term stability is gyrocompassing, - non-magnetic north-finding using a gyroscope. While north-finding is discussed in [3], here we highlight effects of temperature-induced drifts.

The gyrocompassing requires constant bias over temperature for repeatable measurements. In practice, however, bias was timevarying, which resulted in a non-stationary random distribution of azimuth measurement errors, Fig. 8 inset. The detailed analysis confirmed temperature variations to be the primary drift source. Temperature self-compensation using the gyroscope frequency as a thermometer resulted in normal distribution of errors, Fig. 8. The uncertainty of 100 repeated azimuth measurements was 0.04 rad. Filtering of the data produced azimuth estimation with uncertainty diminishing as the square root of the number of measurements, reaching a 4 milliradian precision after averaging, Fig. 9.

\section{CONCLUSIONS}

We demonstrated self-compensation of gyroscope drifts by utilizing its resonant frequency as an embedded thermometer. The approach relied on linear frequency-temperature dependence in the silicon quadruple mass gyroscope, with SNR enhanced by high $Q-$ factor. The measured frequency stability of $0.01 \mathrm{ppm}$ provided a temperature self-sensing precision of $0.0004{ }^{\circ} \mathrm{C}$, on par with the state-of-the-art MEMS resonant thermometers. Self-compensation removed the sense-mode temperature sensitivity and improved the Allan deviation of bias from 0.6 to $0.2 \% \mathrm{hr}$. Most importantly, this method enabled a total bias error of $0.5 \% \mathrm{hr}$ and a total scale-factor error of $700 \mathrm{ppm}$ over temperature variations. When applied to gyrocompassing, self-compensation enabled normal distribution of azimuth measurements, yielding a 4 milliradian precision after

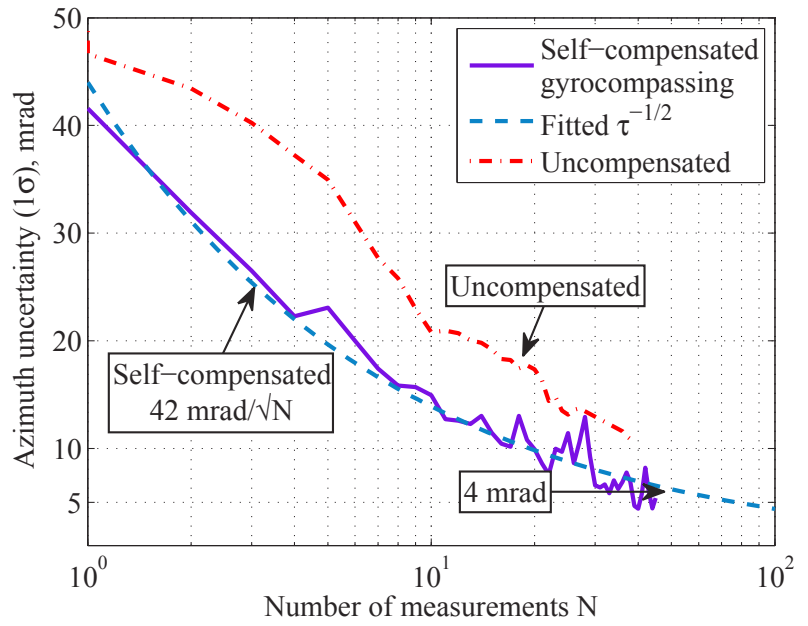

Figure 9: Azimuth precision as a function of number of averages. Self-compensated azimuth precision is 4 mrad after averaging.

averaging. The demonstrated self-compensation method may provide a path for inertial-grade silicon MEMS gyroscopes with proven long-term bias and scale-factor stability.

\section{ACKNOWLEDGMENTS}

The work was supported by ONR and NSWCDD under grants N00014-09-1-0424, N00014-11-1-0483. Authors thank Transducer Research Foundation for travel support, Dr. Heer from ZI and Dr. Zotov for aid in electronics, and D. Senkal for helpful comments.

\section{REFERENCES}

[1] F. Ayazi, "Multi-DOF inertial MEMS: From gaming to dead reckoning," in Proc. Transducers 2011 Conf., Beijing, China, June 5-9, 2011, pp. 2805-2808.

[2] I.P. Prikhodko, S.A. Zotov, A.A. Trusov, A.M. Shkel, "Subdegree-per-hour silicon MEMS rate sensor with 1 million Qfactor," in Proc. Transducers 2011 Conf., Beijing, China, June 5-9, 2011, pp. 2809-2812.

[3] I.P. Prikhodko, A.A. Trusov, A.M. Shkel, "North-finding with 0.004 radian precision using a silicon MEMS quadruple mass gyroscope with Q-factor of 1 million," in Proc. IEEE MEMS 2012, Paris, France, Jan. 29 - Feb. 2, 2012, pp. 164-167.

[4] M. Weinberg, A. Kourepenis, "Error sources in in-plane silicon tuning-fork MEMS gyroscopes," IEEE J. Microelectromech. Syst., vol. 15 (3), pp. 479-491, 2006.

[5] J.R. Vig, "Temperature-insensitive dual-mode resonant sensors - a review," IEEE Sensors Journal, vol. 1 (1), pp. 62-68, 2001.

[6] D.M. Rozelle, "The hemispherical resonator gyro: From wineglass to the planets," in Proc. $19^{\text {th }}$ AAS/AIAA Space Flight Mechanics Meeting, Feb. 2009, pp. 1157-1178.

[7] S.A. Zotov, A.A. Trusov, A.M. Shkel, "Demonstration of a Wide Dynamic Range Angular Rate Sensor Based on Frequency Modulation," in Proc. IEEE Sensors 2011 Conf., Limerick, Ireland, Oct. 28-31, 2011, pp. 149-152.

[8] D.D. Lynch, "Coriolis vibratory gyros," in Proc. Symposium Gyro Technology, Stuttgart, Germany, 1998, pp. 1.0-1.14.

[9] E.J. Ng, H.K. Lee, C.H. Ahn, R. Melamud, T.W. Kenny, "Stability measurements of silicon MEMS resonant thermometers," in Proc. IEEE Sensors Conf., Limerick, Ireland, Oct. 28-31, 2011, pp. 1257-1260.

\section{CONTACT}

*A.A. Trusov, tel: +1-949-824-6314; atrusov@uci.edu. 


\section{ALUMINUM NITRIDE PIEZOELECTRIC MICROMACHINED ULTRASOUND TRANSDUCER ARRAYS \\ Stefon Shelton ${ }^{1}$, Andre Guedes ${ }^{1}$, Richard Przybyla ${ }^{2}$, Reut Krigel ${ }^{2}$, Bernhard Boser $^{2}$, David A. Horsley ${ }^{1}$ \\ ${ }^{1}$ Berkeley Sensor \& Actuator Center, University of California, Davis, CA, USA \\ ${ }^{2}$ Berkeley Sensor \& Actuator Center, University of California, Berkeley, CA, USA}

\begin{abstract}
Air-coupled piezoelectric micromachined ultrasound transducer arrays, operating at $\sim 190 \mathrm{kHz}$, have been fabricated utilizing an aluminum nitride piezoelectric layer. Improved fabrication processes have reduced the frequency variation seen in our previous work with single transducers resulting in arrays of transducers with resonant frequencies $\left(f_{n}\right)$ that match within the fractional half-power bandwidth, $1 / \mathrm{Q}=6.6 \%$, of the transducer. Matching of $\Delta f \mid f<2 \%$ has been achieved across a 20 element 2-D array.
\end{abstract}

\section{INTRODUCTION}

Ultrasound transducers have many applications in medical imaging, ranging, robotics, and velocity sensing. Microelectromechanical systems (MEMS) micromachined ultrasound transducers (MUTs) have been explored for use in many of these applications and both capacitive and piezoelectric transduction methods have been demonstrated [1,2].

The piezoelectric actuation we have chosen for use in our transducers offers several advantages over capacitive drive for inair applications. In order to achieve the large displacement required for effective coupling to the air, cMUTs typically require large DC bias and drive voltages $(>100 \mathrm{~V})$ [3]. Piezoelectric actuation requires much lower drive voltage $(<15 \mathrm{~V})$ [4]. In addition, our pMUTs utilize a simple fabrication process and do not require tight control of the backside gap that is necessary for consistent cMUT performance.

Much of the previous work on pMUTs has focused on lead zirconate titanate (PZT) and zinc oxide $(\mathrm{ZnO})$ piezoelectric materials [2, 5-7]. While PZT has higher piezoelectric constants [8] than the aluminum nitride (AlN) film used in this work, the lower dielectric constant of AIN allows for comparable performance to be achieved. In addition, AlN is deposited in a lowtemperature $\left(<400^{\circ} \mathrm{C}\right)$ sputtering process that is compatible with CMOS processes allowing for future integration with signalprocessing electronics which is particularly attractive for phasedarray applications.

In this work we focus on fabrication challenges and characterization of a well-matched 2D air-coupled AlN pMUT array operating in the $200 \mathrm{kHz}$ region. In our previous work we have developed both ranging and distance sensing systems [4, 9] using single transducers $[10,11]$. By fabricating a frequencymatched array we can extend the utility of these transducers by allowing them to be operated as a phased array, thereby increasing the output sound pressure and creating a narrow, steerable output beam. This allows us to extend our range-finding system to include both distance and angle to an object [12] allowing for simple gesture recognition applications.

\section{TRANSDUCER DESIGN AND FABRICATION}

The transducer, with cross section schematic shown in Fig. 1, is based on a piezoelectric unimorph membrane formed from a patterned $\mathrm{SiO}_{2} / \mathrm{AlN} / \mathrm{Mo} / \mathrm{AlN} / \mathrm{Al}$ layer stack deposited on a $\mathrm{Si}$ wafer.

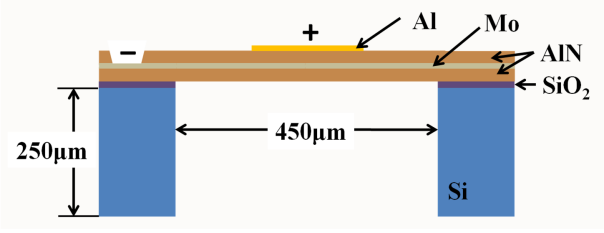

Figure 1: Cross section schematic of pMUT device.

The $450 \mu \mathrm{m}$ diameter circular membrane is actuated by applying an excitation voltage between a $300 \mu \mathrm{m}$ diameter top electrode and a continuous bottom electrode located at the transducer's mid-plane. The applied electric field creates a transverse stress in the active AlN layer which causes the membrane to displace out-of-plane producing a pressure wave in the air.

The transducer dimensions and operating parameters are shown in Table 1. The membrane diameter is chosen to produce the desired natural frequency $(\sim 200 \mathrm{kHz})$ for $1 \mu \mathrm{m}$ thick AlN active and passive layers. Individually-addressed hexagonal 2-D arrays were fabricated with a center-to-center spacing of $0.9 \mathrm{~mm}$ or approximately $\lambda / 2$ for a $190 \mathrm{kHz}$ transducer. Fig. 2 shows an optical micrograph image of the individually-addressed 37 element hexagonal array on a $6.5 \mathrm{~mm}$ die.

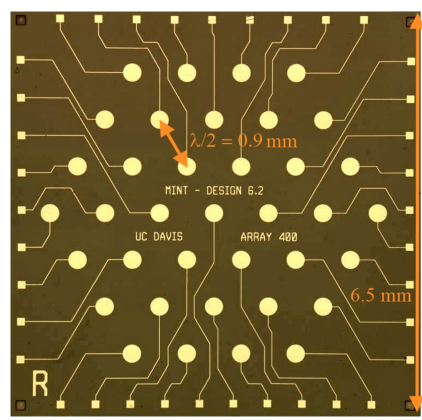

Figure 2: Optical image of a 37 element pMUT die measuring $6.5 \mathrm{~mm}$ square

Table 1: Transducer parameters

\begin{tabular}{|l|c|c|}
\hline Parameter & Value & Units \\
\hline Membrane thickness & 2.1 & $\mu \mathrm{m}$ \\
\hline Membrane diameter & 450 & $\mu \mathrm{m}$ \\
\hline Top electrode diameter & 300 & $\mu \mathrm{m}$ \\
\hline Center-to-center spacing & 900 & $\mu \mathrm{m}$ \\
\hline Natural frequency, $f_{n}$ & 190 & $\mathrm{kHz}$ \\
\hline Quality factor, Q & 15 & - \\
\hline
\end{tabular}

The fabrication process flow is shown in Fig. 3. Fabrication begins with the deposition of a $0.2 \mu \mathrm{m}$ layer of $\mathrm{SiO}_{2}$, after which the structural layers are deposited via sputtering: a $1 \mu \mathrm{m}$ AlN passive layer, the $100 \mathrm{~nm}$ Mo bottom electrode, and the final $1 \mu \mathrm{m}$ active AlN layer. The $200 \mathrm{~nm}$ thick Al top electrode is then deposited and patterned using a lift-off process, after which contacts to the bottom electrode are opened in the AIN using a MF319 wet etch. The wafer is then thinned by grinding to a thickness of $250 \mu \mathrm{m}$ and the backside is patterned and etched using a deep 
reactive ion etch (DRIE), stopping on the thin $0.2 \mu \mathrm{m}$ oxide layer. The oxide layer is removed using a separate plasma etch tool to obtain the final released membrane.

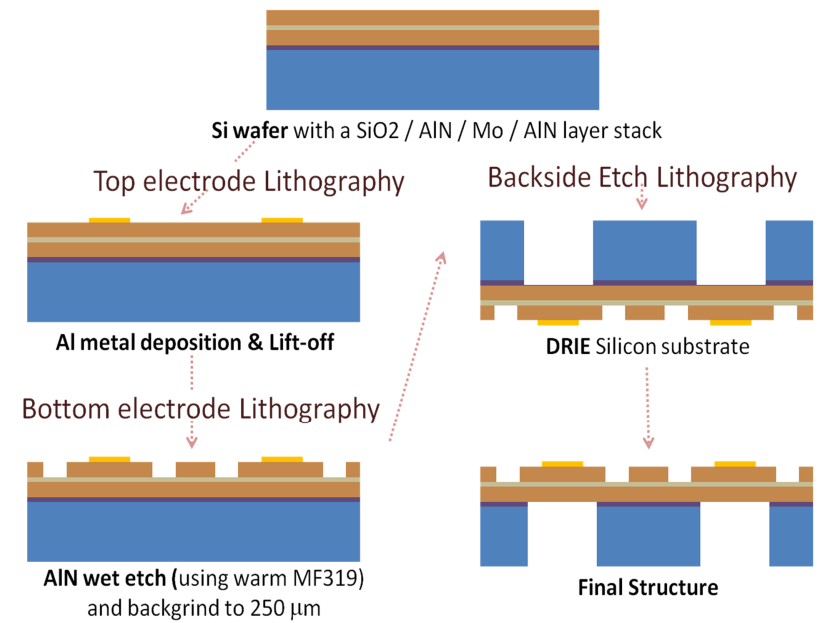

Figure 3: Fabrication process flow

\section{EXPERIMENTAL RESULTS}

\section{a. Acoustic Testing}

Acoustic tests were performed on packaged pMUT arrays using a burst measurement technique. The pMUT array is mounted in the center of a rotating stage and the acoustic axis is aligned with a microphone and is driven using a function generator with a $3.5 \mathrm{~V}_{\mathrm{rms}}, 30$ cycle burst at the natural frequency of the membranes. The burst is generated every $10 \mathrm{~ms}$. The output signal from the microphone is amplified and band pass filtered, then the time domain signal is captured with an oscilloscope. A schematic of the test setup and a representative received burst is shown in Fig. 4. The relative output pressure is determined using the peak to peak value of the received burst.

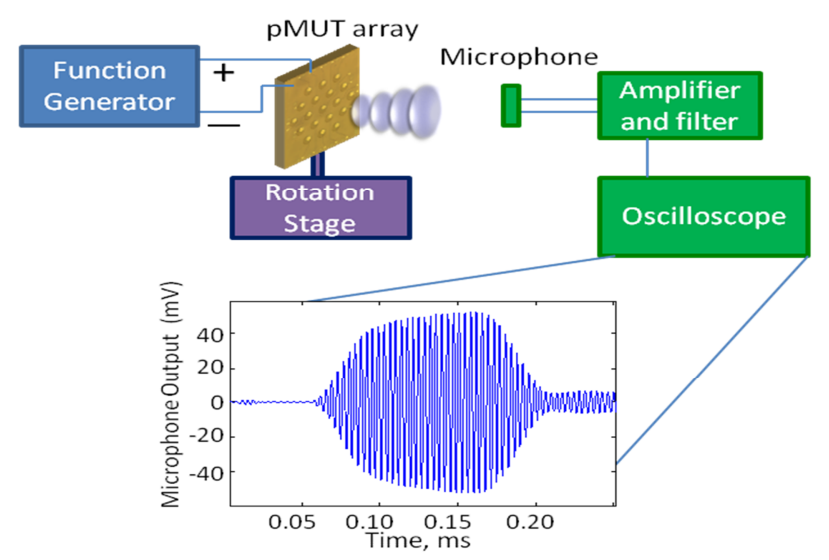

Figure 4: Acoustic test setup diagram and representative received burst

We first explored the relationship between the on-axis output sound pressure and the number of excited transducers. To do this we incremented the number of excited transducers (driven in parallel) and measured the relative output pressure at a fixed distance of $5.8 \mathrm{~cm}$. Fig. 5 shows the results of the experiment for the 20 operative transducers in the array. The linear relation matches well with established acoustic theory [13].

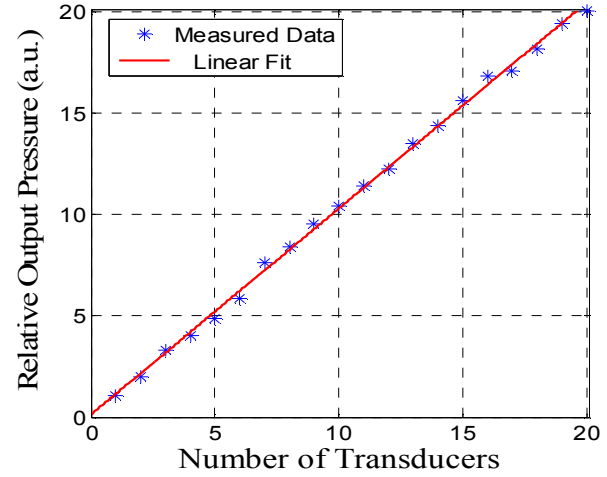

Figure 5: Sound pressure measured on-axis as a function of number of excited transducers, $N$. The sound pressure increases linearly with $N$.

The output pressure directivity pattern of the 20-transducer 2D array was measured using the previously described burst measurement setup and rotating the pMUT array relative to a single pMUT microphone. We scanned $\pm 90^{\circ}$ in both the $\mathrm{X}$ and $\mathrm{Y}$ axes and the result is shown in Fig. 6. As expected, we obtain a narrow main lobe with smaller side lobes. The width of the main lobe is $20^{\circ}$ and agrees well with acoustic modeling.

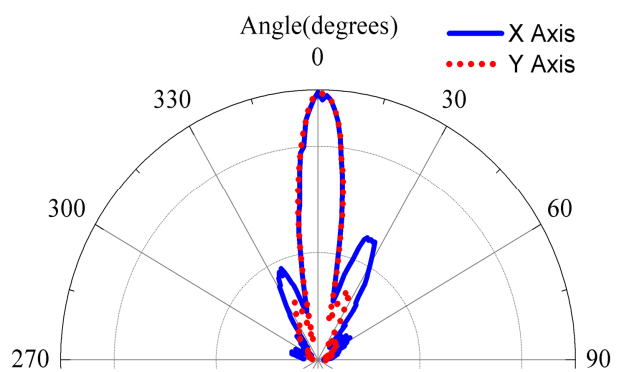

Figure 6: Output sound pressure directivity pattern for a 20element $2 D$ array.

Because sound is emitted from both the front and back sides of the array, the effect of backside reflections was explored using a variation of the burst measurement technique described previously. The microphone was placed at a distance of $1.5 \mathrm{~cm}$ from the front side of the membrane to measure the acoustic output while the distance between an acoustic reflector and the backside of the membrane was varied using a linear translation stage. The results are shown in Fig. 7.

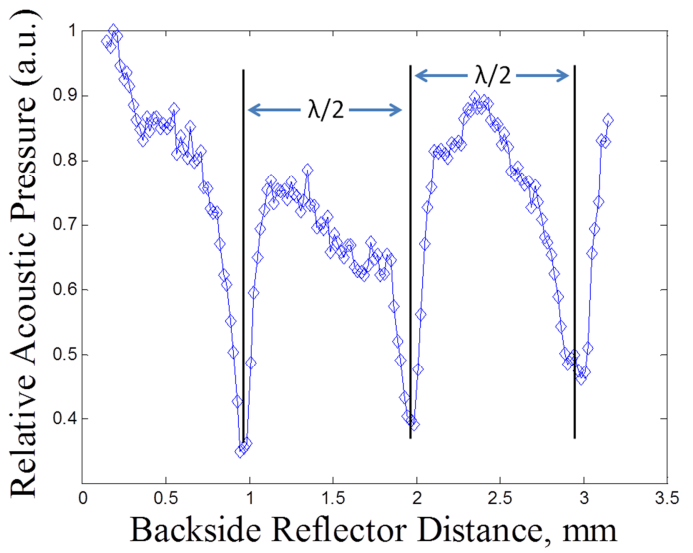

Figure 7: Output sound pressure from the front side of the membrane (normalized to the maximum) for varying distances to a backside acoustic reflector. 
The reflector on the backside of the transducer causes a standing pressure wave to form between the reflector and the transducer. The acoustic output minima correspond with the nodes in the standing wave pattern and occur every $\lambda / 2$ as predicted by acoustic theory.

\section{b. Fabrication Variation}

One of the challenges of fabricating pMUT arrays is ensuring that the pMUTs in the array have matching frequency responses. Our target matching metric is the transducer half-power bandwidth, $1 / Q$, which for our devices is $6.6 \%$.

Across a single die, a main source of mismatch was geometric variation created by the backside DRIE process. Fig. 8 shows a cross-section SEM of a transducer prior to etch optimization and illustrates a $14 \mu \mathrm{m}$ diameter variation due to undercut at the membrane edge. A 3-D FEM model in COMSOL (version 4.2a) predicts a $7 \%$ frequency mismatch for this level of variation. To quantify the frequency spread across a die we measured the frequency response of each transducer using a laser Doppler vibrometer (LDV). The frequency spread for a 17 element array prior to our process improvements is shown in Fig. 9 and illustrates matching of $\Delta f l f \sim 8 \%$ achieved with the early DRIE profile.

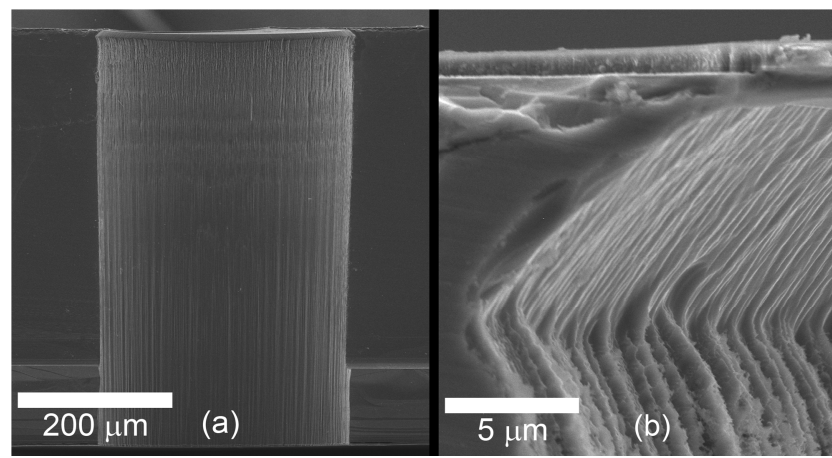

Figure 8: SEM images of pMUT cross-section prior to etch optimization: (a) profile (b) close up of membrane edge showing undesired undercut.

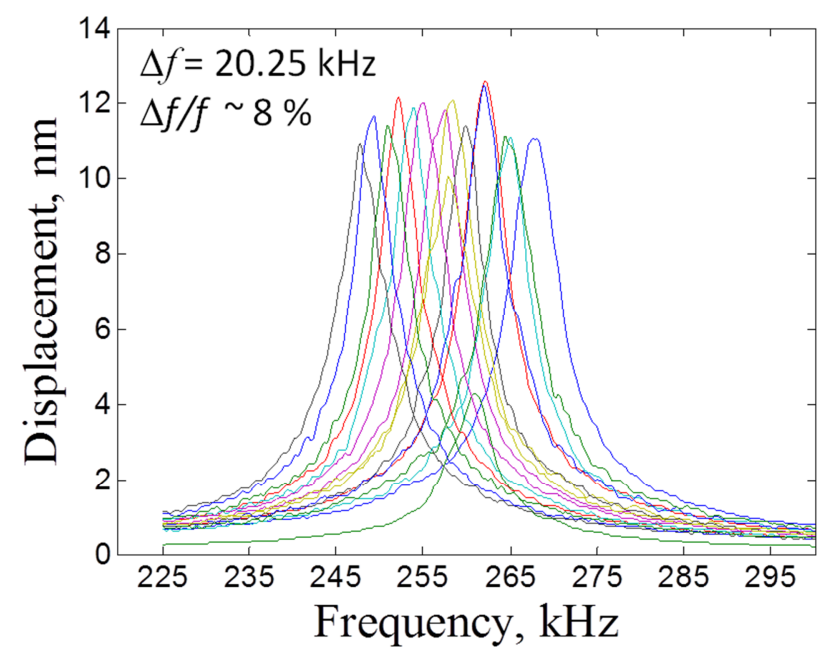

Figure 9: Frequency response of a 17 element array prior to etch optimization.

In order to correct this undercut we optimized the DRIE release step. In the standard DRIE process, the RF power is provided by a $13.56 \mathrm{MHz}$ signal on the electrode (platen). To this process we added a low frequency generator at $380 \mathrm{kHz}$ to bias the substrate platen during the etching cycle, allowing electrons to discharge the ion accumulation on the $\mathrm{SiO}_{2}$ surface, reducing the notching effect. This leads to a more uniform etch profile which translates to less frequency variation across the die. Additionally we also observed better etch uniformity reducing the etch rate by decreasing the gases flow rates. Fig. 10 shows a cross-section of a pMUT etched with the optimized DRIE process in which the diameter variation has been reduced more than threefold to $4.2 \mu \mathrm{m}$. Additionally, as shown in Fig. 11, the frequency matching across an array has improved to $\Delta f / f<2 \%$ which is well within the fractional half-power bandwidth $(6.6 \%)$ requirement.

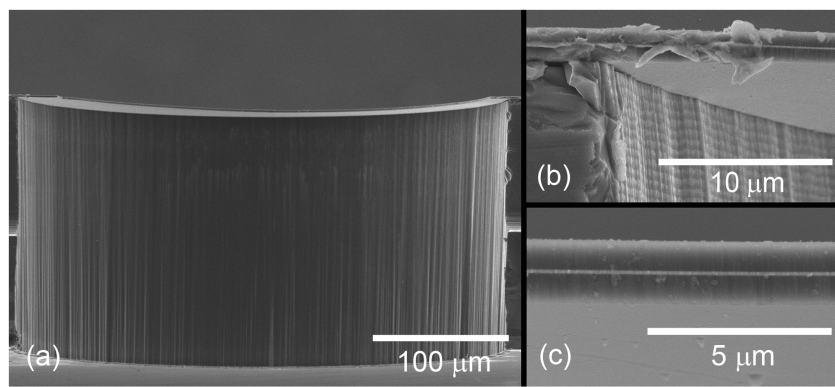

Figure 10: SEM images of pMUT after etch optimization and backgrinding: (a) profile (b) close up of membrane edge profile (c) pMUT cross-section showing well-aligned AlN layers.

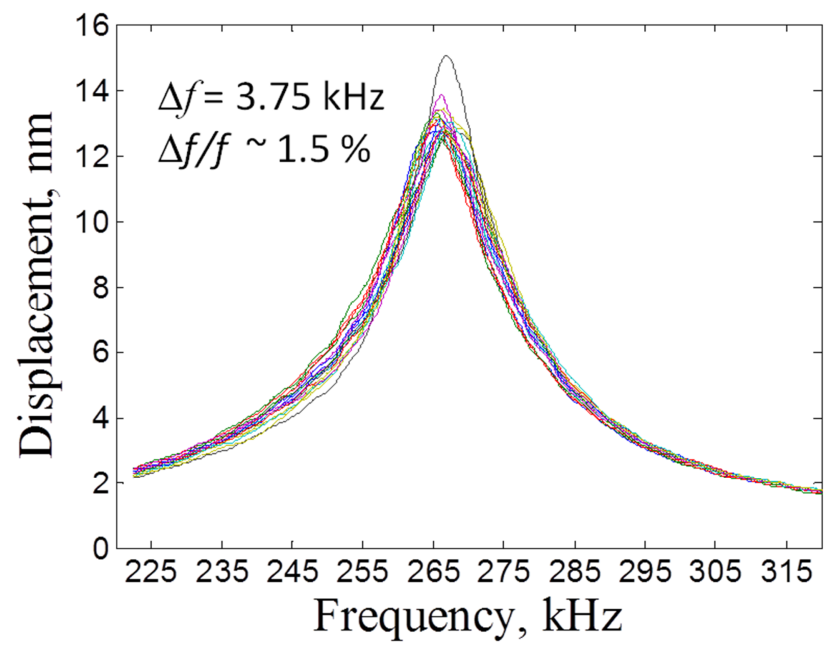

Figure 11: Frequency response of a 20 element array after etch optimization.

A second source of frequency mismatch is the across-wafer residual stress gradient. As the residual stress in the pMUT membrane becomes more tensile, the effective stiffness, and therefore the resonant frequency, increases. To quantify this effect, the frequency response was measured on 234 transducers distributed across a representative $150 \mathrm{~mm}$ wafer using the LDV. From this data we calculated the static displacement per volt and plotted the measured value as a function of the natural frequency, as shown in Fig. 12. The results are in good agreement with FEM predictions and show that the displacement diminishes approximately as $f_{n}^{-2}$. 


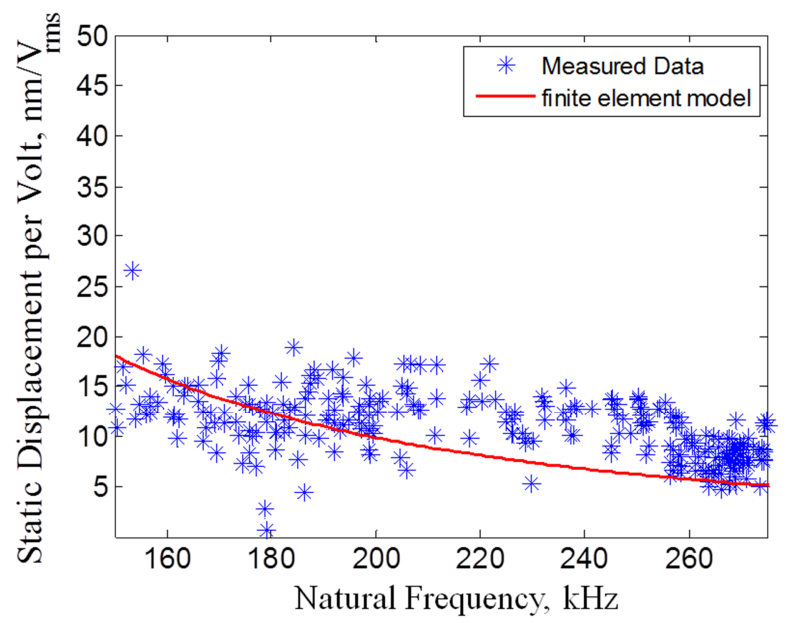

Figure 12: Static displacement per volt as a function of pMUT natural frequency, $f_{n}$.

From the measured frequency response the natural frequency $\left(f_{n}\right)$ was determined and converted into a local stress estimate via a FEM model relating stress and $f_{n}$ as shown in the inset of Fig. 13. Fig. 13 plots the predicted stress distribution across the wafer. Averaging these data points yields an average stress of $-26.6 \mathrm{MPa}$, which matches the $-25 \mathrm{MPa}$ value measured in a thin-film stress measurement tool prior to fabrication.

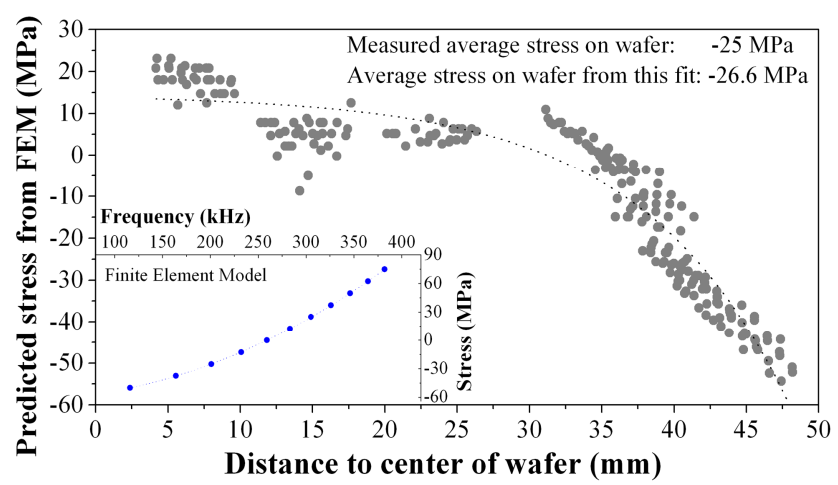

Figure 13: Across-wafer stress variation. Predicted stress was obtained from the measured natural frequency for 234 devices using the inset FEM model.

\section{CONCLUSION}

Air-coupled AIN pMUT arrays have been successfully fabricated using a simple CMOS compatible process. Recent improvements to the backside DRIE process have improved the frequency matching across a 20 transducer die to $\Delta f / f<2 \%$ allowing for improved performance relative to our previous single element transducers. An FEM model to predict the effect of residual stress on the frequency matching has been developed and the across wafer frequency variation due to stress shown. The relation between the number of driven transducers and the on-axis sound pressure level was found to be linear. The directivity of the 2-D array was determined and the main lobe width was found to be $20^{\circ}$.

\section{ACKNOWLEDGMENT}

This material is based upon work supported by the Defense Advanced Research Projects Agency (DARPA) and/or the Space and Naval Warfare Center, San Diego (SPAWAR SSC SD) under Contract No. N66001-08-C2023.

\section{REFERENCES}

[1] I. O. Wygant, N. Jamal, H. Lee et al., "An integrated circuit with transmit beamforming flip-chip bonded to a 2-D CMUT array for 3-D ultrasound imaging," Ultrasonics, Ferroelectrics and Frequency Control, IEEE Transactions on, vol. 56, no. 10, pp. 2145-2156, 2009.

[2] P. Muralt, N. Ledermann, J. Paborowski et al., "Piezoelectric micromachined ultrasonic transducers based on PZT thin films," Ultrasonics, Ferroelectrics and Frequency Control, IEEE Transactions on, vol. 52, no. 12, pp. 2276-2288, 2005.

[3] I. O. Wygant, M. Kupnik, J. C. Windsor et al., "50 kHz capacitive micromachined ultrasonic transducers for generation of highly directional sound with parametric arrays," Ultrasonics, Ferroelectrics and Frequency Control, IEEE Transactions on, vol. 56, no. 1, pp. 193-203, 2009.

[4] R. J. Przybyla, S. E. Shelton, A. Guedes et al., "In-air rangefinding with an AlN piezoelectric micromachined ultrasound transducer," Sensors Journal, IEEE, vol. 11, no. 11, pp. 2690-2697, 2011.

[5] B. Belgacem, F. Calame, and P. Muralt, "5I-2 thick PZT solgel films for pMUT transducers performances improvement." pp. 926-929.

[6] F. Guo-Hua, C. S. Charles, Q. F. Zhou et al., "Fabrication of MEMS $\mathrm{ZnO}$ dome-shaped-diaphragm transducers for highfrequency ultrasonic imaging," Journal of Micromechanics and Microengineering, vol. 15, no. 3, pp. 586, 2005.

[7] Y. Ito, K. Kushida, K. Sugawara et al., "A 100-MHz ultrasonic transducer array using $\mathrm{ZnO}$ thin films," Ultrasonics, Ferroelectrics and Frequency Control, IEEE Transactions on, vol. 42, no. 2, pp. 316-324, 1995.

[8] S. Trolier-McKinstry, and P. Muralt, "Thin film piezoelectrics for MEMS," Journal of Electroceramics, vol. 12, no. 1, pp. 717, 2004.

[9] R. J. Przybyla, A. Flynn, V. Jain et al., "A micromechanical ultrasonic distance sensor with $>1$ meter range," in Transducers, Bejing, China, 2011.

[10] S. Shelton, M.-L. Chan, H. Park et al., "CMOS-Compatible AlN piezoelectric micromachined ultrasonic transducers," in IEEE International Ultrasonics Symposium (IUS), Rome, Italy, 2009, pp. 402-405.

[11] A. Guedes, S. Shelton, R. Przybyla et al., "Aluminum nitride pMUT based on a flexurally-suspended membrane," in Transducers, Bejing, China, 2011.

[12] R. J. Przybyla, S. E. Shelton, A. Guedes et al., "In-air ultrasonic rangefinding and angle estimation using an array of AlN micromachined transducers," in Hilton Head solid-State Sensors, Actuators, and Microsystems Workshop, Hilton Head, SC, June 2012.

[13] D. T. Blackstock, Fundamentals of physical acoustics: Wiley, 2000.

\section{CONTACT}

*S.E. Shelton, tel: +1-510-752-5180; seshelton@ucdavis.edu 


\section{CHARGED PARTICLE DETECTION USING A MICROMECHANICAL ELECTROMETER

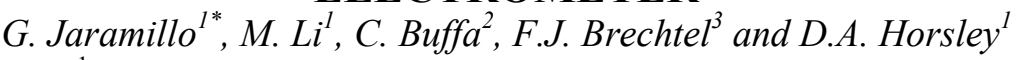 \\ ${ }^{1}$ University of California-Davis, Davis, California, USA \\ ${ }^{2}$ Politecnico di Milano, Milano, Italy \\ ${ }^{3}$ Brechtel Manufacturing, Inc., Hayward, California, USA}

\begin{abstract}
We have developed a MEMS-based electrometer for the detection of small currents from ionized particles in an aerosol particle detection system. We utilize a porous sensing-electrode coupled to a MEMS resonating electrometer. Our electrometer achieved a noise floor below $1 \mathrm{fA}$ rms produced by $10 \mathrm{~nm}$ diameter particles within an airflow of $1.0 \mathrm{~L} / \mathrm{min}$. At this flow rate, the minimum detectable current ( $1 \mathrm{fA}$ ) corresponds to a minimum measurable particle density of $400 \mathrm{~cm}^{-3}$. We compared and calibrated the MEMS electrometer with two commercial units: a Faraday cup electrometer and a MCPC (Mixing Condensation Particle Counter). The MEMS electrometer achieved an experimental sensitivity of $19.68 \mu \mathrm{V}_{\mathrm{rms}} / \mathrm{fA}$ and charge-detection efficiency of $5.476 \times 10^{7} \mathrm{~V} / \mathrm{C}$.
\end{abstract}

\section{INTRODUCTION}

Environmental air quality monitoring is important in assessing the health risks posed by particulate matter (PM). Particles in the ultrafine range, defined as smaller than $100 \mathrm{~nm}$ diameter, have become increasingly important in understanding pollutant health effects. Studies of ultrafine particles have begun to show the hazards associated with indoor and occupational aerosol exposure as well as outdoor exposures in urban areas [1]. These studies can benefit from instruments capable of quantitative measurement of particulate concentration as a function of size. One method of particle detection and classification consists of exploiting the size-dependent electrical mobility of nano-particles. Electrical mobility is a parameter that determines the velocity of a charged nanoparticle moving in an electric field. A differential mobility analyzer (DMA) separates nano-particles as a function of electrical mobility and can be employed for size-sorting over large particle ranges [2]. The size-sorted charged particle stream from the DMA output can then be fed to electrical or optical detectors for counting. One typical electrical detector consists of a Faraday cup electrometer.

Electrometry is a technique for measuring small electrical currents. Electrometer instruments are commonly used in tunneling microscopy, mass spectrometry, surface charge analysis and many other scientific fields. Many types of electrometers exist, including single-electron transistors, nano-mechanical resonators at cryogenic temperatures [3], graphene resonators [4], and units based on the vibrating reed devices. The time-varying capacitance electrometer or vibrating reed can be implemented using MEMS parallel plate sensors and actuators. MEMS sensors allow for the reduction of cost, size, weight, and power while potentially increasing the sensitivity and signal-to-noise ratio.

We present the design and characterization of a MEMS based electrometer for the counting of nanometer-sized aerosol particulate. This device aims to reduce the cost, size, weight and power of current commercial electrometers used in the aerosol scientific field. The MEMS electrometer is based on parallel plate and comb drive capacitive actuators to generate a time varying capacitance, thereby modulating a slowly-varying input charge signal to a frequency $>1 \mathrm{kHz}$ at which the measurement noise is greatly reduced.

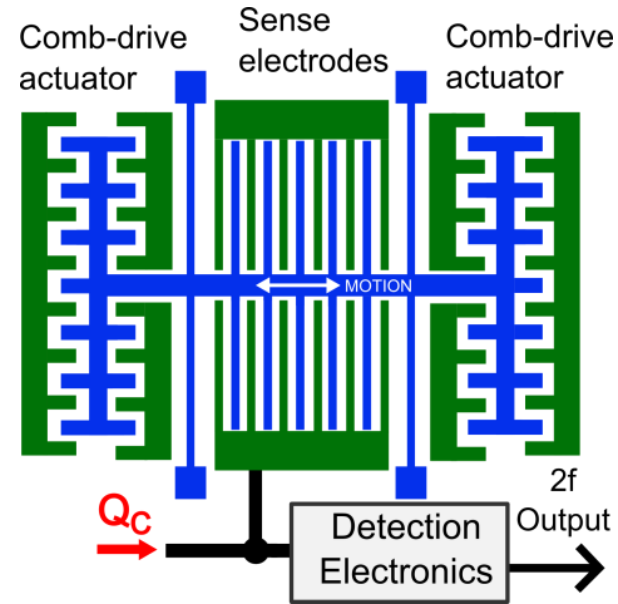

Figure 1: Illustration of the MEMS electrometer. Center shuttle oscillates at $f_{n}$ generating a detection signal at $2 f_{n}$ proportional to input charge $Q_{C}$.

\section{DEVICE DESIGN AND CHARACTERIZATION Sensor Design}

The MEMS electrometer approach was first proposed by Riehl [5]. Sub-10 electron resolution has since been demonstrated for similar MEMS designs at room temperature [6]. In comparison to these earlier demonstrations in which test charges were transferred from a local capacitor, the real-time aerosol-based measurements described here pose the additional challenge of a relatively large porous sampling electrode, introducing additional input capacitance and possible electromagnetic interference. Similar to earlier micromechanical charge sensors, our electrometer measures the induced voltage when an unknown amount of charge $Q_{C}$ is transferred onto the variable capacitor $C(t)=\left(C_{V}+C_{P}\right)$,

$$
v_{i}(t)=\frac{Q_{C}}{C(t)}
$$

Figure 1 shows a schematic of the MEMS device; the $1 \mathrm{~mm} \times 1.2 \mathrm{~mm}$ moving shuttle is supported by four flexures. The MEMS devices were fabricated in $15 \mu \mathrm{m}$ thick epitaxial polysilicon using ST Microelectronics ThELMA (Thick Epitaxial Layer for Micro-actuators and Accelerometers) process and vacuum sealed at the chip level. The device's electromechanical characteristics were presented in [7] and are summarized in Table 1.

Comb drive actuators for push-pull driving are placed at both ends of the shuttle while the parallel plate electrodes that form the measurement capacitor $C_{\mathrm{V}}$ are located in the middle. To maximize the capacitance variation at a given excitation voltage, forcing occurs at the structure's first in-plane natural frequency, which is ideally above the $1 / f$ corner frequency of the detection circuitry. Operating the device as a resonant sensor ensures that the electrostatic force is amplified by the mechanical quality factor $Q$ and allows for the possibility of operating the device as a closed- 
loop resonator. Each moving parallel-plate electrode faces two fixed electrically-connected electrodes, generating a term at the second harmonic when (1) is expanded. This design modulates the sense signal to $2 f_{n}$ and has the benefit of separating the charge signal from the capacitive feedthrough signal at $f_{n}$. The charge-tovoltage conversion gain can be defined by [5]

$$
\frac{d v_{i}}{d Q_{C}}=x_{0}^{2} \frac{C_{0}}{2 \sqrt{2}\left(C_{0}+C_{P}\right)^{2}}
$$

where $C_{P}$ is the parasitic capacitance, $C_{0}$ is the rest capacitance of the parallel plates, and $x_{0}=\hat{x} / g$ is the displacement normalized by the capacitive air gap $g$. Achieving a large conversion gain depends on minimizing $C_{P}$ and maximizing the normalized displacement $x_{0}$.

\section{Electromechanical Characterization}

Preliminary electrical characterization of the MEMS device was conducted to measure the voltage-displacement characteristic and to identify the air gap of the parallel-plate capacitors. Measurements were collected by electrostatically exciting the frame into motion using the comb drives on the sides of the frame. The resulting motion was sensed by applying an AC carrier signal to the moving shuttle and using the parallel-plate measurement electrodes as a capacitive pick-off. The sense current from the pick-offs was amplified by a trans-resistance amplifier and a lockin amplifier (Zurich Instruments HF2LI) was used to both generate drive signal and demodulate the sense signal. Figure 2(A) shows the displacement amplitude at resonance with respect to amplitude of the driving voltage on the comb drive actuators. The device starts showing nonlinear behavior at $x_{0}=0.12$, which is a result of Duffing behavior from spring stiffening in the flexure suspension. The air gap drawn on the mask was $3.0 \mu \mathrm{m}$ but changes due to reduction of line width during device fabrication. The fabricated gap was extracted by measuring the dependence of the natural frequency on the amplitude of the carrier signal applied to moving shuttle, which creates an electrostatic softening spring [8]. Based on this measurement, Figure 2(B), the fabricated air gap was identified as $3.6 \mu \mathrm{m}$.

Table 1: Specifications for the MEMS electrometer and charge calibration parameters. Measured and calculated values correspond to the AC and DC voltage operating point in table.

\begin{tabular}{lcc}
\hline Parameter & Value & Units \\
\hline Spring-beam Length & 428 & $\mu \mathrm{m}$ \\
\hline Spring-beam Width & 2.4 & $\mu \mathrm{m}$ \\
\hline Structural Layer Thickness & 15 & $\mu \mathrm{m}$ \\
\hline Spring Constant & 1.59 & $\mathrm{~N} / \mathrm{m}$ \\
\hline Shuttle Mass & 6.58 & $\mu \mathrm{g}$ \\
\hline Resonance $f_{n}$ & 2.3 & $\mathrm{kHz}$ \\
\hline Comb Drive Gap & 2.6 & $\mu \mathrm{m}$ \\
\hline No. Combs & 96 & \\
\hline$d C / d x$ for Comb Drive & 8.58 & $\mathrm{nF} / \mathrm{m}$ \\
\hline Sense Plate Gap & 3.6 & $\mu \mathrm{m}$ \\
\hline Sense Plate Length & 426 & $\mu \mathrm{m}$ \\
\hline No. Sense Plates & 66 & \\
\hline Sense capacitance $C_{V}$ & 2.1 & $\mathrm{pF}$ \\
\hline Normalized Displacement $\mathrm{x}_{0}$ & 0.17 & $\mu \mathrm{m} / \mu \mathrm{m}$ \\
\hline Quality Factor $Q$ & 180 & \\
\hline Parasitic Capacitance $C_{P}$ & 20 & $\mathrm{pF}$ \\
\hline DC Drive voltage & 2 & $\mathrm{~V}$ \\
\hline AC Drive Voltage & 200 & $\mathrm{mV} \mathrm{V}_{\mathrm{rms}}$ \\
\hline
\end{tabular}
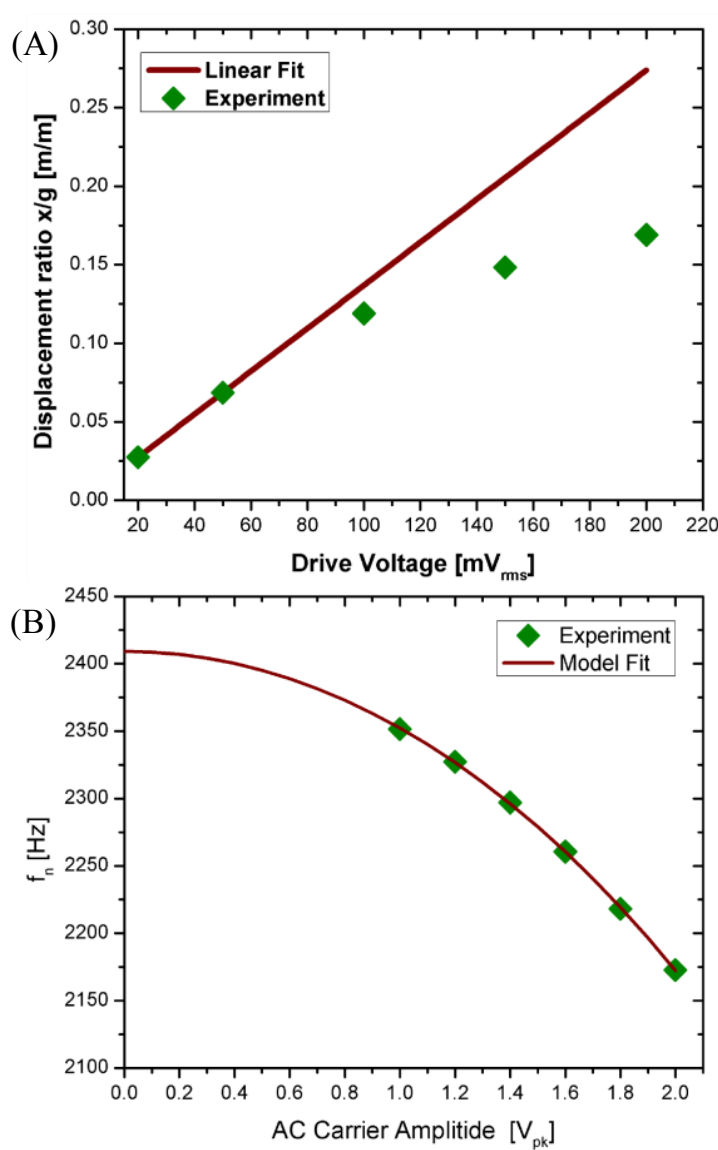

Figure 2: (A) Normalized displacement $x_{0}$ at $f_{n}$ with respect to drive voltage amplitude showing nonlinear response at displacements above $120 \mathrm{~nm}$. (B) Measured dependence of natural frequency on bias voltage amplitude

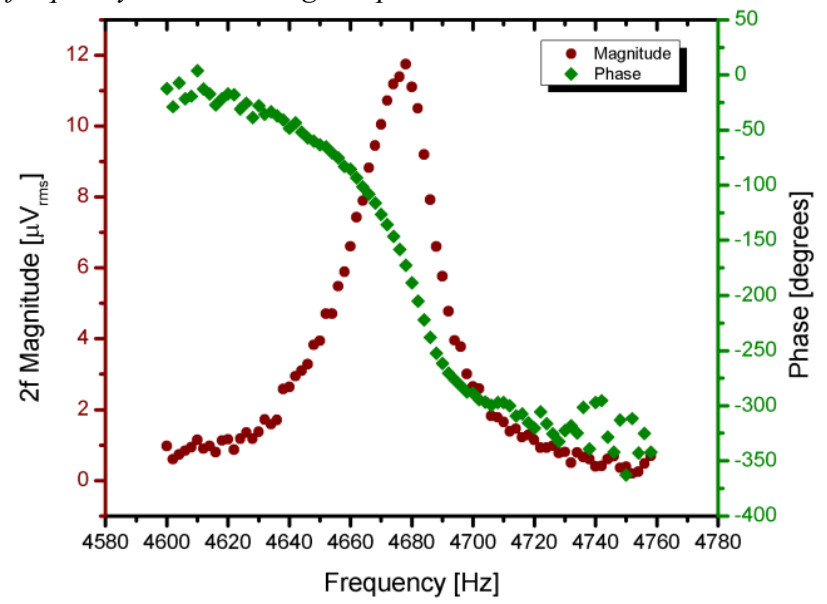

Figure 3: Frequency response around $2 f$ while driving the MEMS

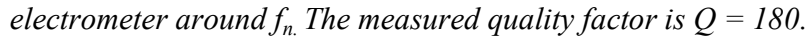

The electrometer's frequency response was also measured by applying a constant charge of $300 \mathrm{pC}$ applied to $C(t)$ and monitoring the $2 f$ component of the output votlage from (1) using a lock-in amplifier. The resulting measurement, in Figure 3, shows that the peak output signal occurs at $2 f_{n}=4.68 \mathrm{kHz}$, in good agreement with the natural frequency $f_{n}=2.3 \mathrm{kHz}$ predicted using FEM. This measurement was recorded using the circuit labelled $\mathrm{G}_{\mathrm{V}}$ in Figure 4 as a high-impedance buffer between the MEMS device and the lock-in amplifier. 

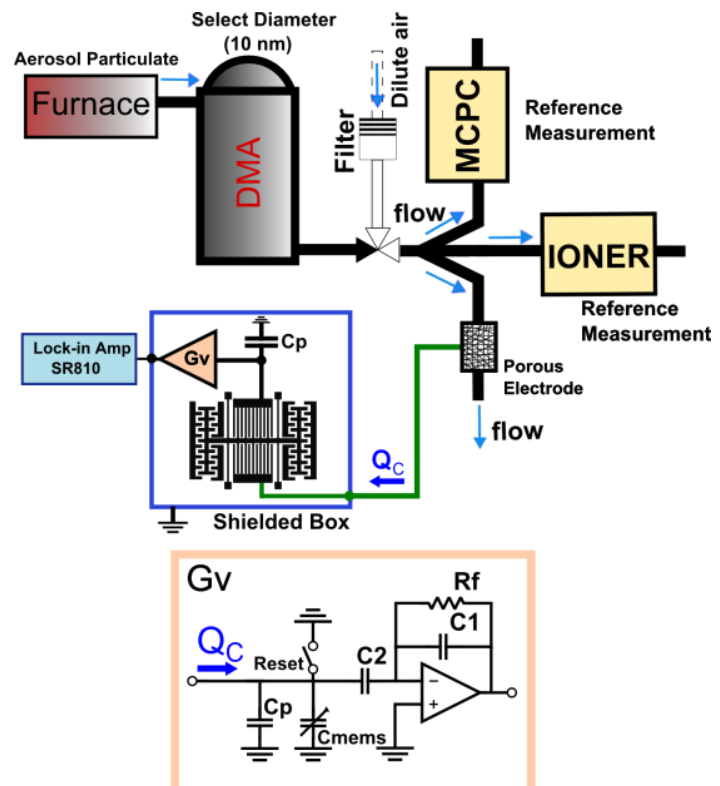

Figure 4: Simplified setup used for aerosol measurements. Particle generation is carried out in a tube furnace and particles of $10 \mathrm{~nm}$ diameter selected using DMA. MEMS device modulates charge input $Q_{C}$ at $2 f_{n}$, with the output voltage detected by LIA amplifier.
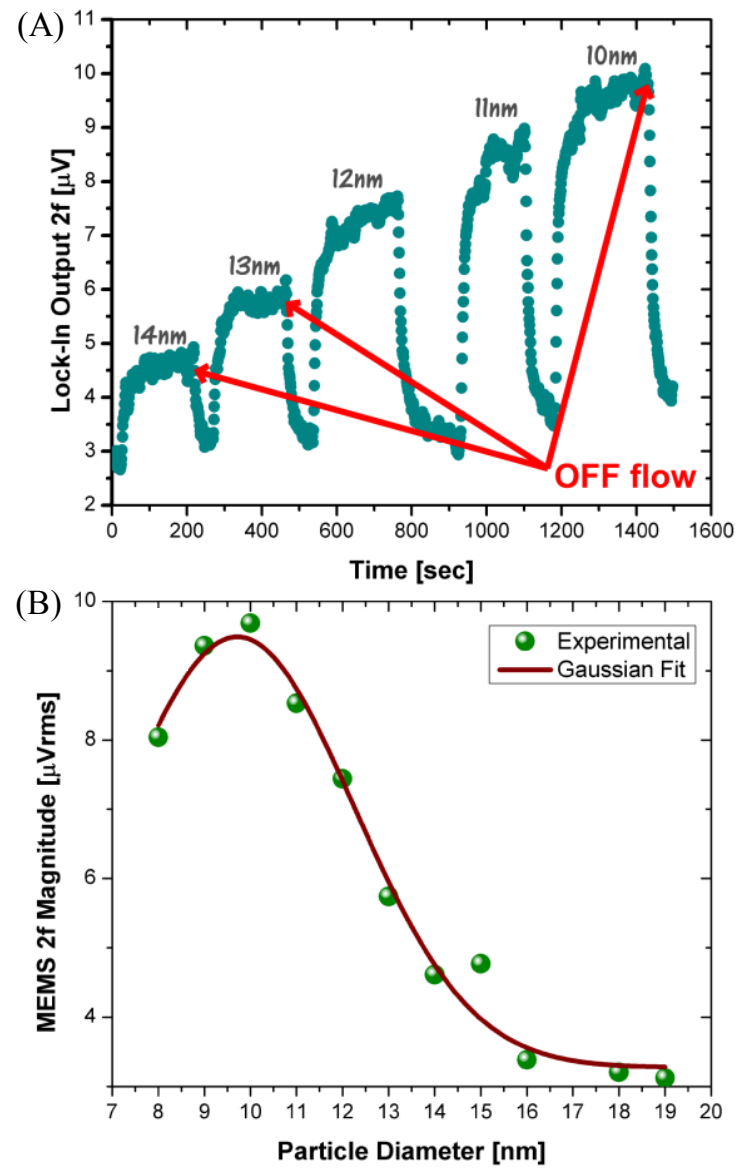

Figure 5: Scanning particle diameter using DMA. (A) Change in MEMS electrometer output as flow of particles is alternated between $\mathrm{ON}-\mathrm{OFF}$ for different particle diameters; (B) Measured MEMS output voltage versus particle diameter and Gaussian fit.

\section{AEROSOL MEASUREMENTS Experimental Setup}

The experimental setup consisted of three detection units: a commercial Faraday cup electrometer (Ioner EL-5030, Ramem), a mixing condensation particle counter (MCPC Model 1710, Brechtel Mfg. Inc.), and the new MEMS electrometer. The two commercial detectors are used as measurement references for the MEMS electrometer. Particle generation and classification is illustrated in Figure 4. The particle source consisted of a tube furnace condensation aerosol generator using a silver source. Particles are passed through a differential mobility analyzer (DMA) to selectively choose specific particle diameters based on their electrical mobility. The airflow of particles was divided and the individual flows delivered simultaneously to the two electrometers and the MCPC. Airflows entering the reference units were processed internally. For the MEMS electrometer, the charged particles were drawn through a porous conductive electrode that collects the charge but allows the particles to pass through. The currents and particle counts from each instrument were recorded using a computer interface. The MEMS electrometer output was recorded using the lock-in amplifier (SR810, Stanford Research Systems) to detect the $2 f$ component of the signal.

For our experiments, first the DMA is used as a monodisperse particle generator to allow different particle sizes to be selected. In a second method a fixed particle diameter is selected and the particle concentration varied by controlling the addition of filtered dilution air to the output flow from the DMA. These two experimental methods allow the MEMS electrometer to determine particle concentration profile and measure the minimum detectable current or particle concentration for any given particle diameter.

\section{Experimental Results}

Particle concentration was measured first by varying the particle diameter coming out of the DMA. The measurements were acquired by turning on the airflow for a specified particle diameter and measuring the MEMS output signal for a period of time long enough to observe complete settling of the output signal, Figure 5(A). The airflow was then completely shut off and the accumulated charge in the sense capacitance $\left(C_{V}+C_{P}\right)$ began to discharge through the high $(>1 \mathrm{~T} \Omega)$ leakage resistance on the interface PCB. The DMA was used to step through a range of diameters from $8 \mathrm{~nm}$ to $30 \mathrm{~nm}$ to determine the concentration distribution. Figure 5(B) shows the measured particle distribution corresponding to a Gaussian distribution centered around $10 \mathrm{~nm}$ average particle diameter.

To measure the sensitivity and sensor efficiency we carried out two experiments. First, the current was set to $I_{\text {test }}=134 \mathrm{fA}$ and steadily fed into the electrometer. Figure 6(A) plots the MEMS output versus accumulated charge. The experimental detection efficiency was $5.48 \cdot 10^{7} \mathrm{~V} / \mathrm{C}$. The sensor's theoretical efficiency in converting charge to voltage can be calculated using (2). From the measured normalized displacement, $x_{0}=0.169$ yields a predicted sensitivity of $4.29 \cdot 10^{7} \mathrm{~V} / \mathrm{C}$ for a parasitic capacitance $C_{p} \sim 20 \mathrm{pF}$, which agrees well with the measured value. Figure 6(B) summarizes the second experiment where the outputs were monitored as the test current was increased from 0 to $\sim 12 \mathrm{fA}$; here the MEMS and Ioner electrometer responses are plotted together. Figure 7 shows the output as charge increases with the test current. Leakage currents in the MEMS electrometer introduce nonlinear response at smaller currents shown on the time response in the inset of Fig. 6(B). Figure 8 shows the measured sensitivities of both the MEMS and Ioner electrometers. Both electrometers are able to detect $1 \mathrm{fA}$ currents $\left(400 \mathrm{~cm}^{-3}\right.$ particle concentration) with 
the Ioner electrometer having a slightly better rms noise resolution of $\sim 0.5 \mathrm{fA}$. The MEMS electrometer sensitivity was measured as $49 \mathrm{nV} / \mathrm{cm}^{-3}$.
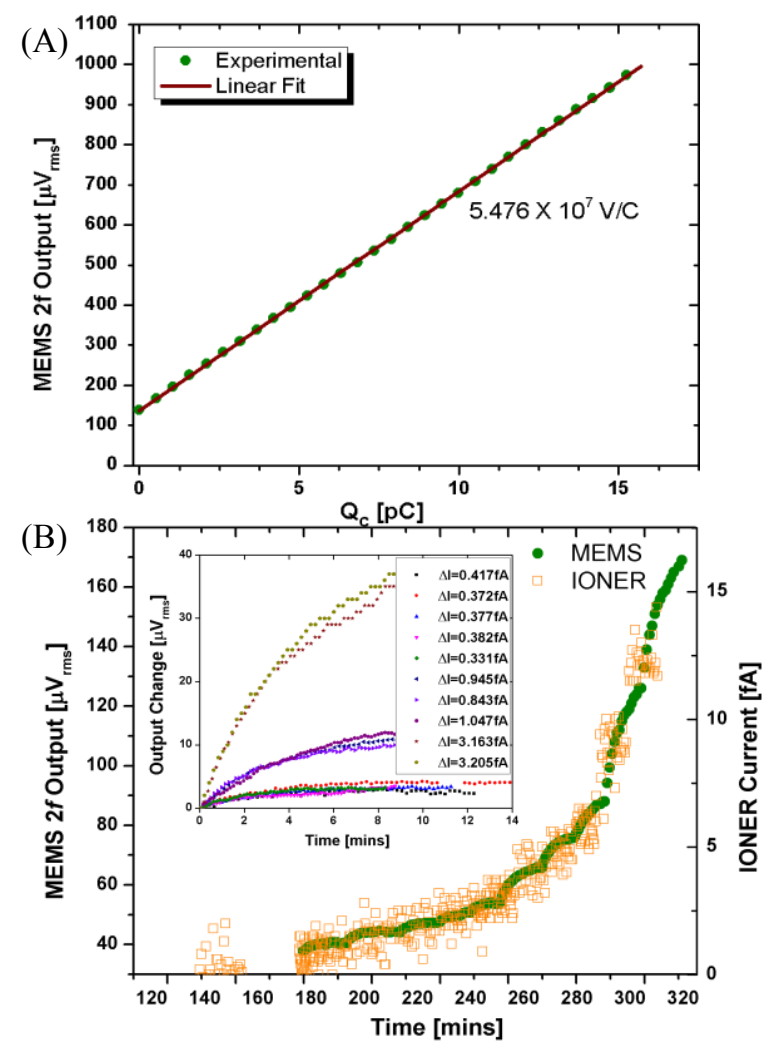

Figure 6: MEMS electrometer output with respect to input charge $Q_{C}$. (A) First experiment showing MEMS output with respect to input charge $Q_{C}$ (Itest $\sim=134 \mathrm{fA}$ ); measured sensitivity of $5.48 \cdot 10^{7} \mathrm{~V} / \mathrm{C}$. (B) Second experiment showing the output rising with time as the test current is increased; inset shows the step change in output due to the step change in test current, $\Delta I_{\text {test }}$.

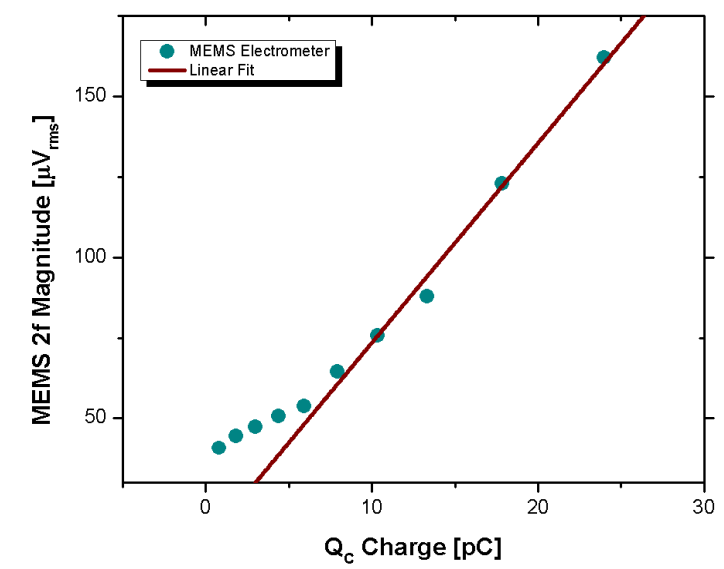

Figure 7: MEMS Electrometer output voltage versus input charge $Q_{C}$. Charge is computed for different test currents at $t=500 \mathrm{sec}$.

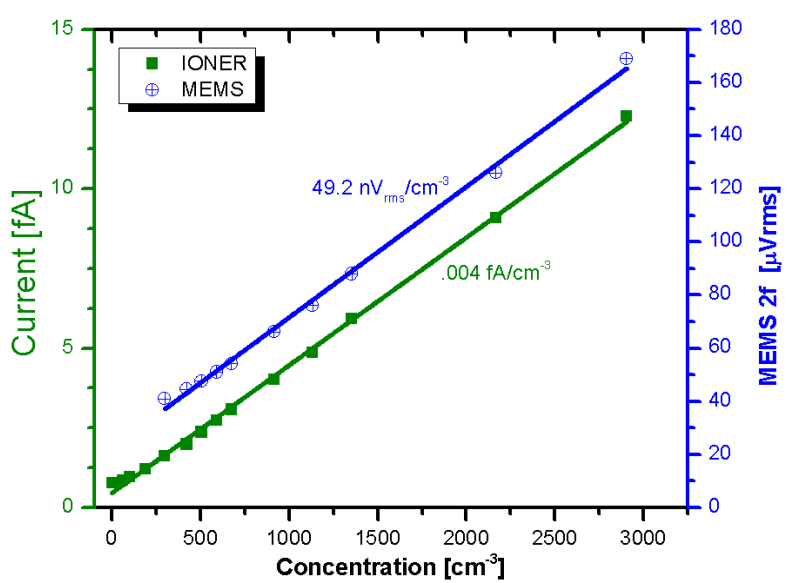

Figure 8: Current and voltage output for the two electrometers with respect to particle concentration. $M C P C$ was used to measure concentrations. MEMS electrometer has a linear sensitivity of $49 \mathrm{nV}_{\mathrm{rms}} / \mathrm{cm}^{-3}$ and Ioner electrometer $0.004 \mathrm{fA} / \mathrm{cm}^{-3}$

\section{CONCLUSIONS}

We demonstrated a MEMS based charge sensor capable of detecting aerosol particles in the nanometer size range at particle concentrations near $400 \mathrm{~cm}^{-3}$. The resolution of the electrometer can be further reduced by improving the noise and parasitic at the interface and readout electronics. The smaller footprint and lower cost of this particle detector could increase the accessibility to better measurement tools by aerosol and environmental scientists.

\section{REFERENCES}

[1] A. Ibald-Mulli, H. E. Wichmann, W. Kreyling, and A. Peters, "Epidemiological evidence on health effects of ultrafine particles," J Aerosol Med, vol. 15, pp. 189-201, Summer 2002.

[2] E. O. Knutson and K. T. Whitby, "Aerosol classification by electric mobility: Apparatus, theory, and applications," $J$. Aerosol Science, vol. 6, pp. 443-451, 1975.

[3] A. N. Cleland and M. L. Roukes, "A nanometre-scale mechanical electrometer," Nature, vol. 392, pp. 160-162, 1998.

[4] J. S. Bunch, A. M. van der Zande, S. S. Verbridge, I. W. Frank, D. M. Tanenbaum, J. M. Parpia, H. G. Craighead, and P. L. McEuen, "Electromechanical resonators from graphene sheets," Science, vol. 315, pp. 490-493, 2007.

[5] P. S. Riehl, K. L. Scott, R. S. Muller, R. T. Howe, and J. A. Yasaitis, "Electrostatic charge and field sensors based on micromechanical resonators," J. Microelectromech. Syst, vol. 12, pp. 577-589, 2003.

[6] J. Lee, Y. Zhu, and A. Seshia, "Room temperature electrometry with sub-10 electron charge resolution," J. Micromech. and Microeng., vol. 18, Feb 2008.

[7] G. Langfelder, A. Tocchio, M. J. Thompson, G. Jaramillo, and D. A. Horsley, "Assessing micromechanical sensor characteristics via optical and electrical metrology," in Sensors, 2010 IEEE, 2010, pp. 1765-1769.

[8] M. Li, V. T. Rouf, M. J. Thompson, D. A. Horsley, "Three-axis Lorentz-force magnetic sensor for electronic compass applications," J. Microelectromech. Syst., accepted.

\section{CONTACT}

*G. Jaramillo, tel: +1-530-752-5180; geomartinez@ucdavis.edu 


\title{
DROPLET-BETWEEN-ELECTRODES FOR ULTRAHIGH INTERFACIAL CAPACITIVE SENSING
}

\author{
B. Nie ${ }^{1}$, S. Xing ${ }^{1}$, J.D. Brandt ${ }^{2}$, and T. Pan ${ }^{1 *}$ \\ ${ }^{1}$ Department of Biomedical Engineering, University of California, Davis, USA \\ ${ }^{2}$ Department of Ophthalmology, University of California, Davis, Health System, USA
}

\begin{abstract}
In this paper we have presented a novel droplet-based interfacial capacitive sensor, achieving ultrahigh mechanical-to-electrical sensitivity $(1.58 \mathrm{~F} / \mathrm{kPa})$ and resolution (1.8Pa) using a simple device architecture. The miniature transparent droplet sensors consisted of two flexible polymer membranes with conductive coating and a separation layer hosting a sensing chamber for an electrolyte droplet. The sensing principle primarily relied on high elasticity of the sensing droplet and large capacitance presented at the electrode-electrolyte interface. Theoretical analyses and experimental investigations on several design parameters were thoroughly conducted to characterize and optimize the overall sensitivity of the device. Finally, the droplet sensor was successfully applied to detect minute blood pressure variations on the skin surface throughout cardiovascular cycles.
\end{abstract}

\section{INTRODUCTION}

Capacitive sensing has received increasing attention from academia, industry, and biomedical application due to its high electrical sensitivity, low power consumption, simple device construction and immunity to temperature fluctuation in comparison with its resistive counterparts. For example, Bao's group recently reported an elastomer-based capacitive sensor to achieve high sensitivity and flexibility [1], while Ezzat, et al., sandwiched a mercury droplet between two electrodes covered by an ultrahigh-permittivity material to detect the change of the contact area under external loads [2]. Moreover, Wise's group devised a capacitive pressure sensor in a wireless configuration to monitor intraocular pressure [3]. The capacitive sensing module, using conventional silicon-based microfabrication integrated with processing circuitry, was fully encapsulated in a transparent glass diaphragm which allowed continuous recording of intraocular pressure once implanted.

Recently, the electric double layer (EDL) between conductive liquid and solid phases has been shown to yield ultrahigh capacitance per unit area, typically on the order of $10 \mathrm{~F} / \mathrm{cm}^{2}$ [4]. The unique EDL interface has been explored for energy storage, interfacial manipulation, and biomolecule sensing. For example, the EDL has been used as an energy hoarder in the field of electrical energy storage and harvesting applications. In the contacts of nanostructured carbon electrodes and ionic liquids, EDL capacitance are able to operate in a wide of temperature range (from $-50^{\circ} \mathrm{C}$ to $100^{\circ} \mathrm{C}$ ) at very high charge/discharge rates (of up to $20 \mathrm{~V} / \mathrm{s}$ ), solving the significant limitation for the batteries relying on redox reactions [5]. In addition, EDL capacitance has been introduced for the detection of human serum albumin (HSA) concentration, according to the changes of the electrical impedances on the anti-HSA-coated gold electrode surfaces [6].

This paper reports a novel droplet-based capacitive sensing mechanism utilizing the highly mechanically deformable elastic and electrically conductive droplet-solid interfaces with ultrahigh unit-area capacitance from the EDL capacitance at the solid-liquid interface. The miniature transparent capacitive pressure sensor achieves an ultrahigh pressure sensitivity of $1.58 \mathrm{~F} / \mathrm{kPa}$ along with ultrahigh resolution of $1.8 \mathrm{~Pa}$, illustrated in Fig. 1, in which the sensing droplets are dyed with various colors for improved visibility.

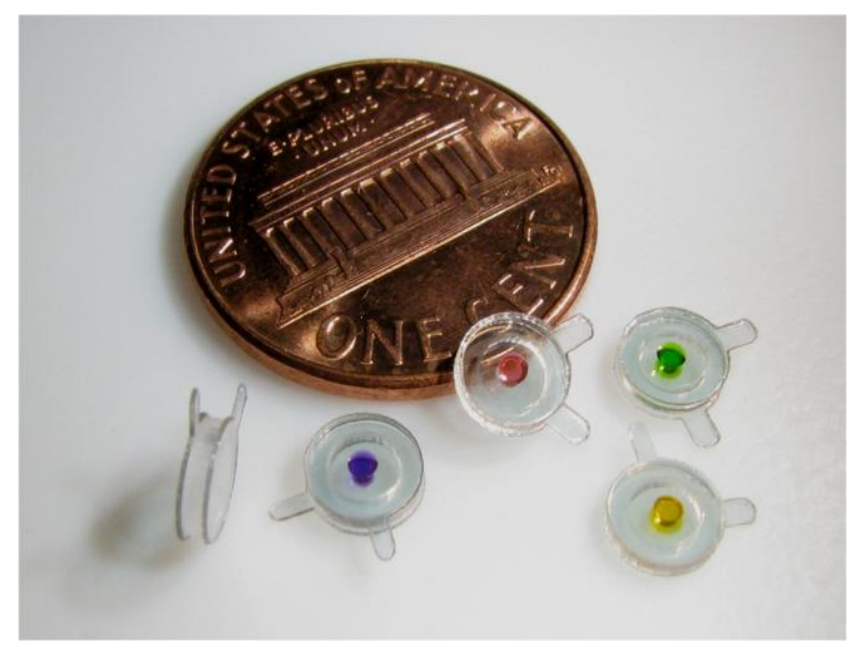

Figure 1: Ultrahigh interfacial capacitive sensors (dyed with different colors).

\section{THEORY}

The interfacial capacitive sensor consists of two flexible polymer membranes with conductive coating and a separation layer hosting an electrolyte droplet, forming a sensing chamber, as illustrated in Fig. 2a. Fig. 2b shows the equivalent circuit diagram, where the interfacial EDL capacitances at the electrolyte-electrode interface are modeled as two capacitors connected in series through the resistive element of the conductive droplet. Under external loads, the suspended membrane and the separation layer deform elastically, and as a result, the interfacial contact area experiences circumferential expansion. Given a relatively constant unit-area capacitance $\left(c_{0}\right)$ of the interfacial EDL, the variation in the contact area will lead to a proportional change in the EDL capacitance $\left(C_{E D L}\right)$ so that the sensor produces a linear capacitive response to pressure changes. The mechanical-to-electrical sensitivity of the sensing device can be analytically expressed as:

$$
\frac{\Delta \mathrm{C}_{\mathrm{EDL}}}{\mathrm{P}}=\mathrm{c}_{\mathrm{o}}\left(\alpha \mathrm{R}^{2}+\beta \mathrm{H}\right) \frac{\mathrm{V}_{\mathrm{d}} \mathrm{R}^{2}}{\mathrm{H}^{2}}
$$

where $P$ is mechanical load. $\alpha$ and $\beta$ represent the membrane deflection and the elastic deformation of the separation layer, and can be determined by the geometrical and mechanical properties of the sensing membrane and the separation layer, respectively. $R$ and $H$ represent the radius and height of the sensing chamber, while $V_{d}$ indicates the volume of the electrolyte droplet. As can be seen, both the membrane deflection (the 1st term) and the separation layer deformation (the 2nd term) contribute to the overall mechanical-to-capacitive sensitivity. It is also worth noting that both the hydraulic pressure of the droplet and the radius of the curvature at the droplet-electrode interface have been ignored in the simplified mathematical expression. In addition, the gravitational effect has been neglected, since the droplet volume is typically confined to the ones smaller than the cube of the capillary length. 


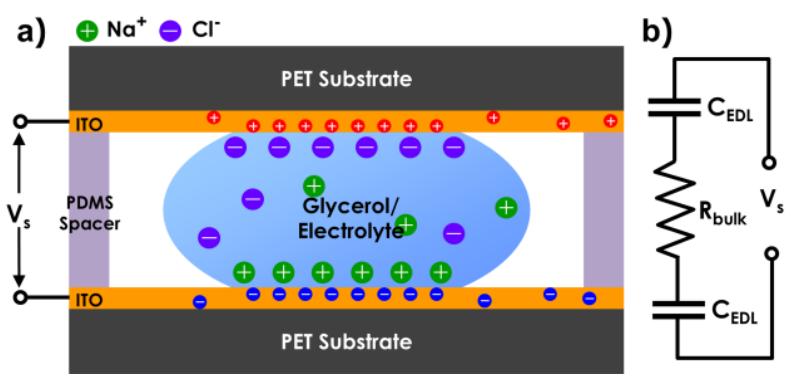

Fig. 2 a) Illustration of the droplet-based interfacial capacitive sensing principle, and b) the equivalent electrical circuit diagram.

\section{METHODS}

\section{Surface Modification}

Indium-tin-oxide (ITO)-coated polyethylene terephthalate (PET) films (Southwall Technology) were used to serve as the pressure sensing membranes. In order to reduce the hysteresis of the droplet deformation, simple surface modification was introduced to the conductive coating. In brief, the ITO-coated PET sheets were exposed to oxygen plasma at $30 \mathrm{~W}$ for $30 \mathrm{sec}$ for surface hydroxylation. Following the plasma activation, a dielectric polydimethylsiloxane (PDMS) stamp was brought into physical contact with the conductive coating enriched with hydroxyl groups for 2 hours, during which a nanometer-thick layer of PDMS oligomers was transferred and immobilized onto the electrode surface [7]. In addition, an indented hole (of $200 \mathrm{~m}$ in diameter) was included in the center of the PDMS stamp, leaving a hydrophilic spot surrounded by the hydrophobic oligomer layer on the electrode surface, by which the sensing droplet could be anchored and stabilized in the middle of the sensing chamber.

\section{Device Fabrication}

To fabricate the device components, ITO-coated PET films and a PDMS layer were laser-micromachined to serve as the sensing membranes and the separation layer, respectively. In the subsequent step, the laser-trimmed PET substrates with ITO coating enriched with PDMS oligomers as aforementioned were bonded to the PDMS separation layer through an oxygen plasma-assisted bonding. Prior to the final assembly of the device, an electrolyte droplet with a desired volume was dispensed by a micropipette in the centre of the sensing chamber.

\section{Electrical and Mechanical Characterization}

External mechanical point loads were applied onto the centre of the sensing membrane through a custom-built motorized force gauge with $1 \mathrm{mN}$ resolution (DFS, Chatillon), driven by a computer-controlled step motor (VT-80, Micos) with a spatial resolution of $0.2 \mathrm{~m}$. The mechanical-to-capacitance responses, measured by LCR meter, were evaluated twice on two identical devices for each parameter. For the resolution measurement, minute droplets (of 50 L volume) dispensed by a micropipette was applied directly onto the sensing membrane till a noticeable capacitive change appeared in the LCR meter, which was evaluated three times for the same device.

\section{RESULTS}

\section{Sensing Droplets}

As a vital part of the interfacial sensing device, physical properties of the sensing droplet have to satisfy following criteria: high ionic concentration (ensuring high electrical conductance and interfacial capacitance), polarized molecular structure (reversible elasticity on the hydrophobic surfaces) and low fluidic viscosity (allowing rapid mechanical response). Aqueous-based electrolyte solution (e.g., $\mathrm{NaCl}$ ) with high ionic concentration can be a natural choice, satisfying all except for moderate evaporation under a room condition. According to a previous study, mixing an aqueous solution with glycerol can effectively reduce evaporation due to decreased vapor pressure [8]. Through examining the physical properties (conductivity, evaporation, and viscosity) of the electrolyte/glycerol $(\mathrm{E} / \mathrm{G})$ mixture (given a $\mathrm{NaCl}$ solution at the concentration of $1.1 \mathrm{~mol} / \mathrm{L})$ at various mixing ratios $(\mathrm{v} / \mathrm{v} \%)$, an optimal mixing ratio of $25 / 75 \% \mathrm{E} / \mathrm{G}$ solution has been chosen as the working fluid for the sensing droplet, considering its highly conductive and non-evaporative under a laboratory condition $(46 \%$ humidity and $24^{\circ} \mathrm{C}$ ).

\section{Surface Modification}

As aforementioned, surface hydrophobic treatment has been applied to the conductive coating, which ensures the reversible and elastic deformability of the sensing interface. The surface energy during the oligomer transfer process is compared by measuring the contact angle of a mixed E/G solution (25/75\%). With an oxygen-plasma treatment, the ITO coating is rendered apparent hydrophilic (the contact angle reduces from $66^{\circ}$ to $15^{\circ}$ ). Following the oligomer transfer step, the PDMS oligmer-coated ITO surface switches the polarity from hydrophilicity to hydrophobicity with the contact angle of $90^{\circ}$, which further enhances the elasticity and reversibility of the droplet-electrode contact by reducing adhesive energy of the liquid to the substrate.

\section{Mechanical-to-Capacitive Sensitivity}

The overall mechanical-to-electrical sensitivity $\left(\Delta C_{E D L} / P\right)$ can be determined by the geometrical confinements (the radius $R$ and height $H$ of the sensing chamber and the thickness of the polymeric membrane), droplet volume $\left(V_{d}\right)$, and the material properties of the construct (Young's modulus and Poisson's ratio), given a fixed unit-area capacitance $\left(c_{o}\right)$. Among those, the radius of the sensing chamber play the most important role, followed by the height of the chamber, as predicted in the Theory. In addition, the system sensitivity is directly proportional to the volume of the sensing droplet. Experimental investigations have been conducted to verify the above theoretical predications.
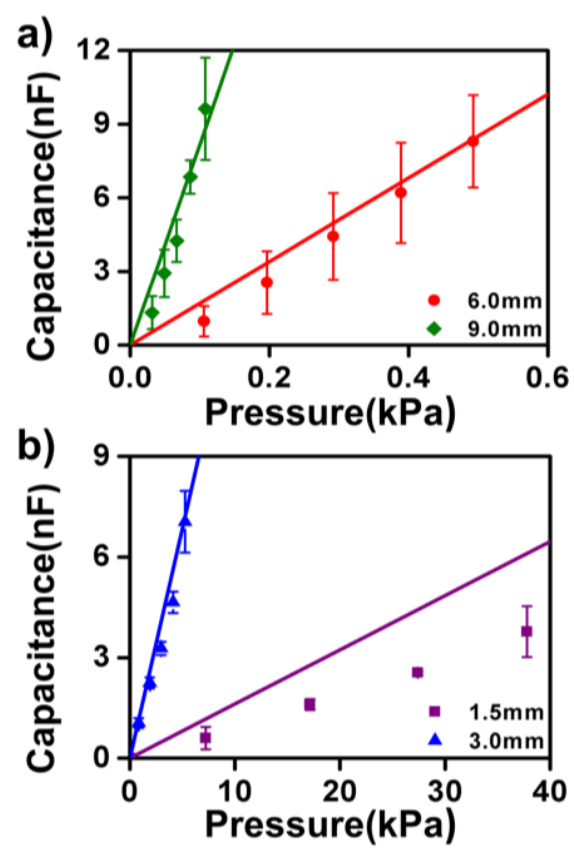

Fig. 3 Influence of different radii of the sensing chambers on the device sensitivity. 


\section{Influence of Chamber Radius}

Fig. 3a and Fig. 3b illustrate the capacitive changes over a wide spectrum of pressure loaded on the droplet sensors with different sizes of the sensing chamber, of which the radius varies from 1.5 to $9.0 \mathrm{~mm}$, given the chamber height of $200 \mathrm{~m}$ and the droplet volume of $0.3 \mathrm{~L}$. The experimental measurements (dots) are plotted in comparison with the values calculated from Eq. 1 (curves), and the slope rate of each device measurement defines the corresponding device sensitivity. As predicted by the sensing theory, the radius exhibits 4th power in membrane deflection and 2 nd power in the elastic deformation. Within the small deflection limit, the capacitive charges change linearly with the external load as one would expect. In the devices with the largest sensing chamber (of $9.0 \mathrm{~mm}$ radius), the highest sensitivity of $90.2 \mathrm{nF} / \mathrm{kPa}$ has been achieved. As the radius is reduced to two thirds of the former one $(6.0 \mathrm{~mm})$, the system sensitivity drops drastically to less than $20 \%$ of that $(17.2 \mathrm{nF} / \mathrm{kPa})$. Those data suggests that the theoretical model fits the experiments reasonably well under the assumptions. In both cases, the contact area between the droplet and the deformed membrane is smaller than $5 \%$ of the whole chamber area, and therefore can be approximated as a flat interface instead of curved. However, in the smallest devices (with a radius of $1.5 \mathrm{~mm}$ ), the measurements deviate considerably (more than 40\%) from the stimulated values. In this case it is highly possible that the small deformation limit, which is associated with the ratio of the maximum deflection to the radius of the membrane, has been exceeded. In addition, it has been observed that the radius of the curvature of the deflected membrane can no longer be ignored in the smallest sensing unit. Overall, given its 4 th power relation, the radius of the chamber is a determinant factor for the overall mechanical-to-electrical sensitivity.

\section{Influence of Chamber Height}

As predicted by the theoretical analysis, the device sensitivity is inversely proportional to the 2 nd power of the height of the sensing as the membrane deflection dominates, and thus, a lower sensing chamber will lead to a larger contact area at a given droplet volume. Fig. 4 a presents the variations of the sensitivity over four different chamber heights ranging from $140 \mathrm{~m}$ to $350 \mathrm{~m}$, provided the chamber radius of $6.0 \mathrm{~mm}$ and droplet volume of $3 \mathrm{~L}$. As can be seen, the lowest chamber $(140 \mu \mathrm{m}$ in height) shows the highest sensitivity $(374 \mathrm{nF} / \mathrm{kPa})$ due to the presence of the largest contact area at the droplet-electrode interface. As the height of the chamber rises from $140 \mu \mathrm{m}$ to $350 \mu \mathrm{m}$, the device sensitivity decreases from $374 \mathrm{nF} / \mathrm{kPa}$ to $54.5 \mathrm{nF} / \mathrm{kPa}$, closely following the negative quadratic relationship between the sensitivity and the chamber height. Moreover, as the chamber height increases, the separation layer becomes increasingly influential on the overall sensitivity. However, in our cases, the maximal deformation of the separation layer is still one order of magnitude smaller than that of the membrane deflection as expected. In brief, all four sets of the sensitivity measurements are in a good agreement with the theoretical analysis presented above.

\section{Influence of Droplet Volume}

Furthermore, the droplet volume is directly proportional to the system sensitivity. In general, a larger droplet covers more interfacial area with a longer circumferential periphery along the contact, which leads to a higher sensitivity given constant dimensions of a sensing chamber. However, the increased droplet size can also lead to non-linear response as the droplet-electrode interface cannot be approximated as a planar surface. Four different droplet sensing units (of $0.3 \mu \mathrm{L}, 0.6 \mu \mathrm{L}, 1.2 \mu \mathrm{L}$ and $3.6 \mu \mathrm{L}$ ) have been characterized and compared under the identical dimensions of sensing chamber $(6.0 \mathrm{~mm}$ radius and $200 \mu \mathrm{m}$ height $)$, as shown in Fig. $4 \mathrm{~b}$. As the droplet volume increases from $0.3 \mu \mathrm{L}$ to $0.6 \mu \mathrm{L}$, and then to $1.2 \mu \mathrm{L}$, the corresponding sensitivity rises in a linearly proportional manner from $17.2 \mathrm{nF} / \mathrm{kPa}$ to $32.1 \mathrm{nF} / \mathrm{kPa}$, and then to $65.7 \mathrm{nF} / \mathrm{kPa}$. The highest sensitivity $(218 \mathrm{nF} / \mathrm{kPa})$ has been achieved on the sensing droplet of $3.6 \mu \mathrm{L}$ in volume. Again, this matched finding re-confirms the applicability of Eq. 1.

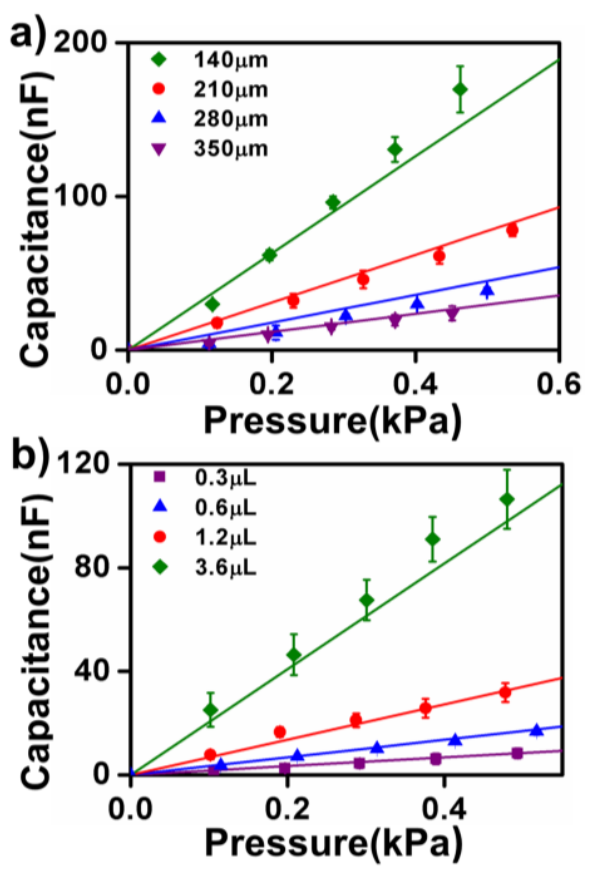

Fig. 4 Influence of (a) heights of the sensing chambers, (b)volume of the sensing droplets on the device sensitivity.

\section{Optimization of Device Sensitivity}

Finally, we have combined the optimal conditions from the above analysis (of $9.0 \mathrm{~mm}$ in radius, $140 \mu \mathrm{m}$ in height and $3 \mu \mathrm{L}$ in droplet volume) and characterized the highest device sensitivity of $1.58 \mathrm{~F} / \mathrm{kPa}$, in comparable to the highest value reported in the literature $(2.24 \mathrm{~F} / \mathrm{kPa}$, using a mercury droplet and an ultrahigh permittivity material), to our best knowledge [2]. Moreover, in the optimal sensing design, the device shows an extremely high pressure resolution of $1.8 \pm 0.4 \mathrm{~Pa}$.

\section{Influences of Temperature and Humidity}

Importantly, the performance of the droplet sensors can be subject to the environmental humidity level. Specifically, the E/G mixture establishes thermodynamic equilibrium with different stable mixing ratios at different humidity levels by either losing (evaporation) or gaining (condensation) aqueous contents to/from the environment [9]. As shown in Fig. 5a, when the humidity level alters, the volume and capacitance of a droplet sensor can substantially deviate from the initial condition (of $25 / 75 \% \mathrm{E} / \mathrm{G}$ ) as the mixture compositions change. However, once a new thermodynamic equilibrium is established, the capacitance will remain stable. Therefore, we can either employ sensing elements with different mixing ratios at different humidity levels or let the sensing element equilibrate with the environment thermodynamically prior to its usage.

In contrast, the environmental temperature fluctuation has only posed minor influence on the interfacial capacitance, in comparison with the resistance-based devices. As shown in Fig. 5b, The electrolyte-electrode interfacial capacitance has been measured at three separate temperatures, $4{ }^{\circ} \mathrm{C}$ (reduced), $25^{\circ} \mathrm{C}$ (regular), and 45 ${ }^{\circ} \mathrm{C}$ (elevated), respectively, from which less than $10 \%$ variation in 
the unit-area capacitance has been observed at the steady state.

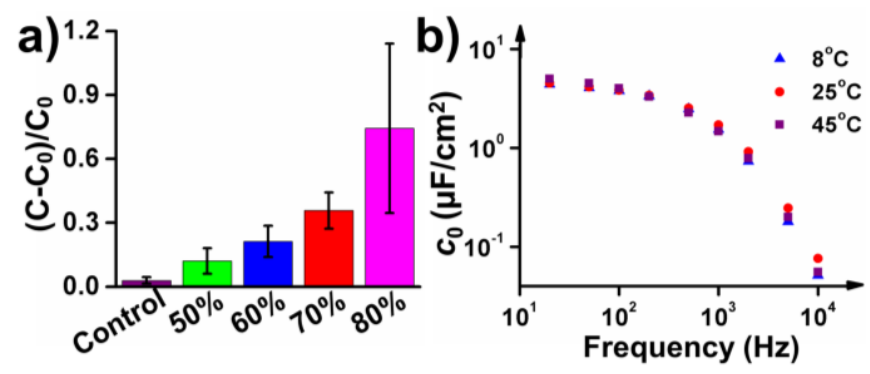

Fig. 5 (a) the humidity influence on the initial capacitance $C_{0}$ and (b) the temperature influence on the unit-area capacitance $c_{0}$.

\section{Demonstration}

To demonstrate the utility of the simply constructed and mechanically flexible droplet sensor with ultrahigh sensitivity and resolution, we applied the device to record blood pressure variations on the skin surface. In this dynamic measurement, a droplet sensor was devised with a built-in chamber size of $6 \mathrm{~mm}$ in radius and $140 \mathrm{~m}$ in height. An electrolyte droplet of $3 \mathrm{~L}$ acted as the sensing element to achieve a facilitated response.

The sensor was attached to the skin surface above the carotid artery (in Fig. 6a) to record the blood pressure wave with a gentle contact force being applied. As shown in Fig. 6b, the minute blood pressure variations were recorded with the maximum value less than $100 \mathrm{~Pa}$ at around $1 \mathrm{~Hz}$. As demonstrated, the ultrahigh sensitivity/resolution, simple configuration and flexible construct of the droplet sensing device could be attractive to a wide range of biomedical applications (e.g., ocular systems, pulmonary, and cardiovascular), where the maximal pressure variations typically range from $2 \mathrm{kPa}$ to $20 \mathrm{kPa}$ and meanwhile, the body conformability and comfortableness are of particular importance for continuous recording [10]-[12].

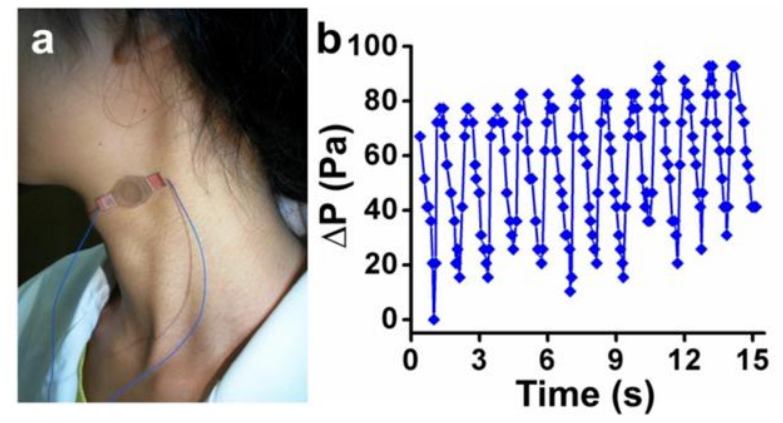

Fig. 6 (a) Applying the capacitive sensor (b) to record the blood pressure variations on the skin surface (above the carotid artery).

\section{CONCLUSIONS}

In this paper, we have presented a novel ultrahigh interfacial capacitive sensor with a very simple architecture and tunable sensitivities. The interfacial sensing principle utilizes the presence of large unit-area EDL capacitance at the elastic droplet-electrode contact. A theoretical model has been proposed to establish the linkage between the geometrical parameters (the radius and height of the sensing chamber and the droplet volume) and the device sensitivity. Taking advantage of ultrahigh sensitivity, simple fabrication, mechanical flexibility and optical transparency, insensitivity to evaporation and thermal noise, the interfacial capacitive sensing devices are expected to be employed in a wide range of biomedical applications where high sensitivity and high flexibility are concurrently required.

\section{ACKNOWLEDGEMENTS}

This work is in part supported the NSF CAREER Award (ECCS-0846502) and EFRI Award (EFRI-0937997) to TP. BN and SX acknowledge the fellowship support from China Scholarship Council (CSC). Authors would also like to acknowledge the samples of ITO-coated membranes generously provided by Southwall Technology. In addition, we would like to thank Mr. Yuzhe Ding for the valuable discussion on the blood pulse measurement.

\section{REFERENCES}

[1] S.C.B. Mannsfeld, B.C.K. Tee, R.M. Stoltenberg, C.V.H.H. Chen, S. Barman, B.V.O. Muir, A.N. Sokolov, C. Reese, and Z. Bao,"Highly Sensitive Flexible Pressure Sensors with Microstructured Rubber Dielectric Layers", Nature Materials, 9, 10 (2010).

[2] E.G. Bakhoum, and M.H.M. Cheng, "Novel Capacitive Pressure Sensor", Journal of Microelectromechanical Systems, 19, 3 (2010).

[3] R.M. Haque, and K.D. Wise, "An Intraocular Pressure Sensor Based on a Glass Reflow Process", Technical Digest of the 2010 Solid-State Sensors, Actuators, and Microsystems Workshop, Hilton Head Isl., SC, 6/6-10/10, Transducer Research Foundation (2010), pp. 49-52.

[4] H. Yuan, H. Shimotani, A. Tsukazaki, A. Ohtomo, M. Kawasaki, and Y. Iwasa, "High-Density Carrier Accumulation in $\mathrm{ZnO}$ Field-Effect Transistors Gated by Electric Double Layers of Ionic Liquids", Advanced Functional Materials, 19, 7 (2009).

[5] R. Lin, P. Taberna, S. Fantini, V. Presser, C.R. Pérez, F. Malbosc, N.L. Rupesinghe, K.B.K. Teo, Y. Gogotsi, and P. Simon, "Capacitive Energy Storage from -50 to $100{ }^{\circ} \mathrm{C}$ Using an Ionic Liquid Electrolyte", The Journal of Physical Chemistry Letters, 2, 19 (2011).

[6] Y. Chuanga, Y. Changa, K. Liua, H. Changb, and T. Yewa, "Electrical Impedimetric Biosensors for Liver Function Detection", Biosensors and Bioelectronics, 28, 1 (2011).

[7] Y. Ding, S. Garland, M. Howland, A. Revzin, and T. Pan, "Universal Nanopatternable Interfacial Bonding", Advanced Materials, 23, 46 (2011).

[8] A.R. Carr, R.E. Townsend, and W.L. Badger, "Vapor Pressures of Glycerol-Water and Glycerol-Water-Sodium Chloride Systems", Industrial and Engineering Chemistry, 17, 6 (1925).

[9] A. K. Ray, R. D. Johnson, A. Souyri, "Dynamic Behavior of Single Glycerol Droplets in Humid Air Streams", Langmuir, 5, 1 (1989).

[10] A.M. Master, L.I. Dublin, and H.H. Marks, "The Normal Blood Pressure Range and Its Clinical Implications", Journal of the American Medical Association, 143, 17 (1950).

[11] J.G. Venegas, R.S. Harris, and B.A. Simon, "A Comprehensive Equation for the Pulmonary Pressure-Volume Curve", Journal of Applied Physiology, 84, 1 (1998).

[12] C.R. Ethier, M. Johnson, J. Ruberti, "Ocular Biomechanics and Biotransport", Annual Review of Biomedical Engineering, 6, 1 (2004).

\section{CONTACT}

*T.Pan, tel: +1-530-754-9508; tingrui@ucdavis.com 


\section{EXPERIMENTAL VERIFICATION OF A CAPACITIVE SHEAR STRESS SENSOR FOR LOW-SPEED WIND TUNNEL APPICATIONS \\ J. Meloy, J. Sells ${ }^{1}$, V. Chandrasekharan ${ }^{2}$, J. Griffin, L.N. Cattafesta ${ }^{3}$, D.P. Arnold, and M. Sheplak ${ }^{*}$ Interdisciplinary Microsystems Group, University of Florida, Gainesville, FL USA}

\begin{abstract}
This paper describes the development of a shear stress sensor system for use in wind tunnels and the comparative performance of the system in two different tunnel facilities. The MEMS sensor uses differential capacitive transduction of a tethered floatingelement plate. The sensor is microfabricated using a silicon-onPyrex fabrication process. An analog synchronous modulationdemodulation circuit is implemented in order to make timeresolved measurements of the mean and dynamic change of the sensor capacitance. The sensor system is packaged in a robust, small form factor enabling convenient installation into multiple test facilities. The final system has a sensitivity of $10.8 \mathrm{mV} / \mathrm{Pa}$ and a minimum detectable signal of $6.5 \mathrm{mPa}$ at an $8 \mathrm{~V}_{\mathrm{ac}}, 100 \mathrm{kHz}$ sinusoidal excitation.
\end{abstract}

\section{INTRODUCTION}

The ability to make time-resolved, continuous, direct mean and fluctuating measurements of skin friction or wall shear stress is currently not available. Wall shear stress characterization has an impact on both the research and commercial communities. Skin friction drag accounts for approximately $50 \%$ of the total drag on an aircraft at cruising speeds [1]. Additionally, wall shear stress is important for the characterization of turbulent flow and is important for the development of turbulence models.

In response to this technology gap, many shear stress measurement techniques have been developed, as outlined in several reviews [2], [3]. Floating-element sensors are the most promising to achieve direct, time-resolved shear stress measurements. Concerns of fabrication complexity, spatial averaging, bandwidth, and cross-sensitivities indicate microelectromechanical systems (MEMS) as an enabling technology for the fabrication of floating-element shear stress sensors. Of the many MEMS floating-element shear stress sensors that have been presented, the most promising for continued development into a robust sensor system is a differential capacitive comb-drive sensor presented by Chandrasekharan et al. [4].

The sensor structure is a floating element suspended over a cavity by tethers. The floating element displaces when a shear stress is applied and the tethers act as restoring springs. On either side of the floating element are interdigitated comb fingers. A schematic of this structure and the equivalent electrical circuit of the structure are shown in Figure 1. The comb fingers form a differential capacitive half bridge and are nominally matched to each other. The modeling of the static and dynamic response of this structure is available in [4]. The dimensions of the device design chosen for this study are shown in Table 1.

\begin{tabular}{|l|l|l|l|l|l|l|l|l|}
\hline $\mathrm{W}_{\mathrm{e}}$ & $\mathrm{L}_{\mathrm{e}}$ & $\mathrm{W}_{\mathrm{t}}$ & $\mathrm{L}_{\mathrm{t}}$ & $\mathrm{g}_{01}$ & $\mathrm{~g}_{02}$ & $\mathrm{~L}_{\mathrm{o}}$ & $\mathrm{W}_{\mathrm{f}}$ & $\mathrm{T}_{\mathrm{t}}$ \\
\hline 1000 & 1000 & 15 & 1000 & 3.5 & 20 & 170 & 20 & 45 \\
\hline
\end{tabular}

Table 1: The dimensions of the floating element structure shown in Figure 1. All in units of [ $\mathrm{\mu m}]$
For a given flow direction, as indicated in Figure 1, capacitance $\mathrm{C}_{1}$, which is formed between electrodes $\mathrm{E}_{1}$ and $\mathrm{E}_{2}$, decreases as the floating element moves. The capacitance $\mathrm{C}_{2}$, formed between electrodes $\mathrm{E}_{2}$ and $\mathrm{E}_{3}$, increases as the floating element moves. These capacitances as a function of shear stress are represented as

$$
\mathrm{C}_{1}(\tau)=\mathrm{C}_{0}-\Delta \mathrm{C}(\tau)
$$

and

$$
\mathrm{C}_{2}(\tau)=\mathrm{C}_{0}+\Delta \mathrm{C}(\tau)
$$

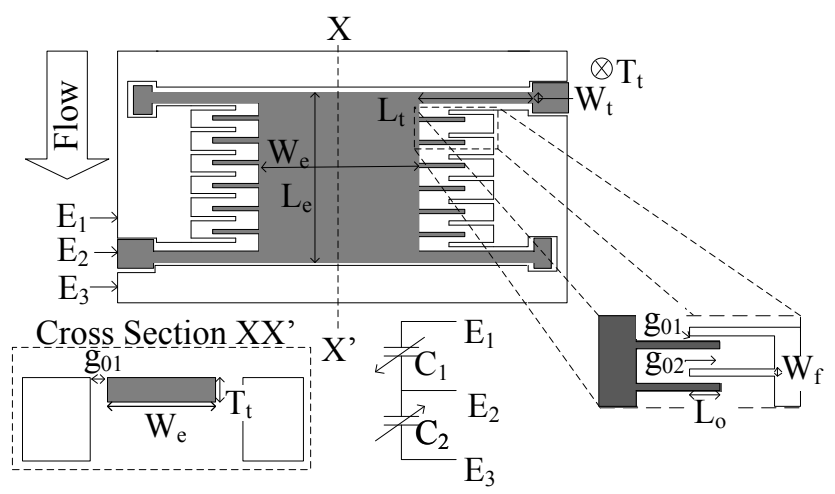

Figure 1: A schematic of the floating element structure with an enlargement of the interdigitated comb fingers, a cross section of the sensor along line $X X^{\prime}$, and an electrical representation of the sensor structure.

\section{FABRICATION}

The sensor is fabricated using a two-mask process, shown in Figure 2. The process begins with a $100 \mathrm{~mm}$ Corning 7740 Pyrex wafer. Chrome is deposited and patterned using the first photomask to create a hard mask for a hydrofluoric acid etch, which creates the cavity beneath the floating-element structure. The chrome is removed, and a silicon-on-insulator wafer is anodically bonded above the cavity. The bulk silicon is removed in a $20 \% \mathrm{KOH}$ bath, followed by the removal of the buried oxide layer in buffered oxide etchant. The remaining device layer is patterned with the second photomask, and the floating element structure is defined using a deep reactive ion etch process. The resulting sensors are diced to a final size of $3.5 \mathrm{~mm} \times 3.5 \mathrm{~mm}$ using a heat release tape from Nitto Denko America, Inc. to protect the floating elements during dicing. Exploiting the reciprocal nature of capacitive transduction, the sensor dice are electrically tested via electrostatic actuation to confirm mechanical release. Fully released die are then packaged. 

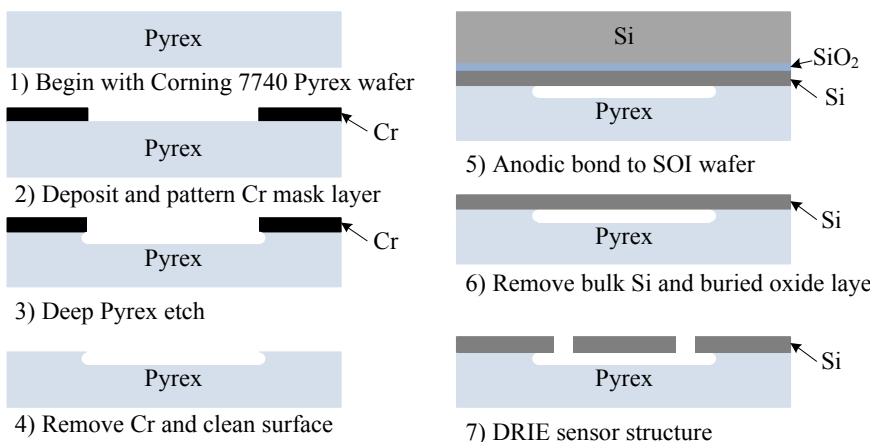

6) Remove bulk Si and buried oxide laye

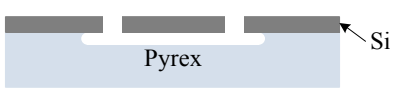

7) DRIE sensor structure

Figure 2: A schematic view of the process flow to create the capacitive floating element sensor structure.

\section{PACKAGING}

In order to be useful as a tool for fluid dynamics measurement the final form factor of the sensor package must be hydraulically smooth [2], robust, and small. In order to achieve this, the package is made of two components: an end cap into which the sensor die is flush mounted and attached to electrical leads, and a cylindrical housing that provides mechanical support for mounting the sensor die into a wind tunnel and contains any interface electronics colocated with the sensor die. The assembly of these two components is shown in Figure 3.

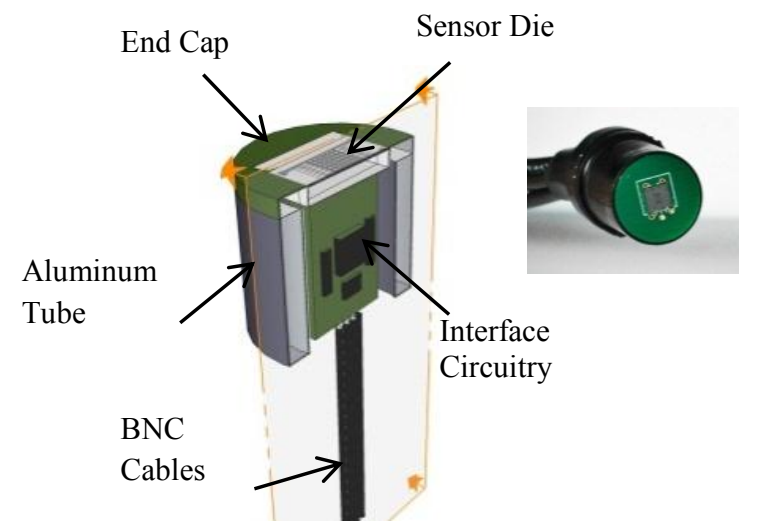

Figure 3: The assembled package showing the end cap, aluminum tube, and flush mounted sensor die (left), and a photo of the $1 / 2$-inch outer diameter assembled device (right).

The end cap is an FR4 circuit board with a die-shaped recess to enable a hydraulically smooth surface. Adjacent to the sensor die are three immersion gold bond pads that fan out to vias, which permit electrical connections to the bias voltages and interface circuitry on the backside of the circuit board. Gold wire bonds are used to connect the sensor die to the circuit board bond pads.

The sensor housing is a $1 / 2$-inch diameter, 3/4-inch long aluminum tube. The aluminum tube serves two purposes: it acts as a mechanical support onto which mounting fixtures can be attached without inducing stress onto the sensor structure directly and it acts as a Faraday cage to protect the interface electronics located within the tube from electromagnetic interference. The small diameter allows for several sensors to be integrated into a single test in an array. The short length allows for integration into small wind tunnel models.

\section{INTERFACE CIRCUITRY}

As shown in Figure 3, there is room in the mechanical support structure to house a small printed circuit board with interface electronics. The complexity of that interface is due to the high impedance of the capacitive transducer and the requirement to make a measurement of a mean capacitance change. The interface circuit is broken into two components; the immediate interface with the sensor die, in the form of a voltage buffer, and the signal conditioning circuitry, which is the frequency translating synchronous modulation/demodulation (synch MOD/DMOD) component.

\section{Voltage Buffer}

The immediate interface to the transducer should preserve the output signal of the transducer, contribute minimal noise to the system, and buffer from a high impedance to a low impedance to prevent contamination from electromagnetic interference. A voltage buffer, shown in Figure 4, is implemented. The overall transfer function of the buffer is

$$
\mathrm{v}_{\text {out }}=\frac{\mathrm{j} \omega \mathrm{R}_{\mathrm{b}}}{\mathrm{j} \omega \mathrm{R}_{\mathrm{b}}\left(2 \mathrm{C}_{0}+\mathrm{C}_{\mathrm{p}}\right)+1} 2 \mathrm{~V}_{\mathrm{b}} \Delta \mathrm{C}(\tau),
$$

where $R_{b}$ is the bias resistance, shown in Figure $4, \Delta C$ and $C_{0}$ are shown in Equation 1, and $\mathrm{C}_{\mathrm{p}}$ is the parasitic capacitance of the packaging and the input capacitance of the operation amplifier chosen to implement the buffer. Equation 3 shows that an overall high pass filter function is implemented with the corner frequency set by the bias resistance and the dominant sensor capacitance. The voltage buffer is implemented with the AD8022 from Analog Devices and a single bias resistor, which was chosen due to a low voltage noise floor and low input capacitance. The output is also only defined when there is an excitation frequency; at dc there is no steady-state output voltage.

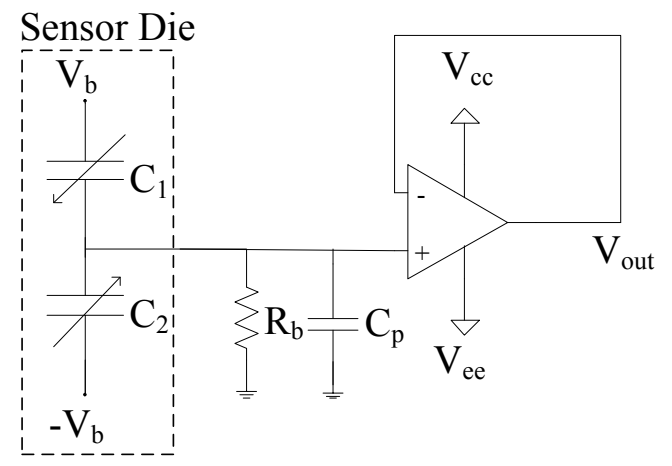

Figure 4: A schematic of the complete voltage buffer circuit, including bias resistor and sensor die.

In order to have a steady-state response to a mean change in capacitance, a sinusoidal bias is applied, making the output of the buffer an amplitude-modulated signal. As the signal is now driven by a low-impedance source, further interface circuitry may be located away from the sensor without raising additional concerns about electromagnetic interference. The voltage buffer, in a single integrated circuit implementation, is co-located with the sensor die in the aluminum housing of the sensor package. The additional interface circuitry can be located near the data acquisition system without consequence. 


\section{Synchronous Modulation/Demodulation}

While it is possible to simply acquire the amplitudemodulated signal and demodulate it in post-processing to obtain flow information, an analog demodulation scheme is implemented to relax requirements on the data acquisition system and extend the use of the system into areas like flow control, which require baseband flow information for feedback. The system is an active envelope detector [5] and is made of five major components: a bias generation, a bandpass filter, an active rectifier, a lowpass filter, and digital control circuitry that control the active rectifier block.

Mathematically, active rectification can be described as the low-pass filtering of the multiplication of an amplitude-modulated signal with a square wave possessing unity amplitude and frequency $\omega_{\mathrm{b}}$. Using a Taylor series expansion of a square wave, the multiplication product, $\mathrm{v}_{\text {rectified }}$, is

$$
\begin{aligned}
\mathrm{V}_{\text {rectified }}= & \frac{1}{2} \frac{2 \Delta \mathrm{C}(\tau)}{2 \mathrm{C}_{\mathrm{o}}+\mathrm{C}_{\mathrm{p}}} \mathrm{V}_{\mathrm{b}}+\frac{2 \Delta \mathrm{C}(\tau)}{2 \mathrm{C}_{\mathrm{o}}+\mathrm{C}_{\mathrm{p}}} \mathrm{V}_{\mathrm{b}} \sin \left(\omega_{\mathrm{b}} \mathrm{t}\right) \\
& +\frac{2 \Delta \mathrm{C}(\tau)}{2 \mathrm{C}_{\mathrm{o}}+\mathrm{C}_{\mathrm{p}}} \mathrm{V}_{\mathrm{b}} \cos \left(2 \omega_{\mathrm{b}} \mathrm{t}\right)+\ldots
\end{aligned} .
$$

Applying a low-pass filter to Equation 4 recovers the demodulated flow information. Any phase errors in the synchronization would be represented by a phase contribution to the square-wave terms and result in more harmonic generation but would not change the final magnitude of the recovered baseband signal or the requirements on the final low-pass filter.

The system is implemented using discrete components. The bias generation block generates two out-of-phase sinusoids at $100 \mathrm{kHz}$ and variable amplitude to serve as bias signals for the sensor. One of the bias signals is fed into the digital control circuit in order to provide phase information to synchronize the demodulation. The output of the sensor package is fed into a bandpass filter. This filter removes any spurious frequencies that have been introduced into the signal path, through electromagnetic interference, harmonic distortion, or any other source. The remaining amplitude-modulated signal is used to derive two signal paths, one inverted and one non-inverted, which are fed into the active rectifier. The control signal for this switch is derived from one of the bias signals and remains in-phase with that bias signal, meaning the rectification is in-phase with a bias signal. The output of the analog switch is the rectified signal given by Equation 5 . This signal is fed into an LTC1653 low-pass filter with a corner frequency of $20 \mathrm{kHz}$. The output of this low-pass filter stage is the baseband flow information over the entire bandwidth of the sensor.

\section{SENSOR CALIBRATION}

With the assembled sensor system and package, characterization is performed in a known shear stress field to verify sensor performance. The sensor is calibrated in an acoustic plane wave tube using a Stokes' layer excitation to generate a known oscillating shear stress [6]. The experimental set up for this is shown in Figure 5.

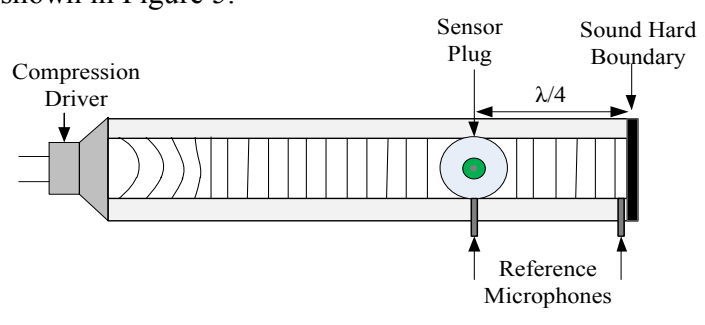

5: Shear stress calibration experimental setup
Increasing the sound pressure level of the incident acoustic wave, the dynamic sensitivity is determined. The ac normalized sensitivity, shown in Figure 6 , is $1.34 \mathrm{mV} / \mathrm{V} / \mathrm{Pa}$. At a bias of $8 \mathrm{~V}_{\mathrm{ac}}$, the sensitivity of the system is $10.8 \mathrm{mV} / \mathrm{Pa}$, and the minimum detectable signal is $6.5 \mathrm{mPa}$. A similar technique is used to measure the frequency response function of the sensor, and the sensor resonance is experimentally determined to be $4.6 \mathrm{kHz}$. With this characterization, the sensor is transitioned into two wind tunnel facilities for validation.

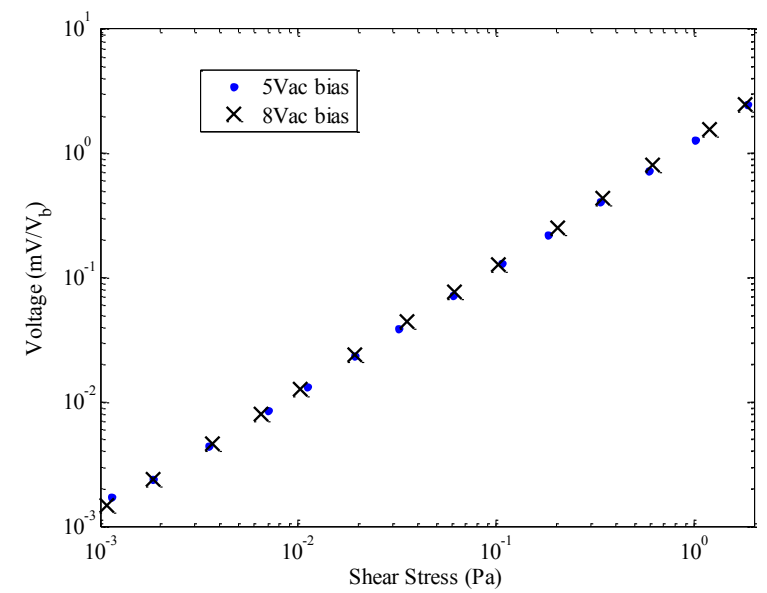

Figure 6: The normalized sensitivity of the shear stress measurement system.

\section{EXPERIMENTAL SETUP}

The sensor is installed into two different wind tunnel facilities; one small-scale wind tunnel at the University of Florida (UF) and the 20" x 28" shear flow control facility at NASA Langley Research Center (LaRC). At UF, the sensor is installed into an elliptical leading-edge flat plate airfoil. The flow over the airfoil is untripped and, for a wide range of Reynolds number, remains laminar. A velocity profile is acquired using particle image velocimetry (PIV) and fit to Blasius' solution [7] in order to educe the mean shear stress acting on the sensor. At LaRC the sensor is again installed into a flat plate, and the flow is tripped, resulting in a zero-pressure-gradient turbulent boundary layer at the sensor location. A traversing hotwire is used to acquire a velocity profile, which is fit to Musker's equation [8] in order to estimate the mean sear stress acting on the surface. Additionally, a hotwire is placed near the wall in the viscous sublayer in order to estimate the dynamic shear stress content of the flow. The result is an estimation of the dynamic shear stress based on the velocity field within the viscous sublayer. This provides a comparison for magnitude and frequency content of the dynamic sensor data.

\section{RESULTS AND CONCLUSIONS}

In Figure 7 , the results of the velocity-profile-based estimation of mean shear stress are compared to the results of the MEMS floating-element based estimation of shear stress, with a line indicating perfect correlation. The sensor shows good agreement to current velocity-profile-based mean shear stress estimation technologies. There are many possibilities that would explain deviations from this line: additional environmental sensitivities, effects of the wirebonds protruding into the flow, and the feed-through of pressure signals into the sensor output. With further bench top and tunnel characterization these effects can be isolated 


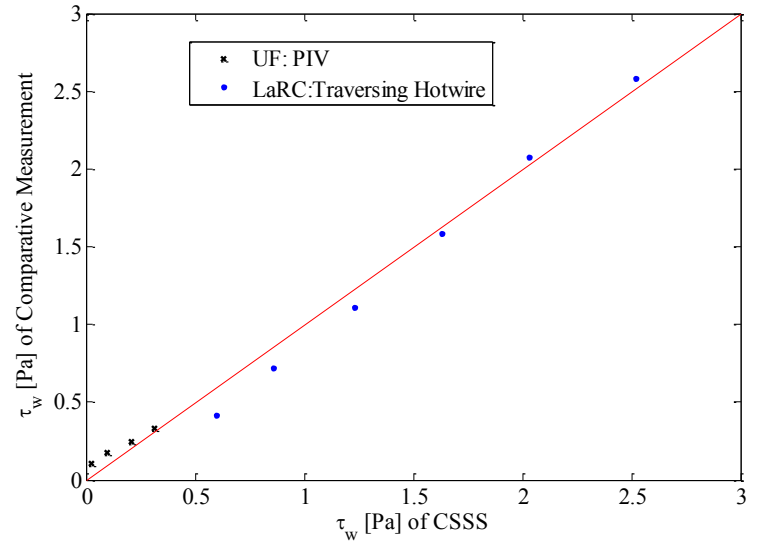

Figure 7: A comparison of the mean shear stress determined by velocity based methods compared to the mean sensor data with a line indicating perfect agreement.

The dynamic shear stress can be estimated by a near wall hotwire. If the hotwire is located within the viscous sublayer of the boundary layer the velocity at a known height can be related to the dynamic shear stress on the surface by

$$
\tau_{\text {ac }}(\mathrm{t})=\frac{\mathrm{v}_{\mathrm{hw}}(\mathrm{t}) v}{\mathrm{~h}}
$$

where $\mathrm{v}_{\mathrm{hw}}$ is the velocity determined by the hotwire, $v$ is the kinematic viscosity, and $\mathrm{h}$ is the height of the hotwire. Figure 8 shows the autospectra of the dynamic shear stress determined by the sensor and the near wall hotwire at a freestream velocity of $43 \mathrm{~m} / \mathrm{s}$ and $\operatorname{Re}_{\theta}$ of 13,500 at NASA LaRC. The apparent offset between the two datasets is most likely an error in the estimation of the hotwire placement, which resulted in the hotwire being just outside of the viscous sublayer ( $<52 \mu \mathrm{m}$ from the wall) at a height of $82 \mu \mathrm{m}$ above the wall.

The agreement in both mean and dynamic performance of the sensor compared to traditional boundary layer measurement techniques demonstrates that the capacitive shear stress sensor system developed in this work is capable of being transitioned into multiple facilities and serving as a direct measurement tool for laminar and turbulent boundary layers.

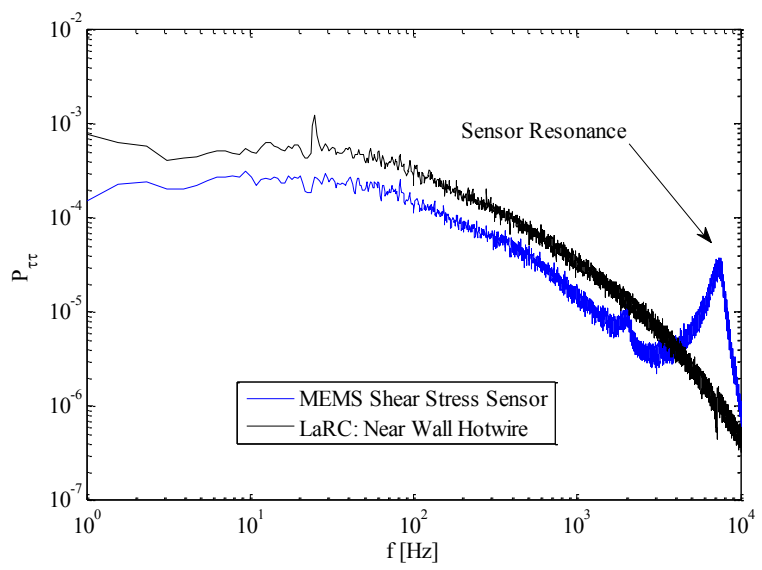

Figure 8: Qualitative comparison of the dynamic shear stress.

\section{ACKNOWLEDGMENTS}

Support for this work has been provided by NASA Langley Research Center Grant NNX07AB27A, monitored by C.B. McGinley, NASA Graduate Student Researchers Program, FCAAP, and AFOSR Grant FA9550-09-1-0257, monitored by D. Smith. The authors would like to thank the Nano Research Facility for help with fabrication and George Beeler and Latunia Melton for assistance at LaRC. Travel support has been provided by the Transducer Research Foundation.

\section{REFERENCES}

[1] M. Gad-el-Hak, "Flow control : passive, active, and reactive flow management," Cambridge, MA: Cambridge University Press, 2000, pp. 209-210.

[2] J. W. Naughton and M. Sheplak, "Modern developments in shear-stress measurement," Progress in Aerospace Sciences, vol. 38, 2002.

[3] J. Haritonidis, "The Measurement Of Wall Shear Stress," Advances in Fluid Mechanics Measurements, pp. 229261, 1989.

[4] V. Chandrasekharan, J. Sells, J. Meloy, D. P. Arnold, and M. Sheplak, "A Microscale Differential Capacitive Direct Wall-Shear-Stress Sensor," Journal of

Microelectromechanical Systems, vol. 20, no. 3, pp. 622635, 2011.

[5] B. P. Lathi, Modern Digital and Analog Communication Systems, 5th ed. Oxford University Press, 1998, pp. 233235.

[6] V. Chandrasekaran, A. Cain, T. Nishida, L. N. Cattafesta, and M. Sheplak, "Dynamic calibration technique for thermal shear-stress sensors with mean flow," Experiments in Fluids, vol. 39, no. 1, pp. 56-65, 2005.

[7] H. Blasius, "Grenzschichten in Flussikeiten mit kleiner Reibung,” Z. Angew. Math. Phys., vol. 56, pp. 1-37, 1908.

[8] A. J. Musker, "Explicit Expression for the Smooth Wall Velocity Distribution in a Turbulent Boundary Layer," AIAA Journal, vol. 17, no. 6, pp. 655-657, 1979.

\section{CONTACT}

* M. Sheplak, tel: +1-352-392-3983; sheplak@ufl.edu

${ }^{1}$ currently with Hewlett-Packard Company, Corvallis, OR USA

${ }^{2}$ currently with Digital Optics Corporation, Arcadia, CA USA

${ }^{3}$ currently with Florida State University, Tallahassee, FL, USA 


\title{
FORCED OSCILLATION AND HIGHER HARMONIC DETECTION IN AN INTEGRATED CMOS-MEMS SCANNING PROBE MICROSCOPE
}

\author{
N. Sarkar ${ }^{1 *}$, R. R. Mansour ${ }^{l}$ and K. Trainor ${ }^{l}$ \\ ${ }^{1}$ University of Waterloo, Waterloo, Ontario, Canada
}

\begin{abstract}
We present the first imaging results obtained with a MEMS scanning probe microscope (SPM) that does not require external scanning hardware, and uses forced oscillation and higherharmonic detection to eliminate thermal coupling. This SPM supports multiple modes of operation including contact AFM (atomic force microscopy), intermittent-contact AFM, and thermal profiling. The focus of this paper is on the intermittent-contact mode, in which we are interested in measuring topology while suppressing thermal effects. The method has been implemented with three SPM-on-a-chip designs, and consistently improves resolution.
\end{abstract}

\section{INTRODUCTION}

\section{Motivation}

The goal of this work is to miniaturize bulky piezoelectric positioning systems that suffer from thermal drift, poor vibration immunity, and creep by replacing them with MEMS electrothermal actuators with integrated position sensors. We also replace laserbased deflection sensing with integrated polysilicon strain sensors. This permits array implementations, and dramatically lowers the cost of SPM's. One of the challenges in the development of our CMOS-MEMS SPM's is that interaction between electrical, thermal and mechanical domains obscures the desired measurement of tip-sample interaction. In particular, the piezoresistive strain sensors that are used to measure tip-sample interaction forces have a large TCR (temperature coefficient of resistivity). This problem is exacerbated by the use of thermal actuation, which results in a large range of operating temperatures. In addition, high temperatures result in unwanted actuation of the strain sensors and this contributes to the tip-sample interaction signal. We therefore seek a method to extract only the tip-sample interaction component of a signal that contains various thermally induced parasitics.

\section{Review}

In [1], we reported the first CMOS-MEMS contact-mode AFM with integrated actuation and position/force sensing in 3-D. The resolution was compromised by thermal coupling from actuation because of the high temperature coefficient of resistivity (TCR) of the polysilicon strain sensors. Frequent thermal calibration and post-processing of the data was required in order to extract the sample topology from the cantilever signal.

The first micromachined STM consisted of a piezoelectric bimorph with patterned electrodes [2]. Another STM was fabricated in single crystal silicon [3]. These works demonstrated open-loop 3-axis positioning only, with no force-sensing capability. More recently the co-fabrication of control electronics with an array of single-axis cantilevers for AFM was demonstrated [4]. Another example of a single-axis cantilever with integrated position sensing is the Akiyama probe [5]. In [6], a thermal profiler with integrated 1-D actuation was presented. Our work was the first to include 3-axis positioning, strain sensors, and position sensors (all the mechanical components required for an SPM to acquire an image) in a single device.

\section{Detection Method}

Figure 1 is a system diagram of the method that is used to separate the tip-sample interaction component from the overall signal. A small-signal sinusoidal input with a DC offset $V$ is applied to the Z-actuator. Heat from actuation couples to the piezoresistors, producing effects in the electrical (due to TCR) and mechanical (due to stress induced by the thermal bimorph effect) domains. The balanced cantilevers are biased with a DC voltage that is also applied to an off-chip trimming potentiometer. This balanced configuration rejects a fraction of the parasitic thermal coupling, but several asymmetries in the design remain and contribute thermal parasitics.

We focus on the case in which the tip is in intermittent contact with the sample due to the periodic excitation of the Z-actuator. At the point of contact, abrupt changes occur in the mechanical and thermal boundary conditions at the tip, generating higher harmonic terms. An instrumentation amplifier follows the bridge, and its output is demodulated using a pair of off-the-shelf modulatordemodulator IC's. The demodulation frequency is chosen at a harmonic that is above the thermal cutoff frequency. We hold the harmonic power constant by adjusting the Z-offset voltage (hence tracking topology) while scanning laterally in $\mathrm{X}$ and $\mathrm{Y}$. In this paper, we do not present imaging results using the phase component of the signal.

This configuration rejects thermal parasitics from the TCR of the piezoresistors. Since we maintain a constant tip-height separation during the scan, the controller intrinsically compensates for the thermal-mechanical coupling between the lateral and

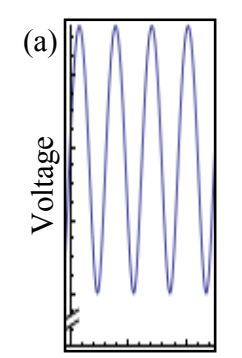

time

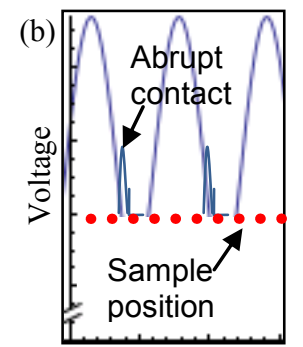

time

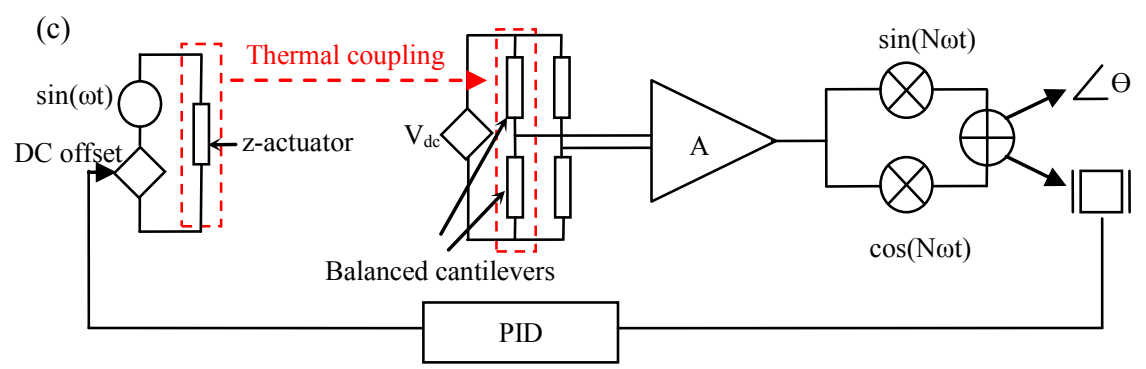

Figure 1: (a) z-actuator driving signal is sinusoidal with a DC offset, (b) piezoresistive signal is clipped by intermittent contact with the sample, and (c) diagram of the measurement setup. MEMS devices are enclosed in red. The z-actuator is oscillated at the fundamental frequency, and a DC offset is used to control the tip-sample gap to control the measured power in the $N^{\text {th }}$ harmonic. 
vertical actuators of the device as well. The result is an image which is tilted on a plane that represents the average temperature of the lateral scanners. This tilt is easily removed with a linear coordinate transformation.

\section{DEVICE DESIGN}

\section{Process}

CMOS-MEMS processes were introduced in the 1980's and the field has grown substantially over the past two decades. [7] These processes are well-suited to integrated SPM device fabrication because they allow for complex signal routing, various types of actuation and sensing methods, and integrated electronics. Some examples of CMOS cantilever sensor systems that exploit the various material properties available in CMOS-MEMS processes are discussed in [8].

Our fabrication process is similar to the post CMOS-MEMS processes, with the optional insertion of two additional maskless process steps. First, grooves are diced or laser-etched into the backside of the wafer to suspend cantilevers over the die edge upon release. The second step removes polymer buildup that occurs during the $\mathrm{SiO}_{2}$ etch using EKC.

There are several process features that are advantageous in the design of CMOS-MEMS SPM's:

In the electrical domain, the designer can choose between conductive (Al), insulating $\left(\mathrm{SiO}_{2}\right)$, and semiconducting (polysilicon) materials. This allows for complex routing of signals in a monolithic beam. It also allows for the judicious placement of resistive heat sources and temperature sensors. The temperature coefficient of resistivity of polysilicon materials can be exploited to measure local temperatures, a feature that is used in our work.

The thermal conductivity of $\mathrm{Al}$ is $191 \mathrm{Wm}^{-1} \mathrm{~K}^{-1}$ while that of $\mathrm{SiO}_{2}$ is $1.4 \mathrm{Wm}^{-1} \mathrm{~K}^{-1}$. This enables the design of thermally isolating members that can be used to separate heat sources from nearby regions that are mechanically and electrically interconnected. Another feature is that the polysilicon-metal contacts in CMOSMEMS processes are thermocouples that can be used as local temperature sensors.

In the mechanical domain, the thermal expansion coefficients (TCE) of $\mathrm{Al}$ and $\mathrm{SiO}_{2}$ are disparate as well. The TCE of $\mathrm{Al}$ is $14.6+/-3.0 \times 10^{-6} \mathrm{~K}^{-1}$ and the TCE of $\mathrm{SiO}_{2}$ is $0.4-1.0 \times 10^{-6} \mathrm{~K}^{-1}$. This feature is desirable in the design of lateral and vertical electrothermal bimorph actuators. Further discussion of the mechanical material properties and how they relate to electrothermal actuator design can be found in [9].

Although a wide variety of material properties are available to the designer, they are seldom optimized to improve SPM performance. For instance, the performance of CMOS electronics improves with low TCR whereas the design of temperature-based position sensors improves with high TCR. Another example is that piezoresistive cantilevers benefit from a high gauge factor, while it is desirable to suppress strain effects in CMOS.

\section{Geometry}

Examples of device geometries are shown in figures 2-4. All three devices contain a balanced pair of piezoresistive cantilevers and various lateral actuator layouts. In figure 2, the lateral actuators are orthogonally arranged and coupled using flexures in an arrangement that cancels the torque applied to the central shuttle. This geometry requires a sample to be lowered onto the chip surface, making it difficult to observe the tip-sample region under an optical microscope. In addition, the flexures are compliant in the z-direction, reducing the strain that is captured by the piezoresistors.

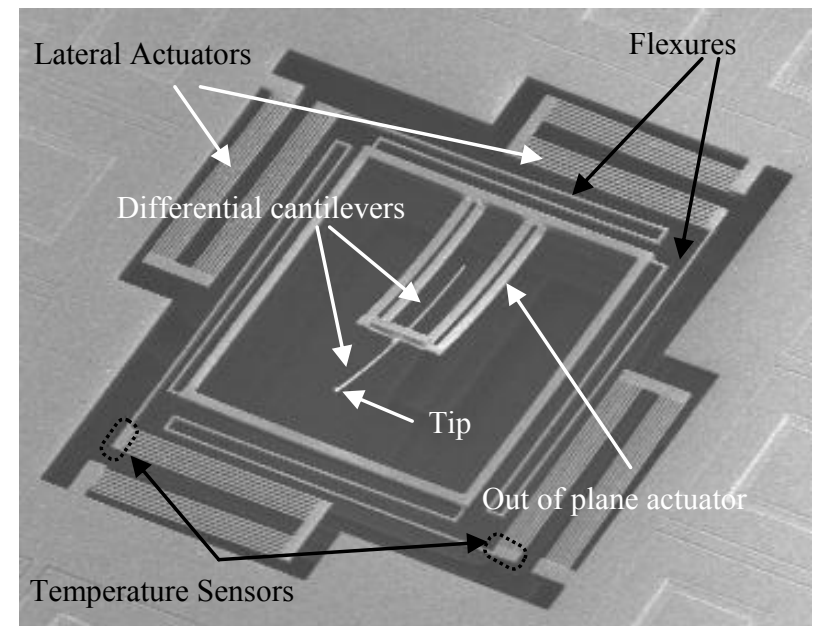

Figure 2: Orthogonally arranged lateral actuators are used to laterally scan the central shuttle which houses the vertical actuator and a balanced pair of cantilevers.

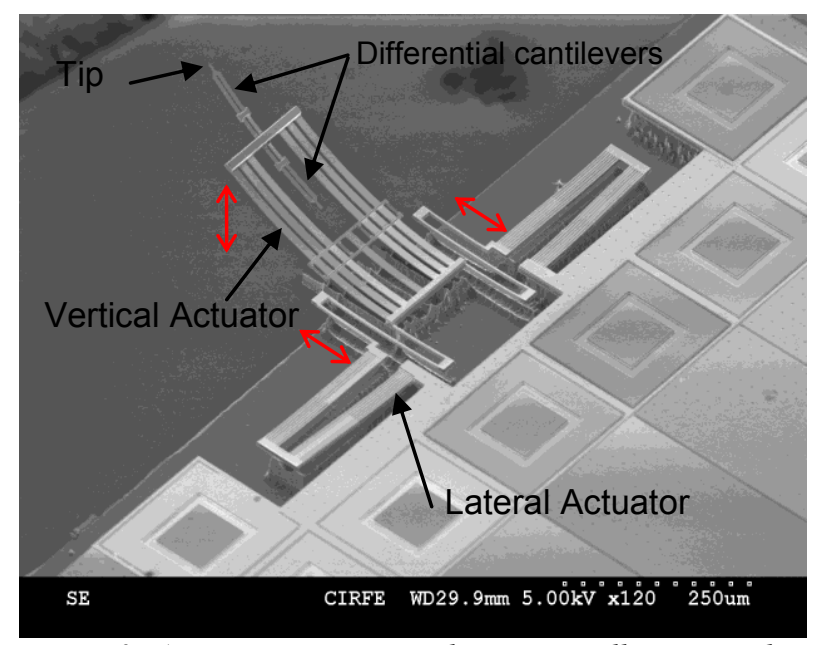

Figure 3: Actuators are arranged symmetrically to scan the tip in spherical coordinates. The tip is suspended over the edge of the chip for observation under an optical microscope.

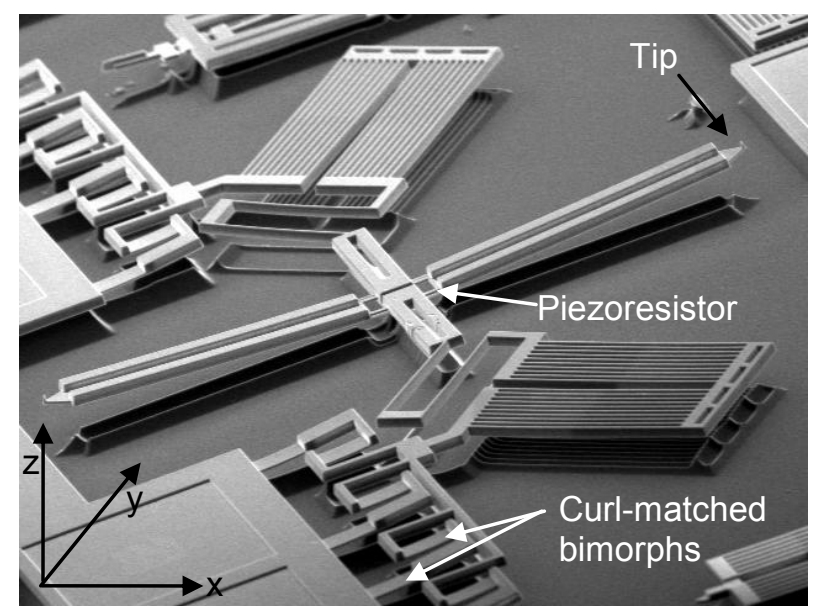

Figure 4: Perspective view of a Cartesian coordinate scanner with an extended cantilever. Z-direction actuation is achieved with a curl-matched bimorph design. 
In figure 3 , the cantilever extends $\sim 300 \mathrm{~m}$ past the edge of the die. This geometry permits observation of the tip-sample region while imaging. Another feature of this geometry is that the average temperature during a lateral scan line can be held constant. This is achieved by applying a DC bias and a differential AC signal to the left and right actuators. A non-linear inversion is applied to the sum of the DC and AC signals in order to compensate for the $\mathrm{V}^{2}$ dependence of the actuators' position and temperature. The result is a spherical coordinate scan $(r, \varphi, \theta)$ in which the angle $\varphi$ is related to the difference between the left and right actuator deflections, the radius $r$ is proportional to the average of the left and right actuator deflectionsand the elevation $\theta$ is provided by the out-of-plane bimorph.

In figure 3, the cantilever is designed to extend $\sim 200 \mathrm{~m}$ past the die edge. The chip presented here has not yet been singulated. This geometry scans in Cartesian coordinates; the Z-actuator is curl-matched to cancel arcuate motion that is usually encountered with out-of-plane bimorph designs.

Figure 5 is a lumped element model (of the device in Figure 2) that considers effects in the electrical, thermal and mechanical domains. The model follows conventions from [10] and is founded on the work described in [11]. Of particular interest in the design of multi degree-of-freedom SPM scanners is the nature of the interconnection between the various actuators in the system. For example, in the thermal domain, the $\mathrm{X}$ and $\mathrm{Y}$ actuators have a direct conductive path to thermal ground, whereas the $\mathrm{Z}$ actuator and balanced cantilevers do not (neglecting air). The non-linearity of the tip-sample interaction is modeled using a voltage-controlled switch, with a threshold corresponding to the sample height.

\section{MEASUREMENTS}

The separation of the tip-sample force component of the signal from the parasitic thermo-electric and thermo-mechanical components is clearly visible in the frequency domain. Figure 6 presents the frequency spectrum of the cantilever signal when there is no sample present. The a.c. component of the driving signal is a square-root-sinusoid at $200 \mathrm{~Hz}$, which is squared by the actuator's transfer function to obtain the single peak visible in the plot. In figure 7, the tip enters intermittent contact with the sample and effectively clips the signal from the balanced piezoresistors. Higher harmonics that are well beyond the thermal cutoff frequency appear in the frequency spectrum, and are used to control the tip-sample separation in practice. The plots were obtained with a Zurich Instruments lock-in amplifier.

Images of a calibration grating were obtained using the device in figure 2. The calibration grating geometry is inset in figure 8 . These images were obtained without the use of a lock-in amplifier, and with no post-processing other than tilt-compensation implemented in realtime in the Labview envirionment.

This approach has several advantages over the contact mode implemented in [1]. Higher-harmonic detection methods are capable of revealing material properties beyond topology [12].

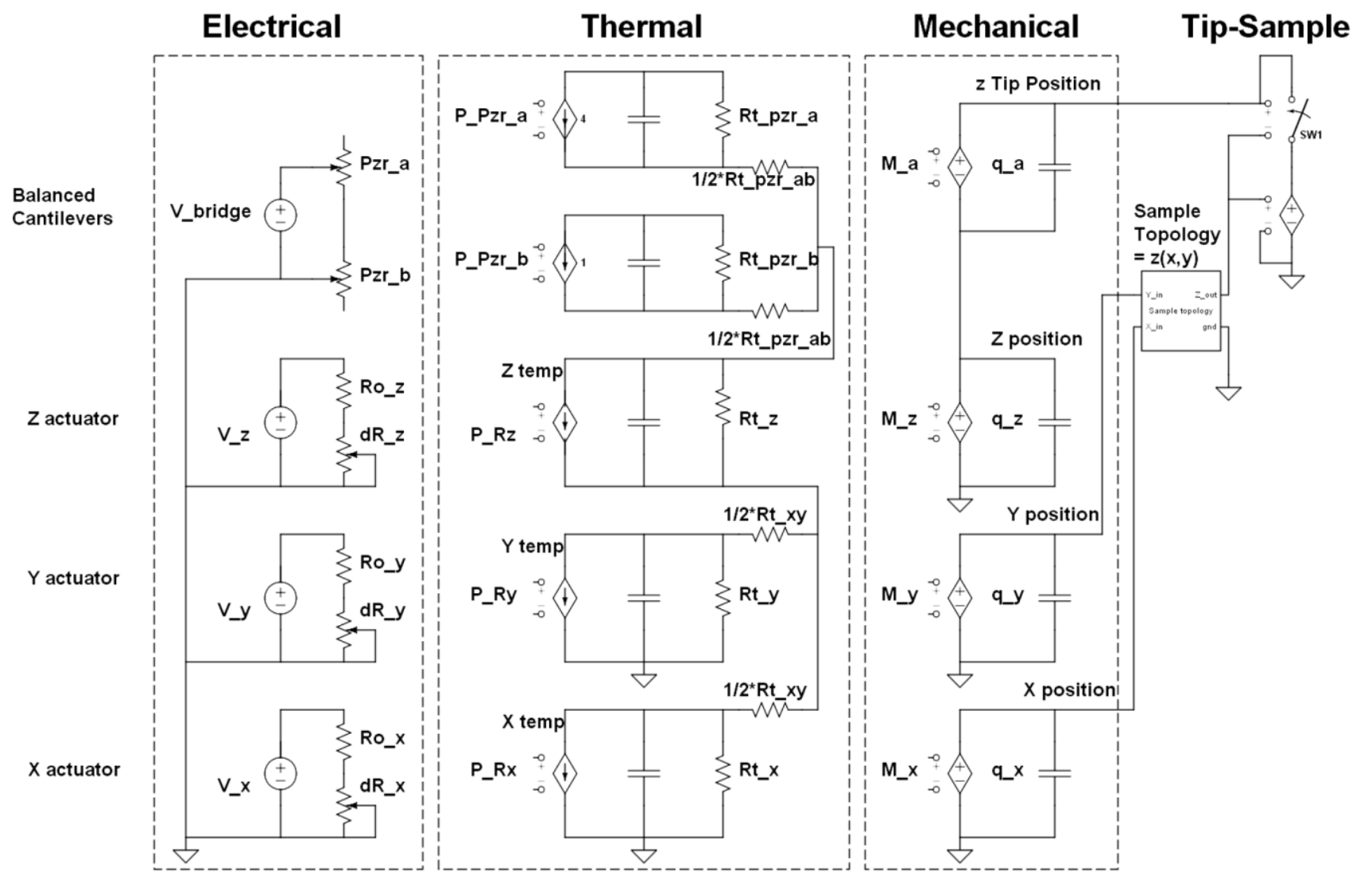

Figure 5: Lumped element model of a CMOS-MEMS SPM in the electrical, thermal and mechanical domains. In the electrical domain, Ro_*labels are electrical resistances and $d R_{-}^{*}$ labels account for the TCR. In the thermal domain, $P_{-}^{*}$ labels represent electrical power converted to heat, and $R t_{-}^{*}$ labels are thermal resistances. In the mechanical domain, $M_{-}^{*}$ labels correspond to thermal moments and $q_{-}^{*}$ labels represent the charge stored on capacitors (analogous to the displacement of beams). The non-linear tip-sample interaction is modeled with a voltage-controlled switch that "shorts" the tip position to the sample's z-height. 


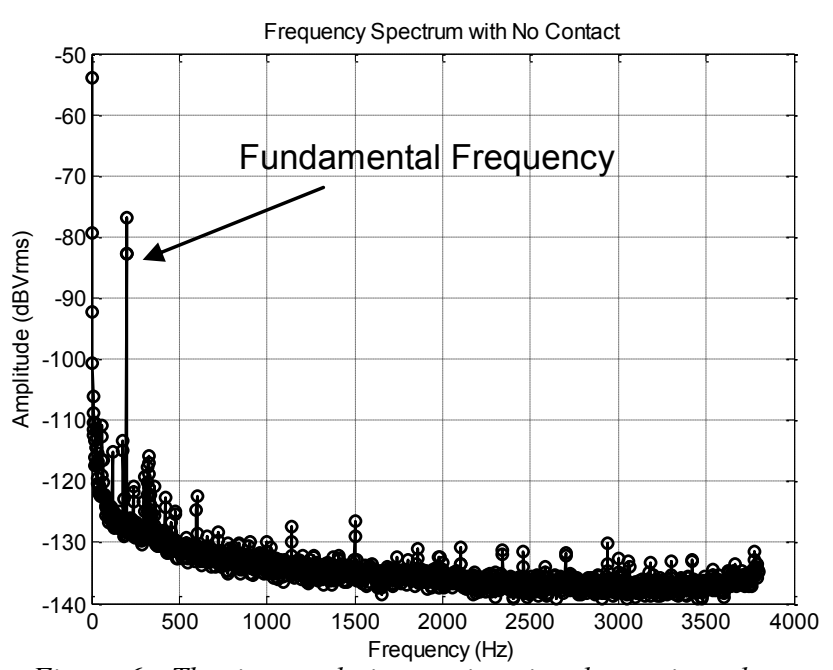

Figure 6: The tip sample-interaction signal contains a large parasitic thermal component at the fundamental frequency.

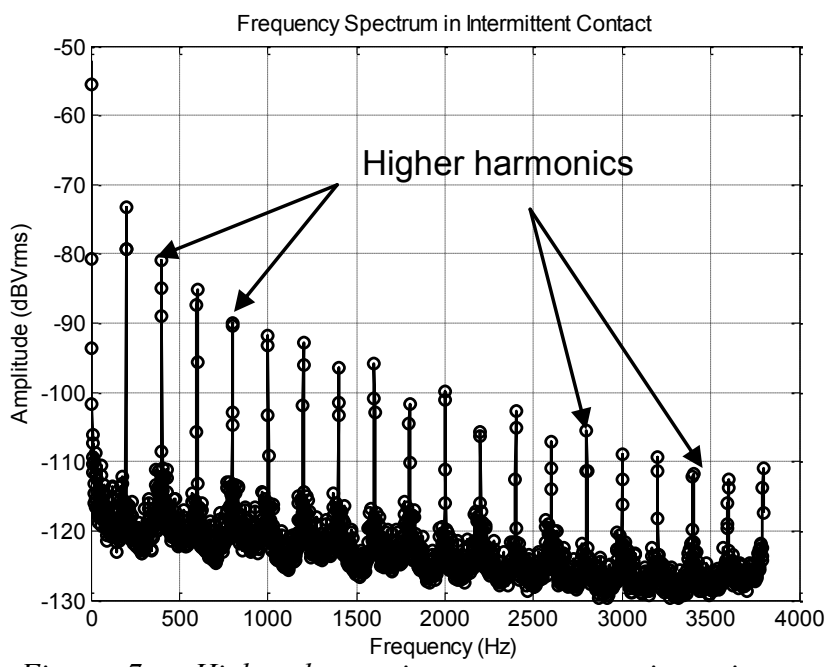

Figure 7: Higher harmonics appear upon intermittent contact.
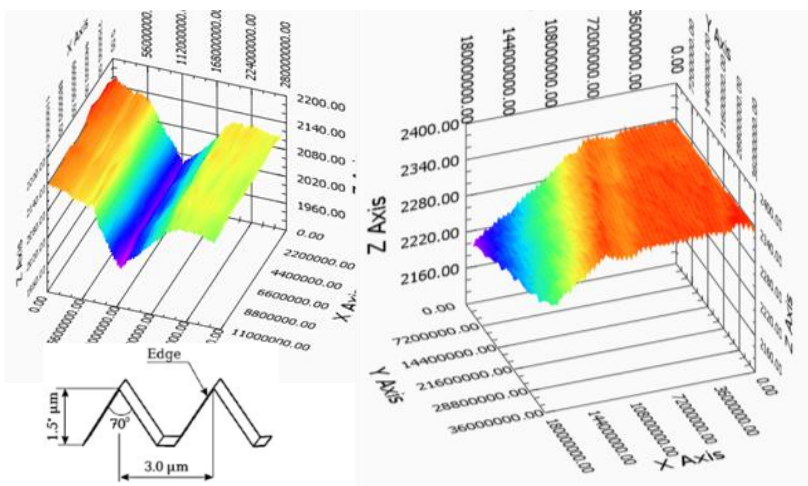

Figure 8: Images of an AFM calibration grating with an inset describing the geometry

This method also permits operation in liquids and does not require Q-control to improve imaging bandwidth [13]. Mechanical modes of the cantilever need not be matched to the harmonic frequencies. The mixer circuit is simple and inexpensive since the cantilever is not in resonance. Tip-sample forces are limited by reducing the higher-harmonic amplitude or adjusting phase to operate in the attractive regime. We have observed that small-amplitude oscillation also eliminates lateral stiction with the sample, which is an issue in the contact mode.

\section{CONCLUSION}

The integration of scanning probe microscopes on-chip has the potential to reduce their cost as well as improve their performance when compared to the state-of-the-art. Electrothermal actuation provides low-voltage (CMOS-compatible) and large scan range; however it introduces parasitics to the tip-sample interaction signal. Higher harmonic detection has been demonstrated as a method to mitigate this issue.

\section{REFERENCES}

[1] N. Sarkar et. al., "CMOS-MEMS Atomic Force Microscope," TRANSDUCERS 2011, pp. 2610-2613.

[2] Akamine, S. et al.,"Microfabricated Scanning Tunneling Microscope," IEEE Electron Device Letters, V. 10, No 11, 1989.

[3] $\mathrm{Xu}, \mathrm{Y}$. et al. "Integrated micro-scanning tunneling microscope," Appl. Phys. Lett. 67 (16), 1995.

[4] D. Barrettino, et al., "CMOS Monolithic Atomic Force Microscope," 2004 Symposium On VLS1 Circuits Digest of Technical Papers, pp. 306-309.

[5] http://www.akiyamaprobe.com

[6] Gianchandani, Y. B. et al, "A Silicon Micromachined Scanning Thermal Profiler with Integrated Elements for Sensing and Actuation," IEEE Trans. Elec. Dev., v 44, no 11, pp 1857-1868.

[7] Baltes, Brand, Fedder, Hierold, Korvink, Tabata, "CMOSMEMS," volume 2, Wiley, 2004.

[8] Lange, Brand, Baltes, "CMOS Cantilever Sensor Systems,' Springer, 2002.

[9] Gilgunn, P.J.et al., "CMOS-MEMS Lateral Electrothermal Actuators," J. Microelectromech. Syst., 17 (2006), pp. 103 114.

[10] Senturia, S.D., "Microsystem Design," Kluwer, 2001.

[11] Todd, S. et. al., "An electrothermomechanical lumped element model of an electrothermal bimorph actuator," J. Microelectromech. Syst., 17, (2008), pp. 213-225.

[12] Sahin, O. et al, Springer Handbook of Nanotechnology, p.717 , 2007.

[13] "High-speed tapping mode imaging with active Q-control for AFM,” T. Sulchek et. al., Appl. Phys. Lett. 76 (1473), 2000.

\section{ACKNOWLEDGEMENTS}

The authors would like to acknowledge the support of TowerJAZZ for their donation of fabrication area, and the Canadian Microelectronics Corporation. This work is supported by DARPA, SPAWARSYSCEN-SD, and the Texas ETF under contract N66001-08-C-2040.

\section{CONTACT}

*N. Sarkar, tel: +1-519-888-4567; nsarkar@uwaterloo.ca 


\section{HIGHLY SENSITIVE III-V NITRIDE BASED PIEZORESISTIVE MICROCANTILEVER USING EMBEDDED ALGAN/GAN HFET Abdul Talukdar ${ }^{1 *}$ and Goutam Koley ${ }^{l}$ \\ ${ }^{1}$ University of South Carolina, Columbia, South Carolina, USA}

\begin{abstract}
In this article, for the first time, a high gauge factor of 3532 is reported using $\mathrm{AlGaN} / \mathrm{GaN}$ Heterostructure Field Effect Transistor (HFET) embedded GaN piezoresistive microcantilever. In addition, deflection transduction signal from the HFET was used to determine dynamic bending (up to $40 \mathrm{~Hz}$ frequency) and $\mathrm{AC}$ response of the cantilever, and also reported for the first time.
\end{abstract}

\section{INTRODUCTION}

Microcantilevers are very attractive for detection of chemicals, explosives, and biological molecules [1-3] due to their high sensitivity and low power consumption. Though optical transduction is widely used and accurate method for transducing mechanical movement of the microcantilever into an electrical signal, piezoresistive and piezoelectric transductions have been demonstrated in order to miniaturize the sensor element. Most of the microcantilever based sensors and their piezoresistive transduction systems are silicon based $[4,5]$, which is not suitable for operating in harsh environments. III-V Nitride semiconductors have attracted wide research interest in optoelectronic and high power microwave devices because of their exceptional physical and chemical properties, which include a wide range of bandgap, high electron mobility, and high breakdown field [6]. Additionally, $\mathrm{GaN}$ related materials exhibit high mechanical and thermal stability coupled with piezoelectric (as well as piezoresistive) properties which make them strong candidates for miniaturized sensors for harsh environments (e.g. automotive, aviation) [7]. The piezoreistive and piezoelectric properties of $\mathrm{AlGaN} / \mathrm{GaN}$ heterostructure is highly attractive for applications in microelectromechanical systems (MEMS), as it can cause large variation in 2-dimensional electron gas (2DEG) at the interface with mechanical strain. The mechanical strain caused by different factors including hydrostatic pressure, biaxial deformation and microcantilever bending [8-10] on 2DEG (and hence the conductivity) and the output characteristics of $\mathrm{AlGaN} / \mathrm{GaN}$ heterostructure field effect transistor (HFET) has already been studied experimentally and theoretically [9-12]. Therefore, $\mathrm{AlGaN} / \mathrm{GaN}$ system can be a good alternative as material and transduction system for developing microcantilever based ultra high detection system. Additionally selective and sensitive detection in a standoff fashion is of great interest at present, with application ranging from homeland security to medical imaging. For example, photoacoustic spectroscopy (PAS) is a standoff method based on detecting acoustic disturbances caused by the infrared absorptions by molecules. PAS can be used to quickly detect very low concentrations. The whole system can be miniaturized to have portable systems capable to in-situ measurements. The photoacoustic system measures absorbed energy and so it is less immune to the background signal in the absence of the sample gas. The sensitivity of the system can be significantly increased by replacing the conventional microphone with a microfabricated cantilever [13]. Previously standard atomic force microscope (AFM) microcantilevers were used as sensitive microscale detectors for photoacoustic signals [14] which seem to be impractical considering AFM setup.

In this article, for the first time, sensing performance of a $\mathrm{GaN}$ piezoresistive microcantilever embedded with $\mathrm{AlGaN} / \mathrm{GaN}$
Heterostructure Field Effect Transistor (HFET) is demonstrated which reports a high gauge factor of 3532. In addition, deflection transduction signal from the HFET was showed for the first time to determine dynamic bending (up to $40 \mathrm{~Hz}$ frequency), AC response of the cantilever, and also acoustic wave resonance which detected vibration amplitude of $22 \mathrm{~nm}$ when the sensor was placed $5 \mathrm{~cm}$ away from an ultrasonic source.

\section{FABRICATION OF MICROCANTILEVER}

A (4" by 4") AlGaN/GaN wafer grown on Silicon (111) substrate was purchased from Nitronex Corporation, USA for this work. The wafer was diced into $32(1.4 \mathrm{~cm}$ by $1.4 \mathrm{~cm})$ pieces. Before dicing, the wafer was spin coated with photo resist (Shipley 1827) and then baked for 5 mins at $110{ }^{\circ} \mathrm{C}$. This is done to avoid contaminating the wafer surface with dust particles generated during dicing. The different layers of the wafer are shown in Fig.1. A $1.1 \mathrm{~m}$ thick layer consisting of $\mathrm{AlN}$ and $\mathrm{AlGaN}$ graded layer was used as a transition layer before growing 1 m undoped GaN layer. This transition layer along with the undoped $\mathrm{GaN}$ form the thickness of our microcantilevers. On the top of the GaN layer, AlGaN barrier layer of $17.5 \mathrm{~nm}$ thickness was grown (see Fig. 1 for different layers). The Aluminum mole fraction was $x=0.26$. A $2 \mathrm{~nm}$ undoped $\mathrm{GaN}$ cap layer was grown on that. $\mathrm{BCl}_{3} / \mathrm{Cl}_{2}$ based dry etching recipe of $\mathrm{GaN}$ in Inductively Coupled Plasma (ICP) etcher to isolate mesa. $150-200 \mathrm{~nm}$ etch was performed to ensure complete isolation. Same recipe was used for both the AlGaN and $\mathrm{GaN}$ etch. The height was verified by Tencor Profilometer. GaN is etched down in the pocket area up to the substrate where silicon acts as the etch stop layer. Here the etching thickness defines the cantilever thickness. To protect the $2 \mathrm{DEG}$ of the mesa, a $1 \mathrm{~m}$ thick PECVD $\mathrm{SiO}_{2}$ was deposited which was eventually etched away by buffer oxide etch (BOE). Ti(20 nm)/Al(100 nm)/Ti(45 $\mathrm{nm}) / \mathrm{Au}(55 \mathrm{~nm})$ metal stack deposition and rapid thermal annealing $\left(800^{\circ} \mathrm{C}\right.$ for $\left.60 \mathrm{~s}\right)$ were done for ohmic contact formation. For gate contact, Ni $(25 \mathrm{~nm}) / \mathrm{Au}(375 \mathrm{~nm})$ Schottky barrier was used. The GaN cantilever pattern was etched down by inductively coupled plasma (ICP) etch process $\left(\mathrm{Cl}_{2}: 32 \mathrm{sccm}, \mathrm{BCl}_{3}: 8 \mathrm{sccm}\right.$, Ar: 5 sccm; $\mathrm{RF}_{1}$ : $70 \mathrm{~W}, \mathrm{RF}_{2}$ : $\left.500 \mathrm{~W}\right)$. Through wafer Si etching was performed by anisotropic silicon etching from the bottom side (Bosch process). The fabricated microcantilever dimension is $350 \times 50 \times 2 \mu \mathrm{m}$.

\begin{tabular}{|c|}
\hline $20 \AA$ i-GaN \\
\hline $175 \AA$ AlGaN, $26 \%$ Al \\
\hline $1 \mu \mathrm{m}$ i-GaN \\
\hline $\begin{array}{c}1.1 \mu \mathrm{m} \text { AIN/(Al)GaN } \\
(\text { Transition Layer) }\end{array}$ \\
\hline $500 \mu \mathrm{m}$ Si(111) \\
substrate
\end{tabular}

Fig. 1: Different layers of the AlGaN/GaN wafer grown on Si (111) used in this work.

The complete package of the fabricated sample is shown in Fig. 3. 

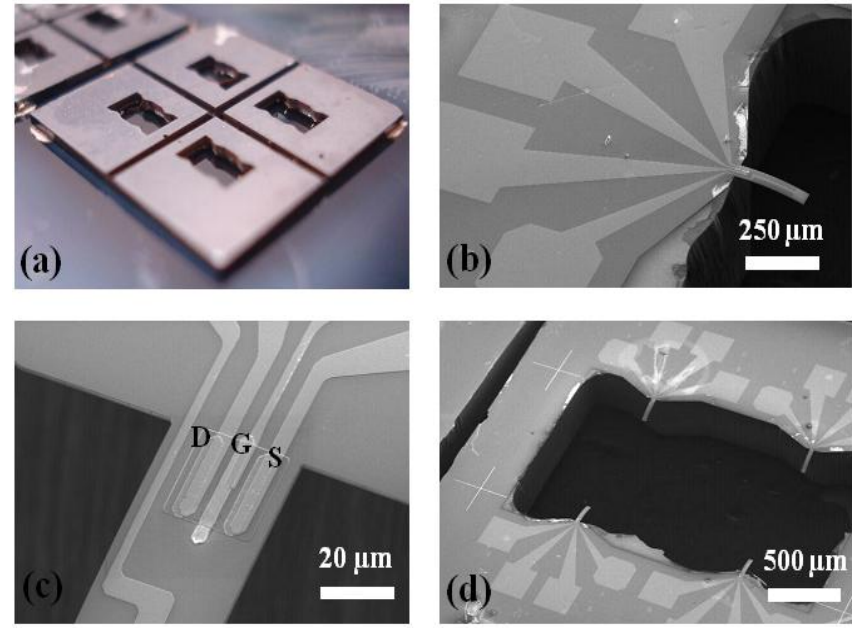

Fig. 2: (a) Image of the sample whic is auto diced into four smaller samples, SEM image of (b) One single microcantilever device with four large bonding pads for drain, gate, source and tip, (c) AlGaN/GaN HFET, (d) a pocket containing four microcantilever devices.

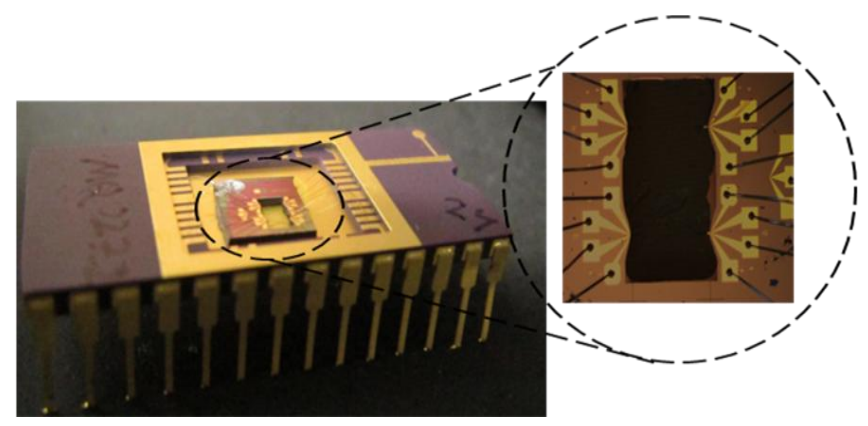

Fig. 3: The complete package of the fabricated sample with wire bonded devices in zoomed view (four microcantilevers).

\section{EXPERIMENTAL SETUP}

The bending experiments on the cantilevers were performed using a Piezo-based Z-axis Nanopositioner purchased from Physik

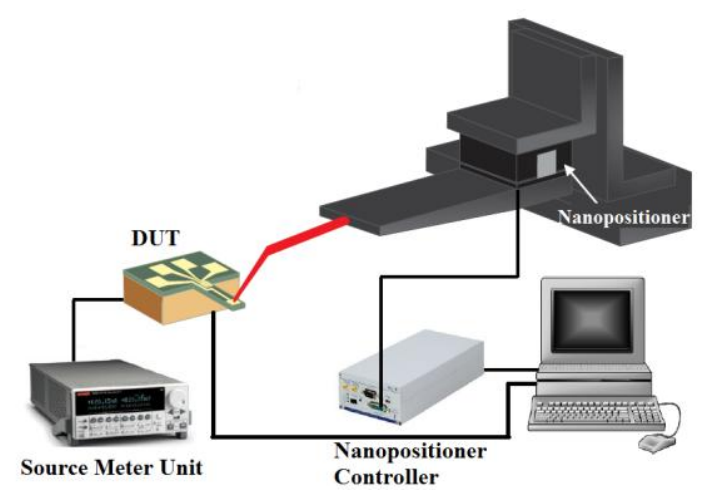

Fig. 4: Schematics of the experimental setup for static and dynamic bending.

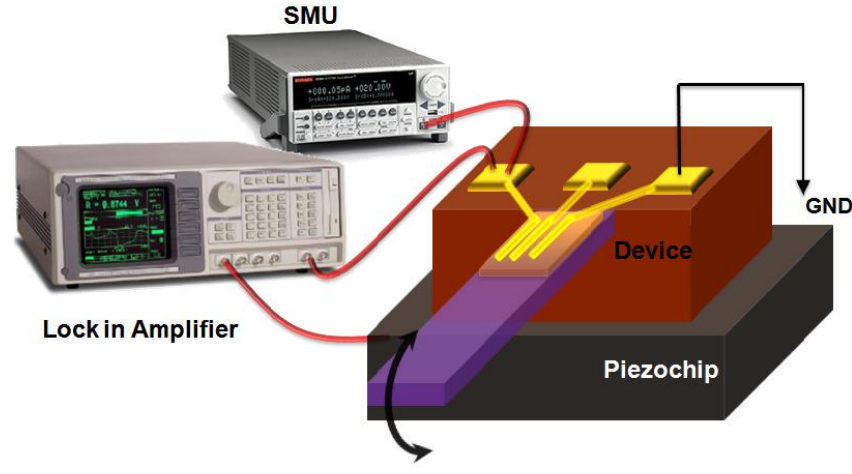

Fig. 5: Schematics of the experimental setup for AC measurement

Instrumente, PI (PI-611 Z) (see Fig. 4). The nanopositioner was attached upside down with a XYZ translation stage (purchased from Thorlabs. Inc.) to allow coarse maneuverability in three axes. The nanopositioner has range of up to $100 \mathrm{~m}$ movement with a closed loop resolution of $2 \mathrm{~nm}$. A micropositioner needle (tip diameter $12 \mathrm{~m}$ ) was attached to the end of the nanopositioner. The nanopositioner is controlled from a computer interface designed in LabView. HFET dc current measurements were performed using Keithley Sourcemeter (2612A). The dc drain current was measured in ambient lighting conditions. We further extended our labview code to perform dynamic bending which helps us to attain low frequency dynamic bending of up to $40 \mathrm{~Hz}$ using the same experimental setup as in Fig. 4. For the AC response of the cantilever a different setup was developed consisting of a miniature peizo actuator (PL055.31 from PI) and a digital lock-in amplifier from Stanford Research Systems (SR850) as shown in Fig. 5. The piezochip has maximum periodic vibration of $2.2 \mathrm{~m}$. Periodic vibration of the pizochip oscillates the cantilver and then the $\mathrm{AC}$ response of the device is extracted by observing the change in HFET current using lock-in amplifier.

\section{RESULTS AND DISCUSSION}

AlGaN/GaN heterostructures has a strain dependent 2dimensional electron gas (2DEG) at the interface, which is sensitive to mechanical load, as well as to chemical modification of the surface, and can be used for novel sensing principles. Both $\mathrm{AlGaN}$ and $\mathrm{GaN}$ will have bending induced polarization at the interface. The $2 \mathrm{DEG}$ carrier density depends on the difference between the polarization of $\mathrm{AlGaN}$ and $\mathrm{GaN}$ at the interface. The whole channel at the $\mathrm{AlGaN} / \mathrm{GaN}$ interface experiences a varying strain along the length. Apart from the change in 2DEG, mobility also gets affected by the change in effective mass. These combined effects of carrier density in 2DEG, and change in mobility offer highly sensitive microsensor with FET embedded GaN microcantilever. The mechanical strain caused by microcantilever bending on 2DEG and the output characteristics of $\mathrm{AlGaN} / \mathrm{GaN}$ HFET have already been studied experimentally [1] and theoretically [2]. At first the DC characteristics of the HFET was recorded and Fig. 6 shows the $I_{d}-V_{d}$ characteristics of one of our best devices for different gate bias. For $\mathrm{AlGaN} / \mathrm{GaN}$ devices, which are normally $\mathrm{ON}$, gate bias can control the channel carrier concentration. The gate modulation helps to reduce the background carrier concentration in 2DEG and thus signifies the deflection induced change of piezoresistivity to increase the sensitivity of such device. We previously reported [15] a gauge factor of $-38\left(\right.$ at $\mathrm{V}_{\mathrm{g}}=0 \mathrm{~V}$ ) and -860 in steady state and transient conditions, respectively. But our second generation devices 


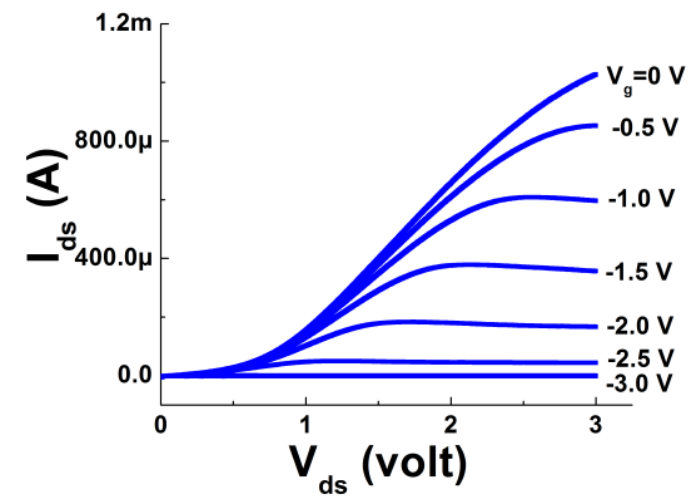

Fig. 6: $I_{d}-V_{d}$ curve for an HEFT device integrated at the cantilever base.

consistently exhibit much higher gauge factor in both static and dynamic bending conditions at zero gate bias. In Fig. 7 the static bending performance of a typical device is shown where the drain current is found change by $1.17 \%$ in magnitude, which gives a gauge factor of 650 . The basic definition of gauge factor is given by: $\mathrm{GF}=(\Delta \mathrm{R} / \mathrm{R}) / \varepsilon$, where $\varepsilon$ is strain, $\Delta R$ is change in resistance, and $R$ is the initial resistance when the cantilever is not bent. The change in drain current is converted to fractional change in resistance of the drain to source contact. Most of our new devices show such higher gauge factor eliminating the need of gate modulation but our best device has exhibited a gauge factor of 3532 which is the highest among its kind ever reported considering zero gate bias to best of our knowledge. We then modified our Labview code for dynamic control of the nanopositioner to bend the microcantilver periodically. Fig. 8 shows the low frequency $(0.5 \mathrm{~Hz})$ response of the same device as in Fig. 7, when the bending magnitude (both downward and upward) was $25 \mathrm{~m}$. We found that the low frequency upward and downward bending does not alter the gauge factor, and the response up to $40 \mathrm{~Hz}$ is also quite similar. Because of the limited response time of the mechanical movement of the nanopositioner, high frequency dynamic bending was not attainable from the setup in Fig. 4. So for extracting the AC response of the devices we used piezochip to attach with the cantilevers as shown in Fig. 5. The piezochip was fed from lock-in amplifier with $1 \mathrm{~V}$ (rms) which generated a periodic vibration on the top surface of the piezochip and thus the cantilever was oscillated. The HFET transduced that oscillation to

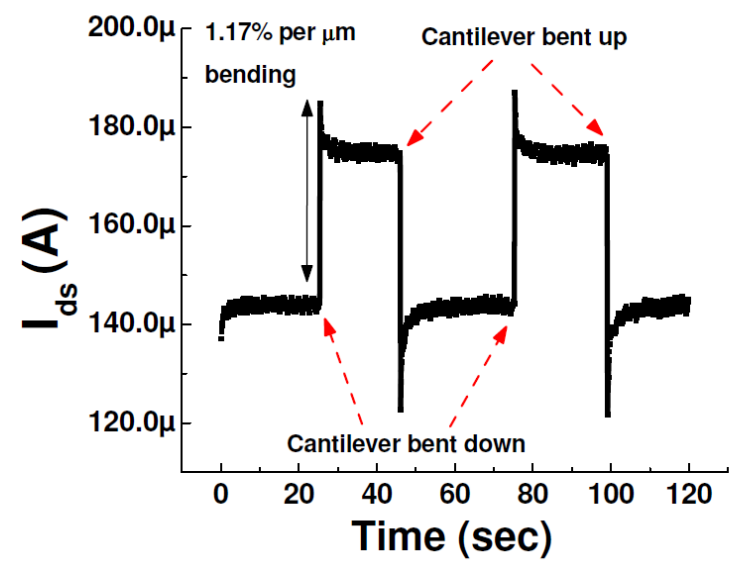

Fig. 7: Static bending response when cantilever is bended (both downward and upward) $25 \mu \mathrm{m}$.

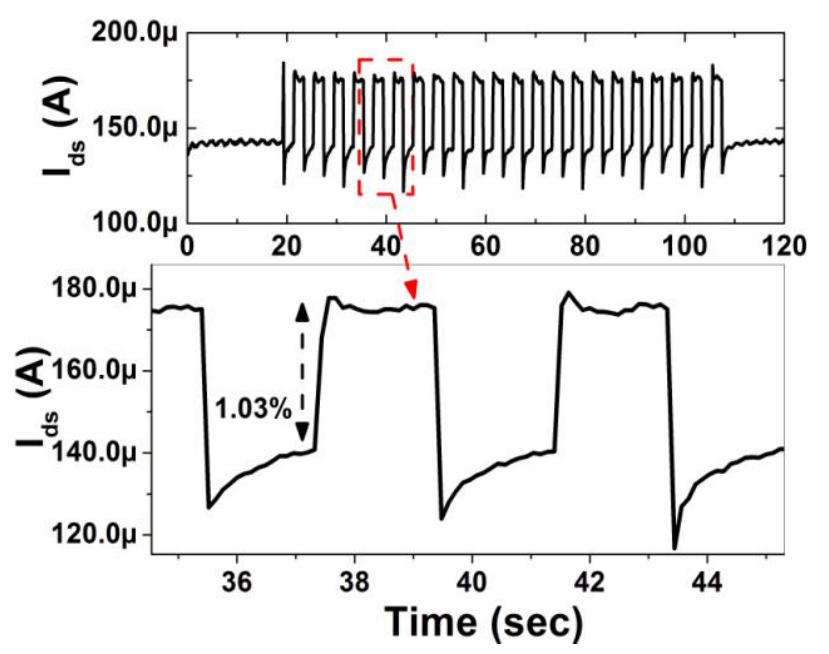

Fig. 8: Static bending response when cantilever is bended (both downward and upward) $25 \mu \mathrm{m}$ and $0.5 \mathrm{~Hz}$ frequency.

resistance change across drain and source which was translated to voltage change in lock-in amplifier. The maximum change in voltage occurred at the resonant frequency of the cantilever which was found $45 \mathrm{KHz}$ (black line graph in Fig. 9). Then the piezochip was held at $1 \mathrm{~cm}$ distance from the device using a micropositioner and was fed again by lock-in amplifier. The periodic vibration of the piezochip generated acoustic wave that propagated by means of adiabatic compression and decompression and eventually oscillated the microcantilever. The resonant peak of the cantilever is found at the same frequency $(45 \mathrm{KHz})$ (blue line graph in Fig. 9). The piezo chip was used as an ultrasonic source and our microcantilever based sensor were able to detect vibrational amplitude of $22 \mathrm{~nm}$ of the ultrasonic generator from $5 \mathrm{~cm}$ distance and $4.6 \mathrm{~nm}$ from $1 \mathrm{~cm}$ distance with a high quality factor of 210 in air. Such HFET transduction setup eliminates the need of conventional laser-photodiode method for acquiring AC response of cantilever. In addition to that, ultrasonic sensing of sub $\mathrm{nm}$ vibration using GaN microcantilever was possible due to the high sensitivity of those sensors.

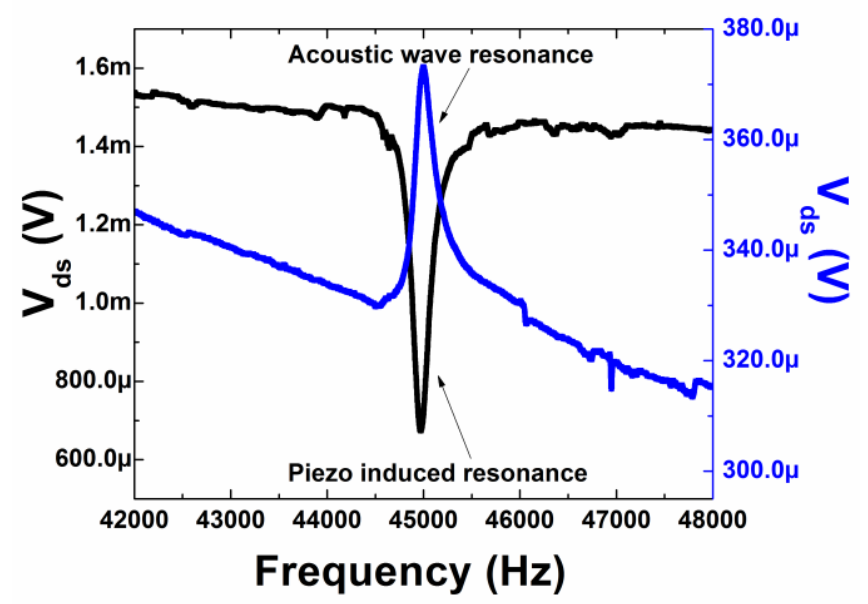

Fig. 9: Resonant peaks for both Piezo induced and acoustic wave induced resonance observed in the $A C$ response of the microcantilevers. 


\section{CONCLUSION}

A high gauge factor of 3532 is reported for the time using GaN piezoresistive microcantilevers embedded with $\mathrm{AlGaN} / \mathrm{GaN}$ HFET without any gate modulation. These sensors have consistent sensitivity in both static and dynamic condition. A novel method of extracting AC response of microcantilevers is also demonstrated. Due to the high sensitivity, those sensors are found efficient as non contact ultrasonic detectors where the preliminary results exhibit the sensing of sub $\mathrm{nm}$ periodic vibration. These results also reveal the capability of using our devices as noncontact ultrasonic remote detector for harsh environment along with sensing different molecular signature using photoacoustic spectroscopy.

\section{ACKNOWLEDGEMENT}

Financial supports for this work from National Science Foundation (Grants Nos. ECCS-0801435 and ECCS-0846898) and Army Research Office (Grant No. W911NF-08-0299) are thankfully acknowledged. We would also like to acknowledge Transducer Research Foundation generously for providing travel support.

\section{REFERENCES}

[1] Z. Y. Hu, T. Thundat, and R. J. Warmack, "Investigation of adsorption and absorption-induced stresses using microcantilever sensors", J. Appl. Phys., 90(1), 427 (2001).

[2] L. A. Pinnaduwage, A. Gehl, D. L. Hedden, G. Muralidharan, T. Thundat, R. T. Lareau, T. Sulchek, L. Manning, B. Rogers, M. Jones, and J. D. Adams, "Explosives: A microsensor for trinitrotoluene vapour", Nature, 425(6957), 474 (2003).

[3] J. Fritz, M. K. Baller, H. P. Lang, H. Rothuizen, P. Vettiger, E. Meyer, H. J. Guntherodt, C. Gerber, and J. K. Gimzewski, "Translating Biomolecular Recognition into Nanomechanics", Science, 288(5464), 316 (2000).

[4] K. Naeli and O. Brand, "Dimensional considerations in achieving large quality factors for resonant silicon cantilevers in air", J. Appl. Phys., 105(1), 014908 (2009).

[5] X. M. Yu, J. Thaysen, O. Hansen, and A. Boisen, "Optimization of sensitivity and noise in piezoresistive cantilevers", J. Appl. Phys., 92(10), 6296 (2002).

[6] H. Morkoc, Handbook of Nitride Semiconductors and Devices, Wiley-VCH, Berlin, 2008.

[7] V. Cimalla, J. Pezoldt, and O. Ambacher, "Group III nitride and $\mathrm{SiC}$ based MEMS and NEMS: materials properties, technology and applications", J. Phys. D: Appl. Phys., 40(20), 6386 (2007).

[8] Y. Liu, P. P. Ruden, J. Xie, H. Morkoc, and K. A. Son, "Effect of hydrostatic pressure on the dc characteristics of $\mathrm{AlGaN} / \mathrm{GaN}$ heterojunction field effect transistors", Appl. Phys. Lett., 88(1), 013505 (2006).

[9] R. Gaska, J. W. Yang, A. D. Bykhovski, M. S. Shur, V. V. Kaminski, and S. M. Soloviov, "The influence of the deformation on the two-dimensional electron gas density in GaN-AlGaN heterostructures", Appl. Phys. Lett., 72(1), 64 (1998).

[10] T. Zimmermann, M. Neuburger, P. Benkart, F. J. HernandezGuillen, C. Pietzka, M. Kunze, I. Daumiller, A. Dadgar, A. Krost, and E. Kohn, "Piezoelectric GaN sensor structures", IEEE Electron Device Lett., 27(5), 309 (2006).

[11] B. Jogai, "Influence of surface states on the two-dimensional electron gas in $\mathrm{AlGaN} / \mathrm{GaN}$ heterojunction field-effect transistors", J. Appl. Phys., 93(3), 1631 (2003).

[12] M. Chu, A. D. Koehler, A. Gupta, T. Nishida, and S. E. Thompson, "Simulation of $\mathrm{AlGaN} / \mathrm{GaN}$ high-electronmobility transistor gauge factor based on two-dimensional electron gas density and electron mobility", J. Appl. Phys., 108(10), 104502 (2010).

[13] N. Ledermann, P. Muralt, J. Baborowski, M. Forster, and J. P. Pellaux, "Piezoelectric $\mathrm{Pb}\left(\mathrm{Zr}_{x}, \mathrm{Ti}_{1-x}\right) \mathrm{O}_{3}$ thin film cantilever and bridge acoustic sensors for miniaturized photoacoustic gas detectors", J. Micromech. Microeng., 14, 1650, (2004).

[14] Brian D. Adamson, John E. Sader, and Evan J. Bieske, "Photoacoustic detection of gases using microcantilevers", J. Appl. Phys., 106, 114510 (2009).

[15] Muhammad Qazi, Nicholas DeRoller, Abdul Talukdar, and Goutam Koley, "III-V Nitride based piezoresistive microcantilever for sensing applications," Appl. Phys. Lett. 99, 193508 (2011)

\section{CONTACT}

*Abdul Talukdar, tel: +1-803-777-3229; talukdaa@email.sc.edu 


\title{
MEMS AIR-MICROFLUIDIC SENSOR FOR PORTABLE MONITORING OF AIRBORNE PARTICULATES
}

\author{
F.L. Doering ${ }^{1,2}$, I. Paprotny ${ }^{1,3 *}$, and R.M. White ${ }^{1,3 \dagger}$ \\ ${ }^{1}$ Berkeley Sensor \& Actuator Center (BSAC), University of California, Berkeley, USA \\ ${ }^{2}$ The Mechanical Engineering Department, University of California, Berkeley, USA \\ ${ }^{3}$ The Electrical Engineering and Computer Science Department, University of California, Berkeley, USA
}

\begin{abstract}
We present the design, fabrication, and experimental results of a MEMS air-microfluidic sensor for measuring the concentrations of airborne particulate matter (PM) such as tobacco smoke or diesel exhaust. Our sensor $(25 \mathrm{~mm} \times 21 \mathrm{~mm} \times 2 \mathrm{~mm}$ in size) consists of an air-microfluidic circuit that separates the particles by size, then transports and deposits the selected particles onto the surface of a mass-sensitive film bulk acoustic resonator (FBAR). The rate of frequency change of the FBAR due to massloading corresponds to the particle concentration in the sampled air-volume. Our sensors exhibit a low-end detection limit of single $\mu \mathrm{g} / \mathrm{m}^{3}$. The small size of our sensor, combined with high sensitivity, enables it to be used in a portable PM monitor that can record personal PM exposure levels.
\end{abstract}

\section{INTRODUCTION}

Particulate matter (PM) is a category of air-borne pollutant that includes dust, smoke, diesel exhaust and smog. Fine particle (PM 2.5) pollution is especially damaging to our health. Due to their small size these particles penetrate the body's natural filtration systems and lodge deep within the lungs, contribute to several health problems such as reduced lung functionality, bronchitis, and heart attacks. The U.S. Environmental Protection Agency (EPA) has been tightening its safe exposure limit criteria for PM 2.5 [1] and in January of 2012, Beijing Municipal Environmental Protection Bureau started publishing PM 2.5 levels in response to high public demand [2]. While there is increasing demand for PM 2.5 monitoring, the prevailing equipment is fairly bulky and expensive [3], which limits the possibilities for widespread, fine granularity sensing. Portable PM sensors are needed to allow for better identification of pollution sources, health awareness, and pollution control.

In this work we present a microfabricated air-microfluidic PM sensor that has a small form factor so as to be easily integrated into portable personal pollution monitors. The sensor is $25 \mathrm{~mm} \times 21$ $\mathrm{mm} \times 2 \mathrm{~mm}$, almost two orders of magnitude smaller than the smallest currently available commercial devices [4], and an order of magnitude smaller than the macro scale predecessor device from our lab [5]. We describe in detail the principles of operation of the key components our microfluidic circuit; an inertial particle size selector (virtual impactor [6]), as well as particle deposition on the mass-sensing film bulk acoustic resonator (FBAR). We then describe the details of the fabrication process that are used to manufacture prototypes of the PM sensors, and experimental results showing the response of the manufactured prototypes to cigarette smoke and diesel exhaust, two common PM sources. Our results indicate that our sensor has a low-end detection limit of single $\mu \mathrm{g} / \mathrm{m}^{3}$, which is an order of magnitude improvement over the performance of our previous macroscale prototype, and is comparable with large, commercially available PM sensors.

\section{PRINCIPLE OF OPERATION}

A schematic drawing of our air-microfluidic circuit is shown in Figure 1. Air containing suspended particles enters the sensor through the inlet (a). Particles with an aerodynamic diameter larger than $2.5 \mu \mathrm{m}$ are then removed from the air-stream through inertial size-selection (virtual impaction) (b). The remaining particulates (PM 2.5) continue down the microfluidic channels and are deposited onto a mass-sensing element, the exposed surface of an FBAR (c), using an induced temperature gradient across the microfluidic channel (thermophoresis). The concentration of PM 2.5 in the sampled air can be inferred from the rate of the (negative) frequency shift for the mass-loaded FBAR. Negative air pressure at the outlet (d), generated by a stack of commercially available miniature fans $(19 \mathrm{~mm} \times 19 \mathrm{~mm} \times 11 \mathrm{~mm}$ in size $)$, is used to drive the air through the device.

(a) (b) (c)

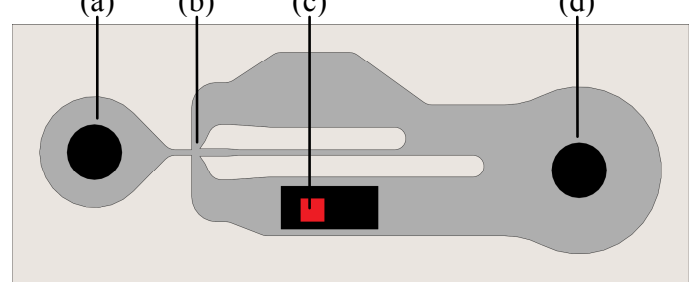

Figure 1: Schematic drawing (top view) of the air-microfluidic channels of our MEMS PM sensor. The channels are shown in dark grey, while the through-wafer holes are in black.

\section{Virtual Impaction (Inertial Size Selectivity)}

The size selectivity of the device is enabled by virtual impaction, where large particles are expelled from the main airstream (major flow) as it is diverted around a tight corner. The momentum of the larger particles cannot be overcome by the drag force of the air, causing these particles to be ejected from the major flow into a minor flow, which exhausts the larger particles. The operation of our virtual impactor is illustrated on the left side of Figure 2, while the right side of Figure 2 shows an SEM of the microfabricated virtual impactor. The inlet jet (a), the major (b) and minor (c) flows are shown.
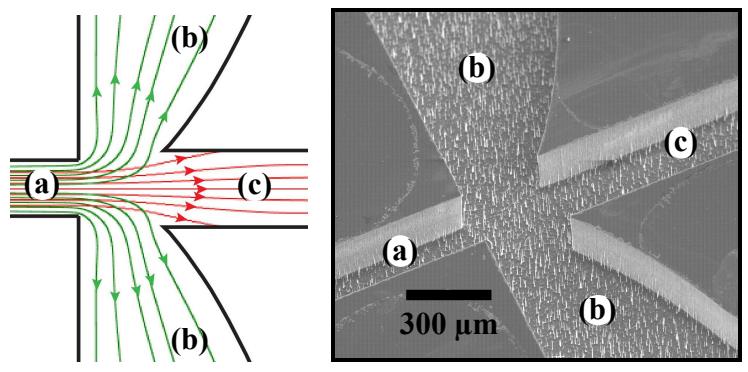

Figure 2: (left) Simulated particle traces for diameters of 5 and 1 $\mu \mathrm{m}$ which both start from the inlet jet (a), but end in the minor flow (c) and major flows (b) respectively. (right) SEM of the microfluidic channels at the size selective region.

The ratio of particles filtered out of the flow increases as their size increases. The aerodynamic diameter at which this ratio is 
one-half is the cutoff size. The cutoff size of an impactor can be described by

$$
d_{50}=\left(\frac{9 \eta W^{2} D\left(S t k_{50}\right)}{\rho_{p} Q}\right)^{1 / 2}
$$

where $d_{50}$ is the particle cutoff diameter, $\eta$ is the dynamic viscosity of air, $W$ and $D$ are the width and depth of the impactor jet respectively, $\rho_{p}$ is the particle density, $Q$ is the jet volumetric flow rate, and $S t k_{50}$ is the stokes number, which is recommended to be 0.59 for rectangular jet impactors [6]. To finely tune the designs of the microfabricated virtual impactor for a cutoff particle diameter of $2.5 \mu \mathrm{m}$, the Finite Element Method (FEM) is used. The simulated collection efficiency (the percentage of particles expelled from the major flow as a function of the particle size) of our virtual impactor is shown in Figure 3.

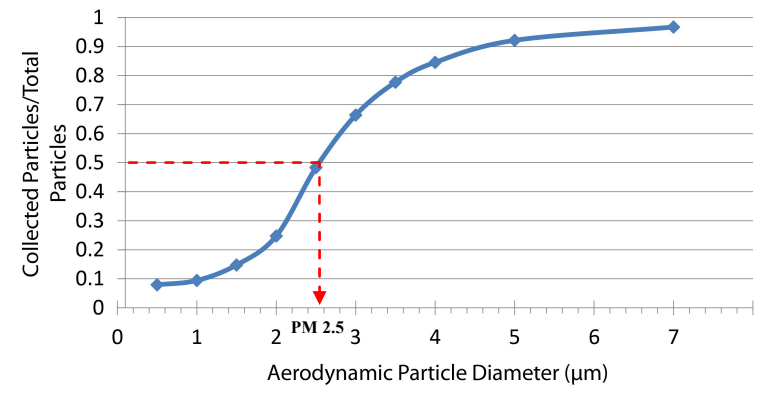

Figure 3: Collection efficiency of simulated particles with aerodynamic diameters near the cutoff diameter for flow through the designed virtual impactor.

Microfabrication techniques allow us to reduce the size of the virtual impactor, which reduces the required airflow and back pressure, lowering the power requirements of the pumping device supplying the negative pressure. To ease fabrication, a rectangular jet impactor is used. A varied channel width is used to affect the viscous pressure loss in each branch of the virtual impactor, thereby regulating the flow rates without valves and the use of only one inlet and one outlet.

\section{Thermophoretic Particle Deposition}

Once the large particles have been removed, a fraction of the remaining particles (PM 2.5) are deposited down-stream from the virtual impactor onto the surface of a mass-sensing FBAR through the process of thermophoresis. Microfabricated heaters create a temperature gradient across the microfluidic channel, which in turn expels the particles from the airflow, and is caused by hot air molecules transferring more momentum to the particle than the cold air molecules. The resulting net thermophoretic force $F_{t h}$ can be approximated by

$$
F_{t h}=\frac{-9 \cdot \pi \cdot d \cdot \eta^{2} \cdot H \cdot \nabla T}{2 \cdot \rho_{g} \cdot T}
$$

where $d$ is the particle diameter, $\eta$ is the dynamic viscosity of the gas, is the temperature gradient, $\rho_{g}$ is the density of the gas, $T$ is the absolute temperature of the gas, and $H$ is the molecular accommodation coefficient defined as

$$
H \cong\left(\frac{1}{1+6 \lambda / d}\right)\left(\frac{k_{a} / k_{p}+4.4 \lambda / d}{1+2 k_{a} / k_{p}+8.8 \lambda / d}\right)
$$

where $k_{a}$ and $k_{p}$ are the thermal conductivities of air and the particle respectively and $\lambda$ is the mean free path length of the gas. The absolute temperature, $T$, is approximated as the average of the temperatures of the heater and the deposition surface, which yields a constant thermal force in the region between the heater and deposition surface. Equating this force with the Stokes drag force yields a thermal particle velocity

$$
V_{t h}=\frac{-3 \cdot \eta \cdot C_{c} \cdot H \cdot \nabla T}{2 \cdot \rho_{g} \cdot T}
$$

where $C_{c}$ is the Cunningham correction factor

$$
C_{c}=1+\frac{2.52 \lambda}{d}
$$

from [6]. The predicted thermal velocity equals $1.36 \mathrm{~mm} / \mathrm{s}$ for our sensor. The deposition rate should be independent of particle size. The thermal gradient is generated by a microfabricated resistive heater as the heat source for the hot side, and a combination of convective and conductive cooling for the cold side. The airstream passing between the heater and the mass sensing element absorbs most of the heat, and the rest is dissipated to the environment by conduction. In order to minimize the power consumed, the heater substrate is quartz, with a low thermal conductivity $(1.3 \mathrm{~W} / \mathrm{K} / \mathrm{m})$. The heaters are released from quartz to further limit the heat transfer.

\section{Mass Sensing}

Our sensor measures the concentration of the particulates by the rate of the frequency shift of the mass-loaded film bulk acoustic resonator (FBAR). As particles are deposited onto the exposed surface of the resonator, the additional mass lowers its resonant frequency. We use FBARs compensated for temperature stability provided by our collaborators at Avago Technologies.

\section{FABRICATION}

Figure 4 shows an exploded CAD drawing of the MEMS PM sensor. The sensor is composed of three wafer die bonded together to form a 3-wafer stack. The top fused quarts wafer serves as a substrate for the thermophoretic heaters as well as the cap for the air-microfluidic circuit. The middle wafer defines the channels of the air-microfluidic circuit, and also contains through-wafer holes for the inlet, outlet, and an insertion opening (a) through which the FBAR is brought into the channel. The bottom fused quartz wafer contains the FBAR (b), its CMOS driver (c), and a polymeric seal (d) for the aforementioned insertion opening.

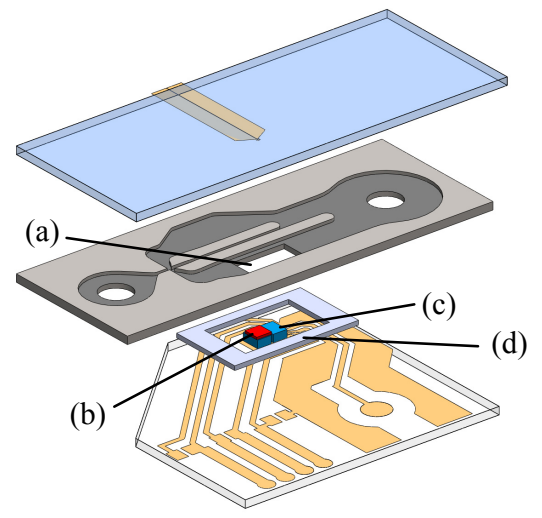

Figure 4: Exploded CAD drawing of the MEMS air-microfluidic PM sensor. 


\section{Top Wafer: Thermophoretic Heaters}

The thermophoretic heaters are fabricated on a fused quartz wafer. The fabrication process is illustrated in Figure 5. First, $\mathrm{a} \approx 2$ $\mu \mathrm{m}$ layer of in-situ doped polysilicon is deposited through an LPCVD process $\left(50 \% \mathrm{SiH} 4 / 50 \% \mathrm{PH} 3,615^{\circ} \mathrm{C}\right)$. In order to avoid the cracking of the quartz wafer, the polysilicon is not annealed. However, the un-annealed polysilicon layer has a sheet resistance of $\approx 40 \mathrm{ohm} / \square$, which is sufficient for our application. A $100 \mathrm{~nm}$ layer of $\mathrm{Cr} / \mathrm{Au}$ is then evaporated using an E-beam evaporator. The $\mathrm{Au} / \mathrm{Cr}$ layer is then lithographically patterned using an iodinebased gold etchant and CR-7, defining the high conductivity leads for the thermophoretic heaters. The doped polysilicon layer is then etched by a dry RIE process $\left(90 \% \mathrm{SF}_{6} / 10 \% \mathrm{O}_{2}, 66 \mathrm{sccm}\right)$, defining the $8 \mu \mathrm{m}$ wide resistive heater elements. The wafer is diced into individual die, and the heaters are released from the quarts wafer by a 10 -minute etch in $49 \% \mathrm{HF}$. An optical micrograph of a fabricated thermophoretic heater, taken through the top quartz wafer is shown in Figure 6. Note the dark area (a) representing the underetched region of the polysilicon layer.

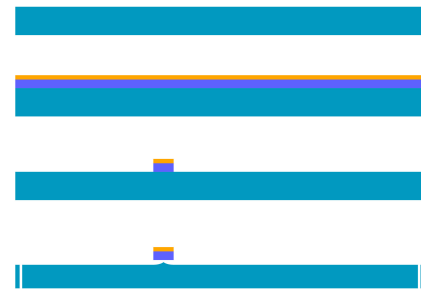

\section{Quartz Wafer LPCVD $2 \mu \mathrm{m}$ PolySi (p-type) $100 \mathrm{~nm} \mathrm{Cr/Au}$ \\ Define heaters, RIE PolySi etch Wet-etch $\mathrm{Cr} / \mathrm{Au}$ \\ Sub-dice \\ Underetch heaters in $\mathrm{HF}$}

Figure 5: Fabrication process for the top wafer, defining the thermophoretic heaters.

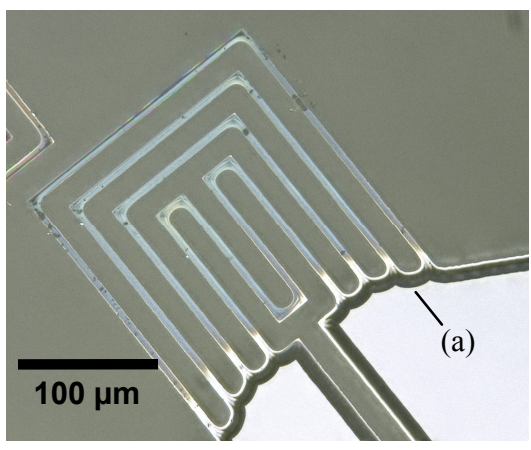

Figure 6: Optical micrograph of a finished heater taken through the quartz wafer.

\section{Middle Wafer: Micro-Fluidic Channels}

The middle wafer defines the microfluidic channels of the MEMS PM sensor. The fabrication process for the middle wafer is shown in Figure 7. The microfluidic channels are lithographically defined using a DRIE process $\left(\mathrm{C}_{4} \mathrm{~F}_{8}\right.$ passivation, $90 \% \mathrm{SF}_{6} / 10 \% \mathrm{O}_{2}$ RIE etch) to etch $200 \mu \mathrm{m}$ deep channels, as well as the throughwafer holes for the inlet, outlet, and FBAR insertion opening. The wafer is then diced into individual die.

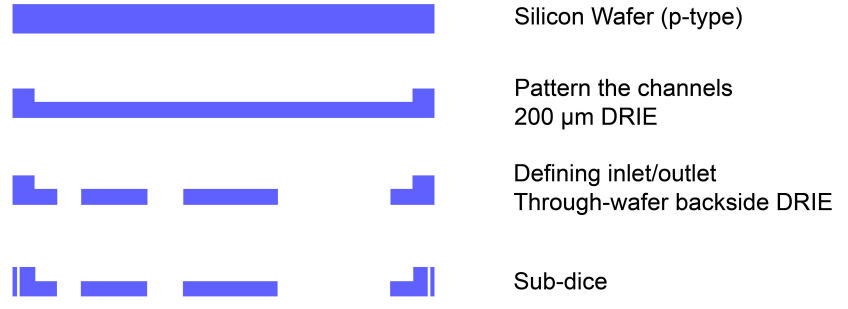

Figure 7: Fabrication process for the middle wafer, defining the channels of the microfluidic circuit.

\section{Bottom Wafer: The FBAR Substrate}

The bottom fused quartz wafer contains the FBAR, its CMOS driver, the gold connection traces, as well as a polymeric spacer seal. The fabrication process for this wafer is outlined in Figure 8. First, $100 \mathrm{~nm}$ of $\mathrm{Cr} / \mathrm{Au}$ is evaporated onto the wafer, and then is lithographically patterned to define the power and signal traces for the driver CMOS. The wafer is then diced. A $475 \mu \mathrm{m}$ tall $1 \mathrm{~mm} \times$ $1 \mathrm{~mm}$ pedestal is attached using cyanoacrylate adhesive at the FBAR location, to ensure the insertion of the FBAR inside the microfluidic channel. The driver CMOS die (a Pierce oscillator) and the FBAR die are then physically bonded to the wafer and the pedestal using the same cyanoacrylate adhesive. The FBAR is then wire bonded to the CMOS, and the CMOS is wire-bonded to the gold traces. Power and RF signal lead are soldered onto the traces. Finally, a $285 \mu \mathrm{m}$ adhesive polymeric seal (made out of a triple stack of dicing tape) is applied around the sensing elements to act as a seal for the insertion opening and to set the distance that the FBAR protrudes into the microfluidic channel.

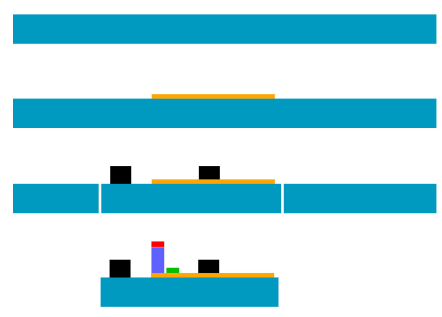

Quartz Wafer

$100 \mathrm{~nm} \mathrm{Cr} / \mathrm{Au}$

Define electrodes by wet-etch

Attach the $285 \mu \mathrm{m}$ seal Sub-dice

Attach FBAR on $475 \mu \mathrm{m}$ pedestal Attach CMOS driver, wirebond

Figure 8: The fabrication process for the bottom wafer, which serves as the substrate for the mass-sensitive FBAR and CMOS driver.

\section{Final Assembly}

Figure 9 illustrates the bonding process of the three wafers. First, a top wafer die is bonded to a matching middle wafer die to form the air-microfluidic circuit, while the heaters are aligned above the insertion opening. To ensure a good seal around the small features of the microfluidic channels, we used adhesive bonding by applying dispenser-printed epoxy (72\% Epon 830 resin, 28\% Epikure 3370 curing agent), and curing overnight on a hotplate at $60^{\circ} \mathrm{C}$. The same epoxy mixture is applied to the polymeric seal, and the bottom wafer die is visually aligned and attached to the top/middle wafer stack, placing the FBAR into the microfluidic channel through the insertion opening. The spacing between the surface of the FBAR and the heater is designed for a nominal spacing of $100 \mu \mathrm{m}$. The sensor is sealed with silicone (Osi Sealants/Henkel Adhesives) around the edges of the bonded die to prevent leaks and increase the structural stability of the final device. 


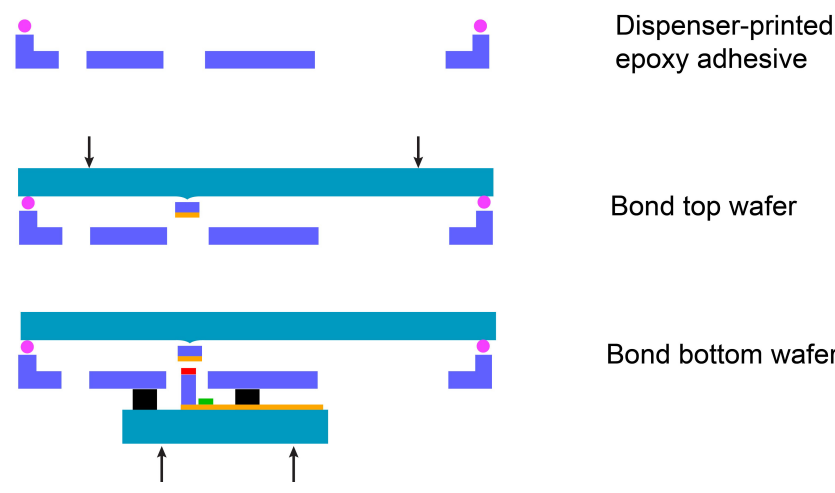

Figure 9: Bonding of the three wafers to complete the assembly of the MEMS PM sensor.

Figure 10 shows a fabricated MEMS PM sensor prototype; labels (a) and (b) correspond to the size-selection and deposition areas respectively. The alignment of all the layers can be seen in the optical micrograph of the sensing area, taken through the top quartz wafer and shown in Figure 11. Two FBARs are visible as pink pentagons, partially obscured by the two heaters. Bond wires connect the CMOS oscillator circuit to one of the FBARs and to the bottom wafer (out of focus).

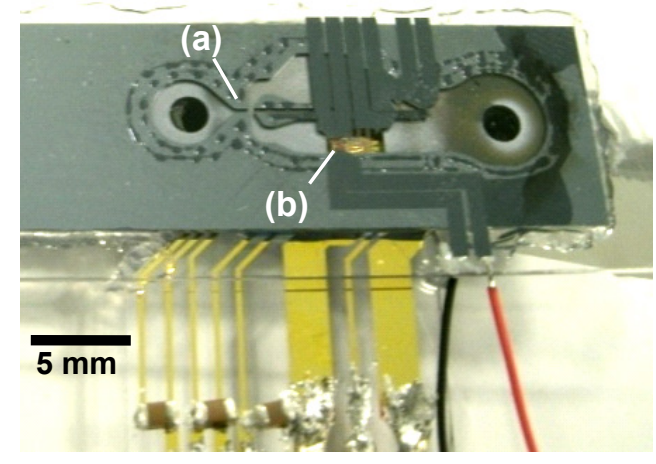

Figure 10: Photograph of a complete prototype of the MEMS airmicrofluidic PM sensor.

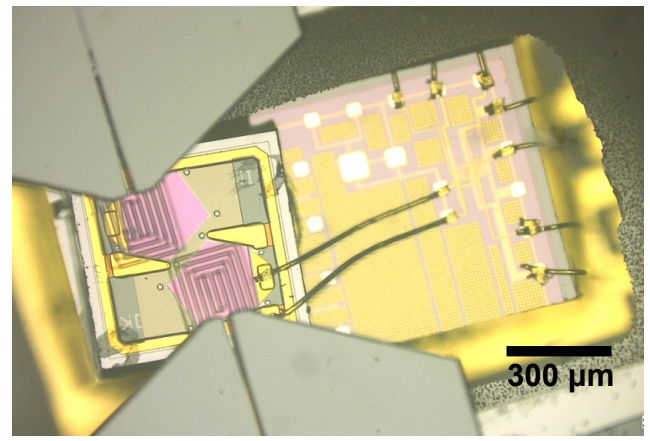

Figure 11: Optical micrograph of the sensing area in an assembled prototype of the MEMS PM sensor (labeled as (b) in Figure 10).

\section{EXPERIMENTAL RESULTS}

Experimental Setup

Two fabricated MEMS PM sensor prototypes (labeled A and B) were tested in an air-quality testing chamber $\left(25 \mathrm{~m}^{3}\right)$ at the Environmental Energy Technologies Division (EETD) of Lawrence Berkeley National Lab. To generate a representative concentration of tobacco smoke, a single cigarette was lit and smoked using a custom built machine, simulating a single cigarette being smoked in a closed room. To generate the diesel exhaust, a portable diesel generator was used. A HEPA filtration unit (Control Resources Systems Inc. 600L) was used to lower the concentration of particles during testing. A DustTrak ${ }^{\mathrm{TM}}$ Aerosol Monitor 8520, calibrated based on [7] and gravimetric analysis [6], was used to provide a reference for the particulate concentration in the test chamber. The resonant frequency of the FBAR was measured using a spectrum analyzer (Agilent 8562EC), and was recorded and tracked from a laptop using a custom-developed LABVIEW (v. 10.0f2) interface.

\section{Results}

The response of the MEMS PM sensor to tobacco smoke and diesel exhaust, during two representative experiments is shown on Figures 12 and 13, respectively. In both cases, good correlation can be observed between our device and the DustTrak reference measurements. The steps in the graphs are the result of periodic activation of the HEPA filtration unit, which causes a rapid drop in $\mathrm{PM}$ concentration.

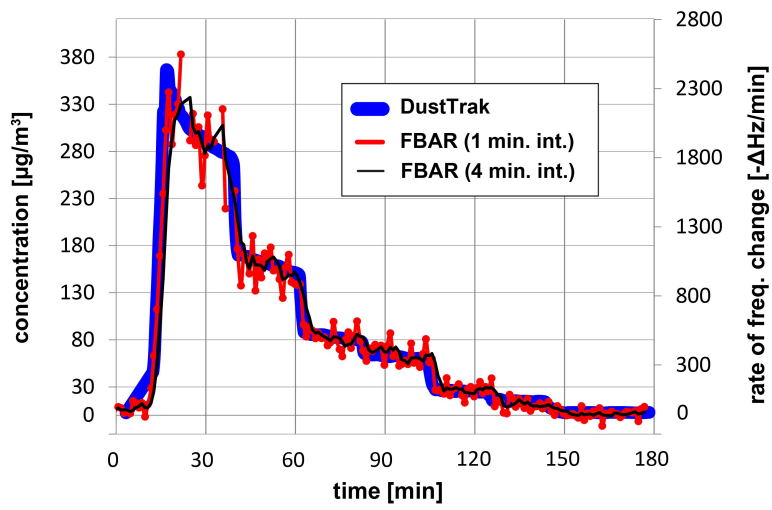

Figure 12: Response of the sensor (prototype A) to cigarette smoke during a representative experiment.

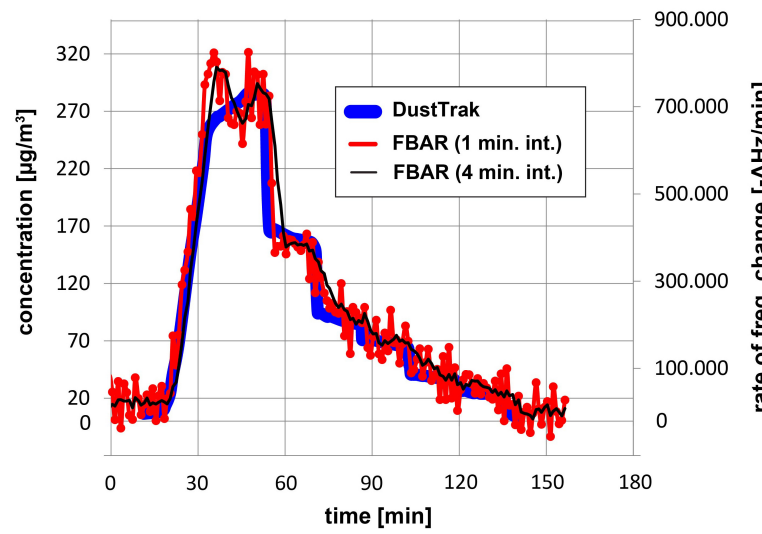

Figure 13: Response of the sensor (prototype B) to diesel exhaust during a representative experiment.

Table I shows the sensitivity coefficient, relating frequency 
shift rate to concentration, for both prototypes. Prototype A failed before the diesel exhaust test was performed; hence sensitivity coefficient for diesel exhaust for that prototype is unavailable. Note the drop in tobacco smoke concentration from 25 to $15 \mu \mathrm{g} / \mathrm{m}^{3}$ around min. 130 in Figure 12. This step is apparent in the $4 \mathrm{~min}$. integration time sensor plot, suggesting a low-end detection limit (with $4 \mathrm{~min}$. integration time) below $10 \mu \mathrm{g} / \mathrm{m}^{3}$ (for prototype A).

Table 1: Average sensitivity coefficient (in $\mathrm{Hz} / \mathrm{s}$ per $\mathrm{mg} / \mathrm{m}^{3}$ ) for sensor prototypes $A$ and $B$ for tobacco smoke and diesel exhaust. Standard deviation is shown in parenthesis.

\begin{tabular}{|l|c|c|}
\hline & $\begin{array}{c}\text { Tobacco } \\
\text { Smoke }\end{array}$ & Diesel Exhaust \\
\hline Prototype A & $96(11.31)$ & NA \\
\hline Prototype B & $28.3(3.82)$ & $40.93(3.76)$ \\
\hline
\end{tabular}

Based on estimated sensor variability, which at low PM concentrations is approximately $50 \mathrm{~Hz}$, we estimate a low-end detection limit of approximately $2 \mu \mathrm{g} / \mathrm{m}^{3}$ with $10 \mathrm{~min}$. integration time (for prototype A).

The sensitivity coefficient of both prototypes is strongly dependent on the rate of the airflow through the microfluidic channel (data in Table 1 is taken at highest flow rate). Such strong dependency indicates that our sensor is depleting the particles in the microfluidic channel above the FBAR, and hence reducing the available particle mass at lower flow rates. This is confirmed by the deposition pattern observed in an optical micrograph of the FBAR after several experimental runs, shown in Figure 14. The air flows from the right (indicated by black arrow). The deposited particles can be observed as a grey fuzzy crescent (outlined by a striped line). The crescent shape, as opposed to an oval, indicates that there are significantly more particles deposited on the upstream half of the heater than the downstream half. This nonuniformity indicates that the particles have been nearly depleted by the time the airflow passes under the right side of the heater.

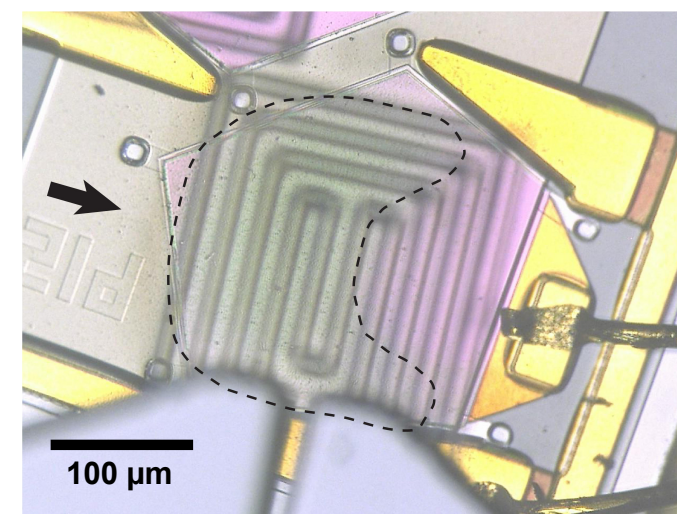

Figure 14: Optical micrograph of the FBAR viewed through the quartz wafer and silicon heater after several deposition runs.

Both tobacco smoke and diesel exhaust contain particles that are much smaller than the cut-off particle diameter of our virtual impactor, preventing us from validating its collection efficiency in our current experimental setup; we leave this validation for future work.

\section{CONCLUSION}

In this paper, we presented the designs, fabrication, and experimental results of an air-microfluidic MEMS PM sensor. The sensor is two orders of magnitude smaller than commercially available PM sensors and an order of magnitude smaller than the previously presented macro-scale prototype by our group. The single $\mu \mathrm{g} / \mathrm{m}^{3}$ low-end detection limit of our sensor, combined with its small size enables our device to be used as a portable, perhaps wearable, personalized PM exposure monitor, which is sufficiently sensitive to monitor compliance with the EPA air quality standard. The use of microfabrication techniques enables us to create complex air-microfluidic circuits with a small footprint. This airmicrofluidic lab-on-a-chip will enable comprehensive and portable air quality monitoring.

The depletion of particulates from the airstream during deposition indicates that we can further increase the low end detection limit by increasing the air flow rate through the microfluidic channel. We will investigate this possibility in subsequent testing.

In this work, we recorded the signal from our sensor using a spectrum analyzer. We are currently working with several collaborators to develop the frequency-tracking electronics and connect our sensor to a cellular radio, enabling massively distributed portable PM sensing applications.

\section{ACKNOWLEDGEMENTS}

The authors gratefully acknowledge Intel Corp. for their generous financial support of this work. The authors would also like to thank Lara Gundel and Dough Sullivan from EETD for their help in testing the sensor prototypes, Allison Woodruff and Alan Mainwaring from Intel Corp., as well as Paul Solomon from the Environmental Protection Agency for their comments and technical discussion. Finally, we would like to thank Rich Ruby and Avago Technologies for providing the FBARs.

\section{REFERENCES}

[1] "Particulate Matter," The United States Environmental Protection Agency (EPA), http://www.epa.gov/oar/particlepollution/, Downloaded on 3/31/12.

[2] "Beijing Releases PM 2.5 Air Quality Readings," Caixin Online, http://english.caixin.com/2012-0121/100350762.html, Downloaded on 3/31/12.

[3] "Met One Aerocet 531 Mass/ Laser Particle Counter," BPA Air Quality Solutions LLC, http://www.breathepureair.com/met_one_gt531.html, downloaded on 3/31/12.

[4] “DUSTTRAK II Aerosol Monitor 8532," TSI Incorporated, http://www.tsi.com/DUSTTRAK-II-Aerosol-Monitor-8532/, Downloaded on 3/31/12.

[5] J. Black, A. Elium, R. White, M. Apte, L. Gundel, and R. Cambie, "MEMS-Enabled Miniaturized Particulate Matter Monitor Employing 1.6 GHz Aluminum Nitride Thin-Film Bulk Acoustic Wave Resonator (FBAR) and Thermophoretic Precipitator," Ultrasonics Symposium, 2007, 10/28-31/07, IEEE, (2007), pp. 476 - 479.

[6] W. C. Hinds, Aerosol Technology: Properties, Behavior, and Measurement of Airborne Particles, 2nd Ed., John Wiley \& Sons, New York, 1999.

[7] S. Liu and Y. Zhu, "A case study of exposure to ultrafine particles from secondhand tobacco smoke in an automobile", Indoor Air, 20: 412-423 (2010).

\section{CONTACT}

* I. Paprotny, igorpapa@eecs.berkeley.edu

† R.M. White, tel: +1-510-642-0540; rwhite@eecs.berkeley.edu 


\section{MEMS AND METAMATERIAL-BASED TERAHERTZ DETECTOR \\ Hu Tao ${ }^{1}$, Emil A. Kadlec ${ }^{2}$, Andrew C. Strikwerda ${ }^{1}$, Kebin Fan ${ }^{1}$, Willie J. Padilla ${ }^{3}$, Richard D. Averitt ${ }^{1}$ Eric A. Shaner ${ }^{2}$, and Xin Zhang ${ }^{1 *}$ \\ ${ }^{1}$ Boston University, Boston, Massachusetts, USA \\ ${ }^{2}$ Sandia National Laboratory, Albuquerque, New Mexico, USA \\ ${ }^{3}$ Boston College, Chestnut Hill, Massachusetts, USA}

\begin{abstract}
We have designed, fabricated and characterized metamaterialsbased far-infrared detector. The detector design, which combines metamaterials (MMs) and MEMS, is frequency selective. The enhanced absorption on the cantilever pixels from split-ring resonators (SRRs) leads to a mechanical deflection which is directly detected with a He-Ne beam. As a demonstration of our first-generation devices, we have fabricated and tested detectors at $95 \mathrm{GHz}$ and $693 \mathrm{GHz}$ with noise equivalent powers (NEP) of $10^{-8}$ $\mathrm{W} / \mathrm{Hz}^{1 / 2}$ and single-pixel responsivity as high as $16,500 \mathrm{~V} / \mathrm{W}$ in a room-temperature and pressure environment.
\end{abstract}

\section{INTRODUCTION}

In the past decades, artificial materials consisting of arrayed subwavelength metallic inclusions have been drawn much attention since their exotic electromagnetic (EM) properties not readily occurring in nature, such as negative index [1-2] and invisible cloaking [3-5]. The scale invariant nature of Maxwell's equations allows tailoring metamaterial elements for a specific EM response at virtually any desired frequency. This is particularly an advantage in the terahertz $(\mathrm{THz})$ spectrum where novel optical devices can impact applications such as chemical sensing and noninvasive real-time imaging [6].

Though progress in $\mathrm{THz}$ detector sensitivity over the past quarter century has been impressive, it is mostly accredited to improvements in thermometer sensitivity. Inefficient absorption of $\mathrm{THz}$ radiation - which yields a low heat-induced temperature variation - remains the performance bottleneck. Furthermore, most $\mathrm{THz}$ microbolometers show broadband response, which can be undesirable for applications such as spectroscopic detection of materials with unique $\mathrm{THz}$ responses [7, 8]. Here we present a $\mathrm{THz}$ detector based on metamaterial resonators. These metamaterials show high absorption, and their sub wavelength nature enables their use as a focal plane array (FPA) to image near the diffraction limit. Furthermore, their narrow band resonance is a salient feature for spectrally selective detection applications. Consequently, metamaterials hold great promise for facilitating the development of a "versatile" $\mathrm{THz}$ detector which can a) strongly absorb $\mathrm{THz}$ radiation; b) operate at room temperature with decent responsivity and sensitivity; c) be scaled up to function as a multi-pixel array for imaging applications; d) operate selectively at any frequency in the $\mathrm{THz}$ regime; and e) be lightweight and low cost.

Here we report on the development of an uncooled metamaterials enhanced $\mathrm{THz}$ radiation detector with optical read-out. Through the incorporation of SRRs, a strong resonant response is obtained that can be optimized for specific design frequencies.

\section{DESIGN}

The cantilever-based $\mathrm{THz}$ detector employs thermally sensitive bimaterial elements as shown in Figure 1a. The detector pixels are comprised of three basic components: 1) a $\mathrm{THz}$ wave absorber consisting of a single gold ( $\mathrm{Au})$ SRR patterned on a silicon nitride $\left(\mathrm{SiN}_{\mathrm{x}}\right)$ thin film serving as the supporting pad; 2) bimaterial cantilever actuation legs composed of layers of $\mathrm{SiN}_{\mathrm{x}}$ and $\mathrm{Au}$ which have a large coefficient of thermal expansion (CTE) mismatch; 3) an optically reflective surface for visible light readout of the thermal deflection of the cantilevers due to the absorption of $\mathrm{THz}$ radiation. On resonance, the incident $\mathrm{THz}$ radiation drives a current in the metallic SRR resulting in ohmic heating. The primary heat flow path is through the supporting bimaterial actuation legs. Due to the CTE mismatch between $\mathrm{Au}$ and $\mathrm{SiN}_{\mathrm{x}}$, the temperature change of the legs results in mechanical deflection of the cantilever which is sensed by optical interrogation of the reflector pad. Figure $1 \mathrm{~b}$ shows the simulation result of the absorption is over $40 \%$ on resonance $(639 \mathrm{GHz})$, as opposed to nearly nothing with the bare $\mathrm{SiN}_{\mathrm{x}}$ pad.

(a)

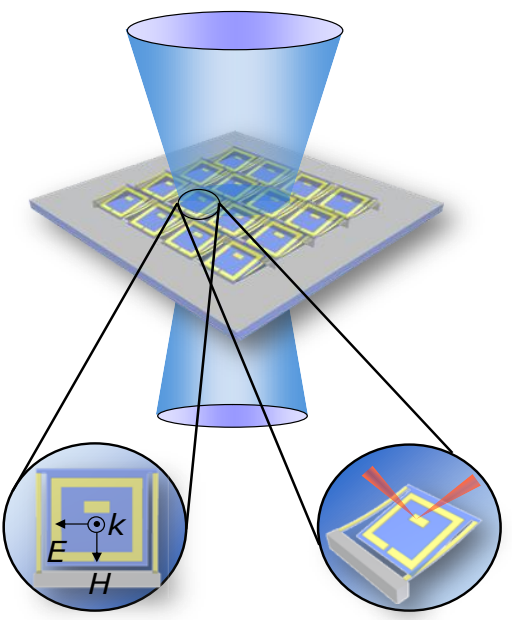

(b)

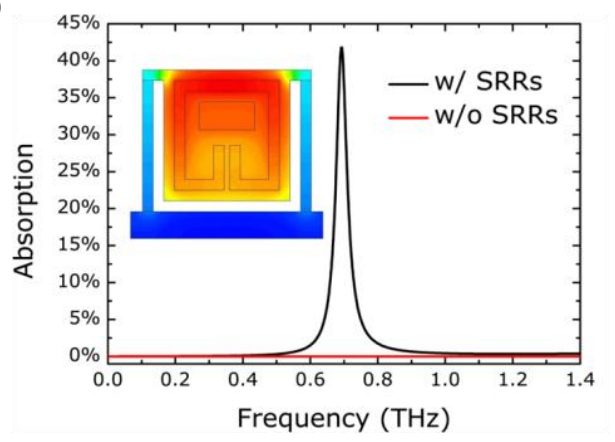

Figure 1: Metamaterial enhanced terahertz detector using optical readout. (a) Schematic of the device showing the metamaterial cantilever array, an individual pixel, and reflective optical read-out. (b) Simulated absorption of the device with (black line) and without (red line) SRR. (Inset) Simulated temperature profile due to the enhanced absorption.

\section{FABRICATION}

Two detectors have been designed with operating frequency at $95 \mathrm{GHz}$ and $693 \mathrm{GHz}$. The dimensions of these two detectors are listed in Figure 2a. Both detectors are fabrication using MEMS technology in a four-step process (Figure 2b). First, a $\mathrm{SiN}_{\mathrm{x}}$ layer 
$(500 \mathrm{~nm})$ is deposited on a silicon substrate. The $\mathrm{Cr} / \mathrm{Au}$ layer $(10 \mathrm{~nm} / 200 \mathrm{~nm})$ defining the SRR absorbers, readout reflector, and cantilever legs is then fabricated using standard lithography and liftoff processes. Next, the $\mathrm{SiN}_{\mathrm{x}}$ layer is patterned using lithography and RIE etching to define the MM supporting membrane as well as the cantilever legs. Finally, the structures are released through backside $\mathrm{KOH}$ etching of the underlying silicon substrate. Figure $2 \mathrm{c}$ and $2 \mathrm{~d}$ show the fabricated and released single pixel of detectors operating at $95 \mathrm{GHz}$ and $693 \mathrm{GHz}$ respectively. (a)

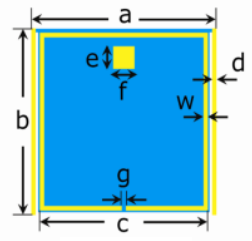

$95 \mathrm{GHz}$

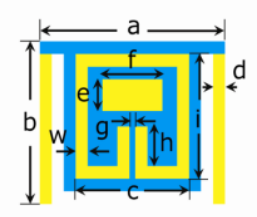

$693 \mathrm{GHz}$

\begin{tabular}{|c|c|c|c|c|c|c|c|c|c|c|}
\hline & a & b & c & d & e & f & g & h & i & w \\
\hline $95 \mathrm{GHz}$ & 435 & 415 & 395 & 10 & 50 & 50 & 10 & - & - & 10 \\
\hline $690 \mathrm{GHz}$ & 80 & 67 & 49 & 5 & 13 & 26 & 2.5 & 16 & 51 & 5 \\
\hline
\end{tabular}

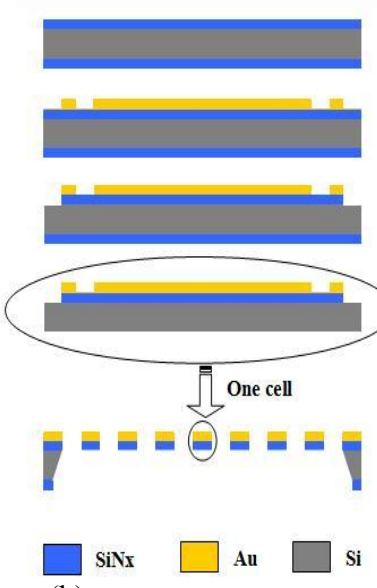

(b)
All units are in microns.
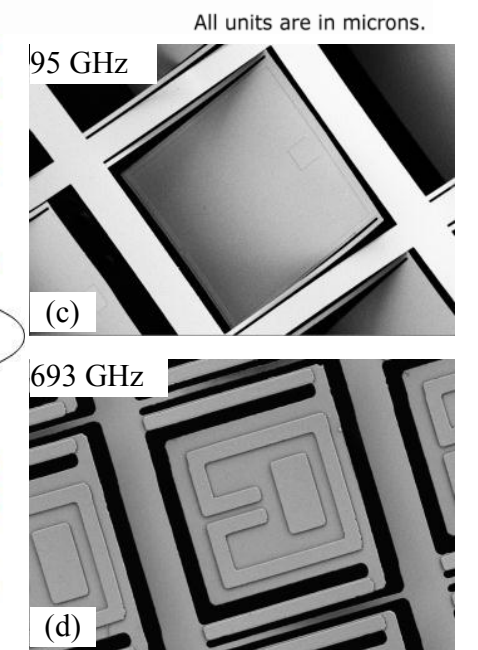

Figure 2: (a) Dimensions of the metamaterial enhanced detectors resonant at $95 \mathrm{GHz}$ and $693 \mathrm{GHz}$, respectively. (b) Fabrication process. (b)\&(c) SEM images of released unit cell of $95-\mathrm{GHz}$ and 693-GHz devices.

\section{CHARACTERIZATION}

Figure 3 shows the schematic of experiment setup for device characterization. The $\mathrm{THz}$ photoresponse is detected by using a position sensitive photodetector (PSD) to measure the change in location of a reflected beam off of the optical pad of a pixel caused by the deflection of the cantilever. An electronically modulated frequency tripled YIG oscillator was used as the microwave source for the $95 \mathrm{GHz}$ detector experiments. The $\mathrm{THz}$ source used for the $693 \mathrm{GHz}$ measurements was an optically chopped $\mathrm{CO}_{2}$ pumped far-infrared laser operating on a formic acid line. A $780 \mathrm{~nm}$ laser diode was focused onto the reflecting pad of the cantilever through a focusing objective and the back reflection was aligned to the PSD. The photoresponse was measured using a lock-in amplifier monitoring the output voltage of the PSD. For time-response measurements the signal was captured on an oscilloscope in place of

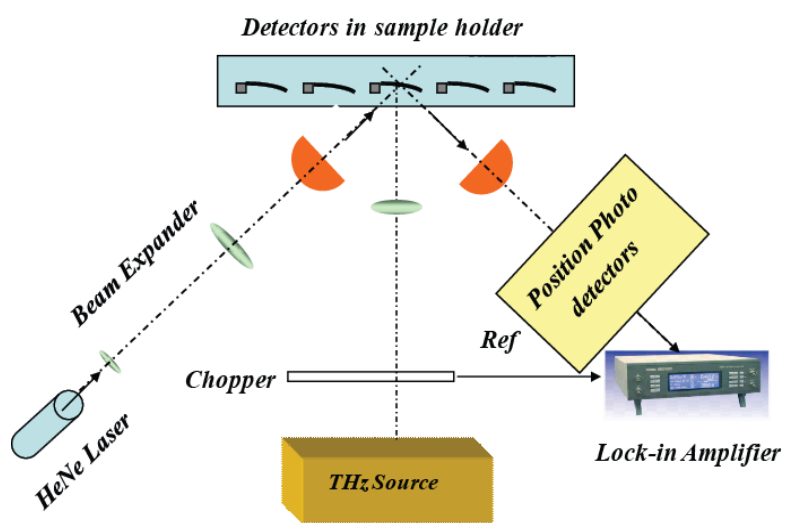

Figure 3: Schematic of the device showing the metamaterial cantilever array, an individual pixel, and reflective optical read-out.

the lock-in. While our fabrication naturally produces an array of cantilever pixels, in our experiment we only characterized the response of single pixels.

All measurements reported in this work were taken at room temperature and pressure. First, we consider the response of the 95 $\mathrm{GHz}$ detector. The spectral characteristics of the array were first verified using THz-TDS, as shown in Figure 4 (left top of inset). The dip in the transmission is associated with absorption (and reflection) of the SRRs. The resonance is centered at $95 \mathrm{GHz}$ with a full-width at half max of $20 \mathrm{GHz}$. The photoresponse of the detector was characterized for both polarizations of the incident microwave radiation by stepping the operating frequency of the source over its full range from $60 \mathrm{GHz}$ to $120 \mathrm{GHz}$. As shown in Figure 4, a strong resonant peak was observed at $95 \mathrm{GHz}$ for the polarization having the electric field perpendicular to the SRR gap which drives the LC resonance. The photoresponse was significantly weaker for the orthogonal polarization with the electric field parallel to the gap. In

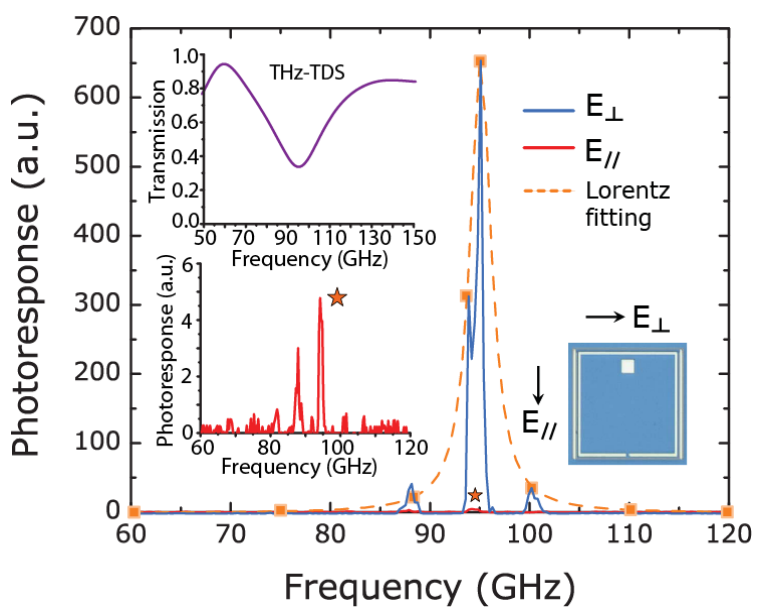

Figure 4: Response of the $95 \mathrm{GHz}$ detector as a function of frequency of the incident radiation at two polarizations. (Inset, left top) Transmission spectra of the detector characterized using THz-TDS with polarization of the electric field normal to the gap $\left(\mathrm{E}_{\perp}\right)$. (Inset, left bottom) Zoom-in view of the detector response with the polarization of the THz electric field parallel to the SRR gap $\left(\mathrm{E}_{\|}\right)$. The response is two orders of magnitude smaller than the response with the polarization of the THz electric field perpendicular to the $\operatorname{SRR} \operatorname{gap}\left(\mathrm{E}_{\perp}\right)$. 
this case, the incident radiation does not couple to the resonance, which is the expected response for this particular SRR design. After verifying the resonant response of the detector, the source was set to $95 \mathrm{GHz}$ and the response of individual pixels were characterized as a function of incident power by attenuating the source with a voltage controlled attenuator. As shown in Figure 5, a nearly linear response of the detector was observed and follows the incident power reasonably well. A signal-to-noise ratio (SNR) of $\sim 1,200$ was obtained by measuring the peak signal $(\sim 380 \mathrm{mV})$ of the detector at the full incident power of $10 \mathrm{~mW}$ with a spot size of $1 \mathrm{~cm}$ in diameter and noise floor of $\sim 0.3 \mathrm{mV}$ with no incident radiation. A responsivity of $16,500 \mathrm{~V} / \mathrm{W}$ with a minimum $\mathrm{NEP}$ of $1 \times 10^{-8} \mathrm{~W} / \sqrt{\mathrm{Hz}}$ was obtained when considering the measurement bandwidth of the lock-in amplifier and the detector area. The temporal response of the detector, which is important for real-time imaging applications, was determined by monitoring the output signal of the PSD on an oscilloscope. The inset to Figure 5 shows the response at $5 \mathrm{~Hz}$ and $25 \mathrm{~Hz}$ showing that the response at $25 \mathrm{~Hz}$ is still about $70 \%$ of the 5 $\mathrm{Hz}$ response. The sweep of modulation frequency in Figure 6 shows that the maximum operation frequency of the detector before signal roll off began was $15 \mathrm{~Hz}$ indicating a time constant of $\sim 10 \mathrm{~ms}$. As shown in Figure 6, the output of photoresponse at $50 \mathrm{~Hz}$ is about half of that at $5 \mathrm{~Hz}$.

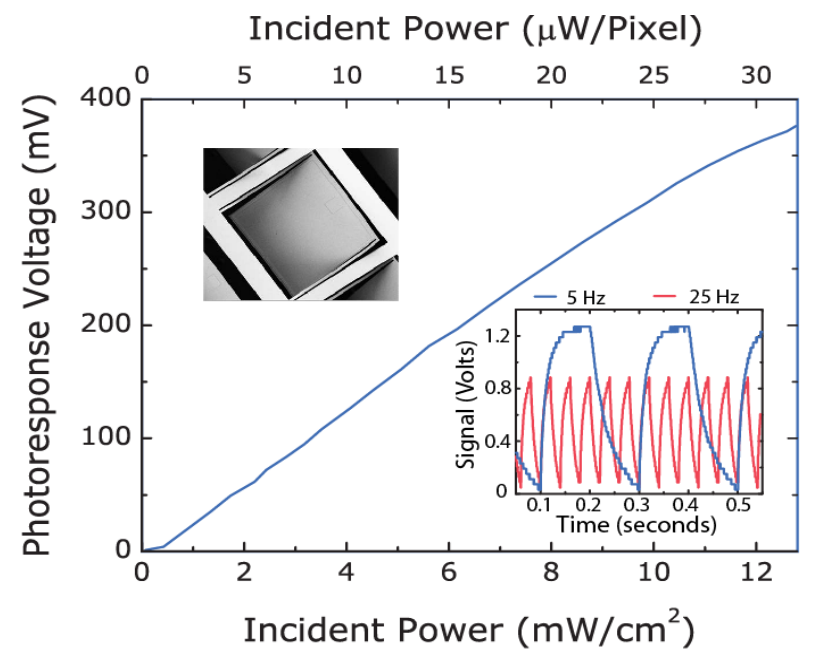

Figure 5: Photoresponse of the $95 \mathrm{GHz}$ device as a function of incident power. (Inset) Oscilloscope observed temporal response of the $95 \mathrm{GHz}$ at $5 \mathrm{~Hz}$ (blue) and $25 \mathrm{~Hz}$ (red), respectively.

The $693 \mathrm{GHz}$ detector pixels were characterized with a similar setup using a far-infrared laser (FIRL) in place of the multiplier source. The resonant response of the $693 \mathrm{GHz}$ detector was again pre-determined using THz-TDS as shown in the inset to Figure 7a. Both the polarization and the power of the FIRL source were controlled using a wire-grid polarizer pair. The FIRL beam was then focused using an off-axis parabolic mirror. The focused beam spot was measured using a pyrocam III beam imager in order to determine the peak intensity of the focused light as well as the beam diameter. A responsivity of $6,800 \mathrm{~V} / \mathrm{W}$ with a minimum NEP of $2.96 \times 10^{-8} \mathrm{~W} / \sqrt{ } \mathrm{Hz}$ was experimentally obtained when considering the power incident on the single pixel. The temporal response (inset Figure 7a showing data at $8 \mathrm{~Hz}$ and $15 \mathrm{~Hz}$ ) of the $693 \mathrm{GHz}$ detector shows a somewhat faster roll-off response in comparison to the 95 $\mathrm{GHz}$ pixel. While the readout approach utilized could be significantly improved, we performed beam profiling measurements with the $693 \mathrm{GHz}$ pixels in order to demonstrate the utility of our detectors, even at this stage, for THz beam diagnostics. In Figure $7 \mathrm{~b}$, we show beam measurements taken using a single pixel while the beam was steered along both axes. To capture the beam along the y-axis, the focusing paraboloid was simply translated. In a similar way, the $\mathrm{x}$-axis data was captured by rotating the focusing optic. The result was a Gaussian profile similar to that measured with the commercial beam imager. While this is by no means an ideal technique for characterizing the beam, it demonstrates that these relatively simple detector elements can immediately find lab applications should an appropriate multi-pixel readout scheme be implemented.

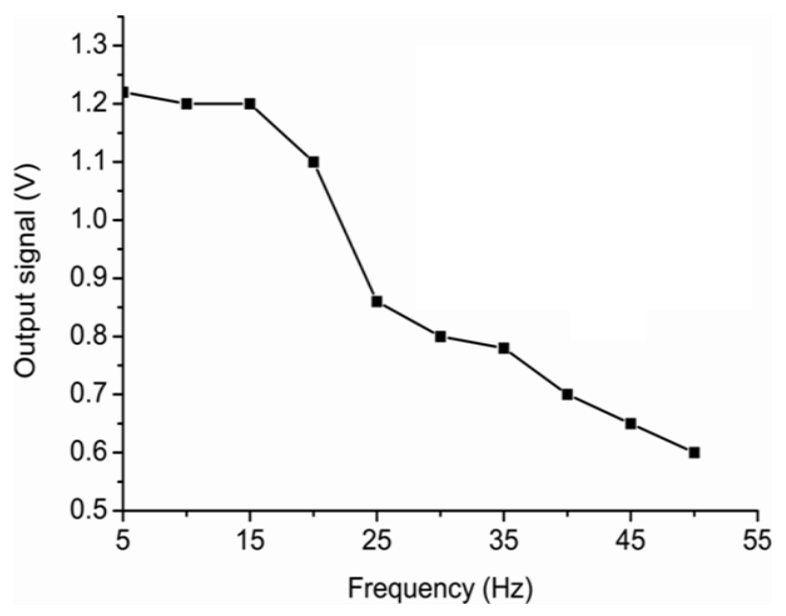

Figure 6: Modulation frequency dependent of photoresponse for detector of $95 \mathrm{GHz}$.

\section{DISCUSSION AND CONCLUSIONS}

It is worth mentioning that the performance of the metamaterial resonant detectors could be significantly improved based on the fact that our characterization was conducted at room temperature and pressure. The $95 \mathrm{GHz}$ and $693 \mathrm{GHz}$ pixels have similar noise which mainly comes from the optical readout system. The noise could be further reduced by using a more stable read-out laser, a higher sensitivity PSD, and further reducing the environmental vibration. Alternative signal addressing methods such as capacitive or resistive readouts could also be implemented. Significant improvements in the thermal engineering should also improve the NEP. Preliminary estimates suggest a reduction of the NEP to $10^{-10} \mathrm{~W} / \sqrt{\mathrm{Hz}}$ (and perhaps lower) is possible with additional engineering effort. In its present form using the experimental setup described in this paper and sub-wavelength pixels, the minimum power detectable in a diffraction limited spot is approximately $1 \mu \mathrm{W}$. Our metamaterial cantilever detectors are naturally fabricated in array form and are therefore suitable for real-time $2 \mathrm{D}$ imaging by simultaneously measuring the deflection of all of the pixels using, for example, a visible CCD/CMOS camera to obtain an intensity map for parallel pixel readout $[9,10]$. Furthermore, it is possible to combine multiple SRRs with different resonant frequencies into a super pixel, thus enabling frequency-sensitive $\mathrm{THz}$ imaging /spectroscopy with a "multicolor" THz illumination source. The optimal design of $\mathrm{THz}$ focal plane imaging detector will also need to consider pixel size for particular imaging needs such as speed and sensitivity. For example, the MM unit cells are typically $\lambda / 5 \sim \lambda / 10$ in size. One may desire to have multiple MM SRRs constructed on a sensing pixel element that is matched to the optics of the overall system for optimum response [11]. 

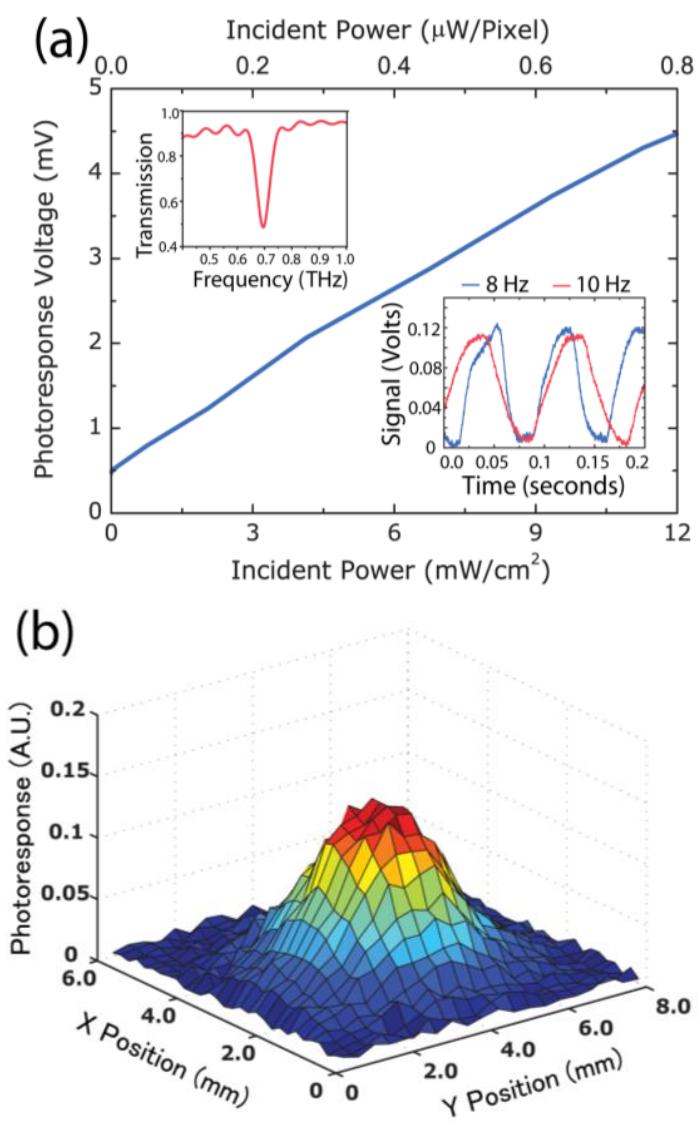

Figure 7: Photoresponse of the $693 \mathrm{GHz}$ pixel. (a) Response of the detector as a function of incident power. (Inset, left) Oscilloscope observed temporal responses of the $95 \mathrm{GHz}$ at $8 \mathrm{~Hz}$ (blue) and $10 \mathrm{~Hz}$ (red), respectively. (Inset, right) THz-TDS characterized transmission spectrum of the detector showing a resonance at $\sim 693$ $\mathrm{GHz}$. (b) Image of the incident $\mathrm{THz}$ beam profile using the metamaterial enhanced $\mathrm{THz}$ detector.

In summary, we have developed metamaterial enhanced resonant detectors with a simple fabrication process for active $\mathrm{THz}$ sensing and detection applications. While we demonstrated detection at $95 \mathrm{GHz}$ and $693 \mathrm{GHz}$, in principle this approach can be applied to other unconventional wavelengths where nature does not provide us with appropriate material resonances amenable to integration with thermal detection platforms. The implementation of low cost thin film $\mathrm{THz}$ absorbers could enable rapid progress in extending the many benefits of uncooled thermal imaging devices to the $\mathrm{THz}$ portion of the spectrum.

\section{ACKNOWLEDGEMENTS}

We acknowledge partial support from NSF under Contract No. ECCS 0802036, AFOSR under Contract No. FA9550-09-1-0708, and DARPA under Contract No. HR0011-08-1-0044. Sandia National Laboratories is a multi-program laboratory managed and operated by Sandia Corporation, a wholly owned subsidiary of Lockheed Martin Corporation, for the U.S. Department of Energy's National Nuclear Security Administration under contract DE-AC04-94AL85000. The authors would like to thank the Photonics Center at Boston University for all the technical support throughout the course of this research.

\section{REFERENCES}

[1] R. A. Shelby, D. R. Smith, and S. Schultz, "Experimental Verification of a Negative Index of Refraction", Science 292, 77 (2001)

[2] J. Valentine, S. Zhang, T. Zentgraf, E. Ulin-Avila, D. A. Genov, G. Bartal, and X. Zhang, "Three-Dimensional Optical Metamaterials with a Negative Refractive Index", Nature 455, 376 (2008)

[3] R. Liu, C. Ji, J. J. Mock, J. Y. Chin, T. J. Cui, and D. R. Smith, "Broadband Ground-Plane Cloak", Science 323, 366(2009).

[4] J. Valentine, J. S. Li, T. Zentgraf, G. Bartal, and X. Zhang, “An Optical Cloak Made of Dielectrics", Nature Materials, 8, 568 (2009).

[5] T. Ergin, N. Stenger, P. Brenner, J. B. Pendry, and M. Wegener, "Three-Dimensional Invisibility Cloak at Optical Wavelengths", Science 328, 337 (2010).

[6] P. U. Jepsen, D. G. Cooke, and M. Koch, "Terahertz Spectroscopy and Imaging- Modern Techniques and Applications", Laser Photonics Review 5, 124 (2011).

[7] T.-L. Hwang, S. E. Scharz, and D. B. Rutledge, "Microbolometers for Infrared Detection", Applied Physics Letters 34, 773 (1979).

[8] E. N. Grossman and A. J. Miller, "Active Millimeter-Wave Imaging for Concealed Weapons Detection", Proceeding SPIE 5077, 66 (2003).

[9] Q. Zhang, Z. Miao, Z. Guo, F. Dong, Z. Xiong, X. Wu, D. Chen, C. Li, and B. Jiao, "Optical Readout Uncooled Infrared Imaging Detector Using Knift-edge Filter Operation”, Optoelectronics Letters 3, 119 (2007).

[10] Y. Zhao, M. Mao, R. Horowitz, A. Majumdar, J. Varesi, P. Norton, and J. Kitching, "Optomechanical Uncooled Infrared Imaging System: Design, Microfabrication, and Performance", Journal of Microelectromechanical Systems 11, 136 (2002).

[11] X. Liu, T. Starr, A. F. Starr, and W. J. Padilla, "Infrared Spatial and Frequency Selective Metamaterial with Near-Unity Absorbance", Physical Review Letters 104, 207403 (2010).

\section{CONTACT}

*X. Zhang, tel: +1-617-358-2702; xinz@bu.edu 


\title{
MEMS-ENABLED RETARDING POTENTIAL ANALYZERS FOR HYPERSONIC IN-FLIGHT PLASMA DIAGNOSTICS
}

\author{
E.V. Heubel, A.I. Akinwande, and L.F. Velásquez-Garcia \\ Massachusetts Institute of Technology, Cambridge, Massachusetts, USA
}

\begin{abstract}
We report the design, fabrication, and preliminary characterization of a microfabricated retarding potential analyzer (RPA) that ensures unprecedented grid alignment accuracy. Through refined manufacturing methods, improved tolerances upon assembly serve to increase the signal to noise ratio (SNR). Furthermore, microfabrication permits smaller features overcoming sensor limitations previously barring RPAs from use in very dense plasmas such as those during reentry. Preliminary results show more than a twofold increase in signal strength compared to conventional RPAs. Finally, a new batch-fabricated RPA design that uses MEMS springs for grid assembly is demonstrated to help drive down cost while improving device reliability.
\end{abstract}

\section{INTRODUCTION}

When a vehicle travels at hypersonic speed in earth's atmosphere, the heat generated through shock waves at leading edges is so intense that gas molecules surrounding the craft dissociate and ionize, generating a quasi-neutral plasma that envelops the spacecraft. These energetic plasma ions collide with the vehicle surface causing material ablation, which generates more ions while damaging and heating the craft. Diagnostic sensors can measure this harsh environment, identify exterior conditions, and while monitoring reentry can be used to help maintain adequate levels through methods such as plasma quenching (e.g. Gemini 3) [1]. Close monitoring of space missions at every step of the process helps ensure crew safety and alert ground control of serious malfunctions.

Plasmas are electrically conductive and as a result, the plasma sheath can block radio frequency communication through signal attenuation. This principle is the cause for the notorious radio blackout first experienced during the Apollo missions. The Space Shuttle and the geocentric Tracking and Data Relay Satellite System (TDRSS) worked around this problem by breaking the sheath aft of the reentry spacecraft and relaying signals back to earth through a complex satellite system $[1,2]$. However, the Shuttle is now being retired after decades of faithful service, leaving the Soyuz capsule as the sole crew return vehicle.

Therefore, in order to regain contact between mission crewmembers, identifying plasma constituents and using adaptive signaling methods is imperative. Yet strict weight requirement for space based missions, and the dense plasmas encountered at high speeds in the suborbital atmosphere, place stringent constrains on the sensor. Thus, we propose the use of microelectromechanical system (MEMS) manufacturing techniques for creating resilient, lightweight, batch-fabricated retarding potential analyzers (RPAs). RPAs measure the energy distribution of plasma ion species directly as a means of determining how harshly the sheath is impacting the craft's exterior surface. Through miniaturization, such devices may also be used in large arrays to form a highresolution sensory skin for the vehicle it equips, detecting both time- and space-resolved conditions.

\section{THEORY OF OPERATION}

An RPA is an electrostatic device that behaves in a manner analogous to a filter. Ions of sufficient energy surpass the established potential hill and penetrate the sensor to a collector plate where their flux is detected as current. Figure 1 shows a schematic of the principle of operation. When plasma of potential $V_{p}$ encounters a conductive surface, it shields itself with a sheath of a thickness proportional to the plasma Debye length. To minimize probe perturbation on the plasma, the first grid of the RPA is usually kept electrically floating to appear as any other conducting boundary surface. The induced potential due to the presence of the plasma is the floating potential, $V_{\mathrm{f}}$.

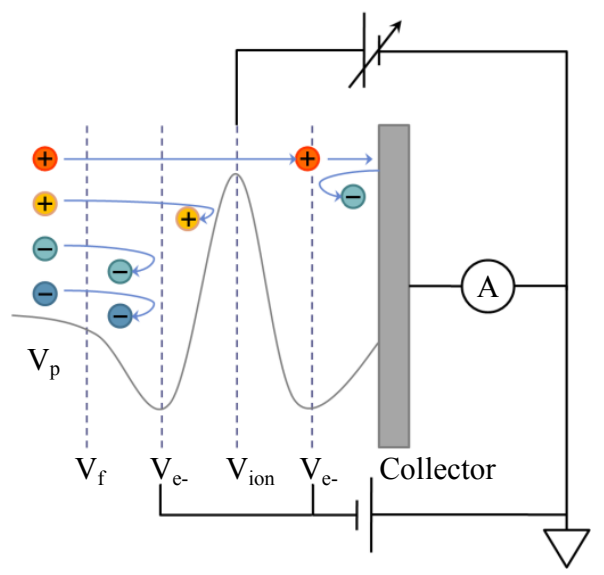

Figure 1: Schematic depicting typical RPA structure and operation

Plasma particles penetrate this self-shielding potential and enter the RPA, where the first negatively charged grid, $\mathrm{V}_{\mathrm{e}-\text {, }}$ repels incoming electrons. The voltage on the ion retarding grid, $\mathrm{V}_{\text {ion }}$, progressively shields more energetic ions as it is swept from a low potential to a high potential. Lastly, the ions make their way to the collector plate through a final negatively charged grid meant to prevent secondary electron emission or photonic emission, which would give a faulty current information [3].

The measured current is representative of the cumulative ion energy distribution. By differentiating this signal with respect to the ion retarding voltage, the transition from complete ion transmission to repulsion becomes the ion voltage distribution through the following equation

$$
-\frac{d I}{d V}=\frac{q_{i}^{2} e^{2} n_{i} A_{c}}{m_{i}} f(V)
$$

with $q_{i}$ as the ion charge state, $n_{i}$ the ion number density, $m_{i}$ ion mass, and $e$ is the elementary charge. The dynamics of RPAs have been extensively studied, yet many relationships established between the signal and the physics at play within the device are empirical in nature. This is due to concerns such as the proper representation of the RPA open area, $A_{c}$. As a result, distributions can be obtained, but their peaks are often normalized to some maximum value, not necessarily representative of the actual ion density.

$$
-\frac{d I}{d V} \sim f(V)=f\left(\frac{E_{i}}{q_{i}}\right)
$$

For a constant charge, then, assuming all atoms are singly ionized, $-d I / d V$ is directly proportional to the energy distribution [4]. 


\section{SENSOR DESIGN}

The most stringent design requirement of the RPA is Debye length, which is reduced with increasing plasma density and decreasing plasma temperature. Through the following relationship

$$
\lambda_{D}=\sqrt{\frac{\varepsilon_{0} k_{B} T_{e}}{n_{e} e^{2}}}
$$

where $\varepsilon_{0}$ is the permittivity of free space, $k_{B}$ is Boltzmann's constant, $T_{e}$ is the electron temperature, and $n_{e}$ is the electron density. To effectively shield plasma, grid openings have to be narrower than two times this critical dimension; otherwise the sheath is broken and particles may flow unobstructed to the next grid in the sensor. Hypersonic reentry experiments carried out in the late 1960s provide a measure of the plasma electron temperature and density in the sheath formed around blunt bodies [5]. The Debye lengths calculated from these parameters (Eq. 2) provide insight into the characteristic plasmas encountered (Figure 2).

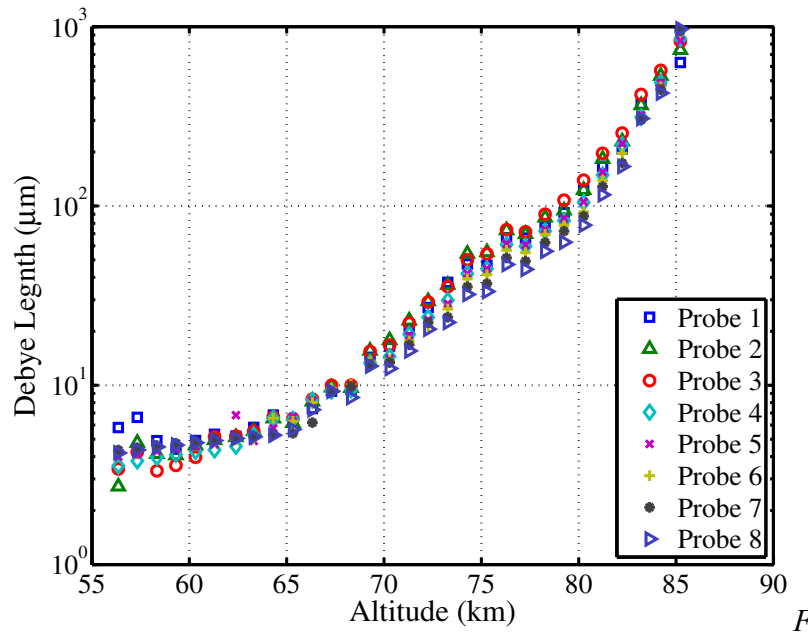

ure 2: Debye length as a function of Altitude from RAM-CII reentry experiments [5].

From Figure 2 we see that during a typical reentry, the Debye length transitions from near a millimeter to only a few microns in size. Radio blackout is usually experienced between $80 \mathrm{~km}$ and $50 \mathrm{~km}$ in altitude for the Space Shuttle, and $85 \mathrm{~km}$ to $25 \mathrm{~km}$ for the RAM-C experiments [1]. At the onset of communication loss, the Debye length is then around $100 \mu \mathrm{m}$, and will be less than $3 \mu \mathrm{m}$ at the end of the blackout period. Grids that are perforated through photochemical etching, or woven wire meshes, are typically used to establish a potential barrier and have apertures of a few hundred microns.

Limitations of present conventional manufacturing methods do not permit effective plasma shielding for these high densities without creative relaxation of the constraints. By utilizing multiple attenuating floating grids or a low transmission micro-channel plate, RPA transparency can be reduced, effectively decreasing the plasma density within the sensor by the time the first electron repelling electrode is reached [6, 7]. However, this method inherently reduces the achievable signal to noise ratio (SNR) by diminishing the flux of ions to the collector. Additionally, these materials do not permit ease of alignment of subsequent grids to better channel the remaining ions to the collector.

Through MEMS processing, micro-scale features on the order of $100 \mu \mathrm{m}$ or less, can be precisely incorporated into a device that ensures high ion transmission by enforcing optical alignment. The other limiting factor in an RPA is the inter-electrode spacing. If the gap between the electron repelling grid and ion retarding grid is too large, space-charge effects can dominate the dynamics of the RPA, i.e., a stronger than prescribed field thus established causes erroneous ion energy distributions to be measured. The acceptable value for this spacing (as derived from equating the Bohm flux to the Child-Langmuir flux) is to be less than four Debye lengths [710]. A brief review of RPAs previously built reveals the present limitations in device dimensions (Table 1).

Table 1: Overview of previous RPA characteristic dimensions. ${ }^{*}$ Note this sensor is an aligned single orifice RPA.

\begin{tabular}{|c|c|c|c|}
\hline Reference & Grid opening $(\boldsymbol{\mu m})$ & Gap $(\boldsymbol{\mu m})$ & Material \\
\hline \hline$[9]^{*}$ & 200 & 457 & Molybdenum \\
\hline$[10]$ & 140 & 500 & Stainless \\
\hline$[11]$ & 279 & 1727 & Copper \\
\hline$[12]$ & 300 & 1700 & Copper \\
\hline This work & 100 & 300 & Tungsten \\
\hline
\end{tabular}

A spacing of less than $500 \mu \mathrm{m}$ between grids is deemed difficult to maintain [8], possibly due to the fact that relatively thin meshes may short as a result of deflection through electrostatic forces when brought into proximity, as well as through thermal expansion. By using a thicker substrate, we reduce the risk of deflection, while at the same time mitigating the cupping observed in the field established by thinner electrodes [7].

As a first-generation RPA, we propose a hybrid design that incorporates micromachined grids with indexing features for alignment (Figure 3). Aspect ratios of 10:1 have been demonstrated in silicon using deep reactive ion etching (DRIE). By using $700 \mu \mathrm{m}$ thick silicon wafers, we were able to create apertures of merely $100 \mu \mathrm{m}$ in diameter. Alignment marks also served as recesses to establish an inter-electrode spacing of $300 \mu \mathrm{m}$ in conjunction with alumina washer. These dimensions are narrower than previously reported RPAs, and MEMS fabrication limitations have yet to be reached (Table 1). Additionally, in utilizing such a thick electrode material, the need for multiple ion retarding grids is averted [9]. With a conventionally machined stainless steel housing, precise assembly is achieved to a few tens of microns. The sensor is kept modular by making electrical contact through pogo pins, eliminating the need to weld wire contacts to each grid. Making use of the $60^{\circ}$ rotational symmetry of the hexagonally packed grid openings, the same pattern can be used for all four grid layers; only the collector varies in shape.

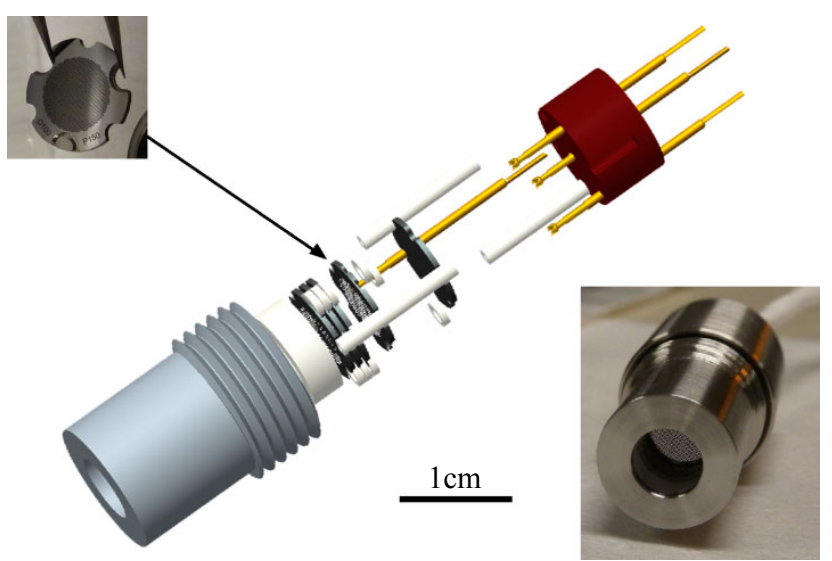

Figure 3: Assembled RPA (bottom right), exploded view of the hybrid-RPA benchmarking sensor (center) and detail of micromachined grid (left). Scale bar common to all three images. 


\section{FABRICATION}

Silicon was selected as a base material for the electrodes due to well-established processing methods, as well as its excellent mechanical performance and resilience. Two masks were used in the manufacturing of the grids to define alignment marks, spacing recesses, and apertures. First, alignment marks and recesses are defined with apertures in $10 \mu \mathrm{m}$ thick photoresist (PR) through contact photolithography and etched through DRIE (Figure $4 a, b$ ). Next, a $4 \mu \mathrm{m}$ hard oxide mask is deposited using plasma enhanced chemical vapor deposition (PECVD) and patterned with apertures and the grid boundary in $10 \mu \mathrm{m}$ PR $(4 \mathrm{c}, \mathrm{d})$. The oxide is removed using reactive ion etching (RIE), and DRIE ensues to finalize the grid pattern $(4 \mathrm{e}, \mathrm{f})$. Finally the PR and oxide are removed, and the grids are coated with tungsten (W) by means of sputtering $(4 \mathrm{~g})$.

a)

b)

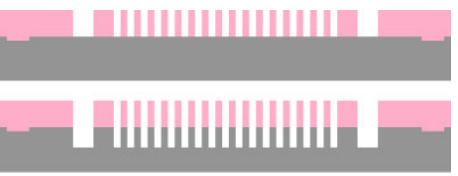

c)

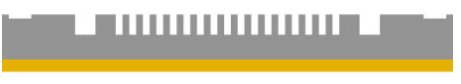

d)

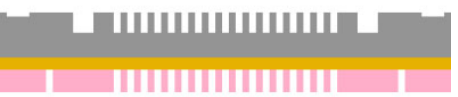

e)

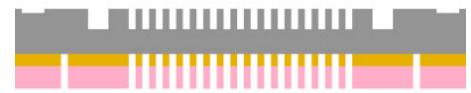

f)

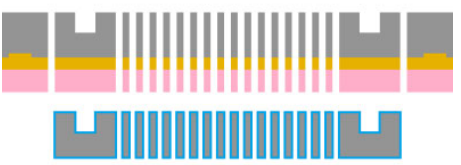

$\mathrm{Si}$

$\mathrm{Ox}$

PR

W

Figure 4: Grid process flow, a) recesses alignment marks and grid apertures defined, b) first DRIE, c) PECVD hard oxide mask deposited, d) aperture and grid edge patterned, e) hard mask RIE, f) second DRIE for release, g) W coating sputtered.

The sensor housing is milled from 316 stainless steel to a thousandth of an inch precision. Alumina rails fit into countersunk features in the main bore of the steel housing. These ensure the alignment of the $\mathrm{W}$-coated grids. The electrode stack is insulated from the device housing by means of an alumina washer and the guide rails. The small washers in Figure 3 are inserted into the DRIE grid recesses and establish an electrode spacing of $\sim 300 \mu \mathrm{m}$.

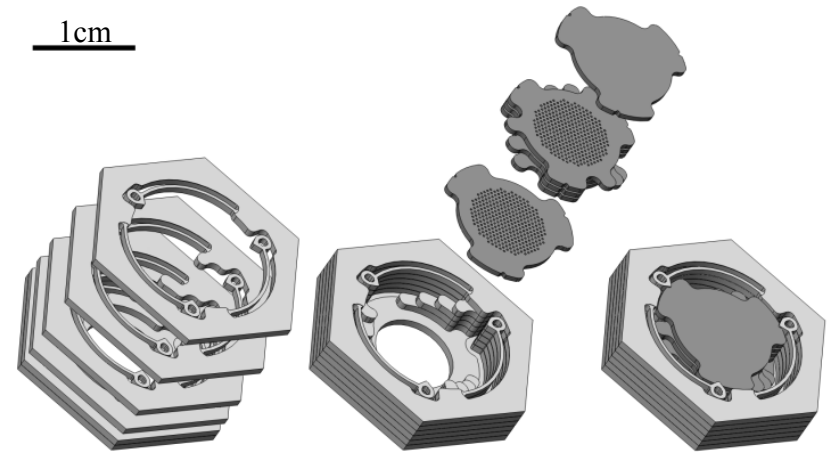

Figure 5: Exploded view of a MEMS-RPA showing housing layers prior to bonding (light), and electrode stack to be housed (dark).
The second-generation RPA will further refine grid alignment by batch microfabricating the device housing out of a silicon wafer stack. Grids will be held in place by means of curved silicon springs, and dimensional tolerances will improve by an order of magnitude to approximately one micron precision. The MEMSRPA concept is shown in Figure 5, where the retaining springs also serve as alignment enforcers. The feasibility of such mechanical assembly using DRIE silicon was demonstrated with a test structure consisting of the first three housing layers (Figure 6).

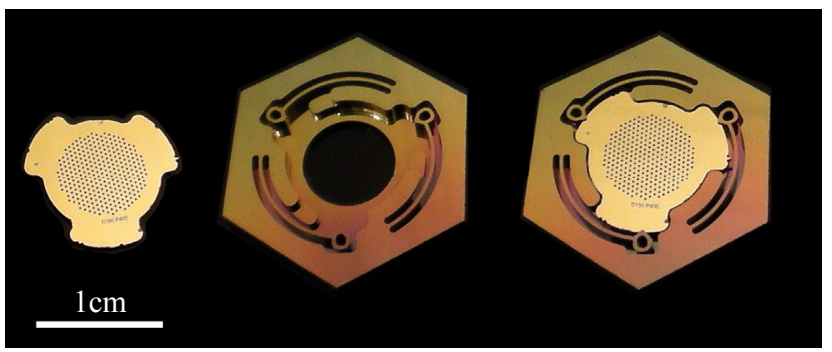

Figure 6: Grid (left), first three layers of the MEMS-RPA housing (center), and assembled grid held by retaining springs (right).

\section{DEVICE TESTING}

Preliminary tests have been carried out with the hybrid-RPA to characterize the operation of the device. The test chamber consists of an Ardara Technologies (Ardara, PA) Slim-Line ${ }^{\mathrm{TM}}$ ionizer mounted in line of sight with the RPA under test downstream of the ion beam. The thermionic ion source is operated at pressures of around $3 \times 10^{-5}$ Torr. The filament generates electrons of $-50 \mathrm{eV}$ energy using an emission current of $0.2 \mathrm{~mA}$. For simplicity, air is used as the ionized gas, and the ion energy is controlled by a potential assigned to a gold cage surrounding their nascent origin.

Since we are using a plasma-less ion source, the floating grid is removed from the RPA. Due to the absence of electrons from the flow, the potential established on a floating grid would effectively repel all impinging ions. Thus, the first grid is the electron repelling electrode, which in our experiments - along with the secondary electron suppression grid - is held at a $-10 \mathrm{~V}$ bias. The ion retarding grid is swept from $-15 \mathrm{~V}$ to $15 \mathrm{~V}$, and currents from these two biasing potentials and the collector plate (at ground) are synchronously recorded. Three Keithley 237 source measure units (SMUs) measure the charge flux to five digits of precision approximately every $500 \mathrm{~ms}$.

\section{EXPERIMENTAL RESULTS}

Data were first collected operating the chamber with a $10 \mathrm{~V}$ ion region. In these conditions, the expected particle energy for singly-charge ions is $10 \mathrm{eV}$. The first recorded trace with the hybrid-RPA shows a peak that is nearly $5 \mathrm{~V}$ lower. Subsequent tests maintained a $4-6 \mathrm{~V}$ discrepancy between the anticipated location of the peak distribution and that measured with our device. Using our modular hybrid design with stainless steel mesh grids instead of our precision grids; a conventional RPA is thus created for benchmarking purposes. This more standard sensor with apertures of $152 \mu \mathrm{m}$ showed a similar shift in peak, while signal strength was cut to less than half (Figure 7).

Comparing the data from the RPA with micromachined grids to an RPA with standard stainless steel mesh, preliminary results show improved signal strength with mechanically enforced optical transparency. A narrower, more prominent peak is observed using our hybrid-RPA. In order to further diagnose the shift in energy, various ion region voltages were tested (Figure 8). Our device was 
shown to reliably follow this increasing energy, and the offset at this time is considered a calibration issue of the instrument. However, we captured an anomaly with the hybrid-RPA data, in the form of a non-physical negative distribution. The apparent negative distribution is believed to result from ion beam focusing and defocusing effects within the device. To better understand this signal oddity, the electron suppression grids were disconnected from one another and measured individually using a fourth Keithley SMU. The second electron repelling grid should be expendable because ion energies presently used are too low (well below $1 \mathrm{keV}$ ) to induce secondary emission [10]. The prognosis is that this grid is not only unnecessary but in fact reduces the ion flux to the collector plate. By combining the collector and secondary electron repelling grid currents (as two collectors), the reported distribution is once again positive (not shown).

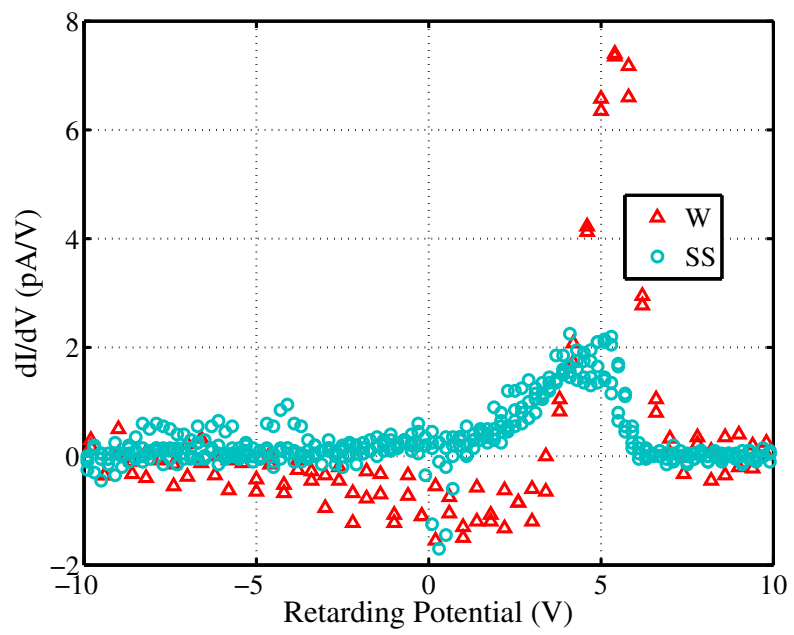

Figure 7: Measured energy distribution comparing tungstencoated aligned micromachined grids (W) to conventional unaligned stainless mesh (SS). A narrow distribution and more intense peak is obtained with the MEMS design.

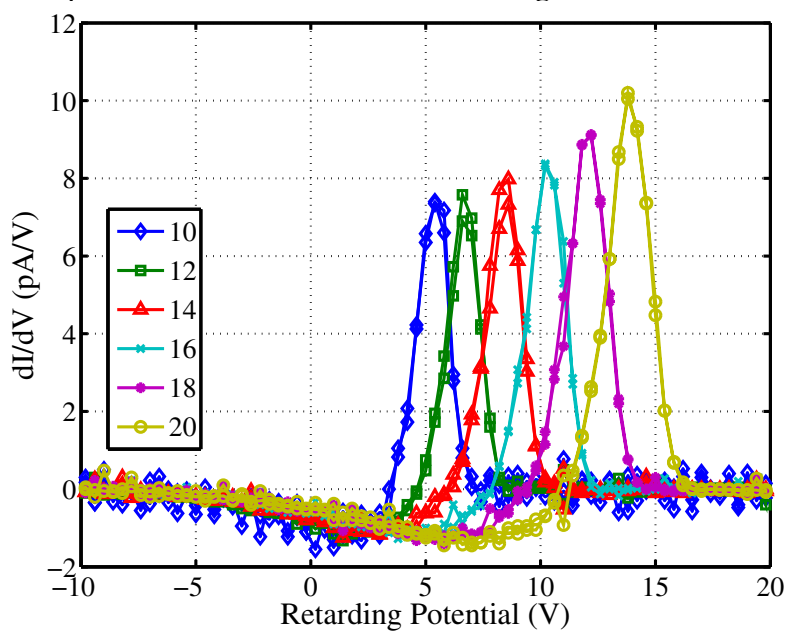

Figure 8: Collected energy distributions using the hybrid-RPA with tungsten-coated grids. Traces demonstrate that the sensor detects the prescribed increase in ion energy.

\section{CONCLUSION}

We preliminarily demonstrated a novel RPA using MEMS machining techniques to enforce grid alignment. With increased optical transparency, signal strength was improved by a factor of more than two over conventional RPAs. By incorporating microscale features and improving construction tolerances, devices can be made compatible with denser plasmas while maintaining large SNR. Thicker electrode material improve the longevity of RPAs in harsh environments, while reducing the effect of electric field cupping, removing the need for multiple ion retarding electrodes. A second-generation RPA is proposed and we demonstrated using a test structure the feasibility of assembling grids using MEMS deflection springs.

\section{ACKNOWLEDGEMENTS}

The microfabrication was conducted at MIT's Microsystems Technology Laboratories. This work was supported by NASA under Award No. NNC08CA58C (program managers R. Manning and T. Wallett). Any opinions, findings, and conclusions or recommendations expressed in this publication are those of the author(s) and do not necessarily reflect the views of NASA and therefore, no official endorsement of NASA should be inferred.

\section{REFERENCES}

[1] R. A. Hartunian, G. E. Stewart, S. D. Fergason, T. J. Curtiss, and R. W. Seibold, "Causes and Mitigation of Radio Frequency (RF) Blackout During Reentry of Reusable Launch Vehicles," The Aerospace Corporation, (2007).

[2] K. M. Lemmer, A. D. Gallimore, and T. B. Smith, "Using a helicon source to simulate atmospheric re-entry plasma densities and temperatures in a laboratory setting," Plasma Sources Science and Technology, 18, (2009), pp. 1 - 8.

[3] M. C. Kelley, The Earth's Ionosphere: Plasma Physics and Electrodynamics, Academic Press Inc., Boston, MA, $2^{\text {nd }}$ edition, 2009.

[4] L. B. King, "Transport-Property and Mass Spectral Measurements in the Plasma Exhaust Plume of a Hall-Effect Space Propulsion System," $\mathrm{PhD}$ thesis, University of Michigan, 1998.

[5] W. L. Jones Jr. and A. E. Cross, "Electrostatic-probe measurements of plasma parameters for two reentry flight experiments at 25000 feet per second," NASA Tech. Report NASA, Langley Research Center, Hampton, VA, (1972).

[6] K. M. Lemmer, A. D. Gallimore, T. B. Smith, and D. R. Austin, "Review of two retarding potential analyzers for use in high density helicon plasma," $30^{\text {th }}$ International Electric Propulsion Conference, Florence, Italy, (2007).

[7] J. M. Partridge, "Development and Implementation of Diagnostics for Unsteady Small-Scale Plasma Plumes," PhD thesis, Worcester Polytechnic Institute, September 2008.

[8] I. H. Hutchinson, Principles of Plasma Diagnostics, Cambridge University Press, New York, NY, $2^{\text {nd }}$ edition, 2002.

[9] C. M. Marrese, N. Majumdar, J. M. Haas, G. Williams, L. B. King, and A. D. Gallimore, "Development of a Single-orifice Retarding Potential Analyzer for Hall Thruster Plume Characterization," Proceedings of the $25^{\text {th }}$ International Electric Propulsion Conference, Cleveland, OH, (1997).

[10] Y. Azziz, "Experimental and Theoretical Characterization of a Hall Thruster Plume," PhD thesis, Massachusetts Institute of Technology, June 2007.

[11] B. E. Beal and A. D. Gallimore, "Energy analysis of a Hall thruster cluster," 28 $8^{\text {th }}$ International Electric Propulsion Conference, Toulouse, France, (2003).

[12] R. R. Hofer, J. M. Haas, and A. D. Gallimore, "Ion voltage diagnostics in the far-field plume of a high-specific impulse Hall thruster," $39^{\text {th }}$ AIAA/ASME/SAE/ASEE Joint Propulsion Conference, Huntsville, AL, (2003). 


\title{
NOVEL READOUT SCHEME FOR MEMS VIBRATORY GYROSCOPES BASED ON SIGNAL PHASE SHIFT
}

\author{
M. Dalal, A.N. Shirazi, W.K. Sung, G. Casinovi, and F. Ayazi \\ Georgia Institute of Technology, Atlanta, Georgia, USA
}

\begin{abstract}
This paper introduces and demonstrates experimentally a new readout scheme for MEMS vibratory gyroscopes that relies on signal phase instead of signal amplitude to measure the rotation rate. When the drive and sense resonance modes of the gyroscope are both excited by forces of equal intensity and $90^{\circ}$ phase difference, the Coriolis force induces a phase shift in the gyroscope response that is proportional to the gyroscope rotation rate. This effect is predicted by theoretical analysis and has been verified through simulation and measurement of two different gyroscopes, a high-frequency bulk acoustic wave (BAW) disk gyroscope, and a low-frequency mode-matched tuning fork gyroscope $\left(\mathrm{M}^{2}-\mathrm{TFG}\right)$. The two gyroscopes report a measured phase sensitivity of 0.597 $\mathrm{mV} / \% / \mathrm{s}$ and $0.148 \mathrm{mV} / \% / \mathrm{s}$, respectively. The linear response observed on both devices supports the use of the phase readout architecture as a valid measurement scheme.
\end{abstract}

\section{INTRODUCTION}

The most common readout architectures currently implemented in Coriolis-based MEMS vibratory gyroscopes rely on signal amplitude to measure the rotation rate of the device. In this type of readout scheme, only one of two resonance modes of the device is electrically excited. The Coriolis force caused by a rotation around the gyroscope axis creates a coupling between the two modes, so that the amplitude of the second resonance mode which is used as the sense signal - is proportional to the angular velocity of rotation $\Omega_{z}$ [1].

This paper introduces a new method of measuring the gyroscope rotation rate based on signal phase instead of signal amplitude. In this scheme, the drive and sense resonance modes are both excited by forces that are of equal intensity but $90^{\circ}$ out of phase. A theoretical analysis of a 2-DOF mass-spring gyroscope model [2] shows that the Coriolis force induces a phase shift in the responses of the two resonance modes of the gyroscopes. For sufficiently small rotation rates, the phase shift is proportional to the angular velocity of rotation $\Omega_{z}$. Therefore, it can be used to measure the gyroscope rotation rate using standard phase-detection circuitry.

A phase-based readout scheme offers several advantages over the more traditional amplitude-based methods mentioned earlier. First, the amplitude of the output signal remains constant, thus minimizing the effect of additive noise. More importantly, it can be shown that a rotating excitation induces a similar phase shift in the responses of the two resonance modes, thus mimicking the effect of physical rotation [2]. This fact can be exploited to devise selfcalibrating gyroscope architectures that do not require the addition of any moving parts to the gyroscope assembly.

\section{THEORETICAL ANALYSIS}

The analysis that follows examines the effect of the Coriolis force on the response of a mode-matched vibratory gyroscope $\left(f_{\text {drive }}=f_{\text {sense }}=\omega_{0} / 2 \pi\right)$ when excitations $90^{\circ}$ out of phase with one another are applied to the two resonance modes of the device with matched frequencies. In this paper, these modes will be designated as $I$ and $Q$, respectively. It is assumed that the gyroscope rotates around its $z$-axis (the sensitive axis) at a constant angular velocity $\Omega_{z}$ with respect to a fixed inertial frame of reference. Then the behavior of the gyroscope can be analyzed in terms of its normal mode model, which consists of an $n$ equivalent 2-DOF mass-spring system, is described by the following set of equations

$$
\begin{aligned}
& \ddot{x}+\frac{\omega_{0}}{Q} \dot{x}-2 \lambda \Omega_{z} \dot{y}+\omega_{0}^{2} x=F_{1} \cos \left(\omega_{0} t\right) \\
& \ddot{y}+\frac{\omega_{0}}{Q} \dot{y}-2 \lambda \Omega_{z} \dot{x}+\omega_{0}^{2} y=F_{2} \sin \left(\omega_{0} t\right)
\end{aligned}
$$

where $x$ and $y$ are generalized coordinates, $\omega_{0}$ is the resonance frequency of the mass-spring system, $Q$ the quality factor, and $\lambda$ a constant that depends on the gyroscope type and on the index of the resonance mode of the device [3]. The steady-state solution to this set of differential equations can be found using phasor analysis, which transforms (1) into the following equivalent set of algebraic equations

$$
j \omega_{0}\left[\begin{array}{cc}
\omega_{0} / Q & -2 \lambda \Omega_{z} \\
2 \lambda \Omega_{z} & \omega_{0} / Q
\end{array}\right]\left[\begin{array}{l}
X \\
Y
\end{array}\right]=\left[\begin{array}{c}
F_{1} \\
-j F_{2}
\end{array}\right]
$$

The solution to these equations is

$$
\begin{aligned}
& X=\frac{1}{j \omega_{0}} \frac{\left(\omega_{0} / Q\right) F_{1}-j 2 \lambda \Omega_{z} F_{2}}{\left(\omega_{0} / Q\right)^{2}+\left(2 \lambda \Omega_{z}\right)^{2}} \\
& Y=-\frac{1}{j \omega_{0}} \frac{2 \lambda \Omega_{z} F_{1}+j\left(\omega_{0} / Q\right) F_{2}}{\left(\omega_{0} / Q\right)^{2}+\left(2 \lambda \Omega_{z}\right)^{2}}
\end{aligned}
$$

which corresponds to the following sinusoidal functions

$$
\begin{aligned}
& x(t)=\frac{Q}{\omega_{0}} \frac{\sqrt{\left(F_{1} \omega_{0}\right)^{2}+\left(2 F_{2} Q \lambda \Omega_{z}\right)^{2}}}{\omega_{0}^{2}+\left(2 Q \lambda \Omega_{z}\right)^{2}} \sin \left(\omega_{0} t-\theta_{1}\right) \\
& =A_{1} \sin \left(\omega_{0} t-\theta_{1}\right) \\
& y(t)=-\frac{Q}{\omega_{0}} \frac{\sqrt{\left(F_{2} \omega_{0}\right)^{2}+\left(2 F_{1} Q \lambda \Omega_{z}\right)^{2}}}{\omega_{0}^{2}+\left(2 Q \lambda \Omega_{z}\right)^{2}} \cos \left(\omega_{0} t-\theta_{2}\right) \\
& =A_{2} \cos \left(\omega_{0} t-\theta_{2}\right)
\end{aligned}
$$

where

$$
\theta_{1}=\tan ^{-1}\left(\frac{2 F_{2} Q \lambda \Omega_{z}}{F_{1} \omega_{0}}\right), \theta_{2}=\tan ^{-1}\left(\frac{2 F_{1} Q \lambda \Omega_{z}}{F_{2} \omega_{0}}\right)
$$

The expressions in (2) are the steady-state solutions of (1), and it can be seen that the Coriolis force introduces phase shifts $\theta_{l}$ and $\theta_{2}$ in the gyroscope response. For small values of $\Omega_{z}$, these phase shifts are directly proportional to $\Omega_{z}$. 


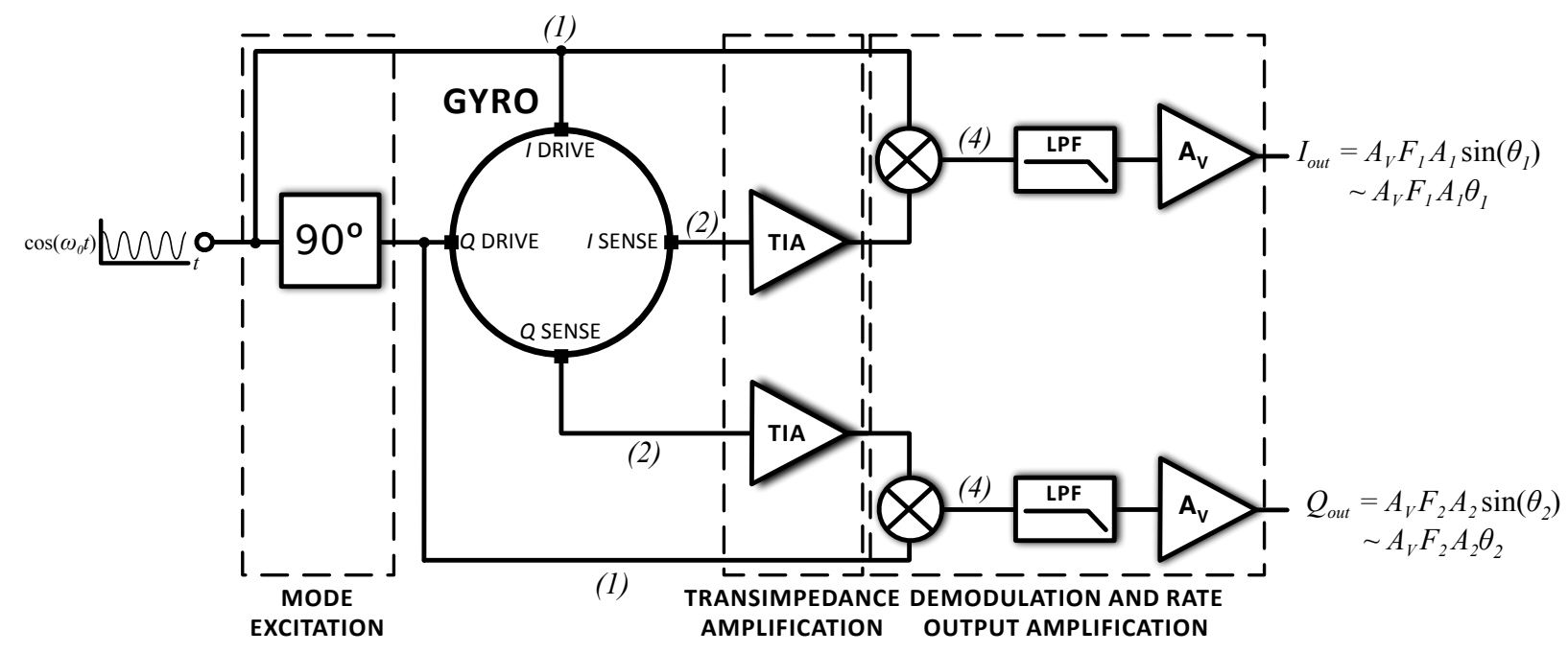

Figure 1: Block diagram of phase-shift readout architecture

A block diagram of the phase readout architecture is shown in Fig. 1, in which the drive forces from (1) are applied to the two degenerate resonance modes of the gyroscope through the electrodes identified as $I_{\text {Drive }}$ and $Q_{\text {Drive }}$, respectively. The gyroscope responses in (2) are seen at output terminals $I_{\text {Sense }}$ and $Q_{\text {Sense, }}$, which are $90^{\circ}$ out of phase with each other as well as their respective inputs. After a Coriolis excitation is applied to the zaxis, the drive and sense signals from each complementary mode are multiplied together, giving

$$
\begin{aligned}
& I_{\text {mult }}(t)=F_{1} A_{1} \cos \left(\omega_{0} t\right) \sin \left(\omega_{0} t-\theta_{1}\right) \\
& Q_{\text {mult }}(t)=F_{2} A_{2} \sin \left(\omega_{0} t\right) \cos \left(\omega_{0} t-\theta_{2}\right)
\end{aligned}
$$

where $A_{1}$ and $A_{2}$ are the coefficients of the sine and cosine terms in (2). Using trigonometric identities, the expressions in (4) simplify to

$$
\begin{aligned}
& I_{\text {mult }}(t)=\frac{F_{1} A_{1}}{2}\left[\sin \left(2 \omega_{0} t-\theta_{1}\right)-\sin \theta_{1}\right] \\
& Q_{\text {mult }}(t)=\frac{F_{2} A_{2}}{2}\left[\sin \left(2 \omega_{0} t-\theta_{2}\right)+\sin \theta_{2}\right]
\end{aligned}
$$

Following the low pass filtering and rate output amplification stages, the frequency components at $2 \omega_{0}$ are removed from (5), reducing the signal of the phase readout scheme output to

$$
I_{\text {out }}(t)=-\frac{F_{1} A_{1}}{2} \sin \theta_{1}, Q_{\text {out }}(t)=\frac{F_{2} A_{2}}{2} \sin \theta_{2}
$$

in which $\sin \theta \approx \theta$ for small values of $\theta$.

\section{SIMULATION RESULTS}

The linear relationship between phase shift in the gyroscope response and its rotation rate was verified using ANSYS numerical simulations of actual designs of a bulk acoustic wave (BAW) disk gyroscope [4,5] and a tuning fork gyroscope [6] (Fig. 2).

The BAW gyroscope consists of a center-supported disk structure fabricated on a [100] silicon wafer with capacitivelycoupled drive, sense, and control electrodes. It operates in the in-
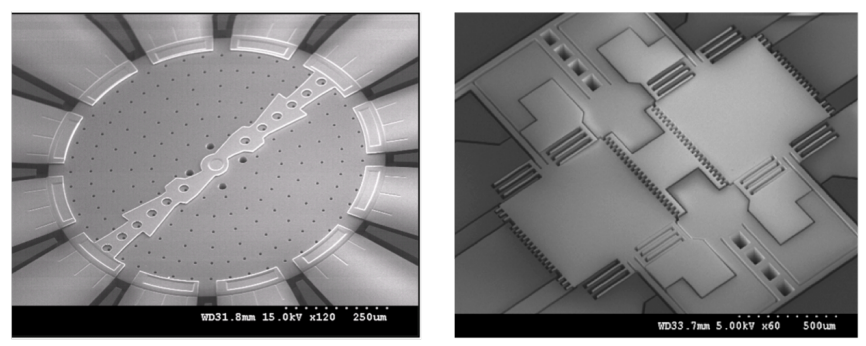

Figure 2: SEM of bulk acoustic wave (BAW) disk gyroscope (left) and tuning fork gyroscope $\left(M^{2}-T F G\right.$, right)

plane resonance mode of index $n=3$, ensuring that both the $I$ and $Q$ modes have the same resonance frequency $(\sim 10 \mathrm{MHz})$ despite the anisotropic nature of single-crystal silicon [4]. Sinusoidal input excitations were applied to the drive electrodes aligned with one of the degenerate resonance modes $\left(I_{\text {Drive }}\right)$, while excitations of equal amplitude but $90^{\circ}$ out of phase were applied to the drive electrodes aligned with its complementary degenerate mode $\left(Q_{\text {Drive }}\right)$. The phases of the output currents at all the electrodes obtained from finite element simulations were recorded at eight different rotation rates, ranging from zero to $2100 \%$ s (Fig. 3). The extracted scale factor sensitivity is $3.81 \times 10^{-4} \%(\% / \mathrm{s})$.

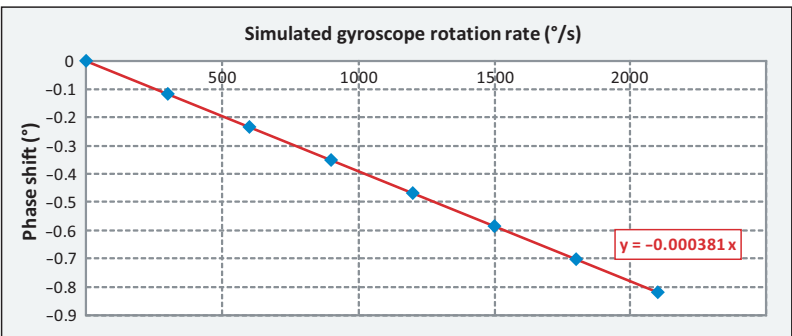

Figure 3: Simulated phase shift in the BAW disk gyroscope response due to Coriolis force, measured relative to its value at zero rotation rate. The solid line shows the theoretical analysis predictions from (3) assuming $F_{1}=F_{2}$.

Similar ANSYS simulations were performed on a modematched tuning fork gyroscope $\left(\mathrm{M}^{2}-\mathrm{TFG}\right)$, a device comprised of two proof masses supported by a network of flexural springs 


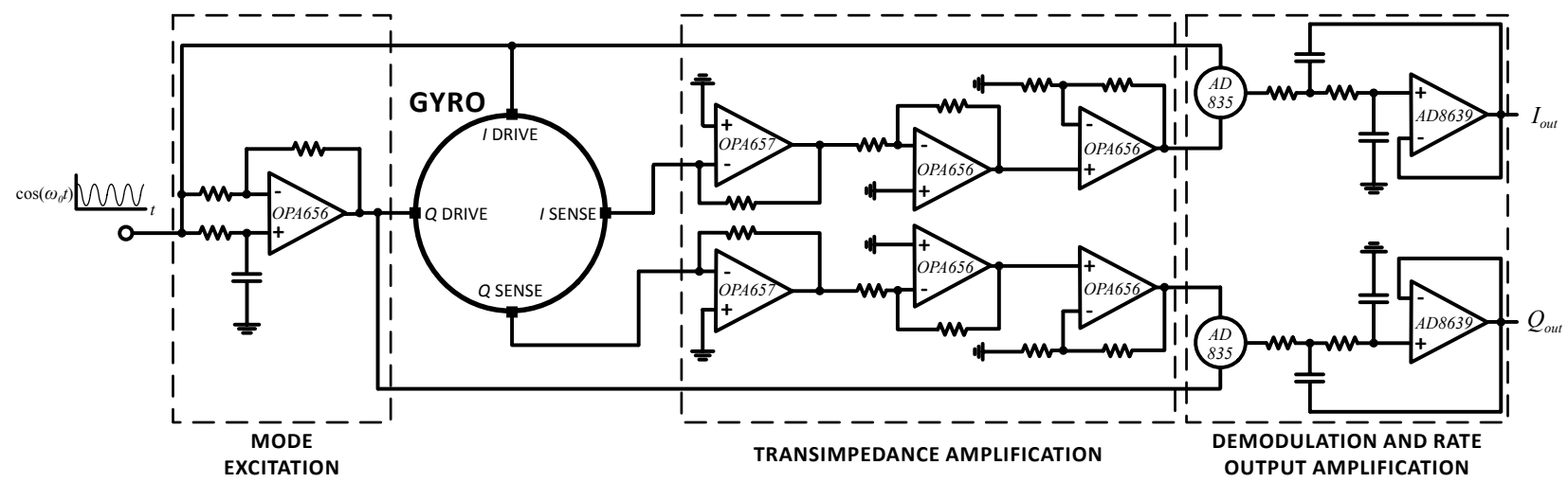

Figure 4: Schematic diagram of phase-shift interface electronics.

anchored to a central post with control electrodes symmetrically distributed around the proof masses [6]. The $\mathrm{M}^{2}-\mathrm{TFG}$ can attain high quality factors because its large proof mass and optimized anchor and flexural mode design reduce device surface and support losses, as well as thermo-elastic damping. However, the large size and flexural actuation of the structure result in a resonance frequency almost 1000 times lower than that of the BAW disk gyroscope.

The results of the ANSYS simulations of the $\mathrm{M}^{2}$-TFG are shown in Fig. 5. Along with Fig. 3, these results confirm that the phase shift is directly proportional to the rotation rate of the gyroscope. The simulated scale factor sensitivity of the $\mathrm{M}^{2}-\mathrm{TFG}$ is $2.51 \%(\%)$, while the equations in (3) with $F_{1}=F_{2}$ yield a value of $2.67 \%(\%)$. This discrepancy can be explained by the fact that in our $\mathrm{M}^{2}$-TFG design, the electrodes corresponding to the $I$ mode have slightly different area from the electrodes corresponding to the $Q$ mode.

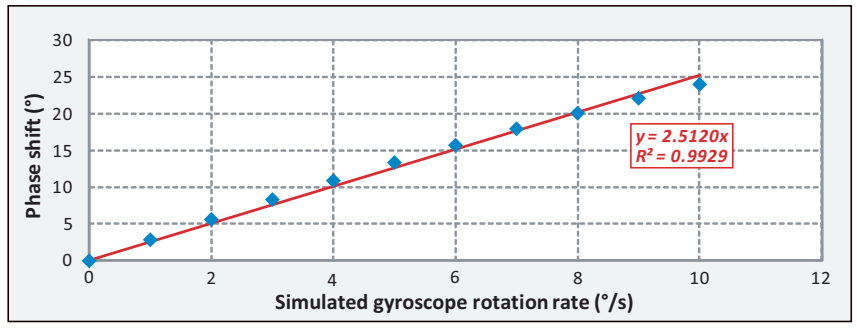

Figure 5: Simulated phase shift in the $M^{2}-T F G$ response due to Coriolis force, measured relative to its value at zero rotation rate. The solid line shows the best linear fit to the data.

\section{MEASURED RESULTS AND DISCUSSION}

In order to validate the results of the theoretical analysis and numerical simulation experimentally, the phase shift readout architecture shown in Fig. 1 was implemented on a printed circuit board (PCB), the schematic of which is shown in Fig. 4. Separate PCBs were made for the BAW (Fig. 6) and $\mathrm{M}^{2}$-TFG so that the unique footprints and traces of each device could be placed without adding too much complexity and size to the PCB design. In both cases, the input $I$-mode drive voltage was generated using an Agilent 4395A network analyzer locked into the resonance frequency of the respective device $\left(\omega_{0}\right)$. This signal was passed to both the gyroscope and an on-board tunable phase shifter that used a discrete op-amp (TI OPA656) to generate the $90^{\circ}$ phase shift for the device $Q$-mode excitation signal.

On both the $\mathrm{M}^{2}-\mathrm{TFG}$ and BAW PCBs, separate amplifier chains were used to read out the $I$-mode and $Q$-mode sense signals.
For the BAW, a trans-impedance amplifier (OPA657) was used at the sense channel input to provide current-to-voltage conversion and amplification of the phase-shifted signal. This signal was then passed to two voltage amplifiers that provided additional amplification and buffering for the mixer (AD835) stages. For the $\mathrm{M}^{2}$-TFG, the input TIA was replaced with an OPA656, which provided higher gain and lower bandwidth than the OPA657. The remaining amplifiers on this board matched their counterpart on the BAW PCB; however, the gain and bandwidth of each stage was adjusted for use with a low-frequency gyroscope.

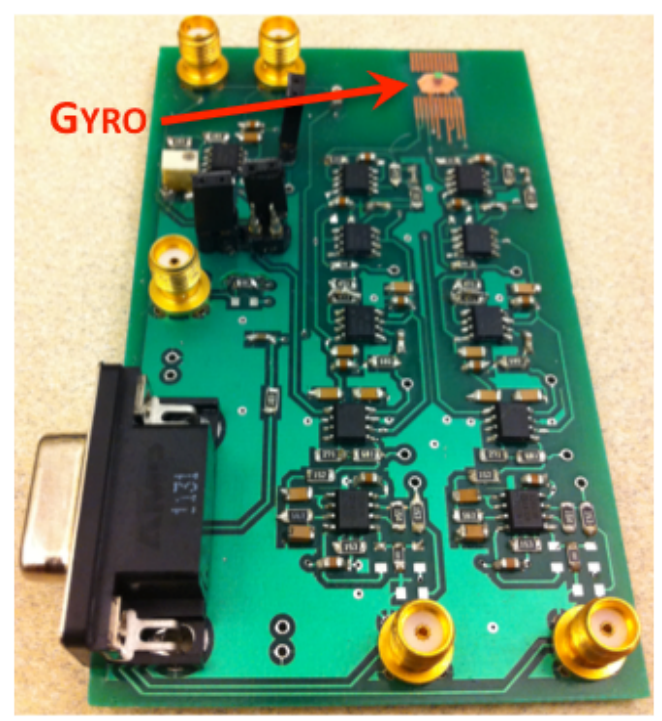

Figure 6: Phase readout $P C B$

For the BAW disk gyroscope, sinusoidally-varying rotation rates ranging from 0 to $10 \%$ were applied via the rate table. Consequently, the output signals denoted as $I_{\text {out }}$ and $Q_{\text {out }}$ in Fig. 1 were also observed to be sinusoids with amplitudes proportional to the applied rotation rate (Fig. 7). At the applied rates, it was derived that $\theta_{1}$ and $\theta_{2}$ would remain small enough to satisfy equation (6).

The BAW gyroscope was tested with an input power of 0 $\mathrm{dBm}$ applied to the $I_{\text {Drive }}$ and $Q_{\text {Drive }}$ terminals of the device. An Ideal Aerosmith rate table was used to generate sinusoidallyvarying rotation rates ranging from 0 to $10 \%$. The corresponding output signal ( $I_{\text {out }}$ in Fig. 4) is shown in Fig. 7. The measured datapoints were closely aligned along a straight line with a slope of 
$0.6 \mathrm{mV} / \%$ s (Fig. 8). The linearity of the collected measurements confirms the response predicted by the theoretical calculations and numerical simulations.

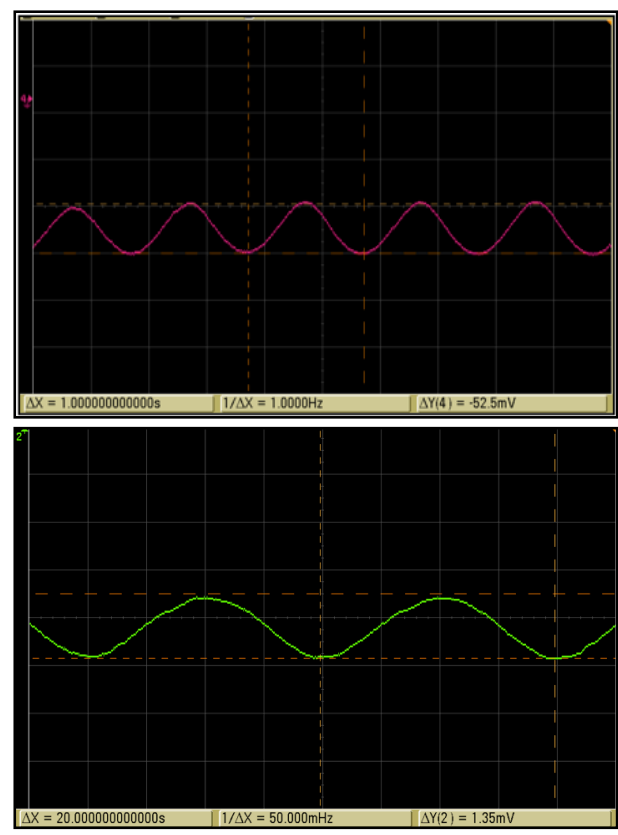

Figure 7: Oscilloscope traces of (top) BAW gyroscope output $\left(I_{\text {out }}\right)$ and (bottom) $M^{2}-T F G$ gyroscope output $\left(I_{\text {out }}\right)$ at $50 \%$ and $8 \%$, respectively.

Measurements were also taken on the $\mathrm{M}^{2}$-TFG using applied rotation rates from 0 to $10 \%$ (Fig. 7); however, the applied input power was reduced to $-4 \mathrm{dBm}$ to prevent the device from saturating. The scale factor measurements (Fig. 8) show a device sensitivity $0.15 \mathrm{mV} / \% / \mathrm{s}$. Like the BAW gyroscope, the $\mathrm{M}^{2}-\mathrm{TFG}$ also exhibits a very linear response to the input excitation.

Table 1 summarizes the simulated and measured performance parameters of both gyroscopes.

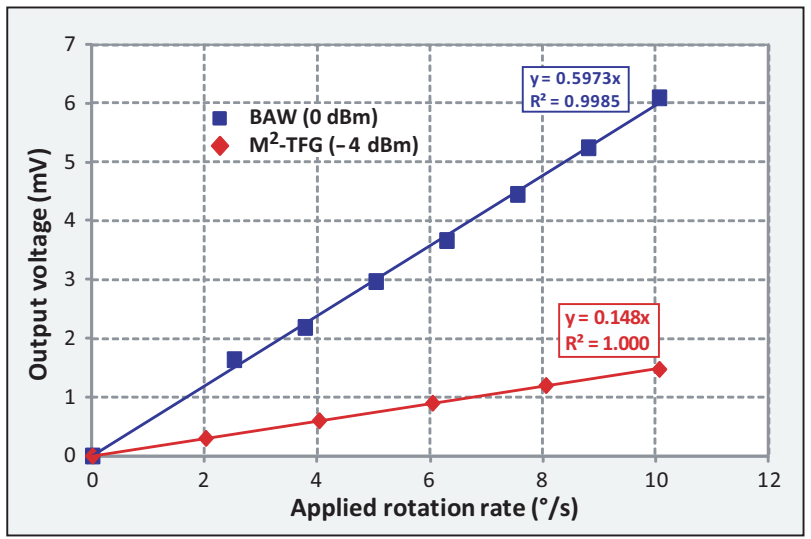

Figure 8: Measured phase shift response due to Coriolis force for the $B A W$ and tuning fork gyroscopes, respectively, relative to its value at zero rotation rate. Each solid line shows the best linear fit to the data.

\section{CONCLUSIONS}

The simulation results and experimental measurements described in the previous sections confirm that the phase-shift readout scheme introduced in this paper is an effective method of measuring the rotation rate of both high-frequency (BAW) and low-frequency $\left(\mathrm{M}^{2}-\mathrm{TFG}\right)$ gyroscopes. In principle, this readout scheme can be applied to any gyroscope that can be modeled by a 2-DOF mass-spring system. As mentioned in [2], the phase readout scheme can be integrated with a calibration scheme that does not require the use of a rotary stage for proper operation. However, the tradeoffs between amplitude- and phase-based readout schemes must be more thoroughly analyzed for a more robust comparison of the two systems and their benefits and challenges.

Table 1: Phase-shift readout performance summary of $B A W$ and $M^{2}-T F G$ gyroscopes

\begin{tabular}{|c|c|c|}
\hline & BAW & M $^{2}$-TFG \\
\hline & \multicolumn{2}{|c|}{ SIMULATION } \\
\hline Q (simulated) & 20,000 & 50,000 \\
\hline$f_{0}$ (simulated) & $10 \mathrm{MHz}$ & $5.95 \mathrm{kHz}$ \\
\hline Phase-Sensitivity & $3.81 \times 10^{-4} \% /(\%)$ & $2.51 \% / \mathrm{s})$ \\
\hline & \multicolumn{2}{|c|}{ MEASUREMENT } \\
\hline Q & 32,000 & 60,000 \\
\hline$f_{0}$ & $9.65 \mathrm{MHz}$ & $11.7 \mathrm{kHz}$ \\
\hline Excitation Power & $0 \mathrm{dBm}$ & $-4 \mathrm{dBm}$ \\
\hline Phase Sensitivity & $0.597 \mathrm{mV} /(\% / \mathrm{s})$ & $0.148 \mathrm{mV} /(\% / \mathrm{s})$ \\
\hline
\end{tabular}

\section{ACKNOWLEDGEMENTS}

Qualtré, Inc. provided research funding for the study described in this paper. Under an agreement between Qualtré and Georgia Tech, Dr. Ayazi is entitled to a share of sales royalty received by Georgia Tech from Qualtré. Under that agreement, the University and Dr. Ayazi also have received Qualtré stock. This study could affect their personal financial status. The terms of this arrangement have been reviewed and approved by Georgia Tech in accordance with its conflict of interest policies. This work was also supported in part by DARPA under contract \#W31P4Q-12-10004.

\section{REFERENCES}

[1] F. Ayazi and K. Najafi, "A HARPSS Polysilicon Vibrating Ring Gyroscope," J. Microelectromech. Syst., 10, 2 (2001).

[2] G. Casinovi, W.K. Sung, M. Dalal, A.N. Shirazi, and F. Ayazi, "Electrostatic Self-Calibration of Vibratory Gyroscopes," IEEE International Conference on Micro Electro Mechanical Systems, Paris, France (2012), pp. 559562.

[3] B.J. Gallacher, J.S. Burdess, A.J. Harris, and M.E. McNie, "Principles of a Three-Axis Vibrating Gyroscope," IEEE Trans. Aerosp. Electron. Syst., 37, 4 (2001).

[4] H. Johari and F. Ayazi, "Capacitive Bulk Acoustic Wave Silicon Disk Gyroscopes," IEEE International Electron Devices Meeting, San Francisco, USA (2006), pp. 513-516.

[5] W.K. Sung, M. Dalal, and F. Ayazi, "A 3MHz Spoke Gyroscope with Wide Bandwidth and Large Dynamic Range," IEEE International Conference on Micro Electro Mechanical Systems, Hong Kong (2010), pp. 104-107.

[6] M.F. Zaman, A. Sharma, Z. Hao, and F. Ayazi, "A ModeMatched Silicon-Yaw Tuning-Fork Gyroscope with Subdegree-Per-Hour Allan Deviation Bias Instability," J. Microelectromech. Syst., 17, 6 (2008).

\section{CONTACT}

*M. Dalal, tel: +1-404-385-3291; mdalal@gatech.edu 


\title{
PARYLENE SUPPORTED UNCOOLED THERMOELECTRIC INFRARED DETECTOR WITH UMBRELLA LIKE ABSORBER
}

\author{
M. J. Modarres-Zadeh", Z. S. Carpenter, and R. Abdolvand \\ Dynamic Microsystems Lab, Oklahoma State University, Tulsa, Oklahoma, USA
}

\section{ABSTRACT}

In this work a very small $(20 \mu \mathrm{m} \times 20 \mu \mathrm{m})$ uncooled surfacemicromachined thermoelectric infrared detector is fabricated and tested. This TE detector features a P-doped polysilicon/Nichrome thermocouple embedded into a layer of Parylene- $\mathrm{N}$ to provide structural support. This sensor also features an umbrella-like IR absorber with the area of $\sim 19 \mu \mathrm{m} \times 19 \mu \mathrm{m}$ resulting in a $90 \%$ fill factor.

At room temperature, a responsivity of $\sim 150 \mathrm{~V} / \mathrm{W}$ with a time constant of $1.3 \mathrm{~ms}$ is measured from the fabricated devices in vacuum when viewing a $500 \mathrm{~K}$ blackbody without any concentrating optics. NEP and $\mathrm{D}^{*}$ are calculated to be $2.6 \mathrm{e}-10$ watt and $7.7 \mathrm{e} 6 \mathrm{~cm}^{*} \mathrm{~Hz}^{\wedge} 0.5 / \mathrm{w}$, respectively.

\section{INTRODUCTION}

Infrared (IR) or thermal imaging cameras have come a long way from their early days of development and MEMS technology has played an important role in the substantial progress made in the last few decades. Today, focal planar arrays (FPA) are fabricated with 100's of thousand detection cells integrated with CMOS interface circuit [1]. The applications of IR cameras have continuously grown with the technology and today it pertains to military, industrial, consumer electronics, and entertainment sectors. However, the relatively high cost of IR cameras is still a limiting factor in their availability into our everyday life.

IR detectors mainly drop into two categories: photon detectors and thermal detectors. Photon detectors have fast responses and they offer very good signal to noise ratio [1]. But, they require cryogenic cooling [1] which greatly increases the system cost/power consumption and limits the mobility of those systems. At room temperature, thermal noises terribly reduce the performance of these detectors. On the other hand, thermal detectors do not have this limitation and operate at room temperature. In these detectors, the incident radiation is absorbed causing a change in a temperature of an element. This change could be measured through several different mechanisms. Bolometer, pyroelectric, and thermoelectric (TE) detectors are examples of such class of IR detectors. In bolometers, a temperature sensitive resistor is used to measure the amount of the absorbed radiation. For these detectors, one limitation is that the instrument (substrate) temperature should be well stabilized [2]. In pyroelectric detectors, internal polarization happens due to temperature change in the pyroelectric material. To create the temperature change, the incoming radiation should be chopped. Adding a mechanical chopper is not favorable for portable and low-power applications. In thermoelectric detectors, Seebeck effect is used to measure the temperature difference between the absorber (hot junction) and the substrate (cold junction). These detectors have relatively low responsivity which makes the readout circuit complicated. However, there is no need for a mechanical chopper or tight temperature stabilization.

In this paper, the focus is to improve the sensitivity of the thermoelectric detectors by improving the thermal isolation resistance between the hot and the cold junctions. There have been many efforts dedicated to developing these detectors in the past $[3$, $4,5,6]$. This work continues the trend of decreasing the detector size, using folded thermal isolation arms, and introduc the application of a high thermally-resistive polymer (i.e. Parylene) to enhance thermal isolation and subsequently improve the responsivity. The physical design of the device and the materials used are presented as well as simulations of the transient thermal response. Lastly, the fabrication of the device is briefly discussed and the experimental results are presented.

\section{DESIGN}

As shown in Figure 1, the detector has an umbrella like absorber that covers most of the device area. This absorber is connected to a membrane through a post. This membrane is made of material with relatively high thermal conductivity and therefore the temperature of the absorber and the post is assumed to be fairly uniform. The TE hot junction is placed on this membrane. This membrane is suspended by two arms that are made of parylene and TE wires where parylene provides the required mechanical strength. On the silicon substrate a thin layer of silicon nitride is deposited to avoid low resistive path between the TE wires.

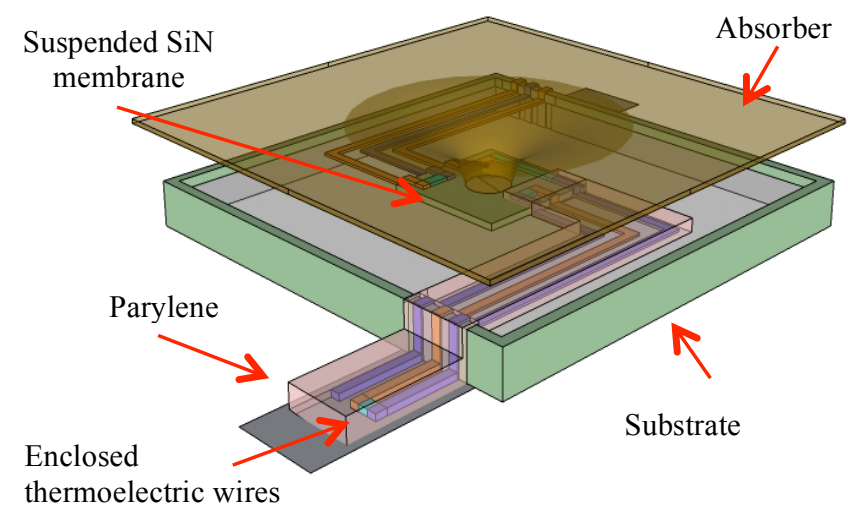

Figure 1: The schematic view of the detector showing the suspended silicon nitride membrane in the center of the device in between the absorber and the substrate, and how the thermoelectric wires are embedded inside the parylene arms.

In a thermoelectric detector, TE wires are the most important components of the detector since they directly generate the output voltage and define the responsivity. To compare different TE materials, ZT $\left(=\frac{\sigma . S^{2}}{k}\right.$ where $\mathrm{S}, \mathrm{k}$, and $\sigma$ are Seebeck coefficient, thermal conductivity and electrical conductivity, respectively) is a relevant figure of merit. For example at room temperature, bismuth telluride exhibits one of the highest ZT values (1.14) [7]. However, this material is not CMOS compliant. In this work polysilicon is chosen as one of the TE wires. ZT of silicon is low (0.01) [8] but it is readily available in the CMOS process which greatly reduces the fabrication cost and complexity. For the second TE wire, nichrome is chosen because of its low thermal conductivity [9] and chemical resistivity to hydrofluoric-based release processes.

To improve the device performance, the thermal resistance between the absorber and the substrate should be increased. This can be realized by looking at the DC responsivity equation [3]:

$$
\mathrm{R}=\mathrm{n} . \mathrm{N} . \mathrm{S} / \mathrm{G}
$$

Where $\mathrm{n}$ is the absorption efficiency, $\mathrm{N}$ is the number of thermocouples, $\mathrm{S}$ is the seebeck coefficient, and $\mathrm{G}$ is the thermal 
conduction ( $1 /$ thermal resistance) from the hot to cold junctions. Since the thermal conductivity of the TE wires is large, the first consideration is to reduce their cross sections and make them as small as possible. In this work, based on the detector size, the 20 $\mu \mathrm{m}$ long polysilicon wire has the thickness and width of $125 \mathrm{~nm}$ and $1 \mu \mathrm{m}$, respectively. This wire does not have the enough mechanical strength to hold the suspended absorber. Another material has to be added to provide structural support for the fragile wires. Silicon dioxide and silicon nitride have been used by others $[4,10]$. In this work, parylene- $\mathrm{N}$ is chosen because of its low thermal conductivity. Comparing the thermal conductivity of parylene $(\sim 0.1 \mathrm{~W} /(\mathrm{m} . \mathrm{K}))[11]$ to silicon dioxide $(>1 \mathrm{~W} /(\mathrm{m} . \mathrm{K})$ for thicknesses larger than $250 \mathrm{~nm}$ )[12] states that using parylene can greatly increase the thermal resistance between the hot and cold junctions. There are different types of parylene. Among them, parylene- $\mathrm{C}$ has the lowest thermal conductivity; however, its melting point is about $290{ }^{\circ} \mathrm{C}$ [11] which limits the processes that can be carried out on the wafer after the film deposition. Parylene$\mathrm{N}$ has slightly higher thermal conductivity $(125 \%)$, but its melting point is close to $420{ }^{\circ} \mathrm{C}$ [11]. Since the absorber is being deposited at high temperature after parylene deposition, parylene- $\mathrm{N}$ was preferred over the other types.

Another consideration in the design of the detector is choosing the number of the TE pairs. This can be determined by looking into the responsivity equation. The objective is to reduce the thermal conduction from the hot to cold junctions $(\mathrm{G})$ which consists of heat loss due to the TE wires, atmosphere, and the supporting parylene arms. By placing the detectors in vacuum, heat loss due to conduction through the gas can be negligible [13]. If the thermal conduction through the supporting arms was higher than the TE wires, increasing the number of the TE pairs could lead to increase in the responsivity. However, in this work, the dimension of the parylene $(2.5 \mu \mathrm{m}$ wide and $0.51 \mu \mathrm{m}$ thick $)$ is chosen in a way that the thermal conduction of the arms is much less than the TE wires. Based on the Fourier law of heat conduction, the product of thermal conductivity $(\mathrm{k})$ and cross section area (A) can be used to compare the heat losses. kAparylene $=1.6 \mathrm{e}-13$ and $\mathrm{kA}_{\mathrm{TE} \text { wires }}=5 \mathrm{e}-12$ are calculated assuming the thermal conductivity of about $40-50 \mathrm{~W} /(\mathrm{m} . \mathrm{K})[14]$ for polysilicon. These results show that the thermal loss due to the TE wires is 30 times than the parylene supporting arms. In this case, adding more thermocouple pairs, does not improve the detector responsivity, therefore, the detectors presented here are designed to contain only one TE pair.

To further improve the sensitivity of a detector, the incoming radiation should be efficiently absorbed by the absorber. There are different ways to achieve high absorption such as using metal black coatings $[15,16]$ such as gold-black or using optical cavities. Among the metal-black coatings, gold-black has relatively low density, low reflection, and high thermal conduction. However, these coatings are not mechanically stable [16]. It has been shown that high absorption can be achieved in metal / dielectric / metal cavities [17]. In this work, the cavity is formed on the absorber. Nichrome and silicon nitride are used as the metal layers and dielectric film, respectively. Since metals have large extinction coefficient, they exhibit high absorption but they also have high reflection due to their high refractive index which greatly reduces the absorption. To lower the refractive index, the metal layer can be made very thin [17]. According to [17]

$$
n=k=\left(\frac{\sigma}{2 * \omega * \varepsilon_{0}}\right)^{.5}
$$

(Where $\mathrm{k}$ is the extinction coefficient, $\sigma$ is electrical conductivity, $\omega$ is the incident light frequency in radians, and $\varepsilon_{0}=$ $8.85 * 1 \mathrm{e}-12$ is the vacuum permittivity), by reducing the metal layer thickness, its refractive index can be lowered. However, if the layer becomes too thin, the IR radiation might pass through without total absorption. The cavity is required so that any ray goes under multiple passes through the metal layer.

\section{SIMULATION}

Finite element analysis (COMSOL 4.1) is used to simulate the heat distribution and find the thermal response of the proposed detector. During the release step in the fabrication process (will be described later), it was observed that the parylene arms tend to bend upward due to the induced stress during the thermal cycles. As shown in Figure 2, two tethers are added to each side of the supporting arms to prevent the arms from deformation.

For the simulation, a constant power is applied to the absorber assuming $100 \%$ absorption and the heat distribution is depicted in Figure 2(a). To measure the transient response, the absorber temperature calculated from the previous part is set as the initial value for the absorber and the time it takes for the absorber to reach the same temperature as the substrate is plotted (Figure 2(b)). As it can be seen, the temperature fall time (or rise time) in the simulation is less than $8 \mathrm{~ms}$ with a time constant of $1.6 \mathrm{~ms}$.

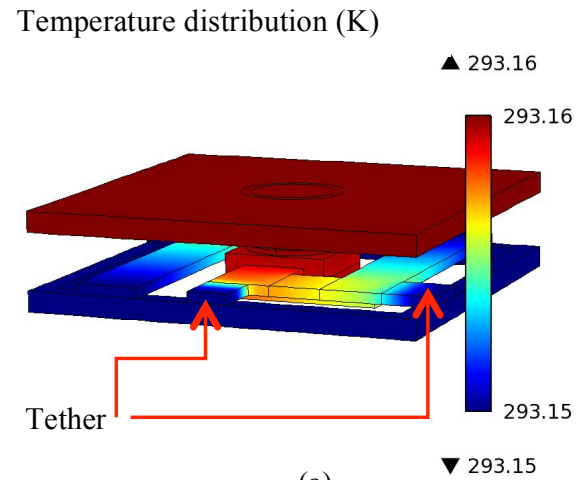

(a)

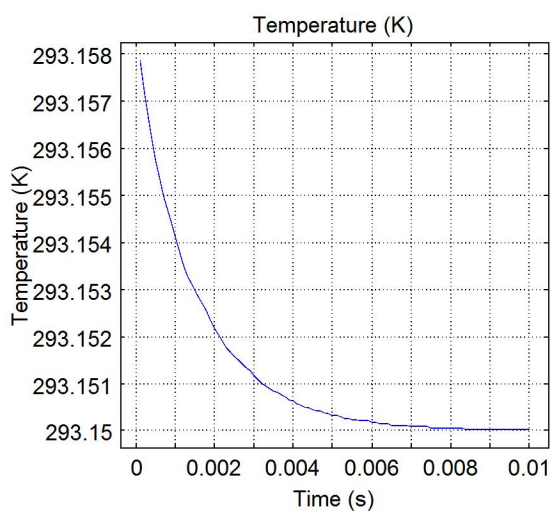

(b)

Figure 2: (a) Heat distribution when a constant power is applied to the absorber assuming $100 \%$ absorption (b) The temperature of the absorber versus time.

\section{FABRICATION}

Figure 3 represents the 7-step surface-micromachining process carried out for fabricating the thermoelectric detectors. The first step of the process is the deposition of a sacrificial PECVD $\mathrm{SiO}_{2}$ layer, which is patterned to serve as the platform for the suspended absorber. Next, LPCVD silicon nitride is deposited and patterned by dry etching to serve as the insulation layer for the electrical connections on top of both the substrate and the absorber post in the middle (Figure 3(a)). The TE junctions are configured with P- 
type polysilicon and nichrome. P-type polysilicon is deposited using LPCVD and then annealed at $950^{\circ} \mathrm{C}$ for $30 \mathrm{~min}$ to activate the dopants. Sheet resistance of $350 \mathrm{Ohm} /$ square was measured using the four point probe technique. Then nichrome is sputtered and patterned by lift-off. To improve the adhesion of nichrome to the substrate a thin layer of chromium is sputtered prior to that.
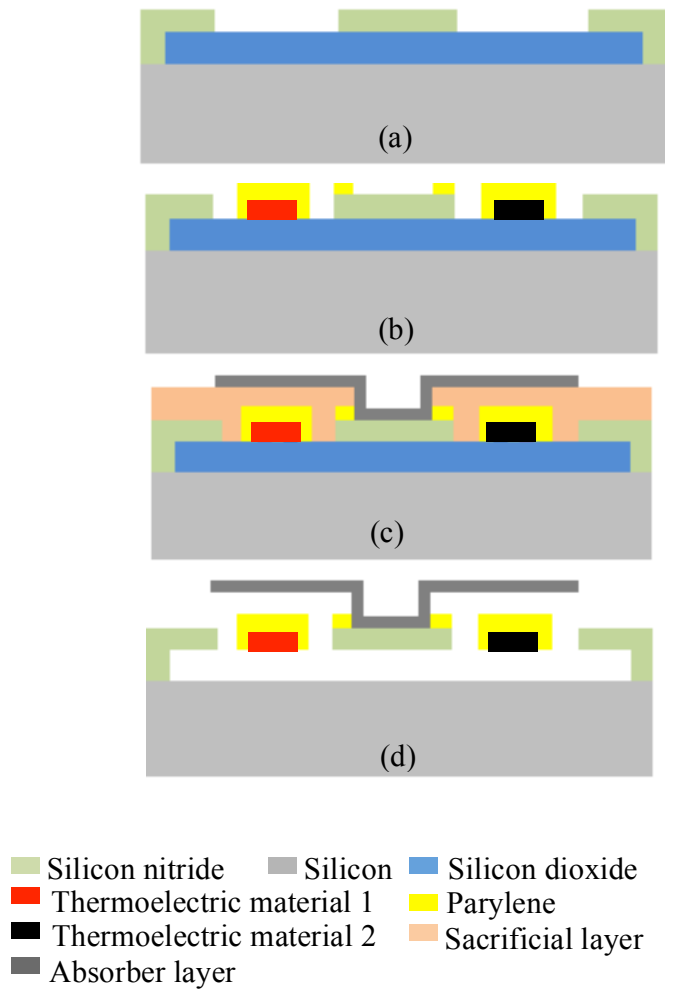

Figure 3: The fabrication process flow, (a) sacrificial $\mathrm{SiO}_{2}$ layer deposition followed by silicon nitride deposition/patterning, (b) TE wire deposition/patterning followed by parylene deposition, (c) second sacrificial layer deposition followed by absorber formation, (d) Releasing the device by removing the sacrificial layers.

Then, supporting arms are formed from the parylene film deposited in a parylene-coating chamber (PDS 2010 system) at room temperature which is patterned in oxygen based plasma (Figure 3(b)). After fabricating the supporting arms, the second sacrificial layer is deposited which is $4 \mu \mathrm{m}$ of PECVD silicon dioxide and patterned to create access to the silicon nitride membrane (Figure 3(c)). Next step is the fabrication of the absorber which is comprised of three layers: (1) the bottom layer is sputtered nichrome with the sheet resistance of $40 \mathrm{Ohm} / \mathrm{square}$. (2) The second layer is $1 \mu \mathrm{m}$ of PECVD silicon nitride. (3) The very top layer is another sputtered nichrome layer with a sheet resistance of $>377 \mathrm{Ohm} / \mathrm{square}$. The absorber is then is anchored to the post through the patterned hole in the sacrificial layer as shown in Figure 3(c). To improve the adhesion of the absorber to its post, a thin layer of silicon nitride (300 nm thick) is deposited prior to the absorber deposition. The process of patterning the absorber is a combination of wet and dry etching: Silicon nitride layers are dry etched while the nichrome layers are wet etched in nichrome TFN. Lastly, both sacrificial layers are removed in an HF solution to completely release the structure (Figure 3(d)). The different layers along with their dimensions are summarized in Table 1 .
Table 1: Layers with their dimensions.

\begin{tabular}{|cccc|}
\hline $\begin{array}{c}\text { Layer } \\
\text { name }\end{array}$ & Thickness(nm) & Width $(\mu \mathrm{m})$ & Length( $(\mu \mathrm{m})$ \\
\hline Polysilicon & 125 & 1 & $\sim 20$ \\
\hline Nichrome & 250 & 2 & $\sim 20$ \\
\hline Parylene & 510 & 2.5 & $\sim 20$ \\
\hline membrane & 345 & 5 & 5 \\
\hline Absorber & 1000 & 19 & 19 \\
\hline
\end{tabular}

For the smallest fabricated device, the device area is $20 \mu \mathrm{m} \times 20$ $\mu \mathrm{m}$ and the absorber area is about $19 \mu \mathrm{m} * 19 \mu \mathrm{m}$ leading to a fill factor of as high as $90 \%$ as depicted in Figure 4. The same fabrication procedure is used to make arrays of $4 \times 4$ (Figure 5).

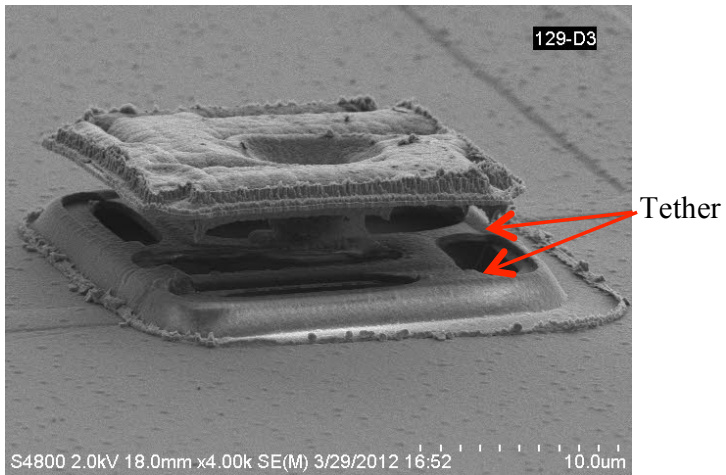

Figure 4: A fully fabricated device. There is an unwanted ring around the device created during the step of absorber patterning.

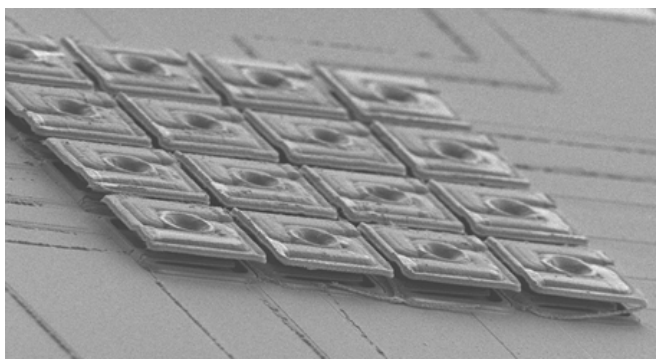

Figure 5: $4 \times 4$ array of detectors.

\section{RESULTS}

For measuring the responsivity, the detector is placed inside a vacuum probe station. On the top of the probing chamber, there is Zinc-Selenide window which let us probe the device by a CCD camera. After probing, a Ge window (3-12 $\mu \mathrm{m})$ is added to ensure blocking of the visible light.

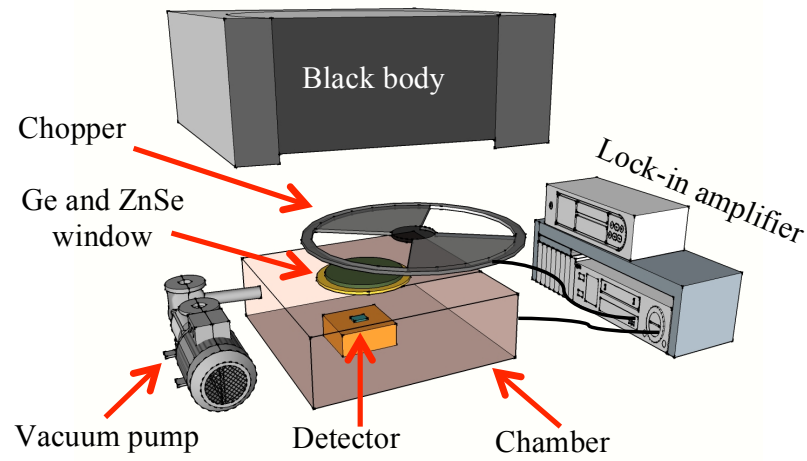

Figure 6: The measurement setup schematic, the vacuum probe station aperture is much smaller than the chopper blade opening. 
A $500 \mathrm{~K}$ black body is then placed in front of the chamber as an IR source. To suppress noise associated with DC measurement and gain a more accurate result, a chopper is placed between the chamber and the black body (as shown in Figure 6). A lock in amplifier is then used to measure the detector output signal. The amplified waveform is then averaged and shown in Figure 7. The responsivity of the device is calculated by dividing the device output voltage by the incoming IR radiation power. To measure the incoming IR power, a NIST calibrated sensor was replaced with the device and the power was measured under the same condition.

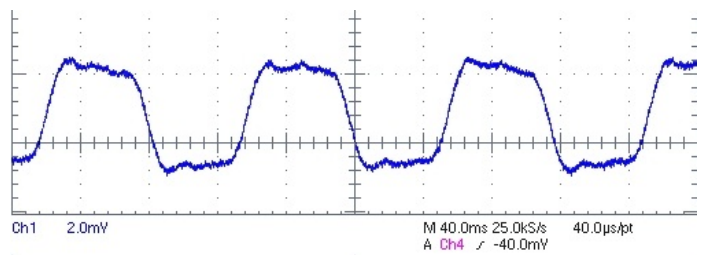

Figure 7: The device amplified and processed output signal.

The device responsivity versus frequency is plotted in Figure 8. It can be understood from the plot that thermal time constant of this detector is about $1.3 \mathrm{~ms}$ which is in agreement with the simulation.

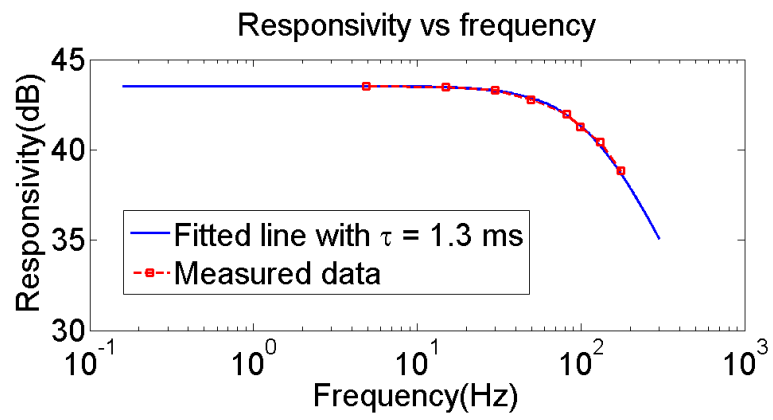

Figure 8: The device responsivity vs. frequency, the red dots are the measured data and the blue line is the fitted response with the time constant of $1.3 \mathrm{~ms}$.

If the devices are measured under open circuit condition, shot noise, flicker noise, and generation-recombination noise are zero and the only noise affecting the measurement is the thermal noise. As a result, the total noise, for the fabricated detector with resistances about $87 \mathrm{KOhm}$ is equal to Johnson noise which is calculated from $V n^{2}=4 K . B . T . R$ and is about $\sim 39 \mathrm{nV} /(\mathrm{Hz})^{\wedge} 0.5$. The $\mathrm{D}^{*}$ is calculated to be $7.7 \mathrm{e} 6 \mathrm{~cm}^{*} \mathrm{~Hz}^{\wedge} .5 / \mathrm{W}$.

\section{CONCLUSION}

Described was the design and fabrication of an uncooled thermoelectric IR detector that features one polysilicon-nichrome thermocouple enclosed in parylene arms. This detector is $20 \mu \mathrm{m} \mathrm{x}$ $20 \mu \mathrm{m}$ with an umbrella like absorber of $19 \mu \mathrm{m} \times 19 \mu \mathrm{m}$. A responsivity of $150 \mathrm{~V} / \mathrm{W}$ was measured in vacuum (1.5e-3 mbar) using a $500 \mathrm{~K}$ black body with a $(3-12 \mu \mathrm{m}) \mathrm{Ge}$ window. Finite element analysis was utilized to simulate the heat distribution and the transient response of the detector.

\section{ACKNOWLEDGMENT}

The work in this paper was supported by Amethyst research incorporated.

\section{REFERENCES}

[1] A. Rogalski, "Infrared detectors: status and trends", Progress in Quantum Electronics, Volume 27, Issues 2-3, 59-210(2003).

[2] M. C. Foote, "Temperature stabilization requirements for unchopped thermal detectors", Proc. of SPIE Conference on Infrared Technology and Applications XXV, 3698, 344-350(1999).

[3] P. W. Kruse, and D. D. Skatrud, Uncooled Infrared Imaging Arrays and Systems, Academic Presse, San Diego, 1997.

[4] M. C. Foote, E. W. Jones, and T. Caillat, "Uncooled thermopile infrared detector linear arrays with detectivity greater than $109 \mathrm{cmHz} / / 2 / \mathrm{W}$ ", IEEE Trans. Electron Devices 45, 18961902(1998).

[5] M. Hirota, Y. Ohta, and Y. Fukuyama, "Low-cost thermoelectric infrared FPAs and their automotive applications", Proc. SPIE 6940, (2008).

[6] D. Kryskowski, and J. Renken, "80 x 60 element thermoelectric infrared focal plane array for high-volume commercial use", Proc. SPIE 7298, (2009).

[7] D. Teweldebrhan, V. Goyal, and A. A. Balandin, "Exfoliation and Characterization of Bismuth Telluride Atomic Quintuples and Quasi-Two-Dimensional Crystals", Nano Letters, 12091218(2010).

[8] C. B.Vining, "Desperately Seeking Silicon", Nature, 451, 132(2008).

[9] P. Majumder, and A. Bhattacharyya, "A computational study of the impact of the Wiedemann-Franz-Lorenz law on the thermal response of nichrome cylinders", Modelling Simul. Mater. Sci. Eng., 16, 015006(2008).

[10] I. L. Choi, and K. D. Wise, "A Silicon-Thermopile-Based Infrared Sensing Array for Use in Automated Manufacturing", IEEE Trans. Electron Devices, 72-79(1986).

[11] N. Majid, S. Dabral, J. F. McDonald, "The ParyleneAluminum Multilayer Interconnection System for Wafer Scale Integration and Wafer Scale Hybrid Packaging", Electron. Mater. 18, 301(1989).

[12] T. Yamane, N. Nagai, S. Katayama, and M. Todoki, "Measurement of the thermal conductivity of silicon dioxide thin films using a 3-omega method", J. Appl. Phys.,91, 9772(2002).

[13] R. Goch, T. Schimert, W. McCardel, and B. Ritchey, "Waferlevel vacuum packaging for MEMS", J. Vacuum Sci. Technol. A, 17, 2295-2299(1999)

[14] A. D. McConnell, U. Srinivasan, M. Asheghi, and K. E. Goodson, "Thermal Conductivity of Doped Polysilicon Layers", J. Microelectromech. Syst. 10, 360 (2001).

[15] M. Hirota, Y. Nakajima, M. Saito, and M. Uchiyama, " $120 * 90$ element thermoelectric infrared focal plane array with precisely patterned Au-black absorber," Sens. Actuators A, Phys., 135, 146(2007).

[16] J. Lehman, E. Theocharous, G. Eppeldauer, and C. Pannell, "Gold-black coatings for freestanding pyroelectric detectors", Meas. Sci. Technol., 14, 916(2003).

[17] P. A. Silberg, "Infrared absorption of three layer films", J. Opt. Soc. Amer., 47, 575-578(1957).

\section{CONTACT}

*M. J. Modarres-Zadeh, tel: +1-918-850-8381;

jeff.modarres_zadeh@okstate.com 


\author{
SIDEWALL SILICON CARBIDE EMITTERS \\ FOR TERAHERTZ VACUUM ELECTRONICS

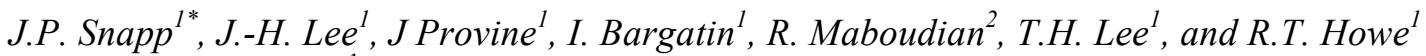 \\ ${ }^{1}$ Stanford University, Stanford, California, USA \\ ${ }^{2}$ University of California at Berkeley, California, USA
}

\section{ABSTRACT}

This paper describes a functional sidewall emitter for future terahertz vacuum electronics. The thermionic emitter is formed by a $1 \mu \mathrm{m}$ thick film of low-stress n-type polycrystalline silicon carbide (SiC), conformally deposited by LPCVD onto high aspect ratio silicon trench sidewalls and subsequently released. The freestanding emitter is resistively heated. We demonstrate lateral thermionic currents of $100 \mu \mathrm{A}$, measured on an integrated collector across a high aspect ratio vacuum gap, for $300 \mathrm{~mW}$ heating power.

\section{INTRODUCTION}

The frequency band from $250 \mathrm{GHz}$ to $2.5 \mathrm{THz}$ is rich in potential applications, but suffers from a lack of efficient sources. This "THz gap" arises from the sharp decrease in the efficiency of solid-state electronic amplifiers at frequencies above $100 \mathrm{GHz}$ and the unavailability of compact, uncooled optical sources for wavelengths longer than the infrared [1]. A micro-fabricated Barkhausen-Kurz ( $\mu$-BK) oscillator has been previously proposed as a promising candidate source [2,3]. The $\mu$-BK oscillator has significant advantages over other vacuum electronic devices, including its capability of operating at harmonics of the electron orbital frequency with large admittances [4]. This paper demonstrates a functional emitter for injecting a sheet electron beam into a silicon $\mu$-BK THz cavity (Figure 1). Our group has previously demonstrated thermionic emitters using poly-SiC films [5]; however, the need for an emitter integrated into the parabolic potential well is a major challenge. We report here a sidewall lateral emitter intended for production of an upright sheet electron beam.

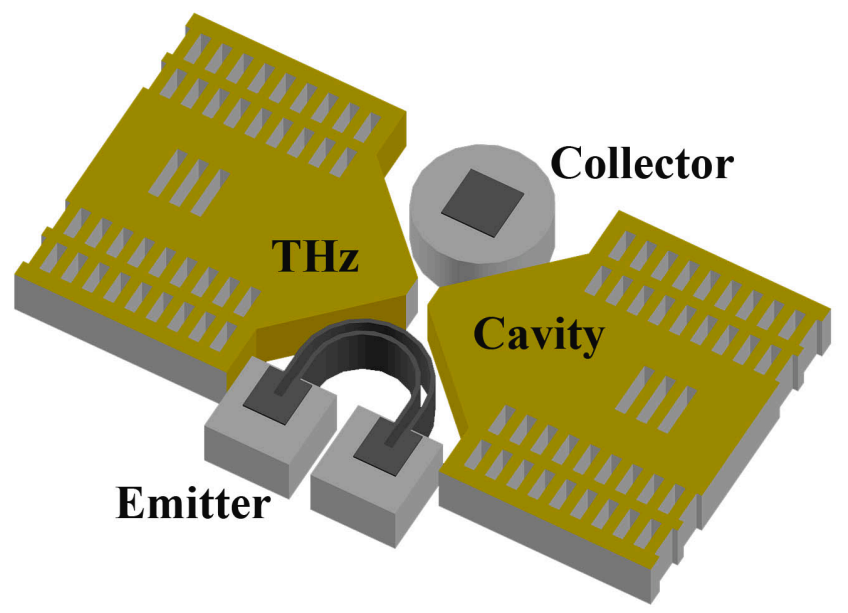

Figure 1: $3 D$ view of the $\mu$ - $B K$ showing quadrupole electrodes surrounding the vacuum interaction region. The emitter, collector, and $\mathrm{THz}$ resonant cavity form the four quadrupole electrodes.

\section{THEORY}

\section{Lateral Emission}

Prior work on micro-fabricated emitters has focused on outof-plane emission for assembly into an external device. To realize a process for terahertz integrated circuits it is desirable to fabricate the entire device on a single substrate. To enable lithographic definition of the quadrupole shaped electrodes, a novel lateral emitter producing a high aspect ratio lateral sheet beam is required. The desired electron orbit frequency limits the electric field strength inside the potential well and precludes the ready use of a field emitter array. A sidewall lateral thermionic emitter is therefore an attractive candidate for future $\mu$-BK oscillators.

\section{Thermionic Emission}

Observation of thermionic emission predates the discovery of the electron. When a conductor is elevated in temperature a significant current of electrons with sufficient energy to escape into vacuum arises. The density of this current is given by the Richardson-Dushman equation:

$$
J=A_{E} T_{E}^{2} e^{-\Phi_{E} / k T}
$$

where $\Phi_{\mathrm{E}}$ is the emitter work function, $\mathrm{T}_{\mathrm{E}}$ the emitter temperature, and $\mathrm{A}_{\mathrm{E}}$ the materials-specific Richardson-Dushman constant. The exponential term is much smaller than unity due to $\Phi_{\mathrm{E}}$ exceeding $\mathrm{kT}$ at temperatures below the material melting point. Thus, emission current rises rapidly with temperature.

\section{Resistive Heating}

Under the idealized assumptions of uniform constant thermal conductivity and neglecting radiation losses, the temperature profile along the length of a resistive heater will be parabolic. When a current is applied, the maximum temperature will occur at the center of the heater and is proportional to the total joule heating generated due to resistive loss:

$$
\Delta T=\frac{V^{2}}{R_{e}} \frac{R_{t h}}{8}
$$

where $\mathrm{V}$ is the voltage across the heater, $\mathrm{R}_{\mathrm{e}}$ is the electrical resistance, and $R_{t h}$ is the thermal resistance. This shows that to get maximum temperature elevation in the heater the thermal resistance should be maximized while simultaneously minimizing the electrical resistance. While the two assumptions stated above are not strictly valid at elevated temperatures, the basic temperature distribution and scaling is illustrative.

Owing to the approximately parabolic profile of temperature distribution, combined with the strong dependence on thermionic emission with temperature, the emitted electron current density will be confined to a narrow central region of the emitter. This is desirable for the formation of a confined sheet electron beam for tight coupling to a $\mathrm{THz}$ resonant cavity. Since thermionic emission is a surface phenomenon, a thin resistive heater film is desired to minimize required electrical heater power. 


\section{FABRICATION}

Fabrication of lateral sidewall emitters starts with an SOI wafer with a $50 \mu \mathrm{m}$ thick device layer. The device layer thickness determines both cavity depth and emitter filament height. Because of the challenges of using spin-coated photoresist for optical lithography after deep etching, a double hard mask process was used. First a $1.5 \mu \mathrm{m}$ thick layer of silicon nitride was deposited followed by a $1.5 \mu \mathrm{m}$ thick layer of silicon dioxide. These layers define the locations for the two critical deep reactive-ion etch (DRIE) steps.

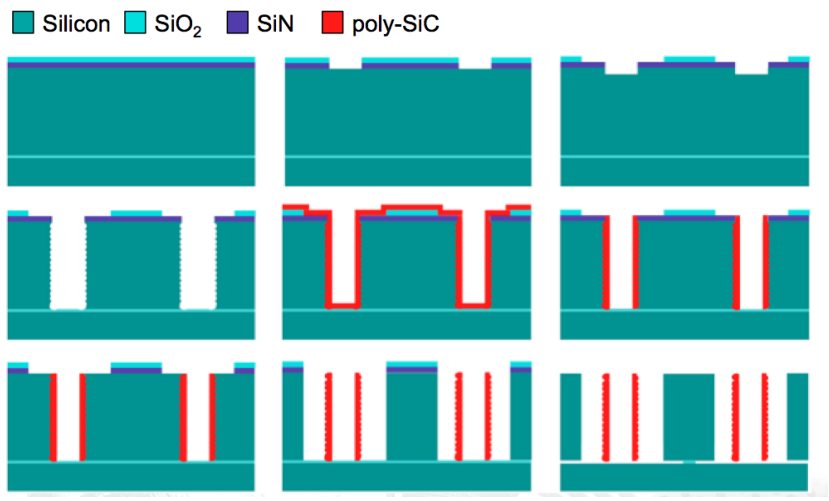

Figure 2: Fabrication process from top left to bottom right: (1) deposit $1.5 \mu \mathrm{m}$ LSN then $1.5 \mu \mathrm{m}$ of LTO on SOI wafer; (2) pattern and RIE etch LSN, LTO for SiC trench definition; (3) pattern and RIE etch LTO, stopping on LSN; (4) DRIE trench to define SiC filament locations; (5) LPCVD $1 \mu$ m thick poly-SiC extremely conformal deposition; (6) blanket RIE etch poly-SiC; (7) etch LSN mask using LTO hard mask; (8) DRIE etch to define vacuum gaps and electrode isolation trenches; (9) etch remaining topside hard mask, release buried oxide, deposit bondpads using a shadow mask in an evaporator.

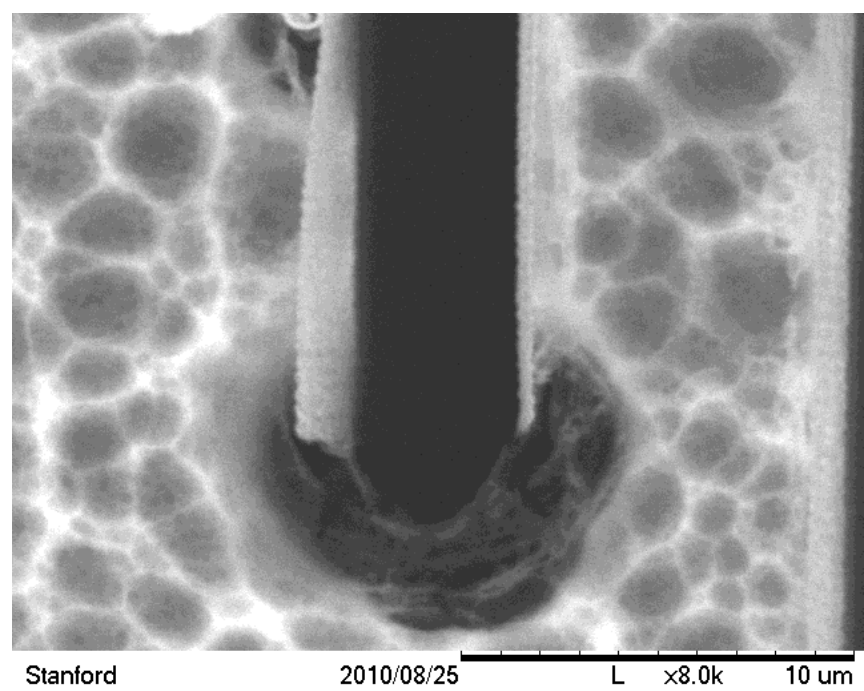

Figure 3: SEM showing 200nm poly-SiC film conformally lining sidewalls of a 4um wide and 100um deep DRIE etched trench in silicon. The $\mathrm{SiC}$ at the bottom of the trench and topside of wafer has been removed during a blanket RIE etch. A short decoration etch in $\mathrm{XeF}_{2}$ has been performed (puckering the cleaved silicon surface) to highlight the thin SiC film.
After hard mask patterning, the first DRIE is performed stopping on a $2 \mu \mathrm{m}$ thick buried oxide. Then, $1 \mu \mathrm{m}$ of $10-20 \mathrm{~m} \Omega-$ $\mathrm{cm}$ n-type polycrystalline $\mathrm{SiC}$ with an intrinsic stress of $325 \mathrm{MPa}$ is deposited using LPCVD [7] to conformally coat the sidewalls of the trench. Poly-SiC was chosen because it is refractory material and because it can be deposited with extremely conformally in the LPCVD reactor. The poly-SiC is then blanket etched from the topside and trench bottoms using an anisotropic $\mathrm{HBr} / \mathrm{Cl}_{2}$ chemistry in a Lam TCP etcher [8]. In characterizing the process, the anisotropic reactive-ion etch was able to remove the poly-SiC material even from the bottom of $100 \mu \mathrm{m}$ deep trenches with an aspect ratio of 25 (Figure 3 ).

The remaining poly-SiC layers on the trench sidewalls form the thermionic filaments. The top $\mathrm{SiO}_{2}$ hardmask is used to reactive-ion etch the $\mathrm{SiN}$ hardmask layer to clear regions for the second DRIE. The poly-SiC filaments are then exposed by a silicon DRIE to define the vacuum gap/cavity. This is followed by final removal of the buried oxide using vapor HF. A shadow mask, fabricated using a silicon wafer, was used to define contacts to the poly-SiC using sputtered gold with a chrome adhesion layer.

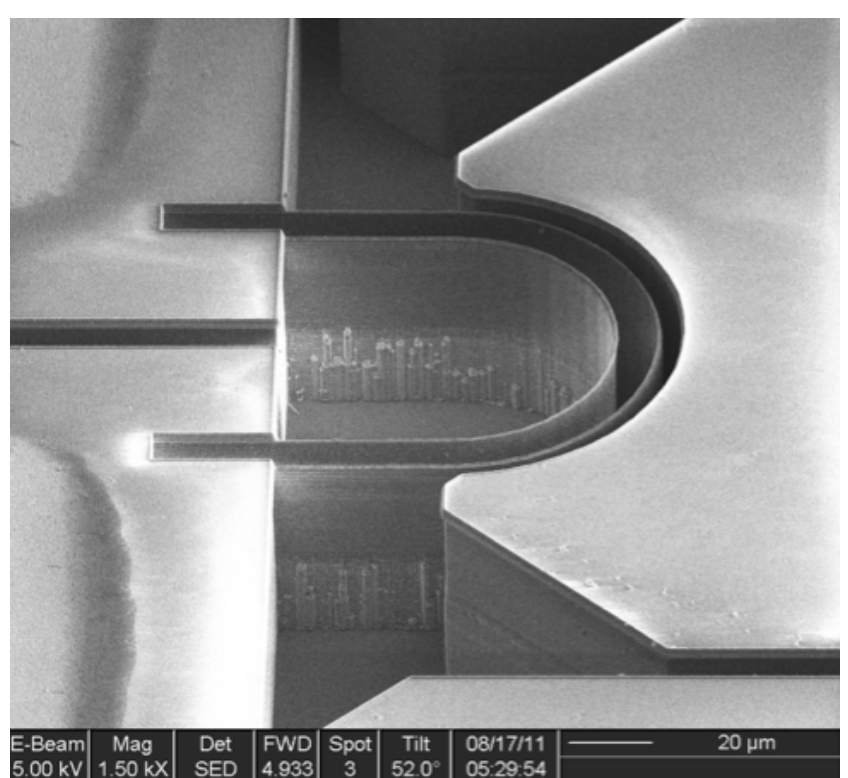

Figure 4: SEM showing a lateral sidewall emitter with integrated collector after fabrication is completed. The transfer of scallops and vertical striations formed during the silicon DRIE into the conformally deposited poly-SiC film are clearly visible.

\section{EXPERIMENTAL SETUP}

After fabrication, individual dies were mounted on a printed circuit board and connected via wirebonds (a single device at a time). The board was placed inside a vacuum chamber with measured pressure below $10 \mu$ Torr. The device under test was connected via electrical vacuum feedthroughs to two Keithley 2400 source meter units. The filament was resistively heated by applying a negative bias voltage, $\mathrm{V}_{\text {emitter, }}$, to one anchor while keeping the other anchor grounded. Thermionic emission current was collected on an adjacent in-plane silicon collector positively biased at $1 \mathrm{~V}$ (Figure 5). The negative bias on the emitter anchor ensures electrons flow efficiently to towards the positively biased adjacent collector. 


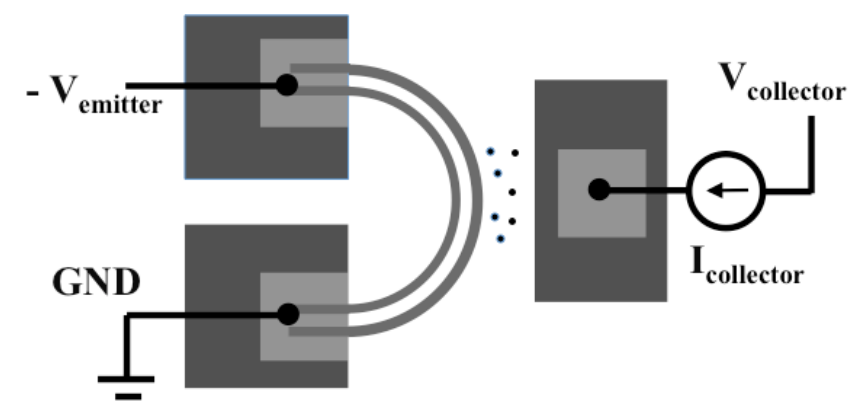

Figure 5. Schematic of emission test setup inside the vacuum chamber

\section{RESULTS}

The measured resistance of the fabricated filaments ranged from 250 to $350 \mathrm{Ohms}$ depending on the amount of overlap of the gold contact onto the SiC filament. The measured thermionic current data (Figure 6) fits the expected relationship between current and heater power. Thermionic emission became detectable above $100 \mathrm{~mW}$ emitter heating power. Over $100 \mu \mathrm{A}$ of collector current was measured for emitter heating power above $300 \mathrm{~mW}$. As the device was kept biased at constant heater power for more than 72 hours, degradation in thermionic emission current was observed. The work function for the $3 \mathrm{C}-\mathrm{SiC}$ film used was previously reported in the range 3.2-3.8 eV [5]. The device degradation over prolonged bias can be attributed to the work function increase due to the graphitization of the emitter surface. Using $\mathrm{BaO}$ coatings to lower the surface workfunction on $\mathrm{SiC}$, a 5-6 orders of magnitude improvement in thermionic current is reported [9].

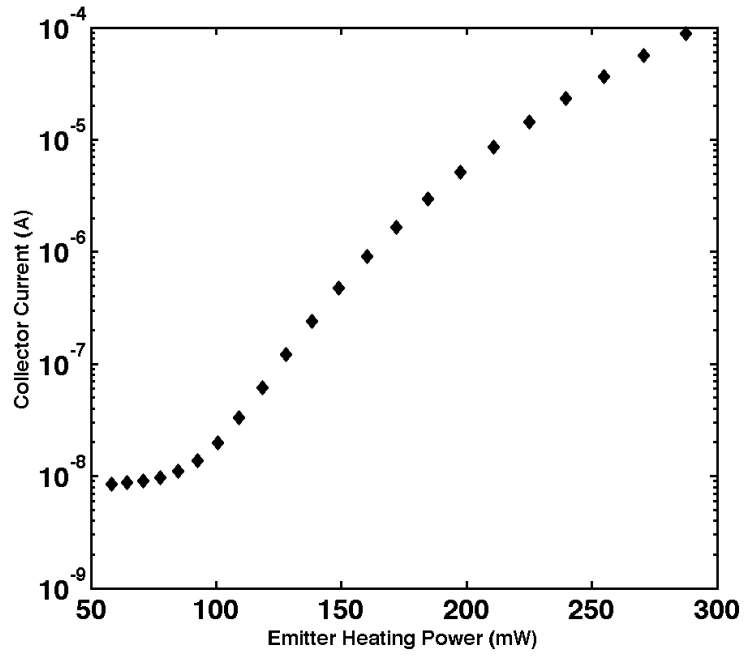

Figure 6. Measured collector current as a function of applied emitter heating power. Over $100 \mu \mathrm{A}$ of collector current was measured for emitter heating power above $300 \mathrm{~mW}$.

At high temperatures $(>1000 \mathrm{~K})$, the optical emissivity of polycrystalline $3 \mathrm{C}-\mathrm{SiC}$ is sufficiently large to enable temperature measurements using an optical pyrometer (PYRO MicroTherm, $550-\mathrm{nm}$ operating wavelength). Work is ongoing to optically measure the emitter temperature during resistive heating.

\section{CONCLUSION}

The demonstrated lateral filament is suitable for integration in a parabolic potential well formed by lithographically defined electrodes. Thermionic emission current exceeding $100 \mu \mathrm{A}$ was measured for emitter heating power above $300 \mathrm{~mW}$. The electron beam's negative admittance at resonance can be measured directly to guide the design of $\mu$-BK oscillators. The demonstrated sidewall filament emitters allow the design of revolutionary vacuum electronics that combine electron emitters, coupled resonant cavities and lithographically shaped electrodes in a single substrate. This will enable a new class of efficient $\mathrm{THz}$ vacuum electronic integrated circuits capable of filling the "THz gap" with portable $\mathrm{THz}$ electronic systems.

\section{ACKNOWLEDGEMENTS}

This work was supported by DARPA Center on Interfacial Engineering in Microelectromechanical Systems (CIEMS) (HR 0011-06-1-0049), Applied Materials, and the NNIN facilities funded by the National Science Foundation. Travel support has been generously provided by the Transducers Research Foundation.

\section{REFERENCES}

[1] T. Crowe, W. Bishop, D. Porterfield, J. Hesler, and R. I. Weikle, "Opening the terahertz window with integrated diode circuits," IEEE Journal of Solid-State Circuits, vol. 40, pp. 2104-2110, 2005.

[2] T.H. Lee, "The Return of the Empty State: Vacuum Nanoelectronics for Terahertz Applications," International Test Conference (ITC), Austin, TX, Oct. 31 - Nov. 5, 2010

[3] R.T. Howe, "Vacuum microsystems for energy conversion and other applications," Solid-State Sensors, Actuators and Microsystems Conference (TRANSDUCERS), 2011 16th International, vol., no., pp.7-11, June 5-9, 2011

[4] E. Boone, M. Uenohara, and D. Davis, "A Barkhausen-Kurz oscillator at centimeter wavelengths," IEEE Trans. on Electron Devices, vol. 5, pp. 196-205, 1958.

[5] J.-H. Lee, I. Bargatin, J. Provine, F. Liu, M.-K. Seo, R. Maboudian, M.L.L. Brongersma, N.A. Melosh, Z.X. Shen, R. $\mathrm{T}$. Howe, "Effect of illlumination on thermionic emission from microfabricated silicon carbide structures," Solid-State Sensors, Actuators and Microsystems Conference (TRANSDUCERS), 2011 16th International, vol., no., pp.2658-2661, June 5-9, 2011

[6] O. Kwon, et al, "Micro-fabricated millimeter wave vacuum electronic devices," $35^{\text {th }}$ Int. Conf. on Infrared, Millimeter, and Terahertz Waves (IRMMW-THz), 2010.

[7] F. Liu, C. Carraro, A.P. Pisano, R. Maboudian, "Characterization of Nitrogen-doped Polycrystalline 3C-SiC Thin Films Deposited from Methylsilane for Harsh Environment MEMS Application", Journal of Micromechanics and Microengineering 20, 35011-35014 (2010).

[8] D. Gao, M.B.J. Wijesundara, C. Carraro, R.T. Howe, R. Maboudian, "Recent progress toward a manufacturable polycrystalline $\mathrm{SiC}$ surface micromachining technology," Sensors Journal, IEEE, vol.4, no.4, pp. 441448, Aug. 2004

[9] J.-H. Lee, I. Bargatin, K. Iwami, K. A. Littau, M. Vincent, R. Maboudian, Z.-X. Shen, N. A. Melosh, R. T. Howe, "Encapsulated Thermionic Energy Converter With Stiffened Suspension", 2012 Solid-State Sensor and Actuator Workshop, Hilton Head Isl., SC, June 3-7, 2012.

\section{CONTACT}

*J.P. Snapp, tel: +1-650-799-3269; jsnapp@stanford.edu 


\section{STRESS SENSITIVE CAPACITIVE TRANDUCERS TO VERIFY PACKAGE MODELS Andrew McNeil and Gary Li}

Freescale Semiconductor Inc., 2100 E. Elliot Rd, Tempe AZ 85254

\begin{abstract}
This paper describes MEMS capacitive transducers modified to characterize package stress. Two transducer types were designed: one to measure uniform in-plane strain, the other to measure uniform z-axis bending ("curvature"). These packagestress transducers had the same sensing mechanism as the MEMS acceleration transducers (capacitive output), so they could be used in existing accelerometers to quantify package-induced deformation. These transducers were fabricated in a production MEMS process (Motorola/Freescale 3u thick polysilicon "two poly" flow) and were characterized with capacitive probe and in accelerometer packages. Test data from the two sensor types provided useful data for verifying package models.
\end{abstract}

\section{INTRODUCTION}

MEMS sensors must meet increasingly stringent accuracy requirements. Some low-cost, mass-market three-axis accelerometers specify typical thermal output changes of 9 milli-g or less $[1,2]$ at specified temperature range. These stringent specifications require accurate modeling; using typical values for proof mass mass and spring constant shows that this corresponds to proof mass displacement of about one Angstrom (1E-10m).

Simulating parameters such as "TCO" (Temperature Coefficient of Offset) requires FEA modeling of package-MEMS interactions. FEA models are required for both package thermalmechanical performance and MEMS electrostatic-mechanical performance. Often the package models and MEMS models can be run separately and then numerically linked [3,4]. Figure 1 shows a cross-section of a typical accelerometer. The packageinduced effects are primarily determined by displacements on the "MEMS mounting surface", as this is where the MEMS structures are fabricated.

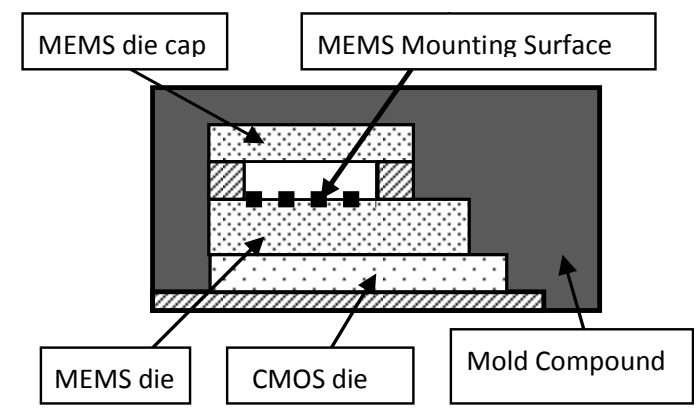

Figure 1: Simplified cross-section of typical MEMS sensor including CMOS die and MEMS die with cap. These die are over-molded with thermoset plastic. MEMS structures are much smaller scale than package features and located on "MEMS mounting surface".

Package FEA models require verification, but this verification can be difficult because the area of interest (displacements on the MEMS mounting surface) is not easily accessible in standard accelerometer packages. Usually the MEMS die is encapsulated by mold compound.

One approach is to use stress sensing silicon dice. An example is to fabricate piezoresistive stress sensors on silicon die
[5]. An array of piezo-resistive stress sensors can be used to estimate the stress state at a location on the stress sensing side.

When used to study capacitive-MEMS products piezoresistive sensors have several potential disadvantages. First they use a different sensing mechanism (piezoresistance vs. variable capacitance) so they can't be coupled with the standard CMOS control die for the accelerometer. A further challenge is that the stress sensing die should be the same physical dimension as the MEMS die, this is because the MEMS die design has the significant effect on the package thermal-mechanical response. It is difficult to achieve the same form factor because not only must the die size be the same, but most MEMS capacitive acceleration die have wafer-level "caps" attached that form a two-die stack as shown in figure 1 [3]. A final problem is that the piezo-resistive effect relies on resistors with fairly large temperature coefficients, and this can complicate multi-temperature testing of packaged devices.

In summary: for validating package stresses in production MEMS products the stress sensing die would have following characteristics:

- Same physical form factor and material as the actual MEMS sensor die

- Compatible with actual CMOS control die so product can be converted into deformation sensor (same sensing mechanism, compatible pin-out, appropriate sensitivity).

\section{PROCEDURE}

Two types of package deformation transducers were constructed: one to sense package-induced strain, the other to sense package induced uniform curvature.

Lateral Device: The lateral strain sensor is a modified version of the lateral acceleration transducer. First the operation of the lateral acceleration transducer will be described.

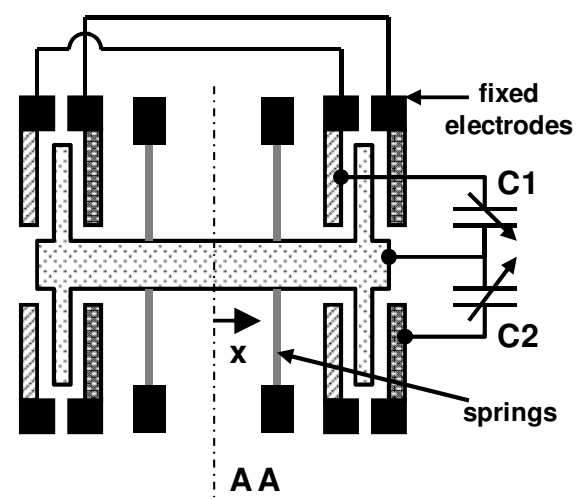

Figure 2: Typical x-axis lateral accelerometer uses proof mass mounted on springs and is symmetric about AA. This produces differential output $(\Delta C=C 1-C 2) . \quad$ Fixed electrodes $x 1, x 2$ form sense capacitors $C 1, C 2$.

An overview of a lateral capacitive acceleration transducer is shown in figure 2. This common type of acceleration transducer uses a proof mass mounted on spring compliant in a lateral (e.g. $\mathrm{x}$ ) 
direction. Acceleration causes proof mass displacement relative to fixed electrodes $\mathrm{x} 1$ and $\mathrm{x} 2$. This causes differential capacitance change, with one sense capacitor increasing and the other sense capacitor decreasing. Typically acceleration output is proportional the difference of the two sense capacitors (C1-C2).

Often acceleration transducers are made with symmetric proof mass/spring design. This allows the transducer to reject a symmetric displacement field such as uniform strain. It can be shown that the differential output $(\mathrm{C} 1-\mathrm{C} 2)$ of the transducer will not be affected by symmetric deformation.

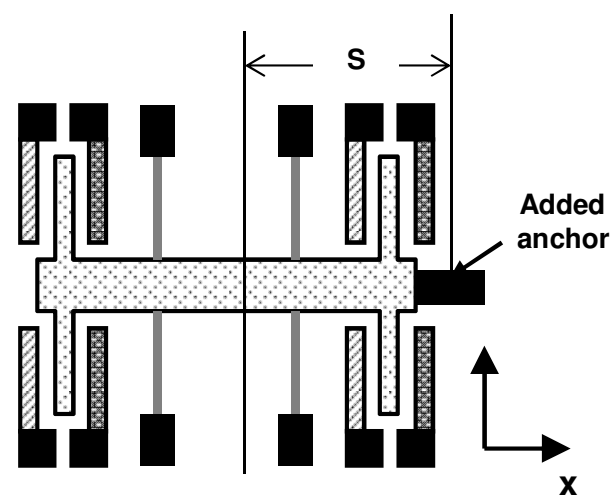

Figure 3: Modified transducer adds anchor to asymmetric location. This anchor virtually eliminates acceleration sensitivity and causes the transducer to produce capacitance signal when subjected to uniform $x$ strain.

Figure 3 shows how the lateral acceleration transducer was modified to convert it into a strain sensor. An extra anchor is attached to the proof mass at distance $S$ from the proof mass center. Assuming a uniform strain $\varepsilon_{\mathrm{x}}$ with zero $\mathrm{x}$-displacement $\Delta \mathrm{x}=0$ at $\mathrm{x}=0$, the displacement at location $\mathrm{x}$ will be:

$$
\Delta x=\varepsilon_{x} x
$$

If the anchor is rigidly attached to the proof mass the proof mass displacement will be equal to the asymmetric anchor displacement. This displacement can be found by substituting $\mathrm{S}$ into eqn. 1 yielding $S \varepsilon_{x}$. The average fixed electrode displacement will be zero because the average electrode location will be zero. $\mathrm{T}$

Assuming the parallel plate equation with capacitor area $\mathrm{A}$, and gap $\mathrm{g}_{0}$, the capacitance $\mathrm{C}$ can be expressed as:

$$
C=\frac{\varepsilon_{0} A}{g_{0}}
$$

Generally thermal-mechanical displacements are a small fraction of the sense gap, in this case the capacitance change can be approximated as linear expression:

$$
\Delta C \approx \frac{-\Delta x}{g_{0}} C_{0}=\frac{\varepsilon_{0} A \Delta x}{g_{0}^{2}}
$$

Thus the average sense capacitor change can be approximated as average gap change, this can be use form the following expression for differential output $\Delta \mathrm{C}$ :

$$
\Delta C=\frac{2 \varepsilon_{x} S C_{0}}{g_{0}}
$$

Equation 4 is an approximation of the differential capacitance output of the lateral strain sensor. The parameter $\mathrm{S}$ can be used to modify the sensitivity of the strain sensor. The goal is to produce $\Delta \mathrm{C}$ of desired level (high enough to achieve good SNR, low enough not to rail CMOS control die). In this case the sensor was made by simply adding an anchor to an existing acceleration transducer.

Curvature Sensor: The die curvature sensor is a modified version of the $\mathrm{z}$-axis acceleration transducer. First the operation of the $\mathrm{z}$ axis acceleration transducer will be described.

Figure 4 shows an overview of how a typical z-axis acceleration transducer works, this device will be referred to as a "teeter totter". This design typically has symmetric design for sense electrodes and springs and an asymmetric portion ("heavy end") to cause rotation under z-axis acceleration. Because of symmetric electrodes a symmetric deformation will cause no differential output $(\Delta \mathrm{C}=\mathrm{C} 1-\mathrm{C} 2=0$ if two capacitors initially equal).

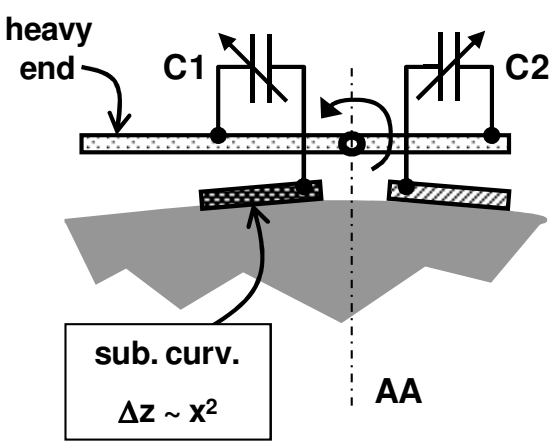

Figure 4: Typical z-axis "teeter totter" accelerometer uses proof mass mounted on torsional suspension and is symmetric about AA. Substrate is shown with uniform curvature such that $\Delta z=K_{c} X^{2}$. Symmetric design of electrodes and spring rejects simple, symmetric curvature.

For z-axis acceleration transducers one important type of deformation is bending of the MEMS die. One type of deformation is uniform, quadratic, $\mathrm{x}$-axis bending,

$$
\Delta z=K_{c} x^{2}
$$

Where $\mathrm{Kc}$ is the curvature constant with units $\mathrm{m}^{-1}$ and $\Delta \mathrm{z}$ is the z-displacement. Another common way of expressing curvature is with a "radius of curvature" Rc. $\quad\left(\mathrm{R}_{\mathrm{c}}=1 / 2 K_{c}\right)$

Figure 5 shows how the teeter totter was modified to form a curvature sensor. The heavy end has been removed, and sense electrodes have been modified such that in-board electrodes form one sense capacitor and out-board electrodes the other capacitor. This sensor now responds to curvature since the in-board gap changes less than the out-board gap. 


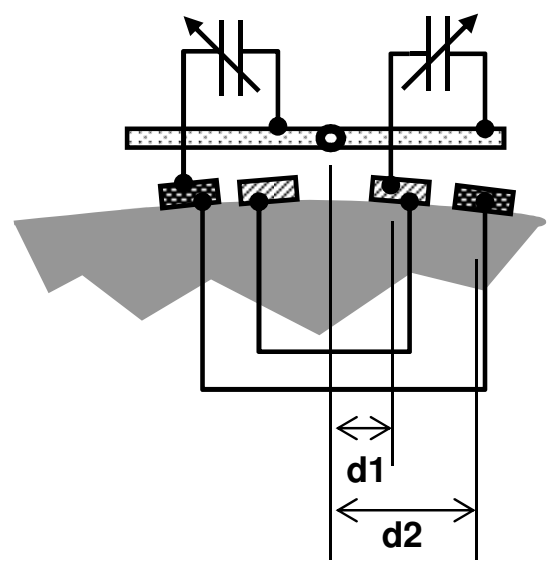

Figure 5: Modified z-axis "teeter totter" accelerometer now functions as deformation sensor. Bottom sense electrodes have each been split into two sections, and re-arranged such that one electrode is in-board and the other electrode is out-board. This device will now respond to uniform substrate curvature. " $d 1$ " and " $d 2$ " are centroid locations of sense electrodes relative to center of transducer.

Using an analysis similar to the lateral device (parallel plate capacitance calculation, small displacement, long narrow proof mass) the following equation approximates the sensitivity of the simple curvature sensor.

$$
\Delta C \approx \frac{K_{c}\left(d_{1}^{2}-d_{2}^{2}\right) C_{0}}{g_{0}}
$$

All stress sensor were manufactured in the standard production process (Motorola/Freescale "two poly" [6]). All stress sensors were virtual "drop in" replacements for the actual acceleration die, with same die size and same pin-out.

For characterization the stress sensors were tested in two ways:

1. Probe: Parts probed using a capacitive LCR meter. This was a room temperature probe to measure the differential capacitive output of bare (unpackaged, un-sawn) MEMS wafer.

2. Sensors were package in standard packages with appropriate CMOS control die, and were testing over temperature $(-40$ to $105 \mathrm{C})$ using accelerometer test systems. In this case the parts were tested in a constant acceleration state $(1 \mathrm{~g})$ so parts have static output.

\section{RESULTS AND DISCUSSION}

A summary of probe data for two lateral stress sensing designs is shown in Figure 6, data is "delta capacitance" $(\mathrm{C} 1-\mathrm{C} 2)$ in $\mathrm{fF}$. This data is from about 600 parts from ten wafers. The two strain sensing designs have opposite location for added anchor, they should show opposite readings of equal magnitude. In this case the probe data is for the bare MEMS wafer so most of the normal package (mold compound, lead-frame, etc) is not present. The small amount of strain (about $-4.8 \mu$ strain) is from thermally dissimilar materials present in the MEMS wafer (glass frit for wafer level packaging, silicon oxide, silicon nitride).

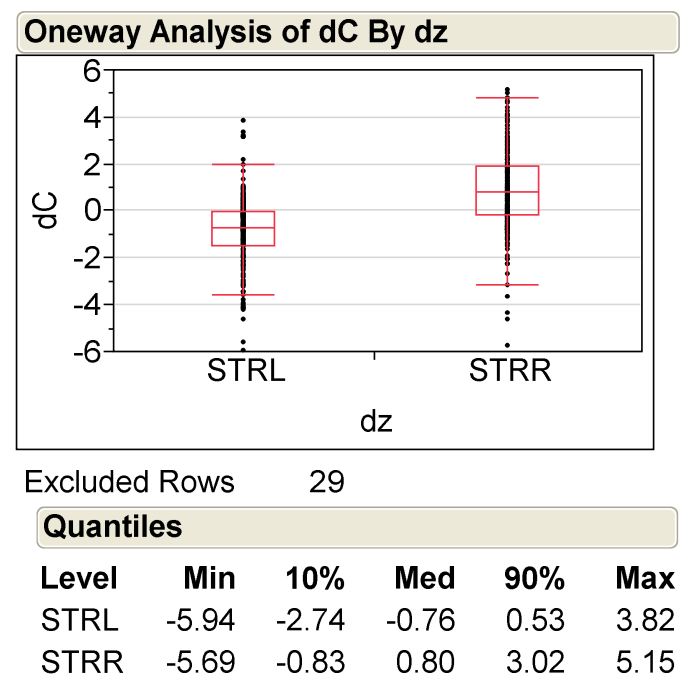

Figure 6: Probe data for $\sim 600$ lateral strain sensors, unpackaged MEMS wafer. The value $d C$ is the delta capacitance between sense electrodes as measured with an LCR meter (fF). The two devices "STRL" and "STRR" have anchors added to left and right side respectively so they should have opposite outputs. In the case the "STRL" has median output of $-0.76 \mathrm{fF}$ and STRR has median output of $+0.80 \mathrm{fF}$

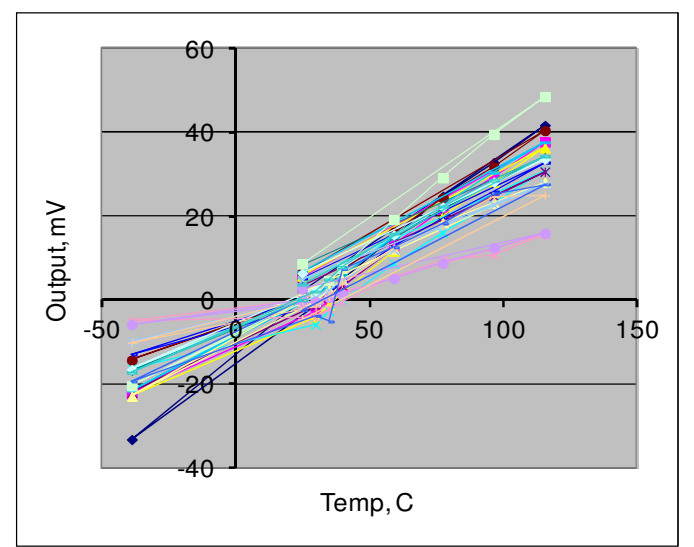

Figure 7: Plot shows output of 20 lateral strain sensing structures measured over temperature. These were used in standard accelerometer package with production control die. This is analog output (in $\mathrm{mV}$ ) of accelerometers with accelerometer die replaced by strain sensing die. The average response was about $0.31 \mathrm{mV} / \mathrm{C}$.

Figure 7 shows a plot of packaged lateral strain sensors measured over temperature. This data was collected with an analog output CMOS control die, so the output shows output in $\mathrm{mV}$ vs. test temperature. The 20 parts show a consistent trend, with about $0.31 \mathrm{mV} / \mathrm{C}$ slope. Using the CMOS control die calculation an estimate was made that this corresponded to approximately $-0.005 \mu$ strain/C. FEA simulations of this package were run and strain was extracted, this yielded thermal strain within $18 \%$ of this value. 


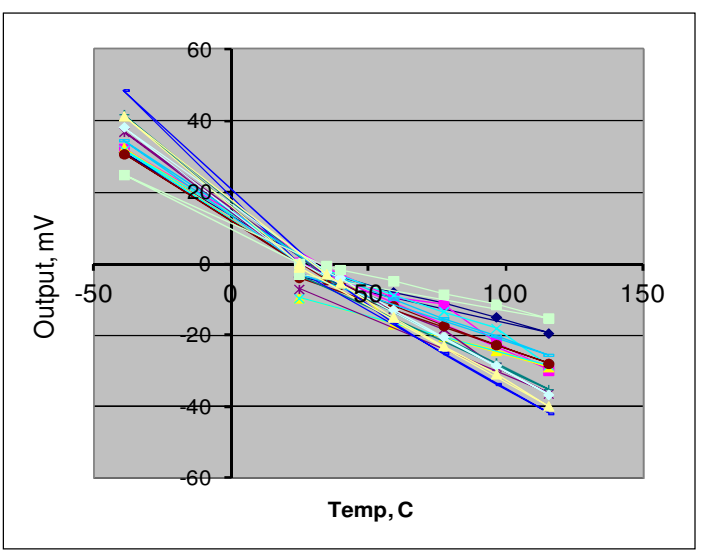

Figure 8: Plot shows output of 12 curvature sensing structures measured over temperature. This is the analogue output (in $\mathrm{mV}$ ) of accelerometers with accelerometer die replaced by strain sensing die. These parts show consistent behavior with about $-0.41 \mathrm{mV} / \mathrm{C}$.

Figure 8 shows a plot of packaged curvature sensors measured over temperature. This data was collected with an analog output CMOS control die, so the output shows output in $\mathrm{mV}$ vs. test temperature. The 12 parts show a consistent trend, with about $-0.41 \mathrm{mV} / \mathrm{C}$ slope. Based on control die characteristics this yields a curvature coefficient $\mathrm{Kc}$ of about $0.085 \mathrm{~m}^{-1}$. This was within about $20 \%$ of curvature from FEA modeling.

These test die were useful in establishing accuracy for an FEA models of a common package. The package used special stress isolating features and thus produced low levels of package stress, and these structures provided a very sensitive measurement of package displacement.

In the future these structures could be modified. These structures could be modified to measure package stress in newer, higher-stress sensor packages. They also could be design to sense other deformation modes. Further multiple sensors could be placed in test die to get deformations in different locations.

\section{SUMMARY AND CONCLUSIONS}

Modified versions of MEMS acceleration transducers can act as displacement sensors, and these sensors can be used to sense several deformation modes (average in-plane strain, out of plane curvature). These sensors allow measurement in the actual MEMS mounting location and can be directly substituted in place of other transducers (e.g. acceleration transducers). These provide a useful method of verifying package models.

\section{REFERENCES}

[1] STmicro-3axis-accelerometer........ LIS331DLH http://www.st.com/internet/analog/product/218132.jsp

[2] Freescale-3axis-accelerometer....... MMA8451Q http://www.freescale.com/webapp/sps/site/prod_summ ary.jsp?code=MMA8451Q

[3] "Design of Transducer and Package at the Same Time," Li G. et al., IPACK05, 8, 497 (2005).

[4] "A Parametric Method for Linking MEMS Package and Device Models," McNeil A.C..., Proceedings of the Solid-State Sensors and Actuators Workshop, Hilton Head Is. SC, June 8-11, 1998

[5] "Design of Silicon Piezoresistive Stress Sensors and Their Application in Electronic Packaging," Suhling J. C. and Jaeger R. C., IEEE Sensor Journal, Vol 1, No 1 (2001)

[6] "Substrate and Annealing Influences on the residual stress of polysilion," Kim T.W. et al., Proceedings of the Solid-State Sensors and Actuators Workshop, Hilton Head Is. SC, June 8-11, 1998

\section{CONTACT}

*Andrew McNeil,

Andrew.moneil@freescale.com telephone: +1-480-413-8857; 


\title{
ULTRA HIGH SENSITIVITY GRAPHENE PIEZORESISTIVE PRESSURE SENSOR
}

\author{
H. Hosseinzadegan ${ }^{*}$, M. Pandey, and A. Lal
}

SonicMEM Lab., School of Electrical and Computer Engineering, Cornell University, Ithaca, NY, USA

\begin{abstract}
We demonstrate the first-ever pressure sensor utilizing graphene piezoresistors demonstrating the sensitivity as high as $323 \mu \mathrm{V} / \mathrm{V} / \mathrm{mmHg}$. This sensitivity is two orders of magnitudes higher than the commercial Omega PX140x series and 36 times higher than that of the reported carbon nanotube based transducers [1]. Our sensor consists of a $400 \mathrm{~nm} \mathrm{Si}_{\mathrm{x}} \mathrm{N}_{\mathrm{y}}$ membrane on silicon substrate with $10 \mathrm{~nm} / 140 \mathrm{~nm}$ thick $\mathrm{Cr} / \mathrm{Au}$ electrodes on top where the graphene layer is transferred on top of this structure. Our pressure sensor highly nonlinear, but can still be useful for applications where ultra-high sensitivity is needed and table lookup can be used.
\end{abstract}

\section{INTRODUCTION}

Piezoresistive pressure sensors are common devices used in biomedical to industrial monitoring applications. The small gaugefactor of the piezoresistive resistors, which are placed in a Wheatstone bridge configuration, limits the sensitivity of a given sensor. The gauge-factor, the membrane thickness, and the required sensitivity for a given application determine the pressure sensor membrane length, width, and thickness. A much higher gauge-factor and transducer sensitivity would enable a smaller sensor for constant sensitivity, or would enable a much more sensitive sensor for constant membrane area (Figure 1). Calculations in this figure are based on the equation (1) [2] for circular membrane pressure sensors where $a$ is the diameter, $h$ is the thickness, $G$ is the gauge-factor, $v$ is the Poisson's ratio, and $\mathrm{E}$ is the Young's modulus.

$$
\frac{\Delta V / V}{q}=G \frac{3}{8} \frac{\left(1-v^{2}\right)}{E}\left(\frac{a}{h}\right)^{2}
$$

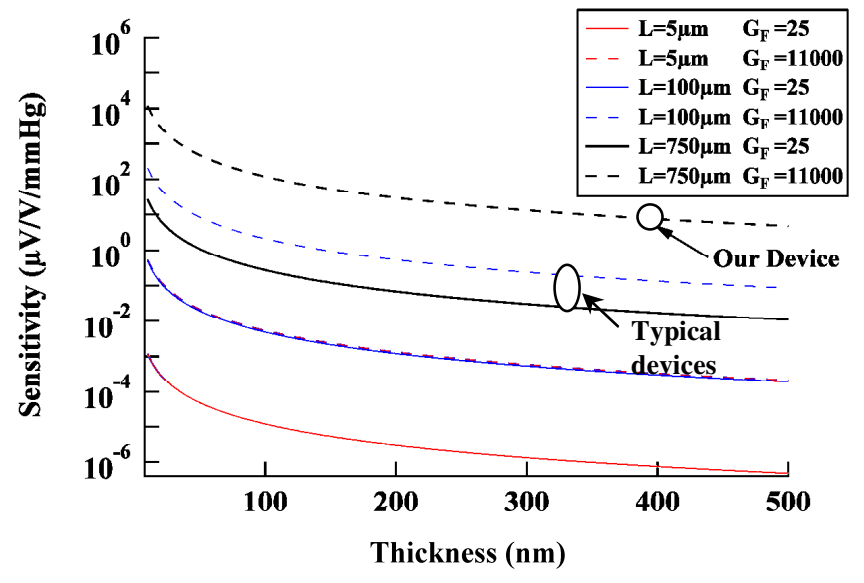

Figure 1: Relative sensitivity of strain transducers versus membrane thickness, for different lateral dimensions and gauge factor. Calculations

Given the $0.335 \mathrm{~nm}$ thickness of single or multi-layer graphene films, the overall reduction of the sensor is possible by reducing the membrane sizes to $100 \mathrm{~s}$ of $\mathrm{nm}$. Hence, graphene films may enable nano-scale pressure sensors that are more sensitive than existing polysilicon and silicon piezoreistor technologies, opening the pathways for using pressure sensors within biological cells, or forming high density pressure sensor arrays.
Tight binding theory for ideal graphene monolayers predicts a gauge factor of 2 for graphene nano ribbons and piezoresistance measurement via in-situ nanoindentation proves this estimation [3]. Similarly, the gauge factor about 11 was obtained at graphene/ epoxy composites cast in mold [4]. We have previously reported an ultrahigh piezo-resistivity coefficients [5]. In contrast to other reported value for graphene our samples are on silicon nitride films, which may result in charge modulation due to mechanical stresses. Another reason for the high value of piezoresistivity could be the grain boundary stretching in graphene films. Practical graphene film often consists of multiple layers of graphene sections with various crystalline grain sizes ranging from a few $\mathrm{nm}$ to 100 s of microns [6-7]. As a graphene film is stressed, the grain boundaries are likely to stretch modulating electron conduction along the boundaries.

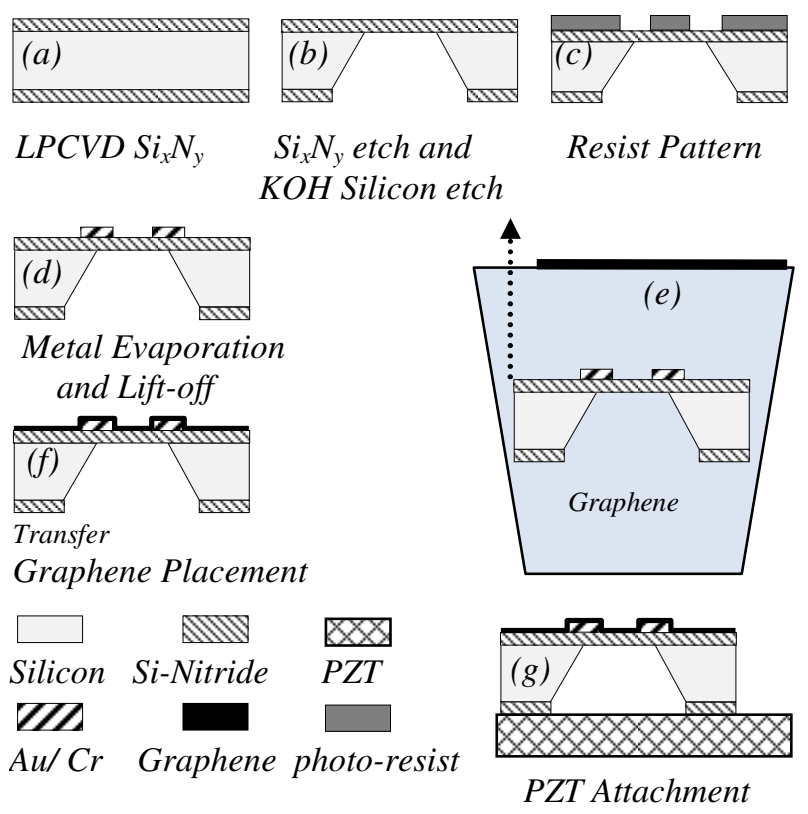

Figure 2. Process flow of graphene piezoresistive membrane type MEMS pressure sensor fabrication: Low stress LPCVD $S i_{x} N_{y}$ deposition (a), Patterning the silicon nitride film and wet etching of the silicon substrate using $\mathrm{KOH}$ solution (b), Patterning SPR220.3.0 photoresist (c), Cr/Au e-beam evaporation and lift-off $(d)$, Transferring graphene on top of the structure (e), Cleaning and drying the sample (f), and PZT attachment for ac actuation $(g)$.

Previous efforts have utilized electrodes or probes applied over the graphene films and then apply stress to the solid substrate to realize strain in the graphene films [8]. In this paper, we placed the graphene films over the electrodes defined over lows-stress $\mathrm{Si}_{\mathrm{x}} \mathrm{N}_{\mathrm{y}}$ membranes using a film transfer method. In this method, the graphene film is poly-crystalline with a grain sizes between 50$800 \mathrm{~nm}$, and can be two or three layers thick. The $\mathrm{Si}_{\mathrm{x}} \mathrm{N}_{\mathrm{y}} /$ electrode/graphene film stack membranes were actuated using a PZT actuator at resonance to obtain high strains, and the resulting change in resistance was used to measure the gauge factor [5]. Optical interferometer was used to measure the displacement 
as a result of PZT actuation, and the displacement was used to determine the strain. A very large gauge factor of 11289 was obtained. This is two to three orders of magnitude higher than that of most other materials which would enable piezoresistive transducer SNR to be higher by the same factor, making piezoresistive transducers much more attractive than other transduction mechanisms such as electrostatic or piezoelectric sense transducers. For example, for electrostatic transduction, the high sensitivity often occurs by decreasing gaps, but that often occurs at the cost of dynamic range and linearity. Piezoelectric films often require thicker films to obtain higher charge for a given film thickness, and do not scale well at the nanoscale.

\section{DEVICE FABRICATION}

Our device fabrication (Figure 2) starts with formation of a $400 \mathrm{~nm}$ low-stress LPCVD silicon-nitride on a 4-inch <100> ptype silicon substrate followed by anisotropic etching of the silicon substrate. A stack of $\mathrm{Cr}(10 \mathrm{~nm}) / \mathrm{Au}(140 \mathrm{~nm})$ is then evaporated and patterned using thermal evaporation to form a four-point probe structure. This structure consists of four wires on each side of the membrane converging from gaps of $10 \mu \mathrm{m}$ to $1 \mu \mathrm{m}$. A $0.5 \mu \mathrm{m}$ thick $\mathrm{SiO}_{2}$ film is sputtered and patterned over the electrodes so only the end section of electrodes are exposed. A CVD deposited [9] graphene-on-copper layer is transferred on top of the electrodes. The process of graphene transfer involves spinning a thin $(300 \mathrm{~nm})$ PMMA on top of graphene followed by wet etching of the copper foil. The silicon wafer which includes the nitride membranes is used to lift the PMMA/graphene bilayer floating on top of the $\mathrm{Cu}$ etch beaker. Acetone is used to remove the PMMA and samples are cleaned within DI water and let to dry for 6 hours. A PZT plate is adhesively attached to the back-side of the silicon die, with two wires soldered to the PZT plate. The Raman spectrum of the graphene films indicates a high quality film owing to the large Gto-D ratio. The D map is associated with defects in graphene [9]. The sheet resistance of the graphene film is $765-1500 \Omega / \square$, measured over 10 samples.

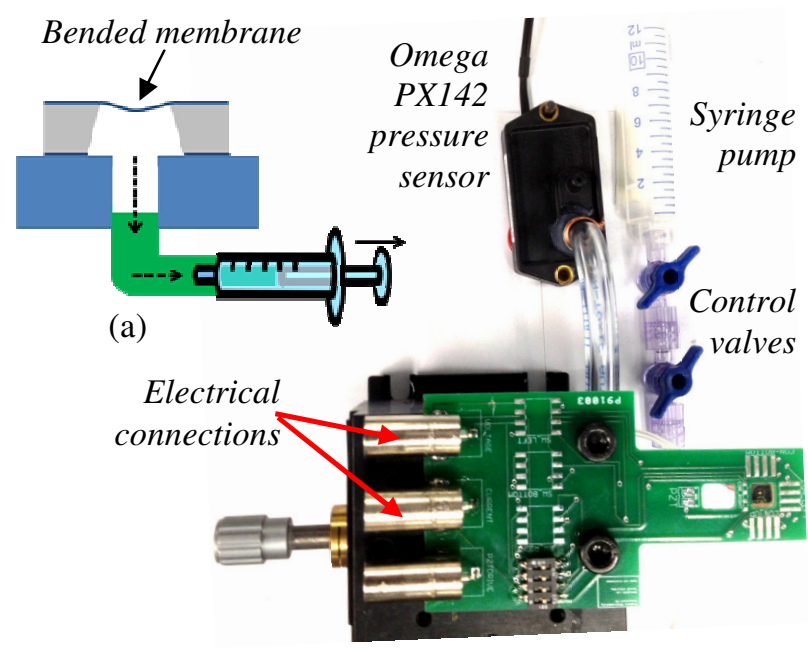

(b)

Figure 3: Schematic (a) and image (b) of measurement system including syringe pump, strain gauge, and leveling stage, and commercial pressure for calibration.

\section{PRESSURE SENSITIVITY MEASUREMENTS}

Measurement system consists of a syringe pump in series with two controlling valves, attached to the backside of the cavity and used to induce constant strain on graphene/ $\mathrm{Si}_{\mathrm{x}} \mathrm{N}_{\mathrm{y}}$ composite plate (Figure 3).
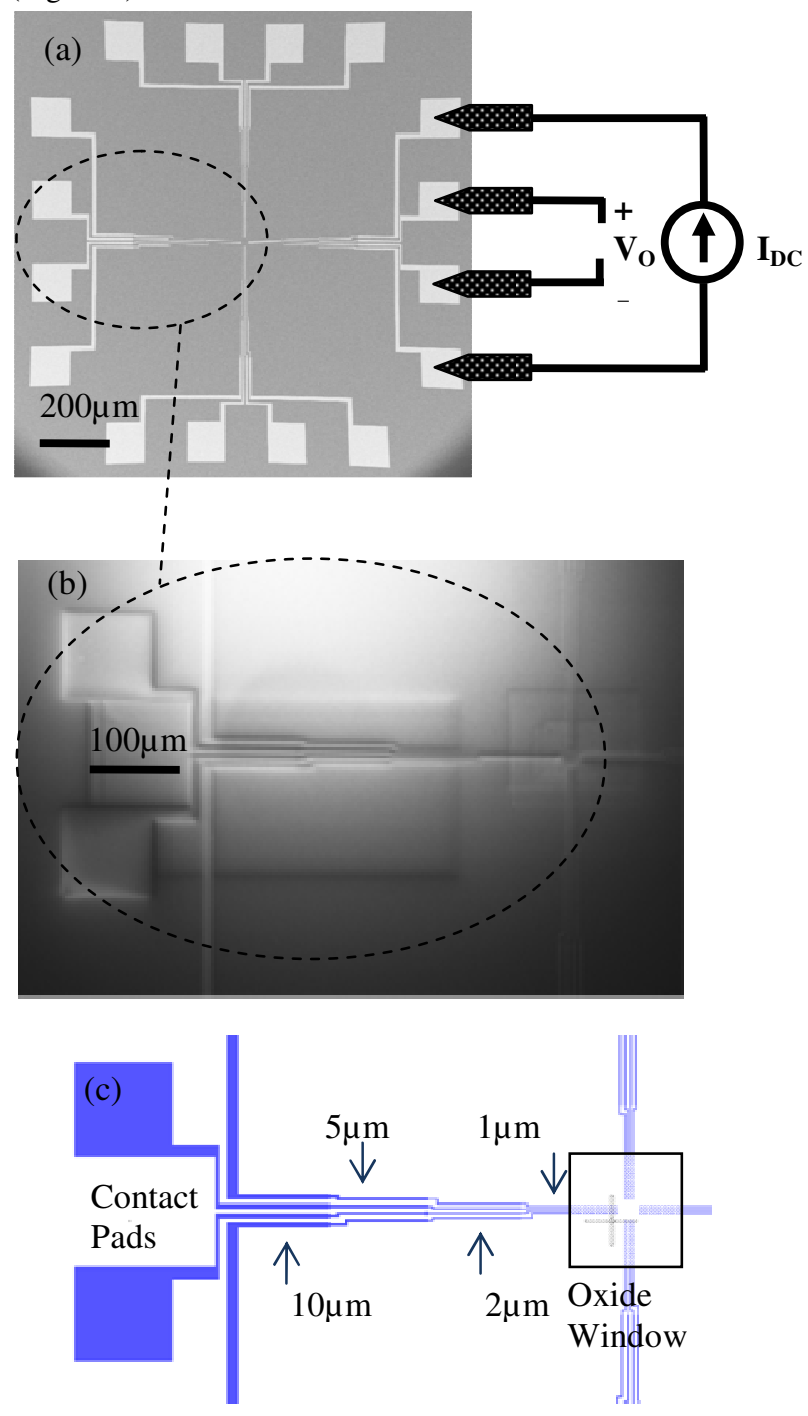

Figure 4: SEM image of 4-point probe electrodes on a silicon nitride membrane to measure pressure response from graphene layer placed in the oxide box ( $a$ and $b$ ), and schematic of this structure is in (c).

Pushing and pulling the pump induces tensile and compressive strains on the graphene/nitride film respectively. A printed circuit board (PCB) is designed to hold our device and carry electrical signals for PZT actuation and resistivity measurements. Entire PCB is mounted on a leveling stage with 0.1 degrees precision in order to make the membrane parallel to the Zygo 7300 optical displacement measurement system. Changes in graphene film resistance are monitored under this steady state strain by measuring the output voltage across the inner electrodes as a function of the applied DC current from the outer two electrodes (Figure 4).

\section{PRESSURE INDUCED STRESS SIMULATION}

Simulations are performed using finite element package ABAQUS for the entire PZT/Silicon/Membrane structure. Plane strain analysis is used here due to the relatively large lateral dimensions of the structure, compared to its thickness, which gives 


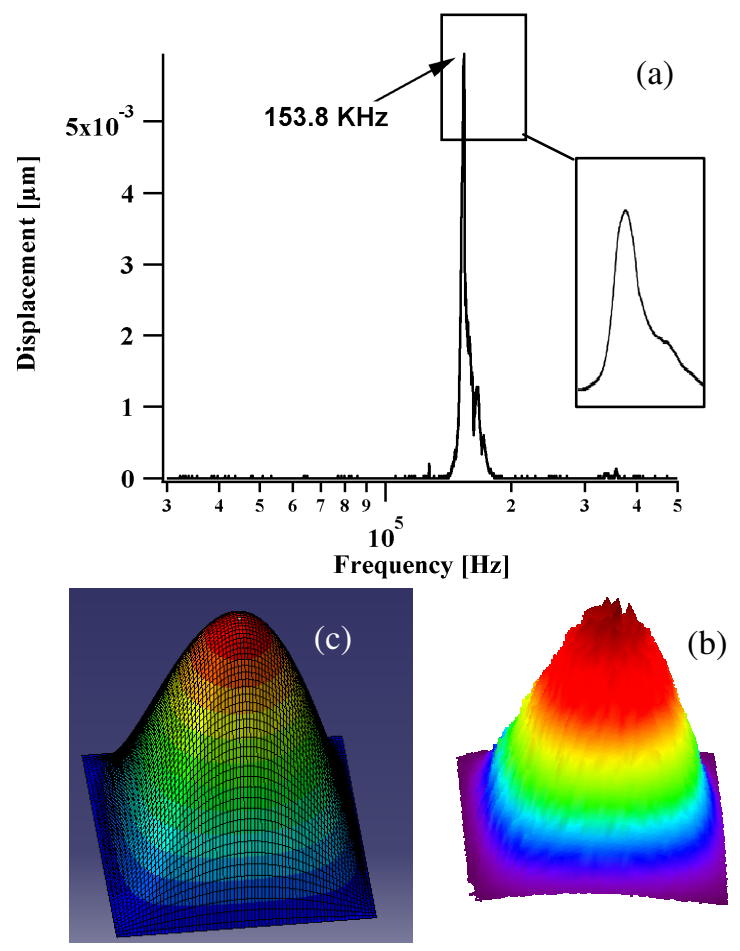

Figure 5. Displacement as a function of frequency at the center of the membrane where the piezoresistivity measuring 4-point probe is placed. (a). The first resonant frequency is at $153.8 \mathrm{KHz}$ with a quality factor of $80.2 \mathrm{in}$ air. Peak-to-peak voltage for the PZT actuation is $100 \mathrm{mV}$. The measured ( $b$ ) and simulated (c) mode shape of this membrane.

good agreement with preliminary 3D simulations.- The mode shape was obtained with assumed in-plane stress in the nitride membrane. A stress of $220 \mathrm{MPa}$ gives good agreement with the experiments and is within the expected range of stress obtained during fabrication. Steady state dynamic simulations were used to obtain resonance response of the structure. While nonlinear geometric effects are considered in the simulations, the PZT is modeled with a built-in linear model.

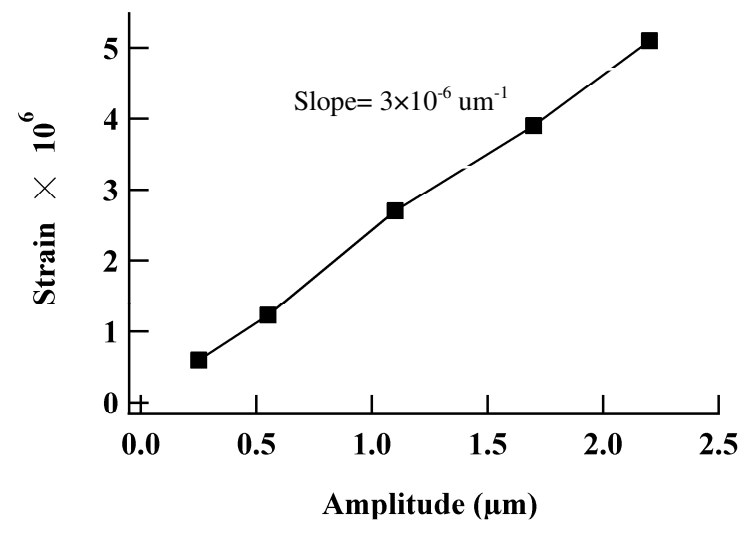

Figure 6. Simulated strain values at different amplitudes shows expected linear relationship

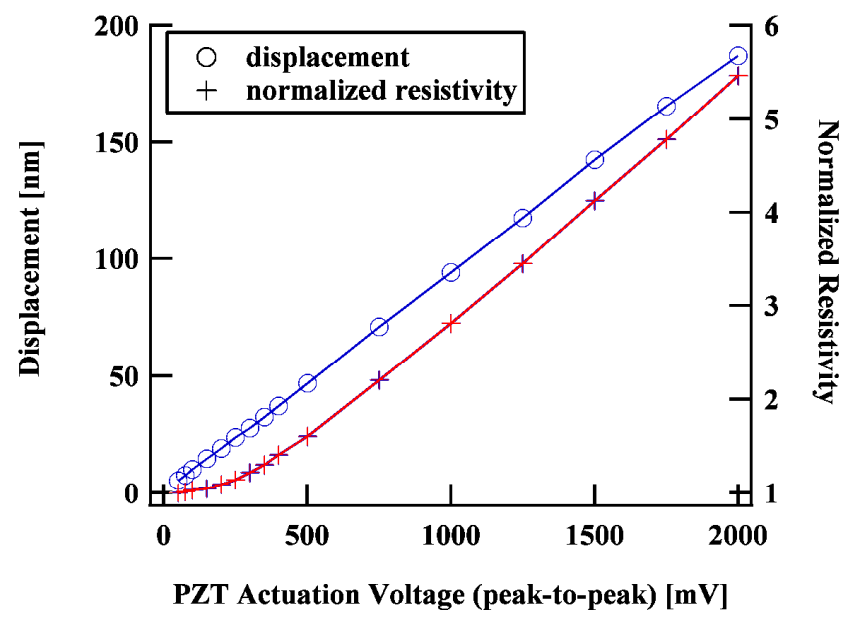

Figure 7. Blue curve is normalized resistivity (resistivity divided by maximum resistivity) as a function of drive amplitude. Red shows displacement at electrode location as a function of drive voltage, from which strain can be calculated.

The experimentally measured value of the quality factor is 80.2 (Figure 5-a), and is used in the simulations. The ABAQUS calculated displacement is in the order of $10 \mathrm{~nm}$ at resonance for a harmonic excitation of $1 \mathrm{~V}$ across PZT at the first resonance frequency. Corresponding strain values along the axis are in the order of $10^{-6}$. An expected linear dependence is obtained between the peak strain and amplitude of vibration (Figure 6). At the same time, the resistivity of the film can be measured as a function of applied PZT voltage (Figure 7). The PZT drive voltage was increased from $100 \mathrm{mV}_{\mathrm{pp}}$ to $10 \mathrm{~V}_{\mathrm{pp}}$.

The minimum limit of $50 \mathrm{mVpp}$ was imposed by the function generator HP-33120A. With this minimum drive voltage, the smallest AC strain that could be applied was $1.25 \times 10^{-6} \%$. Hence, our method is limited to measuring the gauge factors for strain range from $1.25 \times 10^{-6} \%$ to $2.5 \times 10^{-4} \%$. The $\mathrm{RF}$ coupling from the PZT drive to the four point probe was not responsible for the measurement of voltage across the 4-point probe, as the voltage across the inner electrodes also went to zero if the drive current was reduced to zero.

In order to calculate the gauge factor, the voltage between two inner electrodes is measured while actuating the PZT at resonant mode of the membrane with different amplitudes. Corresponding strain at each point is estimated using the linear relationship we came up using ABAQUS simulations (Figure 8).

\section{EXPERIMENTAL RESULTS}

The resistivity increases at both compressive and tensile strains which shows the graphene bends the same way regardless of the pressure direction in our present sensor configuration. Optical interferometer measurement of membrane displacements (Figure 9) indicates that the center part of the membrane moves similarly at positive and negative pressures due to pre-stress from $\mathrm{SiO}_{2}$ layer. The measured graphene gauge factor is orders of magnitude higher than that of most piezoresistive materials used in MEMS such as doped polysilicon and metals. Doped polysilicon can have gauge factors on the order of 30-40, while metals have a gauge factor of 55 [10-11]. 


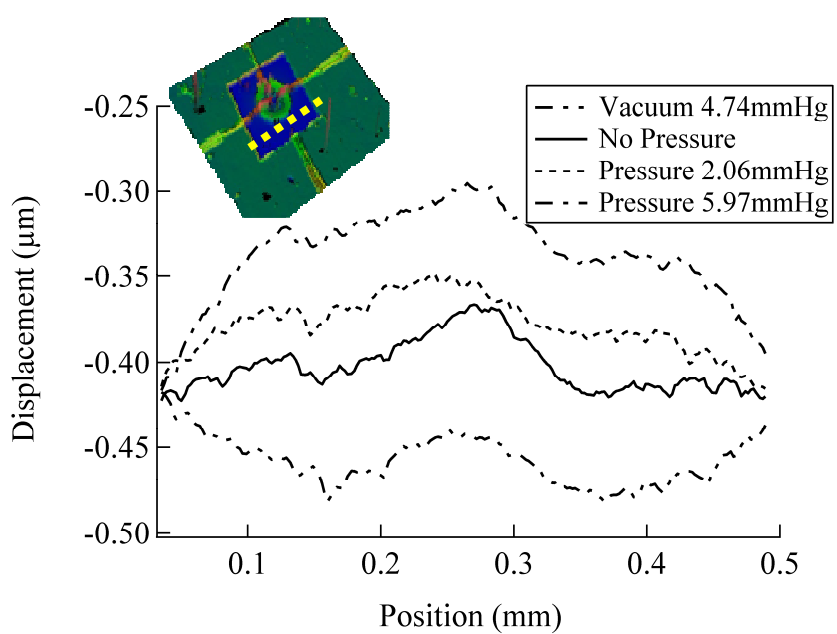

Figure 9: Zygo 7300 displacement profile along the shown the hatched line across the membrane. The dips show a pre-stressed nitride membrane due to the oxide film.

If the measured relative resistance is plotted against the estimated strain, the slope of the line is the gauge-factor (Figure 10).

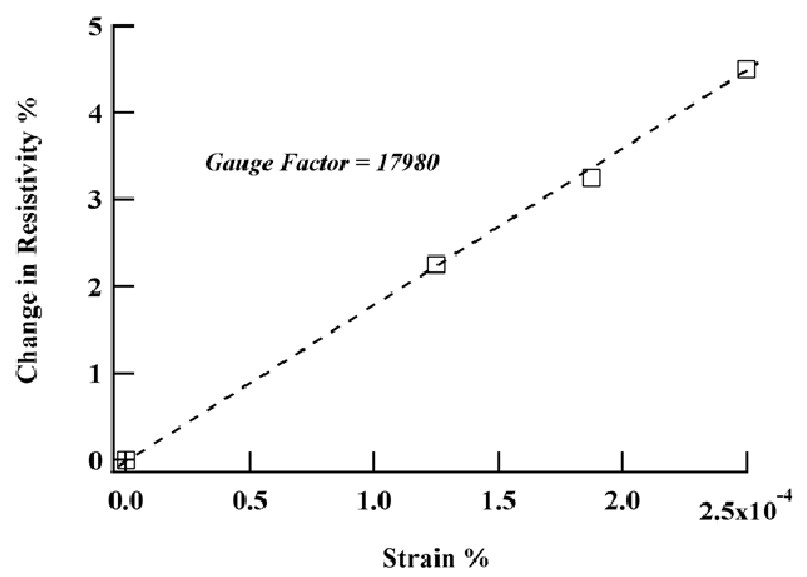

Figure 10. Normalized resistivity change versus applied AC strain at $153.8 \mathrm{kHz}$ membrane resonance.

Resistivity changes of the graphene film as a function of vacuum up to $5 \mathrm{mmHg}$ and positive pressures up to $6 \mathrm{mmHg}$ are plotted in Figure 11. The highly nonlinear behavior may be due to the fact that for either compressive or tensile stress the graphene membrane is stretched in the same direction. Another mechanism could be the generation of $\mathrm{Si}_{\mathrm{x}} \mathrm{N}_{\mathrm{y}}$ polarization charge is of the same variety whether the stress is compressive or tensile.

\section{CONCLUSION}

In summary, we present a pressure sensor process flow and results that pave the way for ultras-high sensitivity pressure sensors. Our estimated gauge-factor for this sensor is 11,300 matching that recently measured with $\mathrm{AC}$ resonance measurement of the graphene film [5]. However, we are exploring the exact reason for the nonlinearity, which maybe tunable for linear devices by adjusting the fabrication process.

\section{ACKNOWLEDGEMENTS}

We acknowledge support for this research from the DARPA TBN program. Travel support has been generously provided by the Transducer Research Foundation.

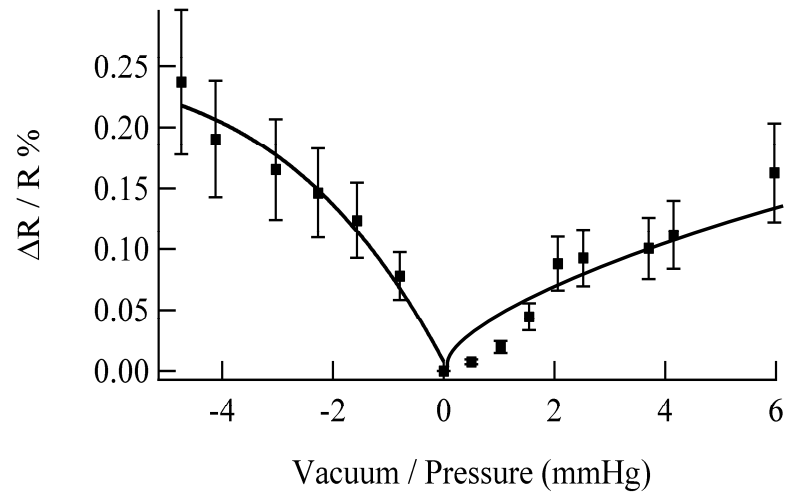

Figure 11: Resistivity change of graphene strain transducer as a function of pressure.

\section{REFERENCES}

[1] J. Cao, Q. Wang, and H. Dai, "Electromechanical Properties of Metallic, Quasimetallic, and Semiconducting Carbon. Nanotubes under Stretching", Physical Review Letter, 90, 157601-157604 (2003).

[2] L. Lin, H. Chu, and Y. Lu, "A Simulation Program for the Sensitivity and Linearity of Piezoresistive Pressure Sensors", Journal of Microelectromechanical Systems, 8, 514-522 (1999).

[3] M. Huang, and J. R. Greer, "Measuring Graphene Piezoresistance via in-situ Nanoindentation", ECS Transactions, Vol. 35 (3), pp. 211-216, 2011.

[4] Y. Kim et al. "Preparation of piezoresistive nano smart hybrid material based on graphene", Current Applied Physics, Vol. 11 (1), pp. S350-S352, 2010.

[5] H. Hosseinzadegan, C. Todd, A. Lal, M. Pandey, M. Levendorf, and J. Park, "Graphene Has Ultra High Piezoresistivie Gauge Factor", The $25^{\text {th }}$ IEEE International Conference on Micro Electro Mechanical Systems, Paris, France, (2012), pp. 611-614.

[6] P. Y. Huang, et al. "Grains and Grain Boundaries n Singlelayer Graphene Atomic Patchwork Quilts", Nature, Vol. 469, pp. 389-392, 2011.

[7] X. Li et al. "Graphene Films with Large Domain Size by a Two-step Chemical Vapor Deposition Process", Nano Lett., Vol. 10, pp. 4328-4334, 2010.

[8] A. B. Frazier and M. G. Allen, "Piezoreslstive Graphite/polyimide Thin Films for MicromachiningApplications", J. Appl. Phys., Vol. 73, 1993.

[9] Xuesong Li, et al., "Large-Area Synthesis of High-Quality and Uniform Graphene Films on Copper Foils", Science, 324, 1312-1314 (2009).

[10] M. le Berre, "Piezoresistive Properties of Boron-doped PECVD Micro- and Polycrystalline Silicon Films", Polycrystalline Thin Films Symposium, pp 733-8, 1994.

[11] M. Huth," Granular Metals: From Electronic Correlations to Strain-sensing Applications", J. of Applied Phys. ,Vol. 107, p.113709, 2010.

\section{CONTACT}

*H. Hosseinzadegan, tel: +1-607-255-1815; hh382@ cornell.edu 


\title{
970 MHZ OVENIZED OSCILLATOR BASED ON AN ALN MEMS RESONATOR WITH MONOLITICALLY INTEGRATED SUSPENDED NANO HOT PLATE
}

\author{
M. Rinaldi ${ }^{*}$, A. Tazzoli $^{2}$, C. Zuniga ${ }^{2}$ and G. Piazza $a^{2,3}$ \\ ${ }^{1}$ Northeastern University, Boston, USA \\ ${ }^{2}$ University of Pennsylvania, Philadelphia, USA \\ ${ }^{3}$ Carnegie Mellon University, Pittsburgh, USA
}

\begin{abstract}
This paper presents the design and experimental verification of the highest frequency (970 MHz) ovenized MEMS oscillator ever reported. Such high frequency of operation along with excellent phase noise $(\mathrm{PN})$ performance $(-83 \mathrm{dBc} / \mathrm{Hz}$ at $1 \mathrm{kHz}$ offset and $-150 \mathrm{dBc} / \mathrm{Hz}$ floor) and efficient ovenization (temperature rise factor of $18.3 \mathrm{~K} / \mathrm{mW}$ ) are enabled by the introduction of a novel technology platform in which a fully anchored Aluminum Nitride (AIN) Lateral Field Excited-Floating (LFE-F) Contour Mode MEMS resonator (CMR) and a monolithically integrated nanoscale heating element are perfectly overlapped and separated by a sub-micron air gap.
\end{abstract}

\section{INTRODUCTION}

Among different MEMS resonator technologies [1], the AlN contour-mode resonator (CMR) [2] has emerged as one of the most promising solutions in enabling the fabrication of multiple frequencies $(10 \mathrm{MHz}-10 \mathrm{GHz})$ and high performance resonators on the same silicon chip [3-4] and has been exploited to synthesize miniaturized reconfigurable CMOS oscillators characterized by low phase noise and low jitter values [5].

Nevertheless, conventional AlN based MEMS oscillators suffer from large temperature dependence and their frequency exhibits a linear dependence on temperature of about $-28 \mathrm{ppm} / \mathrm{K}$ [6]. Such large temperature coefficient of frequency (TCF) is not suitable for modern telecommunication systems, which require oscillators that are stable over a wide range of parameters and especially versus temperature.

In order to overcome this intrinsic issue associated with AlN MEMS resonators different techniques have been proposed. Temperature compensation of AlN MEMS resonators has been demonstrated by adding a compensating layer of $\mathrm{SiO}_{2}$ on the material stack forming the body of the resonator [6-8]. Furthermore, low power heaters have been co-integrated with the resonant devices and few $\mathrm{mW}$ (instead of the 100s of mWs used in crystal based ovenized oscillators) have been used to ovenize them [9-11]. Low frequency ovenized oscillators based on both electrostatic [9] and piezoelectric [10,11] MEMS resonators have been previously demonstrated, but none of the previously proposed design solutions and micro-ovenization techniques allowed the implementation of ovenized frequency sources operating in the $\mathrm{GHz}$ range. $\mathrm{GHz}$ range ovenized AlN CMRs have been recently demonstrated by our group by modifying the conventional layout (continuous plate) of the floating bottom electrode of LFE-F CMRs with a serpentine shaped heater [11]. Although such design solution enabled fast and efficient ovenizaiton of the MEMS resonator it caused a degradation of the device figure of merit $\left(F O M=k_{t}^{2} \cdot Q\right.$, product of the electromechanical coupling coefficient, $k_{t}^{2}$, and quality factor, $Q$ ) compared to the nonovenized case, which prevented connecting the $\mathrm{GHz}$ frequency ovenized MEMS devices to self-sustaining oscillator circuits. Such deterioration of the device FOM is due to the employment of a patterned floating bottom electrode instead of a continuous floating metal plate. In fact the employment of a continuous metal plate as floating bottom electrode enables better confinement of the excitation electric field across the thickness of the piezoelectric layer and also permits to attain an overall better quality of the asdeposited piezoelectric film. This translates to achieving a higher $k_{t}^{2} \cdot Q$.

In this work a stepping stone towards the development of a high frequency, miniaturized, low phase noise and temperature stable frequency source is set by placing the heating element outside the body of the resonator (but suspended over it) (Fig.1). This novel design allowed maintaining the electromechanical properties of the device unchanged (same $k_{t}^{2} \cdot Q$ compared to the non-ovenized case). At the same time, efficient ovenization of the MEMS resonator is achieved by scaling the dimensions of the heating element (i.e. implementing a nano-hot-plate) and minimizing the air gap between the resonator and the heater.

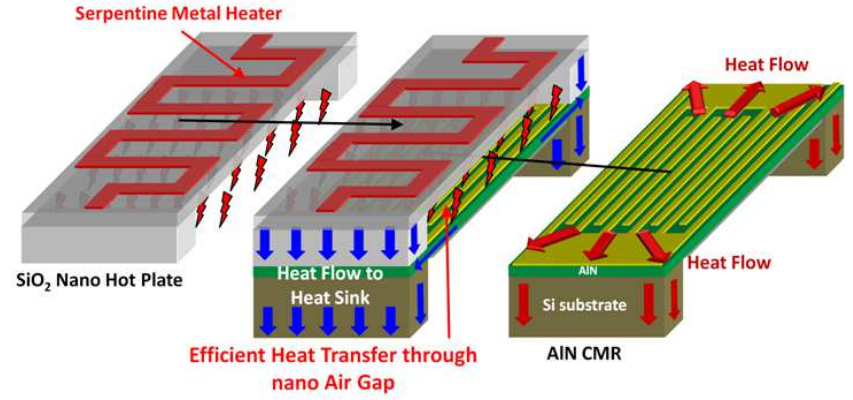

Figure 1: Schematic representation of the innovative technology platform in which an AlN CMR and a monolithically integrated nano-hot-plate are perfectly overlapped and separated by a submicron air gap. Efficient ovenization is achieved by scaling the dimensions of the heating element (i.e. implementing a nano-hotplate) and minimizing the air gap between the resonator and the heater.

\section{DESIGN}

The AlN CMR of this work (Fig. 1) was designed to operate at a frequency of $\sim 1 \mathrm{GHz}$. Therefore, the period, $\mathrm{W}$, of the metal electrode patterned on the AlN plate was set to $4 \mu \mathrm{m}$ while the other geometrical dimensions, thickness, $T$, length, $L$, and number of electrode fingers, $n$, were opportunely scaled in order to maintain a low value of the device equivalent electrical impedance $[2,4](T=500 \mathrm{~nm}, L=80 \mu \mathrm{m}, n=11)$. Lateral field excitation with floating bottom electrode (LFE-F) [5] was employed to excite a higher order contour-extensional mode of vibration in the AlN structure. In addition, the suspended AlN resonant body was fully anchored to the silicon substrate instead of partially anchored through narrow tethers. Such configuration improves power handling and overall robustness of the MEMS resonator. At the same time, since a large number of fingers-electrode $(n=11)$ are used to excite a higher mode of vibration in the AIN plate, the acoustic energy remains confined in the body of the AlN resonator (high $Q$ ) [8]. The simultaneous achievement of high quality factor, $Q$, and high power handling is crucial for the implementation of a low phase noise frequency source [8].

In order to properly design the dimensions of the suspended 
heater and its distance from the resonant device (Fig. 1) a simple one dimensional model was considered which includes heat transfer through conduction, convection and radiation. In this model the metal serpentine is the heat source. For a given power level, $P_{i n}$, supplied to the serpentine, the temperature of the hot plate, $T_{H}$, can be found by solving the following equation:

$$
\begin{aligned}
& P_{i n}=\left(\frac{T_{H}-T_{0}}{R_{P t}}\right)+\left(\frac{T_{H}-T_{0}}{R_{O x 1}}\right)+\left(\frac{T_{H}-T_{0}}{R_{O x 2}+R_{A i r}+R_{A l N}}\right)+ \\
& +\sigma \varepsilon A\left(T_{H}^{4}-T_{0}^{4}\right)+h_{A i r} A\left(T_{H}-T_{0}\right)
\end{aligned}
$$

Where $T_{0}$ is the ambient temperature, $R_{P t}$ is the thermal resistance between the heat source (serpentine) and the heat sink (silicon substrate) thorough the platinum routing, $R_{O x I}$ is the thermal resistance between the heat source and the heat sink thorough the length of the $\mathrm{SiO}_{2}$ suspended plate, $R_{O x 2}$ is the thermal resistance between the heat source and the air gap thorough the thickness of the $\mathrm{SiO}_{2}$ suspended plate, $R_{\text {Air }}$ is the thermal resistance between the the $\mathrm{SiO}_{2}$ suspended plate and the AlN CMR thorough the thickness of the air gap, $R_{A I N}$ is the thermal resistance between the AIN CMR and the heat sink (silicon substrate) thorough the length of the AlN suspended structure, $\sigma$ is the Stefan-Boltzmann constant, $\varepsilon$ is the emissivity of the $\mathrm{SiO}_{2}$ plate ( 0.9), $A$ is the area of the serpentine heater and $h_{A i r}$ is the convective heat transfer coefficient of air (typically between 10 and $\left.100 \mathrm{~W} /\left(\mathrm{m}^{2} \mathrm{~K}\right)\right)$.

Knowing the hot plate temperature, $T_{H}$, it is possible to calculate the AlN resonator temperature, $T_{C M R}$, as function of the input power, $P_{i n}$, by considering the thermal circuit in figure 2 .

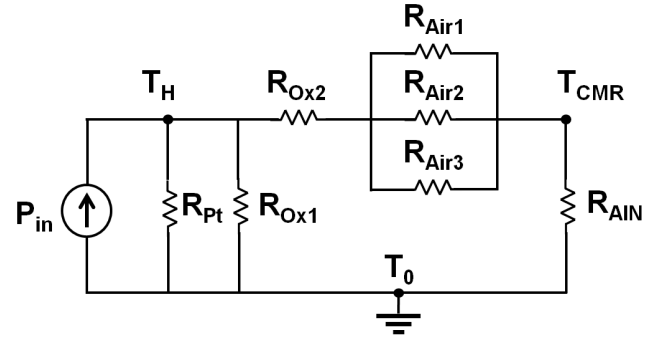

Figure 2: Equivalent thermal circuit describing the heat transfer from the nano hot plate to the AIN MEMS resonator.

In the thermal circuit in figure 2 the thermal resistance associated with the air gap between the hot plate and the AlN CMR is expressed as the parallel combination of conduction $\left(R_{\text {Airl }}\right)$, convection $\left(R_{\text {Air } 2}\right)$ and radiation $\left(R_{\text {Air } 3}\right)$ thermal resistances. Given the geometrical dimensions of the hot plate and the low values of input power level $(10 \mathrm{~s} \mathrm{~mW})$ the convection and radiation thermal resistance values are in the order of $10^{6}-10^{7} \mathrm{~K} / \mathrm{W}$. Therefore, $R_{\text {Air } 2}$ and $R_{A i r 3}$ can be considered as open circuits for the calculation of $T_{C M R}$ (the contribution of convection and radiation mechanisms to the heat transfer is not significant compared to the conduction through such small air gap).

In order to achieve efficient heat transfer between the hot plate and the AlN CMR $\left(T_{H} \approx T_{C M R}\right)$ it is necessary that $\left(R_{O x 2}+R_{\text {Airl }}\right)<R_{A l N}$. For a given geometry of the AIN CMR $\left(R_{A I N} \approx\right.$ $5 \cdot 10^{4} \mathrm{~K} / \mathrm{W}$ for the device of this work) this can be achieved by reducing the values of $R_{O x 2}$ and $R_{\text {Airl }}$, which translates in the following design considerations:

a. Scaling of the hot plate thickness (i.e. implementing a nano-hot plate) to reduce $R_{O x 2}$.

b. Employment of a low thermal conductivity material (i.e. $\mathrm{SiO}_{2}$ ) for the implementation of the nano hot plate (lower $R_{O x 2}$ ). c. Scaling of the air gap between the nano hot plate and the AlN CMR to reduce $R_{\text {Airl }}$.

The implementation of a $\mathrm{SiO}_{2}$ nano hot plate also ensures that a very high value of thermal resistance exists between the heat source and the heat sink (large $R_{O x I}$ ). Therefore, even a small amount of power applied to the serpentine generates a significant temperature increase of the $\mathrm{SiO}_{2}$ nano plate. The generated heat is then efficiently transferred to the MEMS resonator placed right underneath by conduction through the nanoscale air gap.

According to the aforementioned design considerations the integrated nano hot plate of this work was composed of a platinum (Pt $100 \mathrm{~nm}$ thick) serpentine shaped heater deposited on top of a $600 \mathrm{~nm}$ thick $\mathrm{SiO}_{2}$ nano plate. Figure 3 shows the calculaed AlN CMR temperature rise versus the input power level applied to the heater for different values of air gap. Smaller air gaps guarantee significantly larger values of temperature rise factor of the ovenized MEMS resnator. Therefore, the air gap between the nano hot plate and the AlN CMR was set to $250 \mathrm{~nm}$.

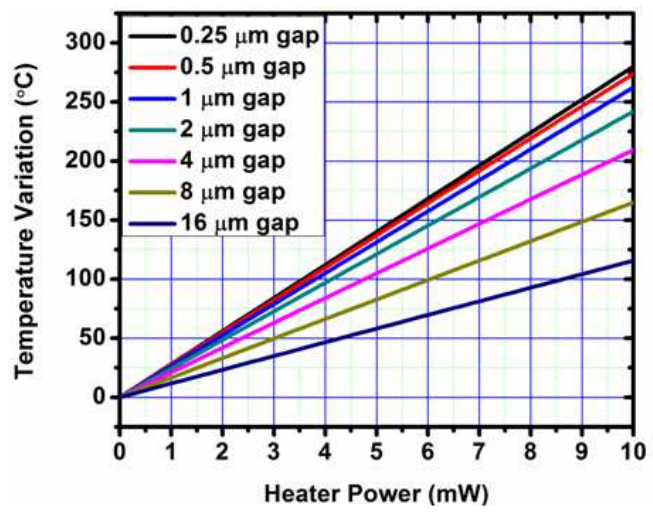

Figure 3: Calculated AlN CMR temperature rise for different levels of input power and different values of air gap. The calculation uses the nominal geometrical dimension of the structure and generic material parameters available in literature. The result of the analysis indicates that higher values of temperature rise factor can be achieved by scaling the air gap between the heating element and the MEMS resonator.

\section{FABRICATION PROCESS}

The AlN MEMS resonator with monolithically integrated nano hot plate was fabricated in a six-mask post-CMOScompatible microfabrication process (Fig. 4). The platinum (Pt) bottom floating electrode (100 nm thick) of the LFE-F device was first patterned by optical lithography, sputtered deposited and lifted off on top of a high resistivity silicon wafer. The $500 \mathrm{~nm}$ thick AlN film was sputtered deposited using a Tegal AMS 2004 SMT (OEM Group, Petaluma, CA) and its quality was optimized to achieve rocking curve values as good as $1.8^{\circ}$. Optical lithography, sputter deposition and lift-off were performed for the definition of the top aluminum (Al) multi-fingered electrode $(100 \mathrm{~nm}$ thick). The inplane dimensions of the AlN resonator were defined by dry etching (Inductively Coupled Plasma, ICP) of the $\mathrm{AlN}$ film in $\mathrm{Cl}_{2}$-based chemistry using photoresist as a mask. A $250 \mathrm{~nm}$ thick polysilicon layer was deposited by RF sputtering and patterned by optical lithography and dry etching (Reactive Ion Etching, RIE) in F-based chemistry in order to create a sacrificial layer covering the surface of the AlN resonator. The thickness of the polysilicon sacrificial layer determines the gap between the nano hot plate and the AlN CMR. A $600 \mathrm{~nm}$ thick $\mathrm{SiO}_{2}$ layer was deposited by Plasma Enhanced Chemical Vapor Deposition (PECVD) and patterned by optical lithography and dry etching (RIE) in F-based chemistry in order to define the structural layer forming the suspended nano hot 
plate. Optical lithography, sputter deposition and lift-off were performed for the definition of the Pt serpentine shaped element acting as heat source for the ovenized MEMS resonator. Isotropic dry etching in $\mathrm{XeF}_{2}$ was finally employed to simultaneously release the MEMS resonator from the silicon substrate and etch the polysilicon sacrificial layer forming the air gap between the nano hot plate and the resonant element. The scanning electron micrographs of the fabricated device are shown in figure 5 .

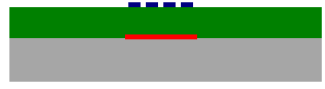

(a)

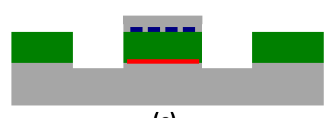

(c)

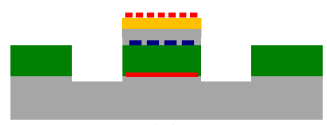

(e)

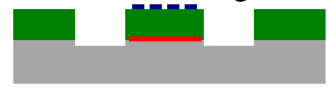

(b)

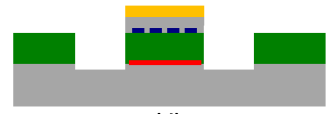

(d)

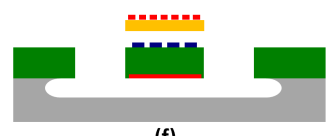

(f)
$\mathrm{Si} \quad$ AIN $\square \mathrm{Pt} \square$ Al $\square \mathrm{SiO}_{2}$

Figure 4: 6 mask fabrication process for the making of the AlN resonators with integrated $\mathrm{SiO}_{2}$ nano hot plate: (a) sputter deposition of Pt $(100 \mathrm{~nm})$ bottom electrode, AlN (500 nm) and Al (100nm) top electrodes; (b) dry etching of AlN in $\mathrm{Cl}_{2}$-based chemistry; (c) deposition and patterning of polysilicon sacrificial $(250 \mathrm{~nm})$ layer forming the airgap between the heater and the CMR; (d) deposition and patterning of $\mathrm{SiO}_{2}$ nano-plate $(600 \mathrm{~nm})$; (e) deposition and patterning of Pt serpentine heater (100 nm); ( $f$ ) $\mathrm{XeF}_{2}$ dry release of the AlN resonator and the air gap between AlN CMR and the nano-hot-plate.

\section{EXPERIMENTAL RESULTS}

The electrical response of the fabricated ovenized AlN MEMS resonator was characterized in a Semiprobe RF probe station and the admittance curve measured by an Agilent N5230A network analyzer after performing a short-open-load calibration on a reference substrate. The measured electrical response of the device was fitted to the Butterworth-van Dyke (BVD) equivalent electrical circuit [5] and showed high values of quality factor, $Q=1805$ and electromechanical coupling coefficient, $k_{t}^{2}=0.9 \%$ (Fig. 6). Such high value of the device figure of merit $\left(F O M=k_{t}^{2} \cdot Q>15\right)$, is higher than what measured for conventional AlN CMR fabricated in the same batch and comparable to what is typically achieved with conventional non-ovenized AIN CMRs (employing a $500 \mathrm{~nm}$ thick AlN film). This value represents the best $F O M$ ever reported for ovenized MEMS resonators operating in the $\mathrm{GHz}$ range. The achievement of a high value of device FOM is of crucial importance for the direct connection of the ovenized MEMS resonator to a compact and low-power oscillator circuit. In fact, the primary power loss in an oscillator circuit is due to the motional resistance, $R_{m}$, of the resonator [5], the value of which is inversely proportional to the device FOM [4, 5]. Given the opportunely designed device geometrical dimensions (device capacitance, $C_{0}=220 \mathrm{fF}$ ) and the achieved high value of $F O M$ a motional resistance, $R_{m}$, of only $56 \Omega$ is obtained for the fabricated $968 \mathrm{MHz}$ ovenized MEMS resonator $\left(R_{m} \propto 1 /\left(C_{0} \cdot k_{t}^{2} \cdot Q\right)\right)$.

The AlN MEMS device with monolithically integrated nano hot plate was directly wire-bonded to a Pierce oscillator implemented with an ATF-551M4 E-pHEMT GaAs transistor. The use of such high gain amplifier (GaAs technology) allowed exploiting the extended dynamic range of the fully-anchored AlN CMR [8].

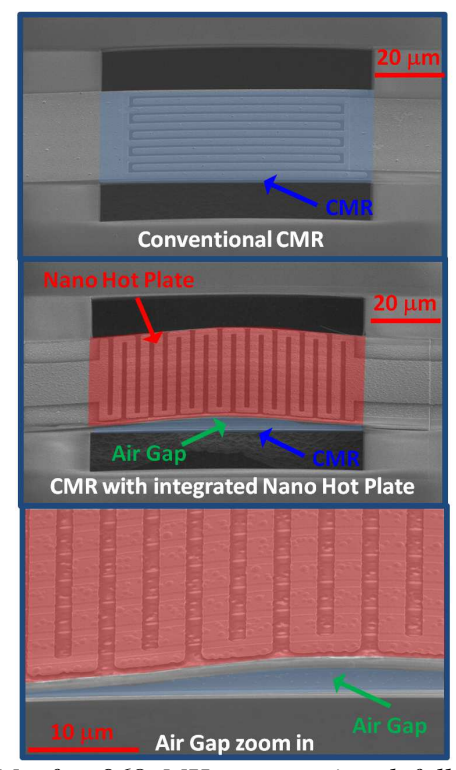

Figure 5: SEM of a $968 \mathrm{MHz}$ conventional fully anchored AlN CMR and SEM of the $968 \mathrm{MHz}$ AlN CMR with integrated suspended nano-hot-plate. The air-gap between the resonant device and the nano-hot-plate (designed to be $250 \mathrm{~nm}$ ) is larger in the center of the device because of residual stress in the SiO2 layer.

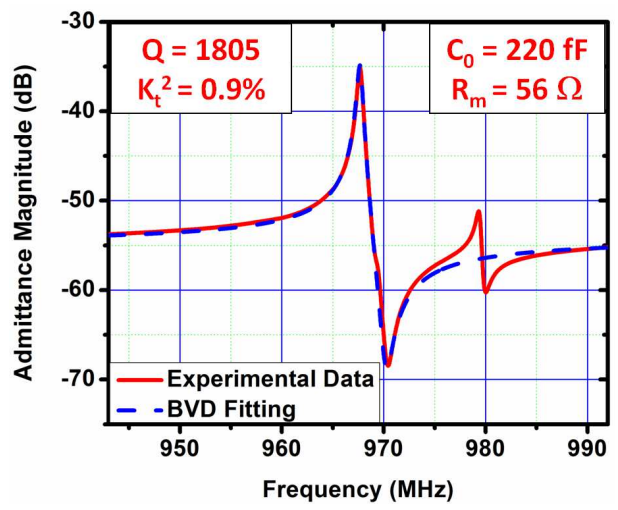

Figure 6: Measured admittance and BVD model fitting of the fabricated $968 \mathrm{MHz}$ ovenized AIN MEMS resonator. The monolithic integration of the suspended nano hot plate does not affect the FOM of the device. A value of motional resistance, $R_{m}$, of only $56 \Omega$ is obtained for the fabricated $968 \mathrm{MHz}$ ovenized MEMS resonator.

The ovenized oscillator was calibrated inside a Tenney TPS climatic chamber and showed a temperature coefficient of frequency (TCF) of $\sim-32.5 \mathrm{ppm} / \mathrm{K}$ with no current flowing in the nano-hot-plate. To test the performance of the ovenized oscillator, the nano hot plate was then turned on and the device response recorded for different levels of power into the heater. The ovenized oscillator frequency and phase noise were monitored with an Agilent E5052B Signal Source Analyzer. The oscillator frequency shifts due to different power levels applied to the nano hot plate were recorded. Taking into account the measured TCF of the oscillator, it was possible to extract the resonator temperature increase versus the power supplied to the nano hot plate and obtain a temperature rise factor of $18.3 \mathrm{~K} / \mathrm{mW}$ (Fig. 7). This value of the temperature rise factor is comparable to what previously achieved using heating elements directly integrated into the body of lower frequency AlN resonators $[10,11]$ and demonstrates the efficiency 
and effectiveness of the proposed innovative design solution.

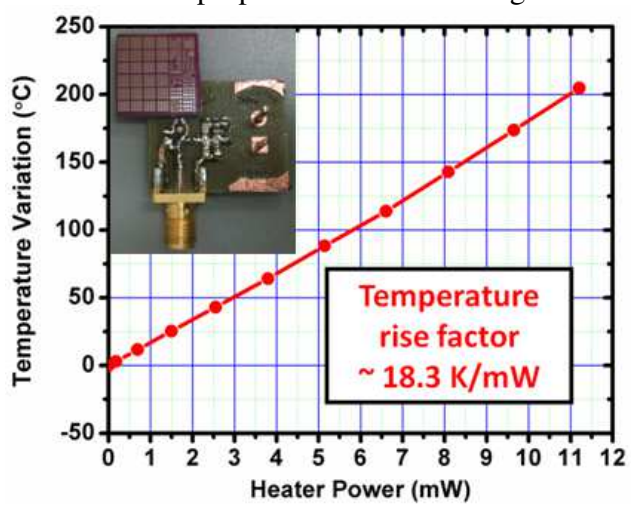

Figure 7: Measured temperature variation of the AlN resonator for different power levels supplied to the nano-hot-plate. The inset shows the AlN MEMS die wire-bonded to the oscillator printed circuit board. The high value of temperature rise factor (18.3 $\mathrm{k} / \mathrm{mW}$ ) demonstrates the efficiency and effectiveness of the proposed innovative design solution.

The phase noise of the ovenized oscillator was also measured for different power levels into the heater, hence different temperature conditions, showing values as good as $-83 \mathrm{dBc} / \mathrm{Hz}$ at $1 \mathrm{kHz}$ offset and $-150 \mathrm{dBc} / \mathrm{Hz}$ for the floor (Fig. 8). No phase noise degradation was recorded for device temperatures up to $\sim 235^{\circ} \mathrm{C}$ (Fig. 9). This means that oscillator temperature variations could be easily compensated over a range of $125{ }^{\circ} \mathrm{C}$ with a power consumption $<7 \mathrm{~mW}$ and without affecting at all the phase noise performance of the oscillator.

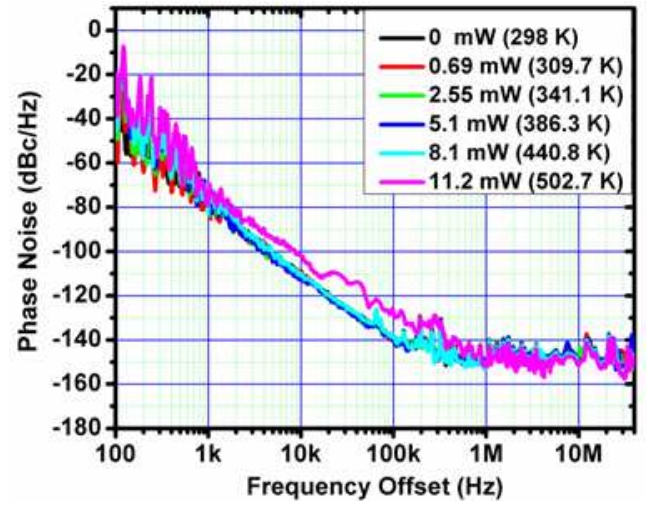

Figure 8: Measured phase noise of the ovenized oscillator for different values of power applied to the nano hot plate. Values of $-83 \mathrm{dBc} / \mathrm{Hz}$ at $1 \mathrm{kHz}$ offset and $-150 \mathrm{dBc} / \mathrm{Hz}$ floor are recorded.

\section{CONCLUSION}

In this paper, a novel technology platform suitable for the implementation of high frequency and high performance ovenized MEMS frequency sources has been introduced. Taking advantage of the unique performance enabled by the proposed technology the highest frequency $(970 \mathrm{MHz})$ ever reported ovenized MEMS oscillator has been demonstrated. A resonator temperature rise factor of $18.3 \mathrm{~K} / \mathrm{mW}$ has been measured demonstrating the efficiency and effectiveness of the proposed innovative design solution. The phase noise of the ovenized MEMS oscillator has been measured for different values of power applied to the nano hot plate showing values of $-83 \mathrm{dBc} / \mathrm{Hz}$ at $1 \mathrm{kHz}$ offset and -150 $\mathrm{dBc} / \mathrm{Hz}$ floor. In addition, no phase noise degradation has been recorded for device temperatures up to $\sim 235{ }^{\circ} \mathrm{C}$. This means that temperature variations of the presented ovenized MEMS oscillator could be easily compensated over a range of $125^{\circ} \mathrm{C}$ with a power consumption $<7 \mathrm{~mW}$ and without affecting at all its phase noise performance. This prototype can be considered a stepping stone towards the demonstration of wafer level packaged ovenized AlN MEMS resonators with a heating element integrated inside the walls of the device package.

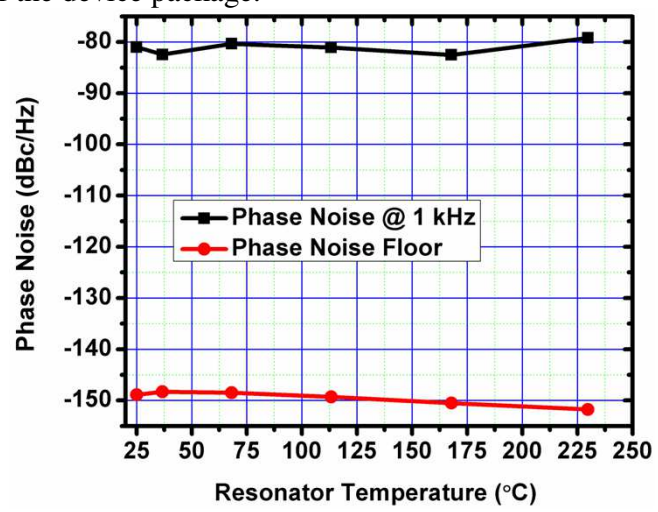

Figure 9: Measured phase noise of the ovenized oscillator for different values of the resonator operating temperature (i.e. different power levels applied to the heater). No phase noise degradation was recorded for temperatures up to $\sim 235{ }^{\circ} \mathrm{C}$.

\section{ACKNOWLEDGEMENTS}

This work was supported by DEFYS DARPA contract number: FA8650-10-1-7030.

\section{REFERENCES}

[1] C. T.-C. Nguyen, IEEE Trans. Ultrason. Ferroelectr. Freq. Control, vol. 54, no. 2, pp. 251-270, 2007.

[2] G. Piazza, P. J. Stephanou, and A. P. Pisano, J. Microelectromech. Syst., vol. 15, no. 6, pp. 1406-1418, 2006.

[3] M. Rinaldi, C. Zuniga, G. Piazza, Proc. 22nd IEEE International Conference on Micro Electro Mechanical Systems pp. 916-919, 2009.

[4] M. Rinaldi, C. Zuniga, C. Zuo and G. Piazza, IEEE Transactions on Ultrasonics, Ferroelectrics, and Frequency Control, vol. 57, no. 1, pp. 38-45, 2010.

[5] M. Rinaldi, C. Zuo, J. Van der Spiegel, G. Piazza, IEEE Transactions on Electron Devices, vol. 58, n. 5, pp. 12811286, 2011.

[6] C-M. Lin, T. Yen, Y. Lai, V.V. Felmetsger, M. A. Hopcroft, J. H. Kuypers, A. P. Pisano, IEEE Transactions on Ultrasonics, Ferroelectrics and Frequency Control, vol.57, no.3, pp.524-532, 2010.

[7] R.H. Olsson, C.M. Washburn, J.E. Stevens, M.R. Tuck, C.D. Nordquist, Proc. IEEE International Frequency Control Symposium 2008, pp.634-639, 2008.

[8] M. Rinaldi, A. Tazzoli, J. Segovia-Fernandez, V. Felmetsger, G. Piazza, Proc. $25^{\text {th }}$ IEEE International Conference on Micro Electro Mechanical Systems pp.696-699, 2012.

[9] J.C. Salvia, R. Melamud, S.A. Chandorkar, S.F. Lord, T.W. Kenny, Journal of Microelectromechanical Systems, vol.19, no.1, pp.192-201, 2010.

[10] Bongsang Kim, R.H. Olsson, K.E. Wojciechowski, Proc. IEEE Ultrasonics Symposium (IUS 2010), pp.974-978, 2010.

[11] A. Tazzoli, M. Rinaldi, G. Piazza, IEEE International Electron Devices Meeting, pp.20.2.1-20.2.4, 2011.

\section{CONTACT}

*M. Rinaldi, tel: +1- 617-373-2751; rinaldi@ece.neu.edu 


\section{APODIZATION TECHNIQUES FOR SPURIOUS MODE SUPPRESSION IN 900 MHZ ALUMINUM NITRIDE CONTOUR-MODE RESONATORS Marco Giovannini ${ }^{1,2}$, Serkan Yazici ${ }^{1}$, Nai-Kuei Kuo ${ }^{1}$, and Gianluca Piazza ${ }^{3}$ \\ ${ }^{1}$ University of Pennsylvania, Philadelphia, Pennsylvania, USA \\ ${ }^{2}$ Politecnico of Milan, Milan, Italy, and \\ ${ }^{3}$ Carnegie Mellon University, Pittsburgh, Pennsylvania, USA}

\begin{abstract}
This paper reports, for the first time, on the application of apodization techniques to $900 \mathrm{MHz}$ MEMS AlN Contour-Mode Resonators (CMRs [1]) to efficiently suppress spurious modes in close proximity of the main mechanical resonance. This concept has been applied with excellent results to a variety of one port resonators formed by patterned top electrodes made out of Aluminum, and a floating bottom electrode made out of Platinum sandwiching the AlN film. As also predicted by 3D COMSOL simulations, a complete elimination of spurious responses in the admittance plot of these resonators is attained without impacting their $Q$ or electromechanical coupling coefficient, $k_{t}^{2}$.
\end{abstract}

\section{INTRODUCTION}

The presence of spurious modes hinders the performance of filters by generating ripples in the pass-band and unwanted responses out-of-band. A spurious free response also could help in reducing phase noise in oscillators. Additionally, the ability to set the device impedance by changing its geometry is limited by the appearance of spurious modes for certain device aspect ratios. Although some methods to suppress out-of-band spurious by means of anchoring techniques [2],[3], or the introduction of dummy electrodes [4] have been implemented, the problem of inband spurious suppression for CMRs has never been addressed.

This paper introduces the concept of apodization for MEMS resonators - a new method to shape the geometry of the electrodes in order to obtain a consistent reduction of spurious modes of vibration in the electrical response of one port CMRs.

The concept of apodization consists of confining the vibration energy in specific regions of the CMR body by shaping its electrodes.

To implement the apodization technique, a standard CMR is used as a reference and its electrodes are modified. Two apodization techniques were studied and applied to one port resonators formed by patterned top electrodes $(25,33,49$ and 65 fingers) made out of Aluminium (120 and $220 \mathrm{~nm}$ thick), and a floating bottom electrode made out of Platinum (90 nm thick) sandwiching the AIN film (500 thick) (see Figure1):

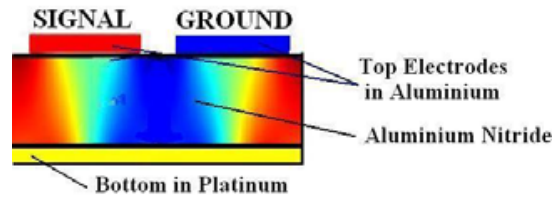

Figure 1. Schematic representation of the finger pair forming the basic element of a lateral field excited CMR.

In the first proposed apodized shape design (shape I, see Figure 2), the electrode overlap progressively decreases when moving from the central part of the resonator towards the edges following a sine like shape. Instead, in the second developed apodized shape (shape II, see Figure 2), the electrode overlap decreases when moving away from the central part of the resonator, but maintains constant for the last set of fingers towards the edges. This variation was implemented with the scope of keeping a larger electrode overlap area, and therefore a higher $k_{t}^{2}$ This concept was applied to a variety of one port CMRs (different geometry and material thickness) in order to prove its effectiveness and repeatability. Each solution has been designed, simulated, fabricated and tested. a)

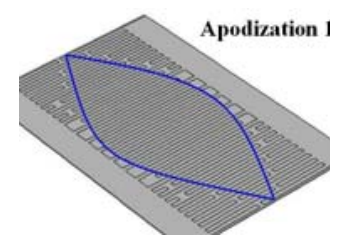

b)

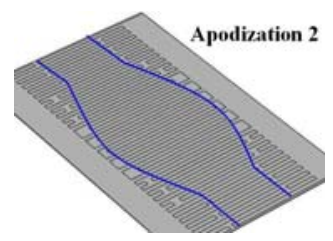

Figure 2. Apodization: shape I (a), shape II (b).

\section{APODIZATION THEORY}

Apodization techniques were first introduced with surface acoustic wave (SAW) transducers in order to improve the sampling of the signal coming from an unapodized SAW device. This technique is the most widely used method for weighting the response of a SAW transducer and pick up exclusively the desired modes of vibration. It relays on properly varying the shape of the electrodes so that the sampled electric signal is properly weighted (Fig. 3) by a prescribed window (sampling) function.

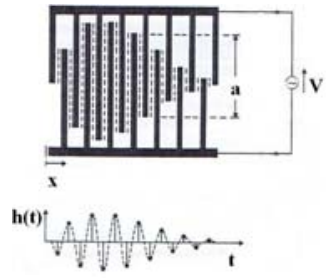

$\mathrm{V}$ : Electric potential a : Overlap electrode length $\mathrm{h}(\mathrm{t})$ : Impulse response

Figure 3. Schematic representation of the apodization technique and its equivalent impulse response.

Although the CMR does not excite a surface acoustic wave, but a lamb wave, the device is formed by a large set of interdigitated electrodes and the same concept of apodization can be implemented to suppress transversal vibrations that cause undesired spurious vibrations in standard, unweighted CMRs. As for SAWs, in the CMRs, the electric field is applied to the AIN body through a set of interdigitated electrodes (also known as fingers). So, shaping of the CMR fingers permits to accurately weigh the electric field distribution that is applied to the CMR. Effectively, the apodization concept consists of finding a method to shape the electrodes so as the electric field is applied in a manner that exclusively the main vibration mode of interest is excited. Apodization techniques are based on the idea that it exists a relationship between the "impulse response" of the device acoustic waveform, the electrodes geometry and its frequency response [5]. Because of this relationship, (due to the nature of the piezoelectric actuation), the device impulse response is seen as a sequence of Dirac functions at times corresponding to the electrode locations, with amplitudes given by the electrode overlap length, $a$ (Fig. 3). The idea of apodization consists in placing and shaping 
the electrodes at those locations corresponding to the peaks and thorough of the waveform (that is propagating) so as to excite the desired mode of vibration. This means that we will maximize the electrode overlap $(a)$ at those locations where the wave amplitude is maximum and shape the electrodes so as to follow the desired frequency response that we want to obtain. Thus, the point is to shape the CMR electrodes layout so as the device impulse response follows the desired frequency response. In the standard case, the impulse response is composed by a series of completely overlapped electrodes. In the case of a 13 fingers one port CMRs, we can identify the electrode layout with a vector (Fig. 4), where the unit number, positive or negative, represents the fact that the electrode (signal or ground) extends over the entire length of the device. The + or - sign is used to distinguish whether the electrode is a signal or ground electrode. A zero represents that no electrode is present in that region (generally in between electrode pairs).

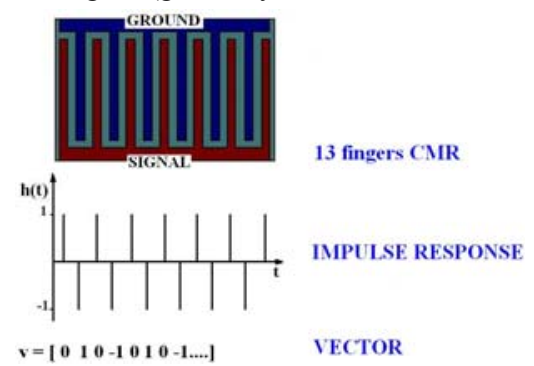

Figure 4. Figure of 13 fingers CMRs with its impulse response and associated vector.

Assuming that this distribution corresponds to the impulse response in time of the resonator, its frequency response can be found by a simple Fourier transform of this vector (Fig.6). It is evident that, in this case, the CMR response is characterized by a relatively spread out lobe for the main frequency, plus other smaller adjacent lobes. Clearly, the energy is not fully confined in a single frequency.

Instead, when we apply apodization to the same CMR, and we adopt, for example, the sine shape window for the electrode design, the same vector (Fig. 4) can be rewritten (Fig.5), so as to follow the desired electrode overlap. In this case, we place the maximum value of the sine function in the middle of the CMR.

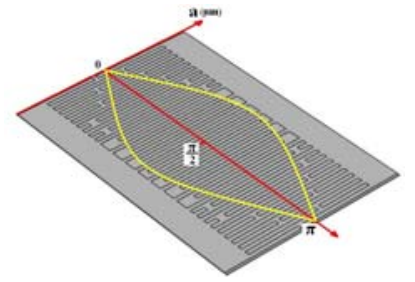

Figure 5. Schematic illustration of the sine shape, the maximum overlap (a) is placed in the middle of the CMR.

For instance, for a 13 fingers apodized CMR, we make the total device width proportional to half period of a sine wave and divide into 14 parts (Fig.6). We evaluate the sine function for each portion comprised between 0 and $\pi$, and design the finger length to be proportional to the value of the sine function at each point along the width.. Therefore, for an apodized 13 fingers CMR that uses the sine wave window, the vector representing the impulse response becomes:

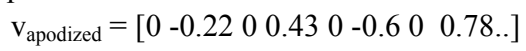

The frequency response (FR) related to the impulse response of the vector in Eq. (1) can be found by a Fourier transform (Fig. 6). As shown in Fig.6, it is clear that the CMR response is characterized by a main lobe centered around the resonance frequency and smaller out-of-band lobes. The reduction in magnitude of the side lobes is proportionally higher than the response at the center frequency. In conclusion, we can affirm that, for the 13 fingers CMR, the application of the sine-function to the shape of the electrodes reduces the spurious vibrations, represented by "smaller secondary lobes", but comes with a drop of the signal amplitude. The signal loss can be recovered by increasing the number of fingers.

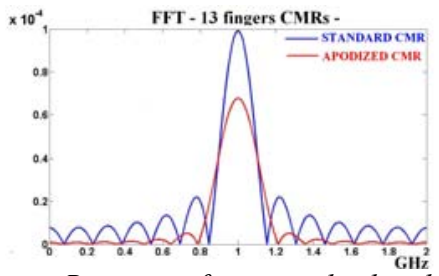

Figure 6. Frequency Response for a standard and apodized CMR.

\section{APODIZED RESONATOR DESIGN}

Beyond the suppression of spurious vibrations, the design of the apodized resonators is driven by the following guidelines: (i) operate at a center frequency around $900 \mathrm{MHz}$, (ii) attain a value of device static capacitance, $\mathrm{C}_{0}$, as compatible as possible with 50 $\Omega$ circuits; (iii) achieve quality factor, $\mathrm{Q}$ higher than 1000 in air and electromechanical coupling coefficient, $k_{t}^{2}$, higher than $1 \%$.

The overall finger width (electroded + unelectroded area) is determined by the frequency of operation. An electrode pitch of $4 \mu \mathrm{m}$ was used to operate around $900 \mathrm{MHz}$ for the given film stack. The remainder of the device geometry is set by the number of electrode pairs, their lengths and the film thickness. These parameters are used to set the device static capacitance. We selected to use an AIN film thickness of $500 \mathrm{~nm}$, finger lengths ranging between 40 and $85 \mu \mathrm{m}$, and a number of fingers between 25 and 65 to yield device capacitance between 180 and $900 \mathrm{fF}$.

The electrode design is mainly constrained by the: (i) electrode coverage ratio, which represents the ratio between the electroded area of the resonator and its overall surface; (ii) finger overlap, which, as shown in Fig. 5, represents the portion of the length of adjacent fingers that are actually overlapping. The coverage ratio was set to $50 \%$ primarily because of fabrication constraints. The electrode overlap was selected to follow, among various available window functions, the sine shape, which represents an optimal compromise to achieve spurious suppression and high coupling. In order to improve the electrical response of the resonator, we have slightly deviated from a perfect sine-shape design and modified the electrode layout in the following way: increase the overlap electrode length at the two ends of the CMR and decrease the overlap electrode length for the central fingers so as to compromise between spurious mode suppression and admittance peak reduction.

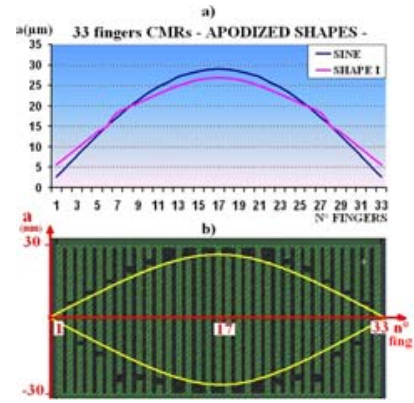

Figure 7. (a) Plot of $1 / 2$ of the finger overlap magnitude (in $\mu m$ ) for the ideal sine shape window and the selected apodization shape I (b), Layout view of the apodized CMR. 
The rationale for this selection was confirmed by COMSOL 3D analysis (Fig. 8), which shows that spurious mode suppression is attained without significantly impacting the series resonance magnitude. Shape I was effectively optimized by an empirical approach based on intuition and COMSOL $3 \mathrm{D}$ analysis. It is evident that the overlap electrode length at the end parts of the CMRs plays a key role, and a slight increase of it permits to obtain best device performance.

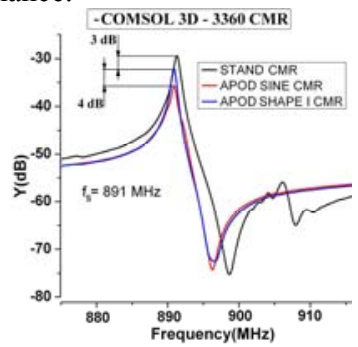

Figure 8. Admittance plot(Y) for different types of $900 \mathrm{MHz}$ AlN resonator (standard, apodized using a sine-wave window and the shape I). Y plot for a standard resonator is characterized by spurious vibrations between the series and parallel resonance. The apodized devices show suppression of the spurious modes and shape I mitigates the reduction in magnitude of admittance.

With the purpose of further improving the device electrical response, a second apodized shape (shape II) was also studied theoretically and experimentally. In shape II, the overlap length towards the ends of the resonator is further increased and made constant for the last group of fingers. The overall electrode layout is similar to shape I, except for the end parts (Fig. 9).

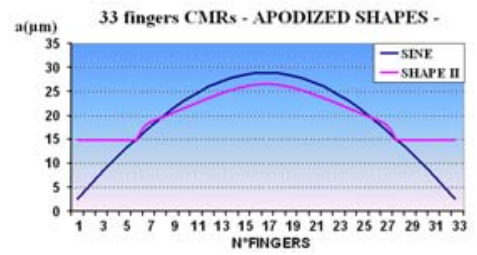

Figure 9. Plot of the finger overlap length in the case of a standard sine wave window and the selected shape II apodization scheme.

Although intuitively this design should strengthen the resonator response at the series resonance, no significant advantages were attained with respect to shape I.

\section{FABRICATION}

The two presented apodization techniques (shape I and shape II) were studied and applied (Fig. 12) to one port resonators formed by patterned top electrodes $(25,33,49$ and 65 fingers) made out of Aluminium (120 and $220 \mathrm{~nm}$ thick), and a floating bottom electrode made out of Platinum ( $90 \mathrm{~nm}$ thick) sandwiching the AlN film $(1 \mu \mathrm{m}$ thick). For comparison, the same identical geometries were laid out and fabricated without apodization (standard resonator).

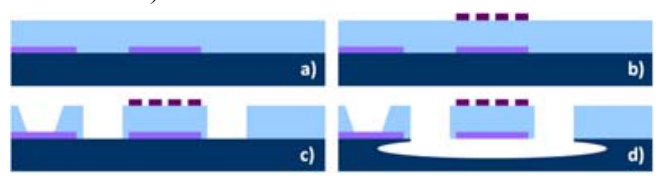

Figure 10. Representation of the 3 mask fabrication process used for the making of the apodized resonator: a) Sputtering and patterning by lift-off of the bottom electrode and sputtering of the thin-AlN film; b) deposition and patterning of the top electrode; c)Etching of AlN; d) Release of AlN resonator in $\mathrm{XeF}_{2}$ atmosphere.
The device fabrication is based on the standard 3-mask manufacturing steps previously reported for AIN CMRs [6] and here schematically reported in Fig. 10. a)

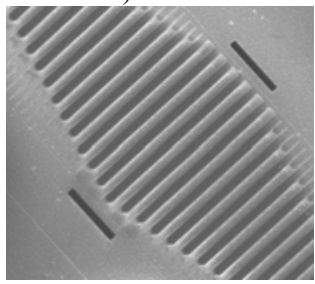

b)

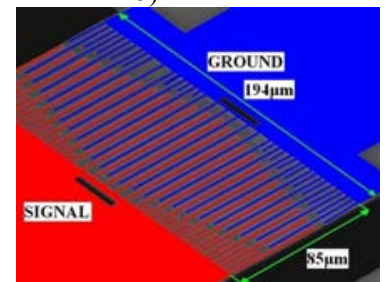

Figure 11. SEM of apodized CMR; with original colours (a), and false colours in order to highlight the apodized shape (b).

\section{EXPERIMENTAL RESULTS}

The devices were tested in a micromanipulated RF probe station under ambient conditions. A short open and load calibration was performed prior to measuring the devices and extracting their admittance response. The apodized device response is compared to standard CMR devices. For each device, admittance peak, electromechanical coupling coefficient and Q were extracted and compared. Table 1 summarizes the results for the two apodization shapes applied to $900 \mathrm{MHz}$ AlN devices. The results are presented as percentage change with respect to the non-apodized case.

Table 1 shows clearly that spurious vibrations are significantly suppressed with a minimal impact on device $Q, k_{t}^{2}$ and admittance peak value. The small value of the standard deviation $(\sigma)$ in the presented data is a sign that this technique can be applied to a large group of devices of different dimensions (and therefore different impedance). Overall, we can state that the introduction of apodization is advantageous and has mostly the following impact on the device performance: (i) complete suppression of the spurious modes; (ii) improvement of the quality factor, likely due to the ability of apodization to focus energy on the main resonance peak; (iii) minimal decrease of the electromechanical coupling coefficient $\left(\mathrm{k}_{\mathrm{t}}^{2}\right)$ and of $\mathrm{C}_{0}$; (iv) increase in the motional resistance $\left(\mathrm{R}_{\mathrm{m}}\right)$ and the consequent decrease of admittance peak (Y).
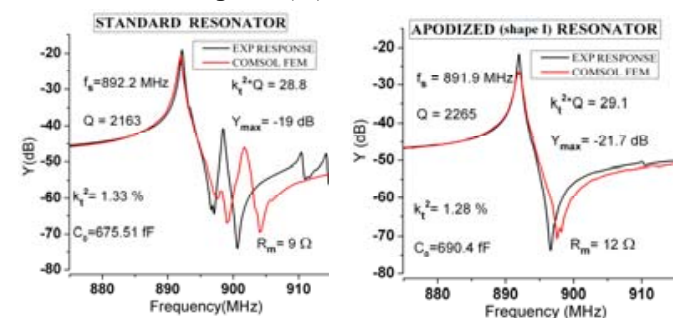

Figure 12. Experimental results for standard and apodized (shape I) $900 \mathrm{MHz}$ AlN CMR formed by $500 \mathrm{~nm}$ of AlN, $120 \mathrm{~nm}$ of Al, 90 $\mathrm{nm}$ of Pt and 49 fingers (finger length of $85 \mu \mathrm{m}$ ).

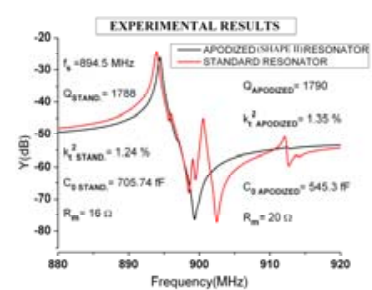

Figure 13. Experimental results for standard and apodized (shape II) $900 \mathrm{MHz}$ AlN CMR formed by $500 \mathrm{~nm}$ of AlN, $120 \mathrm{~nm}$ of Al,90 $\mathrm{nm}$ of Pt, and 33 fingers (finger length of $85 \mu \mathrm{m}$ ). 
It is worth noting that the experimental results are in line with the theoretical expectations as shown in the comparison between the experimental response and the COMSOL FEM model (Fig. 12). The same analysis performed for shape I, was also conducted for shape II (Fig. 13).

From the experimental results, it is evident that, also in this case, the device response for a standard resonator is characterized by large spurious between the series and parallel resonance, while an apodized resonator (shape II) shows complete suppression of the spurious vibrations.

Table 1: Summary of the results for a variety of apodized resonators (shape I and II) operating around $900 \mathrm{MHz}$ and formed by $500 \mathrm{~nm}$ of AlN, $120 \mathrm{~nm}$ of Al and $120 \mathrm{~nm}$ of Pt. The resonators are identified with a code of four numbers; the first two numbers indicate the number of fingers and the last two refer to the length of fingers in $\mu \mathrm{m}$. So, for instance the code "2560" is referred to a resonator of 25 fingers, with a length of $60 \mu \mathrm{m}$. The data are provided as percentage change with respect to the linear value of the non-apodized case. A negative value of $\%$ indicates that a higher value is recorded in the non-apodized case. The decrease of spurious vibrations has been quantified by evaluating the maximum amplitude of the spurious response in the admittance graph (expressed in linear values) before $\left(S p_{\text {stand }}\right)$ and after the application of the apodization technique $\left(S p_{\text {apod }}\right)$. The same concept has been applied in order to calculate the decrease of quality factor $(\Delta Q)$, electromechanical coupling coefficient $\left(\Delta k_{t}^{2}\right)$, and of the admittance peak ( $\triangle Y)$. So, for $\triangle S p=-100 \%$, it means that a total suppression of the spurious vibration has been achieved.

$$
\Delta S p=\frac{S p_{a p}-S p_{s \tan d}}{S p_{s \tan d}} * 100
$$

\begin{tabular}{|c|c|c|c|c|c|c|c|c|}
\hline \multicolumn{5}{|c|}{ Apodization I } & \multicolumn{4}{|c|}{ Apodization II } \\
\hline CMRs & $\Delta \mathrm{Sp}$ & $\Delta Q$ & $\Delta \mathrm{Y}$ & $\Delta \mathbf{k}_{\mathrm{t}}^{2}$ & $\Delta \mathrm{Sp}$ & $\Delta \mathbf{Q}$ & $\Delta \mathbf{Y}$ & $\Delta \mathbf{k}_{t}^{2}$ \\
\hline 2540 & $-69.3 \%$ & $6.7 \%$ & $-6.6 \%$ & $-12.1 \%$ & $-79.3 \%$ & $-11.4 \%$ & $-11.9 \%$ & $-18.9 \%$ \\
\hline 2560 & $-94.0 \%$ & $-2.8 \%$ & $-13.5 \%$ & $-7.1 \%$ & $-95.2 \%$ & $-2.9 \%$ & $-20.4 \%$ & $3.2 \%$ \\
\hline 2585 & $-100.0 \%$ & $-42.1 \%$ & $-27.2 \%$ & $10.2 \%$ & $-100.0 \%$ & $-35.5 \%$ & $-25.6 \%$ & $-1.6 \%$ \\
\hline 3340 & $-62.1 \%$ & $-15.8 \%$ & $-8.9 \%$ & $-13.3 \%$ & $-51.2 \%$ & $-15.7 \%$ & $-10.6 \%$ & $-14.8 \%$ \\
\hline 3360 & $-94.7 \%$ & $-2.8 \%$ & $-13.4 \%$ & $-6.4 \%$ & $-97.9 \%$ & $-6.7 \%$ & $-8.8 \%$ & $6.4 \%$ \\
\hline 3385 & $-100.0 \%$ & $11.1 \%$ & $-10.7 \%$ & $-6.3 \%$ & $-100.0 \%$ & $0.1 \%$ & $-7.4 \%$ & $6.3 \%$ \\
\hline 4940 & $-97.4 \%$ & $-12.6 \%$ & $-15.5 \%$ & $-24.8 \%$ & $-97.4 \%$ & $-32.8 \%$ & $-20.3 \%$ & $-25.5 \%$ \\
\hline 4960 & $-100.0 \%$ & $4.8 \%$ & $-12.3 \%$ & $-12.0 \%$ & $-100.0 \%$ & $4.8 \%$ & $-18.0 \%$ & $-15.5 \%$ \\
\hline 4985 & $-100.0 \%$ & $4.7 \%$ & $-14.2 \%$ & $-8.5 \%$ & $-100.0 \%$ & $4.8 \%$ & $-17.4 \%$ & $-12.7 \%$ \\
\hline 6540 & $-78.8 \%$ & $-7.2 \%$ & $-24.3 \%$ & $-25.0 \%$ & -- & -- & -- & -- \\
\hline 6560 & $-100.0 \%$ & $10.0 \%$ & $-19.9 \%$ & $-31.0 \%$ & -- & -- & -- & -- \\
\hline 6585 & $-100.0 \%$ & $-4.0 \%$ & $-18.0 \%$ & $-27.8 \%$ & -- & -- & -- & -- \\
\hline$\mu$ & $-91.4 \%$ & $-4.2 \%$ & $-15.4 \%$ & $-13.7 \%$ & $-91.2 \%$ & $-10.6 \%$ & $-15.6 \%$ & $-8.1 \%$ \\
\hline$\sigma$ & $13.3 \%$ & $14.7 \%$ & $6.1 \%$ & $11.7 \%$ & $16.4 \%$ & $15.0 \%$ & $6.2 \%$ & $11.9 \%$ \\
\hline
\end{tabular}

Table 2: Summary of performances for a variety of apodized resonators (shape I and II) operating around $900 \mathrm{MHz}$ and formed by $500 \mathrm{~nm}$ of AlN, $90 \mathrm{~nm}$ of Pt and the Al top electrodes of $220 \mathrm{~nm}$.

\begin{tabular}{|c|c|c|c|c|c|c|c|c|}
\hline \multicolumn{9}{|c|}{$120 \mathrm{~nm}$ AI TOP ELECTRODES } \\
\hline & \multicolumn{4}{|c|}{ Apodization I } & \multicolumn{4}{|c|}{ Apodization II } \\
\hline & $\mathbf{Q}$ & $\mathbf{k}_{t}^{2}$ & $\mathbf{Y}(\mathbf{d B})$ & $\mathrm{C}_{0}(\mathrm{fF})$ & $\mathbf{Q}$ & $k_{\mathrm{t}}^{2}$ & $Y(d B)$ & $\mathrm{C}_{0}(\mathrm{fF})$ \\
\hline$\mu$ & 1250.1 & $1.55 \%$ & -32.82 & 418.6 & 1305.2 & $1.27 \%$ & -32.2 & 367.11 \\
\hline $\boldsymbol{\sigma}$ & 423.4 & $0.11 \%$ & 3.51 & 188.7 & 267.7 & $0.12 \%$ & 3.52 & 140.8 \\
\hline \multicolumn{9}{|c|}{$220 \mathrm{~nm}$ AI TOP ELECTRODES } \\
\hline & \multicolumn{4}{|c|}{ Apodization I } & \multicolumn{4}{|c|}{ Apodization II } \\
\hline & $\mathbf{Q}$ & $\mathbf{k}_{\mathrm{t}}{ }^{2}$ & $\mathbf{Y}(\mathbf{d B})$ & $C_{0}(f F)$ & $\mathbf{Q}$ & $\mathbf{k}_{\mathrm{t}}{ }^{2}$ & $\mathrm{Y}(\mathrm{dB})$ & $\mathrm{C}_{0}(\mathrm{fF})$ \\
\hline$\mu$ & 1848.8 & $1.14 \%$ & -27.9 & 454.9 & 1733.1 & $1.22 \%$ & -29.7 & 376.3 \\
\hline$\sigma$ & 367.3 & $0.16 \%$ & 4.95 & 200.3 & 409.3 & $0.10 \%$ & 4.75 & 156.6 \\
\hline
\end{tabular}

Moreover, we verified that the apodized shapes can be successfully applied to CMRs characterized by different thicknesses of the Al top electrode $(120$ and $220 \mathrm{~nm})$. Table 2 reports the statistical performances of samples of the apodized CMRs characterized by $120 \mathrm{~nm}$ and $220 \mathrm{~nm}$ Al top electrodes. Table 3 shows how apodized CMRs characterized by different top electrode thicknesses present similar performances in terms of spurs reduction, $\Delta \mathrm{Q}, \Delta \mathrm{k}_{\mathrm{t}}^{2}$ and $\Delta \mathrm{Y}$. These data further validate the broad applicability of this apodization technique.

Table 3. Summary of results for a variety of apodized resonators (shape I and II) operating around $900 \mathrm{MHz}$; formed by $500 \mathrm{~nm}$ of AlN, $90 \mathrm{~nm}$ of Pt and the Al top electrodes of $120 \mathrm{~nm}$ and $220 \mathrm{~nm}$.

\begin{tabular}{|c|c|c|c|c|c|c|c|c|}
\hline \multicolumn{9}{|c|}{$120 \mathrm{~nm}$ Al TOP ELECTRODES } \\
\hline & \multicolumn{4}{|c|}{ Apodization I } & \multicolumn{4}{|c|}{ Apodization II } \\
\hline & $\Delta \mathrm{Sp}$ & $\Delta \mathbf{Q}$ & $\Delta \mathbf{Y}$ & $\Delta \mathbf{k}_{\mathrm{t}}^{2}$ & $\Delta \mathrm{Sp}$ & $\Delta \mathbf{Q}$ & $\Delta \mathbf{Y}$ & $\Delta \mathbf{k}_{\mathrm{t}}^{2}$ \\
\hline$\mu$ & $-98.9 \%$ & $3.7 \%$ & $-28.7 \%$ & $-19.9 \%$ & $-95.8 \%$ & $9.3 \%$ & $-24.7 \%$ & $-16.3 \%$ \\
\hline$\sigma$ & $2.52 \%$ & $21.61 \%$ & $14.89 \%$ & $12.69 \%$ & $5.6 \%$ & $14.8 \%$ & $18.6 \%$ & $-13.2 \%$ \\
\hline \multicolumn{9}{|c|}{$220 \mathrm{~nm}$ Al TOP ELECTRODES } \\
\hline & \multicolumn{4}{|c|}{ Apodization I } & \multicolumn{4}{|c|}{ Apodization II } \\
\hline & $\Delta \mathrm{Sp}$ & $\Delta Q$ & $\Delta \mathbf{Y}$ & $\Delta \mathbf{k}_{\mathrm{t}}^{2}$ & $\Delta \mathrm{Sp}$ & $\Delta \mathbf{Q}$ & $\Delta \mathbf{Y}$ & $\Delta \mathbf{k}_{t}^{2}$ \\
\hline$\mu$ & $-91.4 \%$ & $-4.2 \%$ & $-15.4 \%$ & $-13.7 \%$ & $-91.2 \%$ & $-10.6 \%$ & $-15.6 \%$ & $-8.1 \%$ \\
\hline$\sigma$ & $13.3 \%$ & $14.7 \%$ & $6.1 \%$ & $11.7 \%$ & $16.4 \%$ & $15.0 \%$ & $6.2 \%$ & $11.9 \%$ \\
\hline
\end{tabular}

\section{CONCLUSIONS}

This paper reported on the application of apodization techniques to $900 \mathrm{MHz}$ MEMS AlN Contour-Mode Resonators (CMRs) to efficiently suppress spurious modes in close proximity of the main mechanical resonance without impacting their $Q$ or $k_{t}^{2}$. The obtained results show that the introduced apodization techniques, until now exclusively applied to SAW devices, have a significant potential for CMRs and could potentially be extended to several other MEMS resonator technologies.

\section{AKNOWLEDGEMENTS}

Travel support has been generously provided by the Transducer Research Foundation. Funding for this project was provided through the DARPA M/NEMS S\&T program.

\section{REFERENCES}

[1] G. Piazza, et al., "Piezoelectric Aluminum Nitride Vibrating Contour- Mode MEMS Resonators", J.MEMS, vol.15, p. 1406-1418, 2006

[2] B.P. Harrignton, et al., "Toward ultimate performance in $\mathrm{GHz}$ MEMS Resonators: low impedance and high Q", J.MEMS, 2010 IEEE 23rd International Conference, p. 707-710, 2010

[3] Y. Xie, et al., "1.52-GHz Micromechanical Extensional WineGlass Mode Ring Resonators", J.MEMS, Ultrasonics, Ferroelectrics and Frequency Control, IEEE Transactions on, vol.55, p. 890-907, 2008

[4] Olsson, et al., "Origins and Mitigation of Spurious Modes in AIN Microresonators", Proc. of the IEEE International Ultrasonics Symposium, p.1272-1276, October 2010

[5] C.S. Hartmann, D.T. Bell, R.C., Rosenfeld, "Impulse Model Design of Acoustic. Surface-Wave Filters", IEEE Transactions on Sonics nd Ultrasonics, vol.20, p. 80-93, 1973

[6] M. Rinaldi, C. Zuniga, C. Zuo, and G. Piazza, "Super-HighFrequency Two-Port AlN Contour-Mode Resonators for RF Applications" IEEE Transactions on Ultrasonics, Ferroelectrics, and Frequency Control, vol. 57, no. 1, 2010.

\section{CONTACTS}

M. Giovannini, Politecnico of Milan (marco.giova23@gmail.com); G. Piazza,CMU (piazza@ece.cmu.edu). 


\title{
MICROMACHINED POLYCRYSTALLINE DIAMOND HEMISPHERICAL SHELL RESONATORS
}

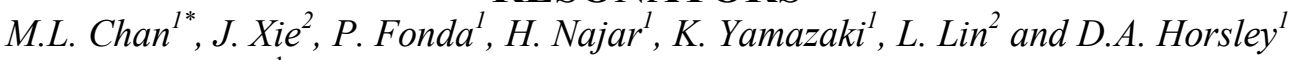 \\ ${ }^{1}$ University of California Davis, California, USA \\ ${ }^{2}$ University of California Berkeley, California, USA
}

\begin{abstract}
We present the development of millimeter scale 3D hemispherical shell resonators fabricated from polycrystalline diamond, a material with low thermoelastic damping and very high stiffness. Resonators are fabricated through a combination of electro discharge machining (EDM) and silicon micromachining techniques, to achieve $1 \mathrm{~mm}$ diameter 3D highly axisymmetric hemispherical shell structures. By using piezoelectric actuation and optical characterization, the elliptical wineglass vibration mode is determined to be at $34.86 \mathrm{kHz}$, with the two degenerate wineglass modes having a relative frequency mismatch of $2 \%$.
\end{abstract}

\section{INTRODUCTION}

The commercial hemispherical resonator gyro (HRG) is a high-precision and highly reliable solid state gyroscope that meets the inertial grade performance [1]. The topology of an axisymmetric shell mounted on a stem minimizes unwanted coupling to the base substrate and, along with the use of highpurity fused-quartz materials, results in high $\mathrm{Q}$ operation. By operating these structures in a rate integrating mode, the sensor can continue to integrate the applied rotation during power interruptions allowing for robust long-term operation.

These characteristics have spurred recent interest in the development of microscale HRGs that can be mass manufactured in a wafer scale level to achieve low-cost inertial grade sensors with a small volume and large dynamic range. To achieve modematching and high-Q performance in a hemispherical resonator, geometric uniformity and symmetry in combination with low thermoelastic damping structural material are critical. Many different methods such as wafer scale glass blowing [2], traditional silicon etching $[3,4]$ and integrating precision machining with standard silicon microfabrication $[5,6]$ have been employed to create 3D spherical or hemispherical shells. Materials such as polysilicon, and glass have also been used as the isotropic structural material for the shell itself.

In this work, we describe the development and characterization of millimeter scale 3D hemispherical shell resonators. Micro-electro discharge machining is first employed to fabricate the silicon mold from which the shell structure can be built. Using traditional silicon micromachining techniques to carry out the subsequent polish, deposition and release steps, we realized a $1 \mathrm{~mm}$ diameter 3D shell resonator. Polycrystalline diamond is used as the structural material due to its unique material properties such as high stiffness and low thermoelastic damping.

\section{FABRICATION}

In order to realize complex 3D shapes such as hemispherical wine-glass resonators, resonators are fabricated through a combination of electro discharge machining (EDM) and silicon micromachining techniques, as shown in Figure 1. By leveraging the advantages of $\mu$ EDM's precision and orientation-independent material removal-rate, highly symmetric and geometrically-precise mold features can be first formed on silicon. Subsequent process steps based on the traditional silicon micromachining technologies are used to define the structural layers, create a free standing shell structure and allowing future integration with electrical actuation and sensing components.

\section{Micro Electro Discharge Machining ( $\mu$ EDM)}

A low resistivity (0.008-0.2 $\Omega$-cm), antimony-doped, (100) silicon substrate is first deposited with a metal hard mask consisting of $50 \mathrm{~nm}$ thick Chromium and $100 \mathrm{~nm}$ thick Gold layers. The hemispherical shell mold is then formed via a two-step EDM process where a hemispherical tool is used to machine $1 \mathrm{~mm}$ diameter hemispherical wells into the silicon substrate. A subsequent isotropic etching step in HNA (Hydrofluoric/Nitric/Acetic acids) etchant chemically polishes the inside surface of the machined hemispherical mold, while the metal hard mask protects the top surface of the silicon substrate. Image analysis demonstrates that the finished shell mold exhibits high symmetry, having a radial standard deviation below $6 \mu \mathrm{m}$ for a $500 \mu \mathrm{m}$ radius shell [6] and a surface roughness of $4 \mathrm{~nm}$ measured at the inside of the silicon mold.

a)

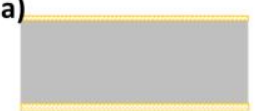

1.Au/Cr mask deposition

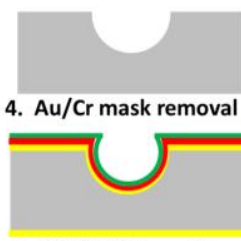

7. PE-TEOS deposition
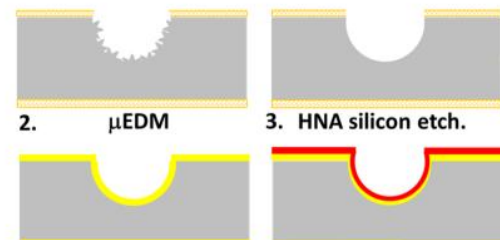

3. HNA silicon etch.
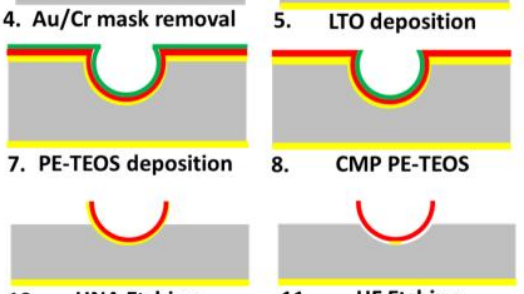

10. HNA Etching

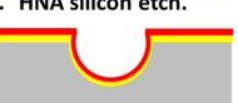

6. CVD diamond deposition

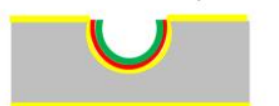

9. Diamond dry etch Legend

Silicon $\quad$ CVD Diamond LTO Oxide $\square \mathrm{Au} / \mathrm{Cr}$ 11. HF Etching

b)

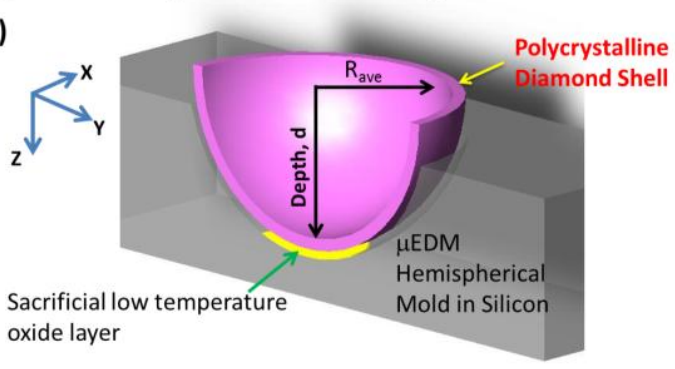

Figure 1: a) Fabrication process flow for creating CVD diamond hemispherical shell resonator, b) cross-sectional schematic of the shell structure.

\section{Silicon Micromachining}

After stripping the etch mask, a $2 \mu \mathrm{m}$ thick LTO $\mathrm{SiO}_{2}$ sacrificial layer is grown conformally on the shell mold. The shell mold is then seeded using ultrasonic seeding suspensions containing nanocrystalline diamond powders with diameter from 5-50 nm . Using methane $\left(\mathrm{CH}_{4}\right)$ concentration of $1.5 \%$ and relative boron concentration of $444 \mathrm{ppm} / \mathrm{CH}_{4}$, a $1 \mu \mathrm{m}$ thick Boron-doped diamond structural layer is deposited via hot-filament chemical vapor deposition (HFCVD, SP3 Diamond Technologies). A $5 \mu \mathrm{m}$ thick plasma enhanced tetraethyl-orthosilicate (PE-TEOS) oxide 
layer is deposited to mask the topside of the wafer. PE-TEOS is selected to ensure conformal oxide mask coverage on the rough microcrystalline diamond surface.

A chemical mechanical polishing (CMP) step (Strausbaugh) removes the oxide mask at a rate of $\sim 3000 \mathrm{~A} / \mathrm{min}$ from the wafer surface but retains the mask within the mold. A subsequent diamond plasma etching (SPTS, APS etcher) is carried out to define the shell. The diamond shells are released in 49\% HF and the top surface of the silicon mold is etched back in HNA to facilitate non-contact optical characterization of the shell structure. SEM micrographs in Fig. 2 show both a totally released diamond shell structure and an array of $1 \mathrm{~mm}$ diameter diamond hemispherical shell that has been etched back to a depth, $\mathrm{d} \sim 370 \mu \mathrm{m}$.

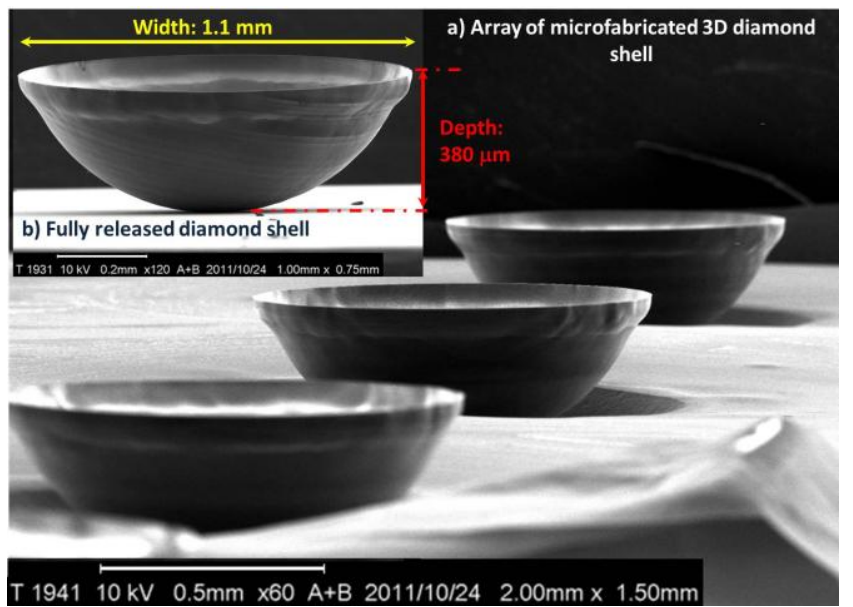

Figure 2: SEM micrographs of the diamond shell, a) shows an array of etched back diamond shells attached to the silicon substrate at the bottom of the well, (b) shows a fully released $1 \mathrm{~mm}$ diameter, 380 um deep shell.

\section{POLYCRSYTALLINE DIAMOND}

Thin-film polycrystalline diamond offers enhanced mechanical properties, including significantly higher stiffness, strength, hardness, thermal conductivity, and chemical robustness, versus silicon and most other thin-film materials commonly used in microfabrication technologies. The excellent strength to density ratio and diamond's superior surface properties make this an ideal material for low surface losses and ultra-low thermoelastic damping, both significant properties required for a resonator.

In this work, the diamond film deposited during the HFCVD process is microcrystalline diamond (MCD). Through control of the deposition conditions, either micro- or nano- crystalline diamond (NCD) films can be deposited. The selection of MCD as the structural material is motivated by the higher sp3 content of the MCD films and reduced density of grain boundaries; both translate into the high Q-factors of 71,400 at $299.86 \mathrm{MHz}$ for a diamond disk resonator [7] and 201,000 at $264 \mathrm{kHz}$ for a diamond double ended tuning fork [8].

The Raman spectra obtained on the Boron doped MCD film is shown in Fig. 3 using an excitation wavelength at $514.5 \mathrm{~nm}$. The spectrum features three different peaks: a) first order Silicon Raman peak at $520 \mathrm{~cm}^{-1}$, b) peaks at $\sim 1230$ and c) $1324.4 \mathrm{~cm}^{-1}$ (Full-width half-maximum, FWHM $=7.0 \mathrm{~cm}^{-1}$ ). The characteristic sp3 single crystal diamond is dominated by the first order Raman line at $1332 \mathrm{~cm}^{-1}$. In our measurement the peak is down shifted to $1324.4 \mathrm{~cm}^{-1}$, consistent with the previous studies on residual stress and effects of boron doping in MCD films [9]. The other peak at
$1230 \mathrm{~cm}^{-1}$ is also broadly consistent with the peaks in the phonon density of states (PDOS) of diamond and attributed to the incorporation of boron in the diamond lattice [10]. The comparison between the diamond and non-diamond components (at 1500-1550 $\left.\mathrm{cm}^{-1}\right)$, ratio of $I(1332) / I(1500)$ shows the purity of the diamond film used in the hemispherical resonator structure.

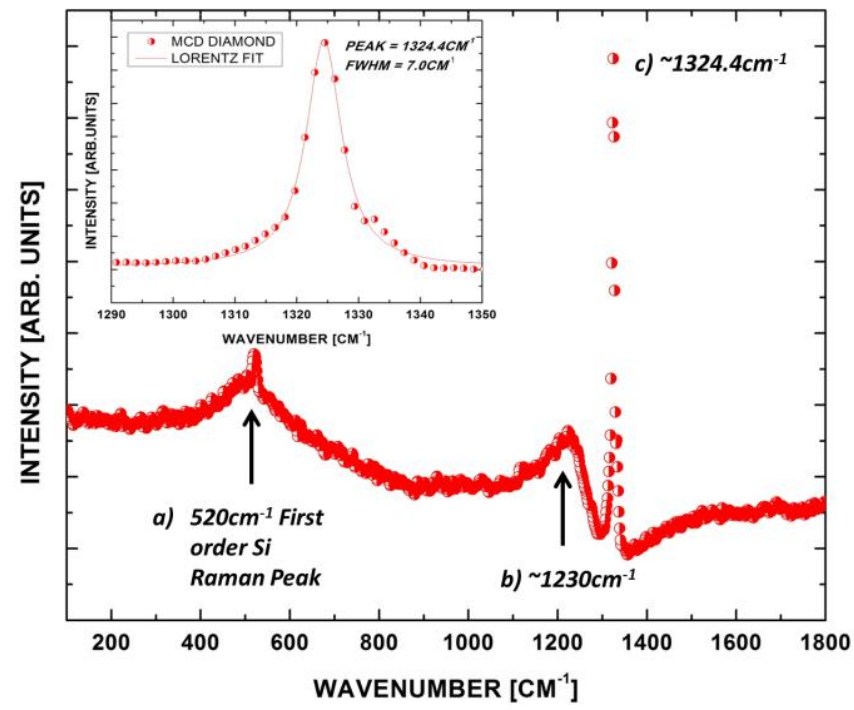

Figure 3: Raman spectra of the Boron doped microcrystalline diamond. Figure inset shows the Lorentzian fit for the first order diamond Raman peak.
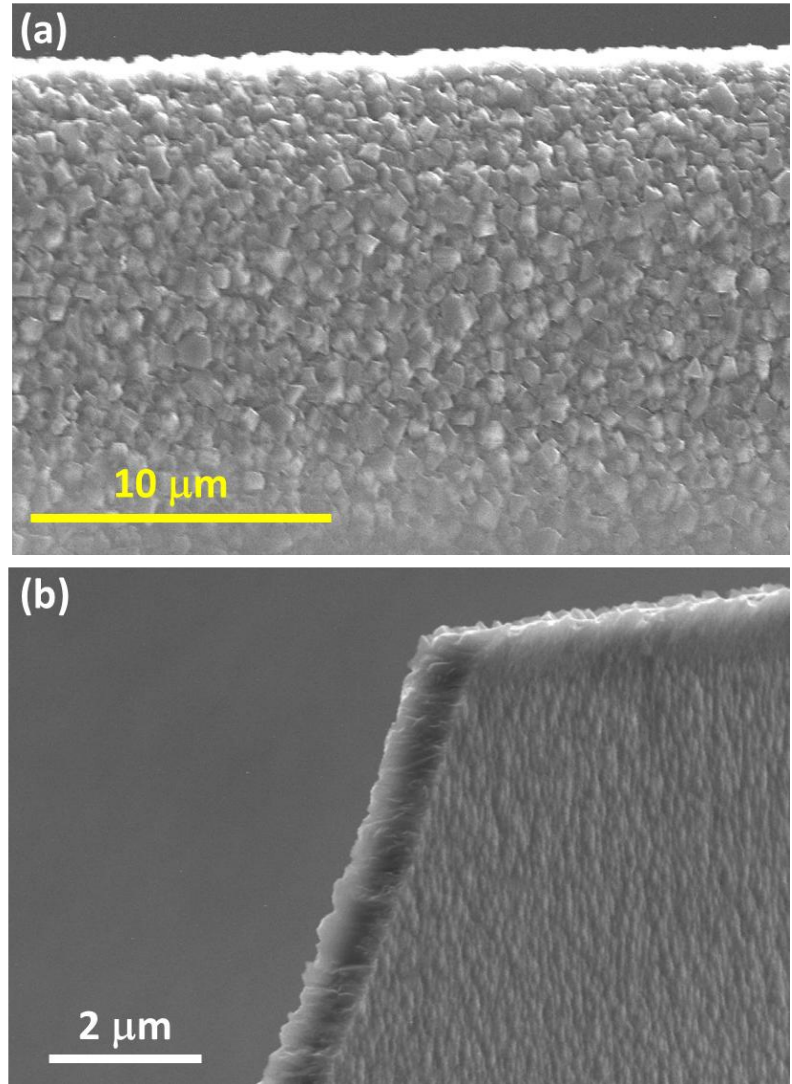

Figure 4: SEM Micrographs of a) top view of the MCD film and b) a representative sample diamond shell with a cracked rim to show the columnar nature of the MCD structural layer. 
The SEM view of the MCD shell resonator shown in Figure 4a illustrates the highly faceted surface with grain size in the range between $0.2-0.5 \mu \mathrm{m}$. Hence, the roughness of shell structure is dominated by the roughness of the MCD film layer. This roughness will increase with increasing film thickness. Fig. 4b shows part of a cracked shell to illustrate the columnar nature of the diamond growth profile from the diamond seeded layer at the base of the silicon mold. The thickness of the diamond shell is between 0.8-1.0 $\mu \mathrm{m}$.

\section{TESTING AND CHARACTERIZATION}

Preliminary prototypes of the diamond hemispherical shell are characterized using a laser Doppler vibrometer (LDV) (Polytec) both in air and in vacuum. Fig. 5 shows the schematic of the testing setup, where the diamond shell was mounted onto a shear mode piezoelectric actuator (Noliac A/S) with a bandwidth of 1.7 $\mathrm{MHz}$ to excite the shell. The single point LDV laser spot is focused through a 10x objective on the rim of the shell to directly measure the displacement of the vibrating shell. A miniature rotation stage and two axis goniometer (OptoSigma) are used to tune the incidence angle of the laser on the shell for maximum signal to noise ratio. The whole setup was also mounted in a vacuum chamber (Janis) to allow measurement at sub-mTorr pressures.

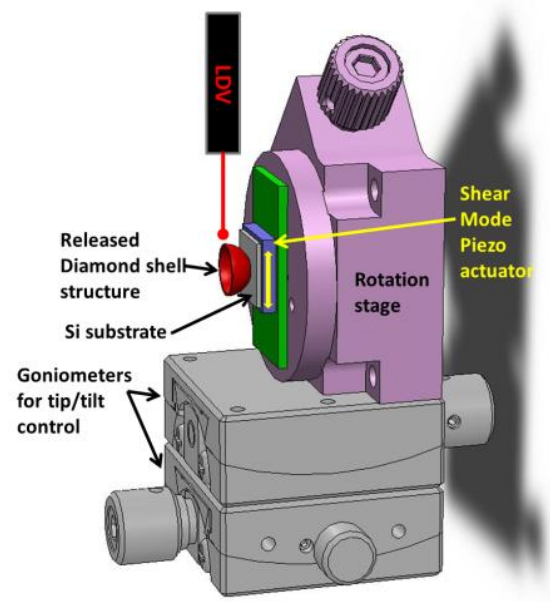

Figure 5: Schematic of experimental setup used to characterize the diamond shell.

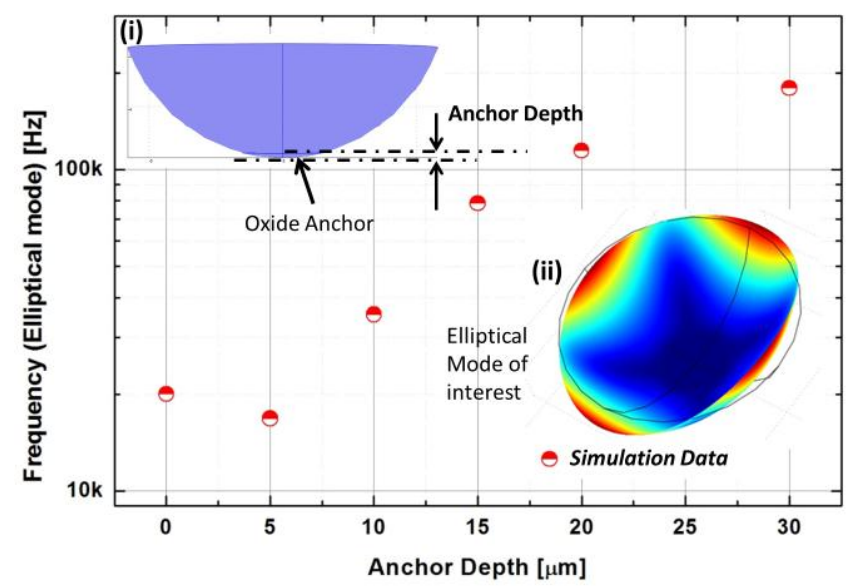

Figure 6: Finite element simulation of the elliptical mode at varying anchor depth. Inset figure: i) illustrates the definition of anchor depth and ii) shows the vibration profile at the elliptical mode.

\section{RESULTS}

\section{Finite Element Simulation}

Finite element modal analysis was carried out using COMSOL $^{\mathrm{TM}}$ to analyze the mode shapes of the hemispherical shell, and compared with the experimental results. The focus of the simulation is on the wineglass/elliptical mode, where the shell shape changes from circular to elliptical and the degenerate modes are spaced $45^{\circ}$ apart from one another. Fig. 6 plots the simulated eigenfrequency at the elliptical mode with varying anchor depth ranging from 5-30 $\mu \mathrm{m}$ and shows a dependence on the anchor depth.

\section{Frequency response}

The frequency response of the hemispherical shell in air and in vacuum at $\mathrm{P} \sim 30 \mu$ Torr are shown in Fig. $7 \mathrm{a}$ and $\mathrm{b}$. The resonance peaks at $f_{1}=34.68 \mathrm{kHz}$ and $f_{2}=35.55 \mathrm{kHz}$ correspond to the two degenerate elliptical modes, where the frequency mismatch $\Delta f / f$ is measured to be $\sim 2 \%$. Lorentzian fit to the response in vacuum extracts a Q-factor of $\sim 3000$ (Fig. $7 \mathrm{c}$ and d).
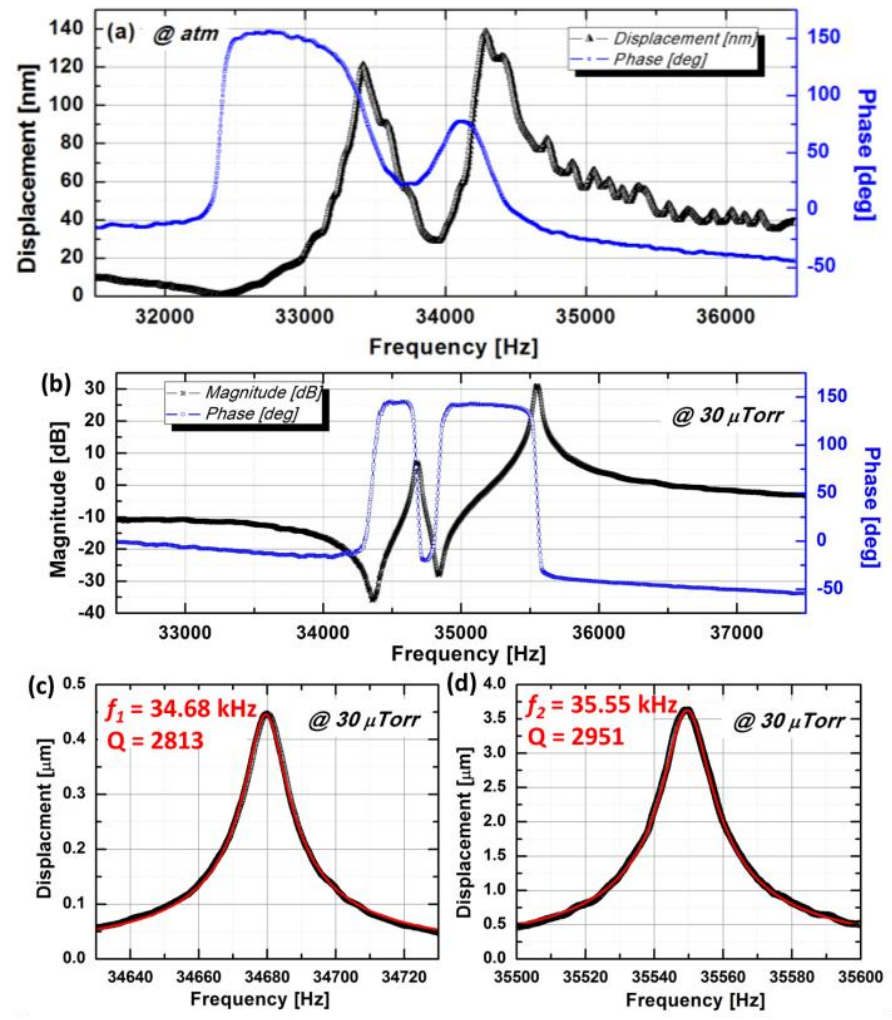

Figure 7: Measured frequency response in (a) air and (b) vacuum. Close-ups at $f_{1}$ and $f_{2}$ are shown in (c) and (d).

In order to verify the actual anchor depth, SEM of the sample is carried out, as shown in Fig. 8. The side view image shows the hemispherical shell profile after the HNA etch back, and the anchor depth is estimated to be $\sim 9 \mu \mathrm{m}$. Hence, the experimental results agree well with the simulation data with an elliptical mode frequency variation of $\sim 5 \%$. The low Q-factor and relatively large frequency mismatch can be attributed to the anchor design and the rim roughness as shown in the SEM image. The anchor is currently controlled by the time etching of silicon during the final HNA and HF etching step and the rim roughness an artifact of the initial HNA polishing etch after EDM. Both issues can be remedied with process optimization. 


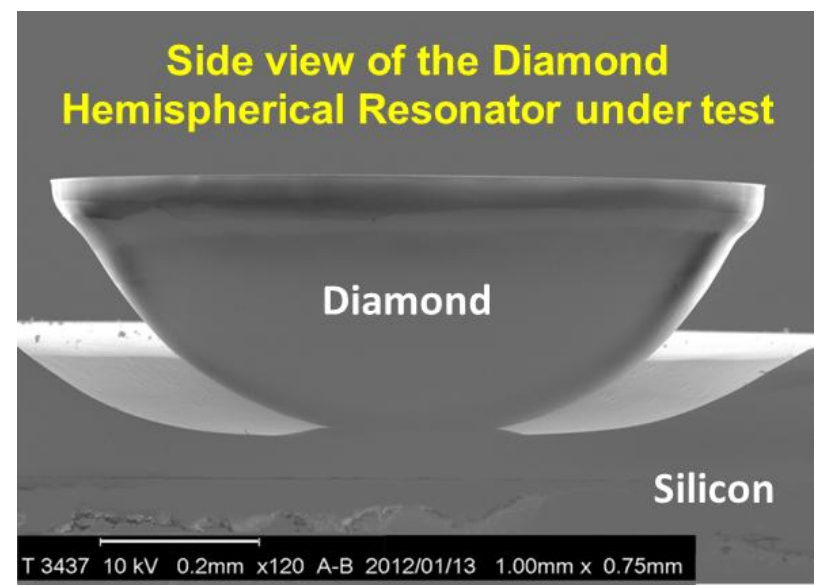

Figure 8: SEM Micrograph showing the side view of the hemispherical shell structure after the HNA floor etch.

\section{Mode Shapes}

The elliptical mode shapes at frequencies $f_{1}$ and $f_{2}$ were identified by measuring the radial vibration around the perimeter of the shell, as the shell is rotated through $360^{\circ}$. At every $10^{\circ}$ interval the frequency response of the structure is measured and the displacement at $f_{1}$ and $f_{2}$ is extracted. Fig. 9 shows the polar plot of the shell displacement as it is excited at each frequency. Each measured mode shape exhibits the 4 nodes which are characteristic of the elliptical modes. By employing a fitting function described by $\left|A_{i} \sin \left(2 \theta+\varnothing_{i}\right)\right|$, the angle between the principal axes of elasticity is extracted to be $\sim 45^{\circ}$ in good agreement with theory.

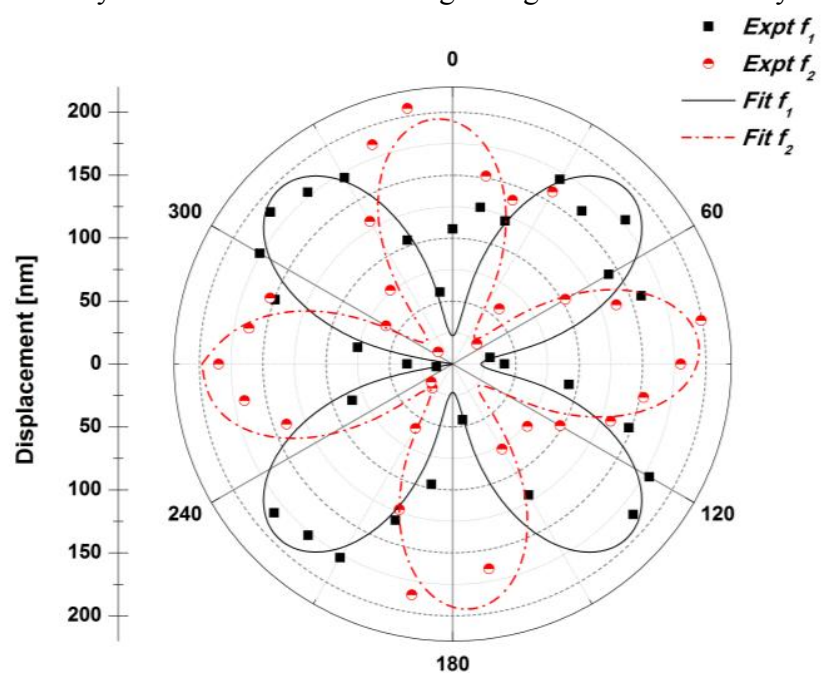

Figure 9: Measured displacement amplitude along the rim of the resonator shell with excitation at $f_{1}(\mathbf{\square})$ and $f_{2}(\theta)$ illustrating the expected elliptical mode shapes.

\section{CONCLUSION}

We developed a 3D hemispherical shaped microcrystalline diamond shell structures, and demonstrated the shells as mechanical resonant structures. Using a combination of $\mu \mathrm{EDM}$ and silicon machining technologies, 3D axisymmetric shell silicon structures can be employed as the molds from which high Q-factor material such as microcrystalline diamond can be deposited. The shells are characterized through piezoelectric excitation at the base and optical detection at the rim of the shell to determine the natural frequencies and mode shapes of the shell structure operating at the wineglass mode. The experimental results agree with the simulation for the anchor depth fabricated. Without any trimming or tuning of the natural frequencies, the frequency mismatch and Q-factor are determined to be $2 \%$ and 3000 at $34.68 \mathrm{kHz}$.

\section{ACKNOWLEDGEMENTS}

This work is supported by DARPA under grant W31P4Q-111-0003. The authors would like to thank the support of Gerry Chandler and Maria Peralta from SP3 Diamond Technologies; Alan Hicklin from UC Davis NEAT facility for Raman spectroscopy; the staff in UC Davis Northern California Nanotechnology Center and UC Berkeley Marvell Nanolab.

\section{REFERENCES}

[1] D.D. Lynch, "Hemispherical Resonator Gyro," R. R. Ragan, ed., Inertial Technology for the Future, IEEE Transactions on Aerospace Electronic Systems, Vol. AES-20, No. 4, pp. 414 444, (1984).

[2] I.P Prikhodko, S.A. Zotov, A.A. Trusov and A.M. Shkel, "Microscale glass-blown three dimensional spherical shell resonators", J. Microelectromech. Syst., 20-3, pp.691-701, (2011).

[3] L.C. Fegely, D.N. Hutchison, S.A. Bhave, "Isotropic etching of 111 SCS for wafer scale manufacturing of perfectly hemispherical silicon molds", 16th International Conference on Solid-State Sensors, Actuators and Microsystems (Transducers 11), Beijing, China, 6/5-9/2011.

[4] X. Gao, L. Sorenson, F. Ayazi, "3-D Micromachined hemispherical shell resonators with integrated capacitive transducers", IEEE $25^{\text {th }}$ Internal symposium on Micro Electro Mechanical Systems 2012, Paris, France, 1/29-2/2/2012, pp. 165-171.

[5] K. Visvanathan, L. Tao, Y. B. Gianchandani, "3D-soule: A fabrication process for large scale integration and micromachining of spherical structures", IEEE $24^{\text {th }}$ Internal symposium on Micro Electro Mechanical Systems 2011, Cancun, Mexico, 1/23-27/2011, pp. 45-48.

[6] M.L Chan, P. Fonda, C. Reyes, J. Xie, H. Najar, L. Lin, K. Yamazaki, D.A. Horsley, "Micromachining 3D hemispherical features in silicon via micro-EDM", IEEE $25^{\text {th }}$ Internal symposium on Micro Electro Mechanical Systems 2012, Paris, France, 1/29-2/2/2012, pp. 289-292.

[7] M. Akgul, R. Schneider, Z. Ren, G. Chandler, V. Yeh and C.T.C. Nguyen, "Hot filament CVD conductive microcrystalline diamond for high $\mathrm{Q}$, high acoustic velocity micromechanical resonators", Frequency Control and the European Frequency and Time Forum (FCS 2011), San Francisco, 5/2-5/2011, pp. 1-6.

[8] H. Najar, M.L. Chan and D.A. Horsley, "Impact of doping and microstructure on quality factor of CVD diamond micromechanical resonators", Frequency Control and the European Frequency and Time Forum (FCS 2012), Baltimore, Accepted.

[9] W.L. Wang, M.C. Polo, G. Sanchez, J. Cifre, J. Esteve, "Internal stress and strain in heavily boron-doped diamond films grown by microwave plasma and hot filament chemical vapor deposition", J. Appl. Phys. 80, pp. 1846 - 1851, (1996).

[10] M. Bernard, A. Deneuville and P. Muret, "Non-destructive determination of the boron concentration of heavily doped metallic diamond thin films from Raman Spectroscopy" Diamond Relat. Mater. 13, pp. 282-286, (2004).

\section{CONTACT}

*M.L. Chan, tel: +1-530-752-5180; cmlchan@ucdavis.edu 


\title{
PIEZOELECTIRC ROTATIONAL MODE DISK RESONATORS FOR LIQUID VISCOSITY MONITORING
}

\author{
E. Mehdizadeh ${ }^{1}$, J. Gonzales ${ }^{2}$, A. Rahafrooz ${ }^{l}$, R. Abdolvand ${ }^{2}$, and S. Pourkamali ${ }^{1}$ \\ ${ }^{1}$ Department of Electrical and Computer Engineering, University of Denver, Denver, CO, USA \\ ${ }^{2}$ School of Electrical and Computer Engineering, Oklahoma State University, Stillwater, OK, USA
}

\begin{abstract}
This work reports on piezoelectric rotational mode disk resonators capable of operation in liquid and their performance as viscosity sensors. Piezoelecrically transduced rotational mode silicon disk resonators with resonant frequencies in the 1.04.0 $\mathrm{MHz}$ range were fabricated and operated in five different liquid alcohols (Methyl, Ethyl, Allyl, Isopropyl and Butyl). A quality factor of up to $\sim 54$ was measured for such devices when immersed in liquid. It is shown through measurements that, as expected by fluid mechanics and vibrations theoretical analysis, both resonant frequency and quality factor of the resonators decrease as the viscosity of surrounding liquid increases. Frequency-viscosity sensitivity in the order of $40 \mathrm{KHz} / \mathrm{cP}$ has been measured for such devices.
\end{abstract}

\section{INTRODUCTION}

Dynamic viscosity, simply known as viscosity, is one of the most important parameters describing fluid characteristics. Viscosity measurements are needed in various industrial, biomedical, and laboratory applications. In automobile industry, the viscosity of oil which is used as an engine lubricant should fall in a specific range to provide the normal operation. In petroleum industry, viscosity measurements are crucial for effective exploration and design/optimization of production strategies especially in biodegraded heavy oil. Viscosity measurement is also one of the steps in quality control of most liquid products such as pharmaceutical drugs, paints, cosmetics, and food. In addition, it can be used as a diagnostic tool to characterize human blood.

Conventional viscosity measurement techniques typically involve complex large machines that require continuous calibration in a laboratory environment. Measurement of viscosity by means of a resonating MEMS structure immersed in a fluid is an interesting alternative gaining attention in recent years. Immersing the resonator in a fluid dampens the resonance which leads to a shift in resonant frequency and quality factor of the device. This technique is beneficial as it only requires a tiny amount of sample liquid for measurement and involves a very straightforward and simple process. It also has the capability of quick and online measurement of viscosity in many complex systems. Li et al. introduced a viscosity sensor using resonant torsional micropaddles with magnetic actuation. Such devices exhibit very low quality factors (in the order of 2-3) in liquid due to their out of plane vibration causing significant damping. Using such devices also involves a complex sensing and actuation mechanism [1]. Puchades and Fuller introduced a thermally actuated resonating plate which senses kinematic viscosity (the ratio of dynamic viscosity and density) [2]. It involves not only a complex procedure to measure viscosity but also relatively low quality factors (less than 18). Etchart et al. used resonating beams with millimetere-scale lengths and micron-scale cross-sections to measure fluid viscosity. They also couldn't achieve quality factors higher than 10 in low viscosity fluids [3].

In order to achieve higher resonator quality factors, thermally actuated rotational mode disk resonators have been previously demonstrated to operate in liquid media with quality factors as high as 300 [4]. Since all the surfaces of such structures in the resonant mode of interest slide in parallel to the liquid interface, it has minimal interaction with the surrounding liquid. Moreover, unlike the previous resonating devices reported in the literature, only viscosity (not the density of fluid) would have an influence on the measured data. That is basically because shear stress, the main factor in rotating devices, merely involves the effect of viscosity while the normal stress involves the effect of fluid density. However, the heat generated by such devices increases the temperature in their surrounding liquid and as a result a precise measurement of the viscosity will not be possible. This is mainly because the viscosity itself is a function of temperature. To avoid this problem, in this work similar resonant disk structures were implemented in a thin-film piezoelectric on silicon (TPoS) technology [5].

\section{RESONATOR FABRICATION}

A TPoS resonator consists of a thin piezoelectric aluminum nitride film which is sandwiched between two metal electrodes. All three layers are deposited on top of the device layer of a silicon-oninsulator (SOI) substrate (Figure 1a). The top metal layer is dry etched to define the top electrodes and signal pads. The Aluminum Nitride is wet etched to reach the bottom ground electrode (Figure 1b). The resonant structure is defined in a stack dry etch (Figure 1c). Backside handle layer of silicon beneath the resonator body is etched and the buried oxide layer of SOI wafer is removed in HF to create free-standing resonant structures (Figure 1d).

(a)
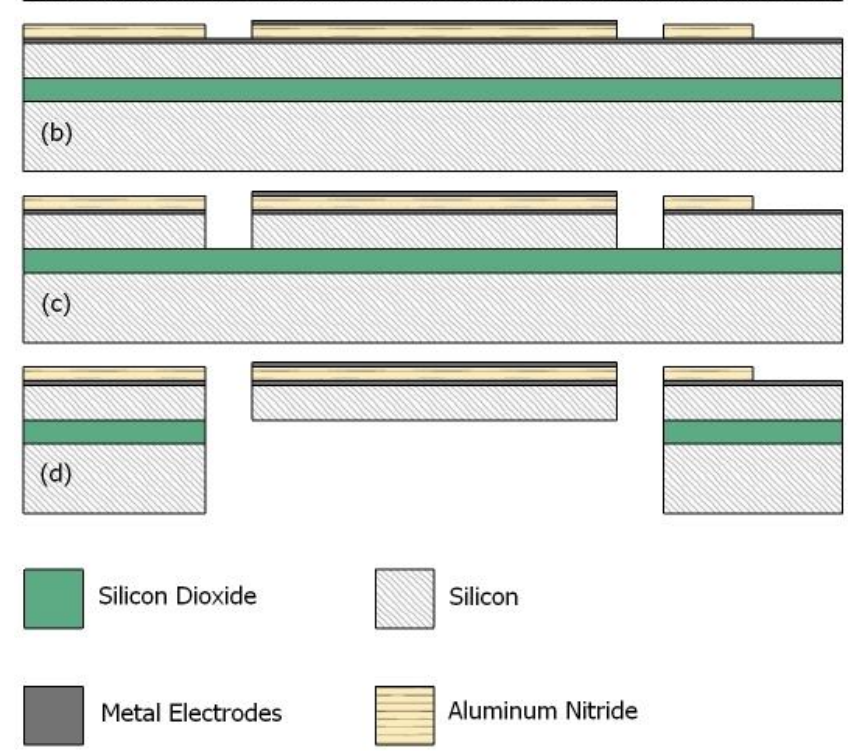

Figure 1: Process flow for TPoS resonators starting with a) thinfilm deposition, b) a top metal etch followed by an aluminum nitride etch, c) device silicon anisotropic dry etch d) and finally handle silicon dry etch with silicon dioxide wet etch release. 
Figure 2 shows a scanning electron microscope view of a $3.44 \mathrm{MHz}$ piezoelectric resonator implemented using the TPoS technology. The fabricated disk resonator is comprised of a central circular mass and two support beams. Resonator actuation and sensing is performed by metal coated aluminum nitride films on the disk support beams. The resonator transduction is performed piezoelectrically. Upon application of an alternating voltage at the right frequency to the metal electrode on one of the support beams, the support beams start vibrating in their first longitudinal mode while the disk vibrates in its rotational mode. The vibration amplitude is sensed through the metal electrode on the second support beam. In TPoS devices, piezoelectric transduction is used to excite the resonant mode of a structure made from a substrate material such as single crystalline silicon. The low-loss single crystalline material provides an improved quality factor over a purely piezoelectric resonator.

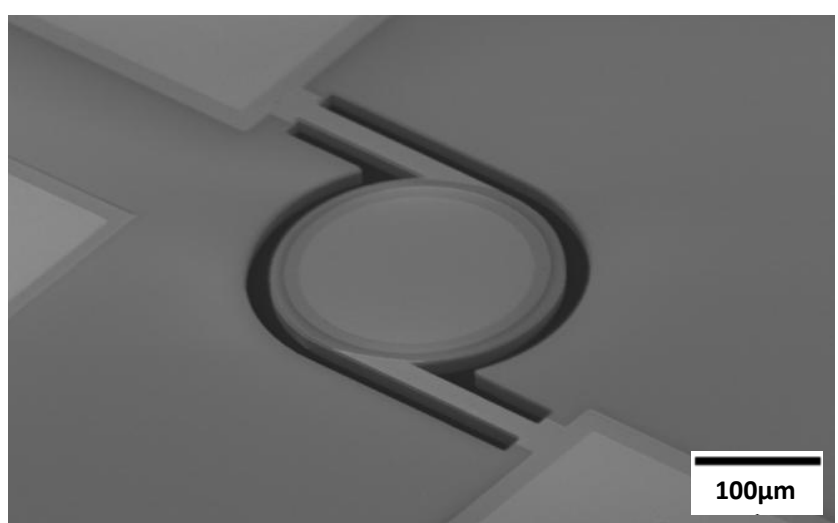

Figure 2: Scanning electron microscope view of a $3.44 \mathrm{MHz}$ thinfilm piezoelectric on silicon (TPoS) rotational mode disk resonator.

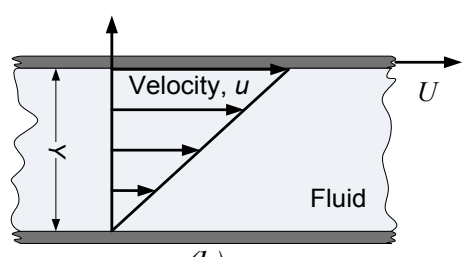

(b)

(c)

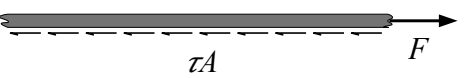

(a)

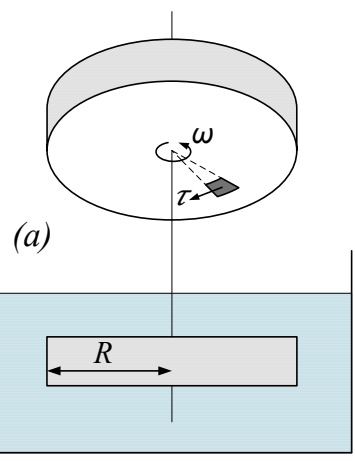

Figure 3: a) Schematic view of the disk resonator rotating in fluid b) Velocity profile of fluid between two plates. c) Forces acting on upper plate.

\section{ANALYSIS}

To analyze the interaction of the resonating element with the surrounding liquid, the basic theory of fluid mechanics is used. Viscosity which is the most important parameter for characterizing specific fluid mechanical behavior, relates the local stress in a moving fluid to the strain of the fluid element. When a fluid is sheared, it begins to move at a strain rate (equal to velocity gradient) inversely proportional to coefficient of viscosity $(\mu)$. For Newtonian fluids (common linear fluids such as water, oil, and alcohols) this shear stress $(\tau)$ can be expressed as:

$$
\tau=\mu \frac{\partial u}{\partial y}
$$

where $\mathrm{u}$ and $\mathrm{y}$ are fluid velocity and distance vertical to the flow of fluid, respectively.

When the flow is induced by relative motion between a fixed lower plate and a moving upper plate at velocity $U$ (Figure $3 \mathrm{a}$ ), the velocity distribution for every instant can be assumed to be linear (as shown Figure 3b). It should be noted that velocity of the fluid layer being in contact with the moving plate is the same as velocity of the plate itself. And as a result, equation (1) can be formulated as [6]:

$$
\tau=\mu \frac{U}{Y}
$$

where $Y$ is the distance between two plates.

Neglecting the shear applied on top surface and side walls of the disk, a fluid element just below the disk surface (as shown in Figure 3a) can be considered to find the torque applied to the disk from the surrounding fluid shear. Thus:

$$
d T=r d F
$$

where $d T$ and $d F$ are the infinitesimal torque and force applied to the element from the liquid. $r$ is the distance between the element and the center of the disk. The force applied to the element can be expressed as below:

$$
d F=\tau d A
$$

where $d A$ is the area of the element which is as follows:

$$
d A=r d r d \theta
$$

where $d \theta$ and $d \mathrm{r}$ are infinitely small angle and distance, respectively. Combining equations (2)-(4), and using the relationship between the linear velocity of the element and the angular velocity of the disk:

$$
U=r \omega
$$

where $\omega$ is the angular velocity of each element at a specific instant. The torque applied from the fluid to the small disk element is therefore obtained as:

$$
d T=\mu \frac{r^{3} \omega}{Y} d r d \theta
$$

Integrating this equation over the whole disk, the equation for damping torque applied from the fluid to the disk is obtained:

$$
T=\frac{\pi \mu R^{4}}{2 Y} \omega
$$

where $R$ is the disk radius. Allowing for this equation to be the damping torque applied to the disk, the system damping coefficient can be easily derived as:

$$
b=\frac{\pi R^{4}}{2 Y} \mu
$$

Considering a resonant damped system, its resonance frequency is as follows: 


$$
f_{r}=f_{n} \sqrt{1-2 \xi^{2}}
$$

where $f_{\mathrm{r}}$ is the resonant frequency of the damped system, $f_{\mathrm{n}}$ is the natural frequency and $\xi$ is the damping ratio of the system which can be defined as:

$$
\xi=\frac{b}{2 \sqrt{K J}}
$$

where $K$ and $J$ are the stiffness of the supporting beams and the disk moment of inertia, respectively. Combing equations (9)-(11), the relationship between viscosity coefficient and resonance frequency can be obtained as:

$$
f_{r}=\frac{f_{n}}{A} \sqrt{C^{2}-\mu^{2}}
$$

in which $C$ is the following constant:

$$
C=\frac{2 y}{\pi R^{4}} \sqrt{2 K J}
$$

Quality factor also could be determined as a function of viscosity coefficient:

$$
Q=\frac{1}{2 \xi}=\frac{B}{\mu}
$$

in which $B$ is the following constant:

$$
B=2 y \frac{\sqrt{K J}}{\pi R^{4}}
$$

As it can be seen, quality factor is inversely proportional to coefficient of viscosity.

\section{MEASUREMENT RESULTS}

When immersed in liquid, damping of the vibrating structure changes as a function of fluid viscosity. This simple principle is used here to measure liquid viscosity based on the change of quality factor and consequently resonant frequency of the piezoelectric resonators. Table 1 shows the viscosity of five different alcohols used in measurements.

Table 1: Viscosity of used liquids in the experiments [7]

\begin{tabular}{cc}
\hline Fluid & Viscosity (mPa s) \\
\hline Methyl Alcohol & 0.55 \\
\hline Ethyl Alcohol & 1.06 \\
\hline Allyl Alcohol & 1.30 \\
\hline Isopropyl Alcohol & 1.95 \\
\hline Butyl Alcohol & 2.60 \\
\hline
\end{tabular}

The fabricated resonators were tested in a two-port configuration and the transmission data were recorded using a network analyzer. The silicon ship containing the resonators was placed on a printed board circuit board (PCB) and electrical connections to the resonators were provided by wedge-bonded aluminum wires.

The PCB was then placed in small containers containing different alcohols successively. Data were recorded at $25{ }^{\circ} \mathrm{C}$ after immersion in each of alcohols when the frequency response got stable after a couple of seconds. Similar set of measurements were performed on different resonators with different dimensions. Table 2 summarizes the measurement results for these resonators operating in different liquids. As expected, with the increase in viscosity of the surrounding liquid, both the quality factor and resonant frequency decrease. Figure 4 shows the frequency response of the device\#1 in five different alcohols.

Figure 5 depicts the changes in quality factor as a function of liquid viscosity for three different devices. The changes in the resonant frequency of the resonators working in liquids with various viscosities are also shown in Figure 6. As it is clearly shown by immersing the resonators in more viscous fluids, their quality factor and resonant frequency further decreases. This is mainly due to the effect of the shear applied from the surrounding fluid to the disk resonator, dampening its vibration.

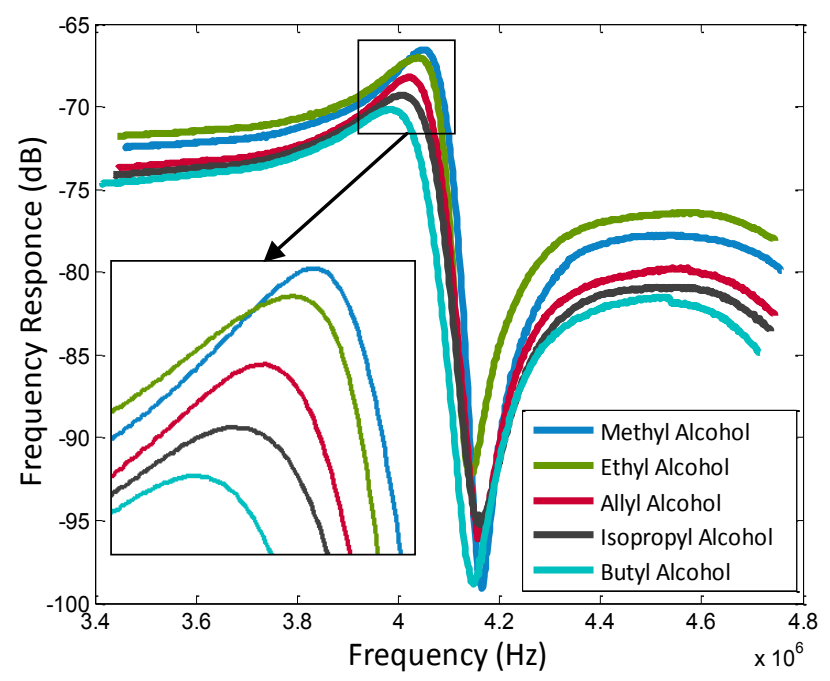

Figure 4: Measured frequency responses for the $4.15 \mathrm{MHz}$ piezoelectric disk resonator operating in different liquids.

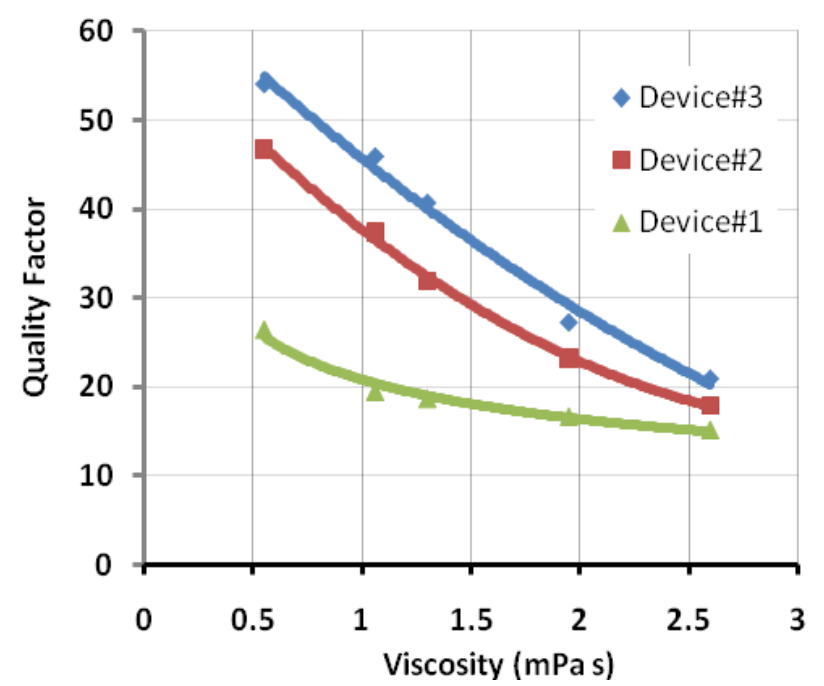

Figure 5: Measured quality factor of the rotational mode disk resonators as a function of liquid viscosity at $25^{\circ} \mathrm{C}$.

Frequency-viscosity sensitivities of 35,40 and $15 \mathrm{kHz} / \mathrm{cP}$, and quality factor-viscosity sensitivities of 5,15 and $17 \mathrm{cP}^{-1}$ were measured for the devices\#1-3, respectively. 
Table 2: Summary of measurement results obtained from piezoelectric disk resonators in different liquids.

\begin{tabular}{|c|c|c|c|c|c|c|c|}
\hline & $\begin{array}{c}\text { Plate } \\
\text { Diameter } \\
(\mu \mathrm{m})\end{array}$ & $\begin{array}{c}\text { Support } \\
\text { Beam Width } \\
(\mu \mathrm{m})\end{array}$ & $\begin{array}{c}\text { Support } \\
\text { Beam Length } \\
(\mu \mathrm{m})\end{array}$ & Liquid/Air & $\begin{array}{l}\text { Quality } \\
\text { Factor }\end{array}$ & $\begin{array}{c}\text { Frequency } \\
\text { (MHz) }\end{array}$ & $\begin{array}{l}\text { Freq. Shift with } \\
\text { Respect to Ethyl } \\
\text { Alcohol (PPM) }\end{array}$ \\
\hline \multirow{6}{*}{ Device\#1 } & \multirow{6}{*}{100} & \multirow{6}{*}{16} & \multirow{6}{*}{50} & Air & 80 & 4.15 & \\
\hline & & & & Methyl & 26.4 & 4.0486 & 2402 \\
\hline & & & & Ethyl & 19.5 & 4.0389 & - \\
\hline & & & & Allyl & 18.7 & 4.0226 & -4036 \\
\hline & & & & Isopropyl & 16.7 & 4.005 & -8393 \\
\hline & & & & Buthyl & 15.2 & 3.9816 & -14187 \\
\hline \multirow{6}{*}{ Device\#2 } & \multirow{6}{*}{200} & \multirow{6}{*}{16} & \multirow{6}{*}{100} & Air & 243 & 3.44 & \\
\hline & & & & Methyl & 46.7 & 3.3967 & 4584 \\
\hline & & & & Ethyl & 37.4 & 3.3812 & - \\
\hline & & & & Allyl & 31.9 & 3.3692 & -3549 \\
\hline & & & & Isopropyl & 23.2 & 3.3586 & -6684 \\
\hline & & & & Buthyl & 17.9 & 3.3236 & -17035 \\
\hline \multirow{6}{*}{ Device\#3 } & \multirow{6}{*}{500} & \multirow{6}{*}{40} & \multirow{6}{*}{300} & Air & 278 & 1.39 & \\
\hline & & & & Methyl & 54 & 1.3808 & 870 \\
\hline & & & & Ethyl & 45.9 & 1.3796 & - \\
\hline & & & & Allyl & 40.7 & 1.3758 & -2754 \\
\hline & & & & Isopropyl & 27.3 & 1.3643 & -11090 \\
\hline & & & & Buthyl & 21 & 1.3552 & -17686 \\
\hline
\end{tabular}
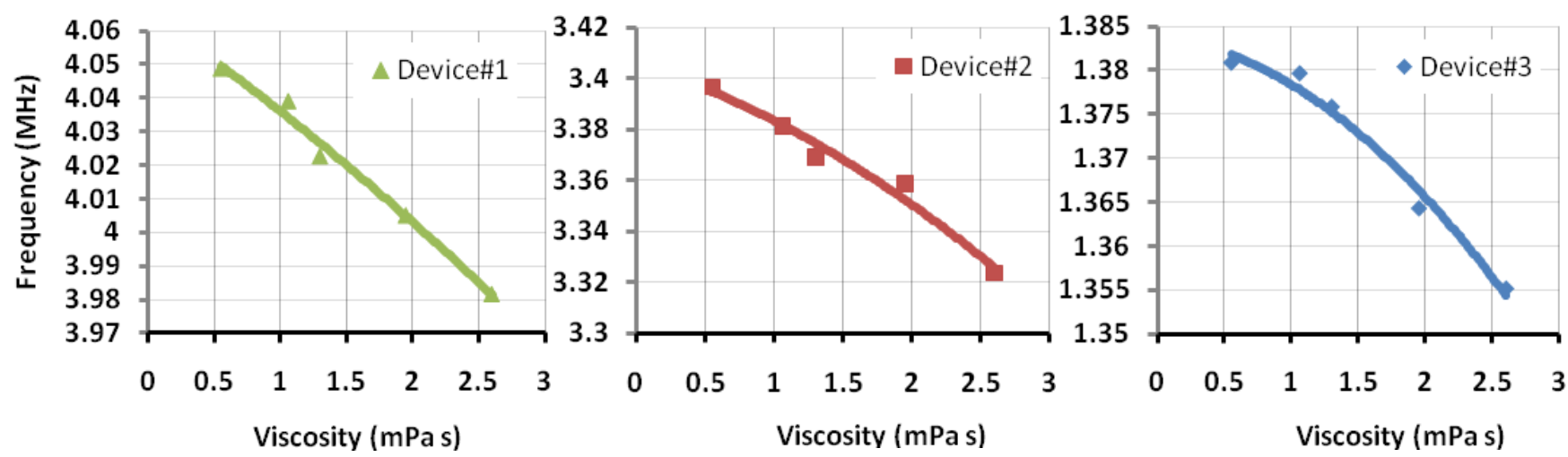

Figure 6: Measured resonant frequency of the rotational mode disk resonators as a function of liquid viscosity at $25^{\circ} \mathrm{C}$.

\section{CONCLUSION}

TPoS rotational mode disk resonators were introduced and utilized as viscosity sensing devices. Due to the minimum interaction with the surrounding liquid and the fact that they do not produce heat during operation, such devices were shown to be capable of reliable viscosity measurements. The frequency response of the fabricated resonators was monitored and recorded in five different liquids using a network analyzer. Quality factors as high as 54 were measured in liquids for such devices. As expected from the formulation, both quality factor and resonant frequency decreased as the viscosity of surrounding fluids was increased. The results show a relatively high frequency-viscosity sensitivity of up to $\sim 40 \mathrm{kHz} / \mathrm{cP}$ for the fabricated resonators.

\section{REFERENCES}

[1] H. Li, J. Wang, X. li, and D. Chen, "Viscosity-Density Sensor with Resonant Torsional Paddle for Direct Detection in Liquid”, IET Nanobiotechnol., 5, 121 (2011).

[2] I. Puchades and L. F. Fuller, "A Thermally Actuated Microelectromechanical (MEMS) Device for Measuring Viscosity", Journal of Microelectromechanical Systems, 20,
$601(2011)$.

[3] I. Etchart, H. Chen, P. Dryden, J. Jundt, C. Harrison, K. Hsu, F. Marty, and B. Mercier, "MEMS sensors for densityviscosity sensing in a low-flow microfluidic environment", Sensors and Actuators A: Physical, 141, 266 (2008).

[4] A. Rahafrooz and S. Pourkamali, "Rotational Mode Disk Resonators for High-Q Operation in Liquid", IEEE Sensors Conference, (2010), pp. 1071-1074.

[5] M. Shahmohammadi, M.J. Modarres-Zadeh, and R. Abdolvand, "Low jitter thin-film piezoelectric-on-substrate oscillators", Proc. IEEE International Frequency Control Symposium (IFCS), (2010), 613-617.

[6] F.M. White, Fluid mechanics, McGraw-Hill, 2001.

[7] D.S. Viswanath, T.K. Ghosh, D.H.L. Prasad, N.V.K. Dutt, and K.Y. Rani, Viscosity of liquids: theory, estimation, experiment, and data, Springer, 2007.

\section{CONTACT}

*E. Mehdizadeh, tel: +1-720-224-4714; emehdiza@du.edu 


\title{
RADIO-FREQUENCY MULTI-MODE MICROMECHANICAL DISK RESONATORS IN 500nm THIN SILICON CARBIDE (SiC)
}

\author{
H. Zamani ${ }^{*}$, J. Lee ${ }^{*}$, S. Rajgopal, C.A. Zorman, M. Mehregany, and P.X.-L. Feng ${ }^{\dagger}$ \\ Electrical Engineering, Case Western Reserve University, Cleveland, OH 44106, USA \\ *Equally contributed authors. ${ }^{\dagger}$ Email: philip.feng@case.edu
}

\begin{abstract}
We report on experimental demonstration of a new type of radio-frequency (RF) multi-mode micromechanical resonators based upon vibrating circular disks made of a 500nm thin $\mathrm{SiC}$ epilayer grown on single crystal $\mathrm{Si}$. These center-clamped microdisk resonators offer multiple flexural-mode resonances at $\mathrm{RF} /$ microwave frequencies, depending on both disk and anchor diameters. We show that simply changing the anchor size can already tune the interesting patterns of the multi-mode frequency spectra. A $\sim 40 \mu \mathrm{m}$-diameter $\mathrm{SiC}$ disk with a slender ( 800nm) anchor exhibits more than a dozen flexural modes in $\sim 2-20 \mathrm{MHz}$ with typical quality factors $Q$ 's $\sim 1000-4000$. A disk with same dimensions but a wide $(\sim 20 \mu \mathrm{m})$ anchor has its first 9 flexural modes within $15-20 \mathrm{MHz}$ with $Q$ 's $\sim 500-2500$. The measurements and analyses show that the frequency spacing between the flexural modes, the splitting of degenerate modes, and their $Q$ 's (or dissipation), can all be tuned by engineering the size and shape of the anchor of a multi-mode flexural thin-film microdisk resonator.
\end{abstract}

\section{INTRODUCTION}

Mechanical disk structures (e.g., rectangular or circular) offer versatile resonance modes due to their many interesting vibrations ranging from flexural (transverse, bending) to bulk (extensional, contour), and also dependent upon their geometries and boundary conditions. Such resonances have hence been widely exploited for signal processing and resonant sensing applications [1,2]. Recent advances in microelectromechanical systems (MEMS) have led to various high- $Q$ microdisk resonators $[3,4]$, in which contour, extensional and other bulk, high-stiffness modes of thick disks are advantageous toward RF/microwave frequency control and timing applications. In today's nanoelectromechanical systems (NEMS) enabled by many emerging nanostructures such as nanowires/tubes and nanobeams/cantilevers, flexural motions (bending, deflection) are prevailing; and the available high- $Q$ flexural resonances are often advantageous for dynamical sensing of ultra-small quantities and effects [5], and for making low voltage and ultra-low power $\mathrm{RF} /$ microwave NEMS [6]. $\mathrm{SiC}$ is an attractive material and proven to be excellent for NEMS toward these goals [5-8], thanks to its exceptional electromechanical properties and nanomanufacturing availability in very thin layers on various sacrificial materials.

In this work, we take a very thin singly-clamped SiC NEMS cantilever, and envisage it to rotate in plane around its clamp and thus extrude into a thin, circular disk (Fig. 1). This new disk is intriguing from both NEMS and MEMS view points. It is much thinner than mainstream bulk-mode MEMS disk resonators; and it has an extremely large surface area in contrast to its original cantilever template (hence a tremendous enhancement on available active surface for capturing, critical for sensing applications). Moreover, the disk derived from the SiC NEMS cantilever would be only anchored and supported by a post/pillar underneath at the center. Here we describe the experimental implementation of this idea, including a novel fabrication process for making the very thin $\mathrm{SiC}$ microdisks, measurement of the $\mathrm{SiC}$ disks' flexural-mode resonance response, and the $\mathrm{SiC}$ disks' multi-mode operations. We focus on exploring the multi-mode characteristics and the effects of varying anchor size upon device performance.

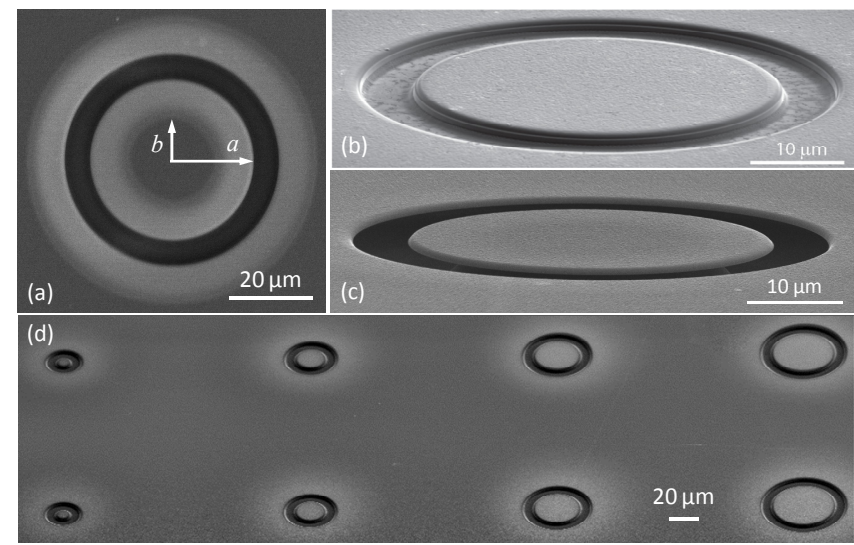

Figure 1: Examples of prototyped 500nm thin SiC circular microdisk resonators and arrays. (a) Top-view scanning electron

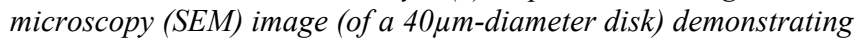
the size of the microdisk (radius a) and the underneath supporting pillar (radius b). (b) \& (c) Titled-view $\left(30^{\circ} \& 75^{\circ}\right.$ respectively) SEM images of the same device before and after Si etch for device suspension. (d) An array of microdisks with various diameters.

\section{DEVICE FABRICATION}

The $500 \mathrm{~nm}$ thin $\mathrm{SiC}$ epilayer is heteroepitaxially grown on single crystal Si (100) by an atmospheric pressure chemical vapor deposition (APCVD) process [9]. As shown in Fig. 2, the device nanomachining process features a simple mask-less design and a resist-less protocol. First, circular rings of width $\sim 5-10 \mu \mathrm{m}$ are directly patterned and nanomachined on the $\mathrm{SiC}$ epilayer, passing the $\mathrm{SiC} / \mathrm{Si}$ interface, by using focused ion beam (FIB) (Figs. $1 \& 2$, specifically, for majority of the devices, with $30 \mathrm{keV}, 47 \mathrm{nA}$, on a state-of-the-art FEI Helios NanoLab 650). The exposed Si is then etched in HNA for various times (depending on desired sizes of the anchoring pillars for the disk resonators) according to calibrated etching rates in this specific process. We prove this to be an excellent protocol in enabling various designs and fast prototyping and testing of thin-film $\mathrm{SiC}$ microdisk resonators and arrays.

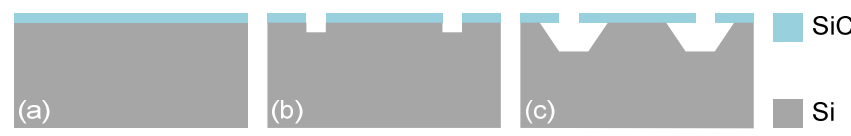

Figure 2: Process flow for fabrication and prototyping of $\mathrm{SiC}$ microdisks. (a) 500nm 3C-SiC epilayer on Si (100) substrate. (b) Mask-less patterning and nanomachining of SiC using FIB. (c) Etching of Si substrate by HNA to yield suspended SiC microdisks.

\section{MICRODISK RESONANCE MEASUREMENT}

The suspended microdisks are tested all at room temperature in a vacuum of $\sim 12 \mathrm{mT}$ Torr to examine their resonance response, by using a home-built apparatus [10] that incorporates a two-port RF network analysis measurement with a sensitive laser interferometry for displacement readout. $\mathrm{SiC}$ microdisk samples are driven by a piezoelectric thin disk actuator. Every observed micromechanical resonance is examined and carefully verified to be from the vibrational modes of the SiC microdisk. To ensure this, we have 
employed a combination of techniques and tests, which generally include examinations of amplitude dependency, linear and nonlinear behaviors at varying drive levels, precise response dependency on laser spot locations on the device surface, control tests of response and dependency when the same laser spots focused upon areas with no devices (Fig. 1d, regions between microdisks), careful calibration and tuning of the spot size (and other specs) of the laser interferometry, dependency on varying laser intensity, examination of thermomechanical fluctuations (Brownian motions) of micromechanical devices, etc. For each device, after convincingly verifying the observed micromechanical modes, the optical detection is further optimized to measure the overall response for each device. The detection is then tuned and optimized for characterizing individual peaks and modes.

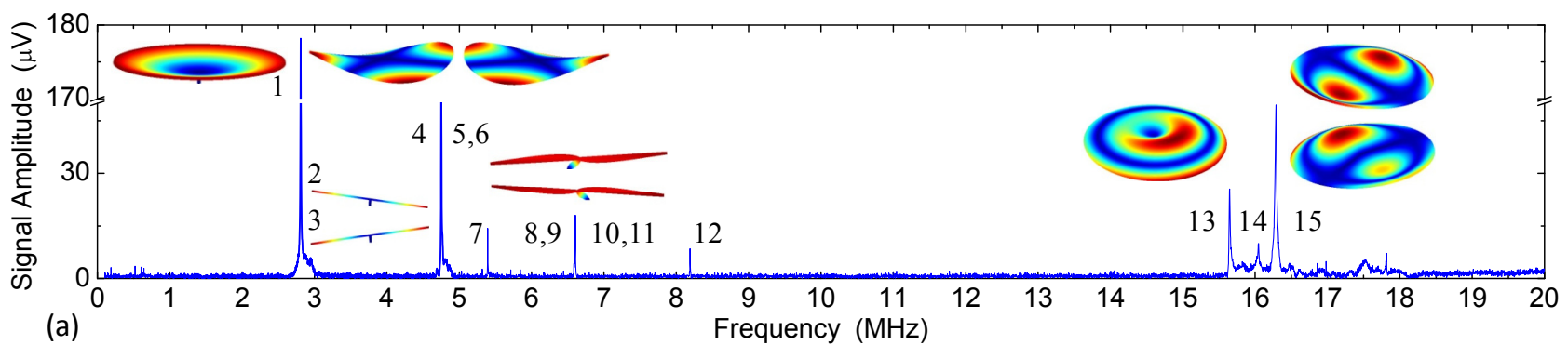

(a)
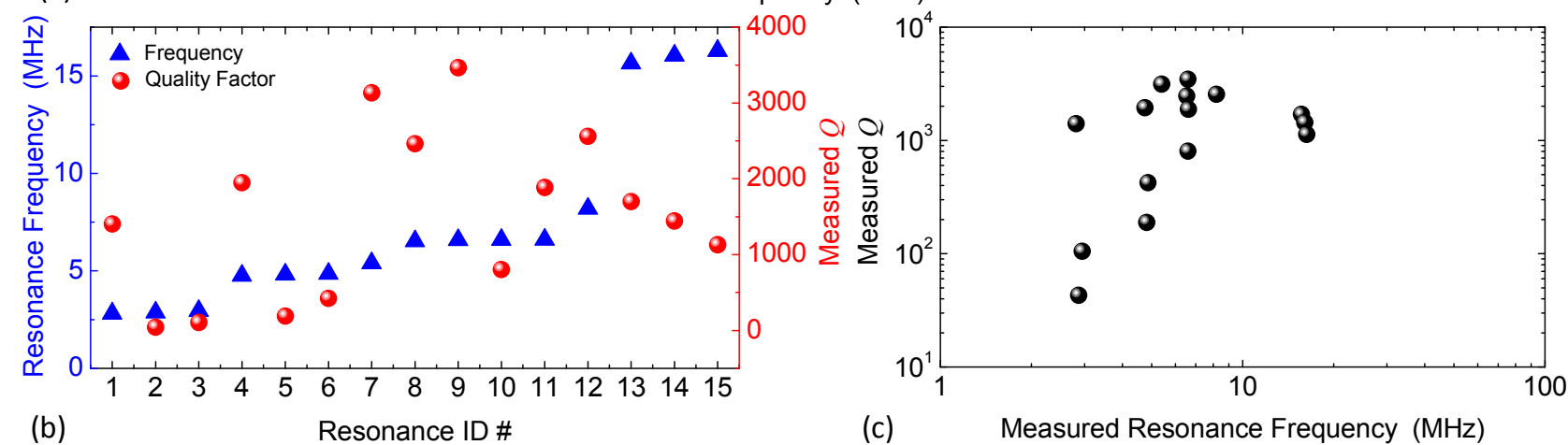

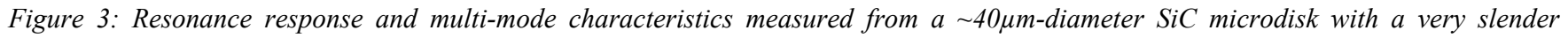
anchoring stem (diameter $\approx 800 \mathrm{~nm}$ ). (a) Wide range $R F$ multi-mode response measured by using two-port RF network analysis with laser interferometric detection techniques. A resonance ID \# is designated to each observed and carefully verified micromechanical resonance, in the order of increasing frequency. Insets: simulated mode shapes. (b) Measured resonance frequency (triangles) and $Q$ 's (circles) versus resonance ID \#. (c) Measured $Q$ versus resonance frequency with a trend defying the conventional $Q$-frequency trade-off.

\section{EXPRIMENTAL RESULTS AND DISCUSSIONS}

\section{SiC Microdisk Resonators with Slender Anchors}

Figure 3 shows the main results of measured flexural response from a 500nm-thick, $\sim 40 \mu \mathrm{m}$-diameter SiC disk with an anchoring stem of only $\sim 800 \mathrm{~nm}$ in diameter. All the verified resonance peaks are from out-of-plane vibrations and a few modes that involve the flexural motions of the thin disk and the slender stem together. Analytical and finite element (COMSOL) modeling are combined to analyze and determine the mode shapes, as illustrated in Fig. 3a.

The interesting step-like pattern of the frequency versus mode number in Fig. $3 b$ clearly indicates the predicted degenerate modes (almost overlapping frequencies) due to the disk's many symmetry possibilities (with abundant theoretical nodal diameters and nodal circles). Structural and fabrication nonidealities break degeneracy and cause mode splitting; hence many of the observed peaks, each in fact is a superposition of two split degenerate modes (Fig. 4b).
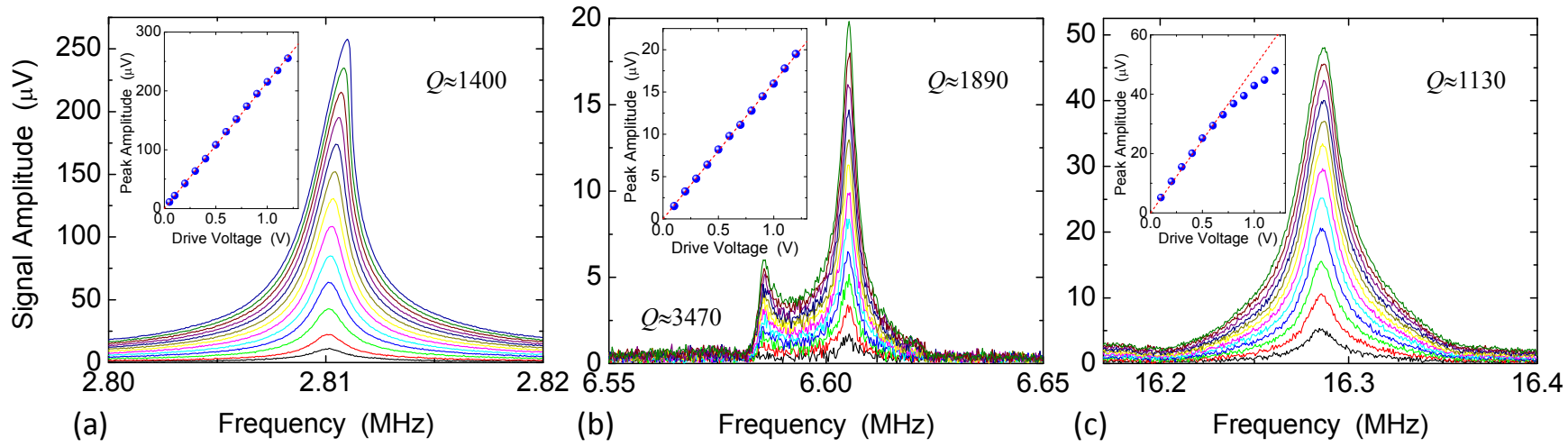

Figure 4: Characteristics of individual resonance modes measured from the SiC microdisk with a very slender supporting stem. (a) The fundamental out-of-plane flexural resonance at $\sim 2.81 \mathrm{MHz}$, with $Q \approx 1400$. (b) A pair of modes at $\sim 6.6 \mathrm{MHz}$ involving flexural vibrations of the slender stem. (c) A high frequency flexural mode at $\sim 16.3 \mathrm{MHz}$, with $Q \approx 1130$, showing nonlinear behavior at high amplitude. Insets: measured peak amplitude of the resonance (or the dominant resonance, in panel (b)) versus drive voltage, along with linear fitting. 

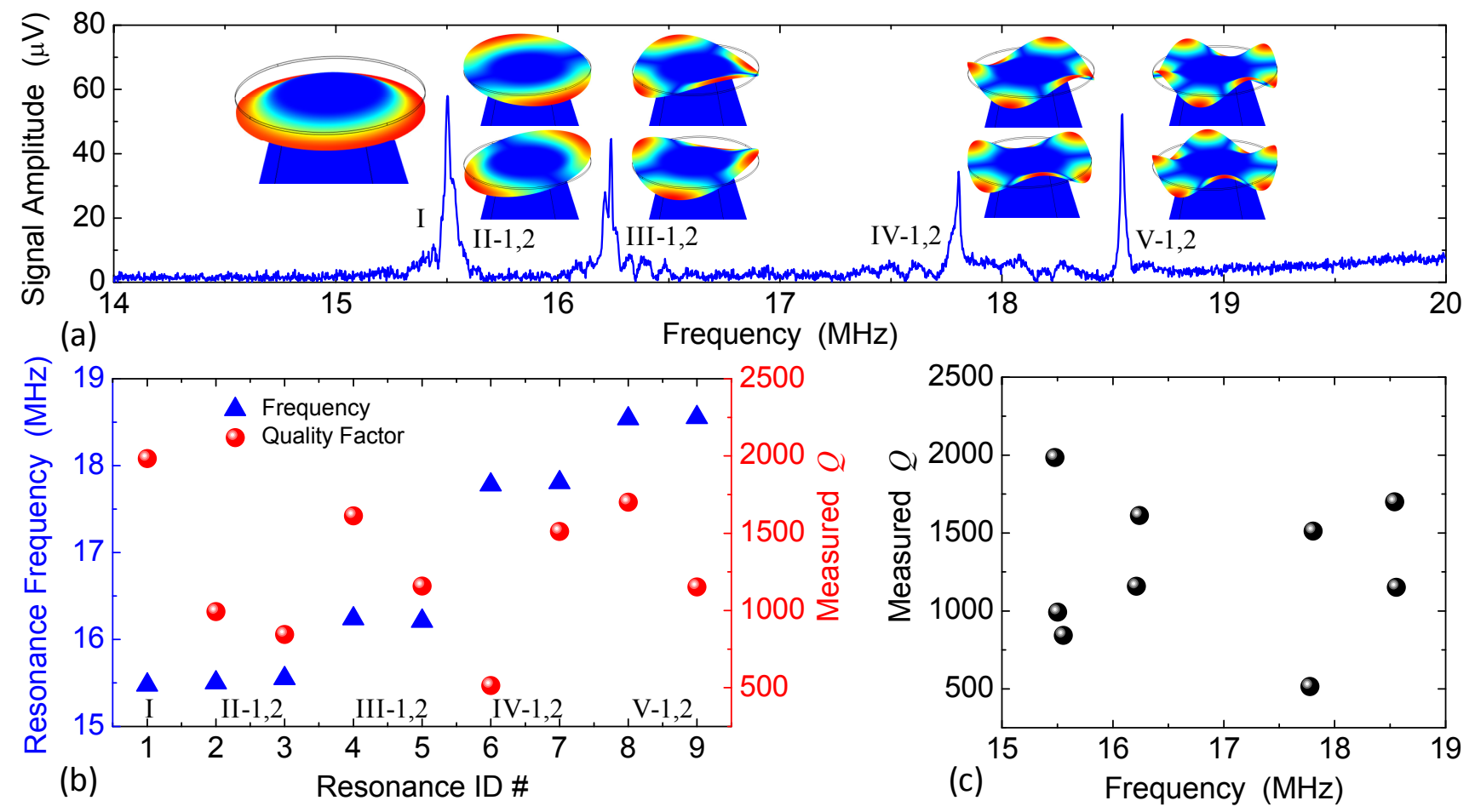

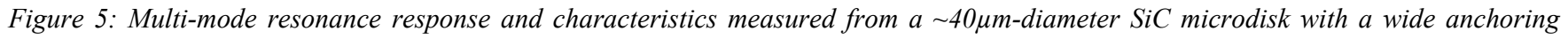
pillar (diameter $\approx 20.9 \mu \mathrm{m}$ ). (a) Wide range RF multi-mode response. The observed major flexural modes are designated I, II, III, IV \& $V$, and the splitting 'degenerate' modes are labeled $1 \& 2$, according to modeling predictions. Insets: simulated mode shapes. (b) Measured vibrational resonance frequency (triangles) and $Q$ (circles) versus resonance ID \#. (c) Measured $Q$ versus resonance frequency.

Figures $3 \mathrm{~b} \& \mathrm{c}$ show, very interestingly, a non-monotonic $Q$ frequency dependency and an overall trend of possibly overcoming the conventional $Q-f$ trade-off. Figure 4a shows the fundamental flexural resonance at $\sim 2.81 \mathrm{MHz}$ from the disk, with its simulated mode shape shown in Fig. 3a close to the peak. Figures $4 \mathrm{~b} \& \mathrm{c}$ demonstrate selected examples of observed mode-splitting, and transition from linear to nonlinear characteristics, respectively.

\section{SiC Microdisk Resonators with Wide Anchors}

When the anchor becomes as wide as $\sim 1 / 2$ of the disk diameter (the images of the specific device shown in Fig. 1a \& c), measurement results in Fig. 5 demonstrate that the first 9 measured flexural modes are all shifted to higher frequencies $(\sim 15-20 \mathrm{MHz}$, compared to results in Fig. 3). This is due to the shortened radial length of the suspended part (rim) of the disk, or corresponding to a shorter cantilever with a wider clamping port that would rotate and extrude into this circular disk.

A compelling step-like pattern of mode frequencies (with counts 1-2-2-2-2) in Fig. 5b clearly shows the degeneracy as predicted. The insets in Fig. 5a illustrate the ideal mode shapes, where each in the II, III, IV, and V pairs has 1, 2, 3, and 4 nodal diameters, respectively. In contrast to the previous device and results shown in Fig. 3, due to the much shorter suspended radial length, in this device the mode shapes with nodal circles will only occur at frequencies much higher than $20 \mathrm{MHz}$. Obviously, in this device the anchor is much more rigid (very high stiffness), and in the measured range, there is clearly no flexural mode that is coupled to either lateral or vertical motions of the anchor.

Figures $5 \mathrm{~b} \& \mathrm{c}$ show a non-monotonic $Q-f$ dependency, similar to that in Fig. 4. The overall trend, however, appears to be toward decreasing $Q$. This suggests clamping loss may be more important in higher frequency disks/modes with wider anchors.

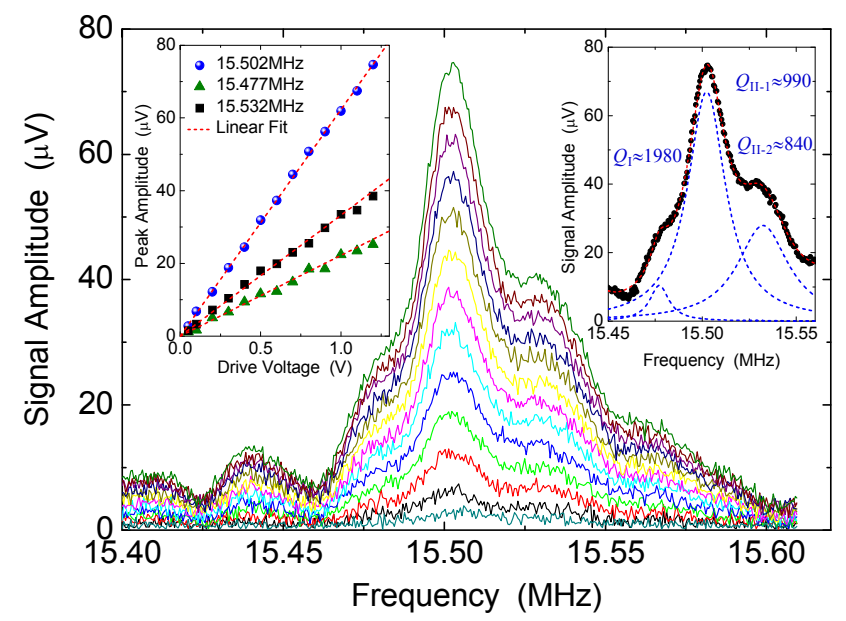

Figure 6: Characteristics of resonance modes at $\sim 15.5 \mathrm{MHz}$. Main plot shows response measured by sweeping $V_{d r v}=0.1-1.2 \mathrm{~V}$ with $0.1 \mathrm{~V}$ steps. Left Inset: Measured peak amplitude of the modes versus drive, with linear fits to the data. Right Inset: Analysis and decomposition of the measured response into three adjacent modes according to careful modeling, with extracted $Q$ values.

Nonidealities cause mode splitting near the ideal degenerate frequencies. Therefore each of the observed peaks II, III, IV, and $\mathrm{V}$ presents a superposition of a pair of split modes. Some nonideal effects not only cause frequency discrepancies, but also strongly change the split modes' amplitudes (e.g., a very small mismatch between the centers of anchor and disk can significantly change the relative amplitudes of the split pairs, and the color-mapped mode 
shapes will be quite different than shown in Fig. 5a). Therefore, while ideally the amplitudes of each pair of modes should be equal and mode shapes be symmetric (Fig. 5a), in fact one split mode can easily have a much higher amplitude response and dominate over the other. Given repeated observations of this imparity, we combine data and multi-Lorentzian analysis to decompose the measured peaks and extract the predicted split modes guided by modeling. Figures 6-9 clearly reveal the detailed characteristics (dominance $\&$ contributions in each pair) resolved by this analysis.

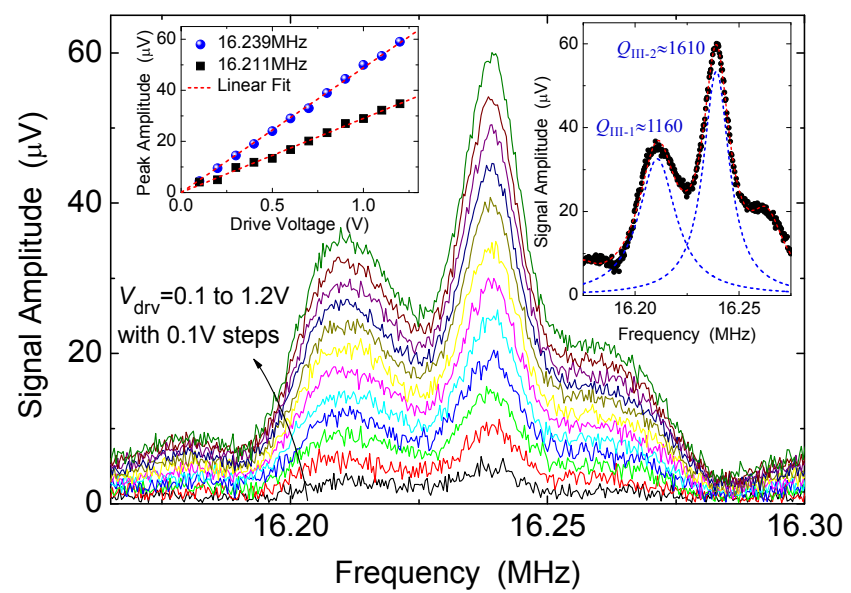

Figure 7: A pair of vibrational modes at $\sim 16.21 \mathrm{MHz}$ and 16.24MHz. Left Inset: Measured peak amplitude of the modes versus drive. Right Inset: Analysis of the contribution from the two split modes to the measured response, and the extracted $Q$ 's.

The very small mode splitting $\left(\Delta f_{N-1,2} / f_{N}<0.2 \%\right.$, for $\left.N=\mathrm{II}-\mathrm{V}\right)$ is just comparable or even smaller than the resonance peak width, further increasing the complications caused by amplitude imparity. While natural (nonidealities induced) mode splitting may help explore intrinsic issues in devices and materials, for specific device applications, sub-resonance-width natural mode splitting may need to be intentionally evaded by design with asymmetries.

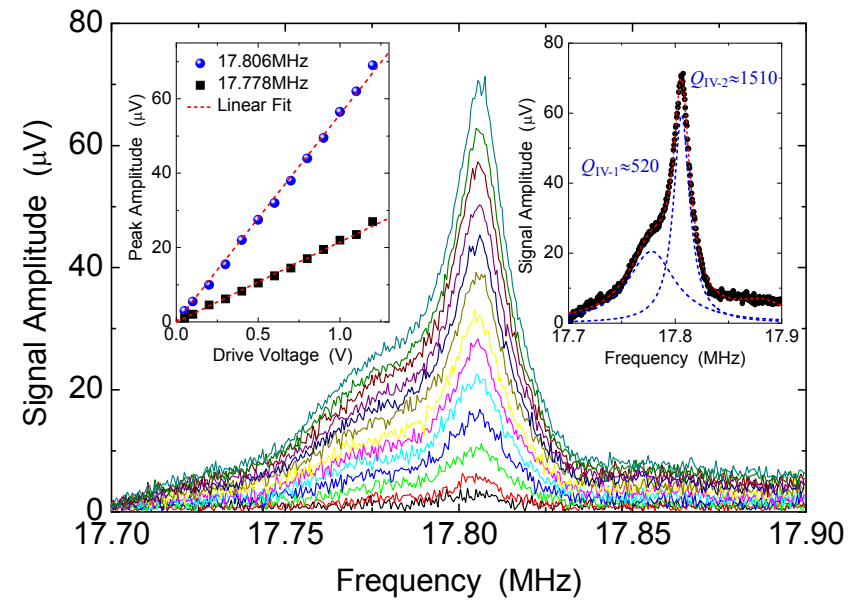

Figure 8: Measured response of an uneven pair of split modes at $\sim 17.78 \mathrm{MHz}$ and $\sim 17.81 \mathrm{MHz}$ (dominating). Left Inset: Peak amplitude versus drive with linear fits. Right Inset: Analysis and revelation of the two split modes, and the extracted $Q$ 's.

We note that the measured multi-mode response is very sensitive to the tuning of the laser interferometry and precise control of the laser spot on the surface of the SiC microdisks. Importantly, each of the full wide-range data traces in Fig. 3a and
Fig. 5a is taken without relocating the spot, simply by optimizing the overall full-range response only. A larger spot is used in obtaining the Fig. 3a data, while a smaller spot is focused on the suspended portion of the microdisk in case of the data in Fig. 5a.

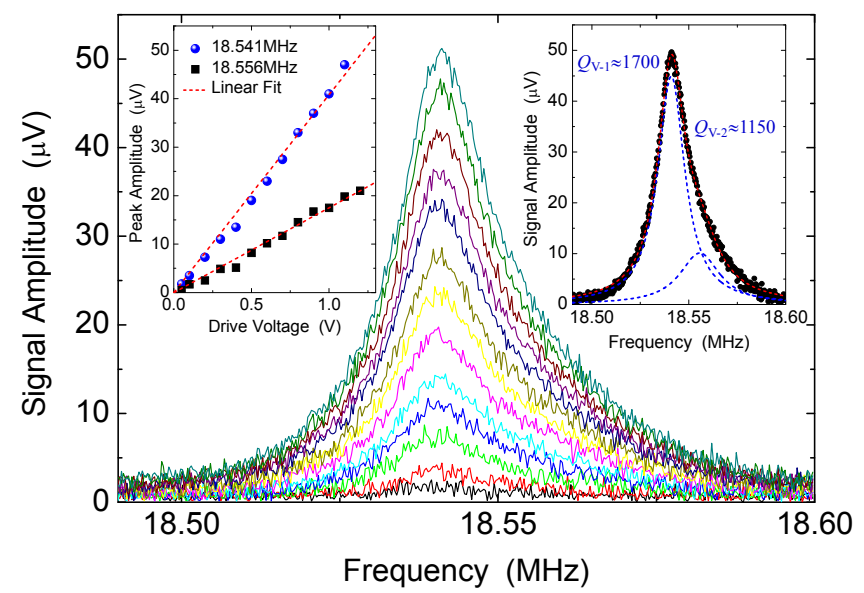

Figure 9: Measured response due to a very uneven pair of split modes at $\sim 18.54 \mathrm{MHz}$ (dominating) \& 18.56MHz. Left Inset: Peak amplitude versus drive with linear fits. Right Inset: Revelation of the contributions from the two split modes, and the extracted $Q$ 's.

\section{CONCLUSIONS}

We have experimentally demonstrated and investigated circular thin-film microdisk resonators nanomachined from a $500 \mathrm{~nm} \mathrm{SiC}$ epilayer grown on $\mathrm{Si}$, which exhibit multi-mode flexural resonances in the range of $\sim 2-20 \mathrm{MHz}$ with typical $Q$ 's 1000-4000. Sensitive interferometric readout and modeling together reveal the characteristics of observed modes, and the effects of varying anchor size upon the frequency spectra and $Q$ 's of the devices. The micromechanical and RF characteristics of these resonators and arrays make them interesting for potential applications including on-chip resonance-based physical sensing elements and multi-mode communication building blocks.

\section{REFERENCES}

[1] R.A. Johnson, Mechanical Filters in Electronics, John Wiley \& Sons, Inc., New York (1983).

[2] M. Rodahl, F. Höök, A. Krozer, P. Brzezinski, B. Kasemo, Rev. Sci. Instrum. 66, 3924-3930 (1995).

[3] J.R. Clark, W.-T. Hsu, C.T.-C. Nguyen, Tech. Digest, IEDM 2000, pp. 493-496, San Francisco, CA, Dec. 11-13 (2000).

[4] C.T.-C. Nguyen, IEEE Trans. Ultrason. Ferroelectr. Freq. Control 54, 251-270 (2007).

[5] Y.T. Yang, C. Callegari, X.L. Feng, M.L. Roukes, Nano Letters 11, 1753-1759 (2011).

[6] X.L. Feng, C.J. White, A. Hajimiri, M.L. Roukes, Nature Nanotechnology 3, 342-346 (2008).

[7] X.L. Feng, C.A. Zorman, M. Mehregany, M.L. Roukes, Tech. Digest, Hilton Head'2006, pp. 86-89, Hilton Head Island, SC, June 4-8 (2006).

[8] X.L. Feng, M.H. Matheny, C.A. Zorman, M. Mehregany, M.L. Roukes, Digest of Tech. Papers, Transducers'2009, pp. 2246-2249, Denver, CO, June 21-25 (2009).

[9] C.A. Zorman, A.J. Fleischman, A.S. Dewa, M. Mehregany, P. Pirouz, et al., J. Appl. Phys. 78, 5136-5138 (1995).

[10] A.C. Barnes, R.C. Roberts, N.C. Tien, C.A. Zorman, P.X.-L. Feng, Digest of Tech. Papers, Transducers'2011, pp. 26142617, Beijing, China, June 5-9 (2011). 


\section{SINGLE TRANSISTOR OSCILLATOR BASED ON PIEZOELECTRIC RESONANT NANOCHANNEL FOR OPERATION IN LIQUIDS \\ Chiara Zuniga $^{l}$, Matteo Rinaldi ${ }^{2}$, Augusto Tazzoli ${ }^{3}$ and Gianluca Piazza ${ }^{3}$ \\ ${ }^{1}$ University of Pennsylvania, Philadelphia, Pennsylvania, USA \\ ${ }^{2}$ Northeastern University, Boston, Massachusetts, USA \\ ${ }^{3}$ Carnegie Mellon University, Pittsburgh, Pennsylvania, USA}

\begin{abstract}
A single transistor oscillator based on an $18 \mathrm{MHz}$ piezoelectric resonant nanochannel is presented as the first demonstration of MEMS acoustic resonators with integrated nanochannels connected to a compact and portable oscillator readout enabling the sensor operation in liquids. This result represents a breakthrough for MEMS resonators operated in liquids as these devices are the first of their kind to be directly connected to a compact electronic oscillator.
\end{abstract}

\section{INTRODUCTION}

In recent years, the demand of miniaturized sensors able to operate in fluids has been steadily growing for a variety of different applications ranging from medical diagnostic to automotive and environmental monitoring.

The medical field, in primis, represents one of the most appealing and fast growing market for such sensors as the need to analyze human fluids recurs in almost every medical assessments. Particularly, the need of personalized and wireless medicine is becoming increasingly urgent.

Recently, miniaturized devices based on micro and nanoelectromechanical systems (M/NEMS) technology have emerged as a viable response to the need for compact, inexpensive and integrable resonant sensors. Their reduced mass allows them to achieve unprecedented levels of resolution [1] and makes them suitable for detecting minuscule concentrations of target analytes in bio-samples. However, the use of these devices for life science applications has been limited because of the challenge of operating them in liquid environments. Viscous damping, efficient transduction and effective fluid-device interaction represent the main issues that acoustic resonators need to overcome in order to be fully exploited for sensing applications in liquids.

Viscous damping, in particular, affects the performance of the NEMS devices depending on their mode of vibration and becomes increasingly detrimental as the size of the resonator is decreased.

Resonant devices vibrating according to flexural or out-ofplane bulk modes have shown greatly damped responses in fluids, with values of $Q$ in the order of 10s - 100s [2-3]. In fact, in a fluid, these modes of vibration cause a pressure force on the displacing surfaces of the resonant device and creates additional damping with respect to shear-like vibrations. As a matter of fact, the latter are preferable for operation in liquids.

Thin Film Bulk Acoustic Resonators (FBARs) [4] have demonstrated similar performance in terms of quality factors but at much higher frequencies of operation, recording Q as high as 130 at $1.5 \mathrm{GHz}$. Therefore, they can provide values of mass sensitivity much higher than QCMs thanks to both their reduced volume and higher frequency of operation ( $\mathrm{GHz}$ range). Nevertheless, for FBAR, as for QCM, the operating frequency is set by the thickness of the piezoelectric layer. This precludes the capability of these devices to provide for multiple frequencies of operation (hence mass sensitivities) on the same substrate and exclude their employment for the fabrication of multi frequency single chip sensor arrays.

The use of laterally vibrating devices $[5,6]$ instead of devices vibrating in a flexural or out of plane bulk modes has shown to partially overcome some of these issues. They can provide for multiple frequencies on the same chip, and their interaction with the fluid is equivalent to shear vibrations, which are less prone to viscous damping as shear waves rapidly decay in fluid media.

The integration of a microfluidic channel inside the body of a resonant cantilever beam sensor $[1,6]$ has been demonstrated as a very effective way to simultaneously address the issue of viscous damping and realizing a compact delivery system that reduces sample volume. Although highly effective in maintaining unaltered mechanical performances, these hollow cantilever beams required a high DC voltage (as high as $120 \mathrm{~V}$ [1]) for their electrostatic actuation and a cumbersome optical readout. In addition, they exhibited a frequency of operation of few $100 \mathrm{~s} \mathrm{of} \mathrm{kHz}$, which limited the device sensitivity for certain applications.

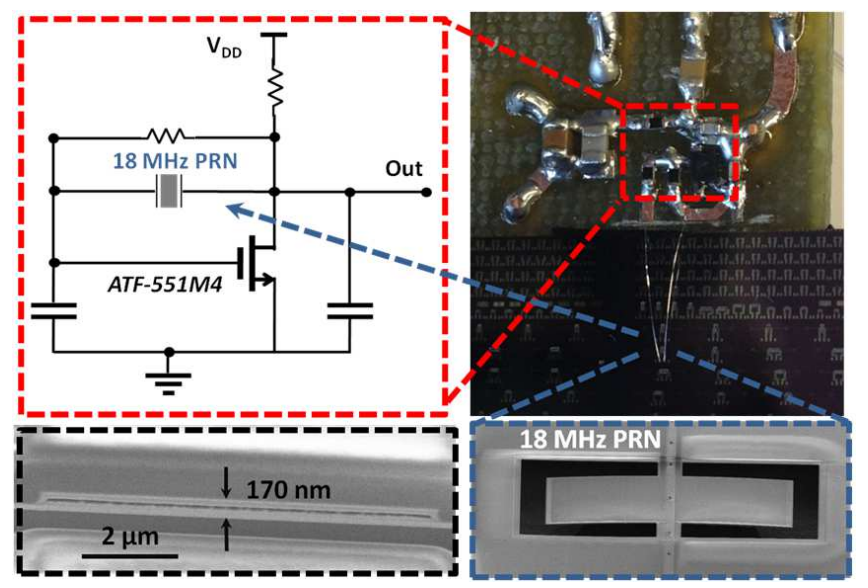

Figure 1: SEM of the $18 M \mathrm{MH}$ PRN and its cross-section and a picture of the device wire-bonded to a Pierce oscillator implemented with an ATF-551M4 E-pHEMT GaAs transistor.

This paper presents a new design and the experimental verification of Piezoelectric Resonant Nanochannels (PRNs). The reduction of viscous damping has been attained through an innovative design solution, in which the fluid is enclosed in the body of the resonant element. In this perspective, the hollow design of a resonant nanochannel implements a high surface to volume sensor that maximizes the sample-device interaction, simultaneously solves the issues related to the transduction efficiency of nanoscale structures in liquids, and addresses the reduction of viscous damping.

PRNs with frequencies of operation ranging from $12 \mathrm{MHz}$ to $1 \mathrm{GHz}$ were designed, fabricated on the same die and tested achieving quality factors as high as 1700 when operated in fluids at $13 \mathrm{MHz}$. Several designs and channels widths have been designed and experimentally tested in order to find the best trade-off between sensitivity and quality factors.

Finally, the AlN PRNs can be efficiently actuated and sensed piezoelectrically on-chip (values of electromechanical coupling, $k_{t}^{2}$, as high as $1 \%$ are achieved despite the operation in fluid 
environment) solving the fundamental transduction issues associated with M/NEMS sensors in fluids and enabling the use of compact and low power CMOS circuits for electronic readout. The capability to connect an $18 \mathrm{MHz}$ PRN to self-sustained, lowpower, single transistor oscillator circuits for direct frequency readout is experimentally demonstrated. The fabricated oscillators show excellent noise performance (phase noise values as low as $-110 \mathrm{dBc} / \mathrm{Hz}$ at $1 \mathrm{kHz}$ offset and phase noise floor values as low as $-154 \mathrm{dBc} / \mathrm{Hz}$ are achieved with a carrier frequency of $\sim 18 \mathrm{MHz}$ ) thanks to the outstanding and unique performance of the nanoscaled resonant elements in terms of figure of merit $(F o M)$, corresponding to a $k_{t}^{2} \cdot Q$ product as high as 7 despite operation in fluids. Because of the unique capability of arraying large number of PRNs with different frequencies of operation in a small footprint, this novel technology lays the foundation for the demonstration of large arrays of Piezoelectric Resonant Nanochannels for highly multiplexed bio-sensing applications that require high throughput and reduced sample volume.

\section{DESIGN}

The device that constitutes the basis of this work consists of a laterally vibrating nanochannel [7, 8]. As explained elsewhere [8], the Piezoelectric Resonant Nanochannel is composed of two layers of AlN enclosing a nano-scaled airgap in between them. Such airgap serves as a nanofluidic channel whose function is to confine a liquid sample within the body of the device instead of having the resonant sensors submersed in it.

A PRN fully displaces into contour extensional vibrations when an electric field is applied across the thickness of the first AlN film as displayed in Figure 2 in the same way as a conventional CMR [9].

The improved design solution presented in this paper delivers the fluid solely on a narrow area of the resonator, a fraction of the total surface of the device in the range from $3.3 \%$ to $30 \%$. Thus, this minimizes the energy radiation and translates to an improved $Q$. The devices are also properly designed to span an extended frequency range from $12 \mathrm{MHz}$ to $1 \mathrm{GHz}$ thus benefiting from the theoretical $Q$ enhancement at both ends of the range. Furthermore, because of the additional confinement of the fluidic sample into a narrower area on the $\mathrm{PRN}$, the expression for the $Q$ in fluids previously computed in [5] needs to take into account that the area in contact with the liquid, where the energy radiation occurs because of the viscous drag, is now much smaller than the PRN top surface as shown in Figure 2.
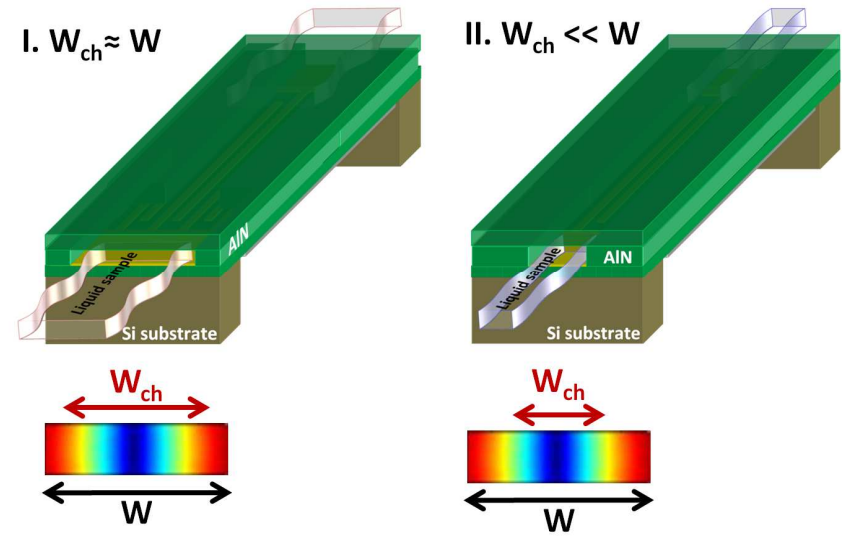

Figure 2: Schematic $c$ of the new comparison design solution of the PRN (II.) that confines the fluid to a narrower area on the device's body
The expression of the $\mathrm{Q}$ in fluids therefore becomes

$$
Q_{f}=\omega_{s} \cdot \frac{\rho_{e q} \cdot T_{e q} \cdot u_{0}}{2 \operatorname{Re}\left[\sigma_{x y}\right]} \frac{S}{S_{\text {channel }}}
$$

where $S_{\text {channel }}$ is the internal area of the nanochannel and $S$ the surface of the PRN, while all the remaining variables $T_{e q}, \rho_{e q}, \sigma_{x y}$ are respectively the equivalent thickness and the equivalent density of the material stack forming the device, and the frictional force acting on the device surface.

Intuitively, it is straightforward to realize that by minimizing the area where the energy dissipation occurs represents an alternative way to enhance the quality factor without further scaling the nanochannel depth to extremely reduced thicknesses. Additional scaling would, in fact, add significant complexity to the fabrication process and would result in nanochannels with values of flow resistance so high to render the connection to a complete flow system almost unfeasible.

Figure 3 is a plot of Eq. 1 and clearly predicts that there is a significant advantage in designing narrow nanochannels operating over the whole frequency range.

The location of the nanochannel within the body of the PRN is an additional design parameter and has a significant weight on the performance of the mechanical resonator. In fact, the induced displacement of the resonator is not uniform over its entire width, since the mode-shape of vibration obeys to a harmonic motion, $u(x)$ [9]. Therefore, designing the nanochannel in the locations of maximum displacement of the PRN translates to higher mass sensitivity, but at the same time higher viscous damping and hence, lower quality factor. Conversely, placing the nanochannel in a nodal point, i.e. where the surface velocity is zero, would noticeably increase the quality factor when the nanochannel is filled, but it would also dramatically decrease the mass sensitivity of the device.

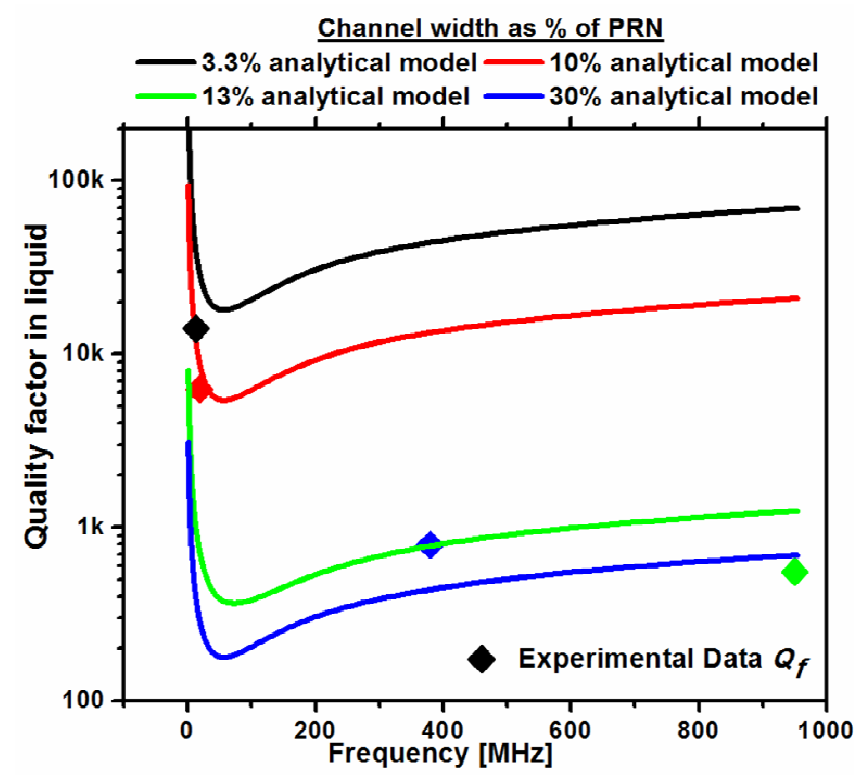

Figure 3: The graph shows the overlap between the experimental Qs in DI-water extracted from the fitted admittance curves and the predicted behavior according to the theory of a laterally oscillating plate in viscous fluids where $1 / Q_{\text {tot }}=1 / Q_{\text {fluid }}+1 / Q_{\text {air }}$ with $Q_{\text {fluid }}$ being the fluidic quality factor and $Q_{\text {air }}$ the quality factor of the PRN in air. The data show good agreement with the theory especially for the lower frequency devices (single finger devices) 
where the hypothesis of uniform velocity of the plate surface represents a good approximation

Based on these considerations, Eq. 1 of the quality factor in fluids should be modified to take into account the location of the channel. As a matter of fact, the lower frequency devices consist of a single finger (for which the width, $W$, is between 200 and 300 $\mu \mathrm{m})$ vibrating in the fundamental extensional mode and the nanochannel runs solely on top of the center of the structure. This channelhas a width, $W_{c h}$, considerably smaller than the one of the device ( $W_{c h}$ is $\left.10-20 \mu \mathrm{m}\right)$ (Fig. 2-II). For this reason, the velocity of the internal surface of the nanochannel cannot be considered to be maximum, but in light of the fact that $W_{c h} / W \rightarrow 0$, it can be approximated as $u\left(W_{c h}\right) \approx u_{0} \frac{\pi W_{c h}}{2 W}$. Hence, the expression for the quality factor is:

$$
Q_{f}=\omega_{s} \cdot \frac{\rho_{e q} \cdot T_{e q}}{\operatorname{Re}\left[\frac{\sigma_{x y}}{u_{0}}\right]} \frac{2 W^{3}}{\pi^{2} W_{c h}{ }^{3}}
$$

The effect of these design changes on sensitivity and limit of detection (LOD) should also be taken into consideration. In fact, the expression of the mass sensitivity for these PRNs vibrating at low frequency $(12-50 \mathrm{MHz})$ needs to take into account the reduction in both velocity and sensing area inside the nanochannel. Therefore, the traditional sensitivity equation [5] can be modified as follows

$$
S=\frac{f_{0}}{2 \rho_{e q} T_{e q}} \frac{\pi^{2} W_{c h}{ }^{3}}{12 W^{3}}
$$

Finally, the ultimate limit of detection, $L O D$, of a PRN given its mass sensitivity, $S$, computed in Eq. (3) can be derived considering the resonator thermomechanical noise as the ultimate source of frequency fluctuations originated from thermally driven random motion of the mechanical device [11]. In this case, the LOD can be expressed as:

$$
L O D=\frac{\Delta f_{\min }}{S}=\sqrt{\frac{k_{B} T(1+F) B}{P_{c}}} \frac{\rho T}{Q} \frac{12 W^{3}}{\pi^{2} W_{c h}{ }^{3}}
$$

where for the higher frequency devices, for which $Q$ is effectively limited by $Q_{f}$ in liquids, it is possible to substitute Eq. 2 in the LOD equation and obtain an expression of the limit of detection that is a function of the dissipative part of the frictional force and no longer dependent on the nanochannel in plane dimensions. At lower frequency, for which the $\mathrm{Q}$ is likely limited by the intrinsic material damping (see Figure 3), there is a net advantage in miniaturizing the lateral dimensions of the channel.

\section{EXPERIMENTAL RESULTS}

The new design has demonstrated performance far superior than the first generation of PRNs effectively proving the enhancement in both the design and fabrication process $[7,8]$. The confinement of the nanochannel over a fraction of the total surface of the device together with a nanochannel thickness comparable to the penetration depth in water $(\sim 160 \mathrm{~nm}$ at $13 \mathrm{MHz})$ allowed the PRNs with very narrow channels' widths $(3.3 \%$ to $10 \%$ of the device total width) to attain $Q s$ as high as 1710 at $13 \mathrm{MHz}$ and equal to 1567 at $18.6 \mathrm{MHz}$ when filled with DI-water. Despite the operation in liquid environment, a high value of the figure of merit, $k_{t}^{2} \cdot Q,(\sim 10$ and 9 at 13 and $18 \mathrm{MHz}$, respectively) have been attained translating to very low values of motional resistance.

Such high values of the device $k_{t}^{2} \cdot Q$ have enabled the direct connection of the fabricated $18 \mathrm{MHz}$ PRN to a compact and low power self-sustained oscillator circuit for direct frequency readout.

The response of an $18 \mathrm{MHz}$ PRN with $10 \%$ channel coverage has been characterized through a RF probe station recording Qs as high as 1600 and 1420, respectively in air and when filled with DIwater. Unchanged values of $k_{t}^{2}$ equal to $0.6 \%$ have been achieved.

The device was directly wire-bonded to a Pierce oscillator implemented with an ATF-551M4 E-pHEMT GaAs transistor (Figure 1). The oscillator was tested for different biases (i.e. different power levels) and its phase noise (PN) monitored with an Agilent E5052B Signal Source Analyzer.

The output of the oscillator in both operating conditions, air and DI-water, was also recorded with a Spectrum Analyzer. Selfsustained oscillations were observed for values of bias voltages, $\mathrm{V}_{\mathrm{DD}}$, in the range of $1.8 \mathrm{~V}$ and $3.4 \mathrm{~V}$.

The oscillator achieved values of $\mathrm{PN}$ as low as $-110 \mathrm{dBc} / \mathrm{Hz}$ at $1 \mathrm{kHz}$ and a noise floor of $-154 \mathrm{dBc} / \mathrm{Hz}$. After a short transient when water initially fills the channel (Figure 5) these values are nearly unchanged whether the nanochannel is filled with air or DIwater.

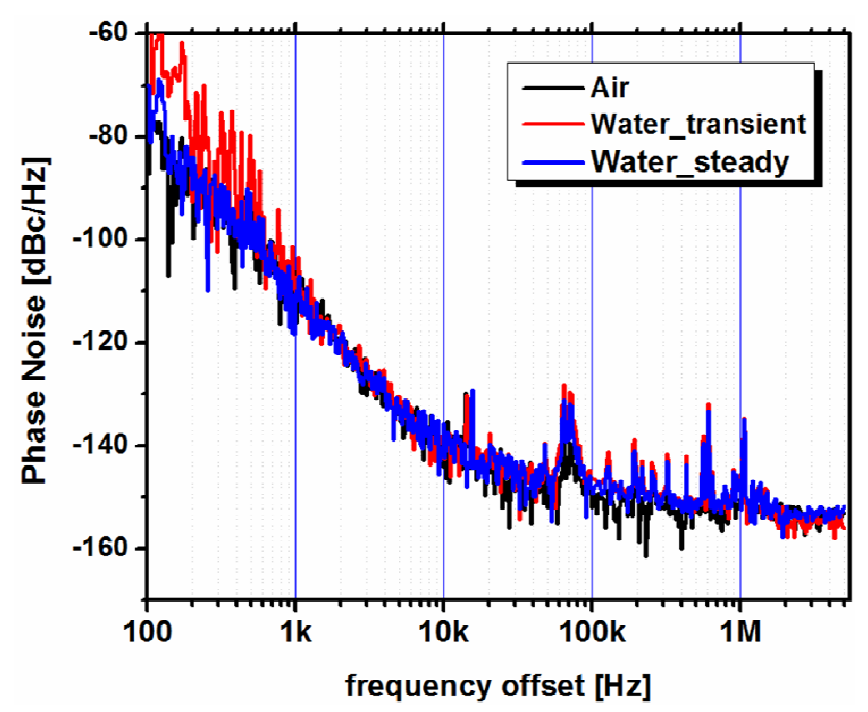

Figure 4: Phase Noise of the 18MHz PRN oscillator when filled with air and water. When water is first filling the channel, a transient response associated to higher close-in phase noise can be noticed. Unchanged performances are shown for both operating conditions when transient associated with water diffusion inside the channel is over.

Given the device phase noise, it is possible to compute through a simple mathematical integration the Allan variance, a statistical tool commonly used to measure the time domain frequency fluctuation of oscillators for a given integration time, $\tau$ (measurement time). Unlike the phase and frequency noise that are defined in the frequency domain, the Allan variance is defined in the time domain as the variance over time in the measured frequency of a source, each measurement averaged over a time interval, $\tau$, with ideally no dead time between measurement intervals.

The Allan variance is related to phase noise through the following relationship:

$$
\sigma_{Y}^{2}(\tau)=\frac{2}{\pi^{2} \tau^{2}} \int_{0}^{\infty} S_{\phi, o}(\omega) \sin ^{4}(\pi \omega \tau) d \omega
$$

where $S_{\phi, o}(\omega)$ is the oscillator phase noise and $\omega$ is the offset 
frequency from carrier. For the form of the close-in phase noise density, the Allan deviation is theoretically described by:

$$
\sigma_{Y}(\tau) \approx \sqrt{\frac{k_{B} T_{0}(1+F) \omega_{0}^{2}}{4 P_{c} Q^{2} \tau}}
$$

Therefore, by applying Eq. (5) to the phase noise experimentally recorded for the $18 \mathrm{MHz}$ PRN oscillator, it is possible to plot the Allan deviation of the oscillator and extract the experimental minimum detectable frequency shift in both operating conditions.

Figure 5 shows the Allan Deviation for the $18 \mathrm{MHz}$ PRN oscillator extracted from the phase noise measurements when the device is operated in air and during the transient response, when water is initially filling the nanochannel. The latter situation represents the worst case scenario, since at steady state the PRN filled with water presents the same noise performance as if operated in air as clearly shown in Figure 5.

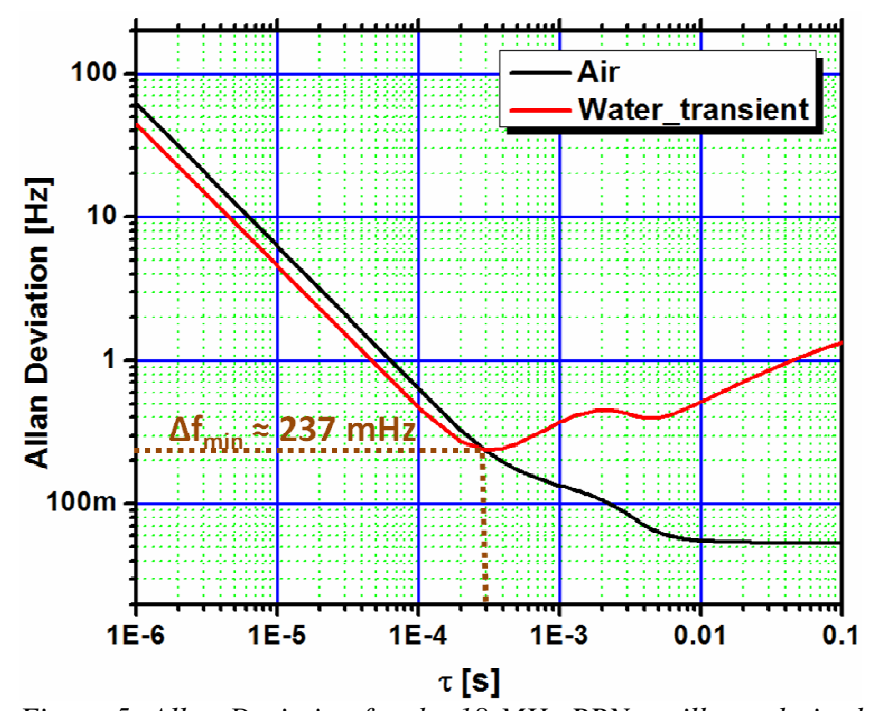

Figure 5: Allan Deviation for the $18 \mathrm{MHz}$ PRN oscillator derived from the phase noise in both operating conditions

A minimum detectable frequency shift equal to $237 \mathrm{mHz}$ has been extracted from the Allan deviation of the water transient curve for a measurement time of roughly $500 \mu \mathrm{s}$. Using Eq. (4) and the sensitivity computed for the $18 \mathrm{MHz}$ PRN, this can be translated to a value of limit of detection equal to $4 \mathrm{ag} / \mu \mathrm{m}^{2}$, which computing the internal surface of the nanochannel of the $18 \mathrm{MHz}$ $\mathrm{PRN}$, is equivalent to an effective absolute mass of $4.8 \mathrm{fg}$. Nevertheless, such experimental value is still slightly higher than the theoretical limit of detection (LOD) for a PRN limited by thermomechanical fluctuations as computed in Eq. 4. The experimentally recorded phase noise and derived Allan Variance are in fact not limited by the thermomechanical noise of the resonator, but the flicker noise of the oscillator. The theoretical thermomechanical limit is instead equal to $0.115 \mathrm{ag} /\left[\mu \mathrm{m}^{2} \cdot \sqrt{\mathrm{Hz}}\right]$, or to $1.6 \mathrm{ag} / \mu^{2}$ for a measurement time of $500 \mu \mathrm{s}$ and $3.0 \mathrm{~V}$ bias voltage $\left(V_{D D}\right)$. This clearly implies that if the read out circuit is properly improved, the LOD could be further lowered. Additionally, the power level $P_{C}$ used to drive the PRN at its center frequency could be also increased to achieve better signal to noise ratio and, hence, lower limit of detection.

\section{CONCLUSIONS}

In this paper, a new and improved design of Piezoelectric
Resonant Nanochannels was experimentally demonstrated. Qs as high as 1710 at $13 \mathrm{MHz}$ and equal to 1567 at $18.6 \mathrm{MHz}$ when filled with DI-water, were attained.

Thanks to such high values of the devices figure of merit, the capability to connect a $18 \mathrm{MHz}$ PRN to low-power single transistor oscillator circuit for direct frequency readout was experimentally demonstrated. The fabricated oscillator shows excellent noise performance: phase noise values as low as $-110 \mathrm{dBc} / \mathrm{Hz}$ at $1 \mathrm{kHz}$ offset and phase noise floor values as low as $-154 \mathrm{dBc} / \mathrm{Hz}$ were achieved for a carrier frequency of $\sim 18 \mathrm{MHz}$ ).

Additionally, a minimum detectable frequency shift equal to $237 \mathrm{mHz}$ has been extracted from the Allan deviation of the transient response of a device filled with DI water and a measurement time of roughly $500 \mu \mathrm{s}$. This can be translated to a value of limit of detection equal to $4 \mathrm{ag} / \mu \mathrm{m}^{2}$, which corresponds to a total mass of $4.8 \mathrm{fg}$.

This result represents a breakthrough for MEMS resonators operated in liquids as these devices are the first of their kind to be directly connected to a compact electronic oscillator.

\section{ACKNOWLEDGMENTS}

The authors wish to thank the staff of the Wolf Nanofabrication Facility (WNF) at the University of Pennsylvania. This work was supported by NTI.

\section{REFERENCES}

[1] J. Lee, W. Shen, K. Payer, T. P. Burg, and S. R. Manalis, "Toward Attogram Mass Measurements in Solution with Suspended Nanochannel Resonators" Nano Letters, 2010, 10, pp. 2537-2542.

[2] S. Choi, W. Xu, X. Zhang and J. Chae, " Characterization of a High-Q in-liquid Longitudinal-Mode Film Bulk Acoustic Resonator for Real-Time in-situ Monitoring of Competitive Protein Adsorption", Proceedings of IEEE MEMS 2010, pp. 739 742.

[3] M. Yue, H. Lin, D. E. Dedrick, S. Satyanarayana, A. Majumdar, A. S. Bedekar, J. W. Jenkins, and S. Sundaram, "A 2-D Microcantilever Array for Multiplexed Biomolecular Analysis", Journal of Microelectromechanical Systems, Vol. 13, No. 2, April 2004.

[4] M. Yue, J. C. Stachowiak, H. Lin, R. Datar, R. Cote, and A. Majumdar, "Label-Free Protein Recognition Two-Dimensional Array Using Nanomechanical Sensors", Nano Letters, 2008 Vol.8, No.2, pp. 520-524.

[5] C. Zuniga, M. Rinaldi and G. Piazza, "Quality Factor of MEMS and NEMS AlN Contour Mode Resonators in Liquid Media", IEEE International Ultrasonic Symposium Proceedings 2009, pp. 2568-2571.

[6] R. Cox, F. Josse1, S. Heinrich, I. Dufour and O. Brand, "Resonant Microcantilevers Vibrating Laterally in Viscous Liquid Media" IEEE International Frequency Control Symposium, 2010.

[7] C. Zuniga, M. Rinaldi and G. Piazza, "High Frequency Piezoelectric Resonant Nanochannel for Bio-Sensing Applications in Liquid Environment" Proceedings IEEE Sensors 2010, pp. 5255.

[8] C. Zuniga, M. Rinaldi and G. Piazza, "Reduced viscous damping in High Frequency Piezoelectric Resonant Nanochannels for Sensing in Fluids", Proceedings of IEEE MEMS 2011, pp. 960963.

[9] M. Rinaldi, C. Zuniga, N. Sinha, M. Taheri, S. M. Khamis, A. T. Johnson, and G. Piazza, "Gravimetric Chemical Sensor Based on the Direct Integration of SWNTs on AlN Contour-Mode MEMS Resonators", Proceedings of the IEEE International Frequency Control Symposium 2008, pp, 443-448. 


\section{TEMPERATURE-COMPENSATED EXTENSIONAL-MODE MEMS RESONATORS ON HIGHLY N-TYPE DOPED SILICON SUBSTRATES \\ M. Shahmohammadi, B. P. Harrington, J. Gonzales, and R. Abdolvand \\ Oklahoma State University, Tulsa, Oklahoma, USA}

\begin{abstract}
For the first time, lateral-extensional micromachined resonators fabricated on highly n-type doped silicon substrates and aligned to the [100] crystalline orientation are shown to exhibit an adjustable turnover temperature. This behavior commonly observed in quartz resonators is a key to achieving exceptional temperature stability in oven-controlled crystal oscillators.

To demonstrate the effect of doping concentration and resonator alignment to different crystalline orientations, the thinfilm piezoelectric-on-silicon (TPoS) platform is utilized. It is shown through combined theoretical analysis and finite element simulation that the turnover temperature is a sensitive function of doping concentration and also can be adjusted by changing the thickness of the resonant structure. In order to experimentally validate these results, similar resonators are fabricated on three silicon-on-insulator (SOI) substrates and the temperature variation of frequency for several devices are measured and the trends are shown to agree with theory. The minimum temperature variation of frequency is measured for a $\sim 25 \mathrm{MHz}$ TPoS resonator aligned to the [100] crystalline orientation showing a maximum frequency variation of $148 \mathrm{ppm}$ over the $-40^{\circ} \mathrm{C}$ to $85^{\circ} \mathrm{C}$ temperature range. This is more than $20 \mathrm{x}$ reduction compared to the earlier results from similar uncompensated devices.
\end{abstract}

\section{INTRODUCTION}

Silicon microresonators are poised to gain widespread use in timing and frequency control applications due to their small size and ease of integration. Significant improvements have been made recently with respect to many critical performance factors including quality factor, operating frequency, phase noise, spurious modes, and insertion loss [1-3]. However, one of the most significant drawbacks to using single-crystal silicon MEMS resonators has continued to be their relatively high and constant temperature coefficient of frequency (TCF) (about $-30 \mathrm{ppm} /{ }^{\circ} \mathrm{C}$ ). This results in more than an order of magnitude larger temperatureinduced frequency variation compared to quartz crystals. In addition, quartz resonators benefit from a turnover temperature at which point the TCF is zero. Therefore, very stable quartz oscillators are attainable within the industrial operating temperature range $\left(-40^{\circ} \mathrm{C}\right.$ to $\left.85^{\circ} \mathrm{C}\right)$ by biasing the temperature of an oven-controlled crystal at the turnover temperature [4].

Previous work has shown that the TCF in silicon resonators can be reduced by including materials with opposing TCF coefficients (i.e. silicon dioxide) [5] or by doping the silicon with boron and aluminum [6]. These methods have demonstrated significant improvement in overall frequency variation but they commonly sacrifice the resonators' quality factor or processing simplicity. In this work, it is shown that n-type doped extensional mode silicon resonators aligned to the [100] direction can exhibit a zero TCF at a certain temperature (i.e. turnover temperature) much like quartz crystal resonators. In addition, it is proved that turnover temperature can be adjusted by doping concentration and the device thickness within a certain temperature range and, therefore, can be designed to produce a minimal frequency shift over a targeted operational temperature range. This approach is very valuable in the sense that it does not require any alteration of the device fabrication process instead the doping concentration of the starting substrate need to be tightly controlled.

We have chosen TPoS resonators [7] to serve as the platform to analyze the effect of thickness, doping concentration and crystal alignment on TCF. TPoS resonators offer low motional impedance while maintaining high quality factors in air due to the singlecrystal silicon resonant structure [1]. A schematic of a typical two port TPoS resonator with input and output pads separated by a ground pad can be seen in Fig. 1. A piezoelectric material is sandwiched between two metal layers and is utilized to both drive a single-crystal silicon structure into resonance and to sense the resulting vibration. A thin metal bottom electrode serves as a ground plane while the patterned, top interdigitated metal electrodes provide the input and output pathways. Using this structure, a $25 \mathrm{MHz}$ resonator aligned to the [100] crystalline orientation is presented to exhibit an overall frequency variation of less than $150 \mathrm{ppm}$ over the $-40-85^{\circ} \mathrm{C}$ temperature range.

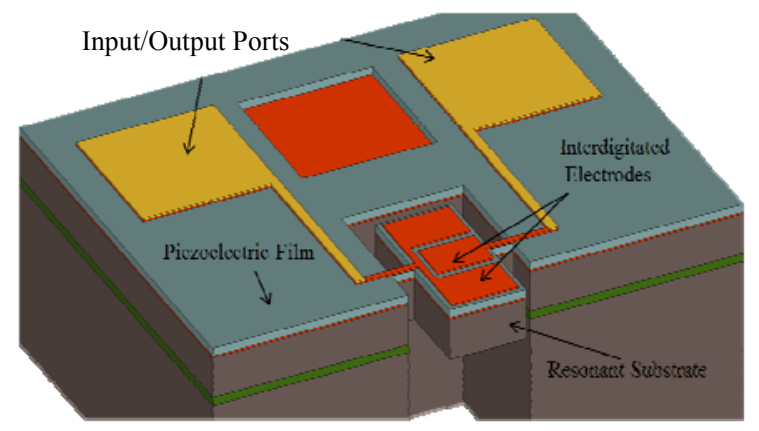

Figure 1: Schematic of a micromachined thin film piezoelectricon-silicon resonator.

\section{THEORY}

The electronic free energy is an important factor in defining the bonding forces between individual atoms and therefore it largely contributes in the elastic properties of solids. The electrons redistribute among the available energy levels in many-valley semiconductors such as silicon so that the total free energy in the crystal is minimized. Since these energy levels vary with the introduction of doping impurities in the crystal, the total free energy of the system also changes. This change in free energy consequently changes the elasticity $\operatorname{matrix}(c)$ of the material according to (1), where $G$ is the total Gibbs free energy, $V_{0}$ is the volume of non-deformed crystal and $S$ is the strain.

$$
c_{i j}=\frac{1}{V_{0}} \cdot \frac{\partial^{2} G}{\partial S_{i} \partial S_{j}}
$$

For free electrons, Keyes has presented several expressions describing the electronic contributions in the elastic coefficients [8]. In the case of n-type silicon, the contribution of free electrons on the elastic coefficients is calculated as [9]:

where,

$$
\delta c_{11}=-\frac{2}{9} N \frac{\Xi_{u}^{2}}{E_{f}} X, \delta c_{12}=\frac{1}{9} N \frac{\Xi_{u}^{2}}{E_{f}} X, \text { and } \delta c_{44}=0
$$

$$
X=\eta F_{1 / 2}^{\prime}(\eta) / F_{1 / 2}(\eta)
$$
Hilton Head Island, South Carolina, June 3-7, 2012 
and $N$ is the electron concentration, $\Xi_{u}$ is the uniaxial deformation potential constant, $E_{f}$ is the Fermi energy, $\eta$ is $E_{f} / k T$ and $F$ is the Fermi integral.

Using (2), the contribution of doping levels on the elastic coefficients, $c_{11}, c_{12}$ and $c_{44}$ (i.e. $\delta \mathrm{c}_{11}, \delta \mathrm{c}_{12}$ and $\delta \mathrm{c}_{44}$ ) at any temperature can be calculated. These values and the temperature dependent elastic constants of pure silicon are then utilized to compute the elastic coefficients of doped silicon at every temperature. Then, the Young's modulus $(E)$ for the directions [100] and [110] are calculated using:

$$
\begin{aligned}
& E_{[100]}=c_{11}-2 c_{12} \frac{c_{12}}{c_{11}+c_{12}} \\
& E_{[110]}=4 c_{44} \frac{c_{11}^{2}+c_{11} c_{12}-2 c_{12}^{2}}{2 c_{44} c_{11}+c_{11}^{2}+c_{11} c_{12}-2 c_{12}^{2}}
\end{aligned}
$$

Once the Young's moduli are calculated, the temperature induced variation of frequency can be plotted assuming that the frequency $(f)$ of the extensional resonator approximately follows the equation below:

$$
f=\frac{1}{2 w} \sqrt{\frac{E}{\rho}}
$$

where $\rho$ is silicon's density and $w$ is the width of the fundamental mode lateral-extensional resonator. The simulated frequency vs. temperature at three different doping concentration are plotted in Fig. 2 for devices aligned to [110] and [100] orientation respectively. From these plots it is clear that TCF for the devices aligned to [110] direction is approximately linear and does not strongly depend on the doping concentrations $(\mathrm{N})$ when $\mathrm{N}$ ranges from $3 \mathrm{e} 19$ to $5 \mathrm{e} 19 \mathrm{~cm}^{-3}$. In contrast for the devices aligned to [100] direction the curves exhibit a quadratic dependency on temperature with a zero TCF at a turnover temperature.
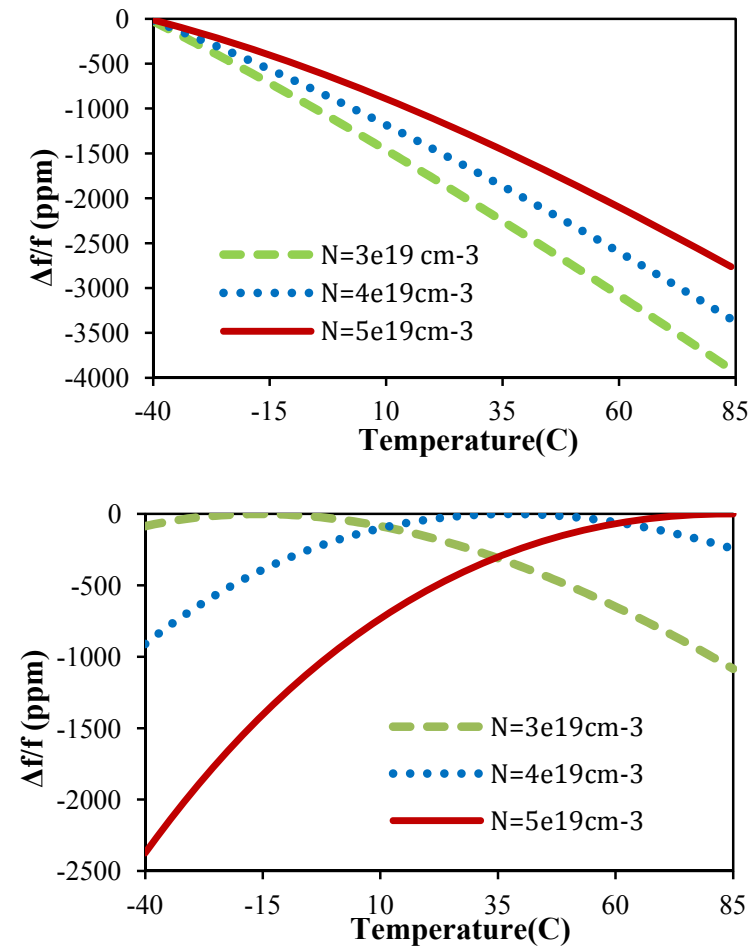

Figure 2: Theoretical temperature-induced frequency variation in two extensional silicon resonators aligned to [110] (top) and [100] (bottom) for three different n-type doping concentrations.
It is observed that the turnover temperature in the [100] plots are a sensitive function of doping concentration. We have used these results to choose a doping concentration for our starting substrates that helps us in validating the existence of turnover temperature in a TPoS resonator aligned to the [100] direction. Although, in preparation of these plots the effect of device thickness and the piezoelectric film (i.e. aluminum nitride) are not considered, the silicon properties are assumed to dominate the overall performance of the device.

\section{SIMULATION}

To better predict the effect of doping concentration in a TPoS resonator and specifically examine the effect of device thickness on the temperature-induced frequency variation, finite element eigenfrequency analysis is utilized. A single-crystal silicon slab with an aluminum nitride layer on top is oriented in the chosen crystalline directions, [100] and [110] in COMSOL. As the temperature is swept from $-40^{\circ} \mathrm{C}$ to $85^{\circ} \mathrm{C}$, the material properties for the single-crystal silicon are changed in accordance to the previously described theory while the AlN Young's modulus is modified as described by Bruls [10].

The plots in Fig. 3 are created for the same three doping concentrations used earlier and show the frequency variation vs. temperature for a TPoS resonator made of $15 \mathrm{um}$ silicon and $1 \mathrm{um}$ AlN. Compared to plots presented in Fig. 2 the TCF appears to be a less sensitive function of doping concentration. This is a more accurate prediction of what is observed in practice considering that the resonance frequency is not a simple function of the unidirectional Young's modulus. Also the turnover temperature has moved to the left for the devices aligned to [100] direction which is expected considering that the TCF of AIN is negative. The turnover temperature is at $-30^{\circ} \mathrm{C},-5^{\circ} \mathrm{C}$, and $40^{\circ} \mathrm{C}$ for the doping concentrations of $3 \mathrm{e} 19,4 \mathrm{e} 19$, and $5 \mathrm{e} 19 \mathrm{~cm}^{-3}$ respectively.
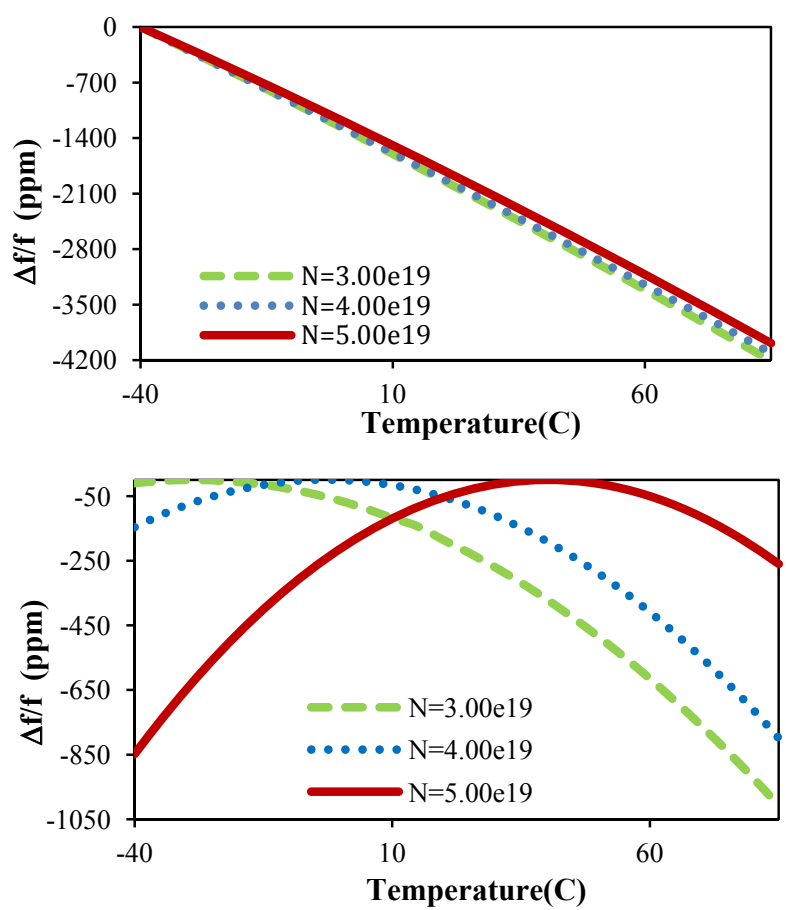

Figure 3: COMSOL-Simulated temperature-induced frequency variation in two TPoS resonators aligned to [110] (top) and [100] (bottom) for three different doping concentration. The silicon and the AlN thicknesses in the device are assumed to be $15 \mu \mathrm{m}$ and $1 \mu \mathrm{m}$ respectively. 
Next, we have also studied the effect of the device thickness on the temperature-induced frequency variation. The two simulated thicknesses, 8 and $40 \mu \mathrm{m}$, are the actual thicknesses of the wafers that are used to fabricated devices. According to the simulated data shown in Fig. 4 the TCF (the slope of the plot) will slightly increase for the devices aligned to [110] direction as the silicon thickness increases. Also, the turnover temperature will gradually shift to the right as the thickness increases for the devices aligned to [100] direction. For example a $20^{\circ} \mathrm{C}$ shift from $20^{\circ} \mathrm{C}$ to $40^{\circ} \mathrm{C}$ is predicted when the thickness is changed from $8 \mu \mathrm{m}$ to $40 \mu \mathrm{m}$ assuming a doping concentration of $4.75 \mathrm{e}^{-3} \mathrm{~cm}^{-3}$. These results suggest that doping levels and the device thickness can be used as design parameters to minimize the overall frequency variation over a given operating temperature range in [100]-aligned resonators.
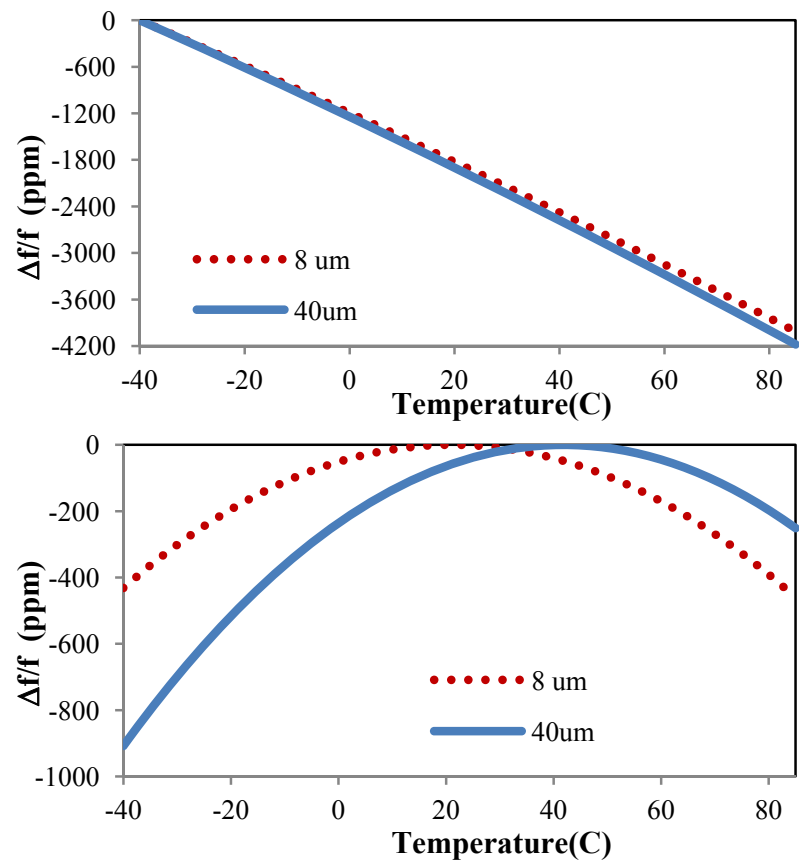

Figure 4: COMSOL-simulated TCF data for two TPoS resonators with the silicon thicknesses of 8 and 40 $\mu \mathrm{m}$ aligned to [110] (top) and [100] directions. The doping concentration for both resonators are assumed to be $4.75 \mathrm{e}^{-19 \mathrm{~cm}^{-3} \text {. }}$

\section{FABRICATION}

The lateral-extensional mode TPoS resonators are fabricated using a five-mask process (Fig. 5). To begin, a stack of molybdenum (Mo), aluminum nitride ( $\mathrm{AlN}$ ), and molybdenum is sputtered on top of the silicon device layer of a silicon-on-insulator (SOI) wafer as seen in Fig. 5a. After deposition, the top Mo layer is dry etched with fluorine-based plasma to form the top electrodes. Access to the bottom electrode is then gained through a wet alkaline etch of the AIN (Fig. 5b). The resonator body is then defined with a series of dry etches through the AlN/Mo and then through the device layer silicon (Fig. 5c). The handle-layer silicon is etched from the backside using a DRIE process. Finally, the structure is released with a buffered oxide etch of the buried silicon dioxide layer (Fig. 5d).

A fabricated resonator can be seen in Fig. 6. Note that the starting substrate in all our experiments are [100] SOI wafers. Therefore resonators aligned to the wafer flat are oriented in the [110] direction. By rotating the resonator $45^{\circ}$ in-plane the device will be aligned to [100] direction.

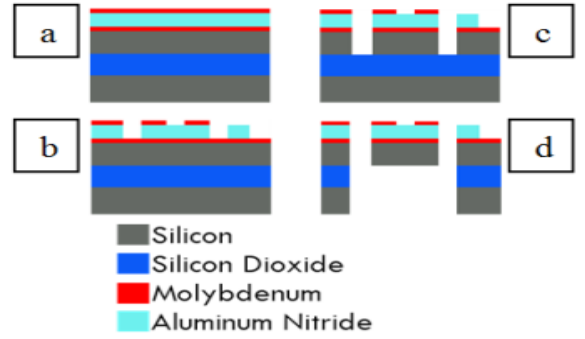

Figure 5: Schematic process flow for TPoS resonators.

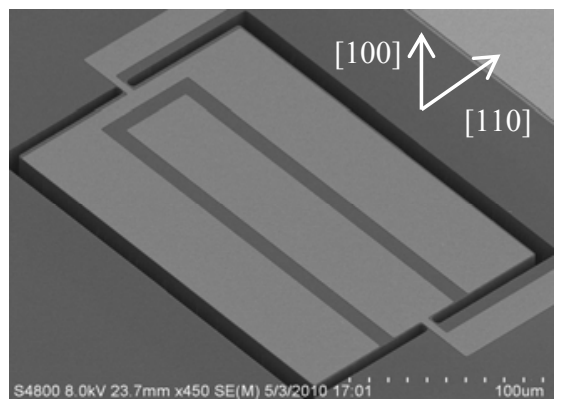

Figure 6: The SEM of a fabricated TPoS resonator aligned to the [110] direction. The arrows in the figure indicate the crystal orientations (this device is a [110]-aligned resonator).

\section{RESULTS}

Three wafers with different device thicknesses, dopants, and dopant concentrations were used in this work (Table 1). As seen in the table the absolute value of the doping concentration is not known for these wafers. However, the $8 \mu \mathrm{m}$ and the $40 \mu \mathrm{m}$ wafers are made from the same starting silicon substrate and their actual doping concentration are expected to be close to each other. Similar resonators were fabricated on each of these wafers oriented in differing crystalline directions. The devices were tested in a Janis cryogenic vacuum probe station using an Agilent network analyzer.

Table 1: The device silicon properties for the utilized SOI wafers.

\begin{tabular}{|l|l|l|l|}
\hline $\begin{array}{l}\text { Thickness } \\
(\mu \mathrm{m})\end{array}$ & Dopant & $\begin{array}{l}\text { Resistivity } \\
(\Omega \mathrm{cm})\end{array}$ & $\begin{array}{l}\text { Doping } \\
\text { Concentration }\left(\mathrm{cm}^{-3}\right)\end{array}$ \\
\hline 8 & $\mathrm{P}$ & $0.001-0.0015$ & $4.97 \mathrm{e} 19-7.85 \mathrm{e} 19$ \\
\hline 15 & As & $0.001-0.003$ & $2.52 \mathrm{e} 19-9.67 \mathrm{e} 19$ \\
\hline 40 & $\mathrm{P}$ & $0.001-0.0015$ & $4.97 \mathrm{e} 19-7.85 \mathrm{e} 19$ \\
\hline
\end{tabular}

Typical frequency responses for two devices fabricated on the $40 \mu \mathrm{m}$ substrate and aligned to [110] and [100] directions are shown in Fig. 7.

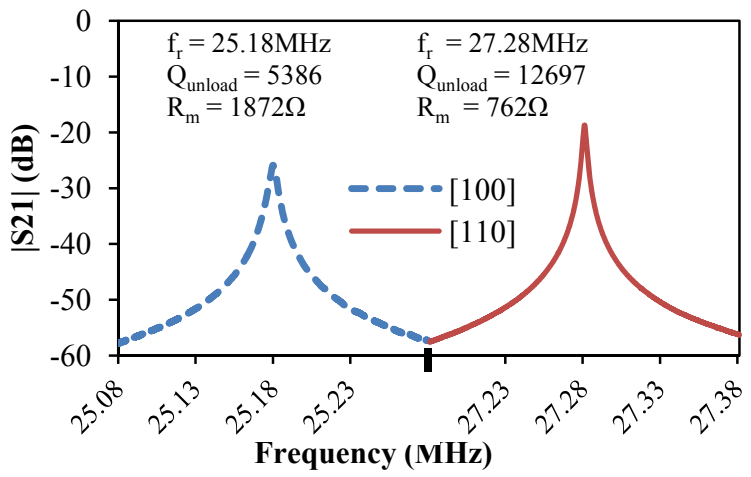

Figure 7: Experimental frequency responses for [100]- (left) and

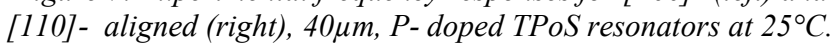


Note that the lower quality factor (and higher motional impedance) of the [100]-aligned resonator is believed to be due to the fact that the dimensions of these resonators are optimized for [110] alignment. As expected, the frequency of the [100]-aligned resonator is lower than the [110]-aligned device.

The resonance frequency is measured over the temperature range of -40 to $85^{\circ} \mathrm{C}$ for the devices fabricated on all three substrates and aligned to both [110] and [100] directions. The results are plotted in Fig. 8. Considering that the effect of thickness on the TCF has been shown to be relatively small, the substantial difference in the measurement from the [100]-aligned $15 \mu \mathrm{m}$ device is considered to be a result of discrepancy in doping concentration. Based on the predictions of Figs. 2 and 3 it can be concluded that the doping concentration of the $15 \mu \mathrm{m}$ wafer is lower than the other two. Also, the difference between the turnover temperatures of the [100]-aligned $8 \mu \mathrm{m}$ resonator $\left(22^{\circ} \mathrm{C}\right)$ and the $40 \mu \mathrm{m}$ device $\left(33^{\circ} \mathrm{C}\right)$ is believed to be a result of the thickness difference. Therefore, the prediction in Fig. 4 is experimentally validated. One may argue that this shift could be a result of a slightly higher doping concentration in the $8 \mu \mathrm{m}$ wafer. This conclusion can be dismissed upon investigation of the results from the [110]-aligned devices. A higher doping concentration should have resulted in a lower TCF for the [110]-aligned $8 \mu \mathrm{m}$ device compared to the $40 \mu \mathrm{m}$ counterpart based on the theoretical analysis shown in Fig. 2 and Fig. 4. This is in contrast with the measurement. Therefore, the data presented in Fig. 8 validates that the turnover temperature shifts to the right as the device thickness and the doping concentration increases. More importantly, the [100]-aligned $8 \mu \mathrm{m}$ thick resonator exhibits an overall frequency variation of $148 \mathrm{ppm}$.
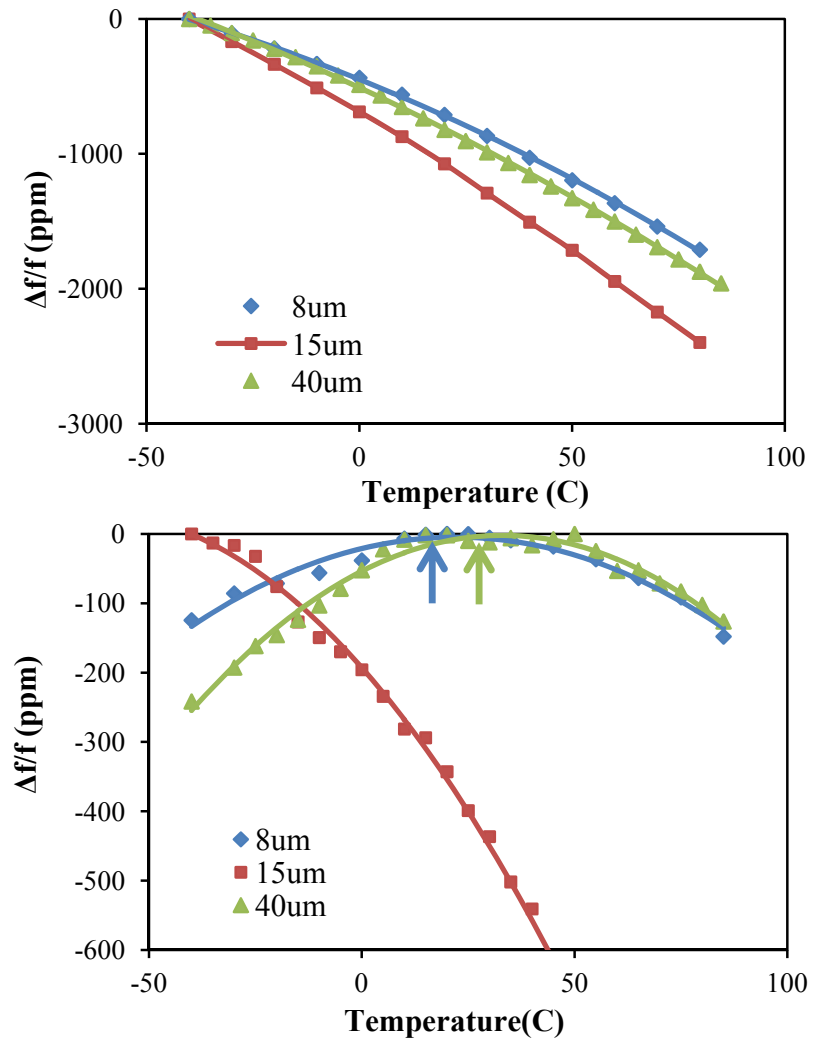

Figure 8: Experimental TCF data for three [110]-aligned and three [100]-aligned resonators fabricated on SOI wafers with varying thicknesses. The results are the first observation of turnover temperature in silicon resonators and agrees with the theoretical prediction about the effect of doping and the device thickness .

\section{CONCLUSION}

In this work, we have investigated the effects of doping levels, device thickness, and device orientation on temperature stability in n-type doped lateral-extensional silicon resonators. The theoretical analysis and simulated data predict that a turnover temperature exists for highly-doped silicon resonators and that the turnover temperature is a function of orientation and doping concentration. We have also shown that for a given doping concentration the turnover temperature can be adjusted by device thickness. Moreover, we have experimentally validated that these theoretical predictions using thin-film piezoelectric-on-silicon (TPoS) resonators as the implementation platform. We have reported a $25 \mathrm{MHz}$ TPoS resonator that exhibits a turnover temperature at $22^{\circ} \mathrm{C}$ and an overall frequency variation of less than $150 \mathrm{ppm}$ over the industrial temperature range $\left(-40^{\circ} \mathrm{C}\right.$ to $\left.85^{\circ} \mathrm{C}\right)$. This is a significant reduction in the previously reported frequency variations from uncompensated silicon resonators which is more than $3000 \mathrm{ppm}$ over the same range of temperature.

\section{REFERENCES}

[1] B. P. Harrington and R. Abdolvand, "In-plane acoustic reflectors for reducing effective anchor loss in lateralextensional MEMS resonators," Journal of

Micromechanics and Microengineering, vol. 21, no. 8, p. 085021, Aug. 2011.

[2] B. P. Harrington, M. Shahmohammadi, and R. Abdolvand, "Toward ultimate performance in GHZ MEMS resonators: Low impedance and high Q," in 2010 IEEE 23rd International Conference on Micro Electro Mechanical Systems (MEMS), 2010, pp. 707-710.

[3] M. Shahmohammadi, "Low jitter thin-film piezoelectricon-substrate oscillators," (FCS), 2010 IEEE, pp. 613-617, 2010.

[4] J. R. Vig, F. L. Walls, "Fundamental limits on the frequency instabilities of quartz crystal oscillators," Proceedings of the IEEE Frequency Control Symposium, pp.506-523, 1-3 Jun 1994.

[5] R. Melamud, B. Kim, S. A. Chandorkar, M. A. Hopcroft, M. Agarwal, C. M. Jha, and T. W. Kenny "Temperaturecompensated high-stability silicon resonators," Applied Physics Letters, vol. 90, no. 24, p. 244107, 2007.

[6] A. K. Samarao and F. Ayazi, "Temperature compensation of silicon micromechanical resonators via degenerate doping," 2009 IEEE International Electron Devices Meeting (IEDM), pp. 1-4, Dec. 2009.

[7] G. Ho, R. Abdolvand, A. Sivapurapu, S. Humad, and F. Ayazi, "Piezoelectric-on-silicon lateral bulk acoustic wave micromechanical resonators," Journal of Microelectromechanical Systems, vol. 17, no. 2, pp. 512520, 2008.

[8] R. Keyes, "Electronic Effects in the Elastic Properties of Semiconductors," Solid State Physics, pp. 37-90, 1968

[9] J. J. Hall, "Electronic effects in the elastic constants of ntype silicon," Physical Review, vol. 161, no. 3, pp. 756 761, 1967.

[10] R. J. Bruls, H. T. Hintzen, G. D. With, and R. Metselaar, "The temperature dependence of the Young's modulus of MgSiN2, AlN and Si3N4," Journal of the European Ceramic Society, vol. 21, pp. 263-268, 2001.

\section{CONTACT}

*Mohsen Shahmohammadi, tel: +1-918-594-8642;

Mohsen.shahmohammadi@okstate.edu 


\title{
ZERO BIAS OPERATION OF THERMAL-PIEZORESISTIVE MICROMECHANICAL RESONATORS VIA INTERNAL ELECTROMECHANICAL MIXING
}

\author{
A. Rahafrooz and S. Pourkamali \\ Department of Electrical and Computer Engineering, University of Denver, Denver, CO, USA
}

\begin{abstract}
This work presents a novel operation technique for single crystalline silicon, thermal-piezoresistive resonators. This transduction technique takes advantage of internal electromechanical mixing of the resonant structure that eliminates the need for application of any bias current for resonator operation. In all previous works, a combination of DC bias and AC actuation signal was used for resonator operation. Here, it is demonstrated that such devices can exhibit resonant peaks in their electrical frequency response at half their mechanical resonant frequency by application of only a single AC actuation without the need for any power supply or biasing components. This allows engagement of such devices as electronic components with zero DC power consumption, which has been one of the main drawbacks of thermal-piezoresistive micro-resonators.
\end{abstract}

\section{INTRODUCTION}

MEMS resonators are emerging as modern frequency selective components gradually replacing the conventional quartz resonators in different markets [1]. The more popular classes of piezoelectric and electrostatic MEMS resonators have been welldeveloped over the past decade. However, the focus on activelytransduced micromechanical resonators, such as thermalpiezoresistive devices, has been quite limited. Such devices have received some attention over the past three years as their potential for high frequency and low power operation has been demonstrated [2]. In addition, they have the advantage of being electrically active devices (similar to transistors) providing unique capabilities such as self-sustained oscillation and self-Qenhancement [3-5]. Such capabilities along with their simple structure and low cost fabrication process make them suitable candidates for a wide range of RF applications. Moreover, unlike electrostatic resonators, thermal-piezoresistive resonators do not require any submicron transduction gaps. Therefore, they can be used as more robust and reliable sensors for particulate sensing applications [6] among others. All these benefits and potentials for improvement trigger interest in their further investigation.

Thermal-piezoresistive resonators are usually operated by applying a combination of DC and AC currents through their structure in order to thermally actuate them at the same frequency as the input signal. In addition, a DC current is required to turn the periodic change in the resistance of their sensing elements into a measurable fluctuating voltage to detect their mechanical vibration. In some thermal-piezoresistive resonators, different electrically isolated parts of the resonator are dedicated to thermal actuation and piezoresistive sensing $[7,8]$ while in others, thermal actuation and piezoresistive sensing are carried out simultaneously by a common part of the resonator [2]. In the former method, two independent bias currents pass through the actuating and sensing elements, whereas the later only requires a single DC bias current. Each of these methods has their own advantages and disadvantages but their common major drawback is the requirement of a DC bias current constantly burning valuable power, even when the resonator is at rest (not vibrating).

Here, a novel operation mechanism for monolithic thermalpiezoresistive resonators with common actuation and sensing element is demonstrated. The new technique takes advantage of the electromechanical mixing inside the resonator structure and waives the need for applying any bias current. In addition, this technique does not require any supporting electronic components such as bias resistors and capacitors. Therefore, it further decreases the overall power consumption and foot-print of the overall circuit. In order to fully investigate the zero bias operation of monolithic thermalpiezoresistive resonators, their conventional operation mechanism [2] is first described. Then, the new operation technique is explained and compared with the conventional one.

\section{OPERATION CONCEPT}

\section{A. Conventional Operation}

Figures 1a shows the required electrical setup to operate thermal-piezoresistive resonators using the conventional technique. In this method, upon application of a combination of AC actuation and DC bias currents $\left(I_{d c}+i_{a c} \cos \left(2 \pi f_{a}\right)\right)$ to the resonator structure an ohmic loss is generated, This ohmic loss has a squared relationship with the applied current and consists of three frequency components at $\mathrm{DC}\left(R_{A}\left(I_{d c}{ }^{2}+i_{a c}{ }^{2} / 2\right)\right)$, actuation frequency of the AC current $\left(2 R_{A} I_{d c} \cdot i_{a c} \cos \left(2 \pi f_{a}\right)\right)$ and twice the actuation frequency $\left(R_{A} i_{a c}{ }^{2} \cos \left(4 \pi f_{a}\right) / 2\right)$ where $R_{A}$ is the electrical resistance of the resonator. Due to the higher resistance of the two narrow beams in the middle of the structure, most of the ohmic power loss happens in them. The three resulting components translate into proportional temperature changes or fluctuations with the same frequencies. Each of the temperature components induces thermal stress in the mechanical structure and therefore mechanical forces at the same frequencies inside the narrow beams (Figures 2a). The alternating temperature of the beams causes their periodic expansion and contraction, actuating the mechanical structure at two different frequencies of $f_{a}$ and $2 f_{a}$. If the frequency of the $\mathrm{AC}$ input current $f_{a}$ matches the first in-plane extensional mode resonant frequency of the structure $f_{0}$, it starts resonating. During resonance, as the length and stress in thermal actuating elements fluctuates, their resistance is modulated due to the piezoresistive effect. Such periodic change in resistance $\left(r_{a c} \cos \left(2 \pi f_{0}\right)\right)$ of the thermal actuators is proportional to the vibration amplitude of the structure. Since a constant DC bias current is passing through the thermal actuators, this change in resistance is turned into a measurable $\mathrm{AC}$ output voltage $\left(I_{d c} \cdot r_{a c} \cos \left(2 \pi f_{0}\right)\right)$. In addition, the AC actuation current $\left(i_{a c} \cos \left(2 \pi f_{0}\right)\right)$ along with periodic changes in resistance of the piezoresistors gives birth to two voltage components at DC $\left(i_{a c}, r_{a c} / 2\right)$ and twice the resonant frequency of the structure $\left(i_{a c} \cdot r_{a c} \cos \left(4 \pi f_{0}\right) / 2\right)$. Among the three output frequency components, only the one with the same frequency (green arrow in figure $2 \mathrm{a}$ ) as the input $\mathrm{AC}$ actuation current is picked up by the network analyzer and shows up in the transmission frequency response.

In terms of power consumption, in order to create a fairly constant DC bias current, the resonator is connected in series with two $(\sim 5 \mathrm{X})$ larger resistors with the whole combination connected to a power supply. The more appropriate way is to use a DC current source. However, both of these configurations consume some DC power which is usually larger than that of the resonator. 
a)

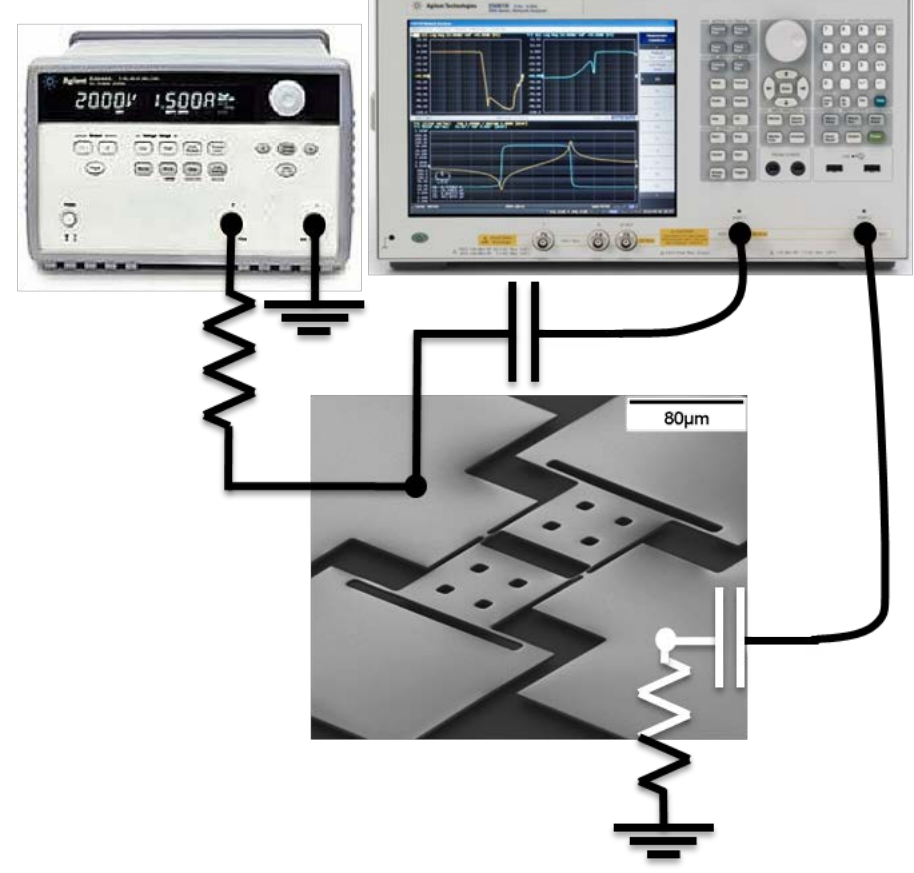

b)

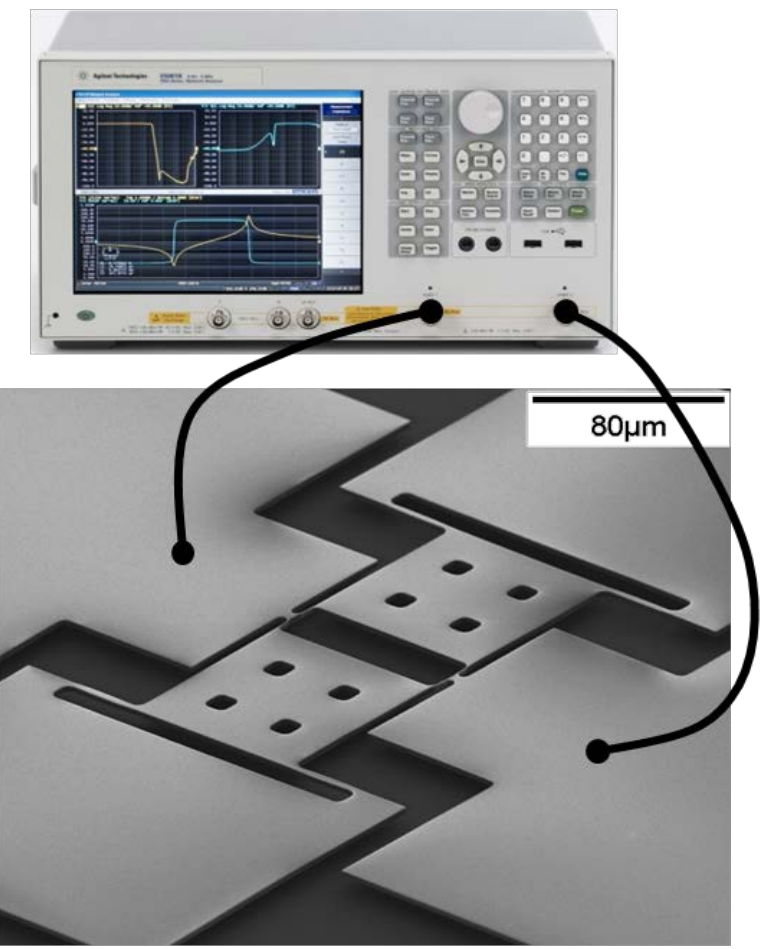

Figure 1: Schematic diagrams of the electrical connections for operation of thermal-piezoresistive resonators by application of a) a combination of DC and AC voltages/currents using a DC power supply, a network analyzer, two bias resistors and two decoupling capacitors and b) a pure AC signal at half the mechanical resonant frequency of the resonator by directly connecting the two terminals of the network analyzer to the resonator pads. In both cases, the network analyzer measurers the transmission data and the scanning electron microscope view is a fabricated $3.47 \mathrm{MHz}, 3.5 \mu \mathrm{m}$ thick resonator tested to demonstrate the concept.

\section{B. Zero Bias Operation}

In the new configuration, the resonator is directly connected to the $\mathrm{AC}$ source (network analyzer in this case) (Figure 1b). Upon application of the $\mathrm{AC}$ actuation current $\left(i_{a c} \cos \left(2 \pi f_{a}\right)\right)$ to the structure, the generated ohmic power $\left(i_{a c}{ }^{2}\left[1+\cos \left(4 \pi f_{a}\right)\right] / 2\right)$ and its resulting temperature and thermal expansion fluctuations will have two frequency components; one at DC and the other at twice the applied input frequency. If the frequency of the input $\mathrm{AC}$ current is half the resonant frequency of the resonator, the thermally generated force will have a component at the right frequency $f_{0}$ to excite the structure. During the mechanical vibration and due to the piezoresistive effect there will be periodic changes in the electrical resistance of the resonator at the same frequency as the resonant frequency of the structure $\left(r_{a c} \cos \left(2 \pi f_{0}\right)\right)$. Such resistance fluctuations are then mixed by the actuation current that has half the resonant frequency $\left(i_{a c} \cos \left(\pi f_{0}\right)\right)$. This modulation gives birth to two frequency components at $f_{0} / 2$ and $3 f_{0} / 2$ (Figure $2 \mathrm{~b}$ ). The two output voltage $\left(v_{\text {out }}\right)$ frequency components can be derived to be:

$$
\begin{aligned}
& v_{o u t}=r_{a c} \cos \left(2 \pi f_{0}\right) \cdot i_{a c} \cos \left(\pi f_{0}\right)= \\
& \frac{r_{a c} \cdot i_{a c}}{2}\left(\cos \left(\pi f_{0}\right)+\cos \left(3 \pi f_{0}\right)\right)
\end{aligned}
$$

Therefore, due to this internal electromechanical mixing there will be an electrical component at half the resonance frequency changing the transmission characteristics of the resonator as seen by the electrical components connected to it at resonance. As a result, in this technique, instead of having a resonant peak at resonant frequency, a peak appears in the electrical frequency response at exactly half the mechanical resonant frequency.
Figure 2 compares the operation details of both transduction mechanisms using block-diagram demonstration of the thermoelectromechanical model of such resonators [2]. It shows all the generated frequency components of the main physical parameters involved in the resonator transduction. Although in both techniques the structure resonates at its resonant frequency, but during zero bias operation the frequency of the input and output electrical signals is half the mechanical resonant frequency of the resonator. Moreover, unlike the conventional operation, in zero bias operation the peak amplitude is dependent on the input signal amplitude making it nonlinear.

\section{DEVICE FABRICATION AND MEASUREMENT RESULTS}

In order to show the new operation concept a dual-plate thermal-piezoresistive resonator was used. The resonator has been fabricated on a low resistivity N-type SOI substrate with the device layer thickness of $3.5 \mu \mathrm{m}$ using a single mask SOI MEMS process. Its thermal actuators were aligned along the $<100>$ crystalline orientation of silicon to obtain the highest piezoresistive coefficient [10] and provide a stronger transduction. Figure 1 shows the scanning electron microscope view of the fabricated $3.47 \mathrm{MHz}$ resonator along with the required electronic connections for both operation mechanisms. In conventional configuration, two bias resistors of $330 \Omega$ and two de-coupling capacitors of $0.1 \mu \mathrm{F}$ were used. DC and AC signals were supplied by a digital power supply and a network analyzer respectively. In both cases the transmission $\mathrm{S}_{21}$ parameters were recorded by the network analyzer.

Figure 3 demonstrates the transmission frequency responses of the $3.47 \mathrm{MHz}$ resonator obtained using both operation 
a)

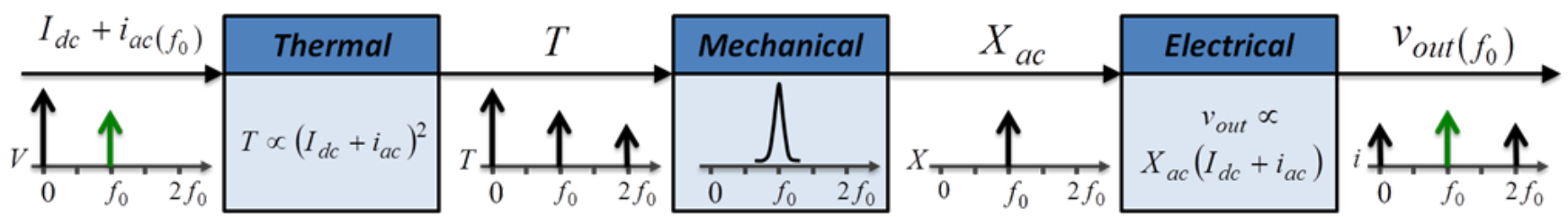

b)

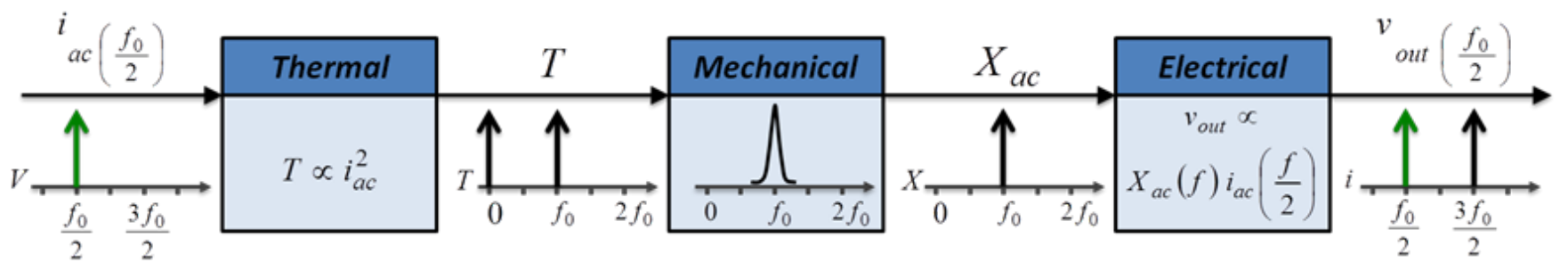

Figure 2: Block-diagram demonstration of thermal-piezoresistive operation of a resonant structure. a) Operation with DC bias: Upon the application of a combination of DC and AC currents, the generated power has a component at the same frequency as the AC input current that can actuate the mechanical structure into resonance. The mechanical vibration of the resonator modulates the resistance of the resonator due to the piezoelectric effect. The fluctuating electrical resistance in the resonator is then modulated by the DC current leading to an output component with the same frequency as the input $A C$ current that can be detected by the network analyzer. b) Operation without DC bias: By applying an AC current at half the resonant frequency of the resonator, there will be a power component at the resonant frequency of the resonator. This power in turn generates a thermal force at the same frequency that actuates the mechanical structure into resonance. During the vibration, the fluctuations of the resistance of the resonator with the resonant frequency of the resonator are modulated by the AC input current providing two different frequency components at the output. The component with half the resonant frequency can be detected. In this case the network analyzer shows a resonant frequency peak at exactly half of the resonant mechanical frequency of the structure. Green arrows show input/output frequency pairs with the same frequencies that are being generated or picked up by the network analyzer to measure the transmission data. In this figure, variables $I_{d c}, i_{a c}$ and $v_{\text {out }}$ are the DC bias current, input $A C$ voltage, input $A C$ current, and $A C$ output voltage of the resonator, $f_{0}, T$ and $X_{a c}$ are the resonant frequency, temperature and mechanical vibration amplitude of the resonator respectively.

techniques. As expected, the frequency response attained from the zero-bias technique (Figure $3 b$ ) shows a resonant peak at exactly half the mechanical resonant frequency of the resonator (Figure 3a). Due to the negative piezoresistive coefficient of the structural material (N-type silicon) [10] in both cases the resonant peaks are pointing downwards. In conventional operation, power of the network analyzer was kept at $0 \mathrm{~dB}$ and the transmission data was recorded at different DC bias currents. On the other hand, in zero bias operation, transmission data were recorded at different applied

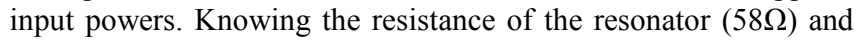
the power output of the network analyzer, the AC current passing through the resonator was calculated for each frequency response (Figure 3b). Figure 3 shows different transmission feedthrough floor levels of $-5.5 \mathrm{~dB}$ and $-3.7 \mathrm{~dB}$ for the conventional and zero bias operation techniques respectively. This change is a result of having different test circuits and consequently different overall impedances between the two ports of the networks analyzer.

It should be noted that in zero bias operation, the only source of power that can thermally actuate the resonator is the AC current (voltage) output the network analyzer. The motional signal for such resonators usually increases by passing more current through them [2]. Therefore, using a highly doped resonator structure can significantly help reduce the electrical resistance of the resonator and increase the current passing through it.

\section{CONCLUSIONS AND FUTURE WORK}

A new operation technique for single crystalline silicon, thermalpiezoresistive resonators was demonstrated that does not require any DC bias current. Due to internal electromechanical mixing, a single AC current is enough to operate the resonator. It is demonstrated that such devices can operate in the electrical domain at half their mechanical resonant frequency without the need for any power supply or biasing components. Therefore, they can potentially be utilized as electronic components without any DC power consumption. In addition, since this technique does not require any supporting electronic components, it further decreases the overall power consumption and circuit foot-print. The demonstrated internal mixing can also be combined with the selfsustained oscillation capability of such resonators to create new opportunities in timekeeping, signal mixing and mass sensing applications.

\section{ACKNOWLEDGEMENTS}

This work was supported by National Science Foundation under grants \#1056068 and \#0923518. 
a)

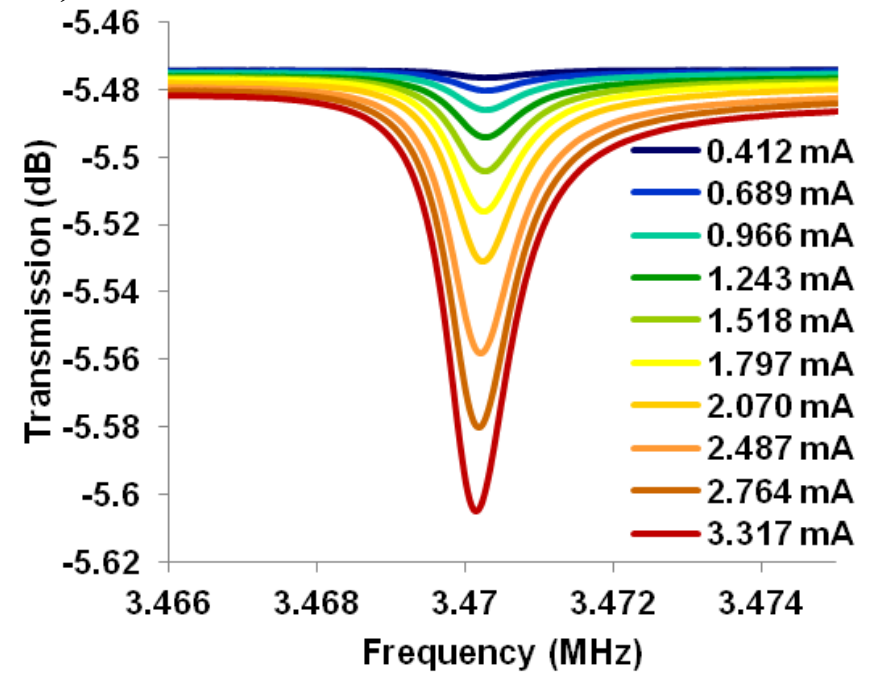

b)

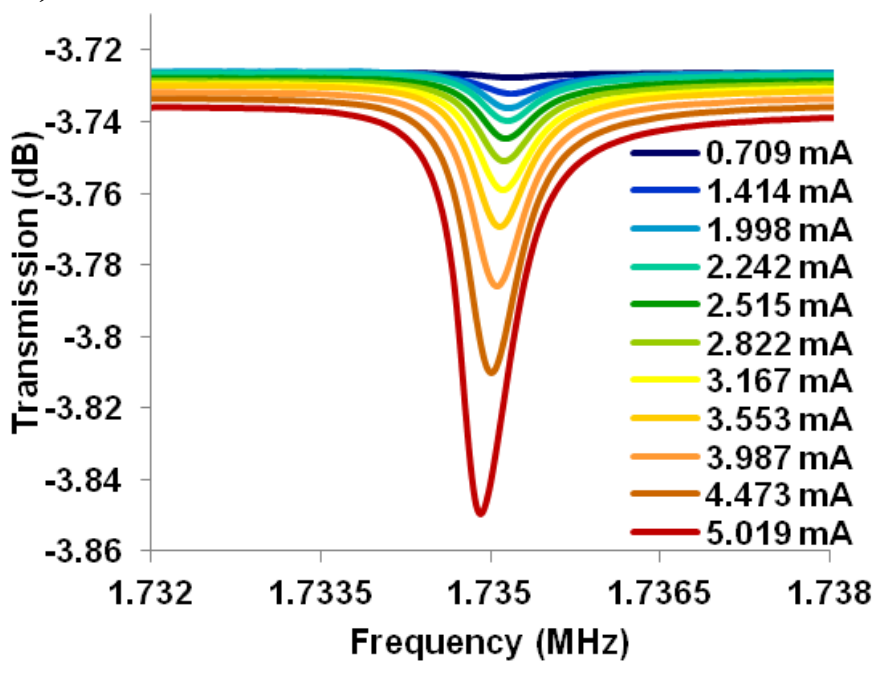

Figure 3: Measured frequency response of the resonator shown in figure 1 directly taken from the network analyzer, a) using the test configuration showed in figure la by application of DC and AC currents at different bias currents (The internal power of the network analyzer was set to $0 \mathrm{~dB}$ which translates into an effective AC current of $2.515 \mathrm{~mA}$ through the resonator), b) using the test configuration shown in figure $1 b$ by applying only an AC signal with different input power levels of -11, -5, -2, -1, 0, 1, 2, 3, 4, 5, and 6 dB that translates into effective AC currents of 0.709 to $5.019 \mathrm{~mA}$ as is shown in the plot. The physical resistance of the resonator is $58 \Omega$. The change in feedthrough level between the two methods is a result of having different testing circuits and eventually different impedances.

\section{REFERENCES}

[1] F. Ayazi, "MEMS for Integrated Timing and Spectral Processing," Invited Paper, Proc. IEEE Custom Integrated Circuits Conference (CICC), pp. 65-72, 2009.

[2] A. Rahafrooz and S. Pourkamali, "High-Frequency Thermally Actuated Electromechanical Resonators With Piezoresistive Readout," IEEE Transactions on Electron Devices, vol.58, no.4, pp.1205-1214, April 2011.

[3] P. G. Steeneken, K. Le Phan, M. J. Goossens, G. E. J. Koops, G. J. A. M. Brom, C. van der Avoort and J. T. M. van Beek, "Piezoresistive heat engine and refrigerator," Natrure physics, Vol. 7, No. 4, pp. 354-350, Apr. 2011.

[4] A. Rahafrooz and S. Pourkamali, "Fully micromechanical piezo-thermal oscillators," 2010 IEEE International Electron Devices Meeting (IEDM), pp.7.2.1-7.2.4, Dec. 2010.

[5] A. Rahafrooz and S. Pourkamali, "Active self-Q-enhancement in high frequency thermally actuated M/NEMS resonators," in Proc. 24rd IEEE MEMS, Cancun, Mexico, Jan, 2011, pp. 760-763.

[6] A. Hajjam, J. C. Wilson, A. Rahafrooz, S. Pourkamali, " Fabrication and characterization of thermally actuated micromechanical resonators for airborne particle mass sensing: II. Device fabrication and characterization," Journal of Micromechanics and Microengineering, vol.20, no.12, Article Number: 125019, Dec. 2010.

[7] J. H. Seo and O. Brand, "High Q-Factor In-Plane-Mode Resonant Microsensor Platform for Gaseous/Liquid Environment," JMEMS 2008, Vol. 17, issue 2, pp. 483-493.

[8] I. Bargatin, I. Kozinsky, and M. L. Roukes, "Efficient Electrothermal Actuation of Multiple Modes of Highfrequency Nanoelectromechanical Resonators," Applied Physics Letters, vol. 90, 2007.

\section{CONTACT}

Amir Rahafrooz, 2390 S. York St., Denver, Colorado, USA, Phone: +1-303-871-2471, Email: amir.rahafrooz@du.edu 


\title{
A WIDEBAND THERMO-ACOUSTIC SOUND GENERATOR ON NANOPOROUS POLYMERIC SUBSTRATE
}

\author{
A. Kim, G. Chitnis, S. H. Song, and B. Ziaie*
}

Birck Nanotechnology Center, Purdue University, USA

\section{INTRODUCTION}

Ultrasonic transducers operating in air are important components in systems used for ranging, surveillance, object identification, and air flow measurement [1]. In ranging and object identification, a wideband transducer is needed in order to achieve a better axial resolution. Some of the most commonly used ultrasonic sources are inherently resonant and hence narrowband. In case of thermo-acoustic sound generator (thermophone) electrical energy is converted to sound waves through Joule heating of a resistor without any mechanical vibration, thus allowing for a wideband operation [2]. Recently, there has been a renewed interest in this mode of sound generation. These efforts include various structures consisting of a thin conductor (e.g., aluminum, carbon nanotubes, ITO, and grapheme) deposited on thermally insulating substrates such as porous silicon, polyethylene-terephthalate, and paper [3-6]. Many of these methods rely on complicated material and fabrication processes, which are not compatible with standard CMOS and other common microfabrication techniques. Here, we report on a thermophone using a thin metallic layer deposited on a nanoporous polymeric substrate. The fabrication method is simple, low-temperature, and compatible with active electronics.

\section{DEVICE STRUCTURE AND FABRICAION}

The thermophone (Figure 1) consists of a mechanical support (silicon, glass, etc.) coated with a thermally insulating nanoporous polymeric layer covered by an electrically conductive thin metal layer. When current is passed through the resistor, temperature of the metal rises due to Joule's heating, thus heating the surrounding air. Since the thin metal layer has very low heat capacity, when excited with alternating current (frequency f), it results in rapid variation of temperature leading to expansion and contraction of surrounding air at doubled frequency $(2 \mathrm{f})$, generating sound waves.

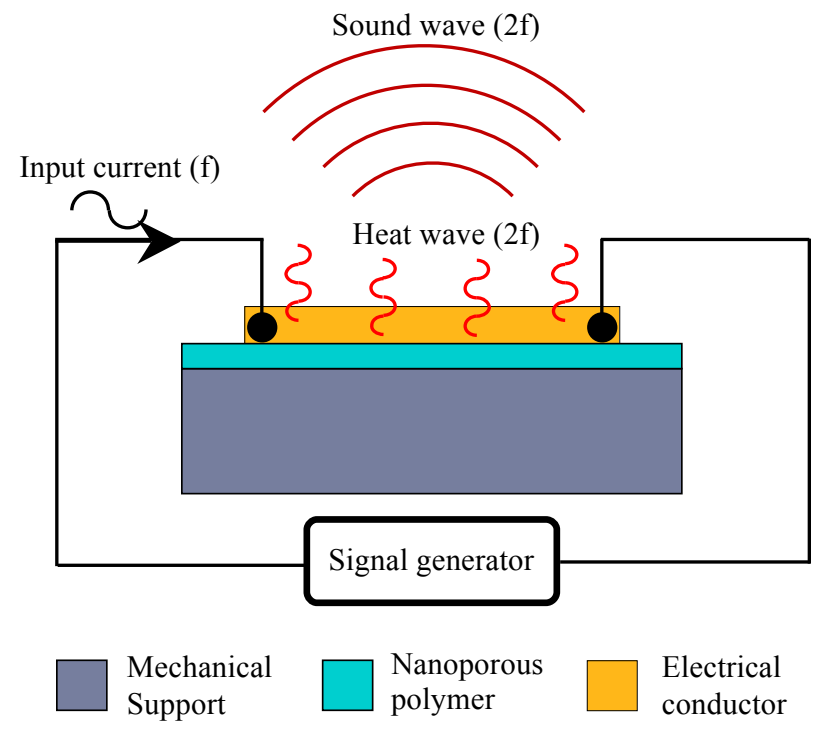

Figure 1: Schematic of the thermo-acoustic ultrasonic sound generator

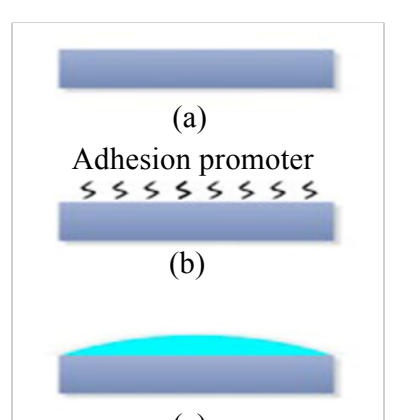

(c)

Glass (pyrex 7740)

Hydrogel

Figure 2: Fabrication process

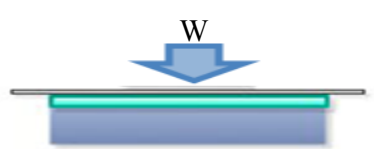

(d)

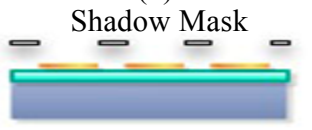

(e)

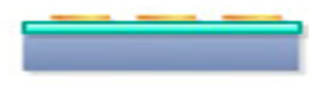

(f)

$\operatorname{Metal}(\mathrm{Au})$

The performance of thermophone is highly dependent on the properties of thermal insulating layer. This layer helps in efficient transfer of heat to the air. A nano-material with adjustable porosity (and thus thermal conductivity) yields a better control over device performance.

Figure 2 shows the fabrication process. For our experiments, we used glass substrate and air-dried hydrogel [Poly(mAA-coAAm)] as the nanoporous layer [7]. One can easily control the porosity of the gel by adjusting the ratio of cross-linker of its precursors. Although air-drying leads to collapse of porous structure, some porosity is expected to be retained (complete retention of porosity can be achieved through critical point drying). The thickness of the hydrogel can be controlled by adjusting the weight applied while curing the gel (a weight of $9 \mathrm{~g}$ resulted in 14 $\mu \mathrm{m}$ thick gel after drying). Finally, a thin layer of gold $(40 \mathrm{~nm})$ was patterned by shadow masking. Figure 3 shows photograph of a fabricated device.

\section{RESULTS AND DISCUSSION}

The hydrogel based thermophone was characterized in an anechoic room using a high-frequency condenser microphone. Figure 4 depicts the experimental set up. A sinusoidal voltage signal $\left(4.5 \mathrm{~V}_{\mathrm{pp}}\right)$ was applied across the serpentine resistor and generated acoustic signal was recorded at a distance of $30 \mathrm{~mm}$.

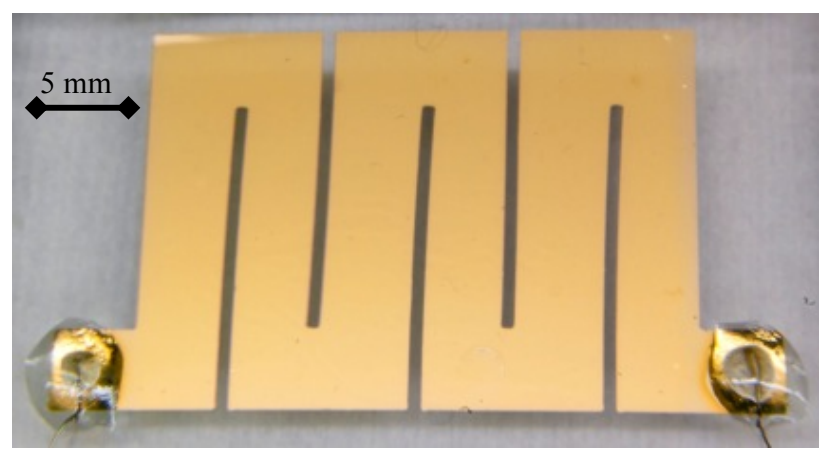

Figure 3: Optical photograph of fabricated prototype Hilton Head Island, South Carolina, June 3-7, 2012 


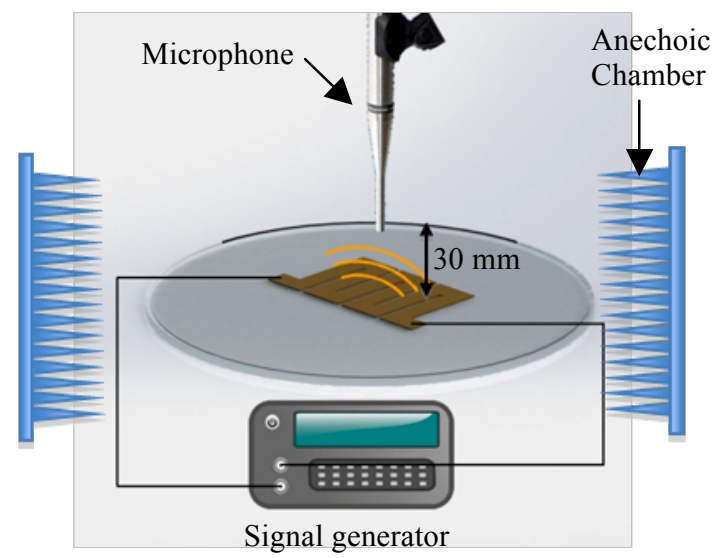

Figure 4: Schematic of experimental set up

Figure 5(a) shows input and output signal on the same plot with the output sound wave double the input signal frequency. Figure 5(b) shows sound pressure level (SPL) obtained at various frequencies. Input power was kept constant at $0.511 \mathrm{~W}$ for all the measurements. The performance of the device remains consistent up to $80 \mathrm{kHz}$ (limited by the microphone used in the experiment).

It is not straightforward to compare the efficiency of our device with other acoustic devices due to difference in powering mechanism. Output SPL of our device is proportional to input power, while that of piezoelectric or electrostatic based acoustic devices is proportional to input voltage. In case of electromagnetic micro-speaker [8], SPL of $93 \mathrm{~dB}$ at $5 \mathrm{kHz}$ has been reported at input voltage of $1.5 \mathrm{~V}$ (320 $\mathrm{mW}$ power). The piezoelectric micromachined ultrasonic transponder shows a peak SPL of $85 \mathrm{~dB}$ at 0.4 $\mathrm{V}$ and $214 \mathrm{kHz}$ [9]. Unlike thermophone, electromagnetic and piezoelectric based devices, which rely on mechanical vibration,

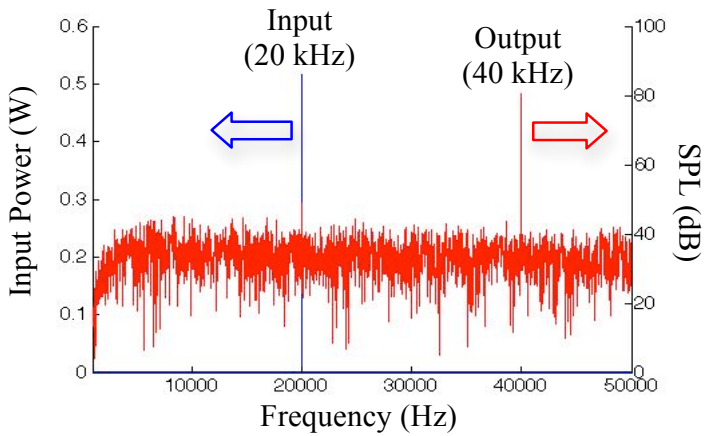

(a)

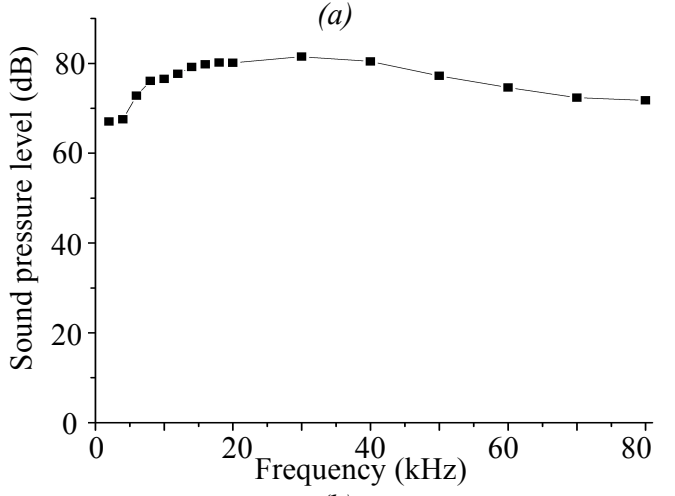

(b)

Figure 5. (a) SPL and Input power relations. Input at $20 \mathrm{kHz}$ generates output at $40 \mathrm{kHz}$, which double the frequency, (b) Frequency response of $S P L$. show a peak performance near the resonance but output signal attenuates before and after the resonant frequency. The performance of thermophone consistent over at least $80 \mathrm{kHz}$ is a major advantage over these conventional ultrasonic transponders used for imaging or in-air ranging applications. Further, the structure and fabrication process presented here is simple enough to be adapted for miniaturization of the device. Although frequency doubling behavior of thermophone is somewhat inconvenient for audible speaker application, one can easily circumvent the problem by using an electronic circuit that reduces input frequency to half before the input [10].

\section{CONCLUSIONS}

We presented a simple, low-temperature method for fabricating a wide-band $(>80 \mathrm{kHz})$ thermo-acoustic sound generator on a nanoporous polymeric substrate. We were able to achieve up to $80 \mathrm{~dB}$ of sound pressure level with input power of only $0.511 \mathrm{~W}$. One can easily control the parameters such as gel thickness, porosity, and metal thickness to further improve the performance. Wide-band ultrasonic performance, simplicity of structure and scalability of the fabrication process make this device suitable for a wide range of ranging and imaging applications.

\section{REFERENCES}

[1] W. Manthey, N. Kroemer, and V. Magori, "Ultrasonic transducers and transducer arrays for applications in air," Measurement Science and Technology, vol. 3, no. 3, pp. 249261, Mar. 1992.

[2] H. D. Arnold and I. B. Crandall, "The thermophone as a precision source of sound," Physical Review, 1917.

[3] H. Shinoda, T. Nakajima, K. Ueno, and N. Koshida, "letters to nature Thermally induced ultrasonic emission from porous silicon," Reactions, vol. 400, no. August, pp. 853-855, 1999.

[4] L. Xiao et al., "Flexible, stretchable, transparent carbon nanotube thin film loudspeakers.," Nano letters, vol. 8, no. 12, pp. 4539-45, Dec. 2008.

[5] H. Tian et al.,"Poly(3,4-ethylenedioxythiophene)poly(styrenesulfonate)-based organic, ultrathin, and transparent soundemitting device," Applied Physics Letters, vol. 99, no. 23, p. 233503, 2011.

[6] H. Tian et al., "Transparent, flexible, ultrathin sound source devices using Indium Tin oxide films," Applied Physics Letters, vol. 99, no. 4, p. 043503, 2011.

[7] Z. Ding, A. Salim, and B. Ziaie, "Squeeze-film hydrogel deposition and dry micropatterning.," Analytical chemistry, vol. 82, no. 8, pp. 3377-82, Apr. 2010.

[8] M. C. Cheng, W. S. Huang, and S. R. S. Huang, "A silicon microspeaker for hearing instruments.," Journal of Micromechanics and Microengineering, vol. 14, no. 7, pp. 859-866. May. 2004.

[9] R. J. Przybyla, S. E. Shelton, A. Guedes, I. I. Izyumin, M. H. Kline, D. A. Horsley and B. E. Boser, "In-Air Rangefinding With an AlN Piezoelectric Micromachined Ultrasound Transducer.," IEEE Sensors Journal, vol. 11, no 11, pp. 2690-2697. Nov. 2011.

[10] R. W. J. Barker, K. P. Corrin, "Sinusoidal-FrequencyHalving Technique.," Electronics Letters, vol. 13, no 7, pp. 201-202, Mar. 1977.

\section{ACKNOWLEDGEMENTS:}

Authors would like to thank Andy Jessop and Prof. Bolton for their help with the acoustic measurements.

\section{CONTACT:}

*B. Ziaie, tel: +1-765-494-0725; bziaie@purdue.edu 


\title{
AN INTEGRATED FIBER-OPTIC MICROFLUIDIC DEVICE FOR DETECTION OF MULTICELLULAR ORGANISM MECHANICS
}

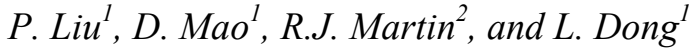

${ }^{1}$ Department of Electrical and Computer Engineering, Iowa State University, Ames, Iowa, US

${ }^{2}$ Department of Biomedical Sciences, Iowa State University, Ames, Iowa, US

\begin{abstract}
A fiber-optic microfluidic device is developed to detect muscular force generated by a nematode worm. The device integrates a single mode (SM) emitting fiber cantilever, a multimode receiving fiber, and a sinusoidal microfluidic channel. The channel is open at multiple troughs and the SM fiber is horizontally placed adjacent to the channel. As a nematode worm swims through the channel, it contacts and deflects the SM fiber, causing to change optical coupling efficiency from the SM to the multimode fiber. The device is capable of detecting worm muscular forces at different troughs of the channel. Multiple force data can be used to verify each other, improving data accuracy and reliability. The minimal detectable muscular force of the device is $0.23 \mu \mathrm{N}$.
\end{abstract}

\section{INTRODUCTION}

Movement of multicellular organisms such as non-pathogenic nematode Caenorhabditis elegans exhibits a sinusoidal pattern, induced by contraction and local bending of cuticle [1]. Study of multicellular organism mechanics is critical for understanding how signals in the neuromuscular system give rise to behavior and how force-sensing organs and sensory neurons work [2]. While many excellent micro-electro-mechanical systems approaches have been developed for sorting, transferring, stimulating, phenotyping, imaging, and screening of C.elegans [3,4], study of multicellular organism mechanics has currently lagged far behind that of cell mechanics. Recently, a piezoresistive cantilever system was developed to analyze muscular force of C.elegans [5], but this microsystem was not able to detect dynamic force of a moving C.elegans nematode. The use of an array of polymeric micropillars overcome this problem [6], but a high resolution and fast speed microscope was required to record dynamic displacement of the pillars. Also, a strain gauge force microsensor was constructed from multiple layers of SU-8 and metal on quartz substrates [7], but substantial room exists for improving force detection resolution and reducing complexity in device structure and fabrication.

\section{DEVICE DESIGN AND PRINCIPLE}

Figure 1 presents the integrated fiber-optic microfluidic device for detecting muscular force generated by a nematode worm. A sinusoidal channel is designed wide enough to allow the worm to move freely, having a minimal physical constraints on the worm's natural movement. A SM optical fiber $(10-\mu \mathrm{m}$ diameter and 1-mm long) is horizontally placed adjacent to the sinusoidal channel. The SM fiber accepts input light at a wavelength of 1310 $\mathrm{nm}$. One end of the SM fiber is anchored, while the other end is free to move. The central axis of the SM fiber is aligned with the central axis of a receiving multi-mode fiber $(65-\mu \mathrm{m}$ diameter) embedded in the device. Multiple troughs of the sine-wave channel provide multiple force detection points, where body contact of a worm with the SM fiber causes to displace the SM fiber away from its original position. This, in turn, reduces optical coupling efficiency from the SM fiber to the multi-mode fiber, and thus, decreasing the transmitted light power received by a detector. Thus, the device can detect muscular forces generated by the worm at different locations along the SM fiber and at multiple time instances in a dynamic manner. These force data can be used to verify each other, largely improving data accuracy and reliability. Furthermore, the horizontal placement of the SM fiber allows us to take full advantage of the high aspect ratio of the fiber $(\sim 100)$, leading to a sensitive response of the fiber to a small force applied normal to the fiber.

Figure 2a shows simulated linear relationship between normal force $F$ applied to the SM fiber at two different detection points of the device and vertical displacement $\Delta x$ at the tip of the SM fiber. Figure $2 \mathrm{~b}$ shows geometric change of the SM fiber under a normal force $(9.16 \mu \mathrm{N})$ applied at the two detection points. In addition, finite-difference time-domain method is used to compute changes in normalized light intensity detected at the multi-mode fiber, as a function of $\Delta x$ (Fig. 2c). By combining Figs. $2 b$ and $c$, we build up a relationship between an applied normal force and a change in normalized light intensity detected.

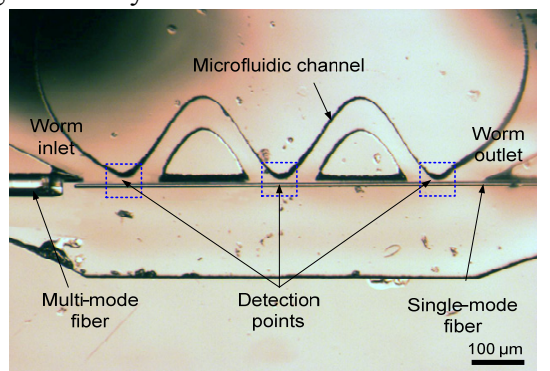

Figure 1: Optical image of the fiber-optic microfluidic device for measuring force generation of a nematode worm.
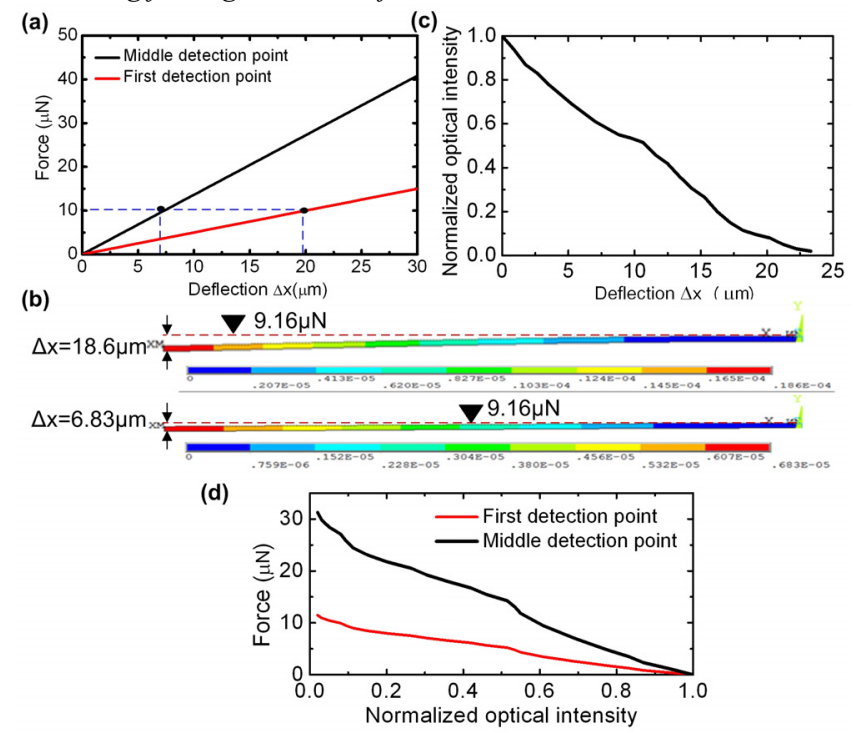

Figure 2: (a) Simulated relationship between applied forces and deflections at the tip of the SM fiber. (b) Computedg deflection of the SM fiber under $9.16 \mu N$ applied to the left and middle detection point. (c) Computed normalized optical intensity as a function of deflection at the free end of the SM fiber (d) Computed force as a function of normalized optical intensity. 


\section{RESULTS AND DISUCUSSION}

Figure 3 describes device fabrication processes. Multiple detection regions are formed along the emitting fiber. Parasitic Oesophagotomum dentatum is used as a model nematode (approximately $20-\mu \mathrm{m}$ diameter and $350-\mu \mathrm{m}$ long).

(a)

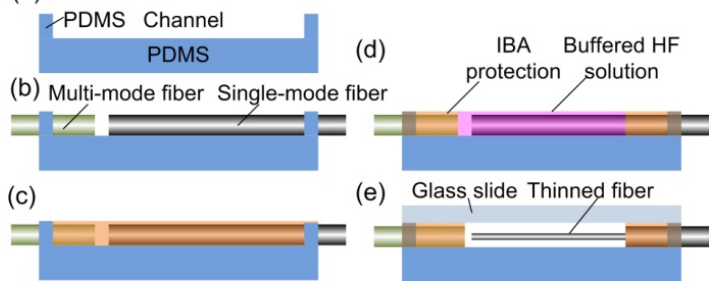

Figure 3: Schematic of fabrication process for the device. (a) A PDMS open channel is formed using soft lithography technique. (b) A multi-mode fiber (CPC6, Corning) and a SM fiber (SMF-28, Corning) are pre-thinned down to $65 \mu \mathrm{m}$ diameter using buffered hydrofluoric acid (HF) solution (49 wt\%). They are then inserted into two aligning slots on two sides of the channel. (c) The channel is filled up with a precursor solution consisting of isobornyl acrylate (IBA), tetraethylene glycol dimethacrylate, and 2,2dimethoxy-2-phenylacetophenone (weight ratio 32:1.7:1). (d) The mixture solution is polymerized under UV light to protect the fibers being etched in the following etching process. (d) Buffered HF solution $(10 \%)$ is used to remove the cladding layer of the SM fiber. (e) The device is capped with a glass slide through oxygen plasma surface treatment.

Figure 4 shows time-lapse images for the bending emitting fiber $(\lambda=1310 \mathrm{~nm})$, as an O.dentatum nematode worm squeezes through the middle detection point (Fig. 1). The maximum deflection at the free end of the fiber caused by the worm movement is about $6.9 \mu \mathrm{m}$. The corresponding optical intensity change is $38.3 \%$, showing a good agreement with the simulation result (Fig. 2). The resulting force generated by the worm is 9.23 $\mu \mathrm{N}$.

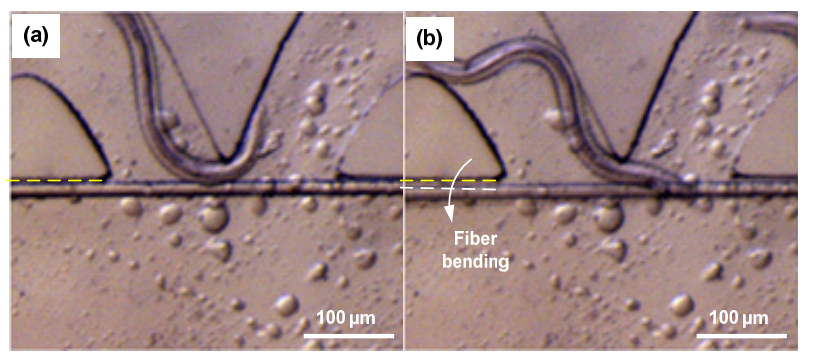

Figure 4: Time-lapse images as a worm interacts with the SM fiber at the middle detection point (see Fig. 1) during force measurement. (a) $t=30 \mathrm{~s}$, and (b) $=35 \mathrm{~s}$.

Figure 5 shows that as the worm swims downstream, the tight interaction with the left detection point results in light intensity change of $90.4 \%$. The corresponding force signal is $8.94 \mu \mathrm{N}$, which is almost similar to the force signal obtained at the middle detection point, demonstrating a high reliability and validity of the present detection method. As shown in the optical measurement result, the background noise intensity is at a level of $2.5 \%$ of the maximum optical signal. The minimal detectable force or the sensing resolution of the device is determined by the signal-tonoise $(\mathrm{S} / \mathrm{N})$ ratio of the detector used, and is calculated as two times the noise floor intensity. A light intensity drop of $5 \%$ from the maximum intensity corresponds to a deflection of $0.48 \mu \mathrm{m}$ at the tip of the SM fiber and thus a normal force of $0.23 \mu \mathrm{N}$ at the first detection point on the left and $0.64 \mu \mathrm{N}$ at the second one in the middle of the device We note in Fig. 2c that the light intensity is almost saturated at a low level of $3 \%$ of the maximum intensity when the SM fiber deflects beyond $25 \mu \mathrm{m}$ from the horizontal direction. Thus, larger bending of the fiber caused by worm-fiber interactions cannot be detected. According to Fig. 2 d, the detection range of the device is up to $12.31 \mu \mathrm{N}$ and $33.53 \mu \mathrm{N}$ at the first the second detection point of the channel.

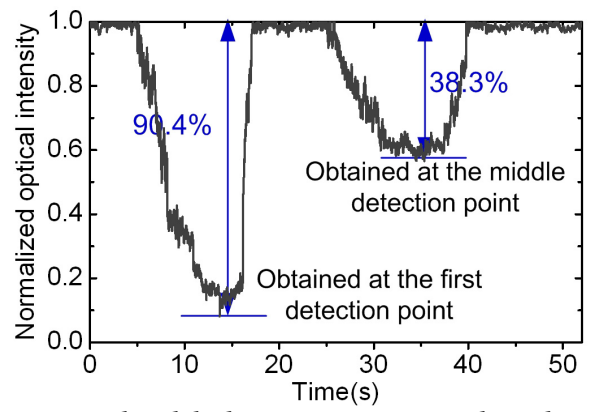

Figure 5: Normalized light intensity received at the multi-mode fiber as a function of time. Two obvious dips demonstrate the significant influence of fiber deflection on reducing optical coupling efficiency from a SM to a multi-mode fiber. Light wavelength used here is $1310 \mathrm{~nm}$.

\section{CONCLUSION}

The integrated fiber-optic microfluidic device offers a simple and effective scheme to measure muscular force of a nematode worm. The method does not involve using complex device structures or a microscopic imaging and recording system. This work can potentially be beneficial to better understand forcesensing organs and neurons of multicellular organisms.

\section{REFERENCES}

[1] R. Waterston, "Genome sequence of the nematode C. elegans: A platform for investigating biology," Science vol. 282, 1998, pp. 2012-2018.

[2] C. Cronin, J. Mendel, S. Mukhtar, Y.-M. Kim, R. Stirbl, J. Bruck, and P. Sternberg, "An automated system for measuring parameters of nematode sinusoidal movement," BMC Genetics vol. 6, 2005, pp. 1-19.

[3] K.H. Chung, M.M. Crane, and H. Lu, "Automated on-chip rapid microscopy, phenotyping and sorting of C. elegans," Nat. Methods vol. 5, 2008, pp. 637-643.

[4] N. Chronis, "Worm chips: Microtools for C. elegans biology," Lab Chip vol. 10, 2010, pp. 432-437.

[5] S.-J. Park, M.B. Goodman and B.L. Pruitt, "Analysis of nematode mechanics by piezoresistive displacement clamp," PNAS vol. 44, 2007, pp. 17376-17381.

[6] A. Ghanbari, V. Nock, W. Wang, R. Blaikie, J.G. Chase, X.Q. Chen, and C.E. Hann, "Force pattern characterization of C. elegans in motion," Proc. 15th International Conference on Mechatronics and Machine Vision in Practice, 2008, pp. 634639.

[7] J.C. Doll, N. Harjee, N. Klejwa, R. Kwon, S.M. Coulthard, B. Petzold, M.B. Goodmanc, and B.L. Pruitt, "SU-8 force sensing pillar arrays for biological measurements," Lab Chip vol. 9, 2009, pp. 1449-1454. 


\title{
SENSITIVE AND SELECTIVE DETECTION OF A CANCER BIOMARKER, THYROGLOBULIN, IN UNDILUTED HUMAN SERUM USING COMPETITIVE ADSORPTION OF PROTEINS AND PATTERN RECOGNITION
}

\author{
Ran Wang ${ }^{l}$, Shuai Huang ${ }^{2}$, Jing Li $^{2}$, and Junseok Chae ${ }^{l}$
}

School of Electrical, Computer, and Energy Engineering, Arizona State University, Tempe, AZ

School of Computing, Informatics, and Decision Systems Engineering, Arizona State University, Tempe, AZ

\begin{abstract}
We report a microfluidic-based Surface Plasmon Resonance (SPR) biosensor for rapid, real-time detection of Thyroglobulin ( Tg, a sensitive indicator of persistent or recurrent differentiated thyroid cancer with abnormal concentration higher than $3 \mathrm{ng} / \mathrm{mL}$ in undiluted human serum, using competitive adsorption of proteins and pattern recognition. The SPR biosensor produces unique patterns from multiple engineered sensing surfaces, and then Linear Discriminant Analysis (LDA) is used to differentiate these patterns. The microfluidic-based biosensor demonstrates the ability to detect $2 \mathrm{ng} / \mathrm{mL}$ Tg in undiluted serum with $91.7 \%$ and $90.0 \%$ classification confidence level for its sensitivity and selectivity, respectively.
\end{abstract}

\section{INTRODUCTION}

Biomarker proteins are often used to assess the progress of diseases and monitor the effects of the treatment as the relative and absolute levels of them are directly associated with specific disease states. Conventional biosensors rely on specific binding interactions between bio-receptors (i.e., antibody, enzyme, aptamer, etc.) and biomarkers. Unlike these biosensors, our work does not require bio-receptors, and instead utilizes competitive adsorption of proteins [1]. Nevertheless, our previously reported SPR biosensor still relied on specific binding interactions to detect a target biomarker in a controlled cocktail [1]. Such specific binding interactions that can be understood as the "lock and key" analogy [2] often do not provide adequate selectivity in a complex mixture, such as undiluted human serum, partially due to nonspecific interferences and weak binding affinity to analyte. To mitigate this challenge, we adopt pattern recognition that relies on cross-responsive interactions [3], rather than a specific interaction. A biosensor array is required to generate a unique composite pattern [4]. For all the bio-receptors in the array, the specific adsorption is not necessary. If only they have different interacting characteristics, the very unique pattern can be generated for discrimination. Without relying on the specific adsorption, the interference from other proteins can be minimized.

In this work, we, for the first time, demonstrate the ability to detect a cancer biomarker with $\mathrm{ng} / \mathrm{mL}$ sensitivity in undiluted serum using competitive adsorption of proteins and pattern recognition. These cross-responsive interactions are dominated by competitive adsorptions of proteins, namely Vroman effect [5], monitored by an optical-based transducer, SPR (Fig. 1). SPR can very sensitively response to minute changes of refractive index occurring adjacent to a metal film, which may results from the biointeractions on the sensing surface. A microfluidic module is mounted on SPR to enclose the gold sensing surfaces in microfluidic channels where the competitive adsorption of proteins occurs [6], which produces unique SPR sensorgram. The weakaffinity proteins initially covering the surface are displaced by strong-affinity proteins [5], yet the reverse sequence does not occur. In Fig. 1, two known affinity proteins (Protein $1 \& 2$ ) are pre-adsorbed on a bare gold and a $\mathrm{COOH}-\mathrm{SAM}$ (Self Assembled Monolayer) modified surfaces via the microfluidic channels. After a stable baseline establishing, a Tg-spiked serum sample is injected into the microfluidic channels and flows across the pre-adsorbed surfaces. The SPR angle shift increases simultaneously as weakaffinity pre-adsorbed proteins are replaced by Tg and other strongaffinity proteins in the sample via the competitive adsorption behavior of proteins. Subtracting the baseline, a final angle shift predominantly caused by the competitive adsorption behavior is obtained. The unique surface interactions generate patterns of realtime SPR angle shifts, which then are statistically processed to discriminate patterns using LDA.

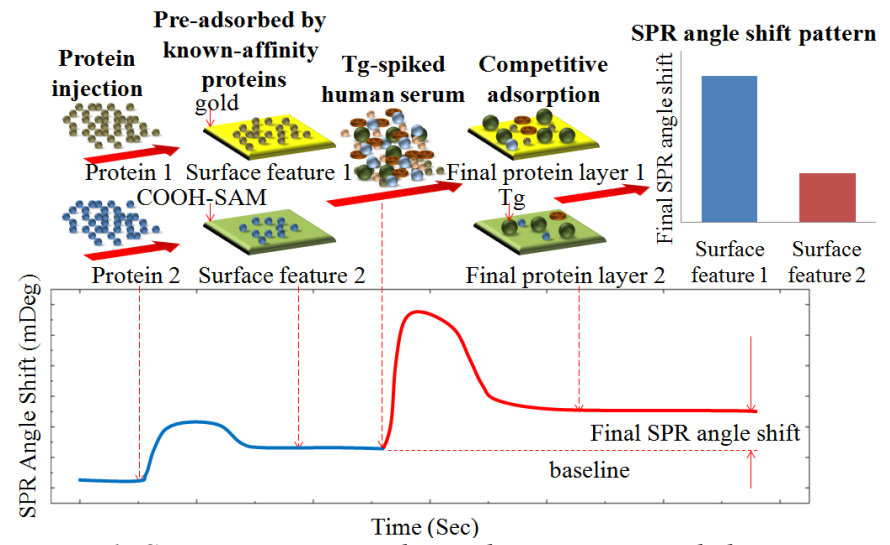

Figure 1: SPR sensorgram with step-by-step proteins behaviors on a sensing surface based on the competitive adsorption of proteins (Vroman effect).

\section{RESULTS}

Sensor surfaces are pre-adsorbed by five known-affinity proteins on bare gold and $\mathrm{COOH}-\mathrm{SAM}$ to form 10 surface features (Table 1). The 10 surface features generate 10 angle shifts, and such process is replicated 6 times. 10 average values of angle shifts form a distinguishing pattern to represent a Tg-spiked sample (Fig. 2(a)). Through LDA, the patterns consisting of different samples can be separated into unique groups of data. Each data point represents a replication generated from the 10 surface features (Fig. 2(b)). As evaluating sensitivity characteristics of our SPR biosensor, 100, 10, and $2 \mathrm{ng} / \mathrm{mL}$ Tg-spiked serums are discriminated from each other and the control. Through sensitivity characteristics analysis by LDA, original classification result, 91.7 $\%$, indicates how well the classification functions predict these samples, while cross-validations result, $66.7 \%$, estimates how well the classification functions derived on given samples predict a subsequent sample.

Table 1: Matrix of ten sensing surface features; 5 different preadsorbed proteins on 2 different surfaces.

\begin{tabular}{|c|c|c|c|c|c|}
\hline & \multicolumn{5}{|c|}{ Pre-adsorbed proteins } \\
\hline & Transferrin & Lysozyme & Albumin & IgM & IgG \\
\hline Bare gold & SF 1 & SF 2 & SF 3 & SF 4 & SF 5 \\
\hline SAM & SF 6 & SF 7 & SF 8 & SF 9 & SF 10 \\
\hline
\end{tabular}

Solid-State Sensors, Actuators, and Microsystems Workshop Hilton Head Island, South Carolina, June 3-7, 2012 
Table 2: Matrix of five samples; control (human serum), and 3 samples of Tg-spiked and one of IgM-spiked serum.

\begin{tabular}{|c|c|c|c|c|}
\hline Sample 1 & Sample 2 & Sample 3 & Sample 4 & Sample 5 \\
\hline $\begin{array}{c}\text { Control } \\
\text { (human } \\
\text { serum) }\end{array}$ & $\begin{array}{c}100 \mathrm{ng} / \mathrm{mL} \\
\text { Tg-spiked }\end{array}$ & $\begin{array}{c}10 \mathrm{ng} / \mathrm{mL} \\
\text { Tg-spiked }\end{array}$ & $\begin{array}{c}2 \mathrm{ng} / \mathrm{mL} \\
\text { Tg-spiked }\end{array}$ & $\begin{array}{c}\text { IgM- } \\
\text { spiked }\end{array}$ \\
\hline
\end{tabular}

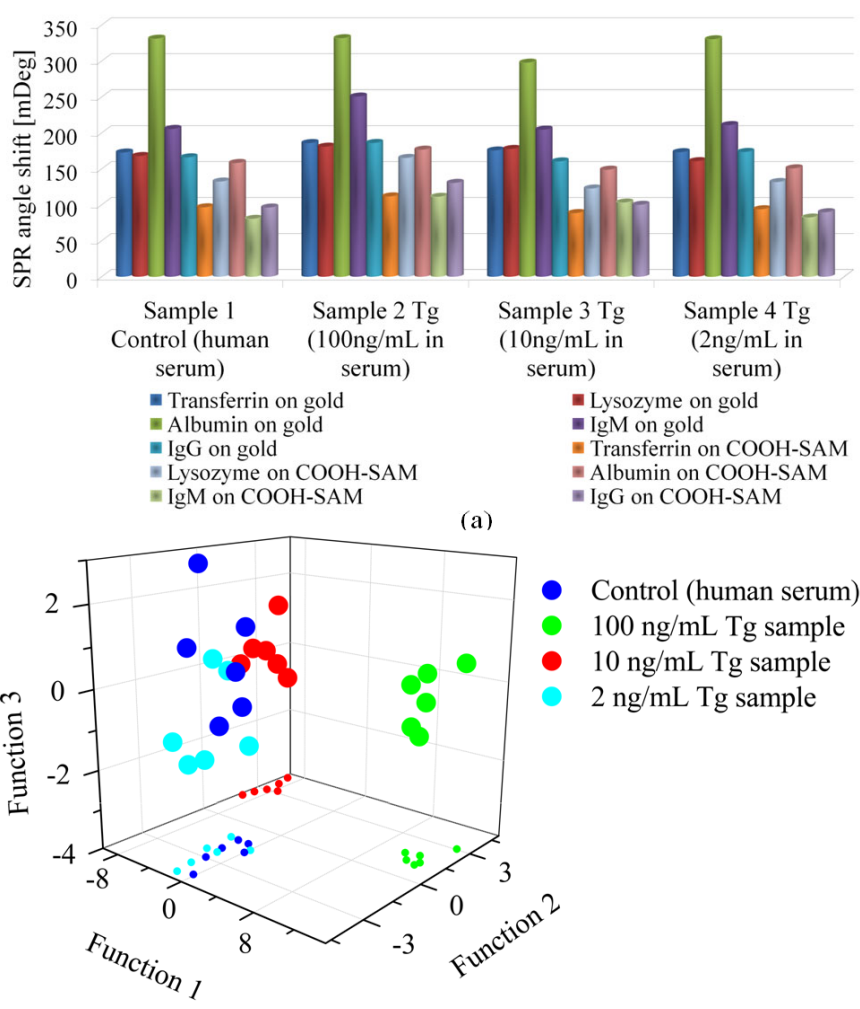

(b)

Figure 2: Sensitivity characteristics: pattern recognition analysis (a) patterns of 4 samples generated from 10 surface features (b) statistical results obtained from LDA (Linear Discriminant Analysis).

We also analyzed the respective discrimination confidence levels for the three Tg-spiked samples (Table 2). 100, 10, and 2 $\mathrm{ng} / \mathrm{mL}$ Tg-spiked samples and the control show the original classification of $100 \%, 100 \%$, and $100 \%$, and the crossvalidation of $83.3 \%, 53.3 \%$, and $41.7 \%$, respectively. In other words, for an unknown sample, higher Tg-spiked concentration provides higher possibility to discriminate the Tg sample from the control, which is shown in Figure 2(b). Data points of 100 and 10 $\mathrm{ng} / \mathrm{mL} \mathrm{Tg}$-spiked samples are far away from those of the control, yet $2 \mathrm{ng} / \mathrm{mL}$ Tg-spiked sample has a small but finite overlap with the control, showing the difficulty of discrimination. Obviously higher concentration $\mathrm{Tg}$ samples and the control result in more distinctively different patterns that are easier for LDA to discriminate.

For selectivity, IgM-spiked serum sample is discriminated from 100, 10, and $2 \mathrm{ng} / \mathrm{mL}$ Tg-spiked serum samples and the control (Fig. 3). IgM is chosen as the affinity strength of $\operatorname{IgM}$ is similar to that of Tg. These 5 samples (Table 2) are injected into the microfluidic system, and then flow across 8 surface features. Selectivity characteristics analysis demonstrates $90.0 \%$ original classification, and $63.3 \%$ cross-validations confidence level.
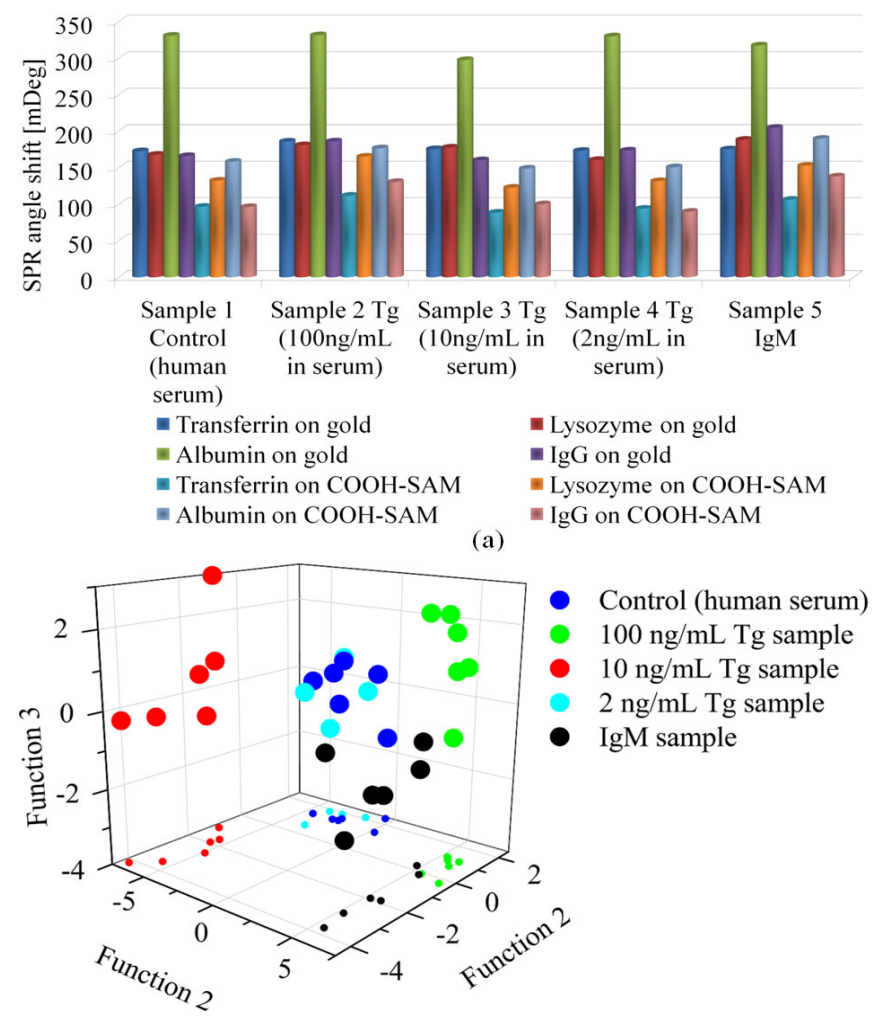

(b)

Figure 3: Selectivity characteristics: pattern recognition analysis (a) patterns of 5 samples generated from 8 surface features (b) statistical results obtained from $L D A$.

\section{ACKNOWLEDGEMENTS}

This project was partially supported by National Science Foundation (\#0846961). Travel support has been generously provided by the Transducer Research Foundation.

\section{REFERENCES}

[1] S. Choi, Y Yang, and J. Chae, "Surface plasmon resonance protein sensor using Vroman effect", Biosensors and bioelectronics, 24, 893 (2008).

[2] M. De, S. Rana, H. Akpinar, O. R. Miranda, R. R. Arvizo, U. H. F. Bunz, and V. M. Rotello, "Sensing of proteins in human serum using conjugates of nanoparticles and green fluorescent proteins", Nature Chemistry, 1, 461 (2009).

[3] P. Gouma, G. Sberveglieri, "Novel materials and applications of electronic noses and tongues", Materials Research Society, 29, 697 (2004).

[4] A. T. Wright, E. V. Anslyn, "Differential receptor arrays and assays for solution-based molecular recognition", Chemical Society Reviews, 35, 14 (2005).

[5] L. Vroman, A.L. Adams, "Findings with the recording ellipsometer suggesting rapid exchange of specific plasma proteins at liquid/solid interfaces", Surface Science, 16, 438 (1969).

[6] R. Wang, A. Lajevardi-Khosh, S. Choi, and J. Chae, "Regenerative surface plasmon resonance (SPR) biosensor: real-time measurement of fibrinogen in undiluted human serum using the competitive adsorption of proteins", Biosensors and bioelectronics, 28, 304 (2011).

\section{CONTACT}

*R. Wang, tel: +1-480-406-5818; rwang32@asu.edu 


\section{A HIGH ON/OFF RATIO MEMS CAPACITIVE SWITCH WITH APPLICATIONS IN SOLAR ENERGY HARVESTING \\ Vikram Thakar ${ }^{*}$, Zhengzheng Wu, and Mina Rais-Zadeh \\ University of Michigan, Ann Arbor, Michigan, USA}

\begin{abstract}
In this paper, a novel electrostatic switch is reported that exhibits an ON/OFF capacitance ratio of 72 , an $\mathrm{ON}$ capacitance value of $90 \mathrm{pF}$, and a pull-in voltage of less than $5 \mathrm{~V}$ within an area of $750 \times 750 \mu \mathrm{m}^{2}$. These characteristics are achieved by utilizing a slit-shape middle metal layer in-between the top and bottom metal plates. This design makes the capacitance ratio and the ON state capacitance tolerant against fabrication and temperature induced residual stress in the suspended metal membrane, without significantly degrading the electrostatic force between the top and bottom metals.

In this three-metal configuration, at the $\mathrm{ON}$ state, the ohmic contact between the top membrane and the middle metal layer enables direct charge transfer between these two electrodes. This characteristic is attractive for device applications in solar energy harvesting. As a proof of concept, charging of an external storage capacitor is shown for an exemplary switch, when a constant current is supplied to the top electrode.
\end{abstract}

\section{INTRODUCTION}

MEMS switches are an attractive alternative to existing switch solutions and have thus been a topic of extensive research in the past few decades [1], [2]. Conventionally, an electrostatic MEMS switch is made using two electrodes separated by an air gap and a suitable dielectric. One of the electrodes is a moving membrane, and it can be pulled onto the fixed electrode with the application of an electrostatic potential. For a capacitive switch with a given device area, the up-state air gap and the down state effective dielectric thickness decide the ON and OFF capacitance, respectively. In many applications where the MEMS device is used as a switched capacitor, the switch is not only required to have a high ON/OFF capacitance ratio but also a sufficient $\mathrm{ON}$ capacitance value. Scaling up the device area to achieve a high ON capacitance is not beneficial as larger devices also exhibit an undesirably high OFF capacitance. In addition, the ON capacitance which is defined by the effective insulator thickness of the switch does not scale linearly with the device size because of warping in the movable membrane (caused by residual or thermal stress) and the surface roughness of the contacts [3]. In order to resolve these issues, a modified switch structure is presented that makes use of an additional metal layer to achieve both a high ON/OFF capacitance ratio and a high $\mathrm{ON}$ capacitance value (Fig. 1). The three-metal switch design makes the $\mathrm{ON}$ capacitance invariant to stress-induced membrane warping. A micromachined capacitive switch exhibiting an ON/OFF capacitance ratio of 72 and an ON capacitance value of $90 \mathrm{pF}$ within an area of $750 \times 750 \mu \mathrm{m}^{2}$ is demonstrated. A low pull-in voltage of less than $5 \mathrm{~V}$ is realized by designing the middle metal electrode in a slit type configuration ensuring sufficient electrostatic actuation force on the top suspended membrane. With such properties, the proposed threemetal switch design compares favorably with the state of the art MEMS switches [4].

In the ON state, the top metal makes an ohmic contact with the middle metal layer, allowing the transfer of electrostatic charge from the top membrane to the un-biased middle electrode. Such charge transfer can be useful when employing the switch as a solar energy harvester. We investigate this application and demonstrate charging of an external capacitor connected to the middle metal layer. Another unique feature of this three-metal switch when used as a harvester is the ability to deliver high-voltage charging pulses under heavy capacitive load without the need for an electrical boost converter. This is a significant advantage over conventional photovoltaic devices [5].

\section{DEVICE CONCEPT AND FABRICATION}

The three dimensional model of the proposed switch is shown in Fig. 1. The MEMS switch is actuated by applying an electrostatic potential between the top and the bottom metal layers, whereas the middle metal layer is left floating. When the top membrane pulls in, it makes an ohmic contact with the middle metal layer. Thus, the ON capacitance of the switch is mainly determined by the capacitance between the middle and bottom metal layers, and is unaffected by the curvature of the top membrane. Therefore, a repeatable and large ON capacitance can be achieved even if the top membrane is warped due to the inevitable fabrication and thermally induced residual stresses.

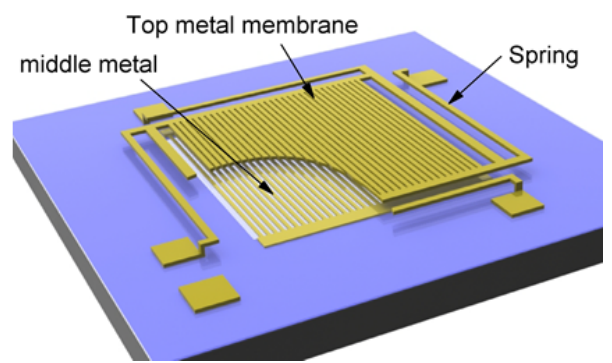

Figure 1: Schematic of the device structure with a top electrode section cut out to reveal the middle metal layer. The slit type design of the top and middle electrode can be clearly seen.

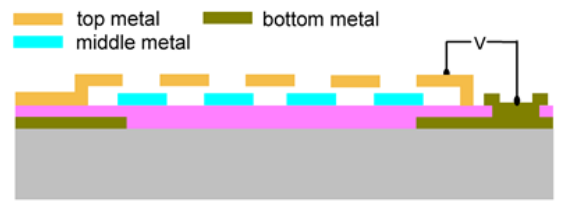

Figure 2: Cross-section of the proposed switch structure. The floating middle metal layer can be used as an additional terminal. Note that in this configuration, the substrate acts as the ground electrode.

To minimally affect the electric field distribution between the top and bottom metal layers, the metal layers are designed in a slit configuration (Fig. 2) and the middle metal layer is shifted laterally (Fig. 3). From the electrostatic simulations shown in Fig. 3, the slit-shaped middle metal layer is seen to have no significant effect on the electric field between the top and bottom metal layers. As a result, the electrostatic force on the suspended top metal remains unaffected. This allows us to design the membrane with a sufficient restoring force to overcome stiction, while still actuating the device with relatively small voltages.

The fabrication process flow for the proposed devices is depicted in Fig. 4. Starting with a nominal silicon wafer, a $500 \mathrm{~nm}$ 
thick aluminum $(\mathrm{Al})$ / chrome $(\mathrm{Cr})$ layer is lift-off patterned as the first metal layer (or ground electrode). A $150 \mathrm{~nm}$ thick aluminum oxide $\left(\mathrm{Al}_{2} \mathrm{O}_{3}\right)$ layer is deposited using atomic layer deposition (ALD) as the dielectric layer. This material and its thickness along with the metal area define the ON state capacitance of the device. The dielectric layer is patterned to enable contact to the bottom metal layer. A $100 \mathrm{~nm}$ thick gold is evaporated and lift-off patterned as the middle floating electrode with $\mathrm{Cr}$ as the adhesion layer. Poly-methyl-methacrylate (PMMA) is spun as the sacrificial layer with a thickness of $1.7 \mu \mathrm{m}$ which defines the air gap between the top and the floating electrode. Using titanium (Ti) as the etch mask, PMMA is patterned using a low power $\mathrm{O}_{2}$ plasma. Post PMMA etching, the Ti mask is removed and a 10/900 $\AA$ $\mathrm{TiW} /$ silver layer is sputtered as a seed layer for electroplating. The plating mold is created using AZ 9260 photoresist and an $8 \mu \mathrm{m}$ thick top electrode is subsequently electroplated. In the final step, the devices are released by removing the PMMA sacrificial layer and dried using critical point drying (CPD) to prevent stiction. The SEM images of a fabricated device are shown in Fig. 5.

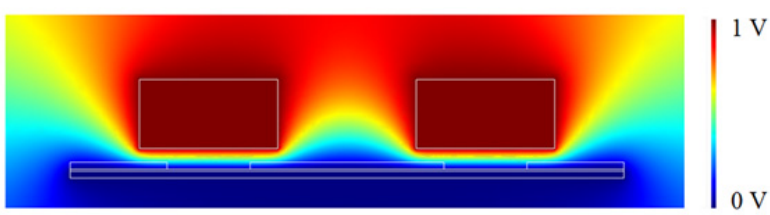

(a)

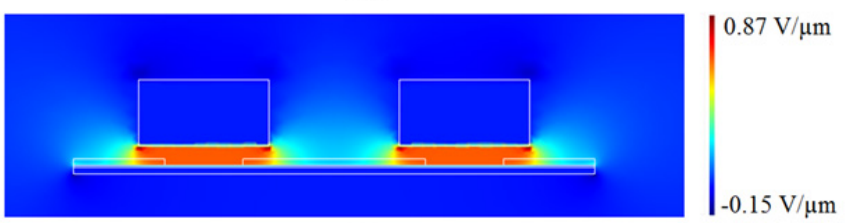

(b)

Figure 3: Simulated (a) electric potential and (b) electric field distribution of the proposed electrode layout for a two finger section. From (b) a uniform electric field is seen in between the top and bottom electrodes. The electric field is not disturbed significantly due to the addition of the middle metal layer. The two scale bars plot the range of voltage and electric field.
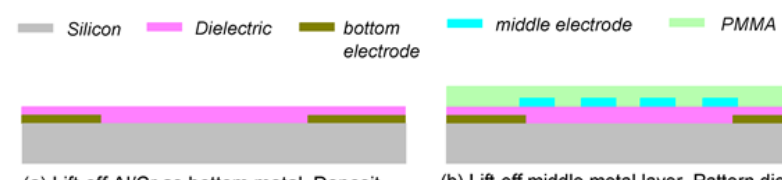

(a) Lift-off $\mathrm{Al} / \mathrm{Cr}$ as bottom metal. Deposit

$150 \mathrm{~nm} \mathrm{Al} \mathrm{O}_{3}$ as dielectric layer.

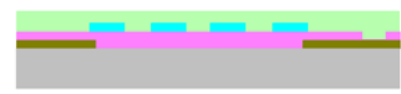

(b) Lift-off middle metal layer. Pattern dielectric to create contact to bottom metal. Spin PMMA as sacrificial layer

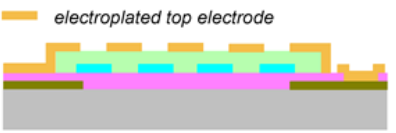

(c) Sputter plating seed layer. Electroplate top metal layer.

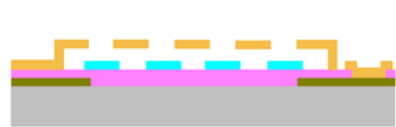

(d) Etch plating seed layer and release structure by removing sacrificial layer.

Figure 4: Process flow of the proposed MEMS capacitive switch.

\section{SWITCH DEVICE DESIGN}

There are two primary design objectives in released membrane type electrostatic switches: low pull-in voltage and large restoring force. Both can be controlled by optimizing the stiffness of the springs, and the membrane shape and area. Using the process shown in Fig. 4, the membrane and the spring

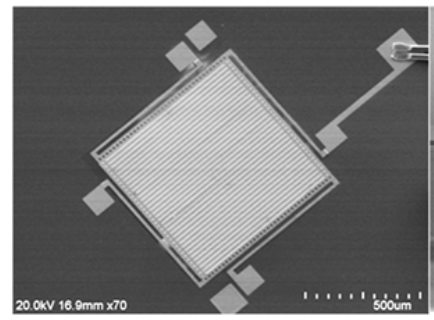

(a)

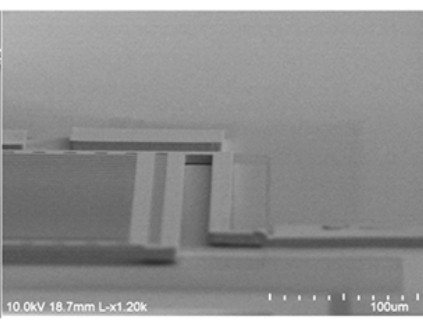

(b)
Figure 5: (a) Top SEM view of a micro-fabricated MEMS switch and (b) incline view of the top membrane revealing the air gap.

thickness is constrained to be the same to reduce the fabrication complexity. The spring stiffness is varied using lithographically defined dimensions, as shown in Fig. 6. A crab leg type spring design is implemented to allow self-compensation against fabrication induced in-plane stresses. While such a design is robust against in-plane stresses, the membrane is prone to bending under a vertical stress gradient commonly observed in most electroplated metals [6]. Figure 7 plots the simulated and measured top membrane bow due to a stress gradient in the top electroplated gold layer. Using a close to optimum plating condition, a membrane bow of $\sim 2 \mu \mathrm{m}$ was measured. From finite element simulations, a stress gradient of $\sim 10 \mathrm{MPa} / \mu \mathrm{m}$ in the top membrane was found to fit the measured bow. Using other plating conditions, higher stress gradients have been measured. With such values of inevitable stress in the top membrane, designing a traditional MEMS capacitive switch with large capacitance values is challenging [7]. In a typical two electrode switch, the membrane bow necessitates application of a voltage higher than pull-in to flatten the membrane and achieve a full area contact [8]. In the proposed design, the capacitance between the middle metal and the bottom metal layer is set by the layout of the two electrodes and the dielectric material thickness in between. As such, a high ON state capacitance is obtained even when the top membrane is only partially in contact with the middle metal layer. Therefore, although warping of the top membrane makes a uniform contact difficult, the ON capacitance value remains unaffected. This is clearly seen from the measurement results shown in Fig. 8.
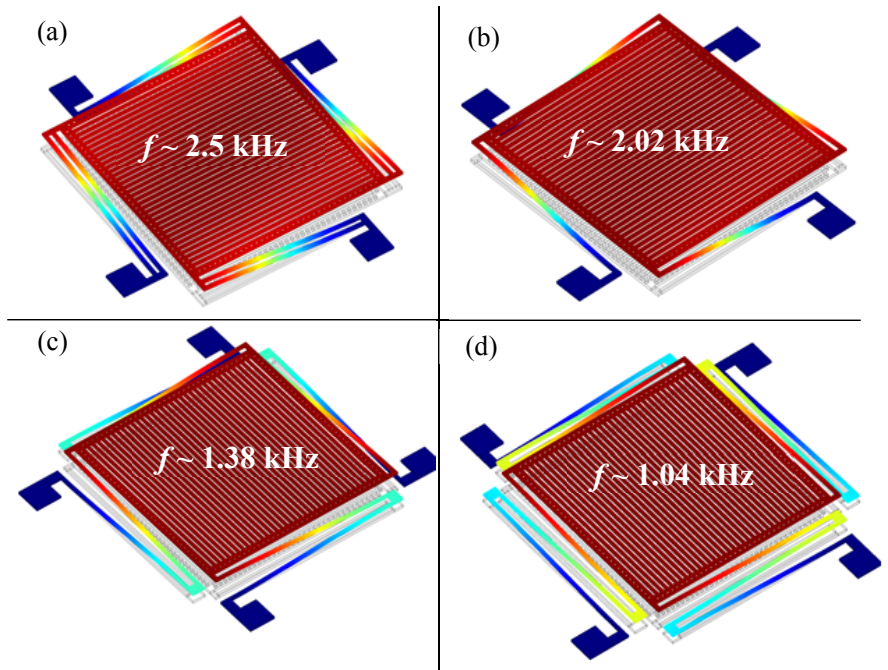

Figure 6: Modal simulation results for (a) double beam (b) single beam (c) single fold beam and (d) double fold beam designs. The color spectrum plots the total displacement. The frequency shown is a measure of maximum switching speed. 

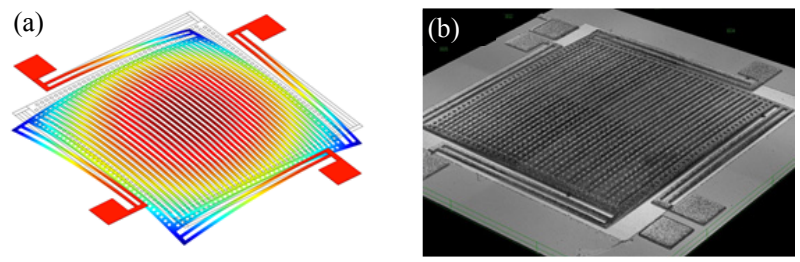

Figure 7: (a) Simulated 2 um top metal membrane bow with a stress gradient of $10 \mathrm{MPa} / \mu \mathrm{m}$ (b) Actual membrane bow measured using optical interferometry. The measured bow is $\sim 2 \mu \mathrm{m}$, indicating the stress gradient in gold is $\sim 10 \mathrm{MPa} / \mu \mathrm{m}$.

\section{SWITCH MEASUREMENTS}

A Keithley 4200 semiconductor characterization system is used to measure the capacitance vs voltage (CV) curves for the fabricated devices. Figure 8 plots the measured $\mathrm{CV}$ curves for a sample device of each spring design. As expected, the pull-in voltage is seen to reduce as the spring compliance is increased.
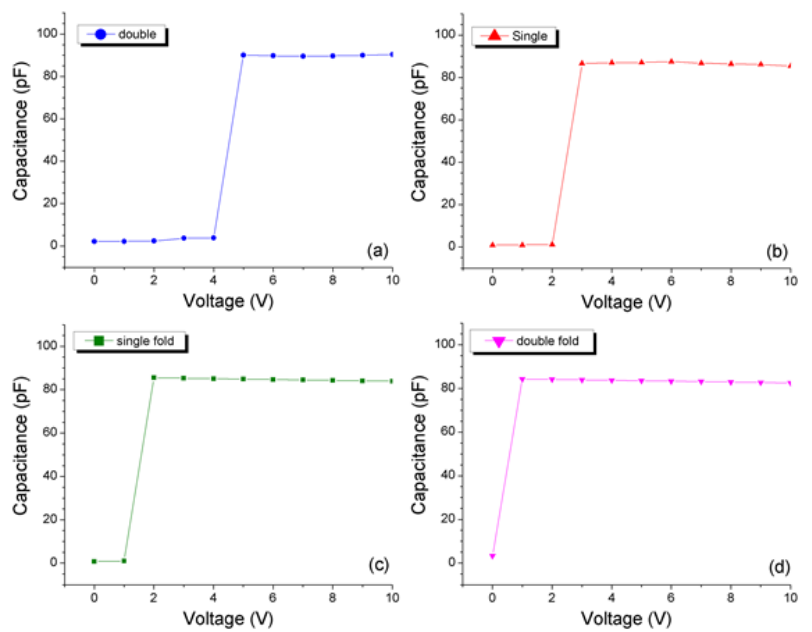

Figure 8: Measured CV curves for the (a) double beam, (b) single beam, (c) single fold beam, and (d) double folded beam spring designs. $C_{O N}$ is similar for all 4 designs. The capacitance of the measurement pads is de-embedded from the measurements.

In order to measure the switching time, DC voltage pulses are applied to the top electrode using an Agilent 81150A signal generator. The output voltage is read from the middle floating metal using an Agilent MSO7104A oscilloscope. The experimental setup is shown in Fig. 9 and the measured results are plotted in Fig. 10. When the input DC voltage is greater than the pull-in voltage, the top metal is pulled onto the middle metal and the output voltage follows the input voltage. Once the input voltage falls below the pull-in potential, the top membrane is pulled away, contact between the top and middle metal layers is broken, and the output voltage goes back to $0 \mathrm{~V}$. From Fig. 10(b), a switching time of $\sim 120 \mu$ s is measured. The results are summarized in Table 1 .

Table 1: Measured switch parameters. The switching time is estimated using equations provided in [1] assuming a mechanical $Q$ of 1.5 and applied voltage $=1.4 \times$ pull-in voltage.

\begin{tabular}{|c|c|c|c|c|c|}
\hline \multirow{2}{*}{$\begin{array}{l}\text { Spring } \\
\text { type }\end{array}$} & \multirow{2}{*}{$\begin{array}{l}\mathrm{C}_{\mathrm{ON}} \\
(\mathrm{pF})\end{array}$} & \multirow{2}{*}{$\mathrm{C}_{\mathrm{ON}} / \mathrm{C}_{\mathrm{OFF}}$} & \multirow{2}{*}{$\begin{array}{c}\text { Pull-in } \\
\text { voltage (V) }\end{array}$} & \multicolumn{2}{|c|}{ Switching time $(\mu \mathrm{s})$} \\
\hline & & & & Modeled & Measured \\
\hline Double & 90.69 & 72.7 & $<5$ & $48-146$ & - \\
\hline Single & 87.48 & 71.7 & $<3$ & $60-180$ & - \\
\hline Single fold & 85.5 & 65.3 & $<2$ & $88-264$ & 120 \\
\hline Double fold & 84.33 & 29.4 & $<1$ & $117-350$ & - \\
\hline
\end{tabular}

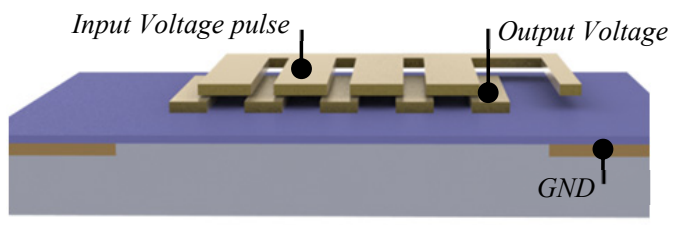

Figure 9: Experimental setup to measure the electrostatic switch response. A DC voltage pulse of $1.2 \mathrm{~V}$ is applied to the top metal membrane. The output is monitored from the middle floating layer using an oscilloscope.
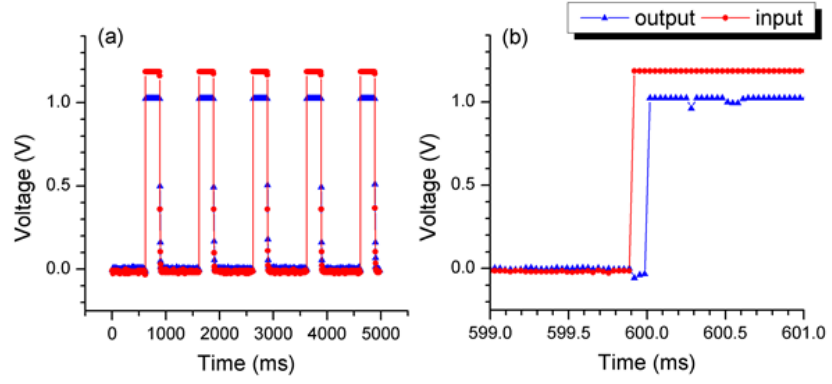

Figure 10: A voltage pulse $\left(V>V_{\text {pull-in }}\right)$ is applied to the top electrode of a single folded beam device to study the pull-in behavior of the membrane. The blue curve shows the detected output from the middle metal electrode. (a) Repeatable operation for multiple cycles and (b) delay of $\sim 120 \mu \mathrm{s}$ is measured between the input and output pulses, indicting the switching time.

While, extensive repeatability testing has not been performed, direct metal to metal contact during pull-in can cause stiction - a typical failure mechanism in ohmic switches. In such cases, the device reliability may be improved by increasing the restoring force but at the cost of increased pull-in voltage. The slit metal layer design and a large device area help reduce the actuation voltage even when the spring stiffness is large. In addition, hard contact metals, such as ruthenium [9], can be sputtered to improve the switch reliability. During high-power operations, large AC signal excitation induces an effective AC driving force on the switch membrane and causes self-actuation [1]. In the proposed switch design, the effective AC force is the electrostatic force between the top and bottom metal layers. Designing a switch with a larger pull-in voltage can make the device more tolerant to $\mathrm{AC}$ self-actuation and can improve the power handling.

\section{ENERGY HARVESTER APPLICATION}

The introduction of a third metal layer in the switch, as presented, opens up novel applications such as incident solar energy harvesting. The three-metal layer switch can be used as a solar energy harvester by coating the top membrane with a photosensitive material [5]. Figure 11 shows the conceptual schematic describing the solar energy harvester architecture.

During operation, the top metal layer accumulates charges due to incident radiation, which in turn causes an increase in the top membrane potential. Once pull-in voltage is reached, the top and middle metal layers will be shorted, and the accumulated charge is transferred to an external capacitor or a rechargeable battery connected to the middle metal layer, as shown in Fig. 11. Once the top metal loses all its charge, its potential drops and the membrane is lifted back up. Such electrostatic charging and discharging cycles will continue as long as energy is incident on the photosensitive material. As the maximum voltage on the top membrane is dependent on the pull-in voltage, high voltage pulses can be directly generated via design of higher pull-in voltage devices. Therefore, the proposed switch behaves as an 
electromechanical DC booster, which overcomes the heavy loading of an ultra-capacitor and improves the electrostatic charge transfer, efficiency. To experimentally verify the application of the device as a solar energy harvester, the photocurrent is replaced by a current source to inject charge on the top metal membrane. The test setup is shown in Fig. 12. In each pull-in cycle, charge is transferred to an external $1 \mu \mathrm{F}$ capacitor. The output voltage across the external capacitor is monitored using an oscilloscope with terminal impedance of $1 \mathrm{M} \Omega$. The measured voltage across the external capacitor when a constant current is supplied to the top electrode is shown in Fig. 13.

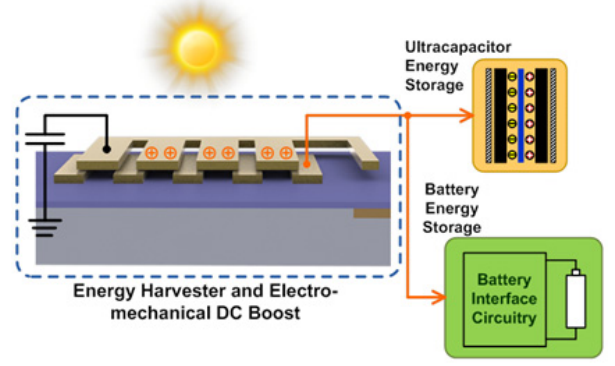

Figure 11: The proposed device architecture as envisioned for solar energy harvesting application. The top electrode should be coated with a photo-sensitive material.

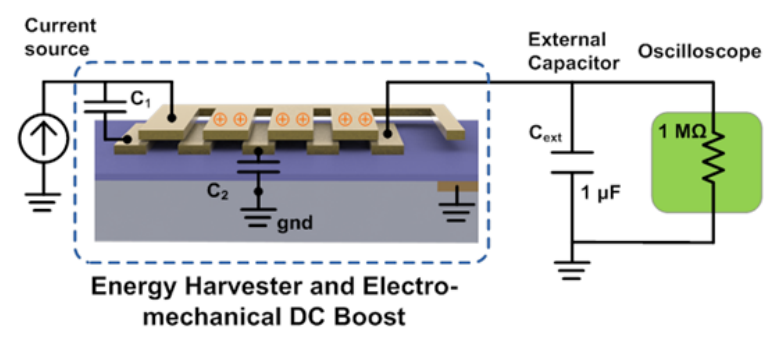

Figure 12: Experimental test setup for measuring the device performance as an energy harvester. A $1 \mu F$ external capacitor is used for collecting charge and the voltage across the capacitor is measured using an oscilloscope (with an impedance of $1 \mathrm{M \Omega}$ ).
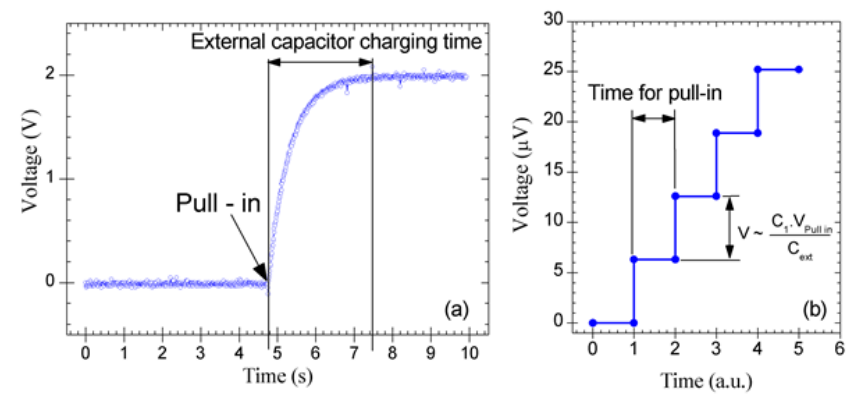

Figure 13: (a) Measured voltage across the external capacitor when a current of $2 \mu \mathrm{A}$ is connected to the membrane. As the current source is switched on, the potential on the top membrane increases, causing the membrane to pull-in and the $1 \mu F$ capacitor to charge. The output voltage is limited by the oscilloscope input impedance. (b) Theoretical plot for the voltage across the external capacitor for the first few cycles. The time step corresponds to the time required to achieve pull-in due to the photocurrent.

Using simple charge balance, the harvester performance can be analyzed. The equations for output voltage, charge collected, and efficiency of charge transfer can be written as
$V_{\text {output }}=\left(\frac{C_{1}}{C_{2}+C_{\text {ext }}}\right) V_{\text {pull in }}$

$Q_{\text {output }}=\left[\frac{C_{1} C_{\text {ext }}}{C_{2}+C_{\text {ext }}}\right] V_{\text {pull in }}$

$\eta_{\text {charge transfer }}=\left(\frac{C_{\text {ext }}}{C_{2}+C_{\text {ext }}}\right)$,

where $C_{1}, C_{2}$, and $C_{\text {ext }}$ are the capacitances in the structure as marked in Fig. 12. Using above equations, the switch design can be optimized for efficient harvester performance. From equations (1) and (2) we can see that the charge collected per cycle and the output voltage can be increased by increasing the pull-in voltage of the switch, and can thus be controlled via the spring design. This is a significant advantage over conventional photovoltaic devices, which suffer from low output voltage levels [10].

\section{CONCLUSION}

We proposed and verified a novel electrostatic switch design with an additional metal layer, which offers an improved ON/OFF capacitance ratio robust against fabrication induced residual stress. As a proof of concept, a switch with a capacitance ratio of 72 and a pull-in voltage of less than $5 \mathrm{~V}$ was demonstrated. We also investigated the use of a switch for energy harvesting applications and demonstrated feasibility of the concept though charging of an external capacitor. With their minimal dead area and the ability to generate high voltage pulses, arrays of such structures may be successfully implemented to power wireless sensor nodes.

\section{ACKNOWLEDGEMENT}

The authors thank Yonghyun Shim and the staff at the Lurie Nanofabrication facility (LNF) for their assistance. This work was supported by NSF under award \# 1055308.

\section{REFERENCES}

[1] G. M. Rebeiz, RF MEMS Theory, Design, and Technology, New York: Wiley, chapter 5, pp. 121-156, 2003.

[2] G. M. Rebeiz and J. B. Muldavin, "RF MEMS switches and switch circuits," IEEE Microwave Magazine, Dec. 2001.

[3] D. Peroulis et al., "Electromechanical considerations in developing low-voltage RF MEMS switches," IEEE Trans. Microwave Theory Tech., vol. 51, pp. 259-270, Jan. 2003.

[4] R. Al-Dahleh; R. Mansour, "High-capacitance-ratio warpedbeam capacitive MEMS switch designs," JMEMS, vol.19, no.3, pp.538-547, June 2010.

[5] Y. Huang et al., "Energy harvesting using RF MEMS," Electronic Components and Technology Conference, 2010.

[6] Y. Shim, Z. Wu, and M. Rais-Zadeh, "A multi-metal surface micromachining process for tunable RF MEMS passives," IEEE J. of Microelectromechanical Systems, 2012.

[7] A. Chinthakindi et al., J. Electrochem. Soc., vol. 149, issue 8, pp. H139-H145, 2002.

[8] Y. Shim, Z. Wu, and M. Rais-Zadeh, "A high-performance temperature-stable continuously tuned MEMS capacitor," IEEE MEMS, Cancun, Mexico, Jan. 2011, pp. 752-755.

[9] C. D. Patel and G. M. Rebeiz, "An RF-MEMS switch with $\mathrm{mN}$ contact forces," IEEE Int. Microwave Symp., May 2010.

[10] J. Christmann et al, "Bringing robustness and power efficiency to autonomous energy harvesting microsystems," Symp. on Asynchronous Circuits and Systems, 2010.

\section{CONTACT}

*Vikram Thakar, tel: +1-734-355-3480; thakar@umich.edu 


\section{ANALOG RF MEMS ATTENUATOR WITH PZT ACTUATORS \\ T.G. Ivanov, R.M. Proie, J.S. Pulskamp, R. G. Polcawich and A. Zaghloul \\ US Army Research Laboratory, USA}

\begin{abstract}
This paper reports on a voltage controlled, analog RF MEMS attenuator with lead-zirconate-titanate (PZT) actuators. Fabrication utilizes a PZT on silicon-on-insulator (SOI) material stack. The RF MEMS device demonstrated attenuation of up to $26.0 \mathrm{~dB}$ at 2.0 $\mathrm{GHz}$ and $9.3 \mathrm{~dB}$ at $25.0 \mathrm{GHz}$. Bias voltage varied from $0.0 \mathrm{~V}$ to $14.5 \mathrm{~V}$ dc. At $0.0 \mathrm{~V}$ the minimum insertion loss ranged from fractions of a dB at low frequencies to $1.16 \mathrm{~dB}$ at $25 \mathrm{GHz}$. This device can be used as part of a beam forming network for phased antenna arrays. Example of an 8 element linear antenna array was considered for $\cos ^{2}$ and Taylor $(-20 \mathrm{~dB})$ excitation distributions.
\end{abstract}

\section{INTRODUCTION}

RF attenuators are an essential part of beam forming networks, test equipment and almost any complex RF systems. There is a big body of experimental work and a variety of technologies have been used to build attenuators.

Classical devices were built with waveguide circuits. Some of the proposed concepts, such as the water loaded waveguide described in [1], were rather exotic. More conventional designs were studied as well [2]. These attenuators have very good RF performance, but tend to be bulky and relatively complicated to manufacture. Adjustment of the attenuation, when possible, was usually accomplished by either manual tuning or with the use of electric motors.

Hybrid solutions based on printed transmission lines and solid-state devices have become available [3], [4]. They offered more compact design and electronic control of the attenuation. Despite being a major advancement in size and weight reduction, these circuits still relied on significant lengths of transmission lines and were relatively large in size. Reference [3] is a typical example of this type of circuits.

The search for more compact solutions and the advances in semiconductor technologies led to the development of monolithic integrated attenuator circuits [5], [6]. These components feature a reduced area and precision voltage control of the attenuation. At the same time, semiconductor solutions require special attention to linearity and uniformity of the attenuation. The attenuation function is still carried out by multiple components operating together as part of the IC.

The ultimate reduction of component count per attenuation function can be achieved with MEMS technologies. In these solutions it is possible to accomplish variable attenuation of the signals using a single mechanical device. MEMS implementation of attenuators is well established in optics [7]. Mirrors, shutters and controlled misalignment of optical fibers can provide attenuation of the optical signals. The dynamic range of these systems is very high and the optical coupling can be controlled over many orders of magnitude. A recent overview of radar technology [8], however, pointed out that MEMS attenuators are still being investigated thoroughly in the RF communication and radar portion of the spectrum.

This work provides initial results for an analog reflection type MEMS attenuator in the $100 \mathrm{MHz}$ to $25 \mathrm{GHz}$ frequency range. The device is fabricated in PZT on SOI MEMS technology. Analog voltage controlled attenuation is achieved with moderate actuation voltages (maximum of $14.5 \mathrm{~V}$ ). The continuous attenuation control, broad bandwidth, moderate actuation voltage and small size of the reported circuit highlight RF MEMS devices as compelling candidates for attenuator solutions in the $\mathrm{RF} /$ microwave frequency range.

In this paper, the underlying MEMS fabrication technology will be described first. Then, the device design, operation and measured results will be reported. Finally, we will discuss the utility of the analog RF MEMS attenuator as a beam forming component for an eight element linear antenna array. To evaluate the device performance against the array requirements, as a function of frequency, uniform, $\cos ^{2}$ and Taylor $(-20 \mathrm{~dB}$ side lobes) excitation distributions will be compared.

\section{FABRICATION}

Detailed fabrication process flow of the PZT-on-SOI technology is presented in Figure 1. The process starts with an SOI wafer with a device layer of $4 \mu \mathrm{m}$ thick and a buried oxide thickness of $0.1 \mathrm{~m}$.

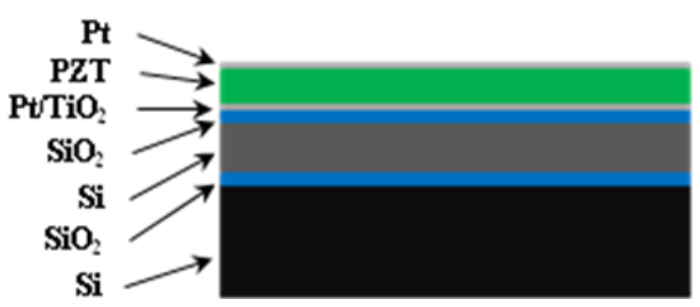

(a)

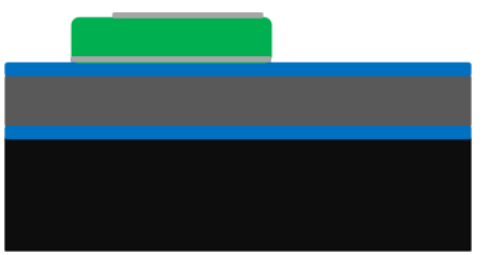

(b)

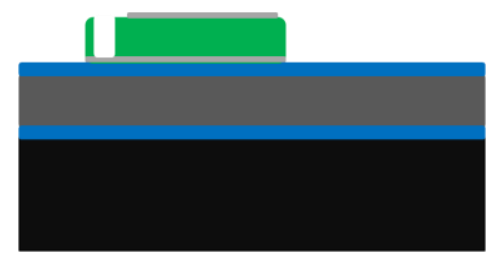

(c)

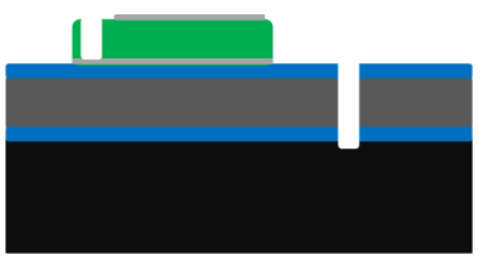

(d)

Solid-State Sensors, Actuators, and Microsystems Workshop Hilton Head Island, South Carolina, June 3-7, 2012 


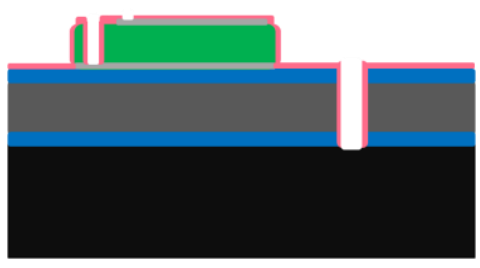

(e)

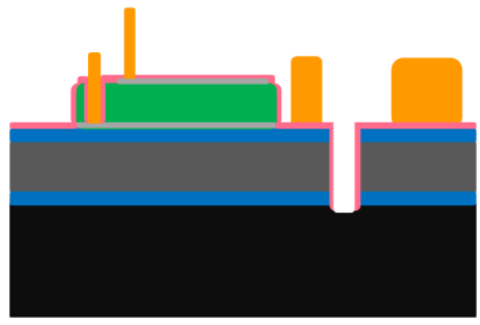

(f)

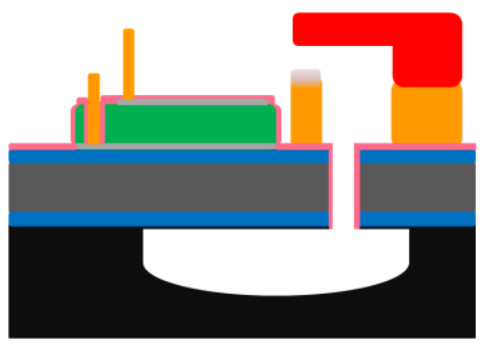

$(\mathrm{g})$

Figure 1. Process flow for fabrication of RF MEMS attenuator.

The actuator materials were deposited next, consisting of a $200 \mathrm{~nm}$ thermal silicon dioxide $\left(\mathrm{SiO}_{2}\right)$ coating, a sputtered titanium thin film annealed to form $33 \mathrm{~nm}$ titanium dioxide, a $100 \mathrm{~nm}$ sputtered platinum $(\mathrm{Pt})$ film, chemical solution deposited PZT with a $\mathrm{Zr} / \mathrm{Ti}$ ratio of 52/48 and a thickness of $1000 \mathrm{~nm}$, and $50 \mathrm{~nm}$ layer of sputtered platinum as shown in Fig. 1a.

The top Pt, PZT, and bottom Pt are then patterned using argon ion-milling, see Fig. $1 \mathrm{~b}$. To access the bottom Pt, a via is patterned into the PZT by ion-milling followed by a wet etch of the residual PZT using $\mathrm{H}_{2} \mathrm{O}: \mathrm{HCl}: \mathrm{HF}(2: 1: 0.05)$, see Fig. 1c. The $\mathrm{SiO}_{2}$ is then etched using reactive ion etching (RIE) followed by a $\mathrm{Si}$ deep reactive ion etch (DRIE) of the device Si layer and another RIE of the buried oxide, see Fig. 1d. Next, a $150 \mathrm{~nm}$ protective coating of alumina is deposited by atomic layer deposition. The $\mathrm{Al}_{2} \mathrm{O}_{3}$ is then patterned with a reactive ion etch to open access to the contact areas of the PZT actuator as well as the bottom of the DRIE etch trench, see Fig. 1e. The co-planar waveguide (CPW) transmission lines consisted of a tri-layer of $\mathrm{Cr} / \mathrm{Pt} / \mathrm{Au}(20 / 20 / 730 \mathrm{~nm})$ and were deposited by electron beam evaporation and patterned via lift-off, see Fig. 1f. In order to control the switch contact surfaces, specific contact materials were deposited on the contacting pads. The contact metallurgy was a bi-layer of $\mathrm{Au} / \mathrm{Ru}(400 / 100 \mathrm{~nm})$ and was deposited by electron beam evaporation and patterned via lift-off. Next, a sacrificial layer of photoresist is deposited and patterned to define the gap for air bridges and contact cantilevers. A $2 \mathrm{~m}$ thick gold air bridge layer is then deposited via electron beam evaporation and patterned using lift-off. Afterwards, the resist layer is removed by an oxygen plasma followed by a wet clean in dimethyl sulfoxide solution. Finally, a short reactive ion etch of the native oxide in the bottom of the DRIE trench is performed in a fluorinated plasma followed by a xenon difluoride etch to fully release the piezoelectric actuator composite, see Fig 1g.

\section{MEASURED RESULTS}

PZT and PZT-on-SOI technology [9], [10] were used to fabricate a variety of MEMS devices and circuits. An optical image of the fabricated analog RF MEMS attenuator is shown in Figure 2.

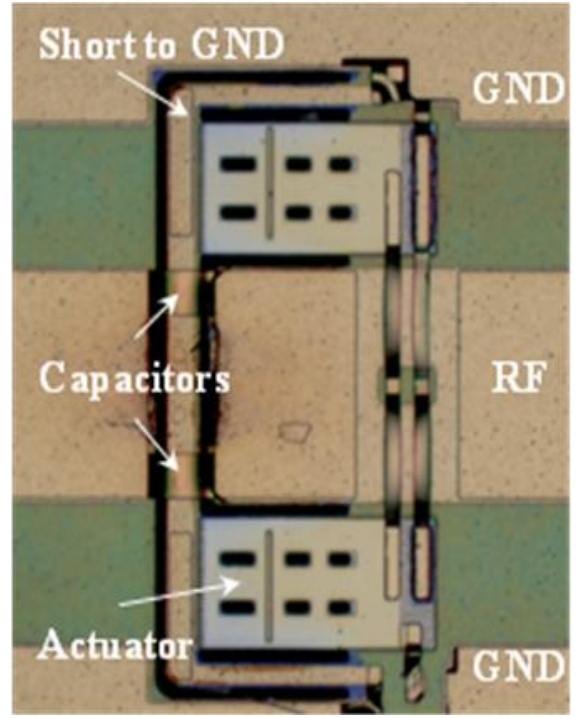

Figure 2. Optical image of analog RF MEMS attenuator.

The MEMS structure is placed in a coplanar waveguide transmission line. The wide line in the middle of Figure 2 is the signal conductor, while the two lines at the top and bottom of the figure are part of the RF ground plane. The transmission line is designed to have a $50 \Omega$ characteristic impedance in the metal/dielectric stack of the technology. RF signals are input in the device from the left side, go through the MEMS attenuator, and exit through the output port on the right hand side. Mechanical motion is accomplished by the use of two PZT actuators; these are the structures with release holes, placed in the dielectric gap of the $\mathrm{RF}$ transmission line. A bias voltage is applied to the PZT capacitors through gold air-bridges located on the anchored side of the actuators (right side of Fig. 2). On the released end of each actuator is a movable beam that mechanically couples them together. The beam extends in a $U$ shape and wraps around the actuators. The top surface of the beam is metalized and serves dual purpose. Under the crossover with the transmission line it forms the bottom movable plate for the coupling capacitors. At the end of the $U$ shape extensions it connects to the ground plane and provides an electrical short circuit for the RF signals.

Two capacitors are formed between bridges in the RF signal line and the movable short to ground. As the actuators change the spacing between the two electrodes of the capacitors, the coupling of the RF signal to ground is changed and different levels of attenuation can be achieved between the input and the output ports. The RF frequency was swept from $100 \mathrm{MHz}$ to $25 \mathrm{GHz}$ and Figure 3 shows the response of the attenuator for different actuation voltages.

The $14.5 \mathrm{~V}$ state determines the maximum attenuation when the two plates of the variable capacitor are in contact, while the 0 $\mathrm{V}$ state represents maximum separation and respectively minimum attenuation. 


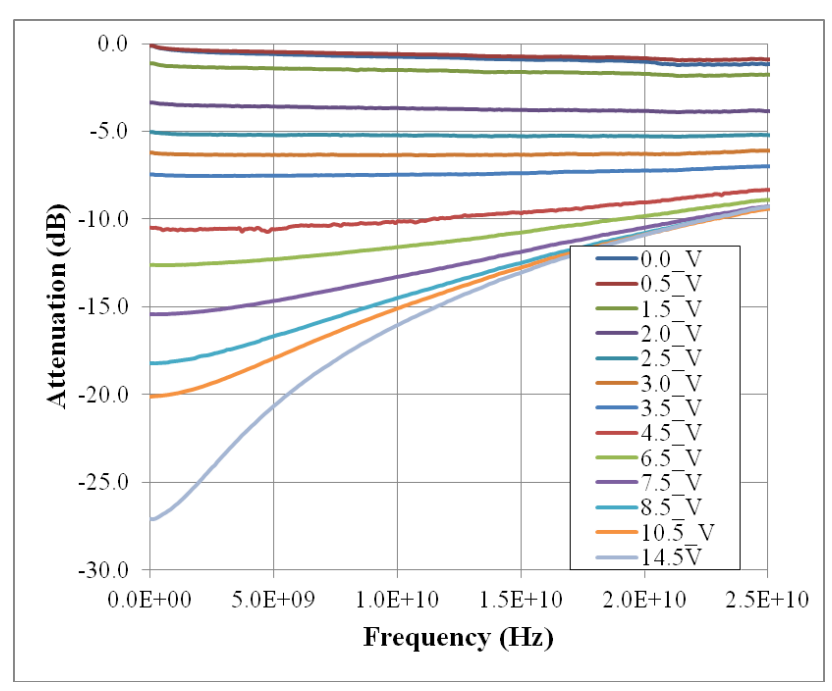

Figure 3. Frequency response of the analog MEMS attenuator for different actuation voltages. The trace for $0 \mathrm{~V}$ is the top most one. As the voltage increases, the attenuation is increased and the bottom most trace shows response for $14.5 \mathrm{~V}$.

These two states constitute a MEMS switch. The rest of the states, which are of particular interest for a variable attenuator, show continuous coverage between the "open" and "closed" conditions.

Piezoelectric devices generate displacement proportional to the voltage applied across the PZT film (not the contact gap), hence they are capable of continuous gap control. Exact numbers of the measured attenuation, for select frequencies, are given in Table 1. Below $2 \mathrm{GHz}$, the attenuation range is from $0.5 \mathrm{~dB}$ to more than $26 \mathrm{~dB}$. At $25 \mathrm{GHz}$ the maximum attenuation is reduced to $9.3 \mathrm{~dB}$. Also, the response is quite frequency independent for attenuation levels of up to $\sim 7 \mathrm{~dB}$, with frequency dependency becoming clearly expressed for higher attenuation levels.

\section{LINEAR ANTENNA ARRAY}

One of the possible applications for continuously tunable RF MEMS attenuators is in beam forming networks for antenna arrays. Schematic representation of an eight element linear antenna array is shown in Figure 4.

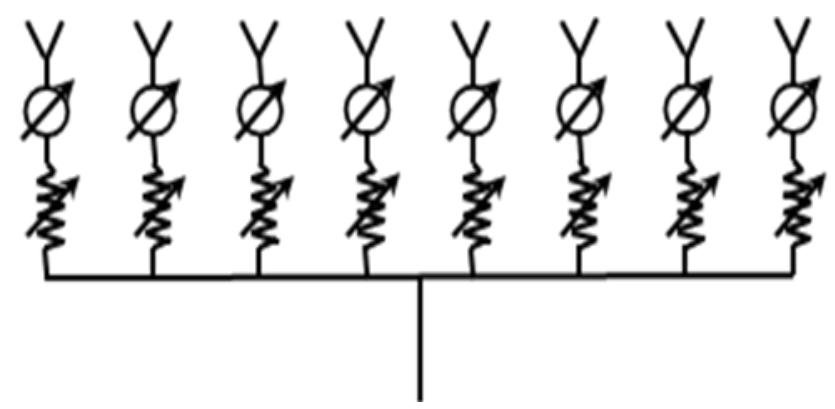

Figure 4. Schematic diagram of beam forming network. Components included are - antenna elements, phase shifters, attenuators and power divider.

The concept of beam steering is relatively straightforward. Input RF power is divided equally among all branches of the beam forming network. In each branch, the amplitude and phase of the radiated signals can be adjusted. The desired outcome is that the main lobe beam width, radiation direction, side lobe levels, nulls locations, etc., can be controlled fast and efficiently through electronic means. Usually, the attenuator, phase shifter and possibly a power amplifier constitute the 'antenna module' for a transmitting array radiator. They are placed behind the individual radiating element and it is desirable for the components to be compact in size and light weight.

Attenuators play a role in adjusting the amplitude of the radiated signals. Creating a non-uniform amplitude distribution (excitation pattern) along the aperture of the array affects the side lobes level of the radiation pattern, the beam width of the main lobe and the gain of the array. There are number of excitation patterns that have been adopted for beam control.

We will consider three of them. Uniform distribution is usually used as a reference for comparison. It provides a narrow main lobe but the peak of the first side lobe is only $13 \mathrm{~dB}$ lower that the main one.

Taylor distribution is commonly used because one can specify the maximum acceptable side lobes level. In this paper we consider a $-20 \mathrm{~dB}$ level for the side lobes. Cosine squared $\left(\cos ^{2}\right)$ is another distribution targeted at reducing the side lobes level. It uses a more aggressive taper. Consequently, the side lobes are reduced more at the expense of some broadening of the main beam and gain reduction. Plots of the radiation patterns calculated based on all three excitation distributions are shown in Figure 5.

Table 1. Attenuation in $d B$ at different actuation voltages and frequencies.

\begin{tabular}{|c|c|c|c|c|c|c|c|c|c|c|c|c|c|}
\hline \multirow{2}{*}{$\begin{array}{c}\text { Freq. } \\
\text { (GHz) }\end{array}$} & \multicolumn{13}{|c|}{ Actuation Voltage (V) } \\
\hline & 0 & 0.5 & 1.5 & 2 & 2.5 & 3 & 3.5 & 4.5 & 6.5 & 7.5 & 8.5 & 10.5 & 14.5 \\
\hline 0.1 & 0.13 & 0.14 & 1.09 & 3.33 & 5.01 & 6.21 & 7.46 & 10.48 & 12.61 & 15.42 & 18.19 & 20.12 & 27.06 \\
\hline 5.0 & 0.60 & 0.50 & 1.40 & 3.56 & 5.21 & 6.33 & 7.54 & 10.58 & 12.27 & 14.66 & 16.65 & 17.92 & 20.63 \\
\hline 10.0 & 0.76 & 0.62 & 1.49 & 3.65 & 5.21 & 6.34 & 7.49 & 10.11 & 11.57 & 13.28 & 14.47 & 15.07 & 16.03 \\
\hline 15.0 & 0.91 & 0.75 & 1.61 & 3.75 & 5.28 & 6.33 & 7.39 & 9.66 & 10.75 & 11.85 & 12.48 & 12.75 & 13.05 \\
\hline 20.0 & 1.04 & 0.85 & 1.74 & 3.81 & 5.28 & 6.28 & 7.22 & 9.04 & 9.81 & 10.46 & 10.76 & 10.86 & 10.90 \\
\hline 25.0 & 1.16 & 0.91 & 1.77 & 3.82 & 5.21 & 6.09 & 6.97 & 8.32 & 8.90 & 9.27 & 9.38 & 9.40 & 9.30 \\
\hline
\end{tabular}




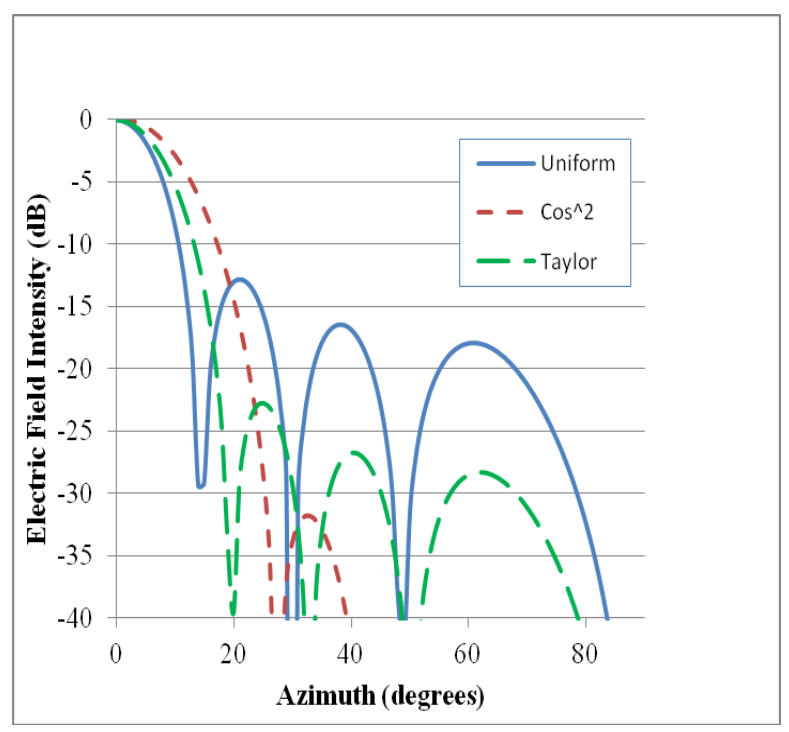

Figure 5 Radiation patterns of 8 element linear array for different amplitude distribution of the signals between individual radiating elements.

The attenuation for every element (both $\cos ^{2}$ and Taylor distributions) is listed in Table 2. Comparing the required attenuation levels from Table 2 with the demonstrated performance of the RF MEMS attenuator from Table 1 allows the following conclusions to be drawn: 1) Uniform distribution can be accomplished with the lowest loss state of the attenuator and the worst case insertion loss penalty will be $1.16 \mathrm{~dB}$ at $25 \mathrm{GHz}$. At lower frequencies the introduced loss is significantly less. 2) Taylor distribution with side lobe peaks suppressed to $-20 \mathrm{~dB}$ can be realized in the whole frequency range from $100 \mathrm{MHz}$ to 25 GHz.

Table 2. Radiating element attenuation in $\mathrm{dB}$ for 8 element array.

\begin{tabular}{|c|c|c|}
\hline element \# & Cos $^{2}$ & Taylor \\
\hline $\mathbf{1}$ & 18.4 & 9.0 \\
\hline $\mathbf{2}$ & 7.4 & 4.0 \\
\hline $\mathbf{3}$ & 2.2 & 1.3 \\
\hline $\mathbf{4}$ & 0.0 & 0.0 \\
\hline $\mathbf{5}$ & 0.0 & 0.0 \\
\hline $\mathbf{6}$ & 2.2 & 1.3 \\
\hline $\mathbf{7}$ & 7.4 & 4.0 \\
\hline $\mathbf{8}$ & 18.4 & 9.0 \\
\hline
\end{tabular}

3) Cosine squared distribution can be supported from 100 $\mathrm{MHz}$ to $7 \mathrm{GHz}$. The broad band performance and low loss of the RF MEMS attenuator exceeds the capabilities of waveguide and hybrid attenuators and rivals the solid state solutions. At the same time the RF MEMS attenuator is very competitive in terms of size, weight and consumed DC power. The presented MEMS attenuator has adequate performance for use in beam forming networks.

\section{CONCLUSIONS}

An analog RF MEMS attenuator with PZT actuators was presented. The device was fabricated using PZT-on-SOI technology. It demonstrated minimum insertion loss of $0.13 \mathrm{~dB}$ and $1.16 \mathrm{~dB}$ for frequencies of $100 \mathrm{MHz}$ and $25 \mathrm{GHz}$, respectively. In the same frequency range, the maximum attenuation was 27.06 $\mathrm{dB}$ and $9.3 \mathrm{~dB}$. Continuous voltage controlled attenuation was achieved for all states between the above two extremes. The attenuation function is achieved with a single MEMS device featuring compact size and with maximum DC voltage of $14.5 \mathrm{~V}$. The analog RF MEMS attenuator was evaluated for use in linear antenna array beam forming network. It was found that uniform distribution can be achieved with minimum insertion loss in broad frequency range $(100 \mathrm{MHz}-25 \mathrm{GHz})$. Taylor distribution with -20 $\mathrm{dB}$ peak level of the antenna pattern side lobes can also be supported across the entire frequency range. The more aggressive $\cos ^{2}$ excitation distribution can be supported from $100 \mathrm{MHz}$ to 7 $\mathrm{GHz}$.

Overall, the proposed analog RF MEMS attenuator with PZT actuators has superior frequency performance compared to waveguide and hybrid solutions and has very competitive size, weight and power consumption, compared to solid state attenuators.

\section{ACKNOWLEDGMENTS}

The authors would like to acknowledge the work of Mr. Brian Power, Mr. Joel Martin, and Ms. Luz Sanchez for their roles in the fabrication of the devices.

\section{REFERENCES}

[1] B.E. Kingdon, "An S - Band Variable Attenuator for High Power Working," Journal of the British Institution of Radio Engineers, 15, pp. 471-478 (1955)

[2] A.B. Giordano, "Design Analysis of a TM-Mode Piston Attenuator," Proceedings of IRE, 35, 545 (1950)

[3] J.P. Starski, "C-Band Attenuator/Phase Shifter Unit," $16^{\text {th }}$ European Microwave Conference, pp. 463-468 (1986)

[4] C.E. Saavedra and Y. Zheng, "Ring-Hybrid Microwave Voltage-Variable Attenuator Using HFET Transistors," IEEE Trans. Microwave Theory \& Tech, 53, pp. 2430-2434, (2005)

[5] K. Miyatsuji and D. Ueda, "A Low-Distortion GaAs Variable Attenuator IC for Digital Mobile Communication System," IEEE Int. Conf. Solid State Circuits, pp. 42-43, (1995)

[6] L. Boglione and R. Pavio, "Temperature and Process Insesitive Circuit Design of a Voltage Variable Attenuator IC for Cellular Band Applications," Microwave and Guided Wave Letters, 10, pp. 279-281 (2000)

[7] A. Unamuno and D. Uttamchandani, "MEMS variable optical attenuator with vernier latching mechanism," Photonics Technology Letters, 18, 88 (2006).

[8] K. Van Caekenberghe, "RF MEMS technology for radar sensors," Radar Conference - Surveillance for a Safer World, 1 (2009).

[9] R. Polcawich, J. Pulskamp, D. Judy, P. Ranade, S. TrolierMcKinstry, and M. Dubey, "Surface Micromachined MicroElectromechancial Ohmic Series Switch Using Thin-Film Piezoelectric Actuators," IEEE Trans. Microwave Theory \& Tech, 55, 2642, (2007).

[10] T.G. Ivanov, J.S. Pulskamp, R.G. Polcawich and R.M. Proie, "Shunt RF MEMS Contact Switch based on PZT-on-SOI Technology," to be published at IEEE IMS 2012

\section{CONTACT}

T.G. Ivanov, tel:+1-301-394-3568; tony.g.ivanov.civ@mail.mil 


\section{HIGH-FREQUENCY LARGE-DEFLECTION ELECTROSTATIC DIAPHRAGM ACTUATORS WITH MAXIMIZED VOLUME DISPLACEMENT}

Seow Yuen Yee, Rebecca L. Peterson, Luis P. Bernal and Khalil Najafi

Center for Wireless Integrated MicroSensing and Systems (WIMS ${ }^{2}$ ), University of Michigan, Ann Arbor, Michigan USA

\section{ABSTRACT}

This paper demonstrates electrostatic (ES) actuators with high frequency diaphragms $(>90 \mathrm{kHz})$, large deflection $(>8 \mu \mathrm{m})$, high volume displacement, and reliable operation using a new fabrication process and device structure. Electrode gaps of up to $9.58 \mu \mathrm{m}$ have been fabricated with a filleted electrode shape which allows the pulled-in diaphragm to follow the curved profile of the electrode, displacing $100 \%$ of the air gap volume. The filleted electrode actuators have been dynamically actuated for over 212 hours without any diaphragms breaking or sticking. In contrast flat electrode diaphragms broke after $<2$ minutes of actuation.

\section{INTRODUCTION}

Many MEMS applications require high-deflection $(>5 \mu \mathrm{m})$, high-frequency $(>50 \mathrm{kHz})$, and low-power diaphragm actuators which displace a large air volume. Examples include micropropulsion systems (high-velocity air jets), micro-pumps (high flow rate), micro-speakers (high sound pressure), and microcoolers (large flow volume). Diaphragm actuation methods include electrostatic (ES) [1-3], electromagnetic [4], piezoelectric [5] and thermo-pneumatic [6]. Typically electromagnetic and piezoelectric actuators face challenges in device miniaturization, while electromagnetic and thermo-pneumatic actuators are limited by slow response time or low actuation frequency. Therefore we pursue ES actuators for high-frequency, high-deflection microactuation.

High frequency operation of parallel plate capacitive ES diaphragm actuators requires small-mass and high-stiffness diaphragms with large intrinsic stress. Large-deflection ES diaphragm actuators induce large extrinsic stress and require large drive voltages, inducing high electric fields. In addition, the microfabrication of large air gaps is not easy. The combination of large mechanical stress (causing fracture), high electric field (degrading reliability and lifetime), and fabrication difficulties has prevented these actuators from being widely utilized. Curved or zipper ES actuators have been made [1-3], but they do not provide the high frequency, large deflection, reliability, and maximum volume displacement needed.

Previous ES actuators fabricated by our group achieved deflection of $6 \mu \mathrm{m}$ [2] and $8.8 \mu \mathrm{m}$ [3], but only a portion of the total air gap volume was displaced. Here we present an ES diaphragm actuator with large deflection $(>8 \mu \mathrm{m})$, maximized volume displacement and reliable actuation, made using a much simpler and more robust fabrication process. We compare the performance of two different electrode designs, flat and filleted.

\section{FABRICATION}

\section{Flat Electrode}

Arrays of ES actuators (Figure 1 (Left)) are fabricated with a simple three-mask process (Figure 2a). First, an oxide-nitrideoxide stack is deposited by LPCVD on a blank silicon wafer. The deposited dielectric stack has a total thickness of $\sim 1.75 \mu \mathrm{m}$ and intrinsic stress of 32 or $74 \mathrm{MPa}$ for membrane resonance frequencies of $64 \mathrm{kHz}$ and $97 \mathrm{kHz}$, respectively. Next, a $\sim 10 \mu \mathrm{m}$ layer of sacrificial photoresist (PR) AZ9260 is spun and patterned
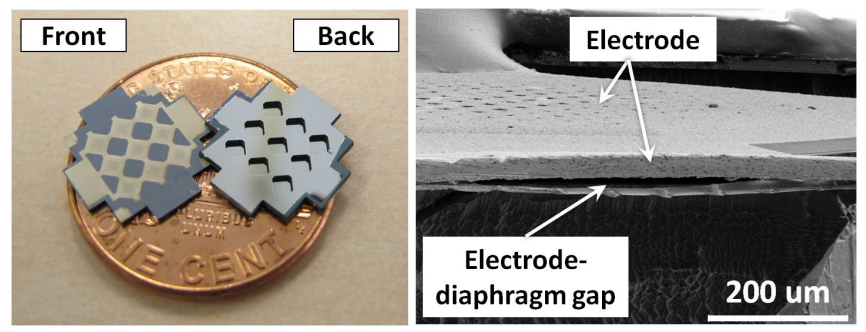

Figure 1: (Left) Fabricated device with an array of nine ES diaphragm actuators, (Right) SEM of flat electrode cross section.

to define the air gap between the diaphragm and electrode. A seed layer of chrome/gold is sputtered over the whole wafer, followed by spinning and patterning of a $>20 \mu \mathrm{m}$ photoresist mold to shape the electroplated nickel electrode. After electroplating, the photoresist mold, seed layer, and sacrificial photoresist are sequentially etched and removed. Finally, the diaphragm is released by deep reactive ion etching (DRIE) from the backside and a thin layer of aluminum is sputtered on backside to form the second electrode of the parallel plate capacitor. Figure 1(Right) shows an SEM of the cross section of a flat electrode actuator.
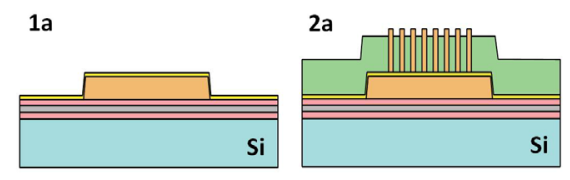

$1 b$
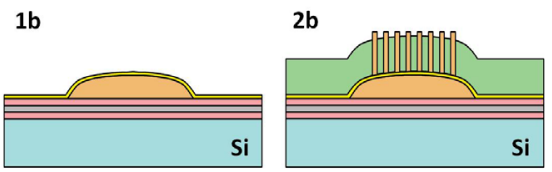

LPCVD ox/ni/ox Pattern sacrificial PR Reflow PR (only 1b)

Pattern PR mold Sputter $\mathrm{Cr} / \mathrm{Au}$
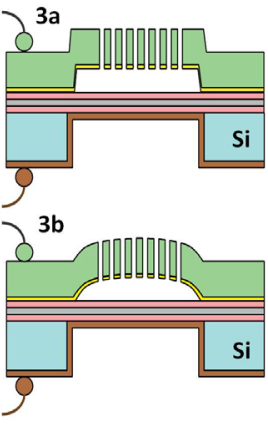

Remove PR mold Etch $\mathrm{Cr} / \mathrm{Au}$ Remove sacrificial PR DRIE backside etch Sputter $\mathrm{Cr} / \mathrm{Al}$ from back

Figure 2: Fabrication process for (a) flat electrode and (b) filleted electrode actuators.

\section{Filleted Electrode}

Filleted electrodes were designed to maximize volume displacement and improve the reliability of the large-gap ES actuator by reducing the large electric field and mechanical stress at the edges of the diaphragm. The filleted electrodes are fabricated with a similar fabrication process but with an extra step: re-shaping of the sacrificial photoresist through a reflow process (Figure 2b). The additional PR reflow step is modified from [7], in which the wafer is turned upside down and exposed to acetone vapor for 8-10 minutes to reshape the PR through gravity and surface tension. The reflow process duration is optimized to shape the PR into a dome while maintaining its base pattern (square, 
hexagon, etc.) as shown in Figure 3. The reflow process increases the PR thickness at the center (Table 1), so the initial PR layer is made thinner to achieve the same final gap as in the flat electrode (Figure 4). Figure 5 shows microscope images of the flat and filleted electrode before and after electroplating. We note that a thicker PR layer could be used in the future for greater volume displacement.
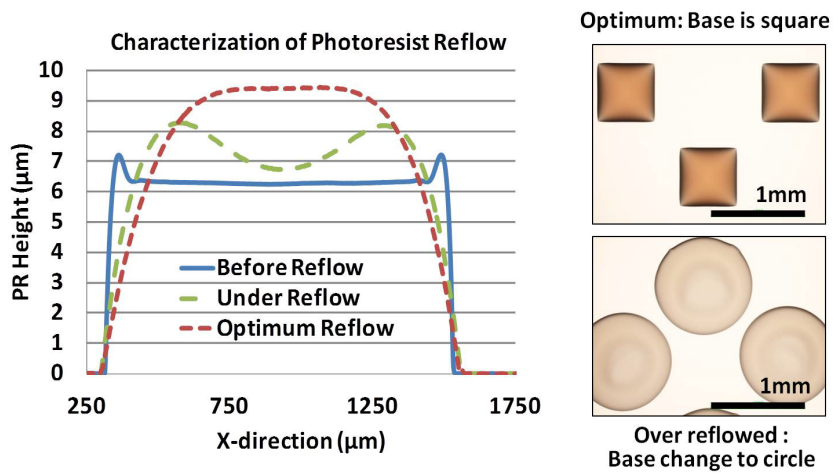

Figure 3: (Left) Filleted sacrificial PR profile under insufficient and optimal reflow; and (right) micrographs showing excessive reflow of base shape from square to circle-like.

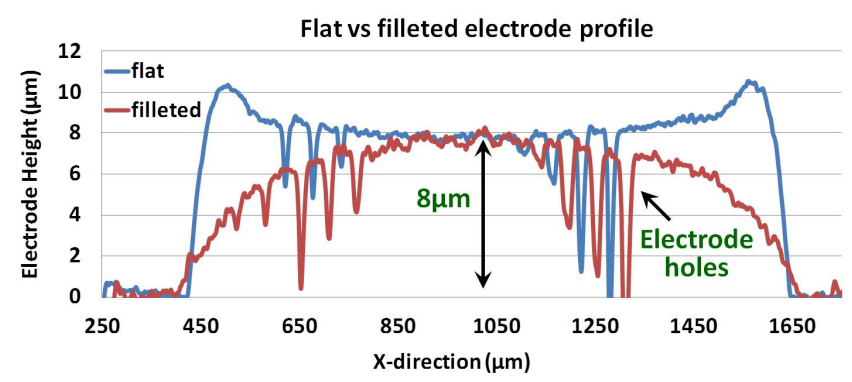

Figure 4: Comparison of flat and filleted electrode profiles with the same center gap spacing.

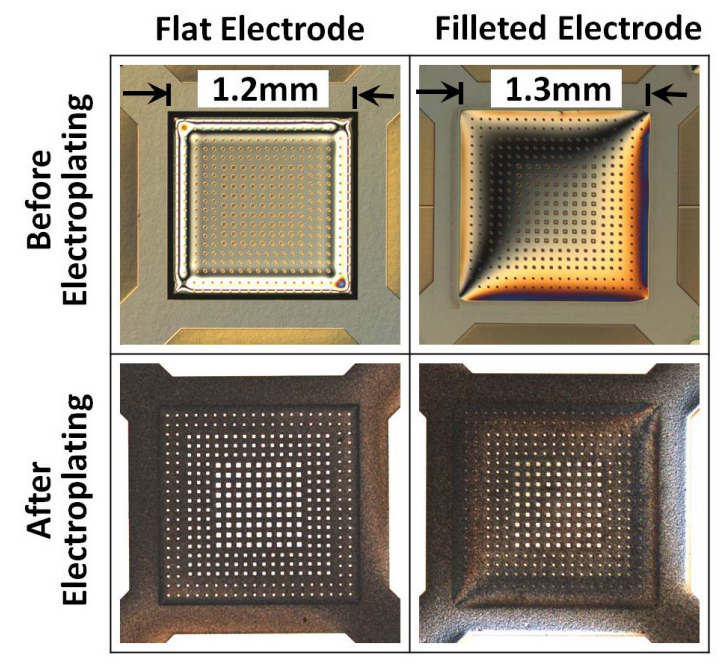

Figure 5: Microscope images before and after electroplating (front view) for flat and filleted electrode.
Table 1: Performance of flat vs. filleted electrode actuators.

\begin{tabular}{|c|c|c|c|}
\hline Device Parameters & \multicolumn{2}{|c|}{ Flat Electrode } & Filleted Electrode \\
\hline Diaphram resonance frequency & $64 \mathrm{kHz}$ & $97 \mathrm{kHz}$ & $97 \mathrm{kHz}$ \\
\hline Diaphragm residual stress & $32 \mathrm{MPa}$ & $74 \mathrm{MPa}$ & $74 \mathrm{MPa}$ \\
\hline Diaphragm area & \multicolumn{2}{|c|}{$1.2 \mathrm{~mm} \times 1.2 \mathrm{~mm}$} & $1.3 \mathrm{~mm} \times 1.3 \mathrm{~mm}$ \\
\hline Diaphragm thickness & $1.46 \mu \mathrm{m}$ & $1.74 \mu \mathrm{m}$ & $1.74 \mu \mathrm{m}$ \\
\hline Sacrificial PR height before reflow & \multirow{2}{*}{$7.9 \mu \mathrm{m}$} & \multirow{2}{*}{$8.33 \mu \mathrm{m}$} & $6.2 \mu \mathrm{m}$ \\
\hline Average gap at center & & & $8.14 \mu \mathrm{m}$ \\
\hline $\begin{array}{l}\text { Applied signal for diaphragm pull- } \\
\text { in }\end{array}$ & $\begin{array}{l}(10 \mathrm{kHz}) \\
\pm 210 \mathrm{~V}\end{array}$ & $\begin{array}{l}\text { (DC) } 337 \mathrm{~V} \\
\text { (AC) N/A }\end{array}$ & $\begin{array}{l}(1 \mathrm{kHz}) \pm 240 \mathrm{~V} \\
(10 \mathrm{kHz}) \pm 279 \mathrm{~V}\end{array}$ \\
\hline Air gap volume (calculated) & \multicolumn{2}{|c|}{$11.52 \mathrm{e}^{-12} \mathrm{~m}^{3}$} & $9.7 \mathrm{e}^{-12} \mathrm{~m}^{3}$ \\
\hline Volume displaced (calculated) & \multicolumn{2}{|c|}{$8.65 \mathrm{e}^{-12} \mathrm{~m}^{3}$} & $9.7 \mathrm{e}^{-12} \mathrm{~m}^{3}$ \\
\hline Reliable actuation & $<2 \min$ & - & $1 \mathrm{hr}$ \\
\hline
\end{tabular}

\section{TEST RESULTS}

\section{Flat Electrode}

The flat electrode $97 \mathrm{kHz}$-diaphragms with a $\sim 8.3 \mu \mathrm{m}$ gap pull-in at $\sim 337 \mathrm{~V} \mathrm{DC}$, and easily break due to the large electric field and mechanical stress at the edges. For the $64 \mathrm{kHz}-$ diaphragms with flat electrodes (7.4-8.22 $\mu \mathrm{m}$ center gap), pull-in happens for pulse waveforms of $\pm 210 \mathrm{~V}$ at $10 \mathrm{kHz}$. The left columns of Figures 6 and 7 show that the collapsed diaphragms displace $75 \%$ of the total gap volume. Only six of the nine diaphragms are pulled down and one or more diaphragms start to break after 2 seconds of actuation, and all of the diaphragms break after only $\sim 2$ minutes of actuation (left column of Figure 8 ).

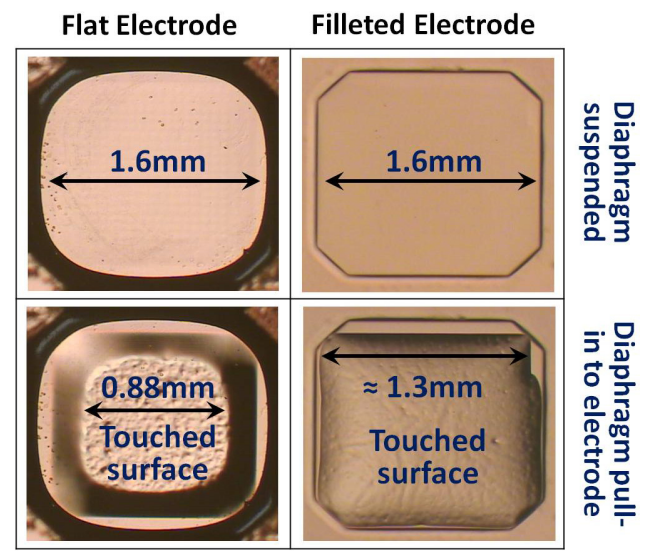

Figure 6: Back view of diaphragm pull-in shape for flat lleft column) and filleted electrodes (right column).

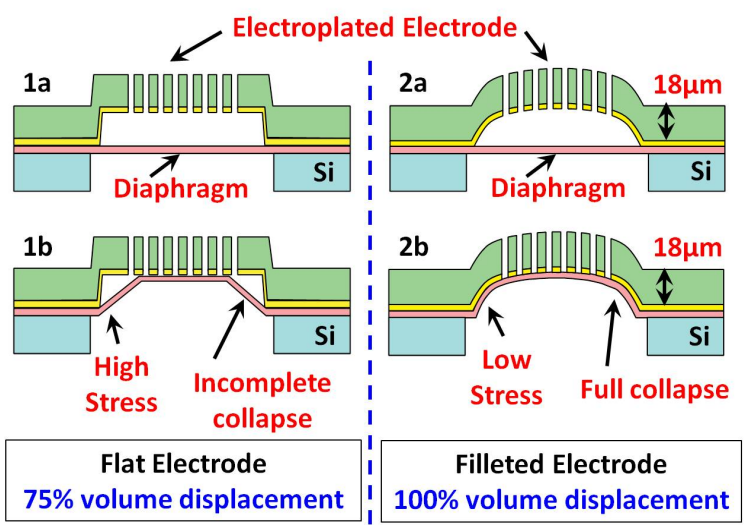

Figure 7: Cross sectional diagram: flat vs. filleted electrode. 


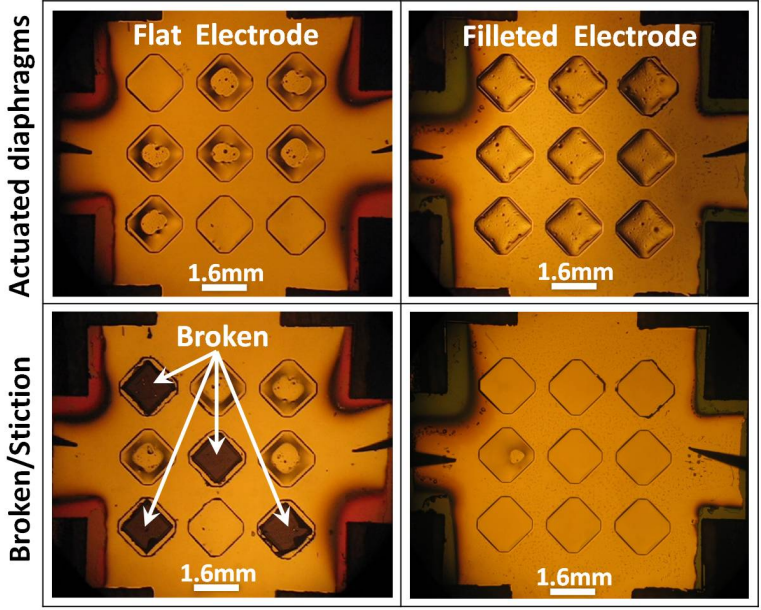

Figure 8: Long Term Test 1: Back view. (Left top) Only six of nine flat electrode diaphragms pull in. (Right to) All filleted electrode diaphragms pull-in and are held against nickel electrode. (Left bottom) One or more diaphragms break after $\sim 2$ minutes actuation. (Right bottom) No diaphragms break after 1 hour actuation.

\section{Filleted Electrode}

With a slightly modified electrode, the $97 \mathrm{kHz}$-diaphragms with filleted electrode $(7.7-9.58 \mu \mathrm{m}$ center gap) pull in at pulse waveform of $\pm 240 \mathrm{~V}$ at $10 \mathrm{kHz}$ (Table 1). The filleted-electrode diaphragm hugs the curved profile of the electrode, displacing $\sim 100 \%$ of the air gap volume (right columns of Figure 6 and 7).

In order to investigate diaphragm deflection, a Polytec laser vibrometer is used to measure dynamic deflection. The laser is focused on the center diaphragm of the actuator array. The diaphragm is actuated from $1 \mathrm{kHz}$ to $14 \mathrm{kHz}$ at varying voltages and duty cycles. (The maximum frequency is limited by the current drive circuitry.) We find that a duty cycle of $20 \%$ provides the best actuation for maximum deflection and triangle-like movement. At voltages greater than the pull-in voltage and with long duty cycles, the diaphragm collapses onto the electrode and sticks to the electrode. Figure 9 shows a drive signal at $10 \mathrm{kHz}$ with $20 \%$ duty cycle and the corresponding diaphragm displacement detected with the laser vibrometer.
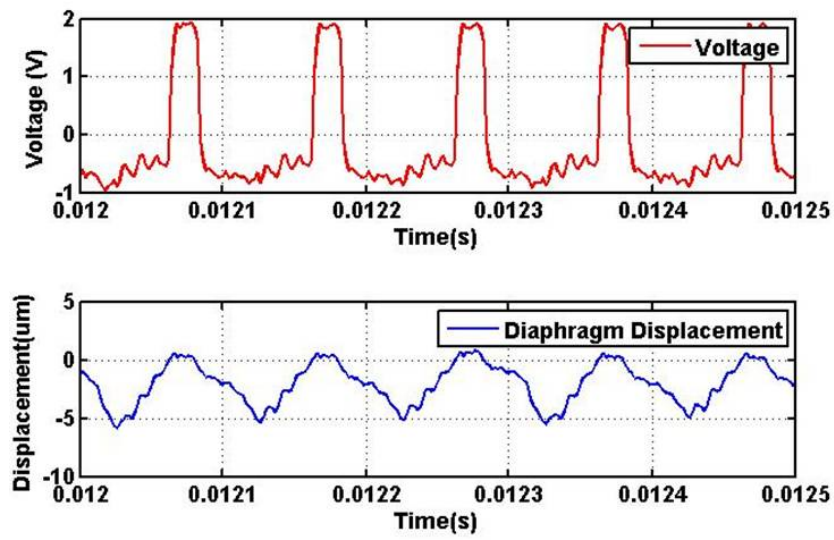

Figure 9: (top) Voltage applied across the actuator array (scaled by a factor of 1/97). (Bottom) Measured displacement of the center diaphragm, as detected by the vibrometer.

\section{Reliability Test of Filleted Electrodes}

Reliability tests with different degrees of mechanical and electrical stress were performed to investigate the device limits. For the worst-case scenario, the diaphragm is collapsed and held to the bottom electrode for a long time. This test injects charge into the dielectric layer from both the bottom and top electrodes, similar to the tests in [8]. In addition, the diaphragm is maximally deflected thus inducing maximum mechanical stress on the diaphragm edges. The diaphragm is held to the electrode with a pulse waveform of $10 \mathrm{kHz}, \pm 279 \mathrm{~V}$ and $50 \%$ duty cycle. The $50 \%$ duty cycle and large actuation voltage cause the diaphragm to be held to the electrode continually. After some time, the voltage is completely removed to investigate whether the diaphragm returns to its release position.

In this test, all nine diaphragms of the actuator array are held against the electrode for $>30$ minutes with no stiction. Only one filleted electrode diaphragm exhibits stiction after 1 hour (right bottom image of Figure 8). This is at least 30 times better than the flat electrode design, where the diaphragm broke beyond repair after $\sim 2$ minutes. Of all three reliability tests conducted, this test shows the shortest reliable actuation time. This test was used to model the worst-case scenario in which charge is injected from both the top and bottom electrodes [9].

The second reliability test involves repeatedly pulling in the diaphragm towards the nickel electrode and then releasing it. This test is designed to investigate its fatigue and DC drift during dynamic operation. In this test, the duration of time when the diaphragm is in contact with the electrode is minimized compared to the first test, so that charging from the nickel electrode is minimized. A $20 \%$ duty cycle is used to minimize the contact time, creating triangle-like dynamic movement for all diaphragms, as shown in Figure 9. For the long-term test, a calibrated microphone is used to measure the acoustic pressure. Tests with various pulse waveforms are performed and sound pressure from the combined movement of the array of diaphragms is measured by a calibrated microphone positioned $\sim 1.5 \mathrm{~cm}$ away from the array. The long-term test is done using the conditions under which maximum sound pressure is detected, with a pulse waveform of $10 \mathrm{kHz}$, duty cycle of $20 \%$ and voltage across the device of $+242.5 \mathrm{~V}$ to $-48.5 \mathrm{~V}$, for minimal charging effects.

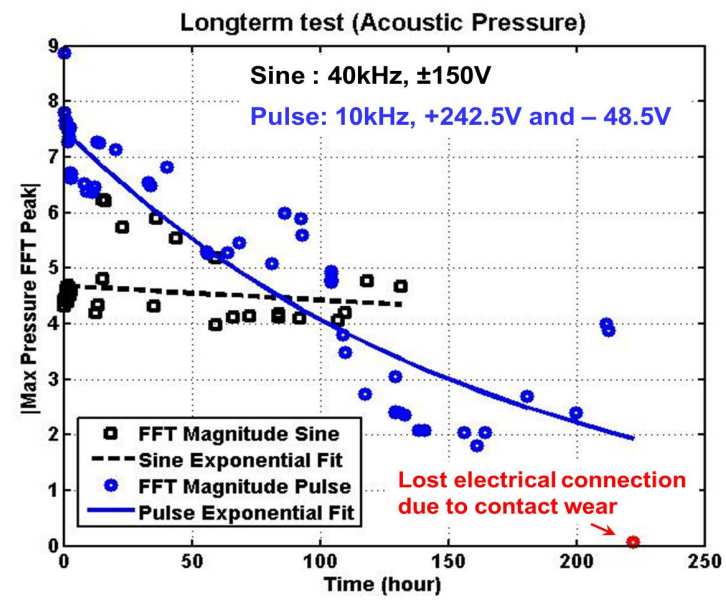

Figure 10: Long term test 2 (blue circle): 20\% duty cycle pulse wave actuation. Sound pressure falls to zero after $\sim 212$ hour due to loss of electrical connection due to damage of the aluminum metal layer (top electrode). Long term test 3 (black square): Sinusoidal actuation, showing a much slower charging effect compared to test 2 . 
The FFT of the acoustic pressure is obtained and the magnitude of the maximum peak of the FFT is plotted with respect to time (Figure 10 (blue circles)). The maximum FFT peak is detected at $110 \mathrm{kHz}$, which is near the $97-\mathrm{kHz}$ resonance frequency of the diaphragm even though it is driven with a $10 \mathrm{kHz}$ pulse waveform. The fluctuations in the detected acoustic pressure might be related to the variable humidity and temperature of the environment during the day or night. For example, higher acoustic pressure corresponding to higher diaphragm deflection is observed at night while lower acoustic pressure is observed during day time. We observe charging effects as the acoustic pressure decays exponentially with time. The charge accumulation in the dielectric of the diaphragm is hypothesized to create DC deflection or DC drift and thus reduces dynamic motion. The time constant for the charging effect is 164 hours. The lower duty cycle used in this test compared with the previous test (in which stiction was observed after $<1$ hour), results in much longer device operation. The lower duty cycle reduces the contact time of the diaphragm to the bottom electrode, which should minimize charging effects as described in [9]. After more than 212 hours, the device stops working when the electrical connection to the top electrode is broken due to damage of the aluminum metal layer under the probe tip. The device can be actuated again if the probe is moved to a different spot, with similar performance, as shown by the first data point in Figure 11. In fact if higher voltage is then applied, the acoustic pressure increases above the initial values before the long-term test began. However, after $\sim 7$ minutes of actuation at this larger voltage, one diaphragm collapses and sticks to the nickel electrode, causing a decrease in acoustic pressure (last point in Figure 11).

In the third reliability test, the diaphragm is driven with a much higher frequency in a sinusoidal fashion $(\sim 80 \mathrm{kHz}$ deflection). A sine waveform at $40 \mathrm{kHz}$ and $\pm 150 \mathrm{~V}$ is applied, plotted in Figure 10 (black squares). The FFT peak frequency is detected at $80 \mathrm{kHz}$. The applied voltage is $\sim \pm 150 \mathrm{~V}$ which is limited by the power amplifier. The array was actuated reliably for greater than 131 hours without significant deterioration in performance. The charging time constant for the sine test is 1754 hours, which is approximately 10.7 times that found using the pulse waveform in the second test. This may be because the second test is performed at higher voltage, close to unipolar waveform and with faster rise and fall time than the sine test, leading to more charging effects during actuation. During the test, there are a couple higher acoustic pressure data points which deviate from the exponential trend. We attribute these jumps in acoustic pressure to partial actuation of certain diaphragm(s) in the actuator array.

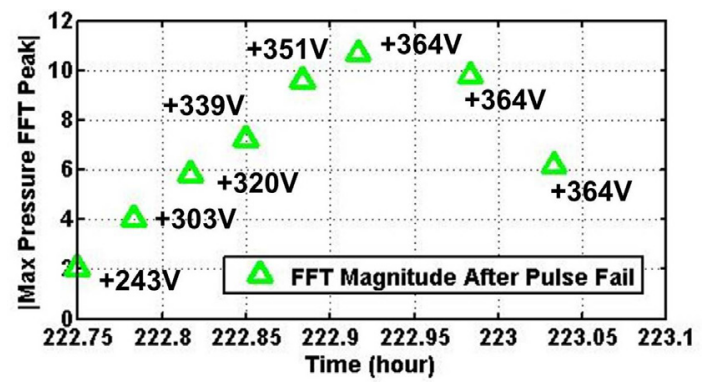

Figure 11: Continuation of long term test 2 after electrical contact is made at a different position. For reference, as plotted in Figure 10, the last measured pressure peak before the probe pad failure was 3.9, at an input voltage of $+243 \mathrm{~V}$.

No diaphragms broke during any of the three reliability tests. The stiction of the diaphragm from the first reliability test releases on its own after a few days. In comparison, the flat electrode diaphragm broke after $<2$ minutes actuation.

\section{CONCLUSIONS}

We have demonstrated a new fabrication process to form flat and filleted large-gap $(>8 \mu \mathrm{m})$ electrostatic actuators. Tests show that the filleted electrodes displace almost $100 \%$ of the air gap volume and are much more reliable than the flat electrodes due to reduction of the electric field and mechanical stress at the edges of the diaphragm. The reliability of the filleted electrode diaphragms has been investigated through several long-term tests. Using a $20 \%$ duty cycle pulse wave to repeatedly pull in the diaphragm, the actuators worked continuously for more than 200 hours until probe pad failure stopped the test. We expect that future tests will show much longer time-to-failure. These high-frequency, largedeflection ES actuators with maximized volume displacement can be used in a variety of microsystems.

\section{ACKNOWLEDGEMENT}

This project is supported by DARPA-CSVMP/HiVac program under grant \#W31P4Q-09-1-0011 and was performed in part at Lurie Nanofabrication Facility (LNF). Travel support has been generously provided by the Transducer Research Foundation.

\section{REFERENCES}

[1] W. van der Wijngaart, H. Ask, P. Enoksson, G. Stemme, “A high-stroke, high pressure electrostatic actuator for valve applications," Sensors and Actuators A: Physical, 100, 264271 (2002).

[2] T.K. Chou, K. Najafi, M.O. Muller, L.P. Bernal, P.D. Washabaugh, "High-density micromachined ejector array for micro propulsion," IEEE Transducers Conference, 2001-2, 890 (2001).

[3] H. Kim, A.H. Jauregui, C. Morrison, K. Najafi, L.P. Bernal, P.D. Washabaugh, "Low-power electrostatic microthruster for propulsion based on Helmholtz-resonance," IEEE MEMS Conference, 2007, 127 (2007).

[4] K. H. Kim, H. J. Yoon, O. C. Jeong, S. S. Yang, "Fabrication and test of a micro magnetic actuator", Sensors and Actuators A 117 (2005) 8-16.

[5] E. E. Aktakka, H. Kim, K. Najafi, "Wafer level fabrication of high performance MEMS using bonded and thinned bulk piezoelectric substrates", Proceedings of Transducers 2009, 849-852.

[6] O. C. Jeong, S. W. Park, S. S. Yang, J. J. Pak, "Fabrication of a peristaltic PDMS micropump", Sensors and Actuators A123-124 (2005) 453-458.

[7] T. Abe, H. Shimamoto, X. Li, "Miniaturization of spherically contoured rectangular AT-cut quartz-crystal resonators by using reactive ion etching," Japanese Journal of Applied Physics, 45, 5283 (2006).

[8] N. Tavassolian, M. Koutsoureli, E. Papandreou, G. Papaioannou, "The effect of silicon nitride stoichiometry on charging mechanisms in RF-MEMS capacitive switches", IEEE Transactions on Microwave Theory and Techniques, 57, 12 (2009).

[9] Z. Peng, X. Yuan, J. C. M. Hwang, D. Forehand, C. L. Goldsmith, "Top vs bottom charging of the dielectric in RF MEMS capacitive switches", Proceedings of Asia-Pacific Microwave Conference 2006, 1535-1538.

\section{CONTACT}

*K. Najafi, tel: +1-734-763-6650; najafi@umich.edu 


\section{HISTOLOGY-EQUIVALENT VERTICAL OPTICAL SECTIONING USING A 2-AXIS MAGNETIC CONFOCAL MICROSCANNER

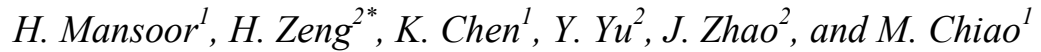 \\ ${ }^{1}$ The University of British Columbia, Vancouver, BC, CANADA \\ ${ }^{2}$ British Columbia Cancer Agency Research Centre, Vancouver, BC, CANADA \\ "hzeng@bccrc.ca}

\begin{abstract}
This paper presents a 2-axis confocal scanner for real-time and direct imaging of vertical cross-sections of biological samples. The device incorporated an out-of-plane lens actuator and a transverse optical fiber scanner. Two-dimensional dynamic scanning of samples using this device has been demonstrated by obtaining a scanning range of $145 \mu \mathrm{m}$ and $190 \mu \mathrm{m}$ in the $\mathrm{X}-\mathrm{Z}$ plane (perpendicular to the surface of sample). Optical resolutions of $4.8 \mu \mathrm{m}$ and $10.7 \mu \mathrm{m}$ have been demonstrated in $\mathrm{X}$ and $\mathrm{Z}$ directions respectively. Images obtained by this scanner are analogous to conventional histology images and can facilitate noninvasive clinical diagnosis and evaluation of diseases such as skin cancer.
\end{abstract}

\section{INTRODUCTION}

Scanning a beam of light in the X-Y plane (parallel to the surface of sample) using micromirrors and optical fibers has been previously demonstrated. Micromirror scanners have been developed using electromagnetic [1], electrostatic [2], electrothermal [3] and piezoelectric [4] techniques. Optical fiber scanners are driven using electromagnetic [5] or piezoelectric [6] actuators. These scanning techniques can be used to obtain confocal images from a horizontal cross-section of samples. Vertical cross-sectional images can also be constructed from stacks of horizontal images obtained from various depths within the sample [7]. However, this method of vertical image reconstruction could be time-consuming for high resolution imaging and therefore may be unsuitable for real-time clinical applications. As a result, a confocal scanner capable of providing direct and real-time vertical cross-sectional images is advantageous. Recently, a group has proposed direct vertical imaging by mounting a micromirror on a stage, actuated in the axial direction using piezoelectric unimorph beams [8]. We have previously reported a magnetic actuator for moving a lens in the axial direction and used it for measuring thickness of biological samples and transparent films [9-11].

In this paper, we demonstrate a 2-axis confocal scanner by coupling a magnetic lens actuator with a transverse fiber optic actuator. The device is capable of two-dimensional scanning of the focal spot of a lens in the X-Z plane. The actuators are fabricated by laser micromachining technique and driven by electromagnetic forces. The system requires a low driving voltage of 6 volts and provides a field of view of $190 \mu \mathrm{m}$ by $145 \mu \mathrm{m}$ in the axial (Z) and transverse $(\mathrm{X})$ directions respectively with imaging frame rate of 2 fps.

\section{METHODS}

A schematic diagram of the optical configuration for light delivery and collection as well as the 2-axis confocal scanner scheme is shown in Fig. 1. A $785 \mathrm{~nm}$ laser beam is directed to a beam splitter and then coupled into a single mode optical fiber (SMF) using a fiber coupler. SMF has numerical aperture (NA) of 0.17 . The output light from SMF is collimated by lens L1. L1 has diameter of $3 \mathrm{~mm}$, focal length of $4.5 \mathrm{~mm}$ and NA of 0.3 . The collimated light is then focused into a sample by lens L2. L2 has diameter of $2.4 \mathrm{~mm}$, focal length of $1.45 \mathrm{~mm}$ and effective NA of
0.35. Two-dimensional imaging is performed by raster scanning of the laser spot in a vertical plane through the sample. This is achieved by actuating L2 in the axial and SMF in the transverse directions. After scanning the sample, the scattered light is sent into a photomultiplier tube (PMT) using L2, L1 and SMF. The intensity signal is converted into image of cross-section of the sample using a data acquisition (DAQ) card and a computer. Driving signals for fiber and lens actuators are also produced and controlled by the DAQ card.

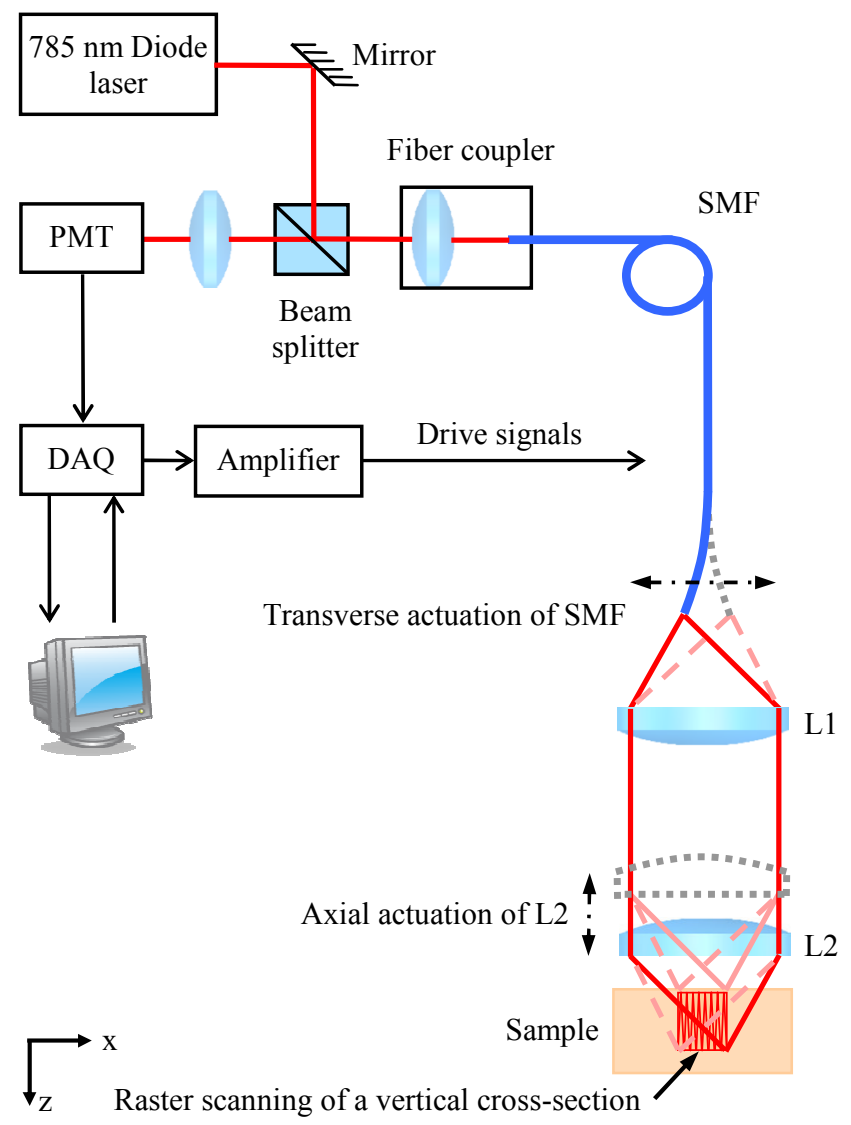

Figure 1: Schematic diagram of the optical configuration for light delivery and collection and the 2-axis confocal scanning system.

\section{SYSTEM DESIGN AND CONSTRUCTION}

Figure 2 shows lens and fiber actuation mechanisms. As shown in Fig. 2(a), L2 is suspended on a nickel flexure that is mounted on an electromagnetic coil (C1). The nickel flexure and L2 are actuated $190 \mu \mathrm{m}$ at resonant frequency of $378 \mathrm{~Hz}$. Collimating lens L1 is placed inside the hollow core of C1. SMF is mounted on a nickel cantilever beam and actuated $450 \mu \mathrm{m}$ by coil $\mathrm{C} 2$ at frequency of $2 \mathrm{~Hz}$ (Fig. 2(b)). The selected lenses provide magnification of 0.32 in transverse direction. As a result, the focal spot into the tissue is moved $145 \mu \mathrm{m}$ in $\mathrm{x}$-direction. 
A commercial laser machining system (QuikLaze 50ST2, New Wave Research Inc.) was used to fabricate the actuators shown in Fig. 3. The selected laser wavelength was green $(532 \mathrm{~nm})$ with energy of $3 \mathrm{~mJ}$, pulse rate of $50 \mathrm{~Hz}$ and cutting speed of 15 $\mu \mathrm{m} / \mathrm{s}$. Thickness of nickel foils (ESPI Metals) used to fabricate lens and fiber actuators were $56 \mu \mathrm{m}$ and $25.4 \mu \mathrm{m}$ respectively. The cantilever beam for fiber actuation has width of $2 \mathrm{~mm}$ and length of $25 \mathrm{~mm}$.

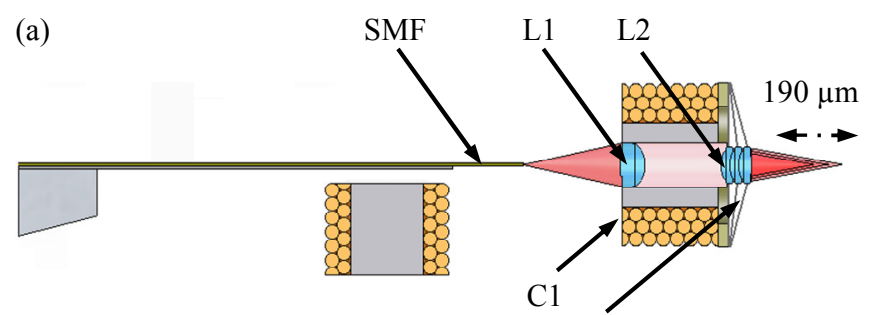

(b)

Nickel flexure for lens actuation

Nickel cantilever beam

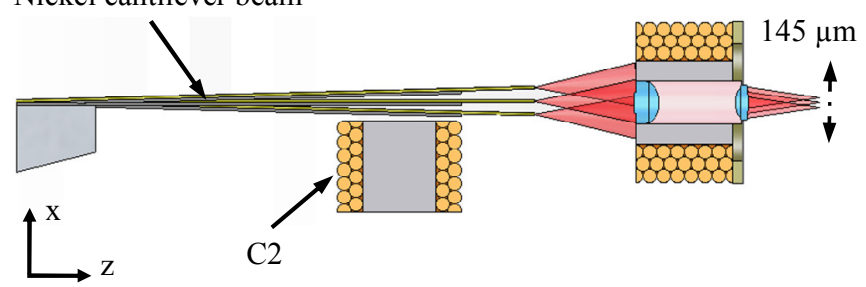

Figure 2: Cross-sectional drawing of 2-axis confocal scanner showing (a) axial scanning and (b) transverse scanning mechanisms.

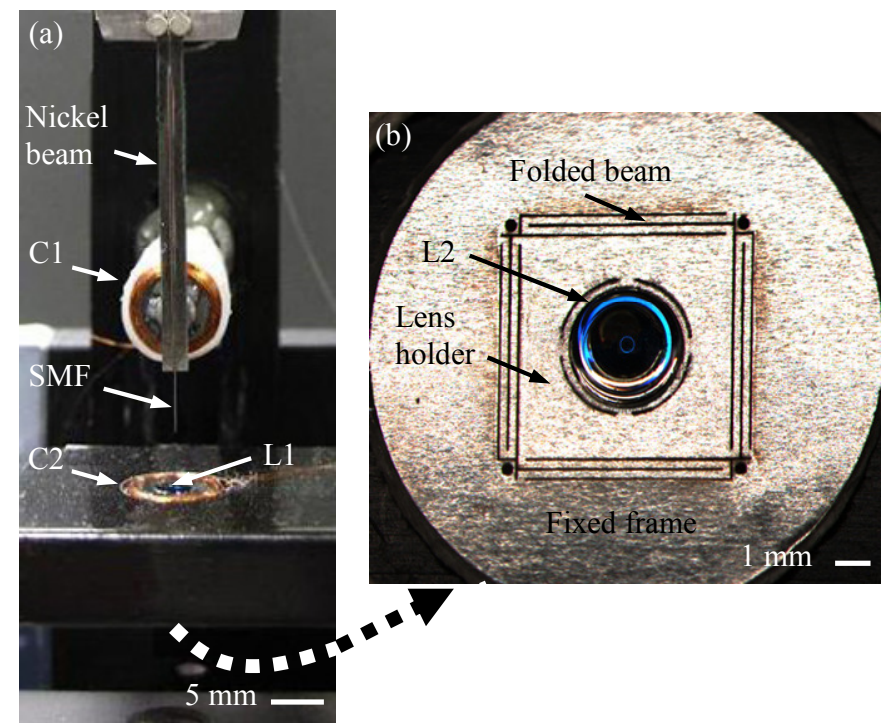

Figure 3: Photo of (a) nickel cantilever beam and (b) nickel flexure, fabricated by laser micromachining.

\section{OPTICAL PERFORMANCE}

Optical resolutions of the scanner in both axial and lateral directions will affect the image quality. The laser beam distribution at the focal point of a lens is shown in Fig. 4. $w(z)$ is beam radius that varies along the z-axis and is calculated using Eq. (1) [12]:

$$
w(z)=w_{0} \sqrt{1+\left(\frac{z}{z_{R}}\right)^{2}}
$$

where $w_{0}$ is the beam waist radius and $z_{R}$ is the Rayleigh range that is related to the beam waist according to:

$$
z_{R}=\frac{\pi w_{0}^{2}}{\lambda}
$$

In Eq. (2), $\lambda$ is wavelength of the laser beam. Optical performance of the system in axial and lateral directions can be assessed by $2 z_{R}$ and $2 w_{0}$ respectively. In our system, from Zemax optical simulation software, the Rayleigh range is $1.63 \mu \mathrm{m}$ and onaxis beam waist is $0.64 \mu \mathrm{m}$.

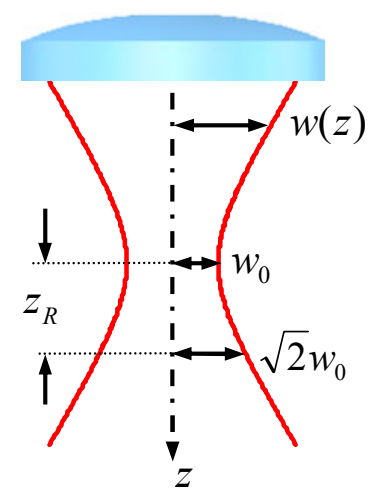

Figure 4: Laser beam profile for focusing a Gaussian beam.

(a)

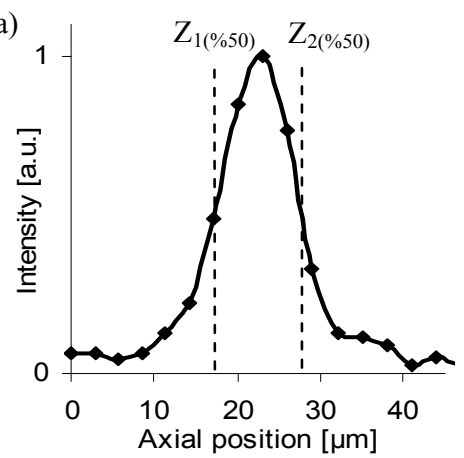

(b)
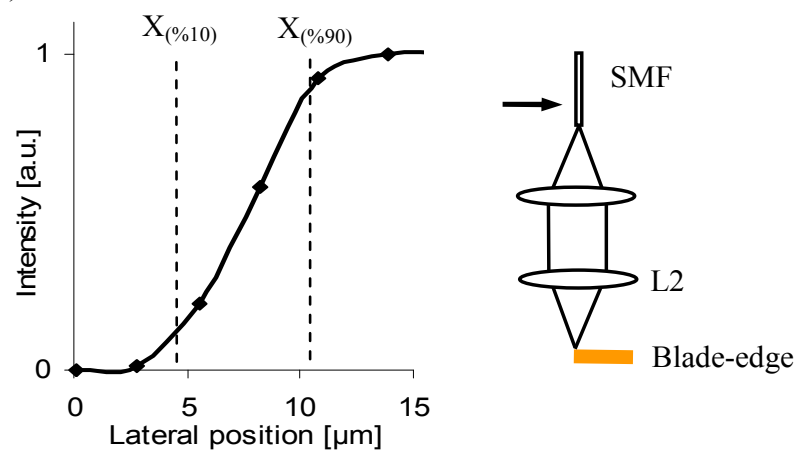

Figure 5: Experimental measurement of (a) axial and (b) lateral resolutions of confocal scanner. 
Axial and lateral resolutions of the scanner were experimentally evaluated from intensity signals obtained by scanning of a flat mirror and a blade-edge respectively [13]. Figure 5(a) is generated by plotting the intensity signal detected by PMT while keeping SMF stationary and actuating L2 toward a flat mirror. The axial resolution can be approximated from the full width at half maximum (FWHM) of the intensity signal:

$$
R_{\text {axial }}=Z_{2(\% 50)}-Z_{1(\% 50)}=10.68 \mu m
$$

Figure 5(b) is an intensity signal obtained from scanning edge of a sharp blade. From this graph, lateral resolution can be calculated according to Eq. 4:

$$
R_{\text {lateral }}=0.78\left(X_{\% 90}-X_{\% 10}\right)=4.84 \mu m
$$

\section{IMAGING RESULTS}

Functionality of the confocal scanner is demonstrated by obtaining vertical cross-sectional images of a photoresist feature on a silicon wafer and an onion epidermal peel. SU-8 photoresist (MicroChem Corp.) was used to fabricate a feature with rectangular cross-section on a silicon wafer. The width and height of the feature are $100 \mu \mathrm{m}$ by $33 \mu \mathrm{m}$. First, an optical profile image of this feature was obtained by Wyko optical profiler (Veeco Instruments Inc.) for comparison (Fig. 6(a)). Cross-section of the feature was then scanned by the confocal scanner (Fig. 6(b)). Confocal image clearly shows surfaces of the photoresist and silicon wafer. However, the sidewalls of the rectangular feature were not detected. This is due to the large incident angle of illumination beam to the side walls.

(a)

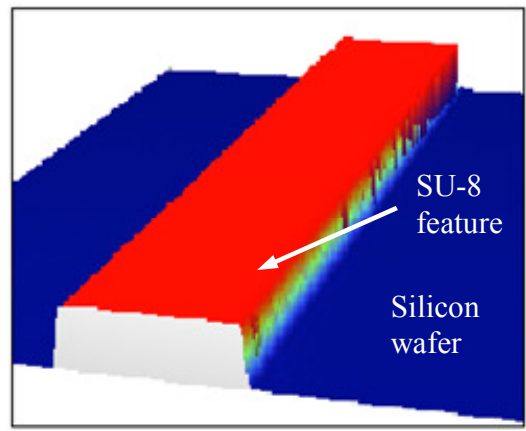

(b)

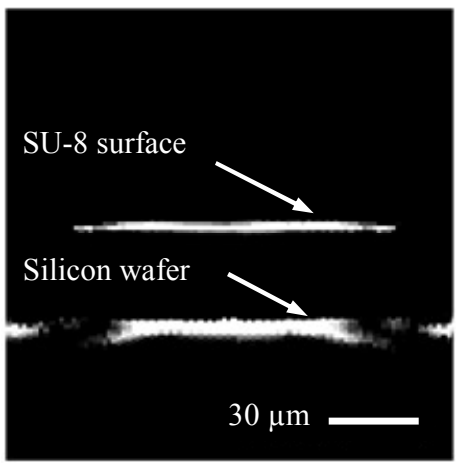

Figure 6: (a) Optical profile of a SU-8 feature with rectangular cross-section measured by an optical profiler; and (b) Confocal image of same feature.

Confocal scanner was also used to image cross-section of an onion epidermal peel layer obtained from a yellow onion. Figure 7(a) shows image of an onion cross-section stained with Alcian blue. This image was obtained using traditional histology imaging techniques. Figure 7(b) is an image obtained by our confocal scanner. Confocal image shows onion epidermal cells and provide information regarding the cell size and position of walls. Image quality of the system has much room for improvement by performing optical optimization to enhance image resolution.

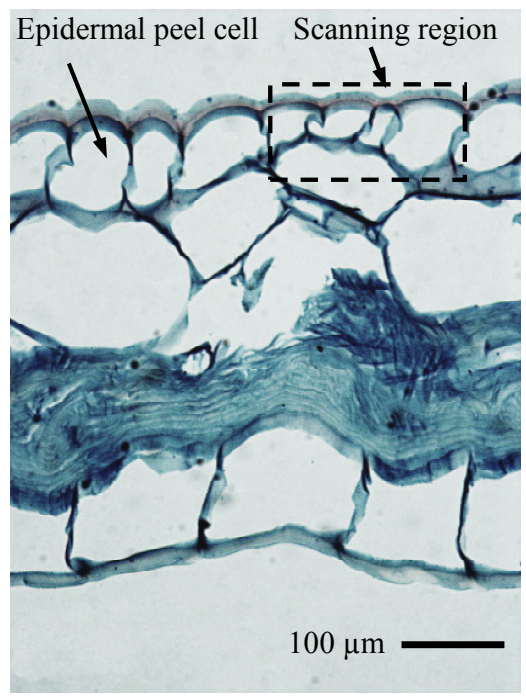

(b)

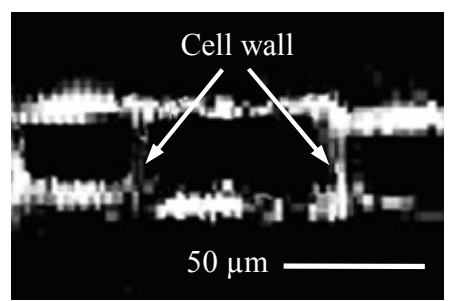

Figure 7: (a) Microscopy image of onion cross-section stained with Alcian blue, and (b) confocal vertical optical section image of onion peel obtained using the 2-axis confocal scanner.

\section{CONCLUSION}

A 2-axis confocal scanner for vertical optical sectioning of samples has been developed and demonstrated by obtaining confocal images of polymer features and onion peel cells. The device has optical resolution of $10.7 \mu \mathrm{m}$ and $4.8 \mu \mathrm{m}$ in axial and transverse directions respectively. Scanning range and speed are $190 \mu \mathrm{m}$ and $378 \mathrm{~Hz}$ in axial and $145 \mu \mathrm{m}$ and $2 \mathrm{~Hz}$ in transverse directions. The device described in this paper has several advantages that make it attractive for further development. The scanner is driven by electromagnetic forces only, hence requires low driving voltages that is desired in clinical applications. The optical configuration of the scanning head has only one optical axis which makes alignment of the system easier. The confocal scanner can potentially be packaged and be used as a hand-held device for various clinical applications such as skin or oral cancer diagnosis. Finally, the scanner design can be further miniaturized using Microelectromechanical Systems (MEMS) technology and microelectromagnets to form a catheter for endoscopic applications.

\section{ACKNOWLEDGMENT}

$\mathrm{Mu}$ Chiao is supported by the Canada Research Chairs Program. This project was supported by the Canada Foundation for 
Innovations (CFI), the Natural Sciences and Engineering Research Council (NSERC) of Canada, and the Canadian Institutes of Health Research (CIHR grant \#: MOP - 102672). Hadi Mansoor is supported by an Alexander Graham Bell Canada Graduate Scholarship.

\section{REFERENCES}

[1] K. H. Kim, B. H. Park, G. N. Maguluri, T. W. Lee, F. J. Rogomentich, M. G. Bancu, B. E. Bouma, J. F. de Boer, and J. J. Bernstein, "Two-axis magnetically-driven MEMS scanning catheter for endoscopic high-speed optical coherence tomography", Optics Express, 15 (26), 1813018140 (2007).

[2] W. Piyawattanametha, and T. D. Wang, "MEMS-based dualaxes confocal microendoscopy”, Selected Topics in Quantum Electronics, 16 (4), 804-814 (2010).

[3] A. Jain, and H. Xie, "A single-crystal silicon micromirror for large bi-directional 2D scanning applications", Sensors and Actuators: A, 130-131, 454-460 (2006).

[4] N. Kikuchi, Y. Hags, M. Maeda, W. Makishi, and M. Esashi, "Piezoelectric 2D micro scanner for minimally invasive therapy fabricated using femtosecond laser ablation", The 12th International Conference on Solid State Sensors, Actuators and Microsystems, Boston (2003), pp. 603-606.

[5] E. J. Min, J. G. Shin, Y. Kim, and B. H. Lee, "Twodimensional scanning probe driven by a solenoid-based single actuator for optical coherence tomography", Optics Letters, 36 (11), 1963-1965 (2010).

[6] T. Wu, Z. Ding, K. Wang, M. Chen, and C. Wang "Twodimensional scanning realized by an asymmetry fiber cantilever driven by single piezo bender actuator for optical coherence tomography”, Optics Express, 17 (16), 1381913829 (2009).
[7] H. Ra1, W. Piyawattanametha, M. J. Mandella1, P. Hsiung, J. Hardy, T. D. Wang, C. H. Contag, G. S. Kino, and O. Solgaard "Three-dimensional in vivo imaging by a handheld dual-axes confocal microscope", Optics Express, 16 (10), 7224-7232 (2008).

[8] T. D. Wang, K. Kurabayashi, K. Oldham, and Z. Qiu, "Targeted dual-Axes confocal imaging apparatus with vertical scanning capabilities" US Patent App. US 2011/0125029 A1, 2011.

[9] H. Mansoor, H. Zeng, and M. Chiao, "Real-time thickness measurement of biological tissues using a microfabricated magnetically-driven lens actuator", Biomed Microdevices, 13, 641-649 (2011).

[10] H. Mansoor, H. Zeng, and M. Chiao, "A micro-fabricated optical scanner for rapid non-contact thickness measurement of transparent films" Sensors and Actuators: A, 167, 91-96 (2011).

[11] H. Mansoor, H. Zeng, K. Chen, Y. Yu, J. Zhao, and M. Chiao, "Vertical optical sectioning using a magnetically driven confocal microscanner aimed for in vivo clinical imaging", Optics Express, 19 (25), 25161-25172 (2011).

[12] YA. Peter, "Micro-optical fiber switch for a large number of interconnects", PhD dissertation, Université de Neuchâtel, 2001.

[13] M. Rajadhyaksha, R. R. Anderson, and R. H. Webb, "Videorate confocal scanning laser microscope for imaging human tissues in vivo", Applied Optics, 38 (10), 2105-2115 (1999).

\section{CONTACT}

*H. Zeng, tel: +1- 604-675-8083; hzeng@bccrc.ca 


\section{IN-PLANE MEMS-BASED FABRY-PEROT FILTER FOR HIGH-SPEED WAVELENGTH-SWEPT SEMICONDUCTOR LASER

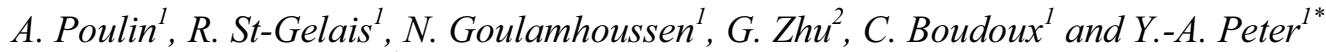 \\ ${ }^{1}$ Department of Engineering Physics \\ ${ }^{2}$ Department of Electrical Engineering \\ Ecole Polytechnique de Montréal, Montréal (Québec) H3C 3A7 CANADA}

\begin{abstract}
This paper reports the development of a MEMS-based wavelength-swept semiconductor laser for biomedical imaging. MEMS-based sources present numerous advantages over the ones based on polygon-scanners. Their non-linearity is however an important drawback for the development of medical applications such as optical coherence tomography where video rate imaging is of great importance. The device we propose overcomes this problem. Its in-plane design enables the push-pull configuration resulting in a linear behavior. Experimental results demonstrate the linearity of the spectral sweep for frequencies up to $30 \mathrm{kHz}$. The laser, centered at $1310 \mathrm{~nm}$, was tuned over more than $20 \mathrm{~nm}$.
\end{abstract}

\section{INTRODUCTION}

Rapidly tunable wavelength-swept sources are of particular interest for optical coherence tomography (OCT). OCT is a technique which consists in acquiring by optical interrogation a series of parallel dept-scans within a semi-transparent medium. The scans are then synthesized in a three dimensional model from which cross sectional views can be extracted. OCT is mainly used as a medical imaging and diagnosis tool [1]. The main advantages over x-rays and ultrasound imagery are its resolution, speed, size and cost. OCT provides information on tissue architecture, but its resolution does not typically allow visualization of subcellular structures. This information is however precious for medical diagnosis of cancers and as a real-time feedback for surgical oncologist [2].

The development of new applications in oncology, and in other fields such as cardiology and gastroenterology, requires OCT systems with real-time imaging capability. The first OCT techniques, based on time-domain low coherence interferometry, required mechanical scanning of a reference arm which limited the acquisition speed. With the development of Fourier-domain OCT techniques, the reference arm remains fixed. Interferometric detection is either performed through a spectrometer or by rapidly tuning the wavelength of the source - a technique called sweptsource (SS) OCT. The reflectivity profile as a function of depth is then recovered by Fourier transforming the detected signal. Spectral scan or spectrometric measurement can be achieved much faster than the equivalent mechanical scan. SS-OCT therefore enables video rate imaging and paves the way for an array of new medical applications. The acquisition rate of OCT images is limited by the sweeping speed of the source and the data treatment needed for image reconstruction. Consequently, the post acquisition treatment must be straightforward and avoid costly steps such as interpolation for relinearization of the spectrum sweep. For these reasons, a laser exhibiting a truly linear sweep over the spectrum at high sweeping rate is of great interest for OCT medical applications.

Various swept-sources have been developed for this specific field [3]-[6]. One of the most challenging aspects has been to reach tuning speeds of tens of $\mathrm{kHz}$. It has first been achieved with sources using a polygon-scanner based filter [3]. This filter exhibits a highly linear response and can achieve tuning speeds faster than $100 \mathrm{kHz}$ [7]. However, the polygon scanner is bulky, expensive, complex to fabricate and involves a free space component prone to misalignments. These drawbacks limit the accessibility of the technology and consequently slow down its use in the development of new medical applications. To address this issue, sources based on micro-electromechanical systems (MEMS) have been developed [4]-[6]. MEMS filters or reflectors are well suited for external-cavity tunable lasers and provide many benefits [8]. They exhibit small dimensions, low fabrication cost and simple designs. They minimize the required optical alignments and the cavity length. In most cases, devices are based on out-ofplane designs [4], [5]. In comparison with in-plane designs, this configuration makes the optical alignment more complex and less robust. In addition, it often involves parallel plate actuators that are intrinsically non-linear and present risks of pull-in. A first inplane MEMS-based swept source was recently demonstrated [6]. The authors approach, based on the Vernier effect, could allow a linear spectral scan at high frequency. However, it involves multiple optical components and requires a complex electronic control. Moreover, the Vernier effect can allow stimulated emission at unwanted resonance orders and therefore generate important instabilities in the laser.

The swept-source design we propose combines the advantages of both polygon and MEMS filters. A tunable FabryPerot (FP) is used in the laser cavity as a wavelength selector. This MEMS filter, unlike the ones used in [4] and [5], is based on an inplane design that provides many benefits. This original design allows the use of comb-drive actuators that do not exhibit pull-in voltage. Moreover, it allows including a third electrode to control the actuator in push-pull configuration [9]. This configuration is used to produce a linear behavior of the comb-drive actuators and overcome the MEMS non-linearity issue. The laser presented in [6] is also based on an in-plane design. The wavelength selection is achieved with a combination of integrated filters and phase modulators. The fabrication process is however complex. It involves multiple materials, deposition and etching steps. The technique we propose is based on a single free-space tunable filter. It requires only one photolithography step and materials are limited to a Silicon On Insulator (SOI) substrate. The in-plane approach we propose was inspired by our previous work on tunable fiber laser for optical communication systems [10]. The filter and the laser designs are however significantly different.

\section{FILTER DESIGN}

The fabricated filter is shown in Fig.1. It is composed of two main components, which are the Fabry-Perot cavity and the actuator. The inset of Fig.1 corresponds to a close-up view of the FP cavity. Two Bragg mirrors are formed by a series of alternating silicon and air layers. Combined together, coplanar and separated by an air gap, they form the FP resonator. This type of cavity, inside the reflection bandwidth of the Bragg mirrors, reflects light except at its resonance frequencies where the transmission is maximal. It can therefore be used as a wavelength selection filter. Various parameters influence the optical response and the performances of such filters. Our design was optimized using a model recently published, taking into account not only the 


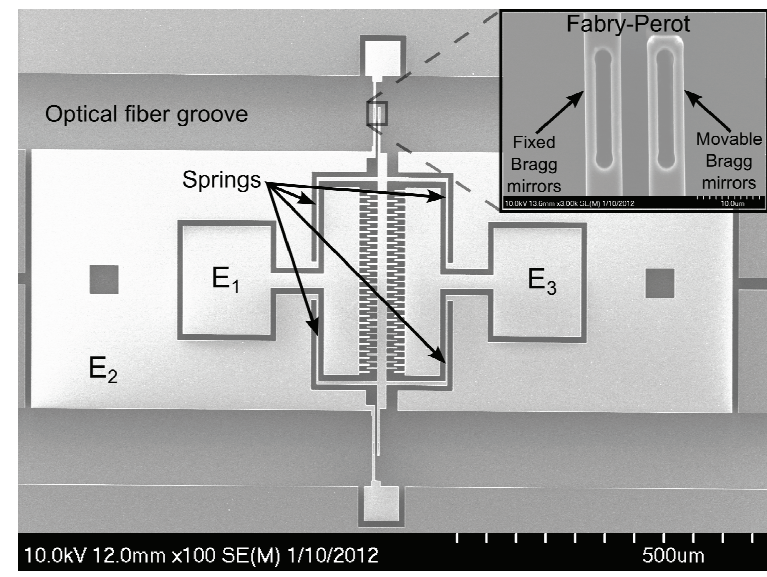

Figure 1: Scanning Electron Microscopy image of the fabricated device. The substrate is a Silicon on Insulator wafer with a $70 \mu \mathrm{m}$ thick device layer.

dimensions and refractive indices of the layers but also the limitations due to Gaussian beam divergence and surface roughness [11].

The second element of importance, the actuator, is composed of two interdigited combs and three electrodes referred as $\mathrm{E}_{1}, \mathrm{E}_{2}$ and $\mathrm{E}_{3}$. The electrode $\mathrm{E}_{2}$, connected to the central suspended section, is grounded. The suspended section is attached to one of the FP Bragg mirrors, which displacement allows spectral tuning of the filter. The use of comb-drive actuators was enabled by the in-plane design. Unlike parallel plate actuators, whose plates collapse for displacements larger than one third of the initial gap, these later allow large displacements and do not exhibit pull-in voltage.

Typical actuators are composed of two electrodes. An alternative voltage $\mathrm{V}_{\mathrm{a}}$ is applied on the fixed electrode while the movable one is grounded. For example, referring at the Fig.1, the electrodes $E_{2}$ would be grounded while the electrode $E_{1}$ would be set at $\mathrm{V}_{\mathrm{a}}$. This type of actuation leads to a quadratic relation between the displacement and the applied voltage. In order to maximize the performance for OCT applications, we look for a device providing a linear scan over the covered spectrum. A linear behavior can be obtained from comb-drive actuators by working in push-pull configuration and by the mean of an additional electrode. It is interesting to note that this third electrode could not be implemented in the design of out-of-plane filters. In the push-pull configuration the movable electrode is surrounded by two electrodes. A bias source $V_{b}$ and an alternative source $V_{a}$ are used to drive the combs. The applied voltage on $\mathrm{E}_{1}$ and $\mathrm{E}_{3}$ are respectively $\mathrm{V}_{\mathrm{b}}+\mathrm{V}_{\mathrm{a}}$ and $\mathrm{V}_{\mathrm{b}}-\mathrm{V}_{\mathrm{a}}$ while $\mathrm{E}_{2}$ is grounded. The value of the bias source determines the slope of the linear relation observed between the displacement and the voltage.

The optimization of the design was not limited to the optical performances of the resonator. The electromechanical response of the device was simulated and optimized using the finite element method (FEM). One of the aspects studied was the frequency and shape of the first resonance mode. We wanted the natural resonance mode to be an in-plane oscillation, parallel to the optical axis of the cavity. We designed the springs in order to get the natural frequency at $40 \mathrm{kHz}$. Figure 2 presents the natural resonance mode of the device obtained through FEM simulation. We can see that its shape corresponds to the one desired. The shape of the first mode was mainly determined by the symmetry of the device with respect to the $\mathrm{x}$ axis and the ratio between its

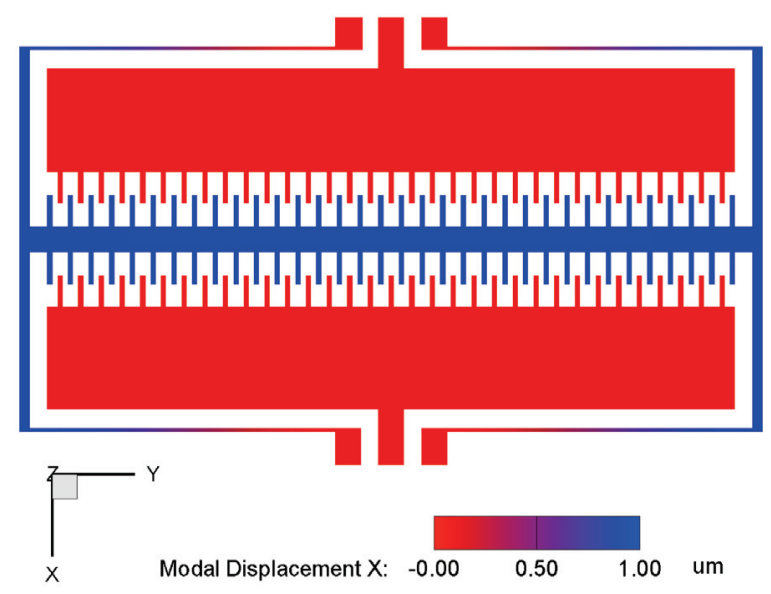

Figure 2: Natural vibration mode of the actuator obtained by finite element simulation. The movement is strictly limited to the $x$ axis.

spring constant along the $\mathrm{z}$ and the $\mathrm{x}$ axis. The stiffness of the central beam has to be high enough in order to avoid its deformation when actuated at high frequencies.

Details of the fabrication process will not be presented in this work. Further information can be found in [10]. It is however important to mention that the device is structured by bulk micromachining of a silicon on insulator substrate and only one photolithography step is required during the process. The original in-plane design enables this simple fabrication process. In addition, it allows the integration of optical fiber grooves which greatly simplify the optical alignment.

\section{LASER DESIGN}

A schematic of the laser design is shown in Fig.3. The gain medium is a semiconductor optical amplifier (SOA) from Thorlabs (BOA1017S, Newton, NJ) centered at $1310 \mathrm{~nm}$ with a spontaneous emission $3 \mathrm{~dB}$ bandwidth of $70 \mathrm{~nm}$. The performance of the SOA was found to be highly influenced by polarization. This component is therefore surrounded by two polarization controllers (PC) which were used to optimize the laser output power. Two circulators are used as isolators in order to avoid back reflections from the different interfaces to propagate in the cavity to the gain medium. These retroreflections could stimulate emission at unwanted frequencies and alter the laser emission spectrum.

The laser cavity is composed of a SOA, two polarization

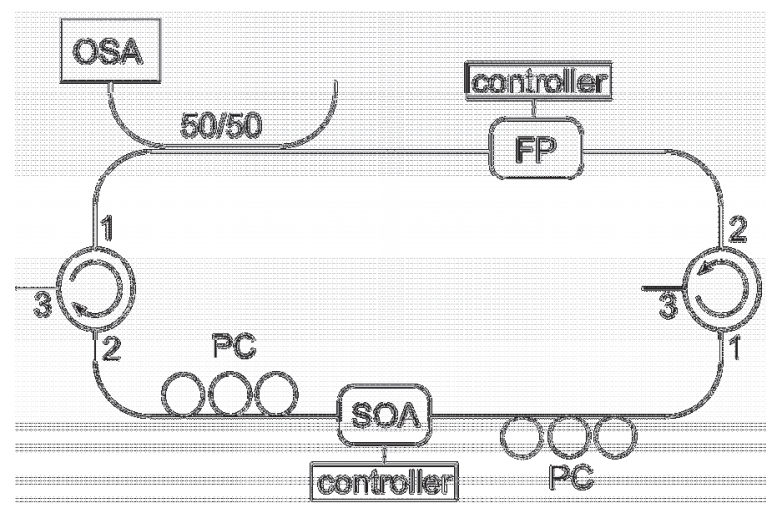

Figure 3: Scheme of the laser setup, connected to an optical spectrum analyzer for characterization. 
controllers, two isolators and the MEMS-based Fabry-Perot filter. All these components are optically connected using optical fiber (Corning SMF-28) in a ring configuration cavity. The gain medium emits a spontaneous emission spectrum which propagates through the cavity in the direction determined by the two isolators. The tunable FP is used to filter this spontaneous emission. It only allows wavelengths near its resonance to complete multiple roundtrips in the cavity, stimulate emission in the gain medium and consequently produce a laser emission. A 50/50 coupler was inserted in the ring cavity to create the laser output. Finally, an optical spectrum analyzer (OSA) was used at the laser output for characterization purposes.

\section{RESULTS}

Using the laser design shown in Fig.3, the fabricated device was used as an intra-cavity filter to form a wavelength sweptsource. The laser, centered at $1310 \mathrm{~nm}$, was tuned over more than $20 \mathrm{~nm}$ as shown in Fig.4. The full-width at half-maximum is lower than $0.06 \mathrm{~nm}$. Its measurement was limited by the resolution bandwidth of the OSA. At this stage, the main limitation of the laser for OCT application is its small tuning range. The tuning range could be increased by a factor of three with the optimization of the fabrication process. Previous work reported in [10] demonstrates that such performances can indeed be reached. Another approach would be to use multiple filters in parallel, covering specific and complementary spectral region. We could therefore expect to cover the whole spectrum of the SOA. The low cost, small size and easy integration of the filter enable this approach.

A white light interferometer (Fogale Nanotech Photomap 3D) was used to characterize the actuator. Its in-plane displacement was monitor for different driving voltage amplitudes and frequencies. First, we applied an alternative voltage between the electrode E1 and E2. The amplitude of the movable Bragg mirror motion was measured in function of the alternative voltage frequency. The results are reported in Fig.5. The natural vibration frequency was observed at $35 \mathrm{kHz}$. It is approximately $13 \%$ lower than the expected value of $40 \mathrm{kHz}$. This shift can be explained by slight variations between the expected and the experimental dimensions of the springs. These variations mainly come from the etching step where we can observe some under-etch and a slight non-verticality of the trenches. We believe these geometrical parameters have a greater influence than the air damping, which have been taken into account during the optimization of the device geometry. It was confirmed by visual inspection that the vibration mode corresponding to this frequency is the one shown in Fig.2. The resonance peak of this vibration mode is narrow and does not affect frequencies lower than $30 \mathrm{kHz}$. The device should consequently be able to follow arbitrary signal waveform with

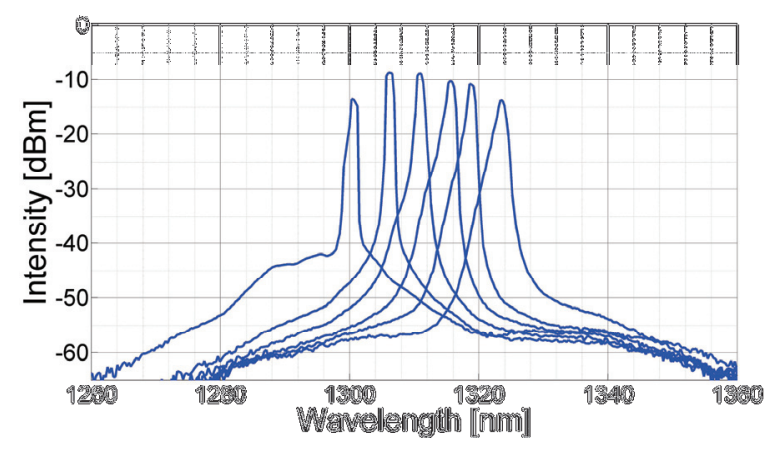

Figure 4: Spectral tuning of the laser over more than $20 \mathrm{~nm}$.

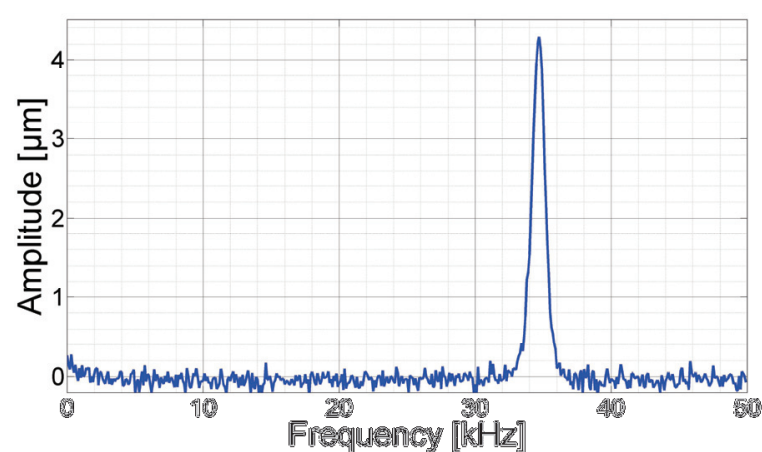

Figure 5: Measured amplitude of the Bragg mirror motion for increasing actuation frequency. The natural vibration mode frequency of the actuator is $35 \mathrm{kHz}$. The actuator must be used offresonance (i.e. below $30 \mathrm{kHz}$ ) to follow non-sinusoidal signals.

frequency component lower than $30 \mathrm{kHz}$. This limit on the actuation frequency could easily be overcome by stiffening the springs supporting the movable combs.

The second step in the characterization of the actuator was to study its in-plane motion in function of the applied voltage. Figure 6 reports the experimental results obtained for both the conventional $\left(\mathrm{E}_{1}=\mathrm{V}, \quad \mathrm{E}_{2}=\mathrm{E}_{3}=0, \quad \mathrm{~V}=2 \mathrm{~V}_{\mathrm{a}}\right)$ and the push-pull $\left(\mathrm{E}_{1}=\mathrm{V}_{\mathrm{b}}+\mathrm{V}_{\mathrm{a}}, \mathrm{E}_{2}=0, \mathrm{E}_{3}=\mathrm{V}_{\mathrm{b}}-\mathrm{V}_{\mathrm{a}}\right)$ configurations. In the push-pull mode $\mathrm{V}_{\mathrm{b}}$ was equal to $200 \mathrm{~V}$. The dots correspond to the experimental results. The lines respectively correspond to a linear and a quadratic fit for the push-pull and the conventional configurations. The results clearly demonstrate that a linear behavior can be obtained from comb-drive actuators by working in push-pull configuration.

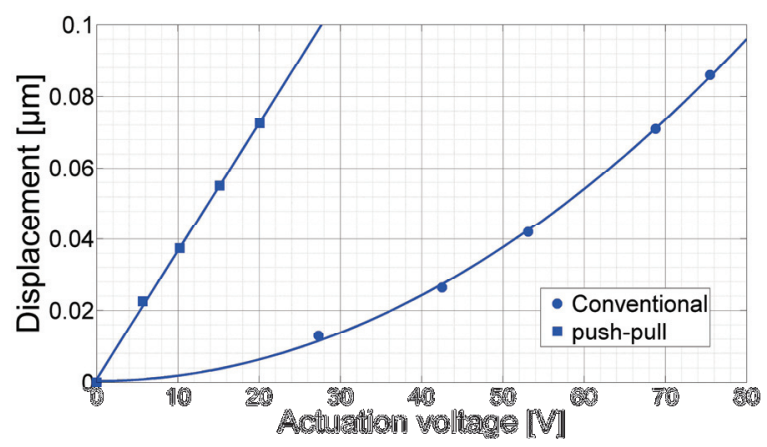

Figure 6: Experimental measurements of the displacement as a function of the applied voltage. Both the push-pull and the conventional actuation techniques were tested.

\section{CONCLUSION}

The device we presented combines the small size, low cost, design simplicity and robustness of MEMS-based sources with the linearity of polygon-scanner based sources. We demonstrated the linearity of the spectral sweep and the $35 \mathrm{kHz}$ natural resonance frequency of the actuator. We can expect the swept-source to follow any arbitrary signal waveform with frequency components lower than $30 \mathrm{kHz}$. This frequency could be easily increased by simple modifications of the device geometry. The laser tuning range, limited to $20 \mathrm{~nm}$, could be further improved by working on the fabrication process or using multiple filters in parallel. We believe the development of a source exhibiting high speed, linearity and low cost is a significant step toward broader uses of OCT for clinical applications. 


\section{REFERENCES}

[1] A. F. Fercher, W. Drexler, C. K. Hitzenberger, and T. Lasser, "Optical coherence tomography - principles and applications", Reports on Progress in Physics, 66, (2003).

[2] J. M. Zara, and C. A. Lingley-Papadopoulos, "Endoscopic OCT Approaches Toward Cancer Diagnosis", Journal of Selected Topics in Quantum Electronics, 14, 1, (2008).

[3] S. H. Yun, C. Boudoux, G. J. Tearney, and B. E. Bouma, "High-speed wavelength-swept semiconductor laser with a polygon-scanner-based wavelength filter", Optics Letters, 28, 20, (2003).

[4] M. Kuznetsov, W. Atia, B. Johnson, and D. Flanders, "Compact Ultrafast Reflective Fabry-Perot Tunable Lasers For OCT Imaging Applications", Proceedings of SPIE: Optical Coherence and Coherence Tomography and Coherence Domain Optical Methods in Biomedicine XIV, (2009), p. 75541F.

[5] K. Isamoto, K. Totsuka, T. Sakai, T. Suzuki, A. Morosawa, C. Chong, H. Fujita, and H. Toshiyoshi, "A High Speed MEMS Scanner for 140-kHz SS-OCT", International Conference on Optical MEMS and Nanophotonics, (2011), pp. 73-74.
[6] B. W. Tilma, Y. Jiao, J. Kotani, B. Smalbrugge, H. P. M. M. Ambrosius, P. J. Thijs, X. J. M. Leijtens, R. Nötzel, M. K. Smit, and E. A. J. M. Bente, "Integrated Tunable QuantumDot Laser for Optical Coherence Tomography in the $1.7 \mu \mathrm{m}$ Wavelength Region", IEEE Journal of Quantum Electronics, 48, 2, (2012).

[7] W. Y. Oh, S. H. Yun, G. J. Tearney, and E. Bouma, " $115 \mathrm{kHz}$ tuning repetition rate ultrahigh-speed wavelength-swept semiconductor laser", Optics Letters, 30, 23, (2005).

[8] A. Q. Liu and X. M. Zhang, "A review of MEMS externalcavity tunable lasers", Journal of Micromechanics and Microengineering, 17, (2007).

[9] W. C. Tang, T.-C. H. Nguyen, and R. T. Howe, "Laterally Driven Polysilicon Resonant Microstructures", IEEE Microelectromechanical Systems Workshop Tech. Dig., (1989), pp. 53-59.

[10] J. Masson, R. St-Gelais, A. Poulin, and Y.-A. Peter, Tunable "Fiber Laser Using a MEMS-Based In Plane Fabry-Pérot Filter", IEEE J. Quantum Electron. 49, 9, (2010).

[11] R. St-Gelais, A. Poulin, and Y.-A. Peter, "Advances in Modeling, Design, and Fabrication of Deep-Etched Multilayer Resonators", Journal of Lightwave Technology, doi: 10.1109/JLT.2012.2191136.

\section{CONTACT}

*Y.-A. Peter, tel: +1-514-340-4711; yves-alain.peter@polymtl.ca 


\title{
SOFTWARE-DEFINED MICROSTRIP ANTENNAS ENABLED THROUGH LARGE VERTICAL DISPLACEMENT ZIPPER MICROACTUATORS
}

\author{
Jason Felder ${ }^{1,2}$, Eugene Lee ${ }^{2}$, Eric Walton ${ }^{3}$, Don L. DeVoe ${ }^{1}$ \\ ${ }^{1}$ Dept. of Mechanical Engineering, University of Maryland, College Park, MD, USA \\ ${ }^{2}$ Syntonics LLC, Columbia, MD, USA \\ ${ }^{3}$ Dept. of Electrical and Computer Engineering, The Ohio State University, Columbus, OH, USA
}

\begin{abstract}
This paper describes a novel large-displacement electrostatic "zipper" microactuator capable of achieving hundreds of microns of out-of-plane deflection, and application of fabricated microactuators to the control of millimeter-scale microfabricated capacitive pixels enabling "software-defined" microstrip antennas. The software-defined microstrip patch antenna concept relies on a reconfigurable array of individually addressed pixels. Current antenna designs demand pixel footprints below $1 \mathrm{~mm}^{2}$, with 300 $\mu \mathrm{m}$ vertical deflections, $1 \mathrm{~ms}$ response times, and low power consumption. To address this challenge we have developed a new technology based on electrostatic zipper actuation, in which a curved beam is switched between bistable states by application of a voltage across a dielectric film separating the beam from a flat electrode surface. This technology is novel in its use of $\mathrm{SiO}_{2}$ as both a high quality dielectric and the stressed layer of the bimorph providing curved actuation, enabling large out-of-plane deflection and large forces.
\end{abstract}

\section{INTRODUCTION}

Despite decades of research on large-displacement out-ofplane microactuation, including electrothermal [1], thin film metallic glass [2], and asymmetric comb drive actuators [3], established technologies are inadequate to meet the required demands of a microstrip patch antenna: footprints smaller than $1 \mathrm{~mm}^{2}, 1 \mathrm{~ms}$ response time, and a tip deflection of at least $300 \mu \mathrm{m}$.

Bistable electrostatic actuators, termed "Zipper" actuators, have been fabricated that are capable of meeting the design requirements [4-6]. The theory and mathematics behind electrostatic zipper actuators has been well documented and can be found in numerous publications such as [7] and [8]. All, however, are metallic bimorph structures, which can become expensive with gold, and which require unnecessarily complex fabrication processes. [5] report a zipper-style bimorph actuator made of 2.3 $\mu \mathrm{m}$ of soft gold and $0.2 \mu \mathrm{m}$ of hard gold, requiring an eleven step fabrication process (photolithography steps were not counted), the final step of which is a release etch of a sacrificial layer, leading to issues with stiction. Dhaubanjar et al. report a poly-Si and gold bimorph beam fabricated using the MUMPS process, and ending in an HF release etch [4]. Chang et al. demonstrate a chromium and aluminum bimorph that requires four materials, five photolithography masks, and nine steps to fabricate, one of which is a timed RIE etch, which can ruin the device if prolonged [7]. Others have shown similar metallic bimorph actuators with comparably complex fabrication processes [6].

We propose a simple new technology based on electrostatic zipper actuation [9], in which a curved beam is switched between bistable states by application of a voltage across a dielectric film separating the beam from a flat electrode surface. Unlike existing thin film zipper microactuators, the present devices comprise bimorph beams fabricated from an SOI wafer with a $10 \mu \mathrm{m}$ active layer $(\mathrm{Si})$ and $2 \mu \mathrm{m}$ thick thermal oxide $\left(\mathrm{SiO}_{2}\right)$. Compressive stress in the $\mathrm{SiO}_{2}$ results in well-defined initial beam curvature, while also serving as a high quality and relatively high-k dielectric for electrostatic actuation. Similarly, the thickness of the beam, relative to most other zipper actuators, provides for high forces at the tip. The novel use of $\mathrm{SiO}_{2}$ for both curvature control and dielectric isolation provides significantly larger deflections than traditional zipper actuators while reducing pull-in voltages. Additionally, since the dielectric layer is part of the actuated beam any flat conductive surface (such as a doped Si wafer) can be used as the underlying electrode without any fabrication. Lastly, using only two common, and well-characterized materials results in a simple, well-defined, four-step fabrication process, that is achieved using only two masks, and could, theoretically be achieved using only one.

\section{DEVICE FABRICATION}

The fabrication of the microactuators is a four step process, illustrated in Fig. 1. A p-type (100) SOI, with a $10 \mu \mathrm{m}$ thick active Si layer, a $1 \mu \mathrm{m}$ thick buried oxide (BOX) layer, and a $500 \mu \mathrm{m}$ thick handle $\mathrm{Si}$ layer, serves as the base for the actuator fabrication, as shown in Fig. 1( $a$ ). To start, $2 \mu \mathrm{m}$ of thermal silicon dioxide is grown to serve as the dielectric and the stressor to induce beam bending (Fig. 1(b)). Next, the frontside $\mathrm{SiO}_{2}$ and $\mathrm{Si}$ are patterned using DRIE to define the actuator beams, as shown in Fig. 1(c). In this 5 minute etch process, the BOX layer serves as an etch stop for the frontside DRIE. Since the etch rate ratio of Si: $\mathrm{SiO}_{2}$ for this particular process was characterized as 50:1 (2.5 $\mu \mathrm{m} / \mathrm{min}$ and $0.05 \mu \mathrm{m} / \mathrm{min}$ ), and the active $\mathrm{Si}$ is only $10 \mu \mathrm{m}$ thick, the Si can be safely etched, with a $25 \%$ over etch without worry of damaging the BOX. The backside $\mathrm{SiO}_{2}$ is removed using RIE and the backside $\mathrm{Si}$ is then etched using the same DRIE process to open backside access down to the BOX, as shown in Fig. 1(d). In

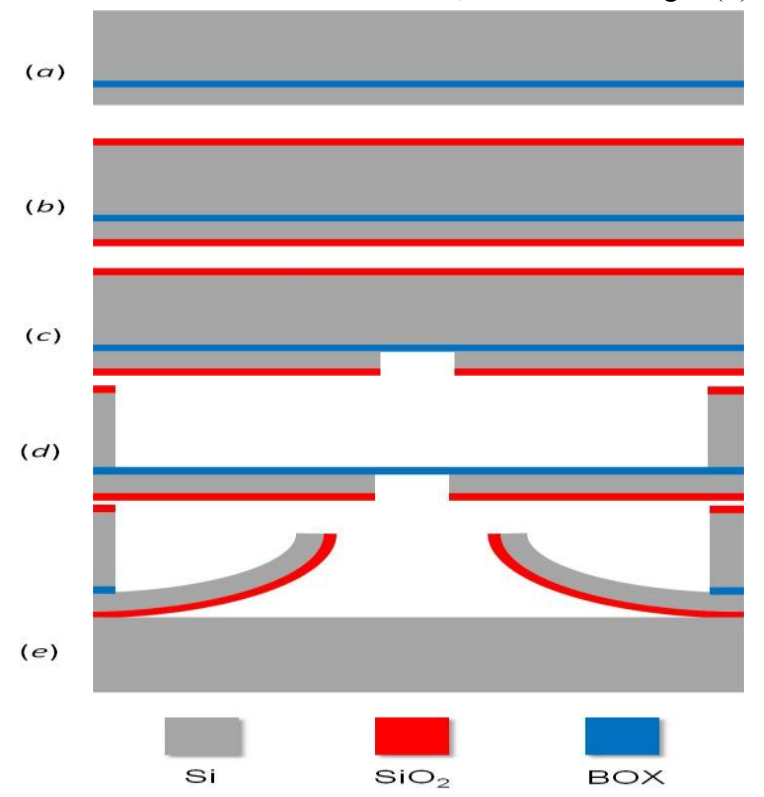

Figure 1: Fabrication process for zipper actuators using one mask and four process steps. 
the fourth and final step, the beams are released in Fig. 1(e) using RIE to remove the BOX and affixed to an underlying doped $\mathrm{Si}$ electrode. The stress induced by the $\mathrm{SiO}_{2}$ causes the beam to curve upon release. In the final iteration of the actuator fabrication, the entire backside will be removed using a buffered oxide etch (BOE) of the BOX layer, and the beams will be thermocompression bonded to the underlying electrode. However, for the purpose of this study, the actuators were mechanically clamped to the electrode.

\section{CHARACTERIZATION OF ZIPPER ACTUATORS}

Both the beam tip deflection, and the voltage necessary to "pull-in" the beam, $\mathrm{V}_{\mathrm{PI}}$, were characterized in this study. It is important to note the positive correlation between the two parameters. Beam deflection is defined by the radius of curvature of the beam. That is, as the radius of curvature becomes small, the beam gains deflection more quickly, achieving a greater tip deflection over a set beam length. $\mathrm{V}_{\mathrm{PI}}$ behaves similarly, requiring a greater voltage bias between the beam and underlying electrode to "pull-in" a beam with a smaller radius of curvature [9].

\section{Deflection Characterization}

The first set of cantilevers was fabricated with an $\mathrm{SiO}_{2}$ thickness of $1.7 \mu \mathrm{m}$. Fig. 2(a) shows a set of cantilevers (upside down) used for characterization and Fig. 2(b) shows a $1750 \mu \mathrm{m}$ fully released double cantilever beam. Optical defocusing, shown in the inset in Fig. 2(b), was used to characterize beam tip deflection. Deflection measurements taken for beams measuring $500,1000,1500$, and $2000 \mu \mathrm{ms}$ correlate well with an analytic model (Fig. 3) originally used to predict beam performance, and a COMSOL model used to corroborate results (Fig. 4). The measured deflections validate both the analytic and COMSOL models for use in future beam designs.

\section{Voltage Characterization}

A high voltage power source and a micro-stage with a digital microscope were used to apply and measure the required pull-in voltage, $\mathrm{V}_{\mathrm{PI}}$. Since the high voltage power source has a digital display accurate to $0.1 \mathrm{kV}$, a multimeter was used to obtain a more accurate voltage reading. The analytic model used above to predict beam deflection was used to predict $\mathrm{V}_{\mathrm{PI}}$ as well. Since $\mathrm{V}_{\mathrm{PI}}$ is a function of the beams radius of curvature, it is independent of the length and width of the beam. Hence, for a given bimorph beam with fixed $\mathrm{Si}$ and $\mathrm{SiO}_{2}$ thickness, $\mathrm{V}_{\mathrm{PI}}$ will be constant for any beam length. Fig. 5 shows measured $\mathrm{V}_{\mathrm{PI}}$ values for various beam lengths plotted alongside the constant $\mathrm{V}_{\mathrm{PI}}$ for three different values of the gap between the beam's base and the underlying electrode. As mentioned above, in this fabrication iteration, the fabricated actuators were mechanically clamped down to the underlying electrode. This can lead to variation in the gap height between the beam base and the electrode. Hence, Fig. 6 shows the analytic model $\mathrm{V}_{\mathrm{PI}}$ values for gap heights of 1,2 and $3 \mu \mathrm{m}$ 's, to demonstrate the validity of the measured $\mathrm{V}_{\mathrm{PI}}$ values. Although the measured values are relatively scattered, they all fall within reasonable values of $\mathrm{V}_{\mathrm{PI}}$ given the variability in gap height that can easily occur through mechanical clamping. In later iterations of actuator fabrication thermocompression bonding will be used to define a uniform gap height (ideally $2 \mu \mathrm{m}$ ) across all actuators. Nonetheless, the model and measured results illustrate respectable and acceptable "pull-in" voltages for beams of large deflections.
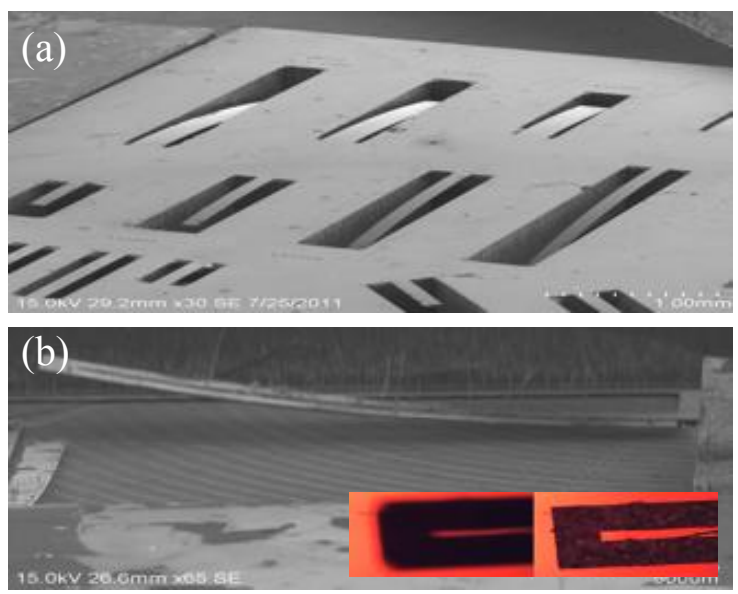

Figure 2: Single- and double-zipper cantilever actuators (a) before and (b) after bonding to the electrode layer. An optical defocusing measurement of beam deflection is shown inset.

Deflection at Tip of the Beam vs. Beam Length

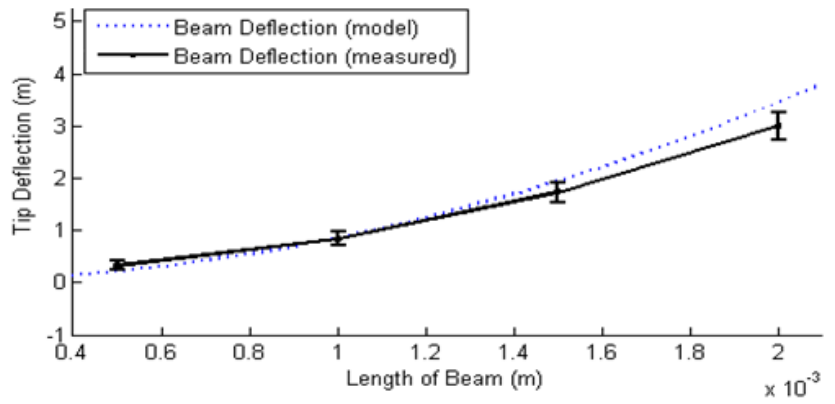

Figure 3: Agreement between analytic model for beam deflection, and actual beam deflection measured using optical defocusing.

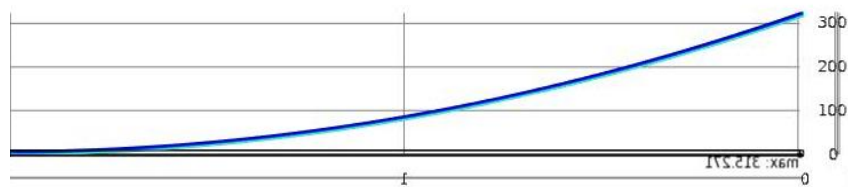

Figure 4: COMSOL model of $2000 \mu \mathrm{m}$ beam with total deflection of $315 \mu \mathrm{m}$, in correlation with analytic model, and measured deflection for 2000 um beam.

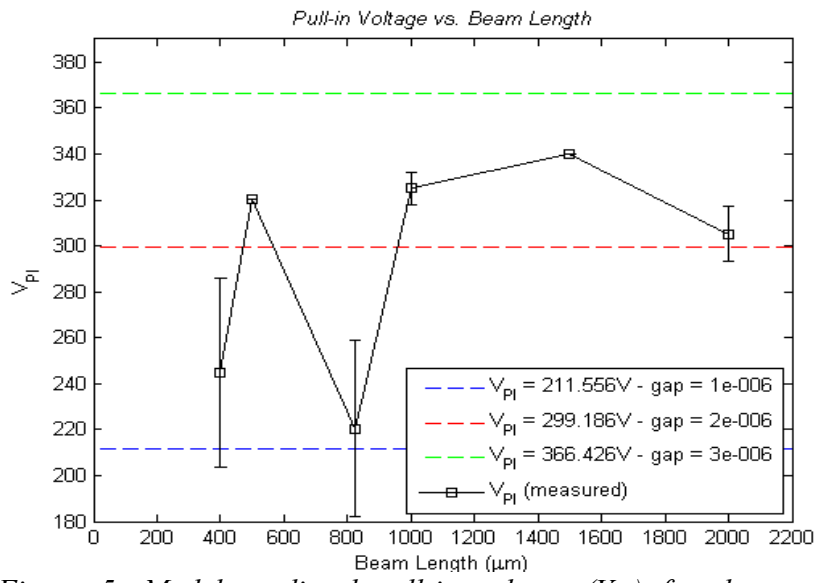

Figure 5: Model predicted pull-in voltage $\left(V_{P I}\right)$ for three gap values (dotted lines) plotted with measured values of $V_{P I}$ for a set of cantilever beam lengths. 


\section{APPLICATION IN MICROSTRIP PATCH ANTENNA}

The software-defined microstrip patch antenna concept relies on a reconfigurable array of individually addressed pixels (Fig. 6). Each pixel comprises a dielectric substrate sandwiched by conductive films, with the upper film serving as a waveguide and the lower film providing a ground plane. Pixels may be actively raised or lowered to define the desired microstrip circuit, enabling the formation of patch antennas that can scan in both frequency and angle by varying the size of the antennas or the feed network structure, or the formation of multiple antennas at different frequencies and polarizations. Current antenna designs demand pixel footprints below $1 \mathrm{~mm}^{2}$, with $300 \mu \mathrm{m}$ vertical deflections, $1 \mathrm{~ms}$ response times, and low power consumption.

Preliminary modeling determined that a linear beam, confined to the $1 \mathrm{~mm}^{2}$ footprint necessitated by the Microstrip Patch Antenna, would not be able to achieve the required $300 \mu \mathrm{m}$ deflection. A logical solution was to fabricate spiral actuators, thereby packing the greatest beam length into a $1 \mathrm{~mm}^{2}$ area. Spiral beams were simulated as linear beams, and the analytic model above was used to arrive at a beam length of at least $2000 \mu \mathrm{m}$ in order to reach $300 \mu \mathrm{m}$ tip deflection.

\section{Geometrical Challenge - Spiral Beams}

After fabrication of various spiral geometries, it became immediately clear that there was a fundamental issue with the

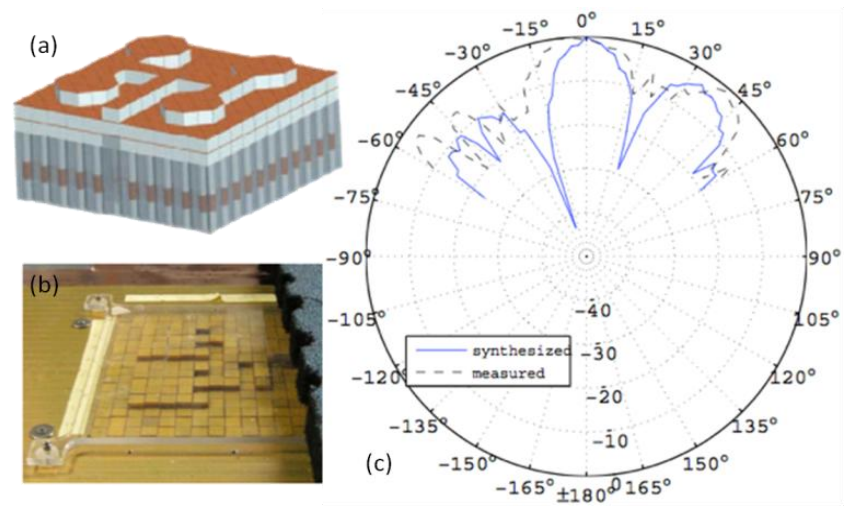

Figure 6: (a) Schematic and (b) photograph of a prototype software-defined antenna, and (c) radiation pattern at $8.3 \mathrm{GHz}$ measured using the prototype device.

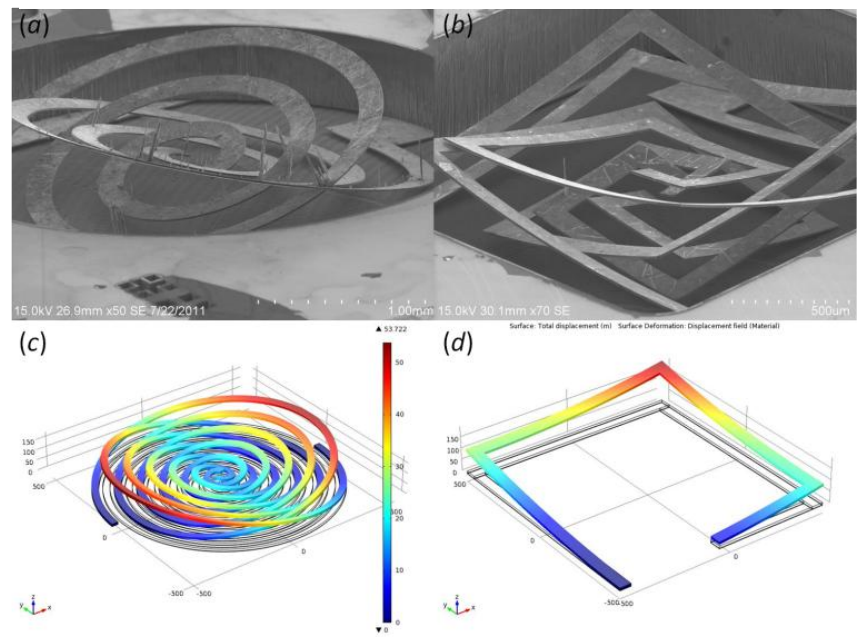

Figure 7: (a) Square spiral (b) round spiral and (c) COMSOL spirals, all exhibiting "bird-nest" phenomena. (d) COMSOL model illustrating the theory behind the bird-nest phenomenon. spiral concept. Unlike the spiral actuators in [2] where spiral actuators achieve a continuously rising slope, the actuators fabricated here achieved a maximum deflection exactly $180^{\circ}$ from the anchor point, no matter the shape (smooth spiral or square spiral) or configuration of the actuator beam. This "bird-nest" phenomenon is shown below in Fig. 7(a) and $(b)$, in SEM's of various spiral actuators, and validated by a COMSOL model of a similar spiral actuator in Fig. 7(c). The cause behind the "birdnest" is best illustrated by Fig. 7(d). Each successive "leg" of the continuous beam has initial conditions that match the tip conditions of the end of the leg to which it is attached. Hence, the third leg, positioned $180^{\circ}$ from the base of the continuous beam has an initial slope down towards the base that is the result of the initial conditions of the previous two legs. This idea can be applied to a spiral, which is essentially an infinite number of small "legs", and can be used to explain why the spiral's maximum deflection occurs $180^{\circ}$ from the base, no matter the configuration of the beams. In [2], the spiral actuators are given a deflection by pulling the spiral out of plane at high heat, a process that induces a natural torque in the spiral, so that as it rises continuously, its torque warps the beam, correcting for its natural downward slope that would occur after $180^{\circ}$. The fabrication of our actuators uses the natural stress of the $\mathrm{SiO}_{2}$ to provide beam deflection. Hence, no beam torque exists to counteract the natural downward slope as the spiral progresses.

\section{Solution to "Bird-Nest" Geometry Challenge}

In order to achieve $300 \mu \mathrm{m}$ deflections in a $1 \mathrm{~mm}^{2}$ footprint, serpentine actuators were fabricated in a modified process involving selective removal of oxide from portions of the beam. In this fabrication process, $2 \mu \mathrm{m}$ of $\mathrm{SiO}_{2}$ was grown atop the $10 \mu \mathrm{m}$ of Si. While a more complex fabrication process is required the resulting designs are capable of achieving exceptionally large deflections within a compact space. Moreover, the geometry lends itself to a symmetric design creating stability and culminating in a flat center area ideal for affixing an object, such as the pixel for the microstrip patch antenna.

A segment of the profile of the "Microstage" actuator can be seen in Fig. 8. The first leg consists of an actuated segment of length $L$ and a linear segment of length $1000-L$. The actuated segment is an $\mathrm{Si} / \mathrm{SiO}_{2}$ zipper actuator of the type presented above, followed by a linear segment of only Si whose slope and deflection amplification are defined by the slope at the end of the actuated segment. The second leg consists of an actuated segment of $2 L$ followed by a linear segment of $1000-2 L$.

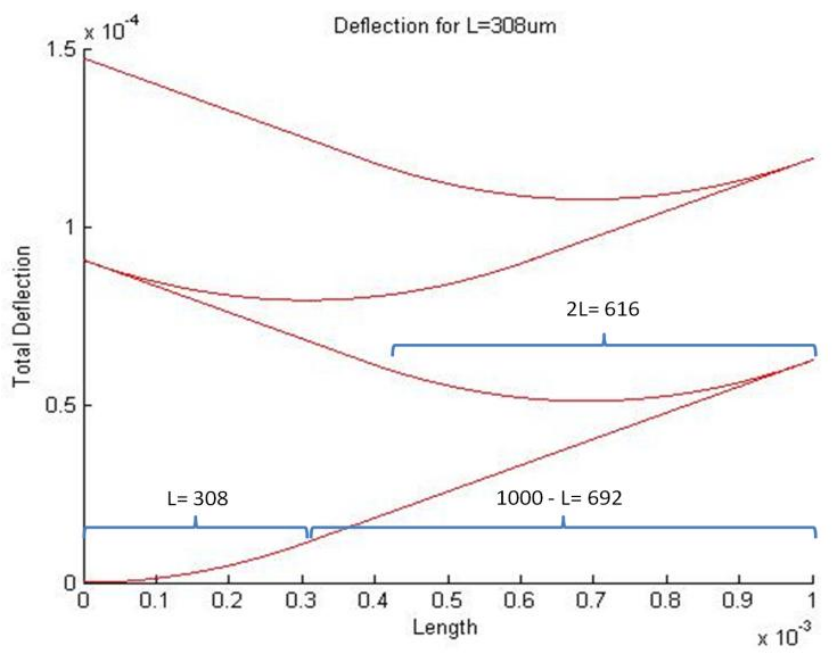

Figure 8: Analytic model of z-profile for a micro-stage actuator. 


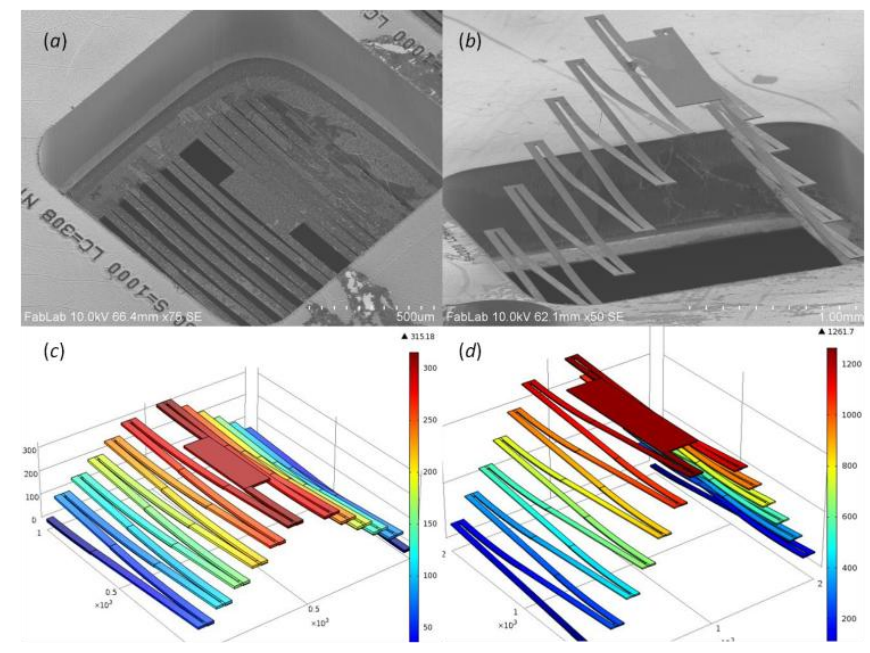

Figure 9: (a) Fully released $1 \mathrm{~mm}^{2}$ Microstage, with a stage deflection of $290 \mu \mathrm{m}$. (b) Fully released $2 \mathrm{~mm}$ Microstage, with a stage deflection of $1243 \mu \mathrm{m}$. (c) $\mathrm{A} \mathrm{1mm}^{2}$ COMSOL model predicting a total deflection of $304 \mu \mathrm{m}$. (d) A $2 \mathrm{~mm} \times 2 \mathrm{~mm}$ COMSOL model predicting a total deflection of $1261 \mu \mathrm{m}$.

By doubling the length of the actuated segment in the second leg, the actuator continues to grow in total deflection for each new segment added. The value of $L$ that maximized deflection for this configuration was found to be $308 \mu \mathrm{m}$. The analytic model predicts the gain in deflection of the first leg to be $62 \mu \mathrm{m}$ and each leg thereafter to be $27 \mu \mathrm{m}$. Various parameters, such as width of the beam, spacing between serpentines, and the number of serpentines were optimized to obtain a $300 \mu \mathrm{m}$ deflection while minimizing the pliancy of the actuator. Since $\mathrm{V}_{\mathrm{PI}}$ of the actuator is based on the actuated segment, the "pull-in" voltage is similar to the actuators characterized above.

\section{Characterization of "Microstage" Actuators}

Microstage actuators with $2 \mathrm{~mm} \times 2 \mathrm{~mm}$ footprints were fabricated alongside actuators with $1 \mathrm{~mm}^{2}$ footprints. A fully released $1 \mathrm{~mm}^{2}$ Microstage can be seen in Fig. 9(a), and a $2 \mathrm{~mm} x$ $2 \mathrm{~mm}$ Microstage can be seen in Fig. 9(b). The $1 \mathrm{~mm}^{2}$ Microstage measured a total deflection of $290 \mu \mathrm{m}$, in good agreement with the COMSOL prediction of $304 \mu \mathrm{m}$ shown in Fig. 9(c), and the $2 \mathrm{~mm}$ Microstage measured $1243 \mu \mathrm{m}$ in good agreement with the COMSOL prediction of $1261 \mu \mathrm{m}$ shown in Fig. 9(d). Optical deflection measurements for the Microstages were taken at each turn in the serpentine, and the results, shown in Fig. 10 correlate well with the COMSOL model. The average deflection gain per leg was found to be $25.6 \mu \mathrm{m}$, which correlates well with the analytic model prediction of $27 \mu \mathrm{ms}$ per leg.

\section{CONCLUSION}

We have presented a simple novel fabrication process for achieving "zipper" actuation through the use of an SOI and thermal silicon dioxide. The deflection and voltage of the actuators were characterized, and the results correlated well with analytic and FEA (Finite Element Analysis) models. Spiral actuators were investigated and insight into their mechanics was presented. "Micro-stage" zipper actuators were conceptualized, modeled and fabricated to achieve $300 \mu \mathrm{m}$ deflection within a $1 \mathrm{~mm}^{2}$, and 1250 $\mu \mathrm{m}$ deflection within a $2 \mathrm{~mm} \times 2 \mathrm{~mm}$ footprint, in order to enable the fabrication of a microstrip patch antenna.

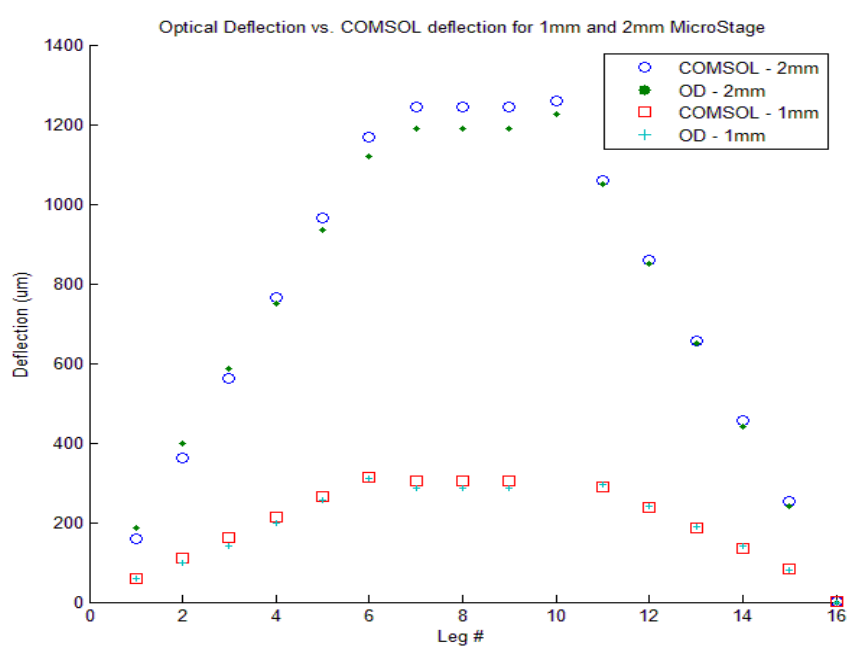

Figure 10: Optical Deflection (OD) measurements at each turn of the Microstage serpentine plotted alongside COMSOL predictions for deflection at each turn. Shown here is the correlation in measurements and predictions for both a $2 \mathrm{~mm}$ and $1 \mathrm{~mm}$ actuator.

\section{ACKNOWLEDGMENT}

Electron microscopy was performed with support from the University of Maryland Nanoscale Imaging, Spectroscopy, and Properties Laboratory (NISPLab).

\section{REFERENCES}

[1] L. Wu and H. Xie, "A Lateral-Shift-Free and Large-VerticalDisplacement Electrothermal Actuator for Scanning Micromirror/Lens," in Transducers \& Eurosensors, Lyons, France, 2007.

[2] T. Fukushige, S. Hata and A. Shimokohbe, "A MEMS Conical Spring Actuator Array," vol. 14, no. 2, 2005.

[3] S. Kwon, V. Milanovic and L. P. Lee, "Large-Displacement Vertical Microlens Scanner," IEEE Photonics Technology Letters, vol. 14, no. 11, 2002.

[4] C. Chang, C.-F. Chiang, C.-H. Liu and C.-H. Liu, "A lobstersniffing-inspired method for micro-objects manipulation using electrostatic micro-actuators," J. Micromech. Microeng., vol. 15,2005 .

[5] N. Dhaubanjar, "The Design and Analysis of Optical Scanners for Optical Coherence Tomography," University of Texas at Arlington, Arlington, Texas, 2006.

[6] A. K. Chinthakindi and P. A. Kohl, "Electrostatic Actuators with Intrinsic Stress Gradient," Journal of The Electrochemical Society, vol. 149, no. 8, 2002.

[7] N. Dhaubanjar, S. M. N. Rao, Y. Cai, D. Popa, M. Chiao and J.-C. Chiao, "A Cantilever-type Electrostatic Zipping Actuator," Smart Structures, Devices, and Systems III, vol. 6414, 2007.

[8] M. A. Rosa, D. De Bruyker, A. R. Volkel, E. Peeters and J. Dunec, "A novel external electrode configuration," Journal of Micromechanics and Microengineering, vol. 14, pp. 446-451, 2004.

[9] R. Legtenberg, J. Gilbert, S. D. Senturia and M. Elwenspoek, "Electrostatic Curved Electrode Actuators," vo. 6, no. 3, 1997. 


\section{LARGE STROKE ELECTROSTATIC COMB-DRIVE ACTUATORS BASED ON A NOVEL FLEXURE MECHANISM \\ Mohammad Olfatnia*l, Siddharth Sood ${ }^{l}$, Jason J. Gorman ${ }^{2}$, Shorya Awtar ${ }^{l}$ \\ ${ }^{1}$ University of Michigan - Ann Arbor, USA \\ ${ }^{2}$ National Institute of Standards and Technology, Gaithersburg, MD, USA}

\section{ABSTRACT}

This paper presents in-plane electrostatic comb-drive actuators with stroke as large as $245 \mu \mathrm{m}$ that is achieved by employing a novel Clamped Paired Double Parallelogram (C-DPDP) flexure mechanism. For a given flexure beam length, comb gap, and actuation voltage, this is currently the largest comb-drive actuator stroke reported in the literature. The C-DP-DP flexure mechanism design offers high bearing direction stiffness while maintaining low motion direction stiffness over a large range of motion direction displacement. The high stiffness ratio between the bearing and motion directions mitigates the on-set of sideways snap-in instability, thereby offering significantly greater actuation stroke compared to existing designs.

\section{INTRODUCTION}

Electrostatic comb-drive actuators have been used in various applications such as resonators [1], and micro/nano positioning [2]. A linear in-plane electrostatic comb-drive actuator, shown in Fig. 1, comprises two electrically isolated conductive combs with $N$ fingers each. While the static comb is fixed with respect to ground, the moving comb is guided by a flexure mechanism so that it can displace primarily in $\mathbf{Y}$ direction (or motion direction) with respect to the static comb. These static and moving comb fingers (length $L_{f}$, in-plane thickness $T_{f}$, out-of-plane thickness $H_{f}$ ) have a nominal inter-digitation gap of $G$ and an initial engagement of $Y_{o}$. In general, the flexure mechanism is designed to provide linear displacement $(Y)$ with relatively small stiffness $\left(K_{y}\right)$ in the $\mathbf{Y}$ direction. Additionally, it provides minimal error motions $\left(E_{x}\right)$ and relatively high stiffness $\left(K_{x}\right)$ in the $\mathbf{X}$ direction, or bearing direction. In an ideal scenario, $K_{y}$ and $E_{x}$ would approach zero while $K_{x}$ would approach infinity. However, in practice, this is never the case given the performance tradeoffs between motion range, stiffness, and error motions that exist in flexure mechanisms [3].

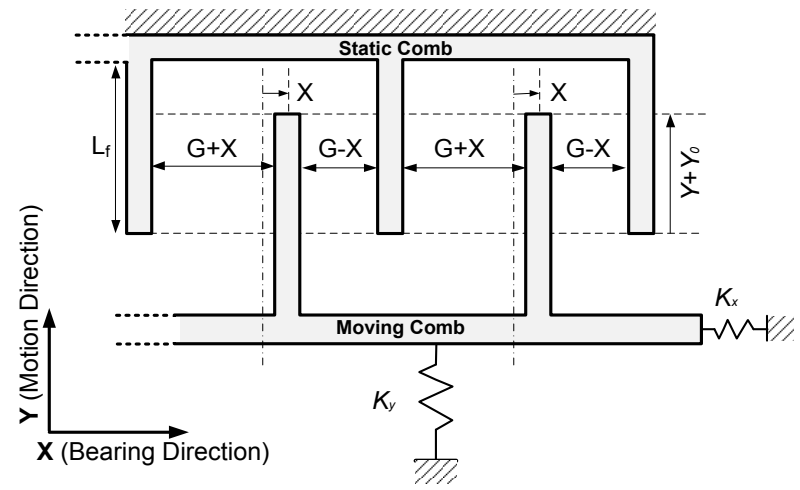

Fig. 1: Schematic of an electrostatic comb drive with the springs representing the flexure bearing.

When a voltage difference $(V)$ is applied between the two combs, they experience a mutual electrostatic attraction, which displaces the moving comb by $Y$ along the motion direction:

$K_{y} \cdot Y=\frac{2 \varepsilon N H_{f} G}{G^{2}-X^{2}} V^{2}$

9780964002494/HH2012/\$25@2012TRF

DOI 10.31438/trf.hh2012.109
Here, $\varepsilon$ is the dielectric constant of air. The bearing direction displacement $X$ can arise due to flexure error motion, fabrication misalignment, electrostatic forces, or disturbances in the $\mathbf{X}$ direction. While the displacement $Y$ is determined by the comb geometry, motion direction flexure stiffness, and the actuation voltage, its maximum value (or actuation stroke) is limited by the snap-in phenomenon, which corresponds to side-ways instability of the moving comb $[2,4]$. For any value of $Y$ displacement, the electrostatic force due the actuation voltage $V$ produces a destabilizing negative spring effect and the flexure mechanism offers a stabilizing positive spring effect in the $\mathbf{X}$ direction. The former increases with increasing stroke, while the latter generally decreases. The moving comb snaps sideways into the static comb at the $Y$ displacement at which the former stiffness exceeds the latter. This condition may be mathematically expressed as $[2,5]$ :

$\left(\frac{K_{x}}{K_{y}}\right) \leq \frac{2 Y\left(Y+Y_{0}\right)}{G^{2}} \frac{\left(1+\frac{3 X_{c}^{2}}{G^{2}}\right)}{\left(1-\frac{X_{c}^{2}}{G^{2}}\right)^{2}}$ where $E_{x}=\frac{4 X_{c}^{3}}{G^{2}+3 X_{c}^{2}}$

This snap-in condition assumes that the comb-fingers are perfectly rigid and all compliance comes from the flexure. Also, the in-plane rotation stiffness is assumed high enough to be ignored. The right hand side represents a "critical stiffness ratio" needed to avoid snap-in, and increases with displacement $Y$ and error motion $E_{x}$. Clearly, to delay snap-in and maximize the actuator stroke, the flexure mechanism has to provide a high $\left(K_{x} / K_{y}\right)$ ratio that is maintained over a large range of $Y$ displacement. Since $E_{x}$ is generally non-deterministic, the stability condition given by Eq.(2) may be mathematically simplified by incorporating a positive Margin of Stability $(S)$. Stable operation is given by:

$\left(\frac{K_{x}}{K_{y}}\right) \geq \frac{2 Y\left(Y+Y_{0}\right)}{G^{2}}(1+S)$

The paired Double Parallelogram (DP-DP) flexure mechanism has been most commonly used in electrostatic combdrive actuators $[1,2,4]$. While this flexure provides a high stiffness ratio $\left(K_{x} / K_{y}\right)$ at $Y=0, K_{x}$ drops precipitously with increasing $Y$ displacement even as $K_{y}$ remains largely constant. This limits the comb-drive actuator stroke due to early snap-in. Pre-bent beams in the DP-DP flexure help shift the value of $Y$ at which $K_{x}$ is maximum, but do not restrict the drop in $K_{x}$ with increasing $Y$ [2]. This results in some improvement in the actuator stroke, but at the expense of robustness [5]. Other designs are successful at restricting the drop in $K_{x}$ with increasing $Y$ by appropriately constraining the secondary stage(s) in a DP or DPDP flexure [6]. However, this also results in an increase in the motion direction stiffness, $K_{y}$, which is undesirable.

This paper presents a new Clamped Paired Double Parallelogram (C-DP-DP) flexure mechanism that offers high $K_{x}$ over a large $Y$ displacement range, while maintaining low $K_{y}$ throughout. This new design is described in Section 2, along with closed-form analytical expressions for its $K_{x}$ and $K_{y}$ stiffness. With these improved stiffness characteristics, the C-DP-DP flexure is well suited for achieving large-stroke in comb-drive

Solid-State Sensors, Actuators, and Microsystems Workshop Hilton Head Island, South Carolina, June 3-7, 2012 
actuators. Micro-fabrication of some representative actuators and associated experimental results are presented in Section 3. With the C-DP-DP flexure, a maximum actuation stroke of $245 \mu \mathrm{m}$ with $1 \mathrm{~mm}$ flexure beam length and $6 \mu \mathrm{m}$ comb finger gap is demonstrated.

\section{FLEXURE MECHANISM DESIGN}

In this section, we first discuss the traditional Paired Double Parallelogram (DP-DP), shown in Fig.2, which has been extensively studied previously $[3,4]$. The advantages and limitations of this design provide the context for the proposed $\mathrm{C}$ DP-DP flexure design that is discussed subsequently.

In all the designs considered here, we assume a general shape for each constituent beam, with two equal end-segments having uniform in-plane thickness $T_{1}$ and length $a_{0} L_{1}$ and a rigid middle section of length $\left(\begin{array}{ll}1 & 2 a_{0}\end{array}\right) L_{l}$. The geometric parameter $a_{0}$ quantifies the degree of distributed compliance; $a_{0}=1 / 2$ represents a uniform thickness beam with highly distributed compliance, while smaller values of $a_{0}$ correspond to increasingly lumped compliance. This parameter allows for subsequent beam shape optimization.

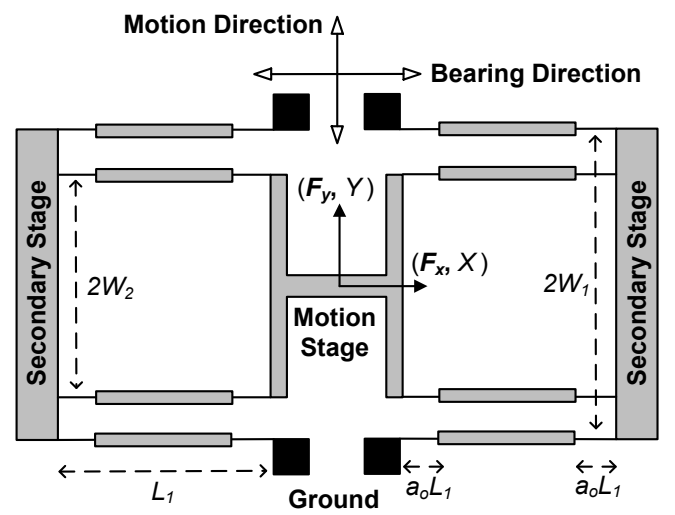

Fig. 2: Paired Double Parallelogram (DP-DP) flexure

Closed-form non-linear stiffness relations for this flexure geometry have been previously derived [3] and are summarized here:

$$
\begin{aligned}
& K_{y}=\frac{2 E I_{1}}{L_{1}^{3}}\left[k_{11}^{(0)}-\frac{1}{4 k_{11}^{(0)}}\left(\frac{\boldsymbol{F}_{x} L_{1}^{2}}{E I_{1}} k_{11}^{(1)}\right)^{2}\right] \\
& K_{x}=\frac{2 E I_{1}}{L_{1}^{3}} \frac{k_{33}}{\left(1+k_{33}\left(k_{11}^{(2)}+\frac{\left(k_{11}^{(1)}\right)^{2}}{k_{11}^{(0)}}\right)\left(\frac{Y}{2 L_{1}}\right)^{2}\right)}
\end{aligned}
$$

Here, the non-dimensional terms $k_{11}^{(0)}, k_{11}^{(1)}, k_{11}^{(2)}$, and $k_{33}$ are all functions of the beam shape $\left(a_{o}\right.$ and $\left.T_{l}\right)$ and are referred to as beam characteristic coefficients [3].

$$
\begin{aligned}
& k_{11}^{(0)}=\frac{6}{\left(3-6 a_{o}+4 a_{o}^{2}\right) a_{o}} \\
& k_{11}^{(1)}=\frac{3\left(15-50 a_{o}+60 a_{o}^{2}-24 a_{o}^{3}\right)}{5\left(3-6 a_{o}+4 a_{o}^{2}\right)^{2}} \\
& k_{11}^{(2)}=\frac{2 a_{o}^{3}\left(105-630 a_{o}+1440 a_{o}^{2}-1480 a_{o}^{3}+576 a_{o}^{4}\right)}{175\left(3-6 a_{o}+4 a_{o}^{2}\right)^{3}} \\
& k_{33}=\frac{6}{a_{0}\left(T_{1} / L_{1}\right)^{2}}
\end{aligned}
$$

As per Eq.(4), the DP-DP flexure provides a low $K_{y}$ stiffness that remains constant with $Y$, and reduces to a simple expression in the absence of significant bearing force $\boldsymbol{F}_{\boldsymbol{x}}$. This motion direction stiffness depends directly on $k_{11}^{(0)}$. This mechanism also provides a high $K_{x}$ stiffness at $Y=0$, as per Eq.(5). However, this stiffness drops precipitously with increasing $Y$ displacement (Fig. $3)$. Analytically, there are two sources of additional compliance, as seen in the denominator of Eq.(5). The first source arises from the elastokinematic effect manifested in the product $k_{33} k_{11}^{(2)}$, which approaches zero with reducing $a_{0}$. However, this is dominated by the second source that is based on the kinematic effect, manifested in the term $\left(k_{11}^{(1)}\right)^{2} k_{33} / k_{11}^{(0)}$. The latter is at least two orders of magnitude greater than the former, is largely insensitive to variation in $a_{0}$, and never approaches zero. Therefore, as seen in Fig. 3, while the initial $K_{x} / K_{y}$ ratio at $Y=0$ can be increased by approaching lumped compliance (i.e. lower value of $a_{o}$ ), the impact of this shape variation is negligible at higher values of $Y$ displacement.

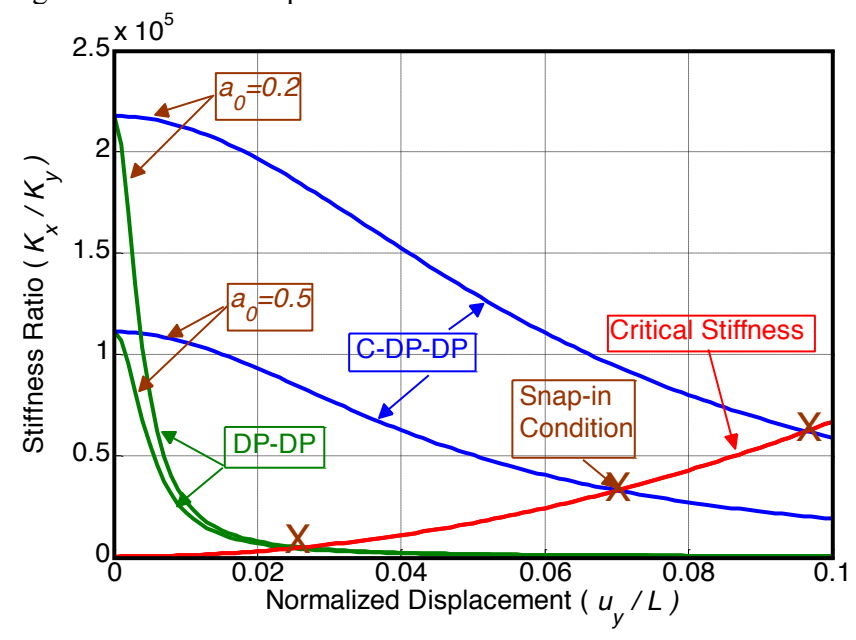

Fig. 3: $\left(K_{x} / K_{y}\right)$ stiffness ratio provided by $D P-D P$ and $C-D P-D P$ flexures for different values of beam reinforcement $\left(a_{0}\right)$. A typical critical stiffness curve is included to demonstrate the effect of stiffness ratio on comb-drive actuator snap-in.

This sharp decrease in the DP-DP flexure's $K_{x}$ stiffness arises due to the mechanism's topology. In this design, the secondary stages of both the DPs are inadequately constrained in the $\mathrm{Y}$ direction. When the $Y$ displacement of the motion stage is held fixed, and a small bearing direction force $\boldsymbol{F}_{\boldsymbol{x}}$ is applied, the two secondary stages move opposite to each other in the motion direction from their nominal displacement of $Y / 2$. This is because the motion direction stiffness of the each of the individual beams within the two DPs changes in the presence of $\boldsymbol{F}_{\boldsymbol{x}}$ due to the loadstiffening effect. Due to this "extra" motion direction displacement of the two secondary stages, the kinematic error of the individual beams in the bearing direction no longer cancels out perfectly, thereby producing an "extra" $X$ displacement at the motion stage and therefore a lower $K_{x}$.

Therefore, in order to avoid the above-described sharp decrease in the $K_{x}$ stiffness, it is desirable to constrain the $\mathbf{Y}$ motion of both the secondary stages such that they always remain at their nominal value of $Y / 2$, which is half the $\mathbf{Y}$ direction displacement of the motion stage. However, any topological feature that is considered to accomplish this should not restrict the small $\mathbf{X}$ direction displacements of each of these secondary stages. Restricting these displacements, which arise from the 
kinematics of beam arc-length conservation, would lead to an over-constraint in the overall flexure mechanism, resulting in an increase in the motion direction stiffness, $K_{y}$.

We propose the Clamped Paired Double Parallelogram (CDP-DP) flexure mechanism that accomplishes this goal via an external clamp, as shown in Fig. 4. In this design, the two secondary stages are connected to an external clamp through additional parallelogram flexures. The high rotational stiffness of these parallelogram flexures minimizes any relative displacement in the $\mathbf{Y}$ direction between the two secondary stages, forcing them to maintain $Y / 2$ displacement at all times. This constrains these stages from responding to an $\mathbf{X}$ direction force on the motion stage. Also, the low $\mathbf{X}$ direction stiffness of the additional parallelogram flexures offers minimal resistance to the kinematic displacement of the secondary stages in the $\mathbf{X}$ direction.

The analytical relations for the motion and bearing stiffness of the C-DP-DP flexure have been separately derived [7] and are summarized here:

$$
\begin{aligned}
& K_{y}=\frac{E I_{1}}{L_{1}^{3}}\left(2 k_{11}^{(0)}+\frac{3 k_{11}^{(0)} k_{11}^{(1)}}{20}\left(\frac{L_{1}^{3}}{L_{3}^{3}}\right)\left(\frac{Y}{L_{1}}\right)^{2}\right) \\
& K_{x}=\frac{E I_{1}}{L_{1}^{3}} \frac{2 k_{33}}{\left.1+k_{33}\left(k_{11}^{(2)}+\frac{\left(k_{11}^{(1)}\right)^{2}}{k_{11}^{(0)}(1+\eta)}\right)\left(\frac{Y}{2 L_{1}}\right)^{2}\right)} ; \eta=\left(\frac{6 W_{3}^{2} L_{1}^{3}}{k_{11}^{(0)} L_{2}^{2} L_{3} T_{3}^{2}}\right)
\end{aligned}
$$

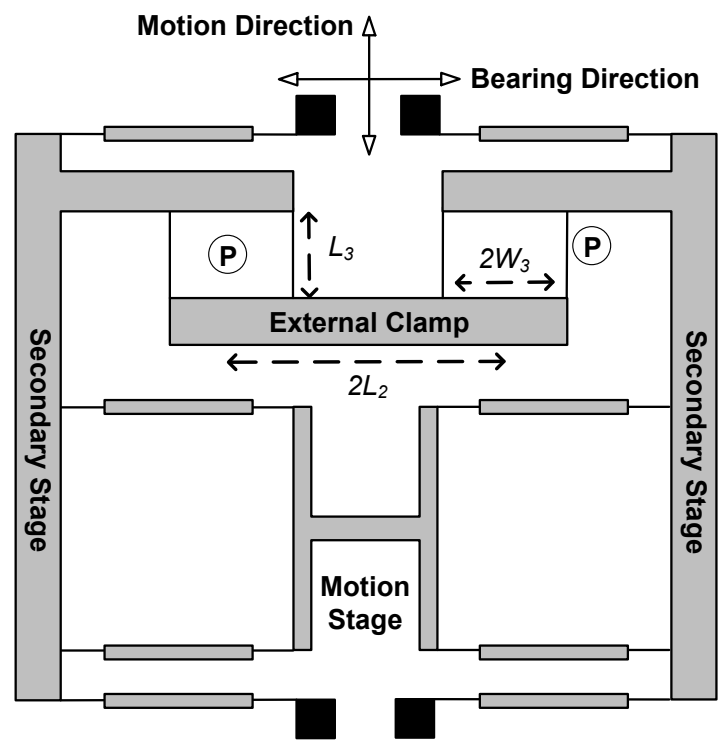

Fig. 4: Clamped Paired Double Parallelogram (C-DP-DP) flexure

Eq.(7) shows that there is a slight increase in $K_{y}$ because of the external clamp. However, this can be mitigated by choosing a large enough beam length $L_{3}$ in the additional parallelograms. Next, the effectiveness of the clamp in restricting the precipitous drop in the bearing stiffness $K_{x}$ is captured via the dimensionless parameter $\eta$ in Eq.(8). The external clamp prevents relative $\mathbf{Y}$ direction motion between the two secondary stages by employing the high rotational stiffness of its constituent parallelograms, which manifests itself in the form of $\eta$. For low values of $W_{3}$, this stiffness and therefore $\eta$ are small. It may be analytically seen that as $\eta \rightarrow 0$, the $K_{x}$ stiffness becomes exactly the same as that for a DP-DP flexure, which corresponds to a completely ineffective clamp. However, as $W_{3}$ increases, the parameter $\eta$ also increases. Eq.(8) shows that as $\eta$ becomes very large, the kinematic term $\left(k_{11}^{(1)}\right)^{2} / k_{11}^{(0)}$ vanishes and only the elastokinematic term $k_{11}^{(2)}$ remains. Since the latter is at least two orders of magnitude smaller than the former, this implies that the drop in $K_{x}$ stiffness now is significantly reduced and that the clamp proves to be effective. Furthermore, because of the sensitivity of the elastokinematic term to beam shape, reinforced beams $\left(a_{0}<0.5\right)$ may be used to produce even greater improvements in the bearing stiffness $K_{x}$ of the C-DP-DP flexure. This is in contrast with the DP-DP flexure for which beam reinforcement produces marginal benefits.

The $\left(K_{x} / K_{y}\right)$ stiffness ratio for the C-DP-DP flexure for two different values of $a_{o}$ is shown in Fig. 3, which demonstrates its superior stiffness characteristics compared to the DP-DP flexure. Also shown is a representative critical stiffness ratio curve for a typical comb-drive actuator, and the resulting snap-in conditions for the flexure designs considered here.

\section{EXPERIMENTAL RESULTS AND DISCUUSION Fabrication and Characterization}

Several comb-drive actuators based on the DP-DP and CDP-DP flexures were fabricated using silicon on insulator (SOI) wafers with a device layer of $50 \mu \mathrm{m}$, a buried oxide layer of 2 $\mu \mathrm{m}$, and a silicon handle layer of $350 \mu \mathrm{m}$. First, the silicon handle layer was patterned and etched by deep reactive ion etching (DRIE); next, the buried oxide was removed by Hydrogen Fluoride (HF) etching; finally, the device layer was patterned and etched using DRIE. A scanning electron microscope (SEM) image of a representative comb-drive actuator is shown in Fig. 5. These actuators were driven using DC voltage applied via probes at the static and moving combs. The response of each actuator for a given voltage was observed with an optical microscope and video-captured with a CCD camera. Subsequently, the displacement of the actuator was extracted from the video using image processing software. The displacement measurements were repeatable within $1 \mu \mathrm{m}$.

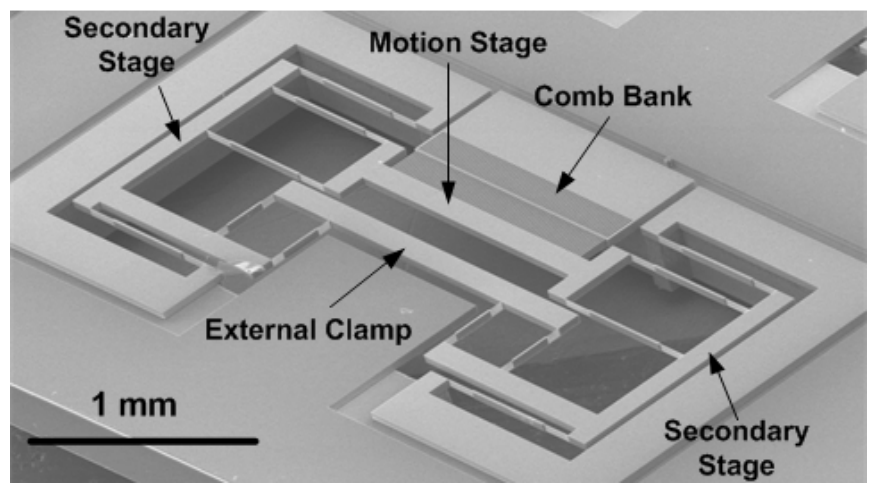

Fig. 5: SEM image of a fabricated $C-D P$-DP based comb-drive actuator.

\section{Voltage-Stroke Curves}

Fig. 6 shows the displacement versus voltage curves for comb-drive actuators based on three different flexures with $G=3$ $\mu \mathrm{m}, L_{l}=1 \mathrm{~mm}$, and $T_{l}=4 \mu \mathrm{m}$. The measured actuator stroke with a conventional DP-DP flexure with these dimensions and $a_{0}$ $=0.5$ was $50 \mu \mathrm{m}$. The actuation stroke increases to $119 \mu \mathrm{m}$ for a C-DP-DP flexure with the same dimensions. This stroke further increases to $141 \mu \mathrm{m}$ by using reinforced beams with $a_{0}=0.2$ in the C-DP-DP flexure. These results clearly highlight the effectiveness of the reinforced C-DP-DP in providing greater 
comb-drive actuator stroke compared to the traditional DP-DP flexure.

By increasing the comb gap, the stroke of the comb-drive actuator can be further improved. This is demonstrated in Fig. 7, where a stroke of $215 \mu \mathrm{m}$ was obtained by a C-DP-DP flexure with $G=4 \mu \mathrm{m}, L_{l}=1 \mathrm{~mm}$, and $a_{0}=0.2$. The benefit of beam reinforcement is also evident here. With an identical design, the experimentally measured stroke is $170 \mu \mathrm{m}$ and $157 \mu \mathrm{m}$ for $a_{0}=0.3$ and $a_{0}=0.4$, respectively. Finally, a large stroke of $245 \mu \mathrm{m}$ at $120 \mathrm{~V}$ for a C-DP-DP flexure with $G=6 \mu \mathrm{m}, L_{l}=1 \mathrm{~mm}$, and $a_{0}$ $=0.2$ is also reported in this figure. For a given flexure beam length, comb gap, and actuation voltage, this is the largest combdrive actuator stroke reported in the literature (Fig. 8), to the best of our knowledge. In general, it is desirable to minimize the product of the device footprint and operating voltage, while maximizing the stroke. This corresponds to the top-left corner of Fig. 8, which is where our present results lie.

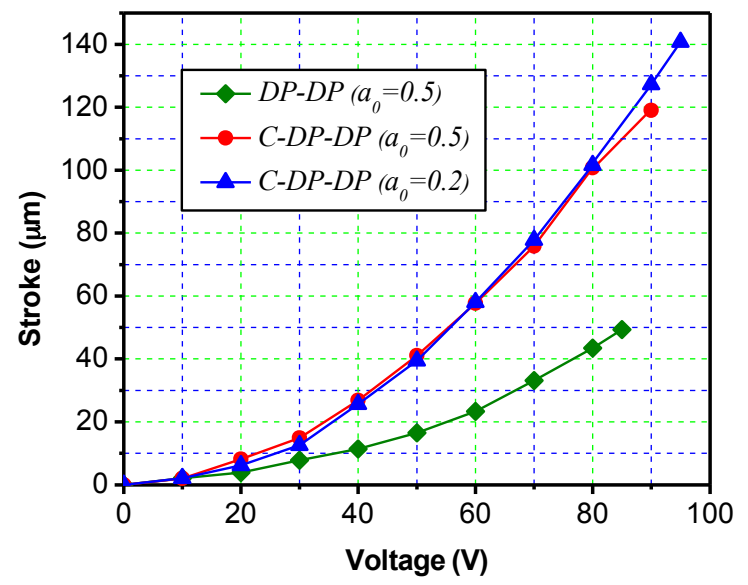

Fig. 6: Displacement measurements for DP-DP and C-DP-DP flexures.

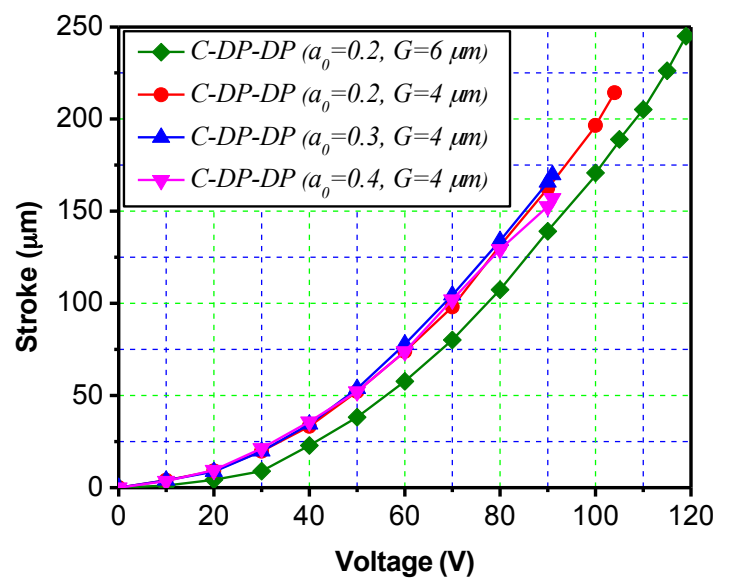

Fig. 7: Displacement measurements for C-DP-DP flexures.

\section{CONCLUSION}

This paper presents the Clamped Paired Double Parallelogram (C-DP-DP) flexure mechanism with reinforced beams, which offers high bearing direction stiffness $\left(K_{x}\right)$ while maintaining low motion direction stiffness $\left(K_{y}\right)$, over a large range of motion direction displacement (Y). It is shown that this flexure mechanism helps delay the on-set of snap-in instability in a comb-drive actuator and therefore provides greater actuation stroke. For $1 \mathrm{~mm}$ flexure beam length and $6 \mu \mathrm{m}$ comb gap, strokes as large as $245 \mu \mathrm{m}$ at $120 \mathrm{~V}$ have been demonstrated. This experimental proof along with the analytical formulation clearly highlights the strength of C-DP-DP flexure for large range combdrive actuators.

\section{ACKNOWLEDGEMENTS}

The experimental portion of this work was performed at the Lurie Nanofabrication Facility, a member of the National Nanotechnology Infrastructure Network, which is supported in part by the National Science Foundation. The second author acknowledges a National Institute of Standards and Technology Measurement Science and Engineering Graduate Fellowship.

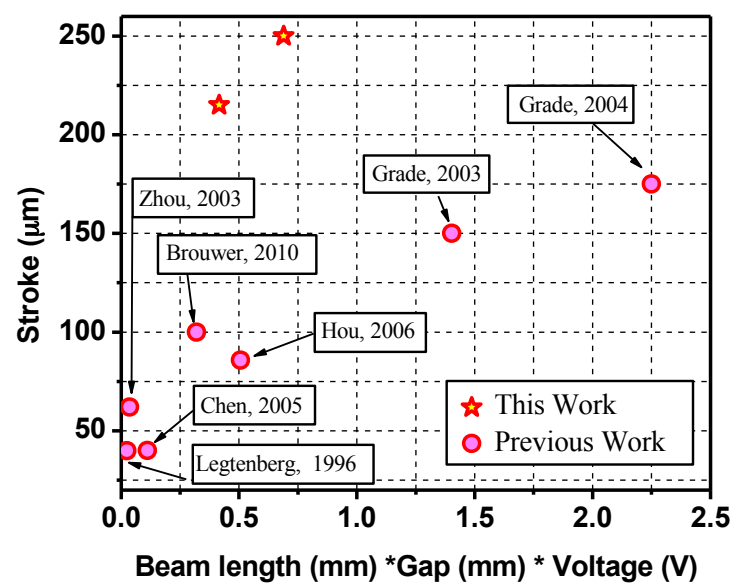

Fig. 8: Comparison of this paper's results with previously reported comb-drive actuator designs.

\section{REFERENCES}

[1] W. C. Tang, T. C. H. Nguyen, And R. T. Howe, "Laterally Driven Polysilicon Resonant Microstructures," Sensors And Actuators, Vol. 20, Pp. 25-32, 1989.

[2] J. D. Grade, H. Jerman, And T. W. Kenny, "Design Of Large Deflection Electrostatic Actuators," Journal Of Microelectromechanical Systems, Vol. 12, Pp. 335-343, 2003.

[3] S. Awtar, A. H. Slocum, And E. Sevincer, "Characteristics Of Beam-Based Flexure Modules," Journal Of Mechanical Design, Vol. 129, Pp. 625-639, Jun 2007.

[4] R. Legtenberg, A. W. Groeneveld, And M. Elwenspoek, "Comb-Drive Actuators For Large Displacements," Journal Of Micromechanics And Microengineering, Vol. 6, Pp. 320-329, 1996.

[5] S. Awtar And T. Trutna, "An Enhanced Stability Model For Electrostatic Comb-Drive Actuator Design," In Proc. Idetc/Cie, Montreal, Canada, 2010.

[6] D. M. Brouwer, A. Otten, J. B. C. Engelen, B. Krijnen, And H. M. J. R. Soemers, "Long-Range Elastic Guidance Mechanisms For Electrostatic Comb-Drive Actuators," In Proceedings of The Euspen International Conference, Delft, 2010.

[7] S. Sood And S. Awtar, "Clamped Symmetric Double Parallelogram Flexure Mechanism With Improved Bearing Performance," In Review Of Scientific Instruments, Ed.

\section{CONTACT}

*M. Olfatnia, tel: +1-734-730-3141; olfatnia@umich.edu 


\title{
MEMS BASED SINGLE DEVICE "XOR" AND "AND" LOGIC GATES FOR LSI MECHANICAL PROCESSORS OPERATING IN HIGH RADIATION ENVIRONMENTS
}

\author{
Faisal K. Chowdhury ${ }^{l}$, Sijing Han ${ }^{2}$, Daniel Saab ${ }^{2}$ and Massood Tabib-Azar ${ }^{{ }^{*}}$ \\ ${ }^{1}$ University of Utah, Salt Lake City, Utah, USA \\ ${ }^{2}$ Case Western Reserve University, Cleveland, Ohio, USA
}

\begin{abstract}
Here we demonstrate the feasibility of MEMS functional devices where a single device functions as a logic gate. This novel approach reduces the number of MEMS devices needed to implement a mechanical processor by $\sim 10 \mathrm{X}$. MEMS processors are suitable for operation in harsh environment in engines and in the presence of ionizing radiations. Generally, MEMS devices have overall lower speed and less reliability than CMOS. By reducing the number of devices needed for a given operation, our approach improves yield, reproducibility, speed and simplifies implementation of MEMS-based circuits such as adders and multiplexers. Specifically, we discuss XOR \& AND gates fabricated on $\mathrm{Si}_{3} \mathrm{~N}_{4}$ \& Polysilicon as bridge materials using $\mathrm{W}$ electrodes. The XOR gates with $\sim 1.5 \mathrm{~V}$ turn-on voltage at $50 \mathrm{MHz}$ with $>10^{9}$ cycles of reliable operations and low operational power consumption (leakage current and power $<10^{-9},<1 \mu \mathrm{W}$ ) were tested. We also present data showing the operation of XOR without deterioration at high temperature and in $90 \mathrm{~kW}$ ionizing radiation for 120 minutes. Related circuits such as 2-bit full adder and multiplexer are also discussed.
\end{abstract}

\section{INTRODUCTION}

Simple micro-electro-mechanical on/off switches have been reported in the past for applications in processors and to address power management in scaled VLSI, programming interconnect in FPGA's, biomedical devices where it is desirable to reduce leakage power to prolong implanted battery life, and other applications in harsh environment where CMOS cannot operate due to high temperature or radiation [1-9]. One such case includes operation in the presence of Ionizing Radiation (I-R) in troubled reactors like Chernobyl and Fukushima or at high temperatures encountered inside combustion engines. In these cases silicon channel in CMOS becomes highly conductive due to thermal generation of carriers or due to lattice defect generation caused by I-R radiation over prolonged exposure [1]. Space applications also require radiationhard devices and materials. In some electronic materials such as $\mathrm{SiC}$, the energy required to produce lattice defects is high enabling these materials to withstand I-R longer than Si. In other materials such as InP, defects heal at relatively low temperatures enabling them to recover quickly.

NEMS/MEMS devices are based on mechanical elements that are inherently insensitive to I-R. The I-R causes lattice defects in these devices but these defects do not alter their characteristics the way they affect channel resistance in CMOS. Eventually large defect densities created over extended I-R exposure leads to embrittlement that may affect NEMS/MEMS electrical and switching characteristics. NEMS/MEMS devices also have very low leakage power making them very desirable in biomedical implant devices or other applications requiring very long battery lifetime.

Despite their very high off-to-on resistance (100 G $\Omega$ to 10 $\mathrm{m} \Omega$ ) ratios, and very low off-state leakage currents $\left(<10^{-14} \mathrm{~A}\right)$, MEMS switches tend to be slow $(<1 \mathrm{MHz})$, large $\left(>40 \mu \mathrm{m}^{2}\right)$, and unreliable with limited lifetime of $\sim 10^{6}$ operation cycles [1-4]. In addition, MEMS/NEMS switches have many interesting and challenging issues including: a) contact reliability, b) stiction problem related to release during fabrication and micro-welding during hot-contact operation, c) reliability of flexure structures usually used as part of the switch and d) particulate problems that occur during repeated operations that can lead to switch failure. To address some of these issues, our approach during the last two years have shifted from the CMOS paradigm that uses individual p- and n-MOSFET (PMOS and NMOS) as complementary switches for implementing logic gates to single device functional structures creating a platform for improved density, reliability, speed and fabrication yield. The devices are based on a composite $\mathrm{Si}_{3} \mathrm{~N}_{4} /$ Polysilicon cross-bridge platform with metal contacts designed to provide AND or XOR capability from a single actuating structure. The following sections elaborate on the functional structure design, fabrication process and the results after testing these novel single device XOR and AND gates.

\section{FUNCTIONAL STRUCTURE DESIGN}

The cross-bridge platform shown in figure 1 forms the basis for both XOR and AND gates. It illustrates the electrode design for an XOR gate where metal traces overlap at the intersection area of the bridges and the bridges are actuated by electrostatic attraction between the gate electrodes.

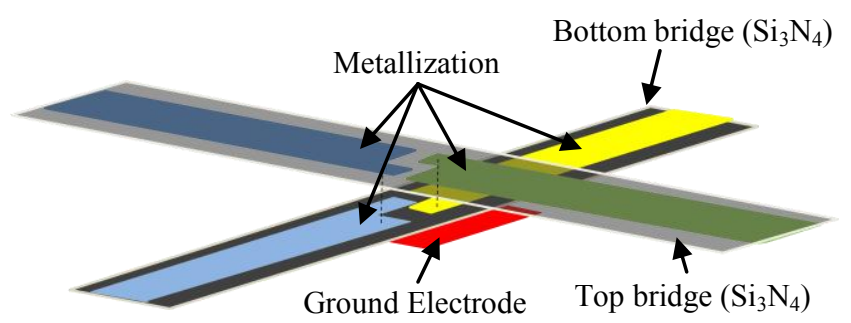

Figure 1: Schematic of MEMS based single device XOR gate. Only the central regions of the bridges are shown. The metallization patterns at the top surface of the bottom bridge and at the bottom surface of the top bridge are matched so that when the two bridges contact each other, the intended function is executed.

Other logic gates can be constructed using similar structures but different metallization/contact patterns as shown in figure 2 for an "AND" gate. In figure 2 the cross sectional view of the XOR and AND device can be seen. The diagram has the two bridges, Gate 1, Gate 2, Drain and Source labeled.

When both gates are low (" 00 ") or when both gates are high ("11") there is no electrostatic attraction (see XOR in figure 2 and figure $3(\mathrm{a})$ ). When either one of the gates are high (" 10 " or " 01 ") electrostatic attraction causes the drain and source to collapse towards each other and thereby make contact. This is the XOR function (ref. to truth table in figure 2). A minor modification of the electrode design produces similar functionality corresponding to AND gate (figure 2 and 3(b)). The AND gate requires that both G1 \& G2 be high at the same time to enable the top bridge to 
experience sufficient electrostatic attraction to cause the bridge to collapse towards the bottom bridge \& thereby make a S-D connection. This is the $\mathrm{ON}$ state. All other conditions translate to OFF state. In the XOR gate, to prevent the drain-source electrodes from causing the attraction between the two bridges their overlapping area is designed to be 4 times smaller than that of the gate electrodes. The same is true for the AND gate with the ratio being slightly lower. The fabrication procedure of these devices is discussed in the following section. Other gates such as NAND and NOT can also be realized in a similar manner. An XOR gate can be converted to NOT gate by fixing one of its inputs at " 1 ". A NAND gate can be produced by NOT(AND) or by using different device structure.

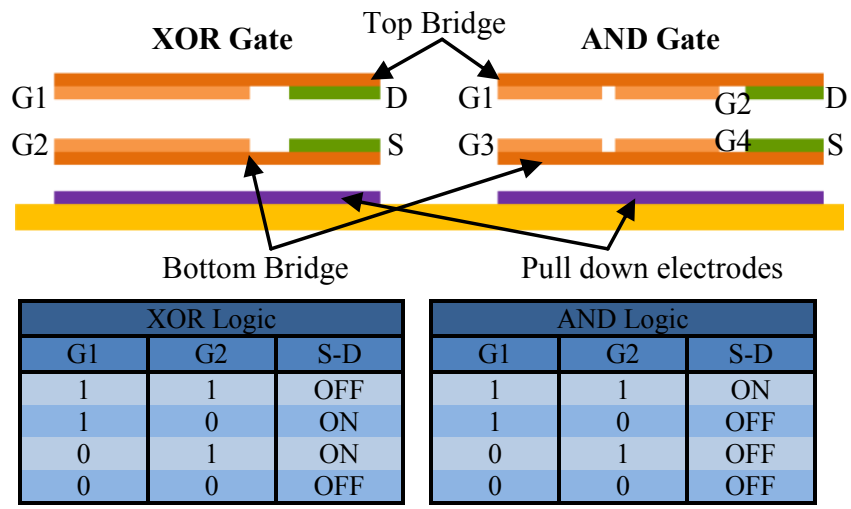

Figure 2: Cross-section of XOR and AND gates. The table shows the truth table corresponding to XOR gate operation

Figure 3 shows optical images of the XOR and AND gates. The common implementation of XOR using 8 individual switches is shown in figure 4 . The factor 8 reduction in device count and associated reduction in number of moving parts and areas lead to 8 times better reliability, at least 4 times faster gate speed and proportionately higher yields. Moreover, multi-input $(>2)$ gates can also be designed using the cross-bridge geometry with two or more metal traces for multiple contact electrodes. A 4-input XOR gate will compress the device count by $\mathrm{x} 24$.
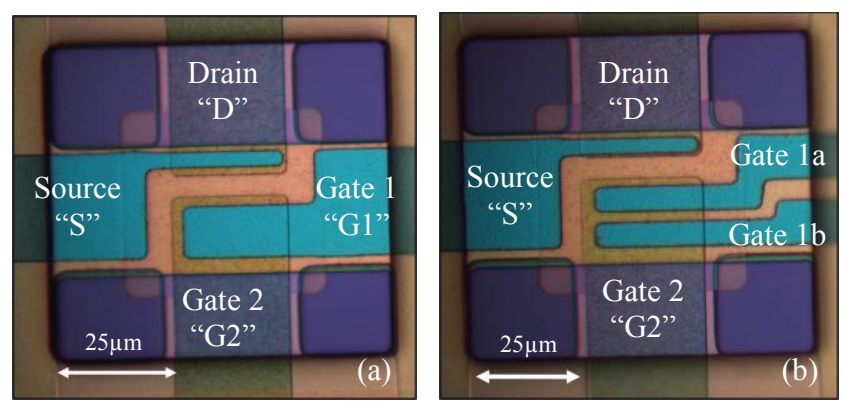

Figure 3: a) Fabricated XOR gate. G1 and G2 are gate electrodes. $S$ and D represent Source/Drain. b) Fabricated AND gate. $S-D$ will connect only if gate $1 a \& 1 b$ are high at the same time.

\section{FABRICATION}

The fabrication process flow is given in figure 5. The conducting silicon wafer was insulated with $100 \mathrm{~nm}$ LPCVD stoichiometric silicon nitride deposited at $780^{\circ} \mathrm{C}$, first tungsten layer (thickness $100 \mathrm{~nm}$ ) sputtered uniformly and patterned to form a "stationary" ground electrode. This electrode was used both as a gate in "MOSFET" like operation of the device as well as to use field effect to separate the two bridges if needed.

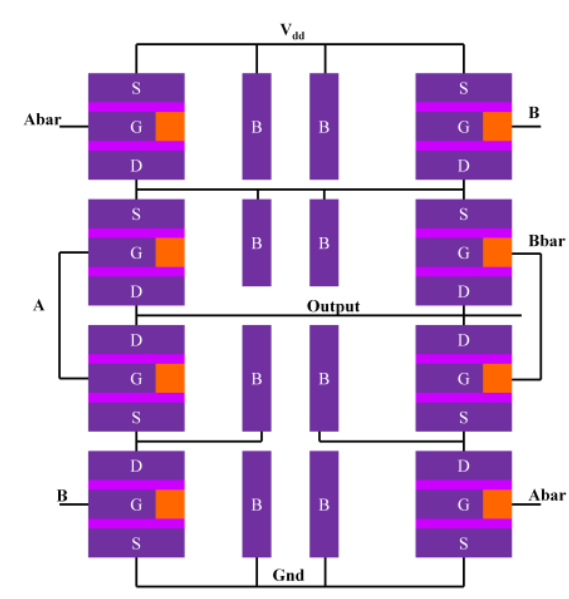

Figure 4: XOR implemented using individual switches require 8 devices each $25 \mu^{2}$ while our single XOR device requires only $25 \mathrm{\mu m}^{2}$ area.

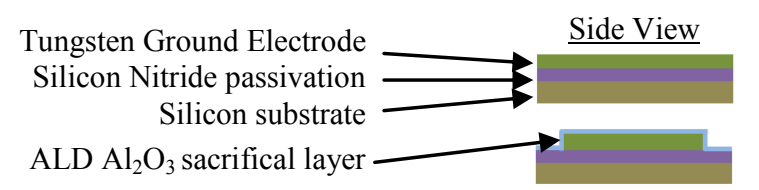

Silicon Nitride Bridge Layer

Tungsten electrode

$\operatorname{ALD~} \mathrm{Al}_{2} \mathrm{O}_{3}$ sacrificial layer

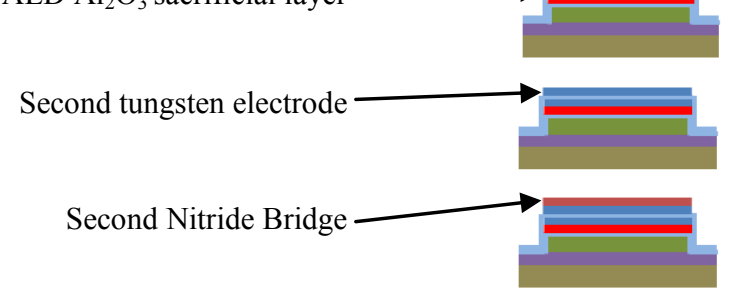

Final device after sacrificial $\mathrm{Al}_{2} \mathrm{O}_{3}$ wet etch

Top View

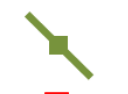

Figure 5: Fabrication process flow for XOR and AND gates. The process flow is the same for other gates.

The $100 \mathrm{~nm}$ tungsten was then capped with 100 layers $(\sim 0.1$ $\mathrm{nm} /$ layer) of thermal $\mathrm{Al}_{2} \mathrm{O}_{3}$ deposited using Fiji Atomic Layer Deposition (ALD) system and a second layer of LPCVD nitride $(100 \mathrm{~nm})$ was deposited and patterned to form the first nitride bridge. In step 6, the second layer of tungsten was sputterdeposited and patterned to form the electrodes on the first nitride bridge. ALD $\mathrm{Al}_{2} \mathrm{O}_{3}$ was subsequently used to cap the patterned tungsten and also serve as the sacrificial gap between bottom and top electrode-bridges. This was followed by the third tungsten metallization and patterning to deposit the electrodes that reside under the second nitride bridge. Then, another patterned nitride layer was used to define the top bridge. After fabricating the whole structure that required 8 masks, the ALD layers were sacrificially etched in BOE etchant to "free" the two bridges from each other 
and from the substrate. $\mathrm{H}_{2} \mathrm{O}_{2}$ at room temperature was used as the wet etchant to pattern tungsten resulting in a clean and uniform etch all over the wafer. Silicon Nitride was pattered using a dry etch recipe of $\mathrm{CF}_{4} / \mathrm{O}_{2}$ at $200 \mathrm{~W}$. This also resulted in a clean etch of the nitride bridges. The ALD $\mathrm{Al}_{2} \mathrm{O}_{3}$ layers acted as an effective etch stop for this dry etch recipe.

\section{TESTING AND RESULTS}

The micro-fabricated XOR gate was tested for switching and reliability using an Agilent $4156 \mathrm{C}$ Precision Semiconductor Analyzer, with the current compliance fixed at $1 \mu \mathrm{A}$, coupled to a probe station. A switching voltage of approximately $1.5 \mathrm{~V}$ was observed over repeated cycling as seen in figure 6 .

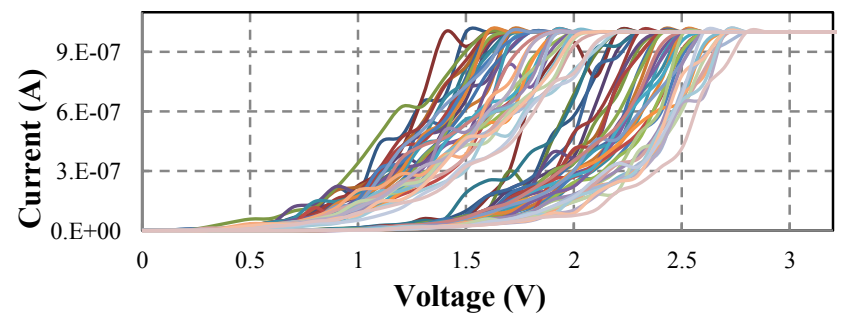

Figure 6: I-V characteristics over a million switching cycles. In this test, the G1 was shorted to D and G2 was shorted to $S$. These contacts are shown in Fig. 2 and the voltage was then applied between the G2-D and G1-S electrodes.

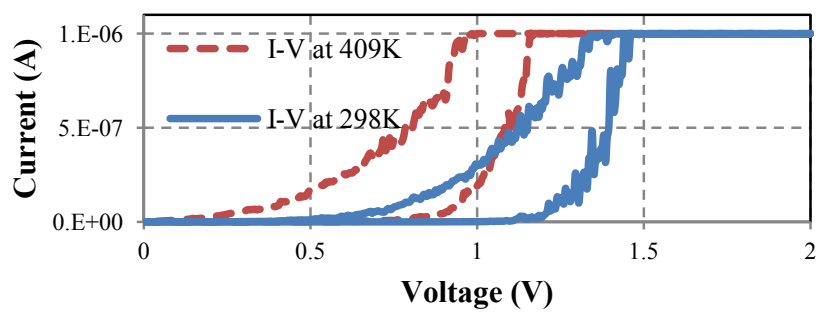

Figure 7: Switching characteristics at elevated temperatures. These tests were performed using the same electrode arrangements used in figure 8.

The devices were also tested under harsh conditions in a chamber with controlled environment where temperature was monitored and maintained as required. The I-V characteristics of the switch at $298 \mathrm{~K}$ and $409 \mathrm{~K}$ are compared in figure 7 . It reveals that the switching characteristics are consistent, even at elevated temperatures, albeit shifted by $\sim 0.5 \mathrm{~V}$.

The setup used to test the device's logic functionality is schematically shown in figure 8 . As seen from the switching characteristics, the two gate regions attract each other only when one of them is high. No actuation was observed when either both gates are low or both gates are high. At the ON state (when the bridges contact each other), the drain and source electrodes contact each other and produce the desired output. This simple structure operates as an XOR gate. The fundamental design of the device with " $D$ " and "S" electrodes situated on separate bridges lead to very small leakage current. The electrodes had dimensions on the order of $\sim 2 \mu \mathrm{m}$ at the points of contact while the leakage current was less than $10^{-9} \mathrm{~A}$. The leakage power is shown in figure 9 .

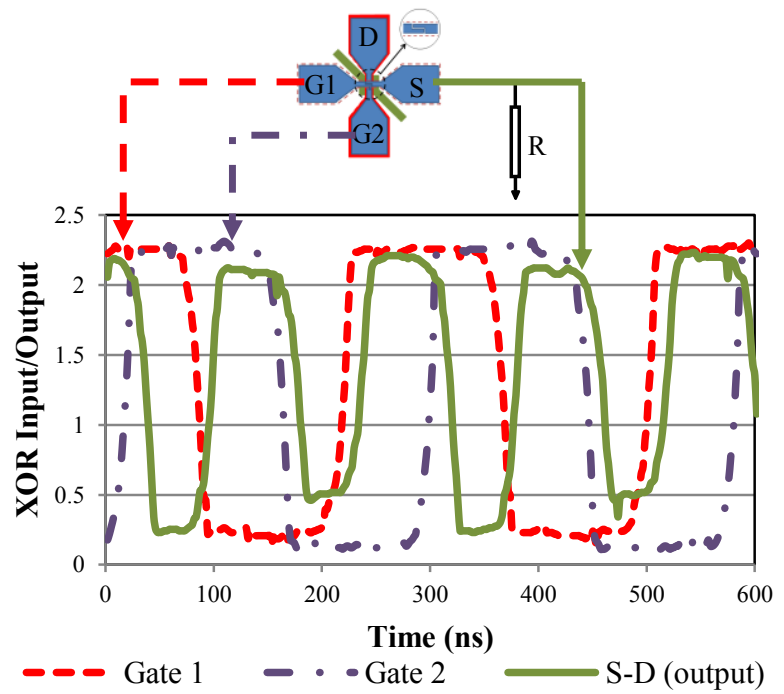

Figure 8: Voltage transitions of a single XOR device. S-D transitions to HIGH only when either G1 or G2 are high. When both $G 1 \& G 2$ are LOW (or HIGH) together, S-D transitions to $L O W$.

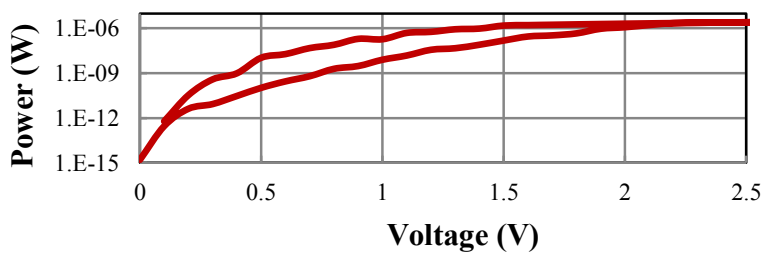

Figure 9: Graph showing leakage power characteristic of the fabricated XOR gate $(\sim 1 \mu \mathrm{W})$.

Figures 10-11 shows the I-V characteristics of a MOSFET and an XOR gate connected as a switch in $90 \mathrm{~kW}$ ionizing radiation environment. After 120 minutes of exposure the MOSFET's I $I_{D S}$ increased by an order of magnitude and its channel became permanently conducting (figure 10 (b)). At this point its gate could no longer control the channel current. In the case of XOR gate, the I-Vs are not affected much by the radiation, as seen in figure 11. However, the switch characteristics changed but the device continued to operate with clear "on" and "off" states.

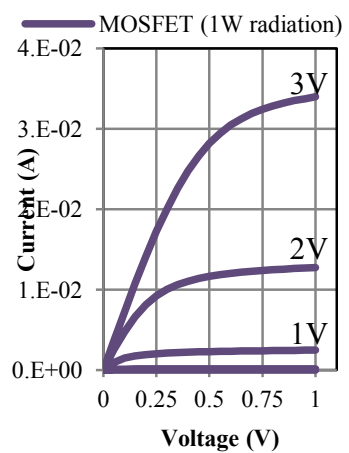

(a)
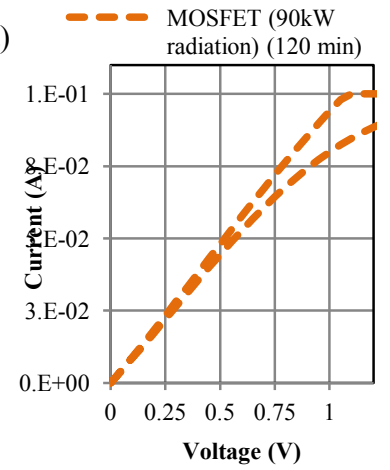

Figure 10: a) MOSFET $I_{d s}-V_{d s}$ at $1 \mathrm{~W}$ ionizing radiation, $\left.1 \mathrm{~min}, b\right)$ its $I_{d s}-V_{d s}$ at $90 \mathrm{~kW}$ ionizing radiation, $120 \mathrm{~min}$ 


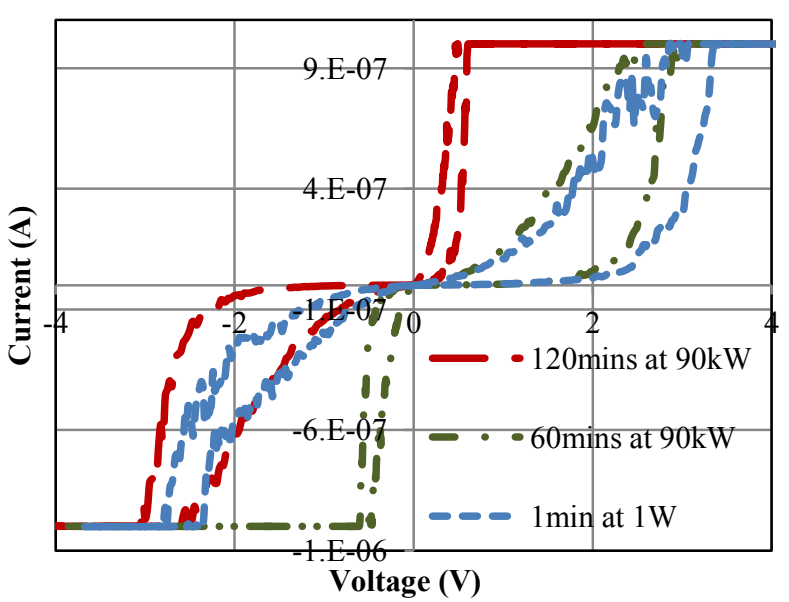

Figure 11: MEMS Switch $I_{d s}-V_{d s}$ at increasing ionizing radiation powers and at prolonged time, (1W/1min to 90kW/120min). Clear "on" \& "off" states are still discernible even after prolonged exposure to I-R.

The TRIGA reactor at University of Utah was used in these experiments. At $90 \mathrm{~kW}$, typical neutron flux is $\sim 3 \times 10^{12}$ neutrons $/ \mathrm{cm}^{2}$-sec. The energy range of the neutrons vary from $0.025 \mathrm{eV}$ to $10 \mathrm{MeV}$, but most of them have neutron energies less than $1 \mathrm{MeV}$. In general, alpha particles do not exist in the reactor core except for inside the fuel element which has an average energy of $6 \mathrm{MeV}$. It is known that Gamma rays exist ubiquitously in the reactor core. Approximate gamma flux is on order of $10^{13}$ gamma $/ \mathrm{cm}^{2}$-sec (or higher). Gamma particles' energy ranges from approximately several $\mathrm{keV}$ to $3 \mathrm{MeV}$. Beta particles' flux at $90 \mathrm{~kW}$ is approximately $10^{13} \mathrm{beta} / \mathrm{cm}^{2}$-sec and typical energy of a beta particle is between $100 \mathrm{keV} \sim 1500 \mathrm{keV}$.

Figure 12 (a)-(c) illustrates optical images of a 1-bit multiplexer chip with 4 AND gates, a 1-bit adder chip with 3 XOR and 2 AND gates and a 2-bit full adder circuit utilizing 6 XOR and 4 AND gates that were fabricated and are being tested.

\section{CONCLUSION}

This article presented functional MEMS/NEMS structures to implement logic gates in a single device instead of using individual switches commonly employed in CMOS. Given that 6-14 switches are typically needed in logic gates, the functional structures reduce the device count leading to better reliability, yield, speed and overall better characteristics (sub-threshold characteristics, smaller turn-on/off voltage variations, etc.). The design, fabrication and characterization of XOR gate as a specific example of a functional structure and AND gates was also discussed. Also, operational characterization data showed a $\sim 2 \mathrm{~V}$ actuation, $<1 \mu \mathrm{W}$ leakage power consumption and an operational lifetime of $\sim 10^{9}$ cycles. Moreover, harsh environment operation at elevated temperatures $(409 \mathrm{~K})$ and high ionizing radiation environment testing also revealed successful and reliable operation of these MEMS devices over CMOS equivalents. Finally, microfabricated circuits such as multiplexers, 1-bit full adder and a 2-bit full adder that employ the XOR and AND gates was also presented here.

\section{ACKNOWLEDGEMENT}

This work is supported by DARPA NEMS grant \#NBCH1090003.

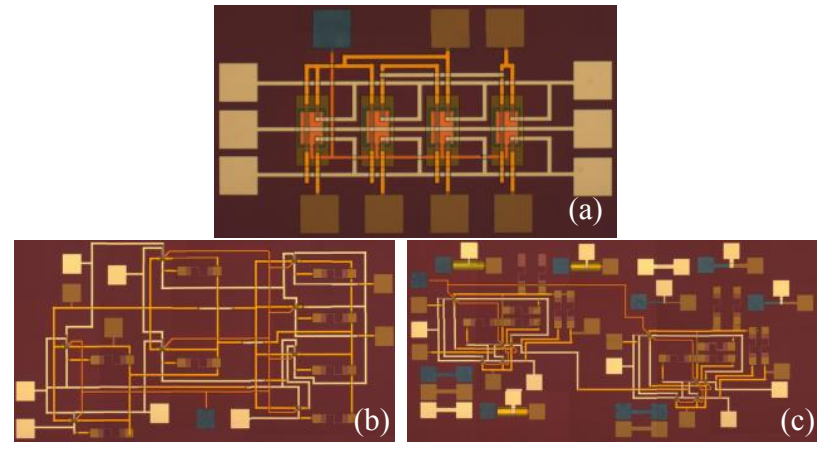

Figure 12: Single-device MEMS logic gates used to fabricate a)1-bit multiplexor using 4 AND gates b)1-bit adder using 3 $X O R \& 2$ AND gates and c)2-bit full adder made using 6 XOR \& 4 AND gates.

\section{REFERENCES}

[1] F. K. Chowdhury, K. N. Chappanda, D. Saab, and M. TabibAzar, "Novel Single-Device "XOR" and "AND" Gates for High Speed, Very Low Power LSI Mechanical Processors " in The 16th International Conference on Solid-State Sensors, Actuators and Microsystems Beijing, China, 2011.

[2] M. Tabib-Azar, S. R. Venumbaka, K. Alzoubi, and D. Saab, "1 volt, $1 \mathrm{GHz}$ nems switches," in Sensors, 2010 IEEE, 2010, pp. 1424-1426.

[3] K. Alzoubi, D. G. Saab, and M. Tabib-Azar, "Circuit simulation for Nano-Electro-Mechanical switches VLSI circuits," in Circuits and Systems (MWSCAS), 2010 53rd IEEE International Midwest Symposium on, 2010, pp. 1177-1180.

[4] V. K. Sirigir, K. Alzoubi, D. G. Saab, F. Kocan, and M. TabibAzar, "Ultra-low-Power Ultra-fast Hybrid CNEMS-CMOS FPGA," in Field Programmable Logic and Applications (FPL), 2010 International Conference on, 2010, pp. 368-373.

[5] M. Tabib-Azar, K. Alzoubi, and D. Saab, "Novel MEMS 900 $\mathrm{MHz}$ electrostatic silicon delay line," in Sensors, 2010 IEEE, 2010, pp. 205-207.

[6] K. Akarvardar, D. Elata, R. Parsa, G. C. Wan, K. Yoo, J. Provine, P. Peumans, R. T. Howe, and H. S. P. Wong, "Design Considerations for Complementary Nanoelectromechanical Logic Gates," in Electron Devices Meeting, 2007. IEDM 2007. IEEE International, 2007, pp. 299-302.

[7] R. Parsa, K. Akarvardar, J Provine, D. Lee, D. Elata, S. Mitra, H.S. P. Wong, and R. T. Howe, "Composite polysilicon-platinum lateral nanoelectromechanical relays " in 14th Solid-State Sensors, Actuators, and Microsystems Workshop Hilton Head, South Caroline, 2010, ,pp. pp. $7-10$

[8] L. Tsu-Jae King, J. Jaeseok, R. Nathanael, K. Hei, V. Pott, and E. Alon, "Prospects for MEM logic switch technology," in Electron Devices Meeting (IEDM), 2010 IEEE International, 2010, pp. 18.3.1-18.3.4.

[9] N. Sinha, T. S. Jones, G. Zhijun, and G. Piazza, "Body-biased complementary logic implemented using AIN piezoelectric MEMS switches," in Electron Devices Meeting (IEDM), 2009 IEEE International, 2009, pp. 1-4.

[10] G. M. Rebeiz, RF MEMS: theory, design, and technology: LibreDigital, 2003.

\section{CONTACT}

*M. Tabib-Azar, azar.m@utah.edu 


\title{
TRAVELING WAVE ULTRASONIC MOTOR USING THIN-FILM PIEZOELECTRICS
}

\author{
Ryan Q. Rudy ${ }^{1,2^{*}}$, Gabriel L. Smith ${ }^{1}$, Don L. DeVoe ${ }^{2}$, and Ronald G. Polcawich ${ }^{l}$ \\ ${ }^{1}$ U.S. Army Research Laboratory, Adelphi, Maryland, USA \\ ${ }^{2}$ University of Maryland, College Park, Maryland, USA
}

\begin{abstract}
The authors report demonstrated bi-directional rotary motion of a millimeter scale traveling wave ultrasonic motor (TWUM) using solution deposited thin film lead zirconate titanate (PZT) and MEMS fabrication techniques. Rotation speeds of 2300 RPM have been achieved at $10 \mathrm{~V}$ and less than $7 \mathrm{~mW}$ in a $500 \mu \mathrm{m}$ thick, $2 \mathrm{~mm}$ diameter rotor. Design, fabrication, and initial testing of the TWUM are described. The low-power, high-torque, zero-power off state rotary motors described here, could enable numerous applications in the fields of small-scale robotics, fuzing, and biomedical technology.
\end{abstract}

\section{INTRODUCTION}

The traveling wave ultrasonic motor (TWUM), reported by Sashida in 1983 [1] holds may advantages over electromagnetic or electrostatic motors including high-torque low-speed operation, and a high holding torque with zero power input [2]. Because the torque output is high at low speeds, the need for gears is eliminated, thus reducing noise and complexity, and creating a more compact design. These benefits can be clearly observed in macro-scale TWUMs used in auto-focus/manual-focus camera lenses [3]. Without the requirement of gears, these near silent TWUMs can be composed of two parts: a stator and a rotor, both of which can be compactly contained within the lens barrel. For miniaturization of motors, the compactness and simplicity of the TWUM is very attractive, especially if one is using MEMS techniques, which offer micron-scale resolution but require largely two-dimensional designs. Because the TWUM exploits friction for the drive mechanism, a high holding torque can be achieved while consuming zero power in an off state. Furthermore, using thin film lead zirconate titanate (PZT) allows for low voltage operation near $5 \mathrm{~V}$ while the high impedance nature of the piezoelectric actuation results in low power consumption when the motor is active.

Large-scale TWUMs, such as the Canon auto-focus lens, have been commercially developed, however effective miniature TWUMs have not been commercialized despite the many advantages they present [4]. Previous work in the area of mm-scale traditional TWUMs has been reported by Flynn [5, 6]. A key improvement between this work and Flynn's is that the devices reported here successfully demonstrate bi-directional rotary motion, whereas the thin-film devices reported by Flynn are unidirectional [5]. The devices reported here are significantly smaller than the assembled bulk PZT devices reported by Flynn in [6]. Kaajakari et al. more recently reported devices which use a bulk PZT driven die to excite parametric resonances which produce motion [7]. These devices however have lost some of the main benefits of traditional TWUM, specifically, a zero-power hold torque, and gearless operation. Our designs maintain these advantages. We have previously reported on our work in characterizing the stator component of the motor [8] and are reporting rotational motion results for the first time here. In the present work we use high quality PZT thin films for wafer-level batch fabrication of hundreds of motors per wafer while exploiting photolithographic patterning for increased manufacturing precision and lower cost. We have demonstrated a compact motor smaller than $3 \mathrm{~mm}$ in diameter with a height of $550 \mu \mathrm{m}$, most of which is inactive substrate. Bi-directional rotor motion has been characterized as well as the effect of input voltage on power consumption and rotational speed.

\section{DESIGN AND FABRICATION \\ Conceptual Design}

The design of our small scale TWUM utilizes solution deposited PZT thin films approximately $1 \mu \mathrm{m}$ thick. Integrated batch fabrication will be realized through wafer bonding of a PZT on silicon-on-insulator (SOI) stator wafer and a rotor wafer. An exploded view of the conceptual design is shown in Fig. 1. In this design, the columns protruding from the stator act to amplify surface motion to increase speed. The preloaded slip ring provides a downward force which increases the torque output by increasing the frictional load. The slip ring also confines the rotor laterally. The stator is supported from the center node point and the electrical connections are made by vias through the base anchor.

\section{Fabrication Process}

Traditional small-scale TWUMs produced using macro-scale manufacturing techniques are bounded in size due precision and accuracy limitations inherent in the fabrication process. Using photolithographic processing and MEMS fabrication techniques these limitations are circumvented and a millimeter-scale TWUM becomes realizable. To demonstrate the rotary motion, stators were fabricated using the process flow in Fig. 2. The process starts with an SOI wafer with a device layer ranging from 20 to $40 \mu \mathrm{m}$ thick and a buried oxide thickness of $1 \mu \mathrm{m}$, shown in Fig. 2 (a). The actuator stack is deposited next, consisting of a 500nm plasma enhanced chemical vapor deposited silicon dioxide thin film, a sputtered titanium thin film annealed to form $33 \mathrm{~nm} \mathrm{TiO}$, a $100 \mathrm{~nm}$ sputtered platinum film, chemical solution deposited PZT with a $\mathrm{Zr} / \mathrm{Ti}$ ratio of $52 / 48$ and a thickness of $1000 \mathrm{~nm}$, and another $100 \mathrm{~nm}$ layer of sputtered platinum as shown in Fig. 2 (b). The top platinum, PZT, and bottom platinum are then patterned using argon ion-milling. To access the bottom $\mathrm{Pt}$, a hole is patterned by

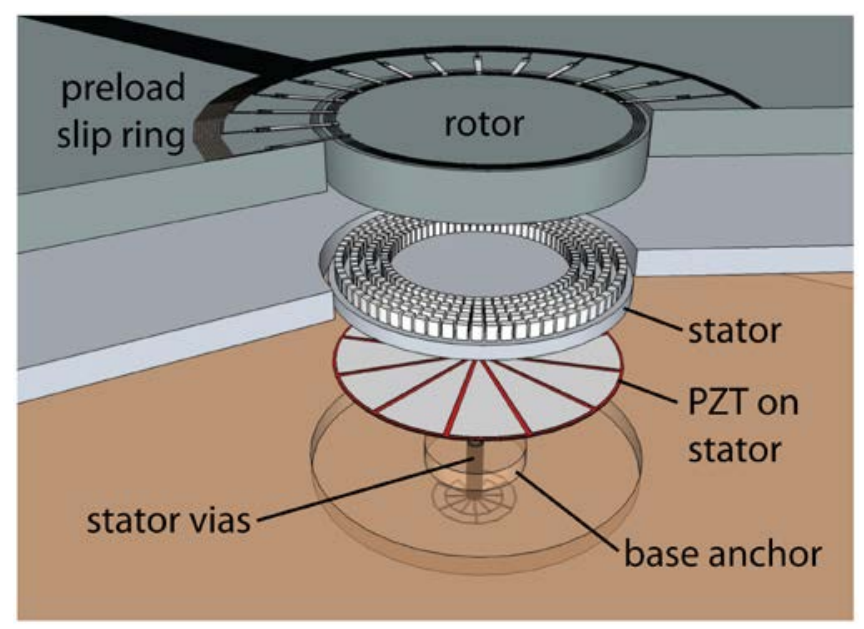

Figure 1: Exploded view of a PiezoMEMS-based TWUM showing the assembled motor. 
a)

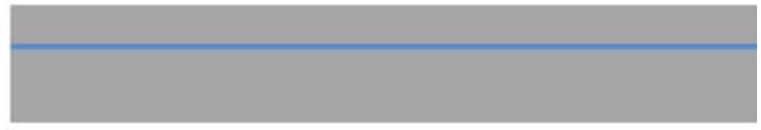

b)

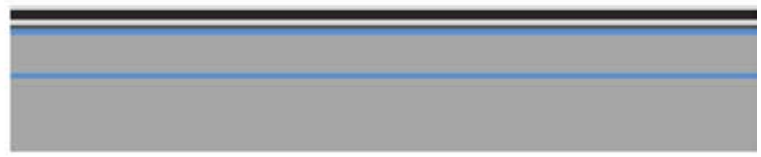

c)

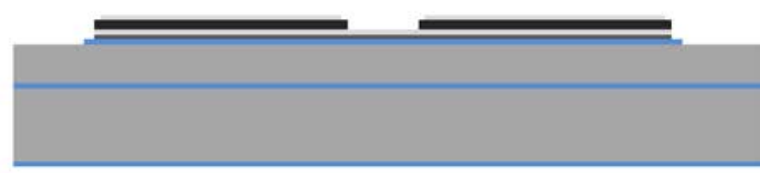

d)

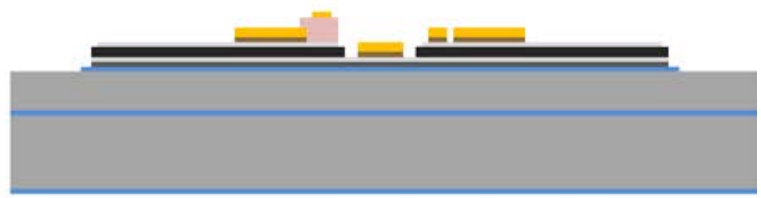

e)

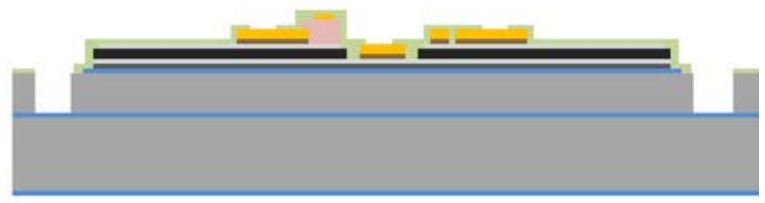

f)

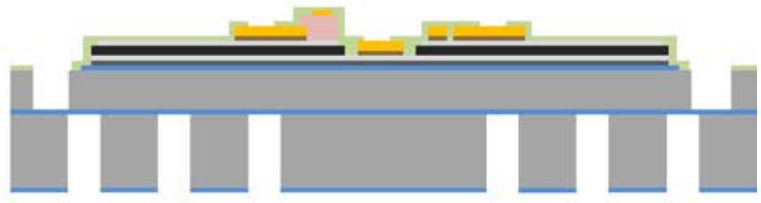

g)

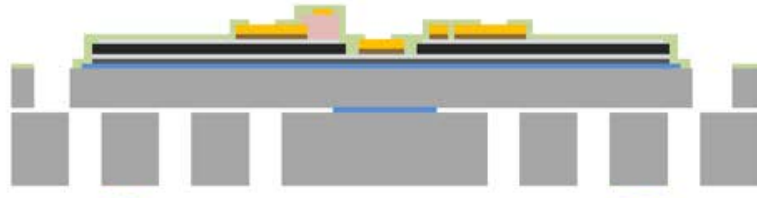

h)

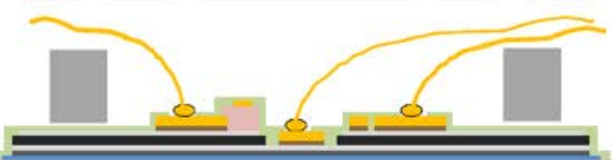

\section{T.}

$\mathrm{Al}_{2} \mathrm{O}_{3}$

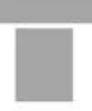

Au

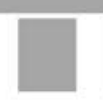

- $\mathrm{Pt}$

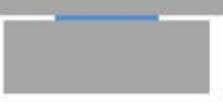

PZT

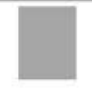

- $\mathrm{Si}$ a lift-off process. A sacrificial layer of photoresist is deposited and patterned to define the gap for gold air bridges. A $2 \mu \mathrm{m}$ thick gold air bridge layer is then deposited via electron beam evaporation and patterned using lift-off, as shown in Fig. 2 (d). The air bridges allow one wirebond to address the three separate drive electrodes of the PZT actuators. A $150 \mathrm{~nm}$ conformal coating of $\mathrm{Al}_{2} \mathrm{O}_{3}$ is deposited using atomic layer deposition to protect the top oxide and PZT during vapor HF release. Following deposition, the $\mathrm{Al}_{2} \mathrm{O}_{3}$ is then etched using Argon ion milling followed by a deep reactive ion etch (DRIE) of the device silicon layer to define the stator device as shown in Fig. 2 (e). Through holes are then etched into the back of the wafer using DRIE to expose the buried oxide as depicted in Fig. 2 (f). The wafer is diced and individual die are released using vapor HF to remove the buried oxide and free the device layer silicon stator as shown in Fig. 2 (g). Once released, the device dies are attached to a package using double-sided Kapton tape. A silicon rotor, fabricated using DRIE, is then placed onto the stator and gold wirebonds from the package are brought through the center of the rotor and attached to the stator electrodes, as shown in Fig. 2 (h). These wirebonds serve two purposes: bringing electrical signal to the stator and constraining the motion of the rotor. A micrograph of the assembled proof of concept system is shown in Fig. 3

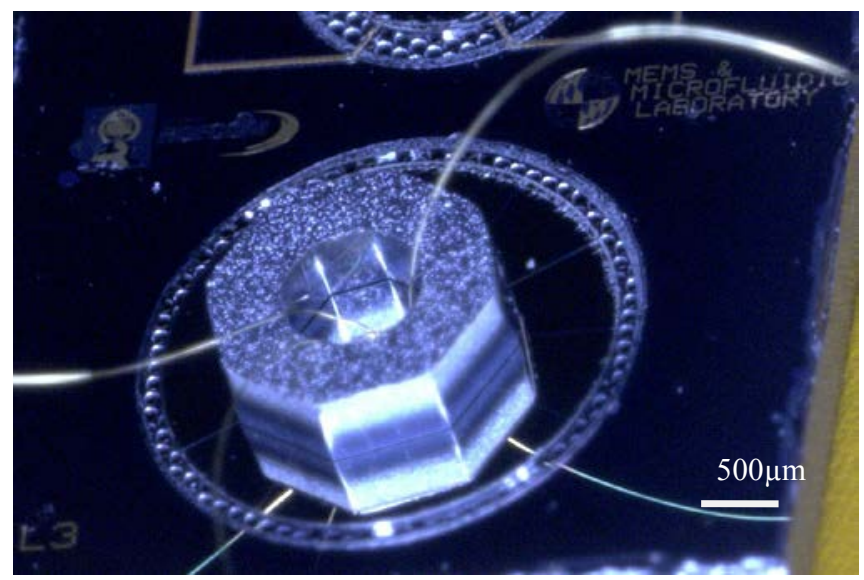

Figure 3: Proof of concept system with a PZT-on-SOI stator with a silicon rotor less than $3 \mathrm{~mm}$ in diameter.

\section{MOTOR OPERATION \\ Operational Theory and Modeling}

TWUMs function by exciting a traveling wave in the stator that propagates around the center-fixed disk at a high frequency. As the wave travels around the disc, the surface points on the disc move in an elliptical trace. This surface motion on the stator then transfers torque through frictional coupling to the free-moving rotor. The traveling wave is created by combining through superposition two identical resonance modes shifted a quarter wavelength in space and offset $90^{\circ}$ in phase. The $\mathrm{B}_{13}$ resonance mode, with one nodal circle and three nodal diameters, was chosen for actuation because the low actuation frequency ensured that we could visualize the wave propagation using a laser Doppler vibrometer (LDV), and the low order mode reduced the number of electrodes that needed electrical connection.

To determine the resonance frequency of the center fixed stator disc, finite element modal analysis was performed. Fig. 4 shows the $\mathrm{B}_{13}$ mode shape simulated using ANSYS, with an associated resonance frequency for a $3 \mathrm{~mm}$ diameter, $30 \mu \mathrm{m}$ thick 


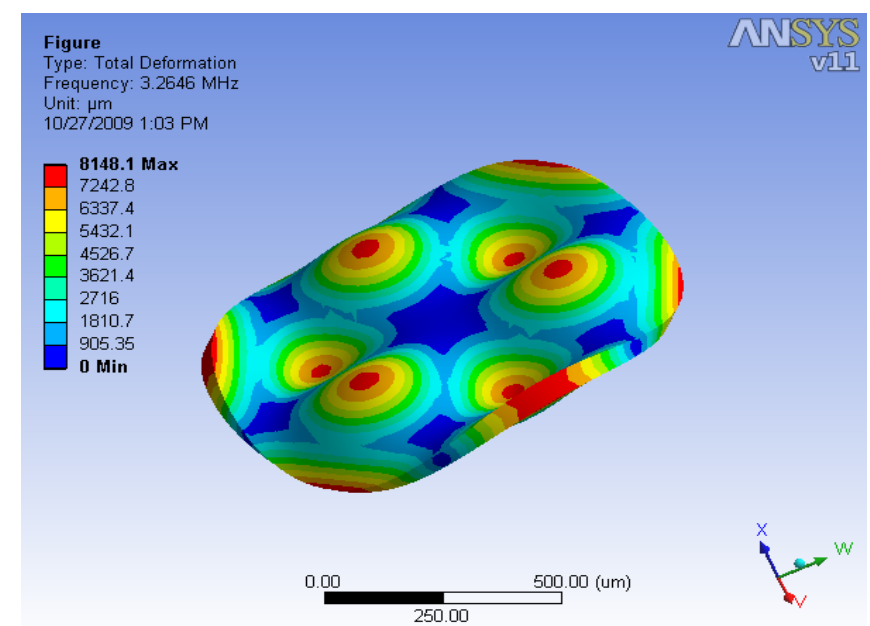

Figure 4: ANSYS modal analysis showing the $B_{13}$ mode.

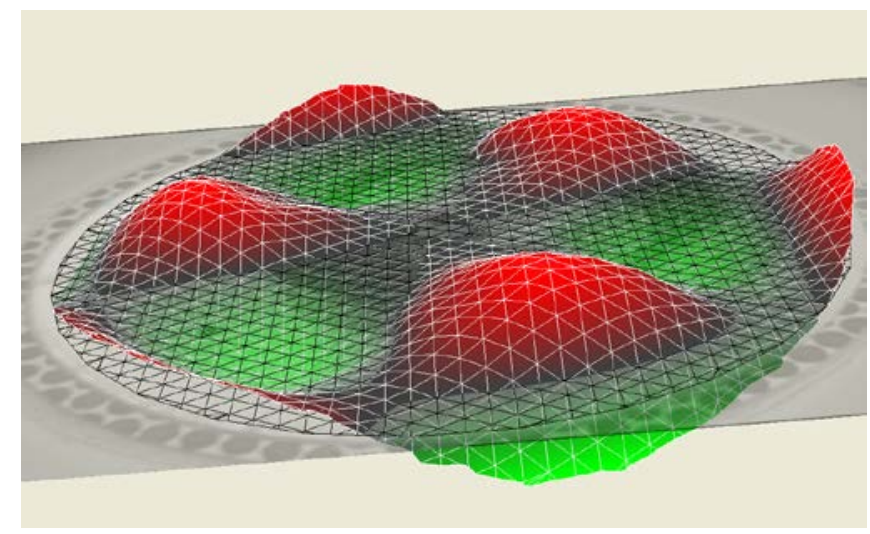

Figure 5: LDV data showing $B_{13}$ mode excited at a frequency of $252 \mathrm{kHz}$.

disc of $284 \mathrm{kHz}$. Experimental results confirmed the model with the $\mathrm{B}_{13}$ resonance mode occurring at $252 \mathrm{kHz}$. The displacement profile of the $B_{13}$ mode, measured using a Polytec LDV, is shown in Fig. 5. According to these measurements, the vibration amplitude of the stator disc is over $1 \mu \mathrm{m}$ at $252 \mathrm{kHz}$. A second set of electrodes excites an identical mode shape with nodal lines at the anti-nodes of the first set. This set-up creates the offset in space to create a traveling wave.

\section{Motor Demonstration and Characterization}

The assembled motor is operated using two actuation electrodes and one ground electrode. The actuation electrodes are offset in phase $90^{\circ}$ and located apart in space to create a propagating wave. Constant rotation begins at $5 \mathrm{~V}$ actuation, where inertial and frictional effects are overcome. Fig. 6 shows the clockwise motion of the rotor in a time-stepped progression. Black dots on the rotor help track the rotation from image to image.

The direction of rotation can be switched by switching the phase offset of the actuation signals from $+90^{\circ}$ to $-90^{\circ}$. Change of direction at $10 \mathrm{~V}$ actuation (1500rpm) has been measured to occur within $30 \mathrm{~ms}$, which highlights the torque output, even without a normal load beyond the weight of the $500 \mu \mathrm{m}$ silicon rotor. Since the motor operates through frictional coupling, increasing the applied normal force increases the torque output of the motor.

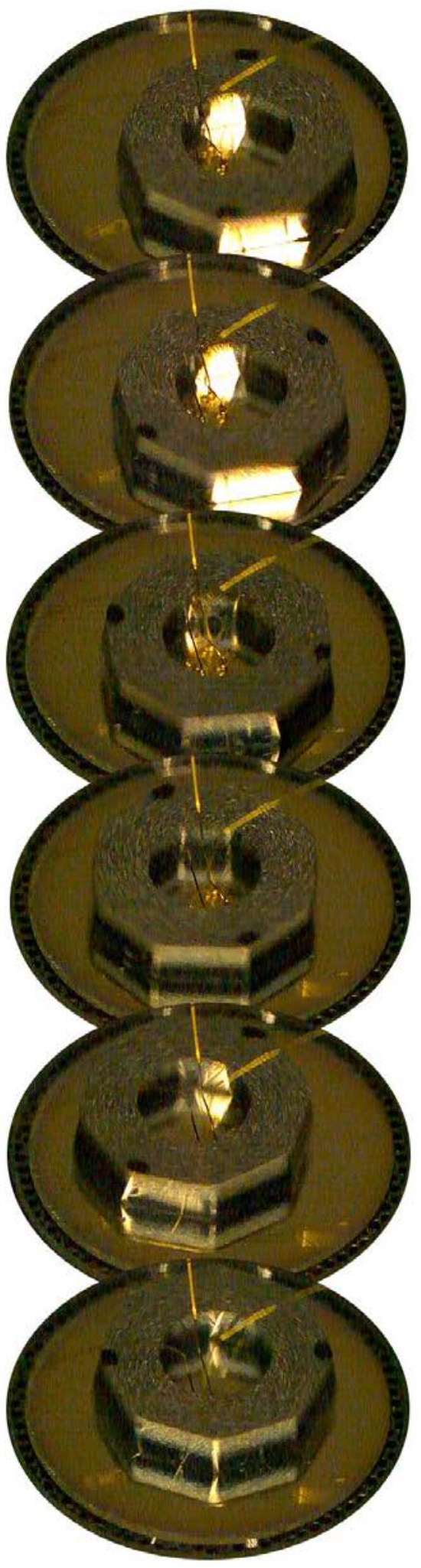

Figure 6: Time-stepped micrographs of $2 \mathrm{~mm}$ rotor spinning at $200 \mathrm{rpm}$. 


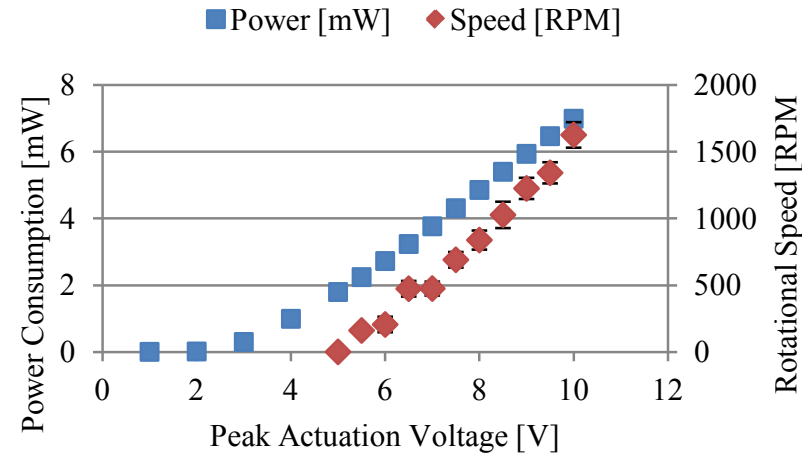

Figure 7: Experimental results showing speed and power as a function of actuation voltage. Error bars on rotational speed represent standard deviation.

At an actuation voltage of $10 \mathrm{~V}$, the wave amplitude is approximately $1 \mu \mathrm{m}$ and current draw from both electrodes totals $0.82 \mathrm{~mA}$ at steady state. Under these conditions, the motor operates near $1500 \mathrm{rpm}$ while drawing $7 \mathrm{~mW}$ of power. Rotational speed and power as a function of actuation voltage are shown in Fig. 7. Maximum rotation speeds, up to $2300 \mathrm{rpm}$, were achieved when the rotor was not in contact with the wirebonds. It should be noted that the breakdown field for the thin-film PZT is significantly higher than applied actuation field, meaning that higher speeds could be achieved with higher applied voltages.

In the assembled motor, the normal force that supports the frictional load is simply the weight of the $500 \mu \mathrm{m}$ thick silicon rotor. Due to the relatively small load applied, estimates of torque are not easily determined, however current designs are being investigated that would allow us to control the normal force and thus tune the output torque.

\section{CONCLUSIONS}

Rotary motion, with bi-directional control, of a $3 \mathrm{~mm}$ diameter TWUM has been demonstrated using thin film PZT and MEMS processing techniques. The characterized proof-of-concept motor shows promise in high rotation speeds, up to 2300rpm and low power, while also providing a substantial lower bound in torque generation and demonstrating the compactness of TWUMs. The stator portion of the motor was modeled and the experimental results compared favorably to this model. This demonstration opens doors to new realms of actuation that have until now been unrealizable, namely high-torques at low-speeds for small-scale actuation. Areas of application include small-scale robotics, low power fuzing, and biomedical devices.

\section{ACKNOWLEDGMENT}

The authors would like to acknowledge Luz Sanchez, Brian Power and Joel Martin of the U.S. Army Research Laboratory for device fabrication. Ryan Rudy would like to acknowledge the SMART fellowship program for support.

\section{REFERENCES}

[1] T. Sashida, "Approach of the Development for the Ultrasonic Motor", Mechanical Automation Japan, vol. 15, no.12, pp.31$35,(1998)$.

[2] T. Maeno, T. Tsukimoto, A. Miyake, "The contact mechanism of an ultrasonic motor," IEEE 7th International Symposium on Applications of Ferroelectrics, (1990), pp. 535-538.

[3] K. Uchino, "Piezoelectric Ultrasonic Motors: Overview", Smart Material Structures, vol. 7, p.273-285 (1998).

[4] K. Uchino, and J.R. Giniewicz, "Micromechatronics", pp.417430 (2003).

[5] A.M. Flynn, et al., "Piezoelectric Micromotors for Microrobots", Journal of Microelectromechanical Systems, vol. 1, no. 1, pp.44-51, (1992).

[6] A.M. Flynn, "Performance of Ultrasonic mini-motors using design of experiments" Smart Material Structures, vol. 7, pp.286-294, (1998).

[7] V. Kaajakari, and A. Lal, "Micromachined Ultrasonic Motor Based on Parametric Polycrystalline Silicon Plate Excitation", Sensors and Actuators A: Physical, vol. 137, no. 1, pp. 120128, (2007).

[8] G.L. Smith, R.Q. Rudy, R.G. Polcawich, and D.L. DeVoe, "Integrated Thin-Film Piezoelectric Traveling Wave Ultrasonic Motors", Sensors and Actuators (2012), 10.1016/j.sna.2011.12.029.

\section{CONTACT}

*R.Q.Rudy, tel: +1-301-394-2324; ryanrudy@umd.edu 


\section{D INTEGRATION USING SELF-ASSEMBLY AT AIR-WATER INTERFACE

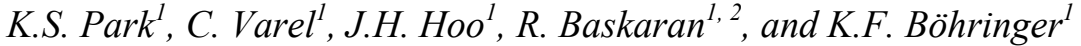 \\ ${ }^{1}$ Department of Electrical Engineering, University of Washington, Seattle, USA \\ ${ }^{2}$ Components Research, Intel Corporation, USA}

\begin{abstract}
This paper presents the first proof-of-concept 3D integration using self-assembly of chip-scale parts (100 $\mu \mathrm{m}$ thickness). 3D integration is achieved by assembling new parts over previously assembled parts. Assembly proceeds as an assembly substrate is pulled up through an air-water interface and electrical and mechanical bonding is achieved through a solder reflow process. Magnetic fields and temporary Faraday waves are introduced for one-to-one part-to-site registration in proper orientation. The maximum number of layers that can be achieved is simulated and analyzed. Via resistance including the effect of degradation of solder over repeated reflow process is measured.
\end{abstract}

\section{INTRODUCTION}

As microelectronics technology approaches its physical limit of CMOS scaling, prevailing growth driven by a continuous reduction in cost per function cannot be sustained by miniaturization (More-Moore technology) alone. Assembly and packaging area is increasingly undertaking this responsibility, and it is doing so based on the principles of densification of the functions. Functional enrichment (More-than-Moore technology) [1] focuses on 3D integration rather than transistor density alone.

Today's smart electronics (GPS, tablets, smartphones, digital cameras, etc.) consist of digital and non-digital components to provide additional functions and increased performances. Non-digital components (RF/MEMS devices, sensors, actuators, etc.) play an equally important role in cost-effective and value-added systems. Thus, integration of digital and non-digital components in small spaces will be another trend of system miniaturization. Moore's law has ruled scaling down of microelectronics for the past decades. However, components for interfacing with the physical world are non-digital and do not scale down at the same rate of Moore's law. Thus, functional enrichment also known as a More-than-Moore technology gains significance to reach beyond the limit of the conventional More-Moore technology.

An integration of multiple electronic components from different fabrication processes in a single unit is known as system in package ( $\mathrm{SiP}$ ). Compared to 2D (horizontal) $\mathrm{SiP}, 3 \mathrm{D}$ integration (stacked and embedded) is emerging due to its advantages; less power consumption, higher performance, smaller area, lower cost and stacking multiple parts from different fabrication processes, which enables the extension of Moore's law and the delivery of smarter and cheaper electronics. Thus, 3D integration gains importance and is already in use in some domains (cell phones and memory). To fully take advantage of $3 \mathrm{D}$ integration, thinner components are preferred.

In addition to $3 \mathrm{D}$ integration, system miniaturization is being done using 0050025 chips $\left(\mathrm{L} \times \mathrm{W}=0.2 \times 0.1 \mathrm{~mm}^{2}\right)$, which has half the height of today's regular micro components (01005 chips, measuring $\mathrm{L} \times \mathrm{W}=0.4 \times 0.2 \mathrm{~mm}^{2}$ ) [2] 0050025 chips are highly fragile and today's pick-and-place assembly still has yield issues even with 0201 chips. For assembly with large numbers of small and thin parts, conventional pick-and-place becomes costly and self-assembly is a viable alternative.

Thus, many research groups have developed self-assembly systems, which employ various driving forces such as gravity [3], surface tension [4-6], electrostatic [7] or magnetic force [8-10] and often require adhesives, liquid solder, shape matching structures or two different liquids [10]. They, however, have lower yield compared to the pick-and-place assembly due to their stochastic nature [11]. Moreover, these assembly methods are not suited for $3 \mathrm{D}$ integration.

In [12], we introduced a novel fluidic self-assembly (FSA) of millimeter-scale thin square parts at the air-water interface. Demonstration of high yield using temporary Faraday waves [13, 14], a comprehensive model and experimental support are also presented. In our previous FSA system, parts are assembled in four orientations $\left(0^{\circ}, 90^{\circ}, 180^{\circ}, 270^{\circ}\right)$ due to their square shape. In [15], we extended our fluidic self-assembly (FSA) repertoire to assembling thin square parts with unique orientation through the use of $\mathrm{Ni}$ patterns and magnetic fields. Here, we present the first proof-of-concept 3D FSA of $2 \mathrm{~mm}$ sized parts (100 $\mu \mathrm{m}$ thick) with experimental data as one of More-than-Moore technologies. The proposed FSA system achieves $3 \mathrm{D}$ integration by assembling parts on top of previously assembled parts.

\section{SYSTEM DESIGN AND FABRICATION Assembly overview}

A 3D FSA system (Figure 1) consists of a water container on a linear electromagnetic shaker (Brüel \& Kjær Type 4809), a stepper motor (Soyo, SY42STH38-0406A) with the controller (Phidgets, 1062), a substrate and parts floating at an air-water interface. The acceleration uniformity over the vibration table is $\pm 10 \mathrm{mg}$ or $0.72 \%$ as measured using a laser vibrometer (Polytec OFV-534 laser unit and OFV-2500 vibrometer controller) when driven at $80 \mathrm{~Hz} / 1.5 \mathrm{~g}(1$ $g=9.8 \mathrm{~m} / \mathrm{s}^{2}$ ).

The substrate has preprogrammed hydrophilic binding sites and a self-assembled monolayer (SAM)-coated hydrophobic Au area. The substrate is tilted at an angle of $45^{\circ}$ relative to the water surface. Test electrodes are patterned on the substrate to measure the resistance of through-silicon vias (TSVs). To attract Ni patterned parts in proper orientation, rare-earth neodymium iron boron magnets $(\mathrm{NeFeB}$, Grade N40, diameter $=$ thickness $=1 / 16$ inch $)$ are placed beneath each binding site. Accurate magnet-binding site alignment is achieved using photolithographically patterned "magnet templates". Magnets are placed in magnet-sized trenches. Then the magnet template is aligned with the assembly substrate.

Standing wave patterns in a layer of liquid enclosed by a vibrating container are known as Faraday waves [13, 14]. Typical yield loss in FSA is due to lack of control of one-on-one part-to-site registration. In our FSA system, Faraday waves are generated by use of a vertical electromagnetic shaker driven at $80 \mathrm{~Hz} / 1.5 \mathrm{~g}$. We avoid multiple parts approaching the same site and guarantee high yield by use of surface waves. Although most parts approach binding sites in proper orientation due to the magnetic field, it is possible that none, multiple or wrongly oriented parts occupy binding sites due to the stochastic character of the approach process. Faraday waves are applied to prevent multiple parts from approaching one site and to achieve one-to-one part-to-site registration in proper orientation [15]. Correctly registered parts experience stronger magnetic force than wrongly oriented ones. Thus wrongly oriented or extra parts are selectively repelled by Faraday waves and other parts have a chance to approach the empty binding sites. 

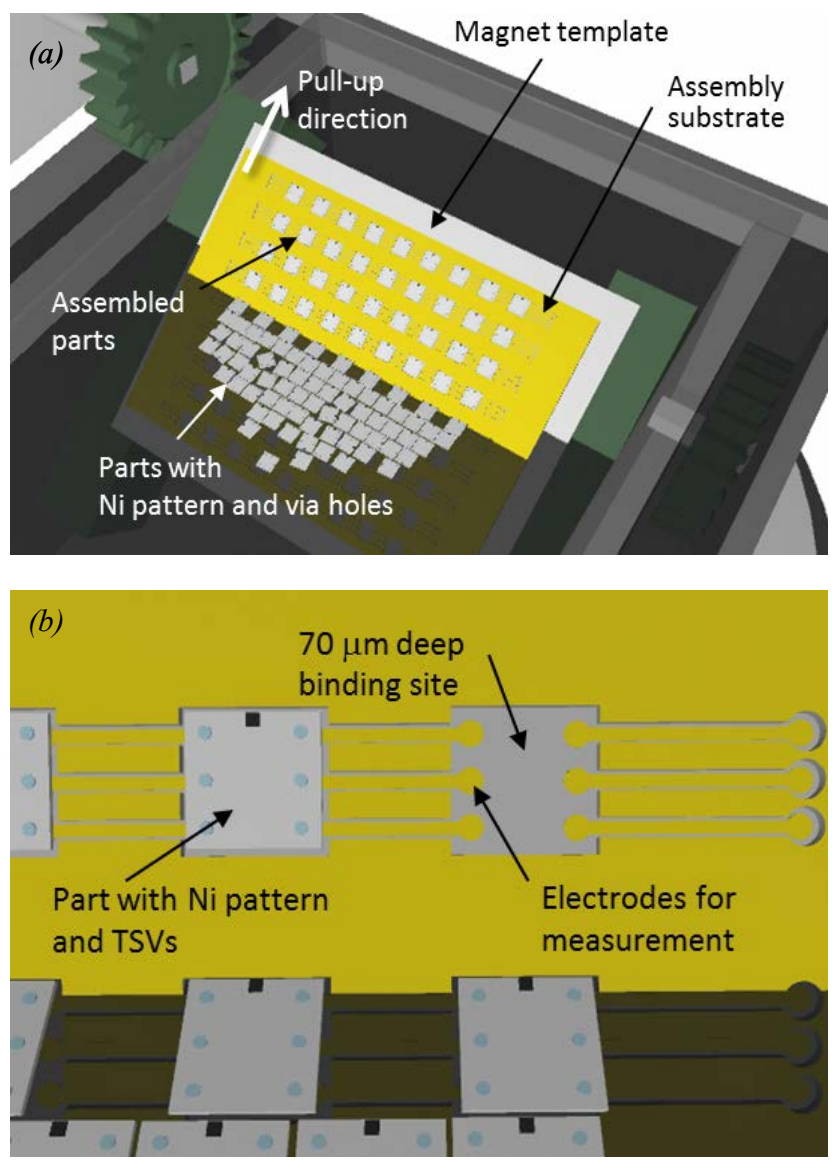

Figure 1. Assembly system. (a) Experimental setup for assembly consists of a water container on a linear electromagnetic shaker, a stepper motor with the controller, a substrate and parts floating at an air-water interface. (b) Design of parts and binding sites. Each part has six via holes and each binding site has six electrodes for electrical measurement.

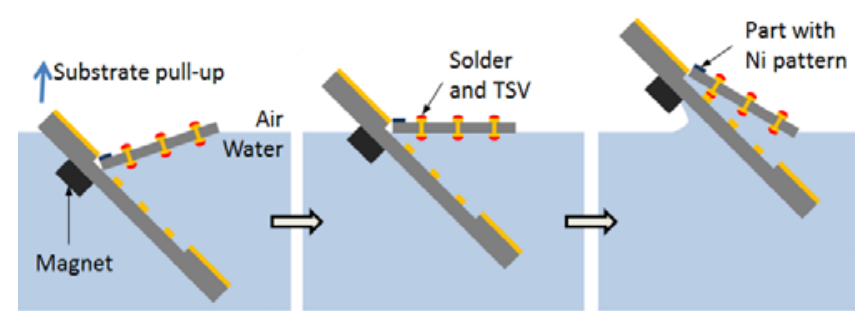

(a) assembly of the first layer

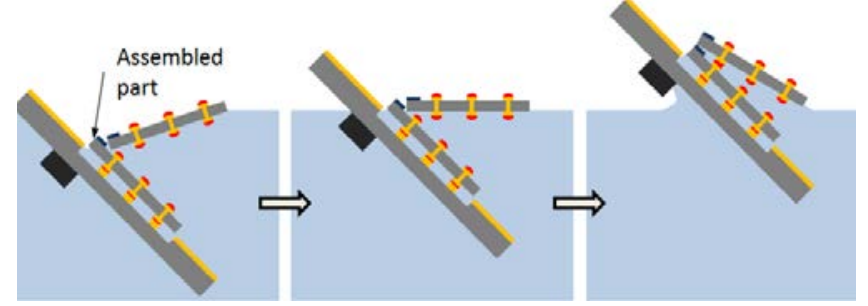

(b) assembly of the second layer

Figure 2. Assembly process. After assembly of each layer of parts, solder reflow process is needed for electrical and mechanical connection. 3D integration of multiple layers is achievable through a layer-by-layer assembly.
The correctly registered parts are assembled by substrate pull-up (Figure 2(a)). The parts are then mechanically and electrically connected to the binding sites by solder $\left(\mathrm{In} / \mathrm{Bi} / \mathrm{Sn}, 60^{\circ} \mathrm{C}\right)$ reflow at $65^{\circ} \mathrm{C}$. The magnetic force and the capillary force between parts help part-to-part alignment.

$3 \mathrm{D}$ integration of multiple layers is achieved by repeating part assembly and solder reflow (Figure 2(b)) processes. Once the first layer of parts is fixed, repeated assembly and solder reflow process achieve 3D multi-layer integration. The maximum number of layers is limited by the magnetic field strength that prevents parts from being withdrawn during substrate pull up.

\section{Fabrication}

The fabrication process includes the assembly template fabrication and part fabrication. A combination of photolithography, deep reactive ion etching (DRIE) and metallization is used for the assembly template. As pictured in Figure 3(a), the process starts with DRIE through a photoresist mask on a Si wafer in order to form the assembly sites and the trenches connecting those assembly sites. The depth of the etched region is limited to $70 \mu \mathrm{m}$ in a timed etch. After that, a metal layer of $10 \mathrm{~nm} \mathrm{Cr}$ and $100 \mathrm{~nm} \mathrm{Au}$ is deposited using electron beam evaporation. This metal layer is patterned for electrical interconnect by metal etching.
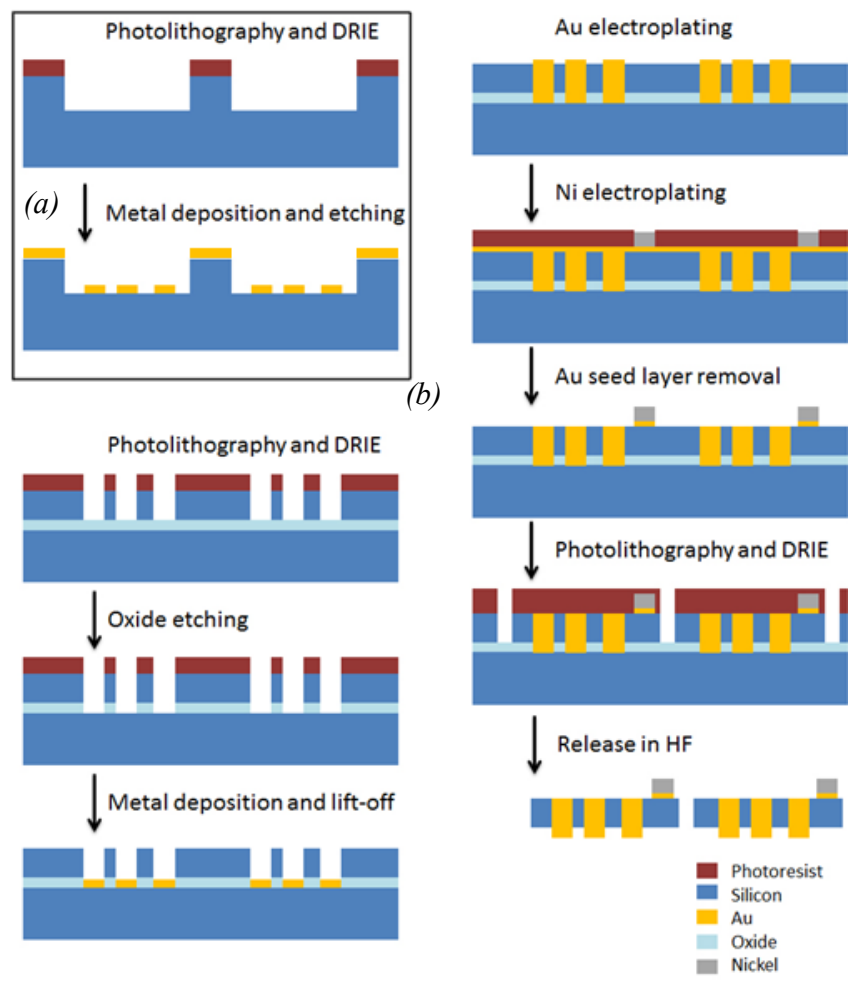

Figure 3. Fabrication process flow for (a) the assembly template and (b) parts.

The fabrication process for parts is more complicated compared to the assembly template. Through silicon vias (TSV) are employed for electrical connection in the z-direction. An unconventional method is employed for that purpose. The fabrication process is divided into three steps; via formation, $\mathrm{Ni}$ pattern deposition and part release. A silicon-on-insulator (SOI) substrate with a device layer of $100 \mu \mathrm{m}$ is used. The SOI wafer has a lightly doped p-type device layer $(100 \mu \mathrm{m}$ thick, 20,000 - 50,000 
$\Omega \cdot \mathrm{cm})$ and highly doped n-type handle wafer (500 $\mu \mathrm{m}$ thick, 0.001 $-0.005 \Omega \cdot \mathrm{cm}$ ) which enables seed layer-less electroplating [16].

Fabrication starts with a photolithography step where via locations are defined as illustrated in Figure 3(b). They are etched with DRIE until the oxide layer is reached. The oxide in openings is removed in $\mathrm{HF}$ in order to expose the underlying $\mathrm{Si}$ layer. Photoresist on the wafer surface is kept throughout the process. TSVs are fabricated by Au electroplating. A $100 \mathrm{~nm}$ Au layer is deposited onto the wafer as the seed layer with e-beam evaporation. $\mathrm{A} \mathrm{Cr}$ adhesion layer is avoided in order to prevent a strong adhesion between TSV and the handle wafer. Metal layer deposited on the top surface is lifted off by removing the photoresist before electroplating. The highly doped handle wafer is used as the conducting medium for electroplating TSV onto Au layer in the hole.

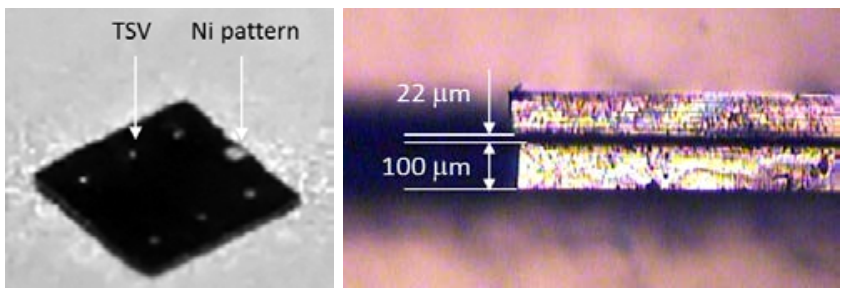

Figure 4. Part with gap between parts. (Left) $6 \mathrm{Au}$ TSVs (diameter $=100 \mu \mathrm{m})$ and a Ni pattern $\left(200 \times 200 \times 13 \mu^{3}\right)$ are patterned. (Right) Measured gap between parts is $22 \mu \mathrm{m}$. Part size is $2000 \times$ $2000 \times 100 \mu^{3}$.

TSV fabrication is followed by the Ni pattern deposition. A metal layer of $10 \mathrm{~nm} \mathrm{Cr}$ and $100 \mathrm{~nm} \mathrm{Au}$ is deposited as the seed layer using e-beam evaporation. The wafer is then spin-coated with $22 \mu \mathrm{m}$ thick AZ 4620 photoresist and patterned by standard photolithography to define $200 \times 200 \mu \mathrm{m}$ square windows. A $13 \mu \mathrm{m}$ tall $\mathrm{Ni}$ layer is electroplated through the exposed $\mathrm{Au}$ areas. $\mathrm{Cr} / \mathrm{Au}$ seed layer used in this process is later removed using the electroplated Ni layer as the mask. Part boundaries are defined in another DRIE step through a photoresist mask. After that, the substrate is left in hydrofluoric acid (HF) in order to release parts from the handle wafer. The released parts are coated with a low-melting point solder $\left(\mathrm{In} / \mathrm{Bi} / \mathrm{Sn}, 60^{\circ} \mathrm{C}\right)$ by dip coating. Figure 4 shows a substrate and parts with solder bumps. Thickness of a part including solder bumps is about $120 \mu \mathrm{m}$.

\section{RESULTS AND DISCUSSION}

\section{The effect of the magnetic field}

It is observed that 4 layers of parts are the maximum achievable limit by our experimental setup (substrate thickness: 430 $\mu \mathrm{m}=70 \mu \mathrm{m}$ deep binding site on $500 \mu \mathrm{m}$ thick Si wafer, magnet diameter/thickness: $1.56 / 0.78 \mathrm{~mm}$ (grade N40), part size: $2000 \times$ $2000 \times 100 \mu \mathrm{m}^{3}$, Ni pattern size: $200 \times 200 \times 13 \mu \mathrm{m}^{3}$ ). Assembly fails if the gravitational force during pull-up is larger than the magnetic force.

Thus, we hypothesize that a critical magnetic force exists that guarantees successful assembly. In our FSA system, the magnetic force on a part is determined by the gap between a magnet and a $\mathrm{Ni}$ pattern. The minimum magnetic force is experimentally determined by assembly test at various gap values. The maximum gap that allows assembly is found to be $0.99 \mathrm{~mm}$ and a corresponding magnetic force is simulated to be $6.6 \mu \mathrm{N}$ using COMSOL Multiphysics $^{\mathrm{TM}}$. Near the threshold magnetic force of $6.6 \mu \mathrm{N}$, assembly becomes vulnerable to inevitable variations such as magnet-to-binding site alignment, part-to-part alignment, Ni pattern thickness and solder thickness. Thus, assembly becomes more reliable below a gap of $0.99 \mathrm{~mm}$.

Figure 5 shows the decrease of the magnetic force with increasing gap between a magnet and a Ni pattern. The gap between parts after solder reflow is measured to be $22 \mu \mathrm{m}$. Thus, the maximum number of layers of parts with successful assembly is predicted to be 4 layers (total thickness $\approx 0.92 \mathrm{~mm}$ including substrate). This limit can be extended by increasing the magnetic force (larger Ni pattern size, stronger magnetic field, thinner substrate, thinner parts, etc.).

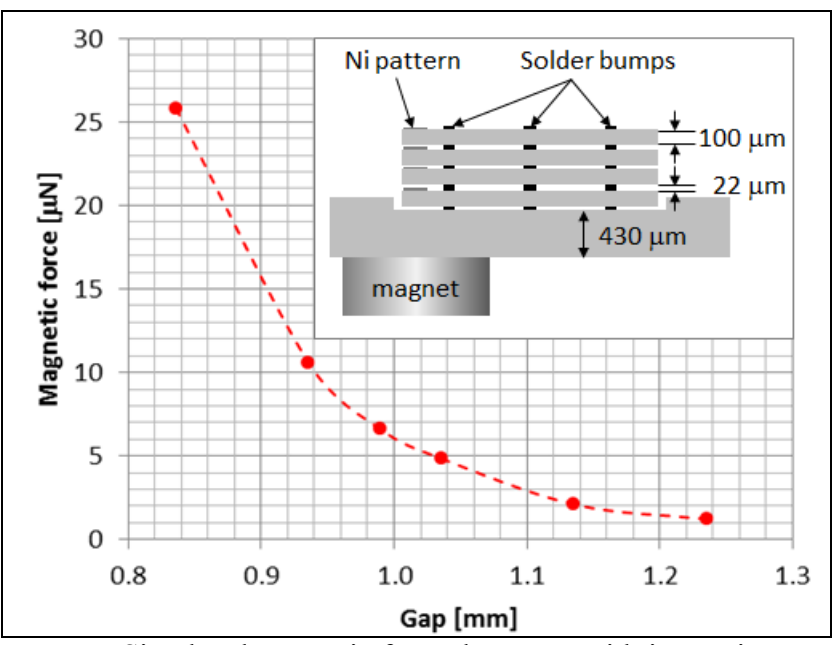

Figure 5. Simulated magnetic force decreases with increasing gap. The minimum magnetic force for successful assembly is $6.6 \mu \mathrm{N}$ (at $0.99 \mathrm{~mm}$ ). $13 \mu \mathrm{m}$ thick Ni pattern is assumed. The total gap between a magnet and a Ni pattern on the 4th layer is $918 \mu \mathrm{m}$.

\section{Electrical tests}

In the proposed FSA system, assembled parts need to be electrically and mechanically fixed through a solder reflow process. The solder bumps on the first layer go through a repeated reflow process as each additional layer is assembled. Thus, degradation of $\mathrm{In} / \mathrm{Bi} / \mathrm{Sn}$ solder over repeated reflow processes needs to be investigated. Resistance through a solder-coated TSV (diameter of $100 \mu \mathrm{m}$ ) is measured by repeating the reflow process at $65^{\circ} \mathrm{C}$ for 20 times. The measured resistance does not show any degradation. Mean and standard deviation are 0.08 and $0.003 \Omega$, respectively.

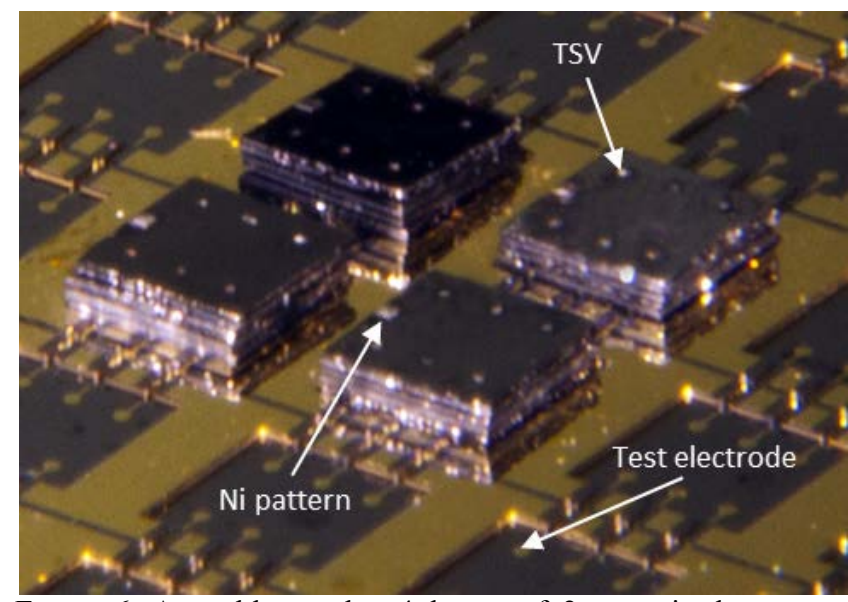

Figure 6. Assembly results. 4 layers of $2 \mathrm{~mm}$ sized parts are assembled on $2 \times 2$ binding sites. 
Next, TSV resistance values are measured after assembling the 1st and 4th layers (Figure 6). Mean and standard deviation of resistance through 4 TSVs in series are measured to be 0.33 and $0.05 \Omega$. Figure 7 shows the comparison between resistance values of single TSV and 4 TSVs in series. Resistance through 4 TSVs is almost 4 times of a single TSV resistance and has wider deviation.

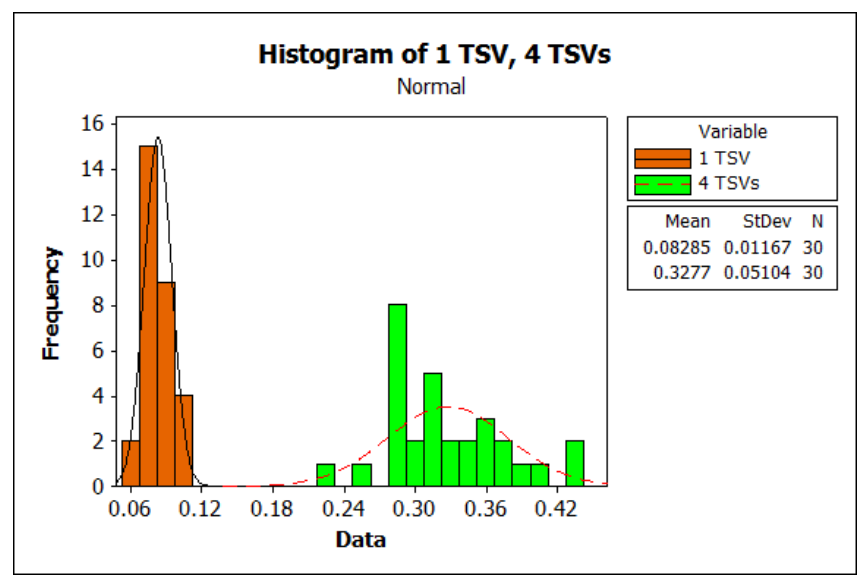

Figure 7. Distributions of measured resistance through (a) single TSV $($ Mean $=0.08 \Omega, \operatorname{StDev}=0.012 \Omega$ ) and $(b) 4 \mathrm{TSVs}$ in series $($ Mean $=0.33 \Omega, \operatorname{StDev}=0.051 \Omega$ ).

Statistical data from these experiments are limited because of low yield in the fabrication process for the parts (Ni patterns on $\mathrm{Au}$ film often peeled off during HF release etch). Assembly is limited to $2 \times$ 2 binding sites due to lack of good parts.

\section{CONCLUSIONS}

The first proof-of-concept 3D multi-layer integration using fluidic self-assembly of chip scale parts $(100 \mu \mathrm{m}$ thick $)$ is demonstrated. 4 layers of parts are assembled through layer-by-layer assembly followed by a solder reflow process for electrical and mechanical bonding.

Thin parts with $\mathrm{Au}$ TSVs with diameter of $100 \mu \mathrm{m}$ are successfully fabricated using electroplating without a seed layer. A $\mathrm{Ni}$ pattern on a part interacting with magnetic field guarantees assembly with unique orientation.

The maximum number of layers that can be achieved using the proposed FSA system is determined by the competition between magnetic force and gravitational force on a part. For given conditions, a threshold magnetic force is experimentally established. Then, the limit of achievable number of layers is predicted using simulation and compared with experimental results.

$\mathrm{In} / \mathrm{Bi} / \mathrm{Sn}$ solder shows no degradation over repeated reflow processes. Measured resistance through a single TSV and 4 TSVs in series are 0.08 and $0.33 \Omega$, respectively.

\section{ACKNOWLEDGEMENT}

This work was supported by the Defense Advanced Research Projects Agency (DARPA) N/MEMS S\&T Fundamentals program under grant no. N66001-10-1-4004 issued by the Space and Naval Warfare Systems Center Pacific (SPAWAR). Part of this work was conducted at the University of Washington Microfabrication / Nanotechnology User Facility, a member of the NSF National Nanotechnology Infrastructure Network.

\section{REFERENCES}

[1] A. B. Kahng, "Scaling: More than Moore's law," Design \& Test of Computers, IEEE, vol. 27, pp. 86-87, 2010.
[2] S. v. Gaste. (2010, Assembling the Next Electronic Equipment Generations. Available: http://www.assembleon.com/about/475874/475882/Future p lacement requirements

[3] S. A. Stauth and B. A. Parviz, "Self-assembled single-crystal silicon circuits on plastic," Proceedings of the National Academy of Sciences of the United States of America, vol. 103, pp. 13922-13927, 2006.

[4] H. O. Jacobs, et al., "Fabrication of a cylindrical display by patterned assembly," SCIENCE, vol. 296, pp. 323-325, 12 APRIL 20022002.

[5] W. Zheng, et al., "Sequential shape-and-solder-directed self-assembly of functional microsystems," Proceedings of the National Academy of Sciences of America, vol. 101, pp. 12814-12817, 2004.

[6] W. Zheng, et al., "Fluidic heterogeneous microsystems assembly and packaging," Journal of Microelectromechanical Systems, vol. 15, pp. 864-870, 2006.

[7] J. Tien, et al., "Microfabrication through electrostatic self-assembly," Langmuir, vol. 13, pp. 5349-5355, 1997.

[8] S. B. Shetye, et al., "Self-assembly of millimeter-scale components using integrated micromagnets," IEEE Transactions on Magnetics, vol. 44, pp. 4293-4296, 2008.

[9] H. Ye, et al., "Integrating nanowires with substrates using directed assembly and nanoscale soldering," IEEE Transactions on Nanotechnology vol. 5, pp. 62-66, 2006.

[10] R. J. Knuesel and H. O. Jacobs, "Self-assembly of microscopic chiplets at a liquid-liquid-solid interface forming a flexible segmented monocrystalline solar cell," Proceedings of the National Academy of Sciences of America, vol. 107, pp. 993-998, 2010.

[11] M. Boncheva, et al., "Millimeter-scale self-assembly and its applications," Pure and Applied Chemistry, vol. 75, pp. 621-630, 2003.

[12] K. S. Park, et al., "Mechanics and scaling of thin part assembly at a fluidic interface," Journal of Micromechanics and Microengineering, vol. 21, p. 025002, 2011.

[13] T. B. Benjamin and F. Ursell, "The Stability of the Plane Free Surface of a Liquid in Vertical Periodic Motion," Proceedings of the Royal Society of London. Series A, Mathematical and Physical Sciences, vol. 225, pp. 505-515, 1954.

[14] S. Douady and S. Fauve, "Pattern Selection in Faraday Instability," Europhysics Letters, vol. 6, pp. 221-226, 1988.

[15] K. S. Park, et al., "Orientation-specific self-assembly at air-water interface using magnetic field," presented at Transducers 2011, Beijing, China, 2011.

[16] T. Fujita, et al., "Seedlayer-less gold electroplating on silicon surface for MEMS applications," Sensors and Actuators A: Physical, vol. 135, pp. 50-57, 2007. 


\section{A NOVEL HIGH-DENSITY CAPACITOR DESIGN AND ITS FABRICATION TECHNIQUE BASED ON SELECTIVE ETCHING

\author{
Victor Farm-Guoo Tseng ${ }^{1 *}$, Khai $\mathrm{Ngo}^{2}$, and Huikai Xie ${ }^{l}$
} \\ ${ }^{1}$ University of Florida, Gainesville, Florida, USA \\ ${ }^{2}$ Virginia Polytechnic Institute and State University, Blacksburg, Virginia, USA}

\begin{abstract}
The design, fabrication, and characterization of a novel high-density metal-insulator-metal (MIM) multilayer capacitor is reported. In order to interconnect the multiple electrode layers without using numerous photolithography steps, a unique process was developed based on depositing MIM layers onto a substrate with two pillars, fine polishing to expose the multilayer cross sections, and then selectively etching the metals on each pillar to form the capacitor electrodes. For demonstration purpose, only capacitors with two dielectric layers were fabricated, with measurement results verified by a theoretical model. With a dielectric thickness of $200 \mathrm{~nm}$, a capacitance density of $0.54 \mathrm{fF} / \mu \mathrm{m}^{2}$ was achieved, which can be easily increased by decreasing dielectric thickness and increasing the number of MIM layers. The capacitor also exhibits low equivalent series resistance (ESR) of $300-700 \mathrm{mohms}$, and can operate up to $63 \mathrm{MHz}$.
\end{abstract}

\section{INTRODUCTION}

There has been a great effort in the recent decade to integrate discrete passive devices into the chip level. As mentioned in the recent ITRS report [1], this trend has mainly been influenced by radio frequency (RF), mixed signal, and system-on-a-chip (SoC) applications, in which on-chip precisely controlled high quality passives are needed for high frequency operation. Similarly, in the field of power electronics, minimization of the bulky power passives, possibly fabricated into a separate passives integrated substrate for further flip-chip bonding or 3D chip stacking [2], is necessary. In the overall system, capacitors and inductors are usually the most space consuming, but are rather desired due to their low power consumption. As a result, it is imperative to develop better chip level integration methods for passive components.

In particular, capacitors are the most widely used passive component. Typical applications are microprocessor decoupling capacitors, analog/filter capacitors, RF bypass capacitors, DRAM storage cell capacitors, and power electronic flying capacitors. Currently, several technologies have been developed to fabricate integrated high-density capacitors, including trench capacitors [3], stacked capacitors [4], hemispherical silicon electrode capacitors [5], and multilayer capacitors [6]. While using multilayer stacks seems straightforward, the interconnection between numerous electrode plates requires numerous photolithography steps (Fig. 1(a)), which is impractical and over expensive. Also, the trench capacitor and hemispherical silicon electrode capacitor methods usually make use of the highly doped silicon substrate as one of the capacitor plates, which is undesirable because of the increased substrate coupling noise and loss, along with higher ESR. Thus, there is an increasing demand for MIM capacitors, and for them to be integrated into the back-end-of-line (BEOL) layers of typical IC processes, farther away from the substrate [1].

In this work, a new method is proposed to resolve the interconnection dilemma of multilayer MIM capacitors. This new method will allow the parallel stacking of several tens of MIM capacitors. Additionally, all the previously mentioned high-density

Travel support has been generously provided by the Transducers Research Foundation (TRF) capacitor fabrication techniques can also be incorporated into this technology.

\section{DESIGN CONCEPT}

Illustrated in Fig. 1(b), the underlining concept of our integrated capacitor design and fabrication is based on the idea of subsequently depositing multiple thin film MIM layers onto pillar structures, then polishing down the pillars, and exposing the multilayer cross sections in a single planar horizontal surface, which virtually "bends" the cross sections upwards. If the alternating capacitor electrode metal plate materials are chosen to have chemical properties that allow them to be selectively etched, two selective etch steps can be carried out, one on either one of the pillars, receding either metal 1 or metal 2 down into the trenches on each pillar, as shown in Fig. 1(c). Finally, by performing dielectric redeposition and etch back, followed by electrode metallization, several tens of electrode plates can be interdigitally connected, forming a single capacitor with only four photolithography steps.

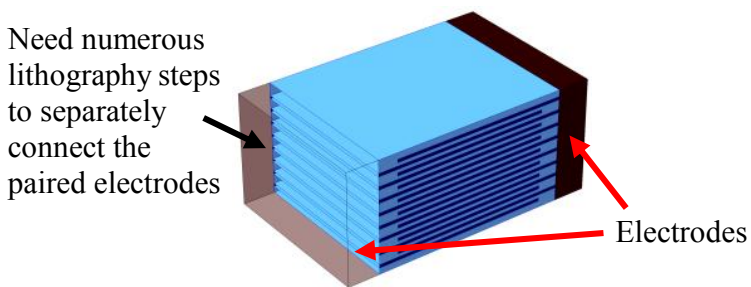

(a)

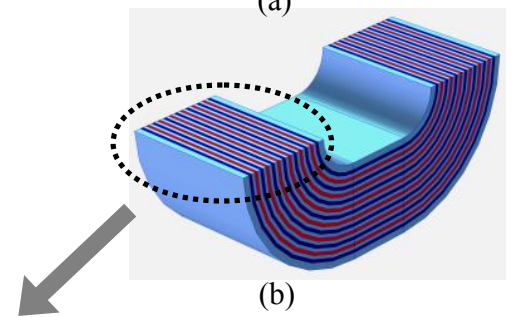

(b)

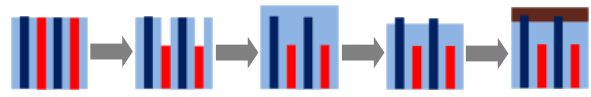

(c)

Dielectric $\quad$ Metal $1 \quad$ Metal 2 Electrode metal

Figure 1. Concept of multilayer capacitor design: (a) typical multilayer capacitors, (b) "bending" the layers upward to access the cross sections while using alternating plate materials, and (c) using selective metal etch, dielectric redeposition and etch-back, and electrode metallization to form the interdigital connections

\section{FABRICATION PROCESS AND RESULTS}

The physical implementation of the fabrication process flow is shown in Fig. 2. First, the pillar structures with height of around $20 \mu \mathrm{m}$ are formed by etching into the silicon substrate. A hydrofluoric, nitric, and acetic acid mixture (HNA) isotropic etch [7] was used to produce smooth pillar sidewalls, which can prevent 
shortage or field concentration at sharp edges. The HNA composition was $\mathrm{HF}(49 \%): \mathrm{HNO}_{3}(70 \%): \mathrm{CH}_{3} \mathrm{COOH}(99.5 \%)=1: 3: 8$, and $\mathrm{Si}_{3} \mathrm{~N}_{4}$ was used as the etch mask. Notice that the pillars can also be made out of the thick low-k dielectrics in typical BEOL layers, but pillars of silicon were chosen for process convenience. Next, a multi-target sputtering system is used to alternatively deposit dielectric, metal 1, dielectric, and metal 2 layers. The dielectric may be $\mathrm{SiO}_{2}, \mathrm{Si}_{3} \mathrm{~N}_{4}$ or other high-k dielectrics. The first and last dielectric layers are deposited by chemical vapor deposition (CVD) to thicknesses around $1 \mu \mathrm{m}$ as isolation/protection layers. The two metals must be chosen to be selectively etched. $\mathrm{Au}$ (with $\mathrm{Ti}$ adhesion) and $\mathrm{Cr}$ were chosen in this work, both sputtered to thicknesses of $500 \mathrm{~nm}$. Then, the pillars are polished down to the first dielectric layer. A combination of polyurethane polishing cloth, $0.3 \mu \mathrm{m}$ alumina lapping film, and $0.05 \mu \mathrm{m}$ colloidal silica/alumina suspension slurry was used to achieve smooth and uniform polishing across the wafer. As shown in Fig. 2(c), photoresist is also spin coated to protect unwanted areas from being attacked during the polishing. Next, the two selective metal etch steps are performed. Gold Etch TFA and Chromium Etch 1020 from Transcene $(\mathcal{C}$ were used respectively for $\mathrm{Au}$ and $\mathrm{Cr}$, and both etchants were shown to be greatly selective to all the other materials. A short Ti etch with a HF based solution is also needed after the Au etch to avoid shortage due to the remaining adhesion layers. The selective etch steps were precisely controlled to recede the metal layers down into the trenches of about $7 \mu \mathrm{m}$. $\mathrm{SiO}_{2}$ is then redeposited by CVD to refill trenches, followed by a short RIE etch back with $\mathrm{CHF}_{3}$ based plasma. Finally, a $300 \mathrm{~nm} \mathrm{Cu}$ seed layer (with $\mathrm{Ti}$ adhesion) is sputtered, molded by photolithography, electroplated to around $10 \mu \mathrm{m}$, and etched back to form the two electrodes. This thick $\mathrm{Cu}$ electrode is needed to achieve lower ESR. For demonstration purpose, only capacitors with two dielectric layers were fabricated: one $200 \mathrm{~nm}$ thick $\mathrm{Si}_{3} \mathrm{~N}_{4}$ dielectric layer, and one $200 \mathrm{~nm}$ thick $\mathrm{SiO}_{2}$ dielectric layer.

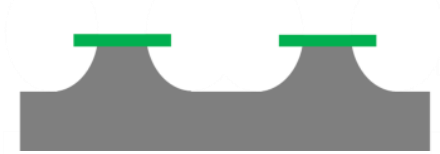

(a)

(b)

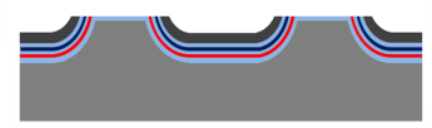

(c)

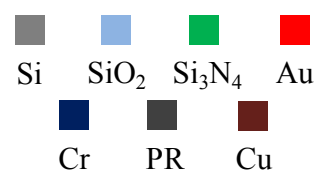

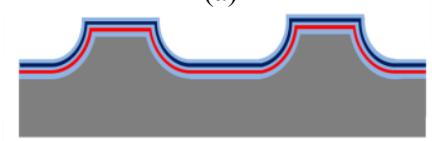

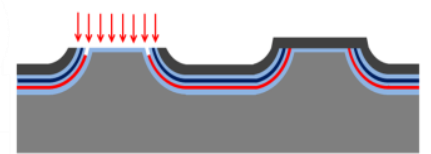

(d)

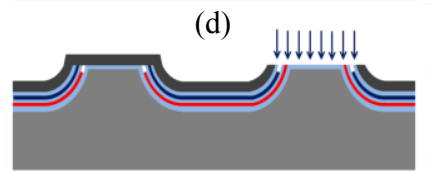

(e)

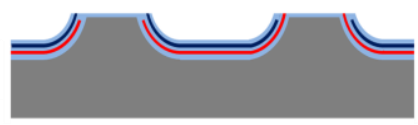

(f)

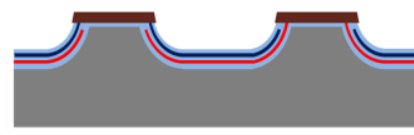

(g)
Figure 2. Complete process flow: (a) HNA etch to form the pillars, (b) multilayer deposition, (c) pillar polishing, (d) selective Au etch, (e) selective $\mathrm{Cr}$ etch, (f) dielectric redeposition and etch back, and (g) sputter and electroplating metallization for $\mathrm{Cu}$ pads

Capacitors were fabricated with areas of $300 \mu \mathrm{m} \times 800 \mu \mathrm{m}$, $800 \mu \mathrm{m} \times 800 \mu \mathrm{m}, 800 \mu \mathrm{m} \times 1800 \mu \mathrm{m}$, and $1800 \mu \mathrm{m} \times 1800 \mu \mathrm{m}$. Fig. 3 shows the two smaller sized completed capacitors. Slight scratches on the first dielectric layer were seen after the polishing process.

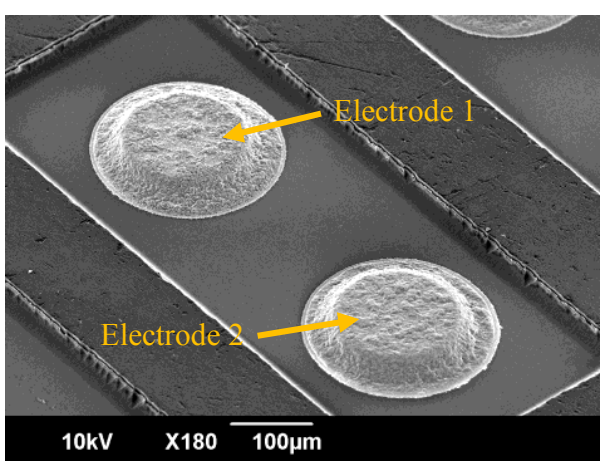

(a)

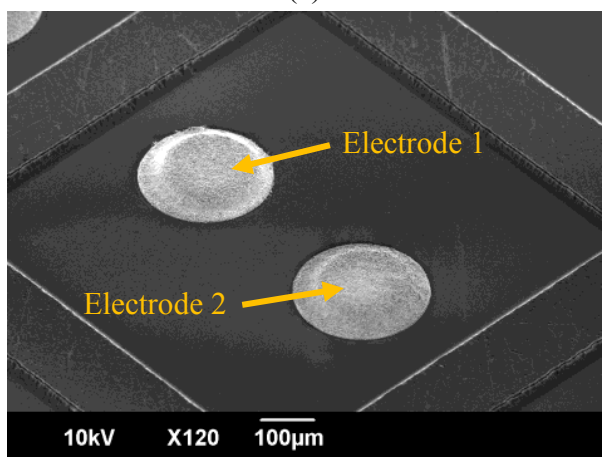

(b)

Figure 3. Completed capacitors with areas of (a) $300 \mu \mathrm{m} \times 800 \mu \mathrm{m}$ and (b) $800 \mu \mathrm{m} \times 800 \mu \mathrm{m}$

Fig. 4 shows an HNA-etched pillar before and after polishing. Clearly, some etching anisotropy exists, and the HNA composition needs to be further tuned to produce a smoother etched surface for the pillars etched out of the high resistivity silicon substrate.

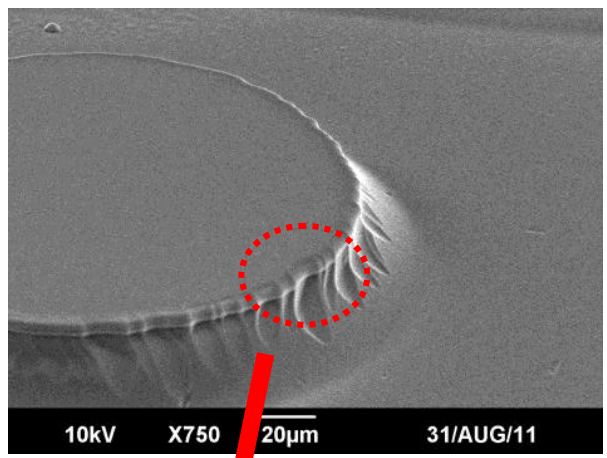

(a)

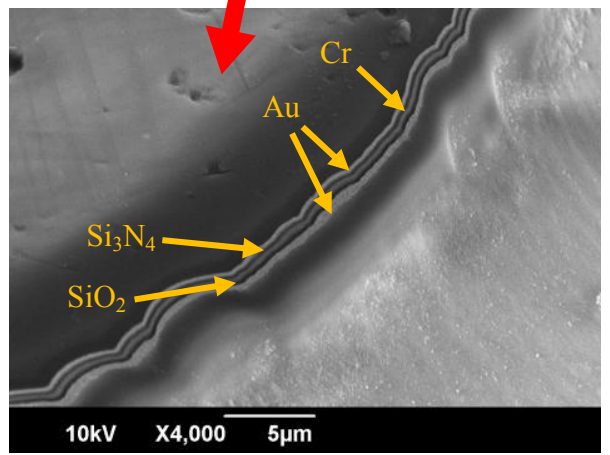

(b)

Figure 4. (a) HNA etched pillar, (b) close-up view showing cross section of a two dielectric layer capacitor after polishing 
Fig. 5(a) shows the selective $\mathrm{Cr}$ etch details. The inner $\mathrm{Cr}$ layer recedes into the cross section, leaving out a trench, while other materials remain intact. Fig. 5(b) shows the results after dielectric redeposition and etch back. The trenches are shown to be refilled and slightly etched. The selective Au etch shows similar results.

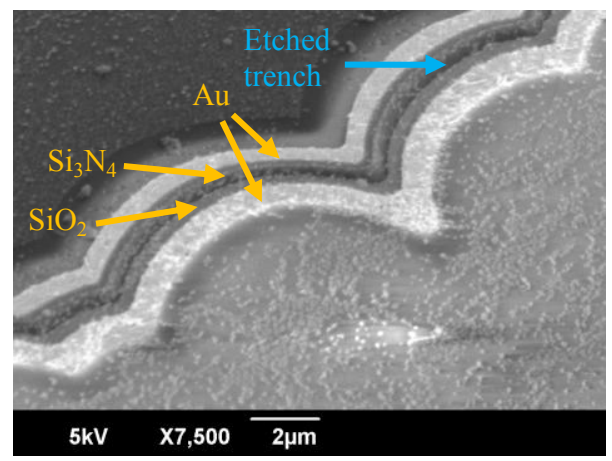

(a)

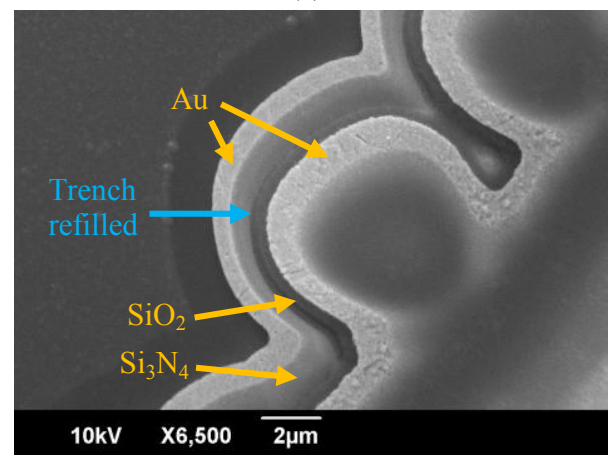

(b)

Figure 5. Close-up view of process details after (a) selective $\mathrm{Cr}$ etch, (b) $\mathrm{SiO}_{2}$ redeposition and etch back

\section{CAPACITOR MODEL}

Shown in Fig. 6 is the typical lumped circuit model for realistic capacitors. $C_{o}$ is the capacitor capacitance, $R_{p}$ is the parallel resistance due to leakage, and $\mathrm{L}_{\mathrm{s}}$ is the equivalent series inductance (ESL) due to the electrodes and plates. $\mathrm{C}_{\mathrm{a}}$ and $\mathrm{R}_{\mathrm{a}}$ are used to fit the response of dielectric absorption, a known result due to polarization hysteresis. $C_{a}$ is usually small compared to $C_{0}$, while $R_{a}$ is large, and both can be neglected in this work. The equivalent series resistance (ESR) includes two components: $\mathrm{R}_{\text {plate }}$, the electrode and plate resistance, which is constant at low frequencies but rises at high frequencies due to skin effect; $R_{d}$, the dielectric loss resistance, which is frequency dependent and has no DC value.

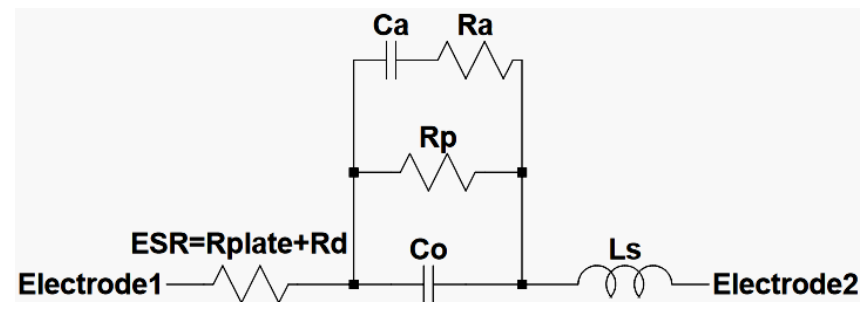

Figure 6. Typical lumped circuit model for realistic capacitor

Assuming a sinusoidal input signal, and defining the complex permittivity as $\epsilon=\epsilon^{\prime}-\mathrm{j} \epsilon^{\prime \prime}$, the loss tangent of the dielectric material then becomes

$$
\tan \delta=\frac{\omega \epsilon^{\prime \prime}+\sigma}{\omega \epsilon^{\prime}} \cong \frac{\epsilon^{\prime \prime}}{\epsilon^{\prime}} \quad \text { (at frequencies above } 500 \mathrm{~Hz} \text { ) }
$$

in which $\sigma$ is the conductivity of the dielectric material. Hence for typical dielectrics, $R_{d}$ can be simplified as

$$
\mathrm{R}_{\mathrm{d}}=\frac{\tan \delta}{\omega \mathrm{C}_{\mathrm{o}}}
$$

Notice that $R_{d}$ takes into account the conductivity of the dielectric, therefore incorporating $R_{p}$ into $R_{d}$. The impedance and dissipation factor (DF) are then expressed as

$$
\begin{gathered}
Z=R_{\text {plate }}+R_{d}+\frac{1}{j \omega C_{o}}+j \omega L_{s} \\
D F=\omega C_{o} \times\left(R_{\text {plate }}+R_{d}\right)
\end{gathered}
$$

\section{MEASUREMENT RESULTS}

Measurements were conducted via a HP 4294A impedance analyzer and an Agilent 4155C parameter analyzer. Measurements of the $300 \times 800 \mu \mathrm{m}$ size capacitors are not shown below due to an existing $\mathrm{k} \Omega$ resistive path through the silicon substrate, mainly caused by over-polishing induced damage to the first dielectric layer. An improved polishing process is needed. The measured capacitances, shown in Fig. 7, were 1 to 1.1 times their expected values. This is due to the increased surface area caused by the HNA etch roughness. The largest capacitance density that is achieved is $0.5 \mathrm{fF} / \mu \mathrm{m}^{2}$, which is comparable to the results in [6]. Shown in Fig. 8, ESR decreases roughly inversely proportional to frequency at first, during which it is dominated by $\mathrm{R}_{\mathrm{d}}$, until it reaches the minimum resistance of $R_{\text {plate }}$. $R_{\text {plate }}$ was found to be around 300 to $700 \mathrm{mohm}$, which is near expectation using equations from [8]. There seems to be an optimal design for capacitor dimensions to minimize $R_{\text {plate}}$, which is still under investigation. More insight can be seen from the DF measurements (Fig. 9). Before around $500 \mathrm{~Hz}$, the higher loss is dominated by the leakage conductivity of the dielectric material. At mid-frequencies, the loss reaches its minimum, dominated by dielectric loss, with $\mathrm{DF} \cong \tan \delta \cong \epsilon^{\prime \prime} / \epsilon^{\prime} \cong 0.003$. At frequencies above several $\mathrm{MHz}$, the loss rises again, dominated by $\mathrm{R}_{\text {plate. }}$. Using (1), the conductivity $\sigma$ of the dielectric material was found to be around $2 \times 10^{-11}(\Omega \times \mathrm{cm})^{-1}$, which corresponds to the $|\mathrm{I}|-\mathrm{V}$ measurements, and is rather large compared to normal dielectric conductivities. In order to improve the dielectric quality, an annealing step can be added. From the impedance magnitude $|\mathrm{Z}|$ measurements (Fig. 10), the self resonances occur at $36.1 \mathrm{MHz}$, $48.6 \mathrm{MHz}$, and $63 \mathrm{MHz}$ respectively, and from (3), $\mathrm{L}_{\mathrm{s}}$ can be found to be $9.6 \mathrm{nH}$. With the extracted model parameters mentioned above, the theoretical response of the lumped circuit model was plotted, and shows close coherence with the measurement results.

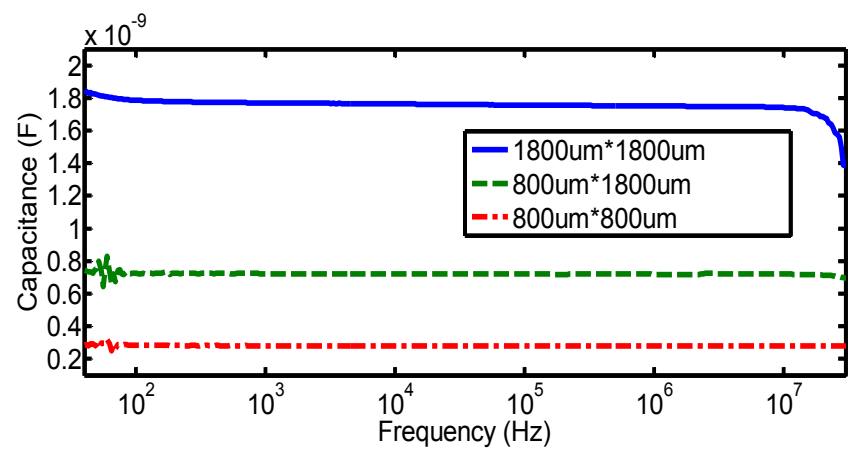

Figure 7. Capacitance measurement results

Fig. 11(a) shows the C-V measurements for the $1800 \mu \mathrm{m} x$ $1800 \mu \mathrm{m}$ size capacitors at different frequencies, showing the capacitance to be constant per bias voltage. Fig. 11(b) shows the $|\mathrm{I}|-\mathrm{V}$ measurements. As mentioned above, a higher than expected 
leakage current due to the higher dielectric conductivity was observed. Occasionally, a premature breakdown voltage at around 2.7 to $3 \mathrm{~V}$ was also observed. Reasons for this might include the inferior dielectric quality, surface roughness induced field concentration, or possible air voids caused by the incompletely filled trenches during the dielectric redeposition step. Further improvements of the dielectric redeposition by using atomic layer deposition (ALD) may help.

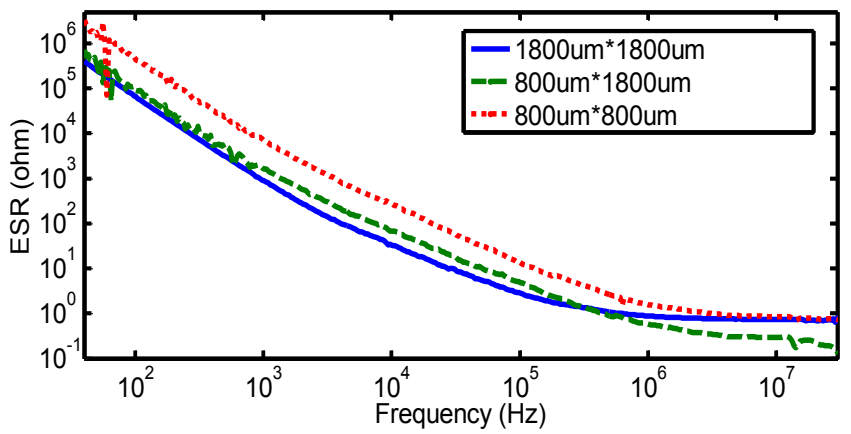

Figure 8. ESR measurement results

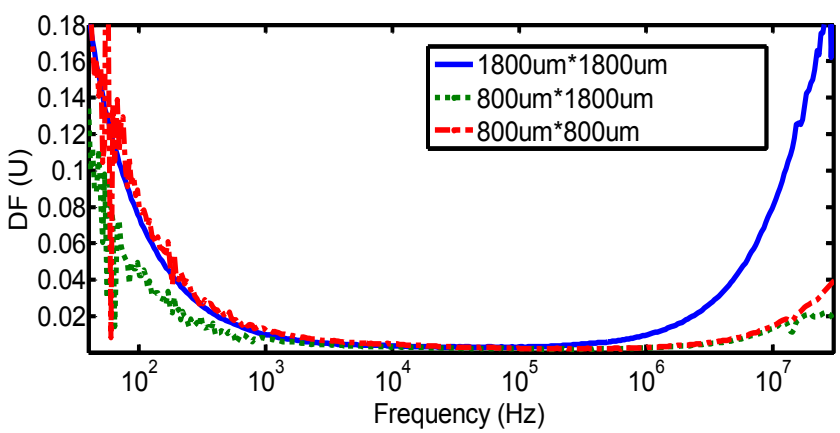

Figure 9. DF measurement results

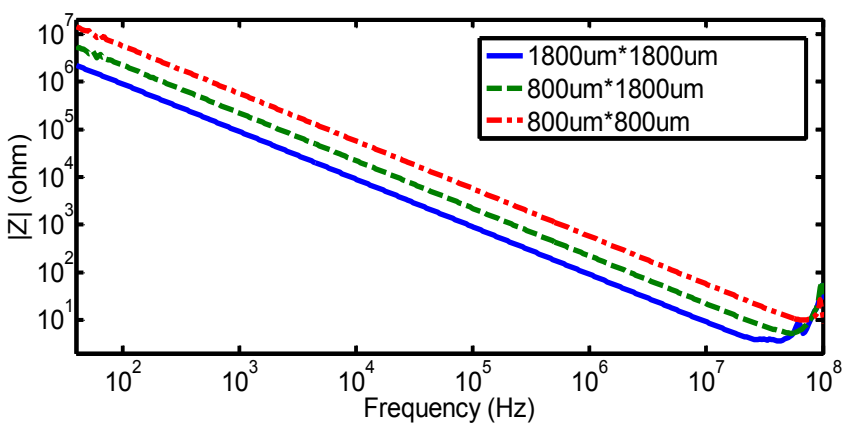

Figure 10. Impedance magnitude measurement results

\section{CONCLUSIONS}

A new method to fabricate high-density multilayer MIM capacitors has been successfully demonstrated, with capacitor performance verified by theory. Further process and design improvements should allow capacitance densities above $12 \mathrm{fF} / \mu \mathrm{m}^{2}$ with lower losses. Since the fabrication is IC-compatible, this innovation should bring benefit to the fields of RF, mixed-signal, power electronics, etc., to achieve the ultimate goal of SoC design.

\section{ACKNOWLEDGEMENTS}

This project is supported by ARPA-E under award \#DE-AR0000105. Device fabrication and characterization was done in the Nanoscale Research Facility of the University of Florida.

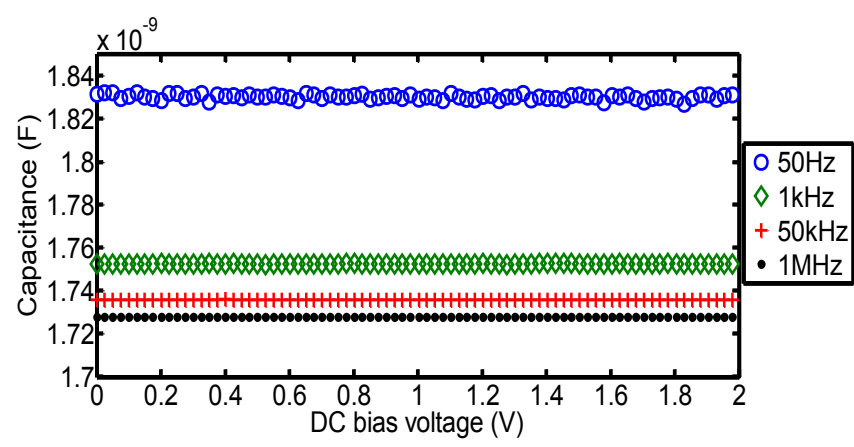

(a)

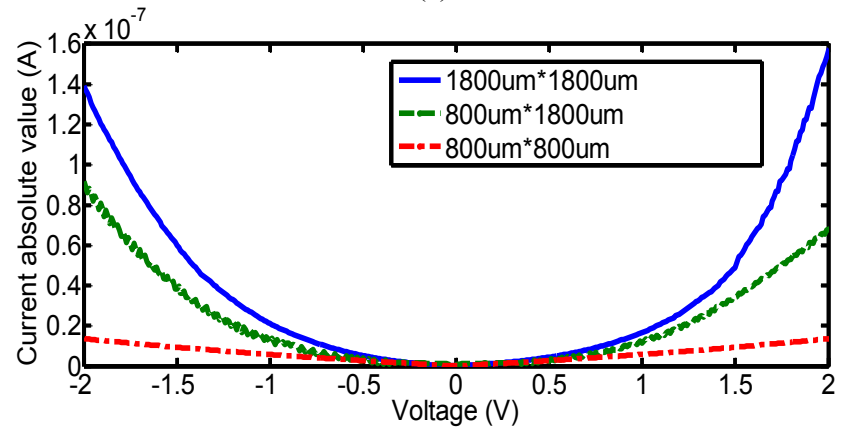

(b)

Figure 11. (a) C-V measurement results for $1800 \mu m \times 1800 \mu m$ size capacitors at various frequencies, (b) $|I|-V$ measurement results

\section{REFERENCES}

[1] The International Technology Roadmap for Semiconductors 2011 edition, Semiconductor Industry Association, 2011.

[2] F. Roozeboom, et al., "Ultrahigh-density trench capacitors in silicon and their application to integrated DC-DC conversion," Proc. 23th Eurosensors Conf., Lausanne, Sept., 2009, pp. 426-430.

[3] J. H. Klootwijk, et al., "Ultrahigh capacitance density for multiple ALD-grown MIM capacitor stacks in 3-D silicon," IEEE Electron Device Letters, vol. 29, no.7, pp. 740 - 742, Jul. 2008.

[4] T. Kaga, et al., "Crown-shaped stacked-capacitor cell for $1.5-\mathrm{V}$ operation 64-Mb DRAMs," IEEE Transactions on Electron Devices, vol. 38, no. 2, pp. 255 - 261, Feb. 1991.

[5] C. T. Black, et al., "Integration of self-assembled diblock copolymers for semiconductor capacitor fabrication," Applied Physics Letters, vol. 79, no. 3, pp. 409-411, Jul. 2001.

[6] T. Tsutsumi, S. Sugitani, K. Nishimura, and M. Ida, "New stacked metal-insulator-metal capacitor with high capacitance density for future InP-based ICs," Japanese Journal of Applied Physics, vol. 50, no. 4, pp. 04DF09-04DF09-4, 2011.

[7] B. Schwartz and H. Robbins, "Chemical etching of silicon II. The system HF, $\mathrm{HNO}_{3}, \mathrm{H}_{2} \mathrm{O}$, and $\mathrm{HC}_{2} \mathrm{H}_{3} \mathrm{O}_{2}$," Journal of The Electrochemical Society, vol. 107, no. 2, pp. 108-111, Feb. 1960.

[8] A. Bajolet, et al., "Low-Frequency series-resistance analytical modeling of three-dimensional metal-insulator-metal capacitors," IEEE Transactions on Electron Devices, vol. 54, no. 4, pp. 742-751, Apr. 2007.

\section{CONTACT}

*Victor Farm-Guoo Tseng, tel: +1-352-328-2344; souldragon227@,ufl.edu 


\section{A SIMPLE SOI-CMOS COMPATIBLE TECHNOLOGY TO MAKE FLEXIBLE ELECTRONICS \\ Hongen $\mathrm{Tu}^{1}$ and Yong $\mathrm{Xu} \mathrm{u}^{1}$ \\ ${ }^{1}$ Wayne State University, Detroit, MI, USA}

\begin{abstract}
Flexible sensors or electronics are expected to play important roles in various healthcare and medical applications. Fabrication techniques like direct coating and patterning organic or inorganic semiconductor materials on flexible substrates are ideal to reduce the cost. However, high temperature processes are not allowed due to the flexible substrate, limiting the choice of materials and reduce the performance of the device. The recently developed transfer printing technology also has limitations due to its incompatibility with mainstream CMOS technology. This paper reports a simple SOI-CMOS compatible technology to make flexible electronics. This new technology takes advantage of $\mathrm{XeF}_{2}$ isotropic gas phase silicon etchant and conformal parylene $\mathrm{C}$ coating, making the process SOI-CMOS compatible.
\end{abstract}

\section{INTRODUCTION}

Flexible sensors or electronics are believed to have significant impacts on wearable health monitoring, medical implants and many other applications. Over the last two decades, many different approaches of making flexible sensors or electronics have been proposed and researched. The most straightforward one is to fabricate directly on flexible substrate. Actually, similar technology has existed for a long time in the electronics industry, for example, the widely used flexible printed circuitry technology and the thin film transistor (TFT) technology on flexible substrates. In reference [1], a flexible large area position sensitive detector was developed by depositing amorphous silicon on Kapton polyimide substrate. In reference [2], a flexible multichannel sieve electrode for interfacing regenerating peripheral nerves was fabricated on polyimide film by using a silicon wafer as a support for a much better dimension control. Simple MEMS structures on plastic substrates, such as amorphous silicon air-gap resonators, have been demonstrated as well [3]. The simple fabrication process and low cost are the main advantages of direct fabrication on flexible substrate. Large area flexible sensors or electronics can be fabricated in this way economically. However, since the process temperature is limited due to the flexible substrate, high temperature processes are ruled out and the material properties are not optimized. Moreover, due to this temperature limit, it will be almost impossible to integrate CMOS circuits and many MEMS transducers to the flexible substrate monolithically.

We have previously reported a flexible skin technology based on silicon island structure [4-6]. The basic structure of the flexible skin is arrays of silicon islands sandwiched by two layers of polymer. The biggest advantage of this technology is its compatibility with MEMS and CMOS since MEMS devices and CMOS circuits can be fabricated on the silicon wafer before the formation of the flexible skin. The minimum bending radius is determined by the size the of the silicon islands. Based on this technology, flexible shear stress sensor skins [4], the integration of CMOS with the flexible skin [5], and intelligent textiles [7] have been demonstrated.

Recently, Dr. John Rogers' group developed a flexible electronics technology using an innovative "transfer printing" method $[8,9]$. Namely, transistors and other devices are fabricated first on SOI wafers and then transferred to flexible substrates by "printing". Many exciting applications have been demonstrated [10, 11]. Nevertheless, the releasing step is generally incompatible with commercial CMOS process. Therefore, this process still cannot fully take advantage of the mainstream CMOS technology. In addition, the metal interconnection among discrete components was formed on the flexible substrate after transferring, limiting the device density and performance.

This paper reports a simple SOI-CMOS compatible technology which can be used to fabricate flexible electronics without the limitations of "printing" technique. The most unique part of our technology is the releasing step. All the electronic components of our device, including metal interconnects and discrete silicon islands, can be fabricated right before the final releasing step. The releasing step includes two parylene $\mathrm{C}$ film depositions. The first parylene layer is used as a mask to release the devices and a supporting layer for released devices. The second parylene layer functions as an encapsulation layer for the devices. $\mathrm{XeF}_{2}$ isotropic gas phase silicon etchant is used here in the releasing step to etch away the bulk silicon in the handle wafer. Parylene $\mathrm{C}$ is selected as the flexible substrate because parylene $\mathrm{C}$ deposition is a stress-free conformal process, and parylene $\mathrm{C}$ possesses excellent properties such as chemical inertness, biocompatibility, low leakage current and low gas permeability. We have previously utilized $\mathrm{XeF}_{2}$ isotropic etching and parylene conformal deposition to fabricate microchannels and individually-addressable microtubes [12, 13]. Here to demonstrate the concept of the new flexible electronics technology, silicon strain gauges were first fabricated.

\section{FABRICATION}

The fabrication process starts with a Soitec SOI wafer with a device layer of $0.36 \mathrm{um}$ and a buried oxide (BOX) layer of $0.5 \mathrm{um}$. As shown in Fig. 1 (a), phosphorus diffusion source was used to dope the Si device layer. Silicon thermal oxide was used as a diffusion mask here. The exposed device layer became highly doped. Then as shown in Fig. 1 (b), individual Si island was patterned and the exposed BOX layer were removed sequentially. $\mathrm{Cr} / \mathrm{Au}$ traces and contact pads were then sputtered and patterned as shown in Fig. 1 (c). Here we chose Au because of its excellent ductility. In the later development, we will use CMOS-compatible metals such as Al. It can be observed that the electronic components can be fabricated via standard CMOS process up to this point since there is no temperature limit of fabrication process.

Next, a few more steps were performed to transfer devices from Si substrate into flexible parylene C substrate. Firstly, a 3 um thick parylene $\mathrm{C}$ layer was deposited as illustrated in Fig. 1 (d). Then arrays of small windows (8um $\times 25 \mathrm{um}) 60 \mathrm{um}$ apart were opened in this parylene layer using $\mathrm{O}_{2}$ plasma (step (e)). Note that all the wide metal traces/pads and silicon islands were also etched selectively to open the windows at this step. Then, $\mathrm{XeF}_{2}$, an isotropic gas phase silicon etchant, was used to completely undercut the silicon underneath through the windows formed in the previous step (step (f)). Note that silicon islands were protected by both parylene $\mathrm{C}$ on the top and $\mathrm{BOX}$ on the bottom. At this point both strain gauges and metal traces/pads were supported by a free-standing perforated parylene $\mathrm{C}$ membrane. Next, a second parylene $\mathrm{C}$ layer (10 um) was conformally deposited to encapsulate the strain gauges and metal traces/pads, and simultaneously seal the 
etching windows as shown in Fig. 1 (g). Oxygen plasma was then used to open bonding pads and shape the outline of the flexible device. Finally the flexible device can be simply peeled off from the Si substrate. Note that there were two physically separated parylene $\mathrm{C}$ layers after step (h). Depending on the application, we can choose to either keep or remove the scalloped bottom parylene $\mathrm{C}$ layer by just modifying the final releasing mask. Because these two parylene $\mathrm{C}$ layers formed an enclosed space, by keeping the bottom parylene layer, we could easily integrate functioning microchannels or microtubes in the system. We cut the bottom layer here to demonstrate a simple strain gauge embedded in top parylene $\mathrm{C}$ layer.

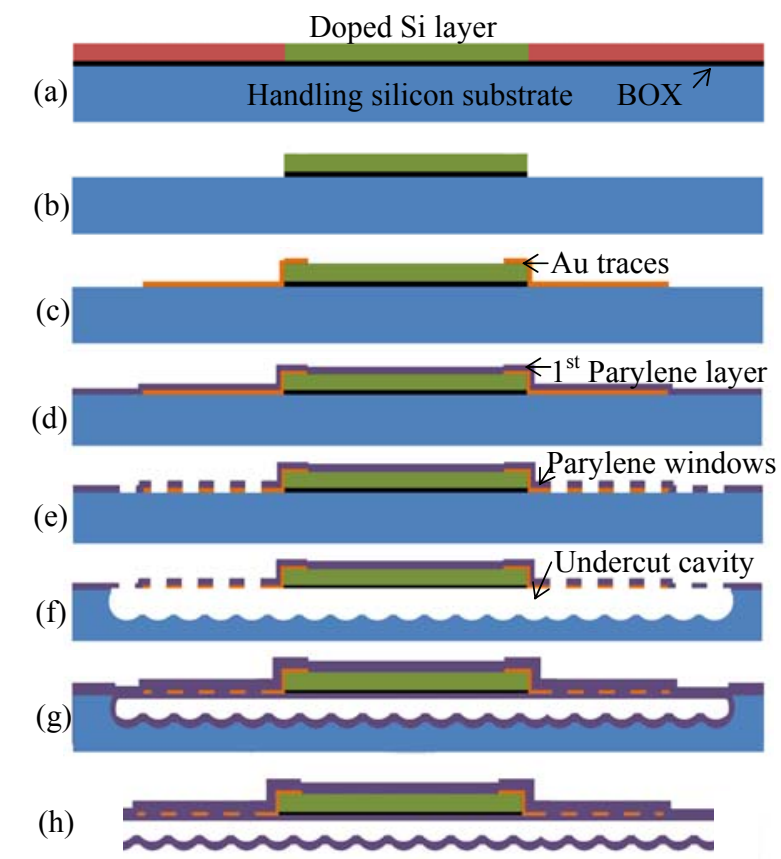

Figure 1: Simplified process flow: (a) Phosphorus diffusion.(b)Pattern the device layer and remove BOX layer.(c)Au deposition and patterning to form traces and pads. (d) $1^{\text {st }} 3 \mathrm{um}$ parylene deposition. (e)Pattern the parylene windows and etch away underneath metal traces.(f) $\mathrm{XeF}_{2}$ etching to release the devices. (g) $2^{\text {nd }} 10$ um parylene deposition. (h) Final released device.

\section{RESULTS AND DISCUSSION}

A fabricated flexible device is shown in Fig. 2, where the flexibility can be clearly observed. All the devices and metal traces/pads are held in position and protected by conformally coated transparent parylene $\mathrm{C}$ films. We have several strain gauges placed across the device which can be electrically accessed by $1 \mathrm{~mm} \times 1 \mathrm{~mm}$ large $\mathrm{Au}$ contact pads to facilitate later testing.

The magnified view of two perpendicular strain gauges can be found in Fig. 3. In this figure, both narrow metal traces and wide perforated metal traces can be observed. Wide metal traces will make our device more durable and they are also required for contact pads. Note that arrays of small dimples, which are resealed parylene $\mathrm{C}$ windows after second parylene $\mathrm{C}$ deposition, can be observed on the surface as shown in Fig. 3.

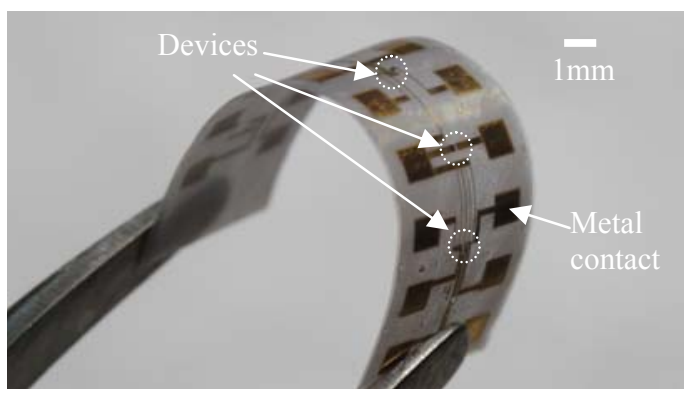

Figure 2: A bent flexible device held by a pair of tweezers.

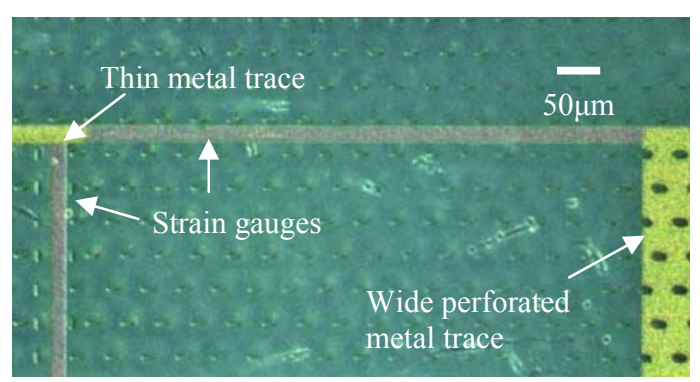

Figure 3: Optical micrograph of two perpendicular strain gauges.

It is also interesting to observe how the $\mathrm{XeF}_{2}$ etches the handle silicon substrate. Figure 4 shows the SEM image of the bottom parylene $\mathrm{C}$ layer after it was peeled off from the substrate. This layer of parylene $\mathrm{C}$ film was formed during the second deposition when the parylene $\mathrm{C}$ windows were sealed. The honey-comb shape reflects the silicon surface resulting from by the $\mathrm{XeF}_{2}$ isotropic etching.

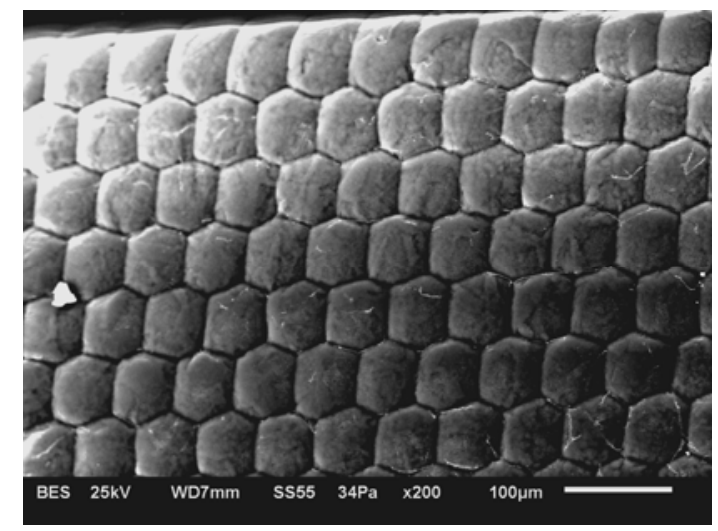

Figure 4: SEM image of the bottom honey-comb shaped parylene layer

More detailed information of sealed parylene windows is provided by Fig. 5. The original opening size of the perforated parylene $\mathrm{C}$ window was only $8 \mathrm{um}$ wide. The second $10 \mathrm{um}$ parylene $\mathrm{C}$ deposition was more than enough to completely seal the windows and provides enhanced strength to the film. The perforated contact metal pads can also be observed. Note that this SEM image was taken without sputtering a thin $\mathrm{Au}$ layer. Consequently, the exposed contact pad was very bright. 


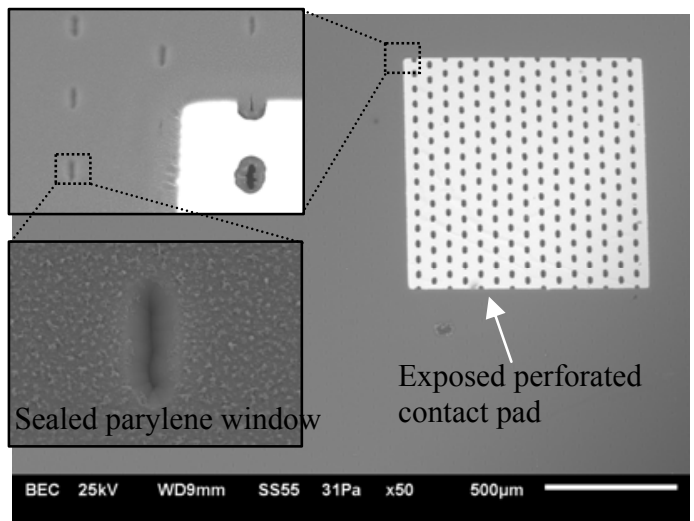

Figure 5: Front side SEM image of the device

Figure 6 shows the cross section of the flexible device. The relative position of the device layer in thickness direction of the parylene $\mathrm{C}$ film can be observed. Note that there was an initial parylene film above the device layer and during the resealing process, there was more parylene deposited on the front side after the windows were sealed. Therefore, the device layer was not positioned at the middle plane of the parylene $\mathrm{C}$ layer. The relative position can be easily adjusted by thinning down either top or bottom side of the parylene $\mathrm{C}$ film via $\mathrm{O}_{2}$ plasma.

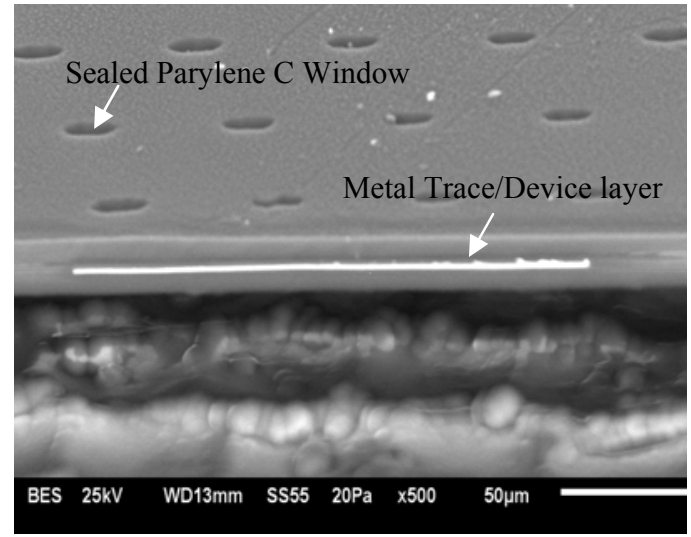

Figure 6: Cross sectional view of one device took by SEM

In order to test the performance of the flexible strain gauge, conductive epoxy was used to connect the testing wires to the exposed $\mathrm{Au}$ contact pads. A simple experiment was carried out by pushing the flexible device in the longitudinal direction (to induce buckling) as illustrated in Fig. 7. The displacement was controlled using a precision micro-manipulator. The resistance change as a function of displacement was recorded. The experiments were repeated 10 times and the averaged result with standard deviation is plotted in Fig. 8. If the gauge factor is 10 (for heavily doped n-type silicon), the strain experienced by the strain gauge is $0.387 \%$ for $1 \mathrm{~mm}$ displacement in the experiment (corresponding to a bending radius of $\sim 3 \mathrm{~mm}$ ). This is actually a sensitive flexible strain gauge because the silicon device layer is not at the neutral plane. It will become even more sensitive if we selectively thin down the bottom side of the parylene $\mathrm{C}$ film and thus move the device layer further away from the neutral plane. For other applications, the device layer can be positioned right on the neutral plane to minimize strains by reducing the thickness of the top parylene $\mathrm{C}$ layer via $\mathrm{O}_{2}$ plasma.

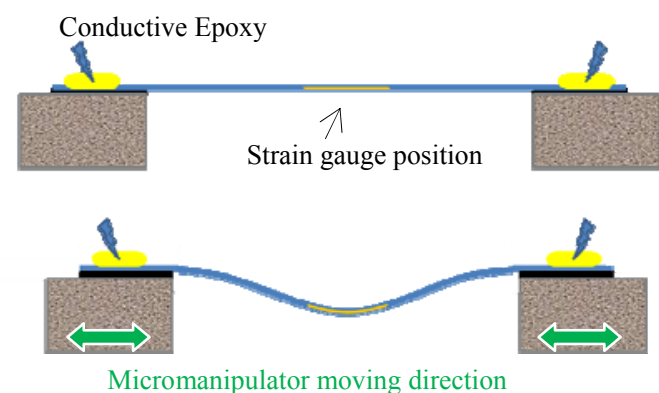

Figure 7: Testing setup with two moving stages to control the displacement.

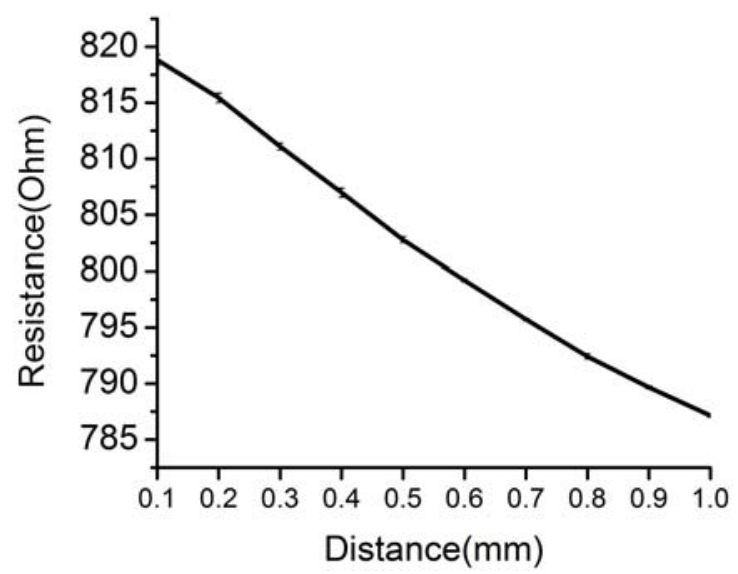

Figure 8: A simple experiment records the resistance change while the device bends when we applied the displacement in the longitudinal direction.

Note for the proof of concept, only simple strain gauges were reported in this paper. We are currently integrating MOSFETs with the flexible substrate to demonstrate more functionalities of our technology.

\section{CONCLUSION}

The biggest advantages of this new flexible electronics technology are its simplicity and compatibility with commercial SOI CMOS process. High-density and high-performance CMOS circuits can be first fabricated using SOI foundry (by slight modifying the layout rules) and readily transferred to flexible substrate. The post-CMOS process only consists of two masks, one is used to form etching holes and the other to open the metal pads and cut the outline. Our proposed fabrication process offers many distinctive benefits for flexible electronics applications. The lamination of the electronics between parylene films offers protection against moistures from the environment. Since there is no transfer printing involved, we can significant increase the device density and improve the yield. Moreover, the electronics or sensors can be positioned at either neutral plane or any other selected planes 
by simply modifying the thickness of the top or bottom parylene $\mathrm{C}$ via $\mathrm{O} 2$ plasma, and therefore allowing more design flexibility.

\section{ACKNOWLEDGMENT}

This material is based upon work partially supported by the National Science Foundation under Grant No. 0747620. Any opinions, findings, and conclusions or recommendations expressed in this material are those of the author(s) and do not necessarily reflect the views of the National Science Foundation. The microfabrication was carried out in the $\mathrm{nFAB}$ cleanroom at Wayne State University and LNF at University of Michigan, Ann Arbor.

\section{REFERENCES}

[1] E. Fortunato, I. Ferreira, F. Giuliani, and R. Martins, "Flexible large area thin film position sensitive detectors," Sensors and Actuators A-Physical, vol. 86, pp. 182-186, 2000.

[2] T. Stieglitz, H. Beutel, and J. U. Meyer, "A flexible, light-weight multichannel sieve electrode with integrated cables for interfacing regenerating peripheral nerves," Sensors and Actuators a-Physical, vol. 60, pp. 240-243, May 1997.

[3] M. Boucinha, P. Brogueira, V. Chu, and J. P. Conde, "Amorphous silicon air-gap resonators on large-area substrates," Applied Physics Letters, vol. 77, pp. 907-909, Aug 72000.

[4] Y. Xu, F. Jiang, S. Newbern, A. Huang, C. M. Ho, and Y. C. Tai, "Flexible shear-stress sensor skin and its application to unmanned aerial vehicles," Sensors and Actuators a-Physical, vol. 105, pp. 321-329, Aug 15 2003.

[5] Y. Xu, Y. C. Tai, A. Huang, and C. M. Ho, "IC-integrated flexible shear-stress sensor skin," Journal of Microelectromechanical Systems, vol. 12, pp. 740-747, Oct 2003.
R. B. Katragadda and X. Yong, "A novel intelligent textile technology based on silicon flexible skins," Sensors and Actuators: A. Physical, vol. 143, pp. 169-74, 2008 .

R. B. Katragadda and Y. Xu, "A novel intelligent textile technology based on silicon flexible skins," Sensors and Actuators a-Physical, vol. 143, pp. 169-174, May 22008. K. J. Lee, M. J. Motala, M. A. Meitl, W. R. Childs, E. Menard, A. K. Shim, J. A. Rogers, and R. G. Nuzzo, "Large-area, selective transfer of microstructured silicon: A printing-based approach to high-performance thin-film transistors supported on flexible substrates," Advanced Materials, vol. 17, pp. 2332-+, Oct 42005.

E. Menard, K. J. Lee, D. Y. Khang, R. G. Nuzzo, and J. A. Rogers, "A printable form of silicon for high performance thin film transistors on plastic substrates," Applied Physics Letters, vol. 84, pp. 5398-5400, Jun 282004.

D. H. Kim, N. S. Lu, R. Ma, Y. S. Kim, R. H. Kim, S. D. Wang, J. Wu, S. M. Won, H. Tao, A. Islam, K. J. Yu, T. I. Kim, R. Chowdhury, M. Ying, L. Z. Xu, M. Li, H. J. Chung, H. Keum, M. McCormick, P. Liu, Y. W. Zhang, F. G. Omenetto, Y. G. Huang, T. Coleman, and J. A. Rogers, "Epidermal Electronics," Science, vol. 333, pp. 838-843, Aug 122011. J. Viventi, D.-H. Kim, J. D. Moss, Y.-S. Kim, J. A. Blanco, N. Annetta, A. Hicks, J. Xiao, Y. Huang, D. J. Callans, J. A. Rogers, and B. Litt, "A Conformal, Bio-Interfaced Class of Silicon Electronics for Mapping Cardiac Electrophysiology," Science Translational Medicine, vol. 2, Mar 242010.

J. John, Y. F. Li, J. S. Zhang, J. A. Loeb, and Y. Xu, "Microfabrication of 3D neural probes with combined electrical and chemical interfaces," Journal of Micromechanics and Microengineering, vol. 21, Oct 2011.

Y. Li, H. Tu, R. Iezzi, P. Finlayson, and Y. Xu, "Development of Individually-Addressable Parylene Microtube Arrays," Journal of Microengineering and Micromechanics, vol. 21, 2011. 


\title{
CHARACTERIZING METAL NANOPARTICLE FILMS: A METHODOLOGY USING SURFACE MICROMACHINING BY DIRECT PRINTING
}

\author{
Eric W. Lam ${ }^{1}$ and Martin A. Schmidt ${ }^{1}$
}

${ }^{1}$ Massachusetts Institute of Technology, Cambridge, Massachusetts, USA

\begin{abstract}
We report a methodology for characterizing the mechanical properties of metal nanoparticle films based on microfabricated structures. Structures are mechanically characterized by beam deflection measurements and nanoindentation. Based on a 2sphere sintering model, and the relationship between particle size and melting temperature, a relationship between the nanoparticle film's elastic modulus and film geometry has been determined to be $\frac{E}{E_{0}}=\left(1-\left(\frac{L}{L_{0}}\right)^{3}\right)^{0.6982}$, where $L / L_{0}$ is the degree of particle fusion. This methodology was used to determine the property-process for silver nanoparticle films but should be transferrable to other nanoparticle films.
\end{abstract}

\section{INTRODUCTION}

Printing-based fabrication methods have emerged as a promising alternative to conventional lithographic processes in a number of applications. These methods are being exploited in display manufacturing [1], flexible electronics [2], and more recently MEMS $[3,4]$. Unlike applications in printed electronics, MEMS devices require comparatively thick layers, typically on the order of microns. In the micron-thickness regime, nanoparticle-based inks are the preferred means for material delivery, in large part due to the ability to deliver high solids content. However, as we previously reported in [3], sintered nanoparticle films possess unique morphology and hence different properties when compared to bulk material or materials deposited through evaporation or sputtering. In this new work, we report on a detailed study of the process-property relationship for nanoparticle-based films with an eye towards optimizing this material for MEMS applications. Our work will enable prediction of mechanical properties for this class of materials, and will provide guidance for optimization of sintering conditions towards a desired mechanical property. The specific results we will report include: i) detailed data which maps modulus versus process conditions and ii) a semiempirical model explaining the data and enabling prediction of resultant properties.

\section{PROCESS OVERVIEW}

The fabrication process contains eight steps as summarized in Figure 1. An overview is provided here with details of the process in the following sections. We have previously reported using a printed PMMA sacrificial layer [3] but in this work we use a conventionally-deposited amorphous-silicon film for simplicity in the process while still maintaining chemical and thermal compatibility with the silver nanoparticle ink. The amorphous-silicon is deposited by PECVD and patterned by photolithography. Etching of the amorphous-silicon is achieved by $\mathrm{XeF}_{2}$ dry-etching. The silver nanoparticle ink is jetted onto the sacrificial layer using our previously reported apparatus [3]. The nanoparticle ink is dried and subsequently sintered, forming a self-supporting film. The structures are laser-trimmed to remove edge effects, such as non-uniform thickness due to the
1) Deposit \& pattern a-Si

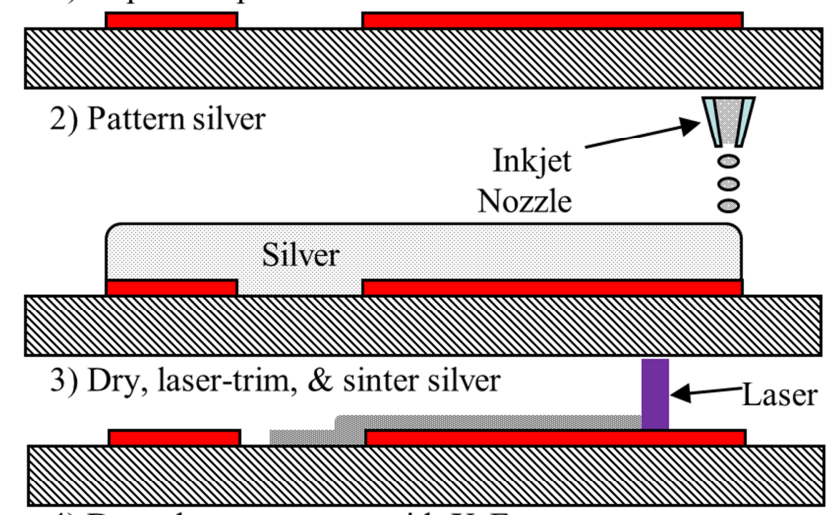

4) Dry release structure with $\mathrm{XeF}_{2}$

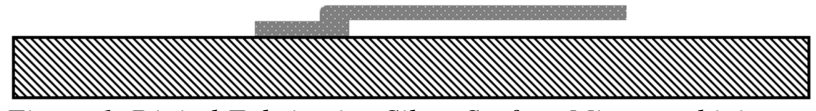

Figure 1. Digital Fabrication Silver Surface Micromachining Process Flow. Laser-trim is optional but results in better defined structures.

coffee-ring effect. The resulting structures are released by another $\mathrm{XeF}_{2}$ dry-etch to complete the surface micromachining process. Figure 2 is an SEM image of a printed silver cantilever.

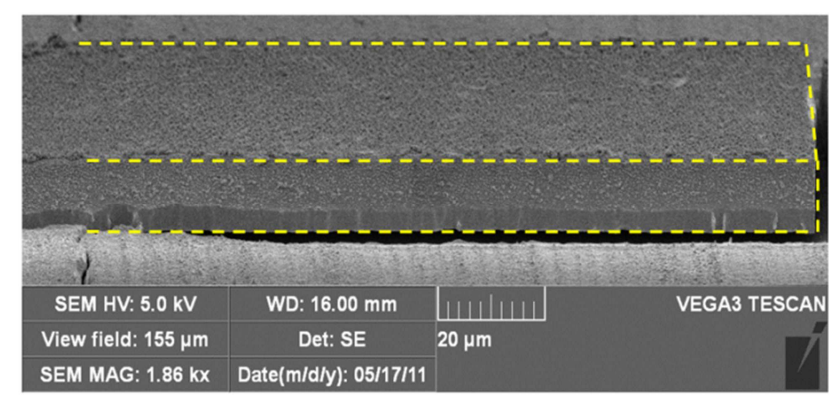

Figure 2. SEM image of surface micromachined cantilever.

Dashed line highlights boundary of the cantilever beam.

\section{MATERIALS}

This process has three layers/materials: substrate, sacrificial layer, and structural layer. Glass was chosen as the substrate material for the process but the process should be compatible with other materials such as polymers, metals, and silicon.

The sacrificial layer was created by depositing amorphoussilicon by PECVD at $200^{\circ} \mathrm{C}$. The amorphous-silicon thickness was $1.1 \mu \mathrm{m}$, but other thicknesses are possible. Patterning of the sacrificial layer consists of $\sim 1 \mu \mathrm{m}$ OCG 825 -20CS photoresist etch-mask and five 60s cycles of $\mathrm{XeF}_{2}$ to etch the amorphoussilicon. The photoresist etch-mask was stripped by cleaning the substrate in a $4: 1 \mathrm{H}_{2} \mathrm{SO}_{4}: \mathrm{H}_{2} \mathrm{O}_{2}$ solution for ten minutes.

The structural layer consists of a silver film created by depositing, drying, and sintering Cabot Corporation's CCI-300 silver-nanoparticle conductive ink. This ink contains $30-40 \mathrm{~nm}-$ 
diameter silver nanoparticles suspended in an ethylene glycol/ethanol solution. Films made from this ink become electrically conductive after a thermal sintering at temperatures greater than $100^{\circ} \mathrm{C}$. The ink's viscosity was lowered by diluting $1: 2$ volumetrically with ethanol to make it more readily printable with thermal inkjet.

\section{INKJET DEPOSITION}

Prior to printing, the droplet diameter for the CCI-300 ink was characterized to improve fabrication reliability and film uniformity. The inkjet printer used a 50pL Thermal InkJet (TIJ) nozzle resulting in a spot diameter of $150 \mu \mathrm{m}$. Deposition occurred in an air environment while the substrate was isothermally held at $50^{\circ} \mathrm{C}$ to assist in ink drying and prevent ink running. While the print head and ink reservoir were not heated, proximity to the substrate heated them to $38^{\circ} \mathrm{C}-40^{\circ} \mathrm{C}$.

\section{DRYING SILVER FILMS}

Silver nanoparticle films were jetted onto the glass substrates and amorphous-silicon steps and then dried immediately after printing. Post-printing, samples are dull and have a purple tint due to the nanoparticles still suspended in ethylene glycol. Samples were considered dry when the ethylene glycol is completely removed by a combination of evaporation or pyrolysis. Upon removal of the ethylene glycol, the films become metallic and reflective. The samples were heated in air to $80^{\circ} \mathrm{C}$ at $3^{\circ} \mathrm{C}-\mathrm{min}^{-1}$, isothermally held overnight ( 12 hours) to ensure the films were completely dried, and then cooled to room temperature at $3^{\circ} \mathrm{C}-\mathrm{min}^{-1}$. Gradual heating/cooling the samples prevents crack formation by reducing the film's thermal shock. It has also been observed that the gradual heating/cooling improves film thickness uniformity [5]. It is likely that the total drying time could be reduced through process optimization, but this was not the focus of the current work.

\section{LASER TRIMMING}

A Resonetics laser milling tool was used to remove nonuniform thickness at the silver film's edges. A $150 \mathrm{~mW}, 248 \mathrm{~nm}$ laser pulse with $15 \%$ attenuation was used to ablate the silver film. The laser spot size and shape was a $100 \mu \mathrm{m}$ square. The laser trimming can be done either after drying or after sintering the nanoparticle film. No discernable differences in mechanical properties have been observed between the nanoparticle films that have been laser trimmed after drying and the films that have been laser trimmed after sintering.

\section{SINTERING SILVER FILMS}

After drying, the samples were sintered on a hotplate to fuse the nanoparticles. Two sintering parameters have been explored: peak sintering temperature and dwell time. For the peak sintering temperature, the samples were heated to the peak temperature of $125^{\circ} \mathrm{C}, 150^{\circ} \mathrm{C}, 250^{\circ} \mathrm{C}$, and $350^{\circ} \mathrm{C}$ at $3^{\circ} \mathrm{C}-\mathrm{min}^{-1}$, isothermally held for 3 hours, and then ramped down to room temperature at $3^{\circ} \mathrm{C}-\mathrm{min}^{-1}$ in an air environment. To explore the dwell time, the samples were heated to $250^{\circ} \mathrm{C}$ at $3^{\circ} \mathrm{C}-\mathrm{min}^{-1}$, isothermally held for $0,1,3$, and 6 hours, and cooled to room temperature at a maximum $3{ }^{\circ} \mathrm{C}-\mathrm{min}^{-1}$ in an air environment.

\section{RELEASING STRUCTURES}

The final step is releasing the structures by removing the remaining amorphous-silicon. The structures were etched in ten $60 \mathrm{~s}$ cycles of $\mathrm{XeF}_{2}$. In a few cases, particularly if the silver structural layer was thinner than $1 \mu \mathrm{m}$, stiction was observed; however this stiction could be overcome by mechanical methods. An SEM of a released silver cantilever is shown in Figure 2.

\section{RESULT AND CHARACTERIZATION}

As a demonstration of the process, cantilevers of $500 \mu \mathrm{m}$ (length) and $50 \mu \mathrm{m}$ or $100 \mu \mathrm{m}$ (width) were fabricated. The thickness of the silver nanoparticle film was typically $2.8 \mu \mathrm{m}$. The amorphous silicon was $1.1 \mu \mathrm{m}$ thick. The amorphous-silicon thickness was measured using optical profilometry prior to printing the silver nanoparticle ink. The silver film thickness was determined by contact profilometry of the anchor region after releasing the structures.

Using a Hysitron Triboindenter, two modes are used to determine the film's mechanical properties: beam-deflection and nanoindentation. During beam-deflection mode, known forceloads are applied to the cantilevers and the resulting mechanical deflections are measured. Repeating these measurements at predetermined spacing between the deflection points, the thirdorder dependence on the cantilever's beam length is removed and the nanoparticle films' elastic moduli is calculated using the cantilever's width and thickness.

In nanoindentation mode, a diamond tip is used to indent the silver film. While the film mainly experiences plastic deformation, a portion of the indent is elastically deformed. By measuring the elastic rebound when unloading the diamond tip, the mechanical properties of the film can be determined. The nanoindentations are made on the cantilever anchors to avoid erroneous measurements caused by beam deflection. Depictions of both modes are summarized in Figure 3.
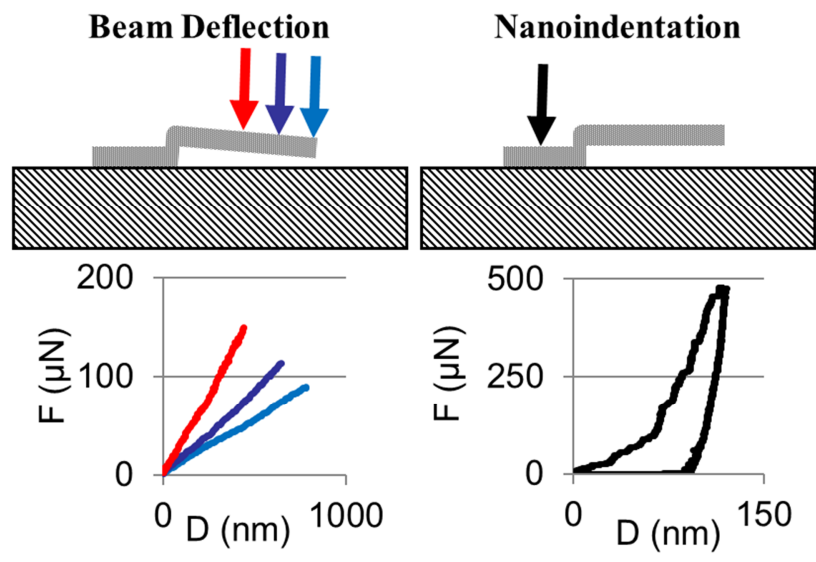

$\mathrm{E}=22.40 \pm 2.76 \mathrm{GPa}$

$\mathrm{E}=\mathbf{2 2 . 4 4} \pm 5.81 \mathrm{GPa}$

Figure 3. Characterization methods and elastic moduli from films sintered at $150^{\circ} \mathrm{C}$ for 3 hours. The elastic modulus is consistent between both methods of characterization. Error depicted in each characterization represents 1 standard deviation from characterizing 5 cantilevers and 49 indents, respectively.

Samples sintered while varying peak sintering temperature and dwell time respectively are summarized in Figure 4 and 5. There is a monotonic relationship between peak sintering temperature and the resulting elastic modulus. Dwell time when sintering at $250^{\circ} \mathrm{C}$ does not seem to significantly affect elastic modulus. The beam deflection calculations assume a clamped boundary condition at the anchor and a rectangular beam dimension where the width and thickness of the beam are uniform. Because the results from both measurement modes are 


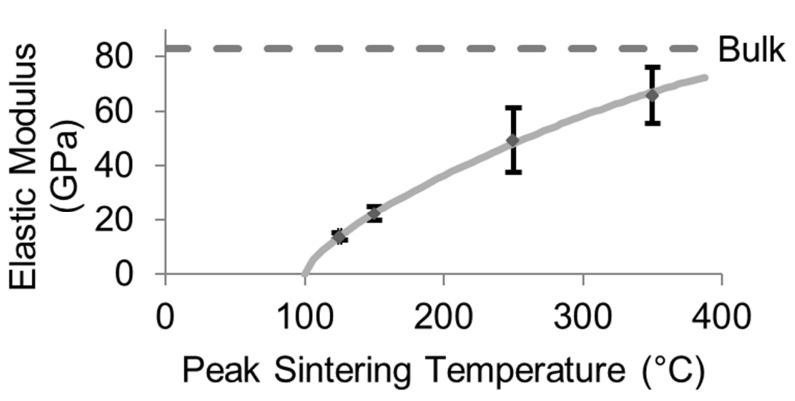

Figure 4. Modulus (beam deflection) vs. Sintering Temperature. Samples were sintered for 3 hours with ramp rate of $3^{\circ} \mathrm{C}-\mathrm{min}^{-1}$. $>3$ cantilevers per test condition were characterized and error bars represent 1 standard deviation of the measurements. Moduli were also measured using nanoindentation and were consistent with beam deflection results. The grey solid line represents values predicted by sintering model.

consistent with each other, it is assumed the characterization methods can be used interchangeably.

\section{ANALYSIS AND MODELING}

Previous silver nanoparticle sintering models have suggested the film's densification corresponds to an increase in cross-sectional area, thus correlating to a monotonic increase in electrical conductivity [6]. Similarly, it can be inferred that an increase in cross-sectional area is proportional to the reduction of the void volume inside the film, which is consistent with SEM microscopy previously presented [3]. As suggested in previous reports, two sintering parameters dominate the processproperty relationship of sintered metal nanoparticle films: sintering temperature and dwell time [6].

\section{Sintering Temperature}

Sintering models in powdered metallurgy suggest the relationship between elastic modulus and void volume is

$$
\frac{E}{E_{0}}=\left(1-\frac{p}{p_{0}}\right)^{f}
$$

where $E_{0}$ is the elastic modulus of the bulk material, $p$ is the pore concentration, $p_{0}$ is the initial pore concentration, and $f$ is a parameter constant dependent on the grain morphology and pore geometry [7]. In this case, $p$ is defined as the fractional void volume between the nanoparticles. As such, if the particles are initially packed in a simple cubic formation, $p_{0}=1-\frac{\pi}{6} \approx$ 0.477 ; similarly, if the particles are packed in a face-centered cubic arrangement, $p_{0}=1-\frac{\pi}{3 \sqrt{2}} \approx 0.260$. As mentioned in [3], it is believed that material and sintering process are directly related to the sintered nanoparticle film's nanostructure and therefore directly affects $f$. Using the standard 2-sphere sintering representation [6,8] and particle melting temperatures, a correlation between the distance between the sphere centers $L$ and melting temperatures can be established. Using equations relating particle size to melting temperature as reported by Buffat et al. [9], it can be assumed that:

$$
\frac{L}{L_{0}}=1-\alpha_{1} r_{l}(T)
$$

where $r_{l}(T)$ is the fractional thickness of the liquid shell surrounding the metal nanoparticle at temperature $T, L$ is the distance between the particle centers, $L_{0}$ is the original distance between the two particle centers, and $\alpha_{1}$ is an empiricallyderived constant to account for deviations in particle boundaries

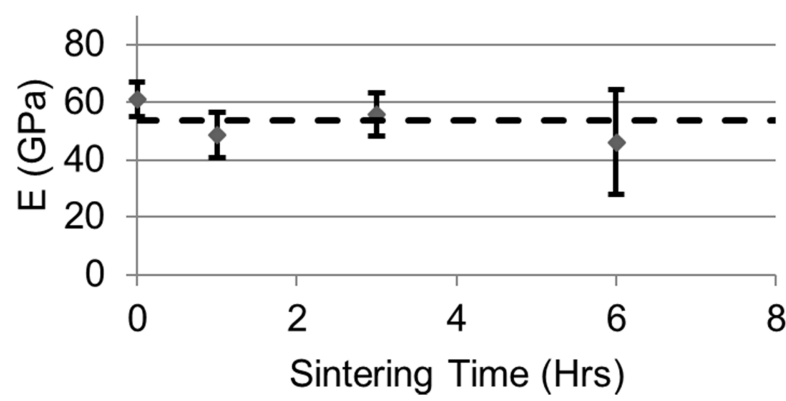

Figure 5. Modulus (nanoindentation) vs. Sintering Time. Samples were sintered at $250^{\circ} \mathrm{C}$ with ramp rate of $3^{\circ} \mathrm{C}-\mathrm{min}^{-1}$. Dashed line represents average modulus of all samples. 49 indentations per test condition were performed and error bars represent 1 standard deviation. Unlike sintering temperature, sintering time does not significantly affect modulus at the time scale investigated.

caused by conservation of volume. Because pores can be seen as void volume between the spherical nanoparticles, we assume that $L$ and $p$ are proportional:

$$
\frac{p}{p_{0}} \propto\left(\frac{L}{L_{0}}\right)^{3}
$$

By substituting (2) and (3) into (1), we obtain

$$
\frac{E}{E_{0}}=\left(1-\left(1-\alpha_{2} r_{l}(T)\right)^{3}\right)^{f}
$$

where $\alpha_{2}$ incorporates both $\alpha_{1}$ and the proportional constant between $p / p_{0}$ and $L / L_{0}$. Fitting the data obtained from varying the peak sintering temperature, $\alpha_{2}$ and $f$ have been determined to be 1.76 and 0.6982 , respectively. The elastic moduli calculated using this sintering model is represented by the grey solid line in Figure 4.

\section{Time Dependence of Sintering}

As shown in Figure 5, no significant relationship between dwell time and resulting elastic modulus has been determined. It is hypothesized that the slow heating and cooling rates used to prevent thermal shock in the silver nanoparticle films causes a quasi-static state in which the film will no longer densify until the sintering temperature increases. As such, it is assumed that the process to date is time-insensitive and dwell time is not a factor in the process-property relationship. This could potentially be different at lower sintering temperatures where silver self-diffusivity is considerably lower [10].

\section{CONCLUSIONS}

In summary, we presented a methodology to characterize the process-property relationship for silver nanoparticles. Printed cantilevers were fabricated while exploring peak sintering temperature and dwell time. Using a 2-sphere representation, we hypothesize the silver nanoparticle films exhibit a nanostructure-elastic modulus relationship of

$$
\frac{E}{E_{0}}=\left(1-\left(\frac{L}{L_{0}}\right)^{3}\right)^{0.6982}
$$

Furthermore we established that peak sintering temperature significantly affects the film's nanostructure and that they have a relationship of

$$
\frac{L}{L_{0}}=1-1.76 r_{l}(T)
$$

Combining equations 5 and 6 , we have established a model explaining the monotonic relationship between peak sintering temperature the sintered nanoparticle film's resulting elastic 
modulus. However, exploration of dwell times at $250^{\circ} \mathrm{C}$ showed no significant relationship between dwell time and elastic modulus, implying dwell time does not play a significant role in the current microfabrication process.

Additional studies will be conducted to further explore the process-property parameter space. One particularly promising investigation is to vary the environment during sintering because increasing partial pressures of oxygen have been shown to change the surface tension of molten silver [11] and thus could potentially affect the melting temperatures of silver nanoparticles. Another proposed study is to explore dwell time at lower sintering temperatures to determine whether the process-to-date remains time-insensitive as silver self-diffusivity decreases. Other studies in consideration include characterizing films made from larger/smaller silver nanoparticles and nanoparticles made out of alternative metals such as gold to validate whether the methodology is transferrable to other metal nanoparticle inks.

\section{ACKNOWLEDGEMENTS}

This work was sponsored by DARPA N/MEMS Fundamentals Program, Hewlett-Packard, and the Iberian Nanotechnology Laboratory-MIT Program and was carried out in part through the use of MIT's Microsystems Technology Laboratories and DMSE NanoLab. We thank Hewlett-Packard for the loan of the TIPS devices. TIPS is a prototype laboratory device used internally at Hewlett-Packard and with selected research partners. Travel support has been generously provided by the Transducer Research Foundation.

\section{REFERENCES}

[1] J. Chen, V. Leblanc, S. H. Kang, P. J. Benning, D. Schut, M. A. Baldo, M. A. Schmidt, V. Bulović, "High Definition Digital Fabrication of Active Organic Devices by Molecular Jet Printing," Adv. Func. Mater., vol. 40, pp. 2722-2727, 2007

[2] V. Subramanian, J.M.J Frechet, P.C. Chang, D.C. Huang, J.B. Lee, S.E. Molesa, A.R. Murphy, D.R. Redinger, S.K. Volkman, "Progress Toward Development of All-Printed RFID Tags: Materials, Processes, and Devices," Procs. IEEE, vol. 93, pp.1330-1338, 2005.

[3] E.W. Lam, H. Li, M.A. Schmidt, "Silver nanoparticle structures realized by digital surface micromachining," IEEE Tranducers Digest of Papers, June 2009, pp. 16981701.

[4] E.S. Park, Y.P. Chen, T.-J. King Liu, V. Subramanian, "Printed Micro-Electro-Mechanical Switches," 2011 International Electron Devices Meeting, Washington D.C., 2011, pp. 665-668.

[5] C. Kim, M. Nogi, K. Suganuma, "Electrical conductivity enhancement in inkjet-printed narrow lines through gradual heating," J. Micromech. Microeng., vol. 22, p. 035016, 2012.

[6] J.R. Greer, R.A. Street, "Thermal cure effects on electrical performance of nanoparticle silver inks," Acta Mater., vol. 55, pp. 6345-6349, 2007.

[7] J. Kováčik, "Correlation between Young's modulus and porosity in porous materials," J. Mater. Sci. Lett., vol. 18, pp. 1007-1010, 1999.

[8] W.D. Kingery, M. Berg, "Study of the initial stages of sintering solids by viscous flow, evaporation-condensation, and self-diffusion," J. Appl. Phys., vol. 26(10), pp. 12051212, 1955.

[9] Ph. Buffat, J.-P. Borel, "Size effect on the melting temperature of gold particles," Phys. Rev. A, vol. 13(6), pp. 2287-2298, 1976.

[10] J. Sommer, C. Herzig, "Direct determination of grainboundary and dislocation self-diffusion coefficienets in silver from experiments in type-C kinetics," J. Appl. Phys., vol. 72(7), pp. 2758-2766.

[11] S. Ozawa, K. Morohoshi, T. Hibiya, H. Fukuyama, "Influence of oxygen partial pressure on surface tension of molten silver,” J. Appl. Phys., vol. 107 p. 014910, 2010.

\section{CONTACT}

*E.W. Lam, tel: +1-617-253-0224; EWLAM@MIT.edu 


\section{CVD HAFNIUM DIBORIDE AS A CONTACT MATERIAL FOR NANOELECTROMECHANICAL SWITCHES \\ W.S. Lee ${ }^{l^{*}}$, A.N. Cloud ${ }^{2}$, J. Provine ${ }^{l}$, N. Tayebi ${ }^{l}$, R. Parsa ${ }^{1}$, S. Mitra ${ }^{l}$, H.-S. P. Wong ${ }^{l}$, J. R. Abelson, ${ }^{2}$ and R.T. Howe ${ }^{l}$ \\ ${ }^{1}$ Electrical Engineering Department, Stanford University, Stanford, California, USA \\ ${ }^{2}$ Department of Materials Science and Engineering, University of Illinois at Urbana-Champaign, Urbana, Illinois, USA}

\section{ABSTRACT}

This paper presents the first usage of hafnium diboride $\left(\mathrm{HfB}_{2}\right)$ deposited via chemical vapor deposition as the contact material for nanoelectromechanical (NEM) switches. $\mathrm{HfB}_{2}$ is an excellent candidate for NEM switches due to its reasonable conductivity, high hardness, and high melting point. $\mathrm{HfB}_{2}$ was deposited conformally at $250^{\circ} \mathrm{C}$ onto the sidewalls of laterally actuated, polysilicon switches. $\mathrm{HfB}_{2}$ coated switches showed sharp switching characteristics when tested in a nitrogen ambient. The contact resistance was initially $29.6 \mathrm{M} \Omega$ but decreased to $1.43 \mathrm{k} \Omega$ by adding a dilute HF dip to remove the surface oxide.

\section{INTRODUCTION}

Complementary metal-oxide-semiconductor (CMOS) scaling has resulted in the mass production and proliferation of microelectronics. Recently, power constraints have become a limiting factor to scaling. Scaling the supply voltage requires simultaneous scaling of the threshold voltage to avoid incurring a delay penalty [1]. Threshold voltage scaling, however, leads to an exponential increase in leakage current. A further reduction in CMOS power consumption requires a device that does not suffer from these same scaling limits such as the nanoelectromechanical (NEM) switch. The NEM switch has zero leakage current, highly scalable actuation voltages, and very sharp switching characteristics $[2,3]$.

A three terminal NEM switch consists of a movable beam electrode, a fixed gate electrode, and a fixed drain electrode. Initially, the gate-to-beam voltage, $V_{G B}$, is zero, and the beam is electrically isolated from the drain so the drain-to-beam current, $I_{D B}$, is zero. As $V_{G B}$ increases, the electrostatic force is balanced by the elastic force of the beam. As $V_{G B}$ continues to increase, the beam can no longer balance the electrostatic force so the beam pulls into the drain, and current flows between the drain and beam. This voltage is called the pull-in voltage, $V_{P I}$, and the switch is in the on-state. As $V_{G B}$ decreases, the electrostatic force also decreases. Due to the reduced gap between the beam and gate in the on state, $V_{G B}$ must decrease below $V_{P I}$ for the beam to pull-out. This voltage is called the pull-out voltage, $V_{P O}$. A five terminal NEM switch has an additional gate and drain electrode that enables bi-directional switching.

Unfortunately, NEM switches suffer from a long mechanical delay. Hybrid systems that combine CMOS with NEM switches have been proposed that take advantage of the low power characteristics of NEM switches while mitigating their long switching delay. Examples include FPGA routing [4], CMOS power gating [5], and SRAM cells [6]. The FPGA routing application showed substantial power and area reductions without a delay penalty by utilizing switches with $2 \mathrm{k} \Omega$ contact resistances [7]. This contact resistance requirement is much higher than the sub- $1 \Omega$ resistances required by RF MEM switch applications [8] and enables the use of harder, more robust contact materials.

We investigate hafnium diboride $\left(\mathrm{HfB}_{2}\right)$ as a contact material for NEM switches. $\mathrm{HfB}_{2}$ is a conductive ceramic with a hardness, elastic modulus, and melting point of $20 \mathrm{GPa}, 400 \mathrm{GPa}$, and $3250^{\circ} \mathrm{C}$

[9], respectively, that can be deposited via chemical vapor deposition (CVD) at back-end-of-line compatible temperatures. Since the deposited film is very conformal, it can be used to coat trenches and form sidewalls [9]. $\mathrm{HfB}_{2}$ has been shown to have higher scratch resistance with a lower coefficient of friction than titanium nitride [10] and has been used as a protective coating on a silicon tip showing negligible wear after $5 \times 10^{9} \mu \mathrm{m}$ of sliding [11]. Hard coatings are desirable for NEM switches because they can suppress plastic deformation of asperities, which leads to variations in contact area, contact resistance, and surface forces.

\section{CONTACT MECHANICS}

NEM switches are actuated either laterally or vertically. We characterize $\mathrm{HfB}_{2}$ with lateral NEM switches because the fabrication process requires a single $\mathrm{HfB}_{2}$ deposition and two mask layers. The fabrication will be further described in the next section. The shape of the contacting surface between the beam and the drain is defined by the sidewall roughness of the polysilicon reactive ion etch. We approximate the contacting surface as a single asperity with a radius, $r$, which is on the order of $10 \mathrm{~nm}$. The contact asperity radius contributes to both the Maxwell resistance, $R_{M}$, and Sharvin resistance, $R_{S}$, components of the total contact resistance, $R_{C}$. They originate from electron lattice scattering and electron boundary scattering, respectively. For a round asperity that undergoes elastic deformation, $R_{C}$ is given by $[12,13]$

$$
R_{C}=\gamma\left(\frac{\lambda}{r}\right) R_{M}+R_{S}=\frac{1+0.83\left(\frac{\lambda}{r}\right)}{1+1.33\left(\frac{\lambda}{r}\right)} \frac{\rho}{2 r}+\frac{4 \rho \lambda}{3 \pi r^{2}}
$$

where $\rho$ is the resistivity of the material, $\lambda$ is the electron mean free path, and $\gamma$ is a fitting function used to transition between the Maxwell and Sharvin resistance regimes. The resistivity of $\mathrm{HfB}_{2}$ was measured to be $303 \mu \Omega-\mathrm{cm}$. Since $\lambda$ is unknown for $\mathrm{HfB}_{2}$, we estimate the contact resistance for two cases. The first case is when the contact asperity is much larger than the electron mean free path (i.e., $\lambda / r \approx 0$ ). The second case is when the contact asperity is roughly the same size as the electron mean free path (i.e., $\lambda / r \approx 1$ ). Figure 1 shows the contact resistance for each of these cases for contact radii ranging from $1 \mathrm{~nm}$ to $50 \mathrm{~nm}$. For a $10 \mathrm{~nm}$ radius, the estimated contact resistance is $150 \Omega$ and $250 \Omega$, for $\lambda / r=0$ and $\lambda / r$ $=1$, respectively. These estimated contact resistances are only based on the contact geometry and do not account for any insulating layer that may appear on the surface of the contact. The measured contact resistance will be higher than these values if there is an oxide or contamination layer on the surface of the contact.

\section{DESIGN AND FABRICATION}

The lateral switch fabrication process flow initially follows the methods presented at Hilton Head 2010 [14] where a $1 \mu \mathrm{m}$ insitu doped polysilicon structural layer is deposited on a $2 \mu \mathrm{m}$ LTO sacrificial layer and annealed. The polysilicon layer is patterned using a photoresist mask exposed with an i-line stepper. The 


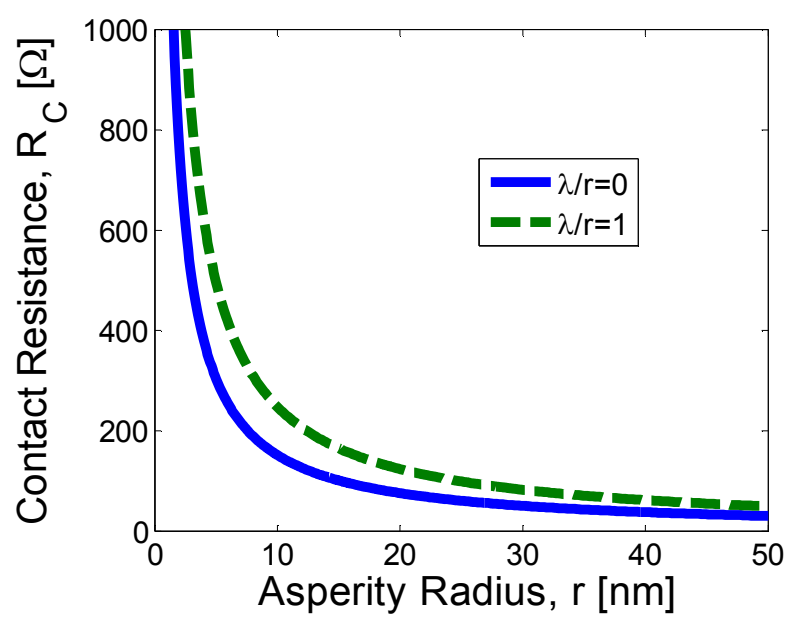

Figure 1: Contact resistance, $R_{C}$, for $\mathrm{HfB}_{2}$ as a function of contact radius for $\lambda / r \approx 0$ (i.e., contact asperity radius is much larger than the electron mean free path) and $\lambda / r \approx 1$ (i.e., the contact asperity radius is approximately the same size as the electron mean free path).

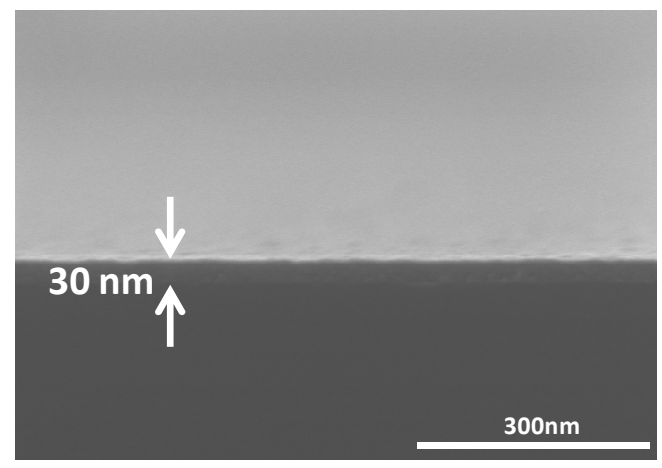

Figure 2: $30 \mathrm{~nm}$ of $\mathrm{HfB}_{2}$ film with subnanometer surface roughness deposited on crystalline silicon by chemical vapor deposition.

pattern is transferred to the polysilicon layer using a reactive ion etch (RIE) (12mTorr, $25 \mathrm{sccm} \mathrm{Cl}_{2}, 140 \mathrm{sccm} \mathrm{HBr}, 3 \mathrm{sccm} \mathrm{O}$ ).

Instead of coating the devices with sputtered platinum as in [14], the devices are coated in $\mathrm{CVD} \mathrm{HfB}_{2}$ at $250^{\circ} \mathrm{C}$ using $\mathrm{Hf}\left(\mathrm{BH}_{4}\right)_{4}$ precursor in an ultra high vacuum chamber as described in [15]. Figure 2 shows an SEM cross section of a typical $30 \mathrm{~nm}$ film deposited on a silicon substrate. A contact aligner is used to define the pads for the devices. The $\mathrm{HfB}_{2}$ is etched using a directional, chlorine-based RIE (5mTorr, 40sccm $\mathrm{BCl}_{3}, 10 \mathrm{sccm} \mathrm{Cl}_{2}, 10 \mathrm{sccm}$ $\mathrm{Ar})$ that removes the $\mathrm{HfB}_{2}$ from the exposed lateral surfaces and electrically isolates the various terminals. A continuous layer of $\mathrm{HfB}_{2}$ remains on the device sidewalls and on the photoresist protected pads of the devices. The photoresist is stripped using an $\mathrm{O}_{2}$ plasma followed by a 10 minute acetone soak and isopropanol rinse.

The switches are released by etching the LTO for 15 minutes in a 5:3 mixture of buffered oxide etch $\left(6\right.$ parts $40 \% \mathrm{NH}_{4} \mathrm{~F}$ to 1 part $49 \% \mathrm{HF}$ ) to glycerin, which obtains a good etch selectivity between the LTO and the $\mathrm{HfB}_{2}$. The switches were dried after the BOE/glycerin etch using a critical point dryer (CPD) to avoid stiction. The final released device is shown in Figure 3. The zoomed-in view of the contacting region in Figure 3 shows the

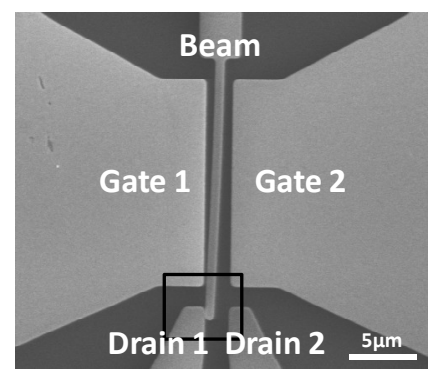

(a)

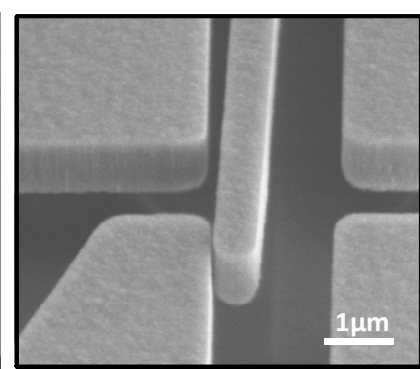

(b)
Figure 3: (a) $\mathrm{HfB}_{2}$ coated relay that is actuated under SEM; (b) zoomed in view of the contact region.

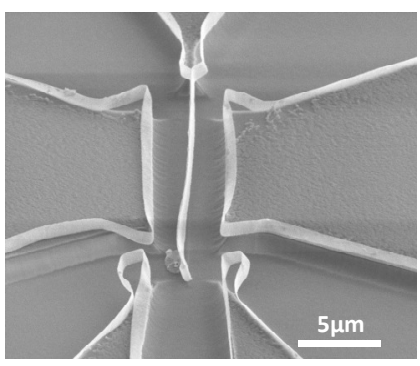

(a)

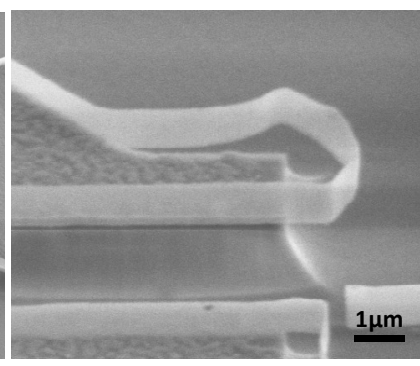

(b)
Figure 4: (a) Zoomed-out view of an $\mathrm{HfB}_{2}$ coated switch that was placed in $\mathrm{XeF}_{2}$ to remove the polysilicon and confirm the presence of $\mathrm{HfB}_{2}$. (b) Zoomed-in view that shows very smooth $\mathrm{HfB}_{2}$ sidewalls.

high conformality of the $\mathrm{HfB}_{2}$ coating. The sidewall surface roughness is equivalent to the bare polysilicon devices and is much smoother than the sputtered platinum coated devices [14]. The presence of $\mathrm{HfB}_{2}$ on the sidewalls was confirmed by etching a device in $\mathrm{XeF}_{2}$ that removed the polysilicon layer without attacking the $\mathrm{HfB}_{2}$ sidewalls as shown in Figure 4.

\section{RESULTS}

The switches were tested in a nitrogen glovebox at room temperature using a Keithley 4200 parameter analyzer. The drainbeam voltage, $V_{D B}$, was set to $1 \mathrm{~V}$, the beam voltage, $V_{B}$, was set to $0 \mathrm{~V}$, and the current compliance was set to $100 \mathrm{nA}$ to protect the contact from welding and Joule heating. $V_{G B}$ was swept from $0 \mathrm{~V}$ to $60 \mathrm{~V}$, and no drain current was measured with the $1 \mathrm{~V} V_{D B}$. The drain-beam voltage was then increased to $5 \mathrm{~V}$, the $V_{G B}$ sweep was repeated, and the current reached the 100nA compliance with sharp switching characteristics as shown in (Figure 5a). To maintain the current compliance, the parameter analyzer adjusts the voltage on both the beam and drain electrodes. By measuring the voltages required to maintain the current compliance, the voltage across the contact and the contact resistance can be calculated. For these initial devices, the contact resistance was $29.6 \mathrm{M} \Omega$, which is much higher than the predicted contact resistance of $250 \Omega$. On the subsequent voltage sweep, the current compliance was increased to $1 \mu \mathrm{A}$ (Figure $5 \mathrm{~b}$ ) while maintaining the $V_{D B}$ at $5 \mathrm{~V}$. The contact resistance decreased to $171 \mathrm{k} \Omega$. For the third voltage sweep, $V_{D B}$ voltage was decreased to $1 \mathrm{~V}$ (Figure $5 \mathrm{c}$ ), the $1 \mu \mathrm{A}$ current compliance was still reached, and the contact resistance decreased to $8.9 \mathrm{k} \Omega$. By passing $1 \mu \mathrm{A}$ of current through the contact, the contact resistance decreased from $29.6 \mathrm{M} \Omega$ to $8.9 \mathrm{k} \Omega$ while the minimum voltage across the contact decreased from $3.0 \mathrm{~V}$ to 


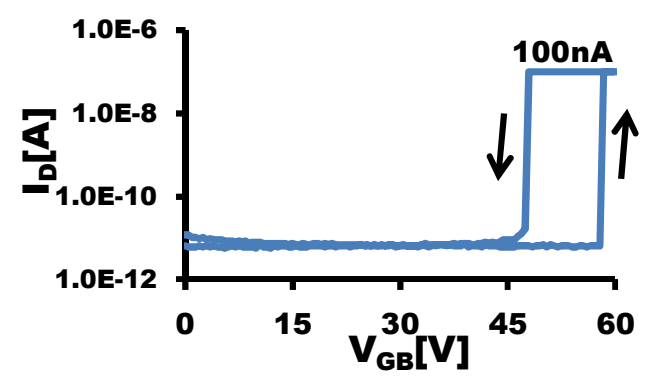

(a)

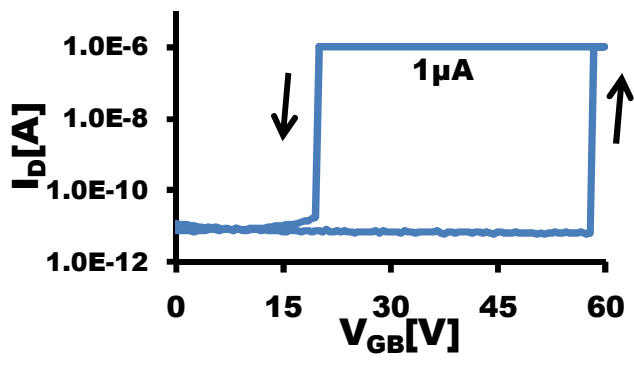

(b)

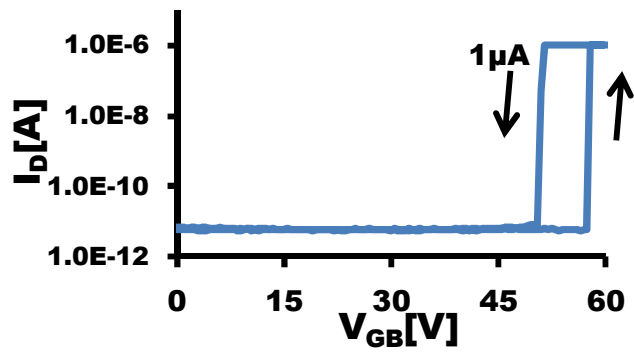

(c)

Figure 5 - (a) Initial IV sweep of $\mathrm{HfB}_{2}$ coated relay that requires high drain voltage to breakdown insulating film $\left(V_{D B}=5 \mathrm{~V}\right.$, Current Compliance=100nA). (b) Subsequent IV sweep with increased current compliance $\left(V_{D B}=5 \mathrm{~V}\right.$, Current Compliance $\left.=1 \mu \mathrm{A}\right)$. (c) Subsequent sweep with decreased drain voltage with the higher current maintained $\left(V_{D B}=1 \mathrm{~V}\right.$, Current Compliance $\left.=1 \mu \mathrm{A}\right)$. Insulating film broken down at contact by high voltage and high current. Actual $V_{D B}$ were less than the setpoint to maintain current compliance.

$8.9 \mathrm{mV}$. These results were indicative of an insulating surface film that experienced breakdown with sufficient current flow.

The $\mathrm{HfB}_{2}$ surface modifications were further characterized by performing a voltage sweep on an unpatterned $\mathrm{HfB}_{2}$ film. The current flow was initially very small. When the applied voltage was $2.8 \mathrm{~V}$, breakdown of the surface was observed, and the current increased significantly (Figure 6a). A second voltage sweep showed only resistive current flow without any breakdown voltage. These results matched the device results showing where the initial contact voltage was measured to be $3.0 \mathrm{~V}$ to observe current flow.

To determine the source of the breakdown voltage, X-ray photoelectron spectroscopy (XPS) was performed on a sample of $\mathrm{HfB}_{2}$ that had been exposed to air for three months. The analysis confirmed the presence of an oxide layer on the surface. As shown in Figure $7 \mathrm{a}$, approximately $80 \%$ of the Hf signal and $50 \%$ of the B

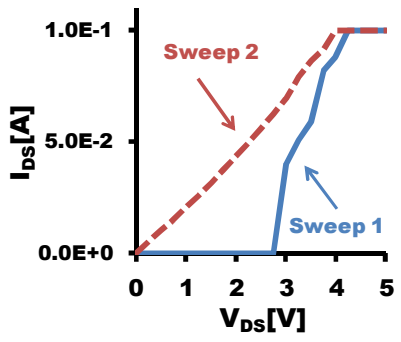

(a)

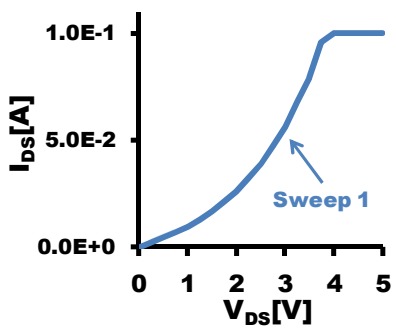

(b)
Figure 6-(a) IV characteristics of $\mathrm{HfB}_{2}$ film without HF dip and (b) with $30 \mathrm{~s}$ dip in 50:1 HF. Two probes were placed on an $\mathrm{HfB}_{2}$ pad, and the voltage between them was swept.

(a)
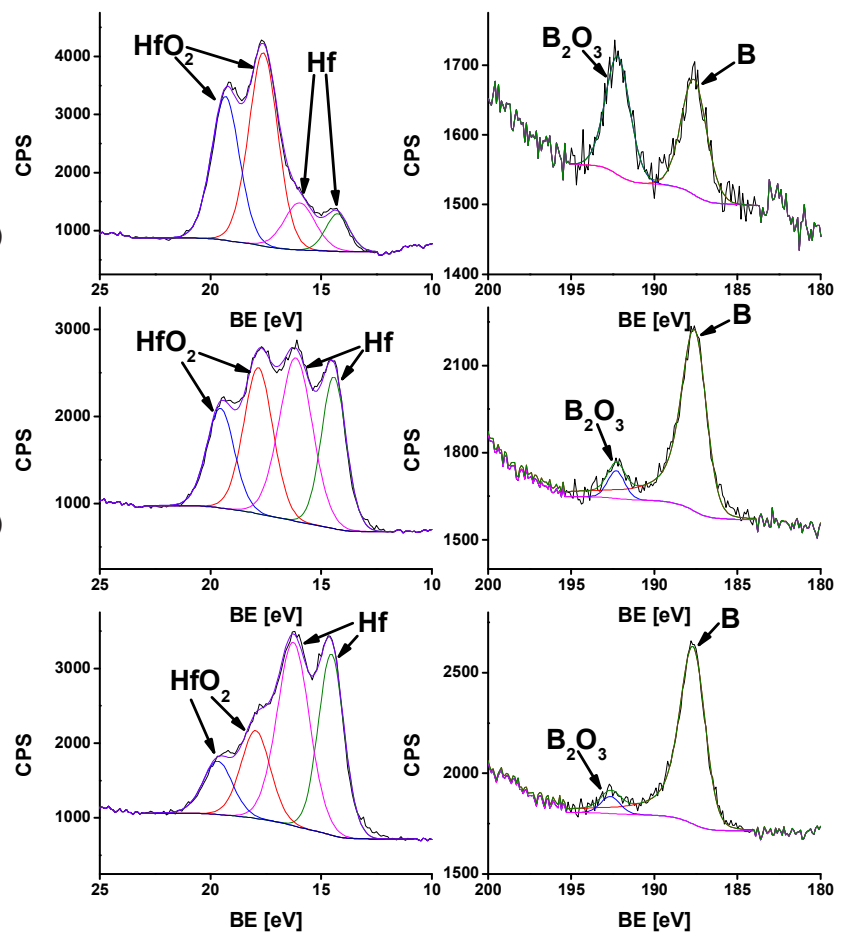

Figure 7 - (a) XPS spectra of a $\mathrm{HfB}_{2}$ film that was exposed to air for 3 months, (middle) Same film after 15 minute etch in glycerin/BOE solution, (bottom) Same film after 15 minute etch in glycerin/BOE and 30s dip in 50:1 HF. The oxygen content of the surface layer is significantly reduced by the etching steps.

signal have binding energy peaks indicative of oxides. The fabricated switches are exposed to a 15 minute etch in the $\mathrm{BOE} / \mathrm{glycerin}$ solution to release the devices that could also affect the surface oxide layer. Therefore, the $\mathrm{HfB}_{2}$ sample was exposed to this same solution for 15 minutes and reanalyzed under XPS. The oxygen content was significantly reduced as shown in Figure $7 \mathrm{~b}$, but it was still present. To further reduce the oxide layer, a 30s dip in 50:1 HF following the BOE/glycerin etch was added. The oxygen content continued to decrease as shown in Figure 7c. The reduced oxygen content is closer to the less than $1 \%$ oxygen content found in the as-deposited film [9]. Based on the XPS results, a $60 \mathrm{~s}$ dip in $50: 1 \mathrm{HF}$ was added to the release process between the BOE/glycerin step and the CPD step. After the CPD step, the samples are immediately stored in nitrogen. The two probe test was repeated and the breakdown was eliminated (Figure $6 b)$. 


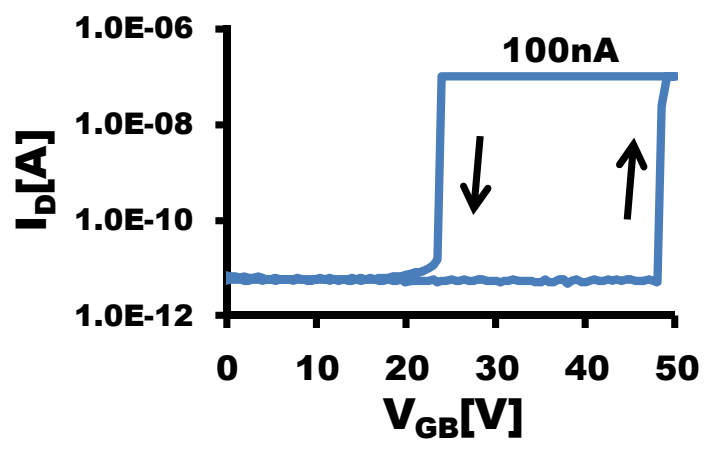

Figure $8-I V$ characteristics of $\mathrm{HfB}_{2}$ coated relay treated with $60 \mathrm{~s}$ etch in 50:1 HF. Required drain voltage is reduced $\left(V_{D B}=1 \mathrm{~V}\right)$ without requiring an initial high drain voltage sweep.

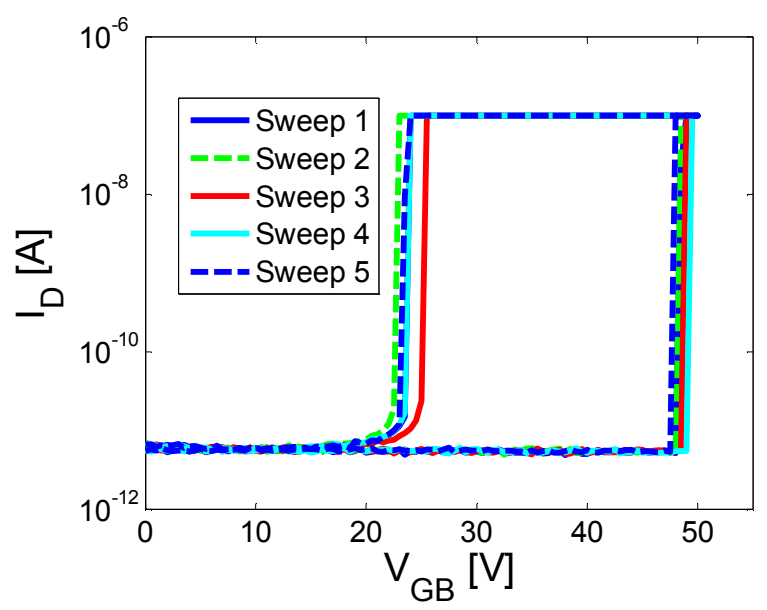

Figure 9 - Five cycles of $\mathrm{HfB}_{2}$ coated switch with $V_{D B}$ set to $1 \mathrm{~V}$ with 100nA current compliance. The contact resistance was measured to be $1.43 \mathrm{k} \Omega$ based on the measured drain-to-beam voltage when actuated.

With the added dilute HF dip, the 100nA current compliance was reached with a $1 \mathrm{~V}$ drain bias without requiring high current to breakdown an insulating film (Figure 8). The measured voltage across the contact was $143 \mu \mathrm{V}$ corresponding to a measured contact resistance of $1.43 \mathrm{k} \Omega$. The switch showed correct operation over five cycles (Figure 9).

\section{CONCLUSION}

$\mathrm{HfB}_{2}$ coated polysilcon switches were fabricated and tested. The switches initially had a contact resistance of $29.6 \mathrm{M} \Omega$, which was attributed to the presence of a surface oxide. By removing this oxide, the contact resistance decreased to $1.43 \mathrm{k} \Omega$, which is close to the $250 \Omega$ calculated contact resistance. The measured contact resistance may be higher due to either the contacting asperity being smaller than the estimated $10 \mathrm{~nm}$ or due to the presence of a thin insulating film on the surface of the contact.

These preliminary results show that $\mathrm{HfB}_{2}$ is a promising NEM switch contact material, but further investigation into long term cycling and device statistics are required. Furthermore, future $\mathrm{HfB}_{2}$ switches should be encapsulated in an oxygen free ambient to prevent oxidation.

\section{ACKNOWLEDGMENTS}

Fabrication work performed at the Stanford Nanofabrication Facility (member of the National Nanotechnology Infrastructure Network) supported by the NSF under Grant ECS-9731293. $\mathrm{HfB}_{2}$ film deposition took place at the University of Illinois at UrbanaChampaign. Materials characterization was carried out in part in the Frederick Seitz Materials Research Laboratory Central Facilities, University of Illinois, which is partially supported by the U. S. Department of Energy under grants DEFG02-07ER46453 and DE-FG02-07ER46471. The work was supported by the DARPA MTO program "Nano Electro Mechanical Computers (NEMS)" (Contract number: NBCH 1090002, Program manager: Dr. T. Akinwande).

\section{REFERENCES}

[1] V. Kursen et al., Multi-Voltage CMOS Circuit Design, John Wiley \& Sons, Ltd., England, 2006.

[2] F. Chen et al., "Demonstration of Integrated Micro-ElectroMechanical Switch Circuits for VLSI Applications", IEEE ISSCC 2010 pp. 150-152.

[3] K. Akarvardar et al., "Design Considerations for Complementary Nanoelectromechanical Logic Gates", IEDM 2007 pp. 299-302.

[4] Y. Zhou et al., "Low Power FPGA Design Using Hybrid CMOS-NEMS Approach", Proc. Intl. Symp. Low Power Electronics and Design, 2007, pp. 14-19.

[5] H. Fariborzi et al., "Analysis and Demonstration of MEMRelay Power Gating”, Custom Integrated Circuits Conference (CICC) (2010) pp. 1-4

[6] S. Chong et al., "Nanoelectromechanical (NEM) Relays Integrated with CMOS SRAM for Improved Stability and Low Leakage", ICCAD 2009, pp. 478-484.

[7] C. Chen et al., "Nano-Electro-Mechanical relays for FPGA Routing: Experimental Demonstration and a Design Technique", IEEE/ACM Design Automation and Test in Europe, Dresden, Germany, March 2012.

[8] G. Rebeiz, RF MEMS: Theory, Design, and Technology, John Wiley \& Sons, Ltd., England, 2004.

[9] S. Jayaraman et al., "HfB ${ }_{2}$ and Hf-B-N Hard Coatings by Chemical Vapor Deposition", Surface and Coatings Technology, 200, 22-23 (2006).

[10] A. Chatterjee et al., "Nanoscratch and Nanofriction Behavior of Hafnium Diboride thin films", Wear, 265 (2008).

[11] N. Tayebi et al., "Ultra Hard $\mathrm{HfB}_{2}$ Metallic Tip-Coatings for Ultra-High Density Probe-Based Storage", ACS Nano [Submitted].

[12] B. Nikolic et al., "Electron Transport Through a Circular Constriction", Physical Review B, 60, 6 (1999).

[13] B. Jensen et al., "Effect of Nanoscale Heating on Electrical Transport in RF MEMS Switch Contacts", JMEMS, 14, 5 (2005).

[14] R. Parsa et al., "Composite Polysilicon-Platinum Lateral Nanoelectromechanical Relays", Technical Digest of the 2010 Solid-State Sensor and Actuator Workshop, Hilton Head Isl., SC, 6/6-10/2010, Transducer Research Foundation, Cleveland (2010), pp. 7-10.

[15] S. Jayaraman et al., "Hafnium Diboride Thin Films by Chemical Vapor Deposition from a Single Source Precursor", J. Vac. Sci. Technol. A 23, 6 (2005).

\section{CONTACT}

*W.S. Lee, tel: +1-650-918-7006; wslee@stanford.edu 


\title{
DIRECT-WRITE NANOLIGHOGRAPHY ON FLEXIBLE SUBSTRATE
}

\author{
Jiyoung Chang ${ }^{l}$, Michael Dommer ${ }^{2}$, Byung Yang Lee ${ }^{3}$ and Liwei Lin ${ }^{l^{*}}$ \\ ${ }^{1}$ Berkeley Sensor \& Actuator Center, U.C. Berkeley, CA, USA \\ ${ }^{2}$ École Polytechnique Fédérale de Lausanne, Switzerland \\ ${ }^{3}$ Department of Bioengineering, U.C. Berkeley, CA, USA
}

\begin{abstract}
This paper presents a mask-less lithography process using direct-write nanofibers via near-field electrospinning on flexible substrate as masking materials in processes such as lift-off, wet-etching and dry-etching. The polymer fibers have diameter of sub-micrometer to micrometer with good adhesion to substrate and chemical sustainability. Key demonstrations successfully realized in this work include sub-micrometer metal gaps, micro heaters and definition of graphene structures on flexible substrates. As such, this direct-write lithography technique could find applications in low-cost microelectronics, including flexible electronics.
\end{abstract}

\section{KEYWORDS}

Mask-less lithography, electrospinning, flexible electronics

\section{INTRODUCTION}

Lithography process is one of the most important and widely used processes in the fabrication of IC (Integrated Circuitry) and MEMS (Microelectromechanical Systems). Large area patterns are generated using photoresist and mask via the conventional optical aligner at moderate cost. Some limitations in the typical lithography processes include low design flexibility and difficulty in processing flexible substrates such as roll-to-roll fabrications. Furthermore, for sub-micrometer features, several advanced lithography solutions have been introduced such as nano imprinting [1], and direct-write femto-second laser mask [2] to realizes small features which are not available in conventional lithography processes. Moreover, e-beam lithography has been used in areas such as electronic circuitry [3] and photonic crystal [4], [5]; AFM (Atomic Force Microscopy) has been used to generate sub-100nm scale features for nanoelectronics [6] and NEMS (Nanoelectromechanical Systems) devices [7]. All of these aforementioned processes are excellent tools in creating ultra-small features in nanolithography processes but they are limited to either very expensive equipment or small area operations.

In this paper, we introduce the direct-write nanofibers on flexible substrates as a "shadow mask" for mask-less lithography in processes such as lift-off, wet-etching and dry-etching with several potential advantages. First, the process does not require photoresist as the hard mask as polymeric fibers are deposited to the substrate by controlling the movement of an $\mathrm{x}-\mathrm{y}$ stage for designated patterns via the near-field electrospinning technique [8]. Second, the process is simple and low-cost without any expensive equipment. The basic setup includes a high voltage power supply and $x-y$ stage to generate continuous fibers with well-controlled deposition precision. Third, the process can deposit fibers on flexible and dielectric substrates for possible applications in new emerging areas such as flexible electronics. Fourth, the diameter of fibers is adjustable with a range from sub-micrometer to $10 \mu \mathrm{m}$. These features make the technology an excellent candidate for mask-less lithography processes. Several proof-of-concept prototype demonstrations have been conducted and characterized in this work and the experimental setup of the details of near-field electrospinning has also been described.

\section{NEAR-FIELD ELECTROSPINNING SETUP Spinning on flexible/dielectric substrate}

Previously, the well-controlled electrospinning process has been conducted on hard and conductive substrate [8] as a strong electrical field between the syringe and substrate is required to generate and control the deposition location of fibers. Here, a modified setup is utilized to enable direct-write of fibers on flexible and dielectric substrates. Figure 1 shows the schematic diagram of the near-field electrospinning process used for flexible or dielectric substrates. In the prototype experiments, a doped silicon wafer or metal coated hard substrate is placed underneath the flexible or dielectric substrate. The conductive substrate is grounded by connecting it to the negative side while the metal syringe tip is connected to the positive side of the power supply, respectively. In contrast to the far-field electrospinning process, a small gap of less than $1 \mathrm{~mm}$ is used in near-field electrospinning process and about $1 \mathrm{kV}$ of voltage is strong enough to generate continuous fibers. The target flexible substrate such as Kapton film is placed on top of the conductive substrate. As illustrated in the electrical field simulation results in the right image of figure 1, the electrical field between syringe and conductive material can be still be established with the existence of the dielectric substrate. Therefore, electrospun fibers can be pulled from the tip of the syringe onto the dielectric substrate.

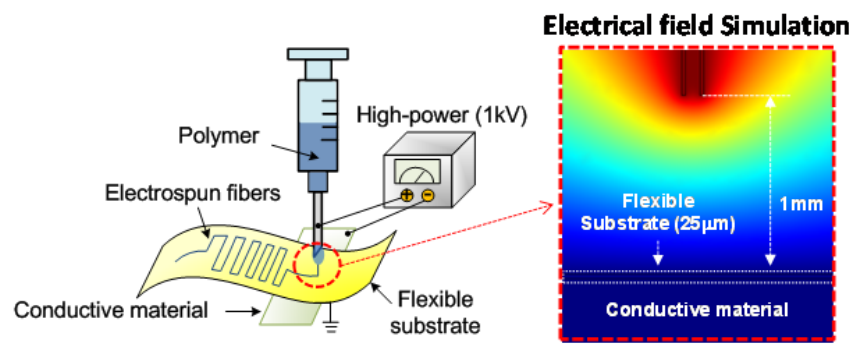

Figure 1. Schematic diagram of the direct-write nanolithography process on a non-conductive flexible substrate. The near-field electrospinning process utilizes high electrical field formed between the metal syringe tip and conductive substrate lying underneath the target dielectric flexible substrate. COMSOL ${ }^{\circledR}$ simulation shows electric field can be established within the flexible dielectric substrate on top of grounded conductive substrate.

\section{DIRECT-WIRTE MASK-LESS LITHOGRAPHY Process demonstrations: Lift-off, Wet-etching, Dry-etching}

Based on the proposed direct-write nanolithography process as shown in figure 1, three lithography-based microfabrication processes including lift-off, wet-etching and dry-etching, are chosen as the demonstration examples in this work. In all experiments, the flexible dielectric film, Kapton, were chosen as the substrate and PEO (Polyethylene Oxide) was selected as the electrospun fiber material. Figure 2 illustrates process flows for lift-off, wet-etching and dry-etching, respectively, using the direct-write mask-less lithography technique. 


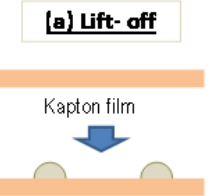

NF electrospinning
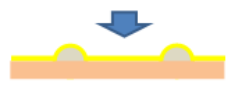

Gold deposition

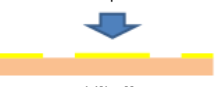

Lift off

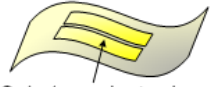

Sub- $1 \mu$ m electrode gap
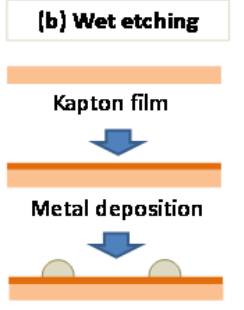

NF-electrospinning

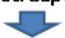
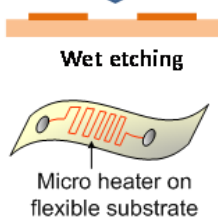

\section{(c) Dry-etching}

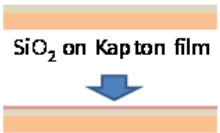

Graphene transfer
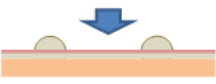

NF-electrospinning ए

\section{$\mathrm{O}_{2}$ plasma etching}

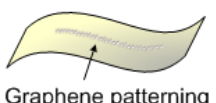

Figure 2. Three basic mask-less lithography and etching processes via the direct-write nanolithography technique: (a) lift-off, (b) dry-etching, and (c) wet-etching to demonstrate (a) sub-micrometer gap, (b) micro heater, and (c) graphene etching processes.

In the lift-off process as shown in figure 2(a), a gap of narrower than $1 \mu \mathrm{m}$ is the target demonstration. First, a $25 \mu \mathrm{m}$-thick Kapton film is placed in the near-field electrospinning setup. Thin PEO fibers are directly deposited on top of Kapton film to form the gap. A 50nm-thick gold layer is deposited using e-beam evaporation and the lift-off process is performed by dipping the sample into DI (Deionized) water for 5 minutes. As the PEO fiber is dissolved in DI water, the sub-micro meter metal gap as defined by the diameter of the electrospun fiber could be easily constructed. Figure 3 shows the optical image of a fabricated sub-micrometer gap at the top and a SEM image at the bottom.
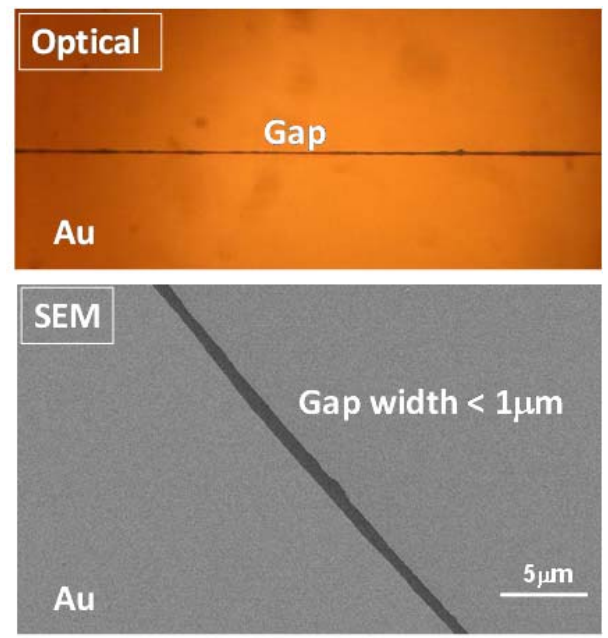

Figure 3. A sub-micrometer gap was formed by using the electrospun PEO nanofiber as the lift-off shadow mask: (a) Optical and (b) SEM images.

For the wet-etching process as shown in figure 2(b), two requirements should be fulfilled for the electrospun fibers to be effectively used as mask. First, the fibers must have good adhesion to the substrate to prevent possible leakage of etchant underneath the fiber during the etching process. The adhesion strength between the fiber and the substrate is generally determined by the choice of polymers and solvent used in the electrospinning process. Solvents such as acetone evaporate fast and result in weak adhesion force. Polymers which use DI water as solvent typically have better adhesion strength with the Kapton substrate. Experimentally, PEO polymer with $20 \% \mathrm{v} / \mathrm{v}$ mixing ratio with DI water have shown good adhesion strength on Kapton and copper substrate in the prototype tests. The second requirement for the electrospun fiber as the etching mask in the wet etching process is their chemical resistance to the etchant as the physical dimension of masking fibers should be maintained throughout the whole wet-etching process. It turns out that the PEO polymer used in the prototype experiments is robust during entire copper wet-etching process. In the process as shown in figure 2(b), a 30nm-thick copper is via the sputtering process on top of the Kapton substrate. The designed device pattern, a micro heater, is then written on to the copper thin film via the near-field electrospinning process. After the wet-etching process, figure 4(a) shows the optical image of a fabricated substrate being deformed under the force between two fingers. Figure 4(b) is the image of the fabricated micro heater under an optical microscope. The width of the heater could be adjustable during the timed etching process. However, as the copper film has many grain boundaries which result in uncontrolled etching in the boundary areas, the overall wet-etching process is challenging with poor pattern accuracy compared with the lift-off and dry-etching process to be discussed in the next session.

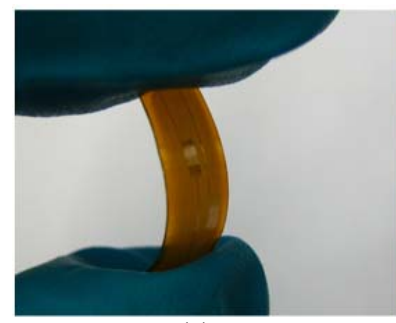

(a)

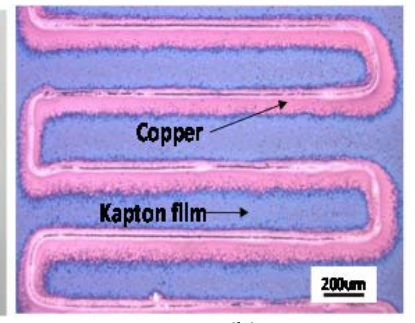

(b)
Figure 4. Optical image of micro-heater fabricated on flexible Kapton film. (a) A fabricated substrate being deformed between two fingers. (b) The image of the fabricated micro heater under an optical microscope. The width of the heater can be adjusted by the time-etching process.

Figure 2 (c) shows the dry-etching process using direct-write electrospun fibers. Similar to the wet-etching process, good adhesion of fiber to substrate is desirable. Moreover, dry-etching is generally used to generate patterns with smaller line width such that even small amount of gas/plasma leak under the fiber can severely impact the process. Multiple tests have been conducted and the conclusions from the prototype experiments indicate that PEO fibers have good adhesion to Kapton substrate and their gas barrier property against oxygen plasma is very good. Therefore, graphene patterning by means of oxygen plasma has been chosen as the demonstration example for the dry-etching process. A silicon dioxide layer is deposited on top of the Kapton film to promote adhesion of graphene. A CVD grown, single layer graphene is transferred on the substrate via the scoop and dry method [9]. The PEO fiber is directly-written on the substrate followed by oxygen plasma etching for 5 seconds at $50 \mathrm{Watt}$ to define a $2 \mu \mathrm{m}$-wide line-pattern of graphene. The fiber is then removed by dipping into DI water with sonication for 5minutes. Figures 4(a) and (b) show: Raman spectroscopy result on the patterned graphene area and the optical photo of the graphene, respectively. Raman spectroscopy has also been examined at the adjacent areas and the responses have shown only background noises (result not shown) as the verification for the successful wet-etching/patterning process. 

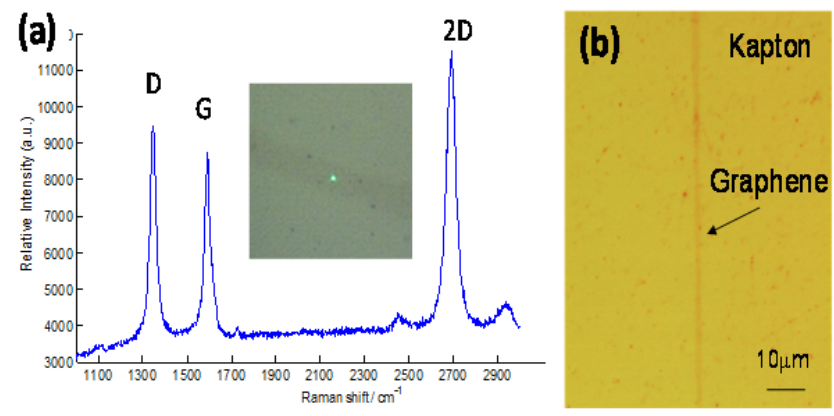

Figure 5. (a) Raman spectroscopy taken on the patterned graphene structure by $\mathrm{O}_{2}$ plasma etching at 50Watt for 5 seconds. (b) The graphene channel was visible under the optical microscope.

Figure 6 shows the capability of using electrospun fibers to make good lithography patterns with an array of structures. Figure $6(\mathrm{a})$ is four $2 \mu \mathrm{m}$-wide electrospun fibers with an equal separation distance of $50 \mu \mathrm{m}$ and figure 6(b) shows the etching result of patterned graphene structure. Clearly, the graphene film has been successfully patterned and PEO fibers have been removed as the successful demonstration of using near-field electrospinning to generate arbitrary patterns as a dry etching masking layer.

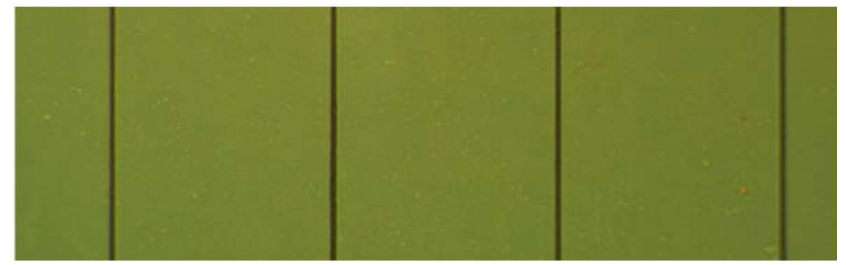

(a)

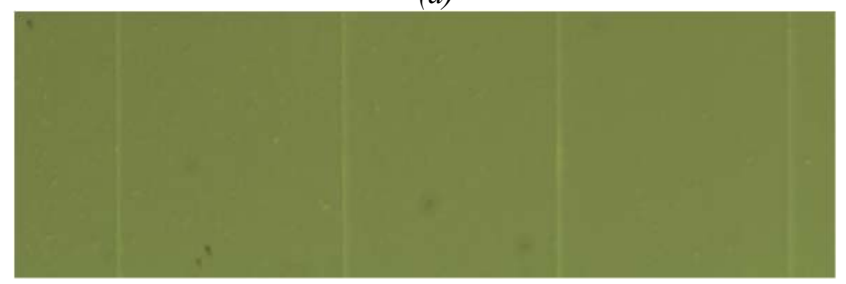

(b)

Figure 6. (a) An array of electrospun fibers with equal seperation distance of $50 \mu \mathrm{m}$ and (b) etching result showing graphene array. The oxygen plasma etching process is followed by fiber removal process in DI water. Arbitrary pattern can be constructed by controlling the movement of an $x$ - $y$ stage.

\section{PROTOTYPE DEVICE TEST}

Mask-less lithography processes using electrospun fibers have been demonstrated in the previous session. In this session, experimental results on fabricated working devices are described.

\section{Micro heater via wet-etching}

The whole micro heater fabricated by wet-etching method and the device is shown in figure 7(a). It is made of 30nm-thick copper with width of $20 \mu \mathrm{m}$ and total length of $20 \mathrm{~mm}$. When an electrical current is applied, the heater can heat up accordingly and an infrared camera is used to monitor the temperature and power during the experiments. Figure 7(b) is a sequence of IR photos showing the micro heater under different input power. It is observed that under an input power of $37 \mathrm{~mW}$, the substrate temperature reaches $92^{\circ} \mathrm{C}$. Figure 7(c) shows the recorded power consumption versus input current and highest temperature versus power consumption.

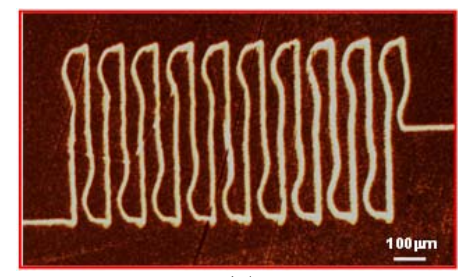

(a)
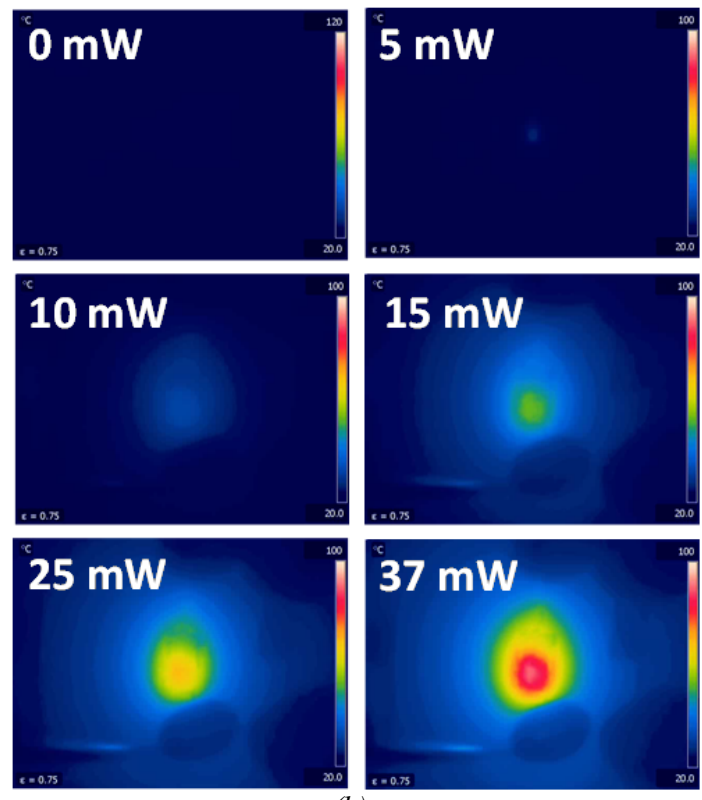

(b)
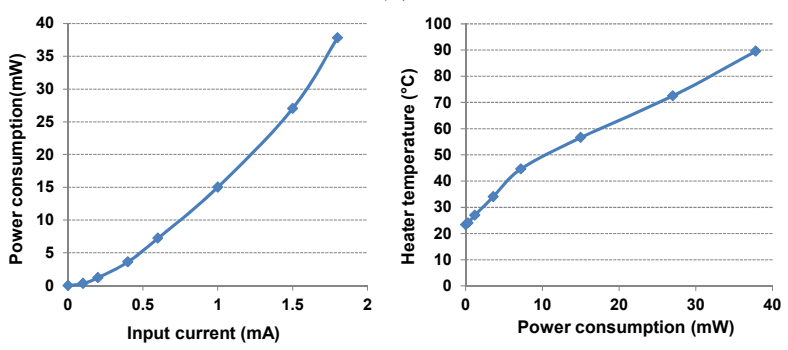

(c)

Figure 7. (a) The micro heater with $20 \mu \mathrm{m}$ in width and $20 \mathrm{~mm}$ in total length is fabricated by the direct-write electrospun fibers with the wet-etching process. (b) Temperature profile of the micro heater with respect to various input currents under an infrared camera. (c) Recorded results of power consumption versus input current and peak heater temperature versus power consumption.

\section{Graphene FET via dry-etching}

Graphene structures fabricated by the direct-write lithography process using dry etching have constructed as channel field effect transistors (FET). In this case, the FET is tested on a p-type silicon wafer with $285 \mathrm{~nm}$ of thermally grown silicon dioxide working as gate oxide instead of a flexible substrate. First, a CVD grown single layer graphene film is transferred onto the substrate and PEO polymer fibers based near field electrospinning are used to construct channel patterns. The width of the fiber is measured to be around $1-2 \mu \mathrm{m}$. Afterwards, oxygen plasma at $50 \mathrm{~mW}$ for 5 seconds is performed to etch graphene. The source and drain regions of the FET are patterned using the conventional lithography process in which OCG825 G-line photo resist is used. An lift-off process with $5 \mathrm{~nm}$-thick palladium and $100 \mathrm{~nm}$-thick gold layer by e-beam 
evaporation is followed by PR removal to complete the device fabrication. Figure 8(a) shows the top view optical image of the graphene channel FET. The patterned graphene channel is clearly visible in the image. The electrical test includes I-V sweep between source and drain. As shown in the top of figure 8(b). It is observed that graphene channel with $2 \mu \mathrm{m}$ in width and $10 \mu \mathrm{m}$ in length has good ohmic contact behavior since graphene is metallic and palladium is used to promote adhesion between gold and graphene.
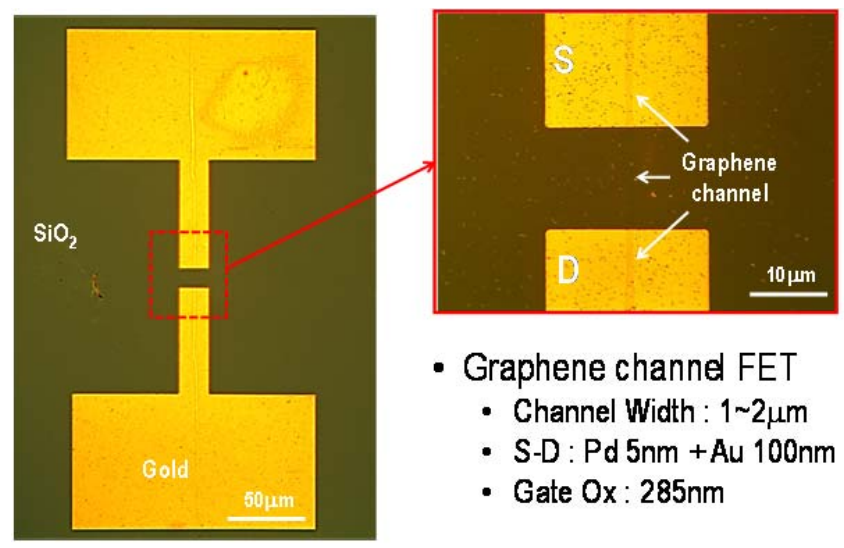

- Graphene channe FET

- Channel Width : 1 2 $\mu \mathrm{m}$

- S-D : Pd 5nm + Au 100nm

- Gate Ox : $285 \mathrm{~nm}$

(a)

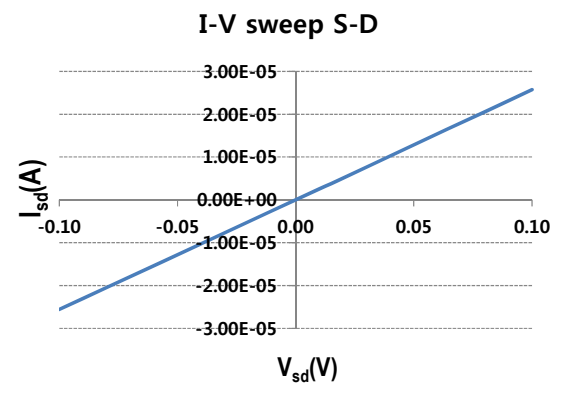

FET channel current

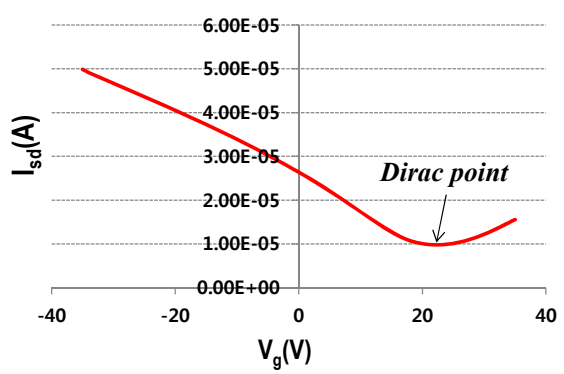

(b)

Figure 8. (a) Optical images of graphene channel FET via the direct-write electrospun fibers using a dry-etching process. The FET is fabricated on silicon wafer with $285 \mathrm{~nm}$ of thermally grown silicon dioxide as gate oxide. (b, top) The graphene is metallic such that I-V sweep between source and drain shows good ohmic behavior. (b, bottom) Gate voltage is swept showing the typical large area graphene channel FET characteristics.

On the other hand, if the gate voltage is swept from $-35 \mathrm{~V}$ to $+35 \mathrm{~V}$ while keeping the SD voltage at $0.1 \mathrm{~V}$, the FET behaves like a p-type transistor where the Dirac point is observed around $+21 \mathrm{~V}$. The Dirac point is where the carrier density is almost zero such that the current flow in graphene channel is minimal. These results show good FET characteristics and imply the patterned graphene of high quality without being damaged by the oxygen plasma process.

\section{CONCLUSION}

Direct-write electrospun polymer fibers on flexible substrate have been successfully utilized as the masking materials in the mask-less lithography processes. The near-field electrospinning on flexible and dielectric substrate was achieved by having conductive substrate underneath to achieve pattern line width from sub-micro to micrometers on flexible substrate. It is found that PEO fibers have good adhesion strength on top of Kapton and copper substrates as well as good wet and dry-etching resistance to wet copper etchant and dry oxygen plasma, respectively. As a result, sub-micrometer gaps on a 30nm-thick gold layer, micro heaters made of copper and graphene patterns have all been successfully demonstrated via the electrospun fibers with lift-off, copper wet-etching and oxygen dry-etching processes, respectively. Testing results also confirms basic operations of micro heaters as well as graphene channel based FET. As such, the direct-write electrospun fiber lithography technique could have versatile application on flexible electronics and wearable electronics with possible simple, low-cost and large area processing capabilities.

\section{ACKOWLEDGEMENT}

The devices have been fabricated in the Marvel Nanofabrication Lab at UC Berkeley. This project is supported in part by NSF grants CMII-10311749 and ECCS-0901864.

\section{REFERENCES}

[1] H. Lee and G.-Y. Jung, "Full wafer scale near zero residual nano-imprinting lithography using UV curable monomer solution," Microelectronic Engineering, vol. 77, no. 1, pp. 42-47, Jan. 2005.

[2] K. Venkatakrishnan, B. K. A. Ngoi, P. Stanley, L. E. N. Lim, B. Tan, and N. R. Sivakumar, "Laser writing techniques for photomask fabrication using a femtosecond laser," Applied Physics A: Materials Science \& Processing, vol. 74, no. 4, pp. 493-496, Apr. 2002.

[3] Y. Matsubara, J. Taniguchi, and I. Miyamoto, "Fabrication of Three-Dimensional Hydrogen Silsesquioxane Resist Structure using Electron Beam Lithography," Japanese Journal of Applied Physics, vol. 45, no. 6, pp. 5538-5541, Jun. 2006.

[4] "Fabrication of high-Q chalcogenide photonic crystal resonators by e-beam lithography," Applied Physics Letters, vol. 90, no. 7, pp. 071102-071102-3, Feb. 2007.

[5] L. Wang, S. Zhang, Q. Wang, J. Chen, W. Jiang, and R. Chen, "Fabrication of three-dimensional (3D) woodpile structure photonic crystal with layer by layer e-beam lithography," Applied Physics A: Materials Science \& Processing, vol. 95, no. 2, pp. 329-334, May 2009.

[6] S. C. Minne, H. T. Soh, P. Flueckiger, and C. F. Quate, "Fabrication of $0.1 \mu \mathrm{m}$ metal oxide semiconductor field-effect transistors with the atomic force microscope," Applied Physics Letters, vol. 66, no. 6, pp. 703-705, Feb. 1995.

[7] Z. J. Davis et al., "AFM lithography of aluminum for fabrication of nanomechanical systems.," Ultramicroscopy, vol. 97, no. 1-4, pp. 467-72.

[8] D. Sun, C. Chang, S. Li, and L. Lin, "Near-field electrospinning.," Nano letters, vol. 6, no. 4, pp. 839-42, Apr. 2006.

[9] X. Li et al., "Large-area synthesis of high-quality and uniform graphene films on copper foils.," Science (New York, N.Y.), vol. 324, no. 5932, pp. 1312-4, Jun. 2009.

\section{CONTACT}

*Jiyoung Chang, changjy@me.berkeley.edu 


\section{GROWTH OF CARBON NANOTUBES DIRECTLY FROM NATURAL PROTEINS \\ Hao-Chieh Hsieh ${ }^{1}$, Md. Mahbubur Rahman ${ }^{1}$, T.C. Shen ${ }^{2}$ and Hanseup Kim ${ }^{1}$ \\ ${ }^{1}$ Electrical and Computer Engineering, University of Utah, Salt Lake City, Utah, USA \\ ${ }^{2}$ Department of Physics, Utah State University, Logan, Utah, USA}

\begin{abstract}
This paper presents a new class of a nano manufacturing method: the synthesis of carbon nanotubes (CNTs) directly from natural proteins, particularly native ferritin, tyrosine, and urease molecules. This manufacturing method enables controlled nanomanufacturing by taking advantages of unique biological properties including nano-scale sizes, self-assembly, selfalignment, large-area coverage, low cost, and diverse metal element availability. In particular, this paper presents the first 'proof-of-concept' results proving its feasibility: single and multiple CNTs were successfully grown from immobilized proteins at the desired spots in predictable lengths up to $10 \mu \mathrm{m}$ in height.
\end{abstract}

\section{INTRODUCTION}

Carbon nanotubes have many superior properties and have been widely applied to various applications including biosensor electrodes, explosive detectors, super-capacitors, and others. For example, CNTs have tensile strength up to $63 \mathrm{GPa}$. which is much stronger than steel $(1200 \mathrm{MPa})$, and demonstrate a wide range of conductivities from metal to semiconductor levels, which is potentially useful for next generation electronic devices.

CNTs can be produced by several methods (Table 1); however, the major trade-off between controllability and manufacture sizes has prevented their practical uses for massproduction. For example, some previous technologies are applicable to either only a small area (nanolithography, fieldassisted nanowire growth), serial and slow (nanolithography); or cover a large area with random control over the numbers and locations of the nanostructures (CVD). Thus, the development of a practical nano-manufacturing technology is clearly necessary that is capable of both accurate control of locations and massproduction over a large area. Such capabilities could be simultaneously achieved by utilizing a biological subject as a CNT growth seed that (1) naturally controls the spacing through the selfassembly and self-alignment, (2) easily grows over an extended area of surface, and (3) provides various metal types after lysis.

Metalloproteins can be an excellent candidate for such a biological seed that provides the foundation for the controlled and wide-area CNT growth. Metalloproteins are specific proteins that contain metal ions inside the structure, such as ferritin, tyrosinase, and urease, and are excellent candidates for the purpose (Table 2). Most of the metalloproteins utilize metal ions as catalyst in specific metabolic functions; some are specially used to maintain body ion concentrations. They also have been providing a wide range of choices for electronic manufacture materials and applications such as biosensors. Recently, a few research groups have reported the use of metalloproteins as catalysts for CNT growth. However, these previous methods involve genetic modification of proteins, which might cause degraded activity or loss of metal ions. Here, we report our first attempt in utilizing natural metalloprotein as the catalysts to provide variety metal sources for massively-parallel and controlled CNT growth.

\section{METALLOPROTEIN}

Metalloproteins play important roles in various chemical reactions using their metal cores from basic chemical reaction (electron transfer and hydrolysis) to complex signal transduction and ion channel control. Several different metalloproteins exist, and each contains specific metal elements as listed in Table 2. Note that these metalloproteins provide a specific number of metal ions per molecule and a specific volume shape. The dimensions of metalloproteins range from several nanometers to a few micrometers, while each metal atom holds the size of 100-200 picometers. A ferritin protein, for example, (Fig. 2) is a sphere shell molecule composed with 24 polypeptide subunits and has a diameter about $9 \mathrm{~nm}$. The inner space contains up to 4500 iron ions. In some research ferritin shells have been utilized as a mold to form metal nanoparticles. Specifically, ferritin, tyrosinase, and urease have been utilized in this work.

\section{Process Flow}

Analyzed Results

1. Surface Modification (3\% APTES in acetone)

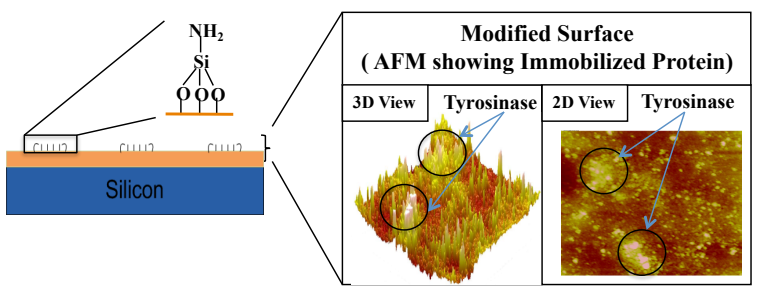

2. Metalloproteins Immobilization

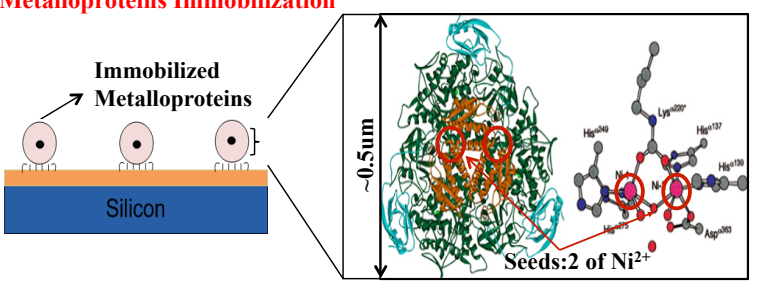

3. Metal Seeding (metal atoms remains after Enzyme lysis)

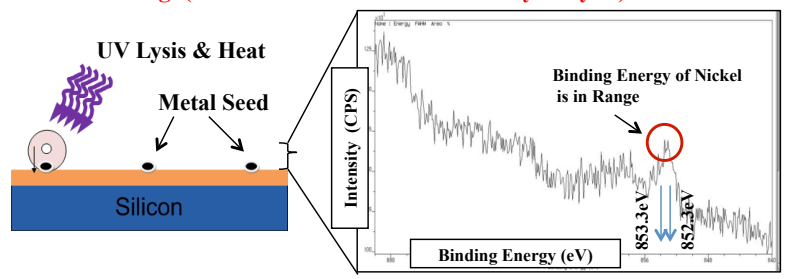

4. Carbon nanotubes induction

$\rightarrow$ (site-specific) $\checkmark$

$\rightarrow$ (large area)

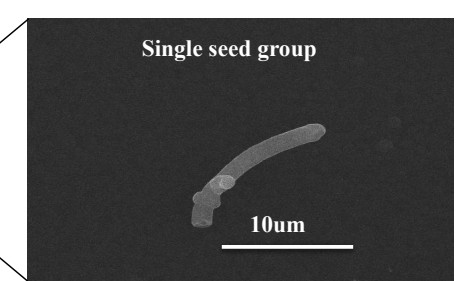

Figure 1: The main concept of the protein-based CNT growth: (Left) the fabrication procedure and (Right) the measurements at each stage. 
Table 1: Advantage comparison of CNT growth methods.

\begin{tabular}{ccc}
\hline Fabrication method & Controllability & $\begin{array}{c}\text { CNT Growth } \\
\text { Area }\end{array}$ \\
\hline Optical Lithography [5] & High & Low \\
Fabrication by Electric Field [6] & Medium & High \\
Chemical Vapor Deposition [7] & Low & High \\
Vectorial Growth by CVD [20] & High & Low \\
Plasma Enhanced CVD [21] & Medium & Medium \\
Pyrolysis by HydroCarbon [22] & Medium & Medium \\
*Bio platform-based CNT growth & High & High \\
\hline *Approach in this paper. & & \\
\hline
\end{tabular}

Table 2: Comparison of metalloproteins.

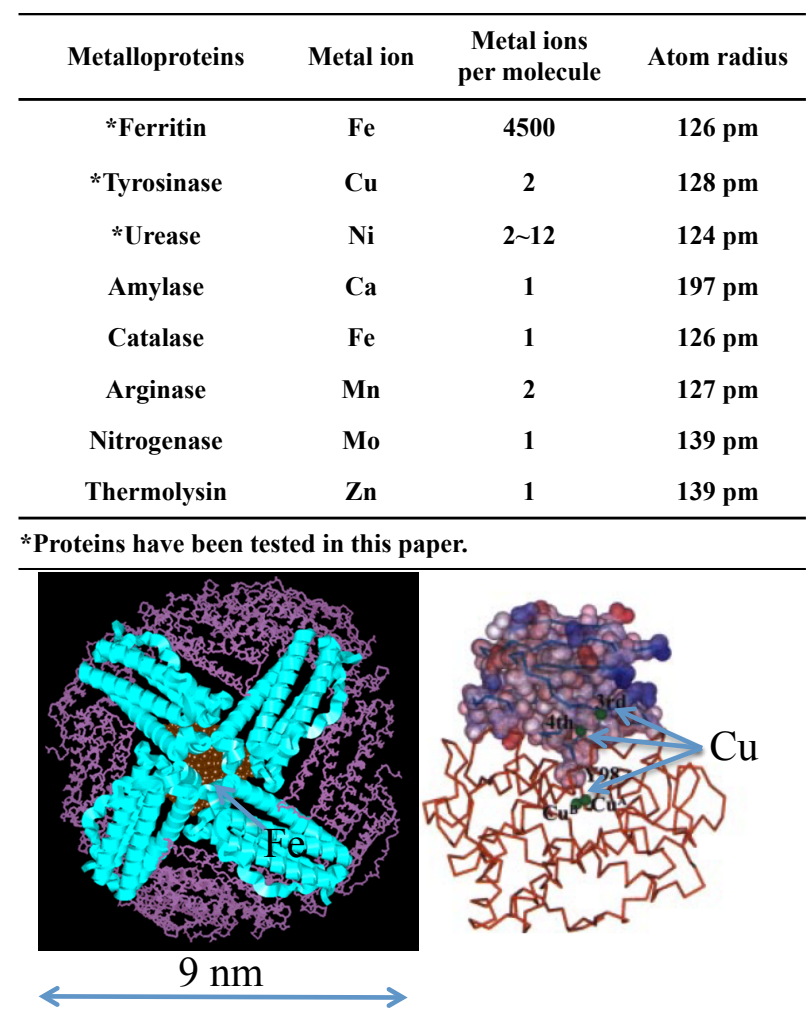

Figure 2: Left: Ferrtitin molecule contains 24 subunits to form a sphere with inner space to contain iron ions. Right: Tyrosinase molecule with copper atoms.

\section{FABRICATION}

The 'proof-of-concept' CNT growth was performed in three stages: metalloprotein immobilization, metal seeding, and CNT induction (in parallel to Fig. 1). The immobilization step overcomes random catalyst distribution, and avoids losing metalloprotein while removing impurities during cleaning process and secures metalloproteins onto the wafer for reliable CNT growth. If not secured, CNT growth is degraded because the impurities, mostly salt, form the crystal cover the surface and cause contamination. The metal seeding step secures only metal atoms by removing the other parts of the metalloproteins including shells thus expose the metal catalysts to feeding gases and also increase the contact area. The CNT induction step grows CNTs from the secured metal atoms in single or multiple CNT configurations.
Surface Modification and Metalloproteins immobilization

Metalloprotein immobilization was performed by modifying the surface of the target wafer diced into $1 \mathrm{~cm} \mathrm{x} 1 \mathrm{~cm}$ utilizing $3 \%$ (v/v\%) 3-Aminopropyltriethoxysilane (APTES, A3648, SigmaAldrich, St. Louis, MO) in acetone. Unbound APTES was removed with acetone in sonicator for 1 minute. For example, ferritin molecules (F4503, Sigma-Aldrich, St. Louis, MO) were secured on the wafer surface by immersing the wafer into a ferritin solution later diluted with DI water for 12 hours on orbital shaker at room temperature. Then the wafer surface with ferritin molecules was washed with static DI water in a beaker to gently remove unbound ferritin molecules.

\section{Metal seeding}

From the immobilized metalloproteins, only metal atoms are needed for CNT growth. Thus this step preserves only metal atoms by removing the protein shell. In order to remove the shell and expose the metal core, the seeded wafers were first dried and air burned by elevating the temperature up to $500^{\circ} \mathrm{C}$ in a furnace (Thermolyne 79400, Thermo Fisher Scientific, Massachusetts) for 10 minutes. Such heating process decomposed the protein structure and left only metal atoms on the substrate. For example, ferritin exposed a certain number (4500) of metal ions (iron) once their protein shell was removed. These irons atoms may start to diffuse on the surface or into the wafer and form bigger cluster with each other, thus the temperature control and time were very critical.

\section{Carbon nanotubes induction}

CNTs were induced to vertically grow by utilizing the standard thermal CVD method that is the most widely accepted. Because the previous seeding process involved air and heat to remove protein shell, it oxidized the metal irons and resulted in the loss of their catalyst activity. Thus, before inducing CNTs, we first chemically reduced iron catalysts by feeding $\mathrm{Ar} / \mathrm{H}_{2}$ gas mixture $(50 \mathrm{sccm} / 45 \mathrm{sccm})$ in the furnace for 10 minutes to assist their activity recovery. Next, the feeding gas, $\mathrm{Ar} / \mathrm{H}_{2} / \mathrm{C}_{2} \mathrm{H}_{4}$ gas mixture, as a carbon source $(50 \mathrm{sccm} / 45 \mathrm{sccm} / 5 \mathrm{sccm})$, was introduced to induce CNT growth at $700^{\circ} \mathrm{C}$ for 10 minutes. Once the $\mathrm{C}_{2} \mathrm{H}_{4}$ was decomposed on the surface of the catalyst, carbon atoms had a chance to connect to each other around the catalyst and started growing and forming carbon tubes. Finally, the samples were cooled down to $50^{\circ} \mathrm{C}$ under $\mathrm{Ar} / \mathrm{H}_{2}$ gas mixture and analyzed by scanning electron microscopes (SEM, Quanta 600 FEG, FEI, Oregon).

\section{TESTING METHODOLOGY}

Surface Modification and Metalloprotein immobilization

To ensure the ferritin molecules were immobilized on the wafer surface through APTES boding. Samples were analyzed with Fourier Transform Infrared Spectroscopy (FT-IR) to confirm the existence of ferritin protein shell. In addition, surface profile was measured by utilizing the AFM-based physical profiler.

\section{Metal seeding}

To ensure the protein shell was completely removed, Fourier Transform Infrared Spectroscopy (FT-IR) was performed to compare samples before and after heat treatment. Immobilized ferritin samples were placed on hot plate (PC-420D, CORNING, Massachusetts) at $500^{\circ} \mathrm{C}$, and then analyzed with FT-IR (Varian 3100, Varian, Inc. California).

To confirm the iron seeds were still remained on the wafer surface after heat treatment, Energy-dispersive X-ray spectroscopy $\left(\right.$ EDAX $^{\mathrm{TM}}$ Genesis system, EDAX Inc, New Jersey) was 
performed to identify metal types on the wafer surface by focusing $\mathrm{X}$-ray beams of $20 \mathrm{kV}$ at $8.6-\mathrm{mm}$ working distance.

\section{Carbon nanotubes induction}

The CNTs growth pattern under immobilization effects was analyzed with SEM at $30 \mathrm{kV}$ in a high vacuum mode. The influence of CNTs growth with or without immobilization and growth pattern were compared from SEM images.

\section{RESULTS}

\section{Surface Modification and Metalloproteins immobilization}

The measured FT-IR spectrum (Fig. 3) indicates that the metal proteins are immobilized on the target wafer surface. The spectrum clearly showed a peak of amide bond (A) from protein shell at $1150 \mathrm{~cm}^{-1}$. This peak indicated that the ferritin outer shell was immobilized in the wafer surface. Note that peaks of protein molecules typically appear at 1650 and $1540 \mathrm{~cm}^{-1}$. We believe that this difference may be caused by the molecular bonding onto wafer surface instead of free molecular status in conventional tests.

\section{Metal seeding}

The combined results of FT-IR and EDX detection confirmed that protein shells were successfully removed and the target metal seeds were exposed on the wafer surface (Fig. 3). As shown in the FT-IR results in Figure 3, the amide bond peak at $1150 \mathrm{~cm}^{-1}$ dramatically shifted between before (A) and after (B) heat treatment. Such reduction indicates the protein shell was removed after the heat treatment. In parallel to FT-IR results, the EDX results (Fig. 3, inset table) clearly showed the FeKa and FeKb Xrays emitted from Fe element and the existence of $29.79 \mathrm{Wt} \%$ of Fe element on the analyzed area, verifying the successful metal seeding process.

\section{Carbon nanotubes induction}

Figure 4 shows the top view of the wafer sample surface after $\mathrm{CNT}$ growth, indicating the influence of immobilization on the CNT growth uniformity. Specifically, immobilization increased the growth uniformity. Without immobilization process, ferritin molecules, which were initially preserved in saline solution, started forming precipitation on the wafer surface during air-dry process and produced a rough surface (A1). The resultant rough surface led to random CNTs growth. In contrast, when the ferritin molecules were immobilized, the CNT grown surface (B1) turned out to be smoother, and ferritin was distributed more evenly. Resultantly, the CNTs were evenly located on the post-immoblization wafer surface (B2).

Figure 5 shows that the higher concentration of seeds led to the higher density of CNT growth. The left photo shows the CNT growth produced from a higher ferritin molecules concentration ferritin $(\mathrm{C} 1: 0.44 \mathrm{mg} / \mathrm{mL})$, and the right photos shown the lowly concentrated CNT growth from a lower ferritn concentration $(\mathrm{C} 2$ : $0.44 \times 10^{-2} \mathrm{mg} / \mathrm{mL}$ ) as expected. It was also observed that the CNT distribution was not so even on the wafer especially at low concentration. This suggested that ferritin molecules form some self-aggregation during immobilization step, causing some areas to have more ferritin molecules than other areas and resulting in nonuniform CNTs distribution. It might be also possible that the iron catalysts may be randomly diffused onto the wafer surface resulting in differently-sized aggregated catalyst. It was also observed that all of higher temperature, heating with longer time and higher ferritin concentration led to forming more severelyaggregated catalysts.

Figure 6 shows the successfully grown CNTs from the natural metalloproteins. The diameters of CNTs were determined by the catalysts sizes and the length was by the period of gas feeding as well as the lifetime of catalyst. The measured height of a single CNT was about $10 \mu \mathrm{m}$ in length and $120 \mathrm{~nm}$ in diameter. By changing the seeding densities of iron catalysts, different types of CNT growth were observed including single carbon nanotubes and CNT forests with the dimension of $5.19 \mu \mathrm{m}$ in height and $9.42 \mu \mathrm{m}$ in width. We believe that the iron catalysts density on the surface and the self-aggregation of ferritin molecules influenced these variations.

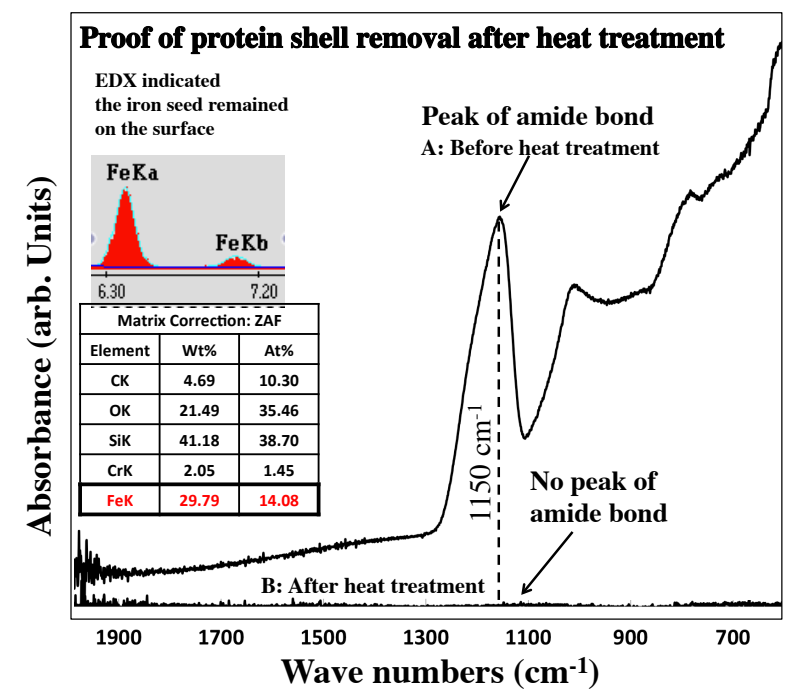

Figure 3: FT-IR spectrum of ferritin molecules (A) before and (B) after heat treatment.

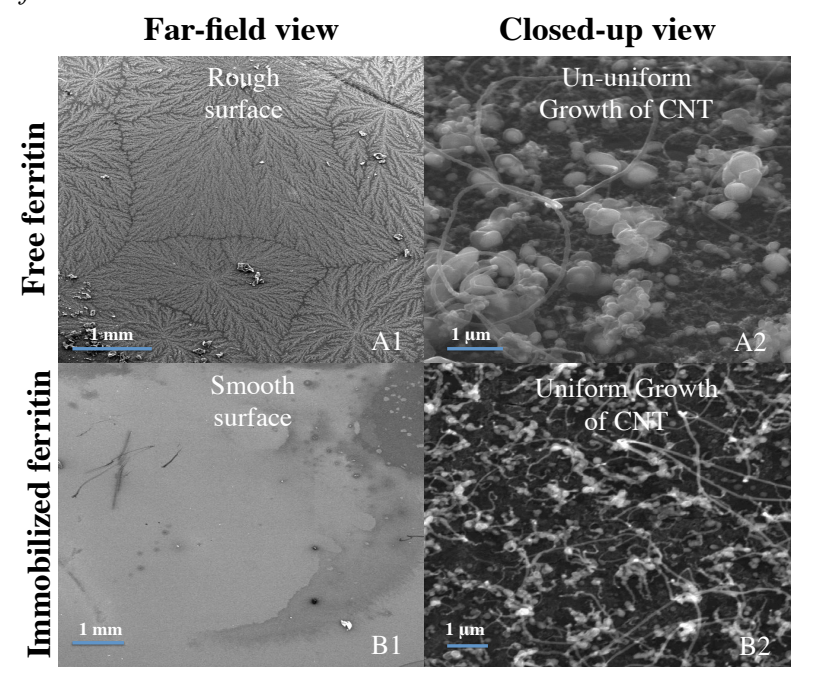

Figure 4: SEMs: A1: wafer with free ferritin. A2: CNTs growth from free ferritin. B1: wafer with immobilized ferritin. B2: CNTs growth from immobilized ferritin.

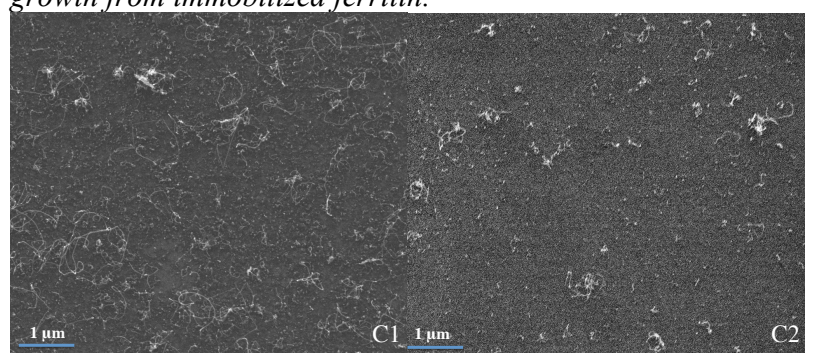

Figure 5: SEMs: C1: wafer with ferritin $0.44 \mathrm{mg} / \mathrm{mL}$. C2: wafer with ferritin $0.44 \times 10^{-2} \mathrm{mg} / \mathrm{mL}$. 


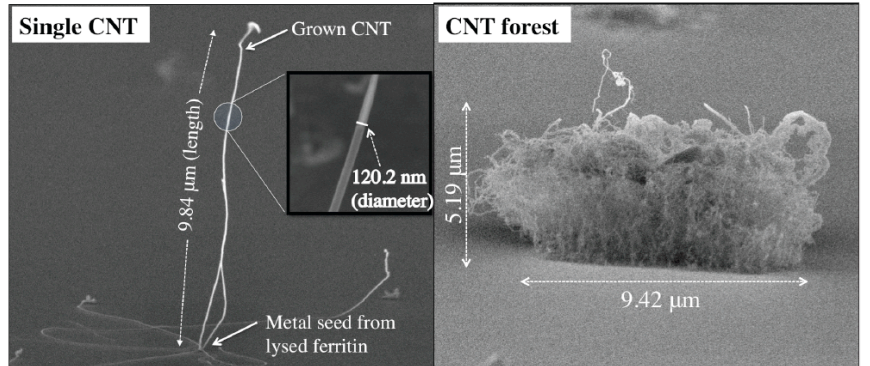

Figure 6: SEMs of (Left) single carbon nanotube and (Right) carbon nanotube forest.

\section{DISCUSSIONS AND CONCLUSIONS}

We have successfully proven the feasibility of producing CNT growth directly from native proteins (ferritin, urease, tyrosinase in this proof-of-concept tests). We have also demonstrated metalloprotein immobilization, metal seeding, and CNT induction. Despite the remaining challenges of regulating the precise location of metal catalysts, the results provide sufficient verification of the proof-of-concept methods as a new class of nanomanufacturing to enable controllable, patternable and massively-parallel CNT production.

\section{ACKNOWLEDGEMENT}

The authors appreciate Dr. Brian Van Devener for his professional help on the SEM image and Mr. Chun-Jen Wu for his help with FT-IR analysis. This project is supported by the USTAR (Utah Science Technology and Research Initiative) Funding and partially by the DARPA Young Faculty Award (through Office of Naval Research: N66001-11-14149). The travel grant is generously provided by the Transducer Research Foundation.

\section{REFERENCES}

[1] M.F. Yu, O. Lourie, M.J. Dyer, K. Moloni, T.F. Kelly, and R.S. Ruoff, "Strength and Breaking Mechanism of Multiwalled Carbon Nanotubes Under Tensile Load", Science, (2000), 287, pp. 637 - 640.

[2] A. Le Goff, M. Holzinger, and S. Cosnier, "Enzymatic biosensors based on SWCNTconducting polymer electrodes“, Analyst, (2011), 136, pp. $1279-1287$.

[3] M. Park, L.N. Cella, W. Chen, N.V. Myung, and A. Mulchandani, "Carbon nanotubes-based chemiresistive immunosensor for small molecules: Detection of nitroaromatic explosives“, Biosensors and Bioelectronics, (2010), 26, pp. 1297 - 1301.

[4] Y.S. Kim, K. Kumar, F. T. Fisher, and E. H. Yang, "Out-ofplane growth of CNTs on graphene for supercapacitor applications", Nanotechnology, (2012), 23, pp. 015301.

[5] S.W. Kang, D. Banerjee, A.B. Kaul, and K.G. Megerian, "Nanopatterning of catalyst by Dip Pen nanolithography (DPN) for synthesis of carbon nanotubes (CNT)", Scanning, (2010), 32, pp. 42 - 48.

[6] O. Englander, D. Christensen, J. Kim, L. Lin, and S.J.S. Morris, "Electric-field assisted growth and self-assembly of intrinsic silicon nanowires", Nano Letters, (2005), 5, pp. 705 708.

[7] G.D. Nessim, "Properties, synthesis, and growth mechanisms of carbon nanotubes with special focus on thermal chemical vapor deposition", Nanoscale, (2010), 2, pp. 1306 - 1323.

[8] J. Min, S.U. Kim, Y.J. Kim, C.H. Yea, and J.W. Choi, "Fabrication of recombinant azurin self-assembled layer for the application of bioelectronic device", Journal of Nanoscience and Nanotechnology, (2008), 8, pp. 4982 - 4987.

[9] T. Lee, S.U. Kim, J.H. Lee, J. Min, and J.W. Choi, "Fabrication of nano scaled protein monolayer consisting of cytochrome $\mathrm{c}$ on self-assembled 11-MUA layer for bioelectronic device", Journal of nanoscience and nanotechnology, (2009), 9, pp. $7136-7140$.

[10] J.J. Davis, D.A. Morgan, C.L. Wrathmell, and A. Zhao, "Scanning probe technology in metalloprotein and biomolecular electronics", IEE Proceedings Nanobiotechnology, (2004), 151, pp. 37 - 47.

[11] Y. Ko, Y. Kim, H. Baek, and J. Cho, "Electrically Bistable Properties of Layer-by-Layer Assembled Multilayers Based on Protein Nanoparticles", ACS nano, (2011), 5, pp. 9918 9926.

[12] J.J. Kim, B.J. Lee, S.H. Lee, and G.H. Jeong, "Size engineering of metal nanoparticles to diameter-specified growth of single-walled carbon nanotubes with horizontal alignment on quartz", Nanotechnology, (2012), 23, pp. 105607.

[13] I. Yamashita, K. Iwahori, and S. Kumagai, "Ferritin in the field of nanodevices" Biochimica et biophysica acta, (2010), 1800 , pp. $846-857$.

[14] P. M. Harrison and P. Arosio, "The ferritins: molecular properties, iron storage function and cellular regulation", Biochimica et Biophysica Acta, (1996), 1275, pp. 161 - 203.

[15] S. Flink, F.C.J.M. van Veggel, and D.N. Reinhoudt, "Functionalization of self-assembled monolayers on glass and oxidized silicon wafers by surface reactions." Journal of Physical Organic Chemistry, (2001), 14, pp. 407 - 415.

[16] H.W. Hellinga, "Metalloprotein design", Current Opinion in Biotechnology, (1996), 7, pp. 437 - 441.

[17] Y. Lu, N. Yeung, N. Sieracki, and N.M. Marshall, "Design of Functional Metalloproteins”, Nature, (2009), 460, pp. 855 862.

[18] D.M. Lawson, P.J. Artymiuk, S.J. Yewdall, J.M.A. Smith, J.C. Livingstone, A. Treffry, A. Luzzago, S. Levi, P. Arosio, G. Cesareni, C.D. Thomas, W.V. Shaw, and P.M. Harrison, "Solving the structure of human $\mathrm{H}$ ferritin by genetically engineering intermolecular crystal contacts", Nature, (1991), 349, pp. 541 - 544.

[19] S. Kumagai, T. Ono, S. Yoshii, A. Kadotani, R. Tsukamoto, K. Nishio, M. Okuda, and I. Yamashita, "Position-Controlled Vertical Growths of Individual Carbon Nanotubes Using a Cage-Shaped Protein", Applied Physics Express, (2010), 3, pp. 015101 .

[20] E. Joselevich, "Vectorial Growth of Metallic and Semiconducting Single-Wall Carbon Nanotubes", Nano Letters, (2002), 2, pp. 1137 - 1141.

[21] P.B. Amama, M.R. Maschmann, T.S. Fisher, and T.D. Sands, "Dendrimer-templated $\mathrm{Fe}$ nanoparticles for the growth of single-wall carbon nanotubes by plasma-enhanced CVD", The journal of physical chemistry. B, (2006), 110, pp. 10636 10644.

[22] Z.D. Hu, Y.F. Hu, Q. Chen, X.F. Duan, and L.M. Peng, "Synthesis and characterizations of amorphous carbon nanotubes by pyrolysis of ferrocene confined within AAM templates", The journal of physical chemistry. B, (2006), 110, pp. $8263-8267$.

\section{CONTACT}

*H.C. Hsieh, tel: +1-801-808-0948; hchsieh1982@gmail.com 


\title{
HIGH FLUX THERMAL MANAGEMENT WITH CMOS COMPATIBLE MICROSTRUCTURED SURFACES
}

\author{
K.-H. Chu ${ }^{l *}$, R. Enright ${ }^{1,2}$, and E. N. Wang $^{l}$
}

${ }^{1}$ Department of Mechanical Engineering, Massachusetts Institute of Technology, Cambridge, Massachusetts, USA

${ }^{2}$ Stokes Institute, University of Limerick, Limerick, Ireland

\begin{abstract}
We demonstrated an enhancement in critical heat flux (CHF) of $\sim 160 \%$ on the microstructured surfaces. The fabrication process used for making the structures is CMOS-compatible and can be integrated into semiconductor processing. A simple force-balancebased model for $\mathrm{CHF}$ was developed and shows excellent agreement with the experimental observations. Based on the model predication, a potential surface design to achieve ultra high CHF is further suggested in the paper. This study shows exciting new insights into achieving high $\mathrm{CHF}$ with microstructures and provides design guidelines for new surface technologies with high heat removal capability for advanced thermal management.
\end{abstract}

\section{INTRODUCTION}

Thermal management with two-phase cooling has received significant interest for high flux applications including concentrated photovoltaics, GaN power amplifiers, and integrated circuits [1-4]. The critical heat flux (CHF) represents the operational limit in a two-phase (boiling) heat transfer system marking the point when a vapor film will begin to cover the heated surface, significantly reducing heat transfer efficiency. Therefore, methods to extend CHF have been studied extensively owing to its significant practical importance in high performance thermal management systems [5-7]. Recent efforts have focused on pushing the limits of CHF by improving surface wettability using decreased feature sizes to nanometer length scales $(\sim 100 \mathrm{~nm})$. While CHF values of $\sim 200 \mathrm{~W} / \mathrm{cm}^{2}$ with water have been reported [8-10], these nanostructures were fabricated with materials (e.g., $\mathrm{ZnO}, \mathrm{Cu}$ ) or required fabrication processes (e.g., anodic oxidation, electroless etching) that are not compatible with CMOS processing. In addition, these nanostructured surfaces were not optimized due to the limited understanding of the role of roughness-augmented wettability on CHF.

In this work, we demonstrate that with silicon microstructures alone $(\geq 5 \mu \mathrm{m})$ fabricated using standard MEMS processing, critical heat fluxes $\mathrm{q}_{\mathrm{CHF}}^{\prime \prime}>200 \mathrm{~W} / \mathrm{cm}^{2}$ are achievable. Furthermore, an analytical force-balance model was extended to explain the CHF enhancement. The excellent agreement found between the model and experimental data supports the idea that roughness-amplified capillary forces are responsible for $\mathrm{CHF}$ enhancement on structured surfaces. The work suggests that the ultra high heat removal capability $\left(>250 \mathrm{~W} / \mathrm{cm}^{2}\right)$ with structured surfaces using CMOS-compatible processing is possible.

\section{FABRICATION AND BOILING SETUP}

Microstructured surfaces with various roughness $r$, defined as the ratio of the true area in contact with the liquid to the projected area, were fabricated using MEMS processing on silicon (Fig. 1). The micropillars were patterned using projection lithography, and etched in silicon with deep reactive ion etching (DRIE). The micropillars with diameter of $5-10 \mu \mathrm{m}$, center-to-center spacings of $5-15 \mu \mathrm{m}$, and heights of $10-20 \mu \mathrm{m}$ were designed to ensure wicking behaviors [11]. Details of the pillar geometries fabricated are listed in Table I. A $300 \mathrm{~nm}$ thick thermal oxide layer was subsequently grown to enhance surface wettability. Finally, the

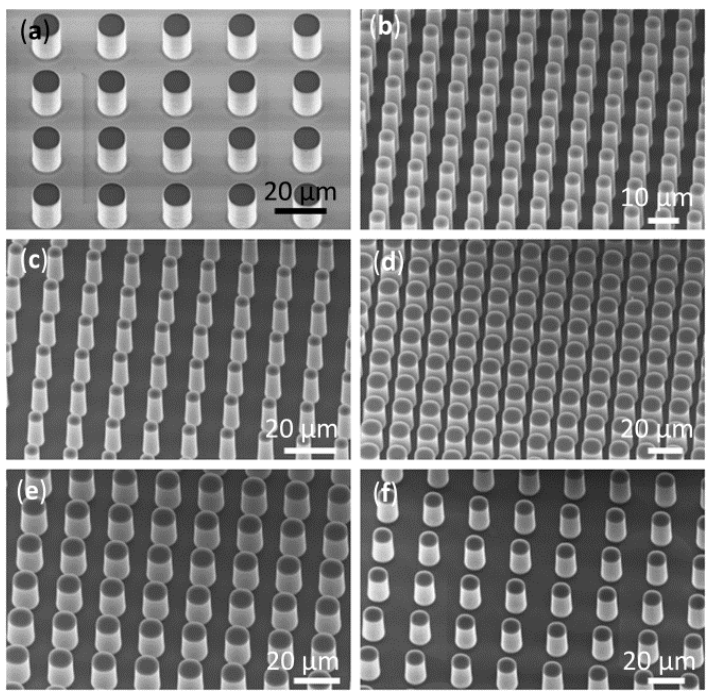

Figure 1: Scanning electron micrographs (SEMS) of the fabricated silicon microstructured surfaces. The pillars have heights of (a) $10 \mu \mathrm{m}$ and (b-f) $20 \mu \mathrm{m}$; center-to-center spacings of (a,f) $15 \mu \mathrm{m}$, $(b, d) 5 \mu \mathrm{m}$, and $(c, e) 10 \mu \mathrm{m}$; and diameters of $(b, c) 5 \mu \mathrm{m}$ and $(a$, d-f) $10 \mu \mathrm{m}$.

etched wafers were diced into samples measuring $2 \times 2 \mathrm{~cm}$, which is large enough to be considered representative of an infinite plate $[12,13]$ and of comparable size to high heat flux electronic components. Smooth oxidized samples were also prepared as benchmarks for comparison.

Fig. 2 shows the pool boiling setup which consists of an oxygen-free copper block and a tempered glass chamber fixed at both ends by Ultem mounts. Five cartridge heaters were imbedded in the copper block allowing for a maximum power of $1400 \mathrm{~W}$ $\left(350 \mathrm{~W} / \mathrm{cm}^{2}\right)$. Five in-line K- type thermocouples (KMQSS-020, OMEGA) were inserted into the center axis of the copper block with the topmost thermocouple located right beneath the sample to accurately determine the heat flux from the linear temperature gradient using Fourier's law. Note that the flux area of the copper block matched the structured sample area $(2 \times 2 \mathrm{~cm})$. A sheathed K-type thermocouple (KQSS-18U-12, OMEGA) was positioned $2 \mathrm{~cm}$ above the mounted sample to monitor the pool temperature. In the experiments, temperature was recorded with a thermocouple logger (18200-75, Cole-Parmer). To minimize losses, the chamber was wrapped in guard heaters and a layer of dense fiber glass insulation allowing the pool to be maintained at saturation temperature during the experiment.

Before experiments, the samples were bonded to the copper block using solder paste (Delta 717D, Qualitek) to ensure good attachment with minimal thermal resistance. Note that a $1 \mu \mathrm{m}$ thick copper layer was deposited on the back side of the samples to facilitate attachment to the boiling setup. For all tests, degassed, high purity water (CHROMASOLV for HPLC, Sigma-Aldrich) was used to avoid premature bubble formation and minimize surface contamination. 


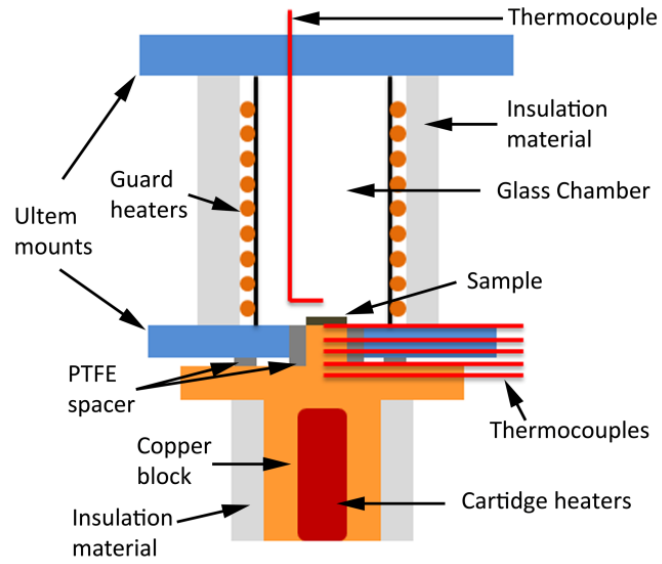

Figure. 2: Schematic of the experimental pool boiling setup with cartridge heaters in the copper block to heat the sample and inline thermocouples to accurately determine the heat flux and surface temperature. The microstructured surfaces were bonded to the copper block using solder to ensure good attachment with minimal thermal resistance.

TABLE I: Geometric parameters of the micropillar arrays. The units of height, diameter and (center-to-center) spacing are in microns. The roughness of contact line, $r$, and solid fraction, $\varphi$, are calculated by: $r=1+\pi d h(\pi / 2) /(d+s)^{2}$ and $\varphi=\left(\pi d^{2} / 4\right) /(d+s)^{2}$, respectively.

\begin{tabular}{c|c|c|c|c|c}
\hline \hline $\begin{array}{c}\text { Sample } \\
\text { No. }\end{array}$ & $\begin{array}{c}\text { Height } \\
(h)\end{array}$ & $\begin{array}{c}\text { Diameter } \\
(d)\end{array}$ & $\begin{array}{c}\text { Spacing } \\
(s)\end{array}$ & $r$ & $\varphi$ \\
\hline S1 & 10 & 10 & 15 & 1.790 & 0.126 \\
S2 & 20 & 10 & 15 & 2.579 & 0.126 \\
S3 & 20 & 5 & 10 & 3.193 & 0.087 \\
S4 & 20 & 10 & 10 & 3.467 & 0.196 \\
S5 & 20 & 10 & 5 & 5.386 & 0.349 \\
S6 & 20 & 5 & 5 & 5.935 & 0.196 \\
\hline \hline
\end{tabular}

\section{EXPERIMENTAL RESULTS}

The heat flux $q^{\prime \prime}$ as a function of wall superheat $\Delta T=T_{w}-$ $T_{\text {sat }}$, where $T_{w}$ is the heated surface temperature and $T_{\text {sat }}$ is the saturation temperature, for the smooth and microstructured $\mathrm{SiO}_{2}$ surfaces are shown in Fig. 3. The maximum uncertainty of the heat flux and temperature measurements was $\sim 5.6 \%$ and $1.8 \mathrm{~K}$, respectively. Note that in the calculation of the roughness, the scalloped features on the sidewall of micropillars were accounted for by multiplying the pillar height by a factor of $\pi / 2$ [14]. Compared with the results on the smooth surface (Sm), the boiling curves showed a significant enhancement in CHF on the structured surfaces (S1-S6). A CHF of $207.9 \pm 9.9 \mathrm{~W} / \mathrm{cm}^{2}$, which is comparable to the highest $\mathrm{CHF}$ value reported in previous studies on nanostructured surfaces [8-10, 13], was achieved with a $\Delta T=39.3 \pm 1.8 \mathrm{~K}$ on $\mathrm{S} 6$. The results demonstrate the positive correlation between the CHF and surface roughness. In addition, the two nearly identical boiling curves for the smooth surface (Sm) demonstrate the consistency and accuracy of the measurements.

\section{CHF MODEL}

To understand and predict CHF on structured surfaces where the liquid wets the surface completely, a model incorporating surface properties (i.e., surface roughness) is required. While a detailed understanding of the CHF mechanism is still lacking [15],

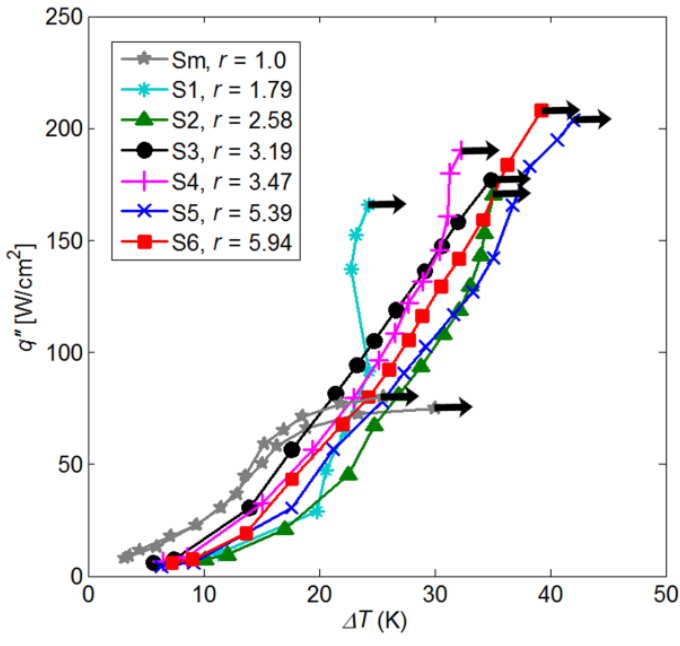

Figure 3: Boiling curves on the smooth $(r=1)$ and microstructured $(r>1)$ surfaces detailed in Table I. The arrows indicate the CHF condition. The consistency and accuracy of the measurements are demonstrated by the two nearly identical boiling curves for the smooth surface. The boiling curves show a clear trend of increasing CHF with surface roughness due to roughnessaugmented capillary forces

it is clear that surface wettability is a key factor dictating CHF [12, 15-20]. Recent works have used the capillary pumping mechanism, which assumes that there is insufficient liquid supply to balance the rate of evaporation, to predict CHF on structured surfaces [8, 16]. However, this model over-predicted $\mathrm{CHF}$ values for our microstructured surfaces $(17-120 \times$ greater than the experimental results), which suggests another mechanism dominates CHF in this case. Kandlikar presented a simplified force-based analysis for smooth surfaces assuming that there is sufficient liquid supply at CHF. Momentum, buoyancy, and surface forces at the liquid/vapor interface of an individual bubble were considered [5]. If the combination of surface and buoyancy forces compensate the momentum force during the growth phase of the bubble, the hot/dry area developed at the base of bubble during growth can rewet upon departure. Otherwise, the hot/dry area will expand irreversibly leading to the CHF condition $[15,20]$. However, in recent studies the data comparing the predictions of the Kandlikar model to the CHF behavior on structured surfaces was typically presented as a function of apparent liquid receding angle $\beta$, which leads to crowding when $\beta \rightarrow 0[9,10]$. This result is attributed to the fact that the model couples bubble geometry and the surface force through the macroscopic contact angle [5]. Thus, on superhydrophilic surfaces the model cannot account for a wide variety of structured surfaces that display no apparent contact angle, i.e., $\beta=0[8-10]$.

To address this issue, we modified the force-balance-based model to predict CHF on superhydrophilic surfaces $(\beta=0)$. In this regime, the microlayer (which includes the structures) underneath the bubble dries out such that a "Wenzel" bubble [21] is formed at CHF [15] as shown in Fig. 4. Therefore, the surface force per unit length maintaining the position of the contact line (i.e., $F_{S, 2}$ in Fig. 4) is amplified due to a longer effective contact line length, which can be estimated, assuming a bubble size larger than the underlying roughness length scale, as the unit length multiplied by the surface roughness,

$$
F_{S, 2}=\sigma_{l v} \times r \cos \theta_{r e c},
$$




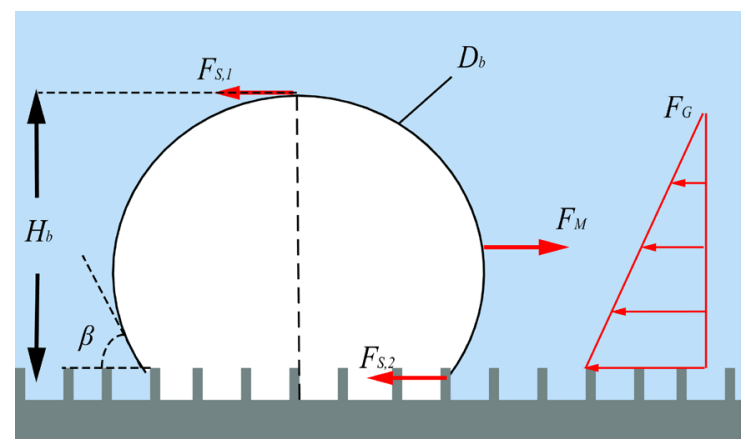

Figure 4: Schematic diagram of horizontal forces acting on the vapor bubble on a structured surface adapted from Kandlikar [5]. At CHF, the vapor film forms within microstructures beneath the bubble, i.e., "Wenzel" bubble. Here $F_{M}$ represents the force due to momentum change while $F_{G}$ represents the buoyancy force, and $F_{S, 1}$ and $F_{S, 2}$ are surface forces. $\beta$ is the apparent liquid receding angle on the structured surface, $H_{b}$ is the height of the bubble and $D_{b}$ is the diameter of the bubble

where $\sigma_{l v}$ is the liquid-vapor surface tension and $\theta_{\text {rec }}$ is the liquid receding angle on the corresponding smooth surface. CHF occurs when momentum force $F_{M}$ is greater than the sum of surface forces $F_{S, 1}, F_{S, 2}$, and buoyancy force $F_{G}$ [5]. Therefore, at CHF, the force balance in horizontal direction yields

$$
F_{M}=F_{S, 1}+F_{S, 2}+F_{G}
$$

Following the set of assumptions introduced by Kandlikar, an expression for $\mathrm{CHF}$ was obtained in the following form:

$$
q_{c}^{\prime \prime}=K \times h_{f g} \rho_{g}^{1 / 2}\left[\sigma_{l v} g\left(\rho_{l}-\rho_{g}\right)\right]^{1 / 4},
$$

where

$$
K=\left(\frac{1+\cos \beta}{16}\right)\left[\frac{2(1+\alpha)}{\pi(1+\cos \beta)}+\frac{\pi}{4}(1+\cos \beta) \cos \psi\right]^{1 / 2},
$$

$\alpha=r \cos \theta_{r e c}, h_{f g}$ is the latent heat, $\rho_{g}$ is the vapor density, and $\psi$ is the inclined angle of surface (i.e., $\psi=0$ for a horizontal upward facing surface). Note that when $\alpha<1$, Eq. 3 simplifies to Kandlikar's model. In this form, the surface force is no longer coupled with the bubble geometry such that Eq. 3 is well-defined in the complete wetting regime where $\cos \beta=1$, but $\alpha>1$.

To demonstrate the applicability of Eq. 3, the predicted CHF as a function of $\alpha$ was overlaid with data from our experiments and previous studies [8-10] in Fig. 5. Also plotted for comparison is the CHF predicted by the classical Kutateladze-Zuber (K-Z) model $[22,23]$ (hydrodynamic instability mechanism) using an empirical factor of $K=0.18$ in Eq. 3 [24]. The $r$ values for Chen et al. [8], Kim et al. [9] and Ahn et al. [10] were estimated based on the reported geometrical parameters and SEMs. These values may be inaccurate due to the fact that the surface roughness was, either, not explicitly reported or the calculation method was not detailed. In addition, contact angles reported in the literature are typically equilibrium values $\left(\theta_{e q}\right)$ measured at room temperature $T_{a}$ [8-10, 13]. Since the surface wettability is a key parameter in determining $\mathrm{CHF}$, the dependence of contact angle and surface tension on temperature should be accounted for $[5,15,19]$. Therefore, to compare the data with our CHF model, estimations for contact angles at the saturation temperature, $T_{\text {sat }}=100{ }^{\circ} \mathrm{C}$, are necessary.
Here, we estimated the variation of $\cos \theta$ with temperature from the Young-Dupré equation [25],

$$
\cos \theta(T)=W_{l s} / \sigma_{l v}(T)-1,
$$

where $\sigma_{l v}(T)$ is the temperature-dependant liquid-vapor surface tension[26] and $W_{l s}$, the work of adhesion between the liquid and solid, was estimated as

$$
W_{l s} \approx 2 \sqrt{\sigma_{s v}^{d} \sigma_{l v}^{d}(T)}
$$

where $\sigma_{s v}$ is the solid-vapor surface tension and the superscript, $d$, represents the dispersive component of surface tension. While $\sigma_{s v}^{d}$ should be only a weak function of temperature, varying by less than $1 \%$ over the investigated range from ambient to saturated temperature, the strong temperature-dependent $\sigma_{l v}^{d}$ is determined from detailed calculations [27].

In Fig. 5, the symbols represent experimental results with estimated $\theta_{\text {rec }}\left(T_{\text {sat }}\right)$ from literature [8-10] and our measurement (solid symbols) and results with $\theta_{\text {rec }}\left(T_{a}\right)$ (hollow symbols) as benchmark values. The difference in $\alpha$ between data from the literature and our estimates demonstrates the potential significance of temperature-dependant liquid contact angle on the amplified surface force (Eq. 1). The error bars for our data along the $\alpha$-axis are based on the uncertainty in the contact angle measurement at $T_{a}$, while the error bars on $\alpha$ for the literature data are due to fact that only $\theta_{e q}$ is reported rather than $\theta_{\text {rec }}$ [8-10]. For these cases, $\alpha$ was estimated using $\theta_{e q} / 2$ (i.e., average between $\theta_{e q}$ and 0 ) where the error in $\theta_{\text {rec }}$ ranged from $\theta_{e q}$ to 0 . The error bars for CHF from Ahn et al. [10] were not reported, and are therefore not shown in Fig. 5. Note that the estimated $\alpha$ values for the data of Kim et al. [9] in the complete wetting regime are shown in the inset of Fig. 5 because of the very large estimated $\alpha$ values $(\alpha \sim 55)$ due to the large reported surface roughness for their nanostructures

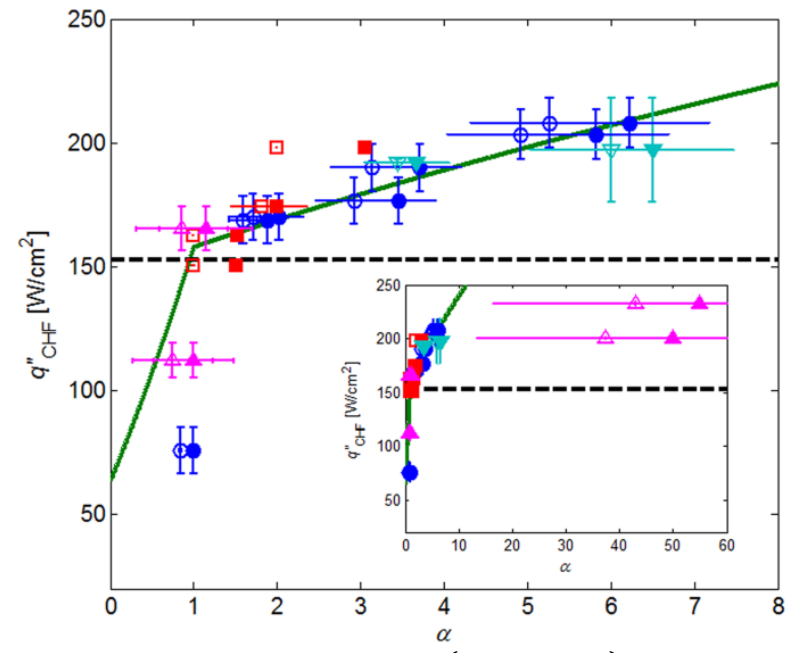

Figure 5: $C H F$ as function of $\alpha\left(=r \cos \theta_{\text {rec }}\right)$. The proposed model (solid line) is compared to the K-Z model [22, 23] with a factor of $K=0.18$ [24] (dashed line) which has been commonly accepted in the past. The symbols show the CHF data from $(\bullet)$ our

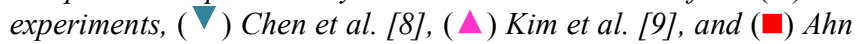
et al. [10] as a function of $\alpha$. The hollow symbols show the data from literature [8-10] while the solid symbols show the results adjusted using our estimation of $\theta_{\text {rec }}\left(T_{\text {sat }}\right)$. The inset shows data from Kim et al. [9] with surface roughness $r \sim 50-55$. 
$(r \sim 50)$ and the approximate nature of our temperature-dependent contact angle analysis. The wide error bars shown in the inset are due to the estimated error of the receding angle (ranging from $\theta_{e q}$ to 0$)$ and the large reported roughness. The result highlights the importance of properly characterizing the wetting properties.

While the value of $\alpha$ has been approximated and simplifications in the extended model exist, the good agreement between the data and model, which does not contain any fitting parameters, suggests that the key physics of the CHF mechanism on these structured surfaces are accounted for. Most importantly, the trend of a small increase in CHF with increasing surface roughness, relative to the regime where $\alpha<1$, is well-captured by the extended model. In addition, the sudden slope reduction predicted by the extended model at $\alpha=1$ can explain how the K-Z model remains well-correlated to $\mathrm{CHF}$ behavior on a range of typical engineering surfaces studied in the past where $\alpha<1.5$ (i.e., native metal oxide). Our extended model also suggests that the $\mathrm{CHF}$ of $\sim 250 \mathrm{~W} / \mathrm{cm}^{2}$ is realizable when $\alpha$ is $\sim 11-12$, which can be achieved by increasing the height of micropillar to $40-50 \mu \mathrm{m}$. However, the reduction in slope of our model for $\alpha>1$ implies that a large increase in surface roughness is required to further enhance $\mathrm{CHF}$, which offers significant fabrication challenges. One potential solution, as demonstrated by Kim et al. [9], is the use of hierarchical structures comprised of multiple roughness length scales. Following the logic suggested by Eq. 1, the effective roughness of a hierarchical surface is estimated as the product of the roughness of each length scale (i.e., $r_{e f f}=\prod_{1}^{N} r_{N}$, where $r_{N}$ is the roughness of each distinct length scale).

\section{CONCLUSION}

An enhancement in $\mathrm{CHF}$ of $\sim 160 \%$ and a $\mathrm{CHF}$ of $\sim 208 \mathrm{~W} / \mathrm{cm}^{2}$, which is comparable to the highest CHF value reported in previous studies on nanostructured surfaces, was demonstrated on the CMOS-compatible microstructured surfaces. To explain the experimental observations, we extended an analytical force balance model to predict CHF in the complete wetting regime. The model shows good agreement with the experimental observations which demonstrates the important effect of roughness-augmented wettability on CHF. The issues of contact angle variation with temperature were also addressed and should be considered in future studies given the nature of the $\mathrm{CHF}$ mechanism presented here. Furthermore, we propose using a hierarchical surface to achieve ultra high CHF based on the model predication. This study shows new insight of the role of structured surfaces in enhancing CHF and suggests opportunities to tailor advanced surface technologies using CMOS-compatible processes to achieve high heat removal for high-power electronics cooling.

\section{ACKNOWLEDGEMENT}

The authors gratefully acknowledge funding support from the Battelle Memorial Institute and the Air Force Office of Scientific Research (AFOSR). R.E. also acknowledges funding received from the Irish Research Council for Science, Engineering, and Technology, cofunded by Marie Curie Actions under FP7.

\section{REFERENCES}

[1] D. C. Price, "A review of selected thermal management solutions for military electronic systems," IEEE Trans. Compon. Packag. Technol., vol. 26, pp. 26 - 39 March 20032003.

[2] T. W. Kenny, et al., "Advanced Cooling Technologies for Microprocessorsf," Int. J. High Speed Electron. Syst, vol. 16, pp. 301313, 2006.

[3] J. R. Thome, "The New Frontier in Heat Transfer: Microscale and Nanoscale Technologies," Heat Transfer Eng., vol. 27, pp. 1-3, 2006.
[4] E. Pop, "Energy dissipation and transport in nanoscale devices," Nano Res., vol. 3, pp. 147-169, 2010.

[5] S. G. Kandlikar, "A Theoretical Model to Predict Pool Boiling CHF Incorporating Effects of Contact Angle and Orientation," J. Heat Transfer, vol. 123, pp. 1071-1079, 2001.

[6] E. Forrest, et al., "Augmentation of nucleate boiling heat transfer and critical heat flux using nanoparticle thin-film coatings," Int. J. Heat Mass Transfer, vol. 53, pp. 58-67, 2010.

[7] C. H. Li and G. P. Peterson, "Experimental study of enhanced nucleate boiling heat transfer on uniform and modulated porous structures," FHMT, vol. 1, p. 023007, 2010.

[8] R. Chen, et al., "Nanowires for Enhanced Boiling Heat Transfer," Nano Lett., vol. 9, pp. 548-553, January 16, 20092009.

[9] S. Kim, et al., "Effects of nano-fluid and surfaces with nano structure on the increase of CHF," Exp. Therm Fluid Sci., vol. 34, pp. 487-495, May 20102010.

[10] H. S. Ahn, et al., "Effect of liquid spreading due to nano/microstructures on the critical heat flux during pool boiling," Appl. Phys. Lett., vol. 98, p. 0719082011.

[11] J. Bico, et al., "Rough wetting," Europhysics Letters, vol. 55, pp. 214$220,2001$.

[12] T. G. Theofanous, et al., "The boiling crisis phenomenon Part I: nucleation and nucleate boiling heat transfer," Exp. Therm. Fluid Sci., vol. 26, pp. 775-792, 2002.

[13] M.-C. Lu, et al., "Critical heat flux of pool boiling on Si nanowire array-coated surfaces," Int. J. Heat Mass Transfer, vol. 54, p. Int. J. Heat Mass Transfer, 2011.

[14] R. Xiao, et al., "Prediction and Optimization of Liquid Propagation in Micropillar Arrays," Langmuir, vol. 26, pp. 15070-15075, 2010.

[15] C. Gerardi, et al., "Infrared thermometry study of nanofluid pool boiling phenomena," Nanoscale Res. Lett., vol. 6, p. 232, 2011.

[16] S. G. Liter and M. Kaviany, "Pool-boiling CHF enhancement by modulated porous-layer coating: theory and experiment " Int. J. Heat Mass Tran., vol. 44, pp. 4287-4311, 2001.

[17] G. P. Narayan, et al., "Mechanism of enhancement/deterioration of boiling heat transfer using stable nanoparticle suspensions over vertical tubes.," J. Appl. Phys., vol. 102, p. 074317, 2007.

[18] S. J. Kim, et al., "Surface wettability change during pool boiling of nanofluids and its effect on critical heat flux," Int. J. Heat Mass Transfer, vol. 50, pp. 4105-4116, 2007.

[19] T. G. Theofanous and T.-N. Dinh, "High heat flux boiling and burnout as microphysical phenomena: mounting evidence and opportunities," Multiphase Sci Technol., vol. 18, pp. 1-26, 2006.

[20] T. G. Theofanous, et al., "The boiling crisis phenomenon Part II: dryout dynamics and burnout," Exp. Therm. Fluid Sci., vol. 26, pp. 793-810, 2002.

[21] R. N. Wenzel, "Resistance of Solid Surfaces to Wetting by Water," Ind. Eng. Chem., vol. 28, pp. 988-994, 1936.

[22] N. Zuber, "Hydrodynamic Aspects of Boiling Heat Transfer," AEC Report AECU-4439, 1959.

[23] S. S. Kutateladze, "On the Transition to Film. Boiling under Natural Convection," Kotloturbostroenie, vol. 3, pp. 10-12, 1948.

[24] W. M. Rohsenow, et al., Handbook of heat transfer fundamentals, 2 ed. New York: McGraw-Hill, 1985.

[25] J. N. Israelachvili, Intermolecular and Surface Forces, 3 ed.: Elsevier, 2011.

[26] E. W. Lemmon, et al., "NIST Standard Reference Database 23: Reference Fluid Thermodynamic and Transport PropertiesREFPROP, Version 8.0," ed. Gaithersburg, MD: U.S. Department of Commerce, Technology Administration, National Institute of Standards and Technology, 2007.

[27] S. Takeda, et al., "Surface $\mathrm{OH}$ group governing adsorption properties of metal oxide films," Thin Solid Films, vol. 339 pp. 220-224, 1999.

\section{CONTACT}

*K.-H. Chu, tel: +1-617-324-3311; khchu@mit.edu 


\title{
HIGH-STRESS FATIGUE EXPERIMENTS ON SINGLE CRYSTAL SILICON IN AN OXYGEN-FREE ENVIRONMENT
}

\author{
V.A. Hong ${ }^{1}$, S. Yoneoka ${ }^{1,2}$, M.W. Messana ${ }^{1,3}$, A.B. Graham ${ }^{1,4}$, J.C. Salvia ${ }^{1,5}$, \\ T.T. Branchflower ${ }^{l}$, E.J. $\mathrm{Ng}^{l}$, and T.W. Kenny ${ }^{l}$ \\ ${ }^{1}$ Stanford University, USA, ${ }^{2}$ now at mCube Inc, USA, ${ }^{3}$ now at Skybox Imaging, Inc, USA, \\ ${ }^{4}$ now at Robert Bosch LLC Research and Technology Center, USA, ${ }^{5}$ now at SiTime Corporation, USA
}

\section{ABSTRACT}

In this paper we present the first fatigue study with stress loadings above $2 \mathrm{GPa}$ applied to single-crystal silicon (SCS) resonators in an inert environment provided by the 'epi-seal' encapsulation process. In total, 6 devices representing a total of 16 experiments were actuated for $>10^{10}$ cycles with stress levels $>3.2$ $\mathrm{GPa}$ at $32^{\circ} \mathrm{C}$. No fatigue-like behavior was observed up to 7.5 $\mathrm{GPa}$, which is far beyond the threshold for fatigue reported by others. High-cycle fatigue in SCS is therefore likely to be a surface or environmentally-assisted phenomenon that can be minimized using better packaging techniques, such as 'epi-seal'.

\section{INTRODUCTION \\ Background}

MEMS-based sensors and actuators made from single-crystal silicon (SCS) are commonly subjected to high-cycle loadings. Understanding and preventing microscale fatigue of SCS therefore plays a large role in the MEMS design process to ensure the longterm stability and reliability of a device. To prevent SCS fatigue, devices are designed to operate below stress levels in which fatigue has been witnessed with the appropriate safety factor. Characterizing these fatigue-inducing stress levels is thus important to allow devices to extract more performance, and has subsequently been a topic of research for the past 20 years.

Since Connally and Brown's seminal work in 1992 [1], there have been many reports of SCS fatigue [2-13]. Several fatigue mechanisms have been proposed; the predominate ones being the reaction layer model from Muhlstein et al. [2] and the subcritical cracking model from Kahn et al. [3]. The reaction layer model asserts that a crack occurs in a native oxide layer and the oxide layer thickens due to SCS exposure to moisture or oxygen. Fatigue of SCS ensues due to repeated cracking and oxide-thickening until the crack has reached a critical length, leading to catastrophic failure of the device. Alternatively, the subcritical cracking model asserts that fatigue cracks occur in the silicon. It is proposed that compressive stresses promote crack extension in the silicon itself due to the increased stress intensity brought on by a wedging effect of native oxide or wear debris. These two fatigue mechanisms center around the effects of surface oxide, humidity and oxygen, and their exact roles remain controversial.

\section{'Epi-seal' encapsulation}

The 'epi-seal' encapsulation process developed at Stanford enables the packaging of an oxide-free MEMS device in an environment that is free of humidity, oxygen, and organics [14], thus eliminating the main factors that other researchers have attributed to long-term fatigue.

Previous experiments performed by Yoneoka et al. using devices fabricated in the 'epi-seal' process confirmed the absence of SCS fatigue using this encapsulation technique, however, the geometry of these devices limited stress levels to $<2 \mathrm{GPa}$ [12]. Devices were unable to be actuated beyond the $3.2 \mathrm{GPa}$ in which other researchers have always witnessed fatigue, as seen in Fig. 6.

In this work wedge-shaped resonators, a design commonly used in MEMS fatigue testing, were fabricated using the 'epi-seal' encapsulation process. The geometry of the comb fingers was modified to accommodate larger stress levels than from Yoneoka et al. Devices were subjected to fully-reversed stresses and resonant frequency change was monitored using an oscillator circuit with automatic gain control (AGC).

\section{DESIGN}

Fatigue test resonators with resonant frequencies between 60 $\mathrm{kHz}$ and $70 \mathrm{kHz}$ were designed and fabricated on a $20 \mu \mathrm{m}$ thick device layer. The test structure consists of a wedge-shaped proof mass connected to the anchor with a cantilever. A V-shaped notch, with depth, $d$, of $7.5 \mu \mathrm{m}$ and radius, $r$, of $0.5 \mu \mathrm{m}$, is placed on the cantilever to concentrate stress. The cantilever, aligned in the $<110>$ direction, has a width, $w$, of $15 \mu \mathrm{m}$ and length, $l$, of $30 \mu \mathrm{m}$. The overall length of the structure, including the proof mass, is 300 $\mu \mathrm{m}$. Because of design rules that limit the structure's release area, the proof mass was designed in a Y-shape to accommodate a support for the cap layer. The proof mass, which has comb fingers, is electrostatically actuated on one side by a stationary drive electrode. On the other side a stationary electrode senses the displacement by measuring the change in capacitance between the proof mass and electrode when the resonator is driven.

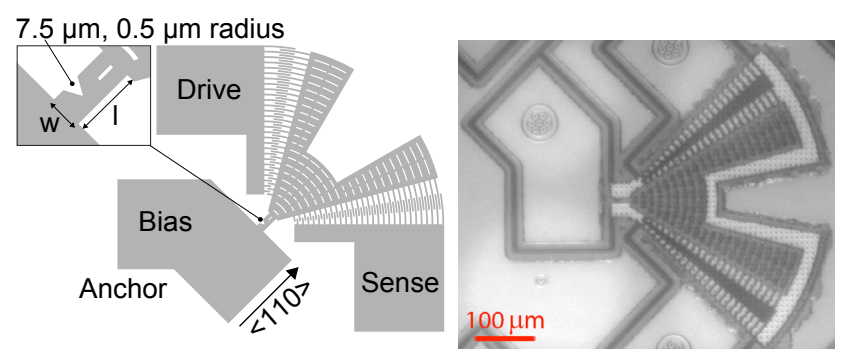

Figure 1: (a) Design of wedge-shaped resonator used as the fatigue structure. (b) Infrared image of a fabricated fatigue structure.

\section{FABRICATION}

The fatigue test resonators were fabricated using a process developed by Messana et al. [15] that combined traditional 'episeal' encapsulation with wafer bonding. Devices were defined with a deep reactive-ion etch and were sealed using a combination of fusion wafer bonding and epitaxially grown silicon, resulting in a SCS cap layer, as shown in Fig. 2. The wafers are sealed at 1130 ${ }^{\circ} \mathrm{C}$ in a hydrogen ambient (20 Torr), resulting in an oxide-free device and cavity free of oxygen and humidity. The wafers were subsequently annealed for more than 100 hours to diffuse out residual hydrogen, thereby reducing cavity pressure and increasing the quality factor of the resonators.

Furthermore, resonators fabricated using this encapsulation technology have demonstrated no discernable inherent aging or drift at the ppm-level under low stresses $(<150 \mathrm{MPa})$ over 416 days of continuous operation [16], providing a robust platform for investigating fatigue-related effects at higher stress levels. 


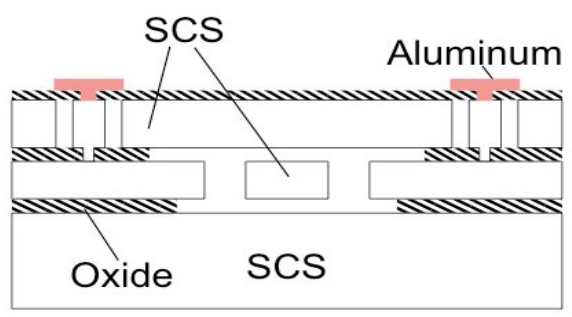

Figure 2: Cross-section schematic of 'epi-seal' encapsulation with SCS device and cap. The device layer thickness is approximately $20 \mu \mathrm{m}$.

\section{EXPERIMENTAL SETUP Oscillator Setup}

The resonators are driven with a fully-reversed $(\mathrm{R}=-1)$, constant amplitude sinusoidal signal in a close-loop oscillator employing AGC, as shown in Fig. 3. $V_{S E T}$ controls the amplitude of the drive signal, $V_{A C}$, and a DC bias voltage, $V_{\text {bias }}$, enables electrostatic actuation; moreover, adjusting this voltage allows for control of the applied stress level. A counter and multimeter are placed after the transimpedance-amplifier (TIA) to measure frequency and RMS voltage of the output signal from the resonator. Any fatigue-like behavior is detected using the measured frequency, as changes in stiffness or mass would immediately be reflected in the resonant frequency; fatigue would be marked by a downward drift in frequency

During experimentation, four resonator-oscillator circuits were placed in a temperature chamber kept at $32 \pm 0.1{ }^{\circ} \mathrm{C}$. A multiplexer enables switching between each resonator-oscillator setup, which allows for monitoring of devices with a single counter and multimeter.

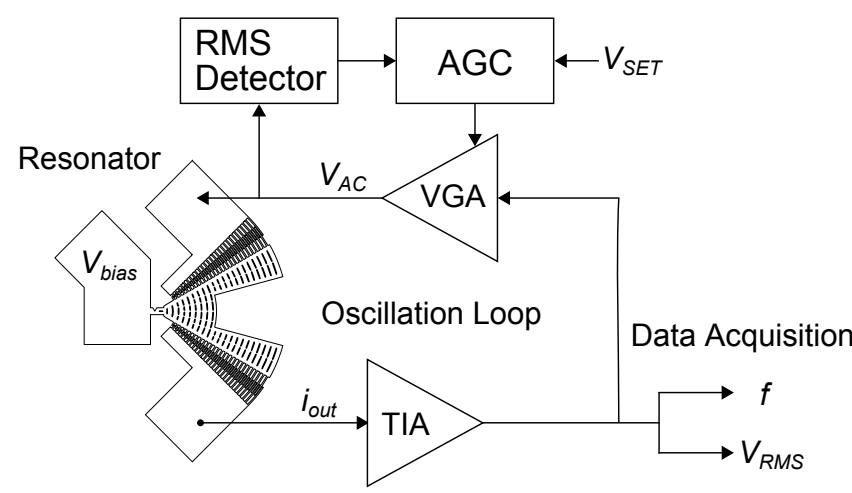

Figure 3: Block diagram of oscillator loop used to drive the resonator. The AGC loop allows for constant AC voltage input by using a variable gain amplifier (VGA).

\section{Stress Calculation}

Though 'epi-seal' provides a robust platform for device packaging, the encapsulation does not allow for direct measurement of stress, therefore stress at the notch must be calculated based on the resonator's electrical output signal, namely the frequency and amplitude.

The measured current arises due to time-varying capacitance at the sense electrode and is given by the following relation, $i_{\text {out }}=\frac{\partial Q}{\partial t}=V_{\text {bias }} \frac{\partial C}{\partial \theta} \frac{\partial \theta}{\partial t}=V_{\text {bias }} \frac{\partial C}{\partial \theta} \theta_{\text {amp }} j \omega e^{j \omega t}$,

where $Q$ is charge, $t$ is time, $\theta$ is angle, $\theta_{\text {amp }}$ is maximum angular displacement, $V_{\text {bias }}$ is bias voltage, $C$ is the total capacitance of the comb fingers, and $\omega$ is angular frequency. The capacitance is calculated by approximating the comb fingers as cylindrical plates and summing over all combs, as shown in the following:

$C=\sum_{k} \frac{\varepsilon h \theta}{\ln \left(r_{o, k} / r_{i, k}\right)}$,

where $\varepsilon$ is permittivity, $h$ is device thickness, $r_{o, k}$ is the outer radius for specific comb finger $k$, and $r_{i, k}$ is the inner radius for specific comb finger $k$.

From Eq. (1), the current amplitude, $i_{a m p}$, is calculated from the measured RMS voltage, $V_{R M S}$, and the current-to-voltage transfer function, $G(j \omega)$ of the TIA, shown as

$i_{\text {amp }}=\frac{\sqrt{2} V_{R M S}}{G(j \omega)}=V_{b} \frac{\partial C}{\partial \theta} \theta_{a m p} \omega$.

$\theta_{\text {amp }}$ is then determined and stress at the notch is subsequently calculated based on a FEA-modeled transfer function of 2.82 $\mathrm{GPa} /{ }^{\circ}$.

\section{RESULTS AND DISCUSSION}

During experimentation, the fatigue structures were subjected to cyclic stress amplitudes of up to $7.5 \mathrm{GPa}$. Fig. 4 is a representative data set that shows frequency change $(\Delta f)$, stress amplitude, bias voltage, and temperature monitored over a period of greater than 10 days. $\Delta f$ is a normalized frequency change, defined as ppm $=10^{6} \times\left(f-f_{0}\right) / f_{0}$, where $f_{0}$ is the initial frequency.
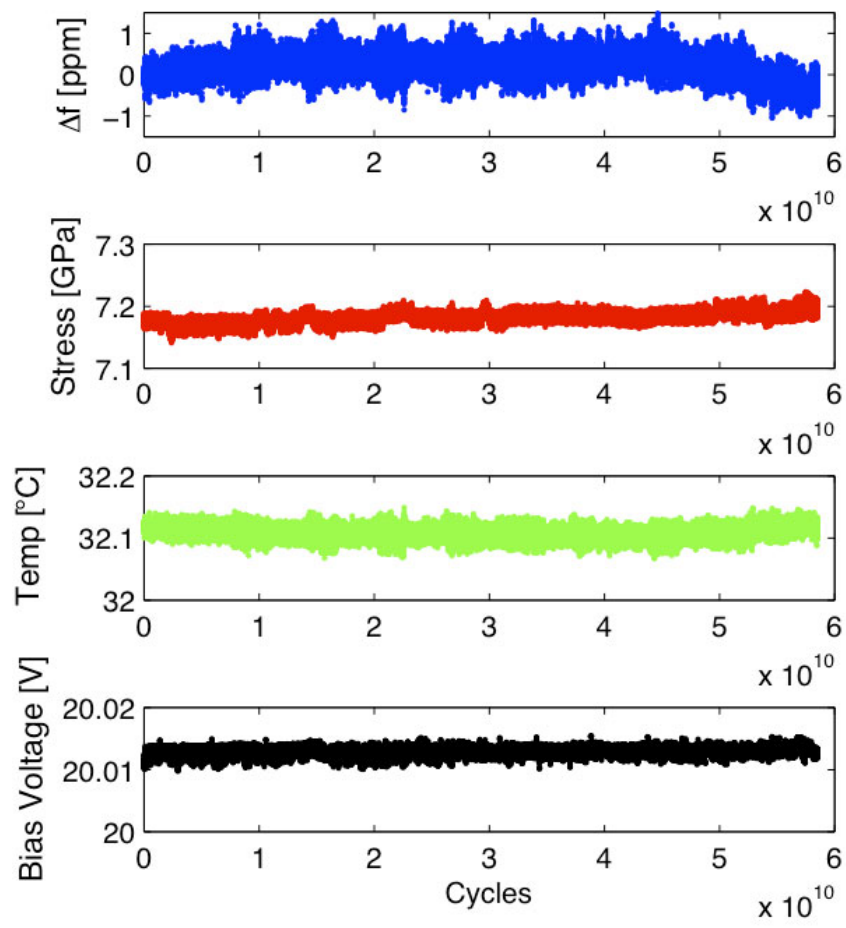

Figure 4: A representative data set, including plot of frequency change in ppm, stress amplitude at notch, temperature, and bias voltage over $6 \times 10^{10}$ actuation cycles (>10 days). 
No fatigue related behavior was observed in any of our experiments. Fluctuations in frequency at the ppm-level can be attributed to temperature or bias voltage noise. Furthermore, a linear fit of $\Delta f$ vs. cycles (normalized to $10^{10}$ cycles) was performed to examine whether device frequencies drifted due to long-term fatigue damage. Fig. 5 shows that although devices exhibited negative frequency drifts, these drifts were smaller than the noise level $( \pm 1.3 \mathrm{ppm})$ at $32^{\circ} \mathrm{C}$. In addition, there was no correlation between drift and stress amplitude, which would be expected in the case of fatigue. These results confirm that SCS is not susceptible to long-term fatigue damage.

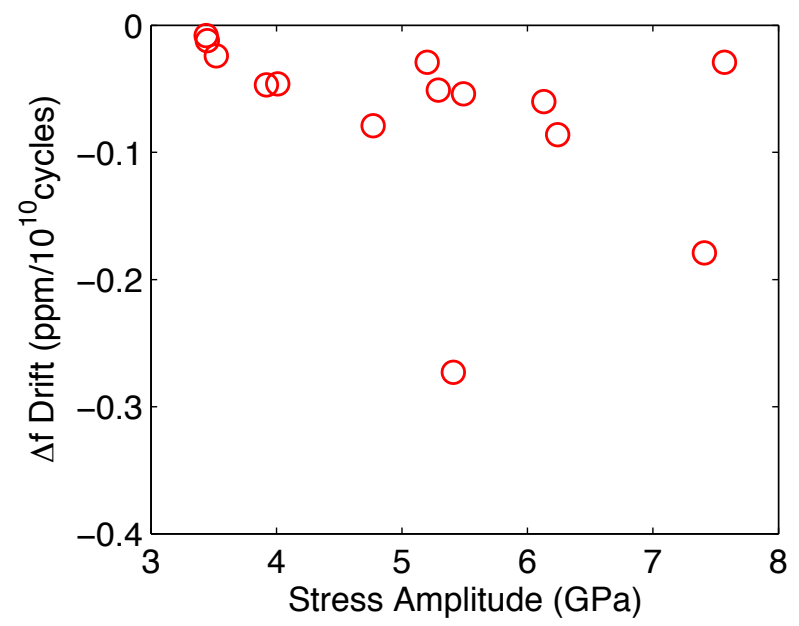

Figure 5: Calculation of frequency drift normalized over $10^{10}$ cycles from various fatigue test experiments with different stress amplitude loadings.

\section{Cross-Comparison Summary}

Fig. 6 shows a cross-comparison between this work and data presented from literature. The 'target' region in the top-right corner marks an area in which other researchers always witnessed fatigue failure, both in terms of stress $(>3.2 \mathrm{GPa})$ and number of cycles $\left(>10^{10}\right)$. These other works witnessed fatigue under varying conditions, but none of these experiments are as controlled as the inert environment and pristine device surface provided by 'episeal' encapsulation.

When compared against other works that reported fatigue [913] at temperatures between $22-30^{\circ} \mathrm{C}$, our devices were subjected to higher stress levels and cycles without onset of fatigue failure. In total, 6 different devices from this work were tested in the 'target' region, making up a total of 16 experiments in which no fatigue failure occurred. Applying higher bias voltages to achieve stress levels above $7.5 \mathrm{GPa}$ resulted in either immediate mechanical fracture or electrical pull-in of the comb fingers; since these results are not due to fatigue failure, they are not presented in Fig. 6.

\section{CONCLUSION}

This work presents a packaging technique that allows for single-crystal silicon devices to be actuated at stress amplitudes up to $7.5 \mathrm{GPa}$ without the onset of high-cycle fatigue. We conclude that fatigue failure at room temperature $\left(<32^{\circ} \mathrm{C}\right)$ witnessed from other works is either a surface or environmentally assisted phenomenon that can be eliminated with the 'epi-seal' encapsulation process. This important result allows for MEMS designers to extract more performance from current designs and allows for greater flexibility in design guidelines for MEMS devices experiencing high cyclic loading.

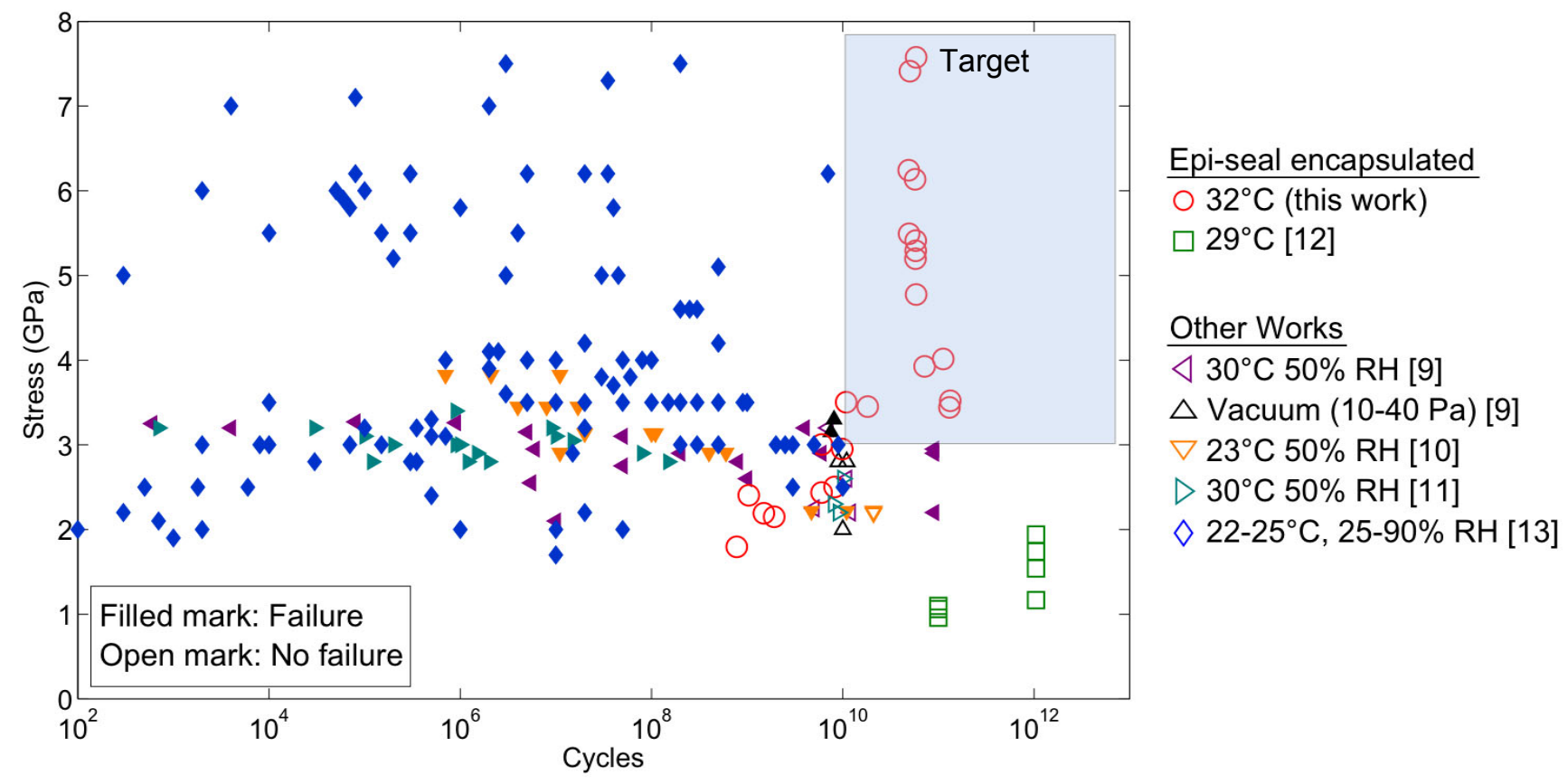

Figure 6: Stress-life ( $S-N)$ plot of data from various studies. All tests from this paper are run-out points (open mark), in which no sign of failure was observed. The "target" region represents stress levels in which other research groups always witnessed failure. We tested 6 different devices constituting 16 experiments in that region with no failure, providing an important design guideline for future MEMS designers. 


\section{ACKNOWLEDGEMENTS}

This work was supported by the Defense Advanced Research Projects Agency (DARPA) thorough the N/MEMS S\&T Fundamentals Program, managed by Dr. Tayo Akinwande. Student support was provided by a National Science Foundation Graduate Research Fellowship (VAH). The authors would like to thank Prof. R.T. Howe and H.K Lee for advice and guidance. Travel support has been generously provided by the Transducer Research Foundation.

\section{REFERENCES}

[1] J.A. Connally and S.B. Brown, "Slow crack growth in singlecrystal silicon," Science, vol. 256, pp. 1537-1539, 1992.

[2] C.L. Muhlstein, E.A. Stach, and R.O. Ritchie, "A reactionlayer mechanism for the delayed failure of micron-scale polycrystalline silicon structural films subjected to high-cycle fatigue loading," Acta Mater., vol. 50, pp. 3579-3595, 2002.

[3] H. Kahn, R. Ballarini, J.J. Bellante, and A.H. Heuer, "Fatigue failure in polysilicon not due to simple stress corrosion cracking," Science, vol. 298, pp. 1215-1218, 2002.

[4] H. Kahn, R. Ballarini, and A.H. Heuer, "Dynamic fatigue in silicon," Curr. Opin. Solid in State Mater. Sci., vol. 9, pp. 7176, 2004.

[5] C.L. Muhlstein, S.B. Brown, and R.O. Ritchie, "High-cycle fatigue of single-crystal silicon thin films," J. Microelectromech. Syst., vol. 10, pp. 593-600, 2001.

[6] T. Ando, M. Shikida, and K. Sato, "Tensile-mode fatigue of silicon films as structural materials for MEMS," Sens. Actuators: A, vol. 93, pp. 70-75, 2001.

[7] A.M. Fitzgerald, R.S. Iyer, R.H. Dauskardt, and T.W. Kenny, "Subcritical crack growth in single-crystal silicon using micromachined specimens," J. Matter. Res., vol. 17, pp. 683692, 2002.

[8] T. Namazu and Y. Isono, "High-cycle fatigue damage evaluation for micro-nanoscale single crystal silicon under bending and tensile stressing," MEMS '04, Maastricht, Netherlands, Jan. 25-29, 2004, pp. 149-152.

[9] O.N. Pierron and C.L. Muslstein, "The critical role of environment in fatigue damage accumulation in deep-reactive ion-etched single-crystal silicon structural films," J. Microelectromech. Syst., vol. 15, pp. 111-119, 2006.
[10] T. Ikehara and T. Tsuchiya, "High-cycle fatigue of micromachined single-crystal silicon measured using highresolution patterned specimens," J. Micromech. Microeng., vol. 18 , pp. 1-7, 2008

[11] P.-O. Theillet and O.N. Pierron, "Fatigue rates of monocrystalline silicon thin film in harsh environments: Influence of stress amplitude, relative humidity, and temperature," Appl. Phys. Lett., vol. 94, 181915, 2009.

[12] S. Yoneoka, Y.Q. Qu, S. Wang, M.W. Messana, A.B. Graham, B. Kim, R. Melamund, G. Bahl, and T.W. Kenny, "High-cyclic fatigue experiments of single crystal silicon in an oxygen-free environment," MEMS '10, Wanchai, Hong Kong, Jan. 24-28, 2010, pp. 224-227.

[13] S. Kamiya, T. Tsuchiya, T. Ikehara, K. Sato, T. Ando, T. Namazu, and K. Takashima, "Cross comparison of fatigue lifetime testing on silicon thin film specimens," MEMS '11, Cancun, Mexico, Jan. 23-27, 2011, pp. 404-407.

[14] R.N. Candler, W.T. Park, H. Li, G. Yama, A. Partridge, M. Lutz, and T.W. Kenny, "Single wafer encapsulation of MEMS devices," IEEE Trans. Adv. Packag., vol. 26, pp. 227232, 2003.

[15] M.W. Messana, A.B. Graham, S. Yoneoka, R.T. Howe, and T.W. Kenny, "Packaging of large lateral deflection MEMS using a combination of fusion bonding and epitaxial reactor sealing," Proc. Hilton Head Workshop, June 6-10, 2010, pp. 70-73.

[16] B. Kim, R.N. Candler, M. Hopcroft, M. Agarwal, W.-T. Park, and T.W. Kenny, "Frequency stability of wafer-scale film encapsulated silicon based MEMS resonators," Sens. Actuators: A, vol. 136, pp. 125-131, 2007.

[17] R. N. Candler, W.-T. Park, M. Hopcroft, B. Kim, and T. W. Kenny, "Hydrogen diffusion and pressure control of encapsulated MEMS resonators," Transducers '05, Seoul, Korea, June 5-9, 2005, pp. 920-923.

\section{CONTACT}

*V.A. Hong, tel: +1-619-885-7081; vuhong@stanford.edu 


\section{LOW POWER STRAIN SENSING USING TUNNELING CURRENT THROUGH MOS CAPACITORS \\ Li Zhu and Shamus McNamara \\ Dept. of Electrical \& Computer Engineering, University of Louisville, Louisville, KY, USA}

\begin{abstract}
Although MEMS pressure sensors and accelerometers have been widely researched and commercialized for decades, the recently increasing requirement for low power sensors is motivating research on new techniques. We present a new technology to make very low power sensors by measuring the tunneling current (Ig) through a MOS capacitor. The tunneling current can be in the nano-amp range, which is a good alternative for low power sensing. We demonstrate a power consumption of couple of nano-Walts (nW) with a minimum detectable strain of $0.0005 \%$. This method can be used to create very low power sensors compared to typical piezoresistive and capacitive sensors whose power is typically in the $\mu \mathrm{W}$ to $\mathrm{mW}$ range. Our fabrication process is very simple and compatible with CMOS processes.
\end{abstract}

\section{INTRODUCTION}

Increasing requirement of low power sensors in the portable electronics and other battery powered devices is motivating research on new technologies. Piezoresistive and capacitive sensing technologies are the most common technologies, which have been widely used to measure strain, pressure and acceleration $[1,2]$. They both typically consume power from $\mu \mathrm{W}$ to $\mathrm{mW}[3]$. Considering the piezoresistive sensors, to achieve low power consumption, we have to decrease doping concentration. For example, to have a minimum detectable strain of $0.0005 \%$ (which is our device's resolution), the doping concentration should be around $10^{16}$ atoms $/ \mathrm{cm}^{3}$ [4]. This doping concentration gives a resistivity of $1 \mathrm{ohm}-\mathrm{cm}$. The tunneling current can be in the nanoamp range and results in a resistivity of $10^{13} \mathrm{ohm}-\mathrm{cm}$, which is a good alternative for low power sensing. Besides adopting tunneling through MOS capacitor, tunneling sensors also employ tunneling current through an air gap between a tip and a plate [5-7]. However, their fabrication process is complex, the response is nonlinear, they require closed-loop control, and the reliability is impacted due to deterioration of the tip. Our technique may overcome these challenges.

Fig. 1 shows the fabrication process of a cantilever beam with a tunneling oxide. The starting wafer is a heavily doped P-type (100) Silicon wafer. A $500 \mathrm{~nm}$ thick thermally grown oxide layer is patterned by wet etching. Then, a thin oxide layer $(3.9 \mathrm{~nm})$ is grown with an area of $1 \mathrm{~mm}^{2}$. Finally, Aluminum is sputtered and patterned as contacts.

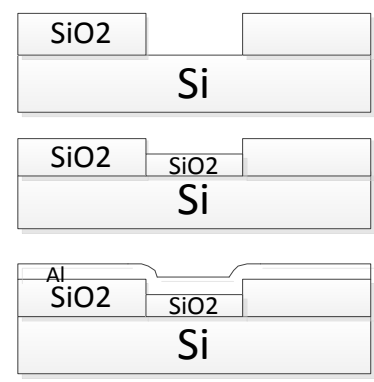

Fig.1 fabrication process

\section{THEORIES}

Since the $\mathrm{SiO}_{2}$ is $3.9 \mathrm{~nm}$ thick, the main tunneling current is direct tunneling when the voltage is lower than $2.5 \mathrm{~V}$. When a positive voltage is applied on Al, the tunneling current comes from the electrons tunneling from $\mathrm{Si}$ to $\mathrm{Al}$ as shown in Fig. 2(a). The MOS capacitor's tunneling current through the Silicon dioxide is determined by (1) the energy barrier height between the Silicon conduction band edge and the $\mathrm{SiO}_{2}$ conduction band edge; and (2) the electrons' out of plane effective mass [8-10]. When a [110] uniaxial tensile strain is induced, the conduction band edge of Silicon is lowered. The six-fold degenerate conduction band valleys are split into two groups, the two-fold degenerate $\Delta 2$ valleys and the four-fold degenerate $\Delta 4$ valleys. $\Delta 2$ valleys have a lower energy which cause the electrons to repopulate in the $\Delta 2$ valleys, and electrons in them have a heavier out of plane effective mass. As a result, both effects decrease the tunneling current. On the contrary, as a [110] uniaxial compressive strain is induced, the energy barrier is decreased and $\Delta 4$ valleys has a lower energy and a lighter out of plane effective mass, so the tunneling current is increased.
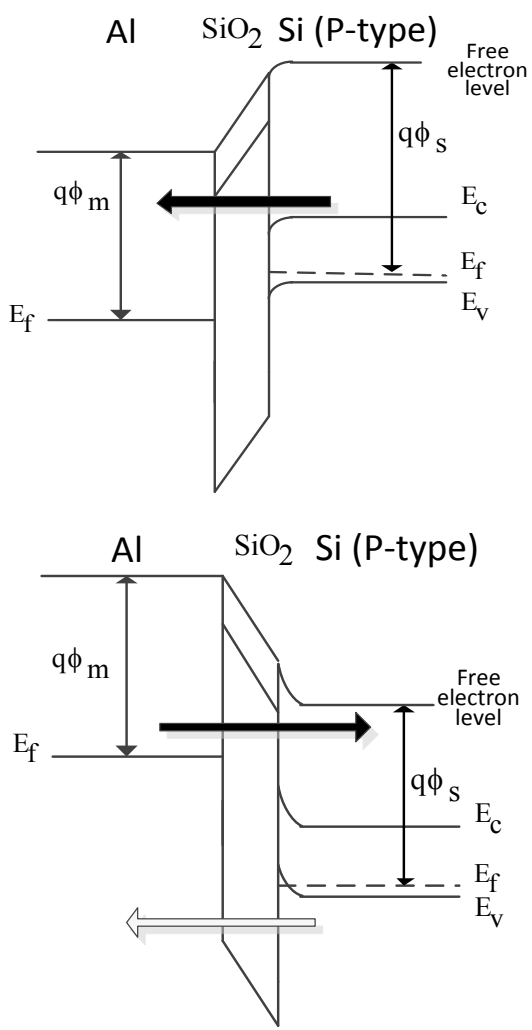

Fig. 2 Tunneling current in the MOS capacitor. (a) electrons tunnel from $\mathrm{Si}$ to $\mathrm{Al}$ with positive applied voltage at $\mathrm{Al}$; (b) electrons tunnel from $\mathrm{Al}$ to $\mathrm{Si}$ and holes tunnel from $\mathrm{Si}$ to Al with negative applied voltage at $\mathrm{Al}$ 
When a negative voltage is applied on Al, the tunneling current is composed by electrons tunneling from $\mathrm{Al}$ to $\mathrm{Si}$ and holes tunneling from Si to $\mathrm{Al}$ as shown in Fig. 2(b). Because the barrier height for holes is higher than that for electrons, the tunneling current mainly depends on the electrons. The tunneling current is then only determined by Al's work function. Uniaxial tensile strain causes Al's work function to decrease, so the tunneling current increases. Uniaxial compressive strain causes Al's work function to increase, so the tunneling current decreases [11].

\section{EXPERIMENT}

Fig. 3 shows the measurement setup. One end of the beam was glued to a metal stage by conductive epoxy, while the other end was deformed by scrolling a micrometer. The micrometer positions are converted to strain. The tunneling current was recorded by an HP4156c Semiconductor Parameter Analyzer. Both uniaxial tensile strain and uniaxial compressive strain are in the [110] direction. The tunneling currents in the accumulation $(-1 \mathrm{~V})$, depletion $(0.5 \mathrm{~V})$ and inversion $(1 \mathrm{~V}$ and $2 \mathrm{~V})$ regions were measured.

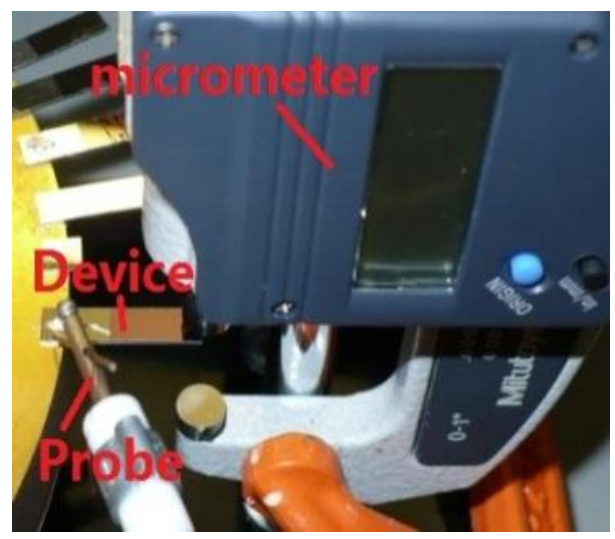

Fig. 3 photograph of measurement setup

All the measurements were done by increasing the strain from $0 \%$ to $0.112 \%$ at the step of $0.016 \%$, and then decreasing the strain from $0.112 \%$ to $0 \%$ at the step of $0.016 \%$. Each step has 25 samples with a sampling time of 2 seconds. The voltage applied on the device is constant voltage.

\section{RESULTS AND DISCUSSION}

Table I analysis of experiment data

\begin{tabular}{|c|c|c|c|c|c|c|}
\hline Voltages & $\begin{array}{c}\text { Avg } \\
(\mathrm{nA})\end{array}$ & $\begin{array}{c}\text { SD } \\
(\mathrm{A})\end{array}$ & $\begin{array}{c}\text { Drift } \\
\text { rate } \\
(\%)\end{array}$ & $\begin{array}{c}\frac{\text { SD }}{\text { avg }} \\
(\%)\end{array}$ & $\begin{array}{c}\Delta \mathrm{Ig} / \mathrm{Ig} \\
(\%)\end{array}$ & GF \\
\hline $2 \mathrm{~V}$ (tensile) & 76.22 & $1.7 \mathrm{E}-11$ & 5.7 & 0.022 & -2.5 & 22.3 \\
\hline $1 \mathrm{~V}$ & 1.415 & $1.0 \mathrm{E}-13$ & 1.55 & 0.007 & -2.2 & 19 \\
\hline $0.5 \mathrm{~V}$ & 0.031 & $2.3 \mathrm{E}-13$ & -1.3 & 0.7 & -0.62 & 5.6 \\
\hline$-1 \mathrm{~V}$ & 0.147 & $3.6 \mathrm{E}-13$ & -6.5 & 0.24 & 0.38 & 3.4 \\
\hline $2 \mathrm{~V}$ (compress) & 75 & $2.0 \mathrm{E}-11$ & 5.2 & 0.026 & 2.1 & 19 \\
\hline $1 \mathrm{~V}$ & 1.085 & $4.4 \mathrm{E}-13$ & 7.6 & 0.04 & 1.8 & 16 \\
\hline $0.5 \mathrm{~V}$ & 0.039 & $1.5 \mathrm{E}-14$ & -5.8 & 0.038 & 1.2 & 11.2 \\
\hline$-1 \mathrm{~V}$ & 0.155 & $1.1 \mathrm{E}-13$ & -18.5 & 0.07 & -0.38 & 3.4 \\
\hline
\end{tabular}

The results of tensile strain and compressive strain are given in the figure $4 \& 5$ and figure $6 \& 7$ respectively. In each figure, strains are shown on the top. Table I gives several analysis for the measurement results. The blue solid lines are the tested currents, which are not constant but drifting with time. Since in the beginning the currents decrease or increase sharply, all the drift rates are calculated from 50 seconds to 800 seconds. Because of the drift, it is uneasy to have a clear vision of how currents change versus strain especially for low voltages. We eliminate the drift of the tested current according to the slope of the current curve. The tested currents after drift correction are plotted by dashed red line. There are some spikes in the tested results of $1 \mathrm{~V}, 0.5 \mathrm{~V}$ and $-1 \mathrm{~V}$. These spikes appear at the time that the strains are changed, so they are introduced by human body and can be ignored in the analysis.

\section{Tensile strain}

In Fig. 4, as the theories predict, increasing tensile strains cause the current to decrease at the positive voltages. In the other hand, increasing tensile strains cause the current tunneling from metal to Silicon to increase at the negative voltages. The $\Delta \mathrm{Ig} / \mathrm{Ig}$ at the strain of $0.112 \%$ at $2 \mathrm{~V}$ (inversion) and $1 \mathrm{~V}$ (weak inversion) are much larger than that at $0.5 \mathrm{~V}$ (depletion). The $\Delta \mathrm{Ig} / \mathrm{Ig}$ at the strain of $0.112 \%$ at $-1 \mathrm{~V}$ is very small.
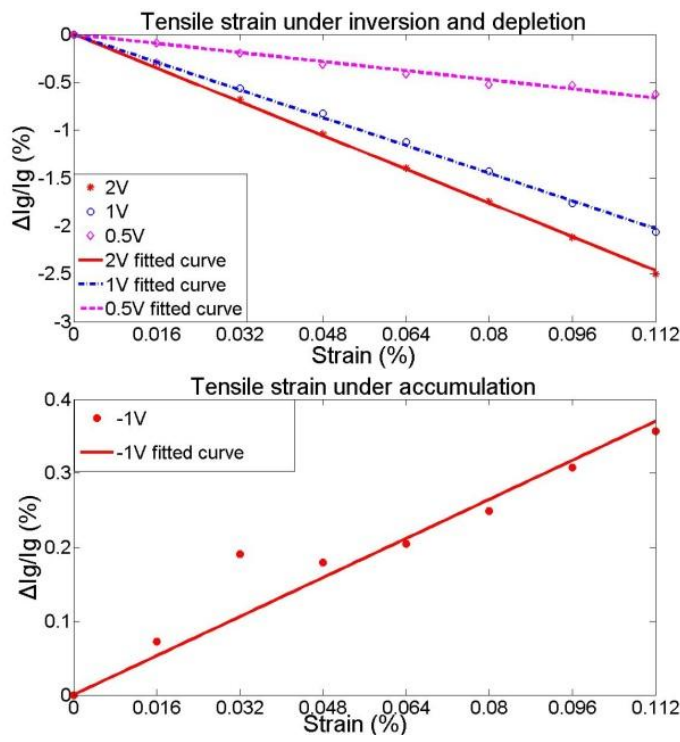

Fig. 4 tunneling current change versus tensile strain

From the analysis in the Table I and Fig. 5, we can see that the average current (avg) without strain decreases as the voltage decreases. The noise or the Standard Deviation (SD) of the currents decreases two orders of magnitude from $2 \mathrm{~V}$ to $1 \mathrm{~V}$, while it keeps the same order of magnitude from $1 \mathrm{~V}$ to $0.5 \mathrm{~V}$. The resolution the device is calculated by the equation of SD/avg. The SD/avg at $2 \mathrm{~V}$ is $0.022 \%$. The $\Delta \mathrm{Ig} / \mathrm{Ig}$ at the strain of $0.112 \%$ at $2 \mathrm{~V}$ is $-2.5 \%$. As a result, the minimum detectable strain at $2 \mathrm{~V}$ is around $0.001 \%$. Likewise, the minimum detectable strain is around $0.0005 \%$ at $1 \mathrm{~V}$. The SD/avg at $0.5 \mathrm{~V}$ and $-1 \mathrm{~V}$ are so close to the $\Delta \mathrm{Ig} / \mathrm{Ig}$ at the strain of $0.112 \%$ that the minimum detectable strains are around $0.112 \%$, which are also obvious from the figures. So the SD/avg and the $\Delta \mathrm{Ig} / \mathrm{Ig}$ at the strain of $0.112 \%$ determine the minimum detectable strain.

The current drifting is observed. The drift rate is defined as 
the normalized current changing from 50 s to 800 s. In the table, the drift rate decreases as the voltage decreases. However, the currents increase at $2 \mathrm{~V}$ and $1 \mathrm{~V}$, while it decreases at $0.5 \mathrm{~V}$ and $-1 \mathrm{~V}$. It is likely that the current may change very slowly at some voltage between $1 \mathrm{~V}$ and $0.5 \mathrm{~V}$.

The gauge factor (GF) also shows that $2 \mathrm{~V}$ and $1 \mathrm{~V}$ have better performance than $0.5 \mathrm{~V}$ and $-1 \mathrm{~V}$, and the GF at $2 \mathrm{~V}$ and $1 \mathrm{~V}$ are in the same range as a piezoresistive sensor.
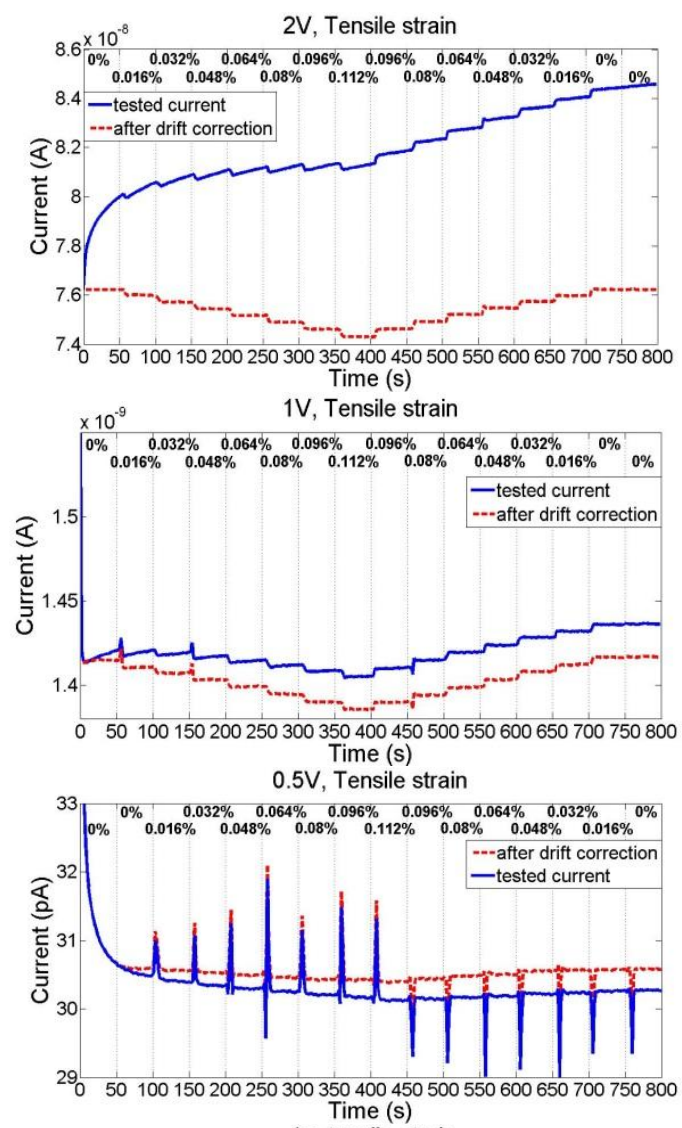

$-1 \mathrm{v}$, tensile strain

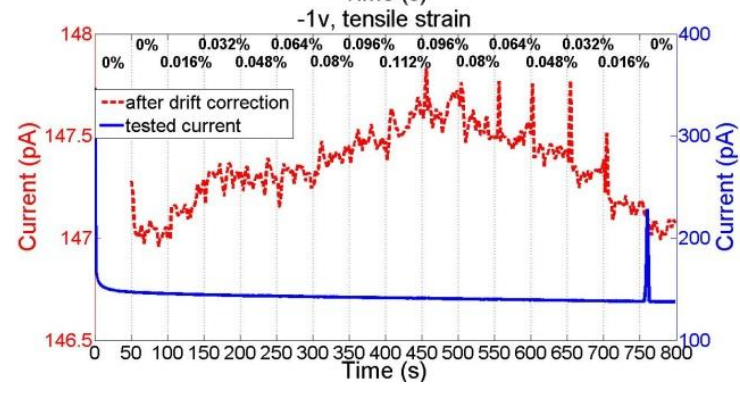

Fig. 5 tunneling current versus time at tensile strain

\section{Compressive strain}

From Fig. 6, we can see that the compressive strain responses are contrary to the tensile strain responses. Increasing compressive strains cause the current to increase at the positive voltages, while increasing compressive strains cause the current tunneling from Aluminum to Silicon to decrease at the negative voltages. Unlike the tensile strain, the $\Delta \mathrm{Ig} / \mathrm{Ig}$ at the strain of $0.112 \%$ doesn't decrease largely from inversion to depletion at positive voltages.
The $\Delta \mathrm{Ig} / \mathrm{Ig}$ at the strain of $0.112 \%$ at the negative voltage is still very small like the tensile strain.

Since the tunneling currents are the same as the tensile strain experiment, the avg and SD should be close to those of tensile strain. However, the SD at $1 \mathrm{~V}$ is 4 times larger than that of tensile strain, while the SD at $0.5 \mathrm{~V}$ is ten times smaller and the SD at $-1 \mathrm{~V}$ is three times smaller. The difference may come from the electrode contact or environment like temperature, since the compressive strain measurement were conducted at different time. As a result, the $\mathrm{SD} /$ avg at $1 \mathrm{~V}$ is $0.04 \%$. Since the $\Delta \mathrm{Ig} / \mathrm{Ig}$ at $1 \mathrm{~V}$ is $1.8 \%$, the minimum detectable strain at $1 \mathrm{~V}$ is around $0.0025 \%$. Likewise, the minimum detectable strain at $2 \mathrm{~V}$ is around $0.0013 \%$.

The drift rate should also be the same as the tensile strain. However unlike drift rate of the tensile strain, the drift rate doesn't become smaller when the voltage goes smaller. The currents still increase at $2 \mathrm{~V}$ and $1 \mathrm{~V}$, while it decreases at $0.5 \mathrm{~V}$ and $-1 \mathrm{~V}$.

The GF at $2 \mathrm{~V}$ and $1 \mathrm{~V}$ are better than those at $0.5 \mathrm{~V}$ and $-1 \mathrm{~V}$.
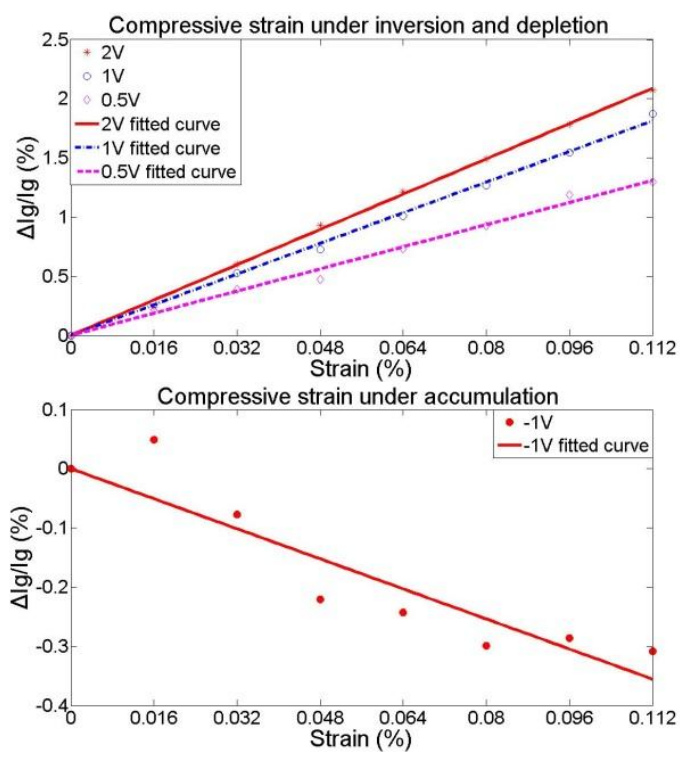

Fig. 6 tunneling current change versus compressive strain

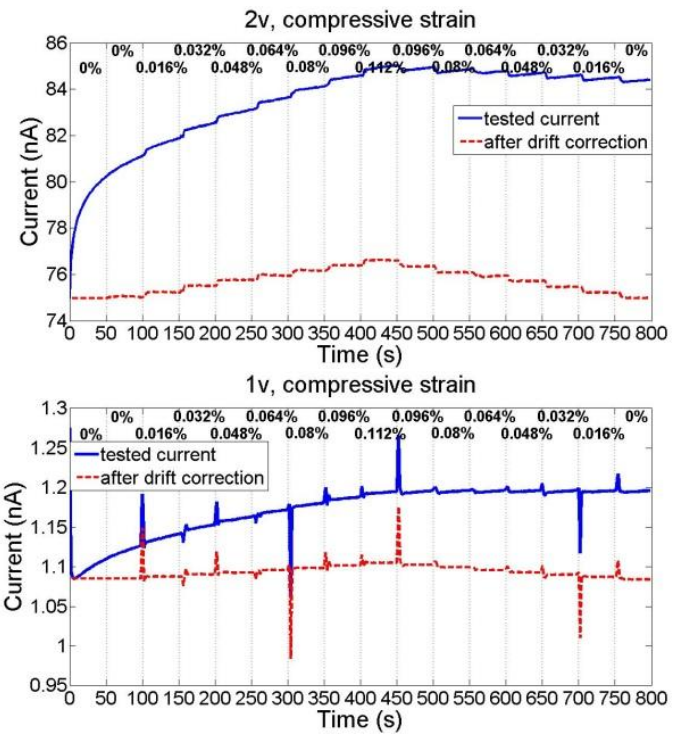




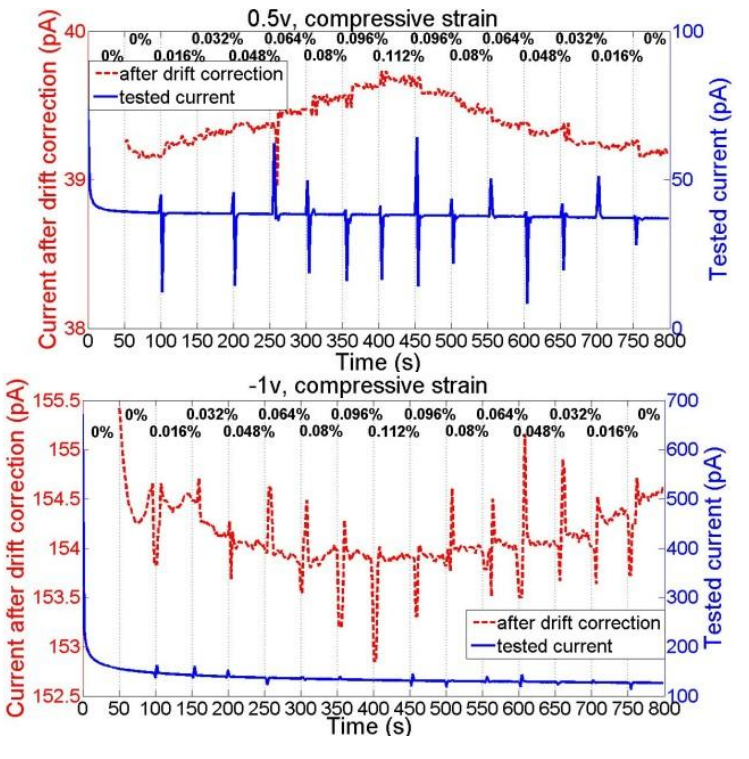

Fig. 7 tunneling current versus time at compressive strain

\section{Discussion}

Considering both tensile and compressive strain, a voltage at $2 \mathrm{~V}$ consumes a power of hundreds of $\mathrm{nW}$ with a minimum detectable strain of $0.001 \%$ for both strains. A voltage at $1 \mathrm{~V}$ consumes only $1 \sim 2 \mathrm{nW}$ with a minimum detectable strain of $0.0005 \%$ for tensile strain and $0.0025 \%$ for compressive strain. However the SD should be the same for both tensile and compressive strain, with better electrode contact, the minimum detectable compressive strain at $1 \mathrm{~V}$ should be also around $0.0005 \%$.

The drift requires further study since it influences the accuracy a lot. If the drift comes from the Fowler-Nordheim tunneling, thinner oxide layer may reduce the drift. If the drift comes from the interface or trap assistant tunneling, better quality oxide should be able to reduce the drift.

\section{CONCLUSION}

We demonstrate a tunneling strain sensor with a low power consumption of several $\mathrm{nW}$ as well as a minimum detectable strain to $0.0005 \%$. The power consumption can be reduced from hundreds of nano-Walts to several nano-Walts as the voltage decrease. Since the SD/avg is getting larger when the voltage decreases, the resolution becomes worse. So there is a balance between the power consumption and the resolution. The device performs best in the inversion region, where the electrons tunnel from Silicon to Al. The gauge factor of our tunneling sensor is in the same range as a piezoresistive sensor. The fabrication process is simple and CMOS compatible.

It seems like that the drift and the noise determine the performance of the tunneling sensor. The drift causes the accuracy to be time dependent, so a further study of the drift is necessary. The noise determines the resolution, so a very low noise read out circuit is demanded.
The authors are grateful to the staffs in the cleanroom of University of Louisville for providing trainings and other necessary help and former labmate, Dr. Kunal Pharas, for being cleanroom buddy and providing helpful suggestions in the experiment. This research was funded by the KY NSF EPSCoR Program (Award \#0814194). We also thank Transducer Research Foundation for the travel support.

\section{REFERENCE}

[1] Barlian, A.A., et al., "Review: semiconductor piezoresistance for microsystems". Proceedings of the IEEE, 2009. 97(3): p. 513-52.

[2] Eaton, W.P. and J.H. Smith, "Micromachined pressure sensors: review and recent developments". Smart Materials and Structures, 1997. 6(5): p. 530-9.

[3] Garcia-Alonso, S., et al. "Low-power MEMS pressure sensor for wireless biomedical applications". in 2011 IEEE 54th International Midwest Symposium on Circuits and Systems (MWSCAS 2011), 7-10 Aug. 2011. 2011. Piscataway, NJ, USA: IEEE.

[4] Mohammed, A.A.S., W.A. Moussa, and E. Lou. "Mechanical strain measurements using semiconductor piezoresistive material". in 2006 International Conference on MEMS, NANO, and Smart Systems, 27-29 Dec. 2006. 2006. Piscataway, NJ, USA: IEEE.

[5] Patra, S. and T.K. Bhattacharyya. "Highly sensitive tunneling accelerometer for low actuation voltage operation". in 9th IEEE Sensors Conference 2010, SENSORS 2010, November 1, 2010 - November 4, 2010. 2010. Waikoloa, HI, United states: Institute of Electrical and Electronics Engineers Inc.

[6] Chingwen, Y. and K. Najafi. "Bulk-silicon tunneling-based pressure sensors". in Technical Digest Solid-State Sensor and Actuator Workshop, 13-16 June 1994. 1994. Cleveland Heights, OH, USA: Transducer Res. Found.

[7] Min, M., et al. "A bulk micromachined Si-on-glass tunneling accelerometer with out-of-plane sensing capability". in 2007 2nd IEEE International Conference on Nano/Micro Engineered and Molecular Systems, 16-19 Jan. 2007. 2007. Piscataway, NJ, USA: IEEE.

[8] Sun, Y., S.E. Thompson, and T. Nishida, "Physics of strain effects in semiconductors and metal-oxide-semiconductor field-effect transistors". Journal of Applied Physics, 2007. 101(10): p. 104503-1.

[9] Yang, X.D., et al., "Strain induced changes in the gate leakage current of n-channel metal-oxide-semiconductor field-effect transistors". Journal of Applied Physics, 2011. 110(1): p.014511-1.

[10] Wei, Z., et al., "Opposing dependence of the electron and hole gate currents in SOI MOSFETs under uniaxial strain". Electron Device Letters, IEEE, 2005. 26(6): p. 410-412.

[11] Kiejna, A. and V.V. Pogosov, "Simple theory of elastically deformed metals: Surface energy, stress, and work function". Physical Review B (Condensed Matter), 2000. 62(15): p. 10445-50.

\section{CONTACT}

Shamus McNamara, tel: +1-502-852-6704;

shamus.mcnamara@louisville.edu

\section{ACKNOWLEDGEMENT}




\title{
MICROFLUIDIC FABRICATION OF MONODISPERSE MICROCAPSULES FOR SELF HEALING MATERIAL
}

\author{
R.J. Lemmens* and D.D. Meng
}

Mechanical Engineering - Engineering Mechanics Department

Michigan Technological University, Houghton, Michigan, USA

\begin{abstract}
We report microfluidic fabrication of microcapsules for use in self-healing composites which results in dramatic reduction of the coefficient of variation $(\mathrm{CV})$ of the microcapsule size from $\sim 40$ to less than 4, as compared to traditional batch emulsification methods. With this fabrication approach the mean microcapsule size can also be accurately controlled through variation of process parameters such as fluid flow rate. The surface morphology, i.e. roughness of the capsule shell, and shell thickness can also be tuned by utilizing fluid shear and controlling temperature.
\end{abstract}

\section{INTRODUCTION}

A self healing material is typically a composite, in many cases polymer, consisting of a bulk matrix with embedded healing agent. In a generic self healing model damage to the composite causes release of healing agent into the crack volume and polymerization of the healing agent results in reestablishment of mechanical properties [1]. Healing agent incorporation schemes include capsules, capillaries, fugitive ink scaffold writing as well as others. Possible intrinsic or extrinsic healing triggers include the addition of heat, pressure, moisture or the presence of catalyst or hardening agent [2]. Taking into account material and fabrication costs, ease of composite fabrication, and production scalability, the use of microcapsules as the means of healing agent incorporation is favored. As a constituent of the composite the properties of these microcapsules have a direct impact on the performance of self healing materials [3] and are therefore a crucial parameter in the engineering of such composites.

The size and surface roughness of the microcapsules can be critical for both the mechanical properties and the self-healing performance of the composite. Rule et al. showed that the degree of healing is directly related to the mass of healing agent delivered to the crack volume and that this mass is dependent only on the size and concentration of microcapsules incorporated into the composite [4] assuming a uniform distribution of microcapsules and a negligible shell thickness. An investigation by Brown et al. found that incorporation of microcapsules into an epoxy matrix resulted in significant fracture toughening [5]. The degree of toughening was seen to have a strong dependence on microcapsule size as well as adhesion between the epoxy matrix and microcapsule shells. These improvements; however, do not always trend in the same direction with respect to the various properties of the microcapsules, e.g. size and shell morphology, requiring an optimization of properties and performance. Such an optimization would be more easily implemented given a microcapsule fabrication technique having precise control over these microcapsule characteristics.

Currently, microcapsules for use in self-healing composites are produced using batch emulsification with an accompanying shell polymerization mechanism. A well established material system utilizes a poly-urea-formaldehyde (UF) capsule shell and dicyclopentadiene (DCPD) as the encapsulated healing agent [6]. In the production process an aqueous solution of the polymerization ingredients is placed in a beaker along with the healing agent which is immiscible in water. A mechanical stirrer is used to create an emulsion of the healing agent and a condensation polymerization is initiated to form the capsule shell.

Attempts have been made with this fabrication approach to control the surface roughness of the microcapsules [6]. Variation of polymerization time showed an increase in the thickness of a rough shell but smooth capsules either could not be obtained or the DCPD diffused out within a couple of days. Smooth capsules were obtained using a constant $\mathrm{pH}$ process but in this case yields were only $\sim 10 \%$. The thickness of the rough shell was also seen to be dependent on the agitation rate as a result of the interplay between the magnitude of the DCPD/water interfacial area and mass conservation of the polymer. The capability of controlling the surface roughness without sacrificing product yield and stability or alteration of the volume of healing agent contained in the microcapsules has not yet been realized.

Additionally, precise size control for the microcapsules is difficult with batch emulsification technology. The mean diameter of the microcapsules produced is directly related to the rotational rate of the mechanical stirrer used to create the emulsions. Increased agitation causes increase shear and therefore, with a given interfacial tension, smaller DCDP droplets on average. The shear forces however are not homogeneous throughout the emulsion bath and DCPD droplet formation is not localized resulting in a broad distribution of microcapsule size. The coefficient of variation (CV) of the size distribution of the as produced microcapsules typically ranges between 20 and 50 . A significant reduction in this distribution would require improved control over droplet formation.

Droplet microfluidics is well known for its reliable and repeatable droplet production. It has been proven to be an invaluable platform for digital fluidics applications, biomaterial encapsulation and handling, as well as producing functional microreactors for studies in reaction kinetics [7]. The unique scaling within microchannels allows for precise control over fluid mixing and dominance of viscous and interfacial forces permitting controlled droplet formation and manipulation. The functionality of the microfluidic platform is adaptable to changes in fluid properties allowing a broad range of materials to be used. This provides optimal conditions for particle and microcapsule fabrication [8]. Incorporation of such advantages into the fabrication process for microcapsules used in self healing material would allot significant improvements in process controllability.

In this work, we report an approach to combine droplet microfluidics, which can produce highly-monodisperse emulsions, with an established condensation polymerization technique for shell formation to produce monodisperse microcapsules for selfhealing material applications. Facile control over shell surface morphology is also demonstrated.

\section{EXPERIMENTAL}

\section{Microcapsules Fabrication}

All materials are used as purchased. Dicyclopentadiene, urea, formaldehyde, ammonium chloride, resorcinol and sodium hydroxide were obtained through Sigma-Aldrich. Ethylene- 


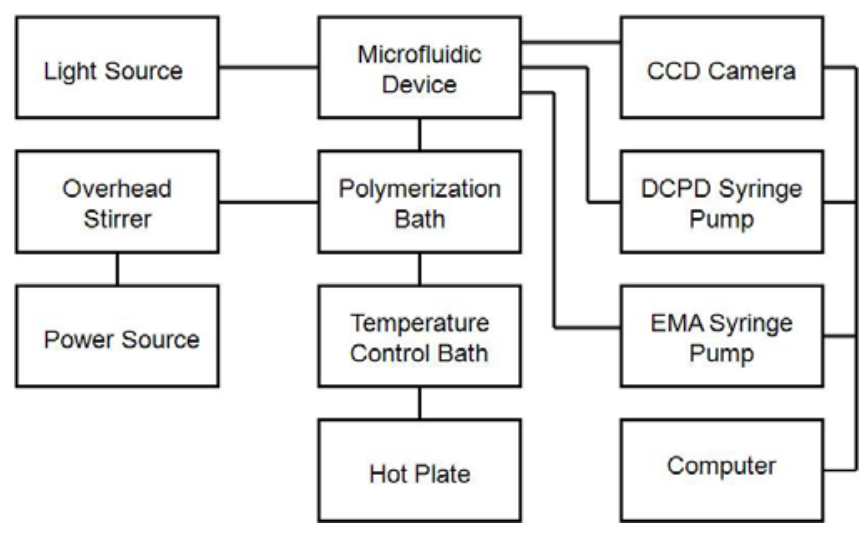

Figure 1: Schematic illustration of the microcapsules fabrication setup including microfluidic emulsification.

maleic-anhydride (EMA) was donated by Vertellus Specialties Inc. Sylgard 184 polydimethylsiloxane (PDMS) elastomer was purchased through Dow Chemicals.

The microcapsules production setup is shown in Figure 1. Two syringe pumps (Harvard Apparatus) are used to supply DCPD and a $2.5 \mathrm{wt} \%$ aqueous solution of EMA to the inlet channels of the PDMS microfluidic emulsifier. A computer is used to control the syringe pumps as well as collect video from a ccd camera (Moticam 2000) used to monitor the emulsification process within the device.

The PDMS device is produced using soft lithography techniques. The cross junction geometry of the microchannels acts as a flow focusing emulsifier [7]. The outlet channel dimensions at the cross junction are $60 \mu \mathrm{m}$ wide by $90 \mu \mathrm{m}$ deep for all emulsifications. Channel dimension are reduced to $60 \mu \mathrm{m} \times 70 \mu \mathrm{m}$ for one device to demonstrate the effects of channel size. The microchannels are oxygen plasma treated (Diener Electronics Femto System) to ensure proper surface wettability and sealed by bonding to a glass slide. An example of a monodisperse emulsification within this device is shown in Figure 2.

Formation of the microcapsule shell occurs in a bath into which the emulsion from the microfluidic device is collected. The chemistry and polymerization mechanism is similar to [6]. Urea (1g), ammonium chloride (100mg), and resorcinol $(100 \mathrm{mg})$ are desolved in $40 \mathrm{ml}$ of water and placed in an $80 \mathrm{ml}$ beaker along with $5 \mathrm{ml}$ of a $2.5 \mathrm{wt} \%$ aqueous solution of EMA. This bath is agitated with an overhead stirrer using a 1 inch, 3 blade impeller. Agitation is performed at a relatively low $200 \mathrm{rpms}$ so as to maintain droplet integrety.

Emulsification is performed with constant EMA and DCPD flow rates for a total of $100 \mathrm{~min}$. prior to shell formation. After collection of the emulsion the $\mathrm{pH}$ of the polymerization bath is increased from $\sim 2.6$ to 3.5 by dropwise addition of sodium hydroxide. Formaldehyde $(2.634 \mathrm{~g})$ is then added to the bath and the temperature is increased from room temperature to $55^{\circ} \mathrm{C}$ in order to initiate polymerization. The temperature is maintained using a hotplate and water bath for $4 \mathrm{hrs}$. after which agitation is stopped, heat is removed, and the bath is strained through a coarse fritted filter. After washing with DI water the product is allowed to dry for $24 \mathrm{hrs}$. This process results in the collection of UF microcapsules containing DCPD in the form of a fine white powder.

For comparison microcapsules are also produced using batch emulsification. This procedure follows that outlined in [6]. Three samples of microcapsules were produced using different agitation rates, 600,650 and $750 \mathrm{rpm}$, in an attempt to produce

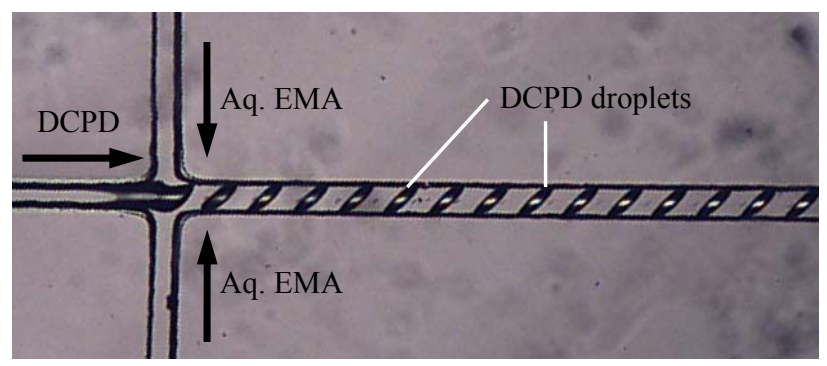

Figure 2: Emulsification of DCPD at the flow focusing cross junction of a microfluidic droplet generator. EMA and DCPD flow rates are $45 \mu \mathrm{L} / \mathrm{min}$ and $15 \mu \mathrm{L} / \mathrm{min}$ respectively.

microcapsules with a mean diameter similar to those produced using the microfluidic emulsification technique.

In order to demonstrate control of surface morphology, post polymerization processing were performed to rapidly quench the polymerization bath to room temperature. Spcificaly, after agitation of the polymerization bath is ceased, the contents of the bath are poured into $1 \mathrm{~L}$ of deionized water. Filtration, washing, and product drying then follow.

\section{Characterization}

Size analysis of the microcapsules is performed using an image analysis software, Image $J^{\circledR}$. Samples of the microcapsules are placed on a glass slide and optically imaged using a ccd camera and Motic Images Plus software. A minimum of 300 microcapsules for each sample are analyzed to find the mean diameter and standard deviation of the distribution.

The surface morphology of the microcapsules is observed by Field Emission Scanning Electron Microscopy. To view the cross section of the shell microcapsules are manually ruptured and the DCPD is allowed to evaporate prior to sample preparation. Microcapsules are also sputtered with $5 \mathrm{~nm}$ of titanium prior to observation.

\section{RESULTS}

\section{Control of Capsule Size}

In this study control over the size of microfluidicly produced microcapsules is demonstrated through variation of fluid flow rates and microchannel dimensions. The size distribution of these microcapsules is also compared against that of those produced using the standard microcapsule production method.

Four samples of DCPD-containing UF microcapsules prepared using the microfluidic fabrication approach described above are presented in Figure 3 as samples A-D. The DCPD flow rate for these four samples was held constant at $15 \mu \mathrm{L} / \mathrm{min}$. Samples A-C were prepared using a PDMS devices whose channel dimensions were $60 \mu \mathrm{m}$ wide and $90 \mu \mathrm{m}$ deep with EMA flow rates of 15,45 , and $75 \mu \mathrm{L} / \mathrm{min}$ respectively. The channel dimensions were altered to $60 \mu \mathrm{m}$ wide and $70 \mu \mathrm{m}$ deep for Sample D and an EMA flow rate of $75 \mu \mathrm{L} / \mathrm{min}$ was used. Sample E was prepared by traditional batch emulsification techniques as a comparison and utilized an agitation rate of $650 \mathrm{rpm}$. Two additional samples were also prepared using batch emulsification with agitation rates of 600 and $750 \mathrm{rpm}$ but images are not shown.

It is found that the samples produced using microfluidics (AD) are monodisperse (typically defined as $\mathrm{CV}<5$ ) while the $\mathrm{CV}$ of control sample (E) is more than eight times greater. Each sample's mean diameter, coefficient of variation (CV), and corresponding normal distributions are shown in Figure 4. The two additional 


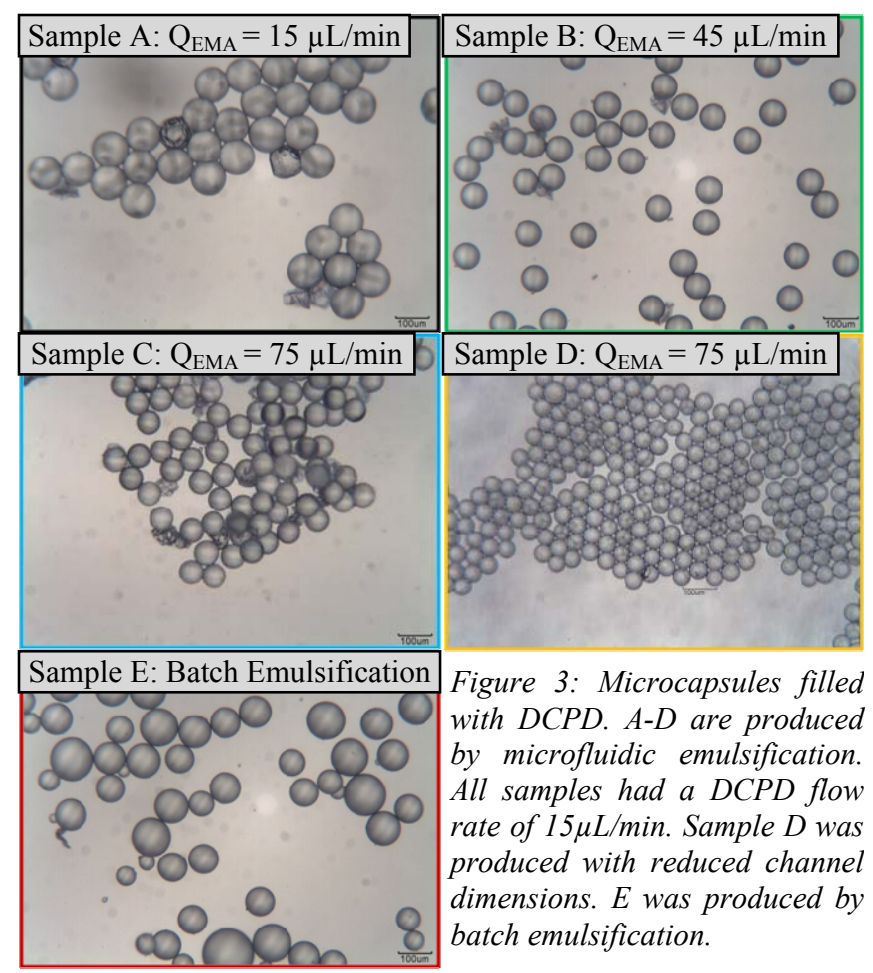

samples produced using batch emulsification were also highly polydisperse. The mean diameters were 88.5 and $65.6 \mu \mathrm{m}$ and the CVs were 25.8 and 45.1 for the 600 and $750 \mathrm{rpm}$ samples respectively.

It has been shown that the size of the microcapsules can be precisely controlled by altering the fluid flow rates and therefore the shear forces within the PDMS device. By tuning $Q_{\mathrm{EMA}}$, the mean capsule sizes of samples A-C have been controlled as 107.4, 80.7 , and $73.2 \mu \mathrm{m}$ respectively. Channel dimensions also play a similar role in control of capsules size as can be seen from Sample $\mathrm{D}$ which has a mean diameter of $53.4 \mu \mathrm{m}$.

The overall size distribution for each sample is attributed to the nature of droplet formation during the emulsification. With batch emulsification, shear forces are heterogeneous and droplet formation is delocalized. This is not the case for microfluidic emulsification. With constant flow rates of input fluids each droplet experiences similar shear and interfacial forces during formation resulting in a significant reduction in size distribution.

In comparing samples $\mathrm{A}$ and $\mathrm{D}$ to samples $\mathrm{B}$ and $\mathrm{C}$ it is seen that their CVs are slightly higher, by a factor of $\sim 2$. The increase seen for sample A can be explained by the coalescence of two or more droplets prior to shell formation. Such droplet coalescence was observed within the outlet channel of the PDMS device. It is conjectured that because the fluid flow rate within the outlet channel was low the tendency for contact between droplets and therefore merging was higher. A decrease of EMA flow rate to $10 \mu \mathrm{L} / \mathrm{min}$ was found to further increase the tendency of droplet coalescences to a point where monodispersity is lost.

The increase in CV seen for sample $\mathrm{D}$ can be explained by a transition into a different flow regime. The decrease in channel dimensions for this sample is accompanied by an increase in fluid velocity and therefore the Weber number of the flow increases. The transition from dripping to jetting in microchannels is strongly dependent on Weber number and the transition consists of a number of distinct regimes. In the initial stages of the transition multiple dripping occurs. In this regime the location of droplet

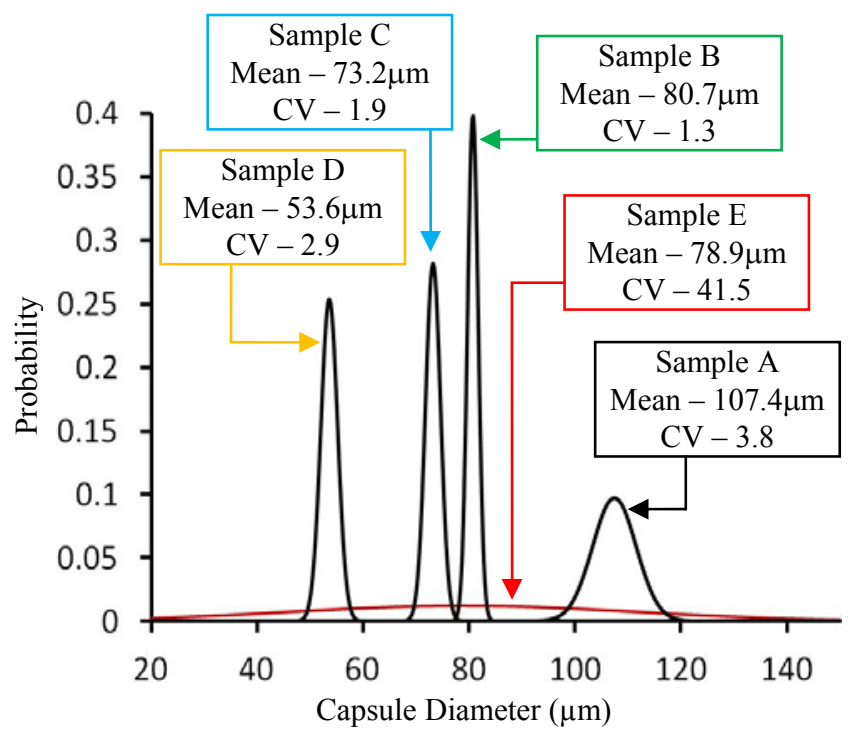

Figure 4: Comparison of the normal distributions of microcapsule diameter of samples produced using microfluidics, $A-D$, and that produced using current microcapsule fabrication techniques, $E$.

formation does not yet move into the outlet channel, as it does for jetting, however a droplet formation event does not produce a single droplet but rather multiple droplets which can vary in size [9].

Although samples A and D seem to have been produced with a broader size distribution the products still fall into the definition of being monodisperse. The observations made for these two sample reveals some of the guidelines which must be followed for this fabrication process. A window of operational flow rates will be specified for given channel dimensions where at the lower limit droplet coalescence is a risk and at the upper limit transition out of the dripping regime will occur. With that said use of a single device still provides the capability of producing microcapsule of various sizes and with fine tuned precision.

\section{Control of Capsule Surface Morphology}

Formation of the microcapsules shell results from a condensation polymerization of urea with formaldehyde which occurs in the continuous, i.e. aqueous, phase of the emulsion [6]. Shell formation begins at the interface between the DCPD droplets and the aqueous phase where in the early stages of polymerization low molecular weight UF is deposited. As the polymerization progresses further, the low molecular weight UF at the DCPD surface continues to crosslink forming a solid, continuous and smooth shell. Simultaneously, high molecular weight UF which is not soluble in water begins to precipitate out of solution in the form of nanoparticles. Deposition of these nanoparticles onto the smooth inner shell results in the formation of a rough outer shell. It was conjectured that during the polymerization and toward the end of the fabrication process a significant amount of UF nanoparticles form and remain in suspension in the continuous phase of the bath. The agitation from the mechanical stirrer keeps these particles as well as the microcapsules in constant motion. The relative motion between the particles causes hydrodynamic lubrication preventing intimate contact from being made. Removal of this agitation and the continued presence of the polymerization reaction permit deposition of the nanoparticles and cross linking to each other and the microcapsules surface. Development of the rough outer surface should therefore be controllable. 

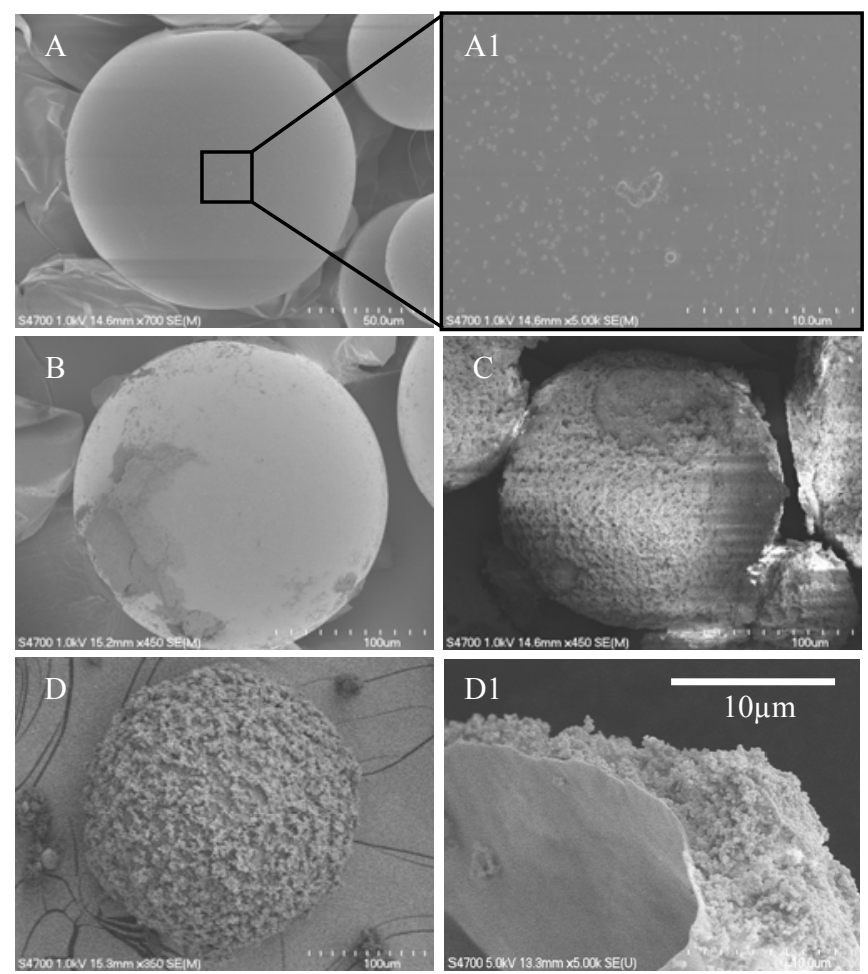

Figure 5: Evolution of microcapsule shell roughness with time elapsed after discontinuation of bath agitation until temperature quenching to room temperature (A) $40 s$ (B) 120 s (C) $200 s$ (D) 280 s (A1) Smooth capsule surface with minimal UF particle deposition (D1) Broken capsule from sample D showing smooth inner surface and representative thickness of rough outer surface.

Control of the microcapsules surface morphology is achieved by tuning the time elapsed between ceasing agitation of the polymerization bath and temperature quenching to minimize further polymerization. Four samples with various degrees of surface roughness are presented in Figure 5. The times used for the study were $40,120,200$, and 280 s for samples A-D respectively. The yields for all samples were between 80 and $90 \%$, where yield is measured as the ratio of the weight of the product capsules to the weight of the DCPD used in the emulsification. Losses are conjectured to be due to a combination of DCPD evaporation prior to shell formation and loss of product during filtration and collection.

Sample A was produced using the shortest time and possesses the smoothest surface. Figure 5A1 shows a magnified view of the surface where minimal UF particle deposition has occurred. The quality of these capsules is good in that they are mechanically stable, form a fine powder after drying, and DCPD does not leak or diffuse through the capsule shell. This quality has been found to remain for a minimum of 3 months with no signs of degradation. The effluent from the initial filtration of these microcapsules from the polymerization bath had a milky white color indicating that UF nanoparticles of high concentration still remain in suspension.

Samples B and C show that the surface roughness does not develop uniformly. This is conjectured to be due to UF particle agglomeration in suspension followed by deposition on the microcapsules surface.

Sample D was produced using the longest time and possesses a rough surface which is uniform over the whole shell and was seen to be consistent for other microcapsules from the same sample. Figure 5D1 shows the cross section of the shell of a microcapsule from this sample. A significant shell thickness has developed for these microcapsules although not uniform due to the textured nature of the surface. The effluent from the initial filtration of these microcapsules from the polymerization bath was clear indicating the absence of UF particles in suspension. Further increase in the time elapsed beyond that used in this sample did not show a significant change in surface morphology.

\section{CONCLUSION}

A microfluidic method of fabrication of microcapsules for use in self-healing material has been demonstrated. Fine tuning of the microcapsule diameter is performed through variation of fluid flow rates and selection of microchannel dimensions. The microcapsules produced are monodisperse representing a significant decrease by a factor of $\sim 10$ of the coefficient of variation when compared to those produced using traditional methods. The surface morphology of the microcapsules has also been controlled. Microcapsules with smooth and rough surfaces are produced with good quality and high yields.

\section{ACKNOWLEDGEMENT}

This material is based upon work supported by the National Science Foundation under Grant No. 0900582. Any opinions, findings, and conclusions or recommendations expressed in this material are those of the authors and do not necessarily reflect the views of the National Science Foundation.

Travel support has been generously provided by the Transducer Research Foundation.

The authors would also like to thank all members of the Multi-Scale Energy Systems Laboratory for their help and support.

\section{REFERENCES}

[1] S.R. White, et al., "Autonomic healing of polymer composites," Nature, vol. 409, pp. 794-797, 2001.

[2] B.J. Blaiszik, et al., "Self-Healing Polymers and Composites," Annual Review of Materials Research, vol. 40, pp. 179-211, 2010.

[3] M. Keller and N. Sottos, "Mechanical Properties of Microcapsules Used in a Self-Healing Polymer," Experimental Mechanics, vol. 46, pp. 725-733, 2006.

[4] J.D. Rule, et al., "Effect of microcapsule size on the performance of self-healing polymers," Polymer, vol. 48, pp. 3520-3529, 2007.

[5] E.N. Brown, et al., "Microcapsule induced toughening in a self-healing polymer composite," Journal of Materials Science, vol. 39, pp. 1703-1710, 2004.

[6] E.N. Brown, et al., "In situ poly(urea-formaldehyde) microencapsulation of dicyclopentadiene," Journal of Microencapsulation, vol. 20, pp. 719-730, 2003.

[7] S.-Y. Teh, et al., "Droplet microfluidics," Lab on a Chip, vol. 8, pp. 198-220, 2008.

[8] J.-T. Wang, et al., "Fabrication of Advanced Particles and Particle-Based Materials Assisted by Droplet-Based Microfluidics," Small, vol. 7, pp. 1728-1754, 2011.

[9] A.R. Abate, et al., "Impact of inlet channel geometry on microfluidic drop formation," Physical Review E, vol. 80, p. $026310,2009$.

\section{CONTACT}

* R.J. Lemmens, tel: 1-920-639-4028; rjlemmen@mtu.edu 


\title{
NONLINEAR DAMPING FOR SHOCK PROTECTION OF MEMS DEVICES USING SHEAR THICKENING FLUID
}

\author{
S. Iyer ${ }^{1 *}$, H. Lee ${ }^{l}$, M. Liger ${ }^{2}, J . J u d y^{l}$ and R. Candler ${ }^{l}$ \\ ${ }^{1}$ Electrical Engineering Department, University of California, Los Angeles \\ ${ }^{2}$ Google, USA
}

\begin{abstract}
This manuscript reports the measurement and analysis of nonlinear viscous damping of MEMS actuators immersed in shear thickening fluids (STFs). The linear second-order system model is modified with a power-law damping term to account for the sheardependent viscosity of the fluid. This model is tested using a torsional microactuator immersed in a STF. At high actuation forces, the vibration velocity amplitude drops sharply. This result reveals the potential to use STFs as adaptive, passive dampers for shock protection of MEMS. To date, this is the only study that uses STFs in the context of dynamic damping for MEMS devices.
\end{abstract}

\section{INTRODUCTION}

Isolation from environmental disturbances is necessary for increased reliability and performance of micro-scale sensors and actuators for space, military and industrial applications. One of the most damaging external disruptions is mechanical shock caused by impact. Microscale devices with fragile mechanical structures have been shown to fracture as a result of large magnitude shocks [1]. Even when complete destruction of the device does not occur, shock can result in reduced performance and lifetime of MEMS devices.

Previous shock studies on MEMS devices focus on failure analysis [1], optimization of the mechanical structure design [1,2] or inclusion of an additional element for increased energy dissipation $[3,4]$. Adjusting device geometry to optimize for shock resistance creates a coupled design problem that results in a performance tradeoff with sensitivity. The inclusion of additional spring elements such as hard stops, soft wall coatings or nonlinear springs does not have this pitfall. However, these additional components do not prevent impact of the mechanical structure. In fact, the effectiveness of these structures for shock protection is limited because they can only dissipate energy when they are in direct, physical contact with the device structure.

Fluidic damping has the advantage that energy dissipation occurs continuously. For large-scale applications such as seismic protectors for buildings and automotive shock absorbers, dedicated, fluid-filled dampers can be used to absorb unwanted energy [5]. In micro-scale systems, however, it is impractical to have an isolated damping element. Instead, damping can be integrated into the device by encapsulating it in a viscous fluid [6]. The main drawback of this approach is that typical fluidic damping increases energy dissipation under all operating conditions, resulting in reduced performance.

In this work, we take advantage of shear thickening fluids (STFs) to create a passively damped system that adapts the damping force to the magnitude of the disturbing force. STFs have the unique property that their viscosity increases with increasing shear rate. Increased viscosity results in increased damping. Thus, STFs allow for normal device operation at low, working velocities, but severely restrict motion at high velocities typical of shocks. Here, we propose the use of STFs for increased energy dissipation in MEMS subject to mechanical shock.

\section{THEORY}

Studies on the mechanical dynamics of MEMS devices typically employ the use of a linear, second-order mass-springdamper model. Equation 1 is the equation of motion for such a system, where $x$ is displacement, $f(t)$ is the forcing function, $m$ is the device mass, $k$ is the spring constant and $b$ is the damping coefficient. The damping coefficient is determined by a number of energy dissipation mechanisms; however, in this case fluidic damping will be the dominant mechanism for energy loss.

$$
m \ddot{x}+b \dot{x}+k x=f(t)
$$

STFs are in a class of non-Newtonian fluids where viscosity is dependent on shear rate. The constitutive relation between viscosity and shear rate for these fluids is given in Equation 2,

$$
\eta=K \cdot \dot{\gamma}^{n-1}
$$

where $\eta$ is viscosity, $K$ is a material dependent constant, $\dot{\gamma}$ is shear rate and $n$ is the power-law index. For STFs, $n$ is greater than 1, so viscosity increases with shear rate. For Newtonian fluids, $n=1$ and viscosity is constant with shear rate. Finally, if $n$ is less than 1 , the fluid has shear thinning properties.

We can incorporate this nonlinearity in the second-order model by replacing the linear damping force that is proportional to velocity with a power-law damping term to account for increased damping under high amplitude actuation $[7,8]$. This substitution is shown in Equation 3. The absolute value and signum functions are

$$
m \ddot{x}+b|\dot{x}|^{n} \operatorname{sgn}(\dot{x})+k x=f(t)
$$

needed to fulfill the viscous damping requirement that the drag force always opposes the direction of motion. For a Newtonian fluid, the power-law index is equal to 1 . Equation 3 can then be simplified exactly to the linear ordinary differential equation presented in Equation 1. However, for shear thickening fluids, the power-law index $n$ is greater than 1. If we assume a sinusoidal input (as in Equation 4) with amplitude $A>0$, then the damping force will have a nonlinear dependence on both amplitude and drive frequency, $\omega$ (Equation 5). This will result in a damping force that has a greater than linear dependence on the magnitude of the actuation force. Additionally, we see that the sinusoid waveform will be distorted by the power-law dependence. Since typical power-law indices fall between 1 and 2, this distortion will result in the emergence of a $2 \omega$ frequency component in the damping force.

$$
\begin{aligned}
& x=A^{*} \sin (\omega t) \\
& F_{d}=-b \cdot(A \omega)^{n}|\cos (\omega t)|^{n} \operatorname{sgn}[\cos (\omega t)]
\end{aligned}
$$

\section{METHODS \\ STF and Preparation}

Nearly all of the well-studied STFs consist of a colloidal suspension of particles in a solvent. Shear thickening results when the suspension transitions from a stable, ordered state to a state of flocculation, where motion causes small groups of particles to interact and form incompressible hydroclusters. Previous studies using STFs focus on characterization of the fluids alone or use them
9780964002494/HH2012/\$25C2012TRF

DOI 10.31438/trf.hh2012.123
Solid-State Sensors, Actuators, and Microsystems Workshop Hilton Head Island, South Carolina, June 3-7, 2012 
in isolated, macro-scale damping components $[9,5]$. This is the first study that employs the use of STFs as damping fluids for MEMS devices.

In this work, the STF used is a colloidal dispersion of 30\% (by weight) fumed silica nanoparticles (Aerosil 200 with $\sim 12 \mathrm{~nm}$ particle diameter) in a polyethylene glycol-200 (PEG) solvent. The silica nanoparticles are dispersed into the PEG solvent using a Thinky AR-100 conditioning mixer. Several hours of mixing are required to break up particle clusters and ensure an ordered dispersion is achieved.

\section{Torsional Device and Actuation}

The nonlinear damping effects of the STF were tested using a magnetically actuated torsional device [10]. The device and principle of actuation are shown in Fig. 1. In a uniform magnetic field, the magnet will try to align with the field, resulting in torsional motion.
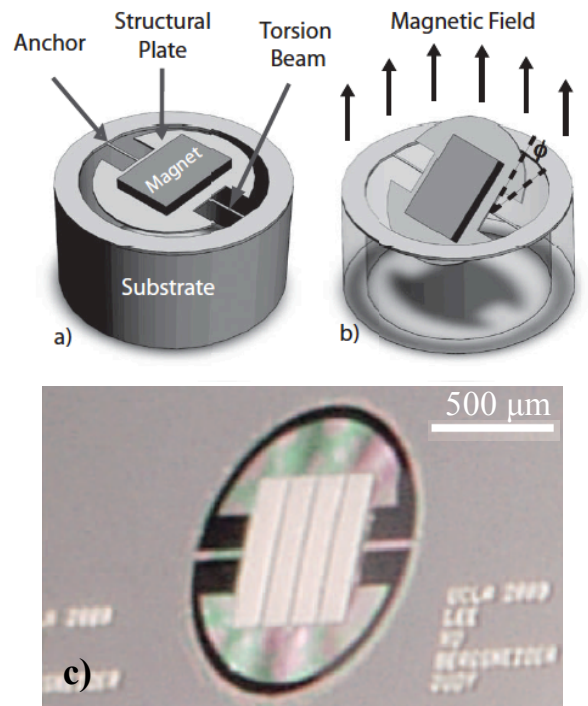

Figure 1: Torsional Si actuator: a) unactuated state, b) actuated by an applied magnetic field, c) released device.

The magnetic field needed to actuate the devices was produced using an external electromagnet. The torque produced by the electromagnet is given in Equation 6, where $M$ is the magnetism of the permanent magnet on the device, $V$ is the volume of the magnet and $H$ is the applied magnetic field strength. Equation 7 shows the expression for the magnetic field strength for a circular, air-core inductive coil, where $I$ is the current through the coil, $N$ is the number of turns, $a$ is the radius of the coil and $L$ is its length.

$$
\begin{gathered}
\tau_{\text {ext }}=(M \times H) \cdot V \\
H=-\frac{N \cdot I}{2 \sqrt{a^{2}+(L / 2)^{2}}}
\end{gathered}
$$

The electromagnet is driven using a custom-built transconductance amplifier from Marchand Electronics with a programmable input. The velocity of the device is then measured using a differential laser Doppler vibrometer. The complete experimental setup is shown in Fig. 2.

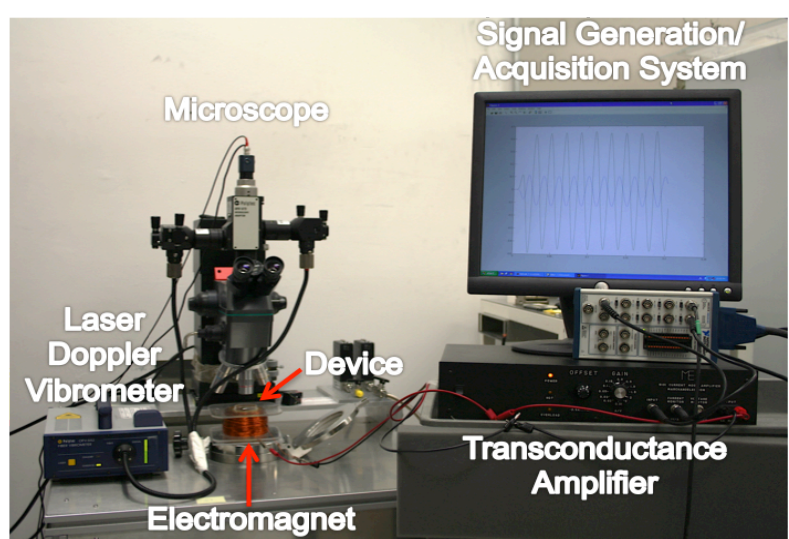

Figure 2: Experimental setup for measuring the velocity amplitude response. A laser Doppler vibrometer is used to measure the velocity of the device.

\section{RESULTS}

Typical studies on mechanical dynamics focus on the frequency response of a system. However, since we are trying to characterize the damping characteristics of the STF instead of the device performance, we are interested in the magnitude response of the damping force. Additionally, changes in the frequency response are difficult to identify and characterize since the system is highly damped.

In order to assess the damping properties of the fluid, we immersed the torsional actuator in the STF and measured the velocity amplitude as the magnitude of the sinusoidal actuation force was increased. The velocity amplitude response was also measured for two Newtonian fluids, PEG and air. Fig. 3 shows the response for a device immersed in STF versus PEG alone. Note that the two data series are plotted in the same velocity range to facilitate comparison of the respective damping forces in each fluid. In PEG, the actuator's velocity is quite linear with the magnetic actuation force, as we expect for a Newtonian fluid with linear viscous damping and power-law index $n$ equal to 1 . In the STF, however, the velocity does not increase linearly with drive force. At very high amplitude actuation, velocity drops of sharply, indicating that the motion of the device is limited despite a large excitation.

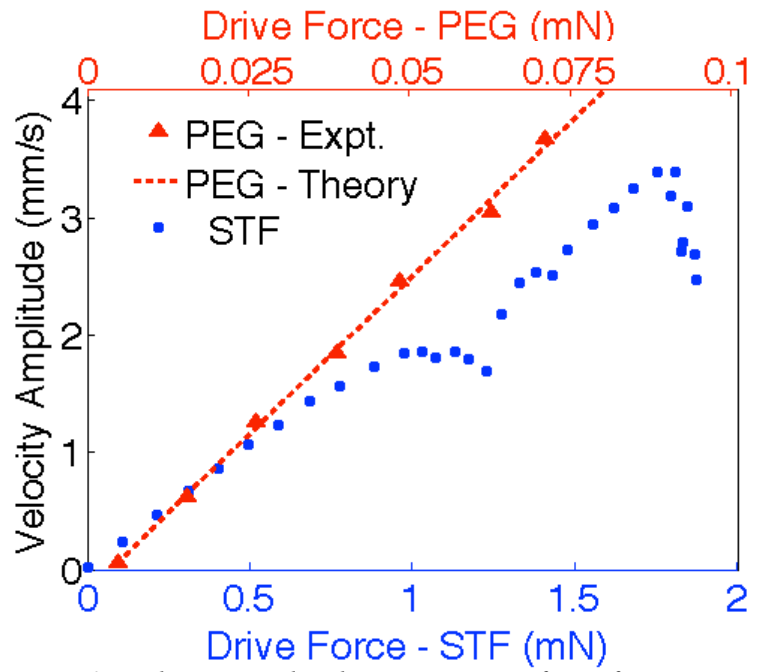

Figure 3: Velocity amplitude vs. actuation force for an actuator in PEG and STF. Two horizontal axes are used to match the velocity range of the device in each material. This facilitates a comparison of the damping, which depends on velocity. 
The Newtonian behavior of PEG alone is again verified in Fig. 4, which compares the velocity response in PEG with that in air, a fluid widely accepted as Newtonian. As expected, to achieve the same velocity in the more viscous PEG as in air, we require nearly twice the magnetic actuation force. The velocity responses in the Newtonian fluids are linearly dependent on actuation force, and we do not see the dramatic roll-off observed in the STF. This is a strong indication that the dispersion of fumed silica nanoparticles induces a shear thickening response in PEG.

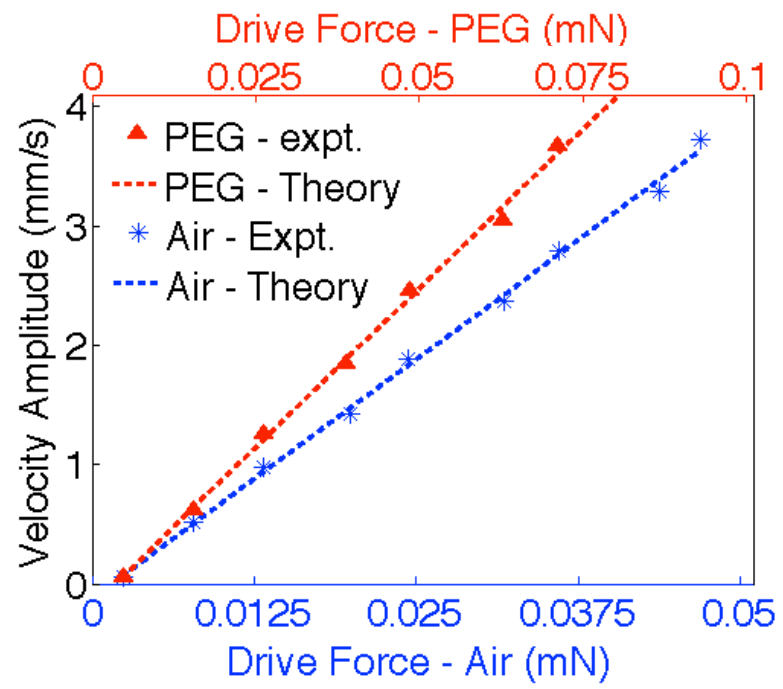

Figure 4: Velocity amplitude vs. actuation force amplitude for Newtonian fluids, PEG and air. The responses are similarly linear with theoretical fits corresponding to $n=1$ for both fluids. This suggests that the STF is responsible for the increased damping power.

The power-law damping model established in Equation 3 suggests that the shear-dependent viscosity of the STF results in a waveform distortion that manifests itself mathematically as an emergence of a frequency component at twice the drive frequency. Fig. 5 shows the relative contribution of the frequency components of the velocity for a torsional device immersed in the STF. Under low amplitude actuation, the $f_{\text {drive }}$ component is dominant.

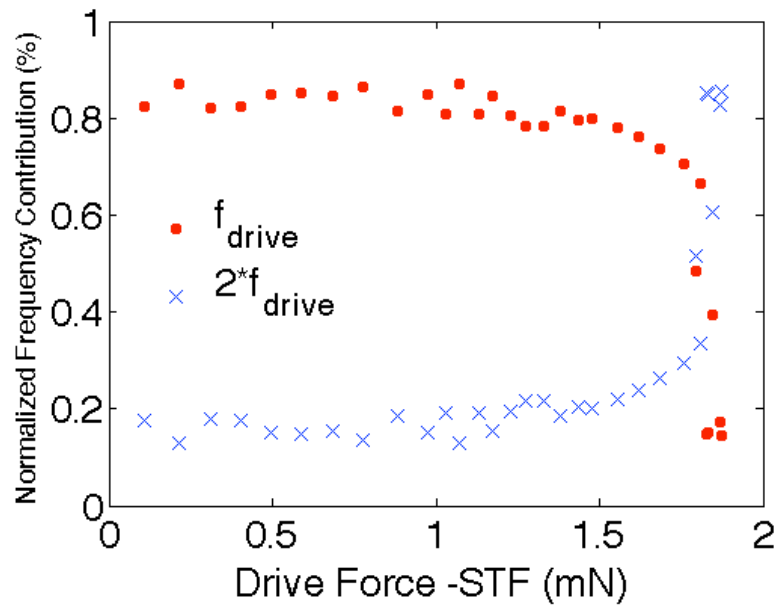

Figure 5: A plot showing the relative contribution of the first and second harmonics of the drive signal as actuation force is increased. In the shear thickening regime, the second harmonic becomes the dominant vibration mode.
However, at the onset of shear thickening behavior, there is a sharp decrease in the $f_{\text {drive }}$ component accompanied by an increase in the component at $2 * f_{\text {drive }}$. At high enough force amplitudes, the strength of the $2 * f_{\text {drive }}$ component surpasses that of the $f_{\text {drive }}$ component and becomes the dominant mode of vibration despite the fact that the forcing function is nearly a pure sinusoid with a single frequency. In fact, at high amplitude actuation forces, the device motion is primarily at twice the drive frequency. This is illustrated in Fig. 6, which superimposes the drive force and the velocity response in the time domain.

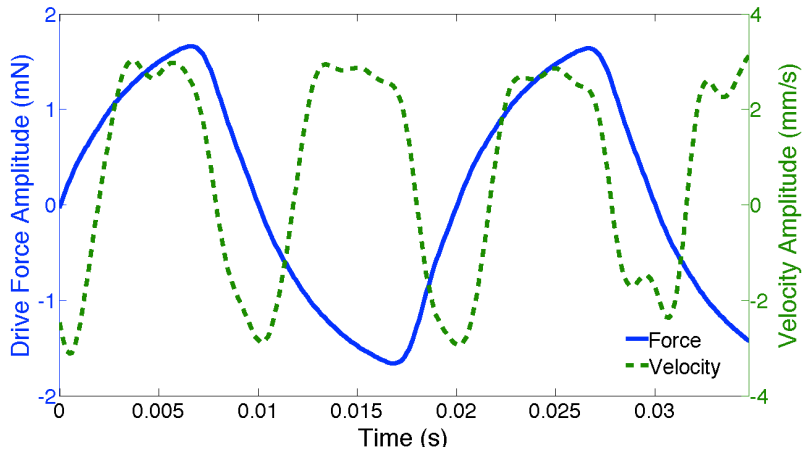

Figure 6: Time domain plots of the drive force and velocity under high amplitude actuation $(\mid$ Force $\mid=1.66 \mathrm{mN})$ at $50 \mathrm{~Hz}$. The velocity profile clearly shows oscillations at $100 \mathrm{~Hz}$.

\section{CONCLUSION}

We have successfully demonstrated a passively damped MEMS device where damping increases sharply when the device is subject to a high amplitude disturbing force. This damping effect is clearly a result of immersion in the STF and reveals the potential to use STFs as adaptive, passive dampers for shock protection of MEMS sensors or actuators. Additionally, we have shown that nonlinear damping due to the shear-dependent viscosity of the fluid can result in the emergence of a second vibrational mode at twice the drive frequency.

\section{ACKNOWLEDGEMENTS}

Student travel support has been generously provided by the Transducer Research Foundation.

\section{REFERENCES}

[1] D.M. Tanner, J.A. Walraven, K. Helgesen, L.W. Irwin, F. Brown, N.F. Smith, and N. Masters. "MEMS reliability in shock environments", Proceedings of the IEEE International Reliability Physics Symposium, San Jose, USA, April 10-3 (2000), pp. 129-138.

[2] J.K. Yee, H.H. Yang, and J.W. Judy. "Dynamic response and shock resistance of Ferromagnetic Micromechanical Magnetometers", Proceedings of the Tech. Digest IEEE Int. Conf. on Solid-State Sensors, Actuators, and Microsystems (TRANSDUCERS '03), Boston, USA (2003), pp. 308-311.

[3] S. Huang, X. Li, Y. Wang, J. Jiao, X. Ge, D. Lu, L. Che, K. Zhang, and B. Xiong. "A piezoresistive accelerometer with axially stressed tiny beams for both much increased sensitivity and much broadened frequency bandwidth", Proceedings of the Tech. Digest IEEE Int. Conf. on Solid-State Sensors, Actuators, and Microsystems (TRANSDUCERS '03), vol. 1, Boston, USA (2003), pp. 91-94 A.B. 
[4] S.W. Yoon, N. Yazdi, N. C. Perkins, and K. Najafi. "Micromachined integrated shock protection for MEMS", Sensors and Actuators A: Physical, Volumes 130-131, 14 August 2006, pp. 166-175.

[5] X.Z. Zhang, W. H. Li, and X. L. Gong. "The Rheology of Shear Thickening Fluid (STF) and the Dynamic Performance of an STF-filled Damper." Smart Materials and Structures 17.3 (2008).

[6] W. Zhang and K. Turner. "Frequency Dependent Fluid Damping of Micro/nano Flexural Resonators: Experiment, Model and Analysis." Sensors and Actuators A: Physical 134.2 (2007): 594-99.

[7] S. D. Dhole, R. P. Chhabra, and V. Eswaran. "Flow of PowerLaw Fluids Past a Sphere at Intermediate Reynolds Numbers." Industrial \& Engineering Chemistry Research 45.13 (2006): 4773-781.
[8] L.S. Jacobsen. "Steady forced vibrations as influenced by damping". Transactions ASME, 1930.

[9] F.J. Galindo-Rosales, F.j. Rubio-Hernández, and A. Sevilla. "An Apparent Viscosity Function for Shear Thickening Fluids." Journal of Non-Newtonian Fluid Mechanics (2011).

[10] H. Lee; S.A. Lee, M. Bergsneider, and J.W. Judy. "Unobstructing magnetic microactuators for implantable catheters." Solid-State Sensors, Actuators and Microsystems Conference (TRANSDUCERS 2009) ,vol., no., pp.857-860, 2125 June 2009

\section{CONTACT}

*S. Iyer, tel: +1-310-825-0585; ssiyer@ucla.edu 


\title{
OIL-INFUSED SUPERHYDROPHOBIC SILICON SURFACES FOR ENHANCED WATER CONDENSATION HEAT TRANSFER
}

\author{
R. Xiao ${ }^{l}$, R. Enright ${ }^{1,2}$, N. Miljkovic ${ }^{l}$, and E. N. Wang $^{l^{*}}$ \\ ${ }^{1}$ Department of Mechanical Engineering, MIT, Cambridge, Massachusetts, USA \\ ${ }^{2}$ Stokes Institute, University of Limerick, Limerick, Ireland
}

\begin{abstract}
Superhydrophobic surfaces have received significant interest for dropwise condensation to increase the efficiency of energy applications such as heat exchangers, power plants, and solar thermal energy conversion systems. While superhydrophobic surfaces can be fabricated using silane self-assembled coatings (SAC) on oxide structures, significant heat transfer enhancements require high nucleation densities that are difficult to achieve due to the high energy barrier for nuclei formation. In this work, we demonstrated that by infusing microstructured surfaces with lowsurface tension oil, nucleation densities can be increased by over an order of magnitude while maintaining low droplet adhesion. This work offers a simple and scalable approach to create surfaces that can be tailored for enhanced heat transfer.
\end{abstract}

\section{INTRODUCTION}

Condensation heat transfer has wide applications in various systems such as heat exchangers, heat pipes and power plants. The heat transfer coefficient of condensation is of great significance to the efficiency of such systems. Dropwise condensation, where the condensate forms discrete droplets rather than continuous films covering the substrate, is considered as one of the most promising approaches to enhance the heat transfer coefficient. Previous work has demonstrated the application of nanostructured superhydrophobic surfaces where condensate can be spontaneously removed via a surface-tension-driven mechanism [1]. However, the nucleation density on these surfaces is relatively low since the phase change process relies on high energy active sites to initiate nucleation at low supersaturations (low $\Delta T$ ), limiting the overall heat transfer performance. Furthermore, air pockets trapped beneath the droplets during growth reduce the contact area between the condensing droplet and substrate, which increases the thermal resistance and reduces the heat transfer coefficient [2].

Recently, Wong et al. [3] demonstrated a liquid-solid composite surface created by infusing a porous fluoropolymer with water-immiscible, low-surface-tension Krytox oil. On such a composite surface, the contact area between droplet and substrate can be large while contact line pinning remains very low allowing easy removal of droplets. These properties make the surfaces potentially suitable for enhanced condensation heat transfer. While such behavior is possible with the proper choice of silane, e.g., dichlorodimethylsilane on $\mathrm{SiO}_{2}$, the nucleation density is limited by the presence of high surface energy defects and contaminants at low supersaturations (low $\Delta T$ ). In this work, we show that the nucleation density on oil-infused, silane-coated structured surfaces can be significantly increased by the use of disordered long-chain silane coatings that result in nucleation sites limited only by the density of pillar structures comprising the surface. We find that the increase in the nucleation density can be explained by heterogeneity in the surface energy of the silane coating and the reduced water-oil interfacial energy. This effect could potentially be used to significantly improve the heat transfer coefficient in condensation by controlling the nucleation density.

\section{FABRICATION}

Well-defined silicon micro/nanopillar arrays with diameters, $d$, ranging from $0.4 \mu \mathrm{m}$ to $5 \mu \mathrm{m}$, periods, $l$, ranging from $4 \mu \mathrm{m}$ to $25 \mu \mathrm{m}$, and heights, $h$ ranging from $10 \mu \mathrm{m}$ to $25 \mu \mathrm{m}$ were used in our experiments. The silicon surfaces were functionalized with three different chemicals: 1) (Tridecafluoro-1,1,2,2tetrahydrooctyl)-1-trichlorosilane (TFTS) [UCT Specialties], which forms a self-assembled coating (SAC) by chemical vapor deposition (CVD) with a relatively long carbon chain $(\mathrm{MW}=$ $481.54 \mathrm{~g} / \mathrm{mol}$ ), 2) Dimethyldicholorosilane (DMCS) [SigmaAldrich], which forms a self-assembled monolayer (SAM) by CVD with a short carbon chain $(\mathrm{MW}=129.06 \mathrm{~g} / \mathrm{mol})$, and 3) Poly $(1 \mathrm{H}, 1 \mathrm{H}, 2 \mathrm{H}, 2 \mathrm{H}-$ perfluorodecyl acrylate) (PFDA) polymer, which was deposited using initiated chemical vapor deposition (iCVD) with a typical film thickness of $35 \mathrm{~nm}$. Goniometric measurements on smooth functionalized silicon surfaces showed advancing and receding contact angles of: $\theta_{\mathrm{a}} / \theta_{\mathrm{r}}=122^{\circ} \pm 1.3^{\circ} / 78^{\circ}$ $\pm 1.3^{\circ}$ (equilibrium contact angle $\theta_{\mathrm{e}} \approx 102.1^{\circ} \pm 0.9^{\circ}$ ); $\theta_{\mathrm{a}} / \theta_{\mathrm{r}}=$ $103.8^{\circ} \pm 0.5^{\circ} / 102.7^{\circ} \pm 0.4^{\circ}\left(\theta_{\mathrm{e}} \approx 103.2^{\circ} \pm 0.3^{\circ}\right) ;$ and $\theta_{\mathrm{a}} / \theta_{\mathrm{r}}=$ $121.1^{\circ} \pm 2.2^{\circ} / 106.3^{\circ} \pm 2.4^{\circ}\left(\theta_{\mathrm{e}} \approx 113.5^{\circ} \pm 1.6^{\circ}\right)$ for deposited films of TFTS, DMCS, and PFDA respectively. A small droplet of Krytox GPL 100 oil [DuPont] was applied to the functionalized silicon pillar arrays. The surface tension of Krytox oil is $\sim 17-$ $19 \mathrm{mN} / \mathrm{m}$, allowing the oil to spread on the surface. A dry nitrogen stream was used to assist spreading and remove excess oil. Typical scanning electron micrographs (SEM) of the silicon pillar arrays without and with the oil are as shown in Fig. 1(a) and (b).

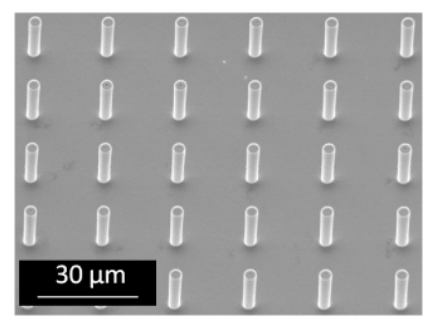

(a)

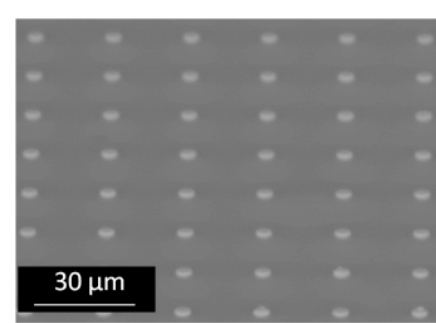

(b)
Fig. 1 Scanning Electron Micrographs (SEM) of a pillarstructured silicon surface $(d=5 \mu m, l=25 \mu m, h=15 \mu m)$ (a) without and (b) with the oil infusion.

We investigated the nucleation behavior on the surfaces with and without the oil under white light optical microscopy (OM). The samples were horizontally mounted on a thermal stage [Instec Inc.] inside an enclosure and cooled to $T_{\mathrm{w}}=283.1 \pm 0.1 \mathrm{~K}$ in a dry nitrogen atmosphere. Following thermal equilibration $(\sim 5 \mathrm{~min})$, nucleation was initiated by flowing water-saturated nitrogen into the enclosure. The supersaturation, defined as the ratio of the vapor pressure to the saturation pressure at the stage temperature $\left(p_{\mathrm{v}} / p_{\mathrm{w}}\right)$, was controlled by the temperature of the water reservoir through which the nitrogen carrier gas was sparged and measured using a humidity probe [Hygroclip, Rotronic] located $\sim 1 \mathrm{~cm}$ above the sample. Typical values of the supersaturation were around $S \approx 1.6$. The nucleation density and subsequent growth behavior was recorded at a frame rate of $30 \mathrm{fps}$ using a CMOS camera [Phantom V7.1, Vision Research] attached to the optical microscope. As 
shown in Fig. 2(a) and (b), the nucleation density on oil-infused surface with long-chain TFTS coating was significantly higher than that on the same surface without oil. We did not observe any nucleation between pillars which can be attributed to the large thickness of oil coverage between pillars that introduces a large diffusion resistance for water vapor. However, a droplet was formed on the tip of almost each pillar where the oil film was thin enough to allow sufficient mass to diffuse to the functionalized silicon surface. Meanwhile, on pillar arrays coated with DMCS and PFDA, we did not observe similar increases in nucleation density, as shown in Fig. 2(c).

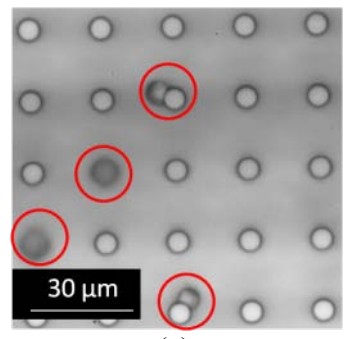

(a)

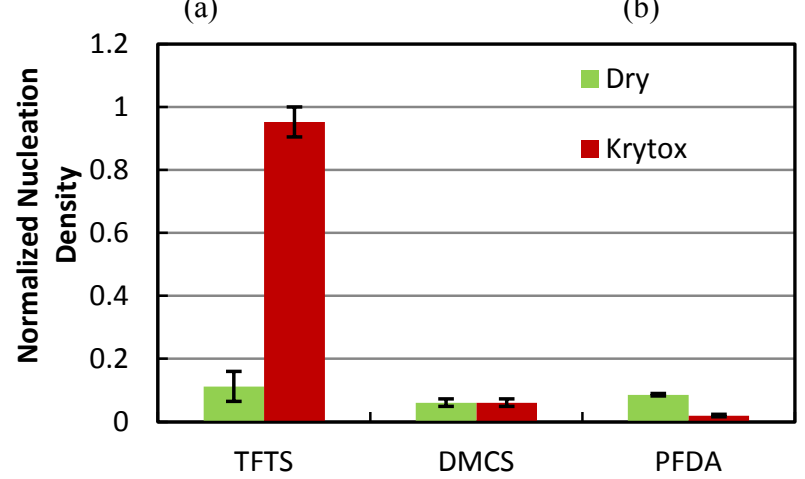

(c)

Fig. 2(a) White-light microscopy image of condensation on a silicon pillar array without oil, with nucleation sites highlighted by red circles. (b) White-light microscopy image of condensation on a composite surface with oil. Nucleation occurred on the tip of almost every pillar. (c) Comparison of the change in nucleation density with various surface coatings. The nucleation densities were normalized against pillar densities for a fair comparison.

\section{AFM IMAGING AND CONTACT ANGLE ANALYSIS}

To investigate the mechanism for this drastic change in nucleation density, we performed atomic force microscopy (AFM) in tapping mode on a smooth TFTS-coated silicon surface and observed the presence of micelle structures, as shown in Fig. 3(a). Such micelle structures have been observed in previous studies and considered as disordered agglomeration of excessive silane molecules [4]. The phase image of the AFM measurement (Fig. 3(d)) showed significantly higher phase angle on the micelle structures, which suggests that the micelles were locally more hydrophilic compared to the background film. Such heterogeneity was also supported by the high contact angle hysteresis $\left(\theta_{\mathrm{a}} / \theta_{\mathrm{r}}=\right.$ $\left.122^{\circ} \pm 1.3^{\circ} / 78^{\circ} \pm 1.3^{\circ}\right)$. However, such micelle structures were not observed on other kinds of surface coatings such as DMCS and PFDA, as shown in Fig. 3(b) and (c).

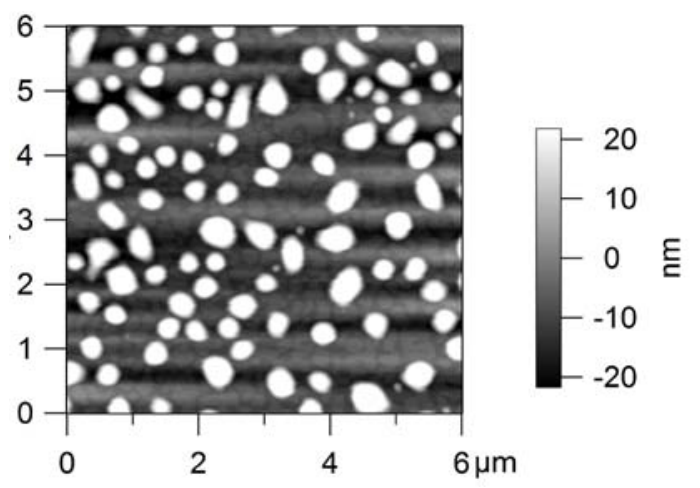

(a)

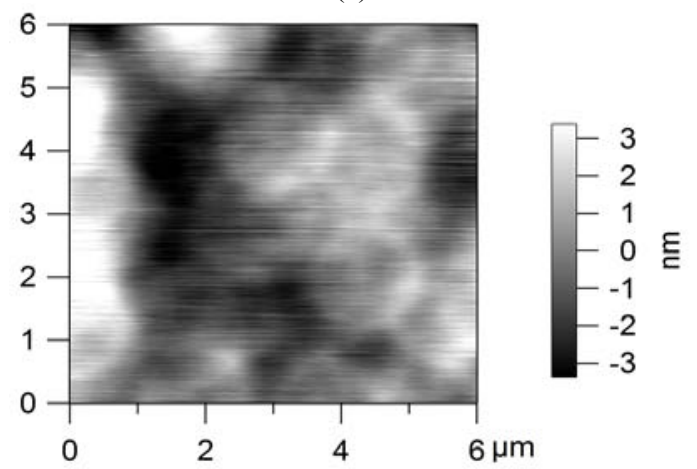

(b)

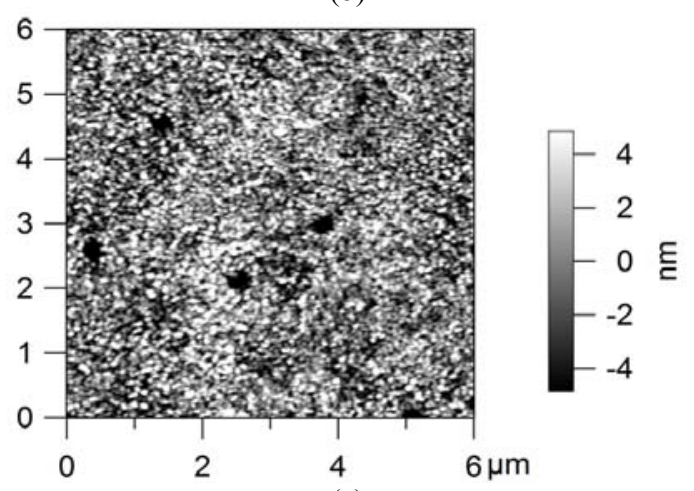

(c)

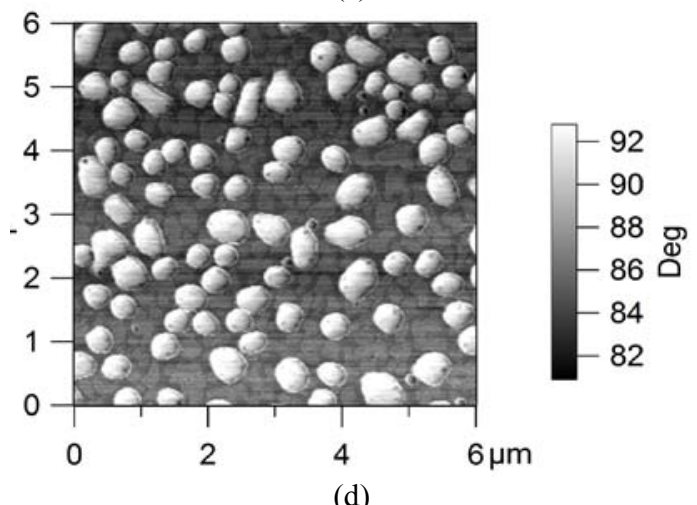

Fig. 3 Atomic Force Microscopy (AFM) height images of smooth silicon surfaces coated with (a) TFTS, (b) DMCS, and (c) PFDA. Micelles structures were only observed on TFTS coated surfaces. (b) AFM phase image of smooth silicon surface coated with TFTS. The local high phase angle indicates higher hydrophilicity of micelles. 
The local contact angles on the hydrophobic substrate and the micelle structures can be determined based on a modified CassieBaxter model. Assuming the local contact angles on the hydrophobic substrate and the micelle structures to be $\theta_{1}$ and $\theta_{2}$, respectively, the macroscopic advancing and receding contact angles are determined as

$$
\begin{gathered}
\theta_{a}=\theta_{1}, \\
\cos \theta_{r}=\sqrt{f} \cos \theta_{2}+(1-\sqrt{f}) \cos \theta_{1},
\end{gathered}
$$

where $f$ is the area fraction of the micelles [5].

Based on the macroscopically measured advancing and receding angles, $\theta_{\mathrm{a}}=122^{\circ} \pm 1.3^{\circ}$ and $\theta_{\mathrm{r}}=78^{\circ} \pm 1.3^{\circ}$, and the fraction of the micelles determined as $f \approx 0.4$ from AFM, the local contact angles on the hydrophobic substrate and the hydrophilic micelles were found to be $\theta_{1}=122^{\circ} \pm 1.3^{\circ}$ and $\theta_{2}=60^{\circ} \pm 1.5^{\circ}$.

\section{CLASSICAL NUCLEATION THEORY}

The nucleation rate, $J$, can be determined by classical nucleation theory (CNT) as [6]

$$
J=z f^{*} \exp \left(-G^{*}\right)
$$

In Eqn. (3), $z$ is the Zeldovich factor and $G^{*}$ is the dimensionless energy barrier, given by

$$
\begin{aligned}
z & =(k T \ln S)^{2} / 8 \pi v_{o} \sqrt{k T \psi(\theta) \gamma^{3}} \\
G^{*} & =16 \pi \psi(\theta) v_{o}^{2} \gamma^{3} / 3(k T)^{2}(\ln S)^{2}
\end{aligned}
$$

where $S$ is the supersaturation and $\psi(\theta)$ is the activity that accounts for the effect of contact angle. $f^{*}$ is the frequency of monomer attachment to the critical droplet nucleus dependent on the nature of the nucleus growth. The main modes of growth during heterogeneous nucleation are limited via surface diffusion or direct impingement of monomers to the nucleus [7, 8]. Volumetric diffusion is a third growth limiting step, which is only considered important for nucleation taking place in liquid or solid solutions [6]. However, we included all three mechanisms when calculating the nucleation rates.

The frequency of monomer attachment due to direct vapor impingement is given by

$$
f^{*}{ }_{i}=\gamma_{\mathrm{n}}\left[\left(1-\cos \left(\theta_{\mathrm{w}}\right)\right) / 2 \psi^{2 / 3}(\theta)\right]\left(36 \pi v_{\mathrm{o}}^{2}\right)^{1 / 3} \operatorname{In}^{2 / 3}
$$

where $\gamma_{\mathrm{n}}$ is the sticking coefficient $\left(0<\gamma_{n}<1\right), I$ is the classical Hertz-Knudsen impingement rate $\left.\left(I=P / \sqrt{2 \pi m_{0} k T}\right)\right), n$ is the number of molecules in the nucleated cluster, and $v_{\mathrm{o}}$ is the volume of an individual water molecule $\left(v_{0}=3 \times 10^{-29} \mathrm{~m}^{3}\right)$. To determine an upper bound on the nucleation rate, a sticking coefficient of one was assumed $\left(\gamma_{\mathrm{n}}=1\right)$.

The frequency of monomer attachment due to surface diffusion is given by $f^{*}{ }_{\mathrm{sd}}=\gamma_{\mathrm{n}} c^{*} \lambda_{\mathrm{s}}{ }^{2} I$, where $c^{*}$ is the capture number due to surface diffusion $\left(1<c^{*}<5\right)$, and $\lambda_{\mathrm{s}}$ is the mean surface diffusion distance of an adsorbed monomer on the substrate. The capture number $c^{*}$ is size independent and approximately equal to 1.9 for heterogeneous condensation of water vapor [9]. The mean surface diffusion distance is dependent on the wettability of the substrate and is given by $\lambda_{\mathrm{s}}=\sqrt{D_{\mathrm{sd}} \tau_{\mathrm{d}}}$ where $D_{\text {sd }}$ is the surface diffusion coefficient $\left(D_{\mathrm{sd}}=d_{\mathrm{s}}{ }^{2} v_{\mathrm{s}} \exp \left[-E_{\mathrm{sd}} / k T\right]\right), \tau_{\mathrm{d}}$ is the desorption time $\left(\tau_{\mathrm{d}}=\right.$ $\left.\left(1 / v_{\mathrm{s}}\right) \exp \left[-E_{\mathrm{des}} / k T\right]\right), v_{\mathrm{s}}$ is the adsorbed molecule vibration frequency determined using the Debye approximation $\left(v_{\mathrm{s}}=\right.$ $\left.V_{D} a / 2\right), d_{\mathrm{s}}$ is the length of a molecular jump along the substrate surface approximated by the lattice constant of the substrate $\left(d_{\mathrm{S}}=5.4 \AA\right)$ [10] and $V_{\mathrm{D}}$ is the speed of sound in the substrate $\left(V_{\mathrm{D}}=8433 \mathrm{~m} / \mathrm{s}\right)$. The desorption and surface diffusion energies are given by $E_{\mathrm{des}}=E_{1}+\sigma_{\mathrm{sv}} a_{\mathrm{o}}$ and $E_{\mathrm{sd}}=0.5 E_{\mathrm{des}}$ [11], respectively, where $E_{l}$ is the binding energy of an $n=1$ sized cluster, $\sigma_{\mathrm{sv}}$ is the solid vapour interfacial energy and $a_{\mathrm{o}}$ is the water molecule surface area $\left(a_{\mathrm{o}}=4.67 \times 10^{-19} \mathrm{~m}^{2}\right)$. The calculated energies of desorption show excellent agreement with that of experiment and molecular dynamics simulations $\left(E_{\mathrm{des}, \mathrm{SiO} 2}=0.9 \mathrm{eV}\right)[12,13]$.

The frequency of monomer attachment due to volumetric diffusion is given by

$$
f^{*}{ }_{\mathrm{vd}}=\gamma_{\mathrm{n}}\left(1-\frac{\cos \theta_{\mathrm{w}}}{\psi^{1 / 3}}\right)\left(6 \pi^{2} v_{\mathrm{o}}\right)^{1 / 3} D C n^{1 / 3}
$$

where $D$ is the self diffusion coefficient of water vapor $(D=$ $\left.\left(3 / 8 \pi n_{\mathrm{o}}{d_{\mathrm{o}}}^{2}\right) \sqrt{k T / \pi m_{\mathrm{o}}}\right), C$ is the equilibrium concentration of monomers $\left(C=\left(1 / a_{0}\right) \exp \left(-W_{1} / k T\right)\right), d_{0}, m_{0}$ and $n_{0}$ are the water molecule diameter $\left(d_{\mathrm{o}}=3.0 \AA\right)[12]$, mass $\left(m_{\mathrm{o}}=3 \times 10^{-26} \mathrm{~kg}\right)$ [6] and number density $\left(n_{\mathrm{o}}=N_{\mathrm{A}} / v_{\mathrm{M}}\right)$, respectively.

By adding the nucleation rate from the three mechanisms together, the nucleation rate, $J$, can be determined as a function of the contact angle and surface tension of the condensate at given supersaturations, as shown in Fig. 4.

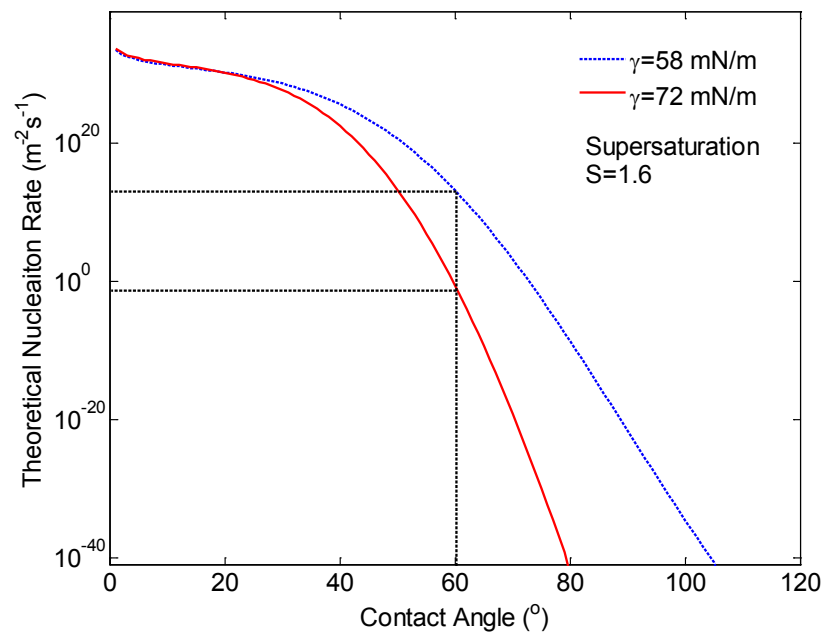

Fig. 4 Nucleation rate predicted by the classical nucleation theory as a function of contact angle and interfacial tension at $S=1.6$.

The surface tension of water in air is $72 \mathrm{mN} / \mathrm{m}$ and the interfacial tension between water and the Krytox oil was found to be $58 \mathrm{mN} / \mathrm{m}$ by measuring the contact angle of water droplet on oil film. From Fig. 4 we can see that for the hydrophilic micelles $(\theta \approx$ $60^{\circ}$ ), the reduced interfacial tension between water and oil leads to a significant increase in the nucleation rate. With the micelles acting as nucleation sites, nucleation was supposed to occur on almost every pillar tip where the oil film was thin enough for water vapor to diffuse through. On surfaces without the micelles, such as DMCS and PFDA-coated surfaces, the contact angles are over $100^{\circ}$ and the nucleation rate was essentially zero even with reduced interfacial tension. 
In order to validate our assumption, we carried out condensation experiments using silicon pillar arrays coated with 3(trimethoxysilyl)propyl methacrylate (3-TMPM). The advancing and receding contact angle of water on a smooth silicon surface coated with $3-\mathrm{TMPM}$ are $65^{\circ} \pm 1.5^{\circ}$ and $53^{\circ} \pm 1.1^{\circ}$, respectively. The contact angle is in the range where the nucleation rate will be almost zero with a surface tension of $72 \mathrm{mN} / \mathrm{m}$ and nucleation should occur on every tip of pillars with an interfacial tension of 58 $\mathrm{mN} / \mathrm{m}$. Optical images of the condensation experiments are as shown in Fig. 5. Similar to the behavior of TFTS-coated surface, a significant increase in nucleation density was observed with the addition of Krytox oil as expected. This result supports well our hypothesis related to the role of the hydrophilic micelle structures in the droplet nucleation process.

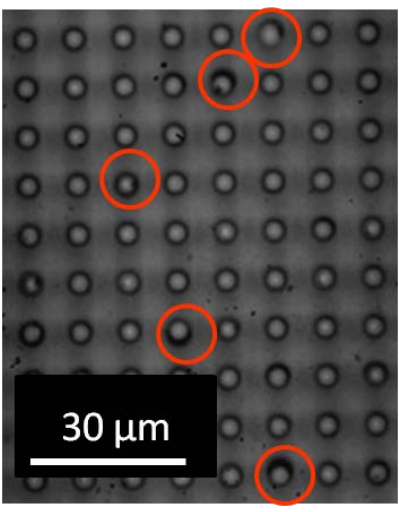

(a)

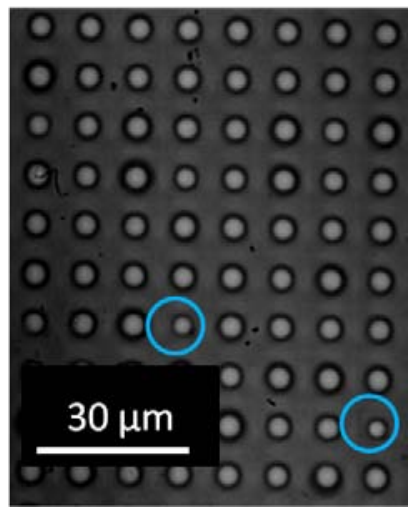

(b)
Fig. 5 Optical microscope images of the condensation experiments on 3-TMPM coated silicon pillar arrays (a) without $\left(N=6.7 \times 10^{8} \mathrm{~m}^{-2}\right)$ and (b) with Krytox oil $\left(N=1.7 \times 10^{10} \mathrm{~m}^{-2}\right)$. Nuclei are highlighted by red circles in (a) and pillars without nucleation were highlighted by blue circles in (b). The pillars have diameters of $2.5 \mu \mathrm{m}$, periods of $7.5 \mu \mathrm{m}$ and heights of $25 \mu \mathrm{m}$ $\left(l^{-2}=1.7 \times 10^{10} \mathrm{~m}^{-2}\right)$.

\section{SUMMARY}

In summary, we observed over an order of magnitude increase in the nucleation density on hydrophobic silicon pillar arrays coated with a long-chain silane molecule when hydrophobic oil was introduced on the surface. AFM imaging revealed the existence of locally hydrophilic micelles despite the overall hydrophobicity of the silane self-assembled coating (SAC). The increased nucleation density is explained in the context of classical nucleation theory as the combined effect of the hydrophilic micelles and the reduction in interfacial energy between water and oil. Control experiments on silicon pillar arrays with hydrophobic coatings without micelles and hydrophilic coatings were performed to support our findings. Such phenomena could potentially be used to create surfaces for enhanced condensation heat transfer for a variety of thermal and energy systems.

\section{ACKNOWLEDGEMENT}

The authors gratefully acknowledge the help of Rong Yang from Prof. K.K.Gleason's group at the Department of Chemical Engineering at MIT for deposition of PFDA polymer. The authors would also like to acknowledge the funding support from the Office of Naval Research (ONR) with Dr. Mark Specter as the project manager. R. Enright also acknowledges funding received from the Irish Research Council for Science, Engineering, and
Technology, co-funded by Marie Curie Actions under FP7. The authors would also like to thank the MIT Microsystems Technology Lab for fabrication staff support, help, and use of equipment.

\section{REFERENCES}

[1] J.B.Boreyko and C.-H. Chen, "Self-Propelled Dropwise Condensate on Superhydrophobic Surfaces", PRL, 2009. 103(18): p. 184501

[2] N. Miljkovic, R. Enright, and E.N. Wang, "Effect of Droplet Morphology on Growth Dynamics and Heat Transfer during Condensation on Superhydrophobic Nanostructured Surfaces", ACS Nano, 2012 6(2): p. 1776-1785

[3] T.-S.Wong, S.H.Kang, S.K.Y.Tang, E.J.Smythe, B.D.Hatton, A.Grinthal and J.Aizenberg, "Bioinspired self-repairing slippery surfaces with pressure-stable omniphobicity", Nature, 2011. 477: p. 443-447.

[4] B. C. Bunker, R. W. Carpick, R. A. Assink, M. L. Thomas, M. G. Hankins, J. A. Voigt, D. Sipola, M. P. de Boer, and G. L. Gulley, "The Impact of Solution Agglomeration on the Deposition of Self-Assembled Monolayers", Langmuir, 2000, 16 (20), pp 7742-7751

[5] R. Raj, unpublished, 2012

[6] D. Kashchiev, Nucleation: Basic Theory with Applications. 1 ed. 2000, Oxford: Butterworth-Heinemann.

[7] G.M.Pound, M.T. Simnad, and L. Yang, "Heterogeneous nucleation of crystals from vapor" J. Chem. Phys., 1954. 22(1215).

[8] R.A.Sigsbee, "Atom capture and growth rates of nuclei", JAP, 1971. 42(10): p. 3904-3915.

[9] D.J. Pocker, and S.J. Hruska, "Detailed calculations of the numberof distinct sites visited in random walk on several twodimensional substrate lattices." J. Vac. Sci. Tech., 1971. 8(6): p. 700-707.

[10] J.P.Hirth, and G.M. Pound, Condensation and evaporation nucleation and growth kinetics. 1963, England: Pergamon Press.

[11] P.A.Thiel, and T.E. Madey, "The interaction of water with solid surfaces: Fundamental aspects." Surface Science Reports, 1987. 7(6-8): p. 211-385.

[12] J.N. Israelachvili, Intermolecular and surface forces. 2nd ed. 1991, Amsterdam: Academic Press.

[13] Y.Ma, A.S. Foster, and R.M. Nieminen, "Reactions and clustering of water with silica surface". J. Chem. Phys., 2005. 122(144709)

\section{CONTACT}

*E.N. Wang, tel: +1-617-324-3311; enwang@mit.edu 


\section{A HIGH DYNAMIC RESTORING FORCE ELECTROSTATIC ACTUATOR Arun Gupta ${ }^{1,2 *}$, Mark Fletcher ${ }^{l}$, Divyanshu Agrawal ${ }^{1}$, Carol McMillen ${ }^{l}$, and J.-B. Lee ${ }^{2}$ \\ ${ }^{1}$ Texas Instruments Inc., Dallas, Texas, USA \\ ${ }^{2}$ The University of Texas at Dallas, Dallas, Texas, USA}

\begin{abstract}
A surface-micromachined parallel-plate MEMS actuator has been fabricated using a modified version of the Texas Instruments Inc. Digital Micromirror Device fabrication process. The actuator employs a unique dynamic restoring force mechanism to overcome stiction and electrostatic hold-down issues commonly faced by surface micromachined actuators. This dynamic boost results in a $\sim 16 \mathrm{x}$ increase in restoring force and a $\sim 4 \mathrm{x}$ increase in the voltage required to hold the actuator down compared with static actuation, without affecting the device's initial pull-in voltage. Furthermore, the actuator utilizes a torsion architecture that greatly reduces contact area and electrostatic field strength, thereby further reducing stiction.
\end{abstract}

\section{INTRODUCTION}

Stiction is one of the biggest reliability challenges in surface micromachined electrostatic contact actuators [1,2]. This challenge is often compounded by the desire to maintain a continuous voltage waveform on the device. Depending on the application, this waveform could serve different purposes. For example, in sensor applications the waveform could be used to sense the position of the moving element. Alternatively, in a micro-mechanical relay, the waveform would be the signal that the device is switching on and off. Regardless of the application, any waveform across the actuator provides its own electrostatic attraction force which can prevent the release of the moving plate when it is landed, creating an unwanted electrostatic hold-down effect.

Both stiction and electrostatic holding force create a need for high spring restoring force. Spring suspensions must be made stiff enough to overcome unwanted forces that are holding the actuator in the closed position. The tradeoff is that stiff suspensions need high amounts of electrostatic force to actuate in the first place, which translates to high actuation voltages and large actuation electrodes. High voltages can create a number of additional challenges, including dielectric breakdown, dielectric charging, arcing, etc [3]. These problems can be alleviated with techniques such as waveform shaping or constant charge biasing [4], in which the high voltages are used only to initiate actuation and are backed down as the structure lands. However, high voltage supplies are generally not available in most MEMS end-applications and must be supplied by SoC or SiP integrated DC-DC converters, which may place practical limitations on the voltage available. Large actuation electrodes can cause fabrication and die area concerns, and they may result in increased stiction due to large surface contact area [1].

To circumvent these challenges, this paper presents an actuation mechanism that dynamically increases spring restoring force without increasing the force needed to actuate the device.

\section{THEORY}

According to basic parallel plate actuator analysis, the electrostatic force $F_{e}$ between two parallel plates with overlap area $A$ resulting from an applied voltage $V$ is

$$
F_{e}=\frac{\varepsilon A V^{2}}{2\left(x_{o}-x\right)^{2}}
$$

where $x_{o}$ is the initial gap between the plates and $x$ is the displacement. As the actuator approaches the closed state and the gap $\left(x_{o}-x\right)$ becomes small, the electrostatic force becomes quite large. However, when the force is applied to a linear restoring spring $F_{s}$, the electrostatic force overwhelms the spring and device "pulls in" when the displacement is a third of the initial gap, at voltage

$$
V_{P I}=\sqrt{\frac{8 k x_{0}^{3}}{27 \varepsilon A}}
$$

From equations (1) and (2), it is easy to see that for a given pull-in voltage, the amount of electrostatic force that exists when the actuator is in the closed position far exceeds the spring restoring force in the closed position. This excess electrostatic force is effectively "wasted" because it does not serve to oppose a restoring force that will help release the actuator from a contact surface.

$$
F_{\text {wasted }}=F_{e}\left(V_{P I}, x_{\text {closed }}\right)-F_{S}\left(x_{\text {closed }}\right)
$$

In a simple example, if we assume air dielectric and further assume that the closed position is $1 / 10^{\text {th }}$ the initial gap and voltage is just above $V_{P I}$, the electrostatic force in the closed position is $\sim 16$ times larger than the spring restoring force.

Ideally this "wasted" force could be recovered by using it to do some useful work, i.e. compress a secondary stiff spring that does not engage until the actuator is nearly closed. However, the amount of energy stored in this hypothetical secondary spring is proportional to the displacement of that spring, so to maximize energy the secondary spring should engage rather early (but after pull-in). However, the spring will then release its energy over its entire range of travel. Since both stiction and electrostatic holding forces are strongest over very small distances, it would be more effective if the secondary spring were to release its energy all at once. This would provide a strong impulse to the moving plate and effectively "knock" it off of the surface on which it was landed. The following section describes a design that accomplishes this goal.

\section{DESIGN AND FABRICATION}

The actuator consists of a metal superstructure suspended above a set of stationary electrodes with torsion and cantilevered springs (Fig. 1a). The superstructure is partitioned into three sections: two paddles and a center plate. The paddles both rotate inwards on a set of torsion springs, while the center plate between the paddles remains parallel to the substrate and eventually lands on the SENSE electrode beneath it. A thin dielectric layer coats all underlying electrodes to prevent shorting. 
(a)

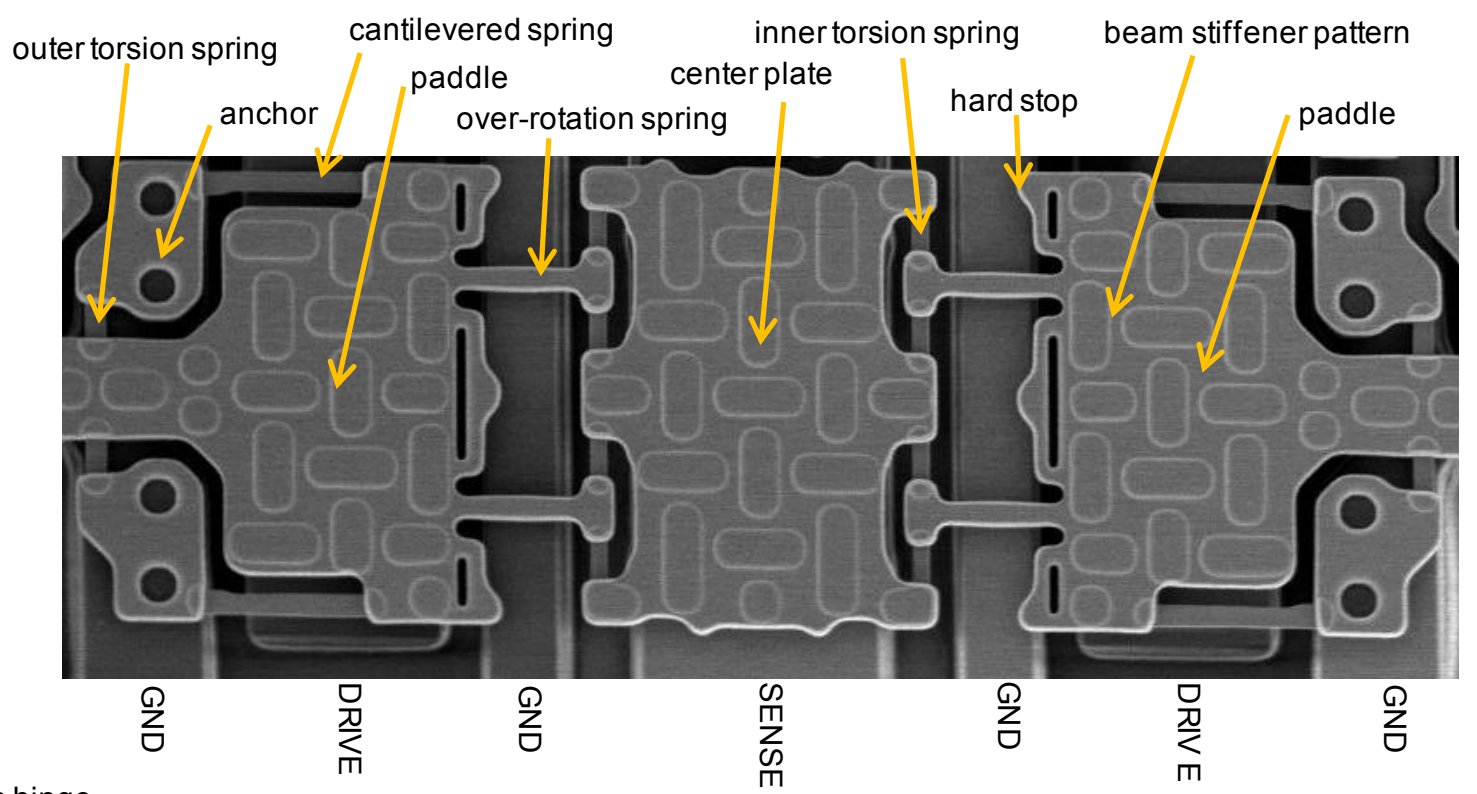

torsion hinge

(b)

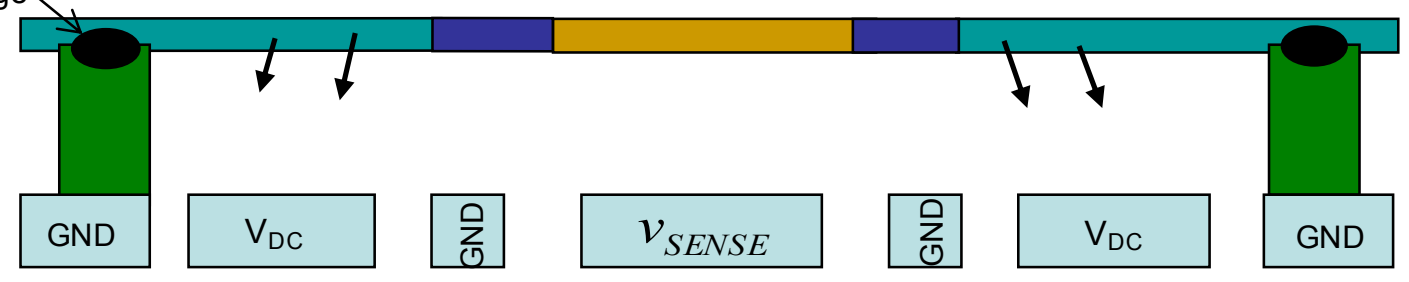

(c)

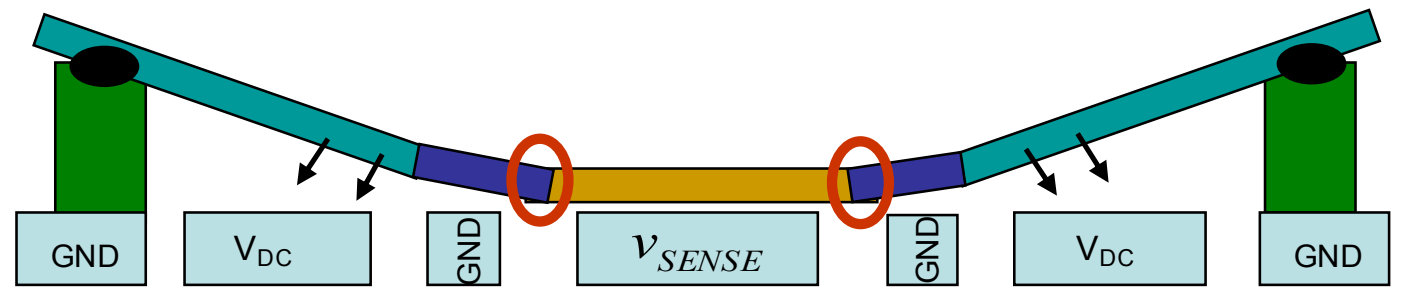

(d)

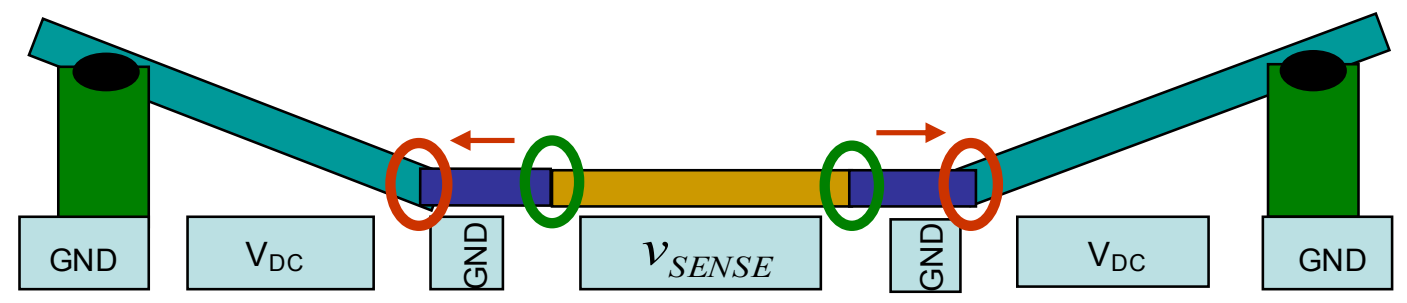

(e)

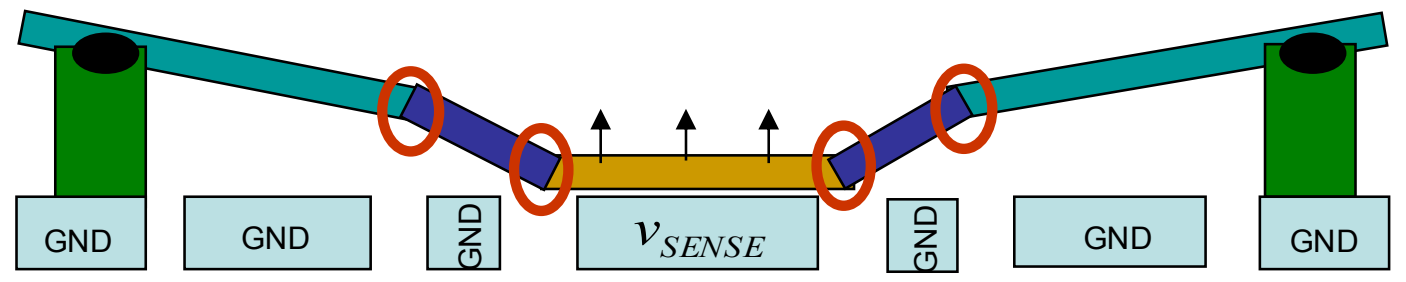

Figure 1: Top view SEM image and side view schematic diagrams are aligned and approximately to scale with each other in the x-axis. (a) SEM image of fabricated actuator with major design fea tures labeled above the image and electrode names labeled below the image. (b) DC potential is applied to DRIVE electrode, center plate moves downward. (c) Inner torsion springs store energy. Center plate lands on SENSE electrode. (d) Paddles continue to rotate until they hit the hard stops. Inner torsion spring relaxes, transfers energy to over-rotation spring. (e) Voltage is removed from DRIVE. Paddles bounce upward, simultaneously loading over-rotation and inner torsion springs. Upward force is transferred to center plate to overcome stiction and electrostatic hold-down forces. 
The actuator is fabricated with a modified version of the Digital Micromirror Device (DMD) process described in [5], with the second sacrificial spacer and mirror metal levels removed. This process produces two thicknesses in the superstructure metal: a thick layer that has been previously described in literature as the "yoke", and a thin layer known as "hinge". The "yoke" layer is used in this design to create the rigid plates that form the paddles and the center plate, as well as the stiff over-rotation springs. Both the paddles and the center plate are further stiffened by a corrugation pattern that can readily be seen in Fig 1a. The "hinge" layer is used to create the more compliant torsion springs and cantilevered springs.

The device operates as follows: A bias is placed on the DRIVE electrodes beneath the paddles, creating an electrostatic force that causes the paddles to rotate inwards (Fig. 1b). Energy is stored in the outer torsion springs, the cantilevered springs, the over-rotations springs, and inner torsion springs. The ratio of the energy stored in the over-rotation spring vs. the inner torsion spring depends on the ratio of their stiffnesses. The inner torsion spring is much more compliant than the over-rotation spring, so it stores a majority of the energy. The role of the cantilevered springs is simply to increase overall restoring force.

Once the center plate lands on the SENSE electrode, the paddles continue to rotate (Fig. 1c), producing so-called "overrotation". This causes the inner torsion spring to relax and transfer energy to the over-rotation spring. The paddles continue to overrotate until the hard stops on paddles land on the GND electrodes that flank the SENSE electrode, producing a stable down-state or closed position (Fig. 1d). Assuming the over-rotation spring is linear, its potential energy is given by

$$
U_{O R}=\frac{1}{2} k x_{O R}^{2}
$$

The over-rotation springs are stiff so their spring constant $k$ is high, and they are also quite long which means that over-rotation travel distance $x_{O R}$ adds substantially to the paddles' overall travel $x_{\text {closed }}$. As such, the over-rotation step significantly increases the total potential energy stored, as shown schematically in Fig. 2. However, since the center plate has already landed before overrotation even begins, this additional energy storage does not affect the pull-in voltage of the device. The high electrostatic force of the parallel plate in the closed position is simply used to do additional work, rather than being "wasted".

\section{(c) Center plate lands}

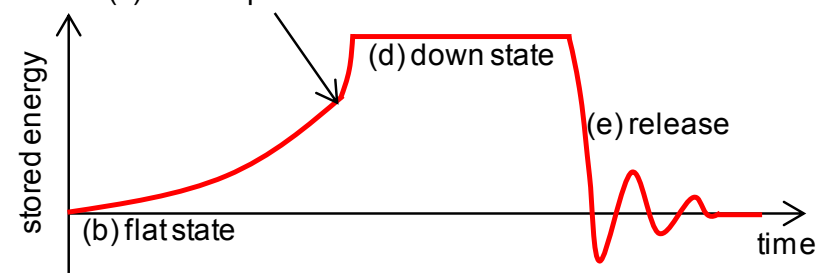

Figure 2: Schematic diagram of stored energy in the various states referenced in Fig. 1.

Upon release, voltage is removed from the DRIVE electrodes with a sharp slew rate. The paddles bounce upwards, and potential energy stored in the springs is converted to kinetic energy in the paddles. Meanwhile, the center plate may remain stuck down due to electrostatic hold-down forces and stiction (Fig. 1e). As a result, both the over-rotation and torsion springs become loaded, but the over-rotation spring deflects in the opposite direction than it does when it lands. This temporarily results in a negative potential energy with respect to equilibrium (Fig. 2) since both springs cannot be simultaneously loaded in equilibrium. Upward force is transferred to the center plate to overcome electrostatic hold-down and stiction, which both operate over small distances.

The combination of the over-rotation springs and the inner torsion springs essentially produces a stiffer spring constant during release than it does during landing. This effect occurs because during the landing over-rotation step, the inner torsion springs relax, which softens the combined spring constant, while during release the inner torsion spring become loaded, which stiffens the combined spring constant. The result is a greater upward impulse delivered to the center plate to "knock" it off the surface on which it was landed.

One of the consequences of this release mechanism is that over-rotation spring will ring and the center plate and paddles will oscillate with respect to each other on their way up. This is an acceptable tradeoff because once the center plate is released off the surface, it is not subject to stiction forces and the electrostatic hold forces diminish rapidly. Thus, the ringing should not cause the center plate to re-land, and it should damp out due to air and anchor damping.

\section{RESULTS}

Figure 3 shows a normalized land and release C-V plot for a parallel array of fabricated actuators. The array is pulled-in at DRIVE $=\sim 35 \mathrm{~V}$. This values match well with Coventorware ${ }^{\circledR}$ FEA simulations after accounting for intrinsic metal stresses. Note that there is less than $1 \mathrm{~V}$ of hysteresis between the land and release curves, evidence that the amount of "wasted" electrostatic force in the landed state has been minimized. This is not only a result of the over-rotation mechanism, but also because the non-parallel relationship between the rotating paddles and the DRIVE electrodes means that the electrostatic force does not grow as quickly with displacement as it would with a parallel plate drive that obeys equation 1 .

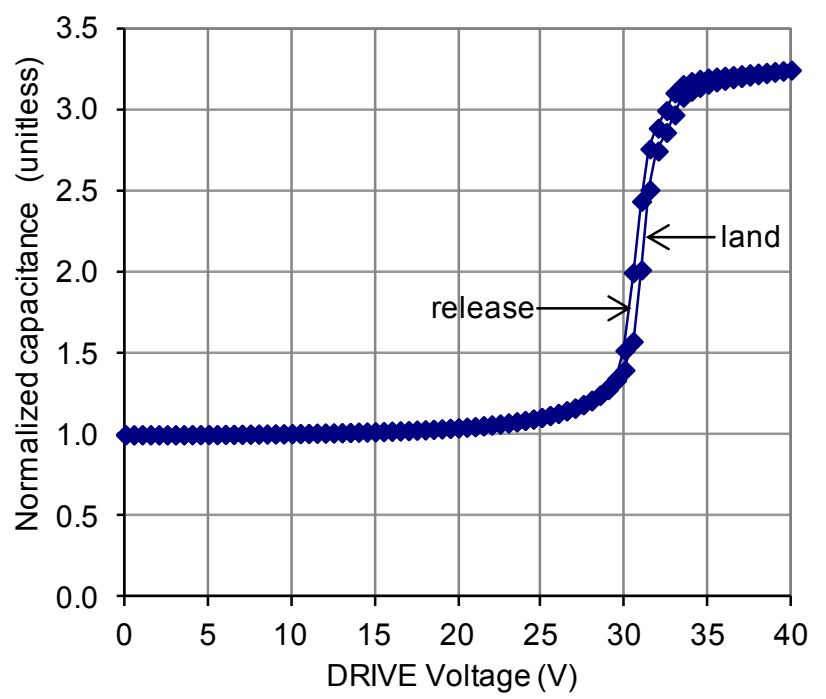

Figure 3: Normalized $C-V$ plot for an array of actuators. Capacitance is measured on the SENSE electrode. Actuation voltage is applied on the DRIVE electrodes.

FEA simulations also confirm that the effective combined spring constant for the inner torsion and over-rotation springs is 
greater when the device is releasing than when the device is landing. Figure 4 shows a stress profile of a quarter-model in the landed state, illustrating the distribution of energy stored in each of different springs.

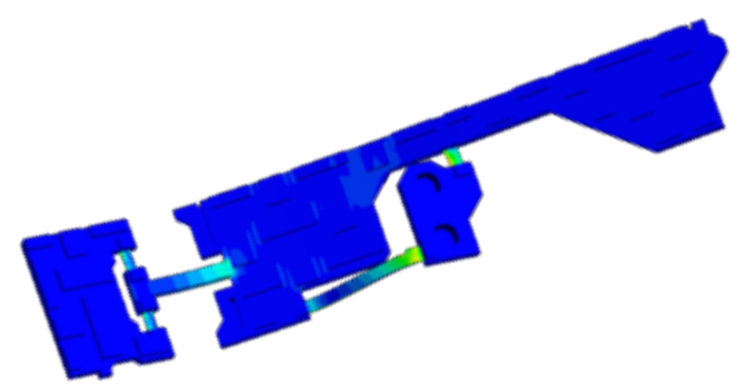

Figure 4: Mises stress profile of a $3 D$ quarter-model actuator in the landed position, simulated with Coventorware ${ }^{\circledR}$ FEA software. Z-axis displacement is exaggerated 10x.

Both static and dynamic hold-down voltages were measured by placing a voltage on the SENSE electrode and attempting to release the actuator. Dynamic electrostatic hold-down voltage is $20 \mathrm{~V}$ and is measured with a DRIVE waveform fall time of $<100 \mathrm{~ns}$ (Fig. 5). This is $4 \mathrm{x}$ greater than the static hold-down voltage of $5 \mathrm{~V}$, measured with a slow fall time, indicating that the dynamic release mechanism is functioning properly. Equation 1 implies that a $4 x$ increase in hold-down voltage results from a $16 x$ increase in restoring force.

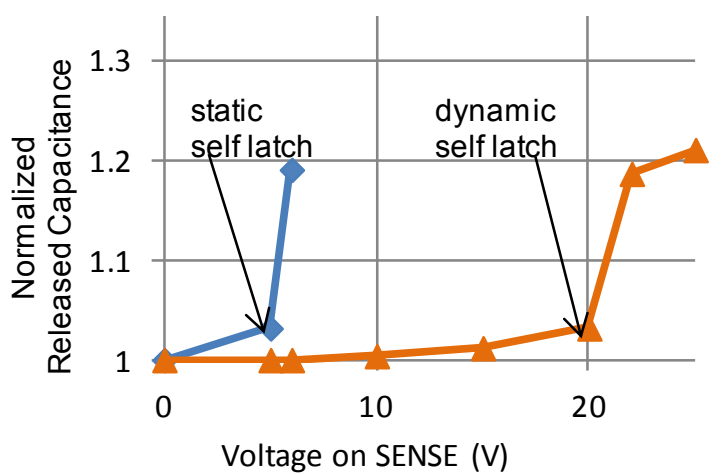

Figure 5: Static electrostatic hold down (5V) and dynamic hold down (20V) plots. Voltage is applied to the SENSE electrode in the down state, the varactor is released, and $C$ is measured. In the dynamic test, the switching waveform on the DRIVE electrode has a fall time of $<100$ ns, while in the static test it is much slower.

Optical measurement of actuator dynamics was taken with a Polytec laser Doppler vibrometer to further verify the over-rotation mechanism. Figure 6 shows displacement vs. time for a point in the center of the center plate and a point on the inner edge of the paddle while the actuator is landing. As expected, once the actuator begins traveling downwards, the displacement of the center plate is slightly greater than that of the paddle edge. Once the center plate makes initial contact with the SENSE electrode (Fig. 6, time a), the paddle continues to over rotate until it hits a hard stop, pulling the center plate flatter and into more intimate contact with the SENSE electrode. After the hard stop is reached (Fig. 6, time b), there are some minor oscillations between the paddles and the center plate that die down within 50ns.

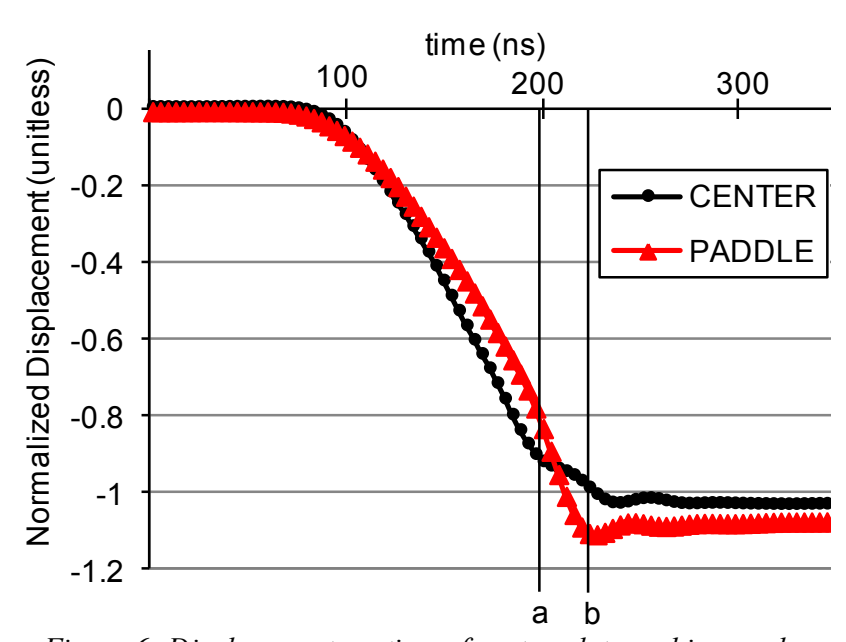

Figure 6: Displacement vs. time of center plate and inner edge of paddle, measured with a laser Doppler vibrometer. The center plate makes initial contact with the SENSE electrode at time (a), while the paddle continues to rotate until it hits a hard stop at time(b).

Also, since the actuator paddles never touch the DRIVE electrodes, contact area under high field is greatly reduced, further reducing stiction. Actuators with this torsion architecture survived room temperature life-test in which the DRIVE voltage was cycled for 5 billion cycles without any observed stiction failures.

\section{CONCLUSION}

A novel surface-micromachined electrostatic parallel plate actuator has been designed and fabricated. The actuator has been shown to require $4 \mathrm{x}$ higher voltage to hold it down under dynamic release conditions than static release. This is a result of a unique over-rotation mechanism that allows the actuator to capture, store, and release more actuation energy without affecting its initial pullin voltage. The extra restoring force is especially useful in applications where stiction and electrostatic holding forces pose a significant challenge, such as micromechanical relays and sensors.

\section{REFERENCES}

[1] N. Tas, T. Sonnenberg, H. Jansen, R. Legtenberg, and M. Elwenspoek, "Stiction in Surface Micromachining", J. Micromech. Microeng., 6 (1996), pp. 385-397

[2] S. J. Jacobs, S. Miller, J. Malone, W. McDonald, V. Lopes, and L. Magel, "Hermeticity and Stiction in MEMS Packaging", 40 ${ }^{\text {th }}$ Annual International Reliability Physics Symposium, Dallas (2002), pp. 136-139

[3] W.A. de Groot, J. Webster, D. Felnhofer, and E. Gusev, "Review of Device Reliability Physic of Dielectric in Electrostatically Driven MEMS Device", IEEE Transactions on Device and Materials Reliability, 9, 2 (2009), pp. 190-202.

[4] J.-B. Lee and C. Goldsmith, "Numerical simulations of novel constant-charge biasing method for capacitive RF MEMS switch," in Proceedings of NanoTech 2003 Conference: Modeling and Simulation of Microsystems, San Francisco, CA, February (2003), pp 396-399.

[5] M. Mignardi and R. Howell, "The Fabrication of the Digital Micromirror Device", Commercialization of Microsystems '96 (1996)

\section{CONTACT}

*Arun Gupta, tel: +1-214-567-9895; arun-gupta@ti.com 


\title{
USING DRIE SILICON AS A CUTTING TOOL FOR HIGH PRECISION MICROMACHINING OF METAL ALLOYS
}

\author{
Tao $\mathrm{Li}^{l^{*}}$, Qing Bai ${ }^{2}$, and Yogesh B. Gianchandani ${ }^{l}$
}

${ }^{1}$ Center for Wireless Integrated MicroSensing and Systems (WIMS ${ }^{2}$ ), University of Michigan, Ann Arbor, MI

${ }^{2}$ Molecular Separations Laboratory, Agilent Research Laboratories, Santa Clara, CA

\section{ABSTRACT}

This paper reports recent advances in batch-mode micro electro-discharge machining $(\mu \mathrm{EDM})$ for high precision micromachining of metal alloys such as stainless steels. Highaspect-ratio and high-resolution silicon microstructures formed by deep reactive ion etching (DRIE) are used as cutting electrodes. These replace the previously-reported, LIGA-defined, electroplated copper electrodes, alleviating complexities associated with the LIGA process. A minimum feature size of $\approx 7 \mu \mathrm{m}$ is demonstrated on stainless steel 304 in batch-mode $\mu$ EDM by using DRIE Si electrodes with a width of $5 \mu \mathrm{m}$. Features with sizes $\geq 15$ $\mu \mathrm{m}$ have shown excellent repeatability and uniformity across a diescale machining area of $5 \times 5 \mathrm{~mm}^{2}$. Machining rates up to $\approx 5$ $\mu \mathrm{m} / \mathrm{min}$. have been demonstrated in micromachining of typical microfluidic structures, including arrays of channels and cavities of different sizes. Other machining characteristics are also discussed.

\section{INTRODUCTION}

Stainless steel and other metal alloys are attractive as structural and packaging materials in microsystems. These materials can typically provide excellent mechanical strength and/or chemical resistance among other desirable properties. There is increasing interest in application of these materials in microsystems, with examples ranging from smart stents [1], micropackages for implantable devices [2], microfluidic devices for gas chromatography [3], high power micro-relays [4], and pressure sensors for oil-exploration [5]. The micromachining of such alloys, however, is typically not amenable to traditional microfabrication approaches. Alternatives include micromilling and other mechanical processes, wet chemical etching, laser machining, laser-induced wet-chemical etching, and electrochemical micromachining [6-8]. Generally these processes provide limited throughput and/or resolution.

Micro electro-discharge machining $(\mu \mathrm{EDM})$ can be used to make microstructures from any electrically-conductive material, including any metal alloy. This approach utilizes sequential spark microdischarges between a cutting electrode and a workpiece while both are immersed in dielectric oil. In serial-mode $\mu$ EDM, a single rotating electrode is used to "write" on a workpiece substrate, giving limited throughput especially for complex patterns. We previously reported a batch-mode $\mu$ EDM process that achieved high throughput and high precision by using LIGAdefined, electroplated copper structures as cutting electrodes [9]. By using x-ray lithography [10], the LIGA process allowed batch fabrication of cutting electrodes that have ultra-fine high-aspectratio microstructures with smooth side-wall surfaces. However, the need for a LIGA-type process adds significant complexity in both lithography and electroplating.

This paper reports the use of silicon as a cutting electrode in batch-mode $\mu \mathrm{EDM}$. The usefulness of $\mathrm{Si}$ in this role was anticipated in a patent [11], for which preliminary results were obtained using wet-etched Si. This paper reports the first results obtained with high-aspect-ratio $\mathrm{Si}$ cutting electrodes that are fabricated by deep reactive ion etching (DRIE). With the development and maturity of the DRIE technology, Si microstructures with high aspect ratio and high resolution can be readily made. The process details are described. Die-scale batchfabrication of several typical forms of microstructures for microfluidic devices is demonstrated on stainless steel 304 (SS304) substrates and the results are reported. The precision that can be achieved with $\mathrm{Si}$ cutting electrodes is assessed. Other process characteristics are also described.

\section{PROCESS DESCRIPTION}

The approach for batch mode $\mu$ EDM using DRIE silicon electrodes is shown in Fig. 1. Preparation of silicon electrodes starts with a heavily boron-doped silicon wafer $(0.001-0.005 \Omega-\mathrm{cm}$ resistivity). A $5000 \AA$-thick $\mathrm{Cr}$ layer is sputtered and patterned on the wafer. This film is intended to help with the initialization of discharge detection during $\mu \mathrm{EDM}$ and is optional. DRIE is then carried out to form high-aspect-ratio silicon structures in the same lithographic pattern as that of the overlay $\mathrm{Cr}$ film. After the silicon microstructures are formed, a Ti/Au layer $(200 \AA / 4000 \AA$ thickness) is evaporated on the backside of the silicon wafer to provide low-resistance ohmic contact to the silicon electrode. The wafer is diced into desired die sizes for use as cutting electrodes in batch-mode $\mu$ EDM.

To transfer the lithographic pattern defined on the electrode to metal alloys, a $\mathrm{Si}$ electrode die is mounted on a custom-made electrode holder; the holder is then installed into the mandrel fixture on a SmalTec ${ }^{\mathrm{TM}}$ EM203 $\mu \mathrm{EDM}$ machine for batch-mode $\mu$ EDM (Fig. 2). The SmalTec $\mu$ EDM machine is an enhanced model based on the technology of a previous Panasonic $\mu \mathrm{EDM}$ machine. It can generate $<100 \mathrm{~nJ}$ discharge energy for ultrafine micromachining. The built-in XYZ motorized stages have $0.1 \mu \mathrm{m}$ resolution. The $\mathrm{Z}$ stage drives the cutting electrode and provides the feeding motion during machining. The XY stages move the workpiece mounted on a worktable; this enables real $2 \mathrm{D}$ patterning with a single rotating electrode in serial mode operation, or allows precision alignment of the workpiece to the cutting electrode in batch mode operation. For batch mode operation, the metal workpiece can be mounted on a vibrator which provides a dithering motion in the feeding $(Z)$ direction of the cutting electrode at a frequency of $100 \mathrm{~Hz}$ and adjustable amplitude. This

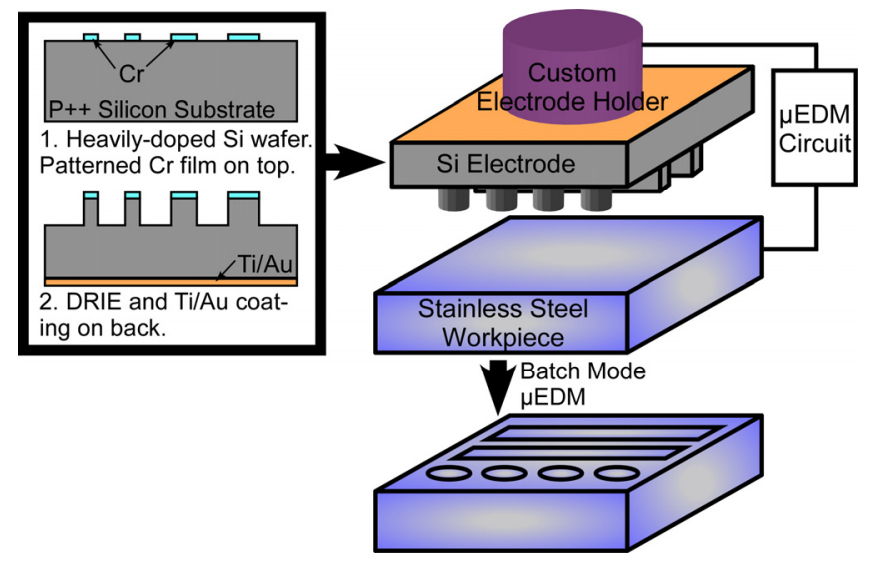

Figure 1: Concept of batch mode $\mu E D M$ for metal alloy workpiece using DRIE silicon electrodes. 


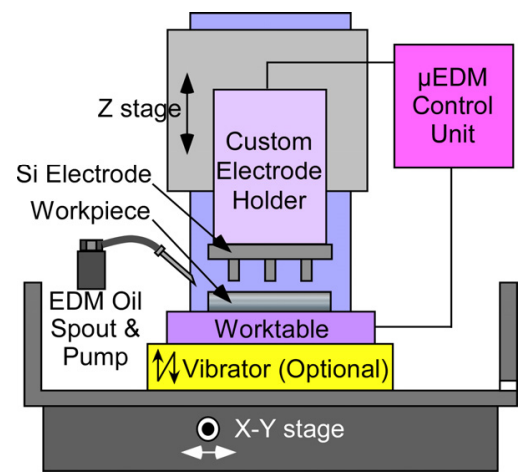

Figure 2: SmalTec EM203 $\mu$ EDM machine adapted for batch mode micromachining of stainless steel with Si electrodes.

vibration can be used to help avoid welding when a short circuit occurs between the electrode and the workpiece, as well as to facilitate removal of machining debris. Batch mode $\mu E D M$ is performed with machining control parameters developed according to desired machining conditions to take advantage of this control capability. These parameters include discharge voltage and capacitance, Z-stage feeding rate, and a set of parameters that determine the Z-stage motion pattern when short-circuit events occur. With proper selection of these parameters, the machining rate can be optimized for the desired feature geometry and dimension.

\section{PROCESS DEMONSTRATION}

The batch mode $\mu$ EDM process with DRIE Si electrodes is demonstrated on SS304 substrates. For these experiments, $5 \times 5$ $\mathrm{mm}^{2} \mathrm{Si}$ electrodes were prepared with various tool structures formed by DRIE, including features representing straight and curved channel arrays, round and square cavity arrays, etc. Three example tool structures, Sil-Si3, are shown in Fig. 3 and summarized in Table I. These structures have a height of $90 \pm 3 \mu \mathrm{m}$ and a minimum lateral feature size of $5 \mu \mathrm{m}$. The etching time for DRIE was $20 \mathrm{~min}$., giving an etching rate of $\approx 4.5 \mu \mathrm{m} / \mathrm{min}$. for the heavily boron-doped silicon.

The electrodes were then mounted on the custom electrode holder using conductive epoxy for this preliminary implementation. For batch mode $\mu E D M$, multiple sets of machining control parameters were developed to accommodate different machining situations due to variations in feature geometry and dimension. For fine features at the micro-scale, a low discharge voltage of 70-80 $\mathrm{V}$ was used with small discharge control capacitance in the range of $10-100 \mathrm{pF}$. The machining rate in terms of cutting depth is typically affected by the effective removal of machining debris and the settings in the control parameters. These have been optimized for each type of geometry and dimension range in the experiments. Selected machining

Table I: Summary of three example structures formed on $5 \times 5 \mathrm{~mm}^{2}$ Si tool electrodes and their resulting workpiece characteristics.

\begin{tabular}{|c|c|c|c|}
\hline $\begin{array}{l}\text { Tool } \\
\text { label }\end{array}$ & $\begin{array}{l}\text { Workpiece } \\
\text { label }\end{array}$ & Workpiece characteristics & Feature \\
\hline Sil & SS1 & $\begin{array}{l}\text { Line width: } 52 \mu \mathrm{m} \text {; length: } 1 \\
\mathrm{~mm} \text {; spacing: } 250 \mu \mathrm{m} / 500 \mu \mathrm{m}\end{array}$ & 三三三三 \\
\hline $\mathrm{Si} 2$ & $\mathrm{SS} 2$ & $\begin{array}{l}\text { Diameter: } 26 \mu \mathrm{m} \text {; spacing: } 250 \\
\mu \mathrm{m} ; 17 \times 17 \text { array }\end{array}$ & $\begin{array}{lllll}\cdot & \cdot & \cdot & \cdot & \cdot \\
\cdot & \cdot & \cdot & \cdot & . \\
\cdot & \cdot & \cdot & \cdot & .\end{array}$ \\
\hline $\mathrm{Si} 3$ & SS3 & $\begin{array}{l}\text { Line width: } 7 \mu \mathrm{m} \text {; length: } 400 \\
\mu \mathrm{m} \text {; spacing: } 25 \mu \mathrm{m}\end{array}$ & 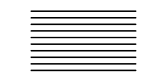 \\
\hline
\end{tabular}
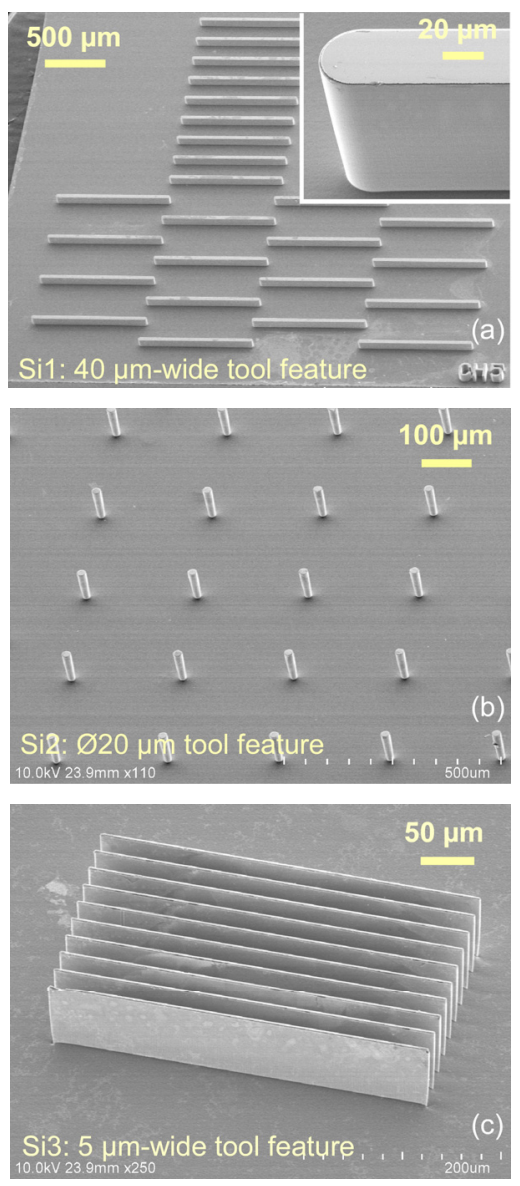

Figure 3: SEM images of three of the $5 \times 5 \mathrm{~mm}^{2}$ silicon electrode dies with high aspect ratio structures formed by DRIE. Feature height: $90 \pm 3 \mu \mathrm{m}$. (a) Tool for array of straight channel segments, $40 \mu \mathrm{m}$-wide, $1 \mathrm{~mm}$-long. (b) $\Phi 20 \mu \mathrm{m}$ tool for $17 \times 17$ array of holes. (c) Tool for array of straight channel segments, $5 \mu \mathrm{m}$-wide, 400 um-long.
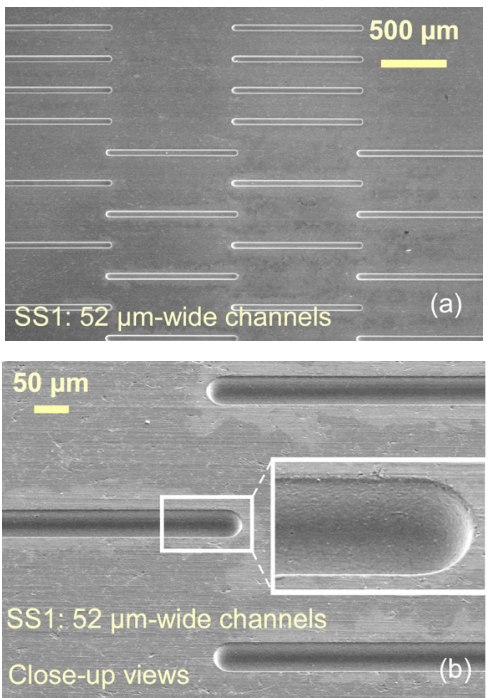

Figure 4: SEM images of array of $52 \mu \mathrm{m}$-wide $1 \mathrm{~mm}$-long straight channel segments formed on SS304 substrate, using the Sil electrode shown in Fig. 3(a). Discharge gap: $\approx 6 \mu \mathrm{m}$; channel depth: $\approx 32 \mu \mathrm{m}$; machining rate: $\approx 5 \mu \mathrm{m} / \mathrm{min}$. (a) Top-down view; (b) perspective view with insert showing close-up details. 

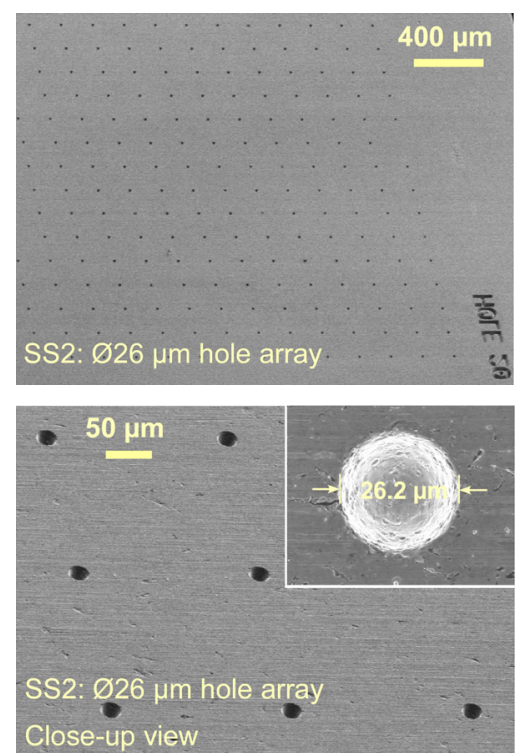

Figure 5: SEM images of $17 \times 17$ array of $\Phi 26 \mu \mathrm{m}$ holes made on SS304 substrate, using the Si2 electrode shown in Fig.3(b). Discharge gap: $\approx 3 \mu \mathrm{m}$; hole depth: $\approx 22 \mu \mathrm{m}$; machining rate: $\approx 3$ $\mu \mathrm{m} / \mathrm{min}$. (Top) Perspective view. (Bottom) Perspective view with insert showing close-up details. Markings on the SS304 surface are from raw material.

parameters are summarized in Table II.

The test patterns were successfully transferred onto SS304 substrates, confirming the feasibility of using silicon cutting electrodes for batch-mode $\mu$ EDM. Figure 4 shows the channelarray pattern that is defined on the cutting electrode Sil (Fig. 3a) and transferred onto SS304. The tool structure has a width of 40 $\mu \mathrm{m}$ and a length of $1 \mathrm{~mm}$ each. After $\mu \mathrm{EDM}$, this becomes a 52 $\mu \mathrm{m}$ width of the channels due to a discharge gap of $\approx 6 \mu \mathrm{m}$. The depth of the machined channels is $\approx 32 \mu \mathrm{m}$ with a machining time of $\approx 6$ min., giving a machining rate of $\approx 5 \mu \mathrm{m} / \mathrm{min}$.

Table II: Some machining parameters for batch mode $\mu E D M$ of stainless steel with DRIE Si electrodes.

\begin{tabular}{|l|l|}
\hline Discharge voltage & $70-80 \mathrm{~V}$ \\
\hline Discharge control capacitance & $10-100 \mathrm{pF}$ \\
\hline Electrode die size & $5 \times 5 \mathrm{~mm}^{2}$ \\
\hline Max. machining rate demonstrated (cutting depth) & $\approx 5 \mu \mathrm{m} / \mathrm{min}$. \\
\hline Min. feature size demonstrated & $\approx 7 \mu \mathrm{m}$ \\
\hline Discharge gap (vary with discharge energy, etc.) & $1-6 \mu \mathrm{m}$ \\
\hline Tool wear ratio (tool height worn vs. cutting depth) & $\approx 1$ \\
\hline
\end{tabular}

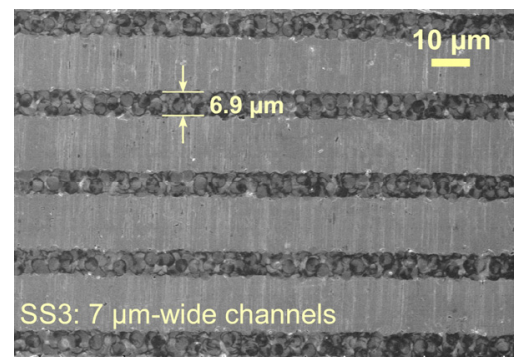

Figure 6: SEM image of $\approx 7 \mu$-wide, $400 \mu$ m-long straight channel segments formed on SS304 substrate, using the Si3

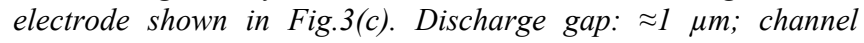
depth: $\approx 5 \mu \mathrm{m}$; machining rate: $\approx 0.5 \mu \mathrm{m} / \mathrm{min}$.
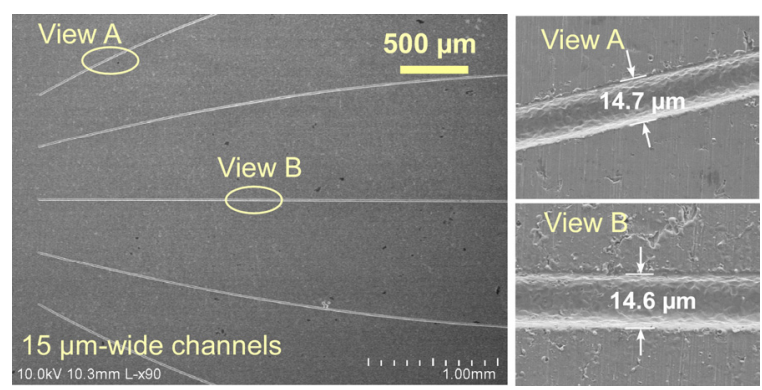

Figure 7: SEM image of $\approx 15 \mu$ m-wide, $>4.5$ mm-long curved channel segments formed on SS304 substrate. Discharge gap: $\approx 2.5 \mu \mathrm{m}$; channel depth: $\approx 15 \mu \mathrm{m}$; cutting rate: $\approx 3 \mu \mathrm{m} / \mathrm{min}$.

Figure 5 shows a $17 \times 17$ array of round cavities formed on SS304 using the Si2 electrode shown in Fig. 3(b). The $20 \mu \mathrm{m}$ tool diameter becomes the $26 \mu \mathrm{m}$ hole diameter on the workpiece, indicating a discharge gap of $\approx 3 \mu \mathrm{m}$. The cavity depth is $\approx 22 \mu \mathrm{m}$ with a machining rate of $\approx 3 \mu \mathrm{m} / \mathrm{min}$.

Figure 6 shows an array of straight channels, each with a width of $\approx 7 \mu \mathrm{m}$. These were formed using the $\mathrm{Si} 3$ electrode shown in Fig. 3(c) with a tool width of $5 \mu \mathrm{m}$ and a length of $400 \mu \mathrm{m}$. The discharge gap is $\approx 1 \mu \mathrm{m}$ and the channel depth is $\approx 5 \mu \mathrm{m}$. The machining rate is $\approx 0.5 \mu \mathrm{m} / \mathrm{min}$.

Other typical microfluidic structures were also evaluated. Shown in Fig. 7 is an array of curved channels with $\approx 15 \mu \mathrm{m}$ width and $>4.5 \mathrm{~mm}$ length formed on SS304. The discharge gap is $\approx 2.5$ $\mu \mathrm{m}$ and the channel depth is $\approx 15 \mu \mathrm{m}$, with a machining rate of $\approx 3$ $\mu \mathrm{m} / \mathrm{min}$.

According to these experimental results, a minimum feature size of $\approx 7 \mu \mathrm{m}$ is feasible in batch-mode $\mu$ EDM using DRIE Si electrodes (Fig. 6); features with sizes $\geq 15 \mu \mathrm{m}$ have shown repeatability and uniformity across the whole machining area (Fig. 4-5\&7). Machining rates up to $5 \mu \mathrm{m} / \mathrm{min}$. have been achieved and can vary according to the machining conditions that are used.

The electrode wear ratio, taken as the electrode height worn
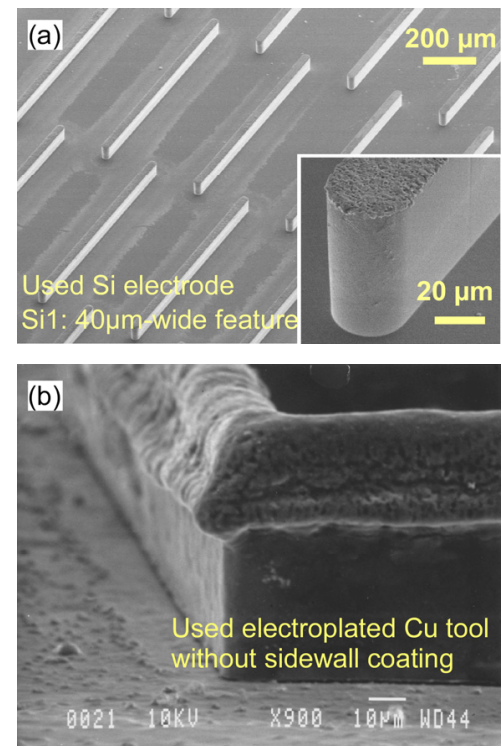

Figure 8: (a) SEM images of the Si electrode shown in Fig. 3(a) after being used for machining. The insert shows close-up details. (b) Previous result showing post-use electroplated $\mathrm{Cu}$ electrode without any coating, for comparison purpose [12]. Note the mushroom-shaped recast top in the uncoated $\mathrm{Cu}$ tool does not appear in the Si tool. 
vs. cutting depth on the workpiece, is $\approx 1$. Figure 8 (a) shows a $\mathrm{Si}$ electrode Sil after being used for machining. For comparison purpose, Figure 8(b) provides a previous result that shows post-use electroplated $\mathrm{Cu}$ electrode without any coating on the sidewalls. It is notable that the mushroom-shaped recast top present in the uncoated $\mathrm{Cu}$ tool does not appear in the Si tool.

Figure 9 plots the cavity diameter distribution for the $17 \times 17$ array of $\Phi 26 \mu \mathrm{m}$ features shown in Fig. 5. The cavity diameter has a standard deviation of $0.97 \mu \mathrm{m}$ and a mean diameter of $26.5 \mu \mathrm{m}$. Because the flushing motion of dielectric oil helps remove debris from the machining region and thus reduce discharge gap, cavities with smaller diameters are located closer to the flushing spout, where flushing is the most effective.

\section{DISCUSSION}

The actual machining rate is reduced from the stage feeding rate by short-circuit events occurring between the electrode and the workpiece, as well as by the short circuits/secondary discharges between these components and the debris that are a normal part of the electro-discharge machining process. To achieve faster machining rate, higher discharge energy can be used at the expense of achievable minimum feature size. For high resolution machining, effective debris removal from the gaps between the electrode and the workpiece is essential for rapid machining and uniformity of feature geometries and sizes. Debris removal can be performed with specially-designed control parameters for Z-stage retraction, and can be further facilitated by effective oil flushing and worktable vibration. To further reduce the effect of debris accumulation on machining and to help reduce the tool wear, techniques such as passivation of electrode sidewalls and hydrodynamic flushing of debris can be applied. These techniques were previously shown to help improve feature geometry quality and reduce tool wear in batch-mode $\mu \mathrm{EDM}$ with electroplated $\mathrm{Cu}$ tools [12]. The effectiveness of these techniques for silicon tools will be investigated in future work.

\section{CONCLUSIONS}

The feasibility of using DRIE silicon cutting electrodes in batch-mode $\mu \mathrm{EDM}$ has been confirmed. The machining process has been successfully demonstrated on SS304 with various feature geometries (channels and cavities) and dimensions $(>7 \mu \mathrm{m})$. Machining rates up to $\approx 5 \mu \mathrm{m} / \mathrm{min}$. have been achieved. These experiments substantiate the potential of the process for a variety of applications, which will be pursued in future effort.

\section{ACKNOWLEDGEMENT}

The research effort was supported in part by a gift fund from Agilent Research Laboratories. Facilities used for this research include the Lurie Nanofabrication Facility (LNF) operated by the Solid-State Electronics Laboratory (SSEL) at the University of Michigan.

\section{REFERENCES}

[1] K. Takahata, Y.B. Gianchandani, and K.D. Wise, "Micromachined antenna stents and cuffs for monitoring intraluminal pressure and flow," IEEE/ASME J. Microelectromechanical Systems, 15(5), pp. 1289-1298, 2006

[2] W.H. Ko, "Packaging of microfabricated devices and

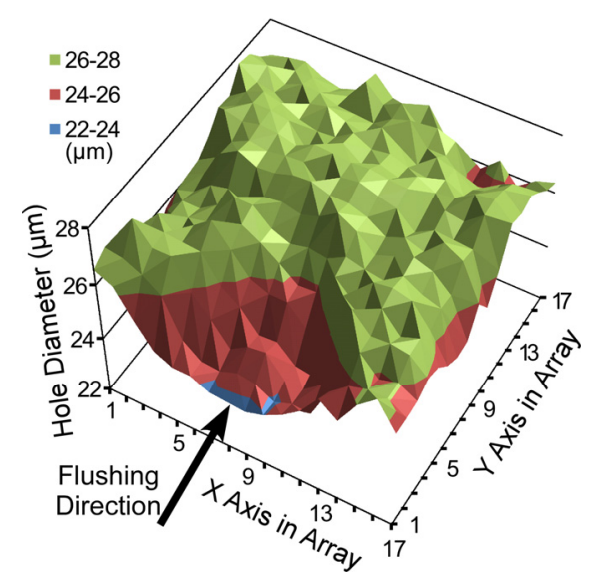

Figure 9: Diameter variation of $\Phi 26 \mu \mathrm{m}$ holes in the $17 \times 17$ array shown in Fig. 5. Mean hole diameter: $26.5 \mu \mathrm{m}$. Standard deviation: $0.97 \mu \mathrm{m}$. Range: 23.8-27.7 $\mu \mathrm{m}$. Flushing helps remove debris and reduce discharge gap, thus smaller holes closer to the flushing spout.

systems," Materials Chem. \& Phys., 42(3), pp. 169-175, 1995

[3] http://www.chem.agilent.com/cag/prod/GC/LCGC_Article.pdf

[4] F. Ozkeskin, S. Choi, K. Sarabandi, and Y.B. Gianchandani, "Metal foil RF micro-relay with integrated heat sink for high power applications," Proc. IEEE Intl. Conf. Micro Electro Mechanical Systems (MEMS 2011), Cancun, Mexico, Jan. 2011, pp. 776-779

[5] S.A. Wright, H.A. Zipperian, and Y.B. Gianchandani, “A 15 atm. pressure sensor utilizing microdischarges in a $1.6 \mathrm{~mm}^{3}$ ceramic package," Tech. Digest Solid-State Sensors, Actuators, Microsystems Workshop (Hilton Head 2010), June 2010, pp. 53-56

[6] R. Nowak and S. Metev, "Thermochemical laser etching of stainless steel and titanium in liquids," Appl. Phys., A63, pp. 133-138, 1996

[7] B.H. Kim, S.H. Ryu, D.K. Choi, and C.N. Chu, "Micro electrochemical milling," J. Micromech. Microeng., 15, pp. 124-9, 2005

[8] P.N. Rao and Deepak Kunzru, "Fabrication of microchannels on stainless steel by wet chemical etching," J. Micromech. Microeng., 17, pp. N99-106, 2007

[9] K.Takahata and Y.B.Gianchandani, "Batch Mode MicroElectro-Discharge Machining", IEEE/ASME J. Microelectromechanical Systems, 11(2), pp. 102-110, Apr. 2002

[10] H. Guckel, "High-aspect-ratio micromachining via deep Xray lithography," Proc. IEEE, 86(8), pp. 1586-93, Aug. 1998

[11] Y.B. Gianchandani and K. Takahata, Micro-electro-discharge machining utilizing semiconductor electrodes, US Patent: $6,586,699 \mathrm{~B} 1,2003$

[12] M. Richardson and Y. Gianchandani, "Achieving precision in high density batch mode micro-electro-discharge machining," J. Micromech. Microeng., 18(1), 015002, 2008

\section{CONTACT}

*Tao Li, 1301 Beal Ave., Ann Arbor, MI, 48109, USA; tel: 1-734615-7983; fax: 1-734-763-9324; email: litz@umich.edu 


\title{
UV-LED LITHOGRAPHY FOR 3-D HIGH ASPECT RATIO MICROSTRUCTURE PATTERNING
}

\author{
Jungkwun 'JK' Kim*, Seung-Joon Paik, Florian Herrault, Mark G. Allen
}

School of Electrical and Computer Engineering, Georgia Institute of Technology, Atlanta, GA, USA

\begin{abstract}
This paper presents a UV lithography method that utilizes a UV-LED (ultraviolet light-emitting diode) array as a micropatterning light source for high aspect ratio polymer microstructure fabrication. The sidewalls of commercial 5-mm-diamater UV-LEDs were coated with an opaque polymer to suppress the side propagation of UV light and to enhance UV collimation, resulting in a maximum flare angle of $15^{\circ}$. The UV-LEDs were then assembled into a 10x10 array to form the light source. Although the flare angle of most conventional UV lithography tools is $2 \sim 3^{\circ}$, resulting in a slightly better light collimation quality, the polymer structures microfabricated with the UV-LED approach showed similar reproduction fidelity and similar exposure times to patterns fabricated using conventional lithography. The UV-LED approach has the potential for cost reduction as well as simplicity of initial system setup and management. This latter feature is of particular importance in advanced, inclined three-dimensional (3-D) lithography schemes to create complex 3-D structures. The UV-LED system has been demonstrated to perform satisfactorily in these advanced 3-D lithography schemes.
\end{abstract}

\section{INTRODUCTION}

UV lithography systems based on mercury lamp sources have traditionally been used in micro/nano patterning [1]. Modifications of these basic systems, such as deep UV lithography [2] and addition of UV filters [3], can further enhance fine resolution patterning. In MEMS, mercury-lamp-based UV lithography has been further extended to encompass unconventional methods including thermal reflow processes [4], multidirectional UV lithography [5], diffuser lithography [6], and timed-development and-thermal-reflow process [7].

Recent advances in light emitting diode technology have resulted in LEDs that emit in the near ultraviolet. Such UV-LEDs have been used not only in conventional applications such as counterfeit detection (bills, credit cards, documents, etc.) and sterilization, but also as a microfabrication tool. Examples include a

Table1: Comparison between UV-LED[10] and Mercury lamp[11].

\begin{tabular}{|l|l|l|}
\hline & $\begin{array}{l}\text { UV LED (100 } \\
\text { LEDs, } \\
\text { RL5-UV0315-380) }\end{array}$ & $\begin{array}{l}\text { Mercury lamp } \\
(6286 \text { Mercury } \\
\text { Arc Lamp) }\end{array}$ \\
\hline Lifetime & $\begin{array}{l}\text { Approximately } \\
2,000 \text { hours @ } \\
30 \mathrm{~mA} / 23^{\circ} \mathrm{C}\end{array}$ & $\begin{array}{l}\text { Approximately } \\
1,000 \text { hours @ } \\
6 \mathrm{~A}\end{array}$ \\
\hline Radiation Power & $\begin{array}{l}8 \mathrm{~mW} / \mathrm{cm}^{2} @ 12 \mathrm{~W} \\
(30 \mathrm{~mW} / \mathrm{LED})\end{array}$ & $\begin{array}{l}\sim 20 \mathrm{~mW} / \mathrm{cm}^{2} @ \\
350 \mathrm{~W}\end{array}$ \\
\hline $\begin{array}{l}\text { Wavelength } \\
\text { characteristics }\end{array}$ & $\begin{array}{l}\text { Single peak (380 } \\
\mathrm{nm})\end{array}$ & $\begin{array}{l}\text { Multiple peaks } \\
(365 \mathrm{~nm}, 405 \\
\mathrm{nm}, 436 \mathrm{~nm})\end{array}$ \\
\hline $\begin{array}{l}\text { Ancillary systems } \\
\text { (power supply, } \\
\text { mirrors, etc.) }\end{array}$ & Simple & Complex \\
\hline
\end{tabular}

9780964002494/HH2012/\$25@2012TRF single UV-LED for direct write lithography [8], and an array of LEDs in which the spatially nonuniform emission of the LEDs was exploited for microlens array fabrication [9]. Since the UV-LED has the advantages of simple operation and relatively low cost, yet produces high quality near-monochromatic light, it shows great potential for use in microfabrication. Table 1 compares the performance of UV-LEDs with a mercury lamp for several key parameters of interest for UV light source designers. It can be seen that the UV-LED shows performance very comparable to the conventional light source.

In this study, the efficacy of a 100-element array of modified UV-LEDs as a light source for conventional and advanced (3-D) UV lithography is assessed. The modification consisted of suppressing the high flare angle light of each UV-LED by means of an opaque blocking layer. The intensity distribution and attenuation as a function of distance of the array are measured. The performance of the UV-LED source is also compared to the performance of a conventional mercury-lamp-based lithography system.

\section{SYSTEM SET-UP AND TEST}

A 5mm through hole LED (RL5-UV0315-380, Superbrightleds, Inc) is adopted for the UV-LED exposure system. This ultraviolet LED has a single wavelength peak centered at $380 \mathrm{~nm}$ with a maximum radiant power of $30 \mathrm{~mW}$. It has a clear epoxy lens situated on top of a transparent cylindrical package, resulting in a viewing angle of 15 degrees. In order to suppress the significant light leakage through the sidewall of the cylindrical package, the sidewall was coated with an opaque epoxy band to block any UV light emitted through the sidewall.

Figure 1 shows a notional ray trace of light emitted from the LED and traversing to a photoresist-coated mask substrate. The photoresist (e.g., SU-8) in Figure 1 is illuminated using backside exposure (although note that the UV-LED technique is not restricted to backside exposure). After the UV light emerges from the LED lens, it has a $15^{\circ}$ viewing angle, which is equivalent to a $7.5^{\circ}$ half-flare angle incident upon the glass photomask. Application of Snell's law results in a final refracted angle at the photoresist layer

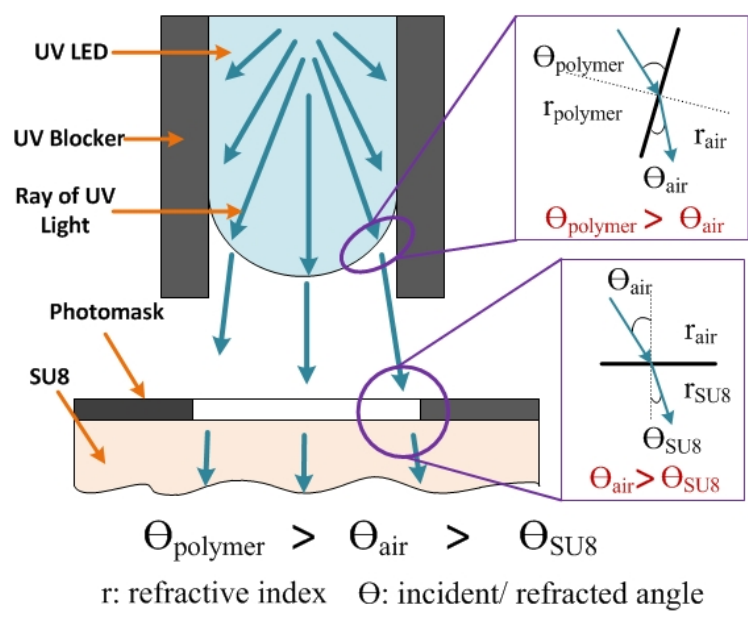

Figure 1: Schematic diagram of UV-LED lithography.

Solid-State Sensors, Actuators, and Microsystems Workshop Hilton Head Island, South Carolina, June 3-7, 2012 


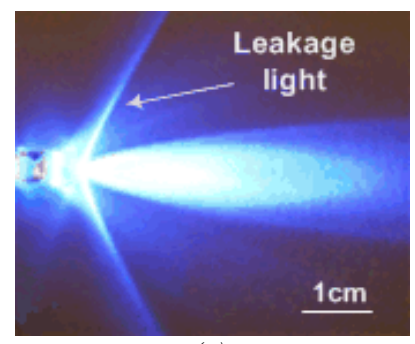

(a)

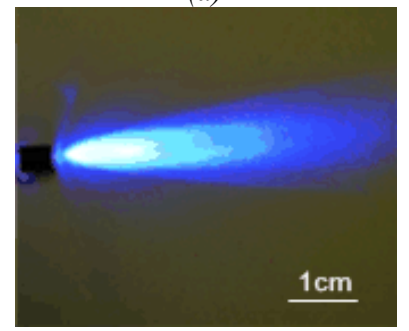

(c)

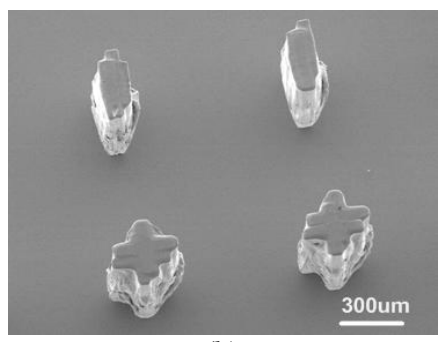

(b)

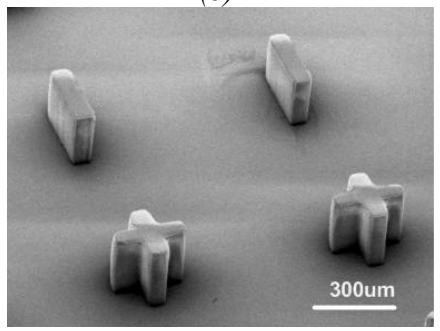

(d)
Figure 2: UV-LED light propagation with and without LED sidewall coating: (a) UV-LED Light emitting without coating, (b) SU-8 patterning with normal LED source, (c) SU-8 pattering with $U V-L E D$ light without sidewall coating, (d) SU-8 patterning with UV-blocking sidewall coating.

of $4.43^{\circ}$ where the refractive indices of air, glass, and SU- 8 are assumed as 1, 1.3, and 1.69 respectively [5]. Since the SU-8 exhibits generally a negatively sloped sidewall (undercut) in top exposure and vice versa in backside exposure caused by diffraction and attenuation, the $4.43^{\circ}$ of the refracted angle enhance the sidewall profile.

Figure 2 shows the profile of the emitted light from a single UV-LED with and without coating the sidewall with opaque material (left), as well as typical SU-8 exposure and development results from each LED (right). The emitted light from a single LED is mostly propagated in the forward direction through the top mounted clear lens. However, there is a considerable amount of leakage light as shown in Figure 2(a). Leakage light results in non-uniform sidewalls and multiple patterning of the fabricated microstructures as shown in Figure 2(b). Figure 2(c) shows the UV-LED with opaque sidewalls and suppressed leakage. As illustrated in Figure 2(d), the corresponding fabricated microstructures exhibit straight sidewalls.

To expose larger areas, a 10x10 element LED array is constructed. This array is realized on a custom printed circuit board (PCB) where the $30 \mathrm{~m}$ thick double sided copper layers were

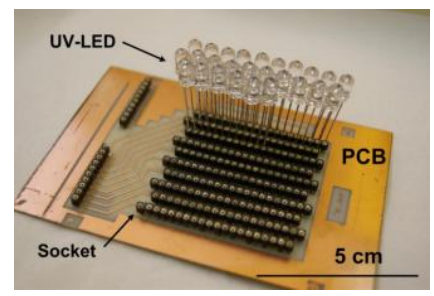

(a)

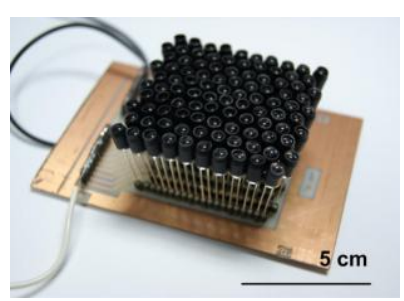

(b)
Figure 3: UV-LED system overview: (a) Circuit board bearing IC socket and UV-LEDs, (b) Completed UV-LED array light source.

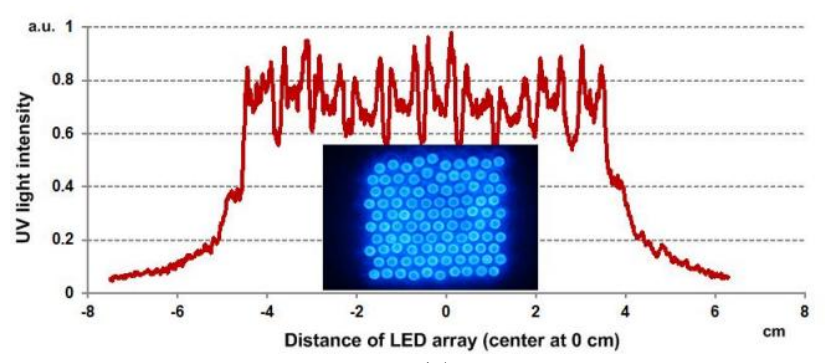

(a)

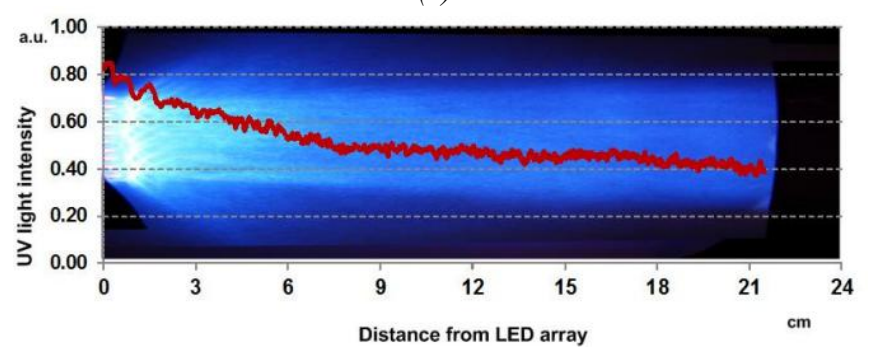

(b)

Figure 4: UV-LED array intensity distribution: (a)Intensity on cross-section view, (b) Intensity attenuation through the distance.

patterned to form LED interconnects. PCB IC sockets were mounted on the PCB to plug in the LEDs instead of directly soldering to the PCB for future possible replacement of LEDs as shown in Figure 3(a). Each LED bulb received the opaque sidewall coating previously discussed. The completed UV light source is shown in Figure 3(b) where the 100 LED array covers an exposure area of 2 by 2 inches.

The areal intensity distribution of the UV-LED array was obtained using the Olympus Wasabi imaging software package [12]. The intensity measurement image of the array is shown in the inset of Figure 4(a), and the intensity of the captured image is plotted as shown in Figure 4(a). The x-axis is the distance in $\mathrm{cm}$ and the $y$-axis is the relative intensity measurement. A reasonable areal uniformity is achieved. The intensity attenuation of the array as a function of distance was also measured and is shown in Figure 4(b). As expected, the intensity decreases as a function of distance. In addition, the intensity distribution as a function of wavelength was measured for the UV-LED light source as well as a conventional UV lithography system (OAI, near UV mercury bulb) with an optical spectrometer (AvaSpec-3648). Figure 5 plots the measured relative intensity from each light source as a function of wavelength.

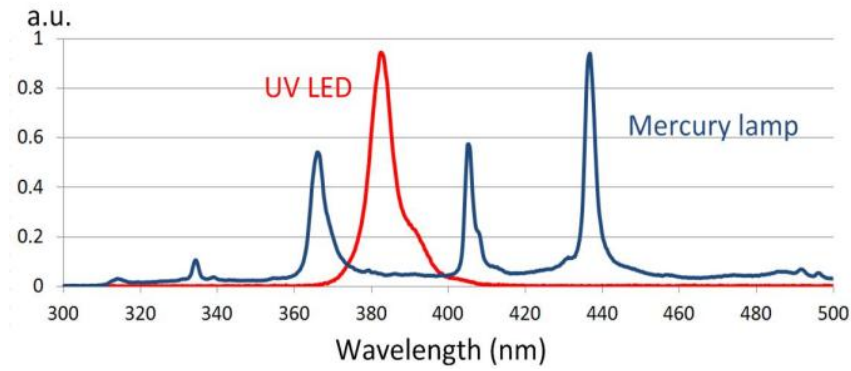

Figure 5: Intensity distribution vs wavelength with UV-LED and conventional mercury lamp. 


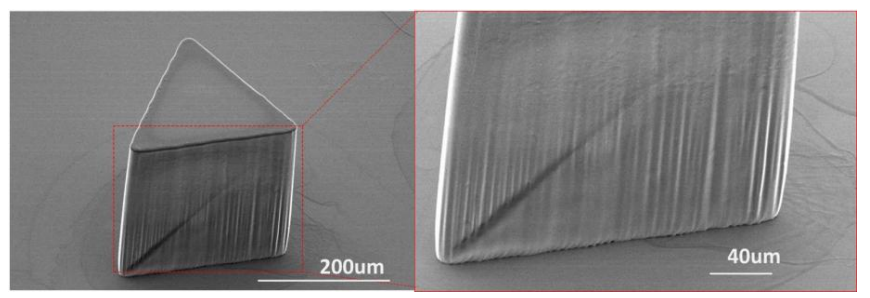

(a)

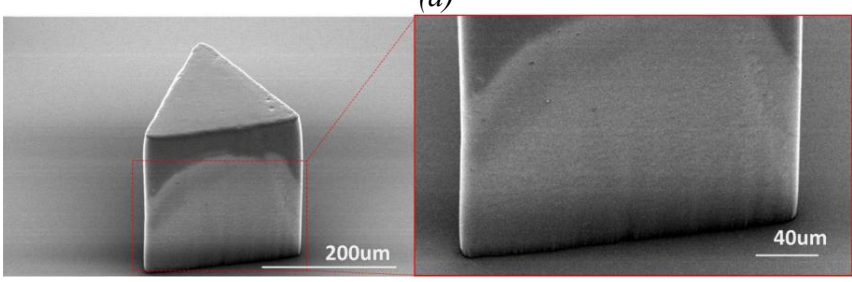

(b)

Figure 6: Comparison of microtriangular pillars fabricated by: (a) mercury lamp exposure scheme (OAI, conventional method), (b) UV-LED lithography scheme.

As expected, the UV-LED source shows a single peak (near-monochromatic) characteristic, while the mercury lamp has typical multiple peaks shown at $365 \mathrm{~nm}, 405 \mathrm{~nm}$ and $436 \mathrm{~nm}$ respectively.

Figure 6 shows a comparison of two triangular columnar SU-8 structures fabricated using a conventional aligner with a mercury lamp source (Figure 6a) and the UV-LED array (Figure 6b). Each was processed under the same soft-bake, post exposure bake, and UV exposure energy $(1440 \mathrm{~mJ})$ conditions. As can be seen, both exposure techniques produce structures with comparable features and resolution. To examine this further, and to demonstrate the utility of the UV-LED system, typical patterns in thicknesses of SU-8 widely used in MEMS processing, namely $15 \mathrm{~m}, 50 \mathrm{~m}$, $70 \mathrm{~m}$, and $100 \mathrm{~m}$, have been exposed, developed, and assessed (Figure 7). The light intensity of the UV-LED system was set at

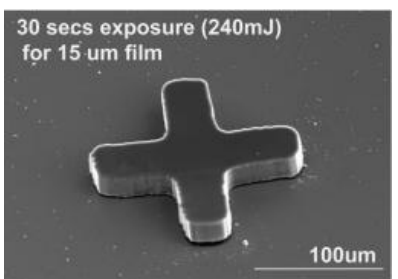

(a)

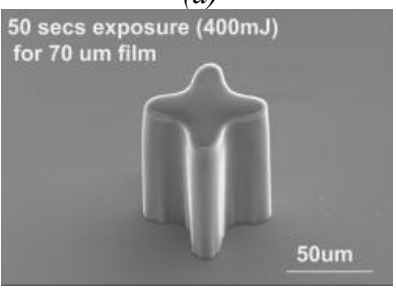

(c)

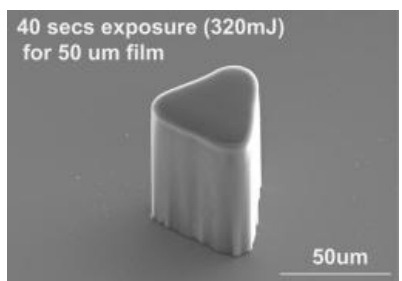

(b)

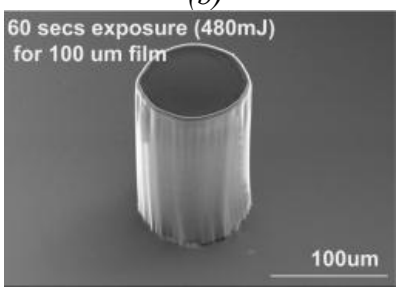

(d)
Figure 7: Microfabricated structures of typical thicknesses of SU-8 by UV-LED lithography (intensity at $8 \mathrm{~mW} / \mathrm{cm}^{2}$ ): (a) $15 \mathrm{~m}$ thick pattern for $240 \mathrm{~mJ} / \mathrm{cm}^{2}$ (30 secs), (b) $50 \mathrm{~m}$ thick pattern for $320 \mathrm{~mJ} / \mathrm{cm}^{2}$ (40 secs), (c) $70 \mathrm{~m}$ thick pattern for $400 \mathrm{~mJ} / \mathrm{cm}^{2}$ (50 secs), (d) $100 \mathrm{~m}$ thick pattern for $480 \mathrm{~mJ} / \mathrm{cm}^{2}$ (60 secs).

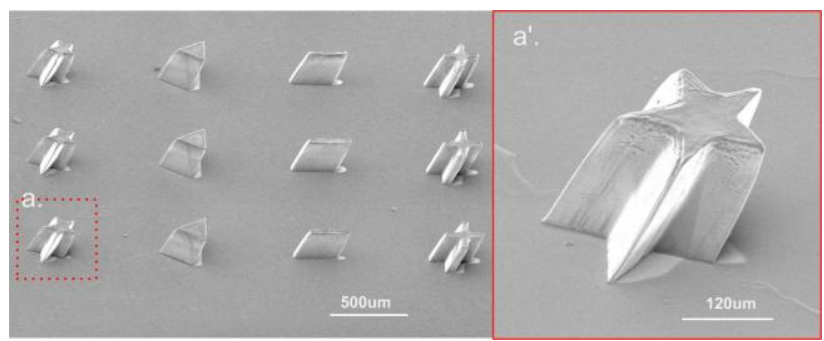

Figure 8: Single static inclined exposure with various geometries; inset ( $(a)$ is the magnified view of (a).

$8 \mathrm{~mW} / \mathrm{cm}^{2}$. The exposure dose of each sample is applied based on the SU-8 datasheet (MicroChem, Inc.), corresponding to $240 \mathrm{~mJ}$, $320 \mathrm{~mJ}, 400 \mathrm{~mJ}$, and $480 \mathrm{~mJ}$ respectively [13]. As can be seen, good reproduction of the patterns is achieved using the UV-LED array system.

\section{ADVANCED UV-LED LITHOGRAPHY METHODS}

One of the potential advantages of the UV-LED system is its ease of use in advanced SU-8 lithography approaches, including multidirectional, rotational, and timed-development-thermal-reflow lithography. Such techniques are being used to create structures in diverse application areas such as microfilters, scaffold structures for cell manipulation, and antenna structures for $\mathrm{GHz}$ and $\mathrm{THz}$ range applications. Previously, modifications to conventional aligners were required to perform these advanced lithographic methods, which may not be desirable in a shared fabrication environment. The UV-LED system is therefore tested in these applications to determine its suitability as a light source for advanced UV lithography.

\section{Multidirectional UV-LED Lithography}

Multidirectional UV lithography is a 3-D microfabrication scheme, based on conventional UV lithography, in which multiple UV exposures with different exposure angles relative to the photomask results in 3-D latent images in the photoresist [5].

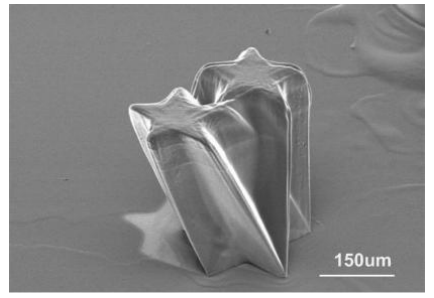

(a)

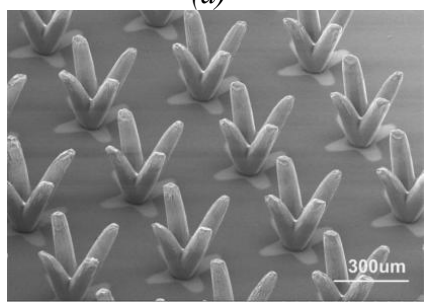

(c)

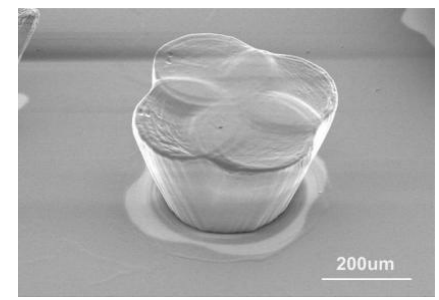

(b)

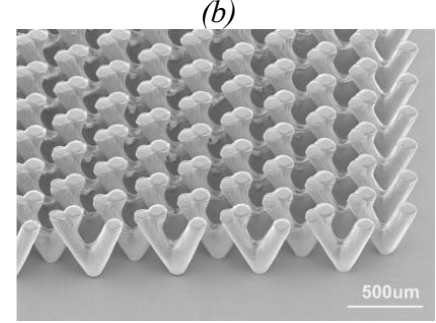

(d)
Figure 9: Multiple static exposure: (a)Micro star shape coupled pillar, (b) Clover horn, (c) Micro cactus field, (d)Anchored micro groove structures. 


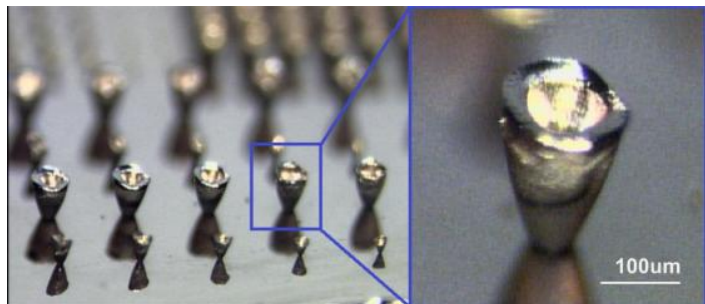

Figure 10: Dynamic mode multidirectional UV-LED lithography for micro 3-D horn array.

A backside exposure scheme is typically utilized, in which the photoresist is coated on a prepatterned $\mathrm{Cr}$ mask to eliminate any air gap between the mask and the thick photoresist. Figure 8 shows various shapes of pillar arrays with $300 \mathrm{~m}$ tall and $40 \mathrm{~m}$ minimum feature size (aspect ratio exceeding 5) and inclined angle of $25^{\circ}$ fabricated from the UV-LED system. $1440 \mathrm{~mJ}$ was applied as an exposure dose which is equivalent to 3 minutes exposure time with UV intensity setting at $8 \mathrm{~mW} / \mathrm{cm}^{2}$. Unlike previous approaches, in which the sample had to be inclined relative to the horizontal exposure tool, the use of the lightweight, compact UV-LED array enabled the tilting of the light source (a $45^{\circ}$ tilt angle) over a horizontally-held substrate. Defining the incident inclined angle of UV light by tilting the UV-LED source eliminates the need of an additional sample stage.

Figure 9 illustrates the multiple-static exposure scheme where multiple UV light exposures are applied with different angles producing complex 3-D microstructures. For example, a four pillar array 'micro cactus field' as shown in Figure 8(c) resulted from a $4 \mathrm{x}$ $\mathrm{UV}$-exposure at a tilt angle of $45^{\circ}$ and a rotation of $90^{\circ}$ between each of the four exposures.

Figure 10 demonstrates a 3-D microhorn array by further utilizing an inclined rotational stage. To expose the horn pattern, the SU-8 coated photomask was rotated with inclined angle of $45^{\circ}$ during the exposure process at a rotational speed of approximately $20 \mathrm{rpm}$.

\section{UV-LED driven timed development and thermal reflow (TDTR) process}

A fabrication process using timed development [7] in the deep via pattern of ultraviolet (UV) lithography with SU-8 and subsequent thermal reflow has been demonstrated to achieve rounded profiles. Concave profile on SU-8 coating was fabricated by time controlled developing on non-exposed area and followed by baking for thermal reflow. Fabricated concave patterns are micromolded by PDMS to produce a convex replica for microlens application as shown in Figure 11.

\section{CONCLUSION}

UV lithography based on an array of UV-LEDs for conventional and three-dimensional exposure of thin and thick photoresists has been developed. The UV-LED approach shows the ability to create structures comparable to those from conventional mercury lamp sources with comparable exposure times. Further, the UV-LED approach has several advantages including simplicity of operation, low power consumption, and reduced system cost. Typical thicknesses of SU-8 resist, namely 15, 50, 70, and $100 \mathrm{~m}$, were successfully patterned. The UV-LED approach was also successfully demonstrated in advanced 3-D lithography, including single and multiple static inclined exposure and dynamic exposure schemes, and timed-development-and-thermal-reflow process.

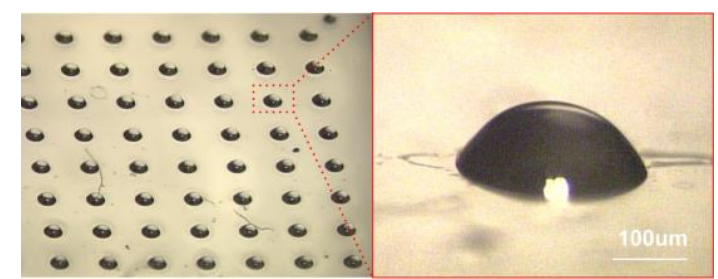

Figure 11: Optical microscopy view of microlens array by TDTR process; inset is the magnified view of a microlens.

Highlighted complex 3-D microstructures such as micro star shape coupled pillar, micro clover horn, micro horn, micro convex lens, micro cactus field, and anchored micro groove structures were successfully fabricated. An advantage of the use of the UV-LED approach in these fabrication schemes is that modification of mask aligners to hold special stages is not required; instead, dedicated equipment for complex lithography can be easily constructed using this light source.

\section{REFERENCES}

[1] Marc J. Madou, Fundamentals of Microfabrication, CRC press, Florida, 2001.

[2] Lin, Burn Jeng, "Deep UV lithography", Journal of Vacuum Science and Technology, Vol 12, Issue 6, (1975), pp1317-1320.

[3] K Jiang, M J Lancaster, I Llamas-Garro, P Jin, "SU-8 Ka-band filter and its microfabrication", J. Micromech. Microeng. 15 (2005) 1522-1526.

[4] Hsiharng Yang, Ching-Kong Chao, Mau-Kuo Wei, Che-Ping Lin, "High fill-factor microlens array mold insert fabrication using a thermal reflow process", $2004 \mathrm{~J}$. Micromech. Microeng. 141197.

[5] Jungkwun 'JK' Kim, Mark G. Allen and Yong-Kyu 'YK' Yoon, "Computer controlled dynamic mode multidirectional UV lithography for 3-D microfabrication," $2011 \mathrm{~J}$. Micromech. Microeng. 21035003.

[6] Hyeon-Don Kim, Gun-Wook Yoon, Jeongho Yeon, Joo-Hyung Lee and Jun-Bo Yoon, "Fabrication of a uniform microlens array over a large area using self-aligned diffuser lithography (SADL)", J. Micromech. Microeng. 22 (2012) 045002 (7pp).

[7] Jungkwun 'JK' Kim, Kangsun Lee, Kwang W. Oh and Yong-Kyu 'YK' Yoon, "Microfabrication of rounded channel and waveguide integrated microlens using timed development and thermal reflow process," uTAS, the 13th international conference, Jeju, Korea, Nov 2009.

[8] Rosanne M. Guijt, Michael C. Breadmore, "Maskless photolithography using UV LEDs", Lab Chip, 2008, 14021404.

[9] S. Suzuki, Y. Matsumoto, "Lithography with UV-LED array for curved surface structure", Microsyst Technol (2008) 14:12911297.

[10] <http://www.superbrightleds.com/moreinfo/component-leds/ 5mm-uv-led-15-degree-viewing-angle-380-nm-30mw/632/> [Accessed: 3 April 2012].

[11] $<$ http://search.newport.com/?q=350\%20watt $\% 20$ mercury $\%$ 20lamp > [Accessed: 3 April 2012].

[12] Hamamatsu Photonics Germany GmbH $<$ www.hamamatsu.com $>$

[13] <http://www.microchem.com/Prod-SU82000.htm> [Accessed: 3 April 2012].

\section{CONTACT}

*Jungkwun Kim: jkim831@gatech.edu 


\title{
MODELING OF THE NEUTRAL AXES OF A CIRCULAR PIEZOELECTRIC MICROMACHINED TRANSDUCER IN TRANSMIT AND RECEIVE MODE
}

\author{
Firas Sammoura, ${ }^{1,2}{ }^{*}$ and Sang-Gook Kim²
}

${ }^{1}$ Microsystems Division, Masdar Institute of Science and Technology, Abu Dhabi, UAE

${ }^{2}$ Department of mechanical engineering, Massachusetts Institute of Technology, Cambridge, MA 02139, USA

\begin{abstract}
A theoretical model that predicts the neutral axes locations of a circular Piezoelectric Micromachined Ultrasonic Transducer (PMUT) in both transmit and receive mode has been successfully developed for the first time. The constitutive vibration equations of plates in polar coordinates were used to analyze the bending of the circular plate forming the PMUT device. A general expression for the neutral axis position, a planar section with zero longitudinal stresses and strains, was derived as a function of the externally applied bending moment, the radial force, and the electric field between the two metal electrodes. Whereas the piezoelectric layer of the PMUT is subjected to an electric field during the transmit mode, the two metal electrodes are grounded during the receive mode. As such, distinct locations of the neutral axes in transmit and receive mode are predicted and verified using Finite Element Modeling (FEM). This unprecedented demonstration opens up new opportunities for accurate modeling of PMUTs and precise extraction of performance parameters.
\end{abstract}

\section{INTRODUCTION}

Ultrasonic sensors have been used as medical diagnostic tools to image anatomy for the past 50 years [1]. Thickness mode piezoelectric resonators operating in a high frequency range (2-15 $\mathrm{MHz}$ ) convert electrical pulses to sound waves and vice versa [2]. In order to design a transducer with high sensitivity and compact impulse response, it is necessary to engineer a piezoelectric composite with enhanced electromechanical coupling and acoustic impedance that closely matches with the tissue [3]. However, the dice-and-fill technique currently used to combine piezoelectric ceramics and passive polymers to form a piezocomposite ultrasonic array is tedious.

With the advancement of microelectromechanical systems (MEMS), Capacitive Micromachined Ultrasonic Transducers (CMUTs) have been introduced as a substitute to the traditional bulk piezoelectric transducers, with enhanced bandwidth and sensitivity [4]. However, CMUTs suffer from limited output acoustic power, high bias voltage, and non-linear plate deflection with applied electromotive force [5].

PMUTs have been lately introduced as an alternative to CMUTs [6]. Unlike CMUT, a PMUT is not subject to the geometric constraints of electrostatic transduction, enabling higher acoustic pressure output and energy efficiency. A typical PMUT is a suspended plate clamped at its edges resulting in reduced device acoustic impedance. The unimorph plate is composed of a structural layer and a piezoelectric material sandwiched between two electrodes. The membrane vibrates with the application of AC voltage due to the piezoelectric phenomenon. Specifically, Shelton et al. have demonstrated an air-coupled PMUT using Aluminum Nitride (AlN) as a piezoelectric material [7], and Muralt et al. characterized a PMUT fabricated with a $2 \mu \mathrm{m}$ thick PZT film [8]. There have also been some efforts aimed at theoretically modeling PMUTs. Bernstein et al. reported simple analytical formulae that predict the resonant frequency of a circular membrane suspended in air or loaded at one-side under simply supported and clamped boundary conditions [9]. Perçin et al. outlined the equations governing the operation of a circular PMUT and attempted to derive a circuit model for piezoelectrically actuated micromachined ultrasound transducers. However, the previous modeling work neither touched on the unimorph nature of a PMUT, nor on the effect of the neutral axis location on the circuit parameters [10].

Determining the neutral axis location is a precursor for evaluating the static and dynamic response of a unimorph PMUT. Recently, DeVoe et al. described a model that predicts the static behavior of a piezoelectric cantilever actuator with arbitrary configuration of piezoelectric and structural layers [11]. Weinberg et al. derived solution to the force, displacements, and charges in piezoelectric beams based on determining the neutral axis location [12]. In contrast to the previous works, this is the first paper that investigates the exact location of the neutral axis of a circular unimorph PMUT and distinguishes between the operation in transmit and receive modes.

\section{(a) Before Actuation}
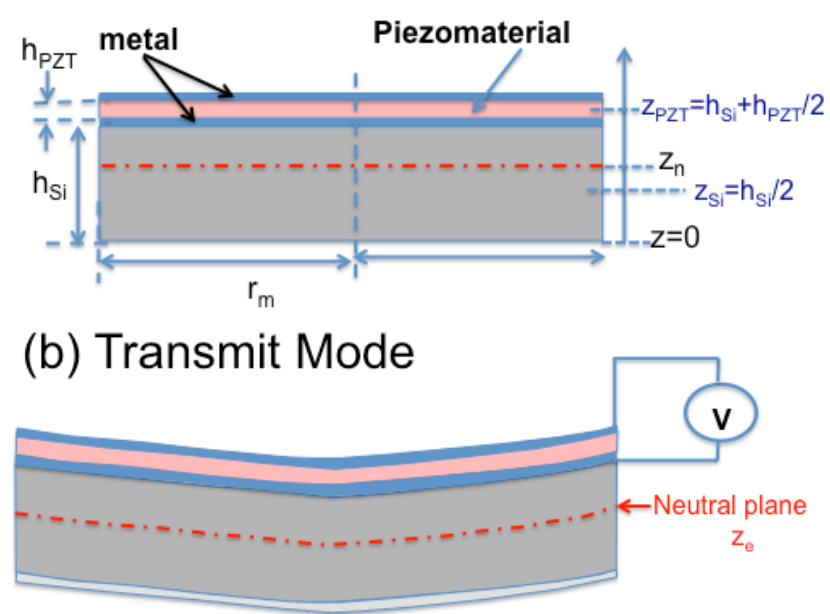

\section{(c) Receive Mode}

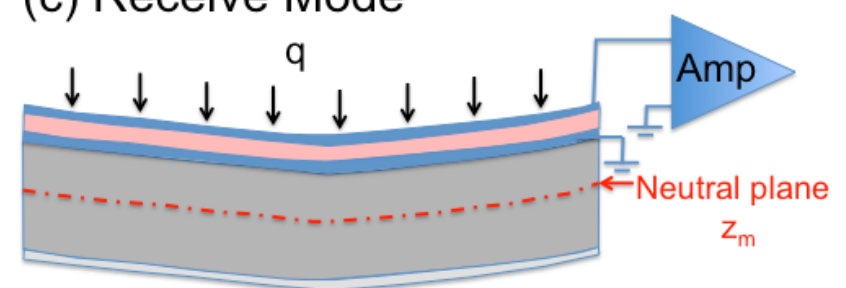

Figure 1: A schematic diagram of a Piezoelectric Micromachined Ultrasonic Transducer (PMUT) composed of a structural layer and a piezoelectric layer sandwiched between two metal electrodes: (a) before actuation, (b) in transmit mode with excitation potential $V$, and (c) in receive mode with an applied pressure of $q$.

\section{THEORY}

Overview of the Unimorph PMUT

Figure 1(a) shows a schematic diagram of the circular 
unimorph PMUT under consideration. The PMUT is composed of a structural layer of thickness $h_{S i}$ and a piezoelectric layer of thickness $h_{P Z T}$. The piezoelectric layer is sandwiched between two metal electrodes of negligible thickness. The plate radius is designated as $r_{m}$ and the neutral axis is located at a position $z_{n}$ from a reference $\mathrm{z}=0$, set at the bottom of the plate. In the transmit mode represented in Figure 1(b), the piezoelectric layer is excited with an electric potential $V$. The strain mismatch between the structural and the piezoelectric layers causes an out-of-plane plate vibration and the generation of an ultrasonic wave. In the receive mode, an echo pressure wave impinges onto the plate as shown in Figure 1(c). The plate deflection leads to charge separation in the piezoelectric layer, which generates a current in the charge amplifier. The neutral axes' locations in transmit and receive modes are denoted as $z_{e}$ and $z_{m}$, respectively.

\section{Working Equations}

Classic plate theory is used to model the circular unimorph PMUT. The two-layer unimorph plate structure shown in Figure 1 is generalized into an n-layer multimorph, each layer of which can be either structural or piezoelectric. As the plate lateral dimensions are much larger than its thickness, in-plane stress conditions dominate. Both the structural and the piezoelectric layers are assumed to be transversely isotropic. The interfaces between the respective layers are considered non-slip surfaces. The strains $\varepsilon_{r r}$ and $\varepsilon_{\theta \theta}$ in the r- and $\theta$-directions, respectively, at a distance $z$ from the neutral axis position are thus continuous and can be measured as follows [13]:

$$
\begin{gathered}
\varepsilon_{r r}=\frac{z-z_{n}}{R_{r r}} \\
\varepsilon_{\theta \theta}=\frac{z-z_{n}}{R_{\theta \theta}}
\end{gathered}
$$

where $R_{r r}$ and $R_{\theta \theta}$ are the radii of curvature in the r- and $\theta$ directions, respectively. The principal strains and stresses in the $i^{\text {th }}$ layer are related to the materials properties and the applied electric field according to Hooke's law [13]:

$$
\left[\begin{array}{c}
\varepsilon_{r r, i} \\
\varepsilon_{\theta \theta, i}
\end{array}\right]=\frac{1}{Y_{0, i}}\left[\begin{array}{cc}
1 & -v_{i} \\
-v_{i} & 1
\end{array}\right]\left[\begin{array}{c}
T_{r r, i} \\
T_{\theta \theta, i}
\end{array}\right]+\left[\begin{array}{l}
d_{31, i} \\
d_{31, i}
\end{array}\right] E_{z, i}
$$

where $T_{r r, i}$ and $T_{\theta \theta,}$ are the in-plane stresses in the $\mathrm{r}$ - and $\theta$ directions, respectively, $Y_{0, i}$ is the Young's modulus, $v_{i}$ is the Poisson's ratio, $d_{31, i}$ is transverse piezoelectric charge constant, and $E_{z, i}$ is the electric field applied along the $\mathrm{z}$-direction of the $\mathrm{i}^{\text {th }}$ layer. The stresses in the $\mathrm{r}$ - and $\theta$-directions can be re-written in terms of the strains by inverting Eq. (3):

$$
\left[\begin{array}{c}
T_{r r, i} \\
T_{\theta \theta, i}
\end{array}\right]=Y_{0, i}^{\prime}\left(\left[\begin{array}{cc}
1 & v_{i} \\
v_{i} & 1
\end{array}\right]\left[\begin{array}{c}
\varepsilon_{r r, i} \\
\varepsilon_{\theta \theta, i}
\end{array}\right]-\left[\begin{array}{c}
d_{31, i}^{\prime} \\
d_{31, i}^{\prime}
\end{array}\right] E_{z, i}\right)
$$

where $Y_{0, i}{ }^{\prime}$ is the equivalent Young's modulus and $d_{31, i}$ is the equivalent transverse piezoelectric charge constant defined as follows:

$$
\begin{aligned}
& Y_{0, i}^{\prime}=\frac{Y_{0, i}}{1-v_{i}^{2}} \\
& d_{31, i}^{\prime}=\left(1+v_{i}\right) d_{31, i}
\end{aligned}
$$

The externally applied force per unit width in the radial direction, $N_{r}$, can be calculated by integrating the radial stresses over the PMUT thickness at any radial position as follows:

$$
N_{r r}=\sum_{i=1}^{n} \int_{h_{i-1}}^{h_{i}} T_{r r, i} d z
$$

By performing the integration as indicated by Eq. (7), the radial force can be expressed as follows:

$$
\begin{aligned}
N_{r r}=( & \left.\frac{1}{R_{r r}}\right)\left[\sum_{i=1}^{n} Y_{0, i}^{\prime} z_{i} h_{i}-z_{n} \sum_{i=1}^{n} Y_{0, i}^{\prime} h_{i}\right] \\
& +\left(\frac{1}{R_{\theta \theta}}\right)\left[\sum_{i=1}^{n} v_{i} Y_{0, i}^{\prime} z_{i} h_{i}-z_{n} \sum_{i=1}^{n} v_{i} Y_{0, i}^{\prime} h_{i}\right] \\
& -\sum_{i=1}^{n} Y_{0, i}^{\prime} d_{31, i}^{\prime} h_{i} E_{z, i}
\end{aligned}
$$

where $z_{i}$ is the distance measured from the z-reference to the middle of the $i^{\text {th }}$ layer. The externally applied moment per unit width in the radial direction, $M_{r r}$, should be balanced by moments generated by the in-plane stresses around the z-reference. The moment is calculated using the following equation:

$$
M_{r r}=\sum_{i=1}^{n} \int_{h_{i-1}}^{h_{i}} T_{r r, i} z d z
$$

The following expression for $M_{r r}$ is obtained upon carrying out the integral of Eq. (9):

$$
\begin{aligned}
M_{r r}= & \left.\frac{1}{R_{r r}}\right)\left[\sum_{i=1}^{n} Y_{0, i}^{\prime}\left(I_{i}+z_{i}^{2} h_{i}\right)-z_{n} \sum_{i=1}^{n} Y_{0, i}^{\prime} z_{i} h_{i}\right] \\
& +\left(\frac{1}{R_{\theta \theta}}\right)\left[\sum_{i=1}^{n} v_{i} Y_{0, i}^{\prime}\left(I_{i}+z_{i}^{2} h_{i}\right)-z_{n} \sum_{i=1}^{n} v_{i} Y_{0, i}^{\prime} z_{i} h_{i}\right] \\
& -\sum_{i=1}^{n} Y_{0, i}^{\prime} d_{31, i}^{\prime} z_{i} h_{i} E_{z, i}
\end{aligned}
$$

where $I_{i}$ is the second moment of area per unit width calculated around each layer's centerline as follows:

$$
I_{i}=\frac{h_{i}^{3}}{12}
$$

If we assume that the Poisson's ratio $v_{i}$ of all the layers, whether structural or piezoelectric, is the same and set its value equal to $v$, the externally applied radial force and moment per unit width can be rearranged using Eqs. (8) and (10) as follows: 


$$
\begin{aligned}
& N_{r r}=\left(\frac{1}{R_{e q}}\right)\left[\sum_{i=1}^{n} Y_{0, i}^{\prime} z_{i} h_{i}-z_{n} \sum_{i=1}^{n} Y_{0, i}^{\prime} h_{i}\right]-\sum_{i=1}^{n} Y_{0, i}^{\prime} d_{31, i}^{\prime} h_{i} E_{z, i} \\
& M_{r r}=\left(\frac{1}{R_{e q}}\right)\left[\sum_{i=1}^{n} Y_{0, i}^{\prime}\left(I_{i}+z_{i}^{2} h_{i}\right)-z_{n} \sum_{i=1}^{n} Y_{0, i}^{\prime} z_{i} h_{i}\right]-\sum_{i=1}^{n} Y_{0, i}^{\prime} d_{31, i}^{\prime} z_{i} h_{i} E_{z, i}
\end{aligned}
$$

where $R_{e q}$ is the equivalent radius of curvature due to the radii of curvature in the r-direction, $\theta$-direction, and the Poisson's ratio as follows:

$$
\frac{1}{R_{e q}}=\frac{1}{R_{r r}}+\frac{v}{R_{\theta \theta}}
$$

By eliminating the equivalent radius of curvature term in Eqs. (12) and (13), the neutral axis position can be finally expressed as a function of the externally applied radial moment and force per unit width as well as the electric fields applied across each piezoelectric layer as follows:

$$
z_{n}=\frac{\left[\sum_{i=1}^{n} Y_{0, i}^{\prime}\left(I_{i}+z_{i}^{2} h_{i}\right)\right]\left(N_{r r}+\sum_{i=1}^{n} Y_{0, i}^{\prime} d_{31, i}^{\prime} h_{i} E_{z, i}\right)-\left[\sum_{i=1}^{n} Y_{0, i}^{\prime} z_{i} h_{i}\right]\left(M_{r r}+\sum_{i=1}^{n} Y_{0, i}^{\prime} d_{31, i}^{\prime} z_{i} h_{i} E_{z, i}\right)}{\left[\sum_{i=1}^{n} Y_{0, i}^{\prime} z_{i} h_{i}\right]\left(N_{r r}+\sum_{i=1}^{n} Y_{0, i}^{\prime} d_{31, i}^{\prime} h_{i} E_{z, i}\right)-\left[\sum_{i=1}^{n} Y_{0, i}^{\prime} h_{i}\right]\left(M_{r r}+\sum_{i=1}^{n} Y_{0, i}^{\prime} d_{31, i}^{\prime} z_{i} h_{i} E_{z, i}\right)}
$$

\section{Neutral Axes Location}

Case I: Transmit mode

For the case of a PMUT in the transmit mode, there are no externally applied radial moments and forces. As such the neutral axis position $z_{e}$ reduces to the following:

$$
z_{e}=\frac{\left[\sum_{i=1}^{n} Y_{0, i}^{\prime}\left(I_{i}+z_{i}^{2} h_{i}\right)\right]\left(\sum_{i=1}^{n} Y_{0, i}^{\prime} d_{31, i}^{\prime} h_{i} E_{z, i}\right)-\left[\sum_{i=1}^{n} Y_{0, i}^{\prime} z_{i} h_{i}\right]\left(\sum_{i=1}^{n} Y_{0, i}^{\prime} d_{31, i}^{\prime} z_{i} h_{i} E_{z, i}\right)}{\left.\left[\sum_{i=1}^{n} Y_{0, i}^{\prime} z_{i} h_{i}\right]\right]\left(\sum_{i=1}^{n} Y_{0, i}^{\prime} d_{31, i}^{\prime} h_{i} E_{z, i}\right)-\left[\sum_{i=1}^{n} Y_{0, i}^{\prime} h_{i}\right]\left(\sum_{i=1}^{n} Y_{0, i}^{\prime} d_{31, i}^{\prime} z_{i} h_{i} E_{z, i}\right)}
$$

If there is only one piezoelectric layer in the PMUT structure, the neutral axis in the transmit mode is simplified to the following:

$$
z_{e 1}=\frac{\sum_{i=1}^{n} Y_{0, i}^{\prime}\left(I_{i}+z_{i}^{2} h_{i}\right)-z_{P} \sum_{i=1}^{n} Y_{0, i}^{\prime} z_{i} h_{i}}{\sum_{i=1}^{n} Y_{0, i}^{\prime} z_{i} h_{i}-z_{P} \sum_{i=1}^{n} Y_{0, i}^{\prime} h_{i}}
$$

where $z_{p}$ is the distance from the z-reference to the center of the piezoelectric layer.

\section{Case II: Receive mode}

When the PMUT operates in the receive mode, there are no electric fields across the respective piezoelectric layers. In addition, there are no radially applied forces. As such the neutral axis position for the receive mode $z_{m}$ reduces to the following:

$$
z_{m}=\frac{\left[\sum_{i=1}^{n} Y_{0, i}^{\prime} z_{i} h_{i}\right]}{\left[\sum_{i=1}^{n} Y_{0, i}^{\prime} h_{i}\right]}
$$

\section{RESULTS AND DISCUSSION}

In order to verify the derived theoretical model in the previous section, a unimorph PMUT structure is considered as shown in Figure 1(a). The PMUT is composed of silicon as a structural layer and PZT as a piezoelectric layer. Table 1 summarizes the material properties and geometric dimensions of the PMUT analyzed in this paper. The theoretically derived neutral axes in transmit and receive modes were calculated as $1.41 \mu \mathrm{m}$ and $2.10 \mu \mathrm{m}$, respectively. It is observed that the neutral axis in the receive mode is closer to the geometric mid-plane scaled by Young's modulus and the thickness of each layer. On the other hand, the neutral axis location in the transmit mode is deeper into the structural layer. Since the piezoelectric layer applies an additional stress to the structural layer, the location of the zerostrain plane is pushed farther into the silicon.

Table 1: Geometric and mechanical properties of the structural layer (Silicon) and piezoelectric layer (PZT), which form the PMUT.

\begin{tabular}{|l|l|c|c|c|}
\hline & \multicolumn{1}{|c|}{ Description } & Silicon & PZT & Units \\
\hline $\boldsymbol{Y}_{\boldsymbol{0}}$ & Young's Modulus & 169 & 63 & {$[\mathrm{GPa}]$} \\
\hline $\boldsymbol{v}$ & Poisson's ratio & 0.3 & 0.3 & \\
\hline $\boldsymbol{r}_{\boldsymbol{m}}$ & Radius & 100 & 100 & {$[\mathrm{\mu m}]$} \\
\hline $\boldsymbol{h}$ & Thickness & 4 & 0.5 & {$[\mathrm{\mu m}]$} \\
\hline $\boldsymbol{d}_{\boldsymbol{3 1}}$ & $\begin{array}{l}\text { Piezoelectric Charge } \\
\text { constant }\end{array}$ & - & 171 & {$[\mathrm{pm} / \mathrm{V}]$} \\
\hline \hline
\end{tabular}

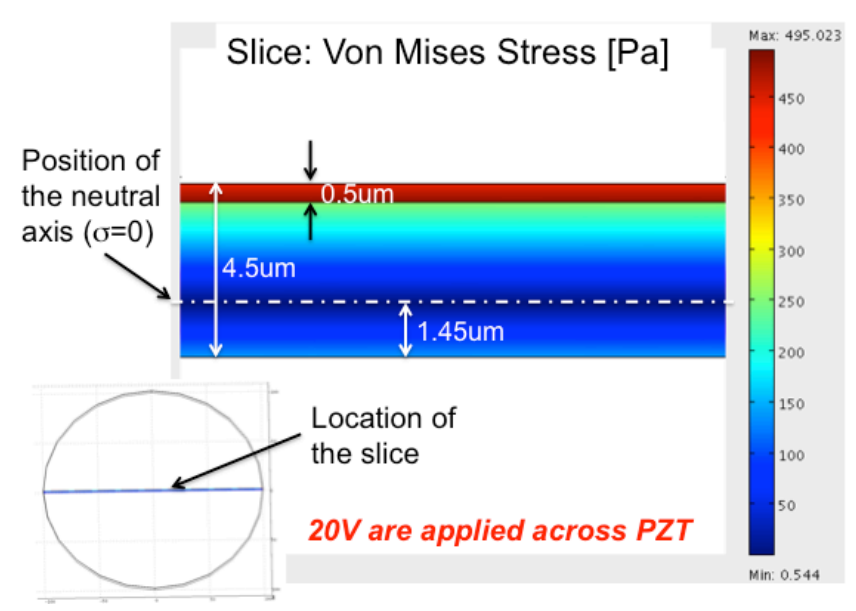

Figure 2: FEM using COMSOL of a PMUT in transmit mode. The PMUT is composed of $0.5 \mu \mathrm{m}$ thick PZT layer on top of $4 \mu \mathrm{m}$ thick Silicon layer. An electric potential of $20 \mathrm{~V}$ is applied across the piezoelectric layer. The Von Mises stresses are measured and the neutral axis is located at $1.45 \mu \mathrm{m}$ using a cross-section at the center of the membrane.

COMSOL Multiphysics was used as the Finite Element Modeling (FEM) tool for the zero-strain plane simulation [14]. The PMUT plate model was developed by extruding the mesh generated from a $2 \mathrm{D}$ circle. The resolution of the vertical mesh layers in the $\mathrm{z}$-direction was set at 10 layers $/ \mu \mathrm{m}$ in order to ensure accurate extraction of the neutral axis position. The Von Mises stresses were calculated across the PMUT cross-sectional area at the center of the plate and the zero-stress location was identified. To simulate a PMUT in transmit mode, only an electric potential of 
$20 \mathrm{~V}$ was applied across the top piezoelectric layer. In the receive mode simulation, both the top and bottom electrodes sandwiching the piezoelectric layer were grounded to ensure that no electric field is developed. An impinging acoustic pressure of $100 \mathrm{kPa}$ was applied. Figures 2 and 3 show the Von Mises stresses extracted from COMSOL simulation of a PMUT plate with the same structure composition and material properties as the theoretically analyzed plate in transmit and receive configuration, respectively. The neutral axes positions were subsequently inferred from the zero-stress locations as $1.45 \mu \mathrm{m}$ and $2.16 \mu \mathrm{m}$. The values extracted from the FEM match closely with the theoretically calculated locations. An error of less than $3.6 \%$ between the theoretical and FEM approach validate the credibility of the developed model.

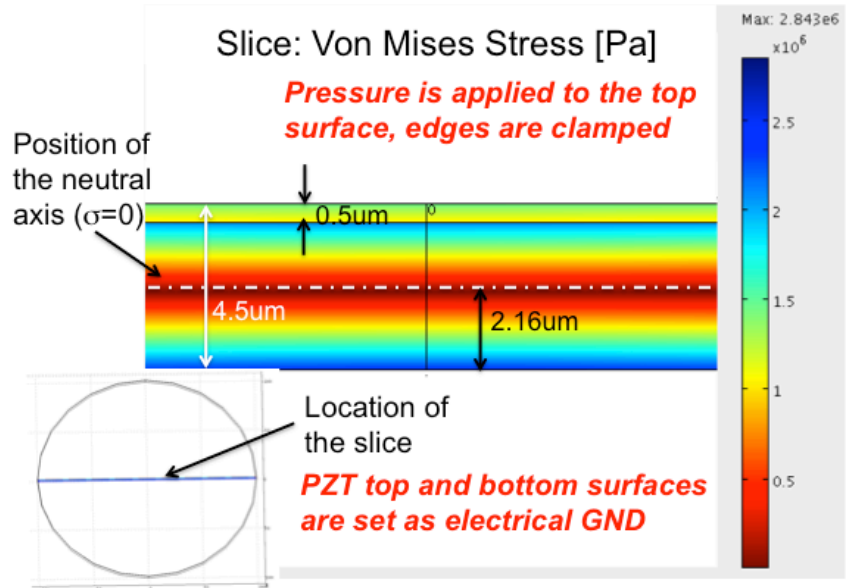

Figure 3: FEM using COMSOL of a PMUT in the receive mode. A pressure level of $100 \mathrm{kPa}$ is applied to the membrane. The Von Mises stresses are measured and the neutral axis is located at $2.16 \mu \mathrm{m}$ using a cross-section at the center of the membrane.

\section{CONCLUSION}

The neutral axis location for a circular PMUT composed of multiple structural and piezoelectric layers was investigated for the first time. The PMUT plate was modeled using the classic plate theory, where in-plane stress conditions, isotropic materials, and non-slip interfaces between the layers were assumed. The externally applied forces and moments per unit width were calculated as a function of the plate radii of curvature, layer geometric and material properties, including the thickness, Young's modulus, and Poisson's ratio, as well as the piezoelectric charge constant and the electric field across each piezoelectric layer. The general position of the neutral axis, or the position of zero bending strain, was derived for any externally applied radial moment and force per unit width, the material properties and layer dimensions, as well as the electric fields applied on each piezoelectric layer. Specific solutions of the neutral axis position were extracted for the transmit case, where only external electric fields are applied, and receive mode, where only external pressure is present. The analytical model was verified using COMSOL Multiphysics with a unimorph PMUT composed of a silicon structural layer and PZT piezoelectric layer. The location of zero Von Mises stress plane was used to locate the neutral axis from the FEM. The agreement between the theoretical and experimental tools was better than $3.6 \%$.

\section{ACKNOWLEDGEMENTS}

This project is supported by Masdar Institute of Science and Technology, Abu Dhabi, UAE grant no. 6923443 under the cooperative agreement between Masdar Institute of Science and Technology and Massachusetts Institute of Technology.

\section{REFERENCES}

[1] G. S. Kino, Acoustic Waves: Devices, Imaging, and Analog Signal Processing, Prentice Hall, 1987.

[2] A. R. Selfridge and S. Gehlbach, "KLM Transducer Model using Transfer Matrices," in Proceeding of IEEE Ultrasonics Symposium, pp. 875-877, 1985.

[3] W. A. Smith, "The Role of Piezocomposites in Ultrasonic Transducers," in Proceeding of IEEE Ultrasonics Symposium, pp. 755-766, 1989.

[4] B. T. Khuri-Yakub and O. Oralkan, "Capacitive Micromachined Ultrasonic Transducers for Medical Imagining and Therapy, "Journal of Micromechanics and Microengineering, vol. 21, no. 5, pp. 1-11, May 2011.

[5] P. C. Eccardt and K. Niederer, "Micromachined Ultrasound Transducers with Improved Coupling Factors from a CMOS Compatible Process," Ultrasonics, vol. 38, pp. 774-780, 2000.

[6] J. H. Mo, A. L. Robinson, D. W. Fitting, F. L. Terry, and P. L. Carson, "Micromachining for Improvement of Integrated Ultrasonic Transducer Sensitivity," IEEE Transactions on Electron Devices, vol. 37, no. 1, pp. 134-140, January 1990.

[7] S. Shelton, M. L. Chan, H. Park, D. Horsely, B. Boser, I. Izyumin, R. Przybyla, T. Frey, M. Judy, K. Nunan, F. Sammoura, and K. Yang, "CMOS-Compatible AIN Piezoelectric Micromachined Ultrasonic Transducers," 2009 IEEE International Ultrasonics Symposium (IUS), pp. 402405, Rome, Italy, September 20-23, 2009.

[8] P. Muralt, N. Ledermann, J. Baborowski, A. Barsegar, S. Gentil, S. Petitgrand, A. Bosseboeuf, and N. Setter, "Piezoelectric Micromachined Ultrasonic Transducers based on PZT Thin Films," IEEE Transactions on Ultrasonics, Ferroelectrics, and Frequency Control, vol. 52, no. 12, pp. 2276-2288, December 2005.

[9] J. J. Bernstein, L. Finberg, K. Houston, L. C. Niles, H. D. Chen, L. E. Cross, K. K. Li, and K. Udayakumar, "Micromachined High Frequency Ferroelectric Sonar Transducers," IEEE Transactions on Ultrasonics, Ferroelectrics, and Frequency Control, vol. 44, no. 5, September 1997.

[10] G. Perçin and B. T. Khuri-Yakub, "Piezoelectrically Actuated Flextensional Micromachined Ultrasound Transducers - I: Theory," IEEE Transactions on Ultrasonics, Ferroelectrics, and Frequency Control, vol. 49, no. 5, pp. 573-584, May 2002.

[11] D. DeVoe and A. P. Pisano, "Modeling and Optimal Design of Piezoelectric Cantilever Microactuators," Journal of Microelectrimechanical Systems, vol. 6, no. 3, pp. 266-270, September 1997.

[12] M. S. Weinberg, "Working Equations for Piezoelectric Actuators and Sensors," Journal of Microelectromechanical Systems, vol. 8, no. 4, December 1999.

[13] T. Ikeda, Fundamentals of Piezoelectricity, Oxford University Press, 1990.

[14] http://www.comsol.com

\section{CONTACT}

*Firas Sammoura, Massachusetts Institute of Technology, 77 Massachusetts Avenue, Building 41-211; Tel: +1-510-529-9142; Fax:+1-617-252-2982; sammoura@MIT.edu 


\title{
APPLICATION OF A PREDICTION METHODOLOGY FOR MEMS RELIABILITY IN SHOCK ENVIRONMENTS
}

\author{
M. Naumann ${ }^{1 *}$,D. Lin ${ }^{2}, A . M c N e i l^{2}, G$. Li $^{2}$ and J. Mehner ${ }^{l}$ \\ ${ }^{1}$ Chemnitz University of Technology, Germany \\ ${ }^{2}$ Freescale Semiconductor, Inc., USA
}

\begin{abstract}
The paper investigates an approach to predict shock induced failure mechanisms of breakage and stiction at MEMS devices. The approach is applied at two surface micro-machined test devices which are exposed to shock accelerations up to $56,000 \mathrm{~g}$ $\left(1 \mathrm{~g}=9.81 \mathrm{~m} / \mathrm{s}^{2}\right)$. Observed failures are correlated to predicted failures in order to validate the proposed methodology. Predictions are based on a comparison between calculated loads obtained from numerical models and permitted loads of utilized device components. Therefore, a detailed characterization of the applied fabrication technology regarding both failure mechanisms is required. The paper presents a selection of data required to predict failures for both test devices.
\end{abstract}

\section{INTRODUCTION}

The adoption of MEMS devices within various automotive, medical and consumer markets products is determined by economical, functional as well as reliability aspects. MEMS devices are required to fulfill their functionality even under harsh environmental conditions. Common sources of failure are mechanical shocks on devices consisting of movable structural components. Depending on the load characteristics, two major failure mechanisms of breakage and stiction can be triggered [1].

As a common practice, rigid travel stops are applied to avoid excessive deflections during overload situations. This often produces very high impact loads at the stops as movable structural MEMS components are stopped almost immediately when they hit a stop. Excessive impact loads can cause breakage of stops and structural components. Furthermore, the probability of stiction at travel stops is increased by higher impact forces as demonstrated in [2]. Latest investigations have indicated that an improvement of device reliability is possible by introducing flexible stops to reduce arising impact loads [3], [4].

A new approach to evaluate the reliability of MEMS devices in shock environments was first proposed in [2]. The approach is intended to assess arising failures at MEMS devices which are exposed to shock accelerations by calculation of their transient system response including arising load conditions. A conclusion on device reliability requires the knowledge of permissible loads which have to be obtained from a detailed characterization of the utilized fabrication technology. The results in terms of fracture strength and adhesion behavior are compared to calculated loads in order to evaluate and predict the device reliability. Once the fabrication technology is characterized, the introduced methodology can be applied to arbitrary designs. The reliability of new MEMS products can be assessed and their design optimized even before first prototypes are fabricated which can be used to experimentally validate device reliability. Furthermore, the methodology is able to improve experimental reliability approaches as device specific critical load situations can be identified and tested purposefully.

In this paper the proposed approach is investigated by surface micro-machined test devices which have been exposed to shock accelerations up to $56,000 \mathrm{~g}$. The observed mechanism of failure and the failure probability are compared to the predictions of the reliability approach at different shock levels.

\section{TEST DEVICES}

Two different test devices have been exposed to shock accelerations parallel to the substrate in order to investigate both failure mechanisms of breakage and stiction.

Figure 1a) shows the SEM image of the test device which was used to investigate the fracture behavior of travel stops. The device consists of a seismic mass suspended at meander shaped springs.

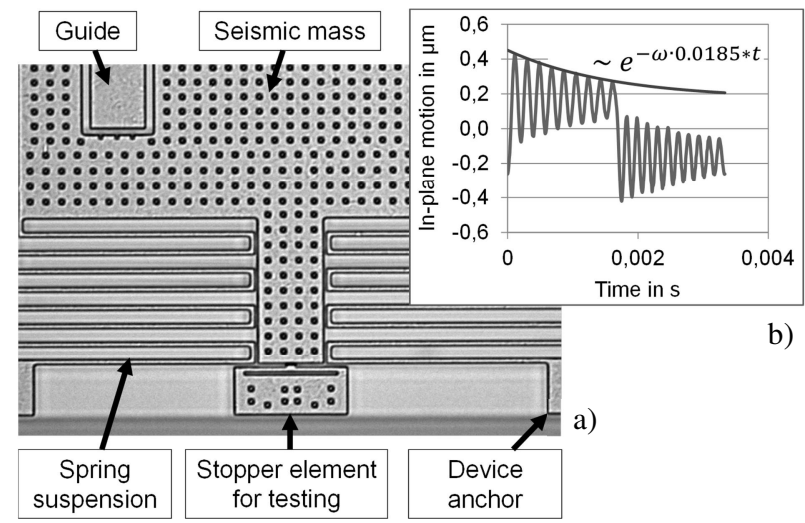

Figure 1: a) Surface micro-machined test device for breakage investigations; $b$ ) Determination of the damping ratio.

The overall dimension of the test device is $320 \mu \mathrm{m} \times 370 \mu \mathrm{m}$ which is small in order to maximize the number of devices on a single chip and therefore to increase the level of confidence of the obtained results. Furthermore, damping has been minimized in order to investigate dynamic impacts when critical loads arise primarily due to the deceleration of the proof mass [4]. The damping ratio of $\mathrm{dr}=0.018$ has been determined by measuring the in-plane decay process (Fig. 1b). Nonlinearities due to the squeeze effect can be neglected for this kind of test devices. Figure 2 shows a small selection of investigated stopper elements.

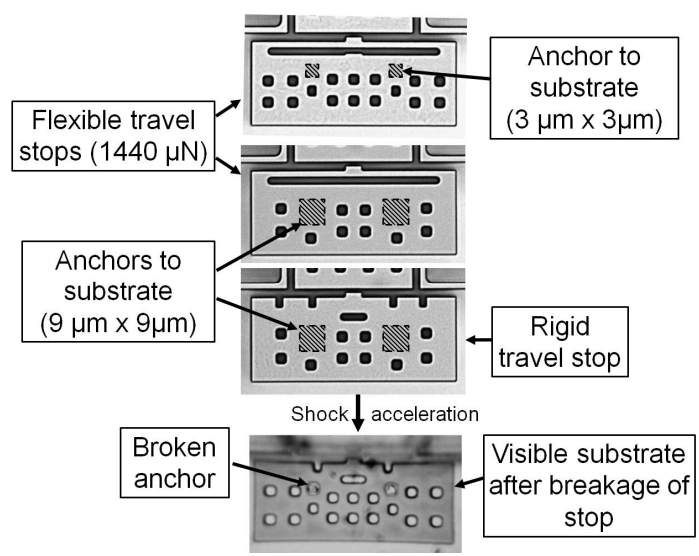

Figure 2: Stopper elements of different stiffness and anchor areas to substrate before and after breakage.

Solid-State Sensors, Actuators, and Microsystems Workshop Hilton Head Island, South Carolina, June 3-7, 2012 
Flexible travel stops of type 720, 1440 and 4350 have been compared to rigid travel stops without any additional flexibility. The type reference of flexible stops corresponds to their stiffness with the unit in $\mathrm{N} / \mathrm{m}$. Each kind of stop has been combined with three different types of anchors to substrate $3 \times 3,5 \times 5$ and $9 \times 9$ corresponding to total anchor areas per stop of $18 \mu \mathrm{m}^{2}, 50 \mu \mathrm{m}^{2}$ and $162 \mu \mathrm{m}^{2}$, respectively.

\section{PROCESS CHARACTERIZATION}

\section{Breakage}

During the applied shock accelerations, stopper elements of the test devices are exposed to forces parallel to the substrate causing a combination of shearing and bending loads. Breakage will occur at the anchors if the load exceeds their fracture strength. In order predict failures for this kind of load condition, characterization results of a special MEMS device have been utilized. The device was used to determine the fracture strength of stopper elements exposed to the described load condition for different anchor areas by static measurements. Figure 3 illustrates the results. Statements on the reliability of a specific stopper element can directly be achieved by comparing the output of the impact models in terms of calculated impact forces with the corresponding measured fracture forces.

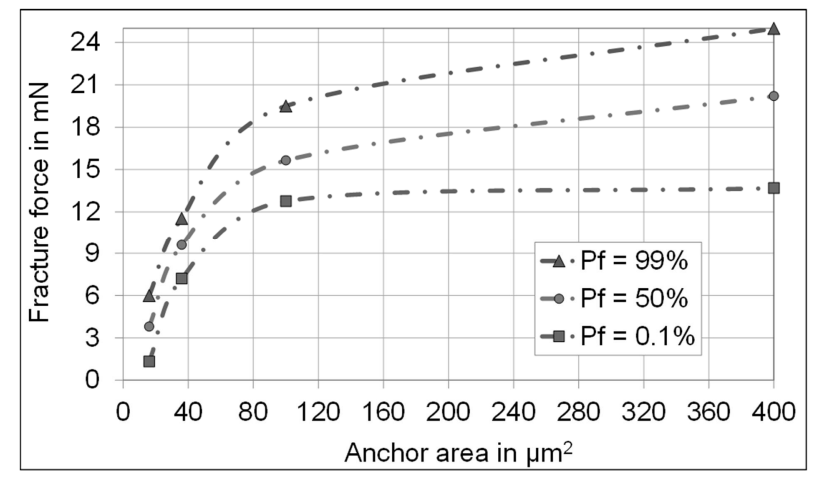

Figure 3: Fracture strength depending on the anchor area for different probabilities of failure.

A closer look on the measurement data revealed the brittle fracture behavior of anchors. Hence, the Weibull distribution was applied to mathematically describe the probability of failure depending on the load. Figure 4 shows the fracture data and the fitted three-parameter Weibull distribution for two anchor sizes of $36 \mu \mathrm{m}^{2}$ (type 6x6) and $100 \mu \mathrm{m}^{2}$ (type 10x10).
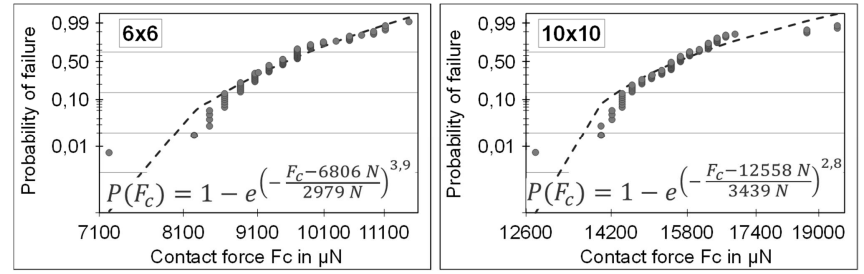

Figure 4: Description of the fracture behavior of anchors of type $6 \times 6$ and $10 \times 10$ by the tree-parameter Weibull distribution.

The diagrams verify the applicability of the Weibull distribution to describe the real fracture behavior of anchors with adequate accuracy. However, the validity of the Weibull theory still needs to be proven as the current evaluation of data links the event of fracture to the acting stopper force without any consideration on the load distribution along the anchor required to determine Weibull parameters like the characteristic stress.

\section{Stiction}

Contacts to travel stops at the test devices occur at side walls of the functional layer as shock accelerations are applied parallel to substrate. The required characterization of adhesion forces to side walls has been performed by a special MEMS device which applied compressive contact forces to stopper elements by electrostatic actuation. After each force step the quantity of sticking devices as well as arising adhesion forces have been determined. The results are illustrated in Figure 5.
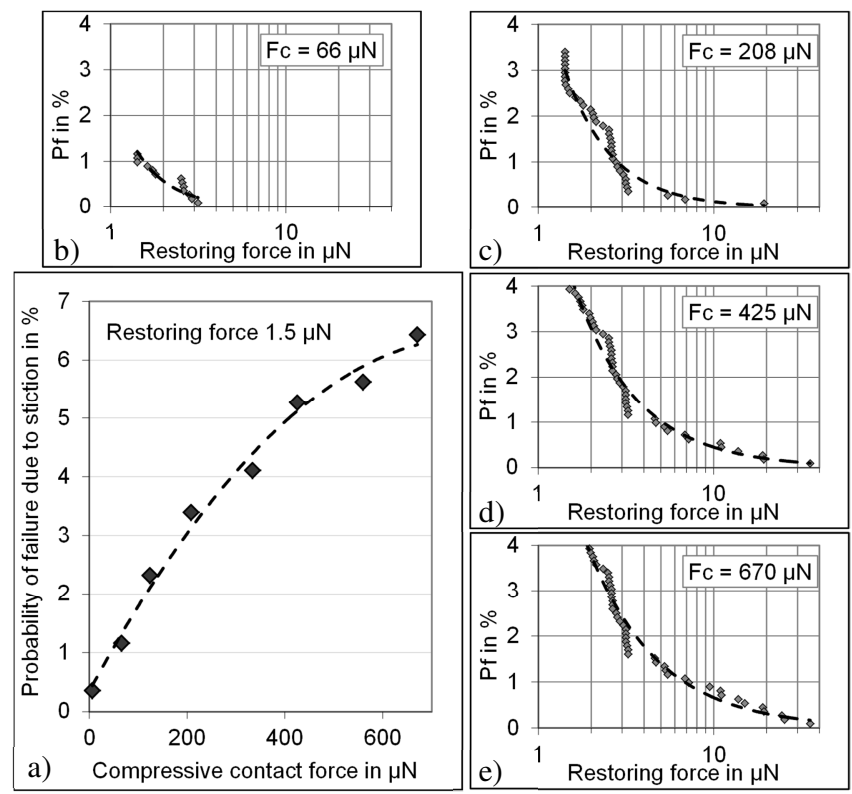

Figure 5: a) Failure rate due to stiction at a stopper element depending on the applied compressive contact force. b-e) Probability of failure Pf due to stiction depending on the restoring force of a device for applied compressive contact forces $F c$ of $66 \mu N(b), 208 \mu N(c), 425 \mu N(d), 670 \mu N(e)$.

In case of the investigated fabrication technology, increased contact forces lead to higher failure rates (Fig. 5a) and thus to larger adhesion forces (Fig. 5b-e). For example, the failure rate of a MEMS device with a restoring force of $5 \mu \mathrm{N}$ would be about $0.5 \%$ after contact to a travel stop with a compressive force Fc of about $208 \mu \mathrm{N}$. Below $\mathrm{Fc}=66 \mu \mathrm{N}$ most likely the device would not stick at all. At $\mathrm{Fc}=425 \mu \mathrm{N}$ the failure rate increases up to about $1 \%$. For contacts with a compressive force of $670 \mu \mathrm{N}$ the failure rate is about $1.5 \%$.

A further investigation on the dependency of adhesion forces on the number of performed contact cycles revealed an increase of adhesion forces by about $20 \%$ after the first cycle. $32 \%$ of the detected sticking incidences are affected by compressive contact forces above $124 \mu \mathrm{N}$.

\section{IMPACT MODEL}

The transient system responses of the test device exposed to shock accelerations are calculated by a lumped element impact model solving the differential equation of motion of the system. The model accounts for nonlinear interactions of the proof mass with travel stops including the resulting adhesion forces and the 
possible event of stiction failure. Figure 6 illustrates the approach. System parameters like device mass and damping as well as the measured shock accelerations are used as input parameters.
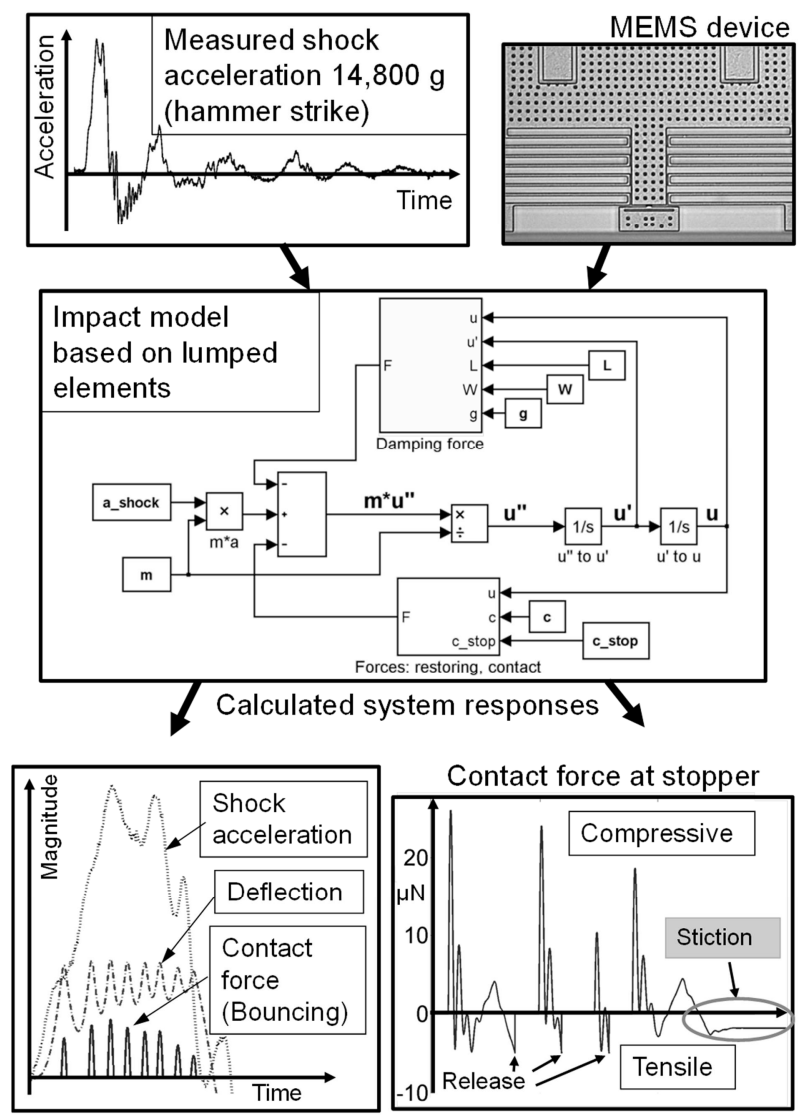

Contact force at stopper

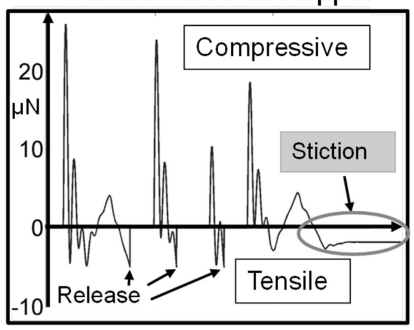

Figure 6: Calculation of the transient system response of the test device for breakage characterization exposed to a measured shock acceleration.

\section{SHOCK TESTS}

\section{Procedure}

Shock tests have been performed for diced silicion chips which have been mounted on a special chip carrier by conductive glue. The applied shock levels have been measured by a reference accelerometer (B\&K 8309) which has been mounted on the chip carrier. In total, three test runs have been performed whereas a hammer strike procedure (run 1 and 2) and a Hopkinson Bar have been utilized. At each test run a series of shocks have been applied to the chip carrier. The shock level has been increased stepwise form low g-level (about 6,000 g) to high g-level (up to 56,000 g). Arising failures have been recorded after each shock from 300 up to 1800 mounted devices.

\section{Breakage}

Figure 7 shows the obtained results in terms of failure rates depending on the applied shock level for different anchor types 3x3 (Fig. 7a), 5x5 (Fig. 7b, 7d) and 9x9 (Fig. 7c). Therefore, the efficiency of different flexible travel stops becomes obvious. For example, considering the results of anchors of type $3 \times 3$ at $14,900 \mathrm{~g}$ (Fig. 7a). The failure rate of rigid travel stops already reached $100 \%$ whereas the failure rate of the flexible stops 720 is only $6 \%$. Similar results can be observed at anchors of types $5 \times 5$ and $9 \times 9$.
The optimum stiffness of flexible stops depends on the utilized anchor area and has been detected to $720 \mathrm{~N} / \mathrm{m}$ of type $3 \times 3$, $1440 \mathrm{~N} / \mathrm{m}$ of type $5 \times 5$ and $4350 \mathrm{~N} / \mathrm{m}$ of type $9 \times 9$.
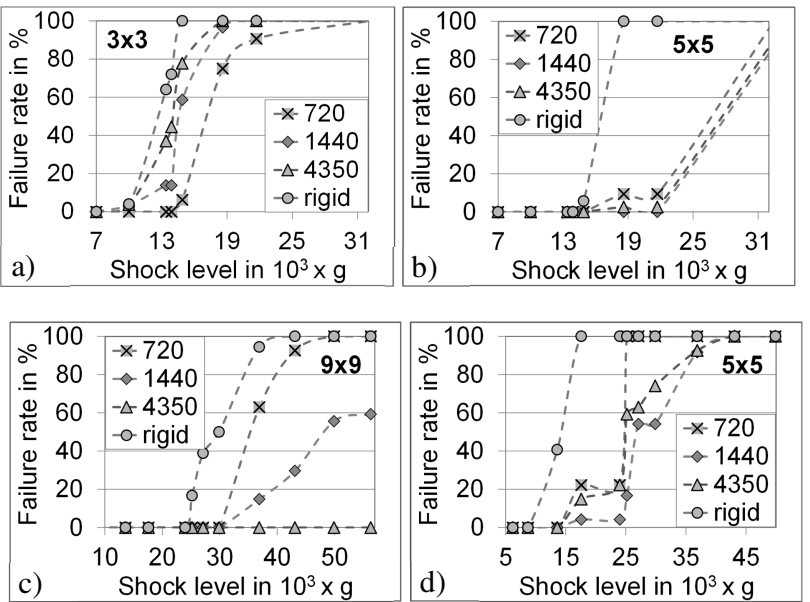

Figure 7: Failure rates of stopper elements of different stiffness and anchor type depending on the applied shock level; anchor types: a) $3 \times 3, b) 5 \times 5$, c) $9 \times 9$, d) $5 \times 5$

A comparison of results between the test runs reveals small differences which can be explained by different shock characteristics in terms of superimposed oscillations and the corresponding frequencies (resulting into deviant loads on the stops for comparable maximum shock levels). Figure $7 \mathrm{~b}$ and $7 \mathrm{~d}$ enable a comparison of results between test run $1(7 \mathrm{~b}$, hammer strike) and test run 3 (7d, Hopkinson Bar) for anchors of type $5 \times 5$. However, the efficiency of flexible travel stops has been verified by all test runs as general trends are comparable.

Shock levels which have been identified to cause damage at stopper elements of the test devices seem to be quite high ranging from $8,000 \mathrm{~g}$ up to $56,000 \mathrm{~g}$. For real MEMS products like gyroscopes or accelerometers, much lower shock accelerations will result into similar failure modes as masses are higher. Therefore, larger anchor size is needed to counter balance for larger mass.

\section{Stiction}

Figure 8 illustrates the observed failure rates due to stiction as a result of the applied shock acceleration for test run 3. For loads up to $30,000 \mathrm{~g}$, the number of sticking devices increases with shock acceleration. For loads greater than $30,000 \mathrm{~g}$, the failure rate remains at an almost constant high level of about $4.6 \%$.

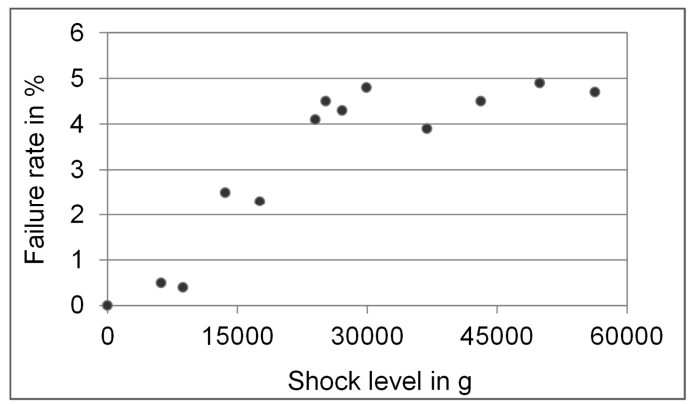

Figure 8: Failure rate due to stiction depending on the applied shock acceleration. 


\section{APPLICATION OF THE IMPACT MODEL Breakage}

The introduced impact model has been used to calculate the transient system responses of the test devices depending on the measured shock accelerations. From the solutions the maximum impact forces on the test stopper elements have been extracted. Figure 9a shows the calculated impact forces for test run 3 at the 9x9 stopper version. Based on the knowledge of the fracture behavior of the utilized anchors the probability of failure has been calculated and compared to the measurement results (Fig. 9b). The results of the impact model represent the general failure trend. However, they do not match the measured behavior exactly which has been observed at all test runs.
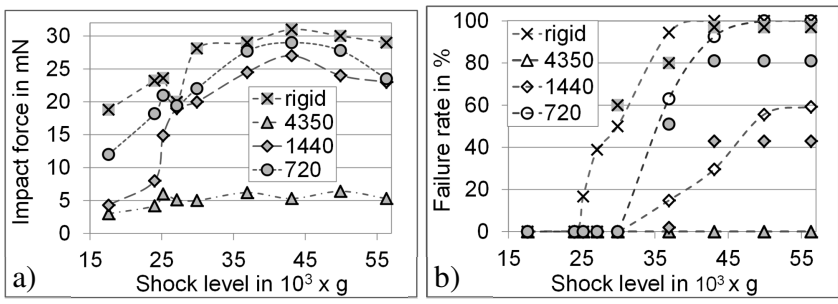

Figure 9: a) Calculated impact forces at stopper type 9x9 for test run 3; b) Comparison between observed and calculated failure rates (filled markers for calculated rates).

The reason for the deviations can be explained by a closer look at the system behavior. Figure 10a illustrates the measured shock acceleration of $43,000 \mathrm{~g}$ at the front side of the Hopkinson Bar (test run 3). Each time the material wave reaches the front side of the bar it is reflected creating an acceleration pulse and superimposed oscillations form the test setup. The calculated impact forces at test stops are illustrated in Figure 10b comparing the flexible stop 4350 with a rigid one. The resulting impact forces are reduced by the flexible stop from up to $31 \mathrm{mN}$ to about $5 \mathrm{mN}$ which correlates to the findings of the shock tests. At $43,000 \mathrm{~g}$ $100 \%$ of the rigid travel stops have been damaged compared to $0 \%$ for type 4350 (compare Fig. 9b).

Due to the low damping and the oscillations of the acceleration load, excessive bouncing occurs between the travel stops of the test devices. For both test setups of hammer strike and Hopkinson Bar, it has been found that maximum impact forces occur during the bouncing process in case when the bouncing frequency matches one of the frequencies contained in the acceleration signal.

A detailed analysis of the bouncing history has shown convergence for a sufficient small simulation time step. However, the consideration of process variations which affect device parameters like mass and stiffness of stopper elements has a significant effect on the time response demonstrated in Figure 10c. The diagram compares calculated impact forces for two versions of rigid travel stops at a shock acceleration of $27,000 \mathrm{~g}$. A maximum impact force of $23.6 \mathrm{mN}$ has been determined for the original stop leading to a predicted failure rate of $0 \%$ compared to $39 \%$ of observed failure rate (Fig. 9b). The deviation can be explained by possible process variations. A reduction of the stopper stiffness by $10 \%$ would increase the impact force to $33 \mathrm{mN}$ which corresponds to a failure rate of $100 \%$. Hence, the strong dependency of the bouncing response on the device parameters can explain the difference between predicted and detected failure rates.

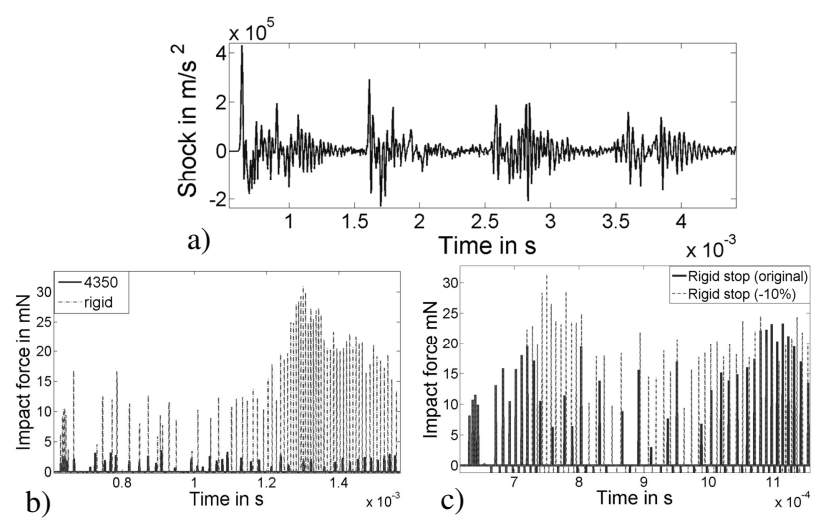

Figure 10: a) Measured shock acceleration (43.000 g) b) Comparison of impact forces between a flexible stop (4350) and a rigid one. c) Comparison of impact forces at rigid stops with $10 \%$ deviation.

\section{Stiction}

Device failure due to stiction occurs when adhesion forces at the travel stops exceed the tensile forces from the spring suspension trying to restore the initial device position. Additional tensile force at the travel stops can arise from the external shock acceleration as well as from the bouncing process. The analysis of the calculated system responses of the utilized test device at different g-levels has shown that stiction is very unlikely as long as the external shock acceleration lasts as tensile forces exceed minimum $100 \mu \mathrm{N}$. First sticking incidences occur in case when the external shock acceleration and bouncing almost decayed. Predicted failure rates range from $1 \%$ up to $3.1 \%$.

\section{SUMMARY}

The paper has demonstrated the ability to describe the observed failures for the test devices by application of the introduced approach for MEMS reliability with adequate accuracy. Limitations result from the unknown distributions of device parameters due to process variations such as mass and stiffness variations which have a significant effect on calculated system responses.

\section{REFERENCES}

[1] V. T. Srikar, S. D. Senturia, "The Reliability of Microelectromechanical Systems (MEMS) in Shock Environments", Journal of Microelectromechanical Systems, 11, (2002), pp. 206 - 214.

[2] M. Naumann, D. Lin, J. Mehner, A. McNeil, T. F. Miller, "Design evaluation of shock induced failure mechanisms of MEMS by correlation of numerical and experimental results", Proc. Transducers, 16, (2011), pp. 2891 - 2894.

[3] S. W. Yoon, "Vibration isolation and shock protection for MEMS", Dissertation, Michigan, 2009.

[4] M. Naumann, D. Lin, J. Mehner, T. Miller, "Design and Application of Flexible Stops for MEMS Devices", Proc. Sensors, 9, (2010), pp. 168 - 173.

\section{CONTACT}

*M. Naumann; michael.naumann@etit.tu-chemnitz.de 


\title{
ENCAPSULATED THERMIONIC ENERGY CONVERTER WITH STIFFENED SUSPENSION
}

\author{
Jae Hyung Lee ${ }^{l}$, Igor Bargatin ${ }^{l}$, Kentaro Iwami ${ }^{1,2}$, Karl A. Littau ${ }^{l}$, Maxime Vincent ${ }^{3}$, \\ Roya Maboudian ${ }^{3}$, Z.-X. Shen ${ }^{1}$, Nicholas A. Melosh ${ }^{1}$, and Roger T. Howe ${ }^{I}$ \\ ${ }^{1}$ Stanford University, USA \\ ${ }^{2}$ Tokyo University of Agriculture and Technology, Japan \\ ${ }^{3}$ University of California at Berkeley, USA
}

\section{ABSTRACT}

This paper reports a prototype of a mechanically and thermally robust encapsulated microfabricated thermionic energy converter ( $\mu$-TEC). We used conformal sidewall deposition of poly-SiC to form stiff suspension legs with U-shaped cross sections, which increase the out-of-plane rigidity and prevent contact with the substrate during the heating of the suspended emitter - a qualitative improvement over the previously reported planar SiC $\mu$-TECs [1]. Furthermore, we tested the new $\mu$-TECs with both barium and barium oxide coatings. The coatings reduced the work function of the $\mathrm{SiC}$ emitter to as low as $\sim 2.14 \mathrm{eV}$ and increased the thermionic current by 5-6 orders of magnitude, which is a key step toward realizing a highly efficient thermionic energy converter. In addition, we have sealed small arrays of $\mu$-TECs using an anodically bonded pyrex wafer.

\section{INTRODUCTION}

Thermionic energy converters (TECs) are unique heat engines that convert heat directly to electricity at very high temperatures, typically $>1000^{\circ} \mathrm{C}$. This energy conversion process is based on thermionic emission - the evaporation of electrons from conductors at high temperatures - and was first proposed as early as 1915. In the simplest case, the converter consists of two electrodes in the parallel-capacitor geometry and uses the thermionically emitted current to drive a useful load (Fig. 1). In the 1950s-1960s, the first practical TECs with conversion efficiencies of $10-15 \%$ were created and, in the subsequent two decades, both NASA and the Soviet space programs developed TECs as power sources for space missions [2,3].

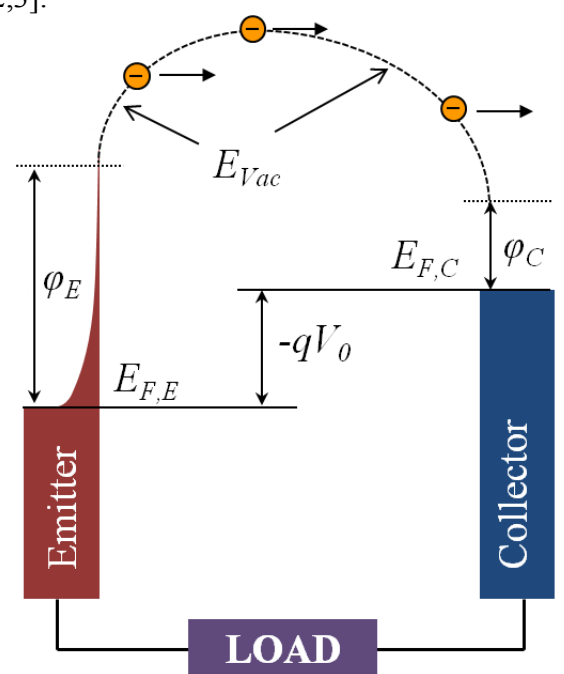

Figure 1: The energy diagram of the thermionic energy conversion process. $E_{F, E}$ and $E_{F, C}$ are the Fermi levels of the emitter and the collector, respectively. $E_{V a c}$ is the position-dependent vacuum level. $\varphi_{E}$ and $\varphi_{C}$ are the work functions of the emitter and the collector. $V_{0}$ is the voltage difference between two electrodes and $-q$ is the electron charge.
In the last two decades, major progress in microfabrication techniques prompted some people to reassess the possibility of making vacuum TECs with micron-scale gaps [3-5]. Microfabricated vacuum TECs are conceptually attractive because of their simpler physics. In addition, their efficiency limits can be very large fractions of the Carnot efficiency (Fig. 2) - exceeding solid state technologies (such as thermoelectric) and competitive with even conventional mechanical heat engines, such as steam turbines or Stirling engines.

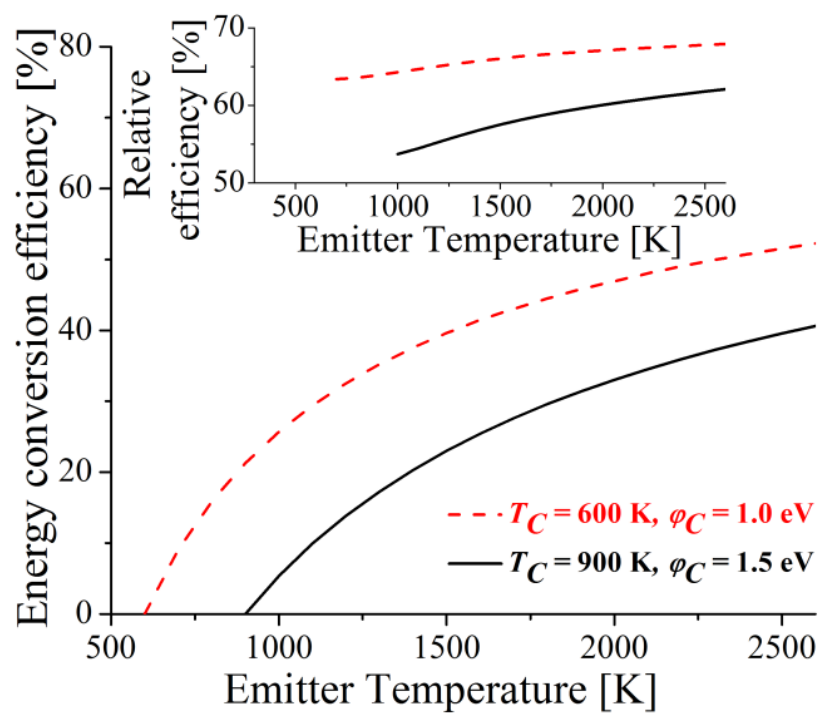

Figure 2: The maximum energy conversion efficiency of $\mu T E C$ versus the emitter temperature for collector work functions of 1.0 and $1.5 \mathrm{eV}$. We assumed the emitter work function proportional to the emitter temperature: $\varphi_{E}=T_{E}[K] / 750 \mathrm{eV}$, and the collector temperature of $T_{C}=\varphi_{C}[\mathrm{eV}] \times 600 \mathrm{~K}$. The inset shows the exergy efficiency, i.e., the energy conversion efficiency divided by the Carnot efficiency, $1-T_{C} / T_{E}$, for the corresponding emitter and collector temperatures. Based on calculations in Ref. [6].

We have recently developed a new approach to microfabricating TECs [1] that uses the substrate wafer as the collector and a plate suspended using flexible crablegs as an emitter (see inset of Fig. 3). Here we report various experiments with a new $\mu$-TECs design that uses stiff suspension legs, which increase the out-of-plane rigidity and help $\mu$-TECs to keep the gap in the optimal range during the operation.

\section{FABRICATION}

\section{Images}

Fig. 3 shows a photograph of an encapsulated die, which contains a parallel-connected $3 \times 3 \mu$-TEC array. The inset of Fig. 1 shows an SEM of an individual U-shape array element. Since all TECs were connected in parallel, only two contacts were needed to measure the I-V characteristic. The suspension width was $30 \mu \mathrm{m}$, 
the sidewall height was $30 \mu \mathrm{m}$, the emitter plate was $500 \mu \mathrm{m} \times 500 \mu \mathrm{m}$, and the poly-SiC film was $2-\mu \mathrm{m}$ thick. Etch holes were used in the emitter plate to facilitate the release. The gap between the poly-SiC emitter and the silicon substrate collector was approximately $10 \mu \mathrm{m}$.

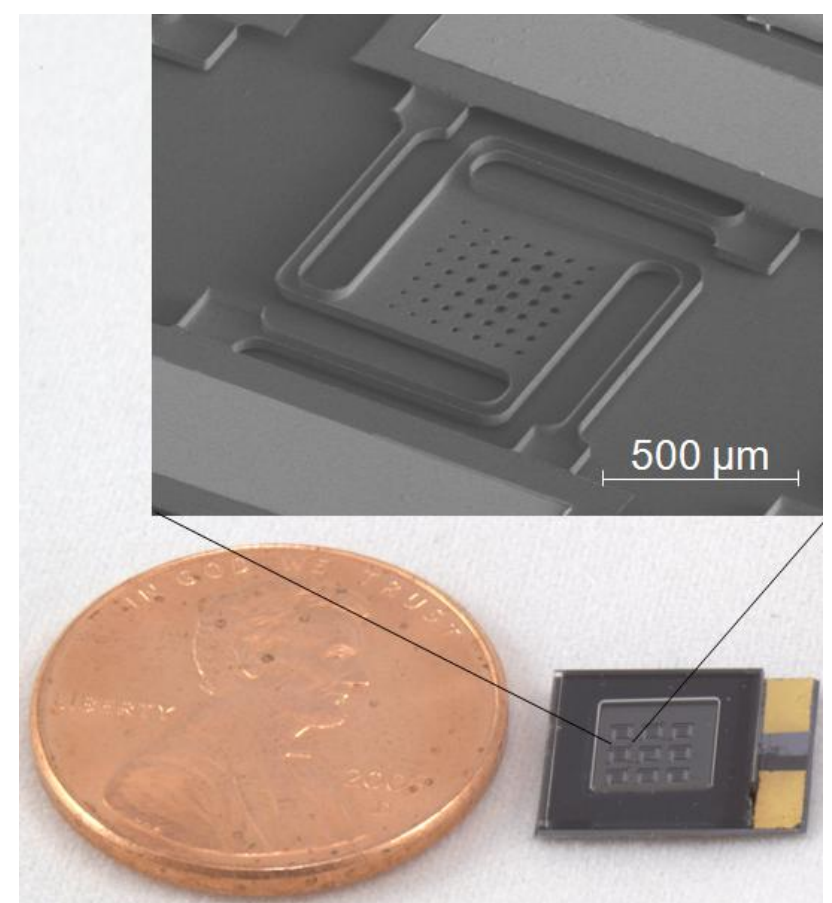

Figure 3: Vacuum-encapsulated die with a $3 \times 3$ array of TECs. A penny is shown to indicate scale. The inset shows a scanning electron micrograph (45 angle $\left.{ }^{\circ}\right)$ of one $U$-shaped TEC element.

Fabrication Process Flow

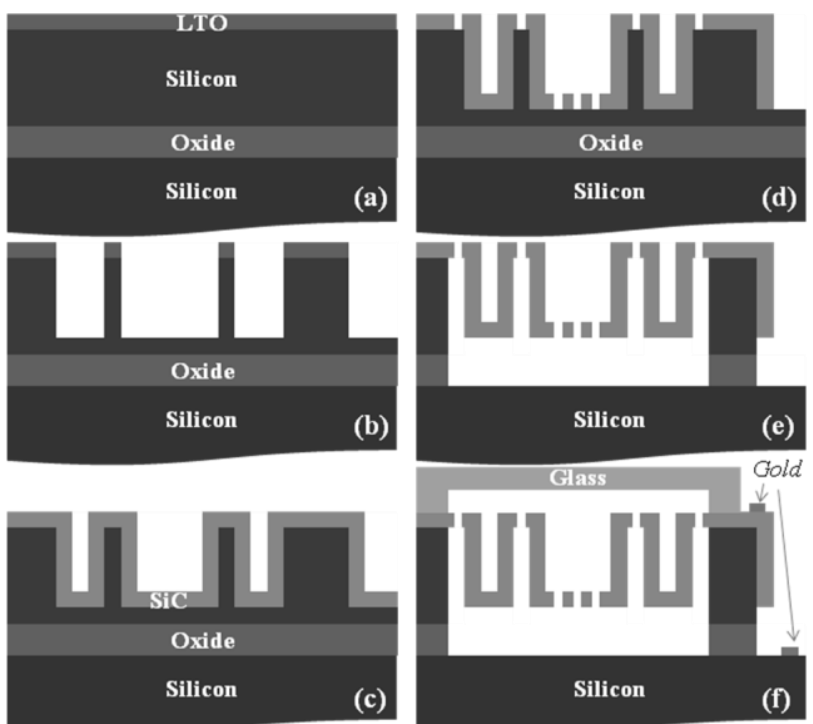

Figure 4: Outline of the fabrication process. (a) Initial 4-inch SOI

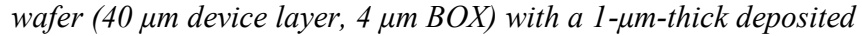
LTO hard mask. (b) After patterning LTO and DRIE etching to define 30-35 $\mu$ m deep silicon trenches. (c) After removing LTO layer and depositing 2- $\mu$ m-thick n-type polycrystalline 3C-SiC. (d) After patterning SiC layer using SPR 220-7 thick resist and anisotropic RIE. (e) After release using $\mathrm{XeF}_{2}$ and vapor HF etching. (f) After encapsulation and deposition of gold contact pads.
Fig. 4 outlines the fabrication process. The 4-inch SOI wafer was patterned to define $30-35 \mu \mathrm{m}$ deep silicon trenches before depositing polycrystalline $3 \mathrm{C}-\mathrm{SiC}$. The buried oxide layer of the SOI wafer was used to define the gap between the suspended emitter and the silicon substrate collector. After the release, the $\mu$-TECs were encapsulated using a 500- $\mu \mathrm{m}$-thick pyrex 7740 wafer with $250-\mu \mathrm{m}$-deep wet-etched cavities. The glass wafer was anodically bonded to encapsulate each $3 \times 3$ array, after which the wafer stack was diced and gold contact pads were deposited.

\section{EXPERIMENT \\ Optical Heating}

A single unencapsulated poly-SiC emitter was optically heated in a vacuum chamber by focusing the output of a blue laser diode (440-455 nm) onto a spot of $\sim 750 \mu \mathrm{m}$ diameter. The emitter was grounded at all times and the collector was biased to measure the current-voltage characteristic. Due to the increased out-of-plane rigidity of the U-shaped structures, we did not observe emitter-substrate shorting during optical heating, allowing us to make the emitter temperature higher than in our previous study [1].

Our $\mu$-TECs converted the estimated $270 \mathrm{~mW}$ of optical power incident on the emitter to $9 \mathrm{nW}$ of electrical power (Fig. 5), which is almost two-orders of magnitude higher than the power conversion efficiency in Ref. [1]. This still very low conversion efficiency $\left(<10^{-7}\right)$ is due primarily to the high work function of bare $\mathrm{SiC}$, which severely limits the thermionic current from the emitter [2]. Judging from the I-V characteristic, the work function of the silicon collector was even higher than that of the $\mathrm{SiC}$ emitter, further reducing the conversion efficiency.

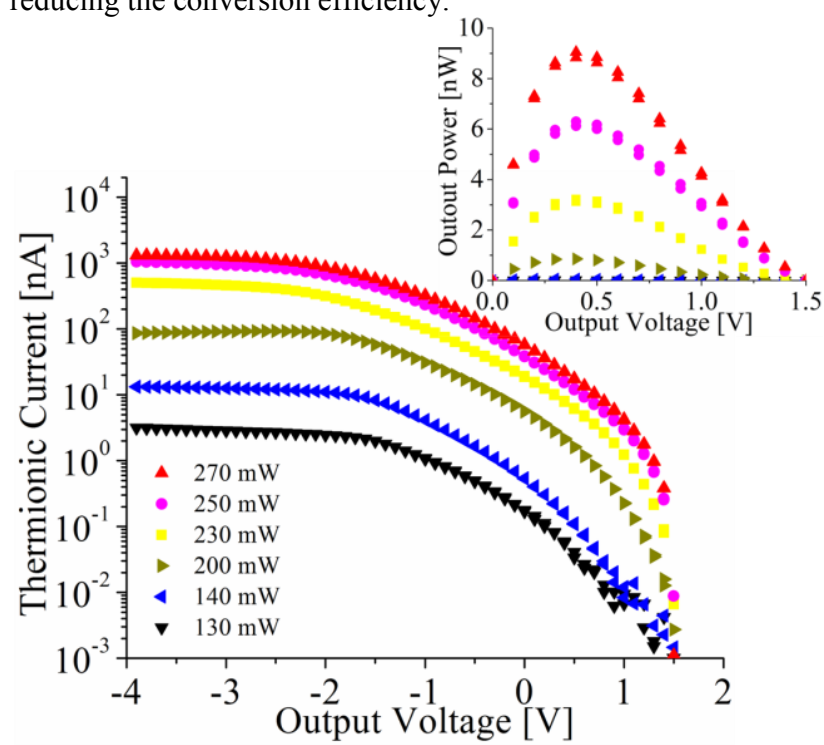

Figure 5: The thermionic current versus the output voltage for different incident power to the SiC emitter. The thermionic current saturated when the output voltage was below $-1.5 \mathrm{~V}$. The inset shows that the maximum output power of this TEC is for an uncesiated $\mathrm{SiC}$ output voltage of $0.3 \mathrm{~V}$.

\section{Work function lowering by barium coatings}

Adsorbed cesium or barium can improve thermionic current from the emitter by reducing the work function of poly-SiC $[1,2,4,5]$. However, cesium reacts chemically with both silicon and silicon oxide, especially at elevated temperatures, which makes barium-based coatings easier to use in $\mu$-TECs made of standard silicon substrates. To investigate the effect of barium coatings on thermionic current, we measured the thermionic current both with 
and without barium coating on the $\mathrm{SiC}$ emitter in a small, dedicated vacuum chamber. After the pressure in the vacuum chamber reached $\sim 10^{-6}$ Torr, a commercial barium dispenser (Alvatec Production and Sales GesmbH) was heated resistively to evaporate the barium on the $\mathrm{SiC}$ emitter surface for a few minutes. Then, we resistively heated the $\mathrm{SiC}$ emitter while applying $40-80 \mathrm{~V}$ bias to the external metal collector, which was $\sim 1 \mathrm{~cm}$ away from the emitter, to collect most of the thermionic current from the emitter. The resulting reduction in the work function of the emitter enhanced thermionic currents from the heated $\mathrm{SiC}$ emitter by 5-6 orders of magnitude (Fig. 6). At high temperatures $(>1000 \mathrm{~K})$, the optical emissivity of polycrystalline $3 \mathrm{C}-\mathrm{SiC}$ is sufficiently large to enable temperature measurements using an optical pyrometer (PYRO MicroTherm, 550-nm operating wavelength). For emitter temperatures ranging from $900-1400 \mathrm{~K}$, we were able to fit our data to the Richardson-Dushman equation: $J=A_{\mathrm{E}} T_{\mathrm{E}}^{2} \exp \left(-\Phi_{\mathrm{E}} / k T\right)$, where $\Phi_{\mathrm{E}}$ is the emitter work function, $T_{\mathrm{E}}$ the emitter temperature, and $A_{\mathrm{E}}$ the materials-specific Richardson-Dushman constant. The work function of the barium-coated $\mathrm{SiC}$ obtained from the fit was approximately $2.14 \mathrm{eV}$ (the inset of Fig. 6).

We repeated the same experiment after we vented the vacuum chamber for a few hours and pumped the chamber back to $\sim 10^{-6}$ Torr to oxidize the barium coating on the $\mathrm{SiC}$ emitter. We observed approximately the same enhancement of thermionic current with the barium oxide coating (Fig. 6). However, the enhancement decreased irreversibly for both types of coatings if the emitter temperature exceeded the critical temperature of approximately $1600 \mathrm{~K}$. We believe that barium and barium oxide decomposed or desorbed from the $\mathrm{SiC}$ emitter above this temperature.

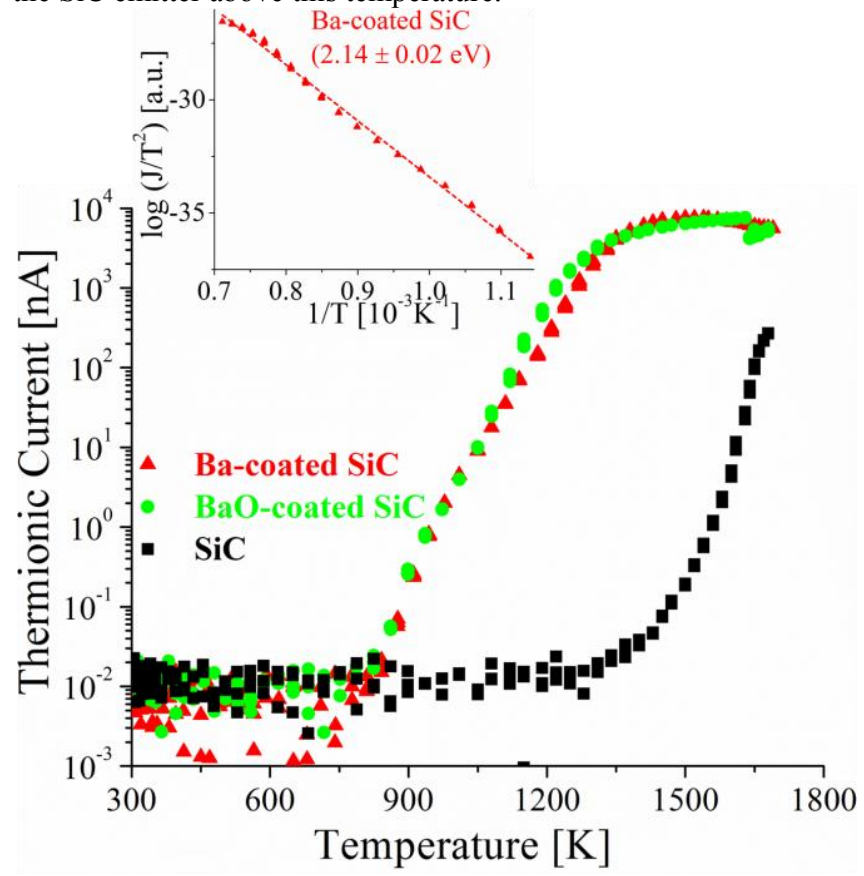

Figure 6: The thermionic current versus the temperature of the $\mathrm{SiC}$ emitter for both before barium coating, after barium coating, and after oxidizing barium which was coated on the SiC emitter. The inset shows the Richardson plot for the barium-coated SiC emitter.

To characterize the activation behavior of both barium coating and barium-oxide coatings, we measured the hysteresis of the thermionic current vs. temperature during upward and downward temperature sweeps (Fig. 6). Interestingly, a pure (unoxidized) barium coating exhibited much less hysteresis than a barium-oxide one, suggesting that pure barium coating does not need activation and can remain thermally stable in very high temperatures. It has been previously reported for barium-oxide coated emitters [7] that the barium atoms are released by the chemical reaction between the barium oxide and the substrate, and that these atoms migrate to the surface of the emitter coating by diffusion through the layer of barium oxide. The migration of barium atoms is facilitated by the high operating temperature of the emitter, and also by the porous, microcrystalline structure of the emitter coating $[7,8]$. This model may explain the difference in the hysteresis of pure barium and barium oxide coatings.

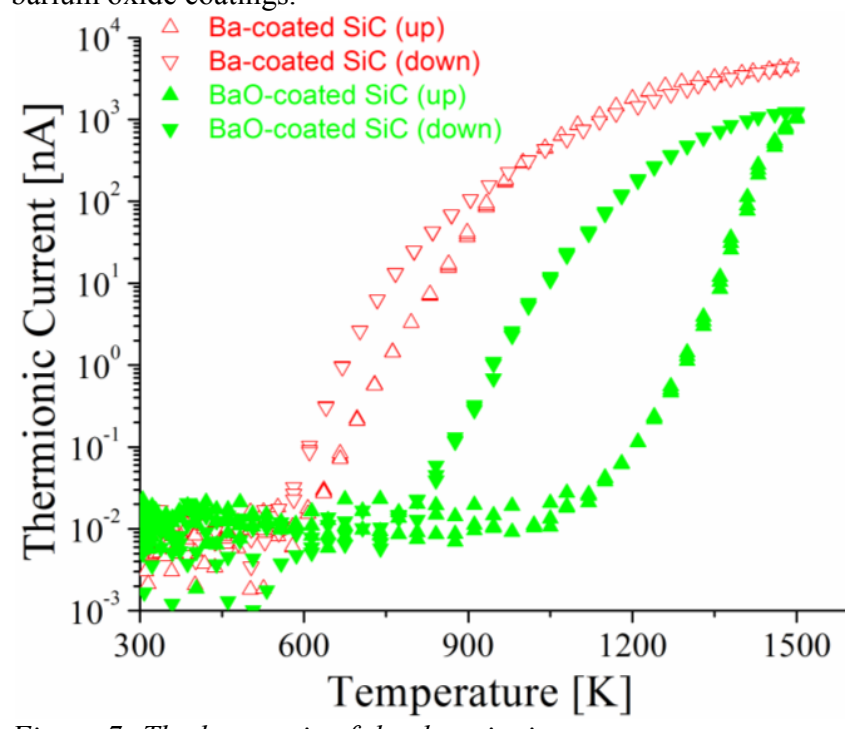

Figure 7: The hysteresis of the thermionic current vs. temperature for the barium and barium-oxide coated SiC emitter.

\section{$\boldsymbol{\mu}$-TEC Encapsulation}

Incorporating cesium into wafer-bonded vacuum packages has been previously demonstrated in the context of chip-scale atomic clocks [9]. $\mu$-TECs would require similar vacuum encapsulation with a work-function-lowering vapor. As an initial step toward achieving this goal, we have encapsulated small $\mu$-TEC arrays (Fig. 3).

We used the quality factor of the out-of-plane mechanical resonance of the suspended emitter to determine the residual gas pressure in the cavity. Toward that goal, we have measured the resonant response of the emitters in a vacuum chamber, which could be filled with a controlled pressure of nitrogen. The out-of-plane motion was actuated capacitively by applying $+3 \mathrm{~V}$ DC voltage to the collector and -15 to $5 \mathrm{dBm}$ of RF power to the emitter. A Polytec laser Doppler vibrometer was used to measure the out-of-plane motion of the suspended emitter inside the vacuum chamber at various pressures of nitrogen. Fig. 8 shows one example measured at a pressure of 0.2 Torr.

The quality factors of the detected mechanical resonance modes of the suspended emitter exhibit a strong dependence on the pressure of the ambient gas. Fig. 9 shows the quality factor of the fundamental resonance mode at $9.25 \mathrm{kHz}$ as a function of the nitrogen pressure. Using this curve, we will be able to calibrate the pressure of residual gas in vacuum encapsulated cavities.

While the bonding was successful, our initial experiments suggest that the packaging was not fully hermetic, and the cavity was eventually filled with ambient air to almost atmospheric pressure. (The quality factor of the encapsulated device was approximately 5.0). We plan to use an improved wafer bonding protocol to achieve hermetic vacuum encapsulation in the near future. 


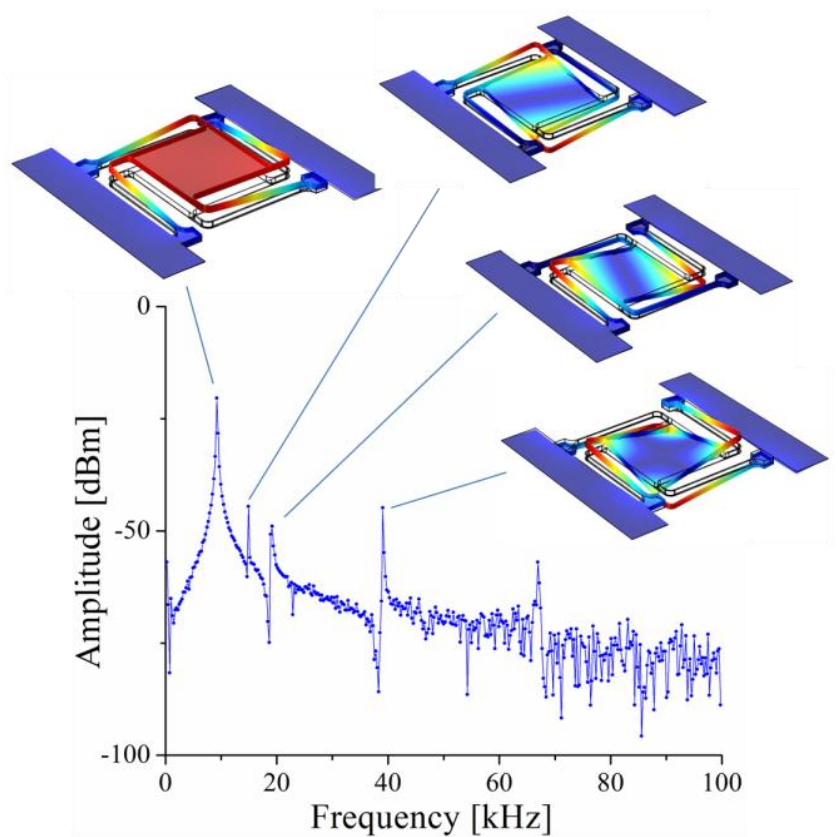

Figure 8: Capacitively actuated out-of-plane resonance modes of the suspended emitter for the nitrogen pressure of 0.2 Torr. We obtained very good agreement between experiment and finite-element simulations: the experimental curve showed resonance frequencies at 9.25, 16.71, 20.08, and $39.25 \mathrm{kHz}$, whereas the COMSOL simulation predicted resonances at 9.55, $14.85,18.99$, and $37.07 \mathrm{kHz}$.

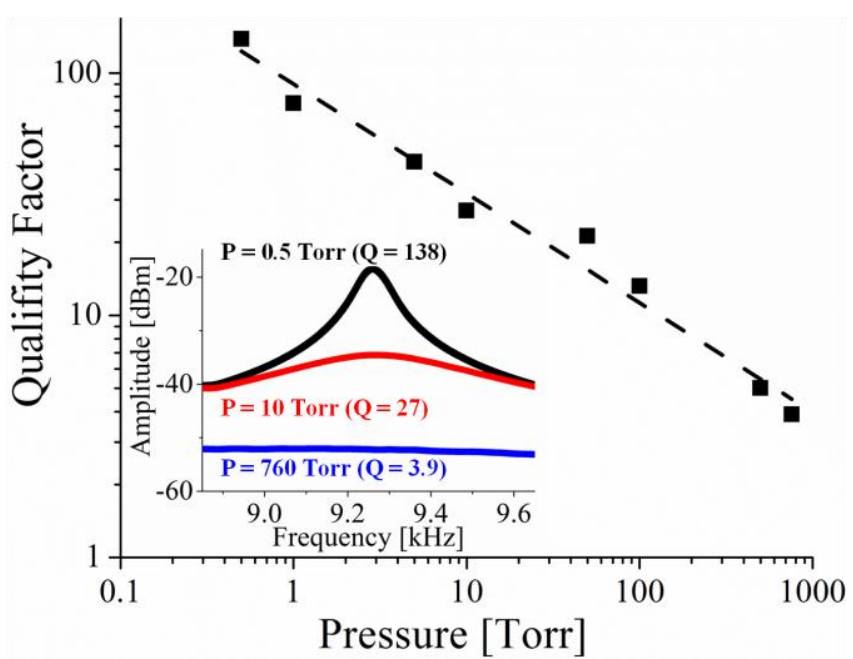

Figure 9: The quality factor versus the nitrogen pressure for the fundamental resonance mode at $9.25 \mathrm{kHz}$. The straight light is a guide to the eye. The inset shows representative resonance curves of the suspended SiC emitter for three different pressures.

\section{CONCLUSION}

We demonstrated a prototype of a mechanically and thermally robust encapsulated $\mu$-TEC. We used conformal sidewall deposition of poly-SiC to form stiff suspension legs with U-shaped cross sections, which increase out-of-plane rigidity and prevent contact with the substrate during the heating of the suspended emitter. We also showed that barium and barium-oxide coatings lowered the work function of the $\mathrm{SiC}$ emitter and enhanced thermionic currents from the heated $\mathrm{SiC}$ emitter by 5-6 orders of magnitude. We are currently working to demonstrate the high conversion efficiency of the $\mu$-TEC with barium or barium oxide coating of both electrodes.

We have thus achieved a number of milestones on the path towards realizing an efficient microfabricated thermionic energy converter. In the future, wafer-scale $\mu$-TECs can play an important role as topping cycles, adding to the efficiency of existing thermal energy converters such as thermoelectric or mechanical heat engines. Concentrated solar thermal power generation is a particularly attractive application for $\mu$-TECs due to the extremely high intrinsic temperature of solar radiation $(\sim 5900 \mathrm{~K})[10]$.

\section{ACKNOWLEDGEMENTS}

This work is supported by the Defense Advanced Research Projects Agency (DARPA) N/MEMS S\&T Fundamentals program under grant no. N66001-10-1-4004 issued by the Space and Naval Warfare Systems Center Pacific (SPAWAR), Robert Bosch North America, and OCI co. through the Center on Interfacial Engineering for MEMS (CIEMS), as well as BASF through the Stanford Energy and Environment Affiliates Program.

\section{REFERENCES}

[1] "Microfabricated Silicon Carbide Thermionic Energy converter for Solar Electricity Generation," J.-H. Lee, I. Bargatin et al., in Digest Tech. Papers MEMS Conference (2012)

[2] "Thermionic Energy Conversion Volume 1: Processes and Devices," G. N. Hatsopoulos and E. P. Gyftopoulos (Cambridge, MIT Press, 1973)

[3] "Thermionics: Quo Vadis", National Research Council, (National Academy Press, 2001)

[4] "Combustion-based Micro Power Generation: Thermoelectric and Thermionic Approaches," C. Zhang, PhD Dissertation, University of Michigan (2003)

[5] "Low Work Function Material Development for the Microminiature Thermionic Converter," D. King, K. Zavadil, D. Jennison, C. Battaile, A. Marshall, Sandia Report SAND2004-0555 (2004)

[6] "Optimal Emitter-Collector Gap for Thermionic Energy Converters," J.-H. Lee, I. Bargatin et al., Appl. Phys. Lett. (submitted, 2012)

[7] "Model of work function of tungsten cathodes with barium oxide coating," K. C. Mishra et al., J. Appl. Phys., 95, 3069 (2004)

[8] "Atomic and electronic structures of barium oxide on $\mathrm{Si}(001)$ studied by metastable impact electron spectroscopy (MIES) and coaxial impact collision ion scattering spectroscopy (CAICISS)," T. Ikeuchi et al., Appl. Surf. Science, 191, pp. 261-265 (2002)

[9] "Wafer-level filling of microfabricated atomic vapor cells based on thin-film deposition and photolysis of cesium azide," L. Liew et al., Appl. Phys. Lett. 90, 114106 (2007)

[10] "Photon enhanced thermionic emission for solar concentrator systems," J.W. Schwede, I. Bargatin et al., Nature Mater., 10, pp. 762-767 (2010) 


\title{
HYBRID ENERGY HARVESTING SYSTEM BASED ON MICROFLUIDICS WITH INTERFACE CIRCUITRY
}

\author{
Arhatha Bramhanand ${ }^{l}$, Md. Mahbubur Rahman ${ }^{1}$, Young-Chul Bae ${ }^{2}$, and Hanseup Kim ${ }^{l}$ \\ ${ }^{1}$ Electrical and Computer Engineering, University of Utah, Salt Lake City, Utah, USA \\ ${ }^{2}$ Electrical, Electronic Communication and Computer Engineering, Chonnam National University, South Korea
}

\begin{abstract}
We present a fluidic-based energy harvesting device that incorporates microfluidic structures, which can hydraulically disperse and accommodate large input force into a micro structure; and power handling circuitry, which converts varying energy to support a commercial wireless link, into a single layer of a shoe insole. The microfluidic structures utilize hydraulic amplification of deflection and de-amplification of force to enhance power generation through electromagnetic induction while preventing potential mechanical fracture of the micro structures. The power regulation circuitry converts AC power into DC at each fluidic channel and then adds them into high voltage output. The fabricated energy harvesting system generated a raw power ${ }_{\text {rms }}$ output of $7.28 \mathrm{~mW}$ under $647.5 \mathrm{kPa}$ pressure and power density of $1 \mathrm{~mW} / \mathrm{cm}^{3}$ from a device volume of $7.2 \mathrm{~cm}^{3}$. The integrated power regulating circuitry demonstrated power conversion efficiency of $62.8 \%$.
\end{abstract}

\section{INTRODUCTION}

Recently wireless systems are finding immensely increasing applications in parallel to the technological advances in low power circuits and digital communication technology $[1,2]$. In order to be truly independent and portable, such wireless systems require highcapacity (long-life) and reliable power sources that can also be small in volume and light in weight. Since the minimum power, required for some sensor applications, becomes sufficiently low within few $\mathrm{mW}$ ranges, an alternative approach of micro-scale energy harvesting has been actively pursued to replace the electrochemical batteries that have been traditionally utilized but require periodic replacement and cause environmental hazards due to harmful chemical contents, such as lead, mercury and cadmium. Micro-scale energy harvesting technology harvests electrical power from ambient environments (vibration, temperature, fluid flows) and thus is permanent and environmental friendly [3-5].

Despite rigorous progress, micro-scale energy harvesting systems have typically demonstrated limited power generation, ranging from a few $\mathrm{nW}$ to $\mathrm{mW}$ with an average power density of $100 \mathrm{~W} / \mathrm{cm}^{3}$ [6,7]. Such low power outputs could stem from conventional uses of relatively weak and mild environmental energy sources due to the limited range of inputs (force and deflection) that a micro device can accommodate under its small and fragile structure. Recently some notable demonstration by producing large output power under heavy body weights $(>60 \mathrm{~kg})$ through piezoelectric materials have been performed particularly for shoes $[8,9]$ in a relatively large scale with some limitations in practicality.

In order to overcome the above issues we utilize a new class of microfluidic-based energy harvesting device that (1) can accommodate relatively high input force and large deflection into micro-scale structures through hydraulic de-amplification and (2) can provide thin and flexible structures appropriate for a shoe insole. A simple proof-of-concept on the operation principle of a microfluidic energy harvesting was previously reported $[10,11]$. Here, we report an integrated system that incorporates both (1) the power handling circuitry and the multiple-channel device.
Particularly, we investigated two different multiple-channel configurations: (2) a bundle structure that has independent and "fluidically non-connected" energy harvesting channels and (3) an all-connected channel device. These multi-channel structures allow electromagnetic amplification proportional to that of the number of channels for enhanced power generation.

\section{MICROFLUIDIC ENERGY HARVESTING SYSTEM Design}

The developed energy harvesting system comprises two major sections: two chambers with deformable elastic diaphragm on the top and fluidic channels interconnecting them (Fig. 1). The chambers and fluidic channels together form a closed system and are filled with a working fluidic medium (DI water) without air bubbles to ensure efficient hydraulic operation. Present inside the channels is a train of $\mathrm{N}$ equally sized cylindrical permanent magnets and N-1 non-magnetic spacers which are alternately placed and free to move within the channel. The movement of the magnet-spacer train is confined by ring shaped stoppers which are placed at either ends of the channel. Conductive coils are wound on the outer surface of each channel. These coils are placed at regular intervals and the spacing between coils is equal to the magnet length, to account for phase-matching.

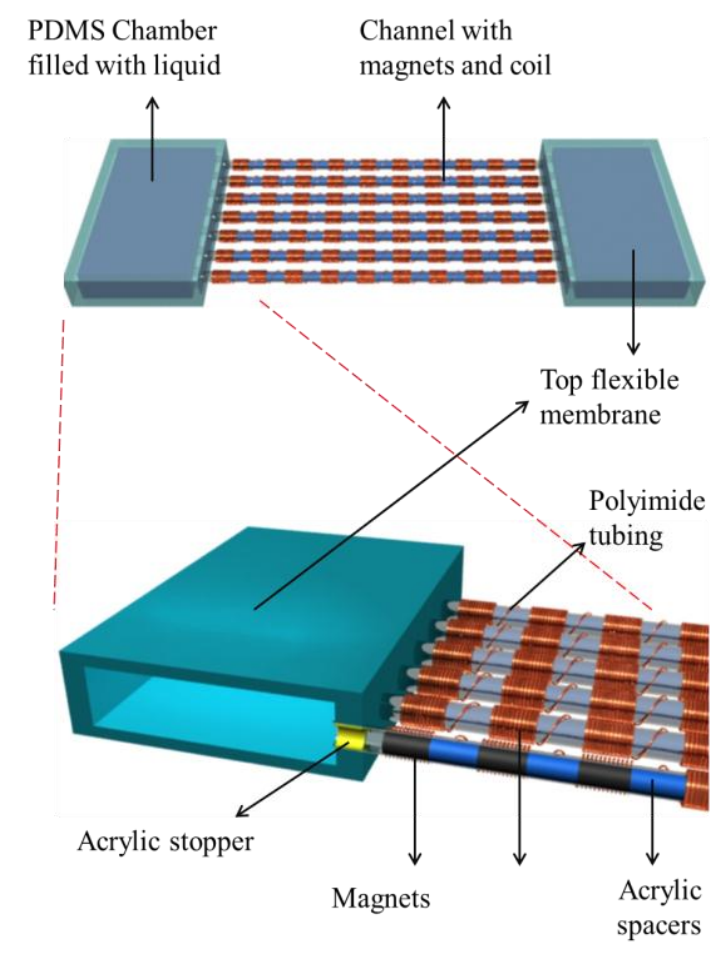

Figure 1: Illustration of the energy harvesting, fluidic based system (Top). A partial cross-section of the PDMS chamber and polyimide channel with stopper, magnets and spacers (Bottom). 


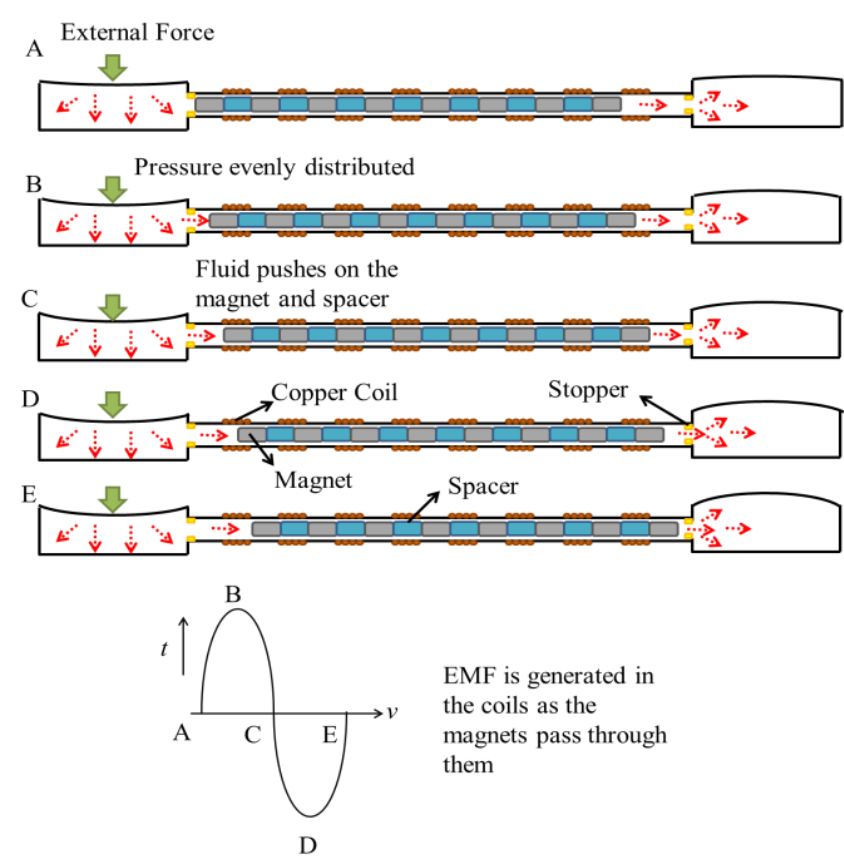

Figure 2: Operation principle of the energy harvesting system.

\section{Operation}

The device, while incorporated into the shoe insole, operates based on the applied pressure at the heel and toes where maximum pressure is available. The applied pressure is accommodated by each diaphragm at both chambers alternatively. The chambers accommodate the high deflection force and transform it into hydraulic pressure that is evenly distributed in accordance with Pascal's law. Since the hydraulic pressure is equal at all points within the chamber and the cross sectional area of the channel is much smaller than the area over which the force was exerted a multiplication of force can be realized. The hydraulic pressure pushes the magnets in the channels. As the magnets pass through the coils emf is induced in the coils and energy is generated (Fig.2). By alternately applying pressure on the two diaphragms the magnets can be continuously shuttled thru and fro in the channels, giving a continuous power output.

\section{Fabrication}

An improved fabrication process is adopted compared to previous reports [10,11] First, polyimide tubes of inner radius of $0.86 \mathrm{~mm}$ are used to form the fluidic micro channels. Inside is a train of $\mathrm{NdFeB}$ neodymium rare earth, grade $\mathrm{N} 52$, axially magnetized permanent magnets $(0.8 \mathrm{~mm}$ radius and $3.13 \mathrm{~mm}$ in length) located, while laser-cut acrylic spacers are assembled between the magnets. Second, insulated copper coils of $0.15 \mathrm{~mm}$ wire with 22turns are wound around the channel. Acrylic laser cut ring like stoppers are placed at either end of the channel. Next the deformable chambers are made of PDMS, Sylgard 184 base and curing agent are mixed in a 10:1 ratio, then poured into molds and cured at $60^{\circ} \mathrm{C}$. Then the channels are inserted into the side walls of the chamber and the two PDMS structures are bonded together using PDMS-PDMS bonding technology. Different PDMS-PDMS bonding methods were tested and the uncured PDMS bonding method proved to be the strongest which could sustain a pressure of up-to $648 \mathrm{kPa}$ for our device. After the bonding a syringe is inserted along the interface between the channel and chamber wall on one side to remove the air and completely fill the system with D.I. water. This opening is later sealed with silicone glue.

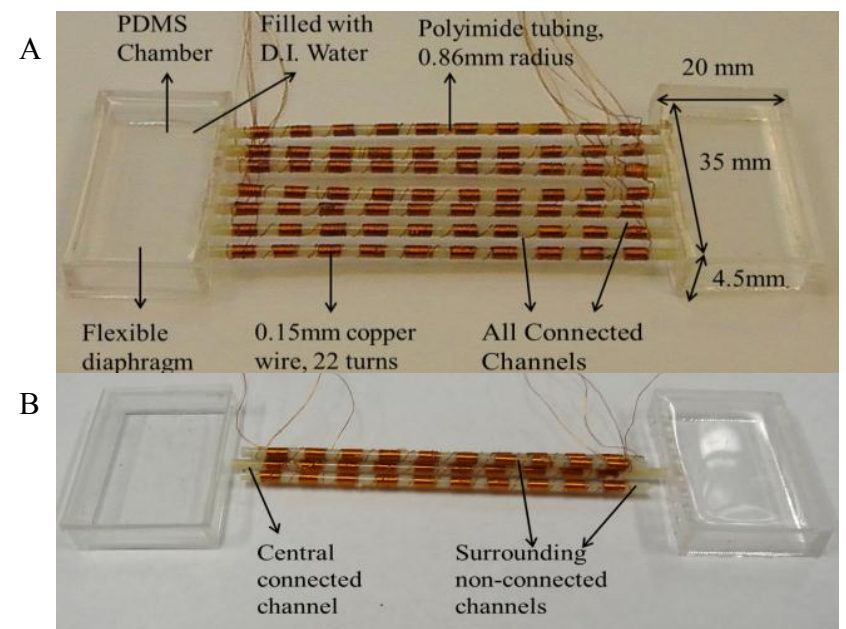

Figure 3: A: Fabricated all-connected multi-channel energy harvesting device. B: Fabricated bundle-channel energy harvesting device.

\section{Multichannel Structures}

The device performance can be improved by having multiple channels between the two chambers. This can be simply analyzed by connecting multiple channels between the chambers such that when an external force is applied the working fluid flows through all the connected channels (Fig 3-A). Thus the displaced working liquid pushes the magnets in all the channels leading to an increase in the total output. Since the increase in the device volume is not large the generated power density increases.

Another approach is the bundle system multi-channel which electromagnetically-amplifies the output for the same amount of work done in pushing the magnets along the channel. The rare earth $\mathrm{NdFeB}$ magnets have high magnetic field flux and as a result when channels are placed close to each other they show strong magnetic field interaction between the magnets in the adjacent channels. This can be used to an advantage by having one main channel connecting the two chambers and independent fludically not-connected channels which are placed around the main channel (Fig 3-B). Thus when an external force is applied the magnets in the main channel move as explained earlier. At the same time due to magnetic interactions between the main channel and surrounding channels, the magnets in the surrounding channel are pulled along. Thus by having additional independent channels higher power can be generated for the same applied pressure without reducing flow velocity or displacement in each channel.

\section{EXPERIMENTAL VALIDATION}

\section{Testing Methods}

The output response of the two multi-channel energy harvesting systems was monitored with respect to the applied external pressure under a single (impulse) actuation condition. The tested systems include an all-connected multi-channel device and a bundle-channel device. The all-connected multi-channel device has seven channels with ten magnet coil pairs in each channel, while the bundle device has one central channel and four surrounding independent channels where in each channel had ten magnet coil pair. Force was applied on one of the diaphragm and monitored by a CSD200 Chatillon dynamometer that has a detection resolution of $1 \mathrm{~N}$ in force. All the coils from each channel were connected in series so that voltage amplification due to phase matching could be obtained, and the resultant sinusoidal output was observed across an optimum load on a Tektronix TDS 2024B oscilloscope. 


\section{Results and Discussion}

Figure 4 shows the produced power output under various input pressure ranges for the fabricated all-connected multichannel device. The output power increases linearly with the increase in the external pressure applied. The device provided a maximum raw power $_{\text {rms }}$ of $7.28 \mathrm{~mW}$ for the input pressure of $647.5 \mathrm{kPa}$. This corresponded to the power density of $1 \mathrm{~mW} / \mathrm{cm}^{3}$. The maximum pressure that could be applied was determined by the maximum PDMS-PDMS bonding strength. Different bonding methods such as plasma bonding, epoxy adhesive, silicone glue, UV curable glue, partial PDMS curing, and uncured PDMS bonding were tested (Table 1) to monitor the maximum burst pressure and select the strongest bonding methodology. It is observed that the uncured PDMS-PDMS bonding method has been most successful which could sustain pressure up to $648 \mathrm{kPa}$.

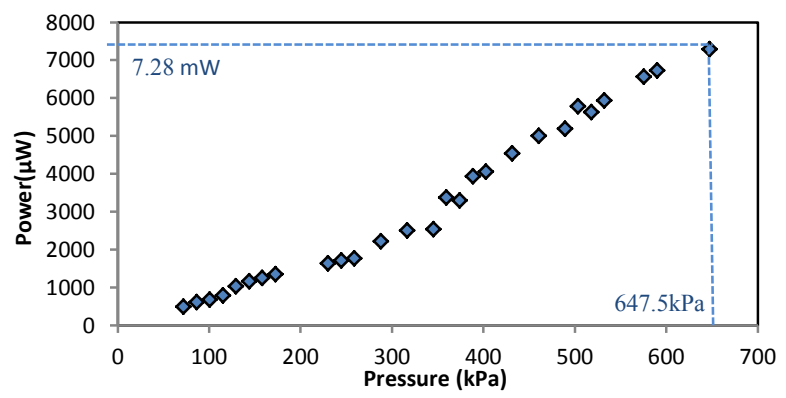

Figure 4: Power(rms) versus pressure for the all-connected multichannel device.
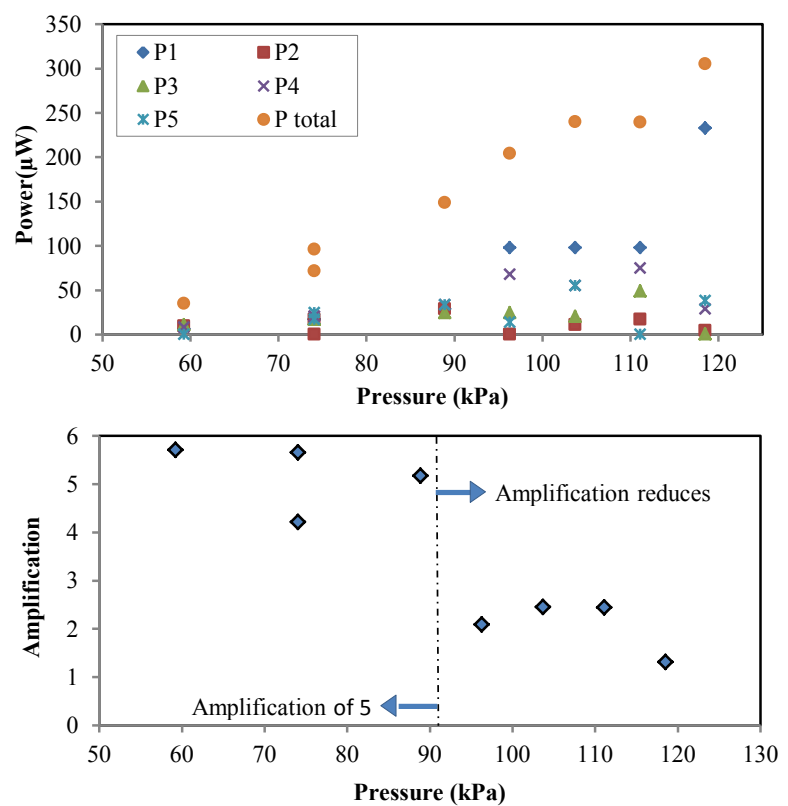

Figure 5: Pressure response obtained for bundle structure device. P1- Power generated in main channel. P2, P3, P4, P5 - Power generated in surrounding channels(top). Resulted in amplification factor proportional to number of channels up-to $90 \mathrm{kPa}$ (bottom).

Figure 5 shows the resultant power generation in a bundledchannel device with 5 channels. In the bundle system, when the magnets in the central channel move, those in the surrounding four independent channels were also induced to move through electromagnetic field. At a lower pressure range $(<90 \mathrm{kPa})$ the power output was amplified proportional to the total number of channels. However, at a higher pressure range $(>90 \mathrm{kPa})$, the amplification factor was reduced (Fig. 5). As pressure increases, the velocity of fluid flow in each channel increases, failing to move magnets in the surrounding channels. In order to enhance the advantages of this approach, optimization of the distance between the channels had to be performed such that they were not too far off which reduces the pull efficiency at higher pressure. The tested device produced a total power output of $305 \mathrm{~W}$ for an input pressure of $118 \mathrm{kPa}$; but at this pressure range an amplification of only 1.5 was observed (Fig. 5). At a lower pressure range $(<90 \mathrm{kPa})$, full amplification of 5 to 5.7 was observed. Thus, it was observed that the bundled-channel device works well only for low pressure applications.

Table 1: Maximum burst pressure for different bonding methods.

\begin{tabular}{lc}
\hline Bonding Method & Max Burst Pressure (kPa) \\
\hline Plasma bonding & unsuccessful bonding \\
Epoxy adhesive & 10 \\
Silicone glue & 254 \\
UV curable glue & 554 \\
Partial curing & 609 \\
Uncured PDMS & $\mathbf{6 4 8}$ \\
\hline
\end{tabular}

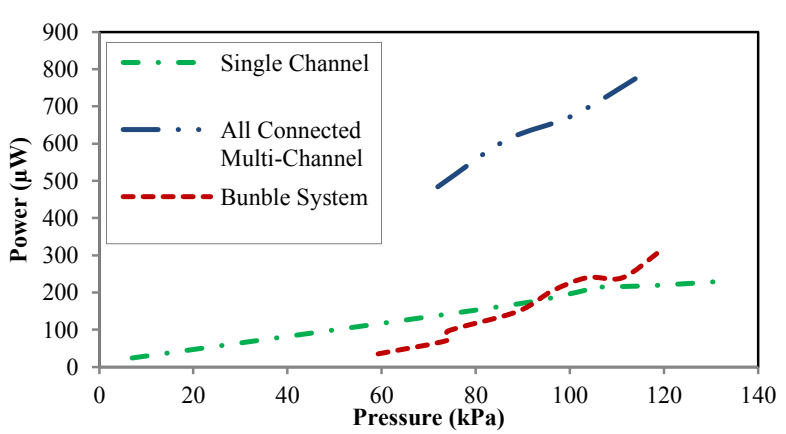

Figure 6: Comparison of the pressure response of the single channel[10], all connected multi-channel and bundle system device.
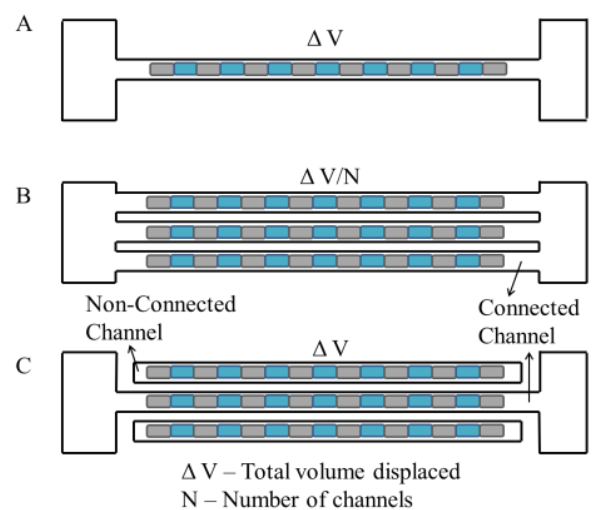

Figure 7: Distribution of displaced liquid in (A)single channel[10], (B)all connected multi-channel and (C)bundle system device.

Figure 6 compares the obtained power outputs for the previously reported single channel device [10] and the tested multi-channel devices under various input pressure ranges. In both single channel and the bundled-channel devices, the total volume of displaced liquid flows only through the central channel. However, in the bundle-channel, such flows and the resultant magnetic movement also induce the movement of magnet in the 
surrounding channel. On the other hand, the total volume of the displaced liquid is distributed through the multiple channels in the all connected multi-channel device (Fig. 7). Although the bundle system theoretically predicts higher power amplification ratio, its output level was measured lower than even that of the single channel device. This is because the magnets move slower than in the single channel configuration. It is also observed that the all connected multichannel device showed better result than the bundle system device because of the enhanced friction in the bundled-channels where dry (air) medium provides greater frictional losses.

\section{Circuitry}

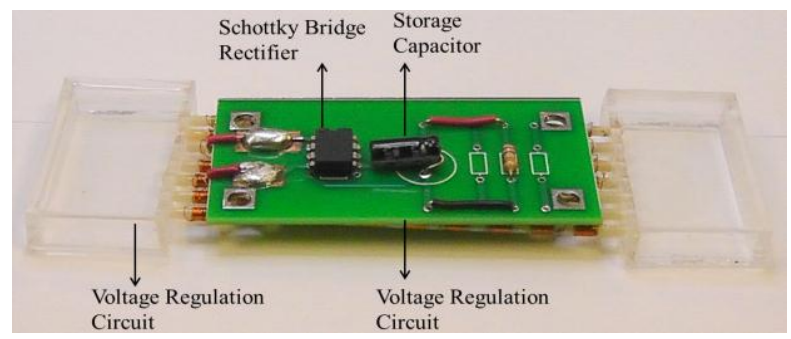

Figure 8: A: View of the 7-channel device with the voltage regulation circuit set for a shoe-embedded application.

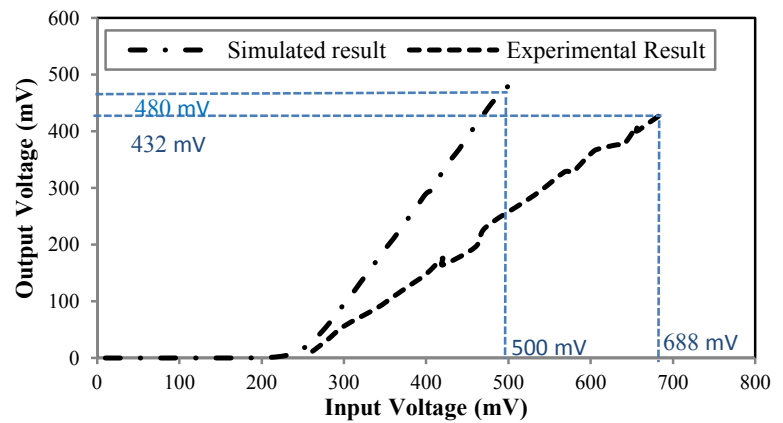

Figure 9: Simulated and experimental results for the performance of the power regulation circuit.

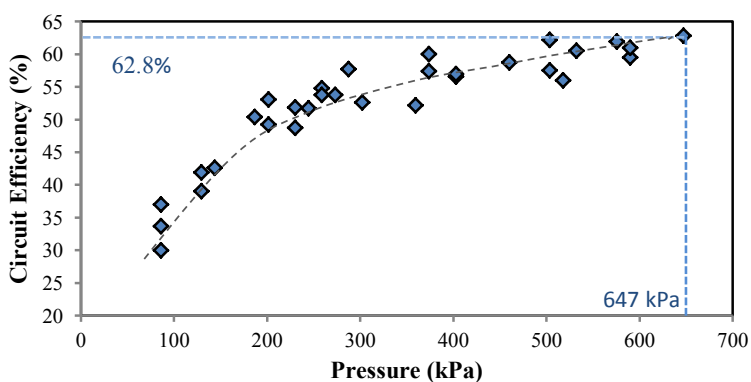

Figure 10: Graph of voltage regulation circuit efficiency verses input pressure.

The produced power was managed by the integrated hybrid circuitry that includes AC-to-DC converter as well as power storage capacity (Fig.8). The power output from each channel is a AC signal, and is first converted to DC Signal before storing in order to make the circuit more energy efficient. The AC to DC conversion is performed using an off-chip single phase Schottky Bridge Rectifier (Digi-key: 296-11231-5-ND) [12] as lesser voltage drop is seen across it. The multiple DC signals from multiple channels after conversion were added and stored across a capacitor. Figure 9 shows the experimentally tested and simulated results of the integrated circuitry with the device. The circuit efficiency (Fig. 10) increases with input pressure as the voltage level increase, giving a maximum efficiency of $62.8 \%$ with the device.

\section{CONCLUSION}

The hydraulic based energy harvesting system has been fabricated with integrated circuitry. The developed device is flexible and has a flat structure, making it convenient to be embedded into the shoe insole and power up commercial wireless devices. The two different approaches to realize a multi-channel device displayed power amplification with increase in number of channels. Further optimization of the distance between the channel for the bundle channel system is necessary. The all-connected showed greater power output with raw power ${ }_{\text {rms }}$ of $7.28 \mathrm{~mW}$ and power density of $1 \mathrm{~mW} / \mathrm{cm}^{3}$ under $647.5 \mathrm{kPa}$ input pressure. The integrated power successfully converted AC-to-DC and stored the power with $62.8 \%$ efficiency.

\section{REFERENCE}

[1] D. Noguet, G. Masera, V. Ramakrishnan, M. Belleville, D. Morche, and G. Asheid, "Considering Microelectronic Trends in Advanced Wireless System Design", Advances in electronics and telecommunications, vol. 1, no. 1, (2010).

[2] K.A. Townsend, J.W. Haslett, T.K.K. Tsang, M.N. El-Gamal and K. Iniewski, "Recent Advances and Future Trends in Low Power Wireless Systems for Medical Applications", 9th International Database Engineering \& Application Symposium, (2005).

[3] S. Basrour, B. Charlot, M. Marzencki, A. Grasso, M. Colin, and L. Valbin, "Design and fabrication of piezoelectric micro power generators for autonomous microsystems", Design, Test, Integration and Packaging of MEMS/MOEMS (2005).

[4] H Kulah, and K. Najafi "An electromagnetic micro power generator for low-frequency environmental vibrations Micro Electro Mechanical Systems", 17th IEEE Conference on MEMS, (2004).

[5] J. Yang, Fuzhi Lu, L.W. Kostiuk, and D.Y. Kwok "Electrokinetic power generation via streaming potential in microchannel: A Mobile-Ion-Drain method to increase streaming potentials", International Conference on MEMS, NANO and Smart Systems, (2004).

[6] S.H. Kim, C.H. Ji, P. Galle, and F. Herrault, " An electromagnetic energy scavenger from direct airflow" PowerMEMS, (2008).

[7] N.N.H. Ching, H. Y. Wong, W.J. Li, P.H. W. Leong, and Z. Wen, "A laser-micromachined vibrational to electrical power transducer for wireless sensing systems" Solid-State Sensors and Actuators (2001).

[8] N.S. Shenck, and J.A. Paradiso, "Energy scavenging with shoe-mounted piezoelectric" IEEE Micro. vol.21, no.3 (2001), , pp.30-42.

[9] S.R. Platt, S. Farritor, K. Garvin, and H. Haider "The use of piezoelectric ceramics for electric power generation within orthopedic implants", IEEE/ASME transactions on mechatronics, (2005).

[10] A. Bramhanand, and H. Kim, "Micro fluidic energy harvesting system for high force and large deflection accommodation", PowerMEMS, (2011).

[11] A. Bramhanand, Y.C. Bae, M.S. Kang, and H. Kim, "Prototype of a fluidics-based Energy Harvesting System", ICEIC (2012).

[12] Horowitz, Paul and W. Hill, The Art of Electronics, Second Ed., Cambridge University Press, (1989), pp. 44-47. 


\title{
SCALING EFFECT ON MEMS-BASED MICROBIAL FUEL CELLS: TOWARD A CARBON-NEUTRAL MINIATURIZED POWER SOURCE
}

\author{
H. Ren and J. Chae
}

School of Electrical, Computer and Energy Engineering, Arizona State University, Tempe, Arizona, USA

\begin{abstract}
We report the scaling effect on a $\mu \mathrm{L}$-scale miniaturized microbial fuel cell (MFC), gearing toward a carbon-neutral miniaturized power source. MFCs have been studied for many years, yet the scaling effect on MFCs has not been addressed effectively in the past. This work studies the scaling effect on mass transfer to improve the power density of a $\mu \mathrm{L}$-scale MFC. As scaling down the characteristic length, Reynolds number decreases and mass transfer coefficient rapidly increases, resulting in a higher power density. Areal and volumetric power densities of $83 \mu \mathrm{W} / \mathrm{cm}^{2}$ and 3.32 $\mathrm{mW} / \mathrm{cm}^{3}$ are obtained, respectively; both of which the highest ever reported among all $\mu \mathrm{L}$-scale MFCs to date. Columbic efficiency (CE) of $79.4 \%$ is marked, more than 2.5 folds of the previously reported maximum $\mathrm{CE}$ in $\mu \mathrm{L}$-scale MFCs.
\end{abstract}

\section{INTRODUCTION}

In the era of energy crisis, renewable power sources, including solar/hydro power, geothermal energy, etc., become very attractive. One of such renewable power sources is a microbial fuel cell (MFC) harvesting electricity from biomass. A MFC is an electrochemical fuel cell harvesting electrons from specific bacteria species, exoelectrogen, via their extracellular electron transfer (EET). Unlike other approaches to convert biomass to electricity, including methanogenic anaerobic digestion, bioethanol, incineration and gasification etc., MFC is unique to have direct electricity generation with high conversion efficiency, to operate at low organic concentration and environmentally unfriendly conditions and to generate reduced amount of sludge. MFCs have been proposed to be used in scaled-up wastewater treatment and renewable energy production [1-3], bioremediation of recalcitrant component [4-6] and power supply for remote sensors in hazard or environmentally unfriendly conditions [7-8]. Miniaturizing MFCs is an interesting approach as scaling effects on the characteristic length largely impact several performance parameters of MFCs. Our prior work [9] presents one of highest areal power density, up to $33 \mu \mathrm{W} / \mathrm{cm}^{2}$, in a $\mu \mathrm{L}$-scale MFC, yet it is still far lower than that of macro-/meso-scale counterparts (up to $680 \mu \mathrm{W} / \mathrm{cm}^{2}$ ).

We report scaling down its characteristic length of a $\mu \mathrm{L}$-scale MFC, resulting in increasing the mass transfer coefficient, consequently enhancing power density and efficiency of MFC. The maximum areal and volumetric power density of a MFC can be written as:

$$
\begin{gathered}
p_{\text {max }, \text { areal }}=\frac{P_{\max }}{A}=\frac{E_{O C V}{ }^{2}}{4 R_{i} \cdot A} \\
p_{\text {max, volumetric }}=\frac{P_{\max }}{V}=\frac{E_{O C V}{ }^{2}}{4 R_{i} \cdot V}=\frac{E_{O C V}{ }^{2}}{4 R_{i} \cdot A} \cdot S A V
\end{gathered}
$$

where $p_{\max , \text { areal }}, p_{\max , \text { volumetric }}, P_{\max }, E_{\text {ocv }}, R_{i}, A, V$ and $S A V$ are the maximum areal power density, maximum volumetric power density, maximum power, open circuit voltage, internal resistance, area, chamber volume and surface area to volume ratio, respectively. For a given type of MFC, as open circuit voltage (OCV) and the product of $R_{i}$ and $A$ are constant, the areal power density is independent of the scaling effect whereas $S A V$ increases as scaling down the characteristic length, resulting in the enhancement of volumetric power density.

In addition to these parameters, miniaturized MFCs benefit from higher mass transfer [11]. Reynolds number, $R_{e}$, and mass transfer coefficient, $k_{c}$, can be written as:

$$
\begin{gathered}
\mathrm{R}_{e}=\rho v L / \mu \\
k_{c}=0.664(\rho v L / \mu)^{1 / 2}(\mu / \rho D)^{1 / 3}\left(\frac{D}{L}\right)
\end{gathered}
$$

where $\rho, v, L, \mu$, and $D$ are the specific density of the fluid, the linear velocity of the fluid, the characteristic length, the viscosity of the fluid, and the diffusivity of the fluid. As shown in figure 1, by scaling down the characteristic length while making other parameters remain unchanged, the Reynolds number decreases and the mass transfer coefficient increases.

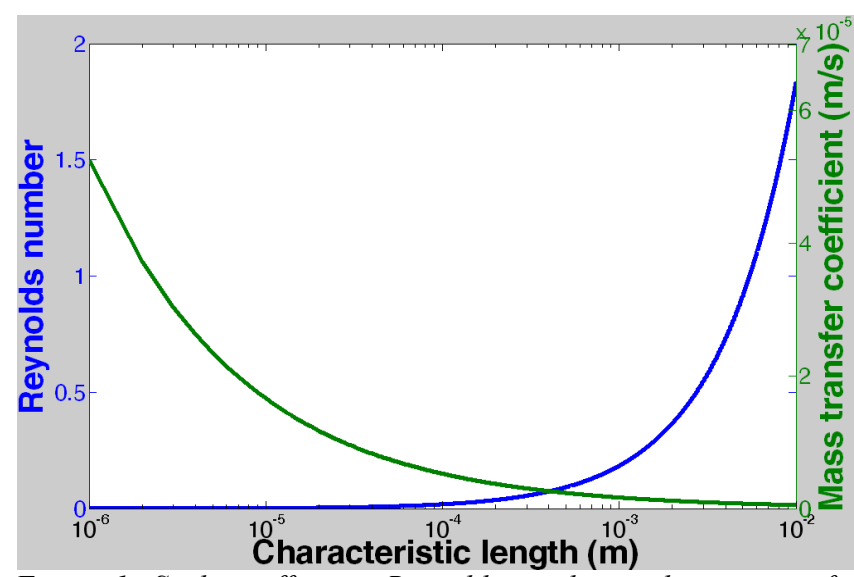

Figure 1: Scaling effect on Reynolds number and mass transfer coefficient; moving from $1 \mathrm{~mm}$ to $1 \mu \mathrm{m}$ in the characteristic length lowers Reynolds number from 0.18 to $1.8 \times 10^{-4}$ and enhances the mass transfer coefficient from $1.7 \times 10^{-6}$ to $5.2 \times 10^{-5} \mathrm{~m} / \mathrm{s}$.

\section{MATERIALS AND METHODS}

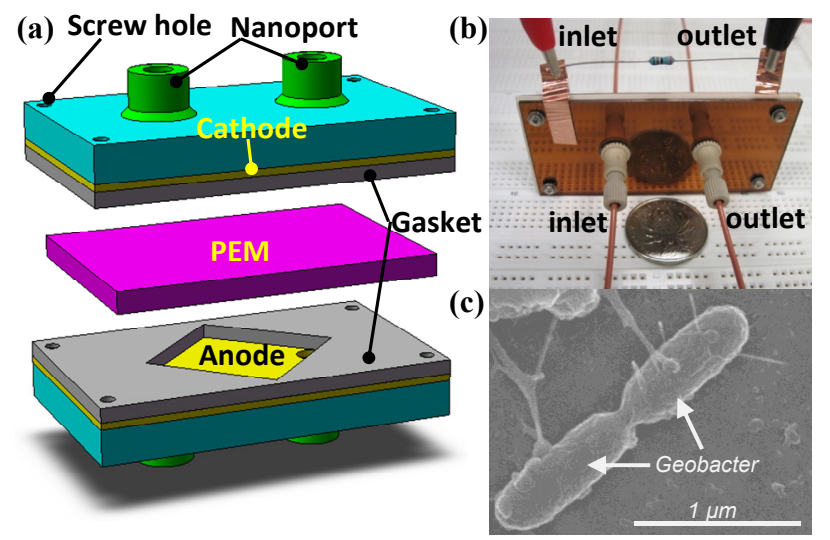

Figure 2: (a) Schematic of a $\mu L$-scale microbial fuel cell (MFC); anode and cathode sandwich an ion exchange membrane and gaskets define the height of anode/cathode chambers. (b) photograph of a fabricated $\mu L$-scale MFC, and (c) SEM of Geobacter on the anode (scale bar: $1 \mu \mathrm{m}$ ).

The schematic of $\mu \mathrm{L}$-scale MFC is illustrated in figure 2(a).

Solid-State Sensors, Actuators, and Microsystems Workshop Hilton Head Island, South Carolina, June 3-7, 2012 
The MFC has a proton exchange membrane (PEM) and two silicone gaskets sandwiched between two glass slides pre-fabricated with gold electrodes. The volume of each chamber is $100 \mu \mathrm{L}$ and the size of electrode is $4 \mathrm{~cm}^{2}$. Two nanoports (10-32 Coned assembly, IDEX Health \& Science) were used to provide microfluidic pathways for the MFC. The fabrication process begins with preparing two glass chips $\left(76 \times 38 \times 0.12 \mathrm{~cm}^{3}, \mathrm{VWR}\right)$ and mechanically drilling six through holes on each chip: one inlet, one outlet, and four for assembly. Afterwards, Cr/Au (20 nm/200 nm) were deposited by sputtering on the anode/cathode. Then, the nanoports and fluidic tubings (PEEK polymer, IDEX Health \& Science) were aligned and glued to the inlets/outlets to supply anolyte and catholyte. Silicone rubber gaskets were cut to define the chamber and electrode and PEM (Nafion 117) was used as a membrane to permit only proton transport and avoid electrical short-circuit and electrolyte cross mix. Finally the MFC was assembled using four screw bolts and nuts to minimize oxygen/electrolyte leakage (figure 2(b)). SEM photograph shows Geobacter on the anode (figure 2(c)), allowing high current density $\left(>10 \mathrm{~A} / \mathrm{m}^{2}\right)[12]$.

The inoculum for the $\mu \mathrm{L}$-scale MFC was obtained from an acetate-fed microbial electrolysis cell (MEC) [14] that had Geobacter-enriched mixed bacterial culture from anaerobic digestion sludge. Clone libraries of the 16S-rRNA gene showed that the inoculum was a mixed bacterial culture dominated by Geobacter sulfurreducens [14]. The anolyte and catholyte are $25 \mathrm{mM}$ sodium acetate medium and $50 \mathrm{mM}$ potassium ferricyanide in $100 \mathrm{mM}$ phosphate buffer solution ( $\mathrm{pH} 7.4)$, respectively. The anolyte and catholyte were supplied into the $\mu \mathrm{L}$-scale MFC using a syringe pump (Cole-Parmers Inc.).

Acetate in the anode chamber is oxidized by Geobacter to carbon dioxide and release electrons and protons:

$$
\mathrm{CH}_{3} \mathrm{COO}^{-}+2 \mathrm{H}_{2} \mathrm{O} \rightarrow 2 \mathrm{CO}_{2}+8 \mathrm{e}^{-}+7 \mathrm{H}^{+}
$$

These electrons are transferred to the anode, pass through an external circuit to arrive the cathode, and are reduced by ferricyanide:

$$
\left[\mathrm{Fe}(\mathrm{CN})_{6}\right]^{3-}+\mathrm{e}^{-} \rightarrow\left[\mathrm{Fe}(\mathrm{CN})_{6}\right]^{4}
$$

The current was monitored every minute by recording voltage drop across an external resistor connected between the anode and the cathode using a data acquisition system (DAQ/68, National Instrument). $148-\Omega$ resistor was used during the start-up and a series of resistors, ranging from $148 \Omega$ to $932 \mathrm{k} \Omega$, was used to obtain the polarization curve of the $\mu \mathrm{L}$-scale MFC, respectively. The current through the resistor was calculated via Ohm's law $(I=V / R)$ and the resulting output power was calculated via Joule's law $\left(P=I^{2} R\right)$.

\section{RESULTS AND DISCUSSION}

The $\mu \mathrm{L}$-scale MFC operated in a semi-continuous mode at 1 $\mu \mathrm{L} / \mathrm{min}$ using anolyte with inoculum (1:1 volume ratio) and catholyte during the start-up process. Figure 3 shows the $\mu \mathrm{L}$-scale MFC reached $>1.8 \mu \mathrm{A} / \mathrm{cm}^{2}, 4$ days after the operation. Then, the current density increased quickly. When the current density reached $20 \mu \mathrm{A} / \mathrm{cm}^{2}$ on the sixth day it began to fluctuate due to cathode-limited MFC condition, which diminished by supplying more catholyte.

Mass transfer is a function of the concentration and linear velocity of electrolyte (Eq. (4)). The concentration of anolyte and catholyte increased from $25 \mathrm{mM}$ to $37.5 \mathrm{mM}$ and $50 \mathrm{mM}$. The concentration increase did not result in enhanced areal power density; contradictory to the prediction that higher concentration induces higher mass transfer, which subsequently resulting in higher power density. A possible reason for this is that a high concentration causes bacteria to dehydrate, which results in lower power densities [15].

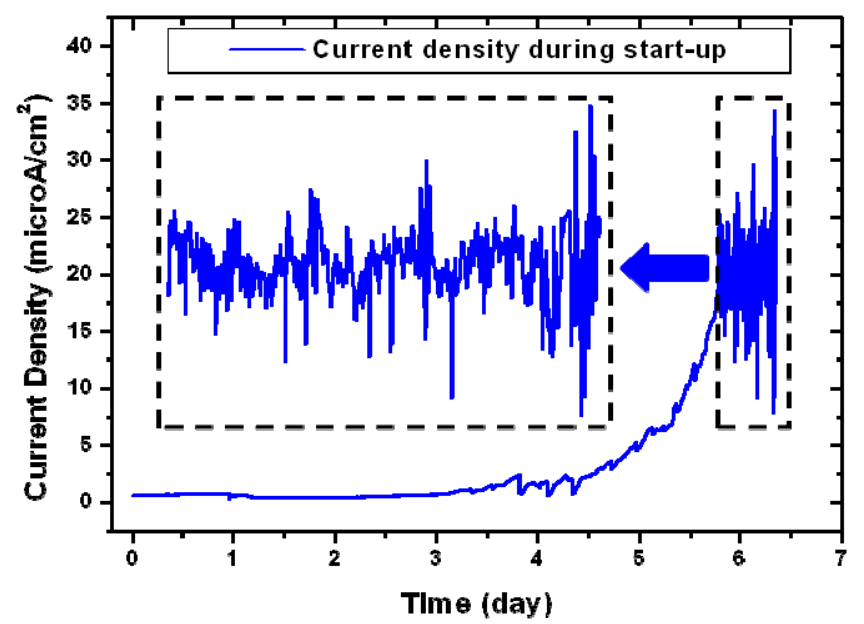

Figure 3: Current density produced by the $\mu L$-scale MFC during the start-up process; the current density began to increase above 1.8 $\mu A / \mathrm{cm}^{2}, 4$ days after adding inoculum, and increased quickly with time, and began to fluctuate once it reached $20 \mu \mathrm{A} / \mathrm{cm}^{2}$. The fluctuation is due to cathode-limited MFC condition, which diminished by supplying more catholyte.

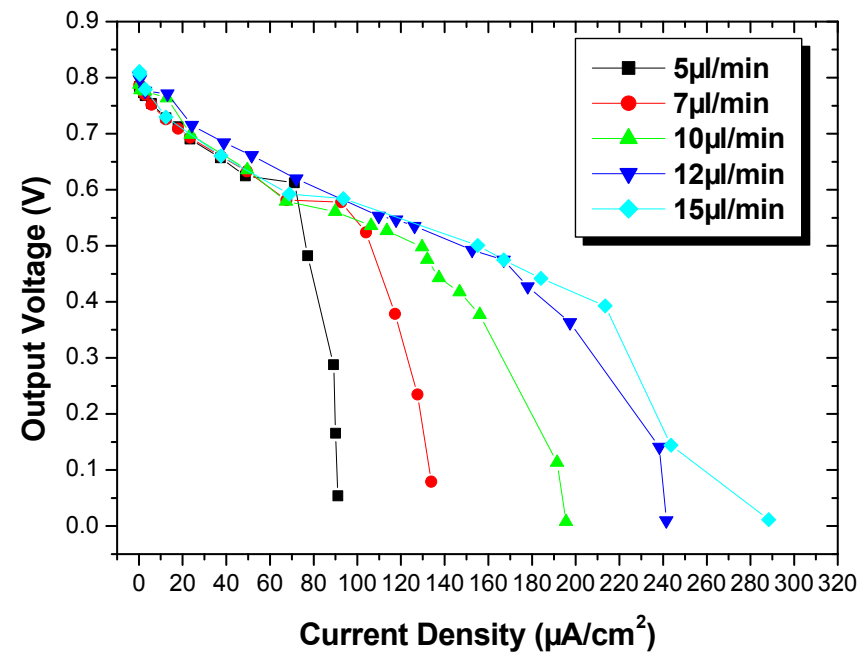

Figure 4: Output voltage versus current density at different flow rates from 5 to $15 \mu \mathrm{L} / \mathrm{min}$. As the flow rate increases the maximum current density increases, suggesting mass transfer improves as the flow rate increases.

The linear velocity of electrolyte was controlled by the flow rate of external syringe pump. The flow rate increased from 1 to 15 $\mu \mathrm{L} / \mathrm{min}$ to study the impact of mass transfer on power densities (figure $4,5 \& 6$ ). The increase in power density can be attributed from higher mass transfer of anolyte and buffer into biofilms, providing more acetate to the bacteria, resulting in a higher power density. The curves of the areal power density as a function of current density for different flow rates, figure 5, suggest that the areal power densities are almost independent of flow rates at low current densities, and increase as the flow rate increases. As the current densities increase, the areal power density of lowest flow rate firstly hit its maximum, which is due to the high concentration 
loss, while the areal power densities of highest flow rate hits its maximum lastly. This suggests the high flow rate keeps the $\mu \mathrm{L}$-scale MFC from suffering concentration loss at high current densities.

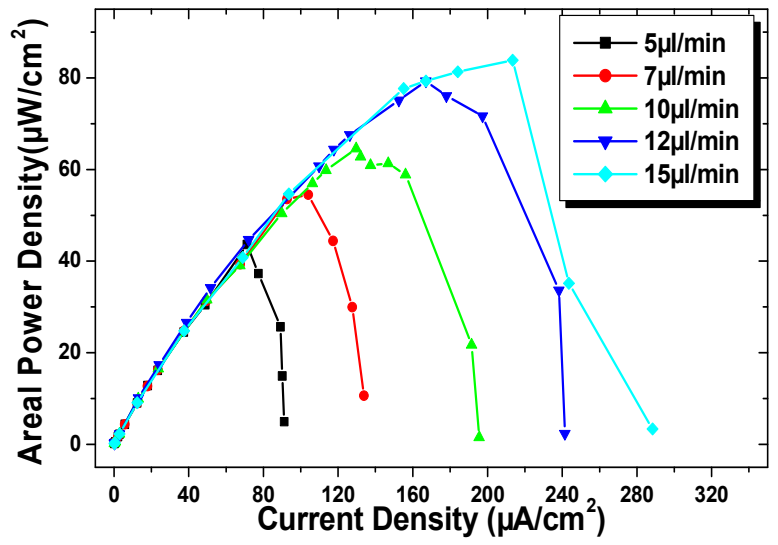

Figure 5: Areal power density versus current density at different flow rates; as the flow rate increased, both the maximum current and power densities improved.

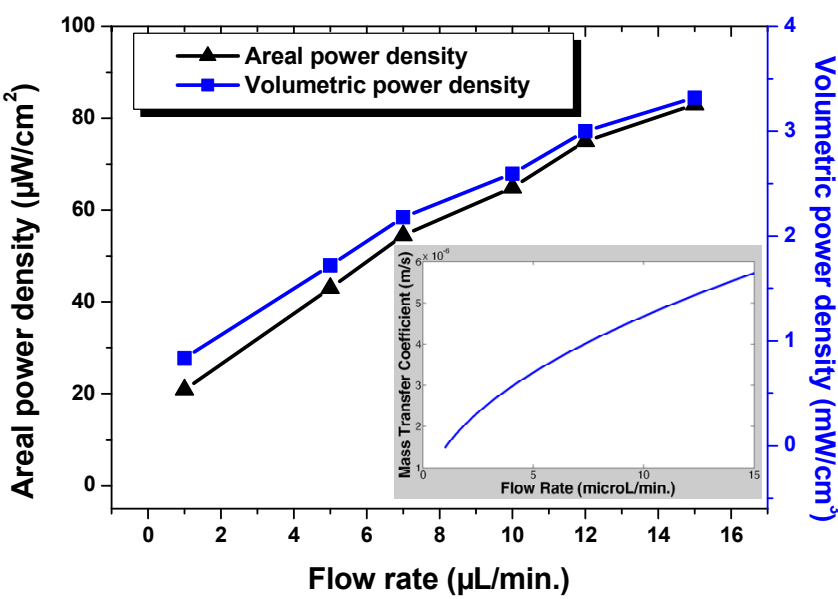

Figure 6: Areal and volumetric power densities as a function of flow rate (inset: calculated mass transfer coefficient versus flow rate)

The internal resistances are calculated by linearly fitting the ohmic region of each output voltage versus areal current density curve in figure 4 , and the internal resistance of $\mu \mathrm{L}$-scale MFCs having flow rate of $5,7,10,12$, and $15 \mu \mathrm{L} / \mathrm{min}$ are $2.76,2.52,1.96$, 1.73 , and $1.39 \mathrm{k} \Omega$, respectively. This also supports higher flow rates incur higher mass transfer, thereby lowering the internal resistance and improving the areal power density. The highest areal and volumetric power densities of $\mu \mathrm{L}$-scale MFCs are to be measured as $83 \mu \mathrm{W} / \mathrm{cm}^{2}$ and $3,320 \mu \mathrm{W} / \mathrm{cm}^{3}$, the highest among all $\mu \mathrm{L}$-scale MFCs to date.

Columbic efficiency (CE) is a measure of how efficiently a MFC harvests electrons. Low CE is another challenge of $\mu \mathrm{L}$-scale MFCs. The maximum CE reported by $\mu \mathrm{L}$-scale MFCs is $31 \%$, significantly lower than that of macro- and meso-scale MFCs, which often reach a $\mathrm{CE}$ of higher than $80 \%$. The low $\mathrm{CE}$ is believed to be primarily due to high oxygen leakage into the anode chamber [9].

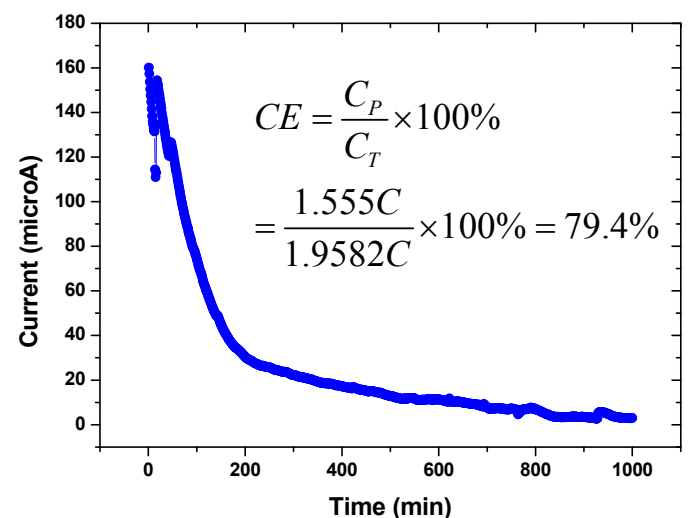

Figure 7: Columbic efficiency (CE) measurement, CE calculation is embedded into the figure.

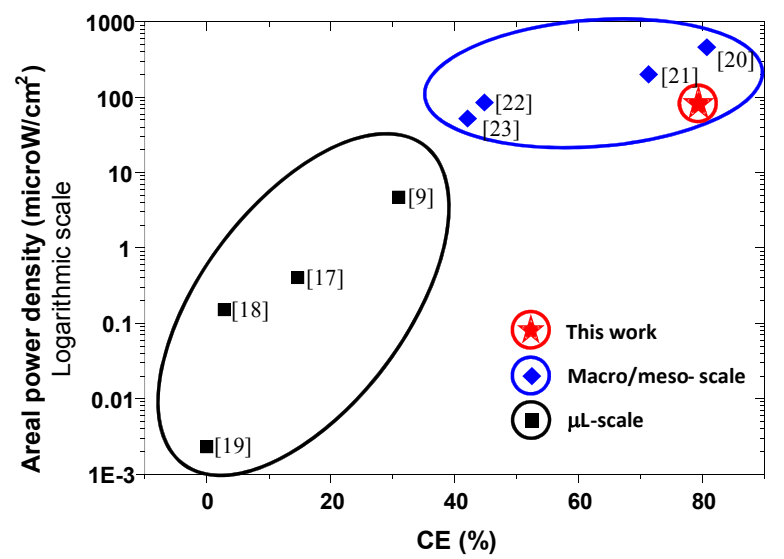

Figure 8: A comparison of areal power density and CE of this work with existing macro-/meso- and $\mu L$-scale MFCs; the power density and $C E$ of this work is substantially higher than those of $\mu L$-scale MFCs and is comparable with those of macro-/meso-scale MFCs.

$\mathrm{CE}$ of the $\mu \mathrm{L}$-scale MFC was measured by integrating current profiles over the time, as shown in figure 7 [16]. The CE is calculated to be $79.4 \%$ (figure 7 inset), significantly higher than that of reported $\mu \mathrm{L}$-scale MFCs, which are generally between $0.03-$ $31 \%$, and it is comparable to that of macro-/meso-scale MFCs (figure 8). Specifications and performance matrix of prior art and this work on $\mu \mathrm{L}$-scale MFCs are summarized in table 1.

Table 1: Summary table of this work and prior art.

\begin{tabular}{|c|c|c|c|c|c|}
\hline & {$[9]$} & {$[10]$} & {$[20]$} & {$[21]$} & $\begin{array}{c}\text { This } \\
\text { work }\end{array}$ \\
\hline Anode volume $[\mu \mathrm{L}]$ & 4.5 & 25 & 0.1 & 1.5 & $\mathbf{1 0 0}$ \\
\hline Anode area $\left[\mathrm{cm}^{2}\right]$ & 2.25 & 1 & 0.12 & 1.2 & $\mathbf{4}$ \\
\hline $\begin{array}{c}\text { Internal resistance } \\
{[\mathrm{k} \Omega]}\end{array}$ & 10 & 32 & 25 & 30 & $\mathbf{1 . 3 9}$ \\
\hline $\mathrm{P}_{\text {areal }}\left[\mu \mathrm{W} / \mathrm{cm}^{2}\right]$ & 4.7 & 33 & 0.4 & 1.5 & $\mathbf{8 3}$ \\
\hline $\mathrm{P}_{\text {volumetric }}\left[\mu \mathrm{W} / \mathrm{cm}^{3}\right]$ & 2333 & 667 & 4.24 & 15.3 & $\mathbf{3 3 2 0}$ \\
\hline $\mathrm{CE}[\%]$ & 31 & $\mathrm{~N} / \mathrm{A}$ & 14.7 & 2.8 & $\mathbf{7 9 . 4}$ \\
\hline
\end{tabular}

One of challenges in our $\mu \mathrm{L}$-scale MFC is that ferricyanide etches the $\mathrm{Cr} / \mathrm{Au}$ cathode, resulting in a short lifetime. After 45 days, a large portion of the cathode and electrical route were etched away, and consequently lowered power and current densities. The etched cathode was replaced and the $\mu \mathrm{L}$-scale MFC was re-assembled to operate. The current profile, figure 9, shows it took five days to recover the maximum current density since the cathode 
replacement.

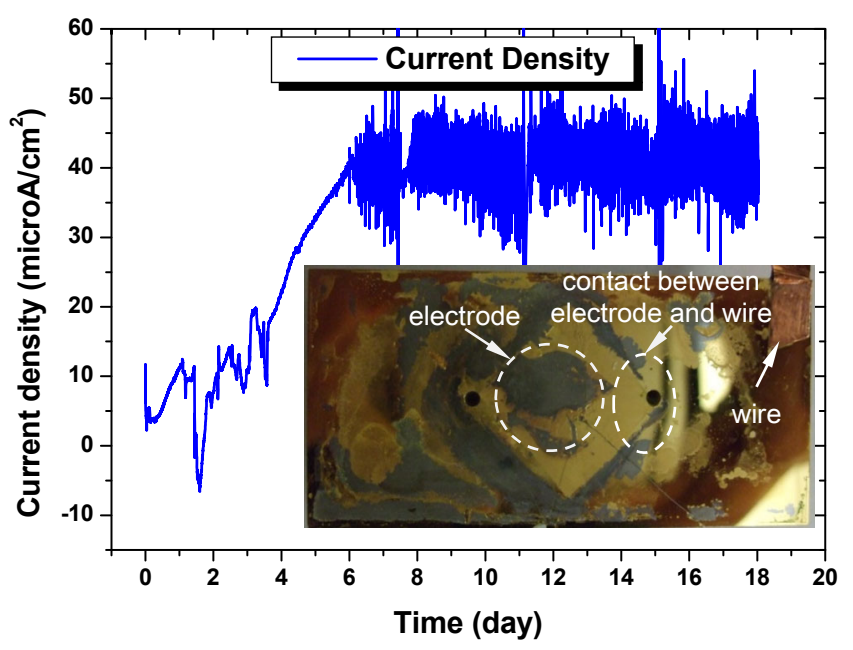

Figure 9: Current density versus time after cathode replacement and re-assembly, it took five days to recover the maximum current density, inset: photograph showing a large portion of cathode and electrical route etched by ferricyanide after 45 days.

\section{CONCLUSION}

In summary, this study presents the scaling effect on the characteristic length of a $\mu \mathrm{L}$-scale MFC. The scaling effect predicts the power density, especially volumetric power density, of a $\mu \mathrm{L}$-scale MFC increases, via improving mass transfer of anolyte/catholyte. A $\mu \mathrm{L}$-scale MFC was built to demonstrate areal and volumetric power densities of $83 \mu \mathrm{W} / \mathrm{cm}^{2}$ and $3,320 \mu \mathrm{W} / \mathrm{cm}^{3}$, respectively and a CE of $79.4 \%$, which are the highest among all previously reported $\mu \mathrm{L}$-scale MFCs.

\section{ACKNOWLEDGEMENT}

The authors thank Dr. Parameswaran for providing Geobacteraceae-enriched culture. Travel support has been generously provided by the Transducer Research Foundation.

\section{REFERENCES}

[1] Y. Ahn and B.E. Logan. "Effectiveness of domestic wastewater treatment using microbial fuel cell at ambient and mesophilic temperatures", Bioresource Technology, 101, pp. 469-475 (2010).

[2] Y. Feng, X. Wang, B.E. Logan and H. Lee. "Brewery wastewater treatment using air-cathode microbial fuel cells", Appl Microbiol Biotechnol, 78, pp. 873-880 (2008).

[3] B.E. Logan. "Scaling up microbial fuel cells and other bioelectrochemical systems", Appl Microbiol Biotechnol, 85, pp. 1665-1671 (2010).

[4] J.M. Morris and S. Jin. "Feasibility of using microbial fuel cell technology for bioremediation of hydrocarbons in groundwater", J. Environ. Sci. Health Part A Tox. Hazard. Subst. Environ. Eng., 43, pp. 18-23 (2008).

[5] T. Catal, Y. Fan, K. Li, H. Bermek and H. Liu. "Effects of furan derivatives and phenolic compounds on electricity generation in microbial fuel cells", J. Power Sources, 180, pp. 162-166 (2008).

[6] J.K. Jang, I.S. Chang, H. Moon, K.H. Kang and B.H. Kim. "Nitrilotriacetic acid degradation undermicrobial fuel cell environment", Biotechnol. Bioeng., 95, pp. $772-774$ (2006).

[7] L.M. Tender, S.A. Gray, E. Groveman, D.A. Lowy, P. Kauffman, J. Melhado, R.C. Tyce, D. Flynn, R. Petrecca and J. Dobarro.
"The first demonstration of a microbial fuel cell as viable power supply: powering a meteorological buoy", J. Power Sources, 179, pp. 571-575 (2008).

[8] Y. Gong, S.E. Radachowsky, M. Wolf, M.E. Nielsen, P.R. Girguis and C.E. Reimers. "Benthic microbial fuel cell as direct power source for an acoustic modem and seawater oxygen/temperature sensor system", Environ. Sci. Technol., 45, pp. 5047-5053 (2011).

[9] S. Choi, H.S. Lee, Y. Yang, P. Parameswaran, C.I. Torres, B.E. Rittmann and J. Chae. "A $\mu \mathrm{L}$-scale micromachined microbial fuel cell having high power density", Lab Chip, 11, 1110 (2011).

[10] S. Choi and J. Chae. "An array of microliter-sized microbial fuel cells generating $100 \mu \mathrm{W}$ of power", Sensors and Actuators: A., 177, pp. 10-15 (2012).

[11] F. Qian and D.E. Morse. "Miniaturizing microbial fuel cells", Trends in Biotechnology, 29, pp. 62-69 (2011).

[12] C.I. Torres, A.K. Marcus, H.S. Lee, P. Parameswaran, R.K. Brown and B.E. Rittmann. "A kinetic perspective on extracellular electron transfer by anode-respiring bacteria", FEMS Microbiol Rev, 34, pp. 3-17 (2010).

[13] N.S. Malvankar, V. Madeline, K.P. Nevin, A.E. Franks, L. Ching, B.C. Kim, K. Inoue, T. Mester, S.F. Kovalla, J.P. Johnson, V.M. Rotello, M.T. Tuominen and D.R. Lovely. "Tunable metallic-like conductivity in microbial nanowire networks", Nature Nanotechnology, 6, pp. 573-579 (2011).

[14] H. Lee, P. Parameswaran, A.K. Marcus, C.I. Torres and B.E. Rittmann. "Evaluation of energy-conversion efficiencies in microbial fuel cells (MFCs) utilizing fermentable and non-fermentable substrates", Water Research, 42, pp. 1501-1510 (2008)

[15] L.M. Prescott, D.A. Klein, and J.P. Harley. Microbiology, $5^{\text {th }}$ edition, The McGraw-Hill Companies, New York, 2002.

[16] B.E. Logan, B. Hamelers, R. Rozendal, U. Schröder, J. Keller, S. Freguia, P. Alterman, V. Verstraete and K. Rabaey. "Microbial fuel cells: methodology and technology", Environ. Sci. Technol, 40, pp. 5081-5092 (2006).

[17] C. Siu and M. Chiao. "A Microfabricated PDMS Microbial Fuel Cell”, J. Microelectromech. Syst., 17, pp. 1329-1341 (2008).

[18] F. Qian, M. Baum, Q. Gu and D.E. Morse. "A 1.5 $\mu 1$ microbial fuel cell for on-chip bioelectricity generation”, Lab Chip, 9, pp. 3076-3081 (2009).

[19] M. Chiao, K.B. Lam and L. Lin. "Micromachined microbial and photosynthetic fuel cells", J. Micromech. Microeng., 16, pp. 2547-2553 (2006).

[20] K. Rabaey, N. Boon, S.D. Siciliano, M. Verhaege and W. Verstraete. "Biofuel cells select for microbial consortia that self-mediate electron transfer", Appl. Environ. Microbiol., 70, pp. 5373-5382 (2004).

[21] Y. Fan, H. Hu and H. Liu. "Enhanced coulombic efficiency and power density of air-cathode microbial fuel cells with an improved cell configuration", Journal of Power Sources, 171, pp. 348-354 (2007).

[22] H. Liu, S. Cheng, L. Huang and B.E. Logan. "Scale-up membrane-free single chamber microbial fuel cells", Journal of Power Sources, 179, pp. 274-279 (2008).

[23] H. Liu and B.E. Logan. "Electricity generation using an air-cathode single chamber microbial fuel cell in the presence and absence of a proton exchange membrane", Environ. Sci. Technol., 38, pp. 4040-4046 (2004).

\section{CONTACT}

*J. Chae, tel: +1-480-965-2082; Junseok.Chae@asu.edu 


\title{
SIC/ALN PIEZOELECTRIC ENERGY HARVESTERS FOR PULSED PRESSURE SOURCES IN HARSH ENVIRONMENT APPLICATIONS
}

\author{
Yun-Ju Lai ${ }^{1 *}$, Wei-Chang Li ${ }^{l}$, Chih-Ming Lin ${ }^{l}$,Valery V. Felmetsger ${ }^{2}$, Debbie G. Senesky ${ }^{3}$, and Albert P. Pisano ${ }^{l}$ \\ ${ }^{1}$ Berkeley Sensor and Actuator Center (BSAC), University of California, Berkeley, California, USA \\ ${ }^{2}$ OEM Group Incorporated, Gilbert, Arizona, USA \\ ${ }^{3}$ Stanford University, Palo Alto, California, USA
}

\begin{abstract}
Piezoelectric micromachined circular diaphragm energy harvesters for pulsed pressure sources have been fabricated and characterized. These devices utilize silicon carbide ( $\mathrm{SiC})$ as the structural material and aluminum nitride (AlN) as the active piezoelectric element to enable operation within harsh environments. In particular, the $\mathrm{SiC} / \mathrm{AlN}$ energy harvesting device yields an output power density of $20 \mu \mathrm{W} / \mathrm{cm}^{2}$ and $\mathrm{rms}$ voltage of $0.2 \mathrm{~V}$ under pressure pulses of $1.09 \mathrm{psi}(\mathrm{rms})$ at $2 \mathrm{kHz}$. Fabricated $\mathrm{SiC} / \mathrm{AlN}$ harvester has been characterized at elevated temperatures as high as $250^{\circ} \mathrm{C}$. This work not only demonstrates the first harsh environment energy harvesters based on $\mathrm{SiC}$ and $\mathrm{AlN}$ for a pulsed pressure environment, but also addresses its potentials for integrating energy harvesting power source with SiC circuitry and enabling self-powered wireless sensors. This type of sensing system can be used for the real-time monitoring of harsh environment energy system such as automotive engines, gas turbines and geothermal wells.
\end{abstract}

\section{INTRODUCTION}

Condition-based monitoring of automotive engines, jet engines and gas turbines requires circuits and sensors, which have stable operation at elevated temperatures $\left(250^{\circ} \mathrm{C}\right.$ to $\left.600^{\circ} \mathrm{C}\right)$ and under harsh chemical environments (oxidizing, hydrocarbon, $\mathrm{CO}$ and $\mathrm{CO}_{2}$ ). This can aid in achieving efficient energy usage, reduced emissions and long lifetime for the combustion processes and critical components. In addition, harsh environment sensors also enable direct subsurface monitoring of geothermal and oil wells leading to enhanced energy recovery. However, at elevated temperatures, power supplies for electronic circuits and sensors are scarce. Current battery technology limits the working temperature range to below $150^{\circ} \mathrm{C}$ and cabled-power wiring dramatically increases the system complexity as well as the installation and maintenance cost.

To address this issue, energy harvesting [1][2] has emerged as promising power source, which can further lead to an autonomous integrated sensing module with reduced cost. Up to date, only a few energy harvesting devices for harsh environments have been reported, converting ambient vibration [3] and UV [4] energy into electrical energy. However, considering the availability of the ambient energies in the potential harsh environment systems, pulsed-pressure sources commonly existing in the combustion processes appears greatly attractive. In fact, the amount of the available energy from pressure pulsation in the target environment can be much larger than vibration sources and in addition, without requiring a UV-rich environment.

This work first demonstrates a piezoelectric SiC/AlN circular diaphragm energy harvester aiming to harvest the energy from pulsed-pressure sources in combustion systems. This micro-scale power source has the potential to achieve system integration with harsh environment circuit and sensor components [5][6]. To enable operation in harsh environments, the structural material selected for the energy harvesting devices consists of epitaxial cubic $\mathrm{SiC}$ (3C-SiC) and $\mathrm{AlN}$. $\mathrm{SiC}$ is used as the diaphragm structural material and AlN serves as the piezoelectric material for power

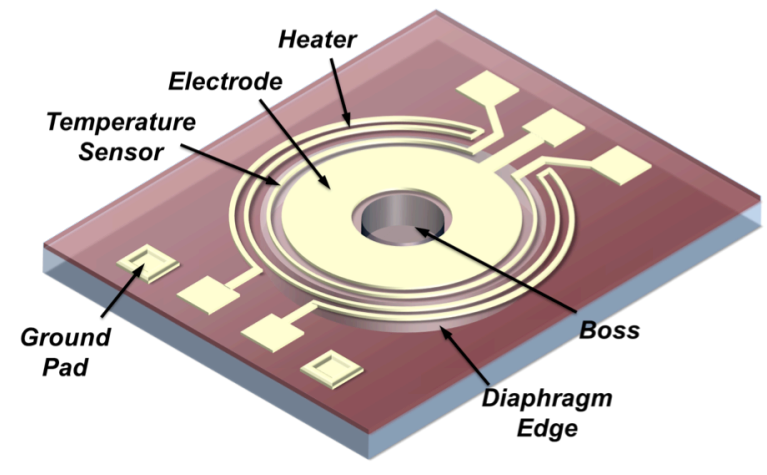

Figure 1: Schematic of a micromachined piezoelectric SiC/AlN bossed-diaphragm energy harvester with an on-chip thermal platform (resistive heaters and temperature sensors) for pulsed pressure sources.

conversion. Both appear favorable due to their excellent mechanical and electrical properties and resistance to chemical attack especially at elevated temperatures compared to their counterparts, e.g., silicon and PZT. By using this material combination, a micromachining process has been developed for fabricating the SiC/AlN composite diaphragm structure. The fabricated energy harvester with $4000-\mu \mathrm{m}$ diameter generates a power output as high as $2.5 \mu \mathrm{W}$, equivalent to a power density of approximately $20 \mu \mathrm{W} / \mathrm{cm}^{2}$, showing promise for providing power to most of the low-power sensor circuits. To investigate the functionality of the harvesters at elevated temperatures, the device equipped with an on-chip thermal platform (resistive heating and sensing elements) is measured and is shown to operate at temperatures above $200^{\circ} \mathrm{C}$. The experimental results not only show a SiC/AlN energy harvesting device with high output power density but also verifies its operation capabilities under high temperature environments. The harvester design, fabrication process, testing setup and measured results are presented as follows.

\section{SIC/ALN ENERGY HARVESTING DEVICE}

Piezoelectric AIN is chosen as the active material for power conversion rather than the commonly used PZT, which possesses large piezoelectric coefficients, for reasons that $\mathrm{AlN}$, which is non-ferroelectric and does not have a Curie point, retains its intrinsic piezoelectric properties even at high temperatures [7]. In fact, AlN has been demonstrated for a Lamb waver resonator to provide stable operation as high as $700^{\circ} \mathrm{C}$ [8]. Moreover, high quality AlN can be sputter-deposited at wafer-level with compatible processing along with $\mathrm{SiC}$. The diaphragm structural material, $\mathrm{SiC}$, on the other hand, in addition to the mechanically robust nature under harsh environments, its superior electrical properties also enable SiC-based circuits and sensors for harsh environment electronics. Plus, SiC and AlN have well-matched mechanical and thermal properties, e.g., thermal expansion coefficients [9]. Platinum (Pt) and molybdenum (Mo) are the two 
(b) Deposit Insulation $\mathrm{SiO}_{2}$

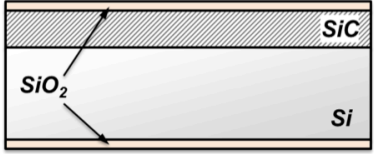

(c) Deposit Mo \& AIN

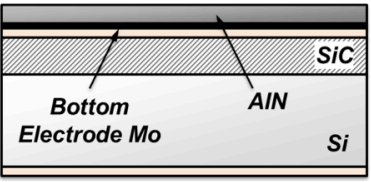

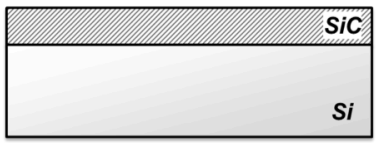

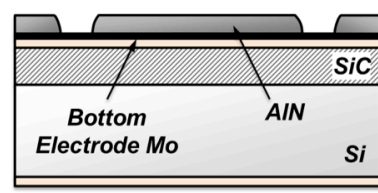

(e) Pattern Top Electrode Pt

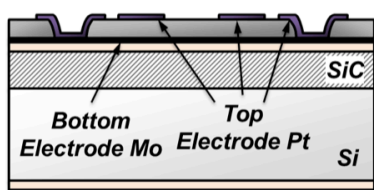

(f) Release Using DRIE

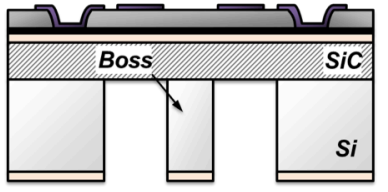

Figure 2: Fabrication process flow for the micromachined $\mathrm{SiC} / \mathrm{AlN}$ bossed-diaphragm energy harvesters.

metal materials utilized for the electrodes considering the high temperature survivability.

Figure 1 illustrates the design of a SiC/AlN energy harvesting device showing a circular bossed-diaphragm with patterned electrodes for electrical outputs as well as an on-chip thermal platform for high temperature measurements. The composite diaphragm is composed of a metal/AlN/metal sandwich layer on top of the structural $\mathrm{SiC}$ with a thin layer of $\mathrm{SiO}_{2}$ for electrical insulation in between. Transduction via $d_{31}$ piezoelectric coefficient is utilized for electrical power generation as the diaphragm deforms in response to the pressure pulses in the environments. The central boss structure adopted in this design can tune the mechanical resonant frequency of the composite diaphragm without modifying the diaphragm sizes. The electrode configurations (size and location) are determined to maximize the deliverable power generated by the piezoelectric AlN layer based on the in-plane strain distribution of the pressure-loaded diaphragm under large deformation. In addition to the electrode geometries, for maximizing the device output power, residual stress in the structural layers (AIN and $\mathrm{SiC}$ ) needs to be well controlled as it significantly reduces the diaphragm compliance [10]. Moreover, excellent crystallinity of the piezoelectric AlN thin film is required to achieve high electromechanical coupling and thus better power conversion.

\section{FABRICATION PROCESS}

Figure 2 depicts the bulk micro-machining process flow that is used to achieve the $\mathrm{SiC} / \mathrm{AlN}$ energy harvesting devices. A $\sim 3.2-\mu \mathrm{m}$-thick structural layer, $3 \mathrm{C}-\mathrm{SiC}$, is first deposited on a $\mathrm{Si}$ (100) substrate by epitaxial chemical vapor deposition with a residual stress below $100 \mathrm{MPa}$ by NOVASiC as shown in Fig. 2(a). A 500-nm oxide is then deposited via low pressure chemical vapor deposition as the insulation layer between $\mathrm{SiC}$ and bottom electrode (Fig. 2(b)). Next, well-textured bottom electrode Mo is sputtered on the insulation oxide with a thin layer of AlN as the seedlayer in order to achieve its high crystallinity for the following AlN growth. Highly $c$-axis-oriented AlN thin film $(\sim 1.0 \mu \mathrm{m}$, rocking curve FWHM $<1.5^{\circ}$, stress $<200 \mathrm{MPa}$ ) [11] is then

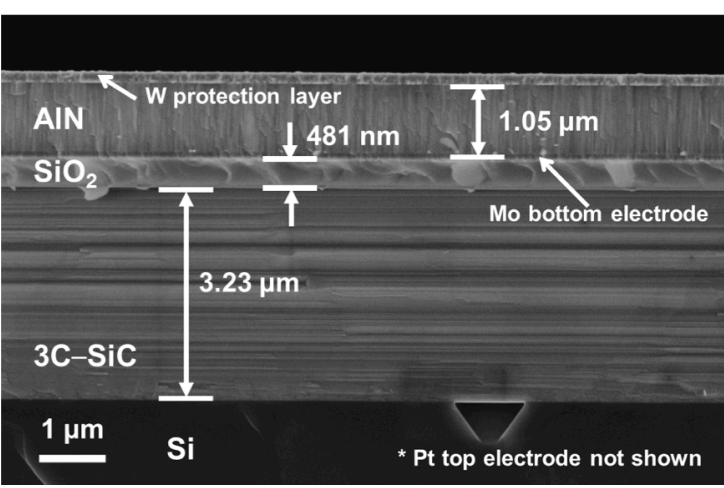

Figure 3: SEM of the cross-section of composite layer including piezoelectric AlN, bottom electrode Mo, insulation $\mathrm{SiO}_{2}$ and structural $3 \mathrm{C}-\mathrm{SiC}$.

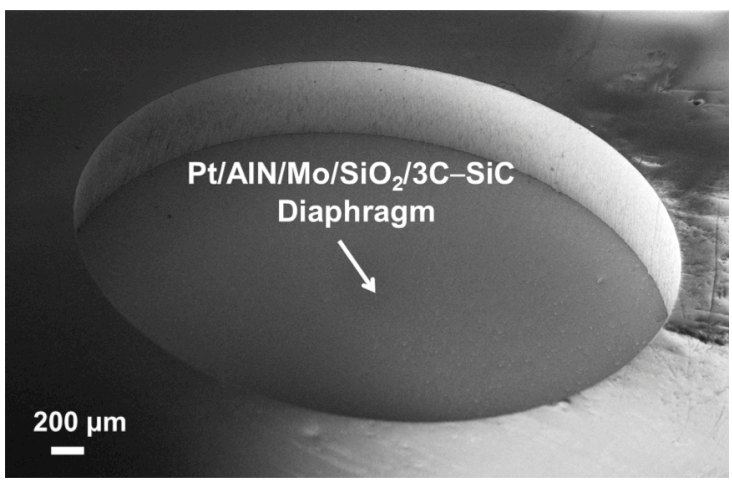

Figure 4: SEM of a released diaphragm structure (without boss) from the backside.

sputtered on blank Mo as shown in Fig. 2(c). A photolithography step followed by wet-etching AlN layer is used to access the bottom electrode (Fig. 2(d)). The top metal, Pt, is patterned on the AlN layer via a lift-off process to obtain the cross-section in Fig. 2(e). A backside photolithography step is then applied to create an etching mask for the diaphragm release step using $\mathrm{SF}_{6}$-based deep reactive ion etching (DRIE) and the final cross-section is shown in Fig. 2(f). The SEM of the cross-section of the composite layers is shown in Figure 3 and Figure 4 shows the SEM of a released energy harvester diaphragm (without boss) from the backside.

\section{MEASUREMENT SETUP}

\section{Pressure pulse generation}

In order to mimic the pulsed-pressure environment in the combustion chambers, an in-house pressure pulse generation unit is built. A commercial chopper (rotating blade with equally spacing slits) is utilized to modulate a static airflow and produce periodic pressure loads. The generated pressure pulse frequencies can be precisely controlled by both the chopper rotation speed and the number of blade slits. Figure 5 shows a time-domain waveform for $1-\mathrm{kHz}, 0.46-\mathrm{psi}$ pressure pulses measured by a commercial pressure sensor IC. The waveform shows the existence of higher frequency tones, which in fact present integer multiples of the prescribed frequency with smaller amplitude. Since the pressure amplitude generated is not constant due to the airflow fluctuations, the amplitude as well as the generated voltage from the harvester indicted in the following experimental results are presented as rms values for more than 100 cycles. 


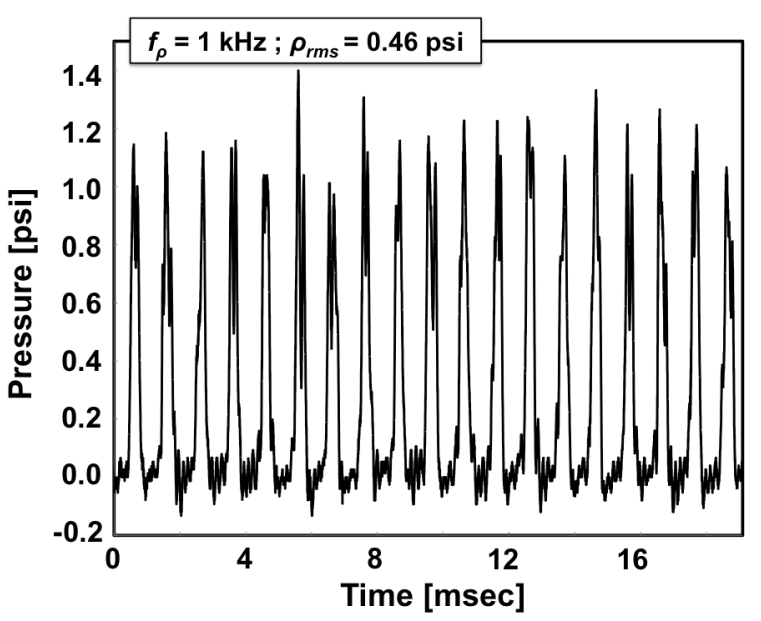

Figure 5: Measured waveform of 1-kHz, 0.46-psi (rms) pressure pulses from the pressure generation unit. .

\section{High temperature vibration testing}

In order to investigate the operation of the SiC/AlN energy harvesting device at elevated temperatures, the on-chip resistive heaters (fabricated along with the top metal electrode) are used to heat up the diaphragm via Joule heating to temperatures higher than $200^{\circ} \mathrm{C}$. The temperature of the diaphragm is then estimated by the variations of the temperature coefficients of resistance from another set of patterned top electrodes, forming the temperature sensor in the thermal platform. A shaker system is utilized in this case to provide vibration sources in order to measure the device responses with thermal elements since the cold pressure pulses generated from the setup discussed above prevent the heating of the device to high temperatures. It should be noted that the mechanical energy obtained from a vibrating shaker by the specific device demonstrated in this study is expected to be much less compared to that from pressure pulses and therefore, this measurement setup is only used to characterize the device operation under elevated temperatures and will not be able to reveal the power generation capabilities of the energy harvesting devices.

\section{EXPERIMENTAL RESULTS}

The devices are first characterized in the pulsed pressure environment as discussed in the previous section at room temperature. By connecting the harvester to a pure resistive load, time-varying voltages across the load are measured from an oscilloscope under various pressure amplitudes and load resistances. Figure 6 and 7 present the generated rms voltage and average power, respectively versus the load resistances from a device of $3500 \mu \mathrm{m}$ in diameter under $1-\mathrm{kHz}$ pressure pulses with varying amplitudes. With the given geometries of the diaphragm without central boss, the device is estimated to operate far from its resonance with $1-\mathrm{kHz}$ pressure pulses. The optimal load resistance, depending on the size of the active area as well as the source and resonance frequency of the device, is required in order to achieve maximum delivered power. A maximum power of $26 \mathrm{nW}$ is shown to be delivered to a $90-\mathrm{k} \Omega$ resistive load under a 0.46 -psi pressure with rms output voltage of $0.049 \mathrm{~V}$. Note that the small power output presented here is due to the low level of pressure amplitude applied as well as the far-from-resonance operation.

Lowering the device resonant frequency by introducing the central boss to the diaphragm with size properly determined, the harvested power can be greatly improved. Figure 8 shows the

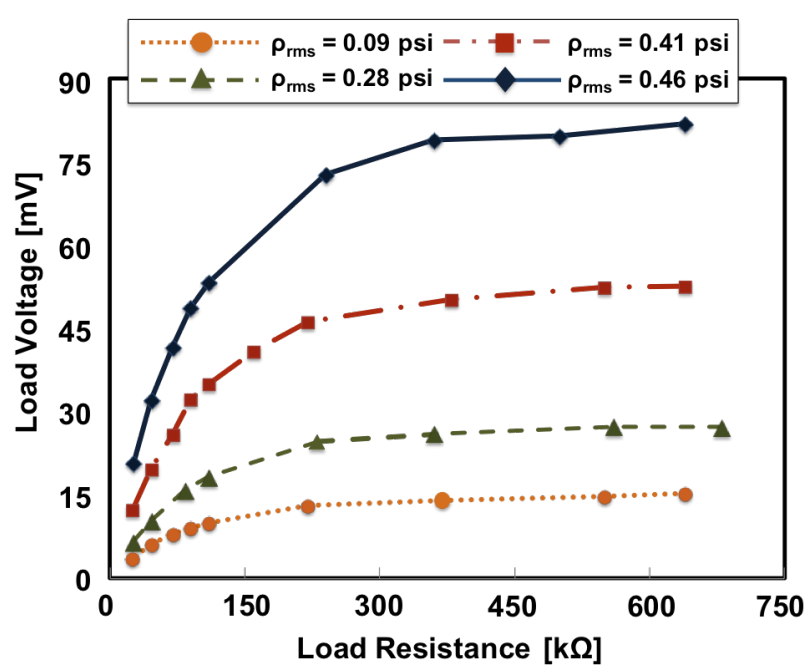

Figure 6: Measured generated voltages versus load resistance under varying pressure amplitude at a fixed $1-\mathrm{kHz}$ frequency. The device is $3500 \mu \mathrm{m}$ in diameter without boss and electrode size is $3100 \mu \mathrm{m}$.

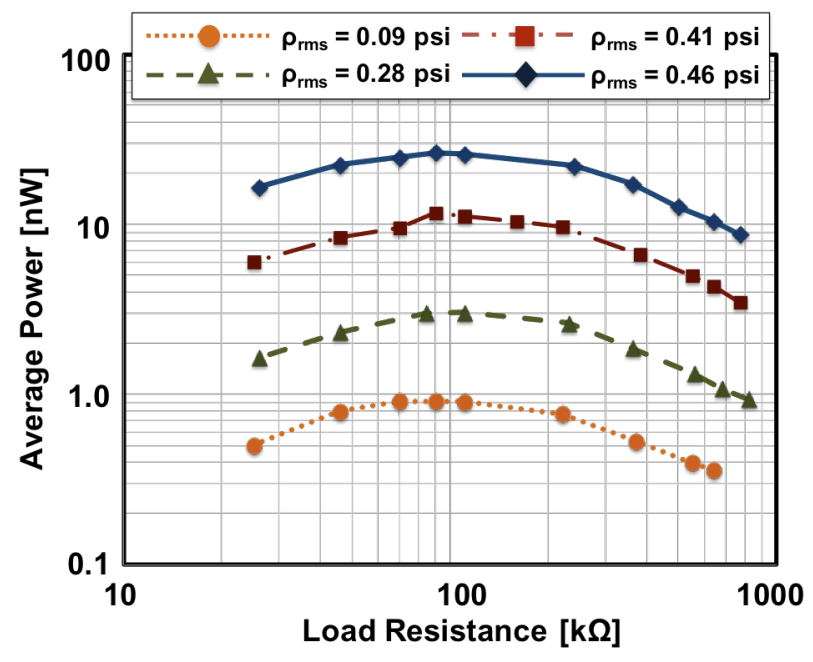

Figure 7: Measured average power versus load resistance under varying pressure amplitude at a fixed $1-\mathrm{kHz}$ frequency. The device is $3500 \mu \mathrm{m}$ in diameter without boss and electrode size is $3100 \mu \mathrm{m}$.

response of a device with a $4000-\mu \mathrm{m}$ diaphragm and a central boss of $1500 \mu \mathrm{m}$ producing rms voltage of $0.2 \mathrm{~V}$ and an average power of $2.5 \mu \mathrm{W}$ with a $26-\mathrm{k} \Omega$ load under $2-\mathrm{kHz}$ pressure pulses with pressure amplitude of $1.09 \mathrm{psi}$ (rms), corresponding to a power density of $\sim 20 \mu \mathrm{W} / \mathrm{cm}^{2}$. Though the prescribed frequency is still lower than the resonance frequency (estimated to be $10 \mathrm{kHz}$ ), the device is excited at resonance by higher frequency tones of the prescribed frequency with reduced amplitude. Based on these results, it can be expected that by matching the device resonant frequency with the pressure source frequency, increased power can be generated with design improvements.

\section{High temperature measurement}

To characterize the energy harvesters' response at elevated temperatures, the devices with central boss, which can be easier to actuate with acceleration from a shaker system, are measured under vibration at temperatures up to $250^{\circ} \mathrm{C}$, achieved by the 


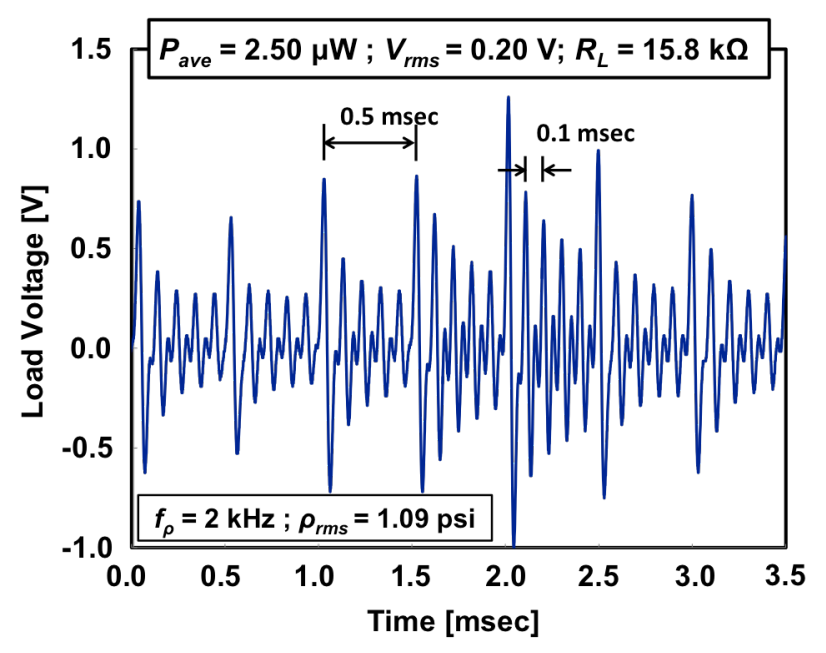

Figure 8: Measured output voltage waveform on a 15.8- $k \Omega$ load under 2-kHz, 1.09-psi (rms) pressure pulses. The device is $4000 \mu \mathrm{m}$ in diameter with a 1500- $\mu \mathrm{m}$ central boss and 1125- $\mu$ m-wide electrode.

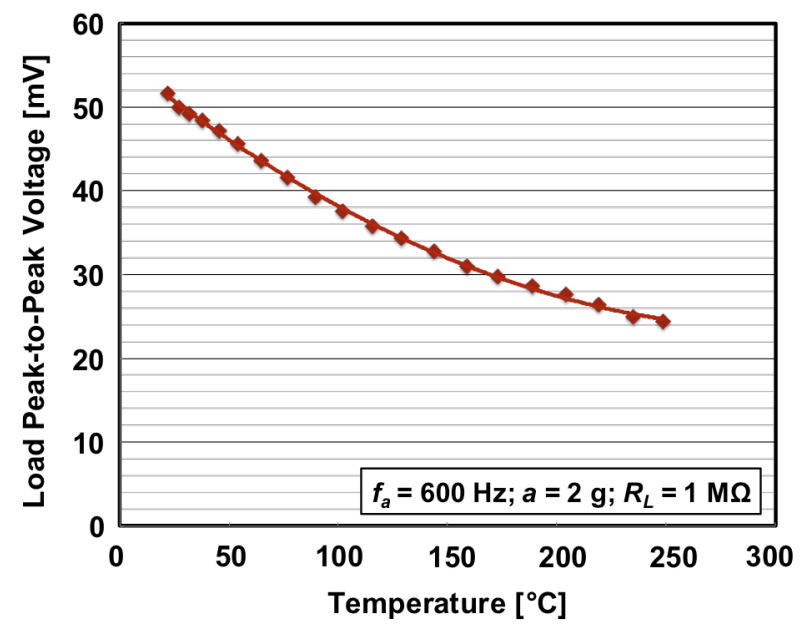

Figure 9: Measured peak-to-peak voltage on a 1-MS load resistor versus temperatures. The device is $3000 \mu \mathrm{m}$ in diameter

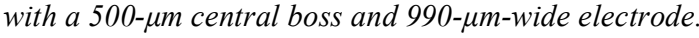

on-chip heater as previously described in the measurement setup section. Figure 9 shows the generated peak-to-peak voltage versus temperatures from $22^{\circ} \mathrm{C}$ to $250^{\circ} \mathrm{C}$ on a $1-\mathrm{M} \Omega$ resistive load for the device under a 2 -g vibration. The presented data is obtained with the harvester experiencing a number of thermal cycling until reaching stable operation. It can be noticed in Figure 9 that the output voltages degrade as temperature increases and possible reasons leading to the degradation are the induced thermal stress in the diaphragm as well as the increased thermal resistances from the metal. More importantly, the device is able to generate power at temperatures above $200^{\circ} \mathrm{C}$ and confirms that the AlN remains piezoelectric under these conditions.

\section{CONCLUSIONS}

An output power density of approximately $20 \mu \mathrm{W} / \mathrm{cm}^{2}$ has been obtained with the micromachined piezoelectric $\mathrm{SiC} / \mathrm{AlN}$ bossed-diaphragm energy harvesters under $2-\mathrm{kHz}$ pressure pulses of 1.09 psi (rms). Although increased electrical power output can be expected by matching the pressure pulse frequency to the diaphragm mechanical resonant frequency, the power density level is already sufficient for the need in most of low-power sensor circuits. Under elevated temperatures up to $250^{\circ} \mathrm{C}$, measurements confirm that the energy harvesting devices are still able to generate electrical power with reduced output levels compared to that of room temperature. This shows that the fabricated SiC/AIN energy harvesting device for pulsed-pressure sources is a promising power source for harsh environment circuits and sensors. Obviously, further investigations are needed to understand the causes of power degradation at elevated temperatures and to ensure functionality in more stringent environments.

\section{ACKNOWLEDGEMENTS}

The authors would like to acknowledge the Marvell Nanofabrication Laboratory staff in the University of California at Berkeley for their processing assistance and the Transducer Research Foundation for the generous travel support.

\section{REFERENCES}

[1] S.P. Beeby, M.J. Tudor and N.M. White, "Energy Harvesting Vibration Sources for Microsystems Applications," Meas, Sci, Technol. 17, pp. 175 - 195, 2006.

[2] P.D. Mitcheson, et al., "Energy Harvesting from Human and Machine Motion for Wireless Electronic Devices," Proc. of the IEEE vol. 96, no. 9, pp. 1457 - 1486, 2008.

[3] S.B. Barker, K.V. Vassilevski, N.G. Wright and A.B. Horsfall, "High Temperature Vibration Energy Harvester System," Proc. IEEE Sensors Conf., pp. 300 - 303,2010.

[4] S.B. Barker, et al., "Silicon Carbide based Energy Harvesting Module for Hostile Environments," Materials Science Forum vol. 645-648, pp. 1093 - 1096, 2010.

[5] A.C. Patil, et al., "6H-SiC JFETs for $450^{\circ} \mathrm{C}$ Differential Sensing Applications," J. Microelectromechanical Systems, vol. 18 , no. 4, pp. 950 - 961, 2009.

[6] R.G. Azevedo, D.G. Senesky, A.V. Jog, B. Jamshidi, D.R. Myers, L. Chen, X.-A. Fu, M. Mehregany, M.B.J. Wijesundara, and A.P. Pisano, "A SiC MEMS Resonant Strain Sensor for Harsh Environment Applications," IEEE Sensors J., vol. 7, No. 4, pp. 568 - 576, 2007.

[7] R. Farrell, et al., "High Temperature Annealing Studies on the Piezoelectric Properties of Thin Aluminum Nitride Films," Proc. of Mater. Res. Soc. Symp., vol. 1052, no. 1052-DD06-18, 2008.

[8] C.-M. Lin, T.-T. Yen, V.V. Felmetsger, M.A. Hopcroft, J.H. Kuypers, and A.P. Pisano, "Thermally Compensated Aluminum Nitride Lamb Wave Resonators for High Temperature Applications," Appl. Phys. Lett., vol. 97, $083501,2010$.

[9] V.V. Luchinin, A.V. Korlyakov, and A.A. Vasilev, "Silicon Carbide-Aluminium Nitride: a New High Stability Composition for MEMS," Proc. SPIE, vol. 3680, pp. 783 791, 1999.

[10] J.H. Jerman, "The Fabrication and Use of Micromachined Corrugated Silicon Diaphragms," Sensors and Actuators, A21-A23, pp. 988 - 992, 1990.

[11] V.V. Felmetsger, P.N. Laptev, and S.M. Tanner, "Innovative Technique for Tailoring Intrinsic Stress in Reactively Sputtered Piezoelectric Aluminum Nitride Films," J. Vac. Sci. Technol. A, vol. 27, no. 3, pp. 417 - 422, 2009.

\section{CONTACT}

*Y.-J. Lai, Tel: +1-510-643-9752; matildal@eecs.berkeley.edu 


\section{Author Index}

Scroll to the author and select a Blue link to open a paper. After viewing the paper, use the bookmarks to the left to return to the beginning of the Author Index.

A

Abdolvand, R.

$332,359,371$

Abelson, J.R.

437

Agah, M.

201, 221

Agasid, E. 94

Agrawal, D. 473

Ahn, C.H. 54

Ahn, C.H.

177,209

Akinwande, A.I

324

Aksyuk, V.

279

Aktakka, E.E 256

Allen, M.G.

$38,149,481$

Almeida, R.

18,90

An, $\mathrm{S}$.

Anand, S.

Arft, C.M 66

Arnold, D.P. $22,117,303$

Arumugam, N. 66

Assaderaghi, F 66

Averitt, R.D. 320

Awtar, S. 409

Ayazi, F.

98, 275, 328

\section{B}

Bae, Y.-C. 497

Bahl, G.

Bai, Q. 477

Baker, M. 157

Bardaweel, H.K.

Bargatin, I.

336, 493

Baskaran, R. 421

Beasley, C. 94

Bedair, S.S.

Bedewy, M.

Ben-Yoav, H.

193, 233

Benito-Lopez, F

Bentley, W.E.

Bernal, L.P.

Beroz, J.

Bhave, S.A.

Bica, L.

Böhringer, K.F.

Bose, S. 
Boser, B.E.

Boudoux, C.

Bramall, N.

Bramhanand, A.

Branchflower, T.T.

Brand, O.

Brandt, J.D.

Brechtel, F.J

Bristol, R.

Brobston, $\mathrm{M}$

Brown, A.D. 100,193

Bryson, K.

Buchman, D.

Buffa, C.

C

Candler, R.N.

217,465

Carmon, T. .....

Carpenter, Z.S.

Carraro, C.

Carron, C.

Casinovi, G.

Cattafesta, L.N.

Chae, J.

137, 383, 501

Chan, M.L. 355

Chandrasekharan, V.

Chang, J.

Chen, K.

Chen, P.C 34

Chen, S.

Chiang, C.-F.

54, 271

Chiao, M.

Chitnis, G.

133, 379

Chittenden, J.

Choi, S.

Choi, S.

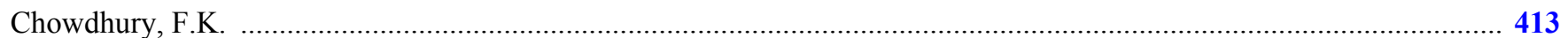

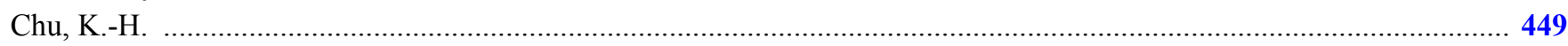

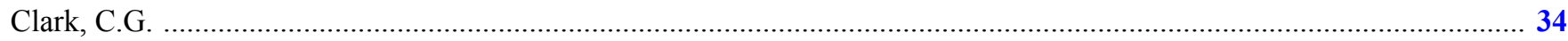

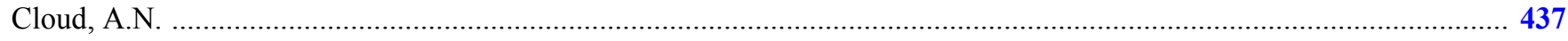

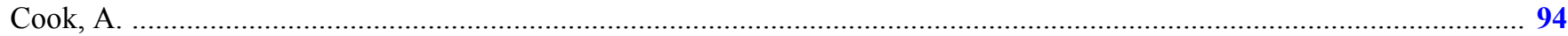

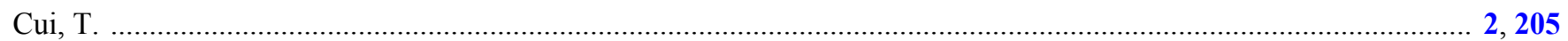

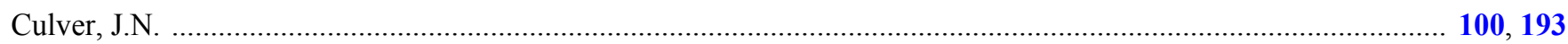

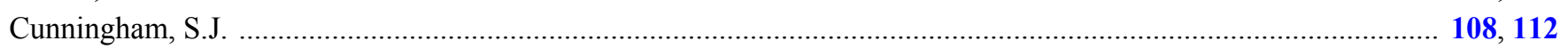

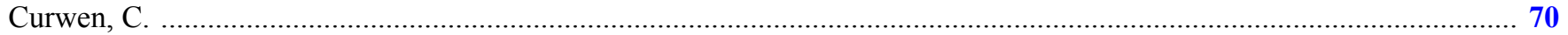

\section{D}

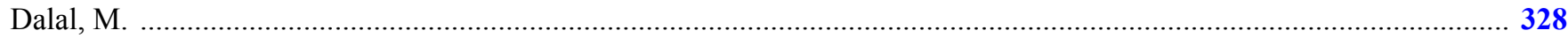

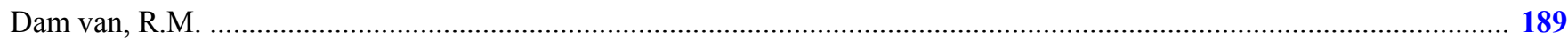

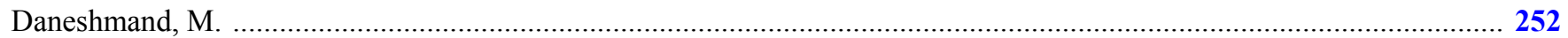




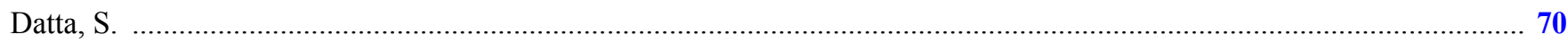

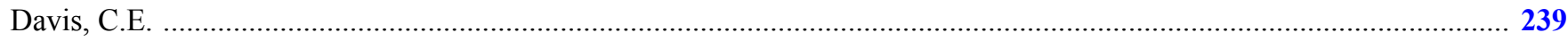

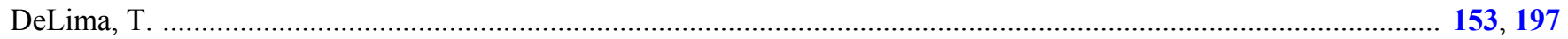

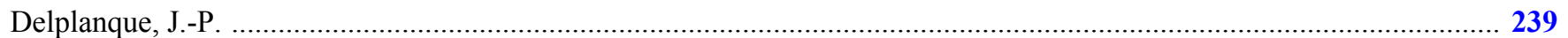

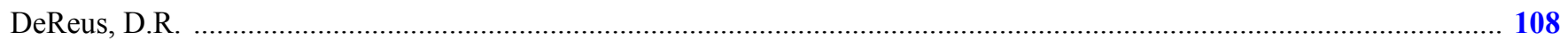

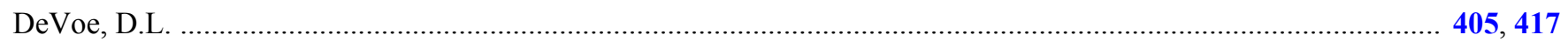

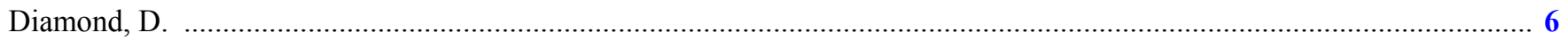

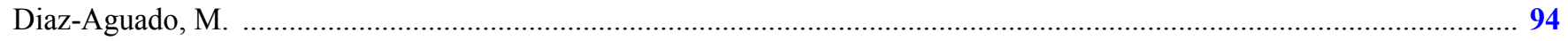

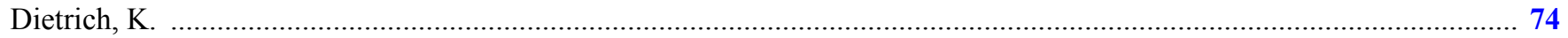

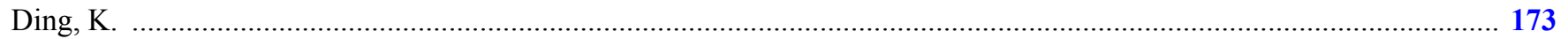

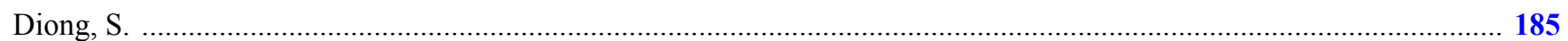

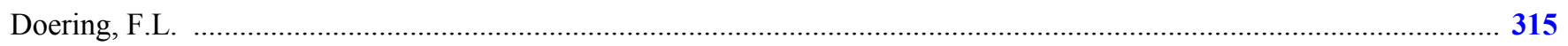

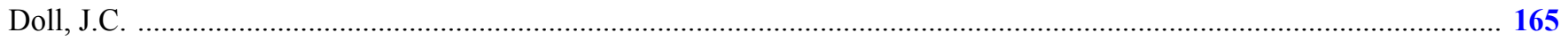

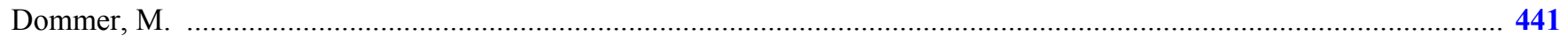

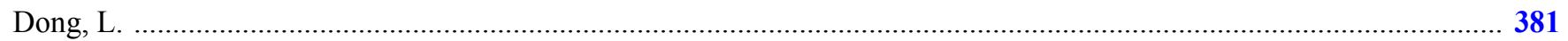

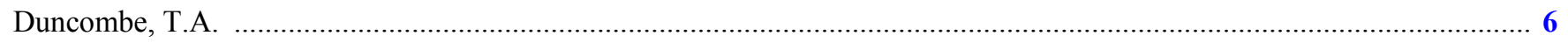

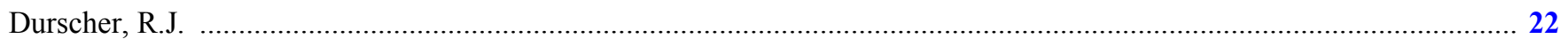

E

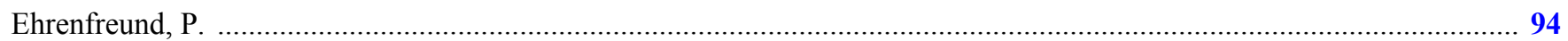

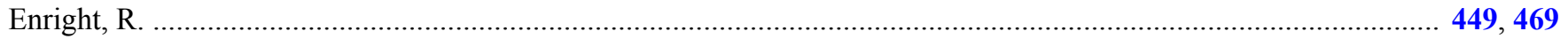

F

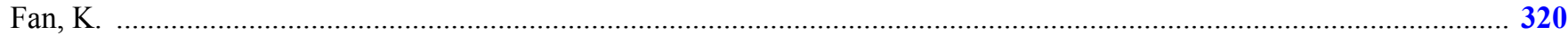

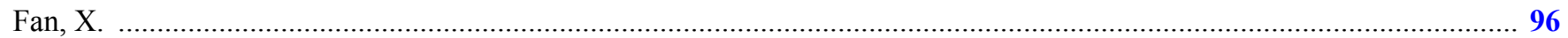

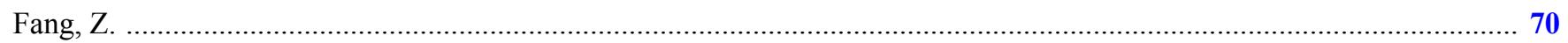

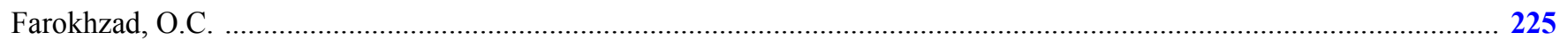

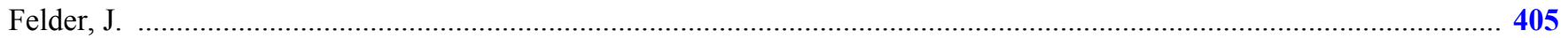

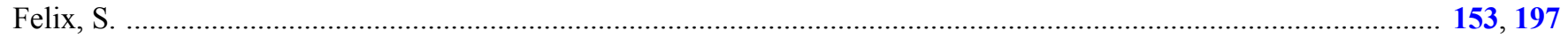

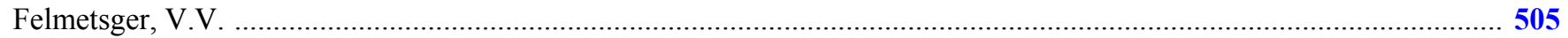

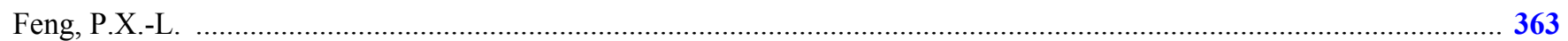

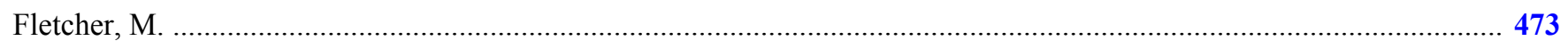

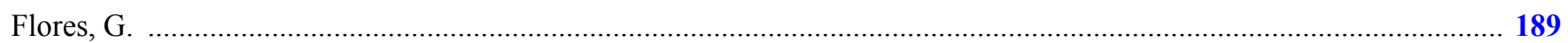

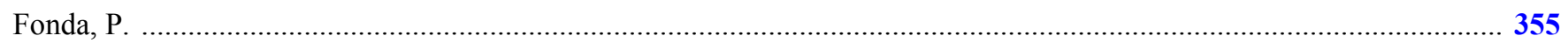

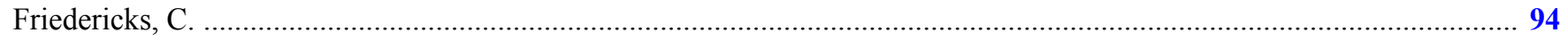

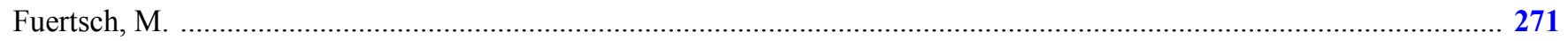

G

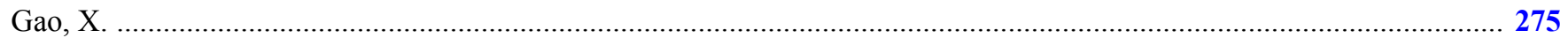

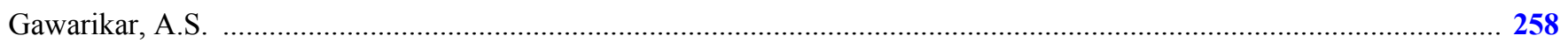

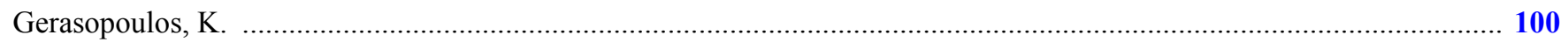

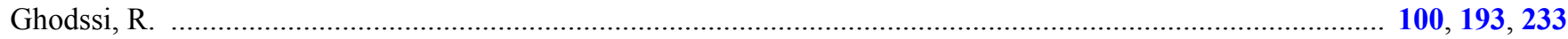

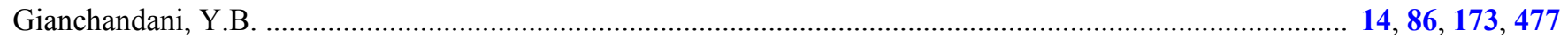

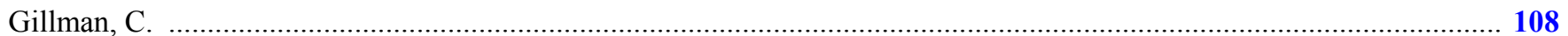

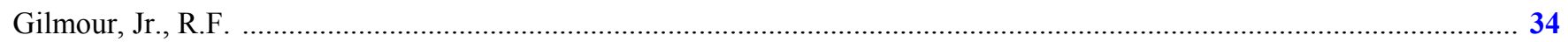

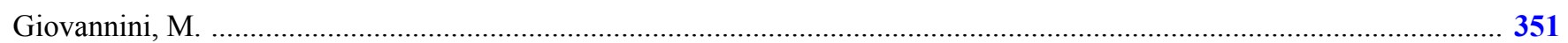

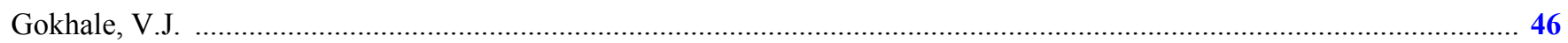

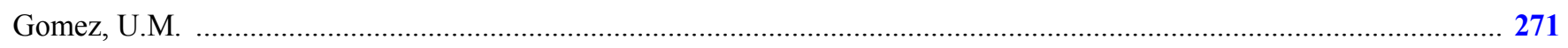

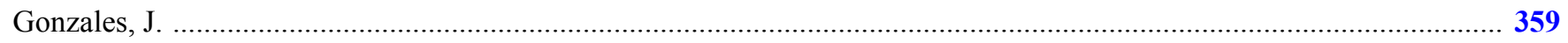

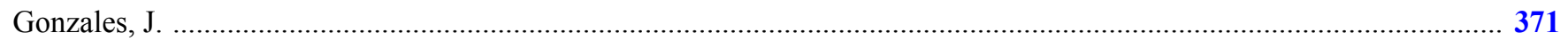

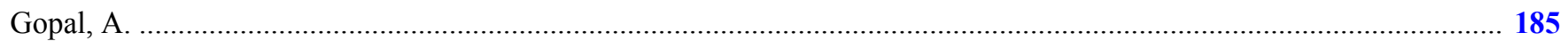


Gordon, M.D.

Gorman, J.J.

Goulamhoussen, N

Graham, A.B.

54,453

Green, S.R.

Griffin, J.

303

Grosjean, C.

Gudeman, C.

Guedes, A.

50, 291

Guo, X.D.

Gupta, A. 473

Gupta, N.K.

Gupta, P.

\section{H}

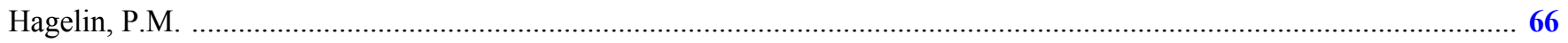

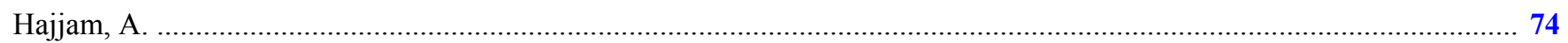

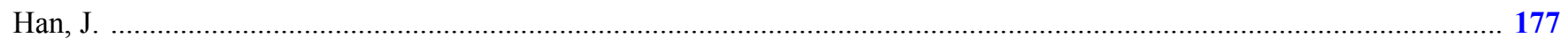

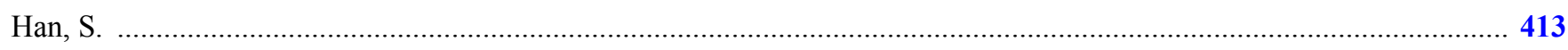

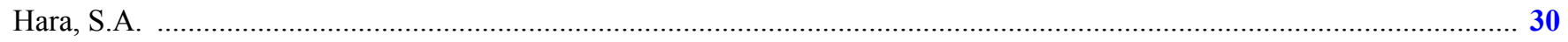

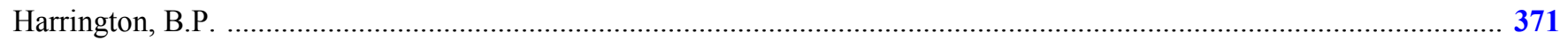

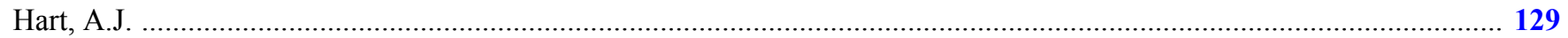

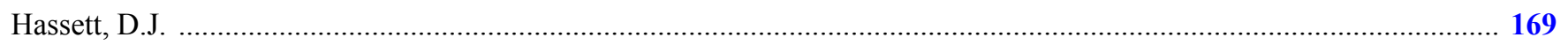

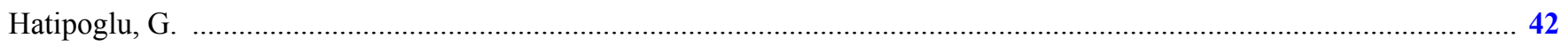

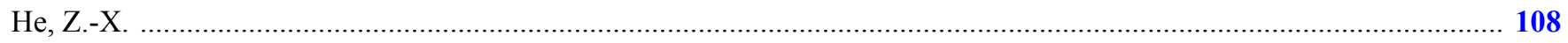

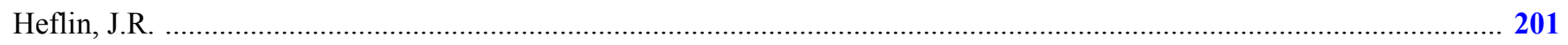

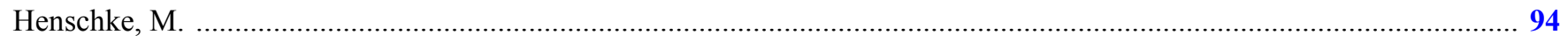

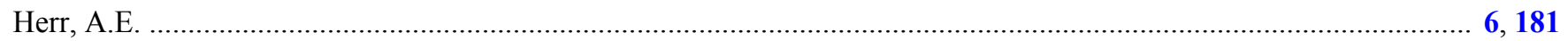

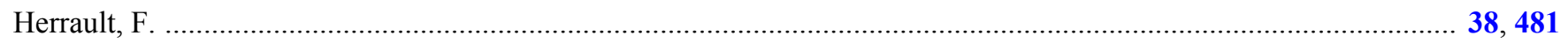

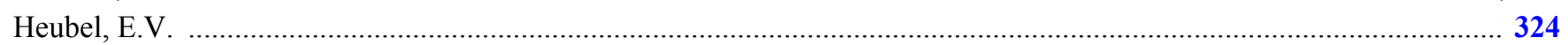

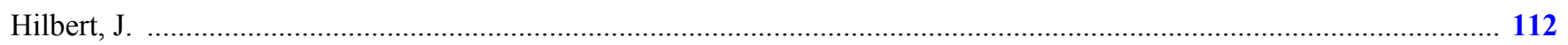

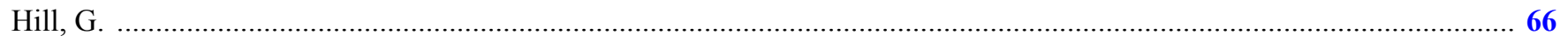

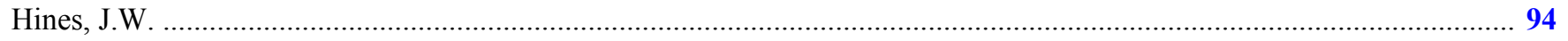

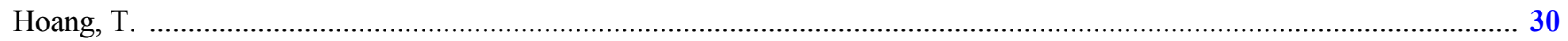

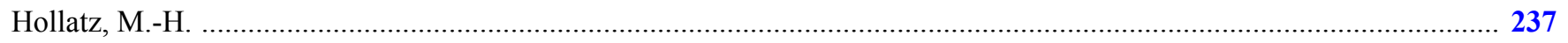

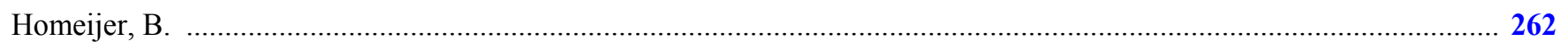

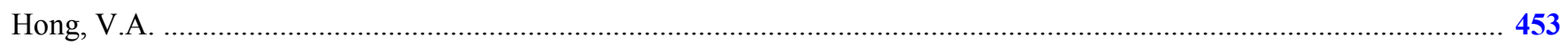

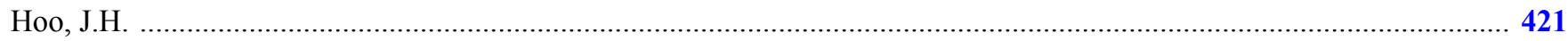

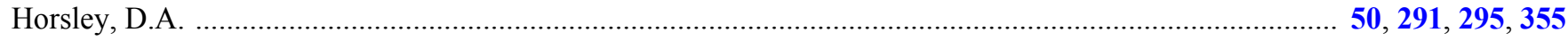

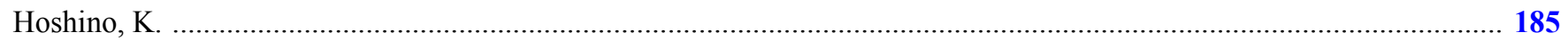

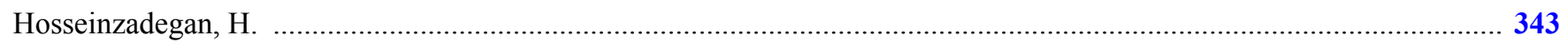

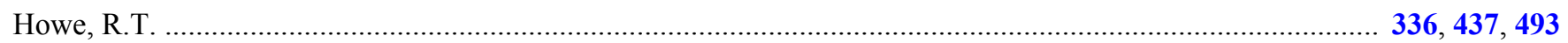

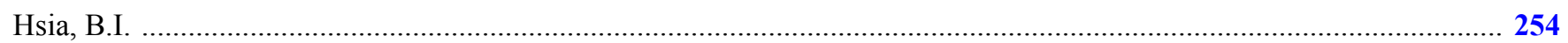

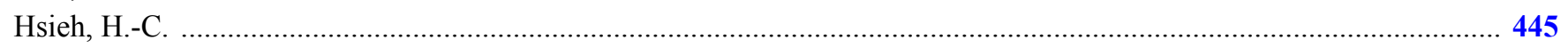

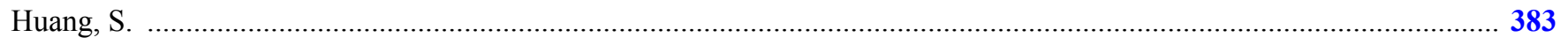

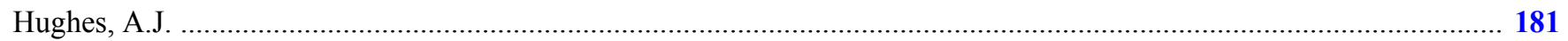

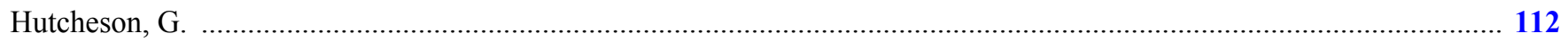


I

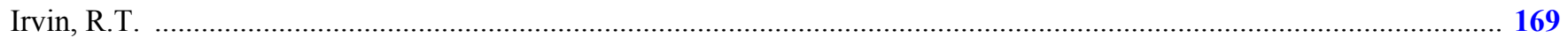

Ivanov, T.G. . .

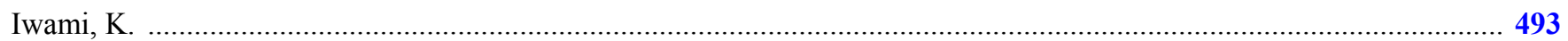

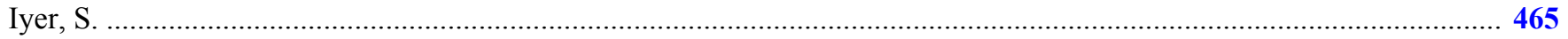

$\mathbf{J}$

Jahnes, C.V. .

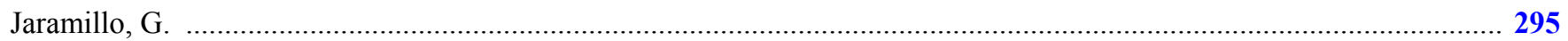

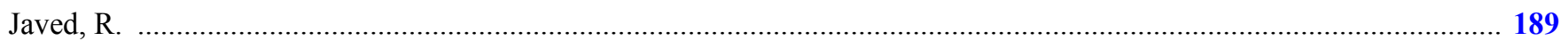

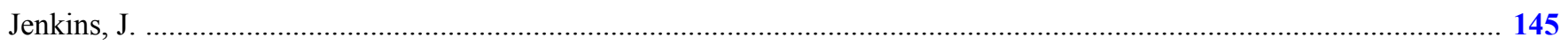

Jiao, J. . .

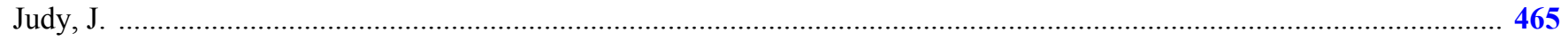

Jug, L. .

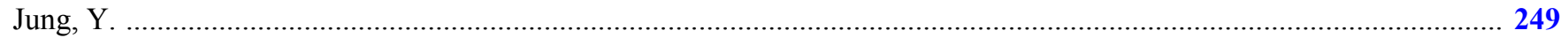

K

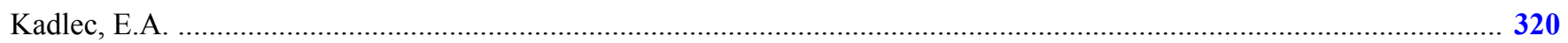

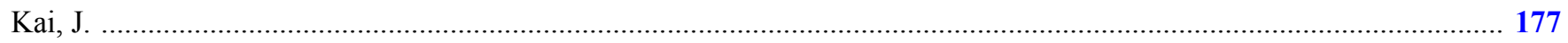

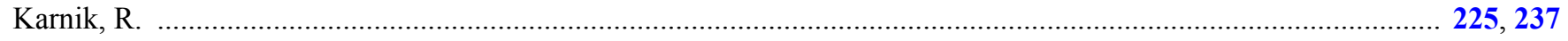

Karp, J.M. .

Keese, B. . . 1 .

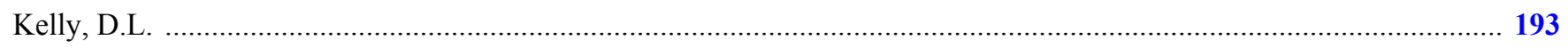

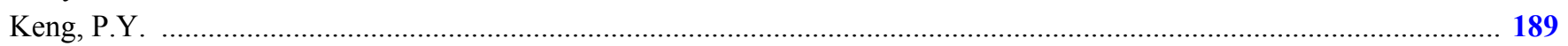

Kenny, T.W. .

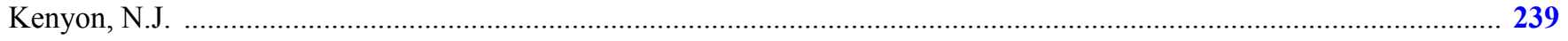

Kim, A. . .

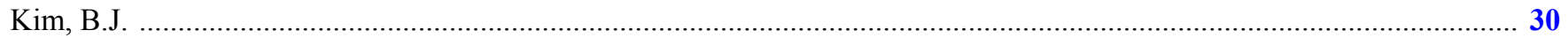

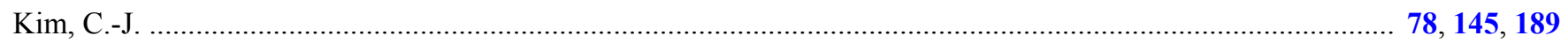

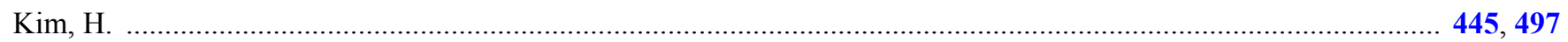

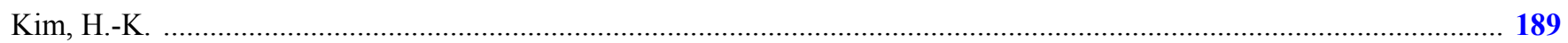

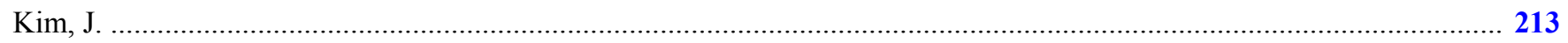

Kim, J.K. .

Kim, K.H. . . -

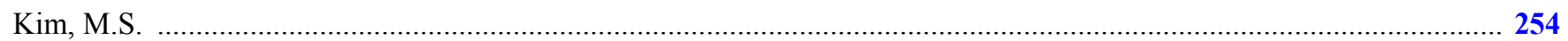

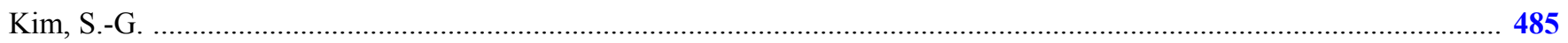

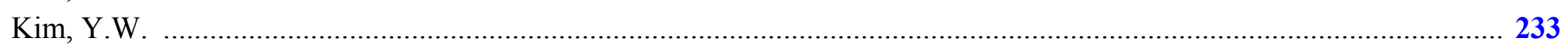

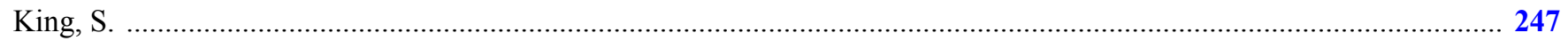

Kitts, C. .

Knutrud, P. . .

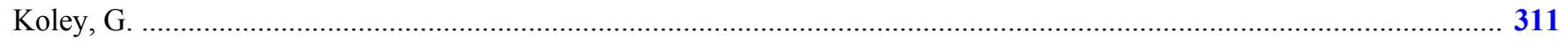

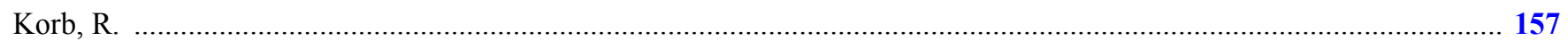

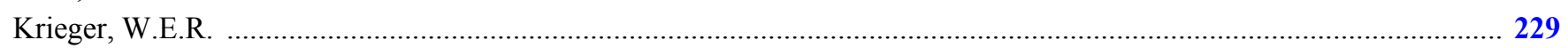

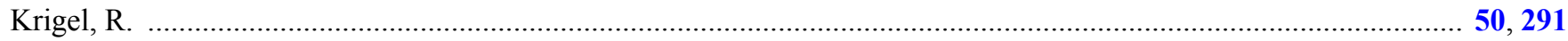

Kuo, J.C. . - w.

Kuo, J.T.W. .

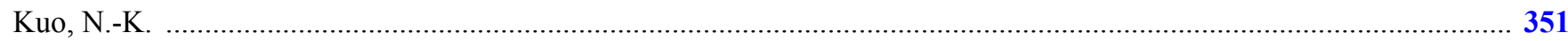

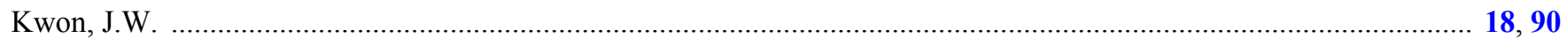


$\mathbf{L}$

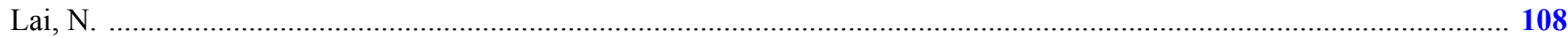

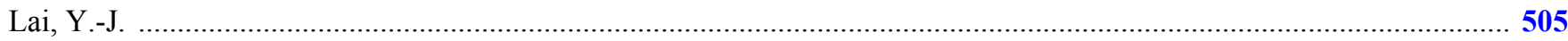

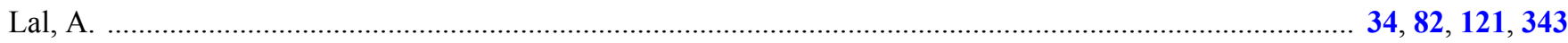

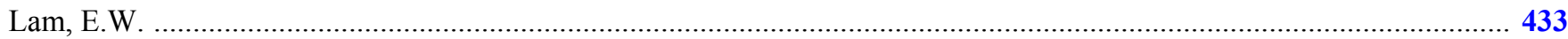

Landis, D. .

Langer, R.S. . . -

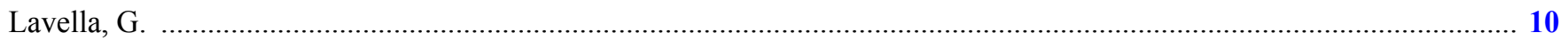

Lee, B.Y. .

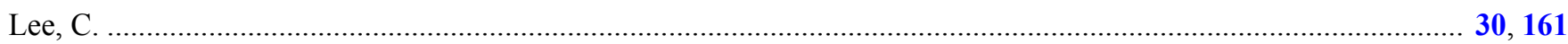

Lee, C.-H. .

Lee, E. .

Lee, H. .

Lee, J. .

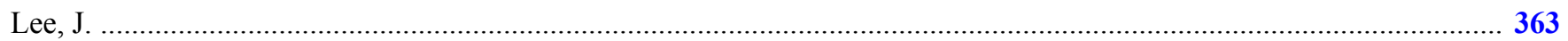

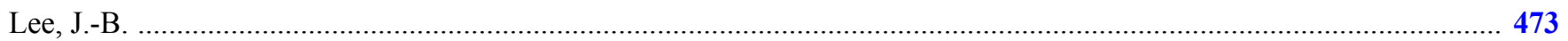

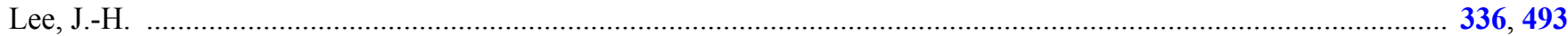

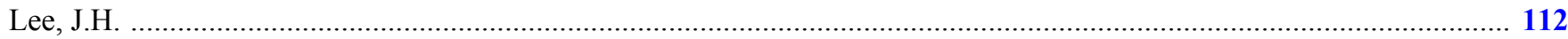

Lee, K.K. .

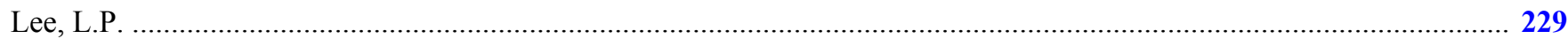

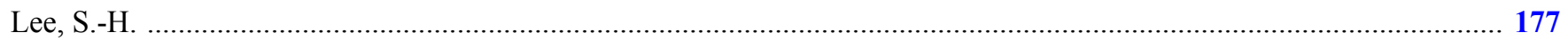

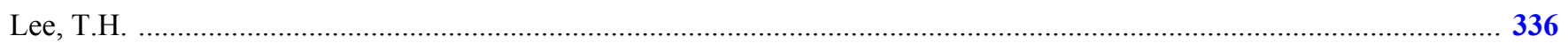

Lee, W. .

Lee, W.S. . 1.

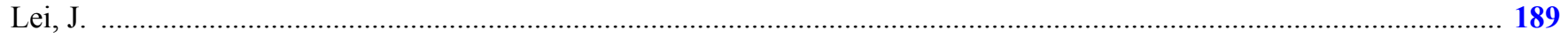

Lemmens, R.J. .

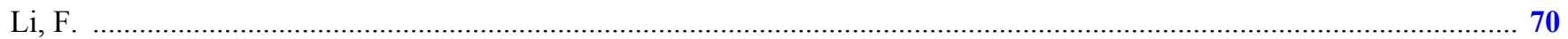

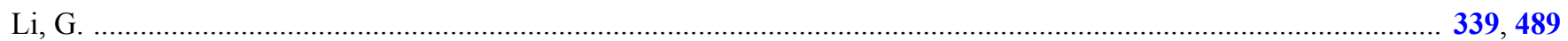

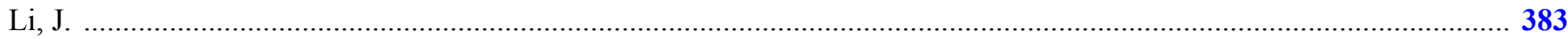

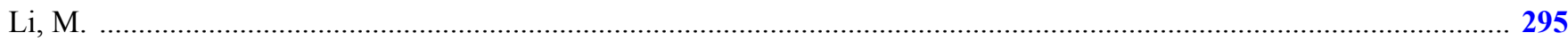

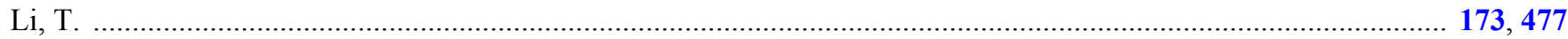

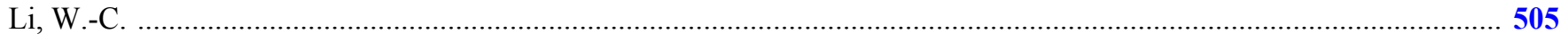

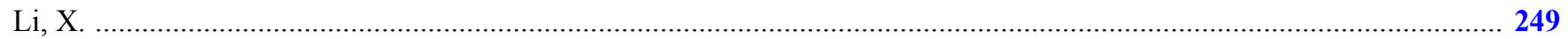

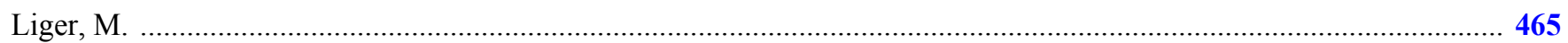

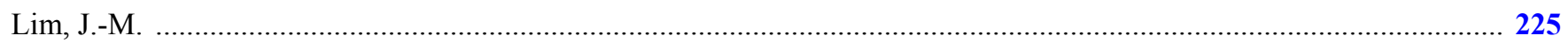

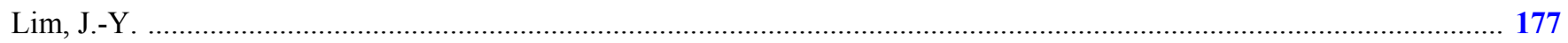

Lin, C.-M. .

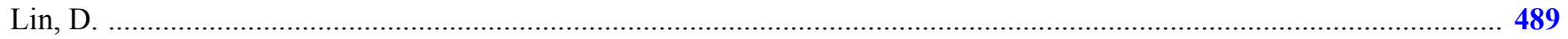

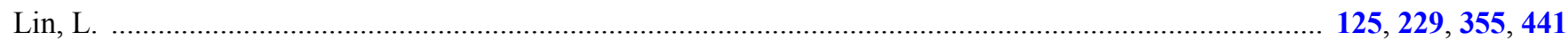

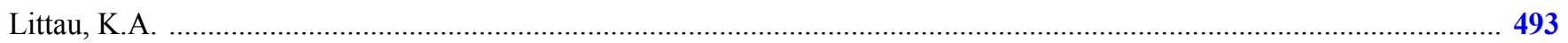

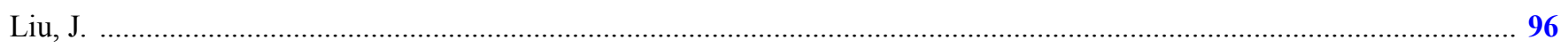

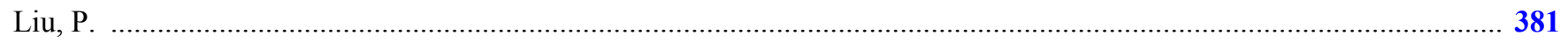

Liu, T. ...

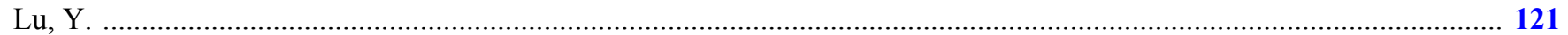

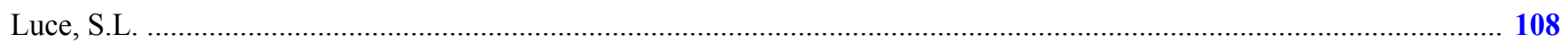

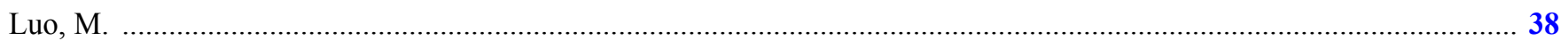

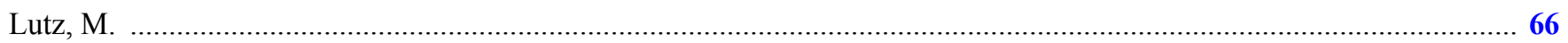

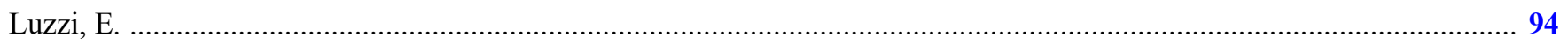

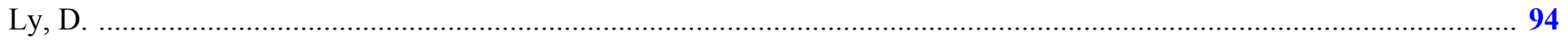


Maboudian, R.

Mahajerin, A.

Maharbiz, M.M.

Mai, N.

Maling, J.

Mancinelli, R

Mansoor, $\mathrm{H}$.

Mansour, R.R

Mao, D.

381

Marek, J.

Martin, R.J.

Mattioda, A.

McCarthy, M.

100, 247

McIntyre, M.

McMillen, C.

McNamara, S.

McNeil, A.

Mehdizadeh, E.

Mehner, J.

Mehregany, M.

Melamud, R.

Melosh, N.A.

Meloy, J.

Meng, D.D.

Meng, E.

30, 161

Meng, G.

Messana, M.W

Meyer, C.D.

Meyer, M.T.

Miao, $\mathrm{H}$.

Miljkovic, N.

Minelli, G.

Misra, R.

Mitra, S.

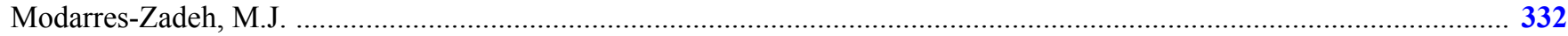

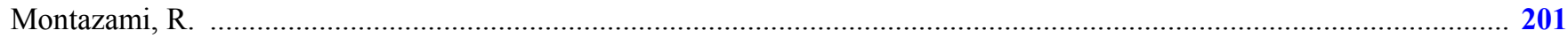

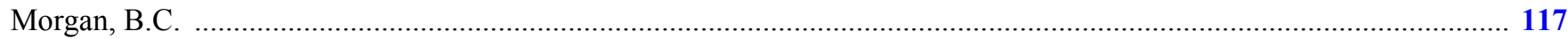

Morris, A.S.

112,108

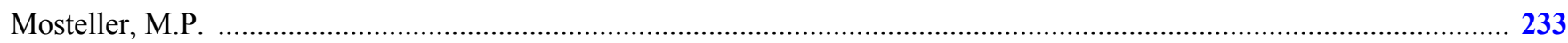

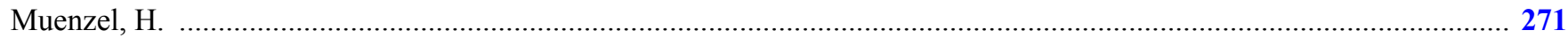

Mukherjee, S. .

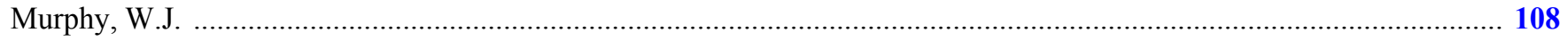

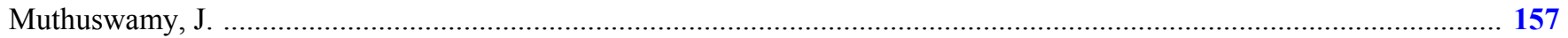

$\mathbf{N}$

Najafi, K. 
Neumann, M.

$\mathrm{Ng}, \mathrm{E}$.

Ng, E.J.

Ngo, K.

Nicholson, W.

Nie, B. 299

Nijjar, B.

\section{O}

O'Brien, G.J.

54, 271

Okandan, M.

Olceroglu, E.

Olfatnia, M.

P

Padilla, W.J

Paek, J.W.

Paik, S.-J.

149, 481

Pan, T.

Pandey, M.

$82,121,343$

Panmanee, W.

Pannu, S.

153, 197

Paprotny, I. 315

Paredes, C.N

Park, K.S.

Park, S.

Parra, M. 94

Parsa, R.

Partridge, A.

Paydar, O.H.

Peterson, R.L.

256, 393

Piazza, G. 347, 351, 367

Piccini, M.

Piratla, S.

Pisano, A.P. 505

Polcawich, R.G.

389, 417

Pomerantseva, E.

100, 193

Potkay, J.A.

Poulin, A.

Pourkamali, S.

Prausnitz, M.R.

Prikhodko, I.P.

Proie, R.M.

Provine, J 336,437

Pruitt, B.L.

Przybyla, R.J.

50, 291

Pulskamp, J.S.

Puntambekar, A. 
Q

R

Rahafrooz, A.

$74,359,375$

Rahman, M.

Rahman, M.M.

445, 497

Rais-Zadeh, M.

46,385

Rajgopal, S.

363

Rasay, R. ....

Raum, C.R.

Reed, M.A.

Ren, $\mathrm{H}$.

Rhee, M.

Ricco, A.J.

94

Rice, G.

Ricks, R.

Rinaldi, M.

Roberts, J.

Roy, S.

\section{S}

Saab, D.

Salvia, J.C.

Sameoto, D.

Sammoura, F.

Samuelson, S.R.

Santos, O.

Sarkar, N.

Schaffer, C.

Schiffer, P.

Schmidt, M.A

Schooley, A.

Schwerdt, H.N

Sells, J.

Sen, P.

Senesky, D.G.

Senkal, D.

Seyfried, Jr., W.E.

173

Shah, K.G.

153, 197

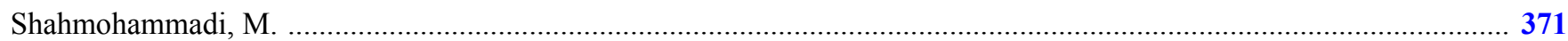

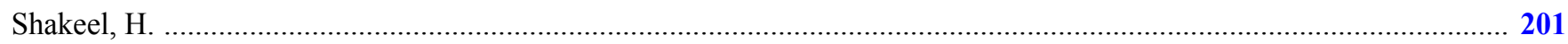

Shaner, E.A. . .

Shao, P. .

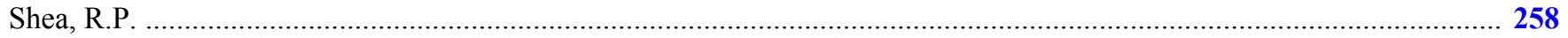

Shelton, S.E. .

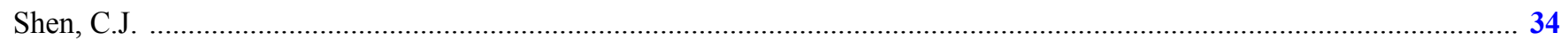

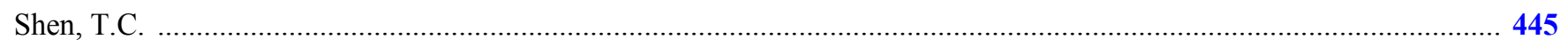

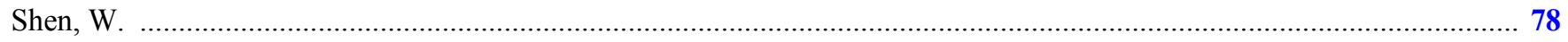




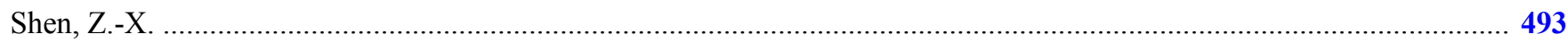

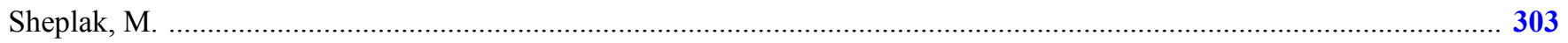

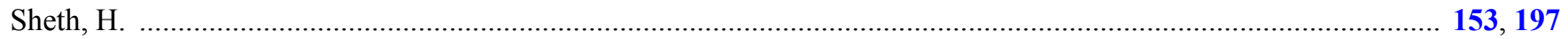

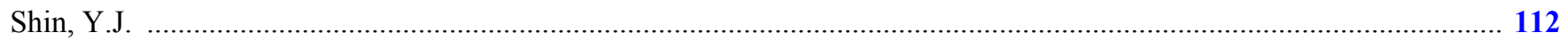

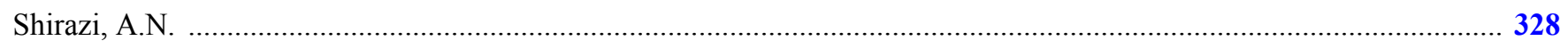

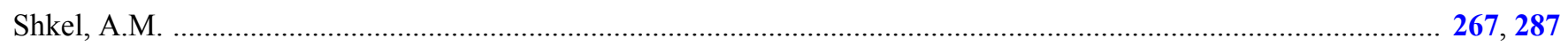

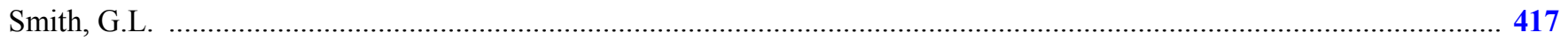

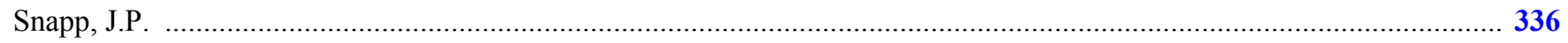

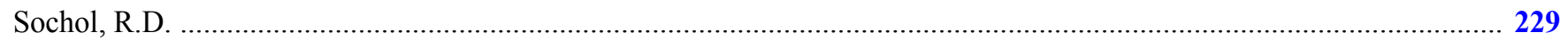

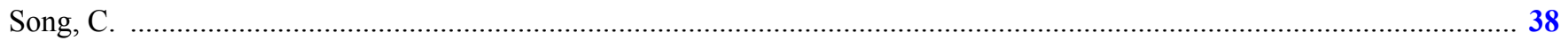

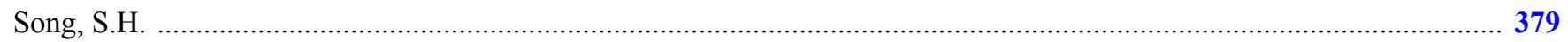

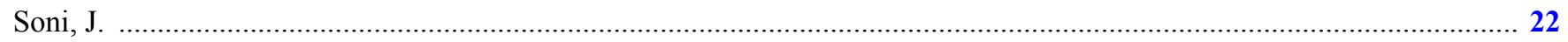

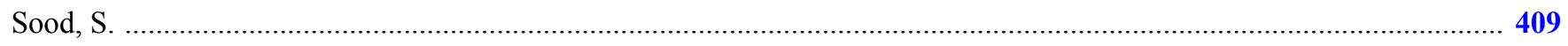

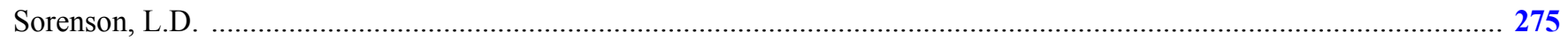

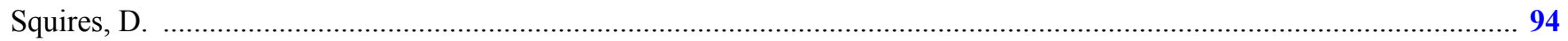

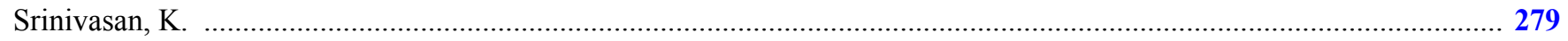

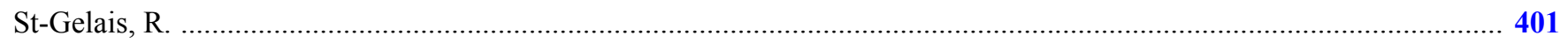

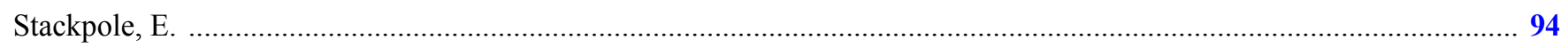

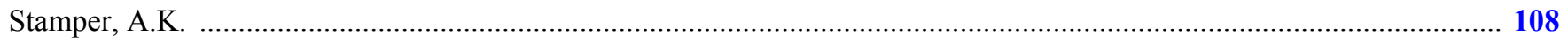

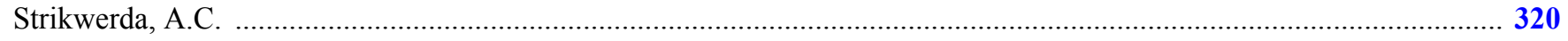

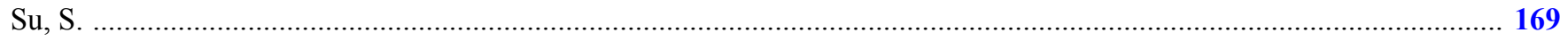

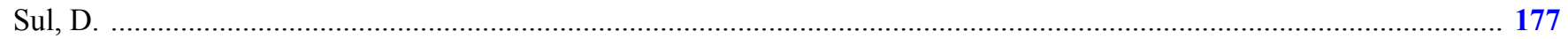

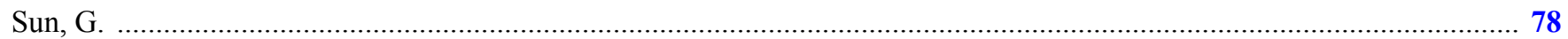

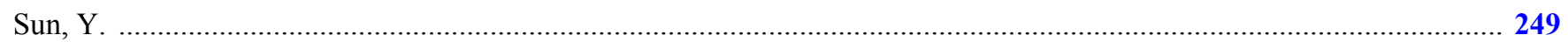

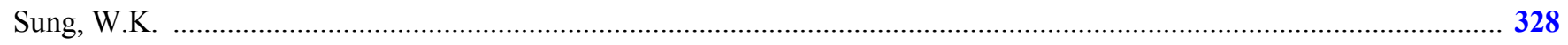

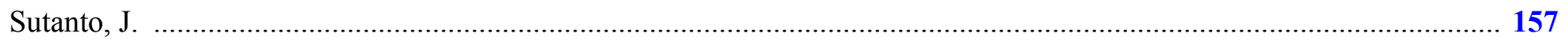

T

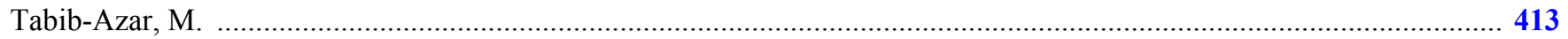

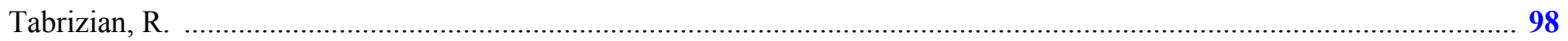

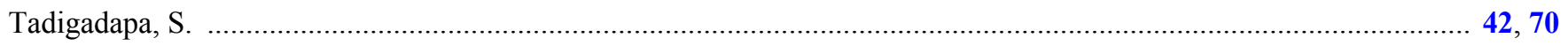

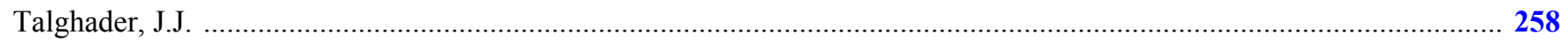

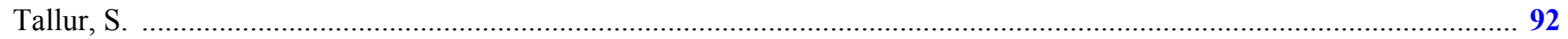

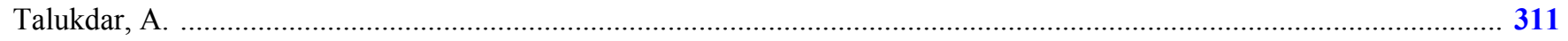

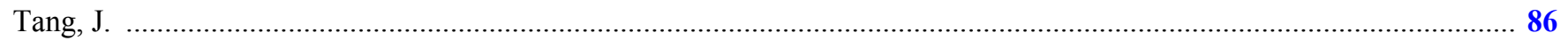

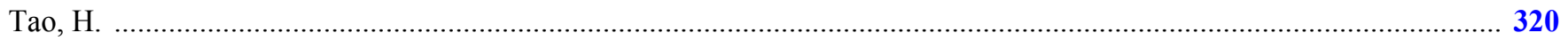

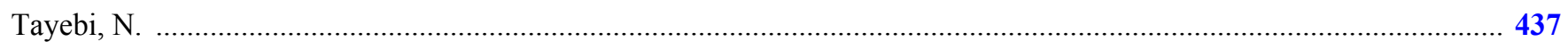

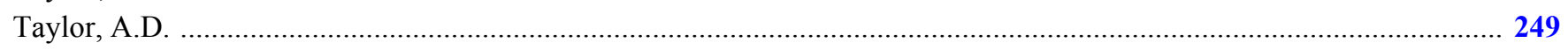

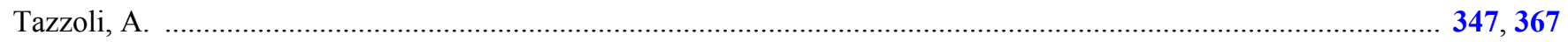

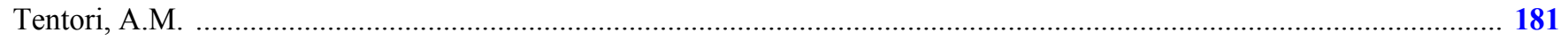

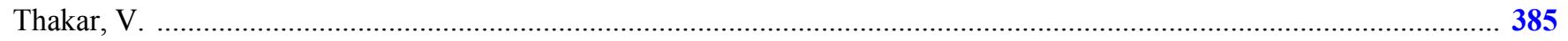

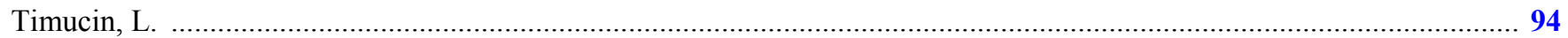

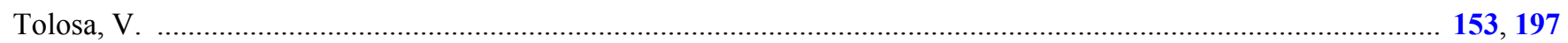

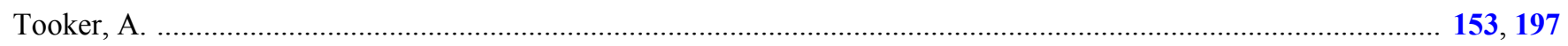

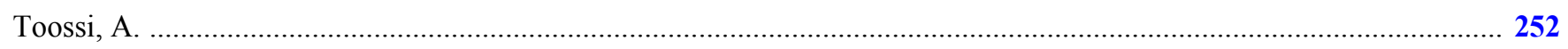

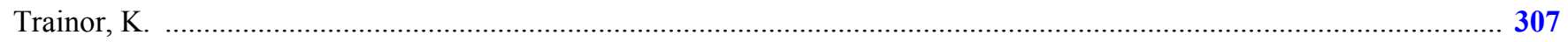

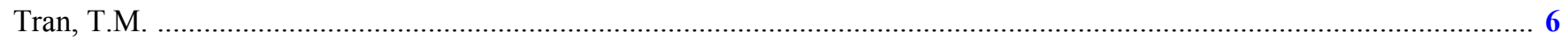

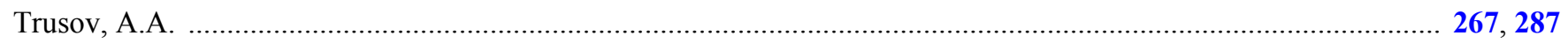

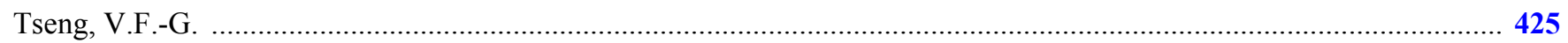

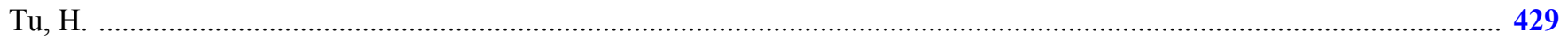


V

Valencia, P.M. .

Varel, C. . .

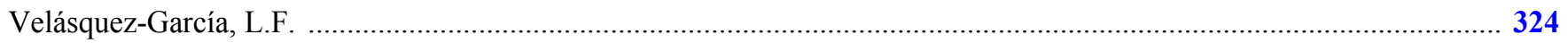

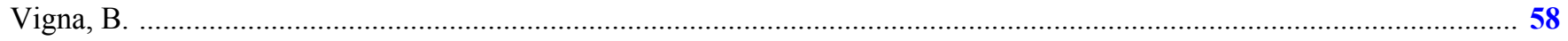

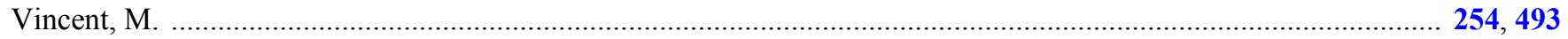

Vinson, A. .

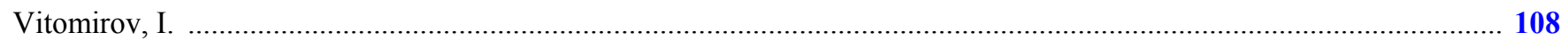

W

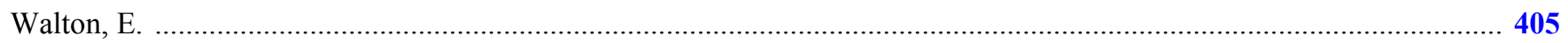

Wang, C. .

Wang, D. .1.

Wang, E.N. . .

Wang, R. .

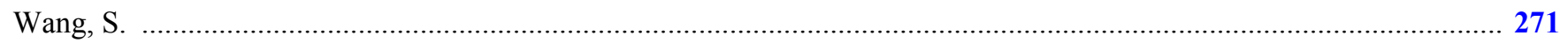

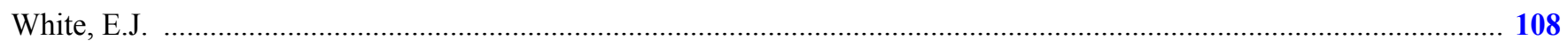

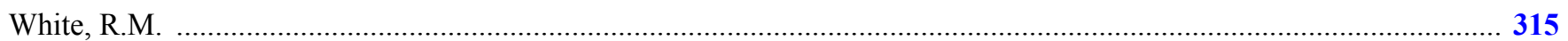

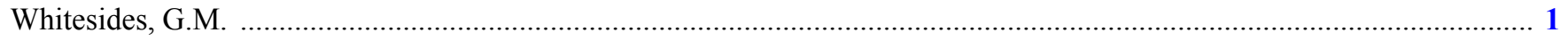

Williams, J.L. . - w

Wise, K.D. .

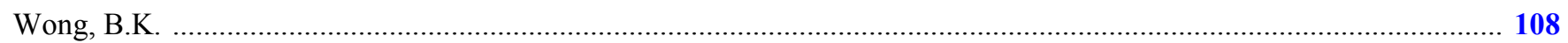

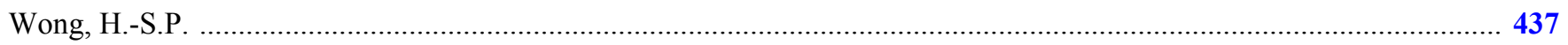

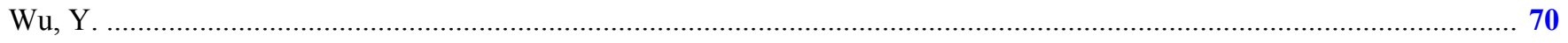

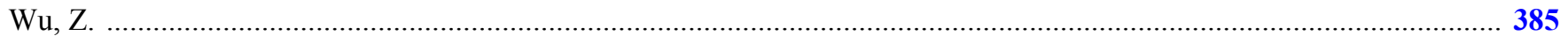

$\mathbf{X}$

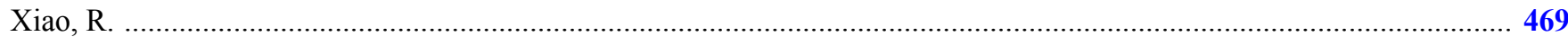

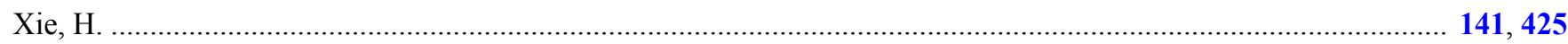

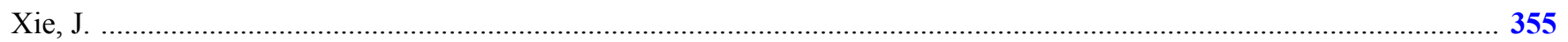

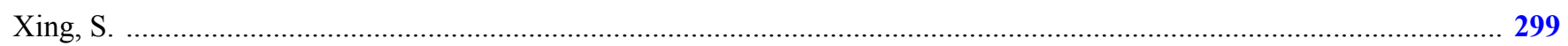

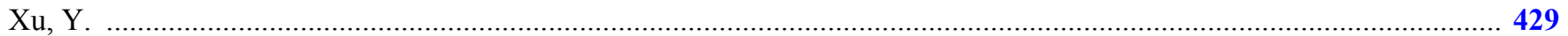

$\mathbf{Y}$

Yama, G. .

Yamazaki, K. . .

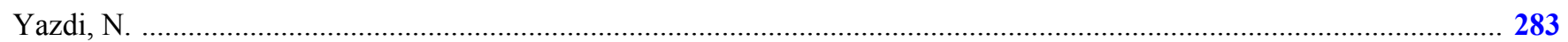

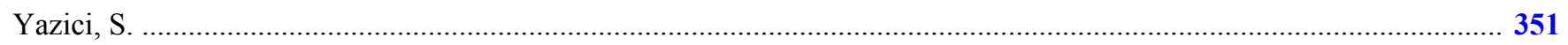

Yee, S.Y. .

Yoneoka, S. .

Yost, B. .

Young, A. . . -

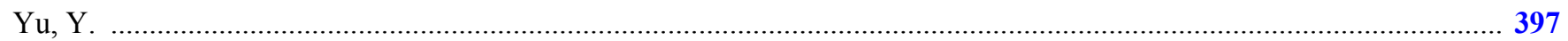

$\mathbf{Z}$

Zaghloul, A. ... -

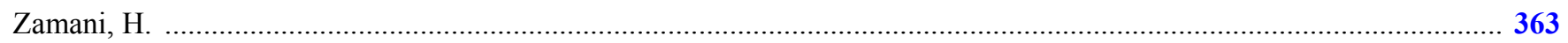

Zamuruyev, K. . .

Zarnitsyn, V. . .

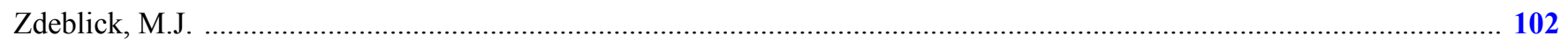

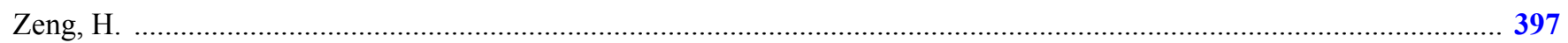


Zhang, B.

Zhang, Q.M.

Zhang, X.

320

Zhang, X.J.

185

Zhang, Y.

283

Zhao, J.

397

Zhou, L. 157

Zhu, G.

401

Zhu, L.

457

Zhu, X.

112

Ziaie, B.

133, 379

Zito, J.C.

22

Zorman, C.A.

363

Zuniga, $\mathrm{C}$. 


\section{Keyword Index}

Scroll to the author and select a Blue link to open a paper. After viewing the paper, use the bookmarks to the left to return to the beginning of the Keyword Index.

\section{A}

Accelerometer 283

Acoustic Wave

Acousto-Optic Modulator

Actuator

141,393

Aerosol 295

Aluminum Nitride $98,291,347,351,505$

Antenna Tuning 108

Astrobiology 94

Atomic Force Microscope 307

Attenuator 389

Axis

\section{B}

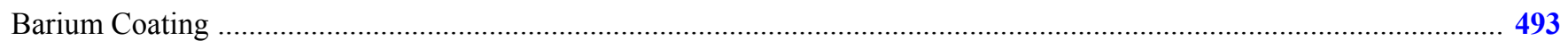

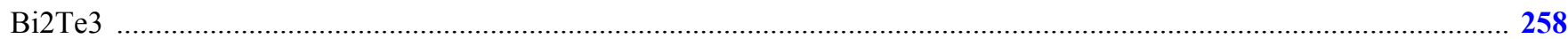

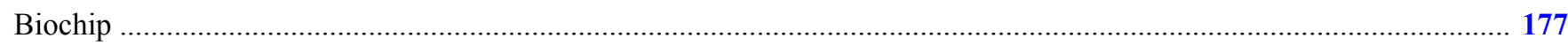

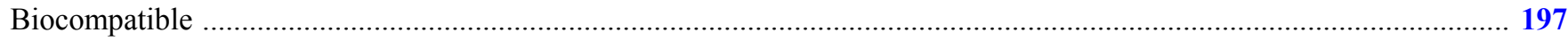

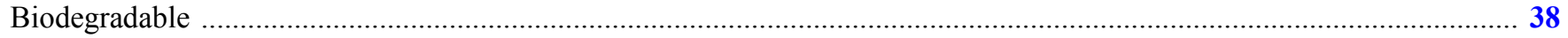

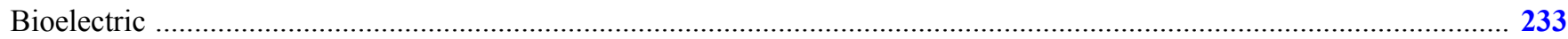

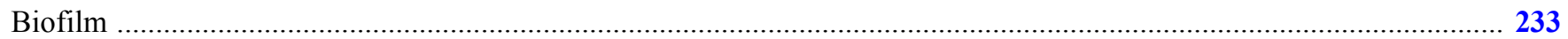

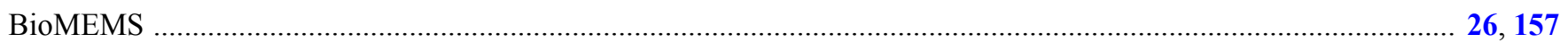

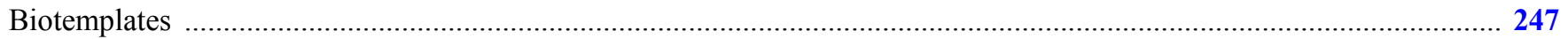

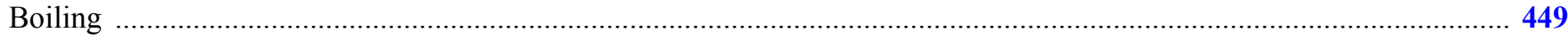

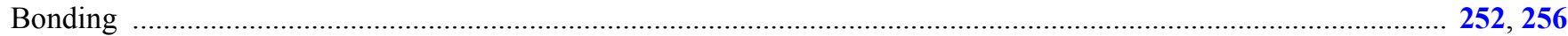

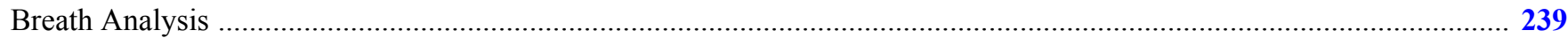

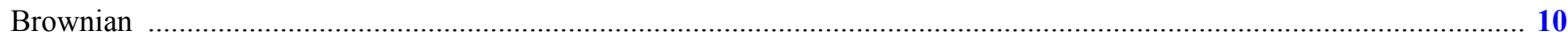

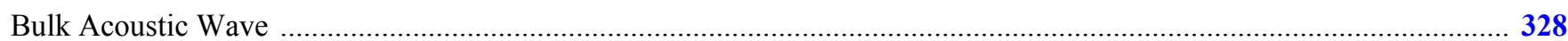

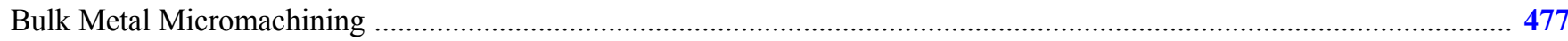

C

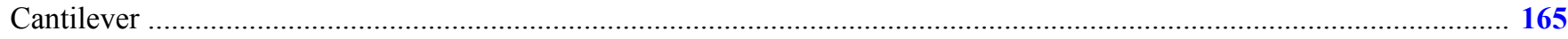

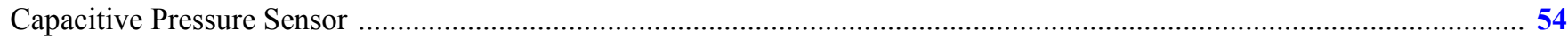

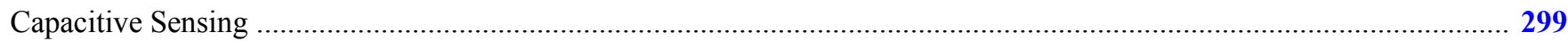

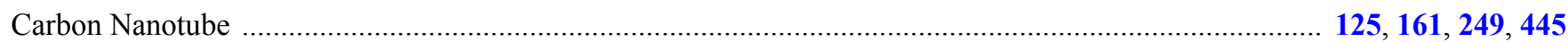

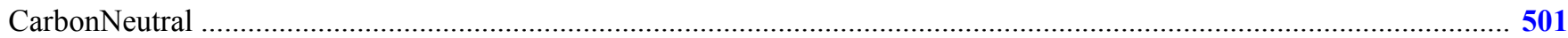

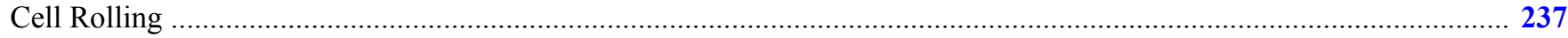

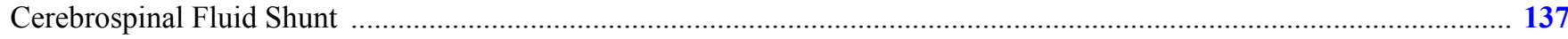

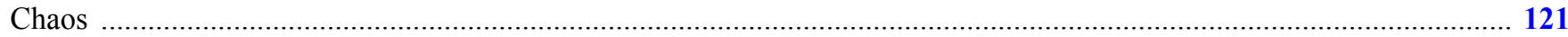

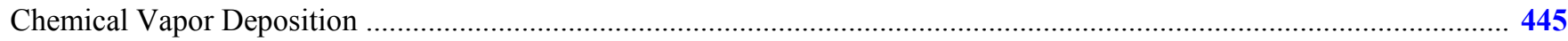




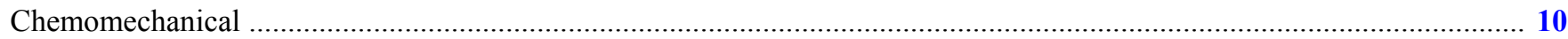

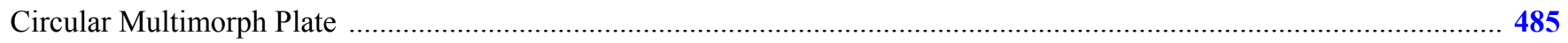

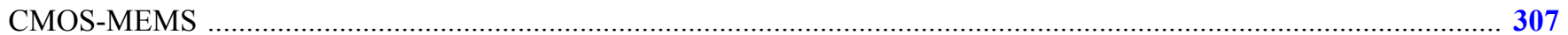

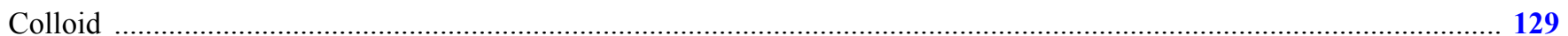

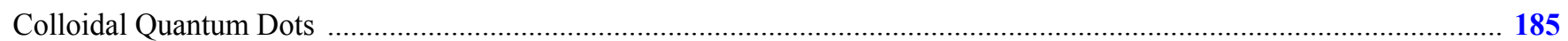

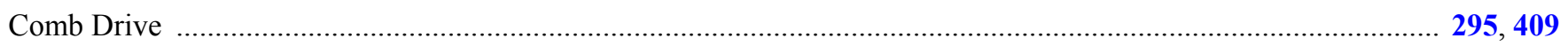

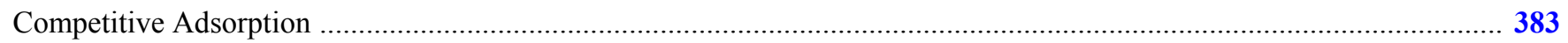

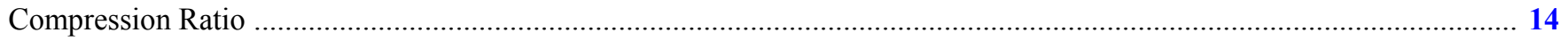

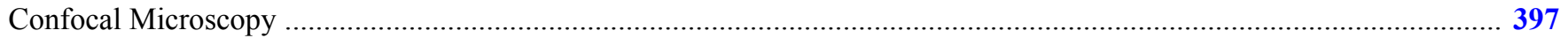

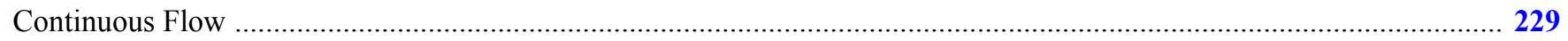

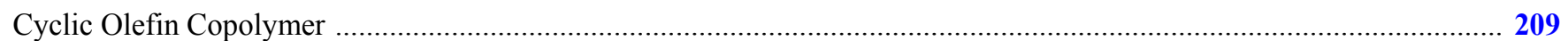

D

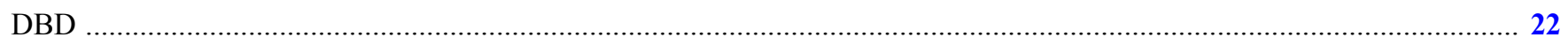

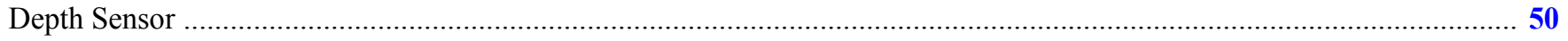

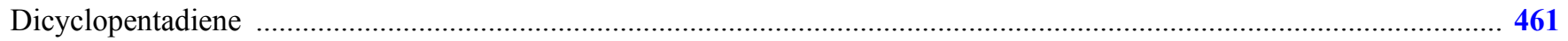

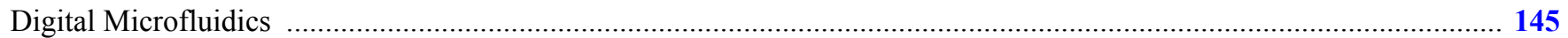

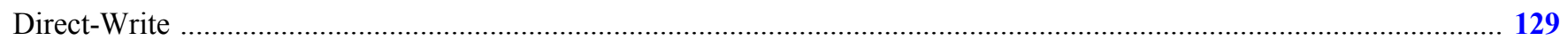

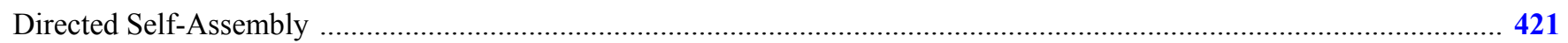

DNA

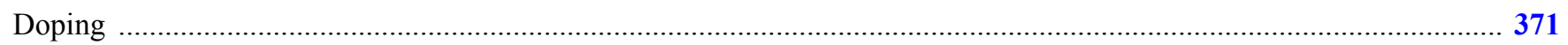

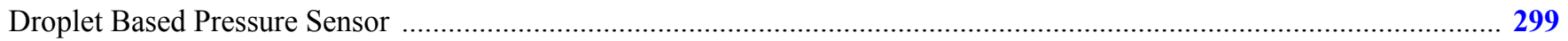

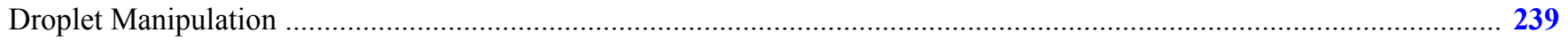

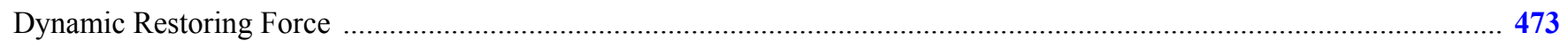

$\mathbf{E}$

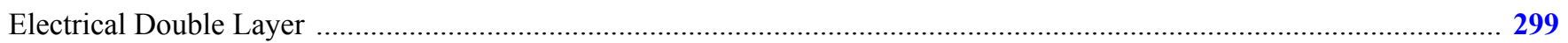

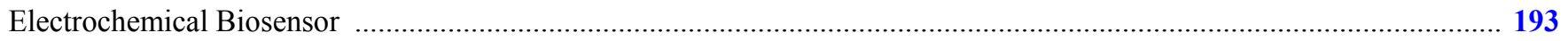

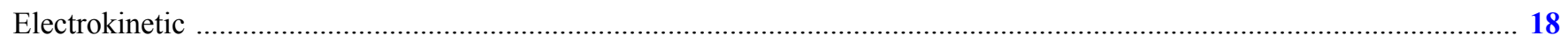

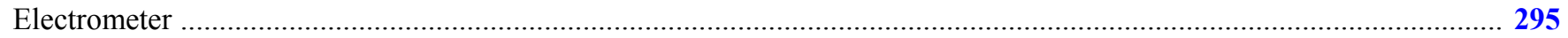

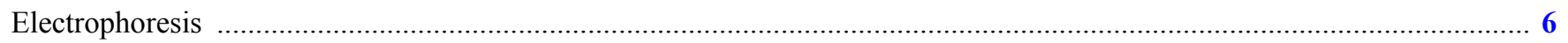

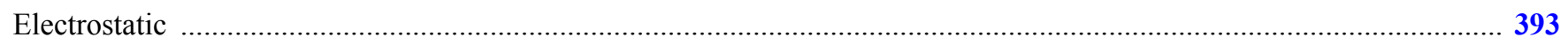

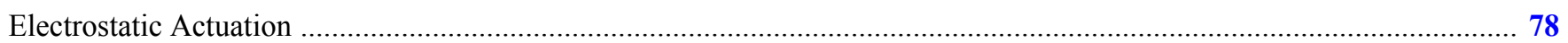

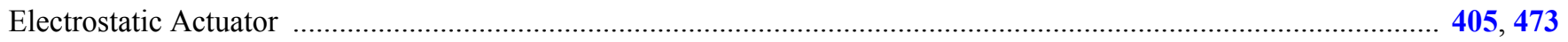

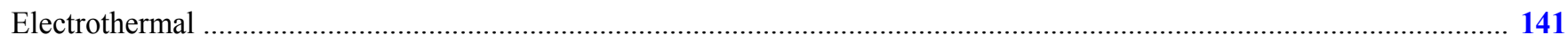

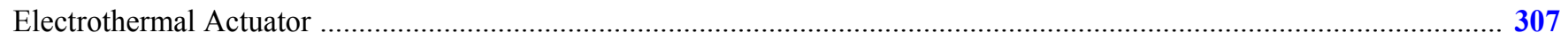

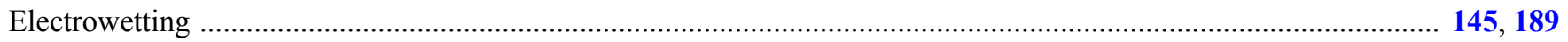

Emulsification

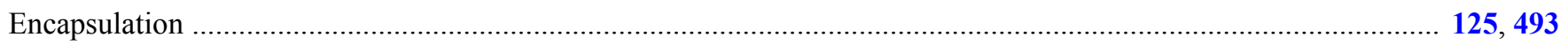

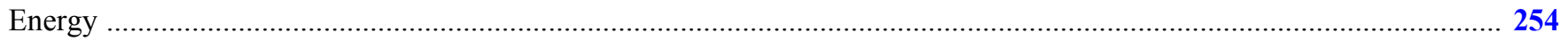

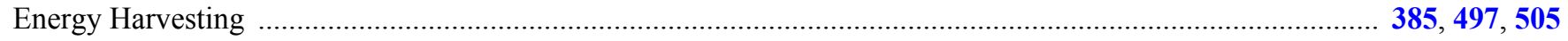

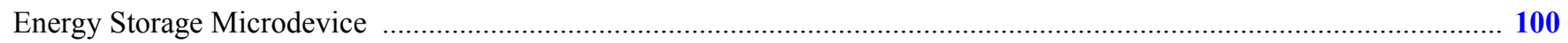

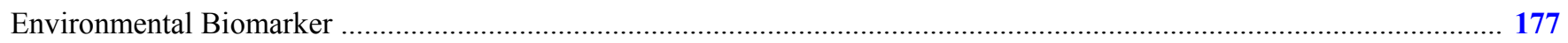

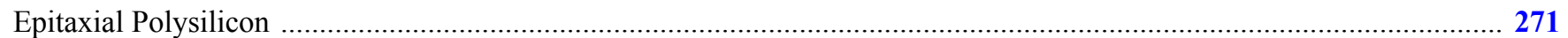

$\mathbf{F}$

Fabrication

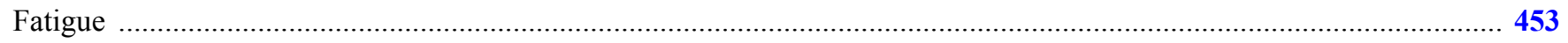

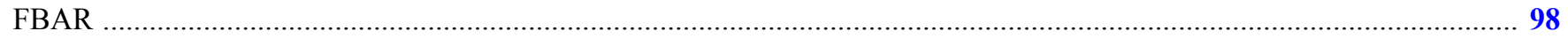

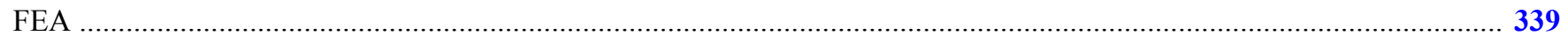

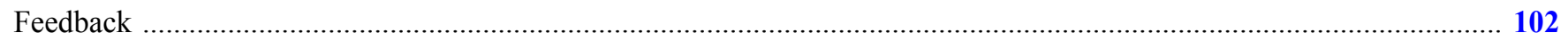




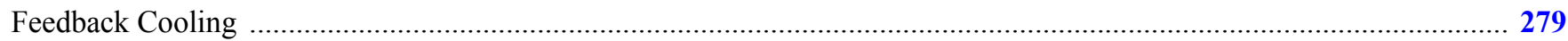

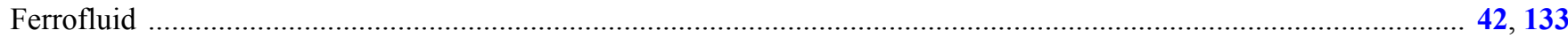

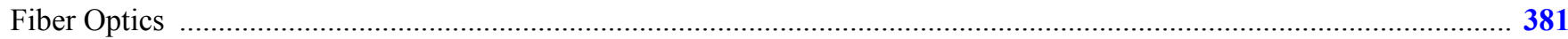

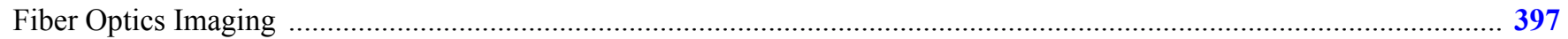

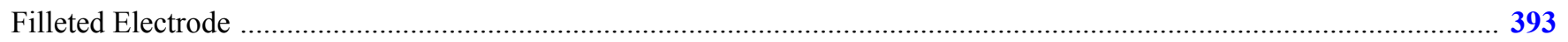

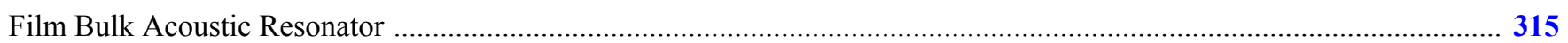

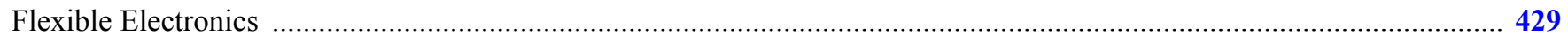

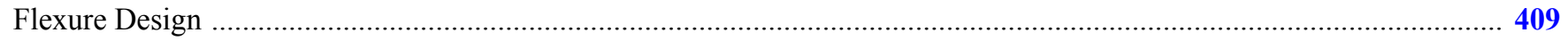

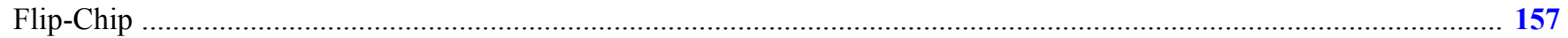

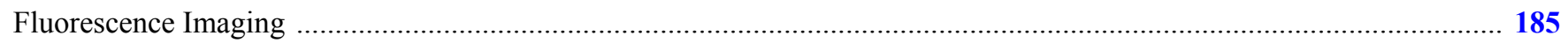

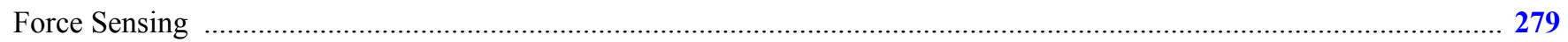

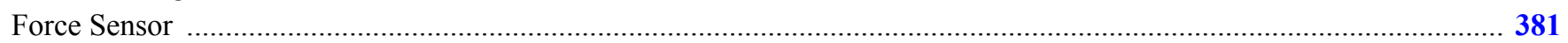

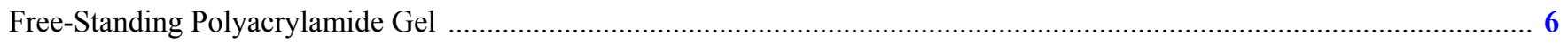

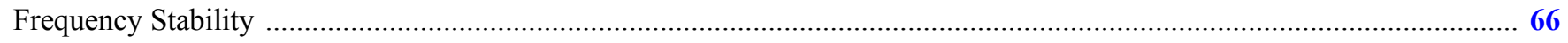

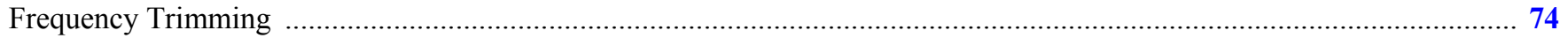

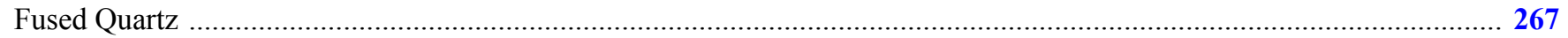

G

$\mathrm{GaN}$

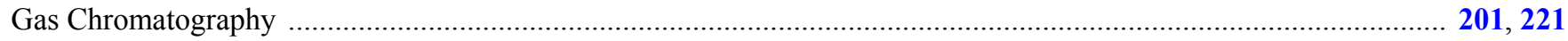

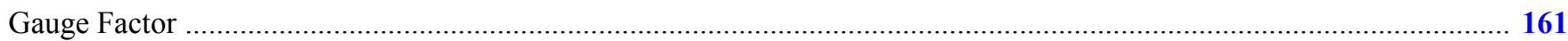

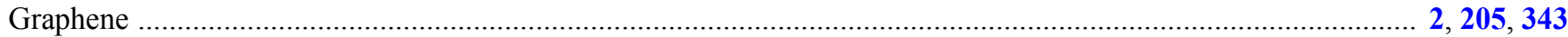

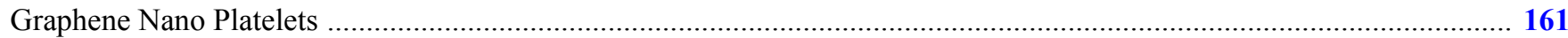

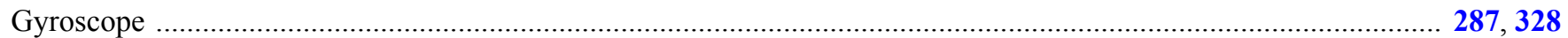

\section{$\mathbf{H}$}

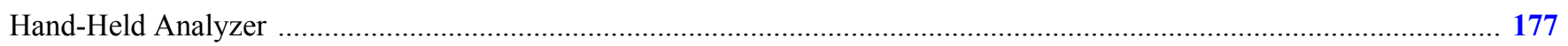

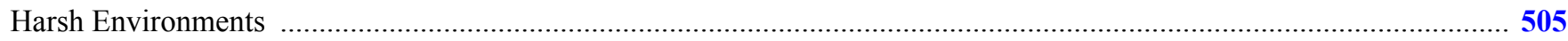

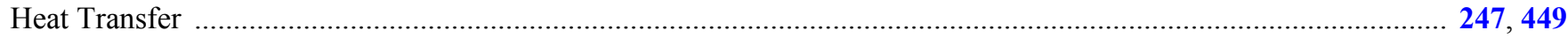

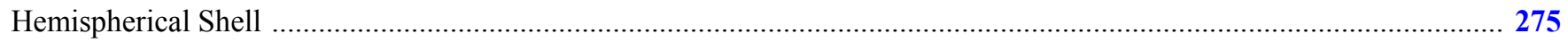

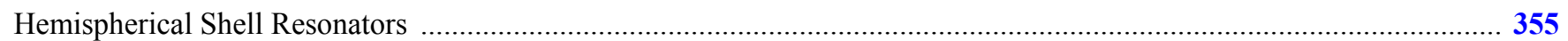

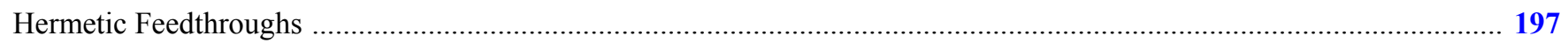

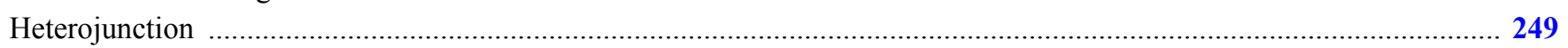

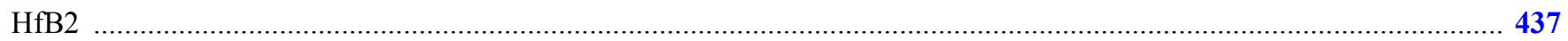

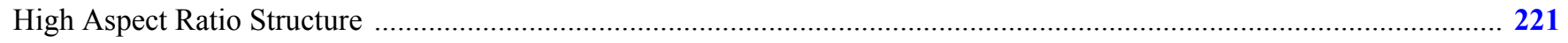

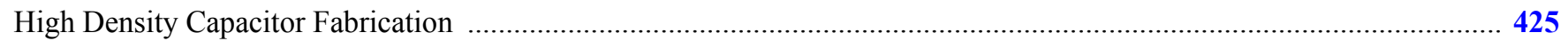

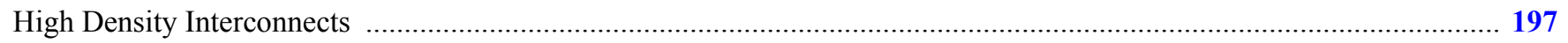

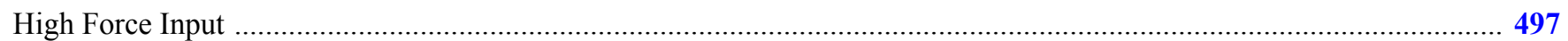

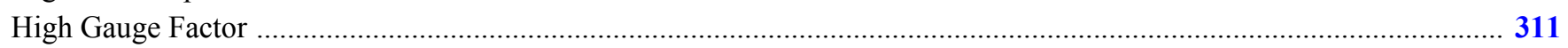

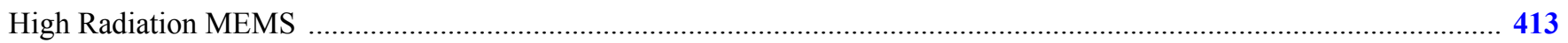

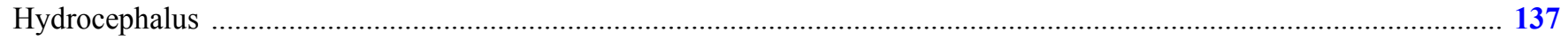

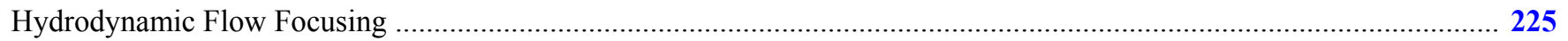

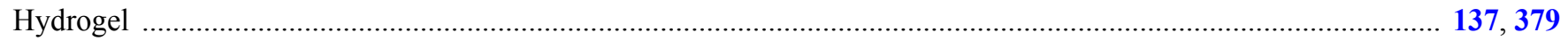

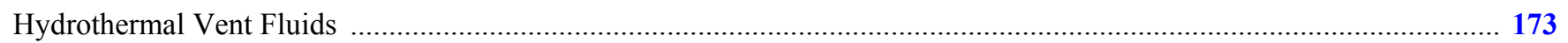

\section{I}

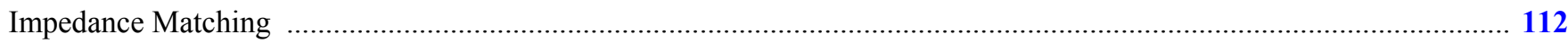

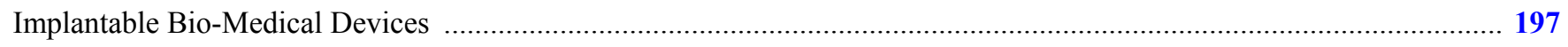

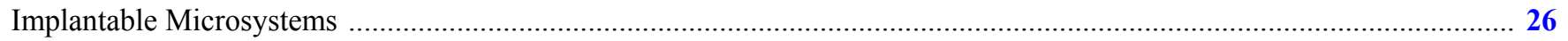

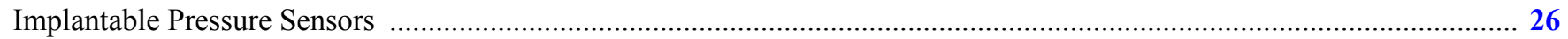

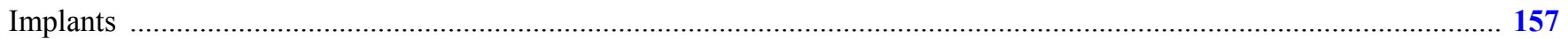




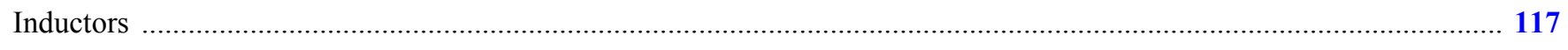

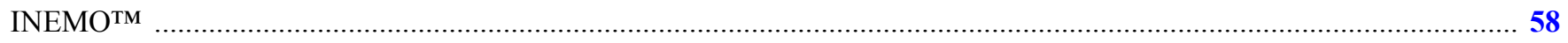

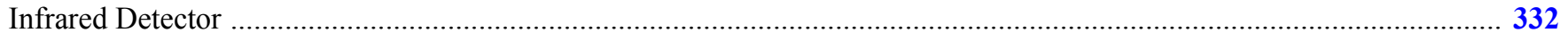

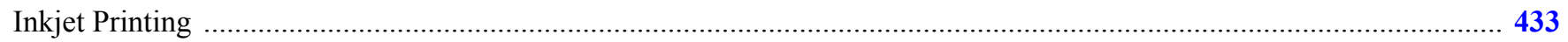

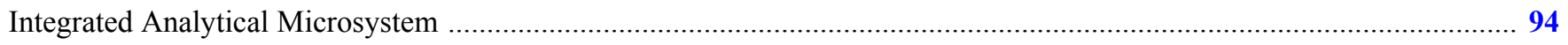

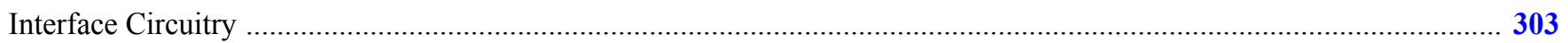

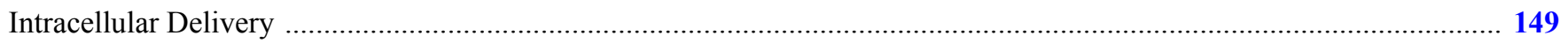

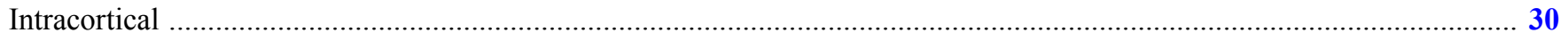

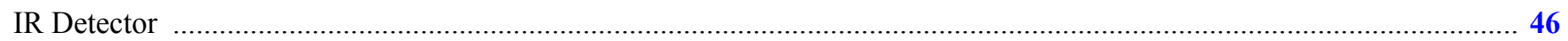

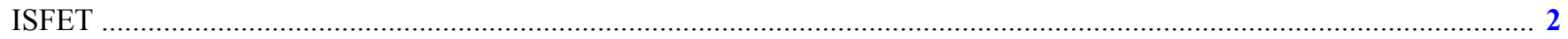

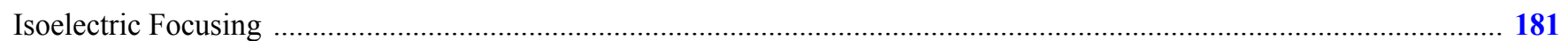

K

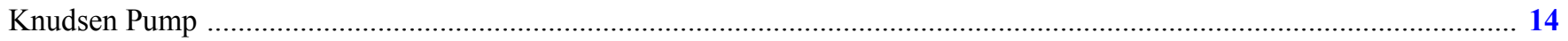

$\mathbf{L}$

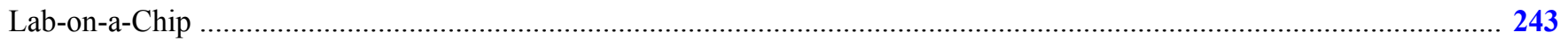

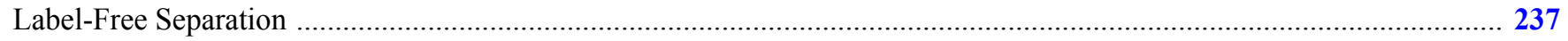

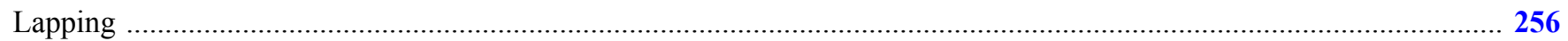

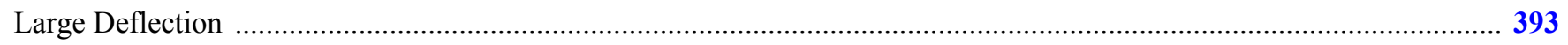

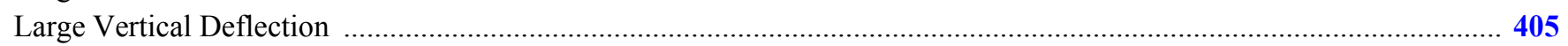

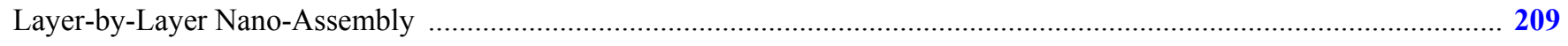

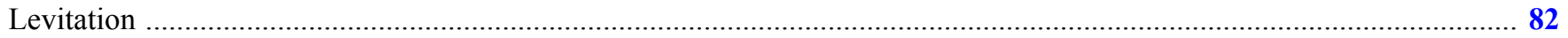

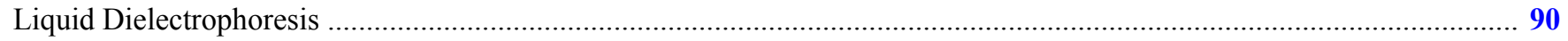

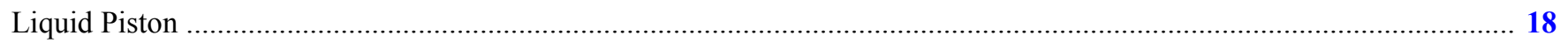

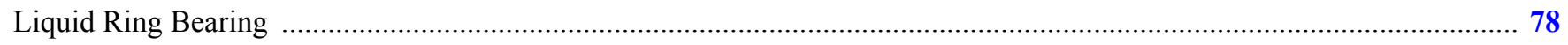

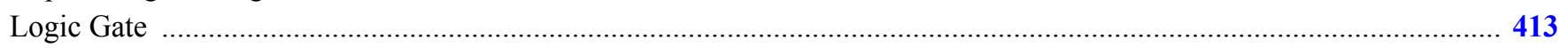

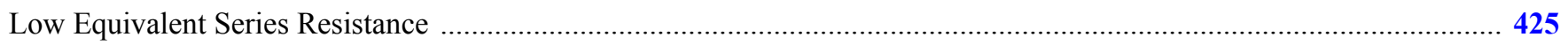

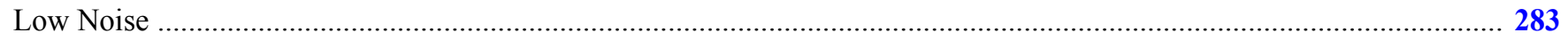

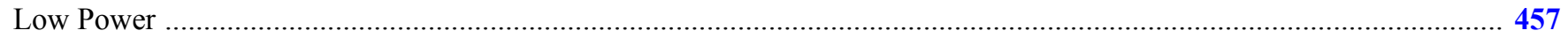

M

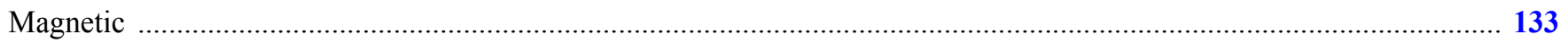

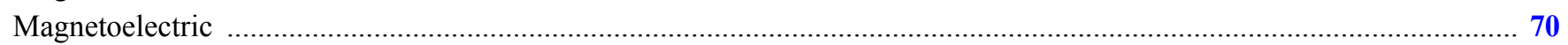

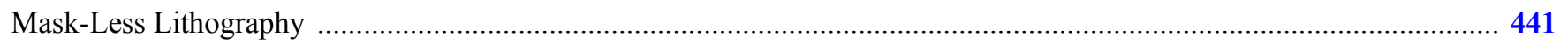

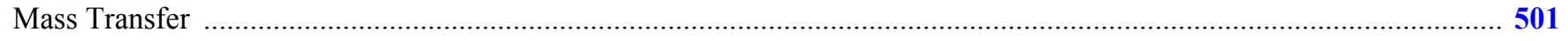

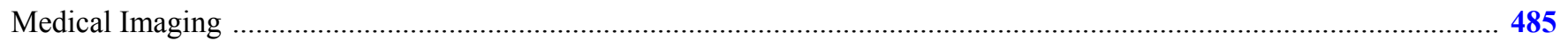

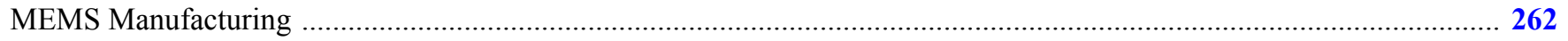

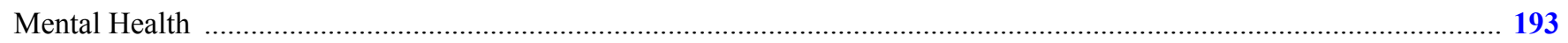

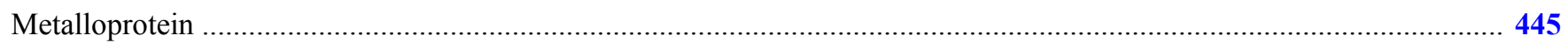

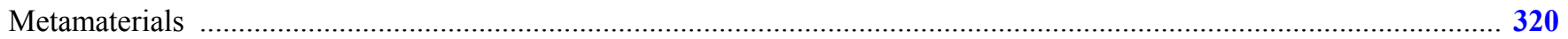

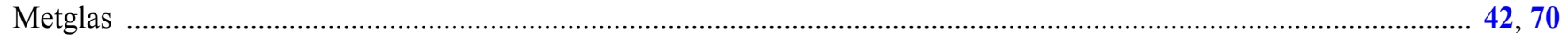

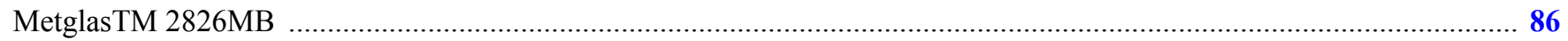

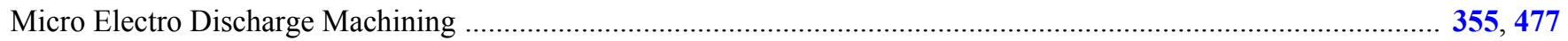

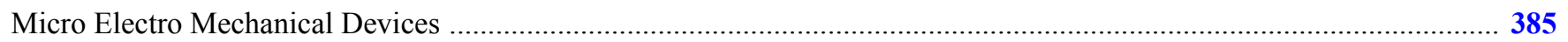

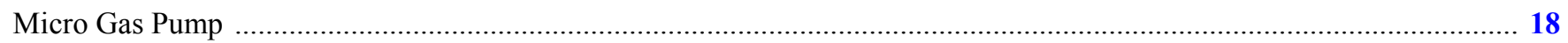

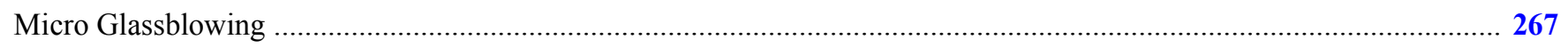

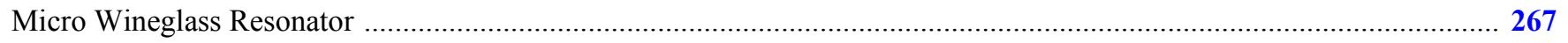

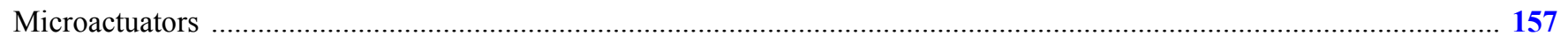

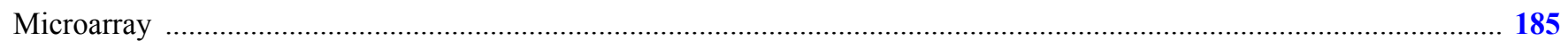

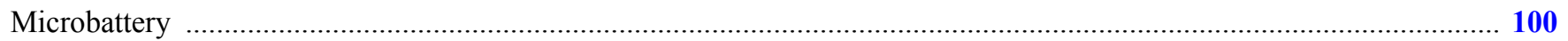


Microbeads

Microbial Fuel Cell

169, 501

Microchannels ....

Microdischarge

Microelectrode 30

Microelectrode Array 153

Microfluidic (s)

Microfluidic Railing

Micromachining .

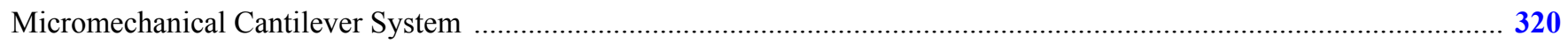

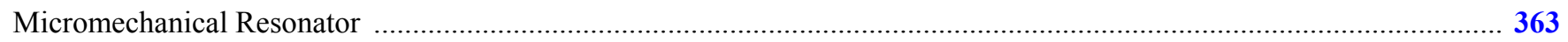

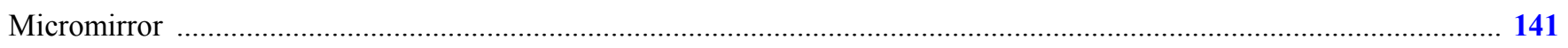

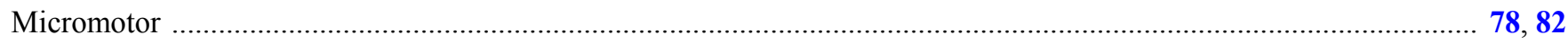

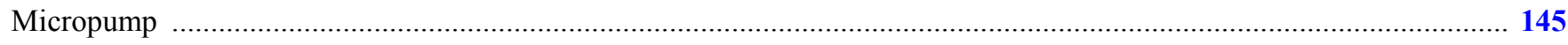

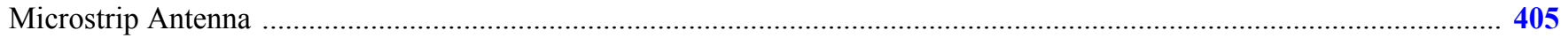

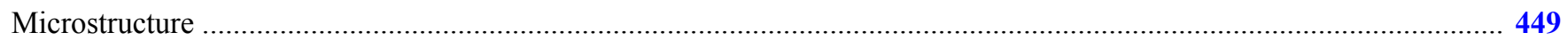

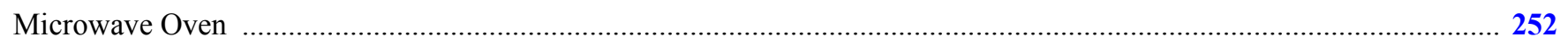

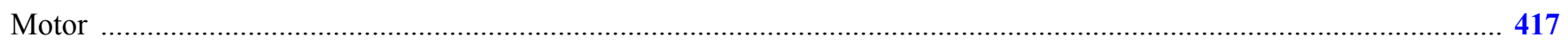

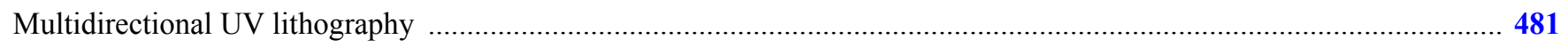

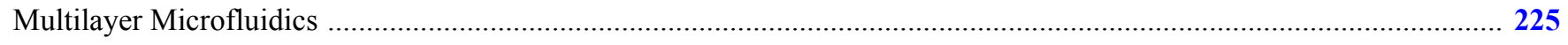

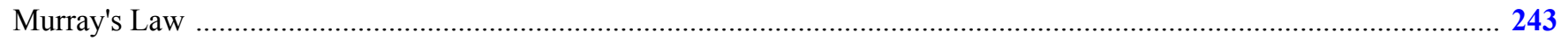

$\mathbf{N}$

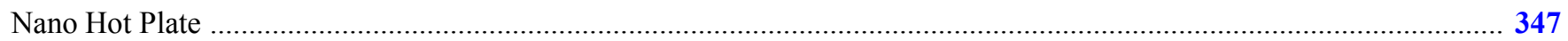

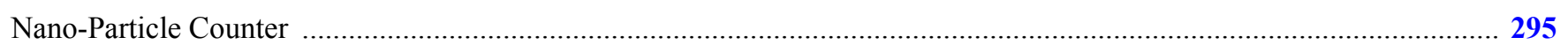

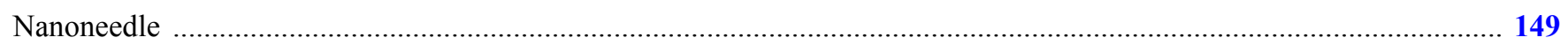

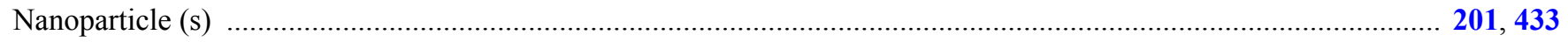

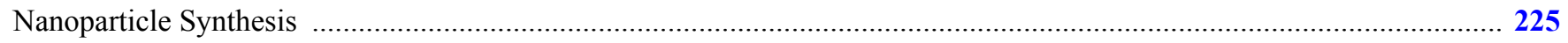

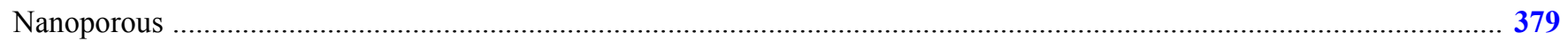

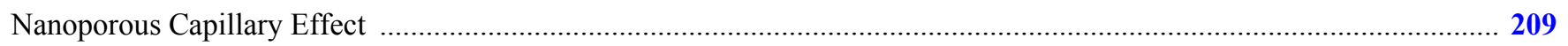

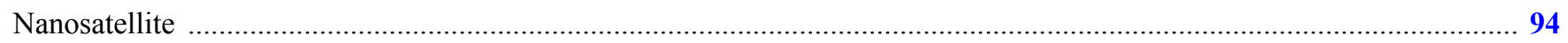

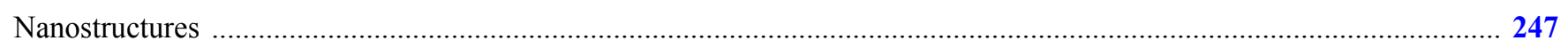

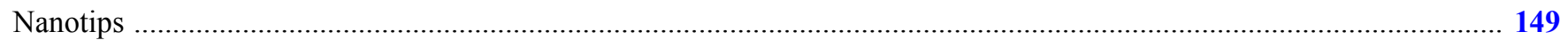

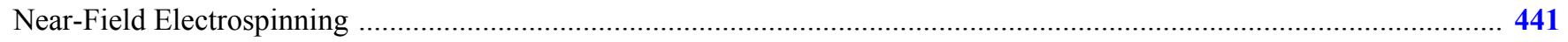

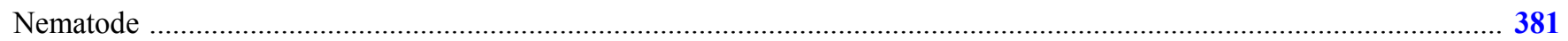

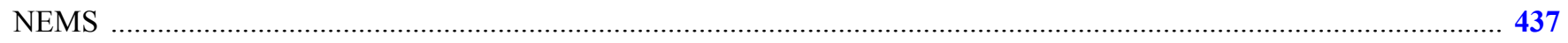

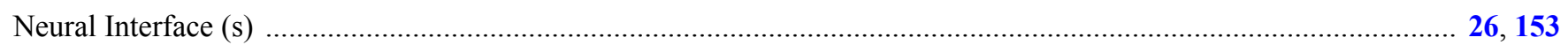

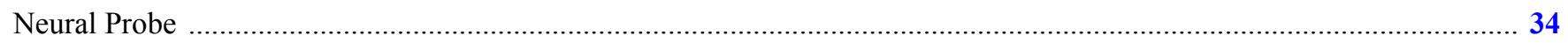

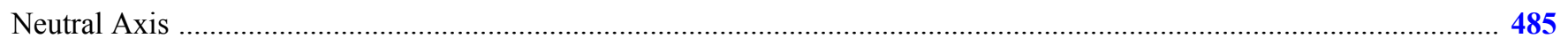

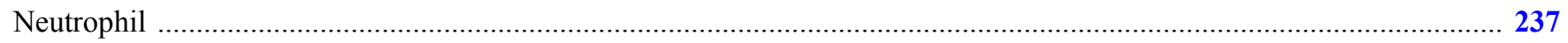

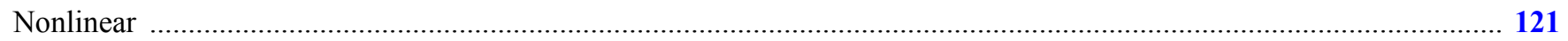

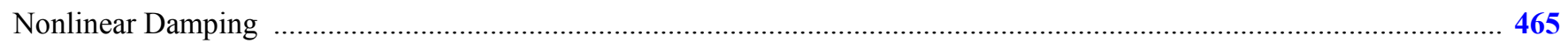

O

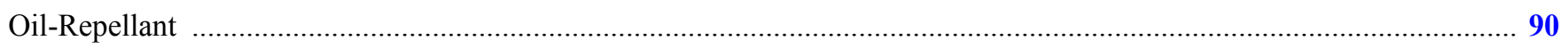

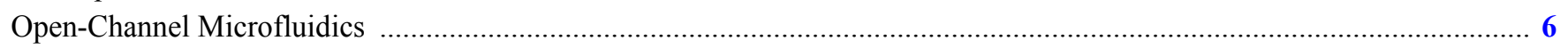

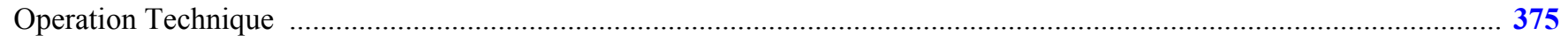

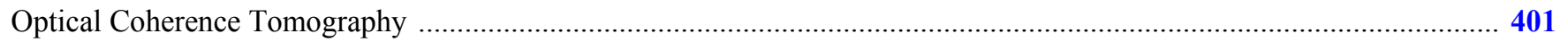

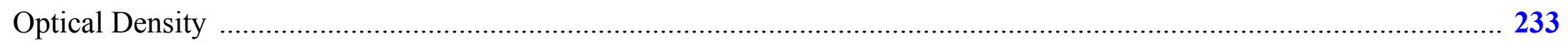

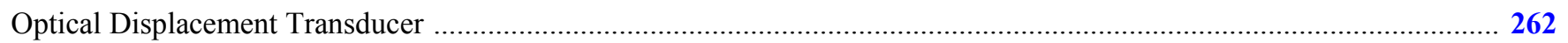




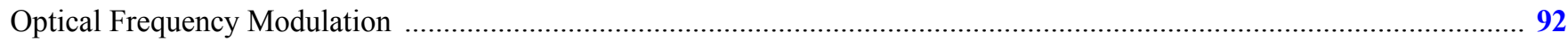

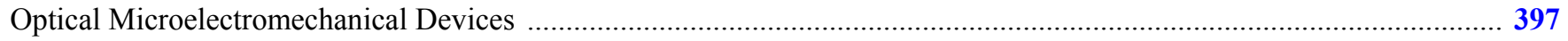

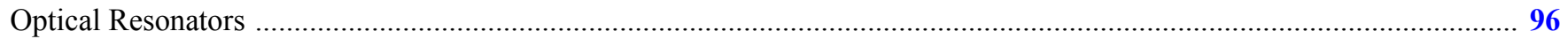

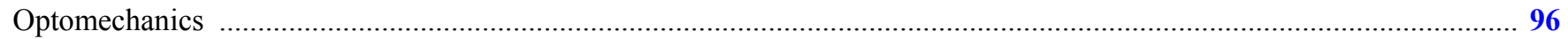

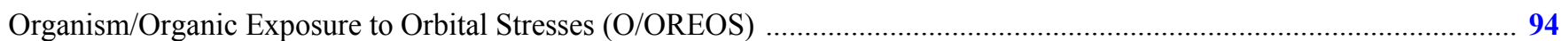

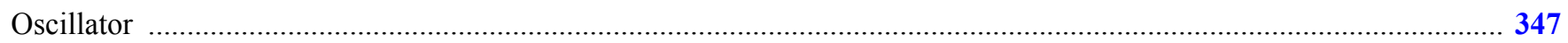

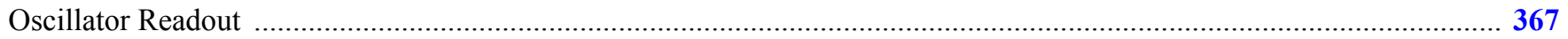

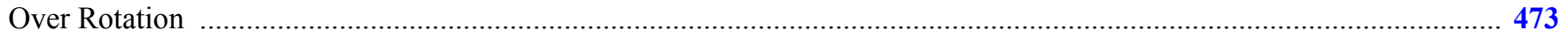

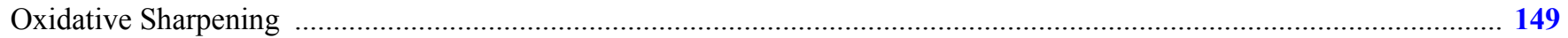

$\mathbf{P}$

Packaging

$117,303,339,453$

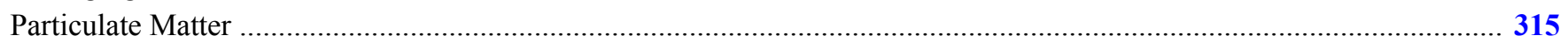

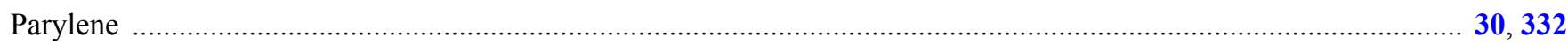

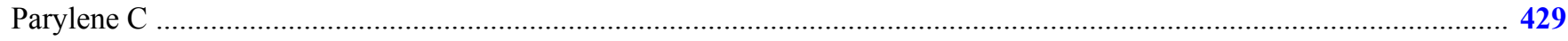

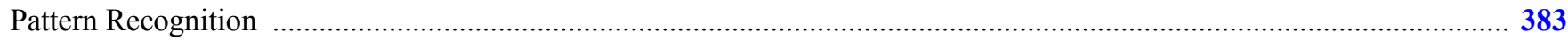

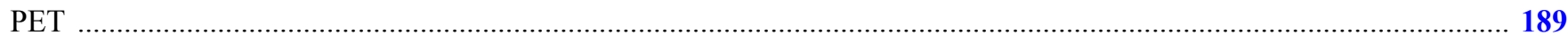

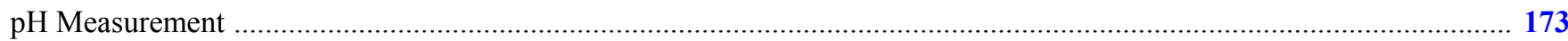

Piezoelectric …................................................................................... 50, 256, 291, 359, 367, 417, 485

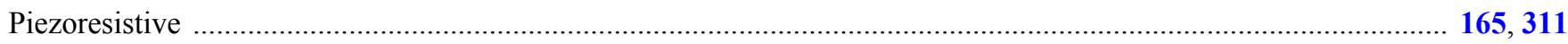

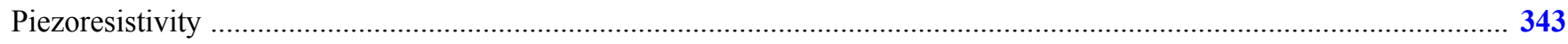

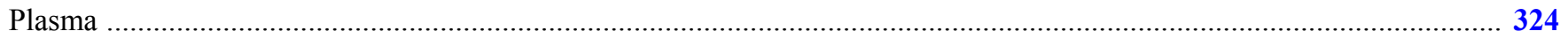

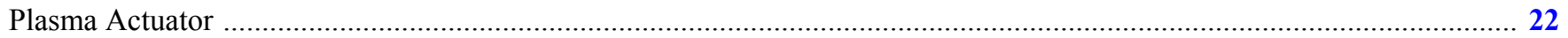

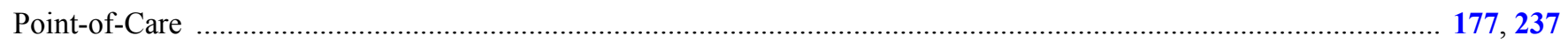

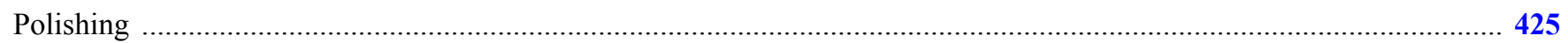

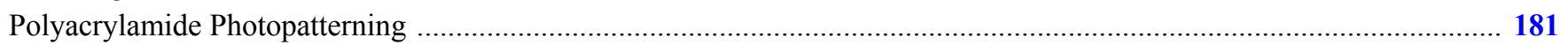

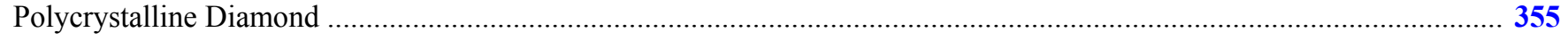

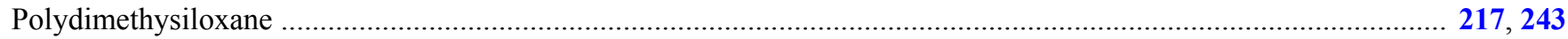

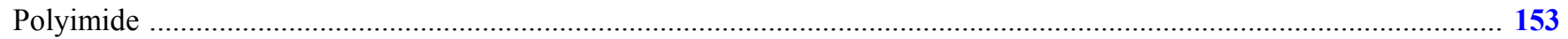

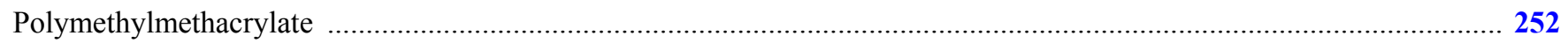

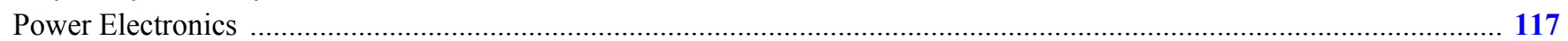

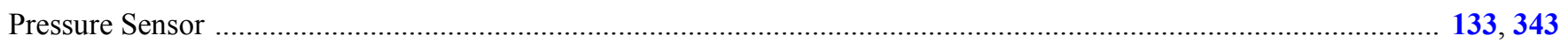

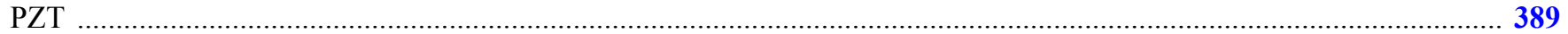

Q

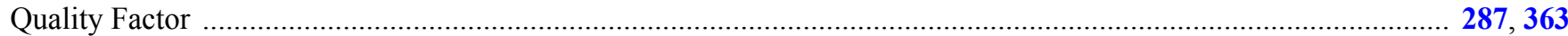

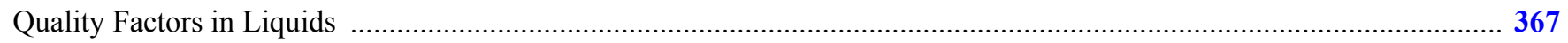

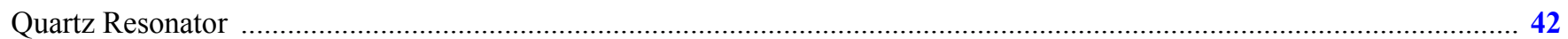

$\mathbf{R}$

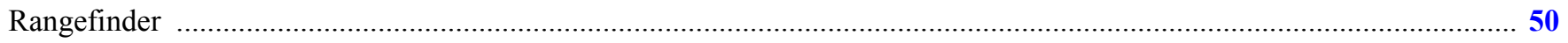

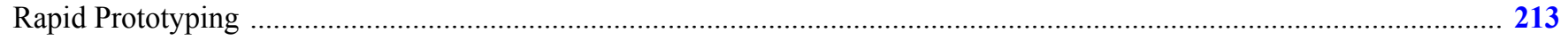

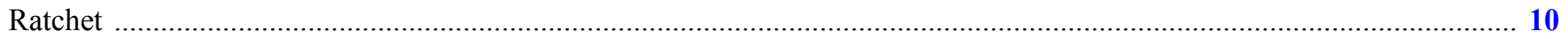

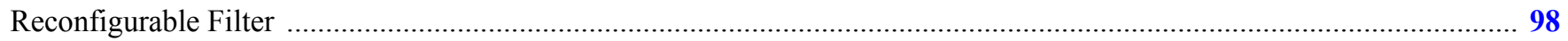

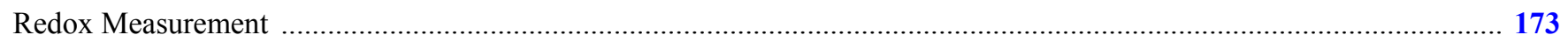

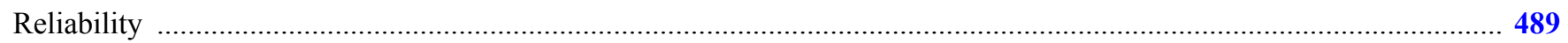

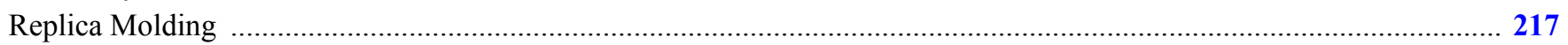

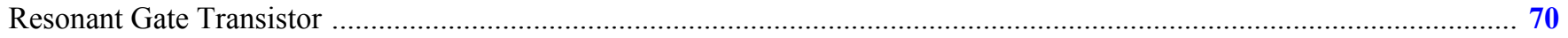

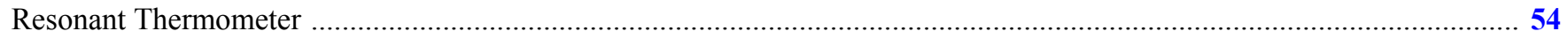

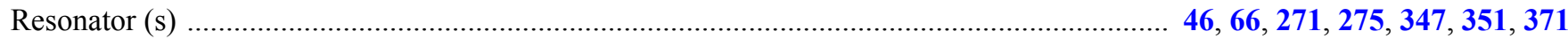

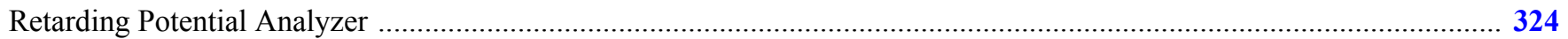




\section{S}

$\mathrm{Sb} 2 \mathrm{Te} 3$

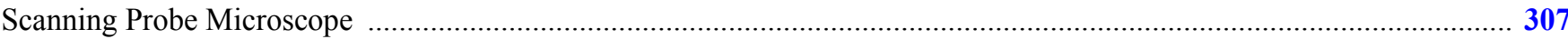

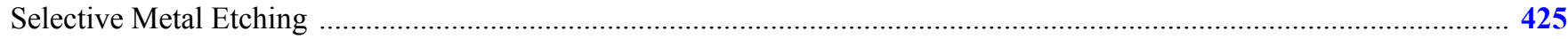

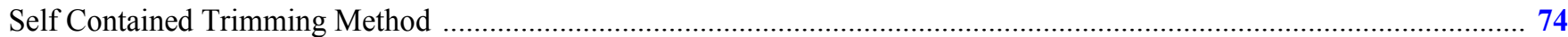

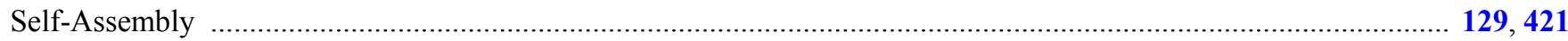

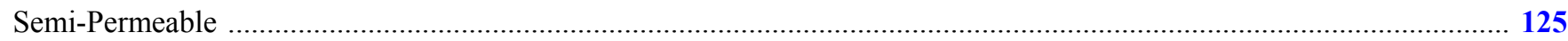

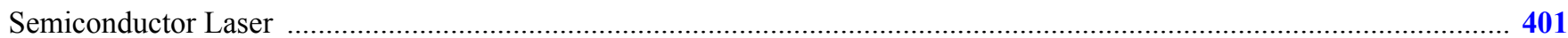

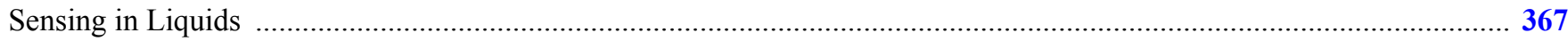

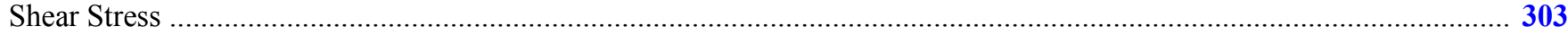

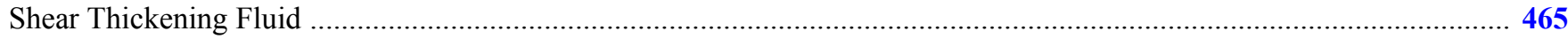

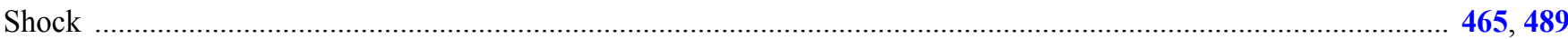

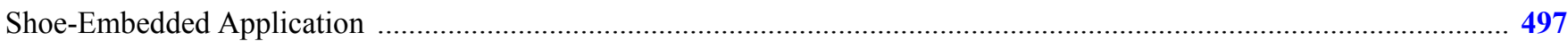

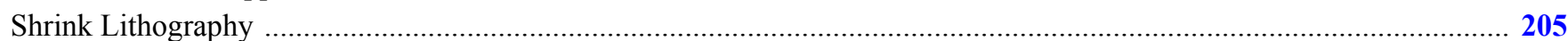

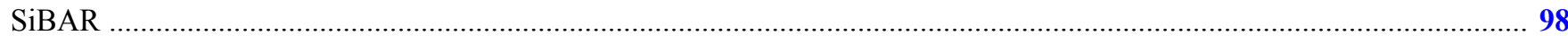

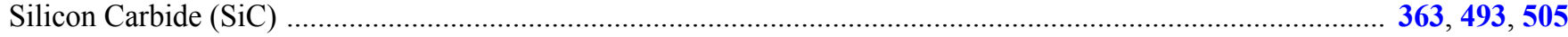

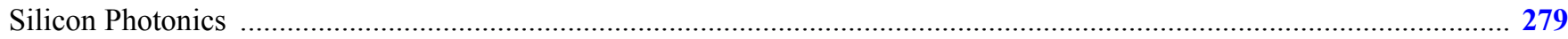

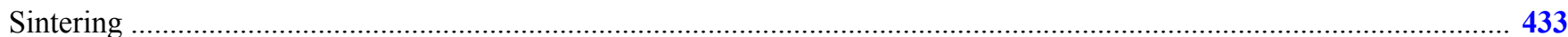

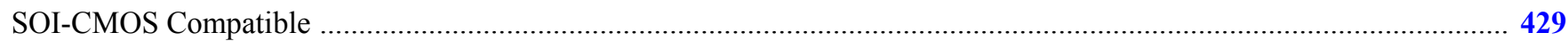

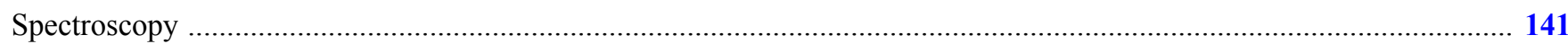

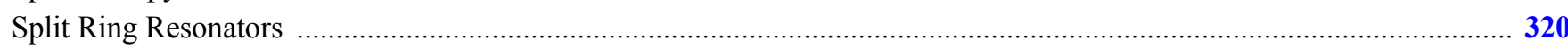

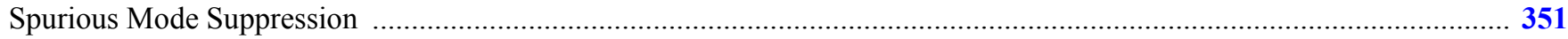

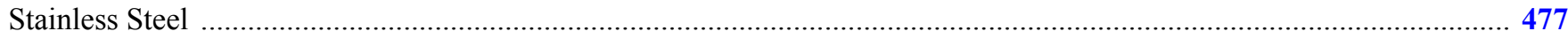

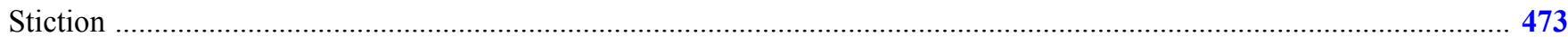

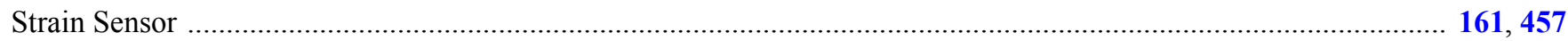

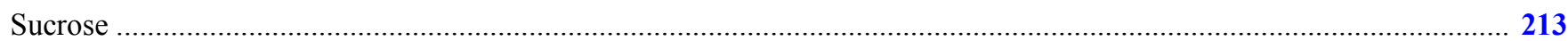

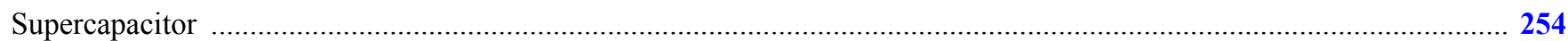

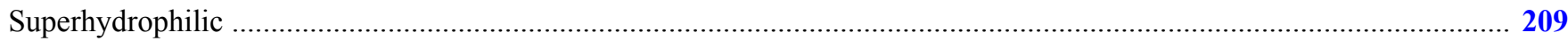

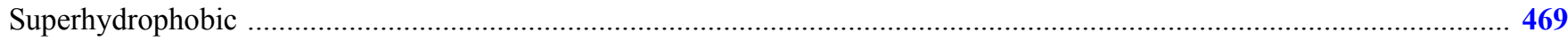

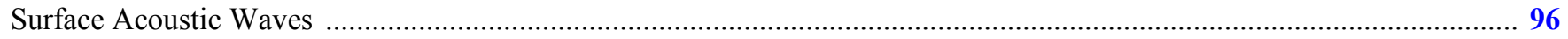

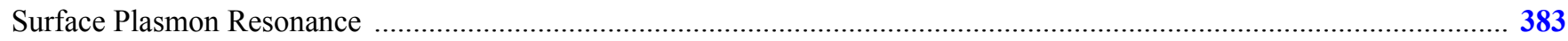

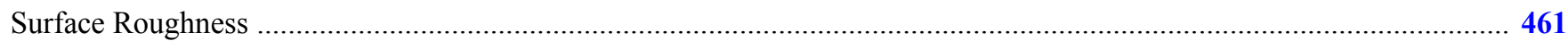

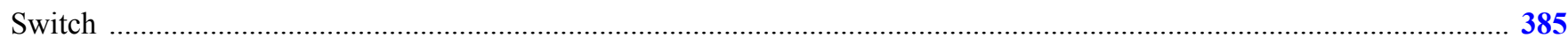

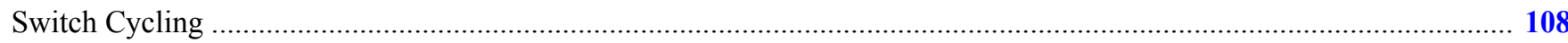

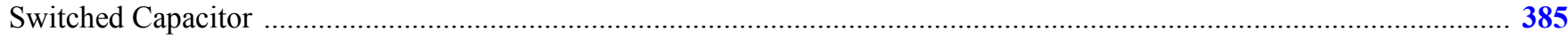

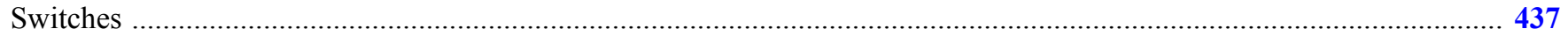

T

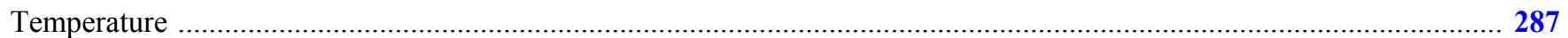

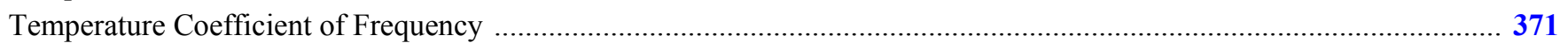

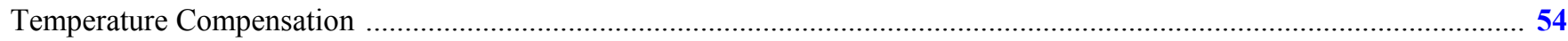

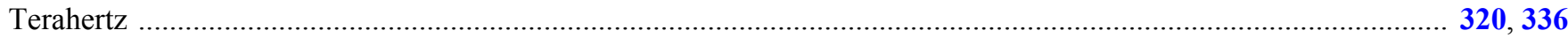

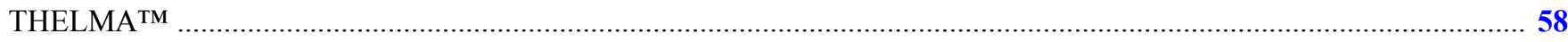

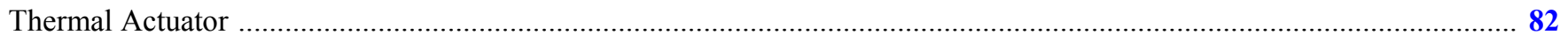

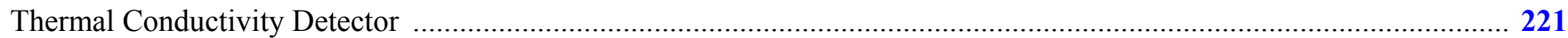




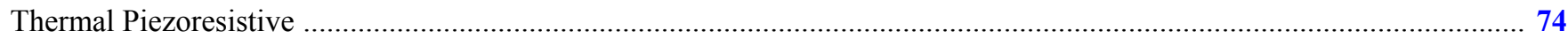

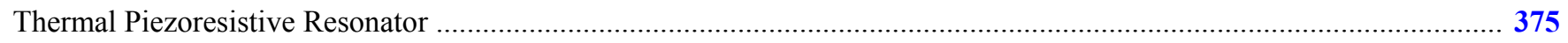

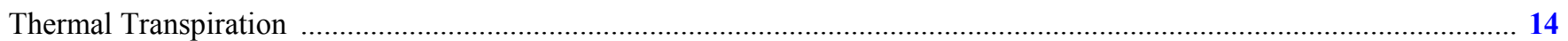

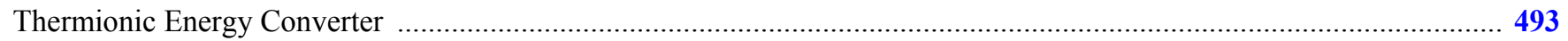

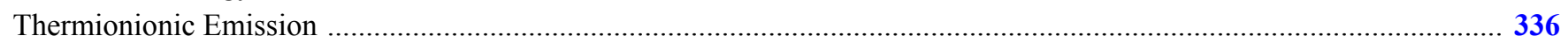

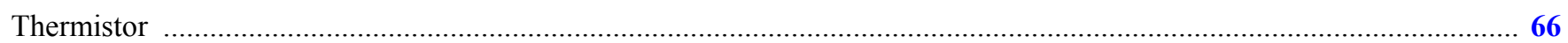

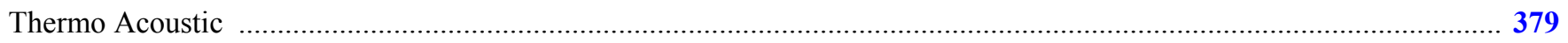

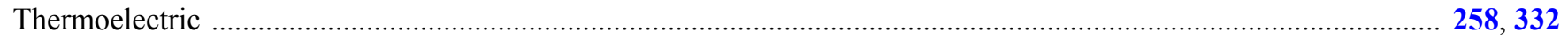

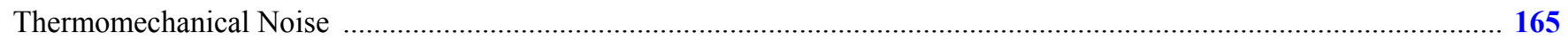

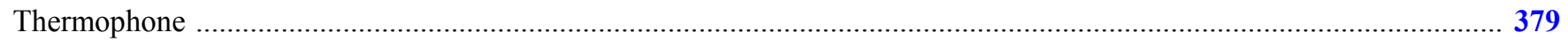

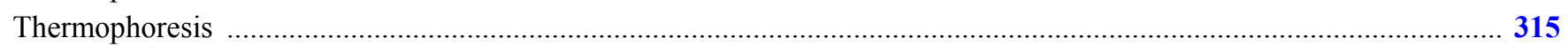

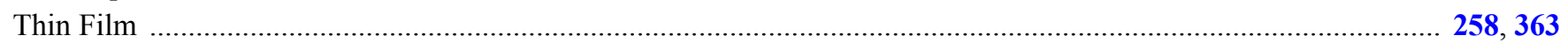

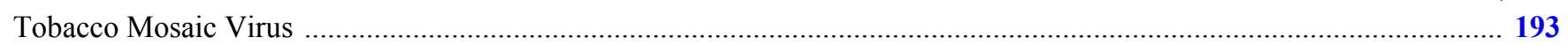

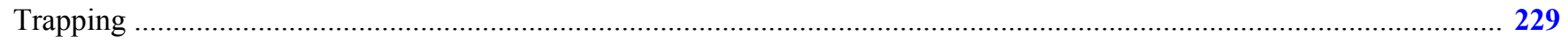

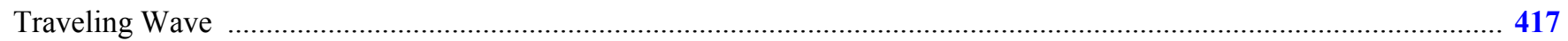

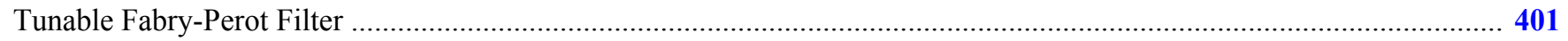

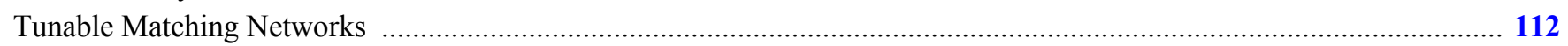

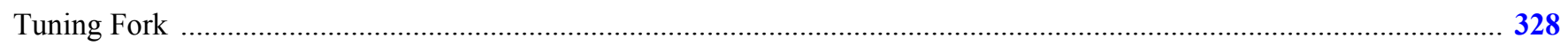

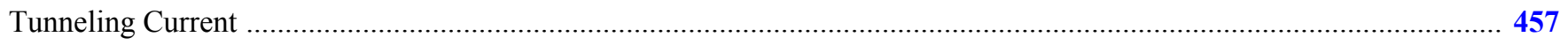

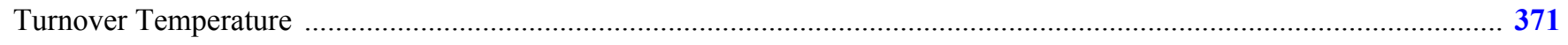

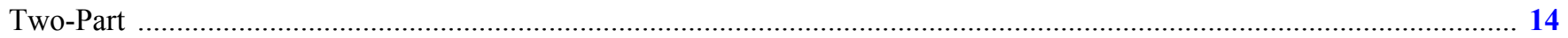

$\mathbf{U}$

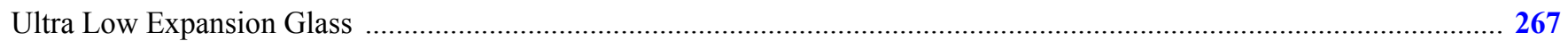

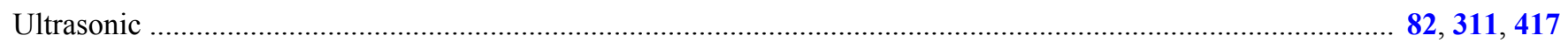

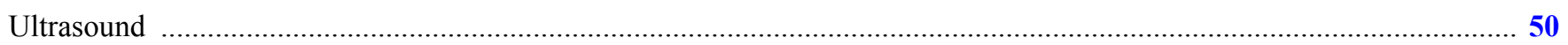

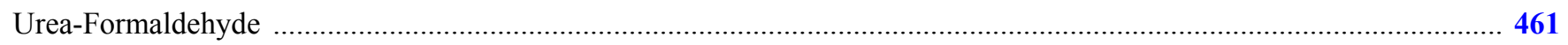

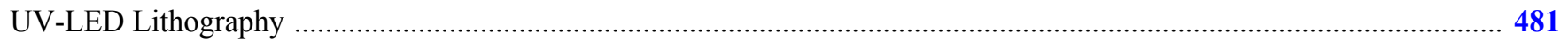

V

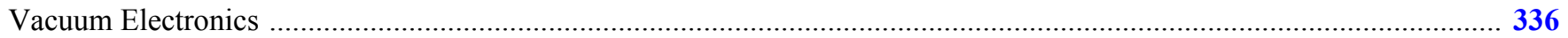

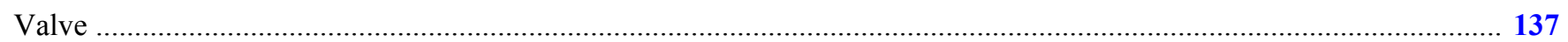

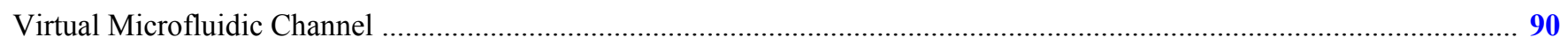

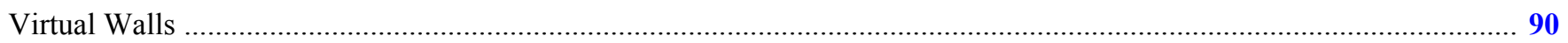

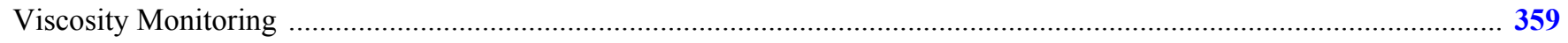

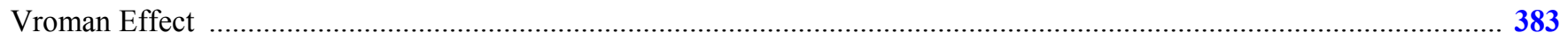

W

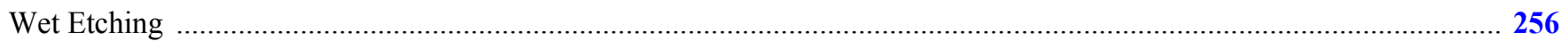

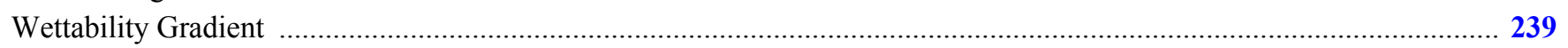

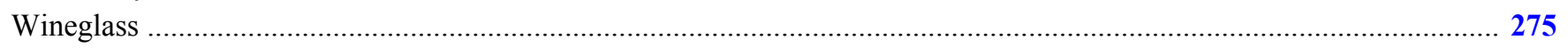

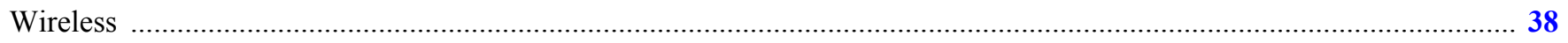

$\mathbf{Z}$

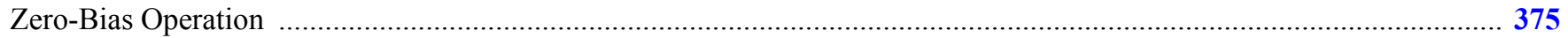


